$\therefore$

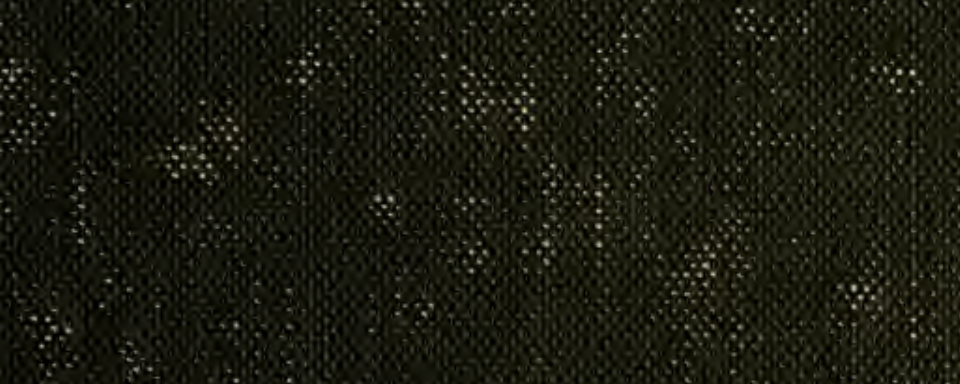





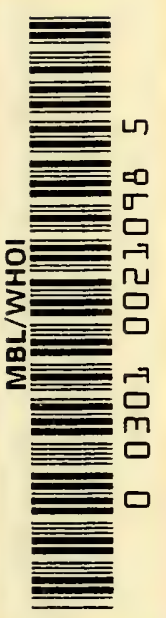




THE NEWER KNOWLEDGE OF BACTERIOLOGY AND IMMUNOLOGY 
THE UNIVERSITY OF CHICAGO PRESS CHICAGO, ILLINOIS

THE BAKER \& TAYLOR COMPANY NEW YORK

THE MACMILLAN COMPANY OF CANADA, LIMITED TORONTO

THE CAMBRIDGE UNIVERSITY PRESS LONDON

THE MARUZEN-KABUSHIKI-KAISHA TOKYO, OSAKA, KYOTO, FUKUOKA, SENDAI

THE COMMERCIAL PRESS, LIMITED SHANGHAI 


\title{
THE NEWER KNOWLEDGE OF BACTERIOLOGY AND IMMUNOLOGY
}

\author{
$\mathrm{Br}$ \\ EIGHTY-TWO CONTRIBUTORS
}

Edited By

EDWIN O. JORDAN AND I. S. FALK

The University of Chicago
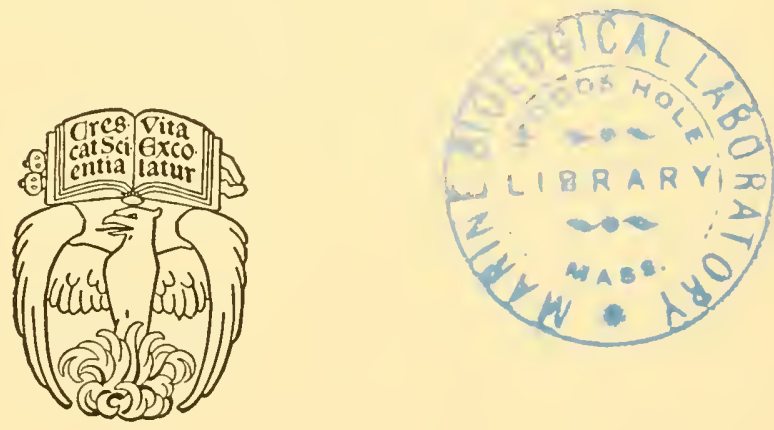

THE UNIVERSITY OF CHICAGO PRESS

CHICAGO - ILLINOIS 
COPYRIGHT 1928 BY THE UNIVERSITY OF CHICAGO ALL RIGHTS RESERVED. PUBLISHED APRIL I 928

COMPOSED AND PRINTED BY THE UNIVERSITY OF CHICAGo PRESS, CHICAGO, ILLINOIS, U.S.A. 


\section{PREFACE}

This book was prepared in an attempt to make the latest results of investigation in various lines of bacteriology and inımunology available for students and active workers. It does not purport in any sense to be a textbook, nor does it pretend to be a comprehensive survey of the whole field. Our object has been primarily to obtain authoritative critical reviews of topics in which at the present time interest is particularly keen or investigation most active. It is our hope that the book will serve to promote research both by furnishing landmarks of progress and by affording suggestions on significant unsolved problems.

With these ends in view, great latitude has been given to the individual contributors, each of whom assumes direct responsibility for the material presented. While the editors have endeavored to avoid serious duplication and overlapping, they have in a number of cases intentionally asked for and included papers with opposing views and interpretations in order to set clearly before the reader the divergences of current opinion.

This independence of treatment has even extended to such a matter as the nomenclature of microbic types and species. It was early found in our preliminary correspondence that many of our contributors had very strong convictions regarding nomenclatorial practice, and that their convictions were widely apart. Since we did not ourselves feel that the time had arrived for insistence upon a uniform and rigid bacterial nomenclature, we chose to give full rein to individual preference. While we are aware that this course is open to criticism, we believe that our decision will at least serve to bring into yet stronger relief the almost hopeless confusion and divergence of opinion into which classification and names of bacteria have fallen.

We considered it an important feature of our undertaking that the individual articles should come to hand as nearly as possible at the same time in order to insure promptness and timeliness of publication. We are greatly indebted to our contributors for their generally hearty response to this request. Many of them have been able to comply with this condition only at serious personal inconvenience and even sacrifice. In a few instances illness has interfered with the preparation of an intended manuscript. Several of our European correspondents who originally promised articles have failed to send anything.

We are under particular obligation to the Board of Trustees of the University of Chicago and to Mr. Gordon J. Laing, director of the University Press, for aiding the publication of this volume with a special fund. Among the many individuals who have given us signal assistance, we wish to acknowledge special indebtedness to $\mathrm{Mr}$. Donald P. Bean and Miss Anabel Ireland, of the University Press, and to Miss Theodora Platt, of the Department of Hygiene and Bacteriology, for their efficient and untiring interest in seeing the book through the press.

THE EDITORS

February 15, I928 



\section{CONTENTS}

CHAPTER

I. The Newer Knowledge of the Morphology of Bacteria Herbcrt C. Ward

II. The Chemical Structure of Bacteria

Traugott Baumgärtel

III. Staining Reactions of Bacteria .

John W. Churchman

IV. Morphological Changes during the Growth of Bacteria . . 38 Paul F. Clark

V. Growth Curves of Bacteria R. E. Buchanan

VI. The Rise and Fall of Bacterial Populations . . . . $\quad 5^{8}$

C.-E. A. Winslow

VII. The Dissociative Aspects of Bacterial Behavior . . . . 84 Philip Hadley

VIII. Bacterial Associations . W. L. Holman

IX. Classification of Bacteria . Roger G. Perkins

X. Atoms, Ions, Salts, ANd Surfaces . . . . . . . 136 William D. Harkins

XI. The Effect of the Surface Tension of the Menstruum upon BaCTERIA AND TOXINS

IV. P. Larson

XiI. Oxidation-Reduction Potentials of Dye Systems and Their SigNIFICANCE IN BACTERIOLOGY W. Mansfield Clark

XiII. Anaerobiosis Ivan C. Hall

XIV. Bacterial Oxidations and Reductions . . . . . . . 2 II James Walter McLeod

XV. Protein (Nitrogen) Metabolism of Bacteria . . . . . . 2 i 8 Leo F. Rettger

XVI. The Utilization of Carbohydrates by Bacteria . . . . . 227 Arthur Isaac Kendall

XVII. Utilization of Aliphatic and Aromatic Compounds by Bacteria 243 S. A. Koser

XVIII. Gas Metabolism of Bacteria M. H. Soule

XIX. Enzymes of Bacteria Selman A. Waksman 


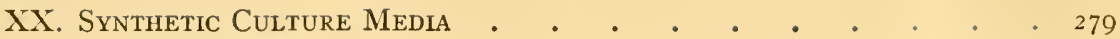

H. W. Schoenlein

XXI. Determinations of Thermal Death-Time

J. Russell Esty

XXiI. The Standardization of Disinfectants and Antiseptics . . . 3oi George F. Reddish

XXili. Nature, Distribution, and Functions of Soll Micro-organisms 310 Selman A. Waksman

XXiV. Autotrophic Bacteria Robert L. Starkey

XXV. The Root-Nodule Bacteria of Leguminous Plants . . . . 332

- Edwin Broun Fred

XXVi. Micro-organisms in Relation to Soll Fertility . . . . . 34I Jacob G. Lipman

XXViI. The Rôle of Bacteria in the Treatment of Sewage . . . . 35I F. W. Mohlman

XXViII. Some Problems in Water Bacteriology . . . . . . . 362 John F. Norton

XXiX. The Action of Ultra-Vrolet Light on Bacteria and Their Products $37 \mathrm{I}$ John F. Norton

XXX. Bacteria in MrLk Robert S. Breed

XXXi. Bacteria in Dairy Products . . . . . . . . . . 395 L. A. Rogers

XXXiI. The Bacterial and Health Aspects of Pasteurization • • . 403 Milton J. Rosenau

XXXiII. Mechanical and Engineering Aspects of Pasteurization . • . 4ig George W. Putnam

XXXiV. Contamination and Deterioration of Food . . . . . . 437 Charles Thom

XXXV. The Bacteria of Food Poisoning Edwin O. Jordan

XXXVI. The Spirochetes Hideyo Noguchi

XXXViI. Current Problems on Yeasts . . . . . . . . . . 498 F. IV. Tanner

XXXViII. The Aspergilli: A Typical Group of Molds . . . . . 509 Charles Thom

XXXIX. Filterable V'iruses Thomas M. Rivers

XL. The Bacteriopiage: Present Status of tire Question of Its Nature AND MODE OF ACTION

J. Bronfenbrenner

Xli. Filterability of Micro-organishis S. P. Kramer

XLII. A Theory of Microbic Virulence I. S. Falk 
Xliil. Elective Localization of Bacteria in the Animal Body a * . 576 Edward C. Rosenow

xliv. Bacteria in Relation to Plant Diseases .

George K. K. Link

XlV. Communicable Diseases of Laboratory Animals K. F. Meyer

XlVi. Bacteria of the Intestinal Tract Leo F. Rettger

XLVII. Bacteria of the Respiratory Tract D. J. Davis

Intestinal Protozoa of Man and Their Host-Parasite Relations 660 Robert Hegner

Xlix. The Immunological Bases for Different Types of Infection by the Blood ProtozoA William H. Taliaferro

L. Antigens and Their Specificity . H. Gideon Wells

LI. The Chemistry of Antigens . Sara E. Branham

LiI. Antigenic Properties of the Bacterial Cell and Antibody ReACTIONS

Hans Zinsser and J. Howard Mueller

LiII. Heterophile Antigens and Antibodies C. G. Bull

LIV. The: Physical Chemistry of Toxin and Antitoxin . Mary E. Maver

LV. The Preparation and Purification of Toxins, 'Toxoids, and AntiTOXINS .

Edroin J. Banzhaf

LVI. The Titration of Toxins and Antitoxins by the Flocculation Method Stanhope Bayne-Jones

LVII. Sublethal Intoxications with Bacterlal Products . • • 772 J. P. Simonds

LVIII. The Mechanism of Agglutination John H. Northrop

LIX. The Functional Rôle of Agglutinins

802

G. Howard Bailey

LX. Bacterial Agglutinins and Their Applications . . . . . 8i I J. G. FitzGerald and Donald T. Fraser

LXi. Precipitins and Their Applications . . . . . . . . . 824 II. M. Powell

LXiI. The Complement Fixation Reaction with Bacterial Antigens ․ 83i Augustus Wadsworth

LxiII. The Complement Fixation Test for Syphilis 
CHAPTER

LXIV. The Kahn Reaction

R. L. Kahn

LXV. The Mechanism of Phagocytosis . . . . . . . . 86

W. O. Fenn

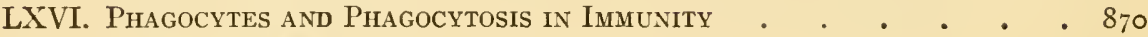
W. B. Wherry

LXVII. Local and Tissue Immunity . . . . . • . . . . 88

Frcderick P. Gay

LXVIII. The Human Blood Groups . • . . . . . . . . 892

K. Landsteiner

LXIX. The Heredity of the Blood Groups . . . . . . . . 909

Reuben Ottenberg and David Beres

Lxx. Antibacterial Sera .

F. M. Huntoon and R. H. Hutchison

LXXi. The Use of Human Serum from Convalescent Cases in Prevention and Treatment of Disease . . . . . . . . . . 934

William H. Park

LXXil. Control and Standardization of Biological Products . . 947 G. W. McCoy

LXXIII. Anaphylaxis and Anaphylactoid Reactions . 966

Howard T. Karsner

LXXiV. The Technique of Experimentation in Anaphylaxis . . gS9

W. H. Manwaring

LXXV. Атору

Arthur F. Cocu

LXXVi. Tuberculin and the Tuberculin Reaction . . . . . . ioi6 Esmond R. Long

LXXVII. Origin of Antibodies

Katharine M. Howell

LXXVIII. The Isolation of Substances with Immune Properties Arthur Locke and Edwin F. Hirsch

Abderhalden's Dialysis Reaction and Theory of the So-Called "Protective" Ferments .

J. Bronfenbrenner

LXXX. Venoms and Antivenins .

I066

Afranio do Amaral

LXXXI. A Critique of the Ehrlich Theory, with an Outline of the Enzyme

Theory of Antibody Formation . . . . . . . . . 1078 W. H. Manwaring

LXXXII. Non-Specific Protein Therapy 1086 William F. Petersen

LXXXiII. Chemotherapy of Bacterial Diseases I IOI John A. Kolmer

Author Index . 


\title{
THE NEWER KNOWLEDGE OF THE MORPHOLOGY OF BACTERIA
}

\author{
HERBERT C. IVARD
}

School of Hygiene and Public Health, Johns Hopkins University

For years bacteriology, dominated by the cellular doctrine of Virchow, has accepted the thesis, more or less completely proved by Cohn and Koch, that each bacterial cell is derived from a previously existing cell of practically the same size and shape. Owing to the fact that a large number of highly diversified forms were included in the group of bacteria, considerable confusion existed before Koch devised his solid media and his plate-pouring methods. The proof that each type of cell, such as the spherical coccus or the elongated bacillus, came from a cell of the same type, a coccus or bacillus, was of enormous benefit in straightening out the confusion and in eventually reconciling conflicting observations and opinions. While it was early recognized that the methods by which the bacterial cells are derived from the pre-existing cells might differ in different species, two regular methods were definitely established: binary fission, where one cell divides transversely into two new cells which eventually attain the size and shape of the original; and spore formation, by which a single spore forms in a bacillus, this spore subsequently giving origin to a vegetative rod like the original rod before sporulation begins. A more complicated cycle of development from conidia was established for certain species which were differentiated from the simple bacteria exhibiting transverse fission or spore formation as the "higher bacteria" and called "streptothrix" or "actinomyces."

Exceptions to these regular methods of reproduction of the bacterial cells were frequently noted. With some species appearances were described which indicated that new cells might rise from old cells by a kind of branching of the cytoplasm, this phenomenon being described as "true branching" to distinguish it from the branching seen in certain plants related to the bacteria, where the cells divide transversely and eventually so crowd the sheath in which they are contained that this itself divides. Knoblike protrusions from the bacterial cells were noted occasionally, and the suggestion was made that these protrusions are in reality buds, capable of growth and development into adult forms. Large, irregular, distorted elements were described in old cultures, especially by Hueppe, who regarded them as true stages in the life-cycle of the bacteria and named them "arthrospores." Occasionally more than one spore was found in a single bacillus, and the idea naturally arose that spore formation may lead to an actual increase in the numbers of the cells. Such double spore formation was admittedly very rare and generally doubted so that the thesis maintained by Kruse was usually accepted, to the effect that spores represent resistant stages of bacteria like the cysts of the protozoa which serve for the perpetuation of the species under adverse conditions, but not for multiplication. Finally, with the flexible spiral organisms, the spirochetes, differing from the ordinary bacteria in many of their characteristics, a pe- 
culiar type of transverse division was early noted and later worked out in great detail by Gross who described it as "multiplication by incurvation." It is of interest in this connection to note that some years ago Gotschlich, in his studies on plasmolysis and plasmoptysis, came to the conclusion that bacterial cells may break up into small bits of protoplasm, resembling in no way the original cell in size or shape but serving as starting-points from which new cells germinate. Gotschlich admitted that no positive evidence exists to show such a kind of reproduction, suggesting it merely as a possibility which cannot be excluded.

During the past few years a number of observations have been made which indicate that many of our earlier ideas in regard to the morphology of the bacterial cells must be subjected to rigid scrutiny, and the new conceptions recently advanced will, if proved, modify our entire point of view with respect to these microscropical organisms.

\section{PLEOMORPHISM}

It has long been recognized that bacterial cells exhibit what we regard as their characteristic morphology under certain favorable conditions which we find in the laboratory, usually in young cultures and on media peculiarly favorable to their growth. Our effort, indeed, is to subject organisms to constant conditions of light, temperature, and nutrition and so maintain constancy of form. Certain species are peculiarly susceptible to environmental influences and prone to exhibit departures from their standard morphology. Thus the cholera vibrio and Vibrio proteus assume in old cultures the most bizarre shapes, swollen spheres, crescents, half-moons, and distorted cells with knoblike protuberances. Similar changes are seen when these species are cultivated on acid media. Transfer of these distorted forms to media of the proper alkaline reaction yields normal vibrios as evidence of their potential viability. Large spherical forms are common in old cultures of the micrococci and are sometimes seen in the streptococci. The plague bacillus is especially likely to produce enlarged distorted forms on certain kinds of media (salt agar), and their production is regarded as practically diagnostic. In the animal body also departures of bacteria from their characteristic morphology have been frequently noted. One can find in streptococcus infections of the throat large cocci (megacocci) bearing little resemblance to ordinary streptococci, yet cultures from such throats may yield only streptococci. With the plague bacillus the appearance of enlarged, distorted forms is not uncommon in both natural and artificial infections.

Organisms exhibiting an atypical morphology are usually called "degeneration" or "involution" forms. A number of investigations bearing upon their origin have appeared within fairly recent years. Thus Wilson ${ }^{\mathrm{I}}$ has found that when Bacillus coli, Bacillus typhosus, Bacillus enteritidis of Gärtner, the Friedländer bacillus, and the plague bacillus are cultivated on media containing urine, a great diversity of forms results. The organisms are large and distorted and filamentous elements are common. $\mathrm{Hata}^{2}$ has shown that the addition of magnesium chloride to agar upon which dysentery and plague bacilli are grown leads to marked variation in the morphology of

'IVilson, W. J.: J. Path. \& Bact., I1, 394. 1906.

'Hata, S.: Centralbl. f. Bakteriol., 46, 289. 1908. 
these organisms. Reed and Orr ${ }^{\mathrm{I}}$ attribute such morphological changes to the influence of the hydrogen-ion concentration in the media. Any acidity or alkalinity close to the limiting reaction for successful cultivation leads to the appearance of these aberrant forms. Changes in morphology cannot be attributed solely to the influence of the hydrogen-ion concentration since $\mathrm{Vay}^{2}$ had previously found that the addition of dyes like dahlia to the media leads to the production of long dye-stained threads by Bacillus typhosus and Bacillus paratyphosus. According to Henrici, ${ }^{3}$ who has studied the involution forms in Bacillus coli especially, variation in morphology seems to correlate with autolysis of dead cells. The rate of autolysis depends upon the degree of acidity or alkalinity of the medium.

That bacteria may have different sizes at different stages has long been suspected. Evidence substantiating this view has been presented by Clark and Ruehl, and by Henrici. Clark and Ruehl ${ }^{4}$ have noted that in general very young bacteria, in the first few hours of growth, are much larger than older organisms. This does not hold for all species, however, since these authors found that this enlargement does not occur with diphtheria and glanders bacilli. Henrici ${ }^{5}$ has found that morphological variations occur in the lag period of bacterial growth. The initiation of growth is marked by a transformation to embryonic or growing cells which are considerably larger than the cells produced during the phase of maximum development.

The production of large, irregular involution forms regarded as characteristic of the plague bacillus has been found by Smillie ${ }^{6}$ in other representatives of the hemorrhagic septicemia group. This work has not thus far been definitely confirmed. Involution forms occur with such species as Bacillus suisepticus, but they are not as marked as are those in Bacillus pestis.

Finally, a new point of view in regard to the branching of bacteria has been brought out by Gardner ${ }^{7}$ who believes that probably many pathogenic bacilli grow by three-point branching from $Y$-shaped forms. If Gardner's observations can be confirmed they will give us a different conception of bacterial multiplication and may explain many obscure points in morphology and physiology.

\section{FILTERABLE FORMS OF BACTERIA}

Under ordinary circumstances the passage of bacteria through the pores of filter material like the siliceous earth in Berkefeld candles and the unglazed porcelain in Chamberland bougies depends on the size of the pores and the size of the bacteria, although other factors of great importance may modify the results. The most important of these are the amount of pressure or suction applied to force the fluid through the filter mass, the composition of the fluid in which the bacteria are suspended, and its reaction. At the same time the type of the micro-organisms has an important influence on their ability to pass through filter material, and it has long been recognized

\footnotetext{
${ }^{1}$ Reed, G., and Orr, J. H.: J. Bact., 8, 103. I923.

2Vay, F.: Centralbl.f. Bakteriol., 55, 193. I91о.

${ }^{3}$ Henrici, A. T.: J. Infect. Dis., 39, 429. I926.

${ }^{4}$ Clark, P. F., and Ruehl, W. H.: J. Bact., 4, 615. I9r9.

5 Henrici, A. T.: J. Infect. Dis., 38, 54. 1926.

${ }^{6}$ Smillie, W. G.: ibid., 27, 378. 1920. $\quad{ }^{7}$ Gardner, A. D.: J. Path. \& Bact., 28, 189. 1925
} 
that the flexible spirochetes can pass filters more easily than other forms of bacteria. On numerous occasions bacteriologists have found that the effluent from the finest filters may yield species like the original on cultures, although organisms cannot be found on microscopic examination. At the same time the effluent from suspensions of pathogenic organisms may produce disease in animals although organisms cannot be found on examination, or obtained in cultures. Unexpected results of this character were usually attributed to defects in the filter material permitting a small number of viable organisms to pass, or to a prolonged time in the process of filtration which permitted motile organisms to penetrate the filter mass, or viable bacteria to grow through. It was eventually recognized that this type of explanation did not always suffice to explain the results and that other possibilities must be considered.

Theoretically, the bacterial cell may at times develop into morphological miniatures of the original but with dimensions only fractions of the standard, or it may produce a kind of spore or seed within its walls, visible, filterable and viable, but morphologically unrecognized except as granules of characteristic size, shape, and staining reaction. Finally, the bacterial cell as we see it ordinarily may be but one stage in a complicated life-cycle, another stage being represented by filterable units, invisible by present methods, but viable in cultures, or viable only in the human or the animal body.

While Schaudinn in his investigations of protozoa and spirochetes anticipated theoretically much of the work of more recent years and expressed his belief that certain species produce units which can pass filters, Gotschlich, as mentioned above, was the first to emphasize the possibility of minute units among the simpler bacteria. In his most recent publication Gotschlich ${ }^{\mathrm{I}}$ accepts filterable forms of bacteria as demonstrated and states that they may be differentiated from the filterable and inanimate products of bacteria by the fact that they are viable, in some instances capable of growth and culture, and in other instances capable of producing a characteristic disease picture in experimental animals. With bacteria obviously too large to pass through filters Gotschlich again emphasizes his earlier opinion that a fractional part of the cell may escape and a normal adult cell regenerate from this portion. A vast amount of work has been carried out to substantiate views of this nature, among which may be mentioned the extensive studies of life-cycles of bacteria by Almquist, Enderlein, Fuhrmann, Löhnis, Mellon, and others. Of especial importance in this immediate connection is the work on the tubercle bacillus.

Much $^{2}$ in 1907 found small gram positive granules in the tubercle bacillus forming an integral part of the cell and which can be recognized outside it by their peculiar appearance. With material containing these granules and no regular tubercle bacilli, Much believed that he had produced tuberculous lesions in experimental animals from which the characteristic acid fast organisms could be obtained. A little later Fontes ${ }^{3}$ pointed out that the Much granules are able to pass through Berkefeld candles. $\mathrm{He}$ inoculated guinea pigs with filtered granules and found no lesions except in the spleen. With spleens from the first series of guinea pigs he inoculated another series, the ma-

\footnotetext{
${ }^{2}$ Gotschlich, E.: Handb. d. path. Mikroorg., 1, 33. 1927.

${ }^{2}$ Much, H.: Beitr. z. Klin. d. Tuberk., 8, 85. 1907.

${ }^{3}$ Fontes, A.: Centralbl.f. Bakleriol., Abt. I, Ref., 51, 244. I91 2.
} 
jority of which showed tuberculous lesions from which the tubercle bacillus was cultivated. On the basis of these experiments Fontes concluded that the Much granules represent a filter-passing stage of the tubercle bacillus. Spengler ${ }^{I}$ noted, in tuberculous sputum, granules somewhat like the Much granules in morphology but acid fast in their staining reactions. In cultures from this material Spengler found the same acid fast granules together with small acid fast bacilli. After some time the cultures revealed acid fast bacilli of the normal size. These minute bacillary types Spengler named "Splitter" and regarded as filterable stages in the life of the tubercle bacillus. Splitter forms were more frequently noted by Spengler in bovine lesions than in human, and this has subsequently been confirmed.

A number of observers, such as Hauduroy and Vandremer ${ }^{2}$ and Valtis, ${ }^{3}$ have employed tuberculous discharges filtered free of adult tubercle bacilli and injected them into animals. These animals later developed tuberculous lesions from which tubercle bacilli were obtained. Valtis was unable to get cultures from his filtrates but found multiple glandular enlargements in his inoculated guinea pigs. Many acid fast tubercle bacilli were present in the visceral and pneumonic lesions. These animals reacted to tuberculin just as did the animals inoculated with the unfiltered tuberculous material. Valtis concluded that the filtrates from the tuberculous material contained the tubercle bacillus in some exceedingly small form capable of passing his filters. His conclusions have recently been confirmed by Arloing 4 who employed tuberculous fluids from a variety of lesions and cultures of the tubercle bacillus. With carefully controlled filters the filtrates were found to be sterile. In animals inoculated with the filtrates the lymphatic glands were enlarged in a characteristic manner, and microscopic examination revealed numerous tubercle bacilli. Finally, De Potter ${ }^{5}$ made use of materials containing the avian type of the tubercle bacillus and controlled his filtrations with the minute organisms which cause chicken cholera. His filtrates were sterile while his experimental animals developed tuberculous lesions from which the avian tubercle bacillus was cultivated.

Observations similar to those made on the tubercle bacillus have been reported for a number of other organisms-for the dysentery bacillus by Hauduroy; for the typhoid bacillus by Almquist, Fijgin, and Bergstrand; and for Bacillus coli by Izar d'Herelle, and Tomaselli. The conception of filterable stages in these organisms does not differ materially from that in regard to the tubercle bacillus, but the experimental results are by no means as clear. For the present we can only state that very strong evidence has been presented in favor of filterable stages in the tubercle bacillus, but that more extensive confirmation of the work is necessary before the question can be regarded as settled.

\section{SPECIAL ORGANS OF REPRODUCTION}

Intimately associated with the question of filterable stages is that of the presence of special, complete morphological structures in the simpler bacteria analogous to the

spengler, C.: Ztschr.f. Hyg. u. Infektionskrankh., 49, 541. I905.

${ }^{2}$ Hauduroy, P., and Vandremer, A.: Compt. rend. Soc. de biol., 89, I276. I923.

3 Valtis, J.: ibid., 90, 74. I924; Ann. de l'Inst. Pasteur, 38, 453. I924.

4 Arloing, F.: Bull. Acad. de méd., Paris, 96, зог. 1926.

5 de Potter, F.: Compt. rend. Soc. de biol., 96, 138. 1927. 
conidia of the actinomyces which are definitely reproductive elements and serve to perpetuate the species. It is debatable whether it is advisable to employ such an analogy since Drechsler has pointed out that the actinomyces form a group closely related to, and possibly forming a subdivision of, the hyphomycetes. Knoblike protrusions have been observed in many species of bacteria, and certain authors have come to regard them as conidia, thereby claiming a complicated cycle of development for the ordinary simple bacteria. Almquist, Enderlein, and Fuhrmann have emphasized this phase of development. Thus Almquist ${ }^{\mathrm{I}}$ has found, in cultures of the typhoid bacillus, the dysentery bacillus, and the cholera vibrio, spherical or globular elements and giant cells, often with distinct internal structure, from which normal forms are regenerated. The transformation of these aberrant forms to the normal has been observed directly under the microscope by Almquist. Similar observations have been made in this country by Löhnis, Mellon, and Bergstrand, with diphtheria bacilli and with streptococci.

Large spherical bodies had previously been observed by Swellengrebel in the larger spirilla but were regarded by him as no longer capable of multiplication. The ability of these knoblike protrusions and spherical bodies to regenerate normal individuals may be regarded as practically settled in view of Almquist's observations, but the interpretation of these bodies as true conidia can hardly be accepted at the present time. Almquist and Löhnis have further described a kind of sexual multiplication, consisting of conjugation or amalgamation of two forms. Interesting as such observations may be, real proof for sexual multiplication in bacteria is still lacking. It should be remembered, however, that if bacteria continue to multiply indefinitely without some kind of change which represents conjugation they form an exception to the laws governing other free-living plants and animals.

\section{INTERNAL STRUCTURE}

The characteristics of the internal structure of the simpler bacteria have been the subject of investigation since the earliest days of bacteriology, with particular reference to the question of nuclear material. Opinions have ranged from the view that the entire cell represents the nucleus of higher cells to the view that no true nuclear material is present in them. Chemical analysis has thrown much light upon the kinds of chemical substances present in the bacteria, and the chemical demonstration of nuclear material has stimulated bacteriologists to approach the question again and make use of the more refined staining methods devised by the cytologists. As a result, a kind of diffuse nucleation was established for the bacteria, nuclear material (chromatin) being distributed through the bacterial cytoplasm. Certain authors like Nakanishi ${ }^{2}$ and Dobell, ${ }^{3}$ however, have always claimed the presence of true morphological nuclei. Thus Nakanishi has described minute spherical bodies in Staphylococcus aureus, in the middle of the cells, which he regards as nuclei. Dobell found true morphological nuclei in a spiral organism which he named Paraspirillum vejdov$s k i$, but which was not cultivated. There is still some doubt whether Dobell's organ-

I Almquist, E.: Ztschr.f. Iyg. u. Infektionskrankh., 83, I. I9 7 .

${ }^{2}$ Nakanishi, K.: Centralbl.f. Bakteriol., 30, I45. I90г.

3 Dobell, C. C.: Arch.f. Protisl., 24, 907. I9I2. 
ism should be included among the bacteria. Douglas and Distaso, ${ }^{\mathrm{T}}$ however, working with a capsulated bacillus easily cultivated by them from cases of respiratory infections, were able to stain successfully certain cellular units closely resembling true nuclei.

Recently Gutstein, ${ }^{2}$ by staining methods, has made out a differentiation of the bacterial cytoplasm into ectoplasm and endoplasm. In the endoplasm he finds a macronucleus and a micronucleus. The ectoplasm of the gram positive bacteria Gutstein $^{3}$ later has shown to contain a basic ground substance demonstrable by malachite green and tannin, and an acid body demonstrable by Victoria blue. This acid substance Gutstein regards as a lipoid. Somewhat similar observations have been made by Churchman ${ }^{4}$ in this country in his valuable studies on the gram reaction. He has found that Bacillus anthracis consists of two distinct parts, a gram positive cortex and a gram negative medulla. The term "cortex" as used by Churchman is equivalent to ectoplasm and the term "medulla" to endoplasm. The cortex may be removed by exposure to acriviolet or gentian violet and sometimes by hydrolysis in distilled water. The material lost is probably protein in character since the Berkefeld filtrate of a suspension of Bacillus anthracis exposed to gentian violet gives a positive ninhydrin test. Bessubet $z^{5}$ believes that the bodies inside bacteria and demonstrable by the use of the Giemsa stain are true morphological nuclei. Similar conclusions have been reached by Schumacher ${ }^{6}$ on the basis of chemical reactions.

In view of the recent investigations on the internal structure of the bacteria we can only say that while true morphological nuclei cannot be regarded as definitely proved, material giving the staining reactions of chromatin may be found in a great many different bacteria. In stained specimens this chromatin is sometimes agglomerated in masses which resemble the nuclei of plant and animal cells.

\section{CAPSULES}

Various new methods for the demonstration of capsules have been devised during the past few years and our ability to fix and stain these structures has been considerably enhanced. In addition to these newer staining methods a number of investigations have been made in regard to the chemical composition of the capsular substance. It should be noted that these investigations relate to the capsules of the true encapsulated bacteria like the pneumococcus, the Friedländer bacillus, and to certain intestinal organisms like Bacillus coli and not to saprophytic bacteria. Such saprophytes as Bacillus subtilis and Bacillus mesentericus under certain circumstances are provided with beautiful capsules, and it is certain that a great many different species may have a capsular material deposited about them under environmental stimuli. With the organisms mentioned above capsules form an integral part of the bacterial cell. With such species as the Friedländer bacillus Toenniessen ${ }^{7}$ has shown that the capsule is a

1 Douglas, S. R., and Distaso, A.: Centralbl.f. Bakteriol., 63, I. I9I 2.

${ }^{2}$ Gutstein, M.: ibid., 95, 357. 1925. ${ }^{3}$ Gutstein, M.: ibid., p. I. I925.

4 Churchman, J. W.: Proc. Soc. Exper. Biol. E Med., 24, 737. 1927.

5 Bessubetz, S. K.: Centralbl. f. Bakteriol., 96, I77. I925.

${ }^{6}$ Schumacher, J.: ibid., 97, 81. 1926.

7 Toenniessen, E.: ibid., 65, 23. I912; ibid., 85, 225. I921. 
secretory product and made up of galaktan, a polysaccharide of galactose, and contains no protein. The investigations of Toenniessen were later extended by Kramár ${ }^{\mathrm{I}}$ who found that the capsule of the Friedländer bacillus consists of galaktan, that of Bacillus anthracis of a glycoprotein, and that of Bacillus radicicola of a dextran. The capsules of different bacteria thus differ among themselves radically in their chemical composition.

Recently Avery, Heidelberger, and Goebel $^{2}$ have approached the question of capsule formation in bacteria from still another angle. From the pneumococcus type II they have obtained the capsular substance and found it to be a nitrogen-free polysaccharide. This substance was non-antigenic. From the bacterial cell they obtained a protein substance which was antigenic but had no type specificity. In order to get type-specific reactions in animals it was necessary to employ both the protein constituent of the cells and the capsular substance before separation of the cell mass into its constituents. A similar carbohydrate, a nitrogen-free polysaccharide, was obtained from a strain of the Friedländer bacillus. The carbohydrate obtained from the type II pneumococcus was the same as the substance found by Dochez and Avery ${ }^{3}$ in the blood and urine in pneumococcus infections in man. Julianelle, ${ }^{4}$ working with rabbits infected with a strain of the Friedländer bacillus, found specific substances in the urine and blood which were practically identical with those in the soluble portion of the organisms. Finally, Smith ${ }^{5}$ has reported biochemical studies on certain encapsulated strains of Bacillus coli. She states that the capsule materials consist of 80 per cent of a special carbohydrate, a hexose, together with a small amount of glycuronic acid. In order to show that this carbohydrate really represents capsule substance, she employed strains of the encapsulated Bacillus coli deprived of their capsules through mutation cultivation (see Theobald Smith). ${ }^{6}$ On comparing the filtrates from the two organisms, she was able to show that the precipitin test was one hundred times less active specifically with the filtrate from the mutant than with the filtrate from the encapsulated strain. These observations on the kinds of substances which may be obtained from capsules are of fundamental importance and must, as they are further amplified, aid materially in the interpretation of the composition and especially the function of capsules. ${ }^{7}$

\section{SPORES}

As already mentioned, the conception of Kruse that spores in the bacteria are resting bodies like the cysts of the protozoa has had wide acceptance. According to this conception, spores are produced under unfavorable conditions, especially as regards food supply, serve purely for the perpetuation of the species, and show no evidence of vital activity. A number of reports have recently been published which must change our interpretation of spore formation. Thus Ruehle ${ }^{8}$ has found that spores

I Kramár, E.: Centralbl. f. Bacteriol., 87, 401. I922.

${ }^{2}$ Avery, O. T., Heidelberger, M., and Goebel, W. F.: J. Exper. Med., 42, 701, 709, 727. 1925.

3 Dochez, A. R., and Avery, O. T.: ibid., 26, 477. 1917.

${ }^{4}$ Julianelle, L. A.: ibid., 46, I 13.1926.

5 Smith, D. E.: ibid., p. 155. 1927.

${ }^{7}$ See chapter lii in this volume.

${ }^{6}$ Smith, T.: ibid., p. I4I. 1927.

${ }^{8}$ Ruehle, G. L. A.: J. Bact., 8, 487. 1923. 
show evidence of enzymic activity even when no evidence of germination is present. Oxidases as well as gelatinase can be demonstrated in spore material. Magoon ${ }^{\mathrm{x}}$ believes that spores are not dormant under ordinary conditions but are sluggishly active. Resistance to heat is not fixed but variable, being influenced by age, temperature, heredity, etc. This resistance to heat may be increased by selective action (Magoon). ${ }^{2}$ In Bacillus mycoides certain organisms surviving after heating seem to have greater resistance to heat than the original spores. Darányi ${ }^{3}$ has recently pointed out that the ability of certain species like Bacillus anthracis, Bacillus subtilis, and Bacillus anthracoides to form spores depends in general upon the same op timum conditions which lead to good vegetative development of the bacteria. Spore formation is brought about primarily by colloidal reactions; the most important of these is a diminution in the water content of the organisms, resulting therefore in a shrinking of the colloids. Under natural conditions spore formation begins when the organisms grow older. This aging is primarily a loss in water on the part of the colloids (hysteresis). The lack of food material for the bacteria has a favorable influence on spore formation only in that the organisms get poorer in water. With artificial dehydration, Darányi was able to bring about spore formation in well-developed young bacilli.

Koser and $\mathrm{McClelland}^{4}$ have added interesting facts in regard to the fate of spores in the animal body. The spores of Clostridium tetani, $\mathrm{Cl}$. putrificum, $\mathrm{Cl}$. chauvei, and $\mathrm{Cl}$. oedematis-maligni are capable of withstanding the deleterious influence of the body tissues and may be transported from the site of inoculation to different organs, where they remain latent. Aerobic bacteria do not apparently suffer the same fate. Such observations are of considerable importance in an explanation of the occurrence of tetanus after wounds in cases where the wound itself is originally uninfected; it is possible that the latter infection is due to the presence of latent spores in other parts of the body. Finally, mention must be made of the newer methods of determining the heat resistance of spores. Esty and Williams ${ }^{5}$ have estimated resistance by heating a large number of tubes containing spores and plotting curves from the results. The rate of destruction corresponds in general to the rate of destruction of bacteria by disinfectants worked out by Chick, by Madsen and Nymen, and by Eijkman.

\section{MULTIPLICATION IN THE SPIROCHETES}

The flexible spirochetes differ considerably in their morphology from the simpler bacteria but not sufficiently to separate them from the group in which the first representative was placed originally by Ehrenberg. In at least three particulars is their morphology interesting to us at the present time-their motility, method of division, and the possession of granules which are occasionally extruded from the cell and which may be reproductive bodies. In regard to motility it has now been satisfactorily proved that the spirochetes possess no organs of locomotion like flagella, as was originally maintained. Flagella-like structures may occasionally be found, attached to the

'Magoon, C. A.: ibid., xI, 253. 1926.

${ }^{2}$ Magoon, C. A.: J. Infect. Dis., 38, 429. 1926.

3 Darányi, J.: Centralbl. f. Bakteriol., Abt. II, 71, 353. 1927.

${ }^{4}$ Koser, S. A., and McClelland, J. R.: J. Hed. Research, 37, 259. I917.

${ }^{5}$ Esty, J. R., and Williams, C. C.: J. Infect. Dis., 34; 516.1924. 
outer rim of the organisms, but it has now been established that these are not flagella but bits of periplast torn off in fixation and staining. Under dark-field illumination no refraction of light can be detected like that seen with the ciliated bacteria. The crista present in some of the larger saprophytic spirochetes, the cristispiras, has been shown to lack independent motility. Motility in the spirochetes must therefore be attributed to streams of protoplasm passing through the bodies of the cells or to the presence of contractile elements within, like the myonemes of certain protozoa. No definite demonstration of myonemes has thus far been effected, although Noguchi has observed bodies which strongly suggest them. It is possible that streams of protoplasm do exist in these organisms and that their motility is due to changes of surface tension. A satisfactory explanation of motility is very much needed.

It is now generally admitted that the simpler spirochetes (treponema, spironema, leptospira) multiply by transverse fission. It is true that appearances suggestive of longitudinal fission have often been noted, but these were explained as the separation of two organisms tightly coiled about each other. The chief evidence that spirochetes divide transversely and not longitudinally depends upon accurate measurements of the diameter of the cells, a method of study carried on especially by Shellack. ${ }^{{ }}$He assumed that the diameter of the newly formed cells must at some time be one-half of the diameter of the cells before division, if longitudinal division takes place. In careful measurements of certain spirochetes (Spironema duttoni, novyi, etc.) Shellack was never able to find organisms which were twice as thick as the thinnest forms. He was, however, always able to find organisms twice as long as the shortest forms. He therefore concluded that the spirochetes always divide transversely and not longitudinally. Lange $^{2}$ has recently called attention to the fundamental misconception in our interpretation of measurements of all morphological types of bacteria. After equal binary fission of spherical organisms the diameter of the daughter-cells is not one-half that of the mother-cell, but equals the cube root of one-half the cube of the diameter of the mother-cell, or over three-quarters of it. With cylindrical organisms the daughtercells are half as long as the mother-cells, after transverse division. After longitudinal division the diameter of the daughter-cells equals the square root of one-half the square of the diameter of the mother-cell, or a little less than three-quarters of it. Since the spirochetes are practically cylindrical organisms, the failure to find organisms whose diameter is one-half the diameter of the thickest cells cannot be regarded as excluding longitudinal division.

A large number of spirochetes, especially the pathogenic varieties, show minute spherical bodies in the interior of the cell, and these are occasionally extruded. These bodies have frequently been regarded as reproductive elements. This conception we owe primarily to Leishman who found numbers of granules in ticks infected with $S p i$ rochaeta duttoni but no spiral organisms. With these ticks he was able to convey spirochetal diseases experimentally. The matter was thoroughly investigated by Hindle ${ }^{3}$ and by Balfour. ${ }^{4}$ Hindle, studying ticks infected with Spirochaeta gallinarum, found

Shellack, C.: Arb. a. d. kais. Gesamle., 27, 364. I908.

${ }^{2}$ Lange, L. B.: J. Bact., 14, 275. 1927.

3 Hindle, E.: J. Parasitol., 4, 463. Cambridge, I9 I .

4 Balfour, A.: J. Trop. Med., ıо, I53. I907; Brit. M.J., 2, İз30. 1907. 
granules and both small and large spirochetes representing every gradation in size from granules to normal adult spirochetes. While he did not observe the actual growth of granules into spirochetes, he concluded from the varying numbers of granules and spirochetes that the change must occur in this direction. Balfour described a special type of spirochete concerned with fowl spirochetosis which he named Spirochaeta granulosa penetrans, and which he believed entered the red-blood corpuscles of the fowl and broke up into granules that were responsible for a peculiar recrudescence of the disease known as the "after-phase." There can be little doubt that granules are produced in a number of both pathogenic and saprophytic spirochetes, and are shed from the cells under certain circumstances. That granules are reproductive elements cannot be regarded as settled since Dutton and Todd have shown that adult spirochetes can sometimes be found on prolonged search in infective ticks along with the granules. Filtrates from spirochetal material showing an abundance of granules may also reveal a few spirochetes which because of their peculiar boring motility are able to pass through the filter substance. Recently Meirowsky ${ }^{\mathrm{I}}$ has observed swollen bodies and coiled forms in Treponema pallidum in secondary syphilis and has suggested that they are stages in the life-history of the organism. Szilvási and Fehér ${ }^{2}$ have found Meirowsky's forms in both primary and secondary lesions in syphilis and agree with him in regarding them as infective stages of the treponema. It may be noted in this connection that Aristowsky and Hölzer ${ }^{3}$ have described peculiar coiled, twisted forms in Spirochaeta obermeieri and believe that this organism also passes through a definite life-cycle.

\section{THE RICKETTSIAE}

Following the discovery of pleomorphic bacteria-like bodies in Mexican typhus (tabardillo) by Ricketts a number of similar organisms were observed in various diseases and in their insect vectors. For this group the term "Rickettsia" was proposed by Da Rocha-Lima4 and the type species named Rickettsia prowaseki in honor of Ricketts who discovered the first representative and von Prowazek who devoted many years to its investigation. The significance of this group of micro-organisms will be considered in chapter xxxix of this volume, but one or two points should be mentioned here in regard to their morphology. Hertig and Wolbach ${ }^{5}$ and Cowdry ${ }^{6}$ in this country have noted their wide distribution in insects and arachnids. According to Arkwright, Atkin, and Bacot, ${ }^{7}$ the rickettsiae are characterized by their minute size, usually being less than $0.5 \mu$ in diameter; their pleomorphism from round, coccus-like bodies and diplococci to minute bacilli and threadlike forms; their resistance to ordinary aniline stains; their loss of gram stain and affinity for Giemsa; their absence of motility; their resistance to cultivation on ordinary media; and their occurrence in very large numbers in the gut of blood-sucking insects. In some cases they may be found

${ }^{1}$ Meirowsky, R.: Mïnchen. med. Wchnschr., 60, I870. I9I3.

'Szilvási, J., and Fehér, D.: Centralbl.f. Bakteriol., 95, 436. I925.

3 Aristowsky, W., and Höltzer, R.: ibid., p. I75. I925.

${ }_{4}^{4}$ Da Rocha-Lima: Mïnchen. med. Wchnschr., 67, г38г. г9г6.

5 Hertig, M., and Wolbach, S. B.: J. Med. Research, 44, 329. I924.

${ }^{6}$ Cowdry, E. V.: J. Exper. Med., 37, 431. I923.

7 Arkwright, J. A., Atkin, E. E., and Bacot, A.: J. Parasitol., 13, 27. I921. 
in other organs. Later Wolbach, Todd, and Palfrey ${ }^{\mathrm{T}}$ emphasized the specificity of the rickettsiae for certain insect hosts. Hertig and Wolbach further amplified the definition of the rickettsiae, suggesting that the term should be limited to proved pathogenic organisms which are pleomorphic, non-motile, gram negative, stain rather lightly with the aniline dyes, and have a tendency to an intracellular habitat.

The rickettsiae have been cultivated in only a few instances. Nöller ${ }^{2}$ has been able to grow Rickettsia melophagi on blood agar inactivated by heating to $57^{\circ} \mathrm{C}$., and Sellards ${ }^{3}$ recently has cultivated a rickettsia-like micro-organism from tsutsugamushi disease. This organism was pathogenic and was named by him Rickettsia nipponica. In consequence it is difficult to come to any definite conclusions as to their nature and proper classification. It is quite clear that they are not mitachondria (Cowdry), ${ }^{4}$ and in many of their characteristics they resemble the bacteria very closely. Their pleomorphism is not greater than that of many bacterial species. In size they correspond to many of the smaller bacteria. They have no definite internal structure although a morphological nucleus has been claimed by Epstein ${ }^{5}$ for Rickettsia prowazeki. They stain badly by the ordinary aniline dyes but no more so than certain bacterial species like the glanders bacillus and the cholera vibrio. When cultivated they grow on media similar to those used for the majority of bacteria. For the present the rickettsiae should probably be included among the bacteria. In this connection the observations of Cowdry and of Wolbach and Schlesinger are of great importance. Cowdry ${ }^{6}$ has described a rickettsia (Rickettsia ruminantium) in Amblyomma hebraeum, the bont tick, which transmits the virus of the disease of sheep, goats, and cattle known as "heart water." These organisms lay in the endothelial cells of the renal glomeruli and in the superficial gray matter of the cerebral cortex. They were uniform coccus-shaped bodies, $0.2-0.5 \mu$ in diameter, sometimes in diplo formation. They stained deep clear blue by Giemsa, and easily by Löffler's methylene blue and other aniline dyes. They were gram negative. Such organisms would naturally be included in the group of rickettsiae as above outlined. Cowdry further described in a number of ticks (Argasidae and Ixodidae) certain non-pathogenic, gram negative organisms characterized by their resemblance to bacteria morphologically, their large size and their intracellular habitat. Obviously such organisms would not meet the exacting requirements laid down by Hertig and Wolbach for the rickettsiae. Size and pathogenicity are somewhat doubtful characteristics for classification, and it is difficult to exclude from any group organisms which possess in the main the character of the group but are larger and endowed with pathogenic action. For the present the non-pathogenic organisms described by Cowdry should probably be regarded as rickettsiae and included with them in a larger group of pathogens and non-pathogens.

' Wolbach, S. B., Todd, J. L., and Palfrey, F. W.: The Etiology and Pathology of Typhus. Cambridge, r 1922.

${ }^{2}$ Nöller, W.: Arch.f. Schiffs- u. Tropen-Hyg., 21, 53. 1917.

${ }^{3}$ Sellards, A. W.: Am. J. Trop. Med., 3, 529. 1923.

${ }^{4}$ Cowdry, E. V.: loc. cil.

${ }^{5}$ Epstein, H.: Centralbl.f. Bakteriol., 87, 553. 1922.

${ }^{6}$ Cowdry, E. V.: J. Exper. Med., 42, 231, 253. 1925.

7 Ibid., 4I, 8I7. 1925 
Finally, Wolbach and Schlesinger ${ }^{\mathrm{T}}$ have brought to cultivation the micro-organisms of Rocky Mountain spotted fever (Dermacentroxemus rickettsi) and typhus (Rickettsia prowazeki) in tissue-plasma cultures. These authors have shown that the parasite of Rocky Mountain fever shows a definite series of morphological changes in ticks, from coccus to bacillary forms. Such observations suggest a somewhat more complicated cycle of development than that accepted for the ordinary bacteria and bring further evidence in favor of the view that binary fission and spore formation are not the only methods by which the bacteria multiply.

${ }^{x}$ Wolbach, S. B., and Schlesinger, M. J.: J. Med. Research, 44, 231. 1923-24. 


\title{
CHAPTER II
}

\section{THE CHEMICAL STRUCTURE OF BACTERIA ${ }^{x}$}

\author{
TRAUGOTT BAUMGÄRTEL
}

Technischen Hochschule, Munich, Germany

Like all plant and animal organisms, bacteria require ten elements-carbon, hydrogen, oxygen, nitrogen, phosphorus, sulphur, potassium, calcium, magnesium, and iron-as indispensable building stones for their body substance.

While bacteria take the metals (potassium, calcium, magnesium, and iron) as well as the metalloids (phosphorus and sulphur) mostly in the form of simple mineral salts and satisfy their need for hydrogen and oxygen chiefly from water, they are able, in the assimilation of carbon and nitrogen, to utilize numerous and widely diverse sources of nutrition. All substances-from complex natural compounds like proteins and polysaccharides down to their simple cleavage and decomposition products and the very elements like nitrogen and hydrogen-can be utilized by the bacteria in their metabolism and modified in many ways. For example, in their carbon nutrition they utilize in this way complex plant as well as animal substances, proteins and their cleavage and decomposition products, carbohydrates, fats, alcohols, acids, carbohydrates, carbon dioxide, carbon monoxide, and methane. Thus, the bacteria, in spite of all advantages and choice of many food substances, show an unparalleled ability for adjustment to the sources of food offered them. Furthermore, the entire process of nutrition in the bacteria is in large part dependent on the hydrogen and oxygen content of the culture medium as well as on the reaction and temperature.

For these reasons, the chemical composition of different kinds of bacteria varies markedly; even in one and the same kind of bacteria there are considerable variations, so that generally valid statements about the chemical composition of the body substance of bacteria can be made only with due consideration of the breadth of their biological variations.

\section{THE WATER CONTENT OF BACTERIA}

In order to determine the water content of bacteria by chemical analysis, the usual procedure is carefully to scrape off the colonies grown on solid medium, centrifugate the liquid growth promptly, and weigh the material thus obtained in its moist, living condition ("fresh weight"); afterward, dry it at $100^{\circ}-110^{\circ} \mathrm{C}$. and weigh it again ("dry weight"). The difference between the fresh weight and the dry weight-expressed per roo gm. of fresh mass of culture-is of course the water content. In this way it has been determined that the water content of most kinds of bacteria is rather high. On the average it amounts to between 75 and 85 per cent of the fresh weight. There are rather wide fluctuations according to the kind of bacteria and the growth of the organism chosen (whether on solid or liquid medium), and also according to the age of the

I For a more extensive summary, cf. Baumgärtel, T.: Grundriss der theoretischen Bakteriologie. Berlin, 1924. 
culture. It is clear that a particular kind of bacteria shows a different water content according to whether one is dealing with a young, fully developed culture in liquid culture medium or an old dying culture on solid medium. Especially rich in water are the mucus-producing kinds of bacteria. Species which store lasting reserve material like volutin, glycogen, or fat, and spores contain proportionately little water.

THE DRY SUBSTANCE OF BACTERIA

Differences in the water content of the bacterial cells lead to differences also in their dry substance, in which the proportions can vary markedly between the combustible ("organic") material and the non-combustible ("mineral") material. In general, a particular kind of bacteria when cultivated on liquid medium contains more water than when grown on solid medium. On the other hand, according to whether an increased content of mineral salts or of carbohydrates is put into the liquid or solid medium, sometimes the mineral part of the dry substance of bacterial growths is greater than usual, sometimes the organic part is greater. In view of the high variability of the bacteria, no general statements can be made beyond this. So far as reliable researches have shown, 70-97 per cent of a bacterium's dry weight is made up of organic substance, and correspondingly $30^{-}-3$ per cent is mineral substance.

THE ORGANIC DRY SUBSTANCE

Doubtless the organic dry substance of bacteria consists of numerous compounds, still in part wholly unknown, which on account of their common properties belong chiefly to the proteins, carbohydrates, and fats. Like the water content of bacteria, the chemical composition of the organic dry substance also varies and is different in any one species according to the cultural conditions under which the colonies chosen for analysis were grown. For example, it has been found that cultures of Bact. prodigiosum grown on potato contain almost 50 per cent more organic dry substance than the cultures raised on yellow turnip, and that the cultures of the same species kept on potato at $33^{\circ} \mathrm{C}$. have more dry substance than similar cultures preserved at $16^{\circ} \mathrm{C}$. It is also demonstrable that four-to-six-day-old cultures on potato show a larger quantity of organic dry substance than cultures thirteen to sixteen days old.

Because in building their organic body substances the bacteria can adapt themselves, within definite but rather wide limits, to the nutrient medium on which they are growing, there can be no generalization as to the results of chemical analysis on the qualitative and quantitative composition of the organic dry substance. According to the findings in many studies, the protein content of the organic dry substance of most bacteria varies somewhere between 40 and 70 per cent, the carbohydrate content between ro and 30 per cent, and the fat or lipoid between I and Io per cent. In general, therefore, proteins make up the greater part of the bacterial body; then follow carbohydrates and fats or lipoidal substances.

As is evident from precipitation and agglutination reactions, different kinds of bacteria grown on the same nutrient medium and under conditions precisely similar in other respects can be differentiated with respect to their proteins, so that with the aid of the serological reactions they can be identified without further difficulty. Chemical analysis, on the other hand, offers at present no method of distinguishing the different 
kinds of bacterial proteins, for the methods are not sensitive enough and do not give unconditionally reliable results; nor do they permit generalization. At least worthy of mention, however, is the hydraulic-press method which produces from moist, living bacterial cultures, without deep-seated changes in the cell substance, protein-rich fluids. For example, in order to obtain in this way the protein material of the cholera vibrio, colonies of this organism are grown on nutrient agar; the thick, growing layer of vibrios is carefully lifted off by means of a platinum spatula; the colonies thus obtained are finely pulverized with diatomaceous earth and quartz sand in a mortar and through the addition of salt solution worked into a pulpy mass. This is thrown into a strong filter cloth and laid under the hydraulic press. By a pressure which is gradually increased to 400 or 500 atmospheres a press fluid is obtained which comes through the thick filter at first as a light, clear, protein-rich liquid, later changing its color in the air to yellow and brownish-the so-called "vibrio plasma." As the chemical investigation shows, the plasma is largely precipitable with acetic acid in the cold; it does not dissolve with excess of acetic acid, i.e., it acts like a nucleoprotein. In other respects the expressed fluid gives the usual protein reactions.

With the help of a number of color reactions the protein chemical differentiation of many kinds of bacteria can be carried out. The best known in this connection is the usual color method of Gram, which depends on the fact that salts of pararosanilin (for example, gentian violet and methyl violet) unite firmly with iodine and that certain kinds of bacteria take the color compounds thus formed very firmly, i.e., when treated with alcohol later, they do not so readily give up the color substance they have taken as do certain other bacteria. In consequence, bacteria may be separated into those "colored by Gram's method" (gram positive) and "not colored by Gram's method" (gram negative). The differential behavior of bacteria in the color test of Gram is due to an unexplained difference in the physico-chemical structure of the protoplasm. ${ }^{\mathrm{I}}$

In analogous ways certain protein or protein-like cell constituents can be detected in the bacteria. Among these may be included the reserve material (volutin) stored up in many bacteria in the form of colorless and strongly light-refracting spheres, visible under the microscope, the presence of which, for example, is utilized practically in the usual bacteriological tests for the diagnosis of diphtheria. Volutin is dissolved by warm water $\left(30^{\circ}\right)$ within two or three days. In water at $80^{\circ} \mathrm{C}$. volutin is dissolved in five minutes. Volutin fixed with heat or alcohol (or formol) is insoluble in boiling water. A 5 per cent or saturated aqueous solution of sodium carbonate dissolves volutin in five minutes, as does also caustic potash. A freshly prepared solution of Javelle water dissolves volutin in five minutes. A 5 per cent sulphuric or hydrochloric acid solution dissolves volutin in from five to ten minutes; a 25 per cent nitric acid solution dissolves it at once; and a I per cent acetic or osmic acid as well as a 5 per cent carbolic acid solution dissolves it slowly. On the other hand, alcohol, ether, chloroform, and carbon tetrachloride do not dissolve volutin. Potassium iodide-iodine solution, Millon's reagent, vanillin hydrochloride, and zinc chloride-iodine give no reaction with volutin; and trypsin and pepsin are also without effect. Moreover, dyestuffs also give a characteristic behavior with volutin. Methylene blue and carbol-fuchsin in Io

'See chap. iii (by Dr. Churchman) in this volume. 
per cent solution color volutin quite intensively, while eosin borax carmine and nigrosin do not stain it; safranin and Bismarck brown give a stronger color to volutin than to the cytoplasm.

As with the protein materials, so with the carbohydrates of bacteria-little definite investigation has as yet been done. Most of the work up to the present has concerned itself with the study of the carbohydrates in the composition of bacterial membranes and mucus. For example, the gelatin formed by Streptococcus mesenterioides has been analyzed. The purified masses of gelatin were extracted with 96 per cent alcohol, then boiled for a rather long time with milk of lime and the thickened masses obtained in this way precipitated with carbonic acid. From the precipitated calcium carbonate the mucous solution was poured off, cleared with hydrochloric acid, and precipitated with alcohol. The substance thus obtained is called "dextran." It appears that neutral lead acetate does not precipitate the concentrated dextran solution, while basic lead acetate produces a pastelike mass. Moreover, it happens that a heatsaturated solution of barium hydroxide precipitates from concentrated dextran solution an oily substance, and Fehling's solution, without itself being reduced, precipitates a mucous substance. When boiled with dilute sulphuric acid, dextran goes over into dextrose slowly; when heated to $120^{\circ} \mathrm{C}$., it goes over in a few hours.

Here may also be mentioned the researches on the carbohydrate content of the pellicle which appears in cultures of Bact. xylinum. The leather-like colonies were first cleansed with water, then boiled with a 20 per cent caustic potash, washed with dilute hydrochloric acid or with water, and finally treated with bromine. In this way there were produced colorless, transparent, thin films which dissolved in ammoniacal copper oxide as well as in concentrated sulphuric acid and, by element analysis, showed the composition corresponding to the formula $\mathrm{C}_{6} \mathrm{H}_{10} \mathrm{O}_{5}$. When the film was dissolved in concentrated sulphuric acid and this solution-after preliminary dilution with water-boiled, neutralized with barium carbonate, and filtered, its reactions in regard to dextro-rotation and reduction power were like dextrose.

The carbohydrates of the bacteria that are demonstrable by microchemistry are glycogen and iogen (granulose), which appear in the protoplasm of many cells as colorless, viscous-flowing masses. Glycogen and iogen differ microchemically in their behavior with iodine. If very dilute potassium iodide-iodine solution is added to bacteria which contain glycogen and iogen, only the iogen will be colored at first (blue); with stronger iodine solution the glycogen will also take (a dark red-brown) color. If the carbohydrate of the bacteria is colored only red brown, then it consists solely of glycogen; if, with a stronger iodine-KI solution it takes only a blue color, then only iogen is present. If carbohydrate-containing bacteria are boiled for five minutes in water, the carbohydrates are still demonstrable by means of iodine-KI solution. If pigmented carbohydrate-containing bacteria are boiled in water, they appear without color when examined on the warm stage of a microscope. On the other hand, they again show color when the iodine-KI solution is cold. If bacteria containing carbohydrates are treated for three minutes with boiling concentrated sulphuric acid, the carbohydrates are completely dissolved. ${ }^{\mathrm{r}}$

r For a discussion of polysaccharides ("specific soluble substances") from bacteria, cf. chap. lii (by Drs. Zinsser and Mueller) in this volume. 
Fat in bacteria appears mostly in the form of strongly light-refracting drops which in the young cells are very finely distributed in great numbers in the protoplasm; with increasing age, however, the drops join to form larger drops which occasionally fill the whole cell interior. Analytical studies on the fat or lipoid content of bacteria have been frequently conducted. From these it appears that the amount and the composition of the detectable fats, lipoids, and waxes vary for the several kinds of bacteria and are dependent upon the conditions of growth. Thorough investigations have been made most frequently with pure cultures of the tubercle bacillus, which has an unusually high fat content. In a very detailed study of this fat, four-to-five-month-old glycerol-bouillon cultures of this organism were killed in the autoclave at $1 \mathrm{ro}^{\circ} \mathrm{C}$., the colonies collected on filter paper and treated with hot water until all the ingredients of the nutrient medium were washed away. The material was then spread on porous earthenware plates and dried at $40^{\circ} \mathrm{C}$. In order to measure the fat contents of the pulverized bacterial masses, these were treated with various fat solvents in the Soxhlet extraction apparatus.

For a more precise determination of the fat of the tubercle bacillus, the same bacterial powder was extracted several times with chloroform, the different extract portions mixed, and, after distilling off the chloroform, dried at $100^{\circ} \mathrm{C}$. In this way there was obtained a dark-brown, semi-solid mass with glassy wrinkles and with the odor typical for tubercle bacillus cultures, like good wax from linden or flower honey. The melting-point of this fatty substance was $46^{\circ} \mathrm{C}$. Further chemical investigation showed that the fat of the tubercle bacillus is a completely homogeneous substance which displays no resemblance to any other fat or wax. It seems to be rather a mixture composed of free fatty acids, neutral fats, fatty-acid esters, and higher alcohols (lecithin, cholesterin), and, in addition, a large quantity of extractives which are insoluble in water and which, when heated with alkalies, disintegrate in part to form products soluble in the water.

The demonstration of bacterial fat is made by the usual microchemical methods. Glacial acetic acid and chloralhydrate dissolve fat; Javelle water does not dissolve it; osmic acid does not blacken it; iodine-potassium iodide solution colors it yellow brown. Caustic potash seems to saponify the fat. The behavior of the bacterial fat toward certain dyes is also notable. While the common stains (methylene blue, gentian violet, and fuchsin) do not color bacterial fat, staining succeeds with Sudan III, naphtol blue, and dimethylamidoazobenzol. The latter stains the fat yellow; Sudan III, red; and napthtol blue, a deep blue.

\section{THE MINERAL DRY SUBSTANCE}

Thorough investigations have been carried out on the quantitative analysis of the ingredients of the ash from many kinds of bacteria. It appears from these studies that the mineral dry substance of the bacterial body always forms only a small proportion of the cell substance, that it differs for the several kinds of bacteria, and that the amount and nature of the mineral substance in any particular species vary with the conditions of cultivation and the age of the culture. ${ }^{\mathrm{I}}$

\footnotetext{
I For a review, cf. Falk, I. S.: Abstr. Bact., 7, 44. I923.
} 
CHAPTER III

\title{
STAINING REACTIONS OF BACTERIA ${ }^{x}$
}

\author{
JOHN W. CHURCHMAN \\ Cornell University Medical College, New York
}

CHEMISTRY OF DYES

Bacteriological stains belong almost entirely to the group known as "aniline dyes." Since a number of these, however, are not derived from aniline and bear no direct relation to it, and since all are derivatives of the hydrocarbon, benzene $\left(\mathrm{C}_{6} \mathrm{H}_{6}\right)$, "coal-tar dyes" is a better term.

Coal-tar dyes are monacid salts of color bases or alkali salts of color acids. "Basic," "acidic," "neutral"- the descriptive terms usually applied to them-are not particularly fortunate terms since the dyes are not necessarily bases or acids, and even if called "basic" may have an $\mathrm{H}$-ion concentration on the acid side of neutrality. Basic dyes are usually encountered as dye salts of a colorless acid such as hydrochloric, sulphuric, oxalic, or acetic acid; acid dyes as sodium, potassium, calcium, or ammonium salts of dye acids. The terms "acidic" and "basic" as applied to dyes really refer to the affinity of the chromogenic radicle for acidic or basic groups as the case may be. The term "chromogenic radicle" leads directly to a consideration of the currently accepted theory as to the molecular structure of the dyes.

The basis of this structure is the benzene ring of Kekule from which-as is well known-an almost infinite number of derivatives can be formed. When the derivatives contain certain groups of elements known as "chromophores," these groups impart the property of color. Benzene derivatives containing chromophore radicles are known as "chromogens." Chromogens, although colored, are not dyes since they may have little or no affinity for fibers or tissues; the color they impart to fibers or tissues is a superficial coat easily removed by mechanical processes-the color does not "take." In order that a chromogen become a dye the chromogen derivative must contain, in addition to the chromophore, auxiliary groups which are known as "auxochromes." These have themselves little or no color and are not the cause of the color of the dye; but they impart to the compound the property of electrolytic dissociation, -furnish it with salt-forming properties, and thus convert it into a dye.

The formation of the yellow dye, picric acid, from the yellow chromogen, trinitro-benzene, by the addition of the auxochrome, hydroxyl $(-\mathrm{OH})$, illustrates present-day conceptions of the chemical structure of coal-tar dyes. When three $\mathrm{H}$ atoms in the benzene ring are replaced with the chromophore $\left(-\mathrm{NO}_{2}\right)$, tri-nitrobenzene results:

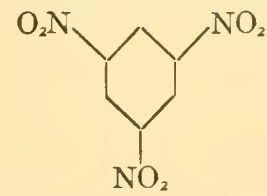

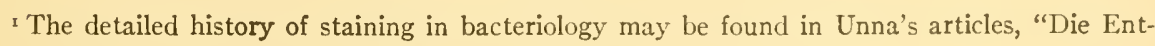
wicklung der Bakterienfärbung," Centralbl.f. Bakleriol., 3, 22-345. I 888. 
This yellow substance, which is insoluble in water, is neither an acid nor a base. Since it cannot dissociate electrolytically, it is incapable of forming salts. It is not therefore a dye. If, however, one more $\mathrm{H}$-atom in the benzene ring be replaced with the auxochrome hydroxyl $(-\mathrm{OH})$, picric acid is formed:

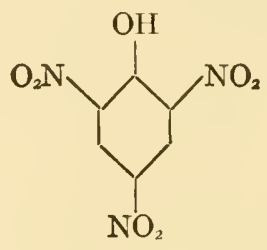

This yellow substance is an acid, capable of electrolytic dissociation and of forming salts with alkalies. It is a dye.

Some auxochromes (e.g., the amine group, $-\mathrm{NH}$ ) are basic; others (e.g., the hydroxyl group, $-\mathrm{OH}$ ) are acidic. The acidity or basicity of a dye-as these terms are used in the expressions "acidic" or "basic" dyes-is determined by the character of its auxochromes. According as the chromogen is united with acidic or basic groups, the dyes are known as "acidic" or "basic." If basic groups are united to an acid chromophore, the dye is more weakly basic than if the same basic groups were united with a basic chromophore. A dye retains its color only so long as its affinities for hydrogen are not completely satisfied. When they are satisfied, reduction occurs and colorless leukobodies are formed.

The polychrome stains stand in a class somewhat by themselves. In principle, all depend upon a combination of eosin and methylene blue, these elements not only staining as units, but acting together in combination. It is assumed that these compound dyes act on the protoplasm as follows: Certain parts of the cell have an affinity for the neutral stain and take it up as such. Others have an affinity for the basic dye and break up the neutral stain so as to obtain the basic portion of it or, if dissociation has taken place, take up the basic ion directly. Other parts of the cell, with an affinity for acid dyes, similarly combine with the acid portion of the stain. These three types of cell structures are known as "neutrophile," "basophile," and "oxyphile" elements, respectively. ${ }^{\mathrm{I}}$ Polychrome stains are used in bacteriology chiefly for the study of spirochetes, Vincent's spirilla, and protozoa; and for the demonstration of chromatin (Zettnow). ${ }^{2}$

Two other types of substance besides dyes are used in staining, namely, mordants and decolorizers. Mordants are chemical substances which have the power of making dyes stain material which they would not stain otherwise. This method of staining, in which the presence of a third substance besides dye and material to be stained is required, is called by Mann the "adjective or indirect" method in contradistinction to the "substantive or direct" method in which the chemical and physical natures of dye and material to be stained are so interrelated that the material acquires the color

${ }^{x}$ Conn, H. J.: Biological Stains. Geneva, N.Y., 1925.

2Zettnow: Ztschr.f. IIyg. u. Infektionskrankh., 30, I. IS89. 
without the addition of a mordant. Pure mordants have a strong chemical affinity both for the substrate and the dye, and are used where an anchorage of dye in substrate is desired.

Decolorizers are used to withdraw stains from certain tissues or organisms or parts of an organism and thus by a process of "regressive staining" to differentiate them. "Regressive staining" is contrasted with the ordinary method of "progressive staining" in which the process is stopped when only those substances with great affinity for the dye are stained.

\section{STRUCTURE OF THE CELL}

To appreciate the significance of staining phenomena and to be able to discuss the mechanism involved it is not only necessary to understand the structure of the dyes but also to be familiar with present-day conceptions of the structure of the living cell. The cell is not to be thought of as a mere random mixture of cell constituents. These constituents have a permanent spatial distribution and physical state. This special structural constitution or organization is responsible for the special peculiarities of chemical behavior. Present evidence indicates that the basic protoplasmic structure has a closer resemblance to an emulsion type of structure than to that of any other simple physical system. The reactions which cells undergo proceed most activelyalthough perhaps not exclusively -at the boundaries of protoplasmic phases; in other words, the surfaces of membranes, fibrils, granules, and other solid cell structures have an accelerating or catalytic influence on these reactions. Many features of the chemical organization and behavior of living protoplasm appear to depend on the presence of thin films (apparently consisting chiefly of lipoid material) by which its structural elements are bounded and inclosed. The entire cell is inclosed by a thin, semipermeable film - the plasma membrane; the internal protoplasm is probably partitioned by similar films. Apparently the intracellular partitions undergo increase of permeability or break down at death so that many chemical reactions which are absent or inappreciable during life proceed rapidly in dead cells. The type of structure characteristic of living protoplasm appears to be one by which free diffusion is prevented or restricted.

Various theories have been advanced to explain cell permeability, most of which, though not all, presuppose a plasma membrane which exhibits differential properties permitting some substances to enter the cell easily (alcohol, ether); others with difficulty (most salts, sugars, etc.); still others not at all (most colloids). While not universally accepted, the membrane hypothesis seems to be the one with which the known facts are best in accord. In the theory of Overton this membrane is supposed to be impregnated with lipoids, probably not ordinary fat but a mixture of lecithin and cholesterol. Others (Ramsden, Loeb, Crozier) emphasize the importance of protein in the membrane, the first calling the structure a "haptogen membrane"; and Nathansohn postulated a membrane composed of a mosaic of both lipoids and proteins. The size of the molecule doubtless plays some part in penetrating power; and if molecular weight be very high, penetration is prevented. But it must not be thought that below a certain point there is any correlation between molecular weight and difficulty of penetration; indeed, the reverse is often the case; fatty acids apparently enter cells 
with increasing ease as molecular weight increases, at least up to a certain po:nt. Molecular weight alone has little to do with cell permeability except as a limiting factor. ${ }^{\mathrm{I}}$

\section{MECHANISM OF STAINING}

The theory of the molecular structure of coal-tar dyes, which I have stated, while perhaps not certainly established in all details, accounts for all the known facts, and its adoption has been so fruitful that it is now generally accepted. About the actual mechanism of staining, on the other hand, there is by no means general agreement. Much of the discussion has been devoted to a debate as to whether the process is chemical or physical. This seems peculiarly futile since a rigorous definition of chemical and physical processes is difficult if not impossible to formulate and since the two processes are ultimately one. Even the ardent adherents of one or the other theory are usually forced in the end to acknowledge the possible identity of the two.

If a chemical process be defined as a reaction between two substances in which a new chemical substance is formed and a physical process as a reaction between two substances in which no new chemical substance is formed, it is clear that both processes occur in staining. A dyestuff as a whole may enter into and be deposited upon a tissue or cell by a process which Michaelis describes as "insorption," in which case the coloring matter may be subsequently extracted by any chemically indifferent solvent. On the other hand, a dye may become chemically united to the cell protoplasm by the formation of a salt, and in such a case the color can be removed only by agents like free acids which are capable of decomposing salts. It is certain that Ehrlich's diagrammatic conception of chemoceptors to account for selective staining can no longer be held. Many observations in recent years point to the fact that other properties of the molecule than its structural formula (upon which the Ehrlich theory laid so much stress) are concerned and are probably of greater importance.

There is good reason to believe that chromophilic protoplasm, so far as it is directly stainable (that is to say, without mordants), is amphophilic or amphoteric, i.e., it possesses basic (amido-) and acidic (carboxyl-) groups side by side in the molecule. It has therefore the structure of amido-carbonic acid. The chemical processes of staining go on best the more auxophoric groups there are in the dye and the greater the number and the adequacy of chromophilic groups in the material to be stained.

The physical processes of staining consist of surface attraction, osmosis, diffusion, adsorption; and the factors which promote these processes are proper size of the dye molecule and adequate pore-volume of the material to be stained.

It cannot be said that a completely satisfactory description of the chemistry of bacterial staining can be given. This is perhaps not strange considering the enormous complexity of the chemical structure of dyes, the complicated structure of bacteria, the difficulties of chemical analysis of bacterial bodies which are constantly changing their composition during life, and the minuteness of the microchemical reactions on which we have to depend.

One thing essential for the process of staining bacteria is water. Water-free, alcoholic solutions of dyes will not stain dehydrated bacteria, nor will water-free alcohol

${ }^{x}$ Lillie, R. S.: General Cytology, p. 167. Chicago: University of Chicago Press, 1924; Jacobs. M. H.: ibid., p. 99. 1924. 
decolorize them. The demonstration that bacteria contain an abundance of nucleoprotein appears to explain their affinity for basic coal-tar dyes, and there are many grounds for the belief that-perhaps in the majority of cases-staining is due to a weak combination between nucleoprotein and basic dye, decoloration being due to dissociation of the dye-protein compound. Bacteria certainly behave in their staining reactions as if composed largely of protein. They were formerly thought to consist entirely of nucleus and cell wall without cytoplasm. Whether this be true or not, one other element in bacterial cells is of great-perhaps of prime-importance in many staining phenomena, namely, lipoids. Bacteria are known to contain fats and lipoids in varying amounts which, because of the marked effect on surface tension, would for purely physical reasons tend to become concentrated at the periphery of such a colloidal system as the bacterial protoplasm. Whether or not there is a morphologically distinct limiting membrane, we can reasonably assume that the surface of the bacterial cell is potentially lipoid. The presence of unsaturated fatty acids in the lipoprotein of the bacterial cell is thought by many to explain the mechanism of the gram stain.

The amount of dye taken up by bacteria and the firmness with which it is held doubtless depends in large part on the H-ion concentration of bacterial protein. Since the $\mathrm{pH}$ of bacterial protein may change with age it might be expected that the staining characteristics of bacteria would change, and it is of course well known that this does occur; the difference between the gram behavior of young and old cultures may be cited as an example.

The chief physical factor of the staining process-emphasized by advocates of the physical hypothesis to explain the phenomena of dyeing, and which must in any event be taken into consideration-is the process of adsorption of the dye by the bacterial substance after it has passed through the wall membrane by osmosis. Adsorption is the property possessed by a solid body of attracting to itself by physical means from a surrounding solution certain compounds or ions present in that solution. In the case of the dyes it is assumed that, when once adsorbed, they remain in the stained tissue in solid solution. There are certain facts which point strongly to a physical explanation of staining. For example, there is no evidence of the formation of a new substance when tissue is stained; the colored tissue merely takes on one of the characteristics of the dye (color). It is usually possible to extract all or nearly all the dye by immersion in water or alcohol. Furthermore, tissue never removes all the stain from the dye solution, no matter how dilute. These facts must be borne in mind, and physical processes must be thought of as playing an important rôle. But more and more evidence is accumulating to indicate that strictly chemical processes are also concerned.

\section{THE GRAMI STAIN}

Dyes are used in bacteriology for four purposes: (I) to make organisms visible, (2) to display their structure, (3) to reveal their chemical nature, and (4) to influence their growth. It is well known that most bacteria are stained easily and a few with difficulty, and that certain parts of the bacterial cell (the spores, capsules, and flagella) are not stained at all by ordinary methods. However, with the exception of the acid fast group, the vast majority of bacteria behave very much alike toward simple 
stains, without mordants. A few instances of elective staining have been describedas, for example, the affinity of picric acid and neutral red for cholera vibrios and kresylechtiviolet for gonococci; but, on the whole, there is so little difference in the behavior of most bacteria to dyes that elective staining methods have not proved of striking value. To this statement there are of course two notable exceptions-the method of Gram and the method of Ziehl-Neelsen.

The method of Gram illustrates well the fact that the progress which results from a new observation is often in a different direction from that in which the observer was at the time searching. Gram was attempting to develop a method for staining microorganisms in tissue. The differential staining which he noticed proved to be of great value for the general identification and classification of bacteria.

The ability to retain dye when stained by the method of Gram is not a property of living cells in general but is almost entirely confined to yeasts and bacteria. All tissue elements appear to be decolorizable save perhaps keratohyalin. Henrici found that sections of vegetable tissue contained no gram positive elements; and in animal tissue -while the nucleus retains the stain somewhat longer than the cytoplasm-all the elements may be ultimately decolorized. Molds stain irregularly, isolated granules in the mycelia retaining the stain, while large areas do not stain at all. Protozoa, spirochetes, and malarial parasites are gram negative. It is well known, of course, that the differentiation of bacteria into gram positive and gram negative is not hard and fast, that the gram characteristic of a given organism, like any of its other characteristics, may change with age or be disturbed by variations in environment and in other ways. But within its well-established limitations the method of Gram is an exact one. It is clear that the differential behavior of gram positive and gram negative bacteria must ultimately depend upon difference in chemical or physical characteristics (or both) of the bacteria themselves. A number of such differences have been proved to exist, and for certain others there is strong, if not conclusive, evidence. Of these differences, the principal ones are shown in Table $\mathrm{I}$.

It can be objected that the facts in nature are not quite so clear cut as a table of this sort suggests. Not all gram positive organisms are equally gram positive, and it has been shown that their gram behavior may in different groups of gram positive organisms rest on an entirely different anatomical basis. They therefore differ among themselves and cannot all be classified together. The same thing is doubtless true of the gram negative organisms. Such a statement, for example, as the one that gram positive organisms are as a rule much more susceptible to the bacteriostatic action of basic triphenyl-methane dyes than gram negative ${ }^{\mathrm{I}}$ is open to a criticism of this kind, and the criticism has in fact been made. ${ }^{2}$ Nevertheless, the statement as originally enunciated is correct: with about Io per cent of exceptions, gram positive aerobes, whether spore bearing or not, are extremely susceptible to the bacteriostatic effect of these dyes and, with about Io per cent exceptions, gram negative aerobes are resistant. The original statement of this parallelism was extended by Smith, Eisenberg, Simon, and Wood $^{3}$ to include about sixty dyes, and the work of these and other investigators

Churchman, J. W.: loc. cil.

${ }^{2}$ Stearns, A. E., and E. IV.: J. Bacl., 9, 493. I924.

3 Simon, C. E., and Wood, M. A.: Am. J. M. Sc., 147, 247, 524. I9I4. 
TABLE I

Gram-Positive Organisms

r. Killed bacteria not digested by trypsin or pepsin (Kantorowiecz)*

2. Only a few are digested by gastric juice (Bürgers) $\dagger$

3. Resistant to alkalies; not dissolved by I per cent $\mathrm{KOH}$ (Kruse, $\ddagger$ Smith§)

4. Lipoids resistant to fat solvents (Jobling and Petersen) $\mid$

5. Degree of dispersion of nucleoproteins high (Hottinger) 9

6. Sensitive to weak electrolytes (dyes) Smith, $\$$ Burke**)

7. Optimum growth at relatively high $\mathrm{pH}$ (Burke) $^{* *}$

8. Resistant to non-electrolytes (Smith) §

9. Limited iso-electric staining range centered about $\mathrm{pH} \quad 2-3$ (Stearns and Stearns) $\dagger \dagger$

ro. For the most part (about ro per cent exceptions) vcry susceptible to bacteriostatic effect of triphenyl-methane dyes (Churchman) $\ddagger \ddagger$

I I. May produce spores

I2. In the living state, more readily permeated by dyes (Benians) $\S$

13. May be acid fast

I4. More susceptible to quinine and hydrocuprein derivatives (Traube) || $\mid$

I5. More susceptible to iodine (Traube) |||

I6. More susceptible to mesohematin, magnesium, and manganese derivatives of mesoporphyrin (Kämmerer) $\llbracket$

17. More readily adsorbed because of surface lipins (Eisenberg) $* * *$

18. Adsorb halogens less strongly (Breinl) $\nmid \dagger \dagger$

19. Unaffected by toluol provided no emulsoid be added (Benians) $+4 \ddagger$

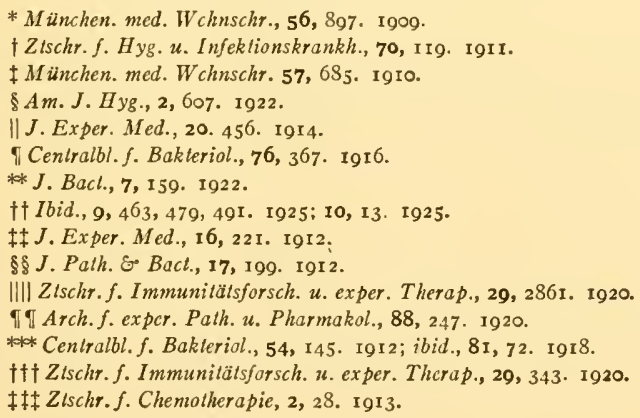

* Munchen. med. Wchnschr., 56, 897. 1909.

$\dagger$ Z Ischr. f. Hyg. u. Infeklionskrankh., 70, II9. I9II.

$\ddagger$ Müchen. med. Wchnschr. 57, 685. I910.

\& Am.J. Hyg., 2, 607. 1922.

\|J. Exper. Med., 20. 456 . I9r4.

ICeniralbl.f. Bakteriol., 76, 367. 19ז6.

** J. Bact., 7, 159. I922.

† Ibid., 9, 463, 479, 49I. 1925; 10, 13. I925.

打. Exper. Med., 16, 221 . I912.

$\S \S$ J. Path. \& Bact., 17, 199. Igr 2.

IIll Ztschr. f. Immunitätsforsch. u. exper. Therap., 29, 286r. 1920.

II Arch.f. expcr. Path. u. Pharmakol., 88, 247. 1920.

* Cenlralbl. f. Bakleriol., 54, 145. I912; ibid., 81, 72. I918.

†† $Z$ tschr. f. Immunitätsforsch. u. exper. Thcrap., 29, 343. 1930.

$\ddagger \ddagger Z$ tschr.f. Chenotherapie, 2, 28. 1913 .

Gram-Negative Organisms

I. Killed bacteria digested by trypsin and pepsin

2. Majority digested by gastric juice

\section{Dissolved by I per cent $\mathrm{KOH}$}

4. Lipoids less resistant to fat solvents

5. Degree of dispersion of nucleoproteins low

6. Resistant to weak electrolytes

7. Optimum growth at relatively low $\mathrm{pH}$

8. Less resistant to non-electrolytes

9. Wider iso-electric staining range centered about $\mathrm{pH} 5$

10. For the most part less susceptible to bacteriostatic effect of triphenyl-methane dyes

II. Never (?) produce spores

12. Less permeable to dyes, in living state

I3. Never acid fast

I4. Less susceptible to quinine and hydrocuprein derivatives

15. Less susceptible to iodine

I6. Less susceptible to these substances

I7. Less readily adsorbed

I8. Adsorb halogens more readily

19. Killed by toluol 
TABLE I.-Continued

Gram-Positive Organisms

20. Less readily subject to auto- and serolysis (Benians) $\$ \S$

2I. Less likely to form demonstrable antibodies in infected host (Benians) $\$ \S \S$

22. Less susceptible to quinine (Graham-

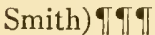

$\S \S \S$. Path. \& Bacl., 23, $4 \mathrm{Ix}$. 1919-20.

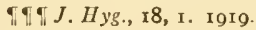

\section{Gram-Negative Organisms}

20. More readily subject to auto- and serolysis

2I. More likely to form antibodies

22. More susceptible to quinine

suggests that perhaps the author's figures for the exceptions in each group are too high This clear-cut but not all-inclusive parallelism between gram behavior and triphenylmethane behavior is striking, in spite of the fact that an exceptional gram positive organism (like the streptococcus) may be resistant and an exceptional gram negative one (like $M$. neisseri) may be susceptible; and in spite also of the fact that within each group there are quantitative differences in susceptibility to dyes.

All the relations stated in the table are doubtless subject to similar qualifications which may be summarized as follows:

I. The division between gram positives and gram negatives is not absolute. Other factors enter in. For instance, the anaerobes form a class by themselves, and, although usually gram positive, may have many characteristics not shared by other gram positives. They are really not included in the table. Again, organisms like the acid fast have peculiarities of structure which separate them chemically from others.

2. The gram positives differ among themselves, and gram positivity does not always depend, in the various bacterial species, on the same mechanism. This point has been established by the observations of Deussen, ${ }^{1}$ and more recently by the author, ${ }^{2}$ who has shown that two strongly gram positive organisms like $B$. anthracis and $M$. freudenreichi differ markedly as to the stability-and probably as to the underlying cause-of their gram positivity.

3. A very interesting and important fact has been established by a number of observers, that there is a group of organisms which-though gram negative-are intermediate between the two groups in most of their other characteristics. M. neisseri, for example, though gram negative is dye sensitive, falls with the gram positives in optimum $\mathrm{H}$-ion test, is quite resistant to lysis by $\mathrm{KOH}$ as compared with other gram negatives, and shows no cytolysis in peptic-digestion tests. There is some evidence (from peptic-digestion tests) that vibrios and spirilla also occupy in some respects a midposition between the two groups.

4. The presence of spores also somewhat complicates the situation. The author ${ }^{3}$ has shown that certain dyes (acriflavine, acid fuchsin) exhibit a reverse selective bacteriostatic action when tests are made between gram positive spore-bearers and the ordinary gram negative bacilli.

\footnotetext{
${ }^{I}$ Deussen, E.: Ztschr. f. IIyg. u. Infektionskrankh., 85, 235. I9r8.

${ }^{2}$ Churchman, J. W.: Stain Technology, 2, No. I. I927.

${ }^{3}$ Churchman, J. W.: J. Exper. Med., 37, I. 1923; ibid., 38, I. 1923.
} 
5. Not even all strains of a given species necessarily behave exactly alike toward dyes. The existence of "strains-within-a-species variants," as measured by the bacteriostatic effect of gentian violet, has been described; and from a given pure culture of $B$. coli two strains have been isolated-one dye sensitive and the other not-which were identical in all cultural, morphological, and tinctorial respects ("strain-within-astrain variant")..$^{\mathrm{r}}$

The facts are that the properties of the two groups stated in the foregoing table are probably to be regarded as "independent variables in the sense that any one of them may be possessed by a particular strain of bacteria independent of the other properties of that group." None the less, as Smith has stated, "this almost clear separation of the families of bacteria on the basis of biochemical reactions cannot be without significance."

It is inevitable that the dilemma as to the physical or chemical nature of staining which we have encountered in discussing the general mechanism of the staining process must also be faced in discussing the method of Gram. On the whole, the tendency is to emphasize the chemical rather than the physical factors of this reaction, and many observers (e.g., Deussen) ${ }^{2}$ regard the process as purely chemical. That physical structure is also involved seems none the less certain. Benians ${ }^{3}$ has shown that bacterial disintegration induced by mechanical measures upsets the gram behavior; Churchman has shown that certain organisms (e.g., B. anthracis) are gram positive only in the cortex, which may be removed by chemical means, exposing a gram negative medulla; and that some sort of bacterial membrane plays a physical part in the process is taken for granted in most of the theories which attempt to account for the phenomenon. Benians has been the chief exponent of a purely physical explanation of the gram stain and has advanced perhaps the most convincing evidence for this view. From experiments in which bacteria were studied after disintegration by crushing, Benians ${ }^{4}$ drew the following conclusions:

I. The gram positive property is inherent in the physical structure of the bacterial cell, and-since mordants are not essential-is not conferred on it by the mordant.

2. Nothing in the nature of chemical fixation of the compound dye to bacterial substance occurs.

3. The effect of the mordant is to dissociate dye from its adsorption compound with the tissues, forming with it a large, compound molecular body which in alcoholic solution does not easily pass out of the gram positive bacteria. This conception of the rôle of the mordant supplanted Benians' earlier idea that it acted by preventing alcohol from entering the cell.

4. Capacity for retaining the compound dye is chiefly dependent on the structure and integrity of the limiting membrane.

5. The essential character of the gram positive cell membrane is that it does not

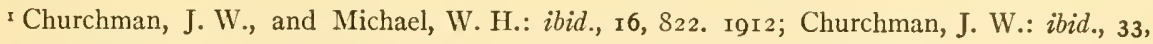
569. I921.

${ }^{2}$ Deussen, E.: loc. cit.

${ }^{3}$ Benians, T. H. C.: J. Path. \&o Bact., I7, I99. I9I 2.

4 Benians, T. H. C.: ibid., 23, 411. 1919-20. 
readily permit the contained large, compound iodine molecule in alcoholic solution to pass through it.

6. Gram negative bacteria are of two types as regards cell membrane: (a) (Represented by $M$. neisseri) allow attachment and probable permeation of the dye; but from them iodine-dye precipitate is readily washed out by the decolorizer. These organisms approximate the gram positives so far as they contain the dye, but the absence of the necessary specific cell membrane does not permit the retention of the dye when alcohol is applied. (b) (Represented by B. coli) allow no penetration of the dye but only peripheral adsorption.

7. Not all bacteria which fail to retain the stain are similar in structure. The apparently permeable gram negatives ( $M$. neisseri) probably have more in common with the gram positives than with the gram negatives.

Burke corroborated Benians' conclusions by demonstrating a reverse gram reaction. ${ }^{\mathrm{I}} \mathrm{He}$ found that an alcoholic solution of the iodine-dye complex stained the gram negatives but not the gram positives, so that gram positives are characterized by permitting the entrance of water-soluble dye but not the egress of the alcoholsoluble iodine-dye complex. The opposite is true of gram negatives.

Brudny, ${ }^{2}$ on the other hand, considered the gram positives more permeable to iodine so that in these organisms a deeper iodine-dye precipitation occurs which is less accessible to the decolorizers. Other observers have laid stress on the chemical constituents of the bacterial surface. Eisenberg found that ether extraction of staphylococcus reverses its gram behavior, concluding that the lipoid-protein compounds on the surface were the important factor; while Dreyer, Scott, and Walker were able to turn B. coli into gram positive organisms by treating them with lecithin.

Stearns and Stearns ${ }^{3}$ called attention to the fact that bacteria exhibit the ampholytic character common to protein and tend to retain acid stains when in acid solution and basic stains when in alkaline solution. They found that gram positive bacteria can be rendered gram negative by increasing acidity, and that the reverse effect is produced by alkalies; at the iso-electric point there is little staining, and the so-called "iso-electric range" is generally wider with gram negative than with gram positive bacteria. Mordanting, they suggest, is due to a mild oxidation which increases acidity and hence the affinity for basic dyes. They suggest further that gram positivity depends on the presence in the compound bacterial lipoproteins of unsaturated fatty acids which are partially oxidized by the mordant, intensifying the acid properties and increasing affinity for basic dyes. The presence of unsaturated fatty acids has been invoked by other observers to explain the phenomenon, but in a different way. Thus Jobling and Petersen ${ }^{4}$ suggested that gram positivity depended on a high fatty acid content and a high affinity for iodine; and Tamura found that the lipoid extract from bacteria contains the element responsible for retention of the dye. Hottinger, ${ }^{5}$ on the

Burke, V.: loc. cil.

2 Brudny, V.: Centralbl.f. Bakleriol., 21, 62. I908.

${ }_{3}$ Stearns, A. E., and E. W.: op. cil., 9, 463, 479, 491. I925; 10, I3. I925.

${ }^{4}$ Jobling, J. W., and Petersen, W. H.: loc. cil.

${ }_{5}$ Hottinger, R.: loc. cil. 
other hand, thought gram positivity is solely dependent on the degree of dispersal of the nucleo-proteins: in gram negatives the stained nucleoprotein forms a colloid of high dispersion; in gram positives it forms an emulsoid; when gram positives become negative the dispersion is increased. Deussen ${ }^{\mathrm{T}}$ concludes his exhaustive study of the subject by stating that the gram reaction depends on the chemical nature of the cell contents which undergo-according to their chemical structure-a greater or lesser degree of hydrolytic splitting of the molecule. The reaction belongs in the complicated field of nuclein combinations. It is chemical and not physical.

It has been for a long time believed on the basis of statements attributed to Unna ${ }^{2}$ that gram positivity depends on the formation of a peculiar iodine-dye-protein compound and that only para-rosanilin dyes could be used for this purpose. That the chemical process involved may not be quite so simple as this was shown by Eisenberg who reported that the reaction could be produced with deeply colored dyes of the acid class as well as with basic dyes and that the use of mordants is not necessary. None the less, para-rosanilin dyes are the best for the purpose, and much the most clear-cut results occur when mordants are used.

Bacteria were for a long time treated as though their constitution were constant, and characteristics were assigned to them which-by implication at least-they were supposed to exhibit always and under all conditions. That they are, as a matter of fact, biological units showing all the changes of metabolism, that they carry on respiratory processes, that they undergo dissociation, that they exhibit polymorphismthese things are now well known. It is clear, therefore, that many of their characteristics are constantly changing and that many of them may be made to change more or less at will by alterations in the environment. Reversibility of the gram reaction by acids (Deussen) ${ }^{\mathrm{r}}$ and by coal-tar dyes (Churchman) ${ }^{3}$ has already been referred to; and it must again be emphasized that the gram reaction of a given organism is constant only when the conditions of examination are held constant. It must also be borne in mind that gram positive organisms differ among themselves as to the stability of their gram positivity. Neide, ${ }^{4}$ for example, used the term Gramdaner to indicate that since a number of factors influenced decolorization by alcohol (among them the concentration and temperature of the alcohol used) gram positive organisms differed among themselves as to the length of time they could retain stain when exposed to decolorizers. Henricis showed that in yeast cells the cytoplasm is not homogeneous as regards gram positivity, certain granules appearing in the decolorizing cell which hold the dye longer than others. The author has recently made very clear the fact that not all gram positive organisms are equally stable in their gram positivity. ${ }^{6}$ Certain of these organisms (e.g., $M$. freudenreichi) are extraordinarily stable in this respect; others (e.g., B. anthracis), though equally gram positive, are less stable. Exposure of

I Deussen, E.: loc. cit.

${ }^{2}$ Unna, P. G.: Monatschr.f. prakt. Dermatol., Supplement No. 6. I887.

${ }^{3}$ Churchman, J. W.: Stain Technology, 2, No. I. 1927.

${ }^{4}$ Neide, E.: Centralbl.f. Bakteriol., 35, 508. I904.

${ }^{5}$ Henrici, A. T.: J. Med. Research, 30, 409. I914.

${ }^{6}$ Churchman, J. W.: Stain Technology, 2, No. I. I927. 
the latter, for example, to certain coal-tar dyes completely reverses their gram reaction although similar treatment is without effect on the gram reaction of the former. Moreover, thorough modifications of the gram technique in which time of exposure to dye and mordant is greatly shortened and time of exposure to decolorizer greatly prolonged decolorize $B$. anthracis in large part but are without effect on $M$. freudenreichi (see Plate I, (c), facing p. 36). In specimens of $B$. anthracis stained by such a modification, beautiful partial decolorizations occur in which a gram negative central rod is to be seen shining through among remnants of undissolved gram positive material which cling to the surface of the bacteria in the form of lumps, plaques, or dots (see Plate I). I have also called attention to the fact that the gram negative forms of $B$. anthracis were much smaller in diameter than the gram positive forms. Filar micrometer measurements of about three hundred organisms showed a reduction in diameter of $0.5145 \mu$ or 42 per cent. Evidence was adduced to indicate that the difference between the gram stability of an organism like $B$. anthracis and that of an organism like $M$. freudenreichi depends on the fact that the latter is composed of an easily destructible gram positive cortex and a gram negative medulla, an anatomical structure which is not possessed by an organism like $M$. freudenreichi.

A critical review of all the known facts leads Smith ${ }^{\mathrm{r}}$ to sum up our knowledge of the mechanism of the gram stain as follows: Gram positivity is due to some property of the bacterial cell which permits the ingress of the water-soluble dye but does not permit the egress of the alcohol-soluble iodine-dye compound. A gram negative cell is not readily penetrated by the water-soluble dye but is easily penetrated by the alcohol-soluble iodine-dye complex. In both cases permeability is conditioned by the physical state of the components of the intact cell membrane. When gram positives become gram negative, what is lost is a particular condition or physical distribution of the constituents of the cell membrane.

The exact technique which Gram himself advised is not now generally used. Immediately after its introduction, modifications of the technique were suggested, identical in general principle with the original method though differing in details. With these every bacteriologist is familiar. In a recent study of this subject involving the examination of nearly fifteen thousand smears, I tried out all the better-known modifications and came to the definite conclusion that Burke's modification ${ }^{2}$ is in every way superior to all others. In this method the stain (methyl violet) is alkalinized on the slide by the addition of sodium carbonate, the mordant is used in strong solution, and acetone-ether, a powerful decolorizer, is employed. The colors in the final result contrast sharply - the pink of the counter-stain (safranine O) with the bluish black of the gram positive forms-and one is never in doubt in which group to place a given organism. The results are constant and clear cut; that is to say, they are constant and clear cut if the conditions of experiment which are known to modify gram behavior be kept in mind and controlled. For it must be emphasized that the method of Gram is an exact one, and irregular results are due, not to the defects of the method but to failure to keep all the conditions of the examination constant. For this reason, since all of the factors are now known, the great variability in results which were obtained in the early work-before these factors were understood-has largely disappeared.

\footnotetext{
${ }^{\star}$ Smith, H.: loc. cit.

${ }^{2}$ Burke, V.: J. Bact., 7, 159. 1922.
} 


\section{STAINING OF ACID FAST BACTERIA}

The phenomenon of acid fastness was first observed in I88 I by Neisser $^{\mathrm{I}}$ in his application of Weigert's staining methods to Hansen's leprosy bacillus. Bienstock and Gottstein in $1886^{2}$ had called attention to the relation between this phenomenon and a high lipin dontent, but it was Hammerschlag ${ }^{3}$ in 1888 who first discovered the high fatty and lipin content. Aronson in I 898 showed that a large part of the ether and alcohol extractable substance was not true fat but a waxy substance. Although acid fastness is not confined to the so-called "acid-fast bacteria" - since spores, the eggs of certain tenia, etc., also possess the property - the phenomenon is of interest in bacteriology, chiefly as a characteristic of B.tuberculosis, B.leprae, B. smegmatis, and the related avirulent species. Of the latter, about forty or fifty strains have been isolated, many of them doubtless identical; there is evidence that the acid fastness of these is associated with a certain common antigenic character, and all are rich in lipins. $B$. tuberculosis differs from the saprophytes in the possession of a higher content of waxy lipins. An analysis of $B$. leprae (Gurd and Denis) ${ }^{4}$ showed the following percentage composition:

$\begin{array}{lr}\text { Fat, fatty acids, and cholesterol..... } & \begin{array}{r}\text { Per Cen } \\ \text { Lecithin } \ldots \ldots \ldots \ldots \ldots \ldots \ldots \ldots \ldots\end{array} \\ & \text { I.7 }\end{array}$

An explanation of the mechanism of acid fastness presents difficulties similar to those encountered in attempting to account for gram staining behavior. The peculiar chemical constitution of acid fast organisms suggested that their distinguishing staining characteristics were due to their fat content. This idea obtained generally and for a long while, although there was disagreement as to whether waxes, alcohols (particularly the "mykol" of Tamura)s, fatty acids, or lipoid proteins were the factors actually concerned. As in the case of the gram stain, however, the mechanism appears to be by no means a simple one, and both chemical and physical factors are doubtless involved. Tubercle bacilli are impermeable to the very fat soluble dyes which readily stain their isolated fats (Sudan III, scarlet R, janus green, etc.). ${ }^{6}$ On the other hand, basic fuchsin (which is only slightly fat soluble), eosin and methylene blue (which are not fat soluble at all), stain the individual organisms deeply in a relatively short time. Corper ${ }^{7}$ showed that tubercle bacilli within tubercles do not become stained when fat soluble dyes are injected into the tuberculous animals, and Sherman ${ }^{6}$ has called attention to the well-known but often overlooked fact that an apparent staining by fat soluble dyes may really be due to the staining of extra-bacillary substances and not to penetration of the organisms. Such facts as these make the rôle of the fatty sub-

INeisser, M.: Virchow's Archiv., 54, 514. I88I.

${ }^{2}$ Bienstock, B., and Gottstein, A.: Fortsch. d. Med., Nos. 6 and 8. 1886.

3 Hammerschlag, A.: Centralbl. f. klin. Med. No. г. I891.

${ }^{4}$ Gurd, F. B., and Denis, W.: J. Exper. Med., I4, 606. I9II.

5 Tamura, S.: Ztschr. f. phys. Chemie, 89, 289. I9r4.

${ }^{6}$ Sherman, H.: J. Infect. Dis., 12, 249. I9г3.

7 Corper, H. J.: ibid., Ir, 373. I9I 2. 
stances somewhat uncertain, to the extent at least that they can hardly be the sole factors concerned in acid fastness. Just as in the case of the gram stain, bacillary integrity is essential for acid fastness. It was pointed out by Koch and later by Benians, and confirmed by others, that disintegrated tubercle bacilli lose the property, from which it would appear that the fatty constituents of the tubercle bacillds are not per se the cause of the staining reaction characteristic of the organism.

Like gram positivity, acid fastness is not an absolutely constant characteristic but is influenced by factors like age of culture and conditions of environment, and may be entirely upset by treatment with chemical agents. It appears established that very young tubercle bacilli may not stain at all by Ziehl's method (Krylow), ${ }^{\mathrm{I}}$ and from the work of Wherry, Mellon, and many other observers there is strong evidence that acid fast organisms go through a varied life-cycle during which their morphology is profoundly altered and their staining characteristics modified. Bienstock and Gottstein were able to make ordinary bacteria acid fast by artificial Einfettung through growth on butter agar. Wherry ${ }^{2}$ was able to render acid fast saprophytes non-acid fast by continual growth under conditions unfavorable to the synthesis of fats. Ritchie $^{3}$ has shown that tubercle bacilli treated with boiling xylene, boiling toluene, boiling benzene, or Aronson's mixture (ether, alcohol, and $\mathrm{HCl}$ ) lose their acid fastness; and Browning and Gulbransen ${ }^{4}$ have shown that the property is rapidly removed by treatment with $\mathrm{CHCl}_{3}$ to which minute amounts of $\mathrm{HCl}$ have been added together with a small amount of $\mathrm{C}_{2} \mathrm{H}_{5} \mathrm{OH}$ to yield a permanent mixture. These observations support the view that the loss of the acid fast property is due to a dissociation of the constituents of the bacillus which is readily effected by the combined action of small amounts of $\mathrm{HCl}$ along with lipoid solvent like $\mathrm{CHCl}_{3}$.

Any amount of extraction of tubercle bacilli with simple fat solvents leaves them acid-fast, probably due to the fact that they continue to contain a small amount of lipin. But if this last trace of lipin be removed by treatment with acid, acid fastness disappears (Long). 5

It is not easy to harmonize completely the observations which have just been cited and to present an entirely satisfactory theory of acid fastness. Three facts seem to be established (Long): ( I) Disintegration of the cell destroys acid fastness. (2) Extraction of the cell body with fat solvents until no more lipin is removed leaves the bacilli acid fast provided they have not been mechanically disintegrated (as by trituration) during the process. (3) The wax of the tubercle bacillus is acid fast. ${ }^{5}$ It remains to reconcile the two chief conflicting explanations: that the phenomenon is due to the presence of an impermeable membrane and that it is due to the content of acid fast wax. Both factors may - as in the case of the gram stain-be of importance. Acid fastness appears ultimately to depend on the small amount of lipin held as an emulsion in the protein substrate and not extractable by fat solvents. But since it is

${ }^{x}$ Krylow, D. O.: Zischr. f. Hyg. u. Infektionskrankh., 70, I35. I91 I-12.

${ }^{2}$ Wherry, W. B.: J. Infect. Dis., r3, r44. r913.

3 Ritchie, W. T.: J. Path. Eo Bacl., 10, 334. 1905.

${ }_{4}$ Browning, C. H., and Gulbransen, R.: ibid., 27, 326. r924.

${ }^{5}$ Wells, H. G., DeWitt, L. M., and Long, E. R.: Chemistry of Tuberculosis. 1923. 
influenced by physical disintegration of the cell wall something more than a chemical process appears to be concerned. Perhaps degree of fineness of emulsification of the acid fast constituent plays a part.

\section{MUCH GRANULES}

Acid fast organisms are also gram positive. But the two characteristics do not necessarily appear at the same stage of bacterial development, and one of them may be upset by processes which do not disturb the other. Krylow, ${ }^{\mathrm{I}}$ for example, showed that very young tubercle bacilli stain neither by the method of Ziehl nor by that of Gram, and that they become gram positive before they become acid fast; Wherry ${ }^{2}$ showed that by growing on suitable media the organisms may be made to lose their acid fastness while remaining gram positive; and Aronson ${ }^{3}$ showed that the gram positivity of $B$. tuberculosis could be reversed by treatment with trichlor ethylene.

In 1907 Much, in examining the tuberculous lesions of a group of animals which had died of the disease, was unable to find organisms which stained by the method of Ziehl but did find granular forms which stained by that of Gram. ${ }^{4}$ He concluded that he was dealing with a cycle of $B$. tuberculosis other than the one usually encountered, and stated that it appeared probable that the method of Ziehl stains substances of the tubercle virus other than those stained by the method of Gram. It is therefore possible to obtain positive results with the method of Gram in cases in which examination by the method of Ziehl is negative. His findings were as follows: (I) There is a granular form of tubercle virus not stainable by Ziehl. (2) This granular form is virulent. (3) It occurs in tuberculous organs either as the only stainable manifestation of the etiological factor of the disease or in company with a fine rod which is also not stainable by Ziehl.

Krylow, ${ }^{1}$ in reviewing Much's findings, came to the conclusion that gram positive material appears in B. tuberculosis much earlier than acid fast material, and this material tends to concentrate in granules. Acid fast material, on the other hand, is spread diffusely throughout the bacterial body. Hence in young cultures a modified method of Gram will demonstrate the Much granules.

The significance of the Much granules is open to debate. Much's findings have been repeatedly confirmed, and there seems little doubt that the bodies he described are actually to be found in many tuberculous lesions. They may, however, be degeneration products (Rosenblat). In any event, demonstration of their presence is almost without diagnostic value since other bacilli form granules of similar appearance.

\section{STAINING ELEMENTS OF BACTERIAL STRUCTURE}

Certain structural portions of some types of bacteria may, under certain conditions of growth, be demonstrable by suitable methods of staining. Here belong the spore, the capsule, flagella, polar bodies, metachromatic granules (the Babes-Ernst

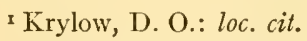

${ }^{2}$ Wherry, W. B.: loc. cit.

${ }^{3}$ Aronson, H.: Berl. klin. Wchnschr., 35, 484. I898; 47, I617. I910.

${ }_{4}^{4}$ Much, H.: Beitr.z. Klin. d. Tuberk., 8, 85. 1907. 
granules), and chromatin. The methods for staining these substances are well known and a discussion of their biological significance will be found in chapter $\mathrm{i}$ of this volume.

STAINING OF BACTERIA IN TISSUES, ETC.

That the immediate environment of bacteria may profoundly influence their behavior toward dyes is well known. Serum, for example, may interfere to some extent with the bacteriostatic action of triphenyl-methanes; the presence of alkalies enhances the staining powers of basic dyes, etc. For these reasons, bacteria in tissues, in milk: soil, sputum, feces, pus, do not necessarily behave toward stains as they do when examined in smears from cultures, and special precautions are necessary in examining these materials to overcome the difficulties presented by the environment. These difficulties are particularly great in examining tissues for bacteria, because the elaborate processes of fixation, hardening, and clearing may profoundly alter the bacteria before they are stained. Recent studies have thrown no particular light on the problems involved, and the classical methods of staining are still in use.

\section{STAINS IN MEDIA}

That certain aniline dyes have the power of affecting bacterial viability has long been known, and their antiseptic properties have been recorded since the days of Koch. Recent observations make it clear that "bacteriostatic properties" better describes the power of many dyes to interfere with the reproductive mechanism (genesistasis, Churchman $)^{\mathrm{I}}$ without necessarily killing or even interfering with their other properties. Genesistatic potency is more striking and is more definitely established than bactericidal power. Probably the most important feature of the bacteriostasis produced by dyes is its highly selective character. This feature was translated into a selective cultural method of great practical value by Conradi and Drigalski ${ }^{2}$ when they introduced a medium for the selective cultivation of $B$. typhosus, by Petroff in his introduction of a gentian-violet medium for the selective cultivation of $B$. tuberculosis, by Krumwiede in his brilliant-green medium, etc. Of a great deal of interest and some theoretical importance as regards the microchemistry of bacteria is the parallelismmarked but not all inclusive-which exists between the selective bacteriostasis of triphenyl-methanes and the gram reaction. Premonitions of this parallelism are to be found in the observations of Stilling, ${ }^{3}$ of Dreyer, Kriegler, and Walker, ${ }^{4}$ and of others; but it was first established as a general relationship by the author ${ }^{5}$ in I9I 2 and later confirmed by Simon and Wood ${ }^{6}$ and others. The mechanism of this selective bacteriostasis is still obscure. The curious fact that it does not entirely parallel selective bactericidal properties has been emphasized by Churchman ${ }^{7}$ and by Burke. ${ }^{8}$

\footnotetext{
I Churchman, J. W.: Proc. Nal. Acad. Sci., 9, 78. I923.

${ }^{2}$ Conradi, H., and von Drigalski, WV.: Ztschr.f. IIyg. u. Infcklionskrankh., 39, 2S3. I902.

3 Stilling, J.: Anilinfarbstoffe als Antiseplica, u.s.w. Strassburg, I 890.

${ }^{4}$ Smith, H. W.: Am. J. IIyg., 2, 607. 1922.

5 Churchman, J. W.: J. Exper. Med., I6, 22 I. I912.

${ }^{6}$ Simon, C. E., and Wood, M. A.: loc. cit.

7 Churchman, J. W.: J. Exper. Med., 37, 543. 1923.

${ }^{8}$ I3urke, V., and Skinner, C. E.: ibid., 39, 6r3. I924.
} 
That certain dyes (acid fuchsin) may exhibit the property in a reverse sense has also been established. These facts are perhaps dependent on the presence of spores in certain bacteria.

Whatever the explanation, it is sufficiently striking that so hardy an organism as B. subtilis is entirely unable to develop in the presence of a minute amount of gentian violet $\left(\mathrm{I}-75^{\circ}, 000\right)$ or that a vigorous and virulent $M$. aureus, when stained with this dye, may be injected into susceptible experimental animals without any untoward effects to the host; while, on the other hand, a sporeless organism like B. prodigiosuseasily killed by slight amounts of heat-will grow vigorously in the presence of this dye in dilutions of 50,000 , or less, and even though deeply stained, when transplanted to agar will grow apparently as well as the untreated controls. That the parallelism between the gram reaction and selective bacteriostasis although striking is not complete, has already been brought out. Reasons have also been advanced why the tempting explanation cannot be accepted that the parallelism depends on the greater ease with which triphenyl-methane dyes penetrate gram positive organisms than gram negative ones. It is quite possible that in these selective reactions some of the dyes may actually stimulate the growth of the organisms which they fail to inhibit.

VITAL STAINING

Much of what has thus far been said applies to bacteria which have been killed during the process of fixation. Since it is well known that profound chemical changes may set in rapidly when protoplasm dies and that disturbance of the cell membrane, as shown particularly by the work of Chambers, ${ }^{\mathrm{r}}$ may induce immediate alterations in the cell, care must be taken not to assume too hastily that the phenomena observed in stained specimens represent the facts of the living cell. Such an experiment as that of Benians, in which the bacterial membrane is deliberately ruptured before examination, is perhaps open to criticism on the ground that the treatment provides a different substance for observation from that present in the living cell. Nevertheless, the examination of bacteria stained in vivo has brought out no facts which are at variance with what has been learned from the staining of fixed smears. Ernst, ${ }^{2}$ Nakanishi, ${ }^{3}$ Amato, ${ }^{4}$ and Pappenheim ${ }^{5}$ have more particularly studied this subject. Methylene blue, brilliant kresyl blue, and toluidin blue have been the dyes chiefly employed.

A special value of the method of vital staining resides in the fact that what is seen may be regarded as characteristic of the living cell and not an artefact, such as might be produced by staining fixed smears. Large spirilla, Vibrio cholerae, B. typhosus, and B. coli have been chiefly studied, and particular attention has been paid to the vital staining of the spore. There is little doubt that some difference exists between the accessibility of dead and living bacteria to certain chemical agents. $\mathrm{Ny}$ -

I Chambers, R.: General Cytology, p. 237. I924.

${ }^{2}$ Ernst, P.: Zlschr.f. IIyg. u. Infektionskrankh., 4, 25. I888.

3 Nakanishi, K.: Mïnchen. med. Wchnschr., No. 6. 1900.

4 Amato, A.: Centralbl. f. Bakleriol., 48, 385 . I908.

${ }_{5}$ Pappenheim, A.: Mionatschr.f. prakt. Dermatol., 37, 429. I903. 
feldt, ${ }^{\mathrm{I}}$ for example, has shown this to be the case for silver nitrate. Many observers have referred to the fact that stained bacilli may remain actively motile, and they have therefore assumed that the bacteria had been actually stained while alive. This raises the question of the possibility of staining living cells without injuring them. So far as the nucleus is concerned, it has long been held that this structure cannot be stained during life although there is a great deal of evidence contrariwise (e.g., Churchman). ${ }^{2}$ That bacteria which are stained, at least in the sense that they appear colored (although it might be objected that the stain was only adhering to the surface and had not penetrated the cell), may grow well has been proved by the experiments of the author. ${ }^{3}$ I have shown that gram negative organisms deeply stained with gentian violet grow luxuriantly when transferred to agar, and by single-cell transplantation have proved that this growth is not due to bacteria which happen to have escaped the stain. Experiments tend to confirm the theory that the penetration of a basic dye into living cells depends on the fact that the dye is dissociated and that, in the form of the free base which predominates at higher $\mathrm{pH}$ values, it penetrates very readily, while in the form of salt its penetration may be so slight as to be negligible. Irwin, ${ }^{4}$ on the basis of experiments with Valonia and Nitella flexilis, has warned against the danger of assuming that the dye which appears to have entered a living cell from a solution is actually the dye present in that solution rather than one of its lower homologues.

Many observers have noted that the gram positive organisms appear to take up dyes in vivo more readily than the gram negative ones. From this fact it might be argued that their greater sensitivity to the staining and bacteriostatic effect of triphenyl-methane dyes was due to their increased permeability. But gram negative and dye-sensitive strains of $B$. enteritidis and $B$. coli have been observed which were

\section{EXPLANATION OF PLATE I}

Photographs, through color screens, of camera lucida drawings in color. The gram positive elements have photographed black; gram negative elements, gray. Magnification $\times_{3200}$. $(a)$. Smear from a suspension of a 4 -hour culture of $B$. anthracis (American Type Culture Collection No. 10) to which acriviolet has been added. Smear made after 45 -minutes exposure to the dye. The organism, sharply gram positive at the beginning of the experiment, has become about 75 per cent gram negative. The gram negative forms are about 40 per cent smaller in diameter than the gram positive. The spore is entirely contained within the gram negative portion. $(b)$. Smears from a 4 -hour culture of $B$. anthracis which has been stained by a modification of Burke's method in which the period of exposure to dye and mordant has been greatly shortened and the period of exposure to decolorizer greatly lengthened ( seen, the gram positive material (cortex) being in some cases entirely removed, in other cases persisting as plaques, or lumps, or dots among which the gram negative medulla shines through. Notice the terminal caps of gram positive material at $a, b$, and $c$. (c). Smear of a mixture of $M$. frcudcurcichi and $B$. anthracis stained by a modification of Burke's method (stain $5 \mathrm{sec}$, iodine $5 \mathrm{sec}$., decoiorizer 5 min.). Almost all the individuals of $B$. anthracis have been completely decolorized; $M I$. freudcrircichi has been unaffected. (Photograph made from colored drawings appearing in article by the author, J. Exp. Med., 56, 1007. 1927.)

${ }^{1}$ Nyfeldt, A.: Nord. med. Arkw., 50, I84. 1917.

${ }^{2}$ Churchman, J. W., and Russell, D. G.: Proc. Soc. Expcr. Biol. Eo Mfed., II, I 20-24. I9I4.

${ }^{3}$ Churchman, J. W., and Kahn, M. C.: J. Expcr. Mcd., 33, 583. I92 I.

${ }_{4}^{4}$ Irwin, M.: Proc. Soc. Expcr. Biol. \& Mcd., 24, 425. 1927. 
PLATE I
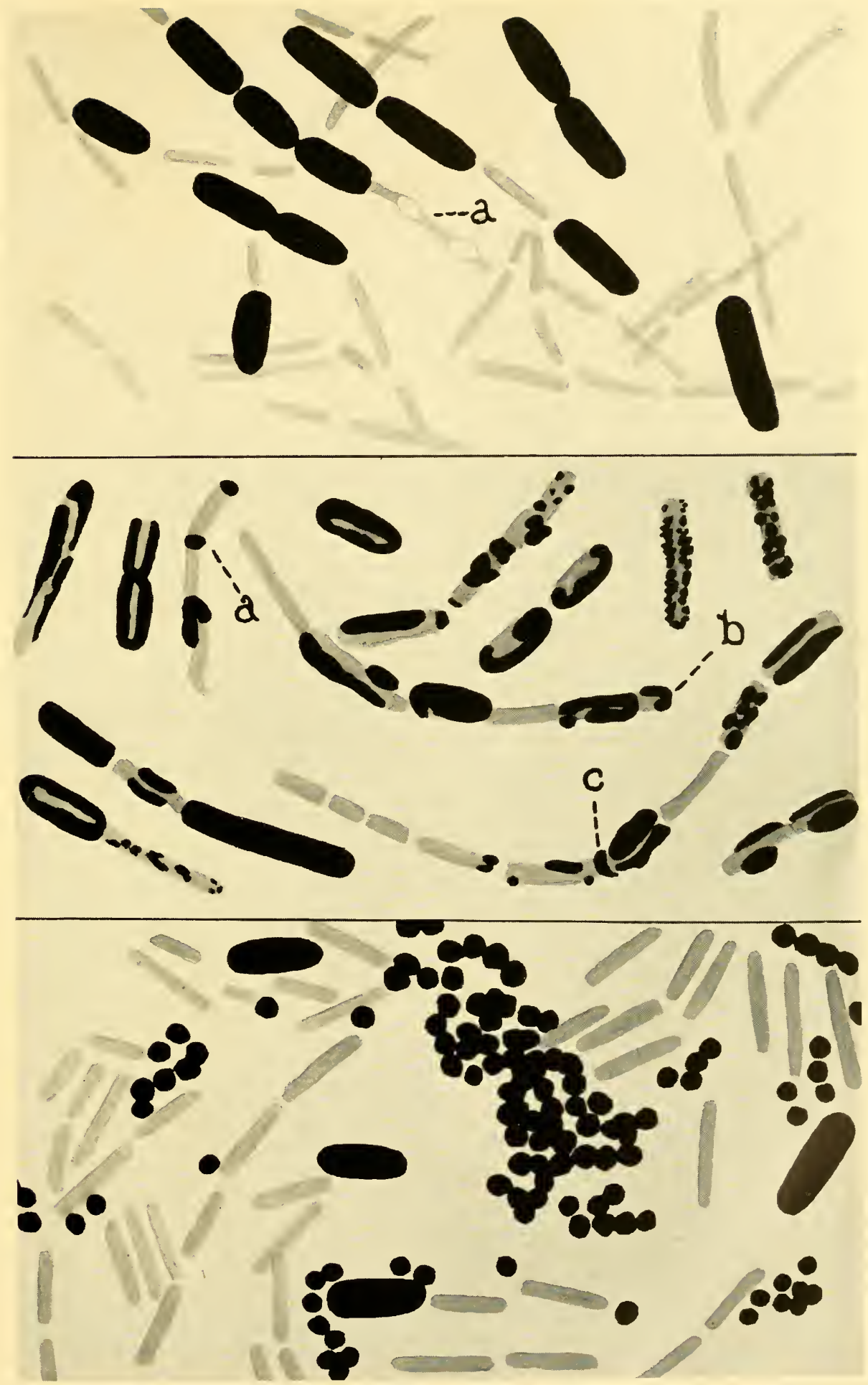

(See Explanation on Page 36) 

not easily permeated by the dyes (Churchman). ${ }^{\mathrm{I}}$ Furthermore, when gram positives become gram negative (e.g., streptococcus), they do not become dye sensitive. For these and other reasons it is not likely that sensitivity is dependent directly on those factors which determine permeability to the reagents of Gram's stain. These two properties are independent variables. In view of their usual association, it may be assumed that they are indirectly associated through some biochemical factor not yet recognized.

\section{SILVER TMPREGNATION}

The method of van Ermengem ${ }^{2}$ for the demonstration of flagella suggests possibilities for the study of the microchemistry of bacteria by methods allied to those of staining. Van Ermengem applied to the study of bacteria the methods of certain photographic processes. The fixed smears were immersed for one to three seconds in a $0.5^{-I}$ per cent solution of silver nitrate, and bacterial structure (particularly the flagella) were in this way rendered clearly visible. The phenomenon was regarded as one not simply of silver precipitation but as due to a true chemical combination pointing to a different chemical structure between flagella (which appear gray black) and bacterial bodies (which appear orange or dark brown). Furthermore, flagella are less easily decolorized by immersion in gold solutions than bacterial bodies. This also points to a stronger affinity of their substance for silver.

${ }^{x}$ Churchman, J. W., and Michael, W. H.: J. Exper. Med., 16, 822. I912; ibid., 33, 569. I921.

${ }^{2}$ van Ermengem, E.: Travaux du laboratoire d'hygiene et de bacteriologie de l'Universitê de Gand. 1, No. 3. 1892. 


\title{
CHAPTER IV
}

\section{MORPHOLOGICAL CHANGES DURING THE GROWTH OF BACTERIA}

\author{
PAUL F. CLARK \\ University of $\mathrm{W}$ isconsin
}

In spite of the efforts of investigators for more than half a century, three general conceptions of the morphology of bacteria still exist. The first assumes that bacteria are simple in form and structure. According to this theory, the size and shape of each species are fixed, varying only within narrow limits, and the organisms multiply only by transverse fission into two daughter-cells of similar size. A second conception at the other extreme has been emphasized especially during the last decade. Several observers have described complicated life-cycles in bacteria including reproduction by budding, and complex mitoses following conjugation; marked changes in form have been reported in presumably pure cultures, even to the extent of the metamorphosis of a diphtheroid into a streptococcus and of filterable viruses into spore-formers. The third position, as one might expect, follows a middle path with the acceptance of a considerable degree of pleomorphism, especially in certain genera such as Corynebacteria and Azotobacter, but with a skepticism concerning the demonstration of proved life-cycles. This conception also includes an emphasis on the importance of observing the changes in form and size during the growth of the organism.

The first-mentioned idea of bacterial morphology exists now chiefly on the pages of the briefer textbooks and in the minds of beginning students. Everyone who has examined ordinary stained preparations of root-nodule bacteria, diphtheria bacilli, streptococci, or members of the Spirillaceae grown on a variety of media will have noted the occurrence of aberrant morphological types to such a degree that the fixed morphology concept must be discarded. Furthermore, some of these forms appear in young cultures (two to eight hours) as well as in those which have been incubated for several days, so that they cannot be dismissed as involution or degeneration forms.

Although this idea of the fixity of bacterial form has been largely discarded in its more precise interpretation, systematic bacteriology is, nevertheless, largely based on this conception; and, in routine, we find it possille, though at times difficult, to work on this foundation. The usual procedures tend to emphasize uniformity; the standardized artificial media select those organisms best fitted to grow under these saprophytic conditions; the crude drying and flaming methods of fixation and the gross overstaining effectively conceal any fine differences in structure; the diurnal rotation of the earth gives us a standard time for the examination of cultures based on our convenience rather than on any physiological cycles in the development of bacteria. Furthermore, bacteria are so fascinatingly varied in their functions and apparently so absurdly simple in morphology - what they do is so vastly more important biologically than what their form and structure may be-that bacteriologists quite generally have become 
physiologists, and have tended to minimize the importance of morphological studies. And if unusual forms are seen, what can be easier than to assume that these are mutants, involution forms, or contaminations?

In spite of this tendency to stress uniformity, the variability of bacteria has forced its way through our methods so that a number of men have been led to make careful cytological observations and eventually to take a position diametrically opposed to the concept of fixed morphology. The work of Nakanishi, ${ }^{1}$ Schaudinn, ${ }^{2}$ Grimme, ${ }^{3}$ Mencl, ${ }^{4}$ Guilliermond, ${ }^{5}$ Ambroz, ${ }^{6}$ Dobell, ${ }^{7}$ Löhnis, ${ }^{8}$ Hort,${ }^{9}$ Mellon, ${ }^{\text {I0 }}$ Bergstrand, ${ }^{\text {II }}$ Enderlein, ${ }^{12}$ Kirchensteins, ${ }^{13}$ Thornton and Gangulee, ${ }^{14}$ and Cunningham and Jenkins, ${ }^{15}$ to name only a few, has been especially noteworthy. It has given foundation for the opinion in the minds of some bacteriologists that bacteria are not simple structureless cells, but fungi with formed nuclei, some of them showing mitotic division and complex life-cycles. Since this conception is philosophically attractive and would co-ordinate bacteria more closely with the rest of the biological world, it is easier for some to accept the evidence as adequate, while others feel that this attractive prospect is an additional reason for maintaining a position of unusually strict skepticism.

The existence of a formed nucleus even in the simpler bacteria (Eubacteriales) has been demonstrated beyond doubt especially by Nakanishi, ${ }^{16}$ Dobell, ${ }^{17}$ Guilliermond, ${ }^{18}$ Meyer, ${ }^{19} \mathrm{Mencl}^{20}$ and Kirchensteins. ${ }^{2 \mathrm{I}} \mathrm{By}$ different technique, with material from a variety of sources, in organisms from young and old cultures, in both the simpler and the higher bacteria, spore-formers and non-spore-formers, nuclei have been demonstrated. The form of the nucleus is variable not only in different species, but also in different stages of the development of one species. In cocci the nucleus is usually more or less spherical, and in fission begins to divide before the division of the cytoplasm. In rods and spirilla the nucleus is more variable and may be seen in the form

s Nakanishi, K.: München. wed.Wchnschr., 47, 187. I900; Centralbl.f. Bakteriol., Abt. I, 30, 97, I45, I93, 225. I90I.

${ }^{2}$ Schaudinn, F.: Arch. Protistenk., 2, 421. 1903.

3 Grimme, A.: Centralbl.f. Bakteriol., Abt. I, 32, 1. I902.

${ }_{4}^{4}$ Mencl, E.: Arch. Protistenk., 8, 259. I907.

${ }^{5}$ Guilliermond, A.: Bull. Inst. Pasteur, 5, 273, 321. 1907.

${ }^{6}$ Ambroz, A.: Centralbl.f. Bakteriol., Abt. I, 51, 193. 1909.

7 Dobell, C. C.: Quart. J. Micr. Sc., 56, 395. 1910-11.

${ }^{8}$ Löhnis, F.: Mem. Nat. Acad.Sci., I6, г. 1921.

${ }^{9}$ Hort, E. C.: Proc. Roy. Soc., B, 89, 468. I9I7; Brit. MI.J., I, 571, 664. I917; ibid., 2, 377. 1917; J. Roy. Micr. Soc., 365. 1917; J. Ityg., 18, 361. 1920; ibid., p. 380. 1920.

${ }^{20}$ Mellon, R. R.: Am. J. M. Sc., 159, 874. 1920.

"I Bergstrand, H.: J. Bact., 8, 365. 1923.

${ }^{12}$ Enderlein, G.: Bakteriensyklogenie. Berlin, 1925.

${ }^{13}$ Kirchensteins, A.: Structure intérieure et Mode de Déreloppement des Bactéries. Riga, I922.

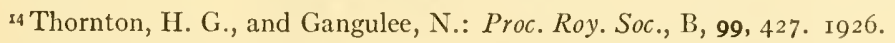

${ }^{15}$ Cunningham, A., and Jenkins, H.: J. Agric. Sc., Part I, I 7, I09. 1927.

${ }^{16}$ Nakanishi, K.: loc. cit. $\quad{ }^{87}$ Dobell, C. C.: loc. cit. $\quad{ }^{18}$ Guilliermond, A.: loc. cit.

${ }^{29}$ Meyer, A.: Centralbl.f. Bakteriol., Abt. II, 6, 339. I900.

${ }^{20} \mathrm{Mencl}$, E.: loc. cit. $\quad{ }^{21}$ Kirchensteins, A.: loc. cit. 
of discrete granules (chromidia), irregular filaments, branched rods, or one or more large masses of nuclear substance. Even different stages of mitosis have been described by Mencl $^{\mathrm{x}}$, Guilliermond ${ }^{2}$, and others.

Beyond this point it is difficult to analyze the large number of observations which various authors have presented as evidence of the existence among the bacteria of the more complex methods of reproduction commonly found among the Protista. Many authors describe various budlike structures, large bizarre forms, minute deeply staining bodies, and imperfectly stained masses, and have read into these observations certain sequences which, it seems to me, have not been adequately proved. The difficulty of demonstrating genetic descent with organisms so minute is apparent. Since, however, the purity of the culture is so completely essential for evaluation of these observations, should we not insist that some method of single-cell isolation be employed as a basis for work of this sort, even though successful transplants can be obtained in a very small percentage of the attempts? Most of the work on "lifecycles" in bacteria has not been carried out with this foundation, and many of the observations are inadequate as experimental proof of developmental cycles, however much the notion may appeal philosophically. A few papers presenting observations in the field of soil bacteriology cause the scales to tip rather sharply toward the "lifecycle" side. Notable recent contributions are those of Thornton and Gangulee ${ }^{3}$ with Bacillus radicicola and Cunningham and Jenkins ${ }^{4}$ with B. amylobacter. Unfortunately, neither of these papers is based on single-cell isolations.

That bacteria, even among the Eubacteriales, do at times reproduce by means other than equal fission seems to me to be definitely proved. Some of the best observations in this connection have been made by Hort, ${ }^{5}$ who has studied a number of the common pathogens, watching the development of the organisms by means of a combination of single-cell technique and warm stage growth. He has demonstrated that, under adverse conditions, some strains of the colon-typhoid group reproduce by budding, by branched $Y$-shaped forms, and by the production of large aberrant forms in which the fragmented chromatin appears as deeply staining granules. These granules are subsequently extruded and are small enough (0.1-0.2 micron in diameter) to pass through the coarser bacterial filters. He watched the subsequent development of these "gonidial bodies" into typical bacilli. He avoided the assumption of any definite life-cycle, but emphasized his demonstration that methods of reproduction other than binary fission occur among bacteria, and that the irregular bodies cannot be considered involution forms since he has shown that they reproduce actively.

The essential details of Hort's observations were confirmed by a specially appointed committee consisting of Leishman, Adami, Farmer, and Harvey. ${ }^{6}$

With a somewhat similar technique Gardner ${ }^{7}$ has also described reproduction at each of the three growing points from $Y$-shaped organisms in six members of the colon-

${ }^{x}$ Mencl, E.: loc. cit. $\quad{ }^{2}$ Guilliermond, A., loc. cit.

3 Thornton, H. G., and Gangulee, N.: loc. cit.

${ }_{4}^{4}$ Cunningham, A., and Jenkins, H.: loc. cil.

${ }^{5}$ Hort, E. C.: see various works cited previously.

${ }^{6}$ Leishman, W. B., Adami, J. G., Farmer, J. B., and Harvey, D.: J. IIyg., 18, 380. 1920.

7 Gardner, A. D.: J. Pall. Eo Bacl., 28, I89. I925. 
typhoid group and $V$. cholerae. He found that this is a common occurrence in the stage of rejuvenation of cultures of pathogenic bacteria, but found no evidence that these $\mathrm{Y}$-forms are part of a complex life-cycle.

The fact that so many of the common pathogens have, in recent years, been passed through infusorial earth and porcelain filters especially after anaerobic growth or through the influence of bacteriophage is additional evidence in favor of the occurrence of minute forms which under suitable conditions are capable of growing into typical organisms. Apparently some nuclear reorganization does occur, but that conjugation is an essential precursor does not seem to have been proved.

Filterability is admittedly not the best basis for judgment as to the size of particles, since electro-physical phenomena play such a large part in the results (Kramer, Mudd) ${ }^{2}$. But when filtrations are carried out under more or less standardized conditions with silica filters and bacteria suspended in physiological saline, the positive results obtained by a number of observers certainly tend to indicate the occurrence among our common pathogens of filterable bodies smaller than the type forms commonly recognized.

Uninterrupted study of the developing organisms by some hanging-block method (Hill, ${ }^{3}$ Orskov, ${ }^{4}$ Hort, ${ }^{5}$ Gardner ${ }^{6}$ ) or by moving pictures (Bayne-Jones, Bronfenbrenner ${ }^{7}$ will continue to be essential to the growth of knowledge of the morphology and development of bacteria. In such studies, however, individual cells quickly become lost in the "log jam" of the dividing bacteria so that clear observation is difficult. Another method which has given some information in regard to the morphological changes during the growth of bacteria is the examination of smears removed at frequent intervals from cultures growing under uniform conditions, the measurement of large numbers of the individual bacteria, and the reconstruction of the life-story largely by statistical methods. The common notion of the "normal" morphology of bacteria is based upon the inspection of material from original habitats or from cultures which have been growing approximately twenty-four hours or some multiple of that. The writer has pointed out the general failure to appreciate this time factor and the resulting errors in our conception of the morphology of the common bacteria. Clark and Rueh ${ }^{8}$ have shown that even on the ordinary standardized media bacteria pass through striking morphological changes which are coincident with the different growth phases described by Lane-Claypon ${ }^{9}$ in the life-history of a bacterial culture. Later, Henrici $^{\text {io }}$ confirmed these findings and worked out many points more completely.

' Kramer, S. P.: J. General Physiol., 9, 8I I. I926.

${ }^{2}$ Mudd, S.: J. Bact., 8, 459. I923.

3 Hill, H. W.: J. Med. Research, 7, 202. I902.

${ }^{4}$ Orskov, J.: J. Bact., 7, 537. I922.

5 Hort, E. C.: see various works cited previously. $\quad{ }^{6}$ Gardner, A. D.: loc cit.

7 Bayne-Jones, S., and Tuttle, C.: J. Bact., 14, 157. I927; Bronfenbrenner, J.: Meeting Soc. Path. Eo Bact. April, I927.

${ }^{8}$ Clark, P. F., and Ruehl, W. H.: J. Bact., 4, 615. r9r9.

9 Lane-Claypon, J. E.: J. IIyg., 9, 239. I909.

ro Henrici, A. T.: Proc. Soc. Exper. Biol. \& Mled., I9, I32. I921; 20, 179, 293. I922-23; 21, 215, 343, 345. 1923-24; 22, 197. 1924; Science, 61, 644. 1925; J. Infect. Dis., 37, 75. 1925. 
During the early latent period, when grow th is slow or completely lacking, no apparent morphological change is seen. Following this, during the logarithmic period when maximum reproduction occurs, the cells from the young cultures of many genera of bacteria attain their maximum size, two to six times larger than the cells from the twenty-four-hour parent-cultures. After four to eight hours the cultures pass gradually into the stationary period, in which the number of organisms remains relatively constant. During this progression to the stationary period, the bacteria become gradually smaller in size until, by the time the cultures are eighteen to twenty-fourhours old, the classical textbook picture is presented. Cultures older than twentyfour and forty-eight hours present more and more of the so-called "involution forms," irregular staining, in many instances bizarre forms, and organisms averaging smaller than those found in the twenty-four-hour cultures predominate.

Clark and Ruehl ${ }^{x}$ studied by this method seventy strains in all, including cultures of the following species:

CocCACEAE Strains

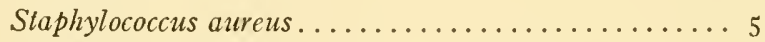

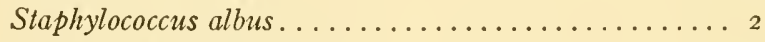

Streptococcus hemolyticus . .................6 6

Diplococcus pneumoniae............... (including types I, II, and III)

Neisseria gonorrheae

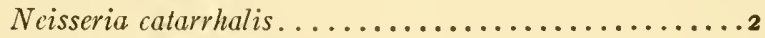

Neisseria intracellularis

Ncisscria mucosis

SPIRILLACEAE

Vibrio comma

Vibrio metchnikovi

Vibrio schuylkilliensis

BACTERIACEAE

Proteus vulgaris

Escherichia coli

Escherichia communior

Eberthella typhi...................... 5

Eberthella dysenteriae

Eberthella paradyscnteriae

Salmonclla paratyphi

Salmonella schottmiillcri

Salmonella suipestifer

Encapsulatus pneumoniac...................2

Pseudomonas pyocyaneus

Serratia marcescens

Pastcurella avicida

Hemophilus influenzae

Hemophilus pertussis

Bacillus anthracis

Bacillus subtilis

Bacillus megatherium

${ }^{x}$ Clark, P. F., and Ruchl, W. H.: loc. cit. 
Bacillus vulgatus

Bacillus mycoides

Mycobacteriaceae

Mycobacterium leprae

Mycobacterium smegmatis

Mycobacterium phlei

Corynebacterium diphtheriae................ 4

Coryncbacterium hoffmanii

Corynebacterium xerosis

Corynebacterium hodgkinii

Pfeifferella mallei

Henrici ${ }^{1}$ studied in detail Vibrio comma, Escherichia coli, Bacillus megatherium, Bacillus cohacrens, and an unidentified member of Corynebacterium.

The increase in size during the "youth" of the cultures occurs in all the organisms studied except the Corynebacteria and $B$. mallei. In these bacilli, more especially in the first named, exactly the opposite progression occurs; the individuals from the younger cultures (two to six hours) are the smallest, averaging in $C$. diphtheriae less than half the size of the rods from a twenty-four-hour culture. The minimum size is reached during the period of rapid reproduction; during the phase of slow growth the size increases, reaching the maximum during the resting period. As the individual organisms become smaller, the metachromatic granules disappear and the bacteria stain uniformly and deeply with Löfler's methylene blue. Not infrequently at this stage members of this genus form coccoidal chains. The peculiar pleomorphism, irregular staining, and metachromatic bars and granules, so characteristic of the diphtheria group, reappear as the cells increase in size again.

The method employed in these studies hardly needs further description save to add that Henricir developed an admirable adaptation of the negative staining method of Benians ${ }^{2}$ to distinguish between the living and dead bacteria in smears. This made it possible to correlate precisely the changes in morphology with the rapidity of cell division in the bacterial cultures.

Members of the colon-typhoid group will serve as a basis for the more detailed consideration of the typical mode of progression. A large majority of the bacteria in a four-hour culture of B. typhosus are 4-6 micra long and $0.7^{-0}-8$ micron wide, so large that they resemble the vegetative cells of the common spore-formers rather than the usual picture of B. typhosus based on the examination of twenty-four-hour growth. The increase in size is greater in length than in breadth, so that the larger cells are relatively more slender. Chain formation occurs commonly at this stage even in those species which in older cultures are characterized by discrete organisms. The bacteria stain more intensely, and the outline is more sharply defined. The time when the maximum average size occurs varies somewhat in different strains of the same or related species. This is dependent largely upon the duration of the period of "lag," which according to Chesney ${ }^{3}$ is an expression of injury to the bacterial cell. By plot-

${ }^{x}$ Henrici, A. T.: see various works cited previously.

${ }^{2}$ Benians, T. H. C.: Brit. M.J., 2, 722. I9r6.

${ }^{3}$ Chesney, A. M.: J. Exper. Med., 24, 387. r9r6. 
ting the projected image of the bacterium divided by the length squared, Henricir ${ }^{r}$ has obtained an index of the variation in form. The coefficient of variation in length of cells, as well as in the area-length index, is increased during the period of increased size.

The various members of the Spirillaceae, because of their marked pleomorphism, offer especially interesting opportunities for the use of this method. The three species studied follow the usual series of changes in size. During rapid growth, the new cells are long and plump and relatively straight save that where several organisms have remained attached they show definite spirals. As reproduction becomes slower, the individuals become more slender, more curved, and distinctly granular in staining. During the period of decline unusual forms are observed-various bulging or budding organisms and coccoid bodies both large and small. Henrici points out that "these latter types are the forms which Löhnis and others have described as extraordinary reproductive cells but that the trend of the growth curve would indicate that this is not the case."

The three members of the Mycobacteria studied need no especial comment save to point out that the "senile" forms are distinctly granular with many coccoid bodies present.

Even the cocci pass through similar cyclical changes in morphology, although the proportional difference between the diameter of the young and the senescent forms is less than with most of the rods and spirilla. The four- to six-hour cultures contain many deeply staining cocci approximately twice the diameter of those usually seen.

In studying $B$. megatherium, Henrici ${ }^{\mathrm{r}}$ has pointed out a number of factors which affect the rate of progression through the period of maximum reproduction and consequently the onset of the phase of decline. If the volume and constituents of the media are constant and if varying numbers of the bacilli are inoculated, the fewer the cells introduced the longer is the period of maximum reproduction and the greater is the maximum size of the organisms. With the same seedings, if the nutrient ingredients of the media are varied, then the richer the media the longer is the period of logarithmic growth and the greater is the maximum size attained. When transplantations are made during the period of increasing size, the organisms in the subculture continue to increase in size, progressing even beyond the maximum reached by the parentculture. Transplantations made immediately after the parent-culture has returned to the original size show no evidence of lag; the subcultures increase rapidly in size again. After two or more hours in the stationary phase, however, subcultures show an appreciable lag and do not progress beyond the curve of the parent-culture. Although other organisms have not been studied with reference to these points, presumably they will follow the same laws.

It is interesting to note that spore formation begins toward the end of the active growth period, so that the factors which lengthen the phase of positive acceleration in growth delay spore formation.

It would appear, then, that even apparently simple bacteria growing under standardized conditions admirably fitted to suppress variation and to increase uniformity pass through a series of cyclical changes which indicate a progression from youth

${ }^{1}$ Henrici, A. T.: see various works cited previously. 
through maturity to old age. These three phases show marked differences in metabolic and reproductive rate, and correlated changes in size and structure. Sherman and Albus ${ }^{r}$ have shown that other physiological correlations exist, in that young bacteria are destroyed by brief exposures to cold and 2 per cent sodium chloride while cells from older cultures are not injured. Differences in agglutinability have been known for many years. There is no evidence that conjugation occurs, but there is ample evidence that, as Child ${ }^{2}$ has shown in higher forms, youth and rejuvenescence may occur apart from sexual processes.

Observations such as the foregoing suggest an interesting correlation between the growth of bacterial cells and that of other living organisms. Protoplasmic changes, similar in kind to those cellular changes we associate with youth, maturity, and senility in metazoan forms, occur even in these supposedly "immortal" single cells. Definite changes in gross morphology and in finer structure are apparent. A logical assumption that bacteria, in common with their more highly organized relatives, regain their youth and vigor of reproduction by the injury and stimulus of conjugation is not borne out by these observations. It must be borne in mind, however, that these observations were all made under conditions admirably suited for growth. Just as numbers of species of fungi have for years been classified with the Fungi imperfecti but later, by more complete studies under varying conditions of growth, have been shown to have definite sexual reproduction and, consequently, have been removed from this family, so it would seem to be highly probable that more detailed study will remove bacteria from their unique position and link them more closely with the rest of the biological world.

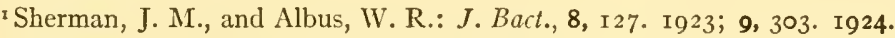

${ }^{2}$ Child, C. M.: Senescence and Rejuvenescence. I9I5. 


\title{
CHAPTER V \\ GROWTH CURVES OF BACTERIA
}

\author{
R. E. BUCHANAN \\ Iowa State College
}

The following discussion of grow th rates of bacteria and their graphical representation in growth curves will be concerned solely with rates of increase.

\section{CHARACTERISTICS OF GROWTH CURVES}

The general characteristics of growth curves may be developed through consideration of the changes in numbers of bacteria which follow inoculation into a medium

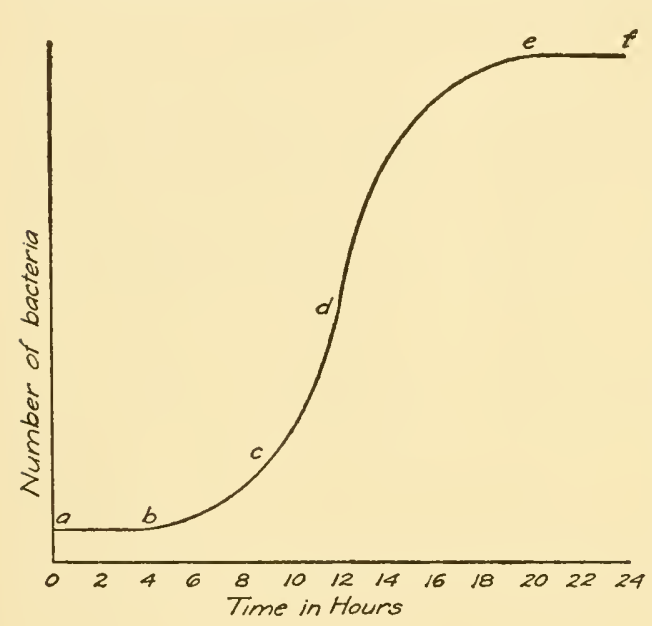

FIG. 1.-Growth curve with various phases

$a-b$. Initial stationary phase

$b-c$. Lag phase

$c-d$. Logarithmic phase

$d-e$. Phase of negative acceleration

$e-f$. Maximum stationary phase

suitable for growth. For the purpose of preliminary discussion it is advantageous to assume that the inoculum consists of bacterial spores, for such material will permit of the maximum opportunity for differentiation of stages or phases of growth.

Examination of such a culture at suitable intervals will show that an appreciable time elapses before any increase in numbers occurs, i.e., some time is required before any of the spores germinate and vegetative cells develop and divide. This may be termed the "initial stationary phase." It is scarcely to be anticipated that all the spores will germinate at the same instant. However, after cell division has been initiated it will proceed with a considerable degree of regularity. Finally, all the viable spores will have germinated, and the culture will have completed its second or "lag phase." For a time thereafter the numbers of cells will increase more and more rapidly, with the rate of growth per cell remaining nearly uniform. This is the third or "logarithmic phase," during which there is a geometrical increase in cells with time. Conditions eventually become less favorable, and the rate of growth decreases. This is the phase of "negative growth acceleration." Finally the bacteria cease to multiply, and the "maximum stationary phase" is instituted. It is thus possible under favorable conditions to differentiate some five different growth phases. 
These facts and relationships may be shown graphically in several ways. The standard growth curve such as that noted above may be graphed (Fig. I) by plotting numbers of bacteria against time. Inspection of the graph shows the existence of some four readily differentiable phases; the distinction between the lag phase and the logarithmic phase is not easily made by examination of this type of curve.

A second method of representing increase in numbers is to plot the total increase in numbers of bacteria in each equal interval of time against time, thus developing a "rate curve." Such a curve corresponding to the growth curve of Figure $I$ is given in Figure 2.

A third type of graph may also be used to illustrate growth rates: one in which the successive rates of increase per cell (or, conversely, the generation

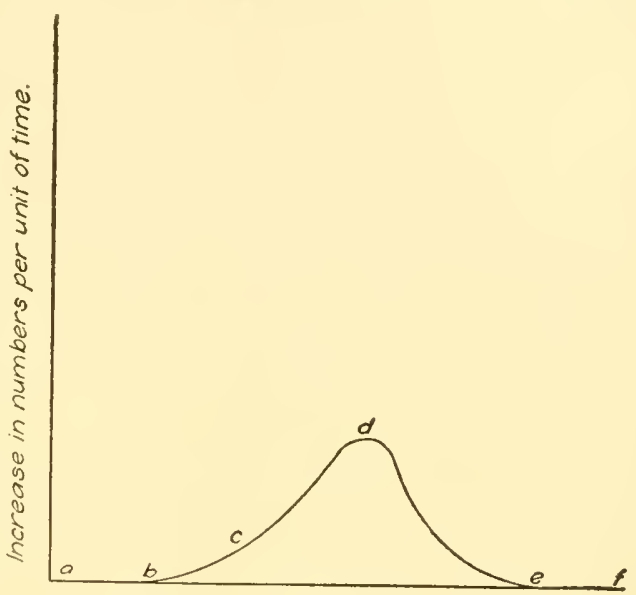

Time in Hours

FIG. 2.-Rate curve corresponding to growth curve of Fig. I.

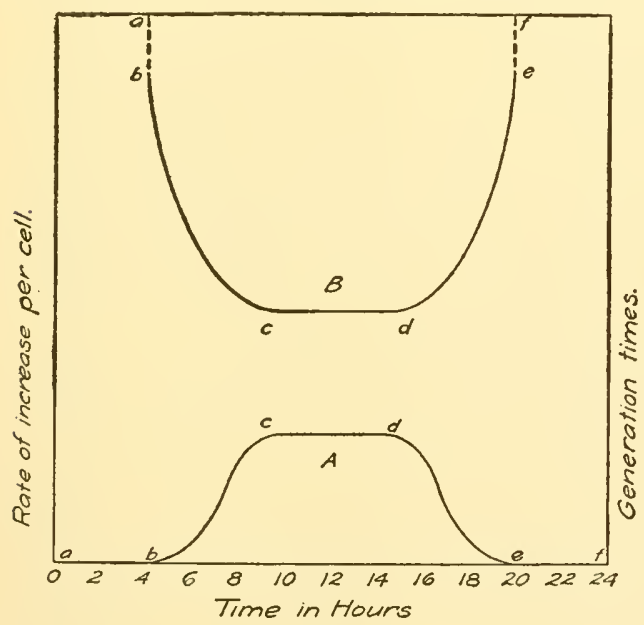

FIG. 3

A. Graph of rate of growth per cell

$B$. Graph of generation times

$b-c$ Lag phase

$c-d$ Logarithmic phase

$d-e$ Phase of negative acceleration

$e-f$ Maximum stationary phase $a-b$ Initial stationary phase

time) may be plotted against time. Curves such as those in Figure 3 may be thus secured. In this graph the identification of the five growth phases is more readily accomplished than in Figures $I$ and 2.

A still clearer differentiation of the various growth phases is to be secured by a fourth type of graph in which the logarithms of the numbers of bacteria are plotted against time, as in Figure 4. During the initial stationary phase $(a-b)$ a straight line with o slope is developed, during the lag phase $(b-c)$ a curved line, during the logarithmic phase a straight line $(c-d)$ with positive slope; during the phase of negative acceleration a curved line $(d-e)$, and finally during the maximum stationary phase $(e-f)$ a straight line of o slope.

A study of the typical growth curve (Fig. I) shows it to be more or less
epend upon the type of organism, its S-shaped. Its exact form in each case will depend upon the type of organism, its immediately antecedent history, and the various environmental influences. 
Two principal types of explanation or interpretation have been used for the form assumed by bacterial growth curves. The first of these (a succession of growth phases) is the one developed above. In its essentials it was apparently first outlined by Lane-Claypon, ${ }^{\mathrm{I}}$ and later expanded by the writer. ${ }^{2}$ A somewhat different method of attack was suggested by McKendrick and $\mathrm{Pai}^{3}{ }^{3}$ and emphasized among others by Robertson ${ }^{4}$ and Lotka ${ }^{5}$. These authors conclude that the sigmoid shape of the growth curve is evidence of the resemblance of growth to the phenomenon of autocatalysis

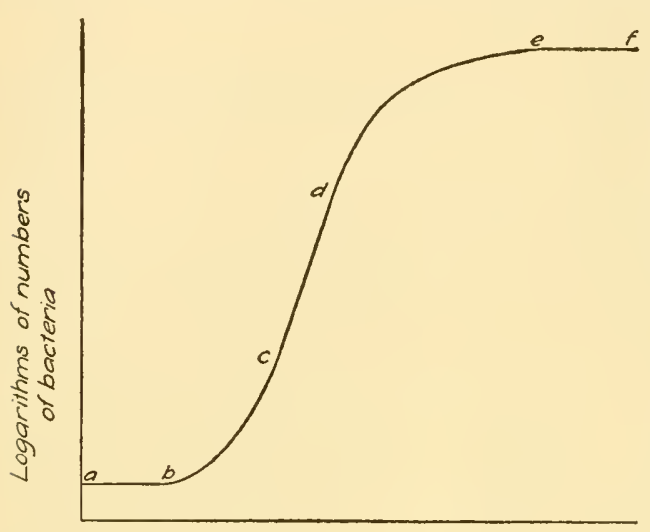

Time in Hours

Fig. 4.-Growth curve graphed as logarithms of numbers of bacteria against time.

$a-b$ Initial stationary phase

$b-c$ Lag phase

$c-d$ Logarithmic phase

$d-e$ Phase of negative acceleration

$e-f$ Maximum stationary phase (or, in the terminology suggested by Ostwald, "autocatakinesis"). While these two interpretations are not essentially antagonistic, they represent somewhat different points of view, and require separate treatment.

GROWTH CURVES CONSIDERED AS A SUCCESSION OF GROWTH PHASES

It was noted above that in some cases at least as many as five different growth phases may be observed in a culture of bacteria. They will be considered in order.

\section{INITIAL STATIONARY PIIASE}

During this phase there is no in. crease in numbers. In the illustration above its existence was ascribed to the time required for spores to germinate. Experience shows, however, that this phase is sometimes evident in a bacterial transfer although the organism is one which does not sporulate. It is apparent that bacterial cells from old cultures (in the maximum stationary and later phases) possess some of the same inertia and slowness to develop under favorable environment usually regarded as characteristic of spores. They may be regarded as in some respects the physiological, though not the morphological, equivalents of endospores. In some cases this phase may be prolonged for days or weeks. Such prolongation has been noted particularly by Esty and Meyer ${ }^{6}$ in heated cultures of the bacillus of botulism. This phase is not found when the inoculum contains any considerable proportion of actively multiplying bacteria.

\footnotetext{
${ }^{x}$ Lane-Claypon, Janet E.: J. Hyg., 9, 239. I909.

${ }^{2}$ Buchanan, R. E.: J. Infect. Dis., 23, 109. 1918.

3 McKendrick, A. G., and Pai, M. Kesave: Proc. Roy. Soc., Edinburgh, 3r, 649. Igr I.

${ }_{4}^{4}$ Robertson, T. B.: J. Physiol., 56, 404. 1922.

5 Lotka, Alfred J.: Elements of Physical Biology. I925.

${ }^{6}$ Esty, J. R., and Meyer, K. F.: J. Infect. Dis., 31, 650. 1922.
} 
During this phase the average rate of growth per cell is increasing to the maximum characteristic of the succeeding (logarithmic) phase. Some authors do not differentiate between this phase and the preceding, terming the two together the "lag phase."

It is evident that during this period the number of bacteria present is a function of the time; and several attempts, both empirical and theoretical, have been made to formulate the mathematical relationships. In the analysis of certain data Ledingham and Penfold ${ }^{\mathrm{I}}$ found that a graph of the logarithms of the logarithms of the numbers of bacteria and the logarithms of the time is a straight line. This leads to the formulation:

in which

$$
b=B e^{k t^{s}}
$$

$b=$ Number of bacteria after time $t$

$B=$ Initial number of bacteria

$k$ and $s=$ Constants which require evaluation for each

particular set of experiments

$e=$ Base of natural logarithms

These authors found the value of $s$ to vary from 1.56 to 2.7 .

\section{LOGARITHMIC PHASE}

During this phase the generation time is a constant, as is also the rate of growth per cell. This phase is the one most susceptible to simple mathematical analysis, and is of major importance in the study of the effect of environment upon bacteria.

Methods for estimating the generation time and number of generations during this phase were apparently first developed by Buchner, Longard, and Riedlin. ${ }^{2}$ If it be assumed that the cells are multiplying regularly by binary fission, and

$$
\begin{aligned}
& B=\text { Initial number of bacteria } \\
& b=\text { Number of bacteria after time } t \\
& n=\text { Number of generations in time } t \\
& g=\text { Length of one generation, i.e., time required for } \\
& \text { the bacteria to double in numbers }
\end{aligned}
$$

then,

and, since $n=\frac{t}{g}$,

$$
\begin{gathered}
b=B 2^{n} \\
b=B 2^{\frac{t}{b}} \\
n=\frac{\log b-\log B}{\log 2} \\
g=\frac{t \log 2}{\log b-\log B}
\end{gathered}
$$

x Ledingham, J. C. G., and Penfold, W. J.: J. Hyg., I4, 242. I9r4.

2 Buchner, H., Longard, K., and Riedlin, G.: Centralbl.f. Bakteriol., 2, т. 1887. 
If the numbers of cells present at any two times during the period of logarithmic growth are determined, the values of $n$ and $g$ may be readily derived. Nomograms for such determination have been developed by the writer. ${ }^{I}$ If the numbers of bacteria are plotted against time a curve will be developed whose equation is (3) (see Fig.5).

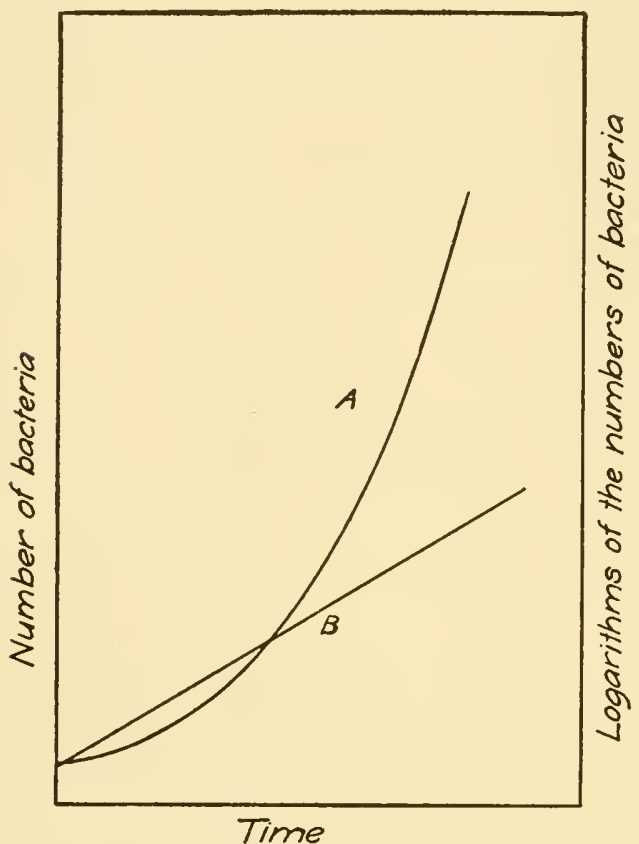

FIG. 5.-A. Growth curve during logarithmic growth phase. $B$. Plot of logarithms of numbers of bacteria against time.
Equation (5) may also be written in the form,

$$
\log b=\frac{t \log 2}{g}+\log B
$$

This indicates that if the logarithms of the numbers of bacteria are plotted against time a straight line will be developed, with slope $\frac{\log 2}{g}$, and with the intercept on the $y$-axis at $\log B$ (Fig. 5). This is a convenient criterion for determining whether or not a culture is in the logarithmic phase.

For some purposes it is advisable to determine the rate of growth per cell. If the cells are increasing regularly in geometrical progression, the rate of increase in the number of cells $\left(\frac{d b}{d t}\right)$ is constantly proportional to the number of cells, i.e.,

$$
\frac{d b}{d t}=k b
$$

in which $k$ is the proportionality constant termed the "velocity coefficient" of the rate of growth. Since

$$
k=\frac{d b}{d t}
$$

the velocity coefficient is the rate of growth per cell. Integration of $(7)$ gives the relationship

$$
l n b=k l+\text { Constant of integration }
$$

When $t=0$, the constant of integration is equal to $\ln B$, and

$$
\begin{gathered}
\ln b=k l+\ln B \\
k={ }_{t} \ln \frac{b}{B} .
\end{gathered}
$$

${ }^{x}$ Buchanan, R. E.: Ione Stale College J. Sc., I, 63. 1926. 
Equation (8) is in the form of an equation of a straight line. It follows, therefore, that if the logarithms (to base $e$ ) of the numbers of bacteria are plotted against time, a straight line will be developed with slope $k$ (rate of growth per cell) and intercept on the $y$-axis at $\ln B$. It follows that

$$
b=B c^{k t} .
$$

This is another form of equation (3), the equation of the logarithmic growth curve.

It is sometimes convenient to evaluate $k$ (rate of growth per cell) in terms of $g$ (generation time). Since the generation time is inversely proportional to the rate of growth per cell,

$$
g=\frac{C}{k}
$$

The value of the proportionality constant $C$ may be determined from equations (5) and (9).

and

$$
g=\frac{\ln 2}{k}
$$

$$
C=\ln 2=2.307 \log _{10} 2=0.692
$$

PHASE OF NEGATIVE GROWTH ACCELERATION

This phase succeeds the logarithmic phase when conditions become progressively more unfavorable to growth, due either to decrease in concentration of nutrients or to the accumulation of toxic products. Mathematical analysis of this phase has been attempted, but the adequacy is questionable.

\section{MAXIMUM STATIONARY PHASE}

This is reached when the cells cease to increase in numbers. A count at this time gives the maximum crop yield. The rate of growth is o.

\section{SPECIAL MODIFICATIONS OF GROWTH CURVES}

In some cases growth curves are found to be more complex than the type indicated above. They may, for example, exhibit more than one logarithmic phase. One may find cultures of organisms which both produce $\mathrm{CO}_{2}$ and are stimulated by it. A small seeding of such an organism (in the logarithmic growth phase) would for a time show a constant rate of growth per cell, later the $\mathrm{CO}_{2}$ would increase to a point where its stimulating action would be manifest, and the value of $k$ would increase with increase in concentration of $\mathrm{CO}_{2}$. Eventually saturation with $\mathrm{CO}_{2}$ would occur and the rate of growth per cell would again become constant. Similarly, more than one of certain other growth phases may be manifested.

\section{GROWTI CURVES INTERPRETED AS AUTOCATAKINETIC PHENOMENA}

McKendrick and Pai (loc. cit) and later Robertson (loc. cit.) and Lotka (loc. cit.) have suggested that the entire growth curve shows marked resemblance to a curve of autocatalysis. The relationship may be derived as follows:

It has previously been shown that under constant environmental conditions the rate of increase of bacteria is constantly proportional to the number of bacteria pres- 
ent. It may be assumed that the rate of increase is also proportional to the concentration of the available nutrients, or to that of some single nutrient which acts as a limiting factor. If concentrations of cells and of nutrients are the only two factors governing the increase, the rate will be jointly proportional to the two. A convenient method of estimating the amount of available nutrient is to determine the total maximum number of bacteria which may be produced in the culture. The difference between the number of bacteria present at any instant and the maximum number of bacteria which may be developed is proportional to the available remaining nutrients. If $\beta=$ maximum bacterial count, then

$$
\frac{d b}{d t}=K b(\beta-b)
$$

Upon integration,

$$
\ln \frac{b}{\beta-b}=K \quad t+C
$$

When $t=0, b=B$, and

$$
\begin{gathered}
\ln \frac{B}{\beta-B}=C \\
\ln \frac{b}{\beta-b}=K t+\ln \frac{B}{\beta-B}
\end{gathered}
$$

Equation (I4) shows a straight-line relationship between $\ln \frac{b}{\beta-b}$ and time, the straight line having a slope, $K$. This relationship may be used to determine whether in any case the growth curve resembles that of autocatalysis, or whether (according to Ostwald) the growth curve is autocatakinetic.

The equation of an autocatakinetic growth curve may be derived from equation (I4):

$$
b=\frac{\beta e^{K t+c}}{\mathrm{I}+e^{K t+c}}
$$

A convenient evaluation of $C$ may be made by taking $t_{1}$ as the time which has elapsed to the instant when $b=\frac{\beta}{2}$, i.e., until the number of bacteria has reached one-half the maximum.

$$
\begin{aligned}
C & =-K t_{\mathrm{s}} \\
\ln \frac{b}{\beta-b} & =K\left(t-t_{\mathrm{I}}\right)
\end{aligned}
$$

and

$$
b=\frac{\mathrm{I}}{\mathrm{I}+e^{-K\left(l-l_{s}\right)}} .
$$

A curve of this type is illustrated in Figure 6. It will be found to be symmetrical, and asymptotic to the lines $b=0$ and $b=\beta$. The point of inflection occurs at $\frac{\beta}{2}$ after time $t_{\mathrm{r}}$. The $\mathrm{y}$-axis is cut at $b=B$. 
Data of bacterial growth as studied by McKendrick and l'ai and by Lotka have been found to conform measurably well to such autocatakinetic curves. Other cases may be cited, however, in which the agreement is not good. This is particularly true when there is manifested a prolonged initial stationary phase or lag phase. Then, too, the rate of growth per cell may not be directly proportional to the concentration of the available nutrients. Other factors may also alter the form of the growth curve, and in consequence it may be unsymmetrical. The equations and relations of such curves are more complex, and are not subject to ready analysis. Even if the data secured are found to fit symmetrical curves, comparisons between curves secured under varying environmental conditions are not as readily made as between corresponding (particularly the logarithmic) growth phases.

EFFECT OF AGE OF PARENT-CULTURE UPON FORM OF GROWTH CURVES

The work of Lane-Claypon (loc. cit.) and others has shown quite clearly that the phase of growth of the culture from which a transfer is made influences markedly the form of the growth curve in the daughter-culture. In most cases the following results will be secured: (I) Transfers from the initial stationary phase will show a continua-

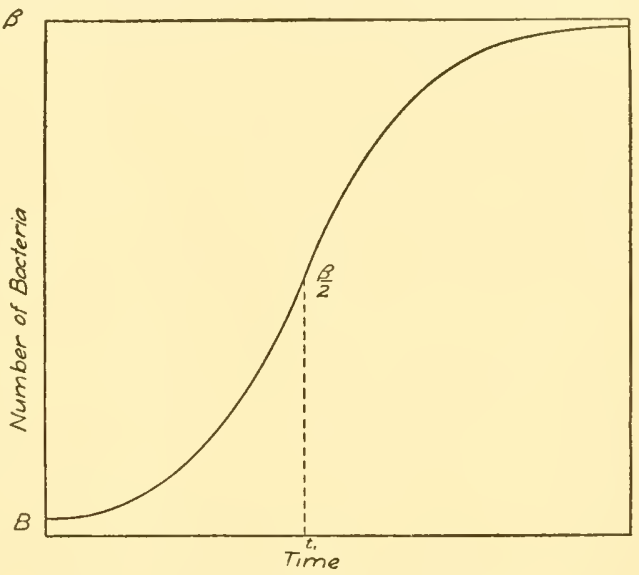

FIG. 6.-An autocatakinetic growth curve tion of this phase, followed by lag phase, etc., in normal sequence. (2) Transfers from the lag phase will usually show a continuation of this phase, followed by the logarithmic phase, etc., in normal sequence. (3) Transfers from the logarithmic phase usually show a continuation of the logarithmic phase. In some cases allelocatalysis (see below) may cause the culture to show an initial lag phase. (4) Transfers from the phase of negative growth acceleration will usually show a lag phase. (5) Transfers from the maximum stationary phase may show an initial stationary phase or a lag phase.

EFFECT OF SIZE OF INOCULUM UPON THE FORM OF THE GROWTH CURVE

It has been shown by Robertson ${ }^{\mathrm{r}}$ that growth in a subculture of certain organisms, particularly protozoa, is stimulated by the presence of other cells of the same type. Single-cell transplants to hanging drops exhibit a much slower initial rate of growth per cell than do seedings of a larger number. This phenomenon of mutual or selfstimulation he terms "allelocatalysis." While in general results with bacteria do not show this effect under the usual conditions of culture, there is evidence that with some forms, as the pneumococcus, single-cell isolations are very difficult. The work of Valley and Rettger ${ }^{2}$ and others seems to indicate that many organisms grow very

I Robertson, T. B.: Biochem. J., r 5, 595. 1915.

${ }^{2}$ Valley, George, and Rettger, Leo F.: J. Bact., I I, 78. 1926. 
slowly or not at all until a certain minimum concentration of carbon dioxide is present. This would lead to the development of a definite lag phase which would be much longer with small than with large seedings. Slator has shown that when very large inocula of yeast are used there may be no logarithmic phase.

\section{EFFECT OF CONCENTRATION OF CONSTITUENTS OF SUBSTRATE UPON GROWTH CURVES}

The various constituents of the culture medium may act either as accelerators or inhibitors of growth. The effect upon rates of increase is in general a function of the concentration. The exact relationship may be most satisfactorily evaluated usually by comparison of the rates of increase per cell $(k)$ in different concentrations during the logarithmic growth phase. In chemical reactions generally it is found that the velocity coefficient of the rate of the reaction varies directly as some constant power of the concentration of the reactant. It is frequently advisable, as a first approximation, to test the hypothesis that a similar relationship holds between rate of increase and concentration of a nutrient or inhibiting agent. This would give the relationship

Integration yields

$$
\frac{d b}{d t}=K C^{n} b
$$

in which

$$
\ln b=K C^{n} t+\ln B
$$

$$
\begin{aligned}
& K=\text { Constant } \\
& C=\text { Concentration of chemical } \\
& n=\text { Constant }
\end{aligned}
$$

It is apparent that the velocity coefficient (rate of increase per cell, $k$ ) is evaluated as

$$
k=K C^{n}
$$

A determination of the validity of this relationship with varying concentrations may be made by plotting the logarithms of the velocity coefficients $(k)$ against the logarithms of the concentrations. Since

$$
\log k=n \log C+\log K
$$

a straight line should be developed with slope $n$. If increase in concentration increases the growth rate, $n$ will be positive; if it inhibits, $n$ will be negative.

\section{EFFECT OF TEMPERATURE UPON THE FORM OF GROWTH CURVES}

The effect of temperature changes upon rates of increase is usually best evaluated by comparisons of the velocity coefficients $(k)$ during logarithmic growth. It is customary to designate the ratio between the velocity constants at the higher and at the lower temperature as the temperature quotient $(Q)$. 'The temperature interval for which determinations are usually made is $10^{\circ} \mathrm{C}$. For this interval the quotient is commonly designated as $Q_{10}$.

It is frequently desirable to determine whether temperature effects upon growth 
rates resemble the effects upon rates of chemical reactions. It is commonly found that chemical reactions are accelerated by rise in temperature, and in ranges of $0^{\circ}-100^{\circ} \mathrm{C}$. frequently doubled or trebled in rate by each increase of $10^{\circ}$. This tendency to double or treble the rate has come to be termed the "R.G.T." (Reaktionsgeschwindigkeit Temperatur) rule. Studies upon rates of bacterial growth in certain ranges have shown values of $Q_{\mathrm{ro}}$ frequently equal to two or three.

It has been shown that in chemical reactions the value of $Q_{\mathbf{r} 0}$ tends to decrease with rise in temperature. This relationship has been developed by van't Hoff and Arrhenius into the generalization: The rate of change in the logarithm of the velocity coefficient of a chemical reaction with temperature is inversely proportional to the square of the absolute temperature, i.e.,

$$
\frac{d \ln k}{d T}=\frac{A}{T^{2}}
$$

It was also shown that the constant $A$ may be substituted by $\frac{\mu}{R}$, in which $\mu$ is a constant characteristic of the reaction (thermal increment) and $R$ the gas constant (numerically equal to 2 ). Integration of equation (22) yields

$$
\ln k=\frac{\mu}{2} \frac{\mathrm{I}}{T}+C
$$

Conformity of a reaction to the relationship of equation (23) may be determined by plotting the values of $l n k$ against the reciprocal of the absolute temperature; agreement is manifest by the development of a straight line, with slope $\frac{\mu}{2}$.

It is of interest to determine whether growth rates (and growth curves) of microorganisms are similarly related to temperature. The work of Crozier et al..$^{\mathrm{I}}$ indicates that results of value may be secured by studies of the values of thermal increments $(\mu)$. It is contended that growth rates are controlled by rates of chemical reactions, and changes in the latter due to temperature changes should produce corresponding changes in the former. Since the growth is probably the resultant, in many cases at least, of a catenary series of reactions, the rate of growth would be controlled by the slowest rate. Changes in temperature may therefore modify the rate of growth in the same manner as they modify the rate of the slowest reaction.

From equation (23) the following relations are evident:

$$
\begin{gathered}
\frac{k_{2}}{k_{\mathrm{I}}}=e^{\frac{\mu}{2}\left(\frac{T_{2}-T_{\mathrm{x}}}{T_{2} T_{\mathrm{x}}}\right)} \\
Q_{\mathrm{I} 0}=e^{\frac{5 \mu}{T_{2} T_{\mathrm{I}}}}
\end{gathered}
$$

in which $k_{2}$ and $k_{1}$ represent the velocity coefficients at the higher and lower temperatures (absolute) $T_{2}$ and $T_{1}$, respectively.

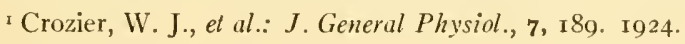




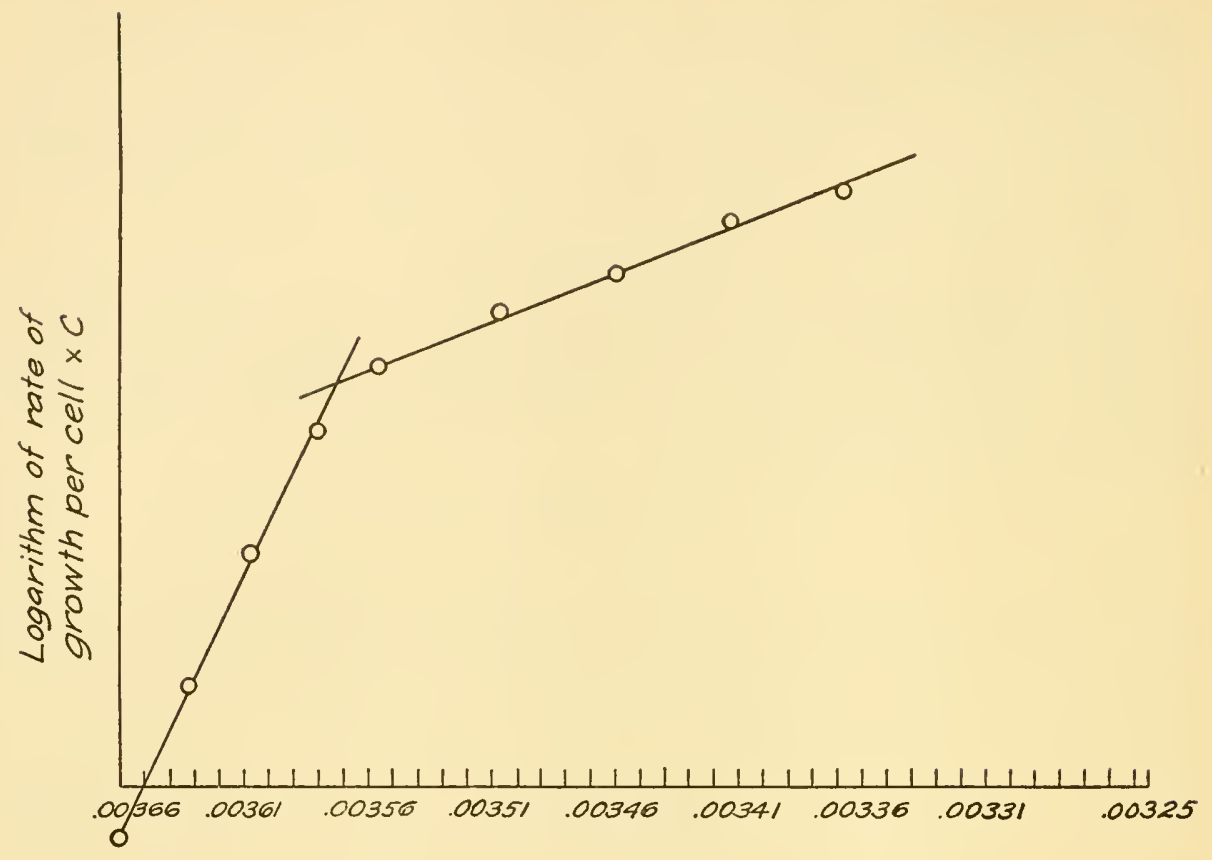

Peciprocal of Absolute Temperature.

FIG. 7

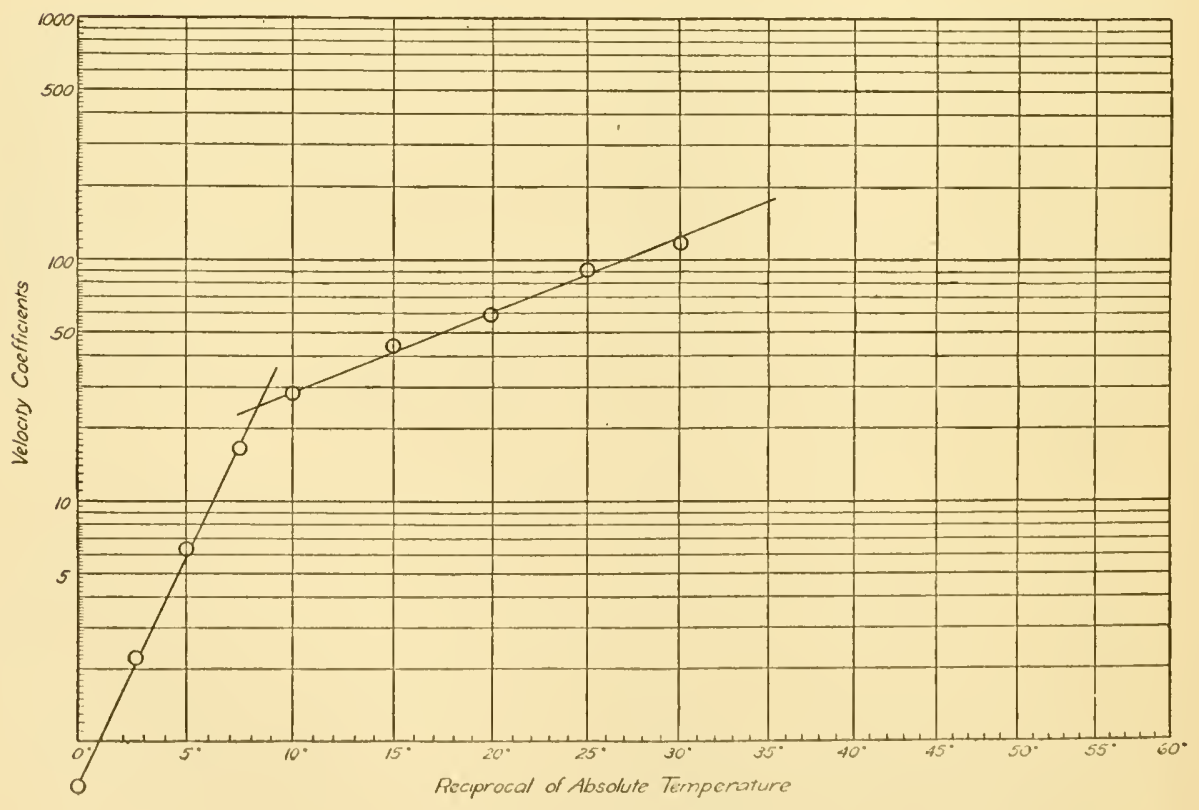

lig. $S$ 
The size and constancy of the thermal increment $\mu$ may be determined either by substitution of values in equation (24) or by plotting the logarithm (base $e$ ) of $k$ against the reciprocal of the absolute temperature. Such a graph is given in Figure 7 for the effect of temperature upon the growth of a bacterial culture between $0^{\circ}$ and $30^{\circ} \mathrm{C}$. It will be noted that the points apparently determine in this case two intersecting straight lines. Crozier and others interpret a finding of this type as indicating a change at a certain temperature from one basic reaction in the catenary series to another as governing the growth rates. Graphs of this type are made most conveniently by using a semilog paper in which the abscissae are indicated as temperatures centigrade but are spaced in proportion to the value of the corresponding reciprocal of the absolute temperature. The data of Figure 7 are plotted on this type of co-ordinate paper in Figure 8.

It is evident that if values of the thermal increment are known, and the relationships outlined above hold, it is possible to predict the form which growth curves will assume at different temperatures. 


\title{
CHAPTER VI
}

\section{THE RISE AND FALL OF BACTERIAL POPULATIONS}

\author{
C.-E. A. WINSLOW
}

Yale School of Medicine

\section{THE LIFE-CURVE OF A BACTERIAL POPULATION}

In a study of the distribution of bacteria in their various natural habitats the bacteriologist is inevitably impressed with a sense of a relatively stable adjustment between a specific environment and the numbers and kinds of bacteria which will generally be found therein. The botanist knows that on a certain kind of soil in a certain climate such-and-such trees and shrubs will be present, about so many to the acre. So, in our microscopical realm, we find that uncultivated sandy soils may yield ro०,००० bacteria per gram while garden soils show I,500,000. A given river in a dry summer will contain from $\mathrm{I}, 000^{-2,000}$ bacteria per cubic centimeter while a lake will contain only $5^{\circ}-150$, and the deep waters of the ocean or those of a driven well will show only 5-Io. From day to day, and from year to year, both the numbers and kinds of microbes in a given habitat will remain extraordinarily stable-provided that the environmental conditions themselves remain approximately constant.

If, on the other hand, the conditions of the habitat change, or if a section of the bacterial population be transferred to a new environment, the balance is upset. A new and active struggle for existence is initiated, such as has occurred among the higher forms of life when a glacial epoch has changed the climate of a continent. With our short-lived forms of life, capable of completing a whole cycle of evolution in twenty-four hours, we can trace the course of such a struggle in a fashion which should be the envy of the ecologist; and as we do so, we find a remarkable degree of constancy underlying even the phenomena of change which characterize such a period of adaptation.

The curve which marks the rise and fall of a bacterial population in a new environment is illustrated graphically and schematically in Figure I; and it may be claimed that this curve is a widely representative one for all conditions, with the limitation that according as the environment is more or less favorable the subdivisions of the curve may be relatively increased or decreased or even suppressed entirely.

\section{THE PHASE OF ADJUSTMENT}

The first phase in the cycle of a bacterial population (if the environment be not too severe) is what may be called the "phase of adjustment." In a medium which is highly favorable this phase will be indicated, as in the solid line $A B$ of Figure $\mathrm{r}$, by a relatively slow increase, the period of lag or dormancy, as described by Rahn (I g06), Barber (I go8), Lane-Claypon (I 909), Coplans (I909), Penfold (I9I4), Chesney (IgI6), and Sherman and Albus (1924).

The first careful studies of the rate of bacterial multiplication and the first de- 
scription of the lag phase in a favorable medium were due to Müller (I895) who introduced the now-familiar formula for generation time:

$$
G=\frac{T \log 2}{\log b-\log a}
$$

where $G=$ minutes per generation, $T=$ elapsed time in minutes, $a=$ initial number of bacteria, and $b=$ final number of bacteria.

He showed that $G$ increased with the age of the primary culture used for inoculating the medium in which generation time was measured. Thus typhoid bacilli inoculated from a $2.5-3$-hour mother-culture gave a generation time of 40 minutes while

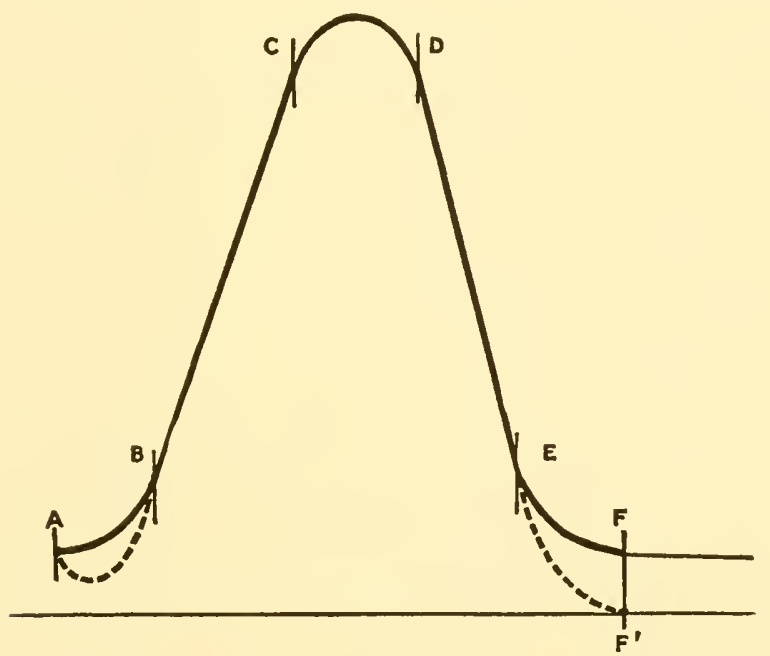
time.

FIg. I.-Ideal curve of a bacterial population cycle. Ordinates = numbers; abscissae =elapsed

$$
\begin{aligned}
& A-B . \text { Phase of adjustment } \\
& B-C \text {. Phase of increase } \\
& C-D \text {. Phase of crisis }
\end{aligned}
$$

$D-E$. Phase of decrease

$E-F$. Phase of readjustment

those from a $6 \frac{1}{4}$-hour culture completed a generation in $80-85$ minutes, and those from a I4-16-hour culture in over 160 minutes. He attributed the slower generation in media inoculated from older cultures to what is now called a "lag effect." Hehewerth (I901) confirmed these results. It was Rahn (I906), however, who first studied the preliminary lag period intensively, using $B$. fuorescens in broth.

He inoculated from (I) 20-hour broth, from (II) 20-hour agar, and from (III) 4month broth, and obtained such results as are shown in Table $I$.

If the medium be less favorable, the phase of adjustment will be marked by a decrease followed by an increase. This latter phenomenon was noted at least as early as I 894 (Fuller, 1895) in studies made at the Lawrence Experiment Station of the Massachusetts State Board of Health which showed that a bottled sample of sewage originally containing I, r90,000 bacteria per cubic centimeter fell off to $1,085,000$ after 2.5 hours, then rose steadily to a maximum of $23,100,000$ after 25.5 hours, and then fell 
steadily to 2,34I,000 after 8 days. Whipple (I9OI) noted the same effect-an initial fall, followed by a rise, as a universal phenomenon in portions of natural waters stored in sample bottles under various conditions. This type of reaction, indicated by the dotted line $A B$ in Figure $\mathrm{I}$, does not appear to be substantially different from the lag phase in a richer culture medium and, indeed, it seems probable that whether the observed net effect be an increase or a decrease there is going on during this period a multiplication of some cells and a death of others, the relative rate of these two processes determining the end-result. The fact that the bacterial count of milk shows an initial decrease was pointed out by Fokker (1890), and a long controversy has been carried on in regard to its cause (excellently summarized by Heinemann, I9I9). Many investigators claim that freshly drawn cow's milk possesses special germicidal properties due to the presence of agglutinins and other bactericidal substances, the presence of living phagocytes, or the restraining action of lecithin. Heating the milk destroys this "bactericidal power," but it must always be remembered that heating also changes the nutritive qualities of a medium and may therefore make it more favorable. In general, the phenomenon seems to be a special case of the lag period in a medium

TABLE I

Generation Time in Minutes

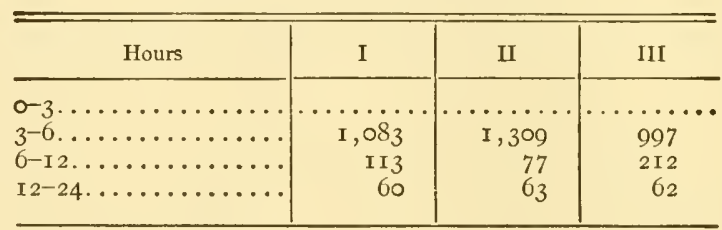

more or less unfavorable to certain types of bacteria which have gained access to the milk.

Ledingham and Penfold (I9I4) and Slator (I9I7) have attempted to formulate mathematical expressions for the multiplication rate even in the highly variable lag period of the population cycle; but if the conclusion be justified that the phase of adjustment may, according to circumstances, be characterized by a decrease followed by an increase or by a gradually accelerating increase, the futility of any attempt at mathematical analysis will be apparent.

The inflection and the slope of the curve of the bacterial population will vary with three general factors: the type of bacteria involved, the medium into which they are introduced, and the temperature. The first two factors may in a sense be reduced to one-the suitability of the particular medium for the particular bacteria in question. If the medium be entirely inadequate, the bacteria simply die off and the phase of adjustment merges into the phase of decrease. On the other hand, as I shall point out, if the medium be ideal and the bacteria in the right condition, the phase of adjustment may be reduced to proportions which are not measurable, and the logarithmic increase will begin at once. There may be an infinite number of gradations between these two extremes, giving a longer or a shorter phase of adjustment. Coplans (r9o9), for example, found that in transferring from peptone water to peptone water the lag period lasted about $\mathbf{I}$ hour while in transfer from one dulcitol medium to another it 
was longer. In fresh unsterilized milk the lag lasted 6 hours, but was apparently abolished by previous heating of the milk. Cohen and Clark (I9r9) note that the onset of the period of maximal increase for Bact. coli was $2-4$ hours in peptone phosphate broth at $\mathrm{pH}$ values between 6.I and 8. I but was increased to $3^{-5}$ hours at $\mathrm{pH}$ values below $5 \cdot 5$, and to ro- 2 hours at $\mathrm{pH} 8.9$ ( $37^{\circ} \mathrm{C}$.).

A high temperature, of course, decreases the length of the phase of adjustment. According to Whipple's data for water bacteria stored in sample bottles, the period of lag averaged about 8 hours at $20^{\circ}-24^{\circ} \mathrm{C}$. and I 7 hours at I $2^{\circ} \mathrm{C}$. Lane-Claypon (I909) reports a lag period in culture media varying from I hour at $42^{\circ}$ to 6 hours at $20^{\circ} \mathrm{C}$.

An extreme case of lag may probably be found in the fact that both spores and vegetative cells occasionally show an exceedingly slow development in entirely favorable culture media. Thus Burke, Sprague, and Barnes inoculated broth and agar tubes with approximately one cell of Bact. coli per tube. While 85 per cent of the 473 tubes which gave growth did so within 2 days and 97 per cent within 6 days, there were Io tubes which developed only on the fourteenth day, 4 only on the fifteenth, and 4 only on the sixteenth. Spores of $B$. subtilis remained dormant under similar conditions for 39 days and spores of B. megatherium for 90 days.

The fundamental causes of the lag phenomenon have been exhaustively discussed, particularly by Rahn (Igo6), Penfold (I9I4), Chesney (I9I6), and Buchanan (I9I8). The phenomenon must be considered in the light of the observation of Müller (I895) and Hehewerth I90I) that the rate of increase of bacteria in a given medium bears a generally inverse relation to the age of the mother-culture from which this medium was inoculated. Hehewerth found that the generation time for Bact. coli in broth, when transferred from a young broth culture, was 2 I-27 minutes while when transferred from an older broth culture it was 43 minutes. It is of cardinal significance to note that lag disappears entirely if transfer is made to an identical medium while the mother-culture is in its phase of logarithmic increase (Penfold, Barber, Chesney). Furthermore, Penfold shows that if the growth in a culture be stopped by chilling for a very short time the growth recommences at a normal rate when the temperature is raised; while more prolonged chilling and subsequent increase of temperature is followed by a lag.

The occurrence of lag cannot in general be due to the presence of inhibitory substances carried over from the mother-culture (as might be concluded from Penfold's finding that centrifugalized cultures showed decreased lag) since we note the same phenomenon in a sample of water transferred from a lake to a sample bottle. Furthermore, Penfold and Chesney show that lag in a secondary culture does not increase with the progress in the mother-culture of the logarithmic phase, and that while it does grow more marked with passage from the logarithmic phase to the phase of crisis there is no further increase with later aging of the mother-culture.

Lag is therefore primarily associated with the biological condition of the cells which are transferred to a new medium (or placed under new environmental conditions, as when a water sample is collected). Under certain conditions this may be associated with definite prior injury to the cells. Sturges (I9I9) found that the development of colonies on plates seeded from sewage disinfected with copper or sulphurous acid was very much retarded (although the fact that chlorine-disinfected sewage ex- 
hibits no such phenomenon suggests that the disinfectants were perhaps carried over to the plates in antiseptic concentration). Allen (1923) and others have ascribed similar slow growths of bacteria in milk after pasteurization as due to attenuation by "temperature shock."

Chesney (19r6) believed this to be the main factor in the lag phenomenon in general, and he cites a striking case in which the generation time of pneumococci was slowed down in a filtrate from an old broth culture of the same organism. That the shock theory cannot be of general application, however, is made clear by the fact that similar phenomena occur in bottled-water samples.

The second possibility which suggests itself is in a sense the converse of the idea that lag is due to a state of injury produced by an earlier environment. It involves the conception that, among the cells carried over from any environment $A$ to another environment $B$, some find themselves ill adapted to the latter and that a process of natural selection must ensue until the less adapted cells are weeded out. Such a condition must apparently be assumed when the lag period is characterized by an actual decrease in number such as occurs in samples of water, milk, or sewage, or in soil to which an antiseptic has been added.

When a pure culture of a single species is transferred to a medium identical with that in which it is already living there must be something still more fundamental involved. We may conclude from the work of Penfold and Barber that an initial period of slow development is an essential necessity whenever bacteria pass to a new environment from one in which they are not multiplying rapidly. Bacteria in active multiplication appear to be in a different biological state from bacteria in other phases of the population cycle, and it takes time to effect this change of state.

We can, however, perhaps go a little further and visualize certain more concrete conceptions of what this difference in state may mean. The work of Sherman and Albus (1922 and 1924) indicates that cells during the early lag period are less sensitive to slightly toxic salts than are cells in the late lag and logarithmic phase, and they find that the sensitiveness to salts appears slightly before multiplication sets in at its most rapid rate. This suggests the possibility that permeability phenomena may play a part. On the whole, however, among the numerous possibilities Penfold's explanation that maximum growth presupposes the existence in the cells of intermediate bodies in the synthesis of protoplasm-intermediate bodies which diffuse out and are lost when growth is checked-seems on the whole most plausible. On such a hypothesis cells in the "resting stage" of an old culture or in a lake water or in any stable environment would be cells lacking these "intermediate bodies" while the "rejuvenated" cells of the logarithmic growth phase would be rich in them.

It is also possible that such "intermediate bodies" may be transferred in the form of dead as well as of living bacterial cells, or in solution in the surrounding menstruum. This would explain the finding of Chesney that cells transferred from a mother-culture during the phase of increase and washed free from the medium by centrifugation show a lag when placed in a new culture, while the cells left in the mother-culture still continue to grow at a logarithmic rate. Such a supposition would also be in accord with the conclusion of Rahn (I906) that maximal multiplication coincides with the presence of heat-stable, non-filterable substances "formed by the bacteria," and with 
the observation of Penfold (I9I4) that generation time decreases with an increase in inoculum. Rettger (I9I8) suggests that lag in culture media may be decreased or eliminated by supplying "satisfactory substitutes for the intermediate bodies in the form of amino acids and perhaps amines of simple composition, and also certain growth-accessory substances."

\section{THE PHASE OF INCREASE}

When the phase of adjustment (whether this involves selection of more readily viable cells or the development within the cells of intermediate products favorable to rapid growth, or both) has been completed in a favorable medium, there next follows a period of rapid and regular increase. During this phase it has been shown by Clark and Ruehl (1919) that the average size of cell is greatly increased as compared with that which is dominant in an older culture. According to Henrici (I92I, I922, I923, I924), the large cells appear toward the end of the lag phase and the beginning of the phase of logarithmic increase, the average size returning to normal as the phase of increase proceeds. In a highly unfavorable medium, this phase will of course be entirely suppressed; but it is a very common phenomenon, by no means limited to the rich culture media of our laboratories. It was perhaps first exhaustively studied by the early water bacteriologists in the case of samples of natural waters which had been placed in a new environment by the mere fact that they had been collected in a sample bottle in the laboratory. Thus Leone as early as r 886 records the following results for Munich water simply stored in flasks without the addition of any foreign material:

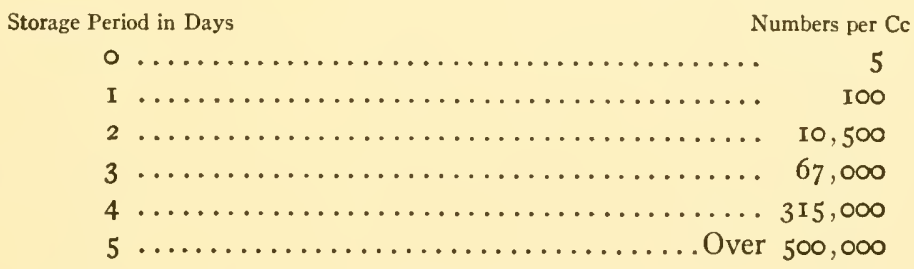

Miquel ( $189 \mathrm{I}$ ) gives the striking curves reproduced in Figure 2 for a series of spring waters stored in flasks at $29^{\circ}-30^{\circ} \mathrm{C}$.

In the phase of logarithmic increase we are dealing with a very simple relationship due to the fact that binary fission carried on at a regular rate leads to a progressive logarithmic increase. In other words (Ledingham and Penfold, I9I4):

$$
t=\mathrm{K} \log b / B
$$

when $t=$ time, $b=$ final number, and $B=$ initial number.

The actual figures obtained for generation times under certain more or less typical conditions are indicated in Table II. The studies of multiplication in soil, feces, and bottled waters did not include counts made at sufficiently frequent intervals to be quite certain that only the phase of logarithmic growth was included, but they are cited in the table as representing the most rapid increases (under this condition) with which the writer is familiar.

It appears from all the more careful work upon this subject that under the most 


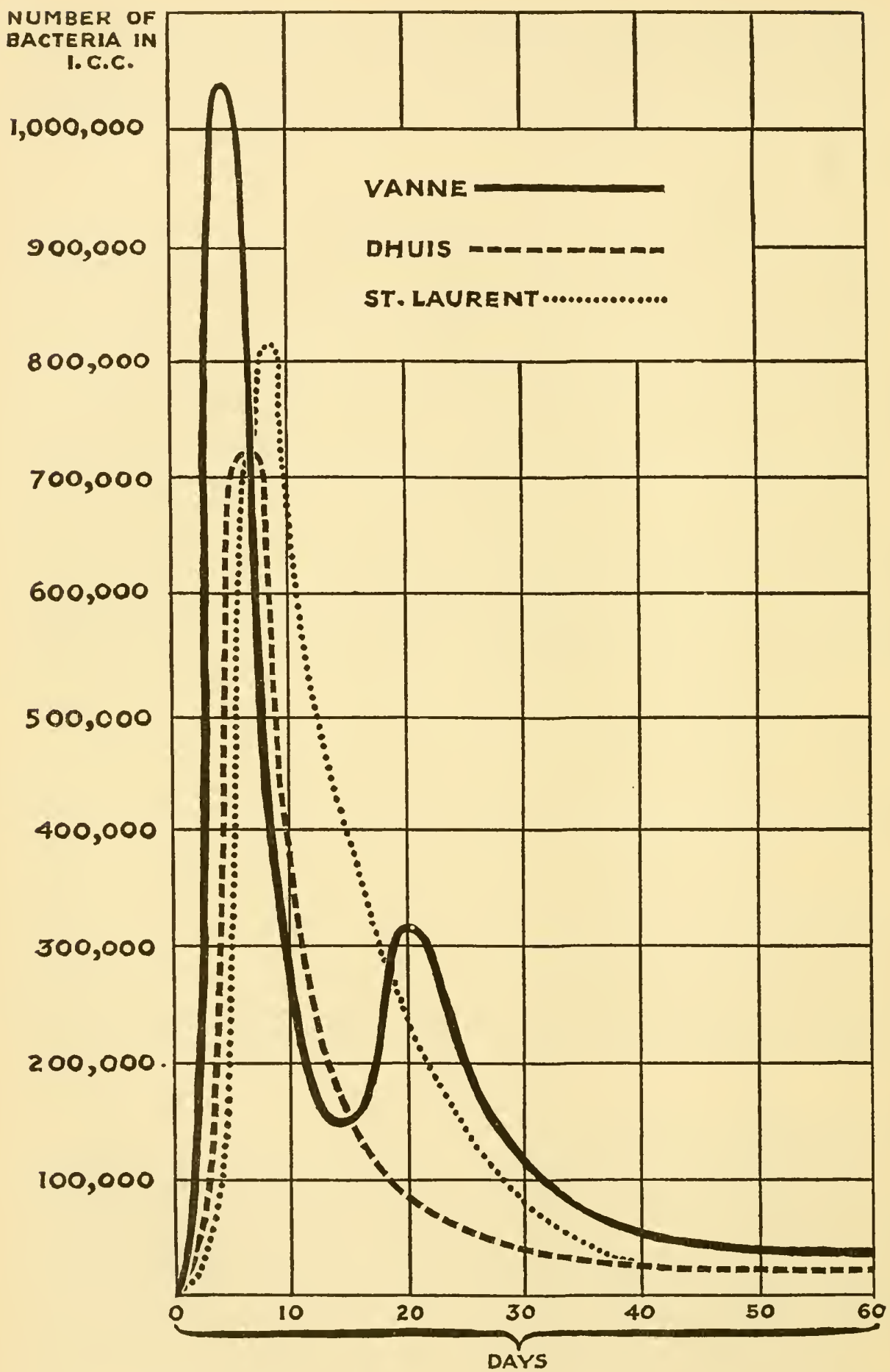

FIG. 2.-Multiplication of bacteria in bottled samples of certain spring waters (Miquel [Frankland, I 894]). 
favorable conditions of medium and temperature the cells of bacteria may divide once in I 7-20 minutes, while under less favorable conditions the rate of multiplication may be slackened to any desired degree.

TABLE II

Typical Generation Times during the Phase of Logarithuic Increase

\begin{tabular}{|c|c|c|c|c|}
\hline Observer & Type of Organism & Medium & Temperature, ${ }^{\circ} \mathrm{C}$ & $\begin{array}{c}\text { Generation Time, } \\
\text { Minutes }\end{array}$ \\
\hline 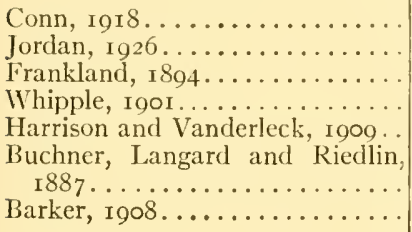 & $\begin{array}{l}\text { Soil bacteria } \\
\text { Bact. coli } \\
\text { Water bacteria } \\
\text { Water bacteria } \\
\text { Bact. coli } \\
\text { V. cholerae } \\
\text { Bact. coli }\end{array}$ & $\begin{array}{l}\text { Manured soil } \\
\text { Feces } \\
\text { Bottled sample } \\
\text { Bottled sample } \\
\text { Milk } \\
\text { Broth } \\
\text { Broth }\end{array}$ & $\begin{array}{l}20 \\
20 \\
20 \\
24 \\
37 \\
37 \\
37\end{array}$ & $\begin{array}{r}840 \\
720 \\
\text { I } 96 \\
\text { I } 15 \\
40 \\
\text { I } 9-40 \\
\text { I } 7\end{array}$ \\
\hline
\end{tabular}

The most obvious of the environmental conditions which determine the generation time - and hence the $K$ in the curve for rate of logarithmic increase-is the temperature (see Ward, I895). The results of certain of the most exhaustive studies on this point are cited in Table III.

\section{TABLE III}

Generation Time at Various Temperatures in Minutes

\begin{tabular}{|c|c|c|c|c|}
\hline 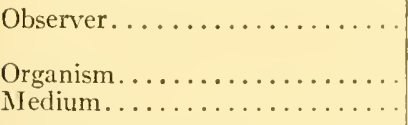 & $\begin{array}{l}\text { Whipple } \\
\text { Water bacteria } \\
\text { Bottled sample }\end{array}$ & $\begin{array}{l}\text { Harrison and } \\
\text { Vanderleck } \\
\text { Bact. coli } \\
\text { Milk }\end{array}$ & $\begin{array}{l}\text { Buchanan } \\
\text { Bact. coli } \\
\text { Broth }\end{array}$ & $\begin{array}{l}\text { Lane-Claypon } \\
\text { Buct. coli } \\
\text { Broth }\end{array}$ \\
\hline 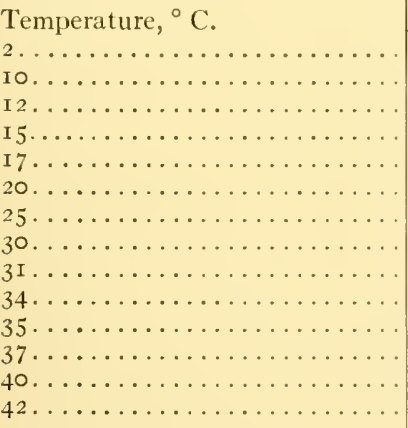 & 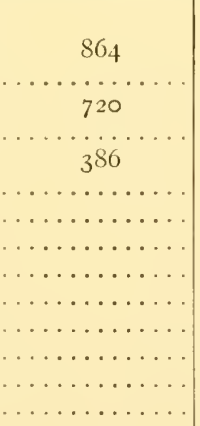 & 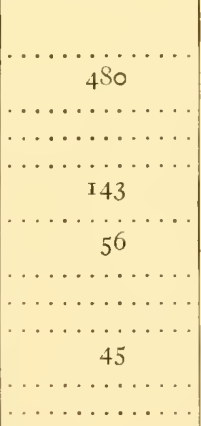 & 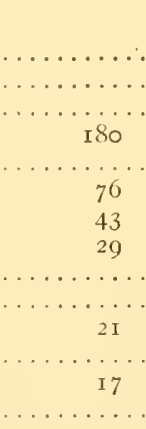 & 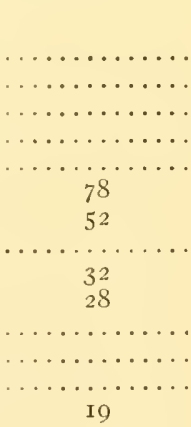 \\
\hline
\end{tabular}

If we plot the logarithms of these generation times against temperature, we obtain a series of straight lines, as was pointed out by Lane-Claypon (I909). The increase in rate of growth is approximately doubled for a $10^{\circ} \mathrm{C}$. rise in temperature, a relationship which Snyder ( I go8, I 9 I I) has shown is as generally characteristic of biological processes as of chemical reactions.

We may next consider briefly some of the other factors which determine the rate of logarithmic increase - a problem by no means easıly elucidated. It is easy enough to understand why bacteria increase when inoculated into a rich, sterile culture me- 
dium but that bacteria in more or less stable equilibrium in the water of a spring or well should increase many thousand fold (see Fig. 2) merely because a portion of the water in which they are living is placed in a sample bottle (even without any increase in temperature, as Whipple's data show) is far more difficult to explain. And the fact reminds us how subtle are the factors which constitute a bacterial environment.

Similarly, it is of interest to note the observation of Jordan ( 1926 ) that fresh human feces (containing already 75,000,000 bacteria per gram) show an enormous further increase on storage, sometimes reaching several hundred times the original figure after a few days. This increase is mainly due to multiplication of Bact.coli and occurs at $10^{\circ}$ and $20^{\circ}$ as well as at $37^{\circ} \mathrm{C}$. It is suggested by Jordan that the multiplication may be due to the loss of specific inhibitory influences present in the lower intestine.

Miquel attributed the rapid growth in a new medium to the absence of toxic products of prior bacterial growth and found that boiling destroyed the "toxicity" of

TABLE IV

Bacterial Content of Normal and of Toluene-treated Soll at Various Temperatures

\begin{tabular}{|c|c|c|c|c|c|c|}
\hline \multirow{3}{*}{ Temperature, ${ }^{\circ} \mathrm{C}$} & \multicolumn{6}{|c|}{ Bacteria, Millions per Gram } \\
\hline & \multicolumn{3}{|c|}{ Untreated Soil } & \multicolumn{3}{|c|}{ Toluened Soil } \\
\hline & 5 Days & 27 Days & 58 Days & 5 Days & 27 Days & $5^{8}$ Days \\
\hline 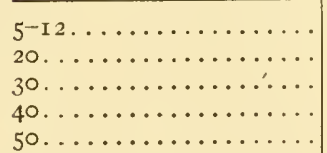 & $\begin{array}{r}\text { I I } \\
8 \\
8 \\
9 \\
4\end{array}$ & $\begin{array}{l}9 \\
5 \\
9 \\
2 \\
\text { I }\end{array}$ & $\begin{array}{l}7 \\
6 \\
6 \\
8 \\
1\end{array}$ & $\begin{array}{r}8 \\
50 \\
43 \\
12 \\
2\end{array}$ & $\begin{array}{r}27 \\
30 \\
24 \\
4 \\
1\end{array}$ & $\begin{array}{r}28 \\
30 \\
3 \mathbf{I} \\
6 \\
\mathbf{I}\end{array}$ \\
\hline
\end{tabular}

water rendered unsuitable for growth by such earlier development. When we transfer from an old culture to a new sterile tube of the same medium in the laboratory, this factor must play a major part, since, as I shall. point out in discussing the phase of crisis, growth is often undoubtedly checked in old cultures by the accumulation of acids or other toxic products. Cohen and Clark (I9I9) found for Bact. coli in broth a generation time of 27 minutes at $\mathrm{pH} 5.0$ while at $\mathrm{pH} 8.9$ the time increased to $46 \mathrm{~min}$ utes and in the acid range at $\mathrm{pH} 4.6$ there was a decrease instead of an increase. For the multiplication following collection of a water sample such an explanation seems, however, clearly inadmissible, and the favorable effect of boiling is probably due chiefly to the alteration which heat produces in certain of the foodstuffs which are present.

The work of Russell and Hutchinson (I9r3), Hutchinson and MacLennan (19r4), and Buddin (rgr4) from the Rothamstead Experiment Station and the similar studies of Truffant and Bezssanoff (1922) in France have given us some important data in regard to the multiplication of bacteria in soils. They found that the treatment of a soil by moderate heat or by mild antiseptics caused a marked secondary increase in bacterial numbers and crop fertility. Thus Table IV (from Russell and Hutchinson, 
I9I3) shows that in an untreated soil bacterial numbers remained constant, unaffected by temperature variations between $5^{\circ}$ and $40^{\circ} \mathrm{C}$.; while in a soil to which a slight amount of toluene had been added marked increases occurred, at $20^{\circ}$ and $30^{\circ}$ and, more slowly, at $5^{\circ}-\mathrm{I} 2^{\circ} \mathrm{C}$.

Table V (from Hutchinson and MacLennan, r9 4 ) is of special interest as demonstrating the influence of lime upon a highly acid soil. It will be noted that o. I per cent $\mathrm{CaO}$ produced a very slight stimulating action. The next three concentrations $(0.2$, 0.3 , and 0.4 per cent) caused progressively increasing stimulation with a maximum count on the ninetieth day. A strength of 0.5 per cent was somewhat less effective but its influence was more prolonged, while a concentration of $\mathrm{I} .0$ per cent proved toxic at first, with a later stimulation giving a higher count after the two-hundredth day than was shown by any other sample.

In this particular instance the effect of the lime upon the reaction of the soil no doubt played an important part in stimulating bacterial multiplication. In general,

TABLE V

Bacterial Content of Acid Soll Treated with Varying Amounts of Lime

\begin{tabular}{|c|c|c|c|c|c|c|c|c|}
\hline \multirow{4}{*}{$\begin{array}{l}\text { Days.............. } \\
\text { Control . . . . . . . } \\
\text { to. per cent } \mathrm{CaO} .\end{array}$} & \multicolumn{8}{|c|}{ Millions of Bacteria per Gram of Dry Soil } \\
\hline & IO & 50 & 90 & I 20 & I 50 & 200 & 232 & 310 \\
\hline & 5 & 5 & 4 & 4 & 4 & 4 & 5 & 5 \\
\hline & 6 & I I & II & 9 & IO & 4 & 5 & 3 \\
\hline .2 per cent $\mathrm{CaO}$. & 23 & 78 & 82 & 68 & 73 & 29 & I9 & I3 \\
\hline .3 per cent $\mathrm{CaO} .$. & 97 & 170 & I 70 & $\mathbf{I} 32$ & I33 & 106 & 77 & 63 \\
\hline .4 per cent $\mathrm{CaO} .$. & I 5 I & 387 & 906 & 345 & I 78 & I 54 & 87 & $7 I$ \\
\hline 0.5 per cent $\mathrm{CaO}$ & 270 & $28 I$ & 470 & 260 & 247 & I 80 & 200 & 77 \\
\hline + I.o per cent $\mathrm{CaO}$. & 2 & 0.2 & 6 & 7 & I I & I76 & 329 & 337 \\
\hline
\end{tabular}

however, Russell and Hutchinson attributed the effect of heat and antiseptics upon soils to the destruction of predatory protozoa. It seems quite possible that the influence of the treatment upon available foodstuffs (whether derived from the bodies of protozoa or from other sources) may have been an even more important factor, as in the case of Miquel's experiments cited above.

In many other instances, it is certainly to the availability of the food supply, rather than to the absence of inhibiting substances, that we must attribute the initiation of the logarithmic growth phase. The amount of food required by the bacteria may of course be exceedingly minute, particularly in the case of prototrophic water forms. Kohn (rgo6) determined the minimal nutrient requirements for certain of these types and found that they could develop in the presence of $198 \times 10-{ }^{\text {ro }}$ to $198 \times$ ${ }^{10}{ }^{13}$ per cent of glucose, $66 \times 1{ }^{10}-{ }^{13}$ to $66 \times 10-{ }^{19}$ per cent ammonium phosphate. With more fastidious organisms, however, a much ampler and more diversified diet is necessary for maximal growth. Thus Penfold and Norris (I 9 1 2) found that the maximum generation time of Bact.typhosum in I per cent peptone at $37^{\circ} \mathrm{C}$. was 40 minutes, but that when the peptone in the medium was reduced below 0.4 per cent the generation time increased and rose quite regularly with decrease in peptone content down to 0.2 per cent. In a 0 . I per cent peptone medium the generation time could be cut in 
half by the addition of 0.17 per cent glucose. The literature is full of comparative studies of media for water and milk analysis or for the isolation of specific organisms which bear upon this point. As an example of such studies we need only cite the recent work on media for milk analysis which has indicated the widely different results obtained with various brands of peptone (Shrader, 1926). Davis and Ferry (I9I9) note that both the growth and the toxin production of the diphtheria bacillus in a beefinfusion medium are dependent on the particular types of amino acids present and suggest that other accessory factors, perhaps of the nature of vitamines, are also essential. The presence of growth hormones was held to be necessary for meningococci and gonococci by Lloyd (I9I6) and Cole and Lloyd (I9I7); and Wildiers (I90I) claimed that a hypothetical substance called "bios" was necessary for the fullest growth of yeast in a synthetic medium.

Devereux and Tanner (I924) and Werkman (I927) have recently reviewed various aspects of this subject and conclude that the evidence as to the influence of true vitamins or growth-promoting substances (other than those of a nutrient character) is very doubtful. ${ }^{\mathrm{r}}$

In addition to those nutrient materials which are directly essential for the upbuilding of bacterial protoplasm the rate of multiplication of bacteria is also governed, like many other biological processes, by the regulative action of mineral salts. This subject has been admirably reviewed by Falk (I923), and we need only point out here that Hotchkiss (1923) and others have shown that a wide variety of salts stimulate bacterial growth in low concentration and inhibit it in a higher concentration. Even such toxic salts as $\mathrm{HgCl}_{2}$ may stimulate growth when present in a dilution of onemillionth of a molar concentration (and inhibit it entirely in a concentration of onehundred-thousandth molar) while $\mathrm{NaCl}$ and $\mathrm{KCl}$ stimulate in $.25^{-\mathrm{M}}$ concentration and inhibit in $2-\mathrm{M}$ concentration (under the conditions of the Hotchkiss study). It seems possible that the multiplication of water bacteria in a sample bottle may in part be due to the stimulant action of minute traces of salts dissolved from the glass during sterilization, since Kohn (I906) has shown that the increase is most marked in bottles of the more soluble types of glass.

Finally, the dissolved gases in a medium affect bacterial multiplication in farreaching ways which we are as yet far from comprehending. Wolfthügel and Riedel (I886) found that multiplication of bacteria in a flask stoppered with cotton was greater than in one closed with a rubber stopper, and Whipple (I 90I) observed that it was greater when a bottle was only partly filled than when it was filled more nearly to the top. Curiously, however, agitation and artificial aeration seemed unfavorable to growth in Whipple's experiments. One of the most important contributions to bacteriology in recent years has been the demonstration by Valley and Rettger (1927) that a small percentage of carbon dioxide is essential to bacterial growth and that when this gas is entirely removed growth ceases completely.

\section{THE PIIASE OF CRISIS}

After a lapse of time, varying with the nature of the organism, the medium, and the temperature, the period of logarithmic increase draws to a close, and after an intermediate phase of crisis a phase of decrease supervenes. This is of course a phenom-

${ }^{x}$ Cf. chapter xxxvii in this volume. 
enon familiar to us in our culture media and in such substrata as soil or milk. It was noted in water by Cramer as carly as ${ }_{1} 88_{5}$ in the following very clear example. Lake Zurich water stored for a period of seventy days:

\begin{tabular}{|c|c|}
\hline Storage Period, Days & Numbers per $\mathrm{Cc}$ \\
\hline o....... & . $\quad$ I43 \\
\hline I ...... & I 2,457 \\
\hline $3 \ldots \ldots$ & - 328,543 \\
\hline $8 \ldots \ldots$ & . $233,45^{2}$ \\
\hline I $7 \ldots \ldots$ & . . I 7,436 \\
\hline $70 \ldots \ldots$ & 2,500 \\
\hline
\end{tabular}

A much less marked but fundamentally similar cycle is given by Conn (I9r 8 ) for the change of bacterial numbers in freshly manured soil.

\begin{tabular}{|c|c|}
\hline \multirow{2}{*}{\multicolumn{2}{|c|}{$\begin{array}{r}\text { Storage Period, Days } \\
\text { O....... }\end{array}$}} \\
\hline & \\
\hline I $\ldots \ldots$ & I $20,000,000$ \\
\hline $2 \ldots \ldots$ & I০০, ,০০, ,০০ \\
\hline $3 \ldots \ldots$ & . I $45,000,000$ \\
\hline $4 \ldots \ldots$ & I $50,000, \infty 00$ \\
\hline $6 \ldots \ldots$ & . I $20,000,000$ \\
\hline $9 \ldots \ldots$ & . $70,000,000$ \\
\hline I6. . . . . & $70, \infty 00, \infty 00$ \\
\hline $60 \ldots . .$. & $75,000,000$ \\
\hline $120 \ldots \ldots$ & $23,000,000$ \\
\hline
\end{tabular}

Jordan (1926), in one of his experiments on stored feces, noted that, taking the initial number of bacteria present as 100 , the relative number rose during storage to between 2,000 and 3,000 on the fifth to the ninth day and then fell to about 1,000 on the eleventh day and to less than 400 on the twenty-first day. For colon-group organisms under similar conditions he gave the following results:

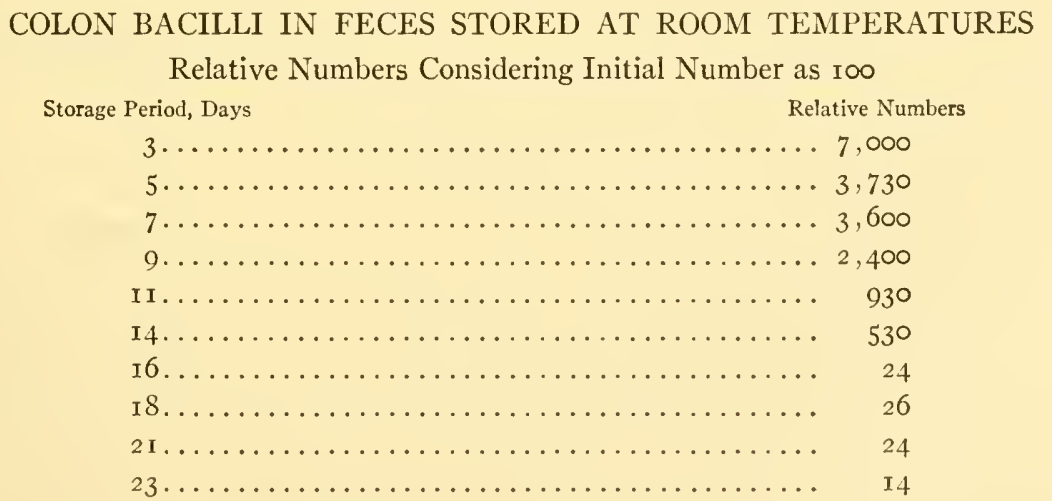

The curves in Figure 3 plotted from averaged results given by Prescott and Baker (I904) for the numbers of colon bacilli and streptococci developing in glucose broth inoculated with polluted water and incubated at $37^{\circ} \mathrm{C}$. illustrate the difference in the 
rate of growth of different species in the same medium, the colon bacilli rising more rapidly at first and the streptococci becoming dominant in the later stages of the cycle. Reed and Reynolds (19r6) give interesting data as to the period of maximum growth for various types of bacteria inoculated into milk in pure culture, the periods at $37^{\circ} \mathrm{C}$ varying from I day for Bact. lactis-acidi and Sarcina lutea to 2 I days for M. citricus and 42 days for Oidium lactis.

In media which are unfavorable the phase of crisis may set in very early. Chick (I9I2) found that the usual relations as to lag period, logarithmic multiplication, and

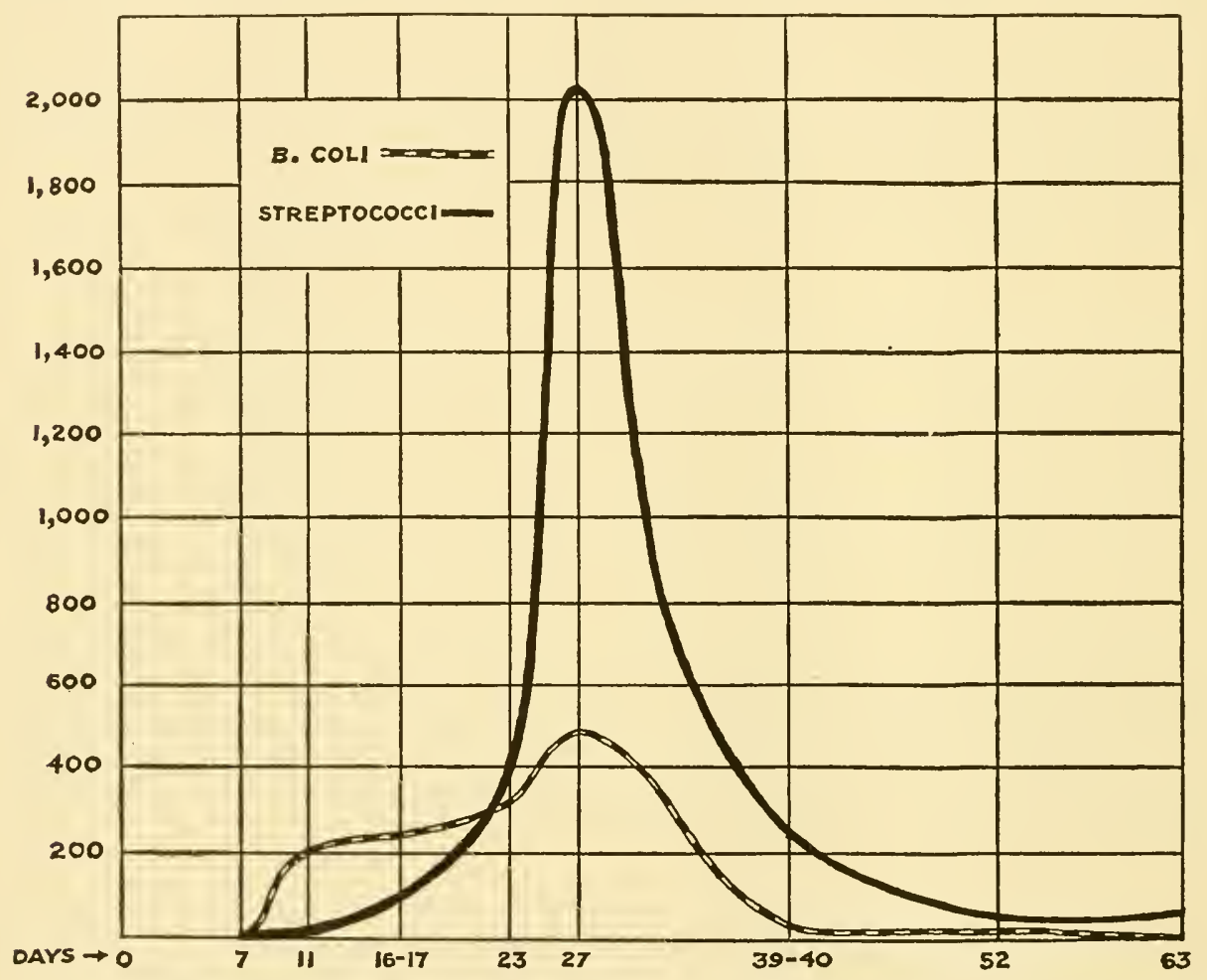

FIG. 3.-Multiplication of Bact. coli and streptococci in glucose broth culture into which they were simultaneously inoculated. (Average of results reported by Prescott and Baker, r904.)

influence of temperature held for the growth of Bact. coli in normal rabbit serum-the only difference being that the period of increase is very brief and leads only to a doubling of bacterial numbers, after which a rapid decline sets in, due to the bactericidal effect of the serum. In rich culture media the initial growth will be rapid, but here too the phase of crisis will usually set in within 24 hours as a result of the formation of inhibitive waste products. In milk containing mixed cultures of many species of bacteria, the bacteria present may continue to increase for clays and may reach enormous figures (several billions per cubic centimeter, for example, in samples held at I $5.5^{\circ} \mathrm{C}$. by Ayers, Cook, and Clemmer, I9I8). In most sturlies on milk bacteriology, indeed, the existence of a period of crisis and subsequent decline has not been apparent since observations have usually not been continued for a long enough period to 
pass the critical point. In the important contribution of Ayers and Johnson (I9ro), however, many individual samples show the beginning of a decline after 4 or 5 days even when held at $10^{\circ} \mathrm{C}$.

So far as the temperature effect is concerned we may note that Lane-Claypon (I909) found the end of the period of logarithmic incres se for Bact. coli in broth to occur after $7 \frac{1}{2}$ hours at $37^{\circ} \mathrm{C}$., after $8-8 \frac{1}{2}$ hours at $30^{\circ} \mathrm{C}$., I $2-I 5$ hours at $25^{\circ} \mathrm{C}$., and $20-24$ hours at $20^{\circ} \mathrm{C}$. In the work of Reed and Reynolds (I9I6) on the growth of pure cultures of thirteen different species of micro-organisms in milk the average period of maximum growth was 7 days at $35^{\circ} \mathrm{C}$. and 20 days at $13^{\circ} \mathrm{C}$.

The explanation of the onset of the phase of crisis must obviously be sought in the changed composition of the medium due to the growth of the bacterial population during the phase of increase. It is clear that there is no essential and invariable lifecycle involved since the results of Penfold and of Barber indicate that transfer of cells from a culture in the early phase of increase to a new tube of the same medium yields a constant and continuous development at a logarithmic rate. The change in the medium which makes it unsuitable for further growth might theoretically be either an exhaustion of essential foodstuffs or the formation of toxic waste products, and both processes may no doubt play a part under certain conditions. In spring-water samples containing but little organic matter it seems very probable that the exhaustion of food may play a predominant rôle (though even Miquel stressed the conception of toxic products). In rich culture media the formation of waste products is probably the factor of major importance. In milk and other sugar media the production of acid is often by itself entirely adequate to explain cessation of growth. Thus Heinemann (I9I5) found that a series of pathogenic bacteria were all destroyed when the acidity of milk reached 0.45 per cent and that even Bact. coli died out when the milk reached an acidity of 0.6 per cent, while Bact. coli itself increased the acidity of milk to 0.5 per cent. Cohen and Clark (I9I9) found the limiting $\mathrm{pH}$ values for growth in peptone broth to vary from 4.4 for Bact. aerogenes to 5.5 for Bact. alcaligenes; but they pointed out the extreme complexity of the phenomena involved, as shown by the fact that while fermentative activity seems to cease in a sugar medium at about the $\mathrm{pH}$ concentration known to limit growth, in previously adjusted media growth in the sugar medium seems to stop at a point corresponding to concentration of the acetic acid formed rather than the $\mathrm{pH}$. Furthermore, in a broth medium free from sugar growth ceases much sooner than in the sugar medium and with a total concentration of cells only one-fifth as great as that which is found in the presence of sugar.

In dealing with the phase of crisis, as in the case of the phase of adjustment, it does not seem particularly profitable to refine our methods of analysis too far until more detailed experimental data are available. If we assume that the cycle of a bacterial population starts from and returns to a stable state there are really but two fundamental processes involved, an increase from the original stable condition and a subsequent decrease to a second level, with phases of transition before the increase, between the increase and the decrease, and before the period of ultimate stability. Clearly, as a result of various factors, such as food supply, toxic products, and temperature, the slope of the curve during the phase of crisis may take any form from a sharp tooth to a flat plateau. 


\section{THE PHASE OF DECREASE}

From the earliest days of bacteriology it has been noted that the decrease in bacterial numbers under the influence of an unfavorable environment (such as is present for one reason or another on the descending side of the curve of the population cycle) followed a gradual and more or less orderly course. This was at first attributed to a process of natural selection, the surviving organisms being assumed to be of a specifically more resistant character. With the work of Koch ( $188 \mathrm{I}$ ), Paul and Krönig (I896), Krönig and Paul (IS97), Ikeda (I897), Madsen and Nyman (1907), and Chick (r908, I9ro) on the action of chemical disinfectants the mortality curve was given a new interpretation as an expression of a more fundamental chemical phenomenon. As summarized by Phelps (I9II), these researches have shown that "the rate of dying, whether under the influence of heat, cold or chemical poison, is unfailingly found to follow the logarithmic curve of the velocity law, if the temperature be constant."

The general slope of the mortality curve during the period of rapid decline is therefore the same as that of the curve for the increase of a bacterial population during its period of rapid multiplication. In the phase of increase the logarithm of the number of new cells formed from a single initial cell in a given time is proportional to the lapse of time; in the phase of decrease the logarithm of the proportion of the cells present which perish in a given interval is proportional to the length of that interval. In other words, increase and decrease alike bear a direct relation to the number of cells present at the beginning of a unit period and a logarithmic relation to any time period of greater duration.

The formula for the rate of decrease is, therefore, in the form used by Chick (1908), as follows:

$$
\frac{\mathbf{I}}{t_{2}-t_{1}} \log \frac{n_{\mathrm{t}}}{n_{2}}=K
$$

where $t_{\mathrm{I}}$ is the initial and $t_{2}$ the final time and $n_{\mathrm{I}}$ and $n_{2}$ the corresponding numbers of bacteria present. Phelps, taking the elapsed time (as $t$ ) instead of the initial and final time readings $\left(t_{2}-t_{1}\right)$ and $B$ as the initial and $b$ the final number of bacteria, expresses the formula as:

$$
\log \frac{B}{b}=K t
$$

which is of course the formula for a monomolecular reaction. ${ }^{\mathrm{I}}$

The results presented by Chick (1908) in regard to the regularity of the process of disinfection were very striking and seemed to justify her conclusion that "a very complete analogy exists between a chemical reaction and the process of disinfection, one reagent being represented by the disinfectant, and the second by the protoplasm of

I This formula is written by Falk and Winslow,

$$
0.434 K_{\mathrm{I}}=\mathbf{1} / t \log \frac{a}{a-x}
$$

where $a=n_{\mathrm{1}}$ of Chick and $a-x=n_{2}$ of Chick because $K_{\mathrm{1}}=\mathrm{I} / l \log _{c} \frac{a}{a-x}$ and $K=\mathrm{r} / / \log _{\mathrm{t} 0} \frac{a}{a-x}$ when $K_{\mathrm{I}}=2.3 K$. 
the bacterium." Later Miss Chick (I9I0) showed that the death of bacteria when dried or exposed to sunlight, or even when killed by moderate heat in water, proceeded in general in accord with the logarithmic law. Cohen (r922) confirmed these general conclusions for the much more gradual death-curve of colon bacilli in water. In all of these latter cases, of course, the reacting agents which cause death must be within the bacterial cells themselves.

This simple concept of the disinfection process has been vigorously challenged by a considerable group of workers such as Loeb and Northrop (I9I7), Brooks (1918), Peters (I920), and Smith (I92I) who attribute the form of the mortality curve to biological differences in the resistance of the individual bacterial cells.

Miss Chick herself invoked this conception of individual-cell variation to explain irregularities in her mortality curves for non-spore-forming organisms such as paratyphoid bacilli and staphylococci. When very young cultures of these organisms were used she obtained constant values of $K$, but with older cultures the rate of disinfection fell off in the later stages of the process. Even Cohen's curves in many instances exhibit a tendency to flatten out toward the end of the periods studied, and Falk and Winslow (1926) present results on the death of Bact. coli in dilute salt solutions which suggest an initial rise in the value of $K$ with the passage of time, followed by a subsequent gradual fall.

It appears certain, however, from a review of all the available literature that when a bacterial population of a reasonably homogeneous character (spores or young vegetative cells) is subjected to an unfavorable environment-whether the unfavorable condition be a chemical disinfectant, heat, sunlight, or merely storage in a dried condition or in an aqueous medium where growth cannot occur-there is a period during which the death of the bacterial cells follows a fairly regular logarithmic rate. This period of regular decrease may be preceded by a brief period of slower decline, representing a sort of lag or adjustment to the unfavorable environment, and seems generally to be followed by a final period of still slower decline. The latter may not occur when death is due to strong disinfectants such as were used by Miss Chick and did not appear in Cohen's studies because they were not prolonged for a sufficient period.

It seems, however, entirely unnecessary to postulate biological variations in the cells of the bacteria to account for these deviations from the logarithmic curve. As Falk and Winslow (I926) have pointed out, it is much simpler to assume that the lethal reactions which go on in bacterial cells dying in an unfavorable environment proceed in accord with a bimolecular reaction or reactions of a still higher order rather than in accord with the monomolecular formula. There is no reason to assume that the decomposition of a single chemical compound is always the determining cause of death. It is shown in the paper cited that the values of $K$ may often be best explained on such an assumption. Just as the lag period in bacterial growth involves the assumption of catenary reactions, so do the variations at the beginning and end of the curve of bacterial mortality. To obtain the values which most nearly approximate a logarithmic curve and represent the period when the lethal process most nearly simulates a monomolecular reaction, Phelps has suggested that $K$ should be determined for the middle portion of the curve- say from a reduction of 75 to one of 25 per cent. 
It is a curious and interesting fact that disinfection by sodium hydroxide follows an entirely different law from that observed in other cases, $K$ increasing progressively with the time of exposure (Levine, Buchanan and Lease, I927).

It may be of interest, in spite of the various factors involved in the problem, to consider some of the absolute values of $K$ which have been observed in certain specific instances and to note their significance in terms of percentage reduction.

At one extreme stand such results as those obtained by Jordan (I926) for the secondary reduction of Bact. coli in stored feces. These give (from the third to the twenty-third day) a $K$ per hour of .oo6. The values which I have cited for the reduction of the numbers of a certain strain of colon bacilli in water (Winslow and Cohen) give a $K$ per hour of .or for the third to the tenth day, corresponding to a reduction in numbers of 2.5 per cent per hour. The reduction of Bact. typhosum in ice for the first three days (Sedgwick and Winslow) is of the same order $(K=.02)$ and involves a reduction of 5 per cent per hour. For Bact.coli dried in sand (Winslow and Abramson) $K=.06$ for the ninth to the forty-eighth hour (a reduction of I3 per cent per hour). The mortality of anthrax spores exposed to 5 per cent phenol at $20^{\circ} \mathrm{C}$. is about thesame (Chick, I908). Falk and Winslow's studies give a $K$-value of about .I per hour for dilute salt solutions ( 20 per cent per hour), and Cohen's data for acid ( $\mathrm{pH} 8$ ) give a $K$ value of 1.0 ( $9 \circ$ per cent reduction per hour). Chick's analysis of Clark and Gage's results on the disinfectant action of sunlight give a $K$ of 2.6, which would involve a reduction of 95 per cent per hour. The death of paratyphoid bacilli exposed to 6 per cent phenol is more rapid still, with a $K$ of about 20 , according to Chick's data (99.4 per cent reduction per hour); while hot water $\left(54^{\circ} \mathrm{C}\right.$.) gives a $K$ of over 60.o. This involves a reduction of 99.75 per cent per hour.

The factors which govern the rate of the mortality of bacteria are essentially the same as those which govern the rate of their multiplication, though of course most of them operate in an inverse sense. When bacteria die out in water or when stored in a dried condition it is the normal katabolic processes of the cell (in the absence of compensating anabolism) which must control the process. It seems doubtful whether drying in itself exerts any specific harmful effect, and (if rapid and complete) it may even slow down vital processes and thus prolong life. Ficker (I898), however, found that alternate drying and moistening accelerated the lethal process. It is of interest to note that Paul, Birstein, and Reuss (I9IO) found the disinfection constant in drying proportional to the square root of the oxygen concentration of the atmosphere, following the law which obtains in the slow oxidation of phosphorus. The whole subject of the longevity of micro-organisms under the influence of desiccation has been well reviewed by Giltner and Langworthy (I916).

The presence of external toxic substances may of course be one important factor in the death of bacteria in water or in soil. Thus Jordan, Russell, and Zeit (1904) found that typhoid bacilli inclosed in collodion sacs survived much longer when the sacs were suspended in pure water than when they were suspended in polluted water. In the presence of known chemical disinfectants this factor of direct toxicity is of course the determining feature. The value found for $K$ may therefore vary within the widest possible limits, depending on the toxicity of the particular disinfectant studied; and the study of the relation between toxicity and chemical composition (as worked out, 
for example, by Schaffer and Tilley [1927] for the various members of the alcohol and phenol group) is a fascinating one.

Chick (rgo8) showed that within certain limits the efficiency of a chemical disinfectant bears a logarithmic relation to its concentration, the expression

$$
\frac{\mathrm{I}}{t_{n}-t_{o}} \log \frac{C_{n} t_{n}}{C_{o} t_{o}}
$$

remaining constant in value when $t_{o}$ and $t_{n}$ are the time periods necessary for disinfection corresponding to concentrations $C_{o}$ and $C_{n}$. Furthermore, it is important to note that disinfectants not only vary in their efficiency at a given concentration but also vary in the degree to which their toxicity increases with increasing concentration. The ordinary carbolic acid coefficient (representing the ratio of the concentration of a given disinfectant to the concentration of carbolic acid which will sterilize in a given time) therefore gives a very incomplete idea of the true relationships. Thus Chick (Igo8) found that mercuric chloride may have a carbolic acid coefficient of I 3 at one concentration and of $55^{\circ}$ at another.

Phelps (I9I I) therefore suggests that values of $K$ ( $K$ and $\left.K^{\prime}\right)$ should be determined at two different concentrations $\left(C\right.$ and $\left.C^{\prime}\right)$. If we express the $K$ of our previous formula (p. 72) by $K C^{n}$ (to allow for this concentration factor) we get

$$
\log \frac{B}{b}=K C^{n t}
$$

and

$$
\log \frac{B^{\prime}}{b^{\prime}}=K^{\prime} C^{\prime n t}
$$

Therefore

$$
n=\log \frac{K^{\prime}}{K} \div \log \frac{C^{\prime}}{C}
$$

From the two determinations of $K$ we can then compute $n$ or the concentration coefficient of the particular disinfectant studied. This figure $n$ shows by what power of 2 the efficiency is increased if the concentration be doubled. For anthrax spores in mercuric chloride at $20^{\circ} \mathrm{C}$. it is 1.08 .

The reaction of the medium is a special case of chemical toxicity which has been studied with particular care. Such a phenomenon occurs in nature in streams receiving acid wastes, and the disinfectant action of carbon dioxide is due to the same cause (Koser and Skinner, I922). As pointed out above, carbon dioxide, aside from its effect upon reaction, is beneficial and indeed essential to bacterial life.

Winslow and Lochridge (IgO6) reported twenty years ago that the toxic effect of mineral acids was in large measure due to dissociated hydrogen. Cohen (I922) has given us one of the most careful recent studies of this problem, and we may cite one of his summary tables (Table VI) indicating the effect of hydrogen-ion concentration upon the death-rate of Bact. typhosum.

The effect of cations other than hydrogen upon bacterial growth and death has been studied by numerous observers (see review by Falk, I923). Those who have worked on the increase of bacterial populations in favorable media, from Richet 
(I892) to Hotchkiss (I923), have found that practically all cations may show either a stimulating or an inhibiting influence, depending on their concentration, and observers

TABLE VI

Average Velocity Constants for the Death of Bacl. typhosum at Different pH Values, $20^{\circ} \mathrm{C}$.

\begin{tabular}{|c|c|c|c|c|c|c|c|}
\hline $\mathrm{pH} \ldots \ldots 3.8$ & 5.0 & 5.4 & 6.4 & 7.1 & 7.6 & 8.7 & 9.5 \\
\hline$K \ldots \ldots \ldots$ I.055 & 0.0134 & 0.0110 & 0.0138 & 0.0437 & 0.1100 & 0.2134 & 0.2855 \\
\hline Relative $K^{*} 95 \cdot 5$ & I. 2 & I.O & I. 5 & 4.8 & I0.0 & 22.4 & $3 \mathrm{I} \cdot 4$ \\
\hline
\end{tabular}

like Winslow and Falk (1923) who have studied the death-rate of bacteria in unfavorable media find that in the same way small amounts of cations favor survival while

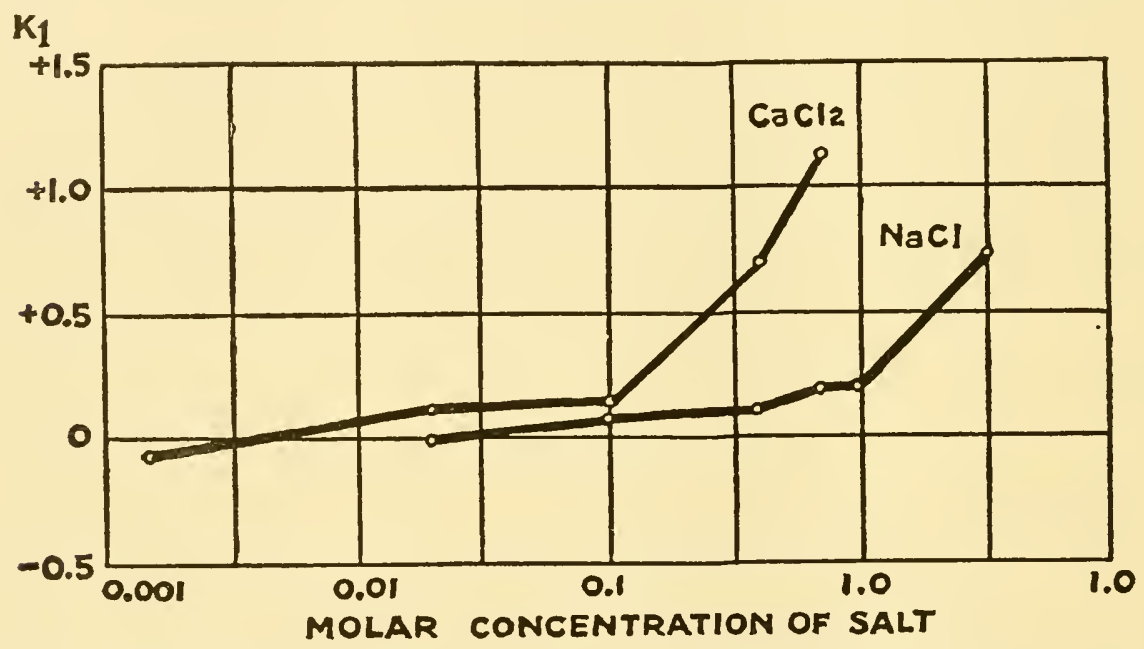
I926).

FIG. 4.- Relation between viability of Bact. coli and salt concentration (Falk and Winslow,

large amounts increase the mortality. Falk and Winslow (1926) pointed out the significant fact that if values of $K$ for the effect of $\mathrm{NaCl}$ and $\mathrm{CaCl}_{2}$ upon Bact. coli in water be plotted against concentration one obtains a reasonably smooth curve for both salts, passing from positive values (indicating toxicity) to negative values (indicating a preservative effect) (see Fig. 4). It will obviously be difficult to harmonize such a phenomenon with any simple chemical assumptions.

It is also important to remember that the net effect of various chemical and physical factors, acting simultaneously, may be an exceedingly complex one. Thus Chick (I9ro) showed that very minute excesses of acid or alkali might enormously accelerate the rate of disinfection by hot water; and the same phenomenon is of great practical importance in connection with heat sterilization. On the other hand, a lethal factor may equally well be neutralized by a favorable one. Sometimes the effect is relatively simple, as in the reduction of the disinfectant power of mercuric chloride due to the presence of organic matter (Chick and Martin, 1908), a fact which makes 
this substance wholly unsuitable for the disinfection of feces, or in the interference of organic matter in water with the action of chlorin. Under other conditions more subthe reactions are involved, as in the results recently reported by Winslow and Brooke (1927). These observers found that several different types of bacteria die out almost immediately when washed free from culture medium and resuspended in distilled water. Salt and sugar solutions will not check the mortality, so we are not dealing merely with a question of osmosis; but the cells can be protected by the presence of peptone or meat extract, serving as "protective colloids" as indicated in Table VII.

TABLE VII

ViabiLIty of $B$. cereus

\begin{tabular}{|c|c|c|c|c|}
\hline & \multicolumn{4}{|c|}{ Percentage Surviving } \\
\hline & Broth & Peptone & Neat Extract & $\begin{array}{l}\text { Ringer-Locke } \\
\text { Solution }\end{array}$ \\
\hline 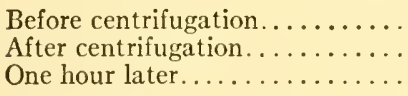 & $\begin{array}{r}100 \\
56 \\
71\end{array}$ & $\begin{array}{r}100 \\
43 \\
53\end{array}$ & $\begin{array}{r}100 \\
51 \\
57\end{array}$ & $\begin{array}{r}100 \\
3 \\
1\end{array}$ \\
\hline
\end{tabular}

A concentration of .005 per cent peptone or of .003 per cent meat extract will protect the cells; but a concentration of .0005 per cent peptone or of .0003 per cent meat extract fails to do so.

Finally, we must consider the influence of temperature upon the course of the curve of decreasing numbers of bacteria. Since the processes of death, like those of life, are essentially chemical in nature, it is of course obvious that an increase of temperature will favor the lethal process when lethal factors are dominant just as it favors the growth process when growth factors are dominant. The classic work of Houston

TABLE VIII

Effect of Temperature on Survival of Typhom Bacilli in Water (Houston)

\begin{tabular}{|c|c|c|}
\hline Temperature ${ }^{\circ} \mathrm{C}$ & $\begin{array}{c}\text { Percentage of Typhoid } \\
\text { Bacilli Surviving } \\
\text { after One Week }\end{array}$ & $\begin{array}{l}\text { Period of Final } \\
\text { Disappearance of } \\
\text { Bacilli (in Weeks) }\end{array}$ \\
\hline$\circ \ldots \ldots \ldots \ldots \ldots$ & 46.00 & 9 \\
\hline $5 \ldots \ldots \ldots \ldots \ldots$ & 14.00 & 7 \\
\hline Iо............... & 0.07 & 5 \\
\hline 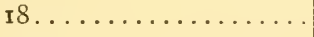 & 0.04 & 4 \\
\hline
\end{tabular}

(IgrI) on the survival of typhoid bacilli in water (illustrated in Table VIII), for example, seems at first sight puzzling, since we find an organism whose optimum for growth lies at $37^{\circ} \mathrm{C}$. dying more rapidly at that temperature than in cooler waters. The explanation lies in the fact that typhoid bacilli in water lack the conditions essential for anabolism; only katabolism can go on, and katabolism is increased by a rise in temperature.

The effect of high temperature in increasing the efficiency of chemical disinfectants was noted by Koch (I88I) in the earliest studies of disinfection, and was first 
carefully studied by Madsen and Nyman (1907), Chick (1908), and Paul (I909). These investigators found that the reaction velocity of disinfection increased with a rise in temperature, according to the formula of Arrhenius, the function

$$
\frac{T_{1} T_{2}}{T_{1}-T_{2}} \log \frac{t_{2}}{t_{\mathrm{I}}}
$$

remaining constant where $t_{1}$ and $t_{2}$ represent times taken for disinfection and $T_{\mathrm{I}}$ and $T_{2}$ represent absolute temperatures.

As the temperature at which such a reaction proceeds increases, the velocity of the reaction increases in geometrical progression. If $K^{\prime}$ and $K$ are the constants of the reaction at the temperatures $T^{\prime}$ and $T$, respectively, and $\theta$ is the temperature coefficient (Phelps),

$$
\frac{K^{\prime}}{K}=\theta^{(T-20)}
$$

By determining the reaction velocity of the lethal process for bacteria at two temperatures $10^{\circ} \mathrm{C}$. apart, we may obtain this temperature coefficient from the formula

$$
K_{T^{\circ}}=K_{20^{\circ}} \cdot \theta^{(T-20)} .
$$

For anthrax spores exposed to 0.5 per cent mercuric chloride $\theta=$ I.I 7 . In general, the mean velocity of disinfection with metallic salts increases two- to four-fold for a $10^{\circ}$ rise in temperature (centigrade), while with other disinfectants the increase may be considerably greater.

The effect of temperature upon the natural death of bacteria in water is essentially the same, although the absolute value of the coefficient seems to be somewhat lower. Table IX, from Cohen (I922), illustrates this phenomenon.

TABLE IX

\begin{tabular}{|c|c|c|c|c|c|}
\hline \multirow{2}{*}{ Temperature, ${ }^{\circ} \mathrm{C}$} & \multicolumn{2}{|c|}{ Bact. typhosum } & \multicolumn{2}{|c|}{ Bact. coli } & \multirow{2}{*}{$\begin{array}{c}\text { RATIO of } K \\
\text { FOR Bact. } \\
\text { typhosum To } \\
K \text { FOR Bact } \\
\text { coli }\end{array}$} \\
\hline & $K$ & $\begin{array}{l}\text { Increase } \\
\text { for } 10^{\circ}\end{array}$ & $K$ & $\begin{array}{l}\text { Increase } \\
\text { for } 10^{\circ}\end{array}$ & \\
\hline $0 \ldots \ldots \ldots \ldots$ & I . I 86 & $\ldots \ldots \ldots$ & 0.0176 & $\ldots \ldots \ldots$ & 67 \\
\hline 10............ & I. 919 & I. 62 & .0373 & 2.12 & $5 \mathrm{I}$ \\
\hline $20 \ldots \ldots \ldots \ldots$ & 2.928 & I. 53 & .1654 & $4 \cdot 36$ & 18 \\
\hline 30............ & 5.176 & I. 77 & 0.6214 & $3 \cdot 76$ & 8 \\
\hline
\end{tabular}

Velocity COefficients for Death of Bact. typhosum and Bact. coli at pH 3.5 at Different Temperatures

It will be noted that the temperature coefficient for Bact. coli is much lower than for Bact. typhosum but rises much more rapidly for a $10^{\circ} \mathrm{C}$. increase.

A peculiarly interesting contribution was made by Chick (I9IO) in the demonstration that the death of bacteria in hot water follows the same general time relation, although of course with an enormously high time factor. In the case of Bact. typhosum the coefficient was $\mathrm{r} .635 \mathrm{per}^{\circ} \mathrm{C}$. The whole phenomenon of cell death 
under the influence of heat is explained by Chick and Martin (I9I0) as a heat coagulation consisting in a reaction between protein and water which is highly accelerated by heat. From the practical standpoint of food preservation, Bigelow (I92I) has shown that the logarithmic relationship between sterilizing time and temperature holds for the destruction of both spores and vegetative cells by high heat.

Phelps (IgII) has given a very valuable analysis of the variations in the effectiveness of a given disinfectant with respect to concentration, time, and temperature, and has shown how its constants can all be fixed by determining its efficiency' in two different dilutions at the same temperature and at two different temperatures in the same dilution (three determinations in all); the values for any other set of conditions can then be obtained from the formulas:

$$
\log \frac{B}{b}=K C^{n} t
$$

and

$$
K_{T^{\circ}}=K_{20^{\circ}} \cdot \theta(T-2 \circ)
$$

when (as before) $B=$ initial number of bacteria present, $b=$ final number of bacteria present, $t=$ elapsed time, $C=$ concentration of disinfectant, $K=$ velocity constant calculated at temperature of experiment, $K_{T^{\circ}}=$ same at any temperature $T^{\circ}, K_{20^{\circ}}=$ same at $20^{\circ}, n=$ concentration exponent, and $\theta=$ temperature coefficient.

\section{THE PHASE OF READJUSTMENT}

Toward the close of the phase of decrease the rate of mortality slackens and the curve passes imperceptibly into the final phase of readjustment. In the work of Chick (I908) on the effect of chemical disinfectants upon vegetative cells, and in the studies by Falk and Winslow (1926) on the death-rate of bacteria in dilute salt solutions, it even appears, as we have seen, that the value of $K$ falls progressively throughout the phase of decrease (see Fig. 4) so that there is no clear distinction between the two processes. In other instances, however, it is often possible to observe two fairly distinct periods, one of rapid and one of slow decline. Thus, Winslow and Cohen (r9r8) in their studies of the life of Bact. coli in water observed values of $K$ ranging from .004 to .020 for the first ten days while from the tenth to the sixtieth day the values varied only between .001 and .002.

The mortality curves given by Sedgwick and Winslow (I902) for the life of typhoid bacilli in ice and in dry earth, by Winslow and Abramson (г91 2) for colon bacilli in water, all show a falling rate of mortality toward the close of the cycle of decrease. Values for $K$ calculated from these data are cited below, obtained by merely subtracting the log of the number present after a given time interval from the log of the number present at the beginning of that interval and dividing by the elapsed time. (It should be noted that in computing these values the reduction in numbers for each period are computed on the basis of the number present at the beginning of that period instead of following the usual procedure of computing $K$ on the basis of reduction from the beginning of the whole experiment. The latter method naturally tends to obscure any differences which may occur.)

The very gradual rate of decrease during the phase of readjustment may end in 
either of two different ways. In a moderately favorable medium, such as water, the cycle usually ends with a new level of stability ( $E F$ in Fig. I) on which the number of bacteria may remain reasonably constant for an indefinite period. Miquel describes an experiment in which a bottle of Seine River water containing originally 4,800 bacteria per cubic centimeter was stored for nine years and showed 220 bacteria per cubic centimeter at the end of that time.

Sometimes, as is shown in Figure 2, there may be one or more secondary waves of increase before the final level of stability is reached.

In his study of stored feces Jordan (I926) found that after the initial multiplication and subsequent decrease a level was reached which remained more or less constant for long periods. The total number of bacteria present after many weeks may be as high as, or higher than, the number initially present. Bact. coli, however, ultimately disappears from the fecal flora under such conditions. In a less favorable medium,

TABLE X

Values of $K$ for Various Periods of Certain Mortality Curves

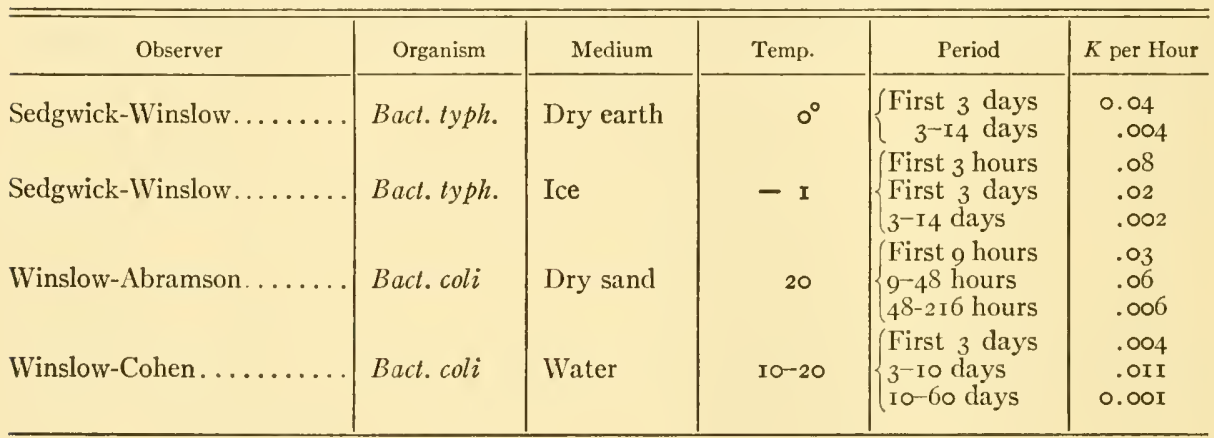

on the other hand, the phase of readjustment ultimately ends in complete sterility, as in disinfection with high heat or strong chemicals ( $E F^{\prime}$ in Fig. I).

In an intermediate case-where conditions are neither sufficiently favorable to permit of the balanced growth and death which maintains a constant stable level nor sufficiently unfavorable to lead to rapid and complete extinction-a small proportion of the original bacterial population may persist for a very long period. Thus Parkes (1903) was able to isolate typhoid bacilli from blankets soiled with feces after more than six months. Konradi (I904) reports Bact.typhosum as surviving in water after seventeen months. Robertson ( 1898 ) isolated the same organism from moistened soil after eleven months. Studies by numerous observers on the drying of typhoid, diphtheria, and tubercle bacilli (summarized by Chapin, I9I2) have shown that all these organisms may survive drying for several months. (Table $\mathrm{X}$.)

Winslow and Kligler (I912) found over 51,000 colon bacilli and 42,500 acidforming streptococci per gram of dust from city streets and 940 colon bacilli and 22,040 acid-forming streptococci per gram of house dust. Winslow and Sanjiyan (I924) conducted an extensive study of the distribution of the acid-forming streptococci, presumably indices of pollution from mouth spray, on objects and surfaces of various kinds. Objects, such as eating utensils, directly exposed to mouth contam 
ination showed these organisms in 62 per cent of the cases studied while objects recently handled (door handles, push buttons, etc.) showed them in 42 per cent of the cases. On locations such as walls six feet above the ground, the undersides of chairs and tables and the like, these organisms were isolated in ro per cent of the cases.

From the standpoint of epidemiology, it is essential to note that the pathogenic bacteria which survive for such long periods as those noted above are so few in number that their presence is of little or no practical significance for disease transmission. Houston (I908), for example, was able to isolate typhoid bacilli from water after nine weeks; but 99.9 per cent of the bacteria originally present had perished after one week. Nothing is of course more certain than the fact that transmission of disease germs occurs in the vast majority of instances only through the rather direct and immediate transfer of fresh body discharges.

In the earlier days of bacteriology it was customary to refer to the few pathogenic bacteria which survive after long periods of time in water or earth as representing a "resistant minority." It is quite possible that in certain instances a selection of more resistant variants may in fact take place. Sedgwick and Winslow (I902) showed that individual strains of typhoid bacteria differ markedly in their ability to survive in ice; and Ayers and Johnson ( $\mathrm{r}^{\mathrm{r}} \mathrm{5}$ ) found that various strains of colon bacilli show great differences in their ability to resist various pasteurization temperatures. There is, however, no direct evidence that the cells which die out toward the end of the cycle of bacterial population derived from a single strain are intrinsically more resistant than those which perish earlier; and it seems probable that the curve for such a cycle is mainly determined by a series of catenary reactions following the ordinary laws of more simple chemical processes.

\section{BIBLIOGRAPHY}

Allen, P. W.: J. Bact., 8, 555. 1923.

Ayers, S. H., Cook, L. B., and Clemmer, P. W.: U.S. Bureau of Animal Industry, U.S. Depariment of Agriculture, Bull. 642 .

Ayers, S. H., and Johnson, W. T.: ibid., Bull. 126.

Ayers, S. H., and Johnson, W. T.: J. Agric. Research, 3, 401. I9I5.

Barber, M. A.: J. Infect. Dis., 5, 379. I908.

Bigelow, W. D.: ibid., 29, 528. I92 r.

Brooks, S. C.: J. General Physiol., r, 6r. 1918.

Buchanan, R. E.: J. Infect. Dis., 23, 109. 1918.

Buchner, H., Langard, K., and Riedlin, G.: Centralbl.f. Bakteriol., 2, I. 1887.

Buddin, WV.: J. Agric. Sc., 6, 4I 7. I9I4.

Burke, V., Sprague, A., and Barnes, L.: J. Infect. Dis., 36, 555. 1925.

Chapin, C. V.: Sources and Modes of Infection. New York, I9I2.

Chesney, A. M.: J. Expcr. Med., 24, 387. I9r6.

Chick, H.: J. IIyg., 8, 92. 1908.

Chick, H.: ibid., ro, 237. I910.

Chick, H.: ibid., 12, 414. ror 2.

Chick, H., and Martin, C. J.: ibid., 8, 654. 1908.

Chick, H., and Martin, C. J.: J. Physiol., 40, 404. I9ro.

Clark, P. F., and Ruehl, IV. II.: J. Bact., 4, 6I5. I9Ig.

Cohen, B.: ibid., 7, 183. 1922. 
Cohen, B., and Clark, W. M.: ibid., 4, 409. I9I9.

Cole, S. W., and Lloyd, D.: J. Path. Bact., 21, 267. I9r7.

Conn, H. J.: Tech. Bull. 64, N.Y. Agric. Exper. Sta. Geneva, N.Y., I9r8.

Coplans, M.: J. Path. Bact., I4, г. I909.

Cramer, W.: Die Wasserversorgung von Zïrich und ihr Zusammenhang mit der Typhusepidcmic des Jahres i 884 . Zurich, I885.

Davis, L., and Ferry, N. S.: J. Bact., 4, 2 I7. г919.

Devereux, E. D., and Tanner, F. W.: ibid., I4, 317. 1927.

Falk, I. S.: Abstr. Bact., 7, 33, 87, I33. I923.

Falk, I. S., and Winslow, C.-E. A.: J. Bact., II, I. 1926.

Ficker, M.: Ztschr. f. Hyg. u. Infcktionskrankh., 29, I. I898.

Fokker, A. P.: ibid., 9, 4r. I890.

Frankland, P.: Proc. Roy. Soc., London (1884-85, 38, 379-93. I 885.

Frankland, Mr. and Mrs. Percy: Micro-Organisms in Water. London, I894.

Fuller, G. W.: Ann. Rep., State Board of Health of Massachusetts for 189.4, 26, 461. 1895.

Giltner, W., and Langworthy, H. V.: J. Agric. Research, 5, 927 . I9 6.

Hadley, P. B.: J. Infect. Dis., 40, I. I927.

Harrison, F. C., and Vanderleck, J.: Rev. gén. du lait, 7, No. I5. 1909

Hehewerth, F. H.: Arch.f. Hyg., 39, 32 I. I90I.

Heinemann, P. G.: J. Infect. Dis., I6, 479. I915.

Heinemann, P. G.: Milk. Philadelphia and London, I9r9.

Henrici, A. T.: Proc. Soc. Expcr. Biol. \& Med., I9, I32, I92 r.

Henrici, A. T.: ibid., 20, I79. 1922.

Henrici, A. T.: ibid., 2I, 215. I923.

Henrici, A. T.: ibid., p. 343. I924.

Hotchkiss, M.: J. Bact., 8, 141. 1923.

Houston, A. C.: First Rept. on Research Work, Mctropolitan Watcr Board. London, rgo8.

Houston, A. C.: Seventh Rept. on Research Work, Metropolitan Water Board. London, r9I I.

Hutchinson, H. B., and MacLennan, K.: J. Agric. Sc., 6, 302. I9I4.

Ikéda, K.: Ztschr.f. Hyg. u. Infcktionskrankh., 25, 95. I897.

Jordan, E. O.: J. Infect. Dis., 38, 306. I926.

Jordan, E. O., Russell, H. L., and Zeit, F. R.: ibid., I, 641. I904.

Koch, R.: Mitt. a. d. Kaiserlich. Gesundheitsamte, I, r. I881.

Kohn, E.: Centralbl.f. Bakteriol., Abt. II, I5, 690. 1906.

Konradi, D.: ibid., Abt. I, Orig., 36, 203. I904.

Koser, S. A., and Skinner, W. W.: J. Bact., 7, Ir I. 1922.

Krönig, B., and Paul, T.: Ztschr. f. IIyg. u. Infektionskrankh., 25, I. I897.

Lane-Claypon, J. E.: J. Hyg., 9, 239. r9o9.

Ledingham, J. C. G., and Penfold, W. J.: ibid., 14, 242. 19r4.

Leone, C.: Arch.f. Hyg., 4, I68. I886.

Levine, M., Buchanan, J. H., and Lease, G.: Iowa State Coll. J. of Sci., I, 379. 1927.

Lloyd, D.: J. Path. Bact., 21, Iг3. г9г6.

Loeb, J., and Northrop, J. H.: J. Biol. Chem., 32, 103. I917.

Madsen, T., and Nyman, M.: Ztschr. f. Hyg. u. Infektionskrankh., 57, 388. r907.

Massachusetts: Twonty-sixth Ann. Rept., State Board of IIealth of Massachusctts for I 89.4 , 1895 .

Miquel, P.: Rev. d'hyg., 9, 737. I887.

Miquel, I'.: Manucl pratique d'analyse bacteriologique des caux. Paris, r $89 \mathbf{r}$.

Müller, M.: Ztschr.f. IIyg. u. Infcktionskrankh., 20, 245. I895. 
Parkes, L. C.: Practitioner, 71, 297. I903.

Parsons, L. B., and Sturges, W. S.: J. Bact., I4, ISI, I93, 20 I. 1927.

Paul, T.: Biochem. Ztschr., I8, I. I909.

Paul, T., Birstein, G., and Reuss, A.: ibid., 25, 367. I910.

Paul, T., and Krönig, B.: Ztschr. f. phys. Chemic, 21, 4I4. 1896.

Penfold, W. J.: J. Hyg., I4, 215. I914.

Penfold, W. J., and Norris, D.: ibid., I2, 527. I912.

Peters, R. A.: J. Physiol., 54, 260. 1920.

Phelps, E. B.: J. Infect. Dis., 8, 27. I9II.

Prescott, S. C., and Baker, S. K.: ibid., I, I93. I904.

Prescott, S. C., and Winslow, C.-E. A.: Elements of Water Bacteriology (4th ed.). New York, I924.

Rahn, O.: Centralbl.f. Bakteriol., Abt. II, I6, 4 I 7. 1906.

Rahn, O.: Tech. Bull. 5, Mich. Agric. Coll. Exper. Sta. I9ıо.

Raju, V. G.: J. Hyg., 2 I, I30. I922.

Reed, H. S., and Reynolds, R. R.: Tcch. Bull. Io, Va. Agric. Exper. Sta. I916.

Rettger, L. F.: J. Bact., 3, го3. I9г 8.

Richet, C.: Compt. rend. Acad. d. sc., I I 4, I 494. Paris, I892.

Robertson, J.: Brit. M. J., I, 69. I898.

Russell, E. J., and Hutchinson, H. B.: J. Agric. Sc., 5, 152. I913.

Schaffer, J. M., and Tilley, F. W.: J. Bact., I4, 259. I924.

Sedgwick, W. T., and Winslow, C.-E. A.: Mcm. Am. Acad. Arts Eo Sc., 12, 47 I. 1902.

Sherman, J. M., and Albus, W. R.: J. Bact., 8, r27. I922.

Sherman, J. M., and Albus, W. R.: ibid., 9, 303. I924.

Shrader, J. H.: Fifteenth Amn. Rept., Int. Assoc. Dairy Eo Milk Inspectors, p. 208. 1926.

Slator, A.: J.Hyg., 16, I00. I917.

Smith, J. H.: Ann. Appl. Biol., 8, 27. I921.

Snyder, C. D.: Am.J. Physiol., 22, 309. I908.

Snyder, C. D.: ibid., 28, 167. I91 I.

Sturges, W. S.: J. Bact., 4, I57. I9I9.

Truffant, G., and Bezssanoff, N.: Science du sol, I, 3. 1922.

Valley, G., and Rettger, L. F.: J. Bact., I4, гог. I927.

Ward, A. R.: Proc. Roy. Soc., 58, 265. 1895.

Werkman, C. H.: J. Bact., I4, 335. 1927.

Whipple, G. C.: Tech. Qutart., I4, 2 I. I901.

Wildiers, E.: La Cellule, 18, 3г3. I90 I.

Winslow, C.-E. A., and Abramson, F.: Proc. Soc. Exper. Biol. E Med., 9, ro7. I9г 2.

Winslow, C.-E. A., and Brooke, O. R.: J. Bact., I3, 235. 1927.

Winslow, C.-E. A., and Cohen, B.: J. Infect. Dis., 23, 82. I918.

Winslow, C-E. A., and Falk, I. S.: J. Bact., 8, 2 I 5 and 237. 1923.

Winslow, C.-E. A., and Kligler, I. J.: Am. J. Pub. Health, 2, 663. I9г 2.

Winslow, C.-E. A., and Lochridge, E. E.: J. Infect. Dis., 3, 547. 1906.

Winslow, C.-E. A., and Sanjiyan, D. H.: J. Bact., 9, 559. I924.

Wolffhügel, G., and Riedel, O.: Arb. a. d. Kaiscrlich. Gesundheitsamte, r, 463. I 886. 


\title{
CHAPTER VII
}

\section{THE DISSOCIATIVE ASPECTS OF BAC'TERIAL BEHAVIOR}

\author{
PHILIP HADLEY \\ University of Michigan \\ INTRODUCTION
}

Two of the most remarkable circumstances relating to the development of bacteriology during the past half-century are: first, that bacteriologists have been so long content to conduct their experiments and to formulate their views in terms of the old monomorphic hypothesis regarding the nature of bacteria and of bacterial reproduction; second, that they have been so active in evolving inadequate schemes for classification before they knew exactly what it was they had to classify. Looking back on the road over which we have traveled, it is impossible to estimate the loss sustained by bacteriology, especially in latter years, through the repressive and misguiding influence of the strict monomorphic conceptions, or to appreciate the often serious biological blunders that are to be laid at its door. In times of festival and jubilee we are accustomed to congratulate ourselves on the significant conquests of modern bacteriological science; and they have, it is true, been considerable. But they should have been greater; and they would have been far greater today if the science, a halfcentury ago, had not become impaled on a false biological conception which has never ceased to influence unfavorably both bacteriological thought and practice. And which, it may be added, even today represents a malicious dogma, accepted by tradition, if not actually embraced by perhaps the majority of bacteriologists.

The doctrine of monomorphism has descended to us from the early conceptions of the nature of bacteria maintained by Cohn, Koch, and others of the early school. Under its influence, in the earliest and most plastic days of the science, there were set up strict notions of "normal" bacterial cell types, "normal" colony forms, and "normal" cultures. Whatever departed from the expected normality was at once relegated to the field of contaminations; or to the weird category of "involution forms," "degeneration forms," or pathological elements possessing neither viability, interest, nor significance. This monomorphic conception found its natural and fundamental support in the assumed mode of reproduction characteristic of the fission-fungi. The dictum was then laid down that "the mode of reproduction of bacteria is by simple fission"- a view which has descended through two generations of bacteriologists and through numerous generations of textbooks, even to the year I927.

Although some early opposition to these views arose, it was probably unfortunate for the beginnings of the science that the first attempts toward modification were made by such extremists as Nägeli and his associates in the Munich group. The extreme plurimorphism which they so eagerly championed through years of bitter controversy was too radical to be accepter graciously as an antidote to strict monomorphism. lior this reason Nïgeli gained few permanent supporters; and, with the final collapse of his views, all views, even those 
espousing a more temperate plurimorphism suffered. The interpretations of the Berlin school triumphed-and to such an extent as to become later the dogma of "normal" colony and culture types that has endured, with hardly a respite, even to the present day.

There did arise, however, at a later date some slight reaction to the monomorphic trend of the science. In the later eighties Gruber and his pupils were demonstrating examples of common and often curiously persistent variability in bacterial cultures. These observations were at variance to the demands of the Berlin group, although the instances concerned also fell far short of conforming to the extreme variability earlier pictured by Nägeli. ${ }^{\mathrm{r}}$ The same was true of the depictions of variability presented in the splendid series of contributions of Eisenberg. "Pathological variants," "involution forms," and "degeneration forms" were for a long time quite adequate to dispose of all such insignificant, though still somewhat bothersome, departures from the "normal" type. In 1906 and 1907 , however, the variationists were fortunate enough to secure a more logical and trustworthy outlet for their explanations of "abnormal" forms of cells or cultures. This was found in the observations of Neisser ${ }^{3}$ and of Massinit on B. coli mutabile, whose pictures of variation they believed involved the phenomenon of mutation. In this way the De Vriesian term, together with many of its connotations, was first introduced into bacteriology. This event was the starting-point for numerous observations and studies on mutating bacterial forms; and the discovery of bacterial "mutants" has continued without appreciable interruption up to the present day. Seldom, however, has the De Vriesian term been used advisedly. Its employment has merely provided a dignified and logical escape from the increasingly unacceptable "involution" hypothesis, as also from the necessity of offering any other more valid explanation of the phenomena concerned. The general result has been to bring into bacteriology many of the terms emp'oyed in genetics - "plain variations," "impressed variations," "hereditary variations," "clones," "biotypes," and "pure lines." But the conceptions apparently supported by the somewhat lavish use of these terms have usually lacked concreteness; and in few instances have the appellations been either appropriate or logical. For the most part, notions of bacterial heredity among bacteriologists have been marked by extreme haziness and uncertainty.

Beginning about 1907 , however, there began to arise among the variationists two groups: first, the larger group of strict variationists who saw in their culture modifications merely a transient, but sometimes permanent (hereditary), departure from the otherwise monomorphic type; second, the cyclical variationists, hardly numerous enough to term a group, and represented by such workers as Führmann, ${ }^{5}$ who believed that they could detect a certain order and direction in the culture modifications. Later supporters in this group have been rare-six only who, since the year 1907 , have combatted to the best of their ability the false but always overwhelming views of bacterial type-stability. These investigators merit naming at this point in our story; they are Führmann, Hort, Almquist, Löhnis, Enderlein, and Mellon. To these workers particularly may be given the credit for directing the current of bacteriological thought into new channels.

${ }^{\prime} \mathrm{v}$. Nägeli, C.: Untersuchungen ïber die niełere P'ilze und ihren Bezichung zu den Infcktionskrankheiten und der Gesundheitspflege. I 877 .

${ }^{2}$ See Bibliography in monograph on microbic dissociation by Hadley, Philip: J. Infect. Dis., 40, I. 1927.

${ }^{3}$ Neisser, M.: Centralbl.f. Baktcriol., Abt. I, Orig., 38, 98. 1906.

${ }_{4}^{4}$ Massini, R.: Arch.f. IIyg., 61, 250. 1907.

${ }^{5}$ Führmann, F.: Verh.d.ges. deutsch. Naturf. u. Artse, p. 278. I906. 
THE DISSOCIATIVE REACTION

As I have pointed out in a previous publication, ${ }^{\mathrm{I}}$ extreme instability of bacterial types has become recognized in recent years as a commonly observed phenomenon. But its significance has been vastly underestimated and its cause or causes a matter of uncertainty. Although descriptions of bacterial "variants" and "mutants" have appeared with increasing frequency in the literature of the past thirty years, it is seldom that they have been regarded as possessing significance outside of that referable to the Darwinian or De Vriesian conceptions. It has, indeed, been only in quite recent times that the striking orderliness and persistence with which these variants are found to appear in different bacterial species and groups have enabled us to relate them to a definite law of variation, widely operative in the bacterial world. Indeed, these observations have led us to the view that, within each bacterial species, there are constantly occurring certain transformations relating to cell morphology, colonial form, biochemical, serological, and immunological characteristics and to virulence; moreover, that these transformations are neither random variations from a "normal" type in the old Darwinian sense nor sudden saltations characteristic of mutations; but that they represent modifications which occur with a certain degree of precision in many different species when confronted with similar changes in environment.

It is only within recent years that serious attempts have been made to correlate any of the different characters of the variants, such as virulence with colony form or serological reaction with cell type. Indeed, it has been the common view that such correlations were seldom possible; or, at least, not sufficiently constant to be of importance. We know today, however, that such correlations are the rule rather than the exception, although they may be partly obscured at times, for a variety of reasons; moreover, that these correlations possess supreme importance for bacteriology, pathology, and medicine.

The results of many observations have thus served to indicate that pure line cultures, of many bacterial species to say the least, are composed of cells all of which are by no means identical. From the same pure line strain may arise, depending on the manner of cultivation and on other environmental conditions, substrains possessing little resemblance either to each other or to the parent-strain. These different culture types may be spoken of conveniently as "dissociated forms" or as "dissociants"; and the phenomenon involved in their production has been termed "microbic dissociation." The terms Umwandlung, Keimumwandlung, and Umformung (of bacterial species), employed by certain German and Swedish investigators, may be regarded as referring to the same phenomenon. Microbic dissociation thus becomes established as a new and highly significant field in the wide province of bacteriology.

But it will be clear that the reactions characteristic of the dissociative phenomenon are, in a sense, superficial. Behind microbic dissociation there must exist a biological mechanisn; and this mechanism, we shall come to see, concerns microbic heredity. Through this medium, therefore, dissociation is intimately related to important studies which have been conducted by a small group of investigators dealing with the socalled "life-cycles" of bacteria.

${ }^{x}$ Hadley, Philip: loc. cit. 
One of the first to suggest that bacterial reproduction was characterized by phenomena more complex than those appertaining to simple fission, and something even more diversified than simple back-and-forth variation between two or three variants, was Führmann ${ }^{\mathrm{r}}$ who, in 1907, proposed his Entwicklungscyclus. This conception of a cyclical development in bacteria was further indicated in a work by $\mathrm{Hort}^{2}$ in England in 1916 , by Löhnis and $\mathrm{Smith}^{3}$ in the United States, and by Enderlein ${ }^{4}$ in Berlin, also in I9r6. It was at this time that the latter worker introduced into bacteriological terminology the term "cyclogeny" (Cyclogenie), implying the cycle through which the microbe passes in leading up to the highest cytological state (Kulminante) and returning to its basal state (Mychit). Many of the papers by Almquist $^{5}$ and Mellon ${ }^{6}$ on microbic heredity have dealt with the problem of life-cycles among bacteria; and in recent years this term, often used rather loosely to indicate certain obscure cellular transformations, has occurred in the literature with considerable frequency.

While it seems probable that the time will eventually arrive when we can speak intelligently regarding definitely cyclical aspects of bacterial reproduction, and even though at the present moment we can of ten detect a certain direction in the serial transformations observed, it has seemed to me that our present knowledge of cyclical development in its details is still too slight to justify a common use of this term as describing the tran formations thus far observed. Until more definite knowledge of the distinctly cyclical development of some one species is at hand, it is perhaps more appropriate to employ a term suggesting merely cultural transformations, often, it is true, apparently directive, but not overemphasizing the cyclical feature. For such a term, "microbic dissociation" will for the present suffice. The ultimate realities on which it depends are those involving the mechanics of microbic heredity. Microbic dissociation might therefore be defined as embracing those distinctly transformatory processes occurring in bacterial cultures, in vitro or in vivo, through which there arise one or more new culture forms which differ from the mother-type, and which (I) may persist for a variable time in an apparently stable state, or (2) may become transformed into still another culture type, or (3) may "revert" to the original form.

The studies that bear on the problems of microbic dissociation may be grouped under two headings: (I) those random observations on culture variations which, even by the authors themselves, were not recognized at the time when the work was conducted as related to the fundamental problem of microbic heredity; (2) those later and more concise studies definitely directed upon the meaning of bacterial variation, its causes and effects. Regarding the first category, little need be said except that there exist in the bacteriological literature of the past thirty years or more numerous isolated citations which, by virtue of our present knowledge of the dissociative process, we are able to translate into the terms of microbic dissociation. Some of these I have brought together in a previous publication. ${ }^{7}$ The second category of studies mentioned above includes those which consciously attack the problem of bacterial variation. These, in turn, are divisible into two groups, each differing from the other in its mode of approach to the fundamental problem. These involve (I) the cytological approach and (2) the cultural approach. In the latter group may also conveniently be included the biochemical and serological characteristics of the variants.

${ }^{2}$ Führmann, F.: loc. cit. ${ }^{2}$ Hort, E. C.: J. Roy. Micr. Soc., p. 11. 1926.

${ }^{3}$ Löhnis, F. and Smith, E. R.: Jour. Agr. Res., 6, 675. 1916. See also Löhnis: ibid. 23, 401. I923; also Men. Nat. Acad. Sc., 16, 252. I921.

${ }_{4}^{4}$ Enderlein, G.: Sitzungsb. ges. naturf. Freunde. Berlin, IgI6.

${ }^{5}$ Almquist, E.: Centralbl.f. Bakteriol., Abt. I, Orig., 60, I67. I91 I; Biologische Forschung über die Bakterien. Stockholm, I925.

${ }^{6}$ See Hadley, Philip: loc. cit.

${ }^{7}$ See ibid. 
THE CYTOLOGICAL APPROACH

The cytological approach to the problem of microbic dissociation has centered mainly on the variable morphology of the bacterial cells and their nuclear apparatus. It has followed (especially in the morphological aspects) the lines laid down in mycology at a much earlier date. Here the attempt has been made to discover, in the peculiar bacterial elements often observed in cultures, the groundwork for an interpretation of microbic heredity, involving modes of reproduction quite different from simple fission. Most of the earlier studies dealt with the microscopical cell changes, but without special reference to nuclear behavior. Later studies, and particularly those of Almquist, Enderlein, and Mellon, have concerned themselves as well with alterations in the nuclear apparatus. In view of the importance of the work dealing with the nuclear changes, a few words must be said regarding later conceptions of the nuclear structure and the "chromatin" of the bacterial cell. The following represents some of the essential features of Enderlein's ${ }^{t}$ view which I believe we may accept as reflecting the best knowledge now available regarding this important organelle.

The nuclear unit $(\mathrm{Mych})$ of the microbic cell possesses a spherical or oval form and, in the coccus, often attaches itself to the inner wall of the cell, against which it may sometimes be flattened. In cocci there is but one nuclear body while in all other forms of bacteria there are two or more. The diameter varies between 0.1 and $0.25 \mu$. It contains no chromatin and, with weak fuchsin, stains hardly any stronger than the cytoplasm of the cell. With methylene blue it may remain practically unstained.

The nuclear body is observable only when the cell containing it holds but little food substance in reserve. The latter commonly exists in the form of ultramicroscopic granules, the trophoconia, and this substance represents the actual "chromatic" material of the cell. It stains strongly because of its high content of nucleic acid and nucleo-proteins. The failure of a cell to stain well with methylene blue is due to the absence of the food-reserve substance. When the trophoconia bodies are abundant, their substance forms a dense aggregation about the nuclear body, and the element so formed stains readily; this is the trophosome. If only a light and thin layer of reserve substance clusters about the nucleus, this body becomes the trophosomelle, which is smaller and more delicate. Neither of these bodies (trophosome or trophosomelle) is the actual nucleus, however; they merely contain the nucleus as a central granule. Sone of the granular bodies earlier described for bacteria, such as the Much granules, the sporogenous granules of Ernst, and the metachromatic granules of Babes, are actually trophosomes or trophosomelles. Other granules appear to be quite different structures - sometimes the gonidia. They are all casily observable and often gram positive. If the trophosome is situated at the end of a rod form, it is a "tclotrophosome"; if at other points in the cell, it is an "ascotrophosome." Moreover, to continue Enderlein's somewhat elaborate but necessary terminology, if a cell is free from reserve substance (trophoconia), it is an "atrophite." If it is merely poor in reserve substance, it is a "metatrophite"; if rich in reserve, it is a "pliotrophite."

When the reserve substance in a cell has been used up, as in bacteria that have been starved (as in clistilled water), the list remnant clings tenaciously about the nuclear body. Large amounts of food-reserve substance, which may conceal not only the nucleus but also the trophosomes, may be removed from the cell by alcohol. Under these conditions, when properly stained, it is observed that, in coccus forms, only a single point takes the stain. In rod forms, on the other hand, two or several such bodies take the stain. These are often at

I Enderlein, G.: Bak'crien-Cyclogenic. Berlin, I925. 
the poles of the cell and represent the true bacterial nucleus or nuclei. After cell division the heavily staining reserve substance soon appears in the daughter-cells.

The first important departure from simple fission is gonidia formation. This form of reproduction was first recognized and named by $\operatorname{Cohn}^{1}$ in 1872 in his study of Crenothrix, but the phenomenon was not carried over to the lower forms of bacteria. Gonidia were also recognized by Lancaster in 1873 , and they were noted the same year in B. lactis by Joseph Lister. They were also indicated in $\mathrm{l}^{*}$. cholerae and in $\mathrm{V}$. proteus (Vibrio finkler-prior) by Finkler and Prior in $\mathrm{s} 885$. Out of the gonidial bodies, which were often regarded as spores, there were seen to arise extremely minute microspiral forms which, through further development, eventually attained normal size. Since these early days, gonidia have doubtless been seen many times without recognition. They have been definitely reported by Jones, ${ }^{2}$ Löhnis, ${ }^{3}$ Almquist, ${ }^{4}$ Mellon, ${ }^{5}$ Enderlein, ${ }^{6}$ Tunnicliff, ${ }^{7}$ and others. Enderlein, who has been able to recognize them in many bacterial species, regards them as the most common seed form (Fruchlform) of bacteria, being homologous with the spores of the fungi (conidia, ascospores). Morphologically and actually, according to Enderlein, they represent the true bacterial spore, which is not true of those elements usually termed "spores" by bacteriologists.

Some of the so-called Much granules are regarded as gonidia, others as trophosomes. In I 870 Cohn differentiated gonidia into the large ("macrogonidia") and the small ("microgonidia"). In the tubercle bacillus the microgonidia are not acid fast but may be gram positive. Enderlein mentions several sorts of gonidia, named according to their point of origin in the cell. If they arise at the end of a rod, they are termed "telogonidia"; if throughout the whole length of a rod, they are termed "ascogoniclia." Apparently compared with such higher forms as Crenothrix, bacteria produce only small numbers of gonidia. That the microgonidia may pass Chamberland filters seems to have been demonstrated by Lourens ${ }^{8}$ for the bacillus of swinepest in 1907 , by Almquist' for B. typhosus in I9I I, by Miehes for a number of bacterial species in 1923, and by Mellon 5 for B. fusiformis in 1926. In other instances of the discovery of filtrable forms of bacteria there is slight basis for an opinion as to the exact nature of the filtrable unit; although, in the experiments of Fontès, ${ }^{5}$ who in I9Io was the first to demonstrate the filtrability of the tubercle bacillus, the description of his cultures suggests that the filtrable forms were microgonidia whose presence, among other small granular bodies, he was unable to recognize. The telogonidia have been recognized in spirochetes (Treponema and Leptospira) and may be concerned with the filtrability of organisms of this class, as was first demonstrated by Novy and Knapp 5 for the relapsing-fever spirochete in I906, although it also appears to be true that fine spiral forms also may pass the Berkefeld filter. According to Enderlein, the filtrable forms of bacteria comprise, not the gonidia alone, but also the gonites, next to be described.

Our knowledge of the bacterial reproductive elements known as the "gonites" is limited to the results of Enderlein's ${ }^{\mathrm{ro}}$ studies, and particularly with reference to the cholera vibrio. His observations, which are highly suggestive, but which naturally demand extended confirmation, are presented forthwith.

I See Hadley, Philip: loc. cit.

${ }^{2}$ Jones, D. H.: J. Bact., 5, 325. 1920.

3 Löhnis, F.: loc. cit.

${ }^{4}$ Almquist, E.: loc. cit.

s'See Hadley, Philip: loc. cit.
${ }^{6}$ Enderlein, G.: loc. cit.

7 Tunnicliff, R.: J. Infect. Dis., 36, 430. I925.

${ }^{8}$ See Hadley, Philip: loc cit.

9 Almquist, E.: loc. cit.

${ }^{10}$ Enderlein, G.: Baleterien-Cyclogenie. Berlin, I925. 
If the gonidia are maintained under conditions involving lack of nutriment, as, for instance, in aged cultures (thus preventing their entrance into a higher cyclostage), or if they are submitted to the influence of prolonged warming at $37^{\circ} \mathrm{C}$., they become transformed into new and smaller elements, the gonites. The number of these forms increases with the gradual decrease of the gonidia. In cholera cultures left standing in the laboratory for a month or more, one finds that a great number of gonites have been produced. They are all extremely small, carry a much reduced amount of cytoplasm, and the smallest are known as the "microgonites." If the original culture is placed in sunlight, the same result occurs in a much shorter time- sometimes within a few days. The gonite is unable further to reproduce itself as such. When cultures that have entered completely the gonite stage are trans. planted to agar, no growth occurs. Such cultures appear to be destitute of living cells. If, however, such a gonite culture is transplanted to broth, the gonites undergo further development, within a period of five to seven hours, into two new forms-namely, the spermite (8) and the oite (क). In a liquid medium copulation occurs, and the fertilized cell is capable of soon regenerating the original cell type. Here, then, we have a strict sexual form of reproduction. Enderlein has followed the details especially in the cholera vibrio.

With further reference to the foregoing considerations, it may be said that the reproductive significance of the gonidia in bacterial reproduction is now beyond a matter of doubt. These bodies have been observed repeatedly, and their subsequent development into the original cell type followed by several competent investigators. As for their further transformation into the gonites, and the subsequent transformation of these into the sex cells - although this phenomenon eventually may be found to occur, and to underlie a true sexual form of reproduction in the bacteria-in so important a matter one is justified in postponing a conclusion until the striking observations of Enderlein can be confirmed in the cholera vibrio and extended to other species. It may be remarked here, however, that many of Enderlein's cytological observations have already been confirmed by Schumacher. ${ }^{I}$ And in this case the confirmation is the more valuable since Schumacher was not acquainted with the work of Enderlein at the time of his own studies.

The cytological aspects of microbic heredity have also been furthered in recent years by the valuable researches of Mellon in a series of contributions extending over many years. Among other matters of importance, Mellon's investigations have dealt especially with a mode of reproduction involving conjugation and zygospore formation, following many of the details of isogamic conjugation in higher forms. These zygospore-like bodies are probably identical with similar forms pictured less clearly by many earlier workers; perhaps with the Pettenkofer bodies described more recently by Kuhn. ${ }^{2}$ Mellon conceives that the origin of the zygospore is through the fusion of adjacent cells of a filament, sometimes indirectly by means of a peduncle. The bodies seem to be formed equally among the small and the large rodlike elements in the culture. They are often small, but in certain diphtheroids, as observed by Massini and by Mellon, ${ }^{3}$ and in B. diphtheriae (Park No. 8), as observed by myself, 4 they may attain a diameter of $6-7 \mu$. By favorable staining they usually reveal a

'Schumacher, Josef.: Centralbl.f. Bakteriol., Abt. I, Orig., 97, 8I. 1926.

${ }^{2}$ Kuhn, Philaethes: Centralbl. f. Bakteriol., Abt. I, Orig., 93, 280.* I924. See also: Arch. f. Schiffs- u. Tropen Hyg., 30, 133. 1926.

${ }_{3}$ Massini, R.: Arch.f. Hyg., 61, 250. 1907; Mellon, R. R.: J. Bact., 2, 81. 1917.

${ }_{4}$ Hadley, Philip: loc. cit. 
cluster of nucleus-like granules. Mellon has also recorded the liberation of large numbers of minute and apparently motile granules from the "giant coccus" forms. This has also been reported by Kuhn, ${ }^{\mathrm{I}}$ who has presented beautiful micro-photographs of these cells (Pettenkofer bodies) both before and after the liberation of the minute granular bodies. From his work it appears that these small forms often fail to stain by the ordinary methods, but that the Giemsa stain is especially favorable. It may be added at this point that Kuhn sees a relation between the presence of these bodies and the ability of the culture containing them to generate the bacteriophage; and this is quite in harmony with the theory of transmissible autolysis which I have proposed in an earlier paper.

Regarding the development of the zygospores, Mellon has observed that in some cases they appear to undergo a double segmentation and yield a large coccus form like that often encountered in dissociating cu tures of $B$. diphtheriae. In such instances Mellon regards the zygospores as transition Anlagen for the development of a new form of culture, the exact nature of which will be determined by the environment surrounding the germinating zygospores.

Finally, with reference to the hereditary mechanism of bacteria, there should be mentioned the formation of symplastic structures such as those first described by Jones $^{2}$ in I913 and 1920 for Azotobacter, and confirmed by both Löhnis ${ }^{3}$ and Enderlein. ${ }^{4}$ Briefly, the reaction involves the fusion of a mass of bacteria into a single group in which the cell boundaries are lost and a union of nuclear elements occurs. From such symplastic structures arise new individual cells.

\section{THE CULTURAL APPROACH}

As early as 1888 observations dealing with several different and more or less permanent culture types arising from pure cultures had been described by Firtsch ${ }^{5}$ for $V$. proteus, and in 1895 Dyar $^{6}$ gave a report on changes of a somewhat similar nature for $B$. lactis erythrogenes. In later years other investigators presented other instances in which striking departures arose from the long-assumed "normal" and constant type. Many of these instances we owe to the splendid researches of Eisenberg. ${ }^{7}$ It remained for Baerthlein, ${ }^{8}$ however, in 19 I 8 to point out the frequent occurence of such variations, their cultural, biochemical, and-to a limited extent-serological reactions. The primary basis for Baerthlein's important study was colony variation, the significance of which had been clearly seen by Firtsch in the case of a single species. Baerthlein showed that plating from old laboratory cultures commonly resulted in the appearance of colony forms quite unlike that of the original culture. Sometimes only one or two colony variants were encountered; at other times, four or five of them. But the most important and significant feature of Baerthlein's study was his demonstration that, commonly associated with colony variation, were variations in other characteristics - morphological, biochemical, and serological.

s Kuhn, Philaethes: loc. cit.

${ }^{2}$ Jones, D. H.: loc. cit.

${ }^{3}$ Löhnis, F.: loc. cit.

${ }^{4}$ Enderlein, G.: Bakterien-Cyclogenie. Berlin, I925.

5 Firtsch, G.: Arch.f. Hyg., 8, 369. 1888.

${ }^{6}$ Dyar, H. G.: Ann. N. Y. Acad. Med., 8, 322, 1895.

7 See Hadley, Philip: loc. cit.

${ }^{8}$ Baerthlein, K.: ibid., 81, 369. I918. 
It is doubtful if Baerthlein appreciated the full significance of his observations. For some time at least he believed that his various culture types represented merely "mutations," such as had been described earlier (especially in secondary colony formation) by Neisser, ${ }^{\mathrm{I}}$ Massini, ${ }^{2}$ Müller, ${ }^{3}$ Penfold, ${ }^{3}$ Thaysen, ${ }^{3}$ Burri, ${ }^{3}$ Eisenberg, ${ }^{3}$ Ledingham, ${ }^{4}$ and many others. It thus remained for Arkwright, ${ }^{4}$ in I $92 \mathrm{I}$, to grasp more fully the significance of Baerthlein's work. Among members of the colon-typhoid-dysentery group Arkwright noted particularly two colony forms which occurred in each species with marked persistency. One was round, regular, opaque, and characterized by a smooth, glistening surface; the other was flat, irregular, translucent, and showed a rough or sandpaper-like surface. The former was termed the "S" type (smooth), the latter the " $\mathrm{R}$ " type (rough). The $\mathrm{S}$ type culture, on aging, transformed readily into the $\mathrm{R}$. The latter, however, held to its new characteristics with considerable tenacity. While the $\mathrm{S}$ type culture grew in broth with a homogeneous clouding, the $\mathrm{R}$ type gave an agglutinative or sedimentary form of growth. These two culture forms were observed by Arkwright in $B$. coli, B. typhosus, and $B$. dysenteriae. His splendid and farseeing work marks the beginning of a new epoch in the study of bacterial variation.

In I92 I De Kruifs also made, independently, a contribution of fundamental importance dealing with dissociation in the rabbit Pasteurella type, Bact. lepisepticum. In this species he observed two forms of culture, differing from each other in colony form, manner of growth in broth, serological and immunological reactions, and particularly in virulence. De Kruif designated these two types " $D$ " and "G," respectively. As we can now see, his D form was analogous to Arkwright's $S$, while his G form was analogous to Arkwright's R. In De Kruif's experience, while the D type culture was highly virulent for rabbits, the $\mathrm{G}$ form possessed little, if any, virulence. Moreover, while the $\mathrm{D}$ form in the killed state was of little value as an immunizing agent, the $\mathrm{G}$ form, living, when injected into rabbits even in small doses, produced immunity to large amounts of virulent culture. Even one dose secured these results.

Since the important works of Arkwright and De Kruif in I92r, the same line of study has been carried into many other fields: to the streptococci by Cowan ${ }^{6}$; to the pneumococcus by Griffith ${ }^{7}$, also later by Reimann ${ }^{8}$ and by Amoss ${ }^{8}$; to B. lyphosus and B. enteritidis by Arkwright and Goyle, ${ }^{9}$ and by Goyle ${ }^{10}$ alone; to the Salmonella forms by White, ${ }^{11}$ and by Topley and Ayrton $^{\mathrm{r2}}$; to B. cholerae suis by Orcutt ${ }^{\mathrm{r2}}$; to the cholera vibrio by Balteanu ${ }^{12}$; to Friedlinder's bacillus by Julianelle ${ }^{13}$; and to $B$. coli quite recently by Dulaney. ${ }^{\mathrm{I} 4}$ De Kruif's work has

\footnotetext{
${ }^{1}$ Neisser, M.: loc. cit. $\quad{ }^{2}$ Massini, R.: loc. cit. $\quad{ }^{3}$ Hadley, Philip: loc. cit.

4 Arkwright, J. A.: J. Path. \& Bact., 24, 36. 1921.

5 de Kruif, P.: J. Exper. Med., 33, 773. 1921; also 35, 631. 1922.

${ }^{6}$ Cowan, Mary: Brit. J. Exper. Path., 3, 187. 1922.

${ }_{7}^{7}$ Griffith, F.: Public Health and Medical Subjects, Ministry of IIealth, Rep. 18. London, I923.

${ }^{8}$ See Hadley, Philip: loc. cil.

9 Arkwright, J. A., and Goyle, A. N.: Brit. J. Expcr. Path., 5, 104. I924.

ro Goyle, A. N.: J. Path. \&o Bact., 29, 149. 1926.

"White, P. B.: Spccial Rep., Med. Research Council, No. 91. London, 1925.

12 Balteanu, I.: J. Path. \& Bact., 29, 251. 1926.

${ }^{13}$ Julianelle, L. A.: J. Exper. Mcd., 44, 683. 1926; also p. 735.

${ }^{14}$ Personal communication.
} 
been followed by Webster into lines of considerable interest. The study of Theobald Smith and Gladys Bryant ${ }^{\mathrm{I}}$ on a "mutating" form of $B$. coli may also be mentioned as an instance dealing with the dissociative reaction, although the authors did not emphasize the relation. Gratia at an earlier date had made somewhat similar, though less detailed, observations on the same species. 'The chief results of all of these studies have been to demonstrate with ever increasing clearness the new characteristics possessed by the recognized variants; also to validate many earlier observations of a similar nature, but manifestly concerned with the same phenomenon. In addition, the more recent studies, besides depicting the $\mathrm{S}$ and $\mathrm{R}$ forms, have presented evidence for the existence of the third significant culture type, the $O$ form (intermediate), also recognized by earlier workers, lying as a transitional form between $\mathrm{S}$ and $\mathrm{R}$.

\section{COLONIAL, CULTURAL, AND MORPHOLOGICAL ASPECTS OF MICROBIC DISSOCIATION}

As has already been pointed out, microbic dissociation manifests itself in variations in colony form, in cultural growth, in comparative cytology, in cell morphology, in biochemical reactions, in immunological reactions, and in virulence. In the present section we shall consider some of the details relating to the first three of these points, with the attempt to indicate that the variations and correlated characters observer are not the result of chance, but depend on certain laws governing the transformations in widely separated bacterial species. ${ }^{2}$

\section{COLONIAL ASPECTS}

As Firtsch ${ }^{3}$ clearly suggested by his remarkable study of variation in $V$. proteus in $\mathrm{r} 888$, colonial variation is the most funclamental, consistent, and clearly observed phenomenon in dissociative variation. Each bacterial species possesses, not one "normal" colony form, but a variety, each of which one must be able to recognize before he can affirm that he knows the "species." Each of these forms is determined by the stage of cyclogeny attained by the individual cells that comprise the colony structure. The time is now past when similarity in colony form must be taken as evidence of the close relationship of the organisms contained; or when dissimilar colony types must be regarded as indicating unrelated species. The fact of the matter is, that the degree of variation in colony form in one and the same species may be, and usually is, greater than the degree of variation in colony form of equivalent cyclostages of clearly distinct species. The diverse colony forms observed within pure lines of $B$. subtilis (Soule), ${ }^{4}$ of B. anthracis (Preisz, ${ }^{5}$ Wagner, Nungester ${ }^{6}$ ), of hemolytic streptococci (Cowan), ${ }^{7}$ of $S$. fecalis (Faith Hadley), ${ }^{8}$ of the meningococcus and gonococcus (Atkin), ${ }^{9}$

I Smith, T., and Bryant, Gladys: J. Exper. Med., 46, I33. I927.

${ }^{2}$ It is impossible to consider the biochemical, serological and immunological aspects within the limits of this chapter.

3 Firtsch, G.: loc. cit.

${ }_{4}^{4}$ Soule, M. S.: Jour. Infect. Dis. 1928, No. 2.

5 Preisz, H.: Centralbl. f. Bakteriol., Abt. I, Orig., 35, 280. Ig04; also 53, 510. IgII.

${ }^{6}$ Nungester, W.: Proc. Soc. Exper. Biol. \& Med., 24, 959. I927.

7 Cowan, Mary: loc. cit.

${ }^{8}$ Personal communication.

$\checkmark$ Atkin, E. E.: loc. cit. 
of the pneumococcus (Griffith, ${ }^{\mathrm{r}}$ Reimann, ${ }^{2} \mathrm{Amoss}^{2}$ ), of $B$. diphtheriae (Corbett and Phillips), ${ }^{3}$ of $S p$. finkler-prior (Firtsch), ${ }^{4}$ and of $V$. cholerae (Eisenberg, ${ }^{2}$ Balteanu ${ }^{5}$ ) differ from one another to such a degree that, on morphological grounds, all of the variants would be regarded as contaminations-indeed, often have been so regarded and treated accordingly. As one illustration of this, and as Soule ${ }^{6}$ has already pointed out, it may be noted that the $\mathrm{R}$ type colony of $B$. subtilis on plates is almost indistinguishable from the $\mathrm{R}$ type colony (Medusa-head type) of $B$. anthracis. On the other hand, one may conclude from the studies of Nungester ${ }^{7}$ that the $S$ type anthrax colony is one that few investigators have ever seen, or at least recognized; and one which would commonly be taken as a contamination, so different is it from the common Medusa-head type. It is thus a rather curious fact that, while it is the $\mathrm{S}$ colony form of $B$. subtilis that has come to be regarded by bacteriologists as the "normal," it is the $\mathrm{R}$ type colony of $B$. anthracis which, during the fifty years of study that this species has received, has become established as the "normal" form of culture.

In most bacterial species for which knowledge is available, the colony of the $\mathrm{S}$ type is smaller and more delicate than the other forms. It is round, even, usually opaque (even when young), and, in certain species such as those of the intestinal group, the streptococcus, the pneumococcus, the proteus, the pneumobacillus, and others, presents a glistening luster or "moist" appearance. In addition, a distinct fluorescent effect by transmitted light is usually seen (intestinal group, pneumobacillus, proteus, and others). The co'ony consistency is commonly soft or butyrous. Such colonies, after four or five days' growth on rich and somewhat alkaline agar, often show pale or translucent, wedge-shaped invaginations where dissociation into the $\mathrm{O}$ or $\mathrm{R}$ types is under way. Culturing from these "blue" areas will give cultures of a quite different type from the original, but usually not well stabilized at this stage. Repeated culturing, accompanied by colony selection, will increase the stability of the (commonly obtained) $\mathrm{R}$ form.

The $\mathrm{R}$ type colony presents, in most species, a quite different appearance from the $\mathrm{S}$ form. It is usually larger, irregular in shape, uneven; when young it is thin and translucent, and reveals a distinctly rough surface, or sometimes merely a dull luster as in B. subtilis (Soule). The fluorescent effect is invariably lacking. Old colonies, however, may become as opaque as those of the $\mathrm{S}$ form. The consistency of the $\mathrm{R}$ colonies is sometimes similar to that of the S form, but often, and when they are well stabilized (as in the "extreme" $\mathrm{R}$ ), they may be hard or even brittle, so that they may be pushed about over the agar surface. These have been observed in the pneumococcus (Griffith) and in the streptococcus (Cowan). One curious feature of the $\mathrm{R}$ type colonies of several species ( $B$. diphtheriae, B. mallci, B. proteus, $V$. cholcrae, V.proteus, B. mesentericus, meningococcus, $S$. fecalis, and probably other species) is that they may take on a yellow or brown chromogenesis. Apparently a similar phenomenon occurs in the fungus of blastomycosis (Mellon). ${ }^{8}$ I have also observed brownish colonies arising in the dissociation of $M$. citreus.

Regarding the colonial features of the type $\mathrm{O}$ cultures, first clearly pictured by Firtsch in 1888 for $V$. proteus, we have less knowledge. In general, the $\mathrm{O}$ colonies are larger than the $\mathrm{S}$, round, even, smooth, glistening, but more fleshy and convex, simulating the colonies of

\footnotetext{
I Griffith, F.: loc. cit.

${ }^{2}$ See Hadley, Philip: loc. cit. $\quad{ }^{6}$ Soule, M. S.: loc, cit.

3 Corbett, L., and Phillips, G.: J. Path. \& Bact., 4, 193. 1897.

${ }^{4}$ Firtsch, G.: loc, cit.

7 Nungester, W.: loc. cit.

s Balteanu, I.: loc. cit.

${ }^{8}$ See Hadley, Philip: loc. cit.
} 
B. aerogenes. They often manifest a mucoid consistency. Cultures of this sort commonly appear in members of the colon-typhoid-dysentery group; also in B. anthracis, S. fecalis, and probably other species. In $B$. anthracis some of these colonies are slimy and readily coalesce on the agar plate. Similar colonies have been reported for various species among cultures resistant to the bacteriophage. In $B$. proteus the equivalent of the $\mathrm{S}$ form yields a spreading growth while the growth of the $\mathrm{O}$ type is restricted. In several species, such as pneumococcus, streptococcus, meningococcus, and gonococcus, the $\mathrm{O}$ type has not been described clearly. In general, it is highly unstable and may sometimes transform with great rapidity into the R. Such cultures have been termed "suicide cultures." They are very difficult to maintain on agar, and it is sometimes impossible to cultivate them in broth. It is reasonable to believe that the $\mathrm{O}$ colony form is at some time present in cultures of all bacterial species, but may pass unobserved. Certain members of the intermediates, such as those representing the Pettenkofer bodies of Kuhn, may play an important rôle in the phenomenon of the bacteriophage, as I have pointed out elsewhere. ${ }^{\text {t }}$

Although it often appears that the three chief colony types (S, O, and R), but particularly $S$ and $R$, are clear cut in their essential features, and usually quite stable on appropriate medium, in other cases there may be observed distinct intergradations; and these may take on various aspects. For example, the $\mathrm{S}$ type colony may show, about its edge, either at a single point or about its entire circumference, an outcropping of the $\mathrm{R}$ type culture. Interesting examples of this have been described by Soule for $B$. subtilis, and observed by Nungester for B. anthracis; also by Faith Hadley for $S$. fecalis. ${ }^{2}$ The fairly smooth and circumscribed S colony, possessing (subtilis) the shallow fringe of filaments extending outward from the border ("bayonet-front" effect), sends out longer outgrowths which soon begin to curl under and to give a marginal appearance which simulates that of the $\mathrm{R}$ type anthrax colony (Soule). This outgrowth is bluish and translucent by transmitted light and rough by reflected light. The result of this reaction is to yield an $\mathrm{S}$ type colony imprisoned within a ring of $\mathrm{R}$ type culture, the breadth of which varies with conditions which cannot be considered here. The type $\mathrm{S}$ anthrax colony may undergo transformations similar to those mentioned above (Nungester). Such outgrowths have been termed the "halo" or "regeneration fringe." In some cases, as in B. proteus for example, the halo may be made up of $\mathrm{O}$ type rather than $\mathrm{S}$ type culture. Indeed, there may be a series of alternate dissociations and recoveries, following each other at intervals of a few hours, in the growth of the colony, the final effect being the production of the typical "ring growth" characteristic of B. proteus.

But the $O$ type colony also may send out such regeneration fringes; and in this case the nature of the fringe growth seems to depend on the degree of stability of the $\mathrm{O}$ colony. If it is in the early intermediate state, it usually forms a halo of normal $S$ culture. If it is in the late intermediate state, it is more likely to send out a fringe of $\mathrm{R}$ type culture. Under these conditions, culturing from the center or from the edge of the colony will yield cultures of two different forms. The natural destiny of the type $\mathrm{O}$ culture is apparently to attain the $\mathrm{R}$; and it usually accomplishes this in the course of time. It is often difficult to maintain the intermediate form in this culture state. The $\mathrm{O}$ form of $B$. proteus seems to be unusually stable, but here the $\mathrm{R}$ form has not been recognized with certainty. It may be added here that it has not been observed clearly that the type $\mathrm{R}$ culture gives regeneration fringes. It may, however, as we shall see later, produce clusters of $\mathrm{S}$ type secondary colonies which usually appear on the free edges of growth; and these are analogous to the fringe, since it can be observed that it is by the coalescence of numerous colonies appearing at the margin of growth that the distinct fringes are produced.

r See Hadley, Philip: loc. cit. Also Arch. of Path. and Lab. Med., I928. In press.

${ }^{2}$ Personal communication. 
Another manner in which dissociative reactions may reveal themselves in cultures is, as just intimated, the generation of secondary or "daughter-colonies" occurring in a background of primary culture. Instances of this phenomenon were given added significance in 1906 and 1907 through the observations of Neisser and Massini on $B$. coli mutabile, and similar observations were soon made on many forms. All these cases, manifesting the spontaneous origin of new culture types within the old culture mass, were quickly seized upon as indications of true mutations among the bacteria; and this false conception persists in the minds of many bacteriologists, even at the present day.

Secondary colonies may be few or numerous; sometimes one only, or again there may be several hundred discrete bodies. As the number increases, however, they blend more and more into the mass of mother culture and thus lose their colonial identity -at least macroscopically. Microscopically they may still be traced as "granulations" of varying size, until the culture mass becomes a fine mosaic of the two or more culture elements. Sometimes the secondary colonies arise near the surface and form the well-known "papillae." Again they lie deeply imbedded in the culture, or even in the medium itself, as in the case of S. fecalis, and do not register on the contour of the primary colony. In some species (S. fecalis - Faith Hadley) ${ }^{\mathrm{r}}$ both forms of secondary colony may be observed, and in this case they appear as entirely different culture types.

The most common forms of secondary colony relate to centers of $\mathrm{O}$ or $\mathrm{R}$ type culture arising in a background of $\mathrm{S}$ culture. On the other hand, it appears from the older work of Preisz ${ }^{2}$ and Pesch $^{3}$ on B. anthracis, and from the more recent studies of Anna Dulaney ${ }^{4}$ on $B$. coli, that secondary, $\mathrm{S}$ type colonies may arise in $\mathrm{R}$ type cultures. In Dulaney's case they were generated particularly at the free margins of the type $\mathrm{R}$ colonies after a prolonged growth. Tertiary colonies arising within the secondary have also been occasionally observed, particularly by Preisz for $B$. anthracis. The whole subject of secondary and tertiary colony formation may possess additional interest in its bearing upon the nature of the bacteriophage reaction, and with special reference to the homogamic theory which I have briefly outlined in a previous publication. ${ }^{6}$ Here it was suggested that the resistant colonies which d'Herelle, Bordet, and others have termed "secondaries" (arising in the lytic sites on agar, or in culture filtrates) may, in reality, be tertiary colonies arising after the disappearance of the secondaries. In this case the characteristic lytic plaques would be regarded as the sites of disappearance of colonies of the secondary type, which itself might represent one of the intermediate forms of culture.

For the present we may leave the subject of the secondary colonies with the conclusion that these formations are of considerable significance as indicating that, hidden in the mass of mother culture, regardless of the type concerned, there may exist certain centers where small or large groups of organisms, quite different from the mother culture in form and physiology, have arisen and where they are carrying on their inde-

${ }^{t}$ Personal communication.

${ }^{2}$ Preisz, H.: Centralbl.f. Bak'criol, Abt. I, Orig., 35, 280. I904; also 53, 510. I9 I I.

${ }^{3}$ See Hadley, Philip: loc. cit. ${ }^{4}$ Personal communication. $\quad 5$ Preisz, H.: loc. cit.

${ }^{6}$ See Hadley, Philip: loc. cit. See also: Arch. of Path. and Lab. Med. 1928. In press. 
pendent activities. When removed and purified by plating methods, they afford new forms of culture which sometimes manifest considerable permanence in their newly acquired characters. It is these forms that have erroneously been regarded as mutants.

\section{CHANGES IN CELL MORPIIOLOGX}

Closely associated with the specific colony type, and in all probability determining, within limits, its characteristics, are to be observed some fairly distinct morphological characteristics of the cells and their organelles. Microscopic study of the type S culture, which usually passes under the appellation of "normal culture," although exceptions have been noted, usually reveals a preponderance of those cell forms which are regarded as characteristic of the "species" in question. The type R culture, on the other hand, is likely to present a different picture; but this, in turn, varies with the species. In the members of the intestinal group the well-stabilized $\mathrm{R}$ type cell is most often coccoid, and a similar shortening is characteristic of the $\mathrm{R}$ forms of the diphtheria bacillus, the plague bacillus, probably of the tubercle bacillus and of several other species. In other cases, as in B. subtilis, B. anthracis, and perhaps in all the sporeformers (none of which, with the exceptions noted, has been carefully studied), the $\mathrm{R}$ type are much more elongated than the $\mathrm{S}$ type cells and are sometimes distinctly filamentous.

The nature of the cell population of the O type cultures is not so clearly recognized. But it may be affirmed that, in comparison with the $S$ and $R$, it is highly diverse. It is particularly in cultures of the $\mathrm{O}$ type that one finds amassed those peculiar cell bodies which, for many years, have been termed "involution forms." They contain long, swollen rods, filaments (often fungoid in nature), giant coccoid bodies, apparently identical with Kuhn's Pettenkofer bodies, zygospore-like bodies, and often numerous minute granules, the exact nature of which is perhaps still in doubt, although it seems probable that some of them arise from the zygospores. The whole picture is extremely bizarre but is fairly constant for the intermediate type cultures of many bacterial species. Such forms have been produced by Kuhn and others by growth on media containing traces of lithium chloride. But it is sufficient to convince us that, under the cover of these bacterial monstrosities, are proceeding reproductive events of which we have, as yet, slight cognizance. Of one point, however, we may be assured. These peculiar forms are not "pathological" nor evidences of degeneration. They have been termed "involution forms"; but, as Mellon has suggested, "evolution forms" would be more appropriate. The details of their production and reproductive behavior constitute at present one of the most important problems in microbic dissociation. It seems possible that in their action is hidden the problem of the filtrable forms of bacteria, if not of the filtrable viruses; also perhaps the mystery of the bacteriophage.

Just as the morphology of the bacterial cell is correlated with the type of culture, so also are correlated motility, capsule formation, and perhaps spore formation. The time is past when we can state with discretion that such and such a bacterial "species" is motile, for both motility and flagellar equipment depend on the cyclostage. Up to the present time, observations seem to indicate that, if an organism shows motility, it belongs to the $\mathrm{S}$ type as opposed to the $\mathrm{O}$ or $\mathrm{R}$, which are commonly non-motile. Ark- 
wright, ${ }^{\mathrm{r}}$ however, has presented certain exceptions. Of this fact we now have evidence in members of the intestinal group, in B. subtilis, B. proteus, and several other species. Motility of a culture therefore loses all significance for species differentiation in systematic bacteriology, unless we can succeed in recoghizing the cyclostage with which we are dealing.

With reference to bacterial capsules, the same situation exists as for motility. So far as we know at present, the capsule is the property of the organism of the S type, the $\mathrm{R}$ form being destitute. With reference to the anthrax bacillus, which presents certain other anomalies, the situation is not yet clear. The correlation is, however. now recognized for $B$. coli, the pneumococcus, Friedländer's bacillus, $M$. tetragenus and some Pasteurella forms. In none of these species does the presence or absenc of capsules possess significance for systematic bacteriology unless we can recogniz: the type of culture under examination.

In addition to the foregoing, microbic dissociation manifests itself in important biochemical and serological differences in the dissociated cultures; also, in a striking manner with certain problems relating to virulence and immunity. These interesting aspects of the subject cannot be discussed within the limits of the present contribution. ${ }^{2}$

\section{THE INCITANTS TO MICROBIC DISSOCIATION}

AND THE PROBLEM OF REVERSION

Although dissociative reactions must be regarded as occurring to some degree in all bacterial cultures, probably beginning in the earliest hours of colony life, and although in some cases they attain spontaneously such a magnitude that they attract the notice of the alert investigator, fortunately, in the study of this phenomenon, we are not dependent upon cultural material showing such spontaneous transformations. The reaction may easily be "forced" as a result of bringing to bear on the young, growing culture certain extraneous influences; and the nature of these influences may be diverse.

Probably the first influence to attract attention was aging, as first pointed out by Firtsch for the spirillum of Finkler-Prior in I888. Most of Baerthlein's colony variations, as also those of Eisenberg, were consequent to aging in broth or on agar, and to the use of alkaline media. Under these conditions, one may often discover in the transition from the $\mathrm{S}$ to the $\mathrm{R}$ forms the presence of the intermediate or transitional $\mathrm{O}$, as clearly depicted by Firtsch. Eisenberg, and many others. Other conditions of cultural growth favoring the reaction, with the subsequent generation of the $\mathrm{O}$ or $\mathrm{R}$ forms of culture, include the following: changes in temperature, various food substances, starvation, the physical state of the medium (solid or liquid), the presence or absence of oxygen, the presence of antiseptic substances or dyes. the reaction of the medium, the volume of the medium, microbic associations, passage through animals, the influence of various kinds of normal animal blood or tissues, normal sera or ascitic fluid, specific immune blood or sera, body excretions or secretions, and finally the metabolic growth products of the same or other bacterial species. To these may be added the influence of the bacteriophage which, in last analysis, is the reagent par excellence for enforcing dissociation upon the sensitive, or even on the partially resistant, culture. Indeed, only one other influence approaches it in degree or speed of action-and that is homologous immune serum, as amply demonstrated by many experiments.

See Hadley, Philip: loc. cil.

${ }^{2}$ See chap. xlii 
Of the influence of these various incitants, only a few brief statements may here be made. First, dissociation cannot occur unless growth occurs; cultures in a state of suspended growth, while still alive, remain fixed in the type in which growth last occurred. This is not necessarily true of old cultures in which growth still takes place slowly. This circumstance reminds us of the conditions limiting the action and regeneration of the bacteriophage. Dissociation, moreover, occurs most freely in liquid media, and most actively at a reaction point of $\mathrm{pH}$ 7.8-8.0. This, it may be noted, is also the optimum reaction for bacterial autolysis in most species. In cultures frequently transferred on favorable solid media, dissociation is more restricted and may even appear to be absent. When dissociation has occurred, and the $\mathrm{R}$ type has once been produced, this type of culture is more stable than the $\mathrm{S}$, and much more stable than the $\mathrm{O}$, both on solid and in liquid media. The presence of phenol, pancreatin or lithium chloride, and other chemical substances, favors the transformation of the $\mathrm{S}$ type to the $\mathrm{O}$ type culture in some species.

The influence of blood serum is of special interest. Sometimes a normal serum of certain species will force the reaction. This is likely to concern the serum of animals which are not susceptible to the organism, or sera supposed to be germicidal. As mentioned above, however, the strongest dissociation-furthering power is possessed by homologous immune serum. This can be demonstrated by growing the $\mathrm{S}$ type culture for a few generations in broth containing about ro per cent of the immune serum, the result being the formation of the $\mathrm{R}$ type culture. This has been demonstrated for the pneumococcus, the streptococcus, B. subtilis, Friedländer's bacillus, B. typhosus, B. paratyphosus $\mathrm{B}\left(\right.$ Soule $\left.^{\mathrm{I}}\right), B$. coli $\left(\right.$ Dulane $\left.^{\mathrm{I}}\right)$, and some other forms. There is some evidence that the same reaction occurs in vivo as well as in vitro; and this suggests the possibility that the chief mechanism of protection of the bacteriotropic antibodies may represent merely the enforcement in the body of a dissociation of the invading organisms, comparable to that observed in the culture tube. This possibility, which was first approached by the researches of Griffith on the dissociation of the pneumococcus in I923, I have considered at greater length in an earlier publication. With reference to the effects of immune sera on the $\mathrm{R}$ and $\mathrm{S}$ culture types, it should be added here that it has been shown for $B$. subtilis by Soule, ${ }^{2}$ for $B$. coli by Dulaney, for the pneumococcus more recently by Avery, and for $B$. paratyphosus $\mathrm{B}$ by Soule, that, when the $\mathrm{R}$ type culture is grown in serum immune to this culture form, a retransformation is enforced to the original S.

When one observes that the dissociative reaction can be precipitated by such diverse substances or conditions of growth as those mentioned above, the question of the actual cause of dissociation seems to be as far removed as at the beginning. But the situation may be simplified if we can discover a common factor among all of these influences. It seems safe to say that there exists such a common factor, and that it may be defined as any condition or substance that is antagonistic to the continued growth of the so-called "normal" culture type. That this factor may not be the same for all bacterial species is a view that we may well accept, but that the same influence will operate in the same manner on different members of the same group of bacteria seems well demonstrated by numerous observations.

\footnotetext{
${ }^{I}$ Personal communication.

${ }^{2}$ Soule, M. S.: loc. cit.
} 
Regarding the permanence of the dissociates, with their newly acquired characters - it has often been stated that they are irreversible; and, for this reason, they have often been described in the literature as mutations. This interpretation has concerned the $\mathrm{O}$ forms much less often than the $\mathrm{R}$, for the former are notably unstable. Even the R types represent a series in which the degree of stability is very variable. It seems to depend, in part, upon the length of time that the $\mathrm{R}$ culture has been propagated continuously under the conditions that gave rise to it. The longer the stimulus is applied, the more stable the type seems to become. There are, however, some exceptions to this. Some R type pneumococcus cultures are apparently irreversible; and the same is true of some $\mathrm{R}$ type Friedländer strains, according to Julianelle. ${ }^{\mathrm{I}}$ The $\mathrm{R}$ form of B. paratyphosus, B. typhosus, B. suipestifer, B. dysenteriae, Bact. lepisepticum, $B$. diphtheriae, $B$. subtilis, and $B$. anthracis have been found highly stable, and have sometimes been reported as permanent variations. But Jordan, ${ }^{2}$ by a special method of cultivation, succeeded in causing the reversion of his $\mathrm{R}$ type of $B$. paratyphosus $\mathrm{B}$; and Soule, by growth of his $\mathrm{R}$ type of $B$. subtilis in an $\mathrm{R}$ immune serum, was finally able to cause a return to the $\mathrm{S}$ type of culture. Later he was able to cause the reversion of an $\mathrm{R}$ type of $B$. paratyphosus $\mathrm{B}$ by similar measures. Mellon ${ }^{3}$ has emphasized the stability of variants of his diphtheroid forms, and I have been unable to effect the retransformation of an $\mathrm{R}$ type of $B$. pyocyaneus even after four years, although $\mathrm{I}$ have not tried the action of $\mathrm{R}$ immune serum. Apparently, the $\mathrm{R}$ forms of culture may remain stable for many years, but I believe there is no justification for the conclusion that they represent hereditary variations or mutations in the strict meaning of the term. It is more likely to mean that we have not yet been able to discover the adequate means for causing their return to the original form of culture.

\section{BIOLOGICAL SIGNIFICANCE OF DISSOCIATION}

In concluding this chapter the question arises, What is the deeper meaning of these phenomena concerned with the separation of bacterial cultures into distinct components whose nature and behavior we have now briefly reviewed? It can mean only one thing: that those living cells that we have commonly regarded in past years as among the simplest of plant forms, and characterized by a correspondingly simple reproductive apparatus, possess in reality a highly complex genetic mechanism, which enables them to reveal, in cultures, pictures of morphological and physiological diversity with which our old and limited notions of "reproduction by simple fission" are utterly unable to deal. Although we may not yet be justified in accepting Enderlein's view of actual sexual reproduction among the bacteria, we must accept the fact that the nuclear equipment and reproductive behavior of bacteria are highly complicated matters. We can no longer doubt that the hereditary mechanism in bacterial cells makes provision for amphimixis, so long denied to these forms; nor can we hesitate in accepting phenomena of gonidia formation, zygospore formation, and perhaps a kind of budding, as common methods of bacterial reproduction. In all of these matters bacteriologists as a class have combined in denying the existence of things that they have not been willing to take the trouble to search for.

'Julianelle, L. A.: loc. cit.

${ }^{2}$ See Hadley, Philip: loc. cit.

${ }^{3}$ Mellon, R. R.: J. Med. Resetrch, 42, 61. 1920. 
The acknowledgment of the existence of definite cyclostages in the development of the bacterial culture naturally concerns our appraisal of the thing that has passed as the "normal" bacterial type. The observations already presented dealing with this subject can lead us only to the view that the older notions of normality and immutability of culture type have determined a highly repressive and dangerous influence on the development of bacteriology - an influence which, even at the time of writing, is still menacing the progress of the science. The present conceptions of "normal type," "normal colony," and "normal" cytology we owe to the influence of monomorphism which, even in most recent textbooks, still clings like a barnacle to modern bacteriology. According to its dictates, whatever departs from the "normal" must be regarded as an "involution form," a degeneration form, a mutant, or a contamination. On the other hand, whatever form of culture the bacteriologist succeeds in causing to develop most freely in his carefully standardized media, and under other standardized conditions of growth which he imposes, are "normal" cultures, while aberrant forms are of little consequence. In order that there may be no lack of means for making the species recognizable, we assiduously fill out data on neatly designed descriptive charts - all dealing with the "normal" type, usually the S. Are we, then, forced to the conclusion that cultures of the $\mathrm{O}$ and $\mathrm{R}$ forms are not normal cultures?

It is indeed time that we revised our notions on "normality" in bacterial species. The results of many studies dealing intentionally or unintentionally with microbic dissociation force the conclusion that there is no such thing as "normal" type in the usual meaning of the term. The $\mathrm{O}$ type cultures are no less normal than the $\mathrm{S}$; nor the $\mathrm{R}$ type cultures less normal than the $\mathrm{O}$. They are all normal, and can be regarded in no other light than that of isolated states or stages of the bacterial species in its progress through the cyclode. And it may be said in passing that this view may no doubt be held equally for the filtrable forms of bacteria. These have often been referred to as "fragments," or as minute "fractions," of the "normal" cell, and endowed with the power of "regeneration" into the original form. We shall perhaps do well to question whether these minute living elements may not represent one or more definite units (cyclostages) in the reproductive history of the species. From this viewpoint, whether we deal with filtrable or non-filtrable forms, it is essential that we should cease to regard "normality" in the old, absolute sense, but should come to regard the characters of bacteria as related to definite stages in their development. Thus we may have a normal growth of $B$. typhosus on plain agar, or on phenol agar, or at $42^{\circ} \mathrm{C}$, or in immune serum, or under reduced oxygen tension. The growth is normal with reference to a certain condition or environment, although it is likely to differ in each of the conditions mentioned above. That "pathological" growih forms may occur cannot be doubted; but at present we are not in a position to recognize them-any more than we are in a position to recognize bacterial mutations, so called-until we have gained a fundamental knowledge of the nature, limits, and sequence of cyclogenic variation. 


\title{
CHAPTER VIII
}

\section{BACTERIAL ASSOCIATIONS}

\author{
W. L. HOLMAN \\ University of 'Toronto
}

\section{INTRODUCTION}

Bacterial association has taken in recent years a much more important place in bacteriological studies than formerly. This increased interest is largely due to the greater attention which is being given to the finer metabolism of bacteria and the interactions which occur between the bacteria and their environment. The idea long held by many that bacteria represent the lowest forms of life and are therefore comparatively simple in their metabolic activity has been replaced by a realization that we are dealing with just as highly specialized and complicated functional activities as in any of the so-called "higher" plants or animals. It is true that we are dealing with unicellular forms of very small size and that these as suggested by Kendall ${ }^{\mathrm{r}}$ should be considered as similar to living colloids in which surface phenomena are so important. Many of the activities in the life of the bacteria can be appreciated better if this complicated metabolism is kept in mind, and the study of mixed or double cultures helps in an understanding of the actual life-processes.

\section{NOMENCLATURE}

It is generally recognized but often forgotten that under natural conditions mixed cultures are the rule, and the earliest work on bacterial associations is to be found largely in the studies which attempted to analyze such natural phenomena. The most variable results may be obtained in these mixed cultures, and there are many factors taking part which determine the final outcome. There may be simple mixtures with no demonstrable effect of one bacterium on another, but this is uncommon since one or the other usually dominates the picture. One microbe may favor the growth and activity of another or both may be benefited by the combination. The latter condition is usually spoken of as "symbiosis," but true examples of this relationship are rare. The term "metabiosis" is sometimes used where one action follows another, and is well illustrated in innumerable examples in nature. Antagonism or antibiosis is often combined with the foregoing, but is mostly employed for the occurrences where there is a clearly demonstrable harmful effect of one micro-organism on another or when a characteristic product fails to be formed or disappears in the mixed culture. Be cause of the impossibility in many cases of determining the actual processes at work these terms must be used with reservations. It is better, I believe, to use the more general word "association" for all these phenomena and "synergism," introduced into bacteriological nomenclature by Kämmerer, ${ }^{2}$ for those in which definite changes are

${ }^{x}$ Kendall, A. I.: Colloid Symposium MIonograph. 2, 195. 1925.

${ }^{2}$ Kämmerer, H.: Klin. U' ${ }^{\prime} h$ hnschr., 2, I1 53. 1923; Dculsches Arch. f. klin. Med., 141, 318. 1923 : ibid., 145, 257. 1924; Klin. Wchnschr., 3, 723. 1924. 
demonstrable which indicate or suggest the combined work of two or more microorganisms. Zoeller ${ }^{1}$ has used "cumulative cultures" to express the results obtained by him in certain biological combinations. Synergism may be conveniently qualified, when one or the other result dominates, into an "antagonistic synergism" and a "beneficent synergism." "Antagonism" alone should probably be retained for outstanding examples of one-sided harmful effect.

\section{GENERAL CONSIDERATIONS}

There are numerous examples of bacterial associations in every field of bacteriology. In man and animals natural infection with more than one bacterium is relatively frequent, and the particular combinations which may occur often determine the course of the disease. There is an enormous literature on this phase of the subject but no very definite conclusions have been drawn. It would be futile to go into the problems of tuberculosis and secondary infections, or those of typhoid fever, gonorrhea, influenza, and many other diseases. Certain phases of these I shall briefly discuss, but this article will deal largely with some of the outstanding phenomena studied experimentally, and I shall not confine myself to the pathogenic bacteria although the greater amount of work has been done on them.

\section{EARLY EXAMPLES OF ASSOCIATION}

Among the early observations we find the recognition by Pasteur $^{2}$ of the harmful effect of "wild" yeast on the normal fermentation processes in the beer and wine industries. He further noted the beneficial effect of aerobic forms which developing a scum on the surface, and using up the oxygen, favored anaerobic growth. Winogradsky ${ }^{3}$ isolated an aerobe which only fixed nitrogen from the air in the presence of other bacteria. Burri and Stutzer ${ }^{4}$ demonstrated that horse feces sp'it nitrate with the production of free nitrogen. He isolated from the feces $B$. coli communis and a strict aerobe, and these two in combination gave the same result. The $B$. coli could be replaced by B. typhosus, and therefore it was the strict aerobe which gave the actual gas production. Previous to this, Marshall Ward $^{5}$ described a yeast and a bacterium which together formed a ginger beer-like product in a saccharine fluid.

\section{ANAEROBES}

Nencki ${ }^{6}$ reported, with a double culture of $B$. paralactici and $B$. chauvoe $i$ the formation from glucose of normal butyl alcohol, a substance not produced by either culture alone. He believed his results might help to clarify certain difficulties in obtaining infections in animals with single pure cultures. Novy ${ }^{7}$ quoted Roger (1889) as the first to show that $B$. prodigiosus added to the bacillus of malignant edema ren-

I Zoeller, C.: Compt. rend. Soc. de biol., 92, 435, 497, 686. I925.

${ }^{2}$ Pasteur, L.: Ocuvres de Pasteur (réunies par P. Vallery-Radot). Paris, I922.

3 Winogradsky, S.: Compt. rend. Acad. de sc., I18, 353. I894.

${ }_{4}$ Burri, R. and Stutzer, A.: Centralbl. $f$. Bakteriol., I, Orig., I6, 8I4. 1894.

5 Ward, Marshall: Phil. Tr. Roy. Soc., B, 187, I 25. London, 1892.

${ }^{6}$ Nencki, M.: Centralbl. f. Bakteriol., I, Orig., I 1, 225. I892.

7 Novy, F. G.: Zischr.f. Hyg. u. Infektionskrankh., 17, 209. I894. 
dered sublethal doses of this anaerobe fatal for rabbits, and he himself found that the injection into guinea pigs of $B$. proteus and his new anaerobe ( $B$. oedematiens) resulted in rapid death and an enormous growth of the anaerobe in the animal body. The overgrowth was absent with pure cultures. He further was able, by adding B. proteus and other aerobes, to grow his anaerobe in the presence of air. Passing over numerous similar results we find Sturges ${ }^{\mathrm{I}}$ devising a method, based on bacterial association, for isolating spore-bearing anaerobes on open plates by growing them with $B$. coli or Staphylococcus aureus. Rhein ${ }^{2}$ used $B$. fecalis alcaligenes in bouillon for anaerobic growth because of the lack of saccharolytic and proteolytic activity in this aerobe. Inoculation of the mixed cultures into animals he considered practical because the aerobe is not toxic, but I believe from the work of many others that this procedure might well give faulty results. Barrieu ${ }^{3}$ noted that $B$. proteus and certain non-pathogenic spore-bearing aerobes found in wounds exalted, by their proteolytic activity, the virulence of pathogenic bacteria. Pringsheim ${ }^{4}$ grew Fränkel's bacillus (B. welchii) with $B$. fccalis alcaligenes for ten transfers on agar slants and could see in the growth of the latter the opaque colonies of the anaerobe. A liquefying sarcina allowed $B$. wclchii and B. butyricus to grow in open tubes. After six days' growth the sarcina had disappeared from the $B$. butyricus culture, and he suggested this as an easy method to obtain a pure culture. Weinberg and Otelescos ${ }^{5}$ considered that many war-wound infections, looked upon as of pure anaerobic origin, may be due to an association with $B$. proteus since this latter organism increased the virulence of $B$. perfringens, $V$. septique, and others. Animals injected with $B$. sporogenes and $B$. proteus did not develop putrid lesions. This combined growth of aerobes and anaerobes on surface cultures I observed on a number of occasions in France while studying the bacterial flora of war wounds. Colonies picked from aerobic plates were not infrequently found to be mixed with anaerobes.

Stillman and Bourn ${ }^{6}$ reported in 1920 the production of gas by 16 of 1 in nonhemolytic strains of $B$. influenwae in I per cent dextrose agar with a little blood extract. Four of 29 hemolytic strains also produced gas. Jordan and Reith 7 also found gas production in certain of their strains. About four years ago, when working with cultures of a tiny anaerobe resembling B. pneumosintes ${ }^{8}$ (probably Staphylococcus parvulus of Veillon and Zuber), I mixed a culture of this anacrobe with a culture of $B$. influcnzae and planted the mixture on blood-agar slants. A good growth of $B$. influenzac occurred, and after five transfers I had no difficulty in recovering the gasproducing anaerobe in cooked-meat media. This anaerobe is practically always present in the oral cavity, and could easily contaminate cultures of $B$. influenzae. It can be

\footnotetext{
i Sturges, Jr., W. S.: Abstr. Bact., I, 63. I9 17.

${ }^{2}$ Rhein, M.: Pressc méd., 27, 504. 1919.

3 Barrieu, A. R.: ibid., 28, 40. I920.

4 Pringsheim, E. G.: Centralb!.f. Baktcriol., II, 51, 72. I920.

5 Weinberg, M. and Otelesco, I.: Compt. rcnd. Soc. de biol., 84, 535. 192 I.

${ }^{6}$ Stillman, E. G., and Bourn, J. M.: J. Expcr. Mcd., 32, 665. I920.

${ }^{7}$ Jordan, E. O., and Reith, A. F.: J. Infect. Dis., 34, 239. I924.

${ }^{8}$ Holman, W. L.: Am. J. IIyg., 3, 487. 1923.
} 
recovered from high dilutions of the saliva, as Hall and Wing ${ }^{\mathrm{r}}$ have shown, and because of its morphological resemblance to forms of $B$. influenzae might very well be overlooked. I would suggest this as a possible explanation of the rather infrequent observation of gas production by $B$. influenzae.

The group of B. botulinus and the effect of associated bacteria particularly on its toxin has received considerable attention. Hall and Peterson ${ }^{2}$ found that certain acidproducing aerobes inhibited toxin production in glucose but not in non-carbohydrate media, and some of these aerobes actually destroyed toxin in glucose broth. It would appear that the acid must be in the nascent state since acid itself was ineffective. Jordan and Dack $^{-3}$ found that a mixture of a large amount of $B$. sporogenes with $B$. botulinus interfered with the development of toxin and might cause its early disappearance. Francillon ${ }^{4}$ studied the same problem. He found that Staphylococcus aureus, $B$. coli, B. proteus vulgaris, and other bacteria permitted the growth of B. botulinus in open tubes of plain and glucose bouillon, but the growth was never as good as under other anaerobic conditions. A moist-meat medium gave somewhat better growth. Toxin was found in the mixed cultures in bouillon and meat, the amount varying with the aerobe. The $B$. pyrcyancus mixture gave no toxin in the bouillon but a strong one from the meat. There was but little effect on the toxin by two weeks' contact with $B$. proteus, $B$. coli, or $B$. pyocyaneus. Dack ${ }^{5}$ reported the gradual destruction of filtered toxin by the growth of $B$. sporogenes and other proteolytic and non-proteolytic anaerobes.

Passini ${ }^{6}$ found that a putrefactive anaerobe $B$. putrificus verrucosus destroyed $B$. tuberculosis in nine days. The effect of similar anaerobes on the survival of anthrax spores in dead animals has been extensively studied. Among a great many other interesting anaerobic and aerobic synergistic phenomena I mention a few. Omeliansky $y^{7}$ studied the fixation of atmospheric nitrogen as Winogradsk ${ }^{8}$ had done years before. He noted that in the surface layers of the soil numerous organisms used the oxygen and created anaerobic conditions for the $B$. clostridium pasteurianum, but in addition some of these accompanying forms also supplied carbon compounds for the anaerobe. The Azotcbacter being alkaligenic used up such products from the anaerobe as butyric acid and thus favored the synergistic process. The other aerobes may at times do harm by depriving the Azotobacter of oxygen. These two nitrogen-fixing forms, one aerobic, the other anaerobic, worked very well together. The work of Kämmerer ${ }^{9}$ and his associates gave interesting examples of synergistic action. They observed that emulsions of human feces reduced pure bilirubin and mesobilirubin to urobilin but had noaction on biliverdin and that the feces of herbivora did not have this action

${ }^{x}$ Hall, I. C., and Wing, H. U.: Am. J. Pub. IIealth, I5, 770. 1925.

${ }^{2}$ Hall, I. C, and Peterson, E.: J. Bact., 8, 319. 1923.

${ }^{3}$ Jordan, E. O., and Dack, G. M.: J. Infect. Dis., 35, 576. 1924.

4 Francillon, M.: Arch.f. Hyg., 95, г 2 I. 1925.

5 Dack, G. M.: J. Infect. Dis., 38, I65. 1926.

${ }^{6}$ Passini, F.: Centralbl.f. Bakteriol., I, 81, 447. 1926. (Ré́.)

7 Omeliansky, V. L.: Arch. de sc. biol., 18, I. I91 5.

${ }^{8}$ Winogradsky, S.: loc. cit.

9 Kämmerer, H.: loc. cit.

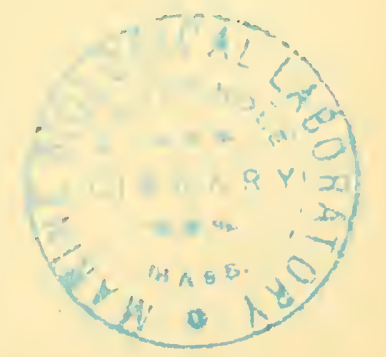


because of its active fermentation. Filtrates had no effect. These changes they believed were due to a synergism between $B$. putrificus and certain aerobes. $B$. coli either helped the bilirubin production or hindered it, depending on the presence or absence of fermentable material. They further demonstrated the development of hematoporphyrin from blood by a similar synergism and that sugar or bile inhibited it. A particularly important instance of bacterial association was reported by Speakman and Phillips. ${ }^{1}$ During the war, acetone and butyl alcohol were produced on a large scale by fermentation of cereals and carbohydrates. Serious difficulties developed in the plants, owing to the contamination of the cultures of $B$. gramulobacter-pectinovorum by the aerobic bacillus $B$. volutans. The acetone yield, as a result of the mixed culture, dropped or disappeared and the development of lactic acid increased. This increase was due to an altered metabolism of the acetone producer so that it formed more lactic acid and less acetone. The resul ts varied with the relative numbers of each organism present. They considered it due to an inhibitory substance from the nitrogen metabolism. In actinomycotic granules there is found a bacterium named by Klinger ${ }^{2} B$. actinomycetum comitans, the presence of which was confirmed by Colebrook $^{3}$ in 80 per cent of his twenty cases. The significance of this associate is not known, but Colebrook suggested a possible genetic relationship. There are many other examples of anaerobic and aerobic associations in the natural metabolism of the sulphur bacteria; in silage fermentation, the heat of which was definitely shown by Hunter ${ }^{4}$ to be due to bacterial action and not to cell respiration; in sewage decomposition and cellulose destruction in which Groenewege ${ }^{5}$ believed a symbiosis occurred but stressed the action of the aerobes and Khouvine ${ }^{6}$ gave chief importance to a strict anaerobe discovered by him, B. cellulosae dissolvens (n.sp.), but pointed out that five times as much cellulose was destroyed when in association as when alone. There are other examples in the natural breakdown of organic materials of all kind; and in innumerable other bacterial activities in nature.

\section{THE ACIDURIC GROUP (AND CERTAIN THERAPEUTIC USES)}

The effects of the aciduric group of bacteria on other bacteria has been closely studied. They are usually facultative aerobes, and their action is considered as chiefly antagonistic. Because of their use for therapeutic purposes there has collected an extensive literature brought together by Rettger and Cheplin ${ }^{7}$ and Kopeloff. ${ }^{8}$ Certain points, however, may be briefly reviewed. Starting from the work of Metchnikoff with $B$. bulgaricus it was soon found that this organism could not be implanted in the intestinal tract and $B$. acidophilus, a normal inhabitant, was substituted. B. bifidus

${ }^{x}$ Speakman, H. B., and Phillips, J. F.: J. Bact., 9, I83. 1924.

${ }^{2}$ Klinger, R.: Centralbl. f. Bakteriol., I, Orig., 62, I9г. I91 2.

3 Colebrook, L.: Brit. J. Exper. Path., 1, 107. 1920.

4 Hunter, O. W.: J. Agric. Research, 10, 75. 1917.

5 Groenewege, J.: reference in J.A.M.A., 76, 279. $192 \mathrm{I}$.

${ }^{6}$ Khouvine, Y.: Ann. de l'Inst. Pastcur, 37, 7 I I. I923.

7 Rettger, L. F., and Cheplin, H. A.: The Intestinal Flora with Special Reference to the Implantation of "Bacillus acidophilus." Yale University I'ress, I92 I.

${ }^{8}$ Kopeloff, N.: "Lactobacillus acidophilus." Williams \& Wilkins Co., I926. 
found in the intestines of breast-fed infants, is responsible for inhibition of the growth of other bacteria, and its acid products are thought to be mild stimulants to the bowel walls. In the vagina, $B$. doederleini is believed to keep the reaction acid, thus inhibiting the growth of contaminating bacteria. Landau ${ }^{1}$ was the first to use fresh beer yeast in the treatment of leucorrhea and considered the anticatarrhal action was due to mechanical overgrowth, the using up of food material and the action of metabolic products in injuring or destroying other bacteria, neutralizing the toxins and changing the reaction to acid. He suggested injecting cultures of this yeast into the bladder in cases of cystitis with alkaline urine. Since this work there have been many suggestions and a variety of bacteria used to obtain such biological inhibitory action. The antagonistic action of $B$. acidophilus has been abundantly proved against putrefactive anaerobes, $B$. coli, and many other bacteria. Schiller, ${ }^{2}$ with a strain of $B$. acidophilus from a dog, found that it rapidly destroyed and dissolved many strains of streptococci in fluid media and suggested this as a useful way to obtain bacteriolysis of cocci. He showed that this action was not due to lactic acid since it occurred in alkaline media and filtrates from a glucose broth culture living or killed (by heat or age) allowed a good growth of the streptococcus. The harmful substance was only formed by the $B$. acidophilus in the presence of the streptococcus or its products. Filtrates of the mixed culture, after thirty-six hours at $37^{\circ} \mathrm{C}$. (when the streptococci are killed), were as toxic as when living cultures of the bacilli were used. Streptococcus cultures killed by heat or age had no toxic effect on living streptococci. He considered the phenomenon an example of induced antagonism and reported other examples in a series of four articles. ${ }^{3}$ In the first he used $B$. mesentericus and forced on it an antagonistic action against streptococci by growing it with the latter in a medium of poor-food value. It secreted a bacteriolytic substance which digested the living bacteria as it would any other insoluble albuminous material, and the amount depended on the number of sensitive streptococci present. It also acted when the $B$. mesentericus had been removed by centrifugation and after evaporation and drying. It was not completely specific. Schiller further showed that yeasts can be made antagonistic against bacteria including $B$. tuberculosis if the medium contained sugars but lacked nitrogenous materials. They acted in the same way as the foregoing, and a more active bacteriolytic substance was secreted in the presence of more resistant forms so that the enzyme induced by $B$. tuberculosis was even capable of attacking beeswax. The reverse was also found. Bacteria (staphylococcus, B. typhosus, B. paratyphosus, et al.) became antagonistic to yeasts in nitrogen-free media, and the secreted cytolytic substance was similar to the foregoing but had no effect on coagulated serum or egg albumin. This method of dissolving the yeast membrane he thought might be of interest in the study of zymase.

Donaldson ${ }^{4}$ during the war used a strain of $B$. sporogenes in the treatment of slowly healing war wounds. The beneficial effects he ascribed, not to direct inhibition, but to the removal by the proteolytic anaerobe of the dead tissue (the pabulum for the

I Landau, T.: Deutsche med. Wchnschr., 25, I 7 I. I 899.

${ }^{2}$ Schiller, I.: Centralbl.f. Bakteriol., I, Orig., 73, I23. I9I4.

${ }^{3}$ Schiller, I.: ibid., 91, 68. I924; 92, I24. I924; 94, 64. I925; 96, 54, I925.

4 Donaldson, R.: J. Path. \&o Bact., 22, 129.1918. 
pathogens in the wound), and, itself producing no harmful products, it further hydrolyzed the toxic bacterial products present or being formed. Bumm ${ }^{\mathrm{I}}$ used a durable preparation brought out by Zeissler called "neocolysin." It was made up of living, albuminolytic bacteria and gave good results in chronic purulent conditions such as osteomyelitis. The bacteria were supposed to function as in Donaldson's method and continued growing as long as there was dead tissue available. Gratia and Dath ${ }^{2}$ discovered an aerobic streptothrix which had a powerful destructive action on a variety of bacteria. It did not act on B. tuberculosis and showed no lipolytic enzyme. Filtrates were equally effective, could act without free oxygen, but were somewhat variable. The active substance was better developed in old cultures and was fairly stable. The dissolved bacteria caused specific response when used for vaccination. They referred to a similar organism reported by Lieske in $\mathrm{I} 92 \mathrm{I}$, but his studies were confined to solid media. Rosenthal ${ }^{3}$ worked with an organism apparently very much like the foregoing, and found it was antagonistic to many bacteria including $B$. diphtheriac. He referred to the report of Gasperini of 1890 on a similar form acting against bacteria. Rosenthal and his associates ${ }^{4}$ found that it could be implanted in the intestines of guinea pigs and that when injected parenterally it was enterotropic. Much and Sartorius ${ }^{5}$ used a strain of $B$. mycoides and showed similar effects, by culture and filtrates, on many bacteria and that the dissolved bacteria had not lost their antigenic properties. A very interesting study by Gratia and Rhodes ${ }^{6}$ proved that living staphylococcus could live on killed suspensions of staphylococci in saline or in saline agar made cloudy with killed staphylococci. Thus we see that bacteria can and do remetabolize their own substances or that of other bacteria, and this helps in the understanding of the antagonistic action of many forms of bacteria. They are indeed cannibalistic.

\section{DIPHTHERIA GROUP}

Because of the pressing problem in carriers of the diphtheria bacillus, special attention has been given to researches for a possible biological method, through bacterial antagonism, which would be effective in treating these cases. Streptococci and $B$. diphtheriae have long been considered mutually helpful in producing severe infections in the throat. There is an extensive literature on this topic. Roux and Yersin, ${ }^{7}$ in studying the problem of the return of virulence in attenuated cultures of $B$. diphtheriae, were successful in accomplishing this by injecting the attenuated culture along with a non-fatal dose of an erysipelas strain of streptococcus. The virulence returned, and it was retained on successive cultures. They therefore warned against the use of Streptococcus erysipelatos to combat diphtheria as had been suggested by

I Bumm: Arch.f. klin. Chir., 138, II I. 1925.

${ }^{2}$ Gratia, A., and Dath, S.: Compl. rend. Soc. de biol., 91, 1442. 1924; 92, 46I, I I25. I925; 93, 451. 1925; 94, 1267. 1926.

\footnotetext{
3 Rosenthal, L.: ilid., 93, 77. 1925.

${ }^{4}$ Rosenthal, L.: itid., 94, 309, 1059, I926; 95, 10. 1926.

5 Much, H., and Sartorius, F.: Mcd. Klin., 20, 347. I924.

${ }^{6}$ Gratia, A., and Rhodes, B.: Compt. rend. Soc. de biol., 90, 640. I924.

7 Roux, E., and Yersin, A: Ann. de l'Inst. Pasteur, 4, 385. I89o.
} 
Babtchinski. Similar results were obtained by Barbier ${ }^{1}$ and Schreider. ${ }^{2}$ Funck $^{3}$ found the fact to be true but did not consider it as striking as had previous workers, and showed that the presence of the streptococci in no way affected the specific action of the diphtheria toxin. Kleint also showed that streptococci enhanced the effect of $B$. diphtheriae. Arnold ${ }^{5}$ found little or no evidence of any increased virulence in the hemolytic streptococci isolated from diphtheria throats, but that there was a decided increase in hemolytic streptococci during diphtheria. These strains showed limiting II-ion concentrations like pathogenic strains, but he believed this change was merely environmental. Gaté, Papacostas, and Billa, ${ }^{6}$ although they found that filtrates of avirulent streptococci stimulated diphtheria-toxin production, reported that the increased virulence was not retained on further transfers. Zoeller ${ }^{7}$ showed it was possible to produce in his cumulative cultures a diphtheria-streptococcus altero-toxin by growing a scarlet fever streptococcus in a diphtheria toxin to which had been added a little horse serum. Stovall, Scheid, and Nichols ${ }^{8}$ reported that the presence of staphylococcus in mixed cultures changed the morphology of virulent $B$. diphtheriae so that they stained more solidly and that the non-virulent pseudo-diphtheria strains became more beaded. Streptococci had no such effect.

The well-known overgrowth of B. diphtheriae by Staphylococcus aureus in cultures led many workers to try such cultures in patients following the report by Schiötz. ${ }^{9}$ Among these, Lorenz and Ravenel ${ }^{10}$ had good results in nine carriers and eight clinical cases of diphtheria although nasal furuncles developed in some of them. Rolleston ${ }^{\text {II }}$ found it helpful in ten carrier cases but ineffective in two cases of nasal infection. He considered it should only be used in chronic cases. There were also a number of unfavorable reports such as that of C. M. Davis ${ }^{12}$ who reported the development of tonsillitis following the use of the staphylococcus spray. Nicholson and Hogan ${ }^{\mathrm{x}}$ were encouraged by the results on nine acute cases, using sprays of $B$. bulgaricus and sour milk. Papacostas and Gatér4 studied the question of the antagonism between the pneumobacillus of Friedländer and $B$. diphtheriae following the observation that clinical cases of such mixed infections were usually mild. Mixed cultures of these two bacteria showed a progressive predominance of the former on serial transfers and the

× Barbier, H.: Centralbl.f. Bakteriol., I, Orig., I I, 382. I892. (Ref.)

${ }^{2}$ Schreider, M. von.: ibid., I2, 289. I892.

${ }^{3}$ Funck, E.: Ztschr.f. Hyg. u. Infektionskrankh., 17, 465. 1894.

4 Klein, E.: Thirty-third Ann. Rep. Loc. Gov. Bd., p. 431. I903-4.

${ }^{5}$ Arnold, L.: J. Lab. Eo Clin. Med., 8, 387, 389 . I923.

${ }^{6}$ Gaté, J., Papacostas, G., and Billa, M.: Compt. rend. Soc. de biol., 9o, 500. I924.

7 Zoeller, C.: loc. cit.

${ }^{8}$ Stovall, W. D., Scheid, E., and Nichols, M. S.: Am. J. Pub. Health, 13, 748. 1923.

9 Schiötz, A.: see Diphtheria, p. 367 . London: Medical Research Council, I923.

${ }^{10}$ Lorenz, W. F., and Ravenel, M. P: J.A.M.A., 59, 690. I9I2.

${ }^{11}$ Rolleston, J. D.: Brit. J. Child. Dis., 10, 298. 1913.

${ }^{12}$ Davis, C. M.: J.A.M.A., 6r, 393. т9г3.

${ }^{13}$ Nicholson, S. T., and Hogan, J. F.: ibid., 62, 510 . I914.

${ }^{14}$ Papacostas, G., and Gaté, J.: Compt. rend. Soc. de biol., 85, 859, 1о38. I92 I. 
morphology of the latter also changed toward a more homogeneous form on staining. By the use of filtrates of each culture they could not discover any evidence that the toxin of the former was able to neutralize diphtheria toxin in vivo or in vitro. If the two are grown together, however, no toxin is formed, nor is there any if the filtrate of the pneumobacillus growth is used to grow the $B$. diphtheriae. They suggested the therapeutic use of filtrates. In later studies on a larger number of clinical cases Gaté et al. ${ }^{\mathrm{I}}$ and Chalier, Gaté, and Grandmaison ${ }^{2}$ confirmed their impressions of the usual mild course of these mixed infections.

Van der Reis ${ }^{3}$, having demonstrated an antagonistic action of B. coli to B. diphtheriae, showed that it was possible, by spraying $B$. coli into the mouth, to have it colonize there. In nine cases it was still present after fifty-four days. A careful study of the antagonistic activity of $B$. col $i$ led him to conclude that there is formed a thermolabile, volatile, non-dialyzable, non-filterable, inhibitory substance not adsorbed by charcoal, not identical with the normal metabolic products of the colon bacillus, but that it may be a special toxic product. It was tried in acute cases of diphtheria by means of sprays of $B$. coli and particles of $B$. coli agar with the result that the $B$. diphtheriae disappeared more quickly than in controls. Carriers could also be rapidly freed of their bacilli. On the other hand, Pesch and Zschocke, ${ }^{4}$ although confirming the crowding out of the $B$. diphtheriae by $B$. coli in cultures, were unsuccessful in treating nasal carriers because the $B$. coli would not grow in the nose. Bloomfield ${ }^{5}$ failed in his attempts to implant Friedländer's bacillus from carriers to non-carriers, and even a foreign strain of the bacillus failed to establish itself in the throat of a carrier of another strain. Pringsheim ${ }^{6}$ studied the inhibiting effect of a strain of $B$. mesentericus vulgatus against a variety of bacteria but particularly against $B$. diphtheriae. $\mathrm{He}$ found that B.typhosus, B. paratyphosus A and B, B. fecalis alcaligenes, B. coli, and streptococcus were without effect on $B$. diphtheriae. $B$. pyocyaneus and an air staphylococcus were strongly inhibitive. Staphylococcus aureus was mildly stimulating as seen in larger colonies as was also a weakly sporing $B$. subtilis strain. On agar plates the effect of his $B$. mesentericus was to produce a circular zone of inhibition and just beyond this a ring of larger colonies. Filtered or heated cultures had no effect. Other proteolytic bacteria had no such action. It was tried on patients but the results were inconclusive. The findings of Zukerman and Minkewitsch ${ }^{7}$ with $B$. mesentericus vulgatus were somewhat different. The antagonism was inherent in the bacillus and was not increased by serial passage. It acted only on diphtheria and pseudo-diphtheria forms and not against a long list of other bacteria. Many other spore-bearers were either negative or but weakly active. Filtrates were very active, killing in four minutes, and were fairly heat resistant.

${ }^{1}$ Gaté, J., et al.: ibid., 86, 929. I922.

${ }^{2}$ Chalier, J., Gaté, J., and Grandmaison, L.: Paris méd., 61, 205.1926.

3 Van der Reiss: Mïnchen. med. Wchnschr., 68, 235. I921; Ztschr. f. d. ges. exper. Med., 30, I922.

4 Pesch, K., and Zschocke, O.: Mïnchen. med. Wchnschr., 69, I276. I922.

5 Bloomfield, A. L.: Johns Ilopkins Hosp. Bull., 32, 10. I92 I.

${ }^{6}$ Pringsheim, E. G.: loc, cit.

7 Zukerman, I., and Minkewitsch, I.: Centralbl.f. Bakicriol., I, 80, 483. I925-26. (Ref.) 


\section{PNEUMOCOCCUS}

The pneumococcus is usually considered a rather delicate organism in culture media, but apparently it may have a striking antagonistic effect on the staphylococcus. Gromakowsky ${ }^{1}$ discovered in eight sputum cultures a coccus which had a definite restraining action on the staphylococcus. This coccus resembled the pneumococcus in morphology, but he considered it different. Mixed with eight different strains of staphylococci and after twenty-four hours' incubation, transfers to agar gave no growth of the staphylococcus. It was irregular in action and also was antagonistic to streptococci from abscesses. Alivisatos, ${ }^{2}$ working with twenty-eight strains of well-identified pneumococci and three strains of Staphylococcus albus, noticed, when the forms were mixed, interesting phenomena on ascites agar plates. Curious clear zones appeared about the colonies of the pneumococci, and the edges of the staphylococcus colonies were irregular and suggested the action of bacteriophage. These zones varied in size, and if enough pneumococci had been added no growth of staphylococcus occurred. He never found mixed colonies. Neither the virulence nor the agglutinating type of the pneumococcus was related to the extent of the phenomena. There was no demonstrable change in the cultures of either of the bacteria after these contacts. Living, growing pneumococci were necessary and filtrates were negative. Eight strains of hemolytic, five of viridans, and one of mucosus streptococci gave negative results. In these cases mixed colonies were frequent, and he suggested that this characteristic might be used to differentiate pneumococci from closely similar streptococci.

\section{COLON-TYPHOID GROUP}

The antagonistic effect of soil bacteria against pathogenic forms has been extensively studied. The early work of Frost ${ }^{3}$ is important and includes the literature to that date. Limitation of space forbids a further discussion of this interesting subject. Fecal bacteriology, particularly of the colon-typhoid group, is replete with examples of supposed antagonism. It has long been held that the presence of slow lactose fermenting $B$. coli, so frequently observed in stool examinations, is due to this phenomenon (von Jeney ${ }^{4}$, and Henningson ${ }^{5}$ gave examples of inhibition of gas production and proposed the name $B$. coli anaerogenes for these. Prell ${ }^{6}$ and many others have studied such defective strains. Nissle, ${ }^{7}$ having observed an inhibitory action in certain stools seeded with $B$. typhosus, studied the antagonistic index of the $B$. coli to $B$. typhosus with various strains of the former. The difference seemed correlated with lactic acid production. The active coli strains also were inhibitory to other coli strains. He therefore gave these active cultures in capsules to persons carrying inefficient $B$. coli strains and reported good results. R. P. Smith ${ }^{8}$ found that B. coli

I Gromakowsky, D.: ibid., Orig., 32, 272. I902.

${ }^{2}$ Alivisatos, G. P.: ibid., 94, 66. I925.

${ }^{3}$ Frost, IV. D.: J. Infect. Dis., I, 599. I904.

${ }^{4}$ von Jeney, A.: Ztschr.f. IIyg. u. Infektionskrankh., 100, 47. I923.

5 Henningson, B.: ibid., 74, 253. I913.

${ }^{6}$ Prell, H.: Centralbl. f. Bakteriol., I, Orig., 80, 225. I9I7.

7 Nissle, R.: Deutsche med. Wchnschr., 42, I 8 1. I916.

${ }^{8}$ Smith, R. P.: J. Path. \& Bact., 26, I 22.1923. 
strains from carrier cases were more active against stock cultures of $B$. typhosus than were stock cultures of $B$. coli. Unfortunately, he did not test the $B$. typhosus from the carrier with its own $B$. coli, but the evidence suggested an inhibition because of the difficulty he had in obtaining the B. typhosus in these cases. Vignati ${ }^{1}$ described the reverse phenomenon in which fresh, actively growing cultures of B. typhosus inhibited the growth of $B$. coli, older cultures not being antagonistic. He explained the facts on Bail's theory of the spatial needs of each bacterium. Lisbonne and Carrère, ${ }^{2}$ by a method suggesting that of Schiller, ${ }^{3}$ forced a bacteriophage to develop by the antagonistic action of $B$. coli against the Shiga bacillus. They found at the end of a series of passages that an active and transmissible lytic principle was developed by what they call a "vitiation" in the metabolism of the Shiga bacillus. B. proteus Xig gave identical results. They considered that this is what occurs in the intestines where antagonistic conditions are always present. They later showed that this principle was not carried by the $B$. coli since the same strain was tested by Beckerich and Hauduroy who suggested such an explanation, and was not found to be lysogenic. It was definitely the result of microbial interactions. Fabry 4 also obtained a principle of the same kind through the antagonistic stimulus of a Staphylococcus albus on $B$. coli which also acted on the Shiga bacillus. Bordet ${ }^{5}$ reported a similar discovery with four primarily non-lytic strains of $B$. coli in which the lytic principle appeared spontaneously and was increased by passage. Gratia ${ }^{6}$ studied an example of antagonism between two races of $B$. coli as Nissle ${ }^{7}$ had shown from another point of view. Filtrates of $B$. coli $\mathrm{V}$. inhibited $B$. coli $\phi$ and caused an agglutinative culture of the latter in fluid media. The same results were obtained with living cultures on agar, and in both cases the secondary colonies were resistant to the action of $B$. col $i \mathrm{~V}$. It resembled the Gratia principle but was not regenerated by the $B$. coli $\phi$, being lost by the third passage, and did not act in high dilutions as bacteriophage does. On agar plates the area about the growth of $B$. coli $\mathrm{V}$. was inhibitive to the growth of $B$. coli $\phi$ but not to $B$. coli $\mathrm{V}$. It was therefore not a vaccination of the medium. It was very resistant to storage, chloroform, and high temperatures $\left(100^{\circ} \mathrm{C}\right.$. for thirty minutes). Bordet ${ }^{8}$ has carefully analyzed the various interactions between the bacteria giving the results that Lisbonne and Carrère ${ }^{9}$ reported, but as this is encroaching on the problem of bacteriophage which is to be presented elsewhere ${ }^{10}$ in this book, I need go no further.

\section{THE THEOBALD AND D. E. SMITH PHENOMENON}

A most interesting example of the inhibitory effect of bacteria in association was reported in 1920 by Theobald Smith and D. E. Smith. ${ }^{\text {II }}$ They found that B. para-

IVignati, J.: Compt. rend. Soc. de biol., 94, 209. 1926.

${ }^{2}$ Lisbonne, M., and Carrère, L.: ibid., 86, 569. I922; 87, I011. 1922; 90, 265. I924.

3 Schiller, I.: loc. cit.

4 Fabry, P.: ibid., 87, 369. 1922; 90, 109. 1924.

5 Bordet, J.: ibid., 90, 96 . I924.

${ }^{6}$ Gratia, A.: ibid., 93, I040. I925. 7 Nissle, R.: loc. cit.

${ }^{8}$ Bordet, J.: Compt. rend. Soc. dc biol., 93, I054. I925.

9 Lisbonne, M., and Carrère, L.: loc. cit.

${ }^{\text {ro }}$ Chapter $\mathrm{xl}$.

"Smith, T., and D. E.: J. Gencral Physiol., 3, 2 I. 1920. 
typhosus B, after it had grown in lactose bouillon for four to six days, prevented the development of gas by B. coli when this was added. Members of the closely related hog-cholera group had no such action in the given time, but after eighteen days' growth they also inhibited the gas production for the $B$. coli added at this time. A fuller analysis of this phenomenon is found in an article by Holman and Meekison.' Besson and De Lavergne ${ }^{2}$ confirmed the results of T. and D. E. Smith and found that $B$. aertrycki gave the reactions of the hog-cholera group. Brutsaer ${ }^{3}$ found the phenomenon most inconstant and variable even in repeated tests of the same strain of bacillus. A hog-cholera type-agglutinating culture inhibited, but as a rule members of this group did not. Moreover, the phenomenon failed if, instead of lactose bouillon, a lactose peptone water were used. He found it too irregular for use in classification. Von Jeney ${ }^{4}$ in studying this question used a bouillon previously freed from glucose by a twenty-four hour growth of beer yeast. (T. and D. E. Smith ${ }^{5}$ presumably used $B$. coli for this purpose.) Both of these procedures may have an important bearing on the results since the effect of these preliminary cultures may be very great as is seen in many of the articles reviewed above and in the studies of Robertson ${ }^{6}$ on food accessory factors in bacterial growth. Although such media may not interfere with gas production by the B. coli per se, it may have an effect on the combined metabolism. Von Jeney investigated the subject very fully. He found five strains of $B$. paratyphosus $\mathrm{B}$ among twenty-six studied which increased the $B$. coli gas production. Also plates from the mixtures at times gave pure $B$. coli. The strains of the $B$. paratyphosus, isolated from the mixture, did not always give the same results on retest. $B$. typhosus also inhibited. He searched for any evidence of bacteriophage action, but was only able to discover suggestions of such and no continuous passage was possible. Kauffmann ${ }^{8}$ carried the work further and used besides human strains, as von Jeney had done, a large number of animal strains. He tested thirty different cultures of $B$. coli and found in pure culture that their gas production was most variable, ranging between $o$ and roo per cent in twenty-four hours, and for no known reason. He also used yeast-treated media. Some $B$. coli strains grown from the same stools as strains of $B$. paratyphosus showed delayed gas production on glucose, but this was not the rule. On the other side he used thirty human B.paratyphosus B strains, Gaertner's bacillus, $B$. typhosus, and others and a further group of fifteen strains from animals. Besides the regular tests he used a number of heterologous and homologous combinations with the cultures from individual stools. A great irregularity was found throughout. After passage, certain strains of the $B$. paratyphosus increased in inhibitory powers, but the $B$. coli did not become more sensitive by such passage. There was, however, no regularity. The animal strains gave the same kind of results as the human. Certain "pseudo-unstable" forms were sometimes seen on plates. In summing

${ }^{1}$ Holman, W. L., and Meekison, D. M.: J. Infect. Dis., 39, I45. 1926.

${ }^{2}$ Besson and de Lavergne: Compt. rend. Soc. de biol., 86, 357. 1922.

3 Brutsaert, P.: ibid., 88, 306. I923.

4 von Jeney, A.: loc. cit. $\quad 5$ Smith, T., and D. E.: loc. cit.

${ }^{6}$ Robertson, R. C.: J. Infcct. Dis., 34, 395. 1924; 35, 311; 1924.

7 von Jeney, A.: loc. cit.

${ }^{8}$ Kauffmann, F.: Zischr.f. Ilyg. u. Infcktionskrankh., ro2, 68. I924. 
up these divergent and variable results, I feel that they tend to confirm the interpretation we have given. The results will depend on many factors such as the relative ability of each bacillus to attack the salts of the organic acids, the effect of alkaline reactions, and others.

\section{THE HEMOGLOBINOPHILIC GROUP}

The association of bacteria with the $B$. influenzae group has received unusual attention. The discussion of this part of the subject, even briefly, would take all my available space. I must merely observe that it has been studied from the earliest days of the discovery of the $B$. influenzae and gives striking illustrations of the importance of the subject. Many bacteria help the growth of $B$. influenzae on media, otherwise inhibitory, such as human blood agar, but they have little or no effect on properly heated blood agar. These beneficent bacteria are most variable in their characters, and include many listed as highly antagonistic such as $B$. pyocyaneus, an organism around whose antagonistic activity there has collected a comprehensive literature. B. influenzae will grow on hemoglobin-free media in association with many bacteria, although Putnam and Gay ${ }^{\mathrm{r}}$ and others were unsuccessful in doing this, and the importance of this and the possible bearing it may have in infection have been repeatedly stressed. Eggerth ${ }^{2}$ was able to grow B. influenzae in plain broth within a collodion sac immersed in cultures of staphylococcus, streptococcus, or pneumococcus and suggested this method as useful for studying symbiosis of bacteria while keeping each in pure culture. It would be of great interest to learn if toxin production may be permanently increased by certain of these combinations. Certainly the virulence is readily raised by mixed injections, as Yanagisawa, ${ }^{3} \mathrm{Hudson}^{4}$ and others have shown. I will not attempt to review the literature because this has been done by $\mathrm{me}^{5}$ and by Kristensen. ${ }^{6} \mathrm{I}$ do not believe there has been anything new on the fundamental ideas of bacterial associations with $B$. influenzae since those reports. I cannot leave the subject of influenza without mentioning the importance Olitsky and Gates, ${ }^{7}$ in a long series of articles, have placed on the relationship between the $B$. pneumosintes and secondary infections. I may say that their controls were quite inadequate to justify the conclusion that this organism is more potent in encouraging secondary infections than a host of others.

\section{B. ANTHRACIS}

In anthrax it has been repeatedly shown that combined injections frequently prevent infection. I have found that guinea pigs did not die after large injections of washings from soil contaminated months before by $B$. anthracis in the slaughtering of

${ }^{1}$ Putnam, J. J., and Gay, D. M.: J. Mcd. Rescarch, 42, I. 1920.

${ }^{2}$ Eggerth, A. H.: J. Biol.Chcm., 48, 203. $192 \mathrm{I}$.

3 Yanagisawa, S.: Kilasato Arch. Exfer. Med., 3, 85. 1919.

${ }_{4}$ Hudson, N. P.: J. Infect. Dis., 34, 54. 1924.

${ }^{5}$ Holman, W. L.: Studies on Epidemic Influenza, p. r6r. University of Pittsburgh, rgrg.

${ }^{6}$ Kristensen, M.: IIacmoglobinophilic Bacteria. Copenhagen, 1922. (In English.)

7 Olitsky, P. K, and Gates, F. L.: J.A.MI.A., 74, 1497. 1920; 78, 1020. 1922; 81, 744, 2 119. 1923; J. Exper. Med., 33, 125, 361, 373, 713. 1921; 34, x. 1921; 35, 1, 553, 81 3. 1922; 36, 501, 685. 1922; 37, 303. 1923 . 
a diseased cow, but gave typical results after injection of the cultures isolated from the soil. I have collected the literature on this subject, but it will not be included here. The explanation of the results rests largely on the fact that the anthrax spores are phagocyted by the leukocytes attracted to the site of injection by the other bacteria before they start developing and then are destroyed or eliminated. A most important discussion on the principles of such infections may be found in an article by Bail. ${ }^{\mathrm{I}}$

\section{GAS SYNERGISM}

Holman and Meekison ${ }^{2}$ reported certain findings in gas production by bacterial synergism and have reviewed the literature on this phase of bacterial association and attempted to show that inhibition and stimulation are both based on the combined metabolism of the bacteria in the mixtures and that the same bacterium may use various methods in acting on the substances offered, depending on the environment. Sears and Putnam ${ }^{3}$ and Castellani ${ }^{4}$ have given many instances of its occurrence. Castellani has further discussed the whole subject of close association of different species and particularly its importance in the causation of certain diseases and their symptoms. He does not, however, review the literature.

\section{ALTERATION BY ASSOCIATION}

Dissociation of bacteria has been gradually attracting more and more attention, and since bacterial association is so important to appreciate the analysis of the results obtained, a word or two may be added here. I have already given a number of examples of important alterations in the biochemical activities of certain bacteria living in association with others. Sometimes such changes were lasting on transfer, at other times fleeting. Rosenow ${ }^{5}$ claimed that he was able to change a hemolytic streptococcus to the viridans type by growth in symbiosis with $B$. subtilis. This $\mathrm{I}^{6}$ was unable to confirm, and I have reviewed the literature on the longevity of streptococci in symbiosis and have shown the many chances of error from mixed cultures, particularly with closely similar forms. Pneumococci may live in intimate contact with non-hemolytic streptococci for long periods, and the demonstration by Alivisatos $^{7}$ of the occurrence of mixed colonies of streptococci with staphylococci gives additional weight to the importance of such sources of error. The more sensitive organism will die out first, and retests may give quite different results from those found with the mixture. This danger was also emphasized by us $^{8}$ for the gram-negative group of aerobic intestinal bacteria. Nevertheless, bacteria are living, reactive beings and as such are subject to alterations of many kinds. Bail ${ }^{9}$ has shown how important this is in the infectiousness of bacteria (the change to the so-called "animal form"), and

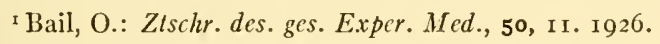

${ }^{2}$ Holman, W. L., and Meekison, D. M.: loc. cit.

${ }^{3}$ Sears, H. J., and Putnam, J. J.: J. Infect. Dis., 32, 270. I923.

${ }^{4}$ Castellani, A.: Brit. M. J., 2, 734. 1925; Proc. Soc. Exper. Biol. Es Med., 23, 481. 1926; J.A.M.A., 87, 15. 1926.

5 Rosenow, E. C.: J. Infect. Dis., r4, г. г914.

${ }^{6}$ Holman, W. L.: ibid., r5, 293. r914. , $\quad{ }^{8}$ Holman, W. L., and Meekison, D. M.: loc. cil.

7 Alivisatos, G. P.: loc. cil.

9 Bail, O.: loc. cit. 
similar adaptive alterations are to be expected in the growth in mixed cultures. How fundamental these changes may be is not as yet determined, and the study of dissociation should help us in our knowledge of the possible range which may occur in the bacteria as they are grouped by our present-day rather crude methods of classification. Bacteria may be forced to metabolize substances in a different way under certain environmental conditions than they would under other conditions, and this induced, but not necessarily new, function may become fairly well fixed by repetitions as a characteristic of the organism. In looking over the examples already given there are many instances to be found of such occurrences apparently brought out by bacterial association. Lommel ${ }^{\mathrm{T}}$ has given an important instance of this. By growing a nonsaccharose fermenting $B$. coli with $B$. typhosus, B. paratyphosus, or the Shiga bacillus after some twenty passages it took on the function of actively attacking saccharose. She does not say how long this induced activity continued under non-associative conditions, as only a plate and agar slant intervened between the tests. She has, however, shown that certain $B$. coli lost their ability to ferment lactose by continued growth on malachite-green media and that this loss remained constant for some, but not for all strains after fifty-six transfers on plain agar.

\section{OTHER APPLICATIONS}

Passing by numerous other well-known examples of bacterial association such as that of B. fusiformis and Spirocheta vincenti, which Rukawischnikoff ${ }^{2}$ and others look upon as only stages in the grow th cycle, and many mixed infections occurring in man and animals, I would give the interesting use made of this phenomenon by Wollman. ${ }^{3}$ He used $B$. coli as an indicator to determine proteolysis by bacteria previously grown in horse serum, egg albumin, and similar substances through its ability, after such primary growths, to produce indol. He thus tested B. anthracis, B. subtilis, Staphylococcus aurcus, and $B$. putrificus and later ${ }^{4}$ determined the proteolytic activity of streptococci by this method. Thompson ${ }^{5}$ was enabled by a symbiotic method with $B$. proteus to isolate an anaerobic $B$. acne-like organism from cultures of $B$. tubcrculosis and suggested its relationship with the latter. I believe it might well have been present as a contaminant and brought to light by this technique.

\section{DISCUSSION}

Before closing I would call attention to the necessity of determining more carefully than has been done the intimate metabolism of the bacteria we are studying and the environmental requirements necessary for the manifestation of their manifold characteristics, before any attempt is made to explain the phenomena I have reviewed, those of bacterial association, or the active metabolism shown by the bacteriophage. It is well known that $\mathrm{d}^{\prime} \mathrm{Herelle}^{6}$ considers the bacteriophage a living, sub-

'Lommel, J.: Compt. rend. Soc. de biol., 95, 7 1 1, 714. I926.

${ }^{2}$ Rukawischnikoff, E.: Cenlralbl. f. Bakleriol., I, Orig., ıоo, 2 18. Ig,26.

3 Wollman, E.: Compl. rend. Soc. de biol., 82, 1263. 1919.

4 Wollman, E.: ibid., 87, I 38 . I922.

5 Thompson, E. T.: Lancet, 2, I86. 1920.

${ }^{6}$ d'Herelle, F.: The Bacteriophage. 1922; Immunity in Natural Infectious Discase. I924; The Bacteriophage and Its Betuvior. I926. 'Translated by G. II. Smith. Williams \& W'ilkins. 
microscopic form, a strict parasite of bacteria, and therefore the most striking example of microbic association. This is discussed in other chapters of this book.

There have been two main explanations offered in the interpretation of most of the phenomena of bacterial association which I have mentioned. The first is the effect of changes in reaction. Usually one of two organisms in a mixture may produce unfavorable $\mathrm{H}$-ion concentration for the continued metabolism of the other organism, and indeed the latter bacterium may be killed. At other times these reaction changes may only alter the degree or kind of metabolism taking place, or the change may be of benefit to one or both. The other explanation is the production of enzymes of various kinds which directly affect the second organism in a mixture. This is undoubtedly a prime factor in many of the examples I have cited.

A distinct advance has been made toward other explanations for the facts given above by M'Leod and Gordon ${ }^{\mathrm{I}}$ in grouping bacteria under their relative sensitivity to, and power to produce, hydrogen peroxide and a corresponding catalase. Certainly, if we take their table, with due consideration for variations in different strains we shall find a rather satisfactory explanation for many of the antagonistic and bencficial results of bacterial associations. Burnet ${ }^{2}$ has analyzed many of these relationships, and his contributions have further made clear their application to our study. Bacteria sensitive to hydrogen peroxide will be inhibited by the presence of a strong hydrogen peroxide producer, and an organism with a well developed catalase production will assist another which forms much hydrogen peroxide to which it is itself sensitive. Anaerobes are very sensitive to this substance, and therefore a variety of aerobes producing catalase will benefit them in this respect as well as do those strongly aerobic forms which help the anaerobic conditions.

A number of workers have stressed the rôle of carbon dioxide in the inhibition phenomena of bacterial cultures. Sierakowski and Zajdel ${ }^{3}$ were able to show, by sealing cultures of various bacteria, that the $\mathrm{H}$-ion concentration alone did not account for the growth inhibition, but they believed it due to the retention in the tubes of carbon dioxide. Valley and Rettger ${ }^{4}$ showed that increasing amounts of $\mathrm{CO}_{2}$ raise the acidity and lessen the oxygen tension, and that the complete absence of $\mathrm{CO}_{2}$ in the atmosphere stops the growth of many bacteria. Other bacteria such as B. acidophilus were benefited by an increase in the atmospheric $\mathrm{CO}_{2}$. I ${ }^{5}$ have shown that anaerobes will form surface colonies on solid media in experiments in which their own gas production displaces the fluid medium in inverted tubes. This may be a factor in wound infections.

A further valuable contribution to our knowledge of these mutual relationships is to be found in the work of Gordon and M'Leod ${ }^{6}$ on the inhibition of growth by some amino acids. They found that the effect differed markedly as tested on different bacteria. B. coli and staphylococcus were not at all affected while other more delicate

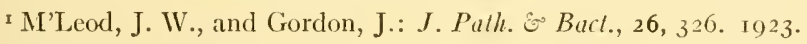

${ }^{2}$ Burnet, F. M.: Australian J. Exper. Biol. \& M. Sc., r1, 65, 77. 1025; J. Path. \&o Bact., 30, 2 I. 1927.

3 Sierakowski, S., and Zajdel, R.: Compt. rend. Soc. de biol., 9o, I ro8. I924.

4 Valley, G., and Rettger, L. F.: Abstr. Bacl., 9, 344. 1925.

${ }^{5}$ Holman, W. L.: Illinois $M . J ., 35,289.36$, ro. 1919.

${ }^{6}$ Gordon, J., and M'Leod, J. W'.: J. Palh. \&o Bact., 29, I3. 1926. 
bacteria were. Tryptophane, as an example, is most toxic and affects the widest variety of bacteria. Indol, a deaminization product of tryptophane, may account for the toxicity of tryptophane since it is more toxic than carbolic acid. Serum prevents to a degree these inhibitory actions. Others are beneficial, such as taurine, aspartic acid, and alanine. It is readily seen that we have here an additional explanation for certain of the phenomena being considered. Various bacteria will produce amino acids harmful or beneficial to others, and on these products will depend the effect on the associated bacteria in proportion to the relative sensitivity of the latter. The marked inhibitory effect of certain proteolytic bacteria on agar plates against the nearby colonies of other bacteria and the stimulating effect at some distance may well be due to differences in diffusibility of the products formed. There are also, of course, direct actions of proteolytic enzymes on the associated bacteria in certain cases as already suggested, and the lack of agreement in many of the examples quoted above may well be due to different factors having been at work. There is a pressing need to correlate the wealth of available material on the various products formed by many bacteria and the effect of these on associated forms. The work of Koser ${ }^{1}$ and many others on the utilization of the salts of organic acids by bacteria makes clear other groups of phenomena and assisis in the explanation of changes in $\mathrm{H}$-ion concentrations as these occur in the reversed reaction of single forms and in bacterial associations. The relative rapidity of growth of two forms, the continual alteration in their activity and sensitivity, the adaptability of bacteria to the form of food material offered at different stages and under different conditions as aerobic or anaerobic, the changed metabolism under acid and alkaline influences, and a host of other factors determine the resultant metabolic products of mixed cultures. The analysis of these factors helps in understanding the metabolic processes involved.

\section{CONCLUSION}

Bacterial association occurs under natural conditions, and it plays an important part in many infections. At one time certain resistant but relatively harmless forms may ward off the body defenses and allow a more sensitive microbe to become established. At other times the reverse may occur and antagonistic bacteria may, and no doubt frequently do, prevent numerous infections. We have in bacterial association, then, a means of studying many natural phenomena and, as has been indicated, we touch on many fields of bacteriological study. In the routine bacteriological diagnosis it must be always in mind that there are antagonisms in our media and method; for preventing them; that pure cultures are essential, and that curious results occur from mixed ones, often quite different from those with either culture alone; that the beneficent associations are to be found and that it must be realized that bacteria coming from varying environments may have been under inhibitory or stimulating influences which alter the results obtained in our test tubes. In artificial animal injections and in natural human and animal infections and diseases bacterial associations as seen in lowered or raised virulence or pathogenicity; the presence of secondary infections from the animal itself leading to faulty conclusions; the changes in bacterial flora as the conditions alter, as seen in war wounds and intestinal infections; and many

$$
\text { ' Koser, S. A.: J. Bacl., 8, 493. } 1923 .
$$


similar considerations must be thought of to avoid error in interpretation of results. In the study of bacteriophage and bacterial dissociation the same considerations are needed. I would finally urge, after reading Claude Bernard's Introduction to the Study of Experimental Medicine ${ }^{\mathrm{I}}$ and in attempting to get working hypotheses for the phenomena of bacterial association, that more and more attention be paid to the physiology of bacteria as reactive, living beings with as complicated metabolisms as our own, and that the study of their pathology, if such we may call it, requires this preliminary knowledge of the normal limits of their physiological activities, alone and together, in the test tube and in the animal body. Thus we may be able to understand better many phenomena which at present cause confusion, and may better appreciate the basic principles in many of the biological activities of bacteria.

× Bernard, Claude: op. cit. Translated by H. C. Greene. Macmillan Co., 1927. 


\title{
CHAPTER IX \\ CLASSIFICATION OF BACTERIA
}

\author{
ROGER G. PERKINS \\ WTestern Reserve University
}

Bacteriological literature is crowded with classifications of bacteria of every sort, prepared by thoughtful workers, with painstaking labor. The literature is also crowded with criticisms of these classifications for reasons which seem good to each author. though at times he is alone in his opinion. Therefore all I dare attempt is a summary of the principles under which these classifications and these honest criticisms have been made, and to present them as far as possible in their relations to one another and to the problem as a whole.

Two main points of view are represented, quite distinct, although closely related. The ordinary student of bacteriology is chiefly concerned with the grouping of bacterial forms to establish their general relations, involving the possibility of keys which shall enable hin to place any new discovery in its approximate place. The taxonomist, the specialist in bacterial classification, concerns himself further with the selection of the correct names for the divisions, and the evaluation of the proper sequence of order, family, genus, species, and varicty, with an cye to the future. Stiles (1927), in a recent address, spoke of taxonomy as the grammar of the science and emphasized its essential value. He comments on the present status that "it has been the exception - not the rule - that pupils who study zoölogy have been taught the grammar of the technical language they are called upon to hear, read, write and speak." This is no less true in bacteriology, and the student who does more than skim the subject of classification outside his own particular interests is rare. This is unfortunate, but perhaps if the grammar were less chaotic it would have more students.

Taxonomists, not only in bacteriology but in general zoölogy and general botany, live in two camps: those who believe that for many reasons all names referring to individuals, varieties, species, etc., should follow the Limnaean law of priority, laid down in I 75I; while the other camp holds that inasmuch as Linnaeus knew only what was known in $\mathrm{I} 75 \mathrm{I}$, and since so much totally new information regarding classification is available, we should no longer be restrained by the dead hand, but should be free to express ourselves. Stiles (I927) presents sharply his idea of these alternatives.

First let every [zoölogist] adopt any technjcal name he wishes, or second let us all agree to follow the Linnaean law of priority. The first alternative is subjective and leads to confusion, the second is objective and makes for uniformity in all objective cases .... In general I would evaluate a failure to apply the law of priority as the second most important formal factor in nomenclatorial confusion.

And perlaps the extreme in the other camp) is Enclerlein, with a complete new classification, anrl indeed a complete new language. Neither side, of course, refuses to arhunit new litles, new defunitions, and new grouphings, nor does it hesitate to accept the fatct that our knowledge is progressive rather than fixed, but the rules of the game differ. 
On the whole it seems as though, in spite of its defects, the work of the last twenty years has established sufficient recognizerl groups, especially of the higher ranks, to enable us, with the appreciation that bacteriology is a young subject, to develop these rationally, without any complete upset. At present there are certainly sufficient shades of difference between the extremes to accommodate anyone.

Another important controversy, more marked perhaps among the adherents to Linnean priority, centers around the question whether a name should be descriptive, giving some indication as to character or the group or individual, or whether this is unimportant, and the name, if historically correct, should be retained even if wrong or confusing. Enlows stated in I920:

It is to be hoped that the many inadequately defined genera here listed may serve as glowing examples of errors to be avoided by future contributors. A plea is made, too, for the introduction of generic names which are descriptive, since many names of this sort define, and in a way, classify. Proper names converted by the addition of -inca, -clla, etc., are very alluring because of the acknowledgment of the debt we owe our leaders, but they are not descriptive terms, and offer no aid whatever to any system of classification.

On the other hand, the Committee of the Society of American Bacteriologists stated in I9I7 that "the name need not be appropriate, it need only be stable. It is an arbitrary description." Stiles stated in I905: "It is essential to recall that names are not definitions; they are merely handles by which objects are known." These last indicate the majority opinion, save in the more independent classifications, such as Orla-Jensen (I92 I), Enderlein (1925), and others, where the attempt at descriptive titles is prominent. In connection with Enlows' (loc. cit.) remarks about the eponymic groupings, it is interesting to note that in medicine there is a growing tendency toward correctly descriptive names for diseases, with corresponding abandomment of proper names.

It seems as though neither the apologists nor the higher critics are satisfactory or satisfied, nor is it surprising in a science in formation. The essential contention appears to center about the proper, relative proportion of a conservatism which objects to the removal of well-known signposts, partly on the basis that their familiarity more than overbalances their misdirections, and a liberalism which wishes to accept only such names and descriptions as fit our present knowledge. What shall be the mixture and who shall mix it?

It is stated with much force of logic that constant change in names and in qualifications is confusing, and that even now the bacteriologist has to have at least one key, if not more, at his elbow when he reads his literature, and that historical articles will soon be quite unusable, a mere hieroglyphic literature, understandable only by a few. It is true also that if in the last thirty years or less the discoveries have been such as to invalidate old classifications, what warrant have we that the next thirty years may not repeat the process? Further advance in technique may reveal details of form yet unknown to us, may crystallize our information, and may yet uncover unsuspected biological relations. On the other hand, it is clear that at present our known details of morphology of any degree of constancy are numbered, and that chemical and physical differentiations have become more and more stabilized within certain group limits. Although it may be true that new information may and probably will necessitate 
changes in another quarter-century and even in a decade, it is likely that the new information will not be morphological.

When acknowledged experts disagree widely on so complex a subject, an ordinary bacteriologist like myself must avoid as far as possible technical taxonomics ind extensive references to authority, of which there is a sufficiency so arranged that any diligent reader can make his own evaluations. To the general reader the main lack is in summaries of opinions which make it possible to omit the individual technical articles.

The recent classifications and keys due to the industry of Bergey (I923), Buchanan (I9I6-25), and the Committee of the Society of American Bacteriologists (I9I72o) with the critical summaries of taxonomic authority by Buchanan (loc. cit.), Enlows (loc. cit.), and others make the entire history of the development of classification readily available. One should note also that not the least service of such attempts at complete classification of a series of organisms, concerning which the authors themselves are the first to admit our information deficient, is their courage in presenting their plans and ideas for criticism. Unless broad plans of this sort are accessible, revision is impossible, since most of us are more particularly concerned with some restricted portion of the whole. It is far easier to criticize than to construct, but only out of a combination of construction and constructive criticism can we hope to reach stability.

At first the whole affair looks extraordinarily discouraging. Each attempt at improvement meets with a volley of objections (Hall, I927), and such attempts as seek to break away from conventions of taxonomy have few adherents. Closer study, however, offers more hope. One finds that there is a great deal of acceptance of main groups, and that most of the trouble comes from disagreement as to the rank of these, and from persons studying a comparatively small group and believing that their arrangement is the only proper one.

To my mind the first essential, as brought forward by many, and ignored by as many more, is that bacteria in comparison with higher plants and with animals are notably unstable, and that such conformity to type as we see in pure cultures of Plymouth Rock chickens, or white rats, or Lima beans is not to be expected.

The more we study them, the less stable appear the characters on which we base much of our classification. If zoölogists find, as they do, that complex multicellular organisms are altered by environment to a point where, had they not been followed through, they would be thought different genera, and the botanists present similar evidence, how much more may we expect environmental modifications of a temporary or permanent character among bacteria? One must admit, of course, that unicellular organisms which divide by simple fission carry heredity in a manner very different from that of more complex forms, each half being supposedly identical with the other; but recent work in variation and in mutations in single-cell cultures has forced a modification of those ideas. There is no a priori reason why a colony of anthrax might not undergo in nature conditions similar to those by which we modify it artificially. Evidence that the citrate-using $B$. coli preceded or followed the non-citrate-using is not yet conclusive. We are told that the development of terminal flagella on a coccus tends to change it to a rod form, and we find that an organism may be changed from 
a gram + to gram - by the use of suitable chemicals. Winslow quotes Wolf as reporting "a considerable number of temporary modifications and some permanent inheritable ones stimulated by exposing bacteria to the action of chemicals. White and dark-red strains were thus produced from a normal $B$. prodigiosus, the resulting modifications breeding in each case true to their new type." Winslow calls these changes "impressed variations," and further on says:

The fact that all cells are potentially reproductive removes any bar against the inheritance of acquired characteristics. Again, the absence of sexual reproduction must operate to preserve variations which arise from within or without . . . . with fission as the normal mode of reproduction every variation which can arise can be handed on unchanged; . . . there are sharp limits to the variability even of the bacteria, and for practical purposes we find the larger groups quite constant in their general properties .... in part at least I am inclined to believe that this is due to the direct or selective action of similar environmental conditions.

Since we are dealing not only with variables, but with variables in a group of organisms susceptible to permanent and hereditary change, we must select as most important those characters which as nearly as possible approach constants, and which seem least susceptible to these permanent changes. One method of approach is well exemplified by the work of Winslow (I906) in his study of the Coccaceae, and the work of Hucker (I924) in a similar study. Believing that improvement might result from a statistical analysis of a variety of characters in the hope of determining correlated groups, these extensive and painstaking studies were undertaken. The fact that the authors are often in marked disagreement shows that the method of procedure, while suggestive, is as yet unsatisfactory.

We must not forget that the actual number of bacteria described is but a small fraction of those which exist. This is of course true in botany and zoölogy as well. Stiles (1927) states that there are hundreds of thousands, possibly millions, of genera and species still to be given technical baptismal certificates. Aldrich (I927) quotes Horn as saying; "Whoever as an entomologist looks into the future knows full well that we are steering into a shoreless sea, no matter whether he estimates the total number of insect species at three, ten or fifteen millions. In the near future any beginner will be greyheaded before he has caught up with what is already known." Although we have not quite reached this status with bacteria, partly because the details of structure and other characteristics are so much less complex, it is already practically impossible for anyone to be an expert in all the lines of bacteriology, and especially in their finer taxonomic relations. The student in medicine interests himself in the nonpathogenic forms only in their relation to the pathogenic (many early classifications, such as Flügge, were of pathogens only), and even neglects the organisms relating to those diseases of animals that are not transferable to man, to say nothing of those which cause disease in plants, while the veterinary and the plant bacteriologists are similarly exclusive. It is only very recently that the workers on filterable viruses have appreciated that the botanist, the zoölogist, and the medical biologist are working on an identical problem and that in their combined effort may lie the key to success.

Students of the non-pathogenic organisms naturally tend to the study and isolation of those groups which have economic relations with soil values, with fermentations, with decompositions, and consider all others as merely annoying contami- 
nations. Yet new perfections in technique constantly cast new organisms on the shores of the bacterial sea, where they become accessible. Workers with the anaerobic group tell us that it is quite possible to describe totally new organisms at the rate of several a week, and it is only when someone gets interested in a special problem that we get a list of the organisms concerned. It is clearly impossible to foresee new discoveries beyond what may be safely prophesied from analogy, and the best we can do is to make and preserve a scheme which we believe will act as a framework on which to hang not only the groups we have, but those which may turn up from time to time.

It seems clear, then, that in common with students of other biological groups we have reached only a small fraction of the varieties which make up the mass, and that it is logical to believe that the changes in our knowledge and beliefs which have followed the lights of informative changes in technique are only an earnest of what is to follow. Bacteriology, as we now understand it, is less than fifty years old, and inasmuch as bacteria are either plants or animals, it fell heir to all the taxonomic literature relating to both. The discovery of the principles of pure-culture study resulted in such a sudden burst of investigation that it was a lost month in which a new organism was not described, catalogued, and laid away, very frequently in the wrong grave.

With this introduction we can enter upon a discussion of the criteria used in past and present times to establish the various groups from order to variety with an attempt at a critical summary of modern opinion.

\section{BASES OF CLASSIFICATION}

In the minds of many, perhaps most persons, the actual definition of the words used in taxonomy are vague, and some definitions may be helpful. Inasmuch as the "genus" is the center, so to speak, the grouping which brings together a collection of species, and itself forms the base for larger groupings, let us begin with it. Agassiz defined genera as "most closely allied groups of [animals] differing . . . . simply in the ultimate structural peculiarities of some of their parts." The Century Dictionary (IgOr) speaks of it as a

classificatory group ranking next above the species, containing a group of species (sometimes a single species), possessing certain structural characters differing from those of any others. The value assigned to a genus is wholly arbitrary, that is, it is entirely a matter of opinion or current usage what characters shall be considered generic . . . . a genus has no natural, much less necessary, definition, its meaning being at best a matter of expert opinion, and the same is true of the species, family, order, class, etc. A genus of the animal kingdom in the time of Linnaeus was a group of species approximately equivalent to a modern family, sometimes even to an order.

Stiles (loc. cit.) defines it as "a taxonomic complex of specimens grouped for the moment (according to our subjective and never absolutely perfect knowledge) around a genotype."

These definitions emphasize that it is impossible to lay down rules which will hold future experts, at least beyond a certain general plan, and also show why it is that in so many cases the desire for revision starts with the study of a genus, and later involves its position in connection with other genera.

Perhaps one of the most important points in the description of genera is the selec- 
tion of the genotype. If this is properly done, the personal equation involved in the selection of various group characters for inclusion is checked and crystallized by the selected type example. This has long been theoretically necessary, but has been much neglected. Buchanan (loc. cit.) and Bergey (loc. cit.) have been the most consistent, not only in their demands for this, but in actually supplying the types.

There are five main headings which influence classification. Beginning with the earliest, "Morphology," we have added "Chemistry and Physiology," "Evolution," "Habitat," and "Immunology." The most successful work has been accomplished with the first two.

\section{MORPIIOLOGY}

In the beginnings of biological classification, this was the only basis, the alpha and omega, and until discoveries multiplied was quite satisfactory. But as more and more individuals were discovered, groups became very large and needed further or different subdivisons. Under the head "Morphology" we generally admit not only form and arrangement, including organelles, capsules, and spores, but also tinctorial distinctions such as gram stain, acid fastness, and granule formation, though of course these might also be considered chemical. But even with these admissions the number of distinctive characters is limited. Moreover, as noted earlier, even form is unstable, and subject to temporary or permanent modification which may readily become inherited.

But whatever may be the changes in morphology under extraordinary conditions, we must still admit that under ordinary conditions, or under readily obtainable standard laboratory conditions, a rod form remains a rod form, a sphere continues as a sphere a spiral form clings to its spirals. Moreover, a coccus with the habit of dividing in certain planes tends to retain this habit with some obstinacy, and a rod form which after division tends to retain two or more individuals in a chain may be reasonably expected to continue this activity. What constants may we add to form and planes of fission? Obviously, the most conspicuous are motility and spore formation.

Migula ( $1897-1900$ ) went so far as to make a major division on the basis of motility, speaking of the motile form as "bacillus," the non-motile as "bacterium." Although this was rather widely accepted for a time, it finds little support at present. Leaving aside the question as to whether motility is a higher or lower divisional point, there is marked variation in this character, to such a degree that insistence upon it will make wide separations of closely allied strains. In this group, moreover, not only is the actual motility a differentiating feature, but inasmuch as in the Eubacteriales (Buchanan) motility is dependent on flagella, the arrangements of these may also be important. Orla-Jensen's two orders are based on such arrangement, separating those with flagella at the end from those with flagella all around. Breed and others (I9I8) state:

In passing, it is of interest to notice that there is a close analogy between the generally recognized groups of bacteria and those of protozoa. Thus the cephalotrichic and peritrichic bacteria find their analogies respectively in the flagellates and ciliates. This analogy goes further than a mere resemblance in the arrangements of organs of locomotion; for the ciliates and peritrichic bacteria are both highly specialized gic ups, while both flagellates and cephalotrichic bacteria contain all gradations between primitive forms and highly specialized human parasites. 
I must confess, however, that on tabulating the one group against the other, save for the fact that more of the peritricheae are pathogenic, the evidence of superiority appears inconclusive. It does not seem to me that our knowledge of bio-chemistry is sufficient to show that the power to utilize or break down certain chemical compounds proves more or less "advancement," and the analogies with the protozoa, while unquestionably fascinating, seem to me to stop at that point.

Without plunging too deeply into controversy, one may say that the division on the basis of spore formation is logical, as showing a major activity, and is widely accepted, though the resultant group is variously placed. Some (Table I) accept this character as a basis for families under the Eubacteriales, others prefer tribal divisions under the family of rod forms, others as divisions of even lower rank. Classifications such as Orla-Jensen's (I921), fundamentally based on physiology and chemistry, are much less interested in spore formation.

How far should the differentiation by morphology carry? It seems to me that this basis can certainly carry us as far as genus, and Hall (I927) goes further, saying: "I submit that morphologic criteria should enable us to identify the genus, and I believe that the definition of orders, families and genera should be based solely upon morphologic data." With this sentiment many but by no means all agree.

\section{CHEMISTRY AND PHYSIOLOGY}

The most extensive changes have been due to attempts to use the activities of the bacteria rather than their form and arrangement as differentiating factors. The changes have ranged from a practically complete substitution, such as Orla-Jensen's (after the original flagellar division), to studies of small fractions of the problem. The general tendency of taxonomists is to emphasize morphology in the larger groupings and to reserve biological activities for smaller collections. Pigment formation, probably the earliest observed of the definite chemical activities, has been graded all the way from family to variety, sometimes in connection with other group characters, sometimes practically alone. No very adequate reason has been adduced why groups of chromogenic organisms with different metabolic activities should be classed by their pigments rather than distributed according to their other characteristics. It is true that we have a very moderately studied collection of pigment-formers with little else by which to classify them and that this procedure may be temporarily convenient. But why the power to use fixed oxygen rather than free oxygen, or to break down sugars, or to form complex toxins or proteolytic ferments is not as remarkable as the power to form pigments, has never been demonstrated to my satisfaction. Hucker (loc. cit.) states in regard to the cocci:

It seems evident that pigment production by the mass-forming cocci is a very important character for use in classification. However, due to its general lack of correlation with other characters, it does not appear that the group should be divided into genera with differences in chromogenesis as the chief diagnostic feature. On the other hand it seems that pigment production is sufficiently constant to warrant its use as a character of importance in differentiating species, and when so used should be interpreted along broad lines rather than to attempt to make fine distinctions in shades of color.

In the same way the "nitro-" bacteria, placed as high as a family by some, a subfamily or tribe by others, seem to be getting more attention than they deserve. As 
will be seen later, even the arguments for early historical precedence are not generally accepted. In other words, are not most of these chemical and physiological characters even less definite and stable than morphology, and should not their use begin where the criteria of morphology alone are inadequate? It has, of course, been frequently brought out that characters important in one group are unimportant in another, as, for instance, fermentations in the cocci and in the bacilli, or character of division in the same groups. It is probably impossible to formulate as a rule whether a given character should always define species or confine itself to varieties.

\section{HABITAT AS A DIFFERENTIAL POINT: PATHOGENICITY}

"Pathogenicity may be taken as a type of those powers of the organism which are easily and profoundly modified by external conditions." This quotation from Winslow in I906 seems incontestable, yet later he uses "habitat" in the separation of his genera in the Coccaceae. This seems rather inconsistent even though he insists upon groups of characters rather than individual ones. Here, again, there is marked disagreement, as Hucker differs from Winslow in a similar paper, as follows:

Due to the fact that the micrococci are found in such a wide variety of sources, and due to their ability of adaptation to various environments, the use of habitat alone as a character in separating the general group is precluded. It is true also that no other character will definitely correlate with the source of the different types and bear out any conclusions that different natural groups of micrococci can be secured from different habitats.

It would seem that if this applies to this group, other groups in which the same objections hold true would be similarly affected.

It has always seemed to me that the selection of pathogenicity as a prime factor of division is only part of the pride of the human race in its superiority, a pride which has from time to time admitted some of the lower animals under the wider cloak of animate, moving life. In the consideration of bacteria, however, why should one metabolic activity be superior to another? We believe that pathogenicity is more or less of an accident, that bacteria are not toxic in order to be pathogenic, but pathogenic because they happen to be toxic. Because some by-product of digestion when applied to a mucous membrane causes death and breaking down of the local cells, whereas other products of digestion can break them down only after cell death, may be a matter of degree rather than character. Non-bacterial poisons may have the same effect, but are not classified on this basis, but rather on the general character of their possible chemical combinations. Should we not therefore consider the chemical activities of bacteria, due to metabolic products with various chemical characters, rather on the basis of these characters alone than on some special reaction, which is, as Winslow (I905) calls to our attention, readily modifiable by environment? Breed, Conn, and Baker (loc. cit.) criticize the Society of American Bacteriologists' Committee (I9I 7) in that "too great weight has been placed on pathogenicity," attacking specially Hemophilus, Pasteurella, and Erwinia. It seems to me dangerous in a general classification to say that a group is generally, or usually, or essentially parasitic, in view of the well-known fact, already recalled, that our knowledge of bacteria is confined to a very small fraction of the total in existence, and since scarcely any groups which contain pathogens do not include forms closely related but without pathogenicity. 
Can we determine habitat by a limited series of findings? Any organism that requires oxygen, moisture, warmth, and broken-down organic matter may develop on the surface of the body and remain there for various periods of time. It will also survive or develop anywhere else under similar conditions, but would not be called parasitic if found on a filthy blanket.

\section{IIABITAT OTHER THAN PATHOGENIC}

The same general criticisms are applicable. It is unquestionably valuable to know that most of a group have been isolated from water or soil or mucous membranes, but the connotation to the average reader is that there is some inherent relation between the organisms and the particular environment. And this may or may not be the case. This is a different question from that of taxonomic names, and whether such a definition is misleading or not is a matter of sharp controversy.

\section{CHRONOLOGICAL CLASSIFICATIONS}

Orla-Jensen (loc. cit.) and Kligler (I9I3) have presented more or less elaborate classifications on this basis, and a large part of the former's grouping, especially that relating to the nitro-bacteria, has been taken over by the Committee of the Society of American Bacteriologists. Breed, Conn, and Baker (loc. cit.) make a well-considered attack on both these systems, emphasizing that we are now dealing with endprocesses of evolution and not the primary forms except in so far as environmental conditions have remained more or less permanent. (It is likely that conditions even in water are not now identical with those of the period of origin of bacteria.) They quote the Committee in their definition of the Nitro-bacteriaceae, and state that if we accept this family, we indorse the theory that its members are modern representatives of the primordial bacteria. They object to this, and indicate that the theory, while interesting, is without adequate proof. (Buchanan [I918] attacks Kligler's paper even more sharply.) The most logical reason adduced for family rank is the close relation of rods and spheres in this group and the inconvenience of separation.

It seems to me also that in this question of primordial relations as a basis for division, too little notice has been taken of the well-recognized fact that characters may be lost as well as acquired. An organism once able to utilize and synthesize simple materials may, under conditions of parasitism or even symbiosis, lose those powers, and in the vast numbers of generations of bacteria it is not far fetched to consider the possibility that strains with certain powers may lose part of them under changed environment, and under subsequent changes may either regain what was lost, acquire new powers, or any combination of these two changes. Orla-Jensen shows that it is quite probable that actual changes in form from sphere to rod may have taken place, and botanical and zoölogical alterations due to environment under short-time experimental conditions are well known. Conclusions drawn from the conditions of light, heat, available food, etc., in those geological periods during which bacteria developed are based on disputable evidence, and must be considered cautiously.

\section{IMMUNOLOGY}

This division of scientific work is still in its infancy, and its application to classification of bacteria has been unsuccessful, save in the differentiation of varieties, and 
rarely in confirmation of species which are already shown to be in close relation. As an aid to differentiation, for such groups as the pneumococci, its value is high.

\section{THE PROBLEM OF A NEW CLASSIFICATION}

With all these difficulties of a classification based on a variable combination of morphology and chemistry, what are the possibilities of a complete revision? I confess it seems to me that unless someone is sufficiently omniscient to foresee the new developments, and to select characteristics which will remain stable for the next hundred years there is little hope for such a classification. Man is conservative and would demand proofs of omniscience and prophecy which would be hard to offer. The boldest now in vicw is that of Enderlein (1925), who believes that morphology is absolutely the only important criterion, especially in its development. He decries monomorphism, mutations, and monorytism, and goes into extensive details as to the minute anatomy of the bacterial cell, with nucleus, sexual and asexual division, reconsideration of the spore, etc. The entire vocabulary is different, so that no comparison can be made.

\section{PRESENT STATUS OF BACTERIAL CLASSIFICATION}

Reviewing the present status of bacterial classification, what hope does it offer for the future? There is practical unanimity in accepting the class Schizomycetes as first brought forward by Naegeli in 1857. Vuillemin gave a good description and Buchanan (1925) modified it somewhat. Vuil'emin stated:

They are simple organisms, formed of a single element without septa and unbranched. The element is circumscribed by a rigid vegetable-like membrane, elastic, but not contractile, sometimes also with a capsule. It may undergo plasmolysis or plasmoptysis. The protoplasm is less differentiated than that of most cells; the chromatin particles do not form an individual nucleus of a permanent type. Division is amitotic. Some forms are motile with flagella which traverse the membrane at points characteristic of the species (polar or diffuse). They are not broader than $5 \mu$ when not in bunches or unimpregnated with metal or colloidal coloring material. The resting stage may be either an arthrospore resulting from a simple modification of the membrane or an endospore. In some cases the spore-bearing telement retains its form or is modified passively by the enlargement of the spore, in other cases it is a specialized element for spore production. Only the latter type of sporulation is satisfactory for generic characters. The amitotic division usually occurs by a pinching transversely with rapid separation of two individuals. These may remain united into families, in chains, layers or packets as determined by the successive planes of deviation.

He emphasizes that all forms showing contractility should be placed in the protozoa, that the myxobacteria constitute a distinct group, and that forms like the tubercle bacillus which show branching should be placed in the mold group Microsiphones.

Buchanan used the following characterization:

Typically unicellular plants, cells usually small and relatively primitive in organization. The cells are of many shapes, spherical, cylindrical, spiral or filamentous; cells often united into groups, families or filaments; occasionally in the latter showing some differentiation among the cells, simulating the organization seen in some of the blue-green, filamentous algae. No sexual reproduction known. Multiplication typically by cell fission. Endospores are formed by some species of the Eubacteriales [see below], gonidia (conidia, arthrospores) 
by some of the filamentous forms. Chlorophyll is produced by none of the bacteria (with the possible exception of a single genus). Many forms produce pigments of other types. The cells may be motile by means of flagella; some of the forms intergrading with the protozoa are flexous, a few filamentous forms (as Beggiatoa) show an oscillating movement similar to that of certain of the blue-green algae (as Oscillatoria).

The Committee used the following description:

Minute, one-celled, chlorophyll-free, colorless, rarely violet-red or green-colored plants, which typically multiply by dividing in one, two or three directions of space, the cells thus formed sometimes remaining united into filamentous, flat, or cubical aggregates. Capsule or sheath composed in the main of protein matter. The cell plasma generally homogeneous without a nucleus. Sexual reproduction absent. In many species resting bodies are produced, either endospores or gonidia. Cells may be motile by means of flagella.

Under the Schizomycetes a number of orders have been proposed, in some of which there is general agreement and in some less. In the discussion following, except where a definite published group is referred to, I shall attempt to avoid the technical terms, using groups, forms, kinds, etc., as well as the common term "bacteria" or "cocci" to indicate rods or spheres.

Among the groups generally known as "higher bacteria" and less important to the medical bacteriologist there is a great deal of agreement.

Under the Schizomycetes the groups of the sulphur (thio-) bacteria, the sheathed (chlamydo-) bacteria, and the pseudoplasmodial ( $m$ yxo-) bacteria are fairly well acknowledged and contain much the same material the world over, though the rank is not always the same. This leaves us the "true bacteria" (Eubacteriales [Buchanan]) with the border-line groups of "actino-" and spiral forms.

Taking up first the border-line groups of thread forms and spiral forms, the summary of their history leading to the present status is about as follows:

Actinomyces-In 1877 Harz described the thread fungi in "lumpy jaw" of cattle as "actinomyces" and started the group on its quarrelsome path. Rivolta in $18 / 3$ preferred "discomyces," and various observers (Blanchard [I895], Brumpt [1910], and Merrill and Wade [1919]) agree with him. Streptothrix (Cohn) is accepted by others, but there is apparently reason to believe this term invalid (Buchanan, p. 497), though it still has plenty of adherents. Study of the group brought out the fact that there are two distinct methods of reproduction within the group, and Trevisan in I899 susgested the name Nocardia for those forming spores, as distinguished from the nonspore-forming "actinomyces," a term accepted by Wright in 1904. Buchanan (p.405) regards it as a synonym. Thus we have four names, each more or less ably supported as the ten pages devoted to the subject by Buchanan indicate. The Committee adopts Actinomycetales as an order, as do also Buchanan and Bergey, while Breed, Conn, and Baker consider them as a family-Actinomycetaceae.

Whatever name we prefer and select, new arguments arise as to the contents of the group (see Table I). Some authors under the prefix "actino-" include the thread fungi and also the tubercle-bacillus group and the diph theria group, others include the same series under the prefix "myco-," while others separate the thread fungi from the "myco-bacteria," placing these last directly among the non-spore-forming rods. The modern tendency, since Lehmann and Neumann, seems to be to place tuberculosis 
and its associates with diphtheria and its associates and to consider them both as related to the thread fungi which have true branching. It is also the prevailing practice where Nocardia is used to confine this to the special group noted above. It is to be hoped that a better agreement may develop in this group.

Spiral forms. - There has naturally been much contest as to the placing of these. One school places all spiral forms, from the cholera organism to the treponema and its associates, in one group, subdivided into: (a) simpler forms long known to us as the cholera group; and $(b)$ the more "protozoan-like" (if that means anything), including treponema, leptospira, spirochaeta, etc. Another school places this second group in a completely separate division, leaving the others in Spirillaceae (q.v. infra). Some believe the "protozoan-like" group to be intermediate between bacteria and protozoa but it is interesting to find recent textbooks on protozoa omitting it entirely (Hegner and Taliaferro, Craig). (See Table I for details). In general, the first of the two groups corresponds to Spirillaceae Migula (I894) emended Committee, Society American Bacteriologists (I9I7), with the following description: "Cells elongate, more or less spirally curved. Cell division always transverse, never longitudinal. Cells non-flexuous. Usually without endospores. As a rule motile by means of polar flagella, sometimes non-motile. Typically water forms, though some species are intestinal parasites." The second corresponds in the same way with Spirochaetaceae (Swellengrebel) described by the Committee as follows: "Free living or parasitic spirilliform organisms with or without flagella, with undulating or rigid spiral twists. Reproduction by transverse division and by 'coccoid bodies,' the equivalent of spores."

This brings us to the group of true bacteria (Eubacteriales, Buchanan) (see Table I).

This vast group of simplified forms, aerobic and anaerobic, spherical and rod shaped, pigment formers or non-pigment formers, fermenters or non-fermenters, motile or non-motile, gram positive or negative, spore-formers or non-spore-formers, and with many other positive and negative characters, has been divided and subdivided on the basis of combinations of these until there is practically no theory which may not find substantiation in published work. The minimum number of divisions under this head is three: the spherical forms, the rod forms, and the spiral forms.

Spherical forms.- This group was recorded as Coccaceae by Zopf in 1884 and is the most widely accepted of all, including all spherical forms of whatever arrangement, with certain minor and not undisputed exceptions.

In spite of possibilities that in the development of bacteria there may be a tendency for cocci, especially when there are one or more flagella, to elongate, it seems agreed by most observers that the spherical forms are worthy of a grouping of their own, and the rod forms likewise. If one selects habitat, or fermentation, or color, or motility, or pathogenicity as prime factors, this grouping will at once disappear. Some observers (Orla-Jensen) believe that the chain-cocci and the chain-rods are to be considered together, but their arguments, though interesting, are necessarily based on theoretical grounds, and, as noted elsewhere, our actual, objective knowledge of the evolution of bacteria from the original, whatever it was, is mostly speculative. Whether the modern work on variation will reach a point from which we may argue 
㝵

曷

\begin{tabular}{|c|c|c|c|c|c|c|c|c|}
\hline & 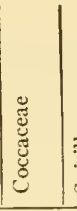 & 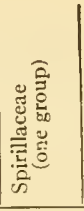 & 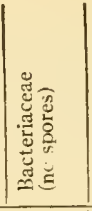 & 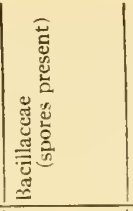 & 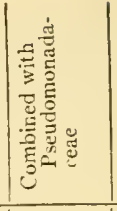 & & 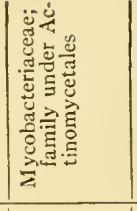 & \\
\hline 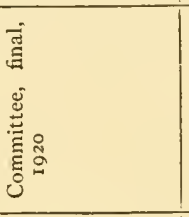 & 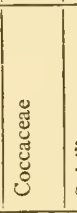 & 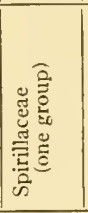 & 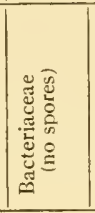 & 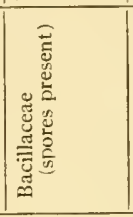 & 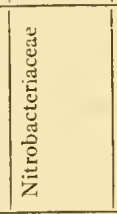 & 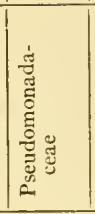 & 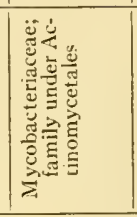 & 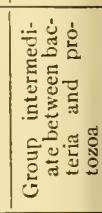 \\
\hline 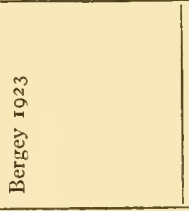 & 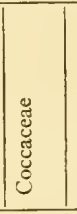 & 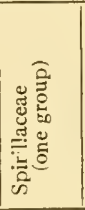 & 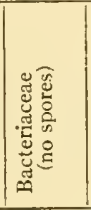 & 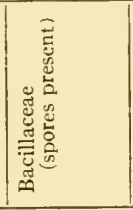 & 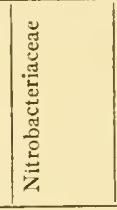 & 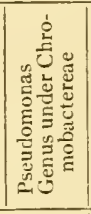 & 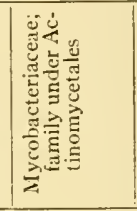 & 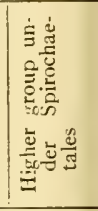 \\
\hline 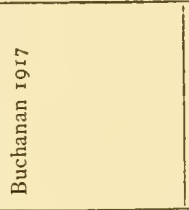 & 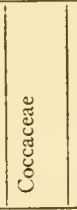 & 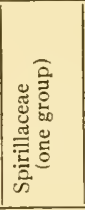 & 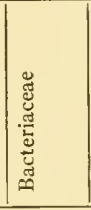 & 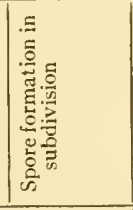 & 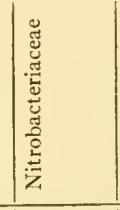 & 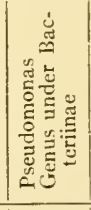 & 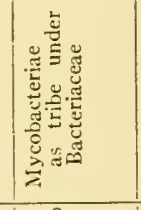 & 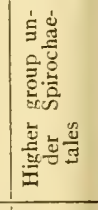 \\
\hline 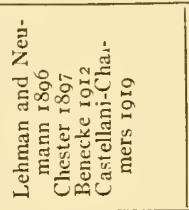 & 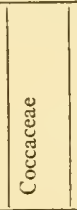 & 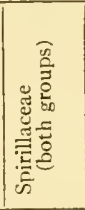 & 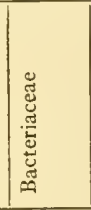 & 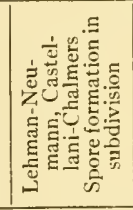 & 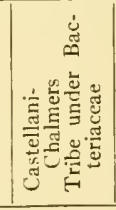 & 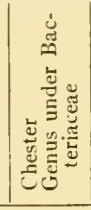 & 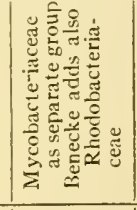 & \\
\hline 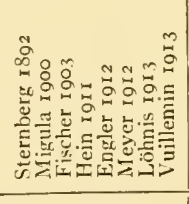 & 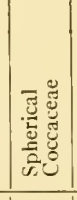 & 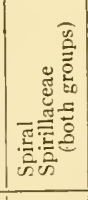 & 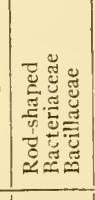 & 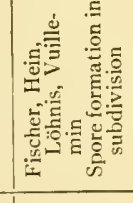 & & 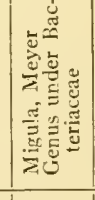 & & \\
\hline 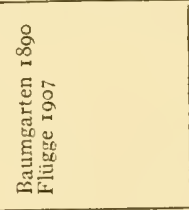 & 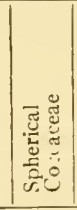 & 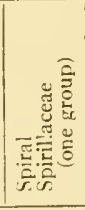 & 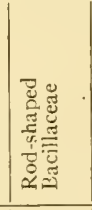 & 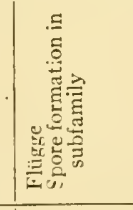 & & & & 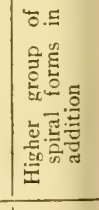 \\
\hline
\end{tabular}

范

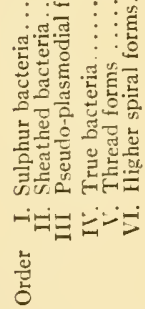

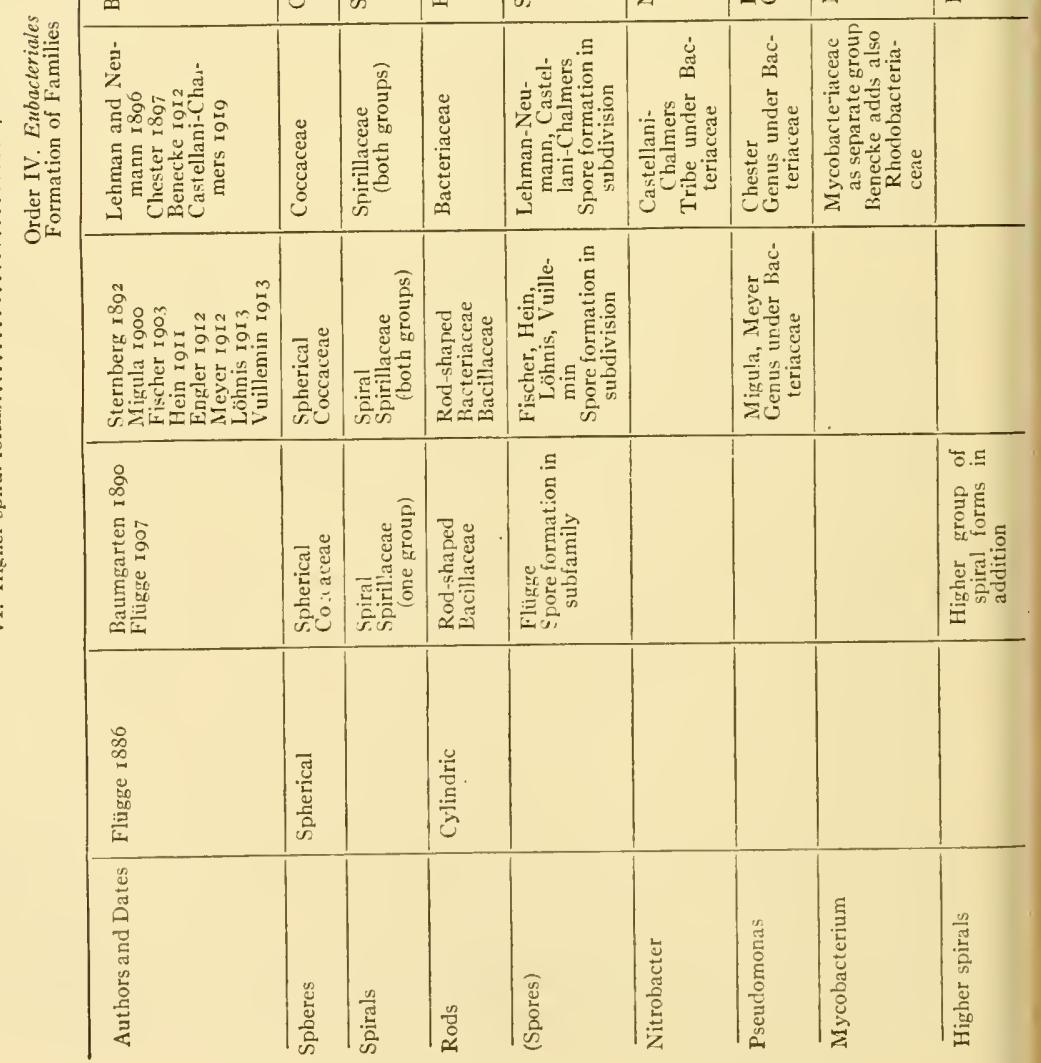


backward is an open question, and in the meantime we find in general that, at least under ordinary conditions, in the laboratory of nature rather than in that of the specialized scientist, the coccus remains coccus. There are two qualifications to the acceptance of a single group for all spherical forms. Some desire to place the so-called "primitive" nitro group together, both the spheres and the rods, on the basis of similar physiological characters regardless of form. In a similar manner there are those who believe that organisms of the lactococcus type should be placed with those of the lactobacillus type. In the first report of the Society of American Bacteriologists Committee in I9I7, Lactobacillaceae was recorded as a family, and in the final report it was placed as a tribe under Bacteriaceae.

Further subdivisions of the family have been made in various ways. Winslow, Bergey, Castellani and Chalmers among recent workers made a primary division on the basis of parasitism, following this by morphology. The Society of American Bacteriologists follows Winslow closely, but many writers feel that the establishment of habitat, or its subdivision parasitism, is likely to be misleading and does not produce natural groups.

Hucker (loc. cit.) considers pigment as a species rather than a generic distinction, and sees no reason for the retention of the red pigment formers as a genus among the cocci, the rest having already been generally abandoned in this group. In general, all the chemical and physiological characters are best used in the differentiation of species and varieties.

Rod forms. - There remains the large group of rod forms about whose classification there is a more or less consistent disagreement. As will be noted by the chart, the tendency of foreign writers is to group all of these under one head, sometimes with one name, sometimes with another, though Orla-Jensen has made much wider separations, from which one of the American expansions has come. This is the group of supposedly "primitive" organisms, mentioned in this paper as the "nitro"- forms, accepted with more or less modification by Buchanan, the Committee, and Bergey and criticized by others, as noted earlier in the paper. An additional family, the Pseudomonadaceae, including pyocyaneus and allied forms, is found as such only in the Society of American Bacteriologists and in Breed's comments (I9I 7 ), elsewhere having a maximum rank of genus. Another American expansion is the elevation to family rank of both the spore-formers and the non-spore-formers, as Bacteriaceae and $\mathrm{Ba}$ cillaceae, a proper division, though there is argument as to the rank which should be given these groups. Buchanan and Castellani, in agreement with most of the European authors, prefer subfamily rank. The variety of further divisions is unending, and, as noted earlier, far more work has been done on species and variety than on genus and higher ranks. It is almost impossible to summarize within any reasonable space the variety of opinions. Primary differentiations, after or before relation to spore formation, are made on the basis of pigment formation, habitat, morphology, and physiology. Bergey, in his eleven tribes of non-spore-formers, names two on the basis of pigment formation, three on parasitism, five on cultural characteristics, and one on morphology. Buchanan makes two divisions of the same group by morphology, with a second division by physiological needs and further subdivisions on different bases for different groups. Castellani and Chalmers divide their family of Bacillaceae 
into ten tribes, primarily on the cultural characters, or physiological activities. This is sufficient to show clearly how little bacteriologists have been able to agree on evaluation of the actual relations of these various characters.

\section{SUMMARY OF "EUBACTERIALES"}

Within the Eubacteriales the final report of the Committee forms the largest number of families, and may consequently be used as a basis. The accompanying chart shows the general relations and the variations in content of most of the European and American classifications, except those which depart so far from the prevailing methods as not to be readily comparable. It will be at once noted that the American group is more detailed. One may summarize the chart somewhat as follows:

The "spherical" group is generally accepted.

The "spiral" group is generally accepted, but the content varies, some writers including both the spirals typified by the cholera organism, and those of the "spirochaete" type, oihers placing the latter group separately, usually in an order by itself.

The "cylindrical" or rod group is the most varied. Under it, however, certain groups are usually put together, such as the tuberculosis-diphtheria combination, unless this is placed with actinomyces.

The "spore-formers" and "non-spore-formers" are usually differentiated, but the rank varies.

The "nitro" -bacteria are generally kept together though again with different ranks and occasionally placed with the pyocyaneus-fluorescens group.

The "pigment formers" are also kept more or less together.

In the various classifications such characters as motility, flagella arrangement, gram stain, fermentation, proteolysis, etc., are mostly well down the line.

\section{REFERENCES}

No attempt has been made to develop or extend a list. Any student of classification must necessarily consult Buchanan, who gives a full bibliography. This is supplemented by Enlows as concerns genera, and the various articles by Winslow and his associates add enough references to occupy the student for some time. Save when otherwise specified, classifications recorded by these writers have been accepted and are referred to in the published articles.

Aldrich, J. M.: "The Limitations of Taxonomy," Science, 65, 38I-85. I927.

Bergey, D. H., et al.: Manual of Determinative Bacteriology, I923.

Breed, R.S., Comn, H. J., and Baker, J. C.: "Comments on the Evolution and Classification of Bacteria," J. Bact., 3, 445-59. I9I8.

Breed, R. S., and Conn, H. J.: "Nomenclature of Actinomycetaceae," ibid., 4, 585-602. I9I9; ibid (Addenda), 5, 480-90. 1920.

Buchanan, R. E.: General Systematic Bacteriology. 1925; "Nomenclature of the Coccaceae," J. Infect. Dis., I7, 528-41. 19г5; "Studies in Nomenclature and Classification of Bacteria," ibid., I-3. 1916-18; "The Evolution of Bacteria," Scicnce, 47, 320-24. I918.

Castellani, Aldo, and Chalmers, A. J.: Manual of Tropical Medicine. 1919; Ann. de l'Inst. Pasteur, 34, 600-621. 1920.

Chester, F. O: Manual of Determinative Bacteriology. rgor.

Enderlein, G.: Baktericn-Cyclogcnie. Berlin and Leipzig, I924. 
Enlows, E. M. A.: "Generic Names of Bacteria." IIyg. Lab. Bull. I2I.

Felt, E. P.: "Nomenclatural Efficiency," Science, 65, 489-9r. 1927.

Hall, I. C.: "Some Fallacious Tendencies in Bacteriologic Taxonomy," J. Bact., r3, 245-53. 1927.

Hitchcock, A. S.: "How the Taxonomists May Utilize the Instructional Committee on Nomenclature." Scicnce, 65, 412-15. 1927.

Hucker, G. J.: "Studies on the Coccaceae," Tech. Bull. 99-ro3. Geneva, N.Y.: New York State Agricultural Experiment Station, 1924.

Kligler, I. J.: "A Systematic Study of the Coccaceae in the Collection of the Museum of Natural History," J. Infect. Dis., 12, 432. 1913.

Lehmann, K. B., and Neumann, R.: English translation of second German edition by G. H. Weaver.

Merrill, E. D., and Wade, H. W.: "Validity of the Name Discomyces, etc.," Philippine J. Sc., 14, 55-69. I919.

Migula, W.: Systcm der Baktcrien. I887-rgoo.

Orla-Jensen, S.: "Hauptlinien des natürlichen bakterien Systems." Cortralbl. f. Bakterio . Abt. II, 22, 305-46. 1909; "The Main Lines of the Natural Bacterial System," J. Bact. 6, 263-73. r92r.

Stiles. C. W.: "International Code of Zoölogical Nomenclatures as Applied to Medicine," Hyg. Lab. Bull. 24; "Underlying Factors in the Confusion in Zoölogical Nomenclature," Scicnce, 65, 195-99. 1927.

Winslow, C.-E. A., ct al. (Committee of the Society of American Bacteriologists on Characterization and Classification of Bacterial Types.) "The Families and Genera of Bacteria" (Preliminary Report), J. Bact., 5, I9I-229. Baltimore, I9I7; "The Families and Genera of Bacteria" (Final Report of the Committee of the Society of American Bacteriologists on Characterization and Classification of Bacterial Types), ibid. Baltimore, I920.

Winslow, C.-E. A., and Rogers, Anne F.: "A Revision of the Coccaceae," Science (N.S.) 2r, 669-72. 1905; "A Statistical Study of Generic Characters in the Coccaceae," J. Infect. Dis., 3, 485-46. I 906.

Winslow, C.-E. A., and Winslow, Anne Rogers: The Systematic Relationships of the Coccaceat. 1908. 


\title{
CHAPTER $X$
}

ATOMS, IONS, SALTS, AND SURFACES

\author{
WILLIAM D. HARKINS \\ University of Chicago \\ ATOMS, IONS, AND SALTS
}

\section{THE ARRANGEMENT OF ATOMS IN SOLIDS}

Several different types of evidence indicate that solid substances are in general built up from minute building blocks called "atoms," whose diameters vary slightly with the element involved, but are of the order of a hundred-millionth of an inch. The atoms attract one another in a way which is suggestive of the action of small magnets;

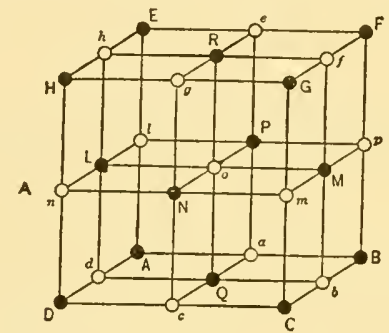

(a)
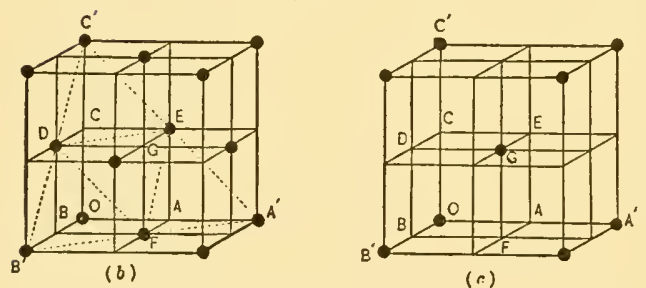

(口)

it is believed that the forces involved arelargely electricalbut partly magnetic. In any crystal the atoms or molecules are arranged in a definite pattern or lattice, and this pattern largely determines the outer form of the crystal. Thus in rock salt (Fig. I $a$ ) sodium and chlorine occupy alternate corners of the cubes of the lattice, and each atom of chlorine is surrounded by six atoms of sodium and each atom of sodium by six atoms of chlorine. Calculations based upon the density of rock salt, its molecular weight and the number of molecules in the molecular weight $\left(6.06 \times 10^{23}\right)$, indicate that the distance between the center of any sodium and any adjacent chlorine atom is $2.8 \mathrm{I}$ hundred-millionths of a centimeter, or 2.8 I A units. ${ }^{\text {. }}$

FIG. I.-(a) Space lattice of sodium chloride, $(b)$ face-centered, and $(c)$ body-centered cubic lattice.

Crystals have 32 types of outer form and 14 types of atomic pattern which may be arranged in 230 different ways.

\section{THE COMPOSITION AND STRUCTURE OF ATOMS}

All known material substances are considered as built up from $9^{2}$ simple forms of matter known as the "chemical elements." Of these 90 have been discovered and 2, with element numbers 85 and 87 , have not been found. Each of these elements was formerly supposed to consist of only one kind of atom of a definite atomic weight, but in recent years it has been found that an element may consist of from one to eleven (probably even more) atomic species which are almost identical in their chemical

${ }^{x} \mathrm{~A}$ glossary of symbols and terms is given on pages 176 and 177 . 



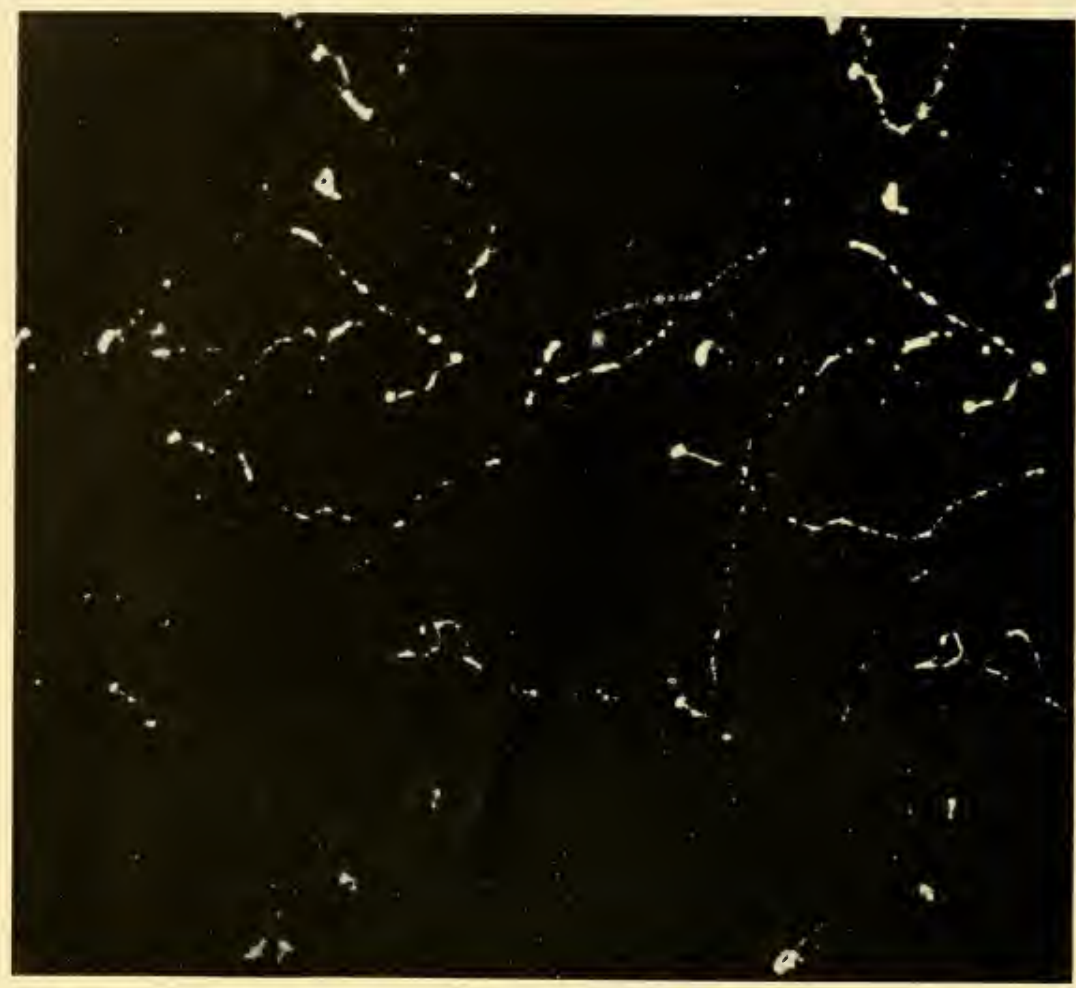

FIG. 2.-Tracks of electrons (C. T. R. Wilson). Note that the tracks are dotted and irregular

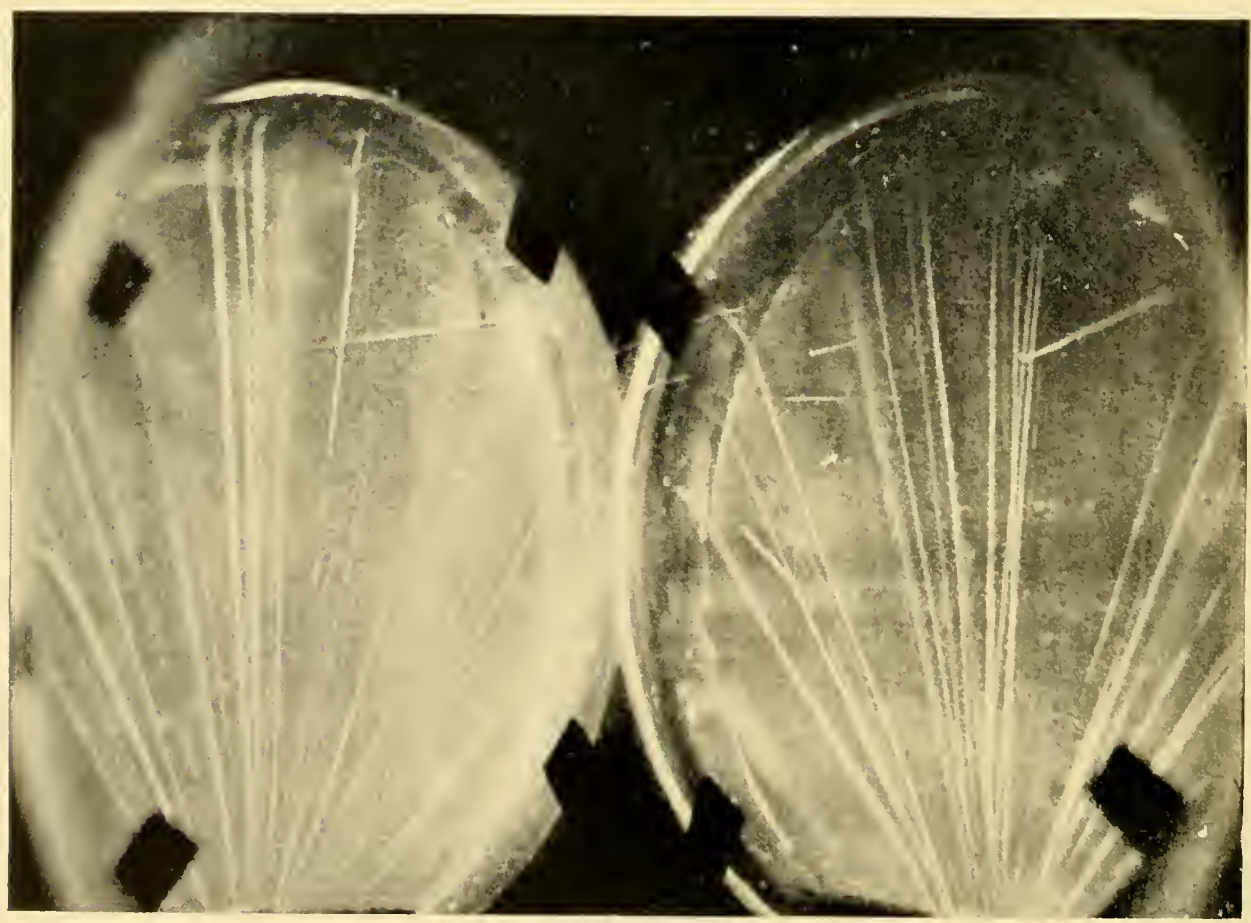

III. $3^{a}$ - (Fig. $3^{b}$ shows how to find the track of a positive electron in this figure.) Tracks of positive helium atoms, and a track of the positive electron (proton or nucleus $[\mathrm{H}+]$ of the hydrogen atom). This photograph illustrates the synthesis of an atom, since a helium atom strikes a nitrogen atom and forms a (heavier) oxygen atom and a hydrogen atom. (Photograph by Harkins and Shadduck.) 
behavior. Since all of the species which constitute an element are given only one place in the periodic system of the chemists, they are called "isotopes" (iso= "same," tope $=$ "place").

The lightest-known atoms are those of hydrogen with a mass of $1.66 \times 10^{-24} \mathrm{gm}$., but the relative or atomic weight is taken as 1.0078 in order that the atomic weight of oxygen may be exactly the whole number 16 .

The mass of a projectile may be determined by the difficulty of deflecting it from its path when moving at any definite speed, since the momentum ( $m v)$ is proportional to the mass at a given velocity. Certain negatively charged particles are thus deflected about two thousand times more easily than hydrogen atoms. These particles are designated as "negative electrons," and their charge is exactly equal in magnitude, within the limits of error of the most exact work, to that carried by positively charged hydrogen in the electrolysis of an acid in water. The tracks of negative electrons in air are shown in Figure 2. It may be noted that these tracks are very irregular, while the tracks given by hydrogen or other atoms are straight.

Thus, this is true of the atom tracks shown in Figure 3. The dim track in the right-hand view is that of positive hydrogen, which is the lightest positively charged particle known, so it is considered as the positive electron, often called the "proton."

All uncharged atoms are built up from equal numbers of protons and electrons. In the hydrogen atom there is one proton, which contains almost all of the mass of the atom and acts as its central sun or nucleus, and one electron which is supposed, in the Bohr theory, to move in an orbit around the proton somewhat as the moon moves around the earth.

It is supposed that the atomic weight of an atomic

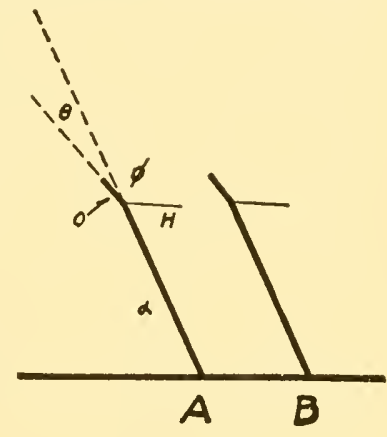

FIG. $3^{b}$.- Shows two views of the synthesis and disintegration of atoms given in Fig. $3 a$. The track marked $H$ represents the track of a positive electron or proton, shown at the left-hand side of the righthand view of Fig. $3 a$. The angles are the same as those in the photograph. species gives the number of protons in its nucleus and also the total number of electrons in the atom. The nucleus of the helium atom consists of four protons and two electrons so that there is a net positive charge of two on the nucleus and there are, therefore, two negative planetary electrons in the outer part of the atom. Helium is the second element in the chemical periodic table, so its atomic number is 2. It may be noted that the atomic number expresses also the positive charge on the nucleus and the number of outer planetary electrons.

\section{MASS, ENERGY, AND FREQUENCY OF VIBRATION}

Mass and energy may be considered as the same but measured in different units. Thus I gm. of mass is equivalent to $\mathrm{I}$ times the square of the velocity of light $(c=3 \times$ $\mathrm{IO}^{\mathrm{I0}} \mathrm{cm}$. a second, so $c^{2}=9 \times 1 \mathrm{O}^{20}$ ), or $9 \times 1 \mathrm{O}^{20}$ ergs. Thus a $\mathrm{I}$-gm. weight (mass $=\mathrm{I} \mathrm{gm}$.) is $9 \times 10^{20}$ ergs of energy.

In I9I 5 Harkins and Wilson showed that, according to the special relativity the- 
ory of Einstein, the conversion of hydrogen into helium should liberate an enormous amount of energy. The reaction may be written:

$$
{ }_{4} \mathrm{H} \rightarrow \mathrm{He}
$$

$4.0312-\mathrm{gm}$. mass $\rightarrow 4.000 \mathrm{gm}$. mass $+0.0312 \mathrm{gm}$. mass in the form of radiation

$0.03 \mathrm{I} 2 \mathrm{gm}$. mass is equivalent to $0.0312 \times 9 \times 10^{20} \mathrm{ergs}=$

$2.8{ } \times 10^{19} \mathrm{ergS}=6.7 \mathrm{I} \times 10^{\text {II }}$ calories

In more ordinary terms, the conversion of I $\mathrm{lb}$. of hydrogen into helium should give off as much energy as radiation-which could be transformed into heat-as would be given by the burning of ro, $\infty$ oo tons of coal.

The frequency of light-vibrations is very great. According to the quantum theory, if this frequency is multiplied by a con-

$\circ$

$\circ$

\section{○迪 0}

O<smiles>O</smiles>

\section{O}

Frg. 4.-Neutral sodium atom (diagrammatic representation in a plane; the structure is unknown).

IONS

Atoms, or molecules which are not electrically neutral, are called "ions," so ions contain either a larger or a smaller number of electrons than of protons. The non'nuclear, or planetary, electrons of an atom are classified in sets. Thus in the sodium atom there are supposed to be three of these sets: the innermost with two, the next with eight, and the third or outermost with one electron. These numerical relations are represented by Figure 4. The chemical properties of an element are supposed to depend to a large extent, but not wholly, upon the number of electrons in the outer set. Thus the atoms of the alkalies, lithium, sodium, potassium, rubidium, and caesium, are all supposed to have one electron in the outer set. They ionize, i.e., lose this outer electron, more easily than any other elements. All of them react violently with water, and in all of their other chemical, and in most of their physical, properties act almost alike.

With helium the outer set of electrons is complete when two electrons are present, but with heavier elements completeness in this set seems to be reached only. when eight electrons are present. 
Sodium, magnesium, and aluminium have, respectively, one, two, and three outer electrons. Such atoms lose electrons to form positive ions. If, however, the outer set of electrons is more than half complete in the neutral atom, there is a much greater tendency to pick up electrons than to lose them, so such atoms usually form negative ions. If the three elements mentioned above are in the gaseous form and the sodium atom loses one $\left(\mathrm{Na}^{+}\right)$, the magnesium two $\left(\mathrm{Mg}^{++}\right)$, and aluminium three electrons $\left(\mathrm{Al}^{+++}\right)$, then ten planetary electrons are present in each of these ions (Fig. 5). This is the number of planetary electrons present in the neutral neon (Ne) atom. Since it is supposed that the arrangement of the electrons is largely conditioned by their number, it is supposed that $\mathrm{Ne}, \mathrm{Na}^{+}, \mathrm{Mg}^{++}$, and $\mathrm{Al}^{+++}$have the same structure except that the electrons are bound somewhat more tightly as the charge on the nucleus changes from $\mathrm{Io}^{+}$for neon to $\mathrm{I}^{+}$for aluminium. That the idea of a common structure is justified is indicated by the fact that in so far as they have been investigated the spectra of these ions upon further dissociation is like that of neon.

0

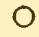

○

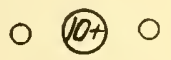

O

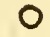

Neutral neon atom
$\mathrm{O}$

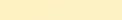

(20)

$\circ$ (13) 0

O

○

Triply positive aluminium ion $(\mathrm{Al}+++)$

Fig. 5. - The composition of $\mathrm{Ne}, \mathrm{Na}+, \mathrm{Mg}++$, or $\mathrm{Al}+++$. The nuclear charges are ro,$+ \mathrm{II}^{+}$, $\mathrm{I} 2+$, and $\mathrm{I}_{3}+$, respectively.

\section{IONS IN SOLID SALTS}

In rock salt the mean distance from the center of a sodium atom to that of any of the six adjacent chlorine atoms is, as has been stated, $2.8 \mathrm{I} \mathrm{A}\left(2.8 \mathrm{I} \times \mathrm{IO}^{-8} \mathrm{~cm}\right.$.). The fact that the analysis of the structure of such a crystal by $\mathrm{X}$-ray methods has given no evidence that the sodium atom is more firmly attached to any one of these atoms than to the five others has led to the hypothesis that no molecules exist in such a crystal, and that the chlorine atoms are attracted to the sodium atom because the former are charged with a negative $\left(\mathrm{Cl}^{-}\right)$and the latter with a positive $\left(\mathrm{Na}^{+}\right)$electrical charge. Thus the assumption is that the crystal is built up from individual positive and negative ions held together by the attraction between charges of like sign. The attraction between the ions of unlike sign of charge is assumed to follow Coulomb's law that attraction or repulsion between electrified particles varies as the inverse of the square of the distance between them;

$$
F=\frac{e_{1} e_{2}}{r^{2} D}
$$


Madelung and Born find that if Coulomb's law is assumed to hold for the attractive forces, it is necessary, in order to accord with the known values of the compressibility of solid salts, to assume that there is in addition a repulsive force between the ions which varies as about the inverse tenth power:

$$
F=\frac{K D}{r^{10}} \text {. }
$$

According to this type of theory, solid salts consist entirely of ions, and in this sense the salt is already completely ionized.

THE IONIZATION OF SALTS IN AQUEOUS SOLUTIONS

The dielectric constant $(D)$ of ordinary solid salts is about 5 , while that of water at ordinary temperatures is about 80 . Since the attraction between charged particles (equation [I]) varies inversely as the dielectric constant, the attractive forces between ions of opposite sign of charge should be very much less in water than in solid salt. If, therefore, it is assumed that the solid salt is completely ionized, it would be unreasonable to assume that the same salts are less than completely ionized when dissolved in water.

The idea that salts in aqueous solution are completely ionized was suggested by Sutherland in 1907. The theory has been put in more definite form by Bjerrum, by Milner, and by Debye and Hückel. Their fundamental idea is that the electrical attractions between ions of unlike sign, and repulsion between those of like sign, give rise to the following effect: on the average any positive ion will be immediately surrounded by more negative than positive ions, while any negative ion will be surrounded by more positive than negative ions. The most important result is that when the solution is diluted the separation of the ions involves the expenditure of energy. ${ }^{\mathrm{I}}$

I Debye and Hückel have calculated this electrical internal energy by assuming that the charges on each ion are concentrated at a point, and that the distribution of these points is determined by probability. They use the probability relation of Boltzmann, developed in connection with the kinetic theory of gases, together with the equation of Poisson derived from the laws of electrostatics, including Coulomb's law.

Suppose that we have an ion of valence $\pm n$ and charge $\pm n E$. In any concentric shell of thickness $d r$, the potential is $P$ and the density of the electric charge is $\rho$.

The average kinetic energy of the molecules at a temperature $T$ is $3 / 2 k T$. The Boltzmann constant $k$ is equal to the gas constant $R$ divided by the number of molecules in a gram molecule $\left(6.06 \times 1 \mathrm{O}^{23}\right)$. According to Boltzmann's principle, if the molecules are distributed in a field of force, such as in an electrical field, the distribution will be such that the number of molecules, instead of being equal to $N$, the number present in the absence of the field of force, will be equal to $N \epsilon^{\frac{-E}{k T}}$. Thus the number present is modified by a factor in which the base of Naperian logarithms is raised by a power equal to the potential energy of the molecule divided by two-thirds of its mean kinetic energy:

The equation of Poisson applies to the variation of the potential $P$ around a point when it is distributed with spherical symmetry. It may be expressed as follows:

$$
\frac{\mathrm{I}}{r^{2}} \frac{d}{d r}\left(r^{2} \frac{d P}{d r}\right)=\frac{d^{2} P}{d r^{2}}+\frac{2}{r} \frac{d P}{d r}=-\frac{4 \pi \rho}{D} .
$$

The electrical density is represented by $\rho$. 
Suppose that two substances, $A$ and $B$, react to form the substances $E$ and $F$, and that the reaction has proceeded until equilibrium has been attained. The chemical reaction may be expressed:

$$
a A+b B=c E+f F .
$$

It was found by Guldberg and Waage that in such a case a definite law, known as the "mass law," determines the condition of the system at equilibrium. If the substances are gases this may be expressed

$$
\frac{p_{E}^{e} \cdot p_{F}^{f}}{p_{A}^{a} \cdot p_{B}^{b}}=K_{p},
$$

in which $K_{p}$ is a constant.

In the case of the simple dissociation

$$
A=B+C,
$$

the mass law becomes

$$
\frac{p_{B} \cdot p_{C}}{p_{A}}=K_{p}
$$

In the ordinary development of the mass law it is assumed that the gas law ( $p v=$ $N R T$ ) is true, so the mass law fails to holt in any case in which the gas law is invalid. The gas law may be written

$$
p=\frac{N R T}{v}=C R T .
$$

If this is substituted in (4) the following expression is obtained:

$$
\frac{C_{B} \cdot C_{C}}{C_{A}}=\frac{K_{p}}{R T},
$$

but since the temperature is constant and $R$ is the gas constant, $K_{p} / R T$ is a constant, or

$$
\frac{C_{B} \cdot C_{C}}{C_{A}}=K_{C} .
$$

\section{DEVELOPMENT OF THE LAW OF MASS ACTION}

The law of mass action may be developed very simply by a consideration of the amount of work necessary to compress a gas reversibly, i.e., in such a way that the same amount of work may be regained when the gas expands. The amount of work done when a force $(F)$ acts through a distance $(S)$ is

$$
W=F \cdot S .
$$

If a gas expands in a cylinder provided with a piston, the force $(F)$ is equal to the pressure $(p)$ times the area $(A)$ of the piston, so

$$
W=p A S=p \cdot \Delta v .
$$


If the increase of volume $(\Delta v)$ is infinitesimal, it is designated by $d v$, so

But by the gas law

$$
d W=p \cdot d v .
$$

$$
p=\frac{N R T}{v},
$$

so

$$
d W=N R T \frac{d v}{v} .
$$

The work $(W)$ done by the reversible expansion of the gas from a volume to a volume $v_{2}$ is

$$
W=\int_{v_{\mathrm{I}}}^{v_{2}} d W=N R T \int_{v_{\mathrm{I}}}^{v_{2}} \frac{d v}{v}=N R T \ln \frac{v_{2}}{v_{\mathrm{I}}} .
$$

Since, however, the volume of a perfect gas varies inversely as its pressure $(p)$,

$$
W=N R T \ln \frac{p_{1}}{p_{2}} .
$$

Consider a chemical reaction

$$
a A+b B \hookrightarrow e E+f F,
$$

in which all of the substances are in the gaseous state. Let the initial pressures of the gases $A$ and $B$ be $p_{A}^{\prime}$ and $p_{B}^{\prime}$ and the final pressures of $E$ and $F$ be $p_{E}^{\prime}$ and $p_{F}^{\prime}$. Assume that a large box contains all of these four gases in equilibrium with one another at pressures $p_{A}, p_{B}, p_{E}$, and $p_{F}$. Let the gases $A$ and $B$ at pressures $p_{A}^{\prime}$ and $p_{B}^{\prime}$ be contained in two cylinders provided with pistons.

The first step in the process is to change the pressure $p_{A}^{\prime}$ to $p_{A}$. The work done by a gas in such a process equals the number of mols $(a)$ of gas times $R T$ times the logarithm of the initial pressure divided by the final pressure, or

$$
W_{\mathrm{I}}=\operatorname{aRT} \ln \frac{p_{A}^{\prime}}{p_{A}} .
$$

By the use of a well-known characteristic of logarithms, this becomes

$$
W_{\mathrm{I}}=R T \ln \frac{p_{A}^{\prime a}}{p_{A}^{a}} .
$$

The similar equation for the gas $B$ is

$$
W_{2}=R T \ln \frac{p_{\mathrm{B}}^{\prime b}}{p_{B}^{b}} .
$$

The gases $A$ and $B$ are now at pressures equal to their partial pressures in the large equilibrium box. Suppose that the cylinders which contain these gases are set on 
the equilibrium box and that at the bottom of each cylinder there is a membrane permeable only to the gas in the cylinder (Fig. 6).

Force the $a$ mols of the gas $A$ and the $b$ mols of the gas $B$ slowly into the box, and as rapidly as these react take out the $c$ mols of the gas $E$ and the $f$ mols of the gas $F$ which are formed.

Since work must be done upon the gases $A$ and $B$ to force them into the box, the work they do is negative, or

$$
W_{3}=p \cdot \Delta v=-a R t
$$

which is obtained from equation (2) by substituting in it the gas law

$$
p \cdot \Delta v=N R T(N=-a) .
$$

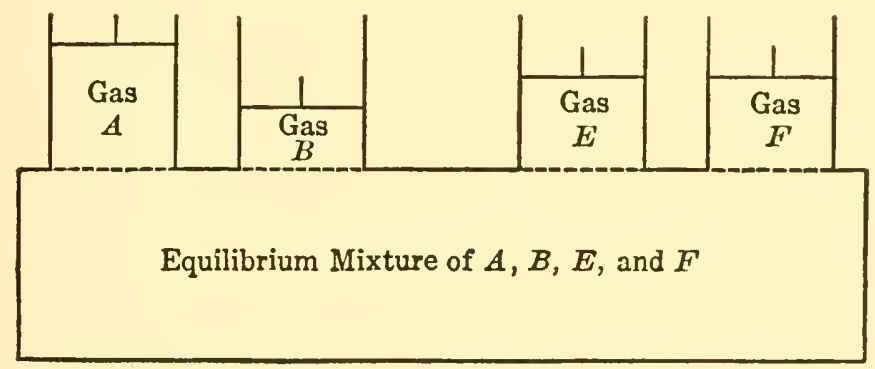

FIG. 6

For the gas $B$

$$
W_{4}=-b R t
$$

Since the gases $E$ and $F$ come out of the box, they do work, and

$$
\begin{aligned}
& W_{5}=e R T, \\
& W_{6}=f R T .
\end{aligned}
$$

The gases $E$ and $F$ are now at pressures $p_{E}$ and $p_{F}$. Let them now be changed to their final state in which the pressures are $p_{E}^{\prime}$ and $p_{F}^{\prime}$ :

$$
\begin{aligned}
& W_{7}=R T \ln \frac{p_{E}^{e}}{p_{E}^{\prime e}}, \\
& W_{8}=R T \ln \frac{p_{F}^{f}}{p_{F}^{\prime} f} .
\end{aligned}
$$

The total work $(W)$ is the sum of these eight quantities of work, or

$$
W=R T \ln \frac{p_{E}^{e} p_{F}^{f}}{p_{A}^{a} p_{B}^{b}}-R T \ln \frac{p_{E}^{\prime}{ }^{e} p_{F}^{\prime} f}{p_{A}^{\prime} p_{E}^{\prime} e}+(e+f-a-b) R T .
$$


Now, $e+f-a-b$ is simply the increase $(\Delta n)$ in the number of molecules when the given reaction takes place, so

$$
W=R T \ln \frac{p_{E}^{e} p_{F}^{f}}{p_{A}^{a} p_{B}^{b}}-R T \ln \frac{p_{E}^{\prime e} p_{F^{\prime}}^{\prime}}{p_{A}^{\prime a} p_{B}^{\prime b}}+\Delta n \cdot R T .
$$

(Term I) (Term 2) (Term 3)

If the reaction takes place at a constant temperature, term 2 is a constant, since the pressures designated by primes are fixed values. Term 3 is also constant, sinc . $\Delta n$, the increase of the number of mols of gas, is fixed by the reaction.

The work done in a series of reversible changes depends only on the initial and final states, and is therefore a fixed quantity for the given reaction. Equation (I $\delta$ ) may now be written:

$$
K_{\mathrm{I}}=R T \ln \frac{p_{E}^{e} p_{F}^{f}}{p_{A}^{a} p_{B}^{b}}+K_{2}+K_{3}
$$

or

Therefore

$$
R T \ln \frac{p_{E}^{e} p_{F}^{f}}{p_{A}^{a} p_{B}^{b}}=K_{1}-K_{2}-K_{3}=K_{4} .
$$

$$
\frac{p_{E}^{e} p_{F}^{f}}{p_{A}^{a} p_{B}^{b}}=K
$$

which is the law of mass action. This equation gives the mass law in a general form.

For a reaction in which only I mol of each substance is involved,

it takes the form

$$
A+B=E+F
$$

$$
\frac{p_{E} \times p_{F}}{p_{A} \times p_{B}}=K
$$

Thus, according to the mass law, the product of the partial pressures in the equilibrium mixture of the substances formed in a chemical reaction, divided by the product of the partial pressures of the substances from which they are formed, is equal to a constant at any given temperature. If more than I mol of any of the substances is involved in the reaction, the corresponding partial pressure must be raised to a power equal to the number of mols.

\section{ESCAPING TENDENCY OR ACTIVITY}

According to the mass law, the effect of any substance present upon the chemical equilibrium is proportional to its concentration. Thus, the activity ( $a$ ) of a substance may be expressed by its concentration, or

$$
\frac{C_{B} C_{C}}{C_{A}}=\frac{a_{B} a_{C}}{a_{A}}=K .
$$


While the activity of a perfect gas, or a substance which in solution obeys the perfect-gas law, is expressed by its concentration, this is not the case if the gas law does not hold. The activity may then be defined as that quantity which, when substituted for the concentration of a substance in the mass-law equation, expresses its effect in determining the equilibrium.

If a small quantity of iodine is shaken with water and carbon disulphide at $25^{\circ} \mathrm{C}$. until equilibrium is attained, it is found that a unit volume of carbon disulphide contains six hundred times more iodine than the same volume of water. Since there is equilibrium, the tendency of iodine to escape from the water is the same as that from carbon disulphide, i.e., the vapor pressure of iodine should be the same over the two solutions, and this is found to be true. The identity of vapor pressures of the iodine indicates that the activities are equal.

If it is considered that the activity of the iodine in the carbon disulphide is equal to its concentration, then the activity in water, which is the same, must be six hundred times the concentration of the iodine in the water. Thus carbon disulphide is a better solvent than water for iodine since it can hold six hundred times as much of the latter and still give no more activity to the iodine. ${ }^{\mathrm{I}}$

I. When the activity of a constituent is the same in two different phases the constituent will not increase its concentration in one phase at the expense of the other unless energy is supplied to effect the transfer.

2. If the activity of a constituent is greater in one phase than another the constituent (if transferred at all) will pass from the phase in which it has the greater into the one in which it has the lesser activity.

THE ACTIVITY COEFFICIENT OF ELECTROLYTES

A solution of common salt which contains $58.5 \mathrm{gm}$. of sodium chloride to $\mathrm{I}, 000 \mathrm{gm}$. of water is considered to be I molal in concentration. If $m$ represents the molality of a solution, its activity coefficient $(a)$ is defined as the ratio of its activity to its molality, or

$$
a=\frac{a}{m} ; \quad \text { so } \quad a=m \alpha \text {. }
$$

The activity coefficient for a salt in its extremely dilute solution is I.0000 by definition. At a molality of 0.0 I the activity coefficient for sodium chloride in its aqueous solution is 0.922 , while at $0.1 m$ it is 0.798 . Now the activity coefficient plays the same

I The escaping tendency may be expressed also in terms of the "fugacity" ( $f$ ), a term introduced by G. N. Lewis. The fugacity of an ideal gas is equal to its pressure. Any gas is practically ideal at low pressures. At higher pressures its fugacity is the geometric mean of the actual pressure $(P)$ of the gas and the ideal pressure $\left(P_{i}\right)$ calculated from the gas law, so that

$$
f=\frac{P_{2}}{P_{i}} .
$$

The activity of a constituent may now be refined as its relative fugacity $(f)$ as compared with its fugacity in some standard state $\left(f_{0}\right)$, or

$$
a=\frac{f}{f_{0}} .
$$


part in the newer work on the ionization of salts in solution as that of the degree of ionization in the older theory. Thus the degree of ionization of sodium chloride in its O. $m$ aqueous solution was given as 0.86 , while the activity coefficient as given above is 0.798 . The activity coefficient is sometimes designated as the "thermodynamic degree of dissociation."

Table I gives the activity coefficients for sodium and potassium chlorides and a few salts of higher types. It may be noted that as the product of the valence of the ions of the salt increases the activity coefficient decreases.

TABLE I

Activity Coefficients of Salts in Aqueous Solution at $25^{\circ}$ C., as Calculated from the Lowering of the Freezing-Point

\begin{tabular}{|c|c|c|c|c|c|}
\hline$\underset{m}{\text { Molality }}$ & $\begin{array}{c}\text { Activity } \\
{ }^{a} \mathrm{NaCl}\end{array}$ & $\begin{array}{c}\text { Coefficient } \\
{ }^{a} \mathrm{KCl}\end{array}$ & ${ }^{a} \mathrm{BaCl}_{2}$ & ${ }^{\alpha} \mathrm{La}\left(\mathrm{NO}_{3}\right)_{3}$ & ${ }^{\alpha} \mathrm{MgSO}_{4}$ \\
\hline $0.001 \ldots \ldots \ldots \ldots$ & 0.966 & $0.9^{6} 5$ & $\cdots$ & $\cdots \cdots$ & $\ldots \ldots \ldots$ \\
\hline $.002 \ldots \ldots \ldots \ldots \ldots$ & .953 & $.95 \mathrm{I}$ & $\ldots \ldots$ & $\ldots \ldots \ldots$ & $\ldots \ldots \ldots$ \\
\hline $.005 \ldots \ldots \ldots \ldots$ & .928 & .926 & $\ldots \ldots \ldots$ & $\ldots \ldots \ldots$ & $\ldots \ldots \ldots$ \\
\hline $.01 \ldots \ldots \ldots \ldots$ & .903 & .899 & 0.716 & $0.57 \mathrm{I}$ & 0.40 .4 \\
\hline $.02 \ldots \ldots \ldots \ldots$ & $.87 \mathrm{I}$ & .865 & .655 & $.49 \mathrm{I}$ & $.32 \mathrm{I}$ \\
\hline $.05 \ldots \ldots \ldots \ldots$ & $.82 I$ & .809 & $\cdot 568$ & $.39 \mathrm{I}$ & .225 \\
\hline. $\mathbf{x} \ldots \ldots \ldots \ldots \ldots$ & $\cdot 778$ & $\cdot 762$ & 0.50 & .326 & . 166 \\
\hline $.2 \ldots \ldots \ldots \ldots \ldots$ & .734 & .715 & $\ldots \ldots \ldots$ & $0.27 \mathrm{I}$ & o. I I9 \\
\hline $0.5 \ldots \ldots \ldots \ldots \ldots$ & $.67 \mathrm{I}$ & .654 & $\ldots \ldots$ & $\ldots \ldots \ldots$ & $\ldots \ldots \ldots$ \\
\hline I. o. . . . . . . & .634 & 0.605 & $\ldots \ldots$ & $\ldots \ldots \ldots$ & $\ldots \ldots \ldots$ \\
\hline $2.0 \ldots \ldots \ldots \ldots \ldots$ & .645 & $\ldots \ldots \ldots$ & $\ldots \ldots$ & $\ldots \ldots \ldots$ & $\ldots \ldots \ldots$ \\
\hline $3.0 \ldots \ldots \ldots \ldots$ & .686 & $\ldots \ldots$ & $\ldots \ldots$ & $\ldots \ldots \ldots$ & $\ldots \ldots \ldots$ \\
\hline $4.0 \ldots \ldots \ldots \ldots$ & .746 & $\ldots \ldots \ldots$ & $\ldots \ldots$ & $\ldots \ldots \ldots$ & $\ldots \ldots \ldots$ \\
\hline $5.0 \ldots \ldots \ldots \ldots$ & $.83 \mathrm{I}$ & $\ldots \ldots \ldots$ & $\ldots \ldots$ & $\ldots \ldots$ & $\ldots \ldots \ldots$ \\
\hline $5.2 \ldots \ldots \ldots \ldots \ldots$ & 0.852 & & $\ldots \ldots$ & & $\ldots \ldots \ldots$ \\
\hline
\end{tabular}

THE IONIC STRENGTH

The activity of ions in a mixture is determined by the concentrations and electrical charges of all of the ions present. Lewis and Randall found that if the molality of each ion is multiplied by the square of its valence, the sum of these quantities (divided by 2 , since both positive and negative ions are included) is an important quantity, designated as the ionic strength $(\mu)$.

It is found that in dilute solutions the activity coefficient of a given clectrolyte is the same in all solutions of the same ionic strength.

The activity coefficient $(\alpha)$ in water of a salt composed of two ions is given by an extremely simple equation:

$$
-\log _{10} a=0.50 Z_{1} Z_{2} 1^{\prime} \bar{\mu},
$$

in which $Z_{1}$ and $Z_{2}$ are the valences of the ions of the salt, and

$$
\mu=\frac{1}{2}\left(m_{1} Z_{1}^{2}+m_{2} Z_{2}^{2}+\ldots\right)=\frac{1}{2} \geq\left(m Z^{2}\right) \text {. }
$$

CALCULATION OF THE ACTIVITY COEFFICIENT FRUM SOLUBILITY

If a solid $A$ is in contact with its saturated solution, the relation may be expressed:

$$
A_{\text {(solid) }}=A_{\text {(dissolved) }},
$$


so the activity of the dissolved substance is equal to the activity of the solid:

$$
a_{\text {(solid) }}=a_{(\text {dissolved } \lambda)}=\text { Const. ; }
$$

but since

$$
\begin{gathered}
a_{(\text {dissolved })}=m a, \\
m_{0} a_{0}=m_{1} a_{1}=m_{2} a_{2},
\end{gathered}
$$

where $m_{0}$ designates the solubility in water and $m_{1}$ and $m_{2}$ refer to the solubility of the substance $A$ in aqueous solutions to which different salts have bcen added. From (6),

$$
a=a_{0} \frac{m}{m_{0}} \text {. }
$$

If the activity coefficient of the pure substance in its aqueous solution is arbitrarily fixed as unity, then

$$
a=\frac{m_{0}}{m}
$$

If the solute (dissolved substance) is a salt, its mean molality ( $m \pm$ ) should be used.

$$
a=\text { Const. } \frac{\mathrm{I}}{m_{ \pm}} \text {. }
$$

The mean molality is a geometric mean as defined by the equation

$$
m_{ \pm}=m\left(Z_{+}^{Z+} Z_{-}^{Z-}\right)^{\mathbf{x} / Z}
$$

and $Z=Z++Z_{-}$.

The extremely simple relation which emerges is that the activity coefficient for a saturating salt in solutions of other salts is inversely proportional to the (geometric) mean molality of its own ions. ${ }^{\mathrm{I}}$

\section{IONIZATION OF SUBSTANCES NOT COMPLETELY IONIZED}

Many acids and bases, and some salts, are not completely ionized. In general, the activities of such substances in aqueous solution have not been determined; but the percentage ionization $\left(a_{i}\right)$ has been calculated from the ratio of the electrical conductance $(\Lambda)$ of the solution at the given concentration to its conductance at zero concentration or from this conductance ratio corrected by the ratio of viscosities $(\eta)$,

$$
a_{i}=\frac{\Lambda \eta}{\Lambda_{0} \eta}
$$

Table II gives the percentage ionizations of a few acids and bases. At the same

${ }^{1}$ This system gives entirely correct values of the activity coefficient, but the values become greater than unity in extremely dilute solutions. It is more common to extrapolate some function of $m_{\mathrm{o}} / m$ to zero concentration. Thus in dilute solutions if $\log \left(\mathrm{m} / \mathrm{m}_{\mathrm{o}}\right)$ is plotted against $\mu^{\frac{1}{2}}$, a straight line is commonly obtained. From (8) $-\log a=\log \left(m / m_{0}\right)$. If for $\mu=0, \log \left(m / m_{0}\right)$ is put eaual to zero, then a becomes unity, and all of the other activity coefficients are on this basis: thus a is unity at zero concentration of the salt. This is the system used in Table I. 
concentration the values indicated are 45 per cent for cadmium chloride and less than o.I per cent for the mercuric halides.

TABLE II

Percentage Ionization of Acids and Bases in Water at o. Normal ANd $25^{\circ} \mathrm{C}$.

Per Cent

Sulphurous acid (into $\mathrm{H}+$ and $\left.\mathrm{HSO}_{3}^{-}\right) \ldots \ldots \ldots \ldots \ldots \ldots . \ldots \ldots$

Phosphoric acid (into $\mathrm{H}+$ and $\mathrm{H}_{2} \mathrm{PO}_{4}^{-}$) $\ldots \ldots \ldots \ldots \ldots 28$

Nitrous or hydrofluoric acid................ 8

Hydrogen sulphide, carbonic acid, and hypochlorous acid... o.r

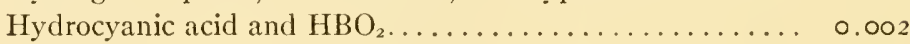

WEAK ELECTROLYTES OR SLIGHTLY IONIZED SUBSTANCES

Slightly ionized substances follow the mass-law relation with respect to ionization at small concentrations, provided strong electrolytes are not present. Thus, with acetic acid (HAC),

$$
\frac{C_{H}^{+} \cdot C_{11}^{-}}{C_{I H A C}}=K_{i}=0.000018 \text {. }
$$

The ionization constants $\left(K_{i}\right)$ for a few weak electrolytes are given in Table III.

TABLE III

Ionization Constants at $25^{\circ} \mathrm{C}$.

Acids

Hydrocyanic

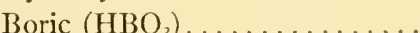

Hypochlorous............

Nitrous................ 400

Hydrofluoric............. 790

Formic................ 210

Acetic............... I8

Propionic................. I3

n-Butyric ............. I

Monochlor acetic.......... I, $55^{\circ}$

Benzoic................ 60

Ortho

Hydroxy benzoic .......... I, 020

Chlor benzoic............ I, 320

Nitro benzoic............ 6, I60 Bases

Ammonium hydroxide.

Phenyl ammonium hydroxide. $\quad 0.0004$
I0 ${ }^{6} \mathrm{~K}$

0.0005

.0017

.044

(1)


The change of state is

$$
\begin{aligned}
& \left.\frac{H_{2}}{2} \text { (Ioft } \mathrm{atm} \text {. }\right) \rightarrow H+(\text { at concn. } x), \\
& \text { Right } \\
& H+(\text { at concn. } x) \rightarrow \frac{H_{2}}{2} \text { ( a atm.). }
\end{aligned}
$$

Therefore, the total change is

$$
\frac{H_{2}}{2} \text { (ro atm.) } \rightarrow \frac{H_{2}}{2} \text { ( I atm.). }
$$

The electromotive force (E) of the cell is an energy quantity and may therefore be calculated as the equivalent of the maximum work which would be obtained if $\frac{1}{2}$ mol of hydrogen gas expands from a pressure of ro atm. to that of $\mathrm{I}$ atm. ${ }^{1}$

Now the amount of work done by a force $F$ in acting through a space $S$ is

$$
W=F S .
$$

But since force equals pressure times area $(A)$,

$$
W=p A S=p \cdot \Delta v,
$$

if $\Delta v$ represents the increase of volume. Thus for a small amount of work

but since

$$
d W=p d v,
$$

$$
\begin{gathered}
p=\frac{N R T}{v}, \\
d W=N R T \frac{d v}{v},
\end{gathered}
$$

or by integration between the limits $v_{2}$ and $v_{1}$,

$$
W=N R T \ln \frac{v_{2}}{v_{1}}=N R T \ln \frac{p_{1}}{p_{2}} .
$$

This gives the amount of mechanical work which is equal to the change of electrical energy $(F) \Re$, if $\Re$ is the number of equivalents of electricity transferred. $F$, the value of one equivalent of electricity, is equal to 96,500 coulombs. So

or

$$
\begin{aligned}
& \text { ๒ฺほ }=N R T \ln \frac{p_{\mathrm{x}}}{p_{2}}, \\
& \mathfrak{E}=\frac{N R T}{\Re F} \ln \frac{p_{\mathrm{r}}}{p_{2}}, \\
& =\frac{N R T 2.303}{\Re 96,500} \log \frac{p_{\mathrm{r}}}{p_{2}} .
\end{aligned}
$$

The small amount of work involved in the change of volume of the liquid is neglected for the sake of simplicity. 
In the special case given above, $p_{1}=\mathrm{IO}$ and $p_{2}=\mathrm{I}$, and $\log \frac{p_{1}}{p_{2}}=\mathrm{I}$, the number of mols of hydrogen $(\mathrm{N})$ transferred is $\frac{1}{2}$ and the number of charges transferred $(9 i)$ is I, so

$$
\leftleftarrows=\frac{1}{2} \frac{R T}{96,500}
$$

If the temperature is $25^{\circ} \mathrm{C}$., then $T=270+25=298^{\circ} \mathrm{K}$., and $\mathrm{F}=\frac{0.059 \mathrm{I} 5}{2}$ volts $=$ 0.02957 volts.

A cell may contain two aqueous solutions with different hydrogen-ion concentrations, such as

$$
\left.M+H_{2} \text { ( } p \text { atm. }\right), H^{+C l-}\left(m_{1} \text { molal }\right), H^{+} C l-\left(m_{2} \text { molal }\right), M+H_{2}(p \text { atm. }) .
$$

Now if I Faraday of positive electricity is passed from left to right, the following changes occur:

$$
\begin{aligned}
& \text { Left Electrode } \\
& \frac{H_{2}}{2}(p \text { atm. }) \rightarrow H^{+}\left(m_{1} \text { molal }\right), \\
& \text { Liquid Junction } \\
& T_{\left(H^{+}\right)} H^{+}\left(m_{1} \text { molal }\right) \rightarrow T_{\left(H^{+}\right)} H^{+}\left(m_{2} \text { molal }\right), \\
& T_{(\mathrm{Cl}-)} \mathrm{Cl}^{-}\left(\mathrm{m}_{2} \text { molal }\right) \rightarrow T_{(\mathrm{Cl}-)} \mathrm{Cl}+\left(m_{1} \text { molal }\right), \\
& \text { Right Electrode } \\
& H^{+}\left(m n_{2} \text { molal }\right) \frac{H_{2}}{2}(p \text { atm. }) \text {. }
\end{aligned}
$$

If the changes at the electrode are alone considered it is found that as much hydrogen $\left(H_{2} / 2\right)$ appears at $p$ atm. as disappears at that pressure, so this change involves no work, and therefore no electromotive force.

If the liquid junction is neglected for the moment, the total remaining change is

$$
H^{+}\left(m_{2} \text { molal }\right) \rightarrow H^{+}\left(m_{1} \text { molal }\right)
$$

and

$$
\mathfrak{E}_{E}=\frac{N R T}{9 i T} \ln \frac{m_{2}}{m_{1}} \quad \text { (Approximate) }
$$

or

$$
\mathfrak{E}_{E}=\frac{N R T}{\mathfrak{M} T} \ln \frac{a_{2}}{a_{1}} \quad \text { (Exact). }
$$

Here $N / \mathfrak{R}=\mathrm{I}$. For the liquid junction potential,

$$
\begin{aligned}
\mho_{L} & \left.=T_{(I I}+, \frac{R T}{\mathfrak{F}} \ln \frac{m_{1}}{m_{2}}+T_{(C l}-\right) \frac{R T}{\Im} \ln \frac{m_{2}}{m_{1}} \\
& =-T_{(H}+\frac{R T}{\mathfrak{F}} \ln \frac{m_{2}}{m_{1}}+T_{(C l}-, \frac{R T}{\Im} \ln \frac{m_{2}}{m_{1}},
\end{aligned}
$$


or since

$$
\begin{aligned}
& T_{C l}-+T_{n}+=\mathrm{I}, \\
& T_{\left(\mathrm{Cl} l^{-}\right)}-\left(\mathrm{I}-T_{C l}-\right)=\left(2 T_{C l}--\mathrm{I}\right), \\
& \mathfrak{E}_{L}=\left(2 T_{C l^{-}}-\mathrm{I}\right) \frac{R T}{\mathfrak{F}} \ln \frac{m_{2}}{m_{\mathrm{I}}}, \\
& \therefore \quad \mathfrak{E}=\mathfrak{E}_{E}+\mathfrak{E}_{L}=2 T_{(\mathrm{Cl}-)} \frac{R T}{\mathfrak{F}} \ln \frac{m_{2}}{m_{\mathrm{s}}} ;
\end{aligned}
$$

or more exactly:$$
\mathfrak{E}=2 T_{\left(\mathrm{Cl}^{-}\right)} \frac{R T}{\mathfrak{F}} \ln \frac{a_{2}}{a_{1}} .
$$

By the use of such concentration cells it is possible to find the hydrogen-ion activity in one solution, provided its activity in some other solution with the same anion is known. If the anion is not the same, difficulties arise in the calculation of the liquidliquid junction potential.

The results of part of the research by biologists upon hydrogen-ion concentration have been based on the assumption that two different liquid-liquid junctions give the same potential, and can therefore be canceled, which is in general not true.

Since the electromotive force, and therefore the maximum work or free energy, varies as the logarithm of the activity (or inexactly as the concentration) ratio, the hydrogen-ion activity or concentration is often expressed as the logarithm. Thus in pure water at $25^{\circ} \mathrm{C}$. the hydrogen-ion concentration is considered to be $10^{-7} \mathrm{molal}$, or the logarithm is -7 . This is often expressed according to the system of Sörensen as pH $=7$. If, for example, $\mathrm{pH}=5.2$, the hydrogen-ion concentration is $10^{-5.2}$ molal, and the solution is on the acidic side of the neutral point $\left(C_{n}+=0.63 \mathrm{Io}^{-5}\right.$ mols per liter).

\section{THE SOLUBILITY PRODUCT}

Suppose that we have two saturated solutions ( $\mathrm{r}$ and 2) of thallium chloride separated by a partition which consists of a single crystal of the salt. Let solution I be on the left and solution 2 on the right.

$$
\mathrm{Tl}++\mathrm{Cl}-\leftrightarrows\left(\mathrm{TlCl} \text { (solid) } \leftrightarrows \mathrm{Tl}^{+}+\mathrm{Cl}^{-} .\right.
$$

The mass-law relation is

$$
a_{T}^{\prime}+\times a_{C l}^{\prime}-=a_{\text {solid }} T l C l=a_{T l}^{\prime \prime}+\times a_{C l}^{\prime \prime}-=K .
$$

This is known as the "solubility product." In the case cited, the solid salt of the middle phase has a constant activity; but even if other salts, such as $\mathrm{Tl}^{+} \mathrm{R}^{-}$or $\mathrm{NaNO}_{3}$, are present, the relations between the activities in the right- and left-hand phases may be expressed:

$$
\frac{a_{T l}^{\prime}+}{a_{T l+}^{\prime \prime}}=\frac{a_{C l-}^{\prime \prime}}{a_{C l-}^{\prime}} .
$$




\section{THE MEMBRANE EQUILIBRIUM}

Let the solid salt be replaced by a membrane, and let one of the salts in the lefthand solution consist of one ion $(\mathrm{Na}+)$, which is, and one $\left(R^{-}\right)$, which is not, diffusible through the membrane. The equilibrium relations are represented below:

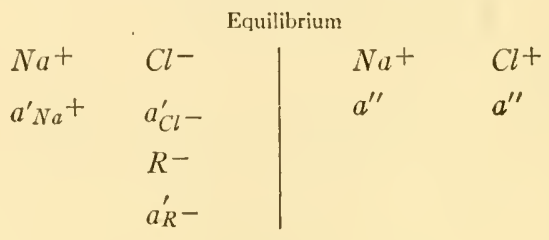

If both solutions are considered infinite in volume, then the transfer of $\mathrm{I}$ mol of sodium chloride from left to right will not disturb the equilibrium. If I mol is thus transferred, the decrease of free energy $(-\Delta F)$ is

$$
-\Delta F=R T \ln \frac{a_{N a}^{\prime}+}{a_{N a}^{\prime \prime}+}+R T \ln \frac{a_{C l}^{\prime}-}{a_{C l}^{\prime \prime}-} .
$$

But the characteristic of an equilibrium process is that the change of free energy $(-\Delta l)$ is zero, so

$$
\ln \frac{a_{N a}^{\prime}+}{a_{N a}^{\prime \prime}+}=\ln \frac{a_{C l}^{\prime \prime}-}{a_{C l}^{\prime}-},
$$

or

$$
\frac{a_{V a}^{\prime}+}{a_{N a}^{\prime \prime}+}=\frac{a_{C l}^{\prime \prime}-}{a_{C l}^{\prime}-}
$$

but this is identical with (3), the relation which expresses ratios obtained from the solubility product. Thus, to this extent the membrane equilibrium is identical with the equilibrium with a solid. Equation (6) may be written

$$
a_{S a}^{\prime}+\times a_{C l}^{\prime}-=a_{N a}^{\prime \prime}+\times a_{C l}^{\prime \prime}
$$

which is the same as the solubility-product relation between the two solutions (2) but differs in that no solid of constant activity is concerned in the equilibrium. In the solubility product the product of the activities of the ions of the saturating salt, in either of the solutions on the two sides of the solid salt, equals the activity of the solid salt which is constant. In the membrane equilibrium the same product is equal to the activity of the salt in the membrane between the two solutions, but this activity is variable.

In order to calculate the equilibrium in any special case, the activities under the conditions of the membrane equilibrium must be known. An attempt was made by Donnan to solve this problem without a knowledge of these activities, but there is no conclusive evidence to indicate that such a solution corresponds with the actual equi- 
librium. However, the method of treatment for a simple case is presented below, where the initial and equilibrium conditions are represented.

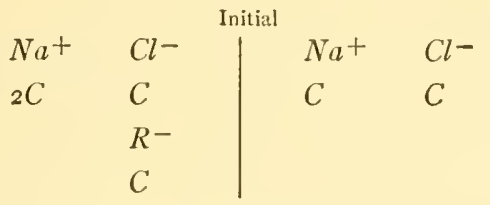

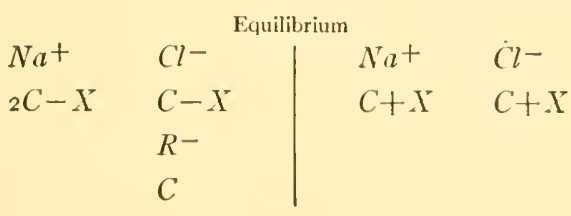

Here $X$ represents the number of mols of $\mathrm{NaCl}$ transferred from left to right. If $\mathrm{X}$ happens to be negative, the equations will reveal that this is the case.

The membrane product relation, written in the inexact form in which the concentration $(C)$ is substituted for the activity, is

$$
(2 C-X)(C-X)=(C+X)^{2},
$$

or

$$
{ }_{2} \mathrm{C}^{2}-{ }_{3} C X+\mathrm{X}^{2}=\mathrm{C}^{2}+{ }_{2} \mathrm{CX}+\mathrm{N}^{2},
$$

so

$$
\begin{aligned}
& X=\frac{1}{3} C \text {. }
\end{aligned}
$$

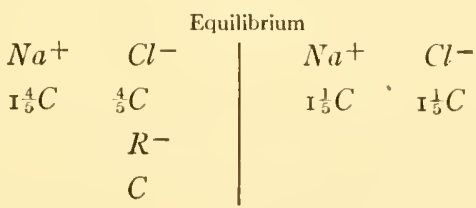

Another simple case is outlined below:

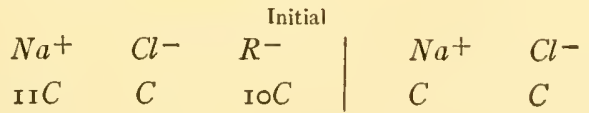

$$
\begin{aligned}
& \text { (II } C-x)(C-x)=(C+x)^{2} \text {, } \\
& \text { I I } C^{2}-\text { I } 2 C x+x^{2}=C^{2}+{ }_{2} C x+x^{2} \text {, } \\
& x={ }_{7}^{5} \mathrm{C} \text {. } \\
& \begin{array}{lll|ll} 
& & & \text { Equilibrium } \\
\mathrm{Na} & \mathrm{Cl}- & \mathrm{R}^{-} & \mathrm{Na}^{+} & \mathrm{Cl}^{-} \\
\mathrm{I} \circ \frac{2}{7} \mathrm{C} & \frac{2}{7} \mathrm{C} & \mathrm{IOC} & \frac{5}{7} \mathrm{C} & \mathrm{I} \frac{5}{7} \mathrm{C}
\end{array} \\
& \text { MEMBRANe "Potential" }
\end{aligned}
$$

The equations for calculating the electromotive force for a liquid-liquid junction are well known in the case in which different concentrations of the same salt are pres- 
ent on the two sides of the junction. The same equations have been applied without change when a membrane has been supposed to be present. Thus, with the cell given below

$$
\begin{array}{lll|ll}
\mathrm{Na}+ & \mathrm{Cl}- & \mathrm{R}^{-} & \mathrm{Na}+ & \mathrm{Cl}^{-} \\
a_{\mathrm{Na}}^{\prime} & a_{C}^{\prime} & a_{R^{-}}^{\prime} & a_{\mathrm{Na}}^{\prime \prime}+ & a_{C C^{-}}^{\prime \prime}
\end{array}
$$

let I Faraday of positive electricity be passed from left to right. The decrease of free energy is

$$
-\Delta F=T_{N a}+R T \ln \frac{a_{N a}^{\prime}+}{a_{N a}^{\prime \prime}+}+T_{C l}-R T \ln \frac{a_{C l}^{\prime \prime}-}{a_{C l}^{\prime}-}=\left(2 T_{N a}+-1\right) R T \ln \frac{a_{N a}^{\prime}+}{a_{N a}^{\prime \prime}+} .
$$

Since the number of equivalents of sodium and chlorine ions which cross the boundary are given by their transference numbers $\left(T_{N a}+\right.$ and $\left.T_{C l}-\right)$ the decrease of free energy is also equal to the electrical work done, or

$$
-\Delta F=\mathfrak{N F}
$$

or, since in the special case given above the number of equivalents of electricity $(\mathfrak{N})$ transferred is one,

$$
\Subset=\left({ }_{2} T_{N a}+-\mathrm{I}\right) \frac{R T}{F} \ln \frac{a_{N a}^{\prime}+}{a_{N a}^{\prime \prime}+},
$$

which is the equation commonly given for the membrane potential. In general,

$$
\S=\left(T_{C}-T_{A}\right) \frac{R T}{F} \ln \frac{a_{+}^{\prime}}{a_{+}^{\prime \prime}} .
$$

Up to the present time no trustworthy experimental verification of these equations has been obtained from any membrane equilibrium, since in the tests of these relations thus far the assumption has been made that other liquid-liquid junction potentials involved are negligible, without proving that this is true.

\section{SURFACES AND SURFACE ENERGY}

The importance of the energy stored up in the surfaces of bodies and in the interfaces between the particles of the bodies is due in part to the influence which the surface tension exerts upon the form of the bodies and the particles. However, the effect of the surfaces upon the chemical composition and action, and upon the electrical phenomena, are of even greater moment.

Interfaces are of particular significance in living organisms, since the motion of an organism as a whole is evidently brought about by transformation of one kind or another of the interfacial energy resident in it. The term "surface" unfortunately implies the entire absence of a third dimension in space, that of thickness, but physical surfaces and interfaces, sometimes designated as "phase boundaries," although they are exceedingly thin, commonly have a thickness as great as the sum of the diameters of several atoms, or a distance of the order of a millionth of a millimeter (ro A), which is by no means negligible. At many interfaces films or membranes collect, and these 
are of particular importance in biological systems, particularly in the human body itself.

If a cubic centimeter of water is sprayed into spherical droplets 0.0 I $\mu$ (IO० A) in diameter, the area of the surfaces thus formed is 600 sq.m. or approximately oneeighth of an acre. The free surface energy $(\gamma \times A)$ for this area at ordinary temperatures is about $2.2 \times 10^{8}$ ergs or 10.5 calories, while the total surface energy $(H)$ is larger and equal to I6.6 calories. This is one-third as large as the total energy of heat vibration of all of the molecules in the water.

Any system in which the area of the surfaces (interfaces) becomes large enough so that the surface energy is appreciable in comparison with the energy of (heat) vibration of the molecules of the disperse phase is considered as a "colloid."

It is commonly observed that water drops on a hot stove or on very dry dust assume a spherical shape, which is the form assumed by a balloon surrounded by a uniform elastic membrane. This suggests that every liquid is surrounded by an elastic film, the tension of which causes the surface to contract to the smallest possible area for the volume of the liquid, provided other forces (such as gravitation) do not act to change the form.

If a faucet with a narrow orifice is turned on very slightly, a drop may be seen to form, and hang for some time, after which it drops very suddenly. The drop is supported before it falls by the vertical component of the surface tension. If a capillary tube is dipped into water, the liquid inside the tube rises higher than that outside. Here, also, the film of liquid on the inside wall of the tube exerts an upward pull. If a camel's-hair brush is dipped into water, the hairs remain spread apart as if they were dry and in the air, but when the

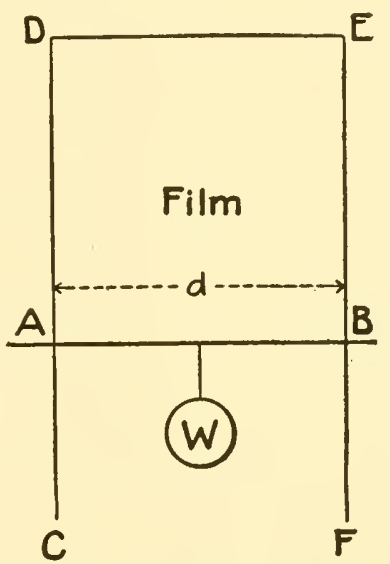

Fig. 7.-Maxwell frame for the determination of the surface tension of a soap film. wet brush is pulled out of the water, the pull of the surface tension of the water binds all of the hairs compactly together.

A soap film stretched on a wire frame such as that shown in Figure 7 has two surfaces. If the distance $A B$ is $\frac{1}{2} \mathrm{~cm}$., then this lower wire is in contact with $\frac{1}{2} \mathrm{~cm}$. of the film or I $\mathrm{cm}$. of the surface. The pull on the film as measured by the weight of the wire and the weights suspended from it at $W$ gives the surface tension of the surface per unit length. This may be expressed in dynes. If the wire is pulled downward I $\mathrm{cm}$., then the surface increases in area by I sq.cm., so the work done is force times area equals $\gamma \times \mathbf{I}=\gamma$ ergs. This energy may again appear as work when the film contracts to its original position, so it possesses the characteristics of free energy. Thus 72.8 dynes per centimeter is the surface tension of water at $20^{\circ}$, and 72.8 ergs per square centimeter is the free surface energy of water at this temperature.

\section{TENSILE STRENGTH AND TENSILE ENERGY}

There are a number of phenomena which indicate that the forces between adjacent molecules in solids and liquids are very high. Tensile-strength tests on bars of 
steel show that it is necessary to apply a force of $100, \infty 00 \mathrm{lb}$. to rupture a bar $\mathrm{I}$ sq.in. in cross-section. If it were possible to carry out a tensile-strength test in an ideal way such that the bar (of I sq.cm. cross-section; see Fig. I 2) would not be deformed before the break occurs, and so that the rupture would give two plane surfaces at right angles to the longitudinal axis of the bar, then the energy used would be equal to twice the free surface energy $(2 \gamma)$ per square centimeter at the temperature of the test. This is true because all that occurs in such an ideal rupture is the formation of a new surface on the steel of 2 -sq.cm. area. This is equal numerically to twice the surface tension of steel per centimeter. The work necessary thus to pull apart a bar of unit cross-section may be designated as the work of cohesion $\left(W_{c}\right)$.

$$
W_{c}=2 \gamma \text {. }
$$

If an endeavor is made to apply such a tensile-strength test to a bar of liquid, it is found that certain experimental difficulties arise. Nevertheless, the numerical value of the work of cohesion is known with considerable accuracy in such a case, since it may be obtained from the surface tension of the liquid.

The surface tension of water at $20^{\circ}$ is 72.8 dynes per centimeter, so its work of cohesion is 145.6 ergs per square centimeter. This small value may seem to indicate a small tensile strength (force of cohesion) in water, but just the opposite is true since the distance to which molecular attraction remains appreciable is very small, and is only of the order of molecular dimensions. Furthermore, it decreases as a moderately high power of the distance. Suppose that the summation of this rapidly decreasing force is equivalent to the action of a constant force through $\mathrm{ro}^{-8} \mathrm{~cm}$. Then the force of cohesion would be

$$
\frac{\mathrm{I} 45.6}{10^{-8}} \text { or } \mathrm{I} .456 \times 10^{\mathrm{Io}} \text { dynes }=\mathrm{I} \cdot 48 \times 10^{7} \mathrm{gm} \text {. per square centimeter, }
$$

or about $\mathrm{r}, \mathrm{,00} \mathrm{atm}$. The theory of van der Waals indicates a value of about i $1, \infty 00$ atm., while other methods of calculation usually give between 10,000 and 15,000.

\section{LATENT HEAT OF A SURFACE}

According to the rule of Le Chatelier, if the state of a system is changed, the system alters in such a way as to oppose a resistance to that change. Thus if the solubility of a salt increases with the temperature, the last amount of salt which dissolves to saturate the solution produces a cooling, since this cooling lowers the solubility, and thus opposes the solution of the salt. Now, since the surface tension decreases with rise of temperature (Fig. 8), a surface must cool if it is expanded, since by cooling the surface tension is increased, and this opposes an extra resistance to the further extension.

That heat should be used up in the formation of a surface is to be expected on other grounds. In the vaporization of a liquid the kinetic energy of molecular vibration of the molecules of the liquid, which determines the temperature, is partly converted into molecular potential energy, i.e., the molecular energy of motion is utilized in the separation of each molecule from its neighbors and against the attraction which they exert. Now since a molecule which is in the interior of a liquid must move into 
the surface against the attraction of the surrounding molecules, as a part of its migration into the vapor phase, it seems probable that in surface formation as well as in vaporization molecular kinetic energy would be utilized and transformed into potential energy of the surface. That heat is actually used in the formation of the surface is shown by the thermodynamic equation of Clapeyron, which gives the latent heat $(l)$ of the surface as

$$
l=-T \frac{\partial \gamma}{\partial T} .
$$

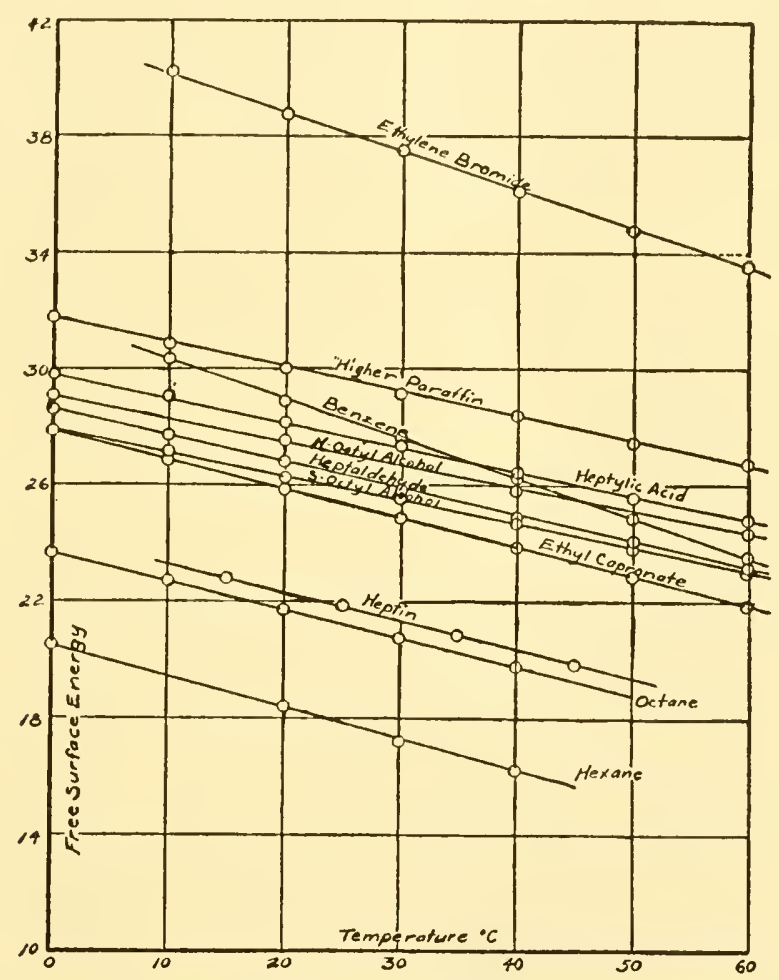

FIg. 8. - The free surface energy (or surface tension) of organic liquids. This equals one-half the tensile work (or work of cohesion $W_{c}$ ) per square centimeter.

Here $l$ gives the amount of molecular kinetic energy which is transformed into molecular energy of position when I sq.cm. of surface is formed.

\section{TOTAL SURFACE ENERGY}

The total energy $(h)$ of a surface is equal to the sum of the free energy $(\gamma)$ and the latent heat $(l)$,

$$
h=\gamma+l=\gamma-T \frac{\partial \gamma}{\partial T} .
$$

In the formation of a surface a part of the energy must be supplied in the form of work in order to give rise to the free surface energy, and a part comes from the kinetic 
energy which the molecules themselves possess. Thus, if a person extends a surface by doing work upon it, the liquid will also contribute its share to the total energy. The Clapeyron equation tells us that the temperature, i.e., the wealth of the molecules in kinetic energy, is an important factor in determining the extent of this contribution.

If a surface is to be formed on a definite liquid at a definite temperature, a definite amount of energy must be contributed and converted into potential energy.

\section{THE ORIENTATION OF MOLECULES IN SURFACES}

The ordinary observation of large-scale objects, such as logs or ships, as they lie upon the surface of a body of water, indicates that these objects exhibit a characteristic orientation with respect to the surface. Thus logs, when not too closely crowded

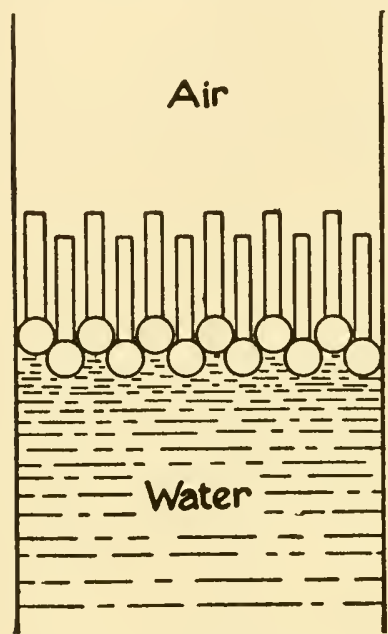

Frg. 9.-Orientation of molecules of an alcohol (or an organic acid) at the surface of its aqueous solution.

together, lie flat upon the water, i.e., the longitudinal axis is parallel to the surface. However, if one end of each log is loaded with a mass of iron or brass of the proper weight, it floats upon the surface and the longitudinal axis becomes vertical. If there is just a sufficient number of logs, the surface becomes covered with a single layer of vertical logs with their sides more or less in contact, while with any greater number, bunches of logs are found raised above the common level in certain places. If the number is smaller, a part of the surface remains uncovered. These phenomena may be illustrated by the use of a large number of cylindrical sticks of wood $3 \mathrm{~mm}$. in diameter and $14 \mathrm{~cm}$. long, weighted by a small cylinder of brass placed at one end. These are thrown upon the surface of the water in a large glass cylinder. This is represented in a diagrammatic way in Figure 9. If one of the vertical sticks is taken from the water, the brass weight removed, the stick dropped upon a vacant space upon a water surface, it at once assumes a horizontal position, thus exhibiting another type of orientation.

It is well known that the molecules or ions which make up a crystalline solid are arranged in an orderly way. A certain type of orderly array of very long and highly symmetrical molecules is found also in certain liquids, which are said to contain liquid crystals. Ordinary liquids are often supposed to be characterized by a complete disorder in the arrangement of their molecules, but it is probable that this disorder has been overemphasized. For example, in organic liquids of the type of acetic acid, the molecule of which consists of the polar carboxyl group, and the "non-polar" methyl group, there is some evidence of molecular association, presumably a type of orientation in which two or more polar groups come close together in the pure liquid as they do in benzene' (Fig. I I, upper part). The theory that the molecules in the surface of a

x The number of molecules which unite in this manner must be large in some groups, as they give definite $\mathrm{X}$-ray patterns. The grouping may be more analogous to that given by the bristles of two brushes which are set with bristles together; this is called the "cybotactic state." 
liquid are oriented in a characteristic fashion is of comparatively recent origin. It seems peculiar that the birth of so obvious a conception should have been so long delayed, but it is probable that this is due to the general habit of considering molecules as spherical, even when their formulas are highly elongated. In such cases the former conception was that it would roll itself up into a sphere. It is obvious that even in a dissymmetrical field of force, such as may be assumed to exist at the surface of a liquid, a molecule which is a perfectly symmetrical sphere could exhibit no orientation. However, even in a uniform gravitational field a perfect sphere may orient itself provided its mass is not uniformly distributed, as will be seen if a sphere is weighted on one side, so that even if all molecules were spherical, molecular orientation would not be at all impossible (Fig. Io).

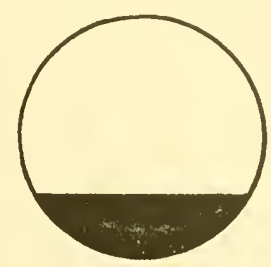

Fig. 10.--Illustrates the orientation of a weighted sphere under the influence of gravity.

\section{SOLUBILITY AND MOLECULAR ORIENTATION IN SURFACES}

The cohesion in liquid ethane,<smiles>C</smiles>

is extremely low, so low that liquid ethane cannot exist at ordinary temperatures. The introduction of one oxygen atom gives rise to ethyl alcohol in which the cohesion is equivalent to a pressure of $3,000 \mathrm{~atm}$. The hydrocarbon molecule (ethane) is nonpolar, but the hydroxyl group $(-\mathrm{OH})$ of the alcohol is polar. The attraction between two such polar groups is very much greater than that between two non-polar groups or that between a polar and a non-polar group.

The solubility of an organic acid, an alcohol, or an amine in water is due to its polar group which is greatly attracted by the water. The solubility of such a substance in hexane is, on the other hand, due to the presence of the hydrocarbon groups. Thus, all saturated hydrocarbons are practically insoluble in water. The old rule is: Similia similibus solvuntur ("Like dissolves like").

If, now, we have a two-phase system consisting of water below and hexane above, and add butyric acid,<smiles>CCC(C)=O</smiles>

the hydrocarbon end, which we may designate by $\square$, is soluble in the hexane, but not in the water, and the carboxyl group $O$ is soluble in water but not in the hexane. However, the carbaxyl group will drag some molecules of the butyric acid into the water, while the hydrocarbon group will drag others into the oil.

At the interface between the water and the oil, however, the hydrocarbon of the 
molecule may dissolve in the oil and the polar group in the water. Thus, each end of the molecule is highly soluble in the liquid toward which il turns. If this is true: (a) butyric acid should be very much more soluble in the interface than in either the water or the benzene; $(b)$ the butyric acid molecules should be oriented with their polar ends toward the water and their non-polar ends toward the hexane. (Fig. II).

\section{GENERAL STATEMENT OF THE THEORY OF ORIENTATION OF MOLECULES IN SURFACES}

The following statement, written in I9I6, gives in concise form the general fundamental principles of the orientation theory:

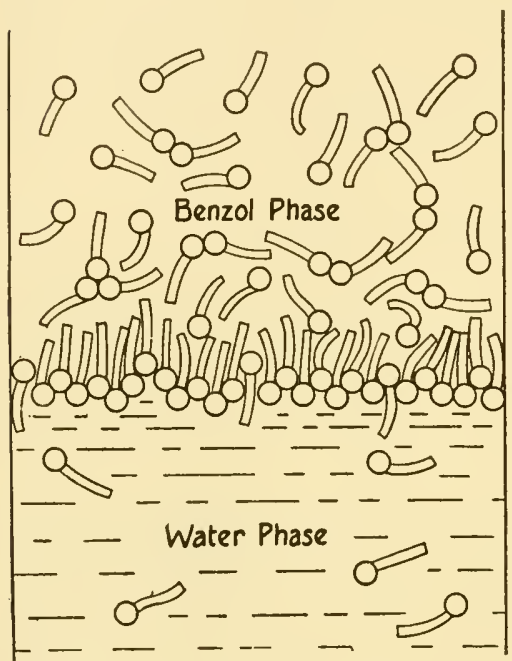

FIG. I I.-A two-phase system of water and benzol which contains butyric acid. The greatest concentration of the acid is at the interface. The acid is more or less associated in the benzol.

I. The molecules in the surfaces of liquids seen to be oriented, and in such a way that the least active or least polar groups are oriented toward the vapor phase. The general law for surfaces seems to be as follows: If we suppose the structure of the surface of a liquid to be at first the same as that of the interior of the liquid, then the actual surface is always formed by the orientation of the least active portion of the molecule toward the vapor phase, AND AT ANY SURFACE OR INTERFACE THE CHANGE WHICII OCCURS IS SUCH AS TO MAKE THE TRANSITION TO THE ADJACENT PHASE LESS ABRUPT. This last statement expresses a general law, of which the adsorption law is only a special case. If the molecules are monatomic, and symmetrical, then the orientation will consist in a displacement of the electromagnetic fields of the atom. This molecular orientation sets up what is commonly called a "double electrical layer" at the surfaces of liquids and also of solids.

This law, if applied to special cases, indicates for a few pure liquids the following orientation: In water the hydrogen atoms turn toward the vapor phase and the oxygen atoms toward the liquid. With organic paraffin derivatives the $\mathrm{CH}_{3}$ groups turn outward, and the more active groups, such as $\mathrm{NO}_{2} \mathrm{CN}, \mathrm{COOH}, \mathrm{COOM}, \mathrm{COOR}, \mathrm{NH}_{2}, \mathrm{NHCH}_{3}, \mathrm{NCS}, \mathrm{COR}, \mathrm{CHO}, \mathrm{I}, \mathrm{OH}$, or groups which contain $\mathrm{N}, \mathrm{S}, \mathrm{O}, \mathrm{I}$, or double bonds, turn toward the interior of the liquid.

If any of these organic compounds are dissolved in water, their orientation in the water surface is the same as that just given, with the active groups inward.

At interfaces between two pure liquids the molecules turn so that their like parts come together in conformity with the general law. With solutions, the solute molecules orient so that the ends of the molecules toward the liquid $A$ are as much like $A$ as possible, and the ends toward $B$ are as much like $B$ as possible. So at interfaces between organic liquids and water, for example, the organic radical sets toward the organic liquid, and the polar group toward the water. 
2. If at an interface the transition from a liquid $A$ to the liquid $B$ is made by a saturated film of solute molecules which we may call $A-B$, i.e., they have one end like $A$ and the other like $B$, then the free surface energy is greatly reduced. For example, with water and benzene with sodium oleate as the solute, the free energy falls as low as 2 ergs per square centimeter.

3. If the solvent is polar, such as water, then solutes will in general be positively adsorbed in the surface if they are less polar than water (or if a part of the molecule of the substance is less polar than water), and the least polar end of the molecule will be turned outward. Solutes more polar than water are negatively adsorbed.

\section{EVIDENCE FOR ORIENTATION OF MOLECULES IN SURFACES} AND INTERFACES

I. Evidence from the energy of rupture.-The orientation theory indicates that if a bar of liquid octyl alcohol (Fig. I2) were to be pulled apart, one of the steps in the process would be for the molecules to orient where the break is to occur (Fig. I3) in such a way that this will become the weakest part of the bar. Evidently this means that the final break will occur between the non-polar ends of the molecules. If octane $\left(\mathrm{C}_{8} \mathrm{H}_{18}\right)$ is ruptured, the work done for a bar of I sq.cm. cross-section $\left(W_{c}\right)$

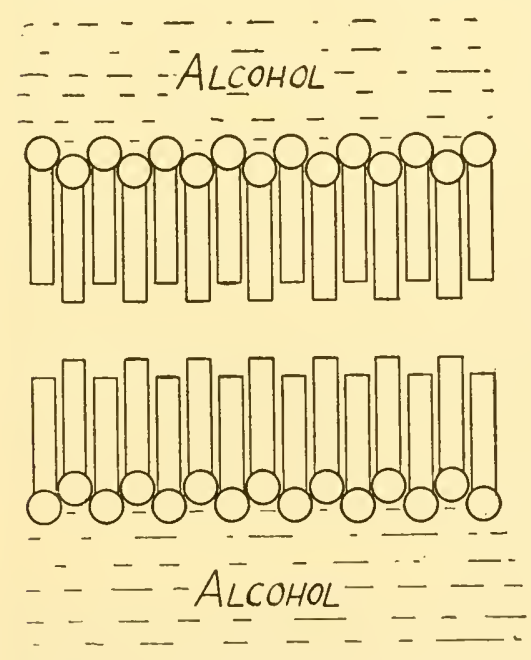

FIG. 13.-Represents the orientation of the molecules which occurs if a bar of alcohol is pulled apart. is 43.5 ergs. When octyl alcohol is pulled apart, additional energy must be utilized in orienting the molecules, so it is not surprising that the work of rupture $\left(\mathrm{H}_{c}\right)$ is slightly higher $(55 \cdot 0)$.

If, however, the octyl alcohol is to be pulled away from water, and the molecules of the alcohol are oriented in the interface, then the funal break must come between the polar hydroxyl (-OH) groups of the alcohol and the polar molecules of water (Fig. I4): therefore (the orientation theory predicts that in this case) the work of rupture (work of adhesion, $W_{A}$ ) should be high. The experimental results show that this prediction of the theory is justified, since the work of adhesion is found to be 92 ergs, or 60 per cent higher than the work required to rupture the alcohol. Even more remarkable is the fact that this is I I p per cent higher than the work required to rupture octane, and I so per cent higher than the amount of work necessary to separate octane from water. The extremely remarkable nature of these results is evident when it is considered that the molecule of octane contains 26 atoms, while that of octyl alcohol contains these same 26 atoms and only one more, an atom of oxygen which gives the polar nature to the molecule. Thus an increase of less than 4 per cent in the number 
of atoms increases the work of attraction for water by r ro per cent, which is suffcient evidence that the oxygen of the alcohol must be oriented toward the surface of the water.

If the alcohol surface is pulled from the water surface at the interface between the two the interface disappears and a water $(A)$ surface and an alcohol $(B)$ surface ap-

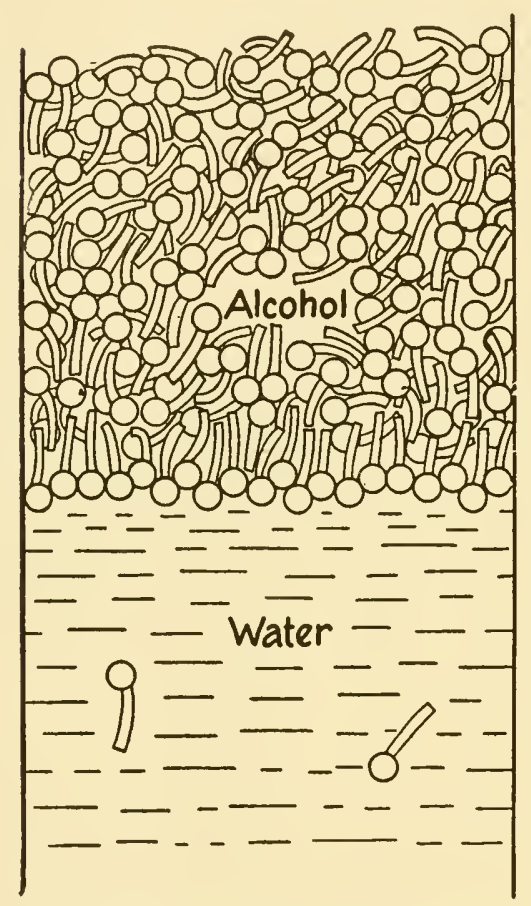

FIG. 14.-Octyl alcohol over water. Illustrates the oreintation of the alcohol molecules at the interface. pear. The work done is aided by the free energy of the surface which disappears, and hindered by those which appear, so

$$
W_{A}=\gamma_{A}+\gamma_{B}-\gamma_{A B} .
$$

Values of the interfacial tension $\left(\gamma_{A B}\right)$ for a number of liquids are plotted in Figure 15 , and for the work of adhesion in Figure $\mathrm{r} 6$.

\section{THE SPREADING OF ONE LIQUID ON THE} SURFACE OF ANOTHER

Most organic liquids will spread on water, but water spreads on almost no organic liquids. It is easy to show that spreading or non-spreading is determined by the work of adhesion $\left(W_{A}\right)$ between the liquids and the work of cohesion $\left(W_{C}\right)$ for the upper liquid (B):

$$
S=W_{A}-W_{C(B)}
$$

If $S$ is positive, the liquid $B$ will spread on the surface of $A$; if $S$ is negative, it will not spread. The presence of polar groups in the organic liquid is not essential for spreading, since hexane and octane, as well as benzene, spread on water. Not only organic liquids, but water as well, spread on a clean surface of mercury:

$$
S=\gamma A-\left(\gamma_{B}+\gamma_{A B}\right) \text {. }
$$

2. Evidence for orientation of molecules in surfaces of pure liquids; comparison of energy of surface formation with heat of vaporization.-If a liquid consists of molecules with one end polar and the other end non-polar, the energy required to lift the nonpolar end (the "light" end from the standpoint of electrical forces) into the surface is much less than for the polar end, so the orientation theory predicts that in the outer layer of molecules the non-polar groups will be at the surface. However, if such a molecule passes into the vapor state, the polar end of the molecule must be separated from the liquid, and this.will require a relatively large amount of energy. Therefore, according to the theory, the energy per molecule of surface formation $(h)$ should be small as compared with the energy of vaporization $(\lambda)$. If the molecule is symmetri- 
cal, then $h / \lambda$ should be much larger, since there is no "light" end which can be lifted in to the surface.

In entire confirmation of these ideas, $h / \lambda$ is very small (o.I8) for the lower alcohols which have highly unsymmetrical molecules, and much larger $(0.50-0.60)$ for highly symmetrical molecules, such as those of oxygen, nitrogen, or mercury. These values are those found at a corresponding temperature of $0.7\left(T=0.7 T_{c}\right)$, but the same general relations are followed at other temperatures.

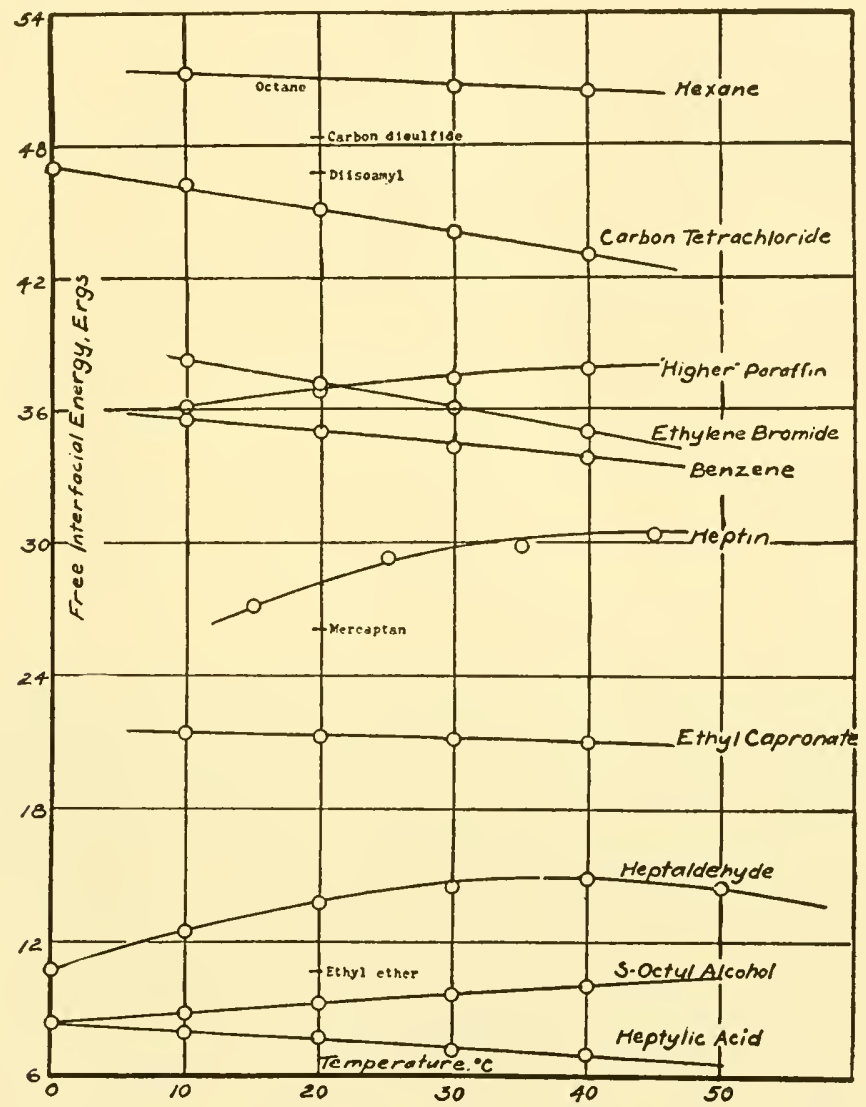

Fig. 15.-The free interfacial energy (or interfacial tension) between organic liquids and water

This is the only evidence thus far found which indicates strongly that the molecules in the surfaces of pure liquids are oriented.

3. Evidence for orientation in relations of monomolecular films.-If a long-chain fatty acid, such as stearic acid, is dissolved in hexane it spreads on water to form a dilute film I molecule thick. This may be compressed between the movable barrier (back of Fig. I 7) and the floating barrier attached to a film balance (front of Fig. I 7). When the film is compressed until it is tightly packed, but still monomolecular, the area per molecule is $19.3 \mathrm{~A}$ units of area (I9.3 $\mathrm{A}^{2}=19.3 \times 10^{-16} \mathrm{sq} . \mathrm{cm}$.) (Fig. I8). The square root of this area, $4.4 \mathrm{~A}$, gives an idea of the diameter of the area occupied by the 
molecule. This area is found to be nearly the same for long-chain compounds of from I6 to 30 carbon atoms long, which indicates that the molecules in the film are oriented. The thickness of such a film, calculated on the basis of the idea that its density is that

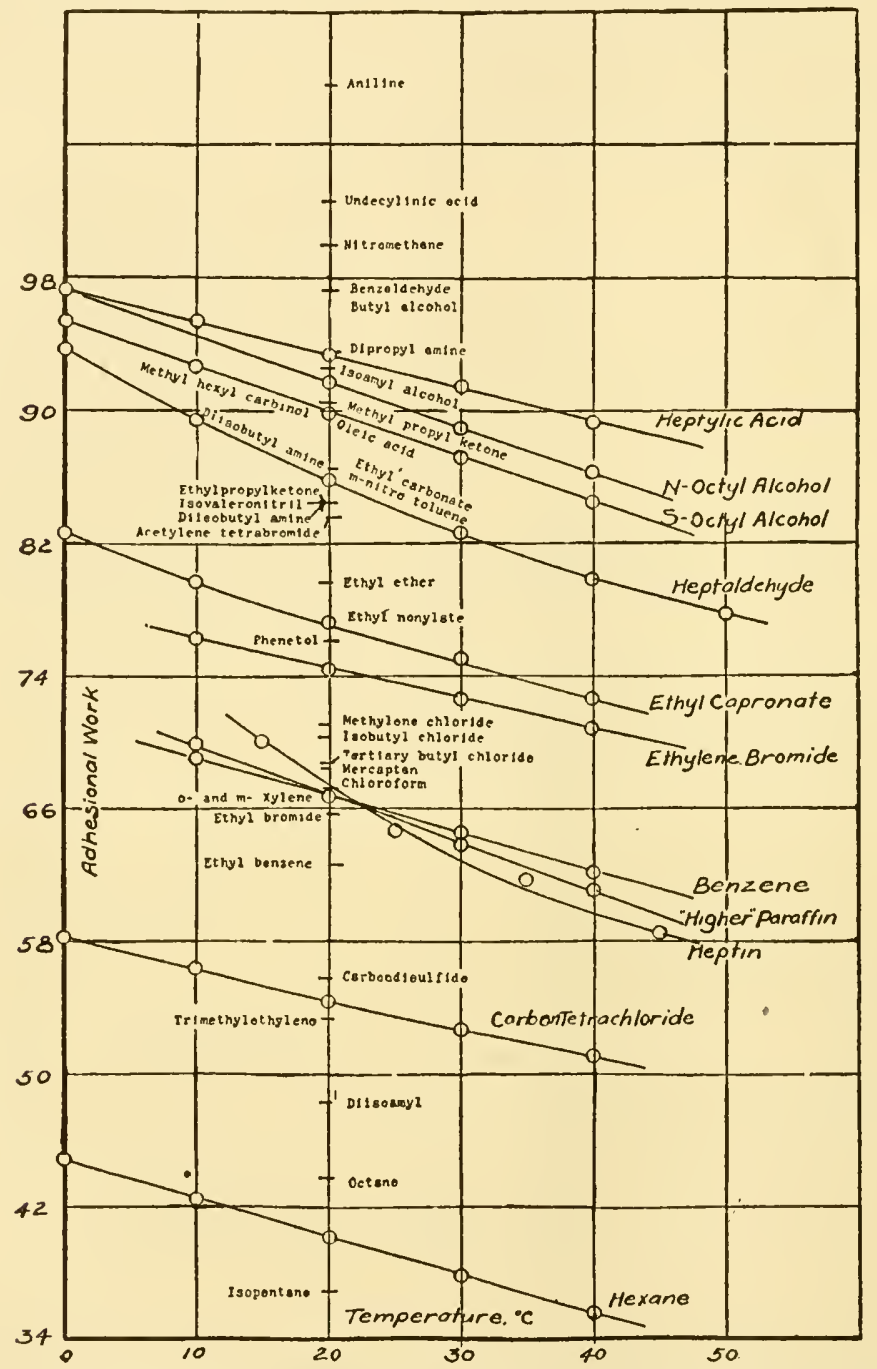

FIG. I6. - Adhesional work, ergs per square centimeter, between organic liquids and water. (The names of the substances represented by the curves are given at the right while the names given in the middle of the diagram represent substances for which the values are given at $20^{\circ}$ only.)

of the pure organic substance, indicates that each $\mathrm{CH}_{2}$ group adds about I.4 A to the thickness of the film, while $\mathrm{X}$-ray measurements on the solid substance give a mean value of about I.I $5 \mathrm{~A}$.

FILMS ABSORBED FROM LIQUIDS

The best evidence at present available indicates that the films on water of butyric acid, amyl amine, octyl alcohol, phenol, resorcinol, and all analogous substances are 
essentially monomolecular at sufficient concentrations of the solutions. With longchain (ro carbon atoms) compounds, only dilute aqueous solutions are obtained, so the time necessary for the diffusion of sufficient organic substance into the surface to
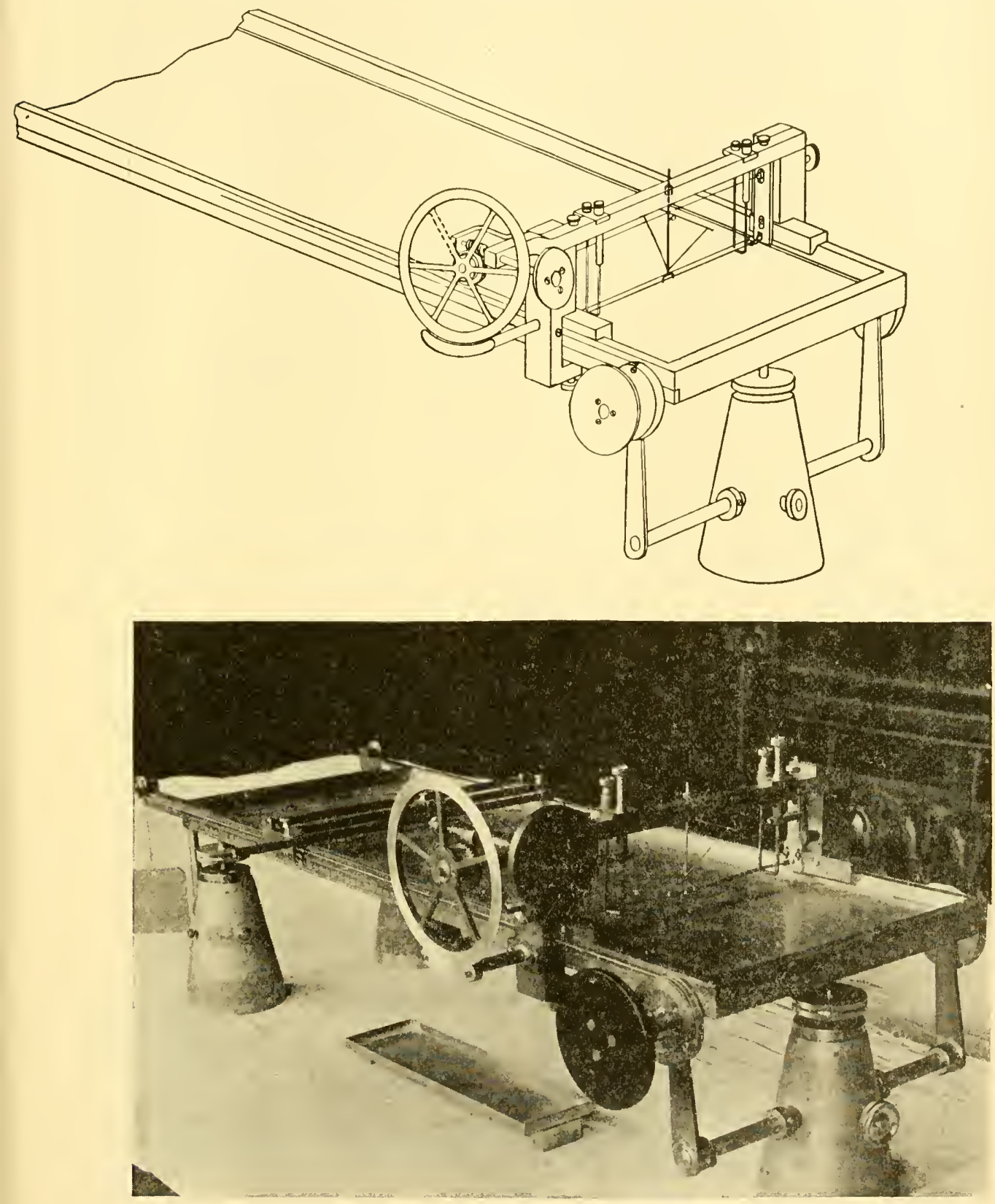

FIG. I 7.-Perspective drawing and photograph of filmometer (design of B. B. Freud, a modification of the filmometer of N. K. Adam). 
give a monomolecular film which is in equilibrium with the solution is considerablemore than thirty minutes in the case of decylic acid (Io carbon atoms) (see Fig. I9).

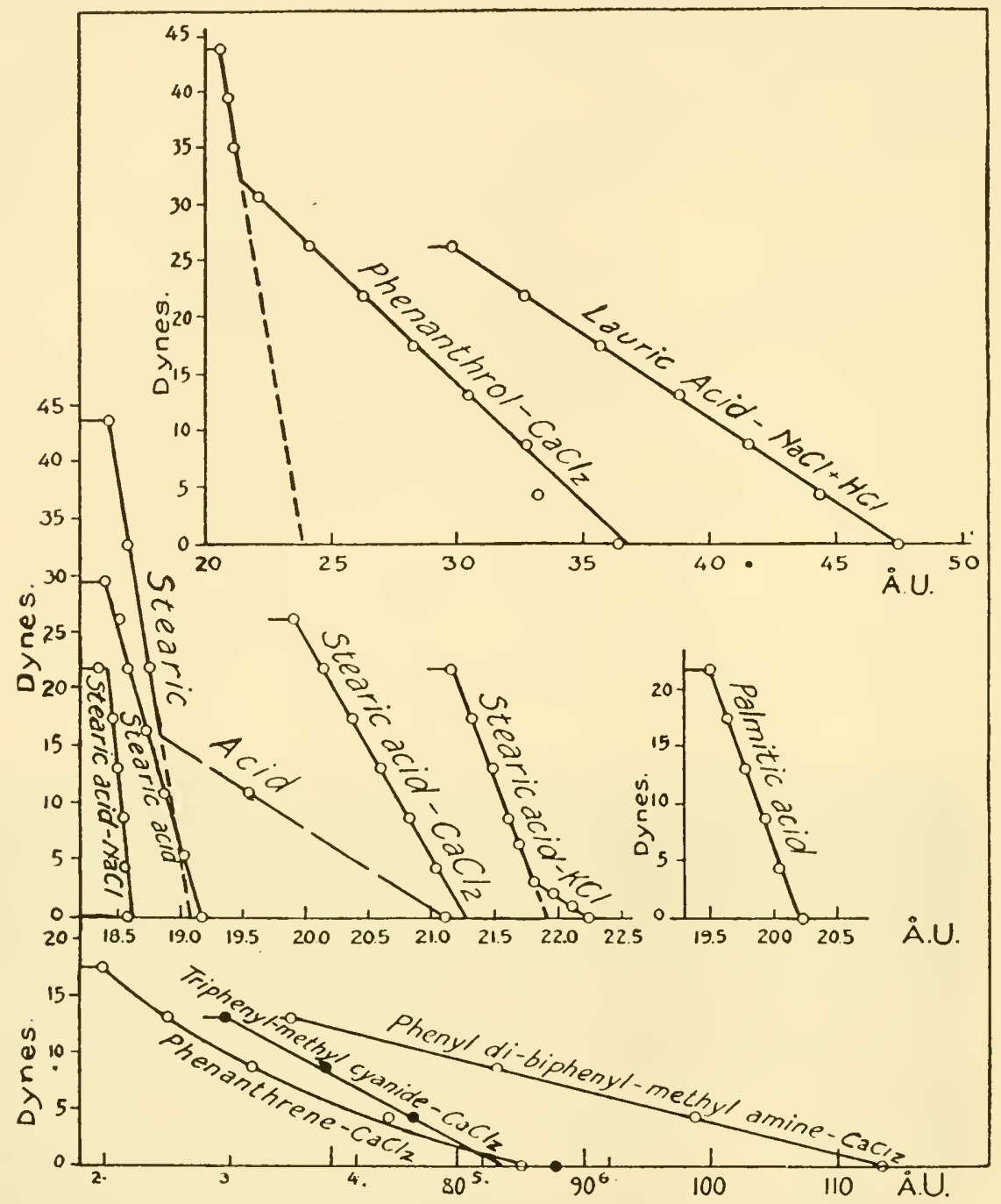

Frg. 18 . - Areas per molecule ( $X$-axis) for monomolecular films on water. The $Y$-axis represents the "force of compression" which is measured by the film balance, and is equal to the surface tension of water minus the surface tension of a water surface covered by a film of the organic substance. The curves for palmitic acid, and for stearic acid alone, are of the more usual type. These films are under high pressures. Under low pressures the curves are similar to the $p, v$ curves for gases. For comparison, two curves for polymolecular films of phenanthrene and triphenylmethylcyanide are given. The areas per molecule for these two substances are from 2 to $6 \mathrm{~A}$, which is too small an area for a monomolecular film. The line for palmitic acid extrapolated to zero pressure gives $20.2 \mathrm{~A}$, and for stearic acid on water alone the two lines give I9.I and I9.2 A of area. Lauric acid forms a dilute monomolecular film. 
The equation of Gibbs, which gives the number of gram molecules ( $u$ ) absorbed per square centimeter of surface formed, may be expressed:

$$
u=\frac{\mathrm{I}}{R T} \frac{\partial \gamma}{\partial \ln a} .
$$

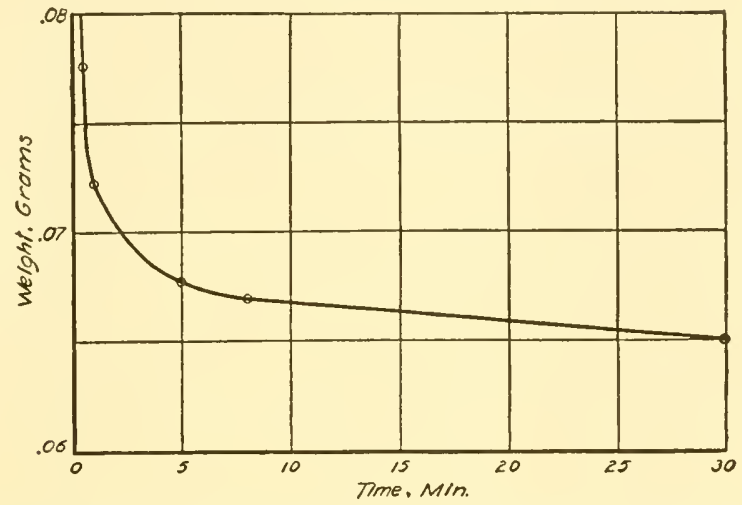

FIG. I9.-Effect of time on the drop weight (surface tension) of decylic acid, 0.0015 N

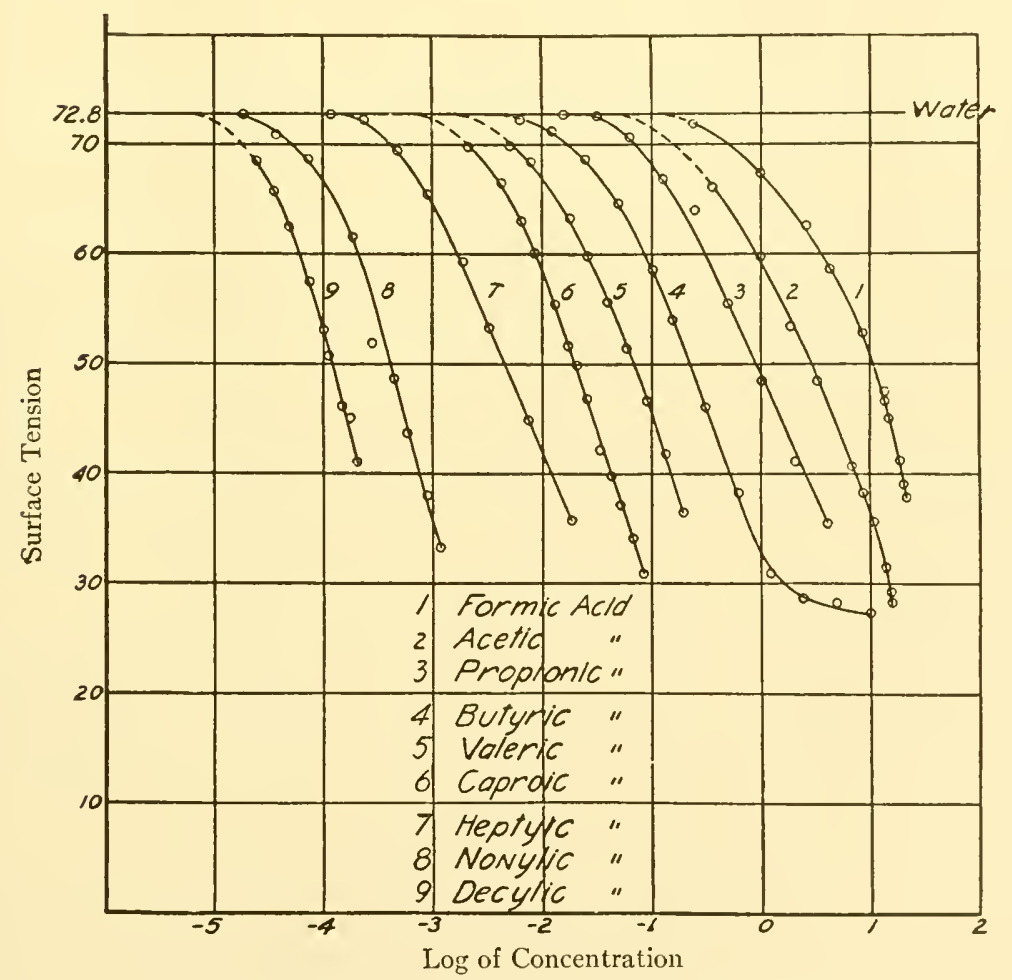

FIG. 20.-Adsorption curves for fatty acids 
The difficulty in the application of this equation is that activities in solution are known for almost no organic substances. Figure 20 shows how the surface tension of solutions of organic acids varies with the logarithm of the concentration $\left(\frac{\partial \gamma}{\partial \ln C}\right)$. The slope of $\frac{\partial \gamma}{\partial \ln a}$ is slightly greater than $\frac{\partial \gamma}{\partial \ln C}$, but the correction is less than roper cent in the case of the condensed monomolecular film of butyric acid. The nearly identical slopes of the $\gamma, \log C$, curves indicate that the adsorption is nearly independent

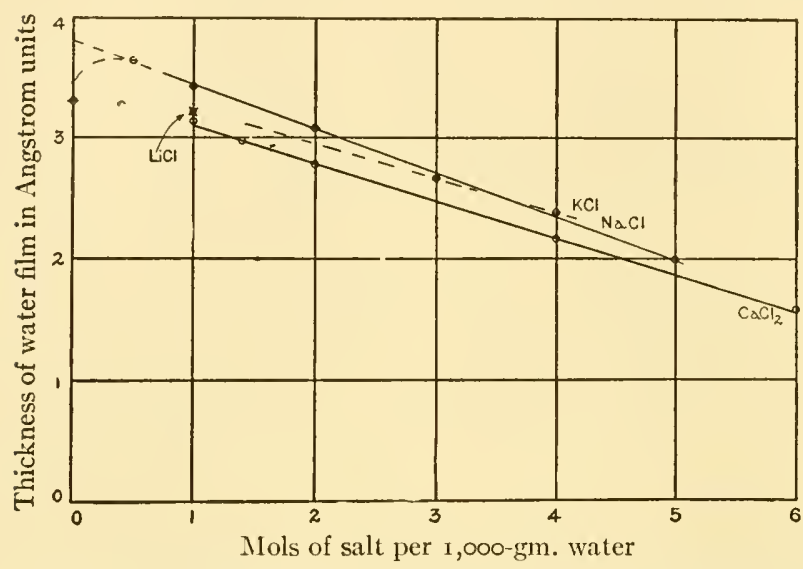

FIG. 21.-Thickness of the water film on salt solutions (Angstrom units). of the number of carbon atoms in the molecule for the tightly packed monomolecular films.

The calculations made thus far indicate that the area per molecule in the film for these soluble substances is of the same order as that for the longer molecules which are insoluble.

Data on the surface and interfacial tensions indicate that between water and benzene, or between water and hexane, the number of butyric acid molecules in unit area of the film is exactly the same, within the limits of experimental error, as between water and vapor. This is additional evidence that the number of molecules in a condensed film depends mainly on the size of the molecules and their orientation.

MEAN THICKNESS OF THE WATER FILM ON SALT SOLUTIUNS

Water is positively absorbed on aqueous salt solutions, i.e., the surface contains much less salt than the solution. It is possible to calculate a mean thickness for the water film if it is assumed that the film consists of pure water, and that just below the film the concentration of the solution is the same as farther inside.

The remarkable relation which emerges from such calculations is that in very dilute solutions the calculated film thickness is almost exactly the cube root of the volume occupied in water by a water molecule (3.I A). Figure 2 I plots the thickness of the water film as a function of the concentration of the salt.

The water film between water and benzene is found also to have practically the same thickness at the same concentration as between water and air.

Thus, either between aqueous solution and vapors, or between the aqueous phase and a non-polar liquid, the water film is monomolecular in dilute solutions, and even thinner in concentrated solutions. Both between salt solution and vapor and between salt solution and benzene the surface tension increases as a linear function of the molality of the salt in the solution. 


\section{ELECTRICAL CHARACTERISTICS OF EMULSIONS AND SUSPENSIONS}

If an emulsion or a suspension is placed between a positive and a negative electrode, it is found that the particles move (with respect to the water) toward one of the two electrodes, and that in general all of the particles of the same material in the same medium move at the same speed, whether they are large or small. This phenomenon of the movement of small particles in the electric field is known as "cataphoresis." It may be considered that this is analogous to the conductance of electricity by the ions of a salt in electrolysis.

If equal parts of hexane and an aqueous solution of a sodium oleate soap are mixed together by shaking or by stirring with an egg-beater, it is commonly found that the droplets vary from less than $0.2 \mu$ to about по $\mu$ in diameter, with the largest number of drops at a diameter of $\mathrm{I}^{-\mathrm{I}} .5 \mu$.

Determinations of the amount of soap absorbed indicate that each droplet of oil in a stable emulsion is surrounded by a monomolecular film of soap. Since soap is partly hydrolyzed, the film should consist of molecules of sodium oleate and of oleic acid. The total number of oleate molecules in the film around a droplet of a diameter of I $\mu$ is of the order of $10,000,000$ or $15,000,000$.

According to the orientation theory, the hydrocarlson groups of the soap are turned toward the oil and the polar groups with their positive sodium ions toward the water. It is to be expected that such a droplet will act like a highly polyzalent salt molecule, and that sodium ions will diffuse off into the solution, leaving a negative charge on the droplet. The question now arises, To what extent is the droplet ionized? Since, however, the different sodium ions diffuse to different distances, the ionization should be expressed as a relation concerning the distribution. However, it is easy to calculate a mean or effective ionization by the use of the law of Stokes for the motion of a spherical droplet in a viscous medium.

In this way it is found that the velocity of such a particle $(4 \mu$ per second per volt per centimeter) corresponds to a negative charge of 2,430 electronic charges on a particle I $\mu$ in diameter, i.e., if all of the $10,000,000$ or $15,000,000$ molecules of the soap except the 2,430 were completely un-ionized, and if the 2,430 molecules of sodium oleate were so completely ionized that the $2,430 \mathrm{Na}+$ ions are at an infinite distance, then the oil droplet should have a negative charge equal to that of 2,430 univalent negative ions. The equation is

$$
N=\frac{6 \pi r \eta}{e} \frac{v}{X}
$$

in which $e$ is the charge on the electron, $N$ is the number of charges, $\eta$ is the viscosity of the solution, and $\frac{v}{X}$ is the mobility or the velocity for unit potential gradient.

The potential of a charged sphere is

$$
\phi=\frac{N e}{D r} ; \quad \text { so } \quad \phi=\frac{6 \pi \eta}{D} \frac{v}{X} .
$$

The use of this potential instead of the fictitious zeta potential was suggested to the writer by Professor A. C. Lunn. According to this equation, the potential $\phi$ is 84 
millivolts for the droplet in question. The potential $\phi$ obtained by the use of the equation of Stokes is always $3 / 2$ the fictitious zeta $(\zeta)$ potential usually given in books on colloid chemistry.

According to the relations given above: (I) the effective ionization $(N)$ of a colloidal particle varies directly as its radius. (2) The effective ionization per unit area $\left(\frac{N}{A}\right)$ varies inversely as the radius of the particle, and therefore directly as the curvature of the surface. (3) The potential $\phi$ for the particle is independent of the radius. Therefore the potential is the same for all spherical particles of the same material in any certain solution. (4) The effective ionization $(N)$ and the potential $(\phi)$ depend upon the nature of the particle and upon the nature of the medium in which it is suspended.

The Helmholtz-Lamb equation for the velocity in cataphoresis is

$$
v=-\frac{\phi_{L} X D}{4 \pi \eta} \bar{l} .
$$

Here $D, \eta$, and $d$ refer respectively to the dielective constant, viscosity, and thickness of the electrical double layer, $l$ is the coefficient of slip, and $X$ is the impressed potential gradient. Smoluchowski simplified this equation to

$$
v=-\frac{\phi_{S} X D}{4 \pi \eta} .
$$

If $\eta$ is taken to be the viscosity of the solution and not of the double layer,

$$
v=-\frac{\zeta X D}{4 \pi \eta}
$$

or

$$
\zeta=-\frac{4 \pi \eta}{D} \frac{v}{X}
$$

gives the value of the fictitious zeta potential, so

$$
\zeta=\frac{2}{3} \phi \text {. }
$$

The equations give the potentials in electrostatic units. The value of $\phi$ in volts is given by

$$
\phi=6 \pi \frac{\eta}{D} \frac{v}{X} \times(300)^{2} \text { volts }
$$

According to Debye and Hückel, the constant of the Helmholtz-Lamb-Smoluchowski equation should be $6 \pi$ for spherical particles. On this basis the value of $\zeta$ should be equal to that of $\phi$.

POTENTIAL AND STABILITY

According to the theory of Hardy, the stability of a colloidal suspension is dependent upon the electrical repulsion of the charges of like sign upon the particles.

${ }^{3}$ Cf. also chaps. xlii and lviii in this volume. 
On account of their Brownian movement the particles would collide, and in some cases unite, if they were not kept apart by this repulsion. However, some of the particles have very high velocities, so even with high potentials the solution may not be permanently stable. If the particles collide there may be an actual union. This is accompanied by a decrease in surface energy. The union of two such particles involves the deorientation of the films between them, and this may require the expenditure of an appreciable quantity of energy. The particles which collide may merely adhere (agglutination), or they may merge and lose their identity completely.

In 1906 Burton made some interesting experiments on the cataphoretic velocity of colloidal gold particles in a gold sol to which different amounts of an aluminum salt were added (see Table IV). Such experiments indicate: $(a)$ that the positive aluminum ions are adsorbed at the interfaces between the gold and the water; $(b)$ that this adsorption may be great enough to change the original negative charge on the particles of gold into a positive charge, the number of ions adsorbed increasing rapidly with the concentration of the aluminum soap; $(c)$ that instability of the colloidal solu-

TABLE IV

Effect of Aluminum Ions $\left(A_{L}+++\right.$ ) upon the Charge of Particles of

Collotdal Gold

\begin{tabular}{|c|c|c|}
\hline $\begin{array}{l}\text { Milligrams Aluminum } \\
\text { per Liter }\end{array}$ & $\begin{array}{l}\text { Cataphoretic Yelocity in } \mu \text { per } \\
\text { Volt per Cm. per Sec. }\end{array}$ & Stability \\
\hline 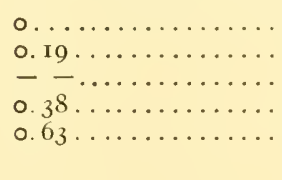 & $\begin{array}{l}330 \text { (toward anode) } \\
\text { I7 I (toward anode) } \\
\text { o } \\
\text { I7 (toward cathode) } \\
\text { I35 (toward cathode) }\end{array}$ & $\begin{array}{l}\text { Indefinitely stable } \\
\text { Flocculated after four hours } \\
\text { Flocculated immediately } \\
\text { Flocculated after four hours } \\
\text { Not completely flocculated } \\
\text { after four days }\end{array}$ \\
\hline
\end{tabular}

tion increases as the charge on the particles (cataphoretic velocity) approaches zero from either the positive or the negative side. The point at which the cataphoretic velocity, and therefore the charge on the particle, becomes zero is called the "iso-electric point."

It is believed that a small concentration of salt is essential for the stability of a colloidal sol (such as mastic). An excess of salt destroys the stability and causes flocculation. If the salt is of the type of $\mathrm{AlCl}_{3}$ and the sol is negative, further addition of the salt produces a positive stable sol, but still further additions of the salt bring about another flocculation. These peculiar phenomena are designated by the technical term "irregular series."

EFFECT OF THE VALENCE OF THE ADSORBED ION

It is impossible to add negative salt ions to a solution without the addition of positive ions, and vice versa. However, it has been found that with negatively charged suspensoids, such as the arsenic trisulphide or the gold sol, the flocculation produced is a function of the valence of the positive ions of the salt, and with positively charged suspensoids it is a function of the valence of the negative ions of the salt.

Thus with an emulsion of a paraffin oil the value of phi $(\phi)$ is reduced from 69 millivolts, the value for water, to 55.5 millivolts by the addition of the specified 
amount of salt (see Table V). Thus it requires twelve hundred times more equivalents of potassium chloride than of thorium chloride to reduce the cataphoretic velocity from 3.I to $2.5 \mu$ (which corresponds with the lowering of $(\phi)$ from 69 to 55.5 millivolts).

TABLE V

Lowering Effect of Salts upon the Cataphoretic Mobility $\left(\frac{v}{\bar{X}}\right)$, the Zeta Potential ( () , or the Phi Potential $(\phi)$ for Droplets of Oil in Water

\begin{tabular}{|c|c|}
\hline Electrolyte & $\begin{array}{l}\text { No. of Milliequivalents of Salt } \\
\text { Required to Lower } 5 \text { from } 46 \text { to } 37 \\
\text { Millivolts (or } \phi \text { from } 69 \text { to } 55.5 \text { Millivolts) }\end{array}$ \\
\hline $\mathrm{KCl}$. & $\ldots \ldots \ldots \ldots 24$ \\
\hline $\mathrm{BaCl}_{2}$. & $\ldots \ldots \ldots 0.90$ \\
\hline $\mathrm{AlCl}_{3}$. & $\ldots \ldots \ldots \ldots . \quad .03$ \\
\hline $\mathrm{ThCl}_{4}$. & $\ldots \ldots \ldots$ \\
\hline
\end{tabular}

The same relationship concerning the valence of the ion of opposite charge is apparent in the flocculation values. Thus, for the negatively charged arsenic trisulphide

TABLE VI

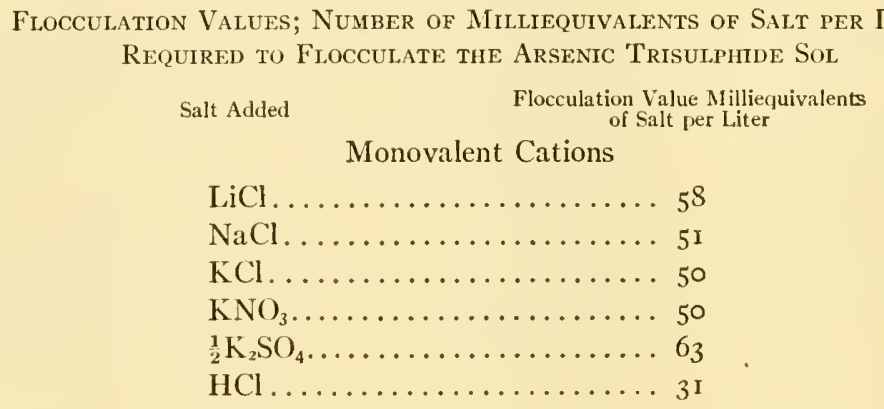

Divalent Cations

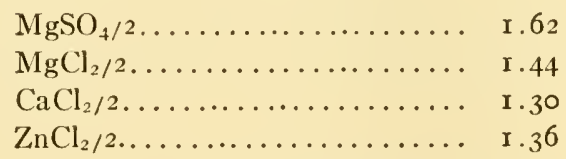

Trivalent Cations

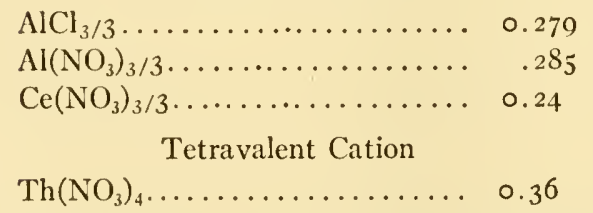

sol the number of millimols per liter of salt required to cause flocculation decreases very rapidly with the valence of the positive ion (Table VI).

The work thus far done upon the carrying down of ions by precipitates shows that with a negatively charged sol flocculation is produced by about the same number of equivalents of one positive ion as another (Table VII). This indicates that inorganic ions of high charge (valence) are much more highly absorbed than those of low charge (valence). 
TABLE VII

Flocculation Values

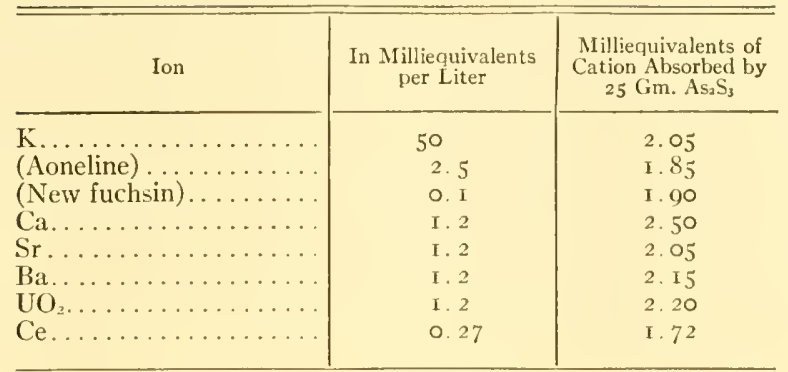

STABILITY OF AN EMULSION AND TIIE MONOMOLECULAR FILM

An emulsion produced from equal volumes of water and oil by the use of sodium oleate (a soap) as an emulsifying agent is found to be stable for a period of years if a o.I-molal solution of soap is used, but changes greatly in a few days if produced by
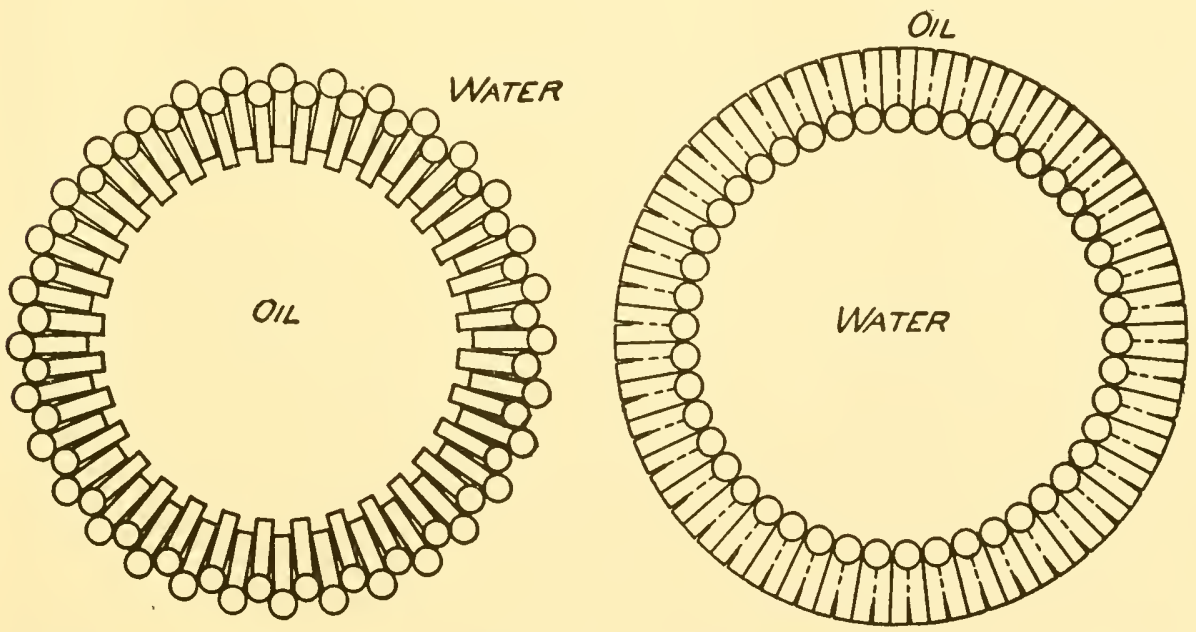

Fig. 22.- Shows how the change from the soap of a monovalent metal, such as sodium, to that of a bivalent metal, such as calcium, may change the emulsion from one of oil droplets in water to an emulsion of water droplets in oil.

0.005-molal soap. In the latter case not enough soap is present to form a condensed monomolecular film around the particles of oil, so the droplets of oil unite in order to decrease the interfacial area and thus increase the concentration of the soap in the film. With o.I-molal soap a condensed film of approximately monomolecular thickness is present, and this gives a considerable degree of stability to the emulsion. If the soap is the salt of a univalent metal, the droplets will be of oil, with water outside; but with bi- or trivalent metals several hydrocarbon chains are present for each single positive ion, and (Fig. 22) the droplets are of water, with oil outside. 


\section{THE DETERMINATION OF SURFACE AND INTERFACIAL TENSION}

The use of what is often called the "drop-number method" of determining the surface or interfacial tension is not only a waste of time but fills the literature of biology with worse than useless data, since they are so extremely deceptive.

Drop-weight method.-The capillary-height and the drop-weight methods are the most accurate now known. The drop-weight method is in general much the more suitable of the two for biological investigations. It depends upon the fact that if a drop of liquid is allowed to fall from a horizontal circular disk (end of a glass capillary tube) with sufficient slowness, the weight of the drop is a definite function of the surface tension, the radius of the tip, and the volume of the drop which falls, so

$$
W=M g=2 \pi r \gamma f,
$$

where $f$ stands for the value of the function. Thus

or if

$$
\gamma=\frac{M g}{2 \pi r f},
$$

$$
\begin{gathered}
F=\frac{\mathrm{I}}{2 \pi f}, \\
\gamma=M \frac{g}{r} F .
\end{gathered}
$$

To calculate the surface tension: (I) The volume (V) of the drop is its mass in grams divided by its density $\left(V=\frac{M}{d}\right)$. (2) Multiply $V$ by $\frac{\mathrm{I}}{r^{3}}$, in which $r$ is the radius of the circular tip. (3) Find $\frac{V}{r^{3}}$ in Table VIII, and note the corresponding value of $F$. (4) Divide $g$ (in dynes per $\sec ^{2}=980.3$ at Chicago) by $r^{3}(r$ in centimeters), and multiply by $M$ in grams, and by $F$. The result gives the surface tension in dynes per centimeter.

In the determination of interfacial tension the volume of the drop is measured directly. The weight of the drop as it hangs in a second liquid is $V\left(d_{1}-d_{2}\right)$, in which $d_{\mathrm{r}}$ is the density of the heavier and $d_{2}$ that of the lighter liquid. The liquid which is dropped should always be the aqueous phase, so if the oil is the heavier the tip should face upward.

Ring method for determination of surface tension. - The pull on a ring just being detached from the surface of a liquid does not give the surface tension, but the pull must be multiplied by a factor $F$, as given by Young and Cheng and the writer in a recent paper in Science. The outline of the method is as follows:

I. Determine the total pull in grams $(M)$ necessary just to detach a circular ring of diameter $R$, of circular wire of diameter $r$, from the surface of the liquid by the use of a chainomatic balance or a torsion balance, such as that of Du Nouy.

2. The surface tension is given by the equation

$$
\gamma=\frac{M g}{4 \pi R} \cdot F .
$$

3. Obtain the value of $F$, the correction factor, from Figure 23. $F$ is plotted on the $Y$-axis. To do this find the value of $\frac{R^{3}}{V}$, the cube of the radius of the ring divided by 
the volume of liquid $(V)$ upheld by the ring. $V=\frac{M}{\rho}$, or the volume of liquid is equal to the weight given by the balance, divided by the density of the liquid.

4. After finding the value of $\frac{R^{3}}{V}$ on the $X$-axis, find a point on one of the curves

TABLE VIII

Drop-Weight Surface-Tension Corrections (Factor for Multiplication $=F$ ), Based on the Value 72.75 as the Surface Tension of Water at $20^{\circ} \mathrm{C}$.

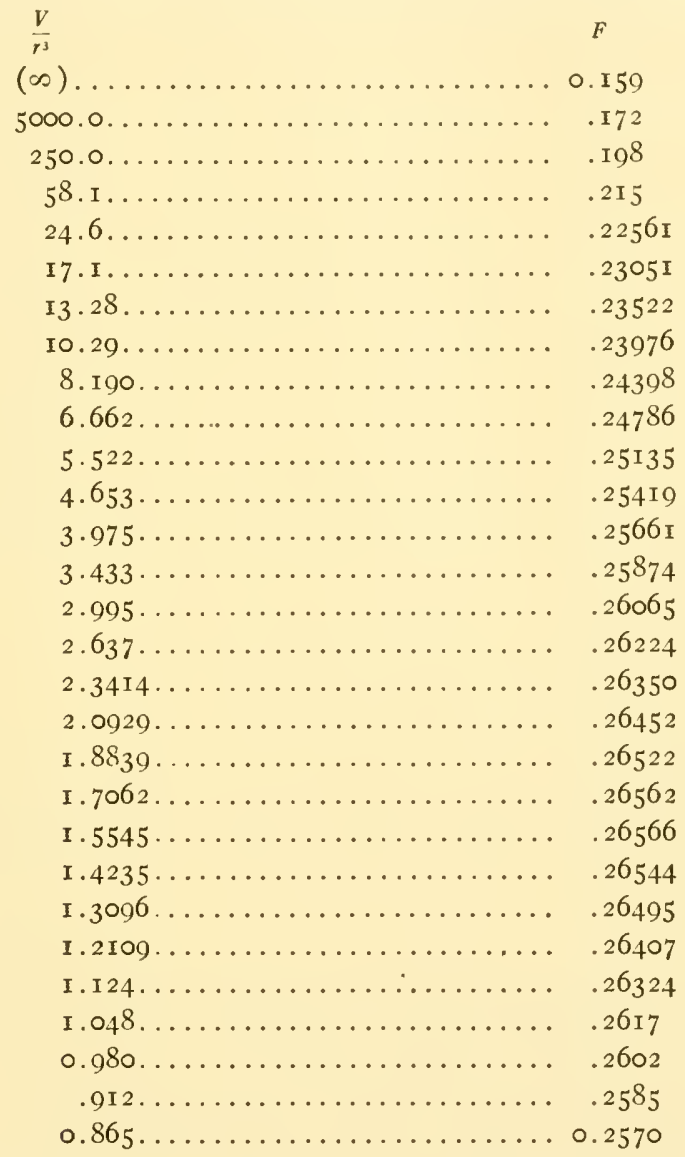

which has the same value of $x$. To do this it is necessary to know $\frac{R}{r}$. Curves are given for values of $\frac{R}{r}$ equal to $29.5,40.2,59.1$, and 78.5 . If the ring corresponds to some other value of this ratio, it is necessary to interpolate.

If the correction factor $F$ is not used, the results will in general be worthless, since they are not even relatively correct, as may be seen from the high curvature of the correction curves. 


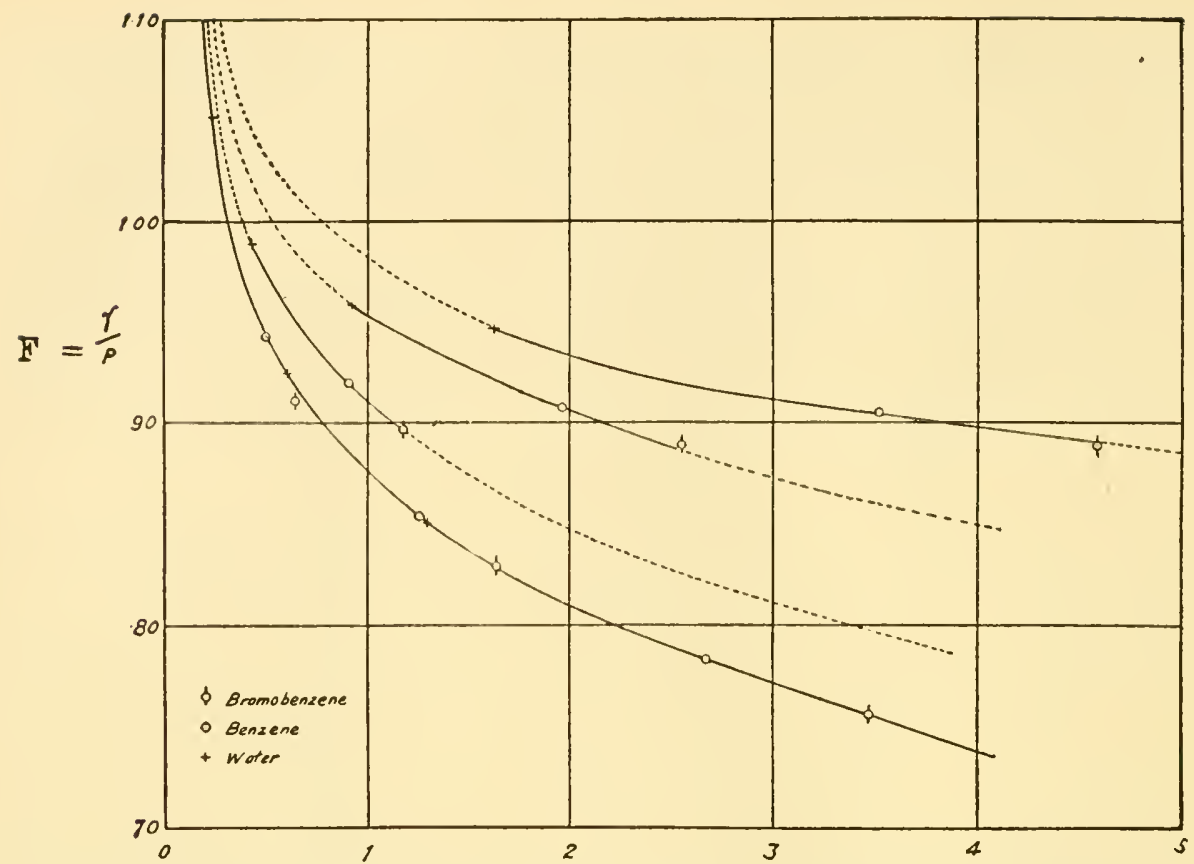

FIG. 23.-The values of $\frac{R}{r}$ for the curves in this figure, beginning with the bottom curve, are: $29.5,40.2,59.1$, and 78.5 .

$A=$ Area

$A=\mathrm{I}$ Angstrom unit of length $=10^{-8} \mathrm{~cm}$.

$\mathrm{A}^{2}=\mathrm{I}$ Angstrom unit of area $=\mathrm{IO}^{-16} \mathrm{~cm}^{2}$

$a=$ Activity

$a=$ Activity coefficient

$c=$ Concentration (respectively, the velocity of light $=2.9986 \times 10^{10} \mathrm{~cm} . / \mathrm{sec}$.

$\delta=$ Partial differential

$D=$ Dielectric constant

$E=$ Energy

$\mathfrak{E}=$ Electromotive force

$e=$ Charge on the electron $=4.774 \times$ ro $^{- \text {ro }}$ electrostatic units

$\epsilon=$ Epsilon, base of Naperian or natural logarithms

$\zeta=$ Zeta, fictitious electrical potential at a phase boundary

$F=$ Force (respectively, a correction factor of multiplication)

$\mathfrak{F}=\mathrm{I}$ Faraday $=96,500$ coulombs

$\Delta F=$ Increase of free energy (zeta function of Gibbs)

$g=$ Acceleration of gravity $\left(=980.278 \mathrm{~cm} . / \mathrm{sec}^{2}{ }^{2}\right.$ at the University of Chicago)

$h=$ Heat function for unit area of surface (total surface energy, respectively, for the area occupied by $\mathrm{I} \mathrm{mol}$ ); quantum constant $h=6.554 \mathrm{erg}$. sec.

$\mathrm{K}=\mathrm{A}$ constant

$k=$ Gas constant per molecule (Boltzmann) $=\mathrm{r} .372 \times 1 \mathrm{ro}^{-16}$ ergs per degree per molecule

$l=$ Latent heat of the surface per unit area

$\lambda=$ Lambda, latent heat of vaporization per molecule 
$\Lambda=$ Lambda, equivalent conductance

$M=$ Mass

$m=$ Molality, or concentration in mols per $\mathrm{I}, 000 \mathrm{gm}$. of solvent

$\mu=\mathrm{Mu}$, ionic strength (respectively, a unit of length $=10^{-4} \mathrm{~cm}$.)

$N=$ Number of mols (respectively, number of electronic charges on a particle)

$\nu=$ Frequency of vibration $=c / \lambda$

$P=$ Potential

$p=$ Pressure

$R=$ Gas constant

$=8.317 \times 10^{7}$ ergs per degree per molecule

$=\mathrm{I} .9885$ calories per degree per molecule

$r=$ Radius

$\rho=$ Rho, density (respectively, electrical density)

$S=$ Spreading coefficient

$T=$ Temperature on the Kelvin (absolute) scale

$T_{c}=$ Critical temperature

$T_{N a}+=$ Transference number of the sodium ion

$v=$ Volume

$\phi=$ Phi, electrical potential at a phase boundary

$\mathrm{I} V=$ Work (respectively, weight)

$V_{A}=$ Work of adhesion

$W_{C}=$ Work of cohesion

$X=$ Ficld strength in electrostatic units

$Z=$ Valence of an ion

\section{BIBLIOGRAPHY}

CRISTAL STRUCTURE

I. Bragg, Sir W.: Concerning the Nature of Things. New York: Harper \& Bros., 1925.

2. Bragg, W. H. and W. L.: X-Rays and Crystal Structure. New York: Harcourt, Bruce \& Co., 1924 .

3. Clark, G. L.: Applicd X’-Rays. New York: McGraw-Hill, I927.

\section{ATOMIC STRUCTURE}

I. Andrade, E. N. da C.: The Structure of the Atom. Lonilon: G. Bell \& Sons, 1927.

2. Lewis, G. N.: Valcuce and the Structure of Atoms and Molecules. New York: Chemical Catalog Co., 1923.

3. Harkins, W. D.: "The Stability of Atom Nuclei, the Separation of Iostopes, and the Whole Number Rule," J. Frankl. Inst., August, 1922, to April, I923.

4. Born, M.: Probleme der Atommechanic. Berlin: Springer, 1926.

5. Schroedinger, E.: "Quantisierung als Eigenwert Problem," Ann. d. Phys., 79, 361, 489. I926; 80, 437. I926; 81, I09. 1926.

IONIZATION OF SALTS IN SOLUTION AND THE ACTIVITY COEFFICIENT

I. Lewis, G. N., and Randall, M.: Thomodynamics. New York: McGraw-Hill, I923.

2. Eucken, A., Jette, E. R., and la Mer, V. K.: Physical Chcmistry. New York: McGrawHill, 1925 .

3. Debye, P., and Hückel, E.: Physikal. Ztschr., 24, 185, 305. I92.3. 


\section{SURFACES AND SURFACE ENERGY}

I. Harkins, W. D.: "Surface Energy and Surface Tension," Colloid Chemistry (ed. J. Alexander), chap. viii. New York: Chemical Catalog Co., 1926.

2. Harkins, W. D.: "Surface Energy in Colloid Systems," ibid. (ed. R. H. Bogue), chap. vi. New York: McGraw-Hill, I924.

3. Harkins, W. D.: "The Orientation of Molecules in the Surfaces of Liquids," Colloid Symp. Mono., Vol. 2, chap. xv. New York: Chemical Catalog Co., ig24; see also Vol. 5,1927 .

4. Debye, P., and Hückel, E.: "Cataphoretic Velocity of Suspended Particles," Physikal. Ztschr., 25, 49. 1924.

5. Willows, R. S., and Hatschek, E.: Surface Energy and Surface Tension. Philadelphia: P. Blakiston's Son \& Co., I923.

\section{COLLOIDS}

I. Kruyt, H. R.: Colloids. New York: John Wiley \& Sons, I927.

2. Freundlich, H.: Colloid and Capillary Chemistry. New York: E. P. Dutton \& Co., 1926.

3. Books edited by Alexander and by Bogue listed in preceding section.

4. Burton, E. F.: The Physical Properties of Colloidal Solutions. New York: Longmans, Green \& Co., I921.

5. Clayton, W., and Churchill, J. and A.: The Theory of Emulsification. London, I923.

\section{DETERMINATION OF SURFACE TENSION}

(Capillary-Height Method)

I. Richards, T W., and Carver, E. K.: J.Am. Chem. Soc., 43, 827. 1921.

2. Harkins, W. D., and Brown, F. E.: ibid., 41, 499. 19 I 9.

\section{(Drop-Weight Method)}

3. Harkins, W. D., and Brown, F. E.: ibid., p. 499. I9rg.

(Drop-Weight Method for Interfacial Tension)

4. Harkins, W. D., and Humphrey, E. C.: ibid., 38, 228, 236. г9ı6.

(Ring Method)

5. Harkins, W. D., Young, T. F., and Cheng, Y. C.: Srience, 64, 333. 1926.

Note.-Acknowledgment is made to Dr. Harry N. Holmes and the Chemical Catalog Company, editor and publishers respectively of Colloid Symposium IInnograph, Vol. II, for permission to reproduce Figs. 7,9, IO, II, I2, I $3, I_{4}$, and 22 from the above-mentioned volume. 


\title{
CHAP'TER XI
}

\section{THE EFFECT OF THE SURFACE TENSION OF THE MEN- STRUUM UPON BACTERIA AND TOXINS}

\author{
W. P. LARSON \\ University of Minnesota \\ INTRODUCTORY
}

Bacteriologists have been slow to recognize the influence of the surface tension of the menstruum upon bacteria and toxins, although surface energy and the phenomenon of adsorption have been studied by physical chemists, and even biologists, for many years. It is not our purpose here to discuss the fundamental problem of surface energy, since a complete treatment may be found in works devoted specifically to that subject. ${ }^{\mathrm{I}}$

It may be recalled that the surfaces of all liquids (and solids) are in a state of stress or tension due to the play of intramolecular forces, the degree of tension being determined by the nature of the fluid and the solutes which it contains.

Surface energy may be defined as the product of the surface area and the surface tension. Since free energy constantly strives toward a minimum, a liquid will assume the form giving it the smallest possible surface area, as the sphere of a raindrop, and many solutes which it contains will be forced-adsorbed-into the surface, provided the attraction of the molecules of the liquid is greater for each other than for the molecules of the solute.

Surface tension may be defined as the force per centimeter, in the plane of the surface, required to overcome the tendency of a liquid to maintain a minimum surface area. The unit of measurement is the dyne. Several methods for measuring the surface tension of a liquid are in use, the best known of which are:

The drop-weight method, the capillary-rise method, the jet method, and the method of measuring the force required to pull a disk or ring from the surface of a liquid.

The physicist, who usually works with pure liquids, seems to prefer the capillary-rise method in preference to others. After having tried out all the foregoing methods I have found the drop-weight method best suited to the work of the bacteriologist who never, or seldom, works with pure liquids but rather with a solution of several substances in water.

Morgan $^{2}$ and Harkins and Brown ${ }^{3}$ have perfected the drop-weight method to a point where most of the errors have been eliminated. In fact, their method is more delicate than the routine bacteriologist requires. Green ${ }^{\dagger}$ has recently developed a torsion balance which is rapid and yet sufficiently accurate for bacteriological work, where a high degree of accuracy is not important. When hundreds of measurements are required, I have found Green's

${ }^{1}$ Harkins, W. D., in Alexander, J.: Colloid Chemistry, New York, 1, I92. 1926. See chap. $x$ by Dr. Harkins in this volume.

${ }^{2}$ Morgan, J. L. R.: Jour. Amer. Chem. Soc., 33, 349. I9I I.

${ }^{3}$ Harkins, W. D., and Brown, F. E.: ibid., 4I, 499. I9I9.

${ }^{4}$ Green, R. G.: Indus. and Eng. Chem., 15, 1024. 1923. 
torsion balance a great convenience. The average of fifty or a hundred readings may be checked with the method of Morgan, or Harkins and Brown. In this way much time may be saved without the sacrifice of accuracy.

Clowes $^{I}$ has shown that it is often desirable to measure the interfacial tension between liquid and oil. Clowes's method consists in immersing the tip of an ordinary stalagmometer beneath the surface of paraffin oil and counting the number of drops in the usual way. This method gives very uniform results.

The surface tension of ordinary bacterial culture media varies from 57 to 63 dynes per centimeter. The surface tension of standard veal infusion broth is approximately $5^{8}$ dynes. That of I per cent peptone solution approximately 63 dynes. Since the surface tension of water is 73 dynes, it may be seen that peptone depresses the air-liquid tension about Io dynes.

The surface tension of culture media may be raised by treating them with charcoal. This raises the surface tension by removing some of the substances from solution. This treatment removes nutritive elements from the medium. The growth of bacteria ${ }^{2}$ may likewise, in some cases, raise the surface tension of the medium in which they are grown.

On the other hand, many things may be used to lower the surface tensions of a liquid. Alcohol which has a surface tension of 22 dynes will lower the tension of a fluid in proportion to the percentage added. However, it does not lower the surface tension by being adsorbed into the surface of the liquid, and, therefore, it should not be regarded as a true surfacetension depressant. Soaps, saponin, and many organic compounds are true surface-tension depressants since they are concentrated in the surface of a liquid, and in this way small quantities exert a marked effect in lowering the surface tension.

A small amount of soluble soap or saponin lowers the surface tension relatively more than larger amounts. The curve expressing such a relationship is that of a parabola.

For bacteriological work I have found the sodium soaps of the unsaturated fatty acids most satisfactory as surface-tension depressants. Potassium soaps are also very effective, but there is no point in using a potassium soap in a medium containing the solium ion, since in this case the sodium ion would invariably replace many of the potassium ions, and the final prorluct would be essentially a sodium soap.

Sodium ricinoleate presents certain advantages over the soaps of other fatty acids from the standpoint of the bacteriologist in that it is very soluble, is readily purified, remains in solution at low temperatures, exerts its maximum action at the $\mathrm{pH}$ optimal for the growth of bacteria and production of toxins, and forms perfectly clear solutions.

It is important to use media which contain no salts of either calcium or magnesium, since these elements form insoluble soaps and would, therefore, remove the latter from solution.

An important step in preparing glassware-only hard glass should be employed for this work - is to wash it with a hot soap solution in orler to remove the surface film usually present. It is desirable to sterilize the medium and soap solution separately and mix when cool.

The air-liquid surface tension of water or broth may be lowered to approximately 32 dynes per centimeter by adding a suitable amount of soap of an unsaturated fatty acid.

In studying the effect of the surface tension of the culture media on bacterial growth, one should not lose sight of the fact that the air-liquid or even the oil-liquid interfacial tension gives no accurate information as to the tension at the interface of the bacteria and water (medium). (Mudd and Mudd $^{3}$ have studied the effect of interfacial tensions of two phase liquids on bacteria.)

${ }^{x}$ Clowes, G. H. A.: Jour. Phys. Chem., 20, 407. 1916.

2 Larson, W. P., and Evans, R. D.: Proc. Soc. Exp. Biol. and M $c d ., 2$ I, I33. 1923.

3 Mudd, S., and Mudd, B. H.: Jour. Exp. Mcd., 40, 647. I924. 
At the present time interfacial tensions cannot be measured or even calculated with any degree of accuracy. The studies of Harkins ${ }^{t}$ and Halvorson and Green ${ }^{2}$ indicate there are all gradations of zones at the particle-water interface. Such zones may vary from one extreme, where they must be well defined, to the other, where they flow together, with all possible intermediate gradations. A pellicle-forming microorganism rich in "fat," like the tubercle bacillus, probably is a good example of the first case, while the pneumococcus in a solution of bile salts represents the latter (not "wet" in one case and dissolved in the other).

\section{SURFACE TENSION AND BACTERIAL BEIIAVIOR}

In discussing the effect of the surface tension of the culture medium on bacterial growth it may be well to begin with the pellicle-forming organism, since its effect is more apparent with this group.

The earlier bacteriologists regarded pellicle formation as an expression of obligate aerobiosis. It is true that pellicle-forming bacteria are aerobic, but they are not all obligate aerobes. Bacteria which are facultative anaerobes may be developed to grow in pellicle. Upon reflection it is apparent that the quality of aerobiosis is not the mechanism which causes bacteria to grow upon the surface of a liquid medium since pellicles are invariably of greater density than the fluid upon which they are growing. Benton ${ }^{3}$ has shown that the pellicle of $B$. subtilis may be sedimented at every stage of its development by centrifugation. It is obvious, therefore, that bacteria growing on the surface of a liquid are supported in this position by some force. Since aerobiosis does not constitute a force, we must look elsewhere for the explanation. It is undoubtedly the tension in the surface of the medium which supports the bacteria in this position. An analogy may be found in the floating steel needle. If a steel needle is coated with a film of oil and carefully placed upon the surface of water it also will remain supported upon the surface. Here there can be no question of aerobiosis. In the language of the physicist, the needle is not "wet" by the water; its weight is not sufficient to break the water surface. It is thus supported by the surface tension of the liquid. Reducing the surface tension by the addition of a little soap or other surface-tension depressant causes the needle to sink promptly. There is little doubt but that pellicle formation is a property of the surface tension of the medium, although the character of the bacterial surface is also an important factor, as we shall see presently.

If the surface tension of broth is depressed to some point below 40 dynes by the addition of sodium ricinoleate and inoculated with the hay bacillus, ${ }^{4}$ it will be observed that the growth is either diffuse throughout the medium, or, as often occurs, it grows at the bottom of the flask. In this connection the question arises as to the relation of the surface tension of a liquid to its ability to dissolve oxygen. If oxygen were more soluble in water of reduced surface tension, this might account for the different type of growth.

${ }^{1}$ Harkins, W. D., in Alexander: op. cit., p. 192. 1926.

${ }^{2}$ Halvorson, H. O., and Green, R. G.: Colloid. Symp. Monograph, 2, 185.

3 Benton, A. G.: Proc. Soc. Exp. Biol. and Med., 20, 513. 1923.

${ }^{4}$ Larson, W. P., Cantwell, W. F., and Hartzell, T. B.: Jour. Infect. Dis., 25, 4I. 1919. 
Green ${ }^{I}$ studied this problem, using standard methods of gas analysis. She found that the dissolved oxygen did not vary with the surface tension. The conclusion, therefore, that pellicle formation is a property of the surface tension of the medium rather than the property of aerobiosis seems to be justified.

Since wetting is a function of the surface tension, it may be stated that bacteria will grow on the surface of a liquid medium if the latter is unable to wet the surface of the organism. Since not all bacteria grow upon the surface of broth of a given surface tension, it follows that some of the factors determining the type of growth are inherent in the bacterial cells.

In discussing this phase of the question it may be assumed that the surfaces of bacteria attract water in varying degrees. In case the water molecules have a greater attraction for each other than for the bacteria, the latter are caught and held in the surface of the fluid, and, finding conditions favorable to growth, develop in pellicle. On the other hand, where there is greater attraction between water and bacteriaas is the case between water and a clean, fat-free, glass surface, the bacteria are drawn into the medium, and, finding conditions favorable to growth, grow diffusely. Thus it is apparent, if this conception be true, that the type of growth in a liquid medium will depend upon two factors, viz.: the liquid-air tension of the medium and the nature of the bacterial surfaces.

Since wetting is a function of the surface tension, it follows that an organism may behave differently in two media of different surface tension, as is the case with the hay bacillus in media with surface tensions of 60 and 35 dynes.

If pellicle-forming bacteria are extracted with acetone and ether and the extract compared with that of non-pellicle formers, it will be found that the former are relatively rich in acetone-ether soluble substances. The tubercle bacillus is a striking example of this group of bacteria, possessing as high as 40 per cent of fatlike substances, compared with about 7 per cent in non-pellicle-growing organisms.

Bacteria, which ordinarily grow diffusely throughout liquid media, will develop in pellicle $^{2}$ if grown on a medium containing a carbohydrate or glycerol, which they will not ferment. The staphylococcus, for example, when grown for a few generations upon 3 per cent glycerol broth, produces a pellicle resembling very much the growth of the tubercle bacillus; the medium beneath the pellicle remains perfectly clear, and, macroscopically, it resembles the growth of the tubercle bacillus. The ether-acetone extract of such a culture represents 39.9 per cent of the weight of the dry matter of the organisms, as compared with approximately 7 per cent of the same strain of the staphylococcus grown upon broth without glycerol.

It has been shown by numerous investigators that surface-tension depressants have a marked effect upon bacterial growth. Indeed, it is the operation of the laws of surface energy which makes it possible for bacteria to obtain food. It has been pointed out earlier in this chapter that many organic compounds are surface-tension depressants; that is to say, that they concentrate in the surfaces and interfaces of the liquid. The nutritive material in culture media must, therefore, be concentrated at the bacteria-water interfaces, which make them immediately available to the bacterial

\footnotetext{
${ }^{x}$ Green, B. S.: Papers from Mayo Found. and MLed. School, Univ. of Minn., p. 578. I921-22.

${ }^{2}$ Larson, W. P., and Larson, L. W : Jour. Infect. Dis., 3r, 407. 1922.
} 
cells. It will perhaps not be considered too theoretical to assume that the rate of growth is influenced by the effect the most immediate surface tension depressants have upon the organism. If it happens to be an ideal food for the particular bacterium, it constitutes an ideal culture medium. If, on the other hand, the surface-tension depressants are toxic, there will be little or no growth. The use of bile in culture media may illustrate the point in question. It has been known for many years that bile salts, when added to culture media, stimulate the growth of the colon-typhoid bacteria, whose normal habitat is the intestinal tract. The pneumococci and streptococci, on the other hand, are very sensitive to bile salts and soluble soaps.

Studies published from this laboratory have shown that relatively low concentrations of sodium ricinoleate deprive pneumococci, ${ }^{\mathrm{I}}$ streptococci, and tubercle bacilli ${ }^{2}$ of their power to infect. Netter and his collaborators ${ }^{3}$ have recently confirmed and somewhat extended this observation. Ayers $e t$ al.,${ }^{4}$ studying the effect of the surface tension of the culture medium on the growth of streptococci, suggested that the surface tension of the medium may be used as a basis for classification. Frobisher ${ }^{5}$ has emphasized the importance of using high-tension medium in growing pneumococci. Albus and $\mathrm{Holm}^{6}$ have further shown that $B$. bulgaricus is more sensitive to surface-tension depressants than $B$. acidophilus. They believe the difficulty of successful implantation of $B$. bulgaricus in the intestinal tract is due to its inability to grow at the low-surface tension created by the bile salts. Hansen, ${ }^{7}$ Frobisher, ${ }^{8}$ and others have shown that the action of some disinfectants is enhanced by surface-tension depressants.

It is an interesting observation that the micro-organisms which thrive well upon-even prefer - a low-tension medium are splendid antigens, and as a rule produce infections which are followed by an immunity which is reasonably permanent, as is the case with typhoid, paratyphoid, and cholera vibrio infections.

Infections by the pneumococci, streptococci, and tubercle bacilli, on the other hand, confer no such degree of immunity. It is not possible at the present time to show a definite relationship between the optimal surface tension for an organism and its antigenic properties. The apparent relationship may be purely incidental, but nevertheless interesting.

The nature of the interfacial zones mentioned above undoubtedly plays an important rôle in antibody reactions. Where bacterial surfaces are of such a nature as to resist being wet by water it might be expected it would have an influence upon the union between antibody and bacterium. Studies in this connection were made by Larson and Greenfield. ${ }^{9}$ A staphylococcus was grown for several generations on glycerol broth until it grew in a pellicle which resembled the growth of the tubercle

${ }^{1}$ Larson, IV. P., and Nelson, E. N.: Proc. Soc. Exp. Biol. and Med., 22, 357. I925.

${ }^{2}$ Larson, W. P., and Montank, I. A.: ibid., 20, 229.1923.

3 Netter, A., André, E., Cesari and Contoni: Compt. rend. Soc. de biol., 96, 184. 1927.

4 Ayers, S. W., Rupp, P., and Johnson, W. T., Jr.: Jour. Infect. Dis., 33, 202. I923.

5 Frobisher, M.: ibid., 38, 66. 1926.

${ }^{6}$ Albus, W. R., and Holm, M. L.: Proc. Soc. Exp. Biol. and Med., 22, 337. I925.

7 Hansen, T.: Comp. rendu. soc. biol., 86, 215. 1922.

${ }^{8}$ Frobisher, M.: Jour. Bact., 13, 163. 1927.

9 Larson, W. P., and Greenfield, R.: Proc. Soc. Exp. Biol. and Med., 20, 348. I923. 
bacillus. The ether-acetone extract of such a culture represented 39.9 per cent of the weight of the dry matter of the organisms, as compared with 7 per cent for the control culture, which was grown on broth without glycerol. Sera prepared against these two strains agglutinated the normal strain of staphylococcus in about two hours, while only 30 per cent, by actual count, of the "fat" strain was agglutinated after thirty hours' incubation.

The data available are not yet sufficient to permit final conclusions as to the mechanism involved, yet in view of our conception of the mechanism of pellicle formation the thought suggests itself that the attraction between the antibody and the water molecule may be greater than between antibody and micro-organism. In other words, if water will not wet the surface of the micro-organism rich in fat or related substances, the antibodies which are dissolved or suspended in water, for which they have greater attraction, cannot come in sufficiently intimate contact with the bacteria to be adsorbed.

The writer is of the belief that by the choice of suitable surface-tension depressants the wetting of bacteria by specific antibodies may be enhanced.

\section{EFFECT OF SOAPS ON TOXINS}

Studies have been conducted in our laboratory on the effect of surface-tension depressants on various toxins. ${ }^{1}$ Many bacterial toxins, snake venoms, ${ }^{2}$ and indeed some of the vegetable toxins are instantly detoxified by soluble soaps, particularly the soaps of the unsaturated fatty acids. Although this work was begun by studying the results from the standpoint of a surface-tension phenomenon, it soon became evident that factors other than the surface tension of the menstrua must be considered. Indeed, some soaps, especially those of the fatty acids of the odd-numbered carbon chain, which have a marked effect in lowering the surface tension, have very little detoxifying action on toxins. The soaps of the saturated fatty acids are far inferior to those of the unsaturated series as detoxifying agents. The double bond of the carbon atom apparently plays a rôle in this reaction, although, as will be pointed out later, the reaction cannot be regarded as a true chemical union.

Diphtheritic, tetanic, streptococcic, and other toxins are instantly rendered nontoxic when mixed with proper amounts of a soap such as sodium ricinolcate.

Soap does not affect all toxins in the same manner, however. While diphtheritic, tetanic, and streptococcic toxins and some of the snake venoms are detoxified by the soaps of the unsaturated fatty acids, other toxins are activated by these soaps. Botulinum toxin, for example, is rendered more toxic when treated with soap. Green and Stoesser $^{3}$ have recently shown that the toxicity of a poisonous mushroomA manita phalloides - is increased many fold by treatment with sodium ricinoleate. So active is this soap upon the Imanita toxin that its effect is apparent in the animal body when injected as much as seventy-two hours in advance of the toxin. This observation will no doubt stimulate further investigation in the field of toxicology.

Superficial examination suggests that toxins which are not detoxified by soaps $\rightarrow$

\footnotetext{
'Larson, W. P., and Nelson, E. N.: Proc. Soc. Exp. Biol. and Med., 21, 27S. 1924.

${ }^{2}$ Carmichael, E. B.: Jour. Phurm. Es Exp. Therap., 31, 445. 1927.

${ }^{3}$ Green, R., and Stoessner, A. V.: Proc. Soc. Exp. Biol. and Med., 24, 913. I927.
} 
are toxic when introduced into the digestive tract. Carmichael, ${ }^{1}$ however, has found an exception to this rule in ricin, which he found was readily detoxified by sodium ricinoleate.

The bile soaps undoubtedly play an important rôle in detoxifying the bacterial toxins of the intestinal tract. The intestinal tract is essentially a thirty-foot culture tube which harbors a large variety of bacteria, many of which, growing under favorable conditions, undoubtedly secrete toxins. Had nature not provided an efficient detoxifying mechanism in the form of the bile soaps, the host probably would not have survived. Since toxins which have been detoxified with soaps are antigenic, it seems reasonable to assume that through absorption of such detoxified toxins man and the lower animals acquire a relative immunity to many of the pathogenic bacteria to which they are daily exposed.

Within certain limits the reaction between soap and toxin is quantitative. ${ }^{2}$ In a study published from our laboratory it was shown that the toxicity of soap-toxin mixtures depends more upon the concentration of the toxin than the actual amount present. It was found, for example, that guinea pigs will tolerate an L + close of diphtheritic toxin in a I per cent solution of sodium ricinoleate provided the total volume injected was at least $5.4 \mathrm{cc}$. Animals injected with this amount of toxin in a I per cent soap solution, but in smaller volumes, died in periods of time varying inversely with the volume given. If, on the other hand, the toxin concentrations were kept constant while varying the soap concentration, it was found that the animals would tolerate an $\mathrm{L}+$ dose of toxin in 3.57 per cent solution of sodium ricinoleate in a total volume of $\mathrm{I} .4 \mathrm{cc}$.

Tables I-III, which are taken from an article by the writer and collaborators in the Colloidal Symposium Monograph, Volume $3,{ }^{2}$ illustrate this point.

These data show that there is a quantitative relationship in the reaction between toxin and soap. The reaction is evidently not a true chemical union since in such case soap which had reacted with one toxin should fail to react with others. This, however, is not the case, as it has been found that, within limits, several toxins may be detoxified with the same soap solutions. Furthermore, if the reaction between soap and toxin were a chemical union, all toxins reacting with a given soap would necessarily be considered identical chemically, and possibly antigenically as well. Such a contention would not be tenable. Wells ${ }^{3}$ found that whenever chemical differences can be shown between two antigens they invariably differ antigenically.

Table III shows the effect of dilution upon a soap-toxin mixture which is nearly neutral. Moderate dilution causes it to become non-toxic, while further dilution causes it to become extremely toxic.

Dissociation of diphtheritic toxin-soap mixture upon dilution may be observed in mixtures which have stood for periods of time up to about ten days. Mixtures which have stood for longer periods are not readily dissociated, but are nevertheless antigenic. It is believed the data given in the tables support the theory cited elsewhere that toxin exists as molecular aggregates. These aggregates are dispersed by a

I Carmichael, E. B.: Proc. Soc. Exp. Biol. and Med., 24, 5. 1927.

${ }^{2}$ Larson, W. P., Halvorson, H. O., Evans, R. D., and Green, R. G.: Colloid Symp. Mono., 3 , 152. 1925 .

3 Wells, H. G.: Chemical Aspects of Immunity. 1925. 
soap solution of proper concentration. After dispersion the toxin is rendered nontoxic by adsorption about the soap molecule.

TABLE I

Effect of Varying the Concentration of the Toxin Using i Per Cent Soap

\begin{tabular}{|c|c|c|c|c|c|}
\hline Guinea Pig No. & $\begin{array}{l}\text { Conc. of } \\
\text { Toxin, L+ } \\
\text { per cc. }\end{array}$ & Dose, L+ & $\begin{array}{c}\text { Conc. of } \\
\text { Soap in Per } \\
\text { Cent }\end{array}$ & $\begin{array}{c}\text { Total Volume } \\
\text { Injected, } \\
\text { cc. }\end{array}$ & Result \\
\hline 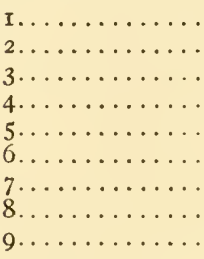 & $\begin{array}{l}.715 \\
.417 \\
.264 \\
.185 \\
.158 \\
.135 \\
.119 \\
.105 \\
.096\end{array}$ & $\begin{array}{l}\text { I } \\
\text { I } \\
\text { I } \\
\text { I } \\
\text { I } \\
\text { I } \\
\text { I } \\
\text { I } \\
\text { I }\end{array}$ & $\begin{array}{l}\text { I } \\
\text { I } \\
\text { I } \\
\text { I } \\
\text { I } \\
\text { I } \\
\text { I } \\
\text { I } \\
\text { I }\end{array}$ & $\begin{array}{l}\text { I. } \\
2.4 \\
3.4 \\
4.4 \\
5.4 \\
6.4 \\
7.4 \\
8.4 \\
9.4\end{array}$ & $\begin{array}{c}\text { Died in } 3 \text { days } \\
\text { Died in } 3 \text { days } \\
\text { Died in } 3 \text { days } \\
\text { Died in } 6 \text { days } \\
\text { Lived } \\
\text { Lived } \\
\text { Lived } \\
\text { Lived } \\
\text { Lived }\end{array}$ \\
\hline
\end{tabular}

TABLE II

EfFect of Varying the Concentration of the Soap

\begin{tabular}{|c|c|c|c|c|c|}
\hline Guinea Pig No. & $\begin{array}{l}\text { Conc. of } \\
\text { Toxin. Lt } \\
\text { per cc. }\end{array}$ & Dose, $\mathbf{L}+$ & $\begin{array}{c}\text { Total Volume } \\
\text { Injected, } \\
\text { cc. }\end{array}$ & $\begin{array}{l}\text { Conc. of } \\
\text { Soap in Per } \\
\text { Cent }\end{array}$ & Result \\
\hline $\begin{array}{l}1 \ldots \ldots \ldots \\
2 \ldots \ldots \ldots \ldots \\
3 \ldots \ldots \ldots \\
4 \ldots \ldots \ldots \\
5 \ldots \ldots \\
6 \ldots \ldots \ldots \\
7 \ldots \ldots \ldots \\
8 \ldots \ldots \ldots \\
9 \ldots \ldots \ldots \\
9 \ldots \ldots\end{array}$ & $\begin{array}{l}.715 \\
.715 \\
.715 \\
.715 \\
.715 \\
.715 \\
.715 \\
.715 \\
.715\end{array}$ & $\begin{array}{l}\text { I } \\
\text { I } \\
\text { I } \\
\text { I } \\
\text { I } \\
\text { I } \\
\text { I } \\
\text { I } \\
\text { I }\end{array}$ & $\begin{array}{l}\text { I. } 4 \\
\text { I . } 4 \\
\text { I. } 4 \\
\text { I. } 4 \\
\text { I . } 4\end{array}$ & $\begin{array}{l}.715 \\
1.413 \\
2.14 \\
2.86 \\
\\
3.57 \\
4.28 \\
5.00 \\
5.71 \\
6.42\end{array}$ & $\begin{array}{c}\text { Died in } 3 \text { days } \\
\text { Died in I } 1 \text { days } \\
\text { Died in } 28 \text { days } \\
\text { Showed paralysis in } \\
40 \text { days. Lived } \\
\text { Lived } \\
\text { Lived } \\
\text { Lived } \\
\text { Lived } \\
\text { Lived }\end{array}$ \\
\hline
\end{tabular}

TABLE III

Effect of Dilution upon a Soap-Toxin Mixture

\begin{tabular}{|c|c|c|c|c|c|c|c|c|}
\hline $\begin{array}{l}\text { Guinea Pig. } \\
\text { No. }\end{array}$ & $\begin{array}{l}\text { Toxin, } \\
\text { cc. }\end{array}$ & $\begin{array}{c}4 \text { Per } \\
\text { Cent } \\
\text { Soap } \\
\text { Sol., cc. }\end{array}$ & $\begin{array}{l}\mathrm{H}_{2} \mathrm{O}, \\
\text { cc. }\end{array}$ & $\begin{array}{l}\text { Total } \\
\text { Yol. In- } \\
\text { jected, } \\
\text { cc. }\end{array}$ & $\begin{array}{c}\text { Conc. } \\
\text { Tox., } \\
\text { L+ per } \\
\text { cc. }\end{array}$ & $\begin{array}{l}\text { Conc. } \\
\text { Soap, } \\
\text { Per } \\
\text { Cent }\end{array}$ & $\begin{array}{c}\text { Dose, } \\
\text { L+ }\end{array}$ & Result \\
\hline $\mathbf{I} \ldots \ldots \ldots$ & .4 & I.O & $0 . \infty$ & I.4 & .715 & 2.86 & I & Died in 41 days \\
\hline $2 \ldots \ldots \ldots$ & .4 & I.O & $1 . \infty$ & 2.4 & .416 & I. 66 & I & Lived \\
\hline $3 \ldots \ldots \ldots$ & .4 & I.O & 2.00 & $3 \cdot 4$ & .300 & 1. I 8 & I & Died in 3 days \\
\hline $4 \ldots \ldots \ldots$ & .4 & I.O & 3.00 & $4 \cdot 4$ & .227 & 0.90 & I & Died in 2 days \\
\hline
\end{tabular}

In view of the fact that a number of bacterial toxins, bacteria, and filterable viruses $^{1}$ are detoxified by soap, without destruction of their antigenic properties, ${ }^{2}$ it should be possible to develop this principle to the point where it would be practicable to immunize against several infections in the same injection.

${ }^{I}$ McKinley, J. C., and Larson, W. P.: Proc. Soc. Exp. Biol. and Med., 24, 297. 1927.

${ }^{2}$ Larson, W. P., and Eder, H.: Jour. Amer. Med. Assoc., 86, 998. 1926. 
The mechanism of the action of soaps upon toxins provides interesting speculation since some toxins are neutralized while others are rendered more active. A theory on the basis of the charge carried by the soap, on the one hand, and the toxins, on the other, may supply a partial explanation of the phenomenon. If it be assumed that soap and diphtheritic toxin are oppositely charged they will attract each other and enter into some relationship which we have chosen to term an "adsorption reaction." If, as may be the case with the mushroom toxins, they bear the same charge, the tendency would be toward dispersion with resultant increased toxicity of the toxin. If we accept the theory of dispersion as the mechanism by which the toxin is rendered more toxic, it will be necessary to assume further that the mushroom toxin normally exists not only as free toxin molecules, but in molecular aggregates as well, otherwise there would be no dispersion.

If this assumption, for which there is some experimental support, be accepted, it may be assumed further that all toxins may exist in the form of molecular aggregates, and that such aggregates act as a unit, or as a single toxin molecule. In the light of this theory, toxins which are charged oppositely to the soap would be dispersed by being attracted to the soap and condensed-adsorbed - about the soap molecule. The like charged toxins, on the other hand, would be repelled by the soap, and in this way the molecular aggregates dispersed. Halvorson and Green have shown by mathematical deductions that the charge on the surface of a particle or molecule must be considered as a part of the surface energy. It is not contended that the charge of the molecules is the only force which brings about the reaction between the toxin and soap molecules. It is undoubtedly only one factor in the interplay of the forces of surface energy. 


\title{
CHAPTER XII
}

\section{OXIDATION-REDUCTION POTENTIALS OF DYE SYSTEMS AND THEIR SIGNIFICANCE IN BACTERIOLOGY}

\author{
W. MANSFIELD CLARK ${ }^{I}$ \\ Hygienic Laboratory, Washington, D.C. \\ INTRODUCTORY
}

Various attempts have been made to trace the history of our knowledge of the decoloration of dyes by bacteria. But let it be remembered that the preparation of several of the older dyes involved fermentative reduction and that the origins of these preparative processes are lost in antiquity. The Chinese, importing synthetic indigo from America, now avoid the necessity of the fermentative splitting of the glucoside of the indigo plant, but still employ the fermentative vat process they imported from India in ancient days. So it was that the decoloration of indigo and also the decoloration of other dyes, during the events known as "fermentations," were common knowledge during the period when the theory of fermentation took shape.

Had a century or two in either direction separated the rise of bacteriology from the rise of modern chemistry, certain important aspects of fermentative dye decoloration might not have been burdened with a nomenclature which somewhat obscures their significance. However, the technical and physiological importance of oxygenation had already established a dominating point of view at the time the theory of the vat process took form. It was the period in which great classes of chemical transformations were formulated in terms of the gain or loss of oxygen. As carbon dioxide is a "higher" oxide of carbon than carbon monoxide, the process

$$
{ }_{2} \mathrm{CO}+\mathrm{O}_{2} \rightarrow 2 \mathrm{CO}_{2}
$$

was naturally called "oxidation," while the reverse process resulting in the reduction of degree of oxidation was called "reduction." So when the cloth came from the indigo vat and was blued obviously by atmospheric oxygen, an oxidation process was said to have occurred, and by the same token the decoloration was regarded as a reduction.

The fact that organic chemistry represents the molecule of indigo as differing from that of indigo white by the loss of two hydrogen atoms does not perturb the formalist. Nothing is simpler than to assume the hydrogen to be removed by combining with oxygen to form water. But when oxidation of indigo white is accomplished by halogens, ferric salts, and other "oxidizing" reagents the postulated chemistry of the reaction is very complex.

It is not the purpose of this chapter to review either the chemistry of dyes, the theory of their oxidative-reductive transformations, or the details of the uses to which these processes are put in bacteriology. There will be described briefly a picture which is useful.

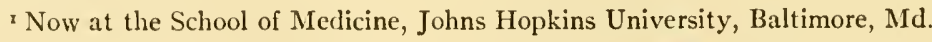




\section{ELEMENTARY OXIDATION-REDUCTION THEORY}

It has been found that a few oxidation-reduction processes occur in such a manner that states in their equilibria may be defined very accurately by certain electrical measurements. For instance, let a solution of hydrochloric acid be divided as shown in Figure I with a liquid connection made narrow to isolate approximately the parts. In one side place a platinum electrode and let there be one atmosphere pressure of hydrogen. In the other side place a platinum electrode and a mixture of ferric chloride and ferrous chloride. These salts dissociate to furnish the ions $\mathrm{Fe}^{+++}$and $\mathrm{Fe}^{++}$. If the electrodes are placed in metallic connection, a current of electrons will flow in the connection from the "hydrogen electrode" to the electrode in the iron solution, and this current will be accompanied by the transformation of hydrogen to hydrogen ions on the one side (an oxidation) and the transformation of ferric to ferrous ions on the other side (a reduction). If the electromotive force of this cell is nicely balanced by an external electromotive force, there is attained a close approach to the operation of the cell under conditions of maximum work, and there can be applied the thermodynamic equation for the change in free energy of the cell process. This free energychange can be factored into two parts: The quantity of electricity determined by the number of chemical equivalents and the intensity factor or electrical potential. The latter, as measured by a potentiometer under the conditions specified above, is a measure of the intensity with which hydrogen, at one atmosphere pressure, restrained by the given hydrion concentra-

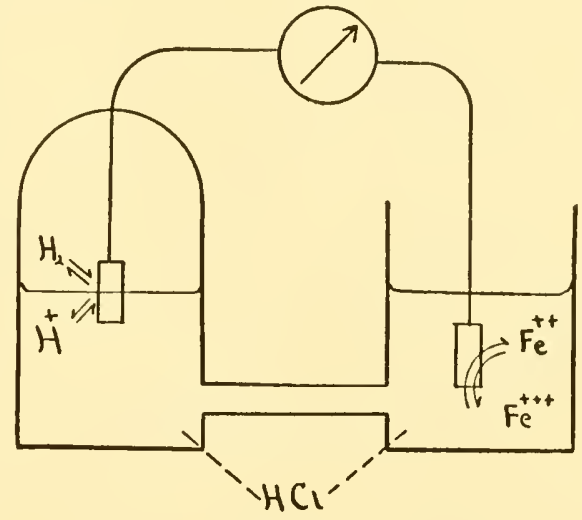

FIG. I tion, tends to transform the given iron solution in the direction of complete reduction.

The measurements are of the intensity factor of a free energy-change, and in the present state of our knowledge we must say that it is a matter of good fortune that the iron and several other oxidation-reduction systems are susceptible to study by this method. It furnishes no secure evidence of mechanism, but it may be inferred from facts which we shall not stop to review that the essential process is one of electron exchange. We may imagine that the ferric-ferrous system is exchanging electrons not only within the system itself but also with the electrode. With a fixed ratio of ferric and ferrous ions the electrode will receive a characteristic charge. In like manner, many dye systems such as methylene blue-methylene white give stable and characteristic electrode potentials.

It is therefore a convenience to assume that relative electron-escaping tendencies determine the ability of one system to reduce another. We shall see presently how, in accordance with this orienting assumption, the hydrogen exchange is taken care of.

For the sake of definiteness let the hydrogen half-cell be that in which the hydrogen is at one atmosphere and the hydrogen-ion concentration is one normal and let 


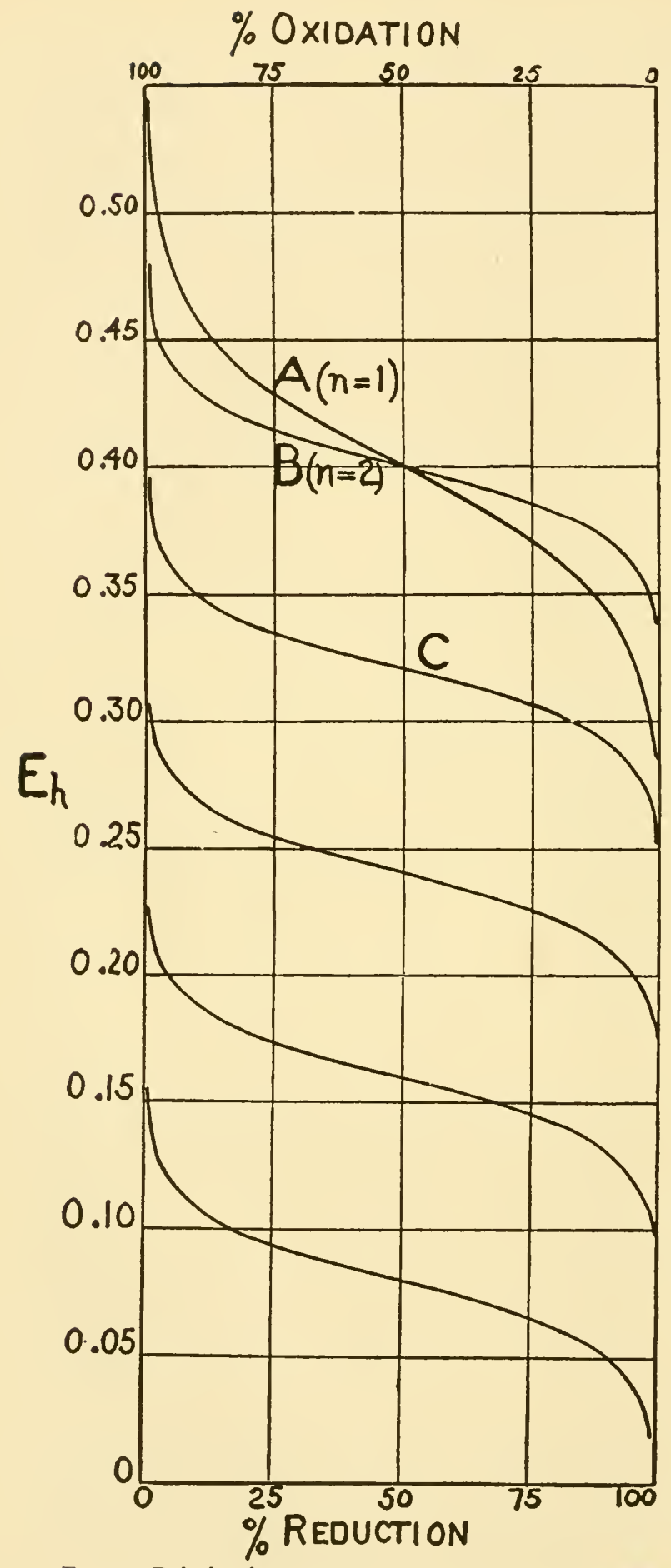

FIG. 2.- Relation between percentage reduction and electrode potential $\left(E_{h}\right)$ for different oxidation-reduction systems. the potential of this halfcell be considered zero. This half-cell will be considered the standard. There may now be substituted for the ferrous-ferric system one or another of several other oxidation-reduction systems. If a half-cell containing each system in turn be joined with the standard hydrogen half-cell and the potentials of the cell at different stages in the reduction of the system be measured, the results when shown graphically will provide a picture like that of Figure 2. An oxidant involving two electrochemical equivalents per mol for complete reduction will give a curve of the slope of $B$ or $C$. All the dye systems to be described furnish the two-equivalent slope. A system involving one equivalent gives a shape like that of curve A. Any such curve is best characterized by its center point-that of 50 per cent reduction or oxidation of the system, the potential of which furnishes a convenient reference point with which to describe quantitatively the relative oxidation or reduction intensity of the system. Each system has such a characteristic point under a given set of conditions to be described later. If it be negative to the characteristic potential of another system, the first is reducing with respect to 
the second. If it be positive with respect to a second, the first system is oxidative with respect to the second.

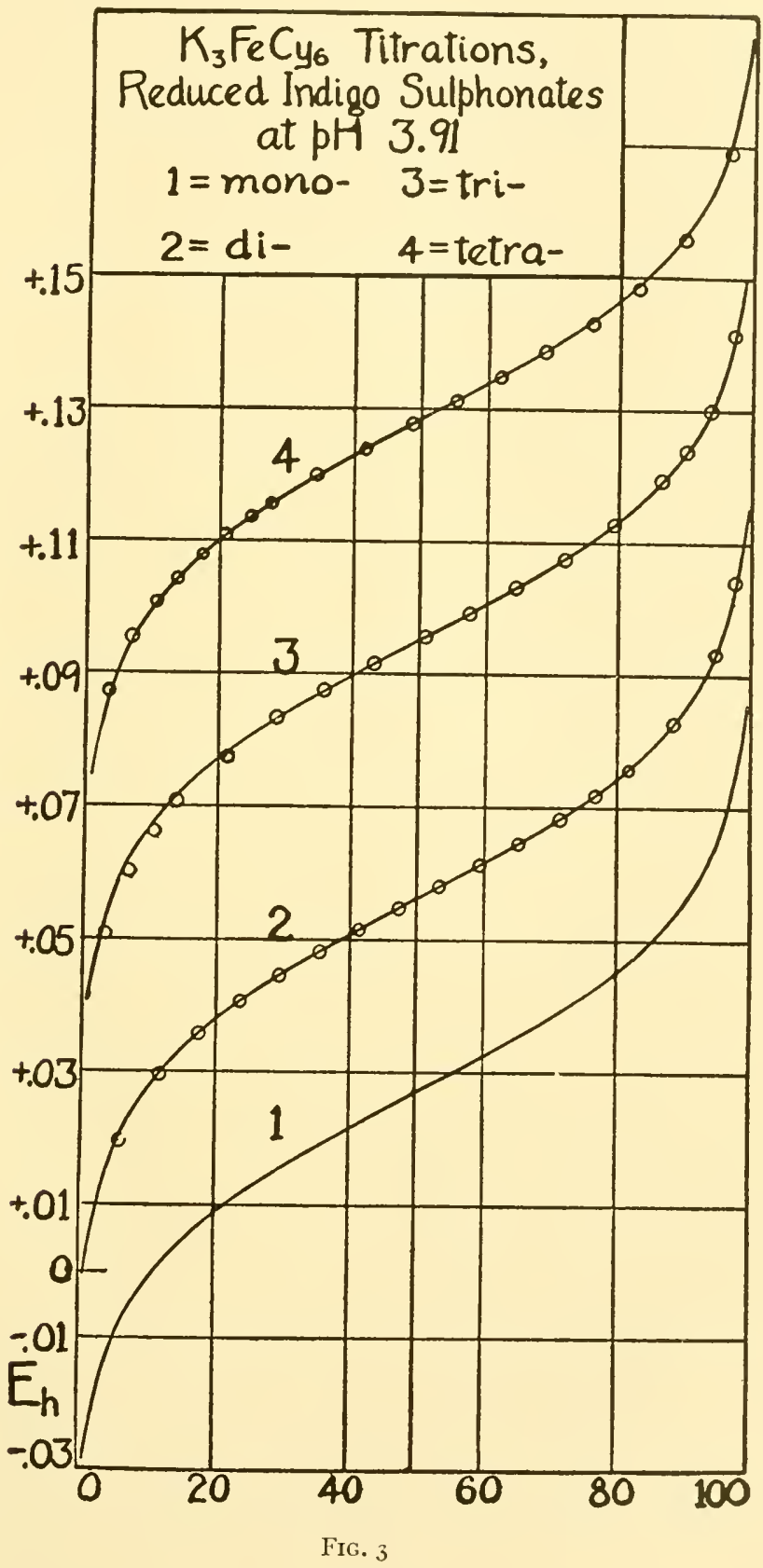

In practical applications the capacity factor must not be forgotten. Thus the ferrous-ferric ion system has an oxidative potential relative to the system methylene 
blue-methylene white; but if too little ferric iron is used to oxidize completely methylene white, there will be left ferrous iron as the only form of iron and a mixture of methylene white and methylene blue. If excess ferric is used, all the methylene white will be oxidized to methylene blue, and there will be left a mixture of ferrous and ferric ions. In the first case the potential will lie on the methylene blue-methylene white curve; and in the second case it will lie in the ferrous-ferric curve.

Figure 3 represents a series of actual measurements with sulphonates of indigo. The experimental data are shown by the centers of circles, and the theoretica! formcurve is shown in each case by the line to which the loci of the experimental data evidentally conform. This theoretical curve is determined by

$$
E=E_{0}^{\prime}-0.03 \log \frac{\text { Total reductant }}{\text { Total oxidant }}
$$

Since all the systems to be described conform to this equation when the hydrion concentration of the solution is fixed and known, and cliffer only in the characteristic constant $E_{0}^{\prime}$, Table I, giving values for the term $0.03 \log \frac{\text { Total reductant }}{\text { Total oxidant }}$ for various percentages of reduction, will be found useful.

Now it is found that a system which has been studied in a solution of constant hydrogen-ion concentration and which has been found to give a curve such as that of

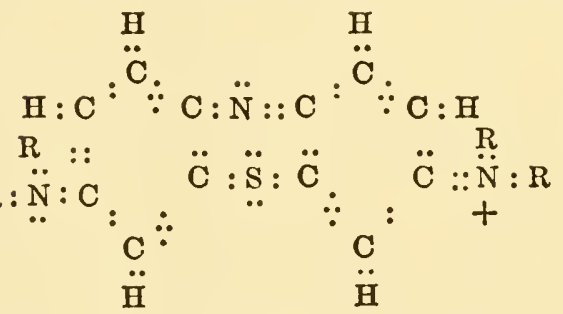

Fig. 4.-Conventional electronic structure of a thiazine.

Figure 3 will give the same form of curve at another value of $\mathrm{pH}$, but the value of $E_{o}^{\prime}$ will depend on the value of $\mathrm{pH}$. Thus the whole curve will be displaced in the potential scale.

Why is this displacement?

Consider the case of methylene bluemethylene white and the conventional electronic structure of a thiazine shown by Figure 4. The terminal substituted amino group at the right is polar and in methylene blue is a very strong base. The opposite dimethyl amino group in methylene blue is very weak. When the compound is reduced, the polarity of the one group is destroyed and this group becomes weakly basic. The other group in the now symmetrical structure is enhanced in strength. At the same time the bridging nitrogen fixes a hydrion. These changes make themselves felt in the thermodynamic measurements of free energy-change because as the several groups ionize, a virtually new oxidant or a new reductant is formed, each differing from the parent by the energy of ionization. Putting the matter another way, we may say that the covering or uncovering of points by hydrogen ions or hydroxyl ions alters the ease of escapement and acquirement of electrons. It is for this reason that the hydrion concentration must be kept rigidly constant if we are to obtain titration curves such as those of Figure 3 .

Remembering that if the hydrion concentration is constant we shall always obtain a curve of the form shown in Figure 3, our interest centers upon the displacement 
TABLE I

Relation of Percentage Reduction to Potential at Constant pH Determined by $E_{\mathrm{h}}=E_{0}^{\prime}-0.03006 \log \frac{[\mathrm{Sr}]}{[\mathrm{So}]}$ at $30^{\circ} \mathrm{C}$.

(Values Rounded to Nearest Millivolt)

\begin{tabular}{|c|c|c|c|}
\hline Reduction & $-0.03006 \log \frac{[\mathrm{Sr}]}{[\mathrm{So}]}$ & Reduction & $-0.03006 \mathrm{log} \frac{[\mathrm{Sr}]}{[\mathrm{Sol}]}$ \\
\hline Per Cent & Volts & Per Cent & Volts \\
\hline I.............. & +0.060 & $55 \ldots \ldots \ldots \ldots$ & -0.003 \\
\hline $2 \ldots \ldots \ldots \ldots \ldots$ & $.05 \mathrm{I}$ & $60 \ldots \ldots \ldots \ldots$ & .005 \\
\hline $5 \ldots \ldots \ldots \ldots \ldots$ & .038 & $65 \ldots \ldots \ldots \ldots$ & .008 \\
\hline $10 \ldots \ldots \ldots \ldots \ldots$ & .029 & $70 \ldots \ldots \ldots \ldots$ & $.01 \mathrm{I}$ \\
\hline $15 \ldots \ldots \ldots \ldots \ldots$ & .023 & $75 \ldots \ldots \ldots \ldots$ & .014 \\
\hline $20 \ldots \ldots \ldots \ldots \ldots$ & .018 & $80 \ldots \ldots \ldots \ldots$ & OI 8 \\
\hline $25 \ldots \ldots \ldots \ldots \ldots$ & .014 & $8_{5} \ldots \ldots \ldots \ldots$ & .023 \\
\hline $30 \ldots \ldots \ldots \ldots \ldots$ & .0 I I & $90 \ldots \ldots \ldots . . . .$. & .029 \\
\hline $35 \ldots \ldots \ldots \ldots \ldots$ & .008 & $95 \ldots \ldots \ldots \ldots$ & .038 \\
\hline $40 \ldots \ldots \ldots \ldots$ & .005 & $98 \ldots \ldots \ldots \ldots \ldots$ & .051 \\
\hline $45 \ldots \ldots \ldots \ldots \ldots$ & +.003 & $99 \ldots \ldots \ldots \ldots$ & -0.060 \\
\hline $50 \ldots \ldots \ldots \ldots \ldots$ & \pm 0.000 & & \\
\hline
\end{tabular}

\section{TABLE II}

$E_{0}^{\prime}$ Values for Several Oxidation-Reduction Indicators, $30^{\circ} \mathrm{C}$. (Values Rounded to Nearest Millivolt)

\begin{tabular}{|c|c|c|c|c|c|c|c|c|c|c|c|c|}
\hline $\mathrm{pH}$ & 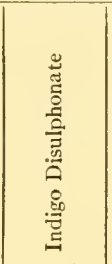 & 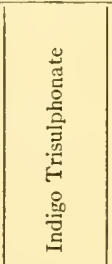 & 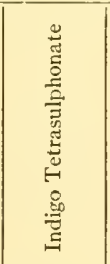 & 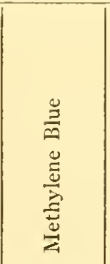 & $\begin{array}{l}\stackrel{0}{\Xi} \\
\frac{0}{0} \\
\frac{0}{3} \\
0\end{array}$ & 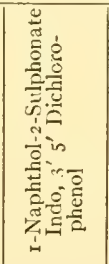 & 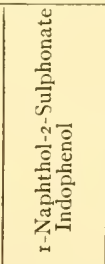 & 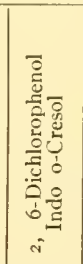 & 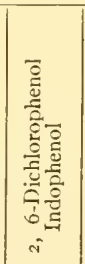 & 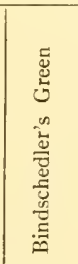 & 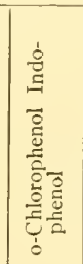 & 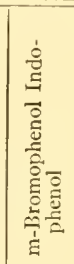 \\
\hline $.0 \ldots$. & -0.010 & 0.032 & 0.065 & 0.101 & 0.221 & 0.262 & & 0.33 .5 & $0.3^{66}$ & 0. & & \\
\hline$; .2 \ldots \ldots$ & .022 & .020 & .053 & .088 & .208 & .249 & & .322 & .352 & .320 & & \\
\hline $4 \ldots \ldots$ & .034 & +.008 & $.04 \mathrm{I}$ & .077 & .196 & .236 & $*$ & .307 & .339 & .307 & * & \\
\hline & .045 & -.004 & .020 & .066 & .184 & .223 & . & .292 & .325 & .293 & & \\
\hline$\cdots$ & .057 & .016 & .017 & .056 & .173 & .210 & $\ldots$ & .277 & .310 & $.28 \mathrm{I}$ & & \\
\hline .0. & .069 & .028 & +.006 & .047 & .162 & .196 & $\circ .18_{3}$ & .261 & .29 .5 & $.27 \mathrm{C}$ & $0.30 \mathrm{I}$ & $\ldots$ \\
\hline .2. & $.08 \mathrm{I}$ & .039 & -.006 & .039 & $.15 \mathrm{I}$ & $18_{1}$ & $.17 I$ & .245 & .279 & .259 & .288 & \\
\hline . 4 & .092 & $.05 \mathrm{I}$ & .017 & .031 & $.14 \mathrm{I}$ & .166 & .159 & .228 & .263 & .249 & .27 .5 & \\
\hline . & .104 & $.06 \mathrm{I}$ & .027 & .024 & .132 & .150 & .147 & .212 & .247 & .240 & .262 & $\ldots$ \\
\hline .8. & . I 14 & .072 & .037 & .017 & .123 & .134 & .135 & .196 & .232 & .232 & .248 & \\
\hline $7.0 \ldots$ & . I 2.5 & $.08 \mathrm{I}$ & .046 & OII & .115 & I I & .123 & $.18 \mathrm{I}$ & .217 & .224 & .233 & 0.248 \\
\hline $.2 \ldots$ & . I 34 & $.09 \mathrm{r}$ & .055 & .004 & .108 & IO & . I I I & .166 & .203 & .217 & .218 & .235 \\
\hline 4. & . I 43 & .099 & .062 & .002 & .101 & .088 & .099 & .152 & . I 80 & .210 & .203 & .22 \\
\hline . & .152 & .107 & .070 &.$\infty 08$ & .094 & .073 & .087 & .138 & .175 & .204 & $.8_{7}$ & .208 \\
\hline & .160 & .114 & .077 & .014 & .088 & .060 & .074 & .125 & .162 & 0.197 & .170 & .19 \\
\hline & .167 & $.12 \mathrm{I}$ & $.08_{3}$ & .020 & .082 & .046 & .062 & .112 & .150 & $\ldots$. & .155 & .17 \\
\hline & .174 & .127 & .090 & .026 & .075 & .034 & .049 & .090 & .137 & & .139 & .16 \\
\hline & .180 & .134 & .096 & .032 & .000 & .021 & .026 & .087 & .125 & & .124 & .145 \\
\hline &. $\mathrm{I} 87$ & .140 & .102 & .038 & .063 & .010 & .023 & .075 & I 13 & $\dagger$ & . 109 & .133 \\
\hline $.8 \ldots \ldots$ & . I93 & .146 & .108 & .044 & .057 & -.002 & +.010 & .063 & . IOI & & .095 & .117 \\
\hline $.0 \ldots \ldots$ & -0.199 & $-0.15^{2}$ & $\mid-0.1 \mathrm{I} 4$ & -0.050 & $0.05 \mathrm{I}$ & -0.012 & -0.003 & 0.051 & 0.089 & & 0.082 & 0.103 \\
\hline
\end{tabular}

* Unstable in this region of $\mathrm{pH}$.

t Decomposes in this region of $\mathrm{pH}$. 
of the curve as a whole when $\mathrm{pH}$ is changed. In Figure 5 is added the new dimension $\mathrm{pH}$, the case illustrated being that of $2-6$ dibromo indophenol (or 2-6 dibromo benzenone indophenol). At a given level of $\mathrm{pH}$ the familiar curve relating percentage oxidation (or reduction) to potential is similar to one in Figure 3 . If any fixed percentage oxidation is carried through changes of $\mathrm{pH}$, the curve is one of those shown running from the upper right-hand to the lower left-hand part of the figure. For convenience the curve of 50 per cent oxidation is chosen for use in two dimensional charts.

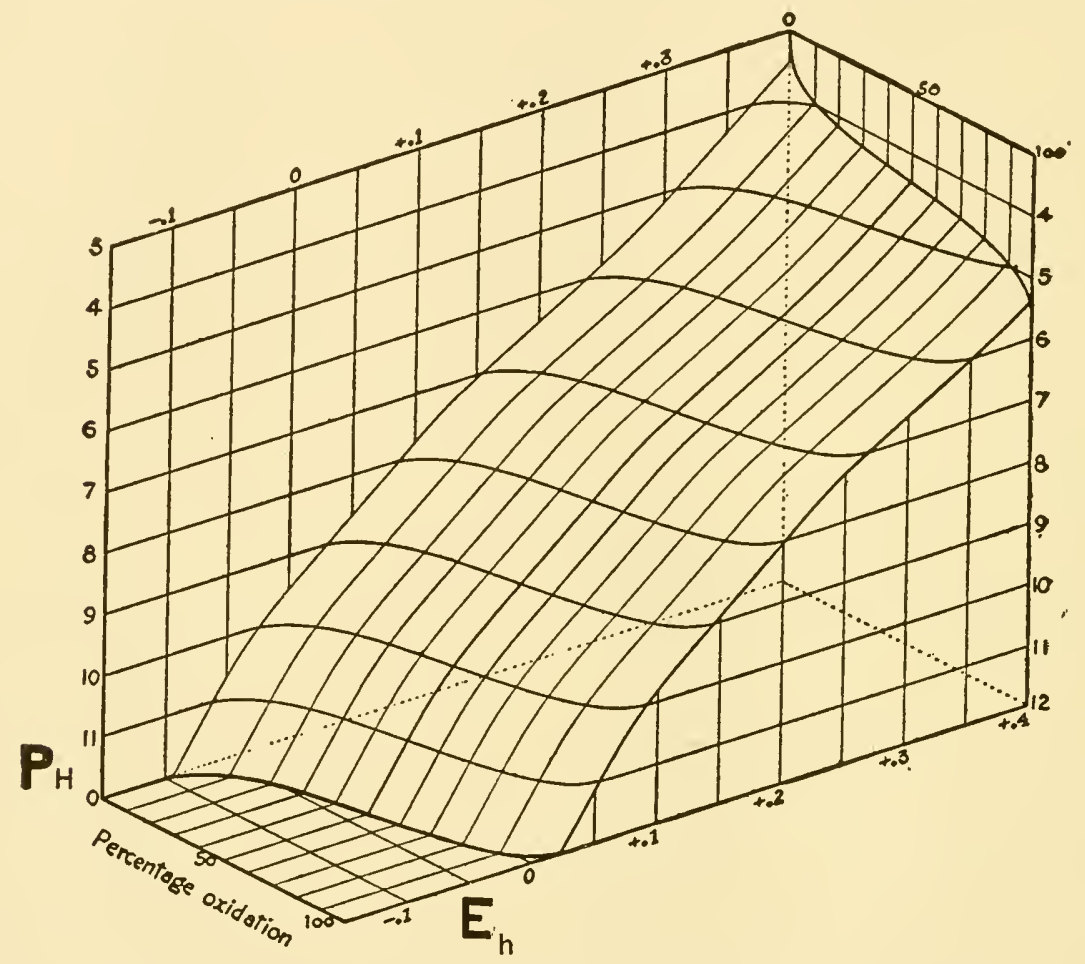

FIG. 5.-Isometric drawing showing the surface descriptive of the system of which 2-6 dibromo indophenol is the oxidant. The co-ordinates are percentage oxidation, $\mathrm{pH}$, and electrode potential.

In Figure 6 are several such curves showing the potentials of half-reduced systems at different levels of $\mathrm{pH}$. Curves $\mathrm{I}-4$ are respectively those of the mono-, di-, tri-, and tetra-sulphonates of indigo; 5 that of methylene blue, 6 that of Lauth's violet, the remainder being curves of indophenols.

In Table II are given the potentials of half-reduced solutions of several indicators at intervals of $0.2 \mathrm{pH}$ unit. This table may be used in conjunction with Table I as follows.

Suppose a culture known to be at $\mathrm{pH}=7.2$ has reduced methylene blue to the extent of 90 per cent. Table II shows that the potential of a 50 per cent reduced methylene-blue solution is +.004 at $\mathrm{pH}$ 7.2. Table I shows that the 90 per cent reduced solution is .029 volts more negative. Hence the culture has a reduction intensity which 
may be described as -.025 volts. If the culture maintains its $\mathrm{pH}$ value at 7.2 and then reduces indigo disulphonate to the extent of 85 per cent, the tables show that the re-

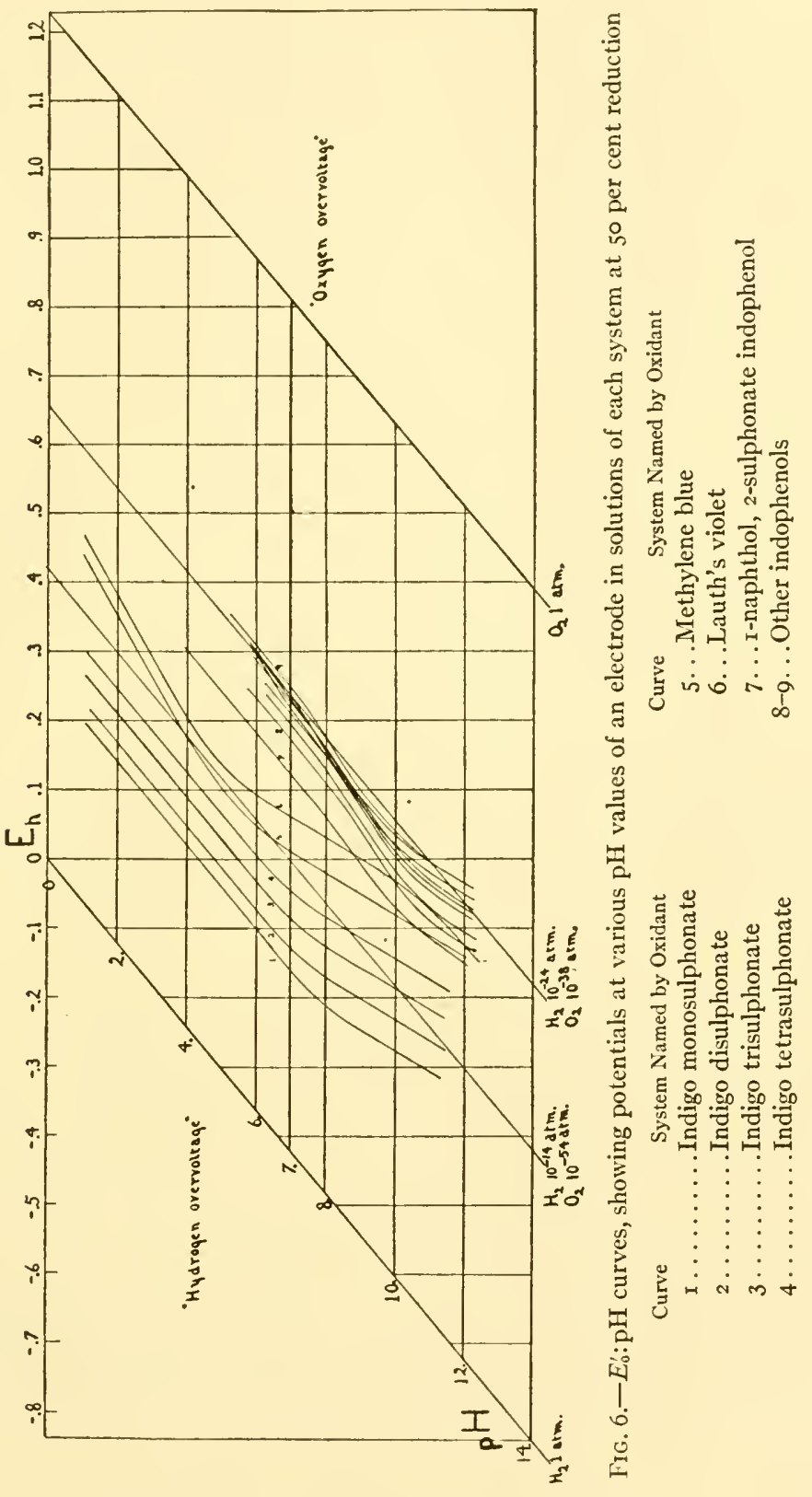

duction intensity may be described as -.157 volts. But if at 85 per cent reduction of indigo disulphonate $\mathrm{pH}$ should have changed to 6.6 , the potential would be -.08 I. 
Thus definite numerical values may be given to the reduction intensities of bacterial cultures.

\section{OXIDATION-REDUCTION AND ANAEROBIOSIS}

Now consider Figure 6 in more detail. The zero of potential at $\mathrm{pH}=0$ is the arbitrary zero of the normal hydrogen electrode. As $\mathrm{pH}$ increases the potential of the hydrogen electrode becomes more negative and follows the straight line at the left. Parallel to this, and about I.23 volts more positive, is the theoretical line of the normal oxygen electrode, an impracticable electrode. Now reverse the point of view. Consider a reducing agent capable of decomposing water with the liberation of hydrogen at one atmosphere. It should give a hydrogen electrode potential. Consider an oxidizing agent capable of decomposing water with the liberation of oxygen at one atmosphere. It should give an oxygen electrode potential. But the hydrogen electrode at any given hydrion concentration becomes more positive by about .03 volt for each power of Io by which the hydrogen pressure (in atmospheres) is lowered. Likewise, the ideal oxygen electrode should become .or 5 volt more negative for each power of Io by which the oxygen pressure (in atmospheres) is lowered. Thus if the oxygen pressure is lowered to $10^{-3^{8}}$ and the hydrogen pressure to Io $^{-24}$ atmosphere, an oxygen electrode and a hydrogen electrode should give a potential on the line of Figure 6 marked $\mathrm{H}_{2} \mathrm{IO}^{-24}, \mathrm{O}_{2} \mathrm{IO}^{-3^{8}}$. Conversely, a half-reduced indophenol system (lying near this line) should indicate a hydrogen or oxygen partial pressure of the values indicated, if a state of equilibrium is attained. The foregoing condition is extremely important. As a matter of fact, many of the indophenols are kept reduced by living cells even though oxygen is bubbled through the suspension. This fact alone is sufficient to suggest not only the relative inertness of atmospheric oxygen but also the caution to be observed in applying equilibrium data to kinetic affairs. It reveals at once the difficulty in obtaining an end-point indicator for oxygen which can be rationally formulated by methods comparable with those used in acidbase titrations.

It was not long after the advent of anaerobic culture of bacteria that Gunning criticized the methods, claiming that they were not adequate to remove the last traces of the oxygen he thought necessary to life. Finally, he appeared before the French Academy to claim that by a better approach to strict removal of free oxygen he had succeeded in stopping bacterial growth. Needless to say, the clain has received little support; but what interests us now is Pasteur's reply to Gunning. He stated that he was convinced of the fact of anaerobiosis because he had observed bacteria to thrive in solutions which maintained indigo in the reduced state.

If it can be shown that in any given case conditions are favorable to the attainment of true equilibrium, calculation shows the following: In a reduced solution of indigo disulphonate at $\mathrm{pH}=7.0$, the potential should be less than $-.125-.060=$ -.185 (Tables I and II). Fronr the formula

$$
-.185=\mathrm{I} .23-.06 \mathrm{pH}+.0 \mathrm{I} 5 \log \mathrm{Po}_{2},
$$

$\mathrm{PO}_{2}=10^{-66.5}$ atmosphere. Since a gram mol of oxygen contains about $10^{23}$ discrete molecules and occupies about 24 liters at one atmosphere, the foregoing condition 
would leave one discrete molecule of oxygen in a cube the order of magnitude of which would be about a million meters to the edge. Therefore, if an approach to an equilibrium condition can be shown to be reasonably attained, Pasteur's intuition is established as correct and anaerobiosis is an indisputable fact.

Since many dye systems give very satisfactory electrode potentials, it is an easy matter to study with this method the kinetics of dye reduction. It has also been possible to determine by the method outlined the dissociation constants of acidic or basic groups in dyes of importance to the art of staining. But even in the absence of the dye, Gillespie (1920) found definite drifts of potential in bacterial cultures. Further work on this was reported by Clark (1920), and was taken up again by Cannan, Cohen, and Clark (1926).

More detail concerning the theory, the measurement of dye systems, and applications to various subjects of interest to bacteriology will be found in the following papers by Clark and his co-workers: ${ }^{1}$

\section{STUDIES ON OXIDATION REDUCTION}

I. Introduction, Pub. Health Rep., 38, 443. 1923. (Reprint No. 823.)

II. "An Analysis of the Theoretical Relations between Reduction Potentials and $\mathrm{pH}$," ibid., p. 666. I923. (Reprint No. 826.)

III. "Electrode Potentials of Mixtures of I-Naphthol-2-Sulphonic Acid Indophenol and the Reduction Product," ibid., p. 933. I923. (Reprint No. 834.)

IV. "Electrode Potentials of Indigo Sulphonates, Each in Equilibrium with Its Reduction Product," ibid., p. r669. I923. (Reprint No. 848.)

V. "Electrode Potentials of Simple Indophenols, Each in Equilibrium with Its Reduction Product," ibid., 39, 3Si. I924. (Reprint No. 904.)

VI. "A Preliminary Study of Indopheno!s: (A) Dibromo Substitution Products of Phenol Indophenol; $(B)$ Substituted Indophenols of the Ortho Type; $(C)$ Miscellaneous," ibid., p. 804. 1924. (Reprint No. 91 5.)

VII. "A Study of Dichloro Substitution Products of Phenol Indophenols," ibid., 40, 649. 1925. (Reprint No. I00I.)

VIII. "Methylene Blue," ibid., p. I13 I. I925. (Reprint No. I0I7.)

IX. "A Potentiometric and Spectrophotometric Study of Meriquinones of the p-Phenylene Diamine and Benzidine Series," Supplement No. 54 to ibid. 1926.

X. "Reduction Potentials in Cell Suspensions," Supplement No. 55 to ibid. I926.

XI. "Potentiometric and Spectrophotometric Studies of Bindschedler's Green and Toluylene Blue," Supplement No. 6r to ibid. I927.

Other papers on the subject are referred to in reviews by $\mathrm{Clark}^{2}$ and by Conant. ${ }^{3}$ A review of particular biochemical interest is that of Joseph and Dorothy Needham. ${ }^{4}$

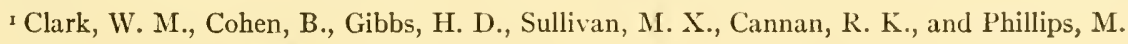

${ }^{2}$ Clark, W. M.: Chem. Rev., 2, I 27. 1925.

3 Conant, J. B: ibid., 3, I. 1926.

4 Needham, J. and D.: Protoplasm, I, 255. 1926. 


\title{
CHAPTER XIII
}

\section{ANAEROBIOSIS}

\author{
IVAN C. HALL \\ University of Colorado Medical School \\ BIOLOGICAL SIGNIFICANCE OF ANAEROBIOSIS
}

Few biological discoveries of more fundamental significance have been made than when Pasteur in I86I proved the existence of micro-organisms that are able not only to live in the complete, or nearly complete, absence of atmospheric oxygen, but which are actually unable to multiply at all under ordinary atmospheric conditions, and require, if not an absolute elimination of free oxygen from their environment, at least a marked reduction in oxygen tension.

Pasteur was brought to this discovery during his famous researches on fermentation, by the observation that certain motile germs in a cover-slip preparation became non-motile on approaching the edge of the cover. Having noted that many products were formed in the so-called "lactic" fermentation, such as butyric acid, mannitol, alcohol, carbon dioxide, and hydrogen, in addition to lactic acid, he was led to investigate the possibility of a special butyric ferment. This he decided was an infusorian, the Vibrion butyrique, living only in the absence of free oxygen, a conclusion in error so far as concerns the animal nature of the organism, but none the less important in its implications and its bearing on the old problem of abiogenesis. It had been generally assumed that air was necessary for the existence of all living things. But Pasteur revealed that the growth of certain micro-organisms is not only not inhibited but is actually dependent upon the practically complete exclusion of air.

Pasteur ${ }^{2}$ called these organisms "anaerobes," as distinguished from "aerobes," which grow in the presence of air; later ${ }^{3}$ three groups were distinguished: obligate anaerobes, obligate aerobes, and facultative aerobe-anaerobes. Holding that all anaerobic life depends upon an ability to satisfy oxygen requirements by sugar cleavage, Pasteur was led to define fermentation as life without air. While the production of "ferments" or enzymes is by no means restricted to the anaerobic micro-organisms, as Pasteur believed, both the most actively fermentative and the most actively putrefactive bacteria are found within this group. They are therefore of great importance in the chemical cycles of nature for the mineralization of organic matter, including processes useful to man, such as the destruction of dead bodies of plants and animals, and the formation of useful chemical solvents (butyl alcohol and acetone), as well as processes which man would like to avoid, notably food spoilage. Some of the obligate anaerobes are dangerous pathogens, producing malignant edema, gaseous gangrene, tetanus, botulism, syphilis, and other diseases in man and animals. The toxins of tetanus and botulism are the most powerful bacterial poisons known.

The first pathogenic anaerobe, Vibrion seplique, was announced by Pasteur and Joubert in 1877 as the cause of a septicemia (churbon symptomatique, symptomatic anthrax, or

\footnotetext{
${ }^{1}$ Pasteur, L.: Compi. rend. Acad. de sc., 52, 344 and I260. I861.

2 Pasteur, L.: ithid., 56, 416 and I192. I863.

${ }^{3}$ Pasteur, L.: Études sur la Biire. 1876.
} 
blackleg) in cattle which they distinguished from anthrax. ${ }^{\mathrm{I}}$ Discoveries of other pathogenic anaerobes have continued from that day until the present, and probably will continue for many years, for this field is still a fertile one. No obligately anaerobic plant pathogens have been discovered, however.

The best-known obligate anaerobes are found among the sporulating bacilli, whose properties and activities have been summarized by von Hibler, ${ }^{2}$ by Jungano and Distaso, ${ }^{3}$ and more recently by various French, ${ }^{4}$ English, ${ }^{5}$ Italian, ${ }^{6}$ and American ${ }^{7}$ investigators. The frequent occurrence of gaseous gangrene during the war, due to the close contact of the soldiers with fertilized (infected) soil, and the extensive uses of high explosives which carried soil particles into deep wounds, served greatly to arouse new interest in wound infections due to the sporulating anaerobes. During the war also, botulism became a conspicuous problem in the United States, possibly due, in part, to an unusual increase in the volume of crude home-canning, although the first cases of botulism were recognized before the war and there is good clinical evidence that botulism has occurred in the United States as in European countries for many years ${ }^{8}$ and a few of the outbreaks were due to commercially canned rather than home-canned products. ${ }^{9}$ Important obligate anaerobes occur also among the cocci, ${ }^{\text {to }}$ the actinomyces, ${ }^{\text {II }}$ the streptothrices, ${ }^{12}$ and the spirochaetes. ${ }^{13}$

What degree of oxygen-tension reduction can be tolerated by higher animals and plants is a problem only recently solved and one which has important practical bearings in the case of man, in connection with aviation. ${ }^{{ }^{4}}$ Bunge $^{15}$ pointed out that various

${ }^{x}$ Pasteur, L., and Joubert: Bull. Acad. de méd., Paris, 6, 78г. 1877.

${ }^{2}$ von Hibler, E.: Untersuchungen iiber path. Anaeroben. 1908.

3 Jungano and Distaso: Les Anaerobies. Paris: Masson, igio.

${ }_{4}$ Weinberg, M., and Seguin, P.: La Gangrine gazeuse. Paris: Masson, 1917.

5 Adamson, R. S.: J. Path. \& Bact., 22, 345. 1919; Medical Research Committee: Reports on the Anacrobic Infections of Wounds and the Bacteriological and Serological Problems Arising Therefrom. 1919.

${ }^{6}$ Desderi, P.: Infezione da germi anacrobi per ferità in guerra. Torino, I919; Fasiani, G. M.: Bolletino dell'Instituto Sièroterapico Milanese, No. 3. Oct., I921.

7 Simonds, J. F.: Monograph of the Rockefeller Institute for Medical Reserch, No. 5. I915; Heller, H.: J. Infect. Dis., 27, 385. 1920; J. Bact., 6, 445, 521. 1921; Kahn, MI. C.: J. Med. Research, 43, 155. 1922; J. Infect. Dis., 35, 423. 1924; Kendall, A. I., Day, A. A., and Walker, A. W.: ibid., 3o, I 41. I922; Hall, I. C.: ibid., p. 445. I922.

${ }^{8}$ Dickson, E. C.: Monograph of the Rockefeller Institute for Medical Research, No. 8. I918.

${ }^{9}$ Dubovsky, B. J., and Meyer, K. F.: J. Infect. Dis., 31, 50I. I922; (see also numerous papers by Meyer and collaborators in succeeding numbers of $J$. Infect. Dis.); Geiger, J. C., Dickson, E.C., and Meyer, K. F.: Pub. Health Bull. 127. 1922.

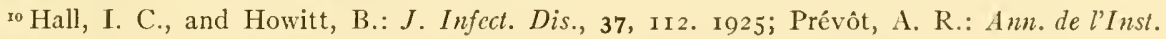
Pasteur, 39, 41 7. 1925; Schwartz, O. H., and Dieckmann, W. J.: Am.J.Obst. \& Gynec., 13, 467. 1927.

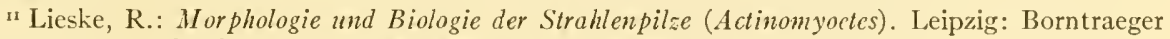
Bros., 1921; Naeslund, C.: Acta path. et microbiol. Scandinav., 2, 110. 1925.

${ }^{12}$ Plaut, R.: Centralbl.f. Bakteriol., Abt. I, Orig., 84, 440. I920.

I3 Noguchi, H.: J. Exper. Med., 14, 99. I9I I; 15, SI and 466. I91 2; 27, 667. I9I8; 28, 559. I9I8.

is Schneider, E. C.: Am.J. Physiol., 34, I and 29. 1914; 36, 380. 1915; also editorial in J.A.M.A., 88, I805. 1927 .

is Bunge, G.: Ztschr.f. phys. Chemie, 8, 48. I88 3 ; 12, 565. I888; 14, 318 . 189o. 
invertebrates such as intestinal worms and mud-dwelling organisms probably live without free oxygen owing to the presence of numerous micro-organisms which reduce the oxygen supply practically to zero, and raised the question as to how the muscles of such organisms secure their energy.

According to Weinland, ${ }^{\mathrm{I}}$ intestinal worms effect a true fermentative process under anaerobic conditions, as follows:

$$
{ }_{4} \mathrm{C}_{6} \mathrm{H}_{{ }^{2} 2} \mathrm{O}_{6}={ }_{9} \mathrm{CO}_{2}+{ }_{3} \mathrm{C}_{5} \mathrm{H}_{10} \mathrm{O}_{2}+{ }_{9} \mathrm{H}_{2} .
$$

Pütter ${ }^{2}$ kept leeches alive ten days without oxygen. During the interval hydrogen was set free with a primary rise in $\mathrm{CO}_{2}$ output followed by a fall.

Packard ${ }^{3}$ showed that the resistance of Fundulus embryos could be increased either by the injection of sodium bicarbonate or of certain carbohydrates. There is a possible analogy in Packard's reasoning to that of Pasteur which suggests that carbohydrates probably present in the organic slimes at the bottom of lakes make anaerobic life among some animals as well as bacteria possible there.

Juday $^{4}$ has noted that while the water from near the bottom of a lake may contain considerable oxygen during the winter months, in summer two factors operate to reduce the oxygen content to so low a value that it cannot be determined, namely, first, decaying organic matter, and second, lack of convection currents due to the warm and therefore lighter layer of water at the surface. In the slime collected from Lake Mendota, Wisconsin, various protozoa as well as higher invertebrates such as rotifers, worms, molluscs, crustaceae, and insect larvae were found frequently. None of the protozoans pumped from the bottom into anaerobic containers was in any way abnormal or inactive. Judays recently recorded an obligately anaerobic ciliate resembling Enchelys. On the other hand, according to Juday and Wagner, "No fish has ever been found which leads either an active or passive life in water that is free from dissolved oxygen." 6 Therefore, while the depths of a given lake may present suitable temperature conditions for the implantation of trout, and the surface layers an ample oxygen and food supply, the separation of these factors by stratification of the water during the summer months may prevent stocking and even account for the death of many fish in a well-stocked lake. Birge and Juday7 state that most fish require an oxygen content in the water of at least 2 cc. per liter (N.T.P.), whereas many protozoa and invertebrates live in water containing less than 0.1 cc. oxygen per liter.

In the tissues of higher animals many of the processes may be considered as essentially anaerobic. Bayliss ${ }^{8}$ referred to lactic acid as the product of anaerobic change in frog muscle, $\mathrm{CO}_{2}$ as that of aerobic change, and Shelford has pointed out that "high respiratory quotients of various animals are further evidence of anaerobic respiration.",

${ }^{1}$ Weinland, E.: Zischr.f. Biol., 42, 55. 1901; 43, 86. 1902; 45, 113 and 517. 1904; 48, 87. 1906.

${ }^{2}$ Pütter, A.: Zlschr. allgem. Physiol., 6, 217. 1907; 7, I6. 1907.

3 Packard, W. H.: Am. J. Physiol., 15, 30. 1905; 18, 164. I907.

${ }_{4}^{4}$ Juday, C.: Tr. Wisconsin Acad. Sc., 16, го. г909.

5 Juday, C.: Biol. Bull., 36, 92. г919.

${ }^{6}$ Juday, C., and Wagner: Tr. Wisconsin Acad. Sc., 16, I 7. I909.

7 Birge, E. A., and Juday, C.: ibid., p. I. Igo8.

${ }^{8}$ Bayliss, W. M.: Principles of General Physiology (4th ed.). London: Longmans \& Co., I924.

9 Shelford, V. E.: chap. ii in Ward, II. B., and Whipple, G. C.: Fresh Water Biology. New York: Wiley, I918. 
Thus the problems of anaerobiosis are not limited to a single group of bacteria but are shared by several groups, by protozoa, various invertebrates, and even by the tissues of warm-blooded animals. While some of the invertebrate metazoa may live as facultative anaerobes during all or part of their existence, the condition of obligate anaerobiosis seems to be limited to single-celled micro-organisms, particularly the bacteria and protozoa. Whether any of the ultra-microscopic viruses are obligately anaerobic must await their certain culture in artificial media. It is of interest to note that no obligately anaerobic yeasts or molds are known.

\section{MECHANISM OF BACTERIAL ANAEROBIOSIS}

PIIYSICO-CHEMICAL CONCEPTIONS

Pasteur's explanation of anaerobiosis was that the organisms secure their oxygen through the fermentation of carbohydrates. Certainly the growth of some obligate anaerobes is dependent upon the presence of fermentable carbohydrates. This seems to be the case with the actively saccharolytic species, B. butyricus, B. multifermentans, $B$. fallax, and B. sphenoides, none of which possesses sufficient lytic ability against nitrogenous compounds to hydrolyze even gelatin. Certain other species, e.g., B. welchii, B. chauvoei, B. septicus, B. novyi, and B. tetanomorphus, grow, but poorly, except in the presence of fermentable carbohydrates; these split gelatin and peptones but not native proteins. But the actively proteolytic bacteria, such as B. bifermentans, B.tyrosinogenes, B. acrofoetidus, B. botulinus, and B. sporogenes, grow quite heavily in sugar-free media though not so heavily as in media containing fermentable carbohydrates (mono-saccharides). Still other species, B. tetani and B. putrificus, though unable to ferment any carbohydrate, still grow anaerobically; both are mildly proteolytic. From these considerations we may conceive that obligately anaerobic bacteria derive the oxygen assumed to be necessary in their metabolism not only through the hydrolysis of carbohydrates but also of nitrogenous compounds.

Unless there is some essential difference in the mechanism of anaerobic growth of facultative and obligate anaerobes, something may be learned about the latter by studying the former. Pasteur ${ }^{1}$ showed that the products of fermentation of molds and yeasts vary according to whether they are provided with oxygen, thus $M$ ucor racemosus in the open air transforms glucose into $\mathrm{CO}_{2}$ and $\mathrm{H}_{2} \mathrm{O}$, but in the absence of air produces alcohol and $\mathrm{CO}_{2}$; in other words, oxidative processes fall short under anaerobic conditions.

Until a few years ago, Hoppe-Seyler's theory of biological oxidation ${ }^{2}$ was most widely accepted. According to him, fermentation results in the liberation of nascent hydrogen which combines with atmospheric oxygen, forming water $\left(\mathrm{H}_{2}+\mathrm{O}_{2}=\mathrm{H}_{2} \mathrm{O}+\mathrm{O}\right)$, setting free nascent oxygen which is directly responsible for oxidation in the protoplasm. But this theory could not account for anaerobic respiration where oxidations occur in the practically complete absence of free oxygen. Armstrong ${ }^{3}$ has shown that in ordinary oxidations free oxygen does not unite directly with carbon to form carbon dioxide or with hydrogen to form water, but any substance that is to be oxidized is first hydroxylated, atmospheric oxygen acting

r Pasteur, L.: Études sur la Bicre. I876.

${ }^{2}$ Hoppe-Seyler, F.: Zischr.f. Physiol. Chemie, I, I2 1. 1877.

${ }^{3}$ Armstrong, H. E.: Chem. News, 90, 25. 1904; Tr. Chem. Soc. London, 83, 1088. 1903. 
as a depolarizer to unite with the nascent hydrogen formed from the water, and upon this basis Mathews has formulated the following hypothesis: "Certain active particles in the protoplasm attack the water which is decomposed into oxygen and hydrogen. The oxygen combines with substances of the protoplasm thus oxidizing them; the hydrogen is either set free in gaseous form or it is united with atmospheric oxygen to form water, or it combines with other substances in the protoplasm." I In the words of Packard:" "Respiration is the dissociation of water with the liberation of hydrogen and the real respiration is brought about not by the oxygen of the air but by that of the water. If atmospheric oxygen is present it unites with the hydrogen set free from the water, thus acting as a depolarizer. According to this theory, aerobic and anaerobic respiration are identical; the only difference is that the anaerobic protoplasm is a powerful enough reducing agent to drive the hydrogen out of the water and let it escape as free hydrogen." Mathews ${ }^{I}$ suggested that other oxidizing substances may replace atmospheric oxygen as a depolarizer and stimulate oxidation in the absence of air. This may, indeed, be the rôle of carbohydrates and of other organic substances in anaerobiosis. Theobald $\mathrm{Smith}^{3}$ showed that some facultative anaerobes behave as obligate aerobes in sugar-free broth, growing only in the open arm of the fermentation tube, while they grow in both the closed and open arms in sugar broth, and Maze $\mathrm{M}^{\mathrm{f}}$ ound that while it is the oxidation by free oxygen that furnishes the energy necessary to the aerobic multiplication of yeasts it is the splitting of sugars into acetic acid that furnishes the energy for their anaerobic growth.

It is by no means certain that obligate anaerobes require an absolute exclusion of oxygen for their maximum growth; but certainly the amount necessary to inhibit growth is very small, according to Matzuschita, ${ }^{5}$ for certain anaerobes about $0.003 \mathrm{I}$ per cent. ${ }^{6}$

Many anaerobes seem to grow best at or near the limit of oxygen tolerance. Englemann, ${ }^{7}$ by observation of the chemotactic behavior of motile bacteria with respect to the oxygen secreted by a living filament, and Beijerinck, ${ }^{8}$ by observing the levels at which bacteria grow in deep media, showed that different anaerobes vary in their oxygen requirements. Chudiakow ${ }^{9}$ and Fermi and Bassu ${ }^{\text {Io }}$ also concluded that even the anaerobic bacteria require some free oxygen, but if this be so the amount is so small for the obligate anaerobes that it is practically impossible to measure it. And no one has ever been able to establish the minimal oxygen requirement, if there is any such thing, for any strict anaerobe.

Yet the conception of maximal, optimal, and minimal oxygen tensions has a distinct place in connection with the "micro-aerophiles" of Beijerinck, ${ }^{8}$ and in the sporulation of aerobes as shown by A. Meyer. ${ }^{\text {Ir }}$

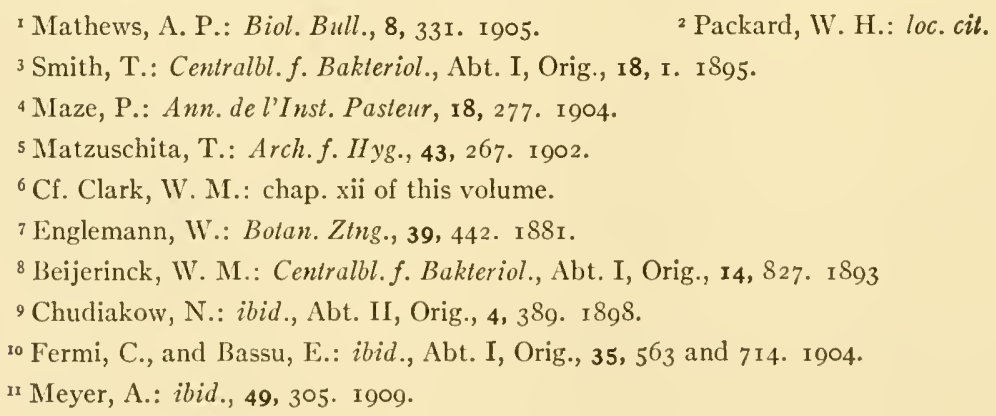


Among the micro-aerophilic aerobes are Bact. abortus (Bang), ${ }^{\mathrm{I}}$ the fowl diphtheria organism of Müller, ${ }^{2}$ and certain pyogenic streptococci of Gräf and Wittneben. ${ }^{3}$ Rosenow ${ }^{4}$ believes that his success in cultivating streptococci with selective pathogenicity depends upon varying oxygen pressures. Wherry and his associates ${ }^{5}$ have concluded from experiments with the gonococcus, Entameba buccalis, Leptothrix innominata, $C$.diphtheriae, and $M$.tuberculosis, that reduced oxygen tension is more important in disease processes than is ordinarily supposed. A slight reduction facilitates the growth of the meningococcus according to Cohen and Markle, ${ }^{6}$ and Cohen and Fleming ${ }^{7}$ have stated that the optimum is represented by an atmosphere of ro per cent $\mathrm{CO}_{2}$ and 90 per cent air. But Kohman ${ }^{8}$ has shown that the buffer action of $\mathrm{CO}_{2}$ is more important than the slight reduction of oxygen tension, and this viewpoint has been extended to other species by Rockwell and his collaborators, ${ }^{9}$ who have shown that carbon dioxide is necessary for the growth, not only of many aerobic but also several obligately anaerobic bacteria.

While some organisms are favored by decreased oxygen, Moore and Williams ${ }^{\text {ro }}$ found that many aerobic bacteria may be cultivated in nearly pure oxygen. But although $M$. tuberculosis and $B$. pestis were apparently oxyphobic, being completely inhibited by $80-90$ per cent of this gas, yet Novy and Soule ${ }^{I I}$ have demonstrated that $M$. tuberculosis grows readily in concentrated oxygen if sufficient moisture be provided.

The physico-chemical conception holds that obligate anaerobes differ from the aerobes by the fact that small amounts of oxygen are inhibitive to their growth and toxic to their protoplasm. It is the toxicity of the oxygen which in practical usage separates them from the aerobes by their failure to grow on the slanted surface of solid media exposed to the air through the cotton plug of a culture tube.

\section{BIOLOGICAL THEORIES OF ANAEROBIOSIS}

Aerobe-anaerobe symbiosis. - The remarkable fact that obligately anaerobic bacteria occur abundantly in soil, water, and decaying substances, apparently in contact with the oxygen of the air, was perhaps first correctly explained by Pasteur ${ }^{2}$ on the

${ }^{x}$ MacNeal, W. J., and Kerr, J. C.: J. Infect. Dis., 7, 469. I910.

${ }^{2}$ Müller, R.: Centralbl.f. Bakteriol., Abt. I, Orig., 4I, 5 I 5 and 62 I. 1906.

3 Gräf, H., and Wittneben, W.: ibid., 44, 97. 1907.

${ }_{4}^{4}$ Rosenow, E. C.: J.A.M.A., 65, I687. I915; J. Infect. Dis., 32, 41, 72, 144, 384. 1923.

sWherry, W. B., and Oliver, W. W.: J. Infect. Dis., 19, 288, 299. 1916; 20, 28. 1917; Wherry, W. B., and Ervin, D. M.: ibid., 22, 194. 1918; Wherry, W. B., and Ray, V.: ibid., p. 554. I918.

${ }^{6}$ Cohen, M. B., and Markle, L.: J.A.M.A., 67, г302. г9г6.

7 Cohen, M. B., and Fleming, J. S.: J. Infect. Dis., 23, 337. 1918.

${ }^{8}$ Kohman, E. F.: J. Bact., 4, 57 r. г9i 9.

9 Rockwell, G. E., and collaborators: J. Infect. Dis., 28, 249 and 352. 1921; 32, 98. 1923; 35, 581. 1924; 38, 92. 1926 .

10 Moore, B., and Williams, R. S.: Biochem. J., 4, 177. 1909; 5, 181. 1910; Brit. M. J., 2, 873 . 1909.

II Novy, F. G., and Soule, M. H.: J. Infect. Dis., 36, I68. 1925.

${ }^{12}$ Pasteur, L.: Compt. rend. Acad. de sc., 56, 4 I6-1 I 92.1863. 
ground that aerobic micro-organisms of various kinds replace the oxygen with carbon dioxide sufficiently to permit the symbiotic growth of the obligate anaerobes. Pasteur's explanation was accepted by Roux ${ }^{\mathrm{I}}$ who utilized the hay bacillus in the cultivation of Vibrion septique by overlaying the latter in deep agar or gelatin with broth cultures of the former, and by $\mathrm{Penzo}^{2}$ who cultivated the "bacillus of malignant oedema," symbiotically with Bact. prodigiosum and Bact. proteus in broth and emphasized the great pathogenicity of such cultures. Novy ${ }^{3}$ did the same thing with his B. oedematis maligni II (B. novyi) and added Bact. acidi lactici and a coccus to the list of successful symbionts.

But Kedrowsky ${ }^{4}$ found that Clostridium butyricum and B. tetani would grow in chloroformed cultures of various aerobes and suggested the production of a reducing agent or ferment by these as a more important means of oxygen reduction than that proposed by Pasteur. Kedrowsky was unable to secure this theoretical substance free from the bacterial bodies by Berkefeld filtration.

Other investigators do not accept Kedrowsky's hypothesis. Thus Scholtz ${ }^{5}$ cultivated $B$. tetani, B. botulinus, "oedem bacillus," and "rauschbrand bacillus" symbiotically with various living aerobes in broth and in the condensation water of agar slants, but failed to secure any growth with chloroformed cultures of aerobes except when large volumes were used, in which, as we now know, growth can be easily secured without other means of anaerobiosis aside from boiling just previous to inoculation.

Bienstock $^{6}$ and Matzuschita ${ }^{7}$ also failed to confirm the idea of a vital ferment, and von Oettingen ${ }^{8}$ found himself at variance both with Pasteur and Kedrowsky because he was unable to secure anaerobic growth when aerobes were closed up with anaerobes but not in actual contact, i.e., in "separated symbiosis," as he called it. But von Oettingen's technique was probably faulty; perhaps his fantastic and complicated containers leaked, because separate growth of aerobes and anaerobes can be secured in devices operating upon this principle, as, for example, those of Salomonson, ${ }^{9}$ Zinsser, ${ }^{10}$ Nichols and Schmitter, ${ }^{11}$ Nowak, ${ }_{1}^{12}$ MacNeal and Kerr, ${ }^{13}$ Smith and Fabyan ${ }^{14}$ Horton, ${ }^{15}$ Giltner, ${ }^{16}$ Wherry and Oliver,${ }^{17}$ and Torrey, ${ }^{18}$ though it must be admitted that

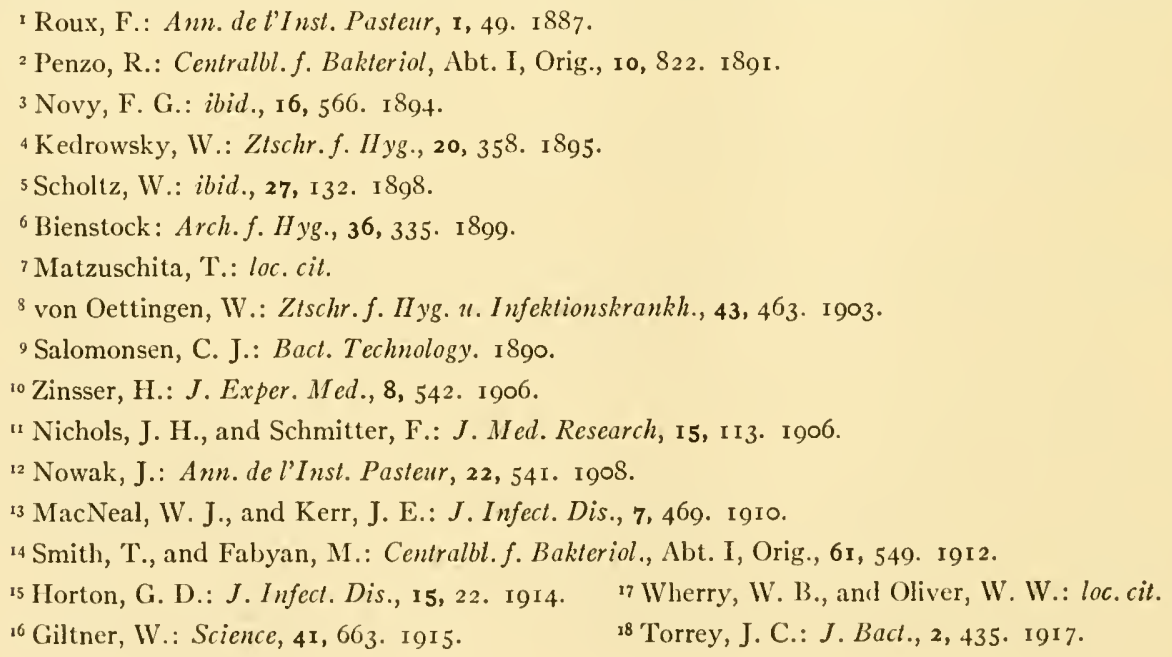


these have been more useful in the cultivation of micro-aerophiles than of obligate anaerobes. Yet the fact that obligate anaerobes can be cultivated on the surface of solid media without actual contact of aerobes is a sufficient rebuttal of Kedrowsky's assumption of a vital ferment and convinces one of the accuracy of Pasteur's original hypothesis of oxygen-tension reduction through bacterial respiration as at least one of the important factors in aerobe-anaerobe symbiosis.

Bienstock ${ }^{1}$ later experimented with several anaerobes in symbiosis with a large variety of aerobes. Heat-killed cultures of acrobes were also tried, but of these only Bact. pyocyaneum in a fibrin medium would support the growth of anaerobes. Control cultures in similar media in which Bact. pyocyaneum had not been cultivated failed. This exception was disturbing to Bienstock as an apparent confirmation of Kedrowsky's view but may be interpreted in the light of present-day information as providing a viscous deep medium sufficiently impervious to air for anaerobic growth. Proca $^{2}$ has since cultivated $B$. tetani and $B$. botnlimus in freshly sterilized liquid cultures of Bact. coli, Buct. typhosum, and Vibrio cholerac. Solid media failed, however, to yield surface colonies, and certain species of aerobes, Staphylococcus doré and Bact. pyocyaneum, seemed unsatisfactory. Such discrepancies are difficult to interpret; one cannot help regarding the results as somewhat accidental.

Many practical considerations in addition to those already mentioned grow out of the phenomena of aerobe-anaerobe symbiosis, as, for example, the necessity of autoclave sterilization of broth intended for the manufacture of diph theria toxin, ${ }^{3}$ the inhibition by certain aerobic bacteria of tetanus ${ }^{4}$ and botulism ${ }^{5}$ toxin formation, the somewhat questionable use of inert aerobes in fermentation tests of anaerobes, ${ }^{6}$ and the use of symbiotic cultures in the isolation of $B$. putrificus. ${ }^{7}$ At the present time most of the technical applications of aerobic symbionts in the cultivation of anaerobes have been supplanted by more precise methods; their significance is mainly academic therefore, but important as confirming Pasteur's conception of the life of anaerobic micro-organisms in nature.

Use of animal and plant tissues in the so-called "aerobic culture" of obligate anaerobes.-In I 877 Gunning, ${ }^{8}$ of the University of Amsterdam, studied the growth of putrefactive anaerobes in media containing meat and coagulated egg, and Nencki ${ }^{9}$ and his pupil Jeanneret were led into the same problem in studying the activity of the pancreas and its ferments in the absence of air. Others, such as Gaffky and Hesse (quoted by Novy), ${ }^{\text {Io }}$ were interested in the isolation of organisms from infected tissues and perhaps did not appreciate that the tissue exerted a favorable influence in an-

r Bienstock: Ann. de l'Inst. Pasteur, 17, 850. 1903.

${ }^{2}$ Proca, G.: Compt.rend. Soc. de biol., 63, 620. 1907.

${ }^{3}$ Smith, T.: J. Exper. Med., 3, 647. I 898 .

${ }_{4}^{4}$ Francis, E.: IIyg. Lab. Bull., No. 95. I9I4.

${ }^{5}$ Hall, I. C., and Peterson, E. C.: J. Bact., 8, 319. 1923.

${ }^{6}$ Wilson, W. J., and Steer, P.: Brit. H. J., 2, 568 . I918.

7 Sturges, W. S., and Rettger, L. F.: J. Bact., 4, г7r. 19r9.

${ }^{8}$ Gunning, J. W.: J.f. prakt. Chemie, I6, 314. I877; 17, 266. I878; 20, 434. I879.

${ }^{9}$ Nencki, M.: ibid., 19, 337. 1879 .

ro Novy, F. G., Jr.: J. Infect. Dis., 36, 343. I925. 
aerobic cultivation. In 1890 Tizzoni, Cattani, and Baquis ${ }^{\mathrm{T}}$ utilized clotted rabbit blood as a means of cultivating $B$. tetani, a change in color indicating reduction. Smith ${ }^{2}$ also in 1890 began the use of fermentation tubes for the cultivation of anaerobes from infected tissues, and in $1899^{3}$ first utilized sterile tissues other than blood to accelerate the growth of $B$.tetani. Smith clearly recognized that the value of tissues lay in their reducing action. Von Hibler' also began the use of sterile raw and coagulated rabbit blood and human brain media about the same time, and Kitt ${ }^{5}$ observed the initial growth of the blackleg bacillus in the immediate vicinity of bits of tissue in liquid media exposed to the air. But it was a mistake on the part of these writers to consider such growth aerobic.

The use of animal tissues was also developed by Italian investigators, apparently without any knowledge of the work already done in other countries. Tarozzi ${ }^{6}$ pointed out that the tissues should be preferably fresh and that heating destroyed their value possibly through oxidation of a reducing agent. Much of the Italian literature is inaccessible, but Jungano and Distaso ${ }^{7}$ and, more recently, Novy ${ }^{8}$ have reviewed it. In 1905 Ori showed that a vegetable tissue, potato, could be used as well as animal tissues, and that sterilization in the autoclave did not destroy its value.

Grixoni cultivated obligate anaerobes in a glycerol extract of liver heated to $55^{\circ} \mathrm{C}$. without other means of excluding air, and Wrzosek ${ }^{9}$ used kidney, liver, and spleen in the culture of certain bacteria from normal organs which would not grow in the absence of the tissues. Later Wrzosek ${ }^{10}$ studied the growth of B. tetani, B. oedematis, $B$. chauvoei, and B. botulinus in tissue broth and emphasized the importance of his supposedly new viewpoint relative to the "aerobic growth of anaerobes."

Harass ${ }^{11}$ found that sterilization of animal tissues did not destroy their ability to support "aerobic" growth of anaerobes in broth. He recognized the desirability of demonstrating aerobic growth on the surface of solid media, but all of his efforts in this direction were fruitless. Bandini ${ }^{12}$ showed conclusively that obligate anaerobes such as B. botulinus, B. oedematis, and B. chauvoei would grow well in broth containing not only various animal and plant tissues sterilized in the autoclave at $120^{\circ} \mathrm{C}$., but also such supposedly inert substances as coal, charcoal, coke, and iron filings.

I Tizzoni, G., Cattani, J., and Baquis, E.: Beitr. z. path. Anat. u. z. allg. Path., 7, 597. I89o.

${ }^{2}$ Smith, T.: Centralbl.f. Baktcriol., Abt. I, Orig., 7, 502. I890.

${ }_{3}^{3}$ Smith, T.: J. Boston Soc. Med. Sc., 3, 340. 1899.

4 von Hibler, E.: Centralbl.f. Bakteriol., Abt. I, Orig., 25, 593. r\$99.

5 Kitt, T.: ibid., I7, I68. I 895 .

${ }^{6}$ Tarozzi, G.: ibid., 38, 619. 1905.

7 Jungano and Distaso: loc. cit.

8 Novy, F. G., Jr.: loc. cit.

9 Wrzosek, A.: Wien. klin. Wchnschr., 18, 1 268. 1905; Centralbl.f. Baktcriol., Abt. I, Orig., 43, I 7. 1907 .

ro Wrzosek, A.: Mïnchen. med. Wchnschr., 53, 2534. r9o6; Centralbl. f. Bakteriol., Abt. I, Orig., 44, 607. I907; 53, 476. 1909.

${ }^{\prime \prime}$ Harass, P.: Mïnchen. wed. Wchnschr., 53, 2237. 1906.

«2 Bandini, P.: Giorn. R. Acad. de. Med. di Torino, 12, 265. 1906. 
These he believed to be reducing agents as shown by their decoloration of methylene blue in broth, but he also emphasized the necessity of heating just prior to inoculation.

Liefmann, ${ }^{1}$ Guillemot and Szczawinska, ${ }^{2}$ and Rosen tha ${ }^{3}$ all recognized clearly that the growth of obligate anaerobes in the presence of such substances is truly anaerobic, not aerobic, and agreed with Wrzosek in attributing such growth to the reducing action supposedly possessed by them.

Hata, ${ }^{4}$ on the other hand, emphasized the importance of the particulate nature of reducing agents and suggested that the best results are to be expected when these agents are in a finely divided condition, as finely minced animal and plant tissues, or iron filings. Douglass, Fleming, and Colebrook ${ }^{5}$ objected that organic substances can scarcely be expected to retain their chemical reducing action after heating. This seems valid. But while a surprisingly large list of organic and inorganic solids may be used in broth cultures to provide anaerobic conditions, including ashes, sand, cardboard, wool, cotton, lint, sponge, charcoal, chalk, cork, cloth, and iron nails, all have a more or less porous or particulate consistency, and the degree of success achieved with them depends upon this and upon the amount of substance used. Finally, the use of small pieces of capillary tubing in broth affords a striking proof of the fact that anaerobic growth begins in the interstices of such substances as afford occluded spaces into which oxygen diffuses with difficulty after once being driven out by heat. At least motile anaerobes find their way into such interstices by reason of a negative chemotactic response to oxygen, and once growth starts, the generation of gas improves the conditions of anaerobiosis through its action in sweeping out oxygen. This has been clearly recognized by Novy, ${ }^{6}$ Wright, ${ }^{7}$ Wolf, McGill, and Harris ${ }^{8}$ and others.

We thus come to the conclusion that physico-chemical factors play an important rôle in the so-called "aerobic cultures" of anaerobes in the presence of plant and animal tissues. The action of such tissues is physical to that degree in which they interfere with the reabsorption of oxygen; it is chemical to that degree in which actual reduction of free oxygen by unheated tissues occurs. It is probable also that certain tissues contribute valuable nutrients to the media and that they serve as important buffers against unfavorable $\mathrm{H}$-ion concentrations. The Italian work started with the biological hypothesis of a reducing ferment, but it ended without proof. And while many were able to demonstrate the growth of obligate anaerobes in broth containing animal and plant tissues and other particulate substances, no one was ever able to secure surface growth of obligate anaerobes in pure culture in contact with air.

Yet the idea that unheated plant and animal tissues may accelerate anaerobic

${ }^{1}$ Liefmann, H.: Munchen. med. Wchnschr., 54, 823. 1907; Centralbl.f. Bakteriol., Abt. I,Orig., 46, 377. 1908 .

${ }^{2}$ Guillemot, L., and Szczawinska, W.: Compt. rend. Soc. de biol., 64, I 7 r. I908.

3 Rosenthal, G.: ibid., 67, 702. 1909.

${ }^{4}$ Hata, S.: Centralbl. f. Bakteriol., Abt. I, Orig., 46, 539. I908.

5 Douglass, S. R., Fleming, A., and Colebrook, L.: Lancet, 2, 530. 1917.

${ }^{6}$ Novy, F. G.: Centralbl.f. Bakteriol., Abt. I, Orig., I4, 590. I893; Ztschr.f. IIyg., 17, 209. 1894.

7 Wright, A. E.: Lancet, I, I. I917.

${ }^{8}$ Wolf, C. G. L., McGill, C. M., and Harris, J. E. G.: ibid., 2, 787. 1917. 
growth through enzymic action cannot be denied; indeed, a most interesting theory of anaerobiosis depends upon this conception.

In 1922 M'Leod and Gordon $^{\mathrm{I}}$ discovered that the early death of the pneumococcus in cultures was apparently due to the accumulation of peroxide (of hydrogen?), for the production of which evidence was found in the green discoloration of "chocolate" blood agar, and in the liberation of oxygen from pneumococcus cultures by liver catalase. Streptococci and other bacteria also produce peroxide, and it was suggested that the well-known beneficial action of fresh tissues upon various bacteria is due to the destruction of detrimental peroxide by catalase. ${ }^{2}$

Neill $^{3}$ holds that the oxidation and reduction reactions of sterile plant tissues are essentially identical with those of pneumococci and anaerobic bacteria and furnish the best explanation, thus far, for the beneficial action of raw plant and animal tissues upon anaerobic growth. On the other hand, Novyt holds that "the inability of anaerobes to grow in the air is not due to the hypothetical production of peroxide and to the absence of catalase" but "that the fundamental difference between obligative aerobes and anaerobes lies in the nature of the respiratory enzymes, which are designated as 'aerase' and 'anaerase,' respectively. 'The potato and the facultative anaerobes possess both types; that present in obligative anaerobes can function only in the absence of oxygen, while that of the aerobe can work only in the presence of oxygen." All living protoplasm respires, and Novy's conception of the beneficial action of plant tissues upon anaerobic growth dispenses with the peroxide-catalase hypothesis and emphasizes the idea that obligate anaerobes grow in the presence of raw plant tissues because the latter reduce the oxygen tension, just as aerobic bacteria do, below the toxic concentration.

\section{AEROBIZATION OF THE ANAEROBES}

Many of the investigations just discussed had for their aim the aerobic cultivation of the anaerobes with the end in view of a better understanding of the nature of anaerobic growth. A secondary consideration has been in some cases that if means were found to cultivate the anaerobes aerobically upon solid media, the technique of isolation might be simplified. But no one has yet succeeded in securing surface growth of any obligate anaerobe in pure culture upon solid media exposed to the air, and the best practical definition of obligate anaerobes separates them from aerobes by their failure to grow in pure culture on the surface of solid media at normal atmospheric pressure. From this standpoint the rather numerous claims of aerobic growth in liquid media through the addition of chemical reducing agents, symbiotic aerophilic micro-organisms, fresh animal or plant tissues, or particulate substances have no standing; all of these are really anaerobic cultivations. Even the symbiotic growth of aerobes and anaerobes upon the surface of solid media fails to comply with the foregoing definition of "anaerobiosis." The same is true of the supposed aerobic culture

${ }^{1}$ M'Leod, J. W., and Gordon, J.: Biochem. J., 16, 499. 1922; J. Path. \& Bact, 25, I39. 1922; 26, I 27. 1923.

${ }^{2}$ See chap. xiv of this volume.

3 Neill, J. M.: J. Exper. Med., 4I, 535. I925.

4 Novy, F. G., Jr.: loc. cit. 
of anaerobes by Larson, Cantwell, and Hartzell ${ }^{1}$ in broth whose surface tension has been reduced by castor-oil soap.

The really important point concerned in the attempts to "aerobize the anaerobes" involves the fundamental validity of differentiating micro-organisms according to their oxygen relation and the stability of this relation.

There have been a few efforts to "aerobize the anaerobes" by acclimation to increasing oxygen tensions. Ferran ${ }^{2}$ claimed that by starting with pure acetylene and, as growth began, mixing more and more air, he could accustom the tetanus bacillus to growth in air on the surface of broth without morphological change. But the formation of a thick pellicle and loss of virulence reported is suggestive of aerobic contamination, for the detection of which no special procedures appear to have merited description in his brief publication. But even if surface growth of a pure culture of tetanus bacillus did occur on the surface of liquid media in a flask, and the writer has seen this without loss of toxicity, it could not be considered correctly as aerobic in view of the sweeping out of oxygen from liquid cultures by other gases evolved; Ferran's claim fails to meet the requirements of our definition of aerobic growth.

The progressive transformation of strict anaerobes into aerobic micro-organisms has received extended attention by Rosenthal, ${ }^{3}$ who held that the distinction between aerobes and anaerobes is not fundamental. By successive cultivation in tubes containing milk of less and less depth or covered with thin layers of lanolin, Rosenthal claimed to have cultivated the anaerobic bacilli of botulism, Achalme (B. welchii), and Legros, under conditions of partial exposure to air, which had hitherto failed to permit growth. No surface colonies on solid media were secured at this time, but later surface colonies were obtained with difficulty with the bacillus of Achalme by more persistently decreasing the depth of milk. Partial evacuation was also used but with somewhat incomplete success. Similar experiments were conducted with $V i$ brion septique, and Rosenthal soon came to believe that there were three steps in the transformation of anaerobes into aerobes as follows:

First, the organisms lost their oxygen intolerance without alteration of biochemical functions (e.g., fermentation, putrefaction) or of biological properties (e.g., virulence). Such cultures were said to grow for one generation only upon the surface of solid media in pure culture and when transferred to deep media displayed immediately their usual characteristics. Repeated transfers in this stage upon solid media were impossible.

Second, after the second or third agar slant the organisms lost their identifying biochemical characteristics, but these might be restored in a few generations by repeated transplantation in suitable media under anaerobic conditions.

Third, cultures lost their identifying chemical properties as well as their pathogenicity permanently, though they were morphologically unchanged.

Reaching the foregoing conclusions in experiments with the bacillus of Achalme,

${ }^{r}$ Larson, W. P., Cantwell, W. F., and Hartzell, T. B.: J. Infect. Dis., 25, 4 I. 1919.

${ }^{2}$ Ferran, J.: Centralbl.f. Bukteriol., Abt. I, Orig., 24, 28. 1898.

${ }^{3}$ Rosenthal, G.: Compt. rend. Soc. de biol., 55, I292. 1903; 60, 828, 874, 928, 957, I116. 1906; 6I, 48, 211, 326, 440. 1906; 62, 438, 578, 784, 1020, 1066, III9. 1907; 64, 398. I 908 . 
Rosenthal shortly claimed to have repeated them with the Vibrion septique and suggested the use of such attenuated cultures of aerobized anaerobes in immunization under the name "allobi-vaccination."

Rosenthal also undertook the progressive anaerobization of $B$. anthracis, and claimed to have accomplished this by the use of deep tubes of media, milk covered with cream, media sealed under lanolin, and the use of decreasing air pressures. The number of required transfers varied with the germ, with the rapidity of multiplication, and with the size of inoculum. But since $B$. anthracis is well known to be a facultative anaerobe, these experiments lacked conclusiveness, and since Rosenthal's main interest lay in the original problem of aerobizing the anaerobes, he next studied the use of decreasing depths of gelatin as formerly of milk, and of tubes of decreasing diameter.

In 1907 he reported results with the tetanus bacillus finally analogous to those already obtained with other anaerobes, but more irregular. In contrast to Ferran, he noted an early appearance of involution forms and loss of sporulation and motility. All three steps of aerobization, he says, were accomplished. The bacilli were found to be shorter, thicker, and less motile than the anaerobic forms. They were gram positive and non-sporulating. Bouillon cultures during the first step of aerobization produced tetanus in animals. The first stage of aerobization continued through five to six generations on slanted agar, and the loss of morphological characters preceded that of biochemical activities and toxicity. The beginning of the second stage was announced in the tardy liquefaction of gelatin and the third by the complete and apparently permanent loss of tryptic activity, sporulation, motility, and toxicity. The last vestige of the parentage of the aerobized tétanus bacillus, as well as of the Vibrion septique, to disappear was the property of specific serum agglutinability. This Rosenthal considered irrefutable proof of the exactness of his researches upon the aerobization of the anaerobes.

Having created several strains of artificial aerobic micro-organisms from various obligate anaerobes, which he called collectively "bacillogenes," specifically "aerovibrion," "aero-bacille de tétanos," etc., Rosenthal next claimed (1907) that a reversal of the process restored the "aero-bacilli" of Achalme and of tetanus wholly to their original state of obligate anaerobes, including their lost chemical functions and power of sporulation.

As a fourth step in the aerobization of the anaerobes Rosenthal (I9o8) claimed the transformation of the aerobized Vibrion septique at the limit of its viability into an organism resembling the entero-coccus, which he found could be reversed again into a typical Vibrion septique.

These remarkable researches have been discussed at some length because they are not widely known and because to my knowledge they have not been confirmed, though confirmation seems most desirable if possible. The length of time, over five years, consumed by Rosenthal in the pursuit of these experiments, together with the logical progress of the results, suggests that a critical study of the problem would be worth while. At least one observer, Szczawinska, ${ }^{1}$ failed to corroborate Rosenthal's findings and concluded that our present distinction between aeroles and anaeroles: is a valid one, and is based upon an essential physiological difference in metabolism.

'Szczawinska, W.: ibid., 69, 15. 1910. 


\title{
CHAPTER XIV
}

\section{BACTERIAL OXIDATIONS AND REDUCTIONS}

\author{
JAMES WALTER MCLEOD \\ University of Leeds, Leeds, England
}

\section{REDUCTIONS}

Clark ${ }^{\mathrm{r}}$ has pointed out recently that the modern conceptions of reduction involve more than the subtraction of oxygen or addition of hydrogen. Much the greater part of the literature, however, dealing with bacterial reductions relates to observations of such changes. A great deal of this work had already been done by the end of the last century. The literature is considerable, and since a comprehensive summary of it would require much space, only the more interesting points and those to which most work has been devoted will be considered. Such are the reductions of compounds of nitrogen and sulphur, of metallic salts such as those of selenium and tellurium, of a variety of dyes, and of certain substances of biological importance, hemoglobin derivatives, cystine or complexes containing it, and bacterial toxins. These various reactions have been studied for a variety of ends, some on account of their economical importance, as the reduction of nitrates and nitrites; others because of their possible value in bacteriological technique, as the reduction of tellurium salts: others again because of their possible significance in explaining the pathogenic action of bacteria, as the reduction of oxyhemoglobin; and almost all have been studied with a view to elucidating the intimate metabolism of the bacterial cell and particularly the mechanism of its respiration.

The reduction of nitrates and nitrites was fully studied by Maassen ${ }^{2}$ twenty-five years ago. His chief conclusions were that the majority of the bacteria which are capable of growing in simple media reduce nitrates to nitrites, that many but not all of these destroy nitrites whereas some of the bacteria incapable of reducing nitrates can yet destroy nitrites, that most bacteria destroy the nitrite by transforming it to $\mathrm{NH}_{3}$ which they utilize almost as rapidly as it is formed, but that one group - the true denitrifying bacteria, B. fuorescens liquefaciens, and species allied to it-utilize the oxygen of the nitrite and give off gaseous nitrogen. Braun and Cahn-Bronner ${ }^{3}$ refer to this last quality in B. pyocyaneus as enabling it to show pseudo-anaerobic growth in the presence of nitrate. Certain of the vibrios are among the group of bacteria forming nitrite without reducing it further, and it is such of these as also produce indol that give the cholera-red reaction.

Not much of importance has been added to our knowledge of this subject since Maassen's work, but some facts have been added with regard to some bacteria which

${ }^{x}$ Clark, W. M.: Pub. Health Rep. (U.S. Public Health Service), 38, 443. I923; cf. chap. xii, this volume.

${ }^{2}$ Maassen, A.: Arbeiten a.d. kuiserlichen Gesundheitsamte, 18, 21. I902.

3 Braun, H., and Cahn-Bronner, C. E.: Centralbl.f. Bakteriol., Abt. I, Orig., 86, 380. I 1902. 
he had not investigated. Logie ${ }^{\mathrm{I}}$ has drawn attention to the fact that the non-mannitefermenting dysentery strains are distinguished from the mannite-fermenting strains by inability to reduce nitrite. Pelz ${ }^{2}$ pointed out that streptococci never reduce nitrates. More attention has been given in recent work to the reduction of compounds of $\mathrm{S}$ generally in the form of studies of $\mathrm{H}_{2} \mathrm{~S}$ production by bacteria.

There is general agreement that most bacteria do not reduce sulphates or form $\mathrm{H}_{2} \mathrm{~S}$ from taurine (Sasaki and Otsuka, ${ }^{3}$ Myers, ${ }^{4}$ Bürger $^{5}$ ). Salkowski ${ }^{6}$ pointed out, however, that whatever may occur in cultural experiment, it is certain that sulphates are reduced in sewage with $\mathrm{H}_{2} \mathrm{~S}$ formation, possibly under the influence of mixed cultures, and Beijerinck ${ }^{7}$ described two anaerobes, capable of reducing sulphates, which are found in the mud of Dutch harbor bottoms.

It is not clear that evolution of $\mathrm{H}_{2} \mathrm{~S}$ from media containing proteins is necessarily evidence of the reduction of S-containing compounds. It is certain, however, that the most vigorous production of $\mathrm{H}_{2} \mathrm{~S}$ is carried on by bacteria which can be shown in other ways to have strong reducing properties as, e.g., B. sporogenes. It has also been shown that such bacteria as tend to produce $\mathrm{H}_{2} \mathrm{~S}$ do so more freely if cystine is added to the medium (Kondo, ${ }^{8}$ Sasaki and Otsuka, ${ }^{3}$ Myers, ${ }^{4}$ Bürger ${ }^{5}$ ), and it is altogether likely that reduction of cystine to cystein is a preliminary to evolution of $\mathrm{H}_{2} \mathrm{~S}$. Bürger, however, pointed out that nascent $\mathrm{H}$ does not liberate $\mathrm{H}_{2} \mathrm{~S}$ from cystine unless in acid solution, whereas bacteria normally grow in slightly alkaline solutions. There is, therefore, need for further investigation on this point.

The statements of the different workers with regard to the capacity of different bacteria to produce $\mathrm{H}_{2} \mathrm{~S}$ from cystine and from protein are conflicting, but there is a general agreement that most coliform bacteria, including the Proteus and B. fuorescens liquefaciens groups, have this property.

It is in connection with the reduction of dyes and biological products such as hemoglobin derivatives that most of the work on bacterial reductions has been done. Theobald Smith, ${ }^{9}$ who was one of the earlier workers in this field, used methylene blue, the dye which has been most used for this purpose, and concluded that all bacteria were alike in their powers of reduring it and that reduction took place only when the bacteria were in actual contact with the dye. Both statements were disputed shortly afterward by Müller, ${ }^{10}$ and although they are generally true in the sense that all bacteria are possessed of reducing powers and that this power is especially exercised within the body or on the surface of the bacteria, the trend of subsequent work has been to justify Müller's position. Von Liebermann, ${ }^{11}$ Wichern, ${ }^{12}$ and Carapelle ${ }^{{ }^{13}}$ all bring forward

I Logie, W. J.: J. IIyg., ro, I43. I910; I1, 36. I9 1 I.

${ }^{2}$ Pelz., E.: Centralbl.f. Bukteriol., Abt. I, Orig., 57, г. т 9 то.

${ }_{3}$ Sasaki,T., and Otsuka, I.: Biochem. Zlschr., 39, 208. I9r 2.

${ }^{4}$ Myers, J. T.: J. Bact., 5, 231. 1920.

5 Bürger, M.: Arch.f. Hyg., 82, 201. I9I4.

${ }^{6}$ Salkowski, E.: Zlschr.f. phys. Chemic, 83, г43. г9г

7 Beijerinck, W. M.: Verzamelde Geschriften, 4, 24.

"Kondo, M.: Biochem. Zlsihr., 136, 198. 1923.

9 Smith, Theobald: Centralbl.f. Bakleriol., Abt. I, Orig., I9, ISr. ISg6.

10 Müller, F.: ibid., 26, 801 . 1899.

"Von Liebermann, Jr., L.: ibid., 51, 440. 1909. ${ }^{22}$ Wichern, H.: Arch.f. IIyg., 72, I. I910.

${ }^{13}$ Carapelle, E.: Centralbl. f. Bukleriol., Abt. I, Orig., 47, 545. 1908. 


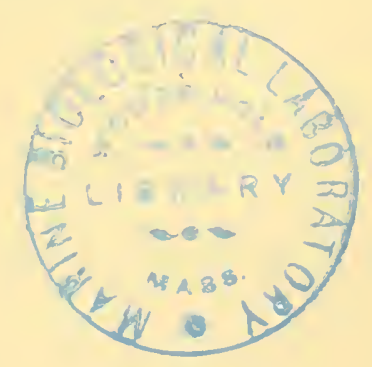




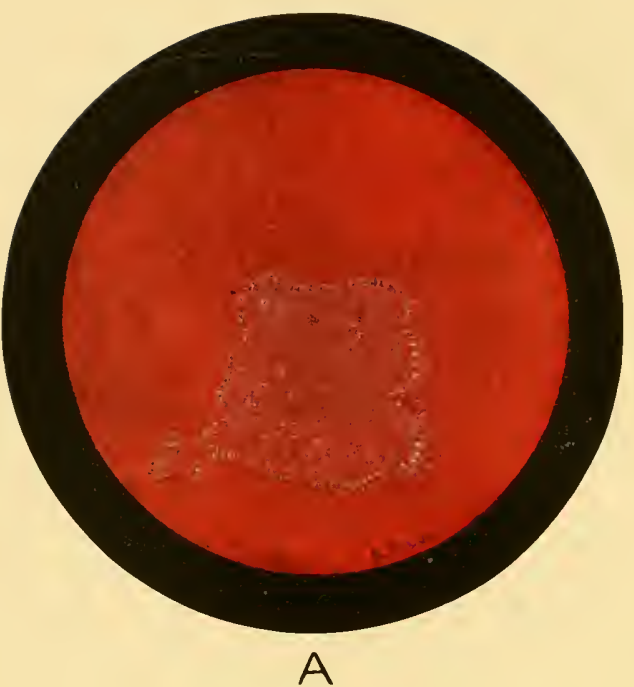

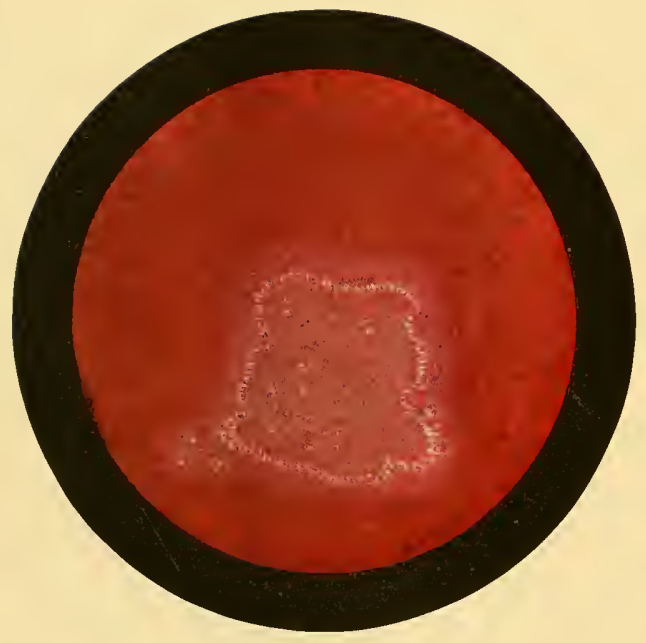

B

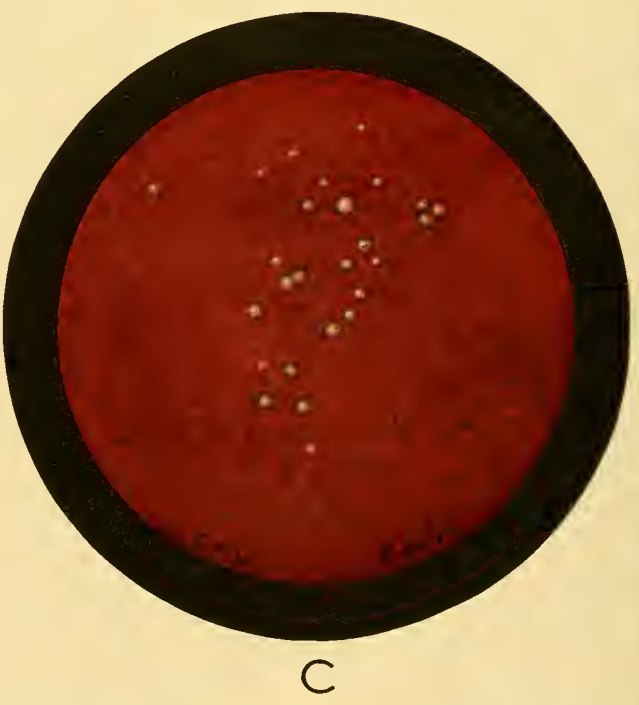

A, Growth of B. welchii on "Chocolate" Agar 5 Minutes after Removal from Anaerobic Jar. B, Growth of B. welchii on "Chocolate" Agar as It Appears Immediately after Removal from ANAEROBIC JAR.

C, Appearance of Culture of $B$. welchii Grown at Reduced Atmospheric Pressure-200-300 Mim. 
evidence to show that products capable of producing reduction diffuse out from bacteria, and the most convincing proof that the bacterial body is not essential has come with the recent demonstration by Avery and Neill' that filtered extracts of pneumococci which are protected from oxidation have definite reducing properties.

Although it is true that all bacteria are alike in reducing methylene blue and the same can be said of oxyhemoglobin $\left(\right.$ Von Liebermann $\left.^{2}\right)$, there exist considerable differences in their activities in this respect and greater differences in respect of other reductions, e.g., in the reduction of anthraquinone. Bieling ${ }^{3}$ has shown that meningococcus, pneumococcus, and B. typhosus are relatively inactive.

The writer and Gordon 4 have shown that when bacteria are grouped according to their power of reducing glutathione they fall into the following order of activity: strcptococci and B. dysentcriae Shiga, very poor reducers; Staphylococcus aurcus and pneumococcus, slight reducers, most bacteria of the coliform group, fairly active reducers; anaerobic bacteria, all active and mostly very active reducers. Another indicator of reduction less easily reduced than methylene blue- and hence more useful in demonstrating differences in reducing activities - is hematin as it occurs in the heated blood agar or "chocolate" medium. Colonies of anaerobic bacteria are surrounded on this medium by a pink halo, due to reduction, which fades very rapidly on exposure to air. This effect is shown in Plate $\mathrm{I}, A$ and $B$. It can be put to practical use in picking out anaerobic colonies in mixed cultures on plates incubated under anaerobic conditions.

A very interesting application of the bacterial reduction of methylene blue has been made by Quastel ${ }^{5}$ and his collaborators by applying Thunberg's method in studying the intimate processes of bacterial metabolism. The bacteria are suspended in a buffered solution containing methylene blue, and the acceleration of the reduction of the dye in the presence of various products which may occur in culture media or arise in the course of the bacterial disintegration of sugars and proteins is investigated. The marked powers shown by $B$. coli for activating the reduction of methylene blue by certain sugars and fatty acids, notably iormic, lactic, and succinic, and its relatively slight powers of activating amino acids are among the interesting results obtained. One other aspect of the work on the reducing action of bacteria has great interest, and that is the suggestion made by Von Liebermann ${ }^{2}$ but followed out with much greater precision by Dibbelt, ${ }^{6}$ that the successful competition of bacteria with the body tissues for the available oxygen of oxyhemoglobin may, especially in the more rapid septicemias, be an important element in the domination of the animal body by the invading bacteria.

${ }^{1}$ Avery, O. T., and Neill, J. M.: J. Exper. Med., 39, 357. I924.

${ }^{2}$ Von Liebermann, Jr., L.: loc. cit.

3 Bieling, R.: Centralbl. f. Bakteriol., Abt. I, Orig., 90, 48. 1923; Ztschr.f. Hyg. u. Infeklionskrankh., 100, 270. 1923.

4 McLeod, J. W., and Gordon, J.: Biochem. J., г8, 937. I924.

${ }_{5}^{5}$ Quastel, J.H., and Whetham, M. D.: ibid., 19, 5 19. I924; ibid., pp. 520 and 645. 1925; Quastel, J. H.: ibid., p. 64I. 1925; Quastel, J. H., Stephenson, M., and Whetham, M. D.: ibid., p. 304. I925; Quastel, J. H., and Stephenson, M.: ibid., p. 660. 1925; Quastel, J. H., and Woolridge, W. B.: ibid., p. $65^{2}$. 1925 .

${ }^{6}$ Dibbelt, W.: Centralbl.f. Bakteriol., Abt. I, Orig., 64, 52. I91 2. 
Neill ${ }^{t}$ has recently made the interesting discovery that various bacterial hemolysins which deteriorate rapidly on exposure to air undergo a reversible oxidation and that their hemolytic activity can be restored equally well by exposing them to a chemical reducing agent or to the reducing action of bacterial suspensions from cultures of B. coli or of an anaerobe.

\section{BACTERIAL CATALASE AND PEROXIDE PRODUCTION BY BACTERIA}

Although the power of bacteria to decompose hydrogen peroxide was observed and investigated independently by Gottstein ${ }^{2}$ and Beijerinck ${ }^{3}$ more than thirty years ago, it has been very little studied by most bacteriologists. Löwenstein, ${ }^{4}$ however, noticed the absence of catalase in cultures of the tetanus organism in I903, and Rywosch and Rywosch ${ }^{5}$ in 1907 noted that it was absent or present in very slight amount in cultures of $\mathrm{Cl}$. tetani and $\mathrm{Cl}$. botulinum. In 1923 the writer and Gordon, ${ }^{6}$ following up earlier work with Govenlock ${ }^{7}$ on the substances inhibitory to bacterial growth diffusing into culture media in the vicinity of growths of pneumococci, found that the inhibition was mainly due to the formation of $\mathrm{H}_{2} \mathrm{O}_{2}$ by the pneumococcus. This result has since been confirmed by Avery and Morgan ${ }^{8}$ and by others. The fact that some bacteria could produce $\mathrm{H}_{2} \mathrm{O}_{2}$ gave an enhanced interest to observations on bacterial catalase and led the writer and Gordon' to investigate a wider range of varieties of bacteria than had been previously examined for their catalase effect. This investigation led to the following classification of the bacteria investigated:

Group I. The anaerobes, devoid of catalase, extremely sensitive to $\mathrm{H}_{2} \mathrm{O}_{2}$, and considered as potential peroxide-producers

Group II. Peroxide-producers, devoid of catalase and only moderately sensitive to $\mathrm{H}_{2} \mathrm{O}_{2}$; the pneumococci, many types of streptococci, the lactic acid bacteria, and some sarcinae Group III. Non-peroxide-producers and devoid of catalase; certain streptococci, dysentery bacilli (Shiga type), and some hemoglobinophilic bacteria

Group IV. Bacteria producing catalase. The great majority of the bacteria capable of growing aerobically or both aerobically and anaerobically

The most vigorous catalase effect is shown by the strictly aerobic bacilli, especially those such as $B$. pyocyaneus which form pigments. Two pathogenic species are also outstanding in this respect-the gonococcus and the $B$. pertussis. Callow ${ }^{\text {to }}$ specially investigated anaerobes and found them devoid of catalase activity. Sherman ${ }^{\mathrm{II}}$ has

${ }^{x}$ Neill, J. M.: J. Exper. Med., 44, 199 and 215.1926.

${ }^{2}$ Gottstein, A.: Virchow's Arch.f. palh. Anat., 133, 295. 1893.

3 Beijerinck, W. M.: Naturwissenschaftliche Rundschau, 8, 671. I923.

${ }^{4}$ Löwenstein, E.: Wien. klin. Wchnschr., 16, I393. 1903. (Quoted by Kluyver, A. J.: Zlschr.f. phys. Chemie, 138, 100. I924.)

s Rywosch, D. and M.: Centralbl.f. Bakteriol., Abt. I, Orig., 44, 295. 1907.

${ }^{6}$ McLeod, J. W., and Gordon, J.: J. Path. and Bact., 25, I39. 1922.

7 McLeod, J. W., and Govenlock, P.: Lancet, I, 900. I921.

${ }^{8}$ Avery, O. T., and Morgan, H. J.: J. Exper. Med., 39, 275 and 2 S9. I924.

9 McLeod, J. W., and Gordon, J.: J. Path. and Bact., 26, 326.1923.

${ }^{\text {ro Callow, A. B.: ibid., p. } 320.1923 .}$

"Sherman, J. M.: J. Bact., 6, 379. 1921; II, 417. 1926. 
drawn attention to an apparent exception to the rule that anaerobes are devoid of catalase in the propionic acid bacteria. These, however, appear to be microacrophilic rather than strictly anaerobic. The further investigation of their metabolism may throw some fresh light on bacterial respiration.

\section{OCCURRENCE OF OXIDIZING FERMENTS IN BACTERIA}

Observations on this subject have been scanty. Lehmann and Sano ${ }^{x}$ claim to have demonstrated tyrosinase in three species of bacteria although they found it absent in the great majority. A brown discoloration in agar media around a colony of mold is not infrequently observed and is probably due to this ferment. Beijerinck ${ }^{2}$ also found tyrosinase in certain vibrios. Roux ${ }^{3}$ observed that B. typhosus produced a green color in extract of artichokes similar to that produced by laccase. The only other observations on the production of direct oxidizing ferments by bacteria that I have been able to find are those of Schultze ${ }^{4}$ and Kramer ${ }^{5}$ who studied the immediate effect of smearing bacteria on agar containing $a$-naphthol and dimethyl-para-phenylene diamine. Briefly stated, their results were: no reaction with anaerobes, coccal forms, B. influenzae, protozoa, and dysentery bacilli; a positive reaction with most other bacteria, especially strong with strict aerobes and pigment-forming bacteria. Stapp, ${ }^{6}$ working with a $\mathrm{H}_{2} \mathrm{O}_{2}$ and the benzidin test, found peroxidase reactions in $B$. coli, Staphylococcus, Sarcina, B. prodigiosus, and B. pyocyaneus. The peroxidase was distinct from catalase in its greater resistance to heat and different sensitivity to iodine and carbon disulphide. He did not find it in streptococcus cultures. In an extremely interesting investigation of Fe containing cell pigments related to hemoglobin Keilin 7 found that these were very widely distributed in nature and that their association with a peroxidase reaction was constant. For such pigment he proposed the name "cytochrome," and he found it to be present in yeast and in the aerobic bacterium, $B$. subtilis, but absent in an anaerobic bacillus, Cl. sporogenes. Callow ${ }^{8}$ has also investigated this subject and found peroxidase in all of a number of bacteria tested, but weak in $B$. acidi lactici and $C l$. sporogenes.

\section{OXIDATIONS PRODUCED BY BACTERIA}

The more the subject of oxidation is studied, the more difficult it becomes to draw any hard-and-fast line between oxidation and reduction. This is true especially of oxidations depending on processes of dehyrogenation and independent of the presence of free oxygen such as have been demonstrated by Wieland ${ }^{9}$ to take place under the influence of a catalyst which may be palladium black or an oxygen-free bacterial powder. The special value of oxygen when taking part in such processes is simply that of taking up the discarded $\mathrm{H}$ with which it forms a product, $\mathrm{H}_{2} \mathrm{O}$, that does not

' Lehmann, K. B., and Sano: Arch.f. Hyg., 67, 99. 1908.

${ }^{2}$ Beijerinck, W. M.: Versamelde Geschriftcn, 5, r.

${ }_{3}$ Roux, M. G.: Compt. rend. Acad. des sc., 128, 693. I899.

${ }^{4}$ Schultze, W. H.: Centralbl.f. Bakteriol., Abt. I, Orig., 56, 544. I910.

5 Kramer, G.: ibid., 62, 394. I9r2. 7 Keilin, D.: Proc. Roy. Soc., B, 98, 312. 1925.

${ }^{6}$ Stapp, C.: ibid., 92, г6r. 1924. $\quad{ }^{8}$ Callow, A. B.: Biochem. J., 20, 247.1926.

9 Wieland, H.: Berichte d. D. Ch. Gesellsch., 46, 3327. 1913; 54, 2353. I92 I. 
interfere with the process of the reaction. A great deal of investigation on this type of oxidation has been done by Quastel ${ }^{1}$ and others, and has already been referred to when discussing the reduction of methylene blue. The actual point chiefly studied is the extent to which various fatty acids, amino acids, and other substances are capable of being induced by bacteria to transfer $H$ to methylene blue, i.e., the capacity of these substances for oxidation by various bacteria. It is this type of oxidation which is apparently responsible for furnishing the energy required by anaerobes or by facultative anaerobes growing in the absence of oxygen by fermentative processes.

Braun and Cahn-Bronner ${ }^{2}$ have drawn attention to the more complex nutriment required by bacteria growing under anaerobic conditions.

A second type of oxidation is that in which oxygen is used freely and the fatty acids which have been present in the medium at the outset, derived from amino acids by deamination or from sugars by fermentative reactions, are oxidized to carbonates. Ayers and Rupp ${ }^{3}$ first drew attention to the fact that such oxidations are associated with the development of an alkaline reaction, and changes of this kind occurring in diphtheria cultures have been the subject of very careful study by Abt and Loiseau 4 and by Abt. ${ }^{5}$ The production of alkali by oxidative formation of carbonates has been noted in cultures of many varieties of bacteria by Sierakowski, ${ }^{6}$ and Phelon, Duthie, and McLeod 7 have drawn attention to its importance in cultures of gonococci and meningococci in which it may be responsible for the rapid death of the bacteria. A third distinctive type of oxidation is that of the types of bacteria described by Winogradsky ${ }^{8}$ as "anorgoxydanten" and by Orla-Jensen ${ }^{9}$ as "autotrophic." These get the energy which they require for the assimilation of the atmospheric $\mathrm{CO}_{2}$ by oxidation of simple elements like $\mathrm{S}, \mathrm{H}$, and Fe or compounds such as nitrites, $\mathrm{CH}_{4}$, or $\mathrm{NH}_{3}$, and are incapable of utilizing complex organic substances for their nutrition. According to Waksman and Starkey, ${ }^{10}$ only certain S-oxidizing bacteria and the nitrifying bacteria are strictly autotrophic in the sense of inability to utilize any complex organic forms of nutritive matter.

A fourth type of oxidative effect produced by bacteria is that due to the production of $\mathrm{H}_{2} \mathrm{O}_{2}$. The association between production of peroxide by bacteria and methemoglobin formation has been specially studied by Neill and Avery ${ }^{11}$ in connection with the pneumococcus, and by Valentine ${ }^{12}$ in connection with the streptococci. The former have brought out the very interesting fact that anaerobic extracts of pneumococci

${ }^{1}$ Quastel, J. H., et al.: loc. cit.

${ }^{2}$ Braun, H., and Cahn- Bronner, C. E.: Centralbl.f. Bakteriol., Abt. I, Orig., 86, I. I921.

3 Ayers, S. H., and Rupp, 1'.: J. Infect. Dis., 23, r88. i9is.

4 Abt, G., and Loiseau, G.: Ann. Inst. de Puste:tr, 39. I I 4. 1925.

5 Abt, G.: ibid., p. 387 . 1925.

${ }^{6}$ Sierakowski, S.: Compt. rend. Soc. de biol., 89, I37r. 1923; Biochem. Ztschr., 15 I, I5. I924.

7 I'helon, II. V., Duthie, G. M., and McLeod, J. WV.: J. P'ath. and Bact., 30, I33. In27.

${ }^{8}$ Winogradsky, S.: Centrulbl. f. Bakteriol., Abt. II, Orig., 57, I. I922-23.

${ }_{9}$ Orla-Jensen, S.: ibid., 27, 305. 1909.

ro Waksman, S. A., and Starkey, R. L.: J. Gencral Physiol., 5, 285. 1923. Sec chap. xxiv in this volume.

Ir Neill, J. M., and Avery, O. T.: J. Exper. Me1., 39, 757. I924.

12 Valentine, E.: J. Infect. Dis., 39, 29. I926. 
in the presence of oxygen and catalase produce much more methemoglobin than any concentration of $\mathrm{H}_{2} \mathrm{O}_{2}$ which is likely to occur on oxygenation of such extracts would do, and they suggest the formation of an organic peroxide insensitive to catalase.

The heated blood agar or "chocolate agar" first introduced by Cohen and Fitzgerald' is an extremely delicate indicator for peroxide formation by bacteria, and by its use the interesting fact is established that anaerobes produce an oxidative effect similar to that produced by bacteria which can be shown to produce $\mathrm{H}_{2} \mathrm{O}_{2}$ by the usual chemical tests. This effect can be elicited either as a green ring at the upper limit of growth of an anaerobe in a deep tube of "chocolate agar" grown at the ordinary atmospheric pressure (McLeod and Gordon ${ }^{2}$ ) or it may appear as shown in Plate I, C, as a faint green coloration around the bacterial colonies when the anaerobe is inoculated as a surface culture and incubated under the greatest tension of oxygen which it can tolerate without complete inhibition of growth.

\section{THE MECHANISM OF BACTERIAL OXIDATION}

It seems likely that all bacteria tend to produce peroxide and that this tendency is proportioned to their reducing activities. Those which can be shown to contain catalase and peroxidase presumably use it in their respiratory processes. It is interesting in this connection that Keilin ${ }^{3}$ found that a reducing mechanism which cannot be separated from peroxidase and may well be identical with it, is essential to the functioning of the respiratory pigment cytochrome.

A reducing mechanism, functioning possibly in combination with a catalyst containing the sulphydryl group such as glutathione, may be responsible for anaerobic respiration independently of any Fe complex. Whether $\mathrm{H}_{2} \mathrm{O}_{2}$ takes any part in respiratory processes of such bacteria as pneumococci is not clear. The lack of catalase and the fact that these bacteria resemble the anaerobes in being relatively insensitive to $\mathrm{KCN}$ (Burnet) ${ }^{4}$ which paralyzes cytochrome by fixing it in the reduced state (Keilin), ${ }^{3}$ suggest that $\mathrm{H}_{2} \mathrm{O}_{2}$ does not at all events function in combination with a peroxidase in these bacteria, and possibly is merely a by-product. The very interesting problem is presented by the Shiga type of dysentery bacilli. These lack catalase, haye slight reducing powers and limited fermentative activities, do not produce detectable amounts of peroxide, give no direct oxydase reaction (Kramer), 5 and although sensitive to cyanides (Burnet), ${ }^{4}$ they diverge in their reaction to this substance from other bacteria tested. I have not found a record of a determination of their peroxidase content. It is clear that none of the mechanisms mentioned could be considered to explain the respiratory processes of this type of bacterium.

It is not unlikely that the dependence of some strictly parasitic bacteria on the presence of blood in the media in which they are cultured indicates their poverty in peroxidase, which they require and which is normally supplied to them from the tissues of the host on which they are parasites (Fildes, ${ }^{6} \mathrm{Webster}^{7}$ ).

${ }^{1}$ Cohen, C., and Fitzgerald, J. G.: Centralbl. f. Baktcriol., Abt. I, Orig., 56, 464. I9ro.

${ }^{2}$ McLeod, J. W., and Gordon, J.: J. Path. and Bacl., 26, 332. I923; ibid., 28, 147. 1925.

${ }^{3}$ Keilin, D.: loc. cil.

${ }^{4}$ Burnet, F. M.: J. Palh. and Bact., 3о, 21. 1927.

${ }^{5}$ Kramer, G.: loc. cit. $\quad{ }^{6}$ Fildes, P.: Brit. J. Exper. Path., 3, 2 то. 1922.

7 Webster, L. T.: Proc, Soc. Exper. Biol. So Med., 22, 139. 1924-25. 


\title{
CHAPTER XV
}

\section{PROTEIN (NITROGEN) METABOLISM OF BACTERIA}

\author{
LEO F. RETTGER \\ Yale University
}

Although single-celled and among the smallest known living organisms, bacteria can effect the most elaborate syntheses, and through a multiplicity of enzymatic and catalytic processes reduce to their elements the most complex organic substances: proteins, fats, and polysaccharides. Their range of activities may be said to be almost unlimited.

\section{PROTEINS AND THE LIVING CELL}

Protoplasm defies analysis, for when it is subjected to chemical study it is no longer protoplasm, but dead tissue. Our knowledge of the internal structure and of the chemical composition of the bacterial cell is as yet very meager. However, sufficient studies have been made of the chemistry of bacteria to leave no doubt of the importance of proteins and protein-like substances, and to show that the bacterial cell is in this respect not very unlike those of the higher forms of life, both animal and vegetable.

According to the observations of Nicolle and Allilaire, ${ }^{\mathrm{r}}$ and of various other investigators, organic nitrogenous material (protein) in bacteria greatly preponderates over the non-nitrogenous. It has been found, however, that the quantitative chemical composition varies markedly among different organisms, and even within the same species.

Cramer ${ }^{2}$ has shown that the amount of total solids, ash, protein, and non-nitrogenous matter present in bacterial cells varies with the composition of the culture medium, though not in direct proportion; also with the age of culture, and incubation temperature. For example, he determined the protein content of the cholera vibrio to be 65 per cent of the total solids, when grown in plain infusion peptone broth, as compared with $45^{-5}$ o per cent in Uschinsky's medium.

It appears to be an established fact that different bacterial species or groups possess certain chemical entities which distinguish them from all other forms. For instance, the acid fast group contains a relatively large proportion of ether-soluble, fat-like substances, and the different pathogens elaborate intracellular toxins and antigenic substances which distinguish them from each other.

Nicolle and Allilaire ${ }^{3}$ determined the nitrogen content of certain organisms to be as follows (in per cent): $V$. cholerae, 9.79; Bact. dysenteriae, 8.89; Bact. typhi, 8.28; Bact. coli, 10.32; P. vulgaris, 10.73; B. anthracis, $9.22 ;$ E. prodigiosus, 10.55; Ps.

I Nicolle, M., and Allilaire, E.: Ann. de l'Inst. Pustcur, 23, 547. 1909.

${ }^{2}$ Cramer, E.: Arch.f. IIyg., 16, 151. 1893; 22, 167. 1895.

3 Nicolle, M., and Allilaire, E.: loc. cit. 
pyocyanea, 9.79; C. diphtheriae, 9.55. These are not very unlike the results obtained by Vaughan and Wheeler.

Quite recently Seibert ${ }^{1}$ isolated from tuberculin a nitrogenous substance in crystalline form which possesses the properties of ordinary protein and which appears to embody the active principle of tuberculin.

The struggle for existence of bacteria means a struggle to furnish for themselves the energy and cell substance necessary to multiply and to reproduce their own kind. Organic carbon is, as a rule, the source of energy, and is supplied in various forms, as, for example, carbohydrates, organic acids, alcohols, $\mathrm{CO}, \mathrm{CO}_{2}$, and $\mathrm{CH}_{4}$. The carbon of amino acids and acid amides also serves the same purpose. Carbon is necessary also to build up organic cell structure, as are nitrogen, hydrogen, oxygen, phosphorus, and sulphur.

The exact nature and composition of proteins generally is still a matter of conjecture. The quantitative determination of the chemical elements in various known proteins has revealed some differences, but the following may be taken as a fair average: Carbon, 50 per cent; nitrogen, I6 per cent; hydrogen, 7 per cent; oxygen, 22 per cent; sulphur, 0.3 per cent; phosphorus, $0.4{\text { per cent. }{ }^{2}}^{2}$

The nitrogen requirements and the means by which these are met constitute a fundamental phase of bacterial, as well as all other plant and animal metabolism, and the problem of nitrogen supply in the best available form is one that greatly concerns all living organisms.

Bacteria may to a large extent be classified on the basis of physiological activities. Jensen believes that this is the only logical criterion. We speak of different groups as the nitrifying, proteolytic, putrefactive, lactic acid producing, etc., groups, or as those of the sulphur or iron bacteria.

With respect to nitrogen utilization alone bacteria often reveal the widest differences, and numerous processes of nitrogen transformations may be observed, even in the same organism. The two main processes, however, are those of katabolism or analysis, and anabolism or synthesis. As this paper deals with protein metabolism, the first of these two processes may be discussed under the following head.

\section{PROTEOLYSIS AND PUTREFACTION}

Cell metabolism involves a complex system of interchange of chemical substances between the cell and the surrounding medium. The work of Lübbert ${ }^{3}, \mathrm{Hesse}^{4}{ }^{4}$ and others, and more recently that of Novy, ${ }^{5}$ has shown that bacterial cells engage in a process of true respiration and that a respiratory coefficient may be established for different organisms. Gotschlich ${ }^{6}$ gives the coefficient as $0.7 \mathrm{I}-0.78\left(\mathrm{CO}_{2}: \mathrm{O}_{2}\right)$ in the absence of fermentable (sugar, etc.) substances. Novy found the coefficient for the tubercle bacillus to be 0.836 , when grown on glycerol agar.

I Seibert, F.: Science, 66, 433, r927.

${ }^{2}$ Mathews, A. P: Physiological Chemistry, p. ro9. New York, ig22.

3 Lübbert,: Biologische Spaltpilz Untersuchungen, p. 38. I886.

${ }^{4}$ Hesse, W.: Ztschr. Hyg. u. Infektionskrankh., I5, r7. I893; 25, 477. 1897.

5 Novy, F. G.: J. Infect. Dis., 36, 168. I925.

${ }^{6}$ Gotschlich, E.: Kolle and Wassermann, Handb. d. Path. Mikroorg., r, 99. rgr 2. 
While it cannot be so easily proved, interchanges of nitrogenous materials must take place in a somewhat analogous manner. Complex nitrogenous compounds must be split into their simple ionizable constituents before they become available for cell nutrition. In the words of Abderhalden, "No cells can directly assimilate and utilize foreign food material. The latter must be prepared for the cell (by enzyme action)." He likens this transformation to the conversion of a church building into a schoolhouse, in which the church must first be reduced to the individual bricks, etc., and then built up again into the new structure.

Abderhalden's statements were directed mainly to animal physiologists. It was he who apparently first showed by actual experiment that man and the higher animals depend upon the hydrolysis of complex nitrogenous food in the stomach and intestine, and a subsequent resynthesis of the digestion products into the tissue and cell substances of the body.

Bainbridge ${ }^{2}$ and Sperry and Rettger ${ }^{3}$ demonstrated that not even the most actively proteolytic aerobes and anaerobes are able to attack native proteins when deprived of accompanying enzymes and when the proteins are the only possible source of nitrogen. Sperry and Rettger employed crystallized egg albumin and edestin, in a medium which furnished all of the other necessary food substances. The addition of a very small amount of commercial peptone (mere traces) was sufficient to initiate growth and the elaboration of proteolytic enzyme which then reduced the peptone to simple products.

Berman and Rettger ${ }^{4}$ demonstrated, further, that proteoses likewise are not directly available to the bacterial cell, and that they and the higher polypeptides at least must first be hydrolyzed by proteolytic or peptolytic enzymes before they are made available. They also showed that many bacteria do not produce the enzymes necessary for preparing proteoses and the higher polypeptides for use, and that these are therefore not utilized at all by these organisms. Bact. coli is one of this group.

According to E. Fisher and others protein is a complex aggregate of amino acids. By the use of strong sulphuric or hydrochloric acid, barium hydrate, superheated steam, or of proteolytic enzymes, these structural units can be broken loose from their combinations, and thus be identified by appropriate methods as individual amino acids.

Besides the numerous monoamino, mono-, and di-carboxylic acids, and the heterocyclic acids (tryptophane, proline, histidine, etc.), two well-known diamino-monocarboxylic acids have been isolated from proteins: arginine and lysine.

The molecular weight of protein is claimed by some to be at least $15,000-18,000$. If the entire protein molecule were made up of amino acids of the average molecular size of alanine $\left(\mathrm{CH}_{3} \cdot \mathrm{CH} \cdot \mathrm{NH}_{2} \cdot \mathrm{COOH}\right)$, it would be a composite of well over $\mathrm{r}_{5} \mathrm{O}$ individual amino acids or amino acid units. One hundred per cent recovery of amino acids from the protein molecule has not been made. Osborne ${ }^{5}$ derived amino acids from

I Abderhalden, E.: Centralbl. f. Bakteriol., Abt. II, 37, 28o. r9r3.

2 Bainbridge, F. A.: J. IIyg., I I, 34 I. I gr r.

${ }^{3}$ Sperry, J. A., and Rettger, L. F.: J. Biol. Chem., 20, 445. I9 5.

4 Berman, N., and Rettger, L. F.: J. Bacl., 3, 367. I9เ8.

s Oslonrne, 'T. B.: The l'egctuble Proteins. Inndon: Longmans, Green \& Co., igon. 
vegetable protein which represented 60 per cent of the protein molecule. The deficiency may be partly explained, according to him, by a loss in the process of hydrolysis and isolation. It may also be due to the occurrence in the protein of modified amino or amide substances which resist isolation and identification.

Amino acids are crystallizable and, with very few exceptions, quite soluble in water. They behave as both weak acids and bases, and form salts with them.

The primary amino acids react with formaldehyde by a process in which the amino groups are condensed and lose their basic character. This constitutes the underlying principle of the Sörensen method of determining the quantity of primary amino acids in any given solution, as, for example, peptone broth cultures of bacteria.

The amino group is readily destroyed by nitrous acid, and by the quantity of nitrogen gas evolved in the reaction the amount of amino acid or acids may be estimated (Van Slyke method).

The occurrence of chromatin or nuclear material in the bacterial cell can no longer be doubted. Nishimura ${ }^{1}$ and Galeotti ${ }^{2}$ demonstrated the presence of nucleins in bacteria. The former also isolated the xanthin bases, xanthin, guanin, and adenin. Nucleic acids were obtained from the tubercle bacillus by Ruppel, ${ }^{3}$ from the diphtheria bacillus by Aronson, ${ }^{4}$ and from Bact. coli by Carega. ${ }^{5}$ More recently Johnson and Brown ${ }^{6}$ isolated thymine and cytosine from the tubercle bacillus as the pyrimidines of the nucleic ("tuberculinic") acid of this organism. At about the same time the purin bases, guanin and adenin, were demonstrated by Long7 in the tubercle bacillus.

\section{ANALYTICAL PROCESSES BROUGHT ABOUT BY BACTERIA}

Through the agency of certain enzymes the complex protein molecule is split up into its numerous amino acids and perhaps some other nitrogenous constituents. The proteolytic enzyme or enzymes concerned differ from ordinary pepsin in that as a rule the hydrolysis is carried beyond the proteose and complex polypeptide stage. They differ also from trypsin of the animal body in that they act over a wide range of $\mathrm{H}$ ion concentration, that is, on the acid as well as alkaline side of $\mathrm{pH} 7.0$. However, the hydrolytic products are, at least in a large measure, like those of trypsin, and are chiefly amino acids, including ordinary tryptophane ( $a$-amino $\beta$-indol propionic acid).

In this process of proteolysis some of the nitrogenous products are again utilized by the organisms to furnish energy for growth and new cell substance. We must assume that a synthetic process goes on simultaneously with the analytic.

Further decomposition of amino acids by bacteria is altogether different from what takes place in the animal body, and an entirely different group of final cleavage products is formed. Indol, skatol, and phenol are common among the strictly bacterial

I Nishimura, T.: Arch.f. Hyg., 18, 318. I893.

${ }^{2}$ Galeotti, G.: Zischr.f. phys. Chemie, 25, 48. г 898.

3 Ruppel, W. G.: ibid., 26, 218 . 1898.

${ }^{4}$ Aronson, H.: Arch.f. Kinderh., 30, 23. 1900.

5 Carega, A.: Centralbl. f. Bakteriol., Abt. I, 34, 323, I903.

${ }^{6}$ Johnson, T. B., and Brown, E. B.: Am. Rev. Tuberc., 7, 285. I925.

${ }^{7}$ Long, E. R.: ibid., 4, 842. 1920. 
products. Indol and skatol result from the action of bacteria on tryptophane, and the phenol has its origin largely in the tyrosine liberated from the protein molecule.

Hydrogen sulphide also is a common product of bacterial action on protein and sulphur-containing derivatives of protein, and mercaptans (methyl and ethyl sulphide) are liberated in the process of anaerobic decomposition by the putrefactive anaerobes. They are derived apparently from the cystin which forms a part of the molecule of most proteins.

The amino acids themselves are physiologically inert. Before they can be of any use to the bacterial cell they require further disruption or rearrangement of the elements, and this is readily accomplished by bacteria, through enzymes and catalysts, presumably within the cell itself. This transformation of the amino acids is one of deaminization in which free ammonia is formed as such out of the amino $\left(\mathrm{NH}_{2}\right)$ groups. This process is commonly one of hydrolysis, with the formation of oxy acid, as is shown in the following simple equation:

\section{$\mathrm{CH}_{2} \cdot \mathrm{NH}_{2} \cdot \mathrm{COOH}+\mathrm{HOH}=\mathrm{NH}_{3}+\mathrm{CH}_{2} \mathrm{OH} \cdot \mathrm{COOH}$ \\ Glycocoll Oxyacetic acid}

Other processes of amino acid change and ammonia liberation are those of reduction, oxidation, oxidation and reduction, and decarboxylation, with the formation, besides ammonia, of amines $\left(\mathrm{R}-\mathrm{NH}_{2}\right)$, alcohols, lower fatty acids, and $\mathrm{CO}_{2}$, as the case may be.

In this transformation of amino acids by bacteria, products are at times constructed which are toxic to the animal organism. By a process of decarboxylation, toxic amines and diamines may be produced, as, for example, tyramine (from tyrosine), histamine (from histidine), and agmatine (from arginine). Other so-called ptomaines are neurin and methyl guanidine. However, not so much credence is given today to the idea of "ptomaine poisoning" as was done several years ago, and bacterial poisons are now generally regarded as the toxic products of specific organisms, like Bact. enteritidis and $\mathrm{Cl}$. botulinum.

Ultimate products of bacterial protein decomposition, if the process is not interrupted, are of the simplest character, and may include ammonia, nitrous oxide, nitrate, nitrogen, hydrogen sulphide, methane, carbon dioxide, hydrogen, and water. The nature of the final products depends, of course, on the amount of available atmospheric oxygen.

The term "putrefaction" has acquired two distinct meanings. In the more general usage it signifies decomposition of protein material through bacterial action, as against "fermentation" or decomposition of carbohydrates, with or without the formation of gas. It has been used by Bienstock ${ }^{\mathrm{r}}$ and by Rettger $^{2}$ in a more restricted sense to mean anaerobic decomposition of protein with the production of foul-smelling products which are characteristic of cadaveric decomposition.

The same observers have maintained that, while many aerobic and facultative anaerobic organisms have the ability to decompose protein, they do so only under aerobic conditions, and that real putrefaction is the work of obligate anaerobes. This

\footnotetext{
${ }^{2}$ Bienstock,: Arch.f. Hyg., 36, 335. I899. $\quad{ }^{2}$ Rettger, L. F.: J. Biol. Chem., 4, 45. 1908.
} 
property is possessed by certain anaerobes only, among the most important of which are $\mathrm{Cl}$. sporogenes, $\mathrm{Cl}$. putrificum, and $\mathrm{Cl}$. aerofetidum.

Some bacterial species a tack casein, without being able apparently to exert any action on serum or egg albumin. Certain staphylococcus and streptococcus forms belong in this class. Gelatin is frequently liquefied by organisms which are non-pro-teolytic. In some instances the gelatin appears to be reduced to the soluble gelatose stage only.

The decomposition of organic waste is participated in by many kinds of bacteria. According to Tissier and Martelly, ${ }^{\mathrm{I}}$ and this is a common observation, aerobes and facultative anaerobes play an important part by preparing a favorable gaseous environment for the proteolytic anaerobes through which rapid destruction takes place.

\section{INFLUENCE OF CARBOHYDRATE ON METABOLISM}

It has long been known that utilizable carbohydrates retard bacterial proteolysis. This principle has in recent years been re-emphasized by Kendall ${ }^{2}$ and his associates, who apparently coined the statement, "Fermentation takes precedence over putrefaction." Their numerous experiments with glucose-utilizing organisms, particularly of the coli-typhi-paratyphi group, have lent further support to the limited observations of earlier investigators.

Kendall and his co-workers showed that when glucose-attacking organisms are grown in nutrient peptone containing I per cent glucose, very little nitrogen metabolism is carried on as indicated by ammonia determinations, in comparison with control cultures which contained no glucose. This inhibition of proteolysis was explained by them to be a sparing action on the proteoses and polypeptides of the medium by the glucose.

Ordinary market milk is prevented from undergoing putrefaction because of the lactose and the lactose-utilizing bacteria (Streptococcus lactis in particular) which are always present. The lactic acid which is formed, even in small amount, retards the development of proteolyzing organisms, as, for example, members of the $B$. subtilis group and the putrefactive anaerobes, and instead of showing evidence of putrefaction. the milk becomes more and more acid, and the casein is precipitated as an acid curd,

Many other examples may be cited, as, for instance, the absence of proteolysis in frozen stored eggs which may contain large amounts (ro per cent) of cane sugar.

It seems to be well established that this inhibition of proteolysis is due to increased $\mathrm{H}$-ion concentration resulting from the sugar fermentation. Berman and Rettger ${ }^{3}$ showed that this inhibition may be prevented by the addition of sufficient buffering agent to regulate the $\mathrm{H}$-ion concentration.

The retardation of proteolysis varies, however, with the different organisms, nitrogenous substances, and carbohydrates employed. For instance, indol production by indol-producing strains of Bact. coli in ordinary peptone broth is prevented by the addition of from 0.5 to $\mathrm{r} . \mathrm{O}$ per cent glucose. In the presence of added tryptophane, some indol may be formed. When lactose is substituted for the glucose, indol formation may be demonstrated readily. Both of these sugars are fermented by Bact.

I Tissier, H., and Martelly: Ann. de l'Inst. Pasteur, r6, 865. I902.

${ }^{2}$ Kendall, A. I., and Farmer, C. J.: J. Biol. Chem., I 2, I3, I9, 21 5, 219, 465, 469; 13, 63. I91 2.

${ }^{3}$ Berman, N., and Rettger, L. F.: J. Bact., 3, 389. 1918. 
coli, but the greater ease with which glucose is broken up enables the organism to draw upon the glucose more easily for most of its carbon supply, and to this extent lcave the nitrogenous substrates intact.

Berman and Rettger ${ }^{1}$ and later Slanetz and Rettger ${ }^{3}$ observed that while B. subtilis readily attacks glucose in ordinary broth, the glucose does not prevent or materially retard nitrogen metabolism of this organism. In fact, the proteolytic activity seems to be accelerated by the glucose. This may be explained by the very active proteolytic property of B. subtilis, and the simultaneous production of acid and alkali in proportions which tend to neutralize each other, and thus permit proteolysis to continue.

Other members of the subtilis group, B. cereus and B. megatherium, are greatly retarded in their nitrogen metabolism by glucose, even in the presence of I per cent phosphate buffer, and in this respect they resemble Bact. coli, Bact. typhosum, and the paratyphoids, to a certain degree.

\section{PROTEIN SYNTHESIS BY BACTERIA}

Chlorophyll-bearing plants synthesize their cell substance with the aid of the sun's energy. Bacteria must obtain their energy by purely chemical action. Synthesis involves energy utilization. Analysis or katabolism is accompanied by the liberation of energy. Carbon plays the chief rôle in these processes; in some instances the necessary energy is provided by the oxidation of nitrogen (nitrification) and of sulphur.

Amino acids serve as a common source of nitrogen for bacteria. The exact nature of the process is still little understood, but it must be assumed that it takes place in the cell, and that the amino acids are deaminized to furnish ammonia directly, and that a very intensive selective action takes place in which the necessary chemical elements are used for energy and the building up of the complex cell substance, particularly proteins, and whereby those elements or atomic groups which are useless are eliminated or rejected and constitute the so-called "metabolic waste products" of the bacterial cell.

Simple amino acids often serve as the only source of organic nitrogen, as, for example, asparagin in the synthetic medium of Jordan ${ }^{3}$ and the same amino acid in Fränkel's modification of the Uschinsky medium. Glycocoll and other simple amino acids have also been used to furnish the necessary conditions. Thus a single amino acid may supply the nitrogen needs for building up the complex nitrogenous substances of the cell which themselves contain simple and complex amino acids of almost endless description, and the purin bases which characterize the nuclear substance.

Purin bases serve as sources of nitrogen in some instances. Koser ${ }^{4}$ found that the aerogenes type of the coli-aerogenes group can attack uric acid and hypoxanthine, whereas the coli type cannot. There is no reason to assume, however, that these purin bases are converted directly into the nuclear substance of the cell.

On the other hand, there are many organisms which require the most complex

s Ibid.

2 Manuscript in preparation.

${ }^{3}$ Jordan, E. O.: J. Exper. Med., 4, 627. 1899 .

4 Koser, S. A.: J. Infect. Dis., 23, 377. 1918. 
mixture of amino acids; and, again, some cannot be made to grow in any known mixture of amino acids, but require for their normal deve'opment special material like hematin (H. influensae) or substances of unknown composition which are present in blood or blood serum (some streptococcus and pneumococcus forms).

UTILIZATION OF AMMONIA AND ATMOSPHERIC NITROGEN

Both ammonia production and ammonia utilization are properties common to most bacteria, if indeed not all. These processes are frequently carried on simultaneously. The ammonia is apparently utilized directly.

Numerous organisms will develop) abundantly in media in which the only source of nitrogen is ammonia, in the form of a soluble ammonium salt. Proskauer and Beck ${ }^{\mathrm{I}}$ found that good growth of the tubercle bacillus (human type) can be obtained in a medium containing ammonium salts of di-basic and tri-basic acids. This observation has been verified frequently. Another example is the meclium of Ayers and Rupp ${ }^{2}$ for the cultivation of the colon-aerogenes group. The nitrogen is supplied here in the form of sodium ammonium phosphate.

Free atmospheric nitrogen can be used by some organisms as a source of nitrogen. The conversion of free nitrogen into ammonia for cell use may be demonstrated easily by the Azotobacter genus and $\mathrm{Cl}$. pastorianum. It appears that non-symbiotic nitrogen fixation among aerobic bacteria is not uncommon; but the action is as a rule so weak and variable as to have little practical significance.

A process of free nitrogen utilization which is of enormous economic importance is that of the Rhizobium or root-nodule type of organism--Rhizobium radicicola. This depends on a close association with the roots of leguminous plants, and takes place within the root tubercles of the legumes. Little is known of the chemical process, but it would seem that ammonia must be an important intermediate product of metabolism.

Denitrification is a property possessed by a considerable number of organisms, particularly of soil origin. Complete denitrification results in the formation of nitrogen or of nitrous oxides. Indirect or incomplete reduction of nitrates gives rise to nitrites or ammonia.

Another process engaged in by a very limited number of organisms is that of nitrification. This involves two separate processes: (I) the oxidation of ammonia to nitrous acid, accomplished by two highly specialized species, the Nitrosomonas and Nitrosococcus of Winogradsky; and (2) the further oxidation of the nitrous acid to nitric through the agency of the Nitrobacter of Winogradsky.

These two processes may be indicated by the following equations:

$$
\begin{aligned}
& { }_{2} \mathrm{NH}_{3}+{ }_{3} \mathrm{O}_{2}={ }_{2} \mathrm{HNO}_{2}+{ }_{2} \mathrm{H}_{2} \mathrm{O}, \\
& { }_{2} \mathrm{HNO}_{2}+\mathrm{O}_{2}={ }_{2} \mathrm{HNO}_{3} .
\end{aligned}
$$

While the nitrification plays an important economic rôle in soil fertility, it would seem that the chief immediate benefit which the bacteria themselves derive from it is

I Proskauer, B., and Beck, M.: Ztschr.f. Hyg. u. Infektionskrankh., r8, I2S. I894.

${ }^{2}$ Ayers, S. H., and Rupp, P.: J. Bact., 3, 433. I9I8. 
the energy supplied by the reactions. Some of the nitrate nitrogen may of course be used for cell structure.

It has been claimed that the entire oxidation process may be brought about by a single organism, but evidence on this point is still quite meager.

The subjects of utilization of free atmospheric nitrogen, and of nitrification and denitrification, are dealt with only very briefly in this chapter because they are discussed at some length in other chapters of this volume. 


\section{CHAPTER XVI}

\section{THE UTILIZATION OF CARBOHYDRATES BY BACTERIA}

ARTHUR ISAAC KENDALL

Washington University School of Medicine, St. Louis, Mo.

Man has been familiar with the results of the fermentations induced in saccharine media from the earliest times, but it was not until i8 io that a serious attempt was made to reduce the reactions of a fermentation process to a definite, balanced chemical equation. In that year Gay-Lussac ${ }^{x}$ published his "Mémoire sur la fermentation," in which he stated that glucose undergoing alcoholic fermentation passes quantitatively into carbon dioxide and ethyl alcohol according to the following equation:

$$
\mathrm{C}_{6} \mathrm{H}_{\mathrm{I}_{2}} \mathrm{O}_{6}={ }_{2} \mathrm{CO}_{2}+{ }_{2} \mathrm{C}_{2} \mathrm{H}_{5} \mathrm{OH} \text {. }
$$

Notwithstanding the fact that this equation is an impossible one, as is readily seen from a consideration of the space formula for glucose, the observation is very valuable not only because two of the most important substances produced from the fermentation of glucose by yeast are thus early identified, but also because this is one of the very first attempts to study a biological reaction in a quantitative way. This observation, furthermore, was made at a time when organic chemistry was a very immature science and long before the discovery of the yeast plant itself.

More than half a century elapsed before the detailed study of micro-organisms and their products of development was resumed. Meanwhile, the compound microscope was brought to a state of perfection compatible with accurate observations, and a violent controversy centering on the doctrine of spontaneous generation had brought to light the part microscopic organisms play in inducing fermentations and putrefactions in decomposable media. Also new and useful methods for culturing and identifying these micro-organisms gradually were developed. The famous controversy between Liebig and Pasteur, which lasted nearly twenty years in the aggregate, terminated with the firmly established thesis of "no fermentation without life," which is one of the great contributions of Pasteur to microbiology. About two decades later Buchner discovered zymase, which in turn opened up a new and very fertile field for further exploration. These three great discoveries - first, the chemical balance sheet of fermentation; second, the organism that incites fermentation; and third, the complex enzymatic nature of the process of fermentation itself-although not fully matured even today-mark a new and highly important epoch in the study not only of microbiology, but of cellular activity in general. While it is undoubtedly true that the nature of the decomposition of carbohydrates by yeast is far better understood than decompositions induced by bacteria, nevertheless considerable progress has been made along bacteriological lines and much valuable information has been obtained.

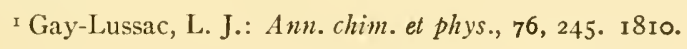


As early as i 862 Pasteur $^{1}$ was studying spontaneously developing butyric fermentation; indeed, he had already isolated and cultivated the anaerobic "vilbrios," as he called them, which would induce the formation of butyric acid from lactates. This is a highly significant phenomenon which has not received the attention it deserves even today, inasmuch as it seems to predicate an extraordinary change of the threecarbon-atom lactate molecule to the four-carbon-atom butyrate molecule. Pasteur also made the highly important discovery that certain microbes attacked the $d$-and $l$-forms of tartaric acid at materially different rates, thus paving the way for the important studies of Fischer and Thierfelder ${ }^{2}$ upon the isomers of glucose and their relations to yeast fermentation. Fischer, whose master-mind opened the great field of carbohydrate chemistry to his successors, did not fail to realize the theoretical importance of these relations between carbohydrate structure and protoplasmic utilizability, and propounded his famous simile of the "key and the lock" 3 in explanation of the reciprocal relations between the two.

Much additional information about the fermentations of carbohydrates by bacteria was afforded by the earlier researches of Nothnagel ${ }^{4}$ and of Brieger ${ }^{5}$ who isolated and identified propionic, lactic, acetic, and formic acids among the products of decomposition of glucose by acetic and lactic fermenting microbes. Perhaps the greatest contribution of all, however, was that of Escherich. ${ }^{6}$ This very careful and thorough investigator not only introduced several of the more important intestinal bacteria into the group of known microbes, but also added much to the technique of bacteriology, including the underlying principle of the fermentation tube. He also improved the anaerobic method of cultivation, and, perhaps most significant of all, started an entirely new chapter in microbic chemistry dealing with the nature of the metabolism of proteins, carbohydrates, and fats by a variety of bacteria, acting alone and in mixture, which he obtained from the intestinal tracts of young children. With the advent of Escherich's important monograph, modern bacteriology may be said to have its origin.

\section{GENERAL CONSIDERATIONS}

With the probable exception of the genera Thiothrix and Beggiatoa, bacteria which appear to utilize inorganic sulphur and sulphur compounds and simple ammonium salts for energy, ${ }^{7}$ require some preformed, that is to say, organic food in their dietary. This food is required in fulfilment of two distinct phases in their lifehistory: the structural or anabolic phase and the energy or katabolic phase. The structural phase comprises those phenomena which are embraced in the separation and maturation of the daughter-cell from the parent-cell, together with those cellular losses incidental to the elaboration of soluble enzymes and other elements. The

${ }^{I}$ Pasteur, L.: The Physiological Theory of Fermentation. An excellent translation appears in "Harvard Classics," 38, 289-381. I9 10.

${ }^{2}$ Fisher, E., and Thierfelder, H.: Ber. d. deut. chem. Gesellsch., 27, 2031. 1894.

3 Fischer, E.: Ztschr.f. phys. Chemie, 26, 60. 1898.

4 Nothnagel, H.: Ztschr f. klin. Med., 3, 275. I88I.

${ }^{5}$ Brieger, L.: Ztschr. f. phys. Chemie, 8, 306. 1883-84; 9, 1. 1885.

${ }^{6}$ Escherich, T.: Die Darmbakterien. Stuttgart. 1886.

${ }^{7}$ See Waksman, S. A.: J. Bact., 7, 231. 1922. 
energy phase comprises those chemical changes which the mature microbic cell induces in its environment in fulfilment of its particular and characteristic chemical activity.

The amounts of substance required for the structural and energy phases respectively, are very unlike. Fifteen millions of bacteria of ordinary size would scarcely balance an ounce weight.' The weight of a simple bacterial cell, therefore, is little indeed, and about 85 per cent of this is water. On the other hand, the amount of substance transformed for energy by bacterial cells is, or may be, relatively great. Hence, the expenditure of foodstuffs in the energy, as contrasted to the structural phase, is frequently in the order of hundreds to one.

Yet it is not a matter of indifference just what the chemical nature of these phases is dependent upon. Bacteria, like other known living things, are nitrogen-containing. Hence some suitable source of nitrogen must be available for structural needs. On the other hand, most bacteria may derive the oxidizable carbon for their energy requirement either from compounds containing nitrogen as well as oxygen, as in the amino acids and their complexes, or from carbohydrates having a suitable configuration. Fats on the whole are probably not particularly suited for the energy requirements of most bacteria.

There is also a group of non-nitrogenous substances, departing somewhat from the carbohydrate configuration, as certain organic acids (tartaric, for example), which are acceptable sources of carbon for energy by many bacteria. A discussion of this problem, however, is without the scope of this chapter, which deals more specifically with carbohydrates.

Carbohydrates frequently have a profound effect upon the character of the substances produced by bacterial action. Thus, to cite a few well-known examples:2

The diphtheria bacillus growing in a suitable nitrogenous medium produces the characteristic, deadly, well-known soluble toxin which makes the organism formidable. If, before the organisms are cultivated, some glucose is added to such a medium, the microbe produces considerable amounts of acid, principally lactic, but no toxin whatsoever.

The proteus bacillus produces large amounts of indol, and forms a soluble, proteolytic enzyme when it is cultivated in a suitable nitrogenous medium. If, however, before the organism is inoculated, some glucose is added to the nitrogenous medium, it no longer produces indol nor the proteolytic enzyme. It forms considerable amounts of lactic acid instead. ${ }^{3}$

In a similar manner the various strains of Bacillus coli produce indol and phenolic bodies from the nitrogenous constituents of ordinary nutrient broth, but the addition of glucose to such a medium prior to inoculation with the microbe changes it into a lactic-acid-producing microbe. These latter observations have some significance in the genesis of indol in the intestinal tract. 4

It appears, therefore, that the addition of utilizable carbohydrate to nitrogenous

${ }^{1}$ Kendall, A. I.: Civilization and the Microbe, p. I7. 1923.

${ }^{2}$ See Kendall, A. I.: Physiol. Rev., 3, 438. 1923.

${ }^{3}$ Kendall, A. I., Cheetham, H. C., and Hamilton, C. S.: J. Infecl. Dis., 30, 251.1922.

${ }^{4}$ Kendall, A. I.: Bacteriology, General, Pathological and Intestinal (2d ed.), p. 70.192 I. 
cultural media in which bacteria are growing affects the character of the products they form very strikingly. In general, it may be said that utilizable carbohydrate protects protein from direct bacterial utilization for energy. On the other hand, it must not be forgotten that, under certain circumstances, the addition of utilizable carbohydrate may indirectly bring about some alteration in the protein constituents of cultural media which might not otherwise take place.

Thus, it appears to be a fact that utilizable carbohydrate, added to cultures of the gas bacillus (Bacillus welchii), indirectly leads to the appearance of a histamine-like substance, which is not produced by the action of the organism upon the protein constituents when carbohydrate is absent. ${ }^{1}$ It seems not improbable that the organism produces an enzyme, of the nature of a carboxylase, which acts upon histidine, or possibly a histidine peptide, in accordance with the equation:<smiles>NC(Cc1c[nH]c(CC(N)C(=O)O)n1)C(=O)O</smiles>

(Histidine)<smiles>NCCc1cc[nH]c1</smiles>

(Histamine)

Only very small amounts of this histamine-like substance are produced in ordinary cultural media, but it is very reactive chemically. One part in five million may cause a very definite contraction in a piece of isolated surviving smooth muscle from a guinea pig. ${ }^{2}$

The observations of Koessler and Hanke ${ }^{3}$ suggest that a somewhat similar reaction may occur among certain strains of other common bacteria. Finally, it must be borne in mind that "resting" bacteria, i.e., actively growing bacteria washed free from cultural medium, and suspended in non-nutritive, isotonic solutions containing appropriate amounts of test substance, can and do bring about chemical changes that do not necessarily take place when the organisms are growing freely. Thus, many "resting" bacteria will change methyl glyoxal to lactic acid, although this reaction does not seem to take place under conditions where the microbes are growing freely. Quastel and his associatest have made several important studies upon this significant, but little studied, aspect of bacterial metabolism.

\section{CARBOHYDRATES AND THE STRUCTURAL REQUIREMENTS OF BACTERIA}

\section{CELL CONSTITUENTS}

The predominance of nuclein compounds in bacteria, described by many investigators, focuses attention upon the nature of the carbohydrate component. Bendix, ${ }^{5}$

${ }^{1}$ Kendall, A. I., and Schmitt, O. F.; J. Infect. Dis., 39, 250.1926.

${ }^{2}$ Guggenheim, M.: Die biogene Amine (II. Aufl.) 1923.

${ }^{3}$ Koessler, K. K., and Hanke, M.: J. Biol. Chem., 50, I3I. 1922.

${ }_{4}^{4}$ Quastel, J., et al.: Biochem J. 1924-27. (Numerous articles).

5 Bendix, E.: Deutsche med. Wchnschr., 27, I8. 1900. 
studying the chemistry of the cellular substance of the tubercle bacillus, claimed to have identified a pentose among the constituents. This, if substantiated, would seem to place this organism at least among the plants, because the animal nucleus contains a hexose. ${ }^{\mathrm{r}}$ The presence of cellulose, and of hemicellulose, is still a matter of discussion, with the balance of evidence as yet unfavorable, although Emmerling, Winterstein, ${ }^{2}$ and others claim to have detected it in the substance of bacteria. On the other hand, chitin, a polymer of glucoseamine ${ }^{3}$ found in the animal kingdom, principally in the carapace of Crustacea, has been reported by several investigators. This is quite important if true, because it leaves the bacteria in an ambiguous position with reference to the usual chemical concepts of classification-having nuclear substance of plant affinity, and at the same time possessing chitin, usually regarded as of animal origin, in their cell membranes.

\section{CAPSULAR CONSTITUENTS}

Certain kinds of bacteria, as pneumococci, pneumobacilli, the gas bacillus ( $B a$ cillus wclchii), and several occurring in the soil, are surrounded with mucoid envelopes. Acetic acid precipitates this mucoid substance in many instances, which has led to the belief that the material may be true mucin. Recently Dochez, Avery, Heidelberger, and others ${ }^{+}$have isolated polysaccharides from the pneumococci, the Friedländer bacillus, and other organisms which are not true mucins but which are precipitated with the homologous-type sera in very high dilutions. These "species specific" polysaccharides are very interesting and important both from the standpoint of immunity and virulence.

\section{EFFECTS OF CARBOHYDRATE UPON THE CHEMICAL COMPOSITION OF BACTERIAL CELLS}

Cramer, ${ }^{5}$ Lyon, ${ }^{6}$ and others have published analyses which seem to indicate that the presence or absence of utilizable carbohydrate in cultural media otherwise of the same composition influences the relative amounts of nitrogenous substance, alcohol and ether extracts, and ash quite materially. Just what significance is to be attached to these data is problematical. They may, however, be of significance in view of the fact that Dochez and Avery" found that the yield of the "species specific" polysaccharides from type-II pneumococci increased from $3^{-4}$ to $35^{-40} \mathrm{gm}$. per 300 liters of culture when glucose was added to the medium.

\section{CARBOLIGASE}

Mention should be made at this point of the important observation of Neuberg? that yeast is capable of bringing about syntheses of organic compounds in the presence

${ }^{1}$ Jones, W.: Nucleic Acids, pp. 21, 29. I924.

${ }^{2}$ Emmerling, O.: Ber. d. deut. chem. Gesellsch., 32, 541. 1897; Winterstein, E.: Ztschr.f. phys. Chemie, 21, I34. I $895^{-966 .}$

3 Viehöver, A.: Ber.d. deut. botan. Gesellsch., 30, 443. I9I 2.

4 See, for an excellent summary, Heidelberger, MI.: "Immunologically Specific Polysaccharides," Chem. Rei., 3, 403. I927; Heidelberger, M., and Goebel, W. F.: J. Biol. Chem., 70, 613. I926; also chap. $\mathrm{x}$ in this volume.

${ }^{5}$ Cramer, E.: Arch.f. Hyg., 16, 151. I893. $\quad{ }^{6}$ Lyon, R. E.: ibid., 28, 30. I897.

7 Neuberg, C., and Kobel, M.: Handb. d. biol. Arbeitsmeth., Abt. IV, p. 625. I927. 
of carbohydrates. Thus, when benzaldehyde is added to a suspension of yeast in glucose solution a condensation product of benzaldehyde with acetaldehyde is formed which seems to have the formula

$$
\mathrm{C}_{6} \mathrm{H}_{5} \cdot \mathrm{CHOH} \cdot \mathrm{CO} \cdot \mathrm{CH}_{3} \cdot
$$

This reaction, according to Neuberg, is brought about by the activity of an enzyme, carboligase. Up to the present time similar studies have not been made with bacteria, but it is not unreasonable to suspect that somewhat similar enzymatic processes may be discovered.

\section{CARBOHYDRATES AND THE ENERGY REQUIREMENTS OF BACTERIA}

THE SUGAR SERIES, AND DERIVED ALCOHOLS AND ACIDS

Mention has been made above of the fact that the addition of utilizable carbohydrates and carbohydrate derivatives of the proper configuration to the nitrogenous constituents of cultural media may very materially affect the character of the products.

Generally speaking, but with some well-known exceptions, as, for example, Bacillus alcaligenes, which apparently does not utilize even glucose for energy, the utilizable carbohydrate is burned for energy, sparing to a very considerable degree thereby the nitrogenous constituents from bacterial attack. ${ }^{\mathrm{I}}$ No authentic instance has been recorded in which a microbe utilizes any carbohydrate for energy that will not utilize glucose. There are, however, organisms that do not seem to be able to use any carbohydrate except glucose for energy. This is reminiscent of the human body, which seems to utilize glucose for energy requirements.

It is rather a striking fact, commented on long ago by Smith, ${ }^{2}$ that the bacteria highly pathogenic for man and for animals are usually less reactive both culturally and chemically than the parasitic types. Thus, typhoid, dysentery, diphtheria, and tubercle bacilli, as well as meningococci and gonococci, are relatively inert culturally, whereas the colon, proteus, and mesentericus groups of bacilli and the staphylococci are characterized by considerable cultural reactivity. This is reflected not only in the respective changes induced in nitrogenous constituents of culture media, but also in the configuration of carbohydrates and carbohydrate-derivatives which these microbes can utilize for energy; which they can ferment. The members of the pathogenic groups above mentioned can generally utilize glucose, and the closely related hexoses, mannose, and fructose and the derived alcohol, mannitol, but cannot apparently break down such biose molecules as lactose and saccharose, or utilize starches.

On the other hand, there are parasitic microbes, as certain members of the mucosus capsulatus group, which can ferment a very considerable variety of carbohydrates, some with $3,4,5,6,12$, and more carbon atoms. The exact explanation for this difference in versatility, aside from Fischer's simile of the "key and the lock,"3 is still to be revealed. Nevertheless, the remarkable specificity of these relations between carbohydrate configuration and protoplasmic utilization is one of the remarkable phenomena of biology.

I See Kendall, A. I.: Physiol. Rev., 3, 438. I923.

${ }^{2}$ Smith, T.: Am. Med., 8, 7I I. Igo4.

3 Fischer, E.: loc. cil. 


\section{GLUCOSIDES AND GLUCOSEAMINE}

There is a large group of substances possessing in common a carbohydrate molecule united to one or more organic compounds, which are resolved into their respective components upon hydrolysis with the addition of $\mathrm{H}$ and $\mathrm{OH}$ ions. These are glucosides and carbohydrate ethers of organic acids, alcohols, or ring compounds, and they correspond in chemical structure to the well-known methyl glucosides. Their significance to the bacteriologist at present lies in the fact that some of these have been used from time to time for purposes of separation and of recognition of certain groups of organisms, notably the streptococci.

The relation of two naturally occurring enzymes, emulsin and maltase, to these glucosides is of great significance. The former cleaves many of those glucose-glucosides which possess the $\beta$-glucose configuration, whereas the latter hydrolyzes many glucose-glucosides which possess the a-glucose configuration. This has been very properly construed as evidence of the $\beta$ or a linkage between the glucose molecule and the associated radicle, alcohol, acid, or otherwise. ${ }^{\mathrm{I}}$

The relationships between the $a$ and $\beta$ glucose-glucosides are indicated in the following diagrams in which " $R$ " indicates the bound radicle:<smiles>[R]O[C@H]1CC[C@H](O)[C@H](O)[C@H]([C@H](O)CO)O1</smiles>

(a Type of glucoside)<smiles>[R]O[C@H]1CCO[C@H]([C@H](O)CO)[C@H]1O</smiles>

( $\beta$ Type of glucoside)

Those glucosides that are commonly used in the identification of bacteriasalicin, amygdalin, and arbutin-together with many others that are occasionaliy utilized for this purpose, are all members of the $\beta$ group, hence, aside from certain minor factors involved in the effects of the radicles upon cleavage, one glucoside of the $\beta$ type is, or theoretically should be, as informative concerning the enzyme equipment of the microbe as another. It would appear that the principal biochemical information to be elicited from the use of one or several members of the $\beta$-glucoside group, therefore, is the presence or absence of an enzyme of the emulsin type. Nevertheless, some practical differentiations between closely related bacteria seem to have been made through the use of some of these $\beta$ glucose-glucosides. ${ }^{2}$ Little or no attention has been paid to $\alpha$ glucosides from this aspect, probably because glucoside deriva-

I See Armstrong, E. F: The Simpler Carbohydrates and Glucosides (4th ed., I924), for a fairly complete chapter on glucosides.

${ }^{2}$ See Gordon, M. H.: Supp. Ann.Rep.Loc. Gov. Bd., p. 388 . London, 1903; Andrews, F. IV., and Horder, T. J.: Lancet, 2, 708, 775, 852. I906; Holman, W. L: J. Med. Reseurch, 34 (N. S., 29), 377. r9ı6; Kendall, A. I., Day, A. A., Walker, A. W., and Ryan, M.: J. Infert. Dis., 25, r89. I9r9. 
tives of the $a$-glucose series have not thus far been obtained from the vegetable kingdom. Also, the glucosides derived from sugars other than glucose, $d$ - and $l$-arbinose, $l$-xylose, galactose, mannose, and fructose do not seem to have been carefully investigated.

Glucoseamine, a cleavage product of chitin, as well as mucin from the submaxillary gland, and from mucous membranes, ${ }^{\mathrm{I}}$ seems to have been isolated from the body substance of certain bacteria. ${ }^{2}$ Meyer $^{3}$ states that this substance may be fermented by a considerable number of bacteria, i.e., it may be a source of energy for these microbes. In the list is Bacillus proteus. If indeed it be true that the proteus bacillus will ferment glucoseamine, then some evidence will have been produced in favor of the glucose rather than the mannose formula for this substance-a point of contention at the present time, because Bacillus proteus does not ferment the mannose configuration. 4

THE CARBOHYDRATE FRACTION OF PROTEINS

Many proteins contain a carbohydrate nucleus in their molecule. Mathews ${ }^{5}$ states that egg white contains 0.5 per cent of glucose. Some evidence of the occurrence of such a carbohydrate-like substance is afforded by a study of the nitrogenous metabolism of active cultures of Staphylococcus aureus in suitable nitrogenous media. It has been found that the deamination induced by this organism is comparatively slight until the Molisch reaction ${ }^{5}$ disappears. Also an acidity develops which increases progressively with the persistence of the Molisch reaction. When the Molisch reaction disappears, however, usually by the end of the fifth day of growth, deamination proceeds rapidly, and concurrently the reaction becomes quite alkaline, due to the accumulation of basic products of the nitrogen metabolism of the organism. It seems very probable that both the initial acidity and low ammonia formation are associated with the utilization of the carbohydrate nucleus of the protein molecule for energy. Little nitrogenous change would be expected under these conditions. When the carbohydrate is used up, the organism attacks the residual nitrogenous fraction for its energy requirements, bringing about the typical evidences of deamination and accumulation of basic substances.

Not many bacteria thus far studied exhibit this phenomenon, however. It seems not unreasonable to explain this difference on the basis of the nature of the peptid linkages in the protein molecule, which are broken by the staphylococcus in such a manner as to liberate the carbohydrate nucleus early in the digestive process, on the one hand, and differently cleaved by most bacteria, on the other hand.

\section{BACTERIAL UTILIZATION OF CARBOHYDRATES}

EFFECT OF CONFIGURATION OF THE CARBOHYDRATE MOLECULE

Seventy years ago Pasteur ${ }^{7}$ noticed that the green mold, Penicillium glaucum attacked the $d$-and $l$-forms of tartaric acid at materially different rates, utilizing the $d$-tartaric acid quite rapidly, leaving the $l$-tartaric acid practically untouched. Not

s Meyer and Jacobson: Lehrb. d. org. Chem. (II. Aufl., Vol. I, Part 2), I9I3.

${ }^{2}$ Viehöver, A.: loc. cit.

${ }^{3}$ Meyer, K : Biochem. Zlschr., 57, 297. I9I3.

4 Kendall, A. I., Cheetham, H. C., and Hamilton, C. S.: loc. cil.

5 Mathews, A.P.: Physiol. Chem. (2d ed.), I5i. r9r6.

${ }^{6}$ Kendall, A I., and Farmer, C. J.: J. Biol. Chem., I2, 2 I5. I9I 2.

7 Pasteur, L.: Compt. rend. Soc. de biol., 46, 615. 1858. 
much attention was paid to this observation until Fischer, the master-chemist of the carbohydrates, and his pupil, Thierfelder, ${ }^{\mathrm{I}}$ exposed various members of the hexose series of sugars to the action of yeasts. They found at once that certain members of the glucose series, always with the $d$-configuration, were consistently fermented by yeast, whereas other members were equally consistently left unattacked. Fischer grasped the meaning of this remarkable relationship between carbohydrate configuration and the ability of the yeast to utilize certain configurations as a very fundamental biological phenomenon. His famous simile of the "key and the lock" is ample evidence of his comprehension of the significance of this very important phenomenon. He states: "Bei dieser Annahme wäre es nicht schwer zu verstehen, dass die Hefezellen mit ihrem asymmetrisch geformenten Agens nur in die Zuckerarten eingreifen und gärungserregend wirken können, deren Geometrie nicht zu weit von derjenigen des Traubenzuckers abweicht."' Fischer and Thierfelder also showed that all the yeasts they studied fermented all those hexoses of the glucose series that had a common enol-namely, glucose, mannose, and fructose. That is to say, their experiments showed very clearly that a yeast culture which fermented any one of these three hexoses fermented the others as well. Armstrong ${ }^{3}$ has reaffirmed this conclusion.

Bacteria are considerably more versatile in their fermentation reactions in the aggregate than yeasts, and it is to be deplored that bacteriology had not developed far enough when Fischer was studying this profoundly interesting "biochemical geometry" to provide him with some of the more active fermenting types to extend this highly important field.

It is now well known that several important groups of bacteria, e.g., members of the Bacillus proteus and Vibrio comma group, do not ferment mannose 4 at all, and several other similar instances are known. Furthermore, such changes of terminal groups in the glucose molecule as are shown below alter the utilization of the resulting compound for many bacteria, thus: 5<smiles>O=CC(O)C(O)C(O)C(O)CO</smiles>

(d-Glucose)<smiles>O=C(O)C(O)C(O)C(O)C(O)C(O)CO</smiles>

(d-Gluconic acid)<smiles>O=C(O)C(O)C(O)C(O)C(=O)O</smiles>

(d-Saccharic acid)<smiles>CC(C)(C)C(O)C(O)C(O)C(O)C(O)C(O)C(O)O</smiles>

${ }^{2}$ Fischer, E.: loc. cit.

${ }^{I}$ Fischer, E.: loc. cit.; Thierfelder, H.: loc. cit.

3 Armstrong, E. F.: op. cit., p. I7 I. I924.

${ }^{4}$ Kendall, A. I., and Yoshida, S.: J. Infect. Dis., 32, 355. 1923.

${ }^{5}$ For convenience the older formula for glucose is used in place of the closed-chain formula, and the acids are not written in the lactone form. 
It has been shown ${ }^{\mathrm{I}}$ that departure from the glucose configuration renders the resulting mono- or di-glucose acid and alcohol progressively less utilizable as a source of energy for bacteria. In general, the more fastidious types are the ones which are the more readily affected by departures from the simple hexose configuration. One of the rather unexpected results which flowed from the study of this phenomenon was the failure of members of the Staphylococcus aureus group and Micrococcus tetragenus to utilize either gluconic or saccharic acid. Glycuronic acid was not available at the time these studies were made. Similar series were studied with mannose and galactose as the starting-point. The members of these series most difficult of utilization again were acids: manno-saccharic and mucic acid. Dulcitol, the hexatomic alcohol of the galactose series, is optically inactive through internal compensation. It is much less readily utilized for energy by most bacteria, and it possesses, largely for this reason, diagnostic value as a reagent for distinguishing certain types of organisms, notably many members of the Bacillus mucosus capsulatus group of bacteria, which are versatile fermenters.

Much more study will be required before the significance of the departure from the glucose configuration in relation to protoplasmic utilization is understood, but at least one rather striking practical possibility has emerged, namely, the ability to identify carbohydrates and their derivatives by the use of microbes. Experiments already published ${ }^{2}$ indicate that not only may certain carbohydrates be detected thus by bacterial means, but also mixtures of sugars and their derivatives may be identified ${ }^{3}$ and even measured with considerable accuracy. As little as one-one-thousandth of a per cent of a sugar in a mixture has thus been detected and estimated, 4 with considerable precision. Not the least interesting possibility inherent in these studies is the bringing together of the field of pure chemistry, biochemistry, and bacteriology in the exploration of one of the most fundamental of problems: the chemistry of vital processes.

\section{BACTERIAL FERMENTATION OF CARBOHYDRATES}

The simplicity of structure and the monotony of structure of bacteria has had even from the earliest pioneer days a peculiarly directing influence upon the lines of development of bacteriology. Coincident with the recognition of the necessity for supplemental criteria to the meager anatomical characteristics of microbes, attention was early directed to chemical changes they might induce in suitable cultural media to afford data upon which to identify them. In this manner Pasteur ${ }^{5}$ recognized butyric acid, and Nothnagel ${ }^{6}$ and Brieger ${ }^{7}$ identified lactic, acetic, propionic, and formic acids as well as carbon dioxide and hydrogen among the products of fermentation of sugars by various microbes, and Escherich ${ }^{8}$ made surprisingly accurate balance sheets for the metabolism of some of the more common bacteria.

Influenced, doubtless, by long familiarity with the yeast plant, by the remarkable

\footnotetext{
${ }^{x}$ Kendall, A. I., Bly, R., and Haner, R. C.: J. Infect. Dis., 32, 377. 1923.

${ }^{2}$ Kendall, A. I., and Yoshida, S.: ibid., p. 362. I923.

3 Kenclall, A. I., and Yoshida, S.: ibid., p. 369 . I923.

${ }^{4}$ Kendall, A. I., and Yoshida, S.: ibid., p. 355. I923.

'Nothnagel, H.: loc cit.

5 l'asteur, l, : loc. cit.

7 Brieger, L.: loc cit.

8 Escherich, T.: loc. cil.
} 
work of Fischer upon the structure of carbohydrates, and by the discovery of hexose phosphate by Harden and Young, ${ }^{\mathrm{I}}$ attention has been paid by later investigators to the mechanism of sugar fermentation by yeast. The obvious complexity of the process and the relative ease with which suitable amounts of yeast may be obtained have together focused attention upon the fermentation activities of the Saccharomycetes to the virtual exclusion of the bacteria.

However, the products resulting from yeast fermentation have at least some qualitative resemblance to those produced by certain types of bacteria and, in the light of studies made in the earlier days of bacteriology by Frankland and Frew, ${ }^{2}$ Frankland and Lumsden, ${ }^{3}$ Harden, ${ }^{4}$ and more recently by the highly significant work of Neuberg and his associates, ${ }^{5}$ much light has been shed upon some of the bacterial fermentations.

The Neuberg equations.-Neuberg ${ }^{6}$ has propounded three principal types of fermentation, aerogenic in character, and relating especially to yeast:

$$
\begin{gathered}
\text { Type I: } \\
\text { (Glucose) } \\
\mathrm{C}_{6} \mathrm{H}_{\mathrm{I} 2} \mathrm{O}_{6}=
\end{gathered}{ }_{2} \mathrm{CO}_{2}+{ }_{2} \mathrm{CH}_{3} \cdot \mathrm{CH}_{2} \mathrm{OH} .
$$

This is the classical Gay-Lussac equation.?

$$
\begin{aligned}
& \text { Type II: } \mathrm{C}_{6} \mathrm{H}_{\mathrm{I} 2} \mathrm{O}_{6}+\mathrm{Na}_{2} \mathrm{SO}_{3}+\mathrm{H}_{2} \mathrm{O}=\mathrm{C}_{3} \mathrm{H}_{8} \mathrm{O}_{3}+\mathrm{CH}_{3} \mathrm{CHO} \cdot \mathrm{NaHSO}_{3}+\mathrm{NaHCO}_{3} \text {. } \\
& \text { (Glucose) (Sodium sulphite) (Glycerol) (Acetaldehyde- } \\
& \text { sulphite compound) }
\end{aligned}
$$

This type is given in the presence of sodium sulphide (Abfangverfahren). The sulphite protects the acetaldehyde from secondary change; the yield of carbon dioxide and of alcohol is diminished and the yield of glycerol is increased.

Type III (in the presence of alkali) is presumed to occur in three stages, as follows:

$$
\begin{aligned}
& \text { Type III: (a) } \mathrm{C}_{6} \mathrm{H}_{12} \mathrm{O}_{6}=\mathrm{CH}_{3} \mathrm{CHOH} \cdot \mathrm{COOH}+\mathrm{CH}_{3} \cdot \mathrm{CO} \cdot \mathrm{COOH}+\mathrm{H}_{2} \\
& (\text { Glucose) (Lactic acid) } \quad \text { (Pyruvic acid) } \\
& \begin{aligned}
& \text { (b) }{ }_{2} \mathrm{CH}_{3} \cdot \mathrm{CO} \cdot \mathrm{COOH}={ }_{2} \mathrm{CH}_{3} \cdot \mathrm{COH}+{ }_{2} \mathrm{CO}_{2} \\
& \text { (Acetaldehyde) } \\
& \text { (c) }{ }_{2} \mathrm{CH}_{3} \cdot \mathrm{CHO}+\mathrm{H}_{2} \mathrm{O}= \mathrm{C}_{2} \mathrm{H}_{5} \mathrm{OH}+\mathrm{CH}_{3} \cdot \mathrm{COOH} \\
& \text { (Alcohol) (Acetic acid) }
\end{aligned}
\end{aligned}
$$

The completed reaction, therefore, becomes:

$$
{ }_{2} \mathrm{C}_{6} \mathrm{H}_{52} \mathrm{O}_{6}+\mathrm{H}_{2} \mathrm{O}={ }_{2} \mathrm{CH}_{3} \cdot \mathrm{CHOH} \cdot \mathrm{COOH}+\mathrm{C}_{2} \mathrm{H}_{5} \mathrm{OH}+\mathrm{CH}_{3} \cdot \mathrm{COOH}+{ }_{2} \mathrm{CO}_{2}+{ }_{2} \mathrm{H}_{2} \text {. }
$$

${ }^{r}$ Harden, A., and Young, W. J.: Proc. Chem. Soc., 21, I89. I905; Proc. Roy. Soc. (Series B), 80, 299. 1908 .

${ }^{2}$ Frankland, P. F., and Frew, W.: J. Chem. Soc., 6r, 254. I 892.

${ }^{3}$ Frankland, P. F., and Lumsden, J. S.: ibid., p. 432. I 892.

4 Harden, A.: ibid., 79, 6г 2. г9ог.

5 See especially Abderhalden, E.: II andb. d. biol. Arbeitsmeth., Abt. IV, pp. 565, 593, 61 5, 625 . 1927; Oppenheimer, C.: Handb. d. Bioch. d. Menschen u. Thiere (II. Aufl.), 2, 422. I924.

${ }^{6}$ Neuberg, C., and Hirsch, J.: Biochem. Ztschr., 100, 304. I919.

7 Gay-Tussac, I. J.: loc. cit. 
The Cannizzaro reaction in step $(c)$, whereby in the presence of alkali two molecules of aldehyde are reduced and oxidized, respectively, into a molecule each of acid and of alcohol, has been termed "dismutation" by Neuberg.

This third type of fermentation is virtually that worked out by Harden ${ }^{\mathrm{t}}$ for B. coli many years ago. Harden believed that at least three separate and distinct enzymatic processes were involved; the first of these results in the change of glucose to lactic acid:

$$
\mathrm{C}_{6} \mathrm{H}_{12} \mathrm{O}_{6}={ }_{2} \mathrm{CH}_{3} \cdot \mathrm{CHOH} \cdot \mathrm{COOH} \text {. }
$$

The second was responsible for a second molecule of glucose being broken down into a molecule each of alcohol and acetic acid, and two molecules of formic acid:

$$
\mathrm{C}_{6} \mathrm{H}_{\mathrm{t} 2} \mathrm{O}_{6}+\mathrm{H}_{2} \mathrm{O}=\mathrm{C}_{2} \mathrm{H}_{5} \mathrm{OH}+\mathrm{CH}_{3} \cdot \mathrm{COOH}+{ }_{2} \mathrm{H} \cdot \mathrm{COOH} \text {. }
$$

The third reaction involved the cleavage of formic acid by the enzyme formiase into carbon dioxide and hydrogen:

$$
{ }_{2} \mathrm{H} \cdot \mathrm{COOH}={ }_{2} \mathrm{H}_{2}+{ }_{2} \mathrm{CO}_{2} \text {. }
$$

The production and subsequent cleavage of formic acid by micro-organisms seems to be a point of much discussion. Schade ${ }^{2}$ and others believe that the fermentation of glucose by yeast involves the production of formic acid, but this view has been unacceptable to many subsequent observers, partly on the ground that the yeast plant cannot decompose formates with the evolution of gas. ${ }^{3}$ On the other hand, there is much evidence that formic acid is produced under widely differing conditions during the utilization of glucose for energy and that this formic acid may or may not be decomposed subsequently into $\mathrm{CO}_{2}$ and $\mathrm{H}_{2}$. The enzyme "formiase" is found in the press juice from muscles ${ }^{4}$ and especially in cultures of certain bacteria. ${ }^{5}$ According to Clark, ${ }^{6}$ one of the chemical differences between bacteria that produce gas from fermenting sugars, and those that produce acid but no gas under parallel conditions, is the presence of formiase in the enzyme armamentarium of the former and its absence among the latter. According to this view, most of the common bacteria produce formic acid during fermentation. Gas-forming bacteria, as the members of the paratyphoid-colon-proteus-mucosus-capsulatus groups, and many anaerobes, which possess formiase in their enzyme equipment, are able to transform formic acid more or less completely into $\mathrm{CO}_{2}$ and $\mathrm{H}_{2}$. Many if indeed not a decided majority of bacteria that do not produce gas also produce formic acid, but it accumulates in the culture. This is certainly true of the typhoid bacillus, ${ }^{7}$ cultures of which, grown in the presence

${ }^{8}$ Harden, A.: loc. cit.

${ }^{2}$ Schade, H.: Biochem. Ztschr., 7, 299. 1908.

3 See Thomas, K.: Compt. rend. Acad. de Sc., r36, ror5. 1903.

${ }_{4}^{4}$ See Stoklasa, J.: Ber.d. deut. Chem. Gesellsch., 38, 607. I905; Battelli, F.: Compl.rend. Acad. de Sc., r 38,65 r. 1904.

${ }^{5}$ See Pakes, W. C. C., and Jollyman, W. H.: J.Chem. Soc., 79, 386, 459. rgor; Franzen, H., and Greve, G.: Zischr.f. physiol. Chemie, 64, г69. г9го.

${ }^{6}$ Clark, W. M.: Science, 38, 669. 19r3.

7 See Franzen, H., and Egger, F.: Ztschr. physiol. Chemie, 79, I77. 1912; 83, 226. 1913. 
of glucose, are found to be quite rich in formic acid even after forty-eight hours' incubation.

According to Frankland and Frew, ${ }^{\mathrm{T}}$ the decomposition of formic acid is more complete under conditions where oxygen is rigorously excluded. It is worthy of note that the equation involving the cleavage of formic acid to carbon dioxide and hydrogen does not involve the interreaction of $\mathrm{H}$ and $\mathrm{OH}$ ions; nearly all true enzymic processes do.

Butyric acid fermentation.-Mention has already been made of Pasteur's discovery $^{2}$ that certain anaerobic "vibrios" could produce butyric acid from lactates. This involves a synthetic process, whereby the four-carbon-atom chain of butyric acid is formed from the three-carbon-atom chain of the lactic acid. Neuberg and Arinstein ${ }^{3}$ have studied the butyric acid fermentation induced by Bacillus butylicus fitzianus, and find by the Anfangverfahren procedure that both butyric acid and butyl alcohol are produced if sulphites are added to the slightly alkaline cultural media. Acetaldehyde was also detected in these cultures, and it seems probable that a synthetic process is involved much like that noted by Pasteur more than sixty years ago.

Acetic acid fermentation.-The industrial production of acetic acid from alcohol has long been practiced, but the process has not been very well understood other than that there was an oxidation of the alcohol to the acid. Recently Neuberg and Windisch ${ }^{4}$ have brought forward evidence which seems to show that the process is somewhat more intricate than a mere addition of oxygen to the alcohol molecule, thus:

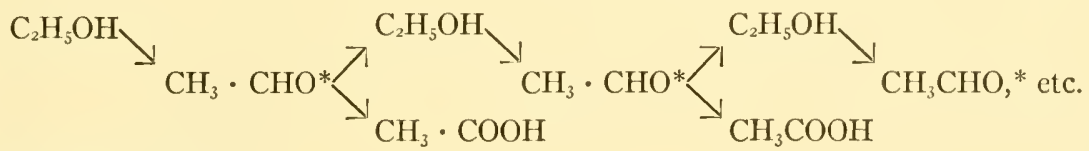

${ }^{*}$ Cannizzaro reaction.

It is of great interest to recall that Liebig, ${ }^{5}$ over fifty years ago, showed that the change of alcohol to acetic acid took place in two steps: (I) removal of hydrogen from the ethyl alcohol molecule to form acetaldehyde, and (2) the addition of oxygen to the acetaldehyde molecule to form acetic acid.

Glycerol fermentation.-Glycerol is fermented by many bacteria; some, like the Shiga type of dysentery bacillus, produce acid from this triatomic alcohol, while many members of the mucosus capsulatus group of bacteria evolve gas during its fermentation. Very little is known as yet of the chemical change involved.

Voges-Proskauer reaction.--The three equations discussed above represent, as well as available information affords, the course of fermentation of simple sugars by the majority of ordinary bacteria, with and without gas production. Nevertheless, they apparently do not provide a complete summary of the products of fermentation

I Frankland, P. F., and Frew, W.: loc. cit.

2 Pasteur, L.: loc. cit.

3 Neuberg, C., and Arinstein, B.: Biochem. Ztschr., I 17, 269. I921.

${ }^{4}$ Neuberg, C., and Windisch, F.: Biochem. Ztschr., 166, 454. 1925.

5 Quoted by Hofmann, A. W.: Faraday Lecture for 1875 , D. I I2. Macmillan and Co., I876. 
produced by some few microbes. Voges and Proskauer $^{1}$ described a color reaction that could be elicited in glucose cultures of certain bacteria by the addition of enough caustic potash solution to render the reaction of the medium very alkaline. Ordinarily, the color develops slowly in fermentation media, requiring from twenty-four to forty-eight hours to reach its maximum. The color thus evolved was reminiscent of the yellowish fluorescence exhibited by alcoholic solutions of eosin.

Harden and Walpole ${ }^{2}$ studied the reaction chemically and found acetylmethylcarbinol $\left(\mathrm{CH}_{3} \mathrm{CO} \cdot \mathrm{CHOH} \cdot \mathrm{CH}_{3}\right)$ was present in such media. They discovered, furthermore, that this compound would not give the color with caustic potash, but that when the oxidization product of acetylmethylcarbinol, diacetyl $\left(\mathrm{CH}_{3} \cdot \mathrm{CO} \cdot \mathrm{CO} \cdot \mathrm{CH}_{3}\right)$ was obtained it reacted with some unknown constituent of peptone in the presence of caustic potash and gave the color very quickly and intensely. The steps involved seem to be:

I $\cdot \mathrm{C}_{6} \mathrm{H}_{12} \mathrm{O}_{6} \rightarrow \mathrm{CH}_{3} \cdot \mathrm{CO} \cdot \mathrm{CHOH} \cdot \mathrm{CH}_{3}$,

(Glucose) (Acetylmethylcarbinol)

$2 \cdot \mathrm{CH}_{3} \cdot \mathrm{CO} \cdot \mathrm{CHOH} \cdot \mathrm{CH}_{3} \rightarrow \mathrm{CH}_{3} \cdot \mathrm{CO} \cdot \mathrm{CO} \cdot \mathrm{CH}_{3}$,

(Diacetyl)

$3 \cdot \mathrm{CH}_{3} \cdot \mathrm{CO} \cdot \mathrm{CO} \cdot \mathrm{CH}_{3}+$ Peptone $+\mathrm{KOH} \rightarrow$ Voges-Proskauer reaction .

Grimbert ${ }^{3}$ found that certain spore-forming bacteria, as Bacillus subtilis and Bacillus mesentericus, gave this Voges-Proskauer reaction, and MacConkey ${ }^{4}$ added Bacillus lactis aerogenes, Bacillus cloacae, and certain members of the Bacillus mucosus capsulatus group to the list. Most strains of Bacillus coli do not give it. ${ }^{5}$

The reaction seems to be elicited quite definitely in cultures of certain bacteria which give a negative methyl-red reaction ${ }^{6}$ (low $\mathrm{H}$-ion concentration) and a gas ratio of $\frac{\mathrm{CO}_{2}}{\mathrm{H}_{2}}=\frac{2-3}{\mathrm{I}}$. This includes the lactis aerogenes and mucosus capsulatus groups of bacteria in addition to Bacillus cloacae. ${ }^{7}$

Miscellaneous fermentations.-A considerable number of fermentations induced by bacteria that have significance in the industries and agriculture have been reported. Some of these, as the organisms which produce mannitol, together with lactic and acetic acids, and carbon dioxide from fructose ${ }^{8}$ are important not only from the viewpoint of their relation to the wine, sauerkraut, and other similar industries, but also because they provide a starting-point for the study of biologically induced

${ }^{1}$ Voges, O., and Proskauer, B.: Ztschr. f. IIyg. u. Infektionskrankh., 28, 20. I898.

${ }^{2}$ Harden, A., and Walpole, S. G.: Proc. Roy. Soc. (Ser. 13), 77, 399. I906; Harden, A.: ibid., p. 424.1906 .

3 Grimbert, L.: Compt. rend. Soc. de biol., 53, 304. rgor.

${ }^{4}$ MacConkey, A.: J. II yg., 5, 349. I905.

${ }^{5}$ Levine, M.: Official Publicution, Iowa State College Agricult. Eo Mech. Arts, 15, No. I4. I9I6.

${ }^{6}$ Rogers, L. A., Clark, W. M., and Evans, A. C.: J. Infect. Dis., 15, 100. 1914; 17, 137. I915: Perkins, R.: ibid., 37, 232. 1925; Paine, I. S.: J. Bacl., 13, 269. 1927.

7 MacConkey, A.: loc. cil.

${ }^{8}$ See Stiles, H. R., Peterson, W. II., and Fred, E. B.: J. Biol. Chem., 64, 643. I925. 
Iransformations in the group of the hexoses and related compounds. Others, as Bacillus granulobacter pectinovorum, are noteworthy because they are used on a large scale to produce acetone and butyl alcohol and other solvents which are indispensable to industry." 'The consideration of these, and other technical fermentation procedures, however, is beyond the scope of this discussion.

\section{BACTERIAL SYNERGISM}

Ten years ago Castellani and 'Taylor ${ }^{2}$ called attention to a phenomenon, which, while it had undoubtedly been noticed before, had not apparently received the attention it deserved. This phenomenon has recently been stated by Fiallos ${ }^{3}$ as follows: "Two bacilli, neither of which causes the production of gas in certain compounds, may do so when artificially mixed together, provided one of them is capable of producing simple acidity (never gas) in these compounds, and the other, though inert to these compounds (i.e., produces neither acid nor gas), is capable of producing gas from glucose." Many observers have studied this phenomenon recently and have amply confirmed the facts, but the explanation is not agreed upon; also, several different types of chemical activity are included in this group of reactions. The most common of these is the one described by Fiallos. ${ }^{3}$ It is quite clear that all of the phenomena thus far discovered are of the nature of coupled reactions.

The following specific instance may be mentioned: a staphylococcus and Bacillus paratyphosus acting together produce acid and gas from nutrient lactose-fermentation media, although the staphylococcus alone merely produces acid, but no gas from this sugar, and Bacillus paratyphosus fails to utilize the sugar at all. Examples might be multiplied almost indefinitely, but in each instance it will be found that one of the coupled reacting organisms must produce acid but no gas from the carbohydrate; the other coupled organism need have no action whatsoever upon the carbohydrate but must be capable of producing $\mathrm{CO}_{2}$ and $\mathrm{H}_{2}$ from formates. All the known bacteria that liberate $\mathrm{CO}_{2}$ and $\mathrm{H}_{2}$ from formates will also produce gas and acid in the usual nutrient glucose media. The addition of nitrates (sodium or potassium nitrate) in small amounts will prevent the formation of gas either in glucose solution or in formate solution.

Pakes and Jollymans long ago showed that bacteria which produce gas from nutrient sodium-formate media also produce gas from nutrient glucose solutions with the exception of many, if not most, yeasts; they also showed the converse to be true -namely, that bacteria which do not produce gas from formate do not produce gas from glucose. They also made extensive studies of the effect of nitrates upon the reaction and found that the nitrates interfere with gas production in accordance with the following equation:

$$
\mathrm{H} \cdot \mathrm{COONa}+\mathrm{NaNO}_{3}=\mathrm{NaHCO}_{3}+\mathrm{NaNO}_{2} .
$$

'See, for details, Fred, E. B., Peterson, W. H., and Mulvania, M.: J. Bact., I I, 323. I926.

${ }^{2}$ Castellani, A., and Taylor, F. E.: Brit. M. J., 2, 855. I9I7; I, I83. I919.

${ }^{3}$ Fiallos, J. M.: J. Trop. Met., 28, 426 . 1925.

4 Much of the later literature is reviewed by Holman, W. L.: J. Infect. Dis., 39, 1 f5. I926. He proposed the term "synergism." Cf. chap. viii in this volume.

5 Pakes, W. C. C., and Jollyman, IV. H.: J. Chem. Soc., 79, 386, 450. I9or. 
It is by no means definitely proved that the mechanism of the reaction in every instance involves the formation of formic acid by the fermenting member, but this certainly happens in at least some of the synergetic couples tried thus far: in fact, formic acid has been separated and measured with considerable precision from several of these synergetic reactions.

This reaction has significance in several fields of bacteriology: It is of prime importance, as Sears and Putnam pointed out ${ }^{\mathrm{I}}$ in the "presumptive test" for $B$. coli in water. It has an important bearing upon the gas formation by intestinal bacteria, and it occasionally leads to false conclusions with respect to gas formation in contaminated and in mixed cultures.

Another type of coupled reaction is one described by $\mathrm{Kendall}^{2}$ in relation to gas formation in milk by $B$. coli and an associated, actively proteolytic organism. Under normal conditions $B$. coli does not produce gas in milk, although this medium is rich in lactose, but does ferment lactose energetically in nutrient fermentation media with a considerable evolution of gas: If, however, $B$. coli be inoculated into milk together with an actively growing, strongly proteolytic strain of microbe which does not ferment lactose, as, for example, B. mesentericus, gas is formed in considerable amounts.

Here the explanation seems to depend upon the cleavage of the casein by the proteolyte with the liberation of amino acid complexes. These in turn, in some manner not well understood, cause the colon bacillus to liberate gas in addition to the acid which it normally produces in milk. Similar results may be obtained by digesting the milk prior to inoculation, with trypsin or by repeated heating of the milk, which of course tends to break down the protein, and thus accomplish the same end as the cleavage by a proteolyte.

Coupled reactions are as yet but little studied, but there is little doubt that some very important results await discovery in this field of bacterial synergism.

${ }^{x}$ Sears, H. J., and Putnam, J. J.: J. Infect. Dis., 32, 270. 1923.

${ }^{2}$ Kendall, A. I.: Boston M. \& S. J., 163, 322. 1910. 


\title{
CHAPTER XVII
}

\section{UTILIZATION OF ALIPHATIC AND AROMATIC COMPOUNDS BY BACTERIA}

\author{
S. A. KOSER \\ University of Illinois
}

Investigations of the carbon and nitrogen requirements of bacteria have directed attention to the relatively simple compounds of these elements which in many cases appear to be readily utilized by the organisms, both as a source of structural material for building new protoplasm and also as a source of energy. The utilization of the commoner carbohydrates which are widely used in the ordinary fermentation tests is well known. In addition to these, however, there are many other chemical groups which are susceptible to bacterial attack. In the amino acids, the purines and extractives, the alcohols, and the organic acids or their salts, we have a wide range of compounds of definite chemical structure in which the nitrogen or carbon is supplied in a great variety of different combinations.

The utilization of these compounds has been studied in several ways, by qualitative or quantitative tests for a certain component of a complex medium and by the use of isolated compounds in chemically definite media. In the latter case an amino acid, for example, may be added to a solution of certain inorganic salts so that it constitutes the sole supply of available nitrogen. By varying the amino acids or other nitrogenous compounds we can determine the ability of the organism to make use of certain chemical groupings. Also, by analysis of cultures developing under these conditions we can determine the products formed and thereby gain an insight into the mode of attack and breakdown which the organism employs. It does not necessarily follow, of course, that the same type of decomposition will take place under other conditions or in the presence of other foodstuffs.

\section{UTILIZATION OF NITROGENOUS COMPOUNDS}

The common observation that most bacteria can develop readily in digests of protein material has led to investigation of the availability of single, isolated amino acids or other simple compounds of nitrogen. Added impetus has perhaps been given to this line of study by the studies on animal nutrition in which it has been shown that certain amino acids are indispensable for growth. In considering the nitrogen requirements of bacteria the question arose whether certain amino acids are also indispensable for the growth and reproduction of these forms. Can the inability of certain pathogens to develop on ordinary media be explained by the lack of certain essential components of nitrogen which are not supplied by the usual laboratory media? May different species or groups of bacteria be distinguished from one another by the ability of one to utilize certain simple compounds which the other is unable to attack? Can the various toxin-producing types make use of simple amino acids and if so is the toxin elaborated in their presence? 
Investigations along this line have been many and varied, and the results at times have been contradictory.' In spite of this, however, certain general conclusions may be drawn. A number of the commoner saprophytic types, B. fluorescens, B. pyocyaneus, $B$. prodigiosus, $B$. protcus, $B$. coli, and probably others, are able to satisfy their nitrogen and carbon requirements when a single mono-amino acid such as alanine is added to a solution of certain inorganic salts, though the development under such conditions will not be as rapid or Iuxuriant as in nutrient broth. If a readily available source of carbon such as lactic acid, glycerol, or dextrose is also supplied, the value of the medium is enhanced and certain organisms which experienced difficulty in appropriating the amino acid for their own uses will speedily break down these more readily available sources of carbon and energy, and at the same time derive their nitrogen from the amino acid. A surprising number of organisms are able to develop under such conditions. Among the better-known forms are the green fluorescent bacilli, $B$. pyocyaneus, B. prodigiosus, B. proteus, the coli-aerogenes group, certain members of the paratyphoid group, and a few spirilla. The tubercle bacillus and other acid fast types are also able to satisfy their nutritive requirements from very simple sources of nitrogen and carbon. ${ }^{2}$ Most of the pathogens, however, are unable to develop under these conditions, and negative results have usually been reported for the typhoid and dysentery bacilli, B. abortus, B. diphtheriae, the pneumococcus, streptococci, and staphylococci of various types, to mention only some of the commoner ones.

The question of the relative values of the various amino acids may be regarded as still an open one, for there is much conflicting evidence. In many instances an organism, if able to satisfy its nitrogen requirements from one of the mono-amino acids, may be expected to make use of the others, although exceptions to this have been reported. Lysine, arginine, histidine, and tryptophane are other amino acids which offer additional nitrogenous groupings. It has been claimed that they possess an added nutritive value in some instances while in others they appear to be of no more value than the simpler mono-amino acids. Dipeptides, also, are susceptible to bacterial attack ${ }^{3}$. Glycyl-glycine, glycyl-tyrosine, glycyl-tryptophane, and other combinations are split by a number of organisms, and the separate amino acids may be recovered if they are not in turn utilized. Finally, in considering the utilization of amino acids or related compounds it should not be overlooked that at least certain of them have been found to exert a toxic or inhibitory effect upon the growth of some of the more "fastidious" bacteria. ${ }^{4}$

The mode of attack and the steps in the breakdown of the amino acids present an interesting though a complex problem, for we may have both nitrogen and carbon

${ }^{x}$ For reviews of earlier work and bibliography see Gordon, M. H.: J. Roy. Army M. Corps, 28, 37r. r9г7; Koser, S. A., and Rettger, L. F.: J. Infecl. Dis., 24, 3оr. r919; Braun, H., and CahnBronner, C. E.: Cenlralbl.f. Bakleriol., Abt. I, Orig., 86, 196 and 380. 1921; Krasnow, F., Rivkin, H., and Rosenberg, M. L.: J. Bact., I2, 385. 1926; den Dooren de Jong, L. E.: Bijdrage tol de kennis van het mincralisatieproces, PP. I-200. Rotterdam: Nijgh and Van 1)itmar, 1926.

2 Long, E. R.: Am. Rev. Tuberc., 3, 86. 1919; 5, 857. I922.

3 Sasaki, T.: Biochem. Zlschr., 4I, I74; 47, 463 and 472. I9r2; Otsuka, I.: Physiol. Abst., 2, 15. 1917 .

${ }^{4}$ Gordon, J., and McLeod, J. W.: J. Puth. \& Bact., 29, I3. 1926. 
supplied in many different groupings. In addition, various methods of decomposition are doubtless brought into play by different organisms and certain other factors exert a pronounced influence upon the process, such as the supply of oxygen, the tonicity of the medium, and the presence of other available nitrogenous and non-nitrogenous compounds.

The initial step in the process of utilization is usually a deamination or decarboxylation. Deamination may be accomplished in a variety of ways: by reduction with the formation of a saturated fatty acid, by oxidation with the formation of a ketone acid, and by hydrolysis with the formation of a hydroxy acid. A simple monoamino acid such as alanine may be broken down as follows:

$$
\begin{aligned}
& \mathrm{CH}_{3} \cdot \mathrm{CH} \cdot \mathrm{NH}_{2} \cdot \mathrm{COOH}+\mathrm{H}_{2} \ldots \mathrm{CH}_{3} \cdot \mathrm{CH}_{2} \cdot \mathrm{COOH}+\mathrm{NH}_{3} \\
& \text { Propionic acid } \\
& \mathrm{CH}_{3} \cdot \mathrm{CH} \cdot \mathrm{NH}_{2} \cdot \mathrm{COOH}+\mathrm{O} \ldots \mathrm{CH}_{3} \cdot \mathrm{CO} \cdot \mathrm{COOH}+\mathrm{NH}_{3} \\
& \text { Pyruvic acid } \\
& \mathrm{CH}_{3} \cdot \mathrm{CH} \cdot \mathrm{NH}_{2} \cdot \mathrm{COOH}+\mathrm{H}_{2} \mathrm{O} \ldots \mathrm{CH}_{3} \cdot \mathrm{CHOH} \cdot \mathrm{COOH}+\mathrm{NH}_{3} \\
& \text { Lactic acid }
\end{aligned}
$$

The ring structures such as those found in tyrosine, proline, histidine, and tryptophane, though not invulnerable, are more resistant to bacterial attack, and in many instances only the side chain is used. In tryptophane, for example, we have nitrogen in the alanine side chain and in the indol ring. The side chain is utilized by many organisms while the ring structure is frequently left intact in the form of indol-propionic or -acetic acid, skatol, or indol. Another type of change has been described by Raistrick, ${ }^{2}$ who found that histidine was converted to urocanic acid, an unsaturated acid, by a number of organisms.

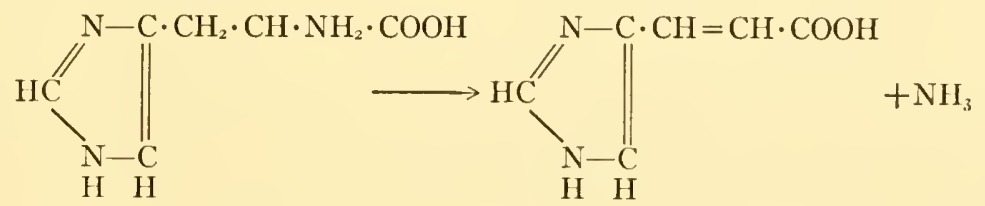

Decarboxylation of an amino acid results in the formation of an amine by the loss of $\mathrm{CO}_{2}$ :

$$
\mathrm{CH}_{3} \cdot \mathrm{CH} \cdot \mathrm{NH}_{2} \cdot \mathrm{COOH} \longrightarrow \underset{\text { Ethyl amine }}{\mathrm{CH}_{3} \cdot \mathrm{CH}_{2} \cdot \mathrm{NH}_{2}+\mathrm{CO}_{2} .}
$$

The ethyl amine formed from alanine may in turn be hydrolyzed to form ethyl alcohol and ammonia:

$$
\mathrm{CH}_{3} \cdot \mathrm{CH}_{2} \cdot \mathrm{NH}_{2}+\mathrm{H}_{2} \mathrm{O} \longrightarrow \mathrm{CH}_{3} \cdot \mathrm{CH}_{2} \mathrm{OH}+\mathrm{NH}_{3} \text {. }
$$

Other amino acids may be changed in a similar way. Histidine is converted into histamine, lysine yields cadaverine, and tyrosine yields tyramine. ${ }^{3}$ It is of special in-

I Raistrick, H.: Biochem. J., 13, 446. I919; Raistrick, H., and Clark, A. B.: ibid., 15, 76. I92r; Hanke, M. T., and Koessler, K. K.: J. Biol. Chem., 50, 131. 1922; Long, E. R.: Am. Rev. Tuberc., 5, 857.1922 .

${ }^{2}$ Raistrick, H.: Biochem. J., II, 71. I9I7.

${ }^{3}$ Hanke, M. T., and Koessler, K. K.: J. Biol. Chem., 39, 539. 1919; 50, 131, 1922; 59, 835. I924. 
terest that certain of these amines are significant from the pharmacological standpoint.

In addition to the amino acids, other compounds may be considered briefly. The purines and their oxidation products, the pyrimidines such as cytosine, uracil, and thymine, the extractive bodies, creatine, creatinine, carnosine, and others present another series of nitrogenous compounds. The utilization of these bodies by various types of micro-organisms has not been studied so extensively as that of the amino acids, and our knowledge concerning them is rather fragmentary. Hypoxanthine, xanthine, and uric acid contain nitrogen only in the purine ring. Evidently nitrogen in such form is of no value to a great many organisms, for many of those which are able to make use of isolated amino acids are unable to develop when the foregoing purines are supplied as the sole source' of nitrogen. On the other hand, allantoin, which is an oxidation product of uric acid and contains a free amino group, frequently yields results quite similar to an amino acid. ${ }^{\mathrm{x}}$ In a similar way, it might be expected that the amino purines, adenine and guanine, could be utilized by a number of organisms which are unable to make use of nitrogen in the purine ring.

\section{NON-NITROGENOUS COMPOUNDS}

Of the non-nitrogenous organic compounds which are used by micro-organisms the best known are the carbohydrates, higher alcohols, and organic acids. Only the last group will be dealt with here since the others are treated elsewhere in this volume ${ }^{2}$. The structure of some of the commoner organic acids is shown below: Here we have

$\begin{array}{ll}\text { Formic } & \mathrm{H} \cdot \mathrm{COOH} \\ \text { Acetic } & \mathrm{CH}_{3} \cdot \mathrm{COOH} \\ \text { Propionic } & \mathrm{CH}_{3} \cdot \mathrm{CH}_{2} \cdot \mathrm{COOH} \\ \text { n-Butyric } & \mathrm{CH}_{3} \cdot\left(\mathrm{CH}_{2}\right)_{2} \cdot \mathrm{COO} \\ \text { Glycollic } & \mathrm{CH}_{2} \mathrm{OH} \cdot \mathrm{COOH} \\ \text { Lactic } & \mathrm{CH}_{3} \cdot \mathrm{CHOH} \cdot \mathrm{COOH} \\ & \\ \text { Pyruvic } & \mathrm{CH}_{3} \cdot \mathrm{CO} \cdot \mathrm{COOH} \\ \text { Oxalic } \quad & \mathrm{COOH}^{\mid} \\ & \mathrm{COOH} \\ & / \mathrm{COOH} \\ \text { Malonic } & \mathrm{CH}_{2} \\ & \backslash \mathrm{COOH}\end{array}$

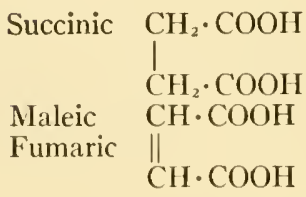

${ }^{3}$ Koser, S. A., and Rettger, L. F.: loc. cit.
Malic

$\mathrm{CHOH} \cdot \mathrm{COOH}$<smiles>[CH]C(=O)O</smiles>

Tartaric

$\mathrm{CHOH} \cdot \mathrm{COOH}$

$\mathrm{CHOH} \cdot \mathrm{COOH}$

Glutaric

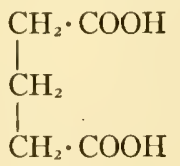

Adipic<smiles>O=C(O)CCCCC(=O)O</smiles>

Citric

$\mathrm{CH}_{2} \cdot \mathrm{COOH}$<smiles>CC(CC(=O)O)C(=O)O</smiles>

Benzoic Salicylic
$\mathrm{C}_{6} \mathrm{H}_{5} \cdot \mathrm{COOH}$

$\mathrm{C}_{6} \mathrm{H}_{4}(\mathrm{OH}) \cdot \mathrm{COOH}$

2 Chapter xvi. 
the fatty-acid series and a few of their hydroxy derivatives, some members of the dicarboxylic and tricarboxylic series, and aromatic acids containing the phenyl radical. The outline will serve to show the wide variety of chemical groupings which are presented.

In the presence of a suitable source of nitrogen many of these acids or their salts are broken down and utilized, though a few appear to be relatively resistant to bacterial attack. Benzoic and salicylic acids, in which the carbon is contained in the carboxyl group and the benzene ring, appear to present a formidable obstacle to most organisms, and very few types are able to make use of them. Oxalic acid with two carboxyl groups joined directly is also resistant to most bacteria. Whether this is due to an inability of the organism to make use of this type of structure or whether it is due to the poisonous character of the compound is not clear. When the two carboxyls are linked by a methyl group, as in malonic acid, the compound is attacked by certain organisms which are unable to make use of oxalic acid, for example, some members of the alcaligenes group and the coli-aerogenes group. Succinic acid appears to lend itself still more readily to bacterial attack, surprising as this may seem, for it is relatively resistant to chemical oxidizing agents. Many of the other acids are readily utilized by the commoner saprophytic bacteria and molds, and in some cases by pathogenic types. ${ }^{x}$ Lactic acid, for example, when supplied as the only source of carbon and with a suitable inorganic nitrogen compound will support development of the coli-aerogenes group, the alcaligenes group, the green fluorescent bacilli, and even certain members of the paratyphoid group.

A comparison of the structural formulas of these acids with their utilization by various types or groups of organisms presents an interesting field though it is impossible to review the results at length here. Suffice it to say that a slight change in the structure of a compound or even the presentation of a different isomer may alter materially its availability for certain organisms though perhaps not for others. Furthermore, we are not always able to predict the utilization of a compound by micro-organisms from a consideration of their behavior toward a similar chemical grouping in another compound.

As might be expected, the utilization of organic acid salts is influenced markedly by the oxygen supply. Under aerobic conditions many bacteria will develop in a much simpler medium than they require for anaerobic life. Coliform organisms can develop in a simple inorganic salt medium with ammonium lactate under aerobic conditions, while under anaerobic conditions other substances such as a fermentable sugar are required. Supposedly, the sugar is required for anaerobic growth because it can be disintegrated without the intervention of oxygen in such a way as to supply energy, while this is not possible with salts of lactic acid. ${ }^{2}$

In the breakdown of the organic acids akaline end-products are frequently formed. These are usually carbonates and bicarbonates. The decomposition of the

I den Dooren de Jong, L. F.: loc. cil.; Ayers, S. H., Rupp, P., and Johnson, IV. T.: U.S. Dept. Agr., Bull. 782. roig; Koser, S. A.: J. Bact., 8, 493. i923; Brown, H. C., Duncan, J. T., and Henry, T. A.: J. Hyg., 23, I. I924.

${ }^{2}$ Braun, H., and Cahn-Bronner, C. F.. loc. cit.; Stephenson, M., and Whetham, M. D.: Biochem. J., 18, 49S. 1924; Quastel, J. H., and Stephenson, M.: ibid., r9, 660. I925. 
calcium and sodium salts of formic acid represents a simple case and may be shown as follows:

$$
\begin{aligned}
& \mathrm{Ca}(\mathrm{COOH})_{2}+\mathrm{H}_{2} \mathrm{O}=\mathrm{CaCO}_{3}+\mathrm{CO}_{2}+{ }_{2} \mathrm{H}_{2}, \\
& \mathrm{Na}(\mathrm{COOH})+\mathrm{H}_{2} \mathrm{O}=\mathrm{NaHCO}_{3}+\mathrm{H}_{2} .
\end{aligned}
$$

The manner of decomposition of other organic acids has been studied in some instances. A review of much of the older work is given by Harden. ${ }^{\mathrm{r}}$ More recently the decomposition of citric acid has been studied by Brown, Duncan, and Henry, ${ }^{2}$ and fumaric, succinic, and pyruvic acids by Aubel ${ }^{3}$ and Quastel. ${ }^{4}$ One example of the type of breakdown accomplished by bacteria may be given, namely, the decomposition of fumaric acid, an unsaturated dicarboxylic acid, by $B$. pyocyaneus. ${ }^{5}$ The main course of fermentation is believed to proceed as follows with acetic acid and carbon dioxide as the final products:

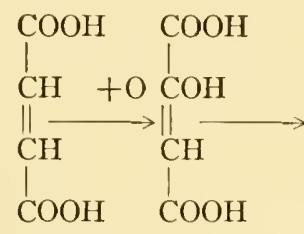

Fumaric
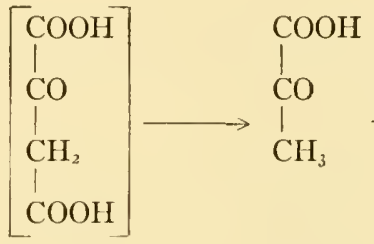

Pyruvic

It has been suggested that the utilization of certain of the simpler aliphatic or aromatic compounds might be used as a basis for the separation of different species or groups of bacteria. A few of the more recent suggestions will serve to illustrate the point. In a study of the alcaligenes group, Ayers, Rupp, and Johnson ${ }^{6}$ used organic acid salts as test substances instead of the usual carbohydrates which in many instances are not attacked by this group of bacteria. It has been further shown by Ayers and Rupp ${ }^{7}$ that sodium hippurate is hydrolyzed to benzoic acid and glycocoll by hemolytic streptococci of bovine origin but not by those of human origin.

The availability of uric acid for certain members of the coli-aerogenes group has been used to distinguish the coli from the aerogenes-cloacae subgroup. ${ }^{8}$ When given this compound as the only source of nitrogen, B. aerogenes and its allies are able to split the purine ring whereas $B$. coli lacks this ability. A somewhat similar distinction has also been made, based on the utilization of citric acid by this group of organisms.? When this acid in the form of its sodium, potassium, or ammonium salt is supplied as the sole source of carbon, fecal strains of $B$. col $i$ are unable to develop. They apparently lack the ability to make use of the citrate radical while closely related types of soil

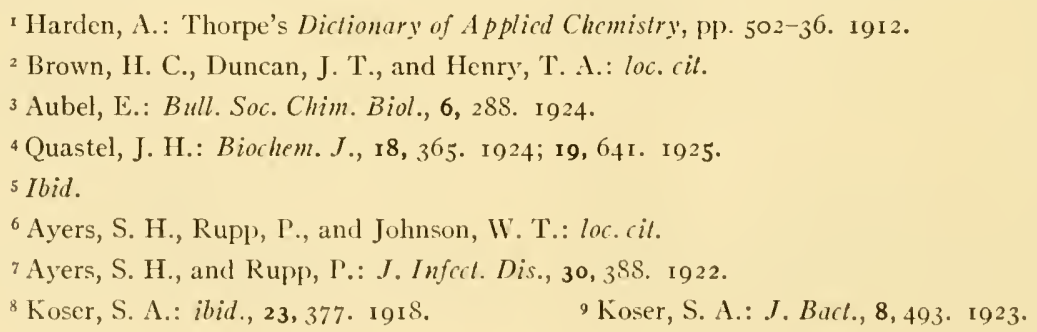


origin - aerogenes and others - are able to break down the citrate and to appropriate it for their own needs. The salts of organic acids have also been suggested for separating different types within the paratyphoid group. By the use of citrate, d-, l-, and $\mathrm{m}$-tartrate, fumarate and mucate, certain distinctions are brought out. ${ }^{\mathrm{I}}$ These may be especially useful in this or other groups of organisms where sugar fermentations at times fail to differentiate certain serologically well-defined types.

A consideration of the salient points in the utilization of aliphatic and aromatic compounds brings out in a striking manner the ability of many bacteria to satisfy their food and energy requirements from the simpler chemical compounds. Many bacteria, like plants, are able to build up their own protoplasmic structure from a variety of non-nitrogenous organic substances and ammonia. One is impressed by the wide range of chemical groupings which may be broken down and appropriated by the micro-organisms for their own needs. Nitrogen may be utilized in various forms: as an inorganic ammonium compound and in the amino, amide, or imino groups. Particularly striking is the ability of some organisms to tear apart complex ring structures such as the imidazole or purine rings. Others apparently are not so well equipped with the necessary tools for making use of such structures and are compelled to satisfy their needs by the utilization of a side chain while the ring structure is left intact. Whatever the form of nitrogenous organic grouping attacked, ammonia is usually liberated. After separation of the nitrogen in this form the carbonaceous residue, an organic acid or perhaps an alcohol, may be further used by the organism. It may be oxidized to supply energy, or perhaps rearranged and combined with ammonia for synthesis of the organism's own particular protoplasm, or if a more readily available source of carbon is present to satisfy these purposes, it may remain as an end-product of metabolism.

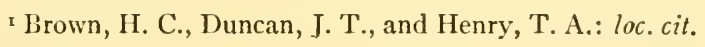




\title{
CHAPTER XVIII
}

\section{GAS METABOLISM OF BACTERIA}

\author{
M. H. SOULE \\ Hygienic Laboratory, University of Michigan
}

INTRODUCTION

It is questionable whether any gas other than oxygen is absolutely necessary for the activities of the cell. The fixation of atmospheric $\mathrm{N}_{2}$, the oxidation of $\mathrm{H}_{2} \mathrm{~S}$ to $\mathrm{S}$ and $\mathrm{H}_{2} \mathrm{O}$, etc., are activities independent of the main function of respiration. The working hypothesis has been laid down that $\mathrm{CO}_{2}$ is essential as a stimulus for the growth of organisms. It will be shown later that this hypothesis probably holds true for all organisms just as this gas is required for the life of the higher animals. It is not utilized as a source of food or energy but it maintains a physico-chemical equilibrium within the cell.

The study of gas changes produced by micro-organisms is of rather recent date. The combustion theory of respiration initiated by Lavoisier ${ }^{\mathrm{I}}$ was extended to all kinds of animals by Spallanzani, ${ }^{2}$ and was developed by Pasteur (I859, I86 I ff.) in his work on fermentations. This was followed by many isolated studies such as those of Buch$n^{3}{ }^{3}$ on the Fitz bacillus and of Escherich ${ }^{4}$ on B. coli and B. lactis aerogenes. Hesse ${ }^{3}$ demonstrated $\mathrm{CO}_{2}$ production and $\mathrm{O}_{2}$ absorption by eight organisms which were not of the aerogenic type. These results were questioned at first by Scheurlen, ${ }^{6}$ who denied that bacteria, like animals, could respire and, with Buchner, he thought that $\mathrm{CO}_{2}$ was the result of the action of acids on the carbonates of the medium. Eventually, however, he confirmed Hesse's work by finding that every one of one hundred and forty-one strains of bacteria which he examined produced $\mathrm{CO}_{2}$. In the meantime, Winogradsky $y^{7}$ had conclusively proved that certain bacteria fix atmospheric nitrogen - a fact indicated many years before by the work of Berthelot ${ }^{8}$ on soils. The presence of $\mathrm{CH}_{4}, \mathrm{~N}_{2}, \mathrm{H}_{2}$, and $\mathrm{H}_{2} \mathrm{~S}$ in intestinal gases had been reported by various observers as mentioned by Escherich, ${ }^{9}$ but exact quantitative determinations of the gaseous metabolism of the organisms producing these substances have not been made.

The early methods employed in the study of gas changes by bacteria were some-

r Lavoisier, A. L.: Hist. et Mém. de l'Acad. de sc. Paris, 1780; Mém., Année, p. I85. 1777.

${ }^{2}$ Spallanzani, L.: Disscrtazioni Varie, Mémorie sulla Respirazione, Vol. 2. I\$26.

3 Buchner, E.: Ztschr. f. phys. Chomie, 8, 367-90. I884.

${ }_{4}$ Escherich, T.: Die Darmbakterien des Säuglings und ihre Bezichungen zur Physiologic der Verdanung, pp. I28-33. Stuttgart: Enke, I886.

${ }^{5}$ Hesse, W.: Ztschr. f. Hyg. u. Infcktionskrankh., 15, 17-37; 183-91. 1893; 25, 477-81. 1897; Arch.f. IIyg., 28, 307-11. I897.

${ }^{6}$ Scheurlen, E.: Arch.f. IIyg., 26, I-29. I896; Intcrnat. Bcitr. 2; inn. Med. zur Feicr 70 jährigen Gcburtslages E. von Leyden, 2, 205-7. Berlin: Hirschwald, 1900.

7 Winogradsky, S.: Compt. rend. Acad. Sc., 116, 1385. 1893.

${ }^{8}$ Berthelot, M.: ibid., 85,178 . I 877 .

9 Escherich, T.: loc. cil. 
what complex and often gave very inexact results. In I9I3 the literature was reviewed in detail by Frieber ${ }^{1}$ and, during the following year, by Rogers, Clark, and Davis ${ }^{2}$ who contributed a valuable supplement to Frieber's study; in both papers the untrustworthy nature of the recorded bacteriological gas analyses was deplored and new methods were suggested. In each of these investigations the metabolism of $B$. coli was studied. This germ, ordinarily considered an aerobe, was grown in broth, in vacuo, under anaerobic conditions, and the gases formed were analyzed. Anderson ${ }^{3}$ modified the technique of Rogers and his co-workers and made accurate quantitative estimations of the gaseous components evolved by several strict anaerobes. The procedure at best has a very limited application.

\section{METHODS}

As already stated, the methods which have been employed in the study of gas changes by bacteria as a rule have been somewhat complex. It is beyond the scope of this article to review these earlier investigations on gas metabolism. The recent studies of Novy, Roehm and Soulet have resulted in methods which are of general applicability. They are equally suitable for the study of the respiration of organisms when grown on solid or liquid media, in tubes, flasks, or on plates, either in an atmosphere of air or in varying concentrations of $\mathrm{O}_{2}, \mathrm{CO}_{2}, \mathrm{~N}_{2}$, or other gases. The technique not only permits of accurate manometric observations over extended periods of time, but also allows the easy withdrawal of samples of the contained gases for the purpose of analysis. These methods have been utilized in the study of a large number of organisms, including various protozoa as well as plant tissue. For full details the reader is referred to the original memoirs.

It is essential in the study of gases to be able to observe the pressure changes which take place within the respiratory chamber. A manometer usually not only reveals whether an organism is alive and growing, but also indicates the point when growth or respiration ceases. Some investigators have made use of ordinary manometers attached to their culture flasks. The instrument devised by Barcroft ${ }^{3}$ is well known and, as modified by Brodie, ${ }^{6}$ has been used for diverse respiration studies such as those on excised tissues by Warburg ${ }^{7}$ and others.

It is desirable to have a manometer which is highly sensitive and yet independent of variations in atmospheric pressure. These conditions are realized in the compensation manometer. This instrument is shown in Figure $\mathbf{I}$, attached to a glass-capped $h$-tube of about roo-cc. capacity; in Figure 2 it is connected to a Novy jar. For details of construction and calibration the original paper should be consulted.

When an experiment is in progress the apparatus is kept at constant temperature and cocks $I$ and 3 on the manometer remain closed. The system is not subject, therefore, to changes in barometric pressure, and any variation in the mercury level is due entirely to the gas exchange of the germs. This is extremely important when an experiment extends over a period of time.

The purpose of stopcock 2 is twofold: (r) to shut off the manometer when the gas pres-

I Frieber, W.: Centralbl. f. Bakteriol., Abt. I, Orig., 69, 437. I9I3.

${ }^{2}$ Rogers, L. A., Clark, W. M., and Davis, B. J.: J. Infect. Dis., 14, 41 I-75. I9I4.

3 Anderson, B. G.: ibid., 35, 213-8I. I924.

${ }_{4}^{4}$ Novy, F. G., Roehm, H. R., and Soule, M. H.: ibid., 36, 109-67. 1925.

${ }_{5}$ Barcroft, J.: Ergebn. der Physiol., 7, 772-75. I908.

${ }^{6}$ Brodie, T. G.: J. Physiol., 39, 391-96. 1909-10.

7 Warburg, O.: Biochem. Ztschr., 100, 230-70. 1919; 1923; 1924. 


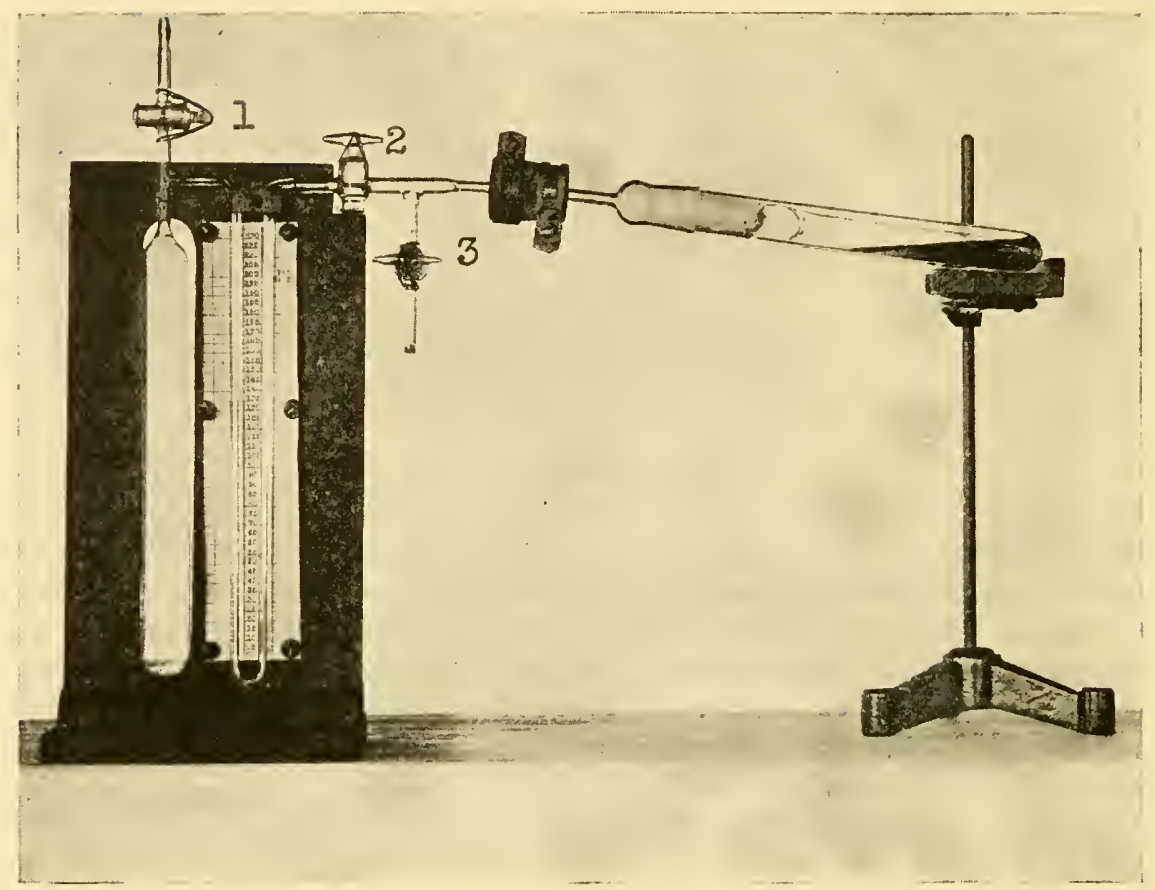

FIG. I.-Manometer and support with $h$-tube

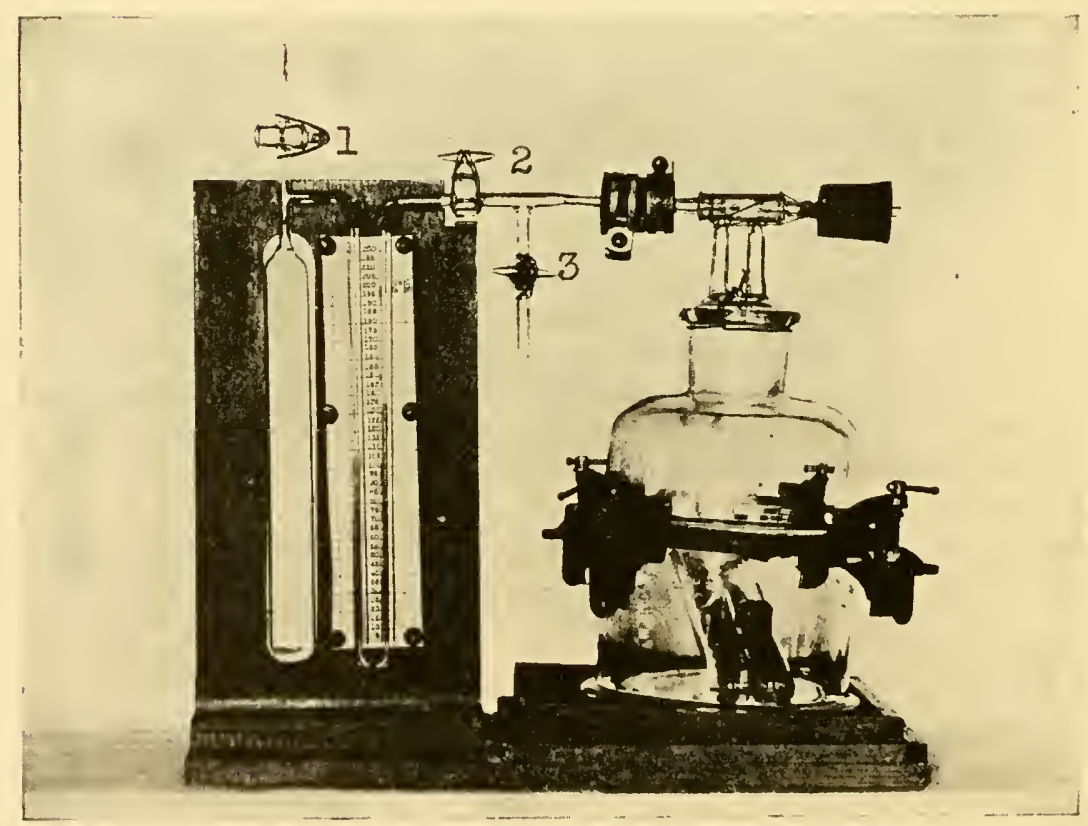

FIG. 2.-The Novy jar as a respiratory chamber, attached to a manometer 
sure is likely to exceed the capacity of the U-tube; and (2) to enable one to withdraw gas from the culture tube without sucking over the mercury column. Likewise, when evacuating the culture tube with the object of refilling it with fresh air, or with $\mathrm{O}_{2}, \mathrm{CO}_{2}$, etc., this cock must be closed. At all other times it is kept open.

Stopcocks I and 3 are used to equilibrate the mercury levels at the beginning of a test, after which both are closed. By means of tailcock 3 a sample of the gas content in the culture tube or jar is easily withdrawn for analysis. It is also the means by which the gas within the culture apparatus is evacuated and replaced with fresh air, or with any desirer gas.

At this place it may be well to indicate the ease with which desired gas tensions may be approximated by the use of an evacuating apparatus which consists of a Chapman water pump, a vertical manometer, and a Woulff bottle. One tubulure of the latter is closed with a rubber stopper through which is inserted one of the main arms of a three-way cock. The mirlarm of this cock, horizontal in position, is joined to a glass $Y$-shaped connector with cocks on each arm. The upper main arm is attached to the descending limb of a vertical manometer. Another tubulure is connected with the pump by means of a three-way tailcock. This serves to disconnect the latter when the desired negative pressure has been obtained.

If it is desired to introduce into a jar a certain percentage of $\mathrm{CO}_{2}$, for example, 40 per cent, this can be readily done. Assuming the barometer to read 750 , and the thermometer $22.3^{\circ}$, the corresponding aqueous tension is $20 \mathrm{~mm}$. of mercury. Hence $B-t=750-20=730$ $\mathrm{mm}$. and 40 per cent of this $=292$. This value represents the partial tension of the gas to be admitted, provided it is dry. Connection is made to the jar by way of one arm of the $Y$; the cock on the other arm is closed. The pump is started and evacuation continued until the manometer registers $292 \mathrm{~mm}$. The three-way cock is now turned so that the connection to the Woulff bottle is closed, and pure $\mathrm{CO}_{2}$ (from a tank or generator) ${ }^{\mathrm{r}}$ is run in through the other arm of the $\mathrm{Y}$-connector until the manometer reads zero. By means of the foregoing procedure, it is possible to introduce into the jar any desired amount of a given gas or various mixtures of gas, and with care the desired tensions may be easily obtained within one-half of I per cent. For exact work a sample of the gas should always be withdrawn and analyzed. Satisfactory manometric readings may be obtained with culture tubes, but they are not suited for the withdrawal of $10-20 \mathrm{cc}$. of gas for analysis.

There is another important consideration, viz., the necessity of providing for a sufficient volume of $\mathrm{O}_{2}$ to permit the full growth of the organisms; for example, it was found that the human variety of the tubercle bacillus required at least $500 \mathrm{cc}$. of air to produce a rich growth in a tube. Hence if the $h$-tube, with a volume of $100 \mathrm{cc}$., is used, little or no visible growth will occur. For this reason it is preferable to make use of the large chamber or jar (Fig. 2) of known volume which can easily be made gas tight. These jars can be obtained in two sizes which have approximate air capacities of 2,400 and 3,400 cc., respectively.

Culture tubes, flasks, or Petri plates may be placed in the jar which can then be used with air or filled with any desired gas. In order to develop quickly the full aqueous tension in the container it is necessary to place a few drops of distilled water on the floor of the jar.

Rubber tubing is not desirable for connecting the respiratory chamber to a manometer. It is well known that $\mathrm{CO}_{2}$ diffuses through a rubber tube, and without doubt it would be preferable to have all-glass connections. However, a satisfactory connection can be made by bringing the end of the manometer into contact with the glass stopper of the respiratory chamber by means of the special No. 25 rubber stopper (Fig. 2). Negative or positive pressures of $200 \mathrm{~mm}$. or more have held for weeks.

The gases may be analyzed by any one of the many standard methods. The modified

s Gases procured in ordinary commercial tanks must be purified before use or the percentage of foreign substances determined and allowed for in the calculations. 
Henderson-Haldane gas apparatus ${ }^{\mathrm{I}}$ is extremely accurate, and has proved very satisfactory. Figure 3 shows the method of connecting the gas burette with cock 3 of the manometer for the purpose of withdrawing a sample of the gas for analysis. The gas sample is then measured

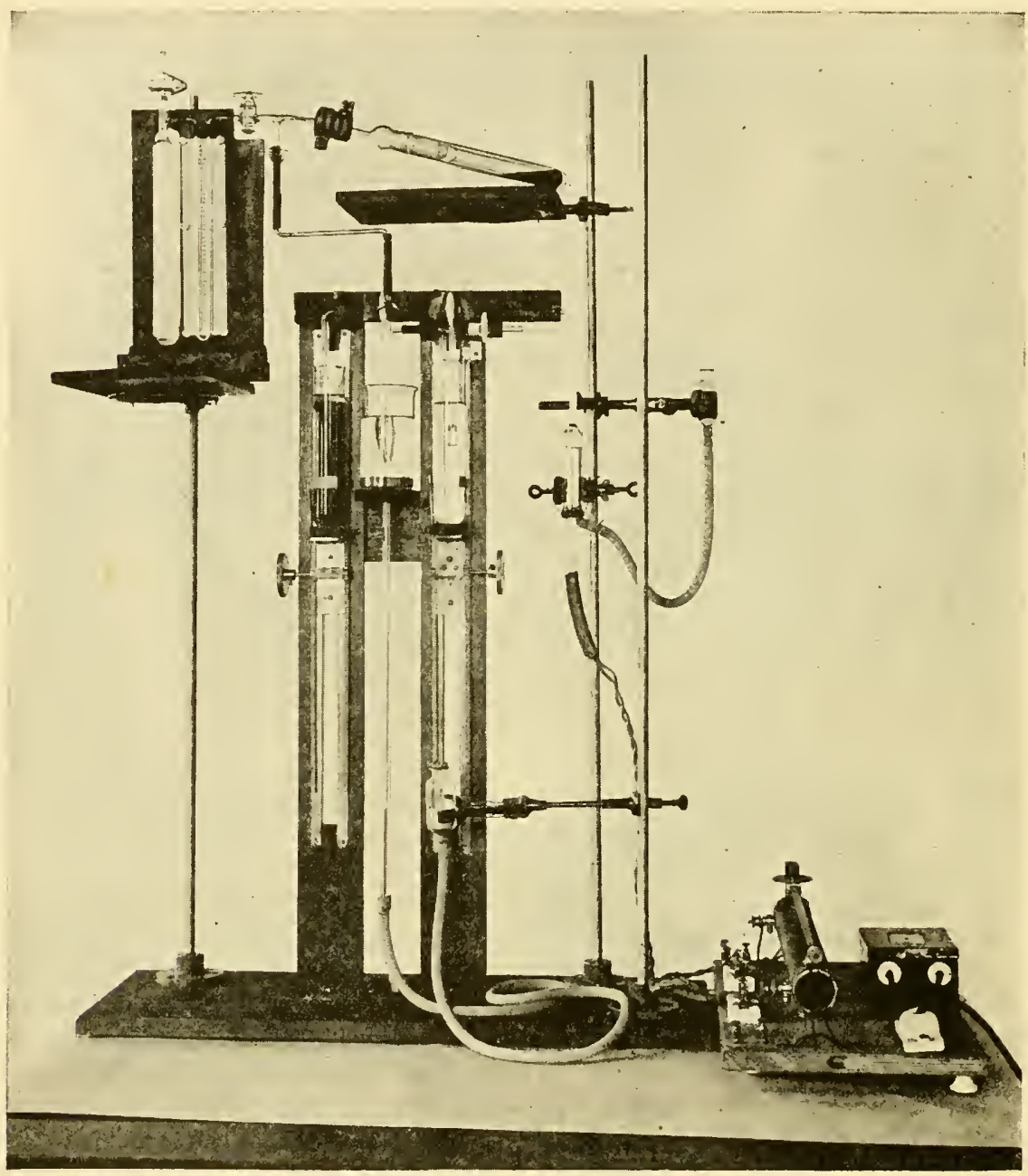

Fig. 3. - The modified Henderson-Haldane apparatus on stand with adjustable platforms to hold the manometer and culture tube or Novy jar. The gas sample is drawn from cock 3 on the manometer, through the capillary connector, in to the burette.

and analyzed. The $\mathrm{CO}_{2}$ is determined by absorption with $\mathrm{KOH}$ after which the $\mathrm{O}_{2}$ is removed by alkaline pyrogallate. If combustible gases are present, a known amount of $\mathrm{O}_{2}$ is now mixed with the sample, the gas passed into the combustion chamber, and the platinum wire heated to a dull-red glow to give a temperature which is sufficient for the ignition of $\mathrm{H}_{2}$. To determine hydrocarbons, such as methane, the wire must be raised to a bright red so that

${ }^{r}$ Novy, F. G., Roehm, H. R., and Soule, M. H.: loc. cit. 
they are burned to $\mathrm{CO}_{2}$ and $\mathrm{H}_{2} \mathrm{O}$. By this method the gaseous components may be determined with an accuracy of 0.02 per cent.

The analysis of the gas over the culture does not give the total gas change since a considerable and varying amount of $\mathrm{CO}_{2}$ is taken up by the medium. The $\mathrm{CO}_{2}$ may be present in mere physical solution, or, reacting with $\mathrm{Na}_{2} \mathrm{CO}_{3}, \mathrm{Na}_{2} \mathrm{HPO}_{4}, \mathrm{Na}$ of proteins, it may form $\mathrm{NaHCO}_{3}$; or combining with the $\mathrm{NH}_{3}$ and amines made by microbic action, it may yield corresponding carbonates. A culture of $B$. subtilis growing on ro $\mathrm{cc}$. of plain agar may produce enough alkali to bind $\mathrm{I}_{5} \mathrm{cc}$. of $\mathrm{CO}_{2}$.

When working with broth cultures, Van Slyke's ${ }^{t}$ apparatus for the determination of $\mathrm{CO}_{2}$ in blood plasma can be used. Rogers and his co-workers, in their study of the colon bacillus, removed the dissolved $\mathrm{CO}_{2}$, but not the fixed $\mathrm{CO}_{2}$, by evacuation. Obviously these methods cannot be used when working with solid media. Satisfactory determinations of the total dissolved $\mathrm{CO}_{2}$ can be made in liquid or solid media by the aeration method which consists in passing $\mathrm{CO}_{2}$-free air through the acidified culture medium and then into known amounts of a standard solution of $\mathrm{Ba}(\mathrm{OH})_{2}$. The medium can be kept at $90^{\circ} \mathrm{C}$. to liquefy the material if a solid substrate, such as agar, is used. The addition of the acid liberates the combined $\mathrm{CO}_{2}$. After sufficient aeration the tubes containing the standard solution are placed in ice water, and the excess of alkali subsequently titrated with standard $\mathrm{HCl}$. The number of cubic centimeters of $\mathrm{N} /$ ro hydroxide neutralized by the $\mathrm{CO}_{2}$ from the medium multiplied by I.I I 29 gives the volume of $\mathrm{CO}_{2}$ in the medium in cubic centimeters at $0^{\circ} \mathrm{C}$. and $760 \mathrm{~mm}$.

The determination of the $\mathrm{CO}_{2}$ content of a blood-agar medium requires a slight modification of the foregoing procedure. It is impossible to liquefy the medium, since the blood coagulates at a relatively low temperature, thus making it difficult to secure proper aeration of the material. The medium is, therefore finely comminuted with a flattened glass rod, and, after adding the $\mathrm{H}_{2} \mathrm{SO}_{4}$, is aerated at a temperature of about $40^{\circ} \mathrm{C}$.

\section{RESPIRATORY QUOTIENTS}

By respiration in its widest sense must be understood all those processes in the cell whereby the potential energy stored up in chemical compounds of high complexity is set free to furnish the energy required by an organism for its vital activities. The object is effected by processes of oxidation; the result is the production of energy with the formation of simple chemical substances such as $\mathrm{H}_{2} \mathrm{O}$ and $\mathrm{CO}_{2}$. The fact that man and animals consume $\mathrm{O}_{2}$ and return $\mathrm{CO}_{2}$ was shown by Lavoisier in 1777 . Dulong, ${ }^{2}$ continuing the work, found a difference in the volume of $\mathrm{CO}_{2}$ returned per volume of $\mathrm{O}_{2}$ consumed in dogs, rabbits, and fowls. He suggested that this might be due to a difference in the character of food. Dulong's suggestion was substantiated by Regnault and Rieset. ${ }^{3}$ The value obtained, by dividing the volume of $\mathrm{CO}_{2}$ produced by the volume of $\mathrm{O}_{2}$ consumed, was later designated as the "respiratory quotient." The ratio of this exchange is the same whether the volumes are expressed in cubic centimeters, or percentage, or as millimeters of pressure.

On the assumption that the oxidation is completed to $\mathrm{CO}_{2}$ and $\mathrm{H}_{2} \mathrm{O}$, the theoretical respiratory quotient of a carbohydrate is $\mathrm{I} .0$, of a fat $0.7 \mathrm{I}$, and of a protein 0.8 . Thus, in the case of glucose, we have the equation

$$
\mathrm{C}_{6} \mathrm{H}_{\mathrm{r} 2} \mathrm{O}_{6}+6 \mathrm{O}_{2}=6 \mathrm{CO}_{2}+6 \mathrm{H}_{2} \mathrm{O} \text {. }
$$

IVan Slyke, D. D.: J. Biol. Chem., 30, 347-68. I917.

${ }^{2}$ Dulong, M.: Ann.d. chim. et d. phys. (3), I, 440. I 84 I.

3 Regnault, V., and Reiset, J.: ibid., 26, 299. 1849. 
It follows, therefore, that the respiratory quotient $=\frac{6 \mathrm{CO}_{2}}{6 \mathrm{O}_{2}}=\frac{6}{6}=\mathrm{I}$. If the respiratory quotient is high, carbohydrate is supposedly being oxidized in the body; and, if low, fat is undergoing change.

The respiratory quotients of micro-organisms, as a rule, have been computed from the analysis of the gases over the cultures, but the values thus obtained can at best be considered only approximate. The direct use of analytical data, uncorrected for the changes in pressure, is wrong, since it gives an apparent respiratory quotient which is usually higher than the real quotient. A further and common error is the failure to determine the amount of $\mathrm{CO}_{2}$ dissolved, free or chemically combined in the medium. Another point which must be considered is the period of incubation, which, with some organisms, may be without appreciable effect; with others, on account of secondary changes in the medium such as decarboxylation, a longer period may result in an increased $\mathrm{CO}_{2}$ production, and hence in a higher final value.

In Table I are presented, by way of illustration, the results of a determination of the respiratory quotient of $B$. subtilis when grown on I per cent agar containing 5 per cent glycerol.

A jar (Fig. 2) received two inoculated tubes ( $20 \times{ }_{1} 50 \mathrm{~mm}$.), very loosely plugged, each containing Io cc. of the medium; also one open tube with Io cc. of boiled distilled water, the latter to supply the requisite aqueous tension. In order to hasten the production of this tension five drops of water were placed on the bottom of the jar. Thereupon the jar was closed, clamped, and attached to a manometer and placed in the hotroom at $32^{\circ} \mathrm{C}$., with stopcocks I and 3 closed. It was equilibrated four hours later.

In ten days the manometer showed a pressure of $-20 \mathrm{~mm}$.; on the thirteenth day it had reached $-2 \mathrm{I}$, and remained at that point until the nineteenth day when its contents were analyzed. It will be seen from the table that the $\mathrm{O}_{2}$ was practically all gone, and evidently this state had been reached about the tenth day. The culture in the two tubes produced $233 \mathrm{cc}$. of $\mathrm{CO}_{2}$ and consumed $275 \mathrm{cc}$. of $\mathrm{O}_{2}$ at $0^{\circ}$ and $760 \mathrm{~mm}$. The close approximation of the respiratory quotient thus obtained with the theoretical oxygen quotient of glycerol should be noted.

The errors often seen in the published work on gas metabolism are brought out in Table I. Thus, the calculated apparent respiratory quotient is the one usually given and is incorreçt since it is based on data uncorrected for temperature and pressure changes and does not include the dissolved $\mathrm{CO}_{2}$. The calculated, real respiratory quotient, corrected for changes in volume, does not include the dissolved $\mathrm{CO}_{2}$. The corrected, real respiratory quotient is based on all of the $\mathrm{CO}_{2}$ produced and all of the $\mathrm{O}_{2}$ consumed, corrected for changes in pressure, and converted to standard conditions.

The value of the manometric reading as a check on the analysis is apparent. When such agreement does not exist, it shows that an error is present which may be in the analysis of the control or of the culture. Apart from this, the error may be due to formation of a gas, such as $\mathrm{N}_{2}$, by the culture. The manometer is therefore a serviceable indicator of the production of such a gas or of products which have high vapor tensions.

The respiratory quotients for a number of organisms are presented in Table II 
To avoid repetition, it may be well to state that in each of these experiments the procedure was essentially the same. Since the protozoa studied do not live in the absence

TABLE I

Sifowing Calculation of Results in Determination of Corrected Real. Respiratory Quotient for B. subtilis Grown on Glycerol Agar, 2 Tubes, ig Days

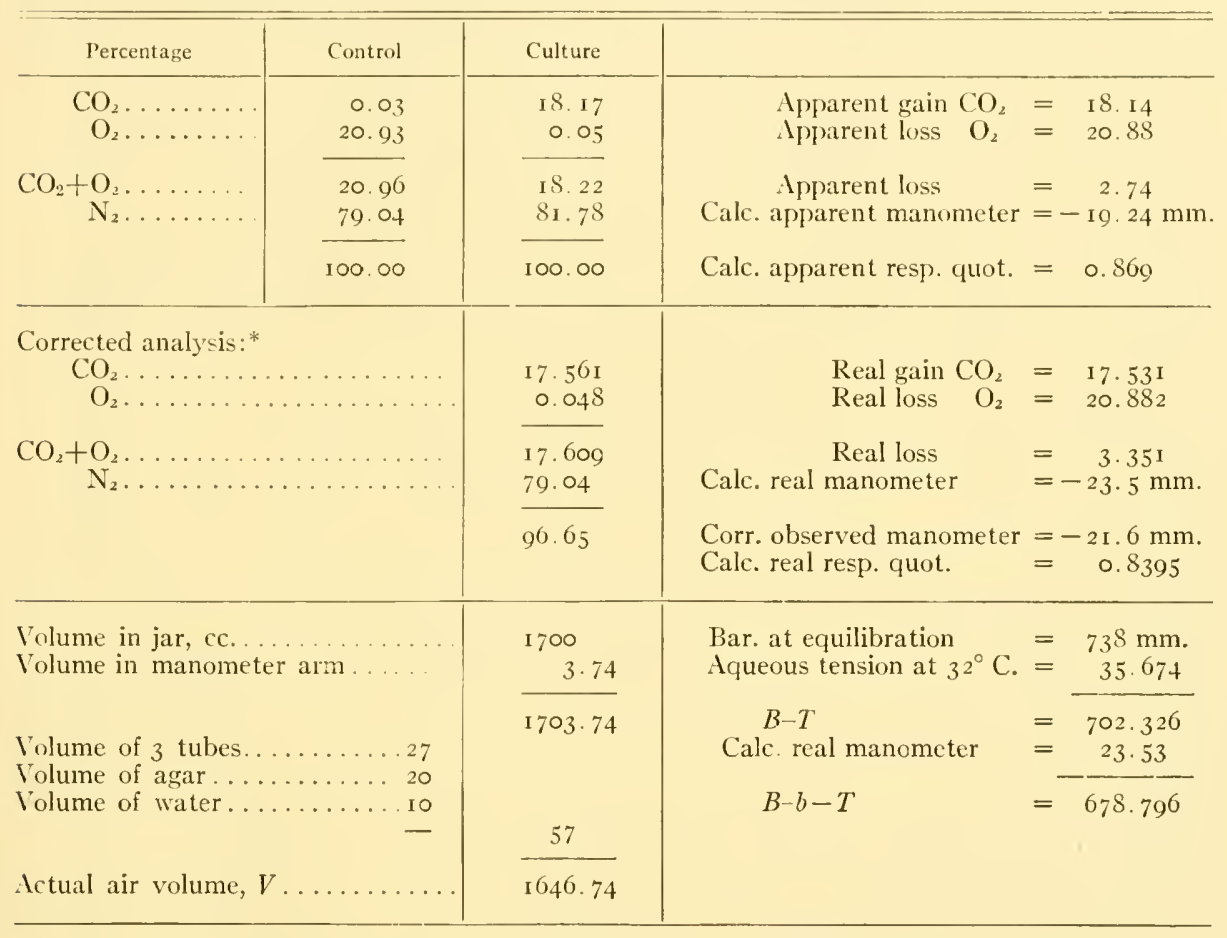

$$
V_{0}=\frac{V}{\mathrm{I}+0.003605 t^{\circ}} \cdot \frac{B-T}{760}=\mathrm{I}_{3} 62.03 \mathrm{cc} \text {. at } 0,760 \mathrm{~mm} \text {. }
$$

C. at $0^{\circ}, 760 \mathrm{~mm}$.

\begin{tabular}{|c|c|c|c|c|c|}
\hline $\begin{array}{l}\mathrm{CO}_{2} \text { in } 2 \text { agar tubes } \\
\mathrm{CO}_{2} \text { in water tube }\end{array}$ & $\begin{array}{l}= \\
=\end{array}$ & $\begin{array}{l}2.398 \\
0.674\end{array}$ & \multirow{2}{*}{\multicolumn{2}{|c|}{ Corr. real resp. quot. $=\frac{24 \mathrm{I} \cdot 249}{284 \cdot 4 \mathrm{I} 9}=$}} & \multirow{2}{*}{ o. $8_{4} 8_{2}$} \\
\hline & & & & & \\
\hline $\mathrm{CO}_{2}$ in 2 agar controls & $=$ & $\begin{array}{l}3.072 \\
0.60\end{array}$ & \multirow{4}{*}{$\begin{array}{l}\text { Resp. quot. of glycerol } \\
\text { l'er cent dissolved } \mathrm{CO}_{2} \\
\text { Tension dissolved } \mathrm{CO}_{2} \\
\text { Tension gaseous } \mathrm{CO}_{2} \\
\mathrm{CO}_{2} \text { quotient }\end{array}$} & \multirow{4}{*}{\multicolumn{2}{|c|}{$\begin{aligned}= & 0.857 \\
= & 0.1875 \\
& 1.32 \mathrm{~mm} \\
= & 127.50 \mathrm{~mm} \\
= & 0.010\end{aligned}$}} \\
\hline $\mathrm{CO}_{2}$ dissolved & $=$ & 2.472 & & & \\
\hline $\mathrm{CO}_{2}$ gaseous & & $3^{8.777}$ & & & \\
\hline $\begin{array}{l}\text { tal } \mathrm{CO}_{2} \\
\text { loss }\end{array}$ & & $\begin{array}{l}241.249 \\
284.419\end{array}$ & & & \\
\hline
\end{tabular}

* Obtained by multiplying the foregoing culture values by the nitrogen factor $\frac{79.04}{81.78}=0.9665$.

of blood, no attempts were made to determine quotients for these organisms on bloodfree media.

A comparison of the quotients determined by experiment with the theoretical values given in the last line is of special interest. In every case, excepting the diph- 
theria bacillus, the addition of glucose to the medium increased the quotient, showing that when glucose is present it is utilized, more or less completely, by the organisms as a source of energy.

If only glucose were oxidized the quotient would be . .o. It will be seen from Table II that this value is very closely approximated by several organisms. On the other hand, it is also clear that some combustion of the proteins or amino acids in the medium is taking place at the same time that glucose is being oxidized and that, as a result, the observed quotient represents the sum of all of the oxidations.

Since the quotient for protein is $0.8 \mathrm{I}$, it follows that complete combustion of a mixture of protein and glucose would yield a value intermediate between 0.8 and 1.0 , depending upon the relative amounts burned.

Furthermore, the utilization of any free amino acids that may be present in the medium may influence the quotient to a marked extent. Thus, the quotient for glycine and asparagine is $\mathrm{I} .33$, while for alanine it is 1.0 and for leucine it is 0.80 . Hence,

TABLE II

Average of Corrected Real Respiratory Quotients Obtained in Jar Experiments*

\begin{tabular}{|c|c|c|c|c|c|c|}
\hline Organism & Plain Agar & Glycerol Agar & $\begin{array}{l}\text { Glucose } \\
\text { Agar }\end{array}$ & $\begin{array}{c}\text { Serum } \\
\text { Agar }\end{array}$ & $\begin{array}{l}\text { Blood } \\
\text { Agar }\end{array}$ & $\begin{array}{c}\text { Glucose } \\
\text { Blood Agar }\end{array}$ \\
\hline 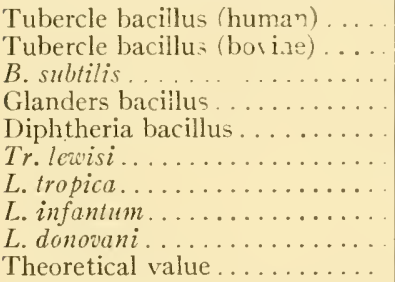 & $\begin{array}{l}0.888(4) \\
.912(\mathrm{I0}) \\
.84 \mathrm{I}(5) \\
.92 \mathrm{I}(4) \\
\ldots \ldots \\
\ldots \ldots \\
\ldots \ldots \\
\ldots .\end{array}$ & 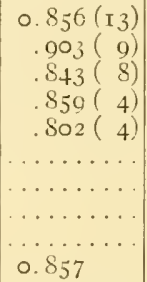 & 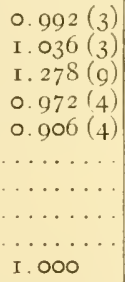 & 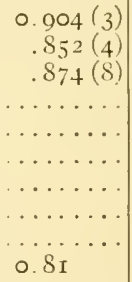 & $\begin{array}{l}\ldots \ldots \\
\ldots \ldots \\
\ldots \ldots \\
0.848(4) \\
.942(4) \\
.822(4) \\
.875(4) \\
.868(4) \\
.862(4) \\
\text { o. } 8 I\end{array}$ & 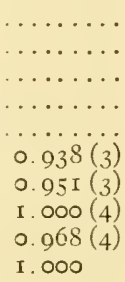 \\
\hline
\end{tabular}

* The figures in parentheses give the number of experiments.

depending upon which amino acid is being utilized, the observed respiratory quotient will be greater or lower than i.o.

Another factor which tends to raise the respiratory quotient above the theoretical value is the decarboxylation of amino and other organic acids, because $\mathrm{CO}_{2}$ is liberated without oxygen consumption.

As shown in Table II, the quotient for B. subtilis, when grown on glucose agar, is unusually high. This may be due solely to the two factors just mentioned. On the other hand, it is possible that a slight reaction, in the nature of alcoholic fermentation, produces an additional excess of $\mathrm{CO}_{2}$ sufficient to give a quotient of $\mathrm{I}_{4} 4$ or even higher.

It appears that glycerol is an important source of energy. The energy thus obtained permits complete utilization of such nutrient substances in the medium as are needed by the organism. It is worthy of note that the human variety of the tubercle bacillus, which is unable to grow on plain agar, multiplies readily on glycerol agar and gives a respiratory quotient corresponding closely to the theoretical value.

On plain agar, serum agar, and blood agar the organisms obtain the required energy by breaking down protein matter or by utilizing the free amino acids present. 
As a rule, the observed quotient is in excess of the theoretical value $(0.8 \mathrm{I})$, and the reason for this must be sought in the factors already discussed.

TIIE GAS EXCHANGE

The jar method serves not only to determine the respiratory quotient but also to supply data as to the extent of the gas changes brought about by the culture in a single tube. With ro cc. of medium in a tube the surface area represents about I5 sq.cm. of culture. Undoubtedly, it would be preferable to have the exact weight of the organisms which have developed in a tube in order to make comparisons of the gas exchange of various germs and the different media. It is more important, however, to determine the total dissolved $\mathrm{CO}_{2}$ in the medium, and to do this the weight of the growth must be sacrificed.

Out of a large mass of data, much as yet unpublished, the values given in Table III have been selected. They show the volumes of $\mathrm{CO}_{2}$ produced and of $\mathrm{O}_{2}$ consumed, per culture tube, by a number of organisms. In the last column of the table is given the volume of air corresponding to the $\mathrm{O}_{2}$ consumed. It will be seen from these values that many of the organisms can and do require relatively large amounts of air.

It has been shown in Table II that an organism when grown on a medium which contains no sugar yields a respiratory quotient of less than I.o. This is because of the fact that the volume of the $\mathrm{O}_{2}$ consumed is greater than that of the $\mathrm{CO}_{2}$ produced. A glance at Table III will serve to bring out this difference in the gas exchange. Failure to appreciate the nature of the chemical change involved has led some writers to misinterpret the meaning of this difference. The fact that the volume of $\mathrm{CO}_{2}$ produced is less than that of the $\mathrm{O}_{2}$ consumed does not mean that the difference represents the volume of $\mathrm{O}_{2}$ assimilated or retained by the organism. The difference represents the volume of oxygen used to oxidize hydrogen to form water.

It will be noted on reference to Table III that ro gm. of sterile raw potato consumed the oxygen present in $55^{2} \mathrm{cc}$. of air in ten days. These data are included because of the importance of this plant tissue in anaerobic culture. Novy ${ }^{\mathrm{T}}$ has furnished conclusive evidence that it is this marked gas exchange that favors anaerobic growth by the rapid and complete removal of the oxygen that may be present.

\section{INCREASED AND DIMINISHED OXYGEN TENSIONS}

The first real tests of the influence of high partial pressures of oxygen on pure cultures of micro-organisms were made by Moore and Williams. ${ }^{2}$ In their work slanted tubes of agar inoculated with the test cultures were placed in a bell jar containing varying percentages of pure oxygen. Samples of the gas in the jar were withdrawn for analysis at the beginning of the experiment and at various intervals during the incubation period. The net result of these investigations, which were extended by Adams ${ }^{3}$ working in the same laboratory, was that out of twenty-six cultures tested in partial pressure of $500 \mathrm{~mm}$. or more of oxygen, the growth of only the tubercle bacillus and $B$. pestis was inhibited. These organisms had not been killed, as subsequent incuba-

${ }^{1}$ Novy, F. G., Jr.: J. Infect. Dis., 36, 343-82. 1925.

${ }^{2}$ Moore, B., and Williams, R.: Biochem. J., 4, I77-90. I909.

3 Adams, A.: ibid., 6, 297-313. I9I r. 
TABLE III

Gas Exchange per Tube of Some Organisms Grown at $37^{\circ}$ C.; Cc. at $0^{\circ}, 760$ Mm.

\begin{tabular}{|c|c|c|c|c|}
\hline Organisms and Media & No. Days & $\mathrm{CO}_{2}$ Made & $\mathrm{J}_{2}$ Lost & $\begin{array}{l}\text { Correspondin } \\
\text { Air Volume } \\
\text { at } 37^{\circ} \mathrm{C} \text {. }\end{array}$ \\
\hline \multicolumn{5}{|l|}{ Human tubercle bacillus: } \\
\hline 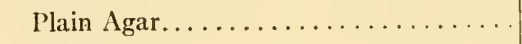 & $\ldots \ldots \ldots$ & $\circ$ & o & $\ldots \ldots \ldots \ldots$ \\
\hline Glycerol agar................ & $\left\{\begin{array}{l}12 \\
27\end{array}\right.$ & $\begin{array}{r}48 \\
\text { I } 24\end{array}$ & $\begin{array}{r}57 \\
149\end{array}$ & $\begin{array}{l}334 \\
874\end{array}$ \\
\hline Glucose agar $\ldots \ldots \ldots \ldots \ldots \ldots \ldots$ & $\left\{\begin{array}{l}26 \\
85\end{array}\right.$ & $\begin{array}{l}26 \\
66\end{array}$ & $\begin{array}{l}50 \\
70\end{array}$ & $\begin{array}{l}293 \\
410\end{array}$ \\
\hline Serum agar. . . . . . . . . . . . & 28 & $2 \mathrm{I}$ & 23 & 135 \\
\hline \multicolumn{5}{|l|}{ Bovine tubercle bacillus: } \\
\hline Plain agar $\ldots \ldots \ldots \ldots \ldots \ldots \ldots$ & $\left\{\begin{array}{l}28 \\
56\end{array}\right.$ & $\begin{array}{l}2 \text { I } \\
24\end{array}$ & $\begin{array}{l}22 \\
29\end{array}$ & $\begin{array}{l}118 \\
170\end{array}$ \\
\hline Glycerol agar . . . . . . . . . . . . . & $\left\{\begin{array}{l}28 \\
56\end{array}\right.$ & $\begin{array}{r}89 \\
155\end{array}$ & $\begin{array}{l}100 \\
174\end{array}$ & $\begin{array}{r}586 \\
1020\end{array}$ \\
\hline Glucose agar $\ldots \ldots \ldots \ldots \ldots \ldots \ldots \ldots$ & $\left\{\begin{array}{l}28 \\
56\end{array}\right.$ & $\begin{array}{l}104 \\
108\end{array}$ & $\begin{array}{r}98 \\
105\end{array}$ & $\begin{array}{l}579 \\
616\end{array}$ \\
\hline Serum agar $\ldots \ldots \ldots \ldots \ldots \ldots \ldots$ & $\left\{\begin{array}{l}28 \\
56\end{array}\right.$ & $\begin{array}{l}19 \\
28\end{array}$ & $\begin{array}{l}22 \\
33\end{array}$ & $\begin{array}{l}\text { I IS } \\
\text { I } 93\end{array}$ \\
\hline \multicolumn{5}{|l|}{ Diphtheria bacillus: } \\
\hline Plain agar. $\ldots \ldots \ldots \ldots \ldots \ldots \ldots$ & 28 & I6 & 27 & $\begin{array}{r}99 \\
\mathbf{2} 29\end{array}$ \\
\hline Glycerol agar.$\ldots \ldots \ldots \ldots \ldots \ldots \ldots$ & $\left\{\begin{array}{r}7 \\
2 \mathrm{I}\end{array}\right.$ & $\begin{array}{l}21 \\
27\end{array}$ & $\begin{array}{l}26 \\
33\end{array}$ & $\begin{array}{l}152 \\
193\end{array}$ \\
\hline Glucose agar ................ & $\left\{\begin{array}{r}7 \\
28\end{array}\right.$ & $\begin{array}{r}6 \\
20\end{array}$ & $\begin{array}{r}7 \\
23\end{array}$ & $\begin{array}{r}41 \\
\text { I } 34\end{array}$ \\
\hline Blood agar.............. & $\left\{\begin{array}{r}7 \\
28\end{array}\right.$ & $\begin{array}{l}\text { I } 8 \\
20\end{array}$ & $\begin{array}{l}19 \\
22\end{array}$ & $\begin{array}{l}\text { I I I } \\
\text { I } 29\end{array}$ \\
\hline \multicolumn{5}{|l|}{ Glanders bacillus: } \\
\hline 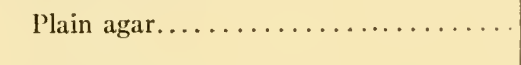 & $\left\{\begin{array}{r}7 \\
21\end{array}\right.$ & $\begin{array}{l}\text { I7 } \\
2 \mathrm{I}\end{array}$ & $\begin{array}{l}21 \\
25\end{array}$ & $\begin{array}{l}123 \\
146\end{array}$ \\
\hline Glycerol agar. $\ldots \ldots \ldots \ldots \ldots \ldots$ & $\left\{\begin{array}{r}7 \\
21\end{array}\right.$ & $\begin{array}{r}S 8 \\
141\end{array}$ & $\begin{array}{l}102 \\
164\end{array}$ & $\begin{array}{l}598 \\
962\end{array}$ \\
\hline Glucose agar $\ldots \ldots \ldots \ldots \ldots \ldots \ldots$ & $\left\{\begin{array}{r}7 \\
21\end{array}\right.$ & $\begin{array}{r}54 \\
\mathrm{I} 22\end{array}$ & $\begin{array}{r}55 \\
126\end{array}$ & $\begin{array}{l}322 \\
739\end{array}$ \\
\hline Blood agar $\ldots \ldots \ldots \ldots \ldots \ldots \ldots$ & $\left\{\begin{array}{r}7 \\
21\end{array}\right.$ & $\begin{array}{l}38 \\
38\end{array}$ & $\begin{array}{l}45 \\
44\end{array}$ & $\begin{array}{l}264 \\
25^{8}\end{array}$ \\
\hline \multicolumn{5}{|l|}{ Hay bacillus, $32^{\circ} \mathrm{C}$. : } \\
\hline 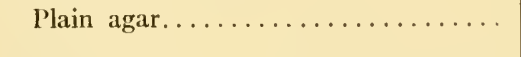 & $\left\{\begin{array}{r}7 \\
56\end{array}\right.$ & 86 & $\mathrm{~S}_{5}$ & $\begin{array}{l}409 \\
4 S_{3}\end{array}$ \\
\hline Glycerol agar............... & $\left\{\begin{array}{r}7 \\
20\end{array}\right.$ & $\begin{array}{r}76 \\
205\end{array}$ & $\begin{array}{r}89 \\
244\end{array}$ & $\begin{array}{r}505 \\
1386\end{array}$ \\
\hline Glucose agar $\ldots \ldots \ldots \ldots \ldots \ldots \ldots$ & 7 & 74 & 62 & $35^{2}$ \\
\hline Serum agar $\ldots \ldots \ldots \ldots \ldots \ldots$ & $\left\{\begin{array}{r}7 \\
2 \mathrm{I}\end{array}\right.$ & $\begin{array}{r}65 \\
142\end{array}$ & $\begin{array}{r}75 \\
159\end{array}$ & $\begin{array}{l}426 \\
903\end{array}$ \\
\hline \multicolumn{5}{|l|}{ Tr. lewisi, 31 ${ }^{\circ} \mathrm{C} .:$} \\
\hline 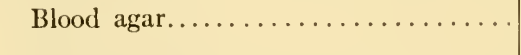 & $\left\{\begin{array}{r}7 \\
2 S\end{array}\right.$ & 44 & $\begin{array}{r}3 \\
49\end{array}$ & $\begin{array}{r}10 \\
277\end{array}$ \\
\hline Glucose blood agar............... & $\left\{\begin{array}{r}7 \\
2 \mathrm{I}\end{array}\right.$ & $\begin{array}{r}9 \\
59\end{array}$ & $\begin{array}{l}\text { I0 } \\
6.3\end{array}$ & $\begin{array}{r}56 \\
354\end{array}$ \\
\hline \multicolumn{5}{|l|}{ L. tropica, $31^{\circ} \mathrm{C} .:$} \\
\hline Blood agar.... & $\left\{\begin{array}{r}7 \\
2 S\end{array}\right.$ & $\begin{array}{l}12 \\
34\end{array}$ & $\begin{array}{l}1.5 \\
.38\end{array}$ & $\begin{aligned} 85 \\
212\end{aligned}$ \\
\hline Glucose blood agar. ............. & $\left\{\begin{array}{r}7 \\
21\end{array}\right.$ & $\begin{array}{l}25 \\
50\end{array}$ & $\begin{array}{l}26 \\
6.3\end{array}$ & $\begin{array}{l}1+7 \\
.354\end{array}$ \\
\hline Raw potato, $34^{\circ} \mathrm{C} . \mathrm{.} \ldots \ldots \ldots \ldots$ & $\left\{\begin{array}{r}3 \\
10\end{array}\right.$ & $\begin{array}{l}70 \\
94\end{array}$ & $\begin{array}{l}60 \\
96\end{array}$ & $\begin{array}{l}396 \\
55^{2}\end{array}$ \\
\hline
\end{tabular}


tion of the test cultures in air permitted excellent growth. However, Adams found later that actinomyces and mycetoma were actually killed by exposure to the high tensions of oxygen.

Recently Karsner, Brittingham, and Richardson ${ }^{\mathrm{I}}$ streaked the surfaces of agar plates with organisms suspended in salt solution. The plates were then stacked in a jar, the atmosphere of which was replaced with high oxygen tensions at normal barometric pressure. The growth of $B$. proteus, hemolytic streptococci, B. typhosus, and B. mucosus capsulatus, four of the nine cultures tested by this method, was inhibited by concentrations of over 80 per cent. Novy and Soule ${ }^{2}$ found that the human and bovine strains of the tubercle bacillus were stimulated by tensions of $40-60$ per cent oxygen, but concentrations of 80 per cent or higher gave partial inhibition. No gas other than $\mathrm{CO}_{2}$ was produced under these conditions, and the respiratory quotients were identical with the values given under ordinary air tensions. The observation was made by Adams, ${ }^{3}$ and confirmed by Novy and Soule, that the colonies of the tubercle bacillus developing in concentrations of oxygen above 80 per cent were isolated and heaped up. It appeared that relatively few organisms were able to grow under this extreme condition. The limiting tension for the growth of L. tropica ${ }^{4}$ and Tr. lewisi was found to be in the neighborhood of 60 per cent. Cleveland ${ }^{5}$ subjected several species of parasitic and free living protozoa to atmospheres of pure oxygen under high pressure, and found that exposure for forty minutes was usually sufficient to kill the organisms.

In making tests to determine the inhibiting tensions of oxygen it is of course necessary to have a large volume of the gas available at the experimental pressure. This will make it impossible for the initial growth of a few resistant cells to lower materially the tension to a concentration that will be easily tolerated by the other organisms planted, and thus lead to erroneous conclusions. Conceivably, the initial grow th in a small container may result in the production of sufficient $\mathrm{CO}_{2}$ to counteract the inhibiting action of the remaining oxygen.

Just how high tensions of oxygen inhibit the growth of organisms it is not possible to state. It may be supposed that the oxidative changes within the cells are increased to a point at which the vitality of the organism becomes exhausted. This may imply inhibition or even destruction of a respiratory enzyme; or, it may mean the formation of oxidative products which are directly injurious to the cell.

Of more interest, perhaps, than the action of bacteria under increased oxygen tensions is the growth of organisms under conditions where the oxygen pressure is below that normally found in the air. ${ }^{6}$

In order to answer the question as to what the effect of decreased oxygen tension may be on a given organism, it is obviously necessary to expose the organisms to known concentrations of this gas. This can be effected by placing the inoculated agar

I Karsner, H. T., Brittingham, H. H., and Richardson, M. I.: J. Med. Reseurch, 44, 83-88. I923.

${ }^{2}$ Novy, I. G., and Soule, M. H.: J. Infect. Dis., 36, 168-232. 1925.

3 Adams, A.: loc. cil.

4 Soule, M. H.: J. Infect. Dis., 36, 245-308. I925.

5 Cleveland, L. R.: Biol. Bull., 48, 455-68. 1925.

${ }^{6}$ For a discussion of anaerobiosis, cf. chap. xiii in this volume. 
tubes or preferably agar plates in containers the atmospheres of which may be varied. It is essential that the absolute amount of oxygen which is present, at the tension under consideration, be sufficient to meet the requirements of the organism. These conditions may be illustrated by the following experiment of Novy and Soule:

It had been determined from a study of the respiratory quotients that the human tubercle bacillus, in order to produce a rich growth, must be provided with about roo cc. of $\mathrm{O}_{2}$ or about $500 \mathrm{cc}$. of air. With this fact in mind, they grew this organism on agar slants in containers which progressively increased in size as the tension of oxygen in the confined gas decreased. Atmospheres of 6, 3, I, and 0.5 per cent were provided in containers of 2,,00, 3,300, 7,700 , and $20,000 \mathrm{cc}$. respectively. Each receptacle had previously received one freshly inoculated agar slant. After drawing a sample of the gas for analysis each chamber was placed at $37^{\circ} \mathrm{C}$. The experiment was conducted over a period of six weeks with analyses at frequent intervals. At the end of this time all of the oxygen in each container had been consumed and $\mathrm{CO}_{2}$ produced. The respiratory quotients were very nearly the same as those obtained under ordinary aerobic conditions. An excellent though varying growth was present in each tube, conclusive evidence that the tubercle bacillus can grow in the culture tube under any decrease in the oxygen tension and that it can utilize this element to the last free molecule. The only limit imposed is the size of the container which, for reasons stated, must be of such capacity as to provide approximately roo cc. of oxygen. It was plainly evident that respiration ceased when the oxygen was gone, as there was no increase in the $\mathrm{CO}_{2}$ content of the jars after this time. The growth of an aerobic organism under diminished oxygen tensions is of necessity slower than under ordinary air conditions.

Several other aerobic organisms have been investigated by the same methods, and all have given similar results. It may be tentatively concluded that a true aerobic organism can utilize free oxygen under any diminished tension, but a visible growth will be obtained only when the absolute volume of this element is present in the amount required under ordinary air conditions.

\section{INCREASED AND DIMINISHED $\mathrm{CO}_{2}$ TENSIONS}

Mention was made earlier in this paper that all growing organisms utilize oxygen, whether free or combined, and liberate $\mathrm{CO}_{2}$. Some writers have felt that this waste product plays an important rôle in the metabolism of bacteria, and that it is required in certain tensions for the growth of the cells. Ample proof has been advanced to show the favoring influence of the presence of this gas when making primary isolations from infected material, but after the organisms have become adapted to cultivation on artificial medium the beneficial action of added $\mathrm{CO}_{2}$ is lost.

Many organisms have been grown in the presence of high tensions of $\mathrm{CO}_{2}$, and by way of illustration one of the experiments of Novy and Soule with the tubercle bacillus may be cited:

Freshly inoculated glycerol agar slants were placed in jars of about 2,000-cc. capacity; the atmospheres of the jars were adjusted to tensions of $30,40,50$, and 60 per cent $\mathrm{CO}_{2}$ with an adequate supply of oxygen present. The jars, after the analyses of the gas contents, were incubated for fifty-nine days and then samples of the gases were withdrawn and analyzed. Excellent cultures were obtained in each tube. The data showed that the growth of the organisms had altered the original composition of the gas by utilizing the oxygen and increasing the $\mathrm{CO}_{2}$ tension. Calculations of the respiratory quotients demonstrated that the presence of the high $\mathrm{CO}_{2}$ tensions had no influence on the respiratory relations. In another ex 
periment, 90 per cent $\mathrm{CO}_{2}$ and ro per cent $\mathrm{O}_{2}$ were used with good results. The high tensions of $\mathrm{CO}_{2}$ necessarily produced marked changes in the acidity of the medium, but it was of extreme interest to note the rich growths obtained under such conditions. In general, the protozoa are unable to tolerate tensions of over 50 per cent of this gas.

Of more interest, perhaps, than the action of high tensions of $\mathrm{CO}_{2}$ on the growth of bacteria is the question of the absolute necessity of this gas for germ life. In all probability a certain tension of this gas is required for the growth of some if not of all organisms, and if this partial pressure is not available, the germs may die. Such a concentration must be extremely small. From the fact that many of the reported experiments designed to test this point cannot be successfully repeated by independent investigators it seems probable that factors other than the presence or absence of free $\mathrm{CO}_{2}$ have influenced the results.

The removal of the free $\mathrm{CO}_{2}$ as fast as it is formed by a rapidly growing culture presents many difficulties. Any method used nuust of necessity be a continuous one to prevent, if possible, the accumulation of the respired gas in close proximity to the cells. In addition, the procedure must alter only the concentration of this gas, for if changes are produced other than in the tension of $\mathrm{CO}_{2}$ the effects noted cannot consistently be attributed to the presence or absence of this substance.

The problem has been attacked in two ways: (I) the freshly inoculated media (broth, agar slants, or agar plates) are placed in close proximity to an alkaline absorbent such as $\mathrm{KOH}$ to absorb the $\mathrm{CO}_{2}$ as fast as formed; (2) a rapid stream of $\mathrm{CO}_{2}^{-}$ free air is passed over the cultures to remove mechanically the free $\mathrm{CO}_{2}$ respired by the cell. In some of the reported experiments the two methods have been combined.

The second method would seem to offer the most interesting possibilities, and it has been extensively used, but the results, in so far as inhibition is concerned, have been uniformly disappointing. If a stream of air is passed over an agar slant by leading the air inlet to the base of the agar, the passage of the air quickly desiccates the medium. Novy and Soule found that, if the vapor pressure of the aerating gas was adjusted to prevent the drying out of the medium, it was impossible to inhibit the growth of the human tubercle bacillus, B. subtilis, or other organisms. In fact, the passage of $500 \mathrm{cc}$. per minute of $\mathrm{CO}_{2}$-free air over the surface of freshly inoculated agar slants or Kitasato plates gave better growth than in controls under ordinary conditions. Analyses of roo-cc. samples of the gas after it had passed over the cultures gave negative tests for $\mathrm{CO}_{2}$. However, these investigators found that by using extract agar $\mathrm{pH} 6.0$ in place of the usual beef infusion agar $\mathrm{pH}_{7.4}$ in their $\mathrm{CO}_{2}$-free aeration experiments, it was possib.e to inhibit the growth of, and eventually kill, $B$. typhosus, B. sultilis, and other organisms.

The first method, in which alkaline absorbents are present to remove the respired $\mathrm{CO}_{2}$, has received a great deal of consideration. Moore and Williams ${ }^{1}$ placed soda lime in the bell jars to remove the $\mathrm{CO}_{2}$ when testing the growth of the tubercle bacillus in high oxygen tensions. Wherry and Ervin ${ }^{2}$ connected freshly inoculated tubes

I Moore, B., and Williams, R.: loc. cit.

${ }^{2}$ Wherry, W. B., and Ervin, D. M.: J. Infect. Dis., 22, 194-97. I918. 
of the tubercle bacillus to containers of $\mathrm{Ba}(\mathrm{OH})_{2}$ and found that the organisms failed to grow. From this they concluded that free $\mathrm{CO}_{2}$ was essential for growth. These authors explained the lag period in the development of cultures as a quiescent interval during which the $\mathrm{CO}_{2}$ was accumulating to the optimum concentration.

Rockwell ${ }^{\mathrm{I}}$ studied the gaseous requirements for the growth of various bacteria and, from a large series of investigations, ${ }^{2}$ has come to the conclusion that all bacteria, yeasts, and molds require free $\mathrm{CO}_{2}$ for their growth and that the gas is used as a source of carbon. He inoculated the surface of agar plates and incubated them over alkali; in many of his experiments the growth of the organisms was inhibited. The lack of growth was not due to the drying of the medium as the use of more vigorous dehydrating agents such as $\mathrm{H}_{2} \mathrm{SO}_{4}, \mathrm{CaCl}_{2}$, and glycerol permitted excellent growth despite marked desiccation. The negative results, i.e., the cases in which no inhibition was evidenced over alkali, were explained by the lack of control of all or any one of five factors, which are: the presence of carbohydrate in the medium, the protein content of the medium, the amount of inoculum, the acidity of the medium, and the concentration of salt. The conclusion that free $\mathrm{CO}_{2}$ is utilized as a source of carbon by all organisms is not warranted from the data presented. In fact, the work on respiratory quotients already mentioned proves that such an utilization does not take place.

A more plausible theory of the influence of $\mathrm{CO}_{2}$ on the growth of organisms is that developed by Novy and Soule, but as yet unpublished. In an attempt to account for the conflicts in the existing data, an extensive series of investigations has been carried out. From these experiments, and based on the fact that bacteria can grow luxuriantly on agar surfaces in the absence of free $\mathrm{CO}_{2}$ when this substance is removed by aeration, and that it is impossible to inhibit the growth of micro-organisms in a liquid medium by either of the methods mentioned, the conclusion has been reached that it is the intracellular $\mathrm{CO}_{2}$ which plays the important rôle in the life of the germ. If this intracellular $\mathrm{CO}_{2}$ is removed by exposure to alkali, or by other means, inhibition, and even death, may result. Any method that will maintain the intracellular $\mathrm{CO}_{2}$ equilibrium will prevent or overcome the inhibiting action of alkali and permit srowth to take place.

\section{GAS METABOLISM UNDER ANAEROIBC CONDITIONS}

No phase of the gaseous metabolism of bacteria has been more thoroughly investigated than the growth of organisms under anaerobic conditions. The bacteria of the colon group were early recognized as gas-producers, and because of their importance have received considerable attention. It was early recognized that $\mathrm{CO}_{2}$ and $\mathrm{H}_{2}$ predominate in the gases produced under anaerobic conditions. Although $\mathrm{CH}_{4}$ las been reported as a by-product of the anaerobic growth of $B$. coli, there is no evidence that such is the case. It is relatively simple to determine the ratio of $\mathrm{H}_{2}$ and $\mathrm{CO}_{2}$ produced when only traces of other gases are formed. An alkali is introduced into the liquid trapping the gas; the $\mathrm{CO}_{2}$ present unites with this substance leaving the hydrogen intact. By measuring the total volume of gas formed and the decrease in volume due

${ }^{1}$ Rockwell, G. E.: ibid., 28, 352-56. I921.

${ }^{2}$ Rockwell, G. E., and Highberger, J. H.: ibid., 40, 438-46. I927. 
to the removal of the $\mathrm{CO}_{2}$, one obtains the ratio of the gases present. Smith ${ }^{\mathrm{T}}$ used the fermentation tube to differentiate three groups of organisms on the basis of the ratios $\frac{\mathrm{H}_{2}}{\mathrm{CO}_{2}}$. (Subsequent workers have usually preferred the ratio $\frac{\mathrm{CO}_{2}}{\mathrm{H}_{2}}$.)

$$
\begin{aligned}
& \text { Group I: } \frac{\mathrm{H}_{2}}{\mathrm{CO}_{2}}=\frac{2}{\mathrm{I}}, \\
& \text { Group II: } \frac{\mathrm{H}_{2}}{\mathrm{CO}_{2}}=\frac{1}{2} \text { or } \frac{1}{3}, \\
& \text { Group III: } \frac{\mathrm{H}_{2}}{\mathrm{CO}_{2}}=\frac{\mathrm{I}}{\mathrm{I} \pm} .
\end{aligned}
$$

Keyes $^{2}$ reviewed the literature of gas analysis and elaborated on the limitation of the Smith tube. The two chief objections to its use are: (1) $\mathrm{CO}_{2}$ is very soluble in the medium and a large percentage of that substance actually produced does not appear as a gas. Hence an analysis of the supernatant atmosphere does not give a true indication of the volume of gas produced nor a true ratio between $\mathrm{CO}_{2}$ and the other gases. (2) The $\mathrm{CO}_{2}$ diffuses from the closed arm through the medium to the open arm and is lost. For these reasons the open fermentation tube has been discarded for use in exact quantitative work.

Rogers, Clark, and Davis, ${ }^{3}$ following Keyes, made an exhaustive study of the gas production by members of the colon group. Their cultures were grown in a vacuum, and the dissolved as well as the supernatant gases were estimated. They made no attempt to determine the fixed $\mathrm{CO}_{2}$, which, however, must have been appreciable in amount in spite of the final acidity of the merlium. The $\mathrm{CO}_{2}$ ratio in two hundred and sixteen determinations varied from 0.95 to 2.72 . In almost all of the analyses there was found to be a small but appreciable amount of "residual gas" which they called $\mathrm{N}_{2}$. The average amount found for the colon group was about 0.7 per cent of the total gas.

The majority of the quantitative data from studies made on strict anaerobes are open to the criticism emphasized before; the workers analysed only the surface gases.

Keyes and Gillespiet employed the exact vacuum method previously described by Keyes, in studying cultures of $B$. welchii. Bushnell ${ }^{5}$ presented the gas changes produced by a saprophytic anaerobe. He removed the dissolved gas by evacuation but made no attempt to estimate the fixed $\mathrm{CO}_{2}$. A more thorough investigation of the gaseous metabolism of strict anaerobes was made by Anderson ${ }^{6}$ who studied twentyfive strains of anaerobic organisms by a modification of the methods of Rogers, Clark, and Davis.

${ }^{x}$ Smith, T.: Centralbl. f. Bakteriol., Abt. I, Orig., I8, I-9. I895.

${ }^{2}$ Keyes, F. G.: J. Med. Research, 21, 69-82. 1909.

${ }^{3}$ Rogers, L. A., Clark, W. M., and Davis, B. J.: loc. cit.

${ }_{4}^{4}$ Keyes, F. G., and Gillespie, L. J.: J. Biol. Chem., 13, 291. r9r3.

${ }^{5}$ Bushnell, L. D.: J. Bact., 7, 373-403. $1922 . \quad{ }^{6}$ Anderson, B. G.: loc. cit. 
Some of the data presented by Anderson are given in Table IV. The general procedure was as follows: $300 \mathrm{cc}$. of medium were inoculated in each case; the culture flasks were evacuated, sealed, and incubated. After seven days the resulting gases were removed by evacuation, measured, and analyzed. No evidence of the presence of $\mathrm{NH}_{3}, \mathrm{~N}_{2} \mathrm{O}, \mathrm{CO}, \mathrm{CH}_{4}$, or other carbonaceous gas was found. The odorous gas fraction was composed mostly of $\mathrm{H}_{2} \mathrm{~S}$. This substance may have been present in concentrations of $2-3$ per cent, but the methods used for its determination were too unsatisfactory to give precise data.

When glucose was added to the peptone broth, B. botulinus, B. sporogenes, and $B$. welchii utilized the carbohydrate with a marked increase in the total volumes of

\section{TABLE IV*}

Shonthg Gas Prgduction at $0^{\circ}$ C., 760 Mim., and $\frac{\mathrm{CO}_{2}}{\mathrm{H}_{2}}$ Ratios; Cultures Grown in $300 \mathrm{Cc}$. of Broth in vacuo, 7 Days; Medium: 2 Per Cent "Difco" Peptone and 2 Per Cent "Difco" Peptone plus i Per Cent Glucose

\begin{tabular}{|c|c|c|c|c|c|c|c|}
\hline Organism & Medium & $\begin{array}{l}\text { Volume } \\
\text { in } \mathrm{Cc} \\
\text { of Gas } \\
\text { Produced }\end{array}$ & $\mathrm{CO}_{2} \%$ & $\mathrm{H}_{2} \%$ & $\mathrm{~N}_{2} \%$ & Total $\%$ & $\begin{array}{l}\text { Ratio: } \\
\frac{\mathrm{CO}_{2}}{\mathrm{H}_{2}}\end{array}$ \\
\hline B. botulimus $97 \ldots \ldots \ldots \ldots$. & $2 \%$ peptone & 70 & 82.0 & 13.6 & 4. I & 99.7 & 6.0 \\
\hline B. botulinus $97 \ldots \ldots \ldots \ldots$ & $\left\{\begin{array}{l}2 \\
\end{array}\right.$ & 510 & $75 \cdot 5$ & 24.0 & 0.7 & IOO. 2 & 3.1 \\
\hline B. sporogenes $46 \ldots \ldots \ldots$. & ${ }_{2}$ (1) peptone & i 18 & 80.5 & 3.5 & 6.0 & 99 & 25.6 \\
\hline B. sporogenes $46 \ldots \ldots \ldots$. & $\left\{\begin{array}{l}2 \\
\text { r peptone }+\end{array}\right.$ & 430 & 82.2 & I 6.8 & 0.9 & 99.9 & 4.9 \\
\hline $\begin{array}{l}\text { B. welchii } 57 \ldots \\
\text { B. welchii } 57 \ldots\end{array}$ & $\begin{array}{l}1, c \text { glucose } \\
2<<\text { peptone } \\
\int_{2}<\text { peptone }+\end{array}$ & $\begin{array}{r}42 \\
645\end{array}$ & $\begin{array}{l}\cdots \\
25 \cdot 9 \\
42.3\end{array}$ & $\begin{array}{l}64 \cdot 5 \\
39 \cdot 2\end{array}$ & $\begin{array}{r}9.5 \\
\mathbf{I} 8.0\end{array}$ & $\begin{array}{l}99.9 \\
99.5\end{array}$ & $\begin{array}{l}\cdots \cdots \\
0.40 \\
\text { I.08 }\end{array}$ \\
\hline & I $\%$ glucose & $\ldots \ldots$ & $\ldots \ldots$ & $\ldots \ldots \ldots$ & $\ldots \ldots$ & $\ldots \ldots$ & $\ldots \ldots$ \\
\hline B. histolyticus W.V... & $2 \%$ peptone & 70 & 92.5 & 0.0 & 7.0 & $99 \cdot 5$ & $\ldots \ldots$ \\
\hline B. histolylicus WV.V... & $\{2$ c peptone $t$ & 63 & 90.7 & 0.99 & $7 \cdot 9$ & 99.6 & $\ldots \ldots$ \\
\hline & I, glucose & & & & & $\ldots \ldots$ & $\ldots \ldots$ \\
\hline
\end{tabular}

* Assembled from Anderson's Tables $3 A$ and $5 B$.

gas produced. B. histolyticus did not decompose glucose, hence its presence had no effect on the quantity of gas produced. By providing only a very small amount of protein material it was possible to force this organism to utilize glucose, but a poor growth was obtained.

The accurate detection and estimation of such gases as $\mathrm{CH}_{4}, \mathrm{CO}$, etc., produced by pure strains of organisms has not been reported. $\mathrm{CH}_{4}$ has been detected and estimated in cultures of B. lactis aerogenes (Baginsky), ${ }^{\mathrm{I}}$ in the gaseous effluent from sewage tanks, and in intestinal gases.

The evolution of large quantities of gases other than $\mathrm{CO}_{2}$ and $\mathrm{H}_{2}$ has been reported when special media have been used for the growth of organisms. The addition of $\mathrm{NO}_{2}^{\prime}$ and $\mathrm{NO}_{3}{ }^{\prime}$ to the nutrient substance frequently results in a marked evolution of $\mathrm{N}_{2}$. However, such reactions have not been thoroughly investigated. Such problems, and the interesting question of gas production by so-called "bacterial synergism," offer new and fascinating fields for investigations.

I Baginsky, A.: Zischr.f. phys. Chemie, I2, 434-62. I888. 
It is apparent that relatively simple apparatus and methods are now available for the exact determination of the gaseous metabolism of bacteria. When applied to aerobic forms, the amount of $\mathrm{O}_{2}$ consumed and of $\mathrm{CO}_{2}$ produced can be readily ascertained. By contrast, the anaerobic organisms yield relatively large volumes of gases which consist essentially of $\mathrm{CO}_{2}$ and $\mathrm{H}_{2}$. The hydrogen quotient, $\frac{\mathrm{CO}_{2}}{\mathrm{H}_{2}}$, may possibly be useful as a basis of classification. 


\title{
CHAP'TER XIX
}

\section{ENZYMES OF BACTERIA}

\author{
SELMAN A. WAKSMAN \\ New Jersey Agricultural Experiment Station, New Brunswick, N.J.
}

DEFINITION AND PROPERTIES OF ENZYMIES

Pasteur was the first to establish the difference between "formed" or "organized" ferments, which bring about various reactions, such as alcoholic and acid fermentations, through the action of the living cell, and "unorganized ferments," such as diastase, pepsin, etc., later designated by Kühne as "enzymes," which are active in the absence of living cells. A distinct difference was thus made between "metabolic" processes and "enzymatic" processes, or between a ferment as an organized living cell and an enzyme as a product of the cell, active inside or outside of the cell. However, further studies, especially the contributions of Buchner on the intracellular enzymes bringing about alcoholic and acid fermentations, finally established the fact that no essential difference exists between enzymes and ferments.

An enzyme is a catalytically active substance which is produced by living cells and the action of which is independent of the life-processes of the cell. Enzymes are capable of accelerating the rate of chemical reactions and remain themselves unchanged. Seemingly the enzyme does not enter into the reaction itself. In certain cases enzymes may accelerate the reaction in one direction more than in another, as in the following illustration:

$$
{ }_{2} \mathrm{H}_{2} \mathrm{O}+\mathrm{O}_{2} \underset{2}{2} \mathrm{H}_{2} \mathrm{O}_{2}
$$

when only the reaction from right to left is accelerated by catalase.

Enzymes act specifically, i.e., they act upon substances of definite structural and stereoisomeric configuration. This specificity of enzymes is independent of the degree of their purity. However, it has not been demonstrated as yet that enzymes are definite chemical substances; no enżyme has been isolated in a pure state. Enzymes must be defined upon the basis of their action rather than their chemical nature.

Enzymes possess certain physical properties, such as being apparently amphoteric electrolytes and colloids, enabling them to form absorptive compounds; they also possess definite structures which give them a specific affinity to the substrates upon which they act.

\section{NATURE OF ENZYMES}

The nature of the structure of enzymes is still imperfectly understood, especially for a number of enzymes not yet separated from the cell constituents, notably the proteins, with which they are bound in the cell. It is possible to obtain aqueous solutions of certain enzymes, such as amylase (diastase), protease, and invertase (saccharase). It has been assumer (Fodor) that yeast invertase is identical with a carbohydrate yeast gum but the removal of this gum does not seem to affect the activity 
of the enzyme; the same is true of Fischer's idea that enzymes possess a protein nature, the work of Willstätter having established that some enzymes at least (lipase, peroxydase, invertase) can be purified to such an extent that the protein reactions disappear completely. Some enzymes were obtained practically free from iron and phosphorus. Nitrogen seems to be an essential constituent, the purest invertase containing I 3 per cent and peroxydase 9.37 to 13.37 per cent nitrogen. According to Euler, this is proof of the protein nature of enzymes. According to Willstätter, the proteins are merely impurities which cannot be readily removed.

Enzymes are thus considered ${ }^{\mathrm{r}}$ to be composed of a specifically active group and a colloidal carrier, the latter varying in its nature but essential for the stability of the active group. A theory has also been advanced ${ }^{2}$ that various enzymatic reactions brought about by bacteria are primarily due to polarizations of substrate molecules induced by electric fields which characterize particular centers of cellular and intracellular surfaces, these being "active centers"; one active center may be able to activate various substrates, the structure of a molecule influencing its activation.

\section{CLASSIFICATION}

According to Neuberg and Oppenheimer, enzymes can be divided into two large groups: (I) Hydrolases, which bring about hydrolytic decomposition. There is only a very small gain of energy in these reactions. Here belong the esterases which act upon fats and esters, the carbohydrases which act upon carbohydrates, the proteases which act upon proteins, the amidases which act upon the amide and amino groupings. (2) Desmolases, which influence the bonds of atoms. They break the carbon chain with the liberation of free energy. They are the enzymes of respiration and of metabolism. Here belong those enzymes which bring about the various oxidation and reduction processes, the fermentation reactions (anaerobic), as well as the important enzymes zymase and catalase.

\section{FORMATION AND SECRETION OF BACTERIAL ENZYMES}

The enzymes of bacteria have been very insufficiently studied. In most instances, analogies must be drawn with animal and plant enzymes, or enzymes of other microorganisms. The recent investigations of Meyerhof and others on respiration and fermentation have shown that such analogies are usually justified.

A bacterial cell is able to elaborate at least two distinct types of molecules, namely, highly specialized molecules which exhibit enzymatic behavior, and non-enzymatic substances; these together make the protoplasmic and histological cell structures, and are inseparably connected with one another. ${ }^{3}$

Many enzymes are produced in an inactive condition, namely, as proenzymes or zymogens, and are changed into the active form by an activator. This activator may be merely an acid or an alkali necessary to adjust the reaction to the optimum for the particular enzyme, as in the case of pepsin or lipase. In some instances, electrolytes $(\mathrm{NaCl})$ are essential, anions being active in the case of diastase, and cations such

I Willstätter, R.: J.Chem. Soc., p. I374. I927.

${ }^{2}$ Quastel, J. H., and Wooldridge, W. R.: Biochem. J., 21, I 224 . I927.

${ }^{3}$ Quastel, J. H., and Wooldridge, W. R.: ibid. 
as calcium being essential for the action of tryptases and thrombase. The zymogen may be a mixture of an enzyme with an inactivating agent or paralyzer; activation then consists in the destruction of the paralyzer. An activator for enzyme A may be the paralyzer for enzyme $B$, which acts destructively upon $A$, as in the action of tryptase of yeast upon zymase. The proteolytic enzyme of the pancreas acts only upon peptone, fibrin, casein, and protamines, but not upon true proteins; when activated by enterokinase, it can act also upon these. This is, however, not a transformation of a zymogen into trypsin, as previously assumed, but a stoichiometric and reversible combination of trypsinogen and kinase to give a new enzyme possessing new characteristics.

The presence of specific nutrients in the medium regulates the quantitative formation of specific enzymes. Thus, the presence of starch will increase the amylolytic power; the presence of proteins, the proteolytic power, etc. The digestive enzymes are usually secreted readily into the medium and are found among the so-called extracellular or exo-enzymes, since the colloidal nutrients (celluloses, starches, proteins) cannot enter the cell; the enzymes of metabolism (respiratory enzymes, zymases, etc.) are found among the so-called endo-cellular or endo-enzymes. The same enzyme may be in a biological sense both an exo- and an endo-enzyme, the difference between these two enzymes being one of degree rather than of kind. In some cases, organisms can be cultured so that they produce a certain enzyme which they do not form otherwise, as in the formation of a zymase capable of decomposing galactose into alcohol. The nature of nutrition influences very markedly the nature and abundance of the different enzymes. This is especially true of bacteria and other micro-organisms.

\section{PREPARATION AND PURIFICATION OF ENZYMES}

The bacterial enzymes which are secreted into the medium can be obtained by filtering the culture free from cells or by using the whole culture as the enzyme preparation. Certain enzymes are obtained only by the destruction of the cell. A slight injury of the cell, resulting in a change in permeability, may be sufficient to allow some of the enzymes to be secreted readily into the surrounding medium. This can be accomplished either: (I) by changing the surface tension of the cell, (2) by bringing about autolysis of the cell, (3) by drying the cells first, then following by a brief autolysis, or (4) by breaking up the cells by mechanical means, as in the use of the Buchner press. The nature of the treatment will depend more upon the nature of the cell than upon the nature of the enzyme. Some enzymes have as yet not been separated from the cell substance, as in the case of lipase of seed or bacterial zymase.

There are various methods available for the separation, purification, and concentration of enzymes. None of these has resulted, however, in the preparation of an enzyme in a pure state. The processes of purification consist in removing as nearly as possible all extraneous matter. Frequently some of the enzyme itself is lost in the process. Since enzymes are very labile, their separation from the accompanying proteins, carbohydrates, and salts is accomplished by physical processes of adsorption, elution, and precipitation rather than by chemical processes such as salt formation. The enzyme is first brought into solution, using as a solvent water, dilute salt or very dilute acid and alkali solutions. A phosphate buffer solution of a definite $\mathrm{pH}$ has been 
used lately with great success. The solution of the enzyme is filtered through paper or through a Chamberland or other convenient filter. Salts can also be removed by dialysis. The enzyme can be precipitated from solution by alcohol or by a definite concentration of a sulphate, preferably ammonium sulphate. The use of different concentrations of salt frequently permits the separation of one enzyme from another. The precipitation is followed by selective adsorption and elution of the enzyme. Positively charged aluminum hydroxide and negatively charged kaolin have been used extensively as adsorbing agents.

The microbial cell begins to liberate the enzyme in to solution only after its death, as in the case of yeast. The autolysis of the cells or the enzymatic degradation of the protoplasm of the microbial cell is accompanied by the passing of the various enzymes into solution. Gentle autolysis accompanied by adsorption processes resulted in the liberation of much more active enzymes than previously obtained. ${ }^{\mathrm{r}}$

For the concentration of bacterial enzymes, the following method has been recommended:2 Pure cultures of bacteria are grown in $x$ liter portions of nutrient media for three days. The culture is then treated with an aqueous emulsion of mastix and acidified with acetic acid. The emulsion is prepared by dissolving mastix in alcohol and diluting with an equal volume of water. The precipitate which is formed as a result of treatment of the culture is allowed to stand twenty-four hours and is then filtered off. The mastix is removed from the precipitate by dissolving with alcohol and ether. The residual precipitate contains the bacterial enzymes.

\section{MEASUREMENT OF ENZYME ACTION}

An enzyme is characterized not by its structure, which is so far unknown, but by its activities. The methods of demonstrating enzyme action are based either upon the disappearance of the specific substrate or the formation of reaction products. The action of diastase (amylase) upon starch is measured, on the one hand, by the disappearance of starch, as shown by the iodine reaction, or by a change in the colloidal condition of the starch, and, on the other hand, by the formation of dextrins and maltose, etc. Unfortunately, we are dealing in most instances with mixtures of enzymes and not with pure enzymes. Hence it is frequently difficult to determine just which products are formed by one enzyme and which are acted upon by another.

\section{INFLUENCE OF ENVIRONMENTAL CONDITIONS}

Enzymes are influenced in various ways by physical and chemical factors, which either favor (activate) or injure (paralyze) their action.

Increasing temperatures, up to a certain region, bring about an increase in enzyme action, according to the laws of chemical kinetics; however, even at relatively low temperature an injurious action sets in. Most enzymes are very sensitive to temperatures above $70^{\circ} \mathrm{C}$, even during a very brief period. Temperatures above $45^{\circ} \mathrm{C}$. prove injurious after a prolonged period of action. The temperature coefficients (Arrhenius' constant A) become smaller with increasing temperatures, finally becoming negative; different enzymes, however, behave differently. The optimum tem-

'Willstätter, R.: op. cit., p. I359. I927.

${ }^{2}$ Schierge, N.: Biochem. Ztschr., I 79, 248. 1926. 
perature for most enzymes is between $35^{\circ}$ and $50^{\circ} \mathrm{C}$. The destructive temperature was defined by Euler as that temperature at which the enzyme is reduced to 50 per cent of its strength in one hour. This temperature also varies for various enzymes, some bacterial proteases resisting for a brief time even boiling temperature.

Sunlight is injurious to enzymes in aqueous solution but not in a dry condition, the ultra-violet rays being especially injurious; the action of visible rays is inappreciable. The destructive action of an electric current is proportional to the current.

The influence of reaction of the medium upon enzyme action should be measured in the actual concentration of hydrogen ions. The optimum reaction varies considerably for the various enzymes, as shown in Table I.

TABLE I

\begin{tabular}{|c|c|c|c|}
\hline Enzyme & Source & $\underset{\mathrm{pH}}{\text { Optimum }}$ & Author \\
\hline 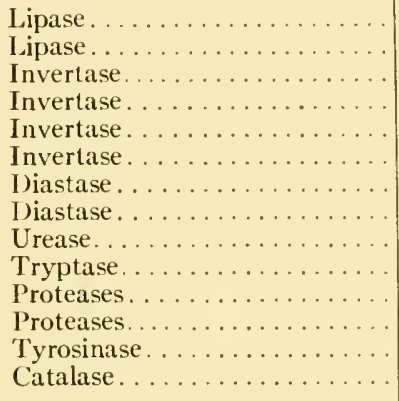 & $\begin{array}{l}\text { Streptococcus } \\
\text { Pneumococcus } \\
\text { Yeast } \\
\text { Asp. oryzae } \\
\text { Penicillium } \\
\text { Bacteria } \\
\text { Asp. oryzae } \\
\text { Pne tmococcus } \\
\text { Bacteria } \\
\text { Yeast } \\
\text { Yeast } \\
\text {...................... }\end{array}$ & $\begin{array}{l}9 \cdot 0 \\
7.8 \\
4 \cdot 4^{-4} \cdot 6 \\
5 \cdot 0^{-5}-5 \\
5 \cdot 0 \\
7.0 \\
4.8 \\
7.0 \\
7 \cdot 2-7 \cdot 5 \\
7.2 \\
6.7-8.5 \\
6.0 \\
6.5 \\
7.0\end{array}$ & $\begin{array}{l}\text { Stevens } \\
\text { Avery } \\
\text { Sörensen } \\
\text { Kuhn } \\
\text { Josephson } \\
\text { Avery, Stevens } \\
\text { Sherman } \\
\text { Avery } \\
\text { Lövgren } \\
\text { E. Meyer } \\
\text { Abderhalden and Fodor } \\
\text { Dernby } \\
\text { Raper and Wormall } \\
\text { Michaelis, Sörensen }\end{array}$ \\
\hline
\end{tabular}

The influence of $\mathrm{pH}$ upon the action of the enzyme may depend upon its purity. A purified enzymatic preparation may show a different optimum reaction than an impure preparation. ${ }^{\mathrm{I}}$

It is frequently difficult to differentiate between the injury caused to the enzyme itself (usually irreversible) and the influence upon the reaction velocity of the process (usually reversible). This is especially true of the action of salts upon enzymes. Salts of heavy metals, especially $\mathrm{Hg}, \mathrm{Ni}, \mathrm{Co}, \mathrm{Zn}, \mathrm{Ag}$, and $\mathrm{Au}$, are distinctly injurious, the action being reversible, because of the formation of a complex between the enzyme and the cation; when the metal is removed, as by treatment with $\mathrm{KCN}$ or with neutral salts, the activity is frequently restored.

\section{ENZYMES AS ANTIGENS}

The problem of the ability of enzymes to bring about the formation of antibodies attracted at one time considerable attention. There is no doubt that on the injection of enzymes antibodies of a specific nature are formed. This phenomenon can be used to separate not only different enzymes, but also enzymes of different origin. However, in view of the fact that each enzyme consists of an active substance possessing a definite structure and a colloidal carrier, and in view of the fact that the nature of this

Willstätter, R.: op. cil., p. I359. I927. 
carrier varies with the source of the enzyme, the nature of the antibody varies accordingly. Enzymes are bound by the specific substrate much as antigen is bound to antibody. In the first case, however, the binding is followed by catalytic action, the substrate is decomposed, the binding is dissolved, and the enzyme is again liberated to combine with more substrate; the combination of antigen-antibody is permanent, except under the influence of special factors such as specific precipitating antibodies for the proteins contained in the antigen or the antibody carriers, alkaline sugar and salt solutions, etc. ${ }^{\mathrm{r}}$

It has been recently suggested ${ }^{2}$ that the whole system of specific antibody formation can be readily explained by enzyme action. The introduction of complex foreign proteins into the animal body learls to a series of hydrolyses, whereby the complex proteins are broken down into simpler components, similar to the processes taking place in gastro-intestinal digestion. Some of the cleavage products presumably have the same specificity as the original proteins. The hydrolytic processes are followed by processes of synthesis, coagulation, conjugation, and adsorption between the foreign proteins or their cleavage products and normal humoral or cellular components. Some of these resulting products may have a specific protective action for the body as a whole; others may increase specific susceptibility; still others may be non-specific or even inert.

\section{OCCURRENCE OF BACTERIAL ENZYMES}

Esterases are enzymes which hydrolyze esters into fatty acids and alcohols; those enzymes which hydrolyze true fats into glycerol and higher fatty acids are usually referred to as lipases; the enzymes which hydrolyze esters of lower fatty acids are frequently spoken of as butyrases. Lecithinase (lecithase), or the enzyme which acts upon lecithin and phosphatides; chlorophyllase, the enzyme acting upon chlorophyll; cholesterinase, acting upon cholesterin ester, and the enzymes which hydrolyze esters of phosphoric acid (phytase, nucleases) and of sulphuric acid (sulphatase), belong to this group.

A number of bacteria, including $B$. prodigiosus, B. pyocyaneus, B. fluorescens, Staph. aurcus, B. typhosus, B. tuberculosis, and other acid fast bacteria were found to form lipase readily. ${ }^{3}$ Lower esterases are produced by a number of bacteria including B. typhosus. ${ }^{4}$

Carbohydrases.-The enzymes which hydrolyze polysaccharides are frequently ${ }^{5}$ divided into two groups: (I) The polyases acting upon the complex polysaccharides. These include amylase (diastase) which acts upon starch and glycogen, cellulase which acts upon cellulose, inulinase (inulase) acting upon inulin, cytases (hemicellulases), including lichenase, which act upon various hemicelluloses, pectinase (pectase) and gelase capable of hydrolyzing pectins and agar-agar, respectively. (2) The hexosidases acting upon di- and tri-saccharides. These include the fructosidases,

${ }^{1}$ See chap. $1 \mathrm{xx}$ in this volume.

${ }^{2}$ Manwaring, W. H.: J. Immunol., 12, I77, 1926; Scient. Month., p. 362. Oct., 1927.

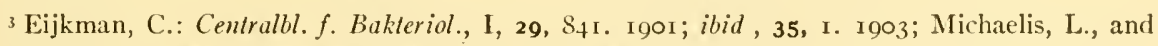
Nakahura, Y.: Ztschr.f. Immunitätsforsch. u. exper. Therap., 36, 449. 1923.

+ Kendall, A. I., and Simonds, J. P.: J. Infect. Dis., I5, 354. I914.

; Oppenheimer, C.: Lehrbuch der Ensyme. Leipzig, I927. 
which hydrolyze fructosides, such as sucrose, raffinose, gentianose, and stachyose; the $a$-glucosidases, which hydrolyze maltose and trehalose; the $\beta$-glucosidases, which act upon cellobiose, gentiobiose, and others; galactosidases, acting upon lactose and melibiose.

The various carbohydrases are produced abundantly by bacteria and other microorganisms. However, considerable specificity exists among the enzymes of different organisms. Cellulase is produced by very few groups of bacteria, namely, those which are capable of utilizing complex celluloses as sources of energy; this is an endoenzyme, although it may also diffuse outside of the cell. ${ }^{\mathrm{r}}$ Hemicellulase or cytase is distributed more abundantly, due to the fact that many more types of bacteria are capable of attacking hemicelluloses and utilizing them as sources of energy. However, neither of these enzymes can be obtained in any great abundance from bacterial cultures.

Amylase (diastase) is produced both by a greater number of organisms and in considerably larger amounts. It is sufficient to mention the bacteria of the B. subtilismesentericus group, which are even utilized for producing the enzyme on a commercial scale. B. anthracis, B. tuberculosis, $Y$. cholerae, pyogenic streptococci, etc., B. coli, $B$. pneumoniae, and various other bacteria produce only traces of this enzyme. ${ }^{2}$ This enzyme can be best demonstrated by growing the bacteria upon a starch agar plate, then covering the plate with a dilute solution of iodine in potassium iodide.

Gelase is produced by some bacteria. ${ }^{3}$ Pectinase is produced by $B$. carotovorns and other bacteria.

Saccharase or invertase is produced by various bacteria, including $B$. subtilis, $B$. mesentericus, $B$. megatherium, B. fuorescens, $B$. pneumoniae, hemolyt ic streptococcus, butyric acid bacteria, etc. The most important source of invertase among microorganisms is found, however, among the yeasts. Maltase and lactase are also produced by a number of bacteria. These also are formed in greater abundance by yeasts and fungi than by bacteria. Emulsin formation has been demonstrated for a number of bacteria, ${ }^{4}$ especially among representatives of the colon-typhoid group. The property of forming this enzyme is frequently utilized for the specific differentiation of bacteria. In the decomposition of glucosides in various natural fermentations (indigo, flax, etc.), bacteria play a prominent rôle.

Nucleases, or the group of enzymes which hydrolyze the nucleic acids, are also found among the bacteria. ${ }^{5}$

The decomposition of the protein molecule to its simplest constituents is carried out by three distinct groups of enzymes, namely, (I) the proteases, which hyrolyze true proteins to proteases and peptones; (2) the peptidases or ereptases which hydro-

${ }^{1}$ Kellerman, K. F., McBeth, I. G., Scales, F. MI., and Smith, N. R.: Centralbl.f. Bakteriol., II, 30, 502. 1913; Pringsheim, II.: Zlschr.f. phys. Chemie, 78, 226. 1912.

${ }^{2}$ Fermi, C.: Centrullh.f. Bukltriol., I 2, 7I 3 -15. IS92; ibid., I, 40, IS7. I905.

${ }^{3}$ Biemacki, W': ibid., II, 29, 166. I91 I.

4 Weintraub, A.: ibid., I, Urig., 91, 273. 1924; Fermi, C., and Montesano, G.: ibid., r5. 722 189.

${ }_{5}^{5}$ Schittenhelm, A., and Schrocter, I.: Ztschr. f. phys. Chemie, 39, 20,3. 1903; 40, 62, 70; 41 $28.4 ; 57,21$. 1908 . 
lyze proteoses, peptones, and various other polypeptides to amino acids; (3) the amidases which attack the amino acid molecule with the formation of ammonia.

Bacteria vary greatly in their ability to form proteolytic enzymes. Some form true proteases, similar to the animal trypsin; others are unable to form enzymes which act upon true proteins, but form ereptases. Although the bacterial proteases are usually classified with animal trypsin, due to the fact that they are sensitive to acids and that they act best at neutral or alkaline reactions, they are not identical with this enzyme. ${ }^{1}$ Some bacteria produce both trypsin-like and erepsin-like enzymes. ${ }^{2}$ The formation of proteolytic enzymes by bacteria in culture media is independent of the presence of proteins in the medium, the gelatin-liquefying enzyme being produced by various bacteria in simple, non-protein media just as abundantly, although not quite so rapidly, as in complex protein media. The reaction of the medium affects enzyme formation only in so far as it influences the growth of the bacteria. ${ }^{3}$

Bacteria vary also greatly in their capacity to form autolytic enzymes. The capacity of bacteria to produce proteolytic enzymes has frequently been used for diagnostic purposes. Here belong the various tests of liquefaction of gelatin, coagulated egg-albumen and blood serum, milk coagulation and clarification, etc. Of special interest in this connection is the rôle of proteolytic enzymes in the formation of toxins by bacteria. Some investigators ${ }^{4}$ consider bacterial toxins to be not specific secretory products of the metabolism of the particular organisms, but biochemical transformation products of the constituents of the medium, as a result of the transformation of the nutrients by bacterial enzymes. The primary non-toxic transformation products give rise, by secondary fermentative decomposition, to very toxic transformation products which may become non-toxic as a result of further decomposition. They are not formed in the cell, since autolyzed cells are only slightly toxic and are not regularly produced in the culture when amino acids are present, but are more frequently produced from albumoses and peptones.

Hemolysin, or the enzyme which "dissolves" red blood cells, is formed by a number of bacteria including B. pyocyaneus, B. tetani, B. coli, staphylococci, and streptococci. This enzyme was first considered to be of the nature of a proteolytic enzyme, but more recent studies have shown these enzymes to be distinctly different. ${ }^{5}$ No definite relationship has been established between the hemolytic power of an organism and its pathogenicity.

The bacteriolytic enzyme of certain bacteria is closely related to the proteolytic and hemolytic enzymes. Pyocyanase, or the bacteriolytic enzyme of B. pyocyaneus, has been studied most extensively. This enzyme or enzyme-like substance is relatively thermostabile, resisting heating at $100^{\circ} \mathrm{C}$. for thirty minutes.

Rennet (lab), the enzyme responsible for milk coagulation, is produced abundantly by bacteria, such as B. prodigiosus, B. pyocyaneus, B. fluorescens, B. amylobacter,

¿Dernby, K. G.: Biochem. Ztschr., ז26, го5. 1921 ; Dernby, K. G., and Blanc, J.: J. Bact., 6, 419. I92I.

${ }^{2}$ Corper, H. J., and Sweany, H. C.: J. Biol. Chem., 29, xxi. I9I4; J. Bact., 3, I29. I9I8.

3 Jordan, E. O.: Biol. Studies, Pupils of IV. T. Sedguick, p. I 24. Boston, Igo6.

4 Dernby, K. G., and Siwa, S.: Biochem. Ztschr., r34, I. I922.

${ }_{5}$ McNeil, A., and Kahn, B. L.: J. Immunol., 3, 295. I9IS; Orcutt, M. L., and Howe, P. E.: J. Exper. Med., 35, 409. I922. 
$V$. cholerae, etc. It is formed by bacteria on casein-containing and casein-free media, and is readily secreted by the cells into the surrounding medium. At first it was thought to be identical with protease but was later found to be a different enzyme.

Among the amidases and deaminases, or the enzymes which hydrolyze the amides and amino acids with the formation of ammonia, it is sufficient to mention urease, histozyme, asparaginase, arginase, and purinamidases. Urease is produced by various bacteria in considerable amounts. The formation of the enzyme is greatly influenced by the composition of the substrate. Histozyme hydrolizes hippuric acid into benzoic acid and glycocoll and is formed by various fungi and bacteria.

A detailed review of the various theories of oxidation-reduction is given elsewhere. ${ }^{2}$ It is sufficient to call attention here to the occurrence of some of the enzymes of bacteria responsible for such reactions. A typical oxidation-reduction reaction can be illustrated as follows:

$$
\begin{aligned}
& \text { R. } \mathrm{CHO}, \mathrm{H}_{2} \text { R. } \mathrm{CH}_{2} \mathrm{OH} \\
& \text { R. } \mathrm{CHO}^{+}{ }_{\mathrm{O}}=\mathrm{R} \cdot \mathrm{COOH}
\end{aligned}
$$

This reaction can also be represented as follows:

$$
\begin{aligned}
& \mathrm{CH}_{3} \cdot \mathrm{CHO}+\mathrm{H}_{2}=\mathrm{CH}_{3} \cdot \mathrm{CH}_{2} \mathrm{OH} \\
& \mathrm{CH}_{3} \cdot \mathrm{CH} \backslash \mathrm{OH}-\mathrm{H}_{2}=\mathrm{CH}_{3} \cdot \mathrm{COOH}
\end{aligned}
$$

One molecule is oxidized and the other is reduced. In the foregoing illustration both molecules ("donator" and "acceptor") are the same. The oxidative phase can be considered as one of dehydration and the reducing phase as one of hydration. It is possible, however, that the acceptor and donator of hydrogen are two distinctly different substances, as in the reduction of methylene blue in fresh milk in the presence of formaldehyde; the latter is the hydrogen donator and the methylene blue the acceptor; nitrate may also act as an acceptor and sulphhydryl group (R.SH) as a donator. The process of dehydration is oxidation and the hydration is reduction. The enzyme which is responsible for the splitting off of the carboxyl group is carboxylase. No atmospheric oxygen is introduced in the oxido-reductase and carboxylase reactions. The same is true of catalase, which brings about the decomposition of $\mathrm{H}_{2} \mathrm{O}_{2}$ to water and oxygen.

According to Bach and Warburg, direct oxidases, which are capable of activating atmospheric oxygen, are to be distinguished from oxido-reductases (perhydridase). Peroxidases, or the enzymes which are active in the presence of peroxides, and zymases, or the enzymes of fermentation, are also included in this group.

Respiration, consisting in the absorption of oxygen and the liberation of $\mathrm{CO}_{2}$, is replaced under anaerobic conditions by fermentation or intramolecular respiration. Both result in the liberation of energy. An anaerobe can be grown in the presence of oxygen provided the medium has a proper reduction potential. ${ }^{3}$

${ }^{x}$ Loeb, A.: Centralbl.f. Bakteriol., I, 32, 47 I. 1902; Gorini, C.: ibid., II, 8, 137. I902; ibid., 24, 369, 470. 1915; ibid., 26, 195, 223. 1917; ibid., 55, 2.70. 1920.

"See Schoen, M.: Le Probleme des fermenlations. Paris: Matsson et Cie, 1926; Nord, F. F.: Chem. Rev., 3, 60. r926; also chaps. xii, xiv, in this volume.

${ }^{3}$ Quastel, J. H.: Biochem. J., 18, 365. 1924; 19, 304, 660. I925. 
Zymases are produced in aljundance by a number of bacteria, especially those forming lactic and acetic acids. The reductases produced by bacteria have been studied extensively in connection with the Schardinger reaction or the reduction of clyes in milk. Among the oxidases ${ }^{1}$ which are produced by bacteria, it is sufficient to mention tyrosinase, luciferase, and various phenolases.

Catalase is produced abundantly by various acrobic bacteria as well as by bacterial spores, the time of maximum formation of the enzyme depending upon the nature of the organism and composition of medium. Anacrobic bacteria produce practically no catalase; this accounts for the sensitiveness of these organisms to $\mathrm{H}_{2} \mathrm{O}_{2}$ and to oxygen. A definite relation was found ${ }^{2}$ to exist between the reducing power of bacteria and their capacity for forming peroxide, anaerobes tending to produce peroxide in the presence of oxygen. The formation or presence of catalase prevents the accumulation of the peroxide and injury to the growth of the bacteria. Hence the ability of an organism to produce catalase may account for its ability to grow in an atmosphere containing free oxygen. ${ }^{3}$

\section{IMPORTANCE OF ENZYMES IN LIFE-PROCESSES}

Most of the nutrients which are available to bacteria, both in artificial culture media and under natural conditions, are not in a form that can be utilized by these organisms for metabolic purposes. These nutrients must first undergo a series of changes, which are largely brought about by means of enzymes produced by the bacterial cells. These changes include the processes of decomposition, which result in the transformation of the complex organic substances into simpler compounds, and the processes of oxidation which lead to the liberation of large quantities of energy. In addition to these changes, which result in the degradation of the complex organic substances, the synthesizing activities of the cell (as well as most processes of reduction) are also carried out by means of enzymes. The simpler substances formed from the complex organic materials by processes of hydrolysis, oxidation, and reduction are utilized as building stones for the synthesis of bacterial protoplasm and other complex organic materials.

Since bacteria vary in the nature of the nutrients which they can utilize and in the conditions under which these nutrients are utilized, the nature of the enzymes produced by the different organisms will also vary.

The enzymes do not increase the energy content of the system nor do they influence the equilibrium conditions. However, they do influence the rapidity with which the equilibrium is reached or the time required to carry out a certain process.

\section{GENERAL REFERENCES}

Bayliss, W. M.: The Nature of Enzyme Action. 5th ed. London, i925.

Euler, H. V.: Chemie der Enzyme. Part I. 3d ed. I925; Part II, Abt. I and 2. 2 d ed. München, I922-27.

${ }^{1}$ Felton, L. D.: J. Exper. Med.. 38, 291. r923; J. Infect. Dis., 34, 407. I924.

${ }^{2}$ M'Leod, J. M., and Gordon, J.: J. Path. \& Bact., 26, 326, 332. I923; 28, I47, I55. I923.

3 See chap. xiv in this volume. 
Oppenheimer, C.: Die Fermente und ihre Wirkungen. 5th ed. Leipzig, 1924-27.

Waksman, S. A., and Davison, W. C.: Enzymes, Properties, Distribution, Methods and Applications. Baltimore, I926.

Waldschmidt-Leitz, E.: Die Enzyme. Braunschweig, 1926.

A detailed review of the formation of enzymes by bacteria is given by:

Fuhrmann, F.: Vorlesungen ïber Bakterienenzyme. Jena, 1907.

Waksman, S. A.: Enzymes of Microorganisms. Abstr. Bact., 6, 265, 331. 1922.

Kruse, W.: Allgemeine Mikrobiologie. Leipzig, I9ı. 


\title{
CHAPTER XX
}

\section{SYNTHETIC CULTURE MEDIA}

\author{
H. IV. SCHOENLEIN \\ Detroit, Mich.
}

Synthetic culture media such as are used in the bacteriological laboratory may be defined strictly as those substrates which contain only ingredients of known composition and purity, and prove useful in the cultivation of micro-organisms. In some cases, the term is used more loosely to include certain media in which the ingredients are of known or specified purity, though not necessarily of known composition. Occasionally the term is (incorrectly?) used for certain media containing materials of unknown or indefinite composition. ${ }^{\mathrm{T}}$

Synthetic media may therefore be grouped under the following headings:

A. Synthetic media in the strict sense

B. Pseudo-synthetic media

I. Media in which there are present compounds of more or less uncertain chemical composition, but of known purity. For example, the exact chemical composition of certain of the proteins is unknown, although they may be secured in a condition of high purity. In such media the materials of unknown composition are nutrients.

2. Media in which all nutrients are of known composition, but in which the non-nutrients may be of unknown or indefinite composition. Agar, for example, may be quite completely freed from materials which might serve as nutrients. Its exact chemical composition is somewhat indefinite, and it is not itself a nutrient for most micro-organisms. Its inclusion in the medium is therefore frequently not regarded as preventing such a medium being classed as synthetic.

It is commonly agreed that a medium is non-synthetic if it includes plant or animal extracts, digests, infusions, or tissues.

In general, the media used by the earlier workers in bacteriology were nonsynthetic; most of the synthetic media have been suggested within the last three decades. A few were noted as useful before I900. Probably the first to be described was that of Cohn. ${ }^{2}$ This investigator developed a synthetic medium containing ammonium tartrate which he found to be particularly useful for the cultivation of fluorescent bacteria. Other early synthetic media were proposed by Naegeli, ${ }^{3}$ Fermi, ${ }^{4}$ Uschinsky, ${ }^{5}$ Beijerinck, ${ }^{6}$ and Winogradsky. ${ }^{7}$ Synthetic media are listed by Krasnow,

I The so-called Waksman's synthetic acid agar contains peptone, an indefinite mixture of unknown composition.

${ }^{2}$ Cohn, Ferdinand: Beitr. s. Biol.d. Pflansen, I, 127. 1875 .

${ }^{3}$ Naegeli,: C. Untersuchungen iiber niedere Pilse aus dem Pfanzenphysiologischem Institut in II ünchen. I882.

${ }_{4}$ Fermi, C.: Arch.f. Hyg., I4, I. I892.

5 Uschinsky, N.: Centralbl.f. Bakteriol., I Abt., 14, 3 16. I893.

${ }^{6}$ Beijerinck, M. W.: ibid., II Abt., I, I. I895. 7 Winogradsky, S.: ibid., II Abt., 2, 425. I896. 
Rivkin, and Rosenberg ${ }^{1}$ in nine groups, based upon the types of organisms for which the media were designed. A review of the bacteriological literature up to I925 shows that approximately twenty-five hundred different combinations, which may be classified as synthetic media in the broader sense, have been described. It is manifestly impossible here even to outline a classification of these media, and certainly impracticable to discuss formulas in detail. It is the purpose of this article briefly to outline the newer knowledge relative to the uses to which synthetic media may be put, and the precautions to be used in the development of a satisfactory or "op imum" medium.

Probably the outstanding advantage of a synthetic medium over a non-synthetic medium is the ease of duplication. It is only when culture media are identical in composition that the cultural characters of two organisms can be compared or the results of studies by different observers on the same organism satisfactorily evaluated. Synthetic media are essential in many cases to a determination of the exact nutrient requirements of an organism, and often constitute the most satisfactory substrates to be used in the isolation and identification of the various products of metabolism. They lend themselves to the determination of ion effects or the necessity for the presence of bios, hormones, auximones, or other growth accelerants. In general, it is advisable, when possible, to use synthetic media for studies of the chemical composition of the cells of micro-organisms or of their products. Particular care is needed in testing the purity of constituents when it is desired to determine the ability of organisms to synthesize vitamines, bios, etc.

A satisfactory synthetic medium should include:

a) All elements essential to normal cell metabolism

b) The elements combined into compounds which may be utilized by the cell

c) Compounds which will act as buffers, poisers, etc., useful in that they tend to maintain a suitable hydrogen-ion concentration, oxidation-reduction potential, etc.

d) Compounds, elements, or ions not strictly nutrients but which may exert a stimulative action

e) All compounds and elements adjusted in concentration to give optimum growth conditions

ELEMENTS ESSENTIAL FOR THE GROWTH OF MICRO-ORGANISMS

The presence of an element, even in considerable amounts, in the cell of a microorganism is not sufficient to prove it essential. It has been repeatedly determined that the chemical composition of the cell of a micro-organism may be markedly influenced by the composition of the medium in which it is grown. Experimental evidence based upon cultural tests is therefore necessary to determine the essential or the nonessential character of a particular element.

Apparently all cells contain proteins, nucleo-proteins, and water. The elenents always found in these compounds, elements certainly essential to cell life, are carbon, hydrogen, oxygen, nitrogen, phosphorus, and probably sulphur. In addition, potassium, chlorine, and iron are generally regarded as necessary. It is common to include in the list sodium, calcium, and magnesium. It may be shown, however, that with certain micro-organisms, at least the last-named elements are either nonessential or need to be present only in traces.

× Krasnow, F., Rivkin, H., and Rosenberg, M. L.: J. Bact., I2, 385. 1926. 


\section{COMPOUNDS OF ELEMENTS ESSENTLAL FOR GROWTH}

It is necessary in a synthetic medium not only that the essential elements be present, but that they be present in types of compounds which may be utilized by the cell. Of special importance are the compounds which contain the elements phosphorus, carbon, or nitrogen.

Apparently phosphates constitute the source of phosphorus for practically all micro-organisms. Salts of phosphoric acid are therefore almost universally added to synthetic media. They are useful not only as nutrients but also as buffers.

Synthetic culture media may be sharply differentiated in to two groups based upon the form in which carbon is supplied. Certain types of organisms are capable of synthesizing organic compounds from carbon dioxide or the carbonates, while others require carbon in "organic" form.

Those micro-organisms which are capable of utilizing carbon dioxide, or the carbonates, must in the synthesis of cell-carbon compounds reduce the carbon partially. Those bacteria which bring about this change have been termed "oligocarbophilous" (Beijerinck) or "autotrophic." For their cultivation, synthetic media are commonly employed.

The reduction of carbon dioxide and the synthesis of organic carbon compounds are endothermic processes, and require available energy. Apparently two sources of such energy have been utilized by different types of autotrophic organisms. The radiant energy of sunlight is absorbed by the pigments of certain types of cells, and is used as an energy source. Such organisms are said to be "photosynthetic." Among the bacteria most of the autotrophic forms are "chemosynthetic." These secure the needed energy by bringing about chemical changes (oxidations) in elements or their compounds.

Synthetic media for the growth of photosynthetic organisms have been found suitable for cer ain of the green algae (Chlorophyceae), blue-green algae (Schizophyceae), and attempts (largely unsuccessful) have been made to secure such a medium for certain of the true sulphur bacteria containing bacteriopurpurin. For the group last named a suitable synthetic medium is highly desirable, as the metabolism is poorly understood. For such would be necessary a medium relatively rich in carbon dioxide or carbonates and a sufficient concentration of hydrogen sulphide to stabilize the oxidation-reduction potential at a point suitable for growth of these microaerophiles. All of these strictly photosynthetic forms require exposure to light.

Synthetic media for the cultivation of chemosynthetic micro-organisms have been elaborated for each of the important known groups. The first were those developed by Winogradsky ${ }^{x}$ for the culture of the so-called "nitrifying bacteria." Essential to the growth of the organisms which oxidize ammonia to nitrites (Nitrosomonas) was found to be the presence of a suitable concentration of an ammonium salt (usually ammonium sulphate), carbon dioxide or carbonates, a high buffer content to prevent the solution from becoming acid, ${ }^{2}$ and a sufficient supply of free oxygen. Buffer and carbonates are usually supplied by adding an excess of magnesium carbonate. Simi-

${ }^{x}$ Winogradsky, S.: Ann. de l'Inst. Pasteur, 4, 21 3; 4, 577. I89o.

${ }^{2}$ If the reaction be written as $\left(\mathrm{NH}_{4}\right)_{2} \mathrm{SO}_{4}+{ }_{4} \mathrm{O}_{2}={ }_{2} \mathrm{HNO}_{3}+\mathrm{H}_{2} \mathrm{SO}_{4}+{ }_{2} \mathrm{H}_{2} \mathrm{O}$, it is evident that from each molecule of the neutral salt there are developed three molecules of acicl. The necessity for the presence of an ample base reserve is evident. 
larly, the essentials for the growth of Nitrobacter (oxidizing nitrites to nitrates) are a suitable salt of nitrous acid (usually potassium nitrite), carbon dioxide or carbonates, and a satisfactory buffer.

Numerous synthetic media have been developed for the group of organisms capable of securing "growth energy" for the assimilation of carbon dioxide by the oxidation of sulphur or its compounds. Those organisms which have been cultured belong to the genus Thiobacillus of Beijerinck. One species, Thiobacillus denitrificans Beijerinck, ${ }^{\mathrm{I}}$ secures its "growth energy" for the assimilation of carbon dioxide under anaerobic conditions by the simultaneous reduction of nitrates to free nitrogen and the oxidation of elementary sulphur to sulphuric acid. Essential to such a medium are therefore sulphur (elementary), nitrates, carbonates (or carbon dioxide), and a suitable buffer.

Other species of the genus Thiobacillus oxidize sulphur or its compounds under aerobic conditions, and a variety of synthetic media have been devised for their study. These contain either elementary sulphur or reduced sulphur in the form of thiosulphates. Carbon is derived from carbon dioxide or the carbonates, and nitrogen usually from ammonia.

Synthetic media for the growth of certain of the autotrophic iron bacteria (Chlamydobacteriales) have been developed by Migula ${ }^{2}$ and Schorler. ${ }^{3}$ Apparently in all cases there must be provided a soluble salt of ferrous iron or manganous manganese, carbon dioxide or carbonates, ammonia, and oxygen. It is claimed that "growth energy" is secured by the oxidation of the metallic ion.

Certain bacteria (as members of the genus Hydrogenomonas) develop in a synthetic culture medium supplied with gaseous hydrogen, oxygen, carbon dioxide, and a suitable source of nitrogen (as ammonia). ${ }^{4}$

Other organisms have been described which develop on a similar culture medium in which the hydrogen is replaced by carbon monoxide as does Carboxydomonas oligocarbophila described by Beijerinck and Van Delden, ${ }^{5}$ or by methane (Methanomonas methanica). ${ }^{6}$

Non-autotrophic (better, non-oligocarbophilous) organisms require for their growth the presence of organic (carbon) compounds. Apparently in all cases studied these carbon compounds serve at least three functions: $(\dot{a})$ they may be oxidized in whole or in part with the freeing of energy of use to the cell, (b) they may be used in the synthesis of protoplasm or essential cell parts, or $(c)$ they may be stored (after more or less modification) as reserve food. Carbon compounds which have been used in the preparation of synthetic media are very numerous. Such synthetic media may conveniently be classified on the basis of the source of the nitrogen supplied.

The nitrogen-fixing bacteria are frequently cultured on synthetic media; in most cases the "energy source" supplied is a carbohydrate or a polyatomic alcohol. The

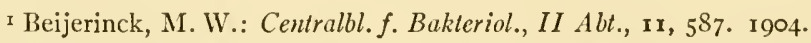

${ }^{2}$ Migula, M.: Arbeilen aus den bakt. Inst. der techn. II ochschule zu Karlsruhe, 1, 235, 238. I 894.

${ }^{3}$ Schorler, B.: Centralbl.f. Bakteriol., II Abt., r2, 691. 1904.

${ }^{4}$ Kaserer, H.: ibid., r6, 681-96. rgo6.

s Beijerinck, M. W., and Van Delden, A.: ibid., 10, 33-47. 1903.

${ }^{6}$ Söhngen, N. L.: ibid., r5, 513-17. 1906. 
sole source of nitrogen is the nitrogen gas of the atmosphere. Such synthetic media are generally used for the cultivation of Azotobacter and Rhizobium.

Synthetic media to which nitrogen compounds are added may be divided into $(a)$ those in which the nitrogen is supplied as ammonia, or some closely related amine, amino-acid, etc., from which ammonia is produced by hydrolysis; (b) those containing nitrites or nitrates; and $(c)$ those containing other nitrogenous compounds such as cyanogen, ring compounds, etc. Ability to utilize these various forms of nitrogen constitutes an excellent criterion for the differentiation of micro-organisms. Most micro-organisms are able to utilize ammonia; a smaller proportion nitrates; and fewer yet, other compounds.

\section{DETERMINATION OF THE OPTIMUM MEDIUM}

It is sometimes advisable to determine the concentration of each of the various ingredients which will produce a medium optimum for the growth of a particular micro-organism. Such media have been frequently elaborated by plant physiologists for the cultivation of plant seedlings. Several investigators have used a somewhat similar technique for the development of a medium for bacteria.

It should be emphasized that there can scarcely be such a thing as an optimum medium in the broad sense for any organism. The addition of one ingredient may alter the optimum concentration of another. For certain materials the optimum concentration is a function of the temperature; in other cases it varies with hydrogen-ion concentration.

It is evident that the term "optimum" as applied to a medium can mean only those concentrations of a given number of ingredients which will give maximum growth with other environmental influences fixed. In the development of such an optimum medium it is usually advisable to determine the materials which must be present for growth, and vary their concentrations one at a time. Such a method, for example, has been used by Fulmer, Nelson, and Sherwood, ${ }^{\mathrm{I}}$ in the development of their Medium $\mathrm{E}$ for the growth of a yeast. It was found that cane sugar, $\mathrm{K}_{2} \mathrm{HPO}_{4}$, $\mathrm{NH}_{4} \mathrm{Cl}, \mathrm{CaCl}_{2}$, and $\mathrm{CaCO}_{3}$ were necessary for growth. The exact concentration of each which would give a maximum rate of growth per cell during the logarithmic growth period was determined. It is of interest to note that the optimum concentration of certain of the ingredients named varied little or not at all with temperature. This was not true of the concentration of the ammonium salts; this varied with the temperature, that is, for each temperature there was a concentration of ammonia which gave maximum growth.

Many studies have been made for the selection of optimum media by other methods. A notable example of such is the effort of Krasnow, Rivkin, and Rosenberg ${ }^{2}$ who studied the suitability of some 67 I different synthetic media to support continuous growth of streptococci without success.

Much interest has been manifested since the work of Wildiers ${ }^{3}$ on the study of the continued growth of organisms in synthetic media. This author stated that yeasts

\footnotetext{
r Fulmer E. I., Nelson V. E., and Sherwood, F. F.: J. Am. Chem. Soc., 43, I9I-99. I921.

${ }^{2}$ Krasnow, F., Rivkin, H., and Rosenberg, M. L.: J. Bact., 12, 385. I926.

3 Wildiers, E.: Cellule, 18, 313. I901.
} 
would not grow when seeded in small numbers in a synthetic medium, but required the presence of a growth stimulant, bios. For a summary of the literature on this topic, the reader is referred to the excellent review by Tanner, Devereux, and Higgins. ${ }^{\mathbf{I}}$

For a synthetic medium to be wholly satisfactory, it should support growth when continuous transfers of small inocula are made.

Certain other difficulties are inherent in the preparation of synthetic media. Particular care must be used in most cases to adjust suitably the buffer content. In many cases distilled water may prove troublesome, for traces of metal from containers may exert a marked inhibitory action; in other cases they may stimulate growth. Distilled water made from city supplies which are chlorinated may contain sufficient chlorine to influence results markedly. In many cases it is necessary to guard against the toxic effects of one ion by the addition of another which antagonizes it. A comprehensive review of the influence of ions on microbial physiology has been given by Falk. ${ }^{2}$

I Tanner, F. W., Devereux, E. D., and Higgins, F. M.: J. Bact., II, 45-64. I926. See also chap. xxxvii in this volume.

${ }^{2}$ Falk, I. S.: Abst. Bact., 7, 33, 87, I33. I923. 


\section{CHAPTER XXI}

\section{DETERMINATIONS OF THERMAL DEATH-TIME}

\section{J. RUSSELL ESTY}

Research Laboratories, National Canners Association, San Francisco

Studies on thermal death-points date back to 1745 , when Needham showed that boiling an infusion of mutton gravy and soup from seeds to kill all living things and sealing it hermetically did not prevent "spontaneous generation" since some flasks became cloudy. About 1760 Spallanzani, believing that Needham had not heated the bottles long enough or plugged them tightly enough, and after heating an extensive series of flasks containing an infusion of peas and almonds, recommended boiling for three-quarters of an hour. In 186 I Pasteur demonstrated that heating at $110^{\circ} \mathrm{C}$. $\left(230^{\circ} \mathrm{F}\right.$.) under pressure may be necessary to prevent "spontaneous germination." In 1870 Ferdinand Cohn showed that irregular results following heating were due to the survival of spores which, according to later workers, were able to resist varying degrees of heat.

\section{DESTRUCTION OF SPORES}

Bredfeld $^{\mathrm{r}}$ reported that spores of $B$. subtilis required 3 hours at $100^{\circ} \mathrm{C}$. $\left(212^{\circ} \mathrm{F}\right.$.) or 5 minutes at $110^{\circ} \mathrm{C}$. $\left(230^{\circ} \mathrm{F}\right.$.) to kill them. Arloing, Cornevin, and Thomas $^{2}$ found that spores of B. chawvoei, if dried, resisted boiling for nearly 2 hours, whereas in the moist state they did not resist boiling for more than 2 minutes. In this connection Zettnow's ${ }^{3}$ observations on the resistance of spores to dry heat are of interest. He reported viable organisms in lime paste from a sugar factory after heating for 30 minutes at $310^{\circ}-320^{\circ} \mathrm{C}$. $\left(590^{\circ}-608^{\circ} \mathrm{F}\right.$.). These organisms grew readily at $37^{\circ} \mathrm{C}$. but did not grow at $58^{\circ}-59^{\circ} \mathrm{C}$. He also claimed that spores from the lime paste which had been dried on silk threads survived from 20 to 25 hours in live steam. On retesting the original material, which had been stored in a paper carton in a sealed room for six months, he observed that the organisms were killed below $199^{\circ} \mathrm{C}$. $\left(390.2^{\circ} \mathrm{F}\right.$.). Similar material, secured from twelve places in the same sugar factory about a year later, was found to be sterile after 30 minutes' exposure at $220^{\circ} \mathrm{C} .\left(428^{\circ} \mathrm{F}\right.$.).

An extensive literature dealing with this subject has accumulated, and it is impossible in this chapter to review adequately many important papers. The reader is, therefore, referred to the bibliographies and summary which have been prepared by Magoon. ${ }^{4}$

Morrison and Tanner ${ }^{5}$ have compiled the thermal relations and thermal deathpoints of a large number of thermophilic spore-bearing organisms which have been described by various investigators. They show a wide variation, some being destroyed

I Cited by Morrison, L. E., and Tanner, F. W.: J. Bact., 7, 35S. I922.

${ }^{2}$ Ibid., p. 359. 1922.

3 Zettnow, E.: Centralbl.f. Bakleriol., Abt. I, Orig., 66, г31. 1912.

${ }_{4}^{4}$ Magoon, C. A.: J. Bact., II, 253. 1926.

${ }^{5}$ MIorrison L. E., and Tanner, F. W.: op. cit., 7, 346-53. 1922. 
in a few minutes at temperatures below $100^{\circ} \mathrm{C}$., while others, notably certain soil organisms, B. cylindricus and B. tostus, studied by Blau, ${ }^{\mathrm{I}}$ are reported to have withstood heating in boiling water at $100^{\circ} \mathrm{C}$. for $\mathrm{I} 9$ hours but were killed in 20 hours. The $y^{2}$ have also determined the thermal death-point of the spores of several aerobic thermophilic bacteria from water.

Spores of certain obligate thermophiles were found by Bigelow and Esty ${ }^{3}$ to withstand boiling at $100^{\circ} \mathrm{C}$. in corn juice, $\mathrm{pH}$ 6.I, for $2 \mathrm{I}$ hours. More recently the same strains, when heated in a phosphate solution, $\mathrm{pH} 7.0$, survived 45 hours' continuous boiling at $100^{\circ} \mathrm{C}$. but were killed in 46 hours. This group of organisms (group Io0) had been isolated by Cameron and Esty ${ }^{4}$ as the causative spoilage agent from certain understerilized canned foods. As such, the foregoing findings are not only of academic interest but are also significant when adequate sterilizing processes are considered.

Numerous articles have been published on the subject of thermal death-points, and in many of these a definite time and temperature have been reported for specific organisms. However, this time-temperature relation has been frequently based upon the study of a single strain or merely a few superficial tests without taking into consideration certain fundamental biological principles involved. As a result, one is confronted with many conflicting statements which make it extremely difficult, if not impossible, to interpret discordant observations. In many cases it is necessary to make additional tests before the results can be evaluated and safely applied to all life-processes.

\section{EXPERIMENTS WITH B. TUBERCULOSIS}

Take, for example, the problem of establishing a safe standard for the pasteurization of milk. This involves a study of the heat resistance of pathogenic organisms which are present in milk, particularly B. tuberculosis, B. typhosus, and the pathogenic streptococci. As regards the tubercle bacillus, at least twenty-five reports were published from $\mathrm{I} 883$ to 1906 .

It appears from the results reported ${ }^{5}$ that under certain conditions the heat resistance of $B$. tuberculosis may vary from $140^{\circ}-\mathrm{I} 56^{\circ} \mathrm{F}$. for 15 minutes, $140^{\circ}-\mathrm{I} 55^{\circ} \mathrm{F}$. for 20 minutes, and at $140^{\circ} \mathrm{F}$. from 10 minutes to 6 hours, and at $212^{\circ} \mathrm{F}$. from less than 30 seconds to 3 hours.

When we consider these variable results in the light of the apparently successful time and temperature requirements for pasteurization that were in effect in 1923 in one hundred of the larger American cities ( $142^{\circ} \mathrm{F}$. for 30 minutes was required for pasteurization in forty of these cities and the maximum in any case was $145^{\circ} \mathrm{F}$. for 30 minutes), ${ }^{6}$ it is evident that some of these observations on the heat resistance of the tubercle bacillus are either in error or are not applicable to the commercial process. In this connection it should be borne in mind that heat-resistance data obtained from

I Blau, O.: Centralbl. f. Bakteriol., Abt. II, I5, 97. I905.

${ }^{2}$ Morrison, L. E., and Tanner, F. W.: Bot. Gas., 77, 2, I7I. I924.

3 Bigelow, W. D., and Esty, J. R.: J. Infect. Dis., 27, 602. I 920.

+ Cameron, E. J., and Esty, J. R.: ibid., 39, 2, 89. 1926.

${ }_{5}$ U. S. Pub. IIealth Bull. I47, Part X, Table I, p. I29. I925. Also cf. chap. xxxii in this volume.

${ }^{6}$ Secured from data collected in an unpublished survey by the American Public Health Association and the U.S. Public Health Service (Am.J. Pub. Health, p. 375. 1927). 
arbitrary laboratory tests apply only to the conditions under which they were made. They are valuable in establishing certain fundamental principles, but in making definite regulations for commercial practice the work must be done under actual existing conditions.

\section{THE DESTRUCTION OF SPORES OF Cl. tetani}

The variations in the heat resistance of the spores of $\mathrm{Cl}$. tetani may be cited as another example. Some of the published reports may be briefly summarized as follows:

Kitasato, ${ }^{\mathrm{I}}$ who isolated the bacillus, stated that the spores survived moist heat at $80^{\circ} \mathrm{C}$. $\left(176^{\circ} \mathrm{F}\right.$.) for $\mathrm{I}$ hour but were destroyed in steam $\left(100^{\circ} \mathrm{C}\right.$.) $\left(212^{\circ} \mathrm{F}\right.$.) in 5 minutes. Vaillard and Vincent ${ }^{2}$ observed that the spores resisted $80^{\circ} \mathrm{C}$. for 6 hours, $90^{\circ} \mathrm{C}$. for 2 hours, $100^{\circ} \mathrm{C}$. for from 3 to 4 minutes, were not always destroyed in 5 minutes, but never resisted more than 8 minutes. Levy and Bruns ${ }^{3}$ report that $\mathrm{Cl}$. tetani spores were not destroyed in $8 \frac{1}{2}$ minutes at $100^{\circ} \mathrm{C}$. and that few survived 5 minutes, but they were killed in 30 minutes. Anderson reports the resistance of one strain isolated from commercial gelatin as $20-30$ seconds at $100^{\circ} \mathrm{C}$. Tuck ${ }^{5}$ claims that no spores of tetanus can resist boiling over 20 minutes. Falcioni ${ }^{-}$ impregnated gelatin with spores of tetanus grown in agar or broth for ro to 12 days and found that these spores survived $2 \frac{1}{2}$ hours but not 3 hours in steam $\left(100^{\circ} \mathrm{C}\right.$.) in 2,5 , and 10 per cent gelatin. Smith's experiments showed that tetanus spores survived $100^{\circ} \mathrm{C}$. for 20 minutes regularly, 40 minutes usually, and 60 minutes occasionally. In one case cultures contained viable spores after 70 minutes at $100^{\circ} \mathrm{C}$. Von Hibler ${ }^{8}$ observed variations in the heat resistance of seven different strains and found that in one case spores survived $2 \frac{1}{2}$ hours at $100^{\circ} \mathrm{C}$. Becker $^{9}$ reported the death-point of two strains as 2 and 3 hours, respectively, in boiling water when heated in brain or alkaline reaction.

Based on a study of twenty-four strains of $\mathrm{Cl}$.tetani, Esty and Meyer ${ }^{\mathrm{ro}}$ found the heat resistance of tetanus spores to vary at $100^{\circ} \mathrm{C}$. from 15 to 90 minutes, the average survival time being 25 minutes. At $105^{\circ} \mathrm{C}$. the heat resistance varied from 3 to 25 minutes and showed an average survival time of 9.2 minutes. The spores were produced either in pea pepticdigest broth, brain medium, double-strength veal-infusion peptic-digest gelatin, or casein broth, and were heated in a $\frac{M}{I_{5}}$ phosphate solution, $\mathrm{pH} 7.0$, with the exception of those produced in the brain medium.

These results indicate, as in the case of $B$. tuberculosis, that strains of $C l$. tetani exist, spores of which vary widely in their resistance to moist heat. Smith, ${ }^{11}$ in reviewing the literature on the thermal death-point of tetanus spores, comments as follows: "It would be necessary to know the tendency to spore formation in different media and at different

${ }^{2}$ Kitasato, O.: Ztschr.f. Hyg., 7, 225. I889.

${ }^{2}$ Vaillard, L., and Vincent, H.: Ann. de l'Inst. Pasteur, 5, 1. I89r.

${ }^{3}$ Levy, E., and Bruns, H.: Grenzgeb. d. Med. in Chir., 10, 235. 1902.

${ }_{4}^{4}$ Anderson, J. F.: U.S. P.H. and Mar. Hosp. Service, Hyg. Lab. Bull. 9. 1902.

5 Tuck, G. L.: J. Path \& Bact., 9, 38. I903.

${ }^{6}$ Falcioni, D.: Ann. d'ig. sperimentale, 14, 319. I904.

${ }_{7}^{7}$ Smith, T.: J.A.M.A., 50, 929. 1908.

${ }^{8}$ von Hibler, E.: Untersuchungen über die pathogenen Anaeroben, p. 2r I. Jena: G. Fischer, Igo8.

9 Becker, L.: Centralbl.f. Bakteriol., Abt. I, 84, 7I. 1920.

ro Esty, J. R., and Meyer, K. F.: J. Infect. Dis., 31, 650. 1922.

"Smith, T.: op. cit., p. 9.32. I908. 
temperatures; the age of the culture at which spores are ripe and, therefore, most resistant; the reaction of the medium in which the spores are boiled or steamed, because all of these variable factors have probably entered into the experiments. In the case of nutrient gelatin, which has been a favorite medium, I find sporulation very feeble and involution forms common. It is quite probable that such cultures would resist boiling but feebly. The use of dextrose in fairly large amounts, such as one or two per cent, has been frequent among bacteriologists, although this amount is inimical to rapid spore formation."

\section{THE DESTRUCTION OF THE, SPORES OF Cl. botulinum}

Equally interesting on account of the many discrepancies in the published data are the recent heat-resistance studies on the spores of $\mathrm{Cl}$. botulinum.

Van Ermengem ${ }^{\mathrm{r}}$ stated that the spores of this bacterium are considerably less heat resistant than are those of the other anaerobic bacilli known to the bacteriologists of his time. They were destroyed at $80^{\circ} \mathrm{C}$. for 30 minutes, $85^{\circ} \mathrm{C}$. for 15 minutes, or by boiling for 5 minutes. Von $\mathrm{Hibler}^{2}$ reported many years later varying resistance to heat, based on a study of three strains of $\mathrm{Cl}$. botulimum produced in different media. In one case a 4 -day brain culture survived 3 hours at $100^{\circ} \mathrm{C}$. $\left(2 \mathrm{I} 2^{\circ} \mathrm{F}\right.$.).

Burke $^{3}$, using ten American strains (probably Cl. parabotulinum Bengtson), ${ }^{4}$ concluded that autoclaving at $15 \mathrm{lb}$. ( $12 \mathrm{I} .3^{\circ} \mathrm{C}$.) for Io minutes is insufficient to destroy the more resistant spores of this anaerobe. Thom, Edmondson, and Giltner ${ }^{5}$ tested the heat resistance of cultures of the Boise asparagus strain and found that they survived $100^{\circ} \mathrm{C}$. for I hour but failed to grow in subcultures from tubes heated for 2 hours. In a series heated under pressure, growth occurred in 50 per cent of the tubes autoclaved at $10 \mathrm{lb}$. for 5 minutes, but no growth was observed after I5 lb. for ${ }^{1} 5$ minutes. Weiss ${ }^{6}$ found that the Boise strain survived 2 ro minutes at $100^{\circ} \mathrm{C}$. $\left(212^{\circ} \mathrm{F}\right.$.) but was killed in 240 minutes, survived 16 minutes at $105^{\circ} \mathrm{C}$. $\left(22 \mathrm{I}^{\circ} \mathrm{F}\right.$.), but was killed in 24 minutes and in 3 minutes at $120^{\circ} \mathrm{C}$. $\left(248^{\circ} \mathrm{F}\right.$.). Working with sixteen strains he concluded that it is evident that under the best conditions for survival the most resistant spores of $\mathrm{Cl}$. botulimum will be destroyed within 5 hours at $100^{\circ} \mathrm{C}$., within 40 minutes at $105^{\circ} \mathrm{C}$., and within 6 minutes at $120^{\circ} \mathrm{C}$., whereas the greater number of them will not survive 3 minutes.

Dickson and his associates ${ }^{7}$ report marked variations in the heat resistance of forty American strains showing a survival period varying from 30 to 375 minutes at $100^{\circ} \mathrm{C}$. Table I gives the maximum thermal death-times for spores heated in sealed tubes in different media and at different temperatures. The times include a lag period of from $2 \frac{1}{2}$ to 4 minutes in each case.

Esty and Meyer, and Esty, ${ }^{8}$ found the spore resistance of I I 2 strains of $\mathrm{Cl}$. botulinum to vary from 3 to 75 minutes at $105^{\circ} \mathrm{C}$. The maximum survival times of such spores heated in a phosphate solution, $\mathrm{pH} 7.0$, was found to be 330 minutes at $100^{\circ} \mathrm{C}$. (21 $2^{\circ} \mathrm{F}$.), I Io minutes at $105^{\circ} \mathrm{C}$. $\left(221^{\circ} \mathrm{F}\right.$.), 33 minutes at $110^{\circ} \mathrm{C}$. $\left(230^{\circ} \mathrm{F}\right.$ ), I I minutes at II $5^{\circ} \mathrm{C}$. (239 F.), and 4 minutes at $120^{\circ} \mathrm{C}$. $\left(248^{\circ} \mathrm{F}\right.$.).

${ }^{1}$ Van Ermengem, E.: IIandb. der path. MI ikroorg. (2d ed.), 4, 909-38. Jena, I9I 2.

${ }^{2}$ von Hibler, E.: loc. cit.

3 Burke, G. S.: J.A.M.A., 72, 88. I9I9.

4 Bengtson, I. A.: IIyg. Lab. Bull. I36. 1924.

5 Thom, C., Edmondson, R. B., and Giltner, L. T.: J.A.M..1., 73, 907. I9I9.

${ }^{6}$ Weiss, H.: J. Infect. Dis., 28, 70. I92 I.

7 Dickson, E. C., Burke, G. S., Beck, D., Johnston, J., and King, II.: J.A.MI.A., 79, 1239. 1922; Dickson, E. C., Burke, G. S., Beck, D., and Johnston, J.: J. Infect. Dis., 36, 472. 1925.

${ }^{8}$ Esty, J. R., and Meyer, K. F.: loc. cil.; Esty, J. R.: Am. J. Pub. Ileallh, г3, то8. 1923. 
Tanner and $\mathrm{McCrea}^{\mathrm{T}}$ also noted variations in the resistance of different strains under controlled conditions. Spores in sealed tubes exhausted to I $7 \mathrm{~mm}$. were destroyed within 5 hours at $100^{\circ} \mathrm{C}$, 2 hours at $105^{\circ} \mathrm{C}$, $1 \frac{1}{2}$ hours at $\mathrm{I} 10^{\circ} \mathrm{C}$, 40 minutes at $115^{\circ} \mathrm{C}$, and 10 minutes at $120^{\circ} \mathrm{C}$. A longer time was required for spores of the same age in open tubes than in tubes exhausted and sealed.

\section{VARIATIONS IN THERMAL DEATII-TIMES}

From the foregoing statements, which are typical examples of all thermal deathpoint studies, there appears to be considerable variation in the results obtained by different investigators on the same group of organisms. The question arises, Why do such differences exist and how can they be interpreted? Several explanations may be offered to account for these discrepancies.

Recent researches have shown that the accurate determination of the thermal death-point of bacteria depends on the careful consideration of several very important factors. It is not, as it was formerly considered, a simple procedure of subjecting the

TABLE I

\begin{tabular}{|c|c|c|c|c|c|}
\hline \multirow{2}{*}{ MEDIUM } & \multicolumn{5}{|c|}{ Time in Minutes At } \\
\hline & $100^{\circ} \mathrm{C}$. & $107^{\circ} \mathrm{C}$. & $115^{\circ} \mathrm{C}$. & I $18^{\circ} \mathrm{C}$. & $12 \mathrm{I}^{\circ} \mathrm{C}$. \\
\hline 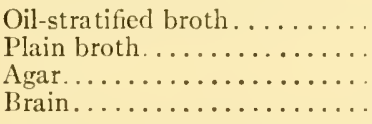 & $\begin{array}{l}375 \\
255 \\
225 \\
285\end{array}$ & $\begin{array}{l}85 \\
45 \\
25 \\
55\end{array}$ & $\begin{array}{r}43 \\
8 \\
6 \\
\text { II }\end{array}$ & $\begin{array}{l}26 \\
\ldots \ldots \\
\ldots \ldots \\
\ldots \ldots\end{array}$ & $\begin{array}{r}23 \\
5 \\
4 \\
8\end{array}$ \\
\hline
\end{tabular}

micro-organisms, vegetative or spore, to heat and then removing a loopful or small portion of the treated material to various sorts of media to determine whether or not the organisms had been killed. Moreover, there is no one time and temperature combination which alone may be defined as the thermal death-point, as the time varies with the temperature at which the organisms are heated. There is, however, a definite time-temperature relationship, the time decreasing as the temperature increases. In view of this, the term "thermal death-time"'2 probably more appropriately designates this relation than "thermal death-point."

It must be emphasized that the resistance of bacteria to moist heat is not a constant but a variable influenced by certain conditions. From previous statements concerning the spore resistance of $\mathrm{Cl}$. tetani and $\mathrm{Cl}$. botulinum, it is apparent that the heat resistance of spores of different strains of the same organism fluctuates within wide limits, even with identical conditions of cultivation, spore production, and heat treatment. Similar variations have been noted with vegetative cells, and the following examples may be cited. Based upon a study of I 74 cultures of $B$. coli, Ayers and Johnson $^{3}$ found that all the cultures survived heating for 30 minutes at $5 \mathrm{I} .7^{\circ} \mathrm{C}$., 54.59 per cent at $60^{\circ} \mathrm{C}$., 6.89 per cent at $62.8^{\circ} \mathrm{C}$., 0.57 per cent at $65.6^{\circ} \mathrm{C}$, and

${ }^{1}$ Tanner, F. W., and McCrea, F. D.: J. Bact., 8, 269. 1923.

${ }^{2}$ Mentioned for the first time by Bigelow, W. D.: J. Infect. Dis., 29, 528. I92 1.

3 Ayers, S. H., and Johnson, W. T., Jr.: J. Agric. Research, 3, 40I. I9I5. 
0.0 per cent at $68.3^{\circ} \mathrm{C}$. On retesting the 6.89 per cent, or I 2 cultures, the following variations were noted in a two-tube series heated at $62.8^{\circ} \mathrm{C}$. for 30 minutes:

\begin{tabular}{|c|c|}
\hline Positive & Positive \\
\hline First test............ I 2 & Fourth test. \\
\hline Second test.......... 4 & Fifth test $\ldots \ldots \ldots \ldots$ \\
\hline Third test.......... 8 & Sixth test........... \\
\hline
\end{tabular}

Thus the results show that cultures of $B$. coli heated for 30 minutes at $62.8^{\circ} \mathrm{C}$. may or may not survive, owing in all probability to the resistance of a few cells. Gage and Stoughton ${ }^{\mathrm{I}}$ also noted that the temperature at which final sterilization occurred with cultures of $B$. coli varied from $60^{\circ}$ to $95^{\circ} \mathrm{C}$. in 18 different tests, while the thermal death-point of the majority lay between $50^{\circ}$ and $55^{\circ} \mathrm{C}$. in $\mathrm{I} 4$ tests and $55^{\circ}-60^{\circ} \mathrm{C}$. in 4 tests.

Any statement on the heat resistance of an organism must of necessity apply to the strains tested, since it is possible that a study of a larger number of strains might give entirely different results. It has been noted that sometimes conditions may develop which affect the resistance of unknown types or even those already tested. Variants may be produced under certain conditions of growth or storage, the spores of which may exhibit varying resistance to heat. As to the comparative resistance of types $\mathrm{A}$ and $\mathrm{B}, \mathrm{Cl}$. botulinum, Esty and $\mathrm{Meyer}^{2}$ found the average survival time of the spores of 78 type A strains to be 40 .I minutes at $105^{\circ} \mathrm{C}$., whereas the average survival time of 30 type $B$ strains at the same temperature was 23.7 minutes. Although this shows that spores of type $B$ are apparently less heat resistant than those of type A, yet from the data presented in the same paper it is evident that under exceptional circumstances strains and spores of a resistance equal to that recorded for type A strains may be encountered in nature or may be produced artificially in the laboratory. Dickson et al. ${ }^{3}$ also observed that in general the average resistance of type A strains was somewhat higher than that of the type B strains, although the maximum survival time of a type B strain (No. Io) when heated in oil-stratified broth

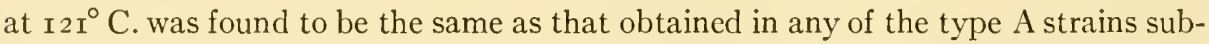
jected to the same temperature. He therefore concluded that many more tests must be made before one may assume that type $\mathrm{B}$ strains of $\mathrm{Cl}$. botulimum are consistently less resistant to heat than type A strains.

Not only the strains and the types but many other factors enter into the production of resistant spores which may influence the resistance to heat. The composition and the acidity of the media, the temperature at which the cultures are incubated, and the anaerobic conditions employed are of primary importance. Attention has already been directed to certain statements by Smith ${ }^{4}$ with respect to the sporulation of $\mathrm{Cl}$. tetani. According to Esty and Meyer, ${ }^{2} \mathrm{Cl}$. botulinum spores formed in certain media were usually of a low resistance. In such cases sporulation was feeble. Von Hibler's ${ }^{5}$ observations that "shyly sporulating" species in ill-adapted media produce spores

'Gage, S., and Stoughton, G. V. E.: Technol. Quart., I9, 41. 1906.

${ }^{2}$ Esty, J. R., and Meyer, K. F.: loc.cit.

3 Dickson, E. C., Burke, G. S., Beck, D., and Johnston, J.: loc. cit.

${ }_{4}^{4}$ Smith, T.: op. cit., p. 932.1908.

${ }^{s}$ von Hibler, E.: loc. cit. 
which would scarcely be differentiated from granular forms of vegetative bacilli have a direct bearing on this aspect of the problem. Furthermore, spores generated in different flasks of the same medium, inoculated with equal amounts of the same stock culture, and incubated for the same period show striking differences in heat resistance. Although the most resistant strains of $\mathrm{Cl}$. botulinum behave fairly consistently, yet in the most suitable medium they may exhibit a low degree of heat resistance. No explanation can be offered for this peculiar behavior. These differences are independent of the final reaction of the culture fluid within the range of $\mathrm{pH} 6-8$, the numbers and the structure of the spores, as demonstrated by Burke's staining reaction. ${ }^{\mathrm{I}}$ However, a well-buffered medium which furnishes abundant food material for "progressive growth" produces spores of average or low resistance. Krönig and $\mathrm{Paul}^{2}$ have noted that the same food does not always induce the production of spores of equal resistance.

Von Hibler ${ }^{3}$ found that the spores from a single species were very constant in heat resistance if they were not too young or too old or had not been exposed too long to incubator temperatures or in an acid medium. Weiss ${ }^{4}$ and Esty and Meyer ${ }^{5}$ found young spores to be the most heat resistant. Dickson et al. ${ }^{6}$ report that the age of botulinum spores which have been kept at room temperature in the culture medium in which they developed does not materially influence the resistance after they reach an equilibrium. They found that the 22 -day-old spores were considerably less resistant than those in a culture 66 days, but that between 66 and 315 days the resistance was practically constant. Magoon, ${ }^{7}$ working with spores of $B$. mycoides grown on sand moistened with broth, concluded that the degree of resistance is influenced by age, temperature, humidity, and possibly other factors. He states that the maximum resistance to heat develops under conditions of moderate temperature and humidity and is probably reached by the time the spores are 60 days old. Furthermore, the change in resistance took place slowly when spores were dry and cold, while a low temperature accompanied by high humidity resulted in the development of resistant spores.

Esty and Meyer ${ }^{8}$ observed that the dehydration of moist spore suspensions of $\mathrm{Cl}$. botulinum lowered the heat resistance, although this remained constant over a period of 347 days. In fact, perfectly dry spores were preserved equally well at $37^{\circ} \mathrm{C}$., room temperature, or $20^{\circ} \mathrm{C}$. indefinitely, and as such were well suited for comparative studies.

Convincing experimental evidence indicates that some of the variations in heat resistance are due to the chemical composition of the solutions in which the organisms are heated, $\mathrm{pH}$ and other so-called "extrinsic" factors. For instance, in some cases vegetative forms and spores were heated in the medium in which they were produced, in others a portion of the suspension was transferred to fresh culture media or to different solutions, while in others the organisms were washed and then resuspended in the test solution. Striking variations have been noted in the resistance of spores heated in different solutions, and Table $\mathrm{II}^{8}$ illustrates the results obtained with peptone solutions as compared to a phosphate mixture.

In view of these and numerous other observations, the spore material should be concentrated by centrifuging and the sediment washed and resuspended in a well-buffered solution, preferably a phosphate mixture of a neutral reaction for comparative heat-resistance tests.

I Burke, G. S.: J. Infect. Dis., 32, 6, 433. I923.

${ }^{2}$ Krönig, B., and Paul, T.: Ztschr.f. IIyg. u. Infektionskrankh., 25, I. I897.

3 von Hibler, E.: loc. cit.

${ }^{6}$ Dickson, E. C., et al.: loc. cit.

4 Weiss, H.: loc. cit.

7 Magoon, C. A.: loc. cit.

5 Esty, J. R., and Meyer, K. F.: loc. cit.

${ }^{8}$ Esty, J. R., and Meyer, K. F.: loc. cit. 
The phosphate mixture is easily duplicated and undergoes practica'ly no change during heating. Experience has shown that the resistance of spore suspensions can be readily standardized on this basis, and this method is highly recommended.

\section{INFLUENCE OF THE HYDROGEN-ION CONCENTRATION}

The effect of the hydrogen and hydroxyl-ion concentration on the heat resistance of spores of $\mathrm{Cl}$. botulinum is shown in Chart I.' In the tests illustrated by the chart, carefully washed spores had been suspended in two different series of solutions of varying $\mathrm{pH}$ values: $(a)$ phosphate mixture series $\left(\frac{\mathrm{M}}{\mathrm{I}_{5}} \mathrm{Na}_{2} \mathrm{HPO}_{4}\right.$ and $\left.\mathrm{KH}_{2} \mathrm{PO}_{4}\right)$ varying

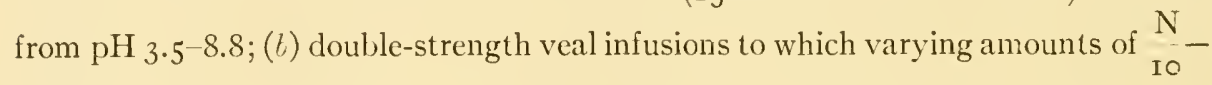
hydrochloric acid, citric acid, and sodium hydroxide had been added. It will be seen

TABLE II

Heat Resistance of Cl. bolulinum Spores in Various Solutions

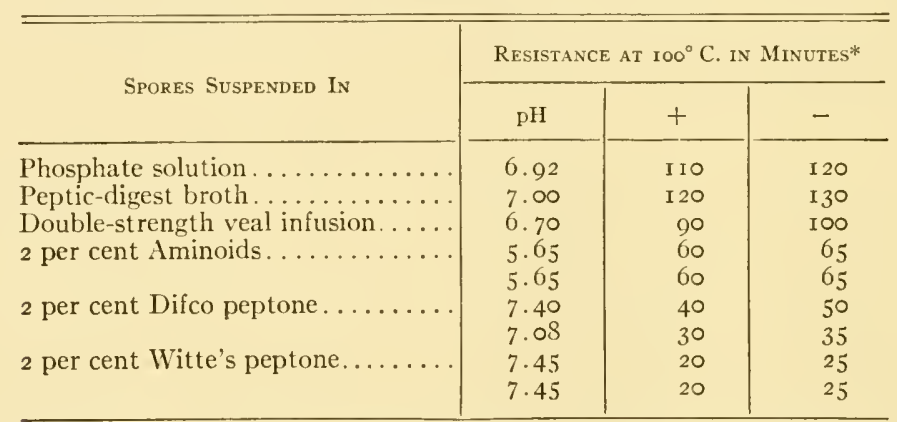

* The figures given in the + column are observed survival times and those in the - column destruction times.

that the maximum resistance in the phosphate series is slightly on the acid side $(\mathrm{pH}$ 6.3-6.9), and in the veal-infusion series the influence of the hydrogen-ion concentration on the heat resistance is striking below $\mathrm{pH} 6.0$ and above $\mathrm{pH}$ 9.०.

Chart I also shows graphically the spore resistance of two different suspensions of $\mathrm{Cl}$. botulinum in juices of different varieties of canned food. The results differed fundamentally from the phosphate and veal-infusion series, indicating that other factors together with the hydrogen-ion concentration affect the thermal death-time. For example, spores heated in ripe olives with a $\mathrm{pH}$ 7.93; corn, $\mathrm{pH} \mathrm{6.35}$; and spinach, $\mathrm{pH} 5.05$ exhibited approximately the same resistance, while in asparagus with a $\mathrm{pH}$ 5.25 and 5.55 sterilization was more readily accomplished. In food juices with a $\mathrm{pH}$ value below 4.5 the hydrogen-ion concentration had a decided influence on the destruction of $\mathrm{Cl}$. botulinum. The results by $\mathrm{Weiss}^{2}$ are also included in Chart I by a broken line, and although the spores appear to be less heat resistant, the same relationship exists. Similar observations, many of which are unpublished, have been made by the writer and his associates with thermophilic organisms both in phosphate solutions and food juices.

Ibid.

${ }^{2}$ Weiss, H.: op. cil., 29, 362. $192 \mathbf{1}$. 
Whitwerth ${ }^{2}$ states that the heat resistance of B. anthracis is influenced by the hydrogen-ion concentration and the composition of the medium in which they are suspended. Anthrax spores suspended in $\frac{\mathrm{M}}{\mathrm{I} 5}$ phosphate mixtures show greater resistance at $85^{\circ} \mathrm{C}$. than those in broth or in broth to which 3 per cent gelatin has been added. CHART I

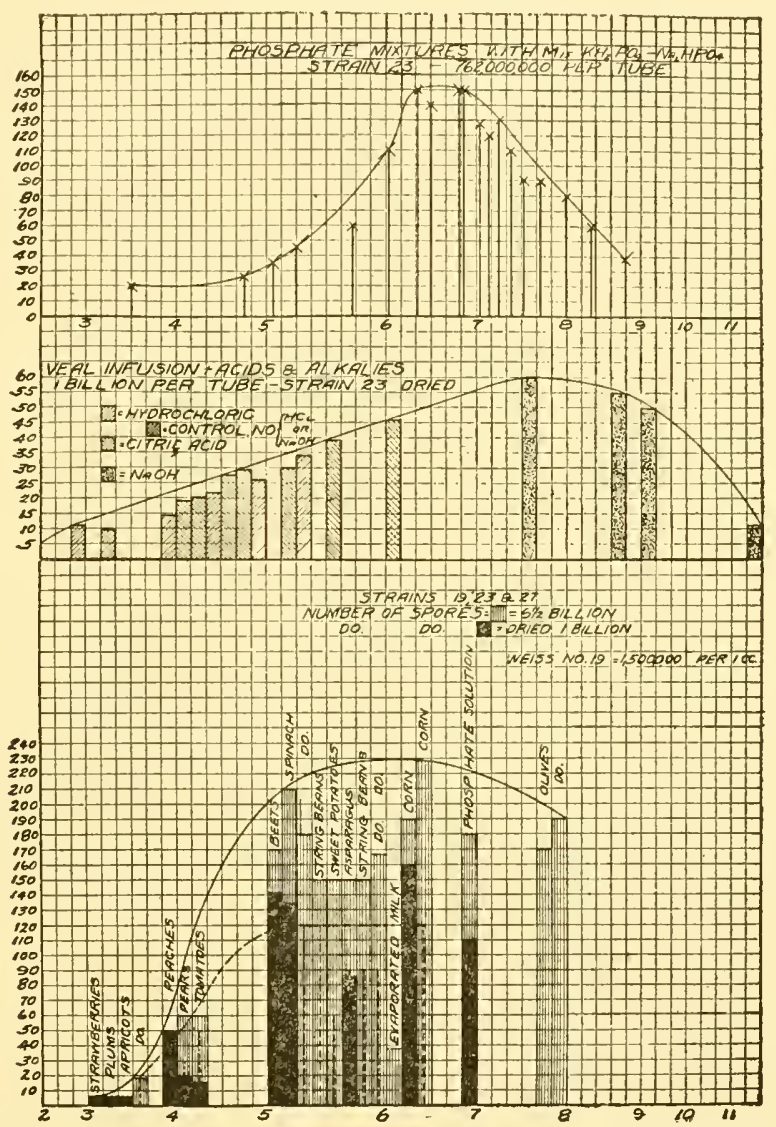

Resistance of $B$. botulimus Spores in Phosphate Mixtures, Veal Infusion of Varying pH Values by the Addition of Acid or Alkali, and Food Juices.

He also noted that the optimum $\mathrm{pH}$ was about 7.0 at $85^{\circ} \mathrm{C}$., and that the decrease of resistance is more marked on the acid side.

Another factor which influences the resistance has been frequently overlooked. Esty and Cathcart ${ }^{2}$ found that heating unbuffered solutions in soft-glass tubes changes the hydrogen-ion concentration. Mixtures of $\mathrm{Na}_{2} \mathrm{HPO}_{4}$ and $\mathrm{KH}_{2} \mathrm{PO}_{4}$ in hard-glass tubes were not altered when subjected to high temperatures, although prolonged heating in soft-glass tubes dissolved alkali in excess of the amount which can be controlled by the buffer salts.

${ }^{x}$ Whitworth, S. H.: Thesis, Inst. Veterinary Pathology, University of Zurich. Jan., I924.

${ }^{2}$ Esty, J. R., and Cathcart, P. H.: J. Infect. Dis., 29, 29. I921. 
The hydrogen-ion concentration of food juices heated in soft-glass changed less than in hard-glass tubes, due largely to the fact that the reaction of the food juices generally decreases during heating. In the case of corn juice, with an initial $\mathrm{pH}$ of 6.0 inoculated with 22,000 spores of a thermophilic organism, the destruction times at $100^{\circ}$ were 25 hours in soft-glass and $2 \mathrm{I}$ hours in hard-glass tubes. Although no general statement can be made regarding the relative merits of these two types of glass in heat-resistance tests, yet the influence should be determined for each solution. It is just as important, however, to know the hydrogen-ion concentration of the solutions during the entire heating period.

\section{INFLUENCE OF NUMBERS OF BACTERIA}

Harrison and Hood ${ }^{\mathbf{r}}$ observed that the thermal death-time depended on the presence or absence of clumps. Spores from filtered material having a thermal deathtime ranging from I to ro minutes in boiling water survived from 30 minutes to 3 hours if unfiltered.

The decided influence of numbers on the heat resistance of bacteria has been pointed out by several investigators. ${ }^{2}$ However, this is observed only when dilutions of a given suspension are used in the tests. In fact, owing to the remarkable variability of the resistance of spores of the same strain produced under identical conditions, cultures containing less than a million may be more resistant than others containing several billion spores.

Our observations have consistently shown that the larger the number of spores in a given suspension the longer the time necessary to destroy them. This statement is supported by experiments involving an extensive series of packs of canned foods, ${ }^{3}$ both artificially and naturally contaminated with varying numbers of spoilage organisms. It was shown that the greater the contamination, the heavier the spoilage losses from understerilization. This appears to be contrary to the statement made by Hastings, Fred and Carroll't to the effect that "certainly one million spores should include all grades of heat resistance."

\section{EFFECTS OF SALTS AND OTHER SUBSTANCES}

The heat resistance is also affected by the presence of varying amounts of sodium chloride in the heating medium. The thermal death-time of $\mathrm{Cl}$. botulinum was not reduced until the salt concentration reached 8 per cent, and a very decided decrease was noted with Io and 20 per cent solutions of sodium chloride. The addition of 0.5 and I per cent sodium chloride made the destruction more difficult than in a 2 or 3 per cent concentration. ${ }^{5}$ Viljoen, ${ }^{6}$ studying the effect of different amounts of sodium chloride on thermophilic spores, reports a protective influence up to a concentration of $3^{-3} \frac{1}{2}$ per cent. A 4 per cent solution showed either no effect or was only

${ }^{1}$ Harrison, F. C., and Hood, E.: Proc. Roy. Soc., Canada, 17. 1923.

${ }^{2}$ Bigelow, W. D., and Esty, J. R.: loc. cit.; Esty, J. R., and Meyer, K. F.: loc. cit.; and Weiss, H.: op. cit., 28, 70. I92I.

${ }^{3}$ Esty, J. R.: Canning Age, 5, I79-81, 236. 1924; Cameron, E. J., Williams, C. C., and Thompson, R. J.: article to appear in early number of $J$. Bact.

${ }^{4}$ Hastings, E. G., Fred, E. B., and Carroll, W. R.: Centralbl. f. Bakteriol., Abt. II, 67, 165. 1926.

${ }_{5}^{5}$ Esty, J. R., and Meyer, K. F.: loc. cit.

${ }^{6}$ Viljoen, J. A.: J. Infect. Dis., 39,286. 1926. 
slightly toxic. The highest protection occurred from I to $2 \frac{1}{2}$ per cent. He also observed that the resistance of the total number of spores is increased in a $I \frac{1}{2}$ per cent salt solution, and that the spores were destroyed according to a logarithmic law. Unpublished data on the heat resistance of thermophilic organisms and non-sporeformers show that different concentrations of sugar, either alone or in combination with salt, also influence the thermal death-time.

The studies of Dickson and his associates ${ }^{\mathrm{r}}$ definitely indicate a protective influence when spores are heated in a medium stratified with oil. Their data leave no doubt that $\mathrm{Cl}$. botulinum spores heated and incubated in broth, covered with a thin layer of mineral oil, exhibited thermal death-times which were very much greater than those ordinarily recorded in identical broth without the covering of oil. Table III illustrates their results, the data having been selected by the writer from Table III, page 479 , of their article. ${ }^{\mathrm{I}}$

TABLE III

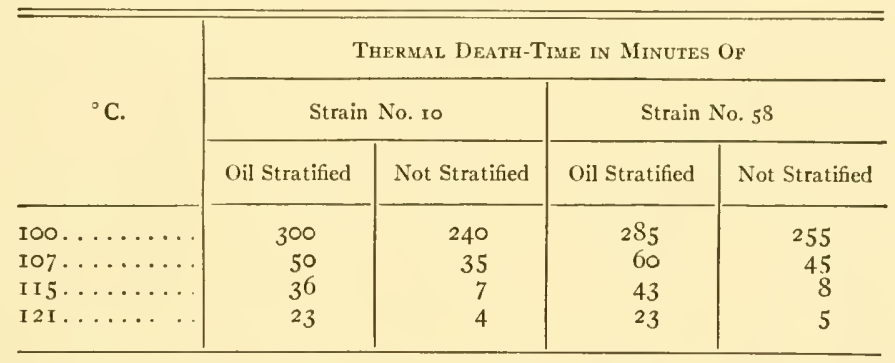

The increased resistance is much more apparent when the spores are heated at higher temperatures ( $\mathrm{I} I 5^{\circ}$ and $\mathrm{I} 2 \mathrm{I}^{\circ} \mathrm{C}$.). According to Dickson, the spores immersed in oil are exposed to the maximum heat for a shorter time than those suspended in broth and, therefore, may not receive the same "sterilizing effect." Although the exact mechanism of this protective influence of oil on the resistance of botulinum spores is not understood, yet it is now appreciated that these observations have a direct bearing on the processing time of all foods which contain oil, as was first pointed out by Dickson.

\section{EFFECTS OF DORMANCY}

Another feature which deserves consideration in the interpretation of heat resistance data is the dormancy factor characteristic for certain organisms. The germination of heated spores of many bacteria when transferred to a favorable environment occurs promptly, whereas with some other organisms, notably $\mathrm{Cl}$. botulinum, the germination of the surviving cells may be markedly delayed even under ideal conditions of subcultivation. Dickson and his associates ${ }^{t}$ report the following maximum dormancies for heated spores of $\mathrm{Cl}$. botulinum:

37 months for spores which were heated and incubated in oil-stratified broth ${ }^{2}$

22 months for spores which were heated and incubated in broth without oil

II months for spores which were heated and incubated in agar

r Dickson, E. C., Burke, G. S., Beck, D., and Johnston, J.: loc. cit.

${ }^{2}$ According to Dickson, since the publication of the foregoing data, observations have been made at frequent intervals on the germination time of spores in the oil-stratified bath series. These show to date a dormancy of sixty-six months for cultures incubated one month at $37.5^{\circ} \mathrm{C}$. and then held at room temperature. 
Esty and Meyer ${ }^{\mathrm{I}}$ report germination of subcultures prepared from heated suspensions after an incubation of 378 days at $37^{\circ} \mathrm{C}$. The cultures produced a virulent toxin and presented typical morphological and biological characteristics. They also ${ }^{\mathrm{I}}$ observed that subcultures prepared from diluted unheated or slightly heated spore suspensions $\left(80^{\circ} \mathrm{C}\right.$. for I hour or $100^{\circ} \mathrm{C}$. for 2 minutes) exhibited the phenomenon of retarded germination. Similar findings have been recorded by Burke. ${ }^{2}$ In view of this, the dormancy of unheated and heated suspensions must be determined for each organism.

Thermophilic organisms and many acrobic types ${ }^{3}$ germinate readily even when only a few viable cells are present. In fact, germination of heated spores of thermophilic organisms occurs from 48 to 120 hours when cultures are incubated under optimum conditions.

It has previously been suggested that another reason for irregularities and variable results may be unsatisfactory technique. The method by Bigelow and Esty ${ }^{4}$ has been used with minor modifications generally by research workers of this country. This method deserves wider recognition and, therefore, is briefly described.

A definite amount of a uniform, preferably strained or filtered, suspension is inoculated into a series of sterile glass tubes (hard or soft) of standard size, approximately $7-\mathrm{mm}$. inside diameter with $\mathrm{I}-\mathrm{mm}$. thickness of wall. The inoculated tubes are sealed in an oxygen flame, preheated in boiling water and then completely immersed in an electrically heated oil bath so adjusted and controlled as to maintain a constant uniform temperature throughout. The preheating and an initial rise in the temperature of the bath of from $2^{\circ}$ to $3^{\circ}$ during the first 2 minutes' exposure of the tubes reduces the lag period to approximately 3 minutes. The bath is so operated that at the end of the lag period the temperature reaches the desired degree and thereafter remains constant.

At definite periods tubes are removed, cooled immediately in a bath of ice water and the entire content subcultured in a favorable medium, if not already in suitable material, and incubated to determine survival.

Weiss ${ }^{5}$ modified the foregoing procedure by using $6^{\prime \prime} \times \frac{3}{4}{ }^{\prime \prime}$ test tubes, in which he found that a lag of about 30 minutes raised the inside of the tubes from room temperature to $99^{\circ} \mathrm{C}$. (with a bath at $100^{\circ} \mathrm{C}$.), of $5_{5}$ minutes to $105^{\circ} \mathrm{C}$. and 8 minutes to $120^{\circ} \mathrm{C}$. It is evident that in the tests reported by Weiss, 3 minutes at $120^{\circ} \mathrm{C}$, the spores were destroyed during the period of lag when the temperature inside the tube was still rising and long before the bath temperature was attained. He also modified the method of subculturing: A loopful of the heated material was inoculated into meat infusion glucose agar tubes, which were finely layered with paraffin oil and incubated at $37 \cdot 5^{\circ} \mathrm{C}$.

Magoon $^{6}$ used thin-walled capillary tubes and introduced them into the culture tubes for sterility tests.

${ }^{\mathrm{I}}$ Esty, J. R., and Meyer, K. F.: loc. cit.

${ }^{2}$ Burke, G. S.: J. Infect. Dis., 33, 274. I923.

3 Bigclow, W. D., and Esty, J. R.: loc. cit.; Esty, J. R., and Williams, C. C.: ibid., 34, 516. 1924.

${ }_{4}^{4}$ Bigelow, IV. D., and Esty, J. R.: loc. cit.

${ }_{5}^{5}$ Weiss, H.: loc. cit.

${ }^{6}$ Magoon, C. A.: loc. cit. 
Dickson and Burke ${ }^{x}$ adopted the so-called "sealed-tube technique" to exclude all possibility of contamination and to obtain accurate information as to the incidence of "skips" (bacterial growth occurring in tubes which have been heated for a longer period than those which frequently remain sterile). The majority of their final tests on the heated resistance of $\mathrm{Cl}$. botulinum spores were made in sealed tubes heated and incubated in I per cent glucose peptic digest broth ( $\mathrm{pH}$ 7.0-7.4) covered by a thin layer of mineral oil. For comparison a smaller series was run in glucose broth without oil, glucose agar, and brain medium. Heating records show a lag period of from $2 \frac{1}{2}$ to 4 minutes at each temperature.

With this technique, Dickson² et al. demonstrated "skips" in all the media and at all the temperatures at which the spores were heated.

Using the Bigelow and Esty ${ }^{3}$ method to determine the spore resistance of numerous strains of the common aerobic soil and water organisms, facultative and obligate thermophiles, $\mathrm{Cl}$. botulinum, and allied anaerobes, "skips" as noted by Dickson were frequently observed by the writer. Cultures heated for periods up to and including a certain time showed growth uniformly when incubated in a favorable environment, but for longer periods the results were irregular. These observations indicated that spore suspensions in a single-tube series occasionally contained viable organisms that had been heated for a considerable time in excess of those in which uniform growth had been observed. To explain these irregularities and to determine more accurately the actual resistance of organisms to moist heat, Esty and Williams ${ }^{4}$ modified the original method by subjecting a large number of tubes, from twenty-five to thirty, instead of one or two, each containing the same suspension, to various temperatures. This procedure conclusively showed that these "skips" are due to the variable resistance of individual spores in any suspension. They occurred in cultures containing different numbers of spores and to some extent in all media. Table IV 5 gives typical results of tests on twenty-five to thirty tubes each containing the same spore suspension, all heated.alike, as compared to those obtained when single tubes were treated. It will be seen that spores in a phosphate solution $\mathrm{pH}$ 5.I in single tubes were viable for 18 minutes at $110^{\circ} \mathrm{C}$. $\left(230^{\circ} \mathrm{F}\right.$ ), while even after $\mathrm{I} 2$ minutes, one of twenty-six tubes failed to show growth. In other words, 96.4 per cent of the tubes showed viable organisms after I 2 minutes and 64.3 per cent after I 5 minutes; and although a single tube indicated complete destruction in 20 minutes, yet 39.4 per cent of the tubes gave growth by th multiple method.

Chart II illustrates graphically the results obtained with phosphate solutions, pH 7.09 and 6.2, corn juice 6.I and pea juice 5.74, as given in Table IV. A straight line is drawn as nearly as possible through the experimentally determined points. These graphs are suggestive of logarithmic relations similar to those found in the mortality curves of bacteria generally and in thermal death-time relations as pointed

x Dickson, E. C., and Burke, G. S.: Proc. Soc. Exper. Biol. E Med., I9, 99. I92I.

${ }^{2}$ Dickson, E. C., Burke, G. S., Beck, D., and Johnston, J.: loc. cit.

3 Bigelow, W. D., and Esty, J. R.: loc. cit.

${ }^{4}$ Esty, J. R., and Williams, C. C.: loc, cit.

5 Esty, J. R., and Williams, C. C.: J. Infect. Dis., 34, 518. 1924. 


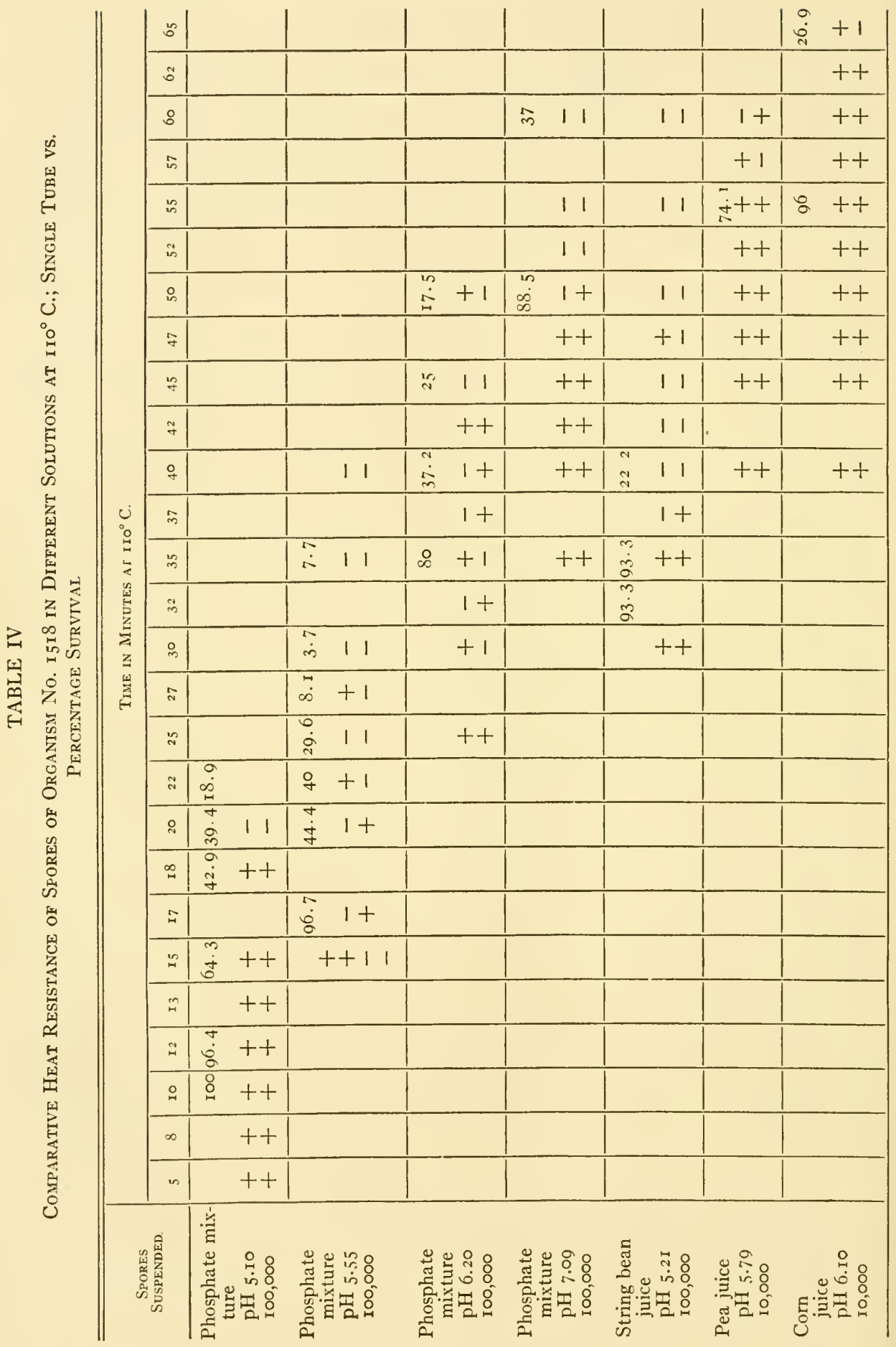


out by Bigelow. ${ }^{\mathrm{I}}$ They are not parallel to each other, indicating that solutions of different chemical composition bear no definite relationship to the heat resistance of bacterial cells. However, the two phosphate solutions appear to be related, and this is

CHART II

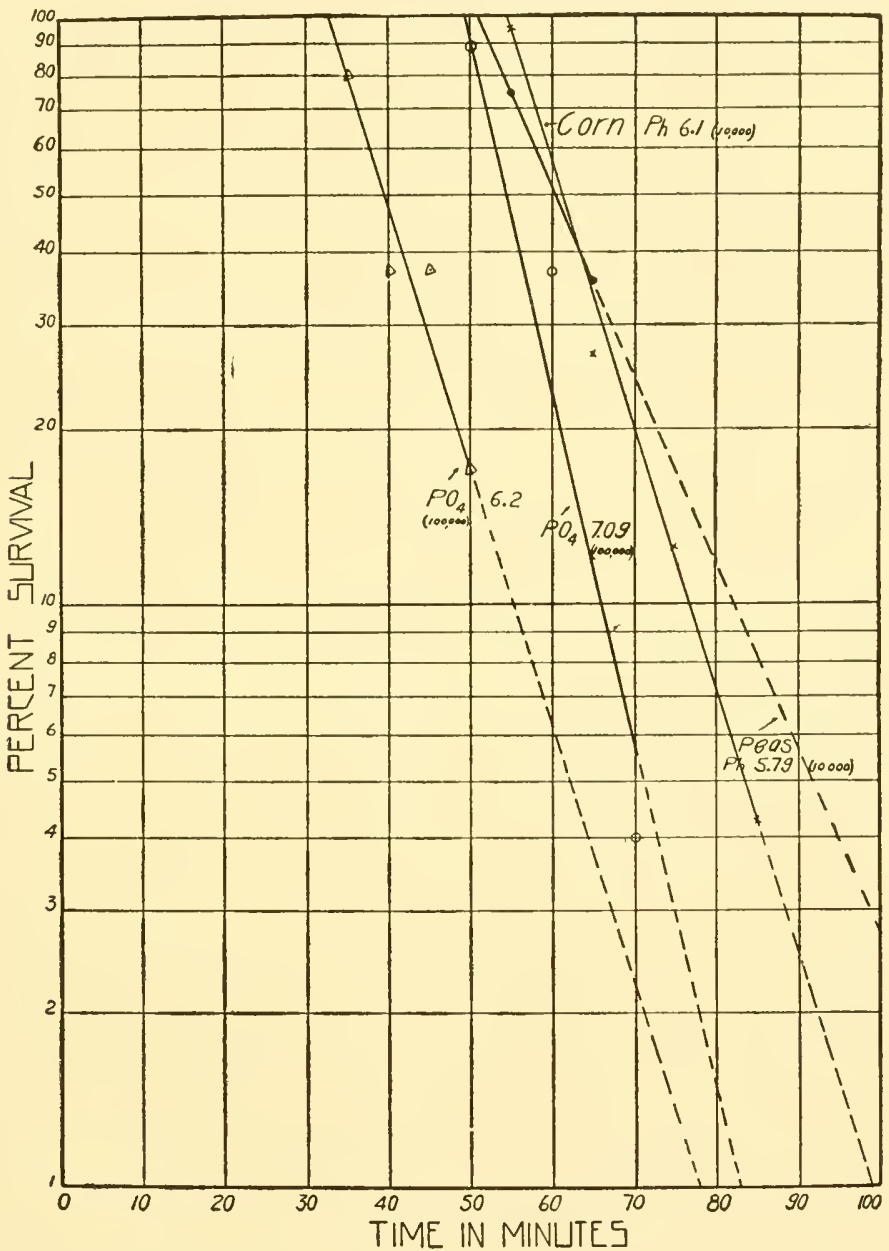

confirmed by other results which are given in this same article. ${ }^{2}$ Data secured in this manner makes it possible to establish standard type curves for a given number of spores in a given solution by determining percentage survivals for at least four wellselected times on a large number of tubes (one hundred to three hundred each time). Such a curve can be used as a type to determine the effect of different numbers of spores in the same solution or the influence of varying hydrogen-ion concentration in

I Bigelow, W. D.: J. Infect. Dis., 29, 528-36. 1921.

2 Esty, J. R., and Williams, C. C.: ibid., 34, 516. I924. 
phosphate solutions by constructing a curve through the percentage survival points that have been experimentally determined.

In general, the percentage survival greatly decreases as the heating progresses, and the results are uniform in proportion to the number of tubes used. The larger the number, the closer the range obtained in duplicate sets. However, for all practical purposes it is believed that twenty-five to thirty tubes of the same suspension heated for at least four different times will give reliable and fairly consistent information. Single-tube results are valuable in preliminary tests to locate the range within which all but the occasional spore is destroyed. A few tubes, all heated alike, determine the approximate percentage survival and serve to establish the range of maximum resistance. The actual death-time of the more resistant spores can be determined only by heating a large number of tubes. This method has a direct bearing on all sterilization processes and permits of the accurate determination of thermal death-times. ${ }^{\mathrm{I}}$

${ }^{I}$ For a discussion of other aspects of this subject, cf. chaps. vi, xxii, and xxxii in this volume. 


\title{
CHAPTER XXII \\ THE STANDARDIZATION OF DISINFECTANTS \\ AND ANTISEPTICS
}

\author{
GEORGE F. REDDISH \\ Baltimore
}

\section{DISINFECT.ANTS}

In I88 I Koch ${ }^{\mathrm{T}}$ produced the first satisfactory method of comparing the germicidal efficiency of disinfectants. Sternberg and Klein, Castro and Wynter-Blyth, had already made a distinct step forward by their use of pure cultures. The test devised by Koch is known as the "thread method"; spores of B. anthracis were used as the test organism. The time necessary to kill spores dried on a silk thread was used to indicate the germicidal value of the substances tested. Krönig and $\mathrm{Paul}^{2}$ modified Koch's method by using small garnets instead of silk thread; this had certain definite advantages in that much less of the germicide being tested was carried over into the subculture medium.

The Rideal-Walker method ${ }^{3}$ was the first really precise test for standardizing disinfectants and is the basis for all standard procedures used up to the present. In this test the following factors are controlled: time, age of culture, choice of medium and its acidity, temperature of medication and incubation, control of resistance of test organisms, specification of a distinct species of test organism, proportion of culture to disinfectant, and the use of a definite standard germicide as control. Space will allow but brief mention of the outstanding points in this test (I92 I revision).

The culture medium is composed of 2 per cent Liebig's meat extract, 2 per cent Witte's peptone, and I per cent sodium chloride in distilled water, adjusted to a reaction of $+\mathbf{r} .5$. Five-tenths of a cc. of a 24 -hour culture of B.typhosus in this broth is added to $5 \mathrm{cc}$. of the diluted disinfectant at $15^{\circ}$ to $18^{\circ} \mathrm{C}$. and transferred with a $4-\mathrm{mm}$. loop into $5 \mathrm{cc}$. of similar broth at $2.5^{-}, 5^{-}, 7.5^{-}$, and ro-minute intervals and incubated at $37^{\circ} \mathrm{C}$. for 48 hours. Only one phenol control is used and this dilution must kill the test organisms in 7.5 minutes but not in 5 minutes. A phenol coefficient is calculated by dividing the dilution of disinfectant which kills the test organism in 7.5 minutes but not in 5 minutes by the dilution of phenol which gives the same result. This figure is called the "Rideal-Walker coefficient."

The Hygienic Laboratory method ${ }^{5}$ differs from the Rideal-Walker test in the following respects (I92I revision):

The culture medium contains 0.3 per cent Liebig's meat extract, I per cent Armour's

${ }^{x}$ Koch, R.: Mitth. a. d. kais. Gsandhtsamte, I, 234. ISS I.

${ }^{2}$ Krönig, B., and Paul, Th.: Ztschr. f. IIyg. u. Infectionskrankh., 25, I. I 897.

3 Rideal, S., and Walker, J. T. A.: J. Roy. Sun. Inst., 24, 424. I903.

${ }_{4}$ Rideal, S., and Walker, J. T. A.: A pproved Technique of the Rideal-Walker Test. London: H. K. Lewis \& Co., I92 r.

5 IIygienic Laboratory Bull. \&2. April, 191 2; Reprint 675, Pub. Health Rep., 36, I 559. I92 I. 
peptone, and 0.5 per cent sodium chloride in distilled water with a reaction between $\mathrm{pH} 6.0$ and 7.0 ; the Hopkins strain of B. typhosus is used as the test organism; the temperature of medication is $20^{\circ} \mathrm{C}$; O.I cc. of culture is added to $5 \mathrm{cc}$. of diluted disinfectant; transfer is made into subculture broth at 5, 7.5, IO, I2.5, and I 5 minutes; a special spiral loop is used; six phenol dilutions are used in each test instead of one; the phenol coefficient is calculated from the weakest dilutions of disinfectant and phenol killing the test organism in 5 , 10, and I5 minutes, the average from these three being taken and the figure obtained called the "Hygienic Laboratory phenol coefficient.",

The "Lancet" method, but little used in America, is also based on the RidealWalker test, differing from it in features somewhat similar to the Hygienic Laboratory method. The use of $B$. coli instead of $B$. typhosus as a test organism constitutes one of the chief points of difference between the Lancet method and the other standard procedures.

In I9r 8 a committee on standardization of disinfectants appointed by the Laboratory Section of the American Public Health Association reported a new method for obtaining a phenol coefficient for disinfectants. ${ }^{2}$ In this "Report" the committee modified the Hygienic Laboratory test in such a way as to get at least theoretically more accurate results. Since this method has not come into practical use in this country a discussion of the procedures will not be given. However, one suggestion was made which is of interest at this time, namely, that phenol coefficients against disease-producing bacteria other than B. typhosus should be determined. Since the majority of bacteriologists conducting phenol coefficient tests agree that a phenol coefficient against one organism only is not of much value, further work on the use of different organisms seemed desirable.

The writer ${ }^{3}$ suggested that representatives of the various groups of pathogens should be used as test organisms for determining the germicidal efficiency of disinfectants. The organisms proposed were: B. typhosus (representative of the gram negative, non-sporing bacilli); $M$. aureus (representative of the suppurative group, and also gram positive cocci); $B$. diphtheriae (representative of granular gram positive group, and diphtheroids); $B$. tuberculosis (representative of the acid fast group); Dip. pneumoniae (representative of the encapsulated, gram positive cocci); Strep. hemolyticus (representative of the septicemic pathogens, scarlet-fever organisms, erysipelas, etc., gram positive chain-forming cocci).

At that time the writer made a thorough study of the resistance of $M$. aureus to phenol and outlined a method for the use of this organism in disinfectant testing. In I 927 the writer ${ }^{4}$ followed out this plan and presented a method for testing disinfectants in which these various test organisms would be used. At this time detailed procedures for $B$. typhosus and $M$. aureus were given and tentative procedures for the other pathogens were outlined. Objections to the Rideal-Walker and the Hygienic Laboratory tests were discussed in detail. Experience with both of these methods has shown weaknesses and inconsistencies which are responsible for inaccuracies. Both

${ }^{\mathrm{I}}$ Reprint 675, Pub. Health Rep., 36, I559. I92 r.

2 "Report of the Committee on Standard Methods of Examining Disinfectants," Am. J. Pub. He.tlh, 7, 506. rgrs.

${ }_{3}$ Reddish, George F.: Am. J. Pub. Ilealth, 15, 534. 1925; ibid., 16, 283. 1926.

${ }_{4}$ Reddish, George F.: op. cit., r6, 283 . 1926. 
methods also require a greater amount of time and material than the results warrant. This led to a phenol-coefficient test using $B$. typhosus as a test organism in which the best features of the Rideal-Walker and Hygienic Laboratory tests were retained and their worst features eliminated. In this way a very simple test was obtained which gave consistent results with a minimum amount of time and material. ${ }^{\mathrm{r}}$

The methods recommended by the writer ${ }^{2}$ and as approved by the American Public Health Association are given below:

\section{B. TYPIIOSUS}

Medium

Liebig beef extract or Lemco $\ldots \ldots \ldots \ldots \ldots \ldots \ldots . \quad 5 \mathrm{~g}$.

Peptonum siccum (Armour) . . . . . .

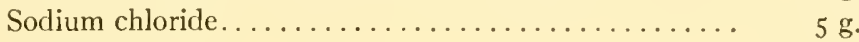

Distilled water...................... I,000 cc.

Place the ingredients in about $1,0<0 \mathrm{cc}$. of distilled water. Boil for 30 minutes and then filter. Add enough sodium hydroxide to bring the broth to a $\mathrm{pH}$ of 6.8 with brom thymol blue. Add enough water to make the total volume $\mathrm{I}, 000 \mathrm{cc}$. Run into test tubes, placing Io cc. in each tube. Sterilize at I5-pound pressure for 30 minutes.

Stock culture.-The stock culture is transferred at least once a month on beef-extract agar slants $\mathrm{pH} 7.0-7.5$, and kept at room temperature in tubes plugged with cotton. The agar should be made as follows:

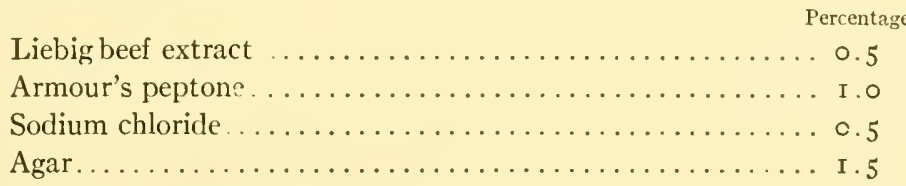

It is transferred in the foregoing broth, incubating at $37^{\circ} \mathrm{C}$, , on three consecutive days before using in the test. Fresh broth culture is started from agar-slant stock culture each month.

Organism.-The organism used in the test is a 22-to 26-hour culture of B. typhosus grown in the specified medium at $36^{\circ}-38^{\circ} \mathrm{C}$. This culture must be capable of resisting dilutions of phenol ranging from I-90 to I-Ioo for at least 5 minutes at $20^{\circ} \mathrm{C}$., and must be killed in 15 minutes by dilutions of phenol ranging up to and including $\mathrm{I}-90$.

In order to obtain the desired results, it is necessary to make daily transfers; however, the Sunday transfer may be omitted and the Tuesday culture will still be satisfactory.

Temperature.-The mixture of culture and diluted disinfectant must be held at $20^{\circ} \mathrm{C}$. during the test.

Phenol.- The phenol used must meet all the requirements of the United States Pharmacopoeia and, in addition, must have a congealing point (point of constant temperature on cooling) not below $40^{\circ} \mathrm{C}$. If the congealing point of the available phenol is slightly below $40^{\circ} \mathrm{C}$. the phenol may be distilled and the middle third of the distillate collected. If the congealing point of this portion is not below $40^{\circ} \mathrm{C}$., it is suitable for use.

Prepare a 5 per cent (by weight) stock solution of the phenol and standardize it by titra-

This method, as well as the procedures which are given below, was approved by the Standard Methods Committee of the Laboratory Section of the American Public Health Association at the fifty-fifth annual meeting, I926.

${ }^{2}$ Reddish, George F.: op. cit., 17, 320.1927. 
tion with deci-normal bromine water as described under "Phenol" in the United States Pharmacopoeia (Ioth rev.), or with sodium bromide and bromate solution as described by Francis Sutton. ${ }^{\mathrm{I}}$ Preserve it in 200-cc. amber-colored tightly stoppered bottles, protected from the light.

Proportion of culture to disinfectant.-Five-tenths cc. of unfiltered culture is added to 5 cc. of disinfectant.

Inoculation loops.-A 4-mm. loop of platinum wire, United States 24 standard gauge, $\mathrm{I} \frac{1}{2}$ inches long, is set into any suitable holder, such as aluminum or glass rod about $0.5 \mathrm{~cm}$. in diameter.

Incubation.-Subcultures are incubated for 48 hours at $37^{\circ} \mathrm{C}$.

Dilutions.--Any series of dilutions which the operator considers advisable may be used, but these dilutions must be made accurately and, within the limitation of the test, must show the maximum dilution capable of killing B. typhosus in so minutes. The method of making the dilutions also is left to the operator, as it is presumed that he has sufficient training to do this accurately.

Seeding tubcs.-Lipped test tubes $5 \times \frac{5}{8}$ inches, plugged with cotton, as used in the Rideal-Walker method, or test tubes of even larger size, according to the preference of the operator, may be used for medication.

Subculture tube racks.-The subculture tube racks specified in Hygienic Laboratory Bull. 82 are convenient.

Method of conducting the test.--Any number of dilutions up to ten may be used. Of these dilutions two must be reserved for the phenol control. Dilutions of $\mathrm{I}^{-}-90$ and $\mathrm{I}-\mathrm{I}$ - 0 phenol are arbitrarily chosen for this purpose. These are accurately made from the stock 5 per cent solution of phenol.

At intervals of 30 seconds, $0.5 \mathrm{cc}$. of culture is run into $5 \mathrm{cc}$. of each dilution from a 5 -cc. pipette. Care must be taken not to contaminate the sides of the tubes with the culture and be sure that no cotton threads adhere to the open ends of the seeding tubes. After inoculating, thoroughly sterilize in the flame the ends of the tubes where the pipette has touched.

At intervals of 5 , Io, and $I_{5}$ minutes transfer from each dilution to the specified broth. This will allow 30 seconds between transfers if ten dilutions are used. Incubate the tubes 48 hours at $37^{\circ} \mathrm{C}$. and read results. If there is any doubt that the growth in the subcultures is B. typhosus, this can be confirmed by agglutination tests.

Calculation of coefficient.-Divide the greatest dilution of the disinfectant capable of killing B. typhosus in to minutes but not in 5 minutes by the phenol dilution which should do this and divide these figures one into another. In order not to convey a false idea of the accuracy of the method the coefficient is calculated to the nearest O.I point if under 1.o, to the nearest 0.2 point if between $\mathrm{I}$ and 5.0 , to the nearest 0.5 point if between 5 and Io, and to the nearest $\mathrm{r} .0$ point if between $\mathrm{I}$ and 20 . For example, if results are read as follows:

DISINFECTANT

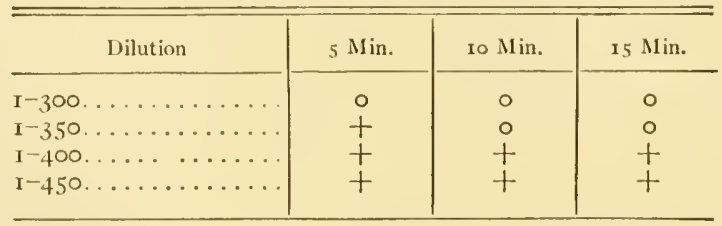

'Sutton, Francis: A Systcmatic Ilandbook of Volumetric Analysis (I th ed.), pp. 40.4-5. I924. 
PHENOL

\begin{tabular}{|c|c|c|c|}
\hline Dilution & $5 \mathrm{M}, \mathrm{n}$. & Io Min. & $\mathrm{I}, \mathrm{Min}$. \\
\hline $\begin{array}{l}I-90 \ldots \ldots \ldots \ldots \\
I-100 \ldots \ldots \ldots \ldots\end{array}$ & $\begin{array}{l}+ \\
+\end{array}$ & $\begin{array}{l}0 \\
+\end{array}$ & $\begin{array}{l}0 \\
+\end{array}$ \\
\hline
\end{tabular}

$350 \div 00=3.89$

Coefficient is 3.8

If the reading is as follows:

DISINFECTANT

\begin{tabular}{|c|c|c|c|}
\hline Dilution & 5 Min. & 10 Min. & Is $\mathrm{Min}$. \\
\hline $1-30 \ldots \ldots \ldots \ldots$ & 0 & 0 & 0 \\
\hline $\mathbf{I}-35 \ldots \ldots \ldots \ldots$ & 0 & 0 & 0 \\
\hline $1-40 \ldots \ldots \ldots \ldots$ & + & + & 0 \\
\hline $1-45 \ldots \ldots \ldots \ldots$ & + & + & + \\
\hline
\end{tabular}

PHENOL,

\begin{tabular}{|c|c|c|c|}
\hline Dilution & 5 Min. & Io Min. & 51 Min. \\
\hline $\begin{array}{l}I-90 \ldots \ldots \ldots \ldots \\
I-100 \ldots \ldots\end{array}$ & $\begin{array}{l}0 \\
+\end{array}$ & $\begin{array}{l}0 \\
+\end{array}$ & $\begin{array}{l}0 \\
0\end{array}$ \\
\hline
\end{tabular}

then estimate the dilution of the disinfectant killing in Io but not in 5 minutes as $1-37.5$ and the phenol as $\mathrm{I}-95(37.5 \div 95=0.395$ or 0.4$)$ giving a coefficient of 0.4 .

In the method just described the figure obtained is named the "B. typhosus phenol coefficient." When any other organism is used, the coefficient could then be named after the organism employed. The coefficient so named will be of service in making specifications for any soluble disinfectant; it is far easier to name a certain figure than it is to enter into a cumbersome, minute description of what is desired, as would be the case if all coefficients were entirely abolished.

\section{AUREUS}

When $M$. aureus is substituted for B.typhosus some changes in the dilutions of the phenol control are found necessary. The same medium and general technique have been found to be satisfactory, but the organism is much more resistant to phenol. At $20^{\circ}$ C. it should survive a $1-70$ dilution of phenol for ten minutes, and may or may not be resistant to a $\mathrm{I}-60$ dilution for five minutes. In routine work both dilutions may be used as controls, and the results obtained are used in calculating the $M$. aureus phenol coefficients. Thus in the following example the figure used as divisor would be 65 .

\begin{tabular}{|c|c|c|c|}
\hline Phenol & 5 Min. & Io $\mathrm{Min}$. & $15 \mathrm{M}$ in. \\
\hline $\begin{array}{l}I-60 \ldots \ldots \ldots \ldots \\
I-70 \ldots \ldots\end{array}$ & $\begin{array}{l}\circ \\
+\end{array}$ & $\stackrel{0}{+}$ & $\begin{array}{l}\circ \\
+\stackrel{\text { or }}{\circ}\end{array}$ \\
\hline
\end{tabular}


If the results were as follows, 60 would be the figure employed.

\begin{tabular}{c|c|c|c}
\hline \multicolumn{1}{c|}{ Pheno! } & 5 Min. & Io Min. & I5 Min. \\
\hline I-60...................... & $\begin{array}{c}+ \\
+\end{array}$ & $\begin{array}{c}0 \\
+\end{array}$ & $\begin{array}{c}0 \\
+\end{array}$ \\
\hline
\end{tabular}

Only resistant cultures are admissible in the test, for the use of weak strains leads to erroneous conclusions. ${ }^{\mathrm{I}}$ As old-stock strains rarely meet these requirements, it is necessary to secure a freshly isolated culture. After isolation, if directions already given for $B$. typhosus are followed, the resistance to phenol should be maintained for at least a year.

\section{B. DIPHTHERIAE}

A preliminary comparison of freshly isolated strains of this organism with a stock culture of Park No. 8 showed that all strains used possessed about equal resistance to phenol. Since Park No. 8 proved so satisfactory as a test organism in a few practical tests and in repeated tests with phenol, it was selected as our stock organism for this purpose, and so long as it continues to retain resistance comparable to that exhibited by new isolations, it will be satisfactory as a test organism.

The method used at the present time is:

Three or four slants of Loeffler's blood serum, made from Difco dehydrated Loeffler's blood serum, are streaked heavily with $B$. diphtheriae and incubated at $37^{\circ} \mathrm{C}$. for 24 hours; this is repeated for three consecutive days. The growth is then taken up in sterile saline and made up to a density corresponding to nephelometer 2. Five-tenths cc. of this suspension is then added to $5 \mathrm{cc}$. of the diluted disinfectant at $20^{\circ} \mathrm{C}$. following the technique given for $B$. typhosus and Staph. aureus. After 5, I0, and 15 minutes, one loopful of the mixture of culture and diluted disinfectant is transferred to the Loeffler's slant, first dipping it into the liquid at the base of the medium and then streaking over the slant. Another transfer is then made from the liquor of the first tube and streaked on to a second tube of Loeffler's medium. In this way further dilution of the disinfectant carried over is assured. Subculture tubes are then incubated at $37^{\circ} \mathrm{C}$. for 48 hours, when they are observed for typical colonies of $B$. diphtheriae. The slants showing growth from the most concentrated dilutions are confirmed by stained microscopic smears. Two phenol controls, I-IOO and I-I 20, are included in each test. $B$. diphtheriae under the conditions outlined above should not be killed by $\mathrm{r}-\mathrm{roo}$ phenol in 5 minutes, nor by $\mathrm{I}-\mathrm{I} 2 \mathrm{O}$ in $\mathrm{I}_{5}$ minutes.

A "B. diphtheriae phenol coefficient" can then be calculated in the usual way from the data obtained with the disinfectant and phenol if desired.

\section{B. TUBERCULOSIS}

Specimens of sputum from clinically active tuberculosis are pooled and evenly mixed by prolonged shaking with broken glass. Stained smears of this material should show one or more tubercle bacilli in nearly every field of the microscope or an average of one to the field.

Four 5-cc. portions of the pooled sputum are then measured out into sterile rubberstoppered bottles, and equal quantities of disinfectant in saline dilutions of $\mathrm{I}-25, \mathrm{I}-50$, $\mathrm{I}-\mathrm{I00}$, and $\mathrm{I}-200$ added, so that the final dilutions are as follows:

${ }^{1}$ Reddish, George F.: op. cil., I 7, 320. 1927 . 
I. Sputum 50 per cent, disinfectant present in $I^{-} 50$

2. Sputum 50 per cent, disinfectant present in $\mathrm{I}-100$

3. Sputum 50 per cent, disinfectant present in $\mathrm{I}-200$

4. Sputum 50 per cent, disinfectant present in $\mathrm{I}-400$

The mixtures are thoroughly shaken at frequent intervals, and at the end of 30 minutes each disinfectant mixture is injected in 2-cc. amounts into the right inguinal region of each of two guinea pigs. With frequent shaking in the following interim, two more pairs of guinea pigs are injected similarly at the end of an hour. Each pig in the first group receives $0.04 \mathrm{cc}$. of disinfectant, in the second group $0.02 \mathrm{cc}$., in the third group $0.01 \mathrm{cc}$., and in the fourth group $0.005 \mathrm{cc}$. of disinfectant.

Four control pairs of guinea pigs are then inoculated with 2-cc. amounts in like manner with pooled sputum alone in the following dilutions: $I-I, I-I O, I-I 00$, and $I-I, 000$. After $6+$ days all surviving animals are chloroformed and necropsied. The results given are based on gross pathological changes only. Histological examination in every case might give positive results in some of the cases reported as negative. It should be pointed out that in this experiment the sputum and disinfectant were very intimately mixed, in a manner which cannot be approximated in the ordinary use of disinfectants.

\section{PNEUMOCOCCUS AND HEMOLYTIC STREPTOCOCCI}

The pneumococcus Type II and hemolytic streptococci may be handled similarly when used as test organisms in examining disinfectants. They require about the same conditions for optimum growths, and the same technique and culture media may be used for both. The technique used for these two organisms is briefly as follows:

If the strain is from a blood-agar plate culture from fresh pathological material, transfer from an isolated colony into ro-cc. glucose broth of the following compositions: x per cent Armour's peptone, 0.5 Liebig's beef extract, 0.5 per cent sodium chloride dissolved in distilled water and to which I per cent glucose is added; the final reaction after sterilization must be between $\mathrm{pH} 7.2$ and 7.4. Incubate at $37^{\circ} \mathrm{C}$. for 24 hours, and then transfer O.I cc. of the culture to ro cc. of plain broth of the foregoing composition without glucose, but with

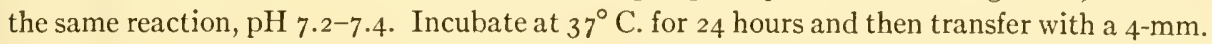
platinum loop into another similar tube of plain broth and then carry out the test as outlined for B. typhosus, using glucose broth for subculture medium. The subcultures in glucose broth are incubated at $37^{\circ} \mathrm{C}$. for 48 hours. Comparisons between the killing strength of the disinfectant being examined may be made with phenol in the usual manner and the results indicated as the "hemolytic streptococcus phenol coefficient" and the "pneumococcus phenol coefficient." Two phenol controls are included in each test. The hemolytic streptococcus should not be killed by $\mathrm{I}-90$ in 5 minutes, nor by $\mathrm{I}-\mathrm{r}$ IO in $\mathrm{I} 5$ minutes, while the penumococcus should not be killed by $\mathrm{I}-\mathrm{IOO}$ phenol in 5 minutes nor by $\mathrm{I}-\mathrm{I} 2 \mathrm{O}$ in $\mathrm{I}_{5}$ minutes. Results with streptococcus strains especially indicate that a somewhat more concentrated dilution of phenol might be selected, but in order that the limits may not be too exacting, the more dilute solutions are suggested. It is certain that if cultures are killed by the dilutions of phenol here given, they will not be acceptable as test organisms.

\section{ANTISEPTICS}

Antiseptics are substances which, when applied to micro-organisms, will render them innocuous, either by actually killing the organisms or by preventing their growth, according to the character of the preparation and the method of application. ${ }^{\mathrm{T}}$ For

r Reddish, George F.: Drug Markets, 20, 495. 1927; J. Am. Phar. Assoc., 16, 50r. 1927. 
testing the efficiency of substances for use as food preservatives, or for the preservation of protein and carbohydrate materials, methods may be employed which prove or disprove the capacity of such substances to prevent the growth of bacteria. Antiseptics for use in treating or counteracting infections or for ridding the skin and mucous membranes of pathogenic micro-organisms should be tested in a manner which simulates as far as practicable the conditions under which these reagents are used in practice. When antiseptics, such as the ordinary liquid preparations, are used in such a manner that short-time contact only is assured, the germicidal test such as described above for disinfectants is used. However, a temperature of $37^{\circ}$ is employed instead of $20^{\circ}$. In this case $M$. aureus ${ }^{1}$ is the test organism, and the ability to kill it in 5 minutes is the criterion of the efficiency of such liquid antiseptics.

The following types of preparations are examined by what may be called the "filter-paper method." The method, briefly, is as follows:

Number 2 Whatman Filter paper is cut into pieces about $0.5 \mathrm{~cm}$. square, placed in a test tube, plugged with cotton, and sterilized in the hot air oven at not over $170^{\circ} \mathrm{C}$. The desired number of these sterile squares are then immersed in a 24 -hour broth culture of the test organism, $M$. aureus. These paper squares, impregnated with the culture of $M$. aureus, are then fished out with a sterile culture wire (bent at the end) and transferred to the antiseptic. They are kept immersed in this preparation for 5 and ${ }_{5} 5$ minutes, and they are then transferred to a tube of broth ( I $_{\mathrm{cc}}$.) of the composition given above. By shaking thoroughly at intervals over a period of 5 to io minutes, the excess of the antiseptic is washed off from the paper squares. The pieces of paper are then fished out and transferred to another tube of broth (Iо cc.) and incubated at $37^{\circ} \mathrm{C}$. for 48 hours. The tubes are then observed for growth.

Preparations that may be tested by this method are antiseptic oils, antiseptic powders, antiseptic toothpaste, antiseptic dyes, shaving cream, and antiseptic soaps. In the case of soaps, a thick lather must be made before the filter-paper squares are used. Lozenges can also be tested in the same way after a concentrated solution has been made.

Antiseptic salves and ointments, however, must be examined by a special procedure which is, briefly, as follows:

M. an reus of normal resistance is grown at $37^{\circ} \mathrm{C}$. in the broth described above and transferred in this medium for three consecutive days. One-tenth of a cc. of a I-Ico dilution of this culture is added to $15 \mathrm{cc}$. melted nutrient agar containing $2 \mathrm{cc}$. normal sterile blood sert:m at $45^{\circ} \mathrm{C}$. ( 1.5 per cent agar in the broth), the culture thoroughly mixed in the agar and poured into a sterile patri dish and allowed to cool at room temperature. As soon as this inoculated agar has hardener, the salves and ointments, previously melted at $37^{\circ} \mathrm{C}$, are streaked over a small surface of the inoculated agar and the plate, inverted, incubated at $37^{\circ}$ C. for 48 hours. A vaseline control streaked in the same way is also included in this test. After being incubated it will be noted that colonies of $M$. aureus grow immediately adjacent to the vaseline control and even under it. There is no active ingredient in pure vaseline which will prevent the growth of $M$. aureus. However, in effective antiseptic salves and ointments a part of the active ingredients contained in them is absorbed into the agar and prevents the growth of the organisms. ${ }^{2}$ Therefore, the plate will show a clear zone around

I The M. aureus uscd must exhibit resistance to phenol as indicated above.

${ }^{2}$ The organisms in the clear zone surrounding the antiseptic preparations are not only inhibited, but usually actually killed except near the edge, as may be proved by subculture into broth. 
the antiseptic salve or ointment which is in marked contrast to the turbidity of the surrounding medium caused by the heavy growth of the organism.

In treating infected surfaces with preparations of this nature it is necessary that the active ingredients leave the inert base and become free to surround the infective organisms. It is only in this way that the preparation will be of benefit in preventing the growth of and even killing these micro-organisms. Serum agar simulates fairly closely the conditions met with in skin and wounds. It is permeable, semi-solid, isotonic, and constitutes a valuable laboratory means of approximating the conditions, found in human and animal tissues, at least so far as the preparations under consideration are concerned.

In testing bunion pads, corn plasters, surgical dressings, and powders for wound dressings for which antiseptic claims are made, the procedure just given is employed. These preparations are placed on top of a poured serum agar plate containing $M$. aureus, and incubated at $37^{\circ} \mathrm{C}$. for forty-eight hours. The antiseptic in such products permeates the agar medium and prevents the growth of the test organism. The amount of antiseptic contained and its solubility, together with the ease with which it leaves the base, are factors in determining the width of the clear zone around these preparations.

In these tests effort has been made to simulate practical conditions of disinfection and antisepsis as closely as possible. In an arbitrary test it is difficult to duplicate exactly the conditions found in practice, but results obtained by these methods give a fair indication of the efficiency of the preparations tested. It is very important that the method of testing should suit the preparation being examined. 


\title{
CHAPTER XXIII
}

\section{NATURE, DISTRIBUTION, AND FUNCTIONS OF SOIL MICRO-ORGANISMS}

\author{
SELMAN A. WAKSMAN \\ New Jersey Agricultural Experiment Station, New Brunswick, N.J.
}

THE SOIL POPULATION

Practically every lump of soil, however small in size, contains numerous microorganisms, varying both in kind and activities. The microscopic plant world is represented in the soil by (I) the algae, including blue greens, grass greens, and diatoms; (2) the fungi, including the actinomyces and yeasts; and (3) the bacteria, including cocci, bacilli, and spirilla. The microsporic animal world is represented in the soil by the numerous protozoa, including various amebae, flagellates, and ciliates, by the nematodes, the rotifers, insects, myriapods, etc.

These various micro-organisms are found in greatest abundance in the upper soil layers, the numbers diminishing with depth of soil. Well-aerated soils, such as cultivated field and garden soils, may contain organisms which are quite different from those found in water-logged soils, such as the peat bogs and acid forest soils. The differences in the flora and fauna of various soils are frequently both qualitative and quantitative in nature. The reaction of the soil, the amount of organic matter, soil temperature, and moisture, are among the factors which modify considerably the nature and distribution of the soil population.

Methods have been developed which enable us not only to count the different organisms present in the soil but also to isolate them from the soil, separate them from one another and study, under controlled laboratory conditions, their physiological activities. This is of great importance, since it enables us to determine the probable rôle of these various organisms in soil processes. One must keep in mind, however, the fact that these numerous micro-organisms act in the soil not in pure culture but in associations. The activities taking place in the test tube or flask, under artificial laboratory conditions, especially in the case of cultures long kept in cultivation, may or may not take place in the soil, in the presence of numerous antagonistic and associative influences from other organisms. In the soil, the products of metabolism of one group of organisms frequently serve as nutrients for other organisms. The substances formed as a result of these activities under natural conditions may be altogether different from those produced in pure culture in the laboratory.

NUMBERS OF MICRO-ORGANISMS IN SOIL

METILOD OF STUDY

The methods used in the study of soil micro-organisms vary considerably, depending chiefly upon the nature of the organisms under consideration. For the determination of the numbers of bacteria and fungi, direct microscopic and cultural methorls are available. For 
the determination of the numbers of protozoa, algae, and specific physiological groups of bacteria, the dilution method has to be employed. The nematodes and other worms can be counted directly in soil, by using a magnifying glass and special methods of suspension. The study of biochemical activities can be carried out either in the soil itself or by the use of pure and mixed cultures of organisms, which have been separated from the soil, under controlled laboratory conditions.

Most of the bacteria live in or upon the surface of the colloidal material which surrounds the inorganic soil particles. It is frequently very difficult to remove the cells from the dead organic and inorganic matter and separate them from one another. As a result of this and also because many of the soil bacteria do not develop upon ordinary agar media, the determination of the number of soil bacteria by the plate method is far from sufficient to give an idea of the abundance of the soil population. The direct microscopic method is more accurate for the determination of the abundance of micro-organisms in the soil. This method consists in spreading a definite amount of a dilute suspension of soil in water upon a definite area of a glass slide, drying, covering with a dilute solution of agar or gelatin, fixing in alcohol and staining with a solution of rose bengal or erythrosine in 5 per cent phenol solution. The bacteria are stained red, while the inorganic particles and the dead organic matter is either not stained at all or is stained only faintly. Only the bacteria, the spores of fungi, and protozoan cysts can thus be counted. The living protozoa, as well as some of the mycelium of fungi and actinomyces, are largely destroyed in the process of staining. It is also somewhat difficult to distinguish, by this method, the living from the dead bacteria, the active from the inactive forms. This method, having the great advantage of giving the absolute number of bacteria present in the soil, has the disadvantage that it does not lend itself to determinations, except within certain broad groups, of the specificity of the relative groups of organisms in the soil. The presence and abundance of fungi in the soil can be determined by adding a drop of methylene blue to a suspension of soil in water placed upon a slide and examined directly.

The cultural methods which are used for the determination of the numbers of microorganisms in the soil are divided into two categories: (I) the plate method, and (2) the dilution method.

I. The plate method has been used most commonly. It consists in diluting the soil with different amounts of sterile tap water, then plating out I-Cc. portions of the final dilutions, using an appropriate agar or gelatin medium. The plates are incubated for a period of time ranging from two to fourteen days, then the number of colonies developing on the plate is determined. This method has a number of very serious disadvantages, which make it almost worthless as far as accurate information concerning the actual abundance of soil organisms is concerned. There is no single medium yet devised which allows the development of all soil organisms. The anaerobic bacteria do not develop at all or only to a very limited extent. The autotrophic bacteria, including the nitrite- and nitrate-forming, sulphur-oxidizing organisms, etc., do not develop at all. Many of the cellulose-decomposing bacteria do not grow on common media; the nitrogen-fixing forms may grow only to a limited extent, etc. However, as far as the organisms that are capable of developing on the particular agar medium are concerned, the plate count gives just as accurate results as the direct microscopic examination. The justification for the use of the plate method is that it permits determination of at least the relative abundance of those organisms which are capable of developing upon the particular medium.

2. The dilution method consists in diluting the soil with sterile water, then adding I-cc. portions of the final dilutions to a medium (liquid or solid) which contains a particular pabulum favorable for the development of the specific organisms. Thus the number of urea- 
decomposing organisms is determined by adding I-cc. portions of several of the final dilutions to flasks of urea medium; cellulose-decomposing organisms in media containing cellulose as the only source of energy; nitrogen-fixing organisms in media containing no combined organic or inorganic nitrogen, but containing a source of energy, such as glucose or mannitol; protozoa on media favorable for the development of bacteria and protozoa, etc. If I cc. of a dilution of $\mathrm{I}: \mathrm{I} 00,000$, or I gm. of soil diluted with $100,000 \mathrm{cc}$. of water, gives no growth, while a dilution of $\mathrm{I}: \mathrm{I} 0,000$ gives growth, the number of specific organisms per I $\mathrm{gm}$. of soil is estimated to be between 10,000 and 100,000. On repeated determination, using narrower dilutions, closer results can be obtained. This method is very convenient not only for determining the abundance of specific organisms, but also for their isolation from soil, and separation from other forms. By selecting carefully a series of dilutions and using two plates with agar medium or two tubes or flasks with liquid medium for each dilution, incubating for seven to twenty-eight days, then determining the number of positive and negative growths, a more or less accurate picture of the abundance of specific protozoa in the soil is obtained.

TABLE I

Numbers of Micro-organisus in Soll, by Direct Microscopic Method

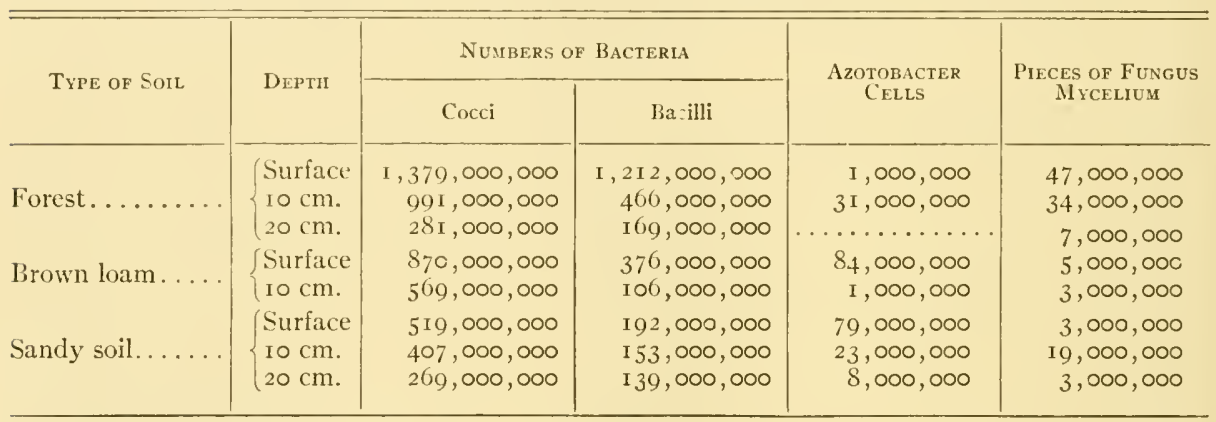

\section{DISTRIBUTION OF MICRO-ORGANISMS IN SOIL}

The numbers of various micro-organisms found in a definite quantity of soil depend upon the nature of the soil, depth of soil, season of year, state of cultivation, and a number of other factors. By the use of the direct microscopic method of Winogradsky, Richter found the numbers shown in Table I in I-gm. portions of different soils.

By the use of the plate method, the number of bacteria found in the soil is considerably less, due to the various limitations of the method. The numbers vary from two or three hundred thousand to a hundred million cells per gram of soil. The organisms developing on the plate consist of actinomyces (10-40 per cent of the colonies), non-spore-forming bacteria ( $50-80$ per cent of the colonies), and spore-forming bacteria (3-10 per cent of the colonies).

The numbers of fungi found in the soil by the plate method range from 10,000 to $\mathrm{I}, \infty \circ,, \infty \circ$ per gram. Acid soils as well as aerated soils rich in organic matter have the highest number of fungi. The most important limitation of the plate methor in this connection is the lack of differentiation between the numbers of spores and vegetative mycelium found in the soil. Yeasts are rare in soil, occurring somewhat more abundantly in acid soils, in vineyards, and in orchards. 
The protozoa occur in the soil in numbers ranging from a few hundred to over a million per gram. The flagellates and amebae are most abundant; the ciliates are less abundant. Nematodes occur in hundreds of millions per acre of soil. The numbers of earthworms and insects also run up to millions per acre.

The maximum number of organisms is found either at or just below the surface of the soil; then the number diminishes rapidly, so that at a depth of $18-30$ inches humid soils contain only very few bacteria and fungi. In arid soils, however, the microflora and microfauna are distributed throughout much greater depths, frequently to ro feet or more, due to better aeration and to a more uniform distribution of the soil organic matter. The numbers of micro-organisms in the soil vary with the different seasons of the year, and frequently they may fluctuate even daily, under field conditions. There is a rapid increase in the number of organisms in the spring, followed by a drop in the summer, then by another increase in the fall and a second drop in the winter. The summer drop is a result of a lack of sufficient moisture and the winter drop because of low temperatures.

\section{ISOLATION AND CULTIVATION OF SOIL MICRO-ORGANISMS}

\section{METHODS OF ISOLATION}

For the isolation of the great majority of soil micro-organisms, the same general methods are employed as for the isolation of bacteria, yeasts, and fungi from any other substrate such as milk, water, sewage, foodstuffs, etc. However, as a result of the fact that the soil harbors a number of specific organisms, which are not found readily in most of the other substrates and which do not develop at all on most of the common media, special methods must frequently be employed. Various enrichment or elective methods have been utilized by Beijerinck and others for the isolation of organisms from soil. These methods consist in preparing a specific medium which allows the development of one particular organism in preference to others. Such a medium is inoculated with soil, and, after successful growth has been obtained, transfers are made to fresh lots of the same medium, with the result that all contaminants, or those organisms which are not able to grow on the particular medium or find conditions unfavorable for their development, are gradually eliminated. Final isolation and purification of the specific organism is accomplished by the use of special plate methods or by employing high dilutions. A number of bacteria have been isolated from the soil by the use of this method. It is sufficient to mention the symbiotic and non-symbiotic nitrogen-fixing bacteria, the sulphur-oxidizing, the urea-decomposing, the sulphatereducing, the cellulose-decomposing, the hydrogen bacteria, the methane bacteria, and a number of others.

Instead of using liquid media to bring about the development of the specific organism, Winogradsky suggested the employment of silica gel media, freed from all traces of impurities, and containing the specific nutrient, which can be acted upon only by the specific organism which is to be isolated. For example, silica gel plates containing the necessary minerals, a source of nitrogen, and some ground cellulose on its surface will allow the growth of practically pure cultures of cellulose-decomposing bacteria, when inoculated with a particle of soil containing these organisms. When a silica plate, containing mannitol or glucose as the only source of carbon and no traces 
of combined nitrogen is inoculated with particles of soil, the development of the aerobic nitrogen-fixing organism Azotobacter will take place. This organism is frequently accompanied by the anaerobic nitrogen-fixing Bact. amylobacter. The isolation of the nitrite- and nitrate-forming bacteria and a number of other organisms that are highly selective in their nutrition can be accomplished by the use of this method.

Those organisms, especially the fungi, actinomyces, yeasts, protozoa, and many heterotrophic bacteria that develop readily upon nutrient agar or gelatin plates can be most readily iso'ated from the respective colonies upon the plates. The protozoa have so far not been isolated in cultures free from bacteria (with certain exceptions, which are still a matter of dispute); however, they can be isolated free from other protozoa and can even be grown on media containing only dead bacterial cells. There are indications that some protozoa at least may be grown on purely inorganic media. The algae can be isolated on media (agar, silica gel, sand) containing the necessary minerals and a source of nitrogen; they are cultivated in the light, since they utilize the energy of the sun.

\section{NUTRITION OF SOIL MICRO-ORGANISMS}

The soil micro-organisms can be generally divided, on the basis of their nutrition, into two groups: (I) The autotrophic organisms, or those which obtain their energy from the oxidation of inorganic elements $\left(\mathrm{S}_{2}, \mathrm{H}_{2}\right)$, inorganic compounds $\left(\mathrm{NH}_{3}+, \mathrm{NO}_{2}\right.$, $\mathrm{S}_{2} \mathrm{O}_{3}-\mathrm{H}_{2} \mathrm{~S}$ ), simple compounds of carbon $\left(\mathrm{CO}, \mathrm{CH}_{4}\right)$; carbon dioxide of the atmosphere is used as a source of carbon for structural purposes. The nitrite- and nitrate-forming, the sulphur-oxidizing, the hydrogen, methane, and iron bacteria belong to this group. All these organisms obtain their energy chemosynthetically. The algae, or the chloorophyll-bearing microscopic plants, which obtain their energy photosynthetically, are also autotrophic organisms. (2) The heterotrophic organisms, or those that obtain both their energy and carbon from complex organic compounds.

There are very few autotrophic bacteria that are obligate in nature; most of them are facultative, being capable of obtaining their energy also from various organic compounds. ${ }^{\mathrm{I}}$

The heterotrophic micro-organisms include a great many forms, which vary considerably both in their morphology and physiology. A number of organisms which are very specific in their nutrition are found among the bacteria. The fungi, actinomyces, and protozoa are all heterotrophic. Some of the bacteria are aerobic; others are anaerobic. Some are capable of utilizing the required nitrogen in the form of gaseous atmospheric nitrogen; others require combined nitrogen, either in an inorganic or in an organic form. Some require celluloses as the only source of energy; others can grow on a great variety of organic compounds. Some decompose paraffins, phenols, fats, lignins; others prefer proteins and carbohydrates.

The composition of the media, to be used for the cultivation of these numerous micro-organisms, is so selected as to have it adapted to the food requirements of the particular organisms. Liquid and solid, organic and inorganic, media are employed.

${ }^{x}$ See chap. xxiv in this volume. 
FUNCTIONS OF SOIL MICRO-ORGANISMS

\section{DECOMPOSITION OF ORGANIC MATTER}

Considerable quantities of organic matter of plant and animal origin are constantly introduced into the soil, in the form of stable manures, green manures, plant roots, plant stubble, and various waste products as well as certain organic fertilizers. The decomposition of these materials is one of the most important functions of the soil organisms. Various bacteria, fungi, protozoa, nematodes, rainworms, and other invertebrate animals take an active part in this process. Some macerate the organic matter mechanically and remove certain constituents for their own nutrition; others decompose a large part of the organic matter, but may leave certain constituents undecomposed. Some attack only certain specific ingredients of the organic matter. All of them build up cell substance, thus tending to replenish the supply of organic matter in the soil.

The organic matter which is commonly added to the soil consists of water-soluble constituents including sugars and amino acids, of pentosans and other hemicelluloses, of true celluloses, of lignins, proteins, fats and waxes, tannins, pigments, etc., and of ash. Among the fungi, for example, the Mucorales can attack the water-soluble constituents, the proteins, and certain hemicelluloses, but not the celluloses, the lignins, the cutins, and the tannins. Other fungi, like various species of Aspergillus, Penicillium, Trichoderma, Fusarium, Cephalosporium, can readily attack the celluloses and hemicelluloses, but not the lignins and the cutins. Some of the wood-destroying fungi can decompose the lignins in preference to the celluloses, while others are unable to decompose the lignins but can decompose the celluloses. Most of the heterotrophic bacteria, including the numerous spore-forming and non-spore-forming bacteria and cocci, attack only the water-soluble constituents, the proteins, and the hemicelluloses. A few highly specific organisms are able to decompose celluloses. Certain anaerobic bacteria decompose celluloses with the formation of organic acids (acetic, butyric), alcohols (butyl, ethyl), and gases (hydrogen, methane, carbon dioxide). The aerobic bacteria decompose the celluloses largely to carbon dioxide and water, with the synthesis of considerable protoplasm and frequently with the formation of slimy substances or gums. The decomposition of lignins by bacteria is still undecided.

The decomposition of organic matter in soil by the mixed-soil population of pure cultures of organisms can be followed by three distinct methods:

I. By determining the evolution of carbon dioxide, which is the most important final product in the metabolism of all aerobic micro-organisms. The more easily the organic matter is decomposed, the larger is the amount of $\mathrm{CO}_{2}$ given off both under controlled laboratory conditions and in the field. Soil itself will give off a constant stream of carbon dioxide, the actual amount depending on the temperature, moisture, and reaction of the soil and the content of organic matter. Under normal temperature, practically all the $\mathrm{CO}_{2}$ is given off as a result of the decomposition of the soil organic matter by micro-organisms. The amount of $\mathrm{CO}_{2}$ given off by the soil itself must be subtracted from the $\mathrm{CO}_{2}$ given off from the soil which has received the particular organic substance, the difference being an index of the decomposition of the latter. 
2. By determining the changes in the nitrogen content. When the organic materials are rich in nitrogen, their decomposition will be accomplished by a rapid evolution of ammonia, which is a waste product in the metabolism of micro-organisms. This ammonia will be rapidly changed, in well-aerated soils, to nitrates. The determination of the formation and accumulation of ammonia and nitrate nitrogen can serve as an index of decomposition of the organic matter. However, with a low nitrogen content, especially when the organic matter contains I per cent or less of nitrogen, the decomposition processes are always accompanied by the assimilation of the nitrogen which has been made available from the decomposition of the soil organic matter, or of the nitrogen which is present in the artificial medium. This nitrogen is required by the micro-organisms for the synthesis of their cell substance. The lower the nitrogen content of the decomposing organic matter, the greater will be the need for additional nitrogen to enable the organisms to decompose the organic matter adderl. There is a very definite relation between the organic matter which is decomposed and the nitrogen (as well as phosphorus) which is required by the organisms for the synthesis of their cell substance.

3. The most accurate method of following the decomposition of organic matter by soil micro-organisms is the complete analysis of this organic matter at the beginning and at the end of the decomposition process. This method requires considerable time and a very careful technique. It involves the determination of the various constituents of the organic matter, especially the celluloses, pentosans, lignins, proteins, starches, sugars, fats, and waxes.

The decomposition of natural organic matter added to the soil results in the formation of soil organic matter or "humus." This so-called "humus" consists of certain constituents of the natural organic matter added to the soil and which resist decomposition, namely, the lignins, the cutins, the fats and waxes, the tannins; of certain synthesized substances, namely, the living and dead cells of the soil micro-organisms; and of a number of substances which are still undergoing decomposition. When one hundred parts of organic matter are composted in the manure heap or are allowed to decompose in soil, there will be left, after two to six months, depending on the acidity, moisture, temperature, and nature of micro-organisms active in the process, nature of organic matter, etc., forty parts of organic matter. The sixty parts that have decomposed comprise practically all the pentosans, celluloses, and proteins. The forty parts remaining comprise largely the lignins and other resistant constituents and synthesized cell substance. The residual organic matter will tend to be uniform, as far as the content of nitrogen and phosphorus is concerned. The organic matter of the soil is more or less constant in composition, containing carbon and nitrogen in a definite ratio which approaches Io: I. The exact nature of this organic matter is still a matter of dispute.

TRANSFORMATION OF NITROGEN IN SOIL

When proteins are acted upon by micro-organisms, they are first broken down to amino acids, and these are sooner or later decomposed with the liberation of a part of the nitrogen as ammonia. A part of the nitrogen will be used by the organisms for the synthesis of their own cell substance, giving rise again to proteins and other complex nitrogen compounds. Since the carbonaceous substances are used by the organisms as sources of energy, and since the assimilation of nitrogen depends on the amount of energy available, the liberation of nitrogen as ammonia will depend on the ratio of carbon to nitrogen in the compound or substance which is decomposed by the 
micro-organisms. Since the carbon content of natural organic materials, including carbohydrates and proteins, ranges between 40 and 50 per cent (with the exception of lignins, which contain $63-64$ per cent carbon, but which are not readily available as sources of energy), and since the nitrogen content varies, we may conclude that the greater the percentage of nitrogen in an organic substance, the more rapid is the liberation of its nitrogen in the form of ammonia. The lower the nitrogen content and the higher the carbohydrate content of the organic matter, the more delayed will be the process of liberation of nitrogen as ammonia.

This process of formation of ammonia can be carried out by practically all soil fungi and actinomyces, and by a large number of bacteria. Some of the bacteria, especially many spore-forming anaerobes and aerobes, are very active in the decomposition of native proteins, while other bacteria, especially certain non-spore-forming organisms and cocci, cannot attack native proteins, but act readily upon protein derivatives, such as the various amino acids. The non-protein nitrogenous substances, such as urea, uric acid, hippuric acid, xanthine, hypoxanthine, etc., are also decomposed by various soil bacteria and fungi, and the nitrogen is sooner or later converted to ammonia.

The ammonia thus liberated may (I) either be assimilated by soil micro-organisms, in the presence of undecomposed carbonaceous materials and changed back into proteins; (2) or be used by higher plants as a source of nitrogen; (3) or be absorbed by the colloidal soil substances and held as ammonia; (4) or be acted upon further by other micro-organisms and changed into nitrates. The last process is carried out by two groups of autotrophic bacteria, ${ }^{\mathrm{I}}$ one changing the ammonia to nitrous acid and the other changing the nitrous acid to nitric acid. The latter is immediately neutralized by the soil bases and changed to nitrates.

The nitrates may again ( $\mathrm{I}$ ) either be used by soil micro-organisms, in the presence of available energy material, and changed into microbial proteins; (2) or be absorbed by the roots of higher plants and used for plant nutrition; (3) or be leached out from the soil; $(4)$ or be reduced by various bacteria to nitrites, to ammonia, to atmospheric nitrogen and simple gaseous oxides of nitrogen. The last process is carried out by various micro-organisms, under partial anaerobic conditions in the absence of atmospheric oxygen, when the nitrates are used as sources of oxygen. The reactions may also take place in the process of assimilation of nitrates as sources of nitrogen.

The nitrogen which is changed into the gaseous form is lost as far as the supply of combined nitrogen in the soil is concerned. However, certain micro-organisms are capable of utilizing the gaseous nitrogen and "fix" it in the soil, thus making it again available for plant growth. This process of fixation of nitrogen is carried out either symbiotically or non-symbiotically. In the first instance, the bacteria ( $B$. radicicola Beij.) can grow in association with leguminous plants, forming nodules on their roots, the plants supplying the bacteria with the necessary energy and the bacteria "fixing" the gaseous nitrogen, changing it into forms available for the leguminous plants. In some instances bacteria form nodules on the leaves of plants. The non-symbiotic nitrogen-fixing bacteria, namely, the aerobic species of Azotobacter and other bacteria and the anaerobic species of clostridia (B. amylobacter), do not need any host plants,

${ }^{2}$ Described in chap. xxiv. 
but are capable of using gaseous atmospheric nitrogen in the presence of available energy. Soil algae, fungi (with the possible exception of some mycorrhiza fungi), and actinomyces are unable to fix atmospheric nitrogen.

True proteins are again synthesized by various soil organisms (including algae, fungi, actinomyces, and bacteria) and by higher plants, all using the nitrogen in the form of ammonia or nitrate; proteins are also produced by the nitrogen-fixing organisms. These proteins may again be transformed when the bacteria, algae, and fungi are used as food by the protozoa and other invertebrate animals.

The processes of nitrogen transformation can be schematically summarized as follows:

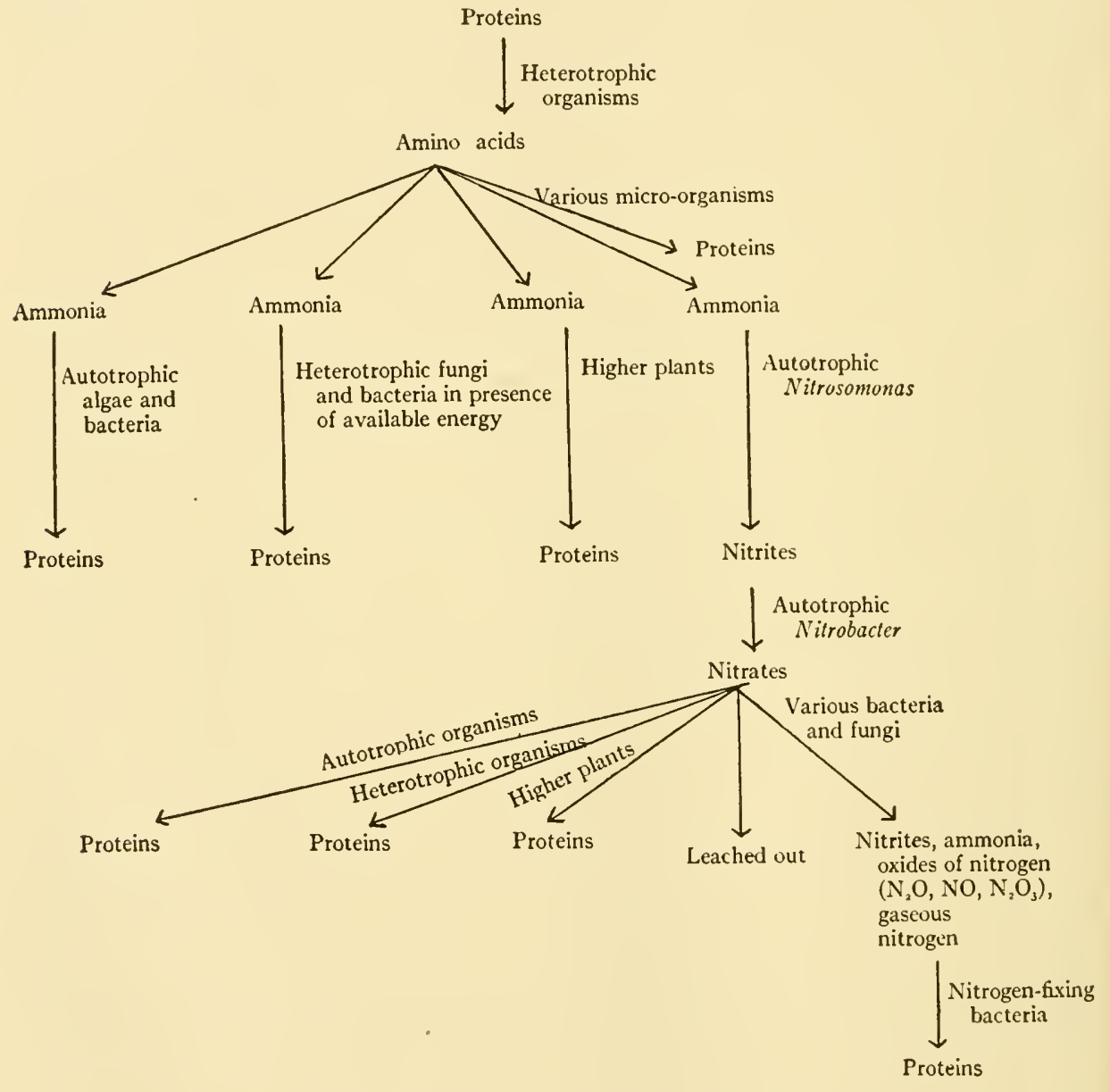

TRANSFORMATION OF MINERALS IN THE SOIL

Among the various mineral elements which are of importance in the growth of plants and which are acted upon in one form or another by micro-organisms, the following may be included: sulphur, phosphorus, potassium, calcium, and iron, and to a less extent, magnesium, zinc, and manganese. 
In the decomposition of proteins, the sulphur is liberated first as cystin and thiocompounds, then as hydrogen sulphide. The latter can be used as a source of energy by certain specific bacteria which oxidize the sulphide first to elementary sulphur, then to sulphuric acid. Elementary sulphur is also oxidized by certain specific bacteria to sulphuric acid. Thiosulphates are oxidized to sulphates and thionates. Some bacteria are capable of reducing the sulphates to hydrogen sulphide, using the sulphate as a source of oxygen. In the metabolism of heterotrophic organisms only a small amount of sulphur is required. This is usually assimilated in the form of sulphate or organic sulphur.

When insoluble phosphates are introduced into the soil they are made readily soluble by interaction with carbon dioxide as well as with the various organic and inorganic acids produced by the soil micro-organisms. When a mixture of sulphur and insoluble phosphate (the tri-calcium form) is added to soil or to a culture solution and inoculated with the proper bacteria, the sulphur will at first be oxidized to sulphuric acid. This acid interacts immediately with the tri-calcium phosphate, changing it to di-calcium, then to mono-calcium phosphate and finally to phosphoric acid. Phosphorus is a very important element in the nutrition of micro-organisms, and considerable quantities of it are utilized in the synthesis of the microbial protoplasm. It has been found that there is a definite ratio between the nitrogen and phosphorus content of bacterial cells such as Azotobacter. This led to the introduction of a method for determining the amount of available phosphoric acid in a given soil, when a certain amount of soil is used as the exclusive source of phosphorus in a medium in which nitrogen fixation takes place. From the amount of nitrogen fixed, the amount of available phosphate is calculated.

Potassium compounds are changed in the soil in an entirely different manner from the phosphorus compounds. This, as well as the fact that potassium is not as indispensable to all micro-organisms as phosphorus, makes the rôle of micro-organisms in the transformation of potassium in soil somewhat different. The small amounts of potassium added to the soil in organic combinations are liberated when the organic matter is decomposed, some of it being again assimilated. Small amounts of potassium may also be liberated from the insoluble silicates when these interact with the various organic and inorganic acids formed by soil micro-organisms.

Calcium is used to a limited extent as a nutrient by soil micro-organisms. It is of greater importance in the neutralization of the acids produced by the various organisms; the reaction of the soil can thus be kept at or near neutrality, a reaction which is essential for the growth of some very important groups of soil micro-organisms. The formation of nitrates from ammonium salts would soon come to a standstill if it were not for the presence of calcium which neutralizes the acids formed. The growth of some nitrogen-fixing bacteria takes place within a narrow range of reaction optimum; this can be attained by the application of calcium in the form of its carbonate or oxide. Asotobacter, for example, will not develop in soil or in solution at a $\mathrm{pH}$ of less than 6.o. The formation of acids by bacteria, notably nitrous, nitric, and sulphuric, and their leaching out from the soil, in the absence of growing plants, also leads to considerable losses of calcium from the soil.

Magnesium plays a rôle similar to calcium although it is likely to be more toxic 
than calcium in high concentrations. Magnesium is also somewhat more essential in the growth of certain organisms, like the fungi, than calcium.

The presence of iron, even if only in very small quantities, is essential for the growth of all micro-organisms. Some organisms refuse to grow when a medium having an alkaline reaction is sterilized under pressure, due to the precipitation of the iron. Iron, in the form of ferrous carbonate, can be used by certain specific bacteria as a source of energy; the iron is thereby precipitated in the form as ferric hydroxide. However, precipitation of iron may also take place as a result of the chemical interaction between the iron salt and products of microbial metabolism. ${ }^{\mathrm{I}}$

\section{SOIL MICROBIOLOGICAL ACTIVITIES AND SOIL FERTILITY}

Soil fertility or the ability of the soil to support the growth of higher plants depends to a large extent upon the activities of micro-organisms.

One of the primary functions of these organisms is the mineralization of the organic matter which is constantly added to the soil in the form of plant residues, including roots, stubble, weeds, and the tree products, green manures, stable manures, various organic fertilizers. These are decomposed, whereby most of the carbon is given off as $\mathrm{CO}_{2}$, the minerals and nitrogen are made again available for plant growth while a part of the organic matter which is more resistant to decomposition remains in the soil, thus tending to improve the soil as a physical and chemical medium for plant growth. When environmental conditions do not favor the activities of most of the micro-organisms, as in the case of water-logged soil, the organic matter tends to accumulate, giving rise to organic soils, such as peats. When conditions favor the activities of micro-organisms, as in well-cultivated and limed soils, organic matter is rapidly decomposed and the soil becomes depleted. This depletion is partly compensated for by the residues of the crops grown on the soil.

Fixation of nitrogen is carried on in the soil to a limited extent by chemical processes. The symbiotic bacteria, in the presence of the host plants, and the nonsymbiotic bacteria, in the presence of available sources of energy, bring about a considerable fixation of atmospheric nitrogen. This valuable element, which is constantly removed from the soil by the growing crops, is thus, at least partly, replaced in the soil.

In view of the fact that numerous micro-organisms carry out their varied activities in the soil simultaneously, stimulating or injuring the growth and activities of one another, the resultant processes may often be unfavorable for plant growth. It has been suggested that the limitation of soil fertility, or the inability of the soil to support a better growth of cultivated plants, and frequently actual soil exhaustion, are due to the destruction of one group of soil micro-organisms by another. This theory was based on the following facts and assumptions: (I) Bacteria are largely responsible for the liberation of plant nutrients from soil organic matter. (2) Protozoa feed largely upon bacteria in the soil. (3) Treatment of soil with volatile antiseptics or with steam or Iry heat makes the soil considerably more fertile. (4) These treatments result in an initial reduction in the numbers of bacteria followed by a very considerable increase greatly in excess of the numbers present in untreated soil. This

${ }^{1}$ See chap. xxiv in this volume by R. L. Starkey. 
increase in bacteria is accompanied by a considerable increase in the evolution of ammonia (and carbon dioxide). (5) These treatments result in the complete or almost complete destruction of the protozoa. The natural assumption was that protozoa by destroying the bacteria in the soil are responsible for the limitation of the fertility of the soil. The destruction of the protozoa by partial sterilization of soil results in the removal of the limiting agents and thus leads to an improvement of soil fertility. ${ }^{\mathrm{T}}$

However, further investigations have shown that many other organisms, especially the fungi, actinomyces, nematodes, and other invertebrates, are also active in the transformation processes in the soil and are also influenced very markedly by partial sterilization of soil.

The soil is a complex medium, harboring, too, many organisms that are responsible for numerous processes. By modifying the conditions of the soil, as by drainage and aeration, liming, and addition of available energy, in the form of organic matter, we can modify markedly not only the nature of the population, but even its activities, and direct them in such a manner as to have the results beneficial to the growth of cultivated crops.

The following references may be consulted for more detailed analyses of soil microbiology:

Russell, J.: The Microorganic Population of the Soil. Longmans, Green \& Co., I924. Waksman, S. A.: Principles of Soil Microbiology. Baltimore: Williams \& Wilkins Co., I927. Waksman, S. A., in collaboration with Barthel, Chr., Cutler, D. W., and Bristol-Roach, B. M.: Methoden der mikrobiologischen Bodenforschung. Abderhalden's Handbuch der biologischen Arbeitsmethoden. Abt. XI, Teil 3. I926.

${ }^{x}$ For a more extensive discussion of micro-organisms in relation to soil fertility, see chap. xxvi by J. G. Lipman in this volume. 


\title{
CHAPTER XXIV
}

\section{AUTOTROPHIC BACTERIA}

\author{
ROBERT L. STARKEY
}

New Jersey Agricultural Experiment Station, New Brunswick, N.J.

Mest of the lower forms of life, including the filamentous fungi, protozoa, and most of the bacteria, derive the energy for their metabolic processes as well as the carbon used for synthesizing their cells from complex organic compounds such as carbohydrates, fats, proteins, degradation products of these, or other compounds of the aliphatic and cyclic series. Such organisms are termed "heterotrophs."

A typical reaction by which such organisms may obtain energy for their nutrition may be represented as follows: ${ }^{\mathrm{T}}$

I.

$$
\begin{gathered}
\left.\left.\mathrm{C}_{6} \mathrm{H}_{12} \mathrm{O}_{6} \text { (solid) }+6 \mathrm{O}_{2} \text { ( } \mathrm{I} \text { atm. }\right)=6 \mathrm{CO}_{2} \text { ( } \mathrm{I} \text { atm. }\right)+6 \mathrm{H}_{2} \mathrm{O} \text { (liquid) } \\
\Delta \mathrm{F}_{298}=-689,800 \text { calories }^{2}
\end{gathered}
$$

From this reaction the complete oxidation of $\mathrm{I} \mathrm{gm}$. molecule or $\mathrm{I} 8 \mathrm{ogm}$. of glucose liberates 689,800 calories which may be available to the organism bringing about such a reaction.

Organisms effecting the synthesis of their own organic compounds from inorganic substances are termed "autotrophs." Of this group by far the most conspicuous forms are the higher plants which contain chlorophyll. Among the microscopic forms of this group would be included the smaller algae. Organic matter is produced within the cells of such organisms by photosynthesis. In this reaction the sunlight furnishes the necessary energy for the elaboration of organic compounds from carbon dioxide.

There is another distinctive group of autotrophs which may live in the absence of light. They are generally microscopic and are indistinguishable from other bacteria in their morphological characteristics. These autotrophic bacteria are distinguished from the heterotrophic forms in that they have the specific ability of obtaining energy for their metabolism by the oxidation of certain inorganic substances. Autotrophic bacteria differ from the autotrophic plants in that this energy, derived from the oxidation of inorganic materials, is utilized by the bacteria for the reduction of carbon dioxide to organic compounds. In the group of sulphur bacteria are found representatives of forms which appear to be intermediate between the autotrophic bacteria and the chlorophyllous plants. These are the purple bacteria which require both hydrogen sulphide and light for their development. It would appear that their nutrition is dependent upon both a photosynthetic reaction and an oxidation of an inorganic substance.

${ }^{1}$ Baas-Becking, L. G. MI., and Parks, G. S.: Physiol. Rev., 7, 85-106. I927.

${ }^{2} \Delta \mathbf{F}_{298}$ refers to the free energy decrease or the maximum amount of useful work obtainable from the process at $25^{\circ} \mathrm{C}$. or $298^{\circ}$ absolute temperature. 'The data reported in these pages on free energy were all obtained from Baas-13ecking and Parks (loc. cit.). 
Obligate autotrophic bacteria and facultative forms existing under autotrophic conditions show certain distinctive physiological characteristics:

I. They thrive on strongly elective, purely mineral media containing the specific inorganic oxidizable substances.

2. Their existence is dependent upon these substances which are oxidized in the performance of the life-processes of the organisms.

3. These oxidation processes furnish the only source of energy for the organisms.

4. They require no organic nutrients as sources of energy.

5. They use carbon dioxide (dissolved) as their exclusive source of carbon. This is reduced by means of the energy obtained from the oxidation of the inorganic foods. ${ }^{\mathrm{x}}$

The number of known organisms which live as obligate autotrophs is quite small. Included in this group are nitrifying bacteria and some of the sulphur bacteria and iron bacteria. There appears to be a greater abundance of forms which can live as facultative autotrophs. These may either derive their energy from the oxidation of inorganic substances and reduce carbon dioxide for synthesizing their organic structures or they may derive their energy, like the heterotrophs, from purely organic substances. The facultative autotrophs have representatives in the groups of sulphur bacteria, iron bacteria, and hydrogen bacteria. The transformations produced by autotrophic bacteria may be represented by the following reaction:

II.

$$
\begin{aligned}
\mathrm{NH}_{4}++\mathrm{I}_{2} \mathrm{O}_{2} & =\mathrm{NO}_{2}-+\mathrm{H}_{2} \mathrm{O}+2 \mathrm{H}^{+}+\left(\mathrm{IO}^{-8}\right) \\
\Delta \mathrm{F}_{298} & =-66,500 \text { calories }
\end{aligned}
$$

The oxidation of $\mathrm{I}$ gram molecule or I 8 grams of ionized ammonia to ionized nitrous acid results in the liberation of 66,500 calories.

This group of organisms, cailed "autotrophic bacteria" (more accurately, autotrophic micro-organisms lacking chlorophyll), is composed of forms varying greatly in morphological appearance as well as cultural habits. Some are minute single cells identical in appearance with the Eubacteriales. Some are large multicellular filamentous forms many micra in diameter. Some appear to be closely related to the algae in morphology (shape and complex mode of division) and may even contain pigments (as the bacteriopurpurin of the purple sulphur bacteria) which function in photosynthetic processes. These organisms compose a physiological group and are not distinctive morphologically from other bacteria. The known autotrophic bacteria may be classified as follows:

A. Bacteria which oxidize compounds of nitrogen

a) Oxidize ammonia to nitrite (Nitrosomonas, Nitrosococcus)

b) Oxidize nitrite to nitrate (Nitrobacter)

B. Bacteria which oxidize sulphur or compounds of sulphur

a) Simple bacteria (genus Thiobacillus)

I. Strictly autotrophic

(a) Aerobic

(I) Develop at reactions close to neutrality (species Th. thioparus Beijerinck)

(2) Jevelop under very acid conditions (species Th. thiooxidans Waksman and Joffe)

(b) Anaerobic (species Th. denitrificans Beijerinck)

I Winogradsky, S.: Centralbl.f. Bakteriol. Abt. II, 57, r-21. 1922. 
2. Facultative autotrophic

(a) Facultative anaerobic (species of Trautwein)

b) Higher bacteria (complex in morphology)

I. Colorless (includes the genera Beggiatoa, Thiothrix, Thioploca, Achromatium, Thiophysa, Thiovulum, and Thiospira)

2. Pigmented-red or purple bacteria (includes the genera Thiocystis, Thiocapsa, Thiosarcina, Lamprocystis, Thiopedia, Amocbobacter, Thiothece, Thiodictyon, Thiopolycoccus, Chromatium, Rhabdochromatium, Thiospirillum, Rhodocapsa, Rhodothece)

C. Bacteria which oxidize ferrous or manganous compounds

a) Simple bacteria

I. Long excretion filaments (genus Gallionclla)

2. Coccoid or oval shapes in masses (genera Siderocapsa and Sideromonas)

b) Filamentous bacteria (genera Leptothrix and Crcnothrix)

D. Bacteria which oxidize hydrogen

Winogradsky was the first to recognize the existence of bacteria with autotrophic habits. The first to be studied were certain of the higher sulphur bacteria ${ }^{\mathrm{I}}$ and later iron bacteria ${ }^{2}$ and nitrifying organisms. ${ }^{3}$

\section{BACTERIA WHICH OXIDIZE COMPOUNDS OF NITROGEN}

Two distinct reactions are performed by organisms of this group: (I) oxidation of ammonia to nitrite (Nitrosomonas and Nitrosococcus) and (2) oxidation of nitrite to nitrate (Nitrobacter). The transformation proceeds according to the following reactions:

III.

$$
\begin{gathered}
\mathrm{NH}_{4}++\mathrm{I}_{2} \mathrm{O}_{2}=\mathrm{NO}_{2}-+\mathrm{H}_{2} \mathrm{O}+2 \mathrm{H}^{+}+\left(\mathrm{IO}^{-8}\right) \\
\Delta \mathrm{F}_{298}=-66,500 \text { calories } \\
\mathrm{NO}_{2}-+\frac{1}{2} \mathrm{O}_{2}=\mathrm{NO}_{3}- \\
\Delta \mathrm{F}_{298}=\mathrm{I} 7,500 \text { calories }
\end{gathered}
$$

IV.

These reactions are produced by different bacteria, and no single species is known which can oxidize ammonia completely to nitrate. ${ }^{4}$ These organisms, called "nitrifiers," are strict autotrophs and are unable to exist in the absence of their specific sources of energy: ammonia for the nitrite formers and nitrite for those which oxidize nitrite to nitrate. The organisms are non-sporulating cocci or short rods. They are very widely distributed in nature, occurring in practically all arable soils and in many bodies of water. These organisms are of particular importance in their natural habitat since they appear to be the agents primarily responsible for the formation of nitrate which is so generally utilized by higher plants as the source of nitrogen. It is an

I Winogradsky, S.: Botan. Zcit., 45, 489-507, 51 3-23, 529-39, 545-59, 569-76, 585-94, 606-1о. I887; Beiträge zur Morphologic und Physiologie der Bakterien. Zur Morphologie und Physiologic der Schwefelbaktericn. I 20 pp. Leipzig: Felix, i 888.

${ }^{2}$ Winogradsky, S.: Bolın. Zeit., 46, 261-7o. I 888.

${ }_{3}$ Winogradsky, S.: Ann. de l'Inst. Pastcur, 4, 213-31, 257-75, 760-71. I 890.

${ }_{4}$ See, however, Kaserer, H.: Zischr. f. d. landw. Versuch. in Ocster, 10, 37. I907 (Contralbl. f. Baktcriol., Abt. II, 20, 170. 190\$); Sach, J.: ibid., 62, 15-24. 1924. 
aerobic process requiring abundance of free oxygen and carbon dioxide. ${ }^{\mathrm{I}}$ The limiting reactions for the process are close to neutrality ${ }^{2}$ (in solution cultures $\mathrm{pH} 7 \cdot 4-8.4$ for Nitrosomonas and pH 6.5- 0.3 for Nitrobacter. Much wider ranges of activity are observed in natural habitats.) Their discovery and isolation by Winogradsky was the climax to a long series of investigations aiming to explain the mechanism of nitrate formation in soils.

\section{BACTERIA WHICH OXIDIZE SULPHUR OR COMPOUNDS OF SULPHUR}

The organisms concerned in these transformations represent a variety of forms concerned in many different types of reactions, some of which appear to be among the most unique physiological processes for deriving functional energy. They are of considerable importance in the oxidation of natural sulphides in waters, and may be active agencies in the transformation of inorganic sulphur compounds originating from organic combinations in decomposition processes. ${ }^{3}$

The known organisms included as the "simple sulphur bacteria" are all nonsporulating small rods. Some are obligate autotrophs and others facultative; some are aerobic, some facultative, and others obligate anaerobes. Among the aerobic forms are two very distinct organisms, both obligate autotrophs, one of which develops at reactions close to neutrality and the other under very acid conditions.

The first-Thiobacillus thioparus Beijerinck-occurs very. widely in soils and natural bodies of water. It oxidizes thiosulphate $\left(\mathrm{Na}_{2} \mathrm{~S}_{2} \mathrm{O}_{3}\right)$, tetrathionate $\left(\mathrm{Na}_{2} \mathrm{~S}_{4} \mathrm{O}_{6}\right)$, sulphur, and sulphide. Some of the reactions may be explained as follows:

V.

$$
\begin{aligned}
& 3 \mathrm{Na}_{2} \mathrm{~S}_{2} \mathrm{O}_{3}+{ }_{5} \mathrm{O}_{2}={ }_{2} \mathrm{Na}_{2} \mathrm{SO}_{4}+\mathrm{Na}_{2} \mathrm{~S}_{4} \mathrm{O}_{6} \\
& \Delta \mathrm{F}_{298}=-260,000 \text { calories (approximate) }
\end{aligned}
$$

VI.

$$
\begin{aligned}
& \mathrm{S}+\mathrm{I}_{2}^{1} \mathrm{O}_{2}+\mathrm{H}_{2} \mathrm{O}=\mathrm{H}_{2} \mathrm{SO}_{4} \\
& \Delta \mathrm{F}_{298}=-\mathrm{I} \mathrm{I} 8,500 \text { calories }
\end{aligned}
$$

As a result of the growth of this organism, precipitated sulphur is formed outside of the cells. It is generally believed that this arises as a product of the fundamental reaction such as expressed by Beijerinck:

VII.

$$
2 \mathrm{Na}_{2} \mathrm{~S}_{2} \mathrm{O}_{3}+\mathrm{O}_{2}={ }_{2} \mathrm{Na}_{2} \mathrm{SO}_{4}+{ }_{2} \mathrm{~S}
$$

Winogradsky, S., and Omeliansky, W.: Centralbl. f. Bakteriol., Abt. II, 5, 329-43, 377-87, 429-40. I899; Godlewsky, E.: ibid., 2, 458-62. I896; Meyerhof, O.: Archiv.f. d. ges. Pliysiol., 164, 353-427; 165, 229-84. 1916; 166, 240-80; I917. Bonazzi, A.: J. Bact., 6, 479-99. 1921; 8, 343-63. I923; Gibbs, W. M.: Soil Sci., 8, 427-8I. I919.

${ }^{2}$ Gaarder, T., and Hagem, O.: Bergens Mus. Aarhok, No. 6, I-31. I 920 . See also Meyerhof, O.: loc. cil.; Meek, C. S., and Lipman, C. B.: J. General Pliysiol., 5, 195-204. I922.

3 Düggeli, M.: Neuj. d. Naturfors. Gesell. Zurich, No. I2 I. 43 pp. I9ı; Bavendamm, W.; Die farblosen und roten. Schwefclbakterien. I 56 pp. Jena: Gustav Fischer, I924; Waksman, S. A., J. Bact., 7, 231-56. I922; Baas-Becking, I. G. M.: Ann. Bot., 39, 613-50. I925.

${ }^{4}$ Nathansohn, A.: Mitt. a. d. zoolog. Station Neapel, 15, 655. I902 (Centralbl.f. Bakteriol., Abt. II, II, I09. I904); Beijerinck, M. II.: Arch. d. Sci. Exacles Nat. Haarlem (2d ser.), 9, I3I-57; Contralbl. f. Bakteriol., Abt. II, II, 592-99. Ig04; Jacobsen, H. C.: Folia Mikrobiol., I, 487-96. I9I 2; 3, I 55-62. I9I4; Waksman, S. A.: Soil Sci., 13, 329-35. I922; J. Agr. Res., 24, 297-305. 1923; Kilpatrick, M., Jr., and M. L.: J. Am. Chcm. Soc., 45, 2132-35. 1923. 
Since the sulphur is precipitated outside of the cells it seems likely that it is produced by some secondary reactions independent of the vital reactions of the cells.

The bacterium developing under acid conditions - Thiobacillus thiooxidans Waksman and Joffe $\mathrm{e}^{\mathrm{r}}$ - is distinctive in that it is able not only to tolerate but to produce higher concentrations of acid $\left(\right.$ also $\mathrm{H}^{+}$) than any other living organism yet known. ${ }^{2}$ Further, this acid is mineral and not organic. Its growth is inhibited in alkaline solutions but may not be injuriously affected in soils at neutral or alkaline reactions. In solutions, growth is most rapid at the very acid range of $\mathrm{pH}$ 2.0-3.0. It may further produce from 5 to ro per cent $(2 \mathrm{~N})$ sulphuric acid from the oxidation of elementary sulphur. It oxidizes sulphur and thiosulphate to sulphate quantitatively with the accumulation of no intermediary products in purely inorganic media. The oxidation of sulphur may be explained by reaction VI, but the thiosulphate oxidation may occur as follows:

VIII.

$$
\mathrm{Na}_{2} \mathrm{~S}_{2} \mathrm{O}_{3}+\mathrm{H}_{2} \mathrm{O}+{ }_{2} \mathrm{O}_{2}=\mathrm{Na}_{2} \mathrm{SO}_{4}+\mathrm{H}_{2} \mathrm{SO}_{4}
$$

This reaction was also suggested by Nathansohn for Th. thioparus, but since the medium did not become acid, he believed that reaction $\mathrm{V}$ better explained the process. With thiosulphate as the source of energy for Th. thiooxidans, some sulphur may become precipitated as the oxidation proceeds but this is further oxidized to sulphate. This sulphur probably originates from some secondary reactions of the sulphuric acid with the thiosulphate.

Th. thiooxidans was obtained from composts of sulphur, soil, rock phosphate, and was probably introduced with the sulphur. It has been noted in soils about sulphur mines but has not been found generally in soils which have not received applications of sulphur. ${ }^{3}$ Its distribution and importance under natural conditions is but little known. It is an obligate autotroph and fails to develop in the absence of its specific energy source in the form of sulphur or incompletely oxidized compounds of sulphur.

Another of the morphologically simple sulphur bacteria is an anaerobic formThiobacillus denitrificans Beijerinck. ${ }^{4}$ It grows only in the absence of free oxygen and

${ }^{1}$ Waksman, S. A., and Joffe, J. S.: Scicnce (N.S.), 53, 216.1921 ; Proc. Soc. Exper. Biol. \&o II ed., I8, I-3. I921; J. Biol. Chem., 50, 35-45. I922; J. Bact., 7, 239-56. I922; Waksman, S. A.: Soil Sci., 329-36. 1922; J. Bucl., 7, 602-16. 1922; Waksman, S, A., and R. L. Starkey: Proc. Soc. Exper. Biol. \&० Med., 20, 9-14. 1922; J. General Physiol., 5, 2S5-310. 1923; Starkey, R. L.: J. Bacl., 10, 135-63, I65-95. I925.

${ }^{2}$ Of interest may be the early observation of Preyer: Sitsungab.Bcr. Mcd. Ges. Natur. Heilkunde in Bonn, pp. 6-9. I 866 (Bayliss, J. M.: Principles of General Physiology, p. 359. Longmans, Green \& Co., 1924). He found in a large mollusc a salivary gland which produced sulphuric acid to a strength of 4-5 per cent.

${ }^{3}$ Joffe, J. S.: New Jersey Agr. Exper. Sta., Bull. 37 . 9ז pp. 1922. See, however, Brown, H. D.: J. Am. Soc. Agron., 15, 350-82. 1923; Jensen, II. L.: Centrulbl. f. Bukteriol., Abt. II, 72, 242-46. 1927.

${ }_{4}^{4}$ Beijerinck, M. W.: Arch.d. Sci., Exactes et Natur. Hatem (2t ser.), 9, I31-57. 1904; Centralbl. f. Bakteriol., Abt. II, I I, 592-99. See, however, Beijerinck, M. W.: Proc. Kon. Akud.v. Wetenschappen, Amsterdam, 22, 899-908. 1920; Gehring, A.: Centralbl. f. Bakleriol., Abt. II, 42, 402-3S. 1915; Lieske, R.: Jahr.f. Wiss. Bolun., 49, 01-I 27. I9I I. 
will not develop upon organic materials. It oxidizes sulphur, thiosulphate, and other compounds of sulphur to sulphate, obtaining oxygen from nitrates by such a reaction as the following:

IX.

$$
\begin{gathered}
{ }_{5} \mathrm{Na}_{2} \mathrm{~S}_{2} \mathrm{O}_{3}+8 \mathrm{KNO}_{3}+\mathrm{NaHCO}_{3}=6 \mathrm{Na}_{2} \mathrm{SO}_{4}+{ }_{4} \mathrm{~K}_{2} \mathrm{SO}_{4}+{ }_{4} \mathrm{~N}_{2}+{ }_{2} \mathrm{CO}_{2}+\mathrm{H}_{2} \mathrm{O} \\
\Delta \mathrm{F}_{298}=-893,000 \text { calories }
\end{gathered}
$$

The organism has been found widely distributed in waters and soils. The results of Lieske indicate that the reaction does not proceed directly to sulphate since other sulphur compounds were present in the media at certain stages of growth. However, none of these intermediary products accumulated. It is likely that in the oxidation of sulphur or sulphur compounds by other sulphur bacteria the process is not as simple as is generally assumed.

An organism obtained by Trautwein ${ }^{\mathrm{T}}$ showed characteristics similar to $T h$. denitrificans but differed in that it could exist either aerobically or anaerobically and either as an autotroph or heterotroph. In the presence of nitrate it developed as an anaerobe, and in the absence of organic compounds could obtain energy from the oxidation of sulphide, sulphur or thiosulphate, but the last was the best source of energy. Like the other forms, it has been found in soils, sewage, and fresh water.

Autotrophic bacteria were first recognized by Winogradsky ${ }^{2}$ in his studies of the more complex sulphur bacteria, some of which are pigmented. These forms are characterized by intracellular globules of amorphous sulphur. They develop as obligate autotrophs and utilize hydrogen sulphide as a source of energy which is oxidized in at least two separate stages, first to sulphur and then to sulphate. The reactions may be represented as follows: ${ }^{3}$

X.

XI.

$$
\begin{gathered}
\mathrm{H}_{2} \mathrm{~S} \text { (aq.) }+\frac{1}{2} \mathrm{O}_{2}=\mathrm{H}_{2} \mathrm{O}+\mathrm{S} \\
\Delta \mathrm{F}_{248}=-4 \mathrm{I}, 500 \text { calories } \\
\mathrm{S}+\mathrm{I} \frac{1}{2} \mathrm{O}_{2}+\mathrm{H}_{2} \mathrm{O}=\mathrm{H}_{2} \mathrm{SO}_{4} \\
\Delta \mathrm{F}_{298}=-\mathrm{I} \mathrm{I} 8,500 \text { calories }
\end{gathered}
$$

In the presence of a continuous supply of sulphide the organisms always contain globules of sulphur. However, if the sulphide is all removed, the globules of sulphur are oxidized to sulphate and disappear from the cells which then die. The oxidation of both the sulphide and sulphur proceeds simultaneously in the presence of sulphide. For the colorless organisms free oxygen and carbon dioxide are indispensable. ${ }^{4}$

These higher sulphur bacteria have been found extensively in both fresh and salt waters but not in soils. The pigmented forms frequently develop to such an extent as to lend very striking red colors to the waters.

Two different pigments have been obtained from the bacteriopurpurin of the purple (red) sulphur bacteria.5 One, a green pigment unlike chlorophyll, is called "bacterochlorin";

I Trautwein, K.: Ccntralbl.f. Bakteriol., Abt. II, 53, 513-48. I921; 61, I-5. I924; see also Klein, G., and Limberger, A.: Biochem. Ztschr., 340, 473-83. I 923.

${ }^{2}$ Loc. cit.

3 See, however, Baas-Becking, L. G. M.: Ann. Bot., 39, 613-50. I925.

${ }^{4}$ Keil, F.: Beitr. z. Biol. d. Pflanzen, 11, 335-72. г912.

5 Molisch, H.: Die Purpurbacterien nach neue Untersuchungen. 95 pp. Jena: Gustav Fischer, I907; Buder, J.: Jahr. f. Wiss. Botan., 58, 525 628. 1919. 
the other, a red pigment of the nature of a carotin, is called "bacterioerythrin." The pigmented bacteria differ in their nutrition from the colorless forms in that they require light in addition to hydrogen sulphide and carbon dioxide, but only little or no free oxygen.' Some photosynthetic reaction appears to be associated with the utilization of the inorganic energy, but the action may be explained in at least two ways. Bavendamm ${ }^{2}$ expressed the opinion that the organism used two reactions as sources of energy, both a photosynthetic one and the oxidation of the sulphide. Since the cells failed to develop in the absence of either light or hydrogen sulphide, but could exist in the absence of oxygen, it seems more likely that the two processes are more closely related. It may be that the light serves as an agent to reduce carbon dioxide to at least intermediary synthetic substances and furnishes oxygen for the oxidation of the sulphide. This reaction is used as a further source of energy for metabolic processes. The following reaction was suggested by Baas-Becking and Parks: ${ }^{3}$

XII.

$$
\begin{gathered}
6 \mathrm{CO}_{2}+\mathrm{r}_{2} \mathrm{H}_{2} \mathrm{~S}=\mathrm{C}_{6} \mathrm{H}_{\mathrm{I}_{2}} \mathrm{O}_{6}+6 \mathrm{H}_{2} \mathrm{O}+\mathrm{I}_{2} \mathrm{~S} \\
\Delta \mathrm{F}_{298}=+2 \mathrm{I}_{2}, 000 \text { calories }
\end{gathered}
$$

Under these conditions the deficiency of energy is supplied by the photosynthetic reaction.

\section{BACTERIA WHICH OXIDIZE FERROUS OR MANGANOUS COMPOUNDS}

Precipitation of large amounts of ferric hydrate has been repeatedly observed from many natural waters such as mineral springs, mines, and flows from other subterranean streams. Associated with such deposits there generally occur cells of filamentous and other bacteria which are incased in sheaths of ferric hydrate. Some are multicellular organisms and others small spherical, oval, or bent cells incased in irregular masses of ferric hydrate or carrying ribbon-like streamers of the substance attached to the cells. In many cases these incrustations have been considered to be sufficient evidence to indicate that these organisms are autotrophic. Pure culture studies $^{4}$ have shown that some of them are obligate autotrophs (Leptothrix ochraceae, L. trichogenes, Gallionella ferruginea, G. minor) and others facultative (Leptothrix crassa, Crenothrix polyspora). Manganese compounds may be substituted for ferrous compounds with some of these bacteria. The reaction by which they may obtain energy may be represented as follows:

XIII.

$$
\begin{gathered}
{ }_{4} \mathrm{FeCO}_{3}+\mathrm{O}_{2}+6 \mathrm{H}_{2} \mathrm{O}={ }_{4} \mathrm{Fe}(\mathrm{OH})_{3}+{ }_{4} \mathrm{CO}_{2} \\
\Delta \mathrm{F}_{298}=-8 \mathrm{I}, 000 \text { (approximate) }
\end{gathered}
$$

Since chemical oxidation of ferric hydrate may be so common in natural waters, the relative importance of the iron bacteria as factors in iron deposition is not known. ${ }^{5}$

s lingelmann, T. WI.: Pfïgers Arch.f. d. ges. Physiol., 30, 95-124. IS83; 42, I83-86. 18S8; Botan. Zeit., 46, 66г. ז8s8.

$=$ Loc. cil.

3 Loc. cil.

4 Winogradsky, S.: Botun. Zcil., 46, 26I-70. ISS8; Centralbl.f. Bakteriol., Abt. II, 57, I-21. I922; Lieske, R.: Jahr.f. II iss. Botan., 49, 91-1 27. I9 I ; Centralbl.f. Bakteriol., Abt. II, 49, 413-25. I919; Molisch, H.: Die Eiscnbukterich. 83 pp. Jena: Gustav Fischer, 1910; Cholodny, N.: Dic Eiscnbaktericn. 162 pp. Jena: Gustav Fischer, ro26.

5 Ilarder, E. C.: U.S. Gcol Survey, Prof. Paper 113. S9 pp. 1919; Gruner, J. II.: Econ. Gcol., I7, 407-60. 1922; IIalvorson, H. O., and Starkey, R. L.: J. Phys. Chem., 31, 626-31. 1927; Starkey, R. L., and Halvorson, H. O.: Soil Sci., 24, 3\$1-402. 1927. 
Even the existence of autotrophic iron bacteria has been questioned on theoretical grounds. ${ }^{\mathrm{I}}$ The precipitation of iron irrespective of its mechanism is of considerable economic importance. This is suggested by the extensive deposits of iron ore of aquatic origin, deposition with rusting of iron pipes, the fouling of drinking waters, and the formation of iron hardpans in soils.

\section{BACTERIA WHICII OXIDIZE HYDROGEN}

Hydrogen arises naturally in considerable abundance as a result of decomposition processes under anaerobic conditions. This is particularly noticeable in stagnant waters. It may also arise from mines and numerous non-biological sources. Biological oxidation may be active in preventing any great accumulation of this gas. It may be oxidized under aerobic conditions by a large number of simple bacteria all of which appear to be facultative autotrophs. ${ }^{2}$ Some of those isolated by Grohmann have endospores. The reaction proceeds to water by the following reaction:

XIV.

$$
\begin{aligned}
\mathrm{H}_{2}+\frac{1}{2} \mathrm{O}_{2} & =\mathrm{H}_{2} \mathrm{O} \\
\Delta \mathrm{F}_{298} & =-5_{5} 6,000 \text { calories }
\end{aligned}
$$

The quotient of $\mathrm{H}_{2}: \mathrm{O}_{2}$ in pure-culture experiments is close to 2. No consistent ratio of $\mathrm{CO}_{2}$ (assimilated): $\mathrm{H}_{2}$ (oxidized) was observed by Ruhland, but from observations on the physiology of other autotrophic bacteria one might infer that in the biological transformation such a relationship exists.

These organisms occur widely in soils and may live upon many different organic compounds as well as hydrogen.

It has been suggested that other organisms have autotrophic habits and utilize such compounds as selenium, ${ }^{3}$ carbon monoxide, ${ }^{4}$ arsenites, ${ }^{5}$ and methane. ${ }^{6}$ As concerns the first three compounds the evidence appears too limited at present to be more than suggestive. Although the bacterial oxidation of methane has been quite definitely established it seems undesirable to classify this process as autotrophic since methane may more logically be considered as an organic compound. Although classification is naturally arbitrary and its distinction is not as sharp in nature as seems to be suggested, it should eliminate confusion and lead toward simplicity and clearness. These advantages do not seem to be gained by including such organisms as methane-oxidizing bacteria among the autotrophic forms.

I Baas-Becking and Parks, loc. cit.

${ }^{2}$ Kaserer, H.: Centralbl.f. Bakteriol., Abt. II, I5, 573-76; I6, 68 I-96. I906; Beijerinck, M. W. and Van Delden, A.: ibid., I0, 33-47. I903; Nikitinsky, J.: ibid., 19, 495-99. I907; Nabokitch, A. J., and Lebedeff, A. F.: ibid., I7, 350-55. I907; Niklewski, B.: ibid., 20, 469-73. Ico8; Jahrb. f. Wiss. Botan., 48, I I3-42. I9 10; Lebedev, A. J.: Ber. d. Deut. Bot. Gesell., 27, 598-602. I910; Grohmann, G.: Centralbl.f. Bakteriol., Abt. II, 61, 256-7I. I924; Ruhland, W.: Jahrb.f. Wiss. Botan., $63,32 \mathrm{I}-89$. I924.

3 Lipman, J. G., and Waksman, S. A.: Science (N.S.), 57, 60. 1923.

${ }_{4}$ Beijerinck, M. W., and Van Delden, A.: loc. cit.; Kaserer, H.: Ccntralbl. $f$. Bakteriol., Abt. II, I6, 68ı-96, 769-75. ıс06; Lantzsch, K.: ibid., 57, 309. I922.

${ }_{5}$ Green, H. H.: Fifth and Sixth Rpts. Vet. Res. Dept. of Agr. of the Union of So. Africa, pp. 595-610. I9 8 .

${ }^{6}$ Söhngen, N. L.: Centralbl.f. Bakteriol., Abt. II, 15, 513-17. I906; Münz, E.: Zur Physiologie der Methanbakterien (Inaug. Diss., Halle). 63 pp. г9г5; Aiyer, P. A. S.: Mem. Dept. Agr. India (Pusa), Chem. Ser., 5, 173-94. I920. 
Although biological oxidation of phosphorus compounds has not been investigated, it is reasonable to believe that autotrophic processes may be concerned in such transformations.

\section{INFLUENCE OF ORGANIC MATERIALS ON AUTOTROPHIC BACTERIA}

The influence which organic compounds exert upon autotrophic bacteria is still a disputed point. These organisms fail to develop where organic materials furnish the sole source of energy in the medium. Further, no organic materials replace carbon dioxide as a source of carbon. However, even in the presence of the specific inorganic sources of energy, organic materials may exert pronounced inhibitory effects upon the development of autotrophic bacteria.

Of the iron bacteria, L. ochracea appeared indifferent to considerable concentrations of organic matter, but $G$. ferruginea was quite sensitive to such substances as peptone, sucrose, and asparagine. ${ }^{\mathrm{I}}$ For the higher sulphur bacteria moderate amounts of organic materials do not appear to be injurious. ${ }^{2}$

Oxidation by $T h$. thiooxidans was not appreciably affected by even 5 per cent glucose. ${ }^{3}$ Considerable citric acid (r per cent) exerted no effects on growth, but lower concentrations were tolerated than of sulphuric acid. It was early noted that some organic materials were toxic to nitrifying bacteria. ${ }^{4}$ Glucose injured growth of nitrate formers at 0.045 per cent and completely inhibited development at 0.27 per cent. The nitrate formers were less susceptible to such injury than the nitrite formers. Coleman $^{5}$ noted no injury to growth of nitrate formers at $0.02-0.05$ per cent glucose, but larger amounts were distinctly toxic. No nitrite formation took place at 0.2 per cent glucose, and even 0.02 per cent was injurious. Toxicity is very different with different compounds. ${ }^{6}$

It has been noted with Th. thiooxidans ${ }^{7}$ as well as the nitrifying organisms ${ }^{8}$ that, although glucose alone does not support growth, it disappears if introduced into cultures containing the specific inorganic energy source.

Although under optimum conditions higher plants may utilize close to 80 per cent of the energy furnished by the light, ${ }^{9}$ more commonly from I to 3 per cent is used. Between 5 and ro per cent of the energy liberated in the oxidation of the inorganic substances is utilized by the autotrophic bacteria to reduce carbon dioxide to organic

${ }^{1}$ Cholodny, N.: loc. cit.

${ }^{2}$ Winogradsky, S.: loc. cit.; Bavendamm, W.: loc. cit.

3 Waksman, S. A., and Starkey, R. L.: loc. cit.; Starkey, R. L.: loc. cit.

4 Winogradsky, S., and Omeliansky W.: Centralbl. f. Baktcriol., Abt. II, 5, 329-43, 377-87, 42940. I 899 .

5 Coleman, L. C.: Ccntralbl. f. Bakteriol., Abt. II, 20, 40I-20, 484-513. I908.

${ }^{6}$ Meyerhof, O.: loc. cit.; see also Beijerinck, M. W.: Folia Mikrobiol. (3d year), 2, 91-113. I914; Winogradsky, S.: Compt. rend. Acad. Sci., 175, 30I-4. I922; Murray, T. J.: Proc. Soc. Exper. Biol. \& Mcd., 20, 30I-3. 1923; Sach, J.: loc. cit.; Fred, E. B., and Davenport, A.: Soil Sci., II, 38c404. I92I.

${ }^{7}$ Starkey, R. L.: loc. cit.

${ }^{8}$ Coleman, L. C.: loc. cil.

9 Warburg, O.: Naturwisscnschaften, 9, 354-58. 1921; 13, 985-93. 1925; Ztschr. phys. Chemic, 106, I9I-218. 1923; Warburg, O., and Negelein, E.: ibid., 102, 235-66. 1922; 108, 101-2. I924; Naturwissenschaften, 10, 647-53. 1922. 
compounds. The energy values for some of the autotrophic bacteria have been calculated by Baas-Becking and Parks as free energy efficiency. ${ }^{x}$

\begin{tabular}{|c|c|c|}
\hline REACTION & Ачтнов & $\begin{array}{c}\text { FrEe ENERGY } \\
\text { EFFICIENCY } \\
\text { Percentage }\end{array}$ \\
\hline $\mathrm{NH}_{4}++\mathrm{I}_{2}^{1} \mathrm{O}_{2}=\mathrm{NO}_{2}-+\mathrm{H}_{2} \mathrm{O}+{ }_{2} \mathrm{H}+\ldots \ldots$ & Meyerhof* & $7 \cdot 9$ \\
\hline $\mathrm{NO}_{2}-+\frac{1}{2} \mathrm{O}_{2}=\mathrm{NO}_{3}-\ldots \ldots \ldots \ldots \ldots \ldots$ & Meyerhof $\dagger$ & $5 \cdot 9$ \\
\hline & $\begin{array}{c}\text { Winogradsky } \ddagger \\
\text { (Beggiatoa) }\end{array}$ & $8 \cdot 3$ \\
\hline $\mathrm{S}+\mathrm{I}_{2}^{\frac{1}{2}} \mathrm{O}_{2}+\mathrm{H}_{2} \mathrm{O}=\mathrm{H}_{2} \mathrm{SO}_{4} \ldots$ & $\begin{array}{l}\text { Waksman and Starkey } \\
\text { (Th. thiooxidans) }\end{array}$ & 8.3 \\
\hline $\left.\begin{array}{c}6 \mathrm{KNO}_{3}+{ }_{5} \mathrm{~S}+{ }_{2} \mathrm{CaCO}_{3}={ }_{3} \mathrm{~K}_{2} \mathrm{SO}_{4} \\
\quad+2 \mathrm{CaSO}_{4}+{ }_{2} \mathrm{CO}_{2}+2 \mathrm{~N}_{2}\end{array}\right\} \ldots \ldots \ldots$ & Beijerinck\| & 5.0 \\
\hline $\begin{array}{l}5 \mathrm{Na}_{2} \mathrm{~S}_{2} \mathrm{O}_{3}+8 \mathrm{KNO}_{3}+{ }_{2} \mathrm{NaHCO}_{3}=6 \mathrm{Na}_{2} \mathrm{SO}_{4} \\
\quad+{ }_{4} \mathrm{~K}_{2} \mathrm{SO}_{4}+4 \mathrm{~N}_{2}+{ }_{2} \mathrm{CO}_{2}+\mathrm{H}_{2} \mathrm{O}\end{array}$ & Lieske & 9.0 \\
\hline $\begin{array}{c}\mathrm{H}_{2}+\frac{1}{2} \mathrm{O}_{2}=\mathrm{H}_{2} \mathrm{O} \ldots \ldots \ldots \ldots \ldots \ldots \ldots \ldots \\
\quad * \text { Loc. cit. } \quad+\text { Loc cit. } \quad \neq \text { Loc. cit. } \quad \text { \& Loc. cit. }\end{array}$ & $\begin{array}{l}\text { Ruhland** } \\
\text { Loc, cit. } * \text { * Loc, cit. }\end{array}$ & 26.4 \\
\hline
\end{tabular}

The mechanism of assimilation of carbon dioxide by autotrophic bacteria shows some striking similarities to the photosynthetic reaction in higher plants. Klein and Svolba $^{2}$ have distinguished between assimilation, respiration, and oxidation in studies of two autotrophic bacteria, one an obligate and the other a facultative autotroph. It was observed that in the assimilation reaction carbon dioxide was reduced, and that in the course of its use for the synthesis of organic compounds formaldehyde was formed. Respiration, or the disintegration of synthesized organic compounds, was accompanied by the formation of acetaldehyde. The energy for these synthetic reactions was derived from the process of oxidation of the specific inorganic compound.

${ }^{1}$ Loc, cit.

${ }^{2}$ Klein, G., and Svolba, F.: Ztschr. f. Botan., 19, 65-100. 1926; see also Loew, O.: Biochem. Ztschr., 140, 324-25. 1923; Kluyver, A. L., and Donker, H. J. L.: Chemie d. Zelle u. Gervebe, r 3, I3490. 1926. 


\section{CHAPTER XXV}

\section{THE ROOT-NODULE BACTERIA OF LEGUMINOUS PLANTS}

\section{EDWIN BROUN FRED}

University of Wisconsin

The growing of leguminous crops for soil improvement is probably one of the oldest applications of bacteriology. More than two thousand years ago the Romans, and, at an earlier date the Chinese, were aware of the fertilizing effect of leguminous plants. Although the Roman farmers made use of these plants to increase the fertility of the soil, they did not know how the beneficial effect was produced. It was not until the early eighties of the last century that investigators demonstrated clearly that this beneficial effect of leguminous plants was due to the fixation of nitrogen within the root nodules.

Outstanding among the early investigators were Hellriegel ${ }^{\mathrm{r}}$ and his associate Wilfarth in Germany, Atwater ${ }^{2}$ in America, and Lawes and Gilbert ${ }^{3}$ in England. As a result of their work, intensive studies were carried out in various countries and the conclusion reached that nodule formation was caused by the associated growth of the leguminous plant and some lower organism, and also that nitrogen fixation takes place in the root nodules. For the first time in 1888 , Beijerinck ${ }^{4}$ in Holland obtained pure cultures of nodule bacteria and demonstrated their ability to produce root nodules.

\section{RELATION OF BACTERIA TO HOST PLANT}

The life of the root-nodule organism outside of the plant and the agencies which lead to its entry into the tissues are not well known. It has been suggested that the bacteria are attracted to the roots by the secretion of a substance of unknown composition. At best, the movement of the bacteria in the soil is slow, perhaps I inch in twenty-four hours. ${ }^{5}$

The bacteria get into the parenchyma of the root through the root hairs or through other epidermal cells. These infected root hairs show a characteristic bending at or near the root tip. Once within the tissues the bacteria multiply rapidly, forming threadlike filaments with many branches throughout the hair and into the parenchyma of the root. Here in the innermost cells of the root cortex, just outside of the endodermis, the bacteria bring about conditions favorable for a rapid multiplication of the surrounding cells and thus the formation of the young nodule begins. As the cells multiply the young nodule soon pushes out the overlying cortical paren-

${ }^{1}$ Helliriegel, H.: Landw. Vers. Stat., 33, 464. 1886.

${ }^{2}$ Atwater, W. O.: Am.Chem.J., 8, 398. 1886.

3 Lawes, J., and Gilbert, J.: Proc. Roy. Soc., London, 47, 85. 1890.

4 Beijerinck, M. W.: Bot. Zlg., 46, 725. I888.

5 Thornton, H. G., and Gangulee, N.: Proc. Roy. Soc., London, B, 99, 427. 1926. 
chyma and epidermis and thus forms a swelling on the side of the root. ${ }^{\mathrm{I}}$ In general, nodules consist of a mass of thin-walled parenchymatous cells, rich in protein, and

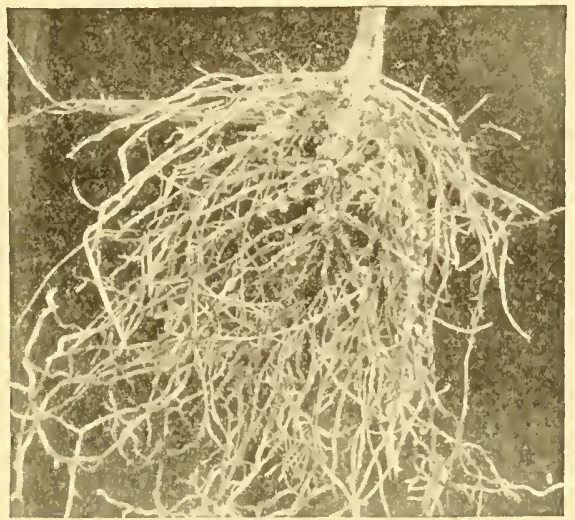

Fig. I.-Young pea plant with nodules

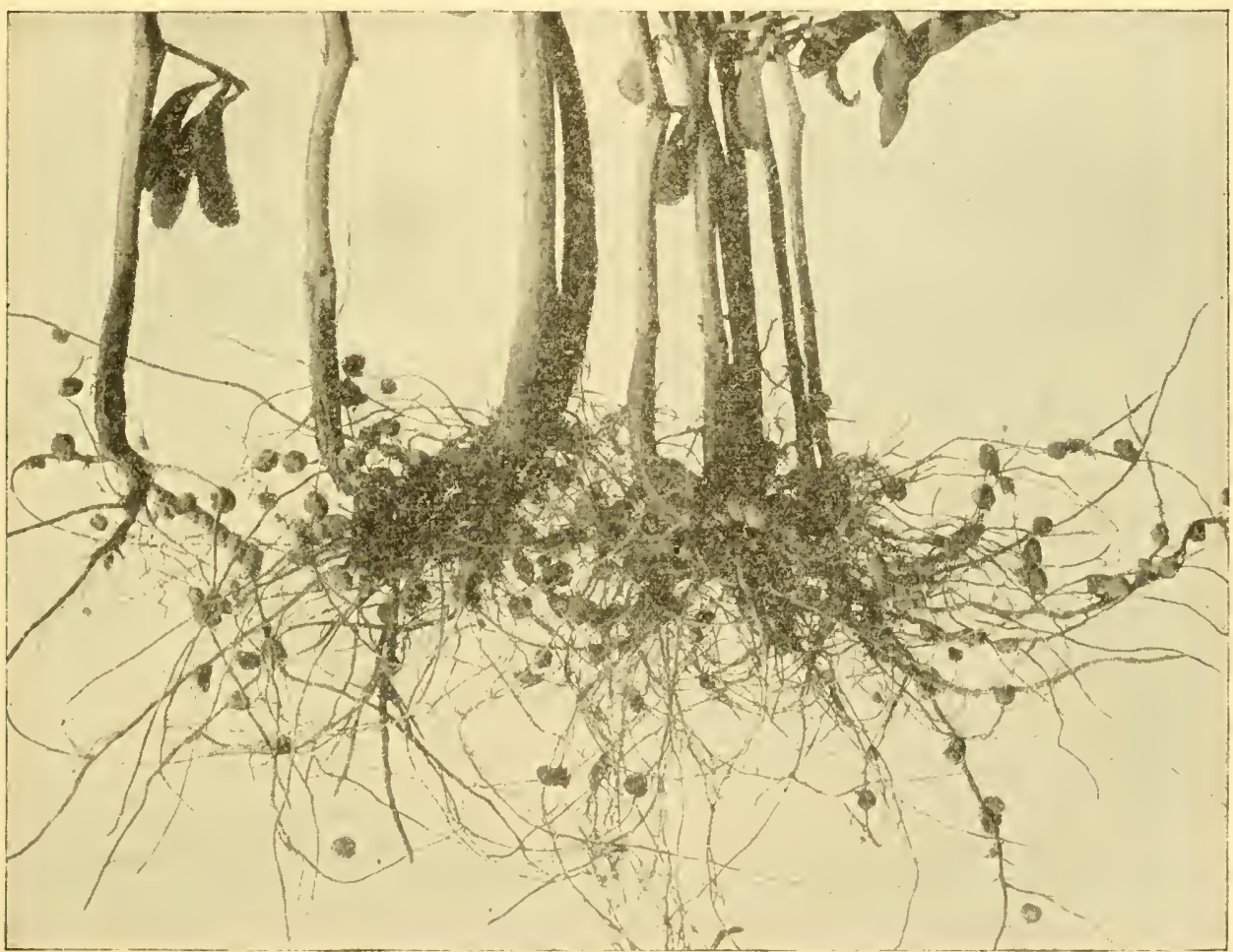

FIG. 2.-Soy bean plants with nodules

usually almost filled with bacteria. These thin-walled swollen cells occupy the greater part of the tissue of the nodule. A layer of cork and branches of a vascular system are

z Brenchley, W. E., and Thornton, H. G.: ibid., 98, 373. I925. 
also present. By means of this system of vascular tubes the plant supplies the bacteria with sugars and other food substances, and in turn takes away the nitrogenous compounds prepared by the bacteria.

The shape, size, and position of the nodules vary with the different leguminous plants, e.g., alfalfa nodules are small, finger-shaped swellings, single or in bunches or clusters; soy-bean nodules are larger, usually spherical, and rarely in clusters. Figures I and 2 show the nodules of pea and soy bean.

Of the ten thousand or more species of leguminous plants described by botanists, all except a very few, like Kentucky coffee tree and wild senna, show root nodules. The bacteria, however, from these various plants are not all alike. In general, plants closely related harbor the same kind of bacteria. ${ }^{I}$ Some idea of the relationship among the bacteria and higher plants is shown in the groups of the more commonly cultivated plants. Below is a list of common leguminous plants divided into groups on the basis of cross-inoculation. The plants of each group have their own specific organism which can inoculate any member of the group but will not interchange with the plants of another group.

\section{CROSS-INOCULATION GROUPS}

I. Alfalfa, white sweet clover, yellow sweet clover, Hubam, bur clover, yellow trefoil, and fenugreek

2. Red, mammoth, alsike, crimson, Egyptian, and white Dutch clovers

3. Garden, canning, and field peas; hairy, spring, and wild vetches; broad bean; lentil; sweet pea; and perennial pea

4. Cowpea, peanut, Japan clover, velvet bean, lima bean, partridge pea, wild indigo, and tick trefoil

5. Garden, field, navy, kidney, wax, and scarlet runner beans

6. Lupines and serradella

7. Soy beans

8. Wood's clover (Dalea)

9. Sanfoin

Iо. Locust

The explanation for the grouping of the plants into the so-called "cross-inoculation" groups has not been determined. Probably some phase of the physiological complex of the plant is responsible. Support for this assumption may be seen in the results of recent precipitin tests with the seed proteins of leguminous plants. ${ }^{2}$ These tests show that the seed proteins of those leguminous plants belonging to any one cross-inoculation group are closely related.

There is some evidence that the bacteria within a given cross-inoculation group are not all alike. The organisms from pea and vetch are interchangeable, and yet the best results are usually secured from pea bacteria on peas and vetch on vetches. All in all, there exists a high degree of specificity. Why this difference should exist is at the present time unknown.

${ }^{x}$ Burrill, T. J., and Hansen, R.: Illinois Agr. Exper. Sta. Bull. 202. I917; Fred, E. B., Whiting, A. L., and Hastings, E. G.: Wisconsin Agr. Exper. Sta. Research Bull. 72. 1926.

${ }^{2}$ Baldwin, I. L., Fred, E. B., and Hastings, E. G.: Bot. Gas., 83, 217. 1927. 
NITROGEN NUTRITION

The process of nitrogen fixation is carried out by the bacteria within the nodule. The plant furnishes the carbohydrate and the bacteria the combined nitrogen. Just

TABLE I

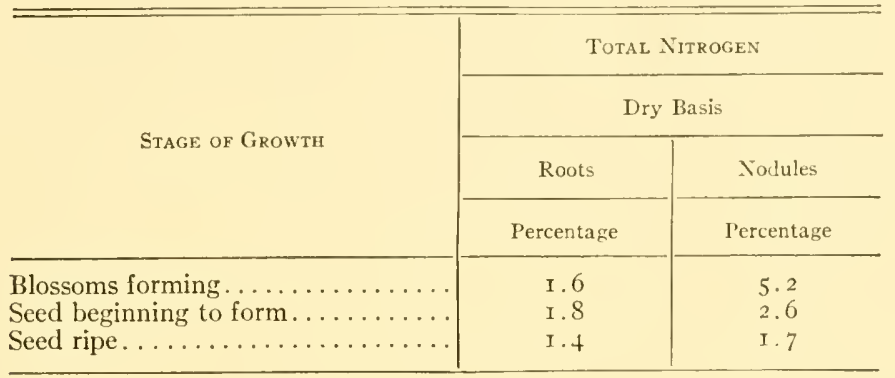

what causes the rapid autolysis of the bacterial cells within the nodule is not definitely known. It is reported that the presence of a bacteriophage brings about the rapid

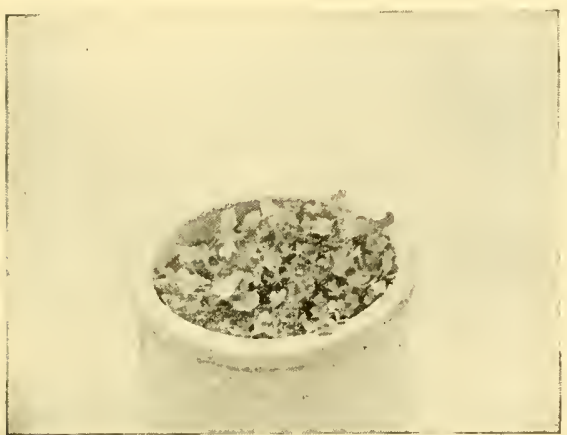

Not inoculated

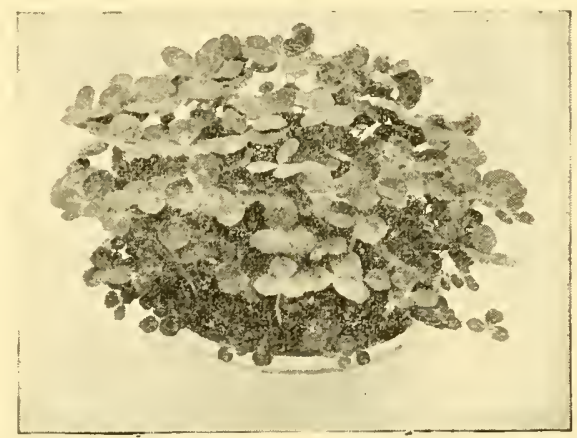

Inoculated

FIG. 3.-Effect of root-nodule bacteria on the growth of clover

dissolution of the bacteria within the nodule, and thus the proper equilibrium is maintained between the higher plant and the bacteria.

The intimate relationship between the nodule and nitrogen content of the plants is shown by the results of chemical analyses. Young nodules of plants are much richer in nitrogen than the rest of the root, while at a later period the nodules are much lower in nitrogen. Analyses of lupine nodules and roots at various stages during the growth of the plant illustrate this point (see Table I). ${ }^{2}$

Concerning the nature of the nitrogenous compounds of nodules, it has been found that about 5 o per cent are soluble in water. ${ }^{3}$ Of the protein-free soluble nitrogen

${ }^{x}$ Gerretsen, F. C., Grijns, A., Sach, J., and Söhngen, N. L.: Centralbl.f. Bakteriol., Abt. II, 6o, 3II. I923; Grijns, A.: Doctor's thesis, p. I04. Wageningen, Holland: Veenman \& Zonen, I926.

2 Stoklasa, J.: Landw. Jahrb., 24, 827. I895.

3 Strowd, W. H.: Soil Sc., I I, I23. I92I. 
about 16 per cent exists as primary amino nitrogen and 20 per cent as amid nitrogen. The nitrogen of the nodule is rapidly transferred from the underground nodule to the

TABLE II

Effect of Inoculation on tile Nitrogen in Soy Beans

\begin{tabular}{|c|c|c|c|c|}
\hline & \multicolumn{4}{|c|}{ Nitrogen in Pounds per Acre } \\
\hline & Tops & Roots & Nodules & Total \\
\hline 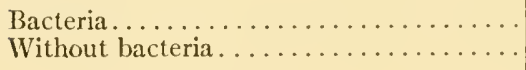 & $\begin{array}{r}57.10 \\
7.46\end{array}$ & $\begin{array}{l}2.40 \\
0.87\end{array}$ & 5.86 & $\begin{array}{r}65.36 \\
8.33\end{array}$ \\
\hline Gain due to bacteria............ & 49.64 & I. 53 & 5.86 & 57.03 \\
\hline
\end{tabular}

seed and stems. Thus the activity of the bacteria results in the formation of a nitrogen supply for the plant. Figure 3 shows the effect of the bacteria on the growth of red clover on poor, sandy soil.

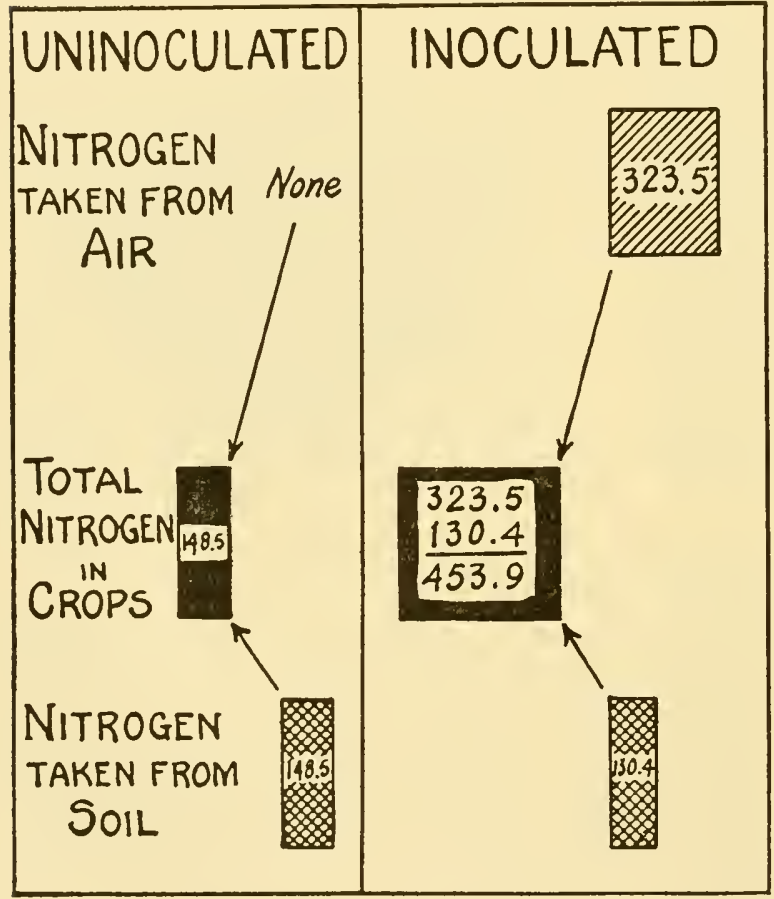

FIG. 4.-Source of nitrogen for inoculated and uninoculated soy beans. Areas in black represent total nitrogen; shaded areas represent nitrogen from air and from soil in pounds per acre.

Since nitrogen may be the element limiting plant growth, it is to be expected that the addition of the proper bacteria to certain leguminous plants will make possible a profuse growth and larger amounts of protein. Some examples of the effect of the nodule bacteria on the host plant are presented in Table II. ${ }^{\mathrm{I}}$ Figure 4 is prepared from the results of field tests of soy beans grown with and without bacteria. Field tests with new crops show that inoculation of the seed with the nodule bacteria has a double advantage to the farmer. There is an increase in the protein and often an increase in the yield. Numerous analyses have been made of alfalfa, clover, peas, and soy beans grown with and without nodule bacteria, some of which are given in Table III. The results are in accord with the general facts brought out by field tests. Aside from the favorable effects of inoculation on nitro-

IFred, E. B.: ibid., p. 469. I921. 
gen content and yield, in the case of canning peas the presence of the nodule bacteria often results in more uniform growth and hence an improvement in the quality of the peas.

\section{FACTORS THAT INFLUENCE NODULE FORMATION}

When leguminous plants are grown in soil abundantly supplied with nitrate nitrogen, the plants may develop luxuriantly and yet fail to show nodules, although the soil and seed have been richly inoculated with the proper bacteria. That this lack of nodules is not due to a toxic effect on the bacteria is well established. From all of the tests carried out it has been found that the suppression of nodule formation is not a direct effect of the nitrates but rather a case of change in the metabolism of the higher plants. ${ }^{\mathrm{x}}$ The addition of certain salts, such as phosphates and sulphates, and also carbon compounds, such as sugars and organic acids, have been found beneficial to nodule production. ${ }^{2}$

TABLE III

\begin{tabular}{|c|c|c|}
\hline & $\begin{array}{l}\text { WEIGHT } \\
\text { PER ACRE }\end{array}$ & $\begin{array}{l}\text { Nitrogen } \\
\text { Per Acre }\end{array}$ \\
\hline & Lb. & Lb. \\
\hline 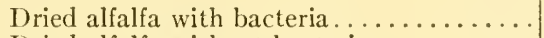 & 2,866 & 87 \\
\hline Dried alfalfa without bacteria. . & $\mathbf{I}, 7 \mathbf{1 5}$ & 46 \\
\hline Green peas (seed) with bacteria....... & $\mathbf{I}, 7 \mathrm{I} 7$ & 43 \\
\hline Green peas (seed) without bacteria.... & 874 & 27 \\
\hline
\end{tabular}

Nodule formation and consequently nitrogen fixation are also greatly influenced by soil reaction, temperature, moisture, and oxygen relations.

\section{CHEMISTRY OF NITROGEN FIXATION}

If the average amount of nitrogen fixed per acre per year is taken as roo lb., then the energy required by the bacteria would be that from about 10,000 lb. of carbohydrate. This estimate is based on the results of pure-culture studies assuming that the nodule bacteria fix I-2 mgm. of nitrogen for roo $\mathrm{mgm}$. of sugar consumed. Obviously this requirement of carbohydrate is far in excess of the amount supplied to the root nodules by the plant. It is highly probable, therefore, that the organisms in the plant nodule function much more efficiently than outside of the host. Christiansen-IVeniger ${ }^{3}$ suggests that the process is exothermic. Just how this fixation takes place is not known. It is probably a reduction process which results in the formation of àmmonia.

\section{PURE-CULTURE STUDIES}

If the roots of a normal leguminous plant bearing nodules are thoroughly washed and the contents of a young nodule pressed out on a glass slide, fixed, and stained, great masses of variously shaped bacterial cells will be seen.

From these active young nodules, it is compartively easy to isolate pure cultures of the nodule-forming bacteria. The ordinary culture media, nutrient or plain agar,

r Fred, E. B., and Graul, E. J.: J. Am. Soc. Agron., 8, 3г6. г9ı6

${ }^{2}$ Wilson, J. K.: Cornell Agr. Exper. Sta. Bull. 386. т9г7.

${ }^{3}$ Christiansen-IVeniger, F.: Centralbl. f. Bakteriol., Abt. II, 58, 41. I923. 
is not very satisfactory for the growth of these organisms. Much better results are obtained if special sugar media almost free of combined nitrogen or if sugar media plus extracts of plants are used. On mannitol phosphate agar at $20^{\circ}-25^{\circ} \mathrm{C}$. welldeveloped colonies are obtained within five to ten days.

The colonies of bacteria vary in appearance depending on the kind of leguminous plant from which they were obtained. From alfalia, clover, and pea nodules the colonies are usually large, raised, opaque, and sticky, while those from the nodules of soy bean are usually small, slightly raised, producing an almost transparent film which slowly spreads over the surface of the agar medium. ${ }^{\mathrm{T}}$

Morphology. - When cultures from a synthetic nitrogen-free agar medium are examined the cells usually appear as short rods. In addition to the rods, cocci and large

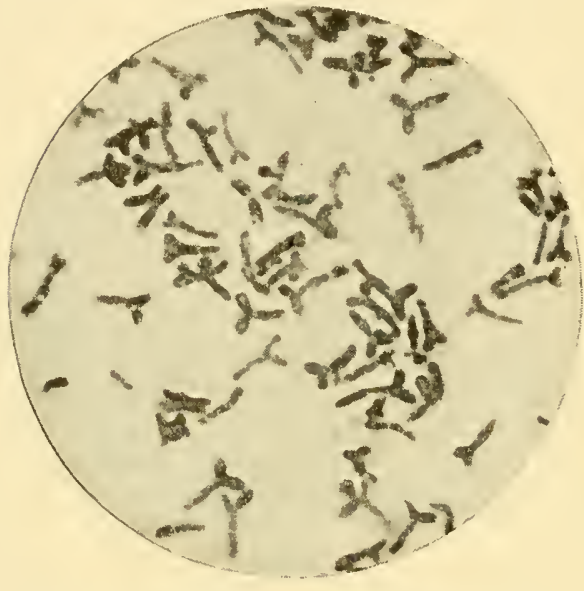

FIG. 5.-Bacteria from root nodule of pea plant showing large vacuolated forms. $X_{1500}$ unevenly stained rods are found. These banded rod forms may in turn become greatly swollen, and are then known as "bacteroids." The coccus forms which arise from the banded mother-cells are at first non-motile, but later become actively motile. As these cocci become older they elongate and return to the rod form, and are often motile. Still later they appear as the banded non-motile rods. According to Löhnis and Smith," Bewley and Hutchinson, ${ }^{3}$ and more recently Thornton and Gangulee, 4 the various forms through which the organisms naturally pass make up the socalled "life-cycle." Figure 5 is a photomicrograph of some of the typical bacteroid forms.

The bacteroid form may not appear on culture media, but in the nodule this greatly enlarged and banded or highly vacuolated form is of common occurrence and somewhat characteristic of the plant species. Small amounts of caffeine, cumarine, and other vegetable alkaloids added to the culture medium promote the formation of bacteroids. ${ }^{5}$ The bacteroids produced in this way are not so numerous as those found in the nodule. Many of the small coccus forms may develop from one of the branched swollen rods. As the nodules disintegrate the root-nodule bacteria pass back into the soil as these small cocci.

Flagellalion.-According to the number and arrangement of the flagella these bacteria may be divided into two great groups. ${ }^{6}$ Those of the alfalfa, clover, pea, and

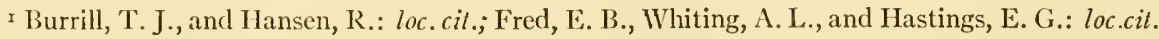

${ }^{2}$ Löhnis, F., and Smith, N. R.: J. Agr. Research, 6, 675. 1916.

3 Bewley, W. F., and Hutchinson, H. B.: J. Agr. Sc., 10, 144. 1920.

${ }_{4}^{4}$ Thornton, H. G., and Gangulee, N.: loc. cit.

5 Barthel, C.: Ann. de l'Inst. Pasteur, 35, 634. 1921.

${ }^{6}$ Shunk, I. V.: J. Bact., 6, 239. 1921; ibid., 5, I81. 1920. 
bean groups possess numerous peritrichous flagella; while those of the cowpea and soy-bean groups possess a single flagellum attached at a corner of the cell (monotrichous).

Acidity rclations.- The two general groups of nodule bacteria established by flagellation may be subdivided according to their behavior toward acidity. The acidity or critical $\mathrm{pH}$ for bacteria differs with the several cross-inoculation groups. In general, the limiting acidity is that known to be injurious to the higher plant. Below is given the critical $\mathrm{pH}$ for the bacteria of several of the more common leguminous plants.

Critical or Limiting $\mathrm{pH}$

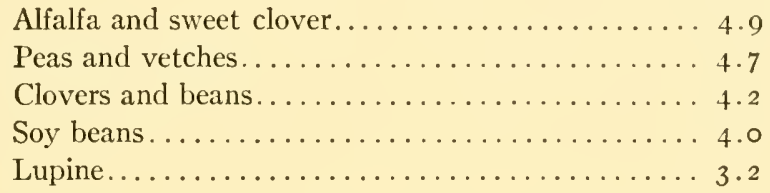

Carbohydrate fermentation.-Differences between the cross-inoculation groups as well as the strains within a given group are easily recognized by differences in the fermentation of carbohydrates. ${ }^{2}$ Since the fermentation of carbohydrates is weak, a medium of low-buffer capacity and a long period of incubation must be used if changes in $\mathrm{pH}$ value are to be noted.

In general, the members of the peritrichous group are much more vigorous fermenters than are those of the monotrichous group. Each of the cross-inoculation groups may be recognized by the use of appropriate carbon compounds, and in several of the cross-inoculation groups subgroups are established. For example, strains of the alfalfa organism produce much acid from mannitol, while others do not.

Scrological reactions.-Because of the high specificity of the agglutination test, it has been found of value in separating strains within a single cross-inoculation group. The sera usually show a high titre, agglutinating homologous antigen in dilutions of $\mathrm{I}-20,000, \mathrm{I}-30,000$, or even higher. Repeated tests indicate that these differences in serological properties remain constant after long cultivation under varying conditions. ${ }^{3}$

Gum production.--One of the most noticeable characters of the nodule bacteria is their pronounced gum formation, especially when grown on plant extracts plus sugar. If pea or clover bacteria are grown on the extract of young pea plants, the formation of gum may be so profuse that the entire culture becomes viscous, like a weak gelatin. The addition of acetone or alcohol to these well-developed cultures precipitates this gum. Great variation in the amount of gum production is observed between the cultures of the different cross-inoculation groups and between strains within each group.

Chemical tests show this gum to be a nitrogen-free carbon compound. It does not reduce Fehling's solution in the natural state but is readily hydrolyzed by acid or heat with the production of a reducing sugar.

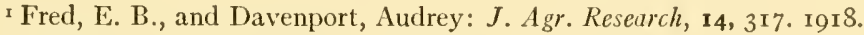

${ }^{2}$ Baldwin, I. L., and Fred, E. B.: Soil Sc., 24, 2 19. 1927.

3 Stevens, J. W.: ibid., 20, 45. I925; Wright, W. H.: ibid., 20, 95. 1925.
} 
Nitrogen fixation.- In spite of numerous investigations on the assimilation of nitrogen in the absence of the host plant, this phase of the root-nodule problem has proved most baffling. The nodule bacteria under proper conditions will combine small amounts of atmospheric nitrogen, $2-3 \mathrm{mg}$. of nitrogen for each Ioo cc. of culture. Compared with the amount fixed in the plant, this is insignificant. A satisfactory explanation for this difference in behavior in the absence of the host plant has not been found.

Strain variations.-Only recently has the importance of the variation between strains within a cross-inoculation group been realized. The relatively small differences between strains in cultural characteristics, carbohydrate fermentation, gum production, and serological properties have been shown to be correlated. The practical importance of this lies in the fact that certain strains in symbiosis with plants have been more efficient in fixing nitrogen, and this activity of the strain has been shown to be related to the behavior of the strain in laboratory tests.

\section{CULTURES OF BACTERIA FOR THE INOCULATION OF LEGUMES}

Since $1887^{1}$ it has been known that the transfer of soil from a well-inoculated field may exert a beneficial effect on the growth of leguminous plants in a new location. The bacteria carried in the soil cause nodule development and thus furnish nitrogen to the plants. More recently much easier and quicker methods have been found for introducing nodule bacteria. Pure cultures of the bacteria are grown in liquid or solid media, and a water suspension of the bacteria is applied directly to the seed. In this way the bacteria are brought in close contact with the young plant. To insure nitrogen fixation the use of bacteria to inoculate the seed has become a well-established practice, especially when a leguminous plant is seeded for the first time.

The property of leguminous plants to accumulate atmospheric nitrogen in large quantities gives them an enviable rôle in economic agriculture.

${ }^{x}$ Kellerman, K. F.: Centralbl.f. Bakteriol., Abt. II, 34, 42. I912. 


\title{
CHAPTER XXVI
}

\section{MICRO-ORGANISMS IN RELATION TO SOIL FERTILITY}

\author{
JACOB G. LIPMAN
}

New Jersey Agricultural Experiment Station, New Brunswick, N.J.

The average cultivated and improved soil is a good culture medium for higher plants and for micro-organisms. A soil is said to be fertile when it manifests ability to produce large crops throughout a series of years. By the same token it is infertile when its crop-producing power is below the average. But a soil fertile or infertile for higher plants is usually also fertile or infertile for micro-organisms. This is borne out by widespread experiments and observations which provide ample proof that an intimate relation exists between the macroflora of soils, on the one hand, and their microflora and fauna, on the other.

THE NATURE OF SOIL FERTILITY

INTERNAL FACTORS

A soil consisting, for the most part, of minute fragments is said to be "finetextured soil," and when made up of particles of relatively large size, it is designated as a "coarse-textured" soil. The relation of the fineness of soil material to texture may be well illustrated by the figures given by Lyon, Fippin, and Buckman, who note that for the average gram of material there will be found, in coarse sand, I,670 particles; in fine sand, I3I,900 particles; in very fine sand, $1,676,500$ particles; in silt, 35,934,000 particles; and in clay, 45,632,000,000 particles. Thus soil texture must necessarily affect the rate of movement of water and air, the temperature, microbiological activities, and the concentration and composition of the soil solution. Obviously, then, the different factors just noted exert a reciprocal influence on one another.

Wide differences exist in the chemical composition of soils. Their origin, their age, and the environment under which they were formed all serve to determine their value as a culture medium. Sandstones, limestones, feldspars, granites, and other rocks when exposed to weathering will give rise to soils of different physical and chemical quality. In humid climates the soil is exposed to constant leaching. Basic ions such as calcium, magnesium, potassium, sodium, and iron are gradually made soluble and carried away. Given a time long enough, everything except the silica skeleton of the soil may be more or less thoroughly removed. In the earlier stages of the process the loss of basic material may not be as clearly reflected in the vegetation, but sooner or later the increased hydrogen-ion concentration is certain to affect the crops, as well as the soil micro-organisms. On the other hand, in arid and semi-arid regions the leaching of the soil is less thorough, an acid reaction does not develop so readily, if at all, the soil tends to become basic in character, and not infrequently an undue accumulation of soluble salts (known as alkali) takes place. Hence the composition of the parent-rock, the length of the weathering period, and environmental factors of temperature, rainfall, glaciation, and aridity have always played a 
prominent rôle in determining the growth and distribution of plants and, in consequence, also of micro-organisms. The fact that the latter carry on their activities largely in the moisture films surrounding the soil particles, that these films represent solutions of varying composition and concentration, that the organic and inorganic colloidal material in the soil is the seat of many chemical reactions, and that the intensity of biological changes is directly reflected in the intensity of chemical changes, is evidence that chemical and biological soil factors are intimately related.

Organic matter is the direct source of food and energy of the heterotrophic soil organisms. It is the indirect source of energy for most of the autotrophic organisms. For this reason too much importance cannot be attached to organic matter as a link in the chain of soil fertility. Aside from the organic matter of recent origin found on the surface of the soil and within its tilled portion, there are older residues in the surface and subsoil of plants, soil-infesting insects, and micro-organisms. The quantity of organic matter will ordinarily vary from less than I per cent in light sandy soils to more than 5 per cent in heavy silt or clay loams. In muck and peat soils the organic matter content may range up to 85 per cent. An acre of surface soil $6 \frac{2}{3}$ inches deep is usually assumed to weigh $2,000,000$ pounds; hence, at 2 per cent there would be present about 20 tons of organic matter in this quantity of soil. In the next layer of the same depth the quantity of organic matter is ordinarily only half as great. It becomes relatively still less in the following layers. There is also a marked difference in the quality of the organic matter at different depths, particularly as to the carbonnitrogen ratio. Everything being equal, the greater the depth from which the organic matter is taken, the narrower its carbon-nitrogen ratio. This is due primarily to variations in the proportions of carbohydrates and proteins brought about by microbiological activities. As would be expected, the older residues in the subsoil represent material that had been used over and over again for the building of microbial cells.

Excessive concentrations of soluble salts injure higher plants and depress the activities of soil micro-organisms. In extremely acid soils soluble salts of aluminium and iron often produce toxic effects. In some abnormal soils compounds of boron, copper, nickel, arsenic, and manganese may be present in unduly large amounts and exert toxic action. Certain organic compounds, toxic to higher plants, have been isolated from soils. Some of these compounds may be injurious also to micro-organisms. In modern fertilizer practice large amounts of chemical fertilizer are often applied in the row or hill. When such treatment increases beyond a certain point, the concentration of the soil solution, seed germination, plant growth, and microbiological activities may be interfered with. Altogether, therefore, toxic or injurious substances may arise in the soil either as the result of the weathering of the rock material or the chemical changes brought about by cropping and the use of commercial fertilizers.

Both positive and negative influences are exerted by micro-organisms in the soil. The transformation by them of various organic substances involves the production of large quantities of carbon dioxide, the formation of ammonia and nitrates, and the mineralization of organic matter. As the end products of decay and fermentation, carbonates, phosphates, sulphates, and other simple salts are again made available for the use of plants. Nitrogen fixing bacteria, both symbiotic and non-symbiotic, may 
add more or less substantial amounts of combined nitrogen to the reserves of this constituent. Under some conditions nitrates may be reduced and thus withheld from the crop. In the presence of sufficiently large amounts of cellulose, starch, sugar, etc., bacteria and fungi may use up available nitrogen compounds fast enough to offer serious competition to higher plants. The kinds, numbers, and distribution of the micro-organisms in the soil are affected by the food supply and the nature of the soil environment. In their turn the micro-organisms modify the soil environment of crops by increasing or decreasing the food supply of the latter, and it is the task of the skilful farmer to treat his soil in such manner as to reduce to a minimum the negative effects and raise to a maximum the positive effects of soil microbiological activities.

\section{EXTERNAL FACTORS}

The amount and distribution of rainfall, the conservation of soil moisture, and the use of irrigation water are related in an important way to the growth of plants. Optimum moisture conditions for plants are established when the pore space in the soil, that is, the space not occupied by the soil particles, is filled with water to the extent of about 60 per cent. When there is too much or too little water in the soil, reduction and oxidation processes and the diffusion of gases may be shifted in the direction unfavorable to normal growth. Micro-organisms, like higher plants, are directly affected by the thickness of the moisture films surrounding the soil particles, the movement of capillary water, the concentration of the soil solution, and the diffusion of soil gases. Fine-textured soils may at times contain an excess of water, while coarse-textured soils may frequently contain an excess of air. Hence in the former the mineralization of organic matter is retarded, and in the latter it is hastened often to the detriment of the farmer. This will account for such agricultural practices as drainage, subsoiling, tillage, liming, green-manuring, etc., intended to speed up the circulation of water and air in fine-textured soils. It will account, also, for marling, claying, liming, green-manuring, and other practices as a means of retarding the movement of water and air in the more open soils. By controlling the water and air supply the farmer can influence the microbiological activities in the direction most favorable for crop growth.

Temperature, like water and air, is a major soil-environment factor for both higher plants and micro-organisms. There is a direct relation between the range of soil temperature and the amount of chemical change produced by the soil microflora. Similarly, there is a direct relation between the intensity of microbiological activity and the growth and yield of crops. In tropical and subtropical regions microbiological activities are never dormant wherever the moisture supply is sufficiently abundant. This accounts for the rapid decomposition of plant residues as an offset to more luxuriant vegetation and the more rapid accumulation of organic matter both in the soil and on its surface. In such environments the circulation of carbon and nitrogen between soil and atmosphere is manifestly more active than it is in regions farther north. On the other hand, in temperate and subarctic regions the rate of both accumulation and dissipation of organic matter is less intense. There must be, therefore, a certain relation between temperature, the microbiological activities in the soil, the supply of carbon dioxide to vegetation, and the rate of growth of the latter. 
Both soil moisture and temperature have a more or less direct relation to soil aeration. As has already been pointed out, the proper circulation of air and water in the soil is essential for the normal activities of both plants and micro-organisms. Temperature affects this circulation by influencing the rate at which inorganic and organic substances become soluble. In its turn, concentration of the soil solution will affect surface tension and the movement of capillary water. Temperature affects also the diffusion of gases and influences the oxygen pressure in the soil solution. Hence we recognize that moisture and temperature both help to determine the prevalence and activities of obligate and facultative aerobes and anaerobes, and the extent and intensity of oxidation and reduction processes. In localities where the winters are long and the spring and summer temperatures relatively low, evaporation is retarded, and the activities of aerobic bacteria are slowed down. Conditions become favorable then for the accumulation of peat as it is found in North America, Siberia, Northern Europe, and elsewhere.

Topography, like moisture and temperature, must receive consideration as a factor capable of influencing soil fertility. Weathered material is constantly being moved to lower levels. The steeper the slope, the more rapid is the downward movement of such material. Hence, topography comes into play as a factor in determining the depth of the soil, not only on the hillsides, but also in the valleys. Soil depth, water storage, qualitative and quantitative differences in the soil solution, and microbiological activities are interdependent and find expression in the size and quality of the crops. The depth of the mantle of soil on the hillsides is a matter of interest not alone for the student of soils, but for the forester, the hydraulic engineer, and the economist. Erosion and its control, floods and droughts, alluvial deposits and their agricultural use, are matters of general interest, yet not too remote from the special interests of the microbiologist.

\section{SOIL TREATMENT}

Aside from the natural forces that directly affect soil productivity, land utilization must reckon with tillage, crop rotations, lime, green manures, animal manures, commercial fertilizers, irrigation, and drainage as important soil-fertility factors. Tillage as an art has evolved from the use of the burned stick by the primitive farmer to the manipulation of the tractor-pulled gang plows. But whether it be human, animal, or mechanical power that furnishes the energy for stirring the surface soil the principle involved is the same. It has been said aptly that the soil is not a simple culture medium, but a collection of culture media. Indeed, it may be claimed with propriety that each soil particle with its surrounding moisture film is a culture medium. Since the solutions surrounding different inorganic and organic fragments in the soil must differ in their composition, they differ also as to the microflora and fauna colonized upon them.

The stirring of the soil tends to establish a greater degree of uniformity in the distribution of its micro-organisms. It modifies the circulation of air and water and, consequently, also the intensity of the oxidation and reduction processes. The varying depth of tillage involves differences in the distribution of plant roots, of organic residues, and of micro-organisms. It should be expected, therefore, that for both micro-organisms and crops a surface layer of 3 inches, on the one hand, and of ro 
inches, on the other, plowed, pulverized, disked, harrowed, and otherwise thoroughly stirred and mixed, would offer culture media markedly different in character. In speeding up microbiological activities, tillage disturbs the balance between the accumulation and dissipation of organic matter. Whereas in forests, prairies, and meadows the gains of organic matter are usually greater than the losses and the accumulation of carbon, nitrogen, and sulphur in the surface soil is the result, in tilled soils the reverse is often true. It is only by establishing suitable crop rotations and by the use of green manures and animal manures that the farmer is able to retard or prevent the depletion of the energy reserves and plant-food resources in the soil.

Crop rotations represent an orderly succession of different plantings. For instance, the five-year crop rotation of corn, oats, and wheat for one year each and of timothy and clover for two years is popular in the general farming sections of the United States. A three-year rotation of corn, oats, and clover is quite common in some localities in the Middle West. The possible number of rotations is almost unlimited. From the standpoint of the farmer, crop rotations are important in that they allow a better distribution of farm labor, lessen the risk of gluts and price declines, allow a better control of insects and plant diseases and the more effective and economical maintenance of soil fertility. From the standpoint of the microbiologist, crop rotations are of interest principally for two reasons. While information on the subject is still meager, there is reason for believing that there is distinct colonization of bacteria and of other micro-organisms on the roots of plants. It may be suspected that there are qualitative differences in the microflora and fauna colonized on the roots of different crops. More definite experimental evidence should be forthcoming, before long, on the direct influence which crops exert on soil micro-organisms. There is also an indirect influence. Crops vary as to their food requirements, water consumption, soilreaction changes, and soil-texture and soil-structure modification. All of these factors are also of significance in so far as they affect the nature of the soil as a culture medium. Moreover, crops belonging to different botanical groups vary sufficiently in composition to influence in a varying degree, by their stubble and roots, the quality of the soil organic matter.

The practice of liming is quite old. Chalk, calcareous marl, sand rich in shell fragments, and wood ashes were recognized many centuries ago as having the ability to ameliorate cultivated soils. The use of burned and slacked lime, and of pulverized limestone, is of more recent origin. Lime has various functions to perform in the soil. When properly used, it improves the texture of heavy, poorly aerated soils by flocculating the fine particles into larger aggregates. The circulation of air and water is thereby affected. Lime enters into base exchange and other reactions and thus modifies the soil solution and the supply of inorganic nutrients to crops. Finally, applications of lime modify the hydrogen-ion concentration of the soil solution, change the reaction of the culture medium for higher plants as well as for micro-organisms, and influence in a more or less specific way the decomposition of organic matter, the formation of ammonia and nitrates, and the fixation of atmospheric nitrogen by symbiotic and non-symbiotic bacteria.

When crops like rye, clover, vetch, cowpeas, etc., are plowed under in a green state for the sake of adding organic matter to the soil they are called "green manures." 
Aside from supplying readily decomposable vegetable material of a wide nitrogencarbon ratio, green manures may conserve plant food, and, under certain conditions, increase the nitrogen resources of the farm. They are usually grown during a portion of the season when the land is not required for one of the principal crops. Hence they may be used in the fall and early spring for protecting the soil against leaching. When legumes are used as green manures the soil's store of combined nitrogen is increased, usually by 25 to roo pounds per acre.

Animal manures are most frequently applied at the rate of 5 to Io tons per acre. Market gardeners employ larger amounts, occasionally up to 30 or more tons per acre. The progressive farmer knows that barnyard manure contains, aside from nitrogen and ash ingredients, 350-450 pounds of organic matter per ton of material and also enormous numbers of bacteria. When added to the soil, manure enriches it in plant food, furnishes energy material for the micro-organisms, and serves as an inoculum, thanks to the mass infection caused by its application. Horse manure containing a large proportion of straw and used at the rate of more than 20 tons per acre may temporarily depress plant growth. This is attributable primarily to the rapid transformation of ammonia, nitrates, and amino compounds into insoluble, microbial cell substance because of the abundant supply of cellulose and other carbohydrates. The competition for available nitrogen compounds between the crop and microorganisms is, then, usually an unfavorable one for the farmer. When the manure is well rotted before application the depressing effects just referred to are not apparent. The practice of composting manure and other refuse materials of plant and animal origin is still quite common in the Far East and in some of the European countries. The significance of animal manure as a soil inoculant is more readily appreciated when we remember that a gram of fresh manure may contain from fifty to several hundred million bacteria; that an adult beef or dairy animal will produce ro to I 2 tons of manure per anmum, and a horse or mule 5 to 6 tons; and that the animal population of the United States, including cattle, horses, mules, sheep, and swine, consists of more than $180,000,000$ units. In recent years there has been developed a process for making "artificial" manure from straw or other waste vegetable materials with the aid of sulphate of ammonia or of other nitrogen salts. The cellulose and carbohydrates in the straw furnish energy for the formation of proteins. The making of artificial manure is, therefore, a composting process.

Commercial fertilizers are made up principally of mono-calcium phosphate, potassium chloride, ammonium sulphate, sodium nitrate, and animal and vegetable by-product materials such as meat scrap, fish scrap, bone meal, cottonseed meal, etc. Other salts furnishing nitrogen, potassium, and phosphorus are used in smaller amounts. The mono-calcium phosphate is derived from phosphate rock by treating the latter with sulphuric acid. The resulting product is known as acid phosphate or superphosphate. It contains, aside from the mono-calcium phosphate, relatively small amounts of di-calcium and tri-calcium phosphate and a very considerable proportion of hydrated calcium sulphate. Since commercial fertilizers consist largely of soluble salts, their application increases the concentration of the soil solution and, among other things, stimulates the activities of the micro-organisms. The quantities of fertilizer applied range from about roo to more than 3,000 pounds per acre. The 
material is most commonly applied in the row or hill with the aid of fertilizer-distributing machinery. The local application of the soluble salts establishes, in the vicinity of the seed or plant, detached spots of soil in which the solution is more concentrated than it is in adjacent spots. All of this has an important bearing on the activities of the soil bacteria and other micro-organisms. It has been shown experimentally that where only small quantities of fertilizer are employed the farmer really fertilizes the soil micro-organisms whose stimulated activities lead to a more rapid decomposition of the soil organic matter and the release of increased amounts of nitrates, phosphate, sulphates, and carbonates for the nutrition of the plants. Where larger amounts of fertilizer are used the plant food removed by the crops may be more or less completely replaced and the microbiological activities intensified at the same time. It has been shown, particularly, that nitrogen salts help the micro-organisms to utilize the more resistant forms of organic matter and to amplify thereby the supply of available plant food. Similarly, when the soil reaction is right the use of monocalcic and di-calcic phosphates makes conditions more favorable for the fixation of atmospheric nitrogen by Azotobacter and other nitrogen fixing bacteria.

The consideration of commercial fertilizers in their relation to soil microbiological activities should reckon also with certain specific influences of individual salts. Thus ammonium salts when employed in relatively large amounts will give us a somewhat different microbiological picture than will equivalent quantities of nitrates. Different fertilizer salts will likewise exert an indirect influence on the micro-organisms by modifying plant growth and through it soil reaction, moisture relations, and the diffusion of gases. On the whole, the relation of commercial fertilizers to soil-microbiological activities is not fully understood. Fertilizer practice is changing, new fertilizer salts are constantly appearing, and new methods of application are being developed. Nevertheless, present-day information is sufficiently accurate and extensive to justify the statement that chemical fertilizers are a potent means of modifying soil-microbiological activities and through them the quantity and quality of the harvested crops.

Irrigation and drainage are both significant soil-fertility factors when considered in their relation to microbiological activities. Like higher plants, bacteria and other micro-organisms will become less and less active as the water supply is reduced below the optimum. In the case of plants, wilting will occur when the thickness of the moisture film on the soil particles is unduly reduced. It has been shown by Briggs and Shantz that in coarse sand, wilting will take place at 0.9 per cent of moisture; in fine sand at 2.6 per cent; in fine sandy loam at 9.7 per cent; and in clay loam at 16.3 per cent. In heavy clay soil, wilting will take place with a still higher percentage of water, while in peat soils there is danger of wilting when the supply of water is reduced below 40 per cent. The differences just noted may be readily accounted for by the extent of the inner surface in soils of different texture. The finer grained the soil, the greater its inner surface and the thinner the moisture film produced by any given quantity of water. Hence a light rain, or a relatively small amount of irrigation water, would start microbiological activities and plant growth in a sandy soil, whereas a much larger supply of water would be required to produce like results in a dry silt or clay soil. On the other hand, during periods of drought, crops on the lighter soils would 
begin to suffer first. It is not difficult to imagine how the drying-out of the soil affects the soil solution and its surface tension. It is obvious that soils rich in soluble salts might develop so concentrated a solution as to cause plasmolysis of bacterial cells. Irrigation water and its content of soluble salts, the local application of fertilizer salts, the removal of the excess of water by drainage, the rainfall-evaporation ratio and moisture conservation by tillage and other methods, must all find a place in the study of the interrelations of soil moisture, micro-organisms, plants, and soil fertility. A more accurate knowledge of these relations will indicate a better control of microorganisms as a factor of soil fertility.

\section{THE MOBILIZATION OF PLANT FOOD}

The foregoing discussion has dealt with the significance of the various internal and external factors in the complex relations of micro-organisms to soils and crops. It has been shown that the soil solution receives contributions from the inorganic and organic soil constituents. It has been shown, likewise, that micro-organisms are essential for the normal changes in the soil, that is, the mobilization of an important portion of the plant nutrients. In new agricultural regions the farmer is chiefly concerned with tillage as a means of mobilizing adequate amounts of available plant food. Later on, tillage alone is found to be ineffective for maintaining the crop yields, and socalled "bare fallows" are resorted to. These were still the basis of the three-field system of husbandry prevalent in Western Europe and in North America well toward the end of the eighteenth century. But bare fallows were only a temporary expedient, for they allowed the sources of food and energy of the soil micro-organisms to run low. The satisfactory mobilization of plant food became impossible. Agricultural chemistry, and later agricultural microbiology, pointed the way to more rational methods. A suitable soil reaction, an adequate supply of organic matter, a sufficiency of certain inorganic constituents, and a reasonably good control of the environmental factors of temperature, moisture, and aeration were indicated as desirable for the proper use of micro-organisms in the mobilization of plant food and the production of large crops. But not content with pointing out the principles that must govern soil practices the microbiologist has contributed certain specific information and methods that are already of large practical value to agriculture.

\section{SPECIAL METHODS AND PRACTICES}

It was observed long ago that the continued production of certain crops on the same land led to falling off in yields and not infrequently to complete crop failures. Soils became "sick" for certain crops and, under given conditions, were said to be "flax sick," "clover sick," "tobacco sick," "potato sick," etc. We know now that pathogenic fungi and bacteria, nematodes and plant-lice, are the cause of most of these soil ills. The continued growing of the favorite host plant allows the pathogens or parasites to survive and flourish. Hence, methods had to be devised for ridding the land of them. Partial soil sterilization has become a well-established practice in greenhouses, and is occasionally resorted to under field conditions. Heat and volatile germicides are employed. Among the latter, carbon bisulphide and formaldehyde are the most popular. Salts of mercury, copper, and nickel are also used, but primarily 
as insecticides and fungicides rather than for partial soil sterilization. Elementary sulphur is employed for the same purpose, for it is rapidly oxidized in the soil to sulphuric acid. By increasing the hydrogen-ion concentration of the soil it renders the latter a less fit medium for the survival and development of certain micro-organisms.

The experience with volatile antiseptics and germicides has given us a better insight into the true character of the soil microflora and fauna. Numerous experimental studies have shown that partial soil sterilization disturbs the microbiological balance, that the initial reduction in numbers is followed by an abnormal increase in the numbers of bacteria, and that partially sterilized soils become very productive for a time at least. More recently the attention of microbiologists has been drawn to the partial soil sterilization that occurs in some arid and semi-arid regions because of the more or less thorough drying of the surface material and its exposure to the intense desert heat. Such land, during the rainy reason, or when supplied with irrigation water, behaves not unlike soil partially sterilized by artificial heat or volatile antiseptics. There is reason to believe that the study of partial soil sterilization will point the way toward better control of soil-fertility factors and the more effective use of microorganisms for the mobilization of plant food.

Soil inoculation is no longer an empirical practice. It is claimed that the scattering of rich garden soil on newly reclaimed peat bogs was practiced in Finland more than a hundred years ago. The preparation of composts and the use of composted earth became a fine art in the Orient long before the beginning of the present era. Manure and other waste materials are still widely used in Europe for the preparation of composts, although high labor costs and the abundance and cheapness of chemical fertilizers have discouraged compost-making in Western Europe and North America. The improvement in the methods of producing activated sludge promises to make this material a means of conserving much of the plant food that is now sewered into the sea. The use of animal manures has already been mentioned. Together with composts and activated sludge, it may be utilized for mass infection, as well as for the addition to the soil of nitrogen and of other plant-food constituents.

In a more particular way soil inoculation is practiced with Thiobacillus thiooxidans and Bact. radicicola. In the case of the former, ground brimstone employed for the control of potato and sweet-potato diseases, the correcting of the sulphur deficiencies of certain soils, or the reclamation of black alkali soils, is inoculated with pure or crude culture of the organisms. It has been observed in many instances that sulphur thus inoculated is more rapidly oxidized when it is applied to the soil.

Inoculation with Bact. radicicola is widely known and practiced. It was noted above that rich garden soil was used in Finland at least as early as the eighteenth century for the improvement of newly drained marshes. This empirical method was observed to favor the establishment of clover. After the announcement by Helliriegel and Wilfarth in I886 that legumes in association with bacteria were able to fix atmospheric nitrogen, Tacke at Bremen introduced the use of earth from clover and other legume fields. Inoculation by means of legume earth is still being practiced in Europe and North America. In the United States soil from old alfalfa fields is still occasionally employed for inoculating new alfalfa fields. On the whole, however, pure cultures of Bact. radicicola are used in preference for inoculating the seeds of 
legumes. Reference to the use of Bact. radicicola for soil inoculation will be found elsewhere in this book. ${ }^{\mathrm{x}}$ It need only be added at this time that the better appreciation of legumes as a means of maintaining the nitrogen resources of our soils and the availability of good inoculating material will not only encourage the more extensive use of legumes, but will help toward a better understanding and control of soil microorganisms as one of the major factors of soil fertility.

\section{BIBLIOGRAPHY}

Lipman, J. G.: Bacteria in Relation to Country Life. New York, I9rr.

Löhnis, F.: Handbuch der landwirtschaftlichon Bakteriologie. Berlin, I9го.

Russell, E. J.: Soil Conditions and Plant Growth. 5th ed. London, 1927.

Waksman, S. A.: Principles of Soil Microbiology. Baltimore, 1927.

Stoklasa, J., and Doerell, E. G.: Handbuch der physikalischen und biochemischen Durchforschung des Bodens. Berlin, I926.

${ }^{x}$ See chap. xxv by E. B. Fred in this volume. 


\title{
CHAPTER XXVII
}

\section{THE RÔLE OF BACTERIA IN THE TREATMENT OF SEWAGE}

\author{
F. W. MOHLMAN \\ Sanitary District of Chicago
}

SEWAGE

The heterogeneous nature of the components of human sewage insures the presence of a complex bacterial life, primarily the major intestinal forms with occasional pathogens. A rich and varied food supply is presented to the organisms, but under new environment, as compared with the temperature and conditions of the intestinal tract. The fate of the bacteria and of the organic and mineral solids depends upon the successive predominance of major groups of organisms and various conditions of symbiosis so complex as to defy analysis.

Because of this complexity there has been little attempt to isolate specific species. Clark and $\mathrm{Gage}^{\mathrm{r}}$ have listed the prevalence of some twenty organisms in Lawrence (Mass.) sewage. The total count at $20^{\circ} \mathrm{C}$. on gelatin, at $37^{\circ} \mathrm{C}$. on agar, and the total number of Bact.coli have been determined on so many sewages that they have become standard procedures in sewage works operation. The $20^{\circ}$ and $37^{\circ}$ counts have little significance unless the age of the sewage is known, since a sewage of low organic content might have an excessive count due to storage and septicity; on the other hand, the Bact. coli count has more stability and is less subject to the vagaries of time and temperature.

Studies have been made by the United States Public Health Service on various polluted rivers where the population contributing pollution has been known. The results shown in Table I have been summarized from their reports. ${ }^{2}$ These data indicate the maximum quantitative units of total bacteria and Bact. coli per capita under conditions of great dilution. The variation between winter and summer values is to be expected for the gelatin and agar counts, but the variation in the Bact. coli count is noteworthy. At the bottom of the table has been added the per capita count at the Des Plaines River Sewage Treatment Works of the Sanitary District of Chicago, where a moderately concentrated sewage, at ro6 gallons per capita per day, is treated. The agar and gelatin per capita counts are only 5 and 3.3 per cent of the river counts, but Bact. coli is 43 per cent of the annual average. The constancy of Bact. coli counts is apparently affected but little by dilution, but with a trend toward increased counts with increased dilution, due either to growth or more effective dispersion.

\section{DECOMPOSITION OF SEIVAGE}

The discharge of excreta into water dilutes the liquids and disperses the solids more or less completely, dependent upon the time and degree of agitation. The water

I Clark, H. W., and Gage, S. DeM.: Bacteriology and Biochemistry of Sewage Purification, 36th Ann. Rep., Mass. State Bd. of Health, pp. 231-33. I904.

${ }^{2}$ Hoskins, J. K.: "Quantitative Studies of Bacterial Pollution," Proc. Am. Soc. Civ. Engin., 5I, 9, 1810-55. Nov., 1925. 
is generally saturated with dissolved oxygen, containing 9.2 parts per million at $20^{\circ} \mathrm{C}$. As bacterial growth progresses in the sewage the dissolved oxygen decreases and disappears in a few hours. This initial aerobic period is negligible in comparison with the subsequent oxygen requirements of the sewage, which may range from roo to several thousand parts per million. Anaerobic species soon predominate, although it has been shown that a comparatively small percentage of the total bacteria are obligate anaerobes. Facultative forms are usually found, and even oxidizing and nitrifying types

TABLE I

Seasonal Changes in Numbers of Bacteria Added to Streams By Sewered Populations

\begin{tabular}{|c|c|c|c|}
\hline \multirow{2}{*}{ CITY } & \multicolumn{3}{|c|}{ Billions of Bacteria per Capita per Day } \\
\hline & $\begin{array}{l}\text { Gelatin } \\
20^{\circ} \mathrm{C} \text {. }\end{array}$ & $\begin{array}{l}\text { Agar } \\
37^{\circ} \mathrm{C} .\end{array}$ & Bact. coli. \\
\hline \multicolumn{4}{|l|}{ Chicago, Ill.: } \\
\hline Summer.............. & 24,800 & 25,650 & 428 \\
\hline 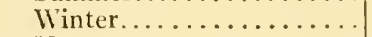 & 4,260 & 847 & 42 \\
\hline 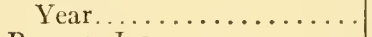 & I 5,300 & I I , I 70 & 230 \\
\hline \multicolumn{4}{|l|}{ PEORIA, ILI.: } \\
\hline Summer............. & I 5,770 & 25,640 & $23 \mathrm{I}$ \\
\hline Winter.............. & 2,130 & 7,650 & $14 \mathrm{I}$ \\
\hline Year................ & I I, 0.50 & I9, 3 I0 & 187 \\
\hline \multicolumn{4}{|l|}{ Cincinnati, O.: } \\
\hline Summer............. & 14,100 & I 8,3 Io & 583 \\
\hline Winter.............. & 2,590 & $\mathbf{I}, \infty \infty$ & II 9 \\
\hline Year................ & I 1,770 & I 2,260 & $35^{8}$ \\
\hline \multicolumn{4}{|l|}{ LoUisvilLe, KY.: } \\
\hline Summer.............. & I 3,560 & I 5,840 & $29 I$ \\
\hline Winter............... & 7,360 & 907 & 193 \\
\hline 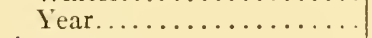 & 10,840 & 7,960 & 222 \\
\hline \multicolumn{4}{|l|}{ AVERAGES: } \\
\hline Summer .............. & 17,070 & 21,360 & $3 S_{3}$ \\
\hline 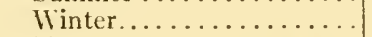 & 4,080 & 2,600 & 124 \\
\hline 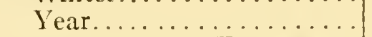 & 12,240 & $\mathbf{1} 2,680$ & 2.49 \\
\hline \multirow{3}{*}{\multicolumn{4}{|c|}{$\begin{array}{l}\text { Des Plaines TreatMent } \\
\text { Works,SANitary District } \\
\text { OF Chicago: }\end{array}$}} \\
\hline & & & \\
\hline & & & \\
\hline & 6 I 2 & 416 & 106 \\
\hline
\end{tabular}

survive, under completely anaerobic conditions, and later develop and complete the work of stabilization.

The unstable organic matter in sewage is suitable for the predominant development of anaerobic or reducing reactions. The food material is rich and complete, including carbohydrates, proteins, fats, cellulose, and mineral salts. Normal sewage has an optimum $\mathrm{pH}$ for bacterial growth, and is usually buffered sufficiently by bicarbonates to permit the inception of vigorous growth. Nevertheless, the limitation of growth and delay of stabilization in the solids is to a large extent caused by change in $\mathrm{pH}$ to a more acid reaction.

If no artificial means are taken to prolong the initial aerobic period by absorption of oxygen, anaerobic conditions develop, characterized by production of hydrogen sulphide, indol, mercaptans, and other foul-smelling compounds. Carbohydrates and 
cellulose pass through organic acids of various stages of saturation to carbon dioxide and methane, protein and urea are ammonified, and fats, which are usually present as soaps, are slowly liberated by increased $\mathrm{CO}_{2}$ production, and gradually gasified, although much more slowly than under aerobic conditions.

This stage of active putrefaction slowly gives way to semi-aerobic conditions due to absorption of oxygen. Colloids are coagulated and deposited, nitrites and nitrates increase, and finally algae and green growths appear. The liquid remains stable during this stage of decomposition, and may even become supersaturated with dissolved oxygen, due to photosynthesis, but the deposited insoluble solids remain anaerobic for long periods, and when the algae die the sewage may again putrefy. This sequence of biological phenomena may be observed in any small, polluted stream in summer when raw sewage is discharged with little opportunity for dilution or re-aeration, and when th: time of flow is several weeks.

When solids are removed by sedimentation from the sewage before discharge and dilution plus re-aeration are sufficient to maintain dissolved oxygen at all times, the first stage of putrefaction is eliminated and decomposition continues aerobically, at least through the algae stage. Secondary putrefaction may follow, however, if the algae die suddenly.

The course of oxygen requirement may be shown by results of biochemical oxygen-demand determinations, which are carried on under aerobic conditions. A typical oxygen-demand curve for diluted sewage taken from the Chicago Drainage Canal at Lockport is shown in Figure I. This curve, which is similar to Theriault's, ${ }^{1}$ follows the first order (mono-molecular) equation quite well up to ten days, but after eleven or twelve days there is a lag, followed by another rise, which continues for many weeks or months. There is some difference of opinion as to the cause of this secondary rise, but the opinion is now rather general that it is the stage of nitrification, and that carbon oxidation is predominant during the first stage.

This curve indicates the course of aerobic decomposition of sewage in nature; but when dilution, re-aeration, or time are lacking, artificial processes must be used to accelerate the process and accomplish in hours what requires weeks in nature. Solids must first be removed by sedimentation, stabilized and dried; proteolytic, ammonifying, and nitrifying bacteria must be concentrated and their functions accelerated; many re-saturations of dissolved oxygen must be obtained; and, finally, pathogenic bacteria must be destroyed. It is the function of sewage treatment to accomplish these requirements by artificial processes which should be economical, as free from odor as possible, and subject to scientific control.

PROCESSES OF SEWAGE TREATMENT

Complete treatment of sewage requires biological action, but there are many preliminary processes which are purely physical or chemical. Coarse and fine screens are used as strainers, sand and cinders are removed in grit chambers. Settling processes do not depend on bacteria-in fact, they should be as free from bacterial growth as

${ }^{1}$ Theriault, E. J.: "The Rate of Deoxygenation of Polluted Waters," Pub. Health Rep., U.S. Pub. Health Serv., 4r, No. 6, 207-I7. Feb. 5, I926. 
possible. Chemical precipitation, now little used, is not bacterial, and the clarification effected by activated sludge may be partly mechanical.

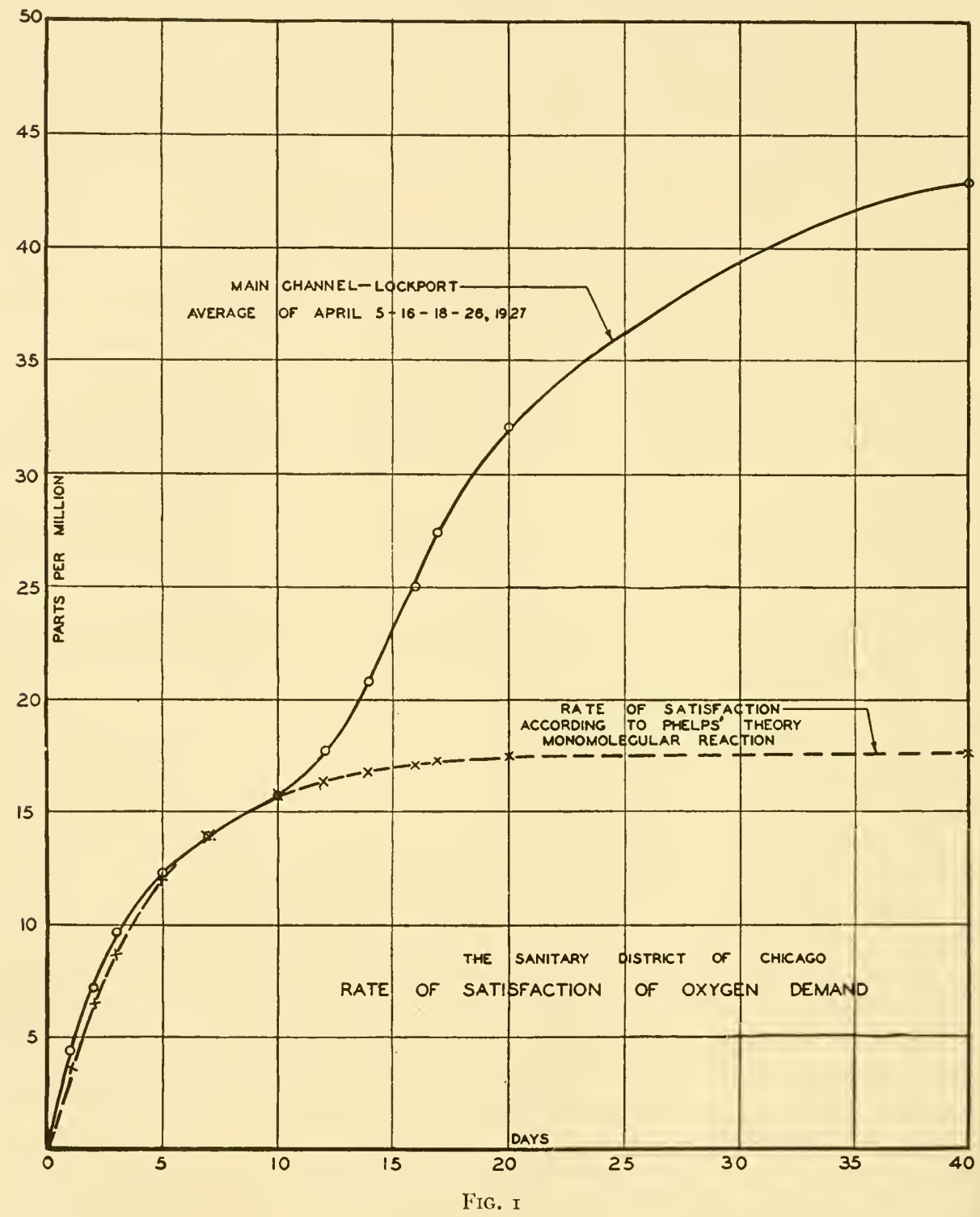

The major work of sewage treatment, however, is done by bacterial processes, including intermittent sand filtration, broad irrigation, contact beds, trickling filters, activated sludge, and digestion of sludge. Septic tanks are biological only because they are not designed to keep the sludge separate from the overflowing sewage. Imhoff tanks and frequently cleaned plain settling tanks do not depend on biological action in the liquid, but only in the deposited solids after removal. It is, of course, true 
that any extension of time even for the liquid sewage permits the progress of proteolysis and ammonification in preparation for oxidizing processes. In sewages containing much carbohydrate, storage in tanks may permit the development of too much acid for satisfactory final treatment. For example, Levine and Soppeland ${ }^{\mathrm{I}}$ have found that dairy wastes containing lactose develop acidities sufficient to prevent proteolysis. The optimum reaction for proteolysis was neutral or slightly alkaline $(\mathrm{pH} 7.0-$ 7.5). Acidities up to $\mathrm{pH} 6.4$ produced no appreciable inhibition under aerobic conditions, but it was felt that under less favorable anaerobic conditions this acidity would have been detrimental. Proteolysis was retarded by higher acidities and frequently stopped if the reaction reached $\mathrm{pH} 5.0-5 \cdot 5$.

General bacteriological surveys have been made from time to time of cultures isolate $\mathrm{I}$ from sewage-treatment plants. In I904 Clark and $\mathrm{Gage}^{2}$ studied the reactions of some thirty colonies isolated from the Lawrence (Mass.) sewage and effluents from septic tanks and intermittent sand filters. Their observations dealt mostly with the nitrogen relations. They found that bacterial growth produced ammonia from organic matter, reduced nitrates to nitrites to ammonia and elementary nitrogen, liberated nitrogen from solutions of organic matter, either with or without the presence of nitrates, and fixed atmospheric nitrogen. Many sewage bacteria also produced the lower oxides of nitrogen as reduction products of nitrates, oxides which appeared to play an important part in the further decomposition of the organic matter in solution, either through katalytic action or by direct chemical action. They noted a compound intermediate between nitrites and nitrates, but apparently did not note an additional reaction, which has since been of interest in activated-sludge studies, namely, the synthesis of insoluble protein from nitrites and ammonia, as discussed by Buswell and Neave. ${ }^{3}$

A year's study of the Plainfield (N.J.) sewage treatment plant was made by Dr. Margaret Hotchkiss, ${ }^{4}$ in I924. This plant consists of a Riensch-Wurl screen, Imhoff tanks, sprinkling filters, and secondary settling tanks. She found that the organisms most important numerically throughout the plant were the nitrate reducers, the hydrogen sulphide producers (from protein), and the albumen digesters. Nitrifying and sulphur-oxidizing bacteria occurred throughout the plant and were consistently found even in the digestion chamber of the Imhoff tank. Nitrifying organisms increased in the filter bed although they never became numerically predominant. Hydrogen sulphide producing organisms were practically eliminated in the trickling filter.

\section{DISPOSAL OF SLUDGE}

The most troublesome and difficult feature of sewage treatment is the disposal of the sludge produced in settling tanks. Fresh sludge is greasy, colloidal, and odor-

${ }^{r}$ Levine, M., and Soppeland, L.: Proteolysis by Bacteria from Creamery Wastes, Engin. Exper. Sta., Iorea State College, Ames, Iowa, Bull. S2. Oct. 13, 1926.

${ }^{2}$ Gage, S. DeM.: "The Bacteriolysis of Peptones and Nitrates," J. Am. Chem. Soc., I7, 327. 1905 .

3 Buswell, A. M., and Neave, S. L.: Bio-chemistry of the Activated Sludge Process, Ill. State Water Surv., Bull. I8, pp. 68-81. 1922.

${ }^{4}$ Hotchkiss, M.: "A Survey of the Bacteriological Flora of a Sewage Treatment Plant," J. Bact., 9, 437-54. Sept., I924. 
ous, usually containing only about 5 per cent solids. When removed from settling tanks it will not dry on sand beds, will not filter in filter presses, and remains a nuisance for an indefinite period. When retained in septic tanks, as formerly practiced, it becomes partially digested to a less objectionable condition, but meanwhile is fouling the effluent and producing odors. Imhoff sludge digests in the lower compartment of the tank, fairly well separated from the upper settling compartment. When digestion is satisfactory the sludge has very little odor, is full of gas bubbles, contains from $\mathrm{I} 2$ to $\mathrm{I} 8$ per cent solids, and dries well on sand beds. When digestion is not satisfactory, foaming and expulsion of solids occur, the sludge is high in moisture, will not dry, and has a bad odor. Difficulties in the digestion of sludge have led to various studies of sludge digestion during the past ten years. The process is purely biological, and when proceeding normally and under control it is the most satisfactory and economical way of disposing of sewage solids.

Scientific work on sludge digestion has been in progress in Stroganoff' ${ }^{r}$ laboratory in Moscow, in the Emscher district in Germany under the direction of Bach and Sierp, ${ }^{2}$ and at Harvard University under Fair ${ }^{3}$; but by far the most extensive work has been done by Rudolfs ${ }^{4}$ and his colleagues at the New Jersey Sewage Experiment Station, New Brunswick (N. J.).

The characteristic feature of the first stage of sludge digestion is the production of increasing amounts of $\mathrm{CO}_{2}$ and organic acids from easily available carbohydrates (sugars, soluble starches, and cellulose). It was formerly believed that cellulose was very resistant to bacterial attack, but Heukelekian ${ }^{5}$ has recently shown that it is digested comparatively rapidly. Approximately 80 per cent was digested in the first three weeks, while the digestion of the nitrogenous material takes several months. The $\mathrm{CO}_{2}$ and acids produced during the first two weeks of digestion of fresh solids at $20^{\circ} \mathrm{C}$. may lower the $\mathrm{pH}$ from 7.0 to 5.5 or less, the acidity depending upon the temperature and the organic matter in the sludge. After this initial acidity the basic products of digestion slowly neutralize the acid, the $\mathrm{pH}$ rises to $6.5-7.0$, and proteolysis becomes active. Gas formation, which increases considerably during the first stage, decreases during this stage, but the percentage of methane increases. Following this stage of proteolysis and digestion of acids comes the final period of intensive digestion of more resistant materials, the more complex proteins, fats, lignins, and soaps. The gas volume and percentage of methane increase, $\mathrm{pH}$ increases to $7.0-$ $7 \cdot 5$, and finally after from six to nine months' storage the sludge is completely digested and ready for drying.

I Stroganoff, S. N.: Anacrobic Decomposition of Scwage Sludge, Sth Rep. Sew. Rescarch Commis. of Moscow, p. 85. 1926. (German abstract.)

${ }^{2}$ Bach, H., and Sierp, F.: "Untersuchungen zur Frage der Sumpfgasbildung aus Abwasserklärschlamm," Centralbl. f. Bakleriol., Abt. II, 60, 318-28. I924.

3 Fair, G. M.: "Sludge Digestion-Reaction and Control," J. Boston Soc. Civ. Engin., I4, No. 2, 82-130. Feb., I927.

${ }^{4}$ Rudolfs, W. M.: 4th Rep. Sew. Substa. New Jersey Agric. Exper. Sta., New Brunswick, N.J. 1925; 5 th Rep. Sew. Substa. New Jersey Agric. Exper. Sta., New Brunswick, N.J. I926.

${ }^{5}$ Heukelekian, H.: "Decomposition of Cellulose in Fresh Sewage Solids," Indust. Eూ Engin. Chem., 19, 928-30. Aug., 1927. 
Two factors, rightly controlled, may greatly decrease this period of digestion. A proper mixture of ripened sludge with fresh solids greatly modifies the initial acid stage; also lime may be added to neutralize the acids and keep the $\mathrm{pH}$ from 7.0 to 7.4. Rudolfs has found that by proper admixture of ripe sludge the digestion period may be cut to fifty days, and more recently that by addition of lime, controlled by $\mathrm{pH}$ determinations, it may be possible to shorten the period to thirty or thirty-five days. Fair and Baity at Harvard also confirm Rudolfs' work. Stroganoff, Bach, and Sierp had also considered the desirability of neutralizing excessive acidity by means of lime, but had not worked out the proper relations or control.

Much work still remains to be done on the bacteria responsible for the various stages of digestion. Carbohydrate fermenters are active in the initial stages, but anaerobic spore-formers are probably of most importance in later stages. Greer ${ }^{1}$ found that $\mathrm{Cl}$. wclchii was present up to $\mathrm{I0}, 000,000$ per gram in Imhoff sludge at the Calumet Sewage Treatment Plants of the Sanitary District of Chicago.

\section{OXIDATION, NITRIFICATION, AND DENITRIFICATION}

Complete treatment of sewage is accomplished by artificial devices which foster the growth of liquefying and oxidizing bacteria. Given these growths, it is necessary to bring all of the sewage into intimate contact with them. In intermittent sand filtration and broad irrigation, there is also a physical straining action, but other processes now in use are not designed to accomplish clarification other than by sedimentation. In all processes it is essential to provide extensive surfaces to which the bacteria may attach themselves; after growths are established it is necessary either to pass the sewage over these growths, as is done in sand filters, trickling filters, and contact beds, or to mix the growths intimately throughout the sewage, as is done in the activated-sludge process. Continuous presence of dissolved oxygen is also essential. The only other requirement is a settling tank for removal of solids before discharge of the effluent.

In all nitrifying devices, organic nitrogen must first be liquefied and ammonified before it can be nitrified. The progress of nitrification, as measured by chemical analyses, is then dependent on the velocity of opposing reactions, nitrification versus denitrification. Winogradsky ${ }^{2}$ first demonstrated that in pure culture organic matter inhibits growth of nitrite- and nitrate-forming bacteria, but that in nature nitrification can occur in the presence of organic matter by symbiotic growth with $B$. ramosus, a common soil bacterium of the subtilis group. Boullanger and $\mathrm{Massol}^{3}$ showed that when an ammoniacal solution, inoculated with both forms of the nitrifying bacteria, was allowed to undergo complete oxidation in contact with clinker and then withdrawn, a further supply of the ammoniacal solution poured on the same clinker did not then interfere with the nitrate-formers, but the oxidation proceeded at once as far as nitrate, and only traces of nitrite could be found. In this case a growth of

'Greer, F. E.: "Anaerobes in Sewage," J. Am. Pub. Health Assoc., 16, 500-501. May, 1926.

${ }^{2}$ Winogradsky, S., and Omeliansky, V.: "The Influence of Organic Matter on the Work of Nitrifying Bacteria," Centralbl.f. Baktcriol., 2, 329-440. 1899.

3 Boullanger, E., and Massol, L.: "Études sur les Microbes Nitrificateurs," Ann. de l'Inst. Pastcur, 17, 492. I903; 18, 181. 1904. 
the nitrate-forming bacteria had been established on the surface of the clinker in a manner which corresponds to what takes place in the "ripening" of a sand or trickling filter. Apparently, ammonia inhibits the initial growth of nitrate-forming bacteria but their growth when once established is not destroyed by additions of ammonia-bearing liquors.

Denitrification has been studied by many observers. Mair ${ }^{1}$ claimed that the denitrifying bacteria are essentially aerobic, but that they can grow under anaerobic conditions in the presence of nitrate, the nitrate supplying the necessary oxygen for their vital activities.

It is well known that there is considerable loss of nitrogen, either as gaseous $\mathrm{N}_{2}$ or as oxides of nitrogen, in filters, especially in contact beds, and in activated-sludge treatment when aeration is vigorous and prolonged. Denitrification probably does not account for all of this loss, but as Clark, Letts, and recently Parisi ${ }^{2}$ have stated, the loss must be explained by the theory that as nitrite is formed it reacts with the amino acids as follows:

$$
\mathrm{R}=\mathrm{CH}=\mathrm{NH}_{2}=\mathrm{CO}_{2} \mathrm{H}+\mathrm{HNO}_{2}=\mathrm{R}=\mathrm{CH}=\mathrm{OH}=\mathrm{CO}_{2} \mathrm{H}+\mathrm{H}_{2} \mathrm{O}+\mathrm{N}_{2} \text {. }
$$

Parisi supports this theory by experiments. If sucrose in sufficient quantity to prevent nitrification is added to a submerged soil containing an $\mathrm{NH}_{4}$ salt and the mixture is aerated, the $\mathrm{NH}_{4}$ salt remains unaltered. Aeration of a soil impregnated with sodium nitrite slowly converts the nitrite to nitrate, because insufficient amino acids are present to react with the nitrite; but if sufficient asparagine is also added, neither nitrites nor nitrates are found, interaction of asparagine and $\mathrm{NaNO}_{2}$ resulting in the liberation of free nitrogen.

In view of the vigorous denitrifying and nitrogen-liberating reactions in oxidizing processes of sewage treatment, it is apparent that considerable energy must be expended and vigorous aerobic conditions maintained in order to build up nitrates in the eflluent. For this reason a recent tendency in activated-sludge development has been to attempt to operate, if possible, without nitrate production, and with minimum amounts of air. It is true that the oxygen demand may be greatly reduced without appreciable amounts of nitrate in the effluent, but it is difficult to maintain well-activated sludge without nitrates. Also, the tendency toward "bulking" or sudden increase of moisture content is intensified, and "filter-pressing" the sludge becomes more difficult.

Very little bacteriological work has been done on the activated-sludge process, mainly because of the difficulty of the problem. The question of fixation of atmospheric nitrogen has been debatable, with claims of evidences of such fixation by Fowler ${ }^{3}$ and Peck $^{4}$, but these claims have been questioned by Richards and Sawyer, ${ }^{5}$

${ }^{1}$ Mair, W.: "On the Rôle of Bacteria in the Biological Methods of Sewage Purification, with Special Reference to the Process of Denitrification," J. Hyg., 8, 609-53. 1908 .

${ }^{2}$ Parisi, E.: "Denitrification in Oxidizing Media," Ann. chim. applic., 16, 40-45. 1926.

3 Fowler, G. J.: "The Nitrogen in Activated Sludge," J. Indian. Inst. Sc., 3, 256-64. Dec., 1920.

${ }_{4}^{4}$ Peck, C. L.: "Fixation of Atmospheric Nitrogen by Activated Sludge," Eng. News-Rec., 90, 487. March 15,1923 .

5 Richards, E. H., and Sawyer, G. C.: "Further Experiments with Activated Sludge," J. Soc. Chem. Ind., 41, 62'T. 1922. 
Pearse and Mohlman, ${ }^{1}$ and Buswell (loc. cit). Bartow and Russell ${ }^{2}$ demonstrated the bacterial basis of the process; Harris, ${ }^{3}$ of Glasgow, states that the presence or absence of protozoa neither increases nor detracts from the efficiency of fully matured activated sludge. Recent experience at the Des Plaines River Activated Sludge Plant of the Sanitary District of Chicago has indicated that larger filamentous bacteria may be very detrimental. A profuse growth of Sphaerotilus natans has produced a slimy, non-settling sludge which will not stabilize the sewage, will not settle, and will not filter-press. It is probably stimulated by the adventitious presence of abnormal amounts of carbohydrate in the sewage, with excessive production of carbon dioxide. Attempts to eliminate the growth by lime treatment have been fairly successful.

\section{INDUSTRIAL WASTES}

Industrial wastes frequently complicate the treatment of sewage by (I) greatly increasing its biological strength, or (2) by inhibiting bacterial action. The most satisfactory evaluation of the first type of waste is based on its biochemical oxygen demand. This procedure has been used by the Sanitary District of Chicago. ${ }^{4}$ Experiments on the treatment of a concentrated starch-factory waste at Argo demonstrated that the undiluted starch and gluten wastes would produce neither activated sludge nor a ripened filter. When diluted with at least three volumes of canal water it was possible to treat the mixture biologically, but activated-sludge treatment was still not uniformly successful. Trickling filters were recommended to operate on a very low rate of filtration.

The inhibitory effect of the second type of waste is injurious to sludge digestion and particularly to activated-sludge treatment. An interesting study has recently been made by Sierp ${ }^{5}$ on the effect of various germicidal wastes in Imhoff tanks. He finds that phenol wastes are particularly detrimental to gas production. The concentration in the sludge used was about 25 parts per million of phenol. Chlorine was quite germicidal, but excessive amounts were used, from 50 to 500 parts per million. Magnesium sulphate, in a concentration of 360 p.p.m. $\mathrm{SO}_{3}$, delayed digestion and produced foul odors of hydrogen sulphide. Sodium chloride in a concentration of 5,500 parts per million did not affect digestion, but 30,000 p.p.m. decreased the gas production about 20 per cent. Sierp states that plasmolysis is not appreciable in wellseeded Imhoff sludge.

The operation of a number of activated-sludge plants has been interfered with by industrial wastes. Gashouse wastes have been detrimental at the Manchester (Davyhulme) and Reading (England) plants. Discharge of mineral oil has seriously

${ }^{2}$ Pearse, L., and Mohlman, F. W.: "Doubt Fixation of Atmospheric Nitrogen by Activated Sludge Process," Eng. News-Rec., 90, 843-941. I923.

${ }^{2}$ Russell, R., and Bartow, E.: "Bacteriological Study of Sewage Purification," Ill. State Water Surv., Bull. I3, pp. 348-58. 19г5.

${ }^{3}$ Harris, F. W., Lockburn, T., and Anderson, T.: "The Biological and Physical Properties of Activated Sludge," Surveyor, 70, 30-31. July 9, I926.

4 Mohlman, F. W.: "Treatment of Packinghouse, Tannery and Corn Products Wastes," Indust. Eo Engin. Chem., 18, 1076-8I. Oct., 1926.

${ }^{5}$ Sierp, F.: "Ueber die Vorgänge im Schlammfaubraum," Techn. Gemeindeblatt, 29, 267-31 2. March, 1927. 
affected the operation of the Milwaukee and Chicago plants. The Calumet (Chicago) plant has received paint-factory wastes containing acids, sulphur dioxide, arsenic, lead, and copper. The cost of treatment (number of tanks and amount of air required) has been doubled by the presence of these wastes. Iron-pickling wastes at Worcester (Mass.) interfered so seriously with the operation of the experimental activated-sludge plant that the activated-sludge process could not be recommended for the treatment of the city sewage.

In general, it appears that one of the most serious problems in biological sewage treatment in the large cities is the effect of inhibitory industrial wastes.

\section{REMOVAL OF BACTERIA}

Most processes of sewage treatment have not been designed primarily to remove pathogenic bacteria or Bact. coli, the main function being to stimulate and promote the growth of saprophytic forms. Nevertheless, a considerable reduction of Bact. coli is effected by settling tanks, filters, and activated-sludge treatment. Clark (loc.

TABLE II

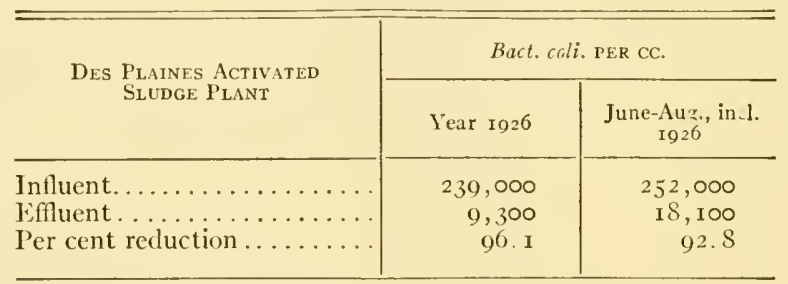

cit.) in I904 reported very high percentage reductions of Bact. coli for experimental devices at the Lawrence Experiment Station, averaging from $\circ$ to 74 per cent for septic tanks, from 49 to 76 per cent for contact beds, from 97 to 99 per cent for trickling filters (stone $\frac{1}{2}-\mathrm{I}$ in. diameter), and from 98 to 99 per cent for sand filters. The removal by trickling filters was undoubtedly greater than could be obtained by more modern, coarser-grained filters. Johnson reported a reduction of 88 per cent of Bact. coli by trickling filters at Columbus, with 35,000 per cubic centimeter remaining in the effluent.

Bacterial reductions are subject to such wide variations that a few results have little meaning. Although a continuous record is kept at many treatment plants of the bacterial counts on agar at $37^{\circ} \mathrm{C}$., very few data are available over a period of months or years concerning the reduction in Bact. coli. A conservative estimate of the reduction in Bact. coli would show about

$$
\begin{aligned}
& \text { 30-5c per cent for Imhoff tanks } \\
& 75^{-90} \text { per cent for trickling filters } \\
& \text { 90-98 per cent for activated sludge } \\
& \text { 95-99 per cent for sand filters }
\end{aligned}
$$

based on the count in the raw sewage.

A year's analyses at the Des Plaines activated-sludge plant showed results as in Table II. 


\section{DISINFECTION}

The discharge of raw or treated sewage into streams, lakes, or the sea near sources of water supply, bathing beaches, or oyster beds usually requires more complete removal of Bact. coli and pathogenic organisms than can be effected by any of the treatment processes now in use. It has been found that chlorination is the most satisfactory method for obtaining bacterial removal. Present practice seems to favor Imhoff-tank treatment followed by chlorination, wherever sufficient dilution is available to satisfy the oxygen requirements of the settled effluent.

The efficiency of chlorine as a bactericide for sewage and sewage effluents has been known for many years. The pioneer work was done in 1907, under Phelps's direction at the Massachusetts Institute of Technology. Calcium hypochlorite was used on raw-sewage and trickling-filter effluent at Boston, septic-tank effluent at Red Bank (N. J.), and trickling-filter effluent at Baltimore (Md.). The amounts of chlorine required were determined, ranging from 5 to 10 parts per million for fresh Boston sewage, from ro to 15 for septic sewage, from 5 or less for tr.ckling-filter effluents. The reductions of Bact. coli for Boston raw sewage with io p.p.m. chlorine were from 35,000 to 24 per cubic centimeter, or 99.9 per cent, and for septic sewage at Red Bank, with II.5 p.p.m. chlorine from 205,000 to 75 per cubic centimeter, or 99.9 per cent. However, when the chlorine was reduced to 7.5 p.p.m., one test showed a reduction of from 220,000 to 16,000 , or only 92.7 per cent. The reductions for trickling-filter effluents were, at Boston with 3.4 p.p.m. chlorine, from 47,000 to 380 per cubic centimeter, or 99.2 per cent, and at Baltimore, with 2.2 parts per million chlorine from 2,000 to 200 , or 90 per cent. In the latter tests residual chlorine of 0.6 was obtained after two hours' storage. The Jackson-bile medium was used for Bact. coli counts at Baltimore.

Many investigations have been made since those of Phelps on the use of chlorine as a bactericide, but liquid chlorine is now used instead of calcium hypochlorite because of the ease of application. The amounts required in operating plants have usually been greater than those determined by Phelps. The amount of chlorine required is now adjusted by determinations of residual chlorine. The chlorine demand varies with the organic matter, sulphides, nitrites, or other reducing compounds present in the sewage. Tiedeman ${ }^{2}$ has recently studied chlorination of an Imhoff effluent. When maintaining residual chlorine of from o.I to 0.5 p.p.m., he obtained more than 99 per cent reduction in Bact. coli. His experiments indicated that the chlorine penetrated the insoluble solids and effected almost as great bacterial reduction as was obtained in the liquid eflluent.

Chlorine is not efficient, however, for sterilization of raw sewage, as the coarser suspended solids are resistant to penetration. When residual chlorine is maintained and Bact. coli is absent from I c.c. or less, the evidence is quite convincing that pathogenic organisms are destroyed. This assurance does not apply to industrial wastes, for example, where enormous amounts of residual chlorine are necessary to eliminate $B$. anthracis from tannery wastes.

x Phelps, E. B.: The Disinfection of Sewage and Sewage Filter Efluents, Water Supply Paper 229, U.S. Geol. Surv., pp. I-73. I909.

${ }^{2}$ Tiedeman, W. v. D.: "Efficiency of Chlorinating Sewage Tank Effluents," Eng. News-Rec., 98, 944-48. June 9, I927. 


\title{
CHAPTER XXVIII
}

\section{SOME PROBLEMS IN WATER BACTERIOLOGY}

\author{
JOHN F. NORTON \\ University of Chicago
}

The accumulation of epidemiological evidence relating drinking water to disease began about the middle of the last century with the investigations of Snow, Budd, and other English sanitarians. With the establishment of the germ theory of disease, it is hardly surprising to find that some of the early bacteriologists were interested in the micro-organic content of waters of all characters and to find them offering biological methods to detect dangerous contamination of water supplies. Emmerich, ${ }^{\mathrm{I}}$ in 1878 , proposed the subcutaneous injection of water, or of extracts of residues left after evaporation, into rabbits. A water dangerous to health was supposed to produce a rise in temperature in the animals and subsequent death. Koch suggested a gelatin-culture method for determining bacteria in water as early as I88I, but it was many years before bacterial methods were sufficiently perfected to be of practical value. Meanwhile, the sanitary chemical examination was regarded as the best means for determining the potability of a water supply. Very early, however, sanitary chemists recognized that biological methods must eventually be of prime importance, although at the present time certain types of information can still be obtained only through chemical methods. William Ripley Nichols, in 1883 , wrote, "There is reason to hope that eventually the decision [i.e., selection or rejection of a drinking water] may be thrown largely upon the biologist."

Between 1892 and 1900 bacteriological technique was developed to an extent to warrant some general agreement among bacteriologists in regard to methods for the testing of water supplies and, perhaps to a less degree, the interpretation of the results obtained. The laboratory procedures were brought together in the first edition of Standard Methods of Water Analysis. ${ }^{3}$ The fifth edition was published in $1925 .{ }^{4}$ While the periodic publication of these standard procedures establishes reasonably satisfactory routine methods for water examination, these methods cannot and should not be regarded as terminating all problems of sanitary bacterial water analysis. A similar statement might be made concerning the establishment of standards for the purity of water supplies ${ }^{5-a}$ necessary and useful procedure but one which must be constantly subject to revision. The development of more satisfactory methods, from the standpoint of both accuracy and speed, represents, however, only one of the present problems confronting the water bacteriologist, although it must be admitted that the

${ }^{1}$ Emmerich, R.: Ztschr.f. Biol., 14, 563. I 878 .

${ }^{2}$ Nichols, W. R.: Water Supply, p. 89. I883.

3 J. Infect. Dis., Supp. I. I904.

4 Standard Methods of Water Analysis. Am. Pub. Health Assoc.: I925.

5 Advisory Committee on Official Water Standards: Pub. Health Rep., 40, 693. r925. 
greater portion of recently published material in this field is concerned directly or indirectly with methods of bacterial water examination or the interpretation of results. Other problems are concerned with the chlorination of water supplies as affecting not only the fate of the typhoid bacillus but of that of other possible water-borne pathogens such as Clostridium welchii; with the survival of organisms of the typhoid-paratyphoid group of bacteria in sewage treatment by means of septic or Imhoff tanks, trickling filters, activated sludge tanks, or chlorination of sewage effluents; or with the very important questions connected with ground-water pollution. I shall attempt to present the present status of these various problems with no pretense toward citing all of the pertinent literature which has accumulated.

\section{BACTERIAL COUNTS}

Much of the earlier work in water bacteriology was concerned with the total number of bacteria which could be recovered from a water by means of a solid nutrient medium under aerobic conditions and at various temperatures (usually $20^{\circ}$ or $37^{\circ} \mathrm{C}$.). At the present time such counts are made in the majority of water laboratories as a matter of routine. Their value appears to be largely in connection with the control of water filtration - as an index of the extent of and variation in the contamination of a raw water and as a measure of the efficiency of a filter. It is universally recognized that these counts do not represent the actual total number of bacteria present. The anaerobic organisms are to a large extent excluded, the colonies obtained on the plates do not necessarily arise from a single cell, and many aerobic bacteria do not grow on the media commonly used. These errors, however, do not detract from the value of the procedure when used under suitable conditions and for suitable purposes. Where comparative results are desired the method will continue to be used. As an index of the purity of a water supply the total count, even at $37^{\circ} \mathrm{C}$., cannot be given serious consideration. It is not included in the standards recommended by an Advisory Committee of the United States Public Health Service. ${ }^{\mathrm{r}}$

The chief problem connected with bacterial counts is that of devising a medium which will give uniform results and can be readily and accurately duplicated. Information on the relation of the chemical composition of meat extracts and peptones to bacterial growth is meager. This is a fundamental problem in the whole field of bacterial nutrition. The water bacteriologist would be satisfied with something less than a final solution. If he could have at his command relatively simple tests which would insure uniformity in the composition of basic nutrient materials, he would be contented. This problem is well recognized and is being attacked. A synthetic medium prepared from pure chemical substances offers another solution. So far, no such medium has been found generally useful.

\section{BACTERIAL SPECIES}

Very few comprehensive investigations have been made of the various species of bacteria found in lakes, streams, and ground waters. Probably the most complete study was that made by Jordan ${ }^{2}$ in connection with his investigations on the Illinois

I Ibid.

${ }^{2}$ Jordan, E. O.: J. Hyg., 3, 1. 1903. 
River. He classified 543 cultures, mostly obtained by isolation on gelatin plates, into I7 groups on the basis of their biochemical characteristics. The Illinois River is a rather highly contaminated stream, and the bacterial flora as found by Jordan cannot of course be regarded as the "natural" flora of a surface water, but rather as representative of conditions existing in a polluted water. As far as the writer is aware, no similar investigation has been made in the past twenty-five years. The attention of water bacteriologists has been focused on a few bacterial groups or species of which the coli-aerogenes group, Clostridium welchii, and the streptococci are the most important.

COLI-AEROGENES GROUP

The coli-aerogenes group of bacteria comprises those organisms which are rod shaped, do not form spores, are facultative anaerobes, ferment dextrose and lactose with production of acid and gas, and do not liquefy gelatin. The use of this group of bacteria as an index of sewage contamination of drinking water is too well known to warrant discussion. There are, however, certain differences of opinion in regard to the laboratory procedures employed in sanitary water analysis and the interpretation of the results obtained in the laboratory. If this bacterial group or any particular member of it is to be used to determine the purity of water supply, there must eventually be unanimity of opinion and practice. The usefulness of standard procedures has already been pointed out.

The appearance of gas in lactose broth seeded with portions of a water to be tested constitutes the so-called "presumptive" test. It is generally conceded that this test is an inadequate basis on which to judge potable waters. Both anaerobic bacteria and spore-forming aerobes appear in such tests, as well as resistant strains of Bact. coli. Omitting for the moment the questions involving specific members of the coli-aerogenes group, it is obvious that spore-forming bacteria, either aerobic or anaerobic, cannot legitimately be used as indexes of the possible presence of typhoid bacilli on the same basis as other members of the intestinal group of bacteria. Various attempts have been made to inhibit these extraneous bacteria. The most popular method has been by the addition of bile or bile salts to the lactose broth. The early work of MacConkey, of Jordan, Russell, and Zeit, ${ }^{\mathrm{I}}$ and of Jackson ${ }^{2}$ on bile salts was not consistent. Hale and Melia $^{3}$ advocated the use of lactose bile in the presumptive test and stated that this medium yielded the most satisfactory results. It was for a time adopted as a standard method. ${ }^{4}$ Jordan, ${ }^{5}$ however, has shown that bile exerts a marked inhibitive action on Bact. coli. Although some laboratories have continuously employed a bile medium in water analysis, this practice has to a large extent been abandoned. Recently, the interest in bile has been revived, particularly in combination with brilliant green. A medium devised by Hale, and containing 5 per cent bile with $\mathrm{I}$ : Io, 000 brilliant green, has been used in laboratories for some time. Dunham and Schoenlein ${ }^{6}$

${ }^{2}$ Jordan, E. O., Russell, H. L., and Zeit, F. R.: J. Infect. Dis., x, 682. r904.

2 Jackson, D. D.: Biological Studies by the Pupils of William Thompson Sedgwick, p. 292. I906.

${ }^{3}$ Hale, F. E., and Melia, T. W.: J. Infect. Dis., 7, 567. r9 10.

${ }_{4}^{4}$ Standard Methods of Water Analysis (2d ed.). 1912.

${ }^{5}$ Jordan, E. O.: J. Infcct. Dis., r 2, 326 . I9r3.

${ }^{6}$ Dunham, H. E., and Schoenlein, H. W.: Stain Technol., r, r29. 1926. 
have suggested the use of 2 per cent bile with $\mathrm{I}: 75,000$ brilliant green in order to avoid the inhibitory action of the former medium. Hale ${ }^{t}$ has pointed out that the medium of Dunham and Schoenlein allows the growth of Clostridium welchii and that the interference of this organism is more significant than the inhibition of "attenuated colon organisms which have little if any sanitary significance." The question at issue involves both laboratory methods and the interpretation of results and has not been satisfactorily solved. The use of a brilliant green bile medium in the isolation of coliaerogenes group organisms will be referred to later. Other methods for inhibiting the growth of anaerobes have been suggested. Among these may be mentioned the use of gentian violet advocated by Hall and Ellefson ${ }^{2}$ for the presumptive colon test.

While the presence of members of the coli-aerogenes group of bacteria has long been recognized as constituting evidence of contamination in a water supply, it has also been evident as a result of the work of Prescott ${ }^{3}$ and others that organisms of this group may be found on grains, grasses, and in other places with sufficient frequency to cast doubt upon the value not only of presumptive tests but of the use of the group as a whole to indicate pollution. The advisability of attempting to distinguish between bacterial strains of coli-aerogenes organisms from intestinal and from other sources is a matter of dispute. It is impossible at the present time to differentiate human and animal organisms. ${ }^{4}$ It is certainly a safe procedure to utilize the group. On the other hand, it is undesirable to condemn a water supply in the absence of other evidence of fecal contamination. More evidence as a foundation for expression of opinion must be produced.

Meanwhile, two lines of investigation are being pursued: One is concerned with the perfection of methods for identification of the coli-aerogenes group, and the other is directed toward procedures for distinguishing bacteria in this group arising from fecal and from non-fecal sources. The latter will be discussed first.

Levine $^{5}$ has summarized our knowledge of the biochemical reactions of the organisms generally regarded as constituting the coli-aerogenes group, and the reader is referred to his publication for details. To this summary must be added Koser's $\mathrm{s}^{6}$ work with sodium citrate. Earlier attempts to differentiate fecal and non-fecal coli-like organisms included reliance upon carbohydrate fermentations and indol production. At the present time these activities must be excluded. The most important correlations are those involving the gas ratio, the methyl-red test, the Voges-Proskauer reaction, and ability to grow in a uric acid or sodium citrate medium. Strains of fecal origin characteristically give a low gas ratio (carbon dioxide $\div$ hydrogen), an acid reaction using methyl red as an indicator, a negative Voges-Proskauer test, and will not grow in Koser's uric acid or sodium citrate medium. Undoubtedly, the correlation between such tests and the source of the organism is not exact and cannot be expected to be.

'Hale, F. E.: ibid., 2, 24. I927.

${ }^{2}$ Hall, I. C., and Ellefson, L. J.: J. Bact., 3, 329. I918.

3 Prescott, S. C.: Biological Studies by the Pupils of William Thompson Sedgwick, p. 208. I906.

${ }^{4}$ See Prescott, S. C., and Winslow, C.-E. A.: Elements of Water Bacteriology (4th ed.), p. 93. I924.

5 Levine, M.: Bacteria Fermenting Lactose and Their Significance in Water Analysis, Iowa State College Engineering Experiment Station Bull.62. I921.

${ }^{6}$ Koser, S. A.: J. Bact., 9, 59. I924. 
Indeed, Bardsley ${ }^{\mathrm{I}}$ has recently reported imperfect correlation between the Koser and other tests on the basis of results from I,44I strains. For those bacteriologists who insist that "fecal" coli are the only significant type, reasonably accurate results are obtainable.

The present methods recommended ${ }^{2}$ for completing the coli-aerogenes group test in water analysis consist in transferring material from a positive lactose-broth tube to Endo medium or eosin-methylene-blue agar. Typical colonies are then tested for lactose fermentation and are also transferred to nutrient agar slants for morphological study. The completion of the test requires from three to five days. If results are based upon finding so-called "fecal" coli, several more days are consumed. It would be of great advantage to the water-works manager if tests could be devised to give results in twenty-four or forty-eight hours. The use of brilliant green bile in place of lactose broth for the initial tests has already been mentioned. At the present time this is a doubtful procedure. However, recent work has indicated that such a medium may be valuable in shortening the time for the completion of the "coli" test. H. E. Jordan, ${ }^{3}$ in collaboration with a number of other laboratory workers, has found a 97 per cent correlation between the fermentations obtained in a medium containing 2 per cent bile and I : 75,000 brilliant green and the "Standard Methods" completed test, when the brilliant green bile medium is used to confirm originally positive lactose fermentation. Ruchhoft has also obtained encouraging results with this method. These original fermentations may be due to members of the coli-aerogenes group, to anaerobic organisms, or to spore-forming, lactose-fermenting aerobes. The significance of these latter organisms has been studied by Norton and Weight $\mathrm{t}^{5}$ and others. What part bacterial "synergism" may play in obtaining fermentation in lactose broth in routine water analysis is problematical. The introduction of brilliant green lactose bile as a confirmatory medium appears to the writer to be a real contribution to sanitary water analysis.

Attempts to improve upon Endo medium and eosin-methylene-blue agar for isolation of Bact. coli do not appear to the writer to represent fruitful research. There appears to be some peculiar fascination to the laboratory worker in devising new combinations of dyes for differential purposes in all bacteriological work. The most recent attempt to improve upon eosin-methylene-blue has been made by Salle. ${ }^{7}$ His medium consists of a mixture of erythrosin, methylene blue, and brom-cresol-purple in a lactose-peptone agar buffered with phosphates.

\section{CLOSTRIDIUM WELCHI}

Since the early work of Klein and Houston, ${ }^{8}$ English bacteriologists have shown interest in the use of Clostridium welchii as an indicator of pollution in water supplies.

\footnotetext{
I Bardsley, D. A.: J. IIyg., 25, II. 1926.

${ }^{2}$ Standard Methods of Water Analysis (5th ed.). Am. Pub. Health Assoc. 1925.

${ }^{3}$ Jordan, H. E.: J.A.W.W.A., 18, 337. 1927.

${ }^{4}$ Ruchhoft, C. C.: J. A.W.W.A., 16, 778. 1926.

${ }_{5}^{5}$ Norton, J. F., and Weight, J. J.: Am. J. Pub. Health, 14, тоr9. 1924.

${ }^{6}$ Holman, W. L., and Meekison, D. M.: J. Infect. Dis., 39, I45. I926.

7 Salle, A. J.: ibid., 4r, I. 1927.

${ }^{8}$ Klein, E., and Houston, A. C.: Suppl. $27 t h$ Ann. Rep. of Loc. Gov. Bd. I8g8.
} 
On the other hand, American workers have not generally favored such an index. Certainly, in dealing with chlorinated waters, it would be unfortunate to use a sporeforming organism as indicative of the possible presence of bacteria of the paratyphoidtyphoid-dysentery groups.

The question of the pathogenicity of strains of Clostridium welchii in water supplies was brought to the attention of water bacteriologists as the result of outbreaks of gastro-enteritis in Montclair, New Jersey, in IgI 8 and I92I. ${ }^{\mathrm{I}}$ More recently, attempts have been made to establish this organism as the etiological agent in pernicious anemia. At the present time more information is needed both on the relation of Clostridium welchii to diarrheal disease and the significance of its presence in filtered and chlorinated waters. The possibility of its interference with presumptive "coli" tests has already been discussed. Recently, Wilson and Blair ${ }^{2}$ have devised a sulphiteiron agar for the detection of sulphite-reducing anaerobic organisms. They claim a good correlation between sulphite reduction and tests for Bact. coli in water. The writer has found the method useful in the study of anaerobes in sewages and sewage effluents, and Greer $^{3}$ has published investigations using Wilson and Blair's method. How useful this will be in sanitary water examination will depend upon the determination of the sanitary status of Clostridium welchii.

\section{STREPTOCOCCI}

Prescott and Winslow 4 have stated that "there seems to be some reason to hope that the streptococci may prove of assistance in the important task of differentiating between human and animal pollution, a task in which all other tests have so far failed." In spite of the enormous literature which has accumulated during the past twenty-five years, and of which the work of Andrewes and Horder ${ }^{5}$ in England, Clemesha ${ }^{6}$ in India, and Winslow and Palmer 7 and Rogers and Dahlberg ${ }^{8}$ in this country may be cited as examples, it is still impossible to correlate biochemical characteristics of streptococci with their source to any degree of certainty. At the present time the search for specific fermentative strains in potable waters is unlikely to reward the water bacteriologist for his effort. Neither can the determination of streptococci (in general) in a water supply be recommended as a satisfactory procedure for routine water analysis.

\section{THE INTERPRETATION OF RESULTS}

The tendency during the past twenty-five years has been toward more rigid standards for water supplies. From a former requirement of not more than one Bact. coli per I cc. of water, the standard has been increased until, if figured on the former basis, a good water must not contain more than one Bact. coli in Ioo cc. The present standards as adopted by an Advisory Committee of the United States Public Health Serv-

${ }^{r}$ Eng. News-Rec., 86, 929. r92 I.

${ }^{2}$ Wilson, W. J., and Blair, E. M. McV.: J. Hyg., 24, I I1. I925.

3 Greer, F. E.: Am. J. Pub. Health, 15, 860. I925.

4 Prescott, S. C., and Winslow, C.-E. A.: op. cit. (4th ed.), p. 720 . I924.

5 Andrewes, F. IV., and Horder, T. J.: Lancet, I 71, 708. 1906.

${ }^{6}$ Cited by Prescott, S. C., and Winslow, C.-E. A.: op. cit. (4th ed.), p. 720. I924.

7 Winslow, C.-E. A., and Palmer, G. T.: J. Infect. Dis., 7, т. rgro.

${ }^{8}$ Rogers, L. A., and Dahlberg, A. O.: J. Agric. Research, I, 79I. I9I4. 
ice are stated in a somewhat different manner. ${ }^{1}$ The agar plate count is not recognized. The following statement includes only the bacteriological requirements.

I. Of all the standard (г сc.) portions examined in accordance with the procedure specified below, not more than ro per cent shall show the presence of organisms of the $B$. coli group.

2. Occasionally three or more of the five equal ( $10 \mathrm{cc}$.) portions constituting a single standard sample may show the presence of $B$. coli. This shall not be allowable if it occurs in more than-

a) Five per cent of the standard samples when twenty (20) or more samples have been examined;

b) One standard sample when less than twenty (20) samples have been examined.

The series of samples must conform to both (I) and (2). The "B. coli group" is defined on the basis of the I923 edition of Standard Methods of Water Analysis. A standard portion is ro cc., and a standard sample is five standard portions.

The foregoing standard seems a reasonable one at the present time. It is not so rigid as to be impossible of attainment. On the other hand, it is sufficiently exacting to protect the public against water-borne bacterial infections while at the same time making allowance for slight variations in laboratory results which are occasionally encountered. It would be unwise to predict how long it will be before even more rigid standards are proposed. Certainly, it is hoped there will be no retrogression.

Attempts have been made to express the results of the colon tests in a mathematical manner on the basis of the theory of probabilities. A discussion of these attempts seems to the writer to be out of place in this summary. It is doubtful if the laboratory methods employed for the detection of Bact. coli or other members of that group are sufficiently accurate from a quantitative standpoint to warrant the application of mathematical theory. The reader is referred to the original articles. ${ }^{2}$

\section{CHLORINATION}

The rapid extension, since I9I I, of chlorination in the treatment of public water supplies has been nothing less than remarkable. In $1924^{3}$ it was estimated that more than 3,750 million gallons were being treated daily. Relatively pure waters, both bacterially and chemically, lend themselves to such treatment. The dosage varies from O.I 5 to I.o part of chlorine per million gallons of water.

The chlorine dosage is a function not only of the number of bacteria present in the water but also of the total amount of organic matter present. Such studies as that recently made by Meadow and Hale 4 on the chlorine absorption of water are likely to be valuable in this connection.

The bacteriological results obtained are, on the whole, satisfactory. One of the

${ }^{I}$ Advisory Committee on Official Water Standards: loc. cit.

${ }^{2}$ McCrady, M. H.: J. Infect. Dis., 17, 183. 1915; Pub. Health J., 9, 201. 1918; Wolman, A.: J.A.W.W.A., 4, 200. I917; ibid., 6, 444. I919; ibid., 7, 927. 1920; Wolman, A., and Weaver, H. L.: J.Infect.Dis., 21, 287. I917; Wells, W. F.: Science (N.S.), 47, 46. I918; J.A.W.W.A., 8, I87. 1921; Stein, M. F.: J. Bact., 4, 243. 1919; J.A.W.W.A., 8, 182. I921.

3 Water Works Practice, p. I74. 1925.

${ }_{4}^{4}$ Meadow, J. R., and Hale, H.: J.A.W.W.A., 18, 75. 1927. 
problems in connection with chlorination is concerned with the prevention of undesirable flavors which are thought to be the result of the action of chlorine on phenols. These enter water supplies with trade-waste contamination. More knowledge is needed as to the proper methods of eliminating such tastes. This is more a chemical and engineering than a bacteriological problem.

The treatment of raw water with chlorine previous to filtration in conjunction with an after-filtration dose is a problem of present day discussion and experimentation. The reduction of the bacterial load on the filter is one object of such treatment. While results available are encouraging, more data are needed.

GROUND WATER

The great mass of both experimental and practical work on drinking water has been concerned with public water supplies. The rural resident, however, is not benefited by the advance in the engineering practice of water purification or by the extension of chlorination or other disinfecting methods, such as ultra-violet light or ozone. The shallow well will remain a source of drinking water for a not inconsiderable number of people. While the factors concerned in the self-purification of streams have been fairly well established as the result of the classical investigations of Jordan and his associates on the Chicago Drainage Canal and the Illinois River, as well as by the more recent investigations under the direction of the United States Public Health Service, our knowledge concerning the pollution of ground waters has been fragmentary and impractical. A recent report from the Hygienic Laboratory ${ }^{1}$ gives almost the first definite information on this subject. No claim is made that the data obtained are generally applicable since only one set of conditions was studied, i.e., sandy soil with slowly moving water. Only a brief summary of the conclusions will be given. Bact. coli was recovered at distances varying from I to 232 feet away from an experimental trench containing excreta. This organism traveled in only one direction-that of the ground-water flow. Wet weather resulted in extension of pollution; dry weather inhibited it. Bact. coli localized in an upper blanket near the ground-water table and tended to filter out into the soil when the ground water fell. This organism gradually disappeared if the soil remained dry. Organisms were recovered as long as two years and eight months after experimental pollution. The movements of the ground-water table upward and downward determined to a large extent the progression of pollution. Pollution did not expand laterally (as previously thought probable) but tended to contract "with the trench representing the base of a truncated section of a cone."

The extension of such investigations is urgently needed if the water bacteriologist or the sanitary engineer is to be in a position to give advice to the users of shallow wells.

\section{THE FATE OF THE TYPHOID BACILLUS}

In connection with all problems involving the purification of polluted water supplies, it is desirable to know with a greater degree of exactness the fate of the typhoid bacillus. To be sure, conclusions are now being drawn on the basis of survival of the organisms of the coli-aerogenes group, and it is argued that if this group has been dis-

Experimental Bacterial and Chemical Pollution of Wells, via Ground Water and the Factors In volved, Hygienic Laboratory Bull. 147. 1927. 
posed of, organisms of the paratyphoid-typhoid-dysentery groups will also have disappeared. From a scientific standpoint such conclusions are not entirely satisfactory. The earlier studies on the viability of the typhoid bacillus in water, of which those of Frost ${ }^{\mathrm{I}}$ and Jordan, Russell, and Zeit ${ }^{2}$ may be mentioned, have been generally accepted. However, considerable advance has been made in laboratory technique for the isolation of these organisms, and the time appears to be ripe for further investigations.

The viability of Bact. coli and Bact. typhosum in stored feces has recently been studied by Jordan. ${ }^{3}$ At room temperature or lower these organisms have been found to survive for considerable periods. For example, typhoid bacilli were recovered from stools of typhoid carriers after fifty-two days' storage at room temperature. Such studies have a direct bearing on the pollution of ground-water supplies from privies. The viability of the typhoid bacillus in the various methods for sewage treatment, including septic and Imhoff tanks, trickling filters and activated sludge tanks, and chlorination, has not been accurately determined. Courmont and Rochaix ${ }^{4}$ have reported the survival of organisms of the paratyphoid-typhoid group in effluents from the activated sludge process. The whole subject, however, needs further investigation.

IFrost, W. D.: J. Infect. Dis., I, 599. I904.

${ }^{2}$ Jordan, E. O., Russell, H. L., and Zeit, F. R.: ibid., p. 64I. I904.

${ }^{3}$ Jordan, E. O.: ibid., 38, 306. 1926.

${ }_{4}^{4}$ Courmont, R., and Rochaix, A.: Rev. d'hyg., 44, 907. 1922. 


\title{
SHAP'TER XXIX \\ THE ACTION OF ULTRA-VIOLET LIGHT ON BACTERIA \\ AND THEIR PRODUCTS
}

\author{
JOHN F. NORTON \\ University of Chicago
}

Light rays at varying positions in the spectrum produce different effects on both inorganic and organic substances - on dead material or on living cells. The visible spectrum extends from a wave-length of about 6,500 A at the red end to about 4,000 A in the violet. (An Angstrom unit equals $10^{-7} \mathrm{~mm}$. or o.I $\mu \mu$.) The near ultra-violet region includes wave-lengths from 4,000 to about $3,000 \mathrm{~A}$, the far ultra-violet extends to about $2000 \mathrm{~A}$, and the extreme ultra-violet (sometimes known as the Schumann region) includes wave-lengths down to about $\mathrm{I}, 200 \mathrm{~A}-$ the lower limit of the metallic arc. X-rays have wave-lengths below $600 \mathrm{~A}$. As will be shown later, these divisions of the wave-lengths of light have a physiological interest.

The action of light on micro-organisms was first described by Downes and Blunt in $1877 .^{\circ}$ Their conclusions are of some interest.

I. Light is inimical to the development of bacteria and the microscopic fungi associated with putrefaction and decay, its action on the latter organisms being apparently less rapid than upon the former.

2. Under favorable conditions it wholly prevents that development, but under less favorable it may only retard.

3. The preservative quality of light, as might be expected, is most powerful in the direct solar ray, but can be demonstrated to exist in ordinary diffused daylight.

4. So far as our investigation has gone it would appear that it is chiefly, but perhaps not entirely, associated with the actinic rays of the spectrum.

5. The fitness of a cultivation-liquid to act as a nidus is not impaired by insolation.

6. The germs originally present in such a liquid may be wholly destroyed and a putrescible fluid perfectly preserved by the unaided action of light.

Downes and Blunt apparently believed that oxygen was essential for the germicidal action of light, in spite of an experiment recorded by them in which bacteria in evacuated tubes were killed by exposure to light.

Nine years later Arloing ${ }^{2}$ and, independently, Duclaux, ${ }^{3,4}$ studied the action of light on both vegetative cells and spores. Arloing showed that anthrax spores germinated more slowly when exposed to gaslight and that vegetative cells gradually lost their virulence. Duclaux noticed a marked difference in the rapidity of action of strong sunlight as compared with diffuse light. Arloing attributed the action to the

I Downes, A., and Blunt, T. P.: Proc. Roy. Soc., 26, 488. I877.

${ }^{2}$ Arloing, S.: Compt. rend. Acad. de Sci., roo, 378. 1885; 1о1, 5 I1. I885.

3 Duclaux, E.: ibid., roo, I19. I88.5.

${ }^{4}$ Duclaux, E.: Ann. de l'Inst. Pasteur, 1, 88. 1887. 
colors of the spectrum. He also agreed with Downe:; and Blunt that oxygen was an essential factor in the germicidal action and that veriations in the germicidal action of light of different character or intensity was due to its effect on oxidizing reactions. Duclaux appears to concur in this conclusion as dous also Roux, ${ }^{1}$ who studied the influence of light and of air on anthrax cells and spoes.

Buchner, ${ }^{2}$ in 1892 , using both suspensions of bacteria and agar plates, demonstrated the germicidal action of direct sunlight on a number of species of bacteria such as the typhoid bacillus, Bact. coli, B. pyocyaneus, B. prodigiosus, and the cholera vibrio. Buchner's papers are of historic interest since we find here the illustration, similar to that in most modern bacteriologies, of an agar plate showing growth of bacteria in those parts protected against sunlight and no growth on the exposed portions.

In 1892 and 1893 Ward $^{3}$ published a series of articles on the action of light on anthrax bacilli and spores. He believed that the action of light was direct and not due to a secondary action involving oxygen. Ward demonstrated by the use of colored glasses and light filters composed of chemical solutions that the active germicidal rays from the sun were in the blue-violet region of the spectrum. By substituting quartz for the glass plates employed by previous investigators he also showed that the ultraviolet region was active in destroying bacteria and their spores. Ward experimented with the carbon arc as well as with solar rays.

During the last thirty-five years an extensive literature has accumulated on the action of ultra-violet light on bacteria. It is possible, here, to refer only to the more important contributions and to present a summary of the present state of our knowledge.

\section{TIIE EFFECTIVE SPECTRAL REGION}

The results of the earlier workers established this region only in a general way. In I 903 Barnard and Morgan ${ }^{4}$ attempted to distinguish between light rays which caused a reaction in living tissue and those which were bactericidal. Although not successful in their first objective, they did obtain fairly accurate data on germicidal action. Wave-lengths between $3,287 \mathrm{~A}$ and 2,265 $\mathrm{A}$ were found to be effective. The longer rays appeared to be physiologically active. A number of investigators, notably Thiele and Wolf, ${ }^{5}$ Bazzoni, ${ }^{6}$ Newcomer, ${ }^{7}$ Browning and Russ, ${ }^{8}$ Cernovodeanu and Henri, ${ }^{9}$ Bayne-Jones and Van der Lingen, ${ }^{10}$ and Coblentz and Fulton, ${ }^{\mathrm{II}}$ have made similar studies.

${ }^{x}$ Roux, E.: Ann. de l'Inst. Pasteur., 1, 445. I887.

2 Buchner, H.: Centralbl.f. Bakteriol., I1, 781. 1892; 12, 217. I892.

3 Ward, M. H.: Proc. Roy. Soc., 52, 393. I892-93; 53, 23. I $893 ; 54,472.1893$; Frankland, P. F., and Ward, M. H.: ibid., 53, 177. 1893 .

${ }_{4}^{4}$ Barnard, J. E., and Morgan, H.: Brit. M.J., 2, 1269. 1903.

5 Thiele, H., and Wolf, K.: Arch.f. Ilyg., 57, 29. 1906.

${ }^{6}$ Bazzoni, C. B.: Am. J. Pub. Ilealth, 4, 975. I9I4.

7 Newcomer, H. S.: J. Exper. Med., 26, 841, I917.

${ }^{8}$ Browning, C. H., and Russ, S.: Proc. Roy. Soc., 90, 3.3. 1917.

9 Cernovodeanu, B., and Henri, V.: Compt. rend. Acad. de Sci., 150, 52. 1910.

ro Bayne-Jones, S., and Van der Lingen, J. E.: Bull. Johns Ilopkins Hosp., 34, Ir. 1923.

"Coblentz, W. W., and Fulton, H. R.: Scientific Papers of the Bureau of Standards, No. 495. 1924. 
While, in general, the results obtained agree fairly c'losely, certain discrepancies should be noted. The active germicidal region of the s'pectrum extends from about 2,950 to I,850 A. From 3,000 to $3,500 \mathrm{~A}$ activity exis'ts but is greatly diminished as compared with shorter rays. Bazzoni ${ }^{1}$ believed that t; hese longer rays were really the active ones but were stimulated by short light waves. There is no evidence to support this contention. Other investigators have reported maximal germicidal action at various wave-lengths between 2,950 and 2,26jo A, but, as Coblentz and Fulton ${ }^{2}$ point out, rapidity of abiotic action is depentient upon two factors-light intensity and wave-length. In most of the experimential work reported in the literature little or no attention has been given to the intensity of the radiation, and it is evident that wave-lengths of, for example, 2,600 A of high intensity might exert a more rapid effect than those of 2,200 A of low intrensity. On the basis of all the data available, it is fair to state that germicidal action of light increases with decreasing wave-lengths in the region through which quart $z$ will transmit light waves.

Coblentz and Fulton ${ }^{2}$ have calculated the energy required to kill bacteria. For

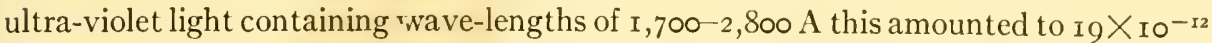
watts per bacterium. Whr.n light waves below 2,200 A were excluded, seven times this amount of energy wris required. This would indicate a high activity for the short radiations. Regardless of the accuracy of these calculations, it is evident that only a minute amount of ligh.t energy is required for the destruction of bacterial cells.

The Schumann region has been the subject of several investigations. Lyman ${ }^{3}$ has criticized some of this work from a physical standpoint. These very short waves are not found in light from the mercury arc. Furthermore, they are absorbed by air and by quartz. Experimental work must be done in vacuo and exposure must be made through fluorite windows. Bovie ${ }^{4}$ has made an extensive study of these radiations, using organisms higher than the bacteria, and has found that they exert a more rapid destructive action on living cells than the longer light waves.

\section{TIIE INFLUENCE OF VARIOUS FACTORS}

Bacterial species.-The extremely rapid germicidal action of ultra-violet light may account for reports showing that but little difference exists in regard to the susceptibility of various bacteria toward ultra-violet light. Even the tubercle bacillus, one of the most resistant of the bacteria to external agents, appears to be almost as easily destroyed by light as are the organisms usually regarded as more sensitive to physical and chemical agents. Reported differences in sensitivity are not consistent. Fluorescent strains are much more difficult to kill than non-fluorescent organisms of the same species. ${ }^{5}$ Bacterial spores have been generally reported as no more resistant than vegetative cells. Higher organisms, such as paramecium, yeasts, and molds, are considerably more resistant than bacteria. Colored mold spores are highly refractory.

Medium.-The various menstrua used to prepare bacterial suspensions for exposure to ultra-violet light may have a marked influence on the results. Broth-peptone media absorb the rays and thus exert a protective action. Pure water, on the

'Bazzoni, C. B.: loc. cit.

${ }^{3}$ Lyman, T.: Nature, 84 , 7т. гого.

${ }^{2}$ Coblentz, W. W., and Fulton, H. R.: loc cil. $\quad{ }^{4}$ Bovie, W. T.: Bot. Gaz., 6r, т. I9r6.

${ }_{5}^{5}$ Burge, W. E., and Neill, S. J.: Am. J. Physiol., 38, 40I, I915. 
other hand, allows the activi? rays to penetrate. Barnard and Morgan ${ }^{1}$ state that fourfifths of the active light will toe absorbed by a layer of water $2.5 \mathrm{~cm}$. thick. The shorter the rays the less the distance of penetration. Lyman found no transmission of waves below I,792 A through $0.5 \mathrm{~mm}$. of water. Kreusler ${ }^{2}$ states that I4.I $\mathrm{cm}$. of water will absorb waves of $2,000 \mathrm{~A}$.

The influence of the $\mathrm{H}$-ion conyentration has been particularly studied by BayneJones and Van der Lingen. ${ }^{3}$ An acid medium is more favorable for the destructive action than an alkaline one. A favorite method of study has been by the use of a seeded agar plate. A slight stimulating action of the medium adjacent to exposed portions has been reported. Coblentz and Fulton 4 found that agar plates exposed for long periods to ultra-violet light were incapable of supporting growth but that short exposures had no effect. It is well known that colcored or turbid waters are difficult to sterilize.

Time.-The time required to kill bacteria varies firom a few seconds to several minutes, depending upon the wave-length of light used and the intensity of the light. Some of the earlier workers maintained that increase of temperature accelerated germicidal action, but Henri and Cernovodeanu ${ }^{5}$ disputed this claim. Bayne-Jones and Van der Lingen, ${ }^{6}$ using temperatures varying from $2^{\circ}$ to $40^{\circ} \mathrm{C}$., were able to detect only a slight increase in the velocity of the reaction with increase in temperature. It is not impossible that differences in velocity may be masked by the rapidity of the reaction itself.

Discontinuous exposure.-The total time required to kill bactieria on an agar plate or in suspension is not changed by intermittent exposure.

Residual action.-It has been claimed that water exposed to bactericidal rays will retain its activity for some time. This was denied many years ago by Cernovodeanu and Henri? but the claim was revived in I92I by Walker and Prye.r. ${ }^{8}$ Their results, using Bacterium paratyphosum B, are somewhat startling and have never been accepted by sanitary engineers. Theoretically, a residual germicidal action is not an impossibility since we know from the work of Steenbock and Daniels, Hess and Windaus,${ }^{10}$ and others that foods containing fresh fats and oils and lipoids such as cholesterol can be activated for the prevention of rickets by exposure to ultra-violet light. It is not outside the range of possibility that water, particularly after contamination by man, might contain absorbing substances.

\section{APPLICATIONS}

Practical use has been made of the bactericidal properties of ultra-violet light in connection with the purification of drinking water. ${ }^{\text {II }}$ Although there are a number of

'Barnard, J. E., and Morgan, H.: loc. cil. $\quad{ }^{2}$ Kreusler: Ann. der Physik., 6, 41 2. I90 I.

3 Bayne-Jones, S., and Van der Lingen, J. S.: loc. cit.

${ }^{4}$ Coblentz, W. W., and Fulton, H. R.: loc. cil. ${ }^{5}$ Cernovoleanu, B., and Henri, V.: loc. cil.

${ }^{6}$ Bayne-Jones, S., and Van der Lingen, J. E.: loc. cil.

7 Cernovodeanu, B., and Henri, V.: loc. cit.

${ }^{8}$ Walker, W. F., and Pryer, R. IV.: Am. J. Pub. Heallh, I I, 703. I92 I.

${ }^{9}$ Steenbock, H., and Daniels, A. L.: J.A.M.A., 84, I093. 1925.

${ }^{10}$ Hess, A. F., and Winlaus, A.: Proc. Soc. Exper. Biol. \& Hcd., 24, 171. 1926; 24, 369. 1927.

"IVater Il'orks Practice, p. 264. Philadelphia, 1925. 
municipal supplies in Europe utilizing this method, it has found little favor in this country. There are certain important difficulties. Water, to be treated satisfactorily, must be relatively pure from a bacterial standpoint and free from suspended matter or color. Prefiltration is therefore almost a necessity. To get the best results a battery of lamps must be used with a consequent high operating cost. It is doubtful whether ultra-violet sterilization will ever become an important means for purification of municipal water supplies in this country.

In the past fifteen years there has been an increasing use of ultra-violet sterilizers for swimming-pool purification. The sterilizer is made part of the recirculating, purifying system. Its value has been seriously questioned.' There are two reasons for such installations. The action of the ultra-violet light in purifying the recirculating water may be additive to that of the filter. Furthermore, the residual germicidal action already referred to may play a part in keeping the pool in good sanitary condition. This action needs extensive and careful investigation before it can be accepted as of any importance.

\section{BACTERIAL PRODUCTS}

In addition to causing death of the bacterial cell, ultra-violet light may exert a destructive action on products of bacterial metabolism - such as toxins and enzymeson the various groups of so-called "antibodies" produced in the animal body as the result of the injection of bacteria or their products - such as antitoxins, agglutinins, and lytic substances - or on the lytic material known as bacteriophage. Light may also influence the ability of bacteria to act as antigens by changing the proteins or carbohydrate-like substances in the cell which appear to be responsible for antigenic power or for reactions with antibodies.

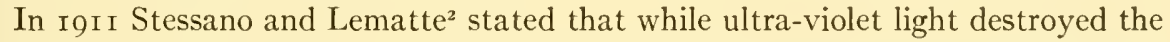
bacterial cell as a living entity, it did not affect the toxins and enzymes in the cell nor did it prevent the power of the cells to cause the production of agglutinins. Irradiation might then be used in place of heat for the production of bacterial vaccines. This has been done but I am not aware that the method has been used extensively. No important studies have been made on the influence of ultra-violet light on the antigenic properties of bacteria. The work of Eberson ${ }^{3}$ has not been generally accepted. Attempts have also been made to modify the action of bacteria by the use of sublethal exposures to ultra-violet light. Mme Henrit claims to have obtained modified strains of the anthrax bacillus, but I cannot find any confirmation of her work. Such effects do not appear to be theoretically impossible.

Almost nothing has been done on the effect of light on bacterial enzymes. If we assume, and I know nothing against such an assumption, that these enzymes do not differ from those produced by body cells, we can fairly well predict the course of events. Pincussen ${ }^{5}$ and his co-workers have studied the effect of ultra-violet light on diastase, amylase, trypsin, urease, etc. The results parallel those with living cells.

I"Swimming Pools and Other Bathing Places," Am. J. Pub. Health, r6, I I86. I926.

2Stessano and Lematte: Compt. rend. Acad. de Sci., 152, 623. I9I I.

${ }^{3}$ Eberson, F.: J. Immunol., 5, 345. I920.

${ }^{4}$ Henri, Mme V.: Elec. Rev., 74, 768. I9I4.

5 Pincussen, L.: Biochem. Zischr., 134, 459. I923. 
Temperature has little effect, but the reaction of the substratum is important. In acid media enzymes are more sensitive than in an alkaline medium. Salts and organic colloids protect enzymes against the action of light. Previously Agulhon ${ }^{\mathrm{r}}$ had worked with such enzymes as sucrose, emulsin, and rennet and noted the destructive action of ultra-violet light. Some enzymes appeared to be acted upon only in vacuo; others were destroyed both in the presence and absence of oxygen. In view of most of the other work with light-waves, the oxygen effect may properly be questioned.

Bacteriophages are gradually destroyed by ultra-violet light. Fisher and McKinle $y^{2}$ have found that the resistance to this agent is proportional to the concentration of the lytic principle.

Two investigations have been made on the effect of ultra-violet radiation on diphtheria toxin-one by Lowenstein ${ }^{3}$ and the other by Hortock, Schurmann, and Stiner. ${ }^{4}$ The latter conducted a series of experiments in which they recirculated a toxin solution through an ultra-violet-light sterilizer and determined the effect on the minimum lethal dose. Very long periods of recirculation (six to ten hours) were necessary to produce any marked change in the toxic action. Direct exposure of toxic broth to ultra-violet light also had little effect except after very long periods of time. Their experimental data are somewhat meager. It is impossible to calculate the actual time of exposure of any particle of toxin in the recirculating experiments on the basis of data presented. In the medium in which bacterial toxins are found or are produced, they appear to be relatively stable toward ultra-violet light.

On the other hand, antibodies are undoubtedly sensitive to irradiation. Heuer's ${ }^{5}$ claim that agglutinins produced against organisms of the typhoid-paratyphoid group react differently has not been substantiated. He states that bacteriolysins are sensitive to light rays. I can find no evidence in regard to the action of ultra-violet rays on antitoxins. Tuberculin is probably affected.

The antibodies to which particular attention has been paid are the so-called "amboceptors" or "sensitizers" against red blood cells. All investigators from Huber ${ }^{6}$ to the present time agree that hemolysins are readily destroyed by ultra-violet irradiation. Friedberger and Scimone ${ }^{7}$ also report that Wassermann positive sera become negative after exposure to these rays. All investigators agree as to the sensitiveness of alexin (complement) to ultra-violet rays. The work of Brooks ${ }^{8}$ in this connection should be particularly noted as well as that of Hill and Schmidt. ${ }^{9}$ The results obtained by the latter indicate that alexin is less resistant than sensitizer and that both are protected by proteins and certain amino acids such as tyrosin and phenylalanin. There

${ }^{r}$ Agulhon, L. H.: Compt. rend. Acad. de Sci., I53, 779. I9I I.

${ }^{2}$ Fisher, R, and McKinley, E. B.: J. Infect. Dis., 40, 399. 1927.

3 Lowenstein, E.: Zischr. exper. path. Therap., 15, 279. I9I4.

${ }^{4}$ Hortock, O., Schurmann, W., and Stiner, O.: Ztschr.f. Immunitätsforsch. u. exper. Therap., 2I, 643. I9I4.

5 Hleuer, G.: Centralbl. f. Buktcriol., Abt. I, 88, 3So. 1922.

${ }^{6}$ Huber, H.: Arch. f. IIyg., 54, 53. 1905.

7 Friedberger, L., and Scimone, V.: Ztschr. Immunitätsforsch. u. exper. Therap., 37, 34I. I923.

${ }^{8}$ Brooks, S. C.: J. General Plyysiol., 3, 160, 185. 1920.

"Hill, F. McC., and Schmidt, C. L. A.: J. Infect. Dis., 25, 335. 19r9. 
are various reports concerning the effect of exposure of animals to ultra-violet rays on their ability to produce antibodies. ${ }^{\mathrm{I}}$ On the whole, however, the results obtained are quite inconclusive. In view of the present furor over heliotherapy, investigations along this line might prove fruitful.

\section{THEORETICAL}

Finally, we may profitably ask, What is the mechanism by which ultra-violet rays of wave-lengths between 3,000 and I, $850 \mathrm{~A}$ exert such rapid action on bacteria? That the presence of free oxygen is essential for the photogermicidal action was disproved many years ago and has already been referred to. $\mathrm{Fair}^{2}$ believes that the formation of hydrogen peroxide cannot account for the effects produced. That the action of light-rays is directly on or in the cell is now generally admitted. However, it should be emphasized that in the presence of certain substances there is a marked protection. ${ }^{3}$ An alkaline reaction in the medium, inorganic salts and aromatic organic compounds are examples of this. Dreyer, ${ }^{4}$ on the other hand, claims to have sensitized bacteria by dyeing with erythrosin. The evidence that bacterial protoplasm and many proteins will absorb ultra-violet rays is indisputable, and the facts concerning lipoids are well known. I think we can safely assume a direct photochemical action on the bacterial cell. But this is hardly a satisfying explanation. What happens when bacterial protoplasm absorbs the rays? Both inorganic and organic colloids undergo changes as a result of exposure to ultra-violet light. These changes are accompanied, perhaps preceded by, change in the electrical charges on the particles. Bacteria, being charged particles, might be first affected in this way. Mrs. Clark ${ }^{5}$ has developed a photo-electrical theory postulating that electrons are given off from proteins under the action of ultra-violet rays and that these electrons attach themselves to other atoms or molecules with the resulting changes in both physical and chemical properties of all substances concerned. For instance, coagulations of proteins may follow exposure to light-rays. Many disinfecting agents are protein coagulants and may depend on this property for their germicidal action. Ultra-violet light would, on this basis, be an agent comparable with known chemical disinfectants. Fair states that the disinfection curve suggests that the action is similar to that of other germicidal agents.

The destruction of bacterial cells by means of ultra-violet light offers an attractive opportunity for the study of the mechanism of disinfection since the action takes place without the addition of extraneous chemical substances. Intensive research in this field is much needed and is likely to be productive of significant results.

${ }^{1}$ Pincussen, L.: loc. cit.; Potthoff, P., and Heuer, G.: Centralbl.f. Bakteriol., Abt. I, 88, 299 I922; Hansen, 'T.: Acta. med. Scandinav., 56, 629. I922.

${ }^{2}$ Fair, G. M.: J.A.W.W.A., 7, 325. 1920.

3 Houghton, E. M., and Davis, L.: Am. J. Pub. IIealth, 4, 224. I914.

${ }_{4}^{4}$ Ellis, C., and Wells, A. A.: The Chemical Action of Ultraviolet Rays, p. 244. Chemical Catalog Co., I925.

${ }_{5}$ Clark, J. H.: Physiol. Rev., 2, 277. I923.

${ }^{6}$ Fair, G. M.: loc. cit. 


\section{CHAPTER XXX}

\section{BACTERIA IN MILK}

\section{ROBERT S. BREED}

New York Agricultural Experiment Station, Geneva, N. Y.

Milk is a secretion produced from the material contained in blood serum or lymph by the glandular epithelial cells lining the alveoli of the mammary glands of mammals. From the moment it is secreted until its final transformation into other forms of matter, it may be, and usually is, acted upon by bacteria.

UDDER BACTERIA

\section{HISTORY OF THEIR DISCOVERY}

Roberts, ${ }^{\mathrm{I}}$ in $\mathrm{I} 874$, was apparently the first to demonstrate that, with aseptic precautions, small portions of sterile milk could be drawn from the udder. Because his work has been frequently overlooked, it may be well to quote his description (p. 469):

From a test-tube thus filled I charged ten empty sterilized tubes in the manner already described, and resealed their capillary orifices. Of these ten, three remained unchanged. When examined from three to six weeks afterwards the milk in them was perfectly sweet to the taste, its reaction was neutral or faintly acid, like that of fresh milk, there was no curdling, and no signs of organisms under the microscope. The other seven changed within ten or twelve days. Some of them curdled and others putrefied; all became highly acid, and, under the microscope, Bacteria, either staff-shaped or spherical, were found in them.

A few years later Lister ${ }^{2}$ succeeded in securing two sterile tubes out of ten tubes in a similar experiment carried out independently. Because of this and other work showing that normal blood, urine, and similar body fluids were sterile, it was generally believed for ten to fifteen years after this that milk as drawn from the udder is sterile. The organisms found in the tubes which did not remain sterile were regarded as air contaminations.

Schulz, ${ }^{3}$ under the direction of K. B. Lehmann in $189 \mathrm{I}$, showed that the first milk drawn contained large numbers of bacteria, while milk drawn midway and at the close of the milking process contained fewer and fewer bacteria. Moore 4 soon after also used the agar-plate technique to study the number of bacteria in milk as drawn from the udder. He reported that

freshly drawn fore milk contains a variable number of bacteria, varying in number from a few individuals to many thousand per cubic centimeter. These are distributed among several species. The last milk drawn at a regular milking contains, as compared with the fore milk, very few micro-organisms. It is the exception, however, to find a sample of milk that is free from micro-organisms unless it is taken during the latter part of the milking process from a single quarter of the udder.

${ }^{1}$ Roberts, W.: Phil. Tr. Roy. Soc., London, 164, 457. 1874.

${ }^{2}$ Lister, J.: Quart. J. Micr. Sc., 18, I77. I874; Tr. Path. Soc., London, 29, 425. I878.

${ }_{3}$ Schulz, L.: Arch.f. Hyg., 14, 260. 1892.

${ }_{4}^{4}$ Moore, V. A.: U.S. Dept. Agric. Ann. Rep. Bur. An. Ind., I2 and 13, 261. I897. 
Later, Moore and Ward, ${ }^{1}$ following up a suggestion previously made by Moore, cultured excised pieces of udder tissue and found that bacteria were present even in the secretory portion of the gland. Bolley, ${ }^{2}$ and Moore and Ward, as well as later investigators, have found that certain species of bacteria become localized in particular quarters of the udder, and that they are able to persist in this habitat for months or years.

\section{TYPES OF BACTERIA FOUND IN THE UDDER}

Even in $1875^{-7} 8$ Lister $^{3}$ found that the milk drawn with aseptic precautions might contain micrococci which produced an orange pigment. Others likewise found micrococci abundant in freshly drawn milk until in I9I3 Harding and Wilson ${ }^{4}$ reported after an extensive investigation: "No organisms producing spores and no motile forms were found. Seventy-five per cent of the forms were micrococci, but only two streptococci were found." Ninety-six per cent of all the types found, whether micrococci or non-spore-forming rods, were gram positive. None of the organisms fermented sugars with the formation of visible gas in fermentation tubes.

Their work and that of later investigators has established the fact that common types of micrococci occurring in normal udders are identical with the classic types known to students of human bacteriology as Micrococcus albus, $M$. aureus, and $M$. citreus, and they are so classified by Evans and by Hucker. ${ }^{5}$ Not all of the udder micrococci belong to these species, however.

\section{ACID-PROTEOLYTIC UDDER COCCI}

Certain types of micrococci and streptococci are found that are both acid and proteolytic in their action on milk. Gorini was the first to recognize these organisms, and he soon came to feel that they play an important rôle in ripening hard rennet cheeses of the Grana, Emmenthal, Edam, and Cheddar types. While Gorini ${ }^{6}$ found some of these to be gelatin liquefiers (Micrococcus casei acido-proteolyticus I), he also found some that do not liquefy gelatin (M. casei acido-proteolyticus $I I$ ). Freudenreich $^{7}$ early in his work found one of these types that was lemon yellow and named it Micrococcus casei amari, as it produced bitter cheese. Bitter cheese was obtained also by Hucker and Marquardt ${ }^{8}$ when they used cultures of acid-proteolytic cocci.

Burri and $\mathrm{Hohl}^{9}$ recognize that some of these cocci are very similar to the ordinary streptococcus of sour milk (Streptococcus lactis) except that they liquefy gelatin. They

${ }^{1}$ Moore, V. A., and Ward, A. R.: Cornell Agric. Exper. Sta. (Ithaca) Bull. I78. 1900.

${ }^{2}$ Bolley, H. L.: Centralbl. f. Bakteriol., Abt. II, I, 795. 1895.

3 Lister, J.: Tr. Roy. Soc., Edinburgh, 27, 319. I875.

${ }^{4}$ Harding, H. A., and Wilson, J. K.: New York Agric. Exper. Sta. (Geneva) Tech. Bull. 27. I913.

5 Evans, A. C.: J. Infect. Dis., I8, 437. I9ı6; Hucker, G. J.: New York Agric. Exper. Sta. (Geneva) Tech. Bulls. 99-103. 1923.

${ }^{6}$ Gorini, C.: Le Lait, 7, 36. 1927. (A review with bibliography.)

${ }^{7}$ von Freudenreich, E.: Landw. Jahrb. d. Schwciz, 8, 136. 1894.

${ }^{8}$ Hucker, G. J., and Marquardt, J. C.: New York Agric. Exper. Sta. (Geneva), Tech. Bull. II7. I926.

9 Burri, R., and Hohl, J.: Landw. Jahrb. d. Schweiz, 31, 315. I9I7. 
speak of them as the "liquefying Güntheri" bacteria. In I9I9 Orla-Jensen ${ }^{1}$ described them under the name of Streptococcus liquefaciens. Rogers and Dahlberg, ${ }^{2}$ in their study of the streptococci of the udder, found eleven cultures of these liquefying streptococci. There is much confusion regarding the identity of these acid-proteolytic types, and it seems probable that much of the conflict in ideas regarding them is due to this confusion.

\section{UDDER STREPTOCOCCI}

Investigators who have studied the udder flora are generally agreed that the ordinary sour-milk organism (Streptococcus lactis) does not occur in the udder. However, pathogenic streptococci do frequently occur, and these are sometimes confused with the saprophytic types that cause the normal souring of milk. On the basis of the work of Avery and Cullen, ${ }^{3}$ who pointed out that the common mastitis streptococcus gives a higher acidity in dextrose broth than the human, so-called Streptococcus haemolyticus (S. pyogenes), and of the work of Ayers and Rupp, ${ }^{4}$ who pointed out that the hemolytic bovine streptococcus was able to hydrolyze sodium hippurate while the human streptococcus did not, it is generally believed that the bovine and human pyogenic streptococci should be recognized as distinct species.

The name Streptococcus mastitidis Migula ${ }^{5}$ is perhaps most commonly applied to the organism causing udder infections. This binomial is sometimes incorrectly attributed to Guillebeau, ${ }^{6}$ who used the descriptive trinomials Streptococcus mastitis sporadicae and S. mastitis contagiosae for two organisms that differed only as indicated in the names, and that are today generally regarded as varieties of the same species. Migula changed the spelling in making the binomial which he applied to the first of Guillebeau's types.

Löhnis ${ }^{7}$ has stated that Guillebeau's organisms were gas-formers, but a careful reading of the original paper does not indicate any characters other than those of typical mastitis streptococci. On the other hand, Adametz $z^{8}$ in his I893 and I894 papers reports cultures of these organisms as causing gassy cheese and as producing gas bubbles in milk cultures. In his 1893 paper Adametz does not refer to Kitt's use of the trinomial $S$. agalactiae contagiosae 1893 , though he quotes this name in his I 894 paper. The binomial $S$. agalactiae appears to have been first used by Lehmann and Neumann ${ }^{9}$ where it is credited to Adametz. As this would appear to give the binomial $S$. agalactiae priority, some writers prefer this name. If, however, Guillebeau is regarded as having named two varieties of the same species, then the names $S$.

s Orla-Jensen, S.: "The Lactic Acid Bacteria." Mém. Acad. Roy. Sc. et Lett. Danemark, Secl. Sc. (8th ser.), 5, 8o. I919.

${ }^{2}$ Rogers, L. A., and Dahlberg, A. O.: J. Agric. Research, I, 491. I9I4.

3 Avery, O. T., and Cullen, E. G.: J. Exper. Med., 29, 215. 1919.

${ }_{4}^{4}$ Ayers, S. H., and Rupp, P.: J. Infect. Dis., 30, 388. 1922; Ayers, S. II., and Mudge, C. S.: ibid., 3I, 40. 1922 .

${ }^{5}$ Migula, W.: System der Baktericn, 2, p. 19. Jena, I9oo.

${ }^{6}$ Guillebeau, A.: Landw. Jahrb. d. Schweiz, 4, 27. 1892; Ccntralbl.f. Bakteriol., 12, Iо1. I892.

7 Löhnis, F.: Mandb. d. landw. Bakterivl., p. 198. Berlin. I9го.

${ }^{8}$ Adametz, L.: J.f. Landw., 42, 231. 1894. Milchseitung, 22, 235-40. I893.

9 Lehmann, K. 13., and Neumann, R. O.: Bakteriologische Diagnostik. (Ist ed.). Munich, I 896 . 
mastitis var. sporadicae and S. mastitis var. contagiosae Guillebeau have priority. Such confusion in names can only be settled by international agreement. If the binomial is attributed to Guillebeau, it should be spelled $S$. mastitis.

Brown, Frost, and Shaw, ${ }^{\mathrm{I}}$ following up the work of Smith and Brown, ${ }^{2}$ have recognized the streptococcus of septic sore throat as an organism that may become localized in the udder. They have characterized this organism by its capsule formation, failure to hydrolyze sodium hippurate, failure to ferment mannite, and fermentation of salicin, and have accepted the name S. epidemicus Davis as the name of the species. In their work, cows were found whose udders were infected with this capsulated streptococcus, but no direct evidence was secured that they had caused cases of human septic sore throat. Frost, Gumm, and Thomas ${ }^{3}$ have later recognized as many as eight species of streptococci found in the udder.

\section{INFECTION OF THE UDDER WITH "BACTERIUM ABORTUS"}

The success of Evans 4 in demonstrating the presence of Bang's bacillus of contagious abortion in milk as drawn from the cow's udder, and her recognition of the resemblance between this organism and the causal organism of Malta fever (Micrococcus melitensis Bruce) isolated from the goat's udder, has directed the attention of several investigators to the possibility that Brucella abortus may cause human disease. The resemblance between these two organisms is so close that no really satisfactory diagnostic methods have yet come into current use, although it is generally believed that the two organisms represent separate species. Carpenter, ${ }^{5}$ Huddleson, ${ }^{6}$ and others have recently found cases of undulant fever in man apparently caused by the use of cow's milk containing Bacterium abortus.

\section{LEUKOCYTES AND OTHER BODY CELLS IN MILK}

The presence of mastitis streptococci in the glandular tissue of the udder has been shown by Baker and Breed ${ }^{7}$ to cause the entrance of only partially elaborated blood serum into the alveoli without the usual change in $\mathrm{H}$-ion concentration from that of normal blood ( $\mathrm{pH} 7.0$ ) to that of normal milk ( $\mathrm{pH} \mathrm{6.5-6.6).} \mathrm{In} \mathrm{spite} \mathrm{of} \mathrm{the} \mathrm{fact} \mathrm{that}$ mastitis streptococci are lactose-fermenters, and must transform this sugar into acid in the individual alveoli, the $\mathrm{H}$-ion concentration of the milk, as drawn from infected quarters, approaches that of normal blood serum. Accompanying this entrance of a serous exudate into the milk, there is an increase in the migration of leukocytes through the walls of the alveoli so that mastitis milk usually contains excessive numbers (millions per cubic centimeter) of these blood cells.

It is probable that infections with other pathogenic bacteria such as Brucella abortus, or even purely physiological disturbances, may cause the same phenomenon.

r Brown, J. H., Frost, W. D., and Shaw, M.: J. Infect. Dis., 38, 38r. I926.

${ }^{2}$ Smith, T., and Brown, J. H.: J. Med. Research, 3 I, 455. I9r 5.

3 Frost, W. D., Gumm, M., and Thomas, R. C.: J. Bact., I3, 6r. I927.

${ }_{4}$ Evans, A. C.: J. Infect. Dis., 23, 354. I9r8; Hyg. Lab., U.S. Pub. Health Ser., Bull. I43. 1925.

${ }_{5}^{5}$ Moore, V. A., and Carpenter, C. M.: Cornell Vet., 16, 147. 1926; Carpenter, C. M.: J. Am. Vet. M. A., 70, 459. I927.

${ }^{6}$ Huddleson, I. F.: J.A.M.A., 86, 943. 1926.

7 Baker, J. C., and Breed, R. S.: New York Agric. Exper. Sta. (Geneva) Tech. Bull. So. 1920. 
Large numbers of these cells occur in milk that has every appearance of being normal (Breed). ${ }^{I}$ Other cellular débris, such as fat-laden gland cells (colostral corpuscles), gland-cell nuclei, and cells from the lining of the ducts, also occur in normal milk. This cellular material usually occurs in abundance only when conditions are abnormal, as at the beginning and end of lactation. There is no apparent relationship between this epithelial-cell débris and bacterial infections of the udder, and it seems to represent normal wastage of worn-out cells.

\section{BACTERIA ADDED TO MILK FROM EXTRANEOUS SOURCES}

As soon as milk is drawn from the udder, it becomes contaminated with bacteria from extraneous sources. These may be washed from the surface of pails, milkingmachine tubes, milker pails, strainers, surface coolers, milk cans, and the like, or they may drop into the milk with floating dust or particles of dirt from the flanks of the cow and the milker's clothing. Extensive studies ${ }^{2}$ have been made to determine the number of bacteria ordinarily found in each of these habitats in order to give practical dairymen and others an idea where to search for contaminations when the number of bacteria in market milk is in excess of the number which might normally be expected. Poorly cleaned, moist utensils that are allowed to stand at temperatures favorable for the development of bacteria have been found to be the most important source of these extraneous bacteria. Under American dairy conditions the two utensils that most frequently cause trouble are milk cans and the rubber tubing of milking machines. Routine laboratory methods used for the control of the sanitary quality of market milk have been standardized by the Laboratory Section of the American Public Health Association. ${ }^{3}$

\section{GERMICIDAI ACTION OF MILK}

It is generally agreed ${ }^{4}$ that fresh milk may contain certain substances derived from the blood that have a germicidal action on bacteria, so that the number of bacteria present in fresh milk usually decreases for a few hours after the milk is drawn. The effectiveness of this action varies with the milk of individual cows, and some claim that the germicidal action may be supplemented by a certain lag in the growth curve produced by the introduction of bacteria in all stages of vitality into a new environment. This delay in the increase in the number of bacteria is utilized in a practical way in the dairy industry where regulations permit dairymen to bring uncooled milk to milk plants if this is delivered within four hours after the milk is drawn.

\section{TYPES OF SAPROPHYTIC BACTERIA IN MILK}

THE BLUE-MILK ORGANISM

Historically, the first species of saprophytic bacteria to attract attention in milk was the one which causes a blue color in milk that has developed a certain acidity.

I Breed, R. S.: ibid., Bull. 380. 1914.

${ }^{2}$ Breed, R. S.: ibid., Circ. 93. 1927.

${ }^{3}$ Laboratory Section of the American Public Health Association: Standard Methods of Milk Analysis (5th ed.). I927.

${ }^{4}$ Jones, F. E., and Little, R. B.: J. Exper. Med., 45, 319. I927. 
This is the Vibrio syncyaneus Ehrenberg ${ }^{\mathrm{I}}$ or Vibrio cyanogenus Fuchs. ${ }^{2}$ Because the bright Berlin-blue chromogenesis is developed in a very characteristic way in milk in which other bacteria have produced acid, and not in sterile milk, it is still recognizable today. ${ }^{3}$

The earlier investigators described spores for this organism; but recent authors (Heim, ${ }^{4}$ Hammer $^{5}$ ) deny their presence and describe the organism as having four polar flagella. It is said to be variable in its liquefaction of gelatin, is gram negative, and resembles in a general way the organism commonly known as Psendomonas fluorescens Migula. Outbreaks of blue milk are uncommon, only two outbreaks being noted by Hammer as having occurred in America. While it is of much historical interest, it has little practical significance.

Other blue-milk organisms are Bacterium coelicolor Müller and the organism described by Mildenberg. ${ }^{6}$ Bergey ${ }^{7}$ has given the unfortunate name Pseudomonas cyanogena to the latter species, having retained the true blue-milk organism in the genus Bacillus under the name B. syncyaneus because of the common statement that it forms spores.

\section{NORMAL SOURING OF MILK}

Quite naturally the second species of milk bacteria to be recognized as distinct was Bacterium lactis Lister (I873). This is now generally placed in the genus Streplococcus, the synonomy of this much-named organism being as follows:

\section{Streptococcus lactis (Lister) Löhnis ${ }^{8}$}

Swnonyms: Bacterium lactis Lister, 1873 , p. 408, 1878, p. 184;? Streptococcus acidi lactici Grotenfelt, I889, p. 185;? Micrococcus acidi paralactici Nencki and Sieber, I889, p. 532; "Bacillus No. I9" Adametz, I889, p. 250; Bacillus acidi lactici Günther and Thierfelder, 1895, p. 195, and Esten, I896, p. 44 (Not "Milchsäurebacterium" Hueppe, I884, p. 340; Not Bacterinm acidi lactici Zopf, I883, p. 60, Grotenfelt, I889, p. I23, Kruse, 1896, p. 357; Not Bacillus acidi lactici Zopf, 1885, p. 87, and numerous others); Bacterium giintheri Lehmann and Neumann, 1896, p. 197; Bacterium lactis acidi Leichmann, I896, p. 778 (Not Bacterium lactis acidi Marpmann, I886, p. I 20); "Der ovaler coccus" Freudenreich, I895, p. 17I, identified as Leichmann's organism by Freudenreich, I899, p. 247, the latter being incorrectly called Bacillus acidi lactici. Correctly named Bacterium lactis acidi, 1902, p. 679, but called B. lactis acidi on pp. 680 ff.; Bacillus lacticus Kruse, I896, p. 356; Bacterium lacticus Chester, 1897, p. 88; Bacillus acidi lactici I Esten, in Conn, 1899, p. 52; Bacillus acidi paralactici Kozai, I899, p. 372; Bacterium lacticum Migula, I900, p. 405; Bacterium truncatum Migula, 1900, p. 407 (Bacillus No. 19, Adametz); Lactococcus

I Ehrenberg, C. G.: Ber. u. Verh. d. Königl. Preuss. Akad. d. Wissens., Berlin, 5, 197. I840.

${ }^{2}$ Fuchs, C. H.: Mag. f. gesam. Thierheilk., 7, I33. I841.

3 The even more striking blood-red chromogenesis of Serratia marcescens Bizio (I823) (Zaogatactina imetrofa Sette [I824], Monas prodigiosa Ehrenberg [I848]) caused this food spoilage bacterium to be recognized earlier than the blue-milk organism, while the packet formation of Sarcina lutea Goodsir (1842) made this a close third in order of recognition.

${ }^{4}$ Heim, L.: Lehrbuch der Bakteriologie (5th ed.). I9I8.

5 Hammer, B. W.: Iowa Agric. Expcr. Sta. (Ames) Research Bull. I5. I9I4.

${ }^{6}$ Mildenberg, H.: Centralbl. f. Bakteriol., Abt. II, 56, 309. I922.

7 Bergey, D. H.: Manual of Determinative Bacteriotogy (2d ed.). I925.

${ }^{8}$ Löhnis, F.: Centralbl. f. Bakteriol., Abt. II, 22, 553. Igog. 
lactis Beijerinck, 1901, p. 213; Streptococcus gïntheri Lehmann and Neumann, I904, p. 242; Bacillus lactis acidi Sewerin, I908, p. 8 (Not Bacillus lactis acidi Marpmann, I886, p. I 20, nor Leichmann, I896, p. 778); Bactcrium lcichmanni Wolff, I909, p. 57.

This long list of synonyms is due to the complicated but interesting history of this inportant milk organism. It is a history that is intimately interwoven with the development of bacteriology as a science.

Pasteur $^{1}$ in 1857 was the first to get a clear conception of the processes involved in the fermentation of sugars by micro-organisms with the production of lactic acid. $\mathrm{He}$ had previously brought about a popular revival of Schwann's theory that alcoholic fermentation was due to living organisms, the yeasts. Because of this, he began his lactic-fermentation studies confident that this fermentation also was caused by microorganisms. Soon he found it to be caused by minute, non-motile organisms, which, because they were non-motile, he regarded as yeasts rather than vibrios.

While he worked with milk fermentations in part, he did more work with sugar bouillons, and reports that in some cases the products of fermentation were $\mathrm{CO}_{2}, \mathrm{H}_{2}$, and butyric as well as lactic acid. Thereby he showed that he probably had an organism of the colon group, mixed cultures, or at least that he did not have the organism causing the normal souring of milk.

Hoffmann ${ }^{2}$ was the first to apply the term "bacteria" to these lactic ferments and to insist that these forms were more closely related to Pasteur's "vibrion butyrique" than to yeasts. The first worker whose descriptions show clearly that he was dealing with the sour-milk organism was Lister. ${ }^{3}$ His work in this field was done between $\mathbf{r} 872$ and 1878 . In the second of a series of papers that he prepared and the first published, he gives a detailed report of his experiments with the lactic fermentation of milk. In this he describes the microscopic appearance of the sour-milk organism and proposes the name Bacterium lactis for it. His attempts to cultivate the organism in Pasteur's solution, urine, and the like resulted in his mistaking contaminating organisms for the sour-milk bacterium. This being in a period before satisfactory pure-culture methods had been developed, and a time when contaminations were popularly regarded as pleomorphic stages in the life of the organism, Lister naturally fell into this error. He was too keen an observer to be misled long, and in his subsequent experiments succeeded in isolating pure cultures by means of the dilution technique. He did this by making a microscopical estimate of the number of bacteria in a small drop of sour milk, and then diluting it with enough sterile water to make the number of bacteria less than four per drop. Drops were then added to tubes of sterile milk whereupon one out of five tubes inoculated with the most highly diluted milk soured normally with a smooth curd while the remaining four showed no change, and no bacteria by microscopical examination.

Thus Lister secured a pure culture of his Bacterium lactis at the period when Schroeter ${ }^{4}$ was securing cultures of chromogenic bacteria by exposing pieces of cooked

x Pasteur, L.: Compt.rend.Acad.de sc., Paris, 45, 913. 1857; Ann. de chem.et phys. (3d ser.), 52, 404. 1858 .

${ }^{2}$ Hoffmann, H.: Bol. Ztschr., Nos. 15-20. r869.

${ }^{3}$ Lister J.: Quart. J. Mficr. Sc., 13, 380. 1873; ibid., 18, 177. 1878.

${ }^{4}$ Schroeter, J.: Bciträge z. Biol. d. Pflanzcn, I, Heft 2, Iog. 1872. 
potato to dust contaminations, and $\mathrm{Koch}^{\mathrm{r}}$ was laboriously preserving pure cultures of the anthrax organism by passage through mice.

Lister's final descriptions of the sour-milk organism are so complete and his studies of its habitat so accurate that it is evident that he was dealing with the organism that is most commonly found in sour milk. A sentence taken from one of his papers ${ }^{2}$ published in 1878 (p. 437) will show this: "The organism is a motionless bacterium, that is to say, exhibiting no movement except a slight jogging, occurring most commonly in pairs, but frequently in chains of three, four or more individuals, each segment being of somewhat rounded form, more or less oval, with the long diameter in the direction of the length of the chain, and often showing, on careful focusing, a line across the central part indicating transverse segmentation." He adds the following illuminating footnote: "In giving these as the characters of the Bacterium lactis, I do not wish to be understood as stating that this species can always be certainly recognized by its morphological features alone."

Fortunately, Lister did his work at ordinary room temperatures, and thereby avoided the pitfall into which Hueppe ${ }^{3}$ fell. Because the latter followed the normal practice in a laboratory dealing with pathogenic organisms and incubated his milk samples at body temperature, he came to regard a short rod bacterium which curdled the milk with extrusion of whey and with the formation of gas bubbles as the organism that caused the normal souring of milk. He utilized the new gelatin-plating technique of his time to isolate pure cultures and quite naturally isolated the common and well developed colonies. In gelatin stabs these organisms grew well along the length of the stab and also on the surface at the point of puncture.

Hueppe reported this as a spore-former, an observation in which he was undoubtedly mistaken, as will be shown later (p. 390). In all his papers he spoke of his organism as the Milchsäurebacterium, but Zopf ${ }^{4}$ and others soon gave it the name $B a$ cillus acidi lactici. For some years after this, confusion reigned because this new organism was regarded by many as the organism causing the normal souring of milk. There was much discussion over the problem as to whether there was more than one organism that could cause lactic acid fermentation. Hueppe's findings indicated that several types of bacteria were concerned in this fermentation, and his conclusions were soon confirmed by Marpmann, ${ }^{5}$ Grotenfelt, ${ }^{6}$ and others. It is difficult to determine what organisms were really studied by these investigators, expecially those studied by Marpmann, for his descriptions were indefinite and his cultures were not preserved and studied later by improved technique as were Hueppe's cultures. The names that Marpmann introduced (Bacillus lactis acidi, Bacterium lactis acidi, Micrococcus lactis acidi, and Sphacrococcus lactis acidi) were likewise destined to increase the confusion, and they have fallen into disuse.

The work by Grotenfelt in 1889 is important as it was done under Hueppe's super-

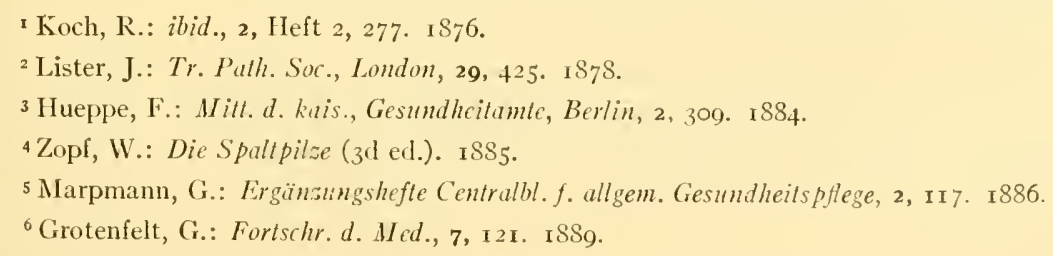


vision, and one of the cultures used was supplied by Hueppe. His observations proved useful in straightening out the confusion in that his descriptions show that he was dealing with organisms of the colon group, not the organism of normal sour milk. Grotenfelt named other cultures that he secured Bacterium acidi lactici and Streptococcus acidi lactici. The latter organism is important, as Lehmann and Neumann accept Grotenfelt's description of this streptococcus as the earliest description of the organism causing the normal souring of milk even as late as the 1927 edition of their Mannal. Inasmuch as the organism causing this souring occurs, as Lister states, in pairs, threes, and fours in milk, whereas Grotenfelt's streptococcus was found "in klumpig gewordener Milch aus Finland" and is described as occurring in milk in long chains, it seems probable that the latter was the mastitis streptococcus rather than Streptococcus lactis.

It was not until five years after Grotenfelt's work that Leichmann first published the statement that the organism that he found in milk souring normally was like Lister's, and not like Hueppe's organism. 'The following year Leichmann' named his organism Bacterium lactis acidi, the sour-milk bacterium, to distinguish it from Bacillus acidi lactici, the lactic acid bacillus. This unfortunate name, which was already preempted by Marpmann, has helped to produce a long list of synonyms.

Günther and Thierfelder, ${ }^{2}$ working independently, likewise found that the sourmilk bacterium did not have the characters ascribed to it by Hueppe, though it did show the characters mentioned by Lister. They continued the use of the name that had been given to Hueppe's organism, however, as did Esten ${ }^{3}$ also in the following year. Esten's work is of interest to us chiefly because it represented the first American work with this organism, and led to confusion in the naming of the sour-milk streptococcus in several papers and books from $\mathrm{H}$. W. Conn' $\mathrm{s}^{4}$ laboratory during the subsequent ten years.

The German bacteriologists were more fortunate in that Lehmann and Neumann ${ }^{5}$ in the first edition of their Manual in I 896 were quick to see the mistake that Günther and Thierfelder had made in regard to the name, and they proposed the new binomial Bacterium giintheri for the sour-milk organism that these authors had found. Independently, Kruse ${ }^{f}$ in Flügge's textbook proposed the binomial Bacillus lacticus. The name Bacterium gintheri came into general use in Germany and nearby countries during the next few years, whereas Leichmann's name came into more general use in America and in some other places. Lister's name was completely ignored during this period.

Adametz ${ }^{7}$ in Austria, following up the work of Duclaux on cheese ripening, had in I889 described a series of bacteria that he found in cheese, among them his "Bacillus

\footnotetext{
${ }^{I}$ Leichmann, G.: Centralbl. f. Bakteriol., Abt. II, 2, 777. I896.

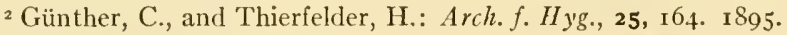

3 Esten, W. M.: Storrs Agric. Exper. Sta. (Conn.) Ann. Rep. for ISg6, p. 44. 1897.

${ }_{4}$ Conn, H. W.: ibid. for 1899 , p. 52. I 900.

${ }_{5}^{5}$ Lehmann, K. B., and Neumann, R. O.: Baktcriologische Diagnostik (2 vols.; ist edl.). 1896.

${ }^{6}$ Kruse, W.: in Flügge's Die Mikroörganismen (2 vols.). Leipzig, 1896.

7 Adametz, L.: Landw. Jahrb., I8 227. I889.
} 
No. 19 " which is described so clearly and his gelatin-stab culture figured so accurately that there can be little question but that he also had the sour-milk streptococcus. Freudenreich, ${ }^{\mathrm{I}}$ working in the same field, early found an organism for which he used the descriptive name of "der ovaler coccus." In I 899 and I 902 he recognized this organism as identical with Leichmann's Bacterium lactis acidi, though he confused the names badly.

Certain things about the souring of milk still puzzled bacteriologists, for, as Leichmann had shown, the sour-milk organism produced dextro-rotary lactic acid while the acid in sour milk was generally reported as inactive. Kozai ${ }^{2}$ was the first to show the explanation of this phenomenon when he found that the acid in milk soured at room temperatures was of the dextro-rotary type while that found in milk soured at body temperature was of the inactive type. He likewise showed that Leichmann's organism grew better at room temperature, while Hueppe's organism grew better at body temperature. Not satisfied with the abundance of names already proposed for the sour-milk organism, he changed its name to Bacillus acidi paralactici.

Because this name is similar to Micrococcus acidi paralactici Nencki and Sieber ${ }^{3}$ given to a very similar organism, which the latter authors state is possibly identical with Grotenfelt's Streptococcus acidi lactici, Nencki and Sieber's name is sometimes given in the list of synonyms of Streptococcus lactis. While it is described as a streptococcus occurring in twos, threes, and fours that produces dextro-rotary lactic acid, their description of its habitat in guinea-pig lesions, presence of capsules, pathogenicity for guinea pigs, and other characters place it in the group of indefinitely described streptococci doubtfully identified as Streptococcus lactis.

The generic relationships of the organism not yet being clear, the period following r 896 saw several changes in the names already proposed. Thus Chester in I 897 changed Kruse's name to Bacterium lacticus, and Migula ${ }^{5}$ in Igoo used the more correct form Bacterium lacticum. The latter likewise gave the name Bacterium truncatum to Adametz's "Bacillus No. 19." This binomial should be regarded as a synonym of Streptococcus lactis.

Beijerinck ${ }^{6}$ meanwhile introduced the name Lactococcus lactis as a more accurately descriptive name, apparently without any reference to the fact that lactis was the specific name used by Lister. If the sour-milk streptococcus is ever placed in a genus separate from the pyogenic streptococci, it should be noted that the generic term Lactococcus has priority.

$\mathrm{Kruse}^{7}$ in 1903 , with a clearer insight into the matter, caught the resemblance between the sour-milk organism and Streptococcus lanceolatus (pneumococcus), and other related streptococci and changed the name he had previously given to Strepto-

${ }^{1}$ von Freudenreich, E.: Centralbl. f. Bakteriol., Abt. II, 1, I68. I895; 5, 241. I899; 8, 674. 1902.

2 Kozai, Y.: Ztschr.f. II yg. w. Infektionskrankh., зг, 337. г\$99.

3 Nencki, M., and Sieber, N.: Monatschr. f. Chem., I o, 532. I $\$ 89$.

${ }^{4}$ Chester, F. D.: Delaware Agric. Exper. Sta. (Newurk) gth Ann. Rep., Pp. 53-145. IS97.

${ }^{5}$ Migula, W.: op. cit., 2, 405. Jena, I900.

${ }^{6}$ Beijerinck, M. W.: Arch. néerl. d. sc. exact. et nat., IIarlem (2d ser.), 7, 212. г9ог.

${ }^{7}$ Kruse, W.: Centralbl. f. Bakteriol., Abt. I, 34, 737. 1903. 
coccus lacticus. His conclusions were soon well supported by the investigations of Hölling ${ }^{1}$ and Heinemann. ${ }^{2}$ Lehmann and Neumann ${ }^{3}$ accepted this conclusion but, because the name that they had previously given had priority, changed their name to Streptococcus güntheri, and later, as already indicated, decided to accept Grotenfelt's still earlier Streptococcus acidi lactici. Sewerin ${ }^{4}$ a few years later carelessly used the name Bacillus lactis acidi, which Leichmann had proposed for long-rod bacilli, for this organism, thus adding to the synonomy.

All of this confusion has been cleared up by the suggestion made by Löhnis ${ }^{5}$ in I 909 that we should return to Lister's original specific name lactis but place the organism in the genus Streptococcus as Kruse had indicated. This practical suggestion met with opposition from Wolff, ${ }^{6}$ who had another name to propose, Bacterium leichmami, and $\mathrm{Kruse}^{7}$ and some others who feel that Lister's original descriptions are too indefinite to be accepted. Nevertheless, the majority of the dairy bacteriologists of Europe and America are already using this binomial; and there is so much that can be said in favor of using this simple and expressive name that it is hoped that it will receive general international approval and use.

A few bacteriologists-e.g., Heim ${ }^{8}$ - not familiar with recommended forms of nomenclatural usage, have written the binomial Streptococcus lactis Lister instead of Löhnis. This is incorrect as it indicates that Lister used this particular binomial, whereas even the thought of the generic name Streptococcus quite certainly never entered his mind. The term S. lactis is also occasionally incorrectly ascribed to Heim.

It has been difficult to determine the natural habitat of this streptococcus. No better summary of the matter can be given than to quote Lister's original remark that reads as follows: "But though the ferment which occasions the souring of milk is present in milk obtained from any dairy, it appears to be by no means common in the world in general." As investigations have been made, it has been discovered that streptococci found in the cow's and in the human mouth, and in cow's and human feces (Enterococcus), all show characters almost identical with those of $S$. lactis so that some have held these habitats to be the original sources of this organism, while others have held that the organisms found in these habitats are closely related to, but not identical with, $S$. lactis. The matter of these relationships is well summarized in recent papers by Ayers, Johnson, and Mudge. ${ }^{9}$ The organisms as they appear in milk show certain definite varieties, i.e., slimy, heat resistant, slow reducing, slow coagulating, and caramel or burnt flavor. Some would regard these as distinct species. They are well described and characterized by Hammer and Baker. ${ }^{\text {To }}$

'Hölling, A.: Inaug. Diss. Bonn, I904; Abstract in Centralbl.f. Bakleriol., Abt. I, Ref., 36, 659. 1905.

${ }^{2}$ Heinemann, P. G.: J. Infect. Dis., 3, 173. 1906.

3 Lehmann, K. B., and Neumann, R. O.: op. cit. (2 vols.; 4th ed.), Munich, 1904.

4 Sewerin, S. A.: Centralbl. f. Bakteriol., Abt. II, 22, 3. I908.

${ }^{5}$ Löhnis, F.: ioid., p. 553. 1909 .

${ }^{6}$ Wolfi, A.: ibid., 24, 55. 1909.

${ }^{7}$ Kruse, W.: Allgemeine Mikrobiologie. Leipzig, 19ro.

${ }^{8}$ Heim, L.: Ztschr. f. IIyg. u. Infektionskrankh., 101, 104. 1923.

9 Ayers, S. II., Johnson, W. T., and Mudge, C.: J. Infict. Dis., 34, 29, 49. 1924.

to Hammer, B. W., and Baker, M. P.: Iow'a Agric. Exper. Sta. (Ames) Research Bull. 99. 1926. 
A streptococcus producing $\mathrm{CO}_{2}$ from dextrose also occurs in milk which Ayers, Johnson, and Mudge report as being found most abundantly in milk of low acidity. This organism in milk is known as $S$. kefir Migula, and may be identical with $S$. paracitrovorus Hammer.

It is of interest that an American bacteriologist, H. W. Conn, ${ }^{\mathrm{I}}$ was the first to prepare lactose-litmus solid media and that he reported that certain of the colonies of $S$. lactis were characteristic on media of this type. He found that the colonies on lactose litmus gelatin were under the surface, and dense red. When examined under the compound microscope certain, though not all, of these colonies presented a burrlike appearance. Heim ${ }^{2}$ has recently pointed out the fact that the agar colonies are also characteristic. Esten, ${ }^{3}$ an associate worker with $\mathrm{H}$. W. Conn, was the first to point out that the reduction of litmus in milk before curdling, with return of color beginning at the top, was an excellent diagnostic character. Others (Hastings ${ }^{4}$; Sherman and Albus ${ }^{5}$; Ayers, Johnson, and Mudge; and Heim) have also found this character useful in separating this organism from pyogenic streptococci. Sherman and Albus feel that reduction of stains, failure to ferment sucrose, and ability of the organism to grow at a temperature as low as $10^{\circ} \mathrm{C}$. are perhaps the most important differential characters, with a tendency to short-chain formation and smooth curd in milk without extrusion of whey as important secondary characters.

\section{ORGANISMS OF THE COLON GROUP FOUND IN MILK}

While the organisms causing the normal souring of milk usually find their environment so favorable that they are almost certain to overgrow all other organisms in milk as ordinarily kept, gas-forming organisms of the colon group frequently become predominant when temperatures are higher than normal. Thus in cheese-making during hot summer weather, floating curds frequently appear. These shrink with a rapid extrusion of the whey.

Organisms of this type were early recognized by Hueppe as already explained (p. 385), and Escherich soon after isolated a closely related organism, Bakterium lactis aerogenes, from the feces of breast-fed infants. These are known today under the names Escherichia acidi lactici (Zopf) Bergey and Aerobacter aerogenes (Kruse) Beijerinck. Because of confusion regarding their identity and the close relationship between the two, they are described together.

\section{Escherichia acidi lactici (Zopf) Bergey, I923, p. I99}

Synonyus: "Milchsäurebacterium" Hueppe, I884, p. 340; Bacillus acidi lactici Zopf, I 885, p. 87 (Not Bacterium acidi lactici Zopf, I884, p. 60); Bacillus acidi lactici Crookshank, I886, p. I69; Bacillus acidi lactici Flügge, I886, p. 293; Bacillus acidi lactici Fraenkel, I887, p. I76; Bacillus acidi lactici I \& II Grotenfelt, I889, p. I 22 (Possibly also Bactcrium acidi lac-

I Conn, H. W.: Practical Dairy Bacleriology. New York, Igo8; Conn, H. W., Esten, W. M., and Stocking, W. A.: Storrs Agric. Exper. Sta. (Conn.) Ann. Rep. for I906, p. 91 . 1907; and several earlier papers.

${ }^{2}$ Heim, L.: Zischr. f. Hyg. u. Infektionskrankh., 101, 104. 1923.

3 Esten, W. M.: Storrs Agric. Exper. Sta. (Conn.) Bull. 59. 1909.

${ }^{4}$ Hastings, E. G.: in Marshall's Microbiology (ist ed.). Philadelphia, I9I I.

s.Sherman, J. M., and Albus, W. R.: J. Bacl., 3, I53. 1918. 
tici I \& II Grotenfelt, I 889, p. I 23); Bacterium acidi lactici Migula, I 895, p. 25 (Not Bacterium acidi lactici Kruse, I896, p. 357, the "Bacterium B" of Peters, I889, p. 422); Possibly also Bacterium grotenfcldtii Migula, I900, p. 408, a synonym of Bactcrium acidi lactici I Grotenfelt; ? Bacillus lacticus Pasteur according to Macé, I913, II, p. $45^{2}$ (Not Bacillus lacticus Kruse, I896, p. 356); Bacterium duodenale Ford, I903, p. I7 (according to Perkins, I925, p. 247); Encapsulatus acidi lactici Castellani and Chalmers, I919, p. 934; (Encapsulata) Bacillus duodenale Perkins, 1925. p. 247.

\section{Acrobacter acrogenes (Kruse) Beijerinck, ${ }^{\mathrm{I}}$ I900, p. I}

Synonyms: Baktcrium lactis acrogenes Escherich, 1885 , p. 520 ; Bactcrium lactis Baginsky, I888, p. 437; Bacterium aceticum Baginsky, ı888, p. 462; Bacillus lactantium Trevisan, I889, p. I5; Bacillus lactis aerogenes Sternberg, I 893, p. 447; Bacillus aerogencs Kruse, I896, p. 340 (Not Bacillus aerogenes Miller, I886, p. I I9); Bactcrium aerogenes Chester, I897, p. 78, and Bacterium aerogenes Migula, I900, p. 396 (Not Bacterium aerogenes Miller, I886, p. I19); Encapsulatus lactis aerogenes Castellani and Chalmers, I919, p. 934; (Encapsulata) Bacillus aerogenes Perkins, 1925, p. 254.

The history of these two micro-organisms has been interwoven from the time when Escherich ${ }^{2}$ stated in his description of Bakterinm lactis aerogenes that this organism showed only minor differences from Hueppe's previously described Milchsäurebacterium. Because of this suggestion, many bacteriologists have regarded the two organisms as identical, the two being given as synonyms even in the latest edition of Lehmann and Neumann's well-known Manual, and in other recent publications by European bacteriologists.

Both Escherich and Hueppe report their organisms as non-motile, while Bacterium coli commune Escherich was motile. In the original description both of the nonmotile organisms were described as forming spores; but there is reason for believing this was a mistake in both cases. During the same period Gaffky described spores for the typhoid organism, spores were described for the blue-milk organism, and other rod-shaped bacteria were generally believed to form spores if grown on suitable media. Migula, using a quince-slime medium, thought he found spores in so many bacteria that he regarded spore formation as such a difficult and uncertain character to determine that he used motility rather than spore formation to separate the genus $B a$ cillus from Baclerium.

Both Escherich and Gaffky later admitted that they were mistaken. While no record has been found that Hueppe did the same, it is worth noting that Grotenfelt, ${ }^{3}$ a student of Hueppe's who used one of Hueppe's cultures, makes no mention of spores, and all later workers who studied probably authentic cultures supplied by Kral or from Koch's laboratory report Hueppe's organism as a non-spore-former. Lehmann and Neumann ${ }^{4}$ state that Migula's deception arose from the fact that spores were already present in the quince slime when it was prepared. In other cases it may be that these reports were caused by contaminated cultures, unstained vacuoles, the presence of swollen tips of pleomorphic forms combined with the uncritical practice of the pe-

${ }^{1}$ Beijerinck, MI. W.: op. cit., 4, I-I8; Centralbl. f. Bakteriol., Abt. II, 6, I93. I900.

${ }^{2}$ Escherich, T.: Fortschr. $d$. Med., 3, 515. 1885; Darmbakterien des Süuglings. Stuttgart, I886.

${ }^{3}$ Grotenfelt, G.: Fortschr. d. Med., 7, I2 1. 1889.

${ }_{4}^{4}$ Lehmann, K. B., and Neumann, R. O.: op. cit. (5th ed.), P. I55. Munich, I9I2. 
riod that frequently did not test the heat resistance of the supposed spores. The common expectation that spores were produced in rod-shaped bacteria under unfavorable conditions may have helped in increasing the illusions. Recent authors who are familiar with this history have generally regarded both Hueppe's and Escherich's organisms as non-spore-formers.

In other respects these two organisms showed the normal characters of bacteria of the colon group in that they fermented dextrose and lactose with the formation of acid and gas, the latter a mixture of $\mathrm{CO}_{2}$ and $\mathrm{H}_{2}$. Unfortunately, no use was made of sucrose as a differential medium. The size of the organisms and the type of growth described as occurring on gelatin media were typical of these two organisms as we know them today. Escherich reports the growth of Bact. lactis aerogenes on potato as frothy, suggesting the fermentation of starch with the production of acid and gas.

During the period between the time when the organisms were first described and I902, the two organisms were sometimes regarded as identical and sometimes as distinct. The name Bacillus acidi lactici apparently became current in Koch's laboratory for Hueppe's unnamed organism, as at least four investigators (Zopf, ${ }^{1}$ Crookshank, ${ }^{2}$ Flügge ${ }^{3}$ Fraenkel $^{4}$ ), in the order named, apparently independently and almost simultaneously published this name. All of these authors were more or less closely associated with this laboratory, and also published other names (Bacillus indicus; see Breed and Breed $)^{5}$ current in this laboratory at the same period. In Zopf's case he notes that the name he proposes is not to be confused with the name Bacterium acidi lactici that he had previously published for another organism. ${ }^{6}$

Grotenfelt used four closely related cultures, regarding two of them as distinct from Hueppe's organism. For the two related cultures he used the names Bacterium acidi lactici I \& II. Migula, ${ }^{7}$ overlooking Zopf's and Grotenfelt's previous use of the term Bacterium acidi lactici, proposed this name for Heuppe's organism, while Kruse ${ }^{8}$ used this name for still another presumably different organism described by Peters. If Grotenfelt's Bacterium acidi lactici cultures were identical with Hueppe's organism, as seems probable, then Bacterium grotenfeldtii Migula likewise becomes a synonym.

Macé, ${ }^{9}$ with a very natural desire to give Pasteur credit for his work, has proposed the name Bacillus lacticus for Pasteur's Levure lactique, regarding this as identical with Hueppe's rather than Lister's organism.

The early synonomy given for Bacterium lactis aerogenes Escherich needs some comment to clarify it. Baginsky ${ }^{\text {I0 }}$ used the binomial Bacterium lactis for this organism in $\mathrm{I} 888$, but repudiated the name in favor of the binomial Bacterium aceticum in the

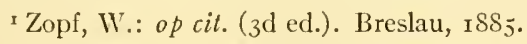

${ }^{2}$ Crookshank, E. M.: Practical Bacteriology (Ist ed.). London, 1886.

3 Flügge, C.: Die Microörganismen (2d ed.). Leipzig, I886.

${ }^{4}$ Fraenkel, C.: Bakterienkunde (ist ed.). Berlin, I887.

${ }^{5}$ Breed, R. S., and Breed, M. E.: J. Bact., xi, 76. 1926.

${ }^{6}$ Zopf, W.: op. cit. (2d ed.). Breslau, I884.

7 Migula, W.: in Engler and Prantl's Natürlichen Pfanzenfamilien, Teil I, Abt. I $a, 25.1895$.

${ }^{8} \mathrm{Kruse}, \mathrm{W} .:$ in Flügge's Die Mikroörganismcn (2 vols.). Leipzig, I896.

${ }^{9}$ Macé, E.: Traité pratique de bactériologic (2 vols.). Paris, I9I3.

io Baginsky, A.: Ztschr.f. phys. Chem., I2, 434. I8ss. 
same article on the ground that the specific name lactis had already been used. Trevisan $^{1}$ the following year proposed the binomial Bacterium lactantium in an obscure pamphlet that has been generally overlooked. By a strict application of the rules of nomenclature, one or the other of Baginsky's names or Trevisan's name has priority depending on the genus in which the organism is placed.

Sternberg ${ }^{2}$ appears to have been the first to use the term Bacillus lactis aerogenes, while Kruse appears to have been the first to shorten this name so as to use the specific name aerogenes for Escherich's rather than Miller's ${ }^{3}$ organism. Usage has so far established this as the specific name for Escherich's organism that it is hoped that it will continue to be used by international agreement. Chester ${ }^{4}$ rather than Migula, 5 as is commonly stated, appears to have been the first to use the binomial Bacterium aerogenes.

Until Durham's ${ }^{6}$ report in I900, very little progress was made in describing additional characters of either Heuppe's or Escherich's organisms that would show their relationship to each other. Durham, working with a culture of $B$. lactis aerogenes labeled "Kosseck" and obtained from Escherich's laboratory, found that this culture fermented sucrose and starch with the production of acid and gas, but did not ferment inulin. This and his related cultures were Voges-Proskauer (methyl-carbinol) positive. In 1904 Perkins, ${ }^{7}$ working with cultures of B. acidi lactici and B. aerogenes obtained from Kral and presumably authentic, reported the first to be a sucrose-nonfermenter, whereas the second fermented sucrose with the formation of acid and gas.

MacConkey ${ }^{8}$ in I905 used Durham's $B$. lactis aerogenes culture and a culture of $B$. acidi lactici from Kral and reports that the first ferments sucrose and is Voges-Proskauer positive, while the latter is a non-sucrose-fermenter and Voges-Proskauer negative. Morgan ${ }^{9}$ also reported that these two organisms differed in regard to sucrose fermentation; and likewise found that neither fermented dulcitol.

These early papers have been followed by a great mass of investigational work on the classification of the colon-typhoid group, the greater part of which has been done in America. It is impossible to review these papers in any detail; the articles by Winslow, Kligler, and Rothberg ${ }^{\mathrm{I0}}$ and Levine ${ }^{\mathrm{II}}$ give extensive kibliographies. While some of the findings are confusing and apparently contradictory, the final result has been to support the idea that the two organisms under discussion are distinct. Recent definitions (Perkins ${ }^{12}$ and Weldin'3) by specialists in the group hold closely to the orig-

\footnotetext{
I Trevisan, V.: I gen. e le spec. delle Batteriacae. Milan, 1889.

${ }^{2}$ Sternberg, G. M.: Manual of Bacleriology. New York, 1893 .

3 Miller: Deutsche med. Wchnschr., г 2, г1 7. I886.

${ }^{4}$ Chester, F. D.: Delaware Agric. Exper. Sta. (Newark) glh Ann. Rep., 53-I 45. 1897.

5 Migula, IV.: op. cil. (2 vols.). Jena, I897-1900.

${ }^{6}$ Durham, H. E.: J. Exper. Med., 5, 353. igoo.

7 Perkins, R. G.: J. Infect. Dis., I, 241. 1904.

${ }^{8}$ MacConkey, A.: J. Hyg., 5, 333. 1905.

9 Morgan, II. de R.: Brit. M. J., I, I257. 1905.

so Winslow, C.-E. A., Kligler, I. J., and Rothberg, W': J. Bacl., 4, 429. 1919.

${ }^{13}$ Levine, M.: Iow State College (Ames) Engin. Exper. Sta. Bull. 6z. 1921.

${ }^{12}$ Perkins, R. G.: J. Infect. Dis., 37, 232. 1925.

${ }_{13}$ Weldin, J. C.: lowa State College (Ames) J. Sir, r, 121. 1927.
} 
inal conceptions of the two species as emended by the series of papers mentioned above.

The former author would, however, do away with the name $B$. acidi lactici and substitute the little-known Bacterium duodcnale Ford $^{\mathrm{I}}$ which he has included, with $B$. acrogenes, under Encapsulata with the new name Bacillus duodcnale. In placing them among the Encapsulata, he follows Castellani and Chalmers, ${ }^{2}$ who include both species in their genus Encapsulatus. Perkins prefers Ford's specific name on the ground that the original description was more definite than that of $B$. acidi lactici. $\mathrm{He}$ feels that later investigators should not emend the original indefinite descriptions even where, as in this case, the principal emendations have been based on the study of apparently authentic cultures. Nevertheless, he uses the studies that he made in I904 to support the view that the original cultures of these organisms were capsulated. Moreover, the emendation of species descriptions is permitted under all internationally recognized codes of nomenclature where it is carried out with regard to certain wellestablished principles.

Weldin places the two colon organisms in question in separate genera, following Bergey ${ }^{3}$ in placing Hueppe's organism in the genus Escherichia, and Beijerinck ${ }^{+}$in making Escherich's organism the type species of genus Aerobacter, the latter on the ground that this name has priority over Encapsulatus.

Few systematic studies of the colon group having been made in Continental Europe, it is not surprising to find many of their bacteriologists conservatively taking the position that the two species are identical. Burri and Düggeli, ${ }^{5}$ however, note the difference in sucrose fermentation as well as other less important differences.

Pfeiffer's Kapselbacillus [Klebsiella capsulatus (Sternberg) Bergey] is regarded as identical with Aerobacter aerogenes by Castellani and Chalmers, and by Weldin; and the names that have been applied to this bacterium are included in their lists of synonyms of Aerobacter aerogenes.

Analyses of the gases produced, made by more accurate methods than were available to Escherich, have shown that Escherichia acidi lactici produces approximately equal quantities of carbon dioxide and hydrogen, while Aerobacter aerogenes produces at least twice as much carbon dioxide as hydrogen.

The former has been established as a methyl-red positive, methyl-carbinol negative organism that produces indol readily. It does not form such an abundant slimy growth as the latter, and capsule formation is questioned by some, though it has been noted by Perkins, and by Castellani and Chalmers.

The latter is methyl-red negative, methyl-carbinol positive, and indol is rarely formed. It forms an abundant, slimy growth, and there is general agreement that the organisms are capsulated. It ferments many of the more complex carbohydrates and alcohols (except dulcitol), usually even attacking starch. It does not liquefy gelatin, however, as does the closely related Aerobacter cioacae (Jordan) Bergey.

' Ford, W. W.: Studies from Roy. Victoria Hosp., Montrcal, 1, No. 5. Pp. 95. 1903.

${ }^{2}$ Castellani, A., and Chalmers, A. J.: Manual of Tropical Medicine (3d ed.). London, I9I9. Ann. de l'Inst. Pasteur, 34, 600. I9zo.

3 Bergey, D. H.: op. cit. (Ist ed.)., Baltimore, I923.

${ }_{4}^{4}$ Beijerinck, M. W.: op. cit., 4, I. I900; Centralbl.f. Bakteriol., Abt. II, 6, I93. I900.

5 Burri, R., and Düggeli, M.: Centralbl. f. Bakteriol., Abt. I, Orig., 49, I45. 1909. 
Further investigations have shown that Aerobacter aerogenes is not commonly present in human feces, though common in the upper intestine of man. Two early habitat studies of colon organisms isolated from milk were made in England. Houston $^{\mathrm{I}}$ determined the characters of 343 cultures of the colon group isolated from milk and found that $22 \mathrm{I}$ non-gelatin-liquefying cultures fermented sucrose with the formation of acid and gas. This result indicates that Aerobacter aerogenes was at least abundant, though the characters determined were not sufficient to establish the identity of the organism. Orr ${ }^{2}$ used the Voges-Proskauer test on 850 cultures of colon organisms from milk and found 333 of them to be positive, again indicating that Aerobacter aerogenes was frequently present.

Rogers, Clark, and Davis ${ }^{3}$ have studied a series of colon cultures from milk by more accurate technique and report that the carbon dioxide and hydrogen ratio that occurred with the greatest frequency was approximately $\mathrm{I}: \mathrm{x}$. The amount of gas produced by these cultures was uniformly less than that produced by high-ratio cultures. The amount of acid produced was frequently masked by secondary alkaline fermentations in which the acid was partially or completely neutralized.

\section{LACTOBACILLI IN MILK}

The long-rod lactic acid bacteria also form an important group in their relationship to milk. They have even more important relationships to fermented milk products and will therefore not be discussed at this place. The most important species are Lactobacillus bulgaricus (Grigoroft) Holland and the closely related, if not identical, L. helveticum (Orla-Jensen) Bergey (Bacillus casei $\epsilon$ Freudenreich). Lactobacillus acidophilus (Moro) Holland is closely related to these species, but is regarded as distinct by those who are most competent to judge the matter.

${ }^{1}$ Houston, A. C.: The Bacteriological Examination of Milk, London Co. Council, No. 933. 1905.

${ }^{2}$ Orr, T.: Investigation as to the Contamination of Milk. Co. Bor. Councils of Bradford, Hull, Leeds, elc. (Beverly). I908.

${ }^{3}$ Rogers, L. A., Clark, W. M., and Davis, B. J.: J. Infect. Dis., I4, 4I I. I9I4. 


\section{CHAPTER XXXI}

\section{BACTERIA IN DAIRY PRODUCTS}

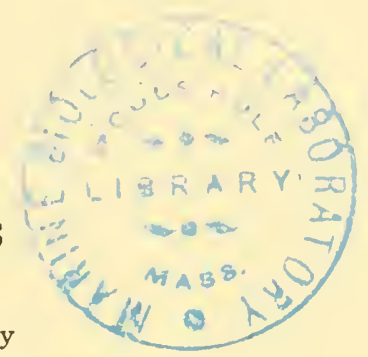

Research Laboratories, Bureau of Dairy Industry

United States Department of Agriculture, Washington, D.C.

FERMENTED MILKS

Primitive people in different parts of the world have developed various types of fermented milks which have become an important part of their diet. The simplest fermented milk is a result of the mild lactic fermentation produced by Streptococcus lactis either spontaneousily or by inoculation of pure cultures into pasteurized milk. Nearly all of the ordinary commercial buttermilk is made by the latter method. In selecting a culture for making buttermilk consideration should be given to the fact that there are decided variations in the flavor and consistency given to milk as well as in the rapidity of coagulation by different strains of St. lactis. An extreme case of variation is found in the "taette milk," a stringy or ropy milk of the Scandinavian countries, produced by a variety of St.lactis which has been described under the name St. taette.

In Bulgaria, Turkey, and other near-eastern countries a fermented milk known as yoghourt is used very generally. This milk undergoes a lactic fermentation due, primarily, to the acid-forming bacilli of which Lactobacillus bulgaricus is the type. Bacteria of this genus in the fermentation of lactose produce lactic acid almost exclusively while the streptococci form a number of acids in addition to lactic acid. The lactobacilli ferment lactose rapidly, especially at temperatures of $35^{\circ}-45^{\circ} \mathrm{C}$., and carry the fermentation to a $\mathrm{pH}$ of 3.8 . Some strains produce a titrable acidity as high as 2.5 per cent lactic acid. The tendency of the lactobacillus group to grow in chains gives the milk a slightly slimy texture which disappears upon agitation. Pure cultures of Lact. bulgaricus are used to prepare a sour skimmed milk which is sold as "Bulgarian buttermilk" and under various trade names. The bulgaricus culture is frequently used in combination with St. lactis to give a product with a milder acid and a less viscous texture.

The sour milk known as matzoon in Armenia and as leben in Egypt are evidently similar to the yoghourt of Bulgaria and Turkey, although leben may contain a lactosefermenting yeast which gives a mild alcoholic fermentation.

In Russia two fermented milks are made by using a combination of a lactobacillus, a streptococcus, and a lactose-fermenting yeast. Kumiss is usually made from mare's milk and is fermented in open vessels or skin bottles. Fresh milk is inoculated from kumiss left in the bottles or by adding kumiss to fresh milk. In addition to the acid fermentation, a small amount of alcohol is produced together with sufficient carbon dioxide to make the milk slightly effervescent. Kefir differs from kumiss in 
that the milk is inoculated with a curious growth known as "kefir grains." These grains resemble miniature cauliflowers and when broken up and put into milk under favorable conditions increase in size, at the same time inoculating the milk with streptococci, lactobacilli, and yeasts. The grains are masses of tangled filaments of these organisms. The fermentation is similar to that of kumiss but is usually produced in closed bottles so that the gas is held and the finished product is more effervescent. A similar product can be made by adding about I per cent of cane sugar to sour milk, inoculating with a bread yeast and fermenting several days in sealed bottles at $20^{\circ}-25^{\circ} \mathrm{C}$.

Great stress has been laid on the therapeutic value of fermented milks, particularly those soured by Lact. bulgaricus, to which the name "bacillus of long life" has been applied. More recent work has shown that such therapeutic efficacy has been over-estimated, due to a misconception of the ability of bulgaricus to establish and maintain itself in the intestines.

Lact. acidophilus is now used extensively in preparing soured milk for therapeutic purposes. Because this organism is much less active than bulgaricus, special precautions are necessary to prepare a milk free from contamination.

\section{CONDENSED MILK}

The commercial term "condensed milk" is applied to milk preserved by the addition of cane sugar with subsequent evaporation to a little less than one-half of its original volume. After condensing, the cane-sugar concentration is between 40 and 45 per cent, giving sufficient osmotic pressure to inhibit the growth of most fermentative bacteria. Owing to the fact that in $100 \mathrm{gm}$. of condensed milk the total amount of water in which $45 \mathrm{gm}$. of saccharose must be dissolved is less than $30 \mathrm{gm}$., of which a part is rendered unavailable by combination with other constituents, the sugar concentration is much higher than is indicated by the percentage composition.

Not all bacterial growth is inhibited at this concentration. Some multiplication of bacteria usually occurs and may continue for a few weeks. If the saccharose content is relatively low, a coccus appears which ferments the sugar, thickening the milk and giving it an unpleasant flavor. ${ }^{\mathrm{I}}$ Such thickening should not be confused with the thickening which frequently takes place spontaneously and is not accompanied by undesirable flavor changes.

The concentration of cane sugar is not high enough to inhibit entirely the growth of yeasts. It is not unusual to find growing yeasts in normal milk, but it is only occasionally that the multiplication is sufficient to produce an appreciable amount of gas. In normal milk the conditions become sufficiently anaerobic in a few days to prevent further multiplication, and it is only when the infection is unusually heavy that enough cells are present to continue an anaerobic fermentation with gas production and spoilage of the product. ${ }^{2}$

\section{EVAPORATED MILK}

"Evaporated milk" is the commercial term applied to milk concentrated under vacuum to a little less than one-half of its original volume and preserved by heat

× Rice, F. E., and Downs, P. A.: J. Dairy Sc., 6, 532. 1923.

${ }^{2}$ Savage, W. G., and Hunwicke, R. F.: (Great Britain) Depl. Sc. Eo Indust. Research, Food Invest. Bd., Spec. Rep. 13. 1923. 
sterilization. The temperature at which the milk may be sterilized without coagulation is affected in some degree by the growth of bacteria in the milk before concentration. A very small increase in the $\mathrm{H}$-ion concentration usually causes a distinct lowering of the coagulation point of the concentrated milk, although in some cases it may have the reverse effect.'

Evaporated milk, like other canned foods, is subject to various types of spoilage due to spore-forming bacteria which survive the sterilization process. Two common troubles are acid fermentations in which there is no gas production and consequently no indication of spoilage before the can is opened. "Flat sour" is caused by an organism to which Hammer ${ }^{2}$ has given the name B. coagrlans. In a somewhat similar fermentation a marked bitter flavor is produced, sometimes without visible change in the milk. Bacteria of the butyric acid group and the spore-forming putrefactive type are responsible for gaseous fermentations causing swelling and sometimes bursting of the cans.

\section{MILK POWDER}

Two distinct types of manufacturing processes have come into general use in making milk powder. In one process the milk is spread in a thin film on the surface of steam-heated revolving drums held either at atmospheric pressure or in a vacuum. The dried film is removed from the drums by scrapers and ground to a powder. In the more generally used spray process the milk is atomized into a current of heated air from which the dry particles of milk are removed by special devices.

In the drum process, particularly drums at atmospheric pressure, the temperature of the film of milk is sufficient to destroy vegetative bacteria, and consequently the bacterial content of the powder is very low. Supplee and Ashbaugh ${ }^{3}$ have shown that the bacteria are usually limited to spore-formers and that the count of powder made on atmospheric drums is not over a few hundreds per gram of powder. Pathogenic bacteria, including the tuberculosis organism, are destroyed by this process. ${ }^{4}$

Since the moisture content of the milk powder is below 5 per cent there is no increase of bacteria on standing, but it has been pointed out that there may be contamination in the process of collection and packing and that the bacterial count may be used as a check on the manufacturing methods. ${ }^{5}$

In the spray process the temperature of the air is high ( $190^{\circ} \mathrm{F}$.) but the evaporation from the surface of the minute particles of milk is so rapid that it is probable the bacteria are not exposed to temperatures above their thermal death-point before they are in a dry state. However, in the operation of this process the milk is almost invariably exposed to pasteurizing temperature or a higher temperature before drying either in precondensing which is commonly practiced or to check bacterial change when it is necessary to hold the milk before drying. There is a gradual falling off in the numbers of bacteria in milk powder in storage. The changes in flavor and

'Benton, A. G., and Albery, H. G.: J. Biol. Chem., 68, 25 г. 1926.

${ }^{2}$ Hammer, B. W.: Yowa Agric. Exper. Sta. Research Bull. 52. I9I9.

${ }^{3}$ Supplee, G. C., and Ashbaugh, V. J.: J. Dairy Sc., 5, 2 I6. 1922.

${ }^{4}$ Hunwicke, R. F., and Jephcott, I.: ibid., 8, 206. 1925.

5 Jephcott, H., Hunwicke, R. F., and Ratcliffe, N.: Proc. World's Dairy Cong., 2, I265. I923. 
physical properties of milk powder are evidently not due to bacterial action but to oxidation and other chemical reactions.

\section{ICE CREAM}

The cream, sugar, condensed milk, gelatin, and other ingredients used in ice cream contain bacteria, sometimes in large numbers. During the manufacturing process the total number may increase and, unless measures are taken to reduce the bacterial content, the frozen product is likely to contain excessive numbers of bacteria. These may be millions per gram. A high bacterial count carries with it a possibility of infection with pathogenic bacteria. Fabian ${ }^{I}$ has compiled records of thirty-five epidemics due to ice cream including typhoid, scarlet fever, and diphtheria. In recent years the manufacturing processes and the sanitary conditions of the factories have been much improved. Pasteurization of the mix is now a common practice, and the better factories produce an ice cream consistently containing less than I00,000 bacteria per gram. ${ }^{2}$ In the freezing there is an apparent increase in numbers due to the violent agitation of the freezer, but in the hardening-room where the product is held at $0^{\circ}$ to $-10^{\circ} \mathrm{F}$. there is a slow decrease in numbers. ${ }^{3}$

\section{BUTTER}

In the customary process of butter-making the cream is soured or "ripened" by inoculation with lactic bacteria to develop acidity and flavor. In the better factories the cream is pasteurized and the cultures are propagated with great care. St. lactis is used for this purpose, but a strictly pure culture forming lactic acid almost exclusively, with only a small proportion of volatile acid, does not give the most satisfactory flavor to the butter. It is now known that the best "starters" are not pure strains but contain, in addition to St. lactis, one or more streptococci which have little effect in the souring of the milk but, through the formation of acetic acid and other volatile products, increase the aroma of the starter and especially that of the butter. ${ }^{4}$

Butter is susceptible to many influences which not only affect its flavor and aroma when fresh from the churn but which also cause it to take on various off-flavors that seriously affect its market value. The natural flavor of butter is due partly to the natural flavor of the cream and partly to volatile compounds produced by bacteria in the milk and especially in the ripening of the cream. Up to a certain point these volatile substances improve the flavor; beyond this they cause a deterioration. The off-flavor may be slight and undefined or it may be pronounced and definite. Thus butter may acquire an oily flavor of varying intensity or it may be metallic and fishy.

${ }^{x}$ Fabian, F. W.: Am.J. Pub. IIeallh, 16, 873. I926.

${ }^{2}$ Fay, A. C., and Olson, N. E.: J. Dairy Sc., 7, 330. 1924; Fabian, F. W.: Michigan Agric. Exper. Sta. Spec. Bull. 158 . I926.

3 Ellenberger, H. B.: Cornell Agric. Exper. Sta. Mem. 18. I9r9.

${ }_{4}$ Boekhout, F. W. J.: Vet. Exploit. Proefzuivelbocrderij IIoorn, Verslag, I9I7, p. 48. 1918; Storch V.: Beret. Forsgslab. K.Vet.og Landbohjskoles (Denmark), p. Io2. I919; Hammer, B. W., and Bailey, D. E.: Iowa Agric. Exper. Sta. Research Bull. 55, 1919; Hammer, B. W.: ibid., Bull. 63. 1920; ibid., Bull.65. 1920; ibid., Bull.67. 1921; Ayers, S. H., and Mudge, C. S.: J. Dairy Sc., 4, 240. 1921. 
The rate of development of these flavors varies greatly in different lots of butter but, other conditions being equal, is proportional to the temperature. While low temperature greatly retards flavor changes, even the very low temperature $\left(0^{\circ} \mathrm{F}\right.$. of commercial storage will not entirely prevent deterioration.

The three factors which may be of importance in the deterioration of butter are the enzymes secreted with the milk, or by bacteria in the milk or cream; bacteria, either by direct action in the butter or by changes brought about in the milk or cream from which the butter is made; and chemical changes which may take place spontaneously in such a complex and unstable product.

The enzymes of the milk, especially the lipase, probably play some part in flavor changes in butter made from unpasteurized cream, but they are nearly or quite inactivated by the temperature of pasteurization. Bacterial enzymes may be a factor under some circumstances.

Butter made from ripened cream contains, in its fresh state, many millions of bacteria per gram. In creamery butter made from pasteurized cream by approved methods these bacteria are almost exclusively the lactic streptococci introduced as starters. These bacteria decrease slowly as the butter stands in cold storage, and more rapidly at higher temperatures.

There is usually little or no multiplication of micro-organisms in salted butter. This is easily understood in view of the fact that butter contains $2 \frac{1}{2}-3 \frac{1}{2}$ per cent of salt dissolved in about $\mathbf{5}$ per cent of water. Sometimes torula yeasts grow for a time, and there may be some multiplication of the salt-tolerant bacteria. Molds may grow on the surface if the butter is not properly packed or is held at or above the freezingpoint of water. The molds cause rapid changes in the butter in the vicinity of the colony but occur only under abnormal conditions. The yeasts and salt-tolerant bacteria have never been shown to have any significant part in the changes causing deterioration.

The direct action of bacteria in the butter as a primary cause of undesirable flavors is excluded by the fact that all of the typical storage flavors appear in the butter held at temperatures at which bacteria cannot possibly grow. On the other hand, the by-products of bacterial growth in the milk or cream may be the precursors of flavor-producing substances which develop later in the butter. Acidity, which was formerly considered essential to good keeping quality, is now known to accelerate deterioration, and butter for storage is made from cream of low acid content or even from pasteurized cream in which no acid is developed.

About Io per cent of the volume of butter is air, incorporated by the working process, in the form of minute bubbles. In a short time the oxygen of this air disappears, presumably through oxidation of some of the constituents of the butter. Moreover, an oxidation of fats may take place without free oxygen, probably through intermolecular rearrangement. ${ }^{1}$ An acid reaction is essential to these changes, and they are further accelerated by such catalysts as the salts of copper, iron, and nickel which are dissolved by milk and cream from cans, pasteurizers, and other equipment.

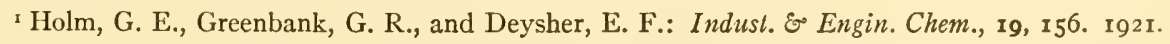




\section{CHEESE}

The characteristic flavor and aroma of each of the great number of cheeses which have been developed by empirical methods are almost entirely due to the growth of bacteria and other micro-organisms. In each type of cheese there is usually a definite sequence of organisms which develop at successive stages of ripening. Some of these organisms have little to do with the flavor production but are of importance in controlling the course of the fermentation and in preparing the ground for the bacteria or molds which give the cheese its characteristic flavor. The nature of this biological sequence and the resulting type of cheese are determined in the making process, especially by the temperatures used, the water retained, the amount of salt, and the method by which it is incorporated, and by the temperature of curing.

The simplest cheeses are those of the cottage or Neufchâtel type which undergo no ripening and depend on a lactic fermentation of the milk for their flavor. The acidity of the curd has a protective action, but deterioration may be caused by surface growth of Oidium lactis or by anaerobic spore-formers which may give sour and bitter flavors.

Hard cheeses such as Cheddar, Edam, Emmental, or Swiss and Parmesan, which are made from milk curdled with rennet, undergo a complicated curing process in which the hard, rubbery texture is materially altered and the insoluble paracasein is converted into soluble products among which are albumoses, peptone, amino acids, ammonia, and carbon dioxide. In this proteolysis the natural enzymes of the milk, the pepsin of the rennet, and possibly the lactic bacteria play an important part.

In Cheddar, which is the cheese most generally made in this country, the manufacturing process encourages the lactic fermentation. The high buffering action of the curd permits a continuation of the fermentation until the sugar is entirely exhausted. In the first few days after the cheese is made the lactic streptococci reach enormous numbers. ${ }^{1}$ As the ripening progresses this type declines followed by a large increase of the lactobacilli and sometimes of liquefying cocci. These bacteria are probably responsible for the active ester production which goes on simultaneously with the proteolysis and is closely associated with the development of flavor. ${ }^{2}$

It has not been definitely proved that any one variety of bacteria is responsible for the characteristic Cheddar flavor, but it is known that this flavor is lacking in cheese made from pasteurized milk. In the Emmental or Swiss cheese the making process includes heating for some time at $50^{\circ}-55^{\circ} \mathrm{C}$. The growth of all bacteria is checked and the greater part destroyed. The large mass of curd cools slowly in the press and the growth of the lactobacilli naturally present in the milk or introduced as starters, or with the rennet is encouraged. These bacteria rapidly ferment the lactose to lactates and produce conditions which tend to inhibit colon-aerogenes and other gas-forming bacteria. In the warm room $\left(70^{\circ}-72^{\circ} \mathrm{F}\right.$.) propionic bacteria develop, converting the lactates to propionic acid and carbon dioxide.

In Cheddar cheese any gas formed escapes through the porous curd, but in Swiss

r Hastings, E. G., Evans, A. C., and Hart, E. B.: Wisconsin Agric. Exper. Sta. Research Bull. 25. 1912.

${ }^{2}$ Suzuki, S. K., Hastings, E. G., and Hart, E. B.: ibid., Bull. II. 1910. 
the gas, held by the rubbery curd, collects at foci to form the eyes. ${ }^{\mathrm{I}}$ The flavor, which is sweet and quite distinct from that of Cheddar, is incidental to the eye formation and is evidently a result of the activities of the propionic acid bacteria. ${ }^{2}$

The Parmesan-cheese process follows in general that of the Emmental, but the fat and moisture are lower and the salt higher. There is the beginning of an eye formation, but at the end of the long ripening period both the texture and flavor are markedly different from that of Emmental. The identity of the bacteria responsible for these changes has not been established.

In cheese of the Limburger type more water is retained in the curd and a rapid and deep-seated splitting of the casein is obtained in which such highly flavored compounds as indol, skatol, and ammonia are found.

Cheese in which the characterist ic flavor is obtained through the activity of molds are of two types. In Camembert, Brie, and similar cheeses the mold grows on the surface and the ripening progresses very distinctly from the outside toward the center. In Roquefort, Gorgonzola, and Stilton the mold grows in the interior and the ripening progresses more or less uniformly throughout the mass. In all of these cheeses there are the preliminary lactic fermentations in which all the lactose is fermented and changes in the protein which follow the same general course as those taking place in the hard cheese. In Camembert and similar cheeses the mold forms a felt of mycelium on the surface and the enzymes which it secretes reduce the curd to a semiliquid condition. Notwithstanding this marked proteolysis, indol, skatol, and other products characteristic of putrefaction do not appear.

The acid reaction of the young cheese is reduced and bacterial growth is resumed. There is no evidence to show that these bacteria have any appreciable influence on the flavor. Oid. lactis is always present on normal cheese, and probably has some part in developing the flavor which Penicillium camembertii is apparently incapable of producing alone. ${ }^{3}$

In Roquefort, which is made from sheep's milk and ripened in very moist cold caves, the growth of bacteria is inhibited by the high salt content ( 4 per cent) and the low temperature of the curing-rooms $\left(48^{\circ} \mathrm{F}\right.$.). There is little growth of the lactic streptococci but some growth of the lactobacilli and some of the cocci more tolerant to sodium chloride. ${ }^{4}$ The essential organism in the ripening of Roquefort is $P$. roqueforti which is added to the curd in the form of a dried culture obtained by growing the mold in loaves of bread until sporulation has taken place, then drying the bread and grinding it to a powder. At the proper time in the ripening, holes are punched in the cheese to admit a small amount of air. Since the Roquefort mold grows at a low oxygen tension it is encouraged, while other molds are unable to develop. ${ }^{5}$ The active lipase secreted by $P$. roquefort $i$ hydrolyzes the fat and liberates fatty acids. Capric, caprylic, and caproic acids give the cheese its peculiar peppery flavor. ${ }^{6}$

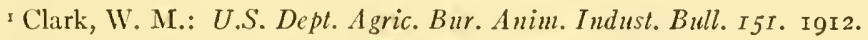

${ }^{2}$ Sherman, J. M.: J. Bact., 6, 379. I92 I.

3 Thom, C.: U.S. Dept. Agric. Bur. Anim. Indust. Bull. 82. 1906.

4 Evans, A. C.: J. Agric. Research, 13, 225. I918.

5 Thom, C., and Currie, J. N.: J. Biol. Chem., 15, 249. I9I3.

${ }^{6}$ Currie, J. N.: J. Agric. Research, 2, I. IgI4. 
Abnormal fermentations of cheese are caused by a great variety of bacteria among which the colon-aerogenes group is perhaps the most troublesome. These bacteria develop while a supply of lactose is still available and cause gassy fermentations while the curd is in the vat in the case of Cheddar or in the press in Swiss cheese. Gassy fermentations which come later are likely to be caused by the we'chii group. A very disagreeable defect of Swiss cheese is caused by the putrificus bacilli. Pigment-forming bacteria sometimes cause red or "rusty" spots in cheese, ${ }^{1}$ and bitter flavors have been traced to activities of torula yeasts. ${ }^{2}$

${ }^{1}$ Connell, W. T.: Canada Commr. Agric. \&o Dairying Rep., I897, Part 16, p. 3. I898.

${ }^{2}$ Harrison, F. C.: Rev. gên. du lait, I, 457, 485. 1902. 


\title{
CHAPTER XXXII
}

\section{THE BACTERIAL AND HEALTH ASPECTS OF PASTEURIZATION ${ }^{\circledR}$}

\author{
MULTON J. ROSENAU \\ Harvard Medical School, Boston, Mass.
}

PASTEURIZATION AND THE BACTERIAL FLORA OF MILK

It is well known that the bacterial population of milk is both large and miscellaneous. The flora often reach the magnitude of incredible numbers. From the standpoint of health, however, it is the kind rather than the numbers that concern us. Milk is pasteurized primarily for the purpose of making it safe for human consumption. There is another benefit, however, namely, the reduction of the miscellaneous bacteria always found in milk. This reduction of the germ life in the milk probably also has some sanitary value, particularly for infants in the summertime. The subject is ably discussed by Prucha in an article which has just appeared. ${ }^{2}$ The most extensive work is that of Rogers $^{3}$ and of Ayers and Johnson, ${ }^{4}$ who include a large bibliography.

Pasteurization ordinarily reduces the bacterial count in milk 99 per cent; however, under certain conditions, the milk may become heavily contaminated with bacteria that are resistant to pasteurizing temperatures. Prucha points out that resistant species of bacteria are likely to get into milk during a heated spell with a prolonged rainy season, making the pastures muddy. In this case, in spite of attempts to keep the cows clean, the milk becomes seeded with fairly large numbers of spore-producing soil bacteria.

It is well known in all bacteriological laboratories that samples of pasteurized milk are encountered with bacterial counts above the legal limits. How to interpret such counts is often puzzling. The bacteria that survive pasteurization in large numbers have a tendency to produce very small colonies on standard agar plates. The term "pin-point colonies" has been coined to describe this phenomenon.

Prucha studied 108 different cultures and found that 45 of these were organisms that usually make up the predominating bacterial flora in raw milk, and all of them were killed at $145^{\circ} \mathrm{F}$. for thirty minutes. Thirty-four cultures invariably present in the raw milk, but usually in very small numbers, survived pasteurization, but none of these would be classed as thermophiles. They probably belong for the most part to the group of bacteria described by Ayers and Johnson as acid-producing, heatresisting bacteria. Most of them are cocci and produce very small pin-point colonies.

I The author has drawn upon his chapter on pasteurization in the fifth edition of Preventive Medicine and Hygiene, and acknowledgment is herewith made to D. Appleton \& Co. The reader is referred to that volume for a more extensive treatment.

${ }^{2}$ Prucha, M. J.: Am. J. Pub. Hcalth, 17, 356. 1927.

${ }^{3}$ Rogers, L. A.: Bur. Anim. II usb., U.S. Depl. Agric., Bull. 73. 1905.

4 Ayers, S. H., and Johnson, W. T.: ibid., Bull. 126. 1910; ibid., Bull. 161. 1913. 
They develop at $37^{\circ} \mathrm{C}$. and are not the same as the true thermophiles occasionally found in pasteurized milk, although they have probably been mistaken for the thermophiles on account of the smallness of the colonies and the resistance to heat. The source of these bacteria has not been disclosed, although there is some evidence that they come from the utensils which are not kept scrupulously clean or thoroughly steamed.

Heat-resisting bacteria do not grow very rapidly in milk, especially when the milk is kept at lower temperatures. They do not seriously affect the keeping quality of the milk at $60^{\circ} \mathrm{F}$. or lower, and while they have no known pathogenic effect, they indicate neglect somewhere along the journey of the milk as it passes from the cow to the final container. There is very little difference in the percentage reduction and the bacterial count between milk pasteurized at $140^{\circ}$ and at $145^{\circ} \mathrm{F}$.

\section{TIIERMAL DEATII-POINTS}

The time and temperature of pasteurization depend upon the time and temperature necessary to kill pathogenic micro-organisms that are a hazard to man if taken in milk. We need not concern ourselves with the spores of tetanus which often get into milk, for these are not harmful when taken by mouth. Fortunately, all the milk-borne diseases that concern man are due to viruses that are relatively thermolabile and have no resistant spores. It has been rightly assumed that any temperature and time that will surely kill the tubercle bacillus will also kill the other microorganisms in question, because the tubercle bacillus, on account of its waxy substance, is somewhat more resistant than the ordinary spore-free bacteria. Much work has therefore been done to determine with precision the thermal death-points of the tubercle bacillus.

Confusion and misunderstanding have resulted from a misapprehension that micro-organisms have a thermal death-point. There are, in fact, an infinite series of thermal death-points, depending upon the time of exposure. Together these points make a curve, from which it is clear that the temperature is a function of the time. This is well shown in Charts I and II.

\section{THERMAL DEATII-POINTS OF THE TUBERCLE BACILLUS}

By common consent, the killing of tubercle bacilli in milk has become the criterion by which the public-health efficiency of the process is judged. Attention therefore has been focused upon the temperature and time necessary to kill this micro-organism.

Since the first report in 1879 by Galtier, eighty-nine separate reports of investigations by bacteriologists in all parts of the world upon the thermal death-points of the tubercle bacillus have appeared in the literature. A detailed and critical review of all the work on this important question is admirably summarized by North and Park $^{1}$ with a complete bilsliography to date.

It was found that only a few of the earlier studies were based upon methods which are now considered reliable. Thus, five of the nine studies marle before Galtier's work in 1879 reported that actual boiling would not kill the "tubercular virus."

' North, C. E., and Park, W. H.: Am. J. IIyg., 7, 147.1927.

${ }^{2}$ Koch discovered the tubercle bicillus in 1882 . 
These and similar bizarre results soon give way to careful work with pure cultures, for it was disclosed by the early bacteriologists that the tubercle bacillus has no spore and is but little more resistant than the ordinary spore-free bacteria.

Schroeder (1894), Theobald Smith (I899), Russell and Hastings (I900), Hesse

CHART I

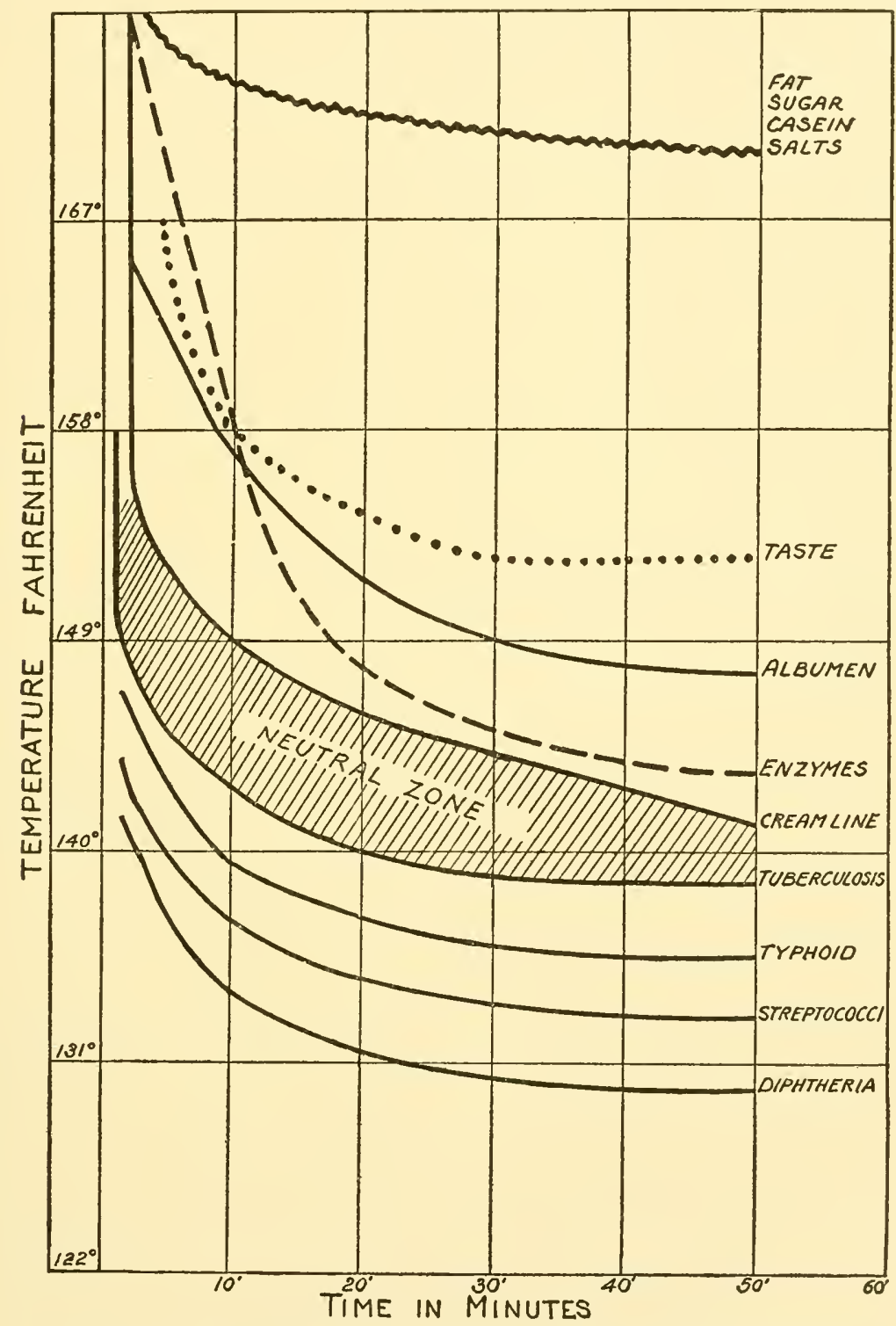

Time and Temperature for Pasteurization in the Neutral Zone Which

Is above the Thermal Deathi-Curves for the Pathogenic Microorganisus with Minimum Injury to the Milk.

(U.S. Pub. Health Bull. 147 , I925.) 
(I90I), McFadyen (I904), Rosenau (I908), Schorer (I9I2), Traum and Hart (I9I6), Traum (I922), and others found that the tubercle bacillus will surely be killed if exposed to $140^{\circ} \mathrm{F} .{ }^{x}\left(60^{\circ} \mathrm{C}\right.$.) for twenty minutes or less, and this has recently been confirmed by North, Park, Moore, Rosenau, Armstrong, Wadsworth, and Phelps ${ }^{2}$ and again by North and Park. ${ }^{3}$

There is nothing in all this work to indicate that the pure cultures are any less resistant than the tubercle bacillus in tissue lesions. North and Park conclude that CHART II

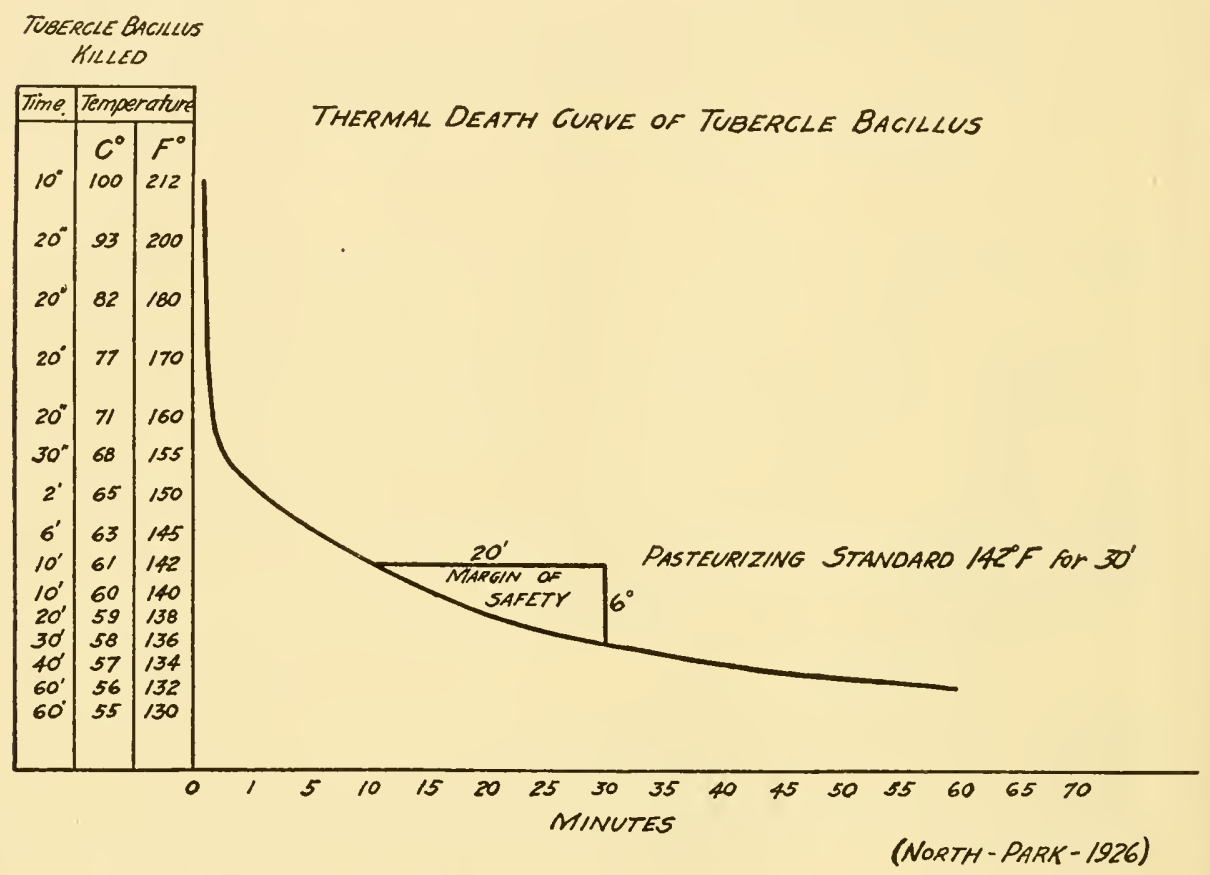

(From "Standards for Milk Pasteurization," $A m . J . I l y g ., 7$, I47. I927.)

artificial cultures of bovine tubercle bacilli show the same resistance to heat as the tubercle bacillus in naturally infected milk.

To determine whether tubercle bacilli are alive or dead, it is necessary to inject guinea pigs. The ordinary cultural and microscopical methods are not applicable. Many of the errors in determining the thermal death-points of this micro-organism were due in part to a misinterpretation of the lesions produced by dead tubercle bacilli, and at times to pseudo-tubercles produced by other pathogenic micro-organisms.

Many of the heating experiments were unreliable on account of imperfect technique, especially when open tubes or flasks were used. The first to realize this was

r Most of the work on pasteurization is reported in the Fahrenheit scale, which is better suited to this purpose because it deals with whole numbers. For convenience, the comparison between the Fahrenheit and Centigrade scales at the temperatures in question are given on Chart II.

${ }^{2}$ North, C. E., et al.: U.S. Pub. Health Bull. I 47 . 1925.

${ }^{3}$ North, C. E., and 1'ark, W. H.: Am. J. IIyg., 7, I47. 1925. 
Yersin, who in I 888 used tapering test tubes. The capillary tubes used by Foster, the sealed test tubes used by Theobald Smith and others, and the sealed boxes used by Bang and others gave effective protection against air. McFadyen and Hewlett used a closed lead coil submerged in a water bath through which the infected milk was passed. This method was elaborated in detail in the technique used by North and Park in their recent work. By this method the cooling effect of air, pellicle, serum, and froth formation are avoided. The pasteurization of milk in glass bottles, such as baby bottles and ordinary milk bottles, has often been advocated, but the air space at the top of such bottles makes possible the formation of pellicle, scum, and froth, which is a refuge to the bacilli, unless the air itself is also heated sufficiently.

Traum and $\mathrm{Hart}^{\mathrm{t}}$ took advantage of an opportunity to test the effect of heat on naturally infected milk pasteurized under commercial conditions such as prevail today in all modern city milk plants. Milk was obtained from a herd of five hundred cattle, all of which were tuberculous, and the milk of which was allowed to be sold after it had been pasteurized. It was found that $140^{\circ} \mathrm{F}$. for twenty minutes killed all the tubercle bacilli in the milk. In a later article and as a result of further work, Traum concludes that pasteurization at a temperature of not less than $142^{\circ} \mathrm{F}$., and for a period of not less than thirty minutes, as required by most health departments at present, should be effective in destroying tubercle bacilli which may be present in the milk. Such exposure leaves a good margin of safety.

The experiments of a group of bacteriologists working at Endicott (N. Y.) in I922-23, the results of which were reported in the Uniled States Public Heallh Service Bullelin 147 , gave special attention to temperatures lower than $140^{\circ} \mathrm{F}$. for a period of thirty minutes with commercial machines. Their conclusions were based upon a large volume of data. A total of 7I 8 samples were tested on 1,436 guinea pigs by Moore, Park, Rosenau, Wadsworth, Armstrong, and North, who found that under practical conditions the tubercle bacillus is killed at $138^{\circ} \mathrm{F}$. in thirty minutes.

This conclusion has been attacked on the ground that the experimental data in the report were insufficient. North and Park therefore carried this work a step farther, using the closed-lead-coil system. They made a total of fifteen separate tests at twelve different temperatures between $170^{\circ}$ and and $130^{\circ} \mathrm{F}$., and found that tubercle bacilli were killed at $136^{\circ} \mathrm{F}$. in thirty minutes. This constitutes the most extensive series of experiments ever made to determine this point, and the careful work carries conviction. Chart II summarizes this work.

It can therefore be asserted with confidence that it has been amply demonstrated that $140^{\circ} \mathrm{F}$. is more than enough to kill tubercle bacilli; in fact, they succumb at $136^{\circ} \mathrm{F}$. in thirty minutes.

\section{TIME AND TEMPERATURE OF PASTEURIZATION}

It has been abundantly demonstrated that heating milk to $140^{\circ} \mathrm{F}$. $\left(60^{\circ} \mathrm{C}\right.$.) for twenty minutes is more than sufficient to kill the bacilli of tuberculosis, typhoid fever, paratyphoid fevers, dysenteries, and diphtheria; the streptococci of scarlet fever and septic sore throat; the micrococcus of Malta fever; the virus of foot-and-mouth disease; and all other non-spore-bearing, milk-borne infections that are of concern to

I Traum, J., and Hart, G. H.: J. Am. Vet. M. A. Aug., igr6. 
man. The temperature is a function of the time; i.e., for every degree above $140^{\circ} \mathrm{F}$. the time may be reduced, so that at $145^{\circ} \mathrm{F}$. all the foregoing pathogenic micro-organisms are killed in six minutes; at $\mathrm{I} 55^{\circ} \mathrm{C}$. within thirty seconds. The exposure under commercial conditions should always be in excess of the minimum requirements so as to provide a factor of safety, for it is of signal health moment in practical pasteurization to be certain that all the milk is uniformly heated to the temperature required. By common consent, therefore, holding methods requiring thirty minutes have uniformly been adopted. Automatic records of the temperature and time should be kept of each run.

A factor of safety is necessary in commercial practice, and it has been found by long experience in great cities handling enormous quantities of milk that $142^{\circ}-\mathbf{I} 45^{\circ} \mathrm{F}$. for thirty minutes is safe and satisfactory, using equipment free from serious engineering defects. Milk thus pasteurized protects the public health and satisfies our knowledge of the thermal death-points of the particular viruses as disclosed by bacteriologists. Most cities and many states have adopted this definition of pasteurization, but some still insist upon a minimum of $145^{\circ} \mathrm{F}$. for thirty minutes. The dairy industry objects to heating milk above $145^{\circ} \mathrm{F}$. because the cream line is affected, but there is no sanitary harm from these temperatures. This, therefore, is clearly a commercial and economic question rather than a health problem.

No pasteurizing device has been made that will automatically heat all the milk passing through under practical conditions to a given temperature and hold it precisely on the mark. There is a plus or minus deviation in the best designed heating apparatus, often of $1.5^{\circ} \mathrm{F}$. To pasteurize milk between $142^{\circ}$ and $\mathrm{I} 45^{\circ} \mathrm{F}$, it is necessary to set the thermoregulator at $143.5^{\circ} \mathrm{F}$. When the sanitary code requires a minimum of $145^{\circ} \mathrm{F}$., the apparatus must be set to at least $146.5^{\circ} \mathrm{F}$., and the milk will then range between $145^{\circ}$ and $148^{\circ} \mathrm{F}$., thereby affecting the creaming ability of the milk.

As the result of years of extensive experience with commercial pasteurization and of recent researches, it has been demonstrated that a temperature of $142^{\circ}-\mathrm{I} 45^{\circ} \mathrm{F}$., with a holding period of 30 minutes, serves the purpose of protecting the public health and preserving the integrity of the milk. This provides a factor of safety of $6^{\circ} \mathrm{F}$. or twenty minutes' time at $142^{\circ} \mathrm{F}$; in fact, New York and other large cities and several states have for some years used this standard for pasteurization with reasonably good results. Massachusetts requires a minimum of only $140^{\circ} \mathrm{F}$. for thirty minutes; other states and cities permit or countenance the newer lime and temperature recommended; Pennsylvania insists upon a temperature of $145^{\circ} \mathrm{F}$. for thirty minutes.

\section{THE EFFECTS OF IIEAT UPON MILK}

The changes produced in milk by heat depend upon the degree of heat and the length of time of exposure. Milk heated to $145^{\circ} \mathrm{F}$. for thirty minutes does not undergo any appreciable physical or chemical change. Higher temperatures, however, produce pronounced changes. In the main these consist of a partial decomposition of the proteins and other complex nitrogenous derivatives; diminution of the organic phosphorus and an increase of inorganic phosphorus; precipitation of the calcium and magnesium salts and the greater part of the phosphates; expulsion of the greater part of the carbon dioxide; caramelization or burning of a certain portion of the milk 
sugar, causing the brownish color; partial disarrangement of the normal emulsion, and coalescence of some of the fat globules; coagulation of the serum albumin, which begins at $167^{\circ} \mathrm{F}$; the ferments are killed; some of the vitamins, notably $\mathrm{C}$, are affected.

Boiled milk has a cooked taste which appears at about $158^{\circ} \mathrm{F}$. This is due perhaps to the decomposition of certain of the proteins in the milk. The loss of certain gases also alters the taste, so that milk heated in closed vessels has a less pronouncerl flavor than if heated in open vessels.

Milk heated in the open air forms a pellicle which renews if it is removerl. This scum forms when milk reaches about $140^{\circ} \mathrm{F}$. It consists of:

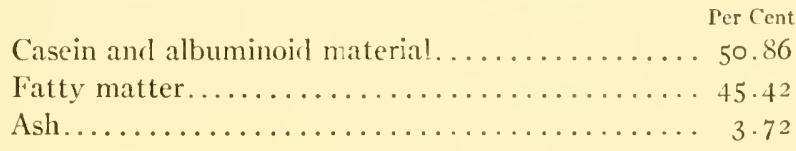

Milk heated in closer vessels does not form a pellicle, even when the temperature reaches the boiling-point. It seems that this pellicle is due mainly to the drying of the upper layer of the liquid. Heat causes a progressive decline in the $\mathrm{H}$-ion concentration of the milk as the temperature rises. Evidently, the heat modifies the balance of colloids in the milk.

It is claimed that heat influences the availability of calcium and phosphorus of the feeding mixture. It appears that the heat of pasteurization throws the calcium salts more or less out of the solution, and thus they are made less readily available. This does not occur to the same degree in quickly boiled milk, which therefore has its advocates both here and abroad for infant feeding.

Milk properly pasteurized at temperatures between $142^{\circ}$ and $145^{\circ} \mathrm{F}$. and then cooled cannot be distinguished from raw milk by taste. Practical tests to determine this point are convincing.

\section{CREAMING ABILITY OR CREAM VOLUME}

By "creaming ability" or "cream volume" is meant the amount of visible cream which rises to the top of the milk on standing. No noticeable effect is produced upon the cream volume when milk is heated up to $145^{\circ} \mathrm{F}$. for thirty minutes. It is first observed at about $146^{\circ} \mathrm{F}$. and is of practical importance at $147^{\circ}-\mathrm{I} 48^{\circ} \mathrm{F}$.

The creaming ability of milk is also influenced by the feed of cattle from which the milk is obtained, the state of lactation of the dairy cow, the percentage of fat in the milk, the size and grouping of the fat globules, the viscosity of the milk serum, the temperature of the milk during creaming, recreaming of the milk, passing of the milk through a separator, and remixing the cream and milk, and finally the agitation of the milk. In the pasteurizing plant the creaming ability is affected by clarification, agitation, cooling, and storing, as well as by the heat.

\section{GERMICIDAL PROPERTY OF MILK}

The germicidal property of milk has been much misunderstood. It is specific and varies in different animals and even in the same animal at different seasons. ${ }^{\mathrm{I}}$ It is destroyed at $167^{\circ} \mathrm{F}$. $\left(70^{\circ} \mathrm{C}\right.$.) for fifteen minutes, or between $176^{\circ}$ and $194^{\circ} \mathrm{F}$. $\left(80^{\circ}-\right.$

I Chalmers: Brit. J. Exper. Path., 5, 27I. I924. 
$90^{\circ} \mathrm{C}$.) for two minutes, but is little influenced at $142^{\circ}-145^{\circ} \mathrm{F}$. $\left(61^{\circ}-63^{\circ} \mathrm{C}\right.$.) for thirty minutes. At most the germicidal action is feeble and transitory and cannot take the place of cleanliness and ice, but may be taken advantage of by prompt use of fresh milk. It is true that bacteria start to multiply more quickly in heated milk than in raw milk, provided the raw milk is fresh; it should be remembered, however, that stale milk, even milk that is a day old, no longer possesses this restraining action. The germicidal property is, therefore, ordinarily absent from market milk.

Rosenau and $\mathrm{McCoy}^{\mathrm{I}}$ have shown that, judged by the number of colonies that develop upon agar plates, the bacteria in milk first diminish, then increase, in number. This occurs only in raw milk during the first eight or twelve hours after it is drawn. Although the bacteria seemingly decrease in numbers, they never entirely disappear. After the initial decrease there is a continuous and rapid increase, until the milk contains almost infinite numbers in each cubic centimeter. The power of milk to restrain the development of bacteria lasts from six to twenty-four hours, depending upon the temperature at which the milk is kept. When the milk is kept warm, $37^{\circ} \mathrm{C}$, the decrease is pronounced within the first eight or ten hours; after this the milk has entirely lost its restraining action. When the milk is kept cool, $15^{\circ} \mathrm{C}$., the decrease is less marked but more prolonged. They further showed that at least part of this decrease is due to agglutination. Chambers ${ }^{2}$ has shown a true germicidal decrease under certain conditions. He finds the action quite specific, some bacteria decreasing while others increase.

\section{DISEASES SPREAD BY MILK}

Diseases known to be conveyed through milk are tuberculosis, typhoid and paratyphoid fevers, diphtheria, scarlet fever, septic sore throat, Malta fever, foot-andmouth disease, and milk-sickness; also some of the summer complaints of children and the diarrhea and dysenteric diseases of adults, which are often referable to infected milk. Epidemic arthritic erythema and infantile paralysis have recently been added to this list. In addition to the specific diseases, milk may be harmful from other causes.

As a rule, milk becomes infected from human sources, sometimes on the farm, sometimes at the dairy, sometimes in transportation, and occasionally in the household. Sometimes the milk becomes infected as a result of disease of the animal, as in the case of bovine tuberculosis, foot-and-mouth disease, streptococcal garget from cows, and Malta fever from goats.

When all the facts are brought together, they make a strong indictment against raw milk. Thus Table I, giving the official record ${ }^{3}$ of milk-borne infections in Massachusetts, gives the facts from 1907 to 1923 ; the story is brought up to date in a report just off the press. ${ }^{4}$ Since the general adoption of pasteurization for the milk supplies of the large cities in Massachusetts, milk-borne outbreaks occur characteristically in the small unpasteurized supplies.

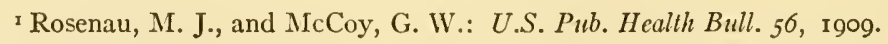

${ }^{2}$ Chambers, W. H.: J. Bact., 5, 527. 1920.

${ }^{3}$ Kelley, E. R., and Webber, W. J.: Am. J. Pub. Health, 14, 963. I924.

${ }_{4}$ Bigelow, G. H., and Forsbeck, F. C.: Am. J. Pub. Health, 17, rorg. 1927.
} 
Practically all the milk (except certified milk) handled by the large dairies in the United States is now pasteurized. This means that most of the milk supplied our

TABLE I

\begin{tabular}{|c|c|c|c|c|c|}
\hline Massachusetts & $\begin{array}{c}\text { Total Cases } \\
\text { Reported }\end{array}$ & $\begin{array}{l}\text { Number of } \\
\text { Cases Traced } \\
\text { to Milk }\end{array}$ & $\begin{array}{c}\text { Cases Traced } \\
\text { to Milk } \\
\text { (Per Cent) }\end{array}$ & $\begin{array}{l}\text { Number of } \\
\text { Outhreaks } \\
\text { Traced to } \\
\text { Milk }\end{array}$ & $\begin{array}{l}\text { Outbreaks } \\
\text { Traced to } \\
\text { Milk } \\
\text { (Per Cent) }\end{array}$ \\
\hline 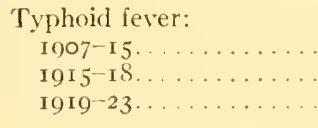 & $\begin{array}{r}23,482 \\
6,331 \\
4,105\end{array}$ & $\begin{array}{r}2,215 \\
496 \\
297\end{array}$ & $\begin{array}{l}9 \cdot 4 \\
7.8 \\
7 \cdot 2\end{array}$ & $\begin{array}{l}50 \\
\text { I } 6 \\
\text { I } 2\end{array}$ & $\begin{array}{l}69 \cdot 4 \\
57 \cdot 1 \\
70.6\end{array}$ \\
\hline Total. .......... & 33,918 & 3,008 & 8.86 & 78 & 66.8 \\
\hline 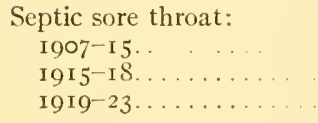 & $\begin{array}{r}\text { I }, 40{ }^{*} \\
829\end{array}$ & $\begin{array}{r}2,5 \text { I } 2 \\
867 \\
\text { I } 18\end{array}$ & $\begin{array}{l}61.9 \\
\text { I } 4.2\end{array}$ & $\begin{array}{l}7 \\
7 \\
3\end{array}$ & $\begin{array}{r}9.7 \\
25.0 \\
17.6\end{array}$ \\
\hline Total ........ & 2,230 & 3,497 & ? & I7 & I4. 6 \\
\hline 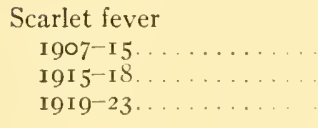 & $\begin{array}{l}70,569 \\
25,328 \\
46,777\end{array}$ & $\begin{array}{r}2,747 \\
140 \\
53\end{array}$ & $\begin{array}{r}3.9 \\
.6 \\
.1\end{array}$ & $\begin{array}{r}10 \\
4 \\
1\end{array}$ & $\begin{array}{r}\text { I } 3.9 \\
\text { I } 4.3 \\
5.9\end{array}$ \\
\hline Total. ....... & 142,674 & 2,940 & 2.06 & I 5 & I 2.8 \\
\hline 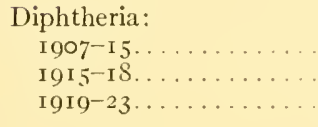 & $\begin{array}{l}69,646 \\
33,807 \\
42,386\end{array}$ & $\begin{array}{r}\text { I3I } \\
30 \\
8\end{array}$ & $\begin{array}{l}0.2 \\
0.1 \\
0.02\end{array}$ & $\begin{array}{l}5 \\
\text { I } \\
\text { I }\end{array}$ & $\begin{array}{l}6.9 \\
3.6 \\
5.9\end{array}$ \\
\hline Total.......... & $\mathrm{I} 45,839$ & 169 & O. II 6 & 7 & 6.0 \\
\hline
\end{tabular}

*Not reported until i9r5.

metropolitan cities is safeguarded. The smaller towns and rural sections mainly use raw milk. Frequently it is not economical for the small dealers to install pasteurizing apparatus. The problem in such places is one of co-operation and education.

TABLE II

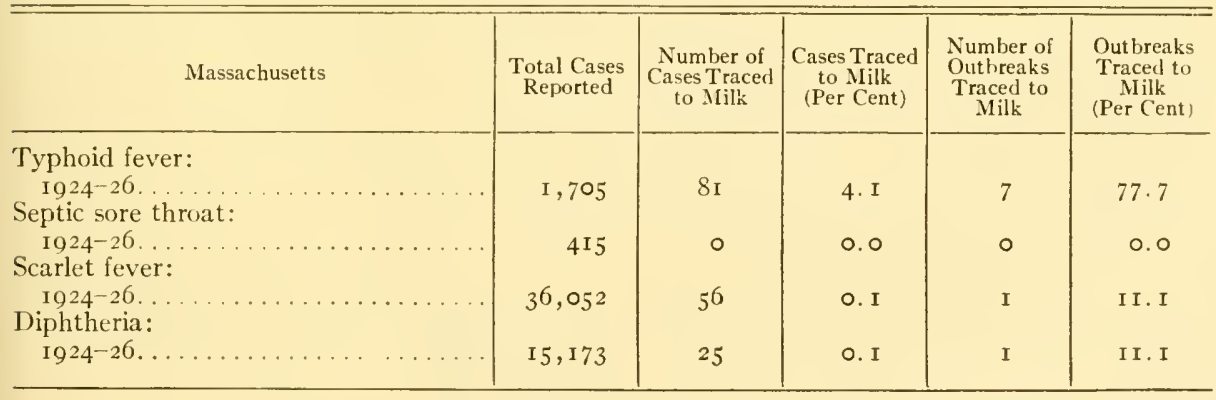

Tuberculosis.-Milk is the chief vector for the conveyance of bovine tubercle bacilli from cow to man. They get into milk either directly as a result of tuberculosis 
of the udder, which occurs in from I to 2 per cent of all tuberculous cows, or indirectly through cow manure. In the latter case the tubercle bacilli are coughed up, swallowed, and passed in the feces. Practically all market milk contains cow feces. Tuberculosis in cattle is very prevalent. In Holland nearly one-tenth of all cattle killed for food are tuberculous; in Berlin, 16 per cent; in Saxony, 30 per cent; in Pennsylvania, from 2 to 3 per cent. The "milk" from a tuberculous udder, when examined under the microscope, may contain as many tubercle bacilli as are ordinarily found in tuberculous sputum. The milk from a tuberculous udder of one cow may contain sufficient bacilli seriously to infect the mixed milk of twenty-five or thirty cows. In one case Ostertag found that $0.00 \mathrm{I} \mathrm{cc}$. of the secretion from a tuberculous udder was sufficient to cause tuberculosis in a guinea pig. In such a case a child would receive myriads in a gill.

We have evidence from four typical American cities. A total of 55I samples of raw milk have been examined in which tubercle bacilli were found in 46 , making a percentage of 8.3. Tonney, in Chicago (I9IO), found ro.5 per cent positive; Hess, in New York (I909), found I6 per cent positive; Anderson in Washington, found 6.72 per cent; and Goler, in Rochester, found 5 per cent of samples examined to contain tubercle bacilli. This may be taken as representative for the entire country.

Wherever these investigations have been carried out similar and sometimes higher results have been obtained, both in Europe and in this country. It is believed that the figures are an underestimate, for the methods used in the laboratory are not sufficiently delicate to detect a few tubercle bacilli in milk. Unless these micro-organisms are present in considerable numbers, they are likely to escape detection, for the tests depend upon infecting guinea pigs. Ordinary cultural or microscopic methods are not satisfactory to disclose the presence of tubercle bacilli in milk. In any event, it is clear that the common, raw, market milk furnished all large cities and probably most small towns very often contains tubercle bacilli. The hazard is real. ${ }^{\mathrm{r}}$

Typhoid fever.-Of milk-borne epidemics, typhoid fever takes the lead. Typhoid bacilli may swarm in milk without appreciably altering its taste, odor, or appearance. In our Washington studies, Io per cent of all the cases of typhoid fever during the four years 1907-Io were traced to milk. The recent figures for Massachusetts are given in Table I. Note that despite the decrease in the number of cases from year to year, the percentage of cases due to infected milk remains about the same. An outbreak of typhoid fever of unusual magnitude, in fact the largest on record, occurred in Montreal, Canada; between March I and June 29, I927, there were 4.755 cases with 453 deaths. ${ }^{2}$ All outbreaks occur on raw-milk supplies. The milk usually becomes infected by a carrier, sometimes by a convalescent or a missed case, and occasionally indirectly through infected water, utensils, etc.

Bolduan estimated in I9I 2 that from 300 to 400 cases of typhoid fever each year come in contact with the milk supplied New York City. He further stated that "the startling total of 90 to 20 typhoid carriers now probably menace the supply of this city." This estimate is based upon the fact that about 200,000 persons come into

${ }^{x}$ Cf. Tonney, F. O., White, J. L., and Danforth, T. F.: ibid., 17, 491. 1927.

${ }^{2}$ Report of the U.S. Public Itealth Service on the Montreal Typhoid Fever Situation. U. S. Pub. Health Rep., 42, I893. 1927. 
more or less contact with the milk from over 40,000 dairy farms. It is estimated that there are I,300 typhoid carriers in Massachusetts, 60 of whom are known (I927). Typhoid fever has also been traced to cream, ice-cream, and other milk products. Milk-borne outbreaks of paratyphoid fever have been described by Levine and Eberson, ${ }^{,}$Williams, ${ }^{2}$ and others.

Scarlet fever.-Milk-borne outbreaks of scarlet fever are sometimes extensive and serious. The milk is practically always infected from human sources. So far as known cows do not have scarlet fever. There is a suspicion, however, that some streptococcal infections of the cow may reproduce a disease resembling scarlet fever in man.

Two outbreaks of scarlet fever due to ice-cream have been reported. One took place in South Kensington, England, in I 875 , following a large dinner where the dessert was frozen pudding. ${ }^{3}$ A clear-cut outbreak due to ice-cream occurred in Flint (Mich.), in July and August, I924, involving forty-one cases extending over seven days. The ice-cream was infected by the maker, who had a mild case of scarlet fever. ${ }^{4}$ Cold does not destroy, but rather preserves, this and other viruses.

Diphtheria.-Diphtheria bacilli in milk practically always come from human sources, either cases or carriers. In a few rare instances ulcers upon the teat of the cow have become infected with diphtheria, and the bacilli are thus transferred to the milk. Such an occurrence, however, is unusual. Even in such instances the ulcers are infected from human sources. As a rule, diphtheria outbreaks caused by infected milk are more limited both as to numbers and area than milk-borne outbreaks of typhoid or scarlet fever.

Septic sore throat.- The first milk-borne outbreak of "septic sore throat" recognized in this country occurred in and about Boston in May, I9I I. Since then similar outbreaks have occurred in Baltimore, in Concord in New Hampshire, in Chicago, and elsewhere. The disease has spread, and our country is now seeded with this infection. Septic sore throat due to infected milk has been well known in Great Britain for thirty years. Swithinbank and Newman state that a year never goes by in which there are not outbrcaks of sore throat or tonsillitis due to milk or cream. The infection usually gets into the milk from human sources, although it is suspected that some streptococci associated with diseases of the udder may be pathogenic for man.

Smith and Brown ${ }^{5}$ have shown that the responsible organism is Streptococcus epidemicus ${ }^{6}$ and furthermore disclose a difference between human (epidemicus) and bovine $(\beta$ type $)$ streptococci. Both are hemolytic. The bovine streptococci produce garget in cows but do not cause tonsillitis in man; on the other hand, the human streptococci produce sore throat in man but have slight pathogenicity for cows.

Smith and Brown studied the streptococci from five milk-borne epidemics at first hand, together with cultures from the big epidemics of Chicago, Baltimore, and Bos-

${ }^{x}$ Levine, M., and Eberson, F.: J. Infect. Dis., I8, г43. г9г6.

${ }^{2}$ Williams, H: J.A.M.A., 84, 25 I. I925.

${ }^{3}$ Buchanan, G. S.: Rep. Loc. Gov. Bd. Suppl. 72 . 1875 .

${ }^{4}$ Ramsey, G. H.: Am. J. Hyg., 5, 669. 1925.

${ }^{5}$ Smith, T., and Brown, J. H.: J. Med. Research, 31, 455. I9I5; also Smillie, W. G.: J. Infect. Dis., $2 \mathbf{I}, 45$. I9I 7 .

${ }^{6}$ Brown, J. H., Frost, W. D., and Shaw, M.: J. Infect. Dis., 38, 381, I926. 
ton. From this work it is now clear that septic sore throat in man is of human origin, even when the disease is contracted through milk infected in the udder, for it was found that while the human streptococcus is but slightly pathogenic for cows, this organism may become implanted in the udder. This may take place through milking, wiping with infected cloths, through passing quills up the milk ducts, and in similar ways. When this takes place garget does not ensue, but the streptococcus becomes seeded in the udder and may remain for six weeks or longer. In other words, the cow may become a passive carrier of the human streptococcus, thus explaining why milk-borne outbreaks of septic sore throat are sometimes long drawn out.

The hemolytic streptococci found in milk show distinct cultural and biological differences from St. epidemicus. No single test, however, can be relied upon to differentiate them. Brown, Frost, and Shaw found hemolytic streptococci in considerable numbers in the certified milk of five dairy herds. These streptococci belonged to several distinct cultural and serological groups. There is no evidence that any of them, whether from gargety cows or from mixed milk, are pathogenic for man. Therefore, to justify condemnation of the milk supply upon bacteriological grounds alone, the streptococcus found should correspond in all respects with the characters of $S t$. epidemicus.

Milk-sickness.-Milk-sickness is an acute non-febrile disease due to the ingestion of milk, milk products, or the flesh of animals suffering from a disaase known as "trembles." The disease is characterized by great depression, persistent vomiting, obstinate constipation, and high mortality. The picture is that of a poisoning rather than an infection.

Milk-sickness is primarily a disease of cattle, secondarily of man. In cattle it is called "slows" or "trembles," and formerly was prevalent in the central part of the United States. The pioneers suffered severely in winning the West. Nancy Hanks, the mother of Lincoln, died from the disease in I8I 8 after an illness of a week. As forests are cleared and pastures fenced the disease becomes less frequent. It is still met with in the valley of the Pecos River, New Mexico, in parts of Tennessee and North Carolina. The cause of the disease has been obscure. Alkali drinking water has been accused; pathogenic micro-organisms have been described; and poisonous plants have been suspected.

Marsh $^{\mathrm{I}}$ studied the rayless goldenrod, A plopappus heterophyllus, and concludes that milk-sickness is a poisoning due to this plant. He produced trembles or alkali disease in horses, cattle, and sheep by feeding sufficient quantities of the rayless goldenrod. The toxic principle is excreted in the milk, and calves and lambs may be poisoned in this manner. Stock eat this weed only where there is little good forage. The obvious remedy is to see that the animals are well fed. It is entirely practical to dig out the weed in fenced pastures.

Malta fever.-Malta fever is a disease primarily of goats, secondarily of man. The infection is transmitted from goats to man through raw milk containing Brucella melitensis. There is an extraordinary relationship between Bruc. melitensis, the cause of Malta fever, and Bacillus abortus, the cause of contagious abortion of cattle. The latter often contaminates milk. Thus, Schroeder and Colton found this bacillus in

${ }^{x}$ Marsh, C. D.: Bull. Cur. Anim. Indust., U.S. Depl. Agric. May, I925. 
eight out of twenty-seven samples of market milk tested. It was long assumed that $B$. abortus could not be pathogenic for man in view of the frequency with which this virus is found in milk and the infrequency of the infection in man. However, it has recently been disclosed that some strains are pathogenic, and about Ioo cases of this disease in man have been recorded. The interesting bacteriological and immunological relationships are being studied.

Epidemic arthritic erythema.-An outbreak of this infection occurred at Haverhill (Mass.), in January, I 926, involving eighty cases, with no deaths. It was studied by Place, Sutton, and Willner. ${ }^{\text {I }}$ The disease resembles dengue clinically, has a sharp onset with a chill, fever, headache, and toxic manifestations. The eruption resembles that of dengue, is measly or rubilliform, and affects especially the extremities. Pain and swelling in the larger joints are common features. The period of incubation is two to three days. All except one of the cases cited drank raw milk from the same dairyman. The source of the infection was not traced. Place and his associates have isolated a gram negative, non-spore-bearing bacillus which seems to be the cause of the trouble. A similar outbreak occurred during May-June, I925, at Chester (Pa.), involving four hundred cases with no deaths. This epidemic was first diagnosed as dengue. $^{2}$

Foot-and-mouth disease.-Foot-and-mouth disease is an infection primarily of cattle and secondarily of man. It is caused by a filterable virus, and is noteworthy for being the first ultramicroscopic virus of animals to be discovered (Loeffler and Frosch, I 898). The infection is transmitted to man through the ingestion of raw milk, buttermilk, cheese, or whey from diseased cows. Children are not infrequently infected by drinking unboiled milk when the disease is prevalent in the neighborhood. In man the disease is mild. The symptoms resemble those observed in animals: There is fever, sometimes vomiting, painful swallowing, heat and dryness of the mouth, followed by an eruption of vesicles in the buccal and mucous membranes, and very rarely by similar ones on the fingers. The vesicles are about the size of a pea; they soon break, leaving small erosions, which rapidly heal. The disease is seldom fatal except occasionally in very weak children.

Diarrheal infections.--Milk may be responsible for gastro-intestinal troubles, some of which are specific. Bacillus enteritidis and its congeners grow well in milk and are the cause of milk-borne outbreaks of food infection. Fresh-milk products are also responsible.

Dysentery has been traced to milk in a number of instances. Dysentery bacilli grow well in milk, andinfected milk is one of the common causes of infantile diarrheas.

Milk is usually too acid for the vibrio of cholera.

One of the chief causes of the high infant mortality is summer diarrheas, but even these are not all due to stale, dirty, and bacteria-laden milk. Many of the diarrheal diseases of infancy are true cases of bacillary dysentery, which is transmitted in a great variety of ways. However, the improvement in the milk supply for babies has directly, and in large part indirectly, resulted in a decrease in infant mortality in recent years.

I Place, E. H., Sutton, L. E., and Willner, O.: Boston M. \&o S. J., 194, 285.1926.

${ }^{2}$ Report (unpublished) by Armstrong, C.: U.S. Pub. Health Serv., July 18, 1925. 
Infantile paralysis (poliomyelitis).-To this long list of milk-borne diseases must now be added infantile paralysis. Two small outbreaks, one at Cortland (N. Y.) and another at Spring Valley (N.Y.), have been described which seem attributable to milk infected by a case of the disease during the acute stage on the dairy farm. Recently, a third and larger outbreak occurred at Broadstairs (England).

Dingman ${ }^{1}$ reported a group of eight cases in and around Spring Valley (N.Y.), all of which had their onset between July 20 and 22, I9I6. All the cases used raw milk from the same two-cow dairy. Three families who used this milk but boiled it escaped. There was a case, a four-year-old child, at the dairy who became ill with the disease on July 4.

The outbreak in Cortland occurred December $14-25,1925 .{ }^{2}$ All the eight cases, which were widely separated, occurred among the users of the same supply, which was only 4 per cent of the milk supplied the city. There was a paralytic case on the dairy farm, and this individual milked the cows and handled the milk for a period of four days (December $7^{-\mathrm{I} I}$ ) while in the acute stage of the disease. The milk was not pasteurized, and no case occurred among those who first boiled the milk.

In October, I926, an epidemic of seventy-two cases of infantile paralysis occurred in Broadstairs (England), which was studied by Aycock. ${ }^{3}$ The explosive nature of the epidemic is shown by the occurrence of seven paralytic cases on October Io and six on October I I fifty-eight of the cases occurred between October ro and 20. Most of them were among the better class. There was clear epidemiological relationship with a particular milk supply, fifty cases occurring among known users of this milk, and nineteen others in individuals supplied by another dealer who was known to have purchased a part of the suspected milk supply.

Summary and discussion of milk-borne diseases.-All the outbreaks have invariably been traced to the use of raw milk. There is no authentic record of a milk-borne epidemic due to properly pasteurized milk. Raw milk is likely to be dangerous milk. Contrary to the common belief, most milk-borne epidemics have been associated with milk of an unusually high grade - even certified milk has been responsible for outbreaks of diphtheria, scarlet fever, and septic sore throat. Most of the serious infections get into milk directly and sometimes indirectly from human sources. Only in occasional instances are the diseases of cattle (other than tuberculosis) transmitted to man. Cream, ice-cream, and other fresh-milk products are quite as dangerous as the milk from which they are obtained.

\section{METHODS OF PASTEURIZATION}

There are five methods of pasteurizing milk: (I) the flash method, (2) the holding method, (3) continuous flow, (4) the vat method, and (5) in the final container. These are discussed in chapter xxxiii of this volume.

\section{DISCUSSION OF ADVANTAGES AND DISADVANTAGES}

Pasteurization prevents sickness and saves lives. Pasteurization is not ideal but only an expedient. It is advocated because milk is likely to convey the viruses of a

${ }^{2}$ Dingman, J. C.: Nerv York State J. Med., 16, 589. 1916.

${ }^{2}$ Knapp, A. C., Godirey, E. S., Jr., and Aycock, W. L.: J.A.M.A., 87, 635. 1926.

${ }^{3}$ Aycock, W. I..: in press. 
number of diseases harmful to man. Pasteurization effectively prevents this hazard. It implies precaution, protection, and prevention. It is the best insurance both for the industry and the consumer, and the simplest, cheapest, least objectionable, and most trustworthy method of rendering infected milk safe.

Pasteurization does not claim to replace sanitation and common decency. It cannot atone for filth and should not be used as a redemption process. Stale, weak, and dirty milk is still stale, weak, and dirty after it has been pasteurized. Theoretically, a pure milk is better than a purified milk. However, no one should drink raw milk that cannot be guaranteed by the health officer as safe and free from danger. Rash, indeed, would be the health officer who would give any raw milk a safe bill of health, for raw milk is likely to be infected by carriers, missed cases, or other unseen means. Furthermore, infection in milk does not disclose its presence by our available tests. Even certified milk or milk of equally high character is not safe without pasteurization. Less than $\mathrm{x}$ per cent of all the milk found upon the market is certified. Therefore, raw milk of this honor class is not a public health problem of any magnitude, although it has been responsible for outbreaks of diphtheria, scarlet fever, and other diseases. There is no authentic record of any milk-borne epidemic caused by properly pasteurized milk.

It is sometimes alleged that pasteurization does not destroy nature's danger signal-souring. Milk pasteurized at the temperatures recommended $\left(\mathrm{I} 42^{\circ}-\mathrm{I} 45^{\circ} \mathrm{F}\right.$.) sours as a result of lactic acid fermentation just as raw milk does, although somewhat more slowly. Nature has no danger signal for infected milk. Milk may be teeming with typhoid bacilli and other pathogenic micro-organisms without its taste, odor, or appearance being changed.

Pasteurization is sometimes objected to because it does not destroy heat-resisting toxins which are supposed sometimes to be in milk. The occurrence of such poisons is a mere assumption. Even if they exist in milk, they would be in the raw milk as well as in the heated milk.

One objection to pasteurization has always been the claim that it will put back the cause of clean milk and good dairy methods, because pasteurization will make cleanliness unnecessary and will put carelessness at a premium. Experience has proved the fallacy of this argument; in fact, the general milk supply of large cities has materially improved despite pasteurization.

Pasteurization is not proposed as a substitute for, but as an adjunct to, inspection. Inspection gives us cleaner and better, but not necessarily safe, milk. Inspectors cannot be present all the time, and, furthermore, even if they were Pasteurs, they could not see missed cases and carriers. Pasteurization destroys the dangers inspection cannot see. The combination of inspection and pasteurization corresponds in all respects to the modern principles of furnishing a safe water supply to a large city. The watershed, through inspection, is kept as clean as practicable, but the water is filtered or purified to protect the consumer.

There can be no more objection to the heating of milk for the use of adults or children above the age of one year than there is to the cooking of meat. Infants should receive breast milk. There is no adequate substitute. When this is not possible, they should have the best and freshest cow's milk that can be obtained. Whether such milk is to be pasteurized, modified, or otherwise treated rests with the pediatrician. 
Pasteurization has the well-nigh unanimous indorsement of sanitarians and pediatricians.

Pasteurization is too important a public health measure to leave to individual caprice. The process should be under official supervision. The sanitary code should clearly define the requirements, and pasteurized milk should be labeled as such, stating the degree of heat and the length of time and the date on which the process was done. Action for misbranding may be taken for milk labeled "pasteurized" but which has been processed by improper or incomplete methods. Milk should not be pasteurized twice.

Only pasteurizing machinery of an approved design should be permitted, and the management and care of the entire procedure should be under skilled supervision and frequent inspection. In addition, bacteriological examination of samples before and after the process tells the story of its efficiency.

Pasteurized milk should be bottled by machinery immediately following the process, kept cold, and delivered promptly. The bottles should first be disinfected with steam or scalding water. All milk, whether raw or pasteuri ed, should be kept clean, cold, and covered.

Milk should be pasteurized at some central station where it may be done scientifically under official surveillance. In other words, it should be done for the householder just as in the case of central water-purification plants. If water needs filtration or chlorination, experience teaches that it is inadvisable and expensive to depend on the householder to carry out the process. The same is true with pasteurization.

There has been a prejudice against pasteurization. To the sanitarian this is unreasonable and unnecessary. Pasteurization as a process does not depend upon faith but upon fact, and its problems cannot be solved by discussion but by the confirmation of the data obtained by careful observation. It is difficult for the sanitarian to understand why so many people oppose so simple and effective a protection against sickness and untimely death. To the health officer pasteurization is a major public health prophylactic measure; in fact, it is now almost universally used by the large dairies in our metropolitan cities. The problem at present is to extend this protection to the milk supplies of the smaller towns and rural sections. This is fraught with practical difficulties largely economic. For smaller towns, co-operation and consolidation of the industry are an advantage.

Pasteur did not think of milk when he advocated the heating of beer and wine, but, as with much of the other work of this genius, he established here a fundamental principle which has signal public health applications. 


\title{
CHAPTER XXXIII
}

\section{MECHANICAL AND ENGINEERING ASPECTS OF PASTEURIZATION}

\author{
GEORGE W. PUTNAMI \\ Department of Health, Chicago, Ill.
}

\section{METHODS OF PASTEURIZATION}

Milk is pasteurized commercially by one of the following methods: (I) the holding method, using the vat, pocket, continuous-flow, and in-bottle pasteurizers; (2) the flash mechod, using steam or electricity.

\section{HOLDING METHOD}

The holding method of pasteurization is practically universally used for milk in the United States. It consists in heating the milk to the legally defined pasteurizing

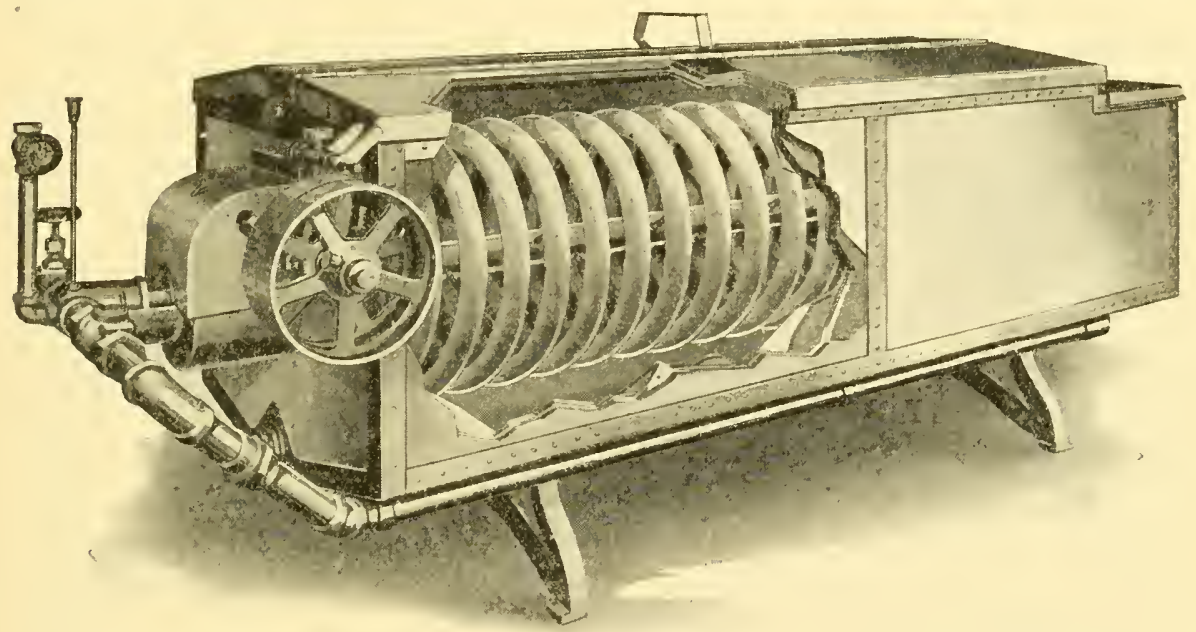

FIG. I.-Vat-type pasteurizer (partial-section view); jacketed type with interior heating coil

temperature (for example, $\mathrm{I} 42^{\circ}$ or $\mathrm{I} 45^{\circ} \mathrm{F}$.), maintaining it at this temperature in a suitable holder for a period of at least thirty minutes, followed by immediate cooling to $50^{\circ} \mathrm{F}$. or lower.

The vat-type pasteurizer consists of an insulated or jacketed vat (see Figs. I and 7) in which the foregoing operations are carried out by one of the following methods: (a) The milk is heated to the pasteurizing temperature by a milk-heater and discharged into one or more vats, which act merely as holders for the milk for the thirtyminute period. The milk is then pumped or flows by gravity from the vat over a cooler. (b) The milk is heated in the vat to the pasteurizing temperature by means 
of heating coils or a heating jacket and agitator, held for thirty minutes, and then run over a cooler. (c) In many instances a combination of the two methods is used, the milk being heated to from $100^{\circ}$ to $140^{\circ} \mathrm{F}$, by a milk heater, discharged into the vat, brought to the exact pasteurizing temperature by means of the heating coil or jacket, held for thirty minutes, and cooled. (d) In a few instances the milk, after being heated and held by one of the foregoing methods, is cooled in the vat by passing cold water or brine through the coils. This usually reduces the cream volume on the bottled milk. In most cases the milk is pumped or flows by gravity over separate coolers. On the other hand, cream, on account of its greater viscosity, is very often heated, held, and cooled in the same vat.

The pocket-type pasteurizer consists of a series of small vats built integrally in a single holder unit (see Figs. 2 and ro). The individual vats or pockets are either insulated or jacketed and may or may not be equipped with an agitator. Milk is pumped continuously through a heater, brought to the pasteurizing temperature, and discharged into the holder pockets in succession by means of one or more automatically operated valves.

Two circular-type holders on the market are designed to rotate at a constant rate so that the sector pockets will be filled in succession by passing under a stationary milk-inlet pipe. In all types of pocket holders the milk, after a thirty-minute holding period, is discharged from the pockets in succession by automatic means, insuring practically continuous flow of milk to the cooler.

The contimuous-flow pasteurizer consists of a holder through which heated milk flows continuously. One holder used extensively consists of a cylindrical tank with nested baffles in which milk, discharged in at the top, flows down through the holder by gravity and is pumped from the bottom over a cooler.

A second holder, termed the "long-distance-flow type," consists of a series of metal tubes, $5 \frac{3}{4}$ or 7 inches in diameter, having a very slight slope, housed in a large boxlike compartment in which the air is heated and maintained at a fairly uniform temperature (see Fig. 3). The milk is heated to the pasteurizing temperature, enters the holder, flows through each of the tubes in succession to the outlet, taking thirty minutes or more for this travel, and is then pumped to the cooler.

The in-bottle pasteurizer, as its name implies, is an arrangement for pasteurizing milk in the bottle in which it is to be delivered to the consumer. Small outfits consist of a combination steam and ice-chest in which cases of bottled milk are placed, using special covers over the bottles, heated with steam to the pasteurizing temperature, held for thirty minutes, and then cooled with water and ice. The cases of milk are then removed and the bottles capped with the ordinary paraffined cardboard cap. In some instances the bottles are capped with crown caps before pasteurizing.

The large, continuous in-bottle machines have traveling carriers for the cases. Bottles are filled with milk, preheated to about $100^{\circ} \mathrm{F}$., and capped with a crown cap. The cases of bottled milk travel on the carriers through a succession of water compartments which first heat the milk to the pasteurizing temperature, hold it for thirty minutes, and then cool the milk.

The in-bottle method has the disadvantage of being somewhat more expensive 
to operate, owing to having to heat and cool successively the glass bottles, cases, and parts of the machine in addition to the milk itself. The consumer must also become

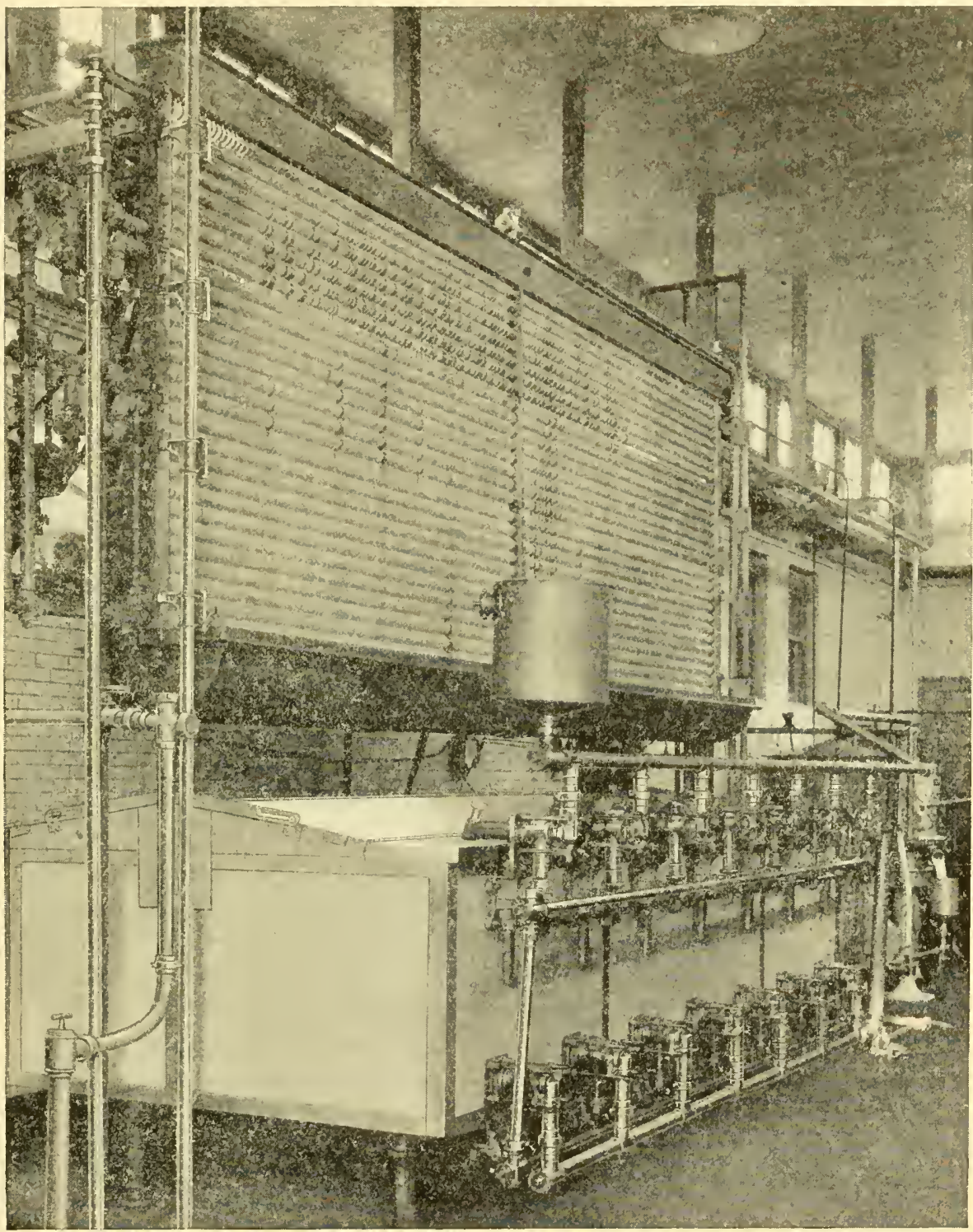

FIc. 2.-Pocket-type pasteurizer; equipped with leak-protector inlet and outlet valves; external surface cooler (above).

accustomed to receiving bottles which are not completely filled, since an empty space, $\frac{3}{4}$ inch deep, remains at the top of the bottle, owing to the difference in volume between the hot milk during pasteurization and the cold milk after cooling. A real ad- 
vantage from the public-health point of view is that this system eliminates the possibility of contamination of the milk between pasteurization and bottling, which may occur with other methods.

\section{FLASH METHOD}

The flash method of pasteurization was extensively used prior to I9I4 and consists in rapidly heating the milk to a temperature of about $160^{\circ} \mathrm{F}$. and immediately

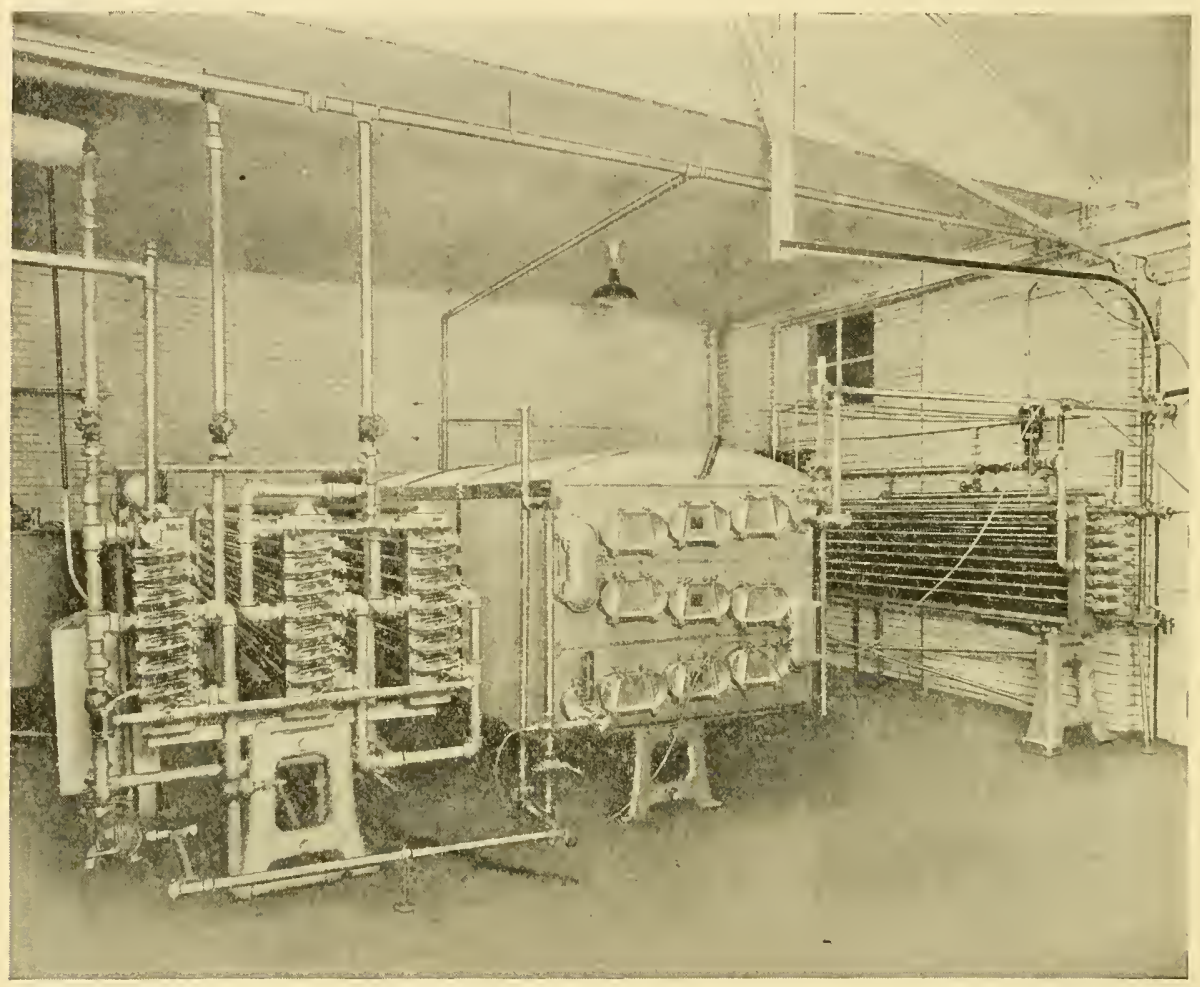

Fig. 3.- Continuous-flow pasteurizer, long-distance flow type; internal tubular cooler (three sections), holder, internal tubular heater.

cooling. This method was condemned by many health officials on account of the fact that, as carried out in commercial practice, there were large fluctuations in the temperature of the heated milk and the temperatures used in a large percentage of cases were considered too low to be effective. ${ }^{\mathrm{I}}$ Several epidemics of disease :were traced to milk treated by this method. It was also objected to by many physicians ${ }^{2}$ on the grounds that practically all the lactic acid or souring bacteria were destroyed and other bacteria left viable with the result that the milk on standing would decompose by putrefaction instead of by souring. The occasional uneven and excessive heating of the milk

I Ayers, S. H. and Johnson, W. T.: U.S. Dept. Agric., Bur. Animal Industry, Bull. I6I, p. 5 S. 1913.

${ }^{2}$ Hyg. Lab. Bull. 56, p. 678. I909. 


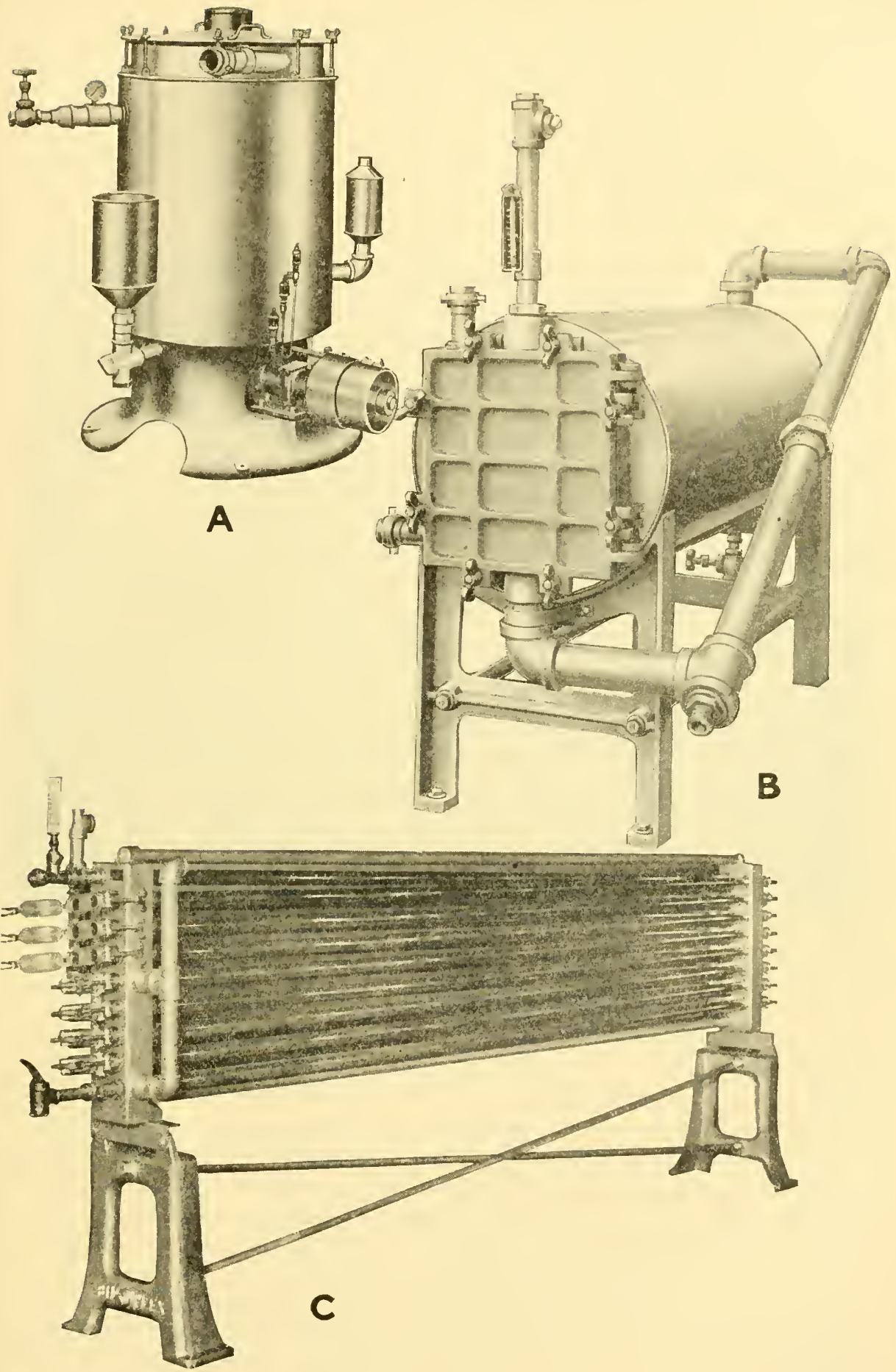

FIG. 4.-Milk heaters: (a) centrifugal, (b) barrel, $(c)$ internal tubular type 
by this method reduced the cream volume on the bottled milk so that many of the dealers themselves became dissatisfied with flash pasteurization.

Practically any type of milk heater, such as the Danish or centrifugal heater, the barrel heater, or the tubular heater, can be used for flash pasteurizing (see Fig.4). The use of a temperature controller insures more even heating of the milk and eliminates the necessity of controlling the hot water or steam, used as the heating medium, by hand-operated valves.

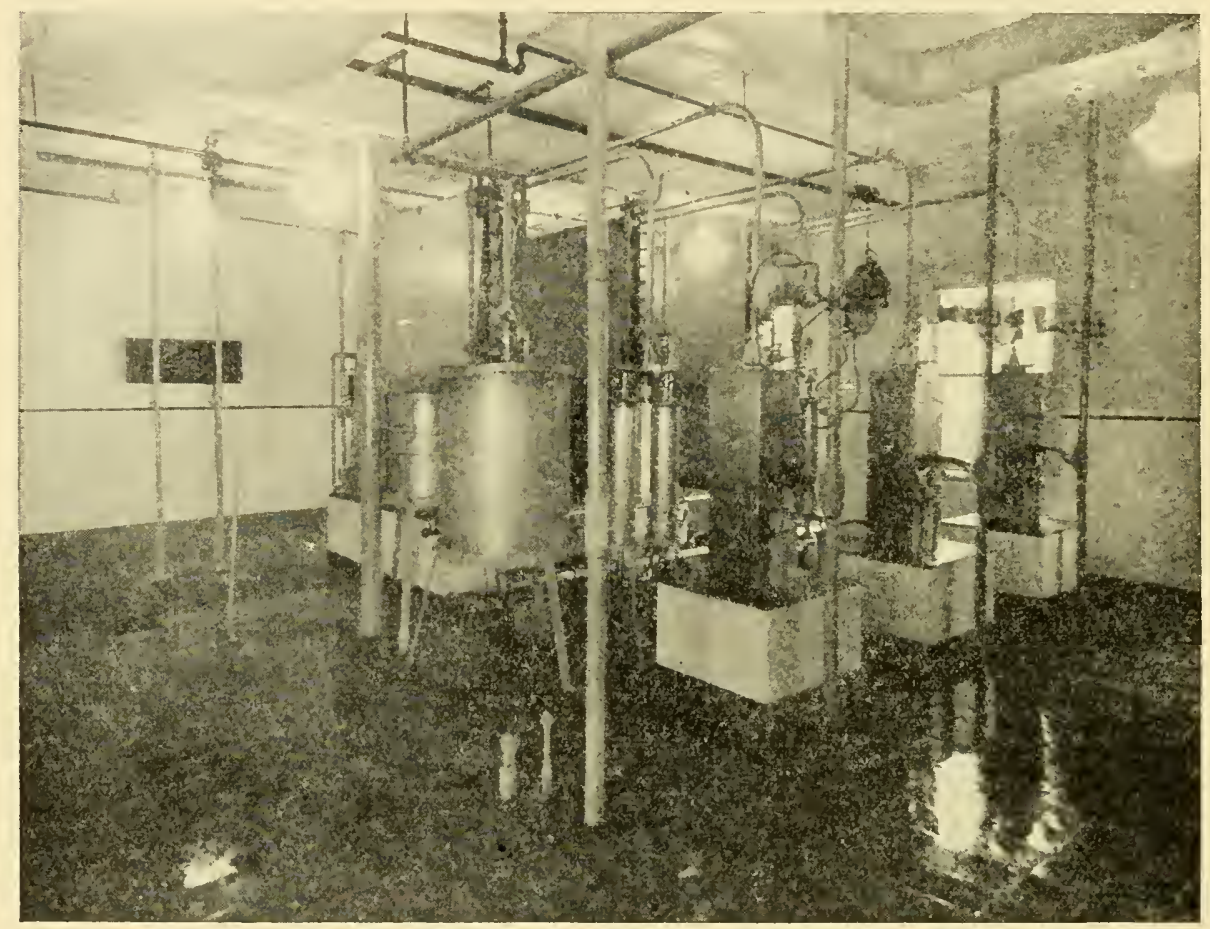

FIG. 5.-Electric-flash pasteurizer (three units); regenerative heater cooler, filter, electrical heating chamber.

The treatment of milk by an electrical method, known as "electropure," is essentially flash pasteurization, using electricity for part of the heating. The raw milk is pumped through a regenerative heater-cooler, bringing it up to about I $20^{\circ} \mathrm{F}$, , through a filter and into an electrical heating chamber in which electricity passes through the milk and heats it by resistance to about $160^{\circ}$ F. (see Fig. 5). The treated milk then passes over the outside of the regenerative heater-cooler and over a second section of cooler using brine. An electrical thermostatic temperature-controller is provided with the bulb located in the outlet pipe from the electrical heating chamber.

PASTEURIZATION DEFECTS AND THEIR CORRECTION

The tremendous benefit of pasteurization of milk to the public health is generally recognized. It has been the greatest single factor in the prevention of thousands of 
deaths which would have otherwise resulted from the use of contaminated raw milk. Only recently we have had a forceful reminder of the sickness and death which can be spread through contaminated raw milk in the example of the Montreal typhoid epidemic of $1927,{ }^{\mathrm{I}}$ the largest epidemic of this disease in many years. A frank discussion of defects preventing proper pasteurization should not be interpreted as belittling in the least the rôle that it has played in the past, but rather as an effort to arrive at means of insuring that commercial pasteurization will give more complete protection against milk-borne diseases.

The publication in February, I925, of the report ${ }^{2}$ on extensive investigations of commercial pasteurization by North and his collaborators marked a milestone in the advancement of milk pasteurization. These studies, known as "The Endicott (N.Y.) Experiments," are the most extensive series of tests on the destruction of pathogenic bacteria in commercial milk-pasteurizing equipment that has ever been undertaken. Although their primary purpose was to establish a safe temperature for pasteurization by the holding method, the main value to milk sanitarians was that they revealed conclusively that mechanical and engineering defects prevented proper pasteurization of all the milk. The finding of living tubercle bacilli in samples of milk collected during numerous test runs on commercial pasteurizers in the Endicott experiments (first series) was proof that the defects in the equipment prevented complete destruction of these organisms under conditions of time and temperature in the main body of the milk which laboratory studies indicated should accomplish this.

The general conclusion made by the authors following the first series of tests with pathogenic bacteria states in part:

The bacteriological examinations confirm the results of the engineers' observations. The engineers had noted defects in the principles, both of the construction and equipment, of all three types of pasteurizers which it was impossible to overcome by even the most skillful supervision of the methods of operation. . . . Everything considered, however, it is proper to observe that the results plainly indicate the importance of better engineering in the building and equipment of these representative types of commercial pasteurizers if they are to be accepted with confidence as proper safeguards of public milk supplies.

It is of interest to note that many of the defects emphasized in this publication were brought to the attention of the public-health profession in the 1920 report ${ }^{3}$ of the Committee on Milk Supply of the Public Health Engineering Section, American Public Health Association. As a result of these revelations, a comprehensive engineering program for the correction of defects in pasteurizing equipment was organized in Chicago in March, I926, by Dr. Herman N. Bundesen, commissioner of health. An outline of this program was recently reported by the writer. ${ }^{4}$ Coincident with this, the United States Public Health Service established its pasteurization-equipment testing station in Chicago, thus effecting a joint research and administrative program.

${ }^{1}$ Am.J. Pub. Health, 17, 783. 1927.

${ }^{2}$ North, C. E., Park, W. H., Moore, V.A., Rosenau, M. J., Armstrong, C., Wadsworth, A. B., and Phelps, E. B.: Commercial Pasteurization, Pub. Health Bull. 147. Feb., I925.

${ }^{3}$ Committee on Milk Supply of the Public Health Engineering Section: Pasteurization of Milk, Am. Pub. Ilealth Assoc. Rep. Aug., 1920.

${ }_{4}^{4}$ Putnam, G. W.: Am.J. Pub. Health. I7, I2 I. I927. 


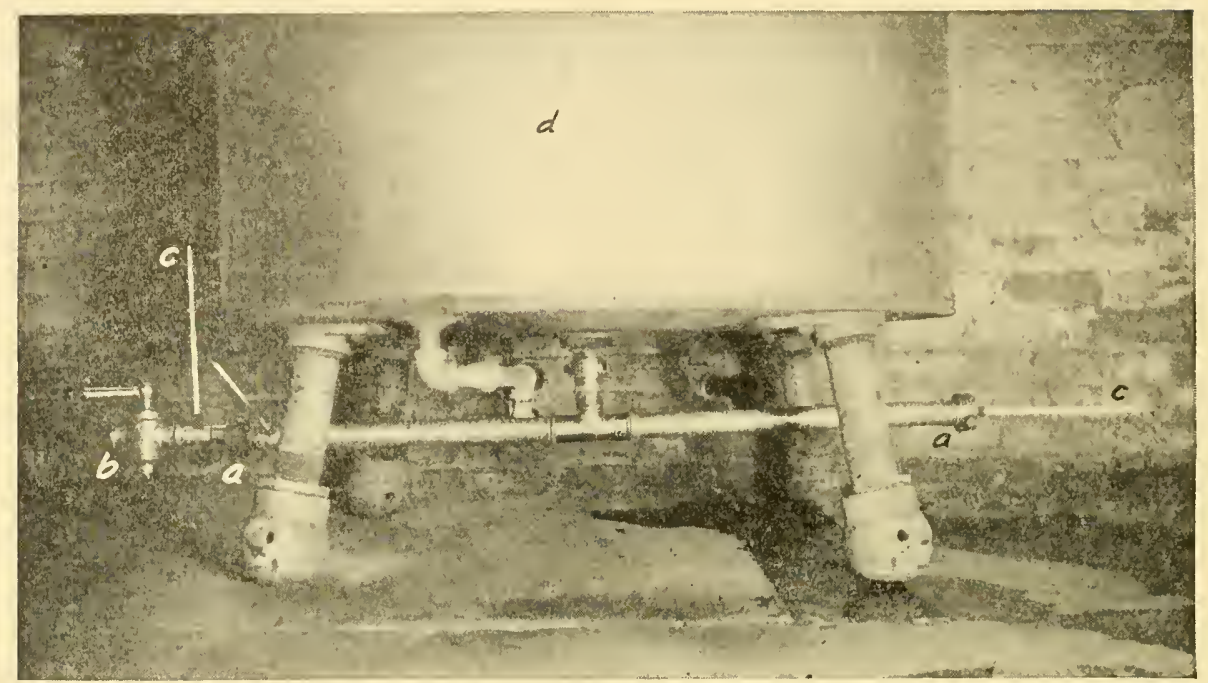

Fic. 6.- Showing dead-end outlet pipe on round vat holder: $(a-a)$ outlet pipe, $(b)$ outlet valve, $(c-c)$ test thermometers, $(d)$ round vat holder.
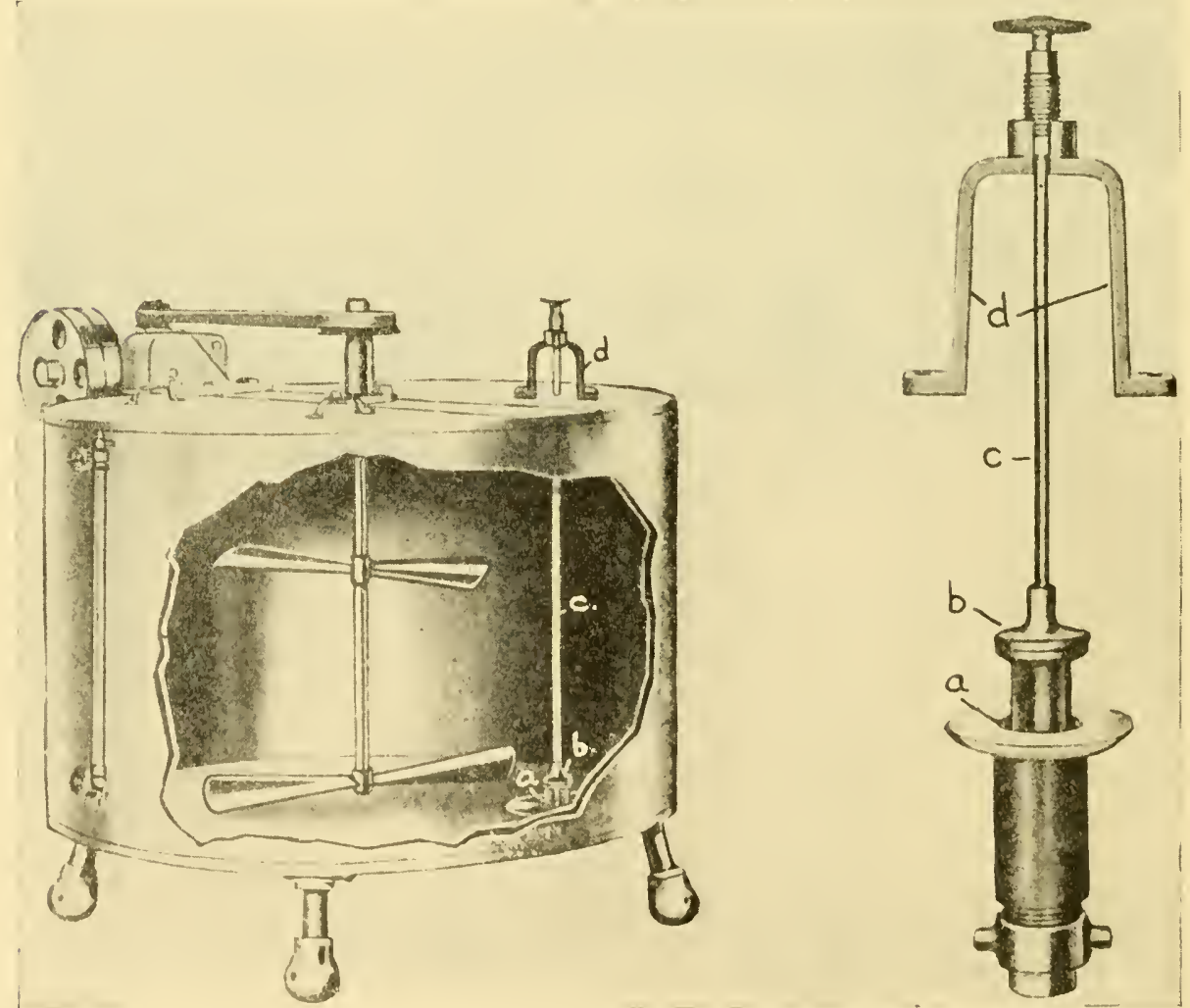

Fig. 7.-Showing round vat holder with heating jacket equipped with bottom-outlet flush-type valve: (a) flush valve seat, (b) valve plug, (c) rod, $(d)$ spindle. 
Tests have been and at this writing are being made on practically every type of pasteurizing apparatus for the purpose of determining the thermal treatment received by the milk under actual plant-operating conditions and locating any engineering defects in the apparatus preventing proper pasteurization of any portion of the milk. When a comprehensive report based on these studies is issued, detailed information will be available to health officers on the various types of commercial pasteurizing equipment. As defects are encountered in these studies they have been brought to the attention of the equipment manufacturers with the result that they have been corrected in much of the new equipment being sold.

Defects found in commercial equipment which will prevent proper pasteurization of all the milk may be grouped as follows: (I) dead ends, (2) leakage through valves, (3) foam and splash, (4) defective continuous-flow units, (5) unsatisfactory thermometers.

The reasons why these defects prevent proper pasteurization and the methods adopted for their correction are outlined below.

Dead ends.-Dead ends are sections of pipe or other pockets in which the temperature drops below that required, preventing the proper pasteurization of the milk held in them. An example of this defect on a vat holder is illustrated in Figure 6. The $2 \frac{1}{2}$ pints of milk in the dead-end outlet pipe $(a-a)$ is entirely outside the zone of agitation and heating and remains cold while the main body of the milk in the vat above is heated and held for thirty minutes. The remedy is obviously the installation of a flush-type valve such as that shown in Figure 7 , which will eliminate the dead end.

In the case of in-bottle pasteurizers, means must be provided to insure that every bottle of milk, and every portion of milk in each bottle, receives the required heat treatment. ${ }^{\mathrm{I}}$

Leakage through valves.-Practically all milk valves will leak to some degree, particularly after they have become warped in sweating on a connection or scored, dented, and worn by usage. Leaky inlet valves permit raw milk to drain into the holder during the holding and emptying period. Similarly, leaky outlet valves permit milk from the holder to escape into the outlet pipe before it is completely pasteurized. In neither case is the leakage subjected to the pasteurizing temperature for the full thirty-minute holding period. Such leakage may be small in some instances, but an amount equal to more than I per cent of the total volume pasteurized has been found in numerous cases.

The remedy for this defect is obviously to divert the leakage of unpasteurized milk. This can be accomplished by disconnecting the inlet piping after each filling of a holder and the outlet piping immediately after each emptying. In order to eliminate labor, wear, and tear on piping and fittings and make this operation more positive, leak-protector inlet and outlet valves have recently been developed which are so constructed that any leakage will not pass through the valve but will be drained out at the bottom.

Figure 8 shows a leak-protector inlet valve consisting of a standard two-way plug valve with a groove in the plug on each side of the discharge opening to carry away leakage. Figure 9 shows a flush-type leak-protector outlet valve for use on a vat holder. When the valve is closed, a leak drain and steam connection are auto-

' Ayers, S. H., and Johnson, W. T.: U.S. Dept. Agric. Bull. 2.qo. I9I5. 
matically opened. Any leakage from the holder past the flush-type seat will escape out the leak drain. Any film of leakage not draining out is sterilized by the small amount of steam continuously passing into the closed valve through the steam connection.

On automatic, pocket-type holders the elimination or remodeling of the multipleport rotary valve has been found necessary on most types. Figure io shows a holder of this type in which the leakage of unpasteurized milk through the valve was found

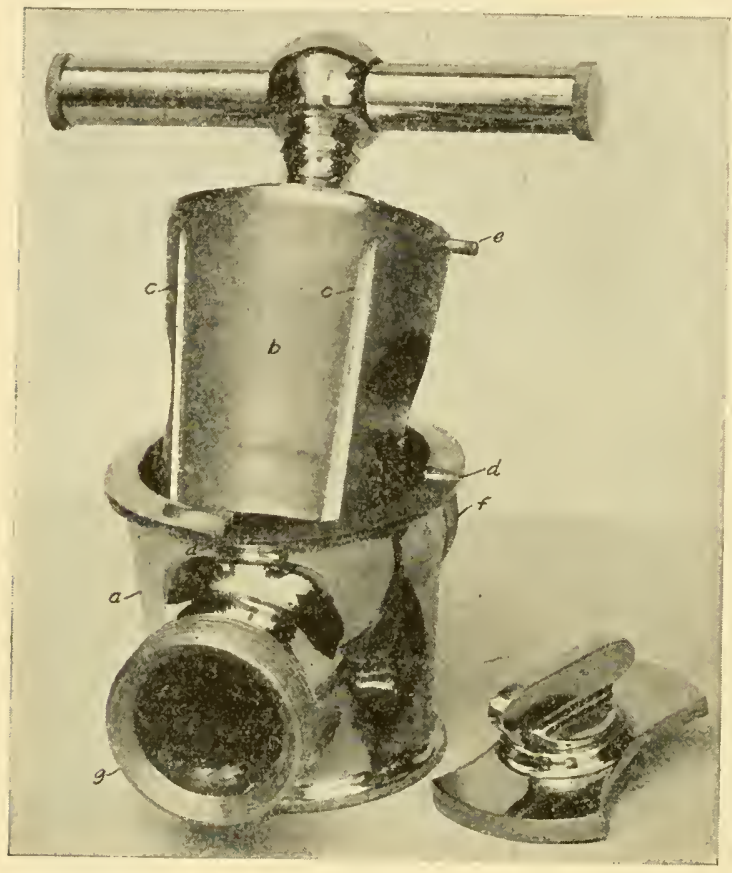

FIG. 8.- Showing leak-protector inlet valve: $(a)$ valve body, $(b)$ valve plug, $(c-c)$ leak-drain grooves, $(d-d)$ stops, (e) stop pin, $(f-g)$ connections to inlet header line and holder, respectively. on test to be $2 \frac{\mathrm{I}}{2}$ per cent. Figure 2 shows this same type of holder satisfactorily equipped with leak-protector inlet and outlet valves.

So-called "valveless system" pasteurizers now on the market eliminate the necessity for an outlet valve on the bottom of the holder, using either pump vacuum or air pressure to force the milk through an outlet pipe extending through the top down to the bottom of the holder.

Foam and splash.-Foam consists of air entrained in milk film forming a mass of bubbles causing it to float on top of the milk. Foam present on the milk in a pasteurizer holder is often cooled by the air to a temperature below that required for pasteurization. Theobald Smith ${ }^{\mathrm{I}}$ first pointed out that any foam or scum on top of milk in laboratory test vessels was cooled by the air so that tubercle bacilli, though destroyed in the liquid by heating and holding at a certain temperature, remained in the froth or scum. Whittaker, Archibald, Leete, and Miller report ${ }^{2}$ that the temperature of foam on milk in a vat during commercial pasteurizing runs, as determined by thermocouple tests, showed an average of $8.3^{\circ} \mathrm{F}$. lower than the milk at the beginning of the holding period on seven runs, where the milk was heated in the vat, and an average of $10.2^{\circ} \mathrm{F}$. lower on twelve runs in which the milk was preheated and pumped into the vat. Comparable differences are also reported in the temperatures at the end of the holding period.

In commercial milk-plant operation the remedy is either to eliminate units caus-

r Smith, T.: J. Exper. $M c d ., 4$, 217. I899.

${ }^{2}$ Whittaker, H. A., Archibald, R. W., Leete, C. S., and Miller, L. F.: Tech. Bull. I S. U.S. Depl. of Agric. Sept., 1927 . 


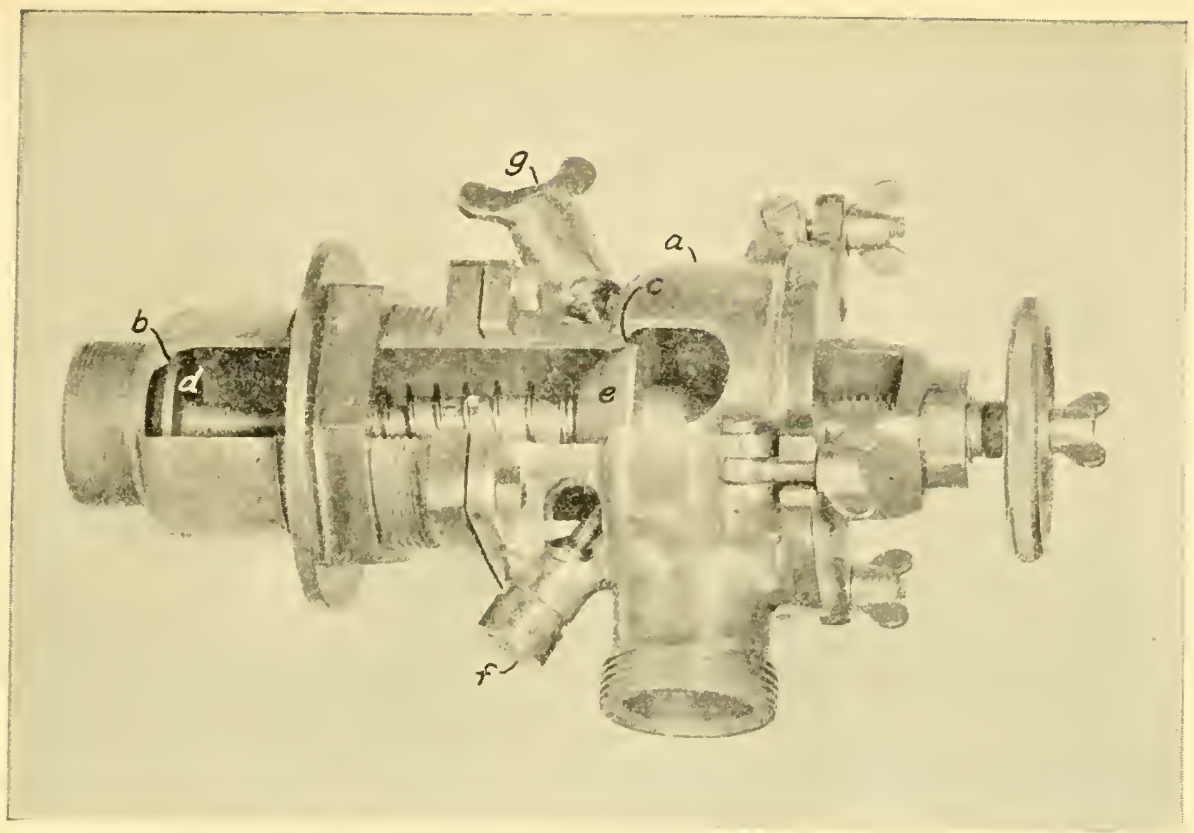

Fig. 9.-Flush-type leak-protector outlet valve (portion cut away-valve in closed position): (a) valve body, $(b-c)$ valve seats and $(d-c)$ corresponding disks, $(f)$ leak drain, $(g)$ steam valve.

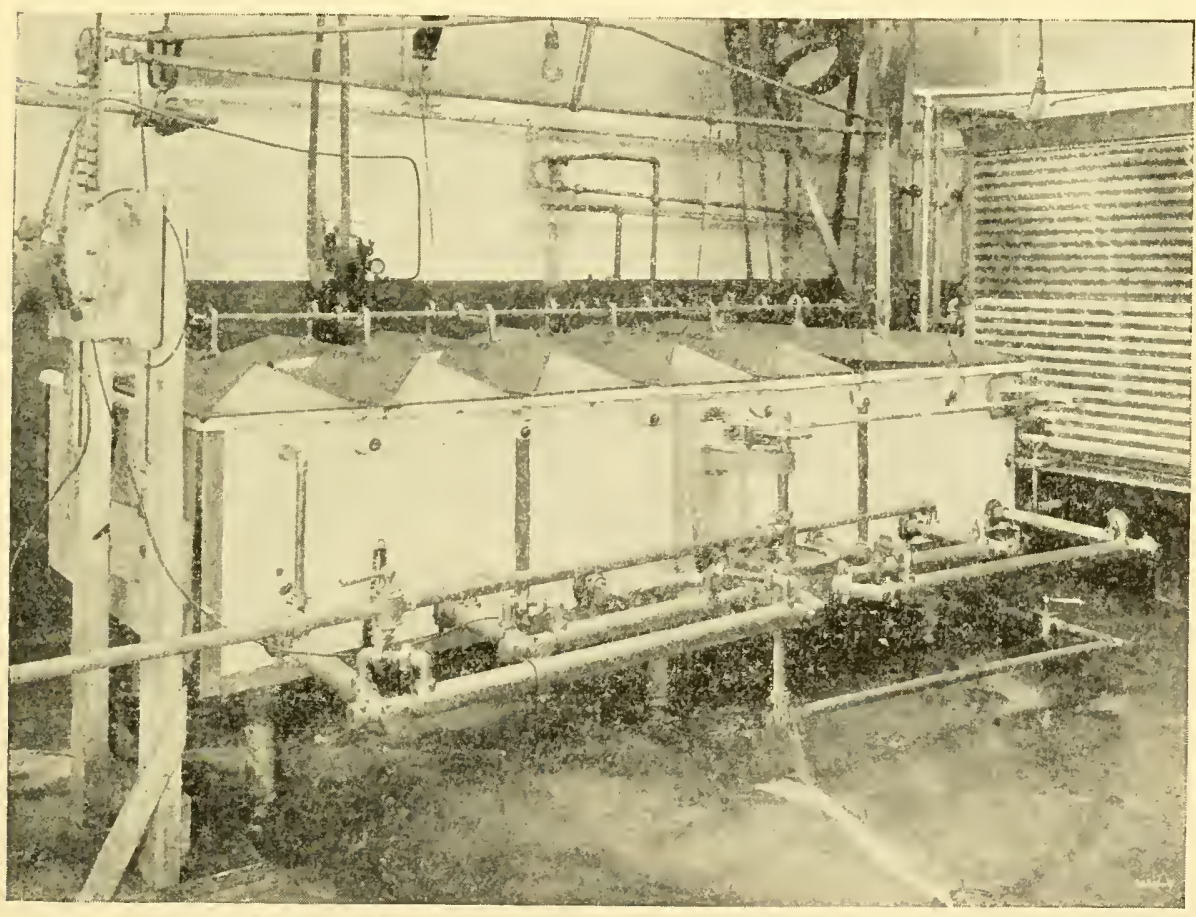

FIG. I0.-Pocket-type pasteurizer with leaky multiple-port rotary valve 
ing the foam, or to keep the foam up to the pasteurizing temperature by heating the air in the holder. The worst offenders as foam creators are the separator, the oldtype clarifier, and the centrifugal-type heater. The barrel-type heater will also cause foam under certain operating conditions. Pumps which suck air will incorporate it in the milk, causing foam. Dropping milk into a storage vat or pasteurizer holder with considerable force and turbulence also creates foam.

Intelligent study and experimenting with the various units will usually be rewarded by complete elimination or marked reduction of foam present during pasteurization. The new type, "non-froth" clarifier when operated properly creates very little, if any foam. Schier reports that foam can be practically, if not entirely, eliminated while using a centrifugal-type heater, by passing the milk through a specially elevated float tank, before discharging it into a holder. Pumps can be repaired or replaced to eliminate foam creation at this point. Inlet pipes extending to the side or bottom of a pasteurizer holder will usually accomplish the smooth, non-turbulent discharge of milk into a holder without foam if none is already present in the milk.

In some instances it does not prove feasible to eliminate foam from the milk during pasteurization. In these cases the foam should be kept up to the pasteurizing temperature by heating the air above the milk in the pasteurizer holder with steam. Steam valves operated automatically by a thermostat and solenoid, or by a sensitive bulb containing a medium of gas or vapor, can be attached to the top of a pasteurizer holder to admit just sufficient steam to keep the atmosphere and foam above the milk at the pasteurizing temperature at all times.

Milk splash which is thrown out of the main body of the milk on the cover and sides of a pasteurizer holder will cool and often drop into the milk again. This defect occurs principally in coil vats owing to too rapid rotation of the coils, and can usually be corrected by reducing the speed of the coil to thirty revolutions per minute or less.

Defective continuous-flow units.-Continuous-flow holders are defective if they fail to hold every particle of the milk at the pasteurizing temperature for thirty minutes. The type consisting of a vertical cylindrical copper tank with nested baffles in which the milk is discharged in at the top and flows down through the holder by gravity has been found to be very unreliable as to holding period. Huelings, Grim, and Horn, ${ }^{2}$ Chilson and Wisler, ${ }^{3}$ and others report instances where part of the milk was held varying intervals as small as three, ten, and twelve minutes in regular installations of this holder in milk plants. Following an extensive study of this holder North and Phelps ${ }^{4}$ outlined certain conditions under which it could be relied upon to give satisfactory results. These appear to be so exacting as to be practically impossible to attain in the average milk plant. This type of equipment, although still on the market, is being rapidly replaced by new holders of improved design.

${ }^{r}$ Schier, O. B.: Proc. Internat. Assoc. Milk Dealers, Plant Scc., p. 69.1926.

${ }^{2}$ Huelings, S. M., Grim, G. W., and Horn, D. W.: 13th Ann. Rep., Internat. Assoc. Dairy \& Milk Inspectors, p. 197. r924.

${ }^{3}$ Chilson, C. H., and Wisler, C. O.: ibid. p. 243.

${ }^{4}$ North, C. E., Park, W. H., Moore, V. A., Rosenau, M. J., Armstrong, C., Wadsworth, A. B. and Phelps, E. B.: loc. cit., p. 86. 
The continuous-flow holder of the long-distance flow type (see Fig. 3) should be checked by flow tests with water at the pasteurizing temperature by adding a chemical, such as starch or chloramine, which can be easily detected to determine that the holder provides a thirty-minute holding period. Slight variations as to holding period or temperature of the milk can be readily corrected in this type when first installed. The holding period can be shortened by the operator to less than thirty minutes when using a steam pump to discharge the milk into the holder by simply opening the steam valve so as to increase the speed of the pump. The remedy is the installation of a piston pump geared to a constant-speed motor which is set at a speed which will fill the holder in approximately thirty-three minutes. In this type of continuousflow holder the holding period during operation is only slightly less than the calculated theoretical holding period.

In order to insure a thirty-minute holding period while emptying the holder at the end of a run, it is often necessary to equip the outlet-drain valve with a stop permitting only partial opening in order that the rate of emptying will be slowed down sufficiently to insure that all of the milk is held thirty minutes.

Temperature defects to be guarded against include the failure of the operator to heat the holder above the pasteurizing temperature before starting. In this case, the first milk to enter gives up part of its heat to the mass of colder metal in the holder tubes until they reach the pasteurizing temperature. Under certain conditions as much as roo gallons of the first milk will flow from the holder, starting as low as I $25^{\circ}$ F. and gradually increasing until the pasteurizing temperature is reached. To minimize the possibility of this occurring in commercial operation, an air thermometer should be placed on the holder air-chamber and the operator instructed to heat the holder above pasteurizing temperature before starting the milk flow. The recording thermometer charts will show if he fails to do this properly. The temperature of the air surrounding the holder tubes must be maintained high enough throughout the run to prevent any drop in the temperature of the milk in passing through the holder.

Continuous-flow milk heaters must be used in connection with holders of the pocket and continuous-flow type, and are frequently used with a series of holders of the vat type. Failure to maintain the flow of milk at the pasteurizing temperature for any considerable interval constitutes a defect. In the case of vat- and pocket-holders a small amount of milk at a temperature lower than that required for pasteurization will mix with the main volume of milk so that the temperature of the entire batch may be lowered only a fraction of a degree. When the resulting temperature is $\frac{1}{2}^{\circ}$ or more lower than that required, it should be raised in the case of holders equipped with heating coils or jackets. This is not possible in the case of holders of the vat or pocket type which are merely insulated, since there is no means for adding heat. In the case of long-distance, continuous-flow-type holders, mixing is limited principally to a single tube as the milk enters, so that milk entering the holder at a temperature lower than that required for even brief intervals often does not become mixed with milk at a higher temperature.

Improved attention and servicing of the steam controllers on heaters are necessary to limit the variation in milk temperature to less than $I^{\circ}$. Automatic shut-off valves 
are also desirable to stop the flow of milk whenever the temperature falls below a certain point which is well within the margin of safety for effective pasteurization with the equipment being used.

Unsatisfactory thermometers.-The use of a recording thermometer alone as a guide for temperature control, as practiced extensively in commercial operation, does not give satisfactory accuracy for positive pasteurization. This is for the reason that recording thermometers frequently get out of adjustment, owing to the delicate mechanism, jarring, and rough handling they receive. It is not uncommon to find one registering from $\mathrm{I}^{\circ}$ to $4^{\circ}$ off. Many of the charts used have small $2^{\circ}$ spacing, and at times the ink line made by the recorder pen may be a full space wide. These factors combine to make the practice of relying on the recording thermometer for temperature control unsatisfactory from the point of view of both the health officer and the milk dealer. If registering too high, the pasteurization temperature is not being reached. If registering too low, the cream volume may be reduced. The use of a small mercury thermometer or floating dairy thermometer to control the temperature of the milk is unsatisfactory also, since it is very difficult to read accurately the fine degree spacings on them when immersed to the proper point in a vat with steam rising from the milk.

The remedy is to provide an accurate mercury-indicating thermometer on each holder for temperature control with the scale at the side or above the holder. Scale divisions of at least $\frac{1}{16}$ inch per degree eliminate guesswork and make reading easy. The scale should be installed and tilted in such a manner that the operator can read the thermometer easily from a standing position.

Both a recording and an indicating thermometer should be provided for each vat used for pasteurizing milk. On continuous-flow and pocket-type holders these two thermometers should be provided on both the inlet and outlet headers. The recording thermometer chart should preferably have wide scale divisions equivalent to at least $\frac{1}{16}$ inch per degree on a twelve-hour chart, so as to give a clear picture for the health officer to judge how satisfactorily the requirements as to time and temperature for pasteurization are being fulfilled. The health officer should insist on the complete pasteurizing operation being shown on each chart. The recording temperature line should start and end at room temperature and should be continuous. An empty space may indicate that the pen was lifted to avoid recording some unsatisfactory point in operation.

\section{FLASH PASTEURIZATION}

As previously mentioned, the flash method of pasteurization consists in rapidly heating milk to the desired temperature followed by immediate cooling without an appreciable holding period. It was used extensively prior to I9I4, but rejected by health officers due to failure of the equipment to heat the milk uniformly to the same temperature.

While bacteriologists have made several determinations showing that a temperature of $160^{\circ} \mathrm{F}$. for sixty seconds would destroy tubercle bacilli, milk-heaters and temperature-controllers had not been sufficiently perfected prior to I9I4 to secure satisfactory results in commercial operation. Whether they have been sufficiently perfected at this time is still questionable, although enormous progress has been made. 
For example, North ${ }^{1}$ and collaborating scientists reported that in a series of tests in which milk was heated in a barrel heater and the temperature reduced slightly in a tubular heater prior to discharging the milk into a holder, all samples from the outlet of the second heater, which had been heated to a maximum temperature of $150^{\circ} \mathrm{F}$. or above, showed destruction of tubercle bacilli in artificially infected milk.

The electrical method of flash pasteurization (see Fig. 5), using hot treated milk to heat raw milk to $\mathrm{I} 20^{\circ} \mathrm{F}$. by means of a regenerator, and electricity to heat from $\mathrm{I} 20^{\circ}$ to $160^{\circ} \mathrm{F}$, is being offered as the equivalent of pasteurization by the holding method. Many health officers are faced with the problem as to whether or not to accept it as such. Numerous tests have been made on this process in various stages of its development in the last twenty years. Many of these have not been reported in literature. In I920 Beattie and Lewis ${ }^{2}$ reported to the Medical Research Committee of Great Britain that the process destroyed tubercle bacilli satisfactorily at $63^{\circ}-64^{\circ} \mathrm{C}$. ( $145.4^{\circ}-\mathrm{I} 47.2^{\circ}$ F.) Recently Prescott reported ${ }^{3}$ that tests made under his direction showed that this electrical method of treating milk destroyed tubercle bacilli satisfactorily when operated at $160^{\circ} \mathrm{F}$. On the other hand, Irwin, Haythorn, and Conover, ${ }^{4}$ testing a commercial unit in Pittsburgh, Pennsylvania, reported on April I3, I926, that four out of twenty-eight guinea pigs injected with portions of samples of milk, artificially infected and then treated by this process at $160^{\circ} \mathrm{F}$., developed tuberculosis of the spleen as well as local lesions. This led to a repetition of the experiments by this group,${ }^{5}$ who reported on December $2 \mathrm{I}$, I926, that three out of twenty-six pigs in four experiments gave similar positives. ${ }^{6}$

It has been suggested that the conflict in results may possibly be due to the fact that the dosage of tubercle bacilli, used to infect the raw milk in the tests by Irwin, Haythorn, and Conover, was considerably greater than in the tests by Prescott and others, and that for this reason a sufficient number of tubercle bacilli remained undestroyed in the portion injected in the former tests to constitute an infecting dosage to a guinea pig. This emphasizes the desirability of (I) agreement among bacteriologists on an approximate standard infecting dosage of tubercle bacilli and other pathogenic organisms for tests on their destruction in milk, and (2) determining as nearly as possible the minimum infecting dose of the raw milk, in order that a better comparison of the results of such tests may be made.

Recent work by Park ${ }^{7}$ reported in October, 1927 , as yet unpublished, indicates that pathogenic strains of hemolytic streptococci are more resistant than tubercle bacilli to destruction by heat. Park reports that hemolytic streptococci commonly found

${ }^{1}$ North, C. E., et al.: ibid. p. 98 .

${ }^{2}$ Beattie, J. M., and Lewis, F. C.: Rep. MIcd. Research Com., Great Brilain. 1920.

3 Prescott, S. C.: Am. J. Pub. Health, 17, 221. 1927.

${ }^{4}$ Irwin, R. E., Haythorn, S. R., and Conover, J. R.: Rep. Com. A ppointed by Sec. of Health (Pa.) to Study the Electropure Process of Milk Treatment. April $13,1926$.

5 Irwin, R. E., Haythorn, S. R., and Conover, J. R.: ibid. Dec. 21, I926.

${ }^{6}$ Another series of tests was run on this process in June, 1927, by the N.Y. State and City departments of health and the U.S. Public Health Service. Results were not available at the time of writing this article.

? Park, W. H.: "Thermal Death Point of Streptococci," Am. J. Pub. IIcalli. (In press.) 
in milk are all killed in laboratory tests by the holding method of pasteurization at a temperature of $140^{\circ} \mathrm{F}$. for thirty minutes. However, their destruction by either steam or electric-flash pasteurization is in considerable doubt. Extensive tests to settle this point will probably be necessary before flash pasteurization will receive any general acceptance by health officials.

\section{STANDARDS FOR PASTEURIZATION}

Pasteurization may be defined as the heating of milk to a temperature sufficiently high to accomplish the complete destruction of disease-producing bacteria found in milk without appreciably altering the taste and nutritional qualities of the milk, followed by prompt cooling. The legal definitions adopted or suggested for ordinances give: (I) the temperature to which the milk must be heated; (2) the time that the milk must be held at this temperature; and usually (3) the temperature to which the milk must be immediately cooled.

Typical legal definitions are given below:

I. Standard Milk Ordinance, United States Public Health Service, as approved by the Conference of State and Territorial Health Officers: "The terms 'Pasteurization,' 'Pasteurized,' 'Pasteurize,' and similar terms shall be taken to refer to the process of heating every particle of milk or milk products to a temperature of not less than $145^{\circ} \mathrm{F}$, and holding at such temperature for not less than 30 minutes in pasteurization apparatus approved by the health officer, the temperature and time being automatically recorded by a temperature and time-recording device approved by the health officer."

2. International Association of Dairy and Milk Inspectors: "Pasteurization is the process of heating milk to a temperature of approximately $145^{\circ} \mathrm{F}$., never lower than $142^{\circ} \mathrm{F}$., holding every portion of the milk at that temperature for a period of at least 30 minutes and then promptly cooling below $50^{\circ} \mathrm{F}$."

3. Food Inspection Decision 200, issued by the United States Department of Agriculture, established a definition which was adopted by the Joint Committee on Definitions and Standards, composed of representatives of the United States Department of Agriculture, the Association of American Dairy, Food and Drug Officials, and the Association of Official Agricultural Chemists, at its meeting January I8-29, 1926. 'The definition states: "'Pasteurized Milk' is milk that has been subjected to a temperature not lower than $\mathrm{I} 45^{\circ} \mathrm{F}$, , for not less than 30 minutes, after which it is promptly cooled to $50^{\circ} \mathrm{F}$. or lower."

In the last few years considerable controversy has arisen over the proper temperature for the pasteurization of milk when using the accepted thirty-minute holding period. It is well recognized that heating higher than $145^{\circ} \mathrm{F}$. reduces the cream volume on the bottle of milk as delivered to the consumer. This is an important commercial factor in the sale of milk in view of the extensive practice of using the cream from the top of a bottle of milk for coffee and cereals in the American home. A reduction in cream line on pasteurized milk is also of indirect public health significance in localities in which it is sold in competition with raw milk, since it may cause consumers to favor the purchase of raw milk having a deeper cream line, although it may have no greater butter-fat content than the pasteurized milk. 
A large number of health officials have demanded $145^{\circ} \mathrm{F}$, while the milk dealers, supported by a number of scientists, have maintained that $142^{\circ} \mathrm{F}$. is a safe temperature. In r92 I the Borden Farm Products Company of New York engaged Dr. Charles E. North to organize and conduct research work on this problem. The second series of these tests, previously referred to as "The Endicott Experiments,"I served to demonstrate that the mechanical defects in commercial pasteurizing equipment prevented the satisfactory destruction of pathogenic bacteria in milk, even when a temperature of $145^{\circ} \mathrm{F}$. was used. Later tests on improved equipment gave more satisfactory results.

The author's final conclusion states in part: "The bovine tubercle bacilli used were destroyed in some tests in three types of improved commercial pasteurizers at ${ }_{1} 38^{\circ} \mathrm{F}$. for thirty minutes. The temperature and time for pasteurization contained in the milk regulations of the cities of New York, Philadelphia, Baltimore, and many other large cities, and the regulations of the states of New York, Connecticut, and others, which is that 'milk or cream shall be heated to a temperature of at least $\mathrm{I} 42^{\circ}$ F. and held at such temperature for 30 minutes or more,' provides an adequate margin of safety, and is recommended as a proper standard for the definition of pasteurization by health authorities." Instead of settling the controversy as to the proper temperature for pasteurization, this admirable report served rather to emphasize the engineering defects preventing proper pasteurization in commercial equipment.

The following difficulties in the enforcement of present-day legal definitions of pasteurization regardless of the temperature standard are pointed out by Frank, Moss, and LeFevre in a recent article: ${ }^{2}$

I. Some definitions which specify by intent a recording thermometer temperature but do not specify approved apparatus permit the use of defective equipment not insuring proper pasteurization; those specifying approved apparatus cannot be generally enforced, because of lack of official information to serve as a basis for approval.

2. Those definitions which require that "every particle of milk" be exposed to the temperature specified imply that the enforcing health officer has the necessary knowledge or information available as to which equipment can be approved as accomplishing this.

3. That some definitions would, if strictly enforced, cause partial or complete destruction of the cream line on the milk, and consequently interfere with the sale of pasteurized in competition with raw milk.

They suggest the re-wording of existing definitions into one of the following forms:

I. Pasteurized milk is milk which has been heated to at least $\left[{ }^{\circ} \mathrm{F}\right.$.] and held thereat for at least [__ $]$ minutes as indicated by its recording device, provided that no apparatus shall be used which has not been approved by the [accepted agency making the official tests] for use under this definition and provided that all apparatus shall be operated in accordance with the directions recommended by the [accepted agency making the official tests.]

2. Pasteurized milk is milk every particle of which has been heated to $140^{\circ} \mathrm{F}$. and held thereat for 30 minutes in apparatus approved by the health officers, provided that the re-

See p. 425 .

${ }^{2}$ Frank, L. C., Moss, F. J., and LeFevre, P. E.: Am. J. Pub. Hcallh, I7, I31. I927. 
cording device shall indicate a temperature and time in excess of $140^{\circ} \mathrm{F}$. and 30 minutes, equal to the safety margin recommended by the [accepted agency making the official tests] for the apparatus in question, and provided the apparatus is operated in accordance with the directions recommended by the [accepted agency making the official tests].

It is apparent that these definitions require detailed knowledge by the health officer secured from his own engineering tests or complete published information from some official agency such as the United States Public Health Service.

Any general agreement on the question of what temperature should be required for pasteurization, whether $142^{\circ}, 145^{\circ}$, or some other temperature, will probably not be accomplished until health officials are satisfied that engineering defects have been eliminated from the commercial pasteurizing equipment under their jurisdiction. Many believe that for the present the maximum factor of safety obtainable without serious cream-line reduction is desirable to offset partially the temperature drops occurring in portions of milk not receiving the full temperature and time of holding due to the defects in construction or operation of the pasteurizing equipment.

When these defects have been corrected, it is possible that a considerable portion of those now favoring $\mathrm{I} 45^{\circ} \mathrm{F}$. will be willing to consider a legal definition which will permit the use of $142^{\circ}$ or $143^{\circ} \mathrm{F}$. as a minimum temperature for pasteurizing milk when held for thirty minutes in apparatus approved by an accepted official agency. 


\title{
CHAPTER XXYIV
}

\section{CONTAMINATION AND DETERIORATION OF FOOD}

\author{
CHARLES THOM
}

Microbiological Laboratory, Bureau of Chemistry, U.S. Department of Agriculture

\section{THE MICROBIC CONTENT OF FOODS}

The ubiquity of bacteria has already been emphasized by others. The surfaces of raw fruits and vegetables as ordinarily handled are in constant contact with currents of air-carrying dust particles, visited by insects and brushed by man and many other animals. Many products are moist from transpired water, often more or less sticky or mucilaginous from the osmotic products deposited by the evaporating juices or exudates. They thus present a lodging-place for bacteria, yeasts, and molds, and hence not infrequently support a large population of micro-organisms of many species measurably influenced in variety by the nature of the exudates.

Some conception of the numbers present is furnished by studies in the Bureau of Chemistry in which a colony count was found to average some 250,000 per gram in spinach and about I35,000 per kernel within the husk and in contact with the individual kernels of corn.

These numbers can be reduced by thorough washing under strictly controlled conditions, but many investigations of routine washing practices show many of them to add to, rather than to reduce, the bacterial content of food. Further, under even good conditions, after washing, contaminated surfaces are wet enough to accelerate the multiplication of the bacteria present.

The findings from fruits and vegetables are repeated when we turn to flesh foods. The healthy flesh of the living animal is believed to be almost free from contamination, but invasion by bacteria from the alimentary canal follows quickly after the animal is killed, while handling processes are notoriously careless from a bacteriological standpoint.

We know comparatively little thus far of the extent of the invasion of tissue areas, for example, the intercellular spaces of plants, by bacteria without the production of evident pathological conditions. Enough has been done to indicate that such invasion through the stomata does occur in many plants, but the extent and nature of the species invading remain largely untested. Similarly with animal foods, entire absence of bacteria is doubtful even in living tissues of apparently healthy food animals, while positive invasion or contamination at time of slaughter is almost inescapalble.

The student of food problems is thus faced with the fact of contamination with micro-organisms in dealing with raw foods. Inquiry as to the relation of this population to fitness of products for human consumption takes sereral forms: microbiology of freshness and soundness in the foods themselves, the changes of flora involved in the handling processes encountered in current practices of the gathering and market- 
ing of these foods, the microbiology of staleness and spoilage, and constructive methods for control.

In our problem of maintaining fitness and soundness of such food we must take note, then, of the initial load carried by the surface, the presence of commensal species within the tissues, and the invading microbes incident to methods of preparation. Soundness and safety call for control based upon intelligent appreciation of all these factors and of the relative values of environmental factors such as cold, heat, evaporation, or pickling upon these organisms.

\section{FRESH SOUND FOOD}

Our knowledge of the bacteriology of fresh sound food is largely empirical. It is the common belief of the race that foodstuffs produced under the usual conditions, gathered or harvested and found to be sound, i.e., to conform to those ideals of appearance, texture, odor, and flavor established as normal by experience, are fit for food. We are just beginning to appreciate the number and variety of bacteria carried upon the surfaces of these foods; these ordinarily prove harmless when the foods are eaten fresh or submitted to methods of preparation dictated by race experience. Even among these foods, we are beginning to find individual substances which occasionally at least carry infections whose source was hitherto unsuspected. Thus our knowledge of the microbic "load" initially present upon fresh food which shows no physical marks of deterioration is actually inadequate for protection. Although race experience has established fairly safe systems of collecting, preparing, and consuming such foods, there are inherent contaminations which are occasionally significant, beside the grosser menace of contaminations due to congested populations-for example, the sewage of great cities discharged into shellfish-producing waters, or the time, temperature, and contact elements involved in long shipment lines followed by complex systems of wholesale, jobbing, and retail distribution.

\section{CONTAMINATIONS OF HANDLING}

So long as the more perishable foodstuffs are largely consumed in the immediate vicinity of production, the slogan of the home canners, "two hours from the garden to the can," will carry the raw materials from the field to the table or to the status of a preserved product practically unchanged. When commercial harvesting processes are added and involve picking, packing in bulk, in baskets, hampers, crates, or bags, transportation by truck or by rail, warehousing, jobbing, and finally the exposure in the retail food stall, the initial bacterial content is supplemented greatly by the contributions of numerous human agents, by the dust and filth of transportation, and by contact with insects and vermin. From the point of view of safety, the bacterial species added during such handling processes may often be far more important than the initial load of micro-organisms.

\section{INCUBATION}

The factor of incubation is, as a rule, fully as significant as contamination. Few natural food products (as distinct from manufactured products) in commercial condition are entirely free from metabolic activities either in the form of enzymic changes 
or actual continuation of the activities of living cells. The rate of such activity varies greatly, however. Some products such as cereal grains in merchantable condition are comparatively stable, and hence can be handled without special control of conditions; some less stable still admit of shipment, storage, and sale with comparatively simple care; other products truly perishable, such as meats, fresh fruits, and vegetables, are enzymically or metabolically active as well as readily subject to the attack of insects, molds, yeasts, and bacteria.

The stable products such as cereal grains are commonly grossly contaminated with micro-organisms, but are so low in water content as ordinarily handled that microbic activity is comparatively negligible. Even among these products a slight addition of moisture leads to spoilage as mustiness, moldiness, or souring.

Among perishable products two general conditions are represented by flesh foods and fresh vegetables or fruits. The flesh foods are high in water content, enzymically active, exceedingly subject to bacterial activity, and usually superficially if not internally contaminated with many types of organisms. In spite of these facts, gross carelessness in handling is constantly observed. Meats - beef, pork, lamb, poultryare handled in open trucks with little more care than coal or firewood and often exposed on hooks, blocks, and counters throughout the business day without protection or icing. Along with the ordinary bacteria of decay or putrefaction which are always present, these foods furnish a favorable substratum for members of the Salmonella group whose infective power is so constantly associated with their growth in meat that they are frequently referred to as meat-poisoning organisms. Our precautions against such contamination are commonly inadequate, hence the main protection of the consumer from greatly increased numbers of such outbreaks lies in the adequacy of the cooking processes applied. Systematic efforts at the correction of negligence in handling would reduce or stop the introduction into our houses of gross infections carrying the constant peril of contaminations which so frequently escape the precautionary knowledge of those who handle food.

During the marketing period vegetable products present another problem. Lettuce, spinach, asparagus, berries, etc., are rapidly growing plants and plant parts. Their growth processes do not stop as the plants are gathered. Bunched in baskets, crates, bags, etc., the heat of metabolism tends to raise the temperature of the mass, and thus to speed up every agency of destruction. Bacteria and molds find warm moist conditions for incubation; the natural enzymes present show increased activity; transpiration furnishes either a highly humid atmosphere in the interstices of the mass or actually wet surfaces in which favorable conditions are offered to micro-organisms. Fabulous numbers of bacteria to the gram develop under such conditions. Spinach, head lettuce, and asparagus become so heavily contaminated with bacteria that they feel slimy to the fingers. Un-iced carloads of such foodstuffs quickly heat, give off steam, and develop offensive odors. Less conspicuous but equally objectionable activities produce the well-known sour flavors of incipient spoilage.

To handle food safely, therefore, we must take account of the initial load of bacteria present, product by product, and of the nature of the product itself, as a basis for practical and economical handling. The practice to be selected, product by product, should be determined largely by the chemical nature of the food mass itself. 
Substances containing free and readily fermentable sugars in small percentage are commonly subject to lactic fermentation. Many species of bacteria may participate in this activity, but the long rod forms (Lactobacilli) constitute the dominant agents in the souring of vegetable products. This tendency in the foods themselves is utilized in making the whole series of foods collectively known as "pickles," which are merely foods soured by the activity of the Lactobacilli under controlled conditions. Ensilage and sauerkraut are simply pickled corn and cabbage. This fermentation is essentially anaerobic. The active species, while able to grow more or less in the presence of oxygen, find their optimum, quickly dominate and suppress other forms under anaerobic conditions and at temperatures near $30^{\circ} \mathrm{C}$.

The organisms when supplied sufficient sugar carry the acidifying process to concentrations varying from about I per cent to, perhaps, 2 per cent or a little higher, calculated as lactic acid. Ordinarily lactic acid forms the major part of the acid present, but it is usually supplemented with acetic, butyric, or other aromatic acids in amount varying with the substratum and the complex of bacteria present. Lactic acid at this concentration reduces other bacterial activity to negligible amount, and hence is an effective preserving agent. The pickle fermentation has been widely and successfully adapted to food preservation, and is effective, when supplemented by the exclusion of air through close covering of the mass, or by hermetic seal, or by a progressive addition of salt. Unsupported by such measures, pickled masses are invaded by the mycoderma yeasts which progressively destroy the acid and release destructive bacteria.

Foods containing larger percentages of fermentable sugars usually develop an alcoholic fermentation if oxygen is present in necessary concentrations. The preservative effect of the alcoholic fermentation is less stable. Unless carefully controlled and protected, the acetic fermentation commonly intervenes before the yeasts have completed their action. The alcoholic fermentation passes directly, therefore, toward the vinegar fermentation unless it is controlled with great care and the resulting liquor concentrated or protected from contamination. The alcoholic fermentation industries are thus very closely specialized in the handling of selected organisms and the attainment of particular combinations of flavor and alcohol concentration. They contribute comparatively little, however, to the maintenance of the food supplies of the world. They are destructive rather than constructive.

Control of bacteria in our food supplies is the basis of a number of large industries. Such control may be attained by drying, by the use of preserving agents, or by cold. Their exclusion as active agents is consistently sought by canning.

\section{DRYING AND IRESERVING}

Drying and preserving are very ancient practices in the preservation of foods. They rest upon a single principle, i.e., that spoilage of a food may be prevented or delayed by rasing the concentration of the osmotic constituents in the water present high enough to render the sohtion unavailable to micro-organisms and to stop, or delaty greatly, enzymic activities. This may be accomplisherl by evaporating the water present to a figure varying with the product; for example, in dried apples to aljout 25 per cent, in clried milk to I.5 per cent, in corn meal to I 2 per cent. Part of the water 
may be withdrawn by the addition of an agent like salt or sugar while the rest becomes a brine or syrup sufficiently concentrated to reduce or stop losses. The combination of reduced water content and salting is the basis of most brining processes with meat and fish. The combination of sugar with the juices of fruit products is used in the manufacture of preserves, jams, jellies, etc. In the development of these industries concentrations capable of controlling the usual processes of decay are readily reached, but along with these developments species of bacteria, yeasts, and molds capable of growing in solutions of salt or sugar verging upon saturation have complicated the processes. Salt-tolerant organisms often invade the tissues of meat and fish, discoloring them and rendering them unfit for use. Molds and yeasts tolerant of high concentrations of sugar break down the sucrose of stored raw sugar, invert maple sugar, and ferment the centers of many forms of confectionery causing tension and bursting which renders them unmarketable.

We are only beginning to appreciate the bacteriological precautions necessary in the handling of foods which depend upon osmotic concentration for extension of their marketing period. The development of this information bids fair to bring about cleaner and more sanitary handling of all these foods as a measure in the prevention of loss, as well as in the interest of the consumer.

\section{PRESERVATION BY COLD}

The whole practice of refrigeration rests upon the progressive slowing of metabolic activities by lowered temperatures. The response of the different species of micro-organisms to low temperature is exceedingly varied. In studies of meat in transit from Australia and New Zealand to London, Brooks ${ }^{\mathrm{r}}$ found that certain molds would develop characteristic colonies at temperatures down to $+22^{\circ} \mathrm{F}$. Pennington and her colleagues in the Bureau of Chemistry, working with bacterial cultures (unpublished), showed that the micro-organisms used would grow slowly at temperatures below the freezing-point as long as crystallization of the substratum did not occur. No growth was found by them in tubes in which crystallization occurred quickly in the cold-storage chamber used. Since the usual temperatures of the refrigerator leave the products unfrozen, we must recognize that storage in such conditions is not indefinite preservation, but such a slowing up of inherent deterioration of the product and of micro-organic activity as will prolong the marketing period for days, weeks, or months according to the conditions furnished. We must recognize further that the products removed from the refrigerator are not fresh products, however good and fit for food they may be, and that they ultimately become unfit even under the most careful handling in the refrigerator. This is well illustrated by the deterioration of eggs in storage. Eggs are stored just above the freezing-point, and show progressive changes differing in rate in the same lot so that there is an increasing percentage of unmarketable eggs in lots removed from storage month by month. These losses become so large as to limit the period of profitable storage to less than a year. Even within that time the discriminating user of eggs recognizes their deterioration within a comparatively short period.

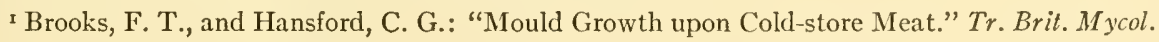
Soc., VIII, Part III (1923), Ir3-42. 
CANNING

The alternative to control of a living flora in food is destruction of the microorganisms present by cooking or processing.

The ordinary procedures used in preparing food for the table destroy the vegetative forms present, but leave the spores of many species of bacteria in viable condition. If such spores are abundant, the cooked food may and usually does spoil much more quickly than the raw products since the tissues of the foodstuffs are usually broken down, or softened, hence more easily penetrable by organisms, and the water content is either actually increased or rendered more immediately available to bacteria by the heating process. The bacteria surviving in good, sound food freshly and thoroughly cooked are not ordinarily a menace to the consumer who uses them at once. Whatever danger there may be either from infection or from toxemia is dependent upon storage with the incubation or reinfection of the cooked product.

To prolong the holding period then, the product must either be sterilized or be put under conditions where any surviving organism will fail to develop, as well as be protected from contamination. Canning has been developed to cover these requirements. Although much canning has not reached true sterility, canning processes have, as a rule, prescribed such methods of washing, precooking or blanching, exhausting, sealing, and cooking or processing in the can as reduces the free oxygen of the product to a negligible minimum, destroys all vegetative forms present, and reduces or destroys the bacterial spores which are nearly always present upon the raw foods.

Extensive tests of canned foods have shown that spores of certain species of aerobic bacteria may be present in canned food without being able to grow there. Such foods keep. Certain species produce highly resistant spores which survive the usual cooking process, but which do not germinate at ordinary storage temperatures. If the foods containing them (for example, canned corn) are quickly cooled and stored under cool conditions, no spoilage results; but if the cans are allowed to cool slowly over a considerable period, these "thermophiles" grow and spoil the food.

The presence of these groups of highly resistant organisms in food has led to extensive studies of the thermal death-points of bacterial spores by the National Canners' Association in their own laboratories and in Harvard University Medical School, the University of California, and the University of Chicago. Such work brings us step by step nearer to a safe food supply.

The space of this article does not permit the discussion of the microbic floras of food. Enough has been said to indicate the fundamental conditions encountered in raw food and the principles of food handling which make possible the gathering and marketing of these varied types of products with their burden of active or inactive micro-organisms. A better knowledge of micro-organisms carried by food into the household or the food factory may be expected to result in wider acceptance of an ideal for the selection of foodstuffs: we should say, not, Is it bad enough to discard? but, Is it right?

${ }^{x}$ For a more extensive discussion, cf. Thom, Charles, and Hunter, Albert C.: Hygienic Fundamentals of Food Handling. Baltimore: Williams \& Wilkins Co., 1924. 


\title{
CHAPTER XXXV
}

\section{THE BACTERIA OF FOOD POISONING}

\author{
EDWIN O. JORDAN \\ University of Chicago
}

The term "food poisoning" has come to have a special technical meaning and is today most commonly applied by public health workers to certain infections or intoxications due to particular micro-organisms. The specific bacteria concerned in food poisoning may be grouped broadly in three divisions: (r) paratyphoid bacilli; (2) Clostridium botulinum, varieties A, B, and C; (3) miscellaneous bacteria. While such a grouping-like classifications generally-is for convenience only, it fairly reflects the present state of our knowledge.

\section{PARATYPHOID BACILLI}

In recent years the chief interest in these organisms has centered in the attempts to unravel the tangled skein of host relationships, in the effort to determine more exactly sources of infection, and in the study of toxin production. In all three lines of investigation significant progress has been made.

Paratyphoid bacilli may be readily distinguished, on the one side, from the $B$. coli group through their inability to ferment lactose; and, on the other, from B. typhosus through their gas production (not, however, absolutely invariable) in dextrose and other carbohydrate media. ${ }^{\mathrm{I}}$ A more constant characteristic differentiating them from typhoid bacilli is their ability to ferment rhamnose. So far as a single cultural character can have weight, rhamnose fermentation affords the readiest means of distinguishing paratyphoid bacilli from typhoid (and dysentery) bacilli. Other cultural characters combine to give the group an independent and distinctive character. ${ }^{2}$ 'The generic name Salmonella was bestowed by Lignières upon the organisms of the "hogcholera group" in 1900 and, although open to objection, appears to be used with increasing frequency. Bergey's $\mathrm{Manual}^{3}$ lists nineteen specifically named kinds.

Within the group or "genus" a gay medley of names and "species" has long existed, partly because of preconceived ideas respecting identification, partly because of the real lack of suitable differential tests coupled with the great variability of many members of the group. One fertile source of error has lain in the institution of exten-

${ }^{x}$ Non-gas-producing strains of various members of the group have been described by a number of observers: Dorset: Isth Ann. Rep., Bur. An. Ind., p. 566. I901; Bock, F.: Arb. a. d. k. Gesundh., 24, 238. 1906; Bainbridge, F. A.: J. Path. \& Bact., 13, 443. 1908-9; Ten Broeck, C.: J. Exper. Iled., 24, 213. I916. I have one such anaerogenic strain that has been under observation for over twenty-five years without change in gas-producing power. Fluctuations in gas-producing power may develop even after a long period of apparent stability. For a recently reported instance cf. Emmy Klieneberger (Centralbl. f. Bakteriol., Abt. I, Orig., I01, 305. 1927).

${ }^{2}$ Jordan, E. O.: J. Infect. Dis., 20, 457. I917.

3 Bergey, D. H., et al: Manual (2d ed.). 1925. 
sive biochemical and immunological comparisons of cultures taken at the face value of the names with which they were labeled. If a culture was labeled B. suipestifer, the characteristics it exhibited were assumed to be those of the organism commonly found in hog cholera. Studies restricted to cultures of known history have cleared up much of the confusion. Two members of the group, B. paratyphosus A (Salmonella paratyphi) and B. paratyphosus B (Salmonella schottmiilleri), are human pathogens biologically akin to the typhoid bacillus, and the infections to which they give rise are communicated chiefly, if not solely, by human agency. They are distinct from those paratyphoid strains ordinarily isolated from outbreaks of food poisoning which belong for the most part to two other and distinct species, B. enteritidis (Salmonella enteritidis) and B. aertrycke (Salmonella aertrycke). These two forms are par excellence the food poisoning bacteria of the paratyphoid group. They can today be quite readily distinguished from other members of the group and from each other by cultural as well as immunological tests. ${ }^{r}$

B. enteritidis was first isolated by Gärtner in I 888 in the classical meat-poisoning outbreak at Frankenhausen. ${ }^{2}$ In this instance the source of infection was meat from a cow slaughtered because the animal was ill from enteritis. One man who ate 800 gm. of raw meat developed gastro-intestinal symptoms in two hours and died within thirty-six hours after partaking of the fatal meal. On necropsy specific bacilli were isolated from the spleen. Bacteria apparently identical with Gärtner's bacillus have been isolated in other outbreaks of food poisoning. The bacilli found by van Ermengem in the Belgian Moorselle and Ghent epidemics, and by Fischer in the Rumfleth and Haustedt epidemics, are generally placed with the B. enteritidis type. ${ }^{3}$ Unfortunately, the term "Gärtner bacillus" came to be used by some writers (e.g., W. G. Savage, Food Poisoning and Food Infections. Cambridge, England, 1920) as a general term synonymous with "paratyphoid bacillus," so that specific identity cannot be inferred from statements regarding findings of Gärtner bacilli. The specific character of $B$. enteritidis, however, is recognized in most recent publications. In one of the more extensive tabulations of outbreaks of food poisoning ${ }^{4} B$. enteritidis is recorded as found in one instance in milk supposed to be the vehicle of infection; in two other instances positive agglutinative reactions for $B$. enteritidis were noted in affected persons, the suspected vehicles of infection being, respectively, brawn and a "Cornish pasty" containing sausage meat; in a fourth instance there was considered to be "some evidence of B. enteritidis toxin" in a frozen oxtail from the Argentine suspected of causing illness in two persons in London. My own collection of cultures contains three strains of $B$. enteritidis isolated from definite outbreaks of food poisoning: one from an outbreak due to beef stew, ${ }^{5}$ one from an outbreak attributed to a foodhandler ${ }^{6}$ and one apparently derived from a commercial rat virus. ${ }^{7}$ Infections in a

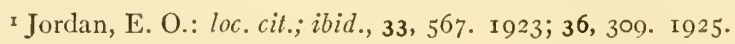

${ }^{2}$ Gärtner, W.: Corr.-Bl. d. allg. ärts. Ver. v. Thuringen. I88s.

${ }^{3}$ Hübener, E.: Fleischergiftungen und Paratyphusinfektionen, p. 204. Jena, I9ro.

${ }_{4}^{4}$ Savage, W. G., and White, B.: Food Poisoning: A Study of Ioo Recent Outbreaks (1Ted. Res. Coun., Spec. Rep. Series No. 92.1925.

${ }_{5}^{5}$ McWeeney, E. J.: Brit. M. J., I, II7I. I909.

${ }^{6}$ Savage, W. G., and Forbes, D.: J. IIyg., 17, 460. 1918.

${ }^{7}$ Spray, R. S.: J.A.M.A., 86, Io9. 1926. 
number of different animal species ${ }^{1}$ may be caused by $B$. enteritidis, but bovine sources of human-food poisoning seem relatively frequent, milk, beef, or veal being the more commonly reported vehicles of infection. Karsten ${ }^{2}$ considers $B$. enteritidis to be the usual cause of paratyphoid disease in calves. Another source of human infection with $B$. enteritidis appears to be accidental contamination of food with the commercial rat viruses which usually contain this organism. ${ }^{\mathrm{I}}$ Several recorded outbreaks have been traced to this cause (e.g., Spray). ${ }^{3}$

$B$. aertrycke is the name now commonly given to an organism with many of the B. schottmiilleri (B. paratyphosus B) characteristics, but of genuinely specific nature. It is not certain that the bacillus isolated by De Nobele in a food-poisoning outbreak in Aertryck was the exact prototype of the organism now called $B$. aertrycke, but there is some evidence that favors this presumption. For a time $B$. aertrycke was definitely identified with $B$. schottmiilleri by many German bacteriologists. ${ }^{4}$ On the other hand, English bacteriologists long regarded it as identical with B. suipestifer. ${ }^{5}$ Schütze, ${ }^{6}$ however, showed clearly that a definite serological difference existed between the Aertryck or "mutton" type and the Schottmüller type. I have elsewhere set forth cogent reasons for regarding $B$. aertrycke as an independent form culturally and immunologically, although closely related to $B$. schottmiilleri. ${ }^{7}$

B. acrtrycke, while often found in spontaneous epidemics in laboratory animals, is not a distinct rodent type, as believed by Krumwiede, but is found frequently in infections of many other animal species such as cattle and sheep. ${ }^{8}$ It has been reported in a relatively large number of human food poisoning outbreaks. ${ }^{9}$ Savage and White, in their study of one hundred recent outbreaks in Great Britain, record the positive isolation of $B$. aertrycke in fourteen instances and consider this organism the more or less probable causal agent in thirteen others. In the same series, as stated above, $B$. enteritidis was isolated but once and suspected in but three other instances. This corresponds with the large number of food poisoning outbreaks due to $B$. aertrycke that have been reported by various German writers, provided we assume that most of the Paratyphosus B bacilli are of the B. aertrycke type.

So far as can be determined from the published accounts, the symptoms produced by $B$. aertrycke and $B$. enteritidis show no noteworthy divergence. Either organism may cause death; whether any difference in case fatality exists cannot be determined from the meager data available.

I Jordan, E. O.: J. Infect. Dis., 36, 309. 1925.

${ }^{2}$ Karsten: Der Paratyphus der Kaller. Pp. ı०9. Berlin, 1921.

${ }^{3}$ Spray, R. S.: loc. cit.

4 Hübener, E.: loc. cit.

${ }^{5}$ Cf., e.g., Bainbridge, F. A., and O'Brien, R. A.: J. Hyg., Ir, 68. Igr r; Savage, W. G.: Rept. to Loc. Gov. Bd. (N.S.), No. 77. 1913.

${ }^{6}$ Schütze, H.: Lancet. 1, 93. 1920.

${ }^{7}$ Jordan, E. O.: op. cit., 33, 567. 1923. $\quad{ }^{8}$ Jordan, E. O.: ibid., 36, 309. 1925.

9 From the following outbreaks: Savage, IV. G., and Gunson, C. H.: J. Hyg., 8, 60r. rgo8; Robinson, G. H.: J. Infect. Dis., 16, 448. 1915; McWeeney, E. J.: op. cit., 2, 451. 1916; Delépine, S.: J. Hyg., 3, 68. 1903; "mutton" strain of English writers: Comrie, J. D., and Bird, G. A.: J. Roy. Army M. Corps, 33, 34. 1919; Young, W. A., and Dawson, G. D.: Lancet, 203, 609. 1922; Winslow, C.-E. A., et al.: Am. J. Hyg., 3, 238. I923; unpublished report to Washington State Board of Health, obtained through Dr. J. C. Geiger; Salthe, O., and Krumwiede, C., Jr.: Am. J. Hyg., 4, 23. 1924 . 
Although $B$. aertrycke has been isolated in typical outbreaks of food poisoning more frequently than any other paratyphoid bacillus, little has been ascertained about its source. In the fourteen instances in which this organism was found by Savage and White, generally in necropsy material, the suspected foods cover a wide range, such diverse articles as canned tomatoes, canned salmon, canned apricots, milk, and shellfish (cockles) being among them. In but three of the fourteen outbreaks did direct bacterial examination of the food give a clue to the origin. In one instance $B$. aertrycke was isolated from suspected steak and liver gravy, in one from beef brawn, in one from ice-cream. In a fourth outbreak, occurring in Glasgow and due to milk, $B$. aertrycke was isolated from an apparently healthy cow at the implicated dairy. In the remaining ten outbreaks not much can be safely inferred regarding origin, especially since in several cases the evidence incriminating particular food articles seems highly conjectural. Many other reports fail to trace infection with $B$. aertrycke to a definite source. I In certain instances investigators have surmised that contamination of food by rats or mice gave rise to the outbreak. ${ }^{2}$

While $B$. aertrycke and B. enteritidis are the organisms of the paratyphoid group that have been most commonly isolated in connection with food-poisoning outbreaks, $B$. suipestifer has been occasionally reported. ${ }^{3}$ It is remarkable that no connection has ever been traced between the relatively frequent outbreaks of hog cholera in which $B$. suipestifer is found abundantly and outbreaks of human disease. This may be due to marked variations in virulence or to the fact that a very massive dosage is necessary to produce infection.

Other bacilli of the paratyphoid group do not appear to have been definitely implicated in food poisoning outbreaks, although they should be regarded as potentially dangerous. Numerous outbreaks attributed to horse meat are reported in Germany, ${ }^{4}$ but do not seem to have been adequately investigated bacteriologically. At least two outbreaks due to horse meat have been described with considerable detail, 5 but in neither case, unfortunately, was the causal organism certainly identified, so that it remains unknown whether the equine paratyphoid strain (B. abortivoequinus) was concerned.

Neither is it known whether the various avian paratyphoid strains, B. sanguinari$u m, B$. pullorum, and B. anatum, are capable of giving rise to food poisoning. As yet they have never been definitely incriminated. These organisms, however, must be looked upon as possible sources of danger. ${ }^{6}$ It is of great interest that $B$. aertrycke has been found to be the cause of epidemics in canaries, parrots, and squabs. ${ }^{7}$

The studies summarized above on the differentiation and distribution of the

${ }^{x}$ E.g., Perry, H. M., and Tidy, H. L.: Med. Research Com., Spec. Rep. Scr., No. 24.

${ }^{2}$ E.g., Savage, W. G., and White, B.: op. cit., Outbreak No. 5 I; Burgess, W. L.: Proc. Roy. Soc. Med., Sec. Epid.\& State Med., r8, 6r. I924-25; Salthe, O., and Krumwiede, C., Jr.: J.IIyg., 4, 23. I924.

${ }^{3}$ Krumwiede, C., Jr., Provost, D. J., and Cooper, G. M.: J. Med. Rescurch, 43, 53. 1922; Scott, W.M.: J. IIyg., 25, 406. 1926; Shaw, F.IW.: J. Lab. E0 Clin. Med., 12, I4I. 1926.

4 Meyer, R.: Reichs-Gesundheitsbl., I, 1027. 1926; Absir., Bull. Hyg., 2, 469. 1927.

5 Federschmidt: Mïnchen. med. Wchnschr., 67, 814. 1920; Harms: ibid., 73, 777. 1926.

${ }^{6}$ Smith, T., and Ten Broeck, C.: J.Med.Researrh, 31, 523. I914-15; Rettger, L. F., Hull, T. G., and Sturges, W. S.: J. Exper. Med., 23, 475. I9I6.

? Beaudette, F.: J. Am. Vet. MI.A., 68, 642, 644. I926. 
paratyphoid group throw light on the ordinary sources of food poisoning with these organisms. First and foremost is the use of meat or other food substance derived from ailing animals, an origin already rendered plausible by epidemiological observations. Since many kinds of food animals are liable to paratyphoid infection and since human paratyphoid infections (or intoxications) are usually sudden, of brief duration, and with low fatality and hence relatively rarely subjected to competent investigation, outbreaks of this type are probably more common than represented by definite records. Another possibility of paratyphoid infection is from the use of food contaminated by animal-carriers of paratyphoid bacilli. Rats and mice are probably the greatest offenders in this respect, and the protection of cooked food from these vermin is a desideratum. The use of bacterial rat viruses containing living paratyphoid bacilli is highly objectionable.

The relative share of living paratyphoid bacilli and of their toxic products in the production of food poisoning outbreaks is quite obscure. There are numerous instances $^{r}$ in which pronounced symptoms have followed within two hours after the ingestion of the incriminated food, and in which formed poisonous substances rather than an infective process are accordingly deemed responsible. Many outbreaks appear to give indications of both toxic action and infection. The toxicity of cultures of various paratyphoid bacilli has been studied by a number of observers. ${ }^{2}$ Ecker, ${ }^{3}$ who worked with broth cultures, was led to believe in the existence of soluble toxic substances, although most previous investigators had considered that the paratyphoid toxins were "endotoxins." Miss Branham, however, regards the paratyphoid toxins as contained within the cell during life and as set free only on cell disintegration. In animal experimentation, culture filtrates have been found toxic for rabbits and mice, though not for guinea pigs, when injected intravenously, but show no effect when given by other routes. Symptoms of intoxication follow the introduction of the toxic filtrate into the blood stream after about forty to forty-five minutes, irrespective of whether the dose is lethal or sublethal. The toxic cultures and filtrates are definitely thermostabile and withstand boiling for a considerable period without loss of toxic power. Cultivation in protein-free medium yields a toxic filtrate which gives rise to definite antibody formation: agglutinins, precipitins, complement-fixing antibodies, and possibly antitoxins. ${ }^{4}$

\section{CL. BOTULINUM, VARIETIES A, B, AND C}

The spindle-shaped, anaerobic, spore-bearing rods classed in the genus Clostridium and distinguished by their unique ability to generate a true toxin that is poisonous when swallowed, constitute a specific group of micro-organisms. The three known varieties differ in various respects, among others with regard to toxicity, Type A producing by far the most potent toxin, the ratio of lethal dose for the three Types $\mathrm{A}, \mathrm{B}$, and $\mathrm{C}$ being given by one author as $\mathrm{I}: 50: \mathrm{I} 25 .{ }^{5}$

I Cf. Savage, W. G.: Rep. Loc. Gov. Bd. (N.S.), No. 77. I913; Savage, W. G., and White, B.: op. cit., No. 92 . 1925 .

${ }^{2}$ Cf. summary of Branham, S. E.: J. Infect. Dis., 37, 29 1. 1925.

3 Ecker, E. E.: ibid., 21, 541. I9I7.

4 Branham, S. E., and Humphreys, E. M.: ibid., 40, 5 I6. I927.

5 Bengtson, I.: Studies on Organisms Concerned as Causutive Factors in Botulism, Bull. I 36, IIyg. Lab. Pp. гог. 1924. A full discussion of the early literature on botulism has been given by Dickson 
Type-A strains and nearly all, if not all, of the strains of Type B are definitely proteolytic. The heat resistance of the spores of Types A and B is greater than that of any other anaerobes yet tested. ${ }^{\mathrm{T}}$ The maximum heat resistance as determined by a study of one hundred and nine strains heated in a phosphate solution of $\mathrm{pH} 7.0$ is as follows: ${ }^{2}$

4 minutes at I $0^{\circ} \mathrm{C}$.
10 minutes at II5
32 minutes at I I0
I00 minutes at 105
330 minutes at 100

The thermal death-point of Type $\mathrm{C}$ strains is reached at temperatures considerably lower, and it is perhaps for this reason that in the outbreaks of botulism in the United States-which are mostly due to heat-preserved foods-Type $\mathrm{C}$ has not been found. The thermal death-point of van Ermengem's original strain (Belgium) was reported to be reached at exposure to only $80^{\circ} \mathrm{C}$. for one-half hour. It seems possible that in Europe, where such foods as sausages and smoked meat and fish have figured largely in botulism outbreaks, Type $\mathrm{C}$ may have been concerned. Besides the differences in heat resistance, toxicity, and proteolytic power, agglutinative and other differences distinguish the three types.

In human food poisoning in the United States Type A botulism seems to be by far the most important. Geiger, Dickson, and Meyer ${ }^{3}$ record the isolation and identification of Type A in nineteen outbreaks, of Type B in three. It is noteworthy also that the majority of reported botulism outbreaks have been in those regions-Rocky Mountain and Pacific Coast states-where $\mathrm{Cl}$. botulinum, Type A, occurs most abundantly in soils. In samples of European soils Meyer found Type B but not Type A. It is remarkable that in the United States the highly toxic Type A is found most abundantly in the uncultivated soils of remote, thinly settled regions. The relation of the three closely allied but immunologically distinct types is not understood. It has been surmised that the B type may be the degenerate descendant of the more highly toxic and resistant $\mathrm{A}$ type, but proof of this connection is still lacking.

Since the spores of $\mathrm{Cl}$. botulinum are relatively common in soil in many parts of the world and must adhere frequently to vegetables used for preservation, and since also the temperatures used in the home canning of food have in the past been often inadequate to insure complete sterilization, it might be expected that botulism would have occurred more commonly than has been the case. How can the rarity of the disease be explained? It is obvious that the factors necessary to bring about the

(Mono. 8, Rockefeller Inst. Med. Research, p. II7. I9I8), who has also given a valuable review of the more recent work on pathogenicity, toxicology, and immunology (J.Prev. Med., I, 7r. 1926). The epidemiology of botulism has been comprehensively summarized with much new material by $\mathrm{J}$. C. Geiger, E. C. Dickson, and K. F. Meyer (Pub. Ilealth Bull. 127, U.S. Pub. IIealth Service, p. II9. I922). Numerous special articles by Meyer and his collaborators have appeared in the Journal of Infectious Diseuses (cf., e.g., 31, 50I-663. 1922).

${ }^{1}$ Esty, J. R.: Am. J. Pub. Health, r3, ro8. 1923.

${ }^{2}$ Esty, J. R., and Meyer, K. F.: J. Infect. Dis., 31, 650. 1922.

${ }^{3}$ Geiger, J. C., Dickson, E. C., and Meyer, K. F.: loc, cit. 
production and persistence of botulinum toxin in canned foods must concur relatively seldom. Numerous instances are on record in which the presence of botulinum toxin has been demonstrated in certain jars or cans of food while other jars or cans of the same lot preserved at the same time and to all appearances in the same manner have proved free from toxin. Among the factors that tend to prevent the production of botulinum toxin are an unsuitable reaction of the culture medium, the maintenance of an unsuitable temperature for germination, the presence of only a scanty number of uninjured spores, and the simultaneous presence of other organisms such as $\mathrm{Cl}$. sporogenes. ${ }^{\mathrm{x}}$ Even when once formed, the toxin of $\mathrm{Cl}$. botulinum may sometimes be destroyed by the growth of $\mathrm{Cl}$. sporogenes and certain other anaerobes. ${ }^{2}$

The opinion of some of the earlier investigators that $\mathrm{Cl}$. botulinum "is absolutely incapable of reproducing itself in the animal body" does not seem to be warranted by recent studies. Coleman and Meyer $^{3}$ and Starin and Dack ${ }^{4}$ have found that under certain conditions detoxified spores are capable of germination in the bodies of laboratory animals, that spores and vegetative forms may become widely disseminated through the tissues, and that toxin may be formed in sufficient quantity to cause death. It is highly improbable, however, that under natural conditions tissue invasion by living botulinum organisms is ever the cause of botulism in man. Enormous doses must be used to produce this effect in experimental animals, and no instance is known of the development of botulism in man except when formed botulinum toxin has been taken into the alimentary tract.

The absorption and mode of action of botulinum toxin are quite obscure. Although botulinum toxin is unique in being the only known exotoxin that is absorbed from the digestive tract, the amount necessary to produce death is much larger by mouth than by other routes. Swine resist enormous doses given orally.

Death from botulism is practically always due to failure of the respiratory muscles or of the heart, more often the former. Dickson and Shevky $y^{5}$ have observed that the toxins of both Types A and B affect the peripheral endings of the motor fibers. There is no demonstrable change in smooth and striated muscles.

In animal experimentation antitoxin may be produced by properly graduated inoculations of botulinum toxin just as with tetanus and diphtheria toxin, and has prover efficacious in preventing the death of animals from experimental botulism. Type A toxin is neutralized by Type A but not by Type B antitoxin; Type B toxin, however, seems to be slightly weakened by large quantities of Type A antitoxin. ${ }^{6}$ The therapeutic value of botulinum antitoxin has never had a fair test with human patients. The early development of symptoms and the fact that the antitoxin (owing largely to the rarity of the disease) is not usually at hand stand in the way of prompt administration.

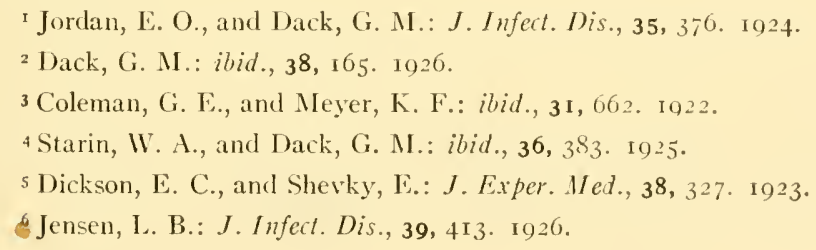




\section{MISCELLANEOUS BACTERIA}

Bacteria of diverse nature have been accused on more or less cogent evidence of causing outbreaks of "food poisoning" either by tissue invasion or by their toxic products.

a) $B$. proteus. - There is no doubt that some members of the large and variable Proteus group are definitely pathogenic. ${ }^{r}$ Whether Proteus bacilli are ever definitely concerned in outbreaks of food poisoning is not so certain. The mere presence of these organisms is not especially significant as they are found generally in decomposing animal matter and are probably not infrequently swallowed with food. Proteus is common in sewage and has been reported by several observers as present in the human intestinal tract, especially in disordered conditions. A number of outbreaks of food poisoning have been attributed by various observers to this organism, but Savage, ${ }^{2}$ after a detailed discussion of nine reported instances, concludes: "From this summary of outbreaks it is evident that for none of them was it established that $B$. protcus was etiologically concerned."

b) Staphylococcus.- One of the most definite instances of food poisoning due to microbic products is that reported by Barber, ${ }^{3}$ who showed that acute attacks of gastro-enteritis were produced by milk in which a toxic substance had been elaborated by a white staphylococcus. The staphylococcus occurred in almost pure culture in the udder of a cow that stood in direct epidemiological relation to the outbreak. The cause of this outbreak was further demonstrated by the reproduction of typical symptoms in a human volunteer who drank milk that had been inoculated with the suspected staphylococcus.

c) Streptococcus.-Linden, Turner, and Thom ${ }^{4}$ have reported two outbreaks at widely separated points in the United States (Biddeford, Maine, and Kansas City, Kansas) which they attributed to a streptococcus found in certain lots of cheese, one an imported Albanian cheese, the other an American Cheddar manufactured in Wisconsin. Savage makes the following comment:

The outbreaks are most interesting, but the proof that they were due to the streptococcus isolated is obviously very incomplete. Apparently this living streptococcus was prevalent in other samples of the fresh cheese, but only one small outbreak resulted. The distribution of this streptococcus in cheese which did not cause illness does not appear to have been investigated. It is unfortunate that essential epidemiological details are omitted. 5

It seems probable that bacteria of various kinds as yet unidentified may by infection or through a preformed poison cause occasional cases or epidemics of food poisoning. Numerous instances are on record where some particular article of food has appeare definitely at fault, yet in which no recognized food poisoning microorganism could be discovered. A number of outbreaks of indeterminate cause are included in the remarkable series of reports in the United States Naval Medical

${ }^{1}$ Larson, W. P. and Bell, E. T.: J. Exper. IIed., 2 I, 629. I915; Warren, S., and Lamb, M. E.: J. 1led. Research, 44, 375, 1923-24.

${ }^{2}$ Savage, W. G.: Food Poisoning and Food Infections, pp. 126-30. Cambridge, 1926.

3 Barber, M. A.: Philippine J. Sc., 9, 515. 1914.

4 Linden, B. A., Turner, W. R., and Thom, C.: Pub. Ilealth Rep., 41, I647. 1926.

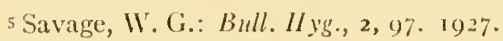


Bulletin (1923-27). Sometimes, however - as in the case of the white staphylococcus cited above-a hitherto undescribed toxigenic microbe is assigned a definitely etiological rôle. Levin , ${ }^{\mathrm{I}}$ for example, attributed six cases of cheese poisoning in Michigan to a toxigenic bacillus of the colon group. No one can review the published reports of "food poisoning" outbreaks, or indeed recall his own experience, without being convinced of our almost complete ignorance of the factors concerned in the production of many epidemics and scattered cases.

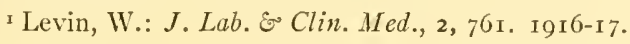




\title{
CHAPTER XXXVI
}

\section{THE SPIROCHE'TES}

\author{
HIDEYO NOGUCHI
}

Rockefeller Institute for Medical Research, New York City

\section{CLASSIFICATION}

Under the name Spirochaeta a large number of micro-organisms has been described, including the variety of free-living spiral forms found in stagnant water, cesspools, and damp soil; the large, actively motile spiral organisms with frilled membranes which live in the crystalline styles of certain shellfish; the minute spiral inhabitants of the normal mouth, genitalia, and intestinal tract of mammals; and, finally, a considerable group of pathogenic micro-organisms, all rather minute, but varying in morphology, which are found in the blood or tissues in certain diseased conditions of man and of animals. All these micro-organisms, notwithstanding their diversity of form, are often indiscriminately referred to as "Spirochaeta," although this genus was created by Ehrenberg ${ }^{\mathrm{I}}$ in $\mathrm{I} 833$ for a saprophytic spiral micro-organism (wurmförmiges Schlingentierchen) which he found in the spring of $\mathrm{I}_{32}$ in some water which had been kept in his house in Berlin through the winter. Recently, however, the problem of the classification of the spirochetes has been taken up by a number of workers with the result that the subject has been considerably clarified.

It is now recognized ${ }^{2}$ that the term Spirochaeta ( $\sigma \pi \epsilon \hat{\imath} \rho a$, "coil"; xair should be reserved for the large free-living species of the type described by Ehrenberg as Spirochaeta plicatilis (Fig. I). ${ }^{3}$ A characteristic axial filament, about which the cell body is spirally wound, has been described for this species, first by Schaudinn (Fig. $4)^{4}$ and more recently by Zuelzer (Figs. 2-3).5 Such a structure cannot be demonstrated in other types of spirochetes, perhaps because of their small size, as Zuelzer maintains, but perhaps also because it is not present in any other type. The writer has attempted to demonstrate it in a Spirochaeta present in the slime of icebox drains (Figs. 5-IO), but without success. Zuelzer maintains that the straight axial filament is the characteristic structure of all true spirochetes. She retains all treponemas and leptospiras in the genus Spirochaeta because she assumes the existence of such a structure in these forms, although it cannot be demonstrated, and excludes the cristispiras from the category of spirochetes because they definitely lack the axial filament. Zuelzer holds this viewpoint almost alone, however, most workers pre-

${ }^{\mathrm{I}}$ Ehrenberg, C. G.: Ablandl. d. Akad. d. Wiss. zu Berlin, I833, p. 313. I834; Die Infusionslierchen. 1838 .

${ }^{2}$ Bergey, D. II., et al.: Manual of Delerminalive Bacleriology. Baltimore, 1925; Buchanan, R. E.: General Systematic Bacteriology. Baltimore, 1925; Wenyon, C. M.: Prolozoülogy. 1926; Stitt, E. R.: Praclical Bacteriology, Blood II'ork, Parasilology (Sth ed.). P'hiladelphia, 1927.

${ }^{3}$ The figures for this chapter follow P. 497.

${ }^{4}$ Schaudinn, F.: Arbeiten. Leipzig and Berlin: Voss, 1911.

sZuelzer, M.: Pronwazck's IIandb. d. path. Protozoen, 3, 1628. I923. 
ferring to classify the organisms on the basis of recognizable morphological differences.

The spirochetes of shellfish were placed by Gross ${ }^{1}$ in a new genus, Cristipira, because of the membranous appendage wound about the body; and another genus, Saprospira, was created by Gross for a type which resembles Cristispira in size and structure, but lacks the membrane.

Opinions still differ somewhat as to whether the parasites of syphilis and those of relapsing fever and smegma should be placed in a single genus or separated. Schaudinn ${ }^{2}$ pointed out that the syphilis organism differs from the saprophytic inhabitant of the smegma, to which he gave the species name refringens, in the rigidity of its spirals. But under cultural conditions, as the writer observed in $19 \mathrm{r} 3{ }^{3}$ the only difference actually existing between the two organisms is in staining property, the refringens having more plasmatic substance and therefore taking stains more readily. Some writers still treat the organisms which cause relapsing fever as a species distinct from the treponemas because they possess certain characteristic biological and cultural properties. Morphologically, however, the parasites differ from the syphilis organism in the same way as does the refringens, but in greater degree, i.e., they have considerably more plasmatic substance. They were first separated from the treponemas by Swellengrebel, ${ }^{4}$ who thought that they all possessed peritrichal flagella. ${ }^{5}$ The publication of Swellengrebel in which he suggested the generic designation of Borrelia for the relapsing-fever group antedated by four months that of Sambon ${ }^{6}$ in which Spiroschaudinnia was suggested; hence, if we regard the group as distinct, we are bound to adopt the name Borrelia, irrespective of the morphological assumption upon which it was based, with anserinum as the type species instead of recurrentis. The writer, in I9I $8,{ }^{7}$ used the name Spironema, which had been adopted by Gonder; 8 but, as has been pointed out, ${ }^{9}$ Spironema was unavailable because preoccupied, and both Borrelia and Spiroschaudinnia have priority. On morphological grounds the separation of the group is not desirable, since the variation between recurrentis and refringens is hardly greater than that between refringens and pallidum and is only a matter of the thickness of the protoplasmic coat. On the whole, it seems best to regard the two groups as one and retain the appropriate generic name of Treponema.

The cause of rat-bite fever (Fig. I28) is more closely affiliated with the Spirilla as are also certain spiral organisms found in the stomach of the dog (Fig. I29) cat, and other animals. ${ }^{10}$ These may be placed in a separate group under Spirella, as first suggested by Duboscq and Lebailly. ${ }^{\text {II }}$

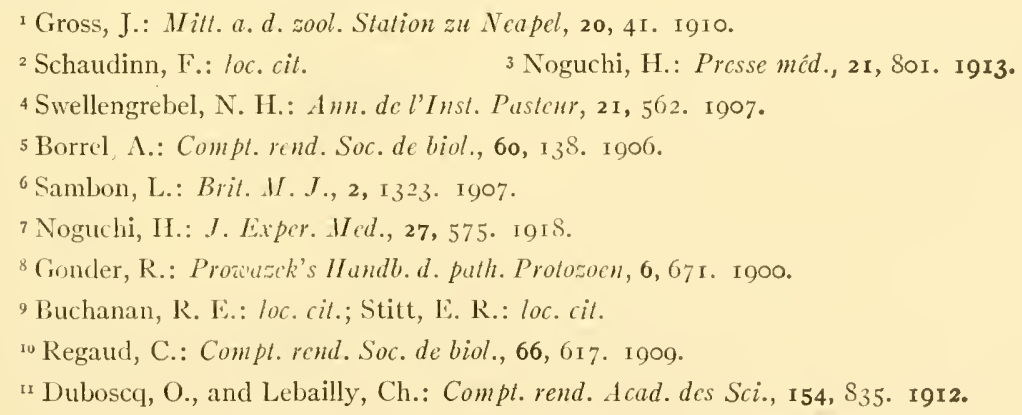


With the discovery of the cause of infectious jaundice in I $9 \mathbf{I} 5$ by Inada and Ido, ${ }^{\mathrm{I}}$ still another morphologically distinct spirochete was added to the family of Spirochaetaceae. That this organism (Fig. I23) possesses certain peculiarities was recognized first by its discoverers, who pointed out the hook formation of one or both extremities and the beaded appearance by darkfield illumination which later proved to be due to the closeness of the spiral windings. The appearance and movements of the organism are so characteristic that the writer $^{2}$ in $I 9 I 7$ introduced the genus Leptospira for it. It developed later, however, that Stimson $^{3}$ had already described this type of spirochete in 1907 (Fig. II9), having discovered it in the kidney from a patient dying of yellow fever and designated it Spirochaeta(?) interrogans because of its hooked extremities. Hence the type species of the genus Leptospira automatically shifts back to Stimson's interrogans, which antedated Inada and Ido's ictcrohacmorrhagiae, and the name Leptospira icteroides, which was given by the writer to the leptospira isolated in ror 8 from yellow-fever patients (Figs. I20-2 I), becomes a synonym of Leptospira interrogans. Other spirochetes belonging to this same group have been discovered, as will be recorded in detail farther on.

The cultural requirements of various groups of spirochetes are quite different. Strict anaerobiosis and the presence of serum and fresh tissue are essential for the pallidum and refringens types. The relapsing-fever spirochetes and the leptospiras are obligatory aerobes. ${ }^{4}$ According to Zuelzer, ${ }^{5}$ Spirochacta plicatilis can be grown in association with bacteria in an air-tight container in water-containing hydrogen sulphide. The cristispiras and saprospiras have never been cultivated.

From the standpoint of pathogenesis, the pallidum is typical of the tissue parasites, which cause chronic manifestations similar to those of a protozoan infection. The relapsing fever organisms are principally blood parasites, while Leptospira interrogans and Leptospira icterohaemorrhagiae invade both the blood and the tissues. None of the organisms of genera I, 3 , and 4 described below is so far known to be pathogenic for man. The classification which the writer suggested in rgi 8 has been modified only as regards the recurrentis group, which has been retained with the treponemas for the reasons already stated.

Genus I: Spirochaeta (Ehrenberg, I 833 )* Type: S. plicatilis (Ehrenberg, I 833 )

Genus 2: Treponema (Schaudinn, I 905$) \dagger$

Genus 3: Cristispira (Gross, I9I I) $\ddagger$

Genus 4: Saprospira (Gross, I 9 I I $) \ddagger$

Type: T. pallidum (Schaudinn and Hoffmann, i905)\|

Type: C. balbianii (Certes, $\left.\mathbf{8} 88_{2}\right) \boldsymbol{\uparrow}$

Type: S. grandis (Gross, I9I0)**

Genus 5: Leptospira (Noguchi, 1917)\$

Type: L. interrogans (Stimson, I907) ††

*Abhandl. d. Akad. d. Wissenseh. zu Berin, I833, p. 313. I834: Die Infusionstierchen. I838.

†Deutsche med. Wchnschr., p. 1728.1905.

+Milt. a. d. cool. Shution zu Veapel, p. I88. I9I r.

\$I. Exper. Med, 25, 755. 1917.

IlArb. a. d. kaiserl. Gesundheitsamte, 22, 527. 1905

- Bull. Soc. zool. de France, 7, 347. 1882.

**Op. cit., 20, $4 \mathrm{I}$. IoIO.

ttU.S. Puh Ileallh Rep. 1907.

Inarla, R., and Ido, K.: Tokyo Iji-shinshi. (Feb.) I9т5.

2 Noguchi, H.: J. Exper. Hed., 25, 755. 1917.

3 Stimson, A. N1.: U.S. Pub. IIeullh Rep., 22, Part 1, 541. 1007.

4. Some authors apparently have the misconception that these organisms can grow without oxygen because they grow under a layer of parafin oil, whereas the oil layer does not interfere with the penetration of oxygen and is used only to delay the evaporation of the medium.

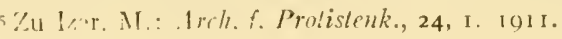


The question whether spirochetes are plants or animals is to my mind still an open one. The absence of polarity and of any definite nucleus and the mode of multiplication (by transverse fission) all point to a relationship with the bacteria. On the other hand, the spirochetes, and particularly those belonging to the pallidum, re currentis, and icterohaemorrhagiae types, appear to form granular elements the significance of which is still under discussion. The observations of Leishman, ${ }^{1}$ Balfour, ${ }^{2}$ Nuttall, ${ }^{3}$ Hindle, ${ }^{4}$ Blanc, ${ }^{5}$ Fantham, ${ }^{6}$ and others on the granule stage of $T$. duttoni in the body of ticks seem to me to prove beyond any doubt that these organisms undergo a granular evolution after ingestion by the tick in the course of which in about ten days there emerges a large number of young, active spirochetes, thinner, smaller, and more difficult to stain than the blood form. Such a phenomenon has never been known to occur in bacterial organisms. Martin and Pettit ${ }^{7}$ have observer granular forms in cultures of $L$. icterohaemorrhagiae. In the case of $L$. interrogans, ${ }^{8}$ cultures containing only granular forms have been proved capable of infecting guinea pigs with reappearance of the spiral form in the animals. A similar phenomenon was recorded by the writer in the case of $T$. pallidum in I9I I-I $2 .{ }^{9}$ The filterability of the spirochetes is another property which distinguishes them from the bacteria. The question of the phylogenetic relationship of the spirochetes is therefore not an easy one to settle.

\section{FAMILY SPIROCHAETACEAE}

Unicellular, spiral, filamentous, highly flexible organisms. Transverse division by fission, usually binary, the daughter-cells being held together for some time to form chains of two or more individuals. In cultures a long thread of several individuals joined by thin bridges is not uncommon. The apparatus of motion consists of an elastic axial filament or a modified fibrillar membrane along the side of the cell body, which serves to produce a propelling effect when contracted and relaxed by successive contraction and relaxation of the contractile bank (myonemes?) imbedded in the outer layer of protoplasm.

Genus Spirochacta (Ehrenberg, I834; Zuelser, I9I0).-Unicellular, flexible, membrane indistinct, long cylindrical filament composed of a straight elastic axial filament surrounderl by a layer of alveolar protoplasm spirally coiling about the axial filament (Figs. 2, 3). Creeping movements. No terminal flagella demonstrated. Volutin granules distributed in pairs at each turn of the protoplasmic spirals. Transverse and multiple division.

Spirochaela plicalitis (Ehrenberg, $18_{34}$ ). - Stagnant water; free living; requires hydrogen sulphide for growth.

Genus Treponema (Schaudinn, 1905).-Long, slender, cylindrical, spirally wound, highly flexible cell body, which exhibits serpentine, corkscrew-like, and sometimes lashing movements. The spiral curves are alternately stretched and relaxed with a certain regularity.

${ }^{1}$ Leishman, W. B.: Lancet, 128, гі. г9го; Tr. Soc. Trop. Med. o Ilyg., 3, 77. 191о; Internat. Cong. Med., 21, 2S2. 1913.

${ }^{2}$ Balfour, A.: Rep. Welleome Research Lab., Khartoum, 3, 35. I908; ibid., 4, 76. I91 I; Internat. Cong. Med., 21, 275. 1913.

3 Nuttall, G. H. F.: Harvey Lectures, p. 28. I9г 2-13.

${ }_{4}^{4}$ Hindle, E.: Ann. Trop. Med., 4, 463. г9гі.

5 Blanc, G. R.: Thèses de Paris, No. +32. г9ro-ix.

${ }^{6}$ Fantham, H. B.: Ann. Trop. Med., 8, 471. 1914.

7 Martin, L., and Pettit, A.: Spirochêtose Icterohaemorragique. Paris, 1919.

${ }^{8}$ Noguchi, H.: J. Exper. Med., 30, 26. $1919 . \quad 9$ Noguchi, H.: Presse méd., 21, 801. 1913. 
When motility is reduced, the organism may rotate along its axis in one and then another direction without changing its curves. The cell body consists of a spiral, elastic axial filament, a layer of protoplasm of varying thickness around the filament, and a delicate flexible membrane covering the whole body. Both ends are sharply drawn to a point. There may be a terminal filament (Figs. 22-29, 43-47) at one or both ends (T. pallidum, T. recurrentis), or peritrichal flagella (Fig. 53) may be present (T. anscrinum). The polar terminal projection may be a smooth or a finely curved elastic spiral appendage of immeasurable thinness, of a length of one-fourth to one-third of the body. The terminal projections are more common in cultures and exhibit a swinging movement around the extremity of the organism. The thickness of the protoplasmic coat varies in different species, is thin in Treponema pallidum, T. portenue, and T. cuniculi; somewhat thicker in T. refringens; and still thicker in $T$. recurrentis. In the organisms with the more plasmatic substance there is often a masking of the elementary curves of the axial filament during active movement. The tendency of the organism to take up stains is proportional to the thickness of the protoplasmic coatthe more protoplasm the greater the tendency to take up the violet nuance of the Giemsa stain. Transverse division. Pathogenic types transmitted by contact (T. pallidum, T. pertenue, T. cuniculi) or by insects (T. recurrentis, T. duttoni, T. anserinum). The tissue-residing forms require anaerobic conditions for growth; the blood-inhabiting species are aerobic. Filterable under certain conditions. Granular phase observed.

Genus Cristispira (Gross, I9II).--Highly active, cylindrical, wavy cell body. There is present a more or less wavy membranous structure composed of numerous fine elastic wavy fibrils resembling bacterial flagella, running spirally along the entire body like a veil (Figs. I9-2I); in a cross-section it is seen to form a ridge, hence the name Cristispira. In stained specimens the protoplasm appears cross-barred or chambered (Figs. 40-42), with alternating deep-staining transverse septa (volutin and chromidial granules) and pale-stained spaces between septa. There is a distinct cell membrane which is elastic, as shown by the marked flexibility of the living organism in its active movements. No terminal flagella or axial filament present. Gross's view as to the multicellular nature of the organism and the occurrence of sporulation has not been confirmed. No definite chambered structure can be demonstrated in the fresh state. Habitat: Crystalline styles of various Lamellibranchs.

Genus Saprospira (Gross, IgII).-Differs from Cristispira in the absence of a crista. The multicellular structure of the organism and the occurrence of sporulation, as described for this genus, await confirmation. Habitat: Foraminiferous sand; saprophytic; a group very little known.

Genus Leptospira. (Noguchi, I9I7).-A long, slender, cylindrical, highly flexible filament, with tightly set, regular, shallow spirals. Both extremities gradually taper to sharply drawn points. In some forms the curves are so closely set that the distance between two spirals is too small to measure; in saprophytic forms there is an appreciable distance between adjacent spirals. A smooth axial filament with hooked ends may be present (Fig. 33) but is not demonstrable by staining. Terminal filaments are sometimes observed (Figs. 30-34, 57-76). The terminal portions of both extremities of the organism are endowed with marked motility in contrast to the middle portion of the body, and they may be bent to a hook or stretched out; the hooked-end portions are characteristic of the species. Perhaps there is a membrane. Transverse division: Transmission by insects or rodents in the case of pathogenic varieties, which invade both tissues and blood. Some species are saprophytic. All are obligatory aerobes. Filterable. Granular phase observed.

Table I is a résumé of the characteristics upon which generic differentiation is made 
TABLE I

\begin{tabular}{|c|c|c|c|c|c|}
\hline & $\begin{array}{c}\text { SPIROCHAETA } \\
\text { (EHRENBERG, } \\
\text { I } 838 \text { ) }\end{array}$ & $\begin{array}{l}\text { SAPROSPIRA } \\
\text { (GROSS, I III })\end{array}$ & $\begin{array}{l}\text { CRISTISPIRA } \\
\text { (GROSS, I9II) }\end{array}$ & $\begin{array}{c}\text { TREPONEMA } \\
\text { (SCHAUDINN, } \\
\text { I9O5) }\end{array}$ & $\begin{array}{c}\text { LEPTOSPIRA } \\
\text { (NoGUCHI, I9I } 7 \text { ) }\end{array}$ \\
\hline & $\begin{array}{c}\text { S. plicatilis* } \\
\left.\text { (Ehrenbere, } 18_{3} 8\right)\end{array}$ & $\begin{array}{l}\text { S. grandis } \\
\text { (Gross, IgII) }\end{array}$ & $\begin{array}{l}\text { C. balbianii } \\
\text { (Certes, } 1882 \text { ) }\end{array}$ & $\begin{array}{c}T \text { pallidum } \\
\text { (Schaudinn, 1905) }\end{array}$ & $\begin{array}{l}L \text { icterohaemor- } \\
\text { rhaciae* (Inada } \\
\text { and Ido, I9ז4) }\end{array}$ \\
\hline Body ............. & $\begin{array}{c}\text { Cylindrical, } \\
\text { lexibl }\end{array}$ & $\begin{array}{l}\text { Cylindrical, } \\
\text { flexible }\end{array}$ & $\begin{array}{l}\text { Cylindrical, } \\
\text { flexible }\end{array}$ & $\begin{array}{l}\text { Cylindrical, } \\
\text { flexible }\end{array}$ & $\begin{array}{l}\text { Cylindrical } \\
\text { flexible }\end{array}$ \\
\hline Extremities......... & Blunt & Obtuse & Obtuse & Pointed & Pointed \\
\hline Length............. & $100-500 \mu$ & $100-\mathrm{I} 20 \mu$ & $45^{-90 \mu}$ & $8-\mathbf{I} 4 \mu$ & $7-9-\mathrm{I} 4 \mu$ \\
\hline Diameter.......... & $0.5^{-0} .75 \mu$ & $\ldots \ldots \ldots \ldots$ & $\mathrm{I}-\mathrm{I} \cdot 5 \mu$ & $0.25-0.3 \mu$ & $0.25-0.3 \mu$ \\
\hline Spiral amplitude... . . & $2 \mu$, regular & $\begin{array}{l}\text { Nospirals but } \\
\text { curves }\end{array}$ & a few wavy & $\begin{array}{l}\text { I } \mu \text {, regular, } \\
\text { rigid }\end{array}$ & $0.45^{-0} .5 \mu$ \\
\hline Spiral depth. ...... & I. $5 \mu$, regular & Inconstant & Inconstant & $\begin{array}{c}0.8-\mathrm{I} \mu \text {, very } \\
\text { constant }\end{array}$ & $0.3 \mu$, regular \\
\hline $\begin{array}{l}\text { Number of spirals or } \\
\text { waves.............. }\end{array}$ & $\begin{array}{l}\text { 5o-25o spi- } \\
\text { rals, said to } \\
\text { remain un- } \\
\text { changed } \\
\text { during } \\
\text { movement; } \\
\text { many un- } \\
\text { dulations } \\
\text { occur }\end{array}$ & $\begin{array}{l}\text { 2-3 loose, } \\
\text { changeable } \\
\text { waves }\end{array}$ & $\begin{array}{l}2-5 \text { loose, } \\
\text { changeable } \\
\text { waves }\end{array}$ & $\begin{array}{c}\text { 8-I4 spirals, } \\
\text { changeable } \\
\text { in very ac- } \\
\text { tive but } \\
\text { rigid in less } \\
\text { active spec- } \\
\text { imens } \\
\text { (coarser } \\
\text { species, re- } \\
\text { fringens, } \\
\text { buccale, are } \\
\text { irregular } \\
\text { except in } \\
\text { culture); a } \\
\text { few undu- } \\
\text { Jations of } \\
\text { whole or- } \\
\text { ganism } \\
\text { may occur }\end{array}$ & $\begin{array}{l}\text { 14-I8-24 spi- } \\
\text { rals which } \\
\text { do not } \\
\text { change } \\
\text { theircurves } \\
\text { even in the } \\
\text { most active } \\
\text { state; long- } \\
\text { er chains in } \\
\text { culture but } \\
\text { spirals of } \\
\text { same di- } \\
\text { mension; } \\
\text { usually } \\
\text { straight ex- } \\
\text { cept that } \\
\text { one or both } \\
\text { ends may } \\
\text { form a } \\
\text { semicircle } \\
\text { or hook }\end{array}$ \\
\hline Axial filament... & $\begin{array}{l}\text { Distinctly } \\
\text { seen in } \\
\text { stained } \\
\text { specimens; } \\
\text { straight, } \\
\text { elastic, and } \\
\text { flexible }\end{array}$ & Absent & Absent & $\begin{array}{l}\text { Demonstrat- } \\
\text { ed easily in } \\
\text { some spe- } \\
\text { cies in de- } \\
\text { generating } \\
\text { specimens; } \\
\text { the whole } \\
\text { body rep- } \\
\text { resents an } \\
\text { axial fila- } \\
\text { ment with } \\
\text { protoplas- } \\
\text { mic coat of } \\
\text { varying } \\
\text { thickness }\end{array}$ & $\begin{array}{l}\text { Smooth fila- } \\
\text { ment with } \\
\text { hooked } \\
\text { ends may } \\
\text { be present }\end{array}$ \\
\hline
\end{tabular}

*Organism for which generic name was created. 
TABLE I-Continued

\begin{tabular}{|c|c|c|c|c|c|}
\hline & $\begin{array}{c}\text { SPIROCHAETA } \\
\text { (EHRENBERG. } \\
\text { I } 838 \text { ) }\end{array}$ & $\begin{array}{l}\text { SAPROSPIRA } \\
\text { (GROSS, IgIr) }\end{array}$ & $\begin{array}{c}\text { CRISTISPIRA } \\
\text { (GROSs, Igrr) }\end{array}$ & $\begin{array}{c}\text { TREPONEMA } \\
\text { (SCHAUDINN, } \\
\left.\text { I } 90^{5}\right)\end{array}$ & $\begin{array}{c}\text { LEPTOSPIRA } \\
\text { (NOGCCHI, IgI } 7 \text { ) }\end{array}$ \\
\hline & $\begin{array}{c}\text { S. p'icatilis* } \\
\text { (Ehrenberg, I } 838)\end{array}$ & $\begin{array}{l}\text { S. grandis* } \\
\text { (Gross, rgII) }\end{array}$ & $\begin{array}{l}\text { C. balbianii* } \\
\text { (Certes, } 188_{2} \text { ) }\end{array}$ & $\begin{array}{c}T \text {. pallidun }{ }^{*} \\
\text { (Schaudinn, I905) }\end{array}$ & $\begin{array}{l}\text { L. icterohaemor- } \\
\text { rhagiae* (Inada } \\
\text { and Ido, I9r4) }\end{array}$ \\
\hline $\begin{array}{l}\text { Protoplasmic sub- } \\
\text { stance......... }\end{array}$ & $\begin{array}{l}\text { Thick alve- } \\
\text { olar layer } \\
\text { coiling spi- } \\
\text { rally about } \\
\text { the axial } \\
\text { flament; } \\
\text { volutin } \\
\text { present }\end{array}$ & $\begin{array}{l}\text { Alveolar and } \\
\text { chambered; } \\
\text { volutin } \\
\text { present }\end{array}$ & $\begin{array}{l}\text { Alveolar and } \\
\text { chambered; } \\
\text { volutin } \\
\text { present }\end{array}$ & $\begin{array}{l}\text { Apparently } \\
\text { homogene- } \\
\text { ous; coils } \\
\text { about the } \\
\text { spiral axis } \\
\text { in extreme- } \\
\text { ly thin } \\
\text { layer; volu- } \\
\text { tin doubt- } \\
\text { ful }\end{array}$ & $\begin{array}{l}\text { The body } \\
\text { seems to } \\
\text { consist of } \\
\text { the spiral } \\
\text { protoplas- } \\
\text { mic sub- } \\
\text { stance; } \\
\text { volutin } \\
\text { doubtful }\end{array}$ \\
\hline Membrane...... & $\begin{array}{l}\text { Not recog- } \\
\text { nized }\end{array}$ & $\begin{array}{l}\text { Distinct, } \\
\text { flexible, } \\
\text { elastic }\end{array}$ & $\begin{array}{l}\text { Distinct, } \\
\text { flexible, } \\
\text { elastic }\end{array}$ & $\begin{array}{l}\text { Distinct, } \\
\text { flexible, } \\
\text { elastic, in } \\
\text { some spe- } \\
\text { cies; diffi- } \\
\text { cult to rec- } \\
\text { cognize in } \\
\text { others, but } \\
\text { probably } \\
\text { present }\end{array}$ & $\begin{array}{l}\text { Difficult to } \\
\text { demon- } \\
\text { strate; } \\
\text { must be } \\
\text { extremely } \\
\text { delicate } \\
\text { and flexible }\end{array}$ \\
\hline Terminal filament. & Absent & Absent & Absent & $\begin{array}{l}\text { Smooth, so- } \\
\text { called "per- } \\
\text { iplastic } \\
\text { terminal } \\
\text { projection" } \\
\text { is present } \\
\text { in some } \\
\text { specimens } \\
\text { while a } \\
\text { finely and } \\
\text { regularly } \\
\text { coiled spiral } \\
\text { filament } \\
\text { (finer than } \\
\text { any known } \\
\text { bacterial } \\
\text { flagellum) } \\
\text { can be } \\
\text { demon- } \\
\text { strated in } \\
\text { some, par- } \\
\text { ticularly in } \\
\text { culture; } \\
\text { peritrichal } \\
\text { flagella de- } \\
\text { scribed } \\
\text { (Borrel) for } \\
\text { some spe- } \\
\text { cies }\end{array}$ & $\begin{array}{l}\text { Not recog- } \\
\text { nizable in } \\
\text { fresh state; } \\
\text { a single } \\
\text { flagellum } \\
\text { at one or } \\
\text { both ends } \\
\text { has been } \\
\text { described } \\
\text { (Martin } \\
\text { Pettit and } \\
\text { Vaudre- } \\
\text { mer) } \dagger\end{array}$ \\
\hline
\end{tabular}

* Organism for which generic name was created.

† Compt. rend. Soc. de bial., 79, ro53. rg16. 
TABLE I-Continued

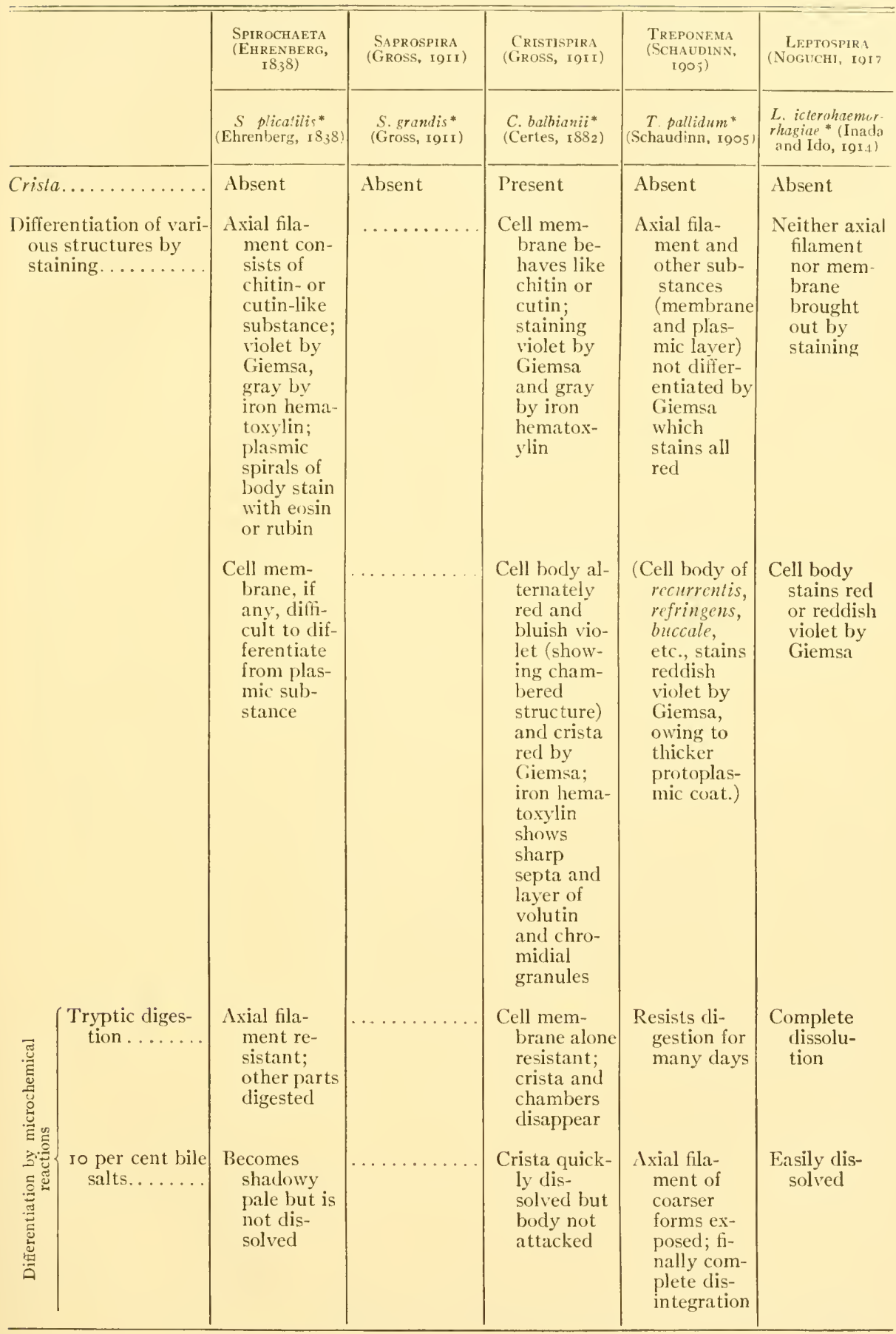

*Organism for which generic name was created. 
TABLE I-Continued

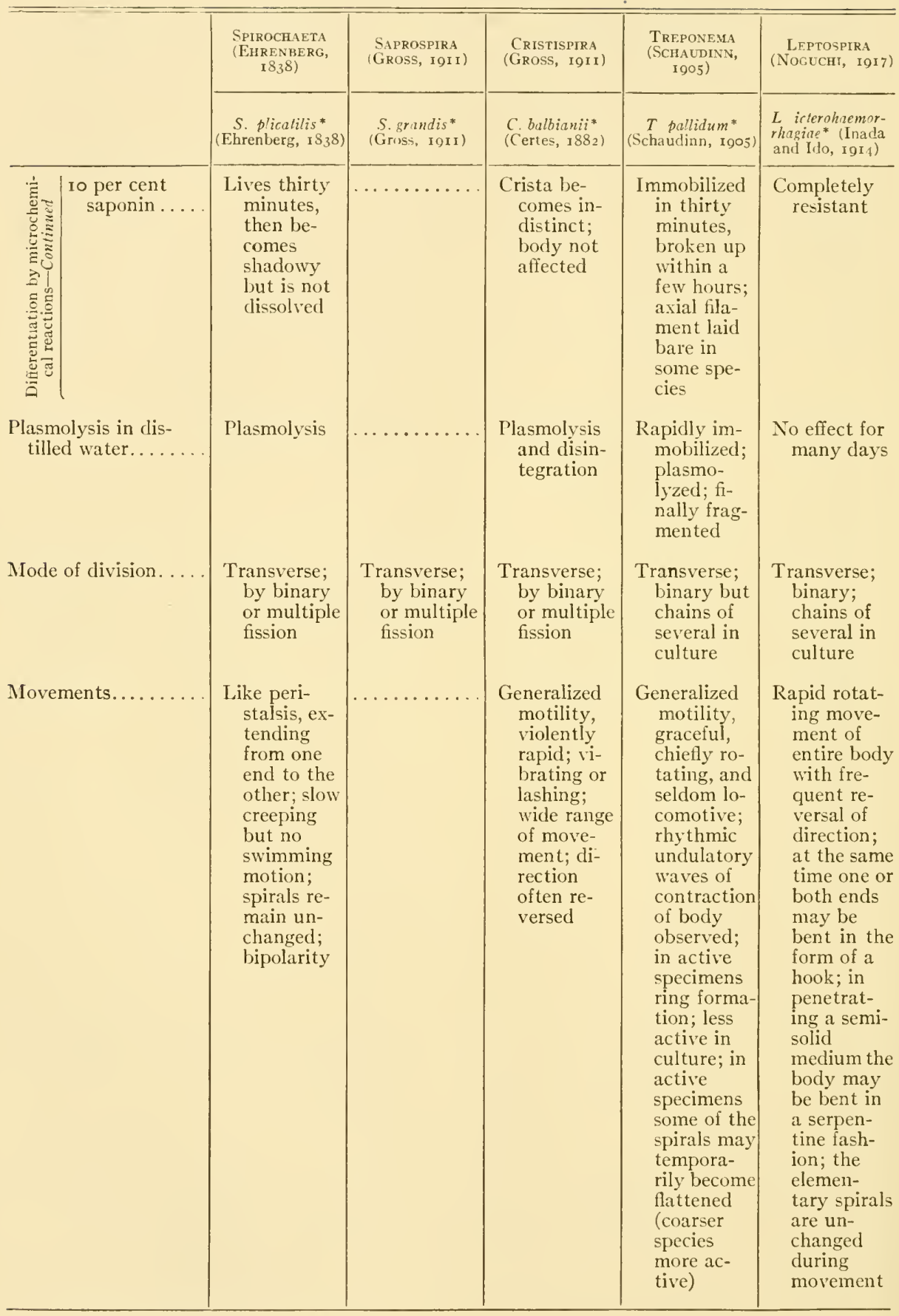

*Organism for which generic name was created. 
TABLE I (Continued)

\begin{tabular}{|c|c|c|c|c|c|}
\hline & $\begin{array}{c}\text { SPIROCHAETA } \\
\text { (EHRENERG, } \\
\text { I8.38) }\end{array}$ & $\begin{array}{c}\text { SAPROSPIRA } \\
\text { (GROSS, IVI I) }\end{array}$ & $\begin{array}{l}\text { CRISTISPIRA } \\
\text { (Gross, Igri) }\end{array}$ & $\begin{array}{c}\text { TREPONEMA } \\
\text { (SCHADINN } \\
\text { (905) }\end{array}$ & $\begin{array}{c}\text { LEPTOSPIRA } \\
\text { (NOGUCHI I } 1917 \text { ) }\end{array}$ \\
\hline & $\begin{array}{c}\text { S. pliculilis }{ }^{*} \\
\text { (Ehrenberg, } 1838 \text { ) }\end{array}$ & $\begin{array}{l}\text { S.grandis* } \\
\text { (Gross, IgII) }\end{array}$ & $\begin{array}{c}\text { C. batbianii** } \\
\text { (Certes, } 188_{2} \text { ) }\end{array}$ & $\mid \begin{array}{c}\text { T. pallidum* } \\
\text { (Schaudinn, I905) }\end{array}$ & $\begin{array}{l}\text { L. iclerohaemor- } \\
\text { rhagiae* (Inada } \\
\text { and Ido, I914) }\end{array}$ \\
\hline Other species.. & $\begin{array}{l}\text { plicati'is } \\
\text { marina, } \\
\text { plicati'is } \\
\text { eustrepta, } \\
\text { steno- } \\
\text { strepta, } \\
\text { daxensis, } \\
\text { etc. }\end{array}$ & nana & $\begin{array}{l}\text { ostreae, } \\
\text { anodontac, } \\
\text { modiolae, } \\
\text { veneris, } \\
\text { tapetos, } \\
\text { chamae, } \\
\text { pinnae, } \\
\text { etc. }\end{array}$ & $\begin{array}{c}\text { pertenue, cu- } \\
\text { niculi, mi- } \\
\text { crodentium, } \\
\text { macroden- } \\
\text { tium, mu- } \\
\text { cosum, cal- } \\
\text { ligyrum, re- } \\
\text { fringens, } \\
\text { genitalis, } \\
\text { eugyratum, } \\
\text { microgyra- } \\
\text { tum, buc- } \\
\text { cale, accu- } \\
\text { minatum, } \\
\text { obtusum, } \\
\text { clusum, } \\
\text { duttoni, } \\
\text { novyi, } \\
\text { kochi, car- } \\
\text { teri, berbc- } \\
\text { rum, acgyp- } \\
\text { licum, thei- } \\
\text { leri, cqui, } \\
\text { oris, an- } \\
\text { serinum, } \\
\text { gatlinarum }\end{array}$ & $\begin{array}{l}\text { biflcxa, int- } \\
\text { tcrrogans, } \\
\text { hebdomadis, } \\
\text { aquatilis, } \\
\text { pseudo- } \\
\text { icterogencs, } \\
\text { trimero- } \\
\text { donta, den- } \\
\text { tale, bovis, } \\
\text { pyrogenes, } \\
\text { hemoglo- } \\
\text { binuriac, } \\
\text { asthenat- } \\
\text { giae } \\
\end{array}$ \\
\hline $\begin{array}{l}\text { Habitat } \\
\text { Parasitism } \\
\text { Pathogenicity }\end{array}$ & $\begin{array}{l}\text { Free living in } \\
\text { fresh or } \\
\text { salt water }\end{array}$ & $\begin{array}{l}\text { Irec living in } \\
\text { foram- } \\
\text { iniferous } \\
\text { sand }\end{array}$ & $\begin{array}{l}\text { Parasitic in } \\
\text { the alimen- } \\
\text { tary canal } \\
\text { of shellfish }\end{array}$ & $\begin{array}{l}\text { Majority par- } \\
\text { asitic in } \\
\text { alimentary, } \\
\text { gastro-in- } \\
\text { testinal, or } \\
\text { urogenital } \\
\text { tracts of } \\
\text { man, ani- } \\
\text { mals, fish, } \\
\text { etc.; some } \\
\text { pathogenic, } \\
\text { causing } \\
\text { chronic } \\
\text { tissue dis- } \\
\text { eases (syph- } \\
\text { ilis, yaws) } \\
\text { or acute } \\
\text { blood in- } \\
\text { fections } \\
\text { (relapsing } \\
\text { fevers) }\end{array}$ & $\begin{array}{l}\text { Blood- and } \\
\text { tissue-in- } \\
\text { vading } \\
\text { varieties as } \\
\text { well as a } \\
\text { few harm- } \\
\text { less para- } \\
\text { sitic or } \\
\text { free-living } \\
\text { species } \\
\text { known; the } \\
\text { pathogenic } \\
\text { species pro- } \\
\text { duce se- } \\
\text { vere, acute, } \\
\text { organ-de- } \\
\text { stroying } \\
\text { febrile dis- } \\
\text { eases } \\
\text { (jaundice } \\
\text { yellow } \\
\text { fever) }\end{array}$ \\
\hline
\end{tabular}

*Organism for which generic name was created. 
TABLE I (Continued)

\begin{tabular}{|c|c|c|c|c|c|}
\hline \multirow[t]{2}{*}{. } & $\begin{array}{c}\text { SPIROCHAETA } \\
\text { (EHRENERG, } \\
\text { 1838) }\end{array}$ & $\begin{array}{l}\text { SAPROSPIRA } \\
\text { (GROSS, IOII) }\end{array}$ & $\begin{array}{c}\text { CRISTISPIRA } \\
\text { (GRoss, I9II) }\end{array}$ & $\begin{array}{l}\text { TREPONEMA } \\
\text { (SCHAUDINN, } \\
\text { T } 905 \text { ) }\end{array}$ & $\begin{array}{c}\text { LEPTOSPIRA } \\
\text { (NoGUCHI, I917) }\end{array}$ \\
\hline & $\begin{array}{c}\text { S. plicatilis* } \\
\text { (Ebrenberg, } 18_{3} 3 !\end{array}$ & $\begin{array}{c}\text { S. grandis* } \\
\text { (Gross, I9II) }\end{array}$ & $\begin{array}{l}\text { C. balbianii* } \\
\text { (Certes, } 188_{2} \text { ) }\end{array}$ & $\mid \begin{array}{c}T . \text { pallidum }{ }^{*} \\
\text { (Schaudinn, 1905) }\end{array}$ & $\begin{array}{l}\text { L. icterohacemor- } \\
\text { rhagiae* (Inada } \\
\text { and Ido, I9I4) }\end{array}$ \\
\hline $\begin{array}{l}\text { Immunity after } \\
\text { infection..... }\end{array}$ & & & & $\begin{array}{c}\text { None recog- } \\
\text { nized in } \\
\text { chronic tis- } \\
\text { sue dis- } \\
\text { eases; com- } \\
\text { plete but of } \\
\text { short du- } \\
\text { ration in } \\
\text { blood dis- } \\
\text { diseases }\end{array}$ & $\begin{array}{l}\text { Prompt, com- } \\
\text { plete, dur- } \\
\text { able for } \\
\text { years }\end{array}$ \\
\hline $\begin{array}{l}\text { Transmission of patho- } \\
\text { genic varieties ..... }\end{array}$ & & & & $\begin{array}{l}\text { By contact or } \\
\text { intermedi- } \\
\text { iate hosts } \\
\text { (lice or } \\
\text { ticks) }\end{array}$ & $\begin{array}{l}\text { Insects and } \\
\text { certain } \\
\text { rodents }\end{array}$ \\
\hline Granular phase. . & & & & $\begin{array}{l}\text { Observed in } \\
\text { culture or } \\
\text { in inter- } \\
\text { mediate } \\
\text { hosts }\end{array}$ & $\begin{array}{l}\text { Observed in } \\
\text { culture; } \\
\text { may also } \\
\text { occur in } \\
\text { insect host } \\
\text { (mosquito) }\end{array}$ \\
\hline Filterability ...... & & & & $\begin{array}{l}\text { Some species } \\
\text { filterable }\end{array}$ & Filterable \\
\hline Sporulation.......... & Absent & $\begin{array}{r}\text { Reported } \\
\text { (Gross) }\end{array}$ & $\begin{array}{r}\text { Reported } \\
\text { (Gross) }\end{array}$ & Absent & Absent \\
\hline Cultural requirements. & $\begin{array}{l}\text { Anaerobic } \\
\text { with } \mathrm{H}_{25} \mathrm{~S} \\
\text { in stagnant } \\
\text { water }\end{array}$ & & & $\begin{array}{l}\text { Blood- } \\
\text { residing } \\
\text { types aero- } \\
\text { bic, tissue- } \\
\text { inhabiting } \\
\text { types ob- } \\
\text { ligatory } \\
\text { anaerobes; } \\
\text { all require } \\
\text { animal- } \\
\text { serum con- } \\
\text { stituents }\end{array}$ & $\begin{array}{l}\text { Obligatory } \\
\text { aerobes; } \\
\text { require } \\
\text { animal- } \\
\text { serum con- } \\
\text { stituents }\end{array}$ \\
\hline
\end{tabular}

*Organism for which generic name was created.

GENERAL FEATURES

\section{MOTILITY AND ITS MECHANISM}

The relation of flagella to motility in bacteria has been well established, and the number and arrangement of flagella are of ten characteristic (Figs. I I-I8). The search for structures in spirochetes analogous to bacterial flagella led to the demonstration in some forms of delicate terminal filaments which were at first interpreted as having a function in locomotion (Figs. 22-29, 43-47). 'There is no evidence, however, 
that these appendages are similar in structure to bacterial flagella, nor does their presence explain the characteristic spirochetal movements.

The tendency in the past has been to attack the problem of the structure of spirochetes by cytological methods, i.e., by the study of individuals fixed and stained in such a way as to preserve the normal structure as nearly as possible. Such methods are less productive in the case of spirochetes than a study of involution or degeneration phenomena. Destruction of the less resistant elements of the body by induced degeneration, e.g., by exposure of the organisms to the action of certain cytolytic agents, bile, or bile salts, gives a clue to the type of skeletal structure present. Changes in the tonicity of the medium serve to indicate whether a periplast is present and of what nature it is. Observation of the morphological sequences in division and the natural process of degeneration also furnish information regarding structure and motility.

From a study of the degeneration phenomena of several types of spirochetes the writer has concluded that the kinetic element in these organisms is the axial filament, which is a kind of modified flagellum. The reasons for this deduction will appear in the separate discussions of the various genera given below.

Cristispira. - This very large, wormlike organism was once known as a trypanosome because of its veil-like membrane. It is found in a rather firm, gelatinous, hyaline structure of shellfish known as the "crystalline style," which is situated in the esophagus. The style, which dissolves rather readily in sea water after extraction from the oyster or clam, often contains a large number of the large, very active spirochetes, swimming by means of a membranous structure, one edge of which is free, the other attached longitudinally along a line which winds about the body spirally (Figs. I9-2I, 40-42). This structure was shown by Gross to be different from the undulating membrane of a trypanosome and was designated by him the "crista." The body itself is not spiral. The periplast is heavy, and the protoplasm is divided into numerous small chambers by transverse septa (Figs. 40-42). The organism is devoid of an axial or terminal filament.

The membrane is thicker at the outer edge than where attached to the body and frilled. Little idea of the structure can be gained from direct observation of living forms (Figs. 35-36) or from stained preparations of normal organisms. When the organisms in the style have died, however, and they do not survive long after the style is removed from the shellfish, many detached cristas are found. They retain their spiral shape (Fig. 38), and when they break up into shorter pieces numerous fibrils are plainly recognizable (Fig. 39). These vary in length and thickness but are all wavy, tapered at the ends, hyaline, and firm in appearance. They lie parallel or may be interwoven in a sort of mesh. The individual fibrils do not fragment or bend sharply but appear to be elastic, and they retain their staining property even after they separate from the mass. Those at the free edge are thicker and longer and form a distinct bundle.

The body form of the resting or dead organism is wavy (Fig. 37), i.e., it conforms to the shape of the spring-like crista. It is difficult to follow the shapes taken by the body in motion, but it seems to straighten and relax in rapid alternation. It is clear that the elastic wavy membrane is stretched at short intervals by the rhythmical 
contraction of the cylindrical body, relaxing between contractions. In a fluid medium the only possible outcome of such contractions is constant rotation of the organism, the spiral membrane propelling the body backward or forward according to the direction of rotation.

From the mechanical and morphological viewpoint the crista is a giant compound flagellum in which innumerable fibrils are united. It is a homologue of the bacterial flagellum but is unique in structure and disposition.

Treponema.- In a fluid medium the movement of these organisms is rotatory; in a semisolid medium it is corkscrew-like. Culture forms, when viewed by the darkfield microscope, usually show at one or both ends a fine, rigid filament with numerous regularly set deep spirals. These tiny filaments may be so loosely attached that they swing about as the protoplasm of the terminal section of the organism contracts, but they have no influence upon the movements of the body; their presence is immaterial.

The filaments may occasionally be smooth instead of wavy (Fig. 46), but they are never motile. The terminal filaments undoubtedly represent portions of the axial filament of the mother-cell, which becomes plastic at the time of division and very finely drawn out (Figs. 23,43). When it divides, each daughter-cell carries away a portion of the maternal axial filament, which subsequently becomes rigid, the material having a tendency to curl when hard. The smooth terminal filaments are probably the drawn-out ends of the periplast, though it may be that the projecting portions of the axial filament occasionally do not become rigid and therefore do not coil. In Figures $43-47$ are shown the appearance of the terminal filaments.

The existence of the axial filament within the body cannot be demonstrated by staining, because it is completely enveloped by a layer of protoplasm which stains far more intensely. Its presence becomes evident, however, during the process of degeneration, whether the latter occurs in the natural course of cultural existence or is brought about by the action of bile or bile salts. The dissolution of the periplast allows the escape of the protoplasm (Fig. 24), and there is left behind the slender hyaline elastic filament with tapering ends and with deep-set and regular windings throughout its length (cf. Figs. 48 and 49, showing appearance before and after treatment with bile). The number of spirals of the exposed filament conforms with that of the resting organism. The relaxed organism takes the shape of its axial filament; it is only vigorously motile organisms in which the body is straightened out in places or shows irregularity of the spirals, the result of contraction of the protoplasmic coat. Stained preparations of organisms fixed while actively motile show the same irregularities which are observed in fresh preparations. Specimens of treponemas kept some days in the refrigerator are non-motile and show beautiful regular spirals, the larger the species the heavier the axial filament and the greater the distance between the spirals.

The existence of a delicate periplast is shown by the effect of hypotonicity. In specimens of $T$. recurrentis, for example, suspended in distilled water, the protoplasm is unevenly distributed, being aggregated at some points into masses which exhibit rhythmic contractions, and absent altogether in other parts, leaving only the axial filament covered by the veil-like periplast (Fig. 25). The periplast finally ruptures, 
and the protoplasm escapes. The appearance of attached spherical bodies (Fig. 24), a common phenomenon in spirochetes, is due to minute extrusions of cytoplasm at points of regional disturbance in the periplast.

The essential structure of a treponema, therefore, is a spring-like axial filament, and a layer of contractile protoplasm, inclosed in a delicate periplast. When suspended in an isotonic medium the body undergoes a rotatory motion whenever the pressure of the contracting protoplasm stretches the axial filament. If the contractions and relaxations of the protoplasm occur in rapid succession, the organism moves in one direction or the other according to the direction of rotation. All varieties of spirochetal movement are readily explained by the zone of operation of the protoplasmic contraction, which may be unipolar, bipolar, median, or irregularly regional.

The ability of Treponema pallidum to bore, corkscrew-like, through a solid medi$\mathrm{um}$, is due to the extreme thinness of the protoplasmic coat and to the closeness of the spirals in the firm, elastic, axial filament. T. recurrentis, which has a thicker protoplasmic layer and fewer spirals, can penetrate a semisolid but not a solid medium.

It is evident that in the treponemas no external flagellar apparatus is present, nor is any necessary. The axial filament, however, has several characteristics in common with bacterial flagella - similar morphology and staining properties, simitar function, i.e., the function of locomotion. The similarity of the axial filament to the giant involution flagella of bacteria is very striking. The only difference in these two types of kinetic elements is that the bacterial flagella are extracellular or exogenous, the axial filaments intracellular or endogenous. Their respective biological properties probably determined their position in the cell.

Leptospira.- The genus Leptospira was created because spiral organisms of this type have several unique biological and morphological characteristics which distinguish them from others of the spirochete family - the extreme closeness and fineness of the spirals, the typical hooking of one or both ends, the apparent absence of terminal filaments, the indifference of the organisms to the action of concentrated saponin solution and to changes in the tonicity of the medium (Leptospira is unaffected either by ro per cent sodium chloride solution or by distilled water). This organism has no axial filament of the type which is so readily demonstrated in treponemas by treatment with bile. Recently, however, in smears of fluid cultures, stained $^{\mathrm{r}}$ by the technique for flagellum-staining, I came across some extremely deli-

I The best way to demonstrate the terminal filaments is by Zettnow's technique (Zettnow, E.: Ztschr.f. Ilyg. u. Infcktionskrankh., 30, 95. I899), or a modification of it. The cultures must be freed from the proteins of the medium by thorough washing (several centrifugations). Leptospiras may be washed with distilled water, but for treponemas it is necessary to use 0.5 per cent sodium chloricle. Slides are more convenient than cover slips because as many as eight rlifferent films can be submitted to identical treatment. To a drop of 2 per cent solution of osmic acid is added a drop of the spirochetal suspension, with gentle mixing. The base of the mordant is a 5 per cent solution of tannic acid, to $100 \mathrm{cc}$. of which is added I cc. of a saturated solution of tartar emetic ('Lettnow's original technique) or some one of the following substances: lead acetate, zinc chloride, zinc sulphate, tin chloride, aluminium chloride, colloilal iron. Lead acetate is usually easiest to obtain and gives excellent results. The mixture is boiled for five minutes and filtered while hot. Ordinary alum may be used in combina tion with the tannic acid; in this case the precipitate is not filtered off but is dissolved by the addition of a little ammonia, followed by boiling. 'The air-dried film is flooded with the mordant and the slide heated a few seconds, washed, then flooded with ammoniacal silver nitrate, made by adding to about 
cate, smooth filaments, hooked at both ends. These elements were at first difficult to account for, being sometimes found end to end with normal heavily stained leptospiras as if they were terminal flagella of these. Careful search, however, revealed specimens in which a portion of the filament was still clothed with deeply stained spirally wound protoplasm (Fig. 63).

The axial filaments of leptospiras are far more delicate than those of treponemas and are not spirally wound. Like finer bacterial flagella, they are invisible under the darkfield microscope. They are apparently firm and elastic.

Leptospira is provided with a thick, almost transparent periplast, which is recognizable by darkfield illumination as a narrow clear zone, or halo, around the organism, and in preparations stained for flagella as a grayish or unstained halo (Figs. 7 I, 74). The indifference of the organism to saponin and to changes in tonicity may be due to the presence of a dense periplast. More prolonged staining results in intense coloration of the periplast, so dark that the spirally wound protoplasmic coat is obscured. Under these circumstances there appears at one or both ends of the body a peculiar, fragile-looking filament which readily becomes entangled with similar filaments of other organisms (Figs. 57-62). It may be as long as the body of the organism. It appears to be of periplastic origin and is no doubt the empty periplastic sheath from which the axial filament and cytoplasm have escaped. Detached entangled filaments are also found. Filaments of this type have been observed previously and interpreted as flagella.

The short spiral terminal elements which are found in degenerated leptospiras, but never in fully developed, healthy specimens, resemble short spirilla. They occur in all the pathogenic species, being usually coarser in L. icterohaemorrhagiae (Figs. 67, 68) and occasionally rather long in L. interrogans (Figs. 64, 65). The origin and nature of these elements is not clear, but in all probability they are involuted forms of the terminal portions of the axial filaments.

To summarize, leptospiras consist of delicate but firm, elastic, smooth, axial filaments with hooked ends; closely wound spiral protoplasmic coats; and dense periplasts. When the bowlike axial frame is alternately straightened and relaxed by the rhythmic contractions of the spiral protoplasmic body, the result, in a fluid medium, is rotation. When only one end is hooked, the organism is propelled in the direction of the straightened end, as is seen by direct observation of active organisms by darkfield illumination. The direction of progression is frequently reversed by alternation in the position of the hooked and straight ends of the organism, or both ends may be simultaneously stretched and bent. Rotation never ceases in the living organism so long as it is in a fluid medium, but the extension of the hooked ends becomes less frequent and effective as the vigor of the organisms diminishes. The curving of the ends is usually greatest in L. interrogans and least in L. hebdomadis. The latter type, while active, keeps both ends nearly straight (Fig. I 22), but when relaxed in death shows the characteristic terminal hooks.

$40 \mathrm{cc}$. of $3 \mathrm{C}_{\mathrm{C}}$ silver nitrate a few drops of ammonia, enough to render the solution slightly opalescent (Fontana's ammoniacal silver nitrate solution; Fontana, 1.: P'athologicu, 5, 205. 1912-13). The color of the film gradually changes from yellow to dark brown and then to steel gray or black, only brief leating being necessary. The films must be covered witls a Canada balsam mount before examination to prevent fading by exposure to cedar oil. 
It seems possible that active leptospiras sometimes become entangled in the threadlike filaments produced by degenerating organisms, for occasionally a vigorous organism appears to be unable to straighten out, as if the ends were held in the bow position by a thread joining them. The diminutive spirillum-like, terminal filaments can hardly be of mechanical significance, since normal leptospiras move without them.

The locomotor mechanism of leptospiras is evidently more complex than that of treponemas. The smooth elastic filament with its hooked ends is undoubtedly the primary factor in locomotion, but the spiral form of the protoplasmic coat, with the dense hyaline periplast which conforms with its windings, has an accessory function in locomotion. The contractile protoplasm operates against the bowlike axis by means of the brace provided by the periplast.

\section{FILTERABILITY}

Novy and Knapp ${ }^{1}$ were able to induce infection with filtrates of blood containing T.novyi, and Breinl and Kinghorn ${ }^{2}$ obtained positive results with duttoni. Torld and Wolbach ${ }^{3}$ confirmed the filterability of these blood spirochetes and were of the opinion that the spirochetes themselves passed the filters, not granular forms of the organisms. The members of the leptospira group are rather readily filterable, as Inada and Ido ${ }^{4}$ and Hübener and Reiter ${ }^{5}$ proved for L. icterohaemorrhagiae and the writer ${ }^{6}$ and Dieterich $^{7}$ for L. interrogans. The common leptospira of water, first described by Wolbach and Binger ${ }^{8}$ as Spirochaeta biflexa, was shown by them to be filterable, and this fact has recently been utilized by Angerer, 9 Dimitroff, ${ }^{10}$ Bauer, ${ }^{11}$ Mochtar, ${ }^{12}$ and others, as a means of obtaining pure cultures. It is interesting to recall that the virus of yellow fever was proved to be filterable ${ }^{13}$ before the discovery of any of the leptospira group, and that Hecker and $\mathrm{Otto}^{\mathrm{I}}$ had foretold from their investigations that the virus of infectious jaundice would be a filter-passer.

Treponema pallidum and $T$. pertenue are unable to pass through any bacteriaproof filters by the ordinary (suction) methods. The pallidum, however, can grow through the pores of Berkefeld filtrates $\mathrm{V}$ and $\mathrm{N}^{15}$ and appear in the filtrate when provided with favorable cultural conditions.

I Nory, F. G., and Knapp, R. E.: J. Infect. Dis., 3, 29 г. 1906.

${ }^{2}$ Breinl, A., and Kinghorn, A.: Lancet, r, 668. I906.

3 Todd, J. L., and Wolbach, S. B.: J. Med. Research, 25, 27. I9I4.

${ }^{4}$ Inada, R., Ido, I., Hoki, R., Kaneko, R., and Ito, H.: J. Exper. Mcd., i 8, 377.1016.

5 Hübener and Reiter: Deutsche med. Wchnschr., 41, I275. I9I5; 42, I. I916.

${ }^{6}$ Noguchi, H.: J. Exper. Med., 30, I3. 1919.

7 Dieterich, F. H.: Am. J. Trop. Med., 4, 553. I924.

8 Wolbach, S. B., and Binger, C. 1.: J. Mcd. Research, 30, 23, I9r4.

9. Angerer, K. v.: Arch. f. II yg., 92, 325. 1924.

In Dimitroff, V. T.: J. Infect. Dis., 40, 50. 1927.

in Bauer, J. H.: Am.J. Trop. IVed., 7, 177. 1927.

I2 Mochtar, 1.: Universitcits Bocklundel. Amsterdam, I027.

iz Reed, II., and Carroll, J. C.: Senute Dox. S2z (6ist Cong., 3t Sess.), P. I 49. I9 I.

${ }^{34}$ Hecker, A., and Otto, R. W.: Verüfentl. a. d. Geb. d. Vilitir-Sanituitsüesens, Heft 40. Igr 1.

15 Noguchi, H.: J. Exper. Med., I4, 99. г9гі. 


\section{MODES OF TRANSMISSION}

The transmission of the blood-inhabiting spirochetes to man is effected through the bite of an infected blood-sucking insect or arthropod. The transmitting agent in each instance is under natural conditions specific. Other blood-suckers may be infected, but the artificially infected insects are not good transmitting agents. Both body lice and bedbugs, for example, can be infected by sucking the blood of a patient with European relapsing fever, but only the lice are able to transmit the disease. The survival of the infecting organism in the bedbug can be demonstrated by inoculation of the crushed body of the insect into a susceptible animal. It is possible, therefore, that bedbugs may bring about infection if crushed on an injured skin surface.

Treponema recurrentis, of European relapsing fever, is transmitted by the body louse, Pcdiculus vestimenti. Infection can be transmitted in experimental animals per os as well as subcutaneously. T. duttoni, of African tick fever, is normally carried by Ornithodoros moubata, ${ }^{2}$ and hereditary transmission occurs in the tick. T. anserinum, of fowl relapsing fever, is transmitted by the tick Argas persicus (miniatus). ${ }^{3}$ L. icterohacmorrhagiae (Weil's disease, or hemorrhagic jaundice) is carried by rats ${ }^{4}$ and thought to be disseminated through the urine; L. hebdomadis (seven-day fever of Japan) is carried by the field mouse. ${ }^{5} L$. icterohaemorrhagiae has, however, been artificially transmitted by insects. ${ }^{6}$ L. interrogans, of yellow fever, is conveyed by the mosquito, Acdes aegypti.7 T. pallidum is usually transmitted directly through contact. It was once thought that excoriation of the mucous membrane was necessary for invasion, but the investigations of Brown and Pearce ${ }^{8}$ show that the organism can penetrate the unbroken skin. Extragenital infections are not uncommon among physicians and nurses, for direct contact with living T. pallidum in any way may lead to infection. Hereditary transmission occurs to the second and even to the third generation. Infection with $T$. pertenue probably occurs through direct contact with the secretion of the yaws lesion. $T$. cuniculi, of spontaneous rabbit venereal disease, is apparently transmitted as a rule by coitus. 9

\section{INMUNITY}

The phenomena of immunity in spirochetal diseases vary with the type of organism. In general, it appears that the spirochetes which produce an acute febrile disease, ending in crisis, or of short duration, leave in the convalescent man or animal a state of more or less complete immunity of variable duration. The organisms of the relapsing fever group confer upon man active immunity lasting about one and a half years, while the leptospiras give an immunity enduring for many years. The tis-

r Nicolle, C., Blaizot, L., and Conseil, E.: Compl. rend. Acad. de sc., I55, 481. I9I 2.

${ }^{2}$ Dutton, J. E., and Todd, J. L.: Brit. MI. J., 2, I259. I905.

3 Marchoux, E., and Salembeni, 1.: Ann. de l'Inst. Pastewr, r 7, 569. I903.

${ }_{4}$ Ido, Y., IIoki, R., Ito, H., and Wani, H.: J. Exper. Med., 26, 3+1. I9I7.

5 Ido, Y., Ito, H., and Wini, II.: ibid., 28, 435. 1918.

${ }^{6}$ Uhlenhuth, P', and Kuhn, I'.: Ztschr. II'g. H. Infektionskrunkh., 84, 5I7. 1917; Blanc, G.: Compl. rend. Soc de biol., 83, 263. 1920 ; Bonne, C.: ibill., 91, 242. 1924.

7 Noguchi, II.: J. Exper. Med., 30, for. I9r9; Koslina, M., Shiozawa, S., and Kitayama, K.: ibid., 42, 873. 1925 .

${ }^{8}$ Brown, W. II., and l'earce, L.: Proc. Soc. Exper. Biol. S Met., r8, 200. I92 I.

9 Fiolle, W., and Ritz, II.: Dermat. Ztschr., 27, 319. 19 I9. 
sue-dwelling treponemas, as a rule, confer no perceptible immunity, so far as natural infection is concerned.

The phenomena of immunity in leptospiral infections were first carefully studied by Inada, Ido, and their co-workers, ${ }^{1}$ in infectious jaundice in Japan. They demonstrated that a person who has had an attack of infectious jaundice may remain immune for many years, and the blood of persons recently recovered contains active immune bodies which will give either Gabritschewsky's or Pfeiffer's phenomenon. In experimental animals similar reactions were shown to follow the infection. Inada and $\mathrm{Ido}^{2}$ produced immune sera of high potency in horses for practical therapeutic purposes and obtained excellent results when the serum was used early in the disease. Similar results were obtained by subsequent workers.

In the case of yellow fever, also, one attack confers complete immunity which last.s for many years; rarely have cases of second infection been reported within a period of nine years. Provided that one lives continually in an endemic locality, where the disease recurs at short intervals, one is protected for life by a single attack, because a mild infection occurs while some immunity is still present, and protection is thus reinforced. Persons born and residing in endemic foci seldom die from yellow fever, and in the majority of instances such persons are unaware of having passed through any infection. They lose their immunity, however, if they live outside the endemic zone for more than ten years.

The immunity phenomena in yellow fever closely parallel those of infectious jaundice. There is a positive Pfeiffer reaction with convalescent yellow fever serum and Leptospira interrogans. The killed culture of the interrogans, in jected into normal guinea pigs, gives the animals an increased resistance to the interrogans infection, and a cubic centimeter of immune serum prepared with the interrogans may protect guinea pigs against several million minimum lethal doses of culture. The efficacy of the interrogans vaccine and the anti-interrogans serum in human yellow fever has been reported in a large number of cases. ${ }^{3}$

\section{CULTIVATION}

ANAEROBIC GROUP

Treponema pallidum was first cultivated from the testicular syphiloma of rabbits in a tall column of ascitic fluid or sheep-serum water containing fresh tissue (rabbit kidney) and incubated at $37^{\circ} \mathrm{C}$. under anaerobic conditions, a method based on the anaerobic principle of Theobald Smith. ${ }^{5}$ For the direct isolation of the parasite from impure human materials, ${ }^{6}$ the medium was made solid by mixing two parts of agar with one part of the ascitic fluid or serum, and purification was accomplished by successive transplants into similar medium until the spirochetes were separated from

I Inada, R., Ido, Y., Hoki, R., Ito, H., and Wani, H.: J. Exper. Mcd., 24, 485. 1916; $27,283$. I9rS.

${ }^{2}$ Inada, R., Ido, Y., Hoki, R., Ito, H., and Wani, H.: ibid., 24, 48.5. I916.

${ }_{3}$ Noguchi, H.: J.A.M.A., 77, 181. I921; J. Trop. Med., 28, 185. 1925.

4 Noguchi, H.: J. Exper. Med., 14, 99. I9гі.

5 Smith, T.: Centralbl.f. Bakleriol., 7, 502. 1890.

${ }^{6}$ Noguchi, H.: J. Exper. Mcd., 15, 90. I9I 2. 
the bacteria. The piece of tissue acts as an oxygen absorber, as first pointed out by Theobald Smith, and also furnishes nutriment. Other treponemas have been grown by the writer by the same methods, including $T$. pertenue ${ }^{\mathrm{I}}$ of yaws, and several organisms which are normal inhabitants in the human mouth ${ }^{2}$ and about the genital region. ${ }^{3}$

Preparation of the fluid medium.-A rabbit kidney (testicle or heart muscle may also be used, but liver is unsuitable because often contaminated with bacteria and containing carbohydrates which give rise to acid), removed under aseptic conditions, is cut into about sixteen pieces, and a piece of the tissue is put into each of several tall tubes ( $14^{-20} \times 200 \mathrm{~mm}$.). The material for inoculation, which must be relatively free from bacteria, as is the case with testicular tissue of the rabbit after several rabbit passages of a strain of pathogenic treponema, is added at this point, and the tubes subsequently two-thirds filled (about I 5 cc. is required) with ascitic fluid or serum water (one part sheep, horse, or rabbit serum and three parts distilled water). A layer of liquid paraffin is added to prevent evaporation of the medium. The tubes are now placed in a sealed jar, in which the air is replaced by hydrogen, 4 and left undisturbed at a temperature of $37^{\circ} \mathrm{C}$. for two to three weeks. Success is by no means constant in the case of the pathogenic treponemas, and when growth is obtained it is usually impure and must be purified either by the use of a filter ( $T$. pallidum is not directly filterable but will grow through a filter into a suitable culture medium before the bacteria are able to pass through, i.e., in about five days), or by subculture on solid medium.

Preparation of the solid medium.-This medium is suitable for impure material, either direct from human lesions or from an impure fluid culture. It is also the medium which the writer uses for the routine subculture of all pure treponema cultures.

The tissue is placed at the bottom of the tube, as before, and to the tubes is added about I 5 cc. of a mixture of 2 per cent nutrient agar (two parts), melted and cooled to about $50^{\circ}$ C., and ascitic fluid (one part), the mixture being kept warm during the course of the distribution. If the material for inoculation is pure, it is best added before the mixture of agar and ascitic fluid; if impure, it is inoculated after solidification of the medium. A picce of tissue from a lesion may be forced to the bottom of the tube with a platinum loop. A fluid inoculum is introduced with a capillary pipette. To furnish the necessary compression force, a heavy metal syringe may be attached to the capillary with a piece of pressure tubing, but care must be taken not to split the medium. A layer of liquid paraffin is always added to prevent evaporation of the medium. The tubes are incubated at $37^{\circ} \mathrm{C}$. for two to three weeks.

The bacteria grow along the stab canal and on the surface of the medium, but if the medium is clear enough a faint haze is seen to radiate from the stab canal toward the sides of the tube. Not infrequently the growth is invisible, however, owing to the quality of the medium, and in this case darkfield examination must be relied on for detection of growth. The surface of the medium is sterilized by sublimate alcohol, which is poured off. The tube is cracked about the center (by making a small scratch and applying a red-hot glass rod), and the upper half removed, exposing the agar column, which is again sterilized with sublimate alcohol, care being taken to absorb excess moisture with sterile gauze. When the agar

I Noguchi, II.: München. med. Wchnschr., 58, 1550.191 I.

${ }^{2}$ Noguchi, H.: J. Exper. Med., I5, 8I. I912; 16, I94. I912.

3 Noguchi, H.: ibid., 15, 466. 1912; 17, 89. 19г3.

4 I used a combination of hydrogen gas, vacuum, and pyrogallic acid to replace the air (ibid., I4, IO2. I9II), but other types of anaerobic apparatus have since been devised, e.g., those of MIcIntosh and Fildes (Lancet, 1, 768. 1916), Brown (J. Exper. Med., 33, 677. 1921), and Boez (J. Bact., 13, 227. I927). 
column is bent, it breaks across and exposes a surface upon which colonies of spirochetes, if present, may be seen more clearly. A capillary pipette may now be introduced into the hazy areas, and if darkficld examination proves that the organisms are present, a number of subcultures are made. Repeated subculturing results finally in bacteria-frec cultures.

In the case of pure material, introduced with the tissue at the bottom of the tube, growth begins about the tissue and gradually extends upward to the border of the acrobic zone, which is about $3 \mathrm{~cm}$. below the surface (cf. Fig. 77). The anaerobic jar is no longer necessary, the requisite anacrobiosis being produced by the tissue, and the entrance of air being excluded by the high column of solid agar.

Animal serum, particularly that of the rabbit, may be used in place of the ascitic fluid. The tissue, however, seems to be essential for growth, at least until the parasites have becn under cultivation for a very long time.

Neither of the media described is ideal for the isolation of fresh strains of treponema, and only a limited number of trials at cultivation is ever successful, hence much patience is required. Once the organism is obtained in culture, however, it grows more readily, and after a period of years on culture medium it becomes saprophytic and may grow without the addition of the fresh tissue. The precautions at first required become less and less important until a great many cultural conditions may prove suitable. Zinsser, Hopkins, and Gilbert ${ }^{\mathrm{r}}$ isolated a strain of $T$. pallidum by the methods outlinerl, and after the tenth generation were able to grow it on various media. Gates ${ }^{2}$ grew some of the author's twelve-year-old strains under anaerobic conditions on the surface of a blood-agar plate. The virulence of the organism is lost very early in the course of cultivation, and pathogenicity tests to determine whether a strain is actually $T$. pallidum must be made soon after isolation.

Treponema pallidum and other anaerobic spirochetes which have been under cultivation for a long time can be grown in fluid medium under a vascline seal, as first recommended by Legros ${ }^{3}$ and recently advocated by Gates and Olitsky ${ }^{4}$ after a careful study of the conditions producing anaerobiosis. A piece of kidney is placed at the bottom of the tube or flask, and removal of the oxygen from the medium may be indicated in a control container to which methylene blue is added. When the last trace of oxygen has been removed from the control tube, the others are inoculated.

The writer used for the isolation of the saprophytic trcponemas (T. microdentium, T. macrodentium, T. refringens, T. mucosum, T. calligyrum, T. genitalis) the same technique as for the pathogenic types, the initial inoculations being made into fluid medium and the impure cultures purified by repeated subculture on solid medium. An anaerobic jar is necessary for the fluid cultures. The saprophytic types grow more rapidly than the pathogenic forms, growth becoming visible within forty-eight to seventy-two hours in the case of $T$. mucosum, and within four to five days in the case of $T$. refringens, T. macrodentium, and $T$. calligyrum. In the case of the larger type of mouth treponema ( $T$. macrodentium), it is desirable to make several transplants of the impure culture on fluid medium before attempting purification. The purification is a tedious process because of the presence of gas-forming and acid-producing bacteria which break up the medium and render it turbid. Smith, 5 in using this medium for the cultivation of spirochetes in sputum, has found that the gas-forming organisms can be eliminated by first inoculating the sputum into the groin of a guinea

× Zinsser, H., Hopkins, J. G., and Gilbert, R.: J. Expcr. Med., 21, 213. I9I5.

${ }^{2}$ Gates, F. L.: ibid., 37, 3I I. I923.

${ }^{3}$ Legros and Besson, A.: Technique microbiologique et serother. (6th ed.), p. 97. I9r I

${ }^{4}$ Gates, F. L., and Olitsky, P. K.: J. Exper. Med., 33, 5I. 1921.

${ }^{5}$ Smith, D. T.: Am. Rev. Tuberc., 16, 584. 1927. 
pig. Sometimes the gas-forming organisms were eliminated by one guinea pig passage; sometimes two or three successive passages were necessary to reduce the number sufficiently for successful cultivation of the spirochetes.

Another organism which grows on the same media as the saprophytic treponemas is the one which I called Spirochacta phagedenis because of its presence in a phagedenic ulcer. ${ }^{\text {I }}$ This organism is not a treponema, and its taxonomic relationships are uncertain.

None of the smegma spirochetes are odor-producing in culture; in this respect they differ from $T$. mucosum and T. microdentium. T. calligyrum grows much more readily than the pallidum and can be cultivated in media not containing fresh tissue. It is easily mistaken for $T$. pallidum, from which it differs in being non-pathogenic and by its presence in nonspecific lesions. In cultures in which $T$. pallidum is associated with calligyrum and refringens, microdentium, or mucosum, it is easy to eliminate the pallidum by subculturing on media not containing fresh tissue and neglecting anaerobic precautions.

Treponema vincenti was cultivated by Tunnicliff ${ }^{2}$ in 1906 under anaerobic conditions on a medium consisting of glucose agar with or without serum. Mühlens and Hartmann ${ }^{3}$ about the same time cultivated $T$. dentium on a similar medium. Repaci, ${ }^{4}$ in 1909 , isolated a number of spirochetes from the human mouth by means of Veillon's medium.

\section{AEROBIC GROUP}

Cultivation of the blood spirochetes (T. recurrentis, T. duttoni, T. kochi, T. novyi, T. (anserinum) was first accomplished 5 in ascitic fluid containing fresh tissue, incubated at $37^{\circ} \mathrm{C}$. under aerobic conditions. If the ascitic fluid is suitable the presence of the tissue will cause the formation of a loose-meshed fibrin net throughout the fluid. The fluid must be free from bile. A small amount of citrated blood from the heart of an infected rat or mouse is added to the tubes either before or after the addition of the ascitic fluid. The organisms multiply steadily until after eight to nine days every field will show numerous motile specimens either singly, in pairs, or in chains of three or more individuals. Sterilization or filtration appears to impair the nutrient quality of the medium and invasion by bacteria kills the spirochetes. In subculturing $0.5^{-1} \mathrm{cc}$. of culture is inoculated into the fresh medium. Subcultures are best made every seven days.

Kligler and Robertson ${ }^{6}$ found that a semisolid serum-agar medium may be used for the blood spirochetes providing the hydrogen-ion concentration is adjusted to a $\mathrm{pH}$ of $7 \cdot 4-7.8$. This medium is analogous to that which the writer used for the cultivation of the leptospira group. The writer's term "aerotropic anaerobiosis"7 was actually an erroneous interpretation of the conditions which exist in the ascitic fluid tissue medium. The presence of the fresh tissue gave rise to the neecssary semisolid condition by causing fibrin formation, and it also brought the reaction to about $\mathrm{pH}$ 7.5. The paraffin-oil cover admitted oxygen enough and at the same time prevented evaporation of the medium.

The simplest and easiest of all spirochetes to cultivate are the members of the leptospira group. Cultivation was first accomplished by Inada, Ido, and their collaborators in $1916,{ }^{8}$ when Leptospira icterohaemorrhagiae, discovered by them in $1914^{-1} 5$ was cultivated on the

\footnotetext{
I Noguchi, II.: J. Expcr. Mcd., 16, 261, I912.

2 Tunnicliff, R.: J. Infect. Dis., 3, I48. 1906.

3 Mühlens, I., and Hartmann, M.: Zlschr.f. IIyg. u. Infcklionskrankh., 55, 8. . I9о6.

${ }_{4}$ Repaci, G.: Compl. rend. Soc. de biol., 69, 784. I91 1.

5 Noguchi, H.: J. Expcr. Mcd., 16, 199, 620. 1912.

${ }^{6}$ Kligler, I. J., and Robertson, O. H.: ibid., 35, 303. 1922.

7 Noguchi, II.: II arvey Lechures (9th ser.), p. 236. I915-16.

${ }^{8}$ Inada, R., Ido, Y., Hoki, R., Kancko, R., and Ito, H.: loc. cil.
} 
same medium as that which I had employed for the blood treponemas. They found, however, that the optimum temperature was $22^{\circ}-25^{\circ}$ in the case of the leptospira, the organisms remaining alive much longer than at $37^{\circ} \mathrm{C}$. Ito and Matsuzaki ${ }^{\mathrm{r}}$ later reported favorable growth of the same organism on solid medium containing agar or gelatin and certain types of whole or defibrinated blood. Reiter and Ramme ${ }^{2}$ found that the German strains grew luxuriantly on a rabbit serum saline, which is composed of one part of serum and five parts of 0.85 per cent saline solution. Beef serum, I cc., to Ringer or Locke solution, 9 cc., may also be used. Uhlenhuth's medium ${ }^{3}$ consists of a mixture of I cc. of rabbit serum and $30 \mathrm{cc}$. of tap water, or I cc. of the infected blood of the guinea pig to $30 \mathrm{cc}$. of tap water. Martin and Pettit ${ }^{4}$ regard the rabbit serum diluted with isotonic salt solution as the best medium for the ictcrohacmorrlagiae, and the writer's experience is that no other animal serum, except perhaps sheep serum, gives as rich a growth of organisms of the leptospira group as does rabbit serum. Wenyon ${ }^{5}$ adds a drop or two of rabbit blood to cach tube. Verwoort ${ }^{6}$ uses horse serum.

An interesting and important phenomenon in the cultivation of leptospiras is their peculiar tendency to multiply more abundantly in a semisolid particle, such as a piece of agar or fibrin, than in clear liquid media. The writer noticed this fact early in his work, and it has been repeatedly confirmed that the addition of melted nutrient agar to the rabbit-serum saline to a final concentration of $0.1-0.3$ per cent furnishes the optinum semisolid condition for abundant growth. Too much agar may not be used, for the organisms are obligatory aerobes, and the depth and rapidity of penetration of oxygen into the medium are less the more agar there is addled. With a concentration of 0.3 per cent or less, the surface layer of $2-3 \mathrm{~cm}$. is easily penetrated by oxygen, as can be demonstrated by using methylene blue as indicator. The leptospira itself may be used as an indicator of the penetration of oxygen into the medium, for it grows only in the aerated zone, the same zone to which the color of methylene blue is found to return after removal of a vaseline seal. A rich culture is casily recognized by the opalescent layer near the surface, sharply demarcated from the clear region below.

When cultures in large quantities are desired to furnish material for the immunization of horses or for preparing material for the vaccination of human beings, the medium may be placed in a flask like that shown in the illustration (Fig. 87 ) to a depth of $\mathrm{I}^{-2} \mathrm{~cm}$. Growth then occurs uniformly throughout the medium, and within two to three weeks the leptospiras are present to a concentration of about I $6,000,000,000$ per cubic centimeter. Rich cultures may be diluted with three parts of saline, killed by heating for one half hour at $55^{\circ} \mathrm{C}$. and used as vaccine. The standardization of the concentration of the vaccine is less easy than in the case of bacteria from fluid media, but darkfield examination of the final product should show large numbers of dead organisms per field.

The medium now used for routine cultivation of all leptospiras in the writer's laboratory is as follows:

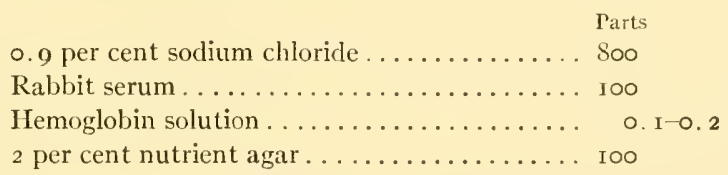

${ }^{x}$ Ito, T., and Matsuzaki, H.: J. Expcr. Med., 23, 557. I9г6.

${ }^{2}$ Reiter, H.: Dcutsche mcd. Wchnschr., 42, I282. I9I6.

3 Uhlenhuth, P.: ibid., 63, 152, 5 11. 1917.

${ }^{4}$ Martin, L., and Pettit, A.: loc. cit.

5 Wenyon, C. M.: Protozoilogy, 2, I 299. I926.

${ }^{6}$ Verwoort, H.: Geneesk. Tijdschr. v. Nederl.-Indie, 62, 697. 1922; 63, 800. 1923; Far Eastern Assoc. Trop. Med., 5th Bienn. Cong., Singapore, p. 683. 1923 (Abstract, Trop. Dis. Bull., 20, 583. I923; 21, 262. I924; 22, I75. I925). 
The agar is melted, cooled to about $45^{\circ} \mathrm{C}$., and added quickly to the saline, hemoglobin, and serum mixture, which has been warmed to about the same temperature. Thorough mixing is important; a medium containing flocculent agar particles does not give as good growth as one of uniformly gelatinous consistency. The hemoglobin is made by laking one part of defibrinated rabbit blood with three parts of distilled water. The addition of the hemoglobin is not essential for the growth of leptospiras, however, although it is probably very important in the cultivation of certain other organisms for which this same medium is used (e.g., Bartonella bacilliformis).

If cultures of spirochetes are to be kept some time, it is desirable to add a layer of paraffin oil, to prevent evaporation of the medium, which is noticeable after six to eight weeks. If transfers are made every two months, however, the paraffin oil is not necessary. The inference drawn by some workers that $L$. iclerohaemorrhagiae is a facultative anaerobe because it can grow under a layer of paraffin oil is based on the erroneous assumption that liquid paraffin can hinder the entrance of oxygen.

The cultural conditions for $L$. icteroides and $L$. hebdomadis are the same as for $L$. icterohaemorrhagiae, and the saprophytic leptospiras of water grow readily on this medium also.

Bauer's technique ${ }^{x}$ for obtaining initial cultures of the water leptospiras is as follows: The water containing the organisms is filtered once through paper, once through a Berkefeld $V$ and twice through Berkefeld $N$ candles. To I $80 \mathrm{cc}$. of the filtrate is added $20 \mathrm{cc}$. of 2 per cent hormone agar and I per cent fresh defibrinated rat's blood, and the mixture is distributed into tall tubes, about 8-1о cc. to a tube, which are kept at room temperature. Bauer obtained thirteen strains of leptospiras and a strain of another water spirochete from samples of water collected in Lagos, on the west coast of Africa. The technique was applied to leptospira containing water from icebox drains in the writer's laboratory, with excellent results. Angerer ${ }^{2}$ and Dimitroff ${ }^{3}$ have also cultivated water leptospiras. Dimitroff first obtained impure cultures on a medium consisting of 2 per cent human feces in tap water, purified these by filtration, and obtained pure growth on a similar medium and also on 4 per cent rabbit serum in sodium chloride. Dimitroff, who obtained thirty-seven strains from drinking and pond waters, puddles, etc., emphasizes the fact that it is well to test the filters used for their ability to pass the leptospiras, only two of a lot of twenty tested by him having been found suitable. The filtration method might well be tried for the isolation of Hoffmann's Leptospira dentium, ${ }^{4}$ which has not yet been cultivated.

\section{INFLUENCE OF CARBOHYDRATES ON THE CULTIVATION OF SPIROCHETES}

Akatsu $^{5}$ tested the effect of a number of carbohydrates on the growth of $T$. pallidum, T. calligyrum, T. microdentium, T. mucosum, and T. refringens. There were no visible alterations in the appearance of media with the growth of the organisms, and no unusual morphological changes of the spirochetes. In the case of the aerobic treponemas, the addition of sugars to the culture medium renders it less suitable for the growth of the organisms.

The leptospiras so far tested are non-fermenters and are indifferent to the presence of carbohydrates in the medium."

s Bauer, J. H.: loc. cit.

${ }^{2}$ Angerer, K. V.: loc. cit.

3 Dimitroff, V. T.: loc. cit.

${ }_{4}^{4}$ Hofimann, E.: Deutsche med. Wehnschr., No. 23. 1920; Centralbl.f. Bakteriol., 86, 134. 1921.

${ }^{5}$ Akatsu, S.: J. Exper. Med., 25, 375. I917.

${ }^{6}$ Noguchi, H.: ibid., 27, 604. I918; 30, г8. г919. 
ALTERATIONS IN SPIROCHETES ON PROLONGED CULTIVATION ${ }^{I}$

The strains of $T$. pallidum cultivated by the writer lost their virulence within four months after their purification; $T$. pertenue became avirulent immediately it was isolated. T. microdcnlium retained its characteristic disagreeable orlor during a year of cultivation, but therafter this property steadily diminished until after two years it had practically disappeared. The characteristic mucin-producing property of $T$. mucosum likewise disappeared after a few months of cultivation.

In the case of the leptospiras, the cultivated organisms may grow to a length of $40-60 \mu$ but show no other striking differences from the naturally occurring forms. Zuelzer $^{2}$ reports a length of $100 \mu$ in cultures of $L$. icterohacmorrhagiae on donkey serum.

$L$. icterohacmorrhagiae and $L$. interrogans soon lose their virulence on cultivation, but infectivity may be retained by passing the strain through guinea pigs every second or third generation.

\section{INDIVIDUAL CIIARACTERISTICS}

THE TREPONEMA GROUP (PLATE V, PLATES IX-XI)

Treponema pallidum (Figs. 88-94).-T. pallidum was first seen by Schaudinn and Hoffmann $^{3}$ in April, I905, in a fresh preparation from chancre under the ordinary oilimmersion objective. Because of its spiral shape and the difficulty of staining, it was given the name Spirocheata pallida by its discoverers. Vuillemin, ${ }^{4}$ in June, I 905 , proposed that the name be changed to Spironema pallidum, on the ground that Spirochaeta was a plant genus, but Spironema proved to be preoccupied, and Schaudinn, 5 in December, I 905, named the organism Treponema pallidum. The generic separation of the organism from Spirochacta is desirable on morphological grounds, irrespective of whether the spirochetes be regarded as plants or animals. The earlier name had, however, taken such deep root in the medical literature that the organism is still called by many Spirochaeta pallida. Taxonomists are rather generally agreed that the correct name for the organism is Treponema pallidum. The name Spironema has been used both in zoölogical and in botanical nomenclature, ${ }^{6}$ and hence is not valid.

Treponema pallidum has been established as the cause of syphilis in several ways. It is always present in syphilitic infections, although not always readily demonstrable, and never in other conditions. The use of the dark-ground illumination for detection in fresh preparations and the introduction of the Cajal-Bertarelli-Levaditi method of silver impregnation for demonstrating its presence in syphilitic tissues have undoubtedly helped much in placing Treponema pallidum in its correct position. The application of the Bordet-Gengou principle of complement deviation to syphilis by Wassermann, Neisser, and Bruck, ${ }^{7}$ as well as by Detre,${ }^{8}$ in 1906 , was another great

\footnotetext{
I Noguchi, H.: Ann. de l'Inst. Pasteur, 30, г. I9I6.

${ }^{2}$ Zuelzer, M.: Centralbl. f. Bakt., Orig. Beihefte, Abt. I, 85, *i41. I92 I.

3 Schaudinn, F., and Hoffmann, E.: Arb. a. d. kaiserl. Gesundheitsamte, 22, 527 . I905.

${ }^{4}$ Vuillemin, P.: Compt. rend. Acad. de sc., 140, 1567. 1905.

5 Schaudinn, F.: Deutsche med. Wchnschr., p. 1728. 1905.

${ }^{6}$ Buchanan, R. E.: loc. cit.; Wenyon, C. M.: loc. cit.; Stitt, E. R.: loc. cit.

7 Wassermann, A., Neisser, A., and Bruck, C.: Deutsche med. Wchnschr., 32, 745. 1906.

${ }^{8}$ Detre, L.: Wien. klin. Wchnschr., 19, 6r9. 1906.
} 
triumph, revealing the existence of a syphilitic infection without demonstrating the organism itself, although the phenomenon proved not to be strictly specific for this disease. Thus syphilis, not only in its usual manifestations, characterized clinically as the primary, secondary, and tertiary stages, but also in its hereditary transmission, is now well understood. The real syphilitic nature of so-called "metalues" or "parasyphilis" was definitely established in I9I3.

Metschnikoff and Roux ${ }^{2}$ in 1903-4 had already transmitted syphilis to apes, and soon after the discovery of the organism in human lesions they found it in experimental syphilis. Schultze, ${ }^{3}$ Bertarelli, ${ }^{4}$ and Parodi ${ }^{3}$ showed that syphilitic infection could be produced in rabbits. For the purpose of maintaining strains of $T$. pallidum the testicular mode of inoculation was formerly employed, but Brown and Pearce have observed that the lymph nodes become involved early in the course of a syphilitic infection of the rabbit and remain infected almost indefinitely. Hata ${ }^{7}$ found Tomasczewski's scrotal chancre most satisfactory in chemotherapeutic experiments. Nichols and $\mathrm{Hough}^{8}$ were the first to isolate a strain of $T$. pallidum from a rabbit with nervous symptoms, the organism being recovered from the cerebrospinal fluid. Graves ${ }^{9}$ succeeded in infecting rabbits by injecting the blood of paretic patients, and Marie, Levaditi, and Banus ${ }^{\text {ro }}$ subsequently confirmed the infectiveness of paretic blood.

The etiological relationship between syphilis and paresis was suspected as long ago as $1857^{\text {II }}$ because of the frequency of syphilis in the history of paretic patients. Ranke $^{12}$ and others demonstrated T. pallidum in the pia and vessel sheaths in congenitally syphilitic brains. Dunlap demonstrated the organism in a case of cerebral syphilis. Moore and the writer ${ }^{13}$ found the parasites in twelve of seventy-two paretic brains stained by the Levaditi method (Fig. 9o). They occurred in all layers of the cortex save the outer or neuroglia layer; a few were found subcortically, but none in the pia or vessel sheaths. Subsequently the writer studied one hundred and thirty other paretic brain $\mathrm{s}^{\mathrm{I}_{4}}$ and in thirty-six of the series was able to demonstrate the parasite by the silver impregnation method. Darkfield examination of the fresh brain tissue was

${ }^{1}$ Noguchi, H., and Moore, J. W.: J. Exper. Med., 17, 232. I913; Noguchi, H.: Mïnchen. med. Wchnschr., 60, 737. 1913.

${ }^{2}$ Metschnikoff, E., and Roux, E.: Ann. de l'Inst. Pasteur, 17, So9. 1903; r8, г, 657. 1904; 19, 673. 1905; 20, 785. 1906.

${ }^{3}$ Schultze, W.: Med. Klin., I, 466. 1905.

${ }_{4}^{4}$ Bertarelli, E.: Centralbl.f. Bakteriol., Abt. I, 4I, 32 I. I906.

5 Parodi, U.: ibid., 44, 428. 1907.

${ }^{6}$ Brown, W. H., and Pearce, L.: J. Exper. Med., 34, IS 5.1921.

${ }^{7}$ Ehrlich, P., and Hata, S.: Die experimentelle Therapie der Spirillosen. Berlin, I910.

${ }^{8}$ Nichols, H. J., and Hough, W. H.: J.A.M.A., 60, ro8. I91 3 .

9 Graves, W. W.: ibid., 6r, 1504. 1913.

1o Marie, P., Levaditi, C., and Banus, G.: Compt. rend. Acad. sci., 170, 1021. 1920.

"Esmarch, F., and Jessen, W.: Allg. z. Psychiat., 14, 20. I857.

${ }_{12}$ Ranke: Z. Erforsch. u. Behandl. jugendl. Schwachsinns, 2, 32, 8I, 211 . I909.

${ }^{13}$ Noguchi, H., and Moore, J. W.: loc. cit.

I4 Noguchi, H.: Mïuchen. med. Wehnschr., 60, 737. 1913. 
made in six instances, and in one the pallidum was demonstrated by this method. The organism was also detected in one of twelve specimens of spinal cord from tabes dorsalis, ${ }^{1}$ being found in small numbers around the nerve cells near the posterior horn. These results were soon confirmed (Marinesco and Minea; Marie, Levaditi, and Bankowski; ${ }^{3}$ and Forster and Tomasczewski ${ }^{4}$ ). General paralysis is therefore a diffuse spirochetosis of the brain affecting chiefly the cortical layers. The presence of the spirochetes in the parenchyma explains a great part of the histopathological changes and nervous symptoms observed in the course of the disease. The infectiousness of the spirochetes found in the brain in general paralysis was demonstrated by transmission of the syphilitic infection to rabbits (Fig. 9I).5

Treponema pertenue (Figs. 95-96).-The organism causing yaws was found by Castellani ${ }^{6}$ in 1905 , not long after Schaudinn's announcement of the discovery of Treponcma pallidum. He called it Spirochaeta pertenuis or Spirochaeta pallidula. With the change in the generic name of the syphilis organism it became Treponema pertenue, Castellani's first-mentioned name having been accepted as the correct specific designation.

The symptoms of yaws (or pean, or framboesia tropica) are somewhat similar to those of syphilis, but the disease is extragenital and is more destructive to the skin. It does not affect the visceral organs or central nervous system as does syphilis. The blood of persons infected with $T$. pertenue gives the Wassermann reaction just as does the blood of syphilitics. T. pallidum and T. pertenue are indistinguishable morphologically, and there is apparently some cross-immunity in experimental animals. In fact, some workers are convinced that the two conditions are different forms of infection due to the same pathogenic agent. ${ }^{7}$

The Nichols strain of yaws, ${ }^{8}$ which I studied, was decidedly heavier than the pallidum strains which I had in rabbits at the same time. Some workers have thought that the pertenue is slightly finer than the pallidum. Both organisms vary $8-\mathrm{I} 2 \mu$ in length and $0.2-0.25 \mu$ in width and have eight to twelve regular, deeply set spirals. In orchitis of rabbits either may be as long as I6-24 $\mu$ with a correspondingly larger number of spirals. In active movements the spirals may be drawn almost straight and rapidly contracted in such a way as to remind one of a spiral spring when alternately stretched and relaxed. The body often bends at the middle as if by a convulsion. Some organisms may progress a short distance in one direction or another, always rotating around the axis. When in a resting or quiescent state the regular deep spirals become beautifully formed and appear rigid. Both ends are sharply drawn,

${ }^{1}$ Ibid.

2 Marinesco, G., and Minea, J.: Bull. Acad. de mêd., Paris (3d ser.), 69, 235. r9r3; Rev. neurol., 2I, 58r. 1913.

3 Marie, P., Levaditi, C., and Bankowski, L.: Bull. et mém. des hôp. de Paris, 29, 88I. I9I3; Compt. rend. Soc. de biol., 74, 794. 1913; Ann. de l'Inst. Pasteur, 27, 577. 1913.

${ }_{4}^{4}$ Forster, E., and Tomasczewski, E.: Deutsche med. Wchnschr., 39, I237. I9г 3.

5 Noguchi, H.: J.A.M.A., 6r, 85. I913.

${ }^{6}$ Castellani, A.: J. Trop. Med., 8, 253. 1905.

${ }^{7}$ Butler, C. S., and Peterson, E.: J. Lab. \& Clin. Med., 12, 670. 1927.

${ }^{8}$ Nichols, H. J.: J. Exper. Med., 12, 616. I910. 
but the filamentous appendages are demonstrable only by special technique. In freshly excised materials, immediately fixed, the pallidum shows irregular spirals, owing to the fact that the organisms were in active movement when suddenly killed by fixation.

Treponema pallidum and $T$. pertenue were obtained on artificial media in r9I I-I 2. The methods are described in another section (pp. 46s, $7 \mathrm{I}$ ).

Treponema cuniculi (Figs. 97-100).-The occurrence of a spiral organism indistinguishable from $T$. pallidum in a spontaneous venereal disease of rabbits was described by $\operatorname{Ross}^{1}$ and by Bayon ${ }^{2}$ in I9I2 and I9I3. The condition was subsequently studied by a number of workers. The names proposed for the organism were $T$. paraluis cuniculi (Jacobsthal) ${ }^{3}$ and $T$. pallidum var. cuniculi (Klarenbeek). ${ }^{4}$ The latter is the valid designation, according to the International Code of Nomenclature, but it seems justifiable to make cuniculi a species rather than a subspecies. ${ }^{5}$

$T$. cuniculi is an organism of great importance to the experimental worker because of the widespread employment of the rabbit in the study of syphilis. Although the organism cannot be differentiated from $T$. pallidum, the spontaneous lesion of the rabbit, which consists of slightly elevated, scaly areas, is not readily confused with the marked induration induced by inoculation of the syphilis organism. ${ }^{6}$ Moreover, the lymph nodes are seldom palpable after inoculation of $T$. cuniculi, nor are chancres, orchitis, or keratitis readily induced by the organism. The histological changes in the affected tissues resemble those of a condyloma. The Wassermann reaction is negative in rabbits infected with $T$. cuniculi, and positive in those having lesions induced by $T$. pallidum or $T$. pertemue.

T. cuniculi, as well as T. pertenue and T. pallidum, is easily destroyed by arsenobenzol, and healing of the lesions follows treatment with the drug.

Treponema (?) argentinesis.--This organism was described by Kuhn and Steiner ${ }^{7}$ in $19 \mathrm{I} 7$, having been found by them in the liver tissue of guinea pigs and rabbits infected with blood or spinal fluid of multiple sclerosis patients. It is pathogenic for rabbits and guinea pigs, inducing paralysis, emaciation, and death. The presence of the organism in human and experimental tissue was confirmed by Siemerling, ${ }^{8}$ Büscher, ${ }^{9}$ Speer, ${ }^{10}$ Marinesco, ${ }^{11}$ and Kalberlah ${ }^{12}$ as well as by Kuhn and Steiner. ${ }^{13}$

In the Levaditi-stained preparations kindly sent me by Professor Steiner, the

I Ross, E. H.: Brit. M. J., 2, I651. 1912.

${ }^{2}$ Bayon, H.: ibid., p. I159. 1913.

${ }^{3}$ Jacobsthal, E.: Dermat. Wchnschr., 71, 569. 1920.

${ }_{4}$ Klarenbeek, A.: Centralbl. f. Bakteriol., Orig., 87, 203. 1921.

${ }^{5} \mathrm{I}$ am indebted to Dr. Stiles for an opinion with regard to this name.

${ }^{6}$ Noguchi, H.: J. Exper. Med., 35, 391. I922.

7 Kuhn, P., and Steiner, G.: Med. Klin., I3, 1007. 1917.

${ }^{8}$ Siemerling, E.: Bcrl. klin. Wchnschr., 55, 273. 1918.

9 Büscher, J.: Arch. f. Psychiat., 62, 426. I920.

${ }^{10}$ Speer, E.: M ïnchen. med. Wchnschr., 68, 425. I921.

It Marinesco, G.: Rev. neurol., 26, 481. 1919.

${ }^{2}$ Kalberlah, F.: Deutsche med. Wchnschr., 47, 102, 192 I.

${ }^{13}$ Kuhn, P., and Steiner, G.: Ztschr. f. Hyg. u. Infektionskrankh., 90, 417. 1920. 
organism appears more like a spirillum than a treponema or leptospira. It looks, in fact, very much like the spirillum of rat-bite fever ( $S$. morsusmuris). It is described by Kuhn and Steiner as being moderately active, somewhat wormlike, seldom rotatory, and as having a short, straight flagellum at either end.

The treponemas causing relapsing fevers (Figs. 48-56).-Relapsing fever was recognized as an independent febrile disease by the British observers of the eighteenth century and the early part of the nineteenth. William Jenner ${ }^{\mathrm{T}}$ and others differentiated it from typhus and typhoid fever, and many recognized its existence in various parts of the world. It was Obermeier, ${ }^{2}$ however, who provided the requisite criterion for recognition of sporadic cases and variant types of the disease by finding the organism, which was named Spirochaeta recurrentis by Leber ${ }^{3}$ in 1874 . The discovery was made in I 868 and announced in I873. Cartert had confirmed Obermeier's finding in India and established the etiological relation of the organism to relapsing fever by reproducing the disease in the monkey (Macacus radiatus). Relapsing fever is of widespread occurrence, and it appears to be the same disease no matter where it occurs. The only difference which has come to be recognized is in the intermediate hosts of the parasites. The so-called "tick fever" of Africa is transmitted by Ornithodoros monbata,${ }^{5}$ the European and East Indian relapsing fevers ${ }^{6}$ are transmitted by lice and perhaps also by bedbugs. The mild type of relapsing fever occurring on the American continent is carried by the tick Ornithodoros talaje. ${ }^{7}$ In the fowl relapsing fever another tick, Argas persicus, is the vector. ${ }^{8}$

All forms of relapsing fever are clinically identical. The incubation period is usually about a week. Onset is sudden, with chills, followed by febrile paroxysms and intense headache. Muscular and leg pains are very characteristic, and arthritic pains without any inflammatory or other joint symptoms not infrequently occur. There is a moderate enlargement and tenderness of the spleen. The fever suddenly ends by crisis. The duration of the initial paroxysm is variable, usually three to four days, and the intermission lasts from four to fourteen days. The period of relapse may be as short as a few hours or longer than the primary fever. The number of relapses varies. The pulse rate is usually high during the paroxysms (90-I IO). Nausea and vomiting are among the most common symptoms. In severe cases slight jaundice may be noticed after each paroxysm. Epistaxis may occur. Spirochetes are usually present during the paroxysm.

The infection is transmissible to monkeys and rats or mice. Rats usually show no signs of illness. All the strains of pathogenic blood spirochetes are maintained in the

${ }^{x}$ Jenner, W.: Lectures and Essays, I849-1879. London, I873.

${ }^{2}$ Obermeier, O.: Berl. klin. Wchnschr., 10, I52. 1873 .

3 Lebert: Ziemsscn's Handb., 2, 267. I874.

${ }_{4}^{4}$ Carter, H. V.: Deutsche med. Wchnschr., 5, I89. 1879.

5 Dutton, J. E., and Todd, J. L.: loc. cit.

${ }^{6}$ Nicolle, C., Blaizot, L., and Conseil, E.: loc. cit.; Sergent, E., and Foley, H.: Ann. del'Inst. Pasteur, 24, 337. I910; Schellack, C.: Arb. a.d. kaiserl. Gesundheitsamte, 30, 351. 1909; Centralbl.f. Bakteriol., 46, 486. 1908.

${ }^{7}$ Bates, L. B., Dunn, L., and St. John, J. H.: Am. J. Trop. Med., I, I83. 1921.

${ }^{8}$ Marchoux, E., and Salembeni, A.: loc. cit. 
laboratory in rats or white mice, being passed from animal to animal at appropriate intervals. The most convenient and economical way to maintain them is to bleed the rats forty-eight hours after inoculation and keep the blood in the icebox for five days before inoculation. The relapsing fever spirochetes live for one to two weeks when the citrated or defibrinated blood is preserved at a low temperature. At $37^{\circ} \mathrm{C}$. they undergo degeneration within three to four days. On suitable culture media they can be maintained if transferred to fresh medium every five to seven days (see p. 472).

The organisms causing fevers of geese $^{r}$ and chickens ${ }^{2}$ were given the names Spirochaeta anserina and $S$. gallinarum, ${ }^{3}$ respectively. Various birds can be infected with one or the other organism, however, and it is not improbable that gallinarum is the same species as anserina. If so, the species name of the fowl spirochete becomes Treponema anscrinum. The organism produces an acute febrile disease, with loss of appetite, diarrhea, and finally emaciation, which usually kills the bird within a few days. It is present in the circulation until death. The spirochetes are taken up by phagocytes toward the end of the attack, as shown by Cantacuzène ${ }^{4}$ in geese and by Levaditi, ${ }^{5}$ Marchoux, and Salembeni ${ }^{2}$ in chickens.

Spirochetes of the relapsing fever type are also found in cattle, sheep, horses, and monkeys, and they closely resemble those causing relapsing fever of man. T. theileri ${ }^{6}$ of cattle is transmissible to horses and sheep, and it is probable that the spirochetes found in the blood of sheep $(T \text {. ovinum })^{7}$ and horses (T. equi) are identical with T. theileri. T. theileri is of doubtful pathogenicity since it produces no symptoms in normal animals.

The difference between $T$. rccurrentis and $T$. anserinum was demonstrated by Gabritschewsky ${ }^{8}$ in $\mathrm{I} 898$, who showed that the blood of immune geese kills $T$. anserimum within a few minutes at $37^{\circ} \mathrm{C}$. but has no effect upon $T$. recurrentis, while the blood of convalescents from relapsing fever kills the recurrentis but not the anserinum. Species differences are also indicated by pathogenicity tests. Novy and Knapp ${ }^{9}$ thought that the organism of American relapsing fever could be differentiated from those of African tick fever and Bombay fever not only by pathogenic properties but also by morphological characteristics. The difference in animal reactions between the American strain (T. novyi) and the European (T. resurrentis) and African (T. duttoni) strains is considerable. The novyi is only mildly pathogenic for monkeys and non-pathogenic for rats, the duttoni is usually fatal to monkeys and may cause death even in rats, and the recurrentis is pathogenic but not fatal to animals.

Schellack, ${ }^{10}$ who carried out serological tests with monovalent immune sera specific for each strain, believed that the organisms causing the European and American re-

I Sakharoff, N.: Ann. de l'Inst. Pasteur, 5, 564. IS91.

2 Marchoux, E., and Salembeni, A.: loc. cit.

4 Cantacuzène, J.: ibid., I 3, 529. I S99.

${ }_{3}$ Swellengrebel, N. H.: ibid., 21, 5 S2. 1907.

5 Levaditi, C.: ibid., I8, 5 I1. I904.

${ }^{6}$ Laveran, A.: Compt. rend. Acad. des sc., 136, 939. 1903.

7 Theiler, A.: J. Comp. Puth. \& Therap., 17, 47. I904.

${ }^{8}$ Gabritschewsky, G.: Anm. de l'Inst. Pastcur, 10, 630. I 896.

9 Novy, F. G., and Knapp, R. E.: loc. cit.

ıo Schellack, C.: Arb. a. d. kaiserl. Gesundhcitsamte, 30, 35 I. I909; Centralbl. f. Bakteriol., Abt. I, 46, 486. I908. 
lapsing fevers are distinct species, and that both in turn differ from the African tickfever spirochetes, of which he also distinguished two species. The American strain was called by him $S$. novyi; the European, S. recurrentis; the West African, S. dnttoni (as first proposed by Novy and Knapp); and the East African, S. kochi. Strong, ${ }^{1}$ however, was able to distinguish only two species, recurrentis and duttoni. Bates, Dunn, and St. John² regard relapsing fever of Panama as a distinct disease and call the parasite $T$. neotropicalis. The species causing relapsing fever in Venezuela and in Spain have been named $T$. venesuelense and $T$. hispanicum, respectively. ${ }^{3}$

SPIROCHETES OF TIIE MOUTH AND RESPIRATORY TRACT (FIGS. IOI-II)

A number of types of spiral organisms occur in the human mouth, their abundance and variety depending on the condition of the mouth and the locality from which the material is obtained. In unclean or diseased mouths the number of spirochetes present is often very much increased, but whether any of them bear a relation to the diseased condition has not yet been adequately demonstrated.

It is not easy to separate and classify the mouth organisms. There are apparently two large types (Treponcma buccale [Figs. IOI-2] and Treponema vincenti [Fig.IoI]), one of intermediate size (Treponema macrodentium [Figs, Io7-8, I Io]), and at least four small types (Treponema microdentium [Figs. 104-5, Io9), Treponema mucosum [Fig. I I I], Treponema dentium or orthodostum of Hoffmann, and Treponema skoliodontum of Hoffmann [Fig. I06]). In addition, the normal mouth may contain a leptospira (L. trimerodonta Hoffmann).

Of all the mouth spirochetes, Treponema vincent $i^{4}$ is the most discussed. It is highly flexible, and active, has three to six rather flat and irregular curves, and sharply drawn ends, belonging to the refringens rather than to the pallidum type. In quiescent forms the curves are more regular. The length and general features are about the same as those of $T$. recurrentis, except that vincenti is perhaps thicker.

Whether the spirochete of Vincent is etiologically related to the ulcerative process or is only an associate like the other organisms found in acute inflammatory conditions has never been determined. It is found in the normal mouth in small numbers, but in ulcerative stomatitis (Vincent's angina) it occurs in abundance. It has been noticed that it is usually associated with the fusiform bacillus, and many believe that the symbiosis is responsible for the diseased condition. Both can be cultivated by anaerobic culture methods on serum agar ('Tunnicliff). 5

Vincent's spirochete (=Treponema schaudinni, q.v.) is also present in certain types of tropical ulcers and has been described as occurring in the sputum in some forms of bronchitis and in the lung tissue in pulmonary abscesses and pulmonary gangrene.

Treponema buccale (Figs. IOI-2) is a long, thick organism with definite double contour and flat curves, wide apart; it is not unlike Treponema refringens, which is a normal inhabitant of the genitalia.

${ }^{x}$ Strong, R. P.: United Fruit Co. Med. Dept., I th Ann. Rep., p. 216.1925.

${ }^{2}$ Bates, L. B., Dunn, L., and St. John, J. H.: loc. cit.

3 de Buen, S.: Ann. de parasitol., 4, I85. 1926; Bull. Acad. de méd., Paris (3d ser.), 95, 294. I926

${ }_{4}$ Blanchard, R.: Arch.f. parasitol., I0, I29. I906. $\quad 5$ Tunnicliff, R.: loc. cit. 
Treponema bronchiale is regarded by some (Pons, ${ }^{1}$ Delamare ${ }^{2}$ ) as indentical with T. buccalc. Others (Strong, ${ }^{3}$ Vincent ${ }^{4}$ ) have expressed the opinion that it is the same organism as $T$. vincenti, while several investigators (Fantham, ${ }^{5}$ Bezançon and Etchegoin $\left.^{6}\right)$ are convinced that it is a distinct species. It was described in 1906 by Castellani, ${ }^{7}$ and a great deal of work has since been done on the relation of spirochetes to the putrid types of bronchitis and empyema, lung gangrene and lung abscess, catarrhal exudate from sinuses, etc., with which are associated not only spirochetes of various types, but also fusiform bacilli and cocci. The problem is a very complex one because of the simultaneous presence of all of these organisms. Putrid empyemas have been induced ${ }^{8}$ by injecting rabbits subcutaneously in the region of the thorax with sputum, pleural exudate, pyorrheal pus, and even with tartar from normal mouths. The several types of organisms are present in the experimental lesions as in the human cases, and their combined activities are believed to be the cause of the diseased condition. The presence of the spirochetes, several types of which are involved, derives some significance from the frequent, though by no means constant,

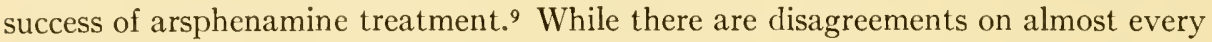
point in connection with this problem, present evidence seems to indicate that mouth hygiene may be an important factor in the prevention of certain lung affections. ${ }^{10}$

Treponema macrodentium, ${ }^{11}$ which the writer cultivated in $19 \mathrm{I} 2$, is usually thicker than $T$. pallidum and resembles more nearly $T$. recurrentis. Thick, definitely doublecontoured forms (Fig. I07), and also forms having only a single line of the body (Fig. I 10), are found, not only under natural conditions, but also in cultures.

Treponema microdentium (Figs. 105-6, I09) and T. mucosum (Fig. I I I), also cultivated in $1912,{ }^{\prime \prime}$ are about the same size, both smaller than T. macrodentium but larger than the smallest types. They are morphologically indistinguishable, and both are odor-producing in culture, but the mucosum differed from the microdentium in its mucin-producing property and in its ability to survive in tissue (rabbit testicle), and exerted a pyogenous action when inoculated together with agar.

Hoffmann ${ }^{12}$ distinguishes the two very minute treponema of the mouth as dentium

${ }^{2}$ Pons, R.: Bull. Soc. path. exot., 17, 170. 1924.

2 Delamare, G.: ibid., 19, 322. 1926.

3 Strong, R. P.: Science, 57, 514.1923.

4 Vincent, H.: Bull. Acad. roy. de méd. de Belg. (5th ser.), 2, 275. 1922; Compl. rend. Soc. de biol., 94, 1143. 1926; Bull. Acad. de méd., 96, 135. 1926.

5 Fantham, H. B.: Ann. Trop. Med., 9, 391. 1915.

${ }^{6}$ Bezançon, F., and Etchegoin, E.: Compt. rend. Soc. de biol., 94, 319. 1926; 95, 475. 1926.

7 Castellani, A.: J. Trop. Mcd., 29, 360. 1926.

${ }^{8}$ Davis, D. J.: Illinois M. J., 47, 200. 1925; Kline, B. S.: J.A.M.A., 77, 1874. 1921; J. Infect. Dis., 32, 48I. 1923; Chalmers, A. J., and O'Farrell, W. R.: J. Trop. Med., 16, 329. r9r3; Ribierre, P., and Kermogant, Y.: Compt. rend. Soc. de biol., 93, I35I. 1925; Smith, D. 'T.: Arch. Surg., 14, Part II, 23I. 1927.

9 Pilot, I., and Davis, D. J.: Arch. Int. Med., 34, 3I3. I924; Davis, D. J.: loc. cit.; Bloedorn, W. A., and Houghton, J. E.: J.A.M.A., 76, 1559. 1921; Smith, D. T.: Am. Rev. Tuberc., 15, 352. 1927; Nolf, P., and Spehl, P.: Arch. mêd. belges, 7r, 1. 1918.

to Kline, B. S., and Blankenhorn, M. A.: J.A.M.A., 81, 719. 1923.

${ }^{11}$ Noguchi, H.: loc. cit. $\quad{ }^{12}$ Hoffmann, E.: Deutsche med. Wchnschr., 46, 257.1920. 
(or orlhodontum) and skoliodontum, the latter being the smallest treponema known [Fig. I06]. These minute forms have not been cultivated. Hoffmann ${ }^{2}$ has also found in many mouths a leptospira which he has named Leplospira trimerodonta. Perrín ${ }^{2}$ has described Leptospira dentale found in the pus of bucco-maxillary gangrene.

SPIROCHETES OF THE GENITALIA (FIGS. I I 2-I I 8)

Analysis of the spirochetal inhabitants of the normal genitalia shows that three distinct types of treponema are present: the large, coarse type seen by Schaudinn and Hoffmann ${ }^{3}$ and given by them the species name refrinzens (Figs. I I $2-I_{3}$ ); the medium-sized type which the writer cultivated from a condyloma ${ }^{4}$ and later ${ }^{5}$ found to be present commonly in smegma, Treponema calligyrum (Figs. I I4-I 5); and a minute type, constantly present, which the writer first named Treponema minutum ${ }^{5}$ but changed to Treponema genitalis ${ }^{6}$ on finding that the specific name minutum was preoccupied. ${ }^{7}$

T. genitalis (Figs. II6-18) is decidedly smaller than T. pallidum and has relatively larger spirals and more of them in a given length. It resembles $T$. microdentium of the mouth in appearance but differs in being non-odor producing and immunologically specific.

T. calligyrum is easily confused with $T$. pallidum, especially in culture (Fig. I I5). It is about the same length but somewhat thicker and has rather deep spirals, not so closely set.

$T$. refringens (Figs. I I 2-I3) is readily differentiated from $T$. pallidum on darkfield examination by its greater thickness and by its tendency to constantly changing curves.

Stoddard ${ }^{8}$ has reported having found leptospira-like spirochetes in the urine of normal persons.

Treponema vincenti was first seen by Vincent in tropical ulcer in 1806 ; later he found it also in ulcerative stomatitis (Vincent's angina). It was named by Blanchard ${ }^{9}$ in I906. Prowazek, who thought the organism was the cause of tropical ulcer, described the organism anew and called it T. schaudinni, ${ }^{\text {10 }}$ in I907. Treponema schaudimi, therefore, is a synonym of $T$. vincenti.

T. noguchii was found by Strong" in a type of skin ulcer which he called "dermal spirochetosis," consisting of nodular, warty growths which are $5 \mathrm{~mm} .-2.5 \mathrm{~cm}$. high, $\mathrm{I}-3 \mathrm{~cm}$. in diameter, and may ulcerate. Fresh preparations from the moist surface under the papules show the spirochetes to be more numerous than are the spirochetes

${ }^{1}$ Hoffmann, E.: Dcutsche med. Wchnschr., 46, 625. I920; Ccntralbl. f. Bakteriol., 86, I34. I92I.

${ }^{2}$ Perrín, T. G.: Rev. mex. de biol., 2, I 71. I922; Gac. méd. de México, 55, I923.

3 Schaudinn, F., and Hoffmann, E.: Deutschc med. Wchnschr., 3 I, I 728.1905.

${ }^{4}$ Noguchi, H.: J. Exper. Med., I 7, 89. I9г3.

5 Noguchi, H.: ibid., 27, 667. Ig I8; 28, 559. I9I8.

${ }^{6}$ Noguchi, H.: Laboratory Diagnosis of Syphilis, p. 260. New York, I923.

7 Dobell, C.: Arch.f. Protistenk., 26, I 7 . 1912.

${ }^{8}$ Stoddard, J. L.: Brit. M. J., 2, 4I6. I9I 7 .

9 Blanchard, R.: loc. cit.

${ }^{\text {10 }}$ von Prowazek, S.: Arch.f. Protistenk., ro, I 29. 1906. ${ }^{11}$ Strong, R. P.: loc, cit. 
in relapsing fever blood. The organisms are also larger $(14-30 \mu)$ and coarser than T. recurrentis. Fusiform bacilli are not present in the lesion. The parasites stain more readily and deeply than $T$. schaudinni ( $T$. vincenti) and are easily differentiated from T. pallidum and $T$. pertemue. Strong obtained an impure culture in sterile Amazon River water containing $I 5^{-20}$ per cent rabbit serum but the spirochetes died out in six days, hence the pathogenicity of the cultures could not be determined.

Treponema aboriginalis (probably a synonym of $T$. vincenti) was described as occurring in granuloma inguinale. ${ }^{\mathrm{T}}$

Treponema acuminatum and Treponema obtusum were described by Castellani ${ }^{2}$ in I905. They occurred together with $T$. pertenue in yaws lesions.

Treponema balanitidis was found by Hoffmann and von Prowazek ${ }^{3}$ in I906 in a case of balanitis erosiva circinata.

Treponema pseudopallidum was described by Mulzer ill $1905 .{ }^{4}$ It was found in ulcers of the genital region.

Rona, in 1907, described Treponema gangraenosa nosocomialis found in ulcers of the genital region. ${ }^{5}$

Treponema urethrae and Treponema vaginalis were the names given by Macfie ${ }^{6}$ to organisms which he found in the urine and the vaginal discharge, respectively, of cases in the Accra Hospital (Gold Coast).

\section{SPIROCHETES OF THE INTESTINAL TRACT}

The presence of spirochetes or spirilla in feces was recorded as long ago as $1884 .^{7}$ Le Dantec ${ }^{8}$ in 1903 observed that the mucus in the feces of a patient with dysentery formed a kind of false membrane consisting of an almost pure culture of spiral organisms which he referred to as "spirilla." Mühlens ${ }^{9}$ described several spirochetes in the intestinal contents, a small one from cholera feces, and several larger ones similar to the spirochetes of the mouth. Werner ${ }^{10}$ named two types of spirochetes found in normal stools, a form with only two flat curves, frequently showing an S-shape, which he called Spirochacta eurygyrata, another with two to six close spirals which he named S. stenostrepta. Broughton-Alcock ${ }^{11}$ thinks that S. eurygyrata has definite significance on certain types of dysentery found in mucus, but that S. stenostrepta is non-pathogenic. Recent work of Sabrazès ${ }^{12}$ lends support to the assumption that pathogenic

I Cleland, J. B.: J. Trop. Med., I2, I 43. I909.

${ }^{2}$ Castellani, A.: loc. cit.

3 Hoffmann, E., and von Prowazek, S.: Centralbl.f. Bakteriol., Orig., 41, 741, 8I 7. I906.

4 Mulzer, P.: Berl. klin. Wclinschr., 42, I144. 1905.

5 Rona, S.: Verh. deutsche dermat. Gessell., 9, 471 . 1907.

${ }^{6}$ Macfie, J. W. S.: Ann. Trop. Med., 10, 305. 1916; J. Parasitol., 9, 274. I917.

7 Escherich, T.: Mïnchen. med. Wchnschr., No. 54. 1884.

${ }^{8}$ Le Dantec: Gaz. hebd. des Sc. méd. de Bordeaux, 31, 196. igoo; Compt. rend. Soc. de biol., $55,617.1903$.

9 Mühlens, P.: Ztschr.f. IIyg. 11. Infcktionskrankh., 57, 405. 1907.

so Werner, H.: Centralbl. f. Bakteriol., Abt. I, 52, 24I. I909.

in Broughton-Alcock, A.: Proc. Roy. Soc. Med., I4, Part III, 46. I923.

I2 Sabrazc̀s, J.: Compt. rend. Soc. de biol., I82, 1052. 1926; Gaz. hebd. des Sc. mêd. de Bordeaux, 47, 196, 226.1926. 
spirochetes may be present in the intestine of man. S. eurygyrata has been cultivated (impure) by Hogue, ${ }^{\mathrm{I}}$ and Sanarelli ${ }^{2}$ has obtained pure cultures of the spirochetes found in the guinea pig intestine.

\section{THE I.EPTOSPIRA GROUP}

When Stimson, in $1907,{ }^{3}$ described a spiral micro-organism, which he found in the kiclney in a case of yellow fever, and gave it the name of Spirochacta (?) interrogans (Fig. I I9), no great interest was aroused among bacteriologists, presumably because it did not seem likely at that time--when filterability connoted invisibilitythat a visible spirochete could have any etiological significance in connection with a disease which had been definitely shown to be caused by a filterable virus. In I9I4 appeared the interesting and very important article by Wolbach and Binger ${ }^{4}$ describing a peculiar spirochete which multiplied in the filtrate from stagnant water taken from a fresh-water pond near Boston. They called the organism Spirochaeta biflexa because of its characteristic hooked ends. This observation also received little notice at the time. A little later (I9I5) the discovery of the causative agent of Weil's disease was announced by Inada and Ido, ${ }^{5}$ and also (I9I6) by Hübener and Reiter ${ }^{5}$ and Uhlenhuth and Fromme. ${ }^{7}$ The hooked ends which had led Stimson to call his organism interrogans and Wolbach and Binger to call theirs biflexa were described as one of the characteristics of the spirochete of Weil's disease, which was named Spirochacta icterohaemorrhagiae. The morphological and biological features of this organism were, in fact, so characteristic that the writer, in $1917^{8}$ was led to propose for it the new genus, Leptospira ( $\lambda \epsilon \pi \tau o$ s $+\sigma \pi \epsilon \hat{\imath} \rho a=$ "fine coil"). It was recognized at the time, from the description given by Wolbach and Binger, that Spirochaeta biflexa was probably an organism of the same type, but the proposal of the new genus was based on careful study of Leplospira icterohacmorrhagiae, which was named as the type species of the genus. To the genus was added, in I9I9, another pathogenic species, L. icteroides (Figs. I 20, I2I), isolated from yellow-fever patients. Subsequently (1920) the writer had opportunity to study Stimson's preparations of Spirochaeta interrogans and became convinced that it also was a leptospira and identical with $L$. ictcroides. Stimson's spirochete is therefore the first leptospira to be described and becomes the type species of the genus (Leptospira interrogans), while Wolbach and Binger's is the first description of a free-living leptospira.

It was not until recently that general interest was aroused in the significance of free-living leptospiras, when Uhlenhuth and Zuelzer ${ }^{9}$ found, in tap water and in ponds and pools in Berlin, the micro-organism which they called Spirochaeta pseudoicterosenes because of its morphological similarity to Leptospira icterohaemorrhagiae (syn-

${ }^{1}$ Hogue, M. J.: J. Expcr. Med., 36, 617. 1922.

${ }^{2}$ Sanarelli, G.: Ann. de l'Inst. Pastcur, 41, r. 1927.

${ }_{3}$ Stimson, A. M.: loc. cit.

5 Inada, R., and Ido, K.: loc. cit.

${ }^{4}$ Wolbach, S. B., and Binger, C. L.: loc. cit.

${ }^{6}$ Hübener and Reiter: toc. cit.

${ }^{7}$ Uhlenhuth, P., and Fromme: Ztschr. f. Immunilütsforsch. u. cxper. Therap., Orig., 25, 3 I 7. I9I6.

${ }^{8}$ Noguchi, H.: J. Expcr. Mcd., 25, 755. 1917.

9 Uhlenhuth, P., and Zuelzer, M.: Klin. Wchnschr., I, 21 24. I922. 
onym Spirochacta icterogenes Uhlenhuth and Fromme). The organisms as they occur in water from the tap differ serologically and immunologically from $L$. icterohacmorrhagiae and are not pathogenic for guinea pigs, but on prolonged cultivation (impure) some strains have gradually acquired the serological properties of the icterohaemorrhagiae and have finally become sufficiently virulent to induce typical hemorrhagic jaundice in the guinea pig. ${ }^{{ }^{2}}$ Immune sera prepared with the pathogenic strain protect guinea pigs against a virulent strain of the icterohaemorrhagiae, and animals which have recovered from an attack of the mutated water leptospiras resist an infection with the ictcrohaemorrhagiac, and vice versa. Other strains of the water leptospiras, which have not been completely converted into pathogenic forms, do not yield antisera protective against the icterohaemorrhagiae, and exhibit serological differences among themselves. It is suggested by Uhlenhuth and Zuelzer that the water leptospiras are taken up by some animal such as the rat, in whose body they are converted into a pathogenic parasite capable of infecting man. The immediate source of infection in bathing pools or slime is, according to this theory, the urine of infected rats.

Numerous investigators have since found free-living leptospiras in both fresh and salt water and in many different countries. The organisms are to be found in any water that comes in contact with objects covered with slime, and they are present in greater numbers in the slime than in the water. They may be very numerous in some specimens-one or two to each microscopic field-and are readily demonstrable by darkfield examination. Coles ${ }^{2}$ recently found that leptospiras, together with flagellated protozoa, may be found in infusions made from decayed leaves in sterilized water, a fact which explains their presence in mountain ditches, where they have been found by the writer (Catskill Mountains of New York). It is not impossible, however, that wild rats or certain tree-climbing rodents might infect such water.

Although most investigators have found the inoculation of water or slime containing free-living leptospiras to give negative results, a pathogenic leptospira may be occasionally present under natural conditions, as shown by the recent experiments of Buchanan, ${ }^{3}$ who infected guinea pigs with slime collected from the roof of a coal mine in East Lothian, Scotland. Moreover, according to Toyama, ${ }^{4}$ and Buchanan, Leptospira ictcrohaemorrhagiae may survive for two months in soil, provided the reaction is neutral or slightly alkaline.

In recent years the water leptospiras have been isolated by a number of workers in pure culture by filtration through bacterial filters, usually Berkefeld $V$ (pores 0.7I $\mu$ ) and $N$ (pores $0.4-0.6 \mu$ ). Bauer ${ }^{5}$ obtained thirteen strains in West Africa; Moch$\operatorname{tar}^{6}$ three strains in Holland; and Dimitroff, ${ }^{7}$ thirty-seven strains in the United States. One strain has been isolated in the writer's laboratory from icebox slime.

${ }^{1}$ ZueIzer, M.: Centralbl.f. Baktericl., Orig., 89, I7I. 1922.

${ }^{2}$ Coles, A. C.: J. Trop., Mcd., 29, I 70. 1926.

3 Buchanan, G.: Spirochetal Jaundice, Mcd. Rcscarch Conncil., Spcc. Rcp. (II 3 th ser.). I927.

4 Toyama, Y.: Sc. Rep., Gov. Inst. for Infcct. Dis., I, I97. Tokyo, 1922; Japan Med. IVorld., 4, 193. 1924.

5 Bauer, J. H.: loc, cit.

${ }^{6}$ Mochtar, A.: loc. cit.

7 Dimitroff, V. T.: loc. cit. 
Angerer, ${ }^{\mathrm{I}}$ who seems to have been the first to obtain pure cultures, used Haen membrane filters (pores I.5 $\mu$ ). None of the pure cultures so far obtained has been pathogenic for animals or serologically related to L. icterohacmorrhagiae. Some strains have a slight group reaction with $L$. hebdomadis. There is undoubtedly more than one distinct strain of leptospira in water. The strain isolated in the writer's laboratory is distinctly larger than any pathogenic leptospira which we know, but in the icebox slime from which this strain was cultivated there seem to be at least two other types: one very fune and short (Fig. I 24), and one of medium size which is similar to L. hebdomadis or L. icterohaemorrhagiae (Fig. I25). Apparently the large type (Fig. I26) is the more readily cultivated. The slime seems to offer ideal conditions for the growth of the organisms, providing it has a neutral or slightly alkaline reaction.

Various names have been proposed for the water leptospiras, Leptospira biflexa (Wolbach and Binger, I9I4), L. pseudoicterogenes (Uhlenhuth and Zuelzer, 1922), L. aquatilis (Hoffmann). It is not yet known whether or not each of these represents a separate species.

Saprophytic leptospiras have been found in the human mouth by Erich Hoffmann, ${ }^{2}$ who called them L. trimerodonta. Perrín ${ }^{3}$ found in an inflammatory buccomaxillary lesion a leptospira to which he gave the name $L$. dentale. The writer ${ }^{4}$ found a very small leptospira in the stomach of the ox (L. bovis [Fig. I27]).

L. icterohacmorrhagiae (Fig. 123).-L. icterohaemorrhagiae, which causes Weil's disease in man and "yellows" in dogs, was discovered by Inada and Ido, ${ }^{5}$ who first found it in the blood, liver, and kidneys of young guinea pigs which had died of hemorrhagic jaundice as a result of the inoculation of the blood of patients with Weil's disease in Japan. About a year later Hübener and Reiter, ${ }^{6}$ working independently in Germany, found a similar spirochete in guinea pigs inoculated with patients' blood, and subsequent investigations showed that the micro-organism is distributed throughout the world. It is rarely seen in the blood during life, ${ }^{7}$ being demonstrated, as a rule, only by inoculation of guinea pigs or implantation on suitable culture medium. During convalescence it is present in the urine over a long period and can readily be recovered by passage through guinea pigs to eliminate associated bacteria. It is readily found postmortem in various tissues, especially in the liver and kidneys.

The severity of the infection with $L$. icterohaemorrhagiae in man varies in different localities, owing no doubt to difference in the virulence of the strains and in individual susceptibility. The disease is often extremely severe in Egypt ${ }^{8}$ and parts of Asia Minor, and is clinically very much like yellow fever. In Japan the mortality is as high as 30 per cent, and there also the disease has been confused with yellow

r Angerer, K. v.: loc. cit.

${ }^{2}$ Hoffmann, E.: loc. cit.

3 Perrín, T. G.: loc. cit.

7 Inada, R., Ido, Y., Hoki, R., Kaneko, R., and Ito, H.: loc. cit.

${ }^{8}$ Valassopoulo, A.: Prem. Cong. Egyp. de méd., I, r67. I904; Kartulis: "A Discussion on Cases of Jaundice in the Military Hospitals at Alexandria," Med. Serv., Mcditcranean Expcd. Force. Alexandria, I915.
${ }^{4}$ Noguchi, H.: New Fork State $M . J ., 22,426.1922$.

5 Inada, R., and Ido, K.: loc. cit.

${ }^{6}$ Hübener and Reiter: $l o c$. cit. 
fever. The majority of cases seen in Europe are mild, ${ }^{1}$ the mortality being only ${ }^{2-4}$ per cent, but during the World War severe cases resembling yellow fever were occasionally reported. ${ }^{2}$ In Scotland, among miners, Buchanan found the mortality to be as high as 30 per cent.

The incubation period is six to twelve days; the initial fever is high and is followed by nausea and vomiting, headache, epistaxis, and muscular pains in the limbs. Bronchitis of moderate severity, jaundice, and mild or severe parenchymatous nephritis develop, and spots of hemorrhage appear on the skin. In most cases there is a second rise of temperature late in the second week of illness. In severe cases the vomitus or stools may contain fresh or altered blood, and hematuria is not uncommon; a moderate degree of anemia is usual. Convalescence is slow, and the patients suffer weakness for months. After several days of illness the spirochetes appear in the urine, being most numerous about the fifteenth day. They have usually disappeared by the fortieth day, though in one case they were excreted as late as the sixtyfifth day. In fatal cases death occurs during the second or third week, occasionally as early as the eighth or ninth day.

One attack prevents reinfection for a long time, immunity being as durable as that from yellow fever. A specific lysin for L. icterohaemorrhagiae appears in the blood and may be detected several years after recovery. ${ }^{3}$

The natural reservoir of infection in the case of $L$. icterohaemorrhagiae is the wild rat, as first pointed out by Inada and Ido and their collaborators, ${ }^{4}$ and substantiated by numerous observers in various parts of the world. 5 The organism may be directly demonstrated in the kidneys of apparently healthy wild rats, or transmitted to guinea pigs by inoculation of suspension of the kidney tissue, and the strains from rats have the same morphological, pathogenic, and immunological properties as those isolated from human cases. The proportion of rats infected varies in different places. In New York 6 it was only 4 per cent, in Guayaquil 7 it was 60 per cent, in England and Scotland it was about 30 per cent. The Japanese workers believed that the occurrence of infectious jaundice among miners was due to contamination of the stagnant water and soil of the mines with rat urine, and their theory was substantiated by the rapicl decline in the incidence of the disease after drainage of the mines and disinfection of the soil. The actual presence of $L$. icterohaemorrhagiae in the water of mines was proved only recently by Buchanan.

There is, however, another theory regarding the source of infection with $L$. ic-

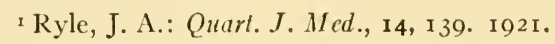

${ }^{2}$ Dawson, B., and Hume, W. E.: ibid., 10, 90. 1916-17.

${ }^{3}$ Inada, R., Ido, Y., Hoki, R., Ito, H., and Wani, H.: loc. cil.

${ }_{4}^{4}$ Ido, Y., Hoki, R., Ito, H., and Wani, H.: J. Exper. Med., 26, 341. I9I7.

5 Courmont, J., and Durant, P.: Bull. ct mém. Soc. méd. d. hôp. de Paris, 41, II5. I9I7; Jobling, J. W., and Eggstein, A. A.: J.A.M.A., 69, I787. I917; Coles, A. C.: J. Parasitol., Ir, I. I918; Ribeyro, R. E.: Crón. méd., Lima, 35, 157. 1918; Smillie, W. G.: Bull. Soc. path. cxot., 13, 56. 1920; Pereiro da Silva, E.: Comp!. rend. Soc. de biol., 86, I043. 1922; Carrieu, M. F., and Sollier, N.: ibid., p. 99. I922; Anigstein, L.: J. Trop. Mcd., 26, 81. 1923; Bonne, C.: Compt. rend. Soc. de biol., 90. 668, 1924 .

${ }^{6}$ Noguchi, H.: J. Exper. Mcd., 25, 755. 1917 .

7 Noguchi, H.: ibid., 30, 95. 1919. 
terohaemorrhagiae. Uhlenhuth and Zuelzer ${ }^{\mathrm{I}}$ found that a leptospira present in tap water in Berlin may produce icterohemorrhagic disease in guinea pigs after cultivation together with bacteria for several generations. The strain was obtained in pure culture from the inoculated guinea pigs and was found to be serologically as well as pathogenically identical with $L$. icterohaemorrhagiae. Uhlenhuth and Zuelzer concluded that the non-pathogenic, serologically distinct, free-living leptospira had been converted into L. icterohaemorrhagiae. Other investigators have found their culture of water leptospiras, whether pure or impure, to be devoid of virulence for guinea pigs. It has long been known that epidemics of infectious jaundice may arise as a result of bathing in river water. It seems probable that the source of infection is the urine of wild rats deposited near the shore, and that the micro-organism enters the body through the skin of the feet. How rats come to act as carriers of a micro-organism which is pathogenic for certain other mammals though harmless to the rat itself is an interesting problem. Uhlenhuth and Zuelzer think that the water leptospira is rendered pathogenic for man by passage through the rat. The writer has injected water containing leptospiras into white rats but has never detected the organisms in the kidneys of the animals.

L. icterohaemorrhagiae passes through Berkefeld filters $V, N$, and $W{ }^{2}$ but can rarely be detected microscopically in the filtrates. Its presence is shown by the infectiousness of the filtrates, or by cultivation by the method described elsewhere (p. 473).

L. hebdomadis (Fig. I22).-L. hebdomadis was first isolated by Ido, Ito, and $W{ }^{3}$ from young guinea pigs inoculated with blood of patients suffering from a disease known in Japan as namıkayami ("seven-day fever"), a non-fatal infection occurring during the summer months among field workers. The disease begins with all the acute symptoms characteristic of infectious jaundice, but only icterus and hemorrhages develop.

L. hebdomadis is present during the febrile period in the blood of guinea pigs. The symptoms in guinea pigs are slight hemorrhage and mild jaundice; the infection is never fatal. The serum of convalescent patients or animals contains lysins for L. hebdomadis but none for L. icterohaemorrhagiae. Guinea pigs which have passed through infection with one of these leptospiras are just as susceptible to the other as are normal animals.

Morphologically, L. hebdomadis is decidedly longer than L. icterohaemorrhagiae and is more closely wound. Living specimens rarely show the hooked ends, but fixed and stained individuals are characteristically hooked. The organism is without doubt a member of the leptospira group.

The natural host of L. hebdomadis is the field mouse, Microtus montebelli, which abounds in certain regions of Japan and probably disseminates the organism through its urine.

L. pyrogenes.-In certain parts of Sumatra an acute febrile disease, resembling a mild form of Weil's disease, or dengue, is endemic. Verwoort ${ }^{4}$ in Deli (1923) found in

r Uhlenhuth, P., and Zuelzer, M.: loc. cit.

${ }^{2}$ Inada, R., Ido, Y., Hoki, R., Kaneko, R., and Ito, H.: lor. cit.

3 Ido, Y., Ito, H., and Wani, H.: J. Exper. Med., 28, 435. 1918.

${ }_{4}$ Verwoort, H.: loc. cit. 
the blood of these cases a leptospira which he called $L$. pyrogenes. The disease he designated Spirochetosis febrilis. Soon afterward similar leptospiras were seen by Kouwenaar ${ }^{\mathrm{I}}$ in the urine of patients, and Baermann ${ }^{2}$ isolated a number of strains and subjected them to serological study. There proved to be at least two serological types, one more or less closely related to $L$. icterohaemorrhagiae, the other allied rather to a strain which had been isolated from rats in Holland. Neither type was related to the hebdomadis. None of the strains was particularly virulent for guinea pigs, being able to infect only very young animals. The experimental infection, like the human one, is seldom fatal, and jaundice occurs only after many passages in guinea pigs.

Leptospira sp. ?-Prausnitz and Lubinski ${ }^{3}$ found a leptospira in the blood of patients during an outbreak of acute febrile disease which affected thousands of persons in the inundated districts along the river Oder in Silesia in the summer of 1926. The disease is known as Schlammfieber, and is characterized by sudden onset, high fever lasting five to seven days, intense pains - especially in the legs-rash, conjunctivitis, mild angina, left shift of leukocytes, and slight albuminuria. Usually there is a relapse. The course is benign, there is no jaundice, but convalescence is slow. Field workers standing in flooded places are chiefly attacked. Insects are not suspected of carrying the infection. The authors found a leptospira in the blood of the patients but could not transmit the disease to animals. The disease resembles nanukayami of Japan, but until the organism is identified it may be regarded as a distinct species.

Leptospira hemoglobinuriae.-This organism was observed by Schüffner ${ }^{4}$ and Snijders in Deli in I9I 8 in the blood of a patient with a disease resembling blackwater fever.

Leptospira biliohemoglobinuriac.-This organism was described but not obtained in cultures by Blanchard and Lefrou, ${ }^{5}$ who found it in the blood in three cases of hemoglobinuria with jaundice in Europeans in the French Congo in I922. Thomson ${ }^{6}$ studied a large number of cases of black-water fever in East Africa but without finding any leptospira.

Leptospira interrogans (syn. L. icteroides) (Figs. I I9-2I).-Stimson, ${ }^{7}$ in I907, observed, in the Levaditi-stained sections of the kidney of a patient who had died of yellow fever in New Orleans during the great epidemic of I905 an organism which he called Spirochaeta? interrogans because of its peculiar hooked ends. In I9r 8 a leptospira was isolated by the writer in Guayaquil, ${ }^{8}$ Ecuador, from blood or postmortem

${ }^{r}$ Kouwenaar: Acad. Procfschr. Amsterdam, i 924.

${ }_{2}^{2}$ Baermann, G.: Gencesk. Tijdschr. Nederl.- Indie, 63, 885. 1923.

3 Prausnitz, C., and Lubinski,: Klin. Wchnschr., 5, 2052.1926.

${ }_{4}^{4}$ Schüffner, W.: Geneesk. Tijdschr. Nederl.-Indie, 58, 352. 1918.

5 Blanchard, M., and Lefrou, G.: Bull. Soc. path. exot., 15, 699. I922.

"Thomson, J. G.: Liverpool Sch. Trop. Med., "Research Memoir Series," 6, 1924.

7 Stimson, A. M.: loc. cil.

${ }^{8}$ Noguchi, H.: J. Exper. Med., 29, 547-96. I919; 30, I-29, S7-107, 401. 1919; 31, I35-68. 1920; $32,3^{81}$. 1920. 
material from six of twenty-seven cases of yellow fever studied, and the name Leptospira icteroides was suggested for it. The writer was at that time unaware of Stimson's previous observation but was subsequently given an opportunity to study Stimson's original preparations in comparison with preparations of $L$. icteroides. Morphologically, the organisms are exactly identical, and, in view of the fact that both have been found in yellow fever, it seems reasonable to assume that they are one and the same. Hence Stimson's interrogans takes precedence over the writer's icteroides as the species name.

L. interrogans was subsequently isolated from cases of yellow fever in Mexico, Peru, and Brazil by various workers, by Kligler and the writer in Mérida and Morropon in 1919 and $1920{ }^{1}$ by Pérez-Grovas ${ }^{2}$ and Le Blanc, ${ }^{3}$ independently, in Vera Cruz in 1920 and 1921 ; by Godofredo Vianna and Bião+ in Palmeiras in 1923; and more recently by Vianna and Vianna, Jr., in Bahia in 1926 . The number of strains isolated is comparatively small: six in Guayaquil, three in Mexico, four in Peru, and four in Brazil.

The morphological and cultural properties of the organism are similar to those of other species of leptospiras except that the interrogans is in general smaller and finer than the icterohaemorrhagiae, pyrosenes, or hebdomadis. As in the case of the other varieties, the interrogans is seldom detected in the blood of patients, and is best demonstrated by means of animal inoculations or cultivation.

The cases from which the micro-organism was isolated were characterized by abrupt onset, chills, moderate or high fever, severe frontal headache, pains in the back and legs, nausea, frequent vomiting, congested gums, puffed face, and general malaise. In the later stages there were hemorrhages into various organs: the stomach, giving rise to black or red vomit; the intestine, causing black or bloody stools; in the bladder (hematuria), the subcutaneous tissues, and the lungs. Albuminuria was severe in most cases, and complete anuria often occurred.

In the experimental disease, the symptoms were similar: the onset acute, the temperature rising abruptly after an incubation period of two to six days and continuing for two to three days. General jaundice, albuminuria (sometimes anuria), and hemorrhages into the subcutaneous tissues, stomach, intestine, and lungs occurred soon after the fall in temperature. The liver showed disseminated foci of necrosis, with extensive fatty degeneration, while the renal epithelia, especially of the convoluted tubules, exhibited fatty as well as necrotic changes. Except for the lesser degree of intermediary-zone necrosis these changes are identical with those found in the human tissues, as shown by W. H. Hoffmann's study ${ }^{6}$ of materials collected by Guiteras in I 899-1900, when the United States Army Yellow Fever Board under Reed was working in Havana, and by Gorgas and Guiteras. Cebus monkeys, inoculated with Bra-

I Noguchi, H., and Kligler, I. J.: ibid., 32, 60I-37; 33, 239-60. I92I.

${ }^{2}$ Pèrez-Grovas, P.: J.A.M.A., 76, 362. I92 I.

3 Le Blanc, T. J.: J. Trop. Med., 28, I69. 1925.

${ }_{4}^{4}$ Torres, O.: Scicncia méd., 2, 219, 313. Bahia, 1924.

5 Vianna, Jr., G., and Vianna, G.: Soc. med. e cirurg. June, 1926.

${ }^{5}$ Hoffmann, W. H.: Scuchenbekämpfung, 2, 279. I925. 
zilian strains of $L$. interrogans showed symptoms and lesions identical with those of human yellow fever. ${ }^{\text {. }}$

The interrogans is decidedly more destructive for the liver and kidneys than the icterohaemorrhagiae, as shown by comparison of the pathological changes in the experimental infections. The difference is an expression of an elective affinity of the interrogans for the liver and kidney, rather than of greater virulence. The icterohaemorrhagiae is invariably more virulent than the interrogans for guinea pigs and dogs, attacking these animals when they are fully grown, while the interrogans is only slightly pathogenic for these animals, even when they are very young.

Repeated successive passages through guinea pigs for a long time alters the pathogenic properties of the interrogans. A strain passed through the guinea pig for many passages no longer produces the original picture of yellow fever but kills the animals, after an incubation period of two to three days, with an acute leptospiremia. At the time of death, which occurs on the fourth or fifth day of illness, the blood is swarming with leptospiras, and the liver and kidneys are less characteristically affected than in animals inoculated with newly isolated strains. There is no question but that the biological and pathogenic properties of the micro-organism undergo a profound modification through continued animal passage. Under normal conditions Leptospira interrogans never passes from man to man but passes alternate generations in the insect-carrier Aedes aegypti, retaining its predilection for the liver and kidney as well as its ability to pass on to the mosquito body.

$L$. interrogans is specifically influenced by the serum of yellow fever convalescents, the relationship being analogous to that existing between $L$. icterohaemorrhagiae and the serum of convalescents from infectious jaundice. This statement applies to strains which have been recently isolated and have not been subjected to repeated animal passages. Serological tests carried out by means of the Pfeiffer reactions and also by cross-protection experiments with freshly isolated cultures yielded clear-cut specific results. The contradictory findings of some investigators ${ }^{2}$ who have recently carried out Pfeiffer reactions and cross-protection tests with old passage strains for the purpose of differentiating Leptospira interrogans from L. icterohaemorrhagiae may have been due to the masking of specificity by a group reaction. The question of strain specificity and group relations is a complex one, and, as observed by Park and Williams, ${ }^{3}$ there is theoretically no reason to believe that apparently complete mutual cross-protection might not occur with two closely related but not identical strains.

The negative Pfeiffer reactions obtained with the sera of yellow fever convalescents in West Africa may be due either to the use of old strains, which have been modified serologically by continued passages in guinea pigs or on artificial medium, or to a difference between the strain causing West African yellow fever and those isolated from cases in Central and South America. The difficulty of cultivation and of animal transmission of the African strain seems to be even greater than in the case

${ }^{x}$ Noguchi, H., Muller, H. R., Torres, O., Silva, F., Martins, H., Ribeiro dos Santos, A., Vianna, G., and Biāc, M.: Rockefeller Inst. for Med. Researeh, Mono. 20. 1924.

${ }_{2}^{2}$ Theiler, M., and Sellards, A. W.: Am. J. Trop. Med., 6, 383. I926; Sellards, A. W.: ibid., 7, 71. 1927; Schüffner, W.: Centralbl. f. Bakteriol., 101, 405. 1927.

${ }^{3}$ Park, W. H., Williams, A. W., and Krumwiede, C.: Pathogenic Microörganisms (Sth ed.), p. 235 . 1924 . 
of the American strains, but the symptomatology and the pathological features of South American and West African yellow fever are essentially alike. That there may exist more than one serological race among the interrogans is made highly probable by the fact that other leptospiras have shown this characteristic. Two or more varieties of $L$. icterohaemorrhagiae and $L$. hebdomadis have been described. ${ }^{\mathrm{I}}$ It is also possible that there is a disease known as yellow fever which is not caused by Leptospira interrogans.

So far as the writer is aware, there are no other two diseases which resemble each other as closely as do yellow fever and infectious jaundice. The fundamental difference between them is epidemiological: one being transmitted by a certain species of insect, the other by infected rodents. This difference may be due to the climatic conditions under which each disease prevails. Tropical temperature and humidity and abundant diptera would favor the arlaptation of the micro-organism to an insect, while in cold climates, where severe winters destroy the insects annually, the parasite must be disseminated in some other way. Numerous trypanosomes are carried by tlies in the tropics, but in cold climates only those species exist which are harbored and transmitted by wingless arthropods parasitic on warm-blooded animals.

L. interrogans has been shown experimentally to be conveyed in a tropical country (Ecuador) from man to animal and animal to animal by itedes aegypti. ${ }^{2}$ Mosquitotransmission experiments made in a colder climate yielded negative results. ${ }^{3}$

Once isolated, L. interrogans is readily maintained on artificial culture media, but the primary cultivation of a strain from human materials has proved extremely difficult. A highly developed human parasitism must account for the resistance of the interrogans to artificial cultivation and transmission to animals. The interrogans is not an unusual organism in this respect, however, since there are many human pathogens which cannot be readily grown or transmitted to animals. Failure to isolate the interrogans in a given case of yellow fever is certainly not proof that it is not present.

For studies of pathogenic as well as of immunological relations it is important to employ recently isolated strains. Apparently the same biological factors which operate to convert a non-pathogenic water leptospira with unrelated serological properties into a new strain indistinguishable from $L$. ictcrohacmorrhagiae in every respect come into play to modify the characteristics of a pathogenic leptospira. Indeed, it is highly probable that if various species of leptospiras are subjected to prolonged successive passages in guinea pigs until they become highly virulent for this animal they will all acquire a new set of common biological properties. It would be desirable to determine this point by experiment; but it has not been possible so far, owing to the low virulence of most leptospiras for the guinea pig. L. interrogans, once rendered virulent, can be carried on for many generations; but L. hebdomadis cannot be maintained through successive generations, and most water leptospiras fail to infect guinea pigs at all.

Leptospira asthenalgiae.-The assertion is often made that yellow fever and dengue are allied infections, owing no doubt to their common intermediate insect host,

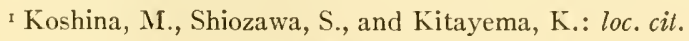

${ }^{2}$ Noguchi, H.: J. Exper. Med., 30, 40г, I9r9.

3 Mochtar, A.: loc. cit. 
and many workers have searched for a leptospira in dengue fever. Couvy, ${ }^{\mathbf{I}}$ for example, described a small spirochete with a few spirals, found in the blood of dengue patients during the incubation period. Carbo-Noboa ${ }^{2}$ has isolated several strains of leptospira from certain cases diagnosed as dengue in Guayaquil and has given them the name Leptospira asthenalgiae. Studies of the pathogenicity and serological reactions of these strains have not yet been published. The American workers, Duval and Harris, ${ }^{3}$ failed to find a leptospira but isolated a filterable, microscopically invisible virus, pathogenic for guinea pigs. Siler, Hall, and Hitchen $s^{4}$ in the Philippines have so far not been able to find a leptospira in their cases.

Dengue resembles yellow fever in symptomatology even less than seven-day fever resembles infectious jaundice. The general features of acute febrile infections at their onset are common to all these infections, but the cardinal symptoms of yellow fevernamely, intense and acute nephritis, hemorrhages, and jaundice - are absent from dengue, which is a non-fatal disease. The skin eruptions so constantly observed in dengue are not seen in yellow fever, and there is little or no immunity from dengue, even when the disease is rather severe, while yellow fever confers durable immunity even when mild. The assumption of a close relation between the two diseases does not appear to be well supported by the clinical and immunological data.

\section{EXPLANATION OF PLATES}

Plate I

FIG. I.-Ehrenberg's drawings of Spirochacta plicatilis. The upper figures were said by him to represent a magnification of 300 times, the lower ones a magnification of 800 times. (From Dic Infusionstierchen.)

FIGS. 2. AND 3.-Spirochacta plicatilis, according to Zuelzer, showing the axial filament; ironhematoxylin stain. Fig. 2, $X_{1,100}$ Fig. $3, X_{1,240 .}$

FIG. 4.-Schaudinn's (see loc. cit.) schematic drawing of a portion of Spirochaeta plicatilis

FIG. 5.-A spirochete of the plicatilis type (S. stenostrcp!a?) from refrigerator slime. Note the resemblance, except in size, to the organisms drawn by Ehrenberg (Fig. I); darkfield view of fresh preparation. $X_{1,000}$

Figs. 6-7.-Water forms, similar to that shown in Fig. 5. Fig. 6 shows also a water leptospira; darkfield view. $X_{1,000}$.

Figs. 8-ro.-Stained preparations of the Spirochaeta shown in Figs. 5-7. Figs. 8 and 9, preparations fixed in osmic acid and stained with Giemsa's solution; Fig. Io, preparation fixed in Schaudinn's fluid and stained with iron-hematoxylin. $X_{I, \infty}$,oo.

\section{Plate II}

FIGs. II-I8.-Drawings showing the types of flagella of various bacteria as brought out by the Zettnow-Fontana stain. Fig. II, B. tctani, type IV; Fig. I2, B. tetani, type III; Fig. I3, E. typhi; Fig. 14, B. pyocyancus; Fig. 15, A. bronchiscpticus; Fig. 16, V. metschnikovi; Figs. 17-18, B. bacilliformis.

Figs. 19-21.- Schematic drawing of Cristispira, showing the structure and dispesition of the membrane or crista, as brought out by darkfield observations of the course of degeneration.

${ }^{1}$ Couvy, L.: Bull. Soc. path. cxot., 14, I9S. I921.

Carbo-Noboa, J. M.: An. dc Soc. méd.-quir. del Guayas, 4. 326. Guayaquil, 1924.

${ }_{3}$ Duval, C. W., and Harris, W. H.: J. Exper. Med., 40, SI 7, 835. 1924.

${ }_{4}$ Siler, J. F., IIall, M. W., and Hitchens, A. P.: Denguc, Its II istory, Epidcmiology, ITechanism of Transmission, Etiology, Clinical Manifestations, Immunity, and Prcvention. Manila: Bureau of Printing, I926. 
FIGs. 22-29.-Drawings illustrating the origin of the terminal filaments of Treponema from the axial filament, as shown by observations of dividing organisms, and of plasmolysis.

Figs. 30-34.-Drawings showing the terminal filaments of Leptospira, as brought by the Zcttnow-Fontana stain. Fig. 33, the denuded axial filaments; Fig. 34, the spiral terminal filaments of unknown significance.

\section{Plate III \\ Cristispira balbianii}

FIGS. 35 AND 36.-Living forms. Note that the crista is difficult to see when the body is stretched in the course of active movement, as in Fig. 36 ; darkfield views. $\times_{1,000}$.

Fig. 37. A dead organism but one which has not begun to degenerate. The body conforms to the shape of the crista; darkfield view. $\times_{\mathbf{r}, 000}$.

FIGS. 38 AND 39.-Degenerating individuals. Only the crista remains of the specimen shown in Fig. 39; darkfield views. $X_{1,000}$.

FIGS. 40 AND 4I.-Preparations fixed with osmic acid and stained with Giemsa's solution, showing the chambered structure and the crista. $X_{1, \infty}$,

\section{Plate IV}

FIG. 42.-Cristispira balbianii, showing the crista and the chambered structure; osmic acid fixation; Giemsa's stain. $\times 2,000$.

Fics. 43-44.-Treponema recurrentis, Zettnow's flagella stain, showing the terminal filaments. Fig. 43 shows how the filaments originate from the axial filament at the time of division. $\times_{2,000}$.

FIGs. 45-47.-Treponema pallidum, Zettnow's flagella stain, showing the smooth (Fig. 46) or wavy (Figs. 45,47 ) terminal filaments. $X_{2,000}$

\section{Plate V \\ (Magnification, $\left.\times_{1}, \infty 00\right)$}

Fig. 48.-Treponema recurrentis in rat blood; darkfield view

Fig. 49. - The same, after it has been acted on by bile. Only the axial filament remains

Fig. 50.-Treponema recurrentis in rat blood; Fontana's stain

FIG. 51.-Treponema anserinum, pure culture; darkfield view

FIG. 52.-Treponema anserinum, pure culture; Giemsa's stain

FIG. 53.-The peritrichal flagella of T. anserinum. (After Borrel)

Fig. 54.-Treponema koclii, culture; Giemsa's stain

Fig. 55.-Treponema novyi in rat blood; gentian-violet stain, after buffered formalin fixation

Fig. 56.-Treponema novyi, culture; Giemsa's stain

\section{Plates VI and VII}

The filaments of degenerated leptospiras, brought out by Zettnow's flagella stain (see text) $\times 2, \infty 00$.

\section{Plate VIII}

(Appearance of Growth of Various Treponemas in Pure Culture)

Fig. 77.-Treponema pallidum

Fig. 78.-Treponema pallidum

FIG. 79.-Treponema pertenue

FIG. 80.-Treponema calligyrum

FIG. 8I.-Treponema macrodentium

Fic. 82.-Treponema refringens (without tissue)

FIG. 83.-Treponema refringens

FIG. 84.-Treponema microdentium (without tissue)

Fig. 85.-Treponema microdentium

Fig. 86.-Treponema mucosum

Fig. 87.-Flask for growing mass cultures of Leptospira for the preparation of vaccine; onethird actual size. This flask is now made (procurable from the A. H. Thomas Co.) with bent neck to facilitate the withdrawal of the contents by a pipette. 


\section{Plate IX}

(Pathogenic Treponemas, Magnification, $\times$ r,,$\infty 0)$

FIG. 88.-Treponema pallidum, preparation from chancre; Giemsa's stain

FIG. 89.-Treponema pallidum, preparation from chancre; stained with fuchsin after fixation in buffered formalin.

FIG. 90.-Treponema pallidum in brain from patient dead of general paralysis; silver impregnation.

FIG. 9I.-Treponema pallidum in testicular tissue of rabbit inoculated with paretic brain tissue; silver impregnation.

Fig. 92.-Treponema pallidum, pure culture; darkfield view

FIg. 93.- Treponema pallid m, pure culture; darkfield view; young culture two weeks old, grown on ascites agar at $37^{\circ} \mathrm{C}$.

FIG. 94.-Treponema pallidum in the testicular tissue of a rabbit inoculated with pure culture; silver impregnation.

Fig. 95.-Treponema pertenue, Giemsa's stain. (After Nichols)

FIG. 96.-Treponema pertenue; pure culture; darkfield view

Fig. 97.-Treponema cuniculi in spontaneous lesion of rabbit; Giemsa's stain

FIG. 95. - The same, fixed in buffered formalin and stained with fuchsin

Figs. 99-100.-Treponema cuniculi as seen by darkfield

\section{Plate X}

(Treponemas of the Mouth, Magnification, $X_{\mathrm{r}, 000)}$

FIG. IOI.-Darkfield view of tartar, showing a specimen of Treponema vincenti (above) and one of Treponema buccale (below); darkfield view.

Fig. I02.-Fontana stain of tartar, showing Treponema buccale (above) and Treponema macrodentium (below).

FIG. I03.-Fontana stain of tartar, showing Treponema macrodentium, Treponema buccale, and Treponema microdentium.

FIG. 104.- Treponema microdentium; darkfield view

FIG. 105.-Treponema microdentium; stained with gentian violet, after mordanting

FIG. I06.-Fontana stain of tartar, showing Treponema microdentitu and Treponema skoliodontum (?), as well as two specimens of Treponema macrodentium.

Fics. 107-8.- Treponema macrodentium, as seen by darkfield, and in Fontana preparation

FIG. 109.-Pure culture of Treponema microdentium; darkfield view

FIG. I ro.-Pure culture of Treponema macrodentium; darkfield view

FIg. III.-Pure culture of Treponema mucosum; darkfield view .

\section{Plate XI}

(Treponemas of the Genitalia, Magnification, $\left.X_{I, \infty}\right)$

FIG. I I 2.-Treponema refringens in smegma; Fontana stain

FIG. I I3.-Treponema refringens, pure culture; darkfield view

Frg. II4.-Fontana stain of smegma, showing several specimens of Treponema calligyrum

FIg. II5.-Pure culture of Treponema calligyrum; darkfield view

Fig. Ir6.-Treponema genitalis in smegma; Fontana stain

FIG. II 7.-Treponema genitalis in smegma; darkfield view

FIG. Ir8.-Pure culture of Treponema genitalis, gentian-violet stain, after mordanting

\section{Plate XII}

(Leptospira Group, Magnification, $X_{1,000}$; All Figures Except I $9^{-20}$ Darkfield Views)

Frg. Ir9.--Stimson's preparation (1907), showing Leptospira interrogans in human kidney; silver impregnation; preparation presented by Dr. Stimson.

FIG. I 20.-Leptospira interrogans in the blood in experimental yellow fever (guinea pig); Giemsa's stain. 
PLATE I

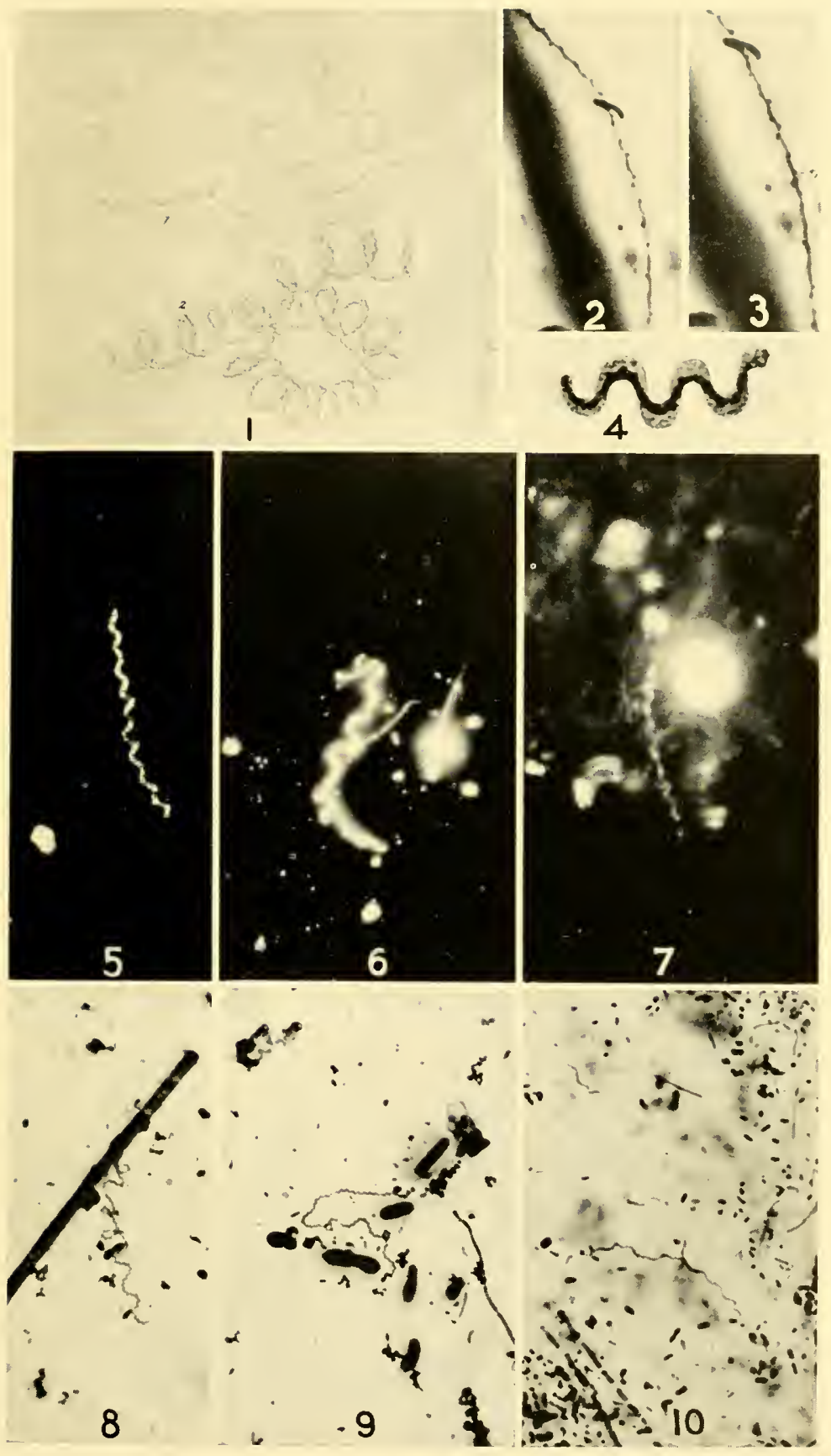





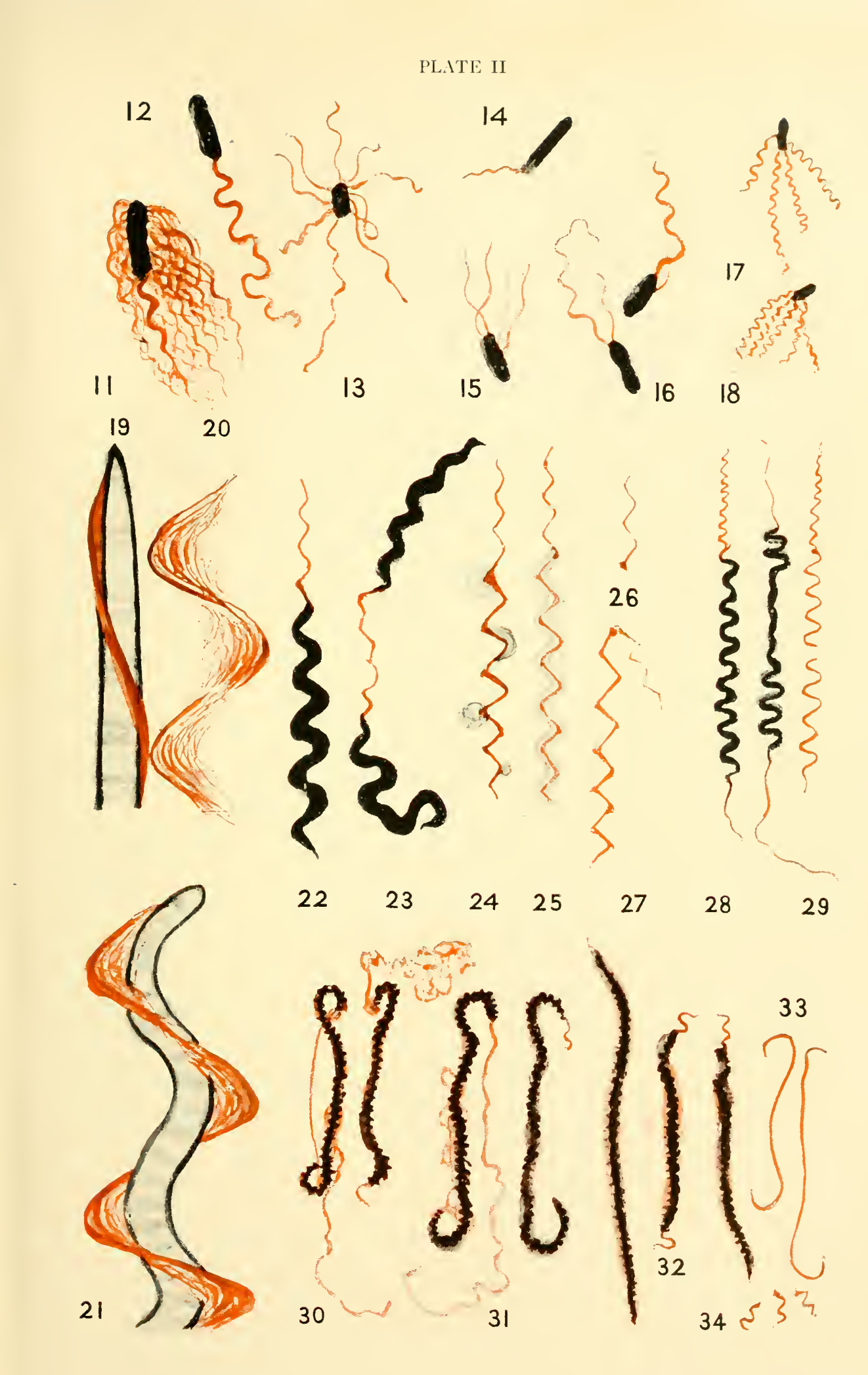



PIATE III

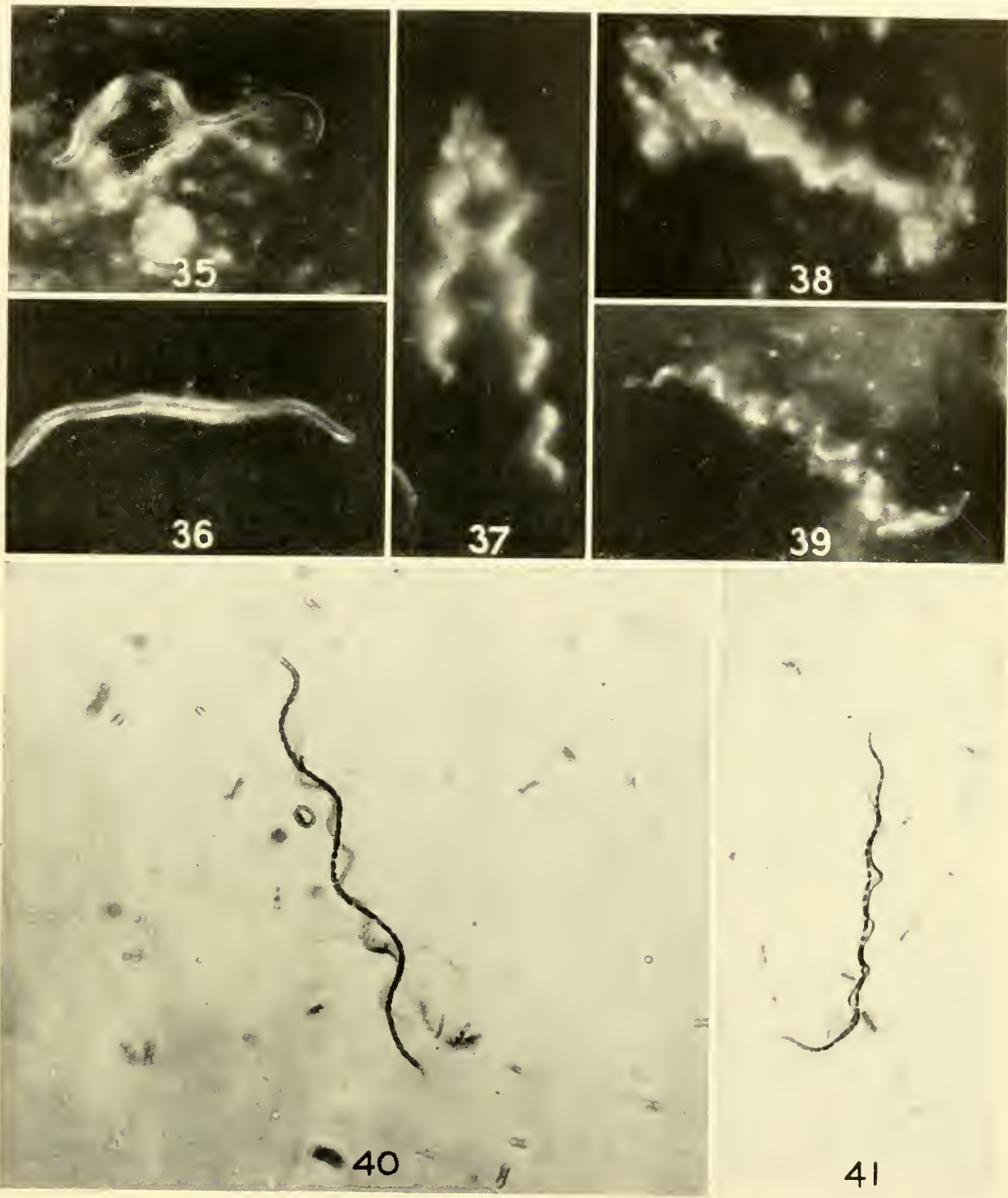



PLATE IV

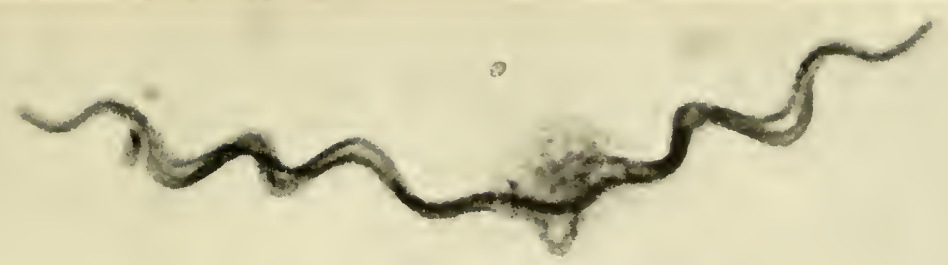

42

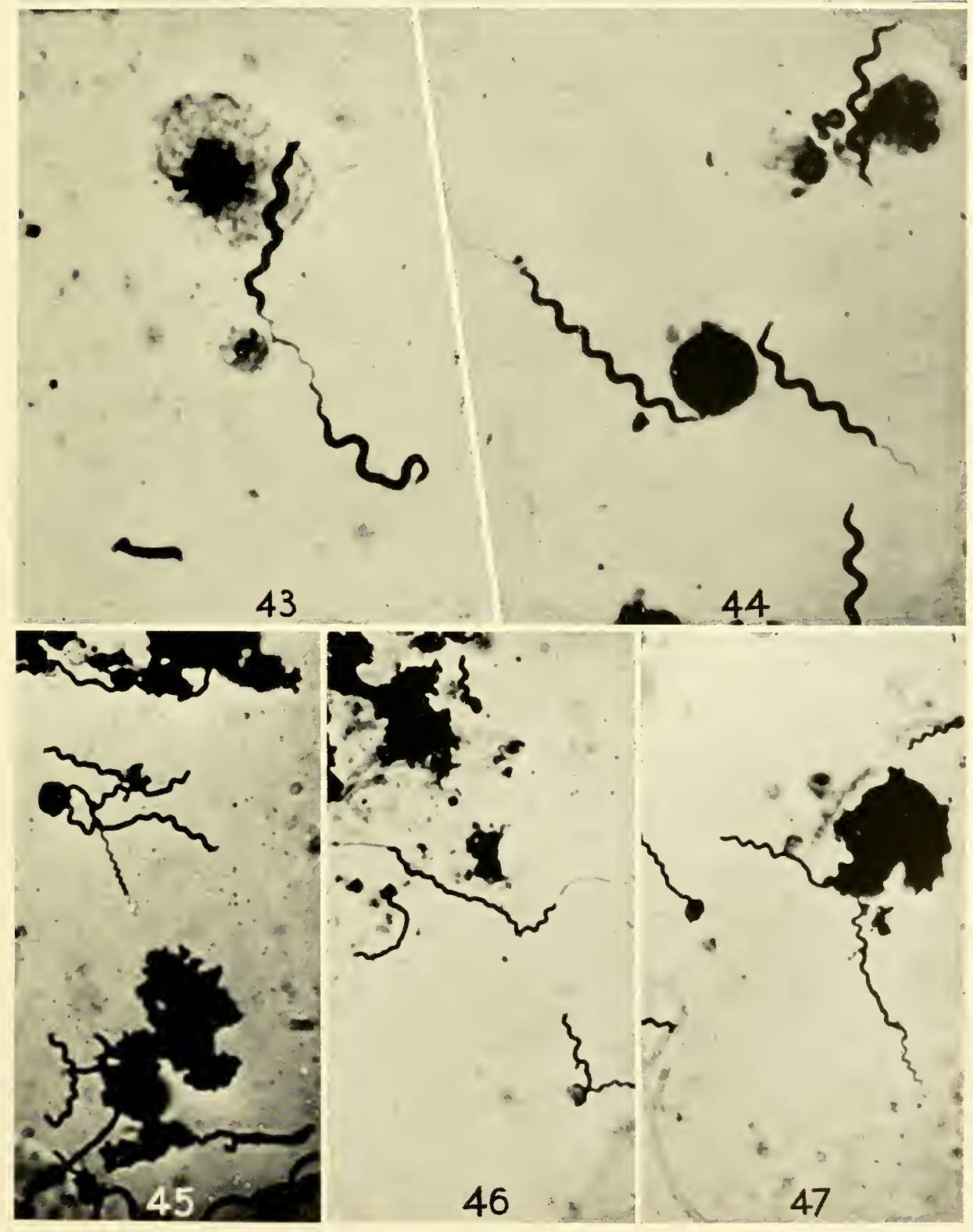




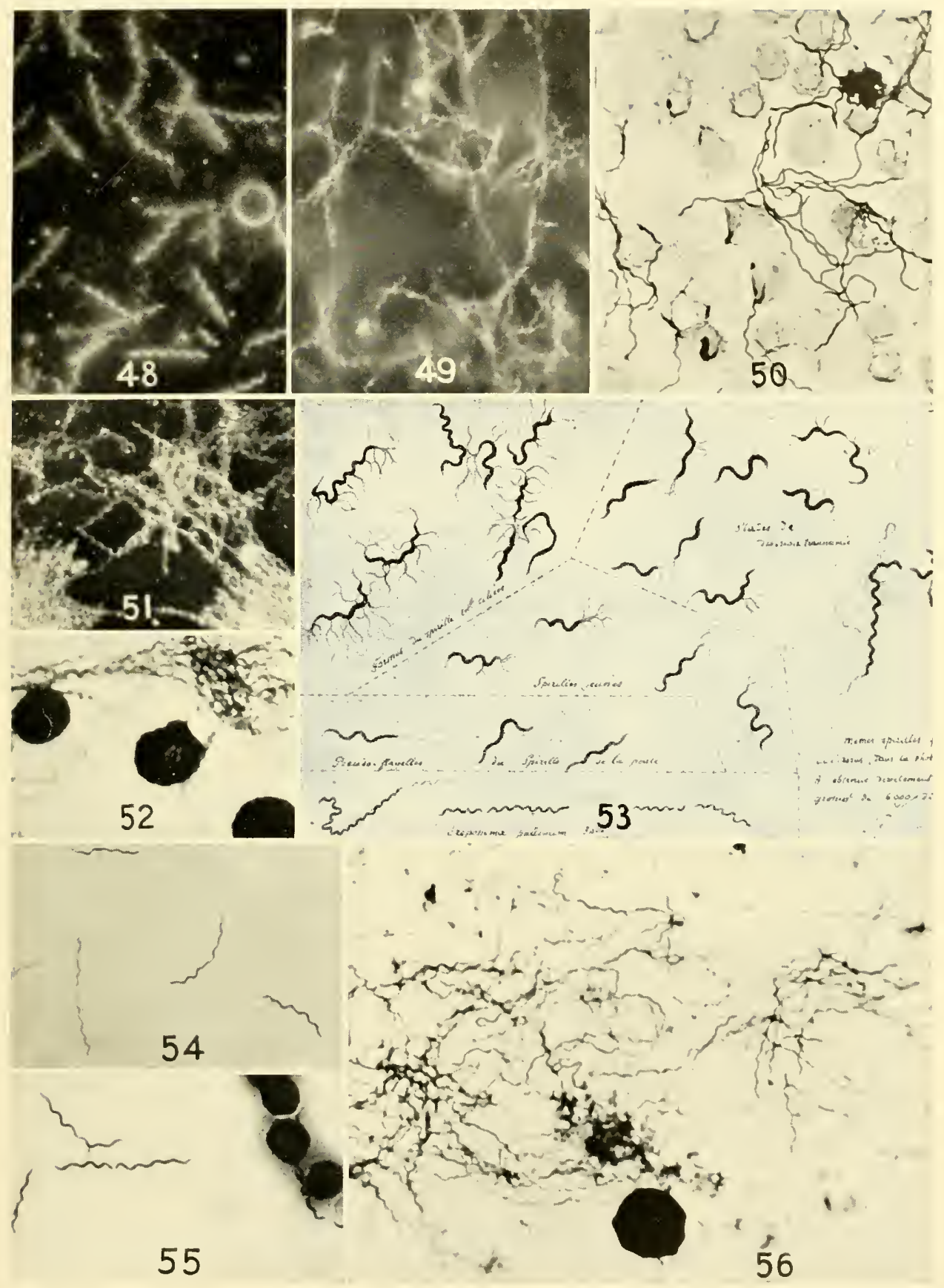



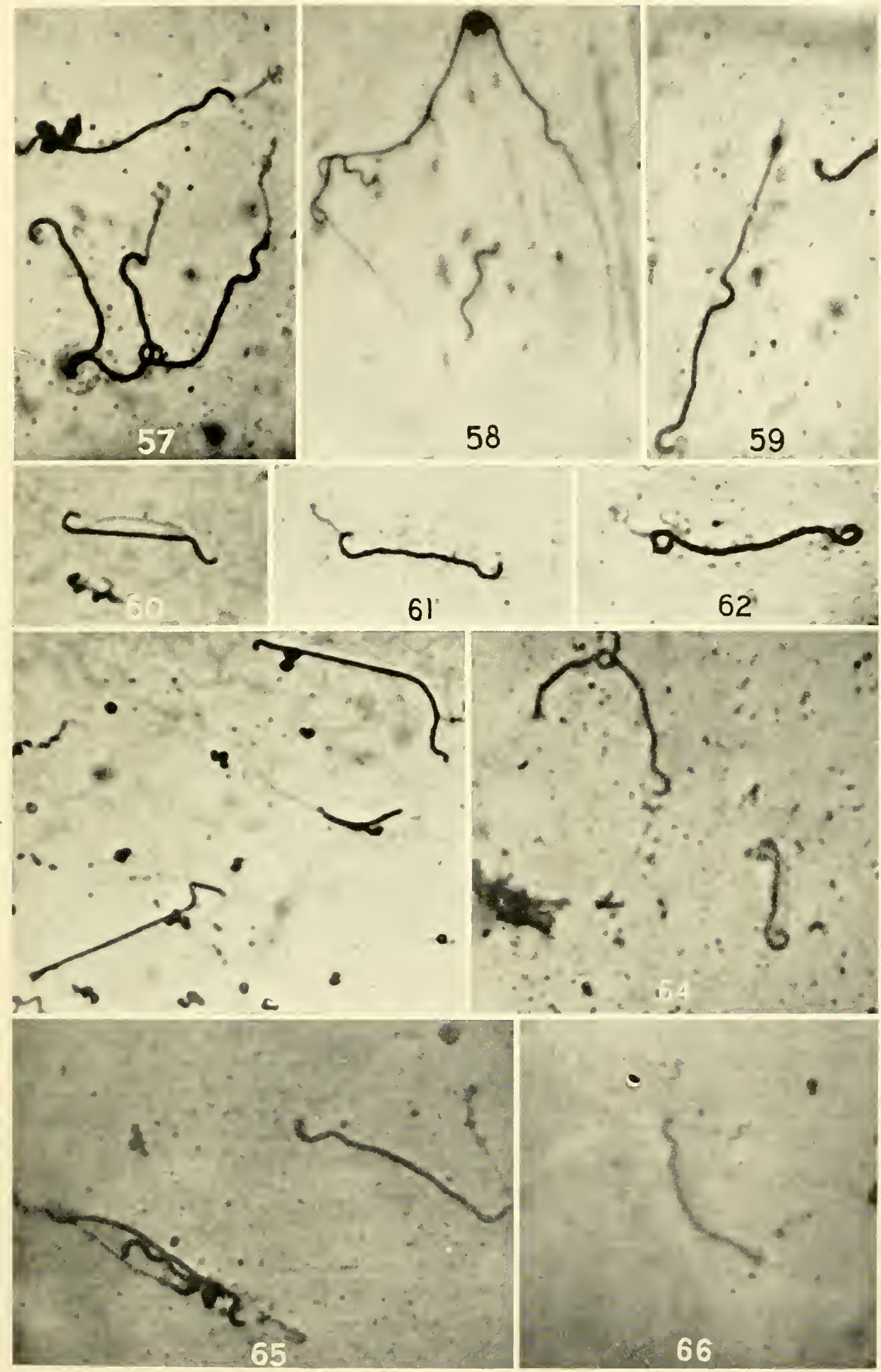





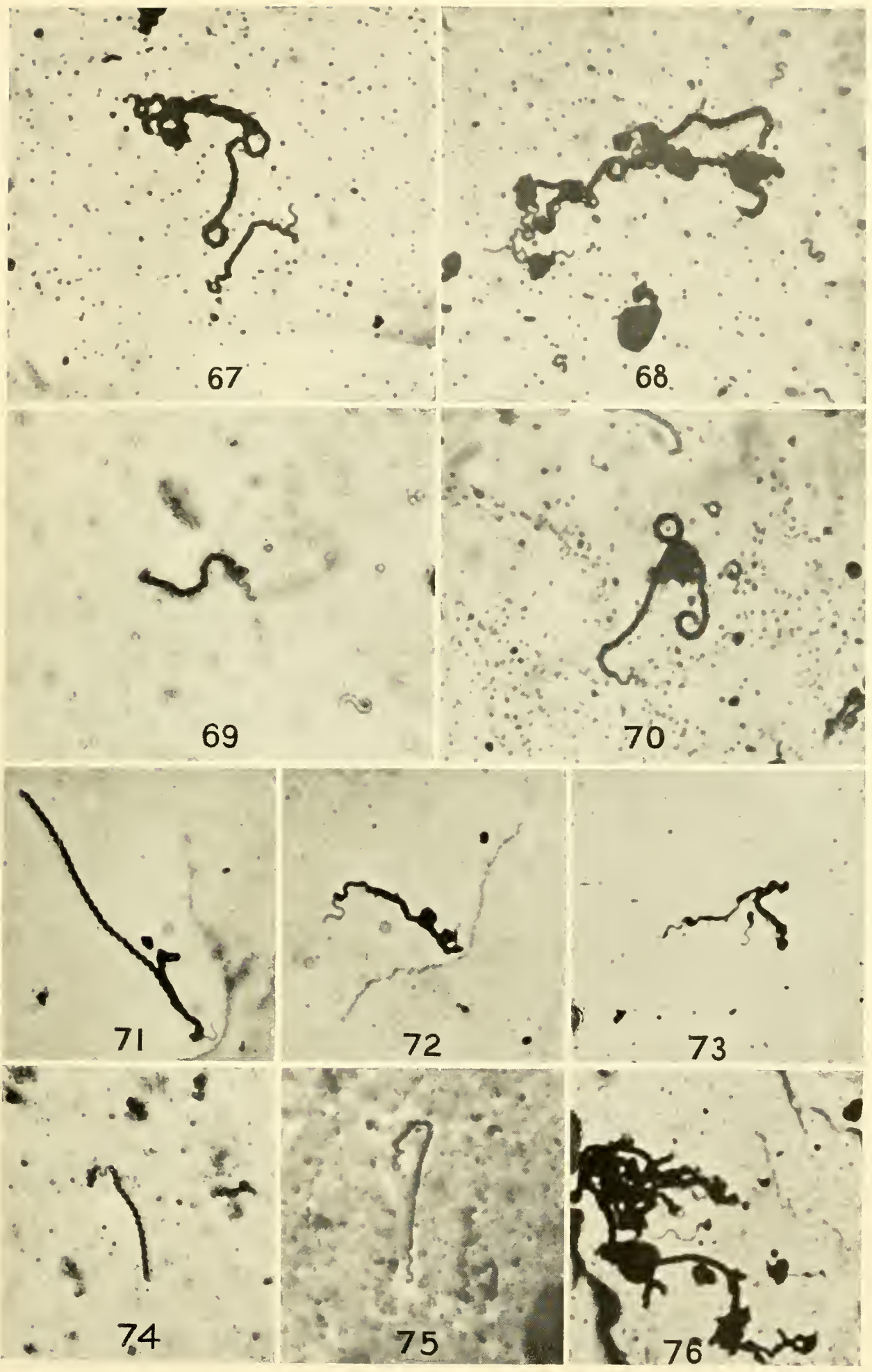



PLATE VIII

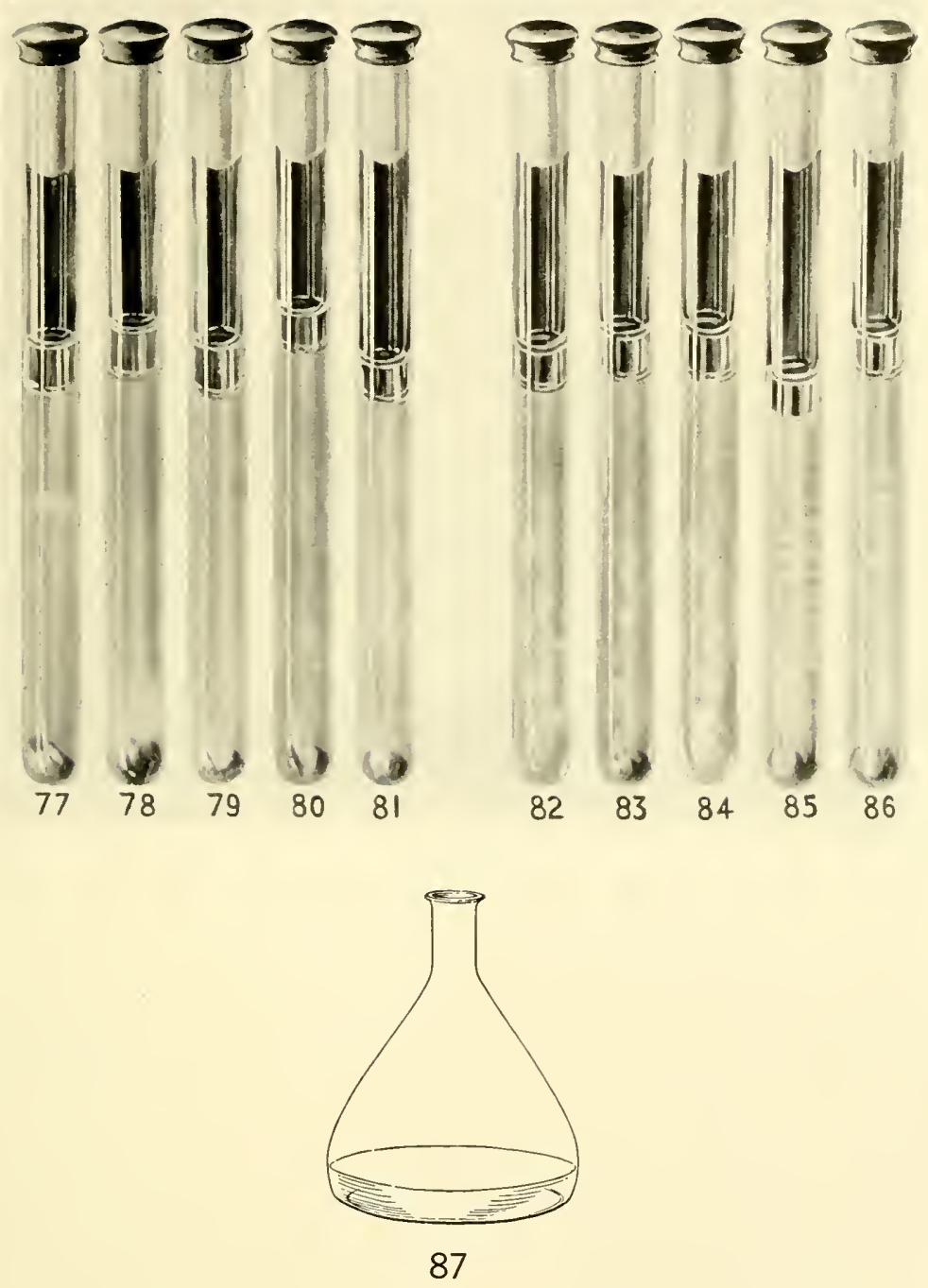



PLATE IX

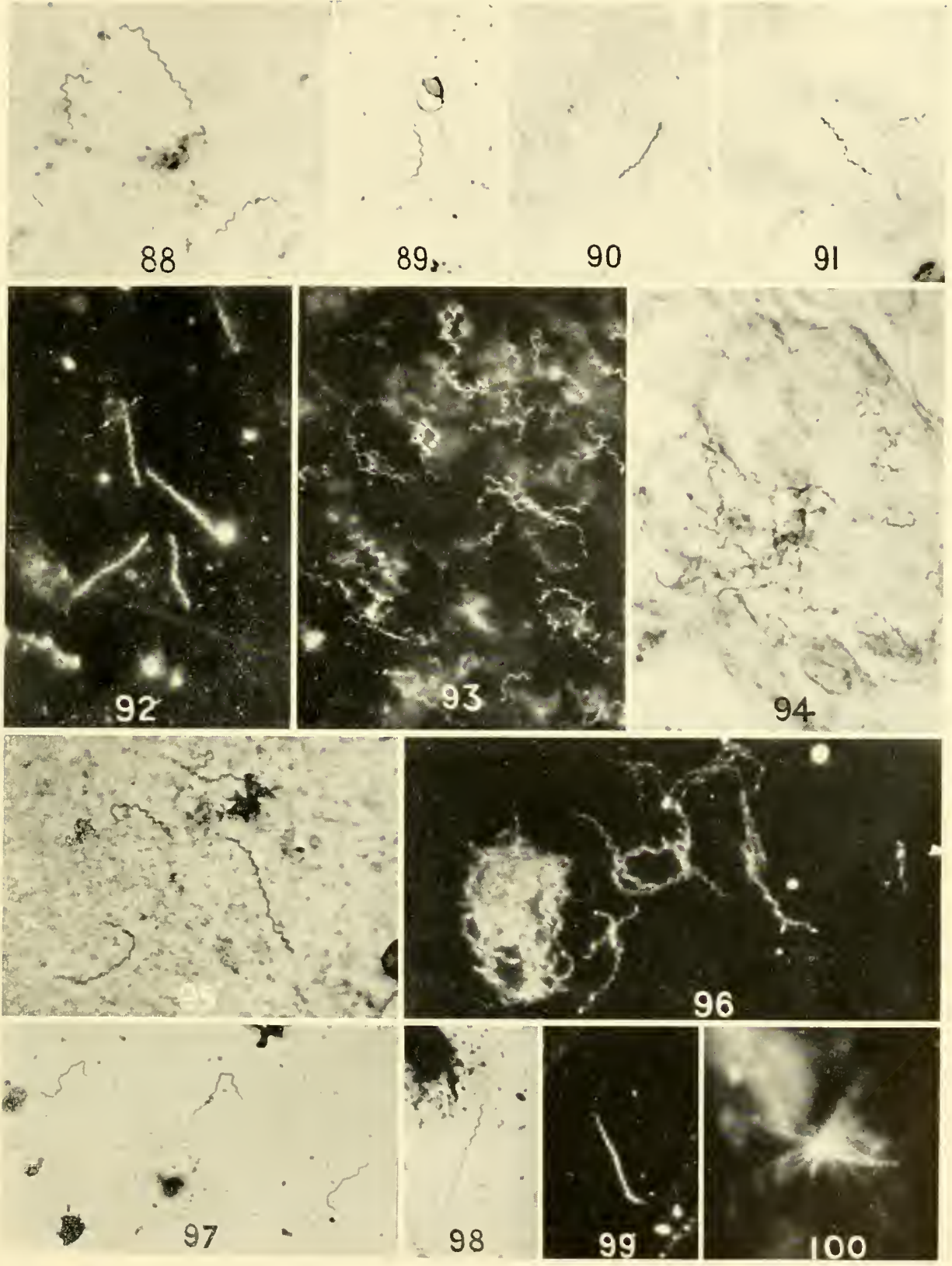



PLATE $\mathrm{X}$

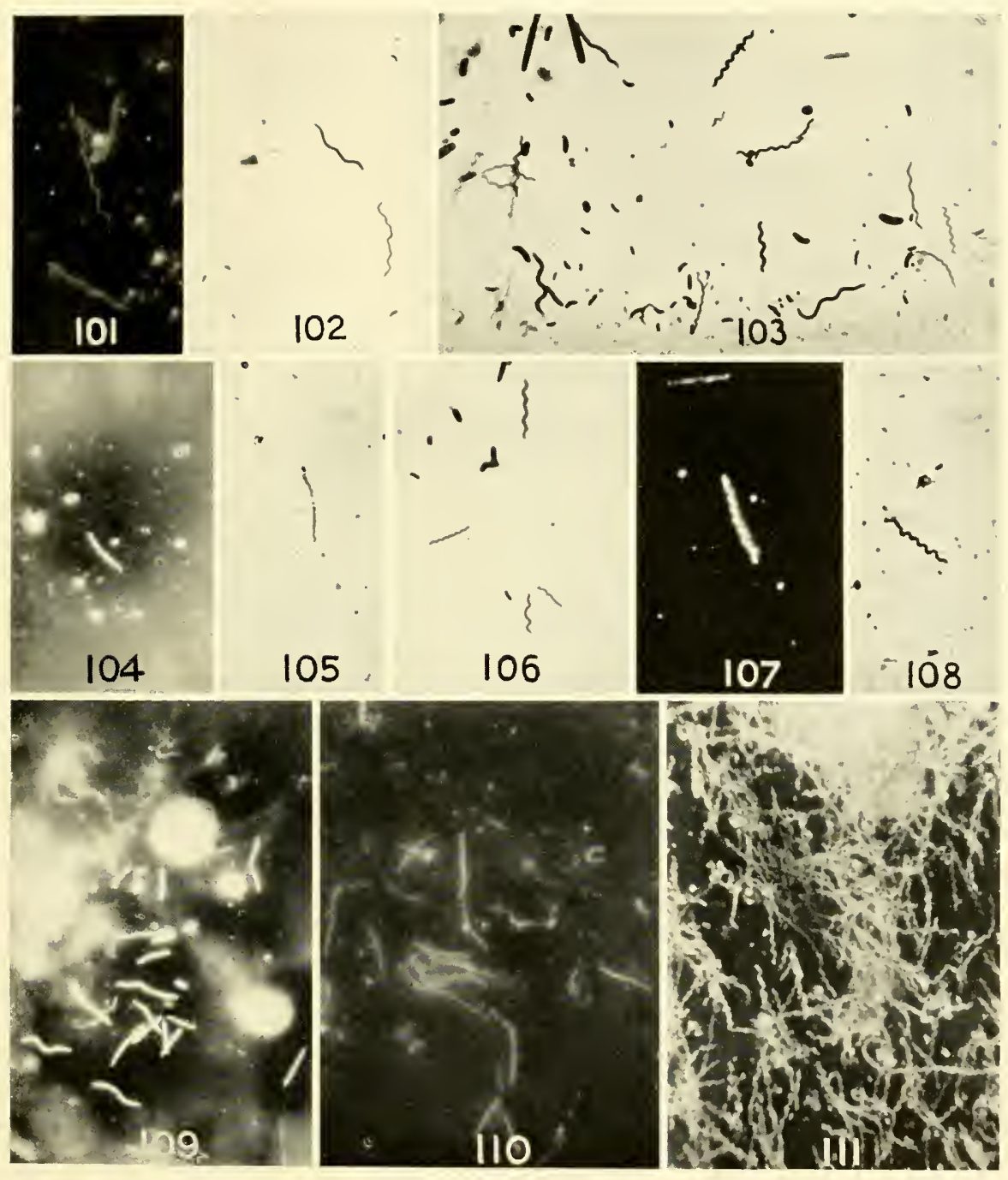



PLATE YI
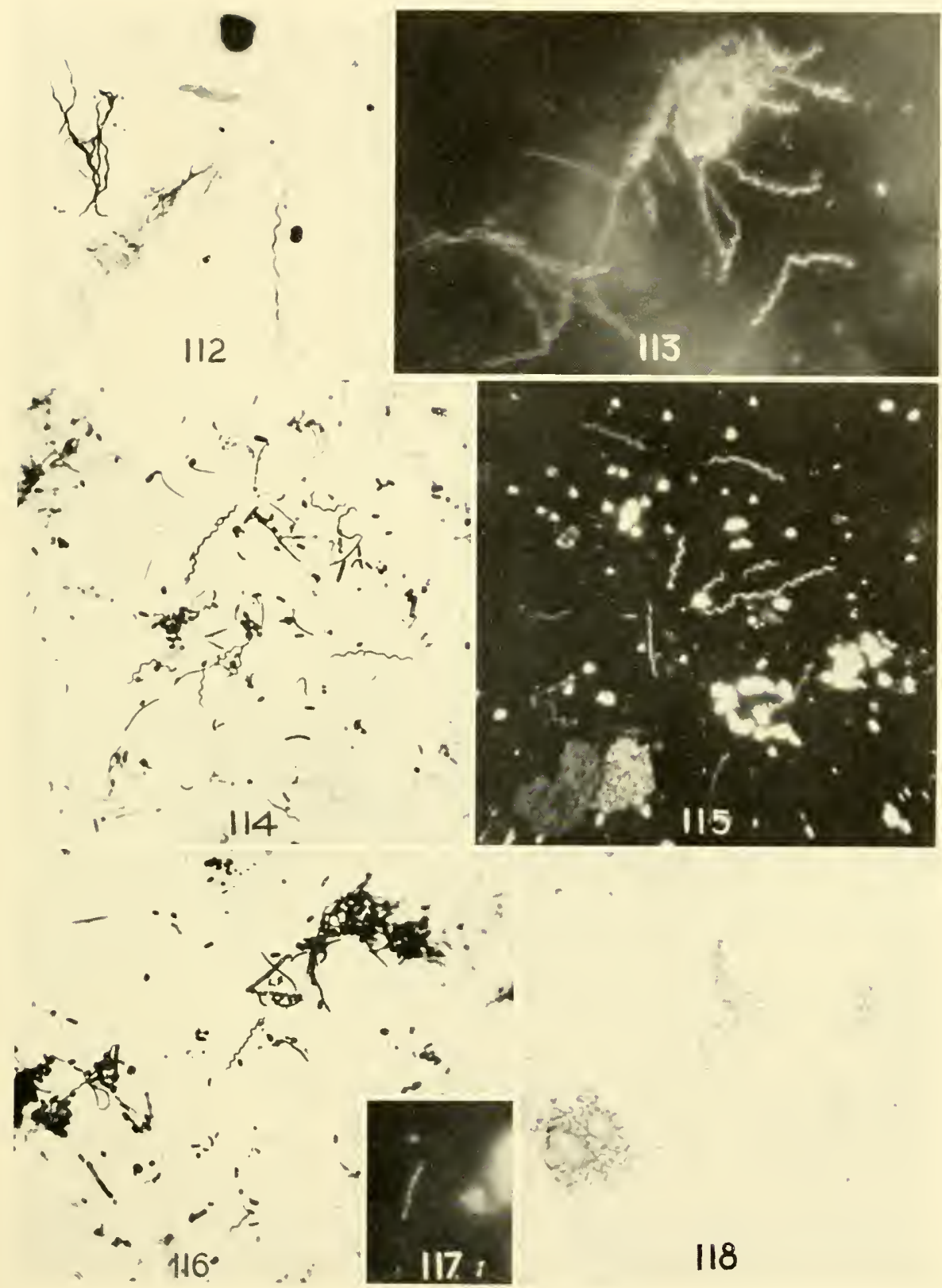

118 

PLATE XII

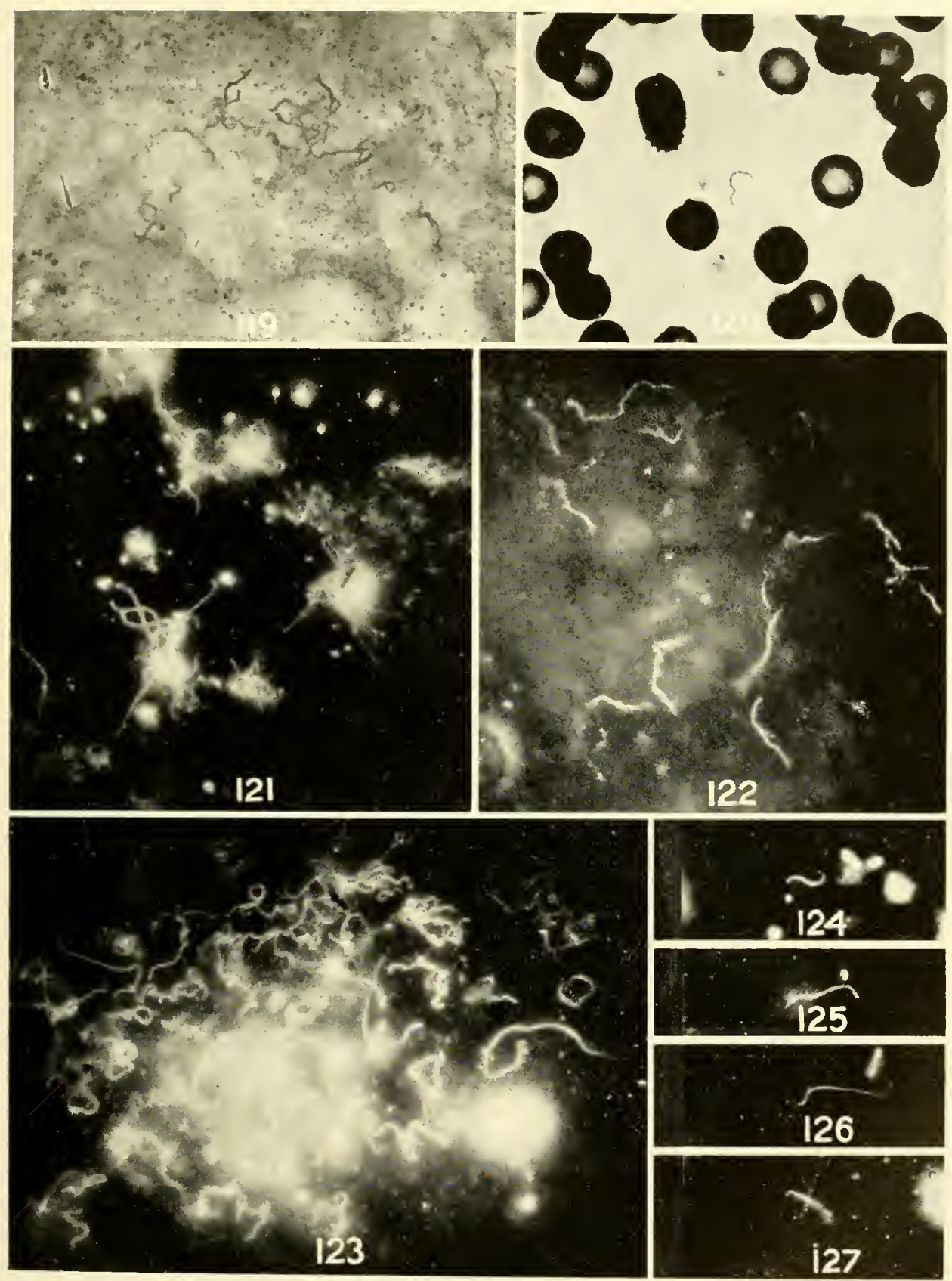



PLATE XIII

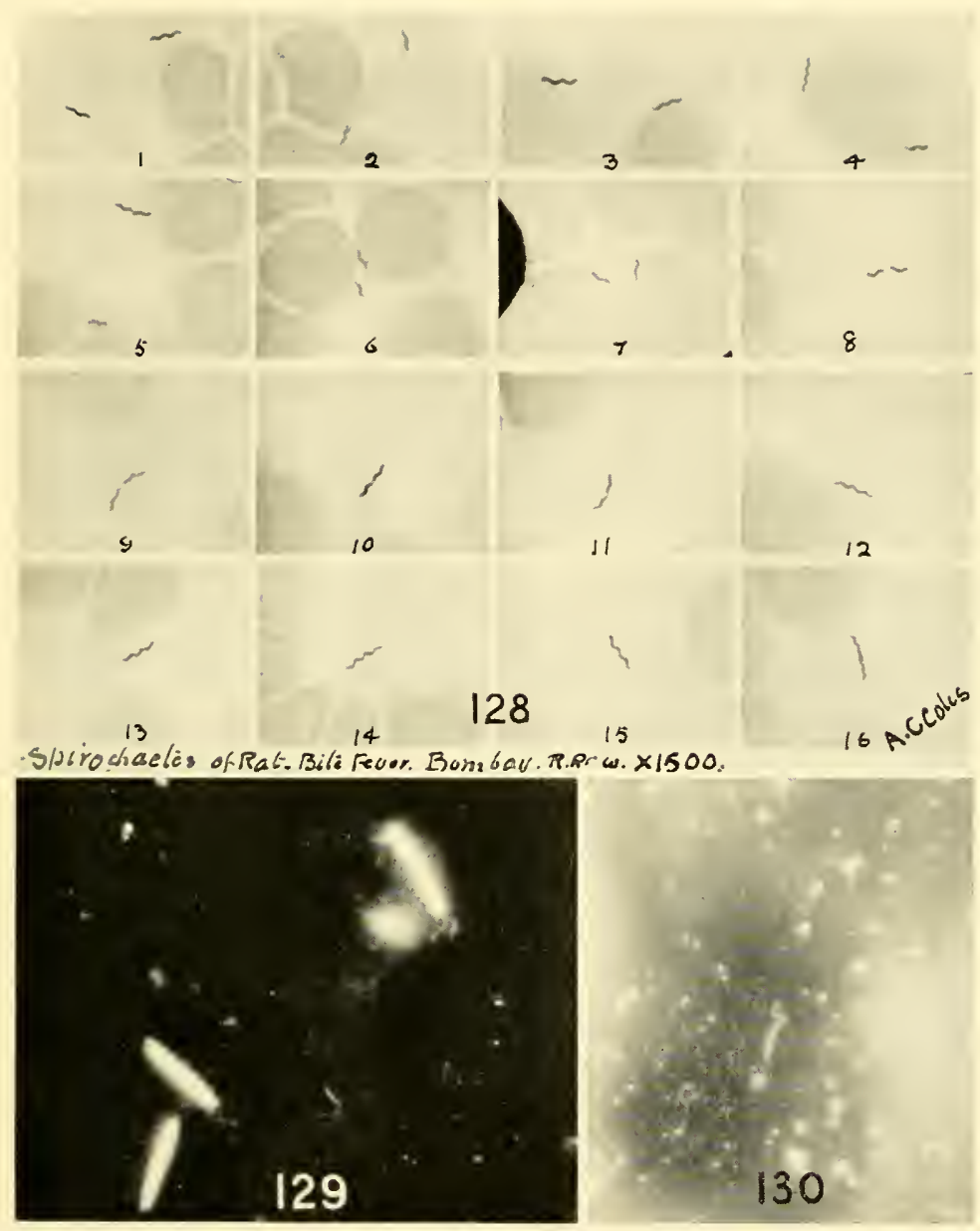



Fig. I21.-Leptospira interrogans, culture, Guayaquil Strain I

Fig. 1 22.-Lcptospira hebdomadis, culture presented by Dr. Inada

FIG. 1 23.-Leptospira icterohaemorrhagiae, cultivated from rats caught in New York City

FIG. I 24.-The very small type of water leptospira

FIG. I 25.-Water leptospira of medium size, resembling Leptospira ictcrohacmorrhagiae

FIG. I 26.-The largest of the varieties of leptospiras in water

Fig. 127.-Leptospira boris, a very small leptospira found in the stomach of the ox

\section{Plate Xili}

Fig. I2S.-Spirella morsusmuris of rat-bite fever. Photographs (Row's strain, Ind.J. Med. Res. 5, 386. 1917) presented by Dr. A. C. Coles, of Bournemouth, England. Giemsa's stain. $X_{1,500}$.

FIG. I 29.-Spirella canis, found in the dog's stomach; darkfield view. $\times_{1,000}$

FIG. I30.-Spirella muris, from the peritoneal cavity of the white mouse; darkfield view. $X_{1,000}$

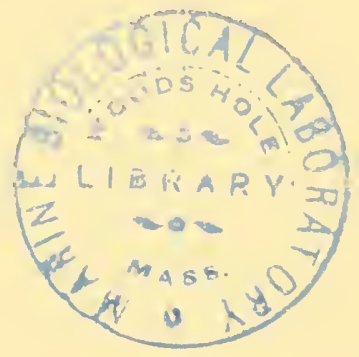




\title{
CHAPTER XXXVII
}

\section{CURRENT PROBLEMS ON YEASTS}

\author{
F. W. TANNER \\ University of Illinois \\ MORPHOLOGY AND ORIGIN OF YEASTS
}

This subject has interested investigators since early times. The earlier literature has been reviewed by Guilliermond and others who have prepared treatises on these micro-organisms. The suggestion that yeasts represent a state of development of higher molds seems to have been first made by Kützing in 1837 . While the idea, according to Fuchs, ${ }^{\mathrm{r}}$ has been widely accepted, good experimental proof has been lacking. He claims to have supplied it by having developed from Aspergillus oryzae an organism having all of the characteristics of true yeasts, i.e., the ability to form ascospores and ferment saccharine materials. His illustrations are quite suggestive.

Fuchs inoculated Freudenreich flasks filled with sterilized hopped wort with conidia of Aspergillus oryzae. Those flasks which were closed with cotton plugs showed normal growth of the yeast. However, those flasks which were closed with a fermentation valve gave normal growth at first but not after the air had been exhausted. When these flasks were shaken the surface growth fell to the bottom of the liquid. In three weeks the contents of these flasks began to ferment with the appearance of a sediment at the bottom. Microscopic examination, after five weeks, showed the presence of budding cells. Fuchs stated that there was little doubt that these budding cells came from the conidia because in some cases the budding cells were still attached to the latter. When these cells were transferred to Pasteur flasks and cultivated as yeasts they retained their yeast characteristics. After a longer time (seven months) hat-shaped ascospores characteristic of the genus IVillia were seen. While the anaerobic conditions and the presence of sugar were important in this transformation, other factors such as composition of the wort were also important. Fuchs has been unable to transform the yeast cells back to the mold type. He suggested that this would be more difficult. Viala and Pacottet, ${ }^{2}$ however, reported the development of Gloeosporium ampelophagum from a few yeast cells.

Some will say immediately that such observations are based upon contaminated cultures. Such an argument, while easy to propose, has been responsible for serious inhibition in the study of the development and forms of microscopic organisms, and should not discourage analysis of Fuch's data. Another attempt to show the close relationship of molds and yeasts was that of Kluyver and Niel, ${ }^{3}$ who reported a new genus Sporobolomyces with three species. After these fungi had multiplied by budding in the usual manner, the cells put out organs much like sterigmata at the tip of

\footnotetext{
I Fuchs, J.: Centralbl.f. Bakteriol., 66, Part II, 490. 1926.

${ }^{2}$ Viala, P., and Pacottet, P.: Compl. rend. Acad. sci., 142, 458-61. 1906.

${ }^{3}$ Kluyver, A. J., and Niel, C. B., van: Cenlralbl. f. Bukteriol., 63, P'art II, I-20. 1924-25.
} 
which conidia were formed. Thus, Sporobolomyces were believed to be Basidiomycetcs, an opinion not concurred in by Lohwag $^{\mathrm{T}}$ and Guilliermond. ${ }^{2}$

Lewis ${ }^{3}$ has reported a filterable yeast under the provisional name of Schizosaccharomyces filtrans. 'This is the first report that filterable stages may occur among the yeasts. Yeasts are many times larger than bacteria. Filterability of yeasts is more surprising than of such a small organism as the Dialister pneumosintes of Olitsky and Gates. In the discussion of his results Lewis considered various explanations for his observations. The fungus reproduces by fission and if placed with the yeasts would be a Schizosaccharomyces. Lewis seems somewhat undecided about the identity of his organism. This is quite proper both from the standpoint of the characteristic which he has presented as well as from the characteristics which have been established for Schizosaccharomyces. To one who has followed some of the more recent contributions to the morphology of bacteria, the evidence presented by Lewis is not convincing. In the first place, the size of Lewis' organism is not as great as that of the average yeast cell, nor are his illustrations definitely demonstrative. The forms portrayed are not at all unlike the transitional forms which have been reported by many of those engaged in the study of life cycles, cytomorphosis, etc., of the Eubacteria.

\section{ACCESSORY SUBSTANCES ("BIOS")}

The question of whether or not yeasts need accessory substances still holds the interest of a number of investigators. The "bios" question was reviewed in 1925 by Tanner. ${ }^{4}$ It was then suggested that the term "vitamin" be not used for the yeast growth-accelerating substance but that discussion of accessory substances for the yeasts center about the term "bios" proposed by Wildiers in I90I. This has been done because the term "vitamin D" is commonly used for the antirachitic vitamin of MacCollum and not for the yeast growth-accelerating substance, if one exists, as was suggested by Funk. ${ }^{5}$ In I925 Funk proposed a new nomenclature for accessory substances, using the term "vitamin D" for the yeast growth-promoting substance. MacDonald ${ }^{6}$ argued that the term "bios" be retained for various reasons: It was proposed ten years before vitamins were recognized; it has not been identified chemically; it is questionable whether bios is a vitamin; and, finally, bios now has a well-established literature of its own.

One of the few announcements of the isolation of a pure bios is that by Eddy, Kerr, and Williams ${ }^{7}$ in 1924 . Their crystalline substance was said to have properties of the bios of Wildiers.

In minute doses ( $0.005 \mathrm{mg}$. per cc.) it stimulated the multiplication of yeast. However, Eddy and his colleagues found a variation of this stimulating ability depending on the species used. It had less effect on top yeasts than on bottom yeasts. The melting point of

${ }^{x}$ Lohwag, H.: Ann. mycol., 24, 194-201. 1926.

${ }^{2}$ Guilliermond, A.: Compt. rend. Acad. sci., r84, 6r7. 1927.

3 Lewis, P. A.: J. Exper. Med., 45, 277-90. 1927.

${ }_{4}$ Tanner, F. IT.: Chem. Rev., 1, 397-412. 1925.

5 Funk, C.: Science, 62, I57-58. 1925.

${ }^{6}$ MacDonald, M. B.: ibid., 63, I87. 1926.

${ }^{7}$ Eddy, IV. H., Kerr, R. W., and Williams, R. R.: J. Am. Chem. Soc., 46, 28,6-55. 1924. 
this substance was $223^{\circ} \mathrm{C}$. Experimental data showed that this crystalline bios lacked antineuritic power and was probably different from vitamin B, but they were not decided whether it was entirely without effect on mammalian growth.

Two years later Kerr, Eddy, and Williams ${ }^{\mathrm{I}}$ published confirmation of their earlier report. They were more concerned in this report, however, in showing that the accessory properties of their "Bios 223" were inherent in the crystals and not due to impurities which adhered to the crystals. They experienced complete inactivation of Bios 223 by forming the crystalline benzene sulphonamide and a recovery of the activity by hydrolyzing this inactive crystalline product. This seemed to convince these authors that the activity of Bios 223 was inherent in the crystals. From the nature of the addition compound it was suggested that the $-\mathrm{NH}_{2}$ group was concerned with the exercise of the accelerating function since the activity was lost when this group was replaced by benzene-sulphon chloride.

The work of Lucas, Miller, and others at the University of Toronto has also been continued by Eastcott, Whiteman, and Sims. Eastcott ${ }^{2}$ hypothecated the existence of inactive "biogens" from which the active bios was formed. The biogens were not isolated. She further postulated the existence of biogenases to explain the irregular behavior of the bioses observed in the production of yeast under controlled experimental conditions. Sims ${ }^{3}$ studied the effect of certain chemical treatments of the bios on its activity. Whiteman ${ }^{4}$ made the interesting statement that a limited development of yeast took place without bios and even without salts or sugar. She assumed that a certain quantity of bios existed in the yeast cell itself.

Zajdel and Funk, ${ }^{5}$ using six species of yeasts propagated from single cells, reiterated the need for vitamin D (bios). They found that reproduction decreased accordingly as the cane sugar was purified, and ceased when the cane sugar was finally purified. Absence of bios resulted in a diminution of granules in the cells. One species, a bakers' yeast, however, grew in the absence of bios, giving always just a little less growth than the culture propagated in the presence of bios. In order to explain this anomaly, Zajdel and Funk suggested that the culture was contaminated or that a wild yeast had been isolated in place of the bakers' yeast. Realizing these possibilitics, why did they not find out the true nature of the culture? Why did they not determine whether their cultures were contaminated? It has been noticed by others that different species of yeasts react differently toward bios. However, they concluded that wild yeasts can synthesize bios while cultivated species cannot. What are the criteria with which to decide whether a strain is a wild or cultivated yeast? Might there not be intermediate strains which would react irregularly to bios if it has any relation to multiplication? Zajdel and Funk's statement is either an explanation of the present chaotic condition of the bios question or a convenient refuge for anomalous data.

Tanner, Devereux, and Higgins ${ }^{6}$ called attention to a number of points which seem to have been overlooked by certain other workers. They stated that many different definitions of the term "growth" have been used by those working in this

${ }^{\mathrm{r}}$ Kerr, R. W., Eddy, W. H., and Williams, R. R.: Proc. Soc. Exper. Biol. \& Med., 23, 416-19. I926.

${ }^{2}$ Eastcott, Edna V.: Tr. Roy. Soc. Canada, Sec. III, 19, 21-24. 1925.

${ }^{3}$ Sims, H. des B.: ibid., 18, I16. 1924. +Whiteman, D.: ibid., 19, 24-25. 1925.

${ }^{5}$ Zajdel, R., and Funk, C.: Compt. rend. Soc. de biol., 92, 1527-28. 1925.

${ }^{6}$ Tanner, F. W., Devereux, E. D., and Higgins, F. AI.: J. Bact., I1, 45-64. 1926. 
field. Different species of budkling fungi have been used. Some of the experiments have been contaminated with bacteria since the inoculum was Fleischmann's yeast. It was also shown that a distinction should be made between growth and multiplication. Eddy and his colleagues reported a variation in response to their Bios 223 between a top and bottom yeast, as did Zadjel and Funk.

Tanner and his colleagues also stated that some yeasts will not grow in certain media because the merlium does not supply the necessary food constituents. The addition of a supposedly rich bios material may supply these necessary constituents and the stimulation in growth which might result from the addition of small amounts of chemically known substances might be attributed to a hypothetical substance. When a yeast cell 5 micra in diameter contains only $0.000,000,009,8$ I $7,5 \mathrm{mg}$. of solid matter, it is easy to recognize that minute traces of organic or inorganic matter might be sufficient to initiate multiplication. After multiplication has started, other factors might enter to cause it to continue.

Werkman ${ }^{1}$ reported that fourteen strains of Saccharomyces ccrevisiac grew and reproduced continuously. The important factor in these experiments was that instead of cane sugar which has frequently been pointed out as the bios-contributing factor, synthetic methose was used. Werkman stated that bios was not necessary for yeast growth. This is a significant report since it is one of the few attempts to utilize synthetic carbon compound in place of sucrose. It refutes decisively the reports that growth in mineral salt-sugar media is made possible by bios in the sugar. Other contributions to the bios question were also made by Susuki and Taira. ${ }^{2}$

\section{CHEMISTRY OF ALCOIOLIC FERMENTATION}

The history of the theories of alcoholic fermentation is well reviewed by Harden, ${ }^{3}$ Schoen, ${ }^{4}$ and Nord. ${ }^{5}$

Neuberg' observed that salts of pyruvic acid $\left(\mathrm{CH}_{3} \mathrm{COCOOH}\right)$ are fermented by yeast to acetaldehyde and carbon dioxide. This change was supposed to be due to an enzyme, "carboxylase," which could remove the C $-\mathrm{O}$ portion of the carboxyl group of pyruvic acid, thus forming acetaldehyde. Carboxylase was believed to be associated with zymase. Consequently, Neuberg was led to believe that pyruvic acid and acetaldehyde were intermediate products in alcoholic fermentation. In order to show this, Neuberg suggested the following equations:

$$
\begin{array}{ll}
\text { I. } \mathrm{C}_{6} \mathrm{H}_{12} \mathrm{O}_{6}-{ }_{2} \mathrm{H}_{2} \mathrm{O}= & { }_{2} \mathrm{CH}_{3}-\mathrm{CO}-\mathrm{COH} \\
\text { Glucose } & \text { Methylglyoxal }
\end{array}
$$

$$
\begin{aligned}
& \text { 2. } \left.\mathrm{CH}_{3}-\mathrm{C}(\mathrm{OH})_{2}-\mathrm{COH}+\mathrm{H}_{2}\right\}=\left\{\mathrm{CH}_{2} \mathrm{OH}-\mathrm{CHOH}-\mathrm{CH}_{2} \mathrm{OH}\right. \text { Glycerol } \\
& \mathrm{CH}_{3}-\mathrm{C}(\mathrm{OH})_{2}-\mathrm{COHI} O \mathrm{CH}_{3} \mathrm{C}(\mathrm{OH})_{2}-\mathrm{COOH} \text { Pyruvic acid (hydrated) }
\end{aligned}
$$

A hudiated methy'glyoxal

I Merkman, C. H.: Science, 62, I15. 1925.

-Susuki, B., and Taira, T.: I. Chem. Soc., Japan, 45, 299-311. 1925.

${ }^{3}$ Harden, 1.: Alioholic Fermenlation. London: Longmans, Green \& Co., I923.

4 Schoen, M.: Le problème des fermentations. Paris: Masson et Cie., 1026.

5 Nord, F. F.: Chem. Rev., 3, 41-79. 1926-27.

${ }^{6}$ Neuberg, C.: Ber., 55, 3624-38. 1922. 
3. $\mathrm{CH}_{3}-\mathrm{CO}-\mathrm{COOH} \rightarrow \mathrm{CO}_{2}+\mathrm{CH}_{3}-\mathrm{COH}$ Acetaldehyde

4. $\left.\begin{array}{ll}\mathrm{CH}_{3}-\mathrm{CO}-\mathrm{COH} & \mathrm{O} \\ \mathrm{CH}_{3}-\mathrm{COH} & +\underset{\mathrm{H}_{2}}{\mid}\end{array}\right\}= \begin{cases}\mathrm{CH}_{3}-\mathrm{CO}-\mathrm{COOH} \text { Pyruvic acid } \\ \mathrm{CH}_{3}-\mathrm{CH}_{2} \mathrm{OH} \quad \text { Ethyl alcohol }\end{cases}$

Some of these reactions have since been verified in the laboratory. The others suggest future experiments. According to this theory, methylglyoxal, the first compound with three carbon atoms, is formed from the sugar. It may be formed by the removal of two molecules of carbon dioxide from the hexose molecule. This requires the intermediate stage of methylglyoxal-aldol. The other equations rest on Cannizzaro $^{r}$ rearrangements on methylglyoxal.

The evidence for the occurrence of the third reaction is afforded by results of fermentations carried out in the presence of alkali bisulphites. The acetaldehyde as it is formed is "fixed" by the sulphite and prevented from undergoing further change. The bisulphite-aldehyde complex then accumulates in large quantities. In comparison with alcohol the aldehyde is an oxidation product, the formation of which must be accompanied by a reduction product. Glycerol is such a product, and it accumulates in amounts equivalent to the amount of aldehyde fixed by the bisulphite. This gives what Neuberg called the "second form of fermentation," which may be expressed:

$$
\begin{aligned}
& \mathrm{C}_{6} \mathrm{H}_{12} \mathrm{O}_{6} \rightarrow \mathrm{CH}_{3} \mathrm{COH}+\mathrm{CH}_{2} \mathrm{OH}-\mathrm{CHOH}-\mathrm{CH}_{2} \mathrm{OH}+\mathrm{CO}_{2} \\
& \text { Glucose Acetal- Glycerol Carbon } \\
& \text { dehyde dioxide }
\end{aligned}
$$

When fermentation is carried out in the presence of alkaline salts (other than bisulphites), there is no fixation of acetaldehyde. It was said to undergo a dismutation into equimolecular amounts of ethyl alcohol and acetic acid by aldehydemutase. Then, for each molecule of aldehyde, there would be produced a molecule of the reduction product, glycerol. Neuberg called this the "third form of fermentation." It was expressed as follows:

$$
\begin{aligned}
& { }_{2} \mathrm{C}_{6} \mathrm{H}_{12} \mathrm{O}_{6}+\mathrm{H}_{2} \mathrm{O} \rightarrow \mathrm{CH}_{3} \mathrm{COOH}+\mathrm{C}_{2} \mathrm{H}_{5} \mathrm{OH}+{ }_{2} \mathrm{C}_{3} \mathrm{H}_{8} \mathrm{O}_{3}+{ }_{2} \mathrm{CO}_{2} \\
& \text { Glucose Water Acetic Ethyl Glycerol Carbon } \\
& \text { acid alcohol dioxide }
\end{aligned}
$$

Neuberg's theory has not been accepted by all investigators. Kluyver and Donker, ${ }^{2}$ for instance, have based an explanation of the processes of fermentation on Wieland's dehydrogenation theory. The protoplasm of the yeast plays the rôle of the hydrogen transporting catalyst. The affinity for hydrogen varies with the protoplasm. Following is the scheme which they proposed for the decomposition of hexoses:

' $A$ Cannizzaro rearrangement is a reaction between two aldehyle molecules in which one is reduced and the other oxidized.

2Kluyver, A. J., and Donker, II. J. L.: I'erslag. Akud. IIetenschappen Imsterdam, 33, 805-9I4. $192+$. 
I. Preparatory reaction:

$\mathrm{C}_{6} \mathrm{H}_{12} \mathrm{O}_{6} \rightarrow{ }_{2} \mathrm{C}_{3} \mathrm{H}_{6} \mathrm{O}_{3}$ (An intermediate hypothetical body)

II. Reactions of the intermediate body:

a) $\mathrm{C}_{3} \mathrm{H}_{6} \mathrm{O}_{3} \rightarrow \mathrm{CH}_{3} \mathrm{CHOH}-\mathrm{COOH}$ (Lactic acid)

b) $\mathrm{C}_{3} \mathrm{H}_{6} \mathrm{O}_{3} \rightarrow \mathrm{HCOOH}+\mathrm{CH}_{3} \mathrm{CHO}$

Formic Acetaldehyde

acid

c) $\mathrm{C}_{3} \mathrm{II}_{6} \mathrm{O}_{3}+$ I'rotoplasm $\rightarrow$ Hydrogenated $+\mathrm{CH}_{3}-\mathrm{CO}$ COOH

protoplasm Pyruvic acid

$\mathrm{CH}_{3}-\mathrm{CO}-\mathrm{COOH} \rightarrow \mathrm{CH}_{3} \mathrm{CHO}+\mathrm{CO}_{2}$

III. Dehydrogenation reactions:

a) $\mathrm{HCOOH}+$ Protoplasm $\rightarrow \mathrm{Hyd}$ drogenated $+\mathrm{CO}_{2}$ protoplasm

b) $\mathrm{CH}_{3} \mathrm{CHO}+\mathrm{H}_{2} \mathrm{O}+$ Protoplasm $\rightarrow \mathrm{Hydrogenated}+\mathrm{CH}_{3} \mathrm{COOHI}$ protoplasm Acetic acid

II. Condensation reactions:

a) ${ }_{2} \mathrm{CH}_{3} \mathrm{CHO} \rightarrow \mathrm{CH}_{3} \mathrm{CO}-\mathrm{CHOH}-\mathrm{CH}_{3}$ Acetyl-methyl carbinol

b) ${ }_{2} \mathrm{CH}_{3} \mathrm{CHO} \rightarrow \mathrm{CH}_{3} \mathrm{CHOHCH}_{2} \mathrm{CHO} \rightarrow \mathrm{CH}_{3} \mathrm{CH}_{2} \mathrm{CH}_{2} \mathrm{COOH}$

Aldol Butyric acid

c) ${ }_{2} \mathrm{CH}_{3} \mathrm{COOH}-\mathrm{H}_{2} \mathrm{O} \rightarrow \mathrm{CH}_{3}-\mathrm{CO}-\mathrm{CH}_{2}-\mathrm{COOH} \rightarrow \mathrm{CO}_{2} \rightarrow \mathrm{CH}_{3}-\mathrm{CO}-\mathrm{CH}_{3}$ Aceto-acetic acid

Acetone

V. Regeneration reactions of protoplasm:

a) Hydrogenated protoplasm $\rightarrow$ Protoplasm $+\mathrm{H}_{2}$

b) Hydrogenated protoplasm + Receptor $\rightarrow$ Protoplasm + Hydrogen receptor

Examples of Reaction V (b)

I. Hydrogenated $+\mathrm{C}_{3} \mathrm{H}_{6} \mathrm{O}_{3} \rightarrow$ Protoplasm $+\mathrm{CH}_{2} \mathrm{OH}-\mathrm{CHOH}-\mathrm{CH}_{2} \mathrm{OH}$ protoplasm Glycerol

2. Hydrogenated $+\mathrm{CH}_{3} \mathrm{CHOH}-\mathrm{COOH} \rightarrow$ Protoplasm $+\mathrm{CH}_{3} \mathrm{CH}_{2}-\mathrm{COOH}$ protoplasm Lactic acid Propionic acid

3. Hydrogenated $+\mathrm{CH}_{3} \mathrm{CHO} \rightarrow$ Protoplasm $+\mathrm{CH}_{3} \mathrm{CH}_{2} \mathrm{OH}$ protoplasm Ethyl alcohol

4. Hydrogenated $+\mathrm{CH}_{3} \mathrm{COCHOICH} \mathrm{CH}_{3} \rightarrow$ Protoplasm $+\mathrm{CH}_{3} \mathrm{CHOHCHOHCH}_{3}$ protoplasm Acetylmethỵl 2, 3-butylene glycol carbinol

5. Hydrogenated $+\mathrm{CH}_{3} \mathrm{CH}_{2} \mathrm{CH}_{2} \mathrm{COOH} \rightarrow$ P'rotoplasm $+\mathrm{CH}_{3} \mathrm{CII}_{2} \mathrm{CH}_{2} \mathrm{ClI}_{2} \mathrm{OH}$ protoplasm Butyric acid

Butyl alcohol 
6. Hydrogenated $+\mathrm{CH}_{3}-\mathrm{CO}-\mathrm{CH}_{3} \rightarrow$ Protoplasm $+\mathrm{CH}_{3} \mathrm{CHOHCH}_{3}$ protoplasm Acetone Isopropyl alcohol

\section{Hydrogenated $+\mathrm{C}_{6} \mathrm{H}_{32} \mathrm{O}_{6} \rightarrow$ Protoplasm $+\mathrm{C}_{6} \mathrm{H}_{14} \mathrm{O}_{6}$}

protoplasm Levulose Mannite

Kluyver and Donker believed that the dehydrogenation theory accounts for the facts better than Neuberg's theory of Cannizzaro rearrangements. Neuberg cannot explain the formation of certain compounds found in fermentation mixtures such as isopropyl alcohol, 2,3-butylene glycol, mannitol, etc. It was also argued that the new theory suggests an explanation for assimilation and dissimilation. In this theory the protoplasm acts as a catalyzer like nickel and platinum in chemical reactions. In fermentation the protoplasm is charged with hydrogen.

\section{GLYCEROL FERMENTATION}

This product is of interest on account of the fact that during times of military emergency it may become scarce. During the World War the nations involved had to develop new sources of glycerol. In a former paragraph in which Neuberg's conception of the chemistry of alcoholic fermentation was shown, the formation of glycerol was explained. While Neuberg and Reinfurth were able to explain the formation of glycerol, Connstein and Lüdecke placed it on a practical basis. Neuberg and Reinfurth ${ }^{\mathrm{I}}$ demonstrated the function of the sodium sulphite.

Connstein and Lüdecke, ${ }^{2}$ seeking to find other sources of glycerol for use by the German army during the World War, found that if the yeast fermentation of sugar was carried out in an alkaline solution, the amount of glycerol could be greatly increased. The early work was done with a number of different alkaline salts. However, on account of the difficulty of preventing the entrance and growth of bacteria, sodium sulphite was added to the mixture. With this salt they secured still greater yields of glycerol. When sodium sulphite amounting to 40 per cent of the sugar present was used, the glycerol was equivalent to 23.I per cent of the sugar present; when the sulphite was increased to Ioo and 200 per cent of the sugar, the glycerol amounted to 30.1 and 36.7 per cent respectively. This method was used by the German military authorities for producing glycerol. Eventually, I, $, \infty, \infty, \infty$ kilos were being produced per month. Twenty-five per cent of the sugar appeared as glycerol; other by-products such as alcohol and acetaldehyde were also secured.

The United States Bureau of Internal Revenue also arrived at this method after learning through Dr. Alonzo 'Taylor that the Germans were making glycerol' by fermentation. Eoff et al. ${ }^{4}$ used Saccharomyces ellipsoideus with diluted black-strap molasses as the substrate. The most favorable concentration of sugar was $\mathbf{7} 7^{-20} \mathrm{gm}$. per $100 \mathrm{cc}$. Between 20 and 25 per cent of the sugar was fermented to glycerol.

r Neubers, C., and Reinfurth, E.: Biochem. Zlschlr., 89, 365. 101S; 92, 23t. I018.

2 Connstein, Wr; and Lüdecke, K.: Ber. d. deutsih. Chem. Gese'lsch.lft, 52, 1385-91. 19r9.

I Ling, A. C.: J. Soc. Chem. Ind., 38, 175-77. 1910.

+ Eoff, J. R., Linder, W. V., and Beyer, G. I.: I. Ind. Fing. Chem., I I, S+2-+5. 19 ro. 


\section{FORMATION OF FAT}

Much work has been done on the fats of the yeast cell, but until quite recently comparatively little has been done with the species which produce larger amounts. The early work is discussed in the several texts on yeasts. Paul Lindner was one of the first to show that fat could be made with yeasts. Among several fungi studied, Lindner isolated Endomyces vernalis, a fungus which, although not a true yeast, may be properly discussed in this chapter. The fungus ordinarily grows with a mycelial vegetation almost devoid of fat. When, however, the hyphae break up into segments which bud like yeasts, fat production begins. By cultivating it in thin layers on the surface of suitable media the fat content may increase to as high as 28 per cent in fifteen days. Fat formation by this fungus was also studied by Nadson and Konokotina, ${ }^{\mathrm{I}}$ members of a commission appointed in Russia to investigate the possibility of making fat with yeasts. Diluted molasses with added ammonium sulphate was found to be a good medium in which to propagate the fungus for fat formation. The fat produced by Endomyces vernalis is a yellowish liquid resembling olive oil in appearance and flavor. The chief constituent is triolein, but free fatty acids are also present.

Another fat-forming yeast from soil was announced by De Jong ${ }^{2}$ in I926. Each cell was found to contain a large globule later demonstrated to be fat. The species was named Torula lipofera. Fat formation was not noticed in the cell until after fourteen days' incubation.

A valuable series of contributions on this subject has come from Maclean ${ }^{3}$ and her colleagues. They reported small amounts of fat in normal yeast; increased fat in yeast which was old and degenerated. This fat was said to be in union with the cell matter and not extractable from it by alcohol or ether. In the second contribution' they showed that fat in the yeast cell was formed from carbohydrate. They shower also that the addition of alkali phosphates to a carbohydrate solution increased the amount of fat which was stored. If the proportion of sugar was varied in a medium containing a certain definite amount of phosphate, the amount taken up by the yeast cell varied with the sugar. Fisher suggested years ago that carbohydrate molecules condensed to form straight chains which by reduction and oxidation were changed into fatty acids. This hypothesis has not been generally accepted. In I 92 I, Lindner suggested that alcohol molecules might condense in the cell to form straight-chain compounds which by subsequent oxidation formed the straight-chain fats. Lindner noticed that when yeast was incubated in a solution containing ethyl alcohol more fat was deposited in the cell.

Maclean and Hoffert studied the availability for fat manufacture of a number of carbon compounds. They found that yeast could utilize a number of compounds in aerated media for building fat but could not utilize other closely related substances. They believed that the evidence obtained from a study of yeast was distinctly against

${ }^{1}$ Nadson, G. A., and Konokotina, A. G.: W'chnsch. Brau., 41, 249-51. 1924.

${ }^{2}$ De Jong, L. E. Den: Nederl. Tÿdschr. v. Hyg. Wicrob., I, I36. 1926.

${ }^{3}$ Maclean, I. S.: Biochem. J., I6, 370-79. 1922.

${ }^{4}$ Maclean, I. S., and Hoffert, C.: ibid., 17, 720-41. 1923; 18, 1273-78. 1924; 20, 343-57. 1926.

${ }^{5}$ Lindner, P.: Zischr. lech. Biol., 9, I00. I92I. 
the view that either acetaldehyde or pyruvic acid was an intermediate stage between the hexose molecule or the fatty acid. Maclean and Hoffert believed that the acetaldehyde was first condensed to hexose, and that this was either converted into stored carbohydrate or directly condensed to form the higher fatty acids without passing through a fatty aldehyde stage, probably by a direct linking of hexose molecules.

Lindner and Unger ${ }^{1,2}$ observed fat formation in many of the common yeasts when exposed to an atmosphere of ethyl alcohol.

Studies on the fat content of yeast have not been without practical application. A German patent (No.320560; April 4, 1920) describes a yeast which ordinarily contains 5 per cent of fat but which, when grown in sugar-mineral salt mixtures in the presence of air, increases its amount of fat to from 40 to 50 per cent.

Hachn $^{3}$ stated that ability to form fat was a vital process of the cell and that it disappeared when the cell was destroyed. This work was continued in collaboration with Kintoff. ${ }^{4}$ Acetaldehyde was suggested as an intermediate product in the conversion of carbohydrates into fats in living cells. The following scheme was suggested:

$$
\begin{aligned}
& \text { Dextrose } \rightarrow \text { Pyruvic } \rightarrow \text { Acetaldehyde } \rightarrow \text { Aldol } \rightarrow \text { Glycerol } \\
& \text { acid ester }
\end{aligned}
$$

Haehn and Kintoff were able to produce evidence that several of these intermediate products were formed.

\section{USE OF YEAST IN THERAPEUTICS}

Different kinds of yeast have been used for ages in the treatment of disease. We are told that the monks used yeast for the treatment of plague and that Hippocrates advised its use in leukorrhea. It has been stated that the administration of brewery yeast $^{5}$ gave beneficial results in cases of boils, anthrax, and skin infections. It was also used as a lotion or injection for malignant discharges. Present-day interest in the therapeutic use of yeast probably began with the publication of Hawk, Knowles, Rehfuss, and Clarke. ${ }^{6}$ They used Fleischmann's pressed yeast and found it to be a useful remedy in the treatment of furunculosis, acne vulgaris, acne rosacea, constipation, and certain other cutaneous and gastro-intestinal conditions. Only one of seventeen cases of furunculosis was not benefited by the yeast treatment; all of the cases (seventeen) of acne vulgaris were improved. All of the eight cases of acne rosacea were improved. Nine out of ten cases of constipation were cured or improved. It was also stated that the yeast treatment was followed by an improvement in the general physical condition of the patient quite unassociated with the improvement of the symptoms associated with the disease treated. Hawk and his colleagues stated that killed yeast was just as efficacious as a laxative as living yeast, an observation not confirmed by later students of the question (Murlin and Mattill). These experiments were, perhaps, not sufficiently controlled to justify the sweeping conclusions. The

\footnotetext{
${ }^{1}$ Lindner, P., and Unger, T.: ibid., No. г. 1919. Chem. Absts., 14, I354. 1920.

${ }^{2}$ Lindner, P.: Ztschr.f. ang. Chem., 35, I I0-14. 1922.

${ }^{3}$ Haehn, H.: Zischr. f. tech. Biol., 9, 217-24. г92т.

${ }^{4}$ Hatehn, 1I., and Kintoff, W.: Berichle, 56, 439-45. I923; Wchnsch. Brau., 42, 213. 1925.

sWchnschr. Brall., 17, 207-8, 1900.

${ }^{6}$ Ilawk, P. B., Knowles, E. C., Rehfuss, M. E., ant Clarke, J. A.: J.A.AI.A., 69, I 243-47. I9I7.
} 
tables, while appearing elucidative, leave something to be desired. In many of the cases the time of the yeast treatment is not given nor is the reader informed of the length of the period of the cure or whether the symptoms appeared again when the yeast treatment was stopped.

Murlin and Mattill ${ }^{\mathrm{T}}$ carried out an extensive study of the laxative action of yeast in which were used both human and animal subjects. They observed a laxative effect in most cases when three cakes a day were eaten. However, they could not confirm the statement of Hawk and his colleagues that killed yeast was as active as living yeast. Intestinal putrefaction was also somewhat diminished. It is interesting to note, also, that as soon as the yeast treatment was stopped, the subjects came back to normal. The subjects of these experiments were young men "some of whom suffered from constipation."

Heintz and Welker, ${ }^{2}$ in using yeast ingestion for certain pathologic conditions, were interested to learn the mechanism of the action of yeast. They used twenty-two young medical students and reported that the ingestion of three cakes of yeast daily caused a marked increase in the leukocyte count. Ten subjects were chosen for establishing a "base line" for comparison with the subjects who were given the yeast. Six of these showed a small variation from day to day, three of the remaining developed respiratory infections, and the remaining one showed a decided rise in the leukocyte count without any apparent reason. This last man was dropped from consideration. Were the authors certain, then, that none of the men to whom yeast was fed did not show the same increase, this increase being attributed to the yeast? Heintz and Welker stated that no infections were observed among the men on the yeast treatment. If the one control subject who was dropped out because his leukocyte count increased for no apparent reason had been left among the controls, of whom six were used, the conclusions appended to the paper would have been considerably modified. Wolf and Lewis ${ }^{3}$ found that eating yeast had no stimulating effect on the production of antibodies. Antibody production was even lower in the controls.

The next question that may be considered is what happens to the yeast in the intestines. This was studied by Rettger and his colleagues. ${ }^{+}$Bakers' yeast was found to undergo a rapid destruction in the alimentary tract. Less than I per cent of the cells appeared alive in the feces. When yeast-feeding stopped, both the dead and living cells disappeared from the alimentary tract. After three days relatively few, if any, living cells remained. No effect on the relative proportion of gram negative and gram positive bacteria was demonstrable, from which we might infer that there was little antagonistic action, if any, between the yeast and intestinal bacteria.

Davison $^{5}$ fed one-quarter to one-half of a yeast cake per day to nine infants for periods of eight to twenty-three days. Such a procedure was reported to be not only without benefit but definitely harmful in some cases. Abdominal distention occurred in some of these infants, and their stools became more frequent. The general tone of

\footnotetext{
${ }^{x}$ Murlin, J. R., and Mattill, H. A.: Am. J. Physiol., 64, 75. 1923.

${ }^{2}$ Heintz, E. L., and Welker, W. H.: Arch. Int. Med., 35, 500. 1925.

3 Wolf, E. P., and Lewis, J. H.: J. Infect. Dis., 25, 3I I-I4. I9I9.

${ }^{4}$ Rettger, L. F., Reddish, G. F., and McAlpine, J. G.: J. Bact., 9, 327. I924.

5 Davison, W. C.: Am. J. Dis. Child., 24, 339. 1922.
} 
Davison's paper is that the yeast treatment was without value and might be harmful. Hawk and his colleagues also stated that in some cases the laxative effect was so pronounced that it was necessary to reduce the dosage.

Very little bactericidal effect of yeast could be observed by Schugt. ${ }^{\mathrm{T}}$ This is in keeping with the results of Rettger and his colleagues who found practically no effect of yeast on intestinal bacteria. Fermenting yeast was said to be germicidal, but the amount usually used would make it of litt'e significance.

I Schugt, P.: Monatschr.f. Gejurtsh. u. Gynäk., 56, 144. I921. 


\title{
CHAPTER XXXVIII
}

\author{
THE ASPERGILLI
}

\section{A TYPICAL GROUP OF MOLDS}

\author{
CHARLES THOM
}

Microbiological Laboratory, Bureau of Chemistry, U.S. Department of Agriculture

The genus Aspergillus' is selected as a group of common molds which may be used to illustrate the place of these organisms in nature, their position in a scheme of plant classification, and a perennial interest as individual organisms. This natural group includes a great series of species and physiological varieties, many of which accompany man closely in all his activities. They are omnivorous, resistant to climatic heat and cold, drought and flood. As producers of a wide range of enzymic activities their growth becomes a help and a menace in many fields of human endeavor.

These molds are mycelial fungi; their bodies consist of branching filaments composed of cells placed end to end, growing mostly at their tips, and extending slowly or rapidly in the substratum. Some ramify deeply throughout great masses of material; others more closely dependent upon immediate access to the free oxygen of the air grow widely over the surface, but penetrate only the outer few millimeters of unbroken surfaces. Even these secrete enzymes which may diffuse widely and cause great changes in large quantities of material.

One spore, lodging favorably in any decomposable product, may germinate and produce a mass of vegetative mycelium which will transform a large volume of organic matter. This mycelium, under favorable conditions of heat and moisture, throws up from the surface within a few days erect branches known as "conidiophores" (stalks). The tips of these stalks swell to form little vesicles from which bud out large numbers of spore-producing cells known as "sterigmata," in some species simple and unbranched, in others again branching to produce a secondary cluster or verticil, and from each ultimate tip spores or conidia are cut off rapidly. These spores or conidia are at first cylindrical segments cut from the tip of a narrow tube. Each is quickly succeeded by a newer cell cut from the same tube. There is thus formed a chain of spores like a string of beads, actively lengthening at the point of origin. Each spore or conidium, growing quickly, swells and assumes the size, shape, and markings characteristic of its species. The whole mass at the tip of the stalk thus forms a head which may vary from globose if its elements are radially directed from the central vesicle, hemispherical when only half the area is covered, to columnar if only a cluster of sterigmata are borne upon its very tip and the chains adhere to form a solid mass. The form assumed is more or less definite for each race.

A selected series of references is appended to this paper. More extensive bibliographies are given in W'ehmer for work antedating $\mathbf{1 9 0}$, and by Thom and Church for the literature up to 1925 . 
CONIDIA OR SPORES AND THE CLASSIFICATION OF ASPERGILLI

Such heads thus produce enormous numbers of spores or conidia, varying according to the species from 2 to 10 micra (roughly $\mathrm{I} / \mathrm{I} 2,000 \mathrm{I} / 2,000 \mathrm{in}$.) in diameter. They are so light that they float readily in every breeze or breath. Some of them are so waterproofed that they will float upon the surface of water a considerable distance before they sink, absorb water and grow. Others are variously sticky, roughened, eroded, or chiseled to favor adherence to moving objects. Thus the widest diffusion by wind, water, and animals is provided for. The molds produc ng their spores in the form of heads as already described are grouped in to the hyphomycete genus Aspergillus (literally "rough head"). Some of them produce perithecia and ascospores as a sexual form of fruit, hence are properly transferred to the Ascomycetes and commonly allocated to the Perisporiales. The vast majority of these molds as encountered in nature are known only as producing heads of conidia or asexual spores, hence must be kept for practical convenience in the old Hyphomycete or "form genus" Aspergillus. In this generic group there is a large number of species, races, and strains; for more than three hundred names have been proposed for the materials studied by various authors during the past two hundred years. Thom and Church split this whole number into sixteen species aggregates which are fairly easily separated where pure cultures are studied with the aid of a compound microscope. This division is shown on page $5 \mathrm{Ir}$.

Within these species aggregates, the task of separation is much more difficult, but they name sixty-six species already described and probably identifiable upon morphological diagnoses.

The problem of identifying species among these molds is complicated by the changes induced by environment upon the structure and appearance of the mold colony. As they appear in nature, moldy masses usually contain more than one species and the individual species may be so far influenced by its associates as to render it scarcely recognizable by gross or even microscopic examination. Before these effects were understood, the students of fungi described a great many species, giving the colors and measurements found in particular collections upon more or less vaguely described substrata. Two tendencies are encountered in such discussions, the description of new species for every change in size of head or length of stalk or shade of color, and, on the other hand, lumping all forms with even a superficial resemblance into heterogeneous aggregates so complex that the names used ultimately came to be meaningless.

\section{BIOCHEMICAL CHARACTERISTICS}

With the development of improved methods of isolation and culture, Aspergilli have been found to grow readily upon a wide range of laboratory media. It has, therefore, been possible to isolate them and work out their life-history and reactions to selected media. In their metabolism they have proved able to utilize inorganic salts of all the usual elements required except carbon. Solutions made by the formulae proposed by Raulin, Cohn, Czapek, and many others have been used as a basis to test the availability of particular nutrients to individual species. The Aspergilli thus furnish favorable species for many forms of physiological and biochemical investigation. 
The first Aspergillus to become well known to botanists was found by the collectors upon partly dried and even mounted specimens in the herbarium. The large heads of gray-green spores were conspicuous wherever the botanist turned his hand

\section{ARTIFICIAL KEY TO GROUPS - PRIMARILY BASED ON COLOR}

A. Conidial heads green or yellow green ............ B

A. Conidial heads never green. ................. I

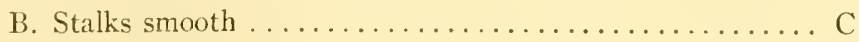

BB. Stalks pitted (often rough as seen with low magnification) $\mathrm{H}$

C. Vesicle cylindrical clavate, stalks coarse............. clavatus

CC. Vesicle flask-shaped or globose, not cylindrical clavate... D.

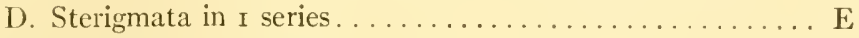

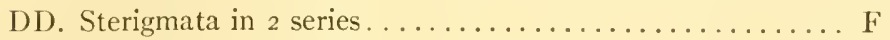

E. Conidia mostly elliptical and more than $4 \mu$ in long axis. Perithecia commonly found, yellow................. glaucus group

EE. Conidia mostly globose $4 \mu$ or less in long axis; conidial chains in narrow, solid columns................ fumigatus group

F. Conidial chains in columns................. A. nidulans

FF. Conidial chains in radiate heads ..............

G. Heads blue green......................... sydowi

GG. Heads glaucus, green, or yellow green to buff ......... versicolor

H. Stalks pitted (often appearing to be rough or asperulate with low magnifications) .................... Alavus-oryzae group

I. Conidial heads never green ............... J

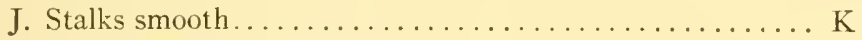

JJ. Stalks pitted (or apparently rough) ............ O

$\mathrm{K}$. Conidial heads avellaneous or in brown shades......... L

$\mathrm{KK}$. Conidial heads white (or slightly yellowed in age) ...... N

KKK. Conidial heads brown to black............... niger group

KKKK. Conidial heads orange to umber................ wentii

L. Conidial heads in columns................. M

LL. Conidial heads radiate, stalks colored ............. A. ustus

M. Stalks colorless or nearly so ................. Aerreus

MM. Stalks yellowed (especially in outer layer)........... Alavipes group

N. Stalks yellowed, conidial chains in columns........... flavipes group

NN. Stalks colorless; heads mostly racliate or globose......... A. candidus group

O. Stalks pitted or rough .................. P

P. Heads yellow to ochre, radiate .............. Q

Q. Heads in bright-yellow colors.................. sulphureus

QQ. Heads in ochraceous shades................... ochraceus group

PP. Heads orange to umber; conidia rough with tubercles and bars of color

A. tamarii and allies

lens. Hence they were called Aspergillus glaucus, while the yellow perithecia scattered over the same areas were long supposed to be a separate fungus which was called Eurotium herbariorum. Only in comparatively recent times have we come to know that there are many species and races with this general morphology; that they are able to maintain themselves more successfully in places of physiological drought than the other common and conspicuous molds. We thus find them as piled masses of red, 
yellow, and green mold on fodder and forage subjected to dampness even from moist or humid air, upon hay and grain which is only slightly above the critical water content, as yellow mold upon jams, jellies, and preserves, upon masses of soft maple sugar as distributed in lines of trade, upon salt fish, and on cured meats, as green mold on leather; in wetter substrata, members of this group, although commonly present, are overgrown by other and more rapidly growing forms. Certain $A$. glaucus strains are active inverters of sugar; they grow well in solutions of 50 per cent of sucrose in water, some of them continue their activity in sugar solutions well up toward 70 per cent, and at times their mycelium is found enmeshing the crystals of stored raw sugar and causing considerable deterioration.

As a genetic group these forms have adapted themselves to growth in highly concentrated media from which few other species can extract the water necessary for metabolism. Correspondingly, while able to develop in wetter situations, they are usually overgrown and contribute little to the decomposition as encountered.

The dark-brown to black Aspergilli (the aggregate species $A$. niger) are more conspicuous, grow under a wide range of environmental conditions, and produce notable changes in the substratum. Investigation of their activities goes back to Van Tieghem, who studied their relation to the fermentation of tannin solutions about I $860-65$, for even then they were used in the manufacture of gallic acid by the fermentation of crude tannin solutions. Wehmer in 1893 investigated the production of oxalic acid from sugar by these organisms. This fermentation, while entirely practicable, cannot compete commercially with other sources of oxalic acid. Following the same lines of study, Molliard, Butkewitch, and Currie investigated citric acid production by A. niger. Currie and Thom went further and showed that certain races or strains indistinguishable morphologically from other members of the series were specially active in producing oxalic acid and others more active in producing citric acid. Thus the way was opened for Currie's successful development of the production of citric acid upon a commercial scale with granulated sugar as its raw material. Citric acid from this source is now a large percentage of the total supply reaching the market. Other by-products of Aspergillus fermentations are being developed at the present time, among them gluconic acid, which has already been experimentally produced by several workers independently.

The diastatic power of the black Aspergilli has been utilized in producing alcoholic liquors, especially in Formosa. Biochemical workers testing for various enzymes find these organisms with enzymes capable of attacking many forms of organic matter. The enzymic picture is a recapitulation of the distribution of the species in nature. An organism limited in nature to a particular substratum or very special conditions shows a limited production of enzymes, whereas a species which grows upon many substrata must be capable of utilizing the wider range of products available in this varied group of substrata.

The members of the $A$. niger series are well adapted for factory exploitation. Their morphological characters and reactions are conspicuous. They grow readily under a widle range of conditions and so vigorously as to swamp minor contaminations. Although their range of biochemical activity is wide, methods of controlling that ac- 
tivity have proved commercially practicable in a sufficient number of cases to encourage the belief that much wider use of these organisms will eventually be made.

Members of the yellow-green series of species or races variously designated $A$. flavus, Link, or 1 . orysae (Ahlberg) Cohn, or the two names hyphenated as the 1 . flavus-oryzac series, are widely used in Japan, China, and the East Indies in fermentation. This utilization takes two forms. The starch of the rice grain is saccharified by the enzyme secreted by the mold, thus furnishing the sugar solution necessary for veast growth and alcohol production. Rice fermentation in this way is marle the first step in the manufacture of saké and related alcoholic liquors. Certain strains selected as favorable for this purpose are propagated upon a large scale and sold as inoculating material. Similarly, other strains were found capable of breaking down the proteins of the soy bean, making possible the production of soy sauce, miso, and many other products which consist primarily of bean proteins more or less completely broken down by the proteolytic ferments of the mold. Extensive investigations of these activities have been made in Japan by T. Takahashi and his associates at Tokio, by Hanzawa, and by Oshima at Sapporo. Oshima, and Osbima and Church, working at Washington, found certain strains to produce large quantities of proteolytic enzyme, and little amylolytic enzyme, others large quantities of amylolytic enzyme and little proteolytic enzyme. Between these extremes they found gradations to forms with both enzymes fairly equally balanced.

Takamine, working in America with other selected races of this group, developed "Taka diastase" as a commercial enzymic preparation. In addition, they and others have developed crude enzyme solutions widely useful in desizing operations in the textile industry. Under rigorous testing such enzyme solutions are found to contain not one but many enzymes which represent the range of metabolic activity of the mold species used in their manufacture.

There remain several species aggregates whose function in nature and whose possibilities of usefulness to man are scarcely known. All are factors in the disintegra$t^{*}$ on of organic matter. As grown in laboratory media, investigators have recorded the source of their cultures and some of the routine reactions obtained, although little systematic effort has been made to follow then to their natural environment and attribute to them specific effects.

The white Aspergilli (A. candidus Link and its allies) are cosmopolitan. Morphologically they are almost identical with the black Aspergilli, lacking only the production of the coloring substance aspergillin. These species are, however, less vigorous growers, somewhat smaller in mass as usually encountered, and appear late in the progress of decomposition after other organisms have ceased to be active. Their presence at this stage in the degeneration of organic matter is strikingly constant. Cereals in the later stages of rot, fodder much disintegrated, and stored manure constantly show fruiting patches of white Aspergilli. Comparative cultures of a few of these forms have indicated active enzyme production. One of them, 4 . okasakii, has been exploited commercially, but no systematic study of organisms representing the whole group has yet been made.

The A. versicolor series of species or races compete with A. candidus series in many 
of the later stages of decomposition. They are green to gray-green forms which produce brilliant yellow, orange, red, and purple-red colors in the substratum. Many races of this group have been reported from soil, from old cheese, from dried salt meats, and from miscellaneous cultures. The range of colors and structures encountered was reported by Thom and Church, but very little of their significance in nature has been determined.

Closely allied to the A. versicolor series, A. sydowi is also widely distributerl, producing delicate stalks, globose blue-green hearls, and usually brilliant orange-tored colors in the substratum. In flask experiments Kopeloff found it exceedingly active in inverting sucrose. Aside from this work very little is known of its activities. Another aggregate of green species, 1. nidulans Eidam, is represented by numerous strains constantly encountered in studies of the soil and readily cultivated. In this group certain strains produce perithecia and ascospores which lend themselves readily to culture. As normally encountered the perithecial walls, asci, and ascospores are purple red. They turn blue with the addition of alkali, then red again with acid. They thus furnish a favorable species for studying the relation of an organism to the hydrogen-ion concentration of the substratum.

Thom and Church described a number of perithecial strains differing somewhat in cultural characters, in the rate of ascospore production, and in the markings of the ascospores themselves. Other cultures have refused to produce perithecia as ordinarily handled in the laboratory. A. nidulans in this way furnishes a favorable species for the investigation of the conditions surrounding ascospore production. Colorless ascopores otherwise closely resembling those of $A$. nidulans are found in A. fischeri Wehmer, which in its conidial form appears to be a close relative of A. fumigatus.

The general morphology of head and conidium production found in A. nidulans is reproduced with differences in color in A.terreus Thom, a brown form common in American soils; in A. ustus Bainier, a gray to fuscous-brown form also constantly met in soil studies; and in A. flavipes Bainier, with its white heads and yellow stalks. Aside from the routine studies made by those who have described these forms, their place in nature is comparatively unknown.

Certain species are used for particular purposes. A. wentii, a brown form, is userl in making a bean preparation in Java. An enzyme preparation from 1. okazakii has been patented in Japan. Such enzyme preparations are used in the United States to clarify jellies and in the manufacture of pectin preparations used in jelly-making. Great possibilities of extension in the utilization of mold enzymes are indicated by the progress already made. Thus far very few species have been studied biochemically, and even these show possibilities as yet undeveloped.

\section{PATIOGENICITY OF ASPERGILLI}

Although the Aspergilli are primarily saprophytes whose function consists in the destruction of organic remains, especially carbohydrates, some of these species are found capable of parasitic habits. These infections of man and other animals have been reported from various countries and in many forms, thus indicating that they are widespread causes of pathological conditions.

The earliest studies of this kind reported various species in ulcerations of the ear. 
Members of the A.nigcr, A. flavus, A. fumigatus, A. nidulans, and A. glaucus groups have been reported from time to time. Most of these reports do not distinguish between organisms present and organisms active in producing disease. Enough studies have been made to show that the black Aspergilli are frequent causes of ulcerations, and that lung infections by A. fumigatus are very common. Destructive outbreaks of Aspergillosis' (A. fumigatus) among domestic fowls have been studied. Losses of birds in zoölogical gardens from this cause occur very commonly. This organism is occasionally reported from the air passages of cattle and even of man. While its paths of infection are uncertain, its frequency and destructive power are great enough to class A. fumigatus among the true pathogenic forms. Many discussions of mold infections in the medical literature lack definiteness in their identification and their use of names. There is great need for systematic and comparative study of these molds wherever encountered in connection with disease, and experimental work to determine their significance. Strains of 1 . fumigatus are found everywhere in soil, in forage, in cereal crops, but the infectivity of these various organisms is so little known that we have made only a beginning in weighing its significance as a cause of disease. Several new species of Aspergilli belonging near A. niger and A. versicolor (Sterigmatocystis) have recently been described from mycotic infections encountered in the Belgian territories of Africa (Mattlet). This work, like the work of Greco in Argentina or Dr. Latham in the mountains of North Carolina, emphasizes the probability that molds are present in many pulmonary diseases and in various ulcerations. Whether they are primary causes of disease or are more commonly secondary contributors to pathological changes already started remains to be proved.

Even in our fragmentary knowledge of a few species in this great group of molds we have already found organisms which can be harnessed to yield results of great value in industry, others whose destructive effects play a large part in the deterioration of our food supplies, and some which are a real menace to human health. In general it must be recognized that cosmopolitan species in any biological group are cosmopolitan because of their wide adaptability to changes in environment. This adaptability commonly manifests itself in accompanying man in his spread over the earth and participating with him in his affairs, for good or ill. Good strategy requires that we know and use them rather than suffer by their activities.

\section{BIBLIOGRAPHY}

Church, M. B.: Soy and Related Fermentations, U.S. Dept. Agr. Bull., I I52, I-27, 2 pls., 6 figs. 1923 .

Currie, J. N.: "The Citric Acid Fermentation of Aspergillus niger," J. Biol. Chem., 3 I, I 5-37, pls. 2, figs. 5. 1917.

De Bary, A.: "Entwickelung und Zusammenhang von Aspergillus glaucus und Eurotium," Bot. Ztg., 12, 425. I 854 .

Molliard, M.: "Production d'acide citrique par le S. nigre," compt. rend. de Acad. Sri (Paris), r68, 360-63. I919.

Oshima, Kokichi: "Studies on the Protease of the 1spergillus oryzue-flavus Group and It. Rôle in Shoyu Brewing," Am. Food J., I 7 (I), 30-31. I022.

Owen, W. L.: Summarizes his many years' stuly" on this sulject in "Mold Fungi in Sugar Inversion," Facts about Sugar, 16, 5 19-21, $546+8$. 1923. 
Sartory, A.: Champignons parasites de l'homme ct des animaux. In fascicles. 1920-23.

Takamine, J.: Process of Making Diastatic Enzyme, U.S. Pat. 525823 , Sept. I I, 189.t; other patents in the same diastatic scries, Nos. 525825, 525971, 562103, 826699, 975656, 991 560, 991 56I, I054626, I054324; for making alcoholic liquor, Nos. $525819,525^{82}$ I, and $525^{822}$; and later for the making of enzyme extracts, No. I52792, Oct. 25, 1920.

Thom, C., and Church, M. B.: The Aspergilli. Baltimore: Williams \& Wilkins Co., I926. Thom, C., and Currie, J. N.: "Aspergillus niger Group," J. Agr. Res., 7, I-15. 1916.

Welimer, C.: "Die Pilzgattung Aspergillus in morphologischer, physiologischer und systematischer Beziehung," Mcm. Soc. de phys. et d'hist. nat. de Genìve, 33, II, I-157. I899I 90 I.

Welimer, C.: "Verlust des Oxalsäure-Bildungsvermögens bei einem degenerierten Aspergillus niger," Centralbl.f. Baktcriol., 2 Abt., 49, 145-48. 1919.

Wilson, J. A., and Daub, G.: "Aspergillus niger, a Common Mold That Causes Black Spots on Leather," J. Am. Leather Chem. A., 20, 400-405, figs. 12. 1925. 


\title{
CHAPTER IXXIX
}

\author{
FILTERABLE VIRUSES \\ THONAS ML. RIVERS \\ Rockefeller Institute for Medical Research \\ New York City
}

For various reasons, many morbid conditions are now referred to as "filterable virus diseases." Certain of these conditions are claimed to be caused by bacteria, protozoa, spirochetes, or self-propagating inanimate substances. Inasmuch as the claims have not been generally accepted, one may consider that the etiological agents of the majority of these diseases are still unknown. Since there is a possibility that many of the agents are not ordinary bacteria, no imperative reason is apparent for discussing them in a book on bacteriology. Nevertheless, the contagious nature of many of the diseases makes it advisable for investigators interested in infection and immunity to be acquainted with certain important facts concerning them.

In Table I are listed most of the diseases which are included by different observers in the group under discussion. The etiological agents concerned in these diseases, or groups of them, have been given a variety of names, e.g., "filterable viruses," "invisible microbes," "ultra-microscopic viruses," "infra-microbes," "protista," "microplasms," "chlamydozoa," and "strongyloplasms." The results of a superficial examination are convincing that none of these names is applicable to all of the etiological agents. Names, however, facilitate the interchange of ideas between individuals. For practical purposes, then, the term "filterable viruses," chiefly because of its wide usage, is as satisfactory as any name suggested. Throughout this chapter the terms "filterable viruses" and "viruses" will be employed in a noncommittal way to designate active transmissible agents which are capable of producing pathological conditions in bacteria, plants, insects, fish, birds, and mammals, and which by general consent are more or less limited for the moment to the etiological agents of the diseases listed in Table I.

\section{FILTERABLE VIRUS DISEASES}

The arrangement of the diseases in Table I is for convenience of discussion and carries no taxonomic significance. In the first place, filterability of the etiological agents does not sharply delimit this group of diseases, as it is well known that the viruses share this characteristic with certain small bacteria and vibrios, and also with some spirochetes and protozoa. Furthermore, in regard to the etiological agents of some of the diseases within the group, e.g., chicken-pox and herpes zoster, no filtration experiments have been recorded. The viruses of still other diseases within the group.

r Parts of this chapter were taken from a paper read by the author before the Society of American Bacteriologists, Dec. 29, 1926, and later published in the Journal of Bacteriology. For a fuller discussion of the subject the reader is referred to the paper mentioned above and also to a book on filterable viruses, to be pullished soon by Williams \& Wilkins Co. 
e.g., typhus fever and vaccinia, are either not filterable or filterable with the greatest difficulty. In the past all attempts to classify these diseases have been unsuccessful, and there is every reason to believe that such attempts are still premature.

TABLE I

\section{Filterable Vipus Diseases}

\section{Bactersophage}

Mosaic diseases of plants (infectious chlorosis)

\section{Sacbrood}

$W_{1} 1$ of European nun moth Wilt of gypsy moth caterpillar Jaundice of silk worms

Epizoötic of gurned pios Hog cholera

Cattle plague (Rinderpest)

Pernicious anemid of horses

Vipus III infection of pabbits

Foot-and-mouth disedse

$$
\begin{aligned}
& \text { 1. Type A } \\
& 2 \text { Type } 0
\end{aligned}
$$

Vesiculap stomatitis of horses

Papavaccinia (No report on fitration)

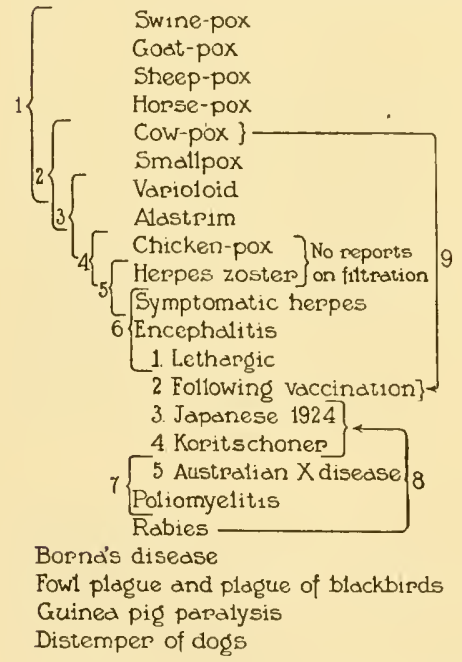

Trachoma and inclusion blenopphed Infectious papular stomatitis of cattle Molluscum contagiosum

Warts

Contagious epthelioma (fow1-pox) 1 Chickens 2. Pigeons

Infectious myxomatosis of pabbits

Rous sarcoma of chickens

Leukemia of chickens

Lymphocystic disease of f1sh

Epitheliom of fish

Capp-pox

No reports on filtration

Mumps (According to Kermorgant, a spipochetal disease) Agalactia (According to Bridre, a bacterial disease) Salivary gland disease of guined pigs

Measles (rubeola)

German measles (rubella) (No peport on fittration)

Grippe (influenza) (According to Olitsky and Gates a Common colds bacterial disease)

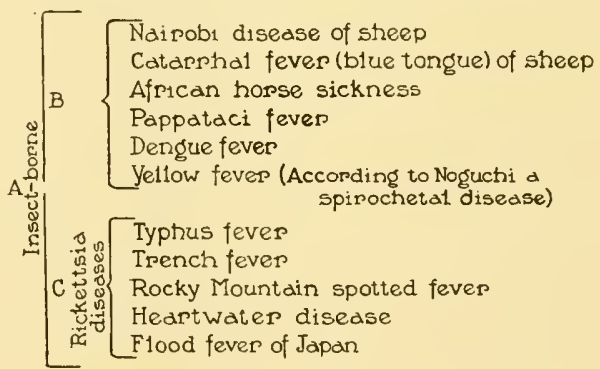

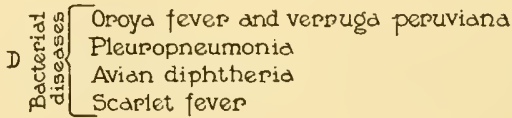

Majopity of the diseases which have been placed in the filterable virus group by different workers.

The diseases listed do not form a homogeneous group and some of them should lee omitted. The evidence that "epizoötic of guinea pigs" is a virus disease is not convincing. There is considerable doubt as to how long the insect-borne diseases will remain on the list. Most olservers no longer consider scarlet fever a virus disease. M'larlyean, ${ }^{1}$ as early as 1908 , suggested that pleuropneumonia be classified with

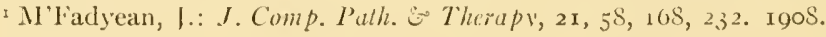


bacterial diseases. Oroya fever and fowl diphtheria, in view of Noguchi's ${ }^{\mathrm{t}}$ work and Bordet's ${ }^{2}$ experiments, respectively, do not belong here. Enough has been said to convince one of the heterogeneity of the diseases listed in the table. In fact, they exhibit so many differences that a discussion of the filterable viruses almost amounts to a separate discussion of each disease. Such a state of affairs is due to the fact that the filterable virus group has been used to a considerable extent for the indiscriminate segregation of infectious diseases of unknown etiology. Therefore, it is not unlikely that some of them will be shown to be caused by small bacteria or protozoa. When this occurs, such diseases should be removed from the filterable virus group and given their correct position in the classification of diseases.

\section{FILTERABILITY AND SIZE OF VIRUSES}

By means of different kinds of filters it has been determined that many diseases are caused by active agents supposedly smaller than ordinary bacteria. Some of them are presumably much smaller and are most likely optically immeasurable. Others, however, do not seem to be so small, and concerning their filterability there is much discussion. Methods of filtration are crude and inaccurate, and the most one can say concerning the viruses is that under given experimental conditions they either pass or do not pass through certain filters. The failure to pass through a filter, however, is certainly not determined in every instance by the size of the virus. The electrical charge on the virus, the electrical charge on the filter, the adsorption of the virus by aggregates of protein or by cell detritus, the amount of protein or other substances in the virus emulsion, the temperature at which the filtration is conducted, the amount of negative or positive pressure employed, the duration of filtration, and other factors not mentioned or not known serve to influence the results of all filtration experiments. Furthermore, sufficient attention has not been given to the possibility that some filters may not only hold back certain viruses but may also inactivate them in some manner so that they can never become active again. ${ }^{3}$

Very little has been recorded in regard to the size of many of the viruses other than that they pass through certain kinds of filters. It is obvious that this method indicates only roughly that the viruses which pass through tight Chamberland candles are very small. No virus has been obtained in an absolutely pure state. Therefore, it is impossible to say that virus alone is being filtered rather than virus attached to aggregates of protein or particles of degraded cells. Nevertheless, attempts have been made in various ways to determine the size of a few viruses. According to D'Herelle, ${ }^{4}$ the diameter of the bacteriophage is $20-30 \mu \mu$. Bechhold and Villa ${ }^{5}$ state that its diameter is more than 35 and less than $200 \mu \mu$. Duggar and Karrer ${ }^{6}$ believe that the

${ }^{1}$ Noguchi, H., and Battistini, T. S.: J. Exper. Mcd., 43, 851. 1926; Noguchi, H.: ibid., 44, 533. I926; 45, I75. I927.

${ }^{2}$ Bordet, J.: Bull. Soc. sc. méd. ct nat., 65, 1 25. Bruxelles, 1907; 67, i96. 1909; Ccntralbl. f. Baktcriol., Abt. I, Orig., 67, 41. I913.

3 Coplans, M.: J. Trop. Mcd., 29, I22, 133. 1926.

${ }^{4}$ d'Herelle, F.: Centralbl. f. Bakteriol., Abt. I, Orig., 96, 385. 1925.

${ }^{5}$ Bechhold, H., and Villa, L.: Biochem. Zlschr., 165, 2.50. 1925; Ccntralbl. f. Bakteriol:, Abt. I, Urig., 97, * 102. 1926; Ztschr.f. IIyg. u. Infektionskrankh., г 05, 601. I926.

${ }^{6}$ Duggar, B. M., and Karrer, J. L.: Ann. Missouri Bot. Gard., 8, 343. I92 I. 
virus of tobacco mosaic is approximately the same size as the colloidal particles of fresh I per cent hemoglobin $(30 \mu \mu)$.

'The size and weight of molecules of crystalline egg albumen and crystalline hemoglobin are not agreed upon. Bechhold ${ }^{\mathrm{r}}$ states that an aggregate of 50 molecules of egg albumen is greater than 4 and less than ro $\mu \mu$ in diameter. According to Du Noüy, ${ }^{2}$ however, I molecule of egg albumen is 4 .I $\mu \mu$ in diameter. If it is difficult to determine the size of molecules of relatively pure crystalline substances, what hope is there at present of ascertaining the size of the viruses which have not been obtained in a pure state? Furthermore, it is useless to pretend to know what is the lower limit in point of size for living things. In general, however, it can be said that many viruses are probably of sufficient size to exist in a living state, and that others are probably small enough to satisfy the demands of those who insist that they are not possessed of life.

\section{VIABILITY OF VIRUSES}

The question as to whether the viruses are animate or inanimate is also an old one, inasmuch as it was propounded simultaneously with the discovery of the filterability of these agents. Beijerinck's ${ }^{3}$ idea of a living contagious fluid called forth many protests. Sanfelice, ${ }^{4}$ working with fowl-pox in I9I4, was led to think of it as an inanimate poison capable of attacking normal cells and producing within them a poison of a similar nature which in turn could attack other normal cells. Thus he described his idea of how a lifeless agent might be passed in series, reproducing itself indefinitely. The work of Twort, D'Herelle, Bordet, and others, concerning the bacteriophage, is familiar to all. The numerous discussions concerning the nature of this active agent have led many investigators to question more closely the living nature of other filterable viruses. ${ }^{5}$ Many tests have been devised to act as criteria for the presence of life but so far no one of them has been found satisfactory. Therefore, it is impossible at present to say whether the viruses are animate or inanimate. Furthermore, it is wise to leave the subject at this point as a further pursuit of it leads one into the sterile discussion of what life is-a problem still in the realm of metaphysics.

\section{VARIATIONS OF VIRUSES}

Mutations of bacteria with concomitant changes in their characteristics are at present of particular interest to bacteriologists. The question arises, then, as to whether viruses can mutate. In the field of filterable viruses, however, this is not a new question inasmuch as it has been under discussion in regard to the relation between vaccine virus and the virus of smallpox since Jenner's time. In spite of all contradictions, it seems that smallpox virus passed through calves for several generations becomes vaccine virus. ${ }^{6}$ Furthermore, if a sufficient number of passages is made in calves, it is impossible for this altered virus to regain the characteristics of smallpox

- Bechhold, H., and Villa, L.: loc. cit.

${ }^{2}$ du Noüy, P. L.: Surface Equilibria of Biological and Organic Colloils. New York: Chem. Cat. Co. ("Im. Chem. Soc. Mono. Series"), 1926.

3 Beijerinck, M. II.: C'ntralbl. f. Bakterial., .bt. II, 5, 27, 310. ISg9.

4.Sanfelice, I:. Ztsihn.f. IIyg. U. Infektionskrankh., 76, 257. 1914.

5 Simon, C. E.: Physiol. Riv., 3, $4 S_{3}$. 1023.

"Cippeman, S. M.: J. P'uth. S Buct., 2, 407. 1804; Proc. Koy. Soc., London, 71, 1 21. I903. 
virus even after repeated passages in man. Observations of a similar nature have been made in regard to other virus diseases, e.g., contagious epithelioma of chickens and pigeons, and mosaic disease of tobacco and cucumbers. ${ }^{\mathrm{T}}$ Whether it is correct to speak of these phenomena as examples of mutation is not known. In any event, when viruses are adapted to alien hosts, their characteristics are frequently altered, as well as those of the diseases produced by them.

\section{CULTIVATION OF VIRUSES}

Following the discovery of the first filterable virus thirty-four years ago, numerous workers claimed to have successfully cultivated in vilro, by means of simple or complex media, one or more of these active agents. The term in vitro will be avoided as there is no agreement in regard to its exact meaning. Therefore, the cultivation of viruses in the presence or absence of living cells will be discussed.

There is no reason to doubt that vaccine virus, herpes virus, typhus-fever virus, the virus of Rous's sarcoma, and the virus of Rocky Mountain spotted fever have been successfully cultivated in the presence of living cells in tissue cultures. ${ }^{2}$ Moreover, Levaditi ${ }^{3}$ has stated that the virus of poliomyelitis either survived or multiplied in fresh spinal ganglia (monkey) placed in plasma. Hardet was able to grow vaccine virus in the presence of living corneal cells, but if the cells were killed by freezing or by hypertonic salt solution the virus failed to multiply. The virus of fowl plague ${ }^{5}$ is cited as one that has been cultivated in vitro. One might ask, however, if it has been cultivated in the absence of living cells, inasmuch as a large amount of blood was added to the medium employed. Furthermore, Landsteiner and Berliner ${ }^{5}$ found that the virus would not multiply if laked or frozen blood was used, and definitely stated in their report that one could not say that growth of the virus had taken place in a lifeless medium. The statement is frequently seen that Bordet cultivated the etiological agent of contagious epithelioma of chickens. Such statements are incorrect. Bordet ${ }^{7}$ claims to have cultivated a small bacterium which causes avian diphtheria. Furthermore, he specifically states that the bacterium does not cause contagious epithelioma and that avian diphtheria and contagious epithelioma are two distinct diseases in spite of the view held by some investigators.

The majority of the attempts to cultivate the viruses have been unsuccessful. No worker has proved that any of the etiological agents of the diseases in the table down to mumps are susceptible of cultivation in the absence of living cells. ${ }^{8}$ A satisfactory explanation of the difficulty experienced in cultivating the viruses on artificial media is not easily found. Their small size alone should not necessarily make them insus-

I Walker, M. N.: Phytopath., r6, 43 I. I926.

${ }^{2}$ Meyer, E.: Arch.f.exper. Zellforsch., 3, 201. I926.

3 Levaditi, C.: Compt. rend. Soc. de biol., 75, 202. I913.

${ }^{4}$ Harde, E. S.: Ann. de l'Inst. Pasteur, 30, 299. 1916.

s Marchoux, E.: Compt. rend. Acad. de sc., I47, 357. 1908.

${ }^{6}$ Landsteiner, K., and Berliner, M.: Centralbl.f. Bukteriol., Abt. I, Orig., 67, I65. I9I3.

7 Bordet, J.: loc. cit.

${ }^{8}$ Dr. Noguchi's report concerning the etiological agent of trachoma has appeared since the construction of this table. 
ceptible to cultivation. Nor does it seem to be a question of delicacy or sensitiveness, because many of them are extremely resistant to chemical and physical agents. Furthermore, no virus has been found multiplying free in nature. Therefore, the viruses appear to be obligate parasites in the sense that their reproduction is dependent upon living cells. Whether this reproduction occurs intra- or extracellularly is a debated question.

In view of the fact that viruses apparently multiply only in the presence of living cells, it is necessary to consider what kinds of living cells best promote their reproduction, and what effect upon the cells is induced by this reproduction. In the first place, a remarkable species specificity is exhibited by many viruses. Rous's sarcoma grows only in chickens. Sanarelli's virus of infectious myxomatosis and Virus III are active only in rabbits. The "salivary-gland virus" described by Cole and Kuttner affects only guinea pigs. A wilt virus that attacks one kind of caterpillar is innocuous for other caterpillars. Second, young cells seem essential for the activity of many viruses. The bacteriophage multiplies only in the presence of young, growing bacteria. The activity of mosaic viruses is manifested only in young leaves. Virus diseases usually attack insects in certain stages of development. In the higher forms of life the activity of many viruses is also best exhibited not in old, undernourished, sickly individuals, but in young, healthy ones. Lastly, some viruses, e.g., the virus of rabies and poliomyelitis, exhibit a remarkable affinity for cells of certain tissues and apparently can neither multiply nor produce signs of disease unless they come into a close relation with these cells.

It has been shown that some viruses multiply only in a restricted number of hosts, that frequently this multiplication occurs only when the virus is in close relation with certain types of cells, and that young, actively growing cells play an important rôle in the infectiousness of many virus diseases.

A word may be said in regard to the effects produced in cells by viruses. At first the involved cells show a remarkable increase in size, often with amitotic division of the nuclei. The increase in size gives one the impression that it is due to growth phenomena and to imbibition of fluid. This process is spoken of as "ballooning degeneration." Eventually the cells die and go to pieces. This process is spoken of as "colliquation." Two forces seem to operate: one stimulating the cell, the other destroying it. Consequently, the picture produced by a virus disease is more or less dependent upon which of these forces predominates. Chicken-pox, foot-and-mouth disease, variola, and lysis of bacteria by bacteriophage are diseases in which destructive agencies predominate. Rous's sarcoma, contagious epithelioma, fowl leukemia, and warts are diseases in which stimulating forces are dominant. Some observers ${ }^{\mathrm{r}}$ have attempted to classify the cytotropic viruses under cytolytic and cytokinetic headings with subdivisions under each according to the type of cells involved. Such a classification is premature.

It is not known whether the viruses multiply intra- or extracellularly. Nevertheless, they have a profound influence upon cells and cause remarkable changes within them. This influence most likely accounts for the fact that in lesions caused by many viruses intracellular changes assume appearances characteristic enough to be spoken

I Philibert, A.: Ann. de mêd., 16, 283. I924. 
of as "inclusion bodies." In this respect many virus cliseases differ from those caused by ordinary bacteria.

IMMUNITY TO FILTERABLE VIRUS DISEASES

With a few exceptions, diseases produced by the filterable viruses, if recovered from, lead to a lasting immunity. In this respect virus diseases differ from those caused by the better-known bacteria. This is not universally true, however, since one attack of typhoid fever produces in the recovered individual a fairly lasting immunity. Many questions have arisen in regard to this kind of immunity, but so far they have not been satisfactorily answered. Nevertheless, a few of the possibilities will be discussed.

In the first place, it is possible that the lasting immunity is due to repeated infections so mild that they attract no attention except in the initial instance. In regard to diseases as prevalent as measles and chicken-pox this might serve as an explanation. On the other hand, one can hardly explain the persistent protection against poliomyelitis and smallpox upon such grounds. Another possible explanation for the lasting immunity is that it is due to a prolonged sojourn of the virus in the body, or perhaps to its persistence in an individual once infected. Winkler, ${ }^{2}$ in his review of immunity to vaccine virus, suggests this possibility. When confronted with the idea one invariably says that it is impossible, because, if it were true, everyone would spread measles and chicken-pox. This would not necessarily be the case, however. Typhoid bacilli have been found in the walls of gall-bladders many years after attacks of typhoid fever. Furthermore, when the mucous membranes lining the gall-bladders are normal, there is little danger of the disease being spread by these carriers. Most human beings carry tubercle bacilli, but only a few spread tuberculosis. Individuals harboring Treponema pallidum are not always infectious, particularly in the latent stages of syphilis. Furthermore, syphilis is an excellent example of a disease in which there is a persistent infection coincident with a refractory state in the host to reinfection. For information concerning discussions of this paradox one should read Chesney's review, "Immunity in Syphilis.",

In regard to virus diseases, is there any evidence (I) of a prolonged or persistent infection, (2) of a coëxistence of infection and refractory state in the host to reinfection from without, and (3) of a causal relation between the prolonged or persistent infection and the lasting immunity? There is considerable evidence 4 that a prolonged infection occurs in some virus diseases and also that this infection can persist for a long time in a host refractory to reinfection, e.g., Cole and Kuttner ${ }^{5}$ have shown that the "salivary-gland virus" of guinea pigs can be obtained at will from immune pigs, and in this particular instance it appears that a pig once infected con-

${ }^{1}$ Findlay, G. Mf., and Ludford, R. J.: Brit. J. Exper. Path., 7, 223. 1926; Lipschütz, B.: Seuchenbekümpfung, 3, 79. I926.

${ }^{2}$ Winkler, W. F.: Ergebn. allgem. Path. u. path. Anat., Abt. I, 21, 45. 1925.

${ }^{3}$ Chesney, A.: Mcdicine, 5, 463. I926.

${ }^{4}$ Cole, R., and Kuttner, A. G.: J. Exper. Med., 44, 855. 1926; Lipschütz, B.: von Prowazek's Ilandb. d. path. Prot., I, 230. Leipzig. I91 2.

${ }^{s}$ Cole, R., and Kuttner, A. G.: loc. cit. 
tinues to harbor the virus indefinitely in spite of a refractory state to reinfection from without. The question as to whether the lasting immunity is dependent per se upon a prolonged sojourn or persistence of viruses in the body cannot be answered at the present time. Nevertheless, such an idea, novel in regard to virus diseases, is worthy of serious consideration.

Another interesting feature concerning immunity to virus diseases is the fact that it is doubtful, with a few exceptions, whether injection of a virus completely inactivated leads to a protection against the same virus in an active state. Furthermore, virucidal properties do not appear in the serum of naturally resistant animals which have received repeated injections of active virus. Therefore, it seems that an actively acquired immunity and evidences in the serum of such an immunity are dependent in some way upon an actual infection with the virus, even though it be so mild at times as to give rise to no symptoms.

The degree of active immunity usually exhibited by individuals recovered from virus diseases seems disproportionate to the amount of passive protection afforded by their sera. This fact has led many observers to believe that the protection against virus diseases is predominantly a tissue immunity rather than a humo:al one. Be that as it may, protective substances do occur in the sera of individuals who have recovered from certain virus diseases. Furthermore, virucidal properties, precipitins, and complement-fixing antibodies have also been demonstrated in the sera of these

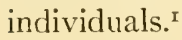

\section{GENERAL REVIEWS AND DISCUSSIONS}

Bayon, H. P.: J. Trop. Med., 29, I 7. I926.

Doerr, R.: Centralbl.f. Bakteriol., Abt. I, Ref., Beih., 50, * I2. I9I I.

Kraus, R.: Med. Klin., 22, 540, 579. Berlin, I926.

Loeffler, F.: Ccntralbl. f. Bakteriol., Abt. I, Ref., Beih., 50, *.. I9I I.

Lucksch, F.: Prag. tierärstl. Arch., Teil A, 5, 83. 1925.

MacCallum, W. G.: Medicine, 5, 59. 1926.

Roux, E.: Bull. de l'Inst. Pasteur, I, 7, 49. 1903.

Twort, F. W.: J. State Med., 31, 351. I923.

Wolbach, S. B.: J. Med. Research, 27, г. I9г2.

'Gordon, M. H.: Med. Research Coun., "Spec. Rep. Ser.," No. 98. London, r925. 


\title{
CHAPTER XL
}

\section{THE BACTERIOPHAGE: PRESENT STATUS OF THE QUESTION OF ITS NATURE AND MODE OF ACTION}

\author{
J. BRONFENBRENNER
}

Laboratories of the Rockefeller Institute for Medical Research, New York City

TIE PARTICULATE NATURE OF BACTERIOPIIAGE

According to D'Herelle, transmissible lysis is the result of an acute infectious disease of bacteria. The etiological factor of this disease is an "autonomous ultra-microscopic corpuscle" - Bacternophasum intestinale. ${ }^{2}$ The corpuscular nature of the active agen $\mathrm{t}^{3}$ of transmissible lysis is claimed by D'Herelle on the basis of the following observations.

If filtrates of cultures subjected to the action of bacteriophage are examined under an ultra-microscope, they show the presence of minute granules. Some of these granules can be seen entering the bacterial cells and multiplying within them. ${ }^{4}$ The identification of these particles with the active agent is supported by the observation that on prolonged standing, or as the result of intensive centrifugation of active filtrates, the particles tend to settle to the bottom of the container, and coincident with this settling, the active agent collects in the lower strata of the solution. ${ }^{5}$ When active filtrate is spread over the surface of agar seeded with susceptible bacteria, it causes the appearance of discrete foci of lysis, and the number of such foci (plaques) is proportional to the concentration of active agent in the solution. ${ }^{6}$

That the active agent is present in the filtrates in the form of indivisible units follows also from the fact that it exhibits no gradual diminution of its activity on serial dilution (such as exhibited by antiseptics, for instance), but becomes at once totally ineffective as soon as dilution is carried beyond the point at which a certain quantum of the agent is present. This quantum (about $\mathrm{I} \times 1 \mathrm{O}^{-10} \mathrm{cc}$.) cannot be subdivided on further dilution. ${ }^{7}$ The question arises as to the nature of these quanta of the active agent.

I EDITORS' NOTE.-It is regretted that a chapter by Dr. d'Ilerelle which had been promised has not been received.

${ }^{2}$ d'Herelle, F.: The Bacteriophage and Its Behavior, pp. 354-55. Williams \& Wilkins, 1926.

${ }^{3}$ Throughout this discussion the terms "bacteriophage," "phage," and "active agent" (of transmissible lysis) are used interchangeably to designate the agent without commitment as to its nature. In referring to the hypothetical living virus of D'Herelle, the terms Backriophagum inlestinale or Protobios bacleriophagus are used.

${ }_{4}^{4}$ d'Herelle, F.: Immunity in Natural Infectious Disease, p. 255. Williams \& Wilkins, r024; The Bacteriophage and Its Behavior, p. 273. Williams \& Wilkins, I926; ibid., p. 370. Williams \& Wilkins, 1926.

${ }^{5}$ d'Herelle, F.: The Bacteriophage and Its Behavior, p. 2So. Williams \& Wilkins, r926.

${ }^{6}$ d'Herelle, F.: Compt. rend. Acad. de Sc., i65, 373. г9г7.

7 The effect of dilution on the distribution of the active agent is therefore analogous to that of bacterial suspensions, for instance. See Bronfenlrenner, J., and Korb, C.: J. Exper. Med., 42, $4 S_{3}$. 1925. 
Since active filtrates consisting of water with a very small percentage of solids (comprising, in addition to active principle, salts, tissue extractives from broth, metabolic products of bacteria, and bacterial débris) can still be diluted Io $^{\text {Io }}$ times before reaching the limit of their activity, it may be supposed that the units of the active agent are free molecules of some inanimate material.

It is possible that the lytic principle is present in the medium in a state of true solution, or as a highly dispersed colloid, and that it is merely adsorbed on bacterial débris and other particles, which thus determine its distribution in the medium. It is possible also that the particles in the filtrate represent the "corpuscles" of autonomous, organized parasites, ${ }^{\mathrm{I}}$ and can be identified with the granules observed by D'Herelle within the infected bacteria under an ultra-microscope. ${ }^{2}$

Later investigations have indicated, however, that these particles do not represent autonomous units of the active agent, but merely serve as a vehicle on which the active principle is adsorbed, and from which, under proper experimental conditions, it can be detached. ${ }^{3}$ For instance, if these particles, carrying the phage, are deposited on an ultra-filter through which they cannot pass, and washed repeatedly with water or buffer solution, they do not give up any of the active agent. But if they are washed with broth, the phage reappears in the filtrate. ${ }^{3}$ Similarly, when phage is adsorbed on bacteria, it cannot be set free by washing with water, but can be freed by broth. ${ }^{4}$

There exists further evidence that the particles observed by D'Herelle ${ }^{5}$ and measured by Bechhold, ${ }^{6}$ Prausnitz, ${ }^{7}$ and others are not likely to represent the autonomous "corpuscles" of the parasite. The lytic principle spreads on agar from a focus radially. This spreading is independent of the progress of "invasion" of bacteria, since it continues at $4^{\circ} \mathrm{C} .{ }^{8}$ (at which temperature the invasive activity of the phage

${ }^{1}$ d'Herelle, F.: Compt. rend. Acad. de Sc., 165, 373. i917; Compt. rend. Soc. biol., 83, 247. 1920.

${ }^{2}$ d'Herelle, F.: Immunity in Natural Infcctious Disease, p. 255. I924; The Bacteriophage and Its Bchavior, p. 273. 1926. According to some investigators (Bechhold, H., and Villa, L.: Ztschr.f. IIyg. u. Infektionskrankh., 105, 601. 1926; Stassano, H., and Beaufort, A. C.: Compt. rend. Soc. de biol., 93, 1378. 1925; Prausnitz, C.: Klin. Wchnschr., I, 1639. I022; von Angerer, K.: Arch.f. IIyg., 92, 312. 1924), these particles are fairly uniform in size, approaching $20 \mu \mu$ in diameter and a calculated mass of about $\frac{4}{3} \pi 1^{-18} \mathrm{gm}$. Prausnitz, C.: Centralbl. $f$. Bakteriol., Abt. I, Orig., Suppl., 93. 148. I 924 .

3 Bronfenbrenner, J.: J. Exper. Med., 45, 873. 1927.

${ }_{4}^{4}$ Eguchi, C.: quoted from Centralbl. $f$. Bakteriol, Ref., 78, 40. I924-25.

${ }^{5}$ d'Herelle, F.: Immunity in Natural Infcctious Discase, p. 255. I924.

${ }^{6}$ Bechhold, H., and Villa, L.: loc. cit. $\quad{ }^{7}$ Prausnitz, C.: Klin. W'chnschr., r, 1639. 1922.

${ }^{8}$ Unpublished experiments of the writer in collaboration with Korb in I924. Agar containing phage and bacteria was poured into plates and incubated at $37^{\circ} \mathrm{C}$. As soon as formation of plaques became visible the plates were placed overnight at $4^{\circ} \mathrm{C}$. The next day they were returned to the thermostat. It will be seen that phage diffused during the night beyond the periphery of original plaques. When, on the next day, the growth of bacteria was resumed (at $37^{\circ} \mathrm{C}$.), a halo of lycis around original plaques resulted (lïg.I). 
is arrested), ${ }^{\mathrm{r}}$ and also in sterile agar. ${ }^{2}$ And finally, the rate of spread of the phage is conditioned by the density of the medium. ${ }^{3}$

If the autonomous particulate nature of the active principle be assumed, such spreading must be accounted for either by postulating that these particles possess lo-

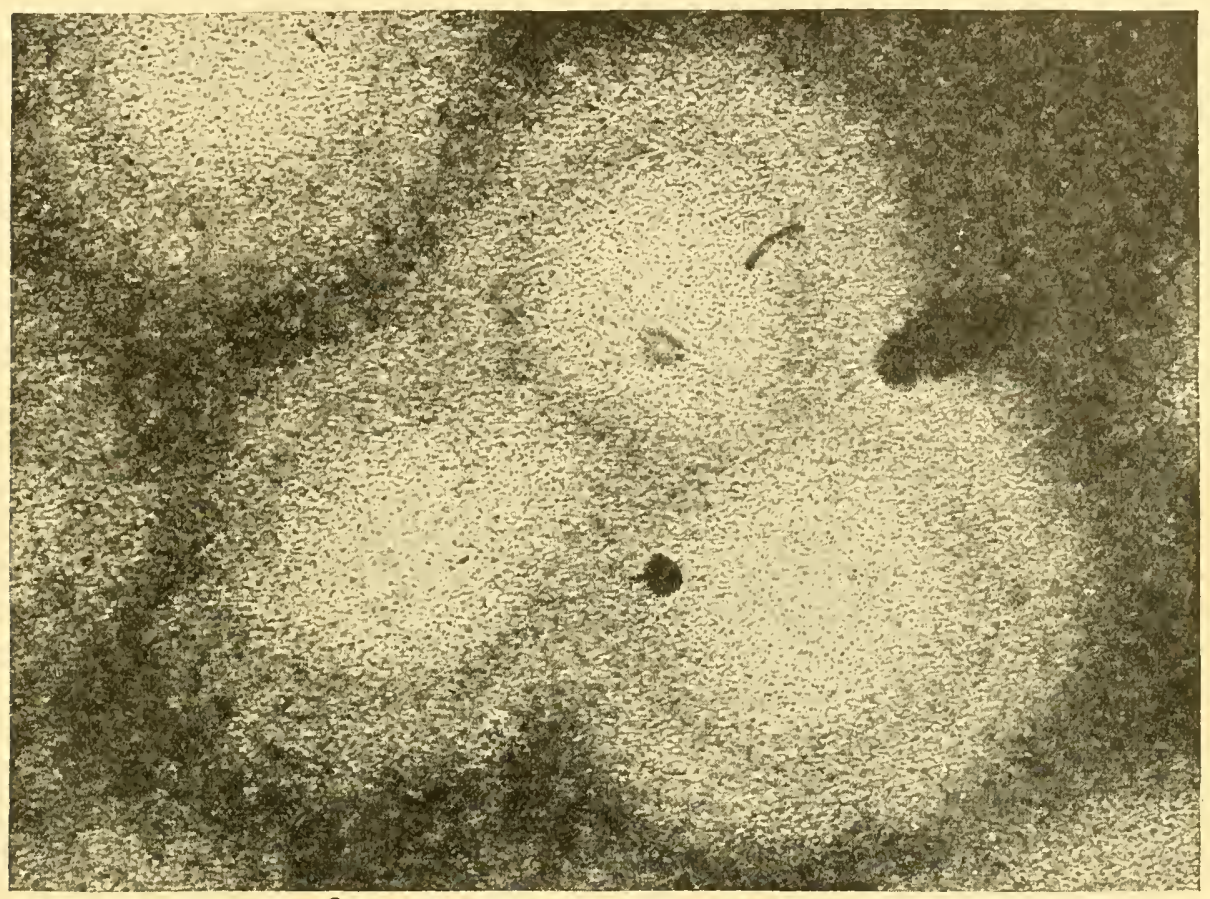

FIG. I. - Zone of diffusion of phage through agar at $4^{\circ} \mathrm{C}$. Magnification $\times 25$

comotion, ${ }^{4}$ or by assuming that they secrete a diffusible enzyme. ${ }^{5}$ But independent locomotion, whether ameboid, flagellate, or ciliate, would account for the transport of a given particle in only one direction at a time, whereas the active principle spreads from the focus in all directions simultaneously. The secretion of the active enzyme by the Bacteriophagum intestinale has been claimed on the basis of evidence ${ }^{6}$ which was later found to be inadequate. ${ }^{7}$

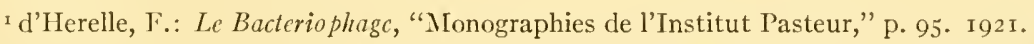

${ }^{2}$ von Angerer, K.: loc. cit.; Bürgers, T. J., and Bachman, H.: Ztschr. f. Hyg. u. Infektionskrankh., Iо I, 350. I92.4; von Preisz, H.: Die Bakteriophagie. Jena: Gustav Fisher, I925; Arnold, L.: J. Lab. Eo Clin. Med., 8, 720. I923.

3 Bronfenbrenner, J., and Korb, C.: Proc. Soc. Exper. Biol. Eo Med., 2 I, 3 I 5. I924.

+ Kabelik, J., and Kukula, K.: Compt. rend. Soc. de biol., 92, I058. I 925.

5 d'Herelle, F.: Le Bacteriophage, "Monographies de l'Institut Pasteur," p. 95. I92 I; Reichert, F.: Centralbl.f. Baktcriol., Abt. I, Orig., 91, 235. I 924; Marshall, M. S.: J. Infect. Dis., 37, I 26. I925.

${ }^{6}$ d'Herelle F.: Le Bacteriophage, "Monographies de l'Institut Pasteur," p. 95. I921.

7 Bronfenbrenner, J., and Korb, C.: J. Exper. Med., 42, 419. 1925; Hauduroy, P., Compt. rend. Soc. de biol., 87, 96.4. 1022; d'Herelle, F.: The Bacteriophage and Its Behavior, p. 372 . I926. 
Such radial spreading could be accounted for by assuming that the observed particulate distribution of phage is only apparent and is due to its ready adsorption on colloidal particles of the medium. This assumption is validated by experiments in which the number of particles endowed with properties of phage in a given volume of filtrate was altered, depending on changes in the composition of the medium. ${ }^{\mathrm{I}}$ It was found that a slight increase in the consistency of the agar from I to 2.5 per cent suppresses 99 per cent of visible plaques. ${ }^{2}$ D'Herelle accounts for this effect of agar by stating that agar interferes with the diffusion of the metabolic products of the Bacteriophagum inlestinale, which in turn inhibits its growth. ${ }^{3}$ But until it has been shown that Bacteriophagum intestinale possesses an independent metabolism, this explanation cannot be accepted.

Moreover, it is difficult to reconcile changes in the size of plaques as brought about by various experimental procedures ${ }^{4}$ with the view of D'Herelle that the plaques represent the "colonies" of bacteriophage. ${ }^{5}$ For instance, if the size of the plaque depends on the progress of "invasion" of bacteria by the parasite, then the more numerous the susceptible bacteria are around the initial particle of Bacteriophagum intestinale the more of them should be "invaded"; each one of the invaded bacteria in turn should become a source from which the next generation of parasites would invade the nearest bacteria, ${ }^{5}$ and in consequence the plaque should be correspondingly larger. As a matter of fact, however, the greater the concentration of susceptible bacteria in the immediate vicinity of the original particle carrying the phage (other conditions being constant) the smaller is the plaque. ${ }^{6}$ Similarly, it would be difficult to explain on the basis of D'Herelle's hypothesis why both number and size of plaques decrease with an increase in the proportion of old bacteria (which are not subject to lysis), whereas an increase in the concentration of resistant bacteria leaves both number and size of plaques unaffected. ${ }^{6}$

An explanation of all these phenomena can be given, if, instead of the hypothesis of "invasion" of bacteria by the "obligate parasite," one takes account of the tendency of the lytic agent to be adsorbed on agar, on old bacteria, etc. This arlsorption interferes with the progress of its diffusion. Since resistant bacteria do not atisorb the lytic principle, ${ }^{7}$ other conditions being equal, the presence of an excess of resistant bacteria among the susceptible ones does not interpose an obstacle for the normal diffusion of the lytic agent.

${ }^{1}$ Nakamura, O.: Arch.f. IIyg., 92, 61. I923; Doerr, R., and Zdansky, E.: Ztschr.f. Hyg. $u$. Infektionskrankh., 100, 79. I923.

${ }^{2}$ Bronfenbrenner, J. and Korb, C.: J. Exper. Med., 42, 483. 1925; Proc. Soc. Exiner. Biol. So Med., 2I, 3I 5. 1924 .

3 d'Herelle, F.: The Bacteriophage and Its Behairior, p. S9. 1926.

${ }_{4}$ Bronfenbrenner, J.; and Korb, C.: J. Exper. Mcd., 42, 483. 1925; Bail, O.: Arch.f. Hyg., 95, I. 1925 .

5 d'Herelle, F.: The Bactcrioplage and Ils Bchavior, p. S7. 1926.

${ }^{6}$ Bronfenbrenner, J., and Korb, C.: J. Expcr. Mcd., 42, 4\$3. 1925.

7 Meissner, G.: Centralbl. f. Bakteriol., Orig., 93, 489. 1924; Kimura, S.: Ztschr. f. Immunitütsforsch. u. exper. Therap., 45, 33. 1926. 


\section{BACTERIOPHAGE AS A SPECIFIC ANTIGLN}

It has just been suggested that the particles seemingly endowed with the power of initiating transmissible lysis may not represent autonomous "corpuscles" of a parasite, but serve merely as a vehicle on which the active principle is arlsorbed. The active agent itself, however, may still be particulate, i.e., may consist of minute aggregates composed of a number of molecules each, and these small aggregates may still possess all the characteristics previously ascribed to larger carrier-particles. These aggregates, though perhaps considerably smaller than $20 \mu \mu$ in diameter, may still represent, according to the definition of D'Herelle, "colloidal micellae which differ from other forms of life only in their size." That the active agent may not be a crystalloid is suggested, for instance, by its behavior with respect to solubility. Once dried, the preparations of bacteriophage cannot be brought to the state of initial dispersion in water. This is particularly true of purified preparations of bacteriophage deprived of all, or nearly all, of the extraneous material. ${ }^{2}$ The colloidal nature of the active agent is further evidenced by the fact that it acts as an antigen and causes the production of specific antibodies when introduced parenterally into experimental animals. ${ }^{3}$ While the antisera thus obtained are capable of neutralizing the activity of the bacteriophage, they usually show simultaneously the presence of agglutinins, opsonins, precipitins, and complement-fixing antiborlies for homologous bacteria. ${ }^{4}$ Moreover, purely antibacterial sera apparently possess the power to inhibit the progress of lysis by homologous bacteriophages. ${ }^{5}$ In spite of the inference suggested by these findings, it has been possible to establish that the active principle possesses independent (autonomous) antigenic value. Thus, when sera prepared by immunization with filtrates of lysed cultures are absorbed with homologous bacteria, they no longer react with bacteria and their products, but retain their power to neutralize the bacteriophage unimpaired. ${ }^{6}$ If the filtrates of lysed cultures are purified by removal or digestion of bacterial protein, leaving only the active principle, the antisera obtained by immunization with such purified preparations do not react with bacteria, while they do neutralize the phage. ${ }^{7}$ Moreover, in the case of phages capable of acting on several related species of bacteria, the antigenic specificity of the fil-

${ }^{1}$ d'Herelle, F.: Centralbl.f. Bakteriol., Orig., 96, 385 . I 926.

${ }^{2}$ Bronfenbrenner, J.: Proc. Soc. Exper. Biol. Eo Med., 24, 372.1927.

3 Bordet, J., and Ciuca, M.: Compt. rend. Soc. de biol., 84, 27S. I921; Watanabe, T.: Ztschr. f. Immunitätsforsch., u. cxper. Therap., 37, I06. I923; Sonnenschein, C.: Deutsche med. WClunselur., 51, 1434. 1925; Asheshov, I. N.: Compt. rend. Soc. de biol., 93, 1327. 1925; Osumi, S.: Ztschr. f. Immunitätsforsch. u. exper. Therap., 40, 261. 1924.

${ }_{4}$ Bail, O.: H'ien. klin. Wchnschr., 34, 447. I92 I; Weiss, E., and Arnold, L.: J. Infect. Dis., 34, 317. 1924 .

5 Bait, O.: loc. cit.; Marcuse, K.: Ztschr. f. IIyg. w. Infektionskrankh., 105, I7. I925; da Costa Cruz, J.: Compt. rend. Soc. de biol., 95, 1457. 1926; Hauduroy, P.: ibid., 87, 966. 1922.

${ }^{6}$ von Preisz, II.: loe. cit.; Heiss, E., and Arnold, L.: loc. cit.; da Costa Cruz, J.: loc. cit.

7 Bordet, J., and Ciuca, M.: Compt. rend. Soc. de biol., 84, 276. I92 I; Arnold, L., and WVeiss, E.: loc. cit., $37,4 \mathrm{I}$. 1925 . 
trate is constant for each phage and is independent of the bacterial substratum which was used to obtain the active filtrate. ${ }^{\mathrm{r}}$

As for the phage-inhibiting activity of antibacterial scra, it has been demonstrated that this action is not directed against the phage, but against the bacteria, rendering them less susceptible to lysis. It has been suggested that this effect is probably due to the saturation by agglutinins of receptors susceptible to union with phage. ${ }^{2}$

It appears then that when filtrates of lysed cultures are used in immunization of animals, the agent of transmissible lysis acts as a specific antigen, independently of other antigens, notably those of bacterial origin, present in the filtrates at the same time. The question naturally arises: Is the active agent therefore to be considered an autonomous, organized being, totally foreign to bacteria and multiplying at their expense, or can it still be a product of bacteria, accumulating in the culture during the growth or during the lysis of bacteria, but antigenically distinct from them?

\section{ASSIMILATION OF BACTERIAL SUBSTRATUM BY BACTERJOPHAGE}

According to D'Herelle, in order that the bacterial substratum, at the expense of which the bacteriophage apparently multiplies, may be thus gradually transformed into antigenically distinct substratum of bacteriophage, the latter must be alive and "must" possess an autonomous metabolism, independent of bacterial metabolism. ${ }^{3}$ In the absence of direct evidence of the existence of such metabolism, D'Herelle relies on the supporting evidence supplied by Wollman, and confirmed by Asheshov, and concludes that "multiplication of bacteriophage corpuscles can take place, at least to a certain degree, even in the absence of the bacterial cell. For this development the corpuscles utilize certain diffusible products present in the culture of susceptible bacteria." 4 However, the findings of Wollman ${ }^{5}$ (since questioned by the author himself ${ }^{6}$ seem to be susceptible to a different and more adequate interpretation, and in the light of the findings of the writer ${ }^{7}$ do not indicate any assimilation of the bacterial products by the bacteriophage.

Jötten ${ }^{8}$ also concluded that bacteriophage may multiply in the absence of actively growing bacteria. His findings suggesting that Bacteriophagum intestinale may litilize dead susceptible bacteria have not been confirmed, however. ${ }^{9}$

The recent evidence of Twort that dead bacteria may undergo lysis, if, in addition

${ }^{x}$ Seiffert, W.: Ztschr.f. Immunitätsforsch.u.exper. Thcrap., 38, 301. I923; Wagemans, J.: Arch. internat. pharmac. et therap., 28, I8I. 1923; Bruynoghe, R.: Arch. internat. méd. cxpér., I, I7. 1924; Bruynoghe, R., and Dubois, A.: Compt.rend. Soc. de biol., 96, 21 1. 1927.

${ }^{2}$ Marcuse, K.: loc. cit.; da Costa Cruz, J.: op. cit., 93, 875. 1925.

3 Reichert, F.: loc. cit.; d'Herelle, F.: Compt. rcud. Soc. de biol., 90, 25. 1924.

${ }_{4}^{4}$ d'Herelle, F.: The Bacteriophage and Its Behavior, pp. 102-3. 1926.

5 Wollman, E.: Compt. rend. Soc. de biol., 84, 3. 1921.

${ }^{6}$ Wollman, E.: Ann. de l'Inst. Pasteur, 39, 7So. 1025.

7 Bronfenbrenner, J., and Muckenfuss, R.: J. E.xper. Hed., 45, 887. 1927.

${ }^{8}$ Jötten, K. W.: Klin. Wilmsshr., I, 2181. 1022.

9 Bronfenbrenner, J., and Muckenfuss, R.: loc, cil.; Otto, R., Munter, I., and Winkler, WV.F.:

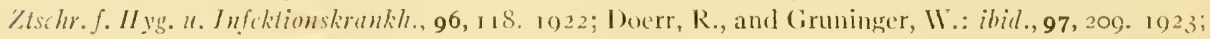
Seiffert, W': Ztschr. f. Immunitutsforsch. u. cxper. Therap., 38, 202. 1923-24. 
to bacteriophage, there are present homologous, live bacteria, seems to have no direct bearing on this question. Even if lysis of dead bacteria were due directly to lytic activity of the phage (which was shown by the writer not to be the case), ${ }^{1}$ so long as live bacteria are present the increase of phage could not be attributed directly to assimilation by phage of dead bacteria.

Apart from the question of the nature of the sulstratum supposedly utilized by the Bacteriophagum intestinale, Asheshov attempted to demonstrate its autonomous metabolic activity by "vaccinating" animals and susceptible bacteria with "ultralsterile" filtrates, presumably free from bacteriophage, but supposedly containing the products of its metabolic activity. ${ }^{2}$ In these experiments, as in those quoted above ${ }^{3}$ and performed in confirmation of experiments by Wollman, Asheshov employed the plaque method for controlling "ultra-sterility" (absence of active phage) in his filtrates. Because of the interference by agar, it is conceivable in the light of our experience ${ }^{4}$ that he might have missed a small amount of phage in the filtrate, particularly as the phage which has been put through a collodion membrane tends to yicld extremely minute plaques. ${ }^{5}$ This assumption seems validated also by Wollman, who attributes his earlier erroneous conclusion, ${ }^{6}$ concerning the impermeability of collodion to phage, to his failure to detect by the plaque method minute amounts of phage present in the filtrate. ${ }^{7}$ In view of these considerations, the findings of Asheshov are probably due to the presence of small amounts of active phage in his supposedly "ultra-sterile" filtrates and cannot, without further evidence, be considered as proof of independent metabolism of the Bacteriophagum intestinale.

It seems to be generally accepted at present that the increase in the concentration of the active agent in the solution takes place exclusively in the presence of live and actively multiplying susceptible bacteria. Therefore the question of the possibility of independent assimilation of the bacterial substratum by the agent still remains open.

THE PRODUCTION OF CARBON DIOXIDE BY THE BACTERIOPHAGE

The question of independent metabolism of the agent of transmissible lysis has been approached from other angles also.

By using a specially constructed micro-respirometer, the writer was unable to detect $\mathrm{CO}_{2}$ production by $\mathrm{IO}^{12}$ active units of bacteriophage, during a period of ninetysix hours. ${ }^{8}$ Owing to the fact that the method does not measure reliably less than $0.005 \mathrm{cc}$. of $\mathrm{CO}_{2}$, it is possible that the agent does respire, but that during the ninety-

I Bronfenbremer, J., and Muckenfuss, R.: loc. cit.

${ }^{2}$ Asheshov, I. N.: Compl.rend. Soc. de biol., 93, 644. 1925; Asheshov, I. N.: ibid., p. 643. 1925. These "ultra-sterile" solutions were obtained by filtration of phage through collodion.

${ }^{3}$ d'Herelle, F.: The Bacterioplage and Ils Bchavior, pp. 102-3. 1926.

4 Bronfenbrenner, J., and Korb, C.: J. Exper. Med., 42, 4 ^3. 1925; Proc.Soc. Exper. Biol. S Hcd., 2I, 315.1924 .

5 Bronfenbrenmer, J.: J. Exper. Med., 45, ISSO n. I927.

${ }^{6}$ Wollman, E.: Compt. rend. Soc. de biol., 84, 3. r92 r.

7 Wollman, E.: Amn. de l'Inst. Pasteur, 39, 7S9. 1925.

${ }^{8}$ I ironfenbrenner, J.: Scionce, 63, 51. Jan. 8, r926; Proc. Soc. F.xper. Bid \& Ifed., 22, 8r. 14? Bronfenbrenner, J., and Reichert, I'.: ibid., 24, 176. 1920. 
six hours of the experiment the amount of $\mathrm{CO}_{2}$ produced was too small to be detected. ${ }^{\mathrm{r}}$ (n the assumption that the bacteriophage did respire, it was calculated that its respiration would have to be at least ten thousand times slower than that of an equal number $\left(\mathrm{IO}^{\mathrm{I} 2}\right)$ of spores not to be detected in ninety-six hours. It is generally observed that the smaller the living organism, and consequently the greater its surface per gram of mass, the higher is its rate of respiration. Since the size of a "corpuscle" of Bactcriophagum intcstinale must be but a small fraction of the size of a bacterial spore, it would be expected that the rate of its respiration should be greater than that of a spore. Hence, the finding that the rate of respiration of the unit (quantum) of phage is at least ten thousand times less than that of the spore seems to indicate that the phage probably does not respire at all. Moreover, when Bacteriophagum intestinale is in the process of active "multiplication," as is assumer to be the case in the presence of susceptible bacteria, the rate of respiration of the whole culture (when corrected for the higher rate of multiplication of bacteria in the presence of bacteriophage) was found to be the same as that of a control culture without the bacteriophage. ${ }^{2}$

Essentially similar conclusions were reached by several investigators who studied the independent metabolism of phage as expressed in terms of its reducing power. The fundings of Fejgin, ${ }^{3}$ of Gozony and Suranyi, ${ }^{4}$ Kauffmann, ${ }^{5}$ and those of Schwarzman, ${ }^{6}$ as well as our own experiments ${ }^{2}$ indicate that the rate of reduction by the culture of susceptible bacteria grown in the presence of bacteriophage is entirely independent of the concentration of the active agent, but rises and falls with the number of intact bacteria present during the various phases of the process.

\section{THE MECHANISM OF INACTIVATION OF PHAGE BY ANTISERUM}

Another type of experiment which might indicate indirectly whether the active agent might be an autonomous corpuscular being is that showing the nature of the reaction which takes place between the agent and its specific antibody. According to Bordet, the active agent is destroyed by antiserum. If the phage is an organized corpuscular virus, one might expect by: analogy with what is known about the mode of action of bactericidal sera that a virucidal action of antibacteriophage serum would require the co-operation of complement for its completion. The results of this inquiry have shown, 7 in accord with earlier findings of Bail, ${ }^{8}$ that inactivated antibacteriophage serum is capable of neutralizing the activity of the phage, just as efficiently as did the same serum previous to destruction of its complement. With the exception of Osumi, ${ }^{9}$ a number of authors have since come to similar conclusions. ${ }^{\text {I0 }}$ They

I Since the last published report, and after a further improvement of apparatus, the experiments were carried over a period of ten ditys, but always without being able to detect $\mathrm{CO}_{2}$ production by the phage.

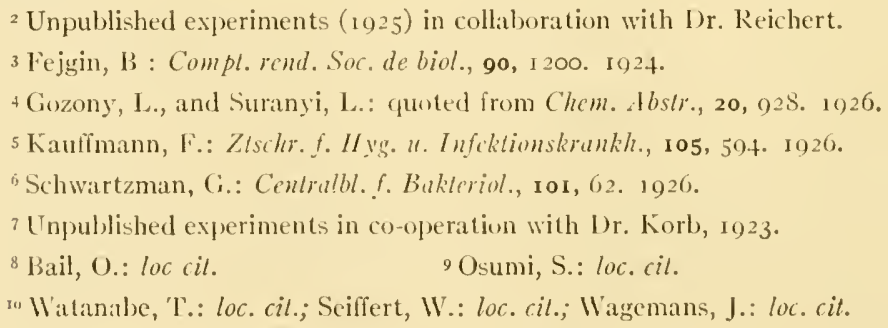


suggest that the phenomenon of neutralization of the bacteriophage by the specific antiserum is analogous to neutralization of toxin by its homologous antitoxin, since the neutralization occurs according to the law of multiple proportions, and since the ingredients can be recovered in an active state by breaking the union by various procedures. ${ }^{\text {I }}$

\section{RESISTANCE OF PHAGE TO CHEMICAL AND PHYSICAL INJURY}

In claiming an autonomous, animate nature for the Bacteriophagum intestinale, its relative resistance to the deleterious influence of various chemical and physical agents has been emphasized. It has been stated that in this respect bacteriophage corpuscles occupy an intermediate position between that of vegetative forms and that of spores of $B$. subtilis. ${ }^{2}$ It has been found, moreover, that, as with bacteria, the suspension of corpuscles of bacteriophage is composed of units having different resistance to these agents. ${ }^{3}$ For example, the resistance to heat of Bacteriophagum intestinale, according to D'Herelle, becomes greater as the corpuscles of the parasite undergo "condensation" or diminution in size during the first few days following their separation from bacteria. ${ }^{4}$ Inversely, the young corpuscles of bacteriophage, according to him, are more resistant to the deleterious effects of repeated freezing and thawing. It seems that slight but consistent differences observed by D'Herelle with respect to heat resistance of phages of different age might be due to some such influences as those observed by Rivers ${ }^{5}$ in freezing of phage in dilutions differing with respect to the concentration of broth or of salts. This seems the more likely since, during the aging, the filtrates may have undergone concentration due to evaporation. In general, however, the effect of heat, of cold, and of repeated freezing and thawing cannot be adduced as decisive evidence of the animate nature of phage since, apart from slight quantitative differences, such inanimate substances as complement and trypsin behave essentially in a similar manner. ${ }^{6}$

Several investigators claim that on repeated or prolonged exposure to these agents, Bacteriophagum intestinale apparently develops a certain degree of tolerance, as do other living organisms. However, the experimental evidence thus far presented does not seem convincing. For example, D'Herelle ${ }^{7}$ has found that a certain concentration of glycerol, if added at once, will surely inactivate the phage. If, however, the sanie total quantity of glycerol is added to the phage in small, repeated doses, at regular intervals, the phage may not be completely inactivated. The failure of complete destruction by the glycerol was not necessarily due to the increased tolerance developed by the Bacteriophagum intestinale, for an analogous phenomenon takes place, for instance, in the case of the coagulation of arsenic sulphide by acid. The amount of acid used up before coagulation of arsenic sulphide takes place is greater when the acid is added gradually than when all the acid is added at once.

r Seiffert, W.: loc. cit.; Weiss, E.: J. Immunol., 13, 301. 1927; d'Herelle, F., and Eliava, G.: Compt. rend. Soc. de biol., 84, 7 г9. I921.

${ }^{2}$ d'Herelle, F.: Le Bacteriophage, "Monographies de 1'Institut Pasteur," p. 95. r92 I.

3 Prausnitz, C., and Firle, E.: Ccnlralbl. f. Bakleriol., Orig., 93, Beiheft, I48. I924.

4 d'Herelle, F.: Compl. rend. Soc. de biol., 83, 247. I920.

5 Rivers, T.: J. Exper. Med., 45, I I. I927. ${ }^{6}$ Rivers, T.: ibid.

${ }^{7}$ d'Herelle, F.: Le Bacteriophage, "Monographies de l'Institut Pasteur," p. 95. I92 I. 
The results of Prausnitz, ${ }^{\mathrm{I}}$ who claims to have observed the adaptation of phage to phenol, $\mathrm{HgCl}_{2}$, and to immune serum, could be confirmed by Munter and Rasch ${ }^{2}$ only so long as they used the strain of bacteria and the phage sent to them by Prausnitz. In all other instances their attempts to demonstrate the adaptation of phage have been unsuccessful. They explain the positive findings of Prausnitz as due to the lysogenic character of the strain of $B$. dysenteriae used, and not to adaptation of the phage.

The adaptation of phage to gradually increasing hydrogen-ion concentration in the experiments of Asheshov ${ }^{3}$ was successful, according to the author himself, with one sample of phage only, and even in this instance the activity of the phage diminished rapidly during the course of the experiment. It is quite evident that in these experiments persistence of traces of phage at a high hydrogen-ion concentration was due not to the "adaptation" of the phage, but to the presence of a small number of bacteria capable of withstanding the acidity of the medium and thus permitting a limited regeneration of phage.

\section{UNICITY (UNIVERSALITY) OF BACTERIOPHAGE}

Quite apart from the indicated possibility of errors in the interpretation of results obtained by these authors, the significance of their findings may also be questioned because the increase in tolerance of the phage, observed by them after adaptation, was usually so small as to be negligible. This is particularly significant in view of their own admissions of the great variability in the behavior of any given sample of phage, when tested at different times. ${ }^{4}$

In the opinion of others, on the contrary, differences in response of various phages to environmental conditions appear to be relatively constant and immutable. Many authors have used these individual characteristics of different phages as a means of identification and even separation of individual phages out of mixtures. ${ }^{5}$ That these differences are essential and not due to minor effects of variability has been demonstrated not only with respect to resistance to chemical agents, ${ }^{6}$ to changes

Id'Herelle, F.: The Bacteriophage and Its Behavior, pp. 354-55. 1926; Prausnitz, C.: Centralbl. f. Bakteriol., Abt. I, Orig., Suppl., 93, r48. 1924; Prausnitz, C., and Firle, E.: ibid.

${ }^{2}$ Munter, H., and Rasch, K.: Ztschr. f. Hyg. u. Infektionskrankh., 105, 205. 1925.

${ }^{3}$ Asheshov, I. N.: Compt. rend. Soc. de biol., 87, I343. 1922; J. Infect. Dis., 34, 536. 1924.

${ }_{4}$ Prausnitz, C., and Fïle, E.: loc. cit.; Asheshov, I. N.: J. Infect. Dis., 34, 536. r924; d'Herelle, F.: The Bacteriophage and Its Behavior, p. 368. r926. For instance, in discussing the attempts of different investigators to interpret differences in the behavior of different samples of plage as indicating the existence of many independent and immutable phages, as opposed to D'Herelle's concept of "unicity" of the Bacteriophagum intestinale, this author says: "As for variability in resistance [of phages], clearly if the resistance of each race were a fixed property, this argument would deserve consideration. But this is not what is observed. The studies of all authors who have considered this question, even including the collaborators of Bruynoghe, show that from one experinent to another a single bacteriophage presents considerable variations,- differences as great as are those observed hetween the different races" (d'Herelle, F.: The Bacteriophage and Its Behavior, p. 368. 1926).

5 Bruynoghe, R.: Arch. internat. méd. expér., x, 17. 1924; Hoder, F.: Centralbl. f. Bakteriol., Orig., 93, 424. 1924; Reichert, F.: ilid., 91, 235. I924.

${ }^{6}$ Wagemans, J.: loc. cit.; Bruynoghe, R.: Arch. internat. méd. expér., r, r7. r924; Brutsaert, P.: .1rch. méd. Belges, 77, 839. 1924; Bronfenbrenner, J., and Korb, C.: J. Exper. Med., 42, S21. 1925. 
of temperature, ${ }^{\mathrm{I}}$ or to size and character of plaques, ${ }^{2}$ but particularly in connection with the strict serological specificity existing even among closely related phages. ${ }^{3}$ This specificity, as stated earlier, is quite characteristic for each phage and, in the case of polyvalent phages, is independent of the nature of the bacterial substratum used for obtaining such phages. ${ }^{4}$ The contrary conclusions of D'Herelle as to the similarity of the antigenic nature of different "races" of Bacteriophagum intestinale were drawn by him on the basis of the complement-fixation tests, and have been shown to be due to a non-specific fixation of complement by the rabbit serum used by him as a source of antibody. Sachs and Klopstock, ${ }^{5}$ Sanderson, ${ }^{6}$ and Flu have independently called attention to the fact that rabbit serum fixes complement in the presence of various phages, as it does also when peptone, broth, or bacterial extracts are employed as antigen.

Moreover, it has been shown repeatedly that the secondary growth of bacteria appearing after the completion of lysis of susceptible bacteria by a given bacteriophage is specifically resistant to this bacteriophage, but is often susceptible to lysis by some of the closely related varieties. ${ }^{8}$ According to D'Herelle, this phenomenon does not prove the plurality of phages, for he has observer a similar phenomenon by using a single polyvalent phage acting on B.typhosus, B. coli, and B. dysenteriae. When grown in the presence of this phage, $B$. typhosus gave resistants which were susceptible to the lytic action of the filtrate obtained by growing $B$. coli or $B$. $d y$ senteriae in the presence of the same phage.

The writer has repeated these experiments, using a similar polyvalent phage, but has failed to confirm the findings of D'Herelle.?

Another characteristic differentiating various phages from one another is their respective specificity or extent of activity ("virulence," in the terminology of D'Herelle). Usually, when recovered from convalescent human beings or animals, the samples of phage are active exclusively or mainly against the species of bacteria concerned in the infection. Often, however, not only recently isolated and thus potentially mixed samples of phage, but also purified (ultra-pure) phages, act on several and

${ }^{1}$ Osumi, S.: loc. cit.; Wagemans, J.: loc. cit.; Bruynoghe, R.: Arch. internat. méd. expér., I, I7. 1924; Gratia, A.: Compt. rend. Soc. de biol., 89, 821. 1923.

${ }^{2}$ Wagemans. J.: loc. cit.; Bruynoghe, R.: Arch. intcrnat. méd. expér., I, I7. I924; Hoder, F.: loc. cit.; Okuda, S.: Arch.f. Hyg., 92, Io9. 1923-24; Matsumoto, T.: Centralbl.f. Bakteriol., Orig., 9I, 4I3. I924.

3 Seiffert, W.: loc. cit.; Wagemans, J.: loc. cit.; Bruynoghe, R.: Arch. internat. méd. cxpér., I, 17. 1924; Gratia, A.: loc. cit.; Bail, O., and Watanabe, T.: Wien. klin. Wchnschr., 35, I69. I922; Bruynoghe, R., and Appelmans, R.: Compt. rend. Soc. de biol., 87, 96. I922.

${ }_{4}$ Seiffert, W.: loc. cit.; Wagemans, J.: loc. cit.; Bruynoghe, R.: Arch. intcrnat. méd. cxpér., I, I7. 1924 .

5 Sachs, H., Klopstock, A., and Takenomata, N.: Klin. Wchnschr., 3, 21. I924.

${ }^{6}$ Sanderson, E. S.: J. Immunol., ro, 625. 1925.

7 Flu, P. C.: Centralbl.f. Bakteriol., 97, 224. 1926.

${ }^{8}$ Bruynoghe, R.: Arch. internat. méd. cxpêr., 1, 17. 1924; Hoder, F.: loc. cit.; Gratia, A.: loc. cit.; Matsumoto, T.: loc. cit.; Wien klin. Wchnschr., 36, 759. I923; Bail, O.: Ztschr. f. Immunitätsforsch.u. exper. Therap., Orig., 38, 57. 1923.

9 Unpublished experiments in collaboration with Muckenfuss in 1926. 
sometimes unrelated bacteria. ${ }^{1}$ Occasionally samples of phage isolated from infected animals may not act at all against the bacteria concerned in the infection, showing activity for unrelated bacteria only. ${ }^{2}$ The fact that bacteriophage may thus act against widely different species of bacteria indicates, according to D'Herelle, that "there is but a single bacteriophage, common to both man and animals, capable by adaptation of acquiring a virulence toward all bacterial species." 3 Thus, under "suitable conditions of the moment," a "Shiga-bacteriophage" may be transformed into a "Staphylo-bacteriophage" or into a "Pestis-bacteriophage" in just the same way as by passages a "horse streptococcus" becomes a "rabbit streptococcus" or a "mouse streptococcus." 4

However, while several authors claim to have observed such a transformation of phage, ${ }^{5}$ others have found that this phenomenon is by no means frequent,${ }^{6}$ and many investigators have been unable to accomplish such an adaptation of phage, even to closely related species of bacteria. ${ }^{7}$ It has been suggested that, in such instances in which the adaptation was apparently successful, the authors have been dealing with polyvalent phages whose potential activity toward new bacterial species existed from the start and merely became more pronounced with repeated passage in new culture, due to the gradual transformation of the latter under the influence of the phage. ${ }^{8}$ Another explanation of the apparent adaptation lies in the possibility that the lytic filtrates used in these experiments were in fact mixtures of several independent phages. ${ }^{9}$ This point of view seems particularly suggestive in view of the fact that several of the authors who apparently succeerled in securing the adaptation of phages to new species of bacteria have noticed that the activity of these phages for the original species of bacteria was greatly weakened or lost (apparently through elimination of corresponding phage through the repeated dilution) during the process of adaptation. ${ }^{\mathrm{IO}}$

${ }^{1}$ d'Herelle, F.: The Bactoriophage and Its Bchavior, p. 366. 1926.

${ }^{2}$ Bronfenbrenner, J., Muckenfuss, R., and Korb, C.: J. Exper. Mcd., 44, 608 n. I926; d'Herelle.

F.: The Bacteriophage and Its Eehavior, p 508. 1926.

${ }^{3}$ d'Herełle, F.: The Bactcriophage, Its Rôle in Immunity, p. I21. Williams \& Wilkins, 1922. According to Reichert (loc. cit.), this capacity of the Bactcriophagum intestinale to adapt itself to various bacteria is due to the fact that it possesses a number of radicals which exhibit different lytic properties.

4 d'Herelle, F.: The Bacteriophage and Its Bchavior, p. 367. 1926.

${ }^{5}$ McKinley, E. B.: J. Lab. \& Clin. Med., 8, 31 I. 1922-23; Ionesco-Mihaiesti, C.: J. Exper. Mcd., 40, 317. 1924; Wollman, E., and Wollman, E.: Compl.rend. Soc. de biol., 96, 332. 1927; Appelmans, R.: Arch. intcrnat. pharm. ct therap., 27, 85. 1923.

${ }^{6}$ Otto, R., Munter, H., and Winkler, W. F.: loc. cit.

7 Marshall, M. S.: loc. cit.; Bail, O.: loc. cit.; Matsumoto, T.: Ztschr. f. Immunitätsforsch. $u$. exper. Therap., 41, I. I924; Epstein, T., and Fejgin, B.: Compt. rend. Soc. de biol., 95, 908. 1926.

${ }^{8}$ Gratia, A.: op. cit., 89, 824. 1923; Seiffert, W.: op. cit., 98, 482.1922.

${ }_{9}$ Matsumoto, T.: ITicn. klin. U'chnschr., 36, 759. I923; Bail, O.: loc. ci.

${ }^{10}$ Ionesco-H] haiesti, C.: loc. cit.; Wollman, E., and Wollman, E.: loc. cit.; Appelmans, R.: loc. cit.; Wolff, L. K., and Janzen, J. W.: Ann. de l'Inst. Pasteur, 37, 1064. I923. By mixing two independent phages (coli-phage and staphylococcus-phage) we have been able to imitate this phenomenon of apparent adaptation of one of the phages and gradual elimination of the other, depending on the species of bacteria used for successive passages with this mixed phage. 


\section{RELATION BETWEEN LYSIS AND PRODUCTION OF PIIAGE}

Dissolution of bacteria and the accumulation of the active agent causing this dissolution are the two characteristics which originally centered the attention of Twort on the phenomenon of transmissible lysis. Although in his original publication Twort ${ }^{1}$ described most of the properties of the active agent (which was later called "bacteriophage"), he did not commit himself on the question of its nature, origin, or mode of action. It is apparent, however, that he considered the disintegration of bacteria as the main part of the process, and the regeneration of the active agent as a necessary consequence. With this basic conception in mind, Twort saw a priori three alternative possibilities for explaining the mechanism of the phenomenon he discovered. Each of these possibilities, formulated by him merely as suggestions, were later developed and supported by others. The first two suggestions were outlined on the assumption that the active agent is derived from the bacteria themselves. The third one is based on the assumption that bacteriophage originates outside the bacteria.

The first possibility is that the active agent represents the filterable stage in the abnormal life cycle of bacteria, capable of inducing a similar deviation in the developmental process of other homologous bacteria. ${ }^{2}$ The second possibility is that the active agent is an autolytic enzyme which destroys bacteria and which is, in turn, set free in increasing amounts as bacteria undergo dissolution. ${ }^{3}$ The third possibility suggested by Twort is that the active agent is not a product of bacteria, but an autonomous virus capable of destroying bacteria by causing an acute infectious disease. ${ }^{4}$

As stated previously, all the views of Twort as well as of those who developed them further, and particularly those of D'Herelle, suggest that lysis of bacteria is the main phenomenon, the increase in concentration of the active agent being only the consequence of the former. ${ }^{5}$ Since the accumulation of phage, according to such views,

r'Twort, F. W.: Lancet, 2, I24I. I9I5.

${ }_{2}$ This conception has been later offered independently by Gamaleia (Gamaleia, N. F.: Prophylakticheskaia medicina, p. 28. 1925) and also by Nicolle (Nicolle, C.: Arch. Inst.Pasteur de Tunis, I4, 105. 1925) as the most logical explanation of the phenomenon of transmissible lysis. No experimental evidence was offered by these authors in support of their views.

3 The view that the active agent is a ferment is held by a large group of workers today. However, they fail to consider the fact that living bacteria are not affected by enzymes applied from without. See Kuttner, A. G.: Proc. Soc. Exper. Biol. \& Med., 18, 222. 192 I; Pico, C. E.: Compt. rend. Soc. de biol., 87, 687. 1922; Prausnitz, C.: Klin. Wchnschr., I, 1639. 1922; Hajós, K.: Ztschr.f.Immunitätsforsch., Orig., 37, 147. I923; Combiesco, D.: Compl. rend. Soc.de biol., 87, I 7. 1922; Davison, W. C.: Abstr. Bact., 6, I59. I922.

4 'This is essentially the same conception which was formulated independently by D'Herelle two years later. Following the progress of the Iysis under a microscope, D'Herelle came to the conclusion that the active agent is an organized corpuscular parasite (protobe) which is attracted to bacteria in virtue of chemotaxis. "The protobe then penetrates the peripheral zone of the bacterium (the openings made remaining open) and multiplies within the bacterial protoplasm, forming a colony composed of a number of elements varying with the conditions of the moment. When the process of multiplication is completed the parasitized bacterium ruptures and undergoes a sudden dissolution. This liberates the young protobes, and each of the latter is then ready to repeat the process and parasitize the nearest bacterium. Thus the cycle begins anew, and continues until all of the bacteria present in the media have disappeared" (The Bacteriophage and Its Behavior [trans. Smith]. p. 370. I926).

s d'Herelle, F.: The Bacteriophage and Its Behavior, p. 74. I926. 
depends entirely on its being set free through the dissolution of bacteria, there can be no accumulation of phage without lysis, and lysis must be accompanied by the increase in the concentration of the active agent in the medium.

However, a number of observers have brought forward evidence showing that such is not the case. Careful experiments, in which the rate of regeneration of the phage was followed at the same time as bacterial counts were made, have shown that increase in the phage concentration is entirely independent of the lysis of susceptible bacteria. It was found that in the presence of undiluted phage the susceptible bacteria undergo complete lysis without increasing the concentration of the phage in the medium. ${ }^{\mathrm{I}}$ On the other hand, if the initial concentration of phage in the medium is low, one can detect the production of new phage long before any lysis of bacteria sets in. ${ }^{2}$ Moreover, under special experimental conditions visible lysis can be entirely prevented in spite of the normal regeneration of the phage to its maximum. ${ }^{3}$

According to these findings, far from being the product of lysis, the active agent is regenerated during the stage of active multiplication of susceptible bacteria preceding the lysis. If the rate of multiplication of bacteria is low, either due to the age of the culture or as a result of experimental conditions, the rate of accumulation of the active agent may be not only materially reduced, ${ }^{4}$ but its concentration in the solution may actually diminish through adsorption on bacteria..$^{5}$ Such is the case, for instance, when bacterial cultures containing the phage are incubated at $43^{\circ}-45^{\circ} \mathrm{C}$. instead of $37^{\circ}$ as ordinarily. ${ }^{6}$

That the failure in production of new phage at this teniperature is due to changes in bacteria, and not in the agent itself, can be inferred from the fact that phage is comparatively resistant to heat, and shows no deterioration until the temperature is raised at least above $60^{\circ} \mathrm{C}$. Moreover, it has been found that bacteriophage regen. erates normally even at higher temperatures than $45^{\circ} \mathrm{C}$. if susceptible bacteria car multiply at these temperatures. While ordinarily the optimum temperature for the regeneration of phage is about $37^{\circ} \mathrm{C}$. (with extreme limits beyond which no traces of regeneration or lysis can be observed, at $8^{\circ}$ and $48^{\circ} \mathrm{C}$., respectively ${ }^{7}$ ), the phage for

r Matsumoto, T.: loc. cit.; Bail, O., and Matsumoto, T.: Mcd. Klin., 19, I579. I923; Meuli, H.: Zischr. f. IIyg. u. Infektionskrankh., 99, 46. 1923.

${ }^{2}$ Doerr, R., and Gruninger, W.: loc. cit.; Saldanha, A.: Arch. do Inst. bact. Camara Pcstana, 5, 266. 1924. A similar observation was made also by Muckenfuss in the laboratory of the writer.

3 da Costa Cruz, J.: loc. cit.; Brutsaert, P.: loc. cit.; Matsumoto, T.: loc. cit.; Saldanha, A.: loc. cit.; Doerr, R., and Berger, W.: Ztschr.f. Hyg. u. Infektionskrankh., 97, 422. 1923; Otto, R., and Munter, H.: ibid., roo, 402. 1923; da Costa Cruz, J.: Mem. do Inst. Oszualdo Crus, 16, 104. I922; Zdansky, E.: Seuchenbekämpfung, 2, I50. 1925.

${ }^{4}$ Doerr, R., and Gruninger, W.: loc. cit.; Zdansky, E.: loc. cit.; d'Herelle, F.: Compt. rend. Acad. sc., I67, 970. I9I8; Otto, R., and Winkler, W. F.: Deutsche med. W'chnschr., 48, 383. I922; Doerr, R.: Schuciz. med. Wrchnschr., 53, 1009. 1923; Scheidegger, E.: Ztschr. f. IIyg. u. Infektionskrankh., 99, 403. May I5, 1923; Bruynoghe, R., and Mund, W.: Compt. rcn.. Soc. de biol., 92, 464. I925.

${ }^{5}$ Otto, R., and Winkler, W. F.: loc. cit.; Doerr, R.: loc. cit.

${ }^{6}$ Doerr, R.: loc. cit.; Kuttner, A. G.: Proc. Soc. Exper. Biol. \& Med., I8, I58. 1921; d'Herelle, F.: The Bacteriophage and Its Bchavior, p. 75. 1926. According to I'Herelle, this phenomenon is not general and can be reproduced with certain strains of phage only (d'Herelle, F.: The Bacteriophage and Its Behavior, p. 6r. 1926).

7 d'Herelle, F.: The Bactcriophage and Its Behavior, p. 75. 1926. 
thermophilic bacteria was found to regenerate best at $45^{\circ}$ to $52^{\circ} \mathrm{C} .^{1}$ At $37^{\circ} \mathrm{C}$. the growth of thermophilic bacteria is retarded, and regeneration of phage is correspondingly less rapid and less complete. Similarly, in the presence of psychrophilic bacteria, multiplying best at a temperature of from $0.05^{\circ}$ to $4^{\circ} \mathrm{C}$., the phage exhibits best regeneration at this temperature range and does not regenerate at all at $37^{\circ} \mathrm{C}$., at which temperature the growth of psychrophilic bacteria is arrested. ${ }^{2}$

Thus, the regeneration of phage takes place only so long as the conditions permit normal multiplication of susceptible bacteria. ${ }^{3}$ Lysis of bacteria is a secondary phenomenon which may or may not follow the accumulation of phage, depending on conditions of environment.

\section{BACTERIAL ORIGIN OF BACTERIOPIIAGE}

The fact that accumulation of active agent in the medium is independent of lysis, but is intimately connected with the active growth of bacteria, strengthens the view expressed earlier that the phage may be some product of bacterial metabolism.

Usually, in order to obtain active bacteriophage, one introduces into a culture of bacteria some material contaminated more or less directly with intestinal contents of higher animals. The nature of this material is such that the possibility of the presence of some living ultra-microscopic agent in it cannot be excluded. If one were able to bring about the appearance and accumulation of the agent of transmissible lysis in the cultures of bacteria by means which would exclude every possibility of introduction of an extraneous living agent, the bacterial origin of phage would be definitely established.

A number of investigators claim to have accomplished this by a variety of procedures. However, their experiments are not free from the criticism that either the material they used to induce the appearance of phage or the bacterial cultures themselves might not have been actually free from phage. From what is known about the wide distribution of phage in the animal body, ${ }_{4}$ it is quite evident that phage obtained by introducing bacteria into the peritoneal cavity of guinea pigs,${ }^{5}$ or by exposing bacteria in vitro to the action of leukocytes, tissue extracts, body fluids, and ferments, ${ }^{6}$

${ }^{1}$ Koser, S. A.: Proc. Soc. Exper. Biol. Eo Med., 24, Iog. 1926.

${ }^{2}$ Elder, A. L., and Tanner, F. W.: ibid., p. 645. I927.

3 The experiments of Wollman (Compt.rend. Soc. de biol., 84, 3. I92I) and those of Jötten (loc. cit.), which suggest that regeneration of phage may take place in the absence of actively multiplying bacteria, do not invalidate this conclusion. These experiments were fully discussed in an earlier part of this paper.

${ }_{4}^{4}$ d'Herelle, F.: Compt. rend. Soc. de biol., 86, 477. 1922; ibid., 85, 767. I921; Hoder, F., and Suzuki, K.: Centralbl. f. Bakteriol., 98, 433. i926; Flu, P. C.: Compt. rend. Soc. de biol., 89, 970. I923; Pico, C. E.: ibid., 91, 31. I924; Wolff, L.: Nederl. Tijdschr. v. Geneesk., 2, I220. I925.

5 Otto, R., Munter, H., and Winkler, W. F.: loc. cit.; Bordet, J., and Ciuca, M.: Compt. rend. Soc. de biol., 83, I293. 1920; Bordet, J., and Ciuca, M.: ibid., p. I 296. 1920; Blair, J. E.: J. Infect. Dis., 35, 401. 1924.

${ }^{6}$ Jötten, K. W.: loc. cit.; Kuttner, A. G.: loc. cit.; Pico, C. E.: loc. cit.; Bordet, J., and Ciuca, M.: loc. cit.; Borchardt, W.: Ztschr.f. Immunitätsforsch. u. exper. Therap., Orig., 37, I. I923; Bürgers, T. J., and Bachmann, W.: ibid., ror, 350. 1924; Pico, C. E.: op. cit., 89, 753. 1923; Lisbonne, M., Boulet, L., and Carrère, L.: ibid., 86, 340. I922; Manoliu, E.: ibid., 92, 724. I925; Gratia, A.: ibid., 85, 25. I921; Putter, E., and Vallen, S.: Klin. Wchnschr., 2, 339. I923. 
cannot be considered as proof of a "spontaneous" appearance of the active agent. All these substances may conceivably be contaminated with phage. ${ }^{I}$ On the other hand, it is known that many strains of bacteria (particularly those of recent isolation) frequently carry phage ${ }^{2}$ which cann ot always be demonstrated, probably because only a few individuals in the culture may be susceptible to its action. Thus, the phage may be carried by a given culture, unnoticed for a time, and may become manifest if the susceptibility of the culture is suddenly changed, or if the filtrates of such "lysogenic" cultures are allowed to act upon more susceptible strains of homologous and occasionally even of heterologous bacteria. This "lysogenic" power of some cultures, manifesting itself against other homologous and heterologous bacteria, has been given as an instance of "spontaneously" arising phage action as the result of bacterial antibiosis. ${ }^{3}$ However, the writer has been able to imitate this phenomenon in every detail by contaminating cultures with phage artificially. Subjecting a phage-free culture of colon bacilli to the action of a polyvalent phage and isolating the overgrowth of resistants, we found it to carry the phage for months without showing any signs of its presence. 4 When, however, this resistant culture was grown in symbiosis with stock cultures of $B$. coli or of $B$. dysenteriae (Shiga), the presence of phage could be readily demonstrated. 5

In view of the fact that cultures of bacteria may thus carry phage without revealing any signs of its presence, one must be very cautious in interpreting the sudden appearance of phage in cultures subjected to various influences of physical or chemical nature, or even in cultures merely kept in the laboratory for a long period of time. ${ }^{6}$ Quite apart from the possibility of occasional direct contamination of these cultures with phage present in the laboratory, such manipulations as, for example, filtration through Berkefeld filters (which is a usual step in the preparation of bacteriophage) have been known to result in contamination of filtrates with phage ${ }^{7}$ re-

I The only promising experiments of this nature have been reported by Doerr (loc. cit.) who obtained bacteriophage by feeding susceptible bacteria to sterilely hatched chickens. These experiments have not been repeated, however, and it is impossible to say at present whether or not the result was merely an accidental occurrence. Somewhat similar experiments were performed by Ebeling in collaboration with the writer, but without obtaining the same result in the preliminary trials. In these experiments bacteria were introduced into the pure cultures of cells of intestinal mucosa of a chick embryo.

${ }^{2}$ Dutton, L. O.: J. Infect. Dis., 39, 48. I926; Manninger, R.: Ccntralbl. f. Bakteriol., 99, 203. 1926.

3 Gildemeister, E., and Herzberg, K.: Contralbl.f. Baktcriol., Ref., 77, 188. 1924; ibid., 93, 402. 1924; Lisbonne, M., and Carrère, L.: Compt. rend. Soc. de biol., 86, 569. 1922; ibid., 87, I0I I. I922; Bordet, J.: ibid., 93, 1054. 1925.

${ }^{4} \mathrm{~A}$ few susceptible bacteria present among the bulk of resistants apparently permitted very slight regeneration of phage to take place at each transfer, thus securing conditions perpetuating the resistance of the majority of bacteria in the culture.

${ }_{5}^{5}$ Unpublished experiments in collaboration with Muckenfuss, 1927.

${ }^{6}$ Jötten, K. W.: loc. cit.; Otto, R., and Winkler, W. F.: loc. cit.; Blair, J. E.: loc. cit.; Gaté, J., and Gardère, H.: Compt. rend. Soc. de biol., 96, 545. 1927; Gildemeister, E., and Herzberg, K.: Centralbl.f. Baktcriol., 91, I2. I923; Ogata, N.: ibid., Orig., 93, 329. 1924.

7 Otto, R., and Winkler, W. F.: loc. cil.; Putter, E., and Vallen, S.: loc. cit.; Weinberg, M., and Aznar, P.: Compt. rend. Soc. de biol., 86, 833. 1922; ibid., 87, 136. 1922; Seiffert, W.: MIcd. Klin., 19, 833. I923; Otto, R., and Munter, H.: Deutsche med. Wchnschr., 48, 382. 1922. 
maining on the filter from previous experiments and not completely removed in washing and sterilization of the candles. ${ }^{\text {I }}$ Furthermore, the "spontaneous" production of phage by addition to the culture of various chemicals" (including distilled water) ${ }^{3}$ may conceivably be the result of making apparent the phage already existing in the culture through suppression of variants most susceptible to a given injurious influence, and permitting the growth of only such variants as happen to be more resistant to it. Such selected bacterial populations may happen occasionally to be more susceptible to the phage carried by the culture all along. No matter how remote such a possibility of eliciting latent phage may seem to be, it should always be kept in mind, particularly as those investigators who were successful in observing the "spontaneous" appearance of phage were not able to obtain this result with every culture, nor repeatedly with the same culture. ${ }^{4}$ Others were never successful in their attempts to reproduce this phenomenon. ${ }^{5}$ During the period of the last four years, the writer has attempted repeatedly, by various procedures, to induce the spontaneous appearance of phage in a variety of cultures known to be free from phage, but thus far the results have been consistently negative.

Although facts concerning the phenomenon of bacteriophagy leave an impression that the active agent of transmissible lysis is a bacterial product, the reports of successful production of phage from bacteria directly without the intermediary of any biological material have thus far not been entirely convincing.

\section{THE MECHANISM OF CLEARING OF CULTURES BY PHAGE}

The investigations reviewed in the preceding pages indicate that D'Herelle's conception that the agent responsible for the phenomenon of transmissible lysis of bacteria is a living organism is not supported by the evidence of later workers. Although the agent represents an autonomous antigenic entity and is probably a colloid, it cannot be identified with the particles which apparently carry it. The agent (bacteriophage) does not exhibit any demonstrable metabolic activity when studied in the absence of living susceptible bacteria, and in the presence of the latter the rate of metabolism depends only on the number of living bacteria present, and not on the concentration of the agent. The conclusion as to the apparent power of the agent to adapt itself to changes in environment rests on inconclusive evidence. The fact that the regeneration of the active agent depends wholly on the presence of living, susceptible bacteria suggests that it may be itself a product of some phase of bacterial activity. Indeed, several investigators have reported their success in obtaining the active agent directly from bacteria. However, their results are not free from possible criticism. At all events, it is clear that when traces of active agents are introduced into a grow-

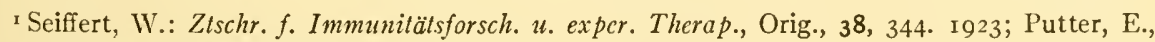
and Vallen, S.: Klin. Wchnschr., 2, 1072. 1923.

${ }^{2}$ Otto, R., and Winkler, W. F.: loc. cit.; Otto, R., and Munter, H.: loc. cit.; Wolff, L. K., and Janzen, J. W., Compl. rcnd. Soc. de biol., 87, 1087. 1922; Botez, A.: ibid., 85, 585. I921; Petrovanu, G.: ibid., 92, 459. 1925 .

3 Otto, R., and Winkler, W. F.: loc. cit.; Weinberg, M., and Aznar, P.: loc. cit.

4 von Preisz, H.: loc. cil.; Otto, R., Munter, H., and Winkler, W. F.: loc. cit.; Flu, P. C.: Centralbl. f. Bakleriol., 90, 362. 1923.

${ }^{5}$ Borchardt, W.: loc. cit. 
ing culture of susceptible bacteria, the regeneration of the agent takes place during the phase of active growth, and precedes the lysis of bacteria (when the latter takes place at all). Thus, the possibility that the active agent is liberated as a result of lysis of bacteria seems to be excluded.

As to the actual nature of the active substance and the mechanism of its production by bacteria, there exists great divergence of opinion. ${ }^{x}$ So far there has been no definite evidence offered to substantiate any of the opinions expressed. However, since the active agent is comparatively stable under ordinary conditions, there is hope that it may be isolated in pure state and its nature may then be determined. ${ }^{2}$

As to the nature of the effect of this hypothetical substance on normal bacteria, resulting on the one hand in its own regeneration and on the other in the disappearance of visible bacterial growth, there exist two views. The majority of workers think that this effect consists in the vitiation of bacterial metabolism accompanied by the accumulation of products which under normal conditions either are not formed at all, or appear only temporarily as intermediary links in the chain of metabolic reactions. The accumulation of these products in turn causes disfunction of new generations of bacteria, and thus perpetuates the disease leading finally to autolysis of the bacteria. ${ }^{3}$ Thus far, however, no proof has been brought forward to identify the

${ }^{1}$ Nicolle, C.: loc. cit.; Otto, R., and Winkler, W. F.: loc. cit.; Bordet, J., and Ciuca, M.: Compt. rend. Soc. de biol., 83, 1293. 1920; Kabeshima, T.: ibid., p. 219. 1920; Doerr, R.: Klin. W chnschr., r, 1489, 1537. 1922; Bail, O.: JVien. klin. Wchnschr., 35, 765. 1922; Eastwood, A.: J. Hyg., 23, 317. 1924; Hadley, P.: J. Infect. Dis., 40, 1. 1927; Rosenthal, L.: Compt. rend. Soc. de biol., 95, 61 2. 1926.

${ }^{2}$ Some of the experiments now in progress in the writer's laboratory indicate that purification of this agent can be carried to a point where it no longer gives any of the protein tests, though its activity or antigenic properties remain unimpaired.

3 According to some investigators, the products of this vitiated metabolism of bacteria possess enzymatic properties (Kuttner, A. G.: loc. cit.; Pico, C. E.: loc. cit.; Prausnitz, C.: loc. cit.; Hajós, K.: loc. cit.; Saldanha, A.: loc. cit.; Otto, R., and Winkler, W. F.: loc.cit.) or are capable of acting as catalysts activating the normal intracellular proferments of the bacteria (Kabeshima, T.: loc. cil.; Burnet, F. M.: J. Path. \& Bact., 28, 407. 1925). The relative specificity of phage action is explained by them on the basis of Salkowsky's findings that ferments in general (and proteolytic ferments in particular) are often most active against the constituents of such cells which originally produced them (Salkowsky, E., quoted from von Preisz, H.: Die Baktcriophagie, p. 9o. Fischer, 1925).

According to others, these products resemble growth hormones (Doerr, R.: loc. cit.) or exhibit cytotoxic properties (Meuli, H.: loc. cit.), and increase the rate of metabolic activities of bacteria to such an extent that lysis occurs as a terminal stage of this trophic disease.

It has also been suggested that with the abnormally rapid rate of growth, the amount of proteolytic enzyme set free by the bacteria is so great that the supply of protein is exhausted and the ferments attack the bacteria themselves (Seiffert, W.: Seuchenbekämpfung, 2, 234. 1925).

According to Bail (Bail, O.: Dentsche med. Wchnschr., 51, 13. 1925), autolysis of bacteria is due to the loss by the bacterial chromosomes of their anabolic function. The cells with imperfect chromosomes, possessing only the function of katabolism, undergo dissolution. The imperfect chromosomes are thereby set free, and are capable of initiating similar vitiation in other bacteria.

Other workers suggest that the abnormality in bacterial development leading to autolysis is transmitted from generation to generation by heredity (Wollman, E.: Ann. de l'Inst. Pasteur, 39, 789. 1925; Bordet, J., and Ciuca, M.: Compt. rend. Soc. de biol., 83, 1293. 1920), or is perpetuated through a continuous effect of bacterial dissociation which results in the tendency of the culture to give off increasing numbers of non-viable, autolyzing variants (Eastwood, A.: loc. cit.; Arkwright, J. A.: Brit. J. Exper. Path., 5, 23. 1924; Hoder, F.: Zlschr. f. Immunitätsforsch. u. expcr. Therap., 42, 197. 1925). 
products accumulating in the culture with those variously assumed to be present by different workers. There is also no explanation of the mechanism responsible for the sudden onset and extremely rapid progress of autolysis of the culture. Besides, repeated chemical analyses of the cultures, after completion of lysis, have failed to indicate any increase in protein split products which would be expected to take place if bacteria were autolyzed. ${ }^{x}$

The opinion of another group of workers is, therefore, that the disappearance of the bacteria is not due to hydrolysis, but is the result either of a simple plasmolysis ${ }^{2}$ or of a special, highly hypothetical type of cleavage not involving chemical disintegration of bacterial cells. ${ }^{3}$ These authors offer no experimental evidence in support of their views.

Some light as to the nature of changes actually taking place in the bacteria under the influence of the active agent seems to come from the direct observation of lysis under the microscope. As has been mentioned earlier, D'Herelle has observed that soon after the exposure of bacteria to bacteriophage the former begin to swell and finally burst, due to internal pressure which he thought was exerted by the parasites multiplying within the cells. ${ }^{4}$ While the swelling of bacteria under the influence of phage has been repeatedly observed by others, ${ }^{5}$ its constancy or its importance as a factor in the disappearance of bacteria has not been generally accepted. The impression gained by several workers from observation of fresh as well as of stained preparations is that only comparatively few bacteria undergo swelling, and that the great majority of bacteria disappear without undergoing any visible change ${ }^{6}$ (Fig.2). Moreover, it has been indicated that swollen bacteria do not end up by bursting, ${ }^{7}$ but, on the contrary, they appear particularly resistant and disappear very slowly, if at all. ${ }^{8}$

However, the extent of the swelling of individual bacteria, the relative proportion of swollen cells, as well as the actual relation between the swelling and the lysis, are difficult to establish by direct observation alone; the swelling and the lysis go on

${ }^{I}$ Weiss, E., and Arnold, L.: loc. cit.; Ionesco-Mihaiesti, C.: J. Exper. Med., 40, 317. 1924: Zdansky, E.: loc. cit.

${ }^{2}$ Ionesco-Mihaiesti, C.: loc. cit.

${ }_{3}$ Some workers postulate the existence of a complex life-cycle in the development of bacteria, during which bacteria may give rise either to functionally incomplete and not self-perpetuating elements ("spermites" [Hadley, P.: loc. cit.] or "ultraspores" [Rosenthal, L.: loc. cit.]) which possess all the properties of bacteriophage. Others believe that bacteria may give rise to living, filterable fragments which are capable of perpetuating this transformation by causing abnormally rapid growth of the culture either directly (Nicolle, C.: loc. cit.) or through the intermediation of ferments secreted by them (Gamaleia, N. F.: loc. cit).

${ }_{4}$ d'Herelle, F.: Compt. rend. Soc. de biol., 84, 339. I921; The Bacteriophage and Its Bchavior. p. II 5 . I 926 .

${ }^{5}$ von Preisz, H.: loc. cit.; Wollman, E.: Ann. de l'Inst. Pastcur, 39, 789. I925; da Costa Cruz, J.: loc. cit.; Zdansky, E.: loc. cit.; Manninger, R.: loc. cit.; Burnet, F. M. J. Path. \&o Bact., 28 , 411. 1925; Twort, F. W.: Brit. M. J., 2, 293. 1922; Hauduroy, P.: Compt. rend. Soc. de biol., 91 I325. 1924 .

${ }^{6}$ von Preisz, H.: loc. cit.; Manninger, R.: loc. cit.; Burnet, F. M.: loc. cit.; da Costa Cruz, J. Compt. rend. Soc. de biol., 95, 1501.1926.

7 Wollman, E.: Ann. de l'Inst. Pasteur, 39, 789. 1925; Burnet, F. M.: loc. cit.

${ }^{8}$ von Preisz, H.: loc. cit.; Burnet, F. M.: loc. cit.; da Costa Cruz, J.: Compt. rend. Soc. de bion. 95, I 501. 1926; Gjфrup, E.: Thesis. Copenhagen, 1925. 
simultaneously and continuously, and at different rates in the case of different bacteria in the same culture (Fig.2). Moreover, the actual disappearance of individual bacteria from the field of observation in fresh preparations is so sudden and rapid that it is difficult to ascertain accurately the morphological appearance of each bacterium just prior to its disappearance. It is evident that such direct observations must lead to highly divergent conclusions when carried out by different investigators, and even by the same investigator at different times. ${ }^{\mathrm{T}}$

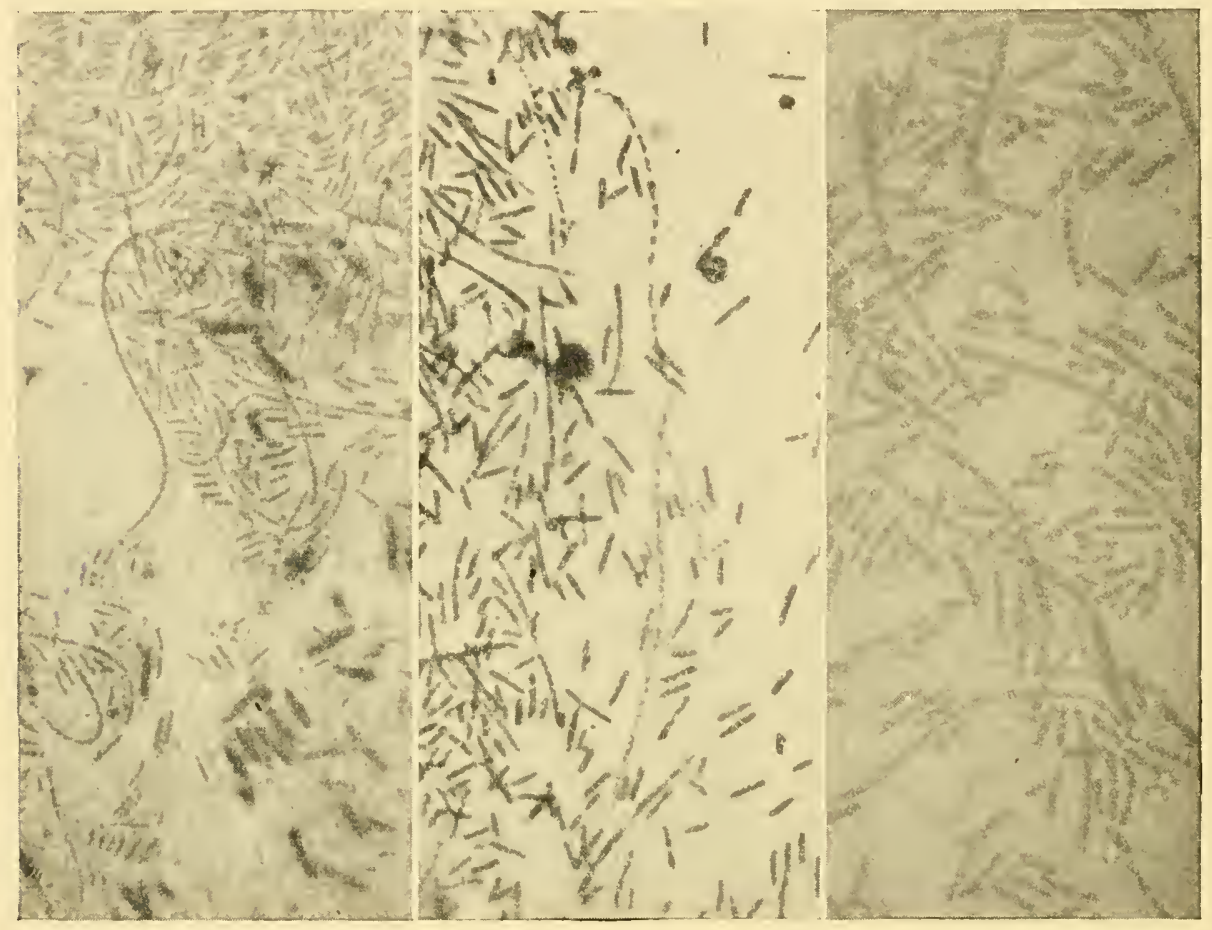

FIG. 2.-A field of active bacteriophagy in early stages, showing simultaneous presence of normal bacteria, threads, and swollen bacteria. Stained with carbol-fuchsin, followed by methylene blue. Eastman $\mathrm{K}_{2}$ yellow filter. Magnification $X_{1,500}$.

FIG. 3.-Granular appearance of the cytoplasm of threads. Stained with carbol brilliant green, counterstained by fuchsin. Photographed through green filter. Magnification $\times 1,500$.

Fig. 4. - Swelling of bacteria and of threads. Stained with carbol-fuchsin, followed by methylene blue. Photographed through green filter. Nagnification $\times 1,500$.

The writer thought that an objective index of degree of swelling, and a definite relation of swelling to lysis, might be established by a study of changes in the relative volume occupied by the entire bacterial population as expressed in the viscosity of the culture. ${ }^{2}$ These measurements have indicated that in about one or two hours ${ }^{3}$ after the addition of bacteriophage to a heavy suspension of susceptible bacteria, the viscosity of the mixture begins to increase slowly up to the time just previous to lysis, when it reaches its maximum. About the time when clearing begins, the viscosity of the mixture diminishes and in a comparatively short time reaches

${ }^{1}$ von Prcisz, H.: loc. cit. ${ }^{2}$ Bronfenbrenner, J.: Proc. Soc. Exper. Biol. Eo Mcd., 23, 635. 1926.

3 This interval varies with the concentration of phage. 
the initial level. If, in place of susceptible bacteria, one employs a culture of a homologous resistant strain, or of heterologous bacteria, or a suspension of susceptible bacteria killed by heat, the viscosity of the mixture remains constant. Similarly, the viscosity of the mixture remains unaffected if heated phage is employed in place of the active preparation. As calculated on the basis of viscosity changes, ${ }^{1}$ the entire bacterial mass occupies, at the time of maximum swelling, from six to twelve (or more) times the volume occupied by a similar number of normal bacteria. The fact that, following visible clearing, the viscosity of the mixture reaches approximately its

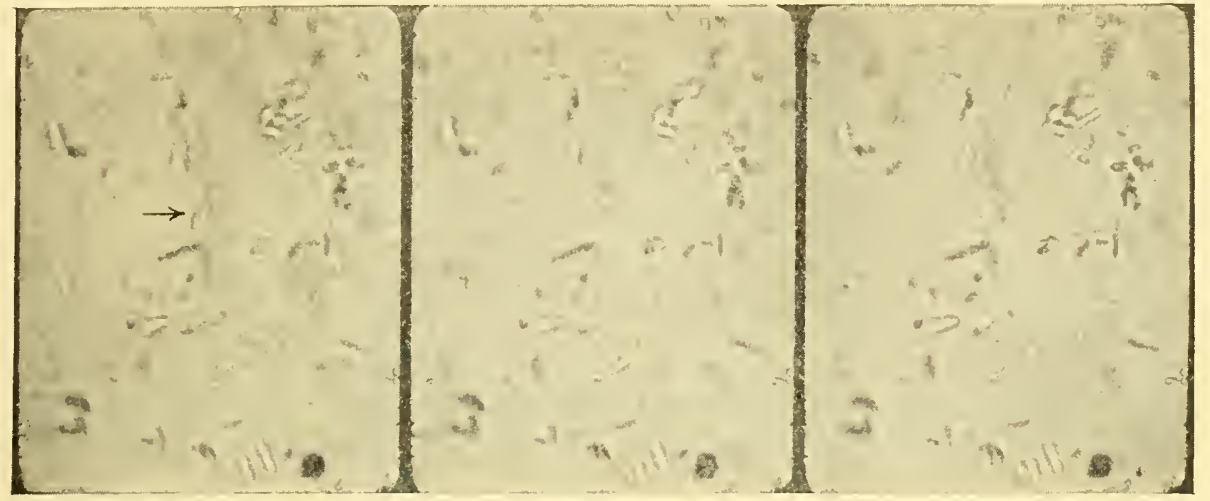

Fic. 5.-Section of a cinematographic record, representing three successive exposures taken at 2.5 seconds' intervals and showing disappearance of two slightly swollen cells. Magnification $\times 1,500$.

original level (or even below it), seems to indicate that the swollen bacteria must be the ones which eventually disappear.

This conclusion was confirmed by a cinematographic record of lysis of $B$. coli on agar, at $37^{\circ} \mathrm{C}^{2}$ As the illustrations show, swollen bacteria with perfect outline in one photograph are completely gone in the next taken less than three seconds later (Figs. 5 and 6). Usually in the space formerly occupied by a bacterium, there remains nothing but the faintest trace of amorphous débris, which, in turn, disappears in less than one minute, leaving only a few more or less refractile granules (Fig. 5). Occasionally the débris left after the disappearance of the bacteria is less easily soluble, and is recorded on the film for a longer period of time (Fig. 6). This happens usually

r Kunitz, M.: J. Gencral Physiol., 9, 715. 1926.

2 Bronfenbrenner, J., Nuckenfuss, R., and Hetler, D.: Am. Jour. of Pathol. (In press.) The progress of lysis was photographed by Mr. Rosenberger by means of an automatic camera at a rate of twenty-five exposures per minute. The record shows that, following a short period of lag, bacteria began to multiply at a rate noticeably exceeding that of normal bacteria photographed under similar conditions but in the absence of phage. (This stimulating effect of phage has been reported by a number of investigators [Schwartzmann, G.: loc. cit.; d'Herelle, F.: The Bactcriophage and Its Behavior, p. 74. 1926; Meuli, H.: loc. cit.; Saldanha, A.: loc. cit.; Doerr, R.: loc. cit.; Hadley, P.: J. Infect. Dis., 37, 35. 1925]). Owing to the rapidity of growth, many cells failed to complete division, and gave rise to filaments having a length of from ten to twenty times that of the average length of a normal cell (Fig. 3). By the end of the first hour of growth, occasional cells began to show signs of swelling, and by the end of the third hour the great majority of cells in the field appeared more or less swollen, a few among them reaching several times their normal dimensions. Filaments swelled as well as ordinary bacteria (Fig. 4). The swelling continued slowly until about the fifth hour, when, one by one, bacteria began to disappear suddenly and quickly. Some of the bacteria disappeared when only moderately swollen. 
in the case of cells which disappear after having undergone only moderate swelling. Extremely swollen cells leave practically no trace after their sudden disappearance.

The rapid melting away of the bulk of the cytoplasm (which under ordinary circumstances is supposed to be semi-gelatinous in consistency) suggests that it probably was liquefied within the cell prior to the disappearance of themembrane. A similarconclusion is suggested also by the appearance of bacteria in the stained preparations. When swollen bacteria are stained by the method of Gutstein, ${ }^{\mathrm{I}}$ the cytoplasm may be differentiated from the ectoplasm. The latter always appears continuous, and even in extremely distended cells it shows no evidence of "holes" described by D'Herelle as resulting from the puncturing of the membrane by the entering parasites. The

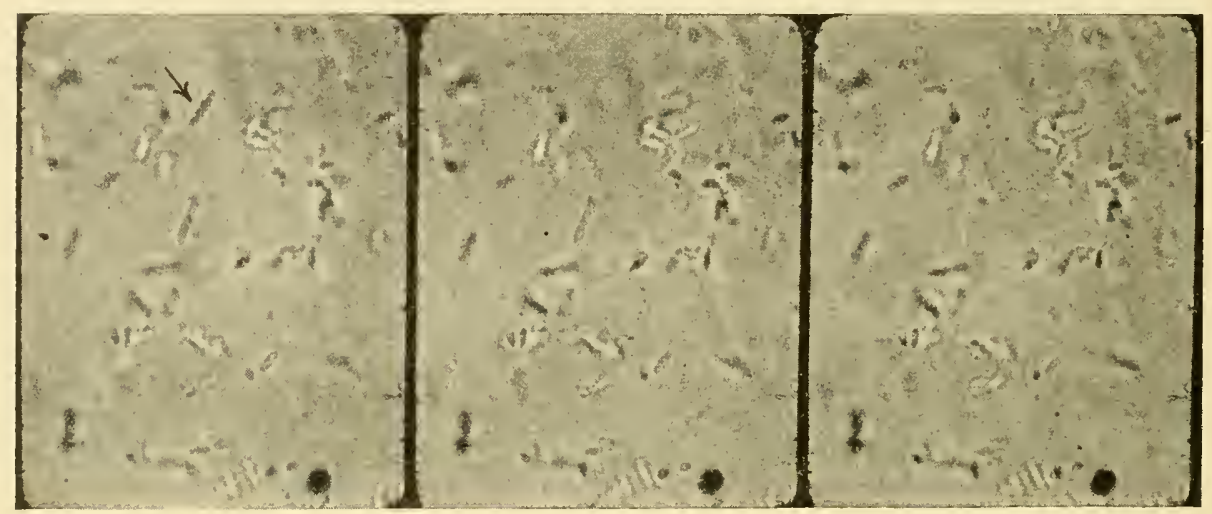

FIG. 6.- Section of a cinematographic record, representing three successive exposures taken at 2.5 seconds' intervals and showing disappearance of a moderately swollen cell. Magnification $\times 1,500$.

cytoplasm, on the contrary, shows marked changes during swelling. It takes the stain less intensely and less evenly as the swelling progresses, so that in many instances it appears segmented or beaded (Fig. 7) ${ }^{3}$ In cells photographed at this stage unstained, by means of ultra-violet light illumination, ${ }^{4}$ the cytoplasm appears to be of uneven density, quite unlike that of normal bacteria. Some of the stained preparations of highly swollen bacteria suggest that the material which normally binds the chromatin has become digested, and the chromatin distributed throughout the cytoplasm in minute dust-like particles (Fig. 8).

If the conclusion drawn from these observations is correct, the cytoplasm must have undergone digestion during the progress of swelling, so that when it was set free, following the disappearance of the cell wall, it went immediately into solution. If such were the case, however, chemical examination of the solution should show the presence of products of digestion. As stated previously, repeated analyses of lysed cultures have failed to indicate the presence of such products. ${ }^{5}$ The failure of these attempts to detect hydrolysis might have been due, however, to the fact that the ma-

IGutstein, M.: Centralbl.f. Baktcriol., Orig., 100, 1. 1926.

${ }^{2}$ d'Herelle, F.: The Bactcriophage and Its Bchavior, p. 115. 1926.

3 Somewhat similar changes were brought out also by the toluidin staining by von Preisz (loc.cil.).

${ }_{4}$ These photographs were secured through the kindness of Dr. F. Gates.

5 Weiss, E., and Arnold, L.: loc. cil.; Ionesco-Mihaesti, C.: loc. cit ; Zdansky, E.: loc. cit. 
terial subjected to analysis consisted of ordinary culture medium too rich in various products of hydrolysis of protein to permit the detection of a possibly small increase due to the hydrolysis of bacteria. The lysis of bacteria in a synthetic medium, devoid of all protein, gave unmistakable evidence of hydrolysis of bacterial protein. ${ }^{\mathrm{r}}$

In the light of these experiments, the clearing of bacterial cultures in the presence of bacteriophage seems to be due to hydrolysis of bacteria. The active agent (bac-
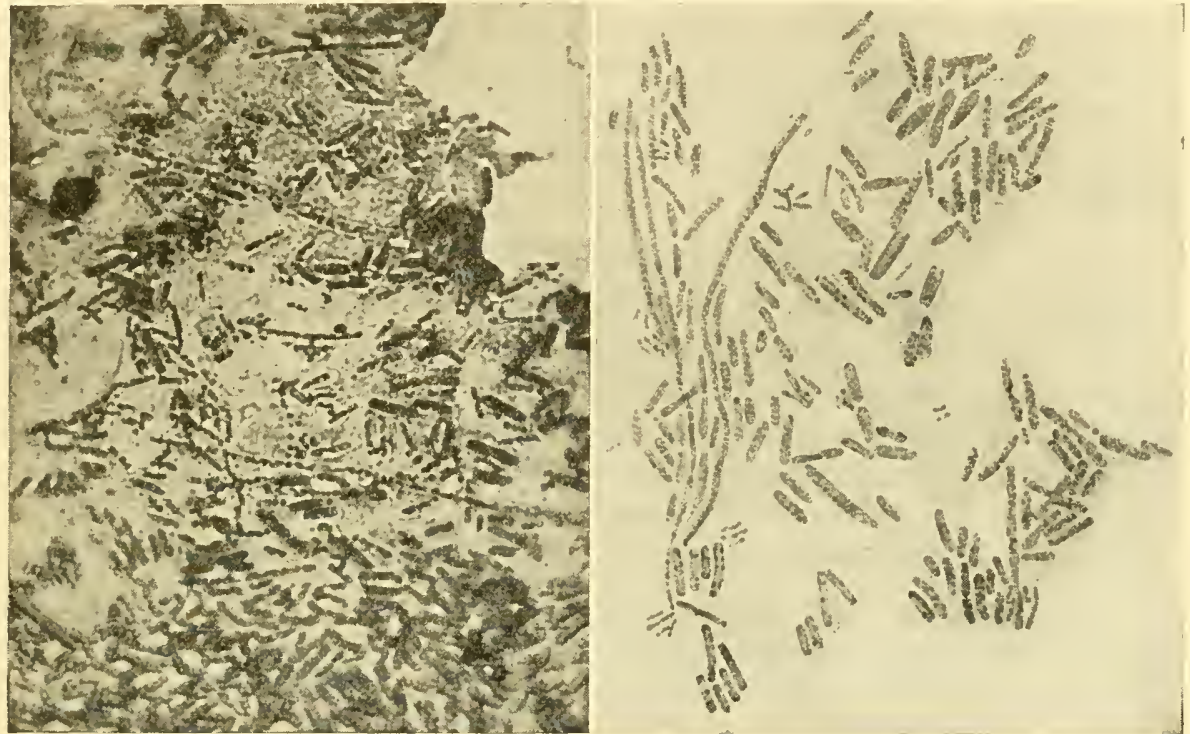

FIG.7.-Advanced stage of disintegration of the cytoplasm with ectoplasm intact. Stained by carbol-fuchsin, followed by methylene blue. Photographed through an orange filter. Magnification $\times 1,500$.

FIG. 8.- Photograph of a drawing, showing distribution of chromatin granules in swollen bac-

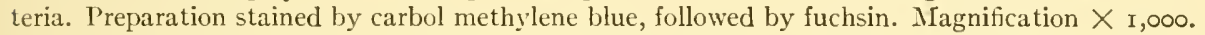

teriophage) plays no part in the actual dissolution. The dissolution is the result of intracellular digestion brought about by normal endoferments. Just how these endoferments become activated remains unknown at present. ${ }^{2}$ The increase of osmotic pressure within the cells, following the beginning of digestion of the cytoplasm, leads to intake of water which probably facilitates further digestion, ${ }^{3}$ and progressive swelling. The degree of swelling of individual bacteria depends on the ability of the membrane to withstand the pressure. When the limit of turgescence of a given cell membrane is reached, it ruptures and the predigested intracellular contents are dissolved in the medium.

The phenomenon of transmissible lysis can thus be divided into two stages. The early stage depends on the presence of the phage, which stimulates bacteria. The

${ }^{\mathrm{I}}$ Bronfenbrenner, J., and Hetler, D.: Jour. Exper. Med. (In press.)

${ }^{2}$ It has been suggested by Warburg (Warburg, O.: Ztschr.f. phy. Chemie, 66, 305. 1910), however, that profound changes may be initiated in a living cell by toxic substances, even in cases where these substances do not penetrate the cell but act on the membrane only.

3 Northrop, J. H.: J. Gencral Plysiol., 4, 245. 1922; Hussey, R. G., and Northrop, J. H.: ibid., 5, 335. 1923. 
mechanism of this stimulation still remains unknown. As a result, the activity of intracellular ferments is increased, and the rate of multiplication is exaggerated. ${ }^{\mathrm{I}}$ Some of the products escape from the cells ${ }^{2}$ into the medium, and in turn produce a stimulating effect on newly formed cells. ${ }^{3}$ These products represent the active agent (bacteriophage). They are toxic only to closely related species of bacteria, hence the multiplicity and relative specificity of different bacteriophages. ${ }^{4}$ The second stage of the process may or may not follow the first, depending on circumstances. It consists in the swelling and eventual bursting of cells due to the intake of water following the increase in osmotic pressure within the cells. Any change in environment which will tend to interfere with the progress of intracellular digestion or with the freedom of passage of water from the medium into the cells may prevent the swelling and disappearance of bacteria.

\section{THE NATURE OF RESISTANCE TO PHAGE}

One of the most interesting and yet comparatively little-understood phenomena connected with the lysis of bacteria by phage is the development of resistant cultures, which takes place in vivo as well as in vitro. ${ }^{5}$ When susceptible bacteria are grown in broth in the presence of bacteriophage, multiplication of bacteria is at first noticeably accelerated, ${ }^{6}$ but later they undergo more or less rapid lysis, and the initial turbidity of the culture disappears. In exceptional cases the culture may be completely and permanently cleared and rendered sterile. In the majority of cases, however, a cer-

'Somewhat similar changes were postulated recently by Armstrong (Armstrong, H. E.: ibid., $8,653.1925^{-27}$ ) in the case of excitation of plant cells.

${ }^{2}$ Some of the experiments on artificial fertilization of eggs suggest that the agents which increase the activity of a cell may cause also an increase in the permeability of the cell membrane, thus permitting the escape of some of the intracellular constituents into the medium (Lyon, E. P., and Shackell, L. F.: Science [N.S.] 32, 249. 1910).

3 It has been recognized for a long time that metabolic products of bacteria are toxic for homologous or closely related species of bacteria (Eijkman, C.: Centralbl. $f$. Bakteriol., Orig., 37, 436. 1904; von Liebermann, L.: Klin IJ'chnschr., 2, I240. 1923). More recently, Osterhout (Osterhout, W. J. V.: J. General Physiol., 7, 561. 1925) has shown that also unchanged intracellular contents may be highly toxic when applied to homologous cells from without. These effects, as ordinarily observed, are depressing in character, whereas in the case of bacteriophage, the effect is that of stimulation. This difference in effect may be due to the difference in the nature of substances concerned, but it is conceivable that it may simply depend on the concentration of injurious agent (Bronfenbremner, J.: Proc. Soc. Exper. Biol. \&o Med., 12, 136. 1915; ibid., 18, 94. 1920).

${ }_{4}^{4}$ The attention of the writer has been called recently to a very interesting paper (Sanfelice, $\mathrm{F}$.: Ztschr.f. IIyg. u. Infektionskrankh., 76, 257. 1914), which explained in I9I4 the mechanism of transmissibility of cpithelioma contagiosum of pigeons on an essentially similar basis. Sanfelice was able to extract from the involved areas of the skin a nucleoprotein-like substance which was regenerated anew when injected into the epithelial cells of the skin of normal birds, and perpetuated the disease in series as if it were a living virus.

5 Dimtza, A.: Centralbl. f. Bakteriol., Orig., เо1, I71. 1927; Sonnenschein, C.: ibid., 97, Beiheft, 312. 1926; Kauffmann, F.: Zischr. f. Hyg. u. Infcktionskrankh., 106, 520. 1926; Grumbach, A., and Dimtza, A.: Zischr.f. Immunitätsforsch. 11. cxper. Therap., 51, 176. 1927.

${ }^{6}$ Doerr, R., and Gruninger, W.: loc. cit.; Schwarzman, G.: loc. cit.; Saldanha, A.: loc. cit.; Hadley, P.: loc. cit.; Doerr, R., and Gruninger, W.: Schwcis. med. Wchnschr., 52, 761. 1922. 
tain number of bacteria fail to undergo lysis and continue to multiply, in spite of the presence of a high concentration of the lytic agent in the medium. This more or less profuse secondary growth consists of bacteria resistant to the action of bacteriophage. Such secondary cultures ordinarily lose their resistance to phage in a short time, if they are transferred repeatedly to new media, to eliminate all traces of phage by dilution, or if they are grown in media to which has been added some specific serum, to eliminate the phage by neutralization. ${ }^{\mathrm{I}}$ Often this loss of resistance takes place slowly, and in its early stages may remain unobserved, unless special effort is made to bring it to light. ${ }^{2}$ Occasionally this property of resisting the action of bacteriophage may be retained by secondary cultures through very long periods of cultivation in the absence of phage. ${ }^{3}$

According to the conception of D'Herelle, appearance of resistant individuals in the susceptible culture is the result of specific adaptation of individual bacteria to the environment. Some cultures, according to him, may exhibit a "natural resistance" to certain races of bacteriophage (although they may be lysed by others). ${ }^{4}$ Other cultures, on the contrary, may be so highly susceptible that all the bacteria are killed by the bacteriophage before they are able to develop any resistance. ${ }^{5}$ Ordinarily, many bacteria in a susceptible culture resist the invasion and "may thus acquire an immunity to the bacteriophage corpuscles which attack them." The phenomena of immunity in bacteria against phage are "strictly comparable with those observed in higher animals." 4 According to Asheshov, this development of resistance is subject to fluctuations entirely analogous to those observed in epidemics among higher animals. ${ }^{6}$ When conditions are suitable, a certain number of bacteria "recover" from infection by directly destroying the bacteriophage corpuscles present within their protoplasm, ${ }^{7}$ or by secreting substances which inhibit the activity of bacteriophage. 8

Apart from the fact that the very possibility of the development of active immunity by the bacteria has been doubted by many, the circumstance that bacteria resistant to one strain of phage may be susceptible to lysis by another homologous

${ }^{2}$ Saldanha, A.: loc. cit.; lMiava, G., and Pozerski, E.: Compt. rend. Soc. de biol., 84, 708. I921; Breinl, F., and Hoder, F.: Centralbl.f. Baktcriol., Orig., 96, I. 1925; Bronfenbrenner, J., and Korb, C: Proc. Soc. Exper. Biol. \& MIcd., 23, 3. 1925; Bronfenbrenner, J., Muckenfuss, R., and Korb, C.: J. Exper. Mcd., 44, 607. 1926; Bordet, J., and Ciuca, M.: Compl.rcnd. Soc. de biol., 84, 748. 192 I.

${ }^{2}$ Failure to recognize this partial return to susceptibility is undoubtedly responsible for the erroneous conclusions of several investigators who believe that the concentration of bacteriophage in the solution may increase at the expense of resistant bacteria (Meissner, G.: loc. cil.; Zdansky, E.: Ztschr.f. IIyg. u. Infektionskrankh., 103, I64. I924; Lepper, E. H.: Brit.J. Exper. Path., 4, 53. 1923).

3 Bronfenbrenner, J., Muckenfuss, R., and Korb, C.: loc. cil.; Bordet, J., and Ciuca, M.: loc. cit.

${ }_{4}^{4}$ d'Herelle, F.: Immunily in-Natural Infectious Disease, p. 259. I924.

5 Asheshov, I. N.: Compl. rend. Soc. de biol., 93, 644. I925; d'Herelle, F.: The Bacteriophage and Its Behavior, pp. IS2-83. 1926.

${ }^{6}$ Asheshov, I. N.: Compl. rend. Soc. de biol., 93, I329. I925.

7 d'Herelle, F.: The Bacteriophage and Its Behavior, p. 239. 1926.

${ }^{8}$ Grumbach, A., and Dimtza, A.: loc. cit.; d'Herelle, F.: The Bucteriophage and Its Behavior, p. 240.1926. 
strain $^{\mathrm{r}}$ indicates that the conception of D'Herelle does not explain the phenomenon satisfactorily. Furthermore, the peculiarities differentiating the secondary from the primary cultures are not limited to the former's resistance to phage. The differences in the behavior of secondary cultures are usually so numerous and often so marked as to complicate identification of the species. ${ }^{2}$ There seems to be no analogy between these changes and those undergone by animals in the course of immunization.

For instance, resistant bacteria may differ from the original cultures in the type of colony, ${ }^{3}$ in morphology, and in size of individual bacteria, ${ }^{4}$ and in motility. ${ }^{5}$ Many authors have observed that resistants may differ from original cultures in their cultural characteristics, involving, for example, marked deviation in the power to ferment carbohydrates. ${ }^{6}$ Although the changes in serological properties are not general ${ }^{7}$ and sometimes variable, ${ }^{8}$ in many instances the serologic identification of resistants is rendered difficult on account of the frequent loss of agglutinability. ${ }^{9}$ Very striking transformations in secondary cultures were observed also in connection with general viability, ${ }^{\mathrm{x} 0}$ thermoresistance, and particularly in connection with virulence which is often increased, ${ }^{\text {II }}$ though sometimes diminished or lost. ${ }^{12}$ In some instances the loss of virulence is only temporary and is regained, in the absence of phage, as the cultures recover susceptibility to lysis during prolonged cultivation. ${ }^{13}$

Although it is evident that the presence of bacteriophage may be responsible for these transformations of the cultures, it is difficult to perceive how such changes could

I Bruynoghe, R.: loc. cit.; Hoder, F.: loc. cit.; Gratia, A.: loc. cit.; Matsumoto, T.: Centralbl f. Bakteriol., Orig., 91, 413. I924; Wicn klin. Wchnschr., 36, 759. I923; Bail, O.: loc. cit. Although D'Herelle admits that this is true exceptionally, in general he claims that "a bacterium which once became refractory to the action of one race of bacteriophage is refractory to the action of all other races" (The Bacteriophage and Its Behavior, p. 239. 1926).

${ }^{2}$ Grumbach, A., and Dimtza, A.: loc. cit.; Seiffert, W.: Seuchenbekämpfung, 2, 203. 1925.

${ }^{3}$ Dutton, L. O.: loc. cit.; Manninger, R.: loc. cit.; Arkwright, J. A.: loc. cit.; Gjørup, E., loc. cit.; d'Herelle, F.; Compt. vend. Soc. de biol., 83, 97. 1920.

${ }_{4}^{4}$ Manninger, R.: loc. cit.; Burnet, F. M.: loc. cit.; Kauffmann, F.: loc. cit.; d'Herelle, F.: Compl. rcnd. Soc. de biol., 83, 97. 1920; ibid., 84, 384. 192 I; d'Herelle, F., and Hauduroy, P.: ibid., 93, I 288. 1925 .

${ }^{5}$ Bordet, J., and Ciuca, M.: loc. cil.; Fejgin, B.: ibid., 89, I38г. 1923.

${ }^{6}$ Fejgin, B.: loc. cit.; Hadley, P.: op. cit., 34, 260. 1924; Gratia, A.: J. Exper. Med. 35, 287. 1922; Ogata, N.: Ztschr. f. Immunitätsforsch. u. expcr. Therap., 45, 405. I925.

7 Bronfenbrenner, J., Muckenfuss, R., and Korb, C.: loc. cit.; Fejgin, B.: loc. cit.; Bruynoghe, R., and I)ubois, A.: ibid., 96, 209. I927.

${ }^{8}$ Gratia, A.: J. Exper. Med., 35, 287. 1922; Brutsaert, P.: Compt. rend. Soc. de biol., 90, 645. 1924 .

9 Marcuse, K.: loc. cit.; Kauffmann, F.: loc. cit.; d'Herelle, F.: Compt. rend. Soc. de biol., 83 , 97. I920; Hoder, F.: op. cit., 44, 423. 1925; Hoder, F., and Suzuki, K.: ibid., 49, 361. I926.

${ }^{10}$ d'Herelle, F.: Le Bacteriophage, "Monographies de l'Institut Pasteur," p. 95. I92 I; d'Herelle. F., and Ilauduroy, P.: Compt. rend. Soc. de biol., 93, I288. 1925; Fejgin, B.: loc. cit.

Ir Bordet, J., and Ciuca, M.: loc. cit.; Dutton, L. O.: loc. cit.; Kauffmann, F.: loc. cit.

${ }^{12}$ Fejgin, B.: loc. cit.; Blair, J. E.: loc. cit.; Bronfenbrenner, J. E., Muckenfuss, R., and Korb, C.: loc. cit.

${ }^{23}$ Bronfenbrenner, J., Muckenfuss, R., and Korb, C.: loc. cit. 
occur as a direct result of the development of active immunity by bacteria. Moreover, similar transformations of normal cultures, including the development of resistance to phage, have been observed in instances where bacteria were not exposed to phage at all. Thus, for example, Hoder ${ }^{1}$ has been able to produce such a transformation by the use of various chemicals, and D'Herelle's own experiments show that the appearance of resistants is greatly influenced by the hydrogen-ion concentration of the medium or by temperature, even in the presence of phage. ${ }^{2}$ Furthermore, similar aberrant types of bacteria have been isolated directly from normal cultures without the intervention of any agent, thus indicating that bacteria potentially resistant to phage exist normally in bacterial cultures. ${ }^{3}$

All these findings may be explained by considering a bacterial culture (as indeed any other "population") as composed of individuals approaching a certain type, but occasionally lacking or possessing exaggerated characteristics. Depending upon the conditions, some of the variants may find themselves favored by the environment and may become quantitatively dominant. On the contrary, if the environment is changed so that it becomes incompatible with normal development of certain types of variants, the latter are eliminated more or less completely, and the predominant character of the bacterial population in the culture deviates accordingly, with corresponding changes in the biological activity of the culture as a whole. That the variants resistant to phage may be obtained from normal cultures by causing them to dissociate under the influence of heat ${ }^{4}$ or of chemicals ${ }^{5}$ seems to suggest that these variants represent the individuals in the population of the culture which survive on account of their relative tolerance to the deleterious influences of the environment in general, and are not the product of specific adaptation to phage.

This simple explanation of the phenomenon of resistance does not, however, explain the variations in susceptibility of different strains of resistants to different closely related phages. This phenomenon cannot be explained until it is known what forces or properties are directly responsible for the higher degree of resistance of certain individuals in a bacterial population of a culture to the injurious agents in general and to phage in particular, and until the specific differences in the phages themselves can be detected by methods other than the lysis of bacteria. The explanations offered thus far are either highly hypothetical ${ }^{6}$ and not susceptible to experimental inquiry, or opposed by the existing experimental evidence. For instance, although it is true that old bacteria are generally more resistant to phage than younger bacteria of the same species, Marshall's ${ }^{7}$ explanation of specific resistance of second-

${ }^{r}$ Hoder, F.: loc cit.; Hoder, F.; and Suzuki, K.: loc cit.

${ }^{2} \mathrm{~d}$ 'Herelle, F.: The Bacteriophage and Its Behavior, pp. 182-83, 1926. These findings are of particular interest in connection with the conclusion of Gratia concerning the effects of hydrogen-ion concentration on the rate of bacterial dissociation (Gratia, A.: op. cit., 84, 275. I921).

3 Gratia, A.: ibid., 89, 821. 1923; Arkwright, J. A.: J. Path. \& Bact., 24, 36. 1921; Brit. J. Exper. Path., 5, 23. 1924; Gratia, A.: op. cit., 84, 750. I921.

${ }^{4}$ Hoder, F.: op. cit., Orig., 92, 197. 1925.

${ }^{5}$ Hoder, F.: ibid., 42, 197. I925; Hoder, F., and Suzuki, K.: loc. cit.; Gratia, A.: op. cit., 84, 275. I92I.

${ }^{6}$ Bail, O.: loc. cit.

7 Marshall, M. S.: loc. cit. 
ary cultures, that they represent the individuals which aged before they underwent complete lysis, seems inadequate. Even very young subcultures from the secondary growths are resistant to lysis. It is also difficult to explain, on this basis, why a given secondary growth will resist the action of the bacteriophage which caused its selection, but will undergo lysis by another closely related phage.

Equally inadequate is the attempt to ascribe the resistance in general to slime or mucus production. Although some cultures under the influence of phage give rise to slime-producing variants which resist the phage action, and although this phenomenon has been considered as a specific protective action on the part of bacteria, ${ }^{\mathrm{I}}$ slime production seems apart from the usual phenomena of resistance to phage, particularly because such cultures are resistant to all lytic agents without discrimination. ${ }^{2}$ This fact, together with the evidence that removal of slime, by bile for instance, renders the bacteria both agglutinable and susceptible to lysis, ${ }^{3}$ seems to indicate that this type of variants differs from usual resistants and owes its properties entirely to the mechanical protection of the slime. Furthermore, since only a few species of bacteria give rise to slime-producing variants, this phenomenon cannot explain the mechanism of resistance to phage in general.

Perhaps a systematic study of the resistance of various bacteria, simultaneously to various chemicals and phages, will open the way to a satisfactory explanation of this question. ${ }^{4}$

\section{SIGNIFICANCE OF BACTERIOPHAGE IN INFECTIOUS DISEASES}

Numerous investigators have shown that the distribution of bacteriophage in nature is as wide as that of bacteria. It is universally agreed that when bacteriophage is removed from its environment and placed under suitable conditions in contact with susceptible bacteria in vitro, it causes their rapid disappearance. The question naturally arises as to its effect on bacteria in the very environment where both exist simultaneously, as in the intestinal canal of animals, or in polluted water or soil. It is evident that if bacteriophage behaves in the human body, for instance, as it does in a test tube, it must greatly influence the course of infection. This is actually claimed by a number of investigators. ${ }^{5}$

The intestinal canal of a fetus does not contain any bacteriophage. ${ }^{6}$ In the first few days of extra-uterine life, however, the intestinal canal becomes infected with bacteria, and some time about the fourth to seventh day after birth phage can be demonstrated in the feces. Usually the phage which shows its appearance at this time is active against $B$. coli only. ${ }^{7}$ It has been claimed that, as result of infection

'Grumbach, A., and Dimtza, A.: loc. cit.

${ }^{2}$ Kimura, S.: Zischr. f. Immunitütsforsch. u. exper. Therap., 42, 507. 1925.

${ }^{3}$ Sonnenschein, C.: Centralbl.f. Bakteriol., Orig., roo, I I. 1926.

${ }_{4}^{4}$ Hoder, F., and Suzuki, K.: loc. cit.

5 d'Herelle calls attention to the fact that phage therapy in its crudest form was practiced in connection with dysentery in the Middle Ages (d'Herelle F.: Proc. Amsterdam Acad. Sc., p. $8_{3} 6$. 1925).

${ }^{6}$ d'Ilerelle, F.: The Bactcriophage and Its Bchavior, p. 423. 1926; Picret, R., and Bilouet, V.: Compt.rend. Soc. de biol., 93, 635. 1925; Sanderson, E. S.: J. Exper. MIcd., 42, 377. 1925.

${ }^{7}$ Surányi, L., and Kramár, E.: Monatschr.f. Kinderh., 28, 330. 1924. 
during life, this phage may adapt itself to a variety of bacteria. If, following an infection, the adaptation of Bacteriophagum intestinale to an infecting bacterium takes place quickly, the latter is destroyed before any symptoms of disease have appeared. ${ }^{\mathrm{r}}$ If it responds to infection more slowly, the adaptation may not be completed early enough to prevent the appearance of first symptoms of disease, but with the development of "virulence," the Bacteriophagum intestinale may still check further progress of infection. The disease can thus develop fully only if the invading organism remains unopposed by the phage. This occurs, for instance, when the invading bacterium happens to be resistant to phage or when it develops resistance in vivo during the process of slow adaptation of phage. In such cases the "refractory" bacteria may give rise to "formation of 'ultrabacteria' with an invasion of all the organs by these filterable forms." In isolated instances of infection, the phage carried by the individual may totally fail to become adapted ("inertia of phage") to the invading bacterium and thus permits unopposed development of infection. According to D'Herelle, during epidemics this does not happen because "Protobios bacteriophagus is transmitted from one individual to another just as is the bacterium itself" and thus "either the bacteriophage has acquired virulence (for the invading bacterium) in the body of the individual who harbors it, or this individual has been 'contaminated' by a bacteriophage which has acquired a virulence in another individual," 3 In short, according to D'Herelle, ${ }^{4}$ Hauduroy, Philibert, and others, the course of infection is determined by the presence and the ready adaptability of phage.

If the invading organism is susceptible to lysis and the phage acting upon it is present, the prognosis of recovery is warranted. In cases ending with death, either bacteria are found to be resistant or the phage fails to become adapted ("inertia").5 On the basis of these findings, clinical use of bacteriophage for therapeutic purposes has been attempted in a great variety of diseases. The results of these attempts are evaluated by D'Herelle as follows:

Phagotherapy represents a specific therapy for the bacillary dysentery and for staphylococcus infections. The results obtained in a few cases of bubonic plague allow us to hope that it is the same for this disease. With respect to the other infectious diseases, the results obtained are encouraging, but as yet a perfect method of treatment has not been attained. ${ }^{6}$

These conclusions of D'Herelle and his collaborators have not been confirmed in many instances. In the first place, several investigators were not able to establish the relation between the presence of active bacteriophage and the progress of infec-

I d'Herelle, F.: Compt.rend. Acad. de sc., I70, 72. I920.

${ }^{2}$ d'Herelle, F.: The Bacteriophage and Its Bchavior, p. 478. 1926.

3 d'Herelle, F.: ibid., p. 508. 1926.

4 "Every time one of the rats survived the infection consequent to the ingestion-and survival is relatively frequent-I could demonstrate in the blood removed by heart puncture on the fourth to sixth day after the infecting meal a bacteriophage virulent for the infecting bacterium" (d'Herelle, F.: ibid., pp. $47 x-72.1926)$.

5 Dutton, L. O.: loc. cit.; Stolz, J.: quoted from J.A.M.A., 85, 2002. I925; de Jong, S., and Hauduroy, P.: Bull. Soc. méd. des hôp. de Paris, 49, I56r. I925; I Hauduroy, P.: Presse méd., 33, 525. 1925 .

${ }^{6}$ d'Herelle, F.: Bacteriophage and Its Behavior, p. 577. I296. 
tion. They have been able to find the phage only in a small percentage of cases, ${ }^{\mathrm{I}}$ and in the cases where phage was present it was often transitory and had no effect on the severity or the outcome of the disease. ${ }^{2}$ In explanation of this discrepancy, Ciuca and Manoliu ${ }^{3}$ call attention to the possibility that consistent findings of phage in convalescents claimed by D'Herelle, Hauduroy, and others may have been due to the fact that fecal filtrates generally display inhibiting effect on the growth of bacteria. This inhibition is not transmissible in series, however, and should not be confused with the effect of phage.

As regards the effect of therapeutic administration of bacteriophage, the review of clinical reports undoubtedly supports D'Herelle's conclusion that in dysentery and in staphylococcus infections the results seem very encouraging. However, it is impossible to say on the basis of this clinical material alone whether the improvement observed by numerous observers was due directly to the effect of bacteriophage or to other causes. ${ }^{4}$ Only a few workers attempted to control the effect of administration of bacteriophages by using bacterial autolysates in their place. In the case of staphylococcus infections, for instance, equally good results are obtained by the use of Besredka's "antivirus." In instances where these control experiments have been instituted, ${ }^{6}$ it was found that administration of filtrates of cultures of bacteria (free from bacteriophage) has caused improvement in cases of dysentery and staphylococcus infections comparable to those obtained through administration of bacteriophage. ${ }^{7}$ Similar conclusions can be drawn also from the observation of Brouardel who reported that the administration of typhoid phage caused improvement in cases of septicemia due to the Friedländer bacillus. ${ }^{8}$

That the improvement of patients under phage therapy is not due to the specific action of bacteriophage is further indicated by almost universal failure to influence the course of experimental infection. ${ }^{9}$ This is particularly significant be-

${ }^{r}$ Larkum, N. W.: J. Bact., I2, 203 and 225. 1926; Ciuca, M., and Manoliu, E.: Compt. rend. Soc. de biol., 91, I225. I924; Schumm, E., and Cooke, R. A.: J. Infect. Dis., 39, 424. 1926.

${ }^{2}$ Gjorup, E.: Acta med. Scandinav., Suppl. 7, p. 365. 1924; Manoliu, E.: Compt. rend. Soc. de biol., 92, 724. 1925; Wagemans, J.: op. cit., 28, 159. 1923; Sickenga, F. N.: Nederl. Maandschr. v. Geneesk., 13, I41. 1925; Cowie, D. M.: Ann. Clin. MIed., 5, 57. 1926. In collaboration with Drs. Korb and Muckenfuss the writer had the opportunity of examining for presence of phage over two hundred mice infected by ingestion of $B$. pestis caviae. The frequency as well as type of phages recovered during these examinations was approximately the same in the animals surviving the infection and in those succumbing to it. Most frequently the phages recovered were active only against $B$. dysenteriae; next in frequency $B$. enteritidis Gaertner; and the least frequently those active against the infecting organism.

${ }^{3}$ Ciuca, M., and Manoliu, E.: loc. cil. $\quad 4$ Zdansky, E.: loc. cit.; Cowie, D. M.: loc. cit.

${ }_{5}$ Besredka, A.: Seuchenbekämpfung, 4, 21. 1927.

${ }^{6}$ Gratia, A., and Doyle, D.: Compt. rend. Soc. de biol., 93, 452. I925.

7 Munter, H., and Boenheim, C.: Ztschr. f. Kinderh., 39, 3SS. I925; Bazy, L.: Compt.rend. Soc. de biol., 92, 4S5. 1925 .

${ }^{8}$ Brouardel, Mr.: Bull. Acad. de méd. de Paris, 96, 240. 1926.

9 Bronfenbrenner, J., and Korb, C.: loc. cit.; Gratia, A., and Doyle, D.: loc. cil.; Seiffert, W.: Zischr. f. Immunitätsforsch. u. exper. Therap., Orig., 38, 350. 1923; Nobechi, K.: Compl. rend. Soc. de biol., 95, 1252. 1926; Levy, M. M.: ibid., 93, 395. 1925; ibid., p. 82. 1925; Clark, P. F , and A. S.: Proc. Soc. Exper. Biol. Eo Med., 24, 635. 1927; Topley, W W. C., Wilson, J., and Lewis, E. R.: J. IIyg., 24, 17. 1925; Topley, W. W. C., and Wilson, J.: ibid., p. 295. 1925. 
cause in these experiments phages which were used were known to be highly potent, having been previously tested against the infecting organisms in vilro. Moreover, the administration of phage in these experiments was not limited to the digestive tract, but in many experiments was supplemented by subcutaneous, intraperitoneal, or intravenous administration. The inquiry into the causes of these failures has shown that they could not be ascribed to the survival of resistants ${ }^{1}$ alone, as postulated by D'Herelle, but undoubtedly are due also to the fact that under the conditions existing in vivo the susceptible bacteria are not subject to lysis as freely as they are in vitro. It was found that, in spite of the presence of numerous susceptible bacteria, bacteriophage does not accumulate in vivo at a rate comparable to that observed in vitro. ${ }^{2}$ This is undoubtedly due in part to the fact that in vivo bacteria multiply more slowly (and thus less phage is produced) and partly to the fact that a considerable portion of the phage which is produced (or introduced for therapeutic purposes) is quickly immobilized by adsorption on colloids of tissues and of body fluids. ${ }^{3}$ Moreover, bacteriophage is continuously and rapidly eliminated from the circulation through the bile and urine, ${ }^{4}$ so that when it is introduced experimentally into animals it may be completely eliminated, according to D'Herelle, in from twenty-four to forty-eight hours, unless susceptible bacteria are present and thus some regeneration of phage can take place. ${ }^{5}$

In view of these findings, limited activity of phage in vivo should not be surprising. The experimental evidence presented in the early part of this paper suggested that in vitro as well the rate of regeneration of phage depends on the rate of multiplication of susceptible bacteria. It was shown also that the presence of some colloids may interfere with the progress of diffusion of phage through adsorption (as in the case of excess of agar) and thus cause lysis in spots only (plaques) instead of diffuse, complete lysis as it occurs in broth where the action of phage on bacteria is unopposed. Furthermore, it was shown that in the presence of colloids (as gelatin, for instance) the phage and the susceptible bacteria may be present, and the phage may even show increase in concentration without causing the complete dissolution of bacteria.

Similar difficulties present themselves in the attempt to utilize bacteriophage for

${ }^{x}$ At least in some instances, as in the case of experimental mouse typhoid caused by B. pcstis caviae, the resistants have been found to be devoid of virulence (Bronfenbrenner, J., Muckenfuss, R., and Korb, C.: loc. cit.).

${ }^{2}$ Doerr, R., and Gruninger, W.: loc. cit.; Meissner, H., and Baars, G.: Centralbl.f. Bakteriol., Beiheft. 93, I3I. I924; Wertemann, A.: Das Verhalten der iiberlragbaren Lysine in der Zirculation von Kalt und Warmblutern. (Dissertation.) r922.

${ }_{3}$ Zdansky, E.: loc. cit.; Seiffert, W.: Seuchenbekämpfung, 2, 234. 1925; Clark, P. F. and A.S., loc. cit.; Meissner, H., and Baars, G.: loc. cit.; Zdansky, E.: Wien. Arch.f. inn. Med., II, 533. 1925.

${ }_{4}^{4}$ Doerr, R., and Gruninger, W.: loc. cit.; Wertemann, A.: loc. cit.; Appelmans, R.: Compt. rend. Soc. de biol., 85, 722. I921; Suzuki, T.: J. Orient. Med., 2, I 25. I 924.

5 d'Herelle, F.: The Bactcriophage and Its Bchavior, p. 538. 1926. The elimination of bacteriophage cannot be counteracted by increasing the therapeutic dose because of the toxicity of many phages (Seiffert, W.: Ztschr.f. Immunnitätsforsch. u. expcr. Therap., Orig., 38, 350. 1923; Meissner, H., and Baars, G.: loc. cit.; Hauduroy, P.: Ann. de méd., I6, 340. 1924). Repeated administration of moderate amounts of phage over a long period is inadvisable because of the development of antiphage which abolishes all phage action (Hauduroy, P.: loc. cit.; Compt. rend. Soc. de biol., 90. 290. 1924; Fabry, P.: ibid., 94, 774. 1926). 
disinfection of polluted surface water. ${ }^{\mathrm{I}}$ While it may be true that in highly polluted water the phage shows limited degree of regeneration and for a time causes dissolution of some bacteria present, ${ }^{2}$ this process is very limited in its significance. ${ }^{3}$ The growth of bacteria is usually slow at best, ${ }^{4}$ and the phage which is present is quickly diluted and dispersed. Often the industrial outflow causes its direct destruction or adsorption in inert organic matter. ${ }^{5}$ Moreover, if any lysis of susceptible bacteria does take place, the resistant bacteria remain unaffected. ${ }^{6}$

The hope of utilizing bacteriophage as an agent for prevention and therapy of infection lies in finding the means of removing the obstacles to its activity in its natural environment and allowing it to act as freely as it does in a test tube.

I Arnold, L.: Am. J. Pub. Ilealth, I5, 950. 1925; Jeney, A.: Centralbl. f. Baktcriol., Orig., 102, 263. г927; Flu, P. C.: ibid., 59, 317 . 1923.

${ }^{2}$ Arloing, I., and Sempé, N.: Compl. rend. Soc. de biol., 94, I9 1. 1926.

3 Zdansky, E.: loc. cil.

${ }_{4}^{4}$ Arloing, F., Sempé, N., and Chavanne, M.: Bull. Acad. de méd., 93, r84. 1925.

5 Arnold, I.: loc. cit.; Nakashima, T.: Centralbl. f. Bukteriol., Orig., 94, 303. 1925; Bilouet, V.: Compl. rend. Soc. de biol., 94, 7 10. 1926.

${ }^{6}$ Zdansky, E.: loc. cii. 


\section{CHAPTER XLI}

\section{FILTERABILITY OF MICRO-ORGANISMS}

\section{S. P. KRAMER}

Cincinnati, Ohio

We have come to divide bacteria and viruses into "filterable" and "non-filterable," and we have come to think that those organisms, visible or invisible, which are smaller than the pores of our filter are filterable. That size, however, cannot be the sole criterion of filterability we have known from the behavior of certain aniline dyes. Thus Victoria blue B, a basic dye, will not pass a Berkefeld filter, while Congo red, an acid dye, will readily pass through the same filter. It happens that the filters which we use in bacteriological practice - namely, sand, porcelain, diatomaceous earth, and asbestos - are all of some form or compound of silicic acid, so that really when we speak of a filterable organism, dye, or other colloid, we should say "filterable through siliceous filters."

The selection of siliceous material for filters seems to have been purely empirical. There had been no realization that these materials were other than porous materials which were chemically neutral. Their use goes back into antiquity. Aristotle in his essay De generatione animalia refers to filtration in the following passage: "Flesh is produced therefore through the veins and pores, the nutriment being deduced in the same manner as water through earthen vessels not sufficiently baked," showing that filters of unglazed porcelain (what we now know as the Chamberland type) were used in the days of Aristotle.

In I9I6 I showed before the Research Society of Cincinnati that any filter made of siliceous material, sand, porcelain, powdered glass, colloidal silica, or diatomaceous earth would absorb basic dyes, and that acidic dyes would pass through. ${ }^{2}$

Dr. Stuart Mudd ${ }^{3}$ described the surface of the pores of a Berkefeld filter as the site of an electrical potential difference, a Helmholtz double layer, in which the wall of the filter carried a negative charge and the liquid a positive charge; and he predicted and found that when suspensions were filtered through such a filter, positively charged particles were absorbed and retained by the filter.

In Figure I the lines $A A^{\prime}$ and $B B^{\prime}$ represent the walls of a capillary or pore of a siliceous filter carrying a negative charge. Minus and plus signs represent particles of matter smaller than the pore of the filter, with their respective charges indicated. The minus particles carrying the same charge as that of the filter pass through the pores. The plus particles carrying a charge of sign opposite to that of the filter are retained by the filter.

${ }^{1}$ Quoted by Bolton: Chem. News, 4I, 55. I 880.

${ }^{2}$ Kramer, S. P.: J. General Physiol., 9, No. 6, 8I I-I2. July, I926; J. Infect. Dis., 40, No. 2, 343-47. Feb., I927.

3 Mudd, S.: Am. J. Physiol., 63, 429. I922. 
Figure 2 visualizes the same thing with the gypsum filter carrying a positive charge. The arrows indicate the direction of the flow of the liquid being filtered.

A filter of any material will of course hold back any rigid particle that is larger than the pores of the filter; but for those particles that are smaller than the pores of the filter, chemical or electrical forces come into play. One may speak of a filter when it is in use as a suspension of the material of which the filter is composed, in the fluid which is being filtered. Silica has a definite negative charge, and it might be that if one

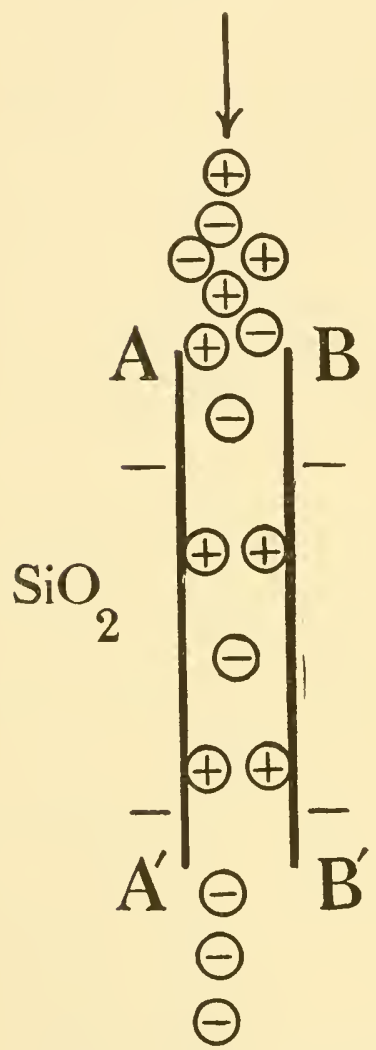

FIG. I.-Siliceous filter with pore of negative charge.

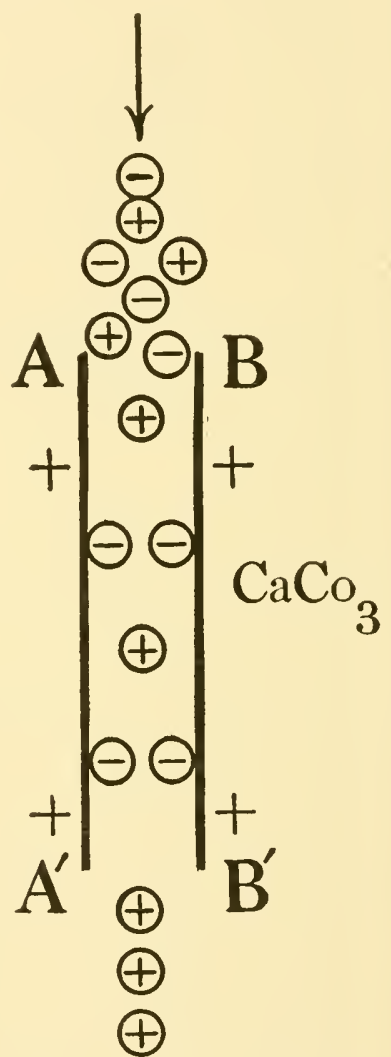

FIg. 2.-Plaster of Paris filter with pore of positive charge.

constructs a filter of a material of charge opposite to that of silica, one might find that bacteria or colloids which are filterable through silica filters are non-filterable through such other filters, and vice versa.

Accordingly, filters were made of plaster of Paris, and experiments with various dyes and viruses were made. Thus Victoria blue B or methyl green, which do not pass a Berkefeld or siliceous filter, readily pass through a filter made of plaster of Paris; Congo red, which readily passes through the Berkefeld filter, does not pass through a plaster of Paris filter. Acidic and basic dyes should be used to test filters. In this work any Berkefeld filter which does not completely remove Victoria blue B from a $\mathrm{I}-20,000$ solution is rejected. The gypsum filter is tested with a similar solution of Congo red. 
When we consider the nature of plaster of Paris a very interesting phenomenon is found. Plaster of Paris is supposed to be calcium sulphate, but when filters were made of calcined chemically pure calcium sulphate, it was found that such filters had no action on either class of colloid dyes. Both the basic Victoria blue B (or methyl green) and the acidic Congo red readily passed through such filters. Calcium sulphate is neutral and without charge. It was found that the plaster of Paris of commerce contained up

FIG. 3.- This is arranged to show the method of making the gypsum filter. $A$ is a heavy glass tube with a $\mathrm{r}$-inch lumen. $B$ is a rubber stopper carrying a glass tube $D$. $C$ is a vaselined glass rod acting as a core.

The plaster of Paris, containing, in some instances, 25 per cent of $\mathrm{MgO}$, is mixed with an equal weight of water, rapidly stirred to get rid of large air bubbles and then poured into $A$ by means of a pipette to avoid air bubbles. As soon as the plaster sets, the core $C$ is withdrawn and the "filter" is allowed to dry out. After testing with a solution of Congo red it is ready for use.

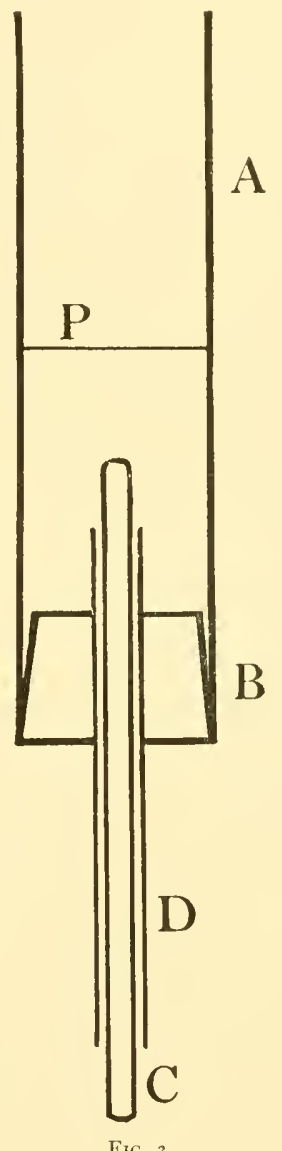

to 5 per cent of calcium carbonate, and when calcium carbonate was added to chemically pure calcium sulphate and filters made from this mixture, such filters acted as did the filters made of commercial plaster of Paris. The calcium carbonate is alkaline and has a positive electrical charge. It is probable that the calcium sulphate in the filters acts as a binder for the calcium carbonate and that it is the calcium carbonate which is the active component.

It was thought that if a filter were made of plaster of Paris and silica or diatomaceous earth intimately mixed before setting, we might produce a filter which would remove both positively and negatively charged dyes and colloids. When this was done, however, it was found that such filters were neutral and that neither suspensoid was 
filtered out. Evidently the calcium carbonate and silica neutralized each other. If, however, the filter was made so that the core was a Berkefeld filter with a cortex of plaster of Paris poured over this, so that the contact of materials was only at the joint surface, it was found that dyes and colloids of both positive and negative charge could be removed by filtration through this apparatus. The outer layer of plaster of Paris removed the acidic or negative particles, the Berkefeld siliceous core removed the basic or positive ones. It was also found that one could increase the efficiency of the basic or gypsum filter by incorporating in the plaster of Paris up to 25 per cent of magnesium oxide, calcined at $1300^{\circ} \mathrm{C}$.

Experiments have been made with so-called "filterable" micro-organisms and viruses that were available, and a concise report of these experiments is appended. The bacteriophage of Staphylococcus aureus which passes through the Berkefeld filter does not pass the filter of plaster of Paris. The Vibrio percolans of Mudd which passes through the Berkefeld filter does not pass the gypsum filter. Vaccine virus and the virus of rabies do not pass through this filter.

The following tests were made by Mr. H. H. Allard, of the United States Department of Agriculture, with the sap of mosaic-diseased tobacco filtered through several types of gypsum filters:

Test I. - Ten plants inoculated with the unfiltered mosaic sap; 9 plants became diseased.

Test 2.- Ten plants inoculated with material passed through a Berkefeld plus plaster of Paris filter; none became mosaic diseased.

Test 3.-Ten plants inoculated with virus passed through plaster of Paris filter alone; none became diseased.

Test 4.-Ten plants inoculated with mosaic sap filtered through a filter made of plaster of Paris plus 2 per cent calcium carbonate $\left(\mathrm{CaCO}_{3}\right)$; none became diseased.

On May 29 these same filtrates were again used with the following results:

Ten plants inoculated with the unfiltered mosaic sap gave 7 plants with mosaic disease.

Ten plants inoculated with the sap filtered through a Berkefeld plus plaster of Paris filter; none became diseased.

Ten plants inoculated with plaster of Paris filtrate; none became diseased.

Ten plants inoculated with sap filtered through a filter made of plaster of Paris plus 2 per cent calcium carbonate $\left(\mathrm{CaCO}_{3}\right)$; none became diseased.

It was also found that we could remove various bacterial toxins by filtration. Diphtheria toxin - and by this I mean standard toxin of the United States Hygienic Laboratory-when filtered through a filter made of a mixture of plaster of Paris with 25 per cent magnesium oxide calcined at $\mathrm{I} 300^{\circ} \mathrm{C}$. had its toxic principle removed. The following series of experiments illustrates this:

390-A (Control).-November 30, 1926, 2:00 P.M., Guinea pig, weight 400 gm., received diphtheria toxin Io M.L.D. subcutaneously. Dead, December I, I926, 6:00 P.M. Autopsy showed diphtheria.

390-B.-November 30, 1926, 2:00 P.M., Guinea pig, weight 460 gm., received subcutaneously I-cc. diphtheria toxin containing two L+ doses or roo+ M.L.D. which had been filtered through a filter made of plaster of Paris +25 per cent MgO.

391.-Guinea pig, weight 420 gm., received 2 cc. $=200+$ M.L.D. or four L+ doses of diphtheria toxin which had been filtered through a filter made of plaster of Paris +25 per cent $\mathrm{MgO}$. 
392.-Guinea pig, weight 550 gm., received 4 cc. $=400+$ M.L.D. or eight L + doses of filtered toxin.

393. - Guinea pig, weight 540 gm., received 5 cc. $=500+$ M.L.D. or ten L + doses of filtered toxin.

All of the guinea pigs receiving toxin filtered through the plaster of Paris-magnesia filters were alive and well December I6, I926, except that each pig had developed an ulcer with denudation of hair at the site of injection. The size of the necrotic area varied directly with the amount of filtered toxin injected.

It was evident that the filter had removed that fraction of the toxin which caused the acute death and neurotoxic symptoms, leaving the fraction which caused the local tissue changes. This tissue toxin was removed by filtration through the Berkefeld filter charged with gelled silicic acid. Animals that received toxin which had been filtered through both filters in amounts up to ten $\mathrm{L}+$ doses developed no symptom whatever-either local or general.

When diphtheria toxin is filtered through siliceous filters alone the animal dies promptly of general intoxication with doses approximating the lethal dose of unfiltered toxin. Botulinum toxin is also rendered innocuous by filtration through filters made of plaster of Paris and magnesia. Abrin, the toxin from jequirity seed, is removed by filtration through siliceous filters. Tuberculin, when passed through a Berkefeld filter or a Berkefeld filter charged with colloidal silica, is still lethal to tuberculous guinea pigs. Tuberculin passed through a filter made of plaster of Paris with 25 per cent magnesium oxide is no longer lethal to tuberculous pigs. This is illustrated by the following experiments:

October I 2, I926.-Guinea pig A, weight 450 gm., received 2-mg. tubercle bacilli culture H. 37 in the axilla.

November 4, r926.-This pig received $5 \mathrm{cc}$. of a solution of tuberculin, ro:30 A.M., equivalent to $0.625 \mathrm{cc}$. of old Koch tuberculin. This pig died during the night and autopsy showed intense tuberculin reaction.

October 12, I926.-Guinea pig B, weight 430 gm., received a like amount of tubercle bacilli.

November 30, I926.-At 10:00 A.M. guinea pig received $5 \mathrm{cc}$. of the above solution of tuberculin which had passed through a Berkefeld filter charged with colloidal $\mathrm{SiO}_{2}$. This pig died at 2:30 P.M. of the same day with a marked tuberculin reaction. bacilli.

October I2, I926.-Guinea pig C, weight 420 gm., received a like amount of tubercle

November 30, 1926.-At 10:00 A.M. guinea pig received $5 \mathrm{cc}$. of the same tuberculin solution which had passed through a filter of plaster of Paris with 25 per cent MgO. The pig was unaffected and remained as before injection.

December 2, I926.-At 9:10 A.M. guinea pig C received $5 \mathrm{cc}$. of the same tuberculin filtered through the Berkefeld filter charged with colloidal silica and died nine hours later with a marked tuberculin reaction.

The gypsum-magnesia filtrate of tuberculin will not produce a skin reaction, nor will it produce a tuberculin reaction in tuberculous guinea pigs in any amount. The $\mathrm{pH}$ before filtration was 6.0 and that of the filtrate was 8.o. Guinea pigs that had received twice weekly for three months I cc. of the gypsum filtrate of a solution of O.T. I- 8 were found to be immune to a fatal dose $-0.5 \mathrm{cc}$. - of unfiltered old tuberculin undiluted. 
Tetanus toxin is unaffected by filtration through any gypsum filter, but, as has long been known, is removed in limited quantities by siliceous filters. If freshly gelled, pure colloidal silica is drawn into a Berkefeld filter, one obtains a siliceous filter through which tetanus toxin may be passed to give a filtrate of which one may inject up to an equivalent of fifty minimal lethal doses for the guinea pig without killing the animal. With the ordinary Berkefeld filter $\mathbf{I}$ have never succeeded in producing a filtrate of which one might inject more than the equivalent of ten minimal lethal doses without killing the pig.

Watery infusions of the various neurotoxic drugs and toxins not of bacterial origin were drawn through both classes of filters with the intention of removing, if possible, the neurotoxic components. A solution of cobra venom containing $2 \mathrm{mg}$. per cubic centimeter was found to be fatal to mice in doses of $0.5 \mathrm{cc}$. by paralysis of respiration within one hour. The filtrate obtained by passage of the solution through the gypsummagnesia filter was equally toxic. The filtrate from the Berkefeld colloidal silica filter was non-toxic.

Solutions of the venom of Crotalus atrox, the western diamond rattlesnake, in normal saline solution $2 \mathrm{mg}$. to the cubic centimeter in doses of $\mathrm{I} \mathrm{cc}$., were found to be fatal to mice within ninety minutes, with paralysis of respiration and a marked hemorrhagic reaction at the site of infection. The gypsum-magnesia filtrate was equally toxic; the filtrate from the Berkefeld colloidal silica filter was not toxic. Solutions of the venom of Crotalus terrificus, the Brazilian rattlesnake, were found to pass through both filters and retained their toxicities in both instances.

Diphtheria antitoxin was found to be removed by filtration through the gypsum magnesia filter as well as through the filter made of a Berkefeld reinforced by colloidal silica. Tetanus antitoxin was not removed by either filter. A 2 per cent solution of standardized adrenal powder was rendered inactive by filtration through the gypsummagnesia filter and passed through the silica filter unchanged. Curare in solution containing I $\mathrm{mg}$. to the cubic centimeter, which readily paralyzes a frog, is totally detoxified when passed through the gypsum filter. Test doses of digitalis, which in the frog arrest the heart in one hour, are rendered non-toxic when filtered through the gypsum filter.

If one extracts $2 \mathrm{gm}$. of powdered opium with $60 \mathrm{cc}$. of water for twenty-four hours and decants the fluid, I cc. of the infusion will paralyze and kill a mouse within fifteen minutes. When filtered through the gypsum-magnesia filter, the extract is no longer toxic. When filtered through the Berkefeld colloidal silica filter, the fluid is toxic. When morphia is added to the gypsum filtrate, I mg. to the cubic centimeter, and again passed through the gypsum filter, the filtrate is again toxic; and when this toxic filtrate is passed through the Berkefeld colloidal silica filter, the filtrate is nontoxic. If morphia be added to the original opium infusion in amounts equal to I mg. to the cubic centimeter, and the solution be passed through the gypsum filter, the filtrate is still toxic. If it be passed through the Berkefeld colloidal silica filter, it is also toxic. But if it be passed successively through both filters, the final filtrate is nontoxic.

Morphia is a base and should be and is removed by the silicic acid, but the narcotic in the powdered opium is not present as a base, but as an acid, and is removed by 
the basic filter. The base, morphia, does not exist in opium, but is an artefact-the product of chemical manipulation.

Thirty gm. of powdered cocoa leaves were extracted with $200 \mathrm{cc}$. of 50 per cent alcohol for twenty-four hours. Of the extract, Ioo cc. were evaporated in a water bath to $30 \mathrm{cc}$. When injected into a 25 -gm. mouse, I cc. of the extract paralyzes the respiration and kills the mouse within one hour. When filtered through the gypsum magnesia filter, the filtrate is non-toxic. The alkaloid cocaine is not removed by the same filter, but is taken out by filtration through the Berkefeld colloidal silica filter.

An infusion of powdered nux vomica, I part in 20 parts of water, was found to be fatal to mice in six minutes, producing typical strychnine convulsions. The filtrate from the gypsum-magnesia filter was equally toxic. The silica gel-Berkefeld filtrate was non-toxic and tasteless. Powdered calabar bean was extracted with Io volumes of

TABLE I

\begin{tabular}{|c|c|c|c|}
\hline Charge "Basophilic" & + Charge "Acidophilic" & Amphoteric \pm & Neutral \\
\hline $\begin{array}{l}\mathrm{SiO} \mathrm{O}_{2} \ldots \text { red }(\mathrm{Collo} \text { idal acid } \\
\text { dyes })\end{array}$ & $\begin{array}{l}\mathrm{CaCO}_{3} \\
\text { Victoria blue } \mathrm{B} \text { (col- } \\
\text { loidal basic dyes) }\end{array}$ & $\begin{array}{l}\text { Diphtheria antitoxin } \\
\ldots \ldots \ldots \ldots \ldots \ldots\end{array}$ & $\begin{array}{l}\mathrm{CaSO}_{4} \\
\text { Tetanus antitoxin }\end{array}$ \\
\hline Diphtheria toxin. ........... & Tetanus toxin & & $\begin{array}{l}\text { Crotalus terrificus ven- } \\
\text { om (Brazil rattle- } \\
\text { snake) }\end{array}$ \\
\hline Botulinum toxin.... & $\begin{array}{l}\text { Crolalus alrox venom } \\
\text { (North American } \\
\text { rattlesnake) }\end{array}$ & . & Inamirta cocculus \\
\hline Jequirity (Abrin)......... & Cobra veno & $\ldots \ldots \ldots \ldots \ldots$ & $\ldots \ldots \ldots \ldots \ldots \ldots$ \\
\hline $\begin{array}{l}\text { Tuberculin . . . . . . . . . } \\
\text { Opium . . . . . . . . . }\end{array}$ & $\begin{array}{l}\text { Cobra venom } \\
\text { Nux vomica }\end{array}$ & 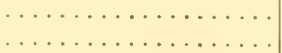 & $\ldots \ldots \ldots \ldots \ldots$ \\
\hline Cocoa leaves............ & Calabar bean & $\ldots \ldots \ldots \ldots \ldots \ldots$ & $\ldots \ldots \ldots \ldots$ \\
\hline 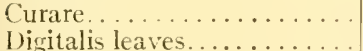 & Tobacco leaves & $\ldots \ldots \ldots \ldots \ldots \ldots$ & $\ldots \ldots \ldots \ldots \ldots \ldots$ \\
\hline 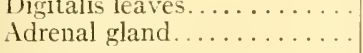 & ${ }^{\prime} \ldots \ldots \ldots \ldots \ldots \ldots$ & $\cdots \cdots \cdots \cdots \cdots \cdots \cdots$ & $\cdots \cdots \cdots \cdots \cdots \cdots$ \\
\hline
\end{tabular}

alcohol, the fluid placed in a water bath and the alcohol driven off. Water was then added up to the original volume. One cc. of this water extract was fatal to mice within five minutes, causing violent tremors and paralysis. The gypsum filtrate was equally toxic. The silica gel-Berkefeld filtrate was non-toxic. The same result was obtained with an infusion of tobacco, I part in ro parts of water, the gypsum filtrate being toxic, the silica gel-Berkefeld filtrate being non-toxic.

In Table I, I have arranged the various substances experimented with and their reactions as found by differential filtration.

It would appear that of those neurotoxic poisons which are removable by filtration those which are positively charged or "acidophilic" are predominately spinal in their action; while those which are negatively charged or "basophilic" are predominately extra-spinal in their action.

If a dry Berkefeld filter be thoroughly saturated with paraffin oil as suggested by Holman, ${ }^{\mathrm{I}}$ it will lose its power of separating out colloid dyes. Both the acidic and basic dyes will pass through. The same thing is true of the gypsum filter provided care is taken that the filter is perfectly dry before saturation with the paraffin oil. In

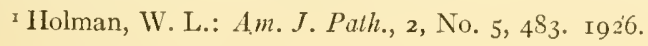


both cases the surfaces of the pores of the filter are "insulated" or their charge is neutralized and so cannot influence the charged particles passing through the filter. When the filters are "cleaned" by running through them alcohol followed by ether, they regain their power of removing colloid dyes of opposite charge. I have tried to picture the action of the two kinds of filters in Figures I and 2.

It may be asked, "What influence has the reaction of the medium on the filterability or non-filterability of suspended particles or colloids?" In general, it may be answered that, assuming that the charge of the filter remains unchanged, whenever a change in the reaction of the medium brings about a change in the charge of the particle or colloid then will the filterability or non-filterability be changed; otherwise not. 


\title{
CHAP'TER XLII
}

\section{A THEORY OF MICROBIC VIRULENCE}

\author{
I. S. FALK \\ University of Chicago
}

\section{INTRODUCTORY}

The term "virulence" is used sometimes loosely to denote the absolute capacity of a parasite to cause disease. On other occasions, when suitably qualified, it is used to describe the severity or lethality of the disease which is induced by a parasite. In almost every case, however, "virulence" refers primarily to a characteristic of the parasite, even as "resistance" or "immunity" denotes a state of the host. For reasons which will be discussed at length, such procedures must be considered unsound.

In any circumscribed series of comparable observations, virulence is never known and probably cannot be known except as a reciprocal measure of resistance. The host for which a particular microbe displays virulence is relatively non-resistant; and the microbe to which a particular host shows high susceptibility is virulent. Such statements as these may seem gratuitous; but they are necessitated by the common, perhaps inadvertent, reference to virulence and resistance as though they were absolute characteristics of parasite and host, respectively. The only precise definition is the following:

$$
\text { Virulence } \propto \frac{\mathrm{I}}{\text { Resistance (or Immunity) }},
$$

i.e., virulence varies reciprocally as resistance or immunity. According to the precision with which virulence is described in terms of the experimental conditions under which it is measured, it takes on proportionally absolute characteristics. A discussion of virulence is then resolved into an analysis of the variables which, by operating primarily upon the parasite, whether in vivo or in vitro, affect the course or the outcome of a particular infection. Correspondingly, immunology is concerned with the analysis, preferably the simultaneous analysis, of resistance.

It is apparent that every precise measurement of virulence, whether expressed in terms of the number of organisms in a minimal infecting or fatal dose, in terms of the size of a lesion produced, or of the elapsed time between infection and the development of a particular diseased state or of death, must always be qualified by the history of the host as well as of the parasite. For a particular host virulence is variable not only for different parasites, or different members of a single genus or a particular species, but even for different strains of the same variety and for the same strain studied at different times, in various subcultures or after purposive modification by experimental procedures.

When variations in virulence occur spontaneously, they must be taken as indicative either of genetic instability in the parasite or of failure to recognize and to hold 
constant and invariate such experimental conditions as affect virulence. The special interest which attaches to spontaneous or induced variations in virulence is the alleged cyclical character of the variation--as in the case of certain examples in epidemiological literature - or, more particularly, the permanence of the variation under laboratory conditions. Insufficient attention has been accorded the general, biological significance of this type of inheritance and persistence of acquired characteristics in such unisexual forms as the bacteria. For the purposes of this analysis 1 wish to direct attention not merely to the relative permanence of modifications in virulence, but to the experimental and theoretical opportunities associated with the fact that the modifications in virulence are generally, if not invariably, associated with concurrent variations in other characteristics of the parasite. In the study of the simultaneously varying characteristics lies the opportunity to integrate a large number of observations into a general theory of the mechanism of virulence.

In epidemiological studies attention has been frequently directed to the problem of variations in the virulence of communicable disease. The cyclical recurrences of epidemic influenza and of other diseases have been explained as easily on the assumption of cyclical changes in the virulence of the etiological agent as in the resistance of the host. Why frank cases of poliomyelitis or of plague show high, and of chicken pox or measles low, fatality rates find no ready answer. ${ }^{x}$ There is little doubt that in scarlet fever and in smallpox-to mention only two diseases of man-there have occurred fairly sharp and perhaps discontinuous variations in epidemicity and in fatality, so far as these characteristics of an infectious disease can be determined by statistical methods. It is only necessary to consult two recent, competent writers on this subject, however, to discover the absence of unanimity of opinion concerning the factors that have caused the changes. ${ }^{2}$ Contrariwise, there are indications in the vital statistics of typhoid fever ${ }^{3}$ and of other diseases, as well as in certain experimental epidemiological investigations, that there are a variety of conditions which tend to make for a constancy of virulence in disease of man and other animals. ${ }^{4}$ Discussions of the problem of the disease carrier which revolve primarily about considerations of microbic virulence or of host resistance are futile. There is not available here or elsewhere any device by which a separation can be made between the two groups of interdependent variables.

Typhoid and yellow fever are primarily diseases of man; mosaic disease is similarly a disease of the tobacco plant. In each case the specificity of microbic virulence is neither greater nor lesser than the specificity of host resistance or immunity. I stress these facts here, as I shall again later, for two reasons: First, the trend of research is to indicate that parasite virulence and host resistance are related and not independent variables. Second, the characteristic of a microbe (P.D. $)^{5}$ which is associated with or determines virulence appears to be identical with that property of antibodies (P.D.) by which the resistance of the host is determined.

\footnotetext{
' Falk, I. S.: Scienl. Monlh., 20, 383.1925.

${ }^{2}$ ('hapin, C. V.: J. Prev. Med., I, 1. 1920; Holst, I'. M.: ibid., J). 270.1927.

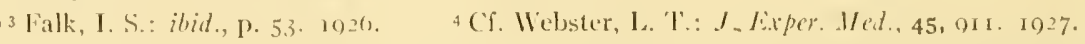

5"P.I)." is used to refer to the electrical potential difference between a particle and its mentrumm, at postulated in the Hembolt\% double-later theory. "Charge" may be used insteal, on the arr unls presented in chapters $x$ and lviii of this volume.
} 
TOXIGENICITY AND INVASIVENESS

Because the dynamics of bacterial virulence still remain essentially obscure, there is no general agreement among writers on this subject as to the scope, the nature, or even the precise implications of the term "virulence." It is often recognized that the capacity to produce specific toxins, to show invasiveness, avidity to fix and localize on specific tissues, and capacity to necrotize are or may be distinct phenomena, and that it is often necessary to distinguish among them in attempting to characterize the virulence of a specific parasite for a particular host.

Virulent diphtheria or tetanus bacilli are notably lacking in high invasive properties. The damage they do in a host is nearly completely due to the specific toxins they excrete. In such cases "virulence" is approximately coterminal with "toxigenicity." The diphtheria bacillus that lacks the capacity to produce toxin is commonly called "avirulent," and some recent experiments by Crowell ${ }^{2}$ indicate that toxigenic capacities are genetically determined. With such organisms as Clostridium welchii or the scarlet fever streptococci, not only toxigenicity, but invasiveness and necrotizing capacities, determine pathogenicity.

In recent years it has been possible to demonstrate toxin production by several groups of pathogenic bacteria which formerly had been supposed to lack toxigenicity. It may be that ultimately it will be established that all bacterial virulence is dependent upon toxigenicity and that the failure of earlier workers to demonstrate toxin production by this or that microbe was due to the inadequacy of the in vitro methods used. But certainly we must say at the present time that the virulence of most pathogenic organisms has not been resolved into toxigenicity. Nor is there any general hypothesis as to the mechanism of virulence when toxin is not demonstrable. On the basis of current theories, it is not easy to explain why a mouse dies some hours after the injection of a few anthrax bacilli, or a rat some days after the introduction of pathogenic trypanosomes. Neither is it known that these micro-organisms produce specific toxins in vivo, nor is immunity against these parasites apparently associated with anything like an antitoxic mechanism. The only easily demonstrable protective mechanism against anthrax bacilli is associated with an enhanced phagocytic capacity of certain fixed tissue and wandering cells. And immunity against trypanosomes may be associated chiefly with the development of trypanolytic and anti-reproducing properties in body fluids.

\section{RELATION OF VIRULENCE TO OTHER CHARACTERISTICS}

Virulence is an expression and a consequence of certain fundamental properties of a parasite in the body of a host. Whether or not it is dependent upon toxin production, the basis for virulence lies in the metabolism of the organism. Hence, it is not surprising that many investigators have called attention repeatedly to the correlated occurrence of virulence and of other characteristic properties that are similarIy associated with metabolism and specific chemical and physical composition.

That this is not entirely accurate, however, may be evidencel by the fact that some cases of so-called "malignant diphtheria" appear to be refractory to antitoxin treatment and that a guinea pig may die after an injection of washed suspensions of non-toxigenic diphtheria bacilli even though a large dose of antitoxin has been injected hefore or after the injection.

${ }^{2}$ Crowell, M. J.: J. Bact., II, 65. I926. 
Among the toxigenic bacteria variations in virulence (toxin production) have not been regularly or frequently associated with variations in other properties. The correlation in the diphtheria group of the capacity to produce toxin and to ferment certain sugars has been repeatedly emphasized and as often demonstrated to be general but not invariable. The identification of toxigenic or non-toxigenic strains of this group by the demonstration of barred or granular structures after special staining has been found similarly untrustworthy.

Among the non-toxigenic bacteria, the correlations of virulence with other properties have been more extensively and more decisively established. That capsulated bacteria are generally more virulent than non-capsulated strains of the same species or genus has long been known. ${ }^{\text {I }}$ When, by animal passage or other treatment, virulence is enhanced, encapsulation becomes more marked; and when, by any of numerous devices, virulence is attenuated, encapsulation is reduced or even abolished. These relations are easily demonstrated with pneumococci, streptococci, anthrax bacilli, members of the Friedländer group, and with many other bacteria. Similarly, it is generally found that virulent or capsulated bacteria are relatively inagglutinable by immune sora and are not easily or readily phagocyted. The direct correlation between virulence and encapsulation, and the inverse correlation of virulence and phagocytability, is extremely common, if not invariable.

It has been argued, and perhaps properly, that the correlation between high virulence and resistance to phagocytosis (and vice versa) is really not a correlation but an identity. A non-toxigenic parasite whose virulence is dependent upon the invasion of tissues and fluids is virulent if it is immune to phagocytosis (and to humoral antibodies), and is avirulent if susceptible. Furthermore, the identity is sometimes extended to include encapsulation. The organism is virulent - it is alleged - because the presence of the capsule renders it insusceptible to engulfment or digestion by the phagocyte (or to destruction and dissolution by a bactericidal or bacteriolytic agency).

According to other views, these correlations are incidental and not determining characteristics of virulence. The real basis of virulence, it is alleged, is the production and excretion by the micro-organism of endotoxins, aggressins, virulins, anaphylatoxins, etc.-metabolic products which injure the defense mechanisms of the host or interfere with their action upon the bacteria. Although the significance of endotoxins and aggressins has been repeatedly and effectively challenged, the rôle of virulins ${ }^{2}$ is still frequently accorded respectful mention. Virulins have been reinvestigated recently in my laboratory by Miss Margaret Pittman. ${ }^{3}$ Except for a slight reduction in the phagocytosis of avirulent pneumococci, extracts prepared from virulent pneumococci after the methods of Rosenow were without demonstrable capacity to affect virulence. The rôle of the acidic and alkaline metabolites, "ptomaines," pressor substances, etc., in the mechanisms of virulence have been adequately discussed in the standard texthooks and lemand no treatment here.

${ }^{x}$ Cf. the review in Zinsser, H.: Infection and Resistance (3d ed.), p. I. New York, 1923.

${ }^{2}$ Rosenow, E. C.: J. Infect. Dis., 4, 285.1907.

3 Pittman, M. J.: Master's dissertition. University of Chicago, I926. 
I have remarked upon the fact that the correlation between virulence and morphological or biochemical characteristics is well established for certain groups of non-toxigenic bacteria. In a search for the mechanism of virulence and an identification of the properties which determine virulence and the correlated characteristics, it seemed logical to start with the study of those phenomena whose operating mechanisms are best understood. For reasons which are presented elsewhere in this volume, ${ }^{\mathrm{r}}$ it has become apparent that under a wide variety of conditions of physiological interest the agglutinability of bacteria is determined by the electrophoretic charge or potential difference (P.D.). Hence, for some years my studies of virulence have been focused upon the P.D. on organisms whose virulence and agglutinability are correlated.

There have been, from time to time, minor and incidental observations on the parallel relations between P.D. and virulence. ${ }^{2}$ However, the first specific demonstrations were made by Northrop and DeKruif ${ }^{3}$ who reported measurements of the higher P.D. on a virulent and relatively inagglutinable strain (type D) of the rabbit septicemia organism and the lower P.D. on a non-virulent and spontaneously agglutinable strain (type G). But they considered that the P.D.--virulence relations might be purely accidental. ${ }^{4}$ With the co-operation of a number of colleagues, I have investigated the relations between P.D. and virulence at some length. For our first series of experiments we elected to work with pneumococci because there are available in that group of organisms type and subtype varieties known to vary in virulence for man and lower animals. After developing a technique to measure virulence for white mice which gives reproducible results and after improving the precision of P.D. measurements, we found particularly:

I. From 76 strains of pneumococci of the three fixed types and of type IV which were isolated from cases of lobar pneumonia, the P.D. and the virulence for white mice were significantly different for the several types and the sequence for P.D. (III $>$ I $>$ II $>$ IV) was identical with the sequence for virulence to mice and the probable sequence for virulence to man.

2. For pneumococci of types II, III, and IV (and not for I), strains which had been isolated from cases of pneumonia which terminated fatally showed higher P.D. than strains isolated from cases that recovered.

3. Variant strains of a type I pneumococcus prepared by Blake and Trask by cultivation in the presence of specific antiserum showed differences in virulence for mice, in agglutinability, antigenic properties, etc., and parallel differences in P.D.

4. The parallel relations between virulence, P.D., and other characteristics were demonstrable on single-cell strains.

${ }^{1}$ Cf. Northrop, J. H.: chap. lviii of this volume.

${ }^{2}$ Cf. Girard, P., and Audubert, R.: Compt. rend. Acad. de sci., 167, 35г. 19r8; Shearer, C.:

Proc. Camb. Phil. Soc., 19, 263. I919; J. Hyg., 21, 77. 1922.

${ }^{3}$ Northrop, J. H., and DeKruif, P. H.: J. General Physiol., 4, 639, 655. 1922.

${ }^{4}$ Personal communication from J. H. Northrop. 
5. The virulence, P.D., agglutinability, etc., showed parallel differences for "Rough" and "Smooth" strains of pneumococci. ${ }^{\text {" }}$

Experiments with cultures of the colon-typhoid-paratyphoid-enteritidis group, hemolytic and green-producing streptococci, bacteria pathogenic for plants, ${ }^{2}$ etc., have given similar parallelisms.

In all cases referred to, the parallelisms between P.D. and virulence obtain within the genus, species, or variety. There is no evidence that P.D., treated as an independent characteristic, is qualitatively or quantitatively different on all organisms which are virulent to a particular host from all organisms which are not virulent for that host. Hence, it is not permissible to assert that P.D. parallels differences in virulence among different micro-organisms, but only that such parallelisms are demonstrable within the groups.

In I9I 8 Baerthlein ${ }^{3}$ reported on the association between variations in colony form and morphological, biochemical, and serological characteristics of bacteria. His observations were first fully appreciated and extended by Arkwright ${ }^{4}$ and by DeKruif, 5 and have led to extensive investigations on "Smooth" and "Rough" strains, so called after the smooth and rough appearance of colonies on agar media. ${ }^{6}$ It has come to be recognized that there are almost invariable parallelisms for " $\mathrm{S}$ " and " $\mathrm{R}$ " strains between variations in virulence, agglutinability, and in other characteristics. Thus, for example, a typical "S" strain is relatively highly virulent, has so-called "normal" morphology, is motile, encapsulated, and is relatively inagglutinable; a typical " $\mathrm{R}$ " strain is slightly or not at all virulent, shows a large proportion of "involution" forms, is non-motile, is without capsule, and is easily or spontaneously agglutinated. Jacobson and I have shown ${ }^{7}$ for pneumococci that when "S"- " $R$ " variations are induced, alterations in virulence are associated with parallel variations in inagglutinability, in production of precipitable (specific soluble) substance, and in the magnitude of the P.D. My observations on the occurrence of " $R$ " and " $S$ " strains in bacteria pathogenic for plants were developed by Sharp and later by Link and Hull. ${ }^{8}$ The parallelism of P.D. and virulence is presented in the report by Sharp. ${ }^{9}$

The indications are that the significance of P.D. in virulence studies on animal hosts extends into similar studies on virulence for plant hosts.

The earlier notions of discontinuity between " $S$ " and " $R$ " variations have latterly

r Falk, I. S., Gussin, H. A., and Jacobson, M. A.: J. Infect. Dis., 37, 481. 1925; Falk, I. S., Jacobson, M. A., and Gussin, H. A.: ibid., Pp. 495, 499. 1925; Falk, I. S., and Jacobson, M. A.: ibid., p. 507; 38, pp. г82, г88. г926; J. Bact., 13, 191. 1927.

${ }^{2}$ Sharp, C. G.: Bot. Gaz., 83, I13. I9I7; Link, G. K. K., and Sharp, C. G.: ibid., p. I45. I927; Falk, I. S., Sharp, C. G., and Link, G. K. K.: Proc. Soc. Exper. Biol. Eo Med., 24, 576. 1927. Cf. also chap. xliv by Dr. Link in this volume.

3 Baerthlein, K.: Centralbl.f. Bakteriol., I. Orig., 8I, 369. r9r8.

4 Arkwright, J. A.: J. Path. \& Bact., 24, 36. I921.

s Dekruif, P. H.: J. Exper. Med., 33, 733. I921; ibid., 35, 631. 1922.

${ }^{6} \mathrm{Cf}$. the extensive review by Hadley, P.: J. Infect. Dis., 40, I. 1927. Also chap. vii by Dr. Hadley in this volume.

7 Jacobson, M. A., and Falk, I. S.: J. Bact., r3, I9I. 1927.

${ }^{8}$ Link, G. K. K., and Hull, K. L.: Bot. Guz., 83, +12. 1927.

9 Loc.cit. 
been modified by the demonstration of intermediate "O" forms. Except for this complication, the variation between " $S$ " and " $R$ " colony form has generally been considered to be qualitative and discontinuous. " Comparisons of virulence between " $\mathrm{S}$ " and " $\mathrm{R}$ " forms are, of necessity, similarly qualitative and discontinuous. It is usually impossible to discover whether or not there occur intermediate gradations in virulence between the extreme "S" and " $\mathrm{R}$ " forms because (when " $\mathrm{S}$ " is being converted to " $R$ " or vice versa) the intermediate cultures are unstable and injections of such cultures into animals to test virulence usually result in reversions to the virulent " $\mathrm{S}$ " variety. But by studying in vitro the electrophoretic potentials (P.D.) on "S" and " $\mathrm{R}$ " forms of pneumococci and on all the intermediate cultures, Jacolsson and I found" that the progressive conversion of " $\mathrm{S}$ " to " $\mathrm{R}$ " is accompanied by apparently gradual (and not discontinuous) changes in P.D. Furthermore, "S" cultures which have been changed with respect to the magnitude of P.D. have not necessarily been sufficiently altered to show " $R$ " colonies, nor have those which have been changed to the " $R$ " forms been, of necessity, sufficiently changed in virulence or P.D. to give sensibly different measurements. I am led to conclude tentatively that:

I. In all the cases which we studied, alterations in the virulence of pneumococci for white mice were accompanied by parallel alterations in P.D., inagglutinability, and in other characteristics; and such alterations occurred in a continuous and not in a discontinuous manner.

2. It is probable that the variations between virulent " $S$ " and avirulent " $R$ " forms represent the fixed extremes of continuous variations in virulence; and that with respect to colony form, as with other characteristics, the variations are continuous.

\section{WHY IS P.D. CORRELATED WITH NON-TOXIGENIC VIRULENCE?}

From evidence of the type which has been briefly summarized, it has appeared that there are parallelisms between virulence and the electrophoretic potential difference (P.D.) on non-toxigenic bacteria which are not merely accidental or occasional. Every device used by which P.D. is changed (without destruction of viability) has resulted in a parallel change in virulence; and every change in virulence studied was accompanied by similar alterations in P.D. Inasmuch as the resistance of the host against the non-toxigenic bacteria studied is principally associated with a phagocytic mechanism of immunity, we turned to a study of the relations between P.D. and phagocytosis for an explanation of the findings. The analysis is still in progress, but enough has already been done to indicate that the phagocytosis of live or dead organisms bears a definite relation to the charges on the bacteria and on the phagocyte. Falk and Matsuda have reported:

I. For the A, B, and C strains of pneumococci, the sequence for P.D. and for virulence to mice is $\mathrm{A}>\mathrm{B}>\mathrm{C}$.

2. In the presence of normal rabbit serum or of immune horse serum, the sequence of the phagocytic index is $\mathrm{C}>\mathrm{B}>\mathrm{A}$.

"Observations in my laboratory are not in accord with this view. We have generally found that the "S" and "R" colonies vary in "smoothness" and "roughness" and grade from one form to the other.

2 Loc. cit. 
3. It had been established that when suspensions of these organisms are washed with water or dilute salt solutions, the P.D. sequence is inverted from $\mathrm{A}>\mathrm{B}>\mathrm{C}$ to $\mathrm{C}>\mathrm{B}>\mathrm{A}$. It was found correspondingly that washing resulted in an inversion of the sequence for the phagocytic indices.

4. If the P.D. on pneumococci be reduced by such a salt as lanthanum nitrate, the phagocytosis of the organisms is increased proportionally. If the P.D. be increased by treatment with sodium oleate, the phagocy tosis is proportionately reduced.

5. The phagocytic indices were modified successively when suspensions of pneumococci were treated with lanthanum nitrate, were washed and then treater with sodium oleate; and, reciprocally, when the procedure was reversed. ${ }^{\mathrm{r}}$

It is apparent that the organisms are more readily and more extensively phagocyted the lower the P.D., and vice versa. Hence, it is not surprising that virulence and P.D. are parallel characteristics.

The precise mechanism by which electrical charges (electrophoretic potential differences) on phagocyte and bacterium affect phagocytosis is still unsolved. It might be considered that the like charges on both phagocyte and bacterium provide merely a repelling force, and the smaller the charges (on either or both), the less the interference with engulfment of the particle by the phagocyte. Or-as Ponder $^{2}$ has indicated recently-bodies with like charges may be attracted to each other when brought close together, if the ratio between their diameters is large, by the attractive effect between original and induced charges. We are investigating this problem further. ${ }^{3}$ Abramson ${ }^{4}$ has shown recently that powerful electrophoretic fields may be set up in vivo following tissue injury.

SPECIFIC SOLUBLE SUBSTANCES, VIRULENCE, AND P.D.

Assuming the validity of the analysis which reduces the parallelism of nontoxigenic virulence, relative inagglutinability, etc., to P.D. and insusceptibility to phagocytosis, the question might be raised: By what constituents or properties of the cell is the P.D. determined? The search for an answer at present lies with permeability phenomena, if the origin of the P.D. is explained on the basis of a Donnan equilibrium; ${ }^{5}$ or with the phenomena associated with the production and behavior of specific soluble substances. ${ }^{6}$

I have already remarked that differences in the production of specific soluble substances parallel differences in P.D. among " $S$ " and " $R$ " variants. Cole? reported that infected exudates and sera which contain the specific, reactive substances of pneumococci possessed the property of neutralizing pneumococcus antibodies. Sia ${ }^{8}$ has presented some evidence to show that the inhibitory action of sera from naturally re-

${ }^{r}$ Falk, I. S., and Matsuda, T.: Proc. Soc. Exper. Biol. Eo Med., 23, 78r. I926.

${ }^{2}$ Ponder, E.: J. General Physiol., 9, 827. 1926.

${ }^{3}$ For a discussion of the mechanism of phagocytosis, see chap. lxv by Dr. Fenn in this volume.

${ }_{4}^{4}$ Abramson, H. A.: J. Exper. Med. 46, 987 . I927.

5 Winslow, C.-E. A., Falk, I. S., and Caulfeild, M. F.: J. General Physiol., 6, 177. I923.

${ }^{6}$ Cf. Heidelberger, M.: Chem. Rev., 3, 403. 1927. Also chap. lii by Drs. Zinsser and Mueller in this volume.

${ }^{7}$ Cole, R.: J. Exper. Met., 26, 453. 1917.

${ }^{8}$ Sia, R. H. P.: ibid., 43, 633. 1926. 
sistant animals on the growth of pneumococci in serum-leukocyte mixtures is annulled by the addition of specific soluble substance. Felton and Bailey ${ }^{1}$ have reported that injections of only partially purified soluble substance into mice renders them more susceptible to partly attenuated pneumococci (or enhances the virulence of the organisms), and that the protective substance of antiserum or antibody solution is neutralized by specific soluble substance. Inasmuch as it is tentatively considered by most investigators that the soluble substances are associated with the capsular structure of bacteria, these observations would harmonize with those on P.D., because of the parallelisms of virulence, P.D., and encapsulation, and because the generally accepted theory of electrophoresis locates the basis of the electrokinetic behavior on the surface layers of the particle.

In apparent contradiction to these views, however, there are observations which we have made repeatedly that:

r. Filtrates from virulent pneumococcus cultures which had been incubated long enough to contain high concentrations of specific, soluble substance do not significantly enhance the virulence of less virulent cultures for mice; and they do not reduce the resistance of mice to such cultures.

2. Preparations of purified soluble substance are not toxic for mice, and are not more active than culture filtrates.

3. Preliminary experiments indicate no significant effects of soluble substances on P.D.

4. The significant differences in suspension stability, motility, etc., between "S" and " $\mathrm{R}$ " cultures appear not to be determined by the menstruum, although when this comes from " $\mathrm{S}$ " type bacteria it contains specific substance, and lacks this reagent when it comes from the "R" type."

It must be considered at this time that the rôle of specific, soluble substances in determining virulence is still uncertain; but the indications are that the secreted substances are probably unimportant, although the specific substances contained in or on the cell may be determining factors. It is also probable that a common mechanism (of a genetic order) determines the production of soluble substances, encapsulation, cell and colony morphology, motility, and P.D.

\section{TOXIGENICITY AND ELECTROPHORETIC POTENTIAL}

The general parallelisms between P.D. and virulence characteristics of nontoxigenic organisms suggest the question whether similar parallelisms obtain for toxigenic organisms. Corynebacteria were chosen for an investigation of this problem in my laboratory.

If it be assumed that the P.D. has its origin in phenomena of semi-permeability of the kind encountered in Donnan membrane equilibria, the a priori argument takes the following form:

I. Assuming that for different strains high toxigenicity grades by imperceptible degrees through low to no demonstrable toxigenicity; ${ }^{3}$

${ }^{2}$ Felton, L. D., and Bailey, G. H.: J. Infect. Dis., 38, 131. 1926.

${ }^{2}$ These experiments are being extended now.

${ }^{3}$ This is entirely in accord with the experimental findings. 
2. Assuming that the ordinary animal test for toxigenicity is a measure of toxin excretion by toxin-producing bacteria;

3. Assuming that the ratio of toxin concentration outside the cell to the toxin concentration inside the cell is a measure of cell semi-permeability;

It follows from the principle of the Donnan equilibrium that:

4. The P.D. between the cell and the menstruum will be minimal when the toxin concentration outside approaches the toxin concentration inside, and will be maximal when the concentration outside is least (because $\pi_{2}-\pi_{\mathrm{I}}=\frac{R T}{n F} \log \frac{C_{\mathrm{I}}}{C_{2}}$ where $\pi_{2}$ and $\pi_{\mathrm{I}}$ are the positive potentials of the solutions, $C_{1}$ and $C_{2}$ the concentrations of the diffusible ion on the two sides of the membrane, and $R, T, n$, and $F$ have the usual thermodynamic significance).

5. And the large P.D. values are to be expected for strains which do not excrete toxin and small P.D. values for strains which freely excrete toxin.

Experimental verification of these expectations has been thoroughly established by L. B. Jensen. ${ }^{2}$ It has even been possible to utilize a simple electrophoresis procedure for the rapid determination of virulence (toxigenicity) in routine diphtheria control. The indications are, further, that small- or large-scale toxin production may be controlled by controlling P.D.

It would appear, then, that even among toxigenic bacteria P.D. parallels virulence (toxigenicity), although it may be for different reasons, and reciprocally from the parallelism which obtains among non-toxigenic bacteria.

\section{ELECTROPHORETIC POTENTIAL AND HOST RESISTANCE}

If it be true that P.D. is a measure of (or a determining factor in) virulence, it follows from the intimate relation between virulence and resistance that was emphasized earlier that certain mechanisms of resistance might be associated with enhanced properties of tissues or body fluids to affect the P.D. on invading organisms or their toxins. And there is a substantial body of evidence in accord with this expectation.

Thus, it has been demonstrated that immune sera not only exceed normal sera in the capacity to reduce P.D. on bacteria, but these enhanced capacities of immune sera are specific. Within the pneumococcus group, the P.D.-reducing capacities of antisera are even type specific. ${ }^{3}$ Furthermore, all of the recent work on specific toxin-antitoxin flocculation ${ }^{4}$ indicates that antitoxin specifically modifies the P.D. on toxin. This follows from the fact that the flocculation of a colloid is intimately related to its P.D. The older notion that the reaction between toxin and antitoxin does not fall into this category of colloid dynamics was based upon the conclusion of Field and Teague (and of Bechhold) that both toxin and antitoxin are electropositive

${ }^{x}$ Donnan, F. G., and Green, G. M.: Proc. Roy. Soc., A, 90, 450. I9I4.

${ }_{2}^{2}$ Preliminary reports of this work have appeared in the Proc. Soc. Exper. Biol. \& Med., 23, 783. 1926; and in the Am.J. Pub. Health, 17, 714. 1927. The detailed reports will appear shortly in the J. Bact.

${ }^{3}$ Shibley, G. F.: J. Exper. Med., 40, 453. 1924; Falk, I. S., and Jacobson, M. A.: J. Infect. Dis., 38, 182. 1926 .

4 Except that of Bronfenbrenner and Reichert. Cf. chap. lvi by Dr. Bayne-Jones in this volume. 
colloids without regard to the acidity or alkalinity of the solution. Maver and $\mathrm{I}^{\mathrm{I}}$ have found that the Field and Teague apparatus is not a true electrophoresis cell and that the migrations of colloidal particles recorded in that apparatus are anomalous. In a true cataphoresis cell, antitoxin is certainly and toxin is probably amphoteric. The indications are that antitoxic action may depend upon a specific capacity to reduce P.D. on toxin and thereby to flocculate the toxin and render it physiologically inert.

The hypothesis that the reactions of immunity are dependent upon (or are closely associated with) alterations of the P.D. on micro-organisms may not be more than casually demonstrable at this time. But, viewed from a particular angle, it is not far fetched. It assumes only that in the study of virulence (or of resistance) we must consider not the microbe alone, but the microbe in an environment. The study of virulence is a special case of the larger study of the organism, a special case in which the universe of discourse is bounded by the confines of the host body. And, whether the microbe be in vitro or in vivo, its P.D. is related to those characteristics which may be measurable in terms of growth, reproduction, toxin production (toxigenicity), virulence, resistance, and immunity.

In concluding, I would say that I have marshaled a portion of the newer evidences on the mechanisms of virulence not so much to prove a new case as to plead for a reanalysis of an old one. I am persuaded that the older notions on virulence and its mechanisms have not been fruitful; a newer one which for the moment places the dynamics of electrical potential differences or charges as a focal point between "virulence" and "resistance" may not be more sound, but it cannot be more sterile.

I Maver, M. E., and Falk, I. S.: J. Immunol., I4, 219. 1927. 


\title{
CHAPTER XLIII
}

\section{ELECTIVE LOCALIZATION OF BACTERIA \\ IN THE ANIMAL BODY}

\author{
EDWARD C. ROSENOW \\ The Mayo Foundation, Rochester, Minnesota
}

The problem of elective localization of bacteria in the animal body embraces an important part of the whole question of virulence and the causation of disease by bacteria. In its broader application, the term "elective localization" has been used to designate specific invasive or localizing power of the causative micro-organisms in various diseases. Besredka ${ }^{x}$ speaks of elective localization of Bacillus dysenteriae and its toxin in the intestines and central nervous system; of Bacillus anthracis and Staphylococcus aureus in the skin in anthrax and furunculosis, respectively. The localization of the Bacillus diphtheriae in the throat, of the virus of rabies in the brain, and of the virus of poliomyelitis in the motor cells of the spinal cord may also be said to be truly elective. The mere entrance of bacteria into the blood stream is not always sufficient to cause metastatic disease. Certain delicately balanced conditions must be fulfilled in order to promote localization and growth so that lesions may be produced in various tissues or organs. Thus, in typhoid fever, the blood is invaded by the typhoid bacillus, yet localization and lesions remain limited to the lymphoid elements of the intestines and spleen. The pneumococcus commonly invades the blood in lobar pneumonia, yet the lesions tend to remain limited to the lung and pleura. An extreme example of the tendency of bacteria to localize and produce lesions electively in the infected host in spontaneous disease is found in subacute bacterial endocarditis. Here green-producing streptococci have been demonstrated in the blood daily or on alternate days for many weeks and yet, in most instances, localization remains limited to the valves of the heart, and when other manifestations of localization occur, such as in the tips of fingers and joints, they are usually temporary. Evidence of similar extreme electivity was found in a series of experiments performed long ago in which it was shown that green-producing streptococci localized and produced lesions in the heart valves and hemolytic streptococci in the joints of animals injected intravenously with known mixtures of these organisms.

In a more limited sense the term "elective localization" has been used to designate the tendency of certain bacteria, especially streptococci, from various localized areas of infection or more normal mucous membranes (such as the nasopharynx) or systemic lesions, to localize and produce lesions in animals corresponding to those in patients or animals from whom the micro-organisms were isolated. ${ }^{2}$ The idea that different diseases might be due to a single member of the pneumococcus-streptococcus group having different elective localizing power had its birth in my experiments from I9I3

${ }^{x}$ Besredka, Alexandre: Local Immunisation. Baltimore: Williams \& Wilkins, I927.

${ }^{2}$ Rosenow, E. C.: J.A.M.A., 65, 1687. I9r5. 
to $\mathrm{rgr}_{4}$ in which mutations of pneumococci into streptococci, and vice versa, were induced. ${ }^{I}$ It was noted then that coincidental with changes in morphological and cultural characters marked changes occurred in the localizing power which was often characteristic of the type. The changes induced experimentally were shown to be complete in the case of a number of representative strains of the group. The new strains corresponded to those obtained from the usual sources, morphologically and culturally, in the Marmorek test, in dextrose-serum broth, in fermentative powers, in bile solubility, in autolysis in sodium chloride solution, in agglutinating properties, and in specific antigenic power. The production of marked changes in cultural character as well as in localizing or infecting power of members of this group has recently been reported by Morgenroth, Schnitzer, and Berger. ${ }^{2}$ I fulfilled the pure-line requirement by obtaining single-cell cultures of different members of the group.

In the work of Morgenroth, Schnitzer, and Berger, as in mine, not all strains yielded to attempts to produce mutations or "dissociation"; 3 a premutational stage, therefore, seemed necessary. The changes which I induced often had the characteristics of true mutations because they appeared suddenly under conditions more or less obscure and the newly acquired properties persisted unless the organisms were again placed under special conditions. In my in vitro experiments, the underlying conditions which tended most to produce changes were, first, favorable media for luxuriant growth and then unfavorable conditions, under stress or strain. Mutations in the animal body have been observed almost exclusively in closed cavities such as joints, peritoneum, pleura, or pericardium, and here, as in vitro, under conditions of stress or when the tissues of the host were gradually getting the upper hand and the organisms were being destroyed. The following observations made during my studies in transmutation had particular bearing on the fundamental questions of elective localization, which have been studied extensively since.

It was found that as non-virulent strains became virulent by successive passage through animals, and highly virulent strains became less virulent by cultivation on artificial media, the site of localization with production of lesions changed markedly. When the virulence was lowest the localization was almost wholly in relatively avascular tissue, such as heart valves, joint structures, and tendinous ends of muscles; when the virulence was moderate, iritis, myositis, ulcer of the stomach, cholecystitis, and focal lesions of the kidneys were more prone to develop; and when it was high from successive passage through animals or on isolation, lesions of the lung and death from bacteremia occurred commonly. Striking as these results were, it was not until the unusual localization in the mucous membrane of the stomach of strains from several sources occurred, producing hemorrhage and ulcer, that the idea of elective localization took definite form.

Since variations in oxygen tension and salt concentration, growth in symbiosis with other bacteria, and injection into closed cavities in animals commonly called forth mutational forms in pneumococci and streptococci, it seemed highly probable that

' Rosenow, E. C.: I. Infect. Dis., r4, I. I9I4.

${ }^{2}$ Morgenroth, J., Schnitzer, R., and Berger, E.: Ztschr. f. Immunitätsforsch. u. exper. Therap., 43, 169, 209. I925.

${ }^{3}$ Hadley, P.: J. Infect. Dis., 40, r. 1927. 
similar changes might occur in various localized but mild inflammatory processes or foci of infection. This suggested that the tonsils, where these conditions prevail, might be a good place to search for causative bacteria possessing elective localizing power. Clinical evidence of the probable presence in these foci of the causative organism was not lacking, for Billings ${ }^{\mathrm{I}}$ had reported good results from removal of such foci in a series of cases. Two other important observations were made (one prior to the work on mutation, the other during that period) which led to the use of proper methods in the newly planned experimental work. The one concerned the importance of injecting freshly isolated strains in order to produce specific lesions, first noted in a series of cxperiments in subacute bacterial endocarditis, ${ }^{2}$ and later in rheumatic fever; ${ }^{3}$ the other concemed the importance of oxygen tension for the successful isolation and maintenance of specific or elective localizing power of streptococci, first noted in my experiments in rheumatic fever. Localization in animals of the freshly isolated streptococcus in these diseases was remarkably specific. The streptococcal strains from tonsils or blood in cases of subacute bacterial endocarditis manifested marked affinity for the valves of the heart and produced, soon after intravenous injection, severe hemorrhage and later huge vegetations. Aside from producing lesions of the glomeruli in the kidney, the strains had little tendency to localize and produce lesions in other structures, such as joints, myocardium, and pericardium. The streptococcal strains freshly isolated by me from tonsils, blood, stool, muscles, lymph nodes draining affected joints, and especially from the joint fluid in rheumatic fever, manifested simultaneous affinity for joints, endocardium, pericardium, and myocardium, and those from diseased muscles affinity for muscles as well. The lesions produced experimentally resembled, in many respects, those noted in cases of rheumatic fever. The early hemorrhage and later vegetations on the valves of the heart were relatively small, and the tendency to the formation of scar was marked. Many joints became involved without suppuration or destruction of tissue. The lesions in the myocardium often resembled Aschoff bodies. The number of organisms in the vegetations, and in joint fluid of injected animals as of patients, was relatively small, and they were difficult to isolate. Lesions such as iritis, appendicitis, pyelonephritis, and ulcer of the stomach, and symptoms resembling chorea occurred in a small number of injected animals, paralleling roughly the incidence of these affections in patients. Requirements for causal relationship of the streptococcus isolated in cases of rheumatic fever appeared fulfilled. After prolonged cultivation on artificial media and successive passage through animals, the characteristic localizing power of both types of strains disappeared. The isolation from cases of rheumatic fever of streptococci possessing characteristic localizing power has been reported since by Clawson ${ }^{+}$and more recently by Small ${ }^{5}$ and by Birkhaug. ${ }^{6}$

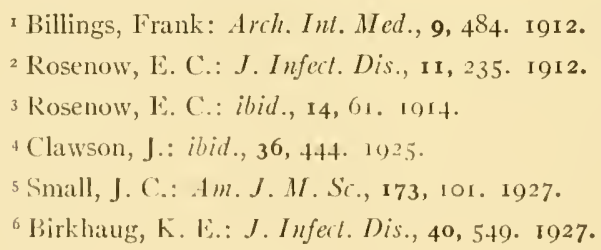




\section{METHODS OF STUDY}

By varying the amount of inoculum, tall columns of ascites-dextrose agar and broth were found especially useful in the isolation of streptococci from the joint exudates in cases of rheumatic fever and, somewhat later, for the isolation of bacteria from emulsions of tissues and the sediment of laked blood even in diseases generally thought to be due to causes other than infection. ${ }^{1}$ The living cells in the inoculum absorbed oxygen and thus afforded a gradient of oxygen tension.

The importance of partial-tension cultures in the isolation of pathogens has since been emphasized, especially by Wherry and Oliver. ${ }^{2}$ In the earlier work on elective localization inoculations in these media sufficed to permit highly sensitive organisms to multiply because the inoculum usually consisted of emulsions of excised tissues containing the metastatic lesions, the sediment of laked blood, the material expressed from tonsils, or pus aspirated from pyorrhea pockets. In later experiments the amount of inoculum was often too small and the bacteria or living cells too few (especially in the case of cultures from devitalized teeth) ${ }^{3}$ to lower the oxygen tension sufficiently in these media to promote growth of the more strictly anaerobic organisms. The addition of fresh sterile tissue is not well adapted for routine work because it is so difficult to exclude contamination. In searching for an inexpensive and easily prepared medium that could be autoclaved and that would furnish a gradient of oxygen tension, and other conditions favorable for growth of highly sensitive organisms, it occurred to me that since anaerobes grow readily in brain-mash medium, the addition of pieces of brain to the bottom of tall columns of glucose broth and agar might suffice to lower the oxygen tension sufficiently to permit anaerobes to multiply in instances in which the inoculum must needs be small and free or relatively free from cells or saprophytic contaminants.

It was found that two or three pieces of brain approximating I cc. each, when added before sterilization to tall columns $(8-10 \mathrm{~cm}$.) of glucose broth $(0.2$ per cent glucose) or agar, contained in test tubes $1.5 \mathrm{~cm}$. in diameter, sufficed after sterilization to decolorize methylene blue in the deeper part of the tubes and keep it so for a long time on standing, and that anaerobic streptococci, tetanus spores, Bacillus wclchii, and Bacillus fusiformis in mixed cultures, and other strictly anaerobic organisms grew readily when inoculated into the bottom of the tubes containing the pieces of brain. On the basis of these observations the brain-containing media were adopted in most subsequent work on elective localization. In special instances ascites fluid or serum or fresh sterile tissue is added and the surface covered with a layer of vascline or paraffin oil; prolonged boiling just before use, to drive off oxygen, is also carried out.

The successful isolation of the causative organisms of various diseases from tonsils, nasopharynx, cervix, prostate, and especially the periapical tissues of pulpless teeth, usually containing a mixture of bacteria, has often been directly attributable to the use of these media. It has happened repeatedly that not only did growth occur in

Rosenow, E. C.: J.A.M.A., 63, 903. I9I4.

2Wherry, W. B., and Oliver, W. W.: J. Infect. Dis., 19, 28S. 1916; 20, 28. 19г7.

${ }^{3}$ Rosenow, E. C.: J. Dent. Research, r, 205. 1919. 
these media but the disease from which the patient was suffering was successfully reproduced in animals with the streptococci isolated, when cultures made according to standard bacteriological methods (low columns of broth on aerobic agar or blood-agar plates) remained sterile or yielded only aerobic saprophytes. Because of these observations and since saprophytic streptococci do not produce lesions and disappear quickly from the blood, we have learned in the study of elective localization to inject into animals the primary (often mixed) culture in glucose brain broth in order not to miss the pathogenic strain or to have it lose specific localizing power during its isolation in pure culture.

The glucose brain broth is prepared by dissolving $8 \mathrm{gm}$. of dehydrated Bactobroth in $\mathrm{I}, 000 \mathrm{cc}$. of distilled water, adding $8 \mathrm{gm}$. of sodium chloride, $2 \mathrm{gm}$. of chemically pure glucose, and, after cooling, ro cc. of Andrade's indicator; it is also prepared from beef infusion, adding 0.2 per cent glucose in the usual way and titrating to $\mathrm{pH}$ 7.4. Several bacteriological peptones on the market have been used with about equally good results. The glucose brain agar is prepared from the broth by adding 4 or $5 \mathrm{gm}$. of powered agar to each liter, just sufficient to set. The media are placed in tubes 20 by $\mathrm{x} .5 \mathrm{~cm}$. To each tube are added three pieces of calf or beef brain, each about I cc., and two or three pieces of calcium carbonate in the form of crushed marble to insure correct reaction. The column in each tube, after the brain and marble are added, is brought to about $\mathrm{I} 2 \mathrm{~cm}$. The media are sterilized for twenty minutes in the autoclave at 17 -pound pressure. Growth in these media, kept at $35^{\circ}-37^{\circ} \mathrm{C}$., usually occurs within twenty-four hours, and in most instances, especially in the case of cultures from pulpless teeth, begins at the bottom of the tube and forces its way to the top as oxygen is consumed. This usually occurs within twenty-four hours in the glucose brain broth, while in the glucose brain agar growth to the top, or more of ten to within I $\mathrm{cm}$. of the top, frequently takes from two to three days. Negative cultures are observed daily for a week or ten days before they are discarded. Colonies in the agar may be exceedingly small or develop only around the pieces of brain at the bottom and may be easily overlooked, or they may be so numerous as to give the appearance of a negative culture.

The animals, chiefly rabbits, were injected usually with eighteen-hour to twentyfour-hour primary cultures in glucose brain broth, sometimes with pure cultures in this medium obtained from single colonies in the soft glucose brain agar, or from inoculated animals and after many rapidly made subcultures (four to eight each twentyfour hours in previously warmed media). Subcultures from aerobic growth on blood agar commonly gave negative results. The number of bacteria injected as a routine in later studies was approximately from one-fifth to one-tenth as great as was used earlier. Depending on the size of the animal, the source of the strain or the particular purpose in mind, 5 or $7 \mathrm{cc}$. of the primary growth in glucose brain broth of material from the focus or infection atria were injected intravenously as a routine into each of two rabbits. The injections were usually not repeated although sometimes several injections were given daily. The animals selected from healthy stock were fed in the usual way and were observed daily. Those that died from the effects of the injections were examined as soon after death as possible, and those that survived were chloroformed in from two to four days after the last injection. Search for lesions was made 
in a strong light. Lesions such as obtained in these experiments practically never occur in the animals that die from intercurrent causes or that are chloroformed as controls. Serving as a further check is the fact that highly specific localizations were obtained consistently when material from different disease entities was injected by myself and co-workers into animals from a common supply. Streak cultures on blood agar and inoculation into tall tubes of glucose brain broth or agar were made, as a routine, from the blood of injected animals and from lesions and (for controls) from other tissues. Blocks of tissue were fixed in ro per cent formalin. Paraffin sections were stained with hematoxylin and eosin for cellular changes, and, by a modified gram method, for bacteria. The latter consists, essentially, of staining the sections more deeply than in the gram method and in decolorizing to a light blue intead of to the end-point. In this way, the nuclei of cells appear light blue, the cytoplasm pale blue, and the bacteria deeply purple. Areas of infiltration, where bacteria are most numerous, can thus be readily identified and random search for bacteria obviated.

Numerous attempts have been made, with only partial success, to find a method of culture that might be relied on to retain elective localizing power and viability for long periods. Preservation of latent life under reduced oxygen tension in tall tubes of meat-mash infusions or in shake cultures containing relatively few colonies in glucose brain agar or ascites glucose agar, in dense suspensions in glycerol (two parts), and saturated sodium chloride solution (one part), have often served to retain viability and elective localizing and other specific properties for a long time. In exceptional instances this was true for as long as eight years when the corresponding strains, grown aerobically, had lost elective localizing power during several daily transfers. Subcultures made rapidly (from four to eight each twenty-four hours) in previously warmed media which afford a gradient of oxygen tension, such as glucose brain broth, often served to maintain specific localizing power and agglutinating properties for many generations. Elective localizing power of streptococci was preserved often for many weeks or months within the pulp chamber or periapical tissues of artificially devitalized teeth in dogs.

The use of proper cultural methods is not the only requirement for the successful application of the principles involved in the study of elective localization, especially as pertains to localized inflammatory processes in different diseases. Here difficult procedures and judgment of elusive factors are often necessary. We have learned that a mere correct diagnosis may not be sufficient. The condition at the time of study should also be considered. A higher incidence of localization and more marked lesions in animals are obtained if cultures are secured at the time of acute systemic manifestations or during exacerbations of symptoms in chronic conditions than during quiescent intervals. Great care is exercised in the collection of material for study both as regards the clinical observations and the prevention of contamination by extraneous organisms. Pus from the depths of tonsils is expressed and cultures made from this instead of swabbings from the surface or from single crypts. Material from the nasopharynx is obtained by swabbings high behind the palate, with cotton swabs on aluminum wire bent to a suitable angle, without touching the tongue. Pus from the depths of pyorrhea pockets or discharging sinuses is aspirated into fine pipettes after the surface has been cleansed with alcohol or some other antiseptic. Pulpless 
teeth are extracted under as nearly sterile conditions as possible, and, in special instances, the apical end is resected for culture before the tooth is extracted. Culture controls are made of the mucous membrane before and after sterilization, and of the overlying bone after the mucoperiosteal flap is resected.

\section{ELECTIVE LOCALIZATION FOLLOWING INTRAVENOUS INJECTION}

Many diseases have been studied from the standpoint of elective localization according to the procedures and methods herein described. Much valuable knowledge as regards the etiology and management of previously poorly understood and closely related diseases, as well as of epidemic diseases, has been revealed. Streptococci that localized and produced lesions electively in the tissues or organs of animals injected intravenously corresponding to those affected in the patient were isolated from one or more foci of infection or from systemic lesions, or both, with great regularity. These reports have been published chiefly in the Journal of the American Medical Association and the Journal of Infectious Diseases during the last ten years. They concern the following diseases: appendicitis, ulcers of the stomach and duodenum, cholecystitis, pancreatitis, epidemic parotitis, acute and chronic arthritis and myositis, acute iritis and allied diseases of the eye, erythema nodosum, herpes zoster and neuraigia, neuritis and sciatica, transverse myelitis, and poliomyelitis.

The incidence of lesions in the specific organ or tissues in the observations reported varied from 60 per cent in cases of ulcer of the stomach to 90 per cent in those of erythema nodosum. Control animals injected with streptococcus strains from patients suffering from miscellaneous diseases or from persons without systemic disease failed to show specific localizations. Specific localization of the streptococcus often occurred despite the fact that often there were few other bacteria in the cultures injected. It may be that localization of the streptococcus was favored by growth in symbiosis with these organisms, but the latter were usually not found in cultures or on microscopic examination of the affected tissues. However, elective localization of mixtures of bacteria has been observed. Thus, in a case of acute appendicitis in which the attack followed closely Vincent's angina, mixtures of streptococci and fusiform bacilli were isolated from the throat and extirpated appendix, and both produced marked lesions of the appendix. Cultures from the lesions in the appendix again revealed the mixtures, and fusiform bacilli and streptococci were found in the lesions in stained sections. Colon bacilli with respective localizing power were isolated in a few cases of acute cholecystitis and appendicitis; and, in one case each of acute cholecystitis and pancreatitis, a staphylococcus was isolated which localized electively on intravenous injection into animals. A striking instance of elective localization of a staphylococcus in the kidney occurred in a case of severe nephritis in which the primary mixed cultures of this organism and streptococcus from an infected maxillary sinus and from tonsils were injected intravenously.

The results of elective localization following intravenous injection have been fully corroborated and extended in important respects by my co-workers in the laboratories of the Mayo Foundation, as well as by independent investigators. Meisser ${ }^{1}$ has reported confirmatory results in a long series of experiments with streptococci isolated

tMeisser, J. G.: J. Am. Dent. A., I2, 554. 1925. 
from dental and other foci of infection of patients suffering from various diseases; Bumpus and Meisser ${ }^{\mathrm{I}}$ have reported on cases of pyelonephritis and submucous ulcer of the bladder and cystitis; and Meisser and Brock ${ }^{2}$ on various forms of chronic arthritis. Working with streptococci isolated from extirpated tonsils in cases of ulcer of the stomach or duodenum, and of arthritis, Nakamura ${ }^{3}$ obtained a much higher incidence of lesions in these respective organs than following injection of strains from tonsils in miscellaneous control cases. Giordano and Barnes ${ }^{4}$ proved the elective localizing power of streptococci isolated from pulpless teeth and systemic lesions after death in cases of perforating ulcer, of gastric hemorrhage, and of perinephritic abscess. Moench, 5 through similar studies in animals, has shown that partial-tension streptococci isolated from the cervix of patients suffering from endocervicitis and arthritis have a marked affinity for the joints of animals. Brown ${ }^{6}$ has corroborated the work on cholecystitis and ulcer of the stomach; and Rosenow and Ashby on myositis. Cantero, in work as yet unpublished, noted similar results in ulcer of the stomach, and he has applied my methods in a study of the etiology of goiter. He has found that certain streptococci isolated from the thyroid in this disease tend to localize and produce lesions in the thyroid of animals. Bargen, ${ }^{8}$ by the use of the same methods, has isolated a diplo-streptococcus from ulcers in the colon and from tonsils and teeth in cases of chronic ulcerative colitis, which localizes electively in the colon of animals and produces hemorrhages, ulcerations, and diarrhea.

Gerdine and Helmholz, ${ }^{9}$ by the use of similar methods, have shown that several epidemics of duodenal ulcer in children were due to streptococci having peculiar affinity for the duodenum. Kennedy ${ }^{\text {In }}$ has reported the finding of large numbers of streptococci in small ulcers of the stomach and duodenum, the source of fatal intestinal hemorrhage in cases of melena neonatorum. Helmholz and Beeler ${ }^{11}$ have demonstrated elective localizing power in strains of Bacillus coli and streptococci isolated from cases of pyelonephritis in rabbits. Nickel, ${ }^{12}$ working with me in a long series of experiments, has corroborated and extended the work on arthritis, on myocarditis and endocarditis, on iritis, on ulcer of the stomach and duodenum, and on abortion.

Especially noteworthy is the independent work by Haden. Not only has he obtained comparable results in diseases such as ulcer of the stomach and duodenum, ${ }^{\mathrm{I}}{ }^{3}$ and

${ }^{x}$ Bumpus, H. C., Jr., and Meisser, J. G.: Arch.Int. Med., 27, 326. 1921; J. Urol., 5, 249. $192 \mathrm{I}$.

${ }^{2}$ Meisser, J. G., and Brock, Sam: J. Am. Dent. A., ıо, г гоо. I923.

3 Nakamura, Tokukichi: Ann. Surg., 79, 29. I924.

${ }_{4}^{4}$ Giordano, A. S., and Barnes, A. R.: J. Indiuna M. A., I5, I. 1922.

5 Moench, L. Mary: J. Lab. \& Clin. Med., 9, 289. I924.

${ }^{6}$ Brown, R. O.: Arch. Int. Med., 23, iS5. I9I9.

7 Rosenow, E. C., and Ashby, Winifred: ibid., 28, 274. I92 I.

${ }^{8}$ Bargen, J. A.: J.A.M.A., 83, 332. 1924.

${ }^{9}$ Gerdine, L., and Helmholz, H. F.: Am. J. Dis. Child., ro, 194. I915.

ro Kennedy, R. L. J.: ibid., 31, 631. 1926.

"Helmholz, H. F., and Beeler, Carol: J. Urol., 2, 395. I9i8.

${ }^{12}$ Nickel, A. C.: J.A.M.A., 87, I I 17.1926.

${ }^{13}$ Haden, R. L.: Arch. Int. Med., 35, 457. 1925. 
pye'onephritis, ${ }^{\mathrm{I}}$ and iritis ${ }^{2}$ studied by me and my co-workers, but he has applied the methods to conditions in which the possibility of focal infection as having etiological significance has not even been considered by clinicians and pathologists. Thus, in cases of persistent infection around the roots of nails of fingers or toes (perionychia) he has isolated streptococci from tonsils or teeth that localized electively, and produced lesions around the roots of claws of rabbits following intravenous injection. ${ }^{3}$ In cases of heart disease the streptococcus cultures from infected teeth produced a high incidence of localization in the heart of animals. Haden paid strict attention to technical details and states that the inability of certain observers to obtain corroborative results in this field is attributable to the use of faulty methods.

Few lines of investigation in medicine have given rise to so much discussion as has the work on elective localization, especially as they pertain to medical and dental practice. That there should be difference of opinion is not surprising. The idea is applicable to a wide range of diseases and calls for radical changes in the concept of their origin. The bacteria, usually streptococci, that proved responsible in many of the diseases studied, are, aside from specific infecting power on isolation, much alike. They occur normally on body surfaces and occasionally in normal tissue or as secondary invaders to other incitants, and hence extraordinary proof of causal relationship is required.

The results obtained in a further study of this question, summarized statistically in the tabulation, go far toward fulfilling this requirement. These experiments were performed during the last five years, not so much for the purpose of determining further the correctness of the elective localization theory as for the purpose of isolating the causative organism from which truly autogenous vaccines might be prepared and used for therapeutic purposes. The methods were essentially alike in the different groups of cases throughout the period of study. Eleven groups of patients suffering from specific diseases and one group having miscellaneous or vague complaints have been studied. The figures indicating the incidence of lesions in the various organs and the incidence of the isolation of the organism injected from the specific lesions or blood are given in percentages and are therefore directly comparable. A higher incidence of lesions in the organs of animals corresponding to the ones affected in patients was found in each of the diseases studied, and thus the results of my previous work are corroborated in an additional large number of cases (see Table I).

While specific invasive or elective localizing power of the streptococci from the different diseases studied was high, the general invasive power or virulence was low, the mortality being only 29 per cent of all the animals injected, no higher than in the control group (3o per cent). In agreement with this are the results of cultures made from the specific lesions and blood of the animals injected. The former yielded the organisms in 66 per cent of the animals in which cultures were made, and the latter in only 43 per cent including the animals that died. Rarely was the blood heavily infected. Moreover, since patients suffering from the diseases studied rarely die from general infection, these lesions would appear to be directly attributable to the elective localizing power of the streptococci isolated.

\footnotetext{
I Haden, R. L.: Am.J. M. Sc., 169, 407. 1925.

${ }^{2}$ Haden, R. L.: Arch. Int. Med., 32, 828. 1923.

${ }^{3}$ IIaden, R. L., and Jordan, W. H.: Arch. Dermut. \& Syph., 8, 3I. 1923.
} 
The figures or statistical representation of the effects obtained in animal experiments as shown in the tabulation, striking as they are, do not represent adequately the observations at necropsy. The lesions produced in the corresponding organ following the injection of specific strains are often much more pronounced than are those in these structures following the injection of strains from other sources. The value, as given in the tabulation, of a small hemorrhage in or around one joint or of slightly turbid joint fluid following injection of a strain from a case other than arthritis, is as

TABLE I

Localization in Anrmals of Streptococci from Patients Suffering From Systemic Disease

\begin{tabular}{|c|c|c|c|c|c|c|c|c|c|c|c|c|c|c|c|c|c|c|c|c|}
\hline \multirow[b]{3}{*}{ Source of Cultures } & \multirow[b]{3}{*}{$\begin{array}{l}\text { D } \\
\text { un } \\
\tilde{U}\end{array}$} & \multicolumn{2}{|c|}{ ANIMALS } & \multirow[b]{3}{*}{$\begin{array}{l}\text { Cases } \\
\text { Yielding } \\
\text { Positive } \\
\text { Results }\end{array}$} & \multicolumn{12}{|c|}{$\begin{array}{c}\text { Percentage of Animals That T)e- } \\
\text { VeLOPED lesions IN }\end{array}$} & \multicolumn{4}{|c|}{ Cultures From } \\
\hline & & \multirow[b]{2}{*}{$\frac{\mathscr{J}}{\mathscr{J}}$} & \multirow[b]{2}{*}{ 1)ied } & & \multirow[b]{2}{*}{$\frac{.5}{v_{2}}$} & \multirow[b]{2}{*}{ 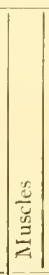 } & \multirow[b]{2}{*}{$\frac{n}{. \Xi}$} & \multirow[b]{2}{*}{ 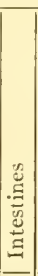 } & \multirow[b]{2}{*}{ 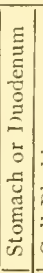 } & \multirow[b]{2}{*}{ 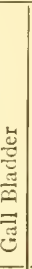 } & \multirow[b]{2}{*}{$\begin{array}{c}n \\
\vdots \\
\vdots \\
\vdots \\
\vdots \\
\vdots \\
\vdots\end{array}$} & \multirow[b]{2}{*}{ 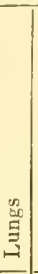 } & \multirow[b]{2}{*}{ 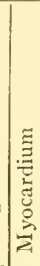 } & \multirow[b]{2}{*}{ 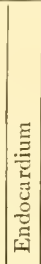 } & \multirow[b]{2}{*}{ 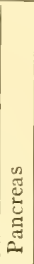 } & \multirow[b]{2}{*}{$\begin{array}{l}2 \\
0 \\
2 \\
2 \\
7\end{array}$} & \multicolumn{2}{|c|}{$\begin{array}{l}\text { Specifir } \\
\text { Lesion }\end{array}$} & \multicolumn{2}{|c|}{ Blood } \\
\hline & & & & & & & & & & & & & & & & & No. & 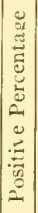 & No. & 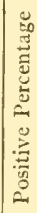 \\
\hline Chronic arthritis........ & I 7 & $4 \mathrm{I}$ & I I & I 5 & 0 & 46 & 66 & o) & I 5 & 이 & 5 & 5 & 5 & 7 & o & o & 27 & 70 & 38 & 58 \\
\hline $\begin{array}{l}\text { Ucer of stomach or duo- } \\
\text { denum ............ }\end{array}$ & 40 & 90 & I 8 & 34 & 0 & 7 & 2 & 3 & 61 & o & 3 & 2 & 7 & $\mathrm{I}$ & of & o & IO & 80 & 60 & 32 \\
\hline Cholecystitis........... & $2 I$ & 57 & 28 & I 8 & 0 & 4 & 0 & 9 & 22 & 65 & I I & 4 & I 2 & o) & I I & o & 42 & 67 & 54 & 72 \\
\hline Myositis. . . . . . . . . . & 3 & 7 & 2 & 2 & O & 71 & 20 & o) & 0 & 0 & I 4 & o) & 0 & 14 & 0 & I 4 & $\ldots$ & . & 6 & I 7 \\
\hline Neuritis.............. & 6 & 26 & 6 & 5 & 0 & I2 & 0 & o & o) & 0 & of & 0 & 8 & 8 & of & 58 & $\ldots$ & . & IO & 60 \\
\hline Pyelonephritis.......... & 5 & 15 & 2 & 4 & 0 & 20 & I 4 & of & 0 & of & So. & 이 & 7 & 71 & of & 0 & 6 & 67 & IO & 20 \\
\hline Endocarditis.......... & 3 & Io & $\mathbf{I}$ & 2 & 0 & 10 & 0 & o & 30 & 0 & 30 & 10 & 10 & 50 & o) & o & I & I & IO & 40 \\
\hline Erythema nodosum..... & $I$ & 12 & 0 & $\mathbf{I}$ & 75 & 67 & 42 & o) & O & 0 & I 7 & 아 & 0 & 0 & o) & o & $\ldots$ & $\ldots$ & I 2 & 0 \\
\hline Herpes zoster.......... & I I & $3 \mathrm{I}$ & I3 & 6 & 35 & I3 & 6 & o) & o & 0 & o) & of & 0 & 0 & O & o & $\cdots$ & $\cdots$ & 29 & 38 \\
\hline Diabetes............. & 8 & 29 & I I & 6 & O & o & 7 & o) & 7 & o) & 3 & o) & O & 9 & 48 & O & II & 37 & $2 I$ & 33 \\
\hline Chorea............. & 6 & I9 & 6 & 3 & 0 & 26 & 26 & 0 & c) & $\mathrm{O}$ & 5 & 0 & $2 \mathrm{I}$ & +7 & o) & o & 5 & 60 & IS & 33 \\
\hline 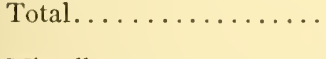 & I 2 I & 337 & $\left(29^{9} \%\right)$ & $\left(\begin{array}{c}95 \\
78,17\end{array}\right)$ & & & & & & & & & & & & & 102 & 66 & 277 & 43 \\
\hline Miscellaneous.......... & 62 & I 29 & $\begin{array}{c}39 \\
(30 \%) \\
\end{array}$ & $\ldots \ldots$ & I & C) & 6 & $c$ & 4 & c & 2 & 3 & 3 & 5 & $c$ & 2 & $\cdots$ & $\cdots$ & 77 & 32 \\
\hline Results exclusive of $\mathrm{s}$ & & & ins & & o) & 9 & 5 & 2 & 8 & c & 6 & 2 & 7 & 3 & 2 & o & $\cdots$ & $\ldots$ & $\cdots$ & $\cdots$ \\
\hline
\end{tabular}

great as one or more greatly swollen joints with numerous hemorrhages containing markedly turbid joint fluid, as commonly follows injection of a strain from cases of arthritis. In a small percentage of animals the occurrence of lesions in organs besides the one affected in the patient from whom the culture was obtained does not minimize the significance of the results since certain patients present transient symptoms referable to other structures besides those chiefly and persistently affected. If the patient had symptoms of more than one disease, such as arthritis and cholecystitis, localization both in the joints and in the gall bladder sometimes occurred.

The methods which have given so much useful information in the diseases studied do not yield positive results in all diseases in which they have been applied. The re- 
sults in diabetes are merely suggestive, and thus far only negative results have been obtained in pernicious anemia, in lymphatic and myelogenous leukemia, in hematuria, in certain lesions of the skin, in chronic diseases of the eye, and in catarrhal and epidemic jaundice.

\section{ELECTIVE LOCALIZATION FOLLOWING INOCULATIONS OTHER THAN INTRAVENOUS INJECTION}

It must not be assumed that it is necessary to inject the bacteria intravenously in order to produce lesions in the tissues for which they have elective localizing power. Characteristic localization has been obtained repeatedly following other methods of inoculation. Marked ulceration of the stomach in guinea pigs occurred following the intraperitoneal injection of the streptococcus from a suppurative sinus draining the infected tooth of a patient with acute ulcer of the stomach. Suppurative pulpitis and hemorrhagic edema of the periosteum opposite the roots of the teeth of animals followed the intraperitoneal injection of the streptococcus from the pulp of a tooth of a patient who had recurring attacks of pulpitis, dental neuritis, and myositis. Localization in the alveolar epithelium of the lungs in guinea pigs occurred after intraperitoneal injection of a highly virulent, green-producing streptococcus from a case of influenza. Suppurative endometritis was found often after intratracheal inoculation of this organism freshly isolated from sputum or lung exudate in cases of severe pulmonary influenza during the epidemic in I9I8. Localization of streptococci, with leukocytic infiltration, was found in the ileum following the intratracheal injection of a small amount of hemorrhagic mucus containing streptococci from a patient with intestinal influenza; hemorrhagic colitis developed after the injection into the stomach of the freshly isolated streptococcus, in oil suspension, from another patient with intestinal influenza.

The extreme specificity for muscles of a streptococcus from a case of myositis was shown in an experiment in which the organism injected intravenously not only localized in the muscles of a pregnant rabbit, but also in the corresponding muscles of the fetuses. A streptococcus isolated from the tooth of a patient with recurring attacks of iritis, myositis, and arthritis produced iritis, myositis, and arthritis on intravenous injection, and also produced iritis, including the uninjected eye, and lesions in muscles when extremely small doses were inoculated into the anterior chamber of one eye.

A staphylococcus from an infected maxillary sinus and tonsils in a patient suffering from severe nephritis, and which localized electively in the kidney of rabbits on intravenous injection, produced nephritis when inoculated into devitalized teeth of dogs. ${ }^{.}$Moreover, characteristic or other symptoms due to elective localization of streptococci inoculated into the teeth of dogs have been reproduced by Rosenow and Meisser $^{2}$ in cases of nephrolithiasis, by Meisser ${ }^{3}$ in cases of ulcer of the stomach, and by Rosenow in cases of chorea ${ }^{4}$ and epidemic hiccup. ${ }^{5}$ The conditions around the teeth in the dogs were similar to those following the devitalization of teeth in human dentistry. The infected teeth became discolored, but remained firmly in place in the

\footnotetext{
x Rosenow, E. C., and Meisser, J. G.: J. Lab. Eo Clin. Med., 7, 707. 1922.

${ }^{2}$ Rosenow, E. C., and Meisser, J. G.: Arch. Int. Med., 31, 807. 1923.

3 Meisser, J. G.: J. Am. Dent. A., 12, 554. 1925.

4 Rosenow, E. C.: Am. J. Dis. Child., 26, 223. 1923.

5 Rosenow, E. C.: Arch. Neurol. \&o Psychiat., 15, 7 I2. 1926.
} 
alveolar sockets; the infection caused rarefaction and absorption of bone in the periapical region without swelling, pain, or tenderness, and the cellular infiltration and distribution of the bacteria and the well-formed granulomas were also similar. The streptococci isolated from the teeth, often months after inoculation, usually had retained respective elective localizing power as measured on intravenous injection into rabbits.

In I9I6 I reported a series of experiments in which lesions in the spinal cord or brain, characteristic in location, were produced by the intravenous injection of cultures containing streptococci from infection atria in cases of multiple sclerosis and transverse myelitis. Similar experiments in cases of neuralgia and multiple neuritis produced, instead, lesions chiefly in the dorsal nerve roots and peripheral nerves, respectively, in a high percentage of animals inoculated. ${ }^{x}$ These experiments suggested strongly that specific or elective localizing power of streptococci, inherent or acquired, might be an important factor in the production of diseases of the nervous system, and led to the isolation of the pleomorphic streptococcus in epidemic poliomyelitis $^{2}$ and a similar streptococcus in epidemic encephalitis. ${ }^{3}$

Owing to the unusual resistance of the central nervous system to bacterial invasion, also well shown in perfusion experiments of Manwaring and Fritschen, ${ }^{+}$the incidence of elective localization following intravenous injection was too low for routine work in this field. It was found that intracerebral injection of small amounts of suspensions in sodium chloride solution of material expressed from tonsils, swabbed from nasopharynx, or aspirated from pyorrhea pockets, and of the corresponding primary cultures in broth or of pure cultures after many rapidly made subcultures, was more likely to produce characteristic symptoms than intravenous injection. It often served to separate the causative streptococcus with neurotropic properties, from contaminating organisms and other streptococci that were without neurotropic power. By this method elective localization of streptococci in different parts of the central nervous system with the production of more or less characteristic symptoms and lesions has been found to occur in lethargic and other forms of encephalitis, in poliomyelitis, epidemic hiccup, ${ }^{5}$ spasmodic torticollis, ${ }^{6}$ and chorea. In cases of lethargic and myoclonic encephalitis, and of epidemic hiccup and spasmodic torticollis, characteristic symptoms followed the injection of sterile filtrates made from cultures and from nasopharyngeal washings during acute attacks, and which were shown to contain the specific streptococcus, and in epidemic hiccup, following injection of the corresponding dead bacteria. The observations in encephalitis have recently been corroborated and extended by Evans, ${ }^{7}$ and Evans and Freeman, ${ }^{8}$ and those in epidemic hiccup by Cadham. 9

\footnotetext{
${ }^{1}$ Rosenow, E. C.: J.A.M.A., 67, 662. г916.

${ }^{2}$ Rosenow, E. C., and Wheeler, G. W.: J. Infect. Dis., 22, 28I. I9r 8.

3 Rosenow, E. C.: ibid., 34, 329. I924.

${ }^{4}$ Manwaring, W. H., and Fritschen, William: J. Immunol, 8, 83. 1923.

${ }^{5}$ Rosenow, E. C.: J.A.M.A., 67, 662. г916.

${ }^{6}$ Rosenow, E. C.: J. Nerv. E Ment. Dis., 59, I. I924.

7 Evans, Alice C.: Pub. Health Rep., 42, I71. 1927.

${ }^{8}$ Evans, Alice C., and Freeman, Walter: ibid., 4r, I095. 1926.

${ }^{9}$ Cadham, F. T.: J.A.M.A., 84, 580. 1925.
} 
A striking example of the extreme specificity of the streptococcus and a shifting of this property under certain conditions was found in experiments performed in a series of cases presenting varying degrees of neuro-myelo-encephalitis which occurred during and after an epidemic of hiccup. ${ }^{3}$ Localization with lesions, in locations strikingly like those in the patient, occurred in animals injected with material from the nasopharynx or tonsil. The study was a striking example in which the changing character in an epidemic was closely simulated in the experimental animal.

In another series of experiments, believed to be of epidemiological importance, the streptococcus of epidemic encephalitis was made to resemble the streptococcus isolated so constantly during the pandemic of influenza of $\mathrm{r} 9 \mathrm{I} 8 .^{2}$ On isolation it had little or no effect on intratracheal insufflation, but had marked neurotropic properties manifested on intracerebral, intravenous, and intraperitoneal injection and following packing of the nose of rabbits and monkeys with gauze soaked in the culture. After successive passage through animals it became highly virulent, produced meningitis on intracerebral injection and the symptoms, with sharp reduction in the leukocyte count and lesions of the lung, of influenza as they occur in epidemic waves (pneumotropic phase). Both neurotropic and pneumotropic properties were lost after prolonged cultivation on artificial media.

\section{GENERAL DISCUSSION AND CONCLUSIONS}

It is uncertain whether the streptococci isolated in each of the many diseases studied, and which manifested such widely different elective localizing powers on isolation, are distinct varieties or modifications of a single strain. They all belonged to the pneumococcus-streptococcus group and were much alike in morphology and cultural reaction. Nearly all produced a greenish zone of hemolysis surrounding small grayish colonies on horse-blood-agar plates. A few were indifferent to blood agar, and a few produced a narrow zone of clear hemolysis. The usual fermentation reactions in sugars were variable and of little value in classification. The cultural features were quite stable under ordinary methods of cultivation. Mutational forms occasionally developed when cultured under conditions of stress, both in vitro and in vivo. Their general virulence was relatively low. Intraperitoneal injections rarely caused death from peritonitis and intravenous injection rarely caused death from bacteremia. Agglutination and precipitation reactions were highly specific with freshly isolated strains in some of the diseases studied. This was often lost soon after cultivation on artificial media as was also the elective localizing power, but not in a strictly parallel manner. Elective localizing power of the streptococci tended to disappear quickly when cultivated on artificial media and more slowly on passage through animals. A shift in the powers of localization was often noted under these conditions, comparable to those noted in the studies on transmutation in the pneumococcus-streptococcus group. Elective localizations of the streptococci isolated were especially marked during acute exacerbations of chronic conditions, and in acute cases, especially during the winter months when respiratory infections were prevalent and an increased incidence of systemic conditions prevailed. The fact that exacerbations and acute attacks such as

I Rosenow, E. C.: Arch. Neurol. EN Psychiat., I6, 2 I. I926.

${ }^{2}$ Rosenow, E. C.: J. Infect. Dis., 33, 531. I923. 
appendicitis, myositis, arthritis, cholecystitis, and ulcer of the stomach usually occur in from one to four weeks after acute infections of the upper respiratory tract have subsided, especially emphasized in appendicitis by Evans, ${ }^{1}$ may, in the light of the experimental findings, be due to the newly acquired elective localizing power of the streptococci responsible for the antecedent respiratory infection.

The results, both with intravenous and other methods of injection, indicate strongly that the streptococci isolated are the causes of the diseases studied. Their power to localize electively; perhaps more than any other single factor, determines the location of the lesions and, hence, the clinical picture. Elective localization occurred, and characteristic changes were produced under experimental conditions like those that pertain to several disease entities. This was possible especially in those instances in which intravenous or intracerebral injection of the respective strains revealed marked elective localizing power.

The underlying physico-chemical conditions that determine elective localization of living bacteria are undoubtedly similar to, yet as obscure as, those that determine the specific pharmacodynamic action of drugs and chemicals. However, the fact that certain streptococci possessing elective localizing power, especially those from ulcer of the stomach, ${ }^{2}$ myositis, epidemic hiccup, and encephalitis, have been shown to produce free poisons or toxins in broth cultures and within the bacterial cell which have the power to injure electively the tissues or organs in which the respective living bacteria localize and produce lesions throws important light on the problem of how the bacteria overcome the inherent resistance of tissues to invasion.

I Evans, J. S.: Wisconsin M.J., I7, 91. г9г 8 .

${ }^{2}$ Rosenow, E. C.: J. Infect. Dis., 33, 248.1923. 


\title{
CHAPTER XLIV
}

\section{BACTERIA IN RELATION TO PLANT DISEASES}

\author{
GEORGE K. K. LINK
}

University of Chicago

Present activity in the field of bacterial diseases of plants may be divided arbitrarily into three categories. The first consists of survey work, i.e., the discovery, diagnosis, and description of new diseases and their pathogens; determination of the host range, method and source of infection, and control of the new pathogens. The second, which is to a great extent incidental to or necessitated by the first, consists of a more detailed restudy of pathogens which have been generally accepted in phytopathological literature. The third consists largely of incidental or isolated exploratory studies of general biological aspects of plant bacteriology. These include studies of life cycles of bacteria, testing of the validity of species iclentification, and of established systems of classification, studies in infection, virulence, immunity-susceptibility phenomena, serological properties of pathogens, and detailed study of the phenomenon of gall formation in crown gall. The relatively late introduction of Koch's method and the small number of workers have left room, even now, for survey and pioneer work in phytobacteriology.

\section{HISTORICAL DEVELOPMENT}

Although a series of observations and experiments by botanists, culminating in the classical work of De Bary on the smut and rust fungi ( $18_{53}$ ), had conclusively demonstrated the association of fungi with diseases and the nature of parasites and infection and De Bary's brilliant researches on the fungus of the late blight of the potato (I86I-63) had conclusively established the causative rôle of a definite microorganism, Phyto phthora infestans, in a specific disease, these researches apparently did not affect the main current of experimentation and of speculation as profoundly as the work of Pasteur and Koch. With the work of these men, especially that of Koch in establishing the science of bacteriology, leadership in the study of infection and disease definitely passed to the animal field.

While study of the rôle of bacteria in animal diseases progressed by leaps and bounds, work in phytobacteriology was slowly getting under way. To be sure, there were studies made in the field, but these, as other bacteriological studies up to Koch's presentation of conclusive evidence that a definite bacterium was the cause of specific disease, consisted of congeries of very important but incomplete observations and experiments. Woronine in $\mathrm{r} 866^{\mathrm{I}}$ had discovered bacteria in the root tubercles of legumes, and Davaine in $1868^{2}$ had inoculated plant tissues with bacteria and obtained

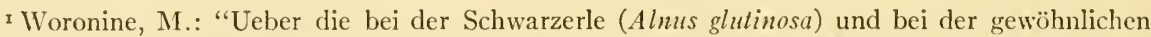
Gartenlupine (Lupinus mutabilis) auftretenden Wurzelanschwellungen," MÍm. de l'Acad. Imp. de St. P'etersbourg (7th ser.), ro, No. 6. г 866.

2 Davaine, C. R.: "Recherches physiologiques et pathologiques sur les Bacteries," Compl.rend hebd. des séunces de l'A cad. des sci., 46, 109-503. I868. 
soft rot. Reinke and Berthold ${ }^{\mathrm{x}}$ indirectly demonstrated that bacteria obtained from rotting potato tubers would rot tubers in the absence of fungi: Prillieux in $1879^{2}$ had described the Micrococcus or rose-red disease of wheat; Comes in $188 \mathrm{O}^{3}$ had recognized the pathogenic rôle of bacteria in plant diseases; and Wakker in I $883^{4}$ had described the yellow disease of hyacinths, and by infection experiments definitely associated the disease with bacteria. It is generally stated that the work of Burrill $(1876-83), 5$ on the nature and cause of fire blight, was the first which conclusively established that a specific bacterium (Micrococcus amylovorus) causes a specific plant disease. This, however, was not completed until I 885 when Arthur $^{6}$ grew the organism in pure culture and produced infection with it.

In the main, however, the botanical profession was so blinded by the luster of De Bary's and other mycologists' successes in establishing causal relations between specific plant diseases and definite species of fungi that it failed to consider seriously the possible rôle of bacteria in plant diseases. Indeed, the consensus was that bacteria could not cause specific diseases of plants. The legume nodules were not considered pathological, and their bacteria were designated as "symbionts." It was contended that if bacteria were at all involved they were secondary to fungi, because bacteria could not enter the stomata of plants, and if they should get access to the tissues through wounds their progress would soon be checked by the plants' development of an excluding layer of cork.

The credit for overcoming this notion, which was most prevalent in Germany, belongs notably to Sorauer, ${ }^{7}$ who in I 886 began to defend the concept that bacteria cause specific plant diseases, but even more to Erwin Smith, ${ }^{8}$ who carried on a spirited polemic with Dr. A. Fischer ${ }^{9}$ of Berlin during $1897^{-1901}$, and convinced the botanical world that certain bacteria cause specific plant diseases.

Relatively few men have worked intensively in phytobacteriology. Smith not only pioneered the field but contributed a considerable part of the literature. The

${ }^{1}$ Reinke, J., and Berthold, G.: Zersetzung der Kartoffel. Berlin, I 879.

${ }^{2}$ Prillieux, Ed.: Maladies des Plantes agricoles et Arbres fruitiers et forestiers canseés par des Parasiles végétaux. Paris, I 895 .

3 Comes, O.: Crittogamia agraria, Vol. I. Naples, I89i.

4 Wakker, J. H.: "Vorläufige Mittheilungen über Hyacinthenkrankheiten," Bot. Centralbl., I4, 315-16. I 883 .

5 Burrill, T. J.: "Pear Blight," Tr. Illinois State IIort. Soc., pp. I14-16. г878; "Fire Blight," ihid., pp. 77-78. 1879; "Anthrax of Fruit Trees," Proc. Am. Assoc. Adv. Sc., 29, 583-97. I880; $4 \mathrm{~m}$. Nat., 17, 319. I 883 .

${ }^{6}$ Arthur, J. C.: "Proof That Bacteria Are the Direct Cause of the Disease in Trees Known as Pear Blight," Bot. Gaz., ro, 343-45. 1885; "Pear Blight," New York Agr. Exper. Sta., Goneva Rept., 5. 1886.

${ }^{7}$ Sorauer, P.: Handbuch der Pflanzenkrankheilen (2d ed.). Berlin, i 886.

${ }^{8}$ Smith, E. F.: "Are There Bacterial Diseases of Plants? A Consideration of Some Statements in Dr. Alfred Fischer's Vorlesungen ïber Bakterien," Centralbl. f. Bakteriol., Abt. II, 5, 27I-78. I 899; "Dr. Alfred Fischer in the Rôle of Pathologist," ibid., pp. 810-17. I899; "Entgegnung auf Alfred Fischer's 'Antwort' in betreff der Existenz von durch Bakterien verursachten Pflanzenkrankheiten," ibid., 7, 88-100, I 28-39, I90-99. I901.

9 Fischer, A.: "Die Bakterienkrankheiten der Pflanzen. Antwort an Herrn Dr. Erwin F. Smith," ibid., 5, 279-87. 1899 . 
Laboratory of Plant Pathology, Bureau of Plant Industry, United States Department of Agriculture, under his direction, was the only one which devoted itself exclusively to the study of bacterial diseases of plants. Three volumes by Smith, Bacteria in Relation to Plant Diseases, ${ }^{\mathrm{I}}$ and a later volume, Bacterial Diseases of Plants, ${ }^{2}$ constitute the most notable contribution in phytobacteriology. A great deal of attention was paid in Smith's laboratory to studies of crown-gall (Bact. tumefaciens), with special reference to the nature and mechanism of tumor formation and the bearing of these on the nature and mechanism of cancer in animals. The Department of Plant Physiology and Pathology of the Imperial College of Science and Technology, London, under guidance of Prof. S. G. Paine is publishing a series of "Studies in Bacteriosis." Otherwise, the literature on phytobacteriology is scattered in journals and research bulletins.

\section{DISTRIBUTION OF BACTERIAL PLANT PATHOGENS}

Practically all serious studies of bacterial diseases of plants have been concerned with economically important plants. Non-cultivated plants are now being given consideration as possible sources of inoculum. ${ }^{4}$ There has not yet been made a complete survey of even the bacterial diseases of economic plants. The field is immense because of the large number of plants which are actually or potentially valuable economically. It is continually growing because man, by selecting and developing new varieties of plants, and by his persistent attempts to grow plants, or to transport or store them or their parts, very often under conditions more or less unfavorable to the plant, is continually creating possibilities of new host and pathogen relations. The fact that the temperature of plants in the main roughly follows that of the air with corresponding changes in metabolism renders them subject to attack by groups of organisms with widely different requirements.

While according to our present knowledge schizomycetes play a greater rôle in animal diseases than do true fungi, their rôle as plant pathogens is subordinate. The reason for this is not known. The inability of most fungi to grow well at $37^{\circ} \mathrm{C}$. probably keeps down the number of fungi attacking animals. The preponderance of fungous versus bacterial diseases of plants is surmised to be in part due to the inhibitory action of acid cell sap on bacterial growth, and in part to the fact that bacteria, especially non-spore-bearing ones, are more subject to vicissitudes of environment than fungi. Furthermore, fungi are better equipped structurally and functionally to effect entry through the protective tissues of plants and when once in, to progress through the tissues.

Smith, ${ }^{5}$ summarizing our knowledge relative to the distribution of bacterial diseases in the plant kingdom, states: "It appears likely that eventually bacterial diseases will be found in every family of plants from lowest to highest," basing this prediction on the fact that while the field is relatively a new one, and there are no workers

ISmith, E. F.: op. cit. (Washington, D.C.), Vol. I, I905; Vol. 2, I9I ; Vol. 3, I9r4.

${ }^{2}$ Smith, E. F.: op. cit. Philadelphia and London, r920.

3 Paine, S. G.: "Studies in Bacteriosis," II Ann. Appl. Biol., 5, 206-r7. I9r8.

4 Gardner, M. W.: "A Native Weed Host of Bacterial Blight of Bean," Phytopath., I4, 341. I924.

5 Smith, E. F.: Bacterial Diseuses of Plants. 
in most parts of the world, nevertheless bacterial diseases have been reported from every continent, and are known to occur in flowering plants of I 50 genera distributed through more than 66 families. This list includes 3 diseases of gymnosperms and 23 of monocotyledons, with the remainder distributed among the dicotyledons. A few bacterial diseases are known to occur among cryptogams.

According to Smith, of Engler's list of 299 families of flowering plants, there still are about 225 families of flowering plants for which no bacterial diseases have been reported. Whether this is due to lack of investigators, time and chance, or natural immunity remains to be determined. Smith lists a total of I 55 described diseases for 66 families; but this does not represent the total of pathogens, because some of them affect members of several families. In Bergey's Mannal of Delerminative Bacteriology 47 species of schizomycetes are listed as plant pathogens. This figure, however, does not include all plant pathogens, for many cannot be included in a determinative manual because of imperfect or non-differentiative descriptions.

It appears at present that the families represented by a large proportion of cultivated plants have a disproportionately large quota of bacterial diseases. Thus, the families in which 5 or more bacterial diseases are reported are: Gramineae, I4; Solanaceae, Io; Leguminosae, Rosaceae, and Crchidaceae, 7 each; Urticaceae, Chenopodiaceae, and Cruciferae, 5 each. Whether this is because the diseases of cultivated forms are more completely known or whether there is a direct correlation between abundance of diseases and growth of plants in culture is not known, and is a question the answer to which lies in the future.

\section{CLASSIFICATION OF BACTERIAL PLANT PATHOGENS}

The schizomycetes which are phytopathologically significant belong with one exception to the Eubacteriales. This exception falls in the Actinomycetales (Actinomyces scabies, cause of potato scab, which is characterized by proliferation of the cortical tissues of the potato tuber). It will be of interest to await further work and see whether all or most of the plant pathogens will fall, as they now do, into the family Bacteriaceae, and whether grouping of these into the separate tribe Erwiniae on the basis of plant pathogenicity will stand the test of time. It is not likely that animal pathogens, with their high optimum temperature, will be found to be plant pathogens, but there is a possibility that some of the plant pathogens with a high temperature range might conceivably become pathogenic to animals. Smith ${ }^{2}$ reported the production of small tumors in trout after inoculation with Bact. lumefaciens (the crown-gall organism). Evidence of continual interest in studying the cross-inoculability of plant and animal pathogens is found in a recent paper by Fygin, Epstein, and Funk, ${ }^{3}$ who report the production of a plant tumor by a bacterium isolated from a human carcinoma. In the past, similar reports have not stood the test of more careful reinvestigation.

The classification and nomenclature used in Bergey's Manual have not been ac-

"Bergey, I). If., ct al.: Manual of Determinatice Bacteriology. Baltimore: Williams \& Wilkins, 1923.

${ }^{2}$ Smith, E. F.: Barterial Disesses of Plants.

3 Fygin, B., Epstein, T., and Funk, Casimir: "Sur une Tumeur végétale provoqué par une Lacterie isolée d'un Carcinome humain," Compl. rend. Soc. de biol., 94 (14), I097-9S. I926. 
cepted by all phytopathologists, e.g., Smith's and Paine's laboratories, maintain the use of names suggested by Smith in his chapter on "Nomenclature and Classification" in Bacteria in Relation to Plant Diseases, ${ }^{\mathrm{I}}$ in which the generic name Aplanobacter was substituted for Bacterium (Migula); Bacterium for Pseudomonas (Migula); and Bacillus (Migula) was retained. Stevens ${ }^{2}$ attempted to return to Migula's system. According to the system proposed in Bergey's Manual, the tribe Erwiniae includes all plant pathogens, the forms with peritrichous flagella falling into the genus Erwinia, while the forms with polar or no flagella constitute the genus Phytomonas. Eleven of the listed forms belong to Erwinia and 36 to Phytomonas.

\section{SEROLOGICAL METHODS IN IDENTIFYING AND DIFFERENTIATING PLANT PATHOGENS}

Uncertainty has existed among phytopathologists as to whether many of the so-called species really deserve such rank, or should be considered subspecics or varieties. In some quarters the attempt to delimit species has been considered futile, and the application of more refined methods in an attempt to differentiate species a senseless waste of effort and time.

Host specificity or association of a pathogen with a definite symptom complex, has been used extensively or even solely to differentiate and diagnose species whenever cultural characters have been unsuited. The situation is becoming steadily more complicated because detailed studies indicate that supposedly well-established and clearcut species may be complexes or aggregates of forms.

Relatively little use has been made by phytopathologists of serological methods in wrestling with the problems of diagnosis and differentiation of plant pathogens. The method has however been used very extensively and successfully in classifying the organisms causing the nodules of legumes. This work, however, done by soil bacteriologists, has gone largely unheeded by phytopathologists. Do:dge, ${ }^{3}$ Jensen, ${ }^{4}$ Paine and Lacey, ${ }^{5}$ Brooks, Nain, and Rhodes, ${ }^{6}$ Takimato, ${ }^{7}$ and Goldsworthy, ${ }^{8}$ had varying success in applying the agglutination test to grouping, identification or differentiation of plant pathogens. Link and Sharp, ${ }^{9}$ Sharp,${ }^{10}$ and Link and Lin $k,{ }^{13}$ working primarily with a group of yellow schizomycetes which are pathogenic to plants and which are

${ }^{x}$ Smith, E. F.: op. cit., I, I 54-77.

${ }^{2}$ Stevens, F. L.: Plant Disense Fungi. New York: Macmillan, i925.

3 Doidge, Ethel: "A Bacterial Spot of Citrus," Ann. Appl. Biol. 3, 53-83. I9г7.

${ }^{4}$ Jensen, C. O.: "Undersogelser vedrorende nogle svulstlignende 1)annelser hos Planter," $K$ gl. Veterinaer-og Landbohojskoles A arsskrift. Copenhagen, I9 8.

5 Paine, S., and Lacey, M.: "Studies in Bacteriosis," Ann. App'. Biol. 1o, 204-9. I923.

${ }^{6}$ brooks, R. St. John, Nain, K., and Rhodes, Mabel: "The Investigation of Phytopathogenic Bacteria, by Serological Methods." Jour. Path. and Bact., 28, 203-9. 1925.

7 Takimato, S.: "Studies on the Putrefaction of Vegetables," J. Pl. Prot., 8, 344-53. I92I. (Japanese) Bot. Abst., I4, 600. I925.

${ }^{8}$ Goldsworthy, . 1. C.: "Studies on the Spot Disease of Caulitower; a Use of Serum Diagnosis," Phytopath., I6, 877 \$,3. 1026.

9Link, Cicurge K. K., and Sharp, C. G.: "Correlation of 1 Iost and Serological Specificity of Bat. campestre, Bacl. flacomfaciens, Bact. phaseoli, and Bact. phaseoli snjeusc," Bot. Gas., 83, I 45 (10. 1927 .

1o Sharp, C. (i.: "Virulence, Serological, and Other Physiological Studies of Bact. flaccumfaciens, Buct. phuseoli, and Bact. phuseoli sojense," ibid., Pp. I13-4t. 1927.

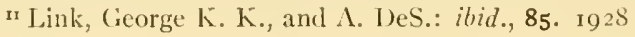


in part so imperfectly differentiated culturally that host specificity has been used as the final diagnostic character, have demonstrated that in the main the method is suited to differentiate species which are not readily distinguishable culturally, but which show some host or symptom specificity. Lacey, ${ }^{\mathrm{T}}$ and Link and Taliaferro ${ }^{2}$ showed that the same situation holds for certain organisms which cause soft rot of vegetables.

In the light of these data, one is justified in venturing the statement that in the main the species which have been created on the basis of even slight difference in cultural behavior and appearance, especially when these are correlated with host and symptom specificity, are probably deserving of species rank. Lacey and Sharp's detailed cultural work with soft rot and bean pathogens also indicates that most serological differences will be found to be correlated with morphological and cultural differences, however slight.

\section{COLONY INSTABILITY OR VARIATION}

The concept of normal culture, colony, or cell, has dominated phytobacteriological researches, and consequently colony variations have received relatively slight attention save mention as disconcerting phenomena, or have been discarded as degeneration or involution forms, or even as contaminations. Recently, Sharp ${ }^{3}$ described variant colonies of Bact. phaseo! $i$ sojense which became umbonate and wrinkled and convoluted in five to eight days. He found, on the one hand, a correlation of the rough ( R) colony form with other properties such as high agglutinability, lesser virulence, and lesser or no motility, and, on the other, correlation of the smooth (S) form of colony, which is the normal colony of the species, with low agglutinability, greater virulence, and greater motility. This study has brought out the striking parallelism between bacteria being studied in the animal and plant fields. ${ }^{4}$ Link and Hulls have reported other plant pathogens which produce smooth and rough colonies. These are Bact. citri, a strain of Bact. tumefaciens which gives rise to colonies that are partly rough and smooth at first, or entirely rough, but which speedily become smooth, and a strain of Eact. medicaginis var. phaseolicola, No. 23 , which makes a rough-and-smooth growth on agar.

Sharp's findings suggest that it will be worth while to devote considerable attention to aberrant colonies. In view of the fact that relatively few investigators describe or illustrate the aberrant colonies, it is impossible to draw any conclusions from the literature as to how generally the phenomenon of rough and smooth colonies occurs among plant pathogens. Fortunately, however, under the leadership of Smith enough investigators have described and illustrated not only the normal but also the variant colonies to enable one to infer that smoothness and roughness and kindred phenomena have been observed before.

Smith $^{6}$ has described for Bact. tumefaciens colonies with smooth, shiny surfaces as

ILacey, Margaret, S.: "Studies in Bacteriosis," XIII. Ann. Appl. Biol., I3, I-II. I926.

${ }^{2}$ Link, George K. K., and Taliaferro, W. H.: ibid., 85. 1928. 3 Sharp, C. (i.: lor. cit.

${ }_{4}^{4}$ [For a review of the earlier studies on $(R)$ and $(S)$ colony forms, cf. chaps. xlii and vii in this volume.-EDITORS.]

5 Jink, George K. K., and Hull, K.: "Smoothness and Roughness, and Spontaneous Agglutination of Buct. citri, Bact. medicaginis var. phuseolicolu, Bact. phuseoli sojense (R), and Bact. tumefaciens," Bot. Gaz., 83, 412-19. I927.

${ }^{6}$ Smith, E. F.: Bacterial Plant Diserses. 
well as some with thin, wrinkled, dull surfaces. Gardner and Kendrick ${ }^{\mathbf{1}}$ described and illustrated aberrant colonies of Bact. viridifaciens which appear similar to the rough colonies of Bact. phaseoli sojense, and Miss Hedges ${ }^{2}$ described and illustrated a colony of Bact. phaseoli sojense with which Sharp's rough colony appears identical. She states that the convolutions are internal. Smith ${ }^{3}$ illustrates a colony of Bact. phaseol $i$ which appears rough but which was described as having internal markings. He describes and figures colonies of Bact. malvacearum which are very suggestive of the erosive phenomena described in some animal pathogens. In discussing the peculiar "windowed" colonies of this organism he writes: "Occasionally the mottling of surface colonies on agar is so conspicuous as to suggest an intruder . . . but inoculation with subcultures produced typical spots on cotton leaves. Moreover, after a few days, such strikingly mottled colonies fill up their thin places." Some of the colonies of Bact. malvacearum appear as rough as the roughest ones of Bact. phaseoli sojense (R). Smith reports and illustrates the development of "liquefaction pits" in colonies of Bact.translucens var. undulosum.

Hadley ${ }^{4}$ has recently reviewed similar or identical observations in the field of general and medical bacteriology, and has grouped them under the unifying concept of species instability or microbic dissociation. He has attempted to show that physiological properties are correlated with such cell and colony changes. Whether the interpretation will prove to be correct either in part or in its entirety is immaterial. It introduces a semblance of order into what appears otherwise to be a chaotic mass of isolated observations, and serves as a working hypothesis. In the main, so far as the very meager data of phytobacteriology on this question go, there seems to be a striking parallelism between some of the phenomena reported from the plant and from the animal field.

\section{BACTERIAL CYCLOGENY}

The controversy which has raged in the other fields of bacteriology relative to cyclogeny of bacteria has hardly been felt in the ranks of the phytopathologists. Under special or abnormal conditions, long filaments and chains are produced, and many of the bacterial phytopathogens lose their normal rod shape and become club shaped, Y-shaped, or otherwise branched. Involution or degeneration forms are reported for Bact. tumcfaciens and the legume nodule bacteria in culture and in the galls. Nevertheless, the general reaction of phytobacteriologists has been that the protagonists of the cyclogeny concept are dealing with involution and degeneration forms, and that there is no good evidence for believing that bacteria pass through cycles. However, Levine $^{\tilde{3}}$ has described morphological changes in Bact. tumcfaciens. He reports the demonstration of amorphous masses of a jelly-like substance, with occasional deeply stained minute spherical bodies in three-month-old cultures stained with Loeffler's

I Gardner, M. W', and Kendrick, J. B3.: "Bacterial Spot of Cowpea and Lima Bean," J. Agr. Research, 31, 841-63. 1925.

${ }^{2}$ Hedges, F.: "I Study of Bacterial Pustule of Soy Bean, and a Comparison of Bact. phaseoli sojense Fledges with Buct. phase.li E.F.s.," iltid., 29, 229-5I. 1924.

${ }^{3}$ Smith, li. F.: Bacterial Diseases of Plants.

4Halley, I.: "Mlicrobic Dissociation," J. Infect. Dis., 40, 1-315. 1927.

5 Levine, M.: "Morphological Changes in Baclerium lumefuciens," Science (N.S.), 42, 424. 1925. 
methylene blue. A transfer from such old cultures to fresh agar gave pearly white growth composed of long rods which not infrequently present a beaded appearance. He considers these forms as an "embryonic" stage. As the culture ages, the rods break up, and finally after twenty days the rods are replaced by small faintly staining cocci with slender bacilli or filaments. Eventually, as the zoc̈gleal mass appears, all cocci disappear. Spores or sporelike bodies also appear in cultures which germinate when transferred to new media, and give rise to rodlike bodies not unlike Bact. tumefacions in size. He suggests that this phenomenon may account for the differences in size of rods noted in this organism by Smith, Brown, and Townsend, ${ }^{1}$ Riker, ${ }^{2}$ and Robinson and Walkden. ${ }^{3}$

A less detailed report of evidence of cyclogeny is the preliminary paper by Nixont on changes of B. amylovorus in the lesions it causes in apple trees. All of this work should be repeated. In the meantime, it will be well for investigators to approach with an open mind the disquieting and troublesome phenomena of degeneration, involution, and contamination of colonies. Much that has seemed incongruous may be clarified.

RÔLE OF ENVIRONIIENTAL FACTORS IN INCITING COLONY OR CELL CHANGES OF PLANT PATHOGENS

Sharp ${ }^{5}$ was not able to correlate the appearance of rough colonies with any environmental factor. Apparently it was spontaneous. This is in line with a great body of data from the animal field. He was, however, able to obtain smooth and rough colonies from rough strains of Bact. phaseo! $i$ sojcnsc after passage through the soy bean. He found that Bact. phaseoli sojense was more rough on potato than on beefdextrose agar.

There is a considerable body of data obtained incidentally, which however sheds light upon the effect of environmental factors upon microbic dissociation. Hadley lists fifteen distinct factors which are reported as inciting dissociation of animal pathogens. Link and Hulli report the apparently spontaneous change of rough to smooth cultures of Bact.citri and of Bact. tumefaciens, and a change from smooth to rough in Bact. medicaginis var. phaseolicola, No. 23. Bact. tumefaciens develops and retains partially rough colonies more freely on beef-dextrose agar than on potato-dextrose agar.

Smith, ${ }^{8}$ incidental to discussions of cultural characters of Bact. tumefaciens, has recorded data which probably are in point here. He points out that the colonies of this organism which he considers characteristically wet, shining, and smooth remain so

'Smith, E. F., Brown, N. A., and Townsend, C. O.: "Crown Gall of I'lants. Its Cause and Remedy," U.S. Dept. of Agr. Bur. Pl. Ind. Bull. 2I 3. I 9 II.

${ }^{2}$ Riker, A. J.: "Some Relations of the Crown Gall Organism in the Host Tissue," J. Agr. Research, 25, I19-32. 1923.

${ }^{3}$ Robinson, IV., and Walkden, H.: “A Critical Study of Crown Gall,” A nn. Bot., 37, 299-324. 1923 .

${ }^{4}$ Nixon, E. L.: "Migration and Transformation of Bacillus amylovorus in Apple Tissue," Phytopath., 16, 77. 1926.

5 Sharp, C. G.: loc. cit.

${ }^{6}$ Hadley, P.: loc. cil.

${ }^{7}$ Link, George K. K., and Hull, K.: loc. cit.

${ }^{8}$ Smith, E. F.: Bacterial Diseases of Plants. 
when transferred from agar to agar, but that upon culture on agar after growth in peptone bouillon they have a thin, wrinkled, dull surface. Furthermore, action of cold, sodium chloride, or acids induces ready conversion into club-shaped, Y-shaped, or variously branched involution forms. Furthermore, these involution forms are reported to occur in tumors. In the bacteria of legume nodules, involution or degeneration colonies appear after a time in the galls of legumes in which a balance seems to have been established between parasite and host; also in culture after a lapse of time.

Gardner and Kendrick, ${ }^{\mathrm{I}}$ in describing what appears to be a typical rough ( $\mathrm{R}$ ) colony of $B$. viridifaciens, point out that drying out of the agar substrate accentuates ridging and piling up of the colony. Whether this is due to agar desiccation has not been determined.

Motility of cells of smooth and rough colonies.-The observation of Sharp ${ }^{2}$ that roughness of Bact. phaseoli sojense is correlated with low motility or no motility of the organism, while smoothness is correlated directly with high motility, is in accord with many observations on bacteria other than plant pathogens.

Virulence.-In consonance with the findings in the animal field, Sharp ${ }^{3}$ observed a correlation of virulence with culture forms. He found that rough colonies of Bact. phaseoli sojense when inoculated into soy-bean leaves by needle pricks are less virulent, as measured by percentage of takes and by size of lesions in a definite time, than the smooth ones. He found further that passage of the rough strain through the plant caused a partial reversion to the smooth type, and that the new smooth forms were as virulent as the original smooth ones.

Smith has taken pathogenicity for the proper host and production of characteristic symptoms as one of the chief criteria in determining the identity of bacterial pathogens. For this reason, certain statements by him ${ }^{4}$ are significant in this place. He cautions workers against using any but smooth translucent colonies of Bact.tumefaciens in making isolations from galls, or in conducting infection tests. In this connection the finding of Link and Hulls for their strain of Bact.tumefaciens is significant. When agar-shake dilutions were made from galls or from agar colonies, this strain produced colonies of which about one half were smooth and the other half rough, or partly rough and smooth. Later all colonies became entirely smooth. Apparently both types are pathogenic.

One of the criteria of the virulence of an organism used by animal bacteriologists is the minimal number of organisms of a given species in a definite state of culture which, by a definite route, will produce infection or death of a definite host, in a definite period of time. Practically no studies using a comparable technique have been made by phytobacteriologists. Smith ${ }^{6}$ in I 9 I I called attention to the observation that often a large amount of bacterial inoculum will produce infection when a smaller amount will fail. He suggested that this phenomenon of mass action should be in-

\footnotetext{
' Gardner, M. W., and Kendrick, J. B.: loc. cit.

${ }^{2}$ Sharp, C. G.: loc. cit.

3 Ibid.

${ }^{4}$ Smith, E. F.: Bacterial Diseases of Plants.

${ }^{5}$ Link, George K. K., and Hull, K.: loc. cil.

${ }^{6}$ Smith, E. F.: Bacteria in Relation to Plant Diseases.
} 
vestigated further. Kotila and Coons ${ }^{\mathrm{I}}$ made a casual determination of the effect of dosage upon infection by B. atrosepticus and found that smaller dosages required longer time and also failed more frequently to produce infection. They found further that whereas larger dosages of this organism cause dissolution of potato-tuber tissue and necrosis of the cells, smaller dosages cause only hypertrophy. The latter observation is in harmony with data relative to the legume-nodule organisms, which when inoculated in large numbers may kill the host cells at once, but when inoculated in smaller numbers first cause hypertrophy.

Virulence and electrophoretic potential in rough and smooth cultures. - Following the work of Falk, Gussin, and Jacobson, ${ }^{2}$ Sharp ${ }^{3}$ and Falk, Sharp, and Link ${ }^{4}$ made measurements of the electrophoretic potential on smooth and rough cultures of Bact. phaseoli sojense. A direct correlation was found between virulence and electrophoretic potential in acid, but not in alkaline menstrua.

Serological characteristics of rormal and abnormal colonies. - Sharp ${ }^{5}$ did not find any difference between the antigenic properties of $\mathrm{S}$ and $\mathrm{R}$ strains of Bact. phaseoli sojense in their reaction against suspensions of the $\mathrm{S}$ strain and heterologous organisms. He was not able to make tests against suspensions of the $\mathrm{R}$ strain because the organism agglutinated spontaneously in 0.85 per cent $\mathrm{NaCl}$ and in distilled water. Link and Link ${ }^{6}$ were able to get a suspension of the rough strain, and were thus able to test the antiserum of Bact. phaseoli sojense $\mathrm{R}$ against homologous and heterologous suspensions of organisms. Their findings indicate that there is no serological difference between the rough and smooth strains of Bact. phaseoli sojense. These authors, in agglutination studies on eleven plant pathogens, obtained surprising results with Bact. tumefaciens. The culture used in the tests was one which was partly smooth and partly rough. The spontaneously agglutinable fraction was allowed to settle out after three washings in saline solution and centrifugation. After standing twelve hours, the suspension was centrifugated at low speed. Apparently all the rough elements had been removed, because there was no more spontaneous agglutination or settling out. When the antiserum of the mixed culture was tested against the suspension of the homologous organism freed of the spontaneously agglutinating $(\mathrm{R})$ element, agglutination was obtained in titres of $\mathrm{I}-\mathrm{IO}, 240$, and cross-agglutination was obtained in dilution I-80 with only one of the ten organisms tested. In other words, the test was specific. However, when heterologous antisera were tested against the suspension of Bact. tumefaciens, cross-agglutination was obtained in all cases. Curiously enough, in one instance the antiserum of one of a pair of animals immunized with one organism gave agglutination whereas that from the second animal failed to agglutinate Bact. tumefaciens. Apparently we have a case of specific agglutination when Bact. tumefaciens

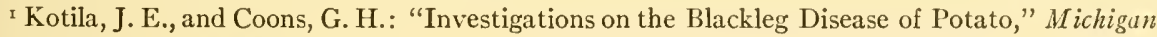
Agr. Exper. Sta. Tech. Bull. 67, 1925.

${ }^{2}$ Falk, I. S., Gussin, H. A., Jacobson, M. A.: "Studies on Respiratory Diseases: XXI. Electrophoretic Potential and Virulence of Pneumococci (types I, 2, 3, and 4)," J.Infect. Dis., 37, 48I. I925.

${ }^{3}$ Sharp, C. G.: loc. cit.

${ }^{4}$ Falk, I. S., Sharp, C. G., and Link, George K. K.: "Relations between pH, Agglutination and P.D. with Bacterium phaseoli sojense," Proc. Soc. Exper. Biol. \& Med., I927.

s Sharp, C. G.: loc. cit.

${ }^{6}$ Link, George K. K. and A. DeS.: loc. cit. 
is used as antigen, but cross-agglutination when the antisera of other pathogens are tested against suspensions of Bact. tumefaciens.

Spontaneous agglutination of plant pathogens.-Berridge ${ }^{\mathrm{I}}$ found that chemical agglutination tests are as reliable as serum agglutination tests for certain soft-rot bacteria. The phytopathological literature contains casual references to instances of self-flocculation of plant pathogens. Sharp ${ }^{2}$ found that the $\mathrm{R}$ strain of Bact. phaseoli sojense agglutinated spontaneously in distilled water even after nine washings, and also in 0.85 per cent $\mathrm{NaCl}$. Link and $\mathrm{Link}^{3}$ were able to get a permanent suspension of this organism by taking up the original flocculum in 0.85 per cent $\mathrm{NaCl}$ and washing and centrifugating it three times. Sharp reports that Bact. phaseoli sojense (R) agglutinates through practically the entire range of $\mathrm{pH}$ 2.0-9.4, little or no agglutination occurring from $\mathrm{pH} 5 \cdot 5-5.8$. The $\mathrm{S}$ strain agglutinated only between pH I.2-4.0. Sharp ${ }^{2}$ and Falk, Sharp, and Link ${ }^{4}$ found further that in the acid zone there seemed to be a correlation between low P.D. (electrophoretic potential difference) and high agglutinability, and between high P.D. and low agglutinability for both the $\mathrm{S}$ and the $\mathrm{R}$ strains, but not in the alkaline range.

Sharp found that Bact. phaseoli sojense (S) produced a scaly pellicle in lactose and levulose broth, whereas Bact. phaseoli sojense (R) produced a very heavy granular precipitate in nearly all sugar broths tested.

Link and Link $^{3}$ and Link and Hulls report that their strain of Bact. tumefaciens which develops colonies that are rough at first, and become smooth when suspended in 0.85 per cent $\mathrm{NaCl}$ or distilled water, always showed spontaneous agglutination for about one-fourth of the suspension. By centrifugation and settling, the two fractions could be separated. Link and Hull found also that flocculation or settling out is by no means a qualitative matter associated or correlated with roughness or smoothness. In the first place, the spontaneous flocculum of Bact. phaseoli sojense (R) could be resuspended. Apparently during the time which elapsed between Sharp's and their tests with the same culture, some change toward lesser agglutinability had occurred in the organism. Furthermore, Bact. citri, which is as rough as Bact. phaseoli sojense and more rough than Bact. tumefaciens, does not agglutinate spontaneously nor does Bacl. medicaginis var. phaseolicola, No. 23. It is apparent that the data are still too few and too diverse to warrant any general conclusions, save the one that further work should be done in this interesting field.

AUtOLysis PIENOMENA: TRANSMISSIBLE LYTIC PRINCIPLE (BACTERIOPHAGE)

Smith $^{6}$ has described and illustrated colonies which suggest the phenomenon of autolysis reported by animal bacteriologists. These are the "windowed" colonies of Bact. malvacearum, and, even more convincingiy, the colonies of Bact. translucens var. undulosum with "liquefaction pits."

'Berridge, Emily M.: "Studies in Bacteriosis." XIV. Ann. Appl. Biol., 13, I2-17. 1926.

${ }^{2}$ Sharp, C. G.: loc. cit.

${ }^{3}$ Link, George K. K. and A. DeS.: loc. cit.

${ }^{4}$ Falk, I. S., Sharp, C. G., and Link, George K. K.: loc. cit.

5 Link, George K. K., and Hull, K.: loc. cit.

${ }^{6}$ Smith, E. F.: Bacterial Diseases of Plants. 
The phenomenon of a transmissible lytic principle (bacteriophage) has been reported by a few investigators as occurring in the plant field. This interesting but highly controversial subject has however been merely touched, and more work will have to be done before any definite conclusions can be drawn. Gerretsen and Sack, and Söhngen and Gryns ${ }^{\mathrm{r}}$ report isolations of lytic principles from nodules, roots, and stems, but not from leaves of leguminous plants bearing nodules, the lytic principles being specific for the bacteria of the plants in question; also from garden and field soil, but not from heath or forest soil. Mallmann and Hemstreet ${ }^{2}$ report the recovery of an inhibitory substance from cabbage decayed by fluorescent organisms, but did not demonstrate lysis. Following their work, Coons and Kotila ${ }^{3}$ report recovery of a lytic principle from rotted carrots, from soil, and from river water which in low dilutions inhibited growth and in higher dilutions lysed B. carotovorus, B. atrosepticus, and Bact. tumefaciens. They report loss of motility, malformation, and agglutination as characteristic of cultures treated with the lytic principle, agglutination being the first evidence of change in the organisms. Kotila and Coons ${ }^{4}$ also report isolation of a lytic principle from $B$. atrosepticus which when placed on potato tubers prevents the rotting normally caused by this organism. They venture the suggestion that this principle is responsible for the rapid decline of $B$. atrosepticus in the soil.

\section{IMMUNOLOGY}

The field of phytobacteriology exhibits a wide range of problems in resistancesusceptibility phenomena, practically all, or all, of which are instances of natural immunity. In the first place, it is an unanswered question why no or little success is had in at tempts of cross-inoculation of plants and animals with animal and plant pathogens. There are among plant pathogens a great number of instances of phylum, order, family, genus, species, variety, and individual specificity. There are also illustrations of tissue specificity so that bacterial diseases of plants are often contrasted as parenchyma and as vascular diseases. As a matter of fact, no sharp line exists and the differences are largely quantitative rather than qualitative. Day ${ }^{5}$ however, reports that Bact. salicis, the cause of the watermark disease of Salix caerulea, is strictly confined to the vessels.

There are abundant illustrations of susceptibility-resistance phenomena directly correlated with age. In some diseases the seedling stage is the one during which the plant is most subject to infection (wilt of maize caused by A planobacter stewarti), and in many others tissues are susceptible only so long as they are immature (fire blightB. amylovorus). The correlation between immaturity and susceptibility makes many

${ }^{1}$ Gerretsen, F. C., Gryns, A., Sack, J., and Söhngen, N. L.: "Das Vorkommen eines Bacteriophagen in den Wurzel-Knöllchen der Leguminosen," Centralbl.f. Bakteriol., Abt. II, 6o, 31 I-x6. I923.

${ }^{2}$ Mallmann, W. L., and Hemstreet, C.: "Isolation of an Inhibitory Substance from Plants," J. I gr. Research, 28, 599-602. I924.

3 Coons, G. H., and Kotila, J. E.: "The Transmissible Lytic Principle (Bacteriophage) in Relation to Plant Pathogenes," Phytopath., I5, 357-70. I925.

${ }^{4}$ Kotila, J. E., and Coons, G. H.: "Investigations on the Blackleg Disease of Potato," Michigan Agr. Exper. Sta. Tech. Bull. 67. 1925.

${ }^{5}$ Day, W. R.: "The Watermark Disease of the Cricket-Bat Willow (Salix caerulea)," Oxford Forest. Mem., 3, 30. I924. 
diseases appear time limited, since they appear unly in the spring and early summer when the plant possesses a considerable amount of growing tissue. On the other hand, there are many illustrations of direct correlation between susceptibility and maturing or ripening tissues (various potato-tuber rots).

There have been innumerable attempts to invoke simple explanations for these phenomena of susceptibility-resistance, and to use them in generalizations. None of them, as is to be expected, has been successful. Juiciness of tissues, presence or absence of food or antagonistic substances such as acids, sugars, amino acids, tannins, anthrocyanins, flavones, and various volatile substances, are some that have been considered. Recently, the factor of acidity of the cell sap has been given much attention. Gardner and Kendrick ${ }^{\mathrm{I}}$ report that Bact. exitiosum, which does not tolerate a $\mathrm{pH}$ less than 5 in culture media, readily attacks seedlings, leaves, and green fruits which have a $\mathrm{pH}$ varying from 6.3 to 5 , but does not infect mature and ripe fruits whose $\mathrm{pH}$ usually is about 4.6 . Berridge, ${ }^{2}$ in testing out twenty-eight species of bacteria, fourteen of which are parasites, determined the points of maximum agglutination of the organisms and their acid limits for growth. She found no consistent direct correlation of these with acidity of the cell sap of plants. Smith and Quirk ${ }^{3}$ were not able to demonstrate that tissues with $\mathrm{pH}$ much less than $5 \cdot 7$, which represents the acid tolerance of Bact. tumefaciens in culture media, are necessarily resistant to attack by the organism. They conclude that possibly the preponderant kind of acid of the tissues is a more important factor than the total acidity. Gillespiet reported that certain soils with a $\mathrm{pH}$ of about 5.2 or less generally produced a scab-free crop, and the assumption became generally current that acidity of the soil was the limiting factor for the pathogenicity of Actinomyces scabies. Sanford, ${ }^{5}$ however, reports that moisture content of the soil is a more important factor than $\mathrm{H}$-ion concentration. It is highly probable that the mechanisms of immunity lie deeper, in the very constitution and activities of the protoplasm itself.

\section{ACQUIRED IMMUNITY}

Attempts to demonstrate an acquired immunity in plants have failed. Brown ${ }^{6}$ unsuccessfully attempted to develop resistance to Bact. tumefaciens in the Paris daisy. Riker $^{7}$ was unable to demonstrate agglutinins or precipitins in plants having large

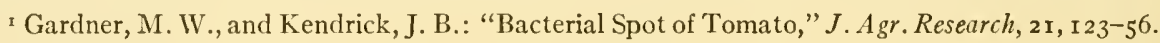
$192 \mathrm{I}$.

${ }^{2}$ Berridge, E. M.: "The Influence of Hydrogen-ion Concentration on the Growth of Certain Bacterial Parasites and Saprophytes," Ann. Appl. Biol., I1, 73-85. 1924.

${ }^{3}$ Smith, E. F., and Quirk, J.: "A Begonia Immune to Crown-Gall: with Observations on Other Immune or Semi-immune Plants, Phytopath., 16, 49I-508. 1926.

${ }_{4}^{4}$ Gillespie, L. J.: "The Growth of the Potato-Scab Organism at Various Hydrogen-Ion Concentrations as Related to the Comparative Freedom of Acid Soils from the Potato Scab," ibid., 8, 257-69. I9I8.

${ }^{5}$ Sanford, G. B.: "The Relation of Soil Moisture to the Development of Common Scab of Potato," ibid., r3, 231-36. 1923.

'Brown, N. A.: "Experiments with Paris Daisy and Rose to Produce Resistance to Crown Gall," ibid., pp. 57-99. I923.

7 Riker, A. J.: "The Influence of Temperature and of Previous Infection in the Development of Crown Gall," ibid., 15, 45. 1925 . 
crown-galls. The report that legume roots become immune to invasion by less virulent strains of bacteria after infection with more virulent strains of nodule bacteria is interesting and deserves reinvestigation. There are many instances of recovery of individual plants or their organs from attack by bacterial pathogens. These may be due to cessation of environmental factors favorable for the host, and not to an acquired immunity. Whether the self-limited spots and cankers that characterize many bacterial diseases are partly or wholly due to acquired immunity is a possibility which should be investigated.

\section{RELATION OF PATHOGEN TO HOST TISSUES AND CELLS}

Only one case of penetration of cell walls and intracellular life of a bacterial pathogen has been established. This occurs in the legume-nodule-producing bacteria. It was contended by Smith $^{1}$ that Bact. tumefaciens was another illustration of an intracellular schizomycete. However, the later findings of Riker ${ }^{2}$ and of Robinson and Walkden ${ }^{3}$ to the effect that Bact. tumefaciens invades mainly through the intercellular spaces, and occasionally through the vascular system, is at variance with the earlier reports of Smith.

In a few diseases which are known as "tracheo-bacterioses," the pathogens are very abundant in the lumina of vessels in the early stages of the disease. Very soon, however, the organisms invade the surrounding parenchymatous tissues. Unless they are directly introduced into the vessels by injuries - for example, insect bites as in bacterial wilt of the cucumber-they must pass through parenchyma before they enter the vessels as in black rot of cabbage. By far the greater number of bacterial pathogens live in the intercellular spaces of the plant.

Route of entry.- Some bacterial pathogens at times, or consistently, have definite routes of entry. B. amylovorus may invade through the unprotected surface of the flower nectary, though it also invades any young tissue through wounds; Bact. campestre invades through the hydathodes and progresses through the vascular system, though it also enters through wounds; many pathogens enter through the stomata or through wounds; some enter principally through lenticels (Actinomyces scabies); and a great number enter through wounds. The legume-nodule bacteria enter through root hairs. Brown 4 has recently shown that Bact. malvacearum gets its first foothold in young seedlings in the injuries made in the cotyledons as these emerge from the seed coat and soil. Insects and cultural practices play an important rôle in getting bacteria past the effective defense mechanism of the epidermal or cork layers of plants.

The mechanism of tissue invasion.-Following the early critical work of Spiecker-

"Smith, E. F.: "Appositional Growth in Crown Gall Tumors and in Cancers," J. Cancer Research, 7, I-105. 1922; "Mechanism of Tumor Growth in Crown Gall," J. Agr. Research, 8, I65-86. I9I 7 ; Smith, E. F., Brown, N. A., and Townsend, C. O.: loc. cit.

${ }^{2}$ Riker, A. J.: "Some Morphological Responses of the Host Tissues to the Crown Gall Organism," J. Agr. Research, 26, 425-36. I924.

3 Robinson, W., and Walkden, H.: loc. cit.

${ }^{4}$ Brown, J. G.: The Influence of Alkaline Soils on the Prevalence of Angular Leaf Spot in PimaEgyptian Cotton. Thesis, Botany Department, University of Chicago, $\mathbf{1} 925$. 
$\operatorname{man}^{\mathrm{I}}$ and later that of Jones, ${ }^{2}$ showing that bacterial pathogens may invade the host tissues by dissolving the cell-wall material by enzymes, a great deal of data has accumulated to show that enzymic dissolution of the pectic or less frequently of the cellulose materials plays a large rôle in the invasion of plant tissues by bacterial pathogens. Coons and Kotila ${ }^{3}$ were able to precipitate out from a culture of $B$. atrosepticus a fraction which dissolved the pectic substances of cell walls. Further advances in this field are dependent upon advances in knowledge of the physiology and chemistry of enzymes.

Casual observation and reflection upon the action of bacterial plant pathogens suggests that bacteria also form or lead to the formation of toxic substances that plasmolzye and necrose the cells of the host, and thus enable them to invade the tissues deeper and deeper. The phytopathological literature is full of references to the rôle of bacterial "toxins" and "toxic" substances in the attack of pathogens upon the host tissues. This elevation of the principle of toxic action of pathogens to a dictum of general application in the field of phytobacteriology is based on comparatively few isolated experiments. Spieckerman ${ }^{4}$ describes experiments presumably carried on with toxic substances, which are thermo-labile. He did not demonstrate, however, that the plasmolysis noted is not associated with a resultant disruption of the tissues. Laurent ${ }^{5}$ and van $\mathrm{Hall}^{6}$ report the demonstration of both enzymic and toxic and plasmolyzing principles from cultures of micro-organisms which are doubtful plant pathogens. Kotila and Coons ${ }^{7}$ report demonstration of both enzymic and toxic action by $B$. atrosepticus.

Smith $^{8}$ contended that Bact. tumefacicns is intracellular and is carried through plant tissues from primary to secondary tumors by metastases. His contention has been challenged latterly by Robinson and Walkden' who contend that the bacteria are intercellular and migrate through the intercellular spaces and protoxylem vessels in the form of zoögleal strands, and stimulate cell division along their path, and by Riker $^{10}$ who contends that the organism is intercellular and is carried through the intercellular spaces when these are suffused with cell sap, following mechanical injury,

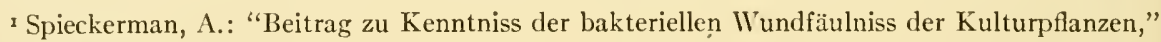
Landw. Jahrb., 3 I, I55-78. I902.

${ }^{2}$ Jones, L. W.: "Pectinase, the Lytolytic Enzyme Produced by Bacillus carotovorus and Certain Other Soft Rot Organisms," Vermont Agr. Exper. Sta. Bull. I 47, pp. 28I-360. I 910.

${ }^{3}$ Coons, G. H., and Kotila, J. E.: "The Transmissible Lytic Principle (Bacteriophage) in Relation to Plant Pathogenes," Phytopath., 15, 357-70. 1925.

${ }^{4}$ Spieckerman, A.: loc. cit.

${ }^{5}$ Laurent, Émile: Recherches expérimentales sur les Maladies des Plantes," Ann. de l'Inst. Pasteur. I899.

${ }^{6}$ van Hall, C. J. J.: "Bacillus subtilis (Ehr.) Cohn und B. vulgatus (Flügge) Mig. als Pflanzenparasiten," Centralbl.f. Bakteriol., Abt. II, 9, 642-52. I902.

${ }^{7}$ Kotila, J. E., and Coons, G. H.: "Investigations on the Blackleg Disease of Potato," Michigan Agr. Exper. Sta. Tech. Bull.67. 1925.

${ }^{8}$ Smith, E. F.: see varicus works cited previously.

9 Robinson, W., and Walkden, H.: loc, cit.

10 Riker, A. J.: "Some Relations of the Crown Gall Organism in the Host Tissue," J. Agr. Research, 25, II9-32. 1923. 
and also along the axis of the plant by rapid elongation of the stem. It has, however, been well established that the legume nodule organisms pass from cell to cell in the form of zoögleal strands. Nixon's ${ }^{\mathrm{I}}$ studies of fire blight indicate that penetration of tissues by zoögleal strands may be more general in bacterial diseases than has been realized.

Effects upon plant tissues. - The cellular responses of the host to the presence of bacteria may be necrotic or hyperplastic or hypoplaspic or a combination of these. The latter type generally is evidenced by suppressed differentiation or suppressed enlargement of cells.

In crown-gall there is a development of xylem elements from cells which usually do not develop xylem. It is doubtful, however, whether this is a real case of metaplasia, because it is not a case of direct change of a permanent cell of one type into another type, but rather an instance of changing tissues, which are more or less permanent, into a meristematic state, and rendering these totipotent whereas the cells in that location usually are not totipotent.

It is contended that the bacteria present in the leaf teeth of Ardisia crispa, and to a lesser extent in the entire plant, even the seed, are real mutualists. ${ }^{2}$ It is assumed that the local injury done in the form of chlorosis of the leaf serratum is more than offset by the nitrogen which they are assumed to contribute. A similar claim is made for the Pavettas by von Faber. ${ }^{3}$ Fred, Whiting, and Hastings ${ }^{4}$ report conclusive proof that the legume-nodule bacteria alone can assimilate atmospheric nitrogen and thus contribute nitrogen to the host plant, thereby offsetting their local injury to the roots.

"EXTRA-VEGETAL" LIFE OF BACTERIAL PLANT PATIIOGENS

This topic is of interest because it involves the phenomena of parasitism and saprophytism and also that of sources of inocula. Every one of the described plant pathogens can be grown on culture media that are not at all specialized. In other words, these organisms are not in the main highly specialized so far as food requirements are concerned. Many, however, lose their pathogenicity after long culture on artificial media. Some of them are known to live in the soil, such as Bact.tumefaciens and the legume-nodule organisms, while it is suspected that many others also are capable of soil life. Others, although they are not spore-formers, can live and endure on the protective surfaces of plants or in or on plant débris even under extremely trying conditions of temperature, desiccation, and illumination. Bact. campestre, for example, can live on the seed coat of cabbage seed and consequently is readily introduced by this route into the seed bed.

\section{INSECT DISSEMINATION}

Insects not only disseminate bacteria mechanically ${ }^{5}$ but there are also two and possibly three experimentally demonstrated cases of internal biological dissemination of various pathogens by insects. The olive fly, Dacus oleae, carries the pathogen of olive knot, Bact. savastanoi, in its salivary glands and intestinal diverticula as a regu-

'Nixon, E. L.: "Fire Blight," Pa. State Coll. Bull. 203, 3-22. 1926.

${ }^{2}$ Miehe, H.: Ber. d. Deutsch. Bot. Gesell., 34, 576-80. I9r6.

${ }^{3}$ von Faber, F. C.: Jahrb.f. wiss. Bot., 54, 243. I9I4.

4 Fred, E. B., Whiting, A. L., and Hastings, E. G.: "Root Nodule Bacteria of Leguminosae," Wisconsin Agr. Exper. Sta. Res. Bull. 72. 1926.

5 Waite, M. B.: "Results from Recent Investigations in Fire Blight," Bot. Gaz., r6, 259. r89r. 
lar occupant. Smith $^{2}$ proved that the striped cucumber beetle, Diabrottica viator, transmits $B$. tracheiphilus, the pathogen of cucurbit wilt, and Rand, ${ }^{3}$ Enlows, and Cash have presented strong evidence that the organism winters over only in the intestinal tract of certain of these beetles. Leach ${ }^{4}$ reports the seed-corn maggot (Phorbia fusciceps) as an agent of dissemination, inoculation, and hibernation of $B$. atrosepticus, and that the organism is necessary for the normal development of the larvae. This work has not been reported in detail, nor has it been repeated by other investigators.

\section{CONTROL OF BACTERIAL PLANT DISEASES}

Investigations in and practice of control of bacterial plant diseases constitute a very considerable part of the activity of plant pathologists. The control measures practiced are determined by the biology of both host and pathogen.

In many instances in which bacteria hibernate on or in plant organs such as seeds, tubers, roots, and stems, disinfection by heat (wet or dry) or chemicals (organic and inorganic) is resorted to. Similar methods are used in sterilizing soils. Selection of disease-free organs or plants is also found useful. Spraying plants with germicidal substances is effective in some cases. Crop rotation, proper timing of the planting so as to avoid environmental factors such as temperature, moisture, etc., optimum for the pathogen, and field sanitation, i.e., destruction of plant débris on which the pathogen can be carried over, are employed with success. Eradication of diseased plants or plant organs is a generally practiced method, and associated with it are local, state, and federal quarantine measures.

In the diseases which are overwintered and disseminated by insects primarily, the main task is to destroy the insect.

Since many plant pathogens enter only through wounds, care to control the agencies that break the protective surfaces of plants becomes imperative.

Finally, the development by selection and crossing of resistant varieties promises to become one of the most important and economical control measures.

I Petri, L.: Centralbl. f. Bakteriol., Abt. II, 26, 357. I910.

${ }^{2}$ Smith, E. F.: Bacterial Diseases of Plants.

${ }^{3}$ Rand, F. V.: "Insects as Disseminators of Plant Diseases." Phytopath., I2, 225-28. 1922.

4 Leach, J. G.: "The Relation of the Seed-Corn Maggot to Potato Blackleg," ibid., r6, 68. 1926. 


\title{
CHAPTER XLV
}

\section{COMMUNICABLE DISEASES OF LABORATORY ANIMALS}

\author{
K. F. MEYER \\ University of California
}

Laboratory animals are frequently called "living test tubes" since they may serve as "culture media" for the isolation of delicate micro-organisms and more often as testing objects for the study of certain properties of bacteria and viruses (pathogenicity, virulence, immunity reactions, pharmacological effect of metabolic products, etc.). Though the importance of animals in bacteriological work is generally admitted, little or no attention is paid to this tool in the majority of the contemporary textbooks. Lengthy dissertations deal with the physical and chemical prerequisites of the test tubes or glassware in general. One may even find a few commentaries on the methods of inoculating and bleeding certain species but nothing is said regarding the breeding, the feeding, and the general care of the animals commonly employed. Even the beginner should be familiar with the normal anatomy and physiology of the guinea pig, rabbit, mouse, rat, pigeon, etc. ${ }^{x}$ Furthermore, the student and advanced worker should be reminded with unremitting insistence that for the successful execution of an experiment of any sort the use of healthy animals preferably with a known hereditary history is an absolute prerequisite. Poorly kept animals or those which are already infected either with a latent or chronic disease may, aside from the great losses entailed in the course of an experiment, furnish exceedingly unpleasant surprises and many misleading observations. Irrespective of this fact, the whole subject is frequently dismissed with the statement that certain animals are often unsatisfactory for experimental purposes owing to the liability to parasitic and intercurrent maladies.

The significance of the infections of laboratory animals has in the past received relatively little attention on account of the hitherto separate flow of research in human and animal diseases. Valuable observations have been reported in scientific and technical journals, but unfortunately no comparative pathology of laboratory animals has as yet been compiled. However, in recent years considerable interest has been aroused by the recognition that some of the communicable diseases of laboratory rodents furnish excellent material for the study of epidemiological problems, the pathogenesis of certain infective agents, the protective mechanism which may follow

'The following books or papers contain valuable information: Bensley, A. B.: Practical Anatomy of the Rabbit (3d ed.). Philadelphia, I92I; Donaldson, H. H.: The Rat (2d ed.). Philadelphia: Wistar Institute, I924; Hunt, H. R.: A Laboratory Manual of the Anatomy of the Rat. New York, I924; Martin, P.: Lehrbuch d. Anatomie der Haussäugetiere (2d ed.), 4, Lief. 3, 303-408. Stuttgart: Schickhardt \& Ebner, r922; Friedberger, E., and Schiff, F.: in Kraus and Uhlenhuth's Handbuch d. mikrobiciogisch. Technik, 2, 1563. I923; Raebiger, H.: Das Meerschweinchen. Hannover: M. and H. Schaper, 1923; Remlinger, P., and Bell, P.: Bull. de l'Inst. Pasteur, 22, 15-29. I924; Ward, A. R., and Gallagher, B. A.: Diseases of Domesticated Birds. New York, 1920. 
vaccination, and the host susceptibility which may be influenced by hereditary and environmental factors, diets, etc. An extensive literature has already accumulated, and it is impossible to do justice to the subject in this brief chapter. Many important data must be omitted, and the reader can only expect a presentation of the essential facts. Then again a number of infections have not been studied with the care they deserve and therefore the conclusions must of necessity be brief and noncommittal. This rather one-sided account must also ignore the various deficiency diseases, such as scurvy, which not infrequently reduce a poorly managed laboratory stock. Particular attention will be paid to the diseases of guinea pigs, rabbits, mice, and rats, and then primarily to those infections which are due to bacterial agents. The presentation considers the various diseases in the order of their importance. Those interested in the parasitic invasions will find some information in a brief summary by the writer. ${ }^{\mathrm{r}}$

\section{DISEASES OF THE GUINEA PIG}

The guinea pig is probably more frequently used for bacteriological studies than any other animal since it is particularly susceptible to infections with human and animal pathogenic micro-organisms. Aside from the artificially produced pathological processes, the rodent is however liable to numerous spontaneous communicable diseases. Everybody who experiments with this animal should be familiar with the clinical and preferably the postmortem findings, since not infrequently morbid lesions are encountered which can only be properly diagnosed when the pathology and the bacteriology of the spontaneous infections are fully recognized. Several years ago Holman ${ }^{2}$ and more recently Raebiger and Lerche ${ }^{3}$ brought together in a concise form the pathologic-anatomical findings of the diseases of guinea pigs with special reference to their etiology. The excellent summaries contain an abundance of important data which the writer will correlate with a number of recent contributions and his personal experiences.

The wavelike outbreaks which may carry off in a short time a large number of animals are due to one of two types of infections: (I) a disease to which Eberth ${ }^{4}$ gave in 1885 the designation "bacillary pseudotuberculosis"; (2) septicemias or respiratory diseases due to a pneumococcus, a pasteurella organism, or the Friedländer's bacillus. Probably the most important one is the pseudotuberculosis caused by representatives of the paratyphoid-enteritis group. To avoid confusion, this communicable disease should be designated as "paratyphoid" in order to distinguish it from those infectious processes which are due to the Bacillus pseudotuberculosis (rodentium) Pfeiffer.

\section{PARATYPHOID}

This disease was recognized as early as 1884 , and a good description of a small epidemic which attacked pregnant females as well as newborn animals was given by

${ }^{1}$ Meyer, K. F.: Practical Bactcriology, Mcdical Zoölogy and Immunology, "University of California Syllabus Series," No. I79, pp. 52-57. Berkeley: University of California Press, i925.

${ }^{2}$ Holman, W. L.: J. Med. Research, 35, I51. I916.

${ }^{3}$ Raebiger, M., and Lerche, P.: Ergcbn. d. allg. Path. u. Anat., Abt. II, 21, 686. Iو26.

${ }_{4}$ Eberth, C. J.: Virchow's Arch.f. path. Anat., Ioo, 15. I885; Pertik, O.: Ergebn. d. allg. Path. u. Anat., Abt. II, 8, II 8. I904. 
Schantyr. ${ }^{I}$ Although the bacillus isolated in the course of this outbreak cannot be properly classified, the epidemiological and anatomical findings leave little doubt that the infection was caused by an organism of the paratyphoid group. The importance of these bacteria to guinea pig infections was first established by Smith and Stewart ${ }^{2}$ who reported a single case of pseudotuberculosis. From the spleen they were able to isolate an organism which was closely related to the hog-cholera bacillus and to the B. typhi murium of Loeffler. Since then numerous publications have dealt with small outbreaks or appalling epizoötics (Wherry, ${ }^{3}$ Weaver, ${ }^{4}$ Eckersdorff, ${ }^{5}$ Kirch, ${ }^{6}$ Dieterlen, ${ }^{7}$ Petrie and O'Brien, ${ }^{8} \mathrm{O}^{\prime}$ Brien, ${ }^{9}$ Bainbridge and O'Brien, ${ }^{10}$ Busson, ${ }^{11}$ Howell and Schultz $z,{ }^{12}$ Trawinski, ${ }^{13}$ Steinmetz and Lerche, ${ }^{14}$ Thomas, ${ }^{15}$ Freund, ${ }^{16}$ Okamoto, ${ }^{17}$ Kittler, ${ }^{18}$ Klein, ${ }^{19}$ Nelson and Smith, ${ }^{20}$ and others); however, a number of workers have recovered the bacilli from guinea pigs which have been treated in various ways or from the organs of apparently healthy animals (Smallmann, ${ }^{21}$ Klein, ${ }^{22}$ Morgan and Marshall, ${ }^{23}$ Bofinger, ${ }^{24}$ Ford, ${ }^{25}$ the writer, and others). One cannot escape the impression that this infection is world wide in its distribution and that its persistence in the past must be largely attributed to the lack of knowledge concerning the spread of the disease and the purchase of guinea pigs casually and indiscriminately at retail. This practice has led in the experience of the writer to catastrophic consequences, particularly when a

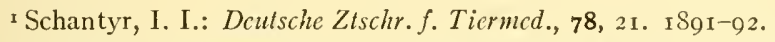

${ }^{2}$ Smith, T., and Stewart, J. R.: J. Boston Soc. Mcd. Sc., I, I 2. 1897.

3 Wherry, W. B.: J. Infect. Dis., 5, 519. 1908.

4 Weaver, G. H.: Bull. Johns Hopkins Hosp., 9, 270. I 898.

5 Eckersdorff, O.: Centralbl.f. Baktcriol., Abt. I, Ref. Beilage, 38, 99. г 906.

${ }^{6}$ Kirch, E.: Arch.f. IIyg., 78, 327. 1913.

7 Dieterlen, T.: Arb. a. d. kaiscrl. Gesundheitsamlc, 30, 429. 1909.

${ }^{8}$ Petrie, G. F., and O'Brien, R. A.: J. Hyg., 10, 287. I910.

${ }_{9}$ O'Brien, R. A.: ibid., p. 231. г9го.

so Bainbridge, F. A., and O'Brien, R. A.: J. Path. Eo Bact., I6, 145. I9I I.

is Busson, B.: Ccntralbl.f. Baktcriol., Abt. I, 86, I01. I921.

${ }^{12}$ Howell, K. M., and Schultz, O. T.: J. Infect. Dis., 30, 516.1922.

${ }_{13}$ Trawinski, A.: Centralbl. f. Baktcriol., 87, 24. I922.

${ }^{14}$ Steinmetz, M., and Lerche, P.: in Raebiger's Das Mccrschwcinchcn, roo, 107. Hannover, I923.

${ }_{15}$ Thomas, B. G. H.: J. Infect Dis., 35, 407. 1924.

${ }^{16}$ Freund, R.: Zischr.f. IIyg. u. Infektionskrankh., 106, 627. 1926.

${ }_{17}$ Okamoto, T.: Klin. Wclinschr., 5, 795. 1926.

${ }^{18}$ Kittler, K.: "Abstract," Centralbl.f. Bakteriol., Ref. I, 81, 137. 1926.

${ }^{19}$ Klein, K.: ibid., 86, 564. I92 1.

${ }^{20}$ Nelson, J. B., and Smith, T.: J. Exper. Mcd., 45, 353. 1927.

2I Smallmann, A. B.: J. Roy. Army M. Corps, 2 r, 636. I9r3.

${ }^{22}$ Klein, F.: Centralbl.f. Baktcriol., Orig. I, 38, 392. 1905.

${ }_{23}$ Morgan, H. de R.: Brit. M. J., 1, 1257.1905.

${ }^{24}$ Bofinger: Dentsche med. Wchnschr., 37, 1063. I911.

${ }^{25}$ Ford, W. W.: Tr. A. Am. Physicians, 15, 389. 1900. 
recent shipment of animals is transferred to the stock without a previous quarantine or search for carriers. Probably owing to subtle differences in microbic virulence and host susceptibility, the frequency with which paratyphoid of guinea pigs may occur in different localities and during certain years varies considerably. Thus during the years $1914-16$ Holman $^{x}$ never encountered paratyphoid bacilli in the organs of guinea pigs, while the studies of Thomas ${ }^{2}$ in 1922 at the same laboratory were seriously handicapped by the endemic existence of a latent paratyphoid infection.

The causative organism of the disease is indistinguishable morphologically, culturally, and biochemically from the representatives of the paratyphoid-enteritidis or Salmonella group. The strains are highly tolerant toward brilliant and malachite green; irregular ionsite fermentation has been reported by Krumwiede, Valentine, and Kohn. ${ }^{3}$ As a rule, normal, smooth colonies have been isolated; according to Raebiger and Lerche, ${ }^{4}$ the "Schleimwall" formation is always missing. Nelson, ${ }^{5}$ however, found early in the endemic stage of an outbreak very carefully studied by him and Smith two cultures which were distinctly mucoid and flocculated spontaneously in broth. These mucoid variants differed neither in agglutinability nor antigenic properties from the normal types. An experimental study failed to reveal the stimuli which were responsible for these variants. The writer has from time to time examined several hundred cultures prepared from paratyphoid-infected guinea pigs and has failed to observe mucoid colonies. Furthermore, the paratyphoid cultures of guineapig origin have not displayed, as the observations of Nelson also indicate, any marked tendency toward variations or reversion of the mucoid to the smooth type.

The agglutinative affinities of the strains which have thus far been studied have not always been definitely established. Krumwiede, Valentine, and Kohn ${ }^{6}$ found a number of guinea pig strains received from various sources to fall into a group which is distinct from the suipestifer and paratyphoid $B$ group, and on the basis of these agglutination differences they applied to these bacilli a new name, B. pestis caviae. Howell and Schultz ${ }^{7}$ found their strains readily distinguishable from $B$. paratyphosus $\mathrm{A}$ and $\mathrm{B}, \mathrm{B}$. enteritidis, and $B$. suipestifcr. but they were unable to reach any conclusion as to the occurrence of a distinct rodent group of paratyphoid organisms. Thomas ${ }^{8}$ identified his cultures as either B. enteritidis or B. paratyphi of animal origin, and Steinmetz and Lerche ${ }^{9}$ state that the guinea pig strains are serologically identical with the Bacterium enteritidis Breslau. According to the careful comparative studies of Jordan, ${ }^{\text {I0 }}$ six of the sixteen strains of paratyphoid bacilli isolated by different workers from guinea pigs in this country are B. enteritidis Gärtner while ten belong to the B. paratyphosus $B$ aertrycke type. These conclusive studies are quite in harmony with those of Savage and White. ${ }^{\text {II }}$ Enteritidis organisms have also been found by Ten Broeck, ${ }^{\text {I2 }}$ Bainbridge and

I Holman, W. L.: loc. cit.

${ }^{2}$ Thomas, B. G. H.: loc. cit.

3 Krumwiede, C., Valentine, E., and Kohn, L. A.: J. Med. Research, 34, 449. Ig19.

${ }_{4}^{4}$ Raebiger, M., and Lerche, P.: loc. cil.

5 Nelson, J. B.: J. Exper. Mcd., 45, 391. 1927.

${ }^{6}$ Krumwiede, C., Valentine, E., and Kohn, L. A.: loc. cit.

7 Howell, K. M., and Schultz, O. T.: loc. cit.

${ }^{8}$ Thomas, B. G. H.: loc. cit.

9 Steinmetz, M., and Lerche, P.: loc. cit.

ro Jordan, E. O.: J. Infect. Dis., 36, 309. I925.

II Savage, W. G., and White, B.: "Medical Research Council Special Report Series," No. 92. 1925 .

I2 'Ten Broeck, C.: J. Expcr. Mcd., 32, 32. I920. 
O'Brien, ${ }^{\mathrm{I}}$ and Trawinski. ${ }^{2}$ I identified fourteen strains of a series of fifty-eight as B. enteritidis and isolated, just as Krumwiede and his associates did, both the $B$. enteritidis and $B$. aertrycke types from the animals of the same epidemic. By means of reciprocal absorption tests a definite identification may be accomplished, and one may therefore conclude that the causative organisms encountered in the natural infections are mostly strains which are either closely related to the $B$. aertrycke or the B. enteritidis group.

Experimental septicemic infections can be produced in guinea pigs, mice, rats, rabbits, and pigeons (Eckersdorff) 3 by subcutaneous and intraperitoneal injections of relatively few organisms (aertrycke type) of recently isolated epidemic strains. The virulence may be preserved in desiccated spleens (Schoenholz) ${ }^{4}$ since it decreases rapidly on agar (Bainbridge and O'Brien). Uchida ${ }^{5}$ concluded from a series of experiments that the peritoneum possesses a greater susceptibility for the infection than the blood-vessel system. Some of the guinea pigs injected intraperitoneally with one to ten organisms died in from two to three days; the same strain did not infect regularly by the subcutaneous route. The majority of workers who attempted to infect guinea pigs per os record absolutely negative or inconclusive results (Dieterlen, ${ }^{6}$ Howell and Schultz, ${ }^{7}$ Okamoto,${ }^{8}$ Freund, ${ }^{9}$ Uchida, ${ }^{10}$ and others). An active disease with a high mortality may be secured in a "clean stock" in rare and irregular instances when enormous numbers of bacteria (6o cc. of broth culture or one-tenth of a Blake flask) are fed repeatedly. Some unpublished observations indicate that the infective dose may be decreased provided the virulence has been exalted by repeated and rapid passage through animals or by continuous cultivation in a special medium. Ordinarily the ingested bacilli persist in the digestive tube (Shaw, Weiner, and Meyer, unpublished observations); fecal excretion may continue for three to four weeks, and at autopsy the organisms may be found in the spleen (Smith and Nelson), mesenteric lymph nodes and the bile (Shaw and Meyer). Occasionally cultures can be secured which are rapidly invasive on intracutaneous injection (Averill and Meyer, unpublished observations). The enteritidis strains tested by the writer behaved on guinea pigs like the acrtrycke cultures. A very important fact is quite often overlooked. Although the causative organisms are discharged in the excreta and have been found by Freund on the hay or the carrots (six times in ten tests) in the cages of the diseased or the dead animals, fatal contact infections are very rarely observed (Petrie and O'Brien, O'Brien, Bainbridge and O'Brien, Freund, Okamoto, Meyer, and others) when healthy guinea pigs are thus exposed to the virus. On the other hand, mice infected with paratyphoid strains of guinea pig origin as a rule spread a rapidly fatal disease among the healthy contacts. In the light of these observations Petrie and O'Brien considered the aertrycke organism merely a secondary invader to a "filter

I Bainbridge, F. A., and O'Brien, R. A.: loc. cit.

${ }^{2}$ Trawinski, A.: loc. cit.

3 Eckersdorff, O.: loc. cit.

${ }^{4}$ Schoenholz, P.: unpublished observations.

${ }_{5}$ Uchida, Y.: Ztschr.f. Hyg. u. Infektionskrankh., 106, 297. 1926.

${ }^{6}$ Dieterlen, T.: loc. cit.

${ }^{7}$ Howell, K. M., and Schultz, O. T.: loc. cit.

${ }^{9}$ Freund, R.: loc. cit.

${ }^{8}$ Okamoto, T.: loc. cil.

to Uchida, Y.: loc. cit. 
passer." Repeated tests by the writer have failed to substantiate this view. In fact careful postmortem examinations accompanied by cultural studies indicate that the paratyphoid bacilli select in all probability the intestinal tract as the portal of entry; they penetrate the digestive tube early in the course of an experinental infection per os and localize in the regional lymph nodes and in the spleen. Unquestionably, various accessory factors, the nature of which is as yet unknown, are required to produce an active disease. The susceptibility of the animals and the dosage of the bacilli are probably subordinate to certain predisposing nutritional, seasonal, or environmental conditions (sudden temperature changes, Freund). ${ }^{\mathrm{I}}$ Since the paratyphoid infections in guinea pigs behave slightly different from those studied by Topley, ${ }^{2}$ Amoss, ${ }^{3}$ Webster, ${ }^{4}$ and others on mice, detailed investigations of spontaneous epidemics are urgently needed. Such a study has recently been reported by Smith and Nelson.5 Their interesting data show that the intestinal carrier state in guinea pig paratyphoid associated with a localization of the organism in the spleen was of relatively short duration. This is of interest since in mice the fecal excretion of bacilli persists over considerable periods of time (Topley and Ayrton). During the epidemic period the individuals of low natural resistance were killed off, and the infective agent maintained itself in the female pigs which acted as chief focus of dissemination to their susceptible young and to the population at large. The majority of the sows excreted the bacilli either by way of the digestive tract, the uterus, or the mammary gland-even in one case intra-uterine transmission was noted. No evidence could be produced that the virulence of the causative organism had decreased. Since the factor or factors which caused the transition from the epidemic to endemic phase in the presence of younger generations could not be established, Smith and Nelson advanced the very acceptable hypothesis that the transition from epidemic to endemic phase was due to a combination of the weeding out of individuals of low natural resistance with a gradual adjustment of the invading organism to the population on a lowered level of virulence. It is a well-known fact that paratyphoid may exist latent in a stock of guinea pigs and may be recognized only during the breeding season on account of the high mortality of pregnant females or when the resistance of the animals is lowered through various experimental procedures. In a number of instances the writer has observed that these occurrences were followed by a series of active and fatal cases of the disease in the same and in the adjacent cages. Although the factors or agencies for these deaths among adult guinea pigs, which had been in contact for many months with the carrier animals, could not be determined, it appears not unlikely that in the light of Lockhart's ${ }^{6}$ observations fluctuations in the virulence of the paratyphoid bacilli must be considered.

The clinical manifestations of paratyphoid are not very characteristic, and various symptoms have been ascribed by the different authors. As a rule, the sick animals show a

Freund, R.: loc. cil.

${ }^{2}$ Topley, W. W. C.: Lancet, I, 477, 531, 645. London, I926.

3 Amoss, H.: J. Exper. Med., 36, 25, 45. 1922.

4 Webster, L. T.: A. J. IIyg., 4, I34. 1924.

5 Smith, T., and Nelson, J. B.: op. cil., 45, 365. 1927.

${ }^{6}$ Lockhart, L. P.: J. IIyg., 25, 50. 1926. 
roughening of the fur, emaciation, and weakness. Diarrhea is noted only in experimental animals fed with highly toxic broth cultures. The autopsy findings vary considerably. The most constant lesions are found in the spleen and liver. The spleen is enlarged and congested $\left(4 \frac{1}{2} \mathrm{~cm}\right.$. long by $2 \mathrm{~cm}$. wide), soft, occasionally covered by a plastic exudate, and studded throughout with minute foci or large yellowish nodules. Grayish foci or necroses similar to those seen in typhoid fever are present in the liver; quite often the gall-bladdler contains a thin, purulent fluid. The intestines are injected and the swollen, grayish Peyer's patches occasionally stippled with yellowish nodules are quite prominent. The mesenteric lymph nodes are always enlarged, medullary, and sometimes transformed into cheesy abscesses. Effusion in the pleural cavities, congestion of the lungs, patchy areas of consoliclation or yellowish foci, or double lobar pneumonia are constant in certain spontaneous outbreaks. The involvement of the uterus (purulent endometritis) is frequently reported (infectious endometritis, Carter's bacillus; see Smith and Reagh). ${ }^{\mathrm{x}}$

Without a thorougb bacteriological study a differential diagnosis between a paratyphoid and $B$. pscudotubcrculosis or pscudopcstis infection is frequently impossible. A number of procedures have been suggested to detect latent infections and carriers; although specific agglutinins can be demonstrated in the blood serum of the animals which present lesions at autopsy (Smith and Nelson ${ }^{2}$ and others), the tests are not practical. Shaw and Meyer ${ }^{3}$ have therefore used with considerable success the intracutaneous hypersensitiveness tests with pure bacterial proteins. Examinations of the feces for paratyphoid bacilli are unreliable.

Since the mortality may vary from 4 to 70 per cent of a laboratory stock (Petrie and O'Brien ${ }^{4}$ lost 479 of 500 animals, and Howell and Schultz ${ }^{5}$ estimated their losses at 500 and Kittler ${ }^{6}$ at 395 guinea pigs; Nelson and Smith ${ }^{7}$ report a mortality of 4 per cent in one month), repeated attempts have been made to control this malady by immunization. Nichols and Stimmel ${ }^{8}$ obtained experimentally some protection against a highly virulent acrtrycke strain, and Howell and Schultz $z^{5}$ apparently controlled an epizoötic by immunization of the guinea pig stock with a polyvalent suspension of the organism. Müller ${ }^{9}$ combatted an outbreak with a polyvalent paratyphoid serum. On the other hand, the reports of Steinmet $z$ and Lerche ${ }^{10}$ and Kittler ${ }^{11}$ confirm the observations of the writer. Neither the use of specific monovalent sera nor various types of vaccines changed the character of an epidemic or protected experimentally a series of guinea pigs subjected repeatedly to heavy infections per os from acquiring a chronic carrier state. In case paratyphoid has broken out in a guinea pig population it is much more economical to adopt strict measures of isolation, detection of the latent infections by means of skin tests, and the destruction of the reactors than to trust the efficacy of immunization. The laboratory stock can be kept free from the disease by judicious selection and purchase of breeding animals

${ }^{\mathrm{I}}$ Smith, T., and Reagh, A. L.: J. Hed. Research, 4, 270.1903.

${ }^{2}$ Smith, T., and Nelson, J. B.: loc. cit.

${ }^{3}$ Shaw, E. B., and Meyer, K. F.: unpublished manuscript. Master's thesis. University of California, I920.

${ }^{4}$ Petrie, G. F., and O'Brien, R. A.: loc. cil.

${ }^{6}$ Kittler, K.: loc. cit.

${ }^{5}$ Howell, K. M., and Schultz, O. T.: loc. cit.

7 Nelson, J. B., and Smith, T.: loc. cit.

${ }^{8}$ Nichols, H. J., and Stimmel, C. O.: J. Exper. Med., 38, 283. 1923.

9 Müller: Ztschr.f. Veterinärk., 29, I15. 1917.

${ }^{10}$ Steinmetz, M., and Lerche, P.: loc. cit.

II Kittler, K.: loc. cit. 
which should never be introduced into the common herd without previous serum or skin tests. Then again a well-trained animal caretaker will take special precautions against cross infections through the excreta of other animals (gray and white mice [Smith and Nelson] and wild rats) and through the agencies of contaminated food (stale bread [Howell and Schultz]). ${ }^{\mathrm{T}}$

\section{PSEUDOTUBERCULOSIS}

The designation of any disease characterized by pathologic-anatomical lesions similar to those produced by the tubercle bacilli as pseudotuberculosis has led to a great deal of confusion (Poppe). ${ }^{2}$ It is well known that the guinea pig responds to various inert substances, parasitic, toxic, and bacterial agents, with the formation of pseudotubercles. In many instances cited in the literatures morbid changes resembling pseudotuberculosis have been produced artificially and such cases have no relationship to a spontaneous communicable disease (Dessy). ${ }^{3}$ In Europe, especially in Italy (Byloff, ${ }^{4}$ Zagari, ${ }^{5}$ Petrie and O'Brien, ${ }^{6}$ Grancher and Ledoux-Lebard, ${ }^{7}$ Bonome, ${ }^{8}$ Cagnetto, ${ }^{9}$ Cipollina, ${ }^{10}$ De Blasi, ${ }^{11}$ Ramon, ${ }^{12}$ Bachmann, ${ }^{13}$ Van Saceghem, ${ }^{14}$ Dessy, ${ }^{15}$ Toschiana, ${ }^{16}$ and others), and less frequently in the United States (Branch, ${ }^{17}$ Meyerand Batchelder ${ }^{18}$ ), destructive epidemics caused by the $B$. pseudotuberculosis (rodentium) Pfeiffer, or B. pseudopestis or Streptobacillus pseudotuberculosis (rodentium), or Corynebacterium rodentium or "Bac. de Malassez et Vignal" have been reported. This organism on account of its close relationship to $B$. pestis as established by GalliValerio, Zlatogoroff, MacConkey, Rowland, and others has been carefully described in the papers by Kakehi ${ }^{19}$ and Römisch. ${ }^{20}$

Microscopically it is demonstrated with difficulty in the typical chronic lesions; the intracellular localization is then the most common. As a gram negative, non-motile, plump, coccoid, distinctly bipolar rod (Wayson's stain) frequently in short chains, the bacterium grows well aerobically in characteristic colonies (Meyer and Batchelder, Kakehi) on plain

Howell, K. M., and Schultz, O. T.: loc. cit.

${ }^{2}$ Poppe, K.: IIandb. d. path. Mikroorg. (2d ed.), 5, 775. Jena, 1913.

3 Dessy, G.: Boll. dell'Istit. sicroterap. milanese, 4, I 23.1925.

${ }_{4}^{4}$ Byloff, K.: Centralbl.f. Bakteriol., 41, 707 and 789. 1906. 42, 5. 1906.

5 Zagari, G.: Fortschr. d. Med., 8, 569. I 890.

${ }^{6}$ Petrie, G. F., and O'Brien, R. A.: loc. cit.

7 Grancher, M., and Ledoux-Lebard: Arch.d. méd. expér. ct d'anat. path., I, 203. ISS9-9o.

${ }^{8}$ Bonome, L.: Arch. perle med. sc., 21, 305. I897.

${ }^{9}$ Cagnetto, J.: Ann. dc l'Inst. Pastcur, 19, 449. 1905.

ro Cipollina, A.: Ann. d'ig., 10, 1. г900. " " de Blasi, D.: ibid., r8, 611. I9o8.

ז2 Ramon, G.: Ann. de l'Inst. Pastcur, 28, 586. I914.

${ }_{3}$ Bachmann, W.: Centralbl.f. Bakteriol., 87, I7 I. I921.

${ }^{14}$ van Saceghem, R.: Compt. rend. Soc. de biol., 86, 281 . I 922.

15 Dessy, G.: op. cit., 4, 133. 1925. ${ }^{16}$ Toschiana, L.: Pathologica, 18, 239. 1926.

${ }_{17}$ Branch, A.: J. Infect. Dis., 40, 533. 1927.

${ }^{8}$ Meyer, K. F., and Batchelder, A. P.: ibid., 39, 383. I926.

${ }^{19}$ Kakehi, S.: J. Path. E。 Bact., 20, 269. Igı́.

Römisch: Ztschr.f. Infcktionskrankh.d. Hanstiere, 21, I38, 212.1920. 
agar. Gelatin is not liquefied; in broth stalactites or flocculent sediment is regularly formed. As a rule, the strains render milk alkaline and ferment, dextrose, galactose, levulose, maltose, arabinose, trehalose, xylose, rhamnose, mannite, and glycerol with the production of acid but no gas. The action on adonite, dextrin, and salicin is irregular (Christiansen, Christensen). ${ }^{x}$ For a definite identification the agglutination tests with specific sera must be used. With the aid of this technique Stockle ${ }^{2}$ has recognized one culture isolated by Branch as a typical strain of $B$. pseudotuberculosis (rodentium).

The experimental infection of guinea pigs, rabbits, mice, and field rats by any route is successful, although the guinea pig strains of B. pseudotuberculosis (rodentium) produce rarely generalized lesions in rabbits. On subcutaneous injection merely local infiltrations and necroses are noted. Guinea pigs succumb in from eight to fifteen days (dosage, 400,000,000 organisms) to an infection per os, by inhalation or by subcutaneous application. White rats are generally considered non-susceptible, but strains have been encountered (Meyer and Batchelder) which produce an active disease by the intraperitoneal route. A toxic substance has been demonstrated in the broth cultures by a number of workers (Dessy, also the writer).

In the course of an epidemic the diseased animals may, according to Ramon, exhibit three types of clinical manifestations: septicemia, fatal in twenty-four to forty-eight hours' classical form with emaciation, diarrhea, and death in three to four weeks, and the glandular form with lymph adenopathy of the cervical and thoracic nodes. Since the differential diagnosis is difficult, serological (agglutination, Pfeiler) or complement fixation (Bachmann) ${ }^{3}$ or intracutaneous hypersensitiveness tests (Bachmann, Dessy) ${ }^{4}$ have been recommended. At autopsy the enlarged mesenteric, iliac, periaortic, and occasionally inguinal lymph nodes are transformed into a mass of thick, yellowish pus, and fibrous tissue together with an enlarged spleen and liver both studded with whitish nodules are generally considered pathognomonic for this infection. The intestines, the omentum, the kidneys, and the uterus may also reveal scattered small abscesses. In a few epidemics the lungs were found similarly involved, but as a rule the abdominal viscera are primarily diseased. It is of interest that histiologically giant cells are usually absent and epithelioidal cells are seen only in rare instances.

The natural mode of infection occurs probably by way of the digestive tract (Ramon, Branch). In a small epidemic started in an isolation room by the introduction of guinea pigs and mice injected with a highly virulent strain, the writer was able to record many fatal contact infections. The primary lesions were found in the intestines and the mesenteric lymph nodes with little or no involvement of the thoracic and subcutaneous nodes. In a number of animals the active disease ended in a chronic carrier state. Nothing definite is known regarding the epidemiology of this infection, and therefore no suggestions concerning its control can be given. Van Saceghem ${ }^{5}$ successfully immunized guinea pigs with I cc. of a broth culture heated to $60^{\circ} \mathrm{C}$. while Dessy was unable to produce an active immunity with killed cultures or toxins, although the treated animals developed a state of allergy.

${ }^{\mathrm{I}}$ Christensen, N. P. C.: reprint from the Kgl. Veierinaer-og Landbohjskoles Aarsskrift. I927.

${ }^{2}$ Stockle, J.: unpublished observations.

3 Bachmann, W.: loc. cit.

${ }_{4}^{4}$ Dessy, G.: op. cit., 4, I33. 1925.

s van Saceghem, R.: loc. cit. 


\section{PNEUMOCOCCUS INFECTIONS}

In recent years considerable evidence has been presented that the pneumococcus is a widely disseminated pathogenic organism among guinea pigs (Smith). ${ }^{x}$ The coccus is not only one of several factors responsible for pneumonias but it is quite often associated with a number of interesting morbid changes. Aside from sporadic cases observed in animals subjected to treatment or inoculation in experiments, epidemic mortalities which occur during the winter or early spring have been noted by many laboratory workers. The association of a pneumococcus with an epidemic disease of guinea pigs was first reported by Stefanski in Odessa. Smith in I9I4 detailed his observations made in I908 and I909 and reviewed the outbreaks reported by Selter, ${ }^{3}$ Ungermann, ${ }^{4}$ Christiansen, ${ }^{5}$ and Richters, ${ }^{6}$ while Holman ${ }^{7}$ in his treatise refers to papers by Tartakowsky ${ }^{8}$ Wittneben, ${ }^{9}$ Salomon, ${ }^{\text {I0 }}$ and Kaspar and Kern. ${ }^{11}$ To these must be added the interesting observations of Chevrel, Ranque, Senez and Gruat, ${ }^{12}$ Freund, ${ }^{13}$ Okamoto, ${ }^{14}$ Branch, ${ }^{15}$ and our own. Most of the writers emphasize the localization of the lesions in the lung and the serous membranes, but Richters and Okamoto encountered outbreaks in which the disease was confined to the female sex organs.

The pneumococcus which can be isolated at autopsy from the heart blood, the lungs, serofibrinous exudates, middle ear, etc., is a gram positive, lancet-shaped, capsulated or non-capsulated (Okamoto), bile-soluble, inulin-fermenting microbe indistinguishable from that obtained from human sources. The cultures studied in different paris of the world were not agglutinated with the standard fixed types I, 2, and 3 sera, although Keegan ${ }^{16}$ reported one and Stillman (quoted by Branch) identified several strains as type $2 C$. Christiansen, Gardner, place them in group 4, and Branch ${ }^{17}$ has shown that they are serologically related by their protein fraction with the human strains. They are inhibited by ethyl hydrocuprein in very high dilutions ( $\mathrm{s}: 600,000$ [Freund]). Their virulence for mice is moderate or very low $(\mathrm{I}, \infty, 0,0,0,000$ organisms intraperitoneally, death in two to four days [Freund and Branch]). Guinea pigs may occasionally succumb to intraperitoneal and subcutaneous injections of

${ }^{1}$ Smith, T.: J. Hed. Research, 29, 291. I913-14.

${ }^{2}$ Stefanski, V.: Ccntralbl.f. Baktcriol., 30, 201. I90г.

3 Selter, H.: Ztschr. f. IIyg. u. Infcktionskrankh., 54, 363. Igo6.

4 Ungermann, E.: Arb. a. d. kuiserl. Gesundheitsante, 34, 286. I910.

5 Christiansen, M.: Ztschr. f. Infcktionskrankh d. Hausticre, 14, Iог. I913.

${ }^{6}$ Richters, E.: ibid., D. I63. 1913.

7 Holman, W. L.: loc, cit.

8 Tartakowsky, M. G.: Ccntralbl. f. Bakteriol., Orig. I, 25, 8I. I899.

9 Wittneben, W.: ibid., 44, 316. 1907.

so Salomon, E.: ibid., 47, 8. I 908.

"Kaspar, F., and Kern, IV.: ibid., 63, 7. 1912.

${ }^{12}$ Chevrel, F., et al.: Compt. rend. Soc. de biol., 82, 74. I9I9.

13 Freund, R.: loc, cit.

14 Okamoto, T.: loc. cit.

15 Branch, A.: loc. cit.

${ }^{16}$ Keegan, J. J.: Arch. Int. Med., 26, 570 . 1920.

${ }_{17}$ Branch, A.: op. cit., 40, 534. I927. 
from 0.I to I cc. of a broth culture (Smith, ${ }^{1}$ Uchida, ${ }^{2}$ Freund, ${ }^{3}$ ) or surviv the injection and die three weeks later when subjected to low temperatures or other environmental changes (Okamoto). In the experiments of Uchida neither the "culture" nor the "body" strains caused infections when fed or applied to the conjunctival sac or by inhalations.

The clinical symptoms are variable and uncertain, although a watery nasal discharge and an enlargement of the cervical lymph nodes has been observed by the writer. Equally inconclusive are the autopsy findings although the frequencies of the principal lesions are recorded as follows: serofibrinous, frequently hemorrhagic pleurisy (Smith, Branch), acute lobar (Christiansen, Freund) or chronic unresolved bronchopneumonia; infarct-like patches of lobular and lobar distribution in the cephalic and caudal lobes with adhesions; enlargement of the spleen, purulent peritonitis with and without extensive inflammatory ulcerative involvement of the uterus (Richters, Okamoto); hemorrhagic or seropurulent pericarditis; otitis media (Branch) and occasionally subcutaneous phlegmonous processes. A bacteriological examination established the diagnosis and proved the septicemic character of the disease in approximately 95 per cent of the cases studied by the writer.

The epidemiology of spontaneous pneumococcus infections in guinea pigs deserves considerable investigation. The reports of Freund and others indicate that the inciting organism may be widely prevalent. At the end of an epidemic he found in per cent and during the endemic period approximately 27 per cent carriers with the pneumococcus either in the nasal passages or in the tracheal lymph norles. It is not unlikely that the percentage would have been increased by an examination of the sinuses and the middle ears as advocated by Branch. The writer has found that apparently healthy animals of certain breeders may show a carrier percentage of as high as 60 per cent provided the examinations for the pneumococcus are made at autopsy. Animals with snuffles can readily distribute the organisms which are, however, of very low virulence. The portal of entry of these organisms is unknown. Some workers suspect the upper respiratory tract (Freund, Branch); others, the uterine cavity. One fact is certain-the exact reproduction of the spontaneous disease is practically impossible. Proved contact infections are very rare although Freund demonstrated the dissemination of pneumococci by exposing blood plates in the animal room. Smith expressed the view that the various guinea pig epidemics reported from different countries were due to pneumococci of widely different degrees of virulence, but it is now generally believed that the epidemic outbreaks of a pneumococcus or any other similar infection is controlled by the host susceptibility and not by the virulence. From the various reports one must conclude that two or three factors affect the susceptibility: pregnancy and parturition, sudden or prolonged changes in the temperature of the environment, and perhaps dietary deficiencies. To these the writer would like to add, from personal observation: crowding in dark and dusty cages, superimposed infections (pasteurellosis), and various experimental procedures. The contributory conditions are probably in many ways similar to those recently elucidated by Webster ${ }^{4}$ in his studies on snuffles in rabbits (see p. 624).

'Smith, T.: loc. cit.

${ }^{2}$ Uchida, Y.: loc. cit.

${ }^{3}$ Freund, R.: loc. cit.

${ }^{4}$ Webster, L. T.: J. Exper. Mel., 45, 9 I I. 1927. 
Chevrel $^{\mathrm{I}}$ and his associates were able to control a small outbreak by using pneumococcus vaccines.

\section{INFECTIONS WITH "BACT. BRONCHISEPTICUM"}

In a noteworthy paper Smith ${ }^{2}$ in I9I 3 called attention to the frequency of chronic pulmonary infections caused by Bact. bronchisepticum (M'Gowan, I9ro). He gave an excellent description of the lung conditions and of the characters of the organism. The pulmonary consolidation which is usually symmetrical varies from a red to a grayish color and involves the small cephalic and ventral and rarely the anterior portion of the caudal lobes. In the chronic cases the atelectatic lobes are grayish and firm. A motile gram negative rod, which grows rarely in the first twenty-four hours on plain agar and fails to ferment carbohydrates but turns milk alkaline and forms a brownish layer on potato, was isolated from these lesions. Several identical observations have been made before (Tartakowsky, ${ }^{3}$ Strada and Traina, ${ }^{4}$ Südmersen, ${ }^{5}$ Selter, ${ }^{6} \mathrm{M}^{\text {'Gowan, }}{ }^{7}$ Ferry $^{8}$ ) and since Keegan ${ }^{9}$ the publication of the paper by Smith. According to the experience of the writer, the Bact. bronchisepticum may be responsible for latent chronic focal lesions in the lungs, and although it may be demonstrated as a common saprophyte of the sinuses and the respiratory tree in certain guinea pig populations (M'Gowan), the bacterium is rarely associated with spontaneous epidemic respiratory infections. The report of Raebiger ${ }^{\text {I0 }}$ that a guinea pig breeder lost during the winter of I9I9 approximately twenty-one hundred animals from Bact. bronchisepticum pneumonia deserves further confirmation. In the light of recent studies, it is evident that the significance of this organism as a factor in such a destructive epidemic may have been overestimated. On the other hand, the relationship to purulent bronchitis is well established (Keegan and others).

\section{INFECTIONS WITH FRIEDLÄNDER'S BACILLUS}

Much more important as the primary cause of epidemic pneumonia and uterine infections is the Friedländer's bacillus. Branch ${ }^{\text {II }}$ has recently reviewed the literature and reports fifty-six deaths attributed to this organism. Schoenholz and Freedlander (unpublished observations) studied seventeen deaths in a small group of guinea pigs purchased from a breeder in California. The animals either died acutely or more often became emaciated, wheezed, and developed a sticky purulent nasal discharge which is, according to Branch, associated with a sinusitis. Death followed the intraperitoneal injection of tuberculin in two animals which had been chosen as controls in a hypersensitiveness experiment. Contact infections were not observed, and the

I Chevrel, F., et al.: loc. cit.

${ }^{2}$ Smith, T.: loc. cit.

3 Tartakowsky, M. G.: Arch. biol. nauk. S.-Petersb., 6, 263. г 897-98.

${ }_{4}$ Strada, F., and Traina, R.: Centralbl. f. Bakteriol., Abt. I, 28, 635. 1900.

5 Südmersen, H. J.: ibid., 38, 59 I and 7I3. I905.

${ }^{6}$ Selter, H.: loc. cit.

7 M'Gowan, M.: J. Path. EN Bact., I5, 372. r910-I I.

${ }^{8}$ Ferry, U. S.: Am.-Vet. Rev., 37, 499. I9Io; J. Path. \& Bact., 18, 445. I9I3-14.

${ }^{9}$ Keegan, J. J.: loc. cil.

ro Raebiger, M.: op. cit., roo, 85. Hannover, 1923.

"Branch, A.: boc. cit. 
fatalities developed among the stock originally purchased and frequently followed experimental inoculations.

The autopsy findings consisted of discrete or confluent lobular patches of pneumonia regularly accompanied by a sticky, gelatinous serosanguineous pleuritic exudate or extensive adhesions. In the majority of cases the bronchi were filled with a gummy secretion. A pericarditis was also recorded. Branch found a sinusitis in 60 per cent, otitis media in 66 per cent, pneumonia in 46 per cent, and pleurisy in 20 per cent of the autopsies. Extradural abscesses, cellulitis, mammary abscesses, peritonitis, serous arthritis, and metritis are relatively infrequent, but Holman ${ }^{\mathrm{I}}$ found the Friedländer's bacillus seventeen times as the cause of endometritis. The causative organism may be cultured from the blood in a high percentage of the fatal cases, and it is always present in the lesions just mentioned. It differs in no way from the typical Friedländer strains, and some cultures belong to the serological type B established by Julianelle. ${ }^{2}$ When grown under proper conditions it produces two types of substances which are toxic for rabbits: one is "heat labile" and the other "heat stable" (Freedlander and Schoenholz). ${ }^{3}$ The virulence for mice is very high; five to twenty bacteria injected intraperitoneally may kill in from twenty-four to thirty-six hours. Slightly larger doses are required to infect guinea pigs by the intraperitoneal route. Subcutaneous injections of o.oI cc. of a twenty-four hour broth culture may produce local necrosis, emaciation, and death within eight days. The intranasal instillation $010.5 \mathrm{cc}$. of an eight-hour broth culture may be followed by death on the third to the fifth day. Extensive pneumonia and pericarditis and enlargement of the spleen are constant findings. The natural epidemiology is unknown. In the outbreak observed by Schoenholz and Freedlander, contributory factors which lowered the resistance played an important rôle.

\section{PASTEURELLOSIS}

Pasteurellosis as a disease of guinea pigs has been reported from Germany by Busson, ${ }^{4}$ by 'Schmidt-Hoensdorf, ${ }^{5}$ and by Freund $;{ }^{6}$ from Italy by Carpano $;^{7}$ from France by Phisalix, ${ }^{8}$ and from Canada by Reed and Ettinger. ${ }^{9}$ Freund described an epidemic in which one hundred and forty-one guinea pigs succumbed in the course of two months. The anatomical lesions resembled those provoked by the pneumococcus; however, the fibrinous exudations on the serous membranes were frequently accompanied by hemorrhages of varying degrees and by a marked enlargement of the cervical and tracheal lymph nodes. Freund found at the end of the epidemic 6 per cent carriers (nasal excretions and tracheal lymph nodes) and during the endemic period only one in thirty-four animals. During the epidemic the distribution of the virus was demonstrated by cultures of the air, by exposure of mice to the emanations of infected guinea pigs, and by contact infections. The virulence of the epidemic strains was very high while the pathogenicity of the carrier strains was irregular and strikingly low. The

${ }^{\mathrm{I}}$ Holman, W. L.: loc. cit.

${ }^{2}$ Julianelle, L. A.: J. Exper. Med., 44, I 13 and 683. 1926.

${ }^{3}$ Freedlander, B. L., and Schoenholz, P.: Proc. Soc. Exper. Biol. \&o Med., 24, 555. 1927.

4 Busson, B.: loc. cit.

${ }^{5}$ Schmidt-Hoensdorf, F.: Arch.f. wiss. u. prakt. Tierheilk., 53, 265. 1925.

${ }^{6}$ Freund, R.: loc. cit.

${ }^{7}$ Carpano, M.: Moderno zooiatro, No. 7. 1915.

${ }^{8}$ Phisalix, C.: Compt. rend. Soc. de biol., ro, s.v. 76 I. I 898.

${ }^{9}$ Reed, G. B., and Ettinger, G. H.: J. Infect. Dis., 4I, 439. 1927. 
possible occurrence of variants of lost pathogenicity was not considered nor investigated. These findings are fully in harmony with those made by Meyer and Batchelder in the course of an investigation which dealt with a similar infection of wild rats. In this connection it is recalled that the epidemic "body strain" was fully capable of causing active, rapidly fatal infections without any contributory factors which would increase the susceptibility of the host. As soon as the individuals of low resistance had been weeded out, the invading organism adjusted itself to the rat population on a lowered level of virulence or disappeared entirely from the nasal passages and the bronchial tree. It seems probable that the observations reported by Freund on guinea pigs are entirely analogous. A long-continued and extensive prevalence of highly virulent pasteurella organisms produced contact infections, a large number of fatalities but few cases of rhinitis and carriers. The experiments of Uchida, which are used by Freund to support his contention that thermal or other factors were necessary to provoke the pasteurella epidemic observed by him, were made with cultures and not with "body" strains and on guinea pigs of an infected population. It is therefore reasonable to suppose that in the pasteurella infections observed by Freund the microbic virulence played a greater rôle than the host factors.

\section{STREPTOCOCCUS INFECTIONS}

In 1907 Boxmeyer $^{\mathrm{I}}$ noted in a caviery belonging to a western antitoxin manufacturer three thousand guinea pigs with enlarged, abscessed lymph nodes of the neck and axilla. He isolated from the lesions a $\beta$ hemolytic streptococcus. The disease, known as "epizoötic lymph adenitis" and in all probability a wound infection, was spread by the pus that escaped when the abscesses ruptured externally or when the virus entered a new host through small abrasions of the upper digestive tract. An identical disease was seen by the writer in I9I 7 in twenty-two guinea pigs of a shipment from a dealer in California. The $\beta$ hemolytic streptococci resembled Str. pyogenes in their carbohydrate reactions; agglutination tests were not made. Young and full-grown guinea pigs were infected by contact through bite wounds. According to Altana, ${ }^{2}$ Kaspar and Kern, ${ }^{3}$ Micrococcus tetragenus may cause a similar contagious disease among laboratory guinea pigs.

\section{TUBERCLE-BACILLUS INFECTIONS}

The significant observations of R. Kocht that guinea pigs exposed in the same room with tuberculous animals for a longer period than four months may not infrequently develop spontaneous tuberculosis have not been adequately appreciated by laboratory workers. Römer, ${ }^{5}$ Roos, ${ }^{6}$ Distaso, ${ }^{7}$ and in particular Feyerabend ${ }^{5}$ have reported isolated spontaneous cases or group infections with the tubercle ba-

${ }^{x}$ Boxmeyer, C. H.: J. Infect. Dis., 4, 657. 1907.

${ }^{2}$ Altana, G.: Centralbl.f. Bakteriol., Orig. I, 48, 42, I909.

${ }^{3}$ Kaspar, F., and Kern, W.: loc. cit.

4 Koch, R.: Gesammelte Werke von R. Koch, r, 512. Leipzig, I9r2.

${ }^{5}$ See Steinmetz, M., and Lerche, P.: in op. cit., roo, 127. Hannover, 1923.

${ }^{6}$ Raebiger, M., and Lerche, M.: op. cit., 21, 687 and 688. 1926.

7 Distaso, A.: Compt. rend. Soc. de biol., 79, 1 r9. 19 r6.

${ }^{8}$ Feyerabend, O.: Beitr. z. Klin. d. Tuberk., 39, I. I9r3. 
cillus. In a shipment of fifty animals Feyerabend found twelve with tuberculosis. From the epidemiological investigations it must be concluded that the disease was contracted from a tuberculous goat. The tubercle bacillus responsible was in all probability a bovine type. Perla, ${ }^{1}$ in a series of very fascinating observations and experiments, has shown that normal guinea pigs exposed to tuberculous cagemates readily contract the same disease. In fact, it was noted that spontaneous tuberculosis acquired from infected cagemates has with few exceptions the character of an infection which has entered by way of the digestive tract, while guinea pigs exposed to tuberculous animals in the same room but not in the same cage may acquire tuberculosis which has the character of a bronchiogenic infection. The sources of the disease are probably the tubercle bacilli which are discharged in the feces and in the urine when the malady has reached its terminal stage.

\section{PROTOZOAN INFECTIONS}

The pathogenic rôle of a number of intestinal protozoan parasites to certain diseases of the guinea pig is not entirely clear. There is no doubt that occasionally emaciated, slightly anemic cadavers may be dissected and thoroughly studied bacteriologically without finding that a microbial cause can be detected. In these cases the writer has always found the inflamed intestinal tube engorged either with Coccidia or Trichomonas or Balantidium. Mostly young animals are the victims, and it seems probable that the protozoa have some relationship to the enteritis and to the emaciation. Strada and Traina ${ }^{2}$ and later Bugge and Heinke ${ }^{3}$ found 73 per cent of their stock animals infected with Coccidia, and they ascribed this prevalence accompanied by sickness and the occasional death to a reduced resistance induced by an inadequate diet. The views are unanimous that in adult animals the infection is without any consequences. Sheather ${ }^{4}$ has described the parasite and given it the name Eimeria caviae. He found that the various stages resembled those of the Coccidia of the rabbit, but occurred only in the large intestines, particularly the proximal portion of the large intestines, and not, as Strada and Traina stated, in the liver. In his feeding experiments one fatal infection resulted on the fourteenth day after the administration of the oöcysts.

Trichomonas infections are very common, and Perroncito, Galli-Valerio, ${ }^{5}$ assumed that they were the inciting cause of a great mortality among the guinea pigs of the Hygiene Institute at Lausanne. However, there is ample evidence that perfectly healthy animals may show ulcerations of the large intestine and cecum with definite invasion of the tissues by the flagellates. Whether these lesions are primarily caused by the Trichomonas has yet to be determined. Entamoeba cobayae and $B a$ lantidium caviae frequently found in the inflamed digestive tract are considered harmless commensals. Klossiella cobayae in the kidney tubules has been described by Pearce. ${ }^{6}$

${ }^{x}$ Perla, D.: J. Exper. Med., 45, 209 and 1025. 1927.

${ }^{2}$ Strada, F., and Traina, R.: loc. cit.

3 Bugge, and Heinke: Deutsche tieräratl. Wchnschr., 29, 4I. I92 I.

${ }^{4}$ Sheather, A. L.: J. Compt. Path. \& Therap., 36-37, 243. 1923-24.

5 Galli-Valerio, B.: Centralbl. f. Bakteriol., Orig. I, 27, 305. I900.

${ }^{6}$ Pearce, L.: J. Exper. Med., 23, 431. I916. 


\section{INFECTIONS WITH FILTERABLE VIRUSES}

Diseases due to filterable viruses have been reported by Petrie and O'Brien, ${ }^{\mathrm{I}}$ Römer, ${ }^{2}$ De Gasperi and Sangiorgi, ${ }^{3}$ and Berge. ${ }^{4}$ The outstanding feature of these infections are nervous symptoms and paralysis. Quite recently Cole and Kuttner ${ }^{5}$ proved that the peculiar acidophilic nuclear changes, which were noted by Jackson ${ }^{6}$ several years ago in the swollen epithelial cells of the salivary glands of 84 per cent of full-grown guinea pigs, were produced by a filterable agent. Transmissions were in part successful; the virus reproduced itself and could be preserved in glycerol for twenty-eight days. The lesions which followed intracerebral injection of the infective agent resembled those produced by Römer with his virus and the possibility that it is identical with the Cole and Kuttner filter-passer should be borne in mind.

\section{DISEASES OF THE RABBIT}

Rabbits are generally considered unsatisfactory for experimental purposes owing to their liability to a number of communicable diseases. In the laboratory or in the rabbitry two infections and one ectoparasitic invasion-snuffles with its complications, coccidiosis and scabies - are probably the most troublesome and costly epidemic-producing maladies with which every worker becomes sooner or later acquainted (Sustmann). ${ }^{7}$ Intestinal infections such as paratyphoid, pseudotuberculosis, etc., are less frequent than in the guinea pig. Various other diseases (spontaneous spirochetosis, encephalitis due to a protozoan parasite, a filterable virus infection, etc.), which may invalidate experiments, have been recognized. The literature is extensive but scattered and confused. ${ }^{8}$ It is evident that multiple infections have frequently not been identified, and the uncertainty regarding the etiological relationship of various bacteria to some diseases persists even in the most recent literature. Several excellent studies have no doubt clarified some of the disputed questions, but even with the knowledge now available it would be unwise to compare the rabbit epidemics of one country or even of one laboratory with those of another. It is desirable that in the future every outbreak be carefully investigated and the findings made accessible for critical analysis. In order to facilitate such an undertaking, the essential facts concerning the communicable diseases of rabbits are set forth in the paragraphs which follow.

\section{RESPIRATORY INFECTIONS}

Respiratory infections are most common among rabbits kept at commercial rabbitries or at laboratories. As early as I $89 \mathrm{I}$ Beck, ${ }^{9}$ and later Kurita, ${ }^{\mathrm{x}}$ Kraus, ${ }^{11}$ Roger

${ }^{2}$ Petrie, G. F., and O'Brien, R. A.: loc. cit.

${ }^{2}$ Römer, P. H.: Deutsche med. Welhnschr., 57, 2685. I910.

3 de Gasperi, F., and Sangiorgi, G.: Centralbl.f. Bakteriol., Orig. I, 7i, 257. 1913.

4 Berge, L.: Deutsche tierürstl. Wchnschr., 32, г го. 1924.

5 Cole, R., and Kuttner, G.: J. Exper. Med., 44, 855. 1926.

${ }^{6}$ Jackson, L.: J. Infect. Dis., 26, 347. 1920.

${ }^{7}$ Sustmann, H.: Deutsche tierärstl. Wchnschr., 29, 247. т921.

${ }^{8}$ See Seifried, O.: "Die wichtigsten Krankheiten des Kaninchen," Frgehn. d. allg. Palh.u. Anat., Abt. I, 22, 432-589. 1927.

9 Beck, M.: Ztschr. f. IIyg. u. Infektionskrankh., 15, 363. I 893 .

ro Kurita, S.: Centralbl. f. Bakteriol., 49, 508. 1909.

"n Kraus, R.: Ztschr.f. IIyg. u. Infektionskrankh., 24, 396. 1897. 
and Weil (I907), ${ }^{\mathrm{r}}$ Volk, ${ }^{2}$ Schwer, ${ }^{3}$ Selter, ${ }^{4}$ Südmersen, ${ }^{5}$ Koppanyi, ${ }^{6}$ Raebiger, ${ }^{7}$ Eberth and Mandry, ${ }^{8}$ Sustmann, ${ }^{9}$ Davis, ${ }^{\text {I0 }}$ Saelhof, ${ }^{11}$ and Baudet ${ }^{12}$ described under different names spontaneous fatal diseases characterized by a serofibrinous pleurisy frequently associated by pericarditis and pneumonia. Clinically the animals show in the acute stage a mucopurulent nasal discharge, dyspnea, loss of appetite, weakness, and emaciation; in the chronic stage, a purulent discharge, coughing, sneezing, and emaciation. A gram negative non-motile coccobacillus, minutely described by Smith ${ }^{13}$ in I886 and later designated by Lignières ${ }^{\mathrm{I}}$ as Pasteurella, was considered by many authors as the bacterial incitant of the epidemic respiratory disease commonly reported as "rabbit septicemia." Recently Ferry and Hoskins ${ }^{15}$ have tried to differentiate etiologically the chronic rhinitis or commonly called "snuffles" from the epidemic pleuropneumonia and septicemia. According to their studies, the ordinary form of snuffles is caused by Bact. bronchisepticum while the fatal cases of septicemic character are due to Pasteurella or Bacterium lepisepticum. Although McCartney and Olitsk ${ }^{16}$ were unable to confirm this differentiation, they expressed the view that typical snuffles is merely an acute exacerbation of a chronic paranasal sinusitis (Ioo per cent in snuffles, 9 per cent in healthy rabbits [McCartney and Olitsky]; 68 per cent and I4 per cent [Tanaka]) ${ }^{17}$ due to Bact. lepisepticum and Bact.bronchisepticum, and that these organisms are not the sole inciting cause. Both workers also emphasize the difficulties which are encountered in the attempts to reproduce the clinical picture of snuffles on laboratory-stock rabbits on account of the pre-existing infection. The resemblance of the rabbit disease to human influenza has prompted Tanaka ${ }^{17}$ in Chicago to search for a filterable virus with negative results. However, he isolated in the majority of cases from the nasal secretions, blood, lung exudates of rabbits with snuffles Bact.lepisepticum, and in fewer cases Bact. bronchisepticum. Furthermore, the etiological relationship of the isolated organisms was clearly indicated by the positive agglutination reactions which were secured with the sera of the diseased rabbits, and finally he was able to produce experimentally snuffles identical with natural snuffles by intranasal inoculations of rabbits with strains of either Bact. lepisepticum or Bact. bronchisepticum. The observations of Tanaka are in harmony with those of Bull and

I Roger, H., and Weil, E.: Arch. méd. expér. et d'anat. path., 13, 545. I901.

${ }^{2}$ Volk, R.: Centralbl.f. Bakteriol., Orig. I, 31, 177. 1902.

3 Schwer: ibid., 33, 4I. I903.

4 Selter, H.: ibid., 41, 432. I906.

5 Südmersen, H. J.: ibid., 38, 343, 59 I, 713. I905.

${ }^{6}$ Koppanyi, E.: Ztschr.f. Tiermed., I I, 429. I907.

7 Raebiger, M.: Ber. d. bakt. Inst. Halle. I907-10.

${ }^{8}$ Eberth, J. C., and Mandry, A.: Fortschr. d. Med., 8, 547. I89o.

9 Sustmann, H.: op. cit., 23, 4I. I9I5.

10 Davis, J. D.: J. Infect. Dis., 21, 314. I917.

"Saelhof, C. C.: ibid., 28, 374. I92 1.

12 Baudet, E. A. R. F.: Tijdschr. v. diergeneesk., 50, 769. I923.

${ }_{13}$ Smith, T.: J. Comp. Med. Eo Surg., 8, 24. I 887.

${ }^{14}$ Lignières, J.: Ann. de l'Inst. Pasteur, 15, 734. I901; Bull. de l'Inst. Pasteur, 5, 249. 1907.

is Ferry, N. S., and Hoskins, H. P.: J. Lab. Eo Clin. Med., 5, 3II. Igig-20.

${ }^{16}$ McCartney, J. E., and Olitsky, P. K.: J. Exper. Med., 38, 591. I923.

${ }_{17}$ Tanaka, A.: J. Infect. Dis., 38, 389, 409. I926. 
McKee. ${ }^{\text {I }}$ These workers found in Baltimore that certain fatal cases of acute snuffles were invariably associated with Bact. bronchisepticum; the Pasteurella organism could not be demonstrated. Since, however, the same organism has been isolated from respiratory infections of dogs, guinea pigs, and white rats, its etiological relationship to acute and chronic snuffles, pleuropneumonia, and septicemia remains obscure.

In subjecting the respiratory infections of rabbits to a detailed experimental and carefully controlled study on specially bred laboratory rabbits with a known history and also on the stock of several commercial rabbitries in New York State, Webster and Burn have shown that Bact. lepisepticum is the primary etiological factor in snuffles and its complications. From personal experience extending over many years, the writer finds himself in agreement with the main observations and conclusions reported by Webster $^{2}$ in a series of valuable papers.

An examination of the nasal secretion of a rabbit population in which snuffles is frequently encountered shows the following bacteria in the order of their frequency: Micrococcus catarrhalis group, So per cent; Bact. lepisepticum, 70 to Ioo per cent; gram negative cocci and Bact. bronchisepticum, 40 per cent; staphylococci, streptococci, and various intestinal bacilli, ro per cent. Tanaka ${ }^{3}$ arranges the varieties in the following order: Micrococcus catarrhalis, Staphylococcus albus, gram negative cocci, Bact. lepisepticum, unidentified organisms, Bact. bronchisepticum, streptococci. In this connection it is important to emphasize that the technique given by Webster must be rigidly followed. A 5 per cent defibrinated rabbit-blood agar $(\mathrm{pH} 7.5$ ) poured shortly before use will give dependable information. The onset of spontaneous snuffles is preceded by the appearance in the nasal passages of Bact. lepisepticum, and during the active stages this organism invariably predominates. Recovery is accompanied by a diminution, the final disappearance from the nasal passages, or the persistence in the paranasal sinuses. The application of a drop of I per cent silver nitrate solution (Freund) ${ }^{4}$ or a $0 . \mathrm{I}-0.3$ per cent brilliant-green solution (Bull and Bailey) 5 may provoke a relapse of clinical snuffles; even pneumococci and Bact.lepisepticum may be isolated in almost pure culture from the lesions. Various other experimental procedures (as, for example, the injection of putrid material [Koch and Gaffky]), ${ }^{6}$ the inoculations of vaccines, toxins, Treponema pallidum, tumor specimens, operations, bleeding, etc. (Webster, ${ }^{7}$ Tanaka), may impair the resistance to spontaneous snuffles.

Without extended infection experiments conducted on rabbits bred with a view to protection from exposure to snuffles in any of its stages, Webster would have been unable to correlate the various clinical and anatomical manifestations which are controlled by the susceptibility of the rabbits and the pathogenicity or the virulence of the different strains of Bact. lepisepticum. Among the many factors which apparently affect the "natural" resistance in a rabbit population the following are in all

I Bull, C.. G., and McKee, C. M.: Am. J.Hyg., 5, 530. I925.

${ }^{2}$ Webster, L. T.: J. Exper. Med., 39, 837, 843, 857. 1924; 40, I09, II 7. I924; 42, I. I925; 43, $55.5,573.1926$.

3 Tanaka, A.: loc. cit.

4 Freund, R.: loc. cit.

5 Bull, C. G., and Bailey, G. H.: Proc. Soc. Exper. Biol. E Med., 24, 183. 1926.

"Koch, R., and Gaffky, G.: Mitl. d. kaiserl. Gesundheitsumte, I, So. I 88 I.

7 Vebster, L. T.: J. Exper. Med., 40, III. I924. 
probability concerned: exposure and temperature fluctuations (Freund); seasonal influences ([Webster]; clinical snuffles in summer 20 per cent, in September and October 60 per cent, followed by a decline to 35 per cent and a second rise in April to 50 per cent); diet; racial, hereditary (Chinchilla Giants showed greater snuffles incidence and mortality per cent than Blue Beverns), ${ }^{\prime}$ and innate non-specific indivirlual differences. Variations in the virulence of different strains of Bact. lepisepticum are well known, but it is interesting to note that, according to Webster, highly virulent D strains (De Kruif classification) ${ }^{2}$ are incapable of vegetating indefinitely in the nasal passages of the rabbits surviving an epidemic, while less virulent, mucoid, and $G$ variants are capable of persisting indefinitely in the nasal passages. The highly virulent strains produce either rapidly fatal infections in susceptible rabbits, or a "healthy" carrier condition with definite inflammatory processes of the nasal mucosa develops in the relatively resistant animals, or they are replaced by variants (type $G$ ) of very low pathogenicity and finally disappear from the respiratory tract. On account of the very low carrier incidence the highly virulent strains are only encountered during very destructive epidemics (Glaue) ${ }^{3}$ or in isolated cases. Under the prevailing conditions and those usually met in a rabbit population the moderately virulent type D (Tanaka) or mucoid variants of moderate and uniform virulence constitute the carrier strains (Webster, Smith). ${ }^{4}$

The question naturally arises, How do cases of snuffles and pneumonia develop? Webster presents convincing evidence that the individual rabbits exhibit a different response to the presence of Bact. lepisepticum in a stockroom. Usually three groups of animals can be distinguished: (I) non-infected, resistant animals; (2) chronic "healthy" carriers; (3) cases with local infections, such as snuffles, parasinusitis (McCartney and Olitsky, ${ }^{5}$ Tanaka ${ }^{6}$ ), otitis media (Smith and Webster), ${ }^{7}$ meningitis (Meyer) ${ }^{8}$ subcutaneous abscesses (Schimmelbusch and Mülısam, ${ }^{9}$ Laven, ${ }^{\text {I0 }}$ Davis, ${ }^{11}$ Tanaka, ${ }^{12}$ Smith $^{13}$ ), pleuropneumonia, and septicemia cases (Davis, Webster, Baudet, and others). Although the percentages of these groups may remain constant in a rabbit population, it is again recalled that various accessory factors (seasonal changes, injection of harmful substances) may lead to an increase in the percentage of snuffles and pneumonia cases and a corresponding decrease in carriers and resistant animals. Sporadic cases of rabbit septicemia are probably the sequelae of such a course of events. When, on the other hand, a high incidence of snuffles and a corresponding

I Webster, L. T.: ibid., 45, 529. 1927.

${ }^{2}$ de Kruif, P. H.: ibid., 33, 773. 1921.

3 Glaue: Centralbl.f. Bakteriol., 60, 176.1910.

${ }^{4}$ Smith, D. T.: Jour. Expcr. Mled., 45, 553. 1927.

${ }^{5}$ McCartney, J. E., and Olitsky, P. K.: loc. cit.

${ }^{6}$ Tanaka, A.: loc. cit.

${ }^{7}$ Smith, D. T., and Webster, L. T.: J. Expcr. MICd., 4I, 275. I925.

${ }^{8}$ Meyer, K. F.: unpublished observations.

${ }^{9}$ Schimmelbusch, C., and Mühsam: Arch.f. klin. Chir., 52, 564. I 896.

xo Laven, L.: Centralbl.f. Baktcriol., Orig. I, 54, 97 . I9ro.

II Davis, J. D.: J. Infect. Dis., I2, 42. I9I3.

r2 Tanaka, A.: loc. cit.

${ }^{13}$ Smith, D. T.: loc. cit. 
high mortality develops among a stock of laboratory animals, it is reasonable to suspect either some environmental change which affected the entire population or the introduction of a very virulent strain (type $\mathrm{D}$ ) or the sudden and wide distribution of virulent organisms among a susceptible population hitherto unexposed.

These interpretations have been tested by Webster and Burn, ${ }^{\mathrm{I}}$ who have succeeded in maintaining a stock of rabbits free from Bact. lepiseptictm infection by proper attention to the "natural" resistance of the animals and by measures which minimized the available dosage of the causative organisms.

In the course of this review special emphasis has been placed on the relationship of Bact. lcpisepticum to snuffles and pneumonia since the studies of Webster permit of no other interpretation. However, it is not unlikely that in some localities Bact. bronchisepticum may assume the same rôle as the Pasteurella organism. Tanaka and Webster produced experimentally with this organism both healthy "carriers" and temporary snuffles, and several cases of pneumonia and otitis media. Sustmann ${ }^{2}$ saw cases of unresolved pneumonias in chronic rabbit septicemia probably due to the same organism. Of interest, however, is the fact that Bact. bronchisepticum is rarely, if ever, found in the pleuropneumonic cases, and certain focalized complications such as otitis media (Smith and Webster) ${ }^{3}$ and subcutaneous abscesses (Tanaka, Meyer). Furthermore, the writer has never isolated a Bact. bronchisepticum from the many cases of pleuropneumonia or rabbit septicemia which developed in the course of an experimental study of typhoid carriers (I9I7-I920) irrespective of the fact that the carrier incidence of this organism was very high (plating of ground lung tissues gave frequently very high counts). ${ }^{+}$

A perusal of the various papers leaves no doubt that a variety of bacteria with bipolar granules and certain definite fermentative reactions have been isolated from cases of snuffles, pleuropneumonia cases, etc. Finer distinctions were not attempted until Webster and Burn ${ }^{5}$ in their papers on the biology of Bact. lcpisepticum showed that at least two types ("D" and "mucoid") with distinctive growth characteristics and antigenic properties may be found. No cross-agglutination between the "D" and "mucoid" types was demonstrable, and avirulent "G" variants were split off from the original strains. Aberrant types which differ, however, from the Webster types have also been isolated by Freund in Berlin. It is evident that the comparative studies must be continued in various parts of the world before one can consider the Bact. lepisepticum (or, according to Bergey, Pasteurella cuniculicida) a host-specific micro-organism.

The autopsy findings of fatal septicemic cases are monotonously uniform. Detailed description may be found in the papers by Smith, ${ }^{6}$ Davis, ${ }^{7}$ Saelhof, ${ }^{8}$ Tanaka, ${ }^{9}$ Webster, and others. Aside from the local purulent inflammatory lesions in the nasal passages, the trachea, and the bronchial tree, confluent areas of bronchopneumonia sometimes including whole

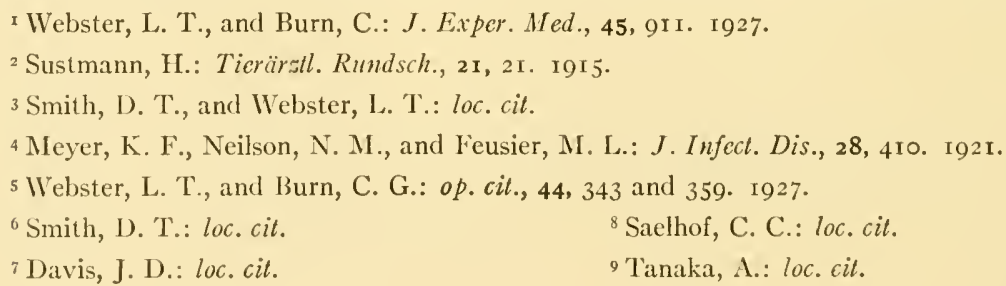


lobes of both lungs with a definite pleural exudate are regularly observed. The pericardium is often involved, but the spleen is rarely enlarged. Webster ${ }^{x}$ divides the pneumonic processes into: (I) an acute, diffuse form with subpleural and perivascular deposition of the exudate; (2) lobar; (3) pleuro- and (4) abscessive form, and concludes that the usual portal of entry of the organism into the lung is by way of the bloodstream. A different view is held by Tanaka. ${ }^{2}$ Based on a comparative study of the morbid lesions produced by Bact. lepisepticum and by Bact. bronchisepticum, he found that suppurative inflammation, bronchopneumonia, purulent pleurisy, and marked lymphangitis are most prominent in the former, while hemorrhage and edema are most marked in the latter. Although he presents neither histopathological nor experimental evidence, he ventures the conclusion that in pneumonia Bact. lepiseplicum may invade the lungs either through the lymphatics or through the respiratory passages. The pathogenesis of the subcutaneous abscesses has not as yet been investigated.

Snuffles in rabbits is a preventable disease not by means of vaccines, serum (Raebiger), ${ }^{3}$ or local treatment (Tanaka), ${ }^{4}$ but by selecting a resistant breed of rabbits, by enhancing their resistance by a careful supervision of the environmental factors, and by systematic elimination of carriers and clinical cases. Under the usual laboratory conditions, which frequently require the purchases of rabbits from unknown sources, the following practice is effective and in the end economical. Isolate the incoming rabbits in a room, and preferably in separate cages. Examine the nasal passages for Bact. lepisepticum. On the third day place one drop of I per cent silver nitrate in each nostril; culture the nasal passages again on the sixth day. Eliminate the carriers and clinical cases. Observe the entire group for fifteen days and then finally segregate in a carrier and in a clean group, which are kept in separate rooms; simple precautions are taken to minimize the transmission of organisms from one room to another. An effort should be made to reduce the carriers in the available rabbit stock by using them in sacrifice experiments. For physiological studies such animals are, however, unsuitable (W. C. Alvarez, unpublished observations).

Sporadic and occasionally benign epidemic infections due to the $B$. pseudotuberculosis (rodentium) have been reported (Messerschmidt and Keller, ${ }^{5}$ Römisch ${ }^{6}$ ). Contact transmissions are either infrequent or the various strains are strictly host specific. The writer placed several young rabbits in a cage with guinea pigs which had been infected with a rat strain of the pseudotuberculosis organism. Although the guinea pigs succumbed in the course of two weeks the rabbits remained well and revealed no lesions at autopsy. A detailed description of two interesting variants of the bacillus appeared in the paper by Römisch.

Isolated instances of spontaneous tuberculosis in rabbits have been reported by Strauss, ${ }^{7}$ Guérin,${ }^{8}$ Coulaud, ${ }^{9}$ and Perla, ${ }^{10}$ while an epizoötic of spontaneous tuberculosis of the bovine type on a rabbit farm was studied by Rothe. ${ }^{\text {II }}$

${ }^{x}$ Webster, L. T.: J. Exper. Med., 43, 555. I926.

${ }^{2}$ Tanaka, A.: loc. cit. $\quad{ }^{3}$ Raebiger, M.: loc. cit. $\quad{ }^{4}$ Tanaka, A.: op. cit., 39, 337. 1926.

${ }^{5}$ Messerschmidt, T., and Keller: Zischr. f. Hyg. u. Infektionskrankh., 77, 289. I9I4.

${ }^{6}$ Römisch: loc. cit.

${ }^{7}$ Strauss, I.: La Tuberculose et son bacille, p. 372. Paris, I895.

${ }^{8}$ Guérin, G.: Hyg. de la vian. et lait, 2, 7. Évreux. 1908.

${ }^{9}$ Coulaud, E.: Ann. de l'Inst. Pasteur, 38, 58г. I924.

זo Perla, D.: loc. cit. $\quad$ in Rothe, E.: Verüfentl. R. Koch Stift. Bekaempf. Tuberk., I, I. I913. 


\section{INTESTINAL INFECTIONS}

Owing to the fact that infection of the rabbit's bile ducts with coccidia leads to the formation of white or yellowish nodules or patches, this condition early attracted the attention of observers. As early as 1854 Kolliker noted that the oöcysts from the intestine of the rabbit were smaller than those from the liver. In fact, it appeared from an extensive series of tests conducted by Lucet, Reichenow, ${ }^{\mathrm{I}}$ and others that the rabbit is liable to infection with two distinct species of Eimeria. Waworuntu, ${ }^{2}$ who made a careful morphological and experimental investigation of the coccidia of the rabbit, finds that the small form, Eimeria perforans, has always ellipsoidal and often asymmetrical oöcysts, while the shape of the Eimeria stiedae is ovoid. The question of individuality of E. stiedae and E. perforans appears to have been placed beyond doubt by Pérard (1924) ${ }^{3}$ who has succeeded in obtaining pure strains of the two species. The former, fed to young specially reared rabbits, produces only liver lesions, which prove fatal to the young animals in twenty-eight to thirty days, while the latter gives rise only to an intestinal infection, which kills the rabbits in nine to fifteen days. He also describes a variety of the usual type, for which he proposes the name $E$. perforans var. magna. The forms found by Bruce 4 in acute and fatal enteritis of young rabbits are either a mixed infection or a distinct species. No final deductions can be drawn from his report. A complete description of the life-cycle of the rabbit coccidium may be found in a paper by Reich (I9I3). ${ }^{5}$

It is well knov'n that young rabbits are specially liable to infection, and they frequently die in large numbers from acute hepatitis, which is caused by the active multiplication of the coccidium in the biliary epithelium. When an outbreak occurs in epidemic form, there may be a high rate of mortality. If the animals survive the acute stage, the infection becomes of a chronic type and remains confined to certain areas of the liver. In very old infections the coccidia disappear from the liver tumors, which become fibrotic or even calcareous. The $E$. perforans remain confined to the small intestines, which in the course of the acute stage are highly inflamed. In both types of infection the virus is spread by oöcysts, which either escape in the intestines or are passed directly in the feces. Dissemination of very resistant oöcysts may occur after the death of the animals. Repeated attempts have been reported to treat coccidiosis chemotherapeutically. Ottolenghi and Pabis ${ }^{6}$ had good results with sodium antimonyltartrate (O.OI gm. subcutaneously). Preventive measures are, however, much more satisfactory. A laboratory stock of rabbits can be kept free from coccidiosis by a systematic examination of the feces of any does which are used for breeding purposes or animals which are brought in from outside. The cane-sugar flotation and concentration method developed by Sheather ${ }^{7}$ gives excellent results. In case the infection already exists among the stock animals, early separation of the young from

\footnotetext{
I Reichenow, E.: Prowasek's IIandb. d. Path. Protosocn, 3, I I36. I92 I.

${ }^{2}$ Waworuntu, F. K.: Procfschr. I'certsenijk Hoogesch. Utrecht, 1924.

3 Pérard, C.: Ann. de I'Inst. Paste:tr, 39, 953. 1925.

${ }_{4}$ Bruce, E. A.: J. Am. Vet. M. A., 55, 620. 1919.

5 Reich, F.: Arch.f. Protistenk., 28, 1. I913.

${ }^{6}$ Ottolenghi, D., and Pabis, E.: Centralbl.f. Bakteriol., 69, 538: 19г3.

7 Sheather, A. L.: J. Comp. Path. Eo Therap., 34, 71. 1923.
} 
their infected mothers is indicated. Frequent removal and disinfection of the feces has, as Pérard ${ }^{\mathrm{I}}$ states, great advantages since the oöcysts can be destroyed much more readily before they have sporulated.

The occurrence of spontaneous paratyphoid-B infections in rabbits has been reported by Uhlenhuth and Hübener, Litch and Meyer, ${ }^{2}$ Smith and Nelson, ${ }^{3}$ von Sarnowslii, ${ }^{4}$ and probably others. The anatomical lesions have been strikingly uniform: necrotic follicles in the appendix and on the Peyer's patches; firm, or pulpy engorged, congested spleen; necrotic foci in the liver; and occasionally areas of pneumonia. It is not unlikely that the pneumonia in the cases observed by Litch and Meyer was due to a superimposed infection with Bact. lepisepticum, although the paratyphoid bacilli were isolated from the consolidated areas in pure culture. The strains studied by Litch and Meyer and by Smith and Nelson are aertrycke types of the paratyphoid B group. Among three cultures submitted by Jordan 5 to a comparative analysis two were aertrycke types and one $B$. enteritidis. The latter was secured from the gall bladder of a rabbit that died after a subcutaneous inoculation with a bacillus of the hemorrhagic septicemia group. This observation, which indicates the occurrence of latent infections or carriers of the paratyphoid-enteritidis group just as noted for guinea pigs and mice, has recently been confirmed by Menten and Manning. ${ }^{6}$ In the course of a study of hyperglycemia, these two workers found that coincident with the glycemia 68 per cent of the rabbits harbored $B$. enteritidis in the macro- and microscopically affected organs. In a subsequent paper Menten ${ }^{7}$ was able to show that soluble toxic substances of the paratyphoid-enteritis organisms produce alterations of the blood sugar and hydropic degeneration in the islets of Langerhans which were identical with those seen in the natural infections.

Concerning the epidemiology of paratyphoid infections in the rabbit very little is known. Nelson and Smith suspect gray field mice as transmitting agencies. The strains isolated by Litch and Meyer regularly infected by oral administration of large doses (2,000,000,000 organisms); those of Menten and Manning gave irregular results on ingestion. Furthermore, the history of the small epidemic observed by the writer indicates a spread by contact, yet healthy rabbits placed in the cages with the experimentally fed rabbits, which excreted paratyphoid bacilli, remained well.

Epidemics in which the outstanding symptoms are swelling of the soft tissues of the upper and lower jaw, intense salivation, occasionally involvement of the external genitalia, diarrhea, and rapid wasting have been reported by Beattie, Yates and Donaldson, ${ }^{8}$ Sustmann, ${ }^{9}$ and Cameron and Williams. ${ }^{10}$ The morbid anatomy with its embolic

I Pérard, C.: op. cit., 38, 953. 1924; 39, 505. 1925.

${ }^{2}$ Litch, V., and Meyer, K. F.: J. Infect. Dis., 28, 27. I921.

${ }^{3}$ Smith, T., and Nelson, J. B.: op. cil., 45, 373. 1927.

${ }^{4}$ von Sarnowski, W.: Vel. Inaug. Disserlation. Hannover, IoIg.

s Jordan, E. O.: op. cit., 36, 317. I925.

${ }^{6}$ Menten, M. L., and Manning, H. M.: J. Infect. Dis., 37, 400. 1925.

7 Menten, M. L.: ibid., 38, 354. 1926.

${ }^{8}$ Beattie, Y. M., Yates, A. G., and Donaldson, R.: J. Palh. S Bact., 18, 34. I9I3-I4.

"Sustmann, H.: Deutsche tierïrall. W'chnschr., 27, 95. I9I9.

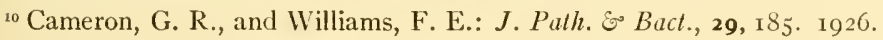


secondary foci in the internal organs and the micro-organisms which have been isolated resemble those generally attributed to B. necroseos or Schmorl's bacillus. However, some of the strains differ in the essential characteristics as referred to in the description given by Césari and Alleaux, ${ }^{1}$ Jensen, ${ }^{2}$ and others. The writer has seen sporadic cases of necrosis-bacillus infection in the buccal cavity of rabbits with injured gums due to the prolonged application of unsuitable gags. In several trials the infected sloughs were rubbed on the nostrils and gums of healthy rabbits with entirely negative results. It is questionable whether or not the bacillus is the causative agent of epidemics or sporadic cases with which it has been associated.

\section{MISCELLANEOUS INFECTIONS}

A number of years ago the discovery of a spontaneous venereal spirochetosis of the rabbit which affected, according to Arzt and Kerl, ${ }^{3} 26.9$ per cent of the animals of a rabbitry, attracted considerable attention. It was disconcerting to find that a spirochete resembled Treponema pallidum sufficiently closely to render a differential diagnosis difficult and to render doubtful a great mass of experimental work on the transmission of human syphilis to the rabbit. Fortunately, the many investigations in Europe (see literature cited by M. Zuelzer) ${ }^{4}$ and in the United States by Noguchis and by Warthin ${ }^{6}$ and his associates show that the invasion of Treponema cuniculi produces a superficial, condylomatous lesion, transmissible by contact, coitus, and inoculation, but no colonization in the internal organs. Rabbits which have recovered from the infection are still susceptible to inoculations with Treponema pallidum. Salvarsan preparations (neosalvarsan $0.25-0.35 \mathrm{gm}$. prodosi) have striking curative properties.

The recent controversial literature on experimental encephalitis in rabbits furnishes an excellent example of the many obstacles which still entangle the workers who are not acquainted with the spontaneous diseases of laboratory animals. Again it is shown that a latent, widespread parasite affecting a variety of species may be responsible for misleading conclusions. The accidental discovery of Encephalitosoon cuniculi must be credited to Bull (I9I7) ${ }^{7}$ and Ten Broeck ${ }^{8}$ who saw it in sections of the brain and the kidneys of rabbits used for experimental purposes. Wright and Craighead 9 observed in 1922 a form of paralysis in young rabbits and found that it was due to an organism which they thought might be an intermediate stage in the life-history of some protozoan parasite. It was noticed in most of the tissues of the body, but it was seen especially in the kidneys, urine, and the nerve cells of the

I Césari, E., and Alleaux, V.: Ann. de l'Inst. Pasteur, 26, 625. 1912.

${ }^{2}$ Jensen, C. O.: Handb. d. path. Mikroorg. (1st ed.), 2,693. 1903.

3 Arzt, L., and Kerl, W.: Wien. klin. Wchnschr., 27, 1053. 1914; Dermal. IVchnschr., 71, 1047. I920.

${ }_{4}$ Zuelzer, M.: Prowazek's Handb. d. path. Prolosoen, Lief. I I, 1). 1784.1924.

s Noguchi, H.: J. Exper. Med., 35, 391. 1922; J.A.M.1., 77, 2052. 1921. Also cf. chap. xxxvi by I)r. Noguchi in this volume.

${ }^{6}$ Warthin, A. S., Buftington, E., and Wanstrom, R. C.: J. Infect. Dis., 32. 315. 1923.

7 Bull, C. G.: J. Exper. Med., 15, 557. 1917.

${ }^{8}$ See Smith, T., and Florence, L.: ibid., 41, 25. 1925.

"Wright, J. H., and Craighearl, k. M.: ibid., 36, I35. 1922. 
spinal cord. The same disease of rabbits has been observed by Oliver ${ }^{1}$ and Twort. $^{2}$ In attempts to reproduce encephalitis lethargica and herpes, Doerr and Zdansky (r923) ${ }^{3}$ saw the protozoan-like parasite which occurred in the inoculated and in the uninoculated animals, and they thought that the bodies were probably parasites responsible for a disease of rabbits which was being confused with encephalitis lethargica. Later in the year Levaditi, Nicolau, and Schoen ${ }^{4}$ also recognized the organism as the cause of an encephalitis of rabbits which had no connection with the human disease. They have given a complete review of the subject and a detailed description of the organism to which they gave the name of Encephalitozoon cuniculi. Furthermore, Levaditi and his associates were able to inoculate the parasite in rabbits, rats, mice, and dogs, and they demonstrated the infectivity of the urine. Since then excellent descriptions have been given by Cowdry and Nicholson, ${ }^{5}$ Da Fano, ${ }^{6}$ Goodpasture, ${ }^{7}$ and others. Smith and Florence ${ }^{8}$ found it associated as a nest infection in a spontaneous epidemic of nephritis among young rabbits. The lesions consist of meningitis of the cortex and septa of the brain, perivascular infiltration of the brain, nodules composed of centrally necrotized cells, and marked changes in the infected epithelium of the renal tubules, liver, and spleen. The parasites are either scattered or inclosed in many so-called "cysts." It is primarily a parasite of the kidneys and is distributed through the bloodstream to all parts of the body. Levaditi and his associates describe sporulating stages (pansporoblasts) and conclude that the parasite is a microsporidium in spite of the fact that these parasites have never been found in warm-blooded animals. The evidence is not conclusive, and it is not unlikely that the organism is not even a protozoon. Spontaneous encephalitis and nephritis is frequent in American, European, Chinese, and Japanese rabbits; but, according to Cowdry, ${ }^{9}$ it does not exist to the same degree in rabbits from certain other localities. Thus far it has not been reported in tropical climates or south of the Equator.

In the course of a series of unsuccessful attempts to reproduce experimental rheumatic fever in rabbits, Miller, Andrewes, and Swift ${ }^{10}$ recovered a virus, which is probably a parasite of the domestic rabbit. It produces an acute orchitis under experimental conditions and is readily propagated from rabbit to rabbit. The natural course of the infection is unknown.

Ectoparasitic diseases due various mites, Notoëdres cuniculi and Dermatocoptes (Psoroptes) cuniculi, may be the cause of sporadic deaths among rabbits. The most

r Oliver, J.: J. Infect. Dis., 3о, 9г. 1922.

'Twort, C. C.: Vet. J., 78, I94. 1922.

${ }^{3}$ Doerr, R., and Zdansky, E.: Schweis. med. Wchnschr., 4, 349, 1189. 1923; Ztschr. f. IIyg. $u$. Infektionskrankh., 101, 239. 1923.

${ }^{4}$ Levaditi, C., Nicolau, S., and Schoen, R.: Ann. de l'Inst. Pasteur, 38, 651. 1924.

${ }^{5}$ Cowdry, E. V., and Nicholson, F. M.: J.A.II.A., 82, 5+5. 192.4.

${ }^{6}$ da Fano, C.: J. Path. \& Bact., 27, 333. 1924.

${ }^{7}$ Goodpasture, E. W.: J. Infect. Dis., 34, 428. 1924. $\quad{ }^{8}$ Smith, T., and Florence, L.: loc. cit.

${ }^{9}$ Cowdry, E. V.: J. Exper. Hed., 43, 725. 1926.

${ }^{10}$ Miller, C. P., Andrewes, C. H., and Swift, H. F.: ibid., 40, 773. 192.4. See also ibid., p. 789. 1924; 38, 673. 1923; and 39, 777.1924 . 
common localization of one of the parasites (Dermatocoptes) is the external ear in which a severe inflammatory process sometimes leads to an otitis media and then to a purulent meningitis. The disease is readily transmitted by contact but can, according to Gmeiner, ${ }^{1}$ be easily cured by removal into coal oil of the scaly crusts which are teeming with the insects and by repeated painting of the denuded skin, fissures, and folds of the external ear with oil of caraway (one part) in almond oil (ten parts), or tincture of larkspur seed (one part) and 90 per cent ethyl alcohol (three parts).

From the published records one must conclude that the rabbit is heir to many other spontaneous diseases, but since it is not unlikely that some of them are only variations of the maladies already described, a detailed account is omitted.

\section{DISEASES OF MICE}

White mice are used extensively as experimental animals. Despite this fact, very little is known regarding the normal physiology and pathology of this rodent. Only quite recently $\mathrm{Knorr}^{2}$ has pointed out that this animal may be the source of many errors (irregular temperature reaction, delay or inhibition of tetanic symptoms, etc., due to starvation). Equally incomplete has been the knowledge regarding its communicable diseases. However, the fascinating studies in the field of experimental epidemiology conducted by Flexner, ${ }^{3}$ Amoss, ${ }^{4}$ Webster, ${ }^{5}$ Pritchett, ${ }^{6}$ Topley and his associates, ${ }^{7}$ Neufeld and his co-workers, ${ }^{8}$ and others have furnished complete information regarding the common disease "mouse typhoid" and the less frequent Pasteurella infection.

Loefller ${ }^{2}$ in 1890 studied the inciting micro-organism of a highly fatal (69 per cent) epidemic among the stock mice of the Institute at Greifswald. He found a motile, gram negative rod for which he proposed the name Bacillus typhi murium. Since the virulence of the bacterium was also very high for field mice, he recommended its use for the extermination of these destructive rodents. Smith ${ }^{10}$ was first to recognize the relationship of the bacillus described by Loeffler with the hog-cholera bacillus and the $B$. enteritidis of Gärtner. Subsequently paratyphoid bacilli have been frequently found in the organs of apparently "healthy" mice (Rothe, Kutscher, ${ }^{\text {II }}$

I Gmeiner: Deutsche tierürstl. Wchnschr., II, 69. 1903; also Jowett, W.: J. Comp. Path. \& Therap., 24, 134. I9II.

${ }^{2}$ Knorr, M.: Centralbl.f. Bakteriol., 99, 576. 1926.

3 Flexner, S.: J. Exper. Med., 36, 9. 1922. 4 Amoss, H. L.: ibid., pp. 25, 45, 107. 1922.

5 Webster, L. T.: ibid., pp. 7I, 97. 1922; 37, 21, 33, 231, 269, and 78I. 1923; 38, 33, 45. 1923; 39, I29, 879, 265. 1924; 40, 397. 1924; 42, I. I925.

${ }^{6}$ Pritchett, I. W'.: ibid., 4I, 195. 1925.

7 Topley, W. W. C.: J. IIyg., 19, 350. 1921; 20, 103. 1921; 21, 10, 20, 226. 1922-23; Topley, W. W. C., and Wilson, G. S.: ibid., 21, 237, 243. 1923; Topley, W. W. C., and Ayrton, J.: ibid., 22, 222, 234, 305. 1924; 23, 198. 1924; Topley, W. W. C., and Wilson, J.: ibid., 24, 295. 1925; Greenwood, MI., and Topley, W. W. C.: ibid., 24, 45. 1925; 25, 336. 1926; Topley, W. IV. C.: Lancet, I, 477, 531, and 645. London, 1926; J. State .Vet., 35, 2, 63. 1927.

${ }^{8}$ Neufeld, F.: Zischr.f. IIyg. u. Infektionskrankh., 101, 466. 1924; 103, 471. 1924.

${ }^{9}$ Loefler, 1.: Centralbl. f. Bakteriol., Orig., I, 11, 129. 1892.

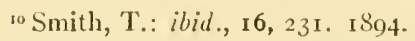

"See Uhlenluth, P'., and Hübener, E.: IIandb. d. path. II ikroorg. (2d ed.), 3, 1073. 1913. 
Okamoto, ${ }^{\mathrm{x}}$ and others). In fact, Zwick ${ }^{2}$ found 28 fecal carriers among $\mathrm{I} 77$ mice, and Heuser $^{3}$ claims that ro per cent of the common laboratory mice may be infected. In this connection it was noted that a severe case of enteritis could be incited in the carrier animals provided certain environmental factors were changed or the animals fed on a protein-high diet. 4

Spontaneous epidemics of mouse typhoid with mortalities from 34 to 95 per cent (Pritchett, Savage and Read, ${ }^{5}$ the writer) although very common have rarely been investigated and the first published account was given by $\mathrm{Lynch}^{6}$ in 1922 . On the other hand, Danysz, ${ }^{7}$ who endeavored to exterminate rodents on a large scale through employment of living cultures of an organism isolated by him from an epidemic among field mice ( $B$. enteritidis), noted that the infective power by ingestion would not extend beyond a very few passages. He therefore conducted a number of experiments which foreshadowed the extensive experimental investigations conducted by Topley, Webster, and others. These fundamental studies are so recent that a detailed account appears unnecessary, although the essential facts may be mentioned. As a rule the anatomical lesions of the infected mice consist of an engorged spleen with or without definite necroses, foci in the liver, enlarged mesenteric lymph nodes with petechiae, and a gastro-enteritis of varying intensity. The causative organism is always a representative of the paratyphoid-enteritidis group. B. aertrycke is probably the most common infecting agent (in the experience of the writer) but $B$. enteritidis has also been isolated (Wherry and Butterfield, ${ }^{8}$ Ten Broeck, ${ }^{9}$ Amoss and Haselbauer, ${ }^{10}$ ) and "suipester variants," both enteritidis and "animal paratyphoids," were encountered by Topley, Weir, and Wilson ${ }^{11}$ in mouse epidemics. The majority of epidemic strains are virulent for mice per os in relatively small doses. The bacteria multiply in the intestinal tube (lower end of the ileum), penetrate the mucosa, invade the bloodstream and the various tissues, or they localize in the spleen (Smith and Tibbets). ${ }^{\mathrm{I} 2}$ The bacilli are discharged from the intestines during the acute disease and from the carriers for some weeks. Contact infections occur among normal mice kept in the same cages and provided a certain degree of uncleanliness is maintained even in the adjacent cages. In this manner interesting epidemics with strikingly constant characteristics may be produced and maintained for an indefinite period of time on the sole condition that susceptible mice are added from time to time to the stock animals. The introduction of fresh individuals from the same or

I Okamoto, T.: loc. cit.

${ }^{2}$ Zwick, X.: Arb. a.d. kaiserl. Gesundheitsamte, 133, 250. 1909-10.

${ }^{3}$ See Uhlenhuth, P., and Hübener, E.: loc. cit.

${ }^{4}$ See also Webster, L. T.: op. cit., 37, 2I. I923.

${ }^{5}$ Savage, W. G., and Read, W. J.: J. Hyg., 13, 343. I913.

${ }^{6}$ Lynch, C. J.: J. Exper. Met., 36, I 5. 1922.

7 Danysz, J.: Ann. de l'Inst. Paste:ır, I4, I93. 1900.

${ }^{8}$ Wherry, W. B., and Butterfield, C. T.: J. Infect. Dis., 27, 315. 1920.

${ }_{9}$ Ten Broeck, C.: J. Exper. Med., 32, I9. I920.

to Amoss, H. L., and Haselbauer, P. P.: ibid., 36, I07. 1922.

II Topley, W. W. C., Weir, H. B., and Wilson, G. S.: J. Hyg., 20, 227. $192 \mathbf{I}$.

${ }^{12}$ Smith, T., and Tibbets, H. A. M.: J. Exper. Med., 45, 337. 1927. 
different dealers into the infected group (laboratory stock) is followed by an epidemic with a typical and remarkably constant mortality curve, and then a return to an endemic distribution of the infection. The survivors of the epidemic may or may not be carriers or immune to subsequent infections. When the factors involved in these occurrences were analyzed it was found by Topley and by Webster that the distribution, the virulence of the microbes, and the host susceptibility were in all probability responsible for the outbreaks. Topley, for example, demonstrated that the preepidemic period was preceded by a marked rise in the fecal excretion of paratyphoid bacilli, and it is reasonable to assume that a certain dosage increases the chance of the mice to ingest the specific bacteria. Regarding the influence of the virulence, the views of Topley are diametrically opposed to those of Webster. According to Lockhart, ${ }^{\mathrm{I}}$ significant variations in the invasiveness of $B$. aertrycke may occur under various conditions, while Webster believes that the inherent virulence of each strain remains constant, uninfluenced by repeated animal passage and varied natural environmental circumstances, and is a relatively fixed quality. However, the fact remains that some strains are highly virulent, others less so. The factors which induce these conditions are unknown. There seems to be ample evidence that the fundamental factors which influence the course of a mouse epidemic is the host susceptibility. Webster ${ }^{2}$ and Pritchet $t^{3}$ have shown that different breeds and strains of mice vary in susceptibility, and that individual variations from extreme susceptibility to complete resistance are influenced by hereditary, acquired immunity (perhaps in consequence of a latent infection ['Topley, Wilson, and Lewis]), ${ }^{4}$ diet (on the McCollum diet the mice are distinctly more resistant than on bread-and-milk diet although the intestinal flora plays no part [Webster and Pritchett]), ${ }^{5}$ seasonal and other environmental factors. In this connection it is interesting to record that the inoculation of killed cultures yields some definite protection against infection per os (Webster, Lange and Yoshio$\mathrm{ka}^{6}{ }^{6}$ Topley and Wilson $\left.{ }^{7}\right)$. However, it is doubtful if vaccination would check a mouse typhoid epidemic in a breeding station (Lynch). Preventive measures are much more effective, but cannot be practiced with any degree of assurance until suitable methods for the detection of carriers and latent infections have been developed.

Although mice are highly susceptible to Pasteurella organisms, spontaneous epidemics are rare. Reference to such infections are, however, found in the papers by Greenwood and Topley (1925); Greenwood, Newbold, Topley, and Wilson; ${ }^{8}$ and Baudet.9 The autopsy findings are not characteristic; pulmonary lesions and en-

I Lockhart, L. P.: J. II yg., 25, 50. 1926.

${ }^{2}$ WVebster, L. T.: op. cit., 36, 7 I, 97. I922; 37, 2I, 33, 23I, 269, and 78I. 1923; 38, 33, 45. 1923; 39, I 29, 879, 265. I924; 40, 397. I924; 42, I. 1925 .

3 Pritchett, I. W.: loc. cit.

${ }_{4}$ Topley, W. W. C., Wilson, J., and Lewis, E. R.: J. IIyg., 23, 421 . I925.

5 Webster, L. T., and Pritchett, I. W.: J. Exper. Med., 40, 397. I924; Webster, L. T.: ibid., $37,21.1923$.

${ }^{6}$ Lange, B., and Yoshioka, M.: Zlschr.f. IIyg. u. Infektionskrankh., ro I, 451. I924.

7 Topley, W. W. C., and Wilson, J.: loc. cit.

${ }^{8}$ Greenwood, M., Newbold, E. M., 'Topley, W. W. C., and Wilson, J.: J. IIyg., 25, 336. 1926. ${ }_{9}$ Baudet, E. A. R. F.: Tijdschr. v. dicrgeneesk., 51, I 5.1925. 
gorged spleens sometimes accompanied by exudations into the cavities have been recorded. The pathogenicity of the epidemic strains is high and infections may be secured by inhalation (Uchida). ${ }^{x}$ Some strains are virulent for fowls, others are not.

Various forms of pseudotuberculosis and pneumonia (Keegan) ${ }^{2}$ due to diplostreptococci (Kutchera, ${ }^{3}$ Fricke) or Bact. bronchisepticum have been reported and incompletely described. Bact. (Erysipelothrix) murisepticum is frequently the inciting agent of highly destructive epidemics among migrating meadow and house mice (Wayson). ${ }^{4}$ Mice are also the host of various intestinal flagellates (Trichomonas, Giardia), which may favor certain intestinal infections (Smith and Tibbets). ${ }^{5}$ Encephalitozoon has been reported by Cowdry and Nicholson ${ }^{6}$ and Anigstein. ${ }^{7}$ Klossiella muris is a common parasite and is not infrequently encountered in sections of the kidneys (Bonne). ${ }^{8}$ An excellent description of the life-cycle has been published by Stevenson. ${ }^{9}$

\section{DISEASES OF RATS}

The maintenance of large rat colonies particularly for studies on nutrition has aroused some interest in the communicable diseases of this rodent (Greenmann and Dühring, ${ }^{\text {Io }}$ Hartwell, Mottram and Mottram ${ }^{\mathrm{II}}$ ), although most researchers still regard them as inevitable. Furthermore, the continuous struggle between man and the rat and the important rôle of the latter in the transmission of disease has prompted many valuable studies and publications on the infections of the wild rat and its hybrid the albino. A summary will be found in a bulletin of the United States Public Health Service entitled The Rat and Its Relation to the Public Health (rgro) and in a recent excellent review by Koehler (1925). ${ }^{\text {I2 }}$

Respiratory infections frequently called "pneumonia" are more prevalent among albino rats. Young animals are rarely affected while the mortality among the old rats may be very high. In fact, all rats after reaching maturity may spontaneously develop "lung disease." As a rule an acute stage marked by loss of appetite, dullness, mucous nasal discharge, conjunctivitis, and a labored, unnatural, noisy breathing is followed by a chronic stage during which the animals appear in a much depressed state of health and finally succumb to the disease. At autopsy various stages of unresolved pneumonia, catarrhal bronchitis with voluminous bronchiectases, abscesses of varying extent from a small mass to the almost complete obliteration of the lung and pleural adhesions are nearly always observed.

\footnotetext{
'Uchida, Y.: op. cit., 106, 303. 1926. ${ }^{2}$ Keegan, J. J.: loc. cit.

${ }^{3}$ Kutchera, F.: Centralbl. f. Bakteriol., 46, 671, r9o8.

${ }_{4}$ Wayson, N. E.: Pub. Health Rep., 42, I491. 1927.

${ }^{5}$ Smith, T., and Tibbets, H. A. N.: loc cit.

${ }^{6}$ Cowdry, E. V., and Nicholson, F. M.: loc. cit.

7 Anigstein, L.: Compt. rend. Soc. de biol., 92, 991. I925.

${ }^{8}$ Bonne, C.: ibid., p. I I go. I 925.

${ }^{9}$ Stevenson, A. C.: Quart. J. Micr. Sc., 61, 127. 1915-16.

Io Greenmann, M. J., and Dühring, F. L.: Breeding and Care of the Albino Rat, p. roo. Phila-

"Hartwell, G. A., and Mottram, E. C., and V. H.: Biochem. J., I 7, 208. r923.

${ }^{12}$ Kochler, G.: Zentralbl.f.d. ges. Hyg., ro, 161. 1925.
} delphia, I923. 
The etiology of these localized infections has not been determined. Various organisms have been cultivated from the lungs. A gram positive bacillus (Klein ${ }^{\mathrm{r}}$ and Mitchell ${ }^{2}$ ), which at least experimentally produced on intrapleural injection a pleurisy and pneumonia, a delicate Streptothrix (Tunnicliff), ${ }^{3}$ Bact. bronchisepticum (Hoskins and Stout), ${ }^{4}$ and an organism resembling Bacillus actinoides (Jones) ${ }^{5}$ has been grown. The writer has not found the Bacillus actinoides and the gram positive B. muris, but has isolated on numerous occasions streptothrices, Bact. bronchisepticum, diphtheroids, streptococci, and fine influenza-bacillus-like rods. A few comparative cultures of the respiratory tract of young and apparently healthy rats revealed, however, a similar flora. Unfortunately, only chronic lesions which give an excellent opportunity for invasion by the organisms of the upper and lower respiratory tract have been examined. It is not unlikely that several types of lung disease exist and that the primary injury may be due to various environmental factors which favor the localization of different micro-organisms. In fact, the writer found that crowding of several animals into one cage or the housing in boxes with sawdust strikingly increased the incidence of respiratory infections. A systematic bacteriological study of the rhinitis which regularly precedes the lung disease is clearly indicated. Furthermore, the streptothrices of the buccal cavity of rats in their relation to certain forms of rat-bite fever (Schottmüller, ${ }^{6}$ Blake, ${ }^{7}$ Tunnicliff and Nayer, ${ }^{8}$ Ebert and Hesse ${ }^{9}$ ) is another problem with many possibilities.

Equally prevalent but less frequently recognized is a suppurative otitis. McCordock and Congdon ${ }^{\mathrm{ro}}$ found pus in the auditory bulla of one or both ears in $22-50$ per cent of the rats examined. Many of the animals showed deafness, and the course of the infection from a purulent rhinitis was traceable through the middle ear. In a number of cases the two workers isolated from the nasal passages and the pus of the ear a gram negative, pleomorphic, motile, short bacillus. The writer failed to cultivate micro-organisms from the pus of four cases of otitis. It seems probable that the ear lesions are the sequelae of the respiratory diseases which are similar to those commonly observed in the rabbit. Moreover, many factors which enhance the susceptibility of the rats, such as vitamin A (Daniels and Armstrong) ${ }^{\mathrm{Ir}}$ or mineral deficiencies (McCordock and Congdon), increase the incidence of the "middle-ear disease" (Greenmann and Dührig).

A pasteurellosis as described by Meyer and Batchelder ${ }^{12}$ for wild rats has been

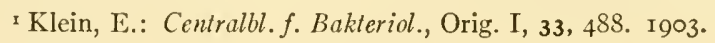

${ }^{2}$ Mitchell, O. W. H.: J. Infect. Dis., 10, 17. 1912.

3 Tunnicliff, R.: ibid., 19, 767. 1916.

${ }_{4}^{4}$ Hoskins, H. P., and Stout, A. L.: J. Lab. E Clin. Med., 5, 207. 1919-20.

${ }^{5}$ Jones, F. S.: J. Exper. Med., 35, 36r. 1922.

${ }^{6}$ Schottmüller, H.: Dermat. Wchnschr., 55, 77. I9r4.

7 Blake, F. G.: J. Exper. Med., 23, 249. I9I6.

${ }^{8}$ Tunnicliff, R., and Nayer, K. M.: J. Infect. Dis., 23, 555. I918.

${ }^{9}$ Ebert, B., and Hesse, E.: Arch.f. klin. Chir., 136, 69. 1925.

${ }^{10}$ McCordock, H. A., and Congdon, C. C.: Proc. Soc. Exper. Biol. \& Med., 22, I50. 1924.

II Daniels, A. L., and Armstrong, M. E.: J.A.M.A., 81, 828. I924.

${ }^{12}$ Meyer, K. F., and Batchelder, A. P.: J. Infect. Dis., 39, 386.1926. 
noted by Jonescu ${ }^{\mathrm{x}}$ in three albino rats. In the typical lesions of the disease a bipolar organism was present; however, the virulence was high for mice and pigeons but low for rats. Successful infections resulted when the Pasteurella organism was injected in combination with streptococci.

Paratyphoid, either in epidemic form with a high mortality (75-100 per cent) or as latent infections, has been described by a number of workers (Loeffler, ${ }^{2}$ Issatschenko, ${ }^{3}$ Danysz, ${ }^{4}$ Schern, ${ }^{5}$ Pappenheimer and Von Wedel, ${ }^{6}$ Trautmann, ${ }^{7}$ Cannon, ${ }^{8}$ Savage and Read, ${ }^{9}$ Ball and Price-Jones, ${ }^{10}$ and others). In the acute infections, diarrhea and the formation of bloody crusts around the eyes are quite characteristic. At autopsy an engorged spleen, multiple focal necroses (not specific for Gärtner infection), enlarged lymph nodes and Peyer's patches, and a blood-tinged intestinal content are rarely absent. The chronic cases may show very few or no lesions. The strains of paratyphoid bacilli cultivated from these rat infections in various parts of the world are predominantly of the $B$. enteritidis type (Jordan). ${ }^{\text {II }}$ However, Herz and Trawinski ${ }^{\mathrm{I} 2}$ claim the isolation of paratyphoid $\mathrm{A}$ and paratyphoid $\mathrm{B}$ strains from wild

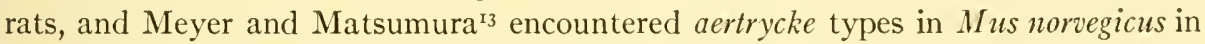
San Francisco. A bacillus similar to but not identical with the representatives of the paratyphoid group has been found by Gheorghiu ${ }^{14}$ in the course of a rat epidemic at Strassburg. Infections per os with a small number of bacteria and contact transmissions to young rats have been successful in a series of experiments conducted by the writer, Price-Jones, ${ }^{15}$ and others. The bacillus can be found in the spleen and liver of nearly all fed animals in forty-eight hours; after ten days it begins to disappear, and at the end of about two months it can be recovered from only about ro per cent. Agglutinins persist long after the animals have lost their infection. "Healthy" carriers can initiate an epidemic among fresh young rats, and in the observations of Price-Jones they caused reinfections. The epidemiology is probably quite similar to that observed in mice. The various intestinal flagellates common to the intestinal tube of the rat in their relationship to the host susceptibility deserve further investigation.

In many rat colonies scab, due to a mite, Notoëdres alepis, which burrows under the skin of the ears and tail, is quite common. It is readily spread in wooden cages

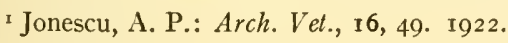

${ }^{2}$ Loeffler, F.: loc. cit

${ }^{3}$ Issatschenko, B.: Centralbl. f. Bakteriot., 23, 873. 1898.

+ Danysz, J.: loc. cit.

${ }^{5}$ Schern, K.: Arb. a. d. kaiserl. Gesundheitsanmte, 30, 575. I909.

${ }^{6}$ Pappenheimer, A. M., and von Wedel, H.: J. Infect. Dis., I4, I80. I914.

7 Trautmann, H.: Ztschr.f. Hyg. u. Infektionskrankh., 54, I04. I906.

'Cannon, P. R.: J. Infect. Dis., 26, 402. I920.

${ }^{9}$ Savage, W. G., and Read, W. J.: loc. cit.

to Ball, N. D., and Price-Jones, C.: J. Path. \& Bact., 29, 27. 1926; 30, 45. 1927.

"Jordan, E. O.: loc. cit.

${ }^{12}$ Herz, A., and Trawinski, A.: Wien. klin. Wchnschr., 30, 524. I917.

${ }^{13}$ Meyer, K. F., and Matsumara, K.: J. Infect. Dis., 41, 395. 1927.

${ }^{14}$ Gheorghiu, I.: Ann. de l'Inst. Pusteur, 39, 71 2. 1925. $\quad$ is Price-Jones, C.: loc. cit. 


\section{COMMUNICABLE DISEASES OF LABORATORY ANIMALS}

and is transmitted to man. Schürmann ${ }^{\mathrm{I}}$ observed a localized parasitic invasion on the right hand of an animal caretaker. The rat disease is easily cured with creosote or flower-of-sulphur preparations. A few years ago it attracted general attention. Rat scab played a rôle in the interpretation of certain rejuvenation experiments since Fiebiger ${ }^{2}$ suggested that the alopecia which Steinach considered indicative of physiological senility was a pathological process caused by the scab mite. In all probability the operative procedures (ether) removed the parasites and the rats were not rejuvenated by the ligation of the spermatic chord but recovered from a chronic skin disease.

In conclusion it may not be out of place to emphasize that the communicable diseases of laboratory animals furnish an abundance of readily procurable material for the study of disease processes. Regular and systematic autopsies may not only prepare the student for his subsequent studies in human pathology but may help to establish the nature of the infection which may cause epidemics among animals and thus serve as an excellent introduction into the field of comparative pathology and epidemiology.

${ }^{\mathrm{I}}$ Schürmann, W.: Centralbl.f. Bakteriol., Orig. I, 48, I67. 1909.

${ }^{2}$ Fiebiger, J.: Wien. klin. Wchnschr., 34, 364. I921. 


\title{
CHAPTER XLVI
}

\section{BACTERIA OF THE INTESTINAL TRACT}

\author{
LEO F. RETTGER \\ Yale University \\ NUMBERS AND QUANTITY
}

The large intestine of man and lower mammals is a veritable culture tube in which definite bacterial types appear to be struggling constantly to gain supremacy. A hasty microscopic examination of the contents from the colon or of the feces is sufficient to indicate that bacteria constitute a large part of the fecal material. No very extensive investigation is required, however, to show that only a very small proportion of the total flora is made up of living micro-organisms.

Strasburger, ${ }^{1}$ by the use of his gravimetric method, arrived at the conclusion that the average normal adult excretes about $8 \mathrm{gm}$. (dry weight) of bacteria daily, or I 28 trillions. MacNeal, Latzer, and $\mathrm{Kerr}^{2}$ estimated the number of bacteria (living and dead) excreted daily by a normal adult as $33 \times \mathrm{IO}^{12}$, and that this number of bacterial cells is equivalent to $5.34 \mathrm{gm}$. of dried bacteria or $0.585 \mathrm{gm}$. of bacterial nitrogen. Matill and $\mathrm{Hawk}^{3}$ place the daily output at $8.27 \mathrm{gm}$. (dry weight), and Sato ${ }^{4}$ at $8.54 \mathrm{gm}$.

Numerous attempts have been made to reduce or control the microbic flora of the intestine by the administration of a sterile diet, but on the whole they were not successful. Sucksdorff ${ }^{5}$ claimed that the number of intestinal bacteria is reduced by the use of sterile food. Somewhat similar results were obtained by Gilbert and Dominici ${ }^{6}$ and by Brotzu. ${ }^{7}$ However, numerous investigators, including Escherich, ${ }^{8}$ Hammerl, ${ }^{9}$ and Belonowsky, ${ }^{\mathrm{ro}}$ were unable to confirm these claims.

The possibility of regulating or completely suppressing bacterial growth and activity in the intestine by the use of chemical antiseptic agents has also received much consideration. Wassilieff, ${ }^{\mathrm{II}}$ Baumann, ${ }^{\mathrm{I} 2}$ and others claimed that calomel lessened the intestinal putrefactive processes, and according to the observations of Fürbringer ${ }^{13}$

IStrasburger, J.: Ztschr.f. klin. Med., 46, 413. I902.

${ }^{2}$ MacNeal, W. J., Latzer, L. L., and Kerr, J. E.: J. Infect. Dis., 6, г 23, 57 I. 1909.

3 Matill, H. A., and Hawk, P. B.: J. Exper. Med., I4, 433. I9I I.

${ }_{4}^{4}$ Sato, T.: Ztschr.f. exper. Path. u. Therap., 7, 427. 1910.

${ }^{5}$ Sucksdorff, W.: Arch.f. Hyg., 4, 355. 1886.

${ }^{6}$ Gilbert, A., and Dominici, S. A.: Compt.rend. Soc. de biol., I17, 277. I894.

7 Brotzu, L.: Centralbl. f. Bakteriol., I7, 726. I895.

${ }^{8}$ Escherich, T.: Fortschr. d. Med., 3, 515. I885.

${ }^{9}$ Hammerl, H.: Ztschr. f. Biol., 35, 355. I 897.

ro Belonowsky, J.: Ann. de l'Inst. Pasteur, 21, 991. г907.

II Wassilieff, N. P.: Ztschr.f. phys. Chemie, 6, I12. I882. I2 Baumann, E.: ibid., 10, т23. I886.

13 Fürbringer, F.: Deutsche med. Wchnschr., 13, 209, 235. I887. 
this agent materially reduced the number of intestinal organisms. Naphthalene, iodoform, chloroform, chlorine water, turpentine, menthol, camphor, magnesium sulphate, and bismuth salicylate have been employed, with apparently some reduction in bacterial numbers and activities.

On the other hand, no diminution in bacterial numbers or in putrefaction products could be demonstrated by Morax, ${ }^{1}$ Biernacki, ${ }^{2}$ Schütz, ${ }^{3}$ Strasburger, ${ }^{4}$ and others. Harris $^{5}$ failed to obtain any reduction in the bacterial count by the employment of beta-naphthol, salol, and guayacol carbonate.

Quite recently Rettger, Valley, and Plastridge ${ }^{6}$ have found that certain resorcinol derivatives, when fed to adult white rats daily in amounts up to $0.3 \mathrm{gm}$., caused a reduction in bacterial numbers, and the almost complete disappearance of all intestinal organisms except Bacterium aerogenes, and that after continued oral administration of these agents, the aerogenes type increased in numbers and constituted almost the entire flora. Further attempts to eliminate this organism were unsuccessful.

The stomach possesses a slightly antiseptic property, owing to the acidity of the gastric juice, but its function as a germ-destroying organ is said to be almost negligible. The usually small bacterial content of the chyle is due very largely to a distinct bactericidal action of the wall of the duodenum and ileum, according to the claims of different investigators (Klein, Landsberger, Kohlbrügge, Moro, and others). Escherich believed that the sparsity of bacteria may be explained by dilution with large amounts of germ-free intestinal juice, which in itself has been shown not to be bactericidal. $^{7}$

The first notable contributions to intestinal bacteriology were those of Escherich,8 in which he described for the first time the organism Bact. coli, which is so characteristically an intestinal bacterium and which has been the object of so much interest as an index to the serious pollution of water supplies, etc.; also its close ally, Bact. aerogenes. Although Escherich noted the predominance of gram positive organisms in nurslings' stools, he was unable to isolate the aciduric types later described.

The digestive tract of normal infants is sterile at birth, as has been shown by Breslauer, Senator, Escherich, Popoff, Tissier, Moro, and others. Adventitious organisms make their entrance through the mouth and anus soon after birth, and constitute a heterogeneous flora which gives way to certain definite types as soon as the infant is placed on an all-milk diet. Tissier ${ }^{9}$ made the observation that the predominating organism of the nursling's flora is a gram positive bacillus, Lactobacillus bifidus (Tissier), which persists as the outstanding organism as long as mother's milk constitutes the entire diet.

I Morax, V.: Ztschr.f. physiol. Chemie, ro, 3 I8. 1886.

${ }^{2}$ Biernacki, E.: Deutsches Arch.f. klin. Med., 49, 87. I892.

3 Schütz, R.: Arch.f. Verdauungskr., 7, 43. I9ог.

${ }_{4}$ Strasburger, J.: Ztschr.f. klin. Med., 48, 49I. I903.

5 Harris, N. M.: J.A.M.A., 59, I344. I9I 2.

${ }^{6}$ Unpublished work.

$7 \mathrm{Cf}$. chap. vi for a further discussion of bacteria in feces.

${ }^{8}$ Escherich, T.: loc. cit.

- Tissier, H.: Thêse. Paris, Igoo. 
Moro $^{1}$ for a while disputed Tissier's claim, and believed that his Lactobacillus acidophilus, which is apparently the same as the "Säureliebender Bacillus" of Finkelstein, is the dominant species. Both the Tissier and the Moro organisms, which are closely related to each other in many respects, are prominent in nurslings' stools. In the change from mother's to cow's milk, L. acidophilus tends to displace the Tissier bacillus. As the child grows older and the diet becomes more varied (milk and eggs, for example) the flora changes from the simple to one that is more complex; and as the daily food more nearly approaches in kind that of the ordinary adult a more or less definite, varied flora becomes established, in which the aciduric organisms of infancy, L. bifidus and L. acidophilus, have all but completely disappeared, in so far as microscopic and cultural observations will determine, and Bact. coli as a rule plays a very dominant rôle.

Tissier noted the presence of the following intestinal organisms in a five-year-old child: L. bifidus, L. acidophilus, Enterococcus (Strept.fecalis), Bact. coli, B. exilis, and $B$. rodella $\mathrm{III}$. These he regarded as the constant, fundamental flora. Besides these he observed the following variable, secondary flora: $B$. perfringens ( $C l$. welchii), Staph. parvulus, B. funduliformis, B. capillosus, B. ventriosus, Coccobac. praccutus and oviformis, Diplococ. arbiculus, and different yeasts.

It is impossible to identify some of the secondary organisms of Tissier with known species today, and their names are perhaps of little significance. It must be conceded, too, that our knowledge regarding the biological entities and relationships of the microbic population of the intestine is still far from complete.

The following groups of organisms are known to be present in the intestine of the ordinary adult:
r. Coli-aerogenes group
4. Anaerobes
7. Spirilla and spirochetes
2. Aciduric types
5. Proteus group
8. Yeasts
3. Micrococci
6. Subtilis group
9. Molds

THE COLI-AEROGENES GROUP

Bact. aerogenes is comparatively rare, while Bact. coli proper is present in large numbers in the intestine of man and the lower animals. In fact, very few species of animals appear not to be natural carriers of this organism. It is, however, seemingly foreign to the intestine of fishes, according to Hunter ${ }^{2}$ and others, and of shellfish, and is found in them only when they occupy waters which contain this organism as the result of pollution by man and the lower animals.

\section{THE ACIDURIC GROUP}

This is represented by L. bifidus and L. acidophilus, both of which are characteristically intestinal organisms. They resemble each other in that they are gram positive, produce and tolerate appreciable amounts of lactic acid, and have high energy requirements. L. bifidus was so called because of its tendency to branch or bifurcate. In this respect it differs from $L$. acidophilus, which reveals branching forms but rarely, in spite of certain claims to the contrary. The two differ also in that L. bifidus is mi-

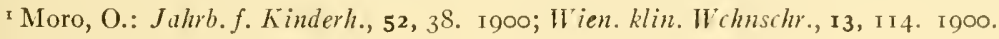

${ }^{2}$ Hunter, A. C.: J. Bacl., 5, 353. 1920. 
croaerophilic, while the other is a facultative anaerobe. Furthermore, agar colonies of the Tissier organism are solid and disk shaped, whereas those of the Moro bacillus are as a rule of the extremely fuzzy " $\mathrm{X}$ " type or the more nearly regular and slightly fuzzy " $Y$ " type. They are always very small, delicate colonies (both are slow to develop in artificial media), and require carbohydrate for growth. Both grow in milk, with the production of lactic acid and, as a rule, a soft curd.

\section{MICROCOCCI}

The most common and best known is the Enterococcus of Thiercelin, or Streptococcus fecalis. A complete description of this coccus form was published recently by Dibble. ${ }^{I}$ It is a common inhabitant of the intestine at almost all ages, and occurs there as a diplococcus, in short chains, and occasionally singly. It produces more or less typical, small, smooth, granular streptococcus colonies on sugar agar, which usually grow to larger size, however, than colonies of pyogenic streptococci. This organism rapidly grows in milk and produces a curd not very unlike that of $L$. acidophilus. Other streptococcus forms may also be present, but their significance as definite fecal organisms needs further study.

Staphylococci also occur in the intestine. These are of much less prominence than the Enterococcus. Schiller found that they are present in the intestine of human adults, but that they are quite rare in infants. Streptococci of the ordinary hemolyticus type may be found occasionally, having come from the mouth.

\section{ANAEROBES}

The most common and best-known intestinal anaerobe is $C l$. welchii. This interesting, actively fermentative organism is often present in enormous numbers, and may almost be said to be ubiquitous in man and animals. It sporulates abundantly in the intestine, and may be isolated readily for this reason. The intestine is believed by many to be a normal habitat of $\mathrm{Cl}$. tetani. Noble found some horses to be carriers, and Ten Broeck observed it to be a common inhabitant of the intestine of Chinese coolies in the Orient.

Other anaerobes are often present; but few, if any of these, carry on a permanent existence in the intestine; in other words, they are adventitious, transient organisms.

Bacteria of the fusiformis and spirocheta types may quite often be seen microscopically, but they appear to resist the ordinary methods of artificial cultivation. Spirochetes have been observed in the intestinal contents by various observers (Kuisl, Miller, Macfie, Wolbach, and Parr). In some instances they were cultured, but there may perhaps be a question as to whether the cultures were absolutely pure.

\section{PROTEUS GROUP}

Organisms of the Proteus vulgaris type may be recovered occasionally from feces. It is doubtful, however, if they are present in large numbers or with any degree of frequency, except here and there perhaps in connection with certain pathological conditions, as some have claimed.

Dilbble, J. II.: J. Path. \& Bact., 24, 3. 1921. 
SUBTILIS GROUP

Members of this group are met with quite frequently, but according to the writer's experience occur in small numbers only, in freshly voided feces. Spores of these organisms, like those of anaerobes, enter the digestive tract freely, to a large degree resist the antagonistic forces of the intestine, and are eliminated as spores. However, some investigators have regarded members of the subtilis group as common invaders and sojourners in the intestine.

\section{YEASTS AND MOLDS}

These are found frequently, but it is doubtful if they multiply in the intestine. Ordinary yeasts may resist the antagonistic forces of the digestive tract and pass through more or less unharmed. Molds may do so also, but probably chicfly in the "spore" stage.

\section{MICROSCOPIC APPEARANCE OF GRAM STAINED SLIDES OF NORMAL FECES OF ADULTS}

While there is, of course, considerable variation in the microscopic pictures presented by slides prepared and gram stained in the usual way, they are as a rule characteristic and may be described briefly as follows:

More or less formless, unorganized matter which takes the counterstain, though often only faintly, may be seen. At times, however, practically the entire picture is that of clearly defined micro-organisms.

Gram negative bacteria predominate, and at times to the extent of 90 per cent or more of the entire field; but as a rule the number varies beween 65 and 85 per cent, and the average lies between 70 and 80 per cent.

Of the gram positive organisms (20-30 per cent, average) the cocci are usually the most numerous, and may outnumber the bacillary forms at least 2 to $\mathrm{I}$.

The large majority of the gram negative organisms are small and short and resemble Bact. coli. There is frequently an appreciable number of gram negative cocci. It is quite probable that most of these are dead and partly autolyzed. Some large gram negative rods may also be seen. A common occurrence is that of large (giant) positive coccus and rod forms.

Another not uncommon picture is that of fusiform gram negative or poorly staining rods or filaments, and of spirochetes. These may occasionally constitute a very large proportion of the gram negative forms. Free spores may also be seen, but they are easily overlooked and not always readily demonstrated.

The foregoing inadequate description applies to feces of adults subsisting on the usual mixed diet, including meat. The use of milk in appreciable quantities or of milk sugar will very materially change the microscopic appearance of the intestinal flora, and may transform it to such an extent as to change the entire character of the slide toward the gram positive aciduric type. Meat and other ligh-protein diet exert the opposite influence.

\section{LIEE WITIIOUT BACTERIA}

Before the days of Pasteur no significance was attached to the occurrence of bacteria in the intestine. They were neither regarded as necessary nor thought to play any harmful rôle in the so-called "normal individual." Pasteur was apparently the 
first to stimulate interest in the question as to whether life without bacteria is impossible, though he left the attempted solution of this problem to others.

Nuttall and Thierfelder ${ }^{1}$ in 1895 concluded, as the result of their observations on guinea pigs, that intestinal bacteria are not necessary. Schottelius ${ }^{2}$ experimented with chicks and arrived at the opposite conclusion. The observations of O. Metchnikoff ${ }^{3}$ on tadpoles supported the claims of Schottelius.

On the other hand, E. Metchnikoff ${ }^{4}$ maintained that scorpions and certain worms do not harbor intestinal bacteria, and that very few are found in the Indian bat. Wollman ${ }^{5}$ experimented with flies which were obtained germ-free from sterilized eggs and kept in sterile glass tubes, and showed that normal growth of the larvae took place without bacteria. He was also successful in rearing tadpoles under aseptic conditions. Cohend $y^{6}$ repeated the experiments of Schottelius on chicks hatched from sterilized eggs, and found that the chicks were in as good condition after two to six weeks as the controls reared in the usual manner.

Levin ${ }^{7}$ reported that the intestines of most of the polar animals examined by him bacteriologically were free from bacteria. These claims were disputed by Chauveau and others.

Küster ${ }^{8}$ removed a young goat aseptically by Caesarean section and kept it germ free for fourteen days. The goat grew and remained normal. However, this same investigator ${ }^{9}$ states that intestinal bacteria are necessary for young animals, and also perhaps for human adults and full-grown animals on account of their fermentative, antagonistic, and physical actions.

The question as to whether bacteria are necessary in the intestine is still an open one, though the preponderance of evidence appears to support the view that they are not essential.

DIET AND INTESTINAL FLORA

The term "intestinal putrefaction" has long been used more or less popularly to designate changes which take place in the intestine through the action of micro-organisms on the more complex nitrogenous substances in the large intestine, with the formation of products which are at least mildly toxic and are in a large measure absorbed from the intestine. Such a process has been described by some as one of "autointoxication."

Whether or not the use of these terms is strictly accurate, it has long been known that different organisms can and do bring about the formation of products characteristic of them, and that the activities of the different types, and indeed most types of

r Nuttall, G., and Thierfelder, H.: Ztschr.f. phy's. Chemic, 2 I, I09. I 895.

${ }^{2}$ Schottelius, M.: Arch.f. IIyg., 67, I 77. 1908.

3 Metchnikoff, O.: Ann. de l'Inst. Pasteur, 15,631. I901.

${ }_{4}$ Metchnikoff, E.: Aml. de l'Inst. Pusteur, 23, 937. 1909; Prolongation of Life. New York, I907.

5 Wollman, E.: Amn. de l'lnst. P'usteur, 24, 79. I01 i 27, 154. 1913.

"Cohendy, M.: ibid., p. Io6. I9I2.

7 Levin.: ibid., 13,558 . 1 Sog.

${ }^{8}$ Küster, I.: Centrall. f. Bakleriol., Abt. I, Beilage, 54, 55. I913; Deatsche mel. Il chnschy 39, I536. 1913 .

9 Küster, E.: Kolle and Wassermann, Ilandb. d. path. Mikroürg., 6, 468. I9I3. 
bacteria, are largely determined by the nature of the food or substrate upon which they subsist. Thus, in a medium containing utilizable nitrogenous substances and no a vailable carbohydrate, intensive nitrogenous metabolism takes place, with the formation of ammonia, amino acids (including tryptophane), amines, indol, etc.

In the presence of fermentable carbohydrate, however, the metabolism assumes a different aspect and becomes essentially fermentative, the degree and exact nature of this process depending largely on the amount and kind of sugar present. In such a metabolic process the nitrogenous products (including the so-called "putrefactive") are relatively few and small in amount.

These conditions are in a large measure reproduced in the large intestine, though many influences are brought to bear here which do not as yet admit of satisfactory interpretation.

As early as I886 Miller and Ostweiler independently demonstrated that the ingestion of carbohydrates tends to decrease so -called "intestinal putrefaction." Kraus observed the same influence in dogs. Hirschler ${ }^{2}$ concluded that particular carbohydrates, lactose, sucrose, dextrin, and starch exerted some inhibiting influence on the type of decomposition in the intestine. Others, including Poehl and Winternitz, ${ }^{3}$ claimed that milk causes a decrease in the amounts of undesirable products of protein decomposition. These observations were confirmed by Herter ${ }^{4}$ and by Leva. ${ }^{5}$ A pronounced decrease in ethereal sulphates of the urine and a shift in the flora toward the aciduric type were noted by Herter and Kendall ${ }^{6}$ after milk and dextrose feeding. Barker $^{7}$ and Torrey ${ }^{8}$ reported changes in intestinal flora and favorable clinical results as the result of administration of lactose to typhoid patients.

Other investigators, among them Solukha ${ }^{9}$ and Kopetski, ${ }^{10}$ concluded that lactose will inhibit putrefaction, when fed by mouth. These claims were not confined, however, by various workers to one or two definite carbohydrates, and g'ucose, maltose, and sucrose were included among the carbohydrates which were claimed to have a more or less direct influence on intestinal putrefaction. Various investigators have found a direct relationship of the intestinal flora to diet, as, for example, Weiss, Settler, De Gasperi, Fischer, Wollstein, etc.

Rettger and Horton ${ }^{\text {II }}$ noted a marked simplification of the intestinal flora of white rats following the administration of a diet consisting of starch, lard, proteinfree milk, and purified proteins, gram positive organisms of the L. acidophilus and L. bifidus types constituting almost the entire flora. Hull and Rettger ${ }^{12}$ found that

× Kraus, E.: Ztschr.f. phys. Chemie, 18, г67. I894.

${ }^{2}$ Hirschler, A,: ibid., 10, 306. IS86. 3 Winternitz, H.: ibid., I6, 460. IS92.

${ }^{4}$ Herter, C. A.: Bril. M.J., 2, 1847. 1897.

${ }^{5}$ Leva, J.: Berl. klin. Wchnschr., 45, 922 . I 908.

${ }^{6}$ Herter, C. A., and Kendall, A. I.: J. Biol. Chem., 7, 203. r909.

i Barker, L. F.: J.A.M.A., 63, 929. I914.

${ }^{3}$ Torrey, J. C.: J. Infecl. Dis., 16, 72. I9I5.

${ }^{9}$ Solukha, I. P.: Dissertation. St. Petersburg, 1896.

ro Kopetski, I. A.: Dissertation. St. Petersburg, I900.

${ }^{11}$ Rettger, L. F., and Horton, G. D.: Centralbl. f. Bakteriol., Hbt. I, Orig., 73, 362. I9I4.

${ }^{12}$ Hull, T. G., and Rettger, L. F.: ibid., 75, 219. 1914; J. Bact., 2, 47. I9I7 
the feeding of milk or lactose to rats, in addition to the regular diet of grain and vegetable feed, causes a profound change in the intestinal population. When as much as $3 \mathrm{gm}$. of lactose was given daily the bulk of the flora consisted of L. bifidus, but when the amount of this sugar was reduced to $2 \mathrm{gm}$. a day L. bifidus gave way to L. acidophilus, which continued as the dominant organism as long as the lactose was fed. They concluded that milk owes its transforming influence to the lactose.

Torrey ${ }^{\mathrm{I}}$ showed that in the administration of lactose, $250-300 \mathrm{gm}$. were required to transform the intestinal flora of typhoid patients from the usual mixed to one dominated by L. acidophilus.

Distaso and Schiller ${ }^{2}$ claimed that of the various carbohydrates employed by them lactose and dextrin alone are.able to transform the flora. These observations were confirmed and extended by Rettger and Cheplin. ${ }^{3}$

\section{TRANSFORMATION OF INTESTINAL FLORA: HYGIENIC AND THERAPEUTIC ASPECTS}

The earlier observations that diet has a large determining influence on the nature and kinds of micro-organisms developing in the digestive tract have been fully confirmed and very materially extended in recent years. The present renewed interest in this field has been stimulated by the investigations conducted in this country, particularly in the laboratories of Yale University. Reference to some of these has already been made in this chapter. Space does not admit of a complete review.

The intestinal flora of white rats can be regulated almost at will. When fed on a diet consisting largely of mixed grain (corn, oats, and sunflower seed), a large proportion of the intestinal population is made up of $L$. acidophilus, as is seen by the direct microscopic and by the plate-culture method. On changing the diet to bread and meat, a more or less complete change in flora to the non-aciduric, and in a large measure gram negative types, occurs within two to three days. A reversion to the aciduric phase may be effected again by the feeding of from I to $3 \mathrm{gm}$. of lactose or dextrin daily, in addition to ground bread or dog biscuit.

In spite of the earlier claims of some investigators, lactose and dextrin alone of the ordinary carbohydrates are able to bring about this transformation. The energy requirements of $L$. bifidus are much greater than those of L. acidophilus, and therefore appreciably more lactose and dextrin must be administered to stimulate the Tissier bacillus than L. acidophilus. Hull and Rettger ${ }^{4}$ found that the flora may be transformed also by milk feeding, though a longer time is required, and the change to the aciduric type is not as nearly complete as when lactose is used.

Human subjects respond to the same influences, though as a rule not so readily. Rettger and Cheplin ${ }^{5}$ were able as a rule to effect pronounced and at times almost complete transformation in the normal subjects employed, by the administration of $300 \mathrm{gm}$. or less of lactose or dextrin daily.

Transformation from the mixed to the aciduric type was further accomplished by

'Torrey, J. C.: loc. cit.

2) istaso, 1., and Schiller, J.: Compl. rend. Soc. de biol., 76, 243. 1914.

3 Rettger, L. F., and Cheplin, II. A.: Intestinal Flora. New Iaven: Yale University Press, igar.

+ Hull, T. G., and Rettger, L. F.: loc. cit.

5 Rettger, L. F., and Cheplin, II. A.: loc. cit. 
the administration of I gm. of lactose or dextrin plus I cc. of fresh broth culture daily to white rats, and $50 \mathrm{gm}$. of lactose plus $\mathrm{I} 50 \mathrm{cc}$. of broth culture to man. It appeared from their experiments also that implantation may be brought about with $2 \mathrm{cc}$. of broth culture alone in the rat and with $300 \mathrm{cc}$. in man.

Perhaps the most far-reaching and practical observations of these authors were those made on milk cultures of $L$. acidoplilus. In some instances $500 \mathrm{cc}$. of a twentyfour-hour acidophilus-milk culture were sufficient to bring about simplification of the flora in man, and the use of $\mathrm{I}, 000 \mathrm{cc}$. taken daily in two or three portions, or of $500 \mathrm{cc}$. of the milk and $100 \mathrm{gm}$. of lactose, proved to be effective in establishing a flora composed largely of L. acidophilus. Since L. acidophilus assumes such prominence in the intestine of normal children subsisting on milk, and since it may be implanted and induced by appropriate diet to grow and establish itself in human subjects of all ages, it has been employed in numerous investigations with persons suffering directly or indirectly from intestinal disturbances (Rettger and Cheplin; ${ }^{\mathrm{I}}$ Cheplin, Post, and Wiseman; $;^{2}$ Reddish; ${ }^{3}$ Kopeloff; ${ }^{4}$ and others), and as a result of these investigations is today widely used as a therapeutic agent, particularly in the form of milk culture or what is generally known as "acidophilus milk."

After several years of intensive effort to produce pure, viable, commercial acidophilus milk under strict laboratory supervision, this product has been made available throughout a large part of the country and is now occupying an important place as a health drink as well as a therapeutic agent among the so-called "cultured milks."

It seems to be quite well established that the viability of the organism is preserved better and for longer periods of time in milk than in other known media. Milk properly ripened with selected strains of $L$. acidophilus has a smooth creamy consistency and a characteristically agreeable odor and flavor. The curd is extremely fine and easily tolerated, and there is little wheying off at best.

Special methods for determining the viability of acidophilus products have been devised. One of these, which has attained very wide usage and has virtually assumed the rôle of a standard method, involves the use of whey agar (Rettger and Cheplin) ${ }^{5}$ and the application of from 5 to ro per cent of carbon dioxide gas to the plates in closed containers (Kulp). ${ }^{6}$

Different strains of L. acidophilus from one and the same, and from different sources, vary among themselves, though they are bound together by group characteristics, and may readily be distinguished from L. bulgaricus in certain important respects; chief among these are a difference in deportment toward maltose (Rahe ${ }^{7}$ and $\mathrm{Kulp}^{8}$ ), a difference in the degree of acid production in definite periods of time, and in the ability to establish themselves in the intestine.

$L$. acidophilus may be distinguished also from $L$. bifidus and L. odontolyticus-

\footnotetext{
${ }^{\mathrm{r}}$ Rettger, L. F., and Cheplin, H. A.: Arch. Inst. Med., 29, 357. 1922

${ }^{2}$ Cheplin, H. A., Post, C. D., and Wiseman, J. R.: Boston M. E S. J., 189, 405. 1923.

${ }^{3}$ Reddish, G. F.: Virginio M. Monthly, 50, 409. I923.

${ }^{4}$ Kopeloff, N.: Lactobacillus acidophilus. Baltimore, I926.

${ }_{5}^{5}$ Rettger, L. F., and Cheplin, H. A.: Intestinal Flora. New Haven: Yale University Press, I92 I.

${ }^{6}$ Kulp, IT. L.: Science, 64, 304. 1926.

7 Rahe, A. H.: J. Infect. Dis., I5, I4I. I9I4. ${ }^{8}$ Kulp, W. L.: J. Bact., 9, 357. I924.
} 
acidophilus of McIntosh and others. The last-named organism is one which is apparently identical with the "B. acidophilus" of Bunting and his associates, ${ }^{\mathrm{I}}$ which was recovered by different investigators from the cavities of decayed teeth and claimed by them to be responsible for dental caries. Recent investigation has shown that $L$. acidophilus as now recognized bears only certain minor resemblances to the organism associated with caries, and that in many important respects the two are entirely different.

\section{METHODS OF FECAL EXAMINATION}

No serious attempts have been made as yet to establish standard bacteriological methods of fecal examination, and there have been almost as many different procedures as investigators. Different aims have led to the use of different methods. Only a few of these will be given here.

MacNeal, Latzer, and $\mathrm{Kerr}^{2}$ were concerned with the following: the numbers and quantity of bacteria, nitrogen determination, and differential count. After mixing with a spatula or mortar and pestle, in order to obtain uniform test samples, I : roo suspensions in saline solution were prepared in 500-cc. glass-stoppered flasks, as a basis for study. The total quantity of bacteria was determined by three different procedures: the Eberle-Klein (plain slide) microscopic method; the method of Winterberg (ThomaZeiss blood-counting chamber); and the gravimetric method of Strasburger (fractional separation by washing and centrifuging, and weighing of dried bacterial residue). The original methods were modified to suit the occasion more adequately. The nitrogen in the bacterial residues was determined by the Kjeldahl method. From the nitrogen factors the average daily bacterial nitrogen in the feces and the percentage of total fecal nitrogen represented were calculated. Differential bacterial counts were made by a modified gram staining method in which the decolorizing was done with anilin-xylol followed with methyl alcohol.

Morris, Porter, and Meyer, ${ }^{3}$ in their investigation of the fecal flora of children, had as their chief aim the establishment of a standard by which the activities of the fecal flora of children could be recognized. This they hoped to do by the use of certain culture media and by microscopic examination. Stock suspensions of the feces were prepared by emulsifying $500 \mathrm{mg}$. of feces with $50 \mathrm{cc}$. of saline solution in small Erlenmeyer flasks.

The following constituted a complete routine examination: (I) microscopic examination and differential counts of gram stained smears; $(2)$ the use of the fermentation-tube method of Herter and Kendall ${ }^{4}$ in which glucose, lactose, and saccharose are employed; (3) cultivation on Loeffler's blood serum and in gelatin stab culture; (4) plate counts on sugar-free agar aerobically, and on lactose agar anaerobically; $(5)$ the use of Endo plates; (6) determination of spore-forming bacteria in lactose agar plates and in milk tubes; and (7) employment of acetic acid glucose broth tubes for aciduric organisms.

Rettger and his associates ${ }^{5}$ had as their chief purpose the determination of the

'Bunting, Nickerson, and Hard: Dental Cosmos, p. 931. Oct., I926.

${ }^{2}$ MacNeal, W. J., Latzer, L. L., and Kerr, J. E.: loc. cit.

${ }^{3}$ Morris, G. B., Porter, R. L., and Meyer, K. F.: J. Infect. Dis., 25, 349. 1919.

${ }^{4}$ Herter, C. A., and Kendall, A. I.: J. Biol. Chem., 10, 7, 203. 1909.

${ }_{5}$ Rettger, L. F., et al.: see various works cited previously. 
numbers and activities of certain types of intestinal organisms, namely, the aciduric (L. acidophilus and L. bifidus). Representative portions of feces (large, well-rounded loopfuls) were vigorously shaken in rubber-stoppered, thick-walled test tubes containing ro cc. of physiological saline solution and broken glass. The suspensions were then diluted to a turbidity of about 8.0 on the McFarland nephelometer scale. These constituted the stock suspensions. For the determination of colonies of the aciduric organisms (particularly L. acidophilus) special agar medium is employed. This may be either whey ${ }^{\mathrm{x}}$ or casein digest ${ }^{2}$ agar, or, as has been shown quite recently, a special tomato-peptone agar. The agar plates are incubated in closed containers in an atmosphere containing $5^{-10}$ per cent carbon dioxide. ${ }^{3}$ For the estimation of colonies of organisms not aciduric, ordinary agar plates are used. For a comparative study of gas production the Veillon tube as described by Veillon and Zuber ${ }^{4}$ is used, with the following modification. Instead of having one end of the 9 -inch tubing permanently sealed, it is left open for the insertion of a rubber stopper. The other end is plugged with cotton. Direct microscopic examination is made of slide films prepared from the stock emulsion and stained by the gram method. The preliminary coating of the slides with a very thin layer of dilute egg albumin enables the films to adhere more permanently to the slides.

Special objects require modification of methods and media. For example, in studying the fate of bakers' yeast in the intestine, the agar medium of Reddish ${ }^{5}$ may be employed. This consists of malt extract (powdered) Ioo gm., agar I $5 \mathrm{gm}$., and water $900 \mathrm{cc}$, and has a $\mathrm{H}$-ion concentration of $\mathrm{pH} 5 \cdot 5-5.6$.

${ }^{1}$ Rettger, L. F., and Cheplin, H. A.: Intestinal Flora. New Haven: Yale University Press, 1921.

${ }^{2}$ Kulp, W. L.: Bact. Abstr., 7, 9. 1923.

${ }^{3}$ Kulp, W. L.: Science, 64, 304. 1926.

${ }_{4}$ Veillon and Zuber: Arch. de méd., го, 517. I898.

${ }^{5}$ Reddish, G. F.: Abstr. Bact., 3, 6. 1919. 


\title{
CHAPTER XLVII
}

\section{BACTERIA OF THE RESPIRATORY TRACT}

\author{
D. J. DAVIS \\ University of Illinois College of Medicine, Chicago \\ ECOLOGICAL RELATIONS
}

In the respiratory passages of the normal newborn, no bacteria are found. Within a few hours organisms of various kinds enter and continue to inhabit these regions in relatively large numbers throughout life. Neither in numbers nor in variety are they uniformly distributed. Certain bacteria tend to localize in certain regions. Anatomical and physiological conditions are no doubt the important ecological factors in this normal distribution. Among such factors we may mention moisture; temperature; the existence of deep pockets or crypts in the mucosa; smooth or rough surfaces with pits, furrows, or grooves; the oxygen supply as determined by anatomic peculiarities in the respiratory passages; acid, neutral, or alkaline reaction of the tissue and secretions; narrow or wide channels; ciliary action; the deposition of food and foreign particles in certain localities; artefacts and alterations resulting from injuries, operations, pathological states, etc.

As a consequence, there have appeared in various regions of the respiratory tract more or less characteristic floras which have adapted themselves to the conditions prevailing there; for example, a nasal flora, buccal flora, tonsil flora, teeth flora, pharyngeal flora, etc. The nasal passages become inhabited chiefly by a moderate number of coccal organisms, both gram positive and gram negative; the buccal mucosa largely by gram positive diplococci; the surface of the tonsil by gram positive diplococci; and the crypts of the tonsils by a variety of organisms both aerobic and anaerobic, quite unlike those of the surface. The pharynx harbors chiefly large numbers of gram positive and gram negative organisms, diphtheroids, cocci, and bacilli. Below the larynx the numbers of organisms rapidly decrease, and in the deeper channels leading to the lungs proper only transients are found normally. After the teeth erupt, the mouth becomes more heavily infected with certain bacteria, especially fusiform bacilli and spirochetes of several kinds. These organs have much to do in maintaining a high bacterial content in the mouth throughout life. Especially is this true in individuals with teeth and gums in that pathological state which in most civilized people is the rule rather than the exception. As the teeth disappear in old age, the mouth becomes less heavily contaminated.

\section{LYMPHOID TISSUE AND BACTERIA}

There appears to be an interesting relation between the distribution of bacteria and the distribution of lymphoid tissue in certain channels of the body, especially the respiratory tract. Lymphoid tissue occurs, generally speaking, only in localities where absorption is taking place; for example, the clusters of lymphatic glands at the 
hilus of the lungs, of the liver, in the mesentery, and in the axillary, inguinal, and cervical regions, etc. The oro-pharyngeal region is especially important from the point of view both of normal and pathogenic bacteria and of lymphatic distribution. Here are prominent accumulations of lymphoid tissue, located around and on either side of the throat and covered by mucous membrane which often, as in the tonsils, dips down into pockets or crypts to a depth of a centimeter or more. Considered by itself, from the point of view of physiology, the oro-pharyngeal lymphoid tissue is not important, nor should we expect it to be. We may look upon it as a small part of the extensive system of lymphatics of the pharyngo-intestinal system, all of which taken together may possess an important function, but a part and even a large part may be removed without serious disturbance so far as we now know.

Lymphatic nodes may be divided into two groups: first, the lymphatic nodes found along the respiratory and intestinal canal located under the mucosa and covered by a layer of loose, modified epithelium, apparently designed for absorption. Bacteria are found normally deep in these nodes (Digby). They have no afferent lymph vessels but an abundant supply of efferent vessels which invariably lead to a second deeper set of nodes. This subepithelial lymphatic tissue is frequently arranged in prominent projecting masses as in the human tonsil and adenoids and in the Peyer's patches, and often the surfaces are corrugated, grooved, or pitted - the result being an increase of absorbing or secreting surfaces. This may be brought about at times by an evagination or tubular depression, as occurs in the tonsil of certain animals like the cow.

Lymphatic glands are recently acquired structures phylogenetically, being apparently limited to birds and mammals. Tonsils are even more recently acquired many mammals like the rat, beaver, porcupine, bat, and some others not possessing them. There is good reason to believe that bacteria and infectious disease in animals preceded the phylogenetic development of lymphatic structures, so that the view is suggested that this striking distribution of lymphatics in the body, and especially in the respiratory tract, may have been determined by the bacterial distribution; the distribution in turn, as stated above, was primarily determined by the anatomical and physiological conditions.

The oro-pharyngeal lymphatic tissues are prominent masses at the portal of two great systems, the respiratory and the gastro-intestinal. We do not know to a certainty that this location is an advantage to the body, but from the standpoint of disease transmission, viruses and bacteria of various kinds evidently find it to their advantage to locate here; for as air, secretions, food, etc., pass back and forth over these structures, aided often by such processes as coughing, sneezing, talking, and breathing, they are readily carried either to the outside of the body where they may be able to enter another body or deeper into the body, thereby extending their field of activities, to the decided advantage of the micro-organisms in their struggle for existence.

When one views the respiratory and alimentary tract from the lips to the rectum, one observes two localities where striking accumulations of lymphoid tissue appear, namely, in the region of the throat, and in the lower small intestine, about the ileocecal valve and appendix. The intervening localities, like the stomach, duodenum, etc., have lymphoid tissue, but it is irregularly distributed and far less in quantity. A priori, this would indicate excessive absorption of dangerous matter in these local- 
ities, and this appears to be true. For in the throat and the region above and below the ileo-cecal valve, we find normally the greatest number and variety of bacteria, as may readily be shown by making smear and culture preparations at intervals along the respiratory and alimentary canal. In these two lymphatic maxima not only is the normal bacterial flora more varied and numerous, but here occurs the greatest number of infections: in the throat, streptococcus, pneumococcus, meningococcus, staphylococcus infections, diphtheria, the viruses of numerous exanthemata, and other diseases; in the lower intestine and colon, typhoid, paratyphoid, dysenteries, tuberculosis, appendicitis, etc. In the intervening localities relatively few infections occur. The pathogenic organisms often invade primarily the lymphoid structures themselves or the parts rich in lymphoid tissue. In other words, it would appear that, in some instances at least, certain organisms become adapted to grow in lymphoid tissue; that is, they attack the very mechanism which the body has apparently designed for bacterial protection. Striking examples of this phenomenon are seen in the hemolytic streptococcus infections of the tonsils. These are acute inflammations of these organs, involving the surface and the crypts and are definitely contagious. Transmission is direct, through droplets or contact. A variety of this infection is septic sore throat, many epidemics of which now have been reported, where the tonsils are infected from drinking milk containing streptococci that found their way there either from a person handling the milk or from the cow whose udder had been infected and then served as an incubator for these organisms. Experimentally, the writer showed some years ago that cows might become carriers of these human hemolytic streptococci when injected into the udder. These streptococci seem to have a very remarkable specific affinity for the pharyngeal lymphoid tissue.

The foregoing observations are quite in accord with a more or less general pathological principle which illustrates strikingly the adaptation that is taking place continually between our bodies and bacteria. The same principle is involved in the formation by staphylococci and streptococci of specific substances called "leukocidins" which destroy the defensive cells in our bodies, the leukocytes. Lymphoid tissue thus may not be equally protective against all bacteria, and in certain infections this mechanism breaks down entirely and instead of being protective furnishes a fertile soil for growth of bacteria and a route for invasion. The germs may directly attack this tissue and successfully thrive there, at least for a time, until the body can marshal defensive mechanisms of another order. It is on account of the prevalence of certain infections in this tissue that it may be to the advantage of the body to remove this mechanism or a part of it as is done in tonsillectomy, appendectomy, etc.

The distribution of plasma cells in the body is suggestive in connection with infections of lymphoid tissue especially of the respiratory tract. Generally speaking, these cells are indicative of chronic inflammation or irritation, and most writers regard them as pathological cells, at least when found in appreciable numbers. They accumulate in masses about centers of chronic inflammation and in general are characteristic of granulation tissue. They appear in many low-grade inflammations of the skin and mucous membranes.

Using the local accumulation of plasma cells as a possible criterion of the absorption of bacteria or their products, the writer studied the time of appearance and 
the distribution of plasma cells in tonsils. ${ }^{\mathrm{I}}$ The results briefly were as follows: 'These cells are not found in the fetus or the newborn. They make their appearance regularly about the second or third week, and are always found thereafter. In children several months old they are constantly found and usually in abundance. They remain present throughout life and even to very old age (eighty-eight years). In pathological states, and especially in hypertrophy, they are very numerous. They occur under the epithelium of the crypts along the strands of connective tissue and clustered about small blood vessels.

In view of the rôle that these cells play in general pathological processes and since they occur so regularly in the throat a short time after the entrance of bacteria, one is led to suggest that their presence here indicates a chronic infective focus, where absorption of irritating products is constantly occurring. Aschoff has noted the same in connection with the appendix. Along the entire gastro-intestinal canal, too, may be observed large numbers of plasma cells under the mucosa and especially in the region of lymphoid follicles. These facts are in harmony with the observations made by Adami and others on the more or less constant penetration of the mucosa by organisms and which they named "subinfection." No doubt, many bacteria are constantly passing through the alimentary wall into the lymphatics and blood stream, there to be disposed of in different ways. In the sense, therefore, that the term "subinfection" has been used in connection with the condition of the so-called "normal tonsil," or in the sense in which Aschoff uses the term "chronic inflammation" in the appendix, so we may regard all throats as chronically inflamed a short time after birth. One should, however, interpret such findings rationally, and when the terms are used as above they should not necessarily convey the idea of a dangerous or serious pathological state requiring surgical intervention. Nor should they be interpreted as a focus of infection in the sense in which that term is now commonly used.

\section{HEMOLYTIC STREPTOCOCCI IN THE RESPIRATORY PASSAGES}

Some years ago, when the bacteriology of extirpated tonsils from certain cases of chronic infection was being studied, a striking difference was noted between the surface flora and the crypt flora of tonsils. ${ }^{2}$ On the surface, the predominant organisms were of the streptococcus viridans type, whereas the predominant organisms in the crypts of the same tonsil were, as a rule, hemolytic streptococci. The exceptions were few. The difference was so striking that at first great significance was attributed to this point, since the hemolytic varieties are so much more virulent, as a rule, than the other varieties. Later it was found that most tonsils, regardless of the associated condition, contained a similar flora. Hypertrophied tonsils, especially, but also others that show no noteworthy pathology, revealed this distribution of streptococci on the surface and in the crypts. Pilot ${ }^{3}$ in our laboratory also examined a series of tonsils after removal, extirpated chiefly for hypertrophy, though many were normal in size, and found on the surface hemolytic streptococci in 6r per cent. These organisms comprised usually less than to per cent of the total number of bacteria. In the same throats from which these tonsils were removed, cultures taken just before extirpation

I Davis, D. J.: J. Infect. Dis., Io, I42. I9I2.

${ }^{2}$ Davis, D. J.: ibid., p. I48. I9I 2.

3 Pilot, I.: ibid., 24, 386. 1919. 
yielded 43 per cent positives. Crypt cultures, however, from these same tonsils yielded 97 per cent positives, and in almost all the hemolytic variety was greatly predominant. Furthermore in another series of cultures from the throat and pharynx $5^{8}$ per cent yielded hemolytic streptococci; and in persons without tonsils cultures similarly made yielded them in $\mathrm{I}_{5}$ per cent, and in these persons were found either bad teeth or tonsil remnants.

It appears that the crypts are an almost constant source of hemolytic streptococci, and this location may be considered in a sense their normal habitat. We have not been able to find any other part of the body that so constantly harbors them. The throat, as we have known for a long time, is their chief source and habitat in the body, and from the throat they may be distributed to various parts of the body by contact and otherwise. Or they may be transferred to other persons through the usual channels of transmission of respiratory diseases. This point deserves emphasis. Nearly everyone is harboring in the tonsils or throat hemolytic streptococci which have not been differentiated from strains that cause serious infections. Some of these strains are less virulent, but not all. Presumably such bacteria may at times cause arthritis, iritis, and other focal infections, but finding them in the tonsil may mean nothing in relation to a possible systemic disease.

The incidence of hemolytic streptococci in the normal throat has been studied by many workers, and the percentages as found range from to to 60 per cent or higher. From the mass of literature on this point the impression may be gained, indeed the conclusion has been drawn by some, that this organism does not occur in the throats of some persons. Others believe that certain persons are definite carriers in that they constantly harbor large numbers of the organisms in the throat. To test this point, I made throat cultures from a large group of adult persons, three or more times, with the following results: All at some time during an interval of about one month showed the presence of hemolytic streptococci in the throat. Probably they might all have been positive in a shorter time had more cultures been taken at shorter intervals. The percentages positive of the different groups varied from 33 to 66 .

On the whole, throat cultures from tonsillectomized persons contain fewer hemolytic streptococci than those made from persons with tonsils. ${ }^{\mathrm{I}}$ It has been shown, too, that in the naso-pharynx the incidence and numbers of these and other bacteria (pneumococci, hemophilic bacilli) are decidedly less in children whose adenoids and tonsils have been removed.

The incidence of hemolytic streptococci in the adenoids has been studied by Pilot and Pearlman ${ }^{2}$ who found them common in the naso-pharynx and its vegetations. From naso-pharyngeal swabs and the surface of the adenoids they were found in 55 per cent; from the depths between the folds of the cryptlike depressions of the adenoids of the same persons in $6 \mathrm{r}$ per cent in larger numbers. The excised tonsils of the same patients revealed them in still larger numbers in 95 per cent. These streptococci agree in their morphology, cultural characteristics, fermentation reactions, and pathogenicity, and are practically identical with hemolytic streptococci from various human sources. The adenoids, therefore, like the pharyngeal tonsils must be considered as common foci harboring hemolytic streptococci.

r Pilot, I.: ibid.

${ }^{2}$ Pilot, I., and Pearlman, S. J.: ibid., 29, 47. 1921. 
Streptococcus infections of the respiratory passages may arise from an endogenous or an exogenous source. The endogenous source is chiefly the tonsil, adenoid, and pharyngeal mucosa, as above stated, and no doubt gives rise to many of our secondary and terminal infections. On the whole, streptococci from this source are not very virulent or contagious, at least for normal persons, but may be highly fatal to those whose resistance is lowered by disease. The exogenous streptococci are those coming from outside sources through milk, contact, droplet infection, etc., and are usually more virulent and may be responsible for epidemics. We should recognize the possibility that the endogenous streptococci may become more virulent and aggressive and thus initiate an outbreak by contact or otherwise. For example, in measles epidemics in military camps, secondary streptococcus infections, at first not so virulent, may later become highly so and ultimately give rise to primary streptococcus pneumonias and empyemas without measles or another disease as a predisposing factor.

\section{FUSIFORM BACILLI AND SPIROCHETES}

The association of these two organisms in the body has been noted by many observers both in normal and diseased states. Certain points remain obscure. Miss Tunnicliff ${ }^{\wedge}$ has offered striking evidence that the bacilli and spirochetes are phases of a single organism, and Mellon ${ }^{2}$ has described various structures in the life-history of these bacilli strongly pointing to wide variations and interesting mutational phenomena. For our purpose, without attempting to establish the identity or non-identity of these organisms, we shall assume their almost invariable association in certain localities and processes. It is now being recognized that these organisms are of great importance both in normal and diseased states, being found constantly in every mouth and throat as opportunists. Under suitable conditions they tend to produce putrid and gangrenous lesions.

Of peculiar interest are their association and morphological relations to other bacteria in connection with two structures, namely, the tonsils and the teeth. Evidently in these two localities they thrive abundantly and from here as centers are disseminated to other organs and tissues of the respiratory passages. In the tonsil crypts frequently occur peculiar granular bodies. ${ }^{3}$ These are cheesy-like particles, foul smelling, small, gray or yellow, single or more often multiple, lying in the crypts, never in the tissues proper. In microscopic sections they are seen to be made up of filaments arranged in raylike fashion, suggesting actinomyces. These structures are remarkably uniform. On analysis, they are seen to be composed chiefly of four kinds of organisms evidently growing together in symbiosis, namely, leptothrix, fusiform bacilli, spirochetes, and streptococci. The leptothrix grows under anaerobic conditions and in the crypts develops into a cluster of filaments some of which radiate to the periphery, forming central stalks about which fusiform bacilli are arranged perpendicularly, closely resembling the structure of a test-tube brush. Scattered throughout this growth are very large numbers of spirochetes and streptococci. The streptococci in these masses are both hemolytic and non-hemolytic. The hemolytic are aerobic and quite

I Tunnicliff, R.: ibid., 33, I47. I923.

${ }^{2}$ Mellon, R. R.: J. Bact., I 2, 279, I926.

3 Davis, D. J., and Pilot, I.: J.A.M.A., 79, 944. 1922. 
like the varieties that occur commonly in the throat. Many of the non-hemolytic streptococci are distinctly anaerobic when first cultivated.

On the teeth along the gum margins appear constantly similar radiating growths made up of the same four varieties of organisms. They compose a considerable part of the tartar on the teeth. When removed, the growth returns again in two or three days.

These organisms may under given manifold conditions cause serious infections. They are prone to develop in tissues which have lost their vitality; for example, when injured by wounds, foreign bodies, vascular changes, tumors, severe infections, anesthetics, etc. As a result of the growth especially of the spirochetes, fusiform bacilli, and streptococci, there arise infections about the throat, gangrene about the mouth or face (noma), growths on the tonsils (pharyngonycosis), putrid infections of the middle ear, and various lung infections characterized by a foul odor of the breath and sputum, such as pulmonary abscesses, putrid bronchitis, and bronchiectasis and empyema. ${ }^{1}$

\section{HEMOPHILIC BACTERIA}

Of all the respiratory bacteria the influenza bacillus of Pfeiffer has attracted most attention recently because of the influenza pandemic of I9r8-i 9 . My own work has been along two chief lines:2 first, a study of the nutrition of this organism; and, second, its distribution and relation to respiratory infections.

Certain points as regards its nutrition are interesting. If blood or hemoglobin media is heated to boiling or less for a short time (a few minutes), it will support a profuse growth of these bacilli. If boiled for a long time (one to two hours) or if heated to a higher temperature in the autoclave for even a few moments, its value as a culture medium for this organism is lost. But its cultural value is regained, or we may say the media may be reactivated, by adding a small amount of any one of a large group of substances, namely, ascites fluid, serum, animal tissues (free of hemoglobin), plant extracts of various kinds (carrot or potato), dead or live bodies, or extracts of bacteria of various kinds or of yeasts, etc. Heating any one of these substances in the autoclave for a short time will render it inert for purposes of blood-media reactivation. Inorganic substances do not so behave. From these experiments it is clear that we are dealing with the interaction of two substances in this nutritive process. Either one alone will not support growth of this bacillus. The two combined will do so. The one is hemoglobin or one of its heat derivatives which is a relatively stabile body. It is an iron-containing substance and has been shown to be hematin or hemin. The second substance is a labile substance destroyed at autoclave temperature in a few moments or at the boiling temperature in one to two hours. It will pass through filter paper and through porcelain filters without appreciable loss. It resists acids, but appears to be susceptible to alkalies. It is found generally in the juices of both plants and animals. Serum, ascites fluid, extracts of heart, liver, brain, kidneys, etc., and also extracts of plants, especially carrot and potato, contain it in abundance. Bacteria

I Pilot, I., and Davis, D. J.: Arch. Int. Mrcd., 34, 313. I924.

${ }^{2}$ Davis, D. J.: J.Infect. Dis., 21, 392. I917; ibid., 29, I71. 1921; J.A.MI.A., 77, 683. 1921: Proc. Chicago Inst. Mcd., 2, I42. I919; J.A.M.A., 64, I814. 1915; ibid., 48, 1563. 1907. 
and various yeasts and related organisms like blastomyces or sporothrix, living or dead, or extracts of these organisms, are rich in this substance. It will appear from the foregoing facts that we are dealing with substances having many of the properties of the vitamines - substances which we now recognize as necessary for the life and growth of animals.

In addition to these two special substances, other nutritive substances, such as protein compounds or peptone, are necessary. Hemoglobin alone, which contains both these bodies, will not support the growth of this organism. This suggests that these two bodies do not themselves serve as food for the bacteria, but somehow through their interaction control other nutritive processes of the organism. As to the real nature of this process we know as much or as little as we know of the mechanism of the action of vitamines in higher organisms. The possibility exists that through the study of such processes in the lower and simpler forms of life we may be able to analyze more clearly analogous or comparable processes in the higher forms. For example, it would seem that in this process, as it concerns the influenza bacillus, the second substance somehow controls the metabolism of iron and through this element which is a vital necessity for life-processes controls other metabolic activities, especially those dependent upon oxidation changes. Similar substances may control metabolic processes dependent on other elements.

As a result of the analysis of the peculiar nutrition of the influenza bacillus it is now possible to explain satisfactorily the phenomenon of symbiosis or satellitism as observed in this organism. When grown with another bacterium or yeast or with plant or animal tissue, especially on plated media, the influenza bacilli near the foreign tissue or organism will grow profusely, often forming large giant colonies. This is explained thus: From the foreign organism or tissue there diffuses into the immediate vicinity this second vitamine substance which in conjuction with the hemoglobin or its derivatives furnishes the proper mechanism for the growth of the influenza bacillus. In the absence of blood or hemoglobin no such stimulating effect is noted in media about the foreign tissues or bacteria.

It is interesting that the influenza organism or its extract will not stimulate itself. This at once suggests the use of this method in the identification and differentiation of related bacteria about which there are disputed points. The pertussis bacillus, the Morax-Axenfeld bacillus, the Ducrey bacillus of soft chancre, and the KochWeeks bacillus all will stimulate the influenza bacillus, and on this basis may be differentiated from this organism. Many different strains of influenza bacilli of Pfeiffer, for example, strains isolated during the epidemic, strains from influenza meningitis, from various respiratory infections, and from normal throats, have been tested, and all fail to reveal this symbiotic phenomenon toward one another.

The distribution of the influenza bacillus is now fairly well known. Because of its hemophilic property it cannot live long except in close association with the animal body. In man it is a very common inhabitant, limited largely to the respiratory tract in normal and pathological states. In its distribution in this tract it is somewhat similar to the streptococcus hemolyticus. In normal throats in both adults and children it occurs roughly in from to to 40 per cent or even higher, and often in large numbers. 
It occurs almost constantly in whooping cough and very commonly in measles, tuberculosis, influenzas and catarrhs, varicella, bronchitis, meningitis, lobar pneumonia, pharyngitis, and sinus infections. In the terminal conditions, especially pneumonias of these different infections, it is common and abundant. On the whole, it appears to be more numerous in the respiratory tract in pathological states than in normal states.

Summing up the evidence at hand from the extensive studies made before, during and after the last epidemic, we may conclude fairly that no specific differences have been made out between the bacilli in the various respiratory infections, including epidemic influenza. As stated, all attempts by different workers to make out a definite epidemic strain of influenza bacilli as the cause of the influenza epidemic have failed. And the slight differences already noted-cultural, morphological, and serological - existing between strains are not known to indicate specificity for a given disease. This point should be emphasized. Because of the prevalence and the very wide distribution of Pfeiffer's bacillus in normal persons and in those suffering with a great variety of respiratory infections, studies upon the incidence of this organism are not of great value in any attempt to determine its relation to a given infection or epidenic. This point was responsible for many erroneous conclusions that have been drawn in connection with earlier studies.

The impression should not be gained that, because the evidence is decidedly against the existence of epidemic strains of Pfeiffer's bacillus, it is a relatively harmless organism. No better example need be given than the occurrence of the so-called "influenza meningitis," caused by pure growths of this bacillus; 90 per cent of these cases are fatal. And it may cause endocarditis, pericarditis, pleuritis, arthritis, and other infections from whose lesions the bacillus may be grown pure. Experimentally, too, for both humans and animals, it may have distinct pathogenic powers. In the human, inoculation with pure cultures will at times cause a definite infection. ${ }^{\mathrm{I}}$ But no one has reproduced a clinical picture that could be called typical influenza.

We may say, then, that the influenza bacillus of Pfeiffer is one of our common throat bacteria found in a high percentage of persons at any time and often in large numbers. It is found more frequently and in larger numbers in respiratory infections. No convincing evidence exists that it is the primary cause of pandemics of influenza or of any other respiratory epidemics. It requires for its existence not only the ordinary constituents of media, but also the presence of a vitamine or vitamine-like body.

\section{EPIDEMIOLOGICAL PROBLEMS}

Before concluding, mention should be made of the numerous epidemiological problems that present themselves in dealing with bacteria of the respiratory passages. Many of these problems remain at the present time extremely obscure and furnish some baffling phenomena. Many respiratory bacteria are opportunists, and because of a long association and close adaptation their relation and behavior to the host are complicated and difficult to analyze and understand. Our great respiratory epidemics appear suddenly, and we are as helpless before them today as were the people hun-

\footnotetext{
I Davis, I). J.: J. Infect. Dis., 3, I. 1)o6.
} 
dreds of years ago before the great epidemics of the Middle Ages. Colds, pneumonias, and virus infections of various kinds are striking examples of such phenomena.

Deserving of special emphasis, also, is the increasing recognition through recent contributions of a close association between respiratory bacteria and viruses and certain epidemic infections of the central nervous system. Enough is known at present to indicate clearly that the solution of at least some of our serious paralytic and encephalitic conditions lies along lines directed to control or combat the growth and dissemination of organisms entering the body through the respiratory passages. 


\title{
CHAPTER XLVIII
}

\section{INTESTINAL PROTOZOA OF MAN AND THEIR HOST-PARASITE RELATIONS}

\author{
ROBERT HEGNER \\ Johns IIopkins School of Hygiene and Public Health \\ PROTOZOA THAT LIVE IN MAN
}

CLASSIFICATION ACCORDING TO HABITAT

At the present time twenty-five species of protozoa are recognized by most authorities as human parasites. Many other protozoa have been recorded from man: most of these are simply well-known species that varied in certain respects from the normal condition; others may be new species but have not yet been sufficiently studied to warrant their inclusion here. These twenty-five species may be separated into three groups according to their habitats. In the first place, the so-called "intestinal protozoa" include six species of amebae, seven species of flagellates, one coccidium, and one ciliate; these live in the mouth, intestine, vagina, or urinary tract. The second group contains nine species of blood-inhabiting protozoa; these include three species of trypanosomes, three leishmanias, and three plasmodia. The last species in the list is a muscle parasite known as Sarcocystis. We know very little about this species in man; it may be a regular inhabitant of some lower animal which on rare occasions succeeds in establishing itself in a human being. In this chapter only the intestinal protozoa will be considered.

THE INTESTINAL PROTOZOA LIVING IN MAN

As indicated above, the intestinal protozoa of man include representatives of all four of the major groups of protozoa. The amebae belong to the class Sarcodina; the flagellates to the class Mastigophora; the coccidium to the class Sporozoa; and the ciliate to the class Infusoria. The morphological characteristics of these various organisms are illustrated in the accompanying figures.

The amebae (Figs. I-5) are characterized by a body that changes its shape frequently and possesses pseudopodia as locomotor organs. The structure of greatest importance in classification is the nucleus. The genus Endamocba (Figs. I, 2, 4) has a spherical nucleus containing a spherical karyosome and a layer of chromatin granules on the nuclear membrane. The members of the genus Endolimax (Fig. 5) have a nucleus with a large and often irregular karyosome but free from chromatin granules on the nuclear membrane. Iodamocbu williamsi (Fig. 3) possesses a nucleus with a large karyosome which is surrounded by a layer of achromatic granules. Dienlamoelua fragilis frequently contains two nuclei each of which has a karyosome consisting of a number of chromatin granules embedled in an achromatic matrix.

The intestinal tlagellates (Figs. 6, 7, and S) belong to five genera. The genus Trichomoms (Fig. 7) is characterized by the presence of an undulating membrane and an axostyle which extends through the center of the body antero-posteriorly. Chilomastix (Fig. 6) 
resembles Trichomonas in shape but lacks the undulating membrane and axostyle and possesses a conspicuous cytostome. Embadomonas intestinalis and Tricercomonas intestinalis are rare species. The human species of Giardia (Fig. 8) is a bilaterally symmetrical organism
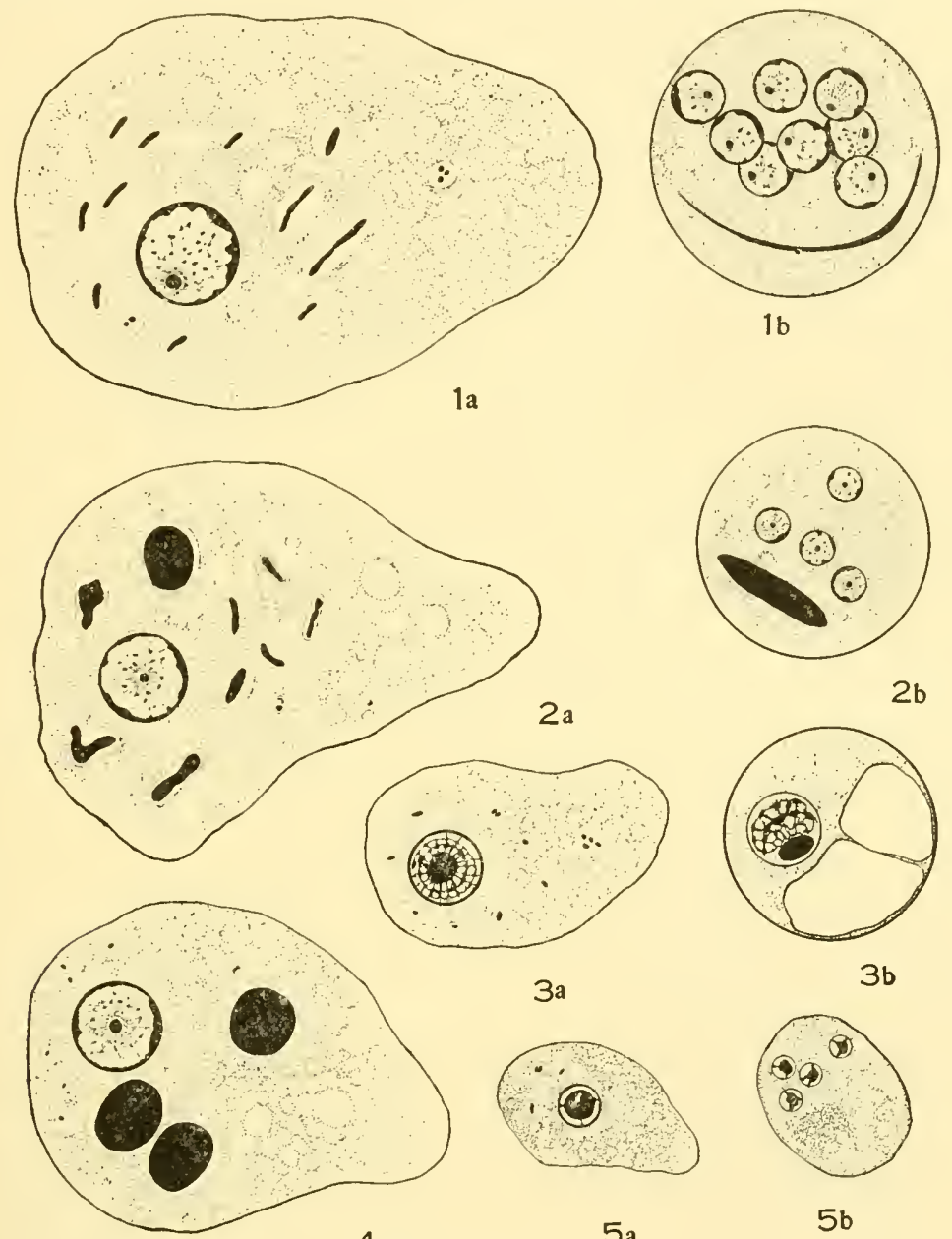

4

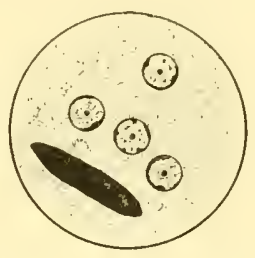

$2 b$

\section{$2 a$}

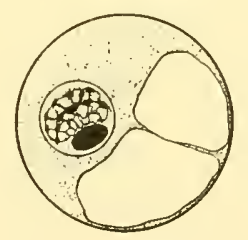

$3 \mathrm{~b}$

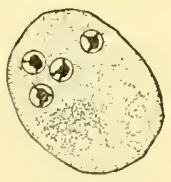

$5 b$

$5 a$

INTESTINAL AMEBAE

FIG. 1a.-Endamoeba coli, trophozoite

Fig. 1 b.-Cyst of same

FIG. 2a.-Endamoeba histolytica, trophozoite

Fig. 2b.-Cyst of same

Frg. 3a.-Iodanoeba williamsi, trophozoite

Fig. $3 b$. - Cyst of same

FIG. 4.-Endamoeba gingivalis

FIG. 5a.-Endolimax nana, trophozoite

Fig. 5b.-Cyst of same

FIGS. I-5 were drawn by Mr. Herbert Ratcliffe with a camera lucida from specimens fixed in Schaurlinn's solution and stained with iron-hematoxylin. All are magnified 2,000 diameters. 

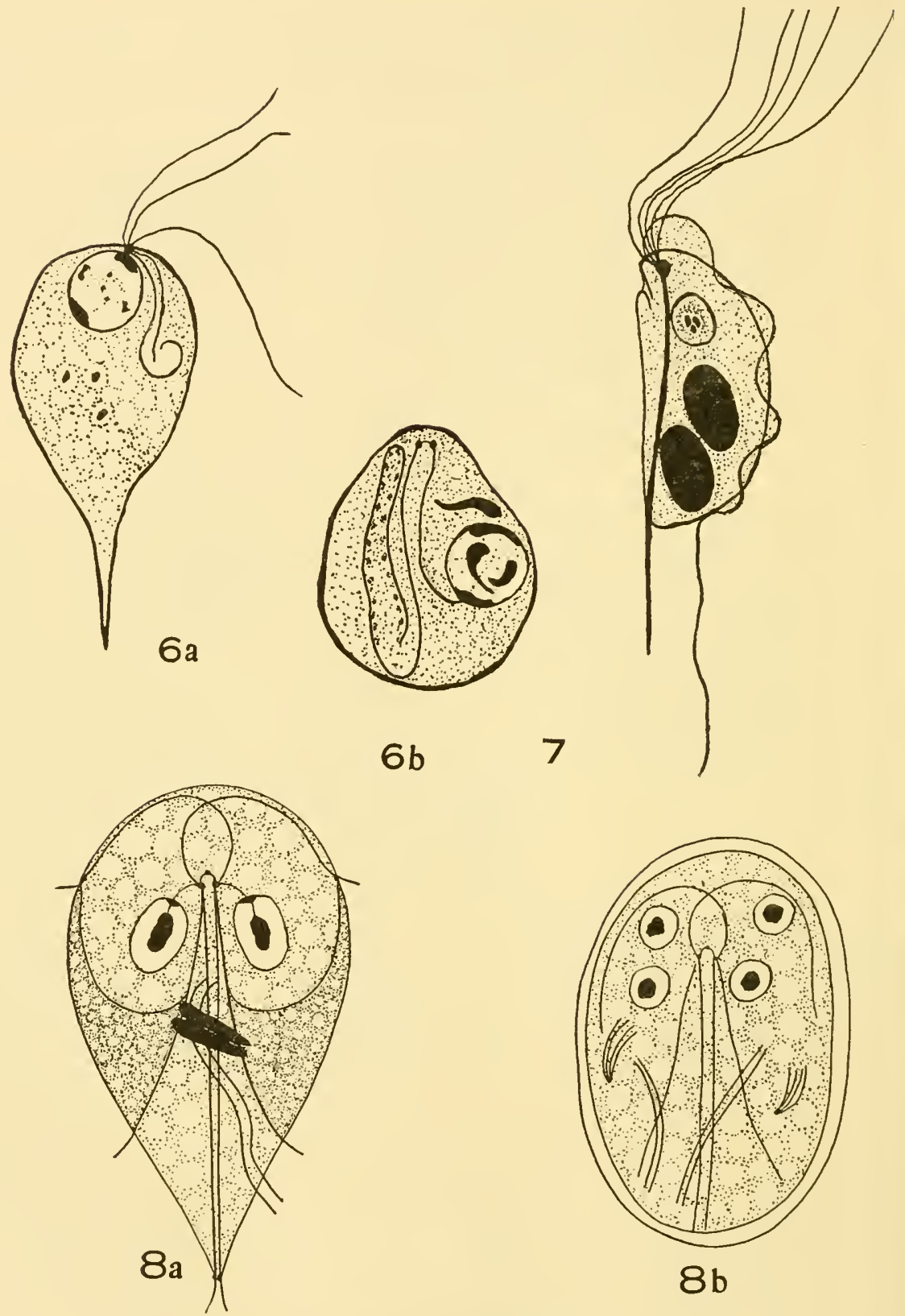

INTESTINAL FLAGELLATES

FIG. 6a.-Chilomastix mesnili, trophozoite

Frg. 6b.-Cyst of same

FIG. 7.-Pentatrichomonas, trophozoite

Figs. $6 a, 6 b$, and 7 were drawn by Mr. Herbert Ratcliffe; Figs. $8 a$ and $8 b$ drawn by the author.

All magnified 2,000 diameters.
Fig. 8a.-Giardia lamblia, trophozoite FIG. $8 b .-$ Cyst of same 
with a large sucking disk occupying the ventral anterior region of the body; it has two nuclei, two axostyles, four pairs of flagella, and two parabasal bodies.

The single human species of the class Coccidia, Isospora hominis (Figs. 9 and ro), is

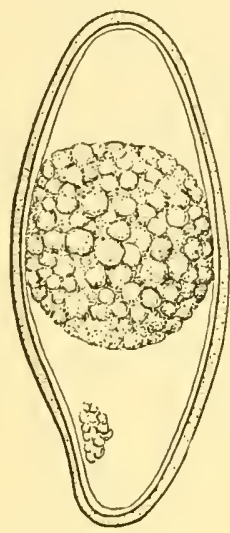

9

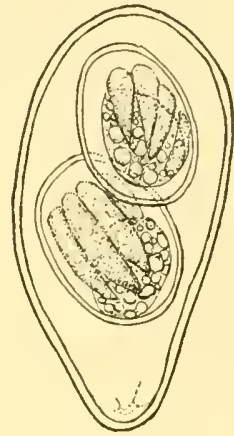

10

INTESTINAL, COCEIDIUM

FIG. 9.-Isospora hominis, young oöcyst

FIG. Io.-Isospora hominis, older oöcyst containing two spores each with four sporozoites.

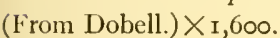
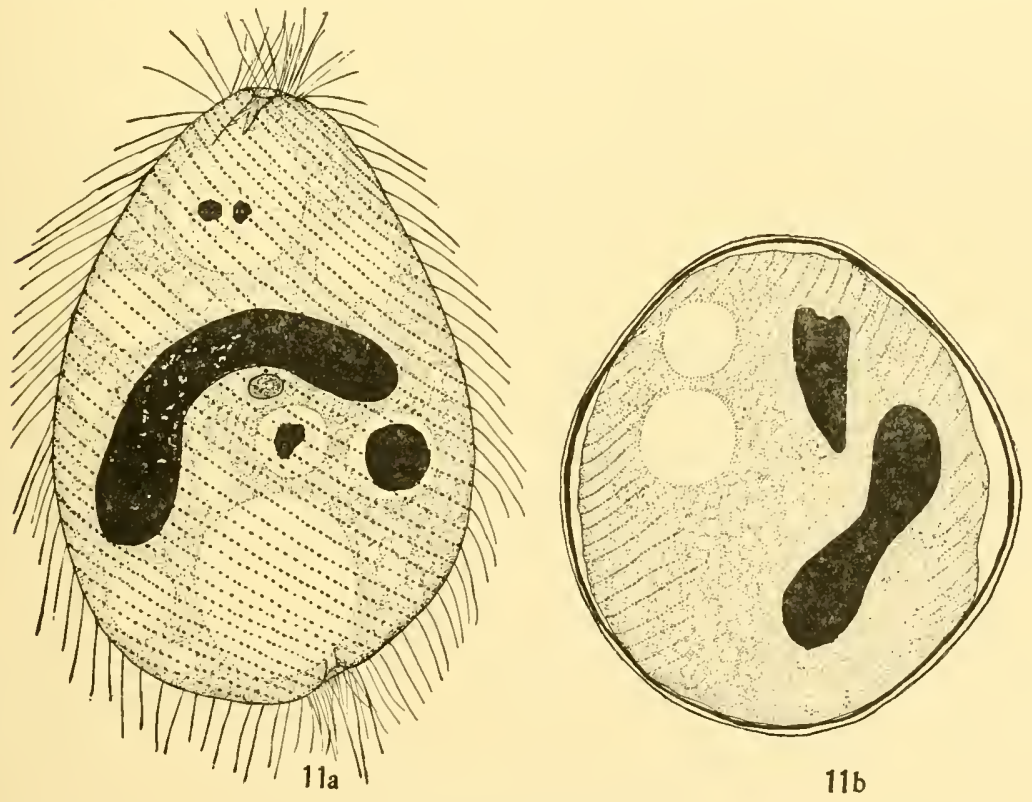

INTESTINAL CILIATE

FIG. I I a.-Balantidium coli, trophozoite. Original

Fig. I 1 b. - Cyst of same. (After Dobell and O'Connor.) $\times 1,000$ 
known only in the oöcyst stage. When fully developed the oöcyst contains two spores each of which has within it four sporozoites.

Balantidium coli (Fig. Ir), the only well-althenticated ciliate that occurs in man, is comparatively large and cannot possibly be confused with any other organism.

These intestinal protozoa are limited to certain definite parts of the body. The mouth is inhabited by Endamocba gingivalis and Trichomonas buccalis; the duodenum by Giardia lamblia; the small intestine by Isospora hominis; the large intestine by five species of amebae, four species of flagellates, and one ciliate as follows: Endamocba histolytica, Endamocba coli, Endolimux nana, Iodamocba williamsi, Trichomonas hominis, Chilomustix mesnili, Embadomonas intestinalis, Tricercomonas intestinalis, and Balantidium coli; and the vagina or urinary tract by Trichomonas viginalis.

The regions indicated may be considered the primary sites of infection of the various species listed. Several of these species, however, have been recorded from other parts of the body, which constitute the secondary sites of infection.

\section{HIOST-PARASITE RELATIONS OF INTESTINAL PROTOZOA}

\section{HOST-PARASITE RELATIONS}

This term is meant to include the relations between host and parasite not only during the period of the infection of the host by the parasite but also during the periof when transmission takes place. It involves studies of the life-cycle of the parasite, especially during the period when it is outside the body of the host; such phenomena as encystment, excystation, the distribution and localization of the parasite within the host, the resistance of the parasite to the defenses of the host, changes in the parasite due to residence in the host, the method of attack of the parasite, and the way in which the parasite escapes from the host so that new hosts may be infected. The reactions of the host to the parasitic invasion are also included. These concern the part played by the host in transmission and the localization of the parasite within the body, the natural resistance of the host to the parasite, and the changes in the host brought about by the attack of the parasite. Besides these subjects the term "hostparasite relations" involves the fascinating problems of host-parasite adjustments and host-parasite specificity. Studies of host-parasite relations are of both scientific and practical importance since any increase in our knowledge of relations between host and parasite adds to the effectiveness of our attempts at prevention and control. Heretofore studies have, for the most part, been made by zoölogists who are primarily interested in the parasite or by medical men who are primarily interested in the host. The present attempt is to bring together these two points of view and add that of the student of hygiene and public health. It is believed progress in prevention and control can be made most rapidly by such a combination.

\section{EPIDEMIOLOGY}

The methods of transmission of intestinal protozoa are fairly well known although exact data are difficult to obtain. Thus we believe, but are not certain, that Endamoeba gingivalis and Trichomonas buccalis, that live in the mouth, are transferred from one host to another by kissing; that Trichomonas vaginalis is transmitted by contamination or cohabitation; and that the other twelve species of intestinal protozon escape from the body in the feces and are ingested by new hosts in food contaminated 
by this infected fecal material. Cysts have been described in the life-cycle of most of these species, and transmission no doubt usually occurs in the cyst stage. Trichomonas, however, apparently exists only in the active trophozoite stage and, therefore, must pass from one host to another in this condition; it is possible that the trophozoites of other intestinal protozoa may also be infective. Successful transmission depends largely on the viability of the cysts and trophozoites during the period when they are outside of the body. It also depends on the behavior of the host rather than on the activity of the parasite, since the latter plays no part in its own distribution but reaches the drinking water or food of man because of man's own carelessness in disposing of infected fecal material. Another point that is of some interest is that protozoa do not increase in numbers while outside of the body of the host although several of them are not infective when passed but develop to the infective stage within a few days.

\section{PARASITOLOGICAL AND CLINICAL PERIODS}

In any discussion of host-parasite relations it is necessary to distinguish between parasitological and clinical periods. The clinical periods are well known. The incubation period extends from the time of the entrance of the parasite until symptoms appear. This is followed by the period of symptoms, and these two periods together constitute what may be called the primary attack. The primary attack is often followed by a latent period which may change to a second period of symptoms which is known as a "relapse." The parasitological periods may be called "prepatent," "patent," and "subpatent." The prepatent period extends from the introduction of the parasites until these organisms can be recovered from the host by a given laboratory procedure. This period is usually shorter than the incubation period, but in at least one case, that of Isospora hominis, it is longer. The patent period, during which parasites can be recovered, follows. Although the parasites cannot be found after the patent period, they may still be present in the body in small numbers; this may be called the "subpatent period." An increase in the number of parasites may again inaugurate a patent period which brings about a relapse.

\section{DISTRIBUTION AND LOCALIZATION OF PARASITES WITHIN TIIE HOST}

The protozoa of the mouth, vagina, and urinary tract that are transmitted by direct contact find themselves in their definitive locations immediately. The other intestinal protozoa are ingested by the host in water or food and passively carried by the host into the stomach. From the stomach they escape into the small intestine where Giardia and Isospora remain, the others being carried by peristalsis through the small intestine and into the large intestine. It is worth noting that this distribution is largely brought about by the host since the parasite, which is in the cyst stage, is inactive during this time. Why Giardia and Isospora remain in the small intestine is not definitely known although various suggestions to account for the primary site of infection of these forms have been made.

NATURAL RESISTANCE OF HOST AND PARASITE

Conditions within the digestive tract of man that are unfavorable for the existence of protozoa may be considered to constitute the natural resistance of the host. No 
doubt large numbers of protozoan parasites, not only characteristic human species but also species that live in lower animals, are frequently ingested by man, but are unable to establish themselves within the human host because of some type of natural resistance. On the other hand, certain protozoan parasites possess means of resisting these natural defenses of the host and, on this account, are successful in their attempts to grow and multiply within the digestive tract and thus bring about an infection.

\section{CHANGES IN HOST AND PARASITE DURING AN INFECTION}

Several of the intestinal protozoa of man are known to injure the host, thus bringing about the production of symptoms and often leading to diseased conditions that may result in the death of the host. On the other hand, other intestinal protozoa are supposed to be harmless commensals that live in the lumen of the intestine on bacteria and other food substances; these species when present in small numbers apparently do not injure the host to any appreciable extent. It is conceivable that enormously large numbers of them may bring about pathological conditions. The symptoms characteristic of the various diseases due to parasitic protozoa are for the most part well known but the genesis of these symptoms is still to be elucidated. Likewise, the injuries due to pathogenic species of intestinal protozoa have frequently been described in detail, but how these injuries are brought about is still in many cases in some doubt. Practically nothing is known regarding immunity to intestinal protozoan infections.

That the parasite itself may undergo changes during residence in the host has been suggested by various investigators. Thus it is supposed that a strain may become more aggressive, especially if it succeeds in passing from host to host in rapid succession. It has been suggested also that an immunity to the defenses of the host may be built up by the parasite. Our knowledge of this subject, however, is too slight to make a discussion of the problems involved profitable.

\section{HOST-PARASITE ADJUSTMENTS DURING AN INFECTION}

Cessation of symptoms in protozoan infections does not mean that the invading parasites have been eliminated from the host. On the contrary, in most cases the organisms remain in the body, perhaps for years, although too few in numbers to bring about the production of symptoms. A host in this condition is known as a "carrier." Such hosts pass infective stages of the organism in their feces and are thus largely responsible for the spread of infection. This carrier condition is due to some adjustment between host and parasite which brings about a sort of equilibrium. The parasite is said to be latent and, when the defenses of the host are weakened sufficiently, may succeed in increasing so rapidly in numbers as to bring about a relapse. Whether the host is sufficiently injured during the carrier period to exhibit observable symptoms is a problem that is at present in dispute.

\section{HOST-PARASITE SPECIFICITY}

Most of the human intestinal protozoa are rather rigidly limited in their habitat to the digestive tract of man. Dogs, cats, and other lower animals can be infected with Endamoeba histolytica but apparently very seldom become infected in nature. 
The Balantidium coli that lives in man is supposed to be the same species as that which occurs in pigs and monkeys. Other intestinal protozoa, however, seem to be more strictly limited to man. On the other hand, man undoubtedly ingests large numbers of the infective stages of intestinal protozoa peculiar to lower animals but does not become infected by them. The factors that make it possible for a certain species of parasite to live in a certain host species and not in others are little known but may be studied experimentally.

\section{HOST-PARASITE RELATIONS OF INTESTINAL AMEBAE}

Very little is known regarding three of the species of human intestinal amebae, namely, Endolimax nana, Iodamoeba williamsi, and Dientamoeba fragilis. Of the other three, Endamoeba histolytica undoubtedly is pathogenic, whereas Endamoeba coli and Endamoeba gingivalis are of doubtful pathogenicity.

ENDAMOEBA HISTOLYTICA

Epidemiology.-A considerable amount of experimental work has been done to determine the reactions of parasitic amebae to factors in the external environment. Most of this work has been done with Endamoeba histolytica but the information obtained is probably for the most part applicable to Endamoeba coli as well. The trophozoites of these amebas die rapidly after their escape from the body and probably seldom reach a new host in a viable condition. The cyst stage, therefore, represents the form capable of resisting exogenous conditions and is responsible for the transmission of the species. Experiments indicate that these cysts are incapable of withstanding desiccation and hence cannot be distributed in dust. Dilution of fecal material is supposed to increase the length of life of the cysts, hence cysts that find their way into contaminated milk or drinking water remain infective longer than in the raw feces. Efforts have been made to determine the resistance of cysts to temperatures with the result that cysts have been found to live at icebox temperatures for many days and still be infective. High temperatures of from $60^{\circ}$ to $70^{\circ} \mathrm{C}$. will kill cysts of Endamoeba histolytica in a few minutes. These temperatures, however, are not ordinarily encountered in nature. The effects of many different kinds of disinfectants on ameba cysts have also been determined. Apparently the best agent for the destruction of cysts is cresol in the strength of $\mathrm{I}: 20$. No practical method has yet been discovered of sterilizing drinking water, but the temperature employed in the pasteurization of milk is sufficient to destroy any cysts present.

The only conceivable method of infection with ameba cysts is the ingestion of them by the host, principally, probably, in contaminated food or drink. Contamination is brought about by insanitary conditions or by the carriage of the cysts by flies or other animals. A number of investigations have been carried on to determine the rôle played by flies in the transmission of intestinal protozoa. These studies have demonstrated that house flies ingest fecal material in nature, that ameba cysts may remain alive for many hours in the intestine of the fly, and that these cysts may be deposited in the feces of the fly in a viable condition after passing through the fly's digestive tract. It is obvious from these results that flies may carry ameba cysts in their bodies and deposit them in a living condition in drinking water or food and 
hence be instrumental in the infection of new hosts. Other animals do not seem to be as important as flies as transmitters of ameba cysts but rats, and possibly other domestic animals, may be infected with human amebae. Cysts are not produced in infected cats and dogs, hence these animals cannot be responsible for the transmission of the amebae. It has been noted that infections with Endamoeba histolytica occur frequently in all of the members of certain families indicating that contamination by association is frequent. It may be pointed out also that transmission is due entirely to the behavior of the host and not to any activities on the part of the parasite. It is man's own carelessness that brings about contamination of food or drink with ameba cysts and transmission can, therefore, be prevented by control measures based on personal hygiene and sanitation.

Parasitological and clinical periods.-It is difficult to determine the prepatent period in infections with Endamoeba histolytica since the time when cysts are ingested is usually unknown. Walker and Sellards (I9I3), however, have provided definite data on this point. These investigators succeeded in infecting eighteen of twenty men who were fed gelatin capsules containing cysts. Many of these men began passing cysts in four or five days, a period which represents the prepatent period. Persons once infected with Endamoeba histolytica apparently remain infected for years and perhaps throughout the rest of their lives, hence the patent period is of long duration. The incubation period as determined by Walker and Sellards ranged from twenty to ninety-five days.

Distribution and localization in the host.-The cysts of Endamoeba histolytica, when swallowed by the host, are passively carried through the small intestine and into the large intestine which is their definitive habitat and the primary site of infection. The cysts are supposed to excyst in the small intestine, but recent investigations prove that they are capable of excysting in the large intestine and may actually do so in normal infections. The process of excystation has recently been observed by Yorke and Adams (1926) in vitro. Apparently the only factors necessary to bring about excystation are moisture and a temperature of about $37^{\circ} \mathrm{C}$. for several hours. Studies of excysted specimens in cultures indicate that the single quadrinucleate ameba that escapes from the cyst wall divides into four uninucleate amebulae.

The primary site of infection.- Up to the year 1925 it was supposed that the trophozoites of Endamoeba histolytica were umable to live without access to tissue elements, but we now know that this species is able to live and reproduce on a diet consisting largely of bacteria. It is, therefore, possible for the amebae to live in the lumen of the large intestine without injuring the tissues of the host. In many cases, however, the amelae attack the intestinal wall. They are supposed to secrete proteolytic enzymes that dissolve the tissues and in course of time bring about the production of ulcers. Studies of autopsy material indicate that these ulcers are most abundant in the dependent portions of the large intestine where movement of the contents is sluggish.

Secondary sites of infection.-When intestinal ulcers are formed many amebae find their way into the capillaries and are carried probably to all parts of the body, where infections may be set up under favorable conditions. The principal secondary site of infection is the liver in which liver abscesses may be produced. Amebic abscesses 
occur less frequently in the lungs, brain, and other organs. Amelae have been recorded from almost all the organs and tissues of the body, the most recent addition to the list being the bones, by Kofoid and his colleagues. Several cases of infection of the ileum have been reported. Why the ileum is not more frequently infected is an interesting but unsolved problem.

Symptoms. - The symptoms due to infection with Endamoeba histolytica are well known. They are diarrhea and dysentery. Most of the human hosts do not exhibit symptoms; presumably the parasites in these cases do not interfere sufficiently with the normal functions of the body to bring about obvious changes. A question of considerable current interest is whether or not symptoms are exhibited by carriers. Kofoid and his colleagues believe that they are and that they are able to distinguish a definite disease entity which they call "chronic amebiasis." Acton and Knowles (I924) and Craig (I927) also believe that the so-called "healthy" carrier may exhibit clinical symptoms.

Pathogenesis.-The pathology of amebiasis is also well known, and descriptions of the lesions are available in a number of textbooks and reference-books on protozoölogy and tropical medicine. The way in which the organism produces these lesions, however, is not entirely clear. The amebae are supposed to secrete enzymes which dissolve away the tissues of the host. Until quite recently it was supposed that they were unable to obtain nutrition except from the hosts' tissues, but it was found that they ingested bacteria in cultures and hence it is possible that some of them may live in the lumen of the intestine and be non-pathogenic. Those that invade the tissues are large and do not appear to ingest red blood cells.

Resistance and susceptibility of the host.-The resistance of the host to infection with Endamoeba histolytica, as noted above, may be natural or acquired. There is some evidence that racial differences exist with respect to resistance and susceptibility. For example, the Chinese seem to be more frequently infected than foreigners in China but their infections are not so severe.

Age likewise seems to have an effect on resistance and susceptibility of the host. It is well known that kittens can be experimentally infected with Endamoeba histolytica more easily than adult cats. The nature of this apparent increase in resistance with age is, however, unknown. Certain experiments reported by Kessel (r923) indicate that infections with amebae of the same or of a different species add to the resistance of the host.

One of the most interesting problems in the study of amebiasis is the apparently greater number of severe infections in the Tropics than in the temperate regions. There may be more aggressive strains in the Tropics but there is no definite evidence of this. The facts observed can better be explained by the favorable conditions resulting from the warm, moist climate and by frequent mass infections due to the insanitary habits of many natives of the Tropics. The largely carbohydrate diet of the inhabitants of many tropical countries may also be favorable for the growth and multiplication of Endamoeba histolytica in the intestine.

Immunological reactions.-Practically nothing is known regarding immunity reactions in amebiasis. Several investigators have attempted to obtain complementfixation reactions; the experiments of Craig (1927) are the only ones that have given 
satisfactory results. Wagener (I924) has reported positive precipitin tests in cats infected with $E$. histolytica, and Scalas (1923) has obtained positive intradermal reactions, but these researches need confirmation.

Acquired resistance of the parasite.-The human experiments with Endamoeba histolytica carried out by Walker and Sellards (1913) prove that one strain of amebae may be more aggressive in one host than in another, thus demonstrating that it is differences in the susceptibility of the host rather than in the aggressivity of the parasite that account for the severity of the infection. The work of Baetjer and Sellards (I9I4) and of Wagener and Thomson (I9I4) with kittens, however, indicates that strains of amebae differ in virulence and that specimens from acute human cases bring about infections in kittens more easily than those from chronic human cases.

Emetin-resistant cases of amebiasis have been reported from time to time which suggest that there may be strains of E. histolytica that are resistant to this drug. At present it is only possible to express an opinion on the subject because of lack of evidence. Apparently in the so-called "emetin-resistant cases" the drug fails to reach the large intestine, and hence the parasite, because of some physiological idiosyncrasy of the host.

The carrier condition, latency, and relapse.-Walker and Sellards (I9I3) separated carriers into those who are infected and pass cysts but have never exhibited symptoms, i.e., contact carriers, and those who have recovered from an acute attack of amebiasis but still carry the organism in the intestine and pass cysts, i.e., convalescent carriers. Apparently when a person is once infected he remains infected for many years, possibly throughout the rest of his life, although what appears to be a continuous infection may be the result of reinfection.

During the carrier period when no symptoms are evident the amebae are said to be latent. Reproduction, however, appears to continue during periods of latency but at a reduced rate, and cysts or trophozoites may be found in the stools if daily examinations are made. Such a carrier may at any time suffer a relapse, a condition that is not uncommon in amebiasis. The relapses are no doubt due to some condition in the host that lowers resistance and enables the parasite to increase more rapidly.

Host-parasite specificity.-Endamoeba histolytica does not appear to be a regular inhabitant of the intestine of any other animal but man. Spontaneous infections have been described in monkeys, cats, dogs, and rats. Amebic liver abscesses have been noted in monkeys, cats, and dogs. Young cats appear to be particularly susceptible to infection with this species of ameba. Various species of laboratory animals, including rats, mice, guinea pigs, and rabbits, have been infected, but usually with difficulty. Infected cats do not pass cysts and hence are not dangerous. Rats, on the other hand, do pass cysts, but are so rarely infected as to be of practically no importance. It is thus evident that although Endamoeba histolytica is able to live in a number of lower animals its true host is man, and only under especially favorable conditions is it able to establish an infection in some other species of host.

Prevention and control.-Amebiasis is a preventable disease just as are typhoid fever, bacillary dysentery, cholera, etc. Contact and convalescent carriers who may pass as many as three hundred million cysts in a single day are more dangerous than hosts suffering from acute infections, since the cysts represent the infective stage, 
whereas only non-infective trophozoites are passed during an acute period of the disease.

Cysts can reach the intestine of man only by way of the mouth and hence efforts to protect food and drink from fecal contamination furnish the best means of prevention. In countries where night-soil is used as fertilizer, for example, in China, uncooked vegetables and fruit should be eaten only after being thoroughly washed, and immersed for ten seconds in boiling water. Infection apparently spreads throughout a family if one member becomes a host, hence particular attention should be directed toward sanitary conditions in the home.

Improvements in water supplies and general sanitation are recommended for the protection of communities, since there is good evidence that infective cysts are carried in water supplies and that flies may transport cysts from infected fecal material to food and drink. The determination and treatment of carriers in the general population has been suggested as a means of preventing the spread of amebiasis, but the cost of such a campaign is at the present time prohibitive. Cresol appears to be the best disinfecting agent for destroying cysts in fecal material.

ENDAMOEBA COLI

This species resembles Endamoeba histolytica in its life-cycle and is no doubt transmitted in the same manner. That it is very successful in gaining access to and establishing infections in man is evident from the fact that about 50 per cent of the general population appears to be infected with this organism.

Endamoeba coli lives in the large intestine where it feeds on bacteria, yeast, starch grains, and all sorts of débris. Cases have been reported of amebae of this type that ingested red blood cells, and there is certain evidence that $E$. coli, under extraordinary conditions, may invade the intestinal wall, but from the data available it is safe to state that only rarely if ever does this species produce intestinal lesions.

OTHER INTESTINAL AMEBAE

Endolimax nana.-This is a harmless species that lives in the large intestine of about 25 per cent of the general population. Its life-cycle is similar to that of Endamoeba coli, and there is no evidence that lesions of any kind are produced by it in man.

Iodamoeba williamsi.-This is another inhabitant of the large intestine; it occurs in about ro per cent of the general population. It is apparently a harmless commensal.

Dientamoeba fragilis.--This species is also an inhabitant of the large intestine but is exceedingly rare and hence no discussion of its host-parasite relations is possible.

Endamoeba gingivalis.-E. gingivalis differs from other endamebae in the absence of a cyst stage in its life-cycle. This is probably correlated with the fact that transmission no doubt takes place by direct contact during kissing, since this organism lives in the human mouth. The incidence of infection is not definitely known, but probably 50 per cent of the general population harbor this species. The tartar of the teeth and materia alba around them and decayed spots are the particular habitats of this species. At one time it was supposed that $E$. gingivalis was responsible for 
pyorrhea alveolaris, but there is no evidence that this is actually the case, and at present this species must be included with the other harmless commensals.

HOST-PARASITE RELATIONS OF INTESTINAL FLAGELLATES

There are seven well-authenticated species of so-called intestinal flagellates that live in man. One species, Trichomonas vaginalis, occurs in the vagina of women and the urinary tract of men, and another, Trichomonas buccalis, lives in the mouth. The other five have been found only in the intestine.

I. Trichomonas vaginalis.-Various investigators have reported from to to 50 per cent of the women examined in various parts of the world to be infected with this organism. Only a few cases have been reported of infections in men. The method of transmission of this species is still in doubt. Both men and women may become infected during coitus. If the intestinal trichomonad and the vaginal trichomonad are one and the same species, a point that is not yet definitely established, the vagina may become infected by contamination with organisms from the intestine.

Very little is known regarding the pathogenicity of Trichomonas vaginalis. It has been reported most frequently from women suffering from some abnormal condition, especially when the reaction of the vaginal mucus is acid. Treatment with sodium bicarbonate has been recommended by certain physicians who have reported favorable results. The only lower animal in which vaginal flagellates have been reported appears to be the monkey, Macacus rhesus.

2. Trichomonas buccalis. - The trichomonad that inhabits the mouths of about one-third of the human population is considered by many protozoölogists to be a distinct species to which the name Trichomonas buccalis is given. Transmission no doubt occurs during kissing. Recent studies by Hogue (1926) and Hinshaw (I926) indicate that persons suffering from pyorrhea, acute gingivitis, or abscessed teeth are more frequently infected than those whose mouths are in a normal condition. There is no real evidence, however, that this flagellate is in any way responsible for the pathological conditions with which it is associated. Trichomonads have recently been reported from the mouths of dogs and cats (Hegner and Ratcliffe [I927a, I927b]) but have not been recorded from any other lower animal.

3. Trichomonas hominis.--The transmission of Trichomonas hominis is of peculiar interest because there is no cyst stage in the life-cycle of this species, and hence new infections must be due to the ingestion of food and drink contaminated with material containing trophozoites. It has been suggested that Trichomonas buccalis that lives in the mouth may be the same as the intestinal species and bring about infection in the intestine when swallowed. This is not true, however, since individuals have been noted who harbored the mouth species but were free from the intestinal form. Furthermore, there are certain morphological differences between these two types. Experiments performed by the writer (Hegner [I924 and I926]) prove that several species of trichomonads are capable of passing through the stomach and small intestine of rats and guinea pigs without apparently affecting their viability, and no doubt $T$. hominis can pass through the stomach and small intestine of man without injury.

The incidence of infection with $T$. hominis in the general population, as reported in the literature, is much lower than actually exists. This is due to the fact that much 
of the fecal material examined is not fresh and the results of examinations are based largely on the finding of cysts, whereas the trophozoites of T. hom inis are not so easy to find. Thus, Boeck and Stiles (r923) noted only 0.07 per cent of this species in their large survey of eight thousand and twenty-nine individuals in the United States. In this survey fecal specimens from twenty-two states were examined many of which were several days old when received. Paulson and Andrews (1927) report an incidence of 4.3 per cent in two hundred and ten persons in Baltimore, from examinations of fresh stools. Hegner (I925) found 20.6 per cent of infection among natives of Tropical America and Hill (I926) obtained similar results in Porto Rico.

The best method of determining the presence of Trichomonas hominis, as first pointed out by Hegner and Becker (I922), is to inoculate cultures with a small sample of fecal material. The best medium seems to be the serum-saline-citrate mixture. This consists of a stock solution containing 0.07 per cent sodium chloride, and $\mathrm{r} . \mathrm{o}$ per cent sodium citrate. The medium is made up by adding to Ioo cc. of this solution $500 \mathrm{mg}$. of Loeffler's dehydrated blood serum. Cultures should be incubated at $37^{\circ} \mathrm{C}$. and examined at the end of forty-eight hours. As in the case of the amebae that are transmitted by fecal contamination, insanitary conditions, flies, and perhaps other animals are largely responsible for the transmission of this flagellate.

The ingested trophozoites of Trichomonas hominis are carried through the stomach and small intestine and set up an infection in the large intestine of susceptible hosts. Here they find conditions favorable for growth and reproduction. In the case of rats, which are infected with a species of trichomonad, T. muris, similar to the human intestinal form, Hegner ( 1923 ) has shown that a carnivorous diet apparently changes the character of the contents of the large intestine so as to react favorably against the parasite. It is suggested that the unfavorable conditions produced are due to a change in the bacterial flora from acidophilous to putrefactive types. A meat diet suggested by the writer (Hegner, I924) for the treatment of flagellate diarrhea has met with success in a number of cases.

Whether or not the trichomonads that live in the human intestine are pathogenic is a problem still unsolved. Frequently enormous numbers are found in cases of socalled "flagellate diarrhea" where no other cause for the diarrheic condition could be determined. The type of trichomonad with five anterior flagella, frequently called Pentatrichomonas, is most frequently associated with diarrheic conditions. This form ingests red blood cells with avidity but has not yet been found guilty of tissue invasion. If trichomonads are not responsible for flagellate diarrhea, they are present in such large numbers when this condition exists, probably because they find the intestinal content at this time particularly favorable for growth and reproduction.

4. Giardia lamblia.-A cyst stage occurs in the life-cycle of Giardia lamblia, and hence this species is no doubt transmitted by the same agencies as is Endamoeba histolytica (see p. 667). The cysts must gain access to the food or drink and are thus ingested by susceptible human beings. Cysts are apparently quite resistant to factors in the environment outside of the body and remain viable for many days. The incidence of infection with Giardia lamblia in the general population appears to be about Io per cent.

Giardia lamblia is the only intestinal flagellate that lives in the small intestine of 
man. It finds its optimum habitat in the duodenum where it clings to the epithelial cells with its ventral sucking disk and thus is able to withstand the downward force of peristalsis. Here large numbers are produced by binary division. Under certain conditions not yet known, the trophozoites encyst and appear in the feces at irregular intervals.

Flagellate diarrhea, also known as "lambliasis" and "giardiasis," is frequently attributed to this species. Most of the infected human beings do not exhibit symptoms but are carriers. As in the case of Trichomonas hominis, the presence of the organisms in diarrheic stools does not convict them of having caused the disturbance and hence the pathogenicity of G. lamblia is still in doubt. Recently a number of physicians have accused this flagellate of causing cholecystitis, but reports are contradictory and no definite conclusion has been reached.

For many years it was supposed that the giardias that occur in human beings and lower animals belonged to the same species and that human beings became infected by swallowing food or drink contaminated with infected feces of rats, mice, etc. More recent work (see Hegner [1927] for discussion) indicates that each host species has its own particular species of giardia, with the exception of rats and mice which are infected with the same species, and that cross-infection is rare or impossible. If this is true, then human beings can be inferted only by ingesting cysts of the human species of giardia, Giardia lamblia.

OTHER INTESTINAL FLAGELLATES

Chilomastix mesnili.-This species is present in about ro per cent of the general population. It lives in the large intestine, has a cyst stage in its life-cycle, and is probably transmitted in the same way as are other intestinal protozoa. C. mesnili is also accused of causing flagellate diarrhea but has not yet been proved guilty.

Embadomonas intestinalis.-This species is apparently rare, having been reported only a few times, and is hence of no practical importance.

Tricercomonas intestinalis. - This species also is rare, having been reported in less than a hundred cases. Its small size and the difficulty of studying it have resulted in much confusion between this and other intestinal flagellates.

\section{HOST-PARASITE RELATIONS OF INTESTINAL CILIATES}

Balantidium coli is the only species of intestinal ciliate that we know with celtainty to be a regular inhabitant of man. This large protozoön lives in the large intestine in the trophozoite stage, and produces cysts, which pass out in the feces and are no doubt responsible for transmission. The trophozoite, as Rees (1927) has found, will live at room temperature in material taken from the intestine of infected pigs for as long as ten days. The cysts are no doubt more resistant, and hence there is considerable opportunity for the contamination of food or drink with viable organisms.

When one considers the fact that the balantidia of man and pig are supposed to be the same species, and the probability that large numbers of human beings frequently ingest specimens from the pig, it seems strange that the incidence of infection should be so low in the general population. Balantidiosis is very rare in temperate regions, 
and nowhere can it be considered common. Some natural resistance of the human host apparently accounts for the small number of infections, but just what factors are responsible for this resistance is not known.

Under extraordinary conditions Balantidium coli may attack the tissues of the intestinal wall, producing ulcers that resemble the lesions caused by Endamoeba histolytica and resulting in balantidial diarrhea or dysentery. It is not certain just how these ulcers are produced. Walker (I9I3) believes that the ciliates bore their way mechanically into the intestinal wall and then dissolve away the surrounding cells by means of ferments which they secrete. Certain other investigators believe that the organisms penetrate the intestinal wall by breaking down the cells, as Endamoeba histolytica is supposed to do. Carriers of Balantidium coli exist among human beings, but Aguilar (1926) has reported evidence indicating that all individuals infected exhibit symptoms at some time during the infection.

According to our present knowledge of Balantidium coli, this species exists not only in human beings but in a large percentage of pigs in various parts of the world, and occurs in several species of monkeys. There is some evidence that the ciliate may be pathogenic to pigs, but this apparently is not the usual condition. Brumpt (I909) and Walker (I9I3) proved that specimens from monkeys were infective to pigs and that specimens from pigs were infective to monkeys. Walker was also able to infect monkeys with specimens from man. According to Scott (I925), the balantidium that occurs in guinea pigs is morphologically indistinguishable from Balantidium coli and may actually be the same species.

Epidemiological studies of balantidiosis in man indicate that in most cases human beings are infected by ingesting organisms from pigs. Strong (I904) found that 25 per cent of the cases described in the literature up to that time had been associated with pigs or had eaten or prepared fresh sausage. Since then many cases have been reported in persons who had worked with pigs. Infection can thus be prevented by the observance of greater cleanliness by those associated with pigs and by the thorough cooking of pork products before their use as food.

\section{HOST-PARASITE RELATIONS OF COCCIDIA}

The human coccidium, Isospora hominis, is known only in the oöcyst stage but it probably resembles in its life-cycle the similar parasite, Isospora felis, that occurs in cats. The oöcysts are extremely resistant and probably remain alive for a considerable period outside the body. During this time they may contaminate food and drink and be ingested by human beings. Human infections, however, are very rare.

It is known that Isospora felis of the cat attacks the epithelium of the small intestine and is thus always pathogenic. Isospora hominis no doubt similarly attacks the intestinal wall of man. The best account of a human infection with this species is that of Connal (I922). The incubation period in this case lasted for six days, at which time diarrhea commenced. Thirty-one days later stools became formed. Oöcysts appeared in the feces twenty-two days after the beginning of the diarrheic condition and were found daily for thirteen days. No more oöcysts were observed and no more symptoms exhibited by the patient after the end of this period. The appearance of symptoms in cases of coccidiosis before oöcysts are present in the feces is a peculiarity 
in the course of infection with coccidia worth pointing out inasmuch as in most protozoan infections the prepatent period is much shorter than the incubation period.

\section{REFERENCES TO LITERATURE}

Acton, H. W., and Knowles, R.: "Of Entamocba histolytica Carriers," Indian M. Gaz., 59, 440-44. I924.

Aguilar, R.: "The Treatment of Balantidium coli," I th A nn. Rep. Med. Dcp. Unitcd Fruit Co., I925, pp. 246-48. 1926.

Andrews, J. M.: "Host-Parasite Specificity in the Coccidia of Mammals," J. Parasitol., I3, I $83-94.1927$.

Baetjer, W. A., and Sellards, A. W.: "The Behavior of Amoebic Dysentery in Lower Animals, etc.," Bull. Johns Hopkins Hosp., 25, 237-4I. I9I4.

Boeck, W. C.: "The Thermal Death-Point of the Human Intestinal Protozoan Cysts," Am.J.Hyg., I, 365-87. I92 I $a$.

Boeck, W. C.: "On the Longevity of Human Intestinal Protozoan Cysts," ibid., pp. 527-40. I $92 \mathrm{I} b$.

Boeck, W. C., and Drbohlav, J.: "The Cultivation of Endamocba histolytica," ibid., 5, 371407. 1925 .

Boeck, W. C., and Stiles, C. W.: Studics on I Tarious Intestinal Parasites (Especially A moebae) of Man, Hyg. Lab., Washington, Bull. 133. Pp. 202. I923. (Contains extensive discussion of the nomenclature of the intestinal protozoa.)

Boyers, L. M., Kofoid, C. A., and Swezy, O.: "Chronic Human Amebiasis," J.A.M.A., 85, I 44 I -47 . I 925 .

Brumpt, E.: "Démonstration du rôle pathogène du Balantidium coli, etc.," Compt. rend. Soc. dc biol., 67, 103. 1909.

Clark, H. C.: "The Distribution and Complications of Amoebic Lesions Found in I86 Postmortem Examinations," Proc. Internat. Conf. H calth Prob. in Trop. Amer., pp. 36579. Boston: United Fruit Co., I924.

Connal, A.: "Observations on the Pathogenicity of Isospora hominis, Rivolta, emend Dobell," Tr. Roy. Soc. Trop. Med. Eo Hyg., 16, 223-45. 1922.

Craig, C. F.: "The Symptomatology of Infection with Endamocba histolytica in Carriers," J.A.M.A., 88, 19-21. 1927 .

Cra:g, C. F.: "Observations upon the Hemolytic, Cytolytic, and Complement-Binding Properties of Extracts of Endamsbul histolytica." Am. J. Trop. Mcd., 7, 225-40. 1927.

Dobell, C.: The Amoebae Living in Man: a Zoölogical Monograph. Pp. I55. London, I9I9. Dobell, C., and O'Connor, F. W.: The Intestinal Protozoa of Man. Pp. 2 I. London, r921. Haughwout, F. G.: "The Tissue-Invasive Powers of the Flagellated and Ciliated Protozoa with Especial Reference to Trichomonas intestinalis," Philippine J. Sc., B, 13, 21 7-59. I9I8. (A critical review.)

Hegner, R. W.: "The Effects of Changes in Diet on the Incidence, Distribution and Numbers of Certain Intestinal Protozoa of Rats," Am. J. Hyg., 3, I8o-200. 1923.

Hegner, R. W.: "Infection Experiments with Trichomonas," ibid., 4, I 43-5 I. $1924 a$.

Hegner, R. W.: "The Relations between a Carnivorous Diet and Mammalian Infections with Intestinal Protozoa," ibid., pp. 393-400. I924b.

Hegner, R. W.: "A Carnivorous Diet in the Treatment of Flagellate Diarrhea," J.A.M.A., $83,23-24.1924 c$.

Hegner, R. W.: "Intestinal Flagellates in Tropical America," Am. J. Trop. Med., 5, 23945. I925a.

Hegner, R. W.: "Trichomonas raginalis Donné," Am. J. Hyg., 5, 302-8. I925b. 
Hegner, R. W.: "The Presence of the Human Intestinal Flagellate, Pentatrichomonas ardindcltcili, in a Healthy Carrier," ibid., pp. 554-55. 1925c.

Hegner, R. W.: "The Biology of Host-Parasite Relationships among Protozoa Living in Man." Quurt. Rev. Biol., I, 393-418. 1926a.

Hegner, R. W.: "Animal Infections with the Trophozoites of Intestinal Protozoa and Their Bearing on the Functions of Cysts," Am. J. IIyg., 6, 593-601. I926h.

Hegner, R. W.: "The Transmission of Human Protozoa," Scicnce, 64, 28-34. I $926 c$.

Hegner, R. W.: Host-Parasite Relations betwecn Man and II is Intestinal Protozoa. P'p. 246. New York: Century Co., I927.

Hegner, R. W., and Becker, E. R.: "The Diagnosis of Intestinal Flagellates by Culture Methods," J. Parasitol., 9, I5-23. I922.

Hegner, R. W., and Ratcliffe, H. L.: "Trichomonads from the Mouth of the Dog," J. Parilsitol., I4, 5I-53. I927a.

Ilegner, R. W., and Ratcliffe, H. L.: "Trichomonads from the Vagina of the Monkey, Mouth of Cat and Man, and Intestine of Monkey, Opossum and Prairie Dog," ib:d., pp. 27-35. $1927 b$.

Hegner, R. W., and Taliaferro, W. H.: II uman Protozoölogy. Pp. 597. New York, 1924.

Hill, C. McD.; "The Successful Application of the Culture Method to the Diagnosis of Intestinal Flagellates in the Field," Am. J. IIyg., 6, 646-58. I926.

Hinshaw, H. C.: "Correlation of Protozoan Infections of the Human Mouth with the Extent of Certain Lesions in Pyorrhea Alveolaris," Proc. Soc. Exper. Biol. Eo Med., 24, 7 I-73. I926.

Hogue, M. J.: "Studies on Trichomonas buccalis," Am. J. Trop. Mcd., 6, 75-S8. I926.

Kessel, J. F.: "Experimental Infection of Rats and Mice with the Common Intestinal Amoebae of Man," Univ. Calif. Pub. Zoöl., 20, 409-30. I923.

Kessel, J. F., and Svensson, R.: "A Survey of Human Intestinal Protozoa in Peking, China," China M.J., 38, 961-82. I924.

Kofoid, C. A.: "Amoeba and Man," Univ. Calif. Chron., pp. 149-74, 291-312. 1923.

Ludlow, A. I.: "Amebic Liver Abscess," China M. J., 40, ir65-89. 1926.

Mackenzie, Sir James: "The Principles of Symptomatology: an Introduction to a New Outlook in Medicine," Lancct, 205, 963-68, 1020-24. 1069-75. 1923.

Mills, R. G., Bartlett, C. L., and Kessel, J. F.: "The Penetration of Fruits and Vegetables and Other Particulate Matter, and the Resistance of Bacteria, Protozoan Cysts and Helminth Ova to Common Disinfection Methods," Am. J. Hyg., 5, 559-79. 1925.

Paulson, M., and Andrews, J. M.: "Detection and Incidence of Human Intestinal Protozoa by the Sigmoidoscope," J.A.M.A., 88, г876-79. г927.

Rees, C. W.: "Ba'antidia from Pigs and Guinea-Pigs: Their Cultivation, Viability and Cyst Production," Scicnce, 66, 89-9I. I927.

Root, F. M.: "Experiments on the Carriage of Intestinal Protozoa of Man by Flies," $A m$. J. Hyg., I, I3 I-53. I $92 \mathrm{I}$.

Scalas, L.: "L’intradermoreazione nella disenterica amebica," Riforma med., 39, 967-69. I923.

Scott, J. M.: "Morphology, Fission, and Conjugation of the Balantidium from the GuineaPig," Anat. Rec., 31, 311. I925.

Sellards, A. W., and Thieler, M.: "Investigations concerning Amoebic Dysentery," Am. J. Trop. Mcd., 4, 309-30. 1924.

Stiles, C. W.: "The Public Health Status of Amoebic Dysentery in the United States as Potentially Influenced by the World War," Boston M. E० S. J., I 86, 377-79. 1922.

Strong, R. P.: "The Clinical and Pathological Significance of Balantidium coli," Bur. Gov. Lab. Bull., 26, Pp. 77. Manila, P.I., I904. 


\section{INTESTINAL PRO'TOZOA AND HOST-PARASITE RELATIONS}

Wagener, E. H.: "A Precipitin Test in Experimental Amoebic Dysentery in Cats," Univ. Calif. Pub. Zoöl., 26, 15-20. 1924.

Wagener, E. H., and Thomson, M. D.: "Experimental Amoebiasis in Cats from Acute and Chronic Human Cases," ibid., pp. 267-80. 1924.

Walker, E. L.: "Experimental Balantidiosis," Philippine J. Sc., B, 8, 333-49. I913.

Walker, E. L., and Sellards, A. W.: "Experimental Entamoebic Dysentery," ibid., pp. 253331. I9I3.

Wenyon, C. M.: Protozoölogy. 2 vols. Pp. 1563. London, I926.

Yorke, IV., and Adams, A.: "Observations on Entamoeba histolytica: I. Deve'opment of Cysts, Excystation, and Development of Excysted Amoebae, in Vitro," Ann. Trop. Med. and Parasitol., 20, 279-302. 1926. 


\title{
CHAPTER XLIX
}

\section{THE IMMUNOLOGICAL BASES FOR DIFFERENT TYPES OF INFECTION BY THE BLOOD PROTOZOA}

\author{
WILLIAM H. TALIAFERRO \\ University of Chicago
}

Certain of the blood protozoa by virtue of their easy accessibility and large size offer the unique opportunity of correlating the type of the infection manifested by the parasite with the immunological resistance acquired by the host. Certain prerequisites however are necessary before a given species can be used in such a study - the parasite must occur in the peripheral blood of the host in all stages and in sufficient numbers to be studied and the host itself must be somewhat tractable and procurable. For these reasons work in this field has been limited to certain of the trypanosomes and malarial parasites in laboratory animals, but it is to be hoped that in the future other infections may become available. Even some species of trypanosomes and malarial organisms are unsuitable. For example, Trypanosoma cruzi, the causative agent of Chagas' disease, passes a large part of its life-cycle in the vertebrate host as an intracellular leishmaniform parasite and Plasmodium falciparum of estivoautumnal malaria undergoes the major portion of its asexual reproduction within the smaller blood vessels of the deeper organs.

The term "resistance" as applied to the defense of an organism against invading parasites has many diverse usages and meanings. In the present review, where a correlation is being developed between the effects of the host's resistance and the types of infection, the term will be used only for those conditions either active or passive which may arise in the body as a result of infection and which directly affect the parasite. Used in this sense it will not include various possible mechanisms such as defense against the act of invasion, regenerative reactions of the host, or the formation of antitoxic substances. Similarly it limits discussion to acquired resistance because, as yet, experimental data are too meager for a systematic study of the effects of natural resistance.

As a matter of convenience in presenting the present résumé I shall give first the various methods which have been developed for analyzing the effects of the host's resistance against the parasite and then under five selected types of infection I shall outline in sequence the course of the infection, the effect of the host's resistance on the parasite, and the immunological bases for the observed effects.

\section{METHODS OF ANALYZING THE EFFECTS OF RESISTANCE}

After protozoa such as are considered in this review have successfully invaded a vertebrate host they reproduce asexually either by binary fission or schizogony. The first method is almost universally found in the trypanosomes (Figs. I and 2), although in Trypanosoma lewisi at the height of reproductive activity complete division of the 
cytoplasm may be retarded so that the progeny from a single trypanosome may be temporarily held together as a "rosette" (Fig. I). Schizogony which is characteristic of the malarial parasites may be looked upon as a modification of binary fission. Here
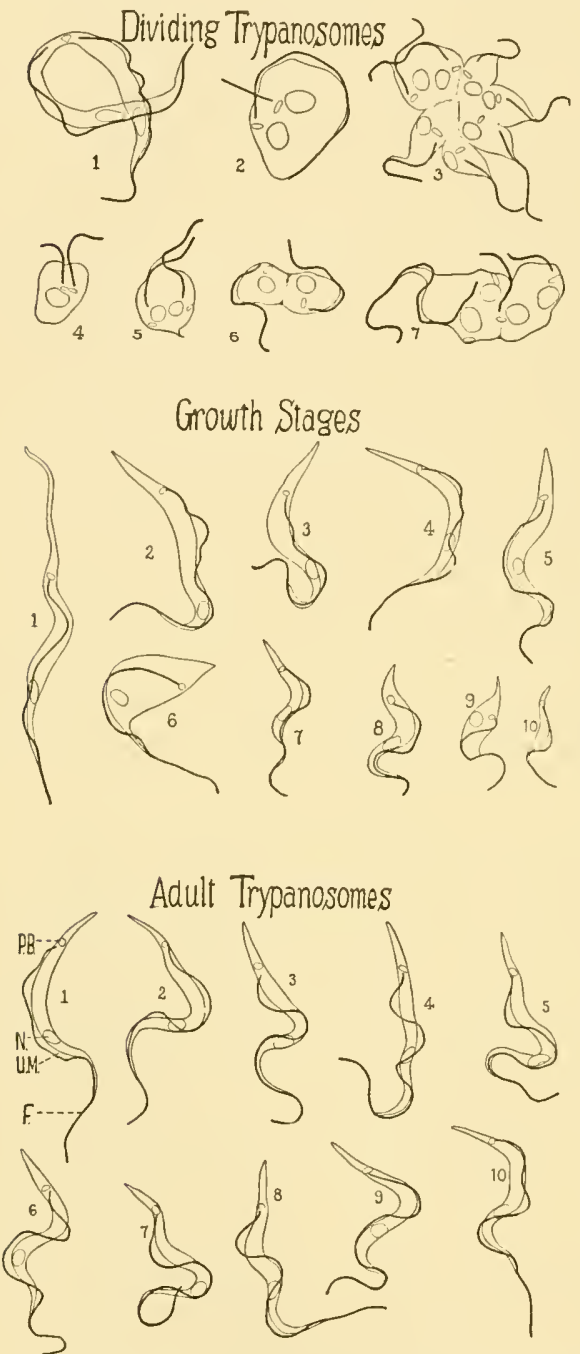

Fig. I.-Trypanosoma lewisi. Top: Dividing trypanosomes such as are found at the height of reproductive activity. Middle: Growth stages, taken from the fourth day of the infection shown in Fig. 7. (These are rarely found after the tenth day of the infection.) Bottom: "Adult" forms, taken from the twenty-eighth day of the same infection. $F$, flagellum; $N$, nucleus; P.B., parabasal body; U.MI., undulating membrane. $X_{\mathrm{I}, 000}$. (Top, after Coventry; others, after the author. the nucleus undergoes a series of binary fissions before the parent subdivides ("sporulates") - thus a number of progeny are produced at one time (Fig. 3).

If there is no resistance operative in the host and reproduction is unimpeded, the parasites whether they are reproducing by binary fission or schizogony should increase according to a geometrical progression series $\left(a, a r, a r,{ }^{2} a r,{ }^{3} a r,{ }^{4} \ldots a r,{ }^{n}\right.$ for example, I, 2, 4, 8, I6, etc.). As will be seen later, some infections follow just this course of number increase, but the

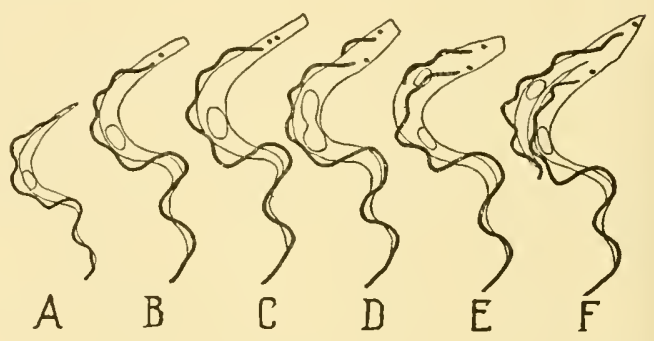

FIG. 2. - Trypanosoma equiperdum. Diagrammatic representation of $A$ and $B$, short and long individuals resulting from binary division; $C, D$, and $E$, stages in growth and division; and $F$, cell division. $X$ about $\mathrm{I}, 500$. (Modified after the author and L. G. Taliaferro.)

majority do not. Sometimes, instead of accumulating uniformly, the number of parasites remains constant for certain periods, or sometimes actually decreases, from which it is immediately apparent that some type of resistance is operative. Number changes such as these demonstrate the first method of analyzing the effects of resistance, i.e., the number curves of different infections indicate whether any resistance is operative and give an approximate measure of this resistance.

The number curve, i.e., the rate of 
accumulation of the parasites, however, may be affected by one or both of two entirely different mechanisms: first, the rate of reproduction of the parasites (cell

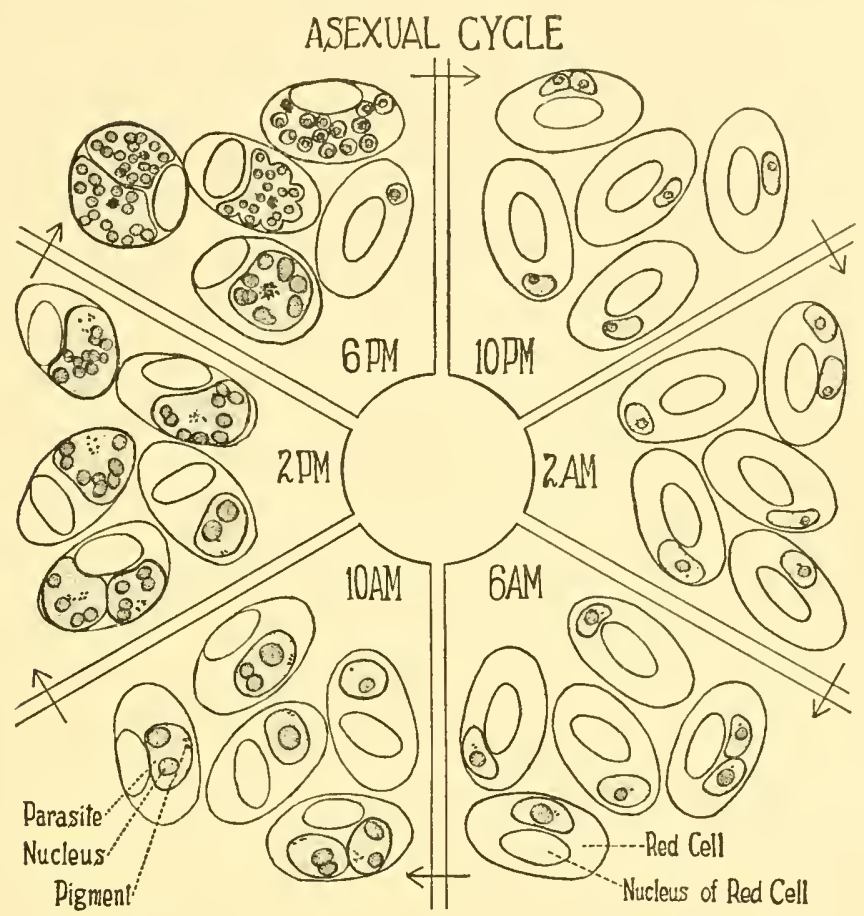

SEXUAL STAGES:present at all hours
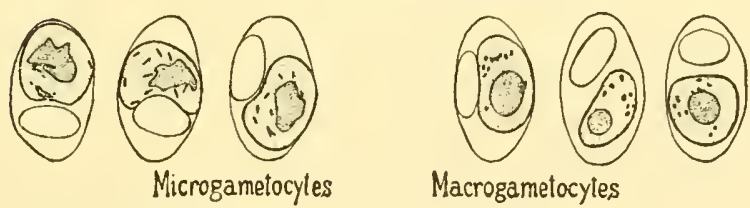

Macrogametocyles

FIG. 3.- Representation of the cycle of reproduction in bird malaria showing changes in size. Outlines of the asexual stages $\left(X_{1}, 500\right)$ of the parasites within the nucleated red cells, showing nuclei and pigment granules, made at four-hour intervals during a consecutive period of twenty-four hours. In addition, outlines of three microgametocytes and three macrogametocytes, which occur in small numbers at all hours throughout the infection. (From drawings by L. G. Taliaferro.)

division) may be retarded or inhibited; or, second, the parasites may be destroyed after they are formed. Briefly, this may be expressed by an equation:

No. of parasites per $\mathrm{cm}$. of blood $=$ No. produced by reproduction - No. destroyed

Hence, for any further analysis, the two mechanisms, represented by terms (2) and (3), respectively, must be difierentiated. The methods devised to attain this end will be outlined in the succeeding paragraphs. 
The members of this equation have not been measured exactly, but fairly accurate conclusions have been made possible by determining the first and second terms and evaluating the third (the author and L. G. Taliaferro [I922], the author [I924] on trypanosomes, and L. G. Taliaferro [ $\mathrm{I}_{92} 5$ ] on malaria). The first term (as previously indicated) is obtained by making frequent parasite counts during the course of the infection. The second term is obtained indirectly, since to be valid it must be independent of both the first and third terms, and hence can in no way depend on number counts. Because reproduction proceeds in cycles in malaria, whereas in trypanosome infections it does not, a different method is used in the two groups, but each method requires that sufficient parasites be in the blood to obtain a statistically valid sample.

An approximate method of measuring the rate of reproduction independent of the number of organisms destroyed is to ascertain the percentage of dividing forms. This is the method used by Robertson (I9I2) and has been used in all of our work as a check on the following method which is considerably more exact. Throughout the trypanosome infections fifty trypanosomes were drawn and measured at frequent intervals and their variability in total length, as expressed in terms of the coefficient of variation, compared. (The coefficient of variation was computed from the actual measurements by means of the usual formula and expresses the variability in terms of percentages of the mean size; the author and L. G. Taliaferro [1922].) For example, in $T$. lewisi, a coefficient of variation of 30 per cent indicates a population in which reproduction is at its height, whereas one of 3 per cent indicates a population of adults in which there is no reproduction (see Fig. I). Relating this to the equation, it is obvious that the number produced by reproduction in the first case would be very high, and in the second case nil. The rationale of this method is based on the wellknown fact that a sample of organisms measured, on the one hand, from a population undergoing rapid reproduction, with the constant production of young forms and intermediate growth stages, will exhibit much greater variability in size than a sample of organisms measured, on the other hand, from a population in which there is little or no reproduction and in which all of the organisms are full-grown adults. (For a fuller discussion of this method as well as a consideration of a number of its possible fallacies see the author and L. G. Taliaferro [1922].)

The coefficient of variation method is applicable to trypanosome infections because the organisms reproduce by binary fission with no periodicity. Thus, if reproduction is going on, a random sample at any time will include all stages of reproduction and growth. In the malarial infections, on the other hand, the asexual forms grow up and sporulate nearly synchronously (Fig. 3). Thus, a sample at one time will contain only small forms (merozoites); at another, only large forms (schizonts), ctc. The length of time it takes for the organisms to complete this cycle of growth and sporulation is, however, actually an expression of how long it takes one merozoite to become fifteen merozoites, and hence is a measure of the rate of reproduction of the parasites, and should it vary, the rate of reproduction may be said to vary. Accordingly, fifty parasites were drawn and their mean size obtained at each two- to four-hour interval during as much of the infection as possible. As can be seen from Figure Io, the data obtained showed a series of cycles, each cycle consisting of a gradual rise and abrupt fall in the mean size of the parasites. Comparing the time it takes this cycle to be completed during the various stages of the infection (acute, 
chronic, relapse) shows whether the rate of reproduction is constant or varying. In one strain of bird malaria, for example, the cycle took twenty-four hours throughout the infection, and therefore the same number of parasites were being produced throughout. This measure of the rate of reproduction presupposes that the average number of young produced by each full-grown parasite does not vary at any time. This assumption has been shown to be valid (I. G. Taliaferro [I925]). Furthermore, this method has the same advantage as the coefficient of variation method used in the trypanosome infections in that it is independent of the other two terms of the equation and can be used as long as sufficient parasites remain in the blood to obtain a statistically valid sample.

\section{CONTINUOUS PROGRESSIVE INFECTIONS OF PATHO- GENIC TRYPANOSOMES IN THE MOUSE}

The simplest type of blood infection is seen when the pathogenic trypanosomes are grown in the mouse. Here, as has been noted by earlier writers on trypanosomiasis, the parasites appear in the blood after a short incubation period and increase in numbers steadily and uniformly until the death of the host. Sometimes, but not invariably, the same type of infection is seen in the rat.

This type of infection is illustrated in Figure 4 when $T$. rhodesiense, the causative agent of one type of human sleeping sickness, is grown in the mouse. The mouse was injected intraperitoneally with some infected blood. Its blood contained trypanosomes four days later, and from then until the animal died, blood smears were made every twelve hours. The data obtained are represented graphically in the figure. In the first place, the variability of the parasites remained fairly high (C.V. $=8.87$-10.46 per cent) throughout the infection with no indication of a progressive change. From this we may conclude that reproduction was occurring at an approximately constant rate. The occurrence of dividing forms in the blood films corroborated these findings. In the second place, the organisms steadily accumulated in the blood, and increased according to a geometrical progression. From the constant rates of reproduction and accumulation in the blood we may conclude

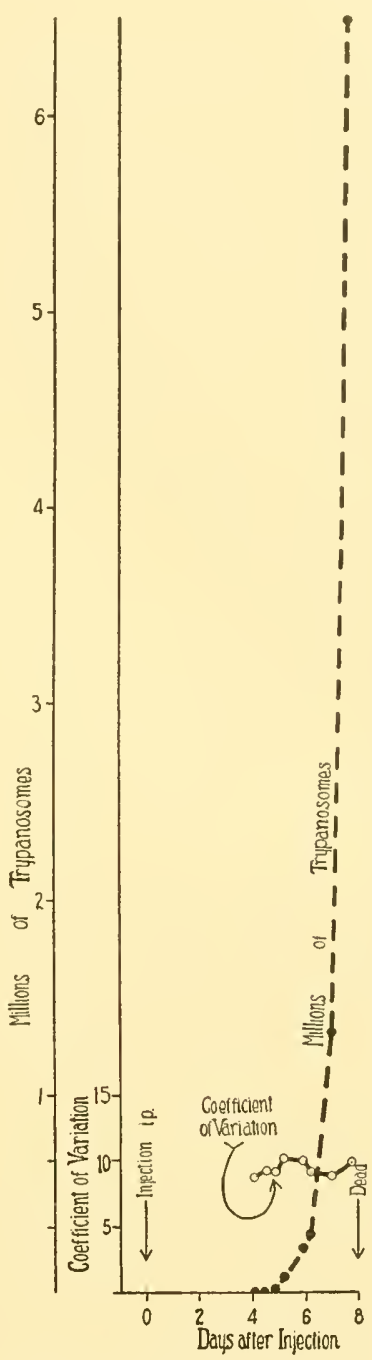

FIG. 4.-Graph showing the course of an infection of $T$. rhodesiense in a mouse. The high reproductive activity (represented by the coefficient of variation curve) and the type of number curve indicate that neither a reproductioninhibiting nor a parasiticidal resistance is developed. (From data by the author and L. G. Taliaferro.) that no resistance whatever is acquired by this host, either affecting the rate of reproduction of the parasites or destroying them after they are formed. 


\section{PATHOGENIC TRYPANOSOME INFECTIONS PROCEEDING BY CRISES AND RELAPSES}

When the same pathogenic trypanosomes as were considered in the preceding section are inoculated into guinea pigs and certain other animals, they exhibit, instead of a continuous and progressive infection, one that is marked by irregular increases and decreases in numbers. The periodic decreases, when the parasites more or less suddenly disappear from the blood, are generally spoken of as "crises," while the subsequent repopulations are termed "relapses." This type of infection has been recognized for many years and is probably very widespread. It is characteristic of $T$. rhodesiense, $T$. gambiense, and $T$. bruce $i$ in guinea pigs, according to the exact enumerative studies of Fantham and Thomson (IgII), and Thomson (IgII); and of $T$. rhodesiense in man, according to Ross and Thomson (I9IO). A number of authors have observed it in the dog, and it generally occurs when $T$. equimum is grown in rats.

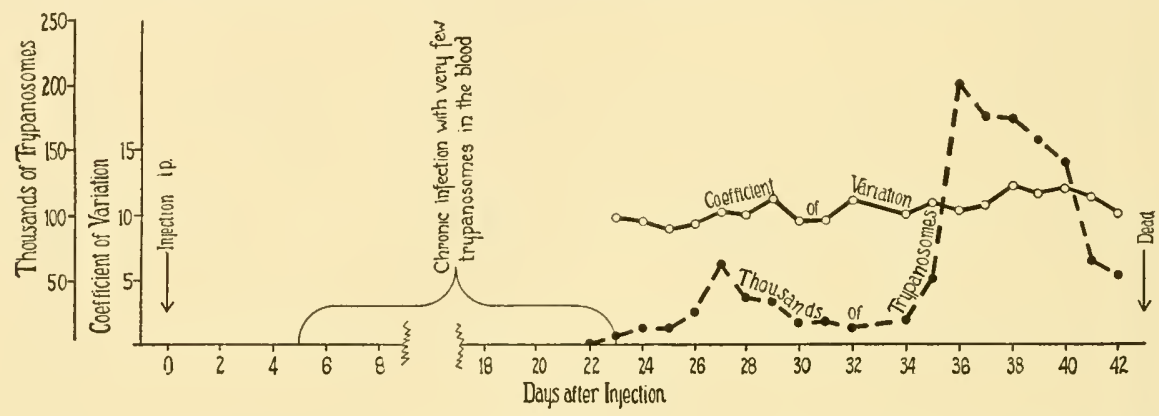

FIG. 5.-Graph showing the course of an infection of $T$. rhodesiense in a guinea pig. The high reproductive activity (represented by the coefficient of variation curve) indicates that no reproduction-inhibiting resistance is developed, but the type of number curve indicates that a parasitical resistance is developed. (From data by the author and L. G. Taliaferro.)

The sudden disappearance of the parasites from the blood, coupled with the in vitro serological studies to be reviewed later, early convinced investigators that the crises were actually periods of wholesale destruction of the parasites. An examination of the number curve in Figure 5, showing T. rhodesiense in the guinea pig, and a comparison with Figure 4 emphasizes how marked this destruction is. Instead of increasing steadily and rapidly, the parasites were very scarce for twenty-two days and subsequently only showed several comparatively small rises and falls in numbers until the death of the host on the forty-second day. Therefore, it may be concluded that a resistance is acquired which destroys the parasites after they are formed. The rate of reproduction, on the other hand, as expressed by the coefficient of variation curve, is strikingly similar in the two figures. Also, daily microscopical examination of the blood films showed cell division of the parasites to be taking place. Essentially analogous results were obtained in the dog. Therefore, this type of infection may be summed up as follows: Once the parasites are introduced into the host, they probably reproduce at a uniform rate during the entire infection. At intervals, however, most of the parasites that have accumulated in the blood are destroyed, but the few which escape destruction, since their rate of reproduction is unchanged, repopulate the blood again and again until the host dies. Expressed in terms of resistance, in this type of 
infection, which proceeds by crises and relapses, one of the two possible effects of resistance is operative, i.e., the host's resistance is directed toward a destruction of the parasites after they are formed but not toward an inhilition of the rate of reproduction per se.

A long series of investigations are in accord in showing that the wholesale destruction of the trypanosomes at each crisis is due to the sudden acquisition by the host of a trypanolytic property of its serum and that the trypanosomes reaccumulate during the relapse, not because the trypanolysin disappears, but because the trypanosomes become biologically altered so that they are no longer susceptible to the trypanolysin.

The first investigators to recognize the phenomenon of trypanolysis were probably Schilling (1902), Lingard (I904), and Franke (1905). The last also noted that the blood of the host could contain both trypanosomes and strong trypanocidal antibodies, which led him to point out that such parasites had evidently become biologically changed from the original strain and resistant to the antibodies. Credit is due Rodet and Vallet (1906) for the first systematic study of the lysins arising during the course of uninfluenced infections and their relation to crises and relapses. Massaglia (1907) showed that a definite correlation exists between the crisis and the lytic property of the serum. Thus, when tested against the original strain of trypanosomes, serum from a guinea pig before the crisis was only slightly lytic, whereas during and after the crisis it was strongly lytic. Furthermore, serum collected during and after the crisis had no deleterious effect on the trypanosomes reappearing after the crisis. This is clear experimental proof that the trypanolysin which effected the crisis remains in the blood (as shown by the activity of the serum against the original passage strain) and that the trypanosomes accumulate during the relapse because they have become biologically altered so that they are no longer susceptible to the lytic property. The work of Massaglia was greatly extended by Levaditi and Muttermilch (I909) who showed that trypanolysis like other immune lytic phenomena is a "complement-amboceptor" reaction, and by Leger and Ringenbach (I9II $a$ and $b$ ) who found the usual group specificity between a given trypanolytic immune serum and different species of pathogenic trypanosomes.

Simultaneously with the studies of these investigators, others, chiefly Ehrlich and his co-workers, were demonstrating the biological difference between the original strain of trypanosomes and the relapse strain in mice incompletely cured with drugs. Previous to their experiments, Ehrlich and Shiga (I904), Halberstaedter (I905), and Franke (1905) had shown that if a mouse is cured of an infection with a pathogenic trypanosome, it is refractory for about twenty days to a second infection with the same strain of parasites. Ehrlich (1909), Ehrlich, Roehl, and Gulbransen (1909), Rosenthal (I9I3), and Ritz (I9I4) found that the original strain of trypanosomes and the relapse strains were so different as to be differentiable by cross immunity tests. Thus, a mouse, infected with the original (passage) strain of trypanosomes and cured, is refractory to a second infection with the passage strain, but can be infected with a relapse strain and vice versa. With the same methods, Ritz (I9r6) differentiated the original and relapse strains arising during the uninfluenced course of an infection in the rabbit. 
It is essential in all of this work to keep the original passage strain of trypanosomes continuously in such an animal as the mouse where no crises occur and where the parasites do not come in contact with lytic antibodies and hence do not become biologically changed as they do after each trypanolytic crisis in such hosts as the guinea pig, dog, rabbit, etc. Failure to do this probably explains the peculiar results of Kligler and Weitzman (I924) who failed to find any evidence of the formation of humoral antibodies in experimental trypanosomiasis. They have, however, added the interesting experimental finding that the injection of olive oil brings about relapses. In view of the numerous experiments I have already cited, it would seem that their contention, that the defense of the body against trypanosome infections lies in cellular responses rather than humoral antibodies, might be modified simply to indicate that humoral antibodies owe their origin to the cellular responses. In this connection it is interesting to note that Muttermilch (I9r I) believes that trypanolysins are formed in the spleen and other hematopoietic organs, and Regendanz and Kikuth (I927) believe that the reproduction-inhibiting reaction product in $T$. lewisi which is described in the next section is formed chiefly by the reticulo-endothelium of the spleen.

The acquisition and inheritance of antibody resistance by the trypanosomes is a biological problem of great interest. Similar resistances to antibodies and lethal drugs have been observed in many parasitic and free-living protozoa. (For a general review of these "induced variations" in the trypanosomes see Dobell [I9I2], and for similar work on a free-living species, Paramecium, see Jollos [I92I].) A remarkable feature of the resistance is that it may be inherited for many asexual generations although in time the strain loses its resistance. Furthermore, a radical change in environment, such as passage through the invertebrate host or through another species of vertebrate host, is followed by the loss of resistance. Trypanosomes, to become resistant, do not have to be in the body, as a few minutes' contact between the organisms and the immune serum produces a resistant strain (Ehrlich, Roehl, and Gulbransen [I909] and Levaditi and McIntosh [I9IO]). Among the explanations offered for the mechanism of this acquisition, the following may be noted: Ehrlich's explanation formed an integral part of his side-chain theory of immunity and is too well known to need discussion. Levaditi and McIntosh (I9IO) believe that it is a process of selection in which the non-resistant strains are killed by the antibody, leaving the resistant ones. If they mean by this the selection of certain genetical strains from a "wild population" of mixed strains, it is not tenable, as the phenomenon is produced within a single cell strain the individuals of which are presumably of the same genetical constitution. Nevertheless, at each crisis when the resistance is acquired, there is obviously a selection, but one selection is effective - a fact in marked contrast to the slow effects of selection observed by various authors working with the free-living protozoa (see Jennings, I920). Finally, Rosenthal (I9I3) believes that there is a substance in the serum which has the specific property of producing relapse strains and which can be differentiated from the lytic antibody by (I) the lack of parallelism between the titre of the two, (2) differences in thermolability, and (3) differences in the fractions of serum in which they are precipitated.

It would seem probable from the work just presented that if lytic serum were 
injected into mice infected with the passage strain of organisms, an artificial crisis should follow similar to that seen in the natural infection in the guinea pig. Strange to say, most earlier investigators failed to obtain such results. Thus, Massaglia (I907) failed to increase the length of life of mice infected with $T$. evansi over that of a control with the injection of $1.0-2.0 \mathrm{cc}$. of serum from guinea pigs during a crisis. Diesing (I905), however, reports the temporary disappearance of parasites and clinical improvement of cattle and horses receiving serum from asses after recovery. Kleine and Möllers (1906) produced artificial crises in mice infected with T. togolense. Recently, the author and Johnson (1926) working with T. equinum have been able to prorluce artificial crises in mice infected with the passage strain by injecting lytic
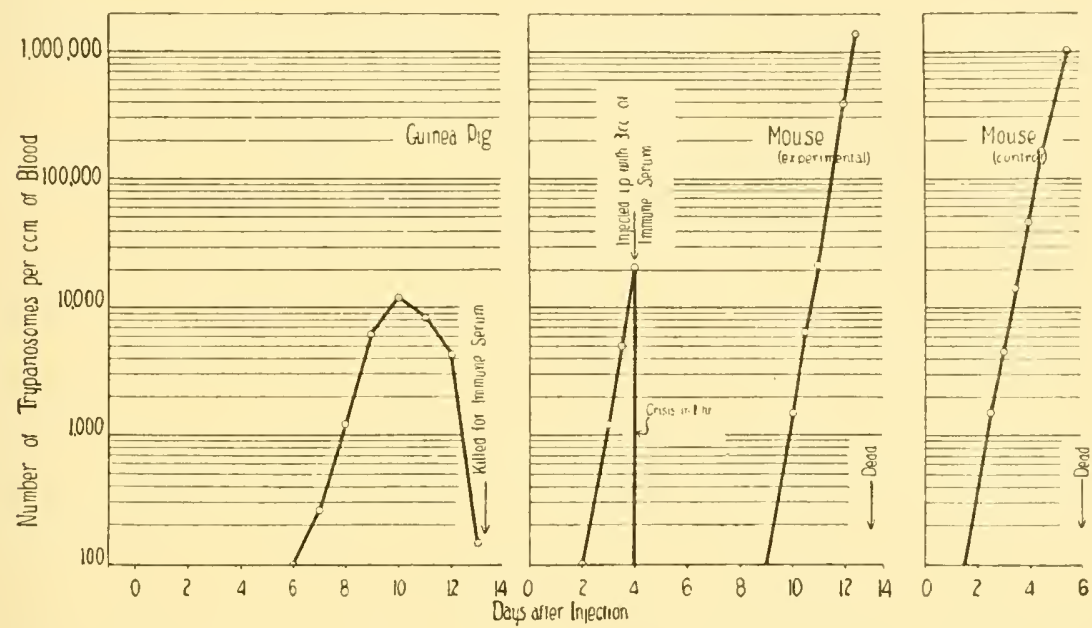

Fig. 6.-Production of an artificial crisis in a mouse with serum taken from a guinea pig after a natural crisis. The first graph shows the course of the infection in the guinea pig and the time of bleeding for immune serum. The second graph shows the crisis resulting from a dose of $0.3 \mathrm{cc}$. of the guinea-pig serum and the relapse which followed. The third graph shows the course of the infection in the untreated control mouse.

serum obtained from guinea pigs and rabbits after a trypanolytic crisis, from a sheep during its chronic infection, and from mice after incomplete cure with drugs. Figure 6 shows an experiment in which lytic guinea pig serum was used. Following infection with the passage strain and a crisis, the guinea pig (first part of figure) was bled and its serum preserved on ice until ready for use. In the meantime two mice had been infected with the same strain, and when the parasites showed up, one (second part of figure) was injected with $0.3 \mathrm{cc}$. of the guinea pig serum while the other (third part of figure) was left untreated as a control. Within an hour no trypanosomes could be found in the experimental mouse, nor did they reappear for six days. On the following day they were found in small numbers, and thereafter increased steadily until the animal died. In marked contrast the control mouse showed an uninterrupted increase of parasites until its death on the sixth day. Thus, an injection of 0.3 cc. of immune (lytic) serum not only produced a six-day crisis but prolonged the mouse's life seven days over that of the control. 
An important development of this work on trypanolysis which has many theoretical ramifications and which may explain some features of relapse phenomena is that there must be a definite relationship between the amount of immune serum and the occurrence of trypanolysis. Thus, paradoxically, if too much immune serum is given, there is no trypanolysis. This is obviously similar to the so-called "zone phenomenon" or the phenomenon of Neisser and Wechsberg (I90I) who, during the estimation of the bactericidal property of immune serum in vitro, made the observa-

TABLE I

Experiment with Immune, Serum from Infected Rabbit (from Taliaferro and Johnson)

Trypanosomes Injected Intraperitoneally into Each Mouse at Beginning of Experiment*

\begin{tabular}{|c|c|c|c|c|c|c|c|c|c|c|c|c|c|c|c|}
\hline \multirow{4}{*}{ Mouse } & \multicolumn{15}{|c|}{ DAY OF INFECTION } \\
\hline & \multirow[t]{3}{*}{ I } & \multirow[t]{3}{*}{2} & \multicolumn{4}{|c|}{3} & \multirow[t]{3}{*}{4} & \multirow[t]{3}{*}{5} & \multirow[t]{3}{*}{6} & \multirow[t]{3}{*}{7} & \multirow[t]{3}{*}{8} & \multirow[t]{3}{*}{9} & \multirow[t]{3}{*}{ I0 } & \multirow[t]{3}{*}{ I I } & \multirow[t]{3}{*}{12} \\
\hline & & & \multirow{2}{*}{ 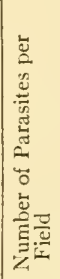 } & \multicolumn{2}{|c|}{$\begin{array}{l}\text { Immune } \\
\text { Serum: } \\
\text { Intra- } \\
\text { peritoneally }\end{array}$} & \multirow[b]{2}{*}{ 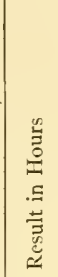 } & & & & & & & & & \\
\hline & & & & 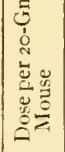 & 总 & & & & & & & & & & \\
\hline І $\ldots \ldots \ldots \ldots \ldots$ & + & + & 20 & 2.5 & 2.9 & -2 & - & $\mathrm{D}$ & $\ldots$ & $\ldots$ & . & $\ldots$ & $\ldots$ & $\ldots$ & $\ldots$ \\
\hline $2 \ldots \ldots \ldots \ldots \ldots$ & + & + & IO & 2.3 & 2.9 & + & + & D & $\ldots$ & $\ldots$ & & & & $\ldots$ & $\cdots$ \\
\hline $3 \ldots \ldots \ldots \ldots \ldots$ & + & + & IO & $2 . I$ & 2.8 & -2 & - & + & + & + & + & $\ddot{+}$ & $\mathrm{D}$ & & $\cdots$ \\
\hline $4 \ldots \ldots \ldots \ldots \ldots$ & + & + & IO & I. 9 & 2.6 & -2 & - & - & - & + & + & + & + & $\mathrm{D}$ & ... \\
\hline $5 \ldots \ldots \ldots \ldots \ldots \ldots$ & + & + & 10 & I. 7 & 2.5 & + & + & $\mathrm{D}$ & . . & $\ldots$ & & $\cdots$ & ... & $\ldots$ & ... \\
\hline $6 \ldots \ldots \ldots \ldots \ldots \ldots$ & + & + & IO & I. 5 & 2.2 & + & + & D & $\ldots$ & $\ldots$ & & $\ldots$ & & $\ldots$ & $\ldots$ \\
\hline $7 \ldots \ldots \ldots \ldots \ldots$ & + & + & 10 & I. 3 & I. 9 & -2 & - & - & - & + & + & + & $\mathrm{D}$ & & $\ldots$ \\
\hline $8 \ldots \ldots \ldots \ldots \ldots$ & + & + & 20 & I. 2 & 1.4 & -2 & - & - & - & + & + & + & + & D) & $\ldots$ \\
\hline $9 \ldots \ldots \ldots \ldots \ldots$ & + & + & 10 & I. I & I. 65 & + & + & $\mathrm{D}$ & $\cdots$ & . & .. & & $\ldots$ & $\ldots$ & $\ldots$ \\
\hline $10 \ldots \ldots \ldots \ldots \ldots \ldots$ & $\dot{+}$ & + & IO & 0.9 & $1 \cdot 35$ & -2 & - & - & + & + & + & $\mathrm{D}$ & $\ldots$ & $\ldots$ & \\
\hline II $\ldots \ldots \ldots \ldots \ldots \ldots$ & + & + & 10 & .7 & I. .08 & -2 & - & - & - & - & + & + & + & + & D \\
\hline І $2 \ldots \ldots \ldots \ldots \ldots \ldots$ & + & + & 10 & $\cdot 5$ & 0.8 & + & + & D & $\ldots$ & $\ldots$ & . & $\ldots$ & $\ldots$ & $\ldots$ & $\ldots$ \\
\hline I $3 \ldots \ldots \ldots \ldots$ & + & + & 10 & .3 & .5 & + & + & D & $\ldots$ & . . & . & ... & $\ldots$ & $\ldots$ & $\ldots$ \\
\hline $14 \ldots \ldots \ldots \ldots \ldots \ldots$ & + & + & IO & 0.1 & o. 17 & + & + & + & 1) & $\ldots$ & . & $\ldots$ & $\ldots$ & $\ldots$ & $\ldots$ \\
\hline I $5 \ldots \ldots \ldots \ldots \ldots$ & + & + & 10 & 0 & 0 & + & + & D & Cor & rol & & & . . & $\ldots$ & $\ldots$ \\
\hline
\end{tabular}

* Plus sign indicates presence of trypanosomes in the blood; minus sign indicates their absence. No attempt is made to indicate the increase in parasites because once they appear in the blood they increase uniformly until death or until an artificial crisis is produced. During the relapse, after the artificial crisis, the same holds true.

tion that in the presence of constant amounts of complement and antigen (Fibrio metchnikovi) moderate amounts of antiserum were completely bactericidal, whereas very large amounts of antiserum were no more bactericidal than small amounts. The phenomenon is of common occurrence in in vitro serological titrations and in various in vivo tests of the protective properties of antibacterial sera.

Furthermore, we found that there are recurring zones of action and inaction of the lytic serum as shown in the experiment in Table I. Here fifteen mice were all infected at the same time with the passage strain. When they showed from ten to twenty trypanosomes per standard field (see third day of infection in table), one was left untreated as a control, while the other fourteen were given various amounts of 
immune-rabbit serum ranging from o. I to $2.5 \mathrm{cc}$. per $20-\mathrm{gm}$. mouse. Within two hours, mouse I I, which received $0.7 \mathrm{cc}$. of serum, showed a crisis, i.e., the trypanosomes disappeared from its blood. Mice I, 3, 4, 7, 8, and Io behaved in a like manner, whereas mice $2,5,6$, and 9 did not have a crisis, did not show a diminution in the number of their trypanosomes, and died as soon as the control. (For many similar experiments see the author and Johnson [1926].)

At first sight one would be tempted to explain such zone phenomena simply on the basis of host variability. Later work by Johnson (1927) indicates, however, that its occurrence is dependent on the number of trypanosomes in the mice at the time the immune serum is given, as Table II shows. The absolute number of trypanosomes at which the zone phenomenon occurs varies with different single cell strains of trypanosomes. These differences are probably induced and of long duration, but not true genetical variations-similar, in other words, to the acquisition of antibody

\section{TABLE II}

Relation between Numbers of Trypanosomes and Zonal Plienonena for a Single Cell Strain of T. cquimum (From Johnson)

Number of Trupanosomes per Standard Microscopic Field at the Time of Treatment With lMaUne SERUM

Occurrence of Zune Phenomens

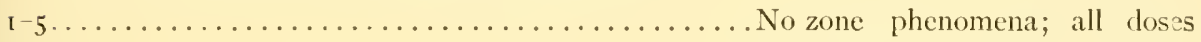
greater than minimal effective dose are effective

$9^{-33}$

\%one phenomena; doses greater than minimal effective dose show alternate zones of effectiveness and non-effectiveness similar to table I

$45^{-50}$

No lysis in any dose up to $4.0 \mathrm{cc}$.

per 20 -gm. mouse

resistance. Thus, Johnson was able to take strain $\mathrm{J}_{4}$, which gave the zone phenomenon with one to five parasites per field, and by subjecting it to immune serum and securing a relapse strain, obtained the zone phenomenon when there were ten to thirty parasites per field.

The results of Johnson on the relationship between the number of trypanosomes and the zone phenomenon are in accord with the recent results of Coventry on the action of antipneumococcus serum on pneumococci in which the serum and virus were introduced simultaneously. The reader is referred to Coventry (I927) for a discussion of similar results in the bacteriological literature and for a consideration of some of the hypotheses advanced to explain it. I am of the opinion that no satisfactory explanation has yet been given for these peculiar results in in vivo work.

\section{INFECTIONS WITH THE NON-PATHOGENIC TRYPANOSOMES}

Trypanosoma lewis $i$ is a representative of a large group of trypanosomes, occurring in various species of rodents, which are very similar in structure and are non-pathogenic to their hosts. Careful enumerative studies of the form by Steffan (I92 I), fully confirmed by the author and L. G. Taliaferro (1922), the author (1924), and Coventry 
(I925), indicate that the course of infection in the blood proceeds as follows (Fig. 7): After an incubation period during which no parasites are found, the organisms increase rapidly in numbers, sometimes attaining several hundred thousand per cubic millimeter of blood, then there is a crisis when most of the parasites are destroyed; those that remain continue to live in the blood (from several weeks to several months) until a second crisis sweeps them too from the blood. Rabinowitsch and Kempner (I899) showed that thereafter the rat is immune to a second infection-an observation which has been repeatedly confirmed by other investigators.

Many years before exact enumerative studies were made on infections with $T$. lewisi, it was noted from microscopical studies by Rabinowitsch and Kempner (I899), Wasielewski and Senn (I900), and Laveran and Mesnil (I90I) that when the organisms are increasing in the blood they are actively reproducing by fission, whereas

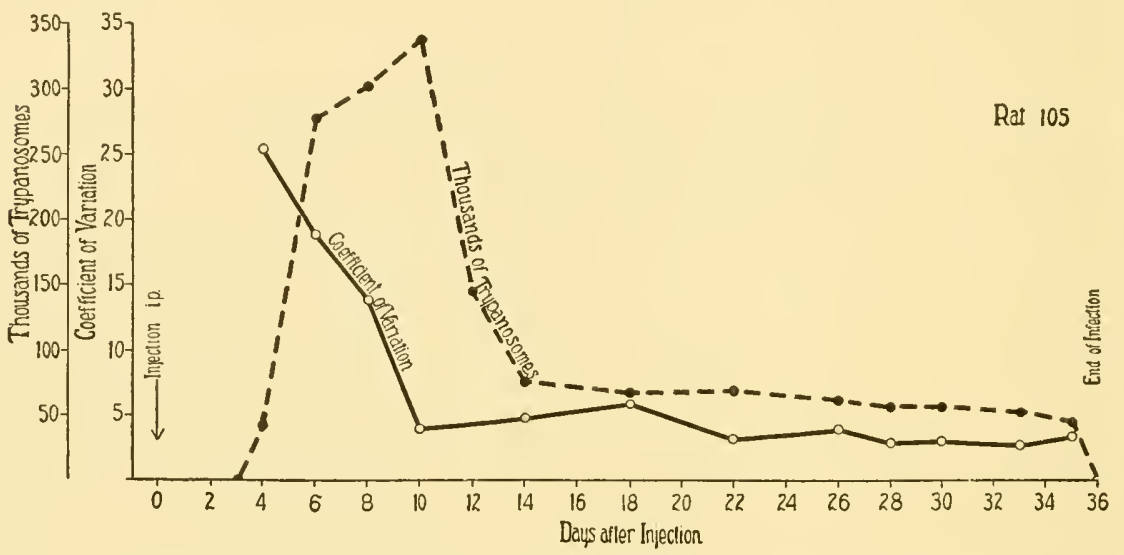

FIG. 7.-Graph showing the course of an infection of T. lewisi in a rat. The changes in reproductive activity (represented by the coefficient of variation curve) and the type of number curve show that both a reproduction-inhibiting and a parasiticidal resistance are developed. (From the author.)

during the latter part of the infection they simply exist in the blood as non-reproducing adults. These facts were studied more intensively by the writer with the assistance of L. G. Taliaferro (1922). From daily blood smears throughout the course of an infection with $T$. lewisi the total lengths of a hundred parasites were drawn and measured and the coefficients of variation computed, as outlined previously. Parenthetically, it may be recalled that the coefficient of variation is an index of the rate of reproduction. From this body of data it was found that when the organisms first appear in the blood (Fig. I and coefficient of variation curve [Fig. 7]), they are reproducing at a very rapid rate, but that their reproduction is quickly but progressively retarded until finally, about the tenth day of the infection, no reproduction is taking place at all, and all of the parasites are in the adult stage.

From this work it may be said that there are three manifestations of resistance: (I) the retardation and final inhibition of reproduction by about the tenth day, (2) the sudden destruction of the majority of the parasites between the eighth and twelfth day, and (3) the eventual total destruction of the parasites which terminates the infection in from a week to several months. 
The immunological basis of the first effect of resistance has been studied by the author (I924) and seems to be due to the acquisition of an immune property by the serum of infected rats which inhibits cell division but which does not kill the parasites. The method of demonstrating the presence of this property is shown in Figure 8

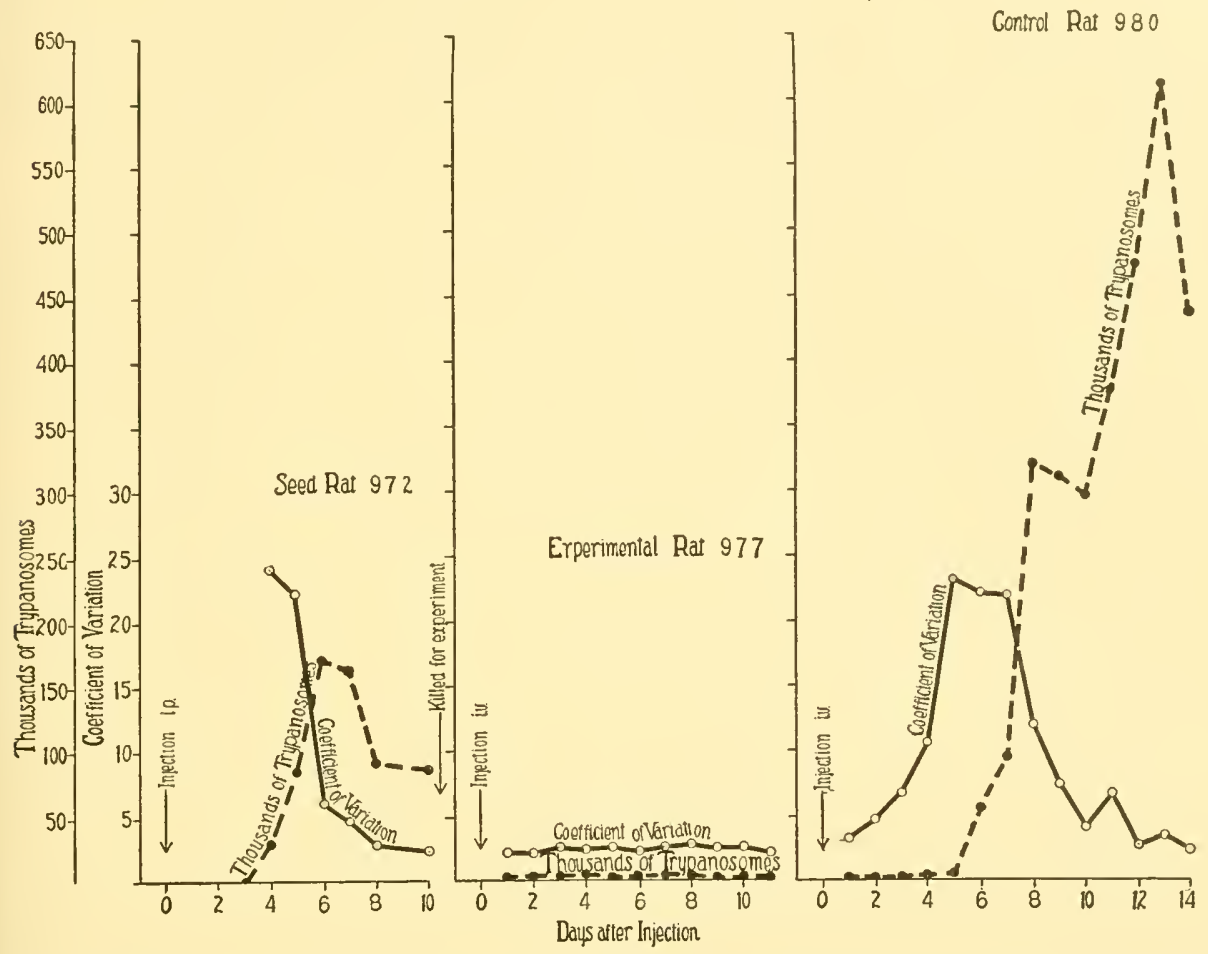

FIG. 8.-Graphs demonstrating the passive immunization of an uninfected rat with the reproduction-inhibiting reaction product which has been formed in an infected rat. Note that after reproduction had been inhibited in seed rat 972 , one half of its adult trypanosomes, plus a suitable amount of its serum, was injected into experimental rat 977 , while the other half of its trypanosomes, plus an equal amount of serum from a normal rat, was injected into control rat 980 . Subsequently, in experimental rat 977 the normal cycle of reproduction was altered-there was a complete inhibition of reproduction as evidenced by the coefficient of variation; whereas in control rat 9 So the normal cycle of reproduction took place. (The peak in the number curve of control rat 980 is so much higher than that of seed rat 972 because it received an enormously larger number of parasites initially.) (After the author.)

which is typical of numerous experiments carried out by the author and confirmed by Coventry (1925) and Regendanz and Kikuth (1927). On the tenth day after infection (when the trypanosomes had reached the adult stage) a rat (seed rat 972 , in figure) was killed and bled and its serum containing the adult trypanosomes collected. Next, the trypanosomes were separated from the serum by rapid centrifugation. Then half of the adult trypanosomes together with $2 \mathrm{cc}$. of the serum (per Ioo-gm. rat) was injected into experimental rat 977 while the other half of the trypanosomes together with a similar dose of normal rat serum was injected into control rat 980. Daily examinations of blood smears and calculations for the coefficient of 
variation for total length for each of these rats showed that the trypanosomes in experimental rat 977 lived in the blood for eleven days (infection died out at this time) without showing any reproduction whatever, whereas in control rat 980 reproduction began on the second day and followed the course of a normal infection. In this experiment it is to be noted that not only did the immune serum, i.e., the serum taken from the seed rat on the tenth day, prevent adult trypanosomes from reproducing in a fresh rat, but normal rat serum failed to prevent adult trypanosomes from reproducing in a fresh rat. This is a clear-cut case of the passive transfer of this type of immunity from an infected to an uninfected rat.

The changes in titre of this immune property of serum through the course of an ordinary infection have been studied in detail by Coventry (1925). She was unable to demonstrate its presence in serum prior to the fifth day of the infection although it is probably present as the rate of reproduction has already begun to decline, but between the fifth and sixth days she found a sudden and very great increase with a subsequent gradual increase until the thirty-fifth day and then a gradual decrease.

As this immune property of serum which inhibits reproduction without affecting the viability of the parasites emphasizes a new effect of resistance, the author has attempted to compare it with the well-established immune bodies (I925 and unpublished work). Like most antiborlies, it is non-ether soluble and is precipitated in the globulin fraction of the immune serum. Strange to say, it comes down with both the euglobulin and pseudoglobulin. It differs from others, however, in its lack of in vitro affinity for its supposed antigen. Thus, when serum containing it is left for twelve hours in contact with large numbers of dividing $T$. lewisi and the parasites are subsequently removed, the serum does not lose any of its titre.

Regendanz and Kikuth (I927) have verified the author's conclusions in regard to the formation of the reproduction-inhibiting reaction product in infections with $T$. lewisi, and similarly found that it exerted no trypanolytic or trypanocidal activity. They have added the important finding that it is not formed in splenectomized rats.

As far as the author is aware, there has been no experimental work nor any explanation advanced to account for the second manifestation of resistance, i.e., the first number crisis which occurs between the eighth and twelfth day of the infection. Regendanz and Kikuth (I927) have not encountered this number crisis, but we have invariably found it in the hundreds of infections studied in this laboratory.

Some work on the immunological basis for the immunity of recovered rats to a second infection, however, probably explains the third manifestation of resistance, i.e., the crisis which terminates the infection. Laveran and Mesnil (Igor) came to the conclusion that the immunity and the final crisis are due to the phagocytosis of living trypanosomes. MacNeal (I904), on the other hand, holds that the crisis and subsequent immunity are due to a trypanolysin, just as has been described for the pathogenic forms. Manteufel (Ig09) also essentially agrees with this view. The author's (1924) investigations and unpublished work of Coventry substantiate the formation of a lysin which terminates the infection, but indicate that the lysin in conjunction with the reproduction-inhibiting property is the basis for immunity to second infection. For example, a rat recovered from an infection of $T$. lewisi receives a few parasites from an infected flea. These find their way eventually into the blood stream, but on 
account of the reproduction-inhibiting property are unable to reproduce. Moreover, they are relatively so scarce that microscopic examination fails to reveal them. Hence, they simply exist there for a time and are unable to set up an infection. The lysin eventually sweeps them away. In the recent work of Regendanz and Kikuth the hypothesis is advanced that there is no lysin formed, but that after reproduction is inhibited, various non-specific agencies, such as phagocytosis by the reticulo-endothelium, continuously remove the adult trypanosomes. In view of the unpublished work of Coventry previously cited, I cannot agree with them in this belief.

COMPARISON OF THE LETHAL AND NON-LETHAL TRYPANOSOMES

Among the trypanosome species, as has been already indicated, there are some that are pathogenic and others that are non-pathogenic. Furthermore, according to a growing mass of evidence, some that are fatal to man and domesticated animals may live harmlessly in their natural vertebrate hosts. For example, T. gambiense causes sleeping sickness in man, but seems to produce no observable symptoms in the infected antelope and other wild game of Africa.

In accounting for these facts, a comparison of the resistance acquired by a host against the pathogenic forms and by a rat against the non-pathogenic T. lewisi is very illuminating. In the first case the host either acquires no resistance (mouse) or periodically forms a trypanolysin (guinea pig, dog, etc.) which is never permanently effective because it does not kill all the parasites and those which remain eventually make good the periodical depopulations. In the second case the host first produces an antibody which completely inhibits reproduction (cell division) in the parasites, and thereafter each number crisis is so much gained by the host since those that remain are incapable of repopulating the blood. It seems possible, then, that whether a given species is pathogenic depends upon the reaction of the host and that the formation of a reproduction-inhibiting antibody, in conjunction with some trypanocidal mechanism, is the immunological basis of non-pathogenicity. Certain experimental findings bear out this hypothesis. Thus, there is evidence that when a rat does not form the reproduction-inhibiting reaction product in infections with $T$. lewisi the parasite produces a lethal infection. Brown (I9I $4 a$ and $b$ ) studied a strain of $T$. lewisi which was pathogenic to rats, and in considering his second paper it is probable that what he considers the chief anomalies of this strain are due to the fact that the parasites reproduced longer than normally. Similarly, the author has observed apparent lethal infections of $T$. lewis $i$ in which reproduction of the parasites was never completely inhibited. In the splenectomized rats of Regendanz and Kikuth (1927), in which no reproduction-inhibiting reaction product was formed, the infection progressed steadily until the death of the host - in other words, the infection resembled an infection with a pathogenic species in the mouse.

In trying to explain the fact that those species of trypanosomes which are lethal to man and domesticated animals can live in the blood of certain wild animals without producing symptons, van Saceghem (1923) postulates a reproduction-inhibiting immune property which causes the pathogenic trypanosomes to become harmless commensals. As yet, however, van Saceghem has not demonstrated such an immune property by suitable immunological experiments as the author and others have done in the case of $T$. lewisi infections. 
MALARIAL INFECTIONS

Investigators have been very much handicapped until recently in the study of the life-cycle of uninfluenced malarial infections in man. With the advent of the use of malaria in the treatment of paresis many advances have been made; but even so, most of our knowledge has been gained from the study of bird malarial parasites, in particular Plasmodium praecox.

In general, the course of infection in the canary is as follows (Fig. 9): After an incubation period $(I)$ during which no organisms can be found, the parasites rapidly increase in the blood (2, acute period) until sometimes every other cell is parasitized, then if the bird does not die, there is a crisis (3) when most of the parasites are killed, but some may remain for a week or more (4, chronic period), and in time all the parasites apparently disappear from the blood (5, latent period). Thereafter, if the bird's resistance is lowered in any way, they may reappear for a week or more in a kind of miniature acute rise and crisis (6, relapse).

Exact enumerative studies of this form have been made by the Sergents (see especially I9I 8), Ben Harel (I923), L. G. Taliaferro (I925), Boyd (I925), and Hartman (I926). L. G. Taliaferro ( 1925 ) found that although the parasites increased at a constant rate according to a geometrical progression during the acute period, out of an average of I5.5 merozoites produced by each mature schizont approximately ro die. The constant rate of increase during the acute period has been verified by Hartman (1927), who finds, however, that the actual rate may vary considerably among different birds. Furthermore, he has made a careful study of the rate of death of the merozoites which perish between each asexual generation and finds that the rate of death is a constant for twenty-one hours of the twenty-four hour period. As pointed out by Taliaferro, this non-viability of the majority of merozoites produced by each sporulation probably represents the suitability of the bird as a culture medium for the parasites and is not in any sense an acquired resistance. In line with the constant rate of increase during the acute period Boyd (I925) has found a definite correlation between the number of parasites injected and the number of parasites at the peak of the infection $(.340 \pm .074)$.

Leaving the consideration of the acute period in which there is no evidence of an acquired resistance and considering the remainder of the infection, it is evident from the wholesale death of the parasites at the crises which terminate the acute, chronic, and relapse periods (Fig. 9) that some type of resistance is acquired which destroys large numbers of the parasites after they are formed. Unlike $T$. lewisi, however, there is no retardation of the rate of reproduction of the parasites, according to $\mathrm{L}$. $\mathrm{G}$. Taliaferro (1925). She found that the asexual cycle which is a measurement of the rate of reproduction, as explained previously, takes the same time (twenty-four hours in one strain) throughout the acute, chronic, and relapse periods (this is shown for the acute period and crisis in Fig. IO), and that furthermore each stage in the cycle takes place at exactly the same time in the relapse as it hart in the acute and chronic periods - a fact which indirectly indicates that the cycle had continued undisturbed throughout the latent perioc. Quoted from her paper, the probalsle picture of an infection with bird malaria is somewhat as follows: 
After the incubation period the asexual stages of the parasites are to be found in the peripheral blood in varying numbers during the entire course of the infection and undergo their cycle of development and reproduction at the same rate throughout. From the very beginning, only a number of the merozoites are viable; this probably represents a natural resistance of the host. During the first part of the infection relatively few parasites are killed, so that they accumulate in the blood and give rise to the acute stage of the infection. Sooner

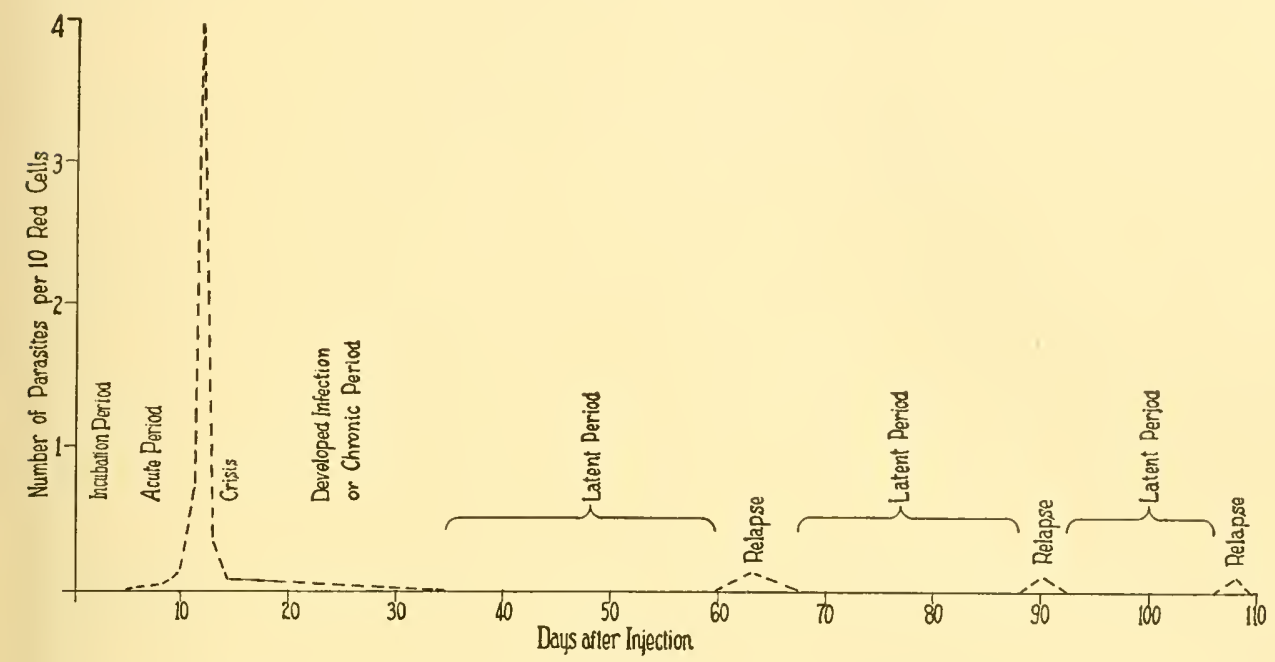

FIG. 9.-Graph illustrating the type of number curve usually encountered in the course of an infection of $P$. praecox in the canary.

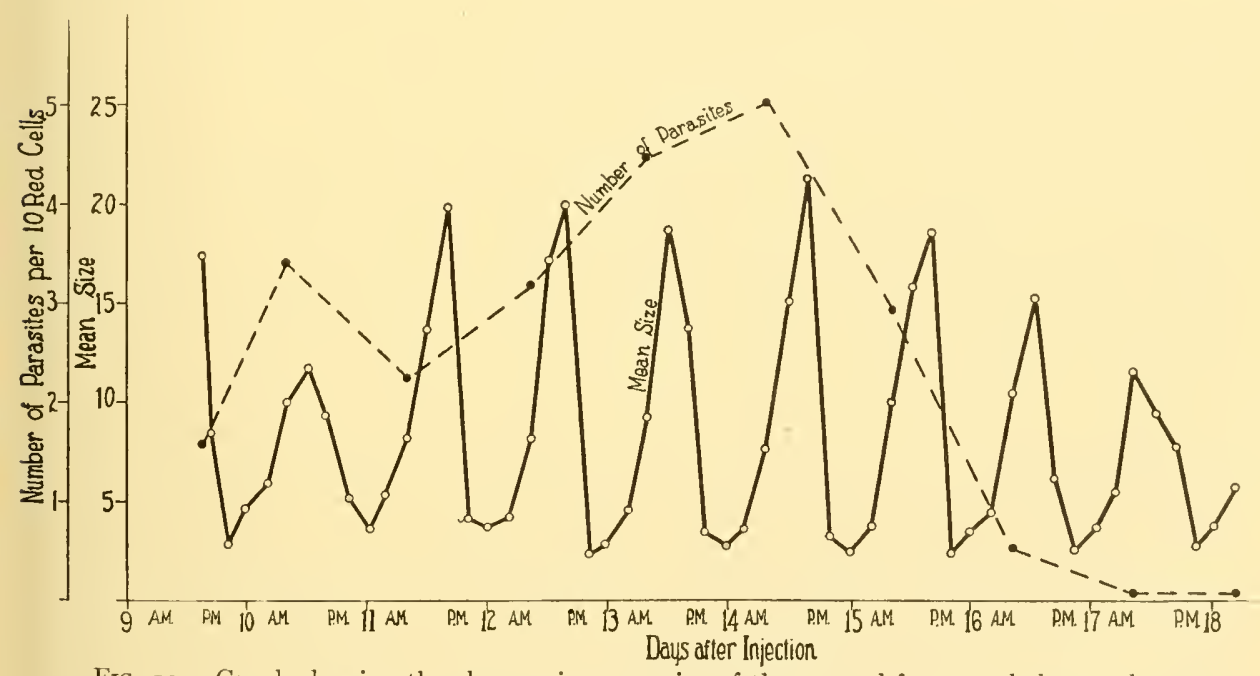

FIG. I0.-Graph showing the changes in mean size of the asexual forms and the number curve through the acute period and crisis in an infection with $P$. praecox in a canary. The uniform rate of reproduction (as shown by the regularity in the mean-size curve) and the type of number curve (as seen in the preceding figure) indicate that there is a parasiticidal but no reproduction-inhibiting resistance developed. (From data by L. G. Taliaferro.) 
or later, however, as the acquired resistance is built up, a large proportion of the parasites are killed. There may then be a temporary relapse, but eventually the destruction of the parasites equalizes or exceeds the number produced by reproduction, and the chronic period or low grade of blood infection ensues. In time, the destruction becomes so great that no parasites can be found in the blood (latent period), but their presence can be demonstrated by Whitmore's technique. This continues until some condition, such as the injection of adrenalin, temporarily stops the destruction and the parasites accumulate again, causing a relapse.

Ross and Thomson (Igrob) have made a very precise enumerative study of infections of human malaria. From their work and the general observations of other workers, it seems probable that the course of infection of both $P$. vivax, the benign tertian parasite, and $P$. malariae, the quartan parasite, is very similar to that of bird malaria. On the other hand, it is difficult to analyze the effects of resistance in $P$. falciparum, the parasite of malignant tertian fever, as well as of certain avian malarial parasites, because schizogony does not occur in the peripheral blood.

Although there is no conclusive evidence as to the defense mechanism involved in the destruction of the malaria parasites to such a great degree at the crisis and to a lesser extent throughout the remainder of the infection, a number of recent investigations on bird malaria have a bearing on this point. It seems very probable from the work of Ben Harel (1923), and from the well-established fact that an enlarged spleen containing many parasites occurs after the crisis, that the wandering mononuclears and fixed-tissue phagocytes of the spleen and other organs ingest the parasites at the crisis and during the latent infection. Furthermore, the author and L. G. Taliaferro have shown (I927) that if a large number of washed parasitized cells are injected intravenously into an infected canary during the latent period, they are quickly removed from the circulation (probably by the phagocytes), whereas in a normal canary they are not removed but steadily increase in numbers until the death of the canary or until a crisis ensues (Fig. I I). One very interesting feature in this work is that the parasites are being removed from the peripheral blood continuously - a fact which is not in accord with Bass and Johns' (I9I2) assumption that the greatest hazard is when the parasite is passing from one cell to another. Evidently, there is some factor in the blood stream of the infected bird which affects either the parasites or the phagocytes or both. All attempts to show that this factor is an antibody have failed. The author and L. G. Taliaferro have been unable either to sensitize infected red cells with serum from infected birds in the latent period or to obtain a passive transfer of the lethal factor. This might be taken to indicate that the parasites simply injure the red cells to such a degree that they are phagocyted, but such an assumption is not tenalle because the same infected cells are not phagocyted by the normal uninfected bird. The failure to obtain evidence of an antibody together with the recent work of Hegner and MacDougall (I926) and MacDougall (I927) suggests that the factor which kills the parasites or which makes the parasite-redcell combination phagocytable is connected with the simpler serum constituents. Hegner and MacDougall have found that increasing the blood sugar by feeding solutions of glucose brings about conditions favorable to the accumulation of the parasites in the blood, whereas decreasing the blood sugar by in jecting insulin probably inhibits 
the accumulation of the parasites. Their experiments are in accord with the work of Bass and Johns (I9I 2) and others who have found sugar necessary for the cultivation of the malarial organisms outside of the body and with the results of Bass and Johns (IgI3) who succeeded in cultivating the parasites in the blood from a case of diabetes without the addition of sugar. Although a great deal more work is needed, it would seem likely in view of all this work that the decrease of blood sugar so injures the parasite or parasite-red-cells combination that it is phagocyted.

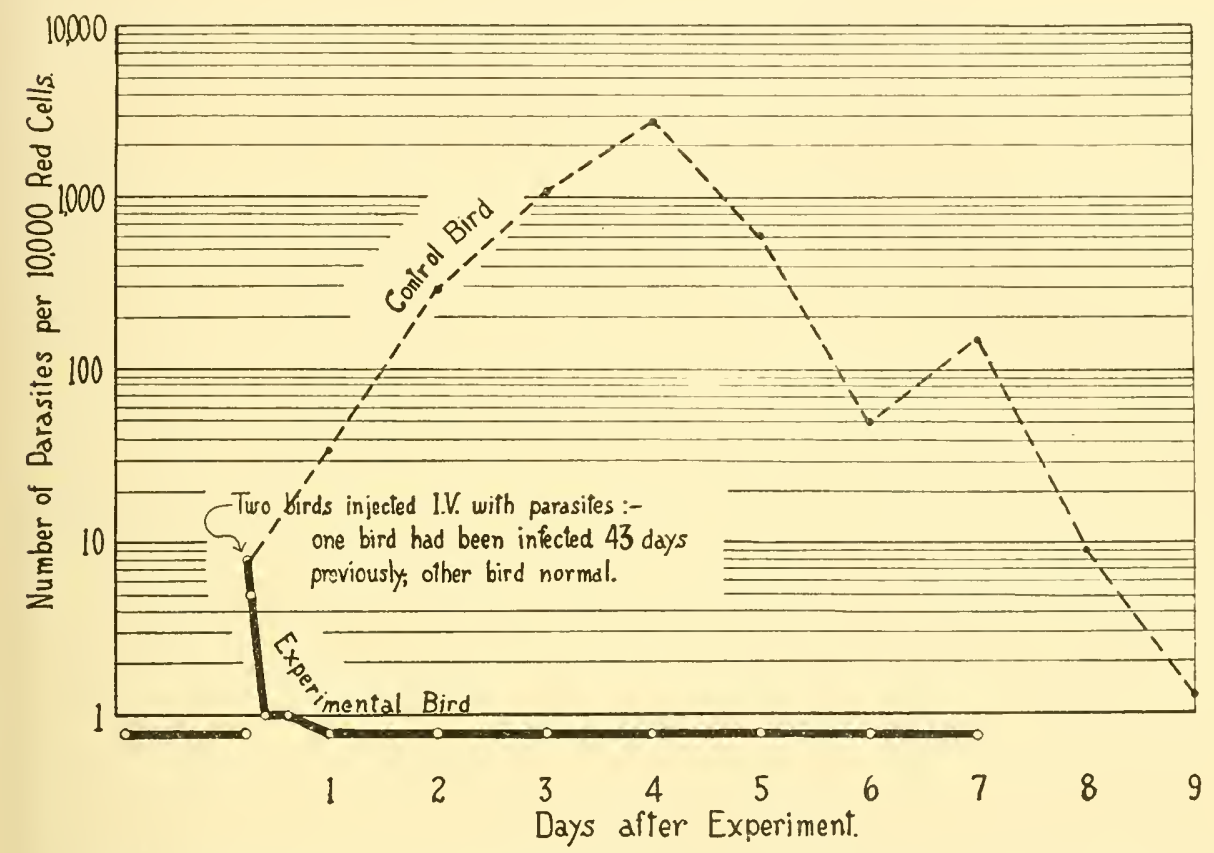

FIG. I I.-Experiment showing the disappearance of washed parasitized cells from the blood of a bird in the latent infection and the survival of the same type of cells in a control uninfected bird. Both birds (one with latent infection and the other uninfected) were injected intravenously with enough washed infected cells so that a few minutes later both showed an infection of about eight parasites per ten thousand red cells. In the bird with the latent infection these were removed from the circulation within twelve hours, but in the uninfected bird they survived and progressively increased in numbers.

The failure to find an antibody basis for the destruction of the parasites in birds suggests the same lack of antibody basis in man, but one hesitates to draw too close a parallelism because no anti-malarial antibodies of any type have been found in the bird whereas they have been found in man. Thus, Maldovan (I9I2) failed to obtain any evidence of protective or complement-fixing antibodies in the avian infection. This has been corroborated by the present author (unpublished work), who in addition found no evidence of a lytic antibody. Fisher (unpublished work) could not demonstrate precipitins. In human malaria, however, both complement-fixing antibodies and precipitins have been demonstrated. (For a review of the literature see the author, L. G. Taliaferro, and Fisher [1927]). Furthermore, the results on 
the relation of sugar to the human infection are rather inconclusive. Working with induced benign tertian malaria in general paresis, Rudolf and Marsh (1927) found glycosuria in a higher percentage of cases of general paresis treated with malaria than untreated. (Also see their paper for a review of previous work on the same subject.) During the induced malaria the blood sugar varied inversely as the temperature, although it may be higher after the fall in temperature than before pyrexia. Administration of glucose gave no effects on objective symptoms nor parasites but apparently relieved subjective symptoms. Administration of insulin produced indefinite results, but in 60 per cent of the cases the fever terminated after administration, and although relapses followed, they were of a lower degree of pyrexia than those following quinine therapy. They also conclude that lowering the blood sugar does not appear to be the cause of the cessation of the fever. In all this work there is the possibility that a diminution of the blood sugar is a concomitant change rather than the basis for the disappearance of the parasites. Thus, in trypanosome infections where there is so much evidence for the rôle of antibodies there may be profound changes in the blood sugar. (For a review of some of this work see Regendanz and Tropp I927].)

\section{REFERENCES}

Bass, C. C., and Johns, F. M.: "The Cultivation of Malarial Plasmodia (Plasmodium vivax and Plasmodium falciparum) in Vitro," J. Exper. Med., 16, 567-79. I9г 2.

Bass, C. C., and Johns, F. M.: "Cultivation of Malaria Plasmodia (Plasmodium falciparum) in Vitro in the Blood of a Diabetic without the Addition of Dextrose," Am. J.Trop. Dis., I, 240-49. I9I3.

Ben Harel, S.: "Studies of Bird Malaria in Relation to the Mechanism of Relapse," Am. J. Hyg., 3, 652-85. г9г3.

Boyd, G. H.: "The Influence of Certain Experimental Factors upon the Course of Infections with Plasmodium praccox," ibid., 5, 818-38. I925.

Brown, W. H.: "A Note on the Pathogenicity of Trypanosoma lewisi," J. Exper. Med., r9, 406-ro. I9г4a.

Brown, W. H.: "Morphological and Developmental Anomalies of a Pathogenic Strain of Trypanosoma lewisi and Their Relation to Its Virulence," ibid., pp. 562-69. I9r4b.

Coventry, F. A.: "The Reaction Product Which Inhibits Reproduction of the Trypanosomes in Infections with Trypanosoma lewisi, with Reference to Its Changes in Titer throughout the Course of the Infection," Am. J. Hyg., 5, I27-44. I925.

Coventry, F. A.: "The Protective and Curative Action of Large Doses of Pneumococcus Antiserum in Mice," ibid., 7, 515-41. 1927.

Diesing: "Ein Immunisierungsversuch gegen die Tsetsekrankheit der Rinder in Kamerun," Arch.f. Schiffs- u. Tropcn-Hyg., 9, 427-31. 1905.

Dobell, C.: "Some Recent Work on Mutation in Microorganisms," J. Gen., 2, 20I-20. I9I 2.

Drensky, K., and Hegner, R. W.: "Periodicity in Bird Malaria," Am. J. IIyg., 6, 312-r4. 1926.

Ehrlich, P.: "Ueber Partialfunktionen der Zelle," München. med. Wchnschr., 56, 21 7-22. I909.

Ehrlich, P., Roehl, W., and Gulbransen, R.: "Ueber serumfeste Trypanosomenstämme," Ztschr. Immunitätsforsch. u. exper. Therap., 3, 296-99. I909.

Ehrlich, P., and Shiga, K.: "Farbentherapeutische Versuche bei Trypanosomenerkrankung," Berl. klin. Wchnschr., 41, 329-32 and 362-65. r904. 
Fantham, H. B., and Thomson, J. G.: "Enumerative Studies on Trypanosoma gambiens" and Trypanosoma rhodesiense in Rats, Guinea-Pigs and Rabbits; Periodic Variations Disclosed," Proc. Roy. Soc., B, 83, 206-II. I9I I.

Franke, E.: Therapcutische Versuche bei Trypanosomenerkrankung (Inaug. Diss.). Giessen, I 905 .

Halberstaedter, L.: "Untersuchungen bei experimentellen Trypanosomenerkrankungen," Centralbl. f. Bakteriol., Orig., 38, 525-32. I 905 .

Hartman, E.: "Certain Interrelations between Plasmodium praccox and Its Host," Am. J. Hyg., 7, 407-32. 1927 .

Hegner, R. W., and MacDougall, M. S.: "Modifying the Course of Infections with Bird Malaria by Changing the Sugar Content of the Blood," ibid., 6, 602-9. 1926.

Jennings, H. S.: Life and Death, Heredity and Evolution in Unicellular Organisms. Pp. 22. Boston, I920.

Johnson, T. L.: In Vivo Trypanolysis with Especial Reference to "Zones of Inhibition," Relapse Phenomena and Immunological Specificity (Thesis). University of Chicago, I927.

Jollos, V.: "Experimentelle Protistenstudien. I. Untersuchungen über Variabilität und Vererbung bei Infusorien," Arch.f. Protistenk., 43, I-222. I92 I.

Kleine, F. K., and Möllers, B.: "Ein für Tryponosoma brucei spezifisches Serum und seinə Einwirkung auf Trypanosoma gambiense," Ztschr.f. Hyg. u. Infektionskrankh., 52, 229237. 1906 .

Kligler, I. J., and Weitzman, I: "Experimental Study of Trypanosomiasis in Palestine," Ann. Trop. Med., I8, 437-59. I924.

Laveran, A., and Mesnil, F.: "Recherches morphologiques et expérimentales sur le trypanosome des rats (Tr. lewisi Kent)," Ann. de l'Inst. Pasteur, 15, 673-7 I4. I90I.

Laveran, A.: "Résistance des chèvres et des moutons aux trypanosomiases; longue durée de l'immunité acquise à la suite de ces maladies," Compt. rend. Acad. de sc., I52, 63-66. I9I I .

Leger, A., and Ringenbach, J.: "Sur la Spécificité de la propriété trypanolytique des sérums des animaux trypanosomiés," Compt. rend. Soc. de biol., 70, 343-45. I9 I I.

Leger, A., and Ringenbach, J.: "Sur la Spécificité de la propriété trypanolytique des sérums des animaux trypanosomiés (deuxième note)," ibid., 72, 267-69. I9I2.

Levaditi, C., and McIntosh: "Mécanisme de la création des races des trypanosomes résistantes aux anticorps," Bull. Soc. path. exot., 3, 368-76. I9ro.

Levaditi, C., and Muttermilch, S.: "Recherches sur la méthode de Bordet et Gengou appliquée à l'étude de trypanosomiases," Ztschr. f. Immunitätsforsch. u. exper. Therap., Orig., 2, 702-22. I909.

Lingard, A.: "The Trypanosoma of Dourine and Its Life History," Centralbl. f. Bakteriol., Orig., 37, 537-47. 1904.

MacDougall, M. S.: "The Effects of Changes in the Sugar Content of the Blood on Bird Malaria," Am. J. Hyg., 7, 635-47. I927.

MacNeal, W. J.: "The Life History of Trypanosoma lewisi and Trypanosoma brucei," J. Infect. Dis., I, 517-43. I904.

Maldovan, J.: "Ueber die Immunitätsverhältnisse bei Vogelmalaria," Centralbl.f. Bakteriol., Orig., 66, 105-10. I912.

Manteufel: "Studien über die Trypanosomiasis der Ratten mit Berücksichtigung der Übertragung unter natürlichen Verhältnissen und der Immunität," Arb. a. d. kais. Gesundh., 33, 46-83. 1909. 
Massaglia, M. A.: "Des Causes des crises trypanolytiques et des rechutes qui les suivent." Compt. rend. Acad. de sc., I45, 687-89. I907.

Mazza, S.: "On the Duration of Relative Immunity in Malaria of Birds," J. Trop. Med. \& Hyg., 27, 98-99. 1924.

Mesnil, F., and Brimont, E.: "Sur les Propriétés protectrices du sérum des animaux trypanosomiés.--Races résistantes à ces sérums," Ann. de l'Inst. Pasteur, 23, I29-54. I909.

Mesnil, F., and Leger, M.: "Documents relatifs au Surra des Caprins et à leur immunité," Bull. Soc. path. exot., 5, 3I-35. I9I 2.

Muttermilch, S.: "Surl'Origine des anticorps chez les cobayes trypanosomiés," Ann. de l'Inst. Pasteur, 25, 776-84. I9II.

Neisser, M., and Wechsberg, F.: "Ueber die Wirkungsart bactericider Sera," Mïnchen. med. Wchnschr., 48, 697-700. I901.

Neuschlosz, S.: "Untersuchungen über die Gewöhnung an Gifte. I," Pflïgers Arch.f.d.ges. Physiol., I76, 223-35. I9I9; "Untersuchungen über die Gewöhnung an Gifte. II," ibid., I 78, 6I-68, 69-79. г920.

Rabinowitsch, L., and Kempner, W.: "Beitrag zur Kenntnis der Blutparasiten, speciell der Rattentrypanosomen," Ztschr. Hyg. u. Infektionskrankh., 30, 251-94. 1899.

Regendanz, P., and Kikuth, W.: "Ueber die Bedeutung der Milz für die Bildung des vermehrungshindernden Reactionsproduktes (Taliaferro) und dessen Wirkung auf den Infektionsverlauf der Ratten-Trypanosomiasis (Tryp. lewisi). Versuche der Uebertragung des Tryp. lewisi auf die weisse Maus," Centralbl. f. Bakteriol., Orig., 103, 271-79. I927.

Regendanz, P., and Tropp, C.: "Das Verhalten des Blutzuckers und des Leberglykogens bei mit Trypanosomen infizierten Ratten," Arch. f. Schiffs- u. Tropen-Hyg., 31, 376-85. 1927.

Ritz, H.: "Ueber Rezidive bei experimenteller Trypanosomiasis, I." Deutsche med. Wchnschr., $40,1355^{-5}$. I9I4.

Ritz, H.: "Ueber Rexidive bei experimenteller Trypanosomiasis. II. Mitteilung," Arch. Schiffs- $u$. Tropen-IIyg., 20, 397-420. I9I6.

Robertson, M.: "Notes on the Polymorphism of Trypanosoma gambicnse in the Blood and Its Relation to the Sexogenous Cycle in Glossina palpalis," Proc. Roy. Soc., London, B, 85, 527-39. I91 2; Reports, Slccping Sickness, Com. Roy. Soc., No. 13, pp. 94-1 Iо.

Rodet, A., and Vallet, G.: "Contribution à l'étude des trypanosomiases. Recherches expérimentales sur le Trypanosoma brucei," Arch. méd. expcr. et anat. path., I 8, 450-94. I906.

Rosenthal, F.: "Untersuchungen über die Genese des Rezidivs bei der experimentellen Trypanosomeninfektion," Zischr.f. Hyg. u. Infcktionskrankh., 74, 489-538. I913.

Ross, R., and Thomson, D.: "A Case of Sleeping Sickness Studied by Precise Enumerative Methods; Regular Periodical Increase of the Parasites Disclosed," Proc. Roy. Soc., London, B, 82, 4II-I 5. I9IOa.

Ross, R., and Thomson, D.: "Some Enumerative Studies on Malarial Fever," ibid., 83, I59-73. I910b.

Rouget, J.: "Contribution à l'étude du trypanosome des mammifères," Ann. de l'Inst. Pasteur, го, 7 г6-28. г 896.

Rudolf, G. de M., and Marsh, R. G. B.: "Some Observations upon Carbohydrate Metabolism in Malaria, with Special Reference to the Effect of Insulin and Glucose upon Benign Tertian Malaria," J. Trop. Mod. \& IIyg., 30, 57-63. I927.

van Saceghem, R.: "Le P'ouvoir empêchant dans les trypanosomiases," Bull. Soc. path. exot., 16, 733-35. I923. 
Schilling, C.: "Bericht über die Surra-Krankheit der Pferde und Rinder im Schutzgebiete Togo," Centralbl. f. Bakteriol., Orig., 31, 452-59. I902.

Sergent, E. and E.: "Sur le Paludisme des oiseaux du Plasmodium relictum (vel Protcosoma)," Ann. de l'Inst. Pasteur, 32, 382-88. г918.

Steffan, P.: "Beobachtungen über den Verlauf der künstlichen Infektion der Ratte mit Trypanosoma lewisi," Arch. Schiffs- $u$. Tropen-Hyg., 25, 24I-47. I921.

Taliaferro, L. G.: "Infection and Resistance in Bird Malaria, with Special Reference to Periodicity and Rate of Reproduction of the Parasite," Am. J. Hyg., 5, 742-89. I925.

Taliaferro, W. H.: "A Study of Size and Variability, throughout the Course of 'Pure Line' Infections, with Trypanosoma lewisi," J. Exper. Zoöl., 37, г 27-67. 1922.

Taliaferro, W. H.: "A Reaction Product in Infections with Trypanosoma lewisi Which Inhibits the Reproduction of the Trypanosomes," J. Exper. Med., 39, I 7 I-90. I924.

Taliaferro, W. H.: "Infection and Resistance in Trypanosome Infections: Studies on the Reproduction-inhibiting Reaction Product in Infections with Trypanosoma lewisi," Proc. Insti. Med., Chicago, 5, 319-20. I925.

Taliaferro, WV. H.: "Host Resistance and Types of Infections in Trypanosomiasis and Malaria," Quart. Rev. Biol., I, 246-69. I926.

Taliaferro, W. H., and Johnson, T. L.: "Zone Phenomena in in Vivo Trypanolysis and the Therapeutic Value of Trypanolytic Sera," J. Prev. Med., I, 85-I23. I 926.

Taliaferro, W. H. and L. G.: "The Resistance of Different Hosts to Experimental Trypanosome Infections, with Especial Reference to a New Method of Measuring This Resistance," Am. J. Hyg., 2, 264-319. I922.

Taliaferro, W. H. and L. G.: "Immunity to Superinfection with Plasmodium relictum (=praccox) in the Canary" (in Proc. Am. Soc. Parasit.), J. Parasitol., 13, 217. 1927.

Taliaferro, W. H., Taliaferro, L. G., and Fisher, A. B.: "A Precipitin Test in Malaria," J. Prev. Med., I, 343-57. I927.

Thomson, J. G.: "Enumerative Studies on T. brucei in Rats and Guinea-Pigs and a Comparison with T. rhodesicnse and T. gambiense," Ann. Trop. Med., 5, 531-36. I9I 2.

von Wasielewski, T., and Senn, G.: "Beitrage zur Kenntniss der Flagellaten des Rattenblutes," Ztschr. Hyg. u. Infektionskrankh., 33, 444-72. I900.

Whitmore, E. R.: "Observations on Bird Malaria and the Pathogenesis of Relapse in Human Malaria," Bull. Johns Hopkins Hosp.. 29, 62-67. I9I8. 


\title{
CHAPTER L
}

\section{ANTIGENS AND THEIR SPECIFICITY}

\author{
H. GIDEON WELLS
}

Otho S. A. Sprague Memorial Institute and the University of Chicago

DEFINITION

An "antigen" is a substance which incites the development of specific reactive agents, "antibodies," when introduced under proper conditions into the circulation or tissues of an animal. These antibodies are demonstrated by their producing recognizable reactions when the serum (or tissues) of the immunized animal is brought into contact with the antigen, the so-called "reactions of immunity," e.g., precipitin, agglutinin, complement fixation, and anaphylaxis reactions. Because of this conventional method of demonstrating the existence of antigens and antibodies, it has sometimes been assumed that all substances giving specific reactions with antibodies are antigens, but this is not always true, and so a deplorable confusion has arisen as to the use of the term "antigen." This is particularly noticeable in the case of the Wassermann test and related reactions used in the diagnosis of syphilis, for the socalled "antigen" in these reactions is not antigenic in the sense of being able to incite the formation of specific antibodies when injected into animals. The essential difference between true antigens and the specific immunological reactive agents which are not antigenic is clearly shown by recent work on the immunological behavior of separated components of pneumococci by Dochez, Avery, Heidelberger, and others. It has been shown that pneumococci, and probably numerous other bacteria, contain varied components with distinctly different immunological behavior. One fraction of a solution of the pneumococcus substance, which seems to be related to nucleoprotein, is strongly antigenic, but the antisera it engenders will react as well with the nucleoproteins of one type of pneumococci as another; i.e., the pneumococcus nucleoprotein is antigenic, but the antibodies it engenders are not specific for pneumococcus types, although specific for the pneumococcus species. Another fraction seems to be composed of a complex carbohydrate, free from protein, which is not antigenic, since it engenders no antibodies when injected into animals; but it does react specifically with antiserum obtained by immunizing with the same type of penumococci that furnishes the carbohydrate. Here is an example of a non-antigenic bacterial substance which reacts with more limited specificity with an immune serum than a true antigenic substance of the same bacteria. ${ }^{2}$

In this discussion the term "antigen" will be used to indicate only substances exhibiting antigenic activity. Non-antigenic substances which react specifically with

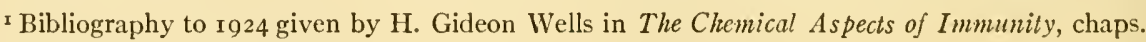
ii and iii, Chemical Catalog Co., r925

${ }^{2}$ See Avery, O. T., and Heidelberger, M.: J. Exper. Med., 38, 8I. I923; 42, 347, 367, 701, 709 , 727. 1925. A good cliscussion of this topic is also given by J. Pryde, in Recent Advances in Biochemistry. Blakiston, 1926. 
antibodies are sometimes referred to as "residue antigens" (Zinsser), and will be discussed in another chapter. ${ }^{\mathrm{I}}$

\section{NATURE OF ANTIGENS}

Practically, antigens are proteins. There are few proteins that are not antigenic, and it is not yet conclusively established that anything else can function as antigens. In order that a protein may exhibit its antigenic effects it must be ( $\mathrm{r}$ ) soluble in the fluids of the injected animal, in order that it may come in contact with the tissue elements that react to form antibodies; (2) foreign to the active tissues of the animal, for tissues are not stimulated to form antibodies by the proteins with which they are normally in contact; (3) a complete or nearly complete protein molecule, possessed of at least a certain minimum size and complexity.

To explain more fully these essentials:

I. Insoluble proteins, and proteins coagulated by heat or other means, which are insoluble in the body fluids, are not antigenic, but proteins that have been coagulated without losing their solubility (e.g., casein precipitated by weak acid, serum albumin coagulated by alcohol) retain their antigenic activity. Therefore the changes induced in the protein molecules during coagulation are not responsible for loss of antigenic activity, but it is only the absence of solubility that renders proteins inactive. This is corroborated by the fact that proteins that are not coagulated by brief boiling (e.g., casein, mucoids) do not lose their antigenic capacity when boiled.

2. As a general rule, the more closely related the animal furnishing the antibodies is to the one furnishing the antigen the less antigenic activity or antibody response will be obtained. Thus, it is difficult or impossible to secure antibodies by immunizing a rabbit with the serum of a hare, or a dog with the blood of a fox. However, even in unrelated species there may be some proteins that are similar, and hence exhibit low antigenic activity (e.g., many tissue proteins). On the other hand, some proteins having structural or physical rather than metabolic functions may be so foreign to the blood stream and the active tissues of the body that they incite antibody formation when introduced into the bloodstream of even the same animal from which they came (e.g., crystalline lens of the eye). Furthermore, chemical alterations in the proteins of an animal may render them sufficiently foreign so that they can react as antigens in that same animal.

3. Certain amino-acid complexes, closely related to typical antigenic proteins, lack antigenic capacity. Gelatin is the best-known example of this group. As gelatin is not a natural protein, but one derived from collagen by hydrolysis, it is possible that it is non-antigenic because it is not a complete protein. Chemically it is characterized by its deficiency in aromatic radicals, for it contains no tryptophan, little or no tyrosine, and not much phenyl alanine, but it is not known whether this deficiency in aromatic radicals is responsible for the absence of antigenic capacity. We do know that proteins may lack some of the usual amino acids of proteins and still be antigenic, e.g., zein, which lacks tryptophan, lysine, and glycine; or casein, which lacks cystine and glycine. On the other hand, such near-proteins as the protamines and histones, which consist chiefly of diamino acids (histidine, lysine, arginine) with but

${ }^{2}$ See chap. lii by Drs. Zinsser and Mueller in this volume. 
a small quantity of the monoamino acids which are the chief constituents of most proteins, are non-antigenic.

\section{FUNDAMENTAL BASIS OF ANTIGENIC ACTIVITY}

As yet we do not know just what structural or chemical features of the protein molecule confer the antigenic function. Probably the colloidal state, or the molecular magnitude which confers non-diffusibility, is a factor, for as far as now known all antigens are colloids. As soon as the protein molecule is disintegrated the antigenic capacity is destroyed. The products of hydrolysis of proteins are almost if not quite devoid of antigenic activity, whether injected alone or all together. ${ }^{\mathrm{I}}$ But if the cleavage products of proteins are resynthesized into larger, colloidal aggregates, the socalled "plasteins," these will be found to be antigenic.

Apparently the colloidal state of proteins is not alone responsible for their antigenic action, for it is possible to destroy the antigenic activity of a protein by racemization without destroying its colloidal character. Racemization is accomplished by treating proteins with alkalies, which alter some of the amino acids so that they lose their optical activity. Dakin believes that the change consists in a rearrangement within the protein molecule whereby the radical<smiles>[R]C(C)N</smiles>

undergoes enolization to the form<smiles>[R]C(C)N</smiles>

thus destroying the asymmetry of the a carbon atom, on which the optical activity depends. Such racemized proteins form colloidal solutions, are soluble, coagulable by heat, and give the usual protein reactions, but they are not antigenic. As they also resist digestion by pepsin and trypsin and are not disintegrated when injected into the body, the inference may be made that antigenic activity is in some way related to digestive proteolysis.

These and other facts have given rise to the hypothesis that antibody formation is a defense reaction against foreign proteins. Under ideal normal conditions no foreign protein gets by the epithelial barriers of the skin and mucous membranes. Whenever these barriers are passed by foreign proteins, whether bacteria, snake venoms, food proteins, or injected therapeutic sera, the body is supposed to protect itself by digestive proteolysis within the tissues, just as ordinarily the foreign proteins are destroyed in the alimentary canal. Zinsser has suggested pertinently that perhaps the reason only colloidal substances serve to arouse the formation of demonstrable antibodies may be their non-diffusible nature. Diffusible foreign substances can enter the cells and be destroyed therein, but to combat non-diffusible foreign substances the cells must excrete their protective agents in order that these may accomplish destruction outside the cells.

${ }^{x}$ See Fink, E. B.: J. Infect. Dis., 25, 97. 1919. 


\section{ACTIVITY OF ANTIGENS}

Whaterer the antigenic function of a protein may depend on, there are marked differences in activity between different proteins which have not yet been explained by anything we know about their composition or structure. In general, the more soluble the foreign protein in the body fluids the better antigen it seems to be, but this is not always true. For example, serum albumin seems to be much less strongly antigenic than the less soluble globulins of the same serum. Casein is less antigenic than many of the other proteins commonly used for experimental immunization. Some toxic proteins, however, possess a most remarkable degree of antigenic activity. For example, ricin, the poisonous protein of the castor-oil bean, injected into rabbits in doses less than one-one-thousandth of a milligram (0.00000I gm.), which is the minimum lethal dose, will lead to antibody formation. Florence Seibert ${ }^{\mathrm{r}}$ has found that some bacteria manage to live in distilled water, and that I 500-I $800 \mathrm{cc}$. of such water concentrated to $20 \mathrm{cc}$. and injected into a rabbit sufficed to produce an agglutinating serum active in a dilution of $\mathrm{I}-8,000$, although it could have contained not more than infinitesimal amounts of bacterial protein.

The anaphylaxis reaction demonstrates that almost any protein may be antigenic in most remarkably small amounts. Thus, crystallized egg albumin has been found to sensitize guinea pigs with a single dose of $0.00000 \mathrm{r}$ gm. or even less, and similar activity has been demonstrated with other proteins.

When a very small amount of one antigen is injected together with a much larger amount of another, the effect of the former may be obscured or lost (concurrence of antigens). ${ }^{2}$ The $\mathrm{pH}$ of the antigenic solution may modify its activity. ${ }^{3}$

\section{TOXINS AS ANTIGENS}

Toxins are also antigens of high potency, although their protein nature has not been fully established. They exhibit colloidal properties, but they diffuse more readily than proteins usually do, and investigators have reported the isolation of active toxins that do not give reactions for proteins. However, as shown by the figures for the size of the lethal dose of a known protein poison, ricin, and the minute amounts of bacterial protein capable of serving as an active antigen, failure to secure chemical tests for protein in toxin preparations that are antigenic and toxic does not prove the absence of proteins. It must be admitted that toxins resemble proteins in many essential respects, especially in being attacked by proteolytic enzymes.

They do not owe their antigenic effect solely to their toxicity, since when detoxicated with formalin or some other agents they retain their antigenic activity, ${ }^{4}$ thus resembling proteins which also are antigenic whether toxic or not. Howevor, when toxins are detoxicated with acids their antigenic power is lost, returning with reactivation of the toxin by neutralizing with alkali. In this process of inactivation and re-

'Seibert, F.: Am.J. Physiol., 71, 621. I925.

${ }^{2}$ See Lewis, J. H.: J. Infecl. Dis., 1 7, 24I. I015; Imai, K.: Zlschr. f. Immunitätsforsch. u. exper-

Therap., 43, 312. 1925 .

${ }^{3}$ Falk, I. S., and Powdermaker, F.: J. Infect. Dis., 37, 514. I925.

${ }_{4}$ Ramon, G.: Ann. de l'Inst. Pasteur, 39, т. I925. 
activation with acid and alkali, toxins behave like peptids in which acids transform cyclic groups to open chains, and alkalies restore the cyclic structure. ${ }^{\mathrm{I}}$

It is possible that toxins consist of toxic radicals attached to proteins, and that the purest toxins, which do not give chemical tests for proteins, represent the toxic radical stripped more or less completely away from the proteins with which it is usually bound. Such isolated radicals could readily reunite with the proteins of an animal into which the toxin was injected, and the resulting complex serve as antigen. Such formation of specific antigenic proteins by union of a protein with nonprotein radicals is a well-known phenomenon. In this way is explained the fact that people sometimes exhibit sensitivity to non-protein substances, such as iodin, formaldehyde, salicylic acid, and other chemicals which are capable of uniting with an individual's own proteins, thus converting them into foreign proteins which serve as antigens. It is possible, therefore, that toxins are proteins to which highly toxic radicals are attached, which radicals may or may not be an essential part of the protein molecule. This possibility is supported by the fact that the venoms of different snakes of a single group (e.g., the vipers) may produce identical physiological and anatomical effects, which suggests that the poisonous elements of each is the same; but the immune serum against each venom shows specific differences, indicating that the protein radicals are different for each species. Landsteiner has also shown that proteins may be combined with various organic radicals, so that the compound proteins act as antigens which produce antibodies reacting with any sort of protein to which the same or similar chemical groups are attached.

\section{NON-PROTEIN ANTIGENS}

The existence of non-protein antigens is still an unsettled question. From time to time reports have been made of successful immunization with materials believed to be free from proteins. Lipoidal mixtures ${ }^{2}$ as obtained from tissues by extraction have been especially mentioned as non-protein antigens, although attempts to secure antigenic action with thoroughly purified lipoids have been unsuccessful. There are two chief difficulties in accepting the idea that pure lipoids can serve as antigens.

First, an antigen must be a substance sufficiently foreign to the injected animal to stimulate the defense reaction of antibody formation. As far as our chemical evidence goes, the lipoids of animals are very few in number, of relatively simple structure, and apparently quite the same among widespread species. The proteins and even the carbohydrates exhibit infinitely greater variation, and proteins from one species have in some instances been demonstrated to be chemically distinct from proteins of similar function in another species. It is difficult to understand how a lipoid injected into an animal whose tissues already contain the same sort of lipoid could incite the formation of antibodies against this lipoid. If such antibodies were formed they would be expected to have disastrous effects on the lipoids and tissues of the immunized animal itself.

Second, it is not easily possible to secure lipoid extracts free from proteins, and

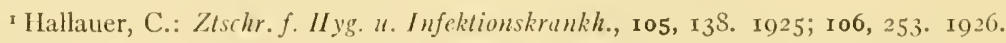

2 For a presentation of the view that lipoids are important as antigens, see Much, H.: frirchow's Arch. f. path. Anat., 246, 292. 1923; Mt ïnch. med. Wchnschr., 72, 2089. I925. 
in the positive experiments such separation has not been demonstrated. When the lipoids are obtained in a high state of purity they have been found to be non-antigenic. Therefore, it is difficult to dismiss the opinion that the observed antigenicity of lipoid preparations depends upon admixed proteins, especially when we consider the infinitesimal amounts of protein that may serve as antigens, as pointed out in previous paragraphs. Lipoids readily take up proteins, and there are experiments suggesting that possibly the lipoidal admixture increases the antigenic efficiency of the proteins.

At present we can say that the antigenic capacity of pure lipoids has not been established. It may be that plants, bacteria, or animal species far removed from the mammals may have lipoids sufficiently foreign to the mammals to incite antibody formation in them, but such an occurrence has not been conclusively demonstrated. ${ }^{1}$

Possibly complex carbohydrates in colloidal state may also be foreign enough to incite antibody formation, for, as Pryde has emphasized, it is possible to secure an enormous variety of carbohydrates. Even as the score of known amino acids of proteins may be built up into an incredibly great number of combinations, sugar molecules may be linked together in a vast number of ways. Two molecules of $d$-glucose may combine in at least twenty-eight ways, and Pryde calculates that I 2 mols of glucose could form somewhere in the order of ${ }^{10}{ }^{18}$ possible different carbohydrates. However, as yet there has been adduced little evidence that carbohydrates do serve as antigens. Even the bacterial carbohydrates, with their strikingly specific reaction with immune sera, have not been found to be antigenic. Ford has reported that by immunizing with extracts of the poisonous fungus, Amanita phalloides, antisera are obtained which neutralize the hemolytic poison of Amanita, and this hemolysin seems to be a glucoside. As yet no one has repeated this experiment, which stands as the chief example of a possible non-protein antigen.

\section{ANTI-LIPOID SERA}

Further support to the idea that antigenic lipoidal mixtures owe their antigenic activity to associated proteins is furnished by demonstration of the readiness with which mixtures of non-antigenic lipoids with proteins produce immune bodies reacting with lipoids. ${ }^{2}$ Landsteiner and Simms ${ }^{3}$ found that the isolated lipoidal element of so-called "heterogenetic antigens" is itself non-antigenetic, but when added to normal foreign serum the mixture functions as an efficient heterogenetic antigen. If the lipoid is injected into a vein of one ear of a rabbit, and the serum into the other ear at the same time, little or no effect is obtained, proving that the lipoid must be in some sort of combination with the antigenic protein to have any effect on the antibody production. Rabbit serum mixed with lipoid will function to some extent as a heterogenetic antigen when injected even into rabbits, although mixtures of foreign serum and foreign lipoids are more potent. The active lipoids in these experiments

I See Schmidt, H.: Ztschr.f. Immunitätsforsch. u. exper. Therap., 38, 5I I. I924; Klopstock, A.: Klin. Winschr., 6, 119. I927.

${ }^{2}$ Review by Klopstock, A.: Ztschr.f. Immunitülsforsch. u. exper. Therap., 48, 97. 1926.

${ }^{3}$ Landsteiner, K., and Simms, S.: J. Exper. Med., 38, 127.1923; see résumé in Klin. Wchuschr., 6. 103.1927 . 
were found to be in the cerebrosid or phosphatid fraction of brain extracts, but, unlike cerebrosids, soluble in water. ${ }^{\mathrm{r}}$

Sachs and Klopstock ${ }^{2}$ have also found that pure cholesterol or pure lecithin mixed with foreign serum constitutes antigens, producing antisera on immunization which serve to react specifically with either of these lipoids. Indeed, brain lipoids can be differentiated from lipoids of other tissues by similar procedures; ${ }^{3}$ i.e., the added lipoids are not of themselves antigenic, but they modify the specificity of the antigens to which they are united. They behave the same way, apparently, as the bacterial carbohydrates which confer group specificity to the proteins of the bacteria. Such radicals which affect the specificity of antigens although themselves not antigenic are called "haptenes" by Landsteiner.

\section{SPECIFICITY}

One of the most striking and important of all the features of immunological reactions is their specific character. This is what gives them most of their practical applications. Early observations first showed that different species of bacteria could be differentiated by their reactions with immune sera, and soon it was found that the blood of different animals could be distinguished readily by the precipitin test. These studies indicated a remarkable correspondence between zoölogical or botanical classifications, although these are based on anatomical features, and immunological relationships, which, as indicated in the previous paragraphs, depend largely on the proteins. Species entirely unrelated to one another were found to show the most marked immunological distinction, whereas species recognized as closely related (e.g., horse and ass, dog and wolf, rabbit and hare, man and anthropoids) ${ }^{4}$ show little immunological difference. Such observations indicated a remarkable situation, for they suggested that structural relations depend primarily on chemical relations of the proteins of the structures.

When immunological studies were undertaken with isolated proteins, instead of such complex antigens as serum, tissue extracts, milk, etc., it was soon found that not all the proteins of a given species are specific for that species. For example, egg white contains antigens specific for the species furnishing the egg, and also common antigens which react with sera obtained from animals immunized with eggs from most varied species. In milk the casein seems not to be specific, anti-casein serum for casein from any one species giving reactions with caseins of many species, but the lactoglobulin of milk is specific for the species; furthermore, the casein is distinct immunologically from any of the blood proteins of the animal furnishing the milk, but the lactoglobulin is identical with the serum globulin.

So it has been found that in a single species of animal or plant there may occur several different antigens readily distinguishable from one another. For example, the several known blood proteins, namely, serum albumin, euglobulin, pseudoglobulin, fibrinogen, and hemoglobin, are all imnunologically separate as well as chemically distinct, even when obtained from the same blood. As each of these proteins obtained

\footnotetext{
${ }^{I}$ Landsteiner, K., and Levene, P. A.: Proc. Soc. Exper. Biol. So Med., 23, 3+3. I926.

${ }^{2}$ Sachs, H., and Klopstock, A.: Biochem. Ztschr., r59, 491. I925.

3 Brandt, R., Guth, H., and Miüler, R.: Klin. Wchnschr., 5, 655. I926.

4See Landsteiner, K.: J. Exper. Med., 42, 84 I, 853, 863. 1926.
} 
from one animal exhibits immunological specificity which distinguishes it from the corresponding proteins of the blood of any other species of animal, as well as the specificity that distinguishes it from the other proteins of its own species, it is apparent that a single protein may exhibit more than one sort of specificity. In hen eggs five different proteins have been separated by chemical means, and these are immunologically distinct from one another. Seeds often show numerous distinguishable proteins in the same species.

On the other hand, a common antigen may occur in many and quite unrelated species. Such common antigens are particularly likely to be proteins that serve only for purposes of structure or food, since they do not need to be specific as are the proteins entering more actively into life-processes. So we find casein and ovalbumin lacking species specificity, likewise some plant-seed proteins. ${ }^{1}$ Lens proteins are immunologically related in many if not all species, although so foreign to the blood of their own species that an animal can be sensitized against its own crystalline lens. In the case of the so-called "heterogenetic" antibodies of Forssman, ${ }^{2}$ the hemolysin antigen effect seems to depend on lipoid radicals common to different species, attached to antigenic proteins which of themselves lack this particular property of inciting hemolysin formation. ${ }^{3}$

\section{BASIS OF SPECIFICITY}

This seems to lie in specific chemical differences in the antigenic proteins, for it has been repeatedly shown that immunological similarity of proteins is associated with chemical similarity, and immunological differences are associated with chemical differences. ${ }^{4}$ For example, the readily isolated proteins of seeds have in many cases been found to be chemically similar although from different seeds, and in this event immunological tests have shown them to be closely related. On the other hand, two chemically distinct proteins from the same seed have been found repeatedly to be immunologically dissimilar. Thus, the crystallizable globulins from the seeds of cantaloupe and squash are chemically, crystallographically, and immunologically identical, ${ }^{5}$ whereas globulin and proteose from squash seeds are chemically dissimilar and immunologically unrelated to one another, despite the fact that both come from the same seed. Or, to pass over to animal proteins, casein from the milk of any species shows a closer biological relation to the casein of any other species than it does to the whey proteins of its own milk, and the same is true of its chemical relations. Only when very particular chemical methods are used can a difference be found in the albumins of hen and duck egss, consisting apparently in a different arrangement of the same amino acids in the two albumins. This agrees nicely with the fact that although by ordinary immunological tests these two albumins seem to be identical, by delicate quantitative methods slight immunological differences can be discerned. ${ }^{6}$

${ }^{1}$ See Wells, II. C.., Lewis, J. II., and Jones, I). B.: J. Infect. Dis., 40, 326. 1927.

${ }^{2}$ Namely, tissue constituents found in most varierl species which, injecterl into rabbits, engender hemolysins for sheep corpuscles.

3 See Doerr, K., and Hallauer, C.: Ztschr. f. Immunitütsforsch. u. exper. Therap., 47, 291. 1926.

+ See Wells, H. G.: J. Immunol., 9, 291. I924.

${ }^{5}$ Jones, 1). B., and Gersdorff, C. E. F.: J. Biol. Chem., 56, 79. I923.

${ }^{6}$ Dakin, H. D., and Dale, H. H.: Biochem. J., 13, 248. 1919. 
These and numerous other observations indicate that the chief factor in determining immunological specificity, at least in immunizing with pure proteins, is the chemical composition of the antigenic protein. In the case of complex antigens the specificity may depend on the composition of the protein molecule itself, but in some cases, e.g., the Forssman heterogenetic antigen, on lipoid radicals attached to the antigenic protein. In the case of some bacteria carbohydrate complexes attached to the protein molecule seem to be important determiners of specificity. ${ }^{\text {I Thus, with }}$ the pneumococcus the bacterial protein seems to determine species specificity, while the group specificity is determined by the carbohydrates of the capsule.

Whether carbohydrate protein complexes also are concerned in the specificity of animal antigens has not yet been determined. Lipoid-protein complexes probably are often important in determining the specificity of animal antigens; for example, the two globulin fractions of serum, euglobulin and pseudoglobulin, are immunologically distinguishable, but seem to differ from each other chemically solely in that the euglobulin has a lipoid radical. The work of Landsteiner indicates that animal cells when used as antigens, as in the production of hemolysin by immunizing with red corpuscles, owe their specificity to complexes of proteins and non-protein radicals, probably lipoidal. ${ }^{2}$

Also, there is reason to believe that the Wassermann reaction may depend on an autoimmunization to lipoid-protein complexes set free by tissue or spirochete disintegration in syphilis. ${ }^{3}$ Apparently, then, when the antigen is a pure protein, specificity is determined by the structure of the protein itself; but when cellular antigens are concerned, specificity depends more on complexes of protein and non-protein radicals. 4

${ }^{x}$ Cf. Pryde, J.: loc. cit.; also chap. lii in this volume.

${ }^{2}$ See Landsteiner, K., and van der Scheer, J.: J. Exper. Me l., 4I, 427. 1925; 42, I23. 1926.

${ }^{3}$ See Weil, A. J.: Ztschr. f. Immunitätsforsch. u. exper. Therap., 46, 8I. I926; Sachs, H., and Klopstock, A.: De:tsche met. Wchnschr., 53, 394. 1927.

${ }^{4}$ For a discussion of complex antigens see Landsteiner, K.: Klin. Wchnschr., 6, I03. 1927. 


\title{
CHAP'TER LI
}

\section{THE CHEMISTRY OF ANTIGENS}

\author{
SARA E. BRANHAM \\ University of Chicago
}

An "antigen" is any substance, which, when introduced into an animal body, causes the appearance of certain new and specific properties in the tissues and circulating fluids. These changes may be variously demonstrated by the phenomena of agglutination, precipitation, complement fixation, hemolysis, anaphylaxis, phagocytosis, and protection against toxin. The reacting factors in the body are commonly termed "antibodies."

It is by no means the rule for all kinds of antibodies to be demonstrable in the same animal, and even those appearing in a single individual may not be made manifest at the same time; frequently a serum that is lacking in agglutinins and precipitins may show marked complement-fixing properties. Nor does a quantitative parallelism exist among those antibodies actually shown to be present; agglutinins may be much more conspicuous than precipitins, complement-fixing antibodies may be more in evidence than either agglutinins or precipitins, and all of these may seem to bear little relationship to the antitoxic strength of a serum used. A discussion of the significance of these variations is not relevant to this chapter.

If a list of all substances which have been employed as antigens were compiled, it would indeed be long. Those most commonly used are very complex materials such as milk, egg white, whole blood, blood serum, blood cells and extracts of blood cells, toxins and venoms, plant pollens, bacteria, bacterial extracts, and tissue extracts of many kinds, even of horse's hoof, cow horn, and human hair!

What is responsible for the antigenic activity of these very complex materials? Are they fundamentally similar in composition? Is there some essential factor which they possess in common? Or may quite different kinds of compounds be able to cause antibody formation in animals? Investigation of these fundamental questions has been approached chiefly from three angles: (I) the fractionation of the commonly used complicated antigens in order to determine which portions are responsible for their activity and to investigate the nature of these component parts; (2) a study of the antigenic activity of simple substances of known composition; (3) deliberate attempts to alter the molecules of materials known to be good antigens in order to study the effect of such changes upon their activity and specificity.

\section{THE FRACTIONATION OF COMPLEX ANTIGENS}

Most studies of this nature have been limited to the various proteins present in antigenic materials. This has been due, no doubt, to the generally accepted idea that only proteins can be antigenic. Nolf, ${ }^{\mathrm{I}}$ in I 900 , first attacked the problem of separating

I Nolf, P.: Ann. de l'Inst. Pasteur, 14, 297. 1900. 
the proteins from blood serum with a view to determining their antigenic activity. He separated a globulin and an albumin fraction, and obtained specific precipitins for the globulin but not for the albumin. The next year Le Blanc ${ }^{\mathrm{I}}$ separated both euglobulin and pseudoglobulin, as well as albumin, from beef serum, and found that all three fractions were immunologically specific, each when injected into rabbits producing precipitins for itself only. In his confirmation of Le Blanc's work Hunter ${ }^{2}$ decided that the specificity of these proteins was relative, or quantitative, rather than absolute, or qualitative. Dale and Hartley, ${ }^{3}$ using the delicate uterine-strip method of demonstrating anaphylaxis instead of the precipitin reaction, concluded that all three of these proteins were independent of one another antigenically, and that the apparent overlapping was due to their incomplete separation.

Still another antigen was demonstrated in serum when Doerr and Berger ${ }^{4}$ obtained a second albumin fraction. They considered all four of these serum proteins to be distinct from one another immunologically. Hektoen and Welker ${ }^{5}$ found these serum proteins to be not only distinct but species specific as well.

To the roster of antigenic plasma constituents already discussed we may add fibrinogen, which Bauer and Engel $^{6}$ found to be antigenically independent of the serum proteins, and seromucoid. In a recent study of the plasma fibrinogen from a number of mammals and fowls Hektoen and Welker ${ }^{7}$ found that whereas, as an antigen, it is distinct from the serum proteins, a strict species specificity seems to be lacking. There even seems to be a relation between the mammalian fibrinogens and those of birds. The anaphylactogenic properties of the seromucoids of various animals have been studied by Lewis and Wells. ${ }^{8}$ They are apparently species specific.

In the formed elements of the blood other antigens have been demonstrated. Bennett and Schmidt ${ }^{9}$ separated a globulin from laked red blood cells, which, injected into rabbits, produced agglutinins and hemolysins for the homologous cells as well as precipitins for the globulin itself.

Antisera containing precipitins for hemoglobin have been obtained by Hektoen and Schulhof ${ }^{\mathrm{ro}}$ by injecting rabbits with either crystallized hemoglobin or an aqueous extract of red blood cells from which all proteins except hemoglobin have been removed by treatment with aluminum cream. They split hemoglobin into globin and hematin, and showed that the globin-free hematin solution contained the antigen, whereas the precipitated globin was inactive. Antisera prepared with the hematin solution were as specific as those obtained with the original extracts, containing pre-

I Le Blanc, A.: La Cellule, r 8, 335. igor.

${ }^{2}$ Hunter, A.: J. Physiol., 32, 327. 1905.

3 Dale, H. H., and Harlley, P.: Biochem. J., ro, 408. I 916.

${ }_{4}^{4}$ Doerr, R., and Berger, IV.: Zischr. f. IIyg. u. Infektionskrankh., 96, I91. I922.

5 Hektoen, L., and Welker, II. II.: J. Infed. Dis., 35, 295. I924.

${ }^{6}$ Bauer, J., and Engel, S. 'T.: Biochem. Ztschr., 42, 399. 1912.

7 Hektoen, L., and Welker, IV. II.: J. Infect. Dis., 40, 706. 1927.

${ }^{8}$ Lewis, J. H., and Wells, H. G.: ibid., 1. 316.1927.

9 Bennett, C. 13., and Schmidt, C. I. A.: J. Immunol., 4, 29. 19 I9.

${ }^{10}$ Ilektoen, L., and Schulhof, K.: J. Infect. Dis., 31, 32. 1922. 
cipitins for the specific hemoglobin only, and not agglutinins, opsonins, or lysins for the red blood cells. Hektoen and Schulhof clid not think that the antigen in question was hematin, but considered it possible that it was adsorbed by the hemoglobin molecule, or that it was a part of it which was split off by the acetic acid used in separating the hematin and globin. Bennett and Schmidt and Hektoen and Schulhof have reminded us that the red blood cells probably contain many antigens. Besides those demonstrated by them in extracts of washed laked cells, there are probably others in the stroma which are yet unaccounted for.

Other complex materials commonly used as antigens have similarly received analytical study. As early as roor several workers separated casein from milk and established its antigenic individuality by means of precipitin reactions. In rgr 8 Osborne and Wakeman ${ }^{\mathrm{r}}$. isolated four chemically different proteins from milk, and Wells and Osborne ${ }^{2}$ showed them to be immunologically distinct by means of the anaphylaxis reaction.

Wells ${ }^{3}$ separated four proteins from egg white: albumin, globulin, ovomucoid, and a substance common to both albumin and globulin; and also a protein from the egg yolk: ovo-vitellin. By producing anaphylaxis in guinea pigs he showed all of these to be good antigens, and quite distinct from one another except for the substance common to both albumin and globulin.

In a series of papers on the biological reactions of vegetable proteins Wells and Osborne, ${ }^{4}$ working with many kinds of seeds, have shown that there may be, in the same seed, a number of proteins which are chemically and immunologically independent. The same seed may contain several globulins besides albumin and the very soluble non-coagulable proteins which have been termed "plant proteoses." All of these are antigenic. Very few ordinary antigens have been overlooked in these analytical studies. Even from horse dandruff two proteins have been isolated. ${ }^{5}$

Most attempts to isolate toxins have not been especially successful, and there is no general agreement upon their chemical structure. Most of the preparations give the color reactions for proteins, but there seems to be no relation between the intensity of these reactions and the activity of the toxin. Some have not given the usual protein reactions, but this may possibly mean that the toxin is powerful enough to show activity in quantities too small to be detected by the color tests used.

Perhaps the most complex of the antigens in common use are bacteria, since unicellular micro-organisms must necessarily contain everything needful for life, growth, and reproduction. Recent investigations have kindled interest in finding out what constitutes the antigenic part of bacterial cells. The micro-organism that has received the most intensive study has been the tubercle bacillus. Its analysis has been approached from various angles, and several fractions for which antigenic activity has been claimed have been derived.

'Osborne, T. B., and Wakeman, A. J.: J. Biol. Chem., 33, 7 and 243. Igr8.

${ }^{2}$ Wells, H. G., and Osborne, T. B.: J. Infect. Dis., 29, 200. 1921.

3 Wells, H. G.: ibid., 9, г 47. г919.

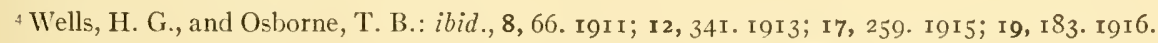

${ }^{5}$ Longcope, W. T., O'Brien, D. P., and Perlzweig, W. A.: J. Immunol., xx, 253. т926. 
As early as I9r 2 Meyer $^{\mathrm{I}}$ demonstrated specific complement-fixing antibodies in the sera of animals immunized with lipoids derived from tubercle bacilli, though he stated that these were much less antigenic than either the whole organisms or those from which all lipoid had been extracted. Thiele and Embleton ${ }^{2}$ confirmed these observations, demonstrating precipitins, complement-fixing and anaphylactic antibodies in sera obtained by injecting phosphatides obtained from tubercle bacilli. Long and Seibert ${ }^{3}$ have separated three distinct proteins from filtrates of cultures of the tubercle bacillus, all of which showed tuberculin activity, though their true antigenic properties were not tested. Zinsser and Tamiya ${ }^{4}$ have separated two distinct active fractions from the tubercle bacillus: so-called "nucleoproteins" which cause antibody production, and are species specific but not type specific; and a part which reacts with striking type specificity in the presence of antiserum, but which does not incite antibody formation in animals. This latter fraction, designated by Zinsser as "residue antigen," has been found by Mueller ${ }^{5}$ and by Laidlaw and Dudley ${ }^{6}$ to consist chiefly of a gum. Dienes and Freund ${ }^{7}$ have described three specific fractions in the tubercle bacillus: (I) a protein, (2) a lipin, and (3) a complex carbohydrate. The first two they report to be truly antigenic in that they induce antibody formation; the carbohydrate is specifically precipitable by antisera, but is not truly antigenic.

Similar studies have been made of other bacteria. The careful studies of Avery and Heidelberger ${ }^{8}$ with the fixed types of pneumococci demonstrated the presence in these of a common protein, species specific and truly antigenic; and in each type a distinctly characteristic carbohydrate, highly specific for its own type alone but incapable of inciting antibody formation.

My own study of the antigens found in filtrates of cultures of some members of the paratyphoid group of bacteria grown in a synthetic medium has resulted in the separation of similar fractions: one, containing at least 9 per cent of nitrogen and giving all of the usual protein reactions, which is specific and truly antigenic; and the other, a stable complex carbohydrate definitely precipitable with antisera. ${ }^{9}$

Little has been done on the number and types of proteins found in bacterial cells. Nelson $^{10}$ has found the globulins in the colon-typhoid group to be antigenic, their specificity within the group being strikingly quantitative.

${ }^{1}$ Meyer, K.: Ztschr.f. Immunitätsforsch. u. exper. Therap., 15, 245. I9I2.

${ }^{2}$ Thiele, F. H., and Embleton, D.: J. Path. \& Bact., 19, 349. 1915.

3 Long, E. R., and Seibert, F. B.: J.A.M.A., 85, 650. 5925.

${ }^{+}$Zinsser, H., and Tamiya, T.: J. Exper. Med., 42, 3 1 1. 1925; Zinsser, H., and Parker, J.: ibid., 37, 275.1923.

${ }_{5}^{5}$ Mueller, J. H.: ibid., 43, I and 9. 1926.

${ }^{6}$ Laidlaw, P. P., and Dudley, H. W.: Brit. J. Exper. Path., 6, 197. 1925.

7 Dienes, L., and Freund, J.: J. Immunol., 12, 137. 1926.

${ }^{8}$ Avery, O. T., and Heidelberger, M.: J. Exper. Med., 38, 81. 1923; 42, 367. 1925; Heidelberger, M., and Avery, O. T.: ibid., 38, 73. 1923; 40, 301. 1924; Heidelberger, M., Goebel, W. F., and Avery, O. T.: ibid., 42, 727. 1925; Heidelberger, M.: Chem. Rev., 3, 403. 1927; cf. chap. lii in this volume.

${ }^{9}$ Branham, S. E.: unpublished work.

ro Nelson, C. I.: J. Infect. Dis., 38, 371. 1926; 40, 4I2. 1927. 
Most of the evidence concerning the antigenic activity of substances that are not proteins has been obtained from bacteria. Nicolle ${ }^{\mathrm{T}}$ reported an antigen in several members of the colon-typhoid group which produced agglutinins in the blood of injected animals, and which was soluble in absolute alcohol and in ether. Perlzweig and Steffen ${ }^{2}$ obtained an actively immunizing antigen which survived digestion with trypsin, was non-lipoidal, and gave Millon, xanthoproteic, and ninhydrin reactions. They believed that this antigen probably adhered to the protein molecule, but was itself not a protein. The apparently protein-free lipoid fraction obtained from the tubercle bacillus by Dienes and Schoenheit ${ }^{3}$ is one of a number of similar lipoidal materials that have been reported. The experiments of Ferry and Fisher ${ }^{4}$ have indicated that the antigenic elements of many bacteria may be more or less loosely bound to the cells. They obtained antisera of high titre by using saline washings of the bacterial cells, whereas they found the washed cells themselves relatively low in antigenic properties. The most active washings gave some protein reactions, but pneumococcus washings containing as little as 0.0298 per cent nitrogen were good antigens.

There have been other reports of antibody production in animals with nonprotein antigens. In many of these instances a negative biuret test has been the criterion for judging the protein-free state of the material used. In others, materials were not considered protein free if they gave any of the typical amino-acid reactions. The biuret test is not very sensitive. Even in the absence of all interfering substances it can detect only about one part of protein in ten thousand parts of water. It is quite conceivable that an amount of protein of antigenic significance may be undetected if such a criterion be employed.

Apparently very little work has been done on the amount of protein necessary to stimulate antibody formation. Wells ${ }^{5}$ succeeded in producing fatal sensitization of guinea pigs with single doses of crystallized egg albumin as small as $0.00000 \mathrm{I} \mathrm{gm}$. Osborne, Mendel, and Harris ${ }^{6}$ reported that, although $0.004 \mathrm{gm}$. of their purified ricin proved fatal to medium-sized rabbits, lesser amounts conferred an immunity to this dose. Seibert ${ }^{7}$ demonstrated agglutinins in the serum of rabbits immunized with an amount of concentrated pyrogenic water which probably contained $0.000062-$ $0.0001 \mathrm{I} 8 \mathrm{gm}$. protein. In our own work ${ }^{8}$ we have succeeded in demonstrating agglutinins, precipitins, and complement-fixing antibodies in the sera from rabbits that received an amount of synthetic medium-culture filtrate from Bacterium enteritidis which we estimated to contain at most $0.000003^{-0.000004}$ gm. of protein. It may

${ }^{1}$ Nicolle, C.: Ann. de l'Inst. Pasteur, I2, г6г. I898.

${ }^{2}$ Perlzweig, W. A., and Steffen, G. I.: J. Exper. Med., 38, 163. 1923; Proc. Soc. Exper. Biol., \&o Med., 20, 378. 1922-23.

${ }^{3}$ Dienes, L., and Schoenheit, E. W.: Am. Rev. Tuberc., 8, 73. 1923.

${ }_{4}^{4}$ Ferry, N. S., and Fisher, L. W.: Brit. J. Exper. Path., 5, 185 and 205. 1924; J. Lab. E Clin. Med., ro, 817. I925.

5 Wells, H. G.: The Chemical Aspects of Immunity, p. 196. I925.

${ }^{6}$ Osborne, T. B., Mendel, L. B., and Harris, I. F.: Am. J. Physiol., 14, 259. 1905.

${ }^{7}$ Seibert, F. B.: ibid., 71, 621. 1925.

${ }^{8}$ Branham, S. E., and Humphreys, E. M.: J. Infect. Dis., 40, 516. 1927. 
be possible that such a minute amount of protein can be exclusively responsible for antibody production. If so, much of the reported work with "non-protein antigens" will need reinterpretation, since these amounts are too small to be detected by the usual methods, in the concentrations used. That such amounts of protein can act as antigens may be questioned by many. This point can be settled only by careful quantitative work with pure materials.

\section{STUDIES WITII PURIFIED MATERIALS}

Extensive investigations on the antigenic properties of purified proteins have grown out of the generally accepted idea that only proteins can act as true antigens. Most of this work has been done with easily available proteins such as casein, ${ }^{1}$ egg albumin, ${ }^{2}$ the serum proteins, ${ }^{3}$ and the plant proteins such as zein, gliadin, hordein, edestin, glutenin, and legumin. ${ }^{4}$ A comparison of their physical properties and chemical composition with their antigenic activity as shown by the precipitin, complement fixation, or the even more delicate anaphylaxis reactions has revealed some interesting facts. A substance may be chemically a protein and yet not be antigenic; as, for example, gelatin which is, however, not a natural but a derived protein, and also proteins that have been racemized. ${ }^{5}$ But nearly all whole native proteins are antigenic, though by no means equally so. They must be soluble in the tissues, and they must be foreign to the animal into which they have been introduced. A study of the chemical composition of many proteins has shown that those that contain aromatic amino acids are antigenic, even though they may lack others, and that the presence or absence of the diamino acids apparently has no influence upon antigenic activity. These findings have led to the suggestion that the aromatic radicals of the protein molecule may be important in determining its antigenic activity, and that the diamino acids are not of importance. Most of the evidence at hand indicates that combinations of amino acids less than the whole protein molecule are not antigenic.

Whether or not lipoidal substances can act as true antigens has been the subject of much discussion and experimentation. Many positive results have been reported with such materials. Much of this work has been done with alcoholic or ethereal extracts, and the possibility of small amounts of protein being present cannot be excluded. Apparently some protein-lipoid combinations are more powerful antigens than the protein alone, and produce antisera reacting with both protein and lipoid. ${ }^{6}$

There is much more variation in the structure and distribution of carbohydrates than in fats. The number of possible combinations of the atomic groups which may enter into their composition is comparable to that of the proteins, and theoretically

${ }^{8}$ Wells, H. G., and Osborne, T. B.: op. cit., 29, 200. I921.

${ }^{2}$ Wells, H. G.: J. Infect. Dis., 5, 449. 1908.

${ }^{3}$ Hunter, A.: loc. cit.; Dale, H. H., and Hartley, P.: loc. cil.; Doerr, R., and Berger, W'.: loc. cit.

4 IVells, H. G., and Osborne, T. B.: op. cit., 8, 66. 1911; 12, 341. I913; 17, 259. 1915; 19, I83. 19I6; Wells, H. G.: ibid., 5, 449. 1908.

s Ten Broeck, C.: J. Biol. Chem., 17, 369. I914.

${ }^{6}$ The relation of the protein and the lipoid to the antigenic activity of such mixtures is more fully discussed in chap. $I$ of this volume. 
there is a possibility of specificity among them. Nevertheless, the types of carbohydrates with which we have been long familiar seem to be entirely devoid of any antigenic activity. The production of anti-hemolysins with a purified hemolytic extract of Amanita phalloides has been reported by Ford. ${ }^{\mathrm{T}}$ This research has often been quoted as the outstanding example in which antibody production may be incited by means of an antigen free from protein. This active component has been described by Abel and Ford ${ }^{2}$ as a derivative falling into the group of saponin-like glucosides. This material, however, can scarcely be classified as a glucoside of the saponin type, not only because such glucosides are non-antigenic, but also because the saponins contain no nitrogen in their molecules. The "glucoside" which Abel and Ford described contained nearly i I per cent of nitrogen.

If one must assume that this unique complex is indeed a nitrogenous pentosecontaining glucoside, as Abel and Ford have classified it, the simplest derivative of this type (the one containing the highest theoretical percentage of nitrogen) contains 8.6 per cent nitrogen, 39.2 per cent oxygen, and 44.2 per cent carbon:

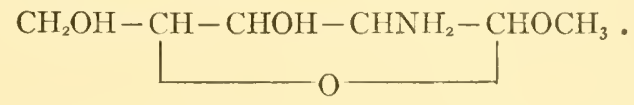

The derivative of Abel and Ford contained 48.9 per cent carbon, Io.8 per cent nitrogen, and 32.3 per cent oxygen. On the basis of these analytical figures it is obvious that the carbon and nitrogen content of their compound is too high, and the oxygen content too low, to permit it to fall into the class of nitrogenous carbohydrate derivatives. ${ }^{3}$

Although Abel and Ford considered their antigenic hemolysin free from protein, they based their contention on the assumption, first, that protein is quantitatively eliminated with metaphosphoric acid and uranyl acetate; and, second, that the resulting filtrate gave no biuret test. Neither of these reagents are quantitative precipitants for proteins, nor can a negative biuret test, sensitive at best to one part of protein in ten thousand parts of water, be accepted as evidence that such filtrates are free from proteins, when one must take into account that antibody response has been secured with amounts of protein as small as $0.00000 \mathrm{Igm}$.

Abel and Ford have shown that when their hemolytic antigen was dialyzed in collodion bags, part of the glucoside passed through the dialyzing membrane. Although the glucosides recovered both from within and without the dialyzing sac appeared to be identical, that which had passed through the membrane was not hemolytic. Unfortunately, no test for antigenicity of this non-hemolytic fraction was made. Hence it is difficult to accept the conclusions of these investigators that the active hemolytic principle of Amanita phalloides is a nitrogenous glucoside capable of producing antibodies. It seems, therefore, that this often-quoted example of a nonprotein antigen should be subjected to a more detailed and rigorous chemical investigation.

${ }^{1}$ Ford, W. W.: J. Pharmacol. \& Exper. Thcrap., 2, I45. I9IO-II.

${ }^{2}$ Abel, J. J., and Ford, W. W.: J. Biol. Chem., 2, 273. 1907.

${ }^{3}$ For consultation, I am indebted to Dr. Milton T. Hanke and Dr. Walther F. Goebel. 


\section{DELIBERATE ATTEMPTS TO MODIFY THE ANTIGEN MOLECULE}

Light has been thrown upon the nature of antigens and upon their structure and the properties necessary for their activity by experiments in which the antigen molecule has been deliberately changed in a definite known way, and the effect of the changes upon activity has been studied. Aside from observing the effect on activity and specificity caused by coagulation, denaturation, and racemization, the chemical composition of the molecule has been changed by introducing or changing atomic groups.

Obermayer and Pick ${ }^{\mathrm{I}}$ introduced iodine, nitro-, and diazo-groups into the aromatic ring of amino acids in beef-serum proteins. These changed proteins completely lost their species specificity as antigens, antisera produced with them reacting not only with the homologous altered protein, but with other proteins such as horse serum, egg white, and even rabbit serum into which the same chemical groups had been introduced; for example, horse serum with nitroprotein gave an antiserum which did not react with normal horse serum or with iodized horse serum, but reacted with horse serum containing nitroprotein and with other sera containing nitroprotein. These results influenced Obermayer and Pick to conclude that the specificity of a protein antigen is determined by the aromatic radicals.

Landsteiner ${ }^{2}$ found that by esterifying proteins with acid alcohols, acetylating with acid anhydride or acid chlorides, or by methylating with diazo methane, such marked changes in specificity were produced that not only, for example, did an antiserum react with acetylated sera from widely different animal species, but also with acetylated proteins from plants as well. These changes in specificity were as great as those reported by Obermayer and Pick, but the chemical changes in the altered proteins were not alike. Those reported by Landsteiner took place in the salt-forming group, and were not made by the substitution of $\mathrm{H}$-atoms in the aromatic nucleus.

Still another type of change was produced when Landsteiner and Lampl ${ }^{3}$ treated rabbit serum with formaldehyde, presumably producing a change in the lysine radical of the proteins. In this case the rabbit serum was so altered that it produced antibodies in rabbits that reacted with the formaldehyde-treated rabbit serum, but not with other formaldehyde-treated sera; rabbit serum had become antigenic for rabbits, but species specificity was retained.

Perhaps the most interesting of Landsteiner's experiments are those which he and his co-workers have performed with azoproteins. ${ }^{4}$ They made a series of thirty-three azoproteins by treating horse serum with diazotized amino compounds. By immunizing rabbits with these, they obtained a number of sera which precipitated all other azoproteins which contained the same azo-compound, no matter whether the serum with which it was combined was horse, chicken, or beef. Some sera were entirely specific, reacting only with antigens containing the homologous compound; others reacted with those made with compounds containing aromatic side-chains chemically

${ }^{2}$ Obermayer, F., and Pick, E. P.: Wien. klin. Wchnschr., 19, 327. I906.

${ }^{2}$ Landsteiner, K.: Biochem. Ztschr., 58, 362. I913; Landsteiner, K., and Jablons, B.: Ztschr. f. Immunitätsforsch, u. exper. Therap., 21, 193. I9I4.

${ }_{3}$ Landsteiner, K., and Lampl, H.: ibid., 26, I33. I917.

${ }_{4}^{4}$ Landsteiner, K., and Lampl, H.: Biochem. Ztschr., 86, 343. I9r8. 
similar to those of the homologous antigen, especially if the diazo-group was similarly located in the aromatic nucleus. Landsteiner later ${ }^{\mathrm{I}}$ repeated some of these experiments, using the anaphylaxis reaction instead of the precipitin test. Guinea pigs were sensitized through injections of one azoprotein (horse serum treated with diazotized para-arsanilic acid) to other proteins containing the same azo-group in chicken serum. Injections of the related simple diazotized components alone did not cause shock, but apparently induced a state of anti-anaphylaxis or desensitization, since subsequent injections of the azoprotein were without marked effect.

Another series of azoproteins was made $^{2}$ by treating many quite unrelated proteins with two diazotized compounds, metanilic and para-arsanilic acids. Immune sera prepared with these were specific, precipitating all proteins which contained the homologous azo-group, whether it were rabbit serum, casein, gliadin, or zein. Compounds made with gelatin or peptones gave only negative results. Here species specificity was apparently entirely lost, all specificity being carried by the introduced groups, the protein part of the molecule serving only to incite antibody formation.

These diazotized compounds are not in themselves antigenic, and when added to immune serum prepared with proteins treated with the homologous azo-compound they cause no precipitate to form. Nevertheless, they seem to unite specifically with the antibodies present in the serum, for when the homologous azo-protein is added later no reaction occurs. This inhibition of the precipitin reaction by the corresponding azo-compounds is shown in an extensive series of experiments with organic acids. ${ }^{3}$ It is probably analogous to the desensitization produced by injecting the compounds alone into guinea pigs sensitized with azoproteins. These experiments led Landsteiner to the conclusion that relatively small portions of the large antigen molecules are responsible for their specificity.

Toxins, too, have been changed by chemical means. Ramon 4 has produced "anatoxins" by treating diphtheria toxin with formaldehyde. The action of the toxic radical in the molecule is completely destroyed. The antigenic properties are, however, unaltered, either in activity or specificity.

\section{SUMMIARY}

Comparatively little is actually known about the chemistry of antigens. Nearly all whole native proteins are more or less antigenic. It appears that astonishingly minute quantities of some of these are sufficient to stimulate antibody formation. That an amount of antigen containing as little as one- to four-millionths of a gram of protein can be entirely responsible for such a biological reaction is surprising. Such findings lead some to question whether or not substances other than protein present in an antigen may have some influence on the production of antibodies. It has not been definitely proved that any other kind of substance, in a pure state, can be truly antigenic. Nevertheless, there is much evidence which indicates that some lipins and

'Landsteiner, K.: J. Exper. Med., 39, 631. 1924.

${ }^{2}$ Landsteiner, K.: Biochem. Ztschr., 93, 106. 1919.

3 Landsteiner, K.: ibid., 104, 280. 1920.

${ }^{4}$ Ramon, G.: Ann. de l'Inst. Pasteur, 39, I. 1925. 
carbohydrates, particularly those found in micro-organisms, are of immunological significance, and perhaps they may exist in the living cells in combinations that make them of antigenic importance. The protein-lipoid combination is frequently reported to be a better antigen than the protein alone; the carbohydrates from many bacteria seem type specific, whereas the proteins from which they are separated are only species specific. The idea that the specificity of an antigen may be determined by a relatively small atomic group in the large antigen molecule becomes much more than conjecture when substantiated by the careful experiments of Landsteiner and his colleagues. The fact that amounts of protein too small to be detected in the concentrations used by any of the methods in general use have been known to be definitely associated with antibody production should be considered in the interpretation of any experiments done with apparently protein-free materials. 


\title{
CHAP'TER LII
}

\section{ANTIGENIC PROPERTIES OF THE BACTERIAL CELL AND ANTIBODY REACTIONS}

\author{
HANS ZINSSER AND J. HOWTARD MUELLER \\ Harvard University Medical School
}

I

The processes of immunity by which the animal body resists invasion and in jury by bacteria are merely specific instances of the operation of general biological laws applicable to a field far wider than that of infectious disease. The elaborately adjusted and complicated mechanism of which we speak as "immunity" is the consequence of a physiological response which, in its broad biological significance, may be stated as follows:

Under conditions of normal metabolism, the tissue cells come in contact with extraneous substances only as the products of digestion or as adventitious, diffusible materials that, as a rule, are readily disposed of. It is almost inevitable, however, that, in the course of life, complex substances-carbohydrate, lipoidal or proteinmay gain entrance without preliminary digestive transformation to assimilable form. As far as the lipoids and carbohydrates ${ }^{\mathrm{r}}$ are concerned, no consequences that fall into the field of the immunologist develop, and it is more than likely that these materials can either be taken care of or eliminated without giving rise to lasting changes in cell reaction. With complex nitrogenous materials (proteins or closely related substances) ${ }^{2}$ this is not the case. Here, contact with the tissue cells sets in motion an emergency mechanism which reveals itself by a specifically changed reaction capacity of the tissue cells. In many cases - those in regard to which we have the most extensive information-this changed reaction capacity is characterized, among other things, by the appearance in the blood stream of specific reaction bodies to which the name "antibodies" has been applied. In other cases, however-notably with foreign substances responsible for the various allergies - profound specific changes in cell reaction may be developed in every fundamental attribute analogous to the former, except that no determinable circulating antibodies can be detected. Thus, while it is of course important to distinguish between foreign materials which call forth antibody formation and those which do not, it is necessary to bear in mind that the appearance of these circulating reagents is purely secondary to a specific cell response and, this having taken place, the development of antibodies in the blood may or may not followdepending upon the chemical and physical peculiarities of the inciting antigen. ${ }^{3}$ Thus,

Except in protein combination as described below.

2 We cannot, in a definition of this kind, cover the entire field by qualifying it for the relatively simple substances concerned in drug idiosyncrasy. This would involve prolonged theoretical discussion not pertinent to our present purposes.

${ }^{3}$ It does not seem pertinent to the purposes of this paper to enter into a complicated discussion on the nature of the circulating antibodies. In former communications we have advanced arguments 
for example, antibody formation is a regular response to the parenteral administration of coagulable proteins and cellular antigens, while no demonstrable true antibodies have so far been detected in many so-called "idiosyncrasies "and "allergies." Fundamentally, however, all of these occurrences are alike in depending upon specific changes in cell response; and in so far as these are concerned, their manifestations show close analogy-differing only in those phases in which the presence of circulating antibodies is'significant (passive sensitization, etc.).

It has seemed to us particularly important to extend the antigen conception in this manner in a discussion involving bacteria; for the bacterial cell, as we shall see, contains a variety of biologically active materials, some of which are truly antibodyinducing substances in the older sense of the word-others, however, like the active substance of tuberculin and its analogues, inciting profound and specific effects upon the tissues without necessarily giving rise to demonstrable circulating antibodies. In speaking of the antigenic materials of the bacterial cells, therefore, we shall employ the word as signifying all substances which arouse specific reactions in the body of the host, whether these involve true antibody formation or not.

In infectious disease, then, we have a specific instance in which the foregoing physiological process is set in motion, but complicated beyond similar phenomena by the fact that the antigen is capable of multiplication within the host and at the host's expense. In the last analysis, all immunological reactions, both those between the antigen and the cell as well as those between the antigen and its antibody, must be dependent upon chemical affinities. Both the facts of specificity and studies upon the modification of antigens by chemical manipulations have made this clear. But, as in enzyme chemistry, ${ }^{\mathrm{I}}$ we are as yet dependent upon indirect experimental methods because no adequate chemical definition of the reacting substances is possible. Nevertheless, it is necessary - even though in a rudimentary manner - to approach the problem by an attempt to correlate the chemical structure of the bacterial cell with the immunological responses of the infected or immunized animal.

in favor of the likelihood of the essential identity of antibodies induced by the same antigen but apparent by different activities in different systems. In connection with this we suggested a purely convenient differentiation between the antigens leading to sensitizing antibodies and those inducing antitoxins. The purpose of establishing a conception of antitoxinogen was merely to simplify a practical bacteriological distinction between antigens that can be expected to produce neutralizing antitoxic substances and those by which sensitizing antibacterial reaction bodies are produced. In a recent paper, Neill, Fleming, and Gaspari have pointed out that there need be no real difference between the mechanism of the. immunity responses induced by the antitoxinogens and sensitizing antigens, which is quite in conformity with our own conception of antibody production as a manifestation of a common biological response to foreign protein. They say: "The usual distinction between the two types of antibodies (antitoxins and sensitizing antibodies) depends upon differences between the properties of the antigen-antibody compounds and the properties of the specific antigens. With the toxin-antitoxin compounds, the prominent change is in plarmacological properties; with the other antigen-antibody compounds, it is usually a change in physical properties." And again, "In a certain sense, 'neutralization' as well as 'agrglutination' can be considered as a phenomenon secondary to the actual combination of antigen and antibody, "both of them representing methods of detecting antigen. antibody union rather than criteria of ileep-seated differences between two types of antigens or two types of antibodies (Neill, J. M., lileming, WV. L., and (Gaspari, E.L.: J. E.xper.Med., Nov., 1927).

${ }^{2}$ For the most recent authoritative discussion of these points see IVells, II. G.: The Chemicu! Ispects of Immunity (IIono. Am. Chem. Soc.). 1925. 


\section{II}

Elementary analysis, or even extraction and fractionation of the bacterial cell body by methods in which immunological properties are "denatured" or destroyed, can be of little service. Insufficient attention to proper correlation of chemical properties with biological activity, therefore, has considerably delayed progress in this field. It is useless, then, to detail the many earlier studies which are of purely biochemical interest, but make no direct contributions to immunology.

In varying proportions, all pathogenic bacteria contain considerable amounts of lipoidal, nitrogenous, protein or protein-like, and carbohydrate material.

\section{THE LIPOIDS}

Concerning the immunological significance of the lipoids, little can be said with certainty. Much has been written about the antigenic properties of the lipoids - especially in connection with alexin fixation reactions, but the issue has here been clouded by the non-specific elements which are involved in tuberculosis and syphilis fixations and, in cases where these considerations can be eliminated, by the probability that the lipoids in question may have been impure, containing traces of protein. Both Landsteiner $^{1}$ and, more recently, Wells ${ }^{2}$ have subjected the evidence to critical analysis, and neither of them is willing to accept without reservation any of the claims of antigenic activity for the bacterial lipoids.

\section{THE BACTERIAL NUCLEOPROTEIN}

The substances which it has lately been the custom to speak of as the "bacterial nucleoproteins" are probably not nucleoproteins in the structural chemical sense. They constitute the bulk of the bacterial cell as judged by quantitative yields of material, and from the method of obtaining them and their gross physical attributessolubility, etc.- they probably consist of a mixture of different things in which, as Wells suggests, there are probably present not only nucleoproteins, but nucleins, nucleo-albumins, mucin, and proteinates formed by the action of the alkali used in their isolation upon proteins. It is important to bear in mind a fact that Wells has repeatedly emphasized, that in most of the methods necessary for the preparation of cell extracts of any kind, relatively severe treatment is necessary, which may denature and alter normal cell constituents; and that it is next to impossible to obtain pure, unaltered proteins. A discussion of the chemical problems involved may be found in the chapter on the nucleoproteins of Wells's book. For our own purposes we may limit ourselves to those phases of the chemical problem which have immunological bearing.

Whether or not the bacterial nucleoproteins have been obtained from centrifugated broth cultures, as in the work of Avery and Heidelberger, ${ }^{3}$ Lancefield, ${ }^{4}$ and others, or from ground, (lried bacteria grown on agar, as in our own work, ${ }^{5}$ the prin-

${ }^{x}$ Landsteiner, K.: Jahresbericht aber die Ergebn. d. Immunitütsforschung, 6, 209. төто.

Wells, H. G.: op. cit. $47-5$ I. 1925 .

3 Avery, O. T., and Heidelberger, M.: J. Exper. Med., 38, 8I. 1923; Avery O. T., and Morgan, H.: ibid., 42, 347. 1925 .

${ }^{4}$ Lancefield, R. C.: ibid., p. 377. 1925.

${ }^{5}$ Zinsser, H.: ibid., 34, 495. I92 I Zinsser, H., and Parker, J. T.: ibid., 37, 275. 1923. 
ciples of manipulation are roughly the same. The method has consisted in extracting with slightly alkaline salt solution, usually about N/IO0 NaOH. Such extracts, produced in various ways, can then be centrifugated to remove all suspended particles, or filtered through a Berkefeld filter, though the colloidal nature of the nucleoprotein mixtures leads to a considerable quantitative loss from filtration. The extract is then precipitated by the addition of acetic acid in the cold, care being taken to avoid an excess of acid. The flocculent precipitate, which is usually most profuse at a hydrogenion concentration in the vicinity of $\mathrm{pH}_{3-4}$, can then be separated by centrifugation. The sediment may be redissolved in alkali and reprecipitated for purposes of purification, but it is questionable whether one can count upon much chemical purification by a repetition of the same method, and such re-solution and re-precipitation always leads to considerable loss of material, the sediment never entirely redissolving. We believe that this may be due to a gradual alteration of some of the materials by the repeated and prolonged treatment with acid and alkali. This opinion is consistent with Wells's statement that the loss of substance is probably due to denaturation of the albumins present in the original precipitate.

It is quite impossible at the present time to make any specific statements about the exact chemical constitution of the so-called "bacterial nucleoproteins" or to separate the constituents into those which are antigenic and those which are not. Probably there are many entirely non-antigenic admixtures which are immunologically negligible. The antigenic constituents of these nucleoproteins, however, act very much like casein, in that very large amounts and persistent injections are necessary for the production of any considerable concentration of antibodies in animals. We have been forced to immunize for months with large amounts of nucleoprotein produced in the foregoing manner from tubercle bacilli and other organisms in order to obtain antibody titres of $\mathrm{I}-\mathrm{I}, \infty 00$, or even less. This indicates that we are dealing with a rather poor antigen, feeble perhaps because of the injury done by the alkali and acid treatment. Indeed, we believe this likely because, for reasons which will become apparent in our section on immunological correlations, the antigen obtained by the simple solutions of pneumococci with bile are qualitatively similar to the nucleoproteins obtained by other methods from the same organisms, but are quantitatively much more potent as antigens. The solution with bile is obviously a much less violent method of disrupting the bacterial body than drying, grinding, and extracting with alkali and precipitating with acid.

THE SPECIFIC SOLUBLE (RESIDUE) SUBSTANCE

The possible importance of carbohydrates in immune phenomena was not recognized until quite recently, though the fact that polysaccharides of the nature of gums were present in certain bacteria has been known since the time of Pasteur. A review of the earlier work dealing with this subject has been published by Buchanan. ${ }^{2}$ In the more recent literature the bacterial carbohydrates have been more particularly associated with the capsular structure, ${ }^{3}$ Jut in none of this work dicl it lecome apparent that the carlohydrate materials had any immunological significance.

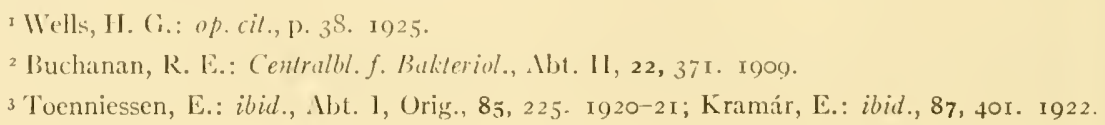


The earliest work dealing with substances of this nature in which their immunological relations were considered was published by Pick in I9I 2. In cultures of typhoid bacilli Pick found a substance not destroyed by pepsin, by trypsin, or by boiling, which failed to exhibit the usual chemical protein reactions but which, nevertheless, was specifically precipitated by typhoid-immune serum. Since this material was described by him as not possessing the power of inducing antibody formation in animals, we feel justified in assuming that Pick's substances were in principle identical with those later described by others.

In I9r 7 , Dochez and Avery ${ }^{2}$ observed in the blood stream and urine of pneumonia patients a substance which reacted specifically with immune serum but was not destroyed by boiling or by tryptic digestion. It was precipitable by alcohol, acetone, and colloidal iron and was not dialyzable. They concluded from nitrogen determinations that it was of protein nature or associated with proteins, undoubtedly an error similar to the one we ourselves made later with tubercle-bacillus residue, and due to the difficulties of purifying the crude carbohydrate extract. They found the same substances in young and rapidly growing broth cultures of pneumococci and some other bacteria, and concluded, therefore, that they were products of cell growth and not of cell decomposition.

In $\mathrm{I} 92 \mathrm{I}$, one of $\mathrm{us},{ }^{3}$ in connection with experiments upon the tuberculin reaction, reported the presence of a similar substance in tubercle-bacillus extracts prepared in a manner calculated to exclude a considerable proportion of the bacterial proteins. These materials, like those previously described by Pick and by Dochez and Avery with other organisms, were left in alkaline extracts after removal of the acid precipitable nucleoproteins, were alcohol precipitable, heat stable, and reacted in vitro with specific sera, both in giving precipitation and alexin fixation reactions. Owing to an insufficient purification of these substances at that time, these "residue materials," as we call them, also gave skin reactions in tuberculous guinea pigs, and it was only by our subsequent studies that it was found that the skin-reactive constituent was an impurity. It was then shown that the specific precipitable substance and the skinreactive fraction in these original residue materials could be separated.

In I923, similar substances were found by one of us with Parker in extracts of pneumococci, staphylococci, influenza and typhoid bacilli, and since then the existence in many other bacteria of these non-protein specific residue materials has been demonstrated. In spite of the earlier difficulties in defining these "residue antigens," as we call them, from other bacteria, they were held to be analogous to the non-protein substances of Pick and to the soluble substances of Dochez and Avery. Like these, they were precipitable from aqueous solution by alcohol; the precipitates were easily soluble in water, were heat stable, and retained their specific properties. Also, in spite of energetic attempts, these residue substances never induced antibody formation upon injection into animals.

Further developments of this work by many investigators have shown that materials similar to those described are present in all bacteria in which suitable efforts have been made to find them. As greater familiarity with these substances was acquired

${ }^{x}$ Pick, E. P., in Kolle and Wassermann: Handb. der path. Mikroorg. (2d ed.), I, 78I. Jena, I92r.

2 Dochez, A. R., and Avery, O. T.: J. Exper. Med., 26, 477. I917.

3 Zinsser, H.: loc. cit.; Zinsser, H., and Parker, J. T.: loc. cit. 
and chemical investigation was carried out on larger quantities, it was found that practically all traces of protein recognizable by available methods could be eliminated and that the material bearing the specific properties consisted largely, or entirely, of polysaccharide. When relatively pure, these carbohydrates in many cases will give specific precipitation and complement fixation reactions in dilution of the dried substance in from $I-I, 000,000$ to $I-6,000, \infty 00$, but fail entirely to produce antibody formation in rabbits, even when immunization is continued for months with large quantities.

The organisms in which the soluble specific or residue substance have been particularly studied are the pneumococcus, the tubercle bacillus, the Friedlinder bacillus, the streptococcus, and yeast cells, and they have been determined and to some extent studied immunologically in the staphylococci, typhoid and influenza bacilli, and meningococcus.

In the case of the pneumococci, Heidelberger and Avery ${ }^{1}$ in 1923 described carefully the substance obtained by them from a type II pneumococcus.

They used eight-day broth cultures in large quantities and separated the specific material by a method of fractional precipitation with alcohol, acetone, ammonium sulphate, and dialysis. The yield was about $\mathrm{I} \mathrm{gm}$. from 75 liters of broth culture. It proved to be mainly a polysaccharide gum, yielding 79 per cent reducing sugar on hydrolysis, at least a part of which was identified as glucose. Specific precipitates were given at a dilution of $1-3,000,-$ $\infty \infty^{\infty}$, and while no claims as to the purity of the material were made, since it still contained I. 2 per cent nitrogen and a trace of phosphorus, it was shown that the activity of the preparation increased progressively with the removal of nitrogenous materials. More recently, completely nitrogen-free preparations have been made.

Since that time types I and III pneumococci have also been investigated. ${ }^{2}$ Substances of widely differing chemical properties but also carbohydrate in nature have been obtained. In the case of type I the gum appears to be made up of monosaccharide units which contain nitrogen as a part of the molecule. The gum itself contains 5 per cent $\mathrm{N}$, half of which is liberated by $\mathrm{HNO}_{2}$, which causes a loss of specific properties. An examination of the products of hydrolysis indicates that it may be composed of an amino-sugar derivative linked to galacturonic acid. It behaves as a strong acid and a weak base, with an iso-electric point at about $\mathrm{pH}_{4}$, at which reaction it is fairly insoluble. The type III compound is produced in larger quantities than the others, and is relatively easy to prepare, since it appears to be a soluble salt of an insoluble acid. It may be precipitated simply by acidification of aqueous solutions of its salts and is readily purified. It is hydrolyzed almost quantitatively to a disaccharide acid which in turn splits with some difficulty into glucose and perhaps glucuronic acid. All three of these specific substances possess acid properties to a greater or lesser degree. Their optical properties vary widely as might be expected. For a more detailed consideration of these substances see Heidelberger. ${ }^{3}$

Yeast has been shown by Mueller and Tomczik ${ }^{4}$ to produce a carbohydrate with specific properties similar in all respects to those shown by bacterial gums. The abundant supply of this material in the form of bread yeast offered a possibility of finding a method of purifica-

\footnotetext{
${ }^{1}$ Heidelberger, M., and Avery, O. T.: ibid., 38, 73. 1923.

${ }^{2}$ Heidelberger, M., and Avery, O. T.: ibid., 40, 301. 1924.

3 Heidelberger, M.: Physiol. Rev., 7, 107. 1927; Chem. Rev., 3, 403. 1927.

4 Mueller, J. H., and Tomcsik, J.: J. Exper. Med., 40, 343. 1924.
} 
tion which might be adapted to further studies on bacterial products available in smaller quantities. Since the work was undertaken before the publication of Heidelberger and Avery's work, the widely differing chemical properties of these substances could not be predicted. It was found that the specific substance of the yeast cell was apparently identical with the "yeast gum" on which many studies had been made without any appreciation of its relation to immunological phenomena. The fact that it was precipitable by alkaline copper solutions facilitated its separation, and further purification was obtained by fractional alcohol precipitation.

Laidlaw and Dudley in 1925 described a specific gum prepared from ground tubercle bacilli by extraction and removal of proteins, etc. A substance with such similar properties as to make it reasonably sure that it represented the same material was obtained by one of us $^{2}$ in 1926 , from concentrated broth cultures of the tubercle bacillus. The purification of this material is rendered more difficult by the fact that it seems to form no insoluble compounds of suitable nature. A considerable proportion of pentose appears to be present in the gum, the remaining fraction being thus far unidentified.

From an unclassified strain of the Friedländer bacillus a gum similar to those already described was prepared by Mueller, Smith, and Litarczek, ${ }^{3}$ using both broth cultures and mass cultures on agar. A high degree of purity was not reached, but a specific precipitation reaction appeared in a dilution of $\mathrm{I}-\mathrm{I}, 000,000$.

Heidelberger, Goebel, and Avery ${ }^{4}$ obtained the specific substance from a type II strain of the Friedländer bacillus in a high degree of purity. Glucose formed a considerable proportion of its hydrolytic products, and in this and other respects it presented certain points of similarity to the material obtained from type II pneumococcus, the immunological and chemical similarities coinciding.

Extracts containing the soluble specific substances of streptococci, both hemolytic and non-hemolytic, have been prepared by Hitchcock,${ }^{5}$ and Lancefield, ${ }^{6}$ and Zinsser and Tamiya. ${ }^{7}$ The hemolytic strains were apparently homogeneous as regards the specificity of their soluble product, while the non-hemolytic strains were heterogeneous.

Zinsser and Parker in their original contribution worked with extracts of Staphylococcus pyogenes aureus, B.typhosus, and B. influenzae, in addition to the pneumococcus and tubercle bacillus. Mueller, Wayman, and Zinsser ${ }^{8}$ included also the meningococcus among others already described. Przesmycki ${ }^{9}$ obtained evidence that similar substances from four type strains of meningococci were here, as in the pneumococcus group, the bearers of type specificity.

It thus appears that every species of micro-organism so far examined produces as a part of its normal metabolism a substance highly characteristic of itself and even differing widely from type to type within the same species. These materials, referred

${ }^{2}$ Laidlaw, P. P., and Dudley, H. W.: Brit. J. Exper. Path., 6, 197. I925.

2 Mueller, J. H.: J. Exper. Med., 43, 9. I926.

${ }^{3}$ Mueller, J. H., Smith, D., and Litarczek, S.: Proc. Soc. Exper. Biol. Eo Med., 22, 373. 1925.

${ }^{4}$ Heidelberger, M., Goebel, W., and Avery, O. T.: J. Exper. MYed., 42, 701 and 727. 1925.

s Hitchcock, C. H.: ibid., 40, 445 and 575. 1924.

${ }^{6}$ Lancefield, R. C.: loc. cit.

${ }^{7}$ Zinsser, H., and Tamiya, T.: ibid., 42, 31 I. 1925.

${ }^{8}$ Mueller, J. H., Wayman, M., and Zinsser, H.: Proc. Soc. Exper. Biol. E MIed., 21, 241. 1924.

9 Przesmycki, F.: J. Infect. Dis., 35, 537. 1924. 
to as "specific soluble substances" or as "residue antigens," are carbohydrate in nature and apparently free from protein, at least in significant amounts. They react with antibodies, but fail to produce them, and hence may represent only a "haptene" group (Landsteiner and Simms) ${ }^{2}$ of an unstable protein-gum complex existing in the intact bacterial cell. They are probably of large molecular size since they do not pass through dialyzing membranes. Their ultimate constitutents are the simple sugars or sugar acids, but not always those best known. As Heidelberger has pointed out, biological specificity, hitherto considered to be an attribute of protein structure alone, may well be determined also by complex carbohydrates of this type. The considerable number of simple sugars, hexoses as well as pentoses and perhaps others, together with sugar acids and nitrogen-containing sugars-several asymmetric carbon atoms in each - and a number of possible modes of linkage, make the possibilities of chemically different gum structure as wide as those in the protein group.

\section{BACTERIAL AUTOLYSATES}

In working with materials as vulnerable to chemical insult as are the biologically active constituents of any cells, it is obvious that injury to antigenic function may easily occur. It is necessary, therefore, to discuss briefly the antigenic properties of bacterial autolysates which represent cell constituents in a state of suspension or solution produced by methods far less destructive than those employed in the manipulations of extraction.

Bacteria do not autolyze readily, and it is only with a few species, like the pneumococcus, meningococcus, and some others, that the effects of autolysis can be studied at the present time. The remarks which follow, therefore, are based for the most part on observations carried out with the pneumococcus.

If one allows thick suspensions of this organism to autolyze, either in slightly alkaline salt solution, in the presence of toluene or carbolic acid, or with the aid of bile-which is probably an accelerator of autolysis ${ }^{3}$ - an antigen is produced which is qualitatively identical, as to the antibodies produced, with the nucleoprotein fraction obtained in the manner above recorded; that is, the antibodies produced by such an autolysate do not react in vitro with the specific carbohydrate substance, but react with autolysate or nucleoprotein. It would seem, however, that though qualitatively there is this resemblance, the autolysate represents the antigen in perhaps a more potent, less denatured form, because of the more gentle method of its liberation. This opinion is based upon the fact that such an autolysate, injected into guinea pigs, will sensitize far more rapidly and vigorously than does similar treatment with extracted nucleoprotein.4 The skin reactions are severe, hemorrhagic, and eventually may ulcerate to a degree exceeding the most severe tuberculin reactions. With nucleoprotein sensitization, on the other hand, nothing more definite than red, edematous lesions

IVhile our original term for these substances was "residue antigen" and we here use the term to aroid confusion, we believe that Dochez and $\Lambda$ very's nomenclature of "soluble specific substance" is most accurately descriptive and should be adopted.

${ }^{2}$ Landsteiner, K., and Simms, S.: J. Exper. Mell., 38, 127. 1923.

3 Atkin, E. E.: Brit. J. Exper. Path., 7, I67. 1926.

${ }_{4}^{4}$ Zinsser, H., and Grinnell, F. B.: J. Bact., 14, 301. 1927. 
of various sizes are obtained. This would seem to indicate that the body can be powerfully sensitized in the course of the disintegration of bacteria, either by autolysis or by the enzymes active in the inflammatory tissues with which they are in contact, with consequent liberation of an antigen far more active immunologically than that which can be obtained by methods of chemical extraction. It seems reasonable to suppose that resistant organisms like the staphylococci, the streptococci, and the various types of tubercle bacilli which we have attempted to subject to autolysis in vitro, so far with only partial success, may undergo lytic changes within the infected body. Evidence obtained from the study of intraperitoneal injections of tubercle bacilli into tuberculous animals points in this direction, and the histological study of tuberculous lesions in many parts of the body has a similar significance. Indirect evidence of this is also obtained from the McJunkin ${ }^{\mathrm{I}}$ experiment, in which an antigen which has sensitizing properties different from and more effective than any that can be obtained from the bacilli in culture or by chemical extraction seems to be liberated in the peritoneum of tuberculous animals injected with tubercle bacilli. Indeed, we are inclined to believe that the secret of the mechanism of the tuberculin reaction lies in this-a matter which, however, cannot be proved until a method of satisfactorily autolyzing tubercle bacilli in vitro can be discovered.

At any rate, the liberation in inflammatory foci of bacterial, autolytic substances gains considerable theoretical importance from the observation that autolysates of bacteria have a sensitizing potency which is wholly or partially destroyed in the manipulations necessary for the preparation of nucleoproteins.

\section{III}

When an animal is infected with living bacteria the processes set in motion are complex and by no means completely understood; for there are responses on the part of the fixed-tissue cells - such, for instance, as those described by Gay and his collaborators ${ }^{2}$ in the pleural cavities of streptococcus-immune animals - which do not find adequate explanation in any of the current immunological conceptions. On the other hand, in so far as the bacterial-cell constituents and products are antigenic in the sense referred to above, considerable understanding has been gained by the correlation of biological function with chemical study.

We are of course omitting in our discussions any reference to the problem of bacterial exotoxins, since this phase of bacterial antigen-antibody reactions involves so many special factors that it must be separately dealt with. ${ }^{3}$ We are confining ourselves, therefore, entirely to the biological activities of the bacterial-body substance.

The bacterial cell consists largely of the so-called "nucleoprotein" material, probably in combination with the carbohydrate structures which Avery and Heidelberger speak of as the "specific soluble substances." When the animal is infected with living bacteria or when dead bacteria are administered without any extensive solution of the bacterial cell, this "complete" antigen arouses the formation of the specific antibodies

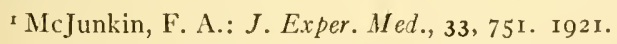

${ }^{2}$ Gay, F. P., and Rhodes, B.: J. Infect. Dis., 21, Ior. I923; and Gay, F. P.: J. Immunol., 8, I. 1923 .

${ }^{3}$ Cf. chaps. liv-lvi, lxxviii. 
with the effects of which we are familiar as agglutination, precipitation, opsonization, etc., effects which we believe to be due to a single sensitizing anti-substance. ${ }^{\text {I The }}$ response to the "complete" bacterial antigen is highly specific, as in the case of the pneumococci, where it is strictly type specific. In vitro these antibodies not only react with the bacteria themselves, but with the carbohydrate soluble specific substances provided from them.

When the bacterial cell is dissolved, either by bile, as in the case of the pneumococci, or, again, by chemical means such as extraction with alkali, and precipitation with acetic acid in the cold, this "complete" antigen is broken up into the nucleoprotein and the soluble specific "residue."

TABLE I

\begin{tabular}{|c|c|c|c|}
\hline Antigen & $\begin{array}{l}\text { Serum H: Rabbit } \\
\text { Injected with } \\
\text { Formalinized } \\
\text { Pneumococci }\end{array}$ & $\begin{array}{l}\text { Serum I: Rabbit } \\
\text { Injected with } \\
\text { Bile-dissolved } \\
\text { Pneumococci }\end{array}$ & $\begin{array}{c}\text { New York State } \\
\text { Antipneumococcus } \\
\text { Serum }\end{array}$ \\
\hline 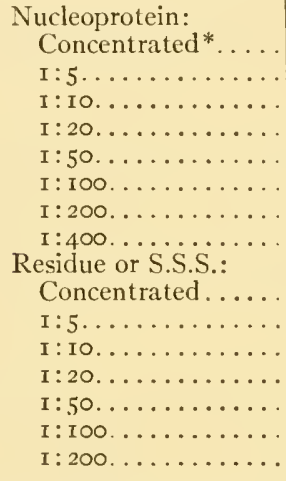 & $\begin{array}{c}++ \\
++ \\
++ \\
++ \\
++ \\
+ \\
+ \\
- \\
++ \\
++ \\
+ \\
+ \\
- \\
-\end{array}$ & $\begin{array}{c}++ \\
++ \\
++ \\
+ \\
\pm \\
\pm \\
- \\
- \\
- \\
- \\
- \\
- \\
- \\
-\end{array}$ & $\begin{array}{c}+++ \\
+++ \\
+++ \\
++ \\
++ \\
+ \\
\pm\end{array}$ \\
\hline
\end{tabular}

* It must be remembered that the so-called "concentrated" solution represents the original solution, probably containing relatively little of the dry material.

The nucleoprotein injected into animals will produce antibodies, but these antibodies react slightly with the whole bacteria and not at all with the soluble specific substance. Their specificity, moreover, is less strictly limited than that of the antibodies produced with the whole antigen.

The "residue" or soluble specific substance is not in itself antibody producing. It represents what Ehrlich would have called the "haptophore group" of the bacterial antigen, in that it reacts, with precise specificity, with the antibodies produced by the "complete" antigen; but injected into the animal in large quantities and for long periods, it induces no antibody formation whatever.

These relations were established by slightly different methods but with identical conclusions at about the same time by Avery and Heidelberger ${ }^{2}$ and by one of us with Tamiya. ${ }^{3}$ Table I, taken from our own paper, illustrates the actual conditions as they are observed experimentally.

'Zinsser, H.: J. Immunol., 6, 289. I921.

${ }^{2} \Lambda$ very, O. T., and Heidelberger, M.: J. Exper. Med., 42, 367. I925.

3 Zinsser, H., and Tamiya, T.: loc. cit. 
These relations are most easily understood from a schematic table such as the following one which we take from the work of Avery and Heidelberger.

A. Intact cell (PS)

Type I

B. Isolated constituents of cell (P), (S)

Protein
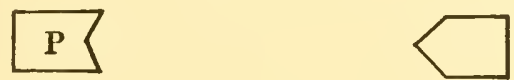

Anti-P serum, not type-specific

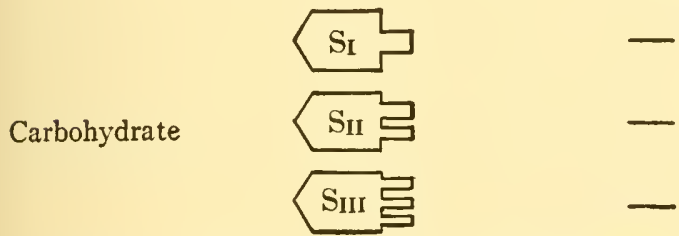

No antibody response

Fig. I. - Under $A$ the three type figures illustrate how the individual type specificity depends upon the conformation of the carbohydrate substance, the antibody produced in each case fitting this part of the complete antigen. Under $B$ the formation of antibody by the separated protein alone is indicated. (Scheme taken from Avery and Heidelberger, J. Exper. Med., 42, 372, I925.)

In order to separate the nucleoprotein and soluble specific substances from each other, it is not necessary to disrupt the cell by chemical extraction and solution, but in organisms like the pneumococcus, in which bile autolysis can be so easily produced, the mere solution of the cell separates the two constituents. This indicates an extraordinarily loose combination between the nucleoprotein nucleus and the carbohydrate associated with it. That the antibodies produced by the separated nucleoproteins are far less strictly specific than is the complete antigen or the carbohydrate soluble specific substance has been demonstrated by Avery and Heidelberger, ourselves, and by Lancefield for the streptococci. The nucleoproteins seem to possess a more general species specificity, overlapping for types of the same organism.

In any injection or immunization in which suspensions of whole bacteria are used a certain amount of solution of the bacterial cells takes place either in vitro or after injection. In consequence, therefore, both types of antibodies will be produced, as is apparent in our table.

In regard to what we consider the special importance of the antigenic properties of autolysates, such as those produced with pneumococci, the point of greatest significance is the fact that they serve to sensitize with a potency far above that of any artificially produced bacterial fractions and are still more noticeably potent when 
used to elicit allergic reactions in sensitive animals. We believe that these facts are essential to the understanding of bacterial allergic reactions of all types.

We are entirely in the dark regarding the property which lends to a chemical complex the power of arousing antibody formation. It is lost in the isolated soluble specific or residue substance and is retained by the protein. It is lost in protein by racemization, is altered by various forms of denaturation, and remains inherent in the protein in spite of modifications of the specificity of the molecule by the introduction, usually into the aromatic amino acids, of methyl and other radicles. We know nothing specific about this property except that it is possessed only by nitrogenous substances, proteins, or protein-like molecules of large size, all of them non-diffusible and colloidal. It appears, therefore, that in order to be capable of antibody production a certain chemical structure, together with non-diffusibility, is indispensable; and since lack of experimental approach occasionally justifies speculation, we may suggest that, teleologically considered, antibody formation represents an emergency mechanism by means of which the body can deal with nitrogenous foreign substances that cannot be taken care of either by intracellular digestion in the fixed-tissue cells or by simple disposal by the excretory organs. Just how the antibodies assist in increasing the ability of the body to get rid of foreign antigenic substances has not been followed in detail, but we do know that in bacterial disease antibodies make possible in some cases lysis, in all cases phagocytosis, and that in such conditions as serum sickness, where an easily determined antigen is in an unformed state, the antigen disappears from the blood stream in an inverse ratio to the appearance of antibodies. 


\title{
CHAPTER LIII
}

\section{HETEROPHILE ANTIGENS AND ANTIBODIES}

\author{
C. G. BULL
}

School of Hygiene and Public Health, Johns Hopkins University

In rgr I Forssman ${ }^{x}$ observed that emulsions of the tissues (other than the blood cells) of the guinea pig, cat, and horse stimulate the production of lysins for sheep blood corpuscles, just as sheep corpuscles themselves do when injected into rabbits. He found the tissues of the ox and rat to be devoid of this property.

It is unnecessary to say that these observations attracted widespread attention and aroused the interest of immunologists in general. The original observations have been abundantly confirmed and greatly extended, thereby revealing one of the most peculiar phenomena of immunity reactions or of general biology.

It has been found that the red blood corpuscles of the sheep possess two distinct antigenic substances, both of which are capable of stimulating the production of lysins for these corpuscles when injected into rabbits. One of these antigens is not destroyed by treatment with alcohol and withstands boiling and higher temperatures. The other is easily destroyed by heat.

Antigenic substances similar to the heat-resistant one of sheep corpuscles occur in various tissues of the guinea pig, horse, cat, and other animals. Because of distinctly peculiar properties common to the group and because the antibodies which they engender do not conform to the laws of species specificity, these substances have come to be designated as "heterophile" antigens. Accordingly, the antibodies produced by them are known as "heterophile" antibodies. The second antigenic substance of sheep corpuscles, which is thermolabile and species specific, as well as the antibodies engendered by it, are distinguished by the term "isophile."

\section{THE OCCURRENCE OF HETEROPHILE ANTIGEN IN NATURE}

Substances having chemical and immunological properties indistinguishable from those of the thermostable - "heterophile"-antigen of sheep red blood corpuscles have been found to occur also in the tissues of the goat (erythrocytes only), guinea pig (not in the erythrocytes), horse, chicken, cat, dog, camel, ostrich, mouse, a number of other mammals and birds, and in certain fish and bacteria. On the other hand, heterophile antigen has not been found in the tissues of man, rabbit, rat, ox, swine, pigeon, goose, etc.

The distribution of the antigen in the various tissues and organs of the animals in which it does occur is of interest. It occurs, for example, in the erythrocytes and spermatozoa of sheep and in the erythrocytes of goats but not in any other tissue, while it has been found in practically all of the tissues of the guinea pig, horse, and dog, with the exception of the blood corpuscles. If heterophile antigen occurs in the

' Forssman, J.: Biochem. Zischr., 37, 78. I9I r. 
blood corpuscles of a given species it does not, as a rule, occur in any other tissue and vice versa, but the domestic fowl is an exception to this rule, the antigen occurring both in the blood and other tissues. The antigen is not equally distributed in the different organs and tissues of "heterophile" animals; the kidney tissue of the horse and guinea pig seems to be particularly rich in heterophile antigen. It has even been found in the urine of certain species-e.g., horse and guinea pig.

It is seen that heterophile antigen is widely and apparently quite promiscuously distributed in nature. It is found in the chicken but not in the pigeon; in mice but not in rats; in certain paratyphoid bacilli, but not in typhoid bacilli; in certain fish (gills only), but not in many others.

\section{PHYSICAL AND CHEMICAL PROPERTIES OF HETEROPIHLE ANTIGEN}

As already stated, heterophile antigen is very resistant to heat. Sheep corpuscles, e.g., can be kept in boiling water for hours with little or no reduction of their content of this antigen. The same is true of emulsions of the kidney tissues of the guinea pig, horse, etc. Even heating in an autoclave at $120^{\circ} \mathrm{C}$. for an hour does not destroy the power of these antigens either to produce or to combine with antibodies. This property alone puts heterophile antigens in a class to themselves, for ordinary antigenic substances are easily injured by heat.

Heterophile antigen seems to be very firmly held by the formed elements of the tissues containing it, since juices obtained from such tissues by mechanical means contain only a very little of it. Organ plasma prepared according to Pohl's method contains the antigen. Treatment of this plasma with a weak solution of acetic acid causes the antigen to be precipitated. Pohl's acetic acid body obtained from the organ plasma is believed to be identical with heterophile antigen.

Chemically, ${ }^{\mathrm{I}}$ heterophile antigen appears to consist of a complex of two fractions. The entire complex is necessary for the stimulation of antibody production in animals, but only one fraction is active in test tube reactions. The latter fraction can be extracted from tissues with alcohol. It is insoluble in acetone but soluble in petroleum ether. It is believed to belong to the lecithin group of lipoids. This fraction is not capable of stimulating the production of antibodies, but it reacts specifically with heterophile antibodies in vitro.

The fraction remaining in the tissue residue after extraction with alcohol is believed to be a protein. This fraction is inactive both in vivo and in vitro. If the two fractions are mixed and injected into animals, no antibodies are produced. Landsteiner and Simms ${ }^{2}$ claim to have restored the antigenic power of heterophile lipoids by treating them with foreign protein, particularly with serum, but the antibody response of the animals treated with these reconstituted heterophile antigens was not very striking.

It seems, then, that one is justified in concluding that the thermostable antigenic substance found in the blood corpuscles of sheep and goats, and in the organs and tissues of various other animals, is a protein-lipoid complex which can be disrupted by treatment with alcohol. The whole complex is necessary for the stimulation of the pro-

${ }^{1}$ Meyer, Kurt: ibid., 122, 225. I921; Taniguchi, T.: J. Path. \& Bact., 23, 364. 1920.

${ }^{2}$ Landsteiner, K., and Simms, S.: J. Exper. Med., 38, 127. 1923. 
duction of antibodies in animals, but only the lipoid fraction takes part in serological reactions in vitro. Disruption of the complex does not alter these latter reactions.

\section{ANTIBODIES PRODUCED BY HETEROPHILE ANTIGENS}

Heterophile antibodies can be obtained by treating rabbits with heterophile antigens. Other animals whose tissues do not contain heterophile antigen can also be used for this purpose. Guinea pigs and other animals whose tissues contain heterophile antigen do not respond to such treatment, presumably because their tissues are already saturated with the antigen.

In order that we may get a clear conception of the nature of heterophile antibodies it will be helpful to discuss a concrete example, e.g., the immunization of rabbits and guinea pigs with sheep corpuscles and with guinea pig or horse kidney. The serum of rabbits treated with unheated sheep corpuscles contains two distinct hemolysins for these corpuscles. One of these hemolytic bodies can be absorbed from the serum with boiled sheep corpuscles or with emulsions of horse or guinea pig kidney without disturbing the other one. Fresh sheep corpuscles will remove both hemolysins. The portion having affinity for the boiled corpuscles is the heterophile antibody. The portion absorbable by the fresh corpuscles only is isophile antibody. The quantitative relations of heterophile and isophile antibodies vary in different specimens of serum. Serum collected early in the course of immunization generally has an excess of heterophile hemolysin, while the isophile variety is usually in excess in sera collected later.

A pure heterophile hemolysin can be obtained by treating rabbits with boiled sheep corpuscles or with emulsions of horse or guinea pig kidney. A pure isophile hemolysin is obtained by absorption of a composite serum with heterophile antigen or by treating guinea pigs or other animals whose tissues contain heterophile antigen with sheep or goat corpuscles.

Heterophile hemolysin has a very strong affinity for its antigen. Sheep corpuscles, for example, will absorb two hundred or more hemolytic units of hemolysin of the heterophile type, while they will scarcely absorb one-tenth this number of units of isophile hemolysin. Also the heterophile antigen-antibody complex is very difficult to disrupt. It is claimed that sheep corpuscles saturated with isophile hemolysin will still absorb heterophile hemolysin, but that corpuscles saturated with heterophile antibody will not absorb any of the isophile type. ${ }^{\text {I }}$

Heterophile antigen binds complement in the presence of heterophile serum. Lecithin prepared from tissues containing the heterophile substance gives positive fixation, while preparations from corresponding tissues of animals not belonging to the heterophile group do not give complement fixation with heterophile serum. It seems that heterophile serum contains little or no agglutinin for fresh sheep corpuscles, but that corpuscles allowed to age for two or three days are readily agglutinated by the serum. Trou-Hia-Hsü ${ }^{2}$ claims that the reaction with old corpuscles is specific, i.e., no such reaction occurs with old ox corpuscles. Corpuscles were treated in various ways but aging was the only thing that rendered them susceptible to the agglutinating action of the serum. The hemagglutinins were absorbed by emulsions of guinea pig kidneys.

${ }^{3}$ Gutfeld, F. V.: Ztschr. f. Immunitätsforsch. u. exper. Therap., 34, 524. I922.

${ }^{2}$ Trou-Hia-Hsü: ibid., p. 507. 1922. 
THE TOXICITY OF HETEROPHILE IMMUNE SERUM

In I909 Friedberger and Hartoch ${ }^{\mathrm{r}}$ observed that the serum of rabbits which had been injected with sheep corpuscles was toxic for guinea pigs, even small quantities of the serum producing death on intravenous injection. It was believed that this toxicity was due to the coexistence of antigen and antibody in the rabbit serum, and there arose a great deal of confusion concerning the toxicity of various immune and normal sera for certain animal species. The discovery of heterophile antigen by Forssman led to observations which made it possible to explain many instances of primary serum toxicity. It has been found, for example, that rabbit serum can be rendered toxic for guinea pigs by injecting the rabbits with sheep corpuscles, emulsions of horse kidneys, guinea pig kidneys, or any tissue containing heterophile antigen. These sera are toxic for any animal whose tissues contain heterophile antigen but they are not necessarily toxic for those animals whose tissues do not contain heterophile antigen.

Intoxication with heterophile-immune serum gives rise to symptoms very similar to those of anaphylactic shock. It is possible that the mechanism underlying the two phenomena is the same. There is, however, one important difference, viz., in anaphylaxis the antibody concerned is in the tissues of the animal and the antigen is supplied by injection, while the reverse is the case in heterophile-serum intoxication, i.e., the tissues of the animal under test contain the antigen and the antibody is supplied from without by injection.

As already pointed out, heterophile-immune serum hemolyzes sheep and goat corpuscles, fixes complement in the presence of the antigen, causes specific precipitation, and finally is toxic for animals whose tissues contain heterophile antigen, particularly the guinea pig. There has been a great deal of discussion as to whether the toxic body is the same as the hemolytic body and whether or not intoxication with heterophile-immune serum is the same as that occurring in anaphylactic shock.

Forssman and Hintze ${ }^{2}$ claim that the toxic substance does not develop during immunization parallel to the other antibodies but that the toxic property of the serum is a product of the process of immunization and is removed from the serum by treatment with heterophile antigen along with the hemolytic and other antibodies. Friedberger and Goretti ${ }^{3}$ also claim that the toxicity of heterophile-immune serum is not directly related to the hemolytic power of the serum. They found further that the hemolysin is more sensitive to heat than the toxic body and that the hemolysin and toxin can be separated by dialysis and treatment with $\mathrm{CO}_{2}$, the hemolysin separating out with the globulin fraction while the albumin fraction holds the toxin.

More recently, Redfern ${ }^{4}$ made a comparative study of the pathological effects of heterophile sera and of anaphylactic shock. This author found some distinctive differences: first, intoxication with heterophile serum causes more extensive edema and hemorrhage in the lungs than anaphylactic shock; second, guinea pigs cannot be de-

${ }^{8}$ Friedberger, E., and Hartoch, O.: ibid., 3, 581. 1909.

${ }^{2}$ Forssman, J., and Hintze, A.: Biochem. Ztschr., 44, 336. I9г 2.

${ }^{3}$ Friedberger, E., and Goretti, G.: Zischr. f. Immunitätsforsch. u. exper. Thera p., 21, 91. r9I4. ${ }_{4}^{4}$ Redfern, W. W.: Am.J. IIyg., 6, 276. I926. 
sensitized to the toxic effects of the serum; third, the uterine muscle of normal guinea pigs fails to contract when exposed to heterophile serum; fourth, heterophile serum injected into the skin of guinea pigs causes a characteristic local necrosis; fifth, the toxic property of a pure heterophile immune serum is directly proportional to its hemolytic power, and the two properties cannot be separated by fractionation with ammonium sulphate, the globulin fraction containing both properties.

In regard to the antibody content of heterophile-immune sera one can state, then, that they apparently contain specific hemolysin, precipitin, hemagglutinin, and complement-fixing antibodies and a specific substance toxic for animals whose tissues contain heterophile antigen. However, one may ask here, as with ordinary antibodies, whether we have five distinct substances or whether one substance gives the five different effects under different conditions.

\section{PRACTICAL ASPECTS}

Besides offering an explanation of many instances of serum intoxication, knowledge of the occurrence of heterophile antigen in the tissues of certain animals and the occurrence of the antibody in the body fluids of other species is of practical importance. For example, the tissues of guinea pigs contain the antigen and, as wil] be recalled, the antigen appears in preparations of lecithin from such tissues. Human serum may contain heterophile antibody. Hence, it would be possible to have falsely positive Wassermann reactions if the antigen were prepared from the heart muscle of guinea pigs, or from any tissue containing heterophile antigen.

The possibility of making use of the heterophile reaction in the identification of meats has also been pointed out. One could determine, for example, if sausage contained horse, dog, or cat flesh, even after it had been cooked, by making a test for the presence of heterophile antigen. However, if sheep or goat blood were present, the test would not be applicable, for these also contain the antigen.

SUMMARY

In IgI I Forssman discovered the substance now known as "heterophile antigen." This substance occurs in the tissues of a number of mammals, birds, fishes, and bacteria, namely, sheep (blood cells and sperm), goat (blood cells), horse, dog, cat. camel; chickens; lobster, carp, pike; Shiga dysentery bacilli, certain paratyphoid organisms, and a certain strain of Bacterium lepisepticum.

Chemically, heterophile antigen seems to be a lipoid-protein complex. It is very resistant to heat. The complex can be disrupted by treatment with alcohol and other lipoid solvents.

When injected into rabbits, or other animals whose tissues do not contain the antigen, heterophi'e antigen stimulates the production of antibodies, namely, hemolysins for sheep and goat corpuscles; precipitins, specific for the antigen; hemagglutinins, especially for aged sheep corpuscles; complement-fixing antibody, also specific for the antigen, and a substance toxic for guinea pigs or other animals whose tissues contain the antigen.

For the stimulation of antibody production the whole lipoid-protein complex is 
necessary. If the complex is disrupted neither fraction gives rise to antibody production. In test tube reactions, however, only the lipoid fraction is necessary, the protein fraction apparently not taking part in these reactions.

The pathological effects of heterophile-immune serum, at least for guinea pigs, are very much like those of anaphylactic shock, but certain essential differences have been pointed out.

In obtaining serological reactions with any kind of tissues, or extracts from them, the presence or absence of heterophile antigen must come into consideration. 


\title{
CHAPTER LIV
}

\section{THE PHYSICAL CHEMISTRY OF TOXIN AND ANTITOXIN}

\author{
MARY E. MAVER \\ University of Chicago
}

The theory of Metchnikoff ${ }^{2}$ which postulated a physiological rôle for antitoxin in stimulating the body to the attack upon toxin was no longer held valid after Ehrlich ${ }^{3}$ demonstrated that the toxin-antitoxin reaction could occur in vitro. Thereafter the problems concerning the nature of toxin and of antitoxin and their reaction were approached by the application of current chemical theories. Since no one has ever succeeded in establishing the chemical identity of either toxin or antitoxin, these reagents have necessarily been studied in their native media. Considering the physical and chemical complexity of the solutions studied, it is not surprising that no theory has been advanced which can explain all of the phenomena exhibited by toxic and antitoxic solutions.

\section{THE THEORIES OF EHRLICH AND ARRHENIUS}

The conception of the chemical nature of the neutralization of toxin by antitoxin was apparently inherent in the discovery of this phenomenon as it was made by von Behring and Kitasato in 1890 . Ehrlich ${ }^{4}$ considered the reaction of toxin with antitoxin to be a simple chemical reaction similar to the practically irreversible reaction of a strong acid with a strong base. However, when experiments were undertaken to standardize toxin and antitoxin it was discovered that, on standing, toxin lost its toxic power but not its ability to combine with antitoxin. To explain this, Ehrlich found it necessary to postulate the presence of such a long series of substances possessing varying degrees of toxic and combining powers that the theory of a simple chemical reaction was lost in the confusion of explanatory details.

At this time Arrhenius, as the leader of the modern ionization theory, undertook to correlate the reaction of toxin and antitoxin with the new theory of solutions which had recently been developed by van't Hoff. Arrhenius ${ }^{5}$ treated the complex toxic and antitoxic solutions as homogeneous solutions of weak electroly tes and applied to these solutions the laws of van't Hoff. He found that toxins and antitoxins, like other substances, diffused with unequal rates into gelatin, and he therefore felt justified in concluding that they obeyed van't Hoff's law of osmotic pressure and consequently the law of mass action. Calculations of velocity constants from reactions in such complex heterogeneous solutions which would call for numerous assumptions

I Fellow under the Douglas Smith Research Foundation.

2 Metchnikoff, E.: Immunity in Infective Diseases. Cambridge, 1905.

3 Ehrlich, P.: Fortschr. d. Med., 15, 41. 1897.

4 Ehrlich, P.: Deutsche med. Wchnschr., 24, 597. 1892.

5 Arrhenius, S.: Immunochemistry. Macmillan, 1907. 
would be difficult and, at best, would shed no light on the real nature of toxin and antitoxin.

\section{THE ADSORPTION THEORY OF BORDET}

The failure of the law of chemical combination in definite proportions in immunological reactions was shown by Danysz ${ }^{\mathrm{I}}$ with ricin and anti-ricin, by Bordet ${ }^{2}$ with alexin and anti-alexin, and by von Dungern $^{3}$ with diphtheria toxin and antitoxin. In these reactions the neutralizing dose was found to vary, depending upon whether the toxin was added in a single or in several doses. Bordet ${ }^{4}$ first characterized these reactions as "combinations in varying proportions" and likened them to the adsorption of varying amounts of dye by cloth. Landsteiner and Jagic ${ }^{5}$ then pointed out the analogy between immunological reactions and the adsorption phenomena of inorganic colloids. The colloidal adsorption theory which was developed from these observations has successfully explained many characteristic phenomena of toxin and antitoxin reactions and is only limited in its application by our meager knowledge of the chemistry of colloidal reactions.

Toxins and antitoxins exhibit the colloidal reactions of large molecular complexes. They can be dialyzed in collodion membranes, and are adsorbed by charcoal and kaolin. ${ }^{6}$ Their sensitive reactions to all of the reagents which are known to alter natural proteins suggest that they might be of protein nature or firmly adherent to, and dependent upon, proteins. Like the proteins, toxins and antitoxins are attacked by enzymes, and they are precipitated by ammonium sulphate and the salts of the heavy metals. They are inactivated on shaking in air. ${ }^{7}$ That the toxin molecule is smaller than the antitoxin molecule is indicated by the fact that it diffuses into gelatin more rapidly than antitoxin. ${ }^{8}$

Antitoxin is always associated with the pseudoglobulin fraction of serum-that fraction which behaves like a true globulin in being precipitated by half-saturation with ammonium sulphate, but which, unlike a true globulin, is soluble in water. Adolf' found that when immune diphtheria serum was electrodialyzed until the supernatant fluid had practically the same conductivity as distilled water the antitoxin was in the globulin precipitate. However, when the electrodialysis was interrupted at intervals before the removal of all electrolytes, the antitoxin was located in the last fractions to be precipitated, corresponding to the pseudoglobulin fraction. This close association of antitoxin with a definite fraction of the immune serum which is commonly ${ }^{10}$ but not necessarily ${ }^{11}$ increased upon immunization of the animal might

'Danysz, J.: Ann. de l'Inst. Pasteur, 16, 33r. r9o2.

2 Bordet, J.: ibid., 1 2, 692 . I 898.

3 v. Dungern: Deutsche med. Wchnschr., 30, 275, 310. I904.

4 Bordet, J.: op. cit., I3, 225. I899; I4, 267. I900; I7, I61. I903.

5 Landsteiner, K., and Jagic, N.: Mïnchen. med. Wchnschr., 50, 764. 1903.

${ }^{6}$ Zunz, E.: Arch. internat. de physiol., 8, 227. r9o9; Eisler, M.: Biochem. Ztschr., 135, 416. 1923.

7 Biltz, W., Much, H., and Siebert, C.: Behring's Beiträge, ro, 30. 1905.

${ }^{8}$ Arrhenius, S.: op. cit., p. 25. 9 Adolf, M.: Klin. Wchnschr., 3, 1214. 1924.

${ }^{10}$ Doerr, R., and Berger, W.: Ztschr. f. IIyg. u. Infektionskrankh., 93, 147. I921.

"Glaesner, K.: Ztschr.f. exper. Path. u. Therap., 2, 154. 1906. 
signify that antitoxin is a definite protein with properties slightly different from the corresponding protein in normal serum. Wells ${ }^{\mathrm{r}}$ has discussed at length the view that the highly specific character of toxin-antitoxin reactions can be most readily accounted for when to these reagents are assigned the complex structures of proteins.

After the colloidal nature of toxins and antitoxins had been established, Biltz, Much, and Siebert ${ }^{2}$ applied to immune sera and toxic broths the electro-colloidal reactions of inorganic and organic colloids. They found that tetanus toxin and antitoxin were precipitated by positive colloids but were unable to demonstrate the cataphoretic migration to the anode. Zunz $z^{3}$ found that animal charcoal adsorbs diphtheria and tetanus toxins and antitoxins, but not the mixture of toxin and antitoxin. He was unable to detect any relation between the adsorption of toxins and antitoxins and modifications of the solution containing them in regard to density, index of refraction, or freezing-point. Toxins lowered the surface tension of the solutions but antitoxins did not alter it. Adsorption studies of the pseudoglobulin fraction from immune sera have yielded interesting results. Reitstotter ${ }^{4}$ has shown that a complex ferric hydroxide-pseudoglobulin sol prepared with immune pseudoglobulin was more sensitive to flocculation by electrolytes than a corresponding sol made with ferric hydroxide and normal pseudoglobulin. Since it is probsble that the particles of the sol are kept from flocculating by their similar charges, this would indicate that the immune pseudoglobulin had caused a greater decrease of the charge on the complex sol than the normal pseudoglobulin.

The neutralization of diphtheria toxin by antitoxin and of ricin by anti-ricin has been studied by Bilt $z^{5}$ as adsorption reactions, and his calculations indicate that these reactions obey Gibbs's adsorption formula. The extent to which the electro-colloidal theory can explain the specificity of immunological reactions depends upon our interpretation of the source of the charge carried by the adsorbing particles. If the charge exists by virtue of the ionization of a characteristically oriented surface molecule ${ }^{6}$ the specificity of the reaction can be explained ultimately upon a chemical basis. In defense of the idea that these specific biological reactions can be adsorption reactions, Freundlich says:

This [specificity] is by no means in contradiction with the view of an adsorption, no matter whether it be regarded as a saturation of the last residual valencies, or whether it be brought into relation with the lowering of interfacial tension; for even in the last case the differential coefficient $\frac{d \sigma}{d c}$ in the Gibbs' formula might depend specifically upon the nature of adsorbent and adsorbed substance. But it is certainly a gap in the theory of these phenomena that up to the present this specific adsorption has been shown only by substances formed in biological processes and not by those prepared in the test tube. The idea suggests itself that only an adsorbent of suitable geometrical form is able strongly to adsorb a sub-

${ }^{2}$ Wells, H. G.: Chemical Aspects of Immunity. I925.

${ }^{2}$ Loc. cit.

3 Zunz, E.: Bull. Acad. roy. méd. de Belg. Oct. 29, r9гі.

4 Reitstotter, J.: Ztschr.f. Immunitätsforsch. u. exper. Therap., 30, 507. 1920.

5 Biltz, W.: Biochem. Ztschr., 23, 37. 1909.

${ }^{6}$ Harkins, W. D., and Beeman, N.: Proc. Nat. Acad.Sc., r1, 631. 1925; Langmuir, I.: Colloid Symposium Monograph, 4, 48. I925. See also chap. lviii, this volume, by Northrop. 
stance which corresponds to it in spatial dimensions. In the biological process adsorbent and adsorbed substance are causally connected in the manner of their formation, so that their shape may very well be fitted to one another. In the laboratory it has not yet been possible to produce an adsorbed substance from an adsorbent which, in consequence of the mode of formation, has a particular spatial arrangement and is preferentially adsorbed. ${ }^{\mathrm{t}}$

The adsorption reactions have been useful in the purification of toxin and antitoxin. Rakusin and Flieher ${ }^{2}$ were able to remove 44 per cent of the unessential proteins from antitoxic diphtheria plasma by the use of dry aluminium oxide. In this case the antitoxin was not adsorbed but was in the filtrate. Botulinum toxin, on the other hand, was adsorbed by this reagent, and by this means Sommer, Sommer, and Meyer $^{3}$ were able to obtain a highly potent toxin. That adsorption reactions of immune bodies with inorganic colloids or other immune bodies are not always smoothly reversible has been explained on the ground that denaturation, involving chemical changes in the protein molecule, takes place when it is discharged by contact with a colloid bearing an opposite charge. ${ }^{4}$

Calmette and $\mathrm{Massol}^{5}$ were the first to measure the antitoxic value of a serum by the precipitate which is formed when the serum and its homologous toxin are mixed. While this in vitro method of standardization, as perfected by Ramon, ${ }^{6}$ Glenny, and others and later by Bayne-Jones, ${ }^{7}$ has been of very great practical value, it is not yet definitely established that the flocculation is produced exclusively by the toxin and antitoxin. ${ }^{8}$

\section{THE CATAPHORESIS OF TOXIN AND ANTITOXIN SOLUTIONS}

In 1907 Field and Teague ${ }^{9}$ published results of experiments which indicated that toxin and antitoxin migrated to the cathode whether the reaction of the toxin and the antitoxin solution was alkaline or acidic. They concluded that toxin and antitoxin were, therefore, not amphoteric proteins, and that the neutralization of toxin by antitoxin was not a chemical reaction since both bore the same charge. Field and Teague used 2 per cent agar arcs as bridges connecting the toxin or antitoxin solutions with the distilled water into which platinum-coil electrodes were dipped. After r ro volts direct current had been applied for from four to six hours, the agar was pushed out of the glass supporting arcs and the toxin or antitoxin was extracted from the agar with water.

Bechhold ${ }^{\text {to }}$ in discussing the work of these authors could find no fault with their experiment, but criticized at length their deductions. His own attempt to determine

' Freundlich, H.: Colloid and Capillary Chemistry, p. 538. I922. 1924 .

${ }^{2}$ Rakusin, M. A., and Flieher, G. D.: Ztschr.f. Immunitätsforsch. u. exper. Therap., 39, 193.

3 Sommer, E. W., Sommer, H., and Meyer, K. F.: J. Infect. Dis., 39, 345. 1926.

4 Samson, K.: Ztschr.f.d. ges. exper. Med., 49, 95. 1926.

${ }^{5}$ Calmette, A., and Massol, L.: Ann. de l'Inst. Pasteur, 23, 155. I909.

${ }^{6}$ Ramon, G.: Compt. rend. Soc. de biol., 86, 711. 1922.

7 Bayne-Jones, S.: J. Immunol., 9, 48I. I924. See also chap. Ivi, this volume.

${ }^{8}$ Bronfenbrenner, J. J., and Reichert, P.: J. Exper. Med., 44, 553. 1926.

${ }^{9}$ Field, C. W., and Teague, O.: ibid., 9, 86, 225. 1907.

so Bechhold, H.: Mïnchen. med. JWchnschr., 39, 1921. 1907. 
the charge carried by toxin and antitoxin had not given unequivocal results, probably because the apparatus he used necessitated the migration of the toxin or antitoxin through either fish bladders or parchment membranes. Of the electric charge carried by antigens and antibodies Bechhold said: "It is so small, however, that in my opinion it cannot be definitely recognized since traces of hydrogen or hydroxyl ions may cause a reversal of charge."

Landsteiner and Pauli, ${ }^{2}$ using a U-shaped cataphoresis tube with adjoining vessels for platinum electrodes, were able to show that abrin, ricin, and the agglutinin for hen serum migrated to the anode in alkaline and to the cathode in acidic solution. They suggested that a potential difference at the boundary between the agar and the solution might be the cause of the different results obtained by Field and Teague.

The results of Field and Teague have been widely quoted, and in some texts toxin and antitoxin are assumed to be positive colloids. Since no careful hydrogen-ion determinations of the solutions used were made by these authors the work was repeated in this laboratory ${ }^{3}$ under their experimental conditions. In our research diphtheria toxin and antitoxin solutions ${ }^{4}$ were electrolyzed in a Field and Teague apparatus at all the hydrogen-ion concentrations compatible with the maintenance of potency in the solutions. The work of Field and Teague was confirmed. Under their experimental conditions antitoxin was found only in the cathode agar in a $\mathrm{pH}$ range of from 2.5 to $\mathrm{I} 3.0$, and, similarly, toxin was found in the cathode agar in a $\mathrm{pH}$ range of from 4.5 to I3.O. In no case was there evidence of any migration of toxin or antitoxin to the anode.

In the antitoxin series it was found that the protein of the antitoxic plasma also was present in the cathode agar in the same $\mathrm{pH}$ range. The protein was determined by nitrogen analyses and by anaphylactic reactions on guinea pigs which had been protected by the extracts of the cathode agar. These results were contrary to those of numerous workers who have all agreed that a protein in a solution alkaline to its isoelectric point migrates to the anode. A great many different kinds of natural and purified proteins in alkaline solution were found in the cathode arc of the Field and Teague apparatus. Since the same substances were found to migrate normally to the anode in alkaline solution in a $U$-shaped cataphoresis tube in which no agar was present, the agar was thought to be responsible for the anomalous migration of the protein in this apparatus.

An investigation of the rôle of the agar in the Field and Teague apparatus led to the conclusion that the proteins in alkaline solution were carried to the cathode agar by the endosmotic streaming of the water. ${ }^{5}$ The agar is always negative, ${ }^{6}$ and affords many small capillaries which favor the endosmotic streaming of the water to the

${ }^{x}$ Bechhold, H.: Colloids in Biology and Medicine, p. 205. I9I9.

${ }^{2}$ Landsteiner, K., and Pauli, W.: Wien. med. Wchnschr., 58, 1010. 1908.

${ }^{3}$ Maver, M. E., and Falk, I. S.: J. Immunol., I4, 219. 1927. (In press.)

${ }^{4}$ We are indebted to Dr. C. R. Hixson, of the United States Standard Products Co., Woodworth, Wis., for a generous supply of diphtheria toxin and antitoxin.

${ }^{5}$ Freundlich, H.: Colloid and Capillary Chemistry, p. 245.

${ }^{6}$ Gyemant, A.: Kolloid-Ztschr., 28, I03. I92 I; Kruyt, H. R., and de Jong, H. G.: Zischr.f. phys. Chemie, 100, 250. 1922. 
cathode. If the velocity of this water-streaming were great enough in comparison to the migration of the protein to the anode, the water would sweep the protein along with it. Direct quantitative proof to substantiate this explanation is very difficult to obtain with an agar membrane $20 \mathrm{~cm}$. thick through which varying amounts of protein and electrolytes are being drawn. In the Field and Teague apparatus an average of I cc. of water per hour is transported through the agar endosmotically when water alone is electrolyzed. A micro-cataphoretic study ${ }^{\mathrm{I}}$ of the velocity of eggalbumin particles, $\mathrm{pH} 7.2$, showed that under a potential difference of 45 volts they migrated at the rate of 8. I $\mu$ per second, or 29. I mm. per hour.

Indirect proof of this explanation is supplied by the fact that factors which effect endosmotic streaming of the water also alter the amount of protein found in the cathode arc. We concluded, therefore, that the protein which has been found in the cathode portion of the agar in the Field and Teague apparatus when alkaline solutions were electrolyzed has not migrated toward the negative pole by virtue of the positive charge upon the protein, but has most probably been carried there by the endosmotic flow of the water in contact with the agar. ${ }^{2}$ This conclusion has been further justified by experiments now in progress in which it is found that antitoxin and toxin migrate with protein to the anode in alkaline solution in a $U$-shaped cataphoresis apparatus. Antitoxin migrates to the cathode in a solution of $\mathrm{pH}$ 4.6. Toxin is more sensitive to acidification, losing its potency at a pH between 5.0 and 4.5 . The results so far obtained are in agreement with earlier work, which indicates that antitoxin, if not of protein nature itself, is closely adherent to a protein of the plasma.

\section{CONCLUSION}

The reaction of toxin with antitoxin cannot be explained upon a chemical basis because the reagents are not known chemical entities. As colloidal protein solutions, or hydrophilic sols, their mutual neutralization can be explained as an adsorption reaction. However, an explanation of the specificity of the union will most probably await their definite chemical characterization as well as an advancement of our knowledge of surface chemistry.

A misinterpretation of the results obtained with their apparatus led Field and Teague to assign a positive charge to toxin and antitoxin. In a $\mathrm{U}$-shaped cataphoresis tube, toxin and antitoxin migrate to the anode in alkaline solution. Antitoxin migrates to the cathode in a solution with a $\mathrm{pH}$ of 4.6 , and can therefore be said to be an amphoteric colloid.

I A simplified micro-cataphoresis cell was used. See Falk, I. S., et al.: Am.J. Pub. IIealth, I 7, 7I4. 1927.

${ }^{2}$ Stary, Z. (Ztsclir. f. phys. Chemie., 126, г73. 1927) has explained the anomalous migration of oxygen and glucose in the Fürth high-potential apparatus upon similar grounds. The "half-conductors" which favor endosmotic streaming in this apparatus are porous clay or wet blotting paper. See Glickhorn, J., Fürth, R., and Blüh, O.: ibid., I23, 344. 1926. 


\title{
CHAPTER LV
}

\section{THE PREPARATION AND PURIFICATION OF TOXINS, TOXOIDS, AND ANTITOXINS}

\author{
EDWTN J. BANZHAF
}

Bureau of Laboratories, New York City Department of Health

\section{SOME PROPERTIES OF BACTERIAL TOXINS}

Bacterial antigens are classified as extracellular and endocellular. Among the extracellular are the exotoxoids. The classical exotoxins are primarily toxic and regardless of the amount injected are not immediately fatal but require an incubation period before fatality ensues. The extracellular toxins produced by the pathogenic anaerobes such as B. welchii (Clostridium welchii) and B. oedematis maligni (Clostridium oedematis) would not under this classification be classed with these exotoxins in that they produce almost immediate symptoms even though a small dose be injected intravenously. They are, however, toxins, and the older definition should be revised to the simple one that toxins are antigenic poisons (Coca); i.e., toxins possess the property of stimulating the production of antitoxins which are specific and which will neutralize the toxins in vitro and in rivo. The endocellular substances possess the property of stimulating the production of agglutinins, precipitins, opsonins, or bacteriotropins and bacteriolytic and bactericidal antibodies. The actual existence of specific endotoxins which are antigenic is still debatable. The best-known exotoxins are those produced by $B$. diphtheriae (Corynebacterium diphtheriae), B. tetani (Clostridium tetani), B. botulinus (Clostridium botulinum), B. welchii (Clostridium welchii), B.anthracis symptomatici (Clostridium chanvei), B.dy'senteriae Shiga (Eberthella dysenteriae), Streptococcus scarlatinae, and Streptococcus erysipelatus.

Toxins are non-crystallizable and have not been obtained in a pure form. They are precipitated by salts of the heavy metals, ammonium sulphate at saturation, alcohol, nucleic acid, and acetic acid. They are considered by Oppenheim as large molecular complexes, probably related to the proteins and classed as "enzymes." They are electropositive, and are not readily dialyzable except through thin membranes. They are readily absorbed by animal charcoal and are destroyed by proteolytic ferments. Sodium chloride at saturation destroys the toxins of $B$. diphtheriae and $B$.tetani. Freezing also destroys about half of these toxins. Toxins are sensitive to oxidation, light, and Roentgen rays. Heating $B$. diphtheriae and $B$. tetani toxins to $50^{\circ} \mathrm{C}$. for twelve hours destroys about half of the acutely toxic fraction (toxophore, Ehrlich's hypothesis) and modifies the rest into toxoid fraction (haptophore, Ehrlich). The toxoids are capable of neutralizing antitoxins and are antigenic. Heating to $56^{\circ} \mathrm{C}$. for two hours and to $58^{\circ} \mathrm{C}$. for one-half hour produces practically the same results as heating to $50^{\circ} \mathrm{C}$. for twelve hours. A notable exception to the lability of the toxins to high dilution and heat is the streptococcus scarlatinal toxin. This toxin is practically stable for years, and even in high dilutions holds its value for at least 
eight months. Its value is retained when brought to the boiling-point, and is not completely destroyed unless boiled for two hours.

\section{STANDARDIZATION OF TOXIN}

Toxins can be detoxified or modified into toxoids (anatoxins, Ramon) by formalin, iodine, and carbon bisulphide. When detoxified by these agents no reversible reaction has been obtained. A reversible reaction can be obtained when soaps are used as detoxifying agents. The use of soaps was described by Raubischek and Russ ${ }^{1}$ in I908. They employed sodium oleate. Nelis ${ }^{2}$ in ${ }^{2} 24$ reported similar observations. Larson $^{3}$ in 1924 recommended the use of sodium ricinoleate, on the ground that a purer and more active soap could be easily prepared from castor oil. Aging toxins, i.e., storing in a cool place for a year or longer, modifies much of the toxin into toxoid. $B$. diphtheriae and B. tetani toxins are very unstable.

Diphtheria toxin.-If the M.L.D. and the L+ value be determined on a freshly prepared $B$. diphtheriae toxin and again determined after one year's aging, one will note a 50-60 per cent decrease in the M.L.D. value and only about a ro per cent decrease in the L+ value. After a year the M.L.D. value remains relatively constant, as does the $\mathrm{L}+$ dose. Advantage is taken of this stability in selecting $B$. diphtheriae toxin for the Schick test (intracutaneous injection to determine antitoxic immunity). It is essential that this toxin for the Schick test remain relatively stable for at least eight months. It is also of importance to use a toxin of high M.L.D. value to reduce the pseudo-reacting substances always present, soluble bacillary products, and other proteins in the toxic broth.

A suitable stable toxin has a value of at least 300 M.L.D.'s to each mil. This value is too great to allow for the very small accurately measured amount of toxin that is placed in the capillary tube or vial, for the Schick "outfit" which is distributed to the physician. It has been found that two or three volumes of pure neutral glycerin may be added to one volume of the aged toxin without upsetting its stability. Diluted to this extent, the required amount of toxin can be accurately measured into the capillary tube or vial. Each capillary tube or vial is accompanied with a vial containing Io mils of physiological salt solution as the diluent for the toxin.

Each 0.2 mil of the dilution should contain I/40 of a guinea pig M.L.D. The Schick test is the intracutaneous injection of this amount in the upper anterior surface of the forearm. In those susceptible to diphtheria an area of erythema develops within twenty-four to forty-eight hours, lasting four to five days, and gradually disappears, leaving a definitely circumscribed scaling area of brownish pigmentation. A pseudo-reaction may occur due to a local hypersensitiveness of the tissue cells to the proteins in the toxin dilution. This reaction is commonly urticarial in character; it appears within eighteen hours and as a rule disappears on the third or fourth day, leaving a poorly defined area of reddish-brown pigmentation without scaling. Indi-

${ }^{1}$ Raubischek, H., and Russ, V. K.: Ztschr.f. Immunitätsforsch. u. exper. Therap., Orig. I, x, 395. 1908-9.

${ }^{3}$ Nelis, P.: Compt. rend. Soc. de biol., gr, rr59. r924.

${ }^{3}$ Larson, W. P., and Nelson, E.: Proc. Soc. Exper. Biol. Eo Med., 21 , 278. r924; Larson, W. P., Evans, R. D., and Nelson, F.: ibid., 22, 194. I924. 
vidual hypersensitiveness to the proteins in the toxin dilution may be tested by means of a control outfit. For this test the toxin should be heated to $75^{\circ} \mathrm{C}$. for ten minutes, which destroys the action of the toxin but leaves unaltered the substances causing the pseudo-reactions.

Scarlatinal toxin.-The streptococcus scarlatinal toxin (Moser, ${ }^{\mathrm{I}}$ Gabritschewsky, ${ }^{2}$ Savchenko ${ }^{3}$ ) was used by the Dicks ${ }^{4}$ in a skin test to determine the presence or absence of immunity to scarlet fever. They also corroborated the work of Savchenko who found that the toxin, when injected in increasing amounts, actively immunized individuals susceptible to scarlet fever. The technique used in making the Dick test is identical with that used in the Schick test, with the exception that the result is noted at the end of twenty-four hours. The reaction disappears in forty-eight to seventy-two hours. A positive reaction consists of a reddish area from I to $3 \mathrm{~cm}$. As in the Schick test, a small percentage of pseudo-reactions occur due to the susceptibility of the individual to the protein substances in the toxin broth. The toxin to be used as a control must be boiled two hours because it is very heat resistant. The Dicks' ${ }^{2}$ standardization of the S.T.D. (skin-test dose) of the toxin is that amount of toxin, which, when injected intracutaneously in non-immunes in a total volume of $\frac{1}{10} \mathrm{mil}$, will cause a reaction of $\mathrm{I}-2 \mathrm{~cm}$. in diameter. The standardization presents difficulties in that at the present time only non-immune human beings and a special breed of rabbits and Swiss goats (Saaren) give a visible reaction (skin reaction) more or less regularly. As a rule, the toxin is quite stable. The Dicks, A. W. Williams, and others have prepared toxins that have retained the larger part of their toxic value for years. The toxin prepared in media, without blood, usually contains about 20,000 S.T.D.'s to each mil. Highly diluted toxins may drop in their S.T.D. content during the first month; after this time the toxin remains constant for at least six months. The Dick-test toxin supplied to physicians is diluted so that each $\frac{1}{10}$ mil contains I S.T.D. For the active immunization of individuals against scarlet fever the Bureau of Laboratories of the Department of Health, City of New York, recommends at the present time four injections subcutaneously at weekly intervals, the following amounts of S.T.D.'s: first injections, 600; second, 2,400; third, 6,000; and fourth, I 2,000 .

The Dicks recommend larger doses of from 50,000 to 70,000 S.T.D.'s, divided in five injections. However, at the present time our evidence shows that four injections are sufficient to confer an immunity to a skin-test dose in about 80 per cent of the individuals. The fifth injection of the Dicks might raise the immunity to 90 per cent. Our method is to test by the Dick method one or more months after the fourth injection and give to those that are still positive a second series of two injections of about ro,000 and 20,000 S.T.D.'s.

It should be emphasized that, when the Dick test is given to determine if active immunity has resulted from this treatment, a control test of heated toxin should be

IMoser: Wien. klin. Wchnschr., 15, 1053. 1902

${ }^{2}$ Gabritschewsky, G.N.: Centralbl.f. Bakteriol., Orig. I, 4I, 7 I9. 1906; Berl. klin. Wchnschr., 44, 556. 1907 .

3 Savchenko, J. G.: Russk. Vrach., 25, 797. 1905.

${ }_{4}^{4}$ Dick, G. F., and Gladys H.: J.A.M.A., 81, 1066. 1923; 83, 84. I924. 
given, as the percentage of pseudo-reactions is considerably increased due to the repeated previous injections of the toxin and its accompanying products.

\section{PREPARATION OF TOXIN-ANTITOXIN}

In preparing the toxin-antitoxin mixture for active immunization against diphtheria of those individuals showing a positive Schick test, an aged toxin is used. During the aging of the toxin, about one-half to two-thirds of the toxin is modified into toxoid and the $\mathrm{L}+$ becomes stabilized.

The toxin selected should contain at least five $\mathrm{L}+$ doses to each mil. The antitoxin used for the partial neutralization of the toxin is purified by concentrating methods and should contain at least 2,000 units of antitoxin to each mil. The toxin is prepared in batches of $16-20$ liters. Each toxin batch should be uniformly mixed before being stored for aging. The o.I $\mathrm{L}+$ toxin-antitoxin mixture is made up in 40-liter preparations. To prepare the 40 liters, 800 mils of toxin (containing five $\mathrm{L}+$ doses to each mil) are placed in a 2-liter flask and 3,000 units of diluted antitoxin are added. These are mixed and added to the diluent of 0.8 per cent salt solution containing 0.5 per cent phenol, again thoroughly mixed and filtered through a Berkefeld candle. It is then injected into guinea pigs to test its active immunizing value and to determine if the preparation is properly balanced. For this purpose, a series of guinea pigs is injected. In each series the following amounts are given (the degree of toxicity as illustrated is that of a properly balanced mixture which will give a high percentage of immunes in susceptible individuals):

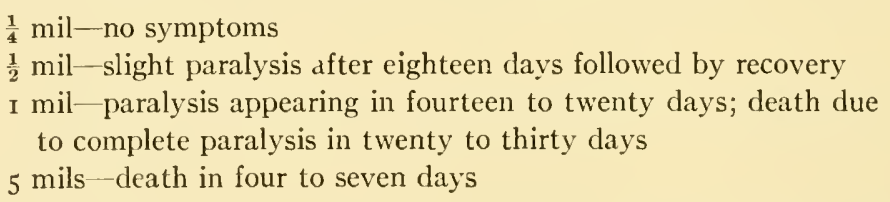

The active immunizing value of the mixture is determined by injecting the surviving guinea pigs with $B$. diphtheriae toxin. Five weeks should elapse before the pigs are tested. The series which received the $\frac{1}{4}$-mil amounts should withstand the injection of five M.L.D.'s; those which received the $\frac{1}{2}$ mil should withstand ten M.L.D.'s. Occasionally a guinea pig which has received the I-mil dose will recover after almost complete paralysis. These animals will withstand twenty to twenty-five M.L.D.'s. A toxin-antitoxin mixture giving the foregoing results is released for active immunization in humans. The amount injected in humans for active immunization is $\mathrm{r}$ mil at weekly intervals for three doses. About 90 per cent of the individuals become immune within three to four months. To ascertain if the individual responded to the treatment and is immune, a Schick test should be done four to six months after the toxin-antitoxin injections. To those who still give a Schick positive test, a second series of toxinantitoxin injections must be given. It should be continually emphasized that no certificate of inmunity should be given until a Schick negative test results.

TOXIN-ANTITOXIN SUBSTITUTE FOR TIIE SCHICK TEST

Advantage has been taken of the toxicity (see above) in the toxin-antitoxin preparations by Dr. W. H. Park. He introduced the method of using the first injec- 
tion of the toxin-antitoxin preparation as an indicator for immunes and non-immunes in children under eight years of age in place of the Schick test. Considerable time is saved, as about 85 per cent of the children of this age group are non-immune. One mil is injected subcutaneously. In the non-immunes an erythema which may be accompanied with a slight tenderness and swelling at the site of injection develops in twenty-four to forty-eight hours and gradually disappears. These non-immunes are given at weekly intervals two more injections to complete the course of treatment. Those that do not react to the first injection are immune and receive no further injections. Each mil of o.r L+ toxin-antitoxin preparation, i.e., each human dose, contains 0.02 of a mil of aged-toxin broth and 0.0000375 of a mil of partially purified antitoxin.

\section{EFFECTS OF FREEZING TOXIN-ANTITOXIN}

A reversible reaction caused by the solid freezing of a toxin-antitoxin mixture was reported by Kelly ${ }^{\mathrm{i}}$ during January, i924.

The toxin in this mixture was prepared and used in the state of Massachusetts to prepare a one $\mathrm{L}+$ toxin-antitoxin mixture. Samples of the mixture bearing the identification No. IоIC, which had not been frozen, were tested at the Hygienic Laboratory in Washington and by the writer, and were found to be perfectly balanced for active immunization, as had been shown by the previous tests of White and Robinson ${ }^{2}$ in Massachusetts.

In the Bureau of Laboratories, New York City Department of Health, samples of this mixture No. IoI $C$ were frozen (Banzhaf and Greenwald) ${ }^{3}$ for eighteen hours at $-8^{\circ} \mathrm{C}$. and on thawing were found to be toxic. Samples frozen for varying periods of time from twentyfour hours to eleven days at a constant temperature of $-26^{\circ} \mathrm{C}$. upon thawing were also found to be toxic. The samples frozen for eleven days at $-26^{\circ} \mathrm{C}$. were considerably more toxic than those frozen for eighteen hours at $-8^{\circ} \mathrm{C}$. On retesting three weeks after freezing and thawing they were found to be non-toxic. Upon refreezing a sample it became toxic again and then again lost its toxicity. The hydrogen-ion concentration in this mixture before and after freezing was $\mathrm{pH}$ 7.9. The sterility tests showed no growth.

A reversible reaction was only obtainable by the writer when the lot of toxin No. $37 \mathrm{Z}$ prepared in Massachusetts was used to prepare mixtures. A noticeable peculiarity of the toxin and the toxin-antitoxin mixture sent to us from Massachusetts was that they had a penetrating and persistent odor which presumably was due to an end-product developing during the preliminary fermentation of the broth. Whether this indicates a possible connection with dissociation cannot be stated. Anderson and Leonard ${ }^{4}$ state that when antitoxic serum or concentrated purified antitoxic serum (Gibson method) was used in balancing the toxin for the toxin-antitoxin mixture, the mixture became toxic after freezing; but that when the antitoxin prepared by the Banzhaf heating method for purifying was used, the mixture did not become toxic after freezing.

This does not clear up or explain the reversible reaction with the Massachusetts toxin. The antitoxin used in balancing this toxin was prepared by the Banzhaf heating method. Kirkbride and Dow ${ }^{5}$ found no increase in toxicity in their toxin-antitoxin mixture after freezing. The

I Kelly, F. L.: J.A.M.A., 82, 567. 1924.

${ }^{2}$ White, B., and Robinson, E.: J.A.M.A., 82, I675. I924.

3 Banzhaf, E. J., and Greenwald, C. K.: "The Effect of Freezing as Regards Toxicity on Diphtheria Toxin-Antitoxin Mixture," read at the Buffalo meeting of the American Society of Immunologists, April, I924.

4 Anderson, J. F., and Leonard, G. F.: J.A.M.A., 82, 1679. 1924.

5 Kirkbride, M. B., and Dow, J. E.: J.A.M.A., 82, I678. I924. 
hydrogen-ion concentration in the toxin-antitoxin mixtures prepared in the Bureau of Laboratories, New York City Department of Health, is $\mathrm{pH} 7.0-7 \cdot 3$. Many preparations of toxin-antitoxin mixtures containing $5 \mathrm{~L}+, 3 \mathrm{~L}+, \mathrm{I} \mathrm{L}+$, and $0 . \mathrm{I} \mathrm{L}+$ values made from different preparations of toxin were frozen at temperatures of $-8^{\circ}$ and $-26^{\circ} \mathrm{C}$. for from eighteen hours to eleven days; all were non-toxic after thawing. The active immunizing value of the frozen products was practically nil.

\section{PREPARATION AND USE OF TOXOID (ANATOXIN)}

Active immunization with a diphtheria toxin which had been modified with formalin was used by Glenny as early as r9o4. Glenny and Sudmerson ${ }^{\mathrm{I}}$ reported their immunizing experiments with formalized toxin (toxoid) which was non-toxic for guinea pigs in 5-mil amounts. Since then Glenny and his co-workers ${ }^{2}$ have reported in a series of publications their work on the antigenic value of toxoids, on the detoxification with various amounts of formalin, and also on a rapid and economical method of determining the detoxification rate. They ${ }^{3}$ call this the "M.R.D. unit," and define it as the smallest quantity of toxin which will produce a reaction when injected into the skin of a guinea pig.

Ramon, ${ }^{4}$ in a series of publications, has reported his work on the preparation of toxoids which he terms "anatoxins" and their antigenic value. Ramon's method of detoxifying is as follows: Diphtheria toxin of high potency is freed from organisms by filtration, and formalin is added up to 0.3 per cent concentration. The formalized toxin is then held at $37^{\circ} \mathrm{C}$. until 6 mils injected into a $300-\mathrm{gm}$. guinea pig gives rise to no early or late local or general reactions. Detoxification to this degree requires about three weeks. If samples are removed from time to time and titrated by Ramon's flocculation method, during the course of modification there is no appreciable change in the flocculation value.

Ramon's results show that these detoxified toxins are valuable immunizing agents. Moloney and Weld and Zingher confirmed these results in animals and humans. Moloney and Weld, 5 in their studies on the preparations of formalinized toxins, show that when toxins containing 0.5 per cent phenol are used, the antigenic value after detoxifying is practically nil. This explains the failure by the writer to obtain a good antigenic toxoid in the early work as all the work was done on phenolized toxin. Moloney and Weld also point out that between $\mathrm{pH} 7$ and $\mathrm{pH}$ ro the rate of detoxification is greater the more alkaline the solution. At $\mathrm{pH}$ ro they find that detoxification is associated with a loss of flocculation value and therefore recommend $\mathrm{pH} 9.0$ as the optimum hydrogen-ion concentration for the rapid production of toxoids. They are able completely to detoxify the formalinized toxin $\mathrm{pH} 8.2$ in five days by incubating at $37^{\circ} \mathrm{C}$. They state the product loses none of its immunizing value when left at room temperature for many months.

${ }^{\mathrm{I}}$ Glenny, A. T., and Sudmerson, H. J.: J. Hyg., 20, I84. 1921.

${ }^{2}$ Glenny, A. T., and Hopkins, B. E.: Brit. J. Exper. Path., 4, 283. 1923; Glenny, A. T., Pope, C. G., and Waddington, H.: J. Path. E० Bact., 28, 29 I. 1925.

${ }^{3}$ Glenny, A. T., and Allen, K.: ibid., 24, 6r. I92 I.

${ }^{4}$ E.g., Ramon, G.: Ann. de l'Inst. Pasteur, 39, I. 1925.

5 Moloney, P. J., and Weld, C. B.: Proc. Roy. Soc., Canada. 1925. 
Several methods have been recommended for standardizing the antigenic (active immunizing value) of toxoids and toxin-antitoxin mixtures. Park and Banzhaf injected $\mathrm{s} / \mathrm{ro}$ of a human dose subcutaneously into guinea pigs, giving three doses at weekly intervals. Three months later they determined roughly the degree of immunity by injecting intracutaneously the guinea pigs with I/I $50,1 / 100$, and I/50 of a fatal dose. A negative reaction with the highest dose was interpreted as a satisfactory product for active immunization. Glenny and his co-workers recommend injecting the guinea pigs with one human dose and three weeks later testing the animals by the Schick method. The products which gave a negative reaction were classed as good, active immunizers. Ramon and others rely to a great extent on the antigenic value as shown by the flocculation test.

During 1923 when I determined the immunizing value of the products of the New York City laboratory, practice was made of injecting the negatively reacting guinea pigs with toxin. It was found that some of these guinea pigs might fail to survive three fatal doses, whereas others would survive twenty fatal doses, it being understood that the twenty fatal doses were given as the first and only injection of toxin.

Further observations on those preparations of toxin-antitoxin mixtures that gave a high percentage of active immunization in humans showed that where $\frac{1}{2}$ mil (one-half the human dose) was injected into guinea pigs, one month later these animals would survive the injection of at least five fatal doses; whereas when the elapsed time was six weeks, the animals would survive at least eight to ten fatal doses.

In determining the active immunizing value of the toxoids, the guinea pigs are injected with $\frac{1}{2}$ mil (the recommended human dose for the first injection), and one month later five fatal doses of toxin are given. Survival is interpreted as indicating that this product will give a high percentage of actively immunized individuals among those treated.

The use of toxin-antitoxin mixtures has been criticized on the assumption that the antitoxin in the mixture, which amounts to about 0.000038 of a mil of partially purified antitoxic horse serum to each human dose, would sensitize the individuals to subsequent injections of horse serum. The observations of Hooker and of Park indicate that a skin sensitivity may develop. There has been no convincing evidence that a systemic sensitization ever develops which would lead to serious or fatal reactions should horse serum or partially purified antitoxins be injected later. In fact, the absence of serum reactions in many cases that had previously received toxinantitoxin indicates the safety of the procedure.

A drawback to the use of the toxoid is that in children over seven years of age a considerable percentage develop annoying reactions due to the proteins (meat extractives, peptone, and endocellular substances) in the toxoid broth. The o.r-L+ toxinantitoxin mixture contains only about one-fiftieth of the amount of these proteins. However, the work of Watson and Wallace, ${ }^{1}$ Moloney and Weld, ${ }^{2}$ and Watson and Langstaff $^{3}$ on the purification of toxoids is encouraging. These workers show that, according to Ramon's flocculation test, the antigenic substances can be obtained over one hundred times purer than in the original toxin broth. If the active immunizing value is retained in these products, the drawback of toxoid mentioned above can be removed. Their method of purifying is essentially as follows: Diphtheria toxin de-

Watson, A. F., and Wallace, U.: J. Path. \& Bact., 27, 27 I. I924.

${ }^{2}$ Moloney, P. J., and Weld, C. B.: loc. cit.

3 Watson, A. F., and Langstaff, E.: J. Biochem., 4, 50. 1926. 
toxified with formalin is precipitated by adding I per cent glacial acetic acid. The precipitate is allowed to settle. The clear supernatant fluid is siphoned off; the remainder, centrifugated. The clear supernatant is again siphoned off and replaced by an equal volume of 0.7 per cent saline or sodium acetate-acetic acid mixture, adjusted to the $\mathrm{pH}$ of the acid precipitation-point $(c a .3 .8)$ to wash the precipitate and again centrifugated. The precipitate is dissolved in physiological saline or distilled water buffered to $\mathrm{pH} 8.0$, using the volume needed to give the desired concentration. They state that the purification of the toxoids can be carried out at room temperature. That there is some loss through absorption on filtering through a Berkefeld is shown by the flocculation value.

The ultra-purification of the purified toxoids (Moloney and Weld) ${ }^{x}$ can be accomplished by adding alcohol to give an 85 per cent final concentration or by the dialysis of the solution for seventy-two hours against water. Purified toxoids, they state, are protein-like substances and give a positive biuret, Millon, xanthoproteic, and Hopkins-Cole tests.

Nicolle, Debains, and Cesari ${ }^{2}$ and Ramon ${ }^{3}$ described the formation of a precipitate when toxin is neutralized with antitoxin, and Nicolle recommended this as a means of determining the unit value of antitoxin. The writer ${ }^{4}$ precipitated two different toxin preparations (Nos. 377 and 517 ) by adding antitoxin to under the neutralizing-point. The toxin-antitoxin precipitate was collected by centrifugating and was then redissolved in saline equal to half the original volume. This was slightly toxic. One-mil doses of the preparation from toxin No. 377 in 250 -gm. guinea pigs caused late paralysis and death in twenty-four to thirty days. One-mil doses of the preparation from toxin of No. $5 \times 7$ caused slight paralysis in some of the guinea pigs with recovery. Both of these precipitated toxin-antitoxin preparations gave a higher percentage of immunity in guinea pigs than the o.I L+ toxin-antitoxin preparations and the toxoid preparations used for comparison. Hartley 5 obtained similar results in his investigations of the antigenic properties of precipitates produced by the interaction of diphtheria toxin and antitoxin. He prepared overneutralized, neutral, and underneutralized (toxic) mixtures, and he obtained from the toxic mixtures precipitates containing only one-five-hundredth ( 0.2 per cent) of the total nitrogen of the original mixture and still these precipitates were very active immunizing agents. Doses containing less than a ten-thousandth of a milligram of nitrogen stimulated the production in six weeks from 0.02 to 0 .I units of antitoxin in guinea pigs. As stated earlier, the purest toxoid obtained by Watson and Wallace was two hundred times ${ }^{6}$ purer than the original toxin from which it was prepared. This is less purification than Hartley obtained.

${ }^{2}$ Moloney, P. J., and Weld, C. B.: loc. cit.

${ }^{2}$ Nicolle, M., Debains, E., and Cesari, E.: Compt.rend. Acad. de sc., I69, I433. I919; Ann. de l'Inst. Pasteur, 34, 709. 1920.

${ }^{3}$ Ramon, G.: Ann. de l'Inst. Pasteur, 37, го0г. 1920; 38 , I. I924.

${ }_{4}^{4}$ Park, W. H., Banzhaf, E. J., Zingher, A., and Schroeder, M. C.: Am. J. Pub. Health, 14, I049. I924.

${ }^{5}$ Hartley, P.: Brit. J. Exper. Path., 6, I1 2. I925; 7, 55. 1926.

${ }^{6}$ Cf. Glenny, A. T., Hopkins, B. E., and Pope, C. G.: J. Path. \& Bact., 27, 26 I n. 1924. 
The studies of Moloney and Weld, ${ }^{x}$ Bronfenbrenner and Reichert, ${ }^{2}$ on the toxinantitoxin flocculation phenomenon indicate that the flocculation is not due to the union of toxin and antitoxin but that it is a specific antibacterial precipitation. The fact remains, however, that even though the flocculation phenomenon may not be due to the union of toxin and antitoxin, these substances are carried down possibly by absorption, with the precipitate caused by the specific bacterial and antibacterial substances. The toxin-antitoxin in the precipitate is insoluble in normal saline solution. Repeated washings do not lessen the active antigenic value of the precipitate (Hartley). ${ }^{3}$

\section{PREPARATION OF ANTITOXINS}

Active immunization for the purpose of obtaining a high antitoxic content in the blood of animals is based on the repeated injections of toxins. The toxins used for the immunization may be modified by physical or chemical means, aging, sunlight, heat, formaldehyde, iodine, or by their specific antitoxins. The toxins are injected either subcutaneously or intramuscularly in any suitable part of the body. During the first stages of immunization the injections of certain toxins may be followed by edema and tenderness at the sites of injections. There may be a rise of temperature, loss of appetite, and general malaise. These conditions may last twenty-four hours or may be prolonged for several days, depending on the amount and source of toxin injected, and upon the sensitivity of the animal to the toxin.

Diphtheria antitoxin.- In general, horses which show a natural antitoxic immunity respond promptly to the stimulating effect of the early injections of toxin and are usually not sensitive during the course of immunization. Those which have no natural immunity respond slowly, as evinced by their extreme sensitivity to the early injections of the toxin. Human beings respond similarly, those with traces of antitoxic immunity responding promptly to toxin-antitoxin injections, and those with no material immunity responding slowly, requiring three to four months before giving a negative Schick test.

Horses selected for active immunization should be reasonably young, vigorous, and healthy. They should be tested for the absence of glanders and for natural antitoxic immunity. All horses, of whatever size or breed, are equally responsive to active immunizations. By appearances one cannot judge whether or not a horse will respond and give a high antitoxic content.

In large establishments, horses are immunized in groups of twenty or more. They are all started with the same amounts of toxin followed by the same increase of amounts (roo per cent increase for the first four injections, then the percentage of increase is reduced). Usually as early as the third or fourth injections, marked differences in sensitiveness will be observed in about 20 per cent of the horses. These will show edema, tenderness at the site of injection, and a rise in temperature dropping to normal next day. If the scheduled increased injections are continued as in the non-reactors, these conditions are aggravated and the rise in temperature will be prolonged. Scheduled injections are then omitted until the temper-

${ }^{1}$ Moloney, P. J., and Weld, C. B.: ibid., 28, 655. 1925.

${ }^{2}$ Bronfenbrenner, J. J., and Reichert, P.: J. Exper. Med., 44, 553. 1926.

3 Hartley, P.: loc. cit. 
ature is normal. On resuming injections a lower percentage of increase usually must be given. These early reactors rarely produce a high antitoxic value; about 50 per cent of the horses after a month's immunization will test five hundred or more units to each mil. From the economic view, those horses that do not have more than three hundred units to each mil after five weeks of immunization are not injected further. Experience has proved that those that are three hundred units or under rarely go to a higher value even though much greater amounts of toxin than the scheduled dose be given. Regular full bleedings of about 8 liters of blood have been tried on these refractory horses on the assumption that during the process of the stimulation of blood formation an indirect stimulation of antitoxin production would occur. The results do not warrant the trouble.

Horses with above three hundred units are listed for the regular full bleedings. The blood is drawn from the jugular vein by means of a suitable-sized canula. An injection of toxin is given the next day after bleeding. Usually two or three daily injections are given between bleedings. Six days after the last injection they are bled again, averaging three bleedings a month. These horses, as a rule, even though full bleedings be taken, respond to the further injections and go to a higher unit content. This increase in unit value may continue for three, four, or more bleedings. They may then remain stationary in unit content for six to twelve or more bleedings and then slowly or rapidly drop in value with each successive bleeding.

A horse may give, after a month's immunization, a test bleeding containing a thousand units to the mil, and on its first full bleeding about two weeks later, test only six hundred units. Because of these occasional instances the practice is to take a regular full bleeding after about twenty-eight days of immunization. If horses are not bled for the full bleedings and the injections continued with the hope of increasing the unit content, the high-unit period may be missed. All horses after seven to eight weeks of intensive immunization with toxin will drop in antitoxic value if full bleedings have not been taken.

There is no doubt that the full bleedings act as a stimulation in most horses and tend to lead to a higher antitoxic content. This holds true only with those horses which respond favorably to the first four or five weeks' immunization. Those which respond poorly may hold their low unit level but usually drop lower with each successive bleeding.

Ramon recommends the use of anatoxin (toxoid) for the immunization of horses. He also advocates the addition to the anatoxin of finely powdered tapioca ( $\mathrm{I} \mathrm{gm}$. to each liter) to delay the absorption of the toxoid, the idea being that a more prolonged stimulus will result in a greater antitoxin response. Madsen injected intravenously Io-mil amounts of a Io per cent manganese chloride solution to augment the stimulation of antitoxic productions. None of these methods have been more successful in our laboratories than the regular methods of toxin injections.

Tetanus antitoxin.-In immunizing horses against tetanus toxin the daily injection method of increased doses cannot be given as with diphtheria toxin. Diphtheria toxin acts on the local tissues, causing in a short time local disturbances and a rise in temperature. These are warnings of a too toxic dose, thereby indicating the amount of toxin for the'next injection. With the injection of tetanus toxin there are none of these early danger signals. During the early injections, muscular rigidity may develop. When this occurs it is due to cumulative toxic action and may be followed by exhaustion and death, even though large amounts of tetanus antitoxin be injected into the spinal canal and by the intravenous route. The early immunity response to injections of tetanus toxin is extremely slow. In order to aid the animal to withstand 
the first weeks of immunization, the practice is to inject about five thousand units of tetanus antitoxin the day before immuni $<$ ation is started. After about three months' immunization a group of ten horses will average about one hundred units of antitoxin to each mil of serum. Those that test one hundred units or more are posted for regular full bleedings. The horses, as in the active diphtheria immunizations, will usually increase in unit value with each subsequent injection and bleeding. The injection of horses showing less than fifty units after three months' immunization is dis continued. The standard tetanus antitoxin unit is that amount which, when mixed with I,000 M.L.D.'s of tetanus toxin and injected subcutaneously into a 350 -gm. guinea pig, will protect the animal four days. Theoretically, the tetanus antitoxin unit is ten times larger than the unit of diphtheria antitoxin.

Botulism antitoxin.-Immunization of horses against B. botulimus toxin (types A and B) was done by the writer. Subcutaneous injections were given every second day. The first injection was one guinea pig M.L.D. The injections were increased roo per cent for each of twelve injections. The temperature rise after injections was usually less than $2{ }^{\circ}$ dropping to normal the following day; the percentage of increase was then lowered to 50 per cent for twelve injections, and thereafter the increase was 25 per cent. After three months' immunization the antitoxin value against the type " $\mathrm{A}$ " strain was one hundred twenty-five units and the value against the type "B" was two hundred units in each mil. The standard B. botulimus antitoxin unit is that amount of serum which when mixed with one hundred M.L.D.'s of toxin will protect a guinea pig four days.

"Staph. aureus" antitoxin.-Parker and Banzhaf ${ }^{\mathrm{I}}$ immunized seven horses against the toxin of Staphylococcus aureus. Four of the serum horses died during the course of immunization, due, they believe, to the injection of the toxin at too short intervais: one- to two-day intervals. A rapid temperature rise of $2^{\circ}$ to $3^{\circ} \mathrm{F}$. occurred frequently after injections, usually disappearing after twenty-four to thirtysix hours. In the successfully immunized horses the first injection was I mil of toxin containing four hundred rabbit S.T.D.'s (skin-test dose). The injections were increased roo per cent at three- to four-day intervals for the first five injections. The percentage of increase was then decreased to 50 per cent. After ten injections the interval was lengthened to from seven to ten days. After two months' immunization the average strength of the sera was such that I mil neutralized twenty thousand rabbit S.T.D.'s of toxin.

Scarlatinal antitoxin.--Immunization of horses against Streptococcus scarlatinae and the resulting bactericidal and antitoxic value was demonstrated by Moser ${ }^{2}$ and Savchenko $\mathrm{o}^{3}$ and improved by the clear-cut work of Dochez $z^{4}$ and the Dicks. Immunization by the Dochez method is done by injecting small amounts of melted nutrient agar subcutaneously in to the horses, and then injecting living scarlatinal streptococci into the agar mass. Small abscesses usually develop. When these have healed, the horses are again injected with melted agar and a larger amount of living scarlatinal

${ }^{\text {I }}$ Parker, J., and Banzhaf, E. J.: J. Immunol., 13, 25. I927.

2Moser: Wien. klin. Wchnschr., 15, 1053. 1902.

${ }^{3}$ Savchenko, G. N.: Russk. Vach., 25, 797. I905.

${ }_{4}$ Dochez, A. R.: Proc. Soc. Exper. Biol. \& Mcd., 4, I84. I924. 
streptococci. After about seven or eight months the serum contains considerable antitoxin and has some antibacterial value. The Dicks ${ }^{\mathrm{I}}$ method of immunizing is by the subcutaneous injections of increasing amounts of toxin from scarlatinal streptococcus cultures which have been passed through a Berkefeld filter. Park and Williams use a modification of Moser's method. They inject intravenously the killed centrifugated twenty-four-hour culture from $50 \mathrm{cc}$. of broth. Two days later $50 \mathrm{cc}$. of a six-day blood-broth culture which had been filtered through a Buchner funnel are injected subcutaneously. The intravenous (dead culture) and subcutaneous (toxin filtrate) doses are continued on alternate days and gradually increased. After eight to nine months I mil of the serum usually contains sufficient antitoxin to neutralize forty thousand S.T.D.'s, and $0.05 \mathrm{mil}$ will protect mice against one hundred M.L.D.'s of living streptococci.

The antitoxin unit of scarlatinal streptococcus has been standardized and defined as the amount of antitoxin which is required to neutralize fully one hundred S.T.D.'s of a standardized scarlatinal toxin.

\section{PARTIAL PURIFICATION OF ANTITOXINS}

It has long been known that antitoxins are precipitated with the pseudoglobulin fraction of horse serum. Gibson ${ }^{2}$ was the first to perfect a practical method of concentrating diphtheria antitoxin for therapeutic use. With his method it was only possible to concentrate about three times, due to the increase of the pseudoglobulin in an antitoxin horse serum over the normal amount. It was thought by some workers that this increase was a new-formed antitoxic pseudoglobulin, as it had all the then-known characteristics of this protein.

Gibson and Banzhaf ${ }^{3}$ showed that the increase of the pseudoglobulin is at its height after one and one-half to three months, depending on the method of immunization. The increase is from 40 to over roo per cent. After several full bleedings there is a gradual decrease of this protein, even though the unit value increases or remains stationary. The writer ${ }^{4}$ was the first to point out that antitoxin could apparently be dissociated from the pseudoglobulin without losing any of its activity. Possibly the results to be described may be due not to dissociation but to a conversion of nonantitoxin-bearing pseudoglobulins. Heating the antitoxic serum or plasma for six to eight hours at $56^{\circ} \mathrm{C}$., or three to four hours at $58^{\circ} \mathrm{C}$., about 30 per cent of the pseudoglobulin is converted into a euglobulin-like substance, i.e., one which has the saltingout characteristics of the euglobulins. Heating for longer periods of time shows but little further conversion of pseudoglobulin and considerable loss of antitoxin. It occurred to the writer ${ }^{5}$ that the conversion curve was lessened, owing to the presence of the greater amount of euglobulin in solution. Heating was then resorted to in the presence of 30 per cent saturated ammonium sulphate solution to precipitate the

× Dick, G. F., and Gladys H.: J.A.M.A., 82, 1 246. 1924.

2 Gibson, R. B.: J. Biol. Chem., I, I6r. I906.

3 Gibson, R. B., and Banzhaf, E. J.: J. Exper. Med., I2, No. 3. 1910.

${ }_{4}$ Banzhaf, E. J.: Proc. Soc. Exper. Biol. \& Med., 5, 8. I9o8; Bull. Johns Hopkins Hosp., 241. 106. I9II.

${ }^{5}$ Banzhaf, E. J.: Coll. Studics, Bureau of Lab. Dept. of Health, New York Cily, 7, II4. Igr2. 
euglobulin-like substance as it was formed. This method showed a further conversion of about ro per cent. During I9I 2 the writer added 0.5 per cent phenol to the antitoxin plasma before adding the ammonium sulphate. The addition of phenol shortened the necessary heating period and aided greatly in the subsequent filtrations. Briefly the method is as follows:

The antitoxin plasma containing 0.5 per cent phenol is diluted with a half-volume of water and 30 per cent of a saturated ammonium sulphate solution is added. The mixture is heated at $57^{\circ} \mathrm{C}$. for one hour and then rapidly brought up to $63^{\circ} \mathrm{C}$. It is cooled to about $50^{\circ} \mathrm{C}$. and filtered. The precipitate contains the euglobulin. The filtrate called " $\mathrm{A}$ " is measured and brought up to a 45 per cent content of saturated ammonium sulphate solution. This will precipitate all the antitoxin and pseudoglobulin. After standing about three hours, the mixture is filtered through hardened filter papers or muslin cloths. The first precipitate containing the euglobulin (see above) is added to a volume of water equal to half the original volume of plasma used. This is precipitated with 32 per cent saturated ammonium sulphate solution. In this "washing" of the euglobulin it is necessary to use a slightly higher percentage of saturated ammonium sulphate solution as the protein content is decreased, which will increase the solubility of the euglobulin. ${ }^{\mathrm{r}}$ After filtering, the washed euglobulin precipitate is discarded. To this second filtrate, called " $\mathrm{B}$," saturated ammonium sulphate solution is added up to 45 per cent. The resulting precipitate is collected by filtration through the hardened filter papers containing the main precipitate from filtrate "A." After filtration is completed, the combined precipitates are pressed to remove as much ammonium sulphate solution as possible and dialyzed to free it from this salt. In the dialysis the accumulated water dissolves the precipitate. One per cent sodium chloride and 0.4 per cent trikresol are added. (The required amount of trikresol is added to a like amount of ether. The mixture can be added to protein solutions without causing any appreciable coagulation.) ${ }^{2}$ The dialyzed antitoxin product is stored in a cold room for about three to four months, to allow for the separation of lipoidal substances which are usually present in the solution. After storing it is clarified by filtering through paper pulp and filtered through a Berkefeld candle to remove bacteria. Practically all the dialyzed antitoxin products concentrated by this method have lipoidal substances in solution. After some months these substances become insoluble and aggregate into a mass which rises to the surface, forming a cream-colored ring on top of the antitoxin solution. The writer's investigations (unpublished work) show that these lipoidal substances are extracted from the euglobulin fraction in the serum by the action of the ammonium sulphate and heat, and are soluble with the pseudoglobulin which is soluble in 30-33 per cent saturated ammonium sulphate solution. When the saturated ammonium sulphate solution is added in sufficient amount to precipitate the antitoxin, these substances are also precipitated. Ammonium sulphate in sufficient amount to precipitate the greater part of the euglobulin fraction has also, but to a lesser degree, a solvent action on the lipoidal substances without the aid of heat. The lipoidal substances may be completely avoided in the antitoxin solution by the following method of concentration:

Antitoxin in plasma containing 0.5 per cent phenol is diluted with an equal volume of water and heated as previously stated. Cooled to below $37^{\circ} \mathrm{C}$., another volume of water is added equal to the amount of original plasma and then saturated with sodium chloride. This will precipitate all the euglobulin and render the lipoidal substances insoluble. Allow to stand four or more hours after complete saturation and filter. To this filtrate " $\mathrm{A}$ " is added 35 per cent saturated ammonium sulphate solution. This amount of sulphate in the presence

${ }^{i}$ Gibson, R. B., and Banzhaf, E. J.: loc. cit.

${ }^{2}$ Krumwiede, C., and Banzhaf, E. J.: J. Infect. Dis., 28, 367 . I92 I. 
of saturated sodium chloride will precipitate all the antitoxin, and the albumen present will remain in solution. This is filtered after three hours' standing. The first precipitate of euglobulin is taken up in water of twice the volume of the original plasma, again saturated with sodium chloride, and filtered. To this second filtrate " $\mathrm{B}$ " saturated ammonium sulphate solution is added up to 35 per cent. The resulting precipitate and solution of salt is filtered through the papers containing the precipitate from filtrate "A." The precipitate is pressed and dialyzed free of ammonium sulphate and salt. This dialyzed product need not be stored as there will be no separation of insoluble substances. The methods given are applicable to all the antitoxins in horse serum.

With other animals the methods of concentration must take cognizance of the differing antitoxin distribution in the globulins. For instance, in goat serum about one-third of the antitoxin is in the pseudoglobulin fraction and two-thirds with the euglobulin fraction. ${ }^{\mathrm{I}}$

${ }^{r}$ Banzhaf, E. J., and Famulener, L. W.: Coll. Studies, Burcau of Lab. Dept. of Health, New York City, 8, 208. I9I4. 


\title{
CHAPTER LVI
}

\section{THE TITRATION OF TOXINS AND ANTITOXINS BY THE FLOCCULATION METHOD}

\author{
STANHOPE BAYNE-JONES
}

University of Rochester

If a physico-chemical titration method could be substituted for, or used largely to supplant, the testing of toxins and antitoxins upon animals, great economies in time and materials would be made, and the precision and character of the procedure would yield new information concerning the nature of these substances. The flocculation method, described in its present form by Ramon in I922, seemed to offer these advantages. Since the publication of his paper, the precipitation of bacterial toxins and antitoxins in appropriate mixtures has been used for the practical titration of toxins and antitoxins, for the estimation of the immunizing values of toxoids and anatoxins, for the preparation of a non-toxic antigen, for the purification of toxin and antitoxin, and for the investigation of their properties and quantitative relationships. The evidence accumulated from these numerous studies substantiates the opinion that a specific flocculation occurs with regularity when a bacterial toxin is mixed in suitable proportions with its antitoxin and that the reaction serves to measure the binding or total antigenic value of the toxic filtrate as well as the neutralizing capacity of the antitoxin. Recent criticisms of the method have again focused attention upon the mixture of bacterial proteins and other residues in these filtrates which react to form precipitates with the antibacterial substances present in the sera obtained from animals immunized by injections of them. While this type of reaction may occasionally introduce confusing zones of precipitation, its occurrence does not exclude the specific mutual flocculation of toxins and antitoxins in mixtures in the region of their neutrality.

Flocculation in approximately neutral mixtures of ricin and antiricin was observed first by Danysz in I902. The precipitate in these "optimum" mixtures contained all the ricin and antiricin originally present in the fluids. In I909 Calmette and Massol measured the protective value of antivenoms by a flocculation method and showed that the precipitate contained both cobra venom and its antivenom in a dissociable combination. Their work was followed by the experiments of Nicolle, Césari, and Debains (1920), in which results conformable with animal tests were obtained by observing the precipitation which occurred at the junction between a layer of antitoxic serum upon a toxin incorporated in gelatin. A simplification of these procedures was made by Ramon in 1922 when he found that an abundant flocculent precipitate occurred in mixtures of diphtheria toxin and antitoxin in equivalent proportions. To a series of tubes containing a constant amount of toxin he added decreasing amounts of antitoxic sera. After one or more hours at room temperature, an opalescence and fine precipitate occurred in several of the tubes. This increased slowly, and at the end 
of a certain time one of the mixtures began to flocculate. The first tube showing definite flocculi was called the precipite indicateur. The toxin-antitoxin mixture in this tube was neutral, while the mixtures as well as the precipitates on either side of the one containing the initial flocculation were either toxic or antitoxic according to whether they contained more or less of the antitoxic serum than the amount in the first tube which flocculated.

\section{TECHNIQUE}

The technique of the procedure, which requires slight modification to suit different conditions, can be described adequately by a summary of the method used by me in 1924 for the titration of diphtheria toxin and antitoxin. Into each of a series of test tubes, 2 cc. of a standardized, rapidly flocculating toxin are placed, and to each tube is added a small amount of antitoxic serum by means of 0.2 -cc. pipettes graduated in

\section{TABLE I}

Standardization of 'Toxin

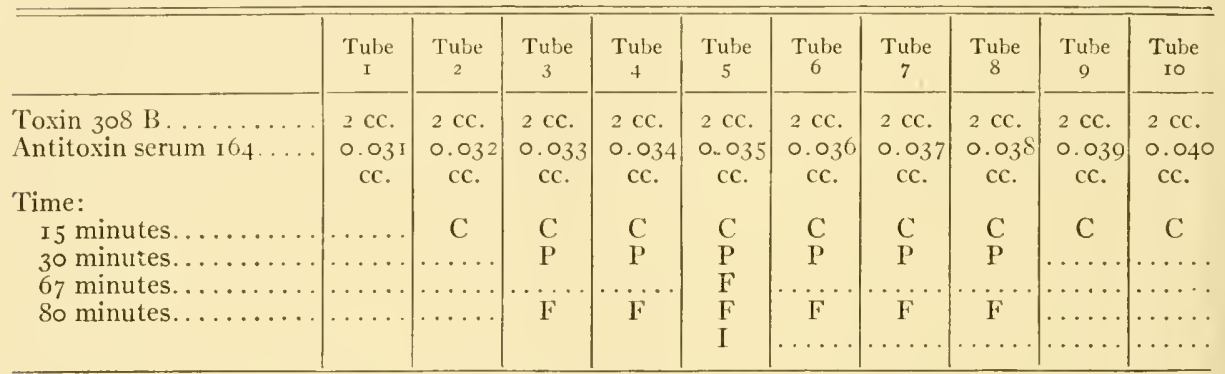

C, cloudy; P, granular precipitate; F, flocculation; I, indicating tube.

Diphtheria toxin: Albany $308 \mathrm{~B}$.

Diphtheria antitoxic serum: Albany 164,430 units per cubic centimeter.

Temperature of water bath: $55^{\circ} \mathrm{C}$.

0.or cc., or by a micro-syringe like that devised by Trevan (I922), capable of delivering $0.00 \mathrm{I} \mathrm{cc}$. with an error of I per cent. The mixtures are then placed in a water bath at $45^{\circ}-50^{\circ} \mathrm{C}$. and observed at frequent intervals for the detection of initial flocculation in the indicating tube.

As long as the Ehrlich unit is used for the evaluation of antitoxin, it must be taken as the point of reference for titrations of toxins as well as antitoxins. Hence, in Ramon's method, the toxin to be employed subsequently for determining the values of antitoxins must be standardized in terms of a known antitoxin, prepared in the usual way. Therefore, the first step to be taken is to determine the amount of toxin which will cause initial flocculation with $\mathrm{r}$ unit of antitoxin. An example of such a standardizing titration is given in Table $\mathrm{I}$. In this titration (Table $\mathrm{I}$ ) the initial flocculation occurred in the fifth tube. This tube, then, contained the indicating mixture composed of $2 \mathrm{cc}$. of toxin and $0.035 \mathrm{cc}$. of the antitoxic serum. As the serum contained 430 units of antitoxin per cubic centimeter, I unit was represented by $0.0023 \mathrm{cc}$. of the serum. Therefore, the amount of serum in the indicating tube $0.035 \mathrm{cc}$. was equal to I 5.3 units of antitoxin. From these figures, the $L_{f}$ amount, which Glenny and Okell 
(I924) define as the amount of toxin equivalent to I unit of antitoxin as determined by flocculation, is O.I I I cc., and I cc. of the toxin is equivalent to 7.65 units of antitoxin.

The titration of antitoxin is made in a similar manner, by noting the initial flocculation occurring when it is mixed with a toxin whose $L_{f}$ value has been established. Methods of rough orientation measurements followed by closer titrations and formulas for calculating values from the results of flocculation tests are given in papers by Ramon (1922-23), Glenny and Okell (I924), Scholz (1924), and H. Schmidt (I925).

In order to obtain dependable results, at least $\mathrm{I}-2 \mathrm{cc}$. of toxin should be placed in each tube, the tubes should be clear and all of the same diameter, and they should be immersed to the same depth in the water bath.

A minimal flocculating value of an antitoxin may be established by mixing an unknown serum with a standardized antitoxin. This method described by Renaux (1924), for the titration of slowly flocculating or non-flocculating antitoxins has been applied with useful results by myself, Ramon (I926), and others to the titration of purified antitoxic sera, composed largely of the pseudoglobulins which Ramon (I922) found to be devoid of flocculating power. The flocculation value thus obtained indicates the total neutralizing properties of the mixed antitoxins. As the same results are obtained whether the fractions are mixed at one time or at intervals, no definite evidence of a Danysz phenomenon has been conclusively educed. Theoretically, it is not to be expected that the Danysz phenomenon which is dependent upon a toxicity factor would be demonstrable by the flocculation method which is an indication of the total combining capacities of the components of the mixtures. But Dr. Elliott Robinson writes me it may be possible to demonstrate by flocculation that a smaller amount of toxin will flocculate a given amount of antitoxin if the toxin is added in several small fractions than if it is added all at once. The experimental difficulties of demonstrating this will be very great.

\section{CONDITIONS AFFECTING THE FLOCCULATION REACTION}

Temperature.-Flocculation may occur after several days at $0^{\circ} \mathrm{C}$. Increase in temperature up to $55^{\circ} \mathrm{C}$. increases the speed of flocculation. Above $55^{\circ} \mathrm{C}$. the results become irregular, and temperatures above $60^{\circ} \mathrm{C}$. often completely inhibit the reaction. Both the toxin and antitoxin can be rendered unsuitable for flocculation by heating them separately at $60^{\circ} \mathrm{C}$. for one hour. The most suitable temperature is $45^{\circ}-50^{\circ} \mathrm{C}$., although especially close watch of the tubes must be maintained to observe the occurrence of initial flocculation. A procedure which permits the observation of a number of titrations in a short time is based upon taking advantage of the slow union of the components at $0^{\circ}-5^{\circ} \mathrm{C}$. and their rapid flocculation when placed at $45^{\circ}-50^{\circ} \mathrm{C}$. The mixtures may be prepared during an afternoon, placed in the icebox over night, and then in the water bath at $45^{\circ}-50^{\circ} \mathrm{C}$. the next morning.

Reaction.-The $\mathrm{H}$-ion concentration of the toxic broth after filtration seemed to have no appreciable effect upon the occurrence or rate of flocculation. The reactions of the toxins have varied from $\mathrm{pH} 6.4-8.4$. Attempts to study the effect of changing the reaction were made by adding $n / \mathrm{I} \mathrm{HCl}$ to toxins in order to bring the $\mathrm{pH}$ to 4,5 , 
and 6 , and by adding $n / \mathrm{I} \mathrm{NaOH}$ to increase the $\mathrm{pH}$ to 8 and 9 . When the toxic broth was made more acid or alkaline than it was at the time of original filtration, nonspecific precipitates were produced in the broth, which obscured any flocculation due to the union of toxin and antitoxin. Removal of the precipitate by filtration or centrifugation reduced the $L_{f}$ value of the toxin. The results of titrations, therefore, at different $\mathrm{pH}$ values beyond the range 6.4-8.4 were irregular and of no significance.

Chemical agents.-Toxoids produced by the action of chemicals flocculate like those produced by age and heat up to $40^{\circ} \mathrm{C}$., retaining substantially the same $L_{f}$ value which the original toxin possessed. Their time of flocculation, however, is prolonged. An excessive amount of such substances as iodine and formaldehyde, which deprive toxins of their poisonous properties while leaving their antigenic qualities intact, may inhibit flocculation. Formaldehyde, in excess of five parts of commercial 40 per cent formalin to $\mathrm{I}, 000 \mathrm{cc}$. of toxin, may prevent flocculation. In this case, the formaldehyde combines with amino acids and other constituents of the toxic broth as well as with the toxin. The properties of the non-specific combinations cannot be predicted. It is, therefore, advisable to control the addition of formaldehyde by the flocculation reaction, as Ramon does in the preparation of the non-toxic immunizing agent which he calls "anatoxin."

Both toxins and antitoxic sera have properties, not understood at present, which influence the rate of flocculation. Usually toxins of high potency and fresh sera flocculate most rapidly. But the sera of some horses flocculate only slowly in spite of a high antitoxic value. Several specimens of antitoxic serum, plasma, and pseudoglobulin may all contain the same number of antitoxic units per cubic centimeter and yet differ by many hours in the time in which they flocculate with the same toxin at the same temperature. These differences may be due to protective colloidal properties of the fluids, or to differences in antigenic potency of the toxins and a quality of the serum known as its "avidity." The rate of flocculation of different toxins, toxoids, and anatoxins, when tested against the same serum, has been shown by Ramon (I922-26), Weinberg and Goy (I924), Descombey (I925), and Glenny, Pope, and Waddington (1925) to give an indication of the immunizing value of the preparation. The best antigens are those which flocculate most rapidly and have a high $L_{f}$. Madsen and Schmidt (I926) have recently published experiments relating the speed of flocculation of antitoxins to their neutralizing speed and curative efficiency. This, therefore, connects the speed of flocculation with the "avidity" of an antitoxin, as sera which flocculated more rapidly were curative in lower doses as measured by antitoxic units than more slowly flocculating sera.

\section{RELATIONSIIIP OF FLOCCULATION VALUE TO OTHER UNITS}

The flocculation reaction is theoretically a measure of the combining value of all the substances in a toxic broth capable of uniting with antitoxin. Most of the comparisons between the various units established by animal tests and those obtained by flocculation have been made with diphtheria toxin and antitoxin, and from them the following generalization may be drawn:

There is no relationship between the actual toxicity of a toxin or of any unit based upon that toxicity and its flocculating value, except possibly in the filtrates of young 
cultures of toxin-producing bacteria. The non-toxic antigenic portion of the filtrate adds a proportional combining capacity to the whole mixture. This proportion may vary from the condition in a fresh toxin where there is little toxoid to that in an old or formalinized product, completely devoid of toxicity while retaining its original flocculating value. Hence, there is no necessary relationship between the M.L.D., $L_{0}, L_{+}$, $L_{f}$, and other doses of toxin. The case of a fresh toxin, however, is a special one which demonstrates that there is a fundamentally close relationship between toxicity and the flocculating value. As the $L_{f}$ value is the amount of toxin equivalent to I unit of antitoxin, a ratio of $L_{0} / L_{f=I}$ should be obtained. Such a unit ratio, however, is rarely found. The $L_{f}$ values are of ten twice or three times as great as the $L_{0}$ values determined by animal tests. Nevertheless, Ramon (I922-23), Scholz (I925), and Glenny and Wallace (I925) have occasionally found $L_{0} / L_{f}$ ratios equal to I with certain toxins and selected antitoxic sera. It seems to be advisable, at present, to follow the example of Glenny and Wallace (I925) and use such provisional terms as "in vitro unit" and "in vivo unit," until the significance of the differences between the results of animal tests and flocculating values has become fully understood. As the measurements made by animal tests indicate only partial neutralization of a toxin, the flocculation method is the only true measure of the combining capacity of a toxin. It has an independent meaning, and can be used to establish an almost self-contained system.

\section{NATURE OF THE REACTION}

Two zones of flocculation occasionally occur in mixtures of toxin and antitoxin and discrepancies as great as 300 per cent between flocculation titrations and animal tests have been observed. These results have cast doubt upon the opinion that the flocculation is a consequence of a specific reaction between toxin and antitoxin. Weinberg, Prévôt, and Goy (1924), Zingher (1924), Moloney and Weld (I925), Bronfenbrenner and Reichert (I926), and Eisler and Kovács (I926) have drawn attention to the agglutinins and precipitins which occur in antitoxic sera, and have again raised the question whether the flocculation reaction is only the long-familiar bacterial precipitin phenomenon. The generally close agreement between the results of animal tests and flocculation reactions which have been obtained by many workers in thousands of titrations of diphtheria toxins and antitoxins, and with other toxins and antitoxins, which will be set forth in sections dealing with these special cases, seems to indicate that there is more than a fortuitous connection between the in vivo and in vitro results. The uniformity of the method of making diphtheria toxin throughout the world and the constant use of the Park No. 8 strain of $B$. diphtheriae are not sufficiently uniform methods to balance the differences in the culture media and the differences in horses under immunization. It is easily demonstrated, however, that an antibacterial serum will precipitate toxic filtrates, and that some antitoxins will agglutinate bacteria and cause precipitates in solutions of bacterial protein which were never specifically toxic. Further examination of these conditions discloses the following:

There is no relationship between the agglutinating titre of an antitoxic serum and its flocculating value when mixed with a toxin. Moloney and Weld (1925), misquoted by Bronfenbrenner and Reichert (I926), found that there was "no obvious relation between the agglutinin titre of a serum and its antitoxic content." Similar results 
have been observed by Ramon (1923), Povitzky (1927), and others. A serum with a high titre of agglutinin may have neither antitoxic nor flocculating power, and a serum devoid of agglutinins may be both antitoxic and capable of causing flocculation. Agglutinins are demonstrable in the supernatant fluid after flocculation, and the antitoxic content may or may not be reduced by absorption of agglutinins from the serum. Moloney and Weld (1926) have shown that diphtheria toxin-antitoxin flocculation is not group-specific according to the serological relationship of the bacterial strains. In this respect, the flocculation results accord with those of animal tests in their indication of the serological unity of diphtheria toxins.

The precipitate which forms when antibacterial precipitin is added to diphtheria toxin does not remove the toxin nor interfere with its subsequent flocculation with antitoxin. A new flocculation occurs when this toxin, from which precipitable bacterial residues have been removed, is added in equivalent proportions to antitoxin. After the removal of the precipitate formed by antitoxin in an "indicating tube," no further flocculation occurs when more antitoxin is added. In repeating these experiments, I have confirmed the results obtained by H. Schmidt (I926), demonstrating the independence of the toxin-antitoxin flocculation with reference to antibacterial precipitins.

Other controls, such as mixtures of toxins with normal sera, and mixtures of toxins with unrelated antitoxins, have been uniformly negative, furnishing additional evidence of the specificity of the toxin-antitoxin flocculation reaction.

The precipitate formed during flocculation is composed of toxin and antitoxin, in a dissociable union from which both substances can be recovered by heat in a slightly acid or alkaline solution, or by the use of solutions of NaI-Calmette and Massol (I909), Ramon (I923), and Madsen and S. Schmidt (I926). The flocculation reaction, therefore, provides a means by which these substances can be isolated in a state of purity hitherto not obtained-Locke and Main (I926). Ramon (I923) obtained a solution containing 60,000 units of antitoxin per gram of dried material from treatment of this precipitate. Abt and Erber (I926) found that the weight of the precipitate increased in proportion to the number of antitoxic units neutralized in mixtures of tetanus toxin and antitoxin. Chemical analyses of the precipitate have been made by Flössner and Kutscher (1924) and by Hartley (1925, 1926). The toxin-antitoxin precipitate contained protein, lipoids, and ammonium magnesium phosphate. Hartley found from 0.00134 to $0.00203 \mathrm{mgm}$. nitrogen per unit of antitoxin in the precipitate from balanced mixtures of diphtheria toxin and antitoxin. These precipitates, as shown by Hartley (1925, 1926) and by H. Schmidt (1925), are antigenic and can be used for the immunization of man and lower animals. Hartley found that an amount of precipitate containing $0.001 \mathrm{mgm}$. $N$ was sufficient to immunize a guinea pig. Precipitates from neutral mixtures were non-toxic and moderately good antigens. Very little antigenic toxin remained in the supernatant fluid in a neutral flocculating mixture. Precipitates from underneutralized mixtures were slightly toxic and very active antigenically. Precipitates from overneutralized mixtures were non-toxic and had small antigenic value. The antigenic properties of these precipitates, their correspondence in weight to units neutralized and their composition reflecting the constitution of the mixtures from which they are derived, strengthen the evidence in favor of the specificity of the toxin-antitoxin flocculation reaction. 
FLOCCULATION WITH DIPIITHERIA TOXIN AND ANTITOXIN

The reports of the number and results of the flocculation titrations of diphtheria toxin and antitoxin are not sufficiently detailed for statistical analysis. It may be inferred from available data that satisfactory agreements between animal and flocculation tests have been obtained with about 90 per cent of the toxins and antitoxins used. The discrepancies which have been observed, together with the significance of the results in general, have been noted in preceding paragraphs. A value within to per cent of the results of animal tests can be expected from flocculation reactions with most sera and toxins. The method has given useful information to those engaged in the production of diphtheria toxin and antitoxin, and contrary to results reported by Zingher (I924-25), most investigators, dealing with toxoids and anatoxins, have found the flocculation values of these modified toxins a useful guide in choosing immunizing agents in diphtheria. Rapidly flocculating toxins with high $L_{f}$ values have been found to be the most efficacious antigens after modification by formaldehyde, and antitoxins with a high speed of flocculation may be found to possess especially valuable curative properties. With diphtheria toxin and antitoxin, the flocculation method has given results of practical utility and, as described in previous sections of this review, has provided a new means for the study of these substances.

\section{SCARLATINAL STREPTOCOCCUS TOXIN AND ANTITOXIN}

The application of the Ramon flocculation method to the titration of scarlatinal streptococcus toxin and antitoxin has given useful results for the standardization of these products (Dyer [1925] and Povitzky [1925]). Povitzky has used the method at the laboratories of the Health Department of New York City to follow antitoxin production in horses during immunization against scarlet fever streptococcus toxin and to measure the potency of this toxin. In the manuscript of her report, which Dr. Povitzky has allowed me to see before its publication, she records a satisfactory correspondence between the results of the flocculation tests and of skin tests in children. She found that the following conditions were essential for the successful result of the test:

(I) A streptococcus toxin standardized for the number of toxic skin doses per cubic centimeter by the Dick test in children. The toxin should be made by growth of the organism in a medium without blood. Phenol, added in crystals to give a concentration of 0.3 per cent, may be used as a preservative. (2) A constant amount of toxin and varying amounts of serum gave best results. For weak sera, $0.5 \mathrm{cc}$. of toxin was used; for strongly antitoxic sera, I cc. of toxin was used in each tube. (3) The optimum temperature was found to be $37^{\circ} \mathrm{C}$. Temperatures above this inhibited the flocculation. Flocculation usually occurred in I 2 to $\mathrm{I} 8$ hours, but occasionally appeared only after 24 to 48 hours.

Scarlatinal streptococcus toxin was not flocculated by diphtheria antitoxin, antipneumococcus serum, or by an antibacterial streptococcus serum. Some specimens of streptococcus antitoxin flocculated with diphtheria toxin. This was thought to be related to the natural diphtheria antitoxin in the blood of some horses. Flocculation in mixtures of tetanus toxin and scarlatinal streptococcus antitoxin may be explicable on a similar basis.

Povitzky found no relationship between the agglutinin content of the antitoxic 
sera and their flocculation value. The serum of one horse having the highest titre in agglutinin for the streptococcus had the lowest antitoxic value as determined both by flocculation and skin-testing methods. Other horses produced serum of high antitoxic quality with no demonstrable agglutinin for the streptococcus. Povitzky and Dyer regard the flocculation reaction in this case as the result of the union in saturating proportions of scarlatinal streptococcus toxin and its antitoxin.

Flocculation titrations with the toxin and antitoxin of Streptococcus scarlatinae have been found to be unsatisfactory by O'Brien, Okell, and Birkhaug (r926) and by Eagles (1927). No constant relationship could be established by them between the skin-test dose and flocculation titre. The bacterial precipitin reaction accounted for the larger part of the precipitates formed.

\section{BOTULINUM TOXIN AND ANTITOXIN}

The evidence of the value of the flocculation method in the titration of botulinum toxin and antitoxin is conflicting. Weinberg and Goy (I925) report that "le phénomène de Ramon peut donc servir également au titrage in vitro, du sérum antibotulinique." In their tests they used $5 \mathrm{cc}$. of diluted toxin in each tube and varied the amount of antitoxic serum. Typical flocculation occurred in three to four hours at $37^{\circ} \mathrm{C}$. in an indicating tube containing an approximately neutral mixture. Botulinum antitoxin, toxin, and a formalinized anatoxin were titrated in this manner. A potent toxin was found to be essential, as the best results were obtained with toxins containing 200,000 to 600,000 M.L.D. per cubic centimeter. Antitoxic sera stored in the icebox for two to four months had a greatly delayed and unsatisfactory flocculating power. These investigators, as shown by their paper published in 1924, were well aware of the precipitation which occurs when agglutinating sera are added to the filtrates of bacterial cultures, but in the case of botulinum antitoxin and toxin they were able to obtain satisfactory results by the flocculation method in spite of that difficulty.

In the section dealing with the nature of the flocculation reaction, the failure of the method to measure the values of botulinum toxin and antitoxin in the experiments of Bronfenbrenner and Reichert ( 1926 ) has been referred to. The evidence presented in this report clearly establishes the fact that an antibacterial serum, devoid of protective power, can cause a precipitate when mixed with a filtrate of an old culture of Cl. botulinum. The tests were made in such a manner, however, as would favor a preponderant bacterial precipitin reaction while masking specific toxin-antitoxin flocculation. The unusually small quantities of toxin and serum used may have contributed to the irregular results obtained. The toxin did not have as high potency as that employed by Weinberg and Goy and was strongly formalinized ( 0.7 per cent) and incubated for a week before its use in the flocculating mixtures. This undoubtedly delayed flocculation. During the long periods of incubation without constant observation of the tubes, the initial flocculation may have been overlooked. Bronfenbrenner and Reichert ( 1926 ) noted that the removal of the precipitate formed by the action of a non-toxic botulinum filtrate upon an antitoxic serum did not materially reduce the antitoxic value of the serum. This is an indication that the bacterial precipitin reaction does not involve the antitoxin. Apparently no attempt was made to determine whether such an absorbed serum would flocculate in a mixture with botulinum toxin. 
These results demonstrate that botulinum toxin and antitoxin present special difficulties from the point of view of flocculation titration, but do not conclusively controvert the evidence on the side of the specificity and usefulness of this reaction.

\section{TETANUS TOXIN AND ANTITOXIN}

Although Descombey (I924, I925) demonstrated that flocculation occurs in a typical manner in mixtures of tetanus toxin and antitoxin, and that formalinized anatoxins (toxoids) having a high flocculating value are more efficacious as immunizing agents than similar preparations with a low flocculating value, other investigators have found the method somewhat unsatisfactory. Scholz (I924) found that the smallest differences measurable by the flocculation titration of tetanus antitoxin were three to four units, whereas he was able to detect differences of o.or unit by animal tests. Abt and Erber (1926) obtained good results with 90 per cent of the preparations tested by them. Of thirty-eight sera, 60 per cent of the antitoxins gave exactly equal values by flocculation and animal tests; in 29 per cent, the flocculating values were somewhat higher than the results of the animal tests, and in I I per cent the values indicated by flocculation were lower than those of the tests on mice. Their opinion was that in view of the irregularity of the results of animal tests when a small number of animals are used the flocculation results were more precise than those of the animal tests. Zone phenomena, the freshness and potency of toxins and antitoxins, the effect of chemicals, heat, and age upon these reagents, and all the factors which influence flocculation with diphtheria toxin and antitoxin come into play in this case also. In their tests, Abt and Erber placed in each tube $4 \mathrm{cc}$. of tetanus toxin containing 20,000 M.L.D. per cubic centimeter, as determined on white mice, and added varying amounts of antitoxin. At $45^{\circ} \mathrm{C}$. in the water bath, the optimum temperature, flocculation usually occurred in two to three hours. Ramon and Descombey (I926) have proved that the mixture in the indicating tube in the flocculating series is a neutral one when injected into mice. They discovered that not all antitoxic sera are satisfactory for this test, but with the serum from one horse, obtained at monthly intervals during the past three years, their flocculation titrations have had the desirable degree of precision and have aided them in the choice of the most efficacious toxins and anatoxins for the immunization of horses. With the proper choice of toxin and antitoxin, this type of titration can be depended upon to yield satisfactory results.

\section{DYSENTERY TOXIN AND ANTITOXIN}

Flocculation in an "indicating" tube has been observed by Dumas, Ramon, and Said Bilal (I926) in mixtures of filtrates of cultures of the Shiga type of dysentery bacillus and the antitoxic sera of horses immunized by injections of this toxin. The mixture in the indicating tube was non-toxic, showing that neutralization had occurred, but in the absence of well-established units of toxicity or protective values, it is difficult to compute the exact significance of the proportions involved. The experiments afforded additional evidence of the specificity of the toxin-antitoxin flocculation reaction, as the antitoxic serum did not contain demonstrable agglutinins for the Shiga bacillus or any other type of dysentery bacillus. Filtrates were deprived of their toxicity by the addition of 0.6 per cent of commercial 40 per cent formaldehyde, being 
converted in this manner into the substance called "anatoxin" by Ramon. The flocculation method served to measure the immunizing value of these anatoxins as well as to give a general indication of the potency of dysentery antitoxic sera.

\section{MISCELLANEOUS TOXINS AND ANTITOXINS}

Toxic filtrates of the organisms associated with gaseous gangrene have been used in flocculation tests by Weinberg, Prévôt, and Goy (1924). Attempts to measure the potency of the toxins of such organisms as $B$. oedematiens, $B$. perfringens, $B$. histolyticus, B. sporogenes, $B$. putrificus, B. bifermentans, and $V^{r}$ ibrion septique by comparison with their antitoxins in flocculation titrations were failures. With the exception of the toxin of $B$. oedematiens, these filtrates were from five hundred to one thousand times less active than diphtheria toxin; they were weak toxins. When weak toxins, and correspondingly weak antitoxins are used, the bacterial precipitin reaction is predominant. But even in these instances, there was no strict concordance between the antitoxic values and agglutinating titres of the sera.

The failure of an antiserum which neutralized the hemotozin of a vibrio to flocculate when mixed with this toxin is considered by Eisler and Kovács to indicate that the phenomenon observed by Ramon is merely the familiar bacterial precipitation. Abundant precipitation of bacterial residues occurred in the mixtures of filtrates and antitoxins studied by them, and some of the toxin and antitoxin was removed by these precipitates. Apparently, the flocculation reaction is unsuitable for the titration of anti-hemotoxin with filtrates of the cultures of this vibrio.

\section{REFERENCES}

Abt, G., and Erber, B.: "Sur le Titrage des antitoxines et des toxines tétaniques par la floculation," Ann. de l'Inst. Pasteur, 40, 659-65. I926.

Bächer, S., Kraus, R., and Löwenstein, E.: "Zur Frage der aktiven Schutzimpfung gegen Diphtherie," Ztschr.f. Immunitätsforsch. u. exper. Therap., 42, 350-68. 1925.

Bayne-Jones, S.: "The Titration of Diphtheria Toxin and Antitoxin by Ramon's Flocculation Method," J. Immunol., 9, 48I-504. I924.

Bronfenbrenner, J. J., and Reichert, P.: "The Nature of the Toxin-Antitoxin Flocculation Phenomenon," J. Exper. Med., 44, 553-65. 1926.

Calmette, A., and Massol, L.: "Les précipitines du sérum antivenimeux vis-à-vis du venim de cobra," Ann. de l'Inst. Pastcur, 23, I55-65. 1909.

Danysz, J.: "Contribution à l'étude des propriétés et de la nature des mélanges des toxines avec leurs antitoxines," ibid., I6, 331-45. I902.

Descombey, P.: "L'Anatoxine tétanique," Compt. rend. Soc. de biol., 91, 239-41. I924.

Descombey, P.: "Vaccination du cheval par l'anatoxine tétanique," Ann. de l'Inst. Pasteur, 39, 485-504. 1925 .

Dumas, J., Ramon, G., and Bilal, Said: "Anatoxine dysentérique," ibid., 40, 134-40. I926

Dyer, R. E.: "Application of the Ramon Flocculation Principle to the Titration of Scarlet Fever Streptococcus Toxin and Antitoxin," Pub. Health Rep. (U.S. Public Health Service), 40, 865-68. 1925 .

Eagles, G. H.: "The Application of the Ramon Flocculation 'Test to the Toxin and Antitoxin of Streptococcus Scarlatinae," Brit. J. Exper. Path., 8, 403-1 2. I927.

Eisler, M., and Kovács, N.: "Untersuchungen über das Verhältnis des Präzipitinogens und Hämotoxins des Vibrio Kadiköj und das Unvermögen dieses Toxins, sein spezifisches Antitoxin zu flocken," Centralbl. f. Bakteriol., Abt. I, 99, 518-45. 1926. 
Flössner, O., and Kutscher, F.: "Zur Kenntnis der Ramonschen Flockungsreaktion," Mï̈nchen med. W'chnschr., 7I, 576. I924.

Glenny, A. T., and Okell, C. C.: "The Titration of Diphtheria Toxin and Antitoxin by Flocculation Methods," J. Path. \& Bact., 27, 187-200. I924.

Glenny, A. T., Pope, C. G., and Waddlington, H.: "The Measurement of the Combining Power of Diphtheria Toxin and Toxoid with Antitoxin in Relation to Their Antigenic Efficacy," ibid., 28, 279-303. 1925.

Glenny, A. T., and Wallace U.: "The Titration of Diphtheria Toxin by the Flocculation Method," ibid., pp. 317-3I. I925.

Glenny, A. T., Pope, C. G., Waddington, H., and Wallace, U.: "Immunological Notes VIII-XVI," ibid., pp. 463-82. 1925.

Glenny, A. T., Pope, C. G., Waddington, H., and Wallace, U.: "Immunological Notes XVII-XXIV," ibid.. 29, 3I-40. 1926.

Hartley, P.: "The Antigenic Properties of Precipitates Produced by the Interaction of Diphtheria Toxin and Antitoxin. Part I," Brit. J. Exper. Path., 6, I I 2-22. I925.

Hartley, P.: "The Antigenic Properties of P'recipitates Produced by the Interaction of Diphtheria Toxin and Antitoxin. Part II," ibid., 7, 55-68. 1926.

Hoen, E., Tschertkow, L., and Zipp, W.: "Die Anwendung der Präzipitationsmethode bei der Auswertung von solchen antitoxischen Diptherieseren, die dem Einfluss phy sikalischchemischer Faktoren ausgesetzt waren," Zlschr. f. Immunitätsforsch. u. exper. Therap., 47, 277-90. 1926.

Hoen, E., Tschertkow, L., and Zipp, W.: "Studien über das Wesen des $L p$ des Diphtherietoxins," ibid., 48, 191-206. 1926.

Locke, A., and Main, E. R.: "The Isolation of Substances with Immune Properties," $J$. Infect. Dis., 39, 482-90. I926.

Madsen, Th., and Schmidt, S.: "Sur 'l'avidité' du sérum antidiphtérique," Ann. de l'Inst. Pasteur, 40, 300-302. 1926.

Moloncy, P. J., and Weld, C. B.: "Diphtheria Toxin-Antitoxin Flocculation (Ramon Test)," J. Path. E。 Bact., 28, 655-72. 1925.

Moloney, P. J.: "The Preparation and Testing of Diphtheria Toxoid (Anatoxine--Ramon)," Am.J.Pu). Heatth, 16, I 208-10. I926.

Nicolle, M., Césari, E., and Debains, E.: "Etudes sur la précipitation mutuelle des anticorps et des antigènes. II. Sérums antitoxiques," Ann. de l'Inst. Pastcur, 34, 596-99. 1920.

O'Brien, R. A., Okell, C. C., and Birkhaug, K. E.: "The Relation of Streptococci to Scarlet Fever and Its Complications," Brit. Mcd. J., 2, 5I3-18. I926.

Povitzky, O. R., and Banzhaf, E. J.: "Diphtheria Toxin-Antitoxin Titration by Ramon Method for Practical Application," Proc. Soc. Expcr. Biol. Eo Mcd., 22, I I-13. I924.

Povitzky, O. R.: "Ramon Flocculation Test for Determining Potency of Antiscarlatinal Serum," ibid., pp. 426-28. I925.

Povitzky, O. R.: "Specificity of Ramon Flocculation Test in Scarlet Fever" (2d paper). (Not yet published.) Reported at meeting of American Association of Immunologists, Rochester, N.Y., April 16, 1927. Manuscript sent me by Dr. Povitzky. 1927.

Ramon, G.: "Floculation dans un mélange neutre de toxine-antitoxine diphthérique," Compt. rcud. Soc. dc biol., 86, 66 I-63. 1922.

Ramon, G.: "Sur une Technique de titrage in vitro du sérum antidiphtérique," ibid., pp. 7II-I 2. I922.

Ramon, G.: " $\bar{A}$ propos du Titrage in vitro du sérum antidiphtérique par la flocuiation," ibid., pp. 813-15. I922.

Ramon, G.: "Dissociation du complexe toxine-antitoxine diphtérique et récuperation d'antitoxine," Compt. rend. Acad. des Sc. (Paris), 176, 267-70. 1923. 
Ramon, G.: "Sur la concentration du sérum antidiphtérique et l'isolement de l'antitoxine," Compt. rend. Soc. de biol., 88, 167-68. 1923.

Ramon, G.: "Pouvoir floculant et pouvoir toxique de la toxine diphtérique," ibid., 89, 2-4. I923.

Ramon, G.: "La floculation dans les mélanges de toxine et de sérum antidiphtérique," Ann. de l'Inst. Pasteur, 37, 100 I-1 I. I923.

Ramon, G.: "Sur la toxine et sur l'anatoxine diphtériques pouvoir floculant et propriétés immunisantes," ibid., 38, I-I0. 1924.

Ramon, G.: "Sur l'anatoxine diphtérique et sur les anatoxines en général," ibid., 39, I-2I. I925.

Ramon, G., and Descombey, P.: "Sur l'appréciation de la valeur antigène de la toxine et de l'anatoxine tétaniques par la méthode de floculation," Compt. rend. Soc. de biol., 95, 434-36. 1926.

Ramon, G., and Grasset, E.: "La réaction de floculation et le dosage du pouvoir antitoxique du sérum antidiphtérique purifié," ibid., pp. 436-38. I926.

Renaux, E.: "Sur la floculation de la toxine diphtérique par le sérum antidiphtérique," ibid., 90, 964-66. I924.

Schmidt, H.: "Die Methoden der Wertbestimmung von Diphtherietoxin und -Antitoxin," Ztschr.f. Kinderh., 39, 214-32. I925.

Schmidt, H.: "Die Schutzimpfung gegen Diphtherie mit einem neuen Impfstoff T.A.F.," Centralbl. f. Bakteriol., Abt. I, Orig., 97, Beiheft, 63-65. 1925.

Schmidt, H., and Scholz, W.: "Studien zur Kenntnis der Eigenschaften von DiphtherieToxin-Antitoxin-Gemischen. I. Die Beziehung zwischen der Neutralisation in vivo $\left(L_{o}\right)$ und der Neutralisation in vitro $\left(L_{f}\right)$ bei Diphtheriegift," Arch.f. Hyg., 95, 308-19. I925.

Schmidt, H., and Scholz, W.: "Studien zur Kenntnis der Eigenschaften von DiphtherieToxin-Antitoxin-Gemischen. II. Über den Einfluss der Temperatur und des Lagerns auf Diphtherie-Toxin-Antitoxin-Gemischen," ibid., pp. 339-50. I925.

Schmidt, H., and Scholz, W.: "Studien zur Kenntnis der Eigenschaften von DiphtherieToxin-Antitoxin-Gemischen. III. Die Beziehung der direkten Giftwirkung des Diphtherietoxins $\mathrm{zu}$ seiner Bindungsfähigkeit mit Antitoxin. Zugleich ein Beitrag zur Vorstellung über die Natur des Diphtherietoxins," ibid., 96, I72-8I. I926.

Schmidt, H., and Scholz, W.: "Studien zur Kenntnis der Eigenschaften von DiphtherieToxin-Antitoxin-Gemischen. IV. Die Bedeutung der Zone bei der Ausflockung von DiT.A. Gemischen," ibid., pp. 185-94. I926.

Schmidt, H., and Scholz, W.: "Studien zur Kenntnis der Eigenschaften von DiphtherieToxin-Antitoxin-Gemischen. V. Die immunisierende Wirkung der bei der DiphtherieToxin-Antitoxinbindung auftretenden Flocken," itid., pp. 25 I-6I. I926.

Schmidt, H., and Scholz, W.: "Studien zur Kenntnis der Eigenschaften von DiphtherieToxin-Antitoxin-Gemischen. VI. Zur Kenntnis des Flockungsvorganges in Di-T.A. Gemischen," ibid., pp. 294-300. 1926.

Schmidt, H.: "Zur Kenntnis der Natur der Diphtherie-Toxin-Antitoxin-Flockung," Ztschr. f. Immunitätsforsch. u. exper. Therap., 48, 217-32. 1926.

Schmidt, S.: "Sur le titrage du sérum antidiphtérique," Compt. rend. Soc. de biol., 88, 1056. I923.

Schmidt, S.: "Remarques sur la technique de titrage du sérum antidiphthérique d'après la méthode de Ramon," ibid., 90, ir 7S-So. 1924.

Scholz, W.: "Über die Brauchbarkeit der Flockungsreaktion für die Auswertung antitoxischer Sera (insbesondere des Diphtherieantitoxins)," Centralbl.f. Bakteriol., Abt. 1, Orig., $91,72-79$. 1924. 
Scholz, W.: "Nachweis und Austitrierung antitoxischer Sera (insbesondere des Tetanusantitoxins) im Reagenzglas," ibid., 92, 434-38. I924.

Sord lli, A., and Serpa, R.: "Titrage du sérum antidiphtérique par la méthode de Ramon," Compt. rend. Soc. de biol., 91, 1043-44. I924.

Trevan, J. W.: "An Apparatus for the Measurement of Small Quantities of Fluid," Lancet, I, 786. I922.

Wcinberg, M., and Goy, P.: "De l'anatoxine botulinique," Compt. rend. Soc. de biol., 9I, I48-49. I924.

Weinberg, M., Prévôt, A.-R., and Goy, P. "Floculation des sérums agglutinants par les filtrats des cultures microbiennes," ibid., 90, 329-3I. I924.

Weinberg, M., and Goy, P.: "Emploi de l'anatoxine dans la préparation du sérum antibotulinique," ibid., 92, 564-65. 1925.

Weinberg, M., and Prévôt, A.-R.: "Nouvelles recherches sur les anatoxines gangréneuses; leur emploi dans la vaccination du cobaye et la préparation des sérums spécifiques," ibid., pp. I484-87. I925.

Zingher, A.: "The Ramon Flocculation Test in Relation to the Antigenic Value of Diphtheria Toxoid (Anatoxin)," Proc. Soc. Exper. Biol. \& Med., 22, 454-59. I924. 


\title{
CHAPTER LVII
}

\section{SUBLETHAL INTOXICATIONS WITH BACTERIAL PRODUCTS}

\author{
J. P. SIMONDS \\ Northwestern University Medical School
}

Sublethal intoxications may result either from a small dose of a powerful bacterial poison which in larger amount would cause the death of the animal, or from the action of bacterial products of relatively low toxicity. In general, sublethal intoxications differ from lethal forms quantitatively rather than qualitatively, in intensity rather than in kind.

Sublethal intoxications may be induced experimentally or occur spontaneously. Experimental intoxications are produced for the purpose of studying the early stages of the diseases for which they are responsible, and in order that, under accurately controlled conditions, the effects of the toxins may be differentiated from those of extraneous causes which under uncontrolled conditions cannot be accurately determined. Spontaneous sublethal intoxications with bacterial products occur in infectious diseases from which the animal or man recovers. Many of the effects of infectious diseases are believed to be due to the action of toxic products of the infecting microorganism and not to the physical effects of the bacteria themselves. (I) Some effects are general, involving the entire body, such as changes in temperature and metabolism. (2) Degenerative changes occur in parenchymatous organs far removed from the actual location of the growing bacteria. (3) Many of the lesions characteristic of infectious diseases differ from those in which bacteria are known to be actually present in the tissues. It has been tersely remarked by Courmont and Rochaix that "the microbe is above all a fabricator of toxins; the infectious disease is an intoxication." I

Toxic bacterial products are of three general types: (I) basic substances resulting from the action of bacteria upon various kinds of organic matter, the ptomaines of Brieger ; ${ }^{2}$ (2) true soluble toxins, definite metabolic products of the micro-organisms, found in the medium in which the bacteria are growing; (3) and toxic substances, the endotoxins, firmly fixed in the bacterial cell and liberated upon disintegration of those cells.

The ptomaines are not true bacterial toxins in that they are not specific products of bacterial metabolism and do not induce the formation of antibodies when injected into animals. Many diseases formerly believed to be due to poisoning with ptomaines are now known to be actual infections. ${ }^{3}$

The soluble toxins require an incubation period for their effects to become mani-

${ }^{x}$ Courmont, J., and Rochaix, A., in Bouchard, Ch., and Roger, G.-H.: Pathologie générale, chap. ii, p. 1074. Paris, r9r4.

${ }^{2}$ Brieger, L.: Die Ptomaine. Berlin, 1885 .

${ }^{3}$ Jordan, E. O.: Food Poisoning. Chicago, I917. 
fest. They have the quality of inducing the formation of antibodies which neutralize the toxin. The pathological changes which they induce are of relatively short duration, for with the development of antitoxins in sufficient quantities they cease to be active. Any damage which they have done to the tissues of the body may be completely repaired. These substances, therefore, do not cause chronic disease nor produce structural changes that become permanent.

The toxic products that are firmly fixed in the bacterial cells must be liberated by autolysis, bacteriolysis, crushing, and grinding or other means before they can become effective. These endotoxins act immediately without the lapse of an incubation period. They do not induce the formation of antibodies which neutralize their toxicity. An animal that has been repeatedly injected with these substances, or a patient who is the subject of a chronic infection with micro-organisms of this type, has not the power of neutralizing the toxic products. The continuous or intermittent presence of these endotoxins in the animal body may, therefore, induce lesions that are chronic.

In addition to the type of toxin and the size of the dose, the lethality of a given bacterial poison depends upon various more or less unrelated factors: (I) Most bacterial poisons are inactive when administered by mouth. They are destroyed by the digestive ferments and bacteria of the gastro-intestinal tract. ${ }^{x}$ The toxin of $B$. botulinus, however, is an exception to this general rule, for it is just as effective and produces as severe symptoms when given by mouth as when injected intravenously. (2) Some animals are not susceptible to the action of certain bacterial poisons. The rabbit is far less susceptible to tetanus toxin than the mouse or the guinea pig. The rabbit's spleen has an affinity for this toxin. In this animal, therefore, a large part of the tetanus toxin is fixed in the spleen and the central nervous system is protected against its action. (3) The effect of tetanus toxin also varies with the body temperature of the animal. A frog injected with this poison and kept in an incubator develops symptoms of tetanus in twelve hours; one injected with this toxin may be kept at a low temperature for a week or more without showing any manifestations of the disease, but, upon then being placed in an incubator, will develop tetanus in an hour. ${ }^{2}$ (4) Animals may be sensitized to bacterial poison so that a dose which, in a normal animal, would be without effect or at most sublethal will cause death in a short time. Koch early showed that a guinea pig infected with tubercle bacilli could be easily killed with a dose of tuberculin that in a normal animal would be quite harmless.

\section{EFFECTS OF SUBLETHAL INTOXICATIONS}

The effects of sublethal intoxications with bacterial products may be (I) local, due to the direct action of the bacterial products upon the tissues with which they come in contact, and taking the form of necrosis and of varying degrees and types of inflammation; (2) general, manifested either by alterations in the blood, or by changes in the temperature and metabolism of the body of the animal; (3) focal or metastatic effects which occur in organs distant from the place of entry of the toxin, usually in an organ which is concerned with the excretion of the poison, and manifesting them-

${ }^{1}$ For detailed data on this point see Courmont, J., and Rochaix, A.: op. cit., pp. 1073-97.

${ }^{2}$ Meyer, H., and Ransom, F.: Arch.f. exper. Path. u. Pharmakol., 49, 369. r903. 
selves as necrotic or inflammatory changes in the organ affected; (4) specific effects, due to the special affinity possessed by the toxic products of certain bacteria for certain organs or tissues in which they accumulate in greater concentration and where they produce effects that are more or less constantly characteristic and specific.

\section{LOCAL EFFECTS}

The local effects of bacterial toxic products vary with the type of poison and with its concentration and dosage. Diphtheria toxin causes a local necrosis accompanied by a marked inflammatory reaction with an exudate rich in fibrin. This is equally true whether the intoxication occurs spontancously or is induced experimentally. In ordinary infections of the throat with the diphtheria bacillus, the surface epithelium becomes necrotic and an exudate of fibrin and leukocytes becomes entangled in this necrotic material. This "membrane" is, by the mechanism of its formation, adherent to the underlying tissues, and bleeding occurs when attempts are made to remove it. When diphtheria toxin in sublethal doses is injected subcutaneously, a local necrosis and exudate results which may slough out leaving an ulcer. In immunizing against diphtheria with toxin-antitoxin there frequently occurs a local inflammatory reaction which, however, rarely goes so far as to induce necrosis.

The toxins of $B$. tetani and $B$. botulinus produce relatively slight local effects. Their action is more definitely specific. The toxin of $B$. welchii appears to be a true soluble toxin, but differs in certain material respects from those just mentioned. It is actively hemolytic both in vitro and in vivo. When injected subcutaneously or intramuscularly it causes a local edema and necrosis with a marked inflammatory reaction in the surrounding tissues. ${ }^{\text {I }}$

The local effects of true soluble bacterial toxins are not therefore strictly specific, that is, they do not produce lesions whereby the type of toxin can be readily identified by the type of changes produced in the tissues. The fixed bacterial toxins, the endotoxins, induce changes which differ in certain respects from those which result from the action of the true or soluble toxins. In general, the exudate induced by the injection of endotoxins is less rich in fibrin, and the necrosis is also somewhat different in type.

The endotoxins of most bacteria have a positive chemotactic effect and induce the formation of an abscess when injected subcutaneously or intramuscularly. Much the same effects are produced when a suspension of living or of killed staphylococci is injected beneath the skin. The bodies of the dead micro-organisms contain the chemotactic substance. The changes which occur in the surrounding tissues are essentially those of an acute inflammation. The products liberated by the disintegration of the staphylococci ( 1 ) attract leukocytes in great numbers into the area of their activity; (2) cause necrosis of that portion of the fixed tissues with which they come in contact in greatest concentration; and (3) kill many of the leukocytes which have been attracted into the region. These three bacterial products of the staphylococcus, namely, the chemotactic, the necrotizing, and the leukocytocidal, are, by their interaction, responsible for the formation of the peculiar type of concentric inflammation, the abscess, which is the characteristic lesion produced by the staphylococcus in tissues in any part of the body. The leukocytes killed by the leukocidin of the staphylococcus liberate, upon their disintegration, their very powerful proteolytic ferments which digest and liquefy the necrotic tissues, thus forming a cavity filled with pus. ${ }^{2}$

I Bull, C. G., and Pritchet, I. W.: J. Exper. Med., 26, I19, 367, and 603. I9I7.

${ }^{2}$ Jochmann, G.: Virchow's Arch.f. puth. Anat., 194, 342. I908; Opie, 1.. 1.. . 1rch. Int. Med., 5. 5.1. 1910 . 
Some bacterial toxins, on the other hand, may repel leukocytes as shown by Vaillard and Rouget ${ }^{\mathrm{r}}$ and others. For example, when tetanus bacilli were injected subcutaneously along with their toxin, leukocytes did not collect at the place of injection; but if these bacilli were washed free from toxin and then injected, the leukocytes did gather and engulf the bacilli.

Tubercle bacilli also give rise to substances which cause local necrosis of tissues. But they do not produce a positively chemotactic substance. They, therefore, induce a characteristic type of coagulative necrosis, known as "caseation." In the absence of leukocytes there are no proteolytic ferments in any abundance and the necrotic material of the tubercle is not digested and liquefied.

Thus although both staphylococci and tubercle bacilli, by the action of their toxic products, cause necrosis of tissues in which they are growing or into which certain of their products have been injected, the type of necrosis in the two cases differs. This is largely because of the difference in their power to attract leukocytes into the tissues affected. The characteristic lesion of the staphylococcus is, thus, a liquefaction necrosis or abscess formation; while the tubercle bacilli induce a coagulative necrosis, or caseation.

Most bacterial toxic products, whether true soluble toxins or endotoxins, cause a dilatation of the capillaries in the tissues with which they come in contact, and probably also alter their walls in such a manner as to render them more permeable. This gives rise to two of the phenomena of the local reaction. In the first place, the dilatation results in a hyperemia or increase in the amount of blood in the affected area. Second, a greater quantity of fluid escapes from these capillaries into the surrounding tissues resulting in edema. Both of these conditions are the basis of the local swelling and redness which follow the injection of bacterial toxic products into the subcutaneous tissues. The irritating effect of these substances on the sensory nerve endings in the inflamed area is one of the causes of the pain which is a constant symptom of inflammation.

The local effects of sublethal doses of toxic bacterial products depend in part upon the degree of sensitization of the animal to the particular product used. The tuberculin test employed for purposes of diagnosis depends upon this principle. ${ }^{2}$

\section{GENERAL EFFECTS}

Sublethal intoxications with bacterial products produce general effects upon $(a)$ the blood, and $(b)$ upon temperature and metabolism.

The injection of an animal with sublethal doses of soluble bacterial toxin or of the bodies of killed bacteria, or of bacterial proteins obtained from them by the various means used for this purpose, is followed sooner or later by an increase in the number of leukocytes in the circulating blood. But the reaction to such injections varies with the size of the dose and, to a less extent, with the type of micro-organism used. Holmes, ${ }^{3}$ Ledingham, ${ }^{4}$ and others have observed this phenomenon after injections of diphtheria toxin. The chemotactic properties of dead bacteria were apparently first observed by Leber in I 888. But it has been shown by Simonds and Baldauf, ${ }^{5}$ Glintschikoff,${ }^{6}$ and others that the first effect of intravenous injections of bacteria is a leukopenia. In sublethal injections this leukopenia is only transitory

IVaillard, L., and Rouget, J.: Ann. de l'Inst. Pasteur, 6, 385. 1892.

2 See chap. Ixxvi in this volume.

${ }^{3}$ Holmes, T. E.: Guy's Hosp. Rep., 59, I 55. I905.

${ }^{4}$ Ledingham, J. C. G.: J. Hyg., 7, 65. 1907.

5 Simonds, J. P., and Baldauf, L. K.: J. Infect. Dis., 6, 38. I908.

${ }^{6}$ Glintschikoff, IV. J.: V'irchow's Ar.h.f. path. Anat., 21 2, 46I. I9I3. 
and is followed in a few hours by a very marked leukocytosis. After lethal doses the leukopenia may persist until death.

Infections, both fatal and non-fatal, with certain bacteria, notably the typhoid bacillus, are characteristically associated with a leukopenia. But the injection of sublethal doses of killed typhoid bacilli is followed by a leukocytosis. There is some evidence to the effect that such an injection into an animal which has been immunized against the corresponding bacteria is followed by a more marked increase in the number of leukocytes than occurs after an injection of a non-immune animal.

Some bacteria produce hemolytic substances. In streptococcal infections from which the patient recovers a marked anemia, often associated with jaundice, is frequently one of the grave clinical manifestations of the disease. Bordet, ${ }^{x}$ Besredka, ${ }^{2}$ and others have described a hemolysin in filtered broth cultures of streptococcus which will dissolve the erythrocytes of laboratory animals and of man. Whether the anemia which accompanies infections with this micro-organism is due to the specific action of this soluble hemolysin is not certain.

Reimann and Julianelle ${ }^{3}$ found that extracts of pneumococci produced a hemorrhagic purpura in mice. This was associated with a marked reduction in the number of blood platelets. Regeneration of the platelets was accomplished by the fourth to the ninth day. There was then an overproduction, and the number did not return to normal until several weeks had elapsed. There was also in these animals a diminution in the number of red cells, regeneration of which was considerably slower than the regeneration of the platelets. They concluded that the pneumococcus extracts were both thrombolytic and hemolytic.

Fever is such a constant accompaniment of infectious diseases that the character of the temperature curve from day to day or from hour to hour is of great diagnostic significance. The effect of injections of toxic bacterial products upon body temperature depends both upon the size of the dose and the micro-organism concerned. Lethal doses and even large sublethal doses cause a fall in temperature. Smaller doses induce a rise in body temperature. Rolly and Meltzer ${ }^{4}$ attempted to reproduce experimentally the conditions that occur in spontaneous infections by the repeated injections of very small amounts of bacteria or their toxins with the result that a rise in temperature always followed. Vaughan ${ }^{5}$ was able to reproduce a temperature curve similar in all essential respects to that of typhoid fever by the injection of animals with the protein split products of the typhoid bacillus in suitable doses at proper intervals. Tuberculin causes fever in normal animals if given in sufficiently large doses, but small doses will induce a rise in temperature in tuberculous animals that are sensitive to this poisonous product of the tubercle bacillus. Jona ${ }^{6}$ produced fever by the injection of a substance extracted from the colon bacillus. Moreschi and Golgi made extracts of typhoid and tubercle bacilli and of staphylococci by treating them with fresh guinea-pig serum. They found no parallelism between the pyrogenic and the anaphylactic value of typhoid "anaphylatoxin." The anaphylatoxin of the tubercle bacillus

× Bordet, J.: Ann. de l'Inst. Pasteur, 9, 462. 1897.

${ }^{2}$ Besredka, A.: ibid., I5, SSo. I901.

3 Reimann, H., and Julianelle, L. A.: J. Exper. Med., 43, 97. 1926.

${ }_{4}$ Rolly, F., and Meltzer, K. S.: Deitsches Lrch.f. Klin. IIca., 94, 335. I 908.

5 Vaughan, V. C.: Protein Split Products, etc. Philarlelphia, igr3.

${ }^{6}$ Jona, J. L.: J. Ilyg., 15, 169. 1916.

7 Moreschi, C., and Golgi, A.: Zlschr. f. Immunitülsforsch. u. exper. Therap., Orig., 19, 623. I913. 
and of the staphylococcus produced no temperature changes in guinea pigs, even in relatively large doses. It may be remarked that failure to induce a rise in temperature by injections of extracts of these organisms was the result of the method employed to liberate the bacterial protein. Neither the tubercle bacillus nor the staphylococcus are readily susceptible to lysis by immune serum. The typhoid anaphylatoxin of Moreschi and Golgi in proper dosage did induce a rise in temperature. Dold and Hanau $^{1}$ report somewhat similar results in which they attempt to differentiate between an anaphylatoxin and an endotoxin in the bodies of these bacteria. But Thiele and Embleton ${ }^{2}$ criticize this work. They contend that in the attempt to disanaphylatoxinize the bacterial protein Dold and Hanau removed from the solution a great part of the more readily attackable protein, and hence the doses which they used were not proportionally large enough to produce the same effects as the untreated bacterial protein.

In the causation of fever it is the products of bacteria and not the bacteria themselves that act as the stimulus. Fever has long been looked upon as a protective mechanism in infections. The manner in which it serves as such is not clear, for the temperature ordinarily reached does not interfere seriously with the growth of bacteria in the body. Rolly and Meltzer ${ }^{3}$ found that animals kept at relatively high temperatures developed antibodies much more rapidly and in larger amounts than when kept at room temperature. This fact has significant possibilities in immunological work.

With the increase in temperature in spontaneous infections and following injections of bacterial toxic products there is also an increase of metabolism. Animals that have been injected with the toxic products of the typhoid bacillus often show a fall in temperature and emaciate and die in a week to ten days. O'ccasionally they recover after a period of such emaciation. Whether this loss of weight is associated with the effect of the bacterial toxic products upon metabolism is not clear.

\section{FOCAL OR METASTATIC EEFECTS}

Bacterial toxic products often produce their effects after they have been absorbed from the focus of infection or from the place where they were injected. These effects usually occur at the site of the elimination of the toxic products from the body.

Flexner and Sweet ${ }^{4}$ found that the toxin of the Shiga dysentery bacillus was harmless when administered by mouth. But when injected intravenously it induced a severe colitis, sometimes with the formation of ulcers. They believed that this toxin was excreted in rabbits and probably in man, by the intestine, chiefly the large intestine, which being injured by the act of elimination reacted by the development of inflammation.

Fahr ${ }^{5}$ observed fatty changes and focal necroses in the visceral organs after infections with the diphtheria bacillus. In guinea pigs, diphtheria toxin affects especially the adrenals causing hyperemia and hemorrhage into these organs, the differences between lethal and

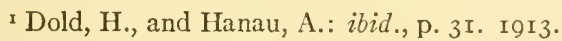

${ }^{2}$ Thiele, F. H., and Embleton, D.: ibid., p. 666. I913.

3 Rolly, F., and Meltzer, K. S.: loc. cit.

4 Flexner, S., and Sweet, J. E.: J. Exper. Med., 8, 5 I 4.1906.

s Fahr, T.: Virchow's Arch.f. path. Anat., 22 1, 38. тот6. 
sublethal doses being quantitative rather than qualitative. Abramow, ${ }^{\mathrm{I}}$ working with rabbits, guinea pigs, and horses, found that under the influence of large doses of diphtheria toxin the secretion of adrenalin ceased, with minimal lethal doses it decreased, and with sublethal doses and in immunization it increased.

Post-diphtheritic paralysis is one of the serious complications of this disease in man, and can be produced experimentally by suitable injections of diphtheria toxin. It is due to the action of the toxin or of the toxon upon the peripheral nerves, giving rise to a peripheral neuritis. This complication occurs in non-fatal cases of this disease in the second or third weeks, after the patient has recovered from the acute symptoms. It is characterized by degeneration of the axis cylinders of the affected nerves with more or less lymphocytic infiltration.

Many bacterial toxic products cause hyperplasia of lymph glands and spleen. In diphtheria not only are the lymph glands of the neck enlarged, but also those within the abdominal cavity and even the spleen are increased in size. Lymph glands which are regional to and drain a localized focus of infection, such as an abscess due to staphylococci, become enlarged. Inasmuch as bacteria cannot usually be demonstrated in them it is believed that the hyperplasia of such glands is due to the action of toxic products absorbed from the focus of infection. The process is a proliferation of the lymphoid elements and not an infiltration with polymorphonuclear leukocytes such as occurs when the staphylococci themselves reach the gland and set up a purulent inflammation. ${ }^{2}$

Tuberculin given in subcutaneous injections, as in the original tuberculin test, causes a focal reaction in the form of a definite and sometimes severe inflammatory reaction with hyperemia and edema in the locally sensitized tissues about tuberculous foci in the lungs and elsewhere. This reaction may sometimes be so severe as to break down the local mechanism of resistance and allow a quiescent focus to become active again.

In many chronic infections, notably in chronic osteomyelitis due to the staphylococcus and in tuberculosis of bones, there is frequently found in the smaller arteries and capillaries of the kidneys, liver, spleen, and other organs a deposit of an insoluble, homogeneous, hyaline substance the exact chemical nature of which is in dispute. This is amyloid degeneration. Attempts to produce this lesion experimentally have yielded confusing results.

Mice have been most frequently used for these experiments. But these animals are especially unsuited for this purpose because of the frequency with which they are found to suffer from spontaneous amyloid degeneration. Thus Simonds ${ }^{3}$ observed amyloid disease in a considerable number of mice from the Slye breeding stock which had never been submitted to experimental procedure of any kind. However, Herzenberg 4 found amyloid degeneration in 70 per cent of mice which she had repeatedly injected with suspensions of staphylococcus. This percentage is probably much too high for all cases to have been of spontaneous origin. Frank ${ }^{5}$ found amyloid disease in an unmentioned number of mice which he had injected with suspensions of $B$. mucosus capsulatus. Bailey ${ }^{6}$ produced amyloid disease in rabbits by repeat-

I Abramow, S.: Zischr.f. Immunitätsforsch. u. exper. Therap., Orig., 15, I2. Igr 2.

${ }^{2}$ Symmers, D.: Arch. Int. Med., 2 r, 237. rgr8.

3 Simonds, J. P.: J: Cancer Reseurch, 9, 329. 1925.

4 Herzenberg, H.: Virchow's Arch.f. path. Anat., 260, 466. 1926.

5 Frank, A.: Beitr. z. path. Anat.u.z. allg. Path., 67, I81. 1920.

${ }^{6}$ Bailey, C. H.: J. Exper. Met., 23, 773. r9ı. 
ed intravenous injections of living cultures of colon bacilli. This condition has also been reported as the result of injections of living cultures of Staphylococcus aureus by Davidsohn ${ }^{\mathrm{r}}$ Lubarsch, ${ }^{2}$ and Green. ${ }^{3}$ Lyon, ${ }^{4}$ however, injected rabbits every second or third day for from one to three months with ten-day-old broth cultures of staphylococcus killed witlı toluol, without the production of amyloid. The belief that amyloid degeneration is due to sublethal intoxications with bacterial products is therefore based largely upon deductions from a study of the condition in human patients in whom it is found associated with some form of chronic infection. But not all cases of such infections are complicated by amyloid disease. Hence there must be some factor other than a bacterial toxic product concerned in its production.

M. B. Schmidt ${ }^{5}$ thought that amyloid degeneration was closely related to the formation of antibodies because it is seen in horses that have been used for long periods for the commercial production of diphtheria antitoxin, and sometimes follows repeated injections of animals with various kinds of bacteria.

The lesions observed in the kidneys in the usual forms of acute and chronic nephritis (Bright's disease) are not those which accompany the growth of bacteria in the tissues. It has long been believed, therefore, that these lesions are due to the action of toxic bacterial products rather than the presence of bacteria themselves. It is known that bacteria injected in to the blood stream may pass through the kidneys without doing them any appreciable harm. ${ }^{6}$ Experimental evidence has been sought by many workers for the possible relation between nephritis and bacterial poisons. These experiments have not yielded uniform results. In general, such attempts have resulted in acute changes only. The successful production of experimental chronic nephritis is always rendered problematical because of the frequency with which laboratory animals are found to have chronic nephritis.

Acute degenerative changes in the endothelium of the arterioles and glomerular capillaries have been produced by the injection of diphtheria toxin by Lyon, ${ }^{7}$ Frothingham, ${ }^{8}$ Bailey, ${ }^{9}$ and others. In addition to the alterations in the endothelium there were frequently found thrombi in the glomerular capillaries, exudate in the glomerular space, and casts in the renal tubules. In no instance were they able to induce a chronic form of nephritis. Fahr ${ }^{10}$ was unable to find in the bodies of persons dead of other diseases long after an attack of diphtheria any evidence that that disease had left any permanent injury in the kidneys.

Faber ${ }^{1 x}$ has reported an important difference between the action upon the kidneys of diphtheria toxin alone and in combination with the endotoxin of the colon bacillus. Diphtheria toxin alone produced damage to the endothelium of the capillary tufts,

I Davidsohn, C.: Virchow's Arch.f. path. Anat., I50, I6. 1897.

${ }^{2}$ Lubarsch, O.: ibid., p. 471.

${ }^{3}$ Green, A. B.: J. Path. \& Bact., 7, 184. 1901.

${ }^{4}$ Lyon, G.: ibid., 9, 400. I904.

5 Schmidt, M. B.: Verhandl. d. dtsch. path. Gesellsch., 7, 2. 1904.

${ }^{6}$ Cabot, H., and Crabtree, E. G.: Surg. Gynec. Obst., 23, 495. 1916. $\quad 7$ Lyon, G.: loc. cit.

${ }^{8}$ Frothingham, C.: J. Med. Reseurch, 30, 365. I9I4.

9 Bailey, C. H.: J. Exper. Med., 25, I09. I9I7.

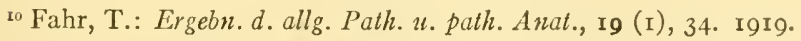

II Faber, H. K.: J. Exper. Med., 26, I39 and 153. I9I7. 
the degree of injury being roughly proportional to the size of the dose. He suggested that the localization of the lesions in these capillaries is due to the concentration of the toxin in the blood which follows the loss of water at this point. By the injection of sublethal doses of colon bacilli into rabbits already suffering from a mild grade of glomerular damage following diphtheria toxin, Faber was able to produce a severe form of glomerulonephritis corresponding to the extra-capillary type of Volhard and Fahr. Similar, though not identical, lesions were produced when diphtheria toxin was followed by Vaughan's split protein (poisonous part) of the colon bacillus. Faber believed that bacteriolysis of colon bacilli in the blood stream liberated toxic substances which induced the glomerulonephritis.

Numerous attempts have been made to produce nephritis by the injection of the toxic products of streptococci, because of the constant presence of these microorganisms in scarlet fever and because nephritis is such a common complication of this disease.

Stoddard and Woods ${ }^{\mathrm{r}}$ injected rabbits with an extract of streptococci and staphylococci prepared by Macfadyen's method and observed acute degenerative changes in the proximal convoluted tubules of the kidneys which persisted for from two to three weeks. Faber and Murray $^{2}$ made repeated intravenous injections of streptococci and staphylococci from cases of scarlet fever, and of $B$. coli, and were unable to produce a typical glomerulonephritis even when immune bodies could be demonstrated in the serum in high dilutions. They were especially interested in this phase of the problem because Volhard and Fahr ${ }^{3}$ had claimed that it is the characteristic of diffuse nephritis to occur at a time when immune processes are active, making possible a "diffuse toxic action." Von Pirquet ${ }^{4}$ and others had attempted to explain the occurrence of nephritis as a complication of scarlet fever on the basis of allergy. Their explanation was as follows: At the beginning of the disease and during the acute symptoms, the virus circulates in the blood and sensitizes the cells of the body. The immune bodies begin to appear at the end of the second week. At this time a reinvasion of the body from various foci occurs, a specific reaction between antigen and antibody takes place, and this, after the latent period common to allergic reactions, leads to active signs and symptoms of the sequelae of scarlet fever in the third and fourth weeks. But Faber and Murray ${ }^{2}$ were unable to verify this view, because they could not demonstrate bacteriolysis of streptococci with the serum of highly immunized animals either in vitro or by the Pfeiffer phenomenon. They therefore conclude that the weight of evidence is against the theory that glomerulonephritis is due to an immune bacteriolysis of streptococci.

Bell, Hartzell, and Clawson ${ }^{5}$ were unable to produce glomerulonephritis in rabbits by the injection of streptococci from human sources. In monkeys similar injections usually induced a severe nephrosis, and in only one instance, a glomerulonephritis. Duval and Hibbard, ${ }^{6}$ however, claim to have produced in rabbits various types of glomerulonephritis, including the epithelial "crescents," endothelial proliferation, hyaline thrombi in the vessels of the glomeruli, hemorrhage into the capsular space, and complete necrosis of the capillary tufts, by the injection of the "toxic principle of the scarlatinal streptococcus of the Dicks."

\footnotetext{
'Stoddard, J. L., and Woods, A. C.: J. Med. Research, 34, 343. I9r6.

${ }^{2}$ Faber, H. K., and Murray, V.: J. Exper. Med., 26, 707. I9I7.

3 Volhard, F., and Fahr, T.: Die Brightsche Krankheit. Berlin, I9I4.

${ }^{4}$ v. Pirquet, C.: Ergebn. d. inn. Med. u. Kinderh., 5, 459. 1910.

${ }_{5}^{5}$ Bell, E. T., Hartzell, T. B., and Clawson, B. J.: Am. J. Path., I, 247. 1925.

${ }^{6}$ Duval, C. W., and Hibbard, R. J.: J. Exper. Med., 44, 567. I926; J.A.M.A., 87, 898. 1926.
} 
From the foregoing review, the experimental evidence of the relation between bacterial toxic products and nephritis may be summarized as follows: (г) soluble poisons like diphtheria toxin produce their effects upon the glomerular capillaries because of their concentration as a result of the loss of water from the blood at this place; (2) bacteria of the colon-typhoid group may give rise to toxic products which will damage the kidneys as a result of bacteriolysis to which this group of bacteria are particularly susceptible; (3) the mechanism by which toxic substances injurious to the kidneys are derived from streptococci is not clear because they are not readily dissolved by bacteriolysis; (4) incontestable evidence of the successful production of chronic nephritis by bacterial toxic products is very scanty if not wholly lacking.

The etiology of nephritis occurring spontaneously in man has been repeatedly sought in the possible absorption of toxic products from areas of focal infection, such as chronically diseased tonsils, abscessed teeth, etc. The mere coexistence of nephritis and focal infection does not prove an etiological relation. Newburgh ${ }^{\mathrm{I}}$ insists that in order to establish a relation between focal infection and the causation of nephritis by toxic products absorbed from such a focus, it must be shown that patients are generally cured or at least greatly benefited by the removal of the focus. In his extensive experience, "The eradication of foci of infection in patients coming to the University Hospital has thus far not resulted in a single cure."

\section{SPECIFIC EFFECTS}

The problem of the specific effects of bacterial toxins is complex. Many of the effects that appear to be specific are not the result of any special affinity of the toxin for a given tissue. For example, the immediate cause of death in pneumonia is frequently failure of the heart. This is not due to any specific action of any product of the pneumococcus upon the heart itself, but to the fact that the myocardium is the site of a parenchymatous degeneration similar to that which occurs simultaneously in other parenchymatous organs, such as the liver and kidneys.

Diphtheria toxin, however, does have a special affinity for nerve tissue and, in the guinea pig at least, for the adrenals. Courmont and Rochaix ${ }^{2}$ have suggested that lipoids, especially those which contain phosphorus, may be concerned in the fixation of this toxin in these locations.

The toxins of $B$. tetanus and of $B$. botulinus have a specific affinity for the central nervous system. They become fixed in brain tissue both in vitro and $i n$ vivo. But the mode of action of the poison is different in the two cases. Tetanus toxin causes convulsions, probably by increasing the activity of the synapses and by upsetting the co-ordinating mechanism of antagonistic muscles. The poison of botulism causes mydriasis and paralysis of the muscles concerned with swallowing and with speech.

The truly specific effects of bacterial toxins are based upon a chemical affinity of these poisons for special tissues as a consequence of which they become concentrated in some particular location. Other organs and tissues are thus protected against their action while the particular organ receives the whole force of their destructive action.

${ }^{x}$ Newburgh, L. H.: Medicine, 2, 77. 1923.

${ }^{2}$ Courmont, J., and Rochaix, A.: loc. cit. 


\title{
CHAPTER LVIII \\ THE MECHANISM OF AGGLUTINATION
}

\author{
JOHN H. NORTHROP \\ Rockefeller Institute for Medical Research \\ Princeton, N.J.
}

The phenomenon of agglutination of suspensions of bacteria in the presence of homologous immune serum was thoroughly described in 1896 by Gruber and Durham. The reaction was then studied by a number of workers and a series of suggestions made as to its mechanism. It was first clearly recognized by Bordet ${ }^{1}$ that the phenomenon was a special case of the flocculation of a colloidal suspension. Subsequent work has tended only to confirm this conclusion, and it is now generally accepted. In the meantime, much progress has been made in the theory of the flocculation of suspensions. In some respects the work with other suspensions is simpler, and a complete picture of the reaction can be obtained only by considering the flocculation of suspensions in general. The literature on the subject is very extensive, and no attempt has been made to refer to individual publications other than those which appear to lead directly to the present theory. More general reviews of the subject may be found in Freundlich, ${ }^{2}$ Burton, ${ }^{3}$ Bancroft, ${ }^{4}$ and Taylor ${ }^{5}$ and recent reviews dealing more specifically with bacterial agglutination in Zinsser $^{6}$ and Wells. ${ }^{7}$

A suspension undergoing the process of flocculation presents a definite series of changes. At first the individual particles cannot be seen except with the microscope. Their presence is shown, however, by a Tyndall cone when light is passed through the suspension. Larger particles then make their appearance and may usually be seen as discrete clumps. These clumps consist of a number of small particles adhering firmly together but still retaining their individual form. More or less rapid settling of these larger particles now occurs, and in the course of time the solid matter forms a precipitate on the bottom of the vessel, leaving a clear liquid above. The suspension is now flocculated or "agglutinated." The appearance under the microscope is similar, except that, in addition, it can be seen that the small particles are in rapid irregular movement - the Brownian movement-whereas the large clumps are nearly station. ary. It can also be usually seen that the particles do not actually coalesce but merely approach one another closely. (In the case of the "breaking" of an emulsion there is actual coalescence; this is a distinct phenomenon and will not be considered here.)

× Bordet, J.: Ann. de l'Inst. Pastcur, 13, 225. r899.

${ }^{2}$ Freundlich, H.: Kapillarchemie (2d ed.). Leipzig, 1922.

3 Burton, E. F.: The Physical Propcrties of Colloidal Solutions (2d ed.). London, New York, Bombay, Calcutta, and Madras, I $92 \mathrm{r}$.

4 Bancroft, W. D.: Applied Colloid Chcmistry. New York and London, I921.

5 Taylor, W. W.: Chcmistry of Colloids (3d ed.). New York, I915.

${ }^{6}$ Zinsser, H.: Infection and Resistance. New York: Macmillan Co., 1925.

7 Wells, II. G.: Chcmical Aspects of Immunity, "American Chemical Society Monographs." New York, 1925 . 
It may be seen from the foregoing brief description that the phenomenon can be divided into two distinct steps: first, the collection of the small particles into larger aggregates; and, second, the settling of these aggregates to the bottom of the vessel. In regard to the latter effect, the small and large particles differ from each other both in the rate of settling and in the final condition of equilibrium, although under ordinary conditions the difference in the rate is of the greater significance.

EFFECT OF THE SIZE OF PARTICLES ON THE RATE OF SETTLING

The formula for the steady rate of fall of a small body in a viscous medium was given by Stokes as

$$
V=\frac{\frac{2}{9} a^{2}(D-d) g}{z}
$$

where $a$ is the radius, $D$ the density of the particle, $d$ the density of the solution. $z$ the viscosity of the solution, and $g$ the acceleration due to gravity. This formula was tested by Perrin ${ }^{\mathrm{T}}$ for small particles by comparing the radius calculated from the rate of fall with that determined by direct measurement or calculated from the weight and size.

RADIUS IN $\mu$ DETERMINED BY

Direct Measurement

$0.37 \mathrm{I}$
Weighing

0.3667
From Stokes's Law

0.3675

The experiment shows that the particles obey Stokes's law with the greatest exactness. This result is of special importance, since the validity of Stokes's law is assumed in all calculations concerning the Brownian movement. It follows, therefore, that the speed of settling of different size particles, other conditions being the same, will increase with the square of the radius and the difference in rate between visible and microscopic particles will be enormous. In Perrin's experiments the rate was a few millimeters a day.

\section{EFFECT OF THE SIZE OF PARTICLES ON THE FINAL EQUILIBRIUM}

The English botanist, Brown, noted that pollen grains as seen under the microscope possessed rapid irregular movements. This peculiar constant motion has become known as the "Brownian movement." It was soon found that the motion was independent of the nature of the particles and could not be ascribed to any outside influence. It is less in viscous liquids and very rapid in gases. The motion is less in large particles. It follows from the doctrine of equipartition of energy that the mean kinetic energy $\left(\frac{1}{2} \mathrm{mv}^{2}\right)$ of the particles must remain constant. The velocity decreases, therefore, as the size increases. ${ }^{2}$ Svedberg has shown that it is not affected by the potential of the particle nor by the addition of electrolytes. ${ }^{3}$ It was suggested by Wiener

${ }^{x}$ Perrin, J.: Die Atome, p. 90. Dresden, I9I4.

${ }^{2}$ Lewis, W. C. McC.: A System of Physical Chemistry, Vol. I, chap. i. London, New York, Bombay, Calcutta, and Madras, i920.

3 For a thorough discussion of the Brownian movement, see Burton, E. F.: op. cit., p. 5o; Perrin, J.: op. cil., p. $8_{3}$. Dresden, 1914; Freundlich, H.: op. cit., p. 469. Leipzig, r922. 
that this motion was due to the bombardment of the particles by the molecules of the solvent. The motion, therefore, becomes strictly analogous to the kinetic motion of the molecules themselves. A quantitative theory for this motion was worked out independently by Einstein and von Smoluchowski and verified experimentally by Perrin. The part of the theory which is of interest in this connection is the prediction regarding the final distribution of the particles at equilibrium. If the Brownian movement is really analogous to the kinetic motion of gases, then the distribution of the particles at equilibrium should be determined by the same law that regulates the density of a gas at different levels. Equilibrium will be established when the effect of gravity exactly equals the osmotic pressure (in this case the Brownian movement) of the particles or molecules. In the case of gases this formula is

$$
h=\frac{R T}{g M} \ln \frac{p_{o}}{p}
$$

where $h$ is the height, $p_{0}$ the pressure at the bottom of the column, $p$ the pressure at height $h, g$ the acceleration due to gravity, and $M$ the molecular weight. ${ }^{.}$Since the osmotic pressure is proportional to the number of particles per unit of volume, the formula, as applied by Perrin to suspensions, becomes

$$
h=\frac{R T}{g N \frac{4}{3} \pi r^{3}(D-d)} \ln \frac{n_{0}}{n}
$$

in which $N$ is Avogadro's number, $D$ is the density of the particle, and $d$ the density of the liquid.

Perrin's experiments leave little doubt that the relation between the size of the particles, the rate of settling, and the final distribution is accurately expressed by formulas (I) and (2). If the necessary data regarding the size of the particles, the viscosity of the solution, etc., are known, it is, therefore, possible to calculate both the rate of fall of the particles and the final state of equilibrium. Briefly, it may be said that if the size alone is varied, the rate at which the particles fall will increase as the square of the radius and that at equilibrium the distance from the bottom, at which the concentration of particles will be halved, will be inversely proportional to the mass.

Perrin's equation and results were confined to the region near the surface of the suspension. Porter and Hedges ${ }^{2}$ have measured at deeper levels, and find the particles distributed in accordance with the equation

$$
\frac{d n}{d y}=K_{n}(\mathrm{I}-b n)^{2}
$$

in which $n$ is the number of particles, $y$ the depth, and $K$ and $b$ are constants.

The rate of settling and the final distribution therefore appear to be on a firm experimental and theoretical basis. Application to bacterial suspensions is difficult,

${ }^{x}$ Freundlich, H.: loc. cit.

${ }^{2}$ Porter, and Hedges: Phil. Mag., pp. 641-51. 1922; Tr. Far. Soc. 1923. 
however, owing to the irregular size of the clumps and the uncertainty as to the surfaces. In any case most bacteria are so large that at equilibrium the individual bacteria as well as the clumps would be collected on the bottom of the vessel. The theory may be of importance, however, in the case of the filterable viruses.

\section{FACTORS CONTROLLING THE FORMATION OF AGGREGATES}

If a stable suspension is observed under the microscope, it may be seen that, a!though the particles approach one another, they do not actually collide. If some substance is now addled which precipitates the suspension, the particles then collide and stick together. Since they sometimes adhere to one another and sometimes remain separate, there must evidently be a force which tends to keep them apart and another force which holds them together. If the repulsive force is greater than the cohesive force, or greater than the momentum of the particles due to their movement, the particles will remain separate, whereas if it is less they will adhere into larger aggregates.

It has long been known that particles or surfaces in contact with liquids are electrically charged, since under the influence of an external e.m.f. the particles move. If the liquid moves while the solid is kept stationary, the phenomenon is known as "electro-endosmosis," whereas if the particles move through the liquid, it is known as "cataphoresis." It was early suggested that it was this repulsion, due to the charge carried by the particles, which prevented their touching one another, and practically all theories of the stability of suspension depend in some way on this potential difference between the particles and the surrounding liquid. In order to trace the connection between this property and the behavior of the particles, it is necessary to touch somewhat on the nature and origin of this potential difference.

\section{METHOD OF MEASUREMENT AND PROBABLE NATURE AND ORIGIN OF THE, CIIARGES OF COLLOIDAL SUSPENSIONS}

All methods of measurement of the charges carried by colloidal suspensions depend on determining the motion of the particles in an external electric field. This may be done either by noting the movement of the boundary of the suspension as a whole in a $U$-tube ${ }^{2}$ or by following the motion of a single particle under the microscope or ultramicroscope. (A convenient type of apparatus for these measurements has been described by the writer. $)^{3}$

The potential between the surface of the particle and the surrounding film of liquid may then be calculated by the Lamb-Helmholtz formula,

$$
\text { P.D. }=\frac{4 \eta v \pi}{K X}
$$

in which $\eta$ is the viscosity, $K$ the dielectric constant of the surface layer, $v$ the velocity in centimeters per second, and $X$ the potential gradient. All electrical units are elec-

${ }^{1}$ Cf. Freundlich, H.: op. cit., p. 326. Leipzig, I922; Burton, E. F.: op. cit., p. I25. I92I.

${ }^{2}$ Burton, E. F.: op. cil., p. I31. 1921 .

3 Northrop, J. H.: J. Gencral Physiol., 4, 629. I921-22; Northrop, J. H., and Cullen, G. E.: ibid., p. 635. I921-22; Northrop, J. H., and Kunitz, M.: ibid., 7, 729. I925. 
trostatic. Substituting the viscosity of water and the dielectric constant at $20^{\circ} \mathrm{C}$. and changing to millivolts, the formula becomes

$$
\text { P.D. in millivolts }=\mathrm{I}_{3} \frac{\mu \text { per second }}{\text { Volts per centimeter }}
$$

This formula was derived on the assumption that each particle acts like a small condenser and is surrounded by a Helmholtz double layer. The charge on the particle as a whole, including the film of liquid, is, therefore, zero. A potential difference exists, however, between the two oppositely charged layers, and it is between these layers that the motion occurs. It follows that the value for the dielectric constant of the liquid between the two layers should be inserted in the formula. There is no way of determining this value, however, so that the dielectric constant of the pure liquid is generally used. It has been found by numerous investigators that this formula is experimentally correct so far as it concerns the relation between the rate of migration and the impressed e.m.f., or the viscosity. ${ }^{x}$ There is, however, no evidence concerning the correctness of the actual value of the potential calculated from the velocity. It was shown by Hardy that the size of the particles was without effect on the observed motion, which also agrees with the theory.

The theory of Helmholtz and Lamb gives us no information as regards the origin of this potential difference, and no satisfactory theory has been suggested up to the present. It has been shown by Haber and Klemensiewicz ${ }^{2}$ that the cataphoretic potential is not the same as the Nernst electrode potential. This has been ascribed by von Smoluchowski to the fact that the P.D. between the interior of the particle and the liquid is the Nernst potential, whereas the cataphoretic P.D. is between the movable and fixed films.

Freundlich ${ }^{3}$ has shown that the discrepancy may be accounted for by assuming that the double layer is of appreciable thickness and that the electrode potential is the total potential across the entire double layer while the electrokinetic potential is that across a part of the layer. Burton ${ }^{4}$ has suggested that the thickness of this double layer may be affected by the electrolytes present in accordance with the Gouy-Debye theory. This point of view accounts for the effect of the valence and concentration of the electrolyte on the potential and hence on the stability of the suspension.

McTaggart's experiments with air bubbles and Lenard's measurements of waterfall electricity indicate that the source of the potential may be entirely in the film of liquid surrounding the particle. ${ }^{5}$

Wilson, ${ }^{6}$ on the other hand, suggested that the P.D. was due to a Donnan equilibrium, and Loeb has found that there is some analogy between the two but that quantitatively they are different. This point of view has been followed by Winslow, Falk, and Caulfeild, who were able to show that the migration of bacteria was similar

\footnotetext{
I Burton, E. F.: op. cit., p. I37. $192 \mathbf{1}$.

${ }^{2}$ Freundlich, H.: op. cit., p. 341. Leipzig, I922.

3 Freundlich, H.: Colloid. Symp., 3, 7. 1925. 4 Burton, E. F.: ibid., 4, 132. I926

5 Ci. Loeb), J.: J. Gencral Physiol., 5, 515. 1922-23.

"Wilson, J. A.: J. Am. Chem. Soc., 8, rgS2. I9i6. 7 Loch. J.: loc. cit.
} 
to that of protain particles and that the observed migration agreed qualitatively with that expected on the basis of a Donnan equilibrium. ${ }^{\mathrm{T}}$

This theory accounts satisfactorily for the observation that the sign of charge changes at the isoelectric point of the particle when the latter is amphoteric, and accounts also for the relation between the ionization and the sign of charge. It is known, for instance, that substances which tend to dissociate as acids are usually negative, and basic substances positive.

Von Hevesy ${ }^{2}$ considers the particles as analogous to large ions. It is true that the rate of migration is about the same. There would seem to be a definite difference, however, between the mechanisn by which the solution as a whole is kept electrically neutral. In the case of an ion there is always an equal number of ions of the opposite charge in the solution, whereas, according to the Lamb-Helmholtz theory, each particle as a whole is electrically neutral. In any case, it is certain that the P.D. of the particles is closely connected with the presence of electrolytes in the solution. It has been found by numerous workers that the more carefully the solution was freed from electrolytes the lower the potential and the more unstable the suspension. ${ }^{3}$

On the other hand, Laing has been able to account for the electrical migration properties of soap solutions on the assumption that the micelles are, fundamentally, large ions differing from ordinary ions merely in that they possess a high valence. This point of view has been accepted by McBain,${ }^{4}$ and there is undoubtedly considerable evidence in its favor. It appears to the writer more probable than the LambHelmholtz theory. In view of this fact, it is evident that the potential calculated in the ordinary way from the Lamb-Helmholtz formula is of very uncertain meaning and may have no real physical significance. It is, however, probably proportional to the charge carried by the particle, and since it serves as a convenient unit it will be retained in the following discussion. If the particles are analogous to large ions then an increase in the rate of migration does not mean an increase in the potential between the surface of the particle and the surrounding liquid but merely an increase in the number of charges per unit of surface. It also follows that according to this theory the charge on the particle should increase by steps corresponding to the loss or gain of one electron while on the basis of the Donnan or Lamb-Helmholtz theory the charge might be expected to increase gradually since it is essentially a statistical result. Unfortunately, the expected change in migration rate owing to the gain of one electron in the case of particles of visible dimensions in water is below the limit of accuracy of the present measurements.

The general opinion at present appears to be that the charge is conferred by the combination of the particle with an ion, although the nature of this combination is uncertain.

I Winslow, C.-E. A., Falk, I. S., and Caulfeild, M. F.: J. General Physiol., 6, 197. 1924; Winslow, C.-E. A., and Shaughnessy, II. J.: ibid., p. 697. 1924; Winslow, C.-E. A., anıl Fleeson, E. H.: ibid.. 8, 195. 1925 .

${ }^{2}$ von Ilevesy, G.: Kolloid-Ztschr., 21, 129. 1917.

${ }^{3}$ Beans, H. T., and Eastlack, H. E.: J. $1 \mathrm{~m}$. Chcm. Soc., 37, 2667 . I9r 5.

4 McBain, J. W.: Colloid. Symp., 4, I. 1925 


\section{STABILITY AND CHARGE}

Whatever the source of the potential on the particles, it follows that similarly charged particles would tend to repel one another and thereby render the suspension stable. If, however, the particle as a whole is electrically neutral, this repulsion would not obey Coulomb's inverse square law, but would become effective only when the particles approached one another so closely that the outside of the double layers overlapped. This conclusion is borne out by Perrin's observation ${ }^{\underline{1}}$ that the distribution

TABLE I*

\begin{tabular}{|c|c|c|c|c|c|c|c|c|}
\hline \multirow{3}{*}{$\begin{array}{l}\text { CONCENTRATION, } \\
\text { MILLIMOL/LITER }\end{array}$} & \multicolumn{8}{|c|}{ SALT } \\
\hline & \multicolumn{2}{|c|}{$\mathrm{KCl}$} & \multicolumn{2}{|c|}{$\mathrm{BaCl}_{2}$} & \multicolumn{2}{|c|}{$\mathrm{AlCl}_{3}$} & \multicolumn{2}{|c|}{$\mathrm{ThCl}_{4}$} \\
\hline & $\begin{array}{l}\text { Oil- } \mathrm{H}_{2} \mathrm{O} \\
\text { Potential, } \\
\text { Mv. }\end{array}$ & $\begin{array}{l}\text { Relative } \\
\text { Stability }\end{array}$ & P. D., Mv. & Stability & P.D., Mv. & Stability & P.D., Mv. & Stability \\
\hline & -46 & I. 00 & -46 & 1.00 & -46.0 & 1.00 & -46.0 & I. 0 \\
\hline o. $005 \ldots \ldots$ & $\ldots \ldots$ & $\ldots \ldots$ & $\ldots \ldots$ & $\ldots \ldots$ & $\ldots \ldots \ldots$ & I. $\infty$ & 39.0 & I. 0 \\
\hline . oro....... & $\ldots \ldots \ldots$ & $\ldots \ldots \ldots$ & $\ldots \ldots \ldots$ & $\ldots \ldots$ & 38.0 & 0.80 & 6.5 & 0.3 \\
\hline $.020 \ldots \ldots$ & $\ldots \ldots$ & $\ldots \ldots \ldots$ & $\ldots \ldots \ldots$ & $\ldots \ldots$ & $\ldots \ldots \ldots$ & .35 & -8.5 & 0.2 \\
\hline $.050 \ldots \ldots$ & $\ldots \ldots$ & $\ldots \ldots$ & $\ldots \ldots \ldots$ & $\ldots \ldots$ & 17.0 & .35 & +29.0 & I. 0 \\
\hline $.100 \ldots \ldots$ & $\ldots \ldots$ & $\ldots \ldots \ldots$ & $\ldots \ldots \ldots$ & $\ldots \ldots$ & $\ldots \ldots \ldots$ & $\ldots \ldots$ & $\ldots \ldots$ & I. 0 \\
\hline $.200 \ldots \ldots$ & 50 & 1.00 & 43 & I. .0 & -8.0 & .30 & $\ldots \ldots$ & I. 0 \\
\hline $0.500 \ldots \ldots$ & $\ldots \ldots$ & $\ldots \ldots \ldots$ & $\ldots \ldots$ & $\ldots \ldots$ & $\ldots \ldots \ldots$ & $\ldots \ldots$ & 52.0 & I. 0 \\
\hline $1.000 \ldots \ldots$ & 59 & I. $\infty 0$ & 30 & 0.75 & +2.5 & .30 & $\ldots \ldots$ & $\ldots \ldots$ \\
\hline $2.500 \ldots \ldots$ & $6 I$ & I. 00 & 25 & .45 & $\ldots \ldots \ldots$ & $\ldots \ldots \ldots$ & $\ldots \ldots \ldots$ & $\ldots \ldots \ldots$ \\
\hline $5.000 \ldots \ldots$ & $5 \mathrm{I}$ & 0.90 & $\ldots \ldots \ldots$ & $\ldots \ldots$ & $\ldots \ldots \ldots$ & $\ldots \ldots \ldots$ & 23.0 & 0.4 \\
\hline $10.000 \ldots \ldots$ & $\ldots \ldots$ & $\ldots \ldots$ & $\ldots \ldots$ & $\ldots \ldots$ & 3.5 & .40 & $\ldots \ldots \ldots$ & $\ldots \ldots \ldots$ \\
\hline $20.000 \ldots \ldots$ & 37 & .60 & $\ldots \ldots$ & 的 & $\ldots \ldots \ldots$ & $\ldots \ldots \ldots$ & 17.0 & .4 \\
\hline $25.000 \ldots \ldots$ & $\ldots \ldots$ & $\ldots \ldots$ & -8 & .40 & $\ldots \ldots \ldots$ & $\ldots \ldots \ldots$ & $\ldots \ldots \ldots$ & $\ldots \ldots \ldots$ \\
\hline $100.000 \ldots \ldots$ & 22 & .60 & $\ldots \ldots$ & .35 & 5.0 & .60 & $\ldots \ldots \ldots$ & $\ldots \ldots \ldots$ \\
\hline $200.000 \ldots \ldots$ & I 2 & .50 & $+I$ & .40 & $\ldots \ldots \ldots$ & $\ldots \ldots \ldots$ & +7.0 & 0.6 \\
\hline $500.000 \ldots \ldots$ & -8 & .25 & $\ldots \ldots \ldots$ & $\ldots \ldots$ & 5.0 & 0.60 & $\ldots \ldots \ldots$ & $\ldots \ldots \ldots$ \\
\hline $700.000 \ldots \ldots$ & $\ldots \ldots$ & $\ldots \ldots$ & +4 & 0. 10 & $\ldots \ldots \ldots$ & $\ldots \ldots \ldots$ & $\ldots \ldots$ & $\ldots \ldots$ \\
\hline $\mathrm{I}, 000.000 \ldots \ldots$ & $\ldots \ldots$ & .30 & $\ldots \ldots \ldots$ & $\ldots \ldots$ & $\ldots \ldots$ & $\ldots \ldots$ & $\ldots \ldots$ & $\ldots \ldots \ldots$ \\
\hline $1,000.000 \ldots \ldots$ & $\ldots \ldots \ldots$ & o. I 5 & 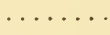 & $\ldots \ldots$ & $\ldots \ldots$ & $\ldots \ldots \ldots$ & $\ldots \ldots \ldots$ & $\ldots \ldots$ \\
\hline
\end{tabular}

* Powis, F.: Zlschr. f. phys. Chemie, 89, 19r. 19r.4-15.

of charged particles is abnormal when the distance beween them is less than about I.7 times the radius. When the charge is removed, this anomalous distribution disappears. This experiment furnishes strong evidence that the particles are held apart by their electric charge.

It was first noted by Hardy ${ }^{2}$ that suspensions of denatured proteins were most unstable at the isoelectric point, and he suggested that this was due to the fact that they were electrically neutral at this point. This conclusion was verified qualitatively by a large number of workers. ${ }^{3}$ A number of cases were found, however, in which there seemed to be no direct connection between the stability and the potential.

Ellis ${ }^{+}$made a number of measurements on oil emulsions and found that the stabil-

\footnotetext{
"Perrin, J.: Compt. rend. Soc. de biol., I58, i I6S. I9I4.

${ }^{2}$ Hardy, W. B.: Proc. Roy. Soc., 66, I Io. I900.

3 Burton, E. I.: op. cit., P. I49. I92 I.

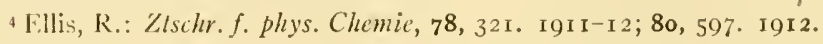


ity was closely connected with the potential. The experiments were then carefully carried out by Powis, ${ }^{1}$ who made accurate measurements of the P.D. between the oil drops and surrounding liquid in a series of electrolyte solutions and found that whenever the potential between the drops and the surrounding liquid was reduced below about $30 \mathrm{mv}$., the particles collected into larger aggregates. A summary of Powis' results is given in Table I. They leave little doubt that in this case the potential is the decisive factor. It will be noted, however, that it becomes necessary to assume a critical P.D. instead of Hardy's zero potential. Powis found later that in the case of arsenic sulphide suspensions this critical P.D. was different for different salts. (This effect will be discussed below in connection with work on bacterial agglutinations.) A number of experiments were carried out by the writer on suspensions of typhoid bacteria.

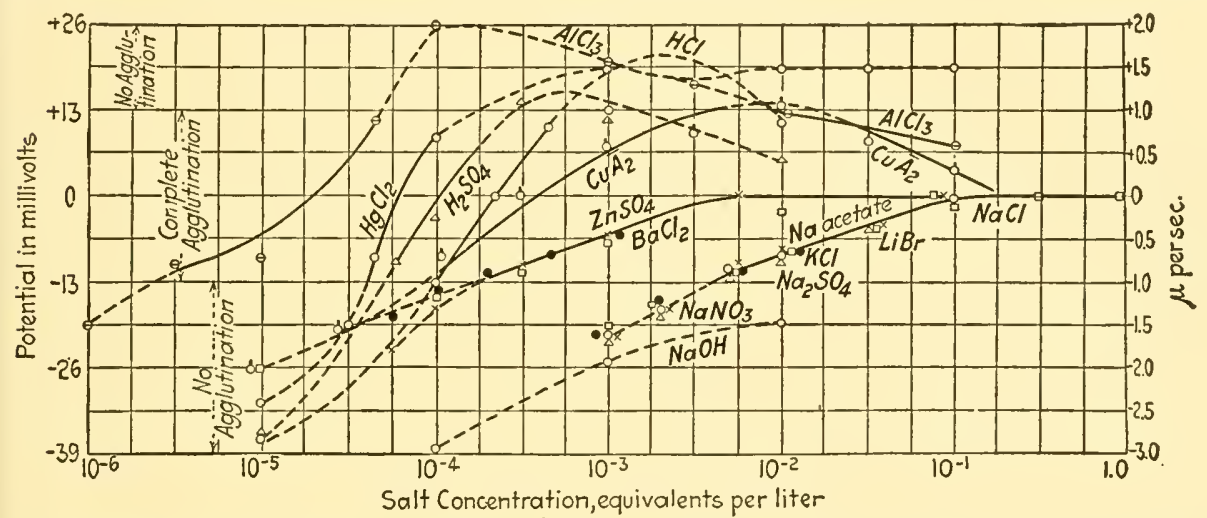

FIG. I.-Effect of various electrolytes on the agglutination of typhoid bacteria sensitized with immune serum; —_ complete agglutination; - - - partial or no agglutination.

It was found that when these had been treated with an excess of antiserum they behaved in the same way as Powis' oil drops, i.e., agglutination always occurred whenever the potential was reduced below a critical value of about I 5 mv. A summary of these experiments is shown in Figure I.

A similar series of experiments was carried out by Loeb ${ }^{2}$ on suspensions of collodion particles. The results are summarized in Table II. Here again there is no doubt that agglutination occurs whenever the P.D. is reduced below about I $5 \mathrm{mv}$. The same result was obtained when the particles were previously treated with egg albumin, and with particles of denatured egg albumin. ${ }^{3}$ These experiments leave little doubt that in these cases the potential is the determining factor for the stability of the suspension. It is possible to predict from a measurement of the cataphoretic P.D. alone whether or not the suspension will remain stable. It also follows that the cataphoretic potential is the decisive one for the prevention of agglutination and, further, that this potential must be directly proportional to the rate of migration in the electric field as predicted by the Lamb-Helmholtz formula.

I Powis, F.: ibid., 89, 9г, I79, х86. I914-15.

${ }^{2}$ Loeb, J.: op. cit., p. I23. I922-23.

3 Loeb, J.: ibid., p. 485. 1922-23. 
PRECIPITATION BY NON-ELECTROLYTES AND THE MUTUAL PRECIPITATION OF OPPOSITELY CHARGED COLLOIDS

It was noted by Linder and Picton ${ }^{1}$ that two oppositely charged suspensions would precipitate each other if they were mixed in proper proportion. If either com-

TABLE II

Cataphoretic Charge and Stability of Suspensions of Particles of COLLODION

\begin{tabular}{|c|c|c|c|c|}
\hline I & 2 & 3 & 4 & $=$ \\
\hline Suspended in Solution of: & $\begin{array}{l}\text { Minimum Concentration } \\
\text { Required for Precipitation }\end{array}$ & $\begin{array}{l}\text { P.D. in } \\
\text { Millivolts }\end{array}$ & $\begin{array}{l}\text { Maximal Concentration } \\
\text { at Which Suspension } \\
\text { Remains Stable }\end{array}$ & $\begin{array}{l}\text { P.D. in } \\
\text { Millivolts }\end{array}$ \\
\hline & \multicolumn{4}{|c|}{ pH 5.8} \\
\hline 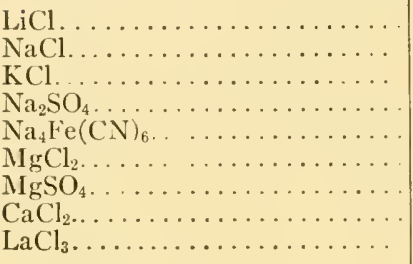 & $\begin{array}{l}M / 2 \\
M / 2 \\
M / 4 \\
M / 4 \\
M / I 6 \\
M / 16 \\
M / 16 \\
M / 32 \\
M / 2,048\end{array}$ & $\begin{array}{l}\text { (10) } \\
\text { I0 } \\
\text { I4 } \\
\text { I3 } \\
\text { I3 } \\
\text { I } \\
\text { I5 } \\
\text { 14 } \\
\text { I4 }\end{array}$ & $\begin{array}{l}\mathrm{M} / 4 \\
\mathrm{M} / 4 \\
\mathrm{M} / 8 \\
\mathrm{M} / 8 \\
\mathrm{M} / 32 \\
\mathrm{M} / 32 \\
\mathrm{M} / 32 \\
\mathrm{M} / 64 \\
\mathrm{M} / 4,096\end{array}$ & $\begin{array}{l}\text { I7 } \\
\text { I } 4 \\
21 \\
\text { I9 } \\
2 \text { I } \\
\text { I } 5 \\
19 \\
\text { I } 7 \\
21\end{array}$ \\
\hline \multirow{3}{*}{ 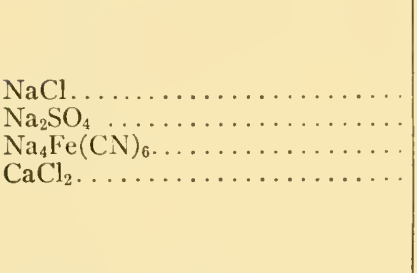 } & \multicolumn{4}{|c|}{ pH II.० } \\
\hline & $\begin{array}{l}\mathrm{M} / 2 \\
\mathrm{M} / 4 \\
\mathrm{M} / \mathrm{I} 6 \\
\mathrm{M} / 32\end{array}$ & $\begin{array}{c}\cdots \cdots \cdots \\
16 \\
\text { I5 }\end{array}$ & $\begin{array}{l}M / 4 \\
M / 8 \\
M / 32 \\
M / 64\end{array}$ & $\begin{array}{l}\text { I } 8 \\
20 \\
24 \\
\text { I9 }\end{array}$ \\
\hline & \multicolumn{4}{|c|}{$\mathrm{pH}_{3.0}$} \\
\hline 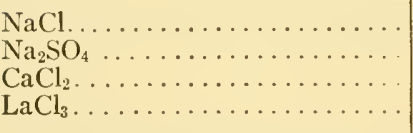 & $\begin{array}{l}\mathrm{M} / 2 \\
\mathrm{M} / 4 \\
\mathrm{MI} / 32 \\
\mathrm{M} / 2,048\end{array}$ & $\begin{array}{l}7 \\
\text { I } 2 \\
\text { I6 } \\
\text { I } 4\end{array}$ & $\begin{array}{l}M / 4 \\
M / 8 \\
M / 64 \\
M / 4,096\end{array}$ & $\begin{array}{l}\text { I4 } \\
\text { (Lost) } \\
\text { I9 } \\
\text { I8 }\end{array}$ \\
\hline $\mathrm{H}_{2} \mathrm{SO}_{4} \ldots \ldots \ldots \ldots \ldots \ldots \ldots$ & $\mathrm{M} / 4$ & $\ldots \ldots \ldots$ & $\mathrm{M} / \mathrm{S}$ & I4 \\
\hline
\end{tabular}

ponent were present in excess, the suspension again became stable. These observations have since been extended and confirmed for a large number of substances.

Webster ${ }^{2}$ has shown that this is also true in the case of mixtures of suspensions of bacteria under such conditions that one suspension was negative and the other positive. It is possible that this phenomenon is connected with the so-called "autoagglutinable" bacteria and may at times interfere with agglutination reactions. Thomas ${ }^{3}$

${ }^{2}$ Picton, H., and Linder, S. E.: J. Chem. Soc., 61, 148. 1892; 67, 63, 1895; 71, 568. 1897; 87 , I906. I905.

${ }^{2}$ Webster, L.: J. General Physiol., 7, 513. 1925.

${ }_{3}$ Thomas, A. W., in Bogue, R. H.: Colloidal Behavior, I, 324. McGraw-Hill Book Co., I924. 
has emphasized the undoubted fact that in certain cases this mutual precipitation is due to removal of the stabilizing ions rather than to any direct reaction between the particles themselves.

In general, suspensions of the same charges do not precipitate one another. It has usually been assumed that the mutual precipitation of oppositely charged suspensions is also an electrical phenomenon, and that agglutination occurs owing to the neutralization of the charges. As Bancroft ${ }^{\mathrm{t}}$ has pointed out, however, the effect is not purely a neutralization one, since the relative order of flocculation of a series of positive suspensions by a series of negative suspensions is not always the same. It is evidently necessary to consider the combination as separate from the neutralization. It is possible, also, that the difference may be partially due to a difference in the critical poten-

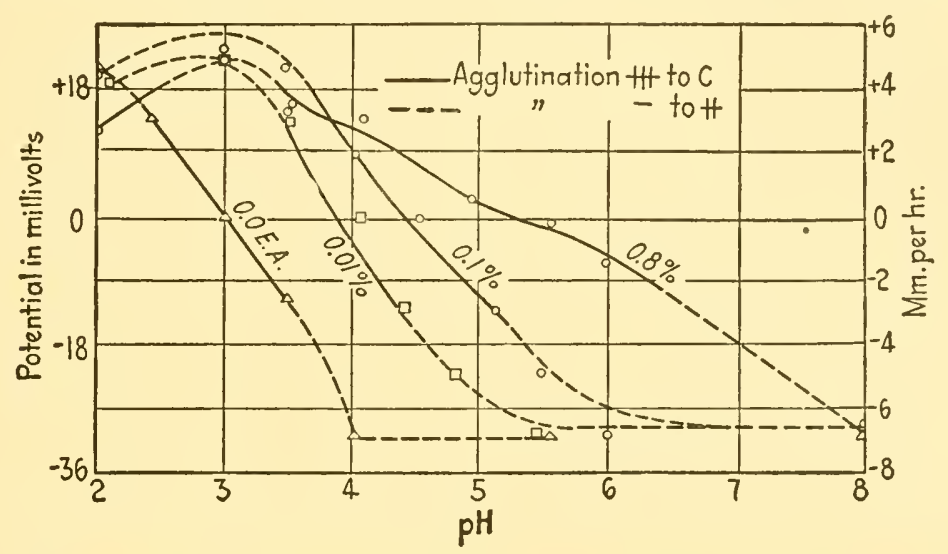

FIG. 2.-Agglutination of bacillus of rabbit septicemia by egg albumin at different $\mathrm{pH}$

tials. Figure 2 represents the results of a series of experiments in which a suspension of bacteria was agglutinated by the addition of egg albumin. The figure shows that the agglutination is again determined solely by the potential. It also shows that the potential of the particles is affected even where the protein has the same sign of charge as the particles, since at $\mathrm{pH}_{3}$ the addition of the (positive) egg albumin renders the organisms still more positive. The fact that the effect of the potential is the result of the combination rather than the cause is clearly shown in Figure 3, in which the amount of "agglutinin" combined with the bacteria at various $\mathrm{pH}$ is compared with the amount required to agglutinate. According to Michaelis, the antibody is positive on the acid side of $\mathrm{pH} 5.0$, so that a maximum effect would be expected between this point and $\mathrm{pH}_{3}$. This is the case with the agglutination, but no difference is noticeable in the amount of antibody combined.

Hitchcock ${ }^{2}$ was able to show by direct measurement that egg albumin was taken up by collodion membranes irrespective of whether the membrane and albumin were of the same or opposite charge.

It will be noted in Figure 2 that the albumin stabilizes the suspension in some $\mathrm{pH}$

r Bancroft, W. D.: op. cit., p. 226. New York and London, I92 I.

${ }^{2}$ Hitchcock, D. I.: J. General Physiol., 8, 6r. I925. 
ranges instead of agglutinating and that this effect is also due to the potential. A protective colloid and precipitating colloid can, therefore, not be separated. ${ }^{\mathrm{I}}$ The addition of proteins, etc., sometimes has a marked effect on the properties of a suspension by affecting the critical potential. This effect will be discussed more fully below.

As far as the writer is aware, there are no complete potential measurements in the case of the mutual precipitation of two oppositely charged suspensions, so that it cannot be determined whether or not this is also purely a question of lowering the potential below the critical value. Linder and Picton, however, showed that in the presence of an excess of one of the components the entire suspension was stable and had the charge (qualitatively) of the excess suspension, and that the flocculated suspension had a lower P.D. than either of the suspensions alone.

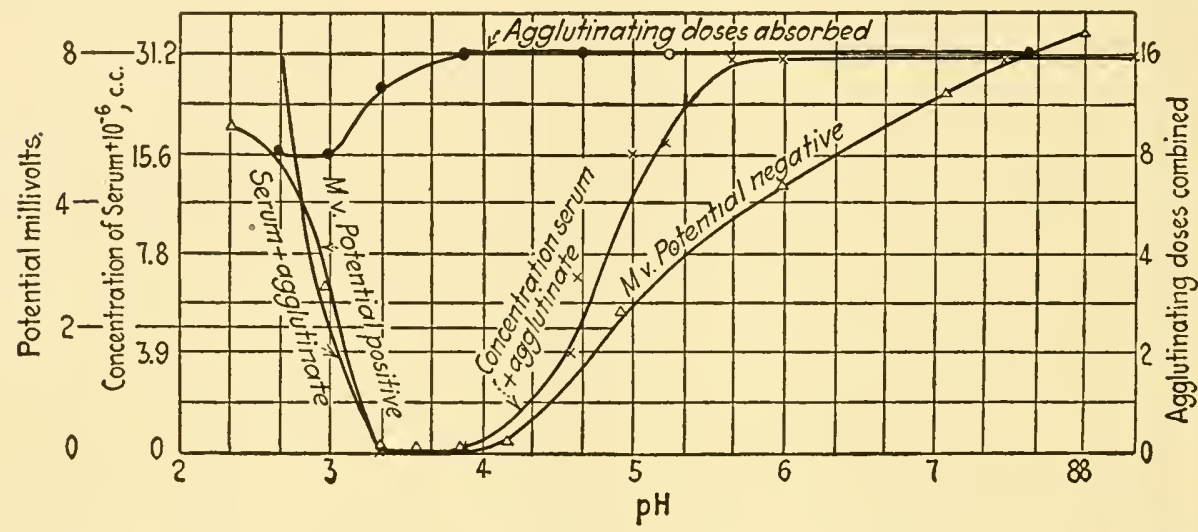

FIG. 3. - The effect of the $\mathrm{pH}$ on the combination of antibody and bacteria and on the potential and quantity of serum required to cause flocculation.

THE COHESIVE FORCE

In the experiments discussed so far it has been possible to predict whether or not the suspension would precipitate simply by measuring the potential after the substance under investigation had been added. There are a number of cases, however, where this is not the case, and it is necessary to measure another variable. This has always been a marked property of bacterial suspensions. It was noted by Neisser and Friedemann $^{2}$ that suspensions of typhoid bacilli were not agglutinated by mono- and divalent salts as were suspensions of the organism treated by immune serum. This observation was confirmed by Buxton. ${ }^{3}$ The experiments were repeated by the writer, ${ }^{4}$ and the potential measurements were made. The results were the same as those found by Neisser and Friedemann. The experiments are shown graphically in Figures 4 and 5 . Figure 5 shows that in all cases where the potential was lowered to less than about I $3 \mathrm{mv}$. by a concentration of salt of less than $0.0 \mathrm{I} \mathrm{M}$, agglutination occurred, as would

${ }^{x}$ Freundlich, H., and Löning, E.: Fest. Kaiser Wilhelm Ges. Förderung Wiss. (Io. Jahr. Jub.), p. 82. 1921 .

${ }^{2}$ Neisser, M., and Friedemann, U.: Mïnchen. med. Wchnschr., No. I9. I904.

3 Buxton, B. H.: Zischr. phys. Chemic, 57, 47. 1907.

${ }_{4}^{4}$ Northrop, J. H., and de Kruif, P. H.: J. General Physiol., 4, 639. I92I-22. 
be the case with the suspensions already discussed. If, however, the concentration of salt required to lower the potential to this value was $0.0 \mathrm{I} \mathrm{M}$ or more, this was no longer true and no complete agglutination occurred, even though the potential was reduced

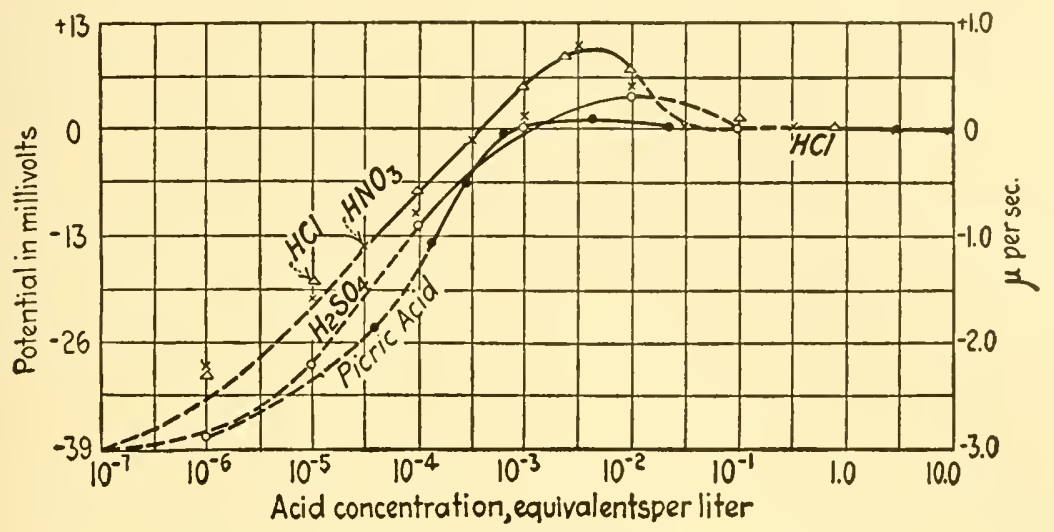

FIG. 4.-Effect of acid concentration on the potential and agglutination of suspension of $B$. typhosus.

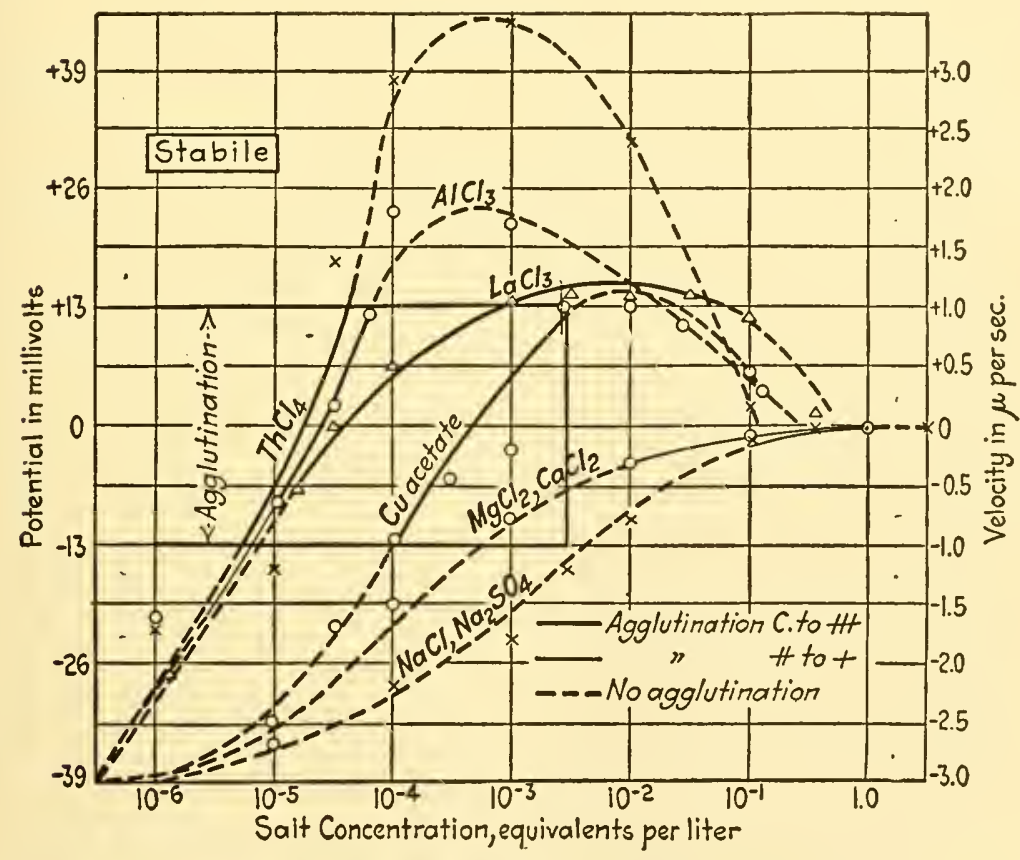

FIG. 5.-Effect of salt concentration on the potential and agglutination of suspension of $B$. typhosus.

to zero. It is evident that either (I) the potential measurements are wrong, or (2) some other factor has to be considered besides the potential, since with this suspension it is no longer possible to predict the flocculation from the potential alone. The fact 
that in the earlier experiments the potential measurements agreed so well with the flocculation results, even in high salt concentrations, renders it very improbable that

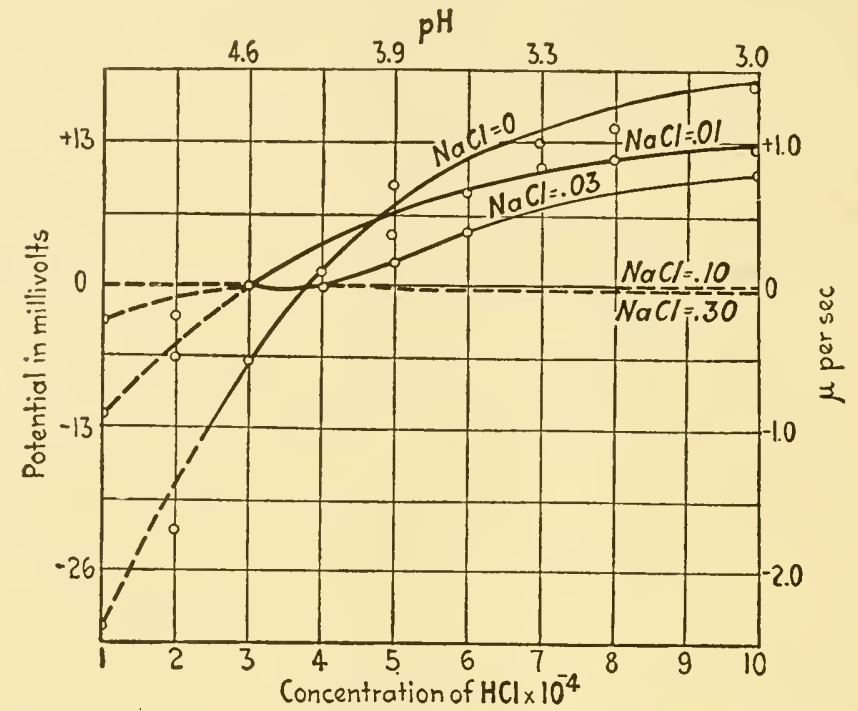

FIg. 6.-Effect of increasing $\mathrm{NaCl}$ concentration on the acid agglutination of B.typhosus

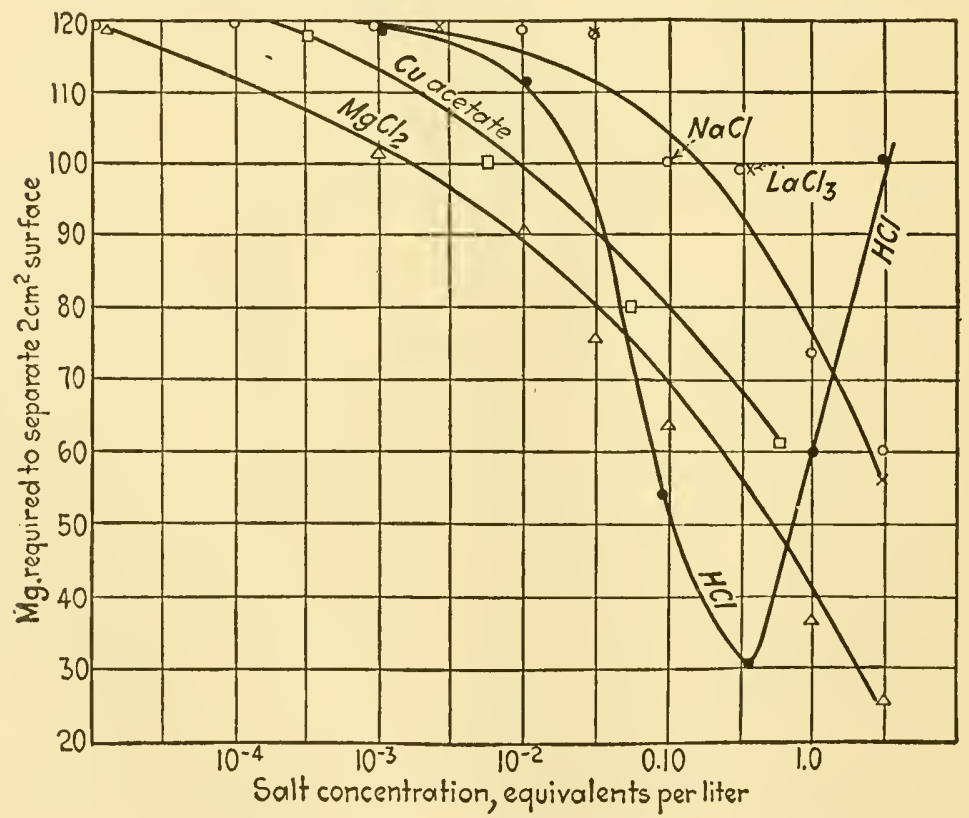

Fig. 7.-Effect of salt concentration on the "cohesive force"

these measurements are at fault. It may be shown directly that this is not the case by suspending the organisms in various concentrations of acid with various total salt 
concentrations. It is found in this experiment that the suspension at one end of the series has a low positive potential and at the other end a low negative potential. There must, then, be a place in between where there is no potential, even though there is a very large error in the method. Nevertheless, no agglutination occurs in any tube in the presence of concentrated sodium chloride. This is shown in Figure 6. The salt evidently acts as though it prevented the particles from sticking together, even though there is no force to hold them apart. It occurred to the writer that it might be possible to measure this sticking or cohesive force by determining the force required to separate two films of the suspension. This turned out to be the case. The measurement was made by coating two pieces of glass with a thick smear of the suspension. The glass was warmed slightly in order to cause the particles to adhere to it, and the two films were allowed to rest together in the solution to be studied. The force required to tear the films apart was then determined by a torsion balance. The measurement is rough and the conditions very different from those existing in the original suspension, but nevertheless the results are surprisingly reproducible and show a very marked effect of concentrated salt solutions on this cohesive force. Furthermore, the effect is in the range of salt concentrations where the potential measurements fail to predict the agglutination. The results of some of these experiments are shown in Figure 7. It will be noted that the only solution which shows a second rise in this value is hydrochloric acid, and this agrees with a second agglutination zone in this acid (cf. Fig. 5). It is evident then that so-called "irregular series" are not always due solely to the potential changes. ${ }^{1}$

It was stated above that the addition of immune serum to a suspension of bacteria caused them to act like collodion or oil particles, i.e., they agglutinate whenever the P.D. is below the critical value. It might be expected, then, that the addition of serum to bacteria whose potential was already below the critical value would cause agglutination without any change in the potential, but an increase in the cohesive force. This is the result obtained, as is shown in Figure 8. The figure also shows that the cohesive force of a smear treated with immune serum is not affected by the salt concentration.

Under the ordinary conditions of bacteriological agglutination in . 075 м salt, therefore, immune serum causes agglutination by increasing the cohesive force between the organisms rather than by decreasing the potential. This effect on the cohesive force is not shown by normal serum nor by ordinary protein solutions. It is possible, however, to agglutinate bacteria either with normal serum or protein solutions provided the solution is at or near the isoelectric point of the protein used..$^{2}$ Under these conditions agglutination may be caused by a very minute amount of the protein or normal serum. The agglutination is caused, however, by a change in the potential of the suspension instead of a change in the cohesive force as is the case in immuneserum agglutination.

The increase in the critical potential of a suspension by the addition of another substance is not confined to immune serum but has been found to occur with various

${ }^{I}$ In saturated salt solutions there is again an agglutination effect (cf. Porges, O.: Centralbl. $f$. Bakteriol., Abt. I, Orig., 40, I33. I905). This is probably a true solubility effect similar to that found by Loeb in the case of gelatin-coated particles.

${ }^{2}$ Eggerth, A. II., and Bellows, M.: J. General Physiol., 4, 669. 1922. 
substances. The effect of egg albumin on suspensions of mastic described by Michaelis and Rona ${ }^{\mathrm{I}}$ is perhaps similar.

Shibley ${ }^{2}$ found that in the presence of phosphate ions the critical potential of sensitized $B$. paratyphosus bacteria was raised to $35 \mathrm{mv}$. instead of the usual $\mathrm{r} 4$. Phos-
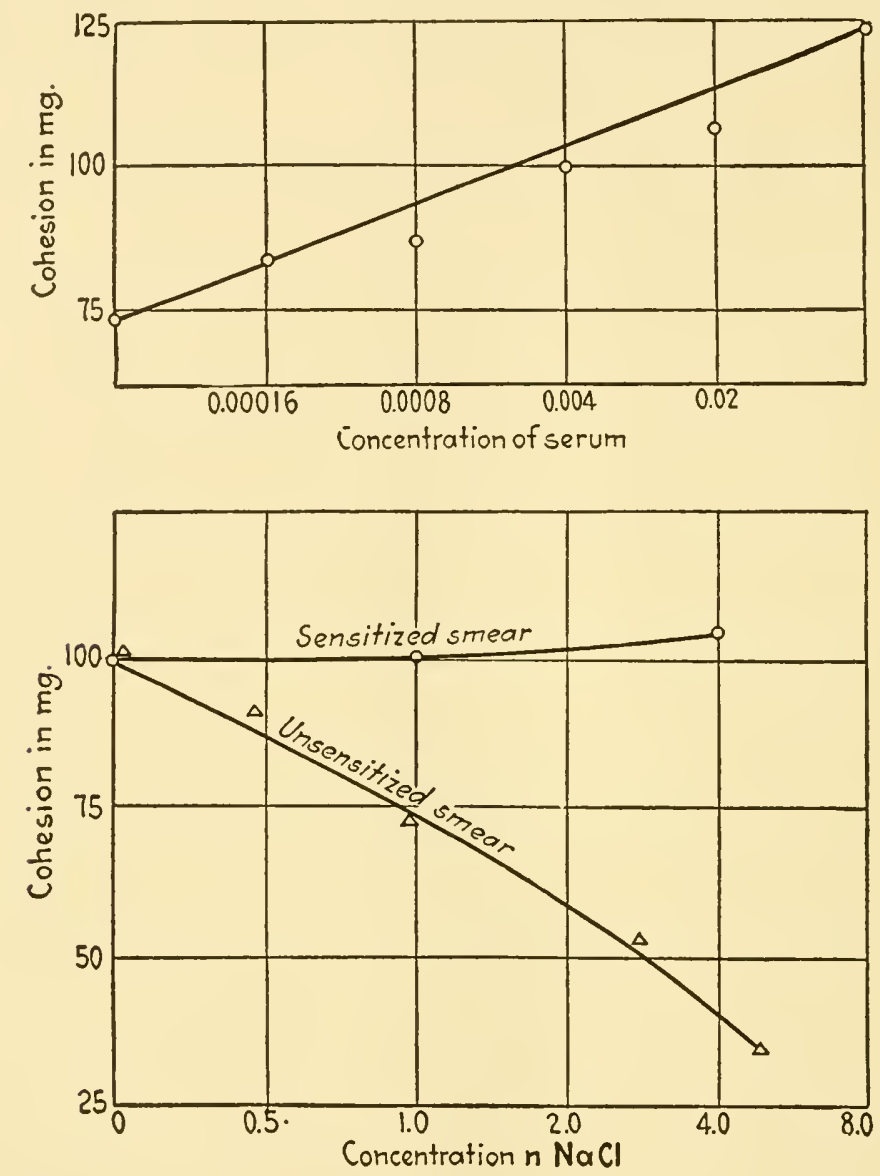

FIG. 8.-Upper curve: Effect of immune serum on cohesive force in r.o is NaCl. Low'er curre: Effect of salt concentration on cohesive force of sensitized and unsensitized smears.

phate ions, then, according to the present hypothesis must affect the cohesive force as well as the potential.

A similar effect had been noted by the writer in the case of $\mathrm{HCl}$ (Fig. 7). Northrop and Freund ${ }^{3}$ found that red cells which had been treated either with specific agglutinin, ricin, colloidal stannic hydroxide, or paraffin oil agglutinated at a critical potential of about I $2 \mathrm{mv}$. while the untreated cells remained in suspension until the charge had

${ }^{x}$ Michaelis, L., and Rona, P.: Biochem. Ztschr., 2, 219.1906.

${ }^{2}$ Shibley, G.: J. Exper. Mcd., 40, 453. 1924.

3 Northrop, J. H., and Freund, J.: J. General Physiol., 6, 603. I924. 
been reduced to $6 \mathrm{mv}$. The action of antiserum is therefore the same as that of other substances except that in the case of the antibody the reaction takes place only with the bacteria used to produce it. The action of the other substances is not so specific.

Shibley found in a study of diffuse and granular strains of streptococci that here also the difference was due to the cohesive force rather than to a difference in potential. ${ }^{x}$ In the autoagglutinable strains agglutination occurred whenever the potential was reduced below a critical value, and measurements of the cohesive force by the method described above showed that this was not reduced in concentrated salt. The diffuse strains are not agglutinated by concentrated salt even though the potential is lowered, and measurements of the cohesive force show that this also is lowered by the salt. The autoagglutinable strains of these bacteria therefore differ from the diffuse just as sensitized B. typhosus differs from the unsensitized.

\section{EFFECT OF ADDED SUBSTANCES ON THE PROPERTIES}

OF THE SUSPENSION

In the cases just considered the critical potential was increased by the addition of a second substance. The reverse condition in which the critical potential is lowered and the suspension thereby made more stable is also well known, and substances which cause this effect are classed as "protective colloids."

Meyer and Lottermoser ${ }^{2}$ stated that a suspension acquires the properties of the added substance, and this statement has been confirmed, in general, by all subsequent investigators. There is little doubt that this is due to the formation of a surface film. It was noted by Bredig, ${ }^{3}$ for instance, that molds grow on the surface of gold particles which had been treated with gelatin. Arkwright ${ }^{4}$ found that a suspension of $B$. coli in water containing soluble substances from $B$. typhosus was agglutinated by antiB. typhosus serum. This is a very sensitive test since the action of immune serum is strictly specific.

An equally striking result has been obtained by Jones ${ }^{5}$ who has found that collodion particles treated with egg albumin are agglutinated with anti-egg-albumin serum. This experiment is the more interesting since it suggests that the "precipitin" reaction is essentially the same as agglutination. In the experiment noted the antiegg-albumin serum would produce the ordinary precipitin reaction if mixed with eggalbumin solutions, but when added to the egg-albumin-coated collodion particles it causes agglutination. The same experiment may be performed with bacteria treated with cow serum and washed. These bacteria are then agglutinated by anti-cow serum. It has also been found by a number of workers that the isoelectric point of a suspension changes to that of the added substance. ${ }^{6}$

I Shibley, G.: J. Expcr. Med., 39, 245. I924.

${ }^{2}$ Lottermoser: Anorganische Colloide, p. 50. I901.

3 Bredig, G.: Anorganische Fermente, p. 15. Leipzig, rgor.

4 Arkwright, J. A.: J. Hyg., 14, 261. I9I4.

${ }^{5}$ Jones, F. S.: J. Exper. Med., 44, 625. I926.

${ }^{6}$ Arkwright, J. A.: loc. cit.; Coulter, C. B.: J. General Physiol., 3, 317. 1920; 4, 403. I92I-22; Loeb, J.: op. cit., p. Iog. I922-23; Northrop, J. H., and de Kruif, P. H.: op. cit., p. 655. 1921-22; Eggerth, A. H., and Bellows, M.: loc. cit. 
It must be noted, however, as pointed out by Loeb, that the behavior of the coated particle may differ in some respects from that of the protective colloid. Collodion particles, for instance, coated with native egg albumin precipitated at the isoelectric point of egg albumin, whereas egg albumin itself does not precipitate under these conditions. Loeb suggests that the protein is denatured by the formation of the film, as has been shown to be the case at the air-liquid surface.

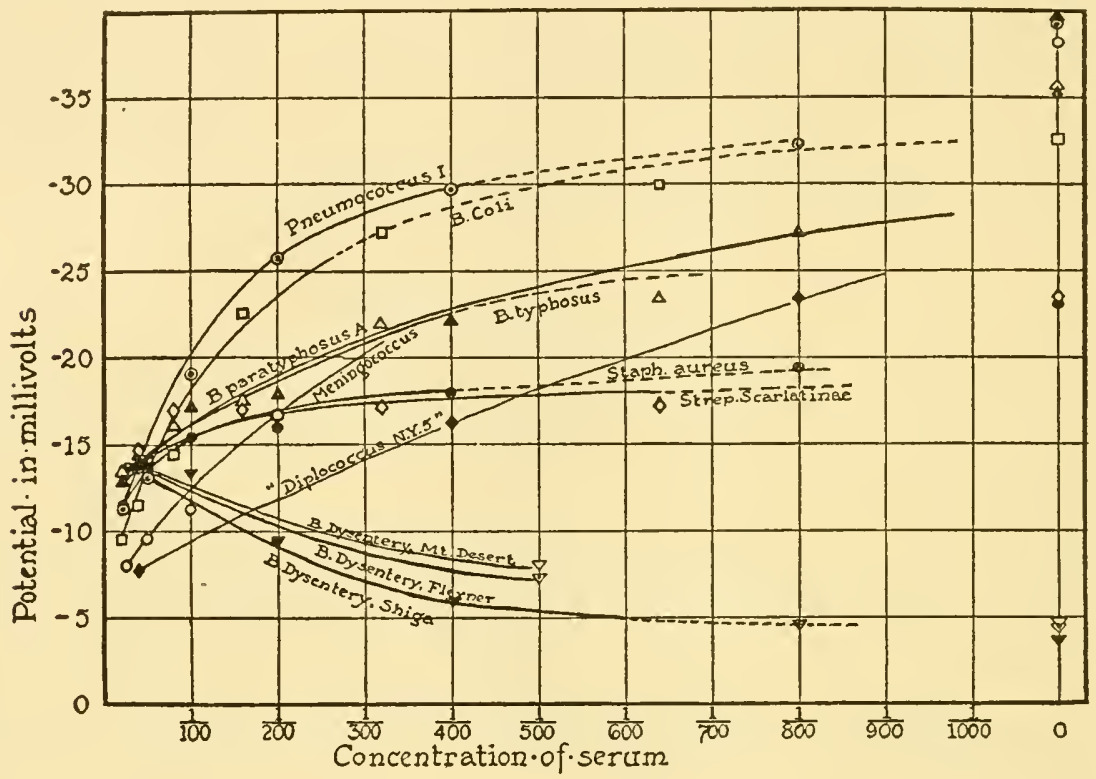

Fig. 9.-Effect of eleven specific agglutinating sera upon the potential and agglutination of their homologous organisms. These experiments were done over a period of about two years and three different cataphoretic cells were utilized in the work. All experiments in $\mathrm{M} / 200$ glycocoll-phosphateacetate buffer mixture, $\mathrm{pH}$ 7.0.

The same point is brought out in the interesting observation of Shibley that all organisms studied when treated with an excess of immune serum tend to reach the same value of the potential, independent of the potential of the bacteria themselves. In some cases it was found that the addition of the immune serum decreased and in others increased the potential. Shibley's results are shown in Figures 9 and ro. They show quite clearly that the sensitized bacteria behave in regard to both cataphoresis and agglutination like particles of denatured globulin.

In most cases Shibley found a marked effect of the immune serum on the potential of the bacteria, and this might be used as a diagnostic test, as Shibley suggests. Mellon and his co-workers ${ }^{2}$ were in fact able to detect a reaction between the antibody and the bacteria by cataphoresis measurements although the agglutination test was negative.

I Shibley, G.: J. Exper. Med., 40, 453. 1925; 44, 667, 1926; Tulloch, W. J.: Biochem. J., 8, 243. I9I4, had suggested that sensitized bacteria were comparable to denatured proteins.

${ }^{2}$ Mellon, R. R., ct al.: J. Immunol., I т, т6r. 1926. 
NATURE OF THE COHESIVE FORCE

The theory of the stability of colloidal suspensions outlined above predicts that coagulation will occur whenever the cohesive force is greater than the repulsive force. There is, in addition, very good evidence that the velocity of migration in an electric field is a measure of the repulsive force. There is every reason to believe that this rate of migration is, in turn, proportional to the potential difference between the surface of the particle and the surrounding liquid, and that the repulsive force is due to the mutual repulsion of this electric charge. The nature of the attractive force, however, is much less certain. Themajority of writers on the subject state that it is a "surface tension" or "capillary" effect, and Billitzer ${ }^{\mathrm{T}}$ assumed that the change in the potential was simply a measure of the change in the surface tension, the latter approaching a maximum as the P.D. approached a minimum. The same view is expressed by Michaelis. ${ }^{2}$

Green and Halvorson ${ }^{3}$ have shown that, hypothetically, changes in surface energy might serve as the cohesive force and could be responsible for the agglutination of suspensions.

Mellon's ${ }^{4}$ experiments are of interest in this connection. $\mathrm{He}$ found that sodium oleate pre-

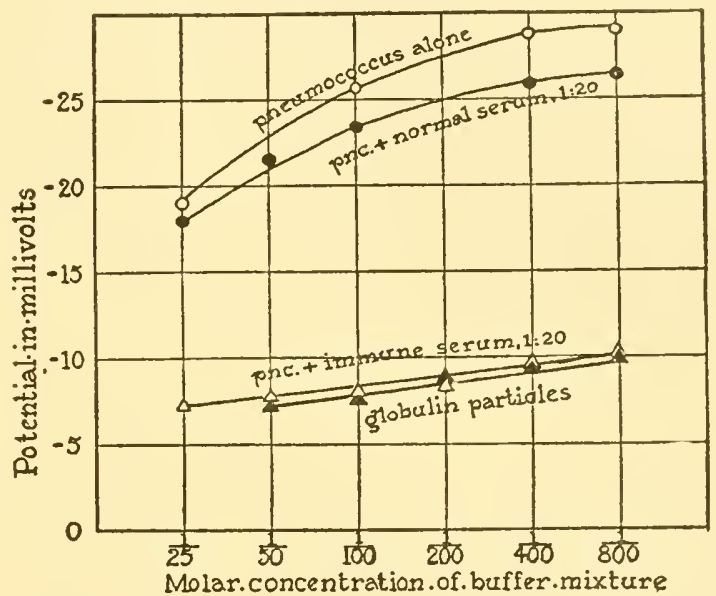

FIG. I0.-Effect of glycocoll-phosphate-acetate buffer, $\mathrm{pH} 7.0$, in varying molar concentration, upon potential of pneumococcus type I, $(a)$ alone, $(b)$ treated with I/20 normal and $(c)$ with $I-20$ specific serum, and $(d)$ of globulin particles obtained from the specific serum.

vented the agglutination while caprylic and nonylic alcohol did not, although the latter have as great an effect on the air-solution interfacial tension as does sodium oleate. The experiment shows, therefore, as might be expected, that measurement at the air-solution interface has no relation with the force at the particle-solution interface.

Von Smoluchowskis also assumes that the attractive force is increased as the potential decreases. Freundlich ${ }^{6}$ assumes the attractive force to be constant. If the writer's measurements of this force are significant, there is no doubt that it does not vary in any way with the potential. There is no valency effect, and the effects of salt are all in concentrations so high that the potential is very low or absent. It must be

I Billitzer, J.: Ztschr. phys. Chemie, 51, I28. I905.

${ }^{2}$ Michaelis, L.: Die Wasserstofionenkonzentration (ist ed.), p. 49. I9I4.

3 Green, R. G., and Halvorson, H. O.: J. Infect. Dis., 35, 5. 1924.

4 Mellon, R. R., et al.: op. cit., p. 365. I924.

5 von Smoluchowski, M.: Physik. Ztschr., I7, 557, 583. I916.

${ }^{6}$ Freundlich, H.: Kolloid-Ztschr., 23, I63. 1918. 
noted that in Powis' experiments with oil drops there was no coalescense of the drops. There is no change in the oil-water surface, therefore, and hence it cannot be the oilwater surface tension which draws the drops together. Whatever force is active must reside in the surface film surrounding the drop, since it is these films that coalesce. There is no evidence of molecular contact of the particles.

\section{THE VELOCITY OF COAGULATION}

The changes in a coagulating suspension or sol take place relatively slowly, and complete coagulation may require several hours. The change in the potential on the addition of the electrolyte, however, is almost instantaneous, at least in the suspensions of bacteria studied by the writer and also in Powis' experiments. The time element, therefore, consists in the time required for two or more particles to meet and stick together. Von Smoluchowski ${ }^{\mathrm{r}}$ has been able to derive a formula for the rate of this reaction based on the probability of collision of the particles. He assumes, as did Zsigmondy, that the particles are uncharged and that the collisions are inelastic, i.e., every collision between two particles results in the formation of an aggregate of two particles. This leads to the equation

$$
k=\frac{\mathrm{I}}{t}\left(\frac{\mathrm{I}}{\mathrm{\Sigma} v}-\frac{\mathrm{I}}{V_{0}}\right)=4 \pi \mathrm{Dr}
$$

in which $V_{o}$ is the number of particles present at the beginning, $\Sigma v$ the number present at time $t, D$ the diffusion coefficient, and $r$ the distance between the particles at which they are attracted. It is assumed, further, that this is not much greater than twice the radius of the particles. It may be noted that the formula is the same as that for a bimolecular reaction, with the exception that all collisions are considered as leading to combination, whereas, as is known, such an assumption will not hold with respect to chemical reactions. The theory predicts the experimental results accurately, as was found by Westgren and Reitstötter and by Kruyt and van Arkel. ${ }^{2}$

In the foregoing derivation it was assumed that the particles possessed no repulsive force. This is the condition at the isoelectric point. Experimentally, however, it is known that agglutination occurs when the particles are slightly charged. Under these conditions it might be supposed that only those particles having sufficient kinetic energy would be able to approach one another within the "attraction sphere." The coagulation would be, therefore, slower but eventually would become complete, as is the case. Freundlich ${ }^{3}$ has taken this effect into account and derived a complete formula which correctly predicts the course of the coagulation.

In certain cases - "slow coagulation" - the rate curve resembles that of an autocatalytic reaction. ${ }^{4}$ According to von Smoluchowski, this is a secondary phenomenon. It seems possible that this effect is due to a slow change in the potential. It was found by Powis, for instance, that there was a very rapid change in the potential immediately on the addition of the electrolyte. This was followed, however, by a second slow

${ }^{2}$ von Smoluchowski, M.: loc. cit.

${ }^{2}$ Freundlich, H.: Kapillarchemie (2d ed.), p. 596. Leipzig, I922.

3 Freundlich, H.: Kolloid-Zischr., 23, I63. 1918.

${ }_{4}^{4}$ Lottermoser, A.: ibid., 15, I45. I914. 
decrease extending over a period of days. If the potential immediately after the addition of the electrolyte were slightly above the critical value, it is readily seen that the flocculation might be caused by the second slow potential change. It is just in these cases that the peculiarity manifests itself. When the potential is reduced at once to zero, ordinary rapid coagulation occurs.

\section{SUMMARY}

The mechanism of agglutination just described makes it possible to analyze in detail the picture of the agglutination process outlined at the beginning of this chapter. In a suspension of bacteria the individual cells are kept apart by the repulsion due to their like charges. If a substance is added which decreases this charge, the cells approach one another more closely and finally become attached unless at the same time the added substance destroys the cohesive force or "stickiness" of the cells. This appears to be the case with concentrated solutions of electrolytes and most unsensitized bacteria. If immune serum or other substances which form films on the bacteria are added the bacteria take on more and more of the nature of the added substance, and this may or may not lead to agglutination. If the potential of the bacteria was originally different from that of the added substance under the same conditions, the potential will be changed and will approach that of the added substance. If this is less than the critical potential of that substance, agglutination will occur. If the potential is greater than the critical potential of the substance forming the film, no agglutination will occur until electrolytes have been added to reduce the potential below the critical value. If the potential on the cells happens to be the same as that of the sensitizer, no change in potential will be noted but the suspension may still agglutinate if this potential is less than the critical value for the sensitizer. The agglutination of bacteria by their homologous antibody (agglutinin) differs from that of agglutination by ordinary proteins or the agglutination of collodion particles by means of egg albumin in that the antibody will form a film only on the bacteria used to produce it. The specific part of the reaction, therefore, lies in the formation of the film. This specificity is of the greatest theoretical and practical importance but does not differ, in the writer's opinion, from the specificity of any chemical reaction. Quantitatively every chemical reaction is specific, and the specificity of immune and enzyme reactions differs only in degree from that of any other reaction. The problem of specificity is one of reactions in general. It is possible with our present knowledge to predict whether or not a given reaction will take place from considerations of the free energy changes involved, but there is no means of predicting the rate at which it will occur. Practically, therefore, it is not possible to predict with certainty the occurrence of even the simplest reaction, so that it is not surprising that such complicated reactions as those involved in biological systems are still unaccounted for. 


\section{CHAPTER LIX}

\section{THE FUNCTIONAL RÔLE OF AGGLUTININS}

\section{G. HOWARD BAILEY}

School of Hygiene and Public Health, Johns Hopkins University

Although several other investigators had previously observed agglutination with a variety of bacteria and immune sera, Gruber and Durham, ${ }^{\mathrm{I}}$ in 1896 , were the first to make an intensive study of the phenomenon during the course of work with the colon bacillus and the cholera vibrio. They pointed out the specificity of the reaction and the fact that it differed in certain essentials from previously studied serum reactions. It was soon noted, however, that there is not absolute specificity, that group reactions occur, and also that blood-cells and other body cells could be agglutinated by specific sera. It was found that agglutinins of various kinds exist normally in certain sera and that these antibodies may be produced artificially by immunization. It was observed also that the property of specifically agglutinating bacteria is developed in man and animals in the course of infectious disease, and this led, a short time after Gruber and Durham's publication, to the discovery of the now widely used Widal test for typhoid fever. Agglutination reactions have been employed for the identification of bacteria and various other diagnostic and clinical purposes, and the study of this reaction in vitro has brought out many facts of both practical and theoretical significance.

A more important consideration, however, is the question of the functional rôle of agglutinins in infectious disease, that is, the part agglutination as such plays as a factor in protecting the body against infection. What is the relation between the presence or production of specific agglutinating and precipitating substances and natural or acquired immunity? For a long time there was much speculation as to the biological significance of this phenomenon, and much difference of opinion has been expressed concerning the influence exerted by agglutinins in the infected organism in warding off a bacterial invasion or in restraining the growth of bacteria after they have once become established.

The agglutinative property is met with in the body fluids of many species of normal and immune animals and is exercised upon many bacteria. It may be demonstrated not only in blood serum but also in transudates and exudates and in certain secretions such as milk, tears, saliva, and urine. The presence of agglutinins in the sera or body fluids of infected individuals has long been considered an index at least of the existence of a greater or lesser degree of immunity. That there should be some relation between agglutinins and immunity would appear certain, if only from the fact that bacteria become immobilized under the influence of the corresponding agglutinins. Yet among the factors to which bacterial immunity is usually ascribed, agglutination has been considered by most immunologists to occupy a subordinate place; for, however

I Gruber, M., and Durham, H. E.: Mïnchen. med. Wchnschr., 43, 285. I 896 . 
valuable the phenomenon may be in vitro in identifying bacteria or in discovering specific forms of infection, it is held that the process plays no essential part in the protection of animals.

EARLY OBSERVATIONS AND VIEWS ON THE FUNCTIONAL SIGNIFICANCE OF AGGLUTININS

Gengou $^{\mathrm{I}}$ was unable to establish any relation between the agglutinative power and the refractory state of animals to anthrax. He found that human serum may contain large amounts of agglutinin for attenuated anthrax bacilli and yet man is far from being immune to the disease. In certain cases agglutinins for a micro-organism may apparently be entirely absent and yet the animal enjoy an immunity. Pigeon's serum is free of agglutinins for pneumococci and yet this animal has a high degree of natural immunity. It has also been pointed out that bacteria exposed to an agglutinating serum are not altered in appearance, viability, or virulence. Certain clinical observations have tended to show that the agglutinating power of a serum gives no indication of the actual degree of immunity that exists.

Metchnikoff ${ }^{2}$ contended that agglutinins play no part, however small, in natural immunity processes since he could establish no relation between the agglutinating power of sera and the refractory state of man or animals to infection. Similarly, he held that the part played by agglutination in acquired immunity is merely accidental and subordinate. He admitted, however, that in certain special cases the immobilization of very motile bacteria and their agglutination into clumps may facilitate the reaction of the animal organism, especially the rapidity of phagocytosis. Besredka ${ }^{3}$ had observed that guinea pigs inoculated with typhoid bacilli which had previously been mixed with the blood serum of normal animals survived. Ox serum heated to $60^{\circ} \mathrm{C}$. was the most active in this respect. The resistance of guinea pigs to bacteria inoculated into the peritoneal cavity was directly proportional to the degree of previous agglutination of the bacilli, the organisms being readily and rapidly phagocyted when in the form of clumps. These facts were interpreted by Metchnikoff as indicating that previous agglutination in vitro may aid in the protection of animals against infection, but at the same time proved that agglutination in vivo, if it occurred at all, was so feeble as to be entirely ineffective.

Working with the cholera vibrio Salimbenit came to the conclusion that bacterial agglutination never takes place within the animal body. In horses, goats, and guinea pigs with high degrees of active or passive immunity it was found that agglutination did not occur when the micro-organisms were injected subcutaneously, small amounts of the exudate being removed at frequent intervals and examined. Nor was it possible to demonstrate the phenomenon when the spirilla were given by intraperitoneal injection into guinea pigs, even though the peritoneal fluid, lymph, or blood serum caused almost instantaneous agglutination when mixed with the vibrios in hangingdrop preparations or in the test tube. The only changes noted in vivo were loss of

\footnotetext{
${ }^{x}$ Gengou, O.: Ann. l'Inst. Pasteur, r 3, 642. I899.

${ }^{2}$ Metchnikoff, E.: Immunity in Infective Diseases, pp. 258, 263. Cambridge, 1905.

${ }^{3}$ Besredka, A.: Ann. de l'Inst. Pasteur, 15, 209. I90I.

+ Salimbeni, A. T.: ibid., II, 277. 1897.
} 
motility followed by the Pfeiffer phenomenon. Salimbeni attributed the apparent inagglutinability of the organisms in the body to lack of access of air which he demonstrated in some way influenced them or their medium.

A considerable number of investigators have contended, however, that intra vitam agglutination is an important mechanism in immunity. Gruber, ${ }^{1}$ in reporting his studies on the specificity and practical application of the phenomenon of agglutination, expressed the opinion that agglutinins were quite essential properties of an immune serum. He believed that agglutination was due to an increased viscidity of bacterial bodies which caused them to adhere to one another. Gruber advanced the idea also that this increased viscidity aided in the englobement of the bacteria by the phagocytes and would probably account for the accumulation of bacteria in the organs when injected into the circulation. It was thought that the agglutinins in modifying the bacterial membranes permit the penetration of the bodies of the bacteria by the alexins which alone bring about the final destruction of the micro-organisms which are then phagocyted.

\section{CLINICAL OBSERVATIONS ON THE RELATION OF AGGLUTININS TO PROGNOSIS AND RECOVERY FROM INFECTION}

The first clinical observations on the activity of agglutinins in vivo were made by Sawtschenko and Melkich. ${ }^{2}$ In an extensive investigation of recurrent fever they made a careful study of the mechanism of immunity to Spirocheta obermeieri, both in patients and experimental animals, and compared the action of immune serum in vitro and in vivo. When the organisms were injected intraperitoneally in to actively or passively immunized guinea pigs and the exudate examined after ten to fifteen minutes many of the spirochetes were non-motile, and after forty minutes it was impossible to find motile organisms. Both fresh and stained preparations showed wellmarked agglutination and phagocytosis.

In recurrent fever in man the agglutinative property is found in the serum or plasma of the patients during apyrexia and especially during convalescence. Metchnikoff ${ }^{3}$ established the fact that the critical fall of temperature and disappearance of the organisms from the blood always coincides with a well-marked phagocytosis in the spleen. An important contributory factor to the phagocytic crisis, as shown by Sawtschenko and Melkich, was the action of the agglutinins, which at this stage of the disease could be easily demonstrated both in vitro and in vivo. The spirochetes could be seen united into small clumps in both fresh and stained preparations made immediately after the blood was drawn. It was suggested that agglutination in the plasma was incomplete because of the rapid movement of the blood. The accumulation of the organisms in the spleen where phagocytosis was most marked was evidently due to filtration of the clumps by this organ and their englobement en masse by phagocytic cells.

Many observers have attached great importance to agglutinins not only in the diagnosis but also in the prognosis of such diseases as typhoid and Malta fever. After

${ }^{1}$ Gruber, M.: Wien. klin. Wchnschr., 43, 206. 1896.

'Sawtschenko, I., and Melkich, A. A.: Ann. de l'Inst. Pasteur, I5, 497. I901.

${ }^{3}$ Metchnikoff, E.: Virchow's Arch.f. palh. Anal., 109, I76. I887. 
an analysis of much clinical and laboratory data Courmont $t^{\mathrm{t}}$ arrived at the following conclusions concerning the relation of agglutinins to prognosis in typhoid fever: (I) a large quantity of agglutinins present in the blood is always favorable (the larger it is the better), especially if this coincides with a remission of the temperature; (2) a small quantity of agglutinins with a rise of temperature is always unfavorable; (3) a strong agglutinating power is good at all times in the course of the disease; a low agglutinating power is usually bad as the latter condition is found in light forms of the disease with a tendency to relapse or in very severe cases. Exactly the same conclusions were reached by Birt and $\mathrm{Lamb}^{2}$ in regard to Malta fever. It is well known that during the early stages of typhoid fever the bacilli may be found in the blood stream, later to localize particularly in the lymphoid tissues of the intestine and spleen. Wright and Lamb ${ }^{3}$ have shown that in cases of typhoid fever examined postmortem the fluids of these tissues are always much poorer in agglutinins than the heart blood, and they thus explained the ultimate localization of the organisms. It was believed that the continued growth in these situations was made possible by the absorption of agglutinins from the surrounding plasma or lymph and the agglutinative power thus reduced below a certain minimum. One hypothesis which has been advanced as an explanation of relapses in this disease in the presence of a relatively high agglutinin titre of the blood is the decrease in agglutinability of the typhoid bacillus after long cultivation in vivo. This phenomenon has not yet been fully explained, but it may depend on an active immunity of the bacteria against the agglutinins, for it can be developed in agglutinable strains by cultivating them in serum containing agglutinins. ${ }^{4}$

\section{EXPERIMENTAL OBSERVATIONS ON AGGLUTINATION in vivo}

Following the interesting clinical and experimental observations of Sawtschenko and Melkich concerning the relation of agglutinins to immunity in recurrent fever, already referred to, a further observation bearing on the question of the influence which is exerted by agglutinins in the infected organism was made by Wright and Lamb. ${ }^{5}$ It was shown that in plague-infected guinea pigs. which had received a sufficient quantity of antiplague serum the plague bacilli were to be found only in discrete clumps or colonies, especially in the spleen. In control animals the bacilli were found in countless numbers uniformly distributed all over the body.

The first extensive experimental investigation, however, on agglutination in vivo was made by Bull. ${ }^{6}$ Working with rabbits and dogs a technique was employed by which specimens of blood were taken from the heart at closely spaced intervals following the intravenous injection of a uniform suspension of bacteria into normal or actively or passively immunized animals. It was found that not only may agglutination occur in the circulation of such animals, but in contradistinction to the slowness with which

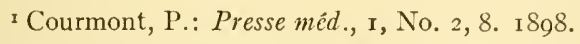

${ }^{2}$ Birt, C., and Lamb, G.: Lancet, 2, 70I. I899.

3 Wright, A. E., and Lamb, G.: ibid., p. 1727.1899.

4 Walker, E. W. A.: J. Path. \& Bact., 8, 34. I903.

5 Wright, A. E., and Lamb, G.: loc. cit. I9I6.

${ }^{6}$ Bull, C. G.: J. Exper. Med., 20, 237. 1914; ibid., 22, 457, 466, 475, 484. 1915; ibid., 24, 7, 25 
the process occurs and becomes evident in vitro is its almost instantaneous occurrence in vivo. Also agglutination within the body seems to take place with dilutions of an antiserum which are wholly ineffective in the test tube, while the specificity of in vivo agglutination is as strict as in vitro. Organisms like Bacillus mucosus capsulatus which are agglutinated only slightly even by highly immune serum in the test tube are rapidly and completely agglutinated in immune animals. Bull studied first the mechanism of bacterial immunity, both natural and acquired, in the rabbit. This animal may be regarded as possessing a high degree of natural immunity to such organisms as typhoid bacilli, dysentery bacilli of the Flexner group, and non-virulent influenza bacilli, while it is very susceptible to virulent pneumococci, dysentery bacilli of the Shiga type, and virulent influenza bacilli. It was shown that the degree of agglutination and opsonization of the bacteria within the body is inversely parallel to the infectiousness of the particular micro-organism for the host. When typhoid bacilli are injected into the circulation of normal rabbits the micro-organisms are promptly agglutinated, quickly removed from the blood stream, and accumulated in the internal organs where they are taken up by the polymorphonuclear leukocytes and fixed-tissue cells, especially in the lungs, liver, and spleen. The rapidity with which the agglutination takes place is remarkable. In fact, it was shown that the bacilli are agglutinated within a few seconds after entering the blood stream. This was proved by making smears from the blood by heart puncture and from the crushed organs. The same was found true for all organisms investigated to which the rabbit is naturally resistant, while slight or no agglutination was observed for bacteria with which the animal may be infected.

It appears, therefore, that agglutination may be an important factor in the foregoing instances of natural immunity. That this is probable is supported by indications that typhoid bacilli, for example, do not undergo intra vitam destruction by bacteriolysis as is the case in vitro. It was shown that the unagglutinated and unphagocyted bacilli in the organs resist longest and stain best. It is known that typhoid bacilli may appear and survive in the blood of typhoid fever patients at a time when the shed blood is highly bacteriolytic for the bacilli. Since the organisms in the blood may also become inagglutinable, they cannot be removed by the organs and hence are not phagocyted and destroyed.

Bull showed that in actively or passively immunized animals agglutination is still more noticeable. His observations in regard to the action of pneumococcus-immune serum in pneumococcus-infected rabbits is particularly interesting. As is well known, virulent pneumococci in the blood stream of normal rabbits soon begin to multiply and a fatal septicemia soon develops from an exceedingly small number of organisms. The virulent pneumococci are neither agglutinated nor opsonized by normal rabbit serum. On the other hand, the non-virulent strains are agglutinated instantly. after injection into the circulation of normal rabbits and are opsonized by the serum of such rabbits. It was found that the injection of a small amount of antipneumococcus serum into septicemic rabbits resulted in a rapid clumping of the virulent pneumococci in the blood stream. An immediate heart puncture revealed clumps of the bacteria which had been evenly distributed in smears prepared just before the injection. Three to five minutes after the serum injection it was impossible to find any 
organisms by the ordinary smear method. Thus the same phenomenon of protection in the normal animal is paralleled by what happens when an effective antiserum is employed to prevent or combat a bacterial infection. The same means which cause clumping and removal of bacteria from the blood and their accumulation in the organs also causes an accumulation in the same organs of large numbers of polymorphonuclear leukocytes which ingest the clumped organisms en masse and bring about their rapid dissolution.

During his investigations of the effects of antisera in vivo, Bull observed an important relation between the degree of agglutination and the curative action of the sera. Thus in heavy septicemias in rabbits very large doses of the specific serum may actually be detrimental owing to the formation of large clumps of bacteria in the lungs and other organs which thus impede the circulation or prevent phagocytosis. On the other hand, very small doses of serum may not reach certain portions of the body, as the cerebrospinal fluid, in sufficient concentration to prevent subsequent infection. The curative action of certain antisera would therefore appear to have a close relationship to maximum and minimum limits of dosage and the degree of agglutination produced, at least in the case of animals with heavy septicemias. The observation would probably have less significance in man.

It has already been stated that the degree of agglutination of bacteria in vivo is inversely parallel to the infectiousness of the bacteria for the host. This was well illustrated by Bull in showing the relatively high virulence of Bacillus avisepticus for rabbits, but not for dogs. It was found that the fresh sera of these animals have no bactericidal effect on this organism, there being a difference in the power of the sera to agglutinate and opsonize the bacteria. A very small quantity of culture produces a fatal septicemia in rabbits, but a subtoxic dose is without effect in dogs.

\section{PHYSICO-CHEMICAL BASIS OF AGGLUTINATION in vivo}

Just why bacteria should agglutinate so much more readily and rapidly in the circulating blood than in serum in the test tube is not definitely known. Bull suggested that some constituent of the blood of the host aids the specific serum in producing those changes in the bacterial bodies which precede agglutination. Recently Wright ${ }^{1}$ has shown in experiments with India ink that this substance remains as an unaltered suspension in sodium chloride, is slowly precipitated by Ringer's solution, more readily by serum and defibrinated blood, and almost instantaneously by whole blood and plasma. Gelatin afforded protection against such precipitation. The more nearly the fluid approaches what is found in the blood stream the more marked is the instability of the suspension and the greater its tendency to precipitate. The most actively precipitating of these fluids (plasma) is probably much less unstable than blood in the vessels with its capacity for clotting uninterfered with by chemical agents. Variation in the effect of blood of different animals has also been noted.

Since a suspension of bacteria behaves much like an emulsoid as regards precipitation, Bordet ${ }^{2}$ considers the effect of agglutinins is to convert the emulsoid suspension into a suspensoid condition which should then behave like ink particles.

I Wright, H. D.: J. Path. Eo Bact., 30, I85. 1927.

${ }^{2}$ Bordet, J.: Traité de l'Immunité, p. 297. Paris, I920. 
Govaerts ${ }^{I}$ and Le Fèvre de Arric ${ }^{2}$ have shown that bacteria flocculate more readily in unheated plasma than in unheated serum, and more rapidly when platelets are present. These observations suggest the possibility of a greater activity of antibodies, especially agglutinins, in vivo than in vitro, as suggested by Bull. It would appear also that the platelets, although not an essential element in the removal of bacteria, yet may assist the agglutinins in the formation of clumps.

\section{FACTORS RELATED TO intra vitam AGGLUTINATION}

The importance of the fixed-tissue cells in relation to agglutination and other factors in the ultimate disposal of invading bacteria has become evident from a number of investigations. As already pointed out, Bull's observations on the intravascular agglutination of various bacteria which are phagocyted by the cells in the lungs, liver, and spleen has thrown light on the mechanism of immunity to many infections and further emphasizes the relationships which must frequently exist between antibodies in the blood stream, notably the agglutinins and opsonins, on the one hand, and the cellular elements, on the other. However, in certain cases it would appear that the latter may be the predominating or only factor in the immunity process. $\mathrm{Kyes}^{3}$ has shown that in the pigeon, a species insusceptible to the pneumococcus, the infecting organisms are rapidly withdrawn from the general blood stream and localized in the liver, and especially in the spleen. In these organs the ultimate localization of the pneumococci is within a type of fixed phagocyte-the hemophage-common to both. This phagocytic destruction of the micro-organisms was found to be so extensive and so rapid that it appeared to be the most important if not indeed the determining factor in the establishment of this instance of natural immunity. It did not appear that agglutination played an important rôle. Instead of a clumping of the bacteria there occurred a filtration by the peculiar structural arrangement of the capillaries of the spleen.

Recently Hopkins and Parker ${ }^{4}$ have also shown the great importance of the fixedtissue cells. These investigators injected streptococci into rabbits and cats and found that the organisms were rapidly removed from the circulation, the removal being largely due to phagocytosis carried on by the endothelial cells in the lungs and by similar cells in the liver and spleen. These investigators were unable, however, to apply Bull's results to the removal of streptococci from the circulation on the basis of agglutination. Other workers have also failed to find evidence of the dominating influence of agglutinins in certain infections. TenBroeck ${ }^{5}$ points out that these antibodies are of little importance in infections with the hog-cholera bacillus.

Some interesting observations bearing directly on the factors concerned in the rapid and complete disappearance of injected pneumococci from the blood of immunized rabbits were made by Manwaring and Coe. ${ }^{6}$ It was shown that, besides the process of agglutination emphasized by Bull, another factor is also operative in the re-

${ }^{x}$ Govaerts, P.: Compl. rend. Soc. de biol., 83, I96. 1920.

${ }^{2}$ Le Fèvre de Arric, M.: ibid., p. гог I. 1920. $\quad{ }^{3}$ Kyes, P.: J. Infect. Dis., 18, 277. I916.

${ }_{4}^{4}$ Hopkins, J. G., and Parker, J. T.: J. Exper. Med., 27, I. I918.

5 TenBroeck, C.: ibid., 26, 44ז. I917.

${ }^{6}$ Manwaring, W. II., and Coe, H. C.: J. Immunol., I, 40г. I9r6. 
moval of the bacteria from the circulation. This is the so-called "endothelial opsonin." By the application of perfusion methods a thermostable opsonin or bacteriotropin was demonstrated in antipneumococcus serum, so altering pneumococci as to cause their adhesion to the endothelial cells lining the hepatic capillaries. This opsonin was operative in the liver capillaries in many hundred times the dilution necessary to cause agglutination of the pneumococci, but was practically inoperative in the extrahepatic capillaries.

It is an interesting fact, then, that in certain instances in which agglutinins in the ordinary sense cannot be demonstrated either in vitro or in vivo, opsonization and phagocytosis of bacteria occur. It is probable, however, that one stage of the process is characterized by a condition of fine aggregation and increased adhesiveness of the sensitized micro-organisms. It has been suggested by Muir and Martin that immune opsonins or bacteriotropins may be identified with agglutinins, inasmuch as they possess resistance to heat and are active without apparent dependence upon alexin. Furthermore, the close similarity of a certain phase of opsonization to agglutination has been emphasized by Ledingham. ' This investigator noted that sensitized organisms congregate at the periphery of leukocytes, and in the course of experiments on the influence of temperature on phagocytosis found that at low temperatures where little or no phagocytosis occurred this ring distribution of the sensitized bacteria was very evident. This attachment phase was regarded as a physico-chemical phenomenon entirely analogous to agglutination. It was shown that the sensitized bacteria were in a state of fine aggregation, and if the sensitizing fluid also had marked agglutinating powers the organisms were frankly clumped.

Such evidence, therefore, would seem to show that in most instances where immunity exists, but agglutination in the body in the ordinary sense cannot be observed, either because of low potency of a normal or immune serum, or scarcity of bacteria, effects physically analogous to agglutination are produced. The sensitized microorganisms are so altered as to become potentially more adhesive to one another, to leukocytes, or to fixed phagocytic cells. Quantitative, and to some extent qualitative, variations in the reaction will depend upon the animal species, the degree of natural or acquired immunity, and the virulence, number, and kind of bacteria.

The condition of affairs, immunity without the presence of demonstrable antibodies such as agglutinins or precipitins, has been referred to as immunity in the absence of antibodies. It is evident, however, that the in vitro tests usually applied are not very sensitive, are somewhat crude, and are certainly artificial. The presence of such antibodies in very small amounts, not demonstrable by ordinary serological methods, may be revealed if the organism be grown in the presence of serum or blood (the thread reaction of Pfaundler). For example, the sera of patients with pneumonia frequently show no agglutinins by the usual methods, but if pneumococci are sown in such sera they grow out in masses with chain formation. It has been shown that growth in immune serum renders pneumococci more agglutinable and less virulent. It is probable that in case of infection with organisms showing such characteristics of growth, phagocytosis would be facilitated and thus the effects of more typical agglutination simulated. Bull has demonstrated this to be the case in pneumococcus in-

r Ledingham, J. C. G.: J. Hyg., 12, 320 . I912. 
fection of the dog, an animal relatively immune to the pneumococcus, in which both typical intravascular agglutination and chain formation occurred, the latter in the cerebrospinal fluid.

Another way in which agglutinins may play a part in defense, over and above that of agglutinating the bacteria, is by the physico-chemical alterations produced by the agglutinins, which are believed to act by digesting or otherwise altering the protective colloidal envelope which interferes with chemical reactions between the bacteria and the surrounding fluids. When this protective layer is destroyed by the agglutinins the permeability of the bacteria for various ions, including bactericidal substances, is increased. It is probable, therefore, that the agglutinins play a considerable part in this way in aiding other serum constituents in the actual destruction of micro-organisms.

It must be concluded that the mechanism of removal of bacteria from the body of an animal lost is a complex one. Most of the evidence seems to show that the humoral part of the process consists in an increased adhesiveness of the micro-organisms by which they agglutinate into clumps of widely varying sizes, and are then either filtered out by the visceral organs or stick to leukocytes, to endothelial cells, or to platelets. In addition, there is active phagocytosis by polymorphonuclear and mononuclear cells of the blood and the cells lining the sinuses of the liver, lungs, and spleen. In the skeletal tissue spaces agglutinins probably do not play as great a part as in the circulating blood. In these situations direct bactericidal action and phagocytosis are probably very important factors.

\section{SUMMARY}

I. Certain clinical observations have shown a parallelism between the power of a serum to cause agglutination of bacteria outside the body and its power to bring about intra vitam agglutination and recovery from infection.

2. Experimentally agglutination can be demonstrated in vivo; the power of the blood to cause agglutination determines largely whether after their direct introduction in an experimental way bacteria are to be removed from the circulation.

3. The power of the blood to produce agglutination in vivo may not be demonstrable in vitro. Agglutination occurs much more readily and promptly in the animal body than in the test tube.

4. The degree of agglutination and opsonization of bacteria within the body is in general inversely parallel to the virulence of the micro-organisms for the host.

5. Bacteria which are not agglutinated in the circulating blood of the normal animal may be agglutinated by the intravenous injection of a specific immune serum. Such serum is many times more active in vivo than in vitro.

6. Agglutination in the infected animal may have a definite protective function through a preliminary concentration of bacteria in the capillaries of the visceral organs whereby phagocytosis is facilitated.

7. Agglutinins, opsonins, and phagocytosis are very closely related, and exert aı important rôle in resistance to, and recovery from, infection. 


\title{
CHAPTER LX
}

\section{BACTERIAL AGGLUTININS AND THEIR APPLICATIONS}

\author{
J. G. FITZGERALD AND DONALD T. FRASER \\ University of Toronto
}

The agglutination of bacteria by immune sera is an immunological or serological reaction. All such reactions are specific, relatively or absolutely. Attempts to explain them upon the basis of analogy with physical reactions which in certain respects they resemble leaves out of account their most important characteristic: specificity.

When agglutinable species of bacteria are appropriately treated with immune sera containing bacterial agglutinins, the individual micro-organisms if suspended in a liquid medium tend to aggregate in clumps or masses and no longer remain discrete. If motile they generally lose their motility. If conditions otherwise are favorable they remain viable. The reaction of bacterial agglutination is relatively specific. It is an antibody-antigen reaction in which the intervention of alexin is unnecessary. It is similar to and perhaps identical with the precipitin reaction.

\section{EARLY OBSERVATIONS ON AGGLUTINATION}

Charrin and Roger, ${ }^{\mathrm{x}}$ in $\mathrm{I} 889$, in a brief communication upon the development of pathogenic microbes in the serum of vaccinated animals, recorded the fact that cultures of Bacillus pyocyaneus when grown in the serum of animals previously injected with cultures of this micro-organism were readily distinguishable from cultures of the same species grown in serum of a normal animal. This difference was described by these observers as follows: "Dans les tubes que nous presentons à la Société et qui ont été resemencés il y a quatre jours les différences sont encore considérables; on peut remarquer que le serum de l'animal réfractaire est clair et transparent; les microbes sont réunis en petits grumeaux, qui s'éparpillent quand on agite le tube, mais retombent au fond quand on le laisse au repos."

The significance of this observation was not, it would seem, understood since the only conclusion recorded in this communication by Charrin and Roger was that bacteria behaved differently depending upon whether they were grown in immune serum (blood of vaccinated animals) or in the serum of normal animals. Metchnikoff, ${ }^{2}$ two years later, observed the clumping action of immune serum upon Vibrio metchnikovii. He did not, however, specifically describe agglutination. In I 893 Isaeff and Ivanoff, ${ }^{3}$ in a study of the pneumococcus, noted that turbid emulsions of this micro-organism were rendered clear by the addition of pneumococcus-immune serum.

Bordet, ${ }^{4}$ in 1895 , approached the problem in a somewhat different fashion. Cultures of the vibrio of Asiatic cholera were grown upon agar slants, and after twenty hours the growth was washed off with physiological salt solution. He noted that a trace of serum obtained from a rabbit immunized against cholera almost immediately immobilized the vibrios, and the

I Charrin, A., and Roger, C.-H.: Compt. rend. Soc. de biol., 2, 667. I889.

${ }^{2}$ Metchnikoff, E.: Ann. de l'Inst. Pasteur, 5, 473. I891.

${ }^{3}$ Isaeff and Ivanoff, M.: Zischr.f. Hyg. u. Infektionskrankh., I7, I I 7. I894.

4 Bordet, J.: Ann. de l'Inst. Pusteur, 9, 462. I895. 
agglomeration into masses visible to the naked eye promptly followed. The serum of a normal rabbit had no such action upon emulsions of cholera vibrios. Bordet observed further that an immune cholera serum heated to $55^{\circ}-60^{\circ} \mathrm{C}$. retained this property, which fact served to distinguish it from bactericidal power, a study of which was at the time engaging the attention of Bordet and many others. The next year Gruber and Durham ${ }^{1}$ continued and extended the observations of Bordet and for the first time described the agglutination reaction as a separate and distinct characteristic of immune sera. In their first joint publication Gruber and Durham wrote: "By mixing the immune sera . . . . with the emulsion of the agar culture .... one can see that the bacteria agglutinate in great clumps, and the characteristic motions come to a standstill. These reactions are in the closest relationship to the protective action of sera. In consequence, the bacteria under the influence of the antibodies contained in the immune sera become sticky or glutinous. For this reason we describe the specific substances of the immune sera 'agglutinins.' " Just prior to the appearance of the joint paper by these authors, one of them, Durham ${ }^{2}$ had presented a note to the Royal Society of London in which he indicated that certain immune sera contained a hitherto incompletely recognized and inadequately described type of antibody which brought about agglutination.

Gruber, ${ }^{3}$ in his first communication, stated that "the essential action of the antibodies of the fluids of immunized animals consists in the fact that they cause the bursting of the covering of the bacteria. This is indicated by the fact that the bacteria which are treated with these fluids become sticky and glutinous, unite in large clumps, and lose their characteristic motility. This fundamental action of immune sera has been completely overlooked by Pfeiffer and his pupils. Metchnikoff and Bordet saw it but did not grasp the importance of it. On account of this fundamental action I shall call the antibodies of specifically immunized animals 'glabrificines.' " This first designation of agglutinins was given by Gruber because he erroneously interpreted their mode of action. This he himself recognized, and in his next publication upon the subject he substituted the term "agglutinin" for "glabrificine." Gruber and Durham extended their study of the phenomenon to various species of bacteria and their homologous immune sera, and emphasized the specific nature of the reaction. Some possible practical applications of the reaction of agglutination were indicated by Gruber and Durham. It was suggested by them that bacteria eliminated in the stools of patients suffering from cholera or typhoid fever might be identified by the employment of appropriate immune sera.

Almost simultaneously publications of Widal $^{4}$ and Grünbaum ${ }^{5}$ described the reaction which occurs when the serum of typhoid-fever patients is added to emulsions of typhoid bacilli. Widal applied the reaction macroscopically and Grünbaum microscopically. The paper by Grünbaum was more extended than that of Widal, but the prior appearance of the latter's note has resulted in this diagnostic procedure being almost universally described as the "Widal or Gruber-Widal test." Grünbaum extended and emphasized the important observation of Gruber that the reaction of agglutination is only relatively specific. He examined thirty-two specimens of sera, nine from normal individuals (three of whom, however, had had typhoid fever previously) and twenty-three from persons suffering from various diseases other than typhoid. Emulsions of cholera vibrios, typhoid bacilli, and colon bacilli were added to varying amounts of sera from different patients. Agglutination of all three species was observed in certain instances; but in higher dilutions sera from normals or from persons

${ }^{1}$ Gruber, Max, and Durham, H. E.: Mïnchen. med. Wchnschr., 43, 285. 1896.

${ }^{2}$ Durham, H. E.: Proc. Roy. Soc., London, 59, 285. 1896.

3 Gruber, M.: Miunchen. med. Wchnschr., 43, 206. 1896.

4 Widal, F.: J. de méd. et chir. prat., p. 533. r 896.

5 Grünbaum, A. S.: Lancet, 2, 806. 1896. 
suffering from diseases other than typhoid fever failed to agglutinate typhoid bacilli. A few months later, further communications by Grünbaum and Durham emphasized additional limitations of the Widal test. However, this method when correlated with clinical findings in doubtful cases of typhoid was a most valuable addition to the physician's diagnostic equipment at a time when typhoid fever was much more widely prevalent and when vaccination against the disease had scarcely been undertaken.

Pfaundler, ${ }^{\mathrm{I}}$ in the year $\mathrm{r} 898$, described his so-called "thread" reaction which is now of historic interest only since it is essentially an agglutination reaction. Bordet ${ }^{2}$ was the first to show that bacterı play a passive rôle when they are agglutinaterl. Vibrios killed by chloroform were shown by Bordet to be still aggiutinable. Bacteria killed by heat or formalin behave in a similar fashion, as pointed out by Widal and Sicard, Van de Velde, and others. Very high temperatures do, however, render bacteria less agglutinable. This may be due to physico-chemical changes in bacterial protoplasm.

It was quickly established that agglutinins usually, but not invariably, appear when animals are immunized. The immunizing agents may be bacteria (vegetative or spore forms), fungi, yeasts, protozoan parasites such as trypanosomes, and the parasites of the sporotrichoses. Considerable variation in capacity to stimu'ate the appearance of agglutinins and, in agglutinability, was early shown to exist among the bacterial species. Generally speaking, motile species were found to be more readily agglutinated than non-motile. While various species of vibrios and members of the typhoid-dysentery-colon group are most readily agglutinable, $B$. pyocyaneus, $B$. pestis, B. tetani, B. pertussis, B. mallei, are also, as a rule, quite agglutinable. On the contrary, such species as $B$. anthracis, $C$. diphtheriae, pneumococci, streptococci, and'various other species of cocci agglutinate less readily. Various types of encapsulated bacilli, such as Friedländer's bacillus and Bacillus mucosus capsulatus, are frequently inagglutinable or but very slightly agglutinable.

\section{AGGLUTINOGENS AND AGGLUTININS}

Different strains or races of the same species of bacteria may show great variations in respect to agglutinability when acted upon by the same immune serum. Various explanations of this, usually speculative in character, have been offered. Bacteria when freshly isolated from the human or animal body are often inagglutinable, but after cultivation upon artificial media may become agglutinable. This very curious fact is difficult to understand. Bacteria which have been killed are not only capable of being agglutinated, as has been pointed out, but are also antigenic. They will, upon injection into animals, stimulate the appearance of agglutinins, as Widal and Sicard and Lévy and Bruns have shown. The former also established the fact that culture filtrates, extracts of bacteria, or fluids in which bacteria have undergone autolysis are antigenic. Arloing showed that tuberculin when injected into animals would produce agglutinins for tubercle bacilli-and mallein, agglutinins for $B$. mallei. This latter observation was made by Bonome. The antigenic element concerned in the production of agghutinins is sometimes described as agglutinogen. It may or may not be the same antigenic element as that which stimulates the appearance of precipitins. After the discovery of agglutinins for bacteria and other micro-organisms, Bordet in 1898 in a study of hemolytic sera showed that these contained in addition to the cellucidal substance a very active hemagglutinin. Furthermore, it was demon-

${ }^{2}$ Pfaundler, M.: Centralbl.f. Bakteriol., 23, 131. I898.

${ }^{2}$ Bordet, J.: op. cit., 10, I93. I 896 . 
strated that there are natural or normal agglutinins and hemagglutinins as well as specific agglutinins for bacteria and red blood cells. The former are present in the blood of normal animals; the latter, in that of immunized animals. The origin of normal antibodies is obscure. Certain types of protective antibodies such as diphtheria antitoxin are found in the blood of at least one species of normal animal, namely, the horse. The reason for the occurrence of such normal antibodies (often in considerable concentration) has not as yet been satisfactorily explained. Agglutinins were soon found to be only relatively specific, i.e., an agglutinating serum for species A was also found to agglutinate species B. Usually it is true only in very low dilutions of the immune serum. When certain species of bacteria are used as antigens, agglutinins in the greatest concentration appear in the blood of the immunized animals for the particular species employed. These are sometimes described as "major" agglutinins. Agglutinins may also be found in the serum for other species and are known as "minor" agglutinins.

It is postulated that in the bacterial protoplasm there is a hypothetical antigenic element, agglutinogen, which in immune animals is responsible for the presence of agglutinins. Many years ago Smith and Reagh, ${ }^{1}$ employing cultures of the bacillus of hog cholera as antigen, found that with this species one type of agglutinin could be produced for the bacterial cell bodies (somatic agglutinins) and another for the flagella. This subject has been again reviewed recently by Arkwright ${ }^{2}$ who has also brought forward some new evidence bearing upon the question as to whether or not there are two distinct antigens or agglutinable substances present at least in motile bacteria of the typhoid-colon group. The experimental evidence supporting the view that there are also two corresponding distinct agglutinins is set forth. The previous work had adduced macroscopic evidence. Arkwright has brought forward microscopic proof of the same thing. His description of the microscopic appearance in flagella and somatic agglutination is as follows:

With flagella agglutination, the flagella become coherent and hampered in their movements; individuals in the clumps are held together by cohering flagella, but the bodies of the bacteria do not lie touching, and, at any rate for some time, they may dance at the end of their tether and occasionally show violent to and fro and up and down movements. On the other hand when somatic agglutinin is in operation the surfaces of the bacteria cohere but the flagella on those surfaces which are not touching the neighboring bacteria are free and move vigorously, thereby bringing about active translation of single bacteria and also of clumps. This movement of clumps is like that seen in a rough motile culture clumped by salt and is a familiar phenomenon in an ordinary broth culture of a rough motile strain. Where much clumping has occurred no free bacteria may be present but nevertheless active motility is easily recognised by the movements of the clumps.

Shortly after the discovery of bacterial agglutinins it was sbown by Malvoz that many suljstances other than serum were capable of clumping bacterial emulsions in a non-specific fashion. Similarly, it was demonstrated that emulsions of certain species of bacteria if allowed to stand would undergo "spontaneous" or "salt" agglutination. This phenomenon is of course not a specific antibody-antigen reaction. Arkwright, in

I Smith, T., and Reagh, A. L.: J. Med. Research, ro, 89. 1903-4.

${ }^{2}$ Arkwright, J. A.: J. Path. Eo Bact., 30, 345. I927. 
I $92 \mathrm{I}$, showed that spontaneous agglutination of some species of bacteria may be prevented by employing $0.4^{2-0.1}$ per cent saline suspensions of micro-organisms. Acid agglutination suggested by Michaelis, Beniasch, and others has not been found to be of any service even in the differentiation of bacterial species which are not readily susceptible to serum agglutination. One of us (J. G. F.) some years ago showed that acid agglutination did not aid in the differentiation of closely related species of bacilli which constitute the encapsulated, gram negative group.

Specific or immune agglutinins appear in the body fluids of man and lower animals during the course of various infectious diseases. They may also be produced by the injection of bacterial and other antigens. In man, vaccination against typhoid and paratyphoid fever, dysentery, and cholera is marked by the appearance of agglutinins which persist for varying lengths of time. Intravenous, intraperitoneal, or subcutaneous injection of emulsions of dead or attenuated bacteria, at suitable intervals, usually repeated on several occasions, will give rise to potent agglutinating sera. As is the case in other types of antibody response to the injection of antigens, some time elapses after the completion of active immunization before the agglutinin concentration is high. The degree of response and the length of this period varies in different animals and with different antigens. Where the injection of emulsions of one species of bacteria results in the appearance of agglutinins for this and other closely related species, dilution may be resorted to, when the major agglutinins will be found in much higher dilutions than the minor. Castellani, ${ }^{\mathrm{I}}$ in $\mathrm{I} 902$, introduced the procedure of absorption of agglutinins which in cases of mixed infection may be employed to remove certain of them while leaving others. This depends upon the fact that, when an animal is immunized against more than one species of bacteria, major and minor agglutinins may be formed for each species.

Agglutinins resist heating to $60^{\circ} \mathrm{C}$., but above this temperature they are gradually destroyed and at $75^{\circ} \mathrm{C}$. destruction is complete. They are not dialyzable and are precipitated in the globulin fraction of serum. They are active in a neutral or slightly acid menstruum. Agglutinins in dried serum will persist for a long time. The isoelectric point of a serum globulin fraction in which the agglutinins are salted out is $\mathrm{pH}$ 5.5. The iso-electric point of agglutinins, according to Szent-Györgi, ${ }^{2}$ lies between $\mathrm{pH} 5.69$ and 5.39 . While agglutination is favored by a temperature of $37^{\circ} \mathrm{C}$, it goes on more slowly at lower temperatures.

A hypothesis to explain the mechanism of practically all immune procesess was formulated by Ehrlich and is known generally as the "side-chain theory." It is now generally agreed that the explanation of bacterial agglutination therein outlined is probably not the correct one. Further reference, therefore, to this conception of the phenomenon seems unnecessary here. ${ }^{3}$

The agglutination of bacteria (and of red blood cells) by their homologous immune sera is a specific antibody-antigen reaction. Bordet, ${ }^{4}$ in 1896 , showed that dis-

${ }^{1}$ Casteỉlani, A.: Ztschr.f. IIyg. u. Infektionskrankh., 40, I. 1902.

${ }^{2}$ Szent-Györgi, A.: Biochem. Ztschr., I 1 3, 36. I921.

${ }^{3}$ Cf. chap. lxxxi, by Dr. Manwaring, in this volume.

${ }^{4}$ Bordet, J.: Traitê de l'immunitê dans les maladies infectienses. Paris: Massons \& Cie, I920. 
sociation of agglutinated red blood cells and their specific serum was possible by heating from $50^{\circ}$ to $60^{\circ} \mathrm{C}$; ; on cooling, the agglutination reappears, to disappear again on heating. If agglutinated bacteria are treated with a dilute soda solution, partial dissociation with liberation of the antibody occurs, according to Hahn and Tromsdorff. ${ }^{\text {I }}$

Bordet has conceived (I899) the reaction of agglutination as consisting of two phases or of two successive phenomena. The first is an immune reaction, an antibodyantigen union. This consists in the formation of a complex resulting from "l'intervention des affinités spécifiques" (Bordet). The second phase is physico-chemicalthe clumping or aggregation of sensitized bacteria. This latter is, according to Bordet, agglutination proper. In 1899 Bordet observed that the higher the concentration of agglutinin in a serum the greater is the susceptibility of the antigen-agglutinin complex to the clumping action of salt (electrolyte). It was further demonstrated by him that this action occurred only in the presence of an electrolyte. Bordet summarized his observations as follows:

En résumé, il résulte de ces faits que l'agglutination, phénomène si frappant et qu'on croirait si caractéristique, est un processus en réalité secondaire. L'essentiel au point de vue de l'immunité c'est union de l'anticorps à l'antigène; c'est à ce moment que la spécificité intervient. Le fait que certains antigènes acquièrent, en se sondant à leur anticorps, cette qualité d'ailleurs très banale d'être flocculables par les électrolytes est théoriquement accessoire. Mais il attire vivement l'attention parcequ'il rend la réaction très perceptible à nos sens, et c'est pourquoi l'anticorps en jeu s'appelle agglutinine lorsqu'il s'agit de pareils antigènes.

The functional significance of agglutinins in infectious processes is probably subsidiary. Certainly in diseases such as anthrax the agglutinating power of the serum of an animal is not an index of the degree of resistance to this infection, as Gengou was the first to show. The earlier view of Gruber and others that agglutinins possess protective or therapeutic properties has been discarded generally. Widal and Sicard showed that relapses occurred in patients suffering from typhoid fever even when the concentration of agglutinins in their blood was greatest. Bull ${ }^{2}$ has shown experimentally that agglutination occurs in vivo. It was observed by this investigator, as it had been earlier by Besredka, ${ }^{3}$ that preliminary agglutination favored subsequent phagocytosis. Bull found that agglutinated bacteria are promptly removed from the circulation in experimental animals, and in consequence bacteremia does not ensue. As a result of the work of Besredka and Bull, it may be argued that agglutination in certain infections at least favors phagocytosis and, if this is the case, is part of the protective mechanism in such infections. This point deserves further exploration. ${ }^{4}$

\section{AGGLUTINATION PROCEDURES}

It is not our purpose to describe the precise details of the technical procedures adopted in carrying out methods of demonstrating agglutination. Such instructions may be found in textbooks on bacteriology or serology. We wish, however, to indicate in outline these methods and to comment upon certain of their salient features.

I Ibid.

${ }^{2}$ Bull, C. G.: J. Exper. Med., 22, $4 \$_{4}$. I915.

${ }^{3}$ Besredka, A.: Ann. de l'Inst. Pasteur, 15, 209. 1901.

${ }^{4}$ See chap. lix, by Dr. Bailey, in this volume. 
The microscopic-slide agglutination test is widely used for determining the presence or absence of a specific agglutinin for micro-organisms, especially those of the enteric-fever group. In collecting blood for the test, a few drops are allowed to dry on the ground surface of a glass slide or other impervious material; or a large amount is collected, sufficient to yield a few drops of serum. Saline or bouillon in appropriate amounts is added to the dried blood with a Pasteur pipette. With small amounts of serum, dilutions may be made with a hemocytometer pipette, either "red" or "white." In order to approximate the desired initial dilution with any degree of accuracy, it is necessary to adopt some color standard with which to match the blood of the specimen. Such a color standard, using defibrinated sheep's blood, has been described by Gilbert and Parsons. ${ }^{\mathrm{x}}$ The dilutions I/IO, I/20, and I/40 are commonly employed. Hanging-drop preparations are made after adding equal amounts of an eighteenhour (incubated at $25^{\circ} \mathrm{C}$.) broth culture of B. typhosus or other desired species. The coverslip margin is streaked with vaseline to insure that the small chamber is sealed to prevent evaporation of the suspended drop. The final dilution after the addition of the bacterial suspension becomes I/20, I/40, and I/80. A control of micro-organisms and diluent is of course essential. The test is incubated at $37^{\circ} \mathrm{C}$., examined at once and at fifteen-minute intervals for one hour. Complete or almost complete agglutination with loss of motility at I/40 dilution of serum is usually considered diagnostically positive for typhoid fever. Normal serum rarely if ever manifests a complete agglutination reaction at I/40 dilution when the microscopic method is employed.

The microscopic-slide agglutination test is widely used, as $\mathrm{Hull}^{2}$ found in answer to a questionnaire relative to the technique employed, sent to state and municipal laboratories. To quote from his paper: "It is safe to say that to-day probably no two laboratories perform the Widal test in exactly the same manner. . . . " The answers received from fifty-three laboratories leave one in no doubt as to the desirability of adopting a definite standard method based upon the control of the known factors involved. In regard to the choice of suspension of bacilli, Gilbert and Moore ${ }^{3}$ and the writers of the Medical Research Council, "Special Report Series," No. 5I, have demonstrated the impractibility of using killed cultures (formalinized o. I per cent) for microscopic agglutination. Further, the culture used must be one of satisfactorily proved agglutinability and employed at a definite density. A barium sulphate standard of opacity as described by McFarland ${ }^{5}$ may conveniently be employed. For microscopic agglutination tests a density of $\mathrm{I}, 000$ million bacilli per cubic centimeter is desirable. Controls with a known standardized serum should be set up. For this purpose the lowest dilution of serum is used which, by microscopic-slide agglutination, definitely agglutinates the homologous organism and agglutinates slightly or not at all the heterologous organism. ${ }^{6} \mathrm{~A}$ uniform system of recording results is essential. Such a system as described in the foregoing publication deserves general adoption.

The microscopic-slide agglutination method possesses certain distinct advantages: It can be carried out with very minute quantities of serum, even with less than a drop; it can be carried out quickly; it requires a minimum of apparatus. Over against these advantages one must balance the limitations. It is quantitatively inaccurate; its end-point is very ill defined; it is qualitative rather than quantitative.

\footnotetext{
${ }^{2}$ Gilbert, R., and Parsons, E. A.: J. Lab. \& Clin. Med., 10, 206. 1924-25.

${ }^{2}$ Hull, T. G.: Am. J. Pub. Health, r6, 901. I926.

3 Gilbert, R., and Moore, A. C.: J. Lab. \& Clin. Med., 7, 547. 1922.

4 "Medical Research Council, Special Report Series," No. 5I. I920.

${ }^{5}$ McFarland, J.: J.A.M.A., 49, I176. 1907.

${ }^{6}$ Wadsworth, A. B.: Standard Methods, p. 174. Williams \& Wilkins, 1927.
} 
The macroscopic methods may conveniently be classified as "qualitative" and "quantitative." In the former category are those methods which are for the most part designed to save time and trouble, where a prompt laboratory opinion is desired as an aid to clinical diagnosis or when a large number of colonies or cultures are to be provisionally identified.

Essentially, a drop of highly potent agglutinating serum is brought into contact on a slide with a homogeneous suspension of micro-organisms and rocked gently to and fro upon a longitudinal axis. If the suspension and serum are homologous, a clumping, visible to the unaided eye, occurs in from three seconds to two minutes. A control of diluent and microorganisms must remain uniformly turbid. The density of suspension should be heavy, approximately 5,000 to 10,000 million per cubic centimeter. Two additional controls should be used; namely, normal serum from the same animal species as that from which the test serum was obtained, used in the same dilution as in the test serum, and the test serum against its homologous culture. The dilution of serum used in the test must be determined by preliminary trial. In addition, it must be sufficiently dilute so that group agglutination will not be manifest and yet sufficiently concentrated for rapidity of action. As a rule, a serum of titre, I in 4,000 , may be used at I in I00. It is frequently desirable to make use of a serum which has been "absorbed" by suspensions of closely related species. Such a serum may be prepared in bulk after the method recommended by Andrewes. ${ }^{x}$ The authors of the Medical Research Council, "Special Report Series," No. 5I, comment upon this rapid method as follows: "In any case it must be understood that this rapid method is merely one for preliminary orientation, and may be wholly misleading; it cannot replace the cultural tests which are requisite for establishing the nature of a bacterium. Neglect of this principle may easily lead to serious errors in diagnosis." Bass and Watson ${ }^{2}$ applied this slide method as an aid to the diagnosis of typhoid fever.

Other macroscopic methods are those of Garrow, ${ }^{3}$ Neisser, ${ }^{4}$ and Wright. ${ }^{5}$

The macroscopic tube agglutination test is certainly to be chosen whenever possible since with this method only is one able to determine with any degree of exactness the actual measure of the agglutinins present in a serum. In the identification of species of microorganisms its use is obligatory. A series of dilutions are prepared, and to unit volumes of these dilutions contained in tubes of uniform caliber is added a unit volume of bacterial suspension. A control tube receives saline and suspension only. After thorough mixing, the tubes are placed in a water bath or incubator at the desired temperature for a definite time. Readings may be made after allowing the test to stand in the icebox or room, usually overnight. Suspension of formalin-(O.I per cent) killed micro-organisms diluted to standard opacity are commonly employed. Within limits, the lower the density the more accurately the titre of a serum may be determined. From 300 to I,000 million bacilli per cubic centimeter is a useful working range for the enteric group. The suspension may be made from a broth culture or of a young agar culture. Repeated subculturing in broth for a period of two or three weeks may be resorted to in the case of strains refractory to agglutination. Spontaneous agglutination ${ }^{6}$ may in certain instances be overcome by employing a buffered broth for the culture and suspension of micro-organisms. Mudd ${ }^{7}$ has described a method of detecting the binding of agglutinins by suspensions which are agglutinated with difficulty.

I Andrewes, F. WV.: J. Path. Eo Bact., 25, 507. 1922.

${ }^{2}$ Bass, C. C., and Watson, J. A.: Arch. Int. Med., 6, 717. I910.

3 Garrow, R. P.: Lancet, 1, 262. I917.

4 Neisser, M.: (Pröscher) Centralbl.f. Bakteriol., Abt. II, Orig., 31, 400. I902.

5 Wright, A. E.: "Medical Research Council, Special Report Series," No. 5I, p. IIO. I920.

${ }^{6}$ Bliss, W. P.: J. Exper. Med., 36, 575. I922. 7 Mudd, S.: J. Immunol., I3, I I3. I927. 
Since two suspensions, prepared in apparently the same way, from two single colonies derived from the same stock culture may evidence wide differences in agglutinability, ${ }^{\mathrm{I}}$ it is often desirable for purposes of uniformity to make up a large amount of suspension at one time. Dreyer ${ }^{2}$ has controlled this variable factor by standardizing the agglutinability of each suspension prepared. The utmost care in pipetting must be observed to insure even a reasonable degree of accuracy. The Committee on Pathological Methods of the Medical Research Council points out that an error in carrying out six to eight doubling dilutions by the pipette method may commonly be in the order of magnitude of 8-10 per cent. Information and instruction regarding the selection and use of pipettes and the measurement by the drop method are contained in its "Special Report Series," No. $5 \mathrm{I}$.

In selecting tubes for carrying out the agglutination reaction it is important to take cognizance of uniformity of size and the fact that the narrower the diameter of the tube the better and more quickly is the agglutination brought about. The optimum temperature for flocculation lies between $50^{\circ}$ and $55^{\circ} \mathrm{C}$. At a temperature of $37^{\circ} \mathrm{C}$. the reaction proceeds more slowly. In addition, at this temperature the growth of micro-organisms may interfere with the interpretation of the reaction. Certain micro-organisms are agglutinated more rapidly than others, one-half to two hours for B. typhosus at $55^{\circ} \mathrm{C}$.; dysentery bacilli, four to six hours; meningococci, twenty-four or even forty-eight hours. In recording the results, complete agglutination with clear supernatant in the highest dilution of serum may arbitrarily be chosen as the titre of the serum. It is preferable, however, to indicate the degree of agglutination in each tube of the test in some manner as illustrated in the manual by Wadsworth. ${ }^{3}$ Dreyer's method incorporates the drop method for all measurements and formalinized cultures of known and constant agglutinability. The agglutinating power of a serum he expresses in definite units. Glynn, ${ }^{4}$ Krumbhaar and Smith, ${ }^{5}$ and Fennel ${ }^{6}$ have reported favorably upon this method. Dryer's technique undoubtedly makes for delicacy and accuracy.

Mention has already been made of absorption of agglutinins. Much confusion has arisen as to the application of this phenomenon in the identification of bacterial species. The matter is further complicated by the introduction of the factor of "variation" later to be discussed. If, to an agglutinating serum, is added a suspension of its homologous micro-organism in appropriate amounts and under suitable conditions, the power of this serum to agglutinate a fresh suspension of its homologous micro-organism will have been reduced almost to zero. The amount of suspension required to effect this reduction is called the "absorbing dose." Commonly this is expressed as the number of micro-organisms, as gauged by opacity standards, per cubic centimeter of undiluted agglutinating serum. In practice it is usual before adding the suspension to dilute the serum I/ IO to $\mathrm{I} / \mathrm{I00}$, depending on its titre. Approximately $25,000-100,000$ million organisms per cubic centimeter of serum will remove all but the last traces of agglutinins. The minimum absorbing dose of the homologous

I Andrewes, F. W.: op. cit., 25, 506. 1922.

2Dreyer, G.: ibid., 13,331. I909; “Medical Research Council, Spec. Rep. Series,” No. 51. I920.

3 Wadsworth, A. B.: op. cit., p. I62. 1927.

4 Gilynn, E.: Lancet, г9г, 877. г916.

${ }^{5}$ Krumbhaar, E. B., and Smith, W. B.: J. Infect. Dis., 23, I26. I918.

${ }^{6}$ Fennel, E. A.: J.A.M.A., 70, 590. 1918. 
micro-organism should be determined for each serum. ${ }^{\mathbf{x}}$ One and one-half to three times this dose is generally found satisfactory for use in absorption experiments. The time and temperature at which absorption is carried out are of importance. Two hours at $50^{\circ} \mathrm{C}$. is adequate. It is obvious that the carrying out of an absorption experiment must be controlled by a tube containing serum alone, diluted to the same degree as the absorbing mixtures and subjected to the same physical conditions.

\section{RECIPROCAL ABSORPTION OF AGGLUTININS}

It is fallacious to assume the identity of two micro-organisms $A$ and $B$ if the agglutinins of A serum are exhausted (or nearly so) by B bacteria, though for practical purposes this assumption is sometimes justifiable. It is absolutely essential in order to establish complete identity that the serum of A be exhausted for agglutinins of $\mathrm{A}$ by the suspension of $\mathrm{B}$, and that the serum of $\mathrm{B}$ be in like manner exhausted for agglutinins of B by the suspension of A. This procedure is termed "reciprocal absorption." In the ultimate analysis there is no more accurate means of species identification than this method when properly carried out and adequately controlled. White, Krumwiede, and others have particularly stressed the importance of reciprocal agglutination as the only legitimate use of the phenomenon of agglutination absorption for establishing the identity of culturally similar species. To quote White: "Any other mode of applying the absorption test to the proof of identity is worse than futile."'z

\section{GROUP AGGLUTININS}

Andrewes $^{3}$ has brought forward interesting data bearing on the problem of group agglutination among the Salmonella. He attempted an analysis of the antigens in the bacteria and of their agglutinins in their respective sera. "Single colony broth cultures picked from a plating of a stock culture do not all behave in an antigenically similar manner. In all mass cultures there exist side by side two types of bacilli, and two only, sharply differentiated in their antigenic structure. The one contains the specific antigen and the other the group antigen, but . . . neither is absolutely pure." It was found impossible to cultivate these as separate entities; each changes readily into the other; they are not identical with the "rough" and "smooth" colonies of Arkwright, later to be mentioned. By carefully selecting. the specific type antigens and producing with these specific sera, Andrewes suggests that the absorption test may be eliminated. He further claims that by using formalinized emulsions of specific typa the sharpness and value of serodiagnosis in human infection would be enhanced. Krumwiede 4 points out the limitation of Andrewes' method as a substitute for absorption.

\section{FURTHER APPLICATIONS OF THE AGGLUTINATION REACTION}

The application of the reaction of agglutination may conveniently be grouped in three categories, namely: identification of micro-organisms, examination of sera, standardization of sera and vaccines. Specific allusion to some of its uses has already

\footnotetext{
${ }^{1}$ Krumwiede, C., Cooper, G., and Provost, D. J.: J. Immunol., Io, 55. I925.

${ }^{2}$ White, P. B.: "Medical Research Council, Spec. Rep. Series," No. 91, p. 12. I925.

3 Andrewes, F. WV.: op. cit., 25, 506. I922.

4 Krumwiede, C., Cooper, G., and Provost, D. J.: loc. cit.
} 
been made in dealing with certain phases of the subject. We wish to give a brief sketch of additional results or uses of this reaction. No attempt has been made to review the voluminous literature bearing upon this phase of agglutination, even were that possible. We have, rather, chosen illustrations for the purpose of showing some of the many and multifarious ways in which the reaction has forwarded the study of bacteriology.

There is no laboratory method of gauging the therapeutic value of antimeningococcus serum comparable in its practical application to the assay of diphtheria or tetanus antitoxin. It has been established that sera having a high agglutination titre against the meningococcus are therapeutically more effective than those with a low titre. In addition, since certain ${ }^{I}$ types or representative strains from groups I, II, and III are used for the immunization of horses, it is important to determine the degree of polyvalency. A polyvalent antimeningococcus serum is therefore tested to determine its agglutination titre against the standard group strains. A polyvalent standard control serum is used for comparison. In a similar manner, polyvalent (mannite fermenters and non-fermenters) antidysentery serum is tested as to its agglutinative titre in addition to its neutralizing value for toxic filtrate from a Shiga strain.

The antigenic power of typhoid vaccine ${ }^{2}$ is tested by the agglutination method by estimating the antibody response of rabbits when injected with the vaccine. The standardization of monotypical sera for diagnostic purposes is also gauged by agglutination.

Mention has been made of the value and use of the agglutination test as an aid to the diagnosis of enteric fevers. It is of definite assistance also in the detection of typhoid carriers. The reaction is positive in from two-thirds to three-quarters of those in whom typhoid bacilli persist, and more frequently in recovered than healthy carriers. ${ }^{3}$ In tularemia, ${ }^{4}$ in suspected cases of Malta fever and $B$. abortus infection, an agglutination test is of diagnostic value; similarly, as evidence of infection with Leptospira icterohaemorrhagiae..$^{5}$ The Weil-Felix ${ }^{6}$ reaction in suspected typhus fever may be carried out.

In veterinary medicine the agglutination reaction is widely used for B. abortus infection of cattle. Fitch, ${ }^{7}$ Gwatkin, ${ }^{8}$ and Schroeder and Cotton ${ }^{9}$ report that all proved carriers of $B$, abortus show a postive reaction in higher dilution than $\mathrm{I} / \mathrm{roo}$. In lacillary white diarrhea of chicks and pullorum infection, Gwatkin ${ }^{10}$ considers any

I Wadsworth, A. B.: J. Exper. Med., 33, 107. I921.

${ }^{2}$ Russell, F. F., Nichols, H. T., and Stimmel, C. O.: Mil. Surgeon, 47, 359. I9zo.

3 Gay, F. P.: Typhoid Ferer, p. I30. Macmillan, r918.

${ }^{4}$ Francis, E., et al.: Bull. Hyg. Lab., No. 130. I922.

5 Wadsworth, A. B., ct. al.: J.A.M.A., 78, I I 20. I922.

${ }^{6}$ Weil, E., and Felix, A.: Wien. klin. Wchnschr., 29, 33. 1916.

7 Fitch, C. P., et al.: J. Am. Tet. W.A., 54, 681. 1919.

${ }^{8}$ Gwatkin, R.: Rep. Ont. I'el. Coll. 1923.

9 Schroeder, E. C., and Cotton, IV. E.: J. Am. I'et. M. A., 64, 479. I924.

so Gwatkin, R.: op. cit. I926. 
degree of agglutination at $\mathrm{I} / 50$ dilution of serum as positive. In dourine $\mathrm{I}^{\mathrm{I}}$ the test is of value.

For the identification of bacterial species the reaction of agglutination and agglutinin absorption is more widely applied than for any other purposes. Differences at present undetectable by an examination of morphological or cultural characteristics are readily manifested. The finding of distinctive subgroups or strains among a great many species is the rule rather than the exception. Thus pneumococci and meningococci are each separable into groups or types. That this finer differentiation is of more than academic interest is attested by the fact that the identification of the group to which the infecting micro-organism belongs has an important bearing upon the choice of serum used in therapy. The toxin elaborated by the various serological strains of $B$. tetani and $C$. diphtheriae are apparently neutralized, respectively, by tetanus and diphtheria antitoxin obtained by immunization with the toxin of any one strain. However, this is not true in the case of B. botulinus. The members of one group produce a toxin which is not neutralized by an antitoxin common to another group.

\section{BACTERIAL VARIATION AND AGGLUTINATION}

One cannot evade the puzzling and very disconcerting problem of bacterial variation or dissociation in its bearing on the application of the phenomenon of agglutination. "The bacteriologist nowadays has not only to define the species of bacterium with which he is dealing, but also the particular form or variant." 2 One must tread lightly and with great caution on the new ground which is being opened for exploration. Certain facts have apparently been established in certain groups of microorganisms. Schütze 3 and Arkwright" have described "rough" variants among the dysentery and Salmonella groups, which behave in a characteristic fashion. The $\mathrm{R}$ ("rough") form from strains of $B$. dysenteriae (Shiga) agglutinates spontaneously in 0.85 per cent sodium chloride solution. Agglutination tests, however, may satisfactorily be carried out with weaker solutions (0.4-0.I per cent). The S ("smooth") form makes a stable suspension in 0.85 per cent saline. The $S$ form on agglutination yields large clumps; the $\mathrm{R}$, small clumps readily shaken up into a turbid suspension. Further, the two forms differ very markedly in their agglutinating, antigenic, and absorbing properties. The $\mathrm{S}$ forms obtained from different strains of $B$. dysenteriae (Shiga) resemble one another serologically, but are distinct from all the $\mathrm{R}$ forms; and the $\mathrm{R}$ forms of different strains appear to have little relation to any $\mathrm{S}$ forms, but to be closely related to one another. The striking fact is that there has apparently been revealed a difference in antigenic properties of $\mathrm{R}$ and $\mathrm{S}$ cultures, both of which were isolated from the same parent culture. Goyle ${ }^{5}$ undertook to investigate the serological relationship between $B$. typhosus and $B$. enteriditis and to discover to what extent the $\mathrm{H}$ and $\mathrm{O}$ forms of Weil and Felix ${ }^{6}$ and the $\mathrm{S}$ and $\mathrm{R}$ forms described by Ark-

"Watson, E. A.: Dominion of Canada Depl. of Agric., Health of Animals Branch. 1920.

${ }^{2}$ Arkwright, J. A.: loc cil. $\quad 4$ Arkwright, J. A.: op. cil., 24, 36. 1921.

${ }^{3}$ Schütze, H.: J. IIyg., 20, 330. т92 I. 5 Goyle, I. N.: J. Path. So Buct., 29, I 49. 1926.

${ }^{6}$ Weil, E., and Felix, 1.: Zlschr. f. Immunitülsforsch. u. exper. Therap., Alst. 1, Orig., $29,24$. 1920. 
wright, and the distinctive antigens of these variants, were concerned in the crossagglutination of the two bacterial species. As interpreted by serological relationships, antigenic structure, if one may justifiably apply a solid term to such a delicate and phantom fabric, has become kaleidoscopic - to use a simile of Andrewes. Tulloch, ${ }^{\mathrm{I}}$ in reviewing the present position of bacterial agglutination, concludes in part:

In the present state of ignorance it is inadvisable to employ the procedure known as receptor analysis in order to investigate bacterial genetics and the antigenic relationship of micro-organisms to one another. The use of the procedure involves the assumption that each reaction observed is referable to a separate constituent of antigen and a separate moiety of antibody. There is no evidence whatsoever that this assumption is valid.

It is largely by means of the phenomenon of agglutination, in respect of the assumed correlation between the in vitro results of in vivo experiments, that a picture of the apparent complexity of antigens is revealed - a picture which perhaps presents today more the aspect of an incompleted jig-saw puzzle than the finished work of art of tomorrow.

\section{SUMMARY}

In viewing the subject, agglutinins and their application, as a whole, certain facts are apparent. First, the reaction is specific in that, when adequately controlled and precisely carried out, differences in the identity of micro-organisms, too slight and too subtle to be appraised by cultural and biochemical methods, may be established. These differences have not only a theoretical interest but may, in certain instances, serve as a basis of more selective and more rational serum therapy, on the one hand, and, on the other, correlate epidemiological data with clinical findings. Further, among certain other species of micro-organisms, strains with distinctive and definite agglutinative characteristics are yet so closely related in their pathogenicity that one measurable antibody response (antitoxin) is identical for all strains. Second, the methods of the technique of measuring agglutination have been developed to an extraordinary degree of delicacy. Third, the fact of bacterial variation, the interpretation of which is still incomplete, introduces a new problem in respect of the application of the phenomenon of agglutination. Fourth, of recent years, much speculative philosophy has been brought to bear in order to correlate the antibody response as revealed by in vitro reactions with the structural components of its antigen. Fifth, the fundamental fact of the specificity of antigen-antibody reaction is as much a mystery as ever.

I Tulloch, W. J.: J. Roy. Army M. Corps, 48, 435. 1927. 


\title{
CHAPTER LXI
}

\section{PRECIPITINS AND THEIR APPLICATIONS}

\author{
H. M. POWELL
}

Eli Lilly and Company, Indianapolis

\section{INTRODUCTORY}

Following closely upon the demonstration of antibody causing the specific clumping or agglutination of cellular antigens came the description of antibodies causing precipitation of unformed protein materials employed as clear solutions. Kraus ${ }^{\mathrm{I}}$ found that cleared bacterial filtrates are antigenic, and that corresponding antibody can readily be obtained from properly treated animals. When suitable mixtures are made of precipitinogen and the artificially incited precipitin, precipitates result. The reaction was found to be relatively specific, and various uses were suggested for the precipitin reaction including species determination of micro-organisms, species sources of proteins, serum diagnosis, etc. There was little hesitancy in assigning a new name, the "precipitins," to the specific agents of such sera. Later work, however, has indicated that the mere precipitation may be a secondary issue and the antibody in question not a "precipitin" but a lytic antibody.

Especially interesting were the observations that each antiserum reacted for the most part only with the homologous antigen. The reaction, then, is in conformity with other antigen-antibody reactions previously described in that specificity, perhaps one of the most fundamental cornerstones of immunity, is a marked characteristic.

It may be supposed that the reactions leading to the appearance of the visible precipitate, the ring or the flakes, are closely similar to those initiating agglutination of cellular antigens. A marked distinction is the degree of fineness of precipitinogen as contrasted with the cellular antigens in agglutination reactions. Extensive lists of substances encountered throughout the field of biology have the property of inciting precipitin on injection into suitable animals. This is true also of the antigen concerned in agglutination.

\section{PRECIPITINOGEN}

The antigenic nature of precipitinogen appears to be closely linked with its protein nature. The material may be desiccated and only extraction will release it, or it may be very old. If it is intact protein it may still act as an antigen. Many attempts toward more or less completely modifying precipitinogen, i.e., so it may no longer give "protein reactions," have resulted in alteration of antigenic power or even in its destruction altogether. Any procedure certainly destroying protein is also destructive of precipitinogen. In a great many experiments in this field it appears doubtful whether non-protein material alone can act as precipitinogen. Ultimately it would be difficult to eliminate in this comection combination between any non-

${ }^{ \pm}$Kraus, R.: Wien. klin. W'chnschr., 10, 736. 1897. 
protein material intended as antigen and the serum proteins of the animal being immunized, thus possibly constituting a sort of synthetic antigen. Also the criteria by means of which the protein nature of a material is judged are very crude compared to the delicacy of the biological reactions. In attempts which have been made toward changing precipitinogen physically, ${ }^{1}$ as by heating or chemically by the addition of various chemical reagents, the changed properties, including those of specificity, seem to be accounted for to a considerable extent by the fact that many precipitinogens are complex materials, in many cases mixtures of several components, and that the various procedures resorted to act as a sorting device through which only some components can pass. Prezone phenomena also are involved as well as the rapidity of production of antibody in the immunized animal. Where questions of altered specificity are involved it is necessary to carry out immunization procedures for a longer period of time, for altered antigen-incited antibody may then be found to give reactions with unchanged native antigen. Also significance is to be attached to the failure to obtain iso-precipitins against altered precipitinogen. It appears very desirable to obtain greater knowledge of the characteristics of precipitinogens which can safely be regarded as single individual substances. Lack of ample accurate knowledge in this respect is probably not common to precipitinogen-precipitin study alone but rather is the case in several fields of immunology. ${ }^{2}$

\section{PRECIPITIN}

The properties of precipitins are like those of the agglutinins. They are fairly heat-resistant antibodies present for the most part in the serum of immune animals only. Their activity does not depend on the presence of complement, and when once inactivated at temperatures of about $70^{\circ} \mathrm{C}$. they cannot be reactivated. Their potency can be most readily estimated by testing the immune serum undiluted against a series of dilutions of precipitinogen. Accurate measurement necessitates learning both antigen and antibody titres through testing a series of dilutions of each, and determining the points of maximum precipitation.

The manner in which precipitins act upon homologous antigens is not clearly understood. Considerable species specificity is shown, although the usual group reactions occur here as elsewhere in immunology. Their appearance is often more marked in testing sera from animals subjected to a prolonged course of immunization. In testtube experiments in which large amounts of precipitinogen are included a prezone may be noticed. ${ }^{3}$ The reaction of the medium and the presence and character of electrolytes markedly influence the precipitin reaction even to the extent of determining in a large measure whether or not a visible reaction will occur. Despite the large amount of work done upon precipitins, their exact manner of action has not been learned. Ordinary precipitins no doubt consist somewhat of mixtures ${ }^{+}$of antibodies since most precipitinogens are mixtures. Therefore much of the work on the proper ties of precipitins must of necessity be repeated with simple antigens.

I Obermeyer, F., and Pick, E.: ibid., r7, 265. 1904; 19, 327. 1906.

${ }^{2}$ Cf. chaps. $1-1 v$, lix, lxx, lxxv-lxxviii of this volume.

3 Opie, E.: J. Immunol., 8, 19. I923.

${ }^{4}$ von Dungern, F.: Centralbl. $f$. Bakteriol., Abt. I, 34, 355. I903. 


\section{SPECIFICITY}

Although the specificity of reactions of precipitins indicates a similarity to other antibodies, group reactions at low titres or high concentrations of antibody are frequent. These may be especially anticipated with low potency sera, with sera which have aged or have been drawn after a prolonged course of immunization. In most cases difficulties in this respect can be avoided by using highly potent antisera, and by carrying out the desired tests at dilutions sufficiently high to eliminate group- or cross-reactions. In routine tests it goes without saying that adequate control mixtures must be arranged in order to gain reliable information from precipitin tests.

Aside from the detection of protein, the determination of species origin is of considerable interest. Group reactions encountered in the precipitin reaction as in other biological reactions have been attributed to close similarity or identity of the various antigens involved, and to close biological relationship of the organic species ${ }^{\mathrm{I}}$ furnishing the antigens.

While this may be true to a considerable extent it should not be surprising to encounter certain protein material several times throughout the animal and plant kingdom when many species are more completely examined. The distribution of so-called "heterophile antigen" indicates that species specificity is not the all-important phase of specificity, and that many overlappings exist in protein distribution which group sometimes very dissimilar and distantly related morphological forms together. This suggests prophylactic immunization with non-toxic varieties of antigens found in disease-producing viruses.

\section{ORGAN SPECIFICITY}

Aside from species specificity of precipitin reactions, considerable interest attaches to organ specificity. It is commonly believed that many organs in an animal organism contain one or more precipitinogens more or less common to the species. The detection of these has been regarded as identifying the species. Also it is commonly believed, and might be anticipated, that certain highly specialized organs such as the eye should contain very similar if not the same material among many species of animals. These substances may be so different from the other antigenic substances of the organism that they may be antigenic (auto-antigenic) when the animal is treated with them. That an animal's own proteins are not generally antigenic for it under normal conditions shows that organ specific antigenic differences are not common.

\section{PRECIPITIN-PRECIPITINOGEN COMBINATION in vivo and in vitro}

On examining the main conditions found in precipitin-precipitinogen reactions there appears a curious apparent coexistence of antigen and antibody in the test tube or the experimental animal. That is, the material or mixture under test reacts with both fresh antigen and fresh antibody. ${ }^{2,3}$ Why such coexisting antigen and antibody do not precipitate each other is hard to understand. Protective accessory substances may be invoked in order to explain tentatively such an anomaly. It may be recalled, on

I Nuttall, G.: Blood Immunity and Blood Relationship. Cambridge University Press, rgo4.

${ }^{2}$ Linossier, G., and Lemoine, G.: Compt. rend. Soc. de biol., 54, 85. 1902.

3 Eisenberg, B.: Bull. de l'Acad. des sc. de Cracou', p. 576, 1902; Centralbl.f. Bakleriol., Abt. I, 31, 773. 1902. 
the other hand, that many antigen-antibody reactions occur rapidly, indicating high affinities, and little probability of coexisting uncombined antigen and antibody.

In a great part of the work with the precipitin reaction, highly complex antigens have commonly been used. These include a wide range of substances such as normal serum and egg albumen. There is little doubt that these materials are in reality mixtures of many individual, simple precipitinogens. When these mixtures are injected into various animals the resulting precipitins are also mixtures, made up of different antibodies. If one animal is used, certain components in the complex precipitinogen may be more antigenic than others and a mixture of unequal amounts of the various simple precipitins may result in the immune serum. If another test animal is treated with the same precipitinogen, certain other individuals in it may be highly antigenic while those most antigenic in the former case may be of little potency or perhaps entirely innocuous. The precipitin incited in the latter animal may differ very much from the former.

Great variability in the constitution of ordinary precipitin in the hands of different experimenters may be expected according to the duration of the course of artificial immunization. Ordinary precipitin serum, which is very often an extensive mixture, drawn early from the animal would contain those individual precipitins in excess which are most easily and quickly incited, while that drawn later would contain the slower-appearing individual antibodies. These conditions, together with different degrees of affinity between the different sets or pairs of antigens and antibodies, would easily permit an apparent coexistence of antigen and antibody with no resulting action. Also instances would occur where supposedly similar immune sera would precipitate on being pooled.

Opie ${ }^{\mathrm{I}}$ has shown that in normal animals an injection of a foreign protein as a precipitinogen brings about a state of affairs in which the introduced antigen may soon be detected in the animal's blood stream. Detection of the antigen in this way may be successful for a considerable time both in experimental animals and in persons who, for example, have received antipneumococcus serum. As soon as precipitin begins to appear in the blood, precipitinogen starts to disappear rapidly. This holds good for both innocuous complex precipitinogens like native serum and also for more purified antigens. The latter, however, tend to disappear more quickly. It appears, then, that as immunization proceeds foreign proteins introduced into the animal body have continually less chance of gaining access to the general circulation, while in the normal animal they may soon easily pass into the circulation in amounts sufficient to be detected.

If the same procedures are applied to a highly immune animal, i.e., one with a high precipitin titre, entirely different results are obtained. The injected dose of antigen, given subcutaneously, may rarely gain access to the blood stream of the treated animal. The animal's precipitin titre may be little affected or again it may fall considerably but it does not disappear completely, provided the antigen is a complex one such as a native serum. The decline in titre would depend largely on the quantity of each individual, simple antigen in the dose injected, and the various amounts of really homologous precipitins existing at this time within the animal. In the case in

I Opie, E.: op. cit., p. 55. I923. 
which a simple antigen, highly purified egg albumen, is introduced into an animal with a high precipitin titre against this substance, a severe drop in titre would be anticipated if the injected dose were large. The fall in the titre may be to zero, and fresh antigen may appear in the blood. At this time there is no simple precipitin left in the circulation. When it reappears, the precipitinogen disappears.

It seems that precipitins are involved, at least in some cases, in the mechanism by means of which the immune animal differs from the normal and is enabled to free itself readily of foreign protein. The immune animal does this quickly; the normal animal does it slowly.

\section{PRODUCTION OF PRECIPITIN}

It is obvious that the precipitin reaction is of value first of all in detecting protein. The sensitivity of the test is such that in some cases protein present in a concentration of one part in one hundred thousand or more can be detected. The superior sensitivity of the reaction over chemical tests is apparent. Satisfactory immune-serum titres can usually be obtained in laboratory animals using most unformed proteins for antigen, and giving a course of three to six doses of antigen so spaced as to avoid shocking the animal. The higher the titre of the precipitin serum the more satisfactory it is for practical use, since confusing cross-reactions may be eliminated more easily. In order to obtain large amounts of immune serum, laboratory animals may be given a very short rest after a bleeding, then desensitized with two or three graded subcutaneous doses during a few hours and given a second or even third series of immunizing injections and subsequent bleedings. Since very clear serum is desirable in precipitin tests, some degree of fasting of the injected animals just previous to bleeding is desirable. Most preservatives have a tendency to cause clouding of serum, especially with older sera. Better results may be obtained by using unpreserved immune serum, handling it under precautions to insure sterility, and storing it at temperatures near freezing.

In preparing a series of tests to detect and identify unknown proteins it is found, in the production of desirable antisera, that not all common proteins are equally antigenic, and variable antiserum titres are obtained against different substances no matter what the method of treatment of animals furnishing the sera may be. The precipitin titres vary much with individual animals and also greatly when different species are used for injection. For instance, rabbit anti-beef precipitin of high titre is rather easy to obtain, while rabbit anti-human precipitin is more often of lower titre. Also for the most part rabbits produce more highly potent precipitin than guinea pigs. The blood of each animal should be kept separate because pooled blood sometimes shows clouding and precipitation.

Bacterial precipitins are not so regularly or so easily obtained as precipitins against unformed, innocuous proteins such as native serum proteins. Generally a longer course of treatment is necessary to incite high bacterial precipitin titres. After the usual injections, the major defensive forces of the treated animal may be involved in producing agglutinin, sensitizer, etc., instead of precipitin, and the latter may appear late. Detoxification of particularly toxic micro-organisms may aid in the preparation of more highly potent bacterial precipitin. 
For careful precipitin tests, clear precipitinogen is necessary. This may be obtained in some cases without any preliminary treatment, or in others by filtration or by extraction with saline. In instances such as the latter it is preferable not to agitate the material while it is being extracted, in order to avoid cloudiness. With bacterial antigens, separation of the filtrate from cultures treated in various ways may be accomplished by centrifugation or by filtration.

\section{TEST PROCEDURE}

The precipitin test may be carried out by setting up mixtures containing constant amounts of antigen and diminishing amounts of (preferably) undiluted immune serum, or by holding the precipitin amount constant and diminishing the precipitinogen. In certain quantitative studies of precipitin and precipitinogen Cromwell ${ }^{\mathrm{I}}$ notes that in tests with the simpler antigens dilution of antigen gives no direct insight into the potency of precipitin. With simple antigens the antigen titre divided by the antigen dilution at maximum precipitation equals the antiserum titre divided by the antiserum dilution at maximum precipitation. That is, the natural units of precipitin and precipitinogen at the point of maximum precipitation are approximately equal. However, in practical precipitin tests complex antigens (mixtures) are used and exact quantitative relations are obscured. It is sufficiently exact here to set up diminishing quantities of antigen against constant amounts of immune serum. Very clear-cut readings of tests may be made when a ring test is used; however, an antigen-antibody mixture may be used and the generally distributed precipitate observed on appearing or after settling out.

\section{SPECIES IDENTIFICATION AND RELATIONSHIP}

The voluminous writings upon the use of the precipitin reaction in identifying blood have been reviewed by Nuttall in his book on Blood Immunity and Blood Relationship. Nuttall's own very extensive tests indicated that the precipitin test is a valuable aid in confirming the biological relationships among species established upon the basis of morphology, comparative anatomy, embryology, etc. Far-reaching conclusions must, however, be made with caution because immunologically very closely allied species, the guinea pig and rat, are shown to be very different according to the distribution of so-called "heterophile antigen." This is true also of the horse and cow. On the other hand, the guinea pig, the horse, and the chicken are "proved" closely related since they contain the same antigenic material.

The identification of blood stains, body fluids, and fixed tissues, either native or modified by heat or other agencies, frequently presents difficulties proportional to the extent to which these materials have been denatured. Generally, proteins which have been dried quickly are easily identified by the precipitin test. However, if materials to be identified in this way have undergone spontaneous decomposition or digestion or have been affected markedly by heat or various chemicals, they may give irregular reactions with native antigen-incited precipitin. It is believed by some investigators that more satisfactory reactions in testing heated proteins may be secured through

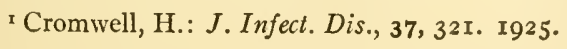


the use of immune sera obtained from animals treated with similar materials, i.e., proteins variously heated. It may be presumed that heating a complex material such as animal flesh would denature some of the simple precipitinogens, and antibody obtained through treatment with what is left unaffected would be a less complex mixture, and the titres of simple precipitins included in such a serum would be higher than were the unaltered antigen injected. These effects, however, do not appear very clearly, and very often more prolonged immunization with altered antigens leads to obtaining immune sera which act very much like those obtained after treatment with native protein. The lability of precipitinogen, like protein in general, is such that the antigen is gradually destroyed or changed as reagents continue to act upon it.

In identification of proteins acting as precipitinogens it is obviously of great importance to use precipitin sera giving highly specific reactions and obtained by as short a course of treatment as possible. Long treatment tends to permit precipitin of a minor nature to appear in response to mildly antigenic precipitinogens, and reactions deviate more and more widely from those showing the highest degree of specificity obtainable.

\section{DIAGNOSIS}

Tests for the presence of bacterial precipitinogens as foreign protein have been carried out, and through the precipitin reaction one may gain information as to the nature of a bacterial species giving rise to inflammatory reactions in animal tissues. It is believed that infecting organisms may liberate protein substances which diffuse more or less into the body fluids, secretions, exudates, etc., and as such may be detected by the use of potent precipitins. In tests such as these, non-specific reactions must be especially guarded against since the fluids tested may contain a large quantity of proteins produced by the infected host. These in high concentration may give false positive reactions. Precipitin tests have been employed in the diagnosis of meningitis by Vincent and Bellot, ${ }^{\mathrm{I}}$ and following this in many other infections. Precipitin tests used in this way add to the evidence obtained by more direct means, but if considered alone they would probably be of questionable value. Carrying such a procedure a step farther, precipitin tests may be performed with the peritoneal fluids from mice injected with pneumonic sputum and type determinations of infecting pneumococci made. The results should parallel the findings with the agglutination test employed for the same purpose.

Tests with known bacterial precipitinogens have been carried out upon sera from infected animals as a procedure in serum diagnosis. The infected host may have developed precipitins due to stimulation by the infecting micro-organisms, and the detection of these may be of value in establishing a diagnosis. In many cases, however, a suitable agglutination test will give very desirable results with less labor and fewer confusing reactions.

In a similar manner, the precipitin test has been used as an index of potency of antiserum intended for therapeutic purposes.

r Vincent, H., and Bellot: Bull. el mém. Soc. méd. de hôp. de Paris, 27, 952. I909. 


\title{
CHAPTER LXII
}

\section{THE COMPLEMENT FLXATION REACTION WITH BACTERIAL ANTIGENS}

\author{
AUGUSTUS WADSWORTH
}

Division of Laboratories and Research, New York State

Department of Health, Albany, N.Y.

The fixation or inhibition of complement by specific immune serum in the presence of a homologous specific antigen is, perhaps, the most sensitive of all the immune reactions, with the possible exception of those reactions in which the hypersensitive animal is utilized. Although the reactions that are based upon the hypersensitivity of the tissues may be more sensitive, they are standardized with greater difficulty and, in general, are not so readily adapted to the routine diagnosis of infection. None of the precipitin tests for the diagnosis of syphilis have yet equaled complement fixation. Corresponding as it does to the Pfeiffer test in principle, and based as it is upon the reactions described by Bordet and Gengou, ${ }^{\mathrm{I}}$ complement fixation is capable of more sensitive adjustment than either the agglutinative or precipitative activities of serum.

It is perhaps interesting to note that the first attempts to adapt the BordetGengou phenomenon to the diagnosis of infection were made by both Bordet ${ }^{2}$ and Wassermann ${ }^{3}$ with antigens of the tubercle bacillus. These early attempts, however, were abandoned on account of unsatisfactory results; and Wassermann ${ }^{4}$ turned to the diagnosis of syphilis by the test which, although primarily based upon what were considered specific relations, has now become so modified by experience, empirically, as to subordinate the specific relations upon which it was originally based. Nevertheless, the practical results with it during the past twenty years must obviously convince one that there are specific relations in it, even as now performed without specific antigens. This is a most important fact to bear in mind.

Meanwhile, the complement fixation reaction has been studied with one after another of the bacterial antigens. In all this application of the test with the bacterial antigens, specific relations have been maintained. The antigens have all been preparations of bacterial culture material. ${ }^{5}$

The earliest antigens were simply suspensions of the bacterial cell, either in the original culture or washed and suspended in physiological salt solution. Similarly, concentrated culture material, such as tuberculin, was also tested. With the serum

${ }^{ \pm}$Bordet, J., and Gengou, O.: Ann. l'Inst. de Pasteur, I5, 289. Igor.

${ }^{2}$ Bordet, J., and Gengou, O.: ibid.; Compl. rend. Acad. de sc., 137, 35 1. 1903.

3 Wassermann, A. P., and Bruck, C.: Deutsche med. W'chnschr., 32, 449. 1906.

${ }_{4}$ Wassermann, A. P., and Neisser, A., and Bruck, C.: ibid., p. 745. 1906.

${ }_{5}$ The observations of Warden (Warden, C. C., and Schmidt, L. E.: J. Lab. Clin. Med., I, 333. I9I5-I6), suggest the possibility of the development of a reaction with non-specific lipoidal antigens. 
of immunized animals, the complement fixation test was easily and readily demonstrated, but certain difficulties arose in the precise standardization of the test, especially when it was applied to the identification of species or the diagnosis of infection. In the identification of species, cross-reactions were obtained with closely related and, less frequently, quite different species of bacteria, and it was evident that these crude antigens possessed non-specific as well as specific activities which must be differentiated if the test was to possess practical, or even academic, significance. When applied to the diagnosis of infection, the reactions occurred with apparently healthy individuals and, not infrequently, failed to detect infection known to exist. Altogether, the results of the early work were not encouraging; in fact, so discouraging that neither Bordet nor Wassermann continued their work with the bacterial antigens. Shortly before his death, Wassermann ${ }^{\mathrm{I}}$ again tried to make practical the test in tuberculosis; how successfully is not even yet quite clear. Thus, the work has been developed very slowly along two slightly different but related lines. Observers who have been interested in the taxonomic study of the identification of species and types of species by delicate, specific immune reactions have turned, in some instances, to the complement fixation reaction. This work covers a large field, including many, or nearly all, of the known common pathogenic bacterial species. Others, more deeply interested in the practical diagnosis of infection, have studied the more common diseases, the clinical diagnosis and prognosis of which might be greatly aided by reliable laboratory tests of the immune reactions that develop during various stages of the infectious processes, either for the purpose of diagnosis or prognosis or to determine cure; for example, such infections as tuberculosis and gonorrhea, on the one hand, and, on the other, meningitis and pneumonia in which the different strains of the species are so important. In determining the etiological relationship of his bacillus to pertussis, Bordet ${ }^{2}$ relied largely upon the complement fixation reaction.

Thus there has been a real interest in the study of complement fixation with bacterial antigens in general, but, on account of the pitfalls with which the study has been and is still beset, attempts to solve the problems have proved inconclusive and have yielded results not satisfactory or convincing.

It is not within the scope of this brief summary to include a review of all earlier work. Reference has been made to the early work of Bordet ${ }^{3}$ and Wassermann. ${ }^{4}$ Widal and Le Sourd ${ }^{5}$ and Camus and Pagniez ${ }^{6}$ might also be mentioned.

Simple suspensions of the bacterial cell in physiological salt solution and crude concentrations of the whole culture, such as tuberculin, were early abandoned for autolyzed culture material or various extractions or residues after extraction. Olmstead and Luttinger found the most reliable antigen of the pertussis bacillus to be an autolysate of an aqueous emulsion. Forty per cent of their cases reacted when in-

\footnotetext{
I Wassermann, A. P.: Deutsche med. Wchnschr., 49, 303. 1923.

${ }^{2}$ Bordet, J., and Gengou, O.: Ann. de l'Inst. Pasteur, 20, 731. I906.

3 Bordet, J., and Gengou, O.: ibid., r5, 289. rgor; Compt.rend. Acad. de si., r37, 351. r903.

4 Wassermann, A. P., and Bruck, C.: Deutsche med. Wchnschr., 32, 449. r906.

5 Widal, F., and Le Sourd, L.: Soc. méd. des hôp., r8, 624. rgor.

${ }^{6}$ Camus, J., and Pagniez, P.: Compl. rend. Soc. de biol., 53, 734. rgor.

7 Olmstead, M., and Luttinger, P.: Arch. Int. Med., 16, 67. I9r5.
} 
active serum was used. Later, Wilson ${ }^{\mathrm{I}}$ described the preparation of the various bacterial antigens which had proved to be most satisfactory. These indicate a further purification; the bacterial cells were suspended in 50 per cent alcohol, washed in absolute alcohol and ether, dried, and then suspended in salt solution. Arkwright ${ }^{2}$ studied different antigens of the gonococcus and meningococcus with their immune sera, and with the most active aqueous extracts which he prepared from these cultures demonstrated cross-fixation between these different species, and also the failure of the reaction occasionally with different strains of the same species. Hirschfelder ${ }^{3}$ prepared antigens from a large number of bacterial species by the action of digestive ferments on the living bacterial cells. It is interesting to note, parenthetically, that he apparently obtained substances with antigenic activity from the living pneumococcus cell and yet the cell proved to be virulent after this treatment. Dombray ${ }^{4}$ reports reactions of diagnostic and prognostic significance in cases of gonorrhea which were very carefully studied by bacterial examination of the urethra. Some of the irregular results that have been recorded, d'Herelle ${ }^{5}$ has suggested, might conceivably be attributed to the interference of bacteriophage or the ultra-filterable viruses. The most extensive study of the bacterial antigens has been made with cultures of the tubercle bacillus.

Much work has also been done with the glanders bacillus in veterinary practice since the early studies of Schütz and Schubert ${ }^{6}$ and Miessner and Trapp, ${ }^{7}$ and the complement fixation test has been developed and has been used extensively. ${ }^{8}$

In all these reports, specific and non-specific reactions are recorded with the different types of antigens used by various observers. This fact was so early recognized that research was directed more extensively to the separation of the different substances by various chemical procedures, extractions, and separations. It was largely on this account, and because of the very practical significance of a reliable test in tuberculosis from the public health standpoint, that we undertook in our laboratory the study of the complement fixation reaction in tuberculosis, ${ }^{9}$ directing attention primarily to careful analysis of the activity of various elements of the tubercle culture in order to differentiate, if possible, their antigenic value. Observations of previous investigators, notably of Meyer ${ }^{\mathrm{ID}}$ and Nègre and Boquet ${ }^{\text {II }}$ on the significance

`Wilson, M. A.: Studies, New Vork City Dept. Ileulth, 9, 486. 1916-19.

${ }^{2}$ Arkwright, J. A.: J. Hyg., 11, 515. 1911.

3 Hirschfelder, J. O.: J.A.M.A., 65, 2073. 1915.

${ }_{4}^{4}$ Dombray, P.: Compt. rend. Soc. de biol., 95, 12. 1926.

${ }_{5}$ d'Herelle, F.: The Bacteriophage, Its Rồe in Immunity (trans. George H. Smith), p. I4I. Baltimore, Maryland: Williams \& Wilkins Co., 1922.

${ }^{6}$ Schütz and Schubert: Arch.f. wissensch. u. prakt. Tierh., 35, 44-83. 1909.

7 Miessner, H., and Trapp: Centralbl.f. Bakteriol., 52, 115.1909.

${ }^{8}$ Mohler, J. R., and Eichhorn, A.: U.S. Dept. Agric., Bur. Anim. Indust., Wushirgton, Bull. 136. April 7, 191 ; McNeil, A.: New York Serological Society Meeting. April 4, 1914; Langdon, F., and O'Toole, S.: J. Met. Reseurch, 28, 333. 1913.

9 Wadsworth, A., Maltaner, F., and Maltaner, E.: J. Immunol., 10, 241. 1925.

${ }^{10}$ Meyer, A.: Zischr.f. Immmitätsforsch. u. exper. Therap., 14, 359. 1912.

${ }^{11}$ Nègre, K., and Boquet, L.: Compt. rent. Soc. de biol., 83, 922. 1920. 
of the acetone insoluble lipoids, and of Calmette and $\mathrm{Massol}^{\mathrm{I}}$ on the quantitative relation between antigen, serum, and complement, were confirmed and extended. The method of securing certain antigenic substances from the tubercle bacillus culture by adsorption on globulin, precipitation of the globulin with carbon dioxide, and subsequent extraction of the antigenic substances from the globulin by alcohol and dispersion in salt solution of the concentrated extract has been recorded. The comparison of the activity of such purified antigens with the various fractions obtained by extraction of cultures with different aqueous and lipoidal solvents is described fully in a recent paper. ${ }^{\text {. }}$

The result of these analyses and separations by various methods of extraction demonstrated the distribution, so to speak, of the antigenic activity of the tubercle bacillus in certain fractions containing lipoid and protein. It has not been possible to separate the lipoid from the protein and thus determine the activities separately. From this work, it is quite evident that when the acetone-insoluble lipoids are separated from the culture material, concentrated, and suspended, a specific and very active antigen is obtained. Similarly, when methyl-and ethyl-alcoholic extracts of the culture material are prepared after removing the acetone-soluble lipoids with the various solvents, an active, specific antigen is obtained. Moreover, simple aqueous extraction also yields an antigen which apparently is quite as specific and active as these other refined preparations. It would seem that the separation of the antigen components has not been carried far enough to isolate the different elements, but the refined product must be considered, from this standpoint, still crude, and it would appear that the specific antigenic activity depends not upon one of these elements, but upon a mixture or combination in very definite relations of a physico-chemical nature, possibly a protein, on the one hand, and lipoid, on the other.

The most important of recent contributions is that of Avery and Heidelberger. ${ }^{3}$ The specific soluble substance first isolated by them from pneumococcus cultures and now known to be produced by other bacteria, ${ }^{4}$ if a carbohydrate, which, however, may still be questioned, must also be considered. To this so-called "polysaccharide" the type specificity is attributed, whereas the protein exhibits species specificity only. Hitherto, specific antigenic activity has been linked up with protein by the great majority of observers. ${ }^{6}$

Apparently some of the simple aqueous or alcoholic extractions yield antigens with quite as satisfactory and specific activity as some of the more highly refined or purified preparations. Yet it is also evident that with the cruder antigens their activ-

${ }^{1}$ Calmette, A., and Massol, L.: ibid., 67, 528. Igog.

${ }^{2}$ Wadsworth, A., Maltaner, F., and Maltaner, E.: loc. cit.

3 Avery, O. T., and Heidelberger, MI.: J. Exper. Med., 38, 73. 1923; ibid., 40, 301. 1924; Heidelberger, M., Goebel, II. F., and Avery, O. T.: ibid., 42, 727. 1925.

${ }_{4}^{4}$ Avery, O. T., Heidelberger, M., and Goebel, W. F.: ibid., p. 709. 1925; Lancefield, R. C.: ibid., p. 377.1925 .

5 Lancefield, R. C.: ibid., p. 397. 1925; Avery; O. T., and Heidelberger, M.: ibid., 38, S1. 1923; Zinsser, H., and Tamiya, T.: ibid., 42, 311. I925.

${ }^{6}$ Wells, H. G.: The Chemicul aspects of Immunity, p. 63. New York: Chemical Catalog Company, 19) 25 . 
ity may be quite complex and only in varying degree specific. The presence of nonspecific and specific activity is taken to indicate complex reactions, as, for example, antigens which fix with the serum of normal or healthy individuals and also with the serum of specifically infected or immunized individuals. In some instances, it is possible to differentiate these reactions, owing to their difference in intensity, by dilution of the antigen and standardization of the test. Striking evidence of how definitely these antigenic reactions differ is to be found in the antigenic activity of antigens prepared from the Treponema pallidum when tested with the serum of syphilitic individuals and the serum of infected and of immunized animals.

Noguchi ${ }^{x}$ early noted the differences in the reaction of the Wassermann antigen as compared with the antigens prepared from spirochetes ground in medium and suspended in salt solution. Recently, Klopstock ${ }^{2}$ has studied the activity of alcoholic extracts of the spirochete, recording much more specific reactions with this antigen than have been obtained by other observers. ${ }^{3}$ Although we have also obtained similar results in our work now in progress and not yet reported, the reactions of the syphilitic serum with such spirochetal antigens are variable and do not parallel those secured with the antigens prepared from heart muscle, sometimes quite the contrary. The presence of agar carried over from the culture medium into the antigen may be a disturbing factor, and one must always bear in mind the fact that many substancesas, for example, animal charcoal, and the like-will absorb or inhibit complement. Not only may the culture material act antigenically in the reaction but, apparently, in one experiment the culture fluid, free from spirochetes, fixed complement with the heart-muscle antigen in the Wassermann test when substituted for patients' serum. The uninoculated serum broth failed to react.

All of this research work indicates that it has been practically impossible to standardize the preparation of bacterial antigens. Some of the crude extracts react as well as the purified extracts. In general, the aqueous extracts are perhaps most satisfactory but there is no scientific basis for their standardization as yet, unless it is done with immune sera which may differ quite materially from the serum of an infected individual. Despite all the extensive methods of chemical analysis that have been used, the elements essential to antigenic activity have not been separated from the bacterial culture, nor have the physico-chemical relations in which they must exist to possess an optimum activity been determined. Further progress thus depends upon the results of future study with more precise methods of distinguishing and separating the different substances that possess antigenic activity.

Bacterial antigens have been so variable that even the attempts to standardize them for the practical diagnosis of infection have not been encouraging. The data available consist of desultory reports in the literature, as above noted. Moreover, although the standardization of the Wassermann antigens has been developed as a

I Noguchi, H.: Laboratory Diagnosis of Syphilis, chap. xiii. New York: Paul B. Hoeber, 1923.

${ }^{2}$ Klopstock, F.: Deutsche med. Wchnschr., 52, 226. 1926.

${ }^{3}$ Craig, C. F., and Nichols, II. J.: J. Exper. Med., I6, 336. I912; Kolmer, J. A., Williams, II. IV., and Laubaugh, E. E.: J. Med. Reseurch, 28, 345. I9I3.

4 Practically all the experiments with the injection of such substances-so-called blocking experiments-lack adequate control to warrant the definite conclusions that have been drawn. 
result of twenty years' experience with the test, it is still crude. The Wassermann technique, with its allowance for so-called "anticomplementary" and "non-specific" activities of the reagents, is only an approximate measure of the specific activity of the sera. Yet this is the form of test which has been used in the majority of the studies of the bacterial antigens, whereas accurate titration of the activity of the specific immune serum is essential. This fact, apparently, has been appreciated by Calmette and Besredka in their application of complement fixation to the diagnosis of tuberculosis. A similar conclusion was forced upon us in our work with the tubercle antigens, and the test was therefore remodeled to provide an accurate titration of the activity of all the sera tested. Thus, only as a result of long experience with the test performed in this manner is any standardization of the antigen possible. The ranges of activity possessed by normal healthy individuals, as compared with those of infected individuals, must be accurately determined with a large number of sera. In fact, it is largely owing to the difficulty of determining these ranges of activity that the practical application of the test to the diagnosis of disease has been so unsatisfactory. Long experience with the Wassermann test was necessary to determine these ranges of activity, and we have only very limited data recorded with the different bacterial antigens, the non-specific fixation of which has so often overlapped the specific activity.

In general, the results of the practical application of the complement fixation test with the bacterial antigens in the diagnosis of infection has been beset with pitfalls which have been avoided only in a very limited number of the researches, and one finds in these reports conclusions invariably so qualified as to destroy the practical value of the test. Thus, positive reactions are obtained in normal healthy individuals or those undergoing other infectious processes, and the infected individual occasionally fails to react. This, however, is also true of the Wassermann test in the diagnosis of syphilis, but experience has so refined the test in its procedure and control as to eliminate such a large proportion of these instances that only a negligibly small percentage of error remains. In the diagnosis of tuberculosis, experience with the better methods is now accumulating so rapidly that results comparable with the Wassermann test in syphilis are assured. It requires a careful, precise technique and, as knowledge increases, the test may acquire prognostic significance.

To sum up, the physico-chemical balance essential to the antigen and the elements concerned in it are still so far from standardized that the specific, refined, purified extracts, although in general superior to the cruder antigens which have been empirically established, are not yet entirely reliable and our knowledge is not yet sufficiently precise to place their standardization upon a scientific basis. Similarly, some of the crude bacterial antigens empirically established may prove even more serviceable but unless carefully controlled and, so far as possible, standardized, there is great danger of obtaining misleading results. The significance of the results really depends upon the precision and extent of the comparisons and controls. Although essent ial, fundamental knowledge regarding the nature and preparation of the antigens is still lacking, material progress has been made in the study of the different extracts and preparations. Research, however, has not very generally recognized the importance of adapting the 
Wassermann technique to the complement fixation test with bacterial antigens, with all factors completely controlled, so that it will provide an accurate titration of the specific activity of the immune serum. Thus it is that, apart from the confirmation and establishment of empirical observations, from whatever angle the student approaches the study of infection and immunity he must ultimately determine the physical and chemical relation between the essential elements concerned in the reactions that take place. 


\title{
CHAPTER LXIII
}

\section{THE COMPLEMENT FIXATION TEST FOR SYPHILIS}

\author{
RUTH GILBERT \\ Division of Laboratories and Research, New York State \\ Department of Health, Albany, N.Y.
}

The development of the complement fixation test for syphilis followed the fundamental work of Bordet and Gengou ${ }^{\mathrm{I}}$ who studied fixation of complement in the presence of suspensions of bacteria and antiserum. They conceived the idea of adding to combinations of complement, antigen, and immune serum a suspension of red blood cells and the inactivated serum of an animal immunized against them so that if the complement in the first combination were fixed or rendered unavailable, the red blood cells would not be hemolyzed in the presence of the inactivated antiserum or amboceptor. This procedure demonstrated the presence of a reaction which had occurred between the antigen and the serum of an animal previously inoculated or infected with the micro-organism from which the antigen had been prepared.

Wassermann and his co-workers ${ }^{2}$ thought that such a reaction might be employed to demonstrate the presence of antibodies in the blood of persons suffering from syphilis. As the incitant of syphilis had not been cultivated, aqueous extracts of the liver of a syphilitic fetus were used as antigen; guinea pig serum, as complement; and serum of monkeys that had developed syphilis after inoculation, as antiserum. After incubation, suspensions of the red blood cells of sheep and the inactivated serum of rabbits that had been inoculated with sheep red blood cells were added. The reagents were titrated so that suitable amounts of the various substances were used and controls were made. However, these apparently did not include the use of extracts made from normal tissues as antigen.

Detre, ${ }^{3}$ working independently, tried a similar test with which he was also able to demonstrate the fixation of complement in the presence of serum from syphilitic patients and extracts from lesions containing Treponema pallidum. Both groups of workers considered the phenomenon due to specific antigen-antibody reactions. In a short time, however, it was shown by Weygandt, ${ }^{4}$ Plaut ${ }^{5}$ and Marie and Levaditi ${ }^{6}$

${ }^{1}$ Bordet, J., and Gengou, O.: "Sur l'Existence de substances sensibilisatrices dans la plupart des serums antimicrobiens," Ann. de l'Inst. Pasteur, 15, 289. 1901.

${ }^{2}$ Wassermann, A., Neisser, A., and Bruck, C.: "Eine serodiagnostische Reaktion bei Syphilis," Deutsche med. W'chnschr., 32, 745. 1906.

3 Detre, L.: "Ueber den Nachweis von spezilischen Syphilisantisubstanzen und deren Antigenen bei Luetikern," Wien. klin. Wchnschr., 19, 619. 1906.

4 Weygandt, W.: "Ueber die Frage syphilitischer Antistoffe in der Zerebrospinaltlüssigkeit bei Tabes dorsalis," HI Hinchen. med. II chnschr., 54, 1557. 1907.

5 P'laut, F.: "Serodiagnostik der Syphilis," Centrallh. f. Nerrenh. u. Psychial., 31, 28g. Igo8.

"Marie, A., and Levaditi, C.: "Les 'Anticorps syphilitiques' dans le liquide céphalorachidien des parraly'tiques généraux et des tabétiques," Ann. de l'Inst. P'ustettr, 21, I3S. I907. 
that extracts from organs which did not contain Treponema pallidum were satisfactory antigens. Landsteiner and Stankovic ${ }^{1}$ demonstrated that alcoholic extracts from the organs of normal animals were even more satisfactory than the aqueous extracts of syphilitic organs which had been used previously.

Almost from the first the complement fixation test was found very helpful as an aid in the diagnosis of syphilis, and it rapidly came into general use in large public health and hospital laboratories. As the technique is somewhat complicated, however, many and diverse procedures for the performance of the test were proposed. In about twenty years, the literature on the subject has become voluminous, and since the fundamental basis for the reaction has not been determined, most of the research work on the subject has of necessity consisted primarily in the comparison of the results of various procedures with the clinical manifestations in the patient, an attempt being made to obtain a large proportion of positive reactions with specimens from syphilitic patients and as few reactions as possible with specimens from patients free from any history or clinical manifestations of the disease. The pendulum has continued to swing between tests which are so lacking in delicacy that specimens from cases of syphilis not infrequently fail to react, and those that give reactions with specimens from patients who present every evidence of being free from syphilis.

The diversity of the procedures used ranges from variations in minor details to marked differences in essential factors. An outline of the methods ${ }^{2}$ employed in some of the large laboratories in this country and abroad may serve to illustrate the degree of this variability.

\section{METHOD OFFICLALLY RECOMMENDED FOR USE IN GERMANY 3}

Each component of the test is so diluted that it will be contained in $0.5 \mathrm{cc}$. The patient's serum is inactivated for one-half hour at from $55^{\circ}$ to $56^{\circ} \mathrm{C}$. A I: 5 dilution is used in the test.

The complement employed is a $\mathrm{I}: \mathrm{I} O$ and $\mathrm{I}: 20$ dilution of fresh guinea pig serum.

Three antigens, or preferably five, are used, one of which is an extract of syphilitic liver. They are prepared by manufacturers and tested by the government during a period of two weeks on at least six days. They are tested against eighty syphilitic and forty control sera.

One hour in the incubator at $37^{\circ} \mathrm{C}$. is a lowed for fixation. After the addition of sensitized cells, there is a secondary incubation period until the controls have hemolyzed.

The amboceptor is titrated daily with $0.5 \mathrm{cc}$. of a $\mathrm{r}$ : 10 dilution of complement, and a similar titration is made with the addition of antigen. The dilution used must contain at least four times the amount necessary for hemolysis without antigen and at least the smallest amount required for hemolysis with antigen.

Sheep cells, washed three times, are used in a 5 per cent suspension.

The results are recorded according to the following scale:

The supernatant fluid colorless $=4+$

The supernatant fluid delicate pink $=3+$

The supernatant fluid somewhat red (one-half cells hemolyzed) $=2+$

The supernatant fluid red (three-fourths or more cells hemolyzed) $= \pm$

The supernatant fluid clear bright red $=-$

I Landsteiner, K., and Stanković, R.: "Ueber die Bindung von Komplement durch suspendierte und kolloid geliste Substanzen," Centralbl. f. Bakteriol., Orig., 42, 353. 1906.

${ }_{2}$ 'The methods outlined have been officially recommended for use in certain countries or were among those used in recent attempts made to standardize the tests, the technique being chosen in each case that was thought to illustrate best the dissimilarity in the procedures in routine use.

3 "Anleitung für die Ausführung der Wassermann'schen Reaktion," Sonderbeilage zu Veröffentlichungen des Reichsgesundheitsamts, No. 46. 1920. 
METHOD USED BY BROWNING, AT THE BLAND-SUTTON INSTITUTE, MIDDLESEX HOSPITAL; ONE OF THE FOUR METHODS RECOMMENDLD IN I9I 8 BY THE MEDICAL RESEARCH COMMITTEE OF GREAT BRITAIN ${ }^{I}$

The patient's serum is inactivated for one-half hour at $56^{\circ} \mathrm{C}$., and $0.025 \mathrm{cc}$. is used in the test.

Guinea pig serum collected eighteen hours previously is used as complement. It is titrated in a $1: 4$ dilution with incubation for one hour at $37^{\circ} \mathrm{C}$. Two, four, six, and eight units are used with constant amounts of patient's serum and antigen.

The antigens are alcoholic "lecithin" solutions of ox liver used with and without the addition of cholesterin. The optimum amount of cholesterin is determined by testing different concentrations in comparison with an antigen that has proved to be satisfactory and is in routine use. Three-tenths of a cubic centimeter of each antigen in a $1: 8$ dilution are used in the tests. Precautions are taken in making the dilution to insure minimum turbidity.

Anti-ox amboceptor is titrated with a 3 per cent suspension of cells, and five units are used in the hemolytic system.

One and one-half hours at $37^{\circ} \mathrm{C}$. are allowed for fixation, and after the addition of amboceptor and cells, there is a secondary incubation at $37^{\circ} \mathrm{C}$. for one hour.

The results are evaluated by comparison with the reactions obtained with specimens that give varying degrees of fixation and are tested for purposes of control.

\section{METHOD OF GRIFFITH AND SCOTT ${ }^{2}$}

All the components of the test are diluted so that $0.25 \mathrm{cc}$. of each are used. The patient's serum is diluted I:5 with physiological salt solution and then inactivated for one-half hour at $56^{\circ} \mathrm{C}$. Both $\mathrm{I}: 5$ and $\mathrm{I}:$ Io dilutions are used in the test.

The complement consists of $0.25 \mathrm{cc}$. of a $\mathrm{I}: 25$ dilution of the serum from male guinea pigs that has been collected on the day the tests are made. It is tested both with and without the addition of antigen to control its lytic activity.

As antigen, an acetone-insoluble fraction of crude organ extract as suggested by Noguchi is recommended. To this is added an equal volume of alcohol, containing I per cent cholesterin. When diluting, six hundred and forty parts of physiological salt solution are added rapidly to one part of the mixture of alcoholic solutions, so that the resulting suspension is clear or faintly opalescent. Patient's serum and complement are mixed and allowed to stand for one hour in the icebox after which the cool antigen suspension is added. They are then replaced in the icebox for from sixteen to twenty-four hours. For the hemolytic system, 6 per cent suspension of sheep cells and an equal volume of antisheep horse serum in a dilution sufficient to furnish maximum sensitization is used.

Reactions are recorded, depending upon the degree of lysis, as follows: o, tr, ?tr, t, ,,,++++++++ ? , and $\mathrm{C}$.

\section{METHOD USED BY MADSEN, STATE SERUM INSTITUTE, COPENHAGEN ${ }^{3}$}

The technique indicated by Thomsen and Boas with an additional antigen prepared from syphilitic liver is used. The serum is inactivated for one-half hour at $56^{\circ} \mathrm{C}$., and amounts of

" "The Wassermann Test," Rep. of Special Committee upon the Standardisation of Pathological Methods, "Medical Research Committee Spec. Rep. Ser.," No. 14, p. 27. London, I9IS.

${ }^{2}$ Griffith, F., and Scott, IV. M.: II. Technique of the IV assemann Resction, Rep. on Pub. Health and Med. Subjects, No. I, p. 7. London: Ministry of Health, 1920.

3 Investigations on the Serodiagnosis of Syphilis, League of Nations, IIealth Organization, C. 5 M. 5 III. I924. 
$0.2,0.1,0.05,0.025$, and $0.01 \mathrm{cc}$. are tested. Even smaller amounts are employed if inhibition of hemolysis still occurs.

Three-quarters of an hour at room temperature followed by three-quarters of an hour at $37^{\circ} \mathrm{C}$. are allowed for fixation.

A 5 per cent suspension of sheep cells with two and one-half units of amboceptor contained in $2 \mathrm{cc}$. are used in the hemolytic system.

As complement, guinea pig serum, titrated in a $\mathrm{I}: \mathrm{so}$ dilution both with and without antigen, is used. The unit is determined after two hours in the incubator at $37^{\circ} \mathrm{C}$. The determination without antigen is made for use in the serum control and the one with antigen for use in the test proper.

Extracts of human heart and syphilitic liver are used as antigens.

After the addition of the hemolytic system, the tests are incubated for two hours at $37^{\circ} \mathrm{C}$. and a hemoglobin scale is used for reading the results.

METHOD USED BY MÜLLER, SERO-DIAGNOSTIC LABORATORY, VIENNA ${ }^{I}$

The patient's serum is inactivated, and four drops are used in the test, a drop corresponding to about $0.05 \mathrm{cc}$.

One drop of guinea pig serum is used as complement. This must be one and one-half times the amount necessary for hemolysis. A unique method of diluting the antigen is recommended. To $20 \mathrm{cc}$. of an alcoholic extract of beef heart tissue is added $4 \mathrm{cc}$. of a 0.5 par cent solution of cholesterin and the mixture evaporated to $8 \mathrm{cc}$. This is poured quickly into a beaker $7 \mathrm{~cm}$. in diameter which contains $5 \mathrm{cc}$. of 0.9 per cent sodium chloride. Next, $20 \mathrm{cc}$. of salt solution which has been kept in a second beaker are added as quickly as possible. This mixture must mature at $56^{\circ} \mathrm{C}$. for from twelve to twenty-four hours. Five drops are used in the test.

One hour in the incubator is allowed for fixation.

The results of the tests are recorded after hemolysis has occurred in most of the tubes containing normal sera, and a final record is made one hour later.

In addition to the usual controls, one control is recommended in which alcohol is used instead of antigen, to insure that fixation will not occur with the amount of alcohol used in the antigen. The test differs from many others in that $2.5 \mathrm{cc}$. of salt solution are put in each tube before the other reagents, which are, with the exception of antigen, added undiluted by dropping them from a pipette.

\section{METHOD USED BY MUTERMILCH IN THE PASTEUR INSTITUTE, PARIS ${ }^{2}$}

The procedure of Bauer-Hecht, modified by Levaditi and Latapie, Weinberg, Mutermilch, and Latapie, is employed.

Human serum that has not been inactivated is tested in o.I-cc. amounts.

The complement and antisheep amboceptor in the patient's serum which is being tested is used. 'The hemolytic index for each serum is determined according to Weinberg's procedure. One-tenth of a cubic centimeter of each serum is tested with amounts of the 5 per cent suspension of sheep cells varying from o.I through I cc. If the sera contain unusually large amounts of amboceptor, they are tested with amounts of the cell suspension varying from $\mathrm{I}$ to $2 \mathrm{cc}$. These tubes are incubated for one hour at $37^{\circ} \mathrm{C}$. In the case of spinal fluids or sera not having natural complement or antisheep amboceptor, active human serum from

I Ibid.

2 "La Technique du séro-diagnostic de la syphilis actuellement employée à l'Institut Pasteur à Paris," Ann. de l'Inst. Pasteur, 38, 827. I924. 
persons free from syphilis is added. This added serum must contain complement and natural antisheep amboceptor which is determined by titration.

The antigen, prepared according to the method of Bordet and Ruelens ${ }^{1}$ from calf heart extracted first with acetone and then with alcohol, is used in 0.I-cc. and 0.2-cc. amounts. The alcohol is not evaporated prior to use.

In accordance with the procedure recommended by Weinberg, one hour at $37^{\circ} \mathrm{C}$. is allowed for fixation.

A 5 per cent suspension of sheep blood corpuscles, washed once or twice and restored to the original volume, is used in the hemolytic system. The amount used varies with each serum, being one-third of the greatest volume which is hemolyzed by o.I cc. of the serum.

METHOD USED BY RENAUX IN THE PASTEUR INSTITUTE, BRUSSELS ${ }^{2}$

The patient's serum is inactivated for twenty minutes at $56^{\circ} \mathrm{C}$. and is used in amounts of 0.1 and $0.05 \mathrm{cc}$.

One drop, approximating $0.04 \mathrm{cc}$., of fresh guinea pig serum is used as complement. This is titrated alone and in 75 per cent and 50 per cent dilutions. These dilutions are tested with cells sensitized with varying amounts of amboceptor. After twenty minutes at $37^{\circ} \mathrm{C}$., the most satisfactory complement dilution is chosen.

One antigen, that described by Bordet and Ruelens, is used. It is an alcoholic extract of the acetone-insoluble lipoids of calf heart. To dilute it, I cc. of extract is evaporated to dryness at $37^{\circ} \mathrm{C}$., after which the residue is suspended in $3 \mathrm{cc}$. of distilled water and then $27 \mathrm{cc}$. of 0.9 per cent salt solution are added. Four drops or $0.16 \mathrm{cc}$. are used in the test.

One hour at $37^{\circ} \mathrm{C}$. is allowed for fixation.

Antigoat rabbit serum inactivated for thirty minutes at $56^{\circ} \mathrm{C}$. is employed as amboceptor. The preliminary titrations are made with blood cell suspensions sensitized with different strengths of the amboceptor varying from $I: 20$ to $I: 60$ dilutions. These dilutions are tested with both diluted and undiluted complement. The most satisfactory dilution of complement is chosen after twenty minutes at $37^{\circ} \mathrm{C}$., and then a second amboceptor titration is performed. This consists of four drops of antigen and one drop of complement with one, two, and three drops of the cells sensitized as previously described. After thirty minutes, the degree of sensitization is chosen which gives complete hemolysis with one drop of corpuscles, almost complete with two drops, and marked hemolysis with three drops. One drop of sensitized cells is used in the test.

Defibrinated goat blood, washed three times, restored to its original volume, and then diluted to twice this volume with 0.9 per cent salt solution, is employed.

\section{METHOD USED BY NOGUCHI, ROCKEFELLER INSTITUTE, NEW YORK CITY 3}

Either $0.02 \mathrm{cc}$. of fresh serum or $0.04 \mathrm{cc}$. of serum inactivated for about fifteen minutes at from $55^{\circ}$ to $56^{\circ} \mathrm{C}$. is used.

The complement consists of guinea pig serum titrated in the presence of fixed amounts of cells and amboceptor. Two units, usually o.I cc. of a 40 per cent dilution, are used.

As an antigen, an alcoholic extract of acetone-insoluble lipoids is used. Antigens are

I Bordet, J., and Ruelens, G.: "L'Antigène syphilitique de l'Institut Pasteur de Bruxelles," Compt. rend. Soc. de biol., 82, 880. 1919.

${ }^{2}$ Investigations on the Serodiagnosis of Syphilis, League of Nations, IIealth Organization, C. 5 M. 5 III. I924.

${ }^{3}$ Noguchi, H.: Serum Diagnosis of Syphilis and Luetin Reaction (3d ed.). Philadelphia: J. B. Lippincott Co., I9I2. Dr. Noguchi furnished antigen only for the standardization tests made in 1925. See Gilbert, R., and Langworthy, V.: "Standardization of the Wassermann Test," $A \mathrm{~m}$. $J$. Syph, I0, 101. 1926. 
tested for anticomplementary and hemolytic properties, as well as antigenic properties. If found to be satisfactory, the antigen is diluted so that O.I cc. contains more than four antigenic units.

The period of fixation is one hour at $37^{\circ} \mathrm{C}$., and after the addition of the hemolytic system, the tests are again incubated for two hours at the same temperature.

Either I cc. of a I per cent suspension or O.I cc. of a ro per cent suspension of human blood cells is used in the hemolytic system with two units of antihuman rabbit serum as amboceptor.

The results are based on the percentage of cells that are hemolyzed, the tinting of the salt solution, and comparison with complete inhibition and complete hemolysis.

\section{METIIOD USED BY KOLMER, IN THE UNIVERSITY OF PENNSYLVANLA, PHILADELPHIA ${ }^{\mathrm{I}}$}

The patient's serum is inactivated for fifteen minutes at $55^{\circ} \mathrm{C}$. Graded amounts, O.I, $0.02,0.004,0.002$, and $0.001 \mathrm{cc}$., are used in a quantitative test.

The complement consists of pooled guinea pig serum titrated with two units of antisheep amboceptor. The amount of amboceptor as well as complement is adjusted each day. Complement in varying amounts of a $\mathrm{r}: 30$ dilution is titrated in the presence of antigen, and the unit is determined after one hour in the water bath at $38^{\circ} \mathrm{C}$. Slightly more than the actual unit is used as the full unit to allow for the difference in the method of fixation. Two units are used in the test.

The antigen is a lecithinized alcoholic extract of beef- or human-heart muscle from which the acetone-soluble substances have been removed and 0.2 per cent cholesterin added. Dilutions are made by slowly adding the extract to salt solution. Titrations for hemolytic, antigenic, and anticomplementary properties are made. Ten antigenic units are used, and twenty times this amount should not be anticomplementary or hemolytic. The antigen is diluted so that the proper amount for the test will be contained in $0.5 \mathrm{cc}$. The serum and antigen are allowed to stand from five to thirty minutes before the addition of complement.

From fifteen to eighteen hours at from $6^{\circ}$ to $8^{\circ} \mathrm{C}$. are allowed for fixation, followed by from five to fifteen minutes in the water bath at $38^{\circ} \mathrm{C}$. Amboceptor and cells are added separately. The period for secondary incubation is one hour at $38^{\circ} \mathrm{C}$. The tests are then placed in the refrigerator to allow the cells to settle, and the results are recorded from one to three hours later.

In determining the reactions, a scale containing antigen, inactivated complement, and varying amounts of hemoglobin solution, and cell suspension is used for comparison.

\section{METHOD USED IN THE NEW YORK STATE DEPARTMENT OF HEALTH, ${ }^{2}$ DIVISION OF LABORATORIES AND RESEARCH}

The total volume of the test is $0.5 \mathrm{cc}$. The patient's serum, after inactivation for onehalf hour at $55^{\circ} \mathrm{C}$., is tested in amounts of 0.05 and $0.02 \mathrm{cc}$.

The complement is a mixture of the fresh serum from six or more guinea pigs. Each of the sera has been found satisfactory by preliminary tests made before the pool is prepared. The mixture is then titrated and diluted so that the two units used in the test are contained in O.I cc.

Two antigens are used: one, an acetone-insoluble antigen prepared and diluted according to the method of Bordet and Ruelens, and an alcoholic extract reinforced with 0.4 per

r Kolmer, J. A.: Infection, Immunity and Biologic Therapy (3d ed.). Philadelphia and London: W. B. Saunders Co., r923.

${ }^{2}$ Wadsworth, A. B.: Standard Methods of the Division of Laboratories and Research of the New Iork State Department of Health. Baltimore: Williams \& Wilkins Co., I927. 
cent cholesterin prepared by a method similar to that of Neymann and Gager. One-tenth of a cubic centimeter of each antigen is used in a dilution that is found to give the most sensitive results in tests of a large number of partially reacting syphilitic sera and that is not even slightly anticomplementary in four times the amount used in the test.

Four hours at from $3^{\circ}$ to $6^{\circ} \mathrm{C}$. is allowed for fixation, and with specimens from cases of possible primary syphilis, or those accompanied by histories indicating the necessity for further study, a second test is made with the cholesterinized antigen with fixation in the water bath for one-half hour at $37^{\circ} \mathrm{C}$. One-tenth of a cubic centimeter of 5 per cent sheep cells and two units of amboceptor contained in an equal volume are used in the hemolytic system. After the addition of sensitized cells, the tests are incubated at $37^{\circ} \mathrm{C}$. for fifteen minutes, or until the serum and antigen controls show complete hemolysis.

The degree of fixation in each tube is then read by comparison with color standards.

\section{STANDARDIZATION OF TECHNIQUE}

The desirability of securing some degree of uniformity in the complement fixation test for syphilis has been recognized for years. The solution of the problem has been undertaken through government regulation in both Germany and Great Britain. In the former country the technique for official tests has been prescribed in detail, and strict regulations have been made for the testing of antigens and amboceptor. In Great Britain, the Medical Research Committee, ${ }^{\mathrm{I}}$ in I9I 8 , recommended minimum standards and a choice of four methods which, on the whole, had been found to give satisfactory results. Two years later the British Ministry of Health indorsed the method developed by Griffith and Scott. ${ }^{2}$ Representative institutions in these and several other European countries have taken part in the work on serum standardization of the League of Nations Health Organization. During $1922^{3}$ certain precipitation reactions were compared with the complement fixation test as performed in the individual laboratories, and during $1923^{4}$ the different methods for complement fixation also were compared. As a result of these studies there was unanimous agreement that the complement fixation reaction could not be replaced by any of the precipitation reactions studied; that the best results were obtained with the complement fixation test when heart extracts were used; and that methods employing several extracts showed no advantage over those employing but one.

A number of attempts at standardization were also undertaken in this country, some of which were initiated by state and federal authorities. In I9I5 the New York City Department of Health ${ }^{5}$ made such an attempt, but the undertaking was abandoned as the members of the committee were unable to agree on technical procedures.

I "The Wassermann Test," Rep. of Special Committee upon the Standardization of Pathological Methods, "Medical Research Committee Spec. Rep. Ser.," No. I4, p. 270. London, I9I8.

${ }^{2}$ Griffith, F., and Scott, W. M.: loc. cit.

3 "Sero-Diagnosis of Syphilis," Reports on Serological Investigations Presented to the Second International Conference of the League of Nations IIealth Organization. Paris, 1922; International Conference on Standardization of Sera and Serological Tests: I. "Sero-Diagnosis of Syphilis," Lancet, 203, 123. 1922.

${ }^{4}$ Investigations on the Serodiagnosis of Syphilis, League of Nations, IIealth Organization, C. 5 M. 5 III. 1924 .

5 Ottenberg, R.: "On the Reliability of the Wassermann Reaction," Arch. Int. Med., 19, 457. 1917. 
The Army and Navy laboratories ${ }^{x}$ have specified the procedures to be used in these departments, and methods followed in certain of the state laboratories have been adopted by many of the local laboratories. ${ }^{2}$ In $1920^{3}$ the United States Public Health Service proposed a uniform complement fixation technique, ${ }^{4}$ and in 1922 the Society of American Bacteriologists appointed a committee of five ${ }^{5}$ for the purpose of considering a standard procedure. The Committee on Standard Methods of the American Public Health Association in 1923 appointed a referee to consider methods for the standardization of the test. Since I 924 a number of the large laboratories in the United States have co-operated with this referee in a further study of the problem. At first merely an outline ${ }^{6}$ for study was made on which forty-six representative laboratories were asked to comment. ${ }^{7}$ After the receipt of the various suggestions, it was decided first to test ${ }^{8}$ representative antigens by the different procedures being used routinely. The comparative tests that were made demonstrated that in general a very sensitive cholesterinized antigen was essential for the detection of the reaction in early and latent syphilis. Conclusions as to the "specificity" of such a sensitive antigen varied, however, according to the method with which it was employed and the nature and amount of the clinical material studied. The possibility was indicated, moreover, that a less sensitive antigen might prove effective if other technical factors were suitably adjusted.

In view of these findings, it was proposed that further study should be directed to the testing of each antigen under the conditions recommended as optimum for its use. To this end, in $1926^{9}$ portions of the same sera were tested, with the antigens and technique routinely employed in eight large laboratories. The results of these tests showed many marked variations, the greatest divergence occurring with specimens from treated cases of syphilis. The most sensitive results were obtained with methods

r At present a precipitation test is being used in the Navy. The Navy adopts the Kahn precipitation test in place of the Wassermann (Mil. Surgeon, 58, 202. I926).

${ }^{2}$ For the past ten years in New York State a satisfactory degree of uniformity has been secured through the limitation of approval to laboratories prepared to perform a fairly large number of tests and that are in charge of properly qualified persons, who submit a description of the technique they plan to employ, agree to use two antigens, one of which is cholesterinized, and to control adequately the test. They also demonstrate their ability to secure reasonably accurate results through the testing of specimens submitted for comparative examination. See Wadsworth, A. B.: "Cooperation between a Central State Laboratory and Local Municipal and County Laboratories," J.A.M.A., 77, 5 I 2. I92I.

3 Cumming, H. S.: Surgeon General-Venereal Disease Division, U.S. Pub. Health Service, Circ. Letter 448. August I2, 1920.

${ }_{4}^{4}$ Neill, M. H.: The Complement-Fixation Test for Syphilis, U.S. Pub. Health Rep., No. 483. I9I8.

${ }^{5}$ Personal communication from Col. Craig to Dr. Wadsworth, as a member of the Committee for Standardization of the Wassermann Reaction, appointed by the Society of American Bacteriologists.

${ }^{6}$ Gilbert, R., and Langworthy, V.: "Standardization of the Wassermann Test," Am. J. Pub. IIealth, 15, 201. 1925.

"Gilbert, R., and Langworthy, V.: "Standardization of the Wassermann Test," ibid., p. 323. 1925.

${ }^{8}$ Gilbert, R., and Langworthy, V.: "Standardization of the Wassermann Test," Am. J. Syph., I0, IOI. 1926.

9 Gilbert, R., and Langworthy, V.: ibid., II, 475. 1927. 
employing cholesterinized antigen with cold fixation and a relatively small amount of complement in proportion to the total volume of the test, the least sensitive results with a method employing water-bath fixation at $37^{\circ} \mathrm{C}$. and relatively more complement. The desirability of using a sensitive cholesterinized antigen was again demonstrated, and there was no indication that such an antigen, if carefully standardized, will give definite reactions with specimens from normal individuals. Two laboratories using identical procedures secured results that agreed remarkably well. If a satisfactory standardization can be secured, therefore, results obtained throughout the various parts of the world should be uniform.

The great variation in procedures that are being employed in different laboratories and the difficulties encountered in efforts made to secure adequate standardization of the test emphasize the importance of determining the fundamental basis for complement fixation. Study directed toward the determination of the nature of the substances in patient's serum, antigen, and complement that take part in the reaction, the nature of the reactions that occur, as well as the effect of physical conditions on these combinations, will be of the greatest value. The solution of these problems will no doubt be most readily obtained through investigation of the reactions that occur between bacterial antigens, immune sera, and complement.

Almost regardless of the method used, there are certain factors essential to the satisfactory performance of complement fixation tests. It is of prime importance that the persons responsible for the work have the proper educational background and adequate training and experience. The apparatus used should be adequate to permit accurate measurement of the reagents, and all glassware should be scrupulously clean. Proper precautions are necessary to insure uniformity of procedure from day to day, and special care should be observed in the performance of the details of the test in order to detect possible technical errors. The patient's serum should be free from evidence of contamination or hemolysis. Complement, if obtained from guinea pigs, should consist of a pool of serum from three or more healthy, non-pregnant animals that have had a comparatively uniform diet consisting in part of leafy vegetables. The refrigeration of the serum used as complement after it has been removed from the clot, as well as its protection from unnecessary agitation, is of importance. The individual sera should be tested for hemolytic activity and fixability with the antigens to be used, and unsatisfactory ones discarded prior to making the pool. The antigen should be prepared and diluted in a uniform manner and tested for anticomplementary and hemolytic activities and for fixability with serum from a large series of cases of syphilis as well as healthy individuals and if possible from those suffering from diseases other than syphilis, to insure that a satisfactory extract and a proper dilution of it has been chosen. The blood cells employed should be tested for fragility and proper control of the density of the suspensions made. Color standards should be prepared for use in reading the results of the test, and there should be uniformity in the volume of the test and the various titrations. The reagents should always be mixed in the same order, preferably-I believe-patient's serum, then antigen, and finally complement, the mixture being shaken after each addition. For control purposes, each serum should be tested without antigen and each series should include tests with antigen and complement without patient's serum and a routine test 
with a serum known to give complete fixation. There should be careful adjustment of the hemolytic system, and the temperature and time for fixation should be uniform.

What has been said relative to tests on blood serum applies equally well to spinal fluid. If the fluid has been freshly collected, however, it does not need to be inactivated unless blood is present. The use of from two to ten times as much spinal fluid as serum is recommended. The reactions of other body fluids have apparently not been extensively studied, but there is no indication that transudates free from bacteria used in properly controlled tests will react, if the patients from whom they are obtained are not syphilitic.

Klauder and Kolmer ${ }^{\mathrm{I}}$ have tested dilutions of fluid removed from chancres and report that fixation is often secured before a reaction can be obtained with serum from the patient. As such material is badly contaminated, it is not satisfactory for submission to a laboratory located at a distance.

The interpretation of the results obtained in the complement fixation test for syphilis of necessity varies, depending on the technique employed and the care with which the test is performed. There are reports of definite fixation occurring with serum from cases of pregnancy and from patients suffering from various diseases including scarlet fever, pellagra, diabetes, tuberculosis, leprosy, malaria, relapsing fever, and yaws. As regards yaws, which is due to Treponema pertenue, an organism almost indistinguishable from Treponema pallidum, the literature indicates that a reaction can be expected with equal regularity as in the case of syphilis. There seems to be an indication also that in rare instances reactions may be secured with blood from cases of malaria, leprosv, and tuberculosis, even when syphilis is not present, but there is little evidence that in the other conditions mentioned there is any likelihood of reactions occurring when syphilis can be ruled out with reasonable certainty. It is not considered desirable to secure specimens of blood when the patient has a fever or is under the influence of alcohol or an anesthetic. Since the test is based on a reaction with a non-specific antigen, it is of course necessary to interpret all reactions in the light of the clinical evidence. If, however, definite fixation is obtained in properly controlled tests, there is strong presumptive evidence of syphilis. The interpretation of partial reactions depends largely on the sensitiveness of the test, but in general should not be considered significant unless there is a history or clinical evidence that the case is one of syphilis. The failure to obtain fixation does not rule out the disease. A reaction is not regularly obtained until from four to six weeks or even longer after the appearance of the lesion and not infrequently specimens from cases of tertiary or latent syphilis fail to react.

In spite of the great variation in the technique used and the many sources of inaccuracy, the complement fixation test for syphilis furnishes information that in the great majority of cases is so in accord with the clinical manifestations that the test is being used more and more generally throughout the world. In fact, there seems to be no other laboratory test that furnishes more valuable information for the clinician.

r Klauder, J. V., and Kolmer, J. A.: "The Wassermann Test Performed with Chancre Fluid as an Aid to the Early Diagnosis of Syphilis," Arch. Dermat. \& Syph., 5, 566. I922. 


\title{
CHAPTER LXIV
}

\section{THE KAHN REACTION}

\author{
R. L. KAHN
}

Bureau of Laboratories, Michigan Department of Health, Lansing, Mich.

INTRODUCTORY

The nature of the precipitation reaction with syphilitic serum and alcoholic extract antigen appears to differ from that of the precipitation reaction with specific immune serum. In the former reaction, the precipitate consists largely of lipoids, while in the latter it consists largely of serum globulins. This apparent difference between the two reactions suggested to the writer the necessity for studying the precipitation phenomenon in syphilis as an entity. Several factors were found to govern this phenomenon, and their recognition made possible the construction of a practical precipitation test for syphilis.

In the latter part of I $22 \mathrm{I}$, the writer observed that within certain limits, concentration of a mixture of antigen-saline suspension ${ }^{\mathrm{I}}$ and serum hastened precipitation, while dilution of the mixture with saline delayed precipitation. It was noted soon that with an antigen of proper concentration of lipoids it was possible to obtain immediate precipitation reactions with syphilitic serum provided the antigen-saline suspension was sufficiently unstable. An antigen suspension was ultimately prepared which was of such instability that it contained coarse lipoid particles which, however, dissolved (dispersed) as soon as they came in contact with serum. Then, if the serum came from a syphilitic patient, a precipitate appeared within a few minutes; if it came from an individual free from syphilis, no precipitate appeared.

The use of an antigen suspension which gave immediate precipitation reactions with syphilitic serum led to the observation of sharp differences in precipitation results due to variation in the proportions of serum and suspension. This could not have been observed readily under conditions of incubation, since some precipitates are enhanced and some redissolved during the incubation period. Ultimately, it was established that optimum precipitation results were obtained only when proper proportions of serum and the suspension were employed. Then it was shown that shaking or agitation of the serum-suspension mixture markedly hastened the formation of precipitates. Strongly potent serum, it is true, required but little agitation; weakly potent serum, however, required from two to three minutes' agitation to bring forth the precipitate.

These four factors which govern the precipitation phenomenon in syphilis, namely, optimum concentration of the ingredients, high instability of an antigen suspension, quantitative relation between the antigen suspension and serum, and agitation of the ingredients, form the foundation on which the writer's test for syphilis was built. The actual evolution of the test required consideration of many

"The more descriptive term "antigen-saline suspension" or "antigen suspension" is used in this chapter in place of the term "antigen dilution" employed in earlier writings of the author. 
practical problems, including the choice of an antigen, the general plan of the test, and the interpretation of the results. Of major importance was the standardization of antigen to assure uniform results in the hands of different workers, for it had been observed that different beef hearts produced antigens of different sensitiveness. This led to the development of methods to correct such differences and to bring all antigens to a uniform scale of sensitiveness. As a result, a practical method has been developed which is highly specific for syphilis, and, in addition, special procedures have been devised which give further assistance in diagnosis and treatment.

\section{PRECIPITATION TESTS PROPOSED BY OTHER WORKERS}

Michaelis was the first to observe precipitates on mixing syphilitic serum with antigen which consisted of a watery extract of syphilitic liver. This antigen was similar to that employed by Wassermann, Neisser, and Bruck in the development of the Wassermann test. Soon Landsteiner, Müller, and Pötzel, also Levaditi and Maria, showed that alcoholic extracts of syphilitic as well as of normal organs might be used as antigens in the Wassermann test. Jacobstahl announced a precipitation method in which an alcoholic extract of syphilitic liver was employed as an antigen and the precipitates were read by darkfield illumination. This author suggested the probable identity of complement-fixing and precipitin substances in syphilis. Precipitation methods by Bruck and Hidaka, Hecht, Meinicke, and Sachs and Georgi followed.

The original Meinicke test was a combined "water" and "salt-solution" method. In the water method, distilled water was added to a given serum-antigen combination, producing precipitates in negative sera and turbidity in positive ones. In the salt-solution method, 2.5 per cent sodium chloride solution was added to a serumantigen-distilled water combination, producing precipitates only in positive sera. Meinicke's "third modification," which he claimed to be a combination of the water and salt-solution method, was published in r9I8. During the same year Sachs and Georgi published the method which bears their name. The antigen was an alcoholic extract of wet heart muscle reinforced with cholesterol. The serum was diluted I : Io with normal saline before its use in the test. The final results in this method and in Meinicke's third modification were read after twenty-four hours' incubation.

In 1922 Meinicke proposed a turbidity reaction involving the use of a cholesterolized antigen containing balsam. Later he devised a similar method with a cholesterolfree antigen, and, more recently, a micro-reaction. Sachs and Klopstock also proposed a method which shortens the incubation period by adding mastic to the extract antigen and shaking the ingredients. For details of technique of these reactions as well as for the precipitation methods proposed by Vernes, Dryer and Ward, Hecht, Dold, Bruck, Wang, and others, the reader is referred to the original publications.

\section{OBSERVATIONS ON PRECIPITATION IN SYPHILIS}

\section{OPTIMUM CONCENTRATION OF THE INGREDIENTS}

As already indicated, the writer's first observation in connection with the precipitation phenomenon in syphilis was that concentration of the reagents that enter

I For references and more complete summary, cf. Bruck, C.: Serodiagnose der Syphilis. Berlin: J. Springer, I924; Kahn, R. L.: Serum Diagnosis of Syphilis by Precipitation. Baltimore: Wiiliams \& Wilkins Co., I925. 
into a precipitation test hastened the formation of precipitates and dilution delayed their formation. Thus, if the same amount, such as O.I cc., of antigen suspensionserum mixture is pipetted into a series of tubes and increasing amounts of saline, let us say, in 0.2 -cc. quantities, are added to each tube, the delay in precipitation is directly proportional to the amount of saline added, and when I cc. is added, precipitation may be inhibited. This observation suggested that for a precipitation test in syphilis the serum should be used in an undiluted form, the antigen employed should have a high concentration of lipoids, and a minimum amount of saline should be used for preparing the antigen suspension. Subsequent studies indicated that excessive concentration of antigen also delayed or inhibited precipitation. This necessitated the preparation of an antigen of optimum concentration-to conform with the desired degree of sensitivity with syphilitic serum.

\section{CHOICE OF AN ANTIGEN-ITS PREPARATION}

An antigen prepared from wet heart muscle was found to be of insufficient sensitivity with syphilitic serum, due undoubtedly to inadequate concentration of lipoids. Furthermore, with an antigen of this type most sera required prolonged incubation before showing precipitation reactions. An antigen prepared by direct alcoholic extraction of dry heart muscle produced non-dispersive suspensions with saline, and could not be used with serum, especially after the addition of cholesterol. Only by adding an excessive amount of saline to the antigen thus producing an opalescent mixture could it be employed with serum. Then, however, the results were of insufficient sensitivity. An alcoholic extract of dry heart muscle following preliminary ether extraction (Neyman and Gager) ${ }^{\mathrm{I}}$ or an acetone-insoluble extract (Noguchi) ${ }^{2}$ prepared from dry heart muscle gave more desirable results. Based on comparative studies with serum, the former antigen appeared superior and ultimately formed the basis for the preparation of a desirable antigen for the writer's test.

In considering the preparation of the antigen, it was observed that the extent of the ether extraction of the beef heart was directly related to the sensitivity of the final product. The ether apparently removes not only the bulk of fat and nonspecific antigenic material, but also some specific lipoids. Therefore, when the ether extraction is excessive, the antigen sensitivity is reduced. When the extraction is insufficient, the antigen-saline suspension may contain lipoid particles which are nondispersive when mixed with serum, thus rendering the antigen unfit for use. After a number of experiments, the following method was adopted for antigen preparation. Twenty-five gm. of powdered heart muscle are extracted in a 250 -cc. Erlenmeyer flask at ten-minute intervals with $100,75,75$, and $75 \mathrm{cc}$. of ether, respectively. After the last extraction, the redried powder is extracted for three days at room temperature with 95 per cent alcohol, in the proportion of $5 \mathrm{cc}$. per gram of powder. To the alcoholic extract is then added $6 \mathrm{mgm}$. cholesterol per cubic centimeter. If the four ether extractions were reduced to one, about four times the amount of ether (about $\mathbf{1 , 2 0 0}$ cc.) would be required for similar results. With regard to the alcolool extraction, at period of several hours gives results similar to that of several days except that by the

${ }^{1}$ Neyman, C. A., and Gager, L. T.: J. Immunol., 2, 57 I. 1017.

${ }^{2}$ Noguchi, H.: Serum Diagnosis of Syphilis. Philadelphia: Lippincott, 19г 2. 
former extraction period the antigen shows a tendency to throw down a precipitate on standing. An extraction period of three days at about $2 \mathrm{I}^{\circ} \mathrm{C}$. was chosen as a standard. Regarding the amount of cholesterol in the antigen, experiments indicated that 0.6 per cent gave optimum results with sera.

\section{PRODUCING AN UNSTABLE ANTIGEN SUSPENSION}

In addition to the proper concentration of antigenic lipoids in a given antigen, the instability of the antigen-saline suspension is of the utmost importance. The suspension employed in the Kahn test contains comparatively large lipoid particles of such instability that they disperse as soon as they come in contact with serum. If the serum is from a syphilitic patient, precipitates appear in a few minutes; if from a nonsyphilitic individual, no precipitate appears.

The following experiment illustrates the antigen-saline relationship. To I-cc. amounts of antigen in a series of tubes are added $0.5,0.7,0.9$, I.I, and I.3 cc. of normal saline, respectively. On mixing the contents of each tube it will be found that all tubes contain coarse lipoid particles, but of unequal power of dispersion. Thus, the particles in the first tube containing the smallest amount of saline are non-dispersive in additional saline. With the increase in salt solution in the other tubes there is a proportional increase in the dispersive power of the particles. If the I cc. of antigen plus I.I cc. of saline mixture contains particles which are completely dispersed in additional saline, then the particles formed in antigen-saline suspensions in which the amount of saline is greater than I.I cc. are also dispersed on addition of saline. When I cc. of antigen is mixed with as much as $3 \mathrm{cc}$. of saline, the mixture is usually opalescent and free from visible particles.

When employed with serum, the criterion for the antigen-saline suspension is to add to I cc. of antigen the minimum amount of saline which will produce coarse lipoid particles which are completely dispersed in additional saline. These particles will also be dispersed in serum and thus will assure clear-cut negative reactions. In syphilitic serum, however, as already indicated, a specific precipitate will appear within a few minutes.

The use of a minimum amount of saline in preparing the antigen suspension is closely related to sensitivity with serum. Increasing the saline amount will, in practically all cases, reduce the sensitivity of the antigen suspension. The only time when the criterion for employing the minimum saline amount in the preparation of the antigen suspension is not adhered to is when the suspension is of greater sensitivity than the standard requirement.

\section{STANDARDIZATION OF ANTIGEN ${ }^{\mathrm{I}}$}

It was essential to establish a standard for antigen sensitivity because experiments have shown that different lots of beef heart may produce antigens of different reactivity. Such a standard was ultimately established as a result of serological and clinical studies. Not a maximum but a conservative degree of antigen sensitivity was chosen and the aim of standardization was to assure uniformity in this characteristic.

I For complete discussion, see Kahn, R. L., Nagle, N., and Kendrick, P. L.: "Studies on Antigen for the Kahn Test: I. Uniformity in Sensitivity of Standard Antigen," J.Infect. Dis., 4I, I I I. I927. 
The first step in standardizing a newly made antigen is to determine the minimum amount of normal saline to add to I cc. of antigen producing lipoid particles that will readily disperse in additional saline. This is spoken of as the "titre" of the antigen, and when determined the second step is to examine a number of negative, positive, and especially weakly positive syphilitic sera with the antigen using this titre, and with standard antigen as a control. If the sensitivity of the new antigen is similar to that of the standard, the new antigen is classed as "standard." If not similar, one of the several methods of antigen correction is employed, depending on the nature of the antigen.

I. An antigen might be more sensitive than the standard-due, probably, to a greater concentration of specific lipoids. Since both excessive dilution and excessive concentration of lipoids reduces antigen sensitivity, the oversensitive antigen may be reduced in sensitivity either by still further increasing its lipoid content by concentration or by decreasing its lipoid content by dilution.

One of two methods of antigen dilution may be used: (I) The antigen may be diluted with alcohol (cholesterolized antigen with cholesterolized alcohol). Ten and 20 per cent dilutions may be tried. Each diluted product is compared in sensitivity with standard antigen. If not comparable, greater or lesser dilution is resorted to. (2) The saline in the antigen suspension may be increased beyond the minimum amount. Thus, if the titre of a given antigen is I cc. of antigen plus I. I cc. of saline, I.2 and I. 3 Cc. of saline may be tried and each antigen suspension compared in sensitivity with standard antigen.

When reducing antigen sensitivity by increasing the lipoid concentration the following procedure is employed: A given amount, such as I cc. of non-cholesterolized antigen is evaporated to dryness. The residue is dissolved in Io cc. of cholesterolized antigen and the sensitivity of the final product tested against the standard. If not comparable, an antigen of a different degree of concentration is prepared and tested.

II. An antigen might be less sensitive than the standard-due either to excessive or to insufficient concentration of lipoids. If it is due to excessive concentration, the method of alcohol dilution, or that of increasing saline in the titre, just discussed, will increase the sensitivity of the antigen. If, on the other hand, insufficient concentration is the cause of lesser sensitivity, either one of two methods may be employed: (I) the concentration of alcoholic extractives, already presented, or (2) the addition of ether extractives to the antigen. This is carried out as follows: The accumulated ether extract from the antigen preparation from $25 \mathrm{gm}$. of beef heart is evaporated to about $50 \mathrm{cc}$. and cleared by filtration. A small amount of this extract $\left(0.5^{-I}\right.$ per cent) is added to the antigen and the final product compared in sensitivity with the standard antigen. If not comparable, a different amount of the ether extract is added to the antigen and the product tested against the standard.

For several years past practically all antigens in the writer's laboratory requiring correction have been brought up to standard requirements by alcohol dilution. The preliminary antigen titration and subsequent tests with serum in comparison with standard antigen furnisher the clue as to the extent of alcohol dilution to employ. Of two antigens requiring concentration, one was corrected by the addition of ether extractives, and the other by the addition of alcohol extractives, apparently with 
equally good results. All antigens, furthermore, were standardized to a titre of I cc. of antigen plus I.I cc. of saline. Antigen once standardized will keep without change at least four years, and perhaps indefinitely.

\section{TREATMENT OF SERUM}

Serum requires heating at $56^{\circ} \mathrm{C}$. for optimum results. Unheated serum gives results markedly weaker than heated serum. Furthermore, the longer a syphilitic serum is heated at $56^{\circ} \mathrm{C}$., up to two hours, the more sensitive are the reactions in most cases. For conservative results, a thirty-minute period of heating was chosen as the standard. Sera that are "anticomplementary" in the Wassermann reaction give specific Kahn reactions. Sera that are hemolyzed (if not excessively) are also satisfactory in the Kahn reaction.

Recent experiments indicate that the precipitin substances are associated with the globulin (euglobulin and pseudoglobulin) fraction and not with the albumin fraction of the serum. ${ }^{\mathrm{r}}$

\section{QUANTITATIVE RELATION BETWEEN SERUM AND ANTIGEN SUSPENSION}

For optimum precipitation results, it is essential that the number of antigenic reacting units correspond to the number of serum reacting units. If the number of antigenic units is excessive, precipitation is inhibited. If the number of serum units is excessive, precipitation is not inhibited, but is somewhat reduced in intensity. Furthermore, the presence of an extra amount of serum over that required to combine with the antigen suspension has the same effect as dilution with salt solution and tends to weaken precipitation. Complete precipitation thus depends on the correct proportion between the antigen (precipitinogen) and serum (precipitin) substances.

In deciding upon the proportion of serum and antigen suspension to be employed in the routine diagnostic test, many factors come into play. Syphilitic sera of unusually marked potency may give precipitation reactions when mixed with as much as an equal amount of antigen suspension. With the majority of syphilitic sera, however, this amount of suspension is inhibitory to precipitation. The same is true when half the amount of suspension in relation to serum is employed. As the amounts of suspension are reduced to one-third, one-fourth, one-eighth, and one-twelfth of the serum amounts, proportionally more precipitation reactions are obtained. A serum which is positive with a comparatively large amount of suspension is also positive with lesser amounts of suspension. Based on clinical and serological studies in comparison with the Wassermann test, a three-tube test was decided upon, employing three proportions of serum to antigen suspension-one part of serum to one-third part of suspension; one to a sixth and one to a twelfth part of suspension. The strongly potent sera produce precipitation in all the three serum-suspension proportions; the moderately potent sera are negative or weakly positive in the proportion of serum to suspension of one to one-third but strongly positive in the remaining proportions, while the weakly potent sera produce precipitation only in the proportion of serum to suspension of one to one-twelfth. To render the final results conservative, the readings in the three tubes are averaged. In order that $0.5 \mathrm{cc}$. of serum be sufficient

\footnotetext{
${ }^{x}$ Kendrick, P. L., and Kahn, R. L.: ibid., 39, 202. 1926.
} 
for a complete test, the quantity of serum chosen for each of the three proportions was $0.15 \mathrm{cc}$, making the quantities of suspension $0.05,0.025$, and $0.0125 \mathrm{cc}$. respectively.

The precipitation reaction between serum and antigen suspension lends itself to the determination of the relative number of reacting substances in syphilitic serum. Since such determinations would be made in practice most frequently in cases undergoing anti-syphilitic treatment, a serum-suspension proportion of somewhat greater sensitiveness than that enployed in any of the tubes in the routine test, namely, I5:I, was chosen for a quantitative procedure. A series of serum dilutions with normal saline-I:I, I:5, I:IO, I:20, and so on up to $\mathrm{I}: 60$-are prepared, and 0.15 cc. of each of the dilutions are tested with $0.0 \mathrm{r}$ cc. of antigen suspension. As will be shown later, if undiluted serum alone gives a precipitation reaction, the final result is four units; if the diluted sera also give precipitation reactions, then the highest dilution multiplied by 4 gives the final result.

\section{THE SHAKING OF THE INGREDIENTS}

Strongly potent serum when mixed with standard antigen suspension in the proper proportion will produce a precipitate after shaking for about ten seconds. Weaker sera may require several minutes' shaking to bring forth a precipitate. The optimum shaking speed was found to be about 275 oscillations per minute. This optimum applies especially to weaker reactions. Most strongly reacting sera show somewhat bulkier precipitates at a speed under 275. When the oscillations extend beyond 300 per minute, the tendency is to break up the particles, making the reading of the tests somewhat more difficult. A shaking period of three minutes is ample for the routine diagnostic procedure, although increased shaking up to seven or ten minutes tends to bring forth more marked precipitation, especially in some weakly positive sera, without apparently producing false reactions. The standard shaking period is three minutes, at 275 oscillations per minute, with a stroke-length of about I. 5 inches. Rapid hand shaking approximates this speed.

The effect of incubation on the test-either at room $\left(2 \mathrm{I}^{\circ} \mathrm{C}\right.$.), icebox $\left(6^{\circ} \mathrm{C}\right.$.), or incubator $\left(37^{\circ} \mathrm{C}\right.$.) temperatures-is inversely proportional to the effect of shaking. With but little shaking, incubation will bring forth many precipitates. The effect of incubation following shaking for three minutes is comparatively insignificant, al. though an occasional positive reaction will be produced following a negative reaction after the shaking period. After five or six minutes' shaking, many reactions following incubation are weaker, the precipitates going into solution-more correctly, into a state of dispersion. To a lesser degree, weaker reactions are also obtained following incubation after shaking for three minutes.

\section{THE READING AND INTERPRETATION OF THE RESULTS}

A definite precipitate suspended in a clear medium is read ++++ . Proportionally weaker reactions are read,,++++++ , and \pm , respectively. After reading the results in each of the three tubes of the routine test, the final result is computed by taking the average of the readings of the three tubes. It is obvious that for a final reaction to be interpreted as ++++ , each of the tubes of the routine test must show 
a definite precipitate. If the readings in the three tubes are: - in the first, ++ in the second, and ++++ in the third, then the final result is ++ . The precipitates in the quantitative procedures are read on a positive and negative scale. A precipitate which is either,+++++++ , or ++ is read "positive," while all others are read "negative." The final result expressed in reacting units is obtained by multiplying by 4 the highest serum dilution giving a positive reaction.

\section{SPECIAL ANTIGEN}

Standard antigen necessarily had to be of a conservative degree of sensitivity. In isolated cases it is desirable to employ a more sensitive antigen than the standard. Since the amount of ether extractives in an antigen is directly related to its sensitivity, a special antigen more sensitive than standard antigen was produced containing a somewhat larger amount of ether extractives than the standard. Thus, in the preparation of special antigen from $25 \mathrm{gm}$. of powdered beef heart, the ether extractions are limited to five minutes, employing $5 \circ, 50,40$, and $40 \mathrm{cc}$. of ether, respectively. In other respects it is prepared like standard antigen. This antigen is employed in the presumptive procedure and may be employed in special cases in other procedures. The sensitivity of this antigen is also standardized to a uniform degree.

\section{RELATION BETWEEN PRECIPITATION AND COMPLEMENT FIXATION ${ }^{\mathrm{I}}$}

There is little doubt but that the precipitation and complement fixation reactions detect the same substance or substances in syphilitic serum. This was suggested by Jacobstahl in I908. In my laboratory the identity of precipitin and complementfixing substances was studied by adding complement to completed Kahn tests, and after incubation, following with amboceptor and washed corpuscles. The results of I,800 such examinations showed 93 per cent absolute agreement, 5 per cent relative agreement, and 2 per cent disagreement. With better understanding of precipitation and complement fixation, closer agreement might be obtained.

The method employed consisted in adding O.I cc. ( 2 units) of complement to $0.2 \mathrm{cc}$. of the serum-antigen suspension mixture of the third tube of the Kahn test which contains the least amount (O.OI $25 \mathrm{cc}$.) of the suspension. This was incubated in the water bath at $37^{\circ} \mathrm{C}$. for thirty minutes after which o.I cc. of antisheep-amboceptor ( 2 units) solution and O.I cc. of a 5 per cent sheep-cell suspension were added and incubated about fifteen minutes, until the serum and antigen control tubes showed complete hemolysis. Complete fixation of complement was read ++++ ; partial fixation,,,++++++ , and \pm , respectively.

The completed Kahn test may be utilized with any complement fixation technique. It is well to use the tube of the Kahn test which contains the least amount of antigen suspension. From this tube an amount of serum-suspension mixture is pipetted off, corresponding to the amount of serum employed in the desired complement fixation technique, complement added, incubated, and the complement fixation test completed in the usual manner. This complement-fixation-Kahn-precipitation method is of special value in presenting this subject to students. It also offers a

${ }^{x}$ Kahn, R. L., Landau, J. L., and McDermott, E.: Proc.Soc. Exper. Biol. \& Med., 24, 775. 1927 
desirable complement fixation procedure in place of the Wassermann test in laboratories where it is employed in parallel with the Kahn test.

When considering the relation between precipitation and complement fixation methods, it is important to keep in mind that the interreaction between serum and antigen is essentially the same in both cases. The end-result of this interreaction being readily visible in the precipitation test, no further steps are necessary. The endresult not being visible in the case of the complement fixation test, a special indicator in the nature of the hemolytic system is employed to render it visible.

\section{NATURE OF THE PRECIPITATION REACTION}

When immune serum is mixed with specific antigen, the resulting precipitate consists largely of globulins from the immune serum. When syphilitic serum is mixed with an extract antigen-saline suspension, the precipitate formed consists largely of lipoids. This was shown as early as igi I by Jacobstahl. The source of the lipoids is possibly the antigen. This would indicate that the precipitation reaction in syphilis is inherently different from the immune reaction. However, the true nature of the reaction-whether the end-result is the formation of a precipitate or the fixation of complement - is still unknown. Originally, the Wassermann reaction was believed to be a specific immunity reaction. This view was abandoned when it was shown by Landsteiner and others that alcoholic extracts of normal tissue can serve as an antigen in this reaction.

Recently, Klopstock ${ }^{1}$ was able to elicit positive Wassermann reactions in rabbits following injections with dead Spirocheta pallida. Landsteiner and Van der Scheer ${ }^{2}$ also recently reported positive Wassermann and Sachs-Georgi reactions in rabbits following injections with dead trypanosomes. This would indicate that these organisms do have antigenic properties. On the other hand, Sachs, Klopstock, and Weil ${ }^{3}$ obtained positive Wassermann reactions in rabbits after injecting into these animals alcoholic extracts of rabbit organs combined with pig serum. As is well known also, apparently normal rabbits frequently give positive precipitation and Wassermann reactions. It cannot be concluded, therefore, that such reactions occur only as a result of spirochetal activity.

As to the mechanism of the precipitation reaction, it is of interest to note that the generalizations deduced from this biological precipitation reaction appear to be closely parallel to some of the essential conditions affecting colloidal precipitation systems of a non-biological character. Thus, in examining the conditions governing the syphilis reaction one may observe several distinct instances of "zone phenomena" typical of colloid reactions. It has, for example, been shown that most sensitive precipitation reactions are obtained with a given antigen when the lipoid concentration is adjusted to a certain optimum value, an increase or decrease in the concentration yielding less sensitive reactions. In this connection it may be noted that little is known of the chemical nature of the antigen excepting that it contains lecithin, cholesterol, and other tissue lipoids. It appears, however, that in its reaction with syphilitic serum the antigen will give desirable precipitation results only after it has

\footnotetext{
I Klopstock, F.: Deutsche med. Wchnschr., 52, 226 and 1460. I926.

${ }^{2}$ Van der Scheer, J.: J. Exper. Med., 45, 465. 1927.

${ }^{3}$ Sachs, H., Klopstock, A., and Weil, A. J.: De.ttsche med. Wchnschr., 51, 589. I925.
} 
been mixed with salt solution in such a way as to produce a lipoid emulsion or suspension. ${ }^{\mathrm{I}}$ Further study of the relationship between the method of mixing antigen with saline and the physical appearance and reactive properties of the resulting lipoid suspension brought to light a second zone phenomenon. Only when the antigen-saline proportion is restricted to a certain zone (determinable by trial for each antigen) are visible particles observable in the mixture. Furthermore, the antigen-saline proportion giving optimum precipitation reactions in the Kahn test is limited to a still narrower zone within the confines of the other. A third zone effect is observed in the relationship between serum and antigen suspension, where excess of antigen suspension over serum and - to a less marked degree - of serum over antigen suspension is inhibitory to precipitation.

Turning from zone phenomena to a consideration of certain other factors, it is found that agitation, which is known to play an important part in hastening the coagulation of precipitates in colloid systems, is an essential feature of the Kahn reaction. Finally, it has been emphasized that dilution of the reacting mixture delays the precipitation reaction, as would indeed be expected from an analysis of the kinetics of precipitation; this observation was among the first to be made in the series of studies that led to the development of this precipitation test. Numerous problems in connection with the nature of the reaction are yet to be solved. It is hoped that the relative simplicity of the Kahn test will permit its ready utilization in connection with further work in this field. ${ }^{2}$

\section{THE KAHN PROCEDURES}

All the procedures in this system are carried out by shaking for three minutes the tubes containing the antigen suspension with the serum, cerebrospinal or other fluid. After the shaking period an arbitrary amount of normal saline is added to the tubes to render the precipitates readily visible, and the results are read.

Routine or diagnostic test with sera.-This is a three-tube test consisting of three different proportions of serum and antigen suspension. The amounts of the latter are 0.05 , 0.025 , and $0.0125 \mathrm{cc}$., respectively, while the amounts of serum are $0.15 \mathrm{cc}$. throughout. Experiments have shown that $0.05 \mathrm{cc}$. is an excessive amount of suspension when used with 0.15 cc. of serum, and will produce precipitation reactions only with serum which is rich in syphilitic reagin. Weaker sera will be negative with the 0.05 -cc. suspension amount and positive either with the 0.025 - or 0.0125 -cc. amounts, depending on the potency of the serum. The less suspension used, the stronger the precipitate with weakly reacting sera. Table I gives an outline of the routine test and the interpretation of the results. The final result of a reaction is the average of the findings in the three tubes, expressed in plus signs.

Clinical application: As an aid in the diagnosis of syphilis and as a check on antisyphilitic treatment.

Quantitative procedure with serum.-This procedure consists of a series of serum dilutions in o.1 5-cc. amounts in which each dilution is tested with o.or cc. of standard antigen suspension. The reactions in the individual tubes are interpreted as positjve or negative, depending on the presence or absence of a definite precipitate. The final result is expressed in syphilitic

I The importance of antigen-salt-solution emulsions in connection with the Wassermann test was pointed out by Sachs and Rondoni as early as I908.

${ }^{2}$ For the application of this precipitation reaction in syphilis to agglutination reactions in other diseases, see Huddleson, I. F., and Carleson, E. R.: J. A. Vet. M. A., 70, 229. 1926; Noble, A.: J. Bacteriol., 14, 287. I927. 
reacting units according to the formula $S={ }_{4} D$, where $S$ is the serum potency in terms of reacting units and $D$ is the maximum dilution of serum giving a positive reaction.

Clinical application: To determine the relative intensity of syphilitic reacting substances in the blood, especially in the cases of patients receiving anti-syphilitic therapy.

Presumptive procedure with serum.-This is a qualitative one-tube test consisting of $0.15 \mathrm{cc}$. of serum with $0.0 \mathrm{I} \mathrm{cc}$. of antigen suspension, employing special antigen-a somewhat more sensitive antigen than standard. The results are interpreted on the basis of positive or negative, depending on the presence or absence of a definite precipitate.

TABLE I

Outline of Kain Test and Interpretation of Results

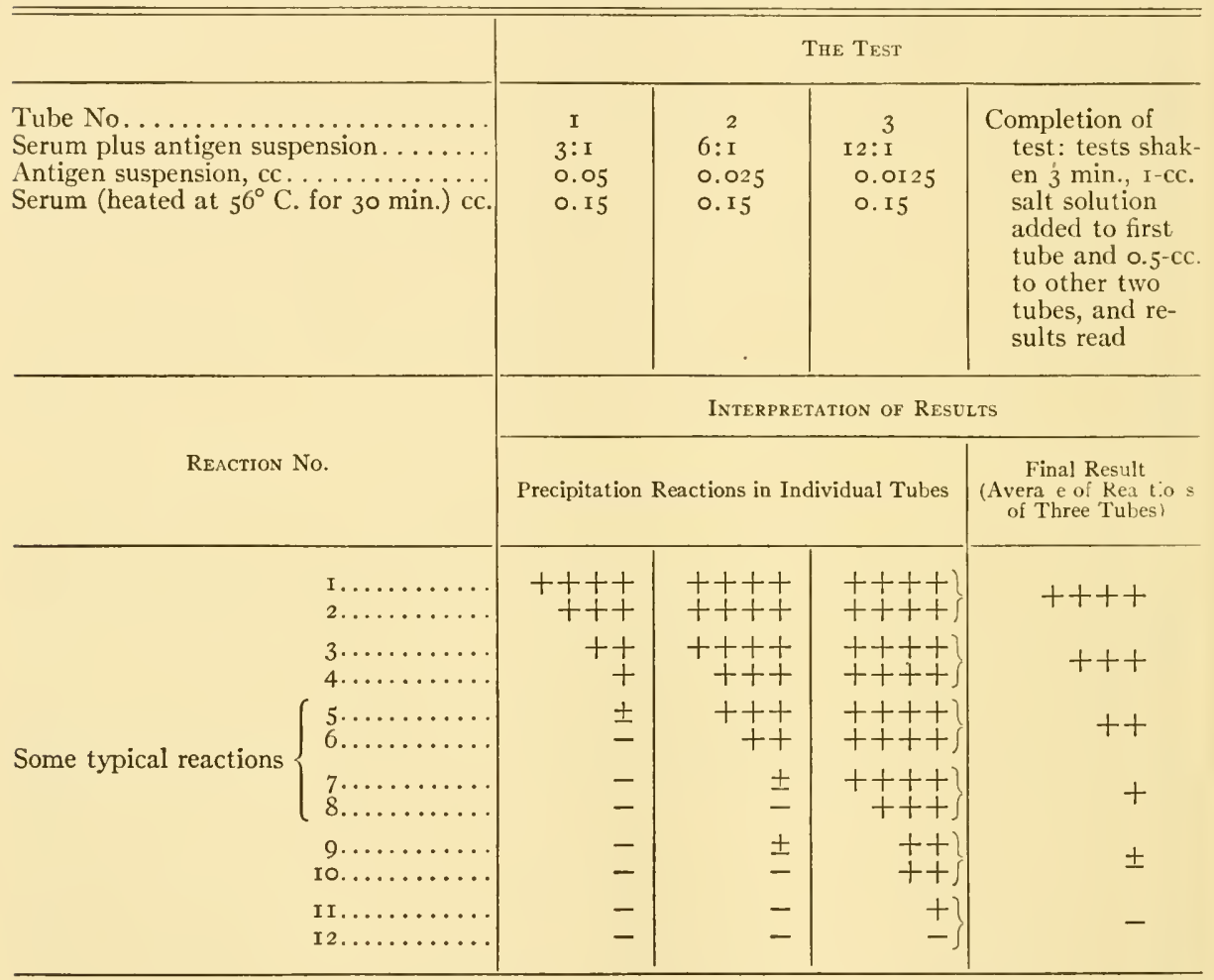

Clinical application: Aid in diagnosis of obscure cases where the routine test might be negative; check on treated cases.

Qualitative spinal fuid procedure.-This method requires precipitating the globulins in spinal fluid with saturated ammonium sulphate solution, producing 40 per cent saturation, separating the globulins by centrifugation, and redissolving them in an amount of normal saline equal to one-tenth of the original amount of spinal fluid. The dissolved globulin, in a o.r 5-cc. amount, is now added to o.or cc. of antigen suspension and the test completed in the usual manner. The results are interpreted on a plus sign basis.

Clinical application: In the diagnosis of neurosyphilis and check on treatment.

Quantitative spinal fluid procedure. ${ }^{\mathrm{I}}$ - This is a continuation of the qualitative procedure,

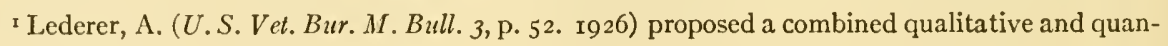
titative spinal-fluid procedure. 
the concentrated globulin solution forming the basis for further quantitative study. This solution is diluted $\mathrm{I}: 5, \mathrm{I}: \mathrm{IO}$, and $\mathrm{I}: 20$ with saline, and $0 . \mathrm{I} 5 \mathrm{cc}$. of each is tested with $0.0 \mathrm{I} \mathrm{cc}$. of antigen suspension. The tests are completed in the usual manner and the results computed as in the case of the quantitative serum procedure according to the formula $S={ }_{4} D$ where $S$ is the potency of spinal fluid in terms of reacting units and $D$ is the highest dilution of the concentrated globulin solution giving a positive precipitation reaction. The original spinal fluid may be used for the I : Io globulin solution and the I: 2 dilution of the spinal fluid for the $I: 20$ g'obulin solution. If a precipitate is obtained in the $I: 20$ dilution, the final reaction is $20 \times_{4}=80$ reacting units. In most cases, however, spinal fluids are negative in this dilution.

Clinical application: As a check on treatment in neurosyphilis.

"Micro-procedures." I- These are special procedures which employ minute amounts of serum or spinal fluid, and are employed only when insufficient quantities are available for regular test. In the case of serum, one-tenth of antigen suspension in relation to serum is employed. Thus, if $0.05 \mathrm{cc}$. of serum is available, $0.005 \mathrm{cc}$. of suspension is employed. In the same way, any measurable amount of serum and suspension may be used. In the case of spinal fluid, the globulins are precipitated in an amount that is available and the "microprocedure" performed with the dissolved globulin solution, employing fifteen parts of this solution to one of antigen suspension. All "micro-results" are interpreted as positive or negative, depending on the presence or absence of a precipitate.

Procedure with fuid from syphilitic lesion.-Chancre or other syphilitic fluids are employed with antigen suspension in the proportion of $10: \mathrm{I}$, as in the case of micro-procedures. Thus, if $0.03 \mathrm{cc}$. of fluid is available, it is employed with $0.003 \mathrm{cc}$. of suspension. In the case of chancre fluid, precipitation reactions have been obtained even after $1: 3$ dilution with saline. The results are interpreted as positive or negative.

Procedure applicable to rabbit syphilis. - The quantitative serum procedure employing the I:I, $\mathrm{x}: 5, \mathrm{I}:$ IO, and $\mathrm{I}: 20$ serum dilutions has been found to be applicable to the serum reaction in rabbit syphilis. The results are interpreted on the basis of quantitative units, as in human syphilis.

Special investigation of a serum.-It is occasionally desired to study a serum giving negative results with the usual Kahn methods, for traces of syphilitic reagin. It is known that the sensitivity of the precipitation reaction can be increased by prolonging the heating period of the serum to one hour, by decreasing the amount of antigen suspension in relation to serum, such as the use of a 20 : I proportion of serum to suspension, and by increasing the shaking period to seven or ten minutes. By applying these steps to a given serum, it is possible, occasionally, to detect a minute amount of reacting substance. Such findings, while of no general diagnostic value, may throw light on the diagnosis of an obscure case.

\section{APPLICATION OF KAHN REACTION}

Studies by different workers on the application of the Kahn reaction to clinical medicine have been limited thus far almost entirely to the employment of the routine diagnostic test. Judging from the results obtained, the test is comparable in specificity and sensitivity to a reliable Wassermann reaction, although some claim the Kahn to be more sensitive in primary syphilis and in treated cases. ${ }^{2}$ It is obvious, however,

${ }^{1}$ Kline, B. S., and Young, A. M. (J.A.M.A., 86, 928. I926) recently suggested a microscopicslide test with very small amounts of serum and antigen dilution.

${ }^{2}$ The reliability of the Kahn reaction is generally accepted (Redfield, Faupel, Giordano, Owen and Cope, Lederer, Dutton, Duemling, Pineda, Houghton, Hunter and Cajigas, Stucky and Huntley, Johnson, Literer, McLean, Maynard, Berry, Ey and Delong, Walker, Hull, etc.). The Michigan De- 
that the employment of the additional Kahn procedures would give clinicians further information regarding the serological status of a patient. Thus, if the routine test with a given serum is positive, the performance of a quantitative procedure would give the number of syphilitic reacting units; if negative, the performance of the more sensitive presumptive procedure might give a clue as to the possibility of syphilitic infection where otherwise syphilis might not be suspected.

The quantitative procedure is of special value in following the serological effect of anti-syphilitic treatment. The blood of a patient before treatment might show, for example, 240 reacting units; after the first course, I80 units; after the second course, 80 units; and after the third course, 20 units. The routine test would be ++++ at each examination, and would give no indication as to the relation between treatment and the true serum reactions. Then again, in another case, the quantitative units might be reduced, let us say, from 400 to 200 units, and not be further reduced until the nature of the therapy is changed. This quantitative reaction, therefore, is an important guide in syphilitic therapy. Furthermore, this reaction combined with the routine and presumptive procedures requires less than I cc. of serum and can be completed within one hour from the time blood is drawn from the patient.

The quantitative spinal fluid procedure should also prove an important aid to clinicians in the treatment of neurosyphilis. Each of the various Kahn procedures has its own distinctive clinical value. The combined use of these procedures should be of greater value to clinicians in the diagnosis and treatment of syphilis than the use of a single procedure.

partment of Health has reported from Oct. I5, I925, to Oct. I5, I927, over I 50,000 Kahn reactions - without Wassermann tests-with satisfactory results. The Kahn test is also standard in the U.S. Navy, State Department of Health laboratories of Illinois, West Virginia, and many other laboratories. 


\title{
CHAPTER LXV \\ THE MECHANISM OF PHAGOCYTOSIS
}

\author{
W. O. FENN
}

School of Medicine and Dentistry, The University of Rochester, Rochester, N.Y.

THE RÔLE OF FREE-ENERGY CHANGES IN PHAGOCYTOSIS

From a very general point of view phagocytosis may be regarded as a problem of cell permeability, there being a continuous and gradual transition from molecules through colloidal particles and fine suspensions to particles nearly as large as the phagocyte. In cases where penetration of molecules involves an approach to a true equilibrium, i.e., a diminution of free energy, an accurate analysis of the factors involved is much facilitated. The question arises, therefore, whether phagocytosis is such a process, or whether the ingestion of particles involves an increased metabolism on the part of the cell, some of this extra energy being stored up as potential energy of position or as surface energy between the cell and the particle. At the outset of an inquiry into the mechanism of phagocytosis it must be admitted that to this most fundamental of all the questions involved we have no unequivocal answer ready. It is not certain whether phagocytes ingest objects because of surface tension or in spite of it. Probably there is truth in both.

There are cases, however, in which it appears certain that the diminution of free energy resulting from ingestion is a limiting factor in the process. Thus in a mixture of polymorphonuclear leukocytes of the rat with equal-sized particles of carbon and quartz, a certain microscopic field was examined ${ }^{\mathrm{T}}$ showing 77 leukocytes, 44 carbon particles, and 38 quartz particles. After twenty-four minutes, 36 chance contacts had been observed between leukocytes and quartz particles and 37 contacts between leukocytes and carbon particles. Yet at the end of this period only i quartz particle was to be seen inside a leukocyte as compared with I 2 carbon particles. In this case, then, the chances of collision for the carbon and quartz particles were equal but the chance of penetration favored the carbon I 2 to I. Between these two insoluble particles the leukocyte is evidently able to detect a difference. This cannot be due, of course, to a chemical difference in the medium, caused, for example, by materials which leach out of the pores in the carbon, for both kinds of particles are incubated together under the same cover slip. It is difficult, therefore, to believe that the difference can be anything but a difference in the surface-energy relationships.

This observation alone, however, does not prove this to be a fact. Amebae have been observed to cut paramecium in two parts in the process of ingesting it. ${ }^{2}$ Surface energy relationships could have made very little difference to this ameba, which was reacting as a whole to some stimulus. A high surface energy between the paramecium

${ }^{3}$ Fenn, W. O.: J. General Physiol., 5, 3 II, 1923; 3, 575. I92I.

${ }^{2}$ Mast, S. O.: J. Morph. \& Physiol., 41, 347. I926. 
and the ameba might have made itself evident as a slight repulsion, but this could not have amounted to much in comparison with the energy being expended by the ameba to cut the paramecium in two. There is the possibility that a leukocyte behaves also in this way-that it receives something in the nature of a stimulus from the particle to be ingested and makes a definite energy expenditure in response. It is conceivable, then, that because of some chemical peculiarity of the carbon surface it acted as a more effective stimulus than the quartz surface. But leukocytes can be shown to carry on very active ameboid movements around quartz particles, sometimes seeming to have partly ingested them, but in the end frequently moving away without them. The activity of the cell is, apparently, no greater in the presence of carbon than in the presence of quartz. Its oxygen consumption would doubtless be no greater. If this is so, the conclusion seems inevitable that carbon particles are better ingested merely because, once they have come into contact with protoplasm, there is less tendency for them to come away again.

Still the observations do not quite prove that surface energy relationships are of importance in determining the greater ease of ingestion of carbon, for it is conceivable that the cell would find the smooth surface of a quartz particle too "slippery," for example, while the microscopical roughness of the carbon afforded a more enduring contact. This possibility can be disproved by a simple and striking experiment. If the same field of leukocytes and particles described above could be examined in an acid medium at $\mathrm{pH} 6,{ }^{\mathrm{r}}$ it would be found that, for the same number of contacts with the leukocytes, quartz was ingested perhaps twice as rapidly as the carbon particles. It cannot be supposed that acid makes the quartz surface rough while the carbon surface becomes smooth. The difference between carbon and quartz particles is evidently something which can be reversed by acid, and this points strongly to surface forces. Confirmatory evidence is derived from the behavior of carbon particles prepared with the aid of gum acacia as a stabilizing agent. At all acidities such particles are taken up more readily than quartz particles, and similarly it is found that the cataphoretic potential can no longer be reversed by acid after treatment with acacia.

With these carbon and quartz particles, however, we are dealing with a peculiarly simple case. It seems to be merely a question of the partition coefficient of carbon and quartz between the two phases-plasma and cells. Similar differences are made use of in methods for separation of ores by flotation, and they can readily be demonstrated by shaking up carbon and quartz particles with phenol and water, in which case 8I per cent of the carbon is found in the phenol and 88 per cent of the quartz is found in the water.

A case which is not so simple is that of the black particles of $\mathrm{MnO}_{2}$ particles and the transparent $\mathrm{Mn}$ silicate particles. Here the former are ingested at least 20 times as rapidly as the latter; but careful observation and counts show that the leukocytes are definitely attracted toward the former, for there are 2.4 times as many contacts per unit time with the $\mathrm{MnO}_{2}$ as with the $\mathrm{Mn}$ silicate, although present in equal num. bers. Here there is evidently something like a stimulus which comes from the $\mathrm{MnO}$, particles and causes an extra liberation of energy from the leukocyte resulting in an apparently "purposeful" progression in a definite direction and prompt ingestion. I have had the good fortune to see some very beautiful and convincing moving pic-

${ }^{\mathrm{I}}$ Fenn, W. O.: loc. cit. 
tures taken by Commandon ${ }^{\mathbf{x}}$ showing leukocytes being attracted toward starch particles from distances many times their own diameter. The moving pictures were speeded up so that the leukocytes seemed to move with great rapidity, pushing erythrocytes violently aside but always moving directly toward the starch grain and finally swarming over it in numbers and apparently "eagerly" devouring it.

Another important complication is seen in studies of the effect of temperature on phagocytosis. At low temperatures it has been observed that particles stick to the cells but are not ingested. ${ }^{2}$ Here the difficulty seems to be that the cell is too rigid to permit the penetration of the particle. As the temperature is raised there comes a point when ameboid movement begins, ${ }^{3}$ spontaneous energy changes begin to occur inside the leukocyte which result in alternate changes of the protoplasm from sol to gel, and the general fluidity of the cell becomes such that large particles can enter. At this transitional point the temperature coefficient of phagocytosis becomes very high $\left(Q_{\mathrm{IO}}=\mathrm{I} 8\right),{ }^{4}$ a small change in temperature causing a large change in fluidity and hence in phagocytosis. Similar changes in consistency have been invoked particularly by Loeb ${ }^{6}$ in explanation of cell behavior and undoubtedly play an important part in phagocytosis.

There are, therefore, many factors other than surface tension which enter into problems of phagocytosis. The cells have structure and show marked changes in their consistency. Like all living matter, they are centers in which free energy is being made available by oxidative reactions at a rate which might dwarf to insignificance the energy changes due merely to transfer of an object from outside the cell to its interior. Without losing sight of this fact, it is nevertheless clear that the cells are exposed to these surface forces and that they are of sufficient magnitude to be of decisive importance in the economy of the cell, at least under some conditions. In addition to the example of the carbon and quartz which demonstrates this fact, two others deserve mention. Thus Falk ${ }^{6}$ and his associates find a correlation between the virulence of pneumococcus strains, their cataphoretic potentials, and their ingestibility by phagocytes. Second, Mudd and Mudd ${ }^{7}$ have observed motile bacteria struggling, mostly in vain, to escape from an oil-water interface in which they were held by surface tension forces. And the higher the surface tension of the interface the more firmly were they held.

Such experiments point the way for future investigations and amply justify a general and inclusive statement of the conditions necessary for equilibrium between three immiscible phases, one of which may be solid.

\section{CONDITIONS NECESSARY IN PHAGOCYTOSIS FOR A MINIMUM OF FREE ENERGY}

The formula for the equilibrium is very simply explained in terms of the contact angle. ${ }^{8}$ When three fluids, or more commonly two fluids, and a gaseous phase are in

${ }^{x}$ Commandon, J.: Compi. rend. Soc. de biol., 82, i г 71. rgг9.

${ }^{2}$ Ledingham, J. C. G.: J. Hyg., 12, 320. 1912; Proc. Roy. Soc., B, 80, 188.1908.

3 de Haan, J.: Arch. néerl. de physiol., 6, 388. 1921. ${ }^{4}$ Fenn, W. O.: op. cit., 4, 331. I922.

${ }^{5}$ Loeb, L.: "Washington University Studies," 8, 3. I920.

${ }^{6}$ Falk, I. S., and Matsuda, T.: Proc. Soc. Exper. Biol. Eo Med., 23, 781. 1925-26.

${ }^{7}$ Mudd, S., and E. B. H.: J. Exper. Med., 40, 647. 1924.

${ }^{8}$ Maxwell, J. C.: Encyc. Brit. (I Ith ed.), 5, 262. I910-rr. 
mutual contact, then the three forces of surface tension acting on the line of mutual contact must be capable of representation both in magnitude and in direction by the three sides of a triangle (the Neumann triangle). When one phase is solid, there is a simplification in that only those components of the forces of surface tension which pull in the plane of the solid surface need be considered. The formula for this condition may be derived simply from the equilibrium diagramed in Figure $\mathrm{I}$ in which one fluid phase, called conveniently the "cell," is represented as in an equilibrium position between a fluid phase and a solid phase, called conveniently the "particle." The interface between the particle and the fluid is represented as flat and infinite, i.e., the particle is infinite in size. At equilibrium the forces of surface tension which pull the point of contact to the left may be considered as equal to those which pull it to the right. Thus

$$
\begin{gathered}
T p f=T p c+\cos A T c f, \\
\cos A=\frac{T p f-T p c}{T c f} .
\end{gathered}
$$

Since it is difficult to conceive of a force of surface tension on a solid surface actually pulling the point of contact, this formula is by no means obviously true. The

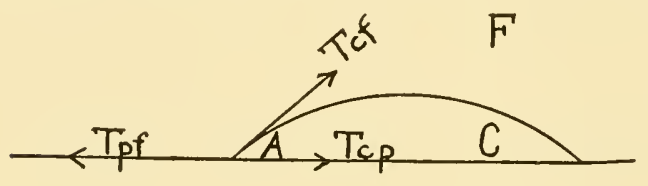

FIG. I same formula may be derived, however, from the more general proposition that the free surface energy must be at a minimum at the point of equilibrium. ${ }^{\mathrm{r}}$ Harkins and Feldman ${ }^{2}$ have also preferred the thermodynamic approach for the derivation of their spreading coefficient. There is some theoretical justification, therefore, for a thermodynamic proof of this equation. Granted the equation, of course, the rest follows simply.

Three general classes of equilibrium are possible according to this equation:

I. If $T p f=$ or $>T p c+T c f: \operatorname{Cos} A$ is then $=$ or $>$ I and $p$ will be completely ingested by $c$, or $c$ will spread to infinity on $p$. This will be the position at which the $p f$ interface with its high surface tension disappears altogether.

2. If $T c p=$ or $>T p f+T c f: \operatorname{Cos} A$ is then $=$ or $<-\mathrm{r}$, and there will be no contact between $p$ and $c$, the values of $A$ being unreal. This will be the position of minimum free energy because the $c p$ interface with its high surface tension is absent. When this is true the force of attraction between the cell and the particle is o or negative (repulsion). Ponder ${ }^{3}$ inadvertently states that when $\cos A=-\mathrm{r}$ the cell would be inside the particle. The correct condition for this equilibrium is that $T c f=$ or $>T p f+T c p$, in which case $\cos A$, according to the formula, must be somewhere between $\mathrm{I}$ and $-\mathrm{I}$. As $T c f$ increases $\cos A$ approaches o and $A$ approaches $90^{\circ}$. Whenever $T c f$ is greater than Tpf the particle may be thought of as "trying" to ingest the cell. The rigidity

I Fenn, IV. O.: op. cit., 4, 373. I922.

${ }^{2}$ Harkins, W. D., and Feldman, A.: J. Am. Chem. Soc., 44, 2665.1922.

3 Ponder, E.: J. General Physiol., 9, 827. 1925-26. 
of the particle prevents this, but the cell nevertheless proceeds partially to ingest the particle because by so doing the $c f$ interface is reduced to a minimum.

3. If neither (I) nor (2) is true, the particle will be partially ingested by the cell or the cell will be partially spread out on the particle. Cos $A$ will be between $+\mathrm{I}$ and - $\mathrm{I}$, and $A$ will be between $\circ$ and $\mathrm{r} 80^{\circ}$.

These three conditions cover all the possibilities. According to the formula, it is not possible for the cell to be inside the particle, and of course it is not possible practically. Such a condition is, however, possible to conceive, and, with certain surface tension values (particularly when Tcf is large), this would be a truer equilibrium than that actually assumed, because the total free surface energy would be less.

Earlier writers on the mechanism of phagocytosis (Rhumbler, ${ }^{\mathrm{T}} \mathrm{Tait}^{2}$ ) have considered only the first and second conditions above, neglecting, as I have pointed out, ${ }^{3}$ the possibility that partial ingestion or partial spreading should occur. Ponder, ${ }^{4}$ while recognizing clearly this possibility, nevertheless doubts whether partial spreading does in fact occur, and states that it does not occur with pure liquids. This is hardly correct, however, for even oils which do not spread on water nevertheless have a definite contact angle against water. Thus the contact angle between water and paraffin is I $00^{\circ}$ not $\mathrm{I} 80^{\circ} .5$ Likewise, many cases are known where solids accumulate at an interface between two liquids, showing that the contact angle must be somewhere between $0^{\circ}$ and $180^{\circ}$ (Reinders, ${ }^{6}$ Hofmann, ${ }^{7}$ Fenn $^{8}$ ). Where air is one phase, in fact, the diffcult condition to find exemplified is where the contact angle is $180^{\circ}$.

Since the formula for the equilibrium can be developed entirely from considerations of the free energy of the interfaces, ${ }^{9}$ it is obvious that it must include all forces which could affect the equilibrium. Electrical forces, therefore, may be regarded as affecting the spreading of cells and phagocytosis by virtue of their effect upon surface tension, surface tension being merely the free energy of a unit area of the interface. Where there is a large electrical attraction between two phases the surface tension of the interface is low, etc.

Much can nevertheless be learned from an accurate analysis, both experimental and theoretical, of the electrical forces between cells and particles, for the charges in such bodies can be measured experimentally. Ponder ${ }^{\text {I0 }}$ has begun the theoretical analysis of these forces. The particle is surrounded by an electrical double layer and as a whole is electrically neutral. There is, therefore, no repulsion between equally charged particles so long as the distance between them is large compared to the distance between the two layers of the double layer (Michaelis). ${ }^{\text {II }}$ When the distance be-

I Rhumbler, L.: Arch.f. Entwcklngsmcchn. d. Organ., 7, 279. IS98.

${ }_{2}$ Tait, J.: Quart. J. Exper. Physiol., I 2, I. I918-20.

3 Fenn, IV. O.: op. cit., 4, 373. I922.

${ }^{4}$ Ponder, E.: loc. cit.

5 Adam, N. K., and Jessop, G.: J. Chem. Soc., I27, 1863. I925.

${ }^{6}$ Reinders, W.: Kolloid-Ztschr., I3, 235. I913.

${ }^{7}$ Hofmann, F. B.: Ztschr. phys. Chemic, 83, 385. I9I3.

${ }^{8}$ Fenn, W. O.: op. cit., 3, 575. I $92 \mathrm{I}$.

9 Fenn, IV. O.: ibid., 4, 373. I922.

10 Ponder, E.: loc. cit.

II Michaelis, L.: chapter in Bogue, R. H.: Colloid Chemistry, Vol. I, chap. ix, p. 251. I924. 
tween them becomes less there is a repulsion due to the redistribution of the charges on the outer layers. Ponder has simplified the situation somewhat in considering only the outer layer of the double layer in his calculations. He calculates the mutual redistribution of the charges and the resulting electrical forces between the cell and the particle (which is considered small in comparison) on the basis of Coulomb's law, and arrives at the conclusion that when the distance between the two bodies becomes very small the repulsion between them (in case they both carry a charge of the same sign) becomes an attraction. This result is true provided his assumptions are true, and a valuable hypothesis is thus provided in the light of which future experimental results can be analyzed. In discussing this result, Ponder has suggested that the preliminary stage of phagocytosis is that the electrical attraction causes the particle to stick to the cell. "Thereafter the early stage of ingestion takes place followed by more and more complete ingestion until an equilibrium is reached." Since electrical forces are included in surface tension forces, this distinction seems unwarranted. The whole process of sticking and ingestion is part of a single process, provided it be admitted that phagocytosis is an approach to an equilibrium. This is of course not necessarily true in all cases.

\section{THE SIZE OF THE PARTICLE AS A TEST OF THE THEORY}

According to the theory outlined, the angle of contact between a cell and a solid particle should be independent of the size of the particle. If a small particle is ingested by a certain cell, a large one will be also. If the particle is very large, ingestion is of course impossible and one speaks instead of a "spreading" of the cell on the particle. Tait ${ }^{\mathrm{I}}$ has drawn attention to numerous instances in which it is found that particles are ingested only by those cells which spread out on glass. This may be regarded as a confirmation of the theory, for in both cases the contact angle would be small between the cell and the solid.

Changes in the physico-chemical environment of the cells affect both their phagocytosis and their spreading. If such effects are caused merely by surface tension changes, then a given change in the environment should affect both spreading and phagocytosis in the same direction, provided the nature of the solid is the same in both cases. I have attempted to test the theory from this point of view but have found mostly a lack of parallelism. ${ }^{2}$

Thus it has been found that while leukocytes ingest quartz particles more readily. than carbon particles in acid solutions, they do not stick to flat surfaces of glass better than to carbon surfaces in similar solutions. Likewise, the addition of serum to a suspension of cells in 0.9 per cent $\mathrm{NaCl}$ increases their ability to phagocytize quartz but decreases their stickiness to glass surfaces. Again the stickiness of leukocytes to a glass slide increases with the alkalinity while the phagocytosis of quartz particles by the same cells increases with the acidity. Finally, the optimum temperature for phagocytosis by mammalian leukocytes is $37^{\circ} \mathrm{C}$. while the optimum for stickiness to glass is at $20^{\circ} \mathrm{C}$. (one to two hours after contact). In the latter case some reason was found for believing that the stickiness was really a maximum at $37^{\circ} \mathrm{C}$. but

× Tait, J.: Quart. J. Exper. Physiol., 13, I. I918-20.

${ }^{2}$ Fenn, W. O.: op. cit., 5, 143 and 169.1922. 
that the cell was so fluid at this temperature that it could pull away, leaving a thin film behind. For the purposes of this comparison, quartz and glass surfaces are considered identical, which may not be strictly true. Some of these discrepancies between observation and the surface tension theory may also be explained by the fact that the changes of shape demanded of the cell for phagocytosis and spreading are very different. Moreover, in the methods which have been used for measuring stickiness, consisting in inverting the surface or in exposing it to a constant flow of water, the force acting to disengage the particle from the surface is greater (at least different) from the forces tending to disengage a particle about to be ingested.

I have attempted to determine experimentally whether the rate of phagocytosis was dependent in any way upon the size of the particles. Only particles up to 5 micra in diameter were used, and no differences could be detected except such as were due to the greater availability of the larger particles (greater chance of collision). Certain experiments did lead to the belief that very small particles (I micron in diameter) were not readily ingested, but the difficulty of counting these very small particles makes this result seem now rather doubtful. In this respect, therefore, the theory is in agreement with such inadequate experiments as have been performed. Particle size would, of course, become important if the capacity of the cells became a limiting factor.

\section{STICKINESS OF CELLS}

Whether or not the theory outlined above is adequate to cover the subject of phagocytosis, it would seem to be adequate to provide a scientific definition of the word "stickiness" which is often confused with viscidity. For a particle to stick to a cell the contact angle must be less than $\mathrm{r} 80^{\circ}$. Water makes such an angle $\left(0^{\circ}\right)$ with glass, and would be difficult to remove from glass if it did not tear away, leaving a thin film behind. Most substances commonly called "sticky," such as glue, owe this property to the fact that they contain water which forms a small angle of contact with solid substances. To take advantage of this property of water, something, such as gelatin, must be added to it to increase its consistency so that it will not tear away. Similar changes in water can be produced by freezing, in which case the ice adheres firmly. The viscidity of glue is independent of the solid phase with which it is in contact, but the stickiness of water depends upon interfacial surface tensions and is, therefore, dependent upon the nature of the solid phase. In defining a cell as "sticky," therefore, it is proper to ask, "Sticky to what?"

Some persons persist in attributing the behavior of cells in solution to their "stickiness" because this seems "simpler" than to attribute it to surface tension. The supposed difficulties of surface tension cannot, however, be avoided by this convenient subterfuge, for whether the force which causes the cells to adhere be described as "adhesion," "cohesion," "electrical," "chemical," or simply as "stickiness," the fact that they do adhere is nevertheless accurately described thermodynamically by the further fact that their separation would involve an increase in the free energy of the system, and surface tension is the intensity factor of the free surface energy. A further analysis of the actual molecular forces involved may of course be made in order to determine the mechanism whereby the surface tension is affected.

Phagocytosis has frequently been considered as taking place in two stages (Kite 
and Wherry, ${ }^{\mathrm{I}}$ Ledingham, ${ }^{2}$ Sawtchenko, ${ }^{3}$ Barikine, ${ }^{4}$ Kanais): (I) a stage in which the particle sticks to the surface, and (2) a stage of ingestion. If phagocytosis were not a process involving a decrease of free energy but rather a process analogous to ingestion by the relatively highly developed organism, the ameba, which probably involves definite energy expenditure (as does any feeding reaction in the higher organisms), then it might be admissible to distinguish an initial stage of adherence between cell and particle. There seems to be no theoretical advantage in making this separation, however, and there is a definite theoretical advantage in considering them two parts of the same process. Stickiness is certainly a matter of surface tension, an approach to an equilibrium; it cannot, for example, demand any extra oxygen consumption on the part of the cell. Phagocytosis in some cases at least is also such a process. Hence they are best regarded as two phases of the same thing. De Haan ${ }^{6}$ has expressed a similar view, believing that phagocytosis is a matter of equilibrium only, because it can take place quite independent of ameboid movement, as he has shown.

\section{CHANCES OF COLLISION AS A FACTOR IN PHAGOCYTOSIS}

Measured differences in rates of phagocytosis may often be attributed to differences in the mechanism of phagocytosis under two given conditions when in reality they are due to differences in the availability of the particles or bacteria to be ingested. The availability of particles in suspension may be measured by their chances of collision with the leukocytes. It has been shown ${ }^{7}$ that this depends upon two factors: (I) the relative speed of settling of the particles, $V_{p}$, with reference to that of the leukocytes, $\mathrm{I}_{c}$ (this shows, for example, the number of particles per minute which a cell is able to overtake, or vice versa, as it settles); (2) a target factor given by the square of the sum of the diameters of the particle, $D_{p}$, and the leukocyte, $D_{c}$. This factor is proportional to the circular area concentric with the particle, which the center of the leukocyte would have to hit in order that the edge of the leukocyte should not miss the edge of the particle, when one overtakes the other in settling through the solution. The chance of collision, $R$, is therefore

$$
\left(V_{p}-V_{c}\right)\left(D_{p}+D_{c}\right)^{2}=R \text {. }
$$

It has been possible to obtain a rough experimental verification of this formula by measuring the relative speeds of ingestion of different sizes of quartz and carbon particles, the measured rates being what was to be expected from their calculated chances of collision with the leukocytes. Further verification of the formula was obtained by rotation of the tubes containing the particles and cells at different speeds without stirring. It was found that the phagocytosis was very low at high speeds because neither particles nor cells had time enough in one revolution of the tube to

\footnotetext{
${ }^{1}$ Kite, G. L., and Wherry, W. B.: J. Infect. Dis., 16, ro9. 1915.

${ }^{2}$ Ledingham, J. C. G.: loc. cit.

${ }^{3}$ Sawtchenko, I. G.: Arch. des sc. biol., 15, I45. 1910; 16, 16г. I911; 17, I28. I912-r3.

${ }_{4}$ Barikine, IV.: Ztschr. f. Immunitätsforsch. 4 . exper. Thcrap., 8, 72. 1910-II.

5 Kanai, T.: Pflüger's Arch.f. d. ges. Physiol., 198, 40г. 1923.

${ }^{6}$ de Haan, G.: loc. cil.

i Fenn, W. O.: op. cit., 3, 439. $192 \mathrm{r}$.
} 
settle down far enough to come into contact with each other. As the tubes are rotated both particles and cells describe circles in the fluid, the circumferences of which are proportional to their relative speeds of settling, and return to their original positions at the end of each revolution. If both cells and particles settled with exactly the same velocity, they could not readily come into contact and there would be very little phagocytosis.

If the number of cells remains constant during an experiment, as well as their capacity for bacteria or particles, then the ingestion of the particles will follow an exponential law. This law will not be strictly followed, however, if the cells become clumped or if the particles are of unequal sizes or if they are aggregated into unequal clumps. In such cases the faster-moving particles will have a better chance of overtaking a cell and will be ingested first, thus changing the chances of collision and hence the rate of the reaction for the suspension as a whole. Some problems of this type have been considered by McKendrick ${ }^{\mathrm{I}}$ from a mathematical point of view. ${ }^{2}$

${ }^{x}$ McKendrick, A. G.: J. Physiol., 60, Proc. p. xxvii. I925; Proc. London Math. Soc. (2d ser.), p. 40I. I914; Science Progr., 8, 497. 1913-I4.

${ }^{2}$ For further discussions of related subjects, cf. in this volume chap. lviii, by Dr. Northrop, and chap. xlii, by Dr. Falk. 


\title{
CHAPTER LXVI
}

\section{PHAGOCYTES AND PHAGOCYTOSIS IN IMMUNITY}

\author{
W. B. WHERRY \\ University of Cincinnati
}

The story of the ardent contest between the champions of the humoral theory of immunity and those who maintained that certain body cells played an equally important rôle has been told by Metchnikoff. ${ }^{\text {. }}$

Briefly, there was established the fact that when foreign bodies of a varied nature, including parasitic micro-organisms, gain entrance to the tissues of the metazoa they are invested by certain cells derived from the fixed tissues or from the circulating blood. In the case of invasion by parasites, when ingestion (phagocytosis) was followed by digestion the host recovered, otherwise it succumbed. Metchnikoff demonstrated that the digestive process within the phagocytes was quite analogous to that which goes on within many protozoa. He distinguished between motile phagocytes, derived from the blood, and fixed phagocytes - certain connective tissue cells, endothelial cells, splenic pulp cells, and phagocytic cells found in the lymph nodes and in the neuroglia. The polymorphonuclear cells of the blood he termed "microphages" and their proteolytic ferment "microcytase." All the other phagocytes were classed as "macrophages" and their digestive ferment "macrocytase."

He clearly recognized the fact that when a host was invaded by certain parasites and responded by exhibiting an "acute inflammation" the microphages were most often concerned in the defense, and that the macrophages only appeared later to clear up the débris and so aid in the process of repair. Where, however, the reaction was of such a nature as to produce "chronic inflammation," the macrophages played a dominant rôle.

Many investigators questioned the ability of the phagocytes to kill the parasites. Some maintained that the parasites were killed first by normal or acquired bactericidal substances present in blood serum and then ingested and removed as so much foreign débris. Metchnikoff was forced to admit the influence of some factors present in blood serum or plasma and termed the substances "stimulins" because he believed they stimulated the leuckocytes directly.

The more important steps in the growth of our knowledge may be summarized as follows: Denys and Le $\mathrm{Clef}^{2}$ noted that the recovery of immunized rabbits from streptococcus infection was apparently due to phagocytes and believed that ingestion was made possible by the antitoxic action of immune substances. Mennes ${ }^{3}$ and many others noted that phagocytosis only occurred to a considerable degree in the presence of immune serum.

I Metchnikoff, Elie: L'Immunité dans les maladies infectieuses. Paris, I901. English trans. Cambridge University Press, 1907.

${ }^{2}$ Denys, J., and Le Clef, J.: Cellule (Lierre and Louvain), I I, I75. I895-96.

${ }^{3}$ Mennes, Fr.: Zischr.f. Hyg. u. Infektionskrankh., 25, 413. 1897. 
A crucial experimental test was devised by Wright and Douglas ${ }^{\mathrm{T}}$ who saw the necessity of testing the phagocytic power of leukocytes outside the animal body and in the absence of body fluids. Employing a modification of the method of measuring the phagocytic power of the blood devised by Leishman ${ }^{2}$ they determined that washed leukocytes could not take up bacteria, or did so very poorly, in the absence of something which was present in normal serum. This substance was thermolabile $\left(55^{\circ} \mathrm{C}\right.$. $)$, was destroyed by Daboia venom and by prolonged exposure to light. This aid to the phagocytes they designated an "opsonic" effect (opsono, "I cater for"). The elements in the blood fluids which they supposed produce this effect they called "opsonins." Experiments in which bacteria and leukocytes were treated separately with serum and then washed and brought together again showed that the opsonic effect was exerted upon the bacteria and not upon the leukocytes. Bulloch and Atkin found that opsonin could combine with bacteria at $37^{\circ} \mathrm{C}$. or $0^{\circ} \mathrm{C}$., and that when such union had taken place even prolonged heating at $60^{\circ} \mathrm{C}$. did not alter the effect. These observations were confirmed by many of Wright's pupils and associates, and in this country especially by Hektoen and his pupils. ${ }^{3}$

Neufeld and Rimpau, ${ }^{4}$ working with sera derived from animals immunized with streptococci and pneumococci and conducting their experiments in vitro, concluded that the heated sera $\left(62^{\circ}-63^{\circ} \mathrm{C}\right.$. for forty-five minutes) contained something which altered the bacteria so as to make them susceptible to phagocytosis. These substances they called "bacteriotropins."

The question as to the identity of the opsonic substances present in normal serum (normal opsonins) and those produced by immunization (bacteriotropins, immune opsonins) has apparently been settled in the affirmative. Normal serum contains small amounts of immune opsonins for various bacteria but this is present in such minute amounts that it requires the aid of complement. When normal serum is heated to $55^{\circ} \mathrm{C}$. the complement is destroyed and the serum appears inactive. But, as shown by Cowie and Chapin, ${ }^{5}$ such heated normal serum may be reactivated by an amount of fresh normal serum which by itself has very little opsonic effect.

While heated immune serum has an opsonic effect, its action is greatly enhanced by the presence of a little normal serum (Dean, ${ }^{6}$ Hektoen $\left.^{7}\right)$. Wright and Reid ${ }^{8}$ had already shown that the apparent relative resistance of immune opsonin to heat or to light was abolished if one diluted it. Therefore the full opsonic action of normal or immune sera is dependent upon the co-operation of two bodies, complement and opsonin.

It seems probable-from the absorption experiments of Bulloch and Western?

${ }^{1}$ Wright, A. E., and Douglas, S. R.: Proc. Roy. Soc., 72, 357. 1903; 73, I28. I904.

${ }^{2}$ Leishman, IV. B.: Brit. M.J., I, 73. 1902.

${ }^{3}$ Hektoen, L., and others: J.A.M.A., 46, 1407. 1906; ibid., 57, I579. I9II.

${ }_{4}$ Neufeld, F., and Rimpau, W.: Deutsche med. Wchnschr., 30, I458. I904.

${ }^{5}$ Cowie, M., and Chapin, W. S.: J. Med. Research, 17, 57, 95, 213. I907.

${ }^{6}$ Dean, G.: Proc. Roy. Soc., 76, 506. 1905.

7 Hektoen, L.: J. Infect. Dis., 6, 67. 1909.

${ }^{8}$ Wright, A. E., and Reid, S. T.: Proc. Roy. Soc., 77, 2 I1. $1905^{-6 .}$

9 Bulloch, W., and Western, G. T.: ibid., p. 53. 
with staphylococci and tubercle bacilli; of Rosenow ${ }^{1}$ with pneumococci, streptococci, tubercle bacilli, and staphylococci; and of Hektoen ${ }^{2}$ with hemopsonins-that normal opsonins, like the immune opsonins, are more or less specific.

The origin of normal opsonins is not known. Wright found them present in the normal blood of man for Staphylococcus pyogenes, pneumococci, Brucella melitensis, B. pestis, B. anthracis, B. coli, B.typhosus, Vibrio cholerae, B. tuberculosis, but absent for $B$. diphtheriae and $B$. xerosis. Tunnicliff ${ }^{3}$ found that the anti-infectious power of the blood of infants, so far as staphylococci, streptococci, and pneumococci were concerned, was at a low level at birth, fell even lower during the first few months of life, and rose to the adult level at about the third year. She also determined that exudate leukocytes are more active phagocytically than leukocytes from the blood of the same animals; as are also those in the blood of cases of acute infection, e.g., pneumonia, scarlet fever.

Attention was called by Wright and Reid ${ }^{4}$ to the fact that in artificial experiments some leukocytes engage in phagocytosis in the absence of opsonin. They called this "spontaneous phagocytosis." They found that the amount of this spontaneous phagocytosis could be increased by diminishing the amount of sodium chloride in the serum or suppressed by increasing the amount.

This points to the necessity of considering the physical consistency of the leukocytes themselves as a factor in phagocytosis. Tunnicliff showed that leukocytes from inflammatory exudates showed greater phagocytic power than those from the normal circulating blood, and Kite and Wherry ${ }^{5}$ found individual differences in their glass adhesiveness. It is probable that leukocytes are more active when they are hydrated.

When bacteria fail to absorb opsonin they show great resistance to phagocytosis as shown by Rosenow, ${ }^{6}$ who was able to extract from virulent pneumococci with physiological salt solution a substance he called "virulin" which inhibited the action of pneumococcus opsonin.

\section{THE PHAGOCYTES AND THEIR ORIGIN}

The white cells of the blood may vary considerably in number even in apparently healthy individuals. The normal average lies between 7,300 and 7,500 cells per cubic millimeter. After the introduction of the technique of cell-staining and differentiation and following the work of Ehrlich came the recognition of six types of cells: the neutrophilic, eosinophilic, and basophilic leukocytes; the small lymphocytes with which were included certain intermediate forms; large lymphocytes or mononuclear forms; and the transitional cells.

There are five types of granulations, only three of which occur in the human

${ }^{1}$ Rosenow, E. C.: J. Infect. Dis., 4, 285. 1907.

${ }^{2}$ Hektoen, L.: ibid., 5, 249. 1908.

${ }_{3}^{3}$ Tunnicliff, R.: ibid., 7, 698. г910; Tr. Chicago Path. Soc., 8, 208. I9 1; J. Infect. Dis., 8, 302. I9II.

4 Wright, $\Lambda$. E., and Reid, S. T.: loc. cit.

${ }^{5}$ Kite, G. L., and Wherry, W. B.: J. Infect. Dis., 16, I09. I9I5.

${ }^{6}$ Rosenow, E. C.: ibid., 4, 285.1907. 
blood: (I) the oxyphilic, eosinophile granule, which is large, refractive, uniform in size, round as in human blood, or in long rods as in birds; (2) the amphophilic granule which takes either a basophilic or acidophilic dye, and is found only in young cells; (3) the metachromatic basophilic granule of the mast cell; (4) the truly basophilic granule which is not found in human blood; (5) the fine neutrophilic granulation of human blood.

Studies on the blood since the time of Ehrlich ${ }^{\mathrm{I}}$ have led to a classification of the white cells into three groups: first, the monocytes, with two different types, large mononuclear forms and the so-called "transitional" cell of Ehrlich; second, the leukocytes with their three different cells (neutrophile, eosinophile, and mast cells); and third, the lymphocytes. It is believed that there is no transition between the cells of these three main groups.

There are three strains of cells of the connective tissues which contribute to the blood cells. All of these strains give more cells to the connective tissues than to the blood.

I. The angioblasts, giving the endothelium, the erythrocytes, and the monocytes.-The monocyte stem is largely extravascular, in small part intravascular, giving the histiocytes which are the monocytes of the blood and the clasmatocytes of the connective tissues. They have developed along the line of the phagocytic power of their parentcell, endothelium, and are especially differentiated to take up and store particulate matter. It has, I think, become clear that certain endothelial cells, like the Kupffer cell of the liver which is a specialized endothelial cell within the capillaries of the liver anchored out into the blood stream by guy ropes of cytoplasm, and the endothelial cells of the veins of the splenic pulp as well as the endothelium of the splenic sinuses have the maximum power of phagocytosis and that they clear the blood stream of foreign particles within a very few minutes (Sabin). ${ }^{\mathrm{I}}$

In the circulating blood two types of monocytes are present- the large mononuclear cell of Ehrlich, which has a large oval nucleus and basophilic cytoplasm, constitutes about 0.2 per cent of the white cells; the other constitutes about 6 per cent of the cells and has a nucleus which varies from indented to horseshoe or S-shape, with a basophilic cytoplasm containing azurophile granules. These are the macrophages of Metchnikoff. These are the histiocytes or monocytes of the blood, the endothelial leukocytes of Mallory. According to Evans, McJunkin, and others, endothelial cells under proper stimulation will leave the vessel walls and enter into the circulation. Sabin, in her studies on the growing blood vessels in the yolk sac of the chick, noted a multiplication of the endothelial cells which wander both into the lumen of the vessel (monocytes) and into the tissues (clasmatocytes).

2. The second strain, the granulocyte.-From these descend the neutrophile leukocyte, the eosinophile leukocyte, and the mast cell.

a) The neutrophile leukocyte.-In the higher animals these are formed only in the marrow. They originate from a myelocyte which has a round vesicular nucleus and neutrophile granules. These myelocytes are grouped in extravascular centers in which development occurs from the center so that the mature cells lie at the periphery; these

'Complete critical reviews by Sabin, F. R.: Physiol. Rev?, 2, 38. I922; Bunting, C. H.: ibid., p. 505; and Foot, N. C.: Anat. Rec., 30, 15. 1925. 
make their way through some unknown chemotactic influence in to the blood stream. The mode by which their numbers is regulated is unknown. The nucleus usually shows three lobes, but apparently, as the result of aging, these increase to four, five, or six. According to Arneth, these changes can be taken as an index of marrow activity. There is much cytoplasm filled with fine neutrophile granules. These cells are actively motile, highly chemotactic, and play the most important part in the phagocytosis of many bacterial parasites. When foreign proteins are injected into the blood stream, there is first a leukopenia, followed by a leukocytosis in which these cells predominate.

b) The eosinophile leukocyte.-The parents of this cell are myelocytes. The large round granules have an affinity for acid dyes. Ordinarily they are present in the circulation as $\mathrm{I}_{-2}$ per cent of the whites but may reach a concentration of 50 per cent or more when the host is infected with animal parasites (e.g., Filaria). They emigrate from the vessels and collect about the site of attachment of worms in great numbers. They are also called forth in response to local or general anaphylactic shock-occur in all "sensitivity" conditions, e.g., asthma, sensitization sinusitis, mucous colitis, and in various inflammations of the skin, pemphigus, urticaria, dermatitis herpetiformis, etc. The nature of the substances which call them forth is unknown, but they are probably certain products of autolysis derived from tissues or from parasites. These cells are concerned but little in the phagocytosis of bacteria under natural conditions but may be made to take them up in in vitro experiments (Weinberg and Séguin).

c) The mast cell.-The mast cells are the least understood. They are granulocytes which develop late and remain extravascular, since the mast cells of the blood have been shown by Weidenreich to be degenerating cells and not true mast cells. Mast cells occur in the bone marrow, along blood vessels, and between muscle fibers. Their function is totally unknown.

3. The third strain, the lymphocyte. - This is the last to develop, but its embryology is uncertain. Originating by mitosis in the lymphoblasts of the germinal centers of the lymph glands, it is widely distributed. It occurs in lymph glands, hemal glands, and spleen, i.e., in a reticulum near lymphatic or vascular sinuses by means of which it enters the blood stream. It also occurs in small follicles near lymphatic capillaries and ducts or even in small clumps without such relation, very widely distributed throughout the organs and in bone marrow as well. Thus it may occur wherever there is reticulum. There are smaller and larger lymphocytes. These have the same type of nucleus. In the larger one the nucleus is surrounded by more cytoplasm of less basophilic character and usually contains some coarse granules. More lymphocytes enter the circulation in twenty-four hours than are present at any one time, and in investigating their fate Bunting and Huston found that they made their way on to the mucous membranes, in particular the intestinal mucosa. Why they do this is not known. They are said to be devoid of autolytic and peptic ferments; are extremely sensitive to $\mathrm{X}$-rays and radium; and apparently have some specific relationship to immunity against tumors as shown by the work of Murphy and his co-workers. ${ }^{2}$

× Weinberg, M., and Séguin, P.: Compt. rend. Soc. de biol., 75, r70. 1913.

${ }^{2}$ Murphy, J. B., et al.: J. Exper. Med., I91 2-21. 


\section{INTRACELLULAR DIGESTION BY PHAGOCYTES}

Tschernoruzki $i^{x}$ determined the presence of the following ferments in the leukocytes of the dog-protease, amylase, dextrinase, catalase, nuclease, peroxydase-but the absence of lipase and oxydase.

The proteolytic enzyme of the polymorphonuclear leukocyte has been named leukoprotease by Opie. ${ }^{2}$ It actively digests denatured blood serum in a neutral or alkaline reaction ( 0.2 per cent sodium carbonate) but is inactive in the presence of acid ( 0.2 per cent acetic acid.) In vitro it acts best at $50^{\circ}-55^{\circ} \mathrm{C}$. It may be preserved in Io per cent formalin and become active again on the removal of the formalin (Jochmann and Ziegler). It is found in those organs which are rich in leukocytes (bone marrow). Most investigators find that its power to digest protein to the peptone and amino acid stage is less than that of trypsin. Its action is only slightly impaired at a temperature of $65^{\circ} \mathrm{C}$.

Opie found that normal blood contains an anti-enzyme for leukoprotease. Its action is not specific; the serum from one animal inhibits the leukoprotease of another. The anti-enzyme is destroyed at $75^{\circ} \mathrm{C}$. in thirty minutes. Its action is favored or retarded by the same amount of alkali or acid that affects leukoprotease. The digestive action of $20 \mathrm{mg}$. of dried leukocytes is arrested by $2.5 \mathrm{cc}$. of serum, but in the presence of more enzyme proteolysis takes place. The anti-enzyme does not come down with the globulins or euglobulins but is precipitated by saturated magnesium sulphate.

There is no evidence that the anti-enzyme is a true antibody. It is antagonistic to trypsin. An increase in the anti-enzyme content of the blood may be brought about by injecting animals with a variety of substances, e.g., extract of dried leukocytes from pus (Jochmann and Kantorowicz), trypsin (Achalme), small doses of chloroform or phosphorus (Opie, Barker, and Dochez), and tissue cells (Braunstein and Kepnow).

The anti-enzyme activity of the blood can be removed by extraction with fat solvents, and experiments seem to show that the enzyme-inhibiting action is due to the presence of compounds of unsaturated, fatty acids (Schwartz, Jobling, and Peterson, etc.).

Metchnikoff believed that the complement concerned in bacteriolysis was derived from the polymorphonuclear leukocytes and named it "microcytase"; and that the complement which combined with antibody to produce hemolysis was derived from the macrophages (macrocytase). The difference in resistance to heat would seem to separate leukoprotease from these substances. It is held by some that the leukoproteases play no direct rôle in the destruction of micro-organisms. Jochmann, Kantorowicz, and others found the extracted ferments without bactericidal action in vitro. Nevertheless, many workers have seen the bacteria taken up by leukocytes in test-tube experiments, dissolve and disappear.

THE PROTEOLYTIC ENZYMES OF THE MONOCYTES

As shown by Opie ${ }^{3}$ in the earlier stages of an inflammation the exudate, composed chiefly of polymorphonuclear leukocytes, is alkaline, a reaction favorable for the ac-

I Tschernoruzki, M.: Ztschr.f. phys. Chemie, 75, 216. I9I .

${ }^{2}$ Opie, E. L.: Physiol. Rev., 2, 552. I922.

3 Opie, E. L.: J. Exper. Med., 8, 410. I906. 
tion of leukoprotease. In the later stages the exudate is composed chiefly of monocytes and is acid in reaction. Experiments show that the enzyme of these cells works best in an acid reaction ( 0.2 per cent acetic acid) but is inactive in a neutral or alkaline reaction. This enzyme is more thermolabile than leukoprotease, being injured at $60^{\circ}-$ $70^{\circ} \mathrm{C}$. It closely resembles the autolytic enzymes of parenchymatous tissues.

Ferments resembling these two have been described by Lord, ${ }^{\mathrm{I}}$ Lord and Nye, ${ }^{2}$ and $\mathrm{Nye}^{3}$ from the pneumonic lung. One digests coagulated blood serum at $\mathrm{pH} .7 \cdot 3^{-}$ 6.7 ; and the other splits peptone to the amino acid stage at $\mathrm{pH} 8.0-4.8$ (most active at $\mathrm{pH} 6.3-5.2)$.

Jobling and Strause ${ }^{4}$ found in fresh pus cells an erepsin-like ferment capable of splitting peptone.

Thus to summarize and give a brief picture of the present conception of phagocytosis: During the acute stage of inflammation the polymorphonuclear cells invest, take up, and attempt to digest the foreign body. In many instances, opsonin-when present in sufficient concentration - greatly facilitates ingestion. If the foreign body be of the nature of a living micro-organism it is presumably first opsonized and then taken up, and digested or not as the case may be. The action of the leukoprotease of the polymorphonuclear cells is usually limited to substances within the cell, for any ferment which is secreted or shed as the result of injury is promptly neutralized by the non-specific anti-ferments of the blood. However, the destruction of the leukocytes often becomes excessive or the local edema dilutes the anti-ferments and more leukoprotease being freed than can be neutralized there is solution of the fixed-tissue cells. In those infections in which the polymorphonuclear cells play a dominant rôle, if the elimination of the infecting agent has been successful, the process becomes chronic and is followed by repair. During these later stages the monocytes play a dominant rôle. It is probable that these cells are mainly derived from pre-existing free phagocytes. That they can multiply has been proved by their cultivation in vitro by Carrell and Ebeling. ${ }^{5}$ Or, also, they may be derived by the multiplication of the endothelial cells of pre-existing capillaries or from newly formed capillary sprouts. ${ }^{6}$ Lewis and Lewis ${ }^{7}$ have seen leukocytes in cultures become transformed into cells identical with clasmatocytes, epithelioid cells, and multinuclear giant cells. They suggest that such transformation may occur in the body wherever the circulation is sluggish, e.g., sinuses of spleen and tissue spaces.

These monocytes are engaged chiefly in the removal of foreign material which is absorbed with difficulty, such as nuclear material, blood corpuscles, and the blood pigments hemosiderin and hemofuscin, malarial pigment, etc. In some parasitic diseases the endothelial cells are stimulated to overgrowth as in tuberculosis, leprosy, Rocky Mountain spotted fever, typhus, typhoid, measles, Leishmaniasis, amebiasis, malaria, etc., and the defense of the hosts seems to be largely dependent on the phagocytic

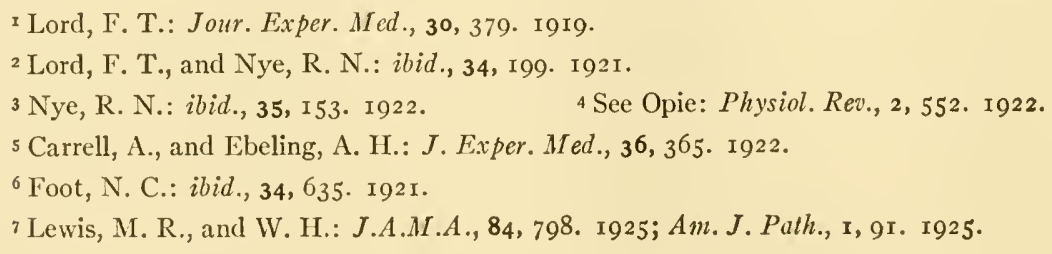


activity of these cells. The disappearance of fibrin from the inflammatory exudate is due in part to leukoprotease and in part to a proteolytic enzyme which acts best in an acid or neutral medium.

\section{PROPHYLACTIC AND THERAPEUTIC IMMUNIZATION}

Prophylactic immunization we owe to Jenner and especially to Pasteur who applied Jenner's idea to the prevention of many diseases of man and animals. It was believed for many years that a living, attenuated virus was necessary in order to produce immunity. The original plan of using an attenuated live virus for immunization (chicken cholera, anthrax, etc. [Pasteur]) has been again revived by Calmette in immunization against tuberculosis. ${ }^{\mathrm{I}}$

The work of Ferran on cholera, of Haffkine on cholera and plague, and of Wright on typhoid fever showed that immunity could be brought about by the injection of a dead virus or antigen; by such means successful immunization has been practiced against typhoid and paratyphoid fever, cholera, plague, Malta fever, bacterial dysentery, pneumonia, etc. By the use of toxins of bacteria, immunization has been accomplished against tetanus, diphtheria, gas gangrene, scarlet fever, eyrsipelas, etc.

To Wright, ${ }^{2}$ however, we owe the conception that immunization may produce therapeutic results even if undertaken after a pathogenic parasite has implanted itself in the tissues of a host. The observations that suitably administered typhoid antigen would lead to a thousand-fold increase in the bactericidal action of the blood, and an increase in its opsonic power, and that this "epiphylactic" response could be measured within twenty-four hours after injection of an antigen, together with reflection on the fact that injection of plague antigen during the incubation period of the disease often markedly benefited the patient, led to that idea-- "The idea that the uninfected and still inactive regions of the body can by applying the stimulus of a vaccine be made to bring succour to the infected regions was, as you know, the mother-idea of vaccine therapy."'3

This direct and logical outgrowth of Wright's studies should have led to the fulfilment of his prophecy- "the physician of the future will be an immunisator"-but baffled by the difficulties and uncertainties of the opsonic technique and perhaps justly fearing the dreaded "negative phase," the physician still fixes his eye on the chemical and physical manifestations of disease and largely ignores the parasites whose destruction is the sine qua non to recovery.

Briefly Wright's techniquet in performing the phagocytosis experiment consisted in mixing in a capillary glass pipette equal volumes of a patient's serum, washed leukocytes, and a homogeneous suspension of bacteria in physiological sodium chloride solution. The mixture was then kept at body temperature for fifteen to thirty minutes, again mixed in order to obtain a uniform sample, and thin smears stained and

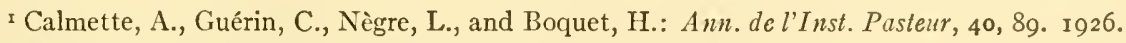

2 Wright, A. E.: Brit. M. J., I, I069. I903.

3 Wright, A. E., Colebrook, L., and Storer, E. J.: Lancet, r, 365. 1923.

${ }^{4}$ Wright, A. E., and Colebrook, L.: Technique of the Teat and Capillary Tube. London: Constable, I921. Jones, Carmalt: Introduction to Therapeutic Inoculation. New York: Macmillan \& Co., I9II. 
examined. The average number of bacteria taken up by some arbitrary number of cells was determined and compared with a control. In the control test he used physiological salt solution in place of serum, or better, the pooled sera of a number of presumably healthy individuals.

Wright determined the "opsonic index" of patients by dividing the average number of bacteria taken up when opsonized by the patient's serum by the average number phagocytized under the influence of normal serum. In a large variety of infections he determined that the index was always below normal. In attempting to raise this index in patients by therapeutic inoculation he found that immediately following the injection of the antigen there was a preliminary drop in the patient's index (negative phase) but that as antibodies were produced the index rose to its former level or even higher. During the negative phase the disease condition became aggravated, but improvement followed upon the rise in the index. As Wright pointed out, this fall and rise in antibody production had already been studied by Brieger and Ehrlich. ${ }^{\mathrm{x}}$

We have had to change our conception of what goes on during these in vitro experiments on phagocytosis, and of their accuracy unless very definite precautions are taken. Kite and Wherry ${ }^{2}$ showed that the phagocytosis is brought about by the mechanical agitation of the mixture, adhesion between the bacteria and leukocytes taking place when they meet in collision. It follows naturally that in order to insure comparable results one must take precautions to use the same number of bacteria and kind of leukocytes and subject then to the same amount of agitation. When such precautions are taken, the final average number of bacteria found within leukocytes in successive experiments made on the same mixture agree very closely. However, the difficulty encountered in eliminating such sources of error makes impracticable the control of therapy by the estimation of the opsonic activity of sera collected at varying intervals. A quantitative estimation of the opsonin content of a serum is best attained by using dilutions up to the point where there is no longer opsonic action. This method is particularly useful when the serum to be tested has a lytic action. ${ }^{3}$

Another group of workers has sought a way out of the difficulty by advocating non-specific protein therapy. ${ }^{4}$ The rapid improvement which sometimes followed upon even a single injection of a foreign protein, especially when this produced fever or shock, was noted as long ago as I 893 when Fränkel treated cases of typhoid fever with typhoid vaccine and Rumpf with $B$. pyocyaneus vaccine. The fever, the initial leukopenia followed by leukocytosis, and the quickened circulation of whatever antibodies the patient possesses through the blood and lymph are the chief physiological factors which aid in combating infection. ${ }^{5}$

It is certain that, in view of the specificity of antibodies, therapeutic immunization must develop along specific lines. We have come to recognize that we must do more than merely inject an antigen. It must be the right antigen, administered in the proper dose at sufficiently frequent intervals; and even if suitable antibodies are formed, we must resort to measures which will overcome the obstacles in the way of

\footnotetext{
${ }^{1}$ Brieger, L., and Ehrlich, P.: Zischr. f. Hyg. u. Infektionskrankh., 13, 336. I893.

${ }^{2}$ Kite, G. L., and Wherry, W. B.: loc. cit.

3 Klien, H.: Bull. Johns Hopkins Hosp., 18, 245. 1907.

${ }_{4}^{4}$ See chapter lxxxii of this volume.

${ }^{5}$ MicIntosh, J.: Lancel, 2, 889. 1926.
} 
these antibodies - obstacles which prevent their coming in contact with the parasites. Here, again, Wright ${ }^{\mathrm{I}}$ has performed pioneer work in analyzing the situation.

Recently Wherry ${ }^{2}$ has suggested that even if a parasite is otherwise equipped to thrive in the body of a host it will not do so and produce disease unless there is some chemical interaction between the parasite and the host which brings about a separation of water from its combination with the tissue colloids, thus putting into solution food for the bacteria. This freeing of water in the tissues (edema) may result from the production of amines. Infections which spread rapidly are accompanied by marked edema. When a series of heat-killed bacterial antigens are injected into man, some give rise to local urticaria, others do not. The production of urticaria is taken to indicate susceptibility.

I may summarize briefly the effect of the local-tissue edema on the further growth of the parasites and upon the defensive mechanism as follows: (a) It provides dissolved food for the bacteria. (b) It dilutes the antibodies. (c) It immobilizes the phagocytes, for leukocytes are dependent on contact for motility. (d) It prevents or delays the absorption of antigenic substances that might lead to immunity. (e) It dilutes anti-leukoprotease, and so permits the freed leukoprotease to digest the fixed tissue, and hastens abscess formation.

Wright ${ }^{3}$ found that there was a loss of anti-tryptic power and a diminution in the alkalinity in the edematous fluid at the site of infection with $\mathrm{Cl}$. welchii, both in man and in animals. In the blood he found a diminution of alkalinity and an increased amount of anti-trypsins. In other words, the lack of the anti-ferment and the reaction at the site of infection were unfavorable to phagocytosis.

Every effort, therefore, should be directed toward reducing the edema by the use of hypertonic salines, glucose, incision, etc. And in the case of open wounds antibacterial substances should be drawn into the focus by establishing an external flow of serum by Wright's method. Wherever possible as many of the parasites as can be reached should be killed by the use of dyes, etc. Often the successful reduction of the edema at the beginning of an acute inflammation may by itself so favor the normal defense mechanism that healing follows promptly.

In attempting immunization against any infection it is most important that the right antigen be selected. This is a simple matter where only one bacterium is concerned, provided that it is certain that the single species found is the only one present. In mixed infections the right antigen can be selected by preparing separate heat-killed suspensions of the species present and testing the patient by intradermal inoculation. Those antigens which give rise to urticaria or urticaria and congestion are chosen as the ones concerned in the infection. Heat-killed bacteria make good antigens, ${ }^{4}$ and subcutaneous inoculation gives rise to the production of opsonins. ${ }^{5}$

The question of dosage should not be based on anyone's personal experience re-

'Wright, A. E., and Colebrook, L.: loc. cit.; Wright, A. E.: Studies on Immunization. London: Constable, I909; Wound Infections. New York: William Wood \& Co., I916; "Treatment of Infected Wounds by Physiologic Methods," Brit. M.J., I, 793. i916; Proc. Roy. Soc., I 14, 576. I927.

${ }^{2}$ Wherry, W. B.: J. Infect. Dis., 41, I77. I927.

3 Wright, A. E.: Lancet, 1, 21. I9I7.

4 Taylor, F. E.: J. Hyg., I5, I63. I9I5.

s Noon, L.: ibid., 9, I81. 1909. 
garding the number of bacteria that may be injected without harm. Wright noted that the effect produced by the same dose, e.g., I, individuals varied greatly according to whether they were well, moderately severely infected, or severely infected. The general knowledge gained by experience concerning the relative toxicity of different bacterial antigens may be considered as of some importance-but it is still more important that one determine the relative sensitivity of the infected individual. This can be done by the intradermal injection of the heatkilled antigen.

The writer has found that the heat-killed saline suspension of bacteria should not contain an excessive number of bacteria; when a too dense suspension is injected intradermally into a very sensitive individual, even in as small a volume as 0.5 minim, this may be followed by local suppuration and necrosis. A rough-and-ready method is to prepare the suspension in a test tube with a diameter of $I_{5} \mathrm{~mm}$. and of such a density that the inoculating needle can be seen plainly through the suspension. One (or $1 \frac{1}{2}$ minims) of such an antigen is injected intradermally. According to the reaction obtained a dose of 0.5 minim or less is injected subcutaneously. The injections are given daily and the dose increased or not by 0.5 minim according to the local reaction produced and according to the rapidity with which the local reaction subsides. In very sensitive individuals it may be necessary to keep to a minute dose for many days; then when the preliminary sensitivity disappears the dose can be increased, and about this time the site of infection shows signs of healing.

We have probably overestimated the amount of antigen necessary to give protection and aid in overcoming infection. Cooper ${ }^{1}$ found that I minim of heat-killed type I pneumococcus suspension injected daily into the submucosa of rabbits for ten days rendered them immune for months.

Recent studies on local tissue immunity, and on the use of detoxicated antigens promise further advances for prophylactic and therapeutic immunization.

${ }^{x}$ Cooper, M. L.: J. Infect. Dis., 38, 49г. г926. 


\title{
CHAPTER LXVII
}

\section{LOCAL AND TISSUE IMMUNITY}

\author{
FREDERICK P. GAY
}

Columbia University

\section{GENERAL CONSIDERATIONS}

Anyone who has carefully followed the evolution of the science of immunology must feel certain that in many of the protective processes there is concerned some third, and as yet not clearly understood, factor in addition to the mobile polymorphonuclear phagocytes of the bloodstream and the antibodies.

Natural or "innate" immunity, which suffices to protect normal animals against slight or moderately virulent infectious agents, may in general be ascribed to the activity of the polymorphonuclear leukocytes of the circulating blood. Acquired immunity, whether expressed actively in a recovered or actively immunized animal, or passively through transfer of the serum of hyperimmunized animals, involves certain new and enhanced physiological activities. These activities are expressed in the reaction products of serum usually designated as "antibodies." 'These antagonistic substances are characterized by their ability to unite in a specific manner with the antigenic substance that has given rise to them. In the case of the antitoxins, this union is followed by a direct neutralization, although usually not by the destruction, of the corresponding toxin. The other antibodies, whether they be regarded as a unit or as multiple, unite with the corresponding antigen, characteristically a bacterium, although they do not in themselves destroy it. Such a "sensitized" antigen is however remarkably susceptible to the ferment-like action of the alexin or complement which is a normal constituent of plasma and serum. And, what perhaps is equally important, a sensitized (tropinized or opsinized) bacterium is rendered readily phagocytable by normal leukocytes, which alone may be ineffective against this particular microbe.

But all these facts, important as they are, do not suffice to explain the numerous phenomena of immunity. They are in particular inadequate in explaining some of the most pronounced instances of acquired immunity. Smallpox immunity and the immunity which follows recovery from typhoid fever, for example, are not to be accounted for either by the activity of the polymorphonuclear leukocytes or yet by the action of antibodies. In spite of the importance of antibodies as indicative of a protective reaction, a very marked immunity may exist without their presence; and their presence alone does not insure protection.

It is evident, therefore, that by a process of exclusion, we are forced to consider the more or less fixed tissues of the body to find an explanation for certain phases of immunity.

I The reader is referred to previous reviews by the author which discuss in fuller detail the historical development and earlier conceptions in this subject: (1) J. Immunol, 8, I. I923; (2) Physiol. Rev., 4, x9I-214. 1924; (3) Arch. Path. \& Lab. Med., x, 590-604. I926. 
The discovery of antibodies, more specifically of antitoxins, in the year I890, was naturally followed by an attempt to explain the mode and locus of origin of these significant reactive substances which are so characteristic of acquired immunity. The earliest observations showed that in spite of the very direct reaction between antigen and antibody, a marked disproportion between the amount of antigen employed and antibody formed is evident. ${ }^{\mathrm{I}}$ Repeated bleedings and consequent mechanical removal of a large proportion of antibody gives only a slight and temporary decrease in this substance, ${ }^{2}$ and indicates that the reservoir of the antibody lies outside the bloodstream; and again, pilocarpin, a cell stimulant, increases antibody production. ${ }^{3}$

In fact no one, from the beginning, has seriously disputed that the cells of the body form the background of immunity. In the process of phagocytosis the more mobile cells, the polymorphonuclear leukocytes, are actively engaged in body defense, although in the case of virulent infections their action to be efficient must be preluded by a sensitizing or tropinizing effect on the invading bacteria, by the action of the reaction antibodies of the body fluids. And these antibodies in turn must originate in cells. In other words, the striking and important new properties of the blood serum in conditions of immunity have to an undue extent distracted our attention from the mechanism through which these properties arise. Too little attention has been paid to cells of the body if we except the polymorphonuclear leukocytes whose function Metchnikoff ${ }^{4}$ and his pupils have so ably exploited. The antibodies of the plasma, important as they are in diagnosis and at times in serum therapy, are at best only a reflection of more fundamental processes that have gone on in the cells.

Studies have of course been undertaken from the very beginning which bear on the origin of antibodies, but it now seems evident that progress has been in no small measure inhibited by the too fully explanatory theory of Ehrlich which for many years has dominated the field. This theory, as is well known, rests on certain assumptions which are probably erroneous, or at all events unproved. The first of these assumptions is that those cells or parts of cells which are particularly fitted to unite with, or, in the case of poisonous substances, be injured by any foreign protein, are the particular "receptors" which through their overgrowth constitute the specific antibodies. We know that every attempt to prove this fundamental proposition has failed. The central nervous system of animals susceptible to tetanus toxin is not equivalent to, nor does it give rise to, tetanus antitoxin as Wassermann and Takakis thought. Metchnikoff found, indeed, that an antispermotoxin from rabbits which neutralizes the spermotoxic antiserum of guinea pigs immunized against rabbit spermatozoa is formed as readily in castrated as in normal rabbits, thus proving that the antispermotoxin is not formed in those cells on which the antigen (spermotoxin) has a specific and harmful effect. In short, Ehrlich's theory, while claiming to offer a complete

${ }^{1}$ Knorr, A.: Forlschr. d. Med., I5, 657. 1897.

${ }^{2}$ Roux, E., and Vaillard, L.: Ann. de l'Inst. Pasteur, 7, 65. I893; Salomonsen, C. J., and Madsen, T.: ibid., 8, 763. I898.

${ }^{3}$ Salomonsen, C. J., and Madsen, T.: loc. cit.

${ }_{4}^{4}$ Metchnikoff, E.: Immunity in Infective Diseases. Cambridgre University Press, 1905; Ann. de l'Inst. Pasteur, 13, 737. I899.

5 Wassermann, A., and Takaki, T.: Berl. klin. Wchnsclr., 35, 5. I898. 
explanation of antibody production, has never demonstrated that any particular organ or tissue is responsible for the formation of any given antibody.

It was quite natural that the scientific world, impressed by the brilliancy of the Ehrlich concept, should expect to find antibody formation in those cells that first fix the antigen and therefore in the leukocytes or the leukocyte-forming organs. We may well question whether phagocytosis and intracellular destruction of a microbe or foreign red blood cell represent the final fixation of the antigenic proteins of which it is composed. In other words, one should prove the persistence of an antigen or, better still, detect the precocious appearance of the corresponding antibody in a given tissue, in order to demonstrate its antibody-producing function. Experiments in this direction have been contradictory. The work of Deutsch, ${ }^{\mathrm{I}}$ Castellani, ${ }^{2}$ Weil and Braun, ${ }^{3}$ and particularly of Kraus and Schiffmann ${ }^{4}$ indicates that agglutinins appear in the serum before they are found in organ extracts. On the other hand, Cantacuzène 5 and Swerew $^{6}$ have noted a hyperleukocytosis preceding the formation of precipitins. Stenstrom ${ }^{7}$ found that more precipitins are produced on injecting the foreign protein with admixture of the leukocytes of the immunized animal. Pfeiffer and Marx, ${ }^{8}$ however, found less antibodies in leukocyte extracts than in the plasma.

Persistent antigens have been sought for by adding organ extracts to a precipitating antiserum. Curiously enough, both precipitinogen and precipitin may concur in the same immune serum. ${ }^{9}$

The hematopoietic organs have also been studied as antibody formers. Pfeiffer and Marx found that cholera lysins are present in spleen and bone marrow before they occur elsewhere. The removal of the spleen may or may not lessen antibody formation. Experiments in injuring the leukopoetic organs by benzene or X-rays ${ }^{\mathrm{Io}}$ seem to lower antibody formation in such animals, but the animals are so debilitated by this treatment that one hesitates to draw conclusions. Other authors have imputed antibody formation to the liver, particularly under stimulation of the iodine content of the thyroid gland. II Gay and Rusk ${ }^{9}$ were not able to repeat these experiments. Manwaring ${ }^{\mathrm{I} 2}$ and Blaizot have suggested more specifically liver endothelium. Kraus and Schiffman, ${ }^{13}$ Eastwood, ${ }^{14}$ and also Siegmund ${ }^{15}$ have attributed antibody formation to the endothelium in general.

${ }^{r}$ Deutsch, L.: Ann. de l'Inst. Pasteur, r3, 689. I899.

${ }^{2}$ Castellani, A.: Ztschr. f. IIyg. u. Infektionskrankh., 37, 381. I9or.

3 Weil, E., and Braun, H.: Biochem. Zischr., I7, 337. 1909.

${ }^{4}$ Kraus, R., and Schiffmann, J.: Ann. de l'Inst. Pasteur, 20, 225. 1906.

${ }_{5}^{5}$ Cantacuzène, J.: ibid., 22, 54. 1908. $\quad{ }^{6}$ Swerew: Russk. Wratsch., 9, Part I, 367. I910.

${ }_{7}$ Stenstrom, O.: Zischr. f. Immunitätsforsch. u. exper. Therap., 8, 483. I9I I.

${ }_{8}^{8}$ Pfeiffer, R., and Marx: Ztschr.f. Hyg. u. Infektionskrankh., 27, 272. 1898.

${ }_{9}$ Gay, F. P., and Rusk, G. Y.: Univ. Calif. Pub. Path., 2, 59. Ig12; ibid., p. 73. I9I 2.

Io Rusk, G. Y.: ibid., p. I39. I9I 4; Simonds, J. P., and Jones, H. M.: J. Med.Research, 33, 183. I9I 5 .

I1 Müller, L.: Centralbl. f. Bakteriol., Abt. I, Orig., 57, 577. I9II; Fassin, L.: Compt. rend. Soc. de biol., 62, 388, 467, 647. 1907 .

${ }^{12}$ Manwaring, W. H.: Ztschr. f. Immunitätsforsch. u. exper. Therap., 8, I. I9I I.

${ }^{13}$ Kraus, R., and Schiffman, J.: Ann. de l'Inst. Pasteur, 20, 225. 1906.

${ }^{14}$ Eastwood, A.: J. Iyyg., 22, 355. 1924. ${ }^{15}$ Siegmund, H.: Klin. Wchnschr., I, 2566.1922. 
This outline of the trend of investigation bearing on antibody formation, schematic as it is, will serve as a necessary preliminary to the statement that in my opinion the outstanding problems of immunity in general are: first, a further understanding of the method of antibody formation; and, second, an appreciation of the importance of fixed-tissue cells in direct defense against micro-organisms through phagocytosis. I believe further that both these problems are being attacked in a new and more effective manner through the recent studies in so-called "local" immunity.

Local immunity rests on the demonstration that a given area of the body may be protected by the topical application of a given antigen without involving a more generalized immunity. The earlier experiments of Wassermann and Citron ${ }^{\mathrm{I}}$ and of Roemer ${ }^{2}$ which awakened the conception of local immunity rested on the unnecessary assumption that such restricted protected areas are dependent on the local production of antibodies. Even admitting that such local protection might be due to localized antibodies, the evidence hitherto adduced would seem to point to the local mobilization of antibodies from the general circulation rather than their strictly local formation. We have elsewhere defined local immunity as "due to a locally superior mechanism for the disposal of a particular micro-organism. ${ }^{3}$ Local immunity may be proved by the local presence of antibodies before their appearance elsewhere in the body, or by their local presence in greater concentration - or again local immunity may be demonstrated, and this we believe most conclusively, by the demonstration of a superior method of direct disposal of bacteria in the particular area in question." I do not believe that the local formation of antibodies has as yet been proved and am now inclined to believe that local immunity is due primarily to an intensified response of cells in the treated area-a true Umstimmung, to revive in a more understandable sense the term proposed by Wassermann and Citron.

\section{THE RECENT HYPOTHESIS OF BESREDKA}

Widespread interest in the phenomena of local immunity has been awakened through the recent work of Besredka. ${ }^{4}$ This author begins his explanation of the mechanism of active immunity with an assumption that we have already expressed, namely, that it cannot be accounted for entirely on the basis of antibodies. This assumption is based on the lack of parallelism between the antibody content of serum and protection; on the fact that in some instances, e.g., anthrax, chicken cholera, swine erysipelas, the known antibodies may be removed by specific absorption and the protective power of the serum remain. "The known antibodies should be without hesitation stripped of their importance; their function in immunity is in reality entirely secondary, or negative in certain cases."4 Active immunity, then, is in essence a cellular phenomenon and depends on rendering insensitive the cells that are sensitive to a particular virus or micro-organism. Besredka believes that the free phagocytes in their process of phagocytosis liberate from bacteria an "antivirus," the precise nature of which is not clear, but which is essentially a non-antigenic toxic

x Wassermann, A., and Citron, J.: Ztschr. Hyg. u. Infektionskrankh., 50, 33I. r905.

${ }^{2}$ Roemer, P.: Arch.f. Ophth., 54, 99. 1902.

${ }^{3}$ Gay, F. P.: Physiol. Rev., 4, I91. 1924.

${ }_{4}^{4}$ Besredka, A.: Locul Immunisation, Specific Dressings (Amer. ed.). Baltimore: Williams \& Wilkins, 1927. (Contains reference to numerous articles of its author.) 
extract of the bacterial cell. This "antivirus" turns at once to the receptive cells in the body which rapidly become saturated with it and thereby desensitized from the further toxic action of the corresponding virus. This process would be essentially similar to desensitization by a small amount of foreign serum in animals that have been rendered susceptible to anaphylactic shock by previous injection of the same protein-in other words, it is analogous to the condition of "anti-anaphylaxis."

When animals are hyperimmunized by repeated doses of a virus as when they are treated to produce an immune serum, the original desensitization of the susceptible cells is followed by an accumulation of the antivirus which is mixed in the serum with the antibodies that have begun to form. The success following the passive transfer of the serum of such immunized animals is due, first, to the tropinizing or lytic effect, already well recognized, of the antibodies on the specific micro-organism. In addition, according to Besredka, passive serum immunity is in no small part due to the vaccinating or desensitizing effect of the antivirus, likewise present in the immune serum, on the susceptible cells of the host.

This interesting and important new concept of immunity, which I have endeavored to present succinctly in its latest expression, has been evolved by Besredka from a series of animal experiments and their clinical application extending over a number of years. The clinical results, which are both preventive and curative in nature, are in themselves increasingly impressive. I do not believe, however, that clinical data in general, and particularly in their initial groupings, are of great value in proving the accuracy of any particular hypothesis that has led to their accumulation. The experimental findings that Besredka has furnished are concerned particularly with bacteria whose pathogenic localization, as he considers them, is either in the skin or in the intestinal canal. The particular bacteria most fully studied have been $B$. anthracis, streptococci, staphylococci, B.typhosus, and B. dysenteriae. The salient features of each of these experimental studies should be rapidly sketched in order to understand their implication.

Anthrax.--Under natural conditions cattle are infected with anthrax through ingestion of anthrax spores. According to Sobernheim, ${ }^{\mathrm{r}}$ a considerable number of these spores is necessary. In the guinea pig or rabbit when a few anthrax spores are introduced per os they are destroyed by the gastric juice. When sufficient numbers are given they soon reach the blood where they may be phagocyted and remain harmless though living. Boquet has found that if the infected blood in such an animal is withdrawn by inserting a needle through the skin, the latter is readily infected and leads to a fatal infection. This proves the relatively great susceptibility of the skin to anthrax infection. If a vegetative or non-encapsulated culture of anthrax is introduced into the guinea-pig peritoneum and great precaution taken to aroid contaminating the skin, the animal recovers. The skin, however, is by no means the only susceptible organ, as Besredka has claimed; the testicle, ${ }^{2}$ the brain, ${ }^{3}$ and the muscles ${ }^{4}$

I Sobernheim, G.: Kolle und IV assermann's Handbuch der Pathogen. Mikroorganism, 3, 583. I9I3.

${ }^{2}$ Sani, L.: Clin. vet., p. 485 . 1925.

3 Adelheim, R., and Kaktin, A.: Klin. Wchnschr., 3, I721. I924; Cernainu, C., and Suhatzanu, C. S.: Compt. rend. Soc. de biol., 90, 869. I924.

4 Basset, J.: ibid., 93, 413. 1925. 
are perhaps equally vulnerable. The reason that these areas are particularly susceptible is simply because they are relatively free from phagocytes. This allows vegetative anthrax bacilli sufficient time to become animalized or encapsulated and thereby virulent. At all events Besredka, by rubbing the attenuated first vaccine of anthrax on the skin or by injecting intradermally, was able gradually to produce a solid immunity in guinea pigs which are almost impossible to immunize by other routes. Enough time would seem to be allowed by this method for the bacteria to multiply sufficiently to immunize but without becoming virulent (encapsulated).

Similar results with intradermal vaccination have been obtained in larger animals. Brocq-Rousseau and Urbain, ${ }^{1}$ and also Nicolas, ${ }^{2}$ have apparently protected horses very successfully in this manner. Very favorable results in cattle and sheep were obtained by a single intracutaneous vaccination by Velu. ${ }^{3}$

It seems quite possible from these findings that intracutaneous inoculation may prove to be the most efficient method of vaccinating against anthrax. They do not, however, prove in my opinion that protection is effected by "closing," as Besredka first expressed it, "a specific portal of entry," nor yet by acting in some desensitizing fashion on specifically receptive cells, to use his later and more reasonable explanation. Besredka may be correct in asserting that the protection he induces by this method is not accompanied by the presence of antibodies, although Gratia ${ }^{4}$ has asserted the contrary, but it does not prove that protection is due to a limited group of particularly sensitive cells.

Streptococci and staphylococci.-Besredka has offered results of interest in the treatment of streptococcus and staphyloccus infections limited to external areas of skin or mucous membranes. Antisera to these two organisms, if we except the treatment of scarlet fever by an antitoxin to an apparently specialized race of the streptococcus, have been wholly inefficient. Vaccine therapy has been questionably successful in preventing the spread of staphylococcus infections. Besredka finds that when guinea pigs are prepared by application in the form of dressings, or by intracutaneous injection, of sterile broth filtrates in which streptococci or staphylococci are grown, subsequent injection of the corresponding micro-organism in the same area twentyfour hours later is relatively harmless, whereas control animals suffer extensive lesions followed by death. This effect he would attribute to a thermostable, specific "antivirus" existing in broth cultures, which inhibits growth of the organism, and which vaccinates within a few hours against it. No protection is afforded against skin infection when the previous injection of "antivirus" has been given intraperitoneally. Plain broth preparation in a similar manner, in Besredka's hands at least, showed little evidence of protection. Distinctly curative results were also obtained by simultaneous injection of culture and filtrate.

Our own experiments, ${ }^{5}$ corroborated by Rivers and Tillett ${ }^{6}$ and by Mallory and

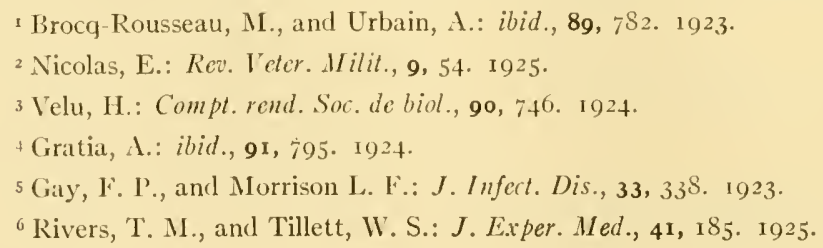


Marble, ${ }^{\mathrm{I}}$ indicate that Besredka's results may be fully explained by the stimulating effect of certain non-specific and even non-antigenic substances such as plain broth, which, as we have shown, stimulate and collect the tissue macrophages in their vicinity. Subsequent study ${ }^{2}$ has shown that collected macrophages have a very remarkable protective value against virulent local streptococcus infections, and within certain limits they may after collection be remobilized in other areas with equally beneficial results. Similar results are offered by Bass. ${ }^{3}$ The importance of monuclear tissue cells in directly combating various infections has been recently shown by Nakahara, ${ }^{4}$ Merklen and Wolf, ${ }^{5}$ Bloom, ${ }^{6}$ Goldzieher and Peck, ${ }^{7}$ and Willis. ${ }^{8}$

Both staphylococcus and streptococcus broth filtrates have been used by Besredka's followers in instances of human infections due to the respective micro-organisms. A number of case reports collected from the literature have been offered by Besredka as indicating a promising field for the use of filtrates in clinical medicine. They range from boils and furuncles to osteomyelitis and puerperal fever. The categorically stated sequence of favorable symptoms which follows the local application of "antivirus" dressings in these cases is uniform and startling. The method certainly seems worthy of a more searching investigation as to the mechanism involved in these apparent cures.

Intestinal infections. - In another series of investigations Besredka has considered a group of human infections which are of intestinal origin, namely, cholera, dysentery, and the typhoidal fevers. These infections are more difficult than the skin infections to study experimentally since they do not produce their human syndromes in the usual laboratory animals.

The Shiga bacillus of dysentery does produce in rabbits, irrespective of the route of introduction, lesions that recall human bacillary dysentery of the most toxic type; hemorrhage in the agminated follicles follows the injection either of small amounts of the living organism, of dead cultures, or of sterile toxic filtrates from its growth. The fact that the lethal dose of Shiga bacilli for the rabbit is infinitely smaller intravenously than when given by the mouth should make us skeptical of the complete analogy between human disease and animal experiments, and again the notable susceptibility of the central nervous system to this organism might equally well designate this locality as the most vulnerable point.

At all events it seems proved by the work of Dopter, ${ }^{9}$ Chvostek, ${ }^{\text {ro }}$ Besredka, and Enlows that mice and rabbits can be protected against the Shiga bacillus by administering killed or living cultures per os. Besredka and Chvostek tested this protection

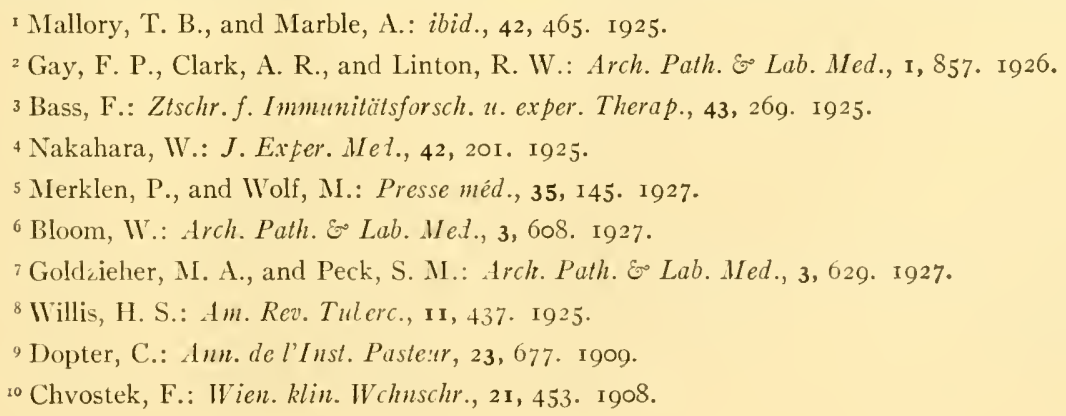


by intravenous inoculation. In other words, to use Besredka's argument, once the susceptible cells are desensitized they are protected from injury which is evident in normal animals, irrespective of the route of introduction of the virus. If it be true that the susceptible cells are reached inevitably wherever the dysentery bacillus is introduced, the whole argument of the desirability of a direct application of the immunizing dose to the susceptible cells falls to the ground since the usual subcutaneous method would equally avail. In the case of dysentery, however, a real advantage for the oral administration lies in the severe reactions produced by other routes. ${ }^{x}$

Of course the possibility of vaccination by the mouth is an already ancient problem and, according to Calmette, ${ }^{2}$ dates from the work of Pasteur on chicken cholera in I880. The repeated attempts to render such a method practicable between the years $1892-1903^{3}$ were largely for the purpose of affording an easy and comfortable method of giving vaccine. In the case of dysentery, then, oral administration is certainly more comfortable and does to some extent at least protect against natural infection, but until it be proved that it protects as well or better than immunization by other routes it certainly cannot supplant them. It is very likely that whatever active immunity may be produced against the dysentery bacillus is antitoxic in nature, and certainly it has not been proved that antitoxic immunity may be local in nature, in spite of the work of Roemer ${ }^{4}$ with abrin and that of Hazen ${ }^{5}$ with ricin.

In the case of the typhoidal fevers, the possibility of oral vaccination by Besredka's method would seem founded on more acceptable and full clinical data, but introduces in its experimental and initial aspects a variation which does anything but confirm the accuracy of his hypothesis of local immunity. The reader should consult Besredka's monograph if he wishes to judge whether or not the oral method of vaccination against typhoid is as good as the subcutaneous method which it may conceivably supplant. Its author himself does not assert that the method is better but simply that it may be as good.

In Germany, between 1906 and I9Io, a number of experimenters, Loeffler, ${ }^{6}$ Kutscher and Meinicke, ${ }^{7}$ Wolf, ${ }^{8}$ and Bruckner, ${ }^{9}$ had demonstrated the possibility of immunizing certain laboratory animals with various strains of paratyphoid bacilli by the oral administration of living or dead bacilli. In I9 I Metchnikoff and Besredka, ${ }^{\text {In }}$ in connection with their interesting production of typhoid fever in anthropoid apes, indicated in a few animals that the previous oral administration of killed cultures might under certain conditions protect them against ingestion of a virulent culture of typhoid bacilli. In attempting to repeat these results in rabbits Besredka met at

I Enlows, E.: Pub. II calth Rep., 40, I4. 1925.

${ }^{2}$ Calmette, A.: Ann. de l'Inst. Paste:tr, 37, 900. I923. $\quad 3$ See various citations.

4 Roemer, P.: Arch.f Ophth., 54, 99. 1902.

5 Hazen, E. L.: J. Immunol., I3, I7 I. I927.

${ }^{6}$ Loeffler, F.: Gedenkschrift of R. V. Leuthold (ed. Schjerming). Berlin, I906.

${ }^{7}$ Kutscher, and Meinicke, E.: Ztschr. f. IIyg. u. Infektionskrankh., 52, 301. I906.

${ }^{8}$ Wolf, K.: Miunchen. med. Wchnschr., 55, 270.' 1908.

9 Bruckner, G.: Ztschr. f. Immunilälsforsch. u. exper. Therap., 8, 439. I91 I.

ro Metchnikoff, E., and Besredka, A.: Ann. de l'Inst. Pasleur, 25, I93. 1911. 
first with complete failure, owing to the resistance of this animal to the typhoid bacillus when administered by the mouth. The previous ingestion of ox bile rendered rabbits so susceptible to both typhoid and paratyphoid bacilli that they died with suggestive symptoms and lesions. These results have since been repeated in rabbits by several investigators (Zingher and Soletsky, ${ }^{x}$ Neri, ${ }^{2}$ Glotoff),${ }^{3}$ and in guinea pigs by Sedan and Hermann. ${ }^{4}$ That this lowered resistance, however, is dependent on epithelial desquamation of the intestinal mucosa which renders the animal at all analogous to the naturally susceptible human being is doubtful. Others, notably Webster, ${ }^{5}$ have shown that bile in itself is very toxic, and how far this may account for the lethal effect is an open question. Moreover, many who have reported on the immunizing effect of bile typhoid vaccines in rabbits, Zingher and Soletsky, Neri, Teale, ${ }^{6}$ and Gye ${ }^{7}$ have failed to confirm the positive results claimed by Besredka.

The prominence we have given to the work of Besredka in the field of local immunization is justified by the stimulus it has given to the more profound study of the possibilities of local immunization rather than from a belief in the correctness of his explanation of the mechanism of local immunity. Besredka's hypothesis, however unsatisfactory it may be in final explanation, has the merit of rather extensive formulation and completeness and therefore furnishes a basis of discussion and experimentation which cannot but carry us forward in a very important and neglected field of investigation.

I believe, in the first place, that there is now overwhelming evidence of the existence of a truly localized form of immunity confined under certain conditions, and in response to certain infectious agents, to localized areas or cell groups of the body, and representing not only the natural resistance enjoyed by many cells, but also the exaggerated and important conditions of true active, acquired immunity. I would list tentatively as representative conditions of active local immunity the marked protection against smallpox and typhoid fever which follows recovery or vaccination, and also in a more restricted sense localized streptococcus and staphylococcus infections. I believe that these conditions are due primarily to "retuning" (Umstimmung) of particular cells or cell groups which may simply acquire new potentialities of reaction that are extremely durable (typhoid, smallpox) or may be due to an increase in numbers and strategic location of certain cells in the more transitory conditions, as in our studies of streptococcus infections. This local or tissue immunity. would not be in the nature of a desensitization, but rather in the nature of a cell immunization. We may revive the original conception of Knorr, that the particular cells concerned in active immunity and antibody formation are the more resistant rather than the

I Zingher, A., and Soletsky, D.: Proc. New York Path. Soc., 20, I33. I920.

${ }^{2}$ Neri, F.: Boll. d. Instit. sieroterap. milanese, 2, 275.1922.

3 Glotoff, E.: Compt. rend. Soc. de biol., 89, 368. I923.

4 Sedan, J., and Hermann, R.: ibid., 90, 567. I924.

5 Webster, L. T.: J. Exper. Med., 37, 33. I923.

"Teale, T. P.: "Discussion" to paper by Besredka, Tr. Roy. Soc. Trop. Med. E॰ IIyg., I7, 346. 1924.

${ }^{7}$ Gye, WV. E.: ibid., p. 346. I924. 
more susceptible cells. At all events I see no evidence for believing with Besredka that certain forms of active immunization "close the specific portal of entry"-a conception that has shifted in his own explanations to "desensitization of the specifically susceptible cells." It should be pointed out that he has confused the two ideas of portal of entry and locus minoris resistentiae which are far from interchangeable.

\section{THE RETICULO-ENDOTHELIAL SYSTEM}

The reticulo-endothelial system of cells seems particularly concerned in active phagocytic opposition to infectious agents. Certain components of this system, the clasmatocytes (histiocytes, polyblasts, rhagiocrine cells, or tissue macrophages), can be selectively marked by vital dyes and shown in the case of localized streptococcus infections to be actively responsible and productive of marked protection when increased in a given area (Gay and Morrison; ${ }^{1}$ Gay, ${ }^{2}$ Gay, Clark, and Linton ${ }^{3}$ ). They may actually be collected in one pleural cavity and attracted for purposes of protection to the other pleural cavity.

These same cells may also eventually be shown to be responsible for more durable conditions of active immunity. Ledingham, ${ }^{4}$ for example, regards them, rather than the epidermal cells, as responsible for vaccine immunity.

The questions of antibody formation and local immunity are closely involved. Local immunity may undoubtedly occur without the demonstrable presence of antibodies, but I do not believe the two conditions are by any means mutually exclusive as Besredka would seem to think. And again, the reticulo-endothelial system seems implicated in the more generalized formation of antibodies, to judge from the evidence that the "blockade" of these cells by dyestuffs (Gay and Clark, ${ }^{5}$ Jungeblut and Berlot $\left.{ }^{6}\right)$ inhibits antibody production. Everything points, then, with increasing emphasis to the reticulo-endothelial system as forming the background of immunity processes; the cells comprising the system are ubiquitous; certain of them, the monocytes and the histiocytes, are mobile; both endothelial and reticular cells have repeatedly been shown to have direct phagocytic activity against bacteria, and those cells which may be stimulated or blocked by dyestuffs are seriously concerned with antibody formation. Blockade furthermore seriously affects the direct protective function of the specific cells in both bacterial and protozoan infections (Meyer, ${ }^{7}$ Jungeblut $\left.{ }^{8}\right)$.

The present tendency in histological studies would seem to indicate that the monocytes of the circulating blood and the histiocytes (clasmatocytes) of connective tissue are not only connected in origin but may actually be transformed into one

${ }^{x}$ Gay, F. P., and Morrison, L. F.: loc. cit.; J. Infect. Dis., 33, 338, 1923.

${ }^{2}$ Gay, F. P.: J. Immunol., 8, I. 1923; Physiol. Rev., 4, I91. 1924; Arch. Path. \& Lab. Med., I, 590. 1926.

3 Gay, F. P., Clark, A. R., and Linton, R. W.: loc. cit.

${ }_{4}^{4}$ Ledingham, J. C. G.: Brit. J. Exper. Path., 8, 12. 1927.

5 Gay, F. P., and Clark, A. R.: J.A.M.A., 83, I296. I924.

${ }^{6}$ Jungeblut, C. W., and Berlot, J. A.: J. Exper. Med., 43, 613. I926.

7 Meyer, H.: Zischr.f. Hyg. u. Infektionskrankh., 106, х24. 1926.

${ }^{8}$ Jungeblut, C. W.: ibid., 107, 357. 1927. 
another (Lewis and Lewis ${ }^{\mathrm{I}}$, Maximow, ${ }^{2}$ Eliot ${ }^{3}$ ). A change in function accompanies the change as demonstrated by the reaction to certain dyestuffs. It is still an open question whether the connection between endothelium and histiocytes is equally direct. ${ }^{4}$ Recent evidence by Fried ${ }^{5}$ would indicate that the so-called "alveolar epithelial cells" of the lungs give rise to the phagocytic cells in inflammatory process of that organ.

It may well be that we shall eventually trace all the fundamental immunity processes to one or another of that group of differentiated cells which we collect loosely under the designation of "reticulo-endothelial system." Antibody formation relatively great resistance, ultimate disposal - not only of bacteria but of polymorpho. nuclears that have already ingested bacteria-we tentatively refer to them. With our attention finally directed in the right direction, progress in immunology should take a new step forward.

${ }^{I}$ Lewis, M. R. and W. H.: Pub. Carnegie Inst. of Washington, No. 363.1927.

2 Maximow, A.: Proc. Soc. Exper. Biol. \& Med., 24, 570.1927.

3 Eliot, C.: Bull. Johns Hopkins Hosp., 39, I49. 1926.

${ }^{4}$ Mallory, F. B.: J. Exper. Med., 3, 6r г. I898.

${ }_{5}^{5}$ Fried, B. M.: Arch. Path. \&o Lab. Med., 3, 751. 1927. 


\title{
CHAPTER LXVIII
}

\section{THE HUMAN BLOOD GROUPS}

\author{
K. LANDSTELNER
}

Rockefeller Institute for Medical Research, New York City

\section{ISOAGGLUTININS AND ISOAGGLUTINOGENS}

After it was established that serological methods can be used for the detection of species differences of animal cells and proteins as well as for differentiating microorganisms, the question presented itself whether the same principle is applicable to the detection of individual variations within a species. The first experiments along this line were made with human blood by mixing erythrocytes with serum of other healthy individuals. ${ }^{2}$ Since it was generally accepted that serum of the same species is the most innocuous medium for cells one would have expected to find either no changes at all or else a great variety of reactions of rather small order. In fact, the tests yielded entirely negative results in certain combinations while in others an agglutination of the red cells took place which was not less pronounced than that brought about by the action of serum on the blood of a foreign species.

Using another method soon afterwards, Ehrlich and Morgenroth ${ }^{3}$ demonstrated antibodies in the serum against cells of the same species-isoantibodies-after injecting goats with the blood of other goats. The further study of the isoagglutination of human blood revealed an unexpected feature of these phenomena. The reactions do not occur at random but follow a definite rule. With regard to isoagglutination all individuals can be divided almost without exception into four well-defined groups, three of which were described by the writer, ${ }^{4}$ the fourth, of rarer occurrence, by von Decastello and Sturli. ${ }^{5}$ The blood-group characteristics were found to be constitutional and to remain unchanged throughout life; the agglutinogens are inherited according to the Mendelian laws (von Dungern and Hirschfeld, Ottenberg). A simple explanation of the reactions can be given by assuming the existence of two agglutinogens $\mathrm{A}$ and $\mathrm{B}$ in the cells and two agglutinins $a$ (or anti-A) and $\beta$ (or anti-B) in the sera.

For the designation of the blood groups necessary for practical purposes numbers have been generally used. Considerable confusion, however, and even accidents, were caused by the existence of two different arbitrary numberings, namely, the classifica-

${ }^{1}$ For a detailed review of the subject cf. Hirszfeld, L.: Ergebn. d. IIyg., 8, 367. I926; Levine, P.: Ergebn.d.inn. Med. u. Kinderh., 34 (in press); Schiff, F.: Die Technik der Blutgruppenuntersuchung. Berlin: Springer, 1926; Doan, C. A.: Physiol. Rev., 7, 1. 1927; Snyder, L. H.: Arch. Path. E Lab. Med., 4, 215. 1927; Lattes, L.: Die Individualität des Blutes. Berlin: Springer, I925.

${ }^{2}$ Landsteiner, K.: Centralbl.f. Bakteriol., 27, 357. 1900.

${ }^{3}$ Ehrlich, P., and Morgenroth, J.: Berl. klin. Wchnschr., 37, 453. I900.

${ }_{4}$ Landsteiner, K.: Wien. klin.Wchnschr., 14, Ir32. I901.

${ }^{5}$ von Decastello, A., and Sturli, A.: Mïnchen. med. Wchnschr., 49, I090. I902. 
tions proposed by Jansky and by Moss.' For that reason several authors prefer a nomenclature by letters as employed in the present article. This designation of the four groups is $\mathrm{O}, \mathrm{A}, \mathrm{B}, \mathrm{AB}$, which, as will be seen, represents the constitution of the corpuscles with respect to isoagglutinogens, the letter $\mathrm{O}$ indicating the absence of both agglutinable substances $\mathrm{A}$ and $\mathrm{B}$.

The correlation of the various nomenclatures and the distribution of the agglutinogens and agglutinins among the groups are shown in Table I. The scheme brings out the rule, which is not self-evident, that the serum regularly contains the agglutinins active for the absent agglutinogens. The other regularity, that corresponding agglutinins and agglutinogens do not coexist in one blood, is plausible a priori. Consequently the characteristics of the groups are as follows: Group O: The corpuscles

TABLE I

\begin{tabular}{|c|c|c|c|c|}
\hline $\begin{array}{c}\text { Jansky } \\
\text { Numbering }\end{array}$ & $\begin{array}{c}\text { Moss } \\
\text { Numbering }\end{array}$ & $\begin{array}{c}\text { Designation by } \\
\text { Letters }\end{array}$ & $\begin{array}{l}\text { Agglutinogens } \\
\text { in the Cells }\end{array}$ & $\begin{array}{l}\text { Agglutinins } \\
\text { in the Serum }\end{array}$ \\
\hline $\begin{array}{r}\text { I. } \ldots \ldots \ldots \\
\text { II. } \ldots \ldots \ldots \\
\text { III. } \ldots \ldots \ldots \\
\text { IV } \ldots \ldots \ldots\end{array}$ & $\begin{array}{l}\text { IV } \\
\text { II } \\
\text { III } \\
\text { I }\end{array}$ & $\begin{array}{r}\mathrm{O} \\
\mathrm{A} \\
\mathrm{B} \\
\mathrm{AB}\end{array}$ & $\begin{array}{r}\ddot{A} \\
B \\
A, B\end{array}$ & $\begin{array}{c}\alpha, \beta \\
\beta \\
a \\
a \ldots \ldots\end{array}$ \\
\hline
\end{tabular}

TABLE II

\begin{tabular}{|c|c|c|c|c|}
\hline \multirow{2}{*}{ SERA of Group } & \multicolumn{4}{|c|}{ Cells of Groups } \\
\hline & o & A & B & $\mathrm{AB}$ \\
\hline $\mathrm{O} \ldots$ & 一 & + & + & + \\
\hline A ... & - & - & + & + \\
\hline B $\ldots$ & 一 & + & - & + \\
\hline$A B \ldots \ldots \ldots$ & 一 & - & 一 & - \\
\hline
\end{tabular}

are not agglutinated by any serum; the serum agglutinates the corpuscles of all other groups (cells A generally more intensely than cells B). Group A: The corpuscles are agglutinated by the sera of groups $\mathrm{O}$ and $\mathrm{B}$; the serum agglutinates the corpuscles of groups $\mathrm{B}$ and $\mathrm{AB}$. Group $\mathrm{B}$ : The corpuscles are agglutinated by the sera of groups $\mathrm{O}$ and $\mathrm{A}$; the serum agglutinates the corpuscles of groups $\mathrm{A}$ and $\mathrm{AB}$. Group $\mathrm{AB}$ : The corpuscles are agglutinated by the sera of all other groups; the serum does not agglutinate the corpuscles of any group. These statements are summarized in Table II where+denotes agglutination, - no agglutination.

It is evident, then, that an unknown blood can easily be grouped by employing either two sera or two sorts of corpuscles known to belong to groups A and B or serum and corpuscles of either group A or B. The technique will be discussed below. The groups can be determined also without the use of standard sera or bloods by crosstesting a sufficient number of blood samples. In this way groups $\mathrm{O}$ and $\mathrm{AB}$ are recognized immediately; but the differentiation of groups A and B is only possible by taking into consideration that group $\mathrm{A}$ is much more frequent than $\mathrm{B}$ among white races.

× See J.A.M.A., 76, г30. г921. 
Another method of identification consists in the use of immune sera against Forssman's antigen, some of which agglutinate intensely human blood of group A, as was discovered by Schiff and Adelsberger.

The assumption of two agglutinins and two agglutinogens as underlying the typical iso-reaction of human blood is, in a general way, supported by the results of absorption experiments. Thus, a serum $\mathrm{O}$ treated with corpuscles $\mathrm{A}$ still contains agglutinins for cells $\mathrm{B}$ and vice versa, and cells $\mathrm{AB}$ absorb from any serum the agglutinins for cells $\mathrm{A}$ and $\mathrm{B}$. The conception of three agglutinins and three agglutinogens, as advanced by Moss, ${ }^{1}$ would require that cells $\mathrm{A}$ or $\mathrm{B}$ absorb the agglutinins from sera $\mathrm{O}$ just as well for cells $\mathrm{A}$ as for $\mathrm{B}$, which is not borne out by the experimental results.

While the hypothesis of two pairs of agglutination elements is practically adequate it cannot be considered as strictly accurate. It was found that by absorbing sera $\mathrm{O}$ with cells $\mathrm{A}$ or $\mathrm{B}$ not infrequently a part of the agglutinins is removed for cells $\mathrm{B}$ or $\mathrm{A}$, indicating that in sera $\mathrm{O}$ a part of the agglutinins $a$ and $\beta$ may exist in combination. ${ }^{2} \mathrm{~A}$ certain relationship of the agglutinogens $\mathrm{A}$ and $\mathrm{B}$ is indicated by the experiments of Hooker and Anderson, ${ }^{3}$ Landsteiner and van der Scheer, ${ }^{4}$ and Doelter. ${ }^{5}$

In regard to corpuscles $\mathrm{O}$ a new point was raised by the genetic theory of Bernstein. ${ }^{6}$ While von Dungern and Hirschfeld explain the inheritance of the blood groups by postulating two dominant Mendelian genes A and B, the absence of which would determine the appearance of group $\mathrm{O}$, Bernstein, by a calculation based on the frequency of the group factors, was led to the assumption of three allelomorph genetic factors $^{7}-\mathrm{A}, \mathrm{B}, \mathrm{R}$. The genetic formulas for the groups are, according to Bernstein's hypothesis, the following:

$\begin{array}{ccccc}\text { Groups........ } & \mathrm{O} & \mathrm{A} & \mathrm{B} & \mathrm{AB} \\ \begin{array}{c}\text { Genetic formulas } \\ \text { of Bernstein. }\end{array} & \mathrm{AA} \text { or } & \mathrm{BB} \text { or } & \mathrm{AB} \\ \text { of } & \mathrm{BR} & \end{array}$

Since $\mathrm{R}$ is a genetic factor, the formula $\mathrm{RR}$ for corpuscles $\mathrm{O}$ does not imply the existence in these corpuscles of a special agglutinogen demonstrable by serological reactions, but in a recent paper Schiff ${ }^{8}$ reports that he obtained fluids reacting by preference on erythrocytes $\mathrm{O}$ by absorbing certain normal beef sera with red cells $\mathrm{AB}$. The corpuscles $\mathrm{O}$ would then seem to be characterized not merely by the absence of the

${ }^{x}$ Moss, W. L.: Bull. Johns Hopkins Hosp., 21, 63. I910.

${ }^{2}$ Landsteiner, K., and Witt, D. H.: J. Immunol., I I, 221. 1926. This phenomenon is possibly of significance for explaining some observations of Dyke, S. C.: Brit. J. Exper. Path., 7, 294. 1926.

${ }^{3}$ Hooker, S. B., and Anderson, L. M.: J. Immunol., 6, 419. I921.

${ }_{4}^{4}$ Landsteiner, K., and van der Scheer, J.: J. Exper. Med., 42, I23. 1925; Proc. Soc. Exper. Biol. Eo Med., 22, 289. I925.

5 Doelter, W.: Ztschr.f. Immunitätsforsch. ul, exper. Therap., 43, 95. I925.

${ }^{6}$ Bernstein, F.: Ztschr. f. indukt. Abstamm. u. Vcrerb., 37, 237. 1925; Bernstein's theory is strongly supported by the recent studies of Schiff, F.: Deutsche Ztschr. d. ges. gcrichll. Led., 9, 360 . 1927; and Thomsen, O., ibid., 10, I. 1927.

${ }^{7}$ For a full presentation of the genetic data see chapter lxix in this volume by Ottenberg and Beres.

${ }^{8}$ Schiff, F.: Klin. Wchnschr., 6, 303. 1927. 
agglutinogens $\mathrm{A}$ and $\mathrm{B}$ but by the presence of a particular agglutinogen for which no agglutinin exists in normal human sera (see p. 902). (If Schiff's agglutinable factor corresponds to Bernstein's genetic R, one might expect that his observation would lead to a serological differentiation of individuals homozygous or heterozygous as to the agglutinogen $\mathrm{A}$ or $\mathrm{B}$.)

Aside from the red corpuscles, group specific substances were found by special procedures in sperm cells, seminal fluid, saliva, and in organ tissues and the blood serum. ${ }^{\text {I }}$ In blood platelets isoagglutinogens could not be demonstrated. ${ }^{2}$ Isoagglutinins are also present in milk. ${ }^{3}$

\section{ISOHEMOLYSINS}

On mixing fresh human serum, which contains complement, with the erythrocytes of other individuals, hemolysis - isohemolysis - can also occur, occasionally masking the agglutination. This isolysis was regarded by some early authors, like isoagglutination, as a pathological phenomenon, but it was shown that it likewise depends on the constitutional group qualities. ${ }^{4}$ That is, isolysis occurs only along with isoagglutination, but the incidence of isolysis is much rarer with the usual technique. By modifying the method, namely, taking large amounts of serum ${ }^{5}$ or making the cells more susceptible by storing the washed corpuscles for several days, ${ }^{6}$ the frequency of hemolysis can be increased considerably so as to approximate the incidence of isoagglutination. In certain pathological cases the titre of isolysis was found to be higher than usual.

Group-specific complement-fixing antibodies in a few normal human sera and also opsonins were described by Schiff and Adelsberger, ${ }^{7}$ antilytic substances by Moss, Jervell, ${ }^{8}$ and others.

\section{DEVELOPMENT OF THE ISOAGGLUTINATION ELEMENTS}

\section{QUANTITATIVE VARIATIONS}

In newborn children the isoagglutinins are often missing or present in small amounts only. In part they are derived from the mother, but agglutinins have been found in infants, that are not present in the mother's serum (von Decastello and Sturli,

r Yamakami, K.: J. Immunol., I2, I85. 1926; Landsteiner, K., and Levine, P.: ibid., p. 415. I926; Ashby, W.: Am. Ass. Immunol., May 4, I925; Witebsky, E.: Ztschr. f. Immunitätsforsch. $u$. exper. Therap., 49, 517. 1927; Schiff, F.: Klin. Wchnschr., 3, 679. 1924; Kritchewski, I. L., and Schwartzmann, L. A.: Klin. Wchnschr., 6, 208г. I927; Ouchi, I.: Ztschr.f. Immunitätsforsch.u. exper. Therap., 53, 462. I927.

${ }^{2}$ Toda, T.: J. Path. \& Bact., 26, 303. 1923.

${ }^{3}$ Landsteiner, K.: Wien. klin. Rundschau, No. 40. I902. Cf. Happ, W. M.: J. Exper. MIed., 31, 313. 1920.

${ }^{4}$ Landsteiner, K., and Leiner, K.: Centralbl. f. Bakteriol., 38, 548. I905; Grafe, E., and Graham, D. A. L.: Mïnchen. med. Wchnschr., 2257, 2338. I9II.

5 Williams, W. C.: J.Exper. Med., 32, I59. I920; Jones, B. B.: Am.J. Dis. Child., 22, 586, 598. I92 I.

${ }^{6}$ Hesser, S.: Akt. med. Skand. Suppl., 9, I. I924.

${ }^{7}$ Schiff, F., and Adelsberger, L.: Centralbl. f. Bakteriol., 93, 172. I924; Ztschr. f. Immunitäts. forsch.u. exper. Therap., 40, 335. 1924; Schiff, F.: Med. Klin., 21, I238. I925.

${ }^{8}$ Jervell, F.: Mitt.d. Grenzgeb. d. Med. u. Chir., 34, 650. 1922. 
Jones, Hirszfeld, Smith, ${ }^{\mathrm{r}} \mathrm{cf}$. Collon $\left.{ }^{2}\right)$. The appearance of the agglutinogens precedes that of the agglutinins so that ordinarily at birth the group of the cells can already be determined although, according to Happ, ${ }^{3}$ agglutinogens may still develop after birth. The groups are definitely established also in the serum within the first or second

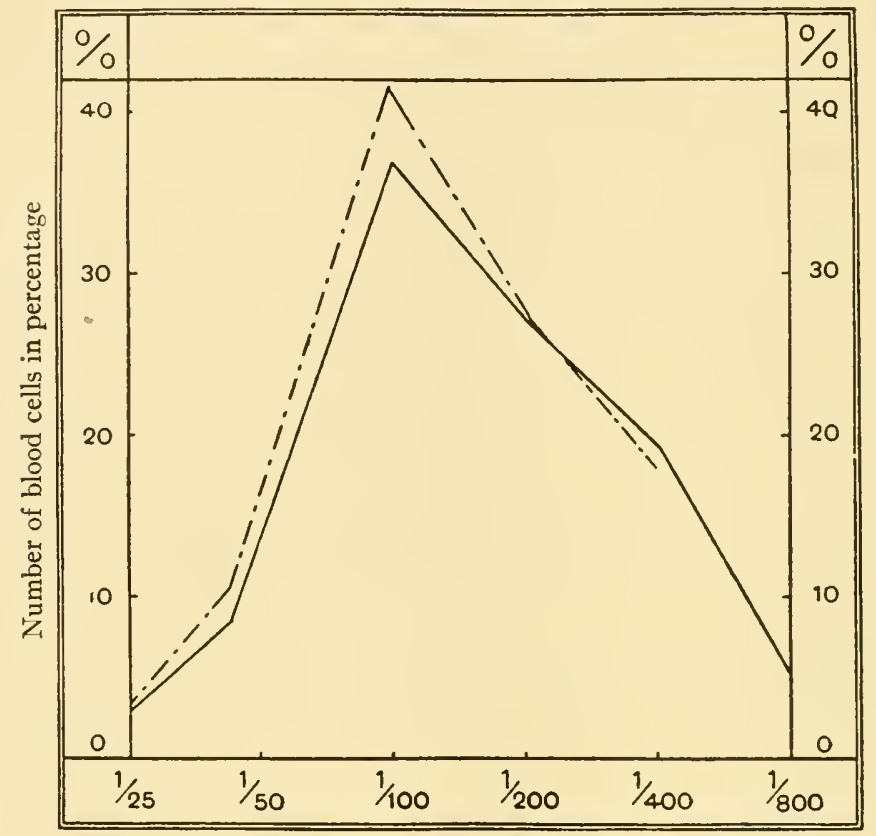

FIg. I.-Sensitivity of various red cells $\mathrm{A}_{\text {(_ }}$ ) and B (--.-). (After Schiff and Hübener)

year of life. The idea that discrepancy of the groups of mother and infant-so-called "heterospecific pregnancy"-may be the cause of pathological conditions such as eclampsia, icterus neonatorum, and of general deleterious effects on the infant has been discussed by various authors (cf. Hirszfeld, ${ }^{4}$ Preger, ${ }^{5}$ Smith) but these investigations did not yield consistent results.

A study of the quantitative variations of the agglutinins and agglutinogens was undertaken by several workers such as Dyke, ${ }^{6}$ Jones and Glynn, ${ }^{7}$ Schiff, ${ }^{8}$ and his collaborators. ${ }^{9}$ Some results obtained by Schiff are presented in Figure I, which show

${ }^{1}$ Smith, C.: Am. J. Dis. Child. (in press).

${ }^{2}$ Collon, N. G.: Arch. int. méd. exper., 3, 237. 1927.

3 Happ, W. M.: J. Exper. Med., 31, 313. I920.

${ }^{4}$ Hirszfeld, L.: loc cit.

5 Preger, A.: Ztschr. f. Immunitätsforsch. u. exper. Therap., 53, I92. 1927.

${ }^{6}$ Dyke, S. C.: Brit. J. Exper. Path., 3, I46. I922.

7 Jones, A. R., and Glynn, E. E.: J. Path. E Bact., 29, 203. 1926.

${ }^{8}$ Schiff, F., and Hübener, G.: Ztschr. f. Immunitätsforsch. u. exper. Therap., 45, 207. 1025; Schiff, F., and Mendlowicz, L.: ibid., 48, I. 1926.

? With regard to the possibility of an increase in agglutinins in pathological conditions see Schneider, P.: Ztschr.f.d.ges. exper. Med., 36, I53. I923; and Mino, P.: Deutsche med. Wchnschr., 
that the sensitiveness of individual corpuscles when tested with the same agglutinating serum varied within a wide range $(\mathrm{I}-32)$. It could not be established whether corpuscles homozygous with regard to $\mathrm{A}$ or $\mathrm{B}$ are on the average more sensitive than heterozygous. However, some observations of Oppenheim and Voigt ${ }^{\mathbf{1}}$ on the blood of Chinese seem to support this view. Also the titre of the isoagglutinins varied considerably in Schiff's experiments (Fig. 2), the extreme values being $\mathrm{I}-\mathrm{I} 6$ and $\mathrm{I}-2,048$. The strength of the agglutinins increases in the first years of life and diminishes in old age.

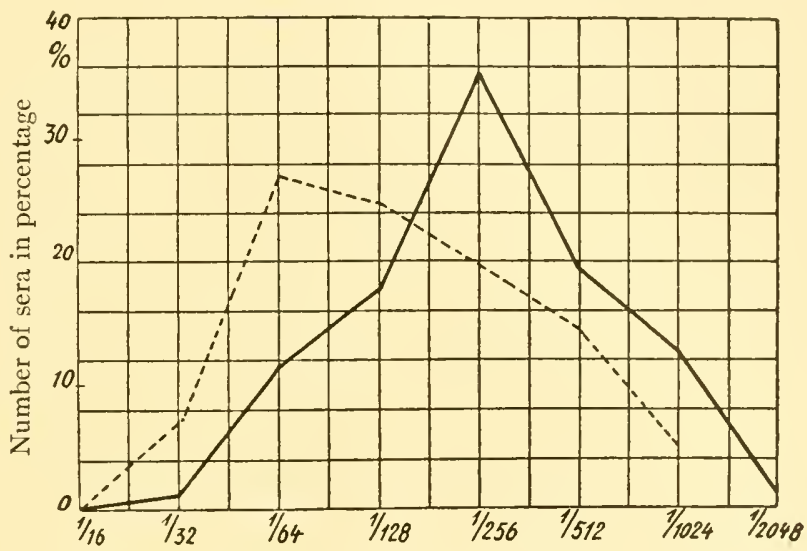

FIG. 2.-Agglutinin titre of various sera $a(-)$ and $\beta$ (..-.). (After Schiff and Mendlowicz).

\section{ATYPICAL REACTIONS ${ }^{2}$}

The existence of individual blood differences aside from the typical group scheme has been recognized since the beginning of the studies on the subject. Doubtless, apparently irregular reactions were obtained not infrequently by improper technique, for instance, by the use of a method not delicate enough for detecting weak agglutinins. Errors in the opposite direction were made by mistaking for isoagglutination other phenomena such as pseudoagglutination and autoagglutination.

\section{PSEUDOAGGLUTINATION}

It was observed long ago that in diseases, especially in inflammatory conditions, the erythrocytes have a great tendency to settle, the blood forming a buffy coat. This phenomenon has attracted much attention, and is also employed in practical medicine since the extensive work of Fahraeus. It is due to an increased capacity of the serum

50, 1533. 1924. For statistical data on blood groups in relation to pathological conditions see Hirszfeld, L., loc. cit.; Thomsen, O.: Acta path. et microbiol. Scandinav., 4, 45. 1927; Johannsen, E. WV.: ibid., p. 175. 1927. There is some evidence that under treatment the Wassermann reaction disappears more readily in individuals $\mathrm{O}$ and $\mathrm{A}$ than in others (Amzel, R., and Halber, W.: Ztschr. f. Immunitätsforsch. 1. exper. Therap., 42, 89. 1925; Gundel, M.: Klin. Wchnschr., 6, 1703. 1927). Oppenhein and Voigt found that group $B$ individuals have a shorter life-span than those of groups $O$ and $A$.

\footnotetext{
${ }^{1}$ Oppenheim, F., and Voigt, K.: Krankheitsforschung, 3, 306. 1926.
}

${ }^{2}$ Cf. Levine, P.: loc. cit.; Landsteiner, K., and Witt, D. H.: loc. cil. 
to agglomerate the erythrocytes of the same or other individuals in the form of rouleaux, or clumps resembling those produced by true agglutination (Shattock). ${ }^{t}$ The phenomenon is also very marked with normal horse blood.

As was shown by Shattock, this form of clumping-pseudoagglutination-disappears on slight dilution ( $\mathrm{I}-2$ or $\mathrm{I}-3)$ of the serum, and can therefore be distinguished readily from isoagglutination. By treating the serum with red cells the active principle is not absorbed in contradistinction to agglutinins. ${ }^{2}$ A phenomenon very similar to pseudoagglutination is brought about when certain colloidal substances such as gum arabic or gelatin are added to blood suspensions.

\section{AUTOAGGLUTINATION ${ }^{3}$}

When animal or human erythrocytes are mixed with a sufficient quantity of serum of the same individual and kept at low temperature (icebox) the cells mostly settle in clumps after some time. The reaction is diminished by an increase in temperature, and, as a rule, disappears entirely at $37^{\circ} \mathrm{C}$. The active principle is absorbed by the cells in the cold and set free by slight warming. When, therefore, the agglutinated cells are washed with ice-cold saline and are resuspended in saline at room temperature, the clumps break up, and after centrifuging the supernatant fluid contains the agglutinin. Its presence can be demonstrated by adding blood cells to the fluid and chilling the mixture. The determining effect of temperature, and also the absorption of the active substances by the cells, distinguishes autoagglutination from pseudoagglutination. The speed of sedimentation due to pseudoagglutination is ordinarily not lower and, indeed, may be higher at $37^{\circ} \mathrm{C}$. than at room temperature; it is not dependent on the content of autoagglutinins in the serum. A high degree of autoagglutination has been observed in certain pathological conditions as in syphilitic or hypertrophic cirrhosis, paroxysmal hemoglobinuria, Raynaud's disease, hemolytic icterus, anemia, spirillosis, and trypanosomiasis of man and animals. In rabbits the formation of powerful agglutinins could be induced by repeated transfusions with rabbit blood or repeated bleedings. ${ }^{4}$ The occurrence of abnormal agglutinins acting on bloods of all groups and due to bacterial products was described by Lacy. ${ }^{5}$

Agglutination reactions produced by sera of any group were described by Hübener, Schiff and Halberstaedter, ${ }^{6}$ and Thomsen, ${ }^{7}$ with blood samples kept for one or several days. In Thomsen's case the agglutinability could be transmitted to other blood specimens by adding some of the peculiar blood, and it was found that the

${ }^{r}$ Shattock, S. G.: J. Path. \& Bact., 6, 303. I900; a detailed study was made by Wiltshire, H.: ibid., 17, 282. 1913.

${ }^{2}$ Meyer, K., and Ziskoven, H.: Med. Klin., 19, 87. 1923.

3 Landsteiner, K.: München. med. Wchnschr., 50, 1818. 1903; Mino, P.: Deutsche med. Wchnschr., 50, 1533. 1924; Alexander, H. L., and Thompson, L. D.: J.A.M.A., 85, 707. 1925.

4 Rous, P., and Robertson, O. H.: J. Exper. Med., 27, 509, 503. I918.

${ }^{5}$ Lacy, G. R.: J. Immunol., I4, I89. I927; cf. Eisenberg, P.: Centralbl. f. Bakteriol., 34, 739. 1903.

${ }^{6}$ Schiff, F., and Halberstaedter, W.: Ztschr.f. Immunititsforsch. u. exper. Therap., 48, +14. 1926

7 Thomsen, O.: Soc. Biol., 96, 556. I927, cf. p. 1079; Ztschr. f. Immunitätsforsch. u. exper. Therap., 52, 85. 1927; Jriedenreich, V.: Soc. Biol., 96, 1079. 1927: 97, 1266. 1927. 
change was brought about by the action of a special bacillus which makes the blood cells sensitive to an agglutinating agent present in human serum. Thomsen's phenomenon is to be kept in mind on testing blood specimens that are not perfectly fresh.

The distinguishing features of pseudo-, auto-, and isoagglutination are represented in Table III.

\section{DEFICIENT REACTIONS}

Among the atypical cases reported there are some in which the agglutinins expected from the rule of the groups were deficient. Although these findings were certainly due in a number of cases to overlooking weak reactions, it is possible that in rare instances there is a temporary or permanent deficiency of agglutinins. Grove, however, did not find such an instance among over two thousand examinations.

TABLE III

\begin{tabular}{|c|c|c|c|}
\hline & Pseudoagglutination & Autoagglutination & Isoagglutination \\
\hline $\begin{array}{l}\text { Absorption of the active prin- } \\
\text { ciple...................... }\end{array}$ & Not absorbable & Absorbable & Absorbable \\
\hline $\begin{array}{c}\text { Influence of temperature } \\
\text { variations............... }\end{array}$ & $\begin{array}{l}\text { Not less, rather more, } \\
\text { active at } 37^{\circ} \mathrm{C} \text {. } \\
\text { than at lower tem- } \\
\text { perature }\end{array}$ & $\begin{array}{l}\text { Generally occurring } \\
\text { only at low temper- } \\
\text { ature }\end{array}$ & $\begin{array}{l}\text { Not much affected by } \\
\text { temperature varia- } \\
\text { tions between } 0^{\circ} \\
\text { and } 37^{\circ}\end{array}$ \\
\hline $\begin{array}{l}\text { Effect of dilution of the } \\
\text { serum } \ldots \ldots \ldots \ldots \ldots \ldots \ldots\end{array}$ & $\begin{array}{l}\text { Disappears on slight } \\
\text { cilution }\end{array}$ & $\begin{array}{l}\text { Stands moderate dilu- } \\
\text { tion }\end{array}$ & $\begin{array}{l}\text { Stands moderate dilu- } \\
\text { tions }\end{array}$ \\
\hline
\end{tabular}

\section{SUBGROUPS OF GROUP A}

It was demonstrated for the first time by von Dungern and Hirschfeld ${ }^{x}$ that after absorbing a group B serum with individual group A corpuscles the supernatant fluid reacted on some and not on other red cells of group A. This experiment, indicating a subdivision of group A, has been confirmed by several authors. ${ }^{2}$ According to Coca, the non-reacting corpuscles amount to about 25 per cent of all samples of group A. Mino and Lattes and Cavazutti attributed these observations to purely quantitative differences in the agglutinability of the corpuscles. This view appeared to be substantiated by their finding that all anti-A agglutinins can be entirely removed from a serum by treating it with the non-reacting corpuscles, particularly at low temperatures, and that these corpuscles are less sensitive toward sera $\mathrm{O}$ and $\mathrm{B}$ than the others. Evidently, then, the assumption seemed warranted that the less sensitive corpuscles would not react with the serum when the amount of agglutinins was diminished by incomplete absorption while the more sensitive cells would still be acted upon. It was possible, however, to separate two fractions of agglutinins from sera $\mathrm{O}$ and $\mathrm{B}$, the one acting on both types of group-A cells, the other chicfly on one of them. ${ }^{3}$ This leads to the conclusion that the corpuscles are qualitatively different. One may consider either two slightly different agglutinogens $\mathrm{A}$ or else an additional agglutinogen in the

Iron Dungern, E., and Hirschfeld, L.: Ztschr. f. Immunitätsforsch. u. exper. Therap., $13,527$. 19 II.

"Schütze, H.: Bril. J. Exper. Path., 2, 26. 1921; Coca, A. F., and Klein, H.: J. Immmnol., 13, 477. 1923; Guthrie, C. G., and Huck, J. G.: Bull. Johns Hopkins Hosp., 34, 37, So, 128. 1923.

3 Landsteiner, K., and Witt, D. H.: loc. cit. 
more sensitive type of cells. However this may be, the hypothetic additionalagglutinogen (termed as $\mathrm{C}$ or $\mathrm{X}$ or $\mathrm{A}^{\mathrm{I}}$ ) should not be put on the same level with the agglutinogens $\mathrm{A}$ and $\mathrm{B}$ as Guthrie and Huck propose, since there is no definite proof yet that $\mathrm{A}^{\mathrm{r}}$ occurs independent of $\mathrm{A}$. If $\mathrm{A}^{\mathrm{I}}$ were an independent mendelizing factor it ought to be found with regularity in groups other than A and AB. The two agglutinins acting on group A cells which may be designated by $\alpha$ and $\alpha^{\mathrm{I}}$ are present in nearly all sera of groups $\mathrm{O}$ and $\mathrm{B}$. Guthrie and Huck and Simson ${ }^{\mathrm{I}}$ described sera containing only $a^{\mathrm{r}}$ or $\alpha$. In the former case the serum agglutinates selectively the cells $\mathrm{AA}^{\mathrm{x}}$.

With regard to other findings of blood differences by means of absorption, ${ }^{2}$ it should be borne in mind that they may be the result of quantitative variations, as has been pointed out by Mino and Lattes and Cavazzuti, and that thorough investigation is needed in every case to exclude this possibility.

\section{"COLD" AGGLUTININS}

It has been mentioned above that at low temperature $\left(0^{\circ}-5^{\circ} \mathrm{C}\right.$.) a serum agglutinates, as a rule, the individual's own corpuscles as well as others of the same species. When a number of corpuscles are tested, differences are noticed in the degree of the reaction, which indicate a certain specificity; ${ }^{3}$ the differences may be more marked with the use of properly absorbed sera. In this way, besides other slight variations, two sorts of group-A corpuscles can be demonstrated corresponding to those discussed in the previous paragraph. ${ }^{4}$ Frequently the sera belonging to the cells $\mathrm{AA}^{\mathrm{I}}$ act more intensely on the other type, which may be termed $\mathrm{AA}^{2}$, than on $\mathrm{AA}^{\mathrm{I}}$, and vice versa; moreover, the former sera react markedly on cells of group O (Table IV).

Using the same method for sera and corpuscles B (and O) a number of individual variations were observed without apparent regularity. A proper classification of all these reactions which indicate the existence of a number of individual blood diferences is not possible as yet and would serve no practical purpose.

The fact that agglutination of red cells tends to disappear on warming and that agglutinins are liberated from their combination with the cells has been established also for normal heteroagglutinins. ${ }^{5}$ This reversion of the agglutinin reaction is well marked at temperatures around $50^{\circ} \mathrm{C}$.; cold agglutination, on the other hand, is much more sensitive to the influence of temperature so that it is rarely encountered under conditions of routine technique, i.e., at room temperature, and practically does not interfere with the group scheme. ${ }^{6}$ But some particularly strong reactions may persist when the tests are made at not-too-high room temperatures. In our opinion, a number of observations on slight atypical isoagglutination-“"minor" agglutination

I Simson, F. W.: J. Palh. \&o Bact., 29, 279. 1926.

${ }^{2}$ Guthrie, C. G., Pessel, J. F., and Huck, J. G.: Bull. Johns IIopkins Irosp., 35, 221 . I924; Bunker, H. A., and Meyers, S.: J. Lab. \& Clin. IIed., 12, 415. 1927.

${ }_{3}^{3}$ Bialousknia, W., and Hirszfeld, L.: Pracgladu Epidemjologicsnego, r, 437. 1921 ; Cuthrie, C. G., Pessell, J. F., and Huck, J. G.: Bull. Johns Hopkins IIosp., 34, 37, 80, 128. 1923; 35, 23, 33, 81, I 26, 221. I924; Landsteiner, K., and Levine, P.: J.Immunol. I2, 44I. 1926.

${ }_{4}^{4}$ Landsteiner, K., and Levine, P.: loc. cil.

${ }^{5}$ Landsteiner, K.: Mïnchen. med. W'chuschr., No. 46. 1902; Jervell, F.: J. Immunol., 6, 445 1921 ; cf. Doelter, W.: Ztschr. f. Immunilätsforsch. u. exper. Therap., 43, 12S. 1925.

'The exceptional cases of unusually strong antoagglutination have been referred to above. 
-recorded in the literature (e.g., Unger) ${ }^{x}$ fall under this head. Whether or not this holds for all such cases cannot be stated definitely since most of ten the effect of changes in temperature is not recorded. That there are transitions between the "cold" and the other agglutinins follows from the fact that heteroagglutinins and probably common isoagglutinins contain fractions similar to cold agglutinins which can be demonstrated by suitable absorption experiments ${ }^{2}$ (see below).

\section{MARKED ANOMALOUS REACTIONS}

Anomalous reactions comparable in intensity to that of atypical isoagglutination are met with only rarely in the usual tests. Most of the published observations are not sufficiently studied to permit of a critical analysis. In all such cases it would be necessary to make cross-tests on an extensive scale with the corpuscles and the sera; the

TABLE IV*

Absorptions ANd Readings at $\circ^{\circ} \mathrm{C}$.

\begin{tabular}{|c|c|c|c|c|c|c|c|c|c|c|c|c|c|c|c|}
\hline \multirow{3}{*}{$\begin{array}{l}\text { SERUM } \\
\text { GROUP } \\
\text { A }\end{array}$} & \multirow{3}{*}{$\begin{array}{c}\text { SERUM } \\
\text { DI- } \\
\text { LUTED }\end{array}$} & \multirow{3}{*}{$\begin{array}{c}\text { AB- } \\
\text { SORBED } \\
\text { WITH } \\
\text { BLOOD }\end{array}$} & \multicolumn{9}{|c|}{ Corpuscles-Group A } & \multirow{2}{*}{\multicolumn{4}{|c|}{ CORPUSCles-GRoup O }} \\
\hline & & & \multicolumn{5}{|c|}{$\mathrm{AA}^{x}$} & \multicolumn{4}{|c|}{$\mathrm{AA}^{2}$} & & & & \\
\hline & & & I & 3 & 5 & 6 & 7 & 2 & 4 & 8 & $\mathrm{r}_{2}$ & I & 2 & 3 & 4 \\
\hline $2 \ldots$ & I : 5 & $2 \mathrm{AA}^{2}$ & + & + & \pm & + & F. tr. & $\circ$ & 0 & 0 & 0 & $\circ$ & F. $\operatorname{tr}$ & $\circ$ & F. tr. \\
\hline I $2 \ldots$ & I : IO & $4 \mathrm{~A}^{2}$ & + & + & + & + & Tr. & \pm & F. tr. & F. tr. & o & o & F. tr. & Tr. & F. tr. \\
\hline $6 \ldots$ & I : IO & $6+A^{1}$ & F. $\operatorname{tr}$. & $\circ$ & E. tr. & 0 & 0 & + & + & + & + & + & + & + & + \pm \\
\hline $7 \ldots \ldots$ & I: IO & $5 \mathrm{~A}^{x}$ & 0 & $\circ$ & F. tr. & o & 0 & + \pm & + \pm & + & + \pm & + & ++ & + \pm & + \\
\hline
\end{tabular}

*The Ggures signify individual bloods.

strength of the reactions and influence of temperature variations should be determined and the method given in detail.

Two fully examined instances are the following: The writer and Witt described two bloods belonging to group $\mathrm{AB}$ with cells lacking the property $\mathrm{A}^{\mathrm{t}}$, the sera of which contained agglutinins of the type $\alpha^{\mathrm{r}}$, thus acting on a number of bloods of group $\mathrm{A}$, namely, those corresponding to the formula $\mathrm{AA}^{I}$. The sensitiveness of the reaction to an increase in temperature was intermediate between cold and common agglutination. In other bloods of group $\mathrm{AB}$, also lacking $\mathrm{A}^{\mathrm{I}}$, either no agglutinins $\alpha^{\mathrm{I}}$ were found or such as behaved like cold agglutinins. The two atypical cases would correspond to a formula $\mathrm{AB}, a^{2}{ }^{2}$ Another indubitable case of an intense anomalous reaction was described by Ottenberg and Johnson. ${ }^{3}$ The blood of a professional donor according to the properties of the cells belonged to group B; the serum contained the agglutinin $\alpha$ as usual but in addition an abnormal agglutinin which reacted on a number of corpuscles of groups $\mathrm{O}$ and $\mathrm{B}$. Transfusion of this blood into an ancmic patient was followed by a grave, presumably fatal, hemolytic accident. An apparently similar instance was noted by Beck. ${ }^{4}$ Examples of doubtful or incomplete observations are the

I Unger, L. J.: J.A.M.A., 76, 9. I921.

${ }^{2}$ Amzel, R., and Hirszfeld, L.: Ztschr. f. Immunitätsforsch. u. expcr. Therap., 43, 526. 1925; Landsteiner, $\mathrm{K}$., and Levine, P.: loc. cit.

3 Ottenberg, R., and Johnson, A. X.: J. Immun ,, I2, 35. 1926.

4 Beck, A.: Ergcbn. d. inn. Mcd. u. Kinderh., 30. I50. 1926. 
cases of Jansky (cells agglutinated by any serum $\mathrm{O}$, not by serum A or B) and Sucker (cells agglutinated by sera of group B, serum-agglutinating cells of group A). Atypical reactions with blood of corpses were described by Oppenheim and Voigt.

\section{HUMAN BLOOD GROUPS AND ANIMAL BLOOD}

Agglutinins specific for the human blood groups are present also in normal animal sera and can be detected directly or by absorption tests. In this manner some evidence was obtained as to the existence of other blood differences. It was possible, moreover, to produce very active specific immune agglutinins by injecting blood $\mathrm{A}, \mathrm{B}$, or $\mathrm{AB}$ into rabbits. ${ }^{\mathrm{T}}$ The effects are not regular but depend largely on the individual response of the rabbit.

In certain immune sera, after absorption with properly selected cells, agglutinins are left behind which distinguish sharply individual bloods regardless of the group. ${ }^{2}$ In this manner three agglutinable factors were found; they differentiate six types of blood within the same group, according to the present results.

The existence in the blood of lower animals of agglutinable substances similar to human isoagglutinins was established by von Dungern and Hirschfeld. According to their results, the isoagglutinin $\beta$ can be absorbed in most cases from human sera by corpuscles, e.g., of rabbits, dogs, and cattle. The animal agglutinogens detected by this method, however, are not quite identical with the human isoagglutinogens. Obviously, an investigation in this direction of the blood of anthropoid apes was of particular interest. Such studies showed that, in contrast to that of lower animals, the blood of anthropoids contains agglutinogens (and agglutinins) indistinguishable from those of human blood. ${ }^{3}$ Consequently, each individual ape can be assigned to one of the four groups. The findings confirm the idea of a close biochemical relationship between apes and man and make it likely that the group agglutinogens appeared at a time before anthropoids and man arose from a common stock. With reference to the group agglutinogens, the blood of lower monkeys resembles that of other mammalians; an element similar to, but not identical with, B was found in the erythrocytes of the New World monkeys, Platyrrhinae and Lemurs, not in the Old World monkeys (Ceropithecidae), a result of some significance for the problem of serological species specificity.

A further interesting relation of the group factors was discovered by Schiff and Adelsberger ${ }^{+}$in tests with immune sera against the so-called "heterogenetic" antigen of Forssman.5 These authors found that human red cells A (and AB) but not corpuscles of the other groups are intensely agglutinated by some such sera. Furthermore, some inmune sera for group A corpuscles contain hemolysins for sheep blood,

I Landsteiner, K.: Ilicn. klin. Rundschau, No. 40. I902; von Dungern, E., and Hirschfeld, L.: Ztschr.f. Immunitätsforsch. u. exper. Therap., 8, 526. I910; Hooker, S. B., and Anderson, L. M.: loc. cit.

${ }^{2}$ Landsteiner, K., and Levine, P.: Proc. Soc. Exper. Biol. So Med., 24, 600, 941. 1927.

3 Landsteiner, K., and Miller, C. P.: J. Exper. Mcd., 42, S41, S53, 863. 1925.

${ }_{4}$ Schiff, F., and Adelsberger, 1.: Ztschr. f. Immunitalsforsch. u. exper. Therap., 40, 335. 1924; I Ialler, W., and Hirszfeld, 1.: ibid., 48, 34. 1926.

s Forssman, J.: Biochem. Zlschr., 37, 78. I91 I. 
and these lysins can be absorbed by the A cells. The results disclose a similarity of the agglutinogen of group A and Forssman's antigen; however, the two substances are not the same since, aside from other reasons, the experiments described did not succeed regularly but only with certain immune sera.

\section{INDIVIDUAL DIFFERENCES OF ANIMAL BLOOD}

As stated already, individual variations in animal blood were first shown with the aid of isoantibodies formed by the injection of blood into other individuals of the same species. This method, used for goat blood by Ehrlich and Morgenroth, was applied to other animals such as dogs, ${ }^{1}$ cattle, chickens, cats. It was believed that such results could be obtained only with isoantibodies. But later work brought out the fact that like reactions may be exhibited by immune sera derived from a foreign species. ${ }^{2}$ Indeed, for human blood this had already been observed. Also normal isoagglutinins and isolysins occur in animals - for instance, in the blood of cattle, sheep, chickens, cats, horses, and pigs. For several species-mice, rabbits, monkeys-no satisfactory evidence was obtained of the existence of individual blood differences.

In some species groupings were found analogous to those of human blood but not quite as regular. ${ }^{3}$ A direct relation of the serological findings to racial differences does not seem to exist; there is only an unequal frequency of the agglutinogens among various races ${ }^{4}$ (see p. 907).

The experiments of Todd and White ${ }^{5}$ with immune isolysins for cattle blood are remarkable in view of the great number of individual differences which they observed. Similar results were obtained with chicken blood. ${ }^{6}$ One may be inclined to correlate these findings with the individual varieties which were established in the studies on transplantation of normal tissues and tumors (cf. L. Loeb).

\section{CHEMICAL NATURE OF THE ISOAGGLUTINOGENS}

The chemical nature of the isoagglutinogens of red cells in general was a matter of much discussion. Recent investigations indicate that these antigens probably consist of proteins combined with other substances which determine at least in part the specificity of the whole complex (cf. Landsteiner and van der Scheer). It was first shown for the heterogenetic antigen of Forssman that by extraction with alcohol a material free from protein could be separated which reacts specifically with heterogenetic antibodies in vitro but has no antigenic activity unless it is injected along with protein. An active fraction obtained from the alcoholic extract was found to contain carbohydrates and fatty acids. Similar observations on the extraction of specific

${ }^{1}$ von Dungern, E., and Hirschfeld, L.: loc. cit.

${ }^{2}$ Landsteiner, K., and van der Scheer, J.: J. Immunol., 9, 2 I 3. I924; Landsteiner, K., and Miller, C. P.: Proc. Soc. Exper. Biol. E Med., 22, I00. I924. Cf. the interesting observation of sheep blood lacking Forssman's antigen (Mutermilch, S.: Ann. de l'Inst. Pasteur, 38, 1002. 1924).

3 Concerning their possible relation to human isnagglutination see Amzel, R., Halber, WV., and Hirszfeld, L.: Ztschr.f. Immunitätsforsch. u. exper. Therap., 42, 369. I925. Cf. Soc. Biol., 94, 204. I926; Witebsky, E.: Ztschr.f. Inmunitätsforsch. u. cxper. Therap., 49, I, 517. 1926-27.

${ }_{4}^{4}$ Bialosuknia, W., and Kaczkowski, B.: J. Immunol., 9, 593. 1924.

5 Todd, C. H., and White, R. G.: $J . I I y g .$, ro, I85. I9 ro.

${ }^{6}$ Landsteiner, K., and Miller, C. P.: loc. cit. 
substances with alcohol were made with a number of different erythrocytes and with the human isoagglutinogens. ${ }^{x}$ In the latter case the extracts were found to give flocculation reactions and complement fixation with group-specific immune sera and to stimulate the production of antibodies when injected in mixture with proteins. These facts support the view that the individual differences which have been brought out by serological tests are due to variations of specific substances not belonging to the class of proteins. The reports on isoprecipitins, perhaps indicating individual protein differences, are few and not quite convincing. ${ }^{2}$

One may hope that further work will ultimately succeed in isolating the agglutinogens $\mathrm{A}$ and $\mathrm{B}$ in a pure state and will bring to light a characteristic chemical difference between them. It is still doubtful, however, whether a special chemical substance corresponds to each of the serological factors which are found when several different antigens are tested with a number of serological reagents.

\section{COMPATIBILITY TESTS PRELIMINARY TO TRANSFUSION ${ }^{3}$}

The use of the isoagglutinin reaction for the selection of proper blood donors was suggested by the present writer in the first publication on the blood groups in I9OI, and this application has been emphasized by Hektoen and others. At the present time the test has become a routine procedure. It can be taken as established that incompatibility as regards isoagglutination is the main cause of untoward events following transfusion, and a number of accidents could be traced directly to this circumstance (Plehn, Brem, Jervell, De Pemberton; ${ }^{4}$ cf. the experiments of Schulz). An experimental confirmation of this view was offered by the work of Ottenberg and his collaborators, on transfusions in dogs and cats. ${ }^{5}$

Ottenberg ${ }^{6}$ called attention to the fact that a difference exists between the transfusion of a blood, the serum of which agglutinates or hemolyzes the corpuscles of the recipient and the reverse case, i.e., the injection of blood cells which are agglutinated by the recipient's serum. In the latter event the erythrocytes come in contact with a large amount of agglutinating serum, while in the former the serum of the donor is in most cases mixed with such an excess of the patient's blood as not to affect it to any appreciable extent.

According to this principle, group O donors (universal donors) are frequently employed for patients of any group. The usefulness of this procedure becomes evident in emergency cases. Similarly, AB patients are transfused with the blood of all other

I Landsteiner, K., and van der Scheer, J.: loc. cit.; Doelter, W.: loc. cit.; Brahn, B., and Schiff, F.: Klin. Wchnschr., 5, 1455. 1926; Witebsky, E.: Ztschr.f. Immunitätsforsch. u. exper. Therap., 48, 369; 49, 1, 517. 1926-27; Halber, W., and Hirszfeld, L.: loc. cit.; Bordet, J., and Renault: Soc. Biol., 95, 888. 1926 .

${ }^{2}$ Cf. Landsteiner, K., and Levine, P.: loc. cil. Brahn and Schiff consider the existence of groupspecific proteins in the erythrocytes.

3 For clinical aspects of transfusions see Jagic, N.: Wien. klin. I1'chnschr., 40, 1565. 1927; Breitner, B.: ibid., 4 I, 77. I928; Rolleston, H.: Brit. M. J., 2, 969. 1926.

${ }^{4}$ de Pemberton, J.: J. Iowa State Med. Soc., ro, I 70. I920.

s Ottenberg, R., Kaliski, A. J., and Friedman, S.: J. Mcd. Rescarch, 28, 141. I913; Ottenberg, R., and Thalhimer, W.: ibid., 33, 213. 19I5.

${ }^{6}$ Ottenberg, R.: J. Exper. Mcd., r3, 425. 19 I I. 
groups. One may assume, however, that the injected agglutinins are not entirely indifferent, and it would seem plausible to prefer donors with the same group as the recipient. Actually, some clinical experiences support this view (e.g., Unger, ${ }^{\mathrm{I}}$ Jones and Glynn, ${ }^{2}$ Kubanyi, ${ }^{3}$ Butka, $\left.{ }^{4} \mathrm{Kraft}\right)^{5}$, and a number of surgeons avoid the use of universal donors, using whenever possible only blood of the recipient's group. A systematic investigation of this aspect is still wanting. The use of universal donors with sera of exceptionally high agglutinin titre is directly contraindicated, as was pointed out by Levine and Mabee $^{6}$ and others. ${ }^{7}$

Two methods are generally employed for the selection of donors, the grouping of the blood of recipient and donor and the direct cross-test. Since isohemolysis does not seem to occur without agglutination it is sufficient to make agglutination tests only; occasionally in such tests hemolysis also may appear. This has the same practical significance as agglutination.

Direct matching is advisable in view of the possible occurrence of exceptional atypical agglutinins and donors with unusually high agglutinin titre. It also serves as a check for the grouping. It is not quite safe to depend on the direct matching alone and to omit the grouping, because in cases of weak agglutinins incompatibility may escape attention in the former test. Moreover, the selection of donors is easier when the group of the patient is determined and donors of known groups are available.

For the agglutination test several methods have been proposed..$^{8}$

The grouping can easily be made by mixing a drop of serum A and serum B, respectively, with a drop of $2-5$ per cent blood suspension on a slide, tipping it repeatedly to hasten clumping. After some minutes the preparation may be covered with a cover slip. Ordinarily the test should be under observation for at least fifteen minutes. The present writer prefers especially when numerous bloods are examined - to set up the tests in small tubes ( 7 -mm. diameter) using one drop each of serum, saline and blood-cell suspension (equivalent to 2.5 per cent normal blood). This dilution of the serum is generally sufficient to prevent pseudoagglutination. The emulsion can be prepared simply by mixing a few drops of blood with the necessary amount of saline solution; citrated blood may also be used, preferably after washing. The tubes are shaken several times and a drop of the mixture is taken up by means of a thin glass rod and examined microscopically with low magnification. The reaction occurs generally within a few minutes. In order to detect unusually feeble reactions the negative tests are re-examined after one hour. Control tests with known cells $\mathrm{A}$ and $\mathrm{B}$ should be included. Special care must be taken to select test sera of known high agglutinating power. Sterile test sera can be kept preferably in the icebox or may be stored with a preservative (e.g., chloroform).

${ }^{x}$ Unger, L. J.: loc. cit.

${ }^{2}$ Jones, A. R., and Glynn, E. E., loc. cit.

3 Kubanyi, A.: Zentralbl. f. Chir., 51, 1503.1924.

4 Butka, H. E.: California \& West. Med., 24, 74. 1926.

${ }^{5}$ Kraft, R.: Arch.f. klin. Chir., 134, 834. 1925.

${ }^{6}$ Levine, P., and Mabee, J.: J. Immunol., 8, 425. i923.

${ }^{7}$ Cf. Freeman, G. C., and Whitehouse, H. J.: Am. J. $M$. Sc., I 72, 664. I926.

${ }^{8}$ A detailed consideration of the technique is given in Schiff, F.: Die Technik der Blutgruppenuntersuchung. Berlin: Springer, 1926; Lattes, L.: Abderhaldens Handbuch der bioch. Arbeitsmeth., Abt. XIII, Teil 2, Heft 5, S. 7 19. I927. 
The technique just described (test tubes or slides) is to be recommended also for the direct matching and will reveal even slight reactions.

The following expeditious method for the direct matching advised by Coca renders the separation of serum unnecessary. It is adapted to the selection of suitable universal donors and to the detection of such as have exceptionally active agglutinins.

Three or four drops of patient's and donor's blood are defibrinated in two tubes. Physiological saline solution is drawn up to the 0.5 mark of a white-cell-counting pipette. The recipient's blood is then drawn up into the pipette, until the upper level of the salt solution is at the "I" mark. This is expelled upon one end of a glass slide and thoroughly mixed, constituting ten divisions of 50 per cent patient's blood. The pipette is rinsed with saline and, in the same way as just described, ten divisions of 50 per cent donor's blood are expelled upon the other end of the slide. Two divisions of this 50 per cent donor's blood are carried over to the ten divisions of the 50 per cent recipient's blood with which they are well mixed. (Avoid bubbles.) The mixture is covered with a cover slip and clumps are looked for with a microscope (low power); it is kept under observation for fifteen minutes.

It is recommended by some authors as an additional check that a small amount of blood be injected first and the transfusion continued only if no untoward symptoms develop.

Aside from the agglutinin tests a thorough general examination of donors is essential, including blood counts and the Wassermann reaction. Mention should be made of the possibility of transmitting allergic conditions and malaria.

With proper preliminary compatibility tests made and performed by experienced men, transfusions are almost free of danger, and several authors report series of hundreds of transfusions without any serious accident (Beck, ${ }^{1}$ De Pemberton ${ }^{2}$ ).

The instances reported of severe transfusion reactions in spite of apparently satisfactory compatibility tests must be viewed with caution, as is exemplified by a recent communication of Forssman and Fogelgren. ${ }^{3}$

Excluding faulty serological tests and inadequate surgical technique, there still remain a certain number of post-transfusion reactions such as chills, fever, and skin manifestations which are of mild character in most cases. Various reasons have been offered to explain the occurrence of these symptoms, viz., extravascular blood changes, the effect of citrate, individual variations of the leukocytes (Doan), ${ }^{4}$ the serum proteins (cf. Hooker and Anderson), ${ }^{5}$ and differences of the red cells not detected by the usual isoagglutination tests. With regard to the agglutinin reactions noticeable only at low temperature there is no definite proof as yet that these are of significance for the outcome of a transfusion. Until such evidence is available it would not seem indicated to resort on that account to a considerable complication of the serum tests (cf. Unger, ${ }^{6}$ Guthrie and Pessel ${ }^{7}$ ).

That in general there will be differences between donor and recipient follows from the facts discussed above (p. 902). This consideration may have a bearing on the results of repeated transfusions in man, in view of the possible formation of immune

${ }^{1}$ Beck, A.: Mïnchen. med. Wchnschr., 74, 398. 1927. $\quad{ }^{2}$ de Pemberton, J.: loc. cit.

3 Forssman, J., and Fogelgren, G.: Klin. Wchnschr., p. I663. I927.

${ }_{4}$ The individual differences between leukocytes found by Doan with the supravital stain do not follow the group rule for erythrocytes. See Doan, C. A., J.A.M.A., 86, I593. 1926.

${ }^{5}$ Hooker, S. B., and Anderson, L. M.: loc. cit.

${ }^{6}$ Unger, L. J.: loc. cit.

7 Guthrie, C. G., and Pessel, J. F.: Bull. Johns Hopkins Hosp., 35, 33. 1924. 
isoantibodies in human beings, in analogy to the experiments in animals. In fact, some cases were reported in which blood clinically compatible at the first transfusion caused disturbances when used repeatedly. This phase, as well as the findings of abnormal isoagglntinins after several transfusions, requires further investigation. At any rate, the crmpatibility tests must be repeated prior to each transfusion. There is little doubt that transfusions could incite the production of isoantibodies when incompatible blood is injected which is tolerated at first by virtue of a low agglutinin content of the recipient's serum.

The question as to the length of life of the injected corpuscles was studied by Ashby. ${ }^{\mathrm{I}}$ After transfusion of group-A individuals with blood $\mathrm{O}$, Ashby counted at various intervals the number of inagglutinable blood cells in the recipient's circulation and found the injected blood still present after thirty to one hundred days. Incompatible blood disappears much more rapidly (Hopkins, Ottenberg, Jervell). ${ }^{2}$

Brief mention may be made of some attempts to apply the principle of the blood groups to skin grafting and transplantation (Davis, ${ }^{3}$ Shawan 4 ).

\section{FORENSIC APPLICATION OF THE ISOAGGLUTINATION TEST}

The isoagglutination test has been employed in medico-legal cases to examine the origin of bloodstains (Landsteiner and Richter ${ }^{5}$ cf. Schiff, ${ }^{6}$ Lattes$^{7}$ ). Of course, it can be determined only that a particular stain does not correspond to a certain blood, and it is not possible to make positive statements as to its identity. The simplest procedure is the test for an agglutinin content in the stain by adding known redcell suspension A or B to the material or to an extract of it. A preliminary precipitin test is needed to show that the stain consists of human blood, and a control with blood $\mathrm{O}$ is useful in order to exclude other phenomena simulating isoagglutination. For the discrimination of iso- and pseudoagglutination an addition of lecithin to the blood suspension is recommended by Lattes. Also the absorption of sera A and B with dried material has been applied for establishing the group. ${ }^{8}$

\section{RACIAL DISTRIBUTION OF THE BLOOD GROUPS}

L. and H. Hirschfeld ${ }^{9}$ discovered that there are significant variations in the incidence of the blood groups among the human races. They stress particularly the fact that the ratio between the factors A and B ("biochemical racial index") is highest $\left(>_{2}\right)$ in Northern European peoples and lowest $\left(<_{I}\right)$ in Asiatic and African races such as Mongolians and Ethiopians. It was pointed out later that the frequency of

${ }^{x}$ Ashby, W.: J. Exper. Med., 29, 267. 1919.

${ }^{2}$ Hopkins, J. G.: Arch. Int. Med., 6, 270. 1910; Jervell, F.: Acta pathol. et microbiol. Scandinav., 3, 201. 1924.

3 Davis, J. S.: Ann. Surg., 66, 88. ISI7.

${ }^{4}$ Shawan, H. K.: Am. J. M. Sc., 157, 503. I919; cf. the experiments of Ingebrigtsen, R.: J. Exper. Med., 16, I69, I912.

${ }^{5}$ Landsteiner, K., and Richter, M.: Ztschr. f. Med.-Beamte, r6, 85. I903.

${ }^{6}$ Schiff, F.: Deutsche Zischr.f. gericht Med., 9, 369. I927.

7 Lattes, L.: Die Individualität des Blutes. Berlin: Springer, 1925.

${ }^{8}$ The application of the isoagglutination reactions for forensic cases of disputed paternity is discussed in the following chapter (by Ottenberg and Beres).

9 Hirschfeld, L. and H.: Lancet, 2, 675. I919; Anthropologie, 29, 505. I918-Ig. 
group $\mathrm{O}$ also shows characteristic differences (cf. Ottenberg). ${ }^{x}$ Studies of this sort have been made upon numerous peoples and, broadly speaking, the observations indicate a relationship between the serological and anthropological data. Moreover, there are examples of very peculiar blood-group distribution. In Indians and Eskimos, e.g., group $\mathrm{O}$ predominates and may occur exclusively in full-blooded individuals (Coca and Deibert, ${ }^{2}$ Snyder, ${ }^{3}$ Heinbecker and Pauli ${ }^{4}$ ); in the Australian aboriginals there prevails a high percentage of $\mathrm{A}$ and $\mathrm{O}$, while $\mathrm{B}$ is absent (Cleland). ${ }^{5}$ In some cases, however, unrelated races were found to be similar in regard to the in-

TABLE $\mathrm{V}$

Percentage of the Blood Groups in Various Peoples

\begin{tabular}{|c|c|c|c|c|c|}
\hline Race & Observer & 0 & A & B & $\mathrm{AB}$ \\
\hline English . ............... & Hirszfeld and Hirszfeld & 46.4 & 43.4 & 7.2 & 3.0 \\
\hline 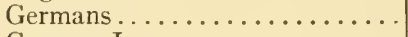 & Hirszfeld and Hirszfeld & 40.0 & 43.0 & 12.0 & 5.0 \\
\hline German Jews............. & Schiff and Ziegler & 42.1 & $4 \mathrm{I} . \mathrm{I}$ & II. 9 & 4.9 \\
\hline 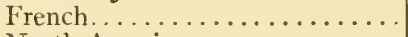 & Hirszfeld and Hirszfeld & 43.2 & 42.6 & II. 2 & 3.0 \\
\hline North Americans............ & Snyder & 45.0 & 42.0 & IO & 3.0 \\
\hline Turks.................... & Hirszfeld and Hirszfeld & 36.8 & 38.0 & I 8.6 & 6.6 \\
\hline 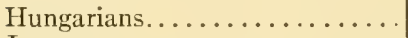 & Verzar and Weszeczky & 31.0 & 38.0 & 18.8 & I 2.2 \\
\hline Japanese ................. & Fukumachi & 26.8 & 40.9 & I 8.4 & I3. 9 \\
\hline Chinese (Schantung)...... & Liang & $2 I . I$ & 31.6 & 36.8 & I0. 5 \\
\hline Chinese (Peking)........ & Liu and Wang & 30.7 & 25.1 & 34.2 & 10 \\
\hline Chinese (Nganhai).......... & Liang & 47.9 & 21.7 & 21.7 & 8.7 \\
\hline 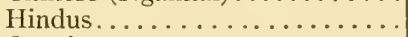 & Hirszfeld and Hirszfeld & $3 \mathrm{I} .3$ & I9. 0 & $4 \mathrm{I} .2$ & 8.5 \\
\hline Gypsies ................. & Verzar and Weszeczky & 34.2 & $2 \mathrm{I} . \mathrm{I}$ & $3^{8.9}$ & 5.8 \\
\hline Negroes (Senegal)............ & Hirszfeld and Hirszfeld & 43.2 & 22.6 & 29.2 & 5.0 \\
\hline American Negroes........... & Snyder & 47.0 & 28.0 & 20.0 & 5.0 \\
\hline Australian aboriginals........ & Cleland & $45 \cdot 54$ & $54 \cdot 46$ & 0 & 0 \\
\hline North American Indians...... . & Snyder & 9 I. 3 & $7 \cdot 7$ & I.O & 0 \\
\hline 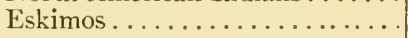 & Heinbecker and Pauli & 80.65 & 12.90 & 2.42 & 4.03 \\
\hline
\end{tabular}

cidence of the groups, and within the same people considerable differences were encountered (cf. Grove, ${ }^{6}$ Lanner $^{7}$ ). Of course, only investigations including a rather large number of individuals are worthy of consideration. Further study is needed to ascertain to what extent conclusions can be drawn from the serological examination as to the origin and relationship of human races, and to determine the relative significance of the blood tests as a supplementary method to the more comprehensive morphological investigations (cf. Hirszfeld, Snyder, ${ }^{8}$ Streng, ${ }^{9}$ Kruse ${ }^{10}$ ). Some characteristic results are summarized in Table V. A graphic representation of the group distribution among various peoples was given by Streng using trilinear co-ordinates. Recent investigations indicate that there are racial differences also with regard to agglutinogens other than $\mathrm{A}$ and $\mathrm{B} .{ }^{\mathrm{II}}$

${ }^{x}$ Ottenberg, R.: J.A.M.A., 84, I393. 1925. For fuller references see Hirszfeld, L.: loc. cit,; Streng, O., and Ryti, E.: Acta soc. med. Fennicae "Duodecim," 8, Part I, p. I. 1927.

${ }^{2}$ Coca, A. F., and Deibert, O.: J. Immunol., 8, 487. I923; Nigg, C.: J. Immunol., r I, 319. 1926.

3 Snyder, L. H.: Am. J. Phys. Anthrop., 9, 233. 1926.

${ }_{4}^{4}$ Heinbecker, P., and Pauli, R.: J. Immunol., 13, 297. 1927.

${ }_{5}^{5}$ Cleland, G. B.: Australian J. Exper. Biol. Eo M. Sc., 3, 33. 1926.

${ }^{6}$ Grove, E. F.: J. Immunol., 12, 25 I. I926.

"Lanner: Klin. Wchnschr., 4, 1477. 1925. 'Snyder, L. H.: loc. cit.

9Streng, O.: Acta. soc. med. Fennicae "Duodecim," 8, Part I, p. ז. 1926.

${ }^{20}$ Kruse, W.: Arch.f. Rassen u. Gesellsch. Biol., 19, 20. 1927.

${ }^{21}$ Landsteiner, K., and Levine, P.: Proc. Soc. Exper. Biol. Med., 24, 600, 94 r. 1927. 


\section{CHAPTER LXIX}

\section{THE HEREDITY OF THE BLOOD GROUPS}

\section{REUBEN OTTENBERG AND DAVID BERES}

Mount Sinai Hospital, New York City

The iso-agglutinable elements in the red blood cells with the complementary agglutinins in the serum, described by Landsteiner in I901, probably offer the most favorable material for the study of inheritance in man. Every person belongs to one of the four blood groups, and every family is a source of data. The problem of the heredity of the blood groups is best considered under two separate phases. The one is

TABLE I

Constitution of the Four Blood Groups

\begin{tabular}{|c|c|c|}
\hline Group & Serum & Cells \\
\hline O............ & $\begin{array}{l}\text { Agglutinates cells of three other } \\
\text { groups; contains agglutinins } \\
a \text { and } \beta\end{array}$ & $\begin{array}{l}\text { Inagglutinable; contain no ag- } \\
\text { glutinogen }\end{array}$ \\
\hline$A \ldots \ldots \ldots \ldots$ & $\begin{array}{l}\text { Agglutinates cells of groups } B \\
\text { and } A B \text {; contains agglutinin } \beta\end{array}$ & $\begin{array}{l}\text { Agglutinated by serum of } \\
\text { groups } \mathrm{O} \text { and } \mathrm{B} ; \text { contain } \\
\text { agglutinogen } \mathrm{A}\end{array}$ \\
\hline B...... & $\begin{array}{l}\text { Agglutinates cells of groups } A \\
\text { and } A B \text {; contains agglutinin } a\end{array}$ & $\begin{array}{l}\text { Agglutinated by serum of } \\
\text { groups } \mathrm{O} \text { and } \mathrm{A} \text {; contain } \\
\text { agglutinogen } \mathrm{B}\end{array}$ \\
\hline$A B \ldots \ldots .$. & $\begin{array}{l}\text { No agglutinative effect; con- } \\
\text { tains no agglutinin }\end{array}$ & $\begin{array}{l}\text { Agglutinated by serum of } \\
\text { groups } \mathrm{O}, \mathrm{A} \text {, and } \mathrm{B} \text {; contain } \\
\text { both agglutinogens } \mathrm{A} \text { and } \mathrm{B}\end{array}$ \\
\hline
\end{tabular}

concerned with the proof that the iso-agglutinable elements are inherited; the other with the analysis of the Mendelian mechanism involved, the relation of the genes.

\section{THE DATA ON INHERITANCE}

a) THE SEROLOGICAL BASIS

Landsteiner ${ }^{1}$ in I90 I demonstrated that human beings may be divided into three groups according to the interreactions of their sera and red blood cells. His pupils, Decastello and Sturli, ${ }^{2}$ the next year added a fourth group. Landsteiner suggested that there exist two sets of factors which are represented by the letters $\mathrm{A}$ and $a$, and $\mathrm{B}$ and $\beta$. A and $\mathrm{B}$ represent the agglutinogens in the erythrocytes; $\alpha$ and $\beta$, the agglutinins in the sera. In a given individual the presence of $\mathrm{A}$ in the cells excludes the presence of $a$ in the serum. The four groups may be represented as shown in Table I. The serological significance of these facts is discussed by Landsteiner elsewhere in this volume. ${ }^{3}$

I Landsteiner, K.: Wicn. klin. Wchnschr., I4, I132. I901.

${ }^{2}$ Decastello, A., and Sturli, A.: Mïnchen. med. Wchnschr., 49, I090. I902.

3 See chap. Ixviii. 
b) THE GENETIC DATA

In I908 Epstein and Ottenberg, ${ }^{,}$in a report of two families, presented the first evidence that the blood groups might be inherited. In one, the mother and seven sons CHART I

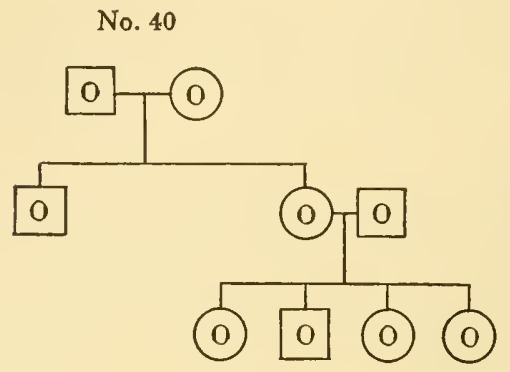

No. $52-53$

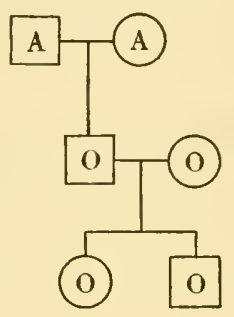

No. 54-64-65

No. $37-67$
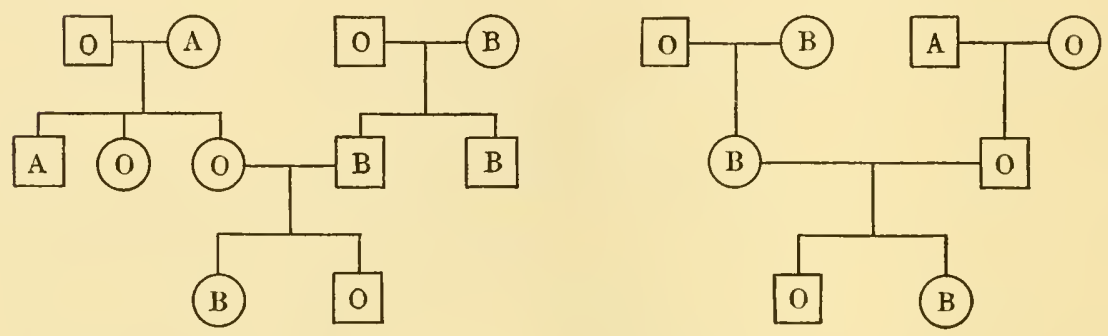

No. 34

No. 46
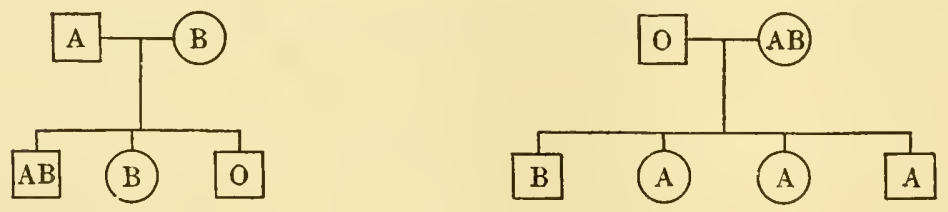

No. $47-48-49$

No. 7
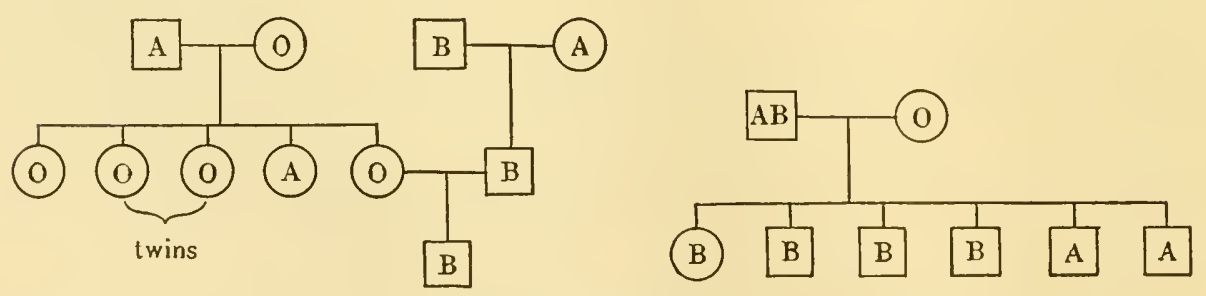

Illustrative Families

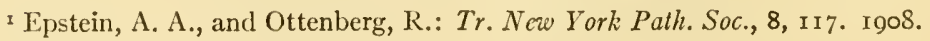


(the father's group was not determined) were all of group A; in the other, the parents and the four sons were all of group B.

Von Dungern and Hirschfeld in I9 Io made the first extensive study of the problem, presenting data on seventy-two families. Out of their observations arose a fundamental relationship which has been repeatedly confirmed by all later workers. They observed that: ( I) A or B never occurs in the red cells of a child if not present in one of the parents. (2) When one of these substances is present in both parents it occurs in most of the children. (3) When only one parent has one of these particular substances some of the children inherit it. (4) When a particular substance is absent from both parents no child ever has it.

TABLE II

Summary of Data on Inheritance of Blood Groups

\begin{tabular}{|c|c|c|c|c|c|c|c|c|c|c|}
\hline \multirow{3}{*}{ Parents } & \multirow{3}{*}{$\begin{array}{c}\text { No. of } \\
\text { FAMILIES }\end{array}$} & \multirow{3}{*}{$\begin{array}{l}\text { No. OF } \\
\text { CHILDREN }\end{array}$} & \multicolumn{8}{|c|}{ Groups of CHILDREN } \\
\hline & & & \multicolumn{2}{|c|}{$\mathrm{O}$} & \multicolumn{2}{|c|}{ A } & \multicolumn{2}{|c|}{ B } & \multicolumn{2}{|c|}{$A B$} \\
\hline & & & Number & Per Cent & Number & Per Cent & Number & Per Cent & Number & Per Cent \\
\hline $\mathrm{O} \times \mathrm{O} \ldots$ & 323 & 839 & 831 & 99.0 & 8 & I.O & & $\ldots \ldots \ldots$ & $\ldots$ & $\ldots \ldots$ \\
\hline $\mathrm{O} \times \mathrm{A}$. & 587 & I, 556 & 620 & 39.8 & $92 \mathrm{I}$ & 59.2 & 8 & 0.5 & 7 & 0.5 \\
\hline $\mathrm{O} \times \mathrm{B} \ldots$ & 222 & $59 \mathrm{I}$ & 261 & 44.2 & 2 & 0.3 & 326 & $\begin{aligned} 5.5 \\
.5\end{aligned}$ & 2 & 0.3 \\
\hline $\mathrm{A} \times \mathrm{A}$. & 253 & 700 & I 29 & I8. 4 & $57 \mathrm{I}$ & $8 \mathrm{I} .6$ & $\ldots \ldots$ & $\ldots \ldots$ & $\ldots \ldots$ & $\ldots \ldots$ \\
\hline $\mathrm{B} \times \mathrm{B} \ldots$ & 48 & 140 & I8 & I 2.9 & $\ldots \ldots$ & $\ldots \ldots$ & I 22 & 87.1 & $\ldots \ldots$ & $\ldots \ldots$ \\
\hline $\mathrm{A} \times \mathrm{B} \ldots$ & 227 & 588 & 97 & I6. 5 & I 74 & 29.6 & I5I & $25 \cdot 7$ & I66 & 28.2 \\
\hline $\mathrm{O} \times \mathrm{AB} \ldots$ & I 12 & 342 & $2 I$ & $6 . \mathrm{I}$ & I 49 & 43.6 & 149 & 43.6 & 23 & 6.7 \\
\hline$A \times A B$. & 89 & 293 & 2 & 0.7 & I 25 & 42.6 & 70 & 23.9 & 96 & 32.8 \\
\hline $\mathrm{B} \times \mathrm{AB} \ldots$ & $4 I$ & I 22 & 5 & 4. I & $2 I$ & 17.2 & 57 & 46.7 & 39 & 32.0 \\
\hline $\mathrm{AB} \times \mathrm{AB}$. & 6 & I6 & $\ldots \ldots$ & $\ldots \ldots$ & 3 & I 2.5 & 5 & $37 \cdot 5$ & 8 & 50.0 \\
\hline Totals. . & I, 908 & 5,187 & I, 984 & $\ldots \ldots$ & I, 974 & & 888 & & $34 \mathrm{I}$ & \\
\hline
\end{tabular}

The families in the chart, taken from the protocols of Ottenberg, ${ }^{\mathrm{I}}$ illustrate these principles (Chart I). Almost two thousand families with a total of over five thousand children have since been analyzed. The investigations were conducted in all parts of the world. In Table II we have summarized all the data available at the time of writing. ${ }^{2}$

We note at a glance the small percentage of exceptions to the rule defined by von Dungern and Hirschfeld, that A or B appear in children only if they are present in one or both parents. Of a total of $5, \mathrm{I} 87$ children cited only 27 , that is, 0.5 per cent, are exceptions. Only I per cent of the children of group $\mathrm{O}$ by $\mathrm{O}$ marriages were not themselves $\mathrm{O}$; only 0.5 per cent of children of $\mathrm{O}$ by $\mathrm{A}$ marriages were of group $\mathrm{B}$, etc. These few exceptions may be expected and explained by errors in technique and by illegitimacy.

\section{Ottenberg, R.: J.A.M.A., 78, 873. I922.}

${ }^{2}$ Table II has been prepared from the data of workers listed in Table VII. A new series of ro7 families studied by K. Landsteiner and P. Levine has very kindly been placed at our disposal by the authors before publication. Only those families were included of which the groups of both parents were known. Studies by F. Schiff (Deutsche Ztschr. f. d. ges. gerichtl. Med., 9, 369. I927), and by N. W. Popoff (ilid., p. 411. 1927) were not included in the table because these authors do not report their data in a way to allow analysis. 
THE MENDELIAN MECHANISM

It is clear that not the groups as such are inherited but the presence or absence of the agglutinogens $\mathrm{A}$ and $\mathrm{B}$. The problem becomes now: Is it possible to describe a simple Mendelian mechanism that will fit the observed facts?

Von Dungern and Hirschfeld noticed that the heredity of the agglutinogen-agglutinin set $\mathrm{A}$ and $a$ (and likewise that of the separately considered $\mathrm{B}$ and $\beta$ ) was similar to that described by Mendel in his first experiments with peas. In the language of

TABLE III

Genetic Formulas of the Four Groups

\begin{tabular}{c|c|c|c}
\hline \multicolumn{3}{c}{ Group } \\
\hline 0 & $\mathrm{~A}$ & $\mathrm{~B}$ & $\mathrm{AB}$ \\
\hline $\mathrm{a}$ & $\begin{array}{c}\mathrm{AAbb} \\
\mathrm{Aabb}\end{array}$ & $\begin{array}{c}\mathrm{a} \text { a B B } \\
\mathrm{aaBb}\end{array}$ & $\begin{array}{c}\mathrm{AABB} \\
\mathrm{AaBB} \\
\mathrm{AABb} \\
\mathrm{AaBb}\end{array}$ \\
\hline
\end{tabular}

Mendelism, the serological elements $\mathrm{A}$ and $a$ are represented in the chromosomes by two allelomorphic genes $\mathrm{A}$ and a, respectively, of which $\mathrm{A}$ is the dominant and a the recessive. In a similar manner, the agglutinogen-agglutinin set $B$ and $\beta$ are repre-

TABLE IV

\begin{tabular}{|c|c|c|}
\hline Parents & Children Possible & $\begin{array}{l}\text { Children Not } \\
\text { Possible }\end{array}$ \\
\hline 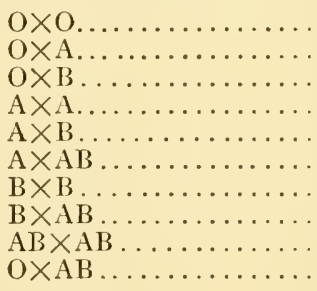 & $\begin{array}{l}\mathrm{O} \\
\mathrm{O}, \mathrm{A} \\
\mathrm{O}, \mathrm{B} \\
\mathrm{O}, \mathrm{A} \\
\mathrm{O}, \mathrm{A}, \mathrm{B}, \mathrm{AB} \\
\mathrm{O}, \mathrm{A}, \mathrm{B}, \mathrm{AB} \\
\mathrm{O}, \mathrm{B} \\
\mathrm{O}, \mathrm{A}, \mathrm{B}, \mathrm{AB} \\
\mathrm{O}, \mathrm{A}, \mathrm{B}, \mathrm{AB} \\
\mathrm{O}, \mathrm{A}, \mathrm{B}, \mathrm{AB}\end{array}$ & $\begin{array}{r}\mathrm{A}, \mathrm{B}, \mathrm{AB} \\
\mathrm{B}, \mathrm{AB} \\
\mathrm{A}, \mathrm{AB} \\
\mathrm{B}, \mathrm{AB} \\
\ldots \ldots \ldots \\
\ldots \ldots \ldots \\
\ldots \ldots \ldots \\
\ldots \ldots \ldots \\
\ldots \ldots \ldots \\
\ldots \ldots \ldots\end{array}$ \\
\hline
\end{tabular}

sented by the genes B and b, of which B is the dominant. Group O without any agglutinogens is thus genetically represented by the double recessive, aabb. Group A with agglutinogen $\mathrm{A}$ and agglutinin $a$ is genetically either AAbb (homozygous) or Aabb (heterozygous). All the genetical possibilities, according to the two-factor theory of von Dungern and Hirschfeld, may be represented as shown in Table III.

It is a simple exercise in genetics to calculate the groups of the children expected from all the possible crosses. This has been done in detail by Ottenberg. ${ }^{\mathrm{I}}$ The summary of all possibilities (on the theory of von Dungern and Hirschfeld) is given in Table IV.

A great deal of attention has been paid to the medico-legal application of the knowledge of the heredity of the blood groups. Reference to Table IV will show the

IOttenberg, R.: J. Immunol., 6, 363. 1921. 
cases in which it is possible to rule out the reputed father as definitely not the father of the child being examined. Thus if the mother is of group $\mathrm{O}$, the reputed father of group A, and the child of group B, it may confidently be stated that the reputed father is not the true one.

\section{THE ANTHROPOLOGICAL DATA}

Until recently the foregoing theory was universally accepted. In 1925 Felix Bernstein $^{\mathrm{I}}$ introduced a new genetic formula; and as this is based essentially on the frequency distribution of the blood groups in different peoples, it will be necessary to consider these first.

\section{TABLE V}

Frequencies of Blood Groups in Different Populations

\begin{tabular}{|c|c|c|c|c|c|c|}
\hline \multirow{2}{*}{ POPULATION } & \multirow{2}{*}{ AUThoR } & \multirow{2}{*}{$\begin{array}{l}\text { NUM- } \\
\text { BER } \\
\text { EXAM- } \\
\text { INED }\end{array}$} & \multicolumn{4}{|c|}{ Percentage in Each Group } \\
\hline & & & 0 & A & B & $\mathrm{AB}$ \\
\hline English. . & L. and H. Hirschfeld* & 500 & 46.4 & 43.4 & 7.2 & 3. I \\
\hline French..... & L. and H. Hirschfeld* & 500 & 43.2 & 42.6 & I I. 2 & 3.0 \\
\hline Germans....... & L. and H. Hirschfeld* & 348 & 40.0 & 43.0 & I 2.0 & 5.0 \\
\hline Russians........ & L. and H. Hirschfeld* & $\mathrm{I}, 000$ & 40.7 & 3 I. 2 & 21.8 & 6.3 \\
\hline Arabs.......... & L. and H. Hirschfeld* & 500 & 43.6 & 32.4 & 10.0 & 5.0 \\
\hline Indo-Chinese....... & L. and H. Hirschfeld* & 500 & 42.0 & 22.4 & 28.4 & 7.2 \\
\hline Chinese........... & Lui-Meng-Wlang† & $I, \infty \infty$ & 30 & 25 & & Io \\
\hline Indians. . . . . . . . . & L. and H. Hirschfeld* & $\mathbf{I}, \infty \infty$ & $3 \mathrm{I} \cdot 3$ & I9.0 & 4 I. 2 & 8.5 \\
\hline American Indians...... & Coca and Deibert $\ddagger$ & 862 & $77 \cdot 7$ & 20.2 & 2. I & $\circ$ \\
\hline Australian aborigines .......... & Tebbutt and McConnel§ & I $4 \mathrm{I}$ & 51.0 & 38.5 & 3 . & I. 5 \\
\hline American Indians (full blooded)... & Snyder & 453 & $9 \mathrm{I} .3$ & $7 \cdot 7$ & I. O & $\circ$ \\
\hline
\end{tabular}

* Lance!, 2, 675. I9I9.

$\dagger$ Cited from Hirschfeld, L.: Ergebn. d. I1yg., Bakleriol., Immunitälsforch. u. exper. Therap., 8, 367. 1926.

$\ddagger J$. Immunol., 8, 478. I923.

$\S M . J$. Australia, 1, 201. 1922.

II Am. J. Phys. Anthropol., 9, 233. 1926.

The study of human blood groups was approached from an entirely new angle by L. and H. Hirschfeld ${ }^{2}$ in I919. These workers, serving on the Balkan front as army physicians, found themselves among large numbers of representatives of many different nationalities. They determined the distribution of the four blood groups among sixteen peoples including over eight thousand persons, and found a difference in frequency which they assumed to be significant.

Similar studies have been conducted by other workers, and there have accumulated data on over one hundred populations. Table $\mathrm{V}$ gives examples of these different kinds of populations. The difference between the frequencies of the blood groups in the American Indians, the Indians of India, and the Europeans is very striking. More complete tables may be consulted in various papers (Bernstein, ${ }^{1}$ Snyder, ${ }^{3}$ Ottenberg, ${ }^{4}$ Hirschfeld, ${ }^{5}$ Lattes $\left.^{6}\right)$.

\footnotetext{
I Bernstein, F.: Ztschr. f. induk. Alstamm.u. Vercrbungs., 37, 2371925.

${ }^{2}$ Hirschfeld, L. and H.: Lancet, 2, 675. I9I9.

${ }^{3}$ Snyder, L.H.: Am. J. Phys. Anthropol., 9, 233. 1926.

${ }_{4}^{4}$ Ottenberg, R.: J.A.M.A., 84, I393. 1925.

${ }^{5}$ Hirschfeld, L.: Ergebn. d. Hyg., Bakteriol., Immunitätsforsch. u. exper. Therap., 8. 367. т926

${ }^{6}$ Lattes, L.: Die Individualität des Blutes. Berlin: Julius Springer, I925.
} 
As Table $\mathrm{V}$ indicates, and as more complete studies have corroborated, the fact seems to be that there is a definite frequency distribution among the various peoples which is related to their geographical distribution. It seems that the peoples of the north and west (English, French, German, Swedish, etc.) have more A individuals than B, while the peoples of the south and east (Indians, Japanese, Koreans, Chinese) on the contrary have more $\mathrm{B}$ individuals and fewer $\mathrm{A}$. The so-called "primitive races"-American Indians, Filipinos, Eskimos, and Australian aborigines-show a very high percentage of group $\mathrm{O}$ as compared to the European and Asiatic peoples.

The conclusion has been drawn that humans may be divided into various anthropological categories on the basis of their blood group frequencies. Various formulae have been proposed for determining these categories and different names assigned to them, but there has been no general acceptance of any formulation.

The high percentage of group $\mathrm{O}$ individuals in the so-called "primitive races" has led Hirschfeld, Bernstein, and others to conclude that man originally was a pure recessive made up entirely of individuals of group $\mathrm{O}$, and that $\mathrm{A}$ and $\mathrm{B}$ arose as recent mutations: the former in the north and west of Europe, the latter in the south and east, probably India. The American Indians and polar Eskimos are assumed to have separated from the rest of the humans before the mutations arose, and their few A and B individuals are taken to indicate later cross-breeding with Europeans and Asiatics.

But a number of considerations arise which require conciliation before these conclusions can be accepted. Foremost among these are the findings of Landsteiner and Miller, ${ }^{1}$ who demonstrated the existence of the human blood groups in the primates. Of twelve chimpanzees examined, the red cells of nine showed agglutinogen A. Of five orangs, two showed A and three B. The only gibbon blood examined showed agglutinogen A. These facts make it difficult to accept the conclusion that the factors A and $B$ are recent mutations. Landsteiner expresses as more probable the hypothesis that "the group specific factors appeared in the phylogeny of the primates prior to the genesis of man." It is furthermore noted that peoples certainly of different anthropological categories, as the Hungarians and the South Chinese, have similar group frequencies, while within the same race diferences have been found as great as those which are supposed to be significant of racial differences. The latter observation was made especially by Grove ${ }^{2}$ in a recent study of the Ainu.

\section{THE THEORY OF BERNSTEIN}

The statistical data outlined above have been accepted by Bernstein ${ }^{3}$ and made the basis of a mathematical analysis from which the conclusion was reached that the blood groups are inherited, not according to two independent pairs of genes as described by von Dungern and Hirschfeld, but according to three multiple allelomorphs lying in the same chromosome.

According to Bernstein, the human race began as pure recessive or group $\mathrm{O}$ with a genetic formula which he gives as $\mathrm{R} R$. The gene $\mathrm{R}$ then mutated to $\mathrm{A}$, giving in-

I Landsteiner, K., and Miller, P.: J. Exper. Med., 42, 863. 1925.

${ }^{2}$ Grove, E. F.: J. Immunthol., 1 2, 251. 1926.

3 Bernstein, L.: loc. cil. 
dividuals $\mathrm{AR}$ and $\mathrm{AA}$, forming group $\mathrm{A}$. A second mutation occurred later in the same gene to $\mathrm{B}$, giving individuals $\mathrm{BR}$ and $\mathrm{BB}$, or group $\mathrm{B}$. The three genes $\mathrm{R}, \mathrm{A}$, and $\mathrm{B}$ form the group of triple allelomorphs of which $\mathrm{R}$ acts as a recessive gene. Intermarriages of the mutated $A$ and $B$ groups gave $A B$ individuals. There are thus in the four blood groups of Landsteiner only six genetic types, according to Bernstein.

TABLE VI

Genetic Formulate of Bernstein

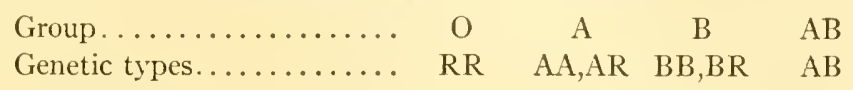

This new terminology does not affect the original observation of von Dungern and Hirschfeld that A and B do not appear in the children if they are absent in the parents. We have here only a new Mendelian mechanism that in the opinion of its author better fits the facts described by all workers. Furuhata and Kishi' recently have independently proposed a formula which is substantially the same as Bernstein's.

Bernstein first attempts to show that the two-factor pair theory is untenable because, by calculation, it seems to require in nearly all races a much larger percentage of group $\mathrm{AB}$ than the statistical data actually show. Bernstein's method of showing this is to represent the frequency of gene $\mathrm{A}$ by the letter $\mathrm{p}$, a by $\mathrm{p}, \mathrm{B}$ by $\mathrm{q}$, and b by $\mathrm{q}$. Then the frequencies of group $\mathrm{O}$ or aabb should $b e \mathrm{p}^{2} \cdot \overline{\mathrm{q}}^{2}$. In a similar manner he calculates the expected frequencies of the other groups according to the two-factor hypothesis. He derives from these the ratio:

$$
(\overline{\mathrm{A}}+\overline{\mathrm{AB}}) \cdot(\overline{\mathrm{B}}+\overline{\mathrm{AB}})=\overline{\mathrm{AB}},
$$

that is, the sum of the frequencies of groups $A+A B$ times the sum of the frequencies of groups $\mathrm{B}+\mathrm{AB}$ should equal the frequency of $\mathrm{AB}$ (on the Hirschfeld theory).

Working with his multiple-allelomorph hypothesis, Bernstein sets $\mathrm{p}$ equal to the frequency of gene $\mathrm{A}, \mathrm{q}$ to the frequency of gene $\mathrm{B}$, and $\mathrm{r}$ to the frequency of gene $\mathrm{R}$. Since these are all the possibilities, it follows that $p+q+r=I$. He then calculates from the observed frequencies of $\mathrm{O}, \mathrm{A}, \mathrm{B}$, and $\mathrm{AB}$ the values of $\mathrm{p}, \mathrm{q}$, and $\mathrm{r}$ to find if their sum is equal to $\mathrm{I}$, as it should be. This Bernstein does as follows:

The frequency of group $O(R R)$ is $r^{2}$

The frequency of group $\mathrm{A}(\mathrm{AR}, \mathrm{AA})$ is $2 \mathrm{pr}+\mathrm{p}^{2}$

The frequency of group $B(B R, B B)$ is $2 q r+q^{2}$

The frequency of group $\mathrm{AB}(\mathrm{AB})$ is $2 \mathrm{pq}$

Therefore

$$
\begin{aligned}
& \bar{O}+\bar{A}=r^{2}+2 p r+p^{2}=(r+p)^{2}, \\
& \bar{O}+\bar{B}=r^{2}+2 q r+q^{2}=(r+q)^{2} .
\end{aligned}
$$

From which

$$
\begin{aligned}
& q=I-\sqrt{\bar{O}+\bar{A}}, \\
& p=I-1 \overline{\bar{O}+\bar{B}}, \\
& r=1 \overline{\bar{O}},
\end{aligned}
$$

${ }^{1}$ Furuhata, T., and Kishi, T.: Japan Med. World, 7, I. I927. 
and, finally, since $p+q+r=I$,

$$
(\mathrm{I}-\sqrt{\overline{\mathrm{O}}+\overline{\mathrm{B}}})+(\mathrm{r}-\sqrt{\overline{\mathrm{O}}+\overline{\mathrm{A}}})+\sqrt{\overline{\mathrm{O}}}=\mathrm{r} .
$$

Bernstein now takes for each population the observed values of $\mathrm{O}, \mathrm{A}, \mathrm{B}$, and $\mathrm{AB}$ and substitutes them in the two formulae:

$$
\begin{aligned}
& (\overline{\mathrm{A}}+\overline{\mathrm{AB}}) \cdot(\overline{\mathrm{B}}+\overline{\mathrm{AB}})=\overline{\mathrm{AB}}, \\
& \left(\mathrm{I}-\mathrm{V}^{\prime} \overline{\mathrm{O}+\overline{\mathrm{B}}}\right)+(\mathrm{I}-\sqrt{\overline{\mathrm{O}}+\overline{\mathrm{A}}})+\sqrt{\overline{\mathrm{O}}}=\mathrm{I} .
\end{aligned}
$$

He finds that in formula (I), which, he asserts, tests the two-factor hypothesis of von Dungern and Hirschfeld, the left side of the equation is always greater than the right. On the other hand, he finds that in formula (2) the calculated value of $p+q+r$ comes out very nearly equal to $\mathrm{I}$. His conclusion is that the two-factor hypothesis is wrong, and that the three-multiple-allelomorph hypothesis is correct. He points out that critical cases for the testing of his theory should occur in the marriages of group AB persons. On his hypothesis such unions (with any of the groups) can never produce group $\mathrm{O}$ children as the recessive gene $\mathrm{R}$ is of necessity absent in group AB. In addition it is evident that marriages of group $A B$ with group $O$ can produce neither group $\mathrm{O}$ nor group $\mathrm{AB}$ children as the individual genes $\mathrm{A}$ and $\mathrm{B}$ here can only unite with the recessive genes $\mathrm{R}$ to produce group $\mathrm{A}$ and group $\mathrm{B}$. On the other hand, on the two-factor hypothesis, unions of group AB may result in children of any group.

Table VII allows us to make immediate comparison of the number of exceptions to the two hypotheses. There are 27 children out of 5,187 in which Group A or B appears though it was absent in both parents, giving against any Mendelian interpretation which assumes the dominance of $\mathrm{A}$ and $\mathrm{B}, .5 \%$ exceptions. With these we may compare those specific exceptions which apply only to the Bernstein theory. There are $5^{\mathrm{I}}$ exceptions in $\mathrm{AB}$ marriages to this theory out of a total of $77 \mathrm{r}$ children from such marriages, giving $6.6 \%$ of exceptions. There are then $\mathrm{I}_{3}$ times as many exceptions proportionately against the Bernstein formula as against the dominance of $\mathrm{A}$ and $\mathrm{B}$ on any hypothesis as to genes.

The genetical evidence requires that judgment be reserved. What can be done with the mathematical computations of Bernstein? Does the failure of the observations to fit the formula $(\overline{\mathrm{A}}+\mathrm{AB}) \cdot(\mathrm{B}+\mathrm{AB})=\overline{\mathrm{AB}}$ mean that the two-factor hypothesis is untenable? Here the question arises: Under what conditions are mathematical formulae binding in the study of the biological phenomena?

When the facts underlying a given phenomenon are thoroughly understood, a mathematical formula may be employed to express concisely the interrelationship of the factors and may then actually lead to the discovery of new laws. This situation does not exist in the blood group investigations. There are other possible, indeed plausible, exp'anations of the failure of calculation to match actual percentages. For example, Hirschfeld ${ }^{1}$ has pointed out the remarkable fact that of the fifty-five recorded cases of children arising from $\mathrm{O}$ mothers and $\mathrm{AB}$ fathers there is not a single $\mathrm{AB}$ child, while there are on record nineteen cases of $\mathrm{AB}$ children from $\mathrm{AB}$ mothers

${ }^{I}$ Hirschfeld, L.: Zischr. f. Immunitätsforschung. u. exper. Therap., 43, 485. 1925; Ergebn. v. Weichlardl, 8, 307. 1926 . 
and $\mathrm{O}$ fathers. He postulates that the child dies during the pregnancy because of the strong incompatibilities of its cells and the serum of its mother. Whether Hirsch-

\section{TABLE VII}

Observed Exceptions to Theories of Mendelian Heredity of the Blood Groups ${ }^{\mathrm{x}}$

\begin{tabular}{|c|c|c|c|c|c|c|c|c|}
\hline \multirow[t]{2}{*}{ Authors } & \multirow{2}{*}{$\begin{array}{l}\text { Total } \\
\text { No. OF } \\
\text { FAM- } \\
\text { ILIES }\end{array}$} & \multirow{2}{*}{$\begin{array}{l}\text { Total } \\
\text { No. OF } \\
\text { CHIL- } \\
\text { DREN }\end{array}$} & \multicolumn{2}{|c|}{$\begin{array}{l}\text { EXCEPTIONS } \\
\text { TO ANY } \\
\text { MENDELLAN } \\
\text { HYPOTHESIS }\end{array}$} & \multirow{2}{*}{$\begin{array}{l}\text { FAM- } \\
\text { ILIES } \\
\text { WITH } \\
\text { AB } \\
\text { PAR- } \\
\text { ENTS }\end{array}$} & \multirow{2}{*}{$\begin{array}{c}\text { Total } \\
\text { No. OF } \\
\text { CHIL- } \\
\text { DREN } \\
\text { WITH } \\
\text { AB } \\
\text { PAR- } \\
\text { ENTS }\end{array}$} & \multicolumn{2}{|c|}{$\begin{array}{c}\text { EXCEPTIONS TO } \\
\text { BERNSTELN'S } \\
\text { HYPOTHESIS }\end{array}$} \\
\hline & & & Families & $\begin{array}{l}\text { Chil- } \\
\text { dren }\end{array}$ & & & Families & $\begin{array}{l}\text { Chil- } \\
\text { dren }\end{array}$ \\
\hline von Dungern and Hirschfeld* ........ & 72 & 203 & 0 & 0 & 9 & 26 & 4 & 9 \\
\hline 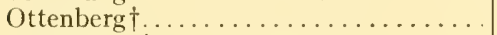 & 69 & 127 & 0 & 0 & 8 & 17 & 0 & 0 \\
\hline 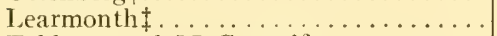 & 40 & 100 & I & I & 6 & I0 & 3 & I 2 \\
\hline Tebbutt and MIcConnel\$............ & I 2 & 45 & 0 & 0 & 2 & I 2 & 0 & 0 \\
\hline 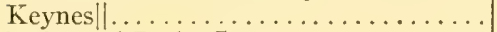 & I 2 & 36 & 0 & 0 & 3 & 9 & 0 & 0 \\
\hline Dyke and Budge $1 \ldots . . \ldots \ldots \ldots$ & 97 & 97 & 0 & ○ & 2 & 2 & I & I \\
\hline Jervell** $\ldots \ldots \ldots \ldots \ldots \ldots \ldots \ldots$ & 32 & 73 & 0 & ○ & 6 & 17 & ○ & 0 \\
\hline 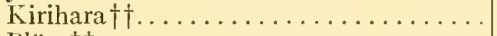 & I 20 & 339 & 0 & 0 & 20 & 55 & 3 & 3 \\
\hline Plüss $t \ldots \ldots \ldots \ldots \ldots \ldots \ldots \ldots$ & $8_{4}$ & 220 & I & I & 10 & 25 & 2 & 2 \\
\hline 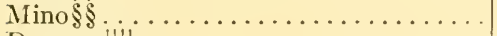 & 90 & 253 & 6 & I 2 & 9 & 25 & $\mathbf{I}$ & 2 \\
\hline Dossena $\mid \| \ldots \ldots \ldots \ldots \ldots \ldots$ & 150 & 150 & o & 0 & 15 & 105 & 2 & 2 \\
\hline Staquet $\ldots \ldots \ldots \ldots \ldots \ldots \ldots \ldots \ldots \ldots \ldots \ldots \ldots \ldots \ldots$ & 56 & 244 & I & I & $\overline{6}$ & $4 \mathrm{I}$ & I & 9 \\
\hline H. and L. Hirschfeld and Prokman *** .. & 62 & I 59 & I & I & 5 & I4 & o & 0 \\
\hline Snydert†t . . . . . . . . . . . . . & 200 & 695 & ० & 0 & 9 & 38 & o & 0 \\
\hline 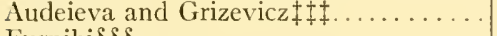 & 84 & I95 & 3 & 6 & 8 & I 8 & 2 & 3 \\
\hline Furnihi $\$ \$ \ldots \ldots \ldots \ldots \ldots \ldots \ldots$ & 9 & IS & 0 & 0 & 9 & I8 & 3 & 4 \\
\hline Furuhata et al. \|\|$\| \ldots \ldots \ldots \ldots \ldots \ldots$ & 399 & I, I 2 I & 4 & 4 & 64 & 2 I 3 & I & $\mathbf{I}$ \\
\hline Thomsen & 2 I 3 & 660 & I & I & 46 & 165 & I & I \\
\hline Landsteiner and Levine ${ }^{* * * *} \ldots . . . \ldots$ & 107 & 4.52 & $\circ$ & ० & IO & 42 & I & 2 \\
\hline 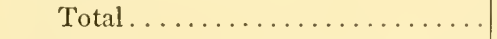 & I , 908 & 5,187 & IS & 27 & 247 & 771 & 25 & 51 \\
\hline
\end{tabular}

$x$ Since the preparation of this article there has appeared a paper by H. Kliewe and R. Nagel (Klin. Wchnschr., 6, 23.32. 1027 ) in which the heredity of the blood groups in 28 families with 60 children is reported. There are no exceptions to the rules of von Dungern and Hirschfeld. There are, however, 4 families of AB by $O$ marriages with ro children, and of these 5 children are exceptions to the Bernstein hypothesis. Among the exceptions there is a group AB child of a group $O$ mother and there are two group $O$ children of a group AB mother. These three exceptions cannot be ascribed to illegitimacy by Bernstein since no matter what the group of the father a group $O$ mother cannot have an AB child, nor can a group AB mother have a group $O$ child on Bernstein's theory. Cases of this type should be very carefully checked up hy examination of serum and cells, since one such case absolutely proven completely invalidates Bernstein's hypothesis. The cases of Kliewe and Nagel are not included in Table V'II.

O. Weszeczky (Biochem. Zfschr., I07, 159. I920) and J. A. Buchanan (J.A.MI.A., 78, 89. I922) have reported respectively three and nine exceptions to any Mendelian hypotheses. Their technique has been so severely criticized and their results are so out of proportion to those of all other workers that one is justified in omitting their families from this table.

* Ztschr.f. Immunitälsforschung. u. exper. Therap., 6, 284. I910.

$\dagger J . A . M . A ., 78,873.1922$.

‡. Gen., 10, I41. I920.

$\S$ Loc. cit.

|| Blood Transfusion, "Oxford Med. Pub." I92r.

I Proc. Roy. Soc. Med., Pathol. Sec., 16, 43. 1923.

** Deutsche Ztsclir. f. d. ges. gerichtl. Med., 3, 42. 1923.

†† Ztschr.f. klin. Med., 99, 522. 1924.

施lhweiz. med. Wchnschr., 54, 544. 1924. Fuller data are given in Lattes, L.: loc. cit.

$\$ \S$ Policlinico, 31, 293. 1924.

III Ann. di. ostet., 46, 355. 1924.

IT Arch. intcrnat. de méd. exper., 2, 71. 1925.

*** Cited from Hirschfeld, L.: loc. cit.

†† Loc. cit.

$\ddagger \ddagger$ Cited from Lattes, L.: loc. cit.

$\S \S$ Cited from Hirschfeld, L.: loc. cit.

|l||| Loc. cit.

II: Compl. rend. Soc. de biol., 96, I496. I927.

*oww. J. Immunol., I2, 415. I926.

feld's opinion is correct cannot yet be said, but the facts are extremely suggestive and the possibility cannot be excluded. 
However, there are four cases where an $\mathrm{O}$ mother is stated to have given birth to an $\mathrm{AB}$ child, but since in each case the father was either an $\mathrm{A}$ or a $\mathrm{B}$ there was either an illegitimacy or an error in technique. Also in the obstetrical material cited by Hirschfeld ${ }^{\mathrm{r}}$ there are seven instances in which an $\mathrm{O}$ mother is stated to have given birth to an $\mathrm{AB}$ child but nothing is said as to the group of the father. It is to be noted that if all of these cases are accepted as correct they would count against Hirschfeld's explanation of a lethal effect of incompatibility between $\mathrm{O}$ mothers and $\mathrm{AB}$ children, but at the same time they would rule out Bernstein's multiple-allelomorph theory. On account of our inability to examine the original description of these cases, they are not included in our table as exceptions to Bernstein's theory. Their existence, however, shows how seriously further accurate work is needed. (It should also be pointed out that in almost none of the recorded instances is mention made of the examination of the serum as well as the cells of each individual.)

TABLE VIII

Differing Values of "R" in Various Races on Bernstein's Assumption

\begin{tabular}{|c|c|c|c|c|c|c|c|c|c|c|}
\hline \multirow{2}{*}{ Population } & \multirow{2}{*}{ INVESTIGATION } & \multicolumn{4}{|c|}{ FREQUENCY OF GROUPS } & \multirow{2}{*}{$\mathrm{p}$} & \multirow{2}{*}{$q$} & \multirow{2}{*}{$\underbrace{10}_{11}$} & \multirow{2}{*}{ 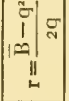 } & \multirow{2}{*}{ 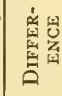 } \\
\hline & & $\mathrm{O}$ & A & $\mathrm{B}$ & $\mathrm{AB}$ & & & & & \\
\hline 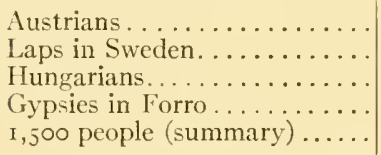 & $\begin{array}{l}\text { L. and H. Hirschfeld } \\
\text { Rietz.............. } \\
\text { Verzar and Weszeczky } \\
\text { Verzar and Weszeczky } \\
\text { Verzar and Weszeczky }\end{array}$ & $\begin{array}{l}42 \\
5 \mathrm{I} \\
3 \mathrm{I} \cdot 8 \\
\mathrm{I} 8 \cdot 3 \\
3 \mathrm{I}\end{array}$ & $\begin{array}{l}40 \\
42 \\
39.7 \\
40.8 \\
38.8\end{array}$ & $\begin{array}{c}10 \\
3 \\
16.1 \\
38.7 \\
18.8\end{array}$ & $\begin{array}{r}8.0 \\
4 \\
\text { I } 2.4 \\
2.2 \\
\text { I } 2.2\end{array}$ & $\left|\begin{array}{c}27 \cdot 9 \\
30 \cdot 8 \\
24 \cdot 5 \\
29 \cdot 4\end{array}\right|$ & $\begin{array}{r}9 \cdot 5 \\
\cdots \\
15 \cdot 5 \\
23 \cdot 1 \\
17 \cdot 0\end{array}$ & $\begin{array}{l}64 \cdot 8 \\
71 \cdot 4 \\
56 \cdot 4 \\
42 \cdot 8 \\
55 \cdot 6\end{array}$ & $\begin{array}{l}47 \cdot 9 \\
39.8 \\
44 \cdot 5 \\
72.2 \\
46.8\end{array}$ & $\begin{array}{l}16.9 \\
31.6 \\
11.9 \\
29.4 \\
8.8\end{array}$ \\
\hline
\end{tabular}

But does not the remarkable fit of the calculations to the formula $p+q+r=I$ command acceptance of Bernstein's hypothesis? Bernstein's formula may be tested by deriving from his assumptions a value of $\mathrm{r}$ in a manner different from his. Thus the probable frequency of group $B$, which is genetically BR or BB, is $\left(2 q r+q^{2}\right)$ as above. 'Then:

$$
\begin{array}{r}
2 q r+q^{2}=\bar{B}, \\
2 q r=\bar{B}-q^{2}, \\
r=\frac{\bar{B}-q^{2}}{2 q} .
\end{array}
$$

Similarly, in terms of $A$ and $p, r$ is found to equal $\frac{\bar{A}-p^{2}}{2 p}$. Now, if we take concrete instances and derive $\mathrm{p}$ and $\mathrm{q}$ by the formulae of Bernstein and then derive $\mathrm{r}$ according to both of the two new formulae which follow from Bernstein's assumptions, we find in many cases a wide divergence from the value of $r$ when it is taken as equal to $\sqrt{\mathrm{O}}$. We find now that $p+q+r$ does not equal $\mathrm{r}$ as frequently as formerly. We append a few such calculations in Table VIII.

In view of these considerations it is perhaps wiser not to accept the multiple-

${ }^{1}$ Hirschfeld, L.: ibid., p. 420. I926. 
allelomorph hypothesis as proved until enough genetical data are accumulated to compel its acceptance. ${ }^{\mathbf{I}}$

\section{HEREDITY OF NEW AGGLUTINABLE FACTORS IN HUMAN BLOOD}

Entirely new vistas of investigation have arisen with the recent discovery of new agglutinable factors in human blood, by Landsteiner and Levine. ${ }^{2}$ By absorbing a number of anti-human-blood immune sera from rabbits with the blood corpuscles of certain individuals, regardless of the group, fluids were obtained from a few sera which gave a sharp differentiation of individual human bloods within the common blood groups. A number of such factors have been found which are arbitrarily designated as $\mathrm{M}$ and $\mathrm{P}$. It is possible to divide humans into $\mathrm{M}$-positive and M-negative, or into P-positive or P-negative, individuals. A somewhat higher incidence of M among colored than white individuals was found. The heredity of the M factor has

TABLE IX

HeredtTy of the "M" Factor

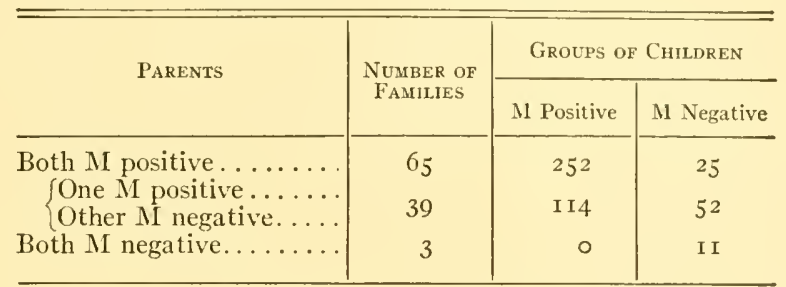

been studied by Landsteiner and Levine ${ }^{3}$ in more than one hundred families. Their results are in keeping with the assumption that $\mathrm{M}$ is inherited as a Mendelian dominant. In Table IX the families are arranged in three classes according to the presence or absence of the factor $\mathrm{M}$ in the parents.

The significance of the facts of the inheritance of the iso-agglutinable blood elements in the biology of man invites consideration. It is the desire of the student of genetics to establish for the organism with which he is working its genetic composition, to draw on a chromosome map the positions of the genes, as T. H. Morgan and his co-workers have done for the fruit fly, Drosophila. Whether the forty-eight chromosomes of man will ever be charted so, no one dare say. But in order that it be done, the linkage relationships of hundreds of characters, whose inheritance will permit simple Mendelian analysis, need to be studied. Thus far, no example of linkage in humans has been described.

So universal a character as the blood group offers a vast field for the study of linkage, and the possibilities are multiplied many times when the new agglutinable

A. A. Mendes-Correa ( $L e$ Sang, I, 322. I927) in a paper which appeared since the preparation of this article claims that Bernstein's mathematical assumptions are based on an identity and are therefore not valid.

${ }^{2}$ Landsteiner, K., and Lavine, P.: Proc. Soc. Exper. Biol. Eo Med., 24, 600. I927.

3 Ibid., p. 941. I927. 
factors described by Landsteiner and Levine are added. The possibility of correlation between blood groups and other inherited characters whose Mendelian mechanisms are known has been sought. Most of the attempts to establish such correlations have given either negative or inconclusive results. There have been several claims of linkage between blood group genes and others. Of these the most important are the claims of linkage with diphtheria susceptibility or immunity. ${ }^{\mathrm{I}}$ Critical analysis has shown the evidence to be insufficient to establish linkage. ${ }^{2}$ But this work should be continued because it offers one of the most fruitful approaches to the determination of the mechanism of heredity in man.

Some new fundamental questions are opened by the work of Yamakami ${ }^{3}$ and of Landsteiner and Levine, ${ }^{4}$ who independently demonstrated the presence of the isoagglutinable substances in human sperm. An interesting situation then arises in the case of a heterozygous man belonging to group A whose sperm though containing agglutinogen A may give rise to offspring of group $\mathrm{O}$ (which cannot contain agglutinogen A). Unless one assumes that there are two kinds of spermatozoa in the individual's semen, the one containing agglutinogen $\mathrm{A}$ and the other not, one must conclude that a spermatozoön can carry genes for a character which is incompatible with a character shown by the spermatozoön itself.

${ }^{x}$ Hirschfeld, L. and H., and Brokman, H.: J. Immunol., 9, 57I. I924.

${ }^{2}$ Levine, P.: J. Immunol., то, 283. 1926; Snyder, L. H.: Ztschr. f. Immunitätsforschung. $u$. exper. Therap., 49, 464. I926.

3 Yamakami, K.: J. Immunol., I2, I85. I926.

${ }_{4}^{4}$ Landsteiner, K., and Levine, P.: J. Immunol., 12, 4I5. 1926. 


\section{CHAPTER LXX}

\section{ANTIBACTERIAL SERA}

\section{F. M. HUNTOON AND R. H. HUTCHISON}

H. K. Mulford Co., Philadelphia, Pa.

Antibacterial sera are those containing immune bodies whose action is directed against bacteria as entities or against the products of their disintegration, or possibly against some product of their metabolism. This action is in contradistinction to the action of antitoxins, in which the antibody acts by neutralizing a toxic principle elaborated by the organism, either as a product of metabolism or as a result of ferment action on some of the substances present in its environment.

The antibacterial immune bodies also apparently differ from the antitoxins in their physico-chemical make-up, since they are found in a different fraction of the serum-one that is precipitated by a comparatively low concentration of ammonium sulphate.

The immune bodies classified as "antibacterial" differ among themselves in their mode of action. Agglutinins and precipitins, which are probably identical, act directly on their antigen without the aid of a third factor. Opsonins or tropins, the so-called "protective" antibody, the complement fixation antibody, and the bacteriotrophic or bacteriolytic antibodies perform their specific function only in conjunction with complement. Since agglutinins and precipitins are believed to have little to do with the curative action of such sera, it follows that the therapeutic value of antibacterial sera depends on those antibodies which require complement, and also, in the case of the tropins, on the ability of the patient's phagocytes to function. In the treatment of disease with antibacterial sera, the time factor is, therefore, a very important consideration since a failure on the part of the patient to supply active complement or active leukocytes involves the failure of the immune bodies to perform their specific function. Many disappointments of specific therapy may be attributed to this cause.

Antibacterial sera as a whole have not had the wide use, nor have the results obtained been comparable to those obtained with diphtheria antitoxin. There are various reasons for this, including failure to use such sera early in the disease, the tendency being to employ such measures only as a last resort.

Emphasis on the importance of early treatment with antibacterial sera is justified by the results that have been obtained in the use of anti-meningococcic serum. This is well illustrated in Table I, compiled by Blackfan ${ }^{\mathrm{x}}$ from the published reports of various observers.

Another, and probably the most important, reason for the lesser efficiency of antibacterial sera is the extreme specificity which is an inherent quality of such sera. For instance, monovalent sera against any fixed type of the pneumococcus have no

I Blackfan, K. D.: Medicine, r, I39. I922. 
influence over any heterologous type, nor are monovalent anti-meningococcus sera of value in treatment of an infection due to strains other than that used in producing the serum.

By way of contrast, we have, in the case of the diphtheria bacillus, at least five immunological types distinguished by the action of the antibacterial immune bodies. All of these produce toxins which are identical in effect. The antitoxin produced in response to the injection of any of these toxins will neutralize all such toxins, regardless of the immunological type of the diphtheria bacillus from which they are derived. This is also true of the tetanus bacillus. Such findings indicate that, since the toxins are identical, they have a common origin, and are in accord with the idea that they are a degradation product of some component of the medium in which they grow, and not a direct product of the organisms themselves.

On the other hand, bacterial antibodies are produced in direct response to stimulation by the bacterial bodies themselves, or their components, and so tend to be highly specific. In a disease process the antigens or bacteria present are highly specific. Since both the serum and the antigens are so specific, a useful serum must

TABLE I

Mortality Percentage Compared with Day of Beginning Therapy

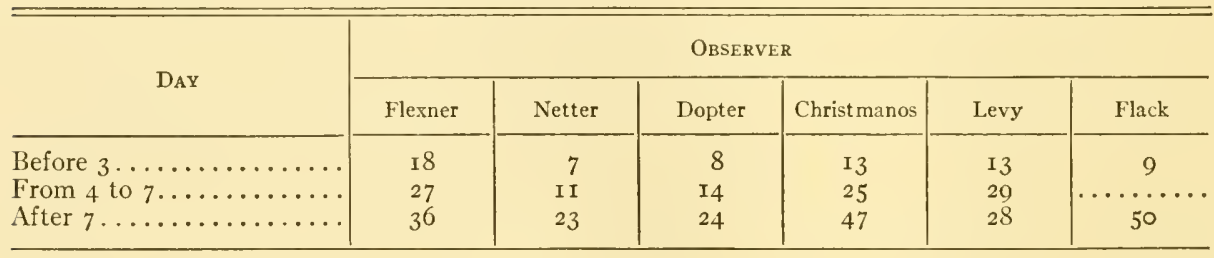

contain antibodies specific for the organisms likely to be encountered, i.e., it must be polyvalent enough to have a wide range. This requisite renders the proper immunization of serum-producing animals a difficult matter.

The best established of the antibacterial sera is anti-meningococcus serum for the treatment of epidemic cerebrospinal meningitis. This serum has had wide usage and is generally accepted as being specific for this disease. But even here, with an organism as homogeneous and true to type as the meningococcus, there exist several well defined immunological subgroups, and it is necessary to include all of them in the immunizing doses in order to produce a generally useful serum. Occasionally, aberrant strains are found which do not fall in the established groups, and as these appear their use is required for immunization. So, at the time of writing, from twelve to fifteen different strains are employed for immunization.

When we attempt to analyze the effects of antibacterial sera we find little information as to the ultimate mode of action of the antibacterial immune bodies as curative agents within the body. We do know that, when specific serum is injected in pneumococcus infections, the organisms present in the bloodstream promptly agglutinate. We know that they presently disappear from the bloodstream and in the presence of sufficient antibody do not reappear. It has also occurred that, in cases of pneumococcus meningitis, the administration intraspinally of pneumococcus antibody 
solution has caused the organisms present to become phagocy tized. This phenomenon also occurs in cases of cerebrospinal meningitis treated with anti-meningococcic serum.

The blood of monkeys suffering from pneumonia, when mixed with pneumococcus antibody solution, is definitely bactericidal in vitro, whereas the blood alone or the antibody solution alone does not have this effect. Anti-pneumococcus serum added to normal rabbit's blood shows this same effect. ${ }^{\text {r }}$

It is probable that the therapeutic result obtained with the various antibacterial sera is due to a summation of the effects of the various antibodies present.

The use of antibacterial sera as therapeutic agents presupposes an accurate diagnosis of the infecting organisms. Bacteriological diagnosis, however, can be carried to an extreme, as in the case of pneumococcus infections where the delay in waiting for an accurate typing of the organism may render the use of any serum of doubtful value. The successful use of antibacterial sera now rests not so much on the question of laboratory production of adequate serum, but upon their clinical use in a proper manner, taking into consideration the time factor and the question of specificity.

\section{ANTI-ANTHRAX SERUM}

Anti-anthrax serum was probably the first antibacterial serum to prove of curative value in the treatment of infectious disease. First developed by Marchoux (1895) in France and in the same year by Sclavo in Italy and later by Sobernheim (1897) in Germany, the serum was widely used in both human and veterinary medicine in Europe and South America with excellent results.

Preparation of the first potent serum in the United States is credited to the Bureau of Animal Industry of the United States Department of Agriculture, and for a time was supplied to the medical profession by the Bureau until it came to be supplied by several biological laboratories. The production of this serum has been recently summarized by Kelser, ${ }^{2}$ as follows:

"Animals used for the production of the serum are first immunized by either the simultaneous or Pasteur method. Two weeks after such treatment each serum animal is given twice the amount of vaccine it received in the immunization treatment. This is usually $2 \mathrm{cc}$. of Pasteur No. 2 vaccine or 2 cc. of spore vaccine of Pasteur No. 2 virulence. After the lapse of one week 4.cc of the vaccine are given and this followed, after a further period of one week, with ro cc. of vaccine. One week following such injection the first dose of living, virulent culture is given. The amount inoculated is one-hundredth of the amount of growth from a 24 -hour agar culture that can be picked up in the loop portion of a standard platinum loop. Injections of virulent culture are then repeated at weekly intervals. For the second dose I loopful of culture is administered." The process is then continued in a series of gradually increasing doses until a high immunity is established, as shown by the presence and increase in specific antibodies-precipitins, agglutinins, and amboceptors.

Acquired immunity to anthrax is not permanent. Recovery from the infection apparently depends upon phagocytosis. This was first emphasized by Metchnikoff, and it was shown by Wright that opsonins in the serum, because of their influence on phagocytosis, are of value in artificial or passive immunity. According to Kolmer,

I Bull, C. G.: personal communication.

${ }^{2}$ Kelser, R. A.: Manual of Veterinary Bacteriology. I927. 
"Anthrax bacilli are also able to produce an aggressin or toxic substance exerting negative chemotaxis; a part of the action of anti-anthrax serum is the neutralization of these substances, which thereby facilitates phagocytosis." I The presence of protective antibodies in the serum can be demonstrated by animal experiment.

Much statistical data has accumulated attesting the therapeutic value of antianthrax serum, but as yet no satisfactory method of standardization has been worked out. The Health Committee of the League of Nations, at its 1924 session, suggested that the time had come for a more thorough investigation of the methods of titration and standardization of the serum, in order to overcome some of the uncertainties now existing on these points.

\section{ANTI-DYSENTERIC SERUM}

The two principal types of dysentery bacilli are differentiated by their toxin-producing properties. The Shiga-Kruse group, which appears to be the principal causative factor in tropical countries, produces a soluble exotoxin having a pronounced neurotoxic effect. The second group includes strains (paradysentery) which produce no soluble exotoxin. These are the so-called Flexner and Hiss types, which are principally responsible for the dysentery in this country. Anti-dysenteric serum prepared for the Flexner type is an antibacterial serum and is without effect in the treatment of the Shiga-Kruse infection. It has proved of little value in the treatment of Flexner-Hiss infections. It is not logical to believe that the introduction into the bloodstream of antibodies directed against the organisms themselves would have any effect on this disease, since the causative organisms are confined to the intestine.

The anti-dysenteric sera supplied commercially are usually polyvalent sera prepared by immunizing horses against the various types of dysentery bacilli, including those of Shiga, Kruse, Flexner, and Hiss. They therefore contain both antibacterial and antitoxic immune bodies. Since the therapeutic value depends principally on the antitoxin content, they have been found of most value in European and Asiatic countries where Shiga-Kruse infections are most common.

Preparation.-Animals must be immunized with great care, owing to the toxic property of the organisms. It is well, for the first few doses, to treat the centrifuged organisms with a weak solution of sodium hydroxide before injection. Afterward, periodic injections of whole culture may be employed, but the condition of the animal must be watched constantly.

Siandardization is done by means of agglutination tests which must conform to the methods and standards required by the Hygienic Laboratory of the Public Health Service. Standardization by means of a toxin-antitoxin reaction has been tried, and it has been found that a good serum in amounts of $\mathrm{I} \mathrm{cc}$. will protect rabbits against Io M.L.D. of the toxic material.

We have used the following procedure in the preparation of toxic material for this test. The bacilli were grown on neutral plain agar for several generations, and finally in Blake bottles on the same media. The growth was washed off in salt solution, centrifuged, washed rapidly with alcohol and ether, and then placed in a desiccating jar and dried rapidly. A weighed quantity of the dried material was mixed with sterile sand and ground in a mortar with a measured amount of salt solution. The resulting mass was centrifugated several times, and the opalescent supernatant was employed for injection purposes. Doses of $0 . \mathrm{x}-0.2 \mathrm{cc}$. injected intravenously in a rabbit resulted, within two or three days, in a progressive paralysis

Kolmer, J. A.: Infection, Immunity and Biologic Therapy (3d ed.). I924. 
affecting the limbs. Where paralysis affected all four limbs the rabbit usually died about the fifth to seventh day. When this material was mixed with the antitoxic serum before injection, not only did the animal live, but no paralysis resulted. Normal serum did not have this effect. The results, however, are rather irregular and do not lend themselves to practical application for the determination of therapeutic power.

\section{ANTI-GONOCOCCIC SERUM}

Specific antibodies have been found in the blood in chronic gonococcus infections. These include opsonins, agglutinins, bacteriolysins, and complement-fixing antibodies. Since recovery from gonococcus infection is usually dependent upon phagocytosis, opsonins or bacteriotropins would appear to be the most important. Antigonococcus serum was first produced by Torrey, ${ }^{\mathrm{I}}$ who showed that it was chiefly antibacterial in nature, containing opsonins, agglutinins, and complement-fixing antibodies, but no antitoxin. It appears to be of some value in the treatment of gonococcus bacteremia and in some of the acute gonorrheal complications, especially arthritis and epididymitis. It is of little or no value in chronic gonorrheal conditions and has no effect on acute urethritis.

Preparation.- Torrey used goats for the production of his serum, giving graduated doses, first of dead and later of living gonococcus cultures, intraperitoneally. In the methods of immunization now commonly used, horses are immunized by methods which closely follow those employed in preparation of anti-meningococcic serum (see p. 926).

Standardization is done by agglutination tests and by complement-fixation tests.

\section{ANTI-MENINGOCOCCIC SERUM}

Some seventeen years after the discovery of the meningococcus by Weichselbaum ( 1887 ), a world-wide epidemic of meningitis developed suddenly, and persisted more or less continuously in various parts of the world from I9O4 to I9IO. The severity of this pandemic, together with the fact that therapeutic measures suggested up to that time had no influence on the death rate, stimulated efforts to develop a specific remedy. While Jochmann of Germany is generally given credit as the first to produce an anti-meningococcic serum, a number of investigators in different countries were attacking the problem at the same time.

During the winters of $\mathrm{I}_{903}$, I904, and I905, a very large number of cases occurred in New York City, and a commission was appointed to study the problem. The fruit of this work was the development by Flexner and Jobling of an anti-meningococcic serum. Park independently at the same time had produced a serum. Another result of this investigation was the publication three years later by Elser and Huntoon ${ }^{2}$ of their "Studies on Meningitis," in which relationships of the meningococcus to the other gram negative cocci were demonstrated. At that time the division of the meningococci into subgroups had not been discovered. Grouping was brought forcibly to the front as a result of the World War experience. In certain epidemics antimeningitis serum proved to be of real value. In others there was a high percentage of failures not accounted for by lack of potency of the sera used. The explanation was supplied during and following the war, when English and French investigators

г Torrey, J. C.: J.A.M.A., 46, 26r. г9o6.

${ }^{2}$ Elser, W. J., and Huntoon, F. M.: J. Med. Research, 20, 373. I909. 
demonstrated the existence of immunological groups. The classification which is usually accepted at this time is that of Gordon, who distinguishes four groups. In the United States the Rockefeller Institute (Flexner and his co-workers) recognized at first two principal strains and one or more intermediate forms. The Second International Conference on the Standardization of Sera and Serological Tests (Paris, I922) recognized two primary groups and a third group of atypical strains. Wadsworth and Kirkbride, ${ }^{\mathbf{I}}$ on the basis of agglutination and absorption tests, recognize three distinct groups, corresponding to Gordon's I, II, and III, and a group of atypical strains including Gordon's group IV. In the authors' opinion, based on experience with a small epidemic in Kansas, Gordon's group IV must be taken into account in this country.

Preparation.-The present method, which has stood the test of long usage, is to inject horses intravenously with gradually increasing doses of killed cultures two to three times a week, and, after two to three weeks, substituting living cultures. These cultures should be handled with rapidity, i.e., an eighteen-hour growth on a solid medium should be used. The growth is washed off with salt solution, rapidly centrifugated, re-emulsified, and injected. Not more than two hours should elapse between washing off the culture and the injection. If this procedure is not followed, rather severe reactions are likely to result. After a period of immunization of two to three months, trial bleedings are taken and the agglutination test applied. Based on the results of these tests, the amount of the different strains employed can be varied so as to balance the serum and eventually to produce a well-distributed polyvalent serum.

Standardization.-The testing and standardization of anti-meningococcic serum has offered many difficult problems. In efforts to find a laboratory test that has relationship to therapeutic value, the complement fixation test of Schwartz and McNeil, as employed by the New York City Board of Health, the bacteriotropin test as employed by Miss Evans of the Hygienic Laboratory and checked in the authors' laboratory, the protection tests of Flexner, of Amoss and Wollstein, of Hitchens and Robinson, and of Huntoon have all been eventually discarded as either being too complicated for routine use or of too difficult technique, leaving, at the present time, only the agglutination test in general use. This test is recognized merely as an indication that the horses have been under immunization for a considerable time.

Method of performing agglutination test.-See chapter Ixxii, by Dr. McCoy, in this volume, pp. 958, 959 .

THERAPEUTICS OF ANTI-MENINGOCOCCIC SERUM

It is usually possible to discover meningococci in the throats and nasopharynges of individuals who have been in contact with cases. That these individuals are actually carriers and responsible for the development of cases in others is not proved. However, in the army experience of one of us (F. M. H.), isolation of meningococcus carriers in an organization resulted in eliminating the disease. These meningococcus carriers are extremely resistant to all known forms of local treatment, the organisms persisting sometimes for many weeks.

Practically all cases of meningitis, if investigated at an early stage, show organisms in the blood, and cases have been known of true meningococcus septicemia which did not develop meningeal symptoms. These facts have a direct bearing on treatment,

I Wadsworth, A., and Kirkbride, M. B.: Am. J. Hyg., 6, 4, 507. July, I926. 
since early in the disease the cases should be treated intravenously as well as intraspinally.

In the third or meningeal stage, specific serum therapy by intradural administration is necessary. Two avenues for the introduction of serum have been commonly used-lumbar puncture and through the fontanel into the ventricles. Lumbar puncture is performed with little danger, and serum given by this route is effective in a considerable percentage of cases, particularly in those treated early. Ventricular puncture is apparently harmless, and it is a comparatively easy method in infants. After closing of the ventricle trephining is necessary. To these methods must now be added a third avenue, namely, cistern puncture. The discouraging results often noted in late cases are sometimes due to spinal subarachnoid or ventricular block, which prevents serum introduced by lumbar puncture from reaching the cerebral meninges. Puncture of the cisterna magna gives the optimal route for overcoming the effects of spinal block. It has been shown to be a safe and efficient procedure. Some authors go as far as to recommend cistern puncture as the routine method, discarding lumbar puncture as less efficient, but probably the more conservative attitude is to use the cistern puncture only in cases which do not show satisfactory progress under lumbar therapy. In the presence of spinal subarachnoid block, cistern puncture is not only indicated but imperatively demanded.

It should be noted that, after intraspinal injection in the treatment of meningitis, the individual becomes actively sensitized to horse serum, and if a recrudescence of the attack occurs, such cases must not be treated by intravenous injection.

The influence of specific therapy in the treatment of meningitis as compared with the results of the non-specific treatment of the pre-serum period is well covered in Blackfan's monograph. ${ }^{1}$ The average mortality for meningococcus meningitis untreated by serum is given as from 60 to 80 per cent, whereas the mortality rate for serum-treated cases reported by different observers ranges anywhere from I6 to 37 per cent. The beneficial effects of serum treatment are especially noted in cases of children under two years of age, in which age group the mortality is nearly Ioo per cent without serum treatment. These striking results mark anti-meningococcic serum as the most efficient of the anti-bacterial sera.

\section{ANTI-PLAGUE SERUM}

Sera have been produced against the plague bacillus (Pasteurella pestis)-the causative organism of bubonic plague. Their chief value depends upon their antibacterial properties. The plague bacillus produces a small amount of exotoxin and some sera have shown limited antitoxic value, but this property is apparently unimportant. Potent sera contain opsonins or bacteriotropins, agglutinins, and possibly bacteriolysins. As with other antibacterial sera, its best therapeutic results are obtained when given as early as possible and in large doses.

Preparation.- Owing to the dangers connected with handling the plague organism, commercial laboratories are required to use only non-virulent strains. Horses are immunized by injecting cultures intravenously in gradually increasing doses. The serum recovered can be shown to contain various antibacterial immune bodies, but the direct potency test on rats is

r Blackfan, K. D.: loc. cit. 
the only method which seems to have any relation to its therapeutic value. Since virulent strains must be employed, this test can be done only in isolated laboratories by experts and cannot be employed as routine in the usual laboratory.

\section{ANTI-PNEUMOCOCCIC SERA}

The pneumococci of the three fixed types, I, II, and III, are highly specific in their immune reactions, so that a serum effective against one type has little or no value against the others or against the miscellaneous group IV. Sera have been produced against type I and type II pneumococci, both as monovalent and as polyvalent sera. Of these, type I serum is the easier to prepare in high potency, and is the one with which most clinical work has been done. The type I serum was the original serum prepared at the Rockefeller Institute. Because of the difficulties in preparing type II and type III sera, it has been advised that typing be done in each case and that the serum be administered only in type I cases. Later, Park, of the Bureau of Laboratories, Department of Health of New York City, produced a potent type II serum which, in a series of clinical tests, has shown definite therapeutic value. Up to the present, no one has succeeded in preparing a therapeutically effective type III serum.

Preparation.--The methods of immunization are the same for the monovalent fixed type of sera and for the polyvalent serum. The procedure for all may be described under one heading, with the understanding that the end-product depends upon the antigen used.

The usual method is to inject horses intravenously with small doses of killed organisms, followed later by emulsions with living organisms. It is thought necessary to start with virulent strains, but it is unwise to grow the organisms in media which retain the virulence, since this is unnecessary and destructive to the horses. The method of one of us (F.M.H.) is to grow the organisms eighteen hours in hormone gelatin broth, centrifuge, re-emulsify the organisms in salt solution, and kill by heating to $55^{\circ} \mathrm{C}$. for thirty minutes. Sodium bicarbonate to the amount of 0.25 per cent is added to the emulsion before heating. It has been found that after this addition the horses tolerate the injections much better and that the immunity rises faster. After treatment with killed organisms for two weeks, living organisms are employed, giving from one to three injections per week, gradually increasing the dosage until the animals receive 3,5 , and Io cc. of an emulsion containing 50,000 million organisms per cubic centimeter. At the end of six weeks, trial bleedings are made and the serum is tested for potency according to the method specified by the Hygienic Laboratory.

Method of testing.- - The protection test, prescribed by the Hygienic Laboratory of the United States Public Health Service, is described in chapter lxxii of this volume (see pp. 958, 959).

When type I serum has reached a point where $0.2 \mathrm{cc}$. protects white mice against $0.0 \mathrm{I}-0 . \mathrm{I}$ cc. of a culture of type I pneumococcus, of which $0.0000000 \mathrm{I} \mathrm{cc}$. will kill a white mouse in forty-eight hours, the serum is then regarded as potent. In the production of type II serum, the usual potency found is a serum of which $0.2 \mathrm{cc}$. protects a white mouse against $0.0 \mathrm{I}$ Cc. of a type II culture which kills in $0.0000000 \mathrm{I}$ cc. Park has produced a serum protecting against $0 . x$ cc., and the authors have recently produced a similar serum. The type III potency is that of a serum of which $0.2 \mathrm{cc}$. protects against $0.0 \mathrm{I} \mathrm{cc}$. of a culture which kills in $0.000000 \mathrm{I} \mathrm{cc}$.

In the authors' opinion, however, a more reliable criterion of the value of an 
anti-pneumococcic serum is found in the method of Felton, in which o.or cc. of a culture killing in $0.0000000 \mathrm{I} \mathrm{cc}$. is taken as a standard, and varying dilutions of the serum are tested against that standard. There are both theoretical and practical reasons for a preference for this method. In the testing of antibacterial sera by the protection method against living virulent organisms, a third component of the reaction must be furnished by the animal. Where $0.0 \mathrm{I} \mathrm{cc}$. of culture is employed, one may be certain that there is an excess of this component present, and the results obtained by this method are much more sharp cut and regular than by the standard method.

The presence of agglutinins is no indication of the protective value of the serum, and is useful only where diagnostic sera are desired.

THE VALUE OF ANTI-PNEUMOCOCCIC SERA

The accumulated reports have been critically examined by Locke, ${ }^{x}$ with a view to estimating more accurately the clinical value of this mode of treatment. Limiting his analysis to the type I serum, which is admittedly the most potent, Locke states that "a final estimate of the value of this serum in Type I lobar pneumonia, based on the figures thus far published, is impossible. . . . Granted that the serum is given every eight hours and in sufficient doses, the most vital consideration is unquestionably the stage of the disease when serum injections are begun." While it is impossible to foretell the results of serum therapy in individual cases, yet there is some reason to believe that anti-pneumococcic serum of high potency, properly administered in adequate dosage, is of definite value in the treatment of type I pneumonia.

\section{CONCENTRATED ANTI-PNEUMOCOCCIC SERA}

Probably the first instance of the successful concentration of antibacterial immune bodies from sera is recorded in the announcement by Izar and Caruso. ${ }^{2}$ In I92I they concentrated antibodies by dilution of the serum with double distilled water. In 1924 Felton $^{3}$ announced success with a practically identical method. With close attention to the hydrogen-ion concentration and the proportion of distilled water, a precipitate was obtained, apparently of euglobulins, and roughly proportionate in volume to the potency of the sera.

Felton's serum is therefore essentially a concentrated anti-pneumococcic serum, and it contains probably about $\mathbf{I} \cdot 5^{-2} .5$ per cent serum proteins. Banzhaf ${ }^{4}$ has also prepared a concentrated serum by a modification of Felton's method.

\section{ANTIBODY SOLUTION}

As early as December, I9I $9,,^{5}$ one of us (F. M. H.) announced success in preparing a solution of pneumococcus protective antibodies, "approximating in antibody content the best immune sera and containing so low a serum protein content that $5 \mathrm{cc}$.

I Locke, E. A.: J.A.M.A., 80, I507. May 26, I923.

${ }^{2}$ Izar, G., and Caruso, G.: Riforma med., 38, 145. Feb. I3, I922.

3 Felton, L. D.: Boston M. E. S. J., I90, Sig. May 15, I924.

4 Banzhaf, E. J.: Weekly Bull. New York Dept. of Health, r6, I7. Jan. 29, I927.

s Huntoon, F. M.: Tr. Philadelphia Path. Soc. (N.S.), 22, 75. 1920. 
amounts sensitize guinea pigs only irregularly to subsequent injections of horse serum." I

This solution is obtained by a biological process rather than by a chemical reaction, and is made possible through the capacity of living pneumococci for the adsorption of their specific immune bodies when treated with a specific serum, and by the development of a practical method of separating or dissociating the pneumococcus protective antibody from its antigen. By this method, protective antibodies of types I, II, and III have been removed from polyvalent anti-pneumococcus serum and resuspended in salt solution, giving a solution remarkable for its extremely low serum protein content.

This substance has had a rather extensive clinical trial, and when used early has shown definite therapeutic value in type I and type II cases. This has been confirmed by experimental work on monkeys with lobar pneumonia. An occasional case of type III infection has responded favorably to treatment with this solution, but experimental work on monkeys shows no effect on type III. This is doubtless due to the fact that type III antibodies are present to a much less extent than antibodies of type I or II. It is probable that if a type III serum can be produced with potency equivalent to the best type I sera, the therapeutic results will also be assured in cases caused by type III pneumococcus.

\section{ANTI-STAPHYLOCOCCIC SERUM}

- No anti-staphylococcic sera have been regularly or generally prepared, but their occasional use has been attended with some beneficial effects in certain cases.

The preparation of anti-staphylococcic serum involves the immunization of horses for an extended period, using as antigens several representative strains of staphylococci, both the $S$. aureus and the $S$. albus. The only method of standardization applied in the routine production of this serum on a large scale is the agglutination test, the serum being required to agglutinate each strain in a dilution of 100 or better.

Parker $^{2}$ has reported that a considerable number of the strains of staphylococcus produce a soluble exotoxin in sufficient amounts to be demonstrable by intradermal tests on rabbits. Parker and Banzhaf ${ }^{3}$ showed that horses could be immunized to this toxic substance, with the production of a rather high-potency antitoxic serum. The therapeutic use of this serum has not as yet been reported, so that its value in the treatment of such diseases as staphylococcus septicemia, etc., is not known.

\section{ANTI-STREPTOCOCCIC SERUM (POLYVALENT)}

From the early studies on the streptococci it was quickly apparent that almost all streptococci responsible for acute infections possessed hemolytic properties. While they were found to exhibit varied activities, they were regarded as one species under the name of Streptococcus hemolyticus. Later, it was shown that the hemolytic streptococci could be subdivided as to their sugar reactions, but that these reactions had little relation to pathogenic activity. After the introduction of immunological technique it began to be apparent that hemolytic streptococci causing similar lesions

I Huntoon, F. M.: J. Immunol., 6, 2. March r9, I920.

${ }^{2}$ Parker, J. T.: Proc. Soc. Exper. Biol. E Med., 23, 344. Feb., 1926.

3 Parker, J. T., and Banzhaf, E. J.: J. Immunol., 13, 25. Jan., I927. 
had an immunological relationship, and that several strains or groups, while morphologically and culturally indistinguishable, are serologically distinct. This was strikingly brought out by the work of Tunnicliff and others.

The work of Dochez and Avery and of Havens on the group classification, of the Dicks on scarlet fever streptococci, of Rivers, Amoss, and Birkhaug on streptococci of erysipelas, all support the idea of distinct strains or varieties within the species. Some more recent work indicates that possibly the organisms causing puerperal septicemia may also form a distinct immunological group.

One may now assume that the hemolytic streptococcus covers a number of groups, each of which tends to set up a characteristic disease entity. It may be possible that the streptococcus under certain conditions changes its character, and then tends to reproduce the same type of lesions. For example, the streptococcus of erysipelas, when conditions are favorable, will continue to produce erysipelas, but this does not preclude the possibility that under different conditions it may cause some of the manifestations of other streptococcus infections, such as phlegmons, etc. It has recently been shown capable of causing "acute anginas resembling ordinary sore throat or tonsillitis without the skin manifestations of erysipelas." However the facts may be interpreted, the tendency of the streptococcus to select certain tissues and to reproduce a certain type of disease renders the problem of producing an adequate anti-streptococcic serum for these diseases much more simple. The brilliant results obtained with scarlet fever streptococcus antiserum and with erysipelas streptococcus antiserum substantiate this theory.

Preparation of anti-streptococcic serum (polyvalent). - Various methods have been used in preparing anti-streptococcic serum. Space will permit only an outline of one of the more recent.

A method in common use is as follows. Cultures of the various strains used as antigens are grown in bottles containing hormone gelatin broth, and incubated until maximum growth is obtained. After centrifugation the supernatant liquid or bouillon is rejected and the mass of streptococci is made up into a standardized emulsion containing 50,000 million organisms per cubic centimeter and heated to $55^{\circ} \mathrm{C}$. for thirty minutes. This is reemulsified, and doses are prepared for the treatment of horses by mixing the emulsions of different strains in proper portions. Normal full-grown horses are treated by giving intravenous injections twice a week, beginning with a dose of $0 . \mathrm{x}$ and $0.5 \mathrm{cc}$. for the first week, two doses of I cc. in the second week, and thereafter increasing the weekly doses by 2 cc. until ro cc. are given at a time. This dose is repeated until trial bleedings show that the titre of the serum has reached the required level. Trial bleedings are begun after the treatment has been in progress four weeks and the serum is tested for agglutinins against the antigens used in its preparation, and against other strains. As soon as it agglutinates all strains in a dilution of I : 200 or better, regular bleedings are made and the serum prepared and tested for sterility, safety, and potency. Good sera show an agglutinin titre from $\mathrm{I}: 200$ to $\mathrm{r}: 800$.

Other means of standardizing anti-streptococcic serum have been used, including a protection test similar to that employed for anti-pneumococcic serum, a bacteriotropin test, and a complement fixation test. All of these are more or less cumbersome and apparently give no better indication of the therapeutic value of the serum than the agglutination test.

${ }^{1}$ Dick, G. F., and Dick, G. H.: J.A.M.A., 89, II35. Oct. I, I927. 


\section{ENCEPHALITIS STREPTOCOCCUS ANTISERUM}

Rosenow, ${ }^{\mathrm{I}}$ in I922, reported isolating a somewhat peculiar non-hemolytic streptococcus from cases of encephalitis, and with this organism he reproduced in animals typical symptoms and lesions of encephalitis. He also produced a serum by repeatedly injecting horses with these streptococci. Other investigators have cultivated streptococci from cases of encephalitis. Notable among these is the work of Evans and Freeman, ${ }^{2}$ who isolated a pleomorphic streptococcus from nasal washings, heart blood, and midbrain of a case of epidemic encephalitis. At the request of Miss Evans and Dr. Freeman, the authors have co-operated in the production of an encephalitis antiserum, the method of preparation being similar to that used for polyvalent anti-streptococcic serum. The serum has been used by Dr. Freeman and distributed in limited quantities to other clinicians for trial, with results that are at least encouraging.

\section{ERYSIPELAS STREPTOCOCCUS ANTISERUM}

Erysipelas is a streptococcus infection, the knowledge of which has recently been considerably extended. Polyvalent streptococcus sera have heretofore been used in the treatment of erysipelas with comparatively indifferent results. Following the work of Rivers, Amoss, and Birkhaug on the specific strains of the streptococcus associated with this disease, and with the knowledge that the erysipelas streptococcus produces a toxin, specific anti-erysipelas sera have been prepared, special effort being made to develop antitoxic as well as antibacterial properties.

Preparation.-A number of strains isolated from erysipelas lesions are employed. The whole living culture is used, the method of one of us (F. M. H.) being to start immunization by intracutaneous inoculations. The doses range from I to Io cc., the larger of which sometimes causes rather extensive local reactions. These, however, subside quickly, without the production of abscesses. Later, the horse is injected not only intracutaneously but also subcutaneously.

There is no laboratory test at present which gives a true index of the value of the serum. That produced in the authors' laboratory is given a clinical test on actual cases of erysipelas. In early cases the results are very striking, the first effect being the clearing up of edema and a rapid fall in the albumin content of the urine. This is accompanied by a drop in the leukocytic curve and temperature curve and the fading of the lesion. It is necessary to emphasize that adequate doses must be employed to obtain therapeutic results.

Recently one of us (F.M.H.) has succeeded in concentrating erysipelas streptococcus antitoxin by a method which retains both antibacterial immune bodies and the antitoxin in the final product. Both the unconcentrated and the concentrated serum have proved of much value in the treatment of erysipelas.

\section{POLIOMYELITIS STREPTOCOCCUS ANTISERUM}

In I9I 7 Rosenow reported the production of an anti-poliomyelitis serum in horses by inoculations of a pleomorphic non-hemolytic streptococcus isolated from poliomyelitis cases, and came to the conclusion that the immune serum, thus prepared by repeated injections of increasing doses of freshly isolated strains of the organism, had curative power, especially when given in the early stage of the disease. The specificity

${ }^{1}$ Rosenow, E. C.: ibid., 79, 443. Aug. 5, I922.

${ }^{2}$ Evans, A. C., and Freeman, W.: U.S. Pub. Ilealth Rep., 41, 1095. June 4, 1926. 
of Rosenow's serum depends upon the hypothesis that a streptococcus, having peculiar neurotropic properties and isolated from cases of epidemic poliomyelitis, may stand in causative relation to the disease.

\section{RHEUMATIC FEVER STREPTOCOCCUS ANTISERUM}

Small ${ }^{\mathrm{r}}$ has reported the isolation of a strain of streptococcus from blood cultures of patients suffering from acute rheumatic fever. This is a non-hemolytic streptococcus repeatedly found in cases of this kind and is so distinctive as to warrant its designation under a specific name $-S$. cardioarthritidis. A serum has been prepared which apparently has considerable antibacterial value, as shown by the agglutinin titre and the opsonic index. While still in the experimental stage, laboratory and clinical work now in progress give every indication of confirming Small's observations and of giving the serum a definite place in biological therapy.

\section{SCARLET FEVER STREPTOCOCCUS ANTISERUM}

A number of workers have demonstrated that the streptococci associated with scarlet fever form a distinct serological group. When the Dicks demonstrated the etiological relation of the scarlet fever strains of the streptococcus to the disease and that this organism forms a soluble toxin which appears to be responsible for the symptoms of scarlet fever, the necessity of producing sera of high antitoxic properties was apparent.

Preparation.-Two basic methods are at present employed. The first is that advocated by the Dicks-namely, the subcutaneous injection of scarlet fever streptococcus culture filtrates, which have been shown to produce a skin test in high dilution in susceptible individuals who have not had scarlet fever. After a period of several months the serum from the animals can be shown to neutralize the substance which causes the skin reactions. The final product is strictly an antitoxic serum, any antibacterial properties being lost during concentration.

The second is the method advocated by Dochez. A certain amount of semifluid agar is injected under the skin of the animal. After the agar has solidified, a culture of living scarlet fever streptococcus is injected into the center of the mass. The organisms proceed to grow in this medium and give off their toxic products into the circulation. This method, however, is likely to cause abscesses in the animals, and a modification proposed by Zinsser is more useful. In this method citrated or oxalated horse blood is mixed with a culture of the streptococcus, and just before injection enough calcium chloride is added to cause the coagulation of the blood. The whole mass is rapidly injected under the skin, where clotting takes place. This method has the advantage that it is much less likely to cause abscesses. Either of these methods will produce an adequate therapeutic serum.

In the beginning of the work on scarlet fever serum one of us (F.M.H.) preferred to combine subcutaneous injections of the filtrate with intravenous injections of the centrifuged organisms. This produces a combined antibacterial and antitoxic serum, but the antibacterial portions of the serum are lost in the process of concentration.

A detailed description of the method of immunization of horses for production of this serum will be found in an article by Anderson and Leonard. ${ }^{2}$ The serum is standardized on its antitoxic properties.

\footnotetext{
s Small, J. C.: Am.J. M.Sc., r73, гог. Jan., г927.

${ }^{2}$ Anderson, J. F., and Leonard, G. F.: ibid., 172, 334. Sept., I926.
} 


\title{
CHAPTER LXXI
}

\section{THE USE OF HUMAN SERUM FROM CONVALESCENT CASES IN PREVENTION AND TREATMENT}

\author{
WILLIAM H. PARK \\ Bureau of Laboratories, Department of Health, City of New York
}

It is now well known that persons who recover from any one of a number of the infectious diseases develop antibodies which accumulate in the blood. In at least several of these diseases the accumulation is great enough in the blood to transfer through injections of a few cubic centimeters a considerable immunity to others and possibly to transmit sufficient to be used therapeutically.

The advantages of human antibodies over those of the horse or any other animal are several. Since they are used to confer immunity in a member of the same species they persist longer. Thus, a guinea pig receiving ten units of diphtheria antitoxin prepared in an immunized guinea pig remains immune for from six to eight months, while a guinea pig receiving ten units of antitoxin made in an immunized horse remains immune for only two or three weeks. The disadvantages are several. The potency of the serum in the antibacterial or antitoxic antibodies from convalescent cases is very variable, is often low, and is usually unknown, the amount of serum being frequently so small and the need for its immediate use so great that tests are not made. There is the great difficulty also of getting a supply at the time needed, for it is impossible to count in an emergency on a donor who has recently had the disease. The greatest concentration of the antibodies in the serum is usually one or two weeks after the recovery. There is then a gradual decline. Many persons after recovery retain appreciable amounts for a lifetime. Because of the drawbacks, convalescent serum is rarely used when there is available a horse serum of much greater antibody potency. The serum can be stored for months because the antibodies are fairly stable.

The serum from many persons who have never had certain communicable diseases possesses antibodies against the micro-organisms causing these diseases. Thus we know that many persons who have never had diphtheria are not only immune to diphtheria, but that in the blood they have appreciable concentrations of diphtheria antitoxin. We know that the same is true as to scarlet fever antitoxin and antibacterial antibodies in many who have never had scarlet fever. People who have never had pneumonia frequently have very considerable quantities of antibodies against one or the other of the different pneumococcus types. The same is true for poliomyelitis and many other infections. Our only explanation is that these persons at some time had a very light, unrecognized sickness or that they were for a time healthy carriers of micro-organisms. It is important to remember this fact; otherwise we may make misleading conclusions, as, for instance, when the convalescent serum from a case of epidemic encephalitis neutralizes the virus of poliomyelitis the probabil- 
ity is not that the encephalitis infection caused the development of antibodies against the virus of poliomyelitis but rather that these antibodies were already there. So far as can be determined from a study of the antibodies in the convalescent sera from cases of pneumonia and scarlet fever, they are about as stable as those developed in animals after immunizing injections.

\section{VALUE OF CONVALESCENTS' SERUM IN PROTECTION AGAINST VARICELLA}

In 1923, Blackfan Peterson and Conroy ${ }^{I}$ reported that a temporary passive immunity to varicella could be produced in the majority of cases by the intramuscular injection of convalescents' serum. Of forty-two exposed children who were given $5 \mathrm{cc}$. of serum within five days after exposure to the disease only seven contracted it, and then in a mild form. Later, Weech, ${ }^{2}$ by injecting from 3 to $4.5 \mathrm{cc}$. of convalescents' serum within from one to six days after exposure, was able to protect nine out of ten infants, the only infant who contracted the disease having an exceptionally mild attack with an unusually long incubation period. Schmidt, ${ }^{3}$ however, was unable to prevent varicella by the injection of 7-8 cc. of convalescents' serum during the incubation period. These reports are the only ones I have found in the literature available to me, although it seems quite certain that the procedure is more widely employed than is indicated.

Mitchell and Ravenel ${ }^{4}$ gave sixty-eight young and older children the serum from a convalescent varicella patient. Only three of these developed varicella, the others showing no symptoms for at least thirty days after exposure. Of these three patients, two were observed by them during the period of the disease, but the other one was diagnosed by his family physician after he had left the hospital. A fourth patient who had escaped contracting the disease on the first exposure was re-exposed twentytwo days after his injection. Sixteen days following the second exposure, this patient leveloped a rash which somewhat resembled varicella. There were no symptoms except the rash, which was of a type the child had had on two other occasions during a period of five months' observation. These facts make it very doubtful whether the condition should be regarded as varicella. The incubation period in the three patients developing the disease was rather long, being twenty-one, twenty-two, and twentythree days, respectively - thus corresponding to Weech's case, in which the incubation period was twenty-three days. However, in contrast to the observations of Blackfan, Peterson, and Conroy and of Weech, who found that the disease after injection assumed a mild form, two of their patients presented a rather profuse rash and moderately severe general symptoms.

In a group of twenty-two children, one failed to receive convalescents' serum, and he alone of this group developed varicella on the nineteenth day. Twelve of this same group were re-exposed on the twenty-first day after injection. Although none of these children received a second injection, only one developed the disease, his rash appearing nineteen days after this second exposure. This patient at the time of the

I Blackfan, K. D., Peterson, M. F., and Conroy, F. C.: Ohio State M. J., I9, 97. I923.

${ }^{2}$ Weech, A. A.: J.A.M.A., 82, 1245. I924. $\quad 3$ Schmidt, W.: Med. Klin., 20, 642. I924.

${ }_{4}$ Mitchell, A. G., and Ravenel, S. F.: Arch. Ped., 42, 709. I925. 
development of varicella re-exposed three children of this same group of whom none developed the disease, although this represented their third exposure forty days following the single injection of convalescents' serum.

They were able to make some other interesting observations on the possible length of time during which convalescents' serum was protective. A group of six children was accidentally re-exposed to a child with varicella thirty-two days after the injection. Later, four of this same group had a third accidental exposure fifty days after injection. None of them, however, contracted the disease in spite of three exposures, the last being fifty days after their one injection of convalescents' serum. The protection from the serum from convalescent varicella is apparently longer than that from measles convalescents.

The amount of serum used in fifty-four patients was between 4 and 5 cc.; one patient received Io cc.; four received $6 \mathrm{cc}$.; seven received $3 \mathrm{cc}$.; two received $2 \mathrm{cc}$. All but nine of the patients had received the serum within from one to three days after exposure. Of these nine patients, seven had received serum within five days, one within six days, and one within seven days. The three patients developing the disease all had received $4 \mathrm{cc}$. of serum, two of these not until five days after exposure. It is problematical whether more serum injected at a shorter interval after exposure would have protected these patients.

The serum used was obtained within from ten to fourteen days after the onset of varicella from adults who had negative Wassermann reactions. It was preserved by the addition of Io per cent by volume of 0.7 per cent sodium chloride solution containing I per cent phenol, and it was kept in sealed glass tubes in an icebox. Serum as old as eleven months was apparently successful in protection. The injections were given deep into the anterior thigh muscles, and in no case was there a local reaction. One patient developed definite serum sickness with fever and urticaria nine days after the injection of varicella convalescents' serum. Three weeks previously he had been given scarlet fever convalescents' serum intramuscularly.

The percentage developing varicella in the total group of sixty-eight patients was 4.4. Excluding thirteen patients, who were fifteen years of age or over, and eleven patients who were six months of age or younger, the percentage developing the disease in the remaining forty-four was 6.8 .

\section{PROPHYLACTIC USE OF CONVALESCENT SERUM FROM PERSONS HAVING HAD MUMPS}

In view of these considerations it is interesting to consider what may be accomplished in preventing mumps, or epidemic parotitis, especially in institutions in which a large number of cases arise from time to time. Mumps is still to be ranked among the highly contagious infective diseases of unknown etiology, the specific causative microbic agent not having been discovered. The disease is, however, one in which one attack yields protection that is general and persistent. The question arises, therefore, whether the blood of persons who have recovered from one attack of the disease does not contain immunizing and protective principles which can be employed in the protection of other exposed persons. Hess in I9 5 utilized the blood of convalescents in an attempt to control an epidemic of mumps in the Home for Hebrew Infants, which for the second time in two years was visited by a severe epidemic. The epidemic 
was well under way when he determined to undertake this prophylactic therapy. At the time treatment was begun, about forty cases of mumps had broken out in the institution within the past month, and new cases were developing almost daily. The conditions surrounding an institution of this kind are peculiarly favorable for judging the effect of prophylactic treatment of the various infectious diseases, as most of the children are admitted during infancy, and we have complete knowledge of the infectious diseases which they have contracted while under our care.

Twenty children were given protective injections, but three were probably not exposed. This leaves seventeen who had entered the institution before the age of one year. Naturally, none was injected who had had mumps in the course of the epidemic in the winter of I9I2-I3 and who had acquired immunity in this way. The ages of these children were from one and one-half to five and one-half years. Whole blood was used and injected at once intramuscularly. The donors form three groups: The first includes children, four in number, who were just recovering from mumps; the second comprises ten children who had recovered from the disease about ten days previously; the third is composed of six children who had had mumps one or two years before. It should be added that the results of the treatment in the last group cannot be considered convincing, in that three of the children treated ( ${ }_{5}$, I6, and I 7) must be left out of consideration, as they did not happen to come in contact with active cases of the disease, and the other three (18, 19, and 20) had been in the institution but six weeks, so that we did not possess first-hand knowledge as to their susceptibility to mumps. As was to be expected from the experience of others, there were no disagreeable manifestations following the injections-neither a rise of temperature nor local reaction. The epidemic was so widespread that only three of the children under treatment did not come into contact with the disease. There are therefore seventeen who must be regarded as having been not only susceptible, but also exposed to mumps. It is therefore striking to note that among these seventeen none contracted the disease. That the injections were effective in bringing about this result may be seen from a glance at Table I, which shows that in the wards where the children were not protected by injection fully one-third, or even one-half, came down with the disease.

Our results hardly seem to require extended comment. We may add, however, that by means of these injections we were able, in a large measure, to check the epidemic. It would seem that preventive treatment of this kind could well be carried out in institutions for children, and that this simple procedure might likewise be resorted to in the home where one or more children are exposed to infection. Our experience seems to indicate that the blood may, with advantage, be obtained from the donor even before the parotid swelling has disappeared, and that it possesses protective principles at this early stage. As is well known, the incubation period of mumps is long, about eighteen days, so that it is possible to resort to protective injection some days following exposure. It is impossible to state the duration of immunity acquired in this way; but it seems reasonable to believe that it well outlasts the usual danger period of infection.

Joseph C. Regan ${ }^{\mathrm{I}}$ reports a successful use of the serum. Six groups of children

${ }^{\prime}$ Regan, J. C.: J.A.M.A., 84, 279. 1925 . 
comprising eighty-one in all, exposed to epidemic parotitis at different times, were immunized with an average injection of $3 \mathrm{cc}$. of convalescent serum within a period varying from the first to the sixth day after exposure. Only one of these patients developed mumps; but eleven were discharged from observation before their possible incubation period was over, and the results in these eleven immunized patients are not known. The remaining sixty-nine showed no signs of the disease. The convalescent blood used for this immunization was taken from healthy adult donors, who were Wassermann negative and clinically free from any active signs of tuberculosis, between the extremes of the tenth and twentieth day, usually on the fourteenth or sixteenth day of their disease.

TABLE I

Incidence of Mumps among Injected and Non-Injected Children (Hess)

\begin{tabular}{|c|c|c|c|c|c|c|c|}
\hline \multirow{2}{*}{$\begin{array}{l}\text { WARD } \\
\text { No. }\end{array}$} & \multirow{2}{*}{$\begin{array}{l}\text { Total } \\
\text { CENSUS }\end{array}$} & \multirow{2}{*}{$\begin{array}{l}\text { No. of } \\
\text { SUSCEP- } \\
\text { TIBLES } \\
\text {. }\end{array}$} & \multirow{2}{*}{$\begin{array}{c}\text { Inoculated } \\
\text { CaSES }\end{array}$} & \multirow{2}{*}{$\begin{array}{c}\text { DATE } \\
\text { TRANSFERRED } \\
\text { TO WARD }\end{array}$} & \multicolumn{2}{|c|}{$\begin{array}{c}\text { No. CASES DEYELOP- } \\
\text { IN. NIUMPS } \\
\text { SUBSEQUENTLY }\end{array}$} & \multirow{2}{*}{ REMARKS } \\
\hline & & & & & $\begin{array}{c}\text { Not } \\
\text { Inoculated }\end{array}$ & Inoculated & \\
\hline $4 \ldots \ldots$ & 25 & 22 & $\left\{\begin{array}{c}3 \\
(\mathrm{Nos}, \mathrm{I} \\
\mathrm{I} 2,14)\end{array}\right\}$ & $2 / 1_{5} / \mathrm{I}_{5}$ & I I & 0 & $\begin{array}{l}\text { Nine cases of mumps re- } \\
\text { moved from this ward } \\
\text { within previous three } \\
\text { weeks }\end{array}$ \\
\hline $6 \ldots$. & 30 & I9 & $\left\{\begin{array}{c}\mathbf{I} \\
(\text { No. I } 3)\end{array}\right.$ & $2 / \mathrm{II} / \mathrm{I} 5$ & 8 & $\circ$ & Twelve cases removed \\
\hline IO. . & 30 & 27 & $\left\{\begin{array}{c}\mathrm{I} \\
(\text { No. 20) }\end{array}\right.$ & $2 / I_{5} / I_{5}$ & 5 & $\circ$ & $\begin{array}{l}\text { Four cases removed from } \\
\text { this ward within previ- } \\
\text { ous three weeks }\end{array}$ \\
\hline II .... & 34 & 32 & $\left\{\begin{array}{c}2 \\
(\text { Nos. } 18 \\
19)\end{array}\right\}$ & $2 / I_{5} / I_{5}$ & 7 & $\circ$ & $\begin{array}{l}\text { Six cases removed within } \\
\text { previous three weeks }\end{array}$ \\
\hline I $2 \ldots$ & 34 & 34 & $\left\{\begin{array}{c}\text { 10 } \\
(\text { Nos. } 2 \\
3,4,5,6 \\
7,8,9 \\
\text { Io, I I })\end{array}\right\}$ & $2 / 12 / 15$ & I3 & $\circ$ & $\begin{array}{l}\text { Ten cases removed within } \\
\text { previous three weeks }\end{array}$ \\
\hline
\end{tabular}

THE PROPHYLACTIC USE OF MEASLES CONVALESCENT SERUM

Some thirty years ago, a German physician used large amounts of serum from convalescents in the treatment of several cases in which the symptoms were just beginning. The results were favorable, and he suggested that it might be used as a preventive measure. The first results published on the use of convalescent measles serum as a preventive were by Nicolle and Conceil from the Institute of Pasteur in Tunis. In the same year, 1916, Park and Zingher injected forty-one very recently exposed children at the Metropolitan Hospital. Twenty of these children received $8 \mathrm{cc}$. of serum, and none of them developed measles. Twenty-one received $4 \mathrm{cc}$. and three developed the disease, one on the fifteenth day, one on the seventeenth day, and one on the twenty-fifth day after the serum was given.

Since then, a number of articles have been published on the use of whole blood, 
serum, and plasma obtained from measles convalescents. ${ }^{\mathrm{I}}$ Degwitz, in I920, I92I, and 1922 , published the results obtained by him and those working with him in more than one thousand cases. The results were on the whole quite favorable in that if the disease was not prevented in some, it was lessened in its severity. His routine method was to draw the blood between the seventh and seventeenth day after convalescence, allow it to clot, and use the serum intramuscularly after sterility and Wassermann tests had been done. Degwitz does not believe that the results obtained warrant the use of serum after the seventh day of exposure. He reports among his own series 85 per cent complete protection. This is more favorable than the results in other large series. Probably some of his children were not very markedly exposed.

Since I92 I the convalescent serum has been utilized with excellent results in the hospitals for contagious diseases in New York City in children who have been exposed by the development of measles in the wards. A grant of money by the Metropolitan Life Insurance Company made it possible to attempt to provide enough serum for all exposed inmates in children's institutions in New York City, and also for all the children in the city who were under three. These classes of children were chosen be-

TABLE II

\section{Amounts of Serum Used by Degwttz}

$2.5 \mathrm{cc}$. in those exposed 4 days or less

$5.0 \mathrm{cc}$. in those exposed 6 days or less

$7.0 \mathrm{cc}$. in those exposed 7 days or less

cause institutional children and those under three years of age are the most liable to pneumonia.

The use of convalescent serum in an infected child may prevent the development of the disease, modify the severity of the attack, or fail entirely of any effect. So far as the interests of the child are concerned, the best result is the development of a very mild attack. Under such conditions there is practically no danger of complicating pneumonia or of any other complication. Recovery is practically certain, and the child has the advantage of an active immunity which may be considered lifelong. Those children have as a rule a very slight rise of temperature, perhaps to $100^{\circ}$ or Ior $^{\circ}$ for a day or so, and a slight coryza, and a very slight to a moderate rash. Koplik spots may or may not be present. Those who are absolutely protected have only a passive immunity so long as sufficient serum remains, and this varies from about two to four weeks, and depends on the individual and the amount of serum injected. The child is after this period as susceptible as before.

The great problem in the prevention of measles by this method has been the difficulty of obtaining a continuous supply of blood large enough to meet the demands. When we realized the value of the serum in New York, as far as possible, every adult measles convalescent was asked to give some blood, for which service a suitable reward was offered. Because of the small incidence of measles in adults in New York

r A bibliography is given by Zingher, A.: "Convalescent Whole Blood, Plasma, and Serum in Measles," J. A. M. A., 82, rr8o-87. April r2, r924. 
City and the usual refusal of patients to give their blood, this source of supply proved to be inadequate. It then came to the attention of the department that there was an epidemic of measles of considerable extent among the young adult students of a southern university, all of whom came from rural districts. The students volunteered in large numbers to give their blood, and as they had all been treated in the university hospital, the diagnosis of measles was certain. It is in some such way as this that a supply must be obtained.

The method of drawing the blood was as follows: At the Willard Parker Hospital, blood was drawn on the ninth day after the defervescence of fever up to the twentyfirst day, but at the southern university the time was more varied, and some was drawn as late as five months after the disease. In the case of adults, all the blood was drawn through a 16 -gauge Luer needle into a 500-cc. bottle containing $20 \mathrm{cc}$. of 25 per cent sodium citrate solution and $0.3 \mathrm{gm}$. of oxyquinolin sulphate (chinosol) as a preservative. This preparation was used because it was found that in the dilution in which it was used it was an efficient bactericidal agent; the red cells were not destroyed, and it was not irritating when injected intramuscularly. The bottles were then put in the icebox and the cells allowed to settle, or were centrifugated, and the plasma pipetted off. Blood for a Wassermann test was taken at the time of bleeding, and a sterility test was done at the time the plasma was put up into small bottles.

Plasma was used instead of serum, because of the greater yield for the amount of blood drawn and the greater ease of handling. All the blood drawn within six days of the same period in convalescence was pooled. Thus far, the figures indicate that the efficiency of the plasma is not markedly diminished by postponing bleedings for three months after the occurrence of the disease, and that storage in the icebox for six months does not appreciably alter its usefulness. The plasma was put up in 3-cc. and 6-cc. vials and 30-cc. bottles. The vials were distributed to private physicians with printed directions for use, and a card was given which was filled out by the physician and mailed back to the laboratory. This card contains questions concerning the number of children exposed, the date and type of exposure, the date of injection, and the lot number of plasma. Twenty-one days after injection, each case was investigated by a member of the department staff. The 30-cc. bottles were kept for institutional use when a large number of children were to be injected.

RESULTS OBTAINED IN NEW YORK CITY

More than I, 500 children received preventive injections between December I and June I, 1925. From 979 we have fairly accurate data as to the date and degree of exposure, the amount of plasma injected, and the success attained. Of these 979 children, 753 were exposed in institutions and 226 in private families. In 404 of the institutional children and in all of the children in families, the exposure was direct. In 349 it was indirect in that actual personal contact with a measles patient was not proved.

No bad effect followed the injections in any case. Only one of the children died and this one had an empyema before contracting modified measles and was only two years of age. We do not think that the attack of measles appreciably hastened its death.

${ }^{1}$ Park, W. H., and Freeman, Jr., R. J.: ibid., 87, 556. Aug. 2 I, I926. 
CONVALESCENT SERA IN THE TREATMENT OF EPIDEMIC POLIOMYELITIS

Following the discovery by Flexner and Lewis ${ }^{\mathrm{T}}$ and by Levaditi and Landsteiner ${ }^{2}$ that the serum of monkeys convalescent from an attack of poliomyelitis contained antibodies which were capable of neutralizing the filterable virus, it was found that the serum of human convalescents was equally efficacious. The serum from slight abortive cases is on the average as potent as that from pronounced cases. The serum of many persons who have never had poliomyelitis contains antiborlies, but these are not so potent as in the serum of convalescent cases. The antibodies remain in the blood of convalescents for a long time.

Netter ${ }^{3}$ utilized this information to test the value of the serum therapeutically in human cases. The serum should be free from hemoglobin as this causes irritation when the serum is injected intraspinally. At first it was given only intraspinally but, owing to the fact that the disease affects the tissues of the brain and cord rather

TABLE III

Institutional Children Receiving Preventive Injections Against Measles.

\begin{tabular}{|c|c|c|c|c|c|}
\hline Amount of Plasma Injected & $\begin{array}{l}\text { Number } \\
\text { of } \\
\text { Children }\end{array}$ & $\begin{array}{l}\text { Modified } \\
\text { Measles } \\
\text { Per Cent }\end{array}$ & $\begin{array}{l}\text { Unmodified } \\
\text { Measles, } \\
\text { Per Cent }\end{array}$ & $\begin{array}{l}\text { Complete } \\
\text { Success, } \\
\text { Per Cent }\end{array}$ & $\begin{array}{l}\text { Incomplete } \\
\text { Success, } \\
\text { Per Cent }\end{array}$ \\
\hline 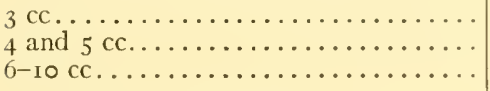 & $\begin{array}{l}2 \text { I } 9 \\
\text { I } 90 \\
243\end{array}$ & $\begin{array}{r}20 \\
8 \\
6\end{array}$ & $\begin{array}{l}8 \\
2 \\
2\end{array}$ & $\begin{array}{l}72 \\
90 \\
92\end{array}$ & $\begin{array}{l}92 \\
98 \\
98\end{array}$ \\
\hline $\begin{array}{l}\text { Total in institutions . . . . . . . . . . . } \\
\text { Total in families. . . }\end{array}$ & $\begin{array}{l}753 \\
226\end{array}$ & $\begin{array}{l}\text { I I } \\
42\end{array}$ & $\begin{array}{l}5 \\
5\end{array}$ & $\begin{array}{l}84 \\
52\end{array}$ & $\begin{array}{l}95 \\
95\end{array}$ \\
\hline
\end{tabular}

than the covering membranes, and also to the discovery by Flexner and Amoss ${ }^{4}$ that the aseptic meningitis set up by the intraspinal injection promotes the passage of antibodies from the blood to the cerebrospinal fluid, the serum is now usually given both intraspinally and intravenously. On the first treatment injections are given both intraspinally and intravenously and later injections for one to three days intraspinally.

Amoss and Chesney ${ }^{5}$ state that the following conditions should be observed: ( $I$ ) early and prompt diagnosis and treatment, (2) intraspinal injection of immune serum, (3) intravenous or intramuscular injection, (4) the serum injected should have been collected from cases which have recently passed an attack of poliomyelitis. The serum is preserved by the addition of 0.2 per cent tricresol. In children they advise that $10-20$ cc. be given intraspinally and 40-80 cc. intravenously. If given before the development of paralysis, these injections may be sufficient; if not, the intraspinal injection may be repeated.

In the r9I6 epidemic in New York Neal and Zingher treated a large number of cases of poliomyelitis. In those seen early before paralysis had developed the results were very good whether serum was used or not. Thus in twenty-five early cases treated by Zingher at the Willard Parker Hospital with intraspinal injections;

I Flexner, S., and Lewis, P. A.: ibid., 54, 45. I9ro.

${ }^{2}$ Levaditi and Landsteiner, K.: Compt. rend. Soc. de biol., 68, 3 I I. IgIo.

3 Netter, A., et al.: ibid., 70, 625, 707, 739. I9Ir.

4 Flexner, S., and Amoss, H.: J. Exper. Med., I9, 4 II; 20, 249. I9I4.

${ }^{5}$ Amoss, H., and Chesney, A. M.: ibid., 25, 58I. I9I7. 
only two developed paralysis and none died, while those treated after paralysis had developed had a high mortality. Zingher believed the serum was very beneficial in the early cases and of doubtful value in those in whom paralysis had developed. Neal, with even more experience with cases treated by intraspinal injections, was very doubtful as to its value. Personally I do not believe in the streptococcic origin of the disease or in the antistreptococcic serum. As we have no reliable potent antivirus serum from horses, I should be inclined to use the convalescent serum. I think it should be given both intraspinally and intravenously.

\section{USE OF SERUM FROM CASES OF CONVALESCENT INFLUENZA IN INFLUENZA PNEUMONIA}

The fact that an attack of influenza develops in the patient an immunity to the disease, and probably also an immunity to complicating infections suggested the use of the blood or serum of convalescent cases in the treatment of severe and complicated cases. Ross and Hund ${ }^{I}$ treated a group of cases with transfusion or intravenous injections of $250-500 \mathrm{cc}$. of citrated blood taken from compatible donors among convalescent cases of influenza; the mortality rate was 2 I.4 as against 42.8 per cent among cases treated symptomatically. It seems to the writer that the results published indicate that the serum from convalescent cases or from immunes who did not contract the disease when exposed is of value. In influenza pneumonia we are treating the complicating infections more than that caused by the influenza virus.

\section{USE OF CONVALESCENT SERUM IN LETHARGIC ENCEPIIALITIS}

This is still wholly experimental; as we do not know the germ we have no way of testing its potency. Neustaedter, Larkin, and Banzhaf ${ }^{2}$ report that monkeys were protected against poliomyelitis by the administration of sera from human convalescent cases of encephalitis. If these antibodies were developed against the virus of poliomyelitis, it would seem certain that they were developed against the virus of epidemic encephalitis, but Amoss $^{3}$ found that in his test monkeys the sera of recently recovered human cases of encephalitis were unable to neutralize the virus of poliomyelitis and regards the two diseases as two entirely separate entities.

The probability is that a considerable percentage of healthy human beings have antibodies against the poliomyelitis virus and that they were not developed because of the encephalitis.

Gruenwald ${ }^{4}$ has injected $80-100$. cc. of convalescent serum intragluteally in the treatment of the disease, possibly with some success. If sufficient serum is available it would appear advisable to administer it intraspinally and intravenously in the same manner as described for the treatment of poliomyelitis.

${ }^{x}$ Ross, C. W., and Hund, E. J.: J.A.M.A., 72, 640. i9i9.

${ }^{2}$ Neustaedter, M., Larkin, J. H., and Banzhaf, E. J.: Am.J. MI. Sc., I62, 715. I921.

3 Amoss, H. L.: J. Exper. Med., 33, 187. I921.

${ }_{4}^{4}$ Gruenwald, E. A.: Deutsche med. Wchnschr., 46, I 243 . 1920. 
TREATMENT OF TYPHOID FEVER BY SERUM FROM CONVALESCENT CASES

Nicolle and Conseil ${ }^{\mathrm{I}}$ have treated five patients with the serum of convalescent typhoid patients. The dose was about I 30 cc.; in their experience the duration of the disease was not shortened. A few others have used the serum with possillly favorable results. It is seldom used.

\section{USE OF SERUM FROM CONVALESCENT CASES IN THE TREATMENT OF WHHOOPING COUGH}

It is now usually thought that whooping cough is a specific infectious disease due to the Bordet-Gengou bacillus; during the course of the second or third week, antibodies appear which are specific by agglutination and by complement tests. For the purpose of determining the value of this method, Bleyer ${ }^{2}$ carried out injections of human blood in the early weeks of this disease in forty-five cases. These were divided into three groups of fifteen cases each. In group A the blood injected was from persons who were convalescent or who had recovered from whooping cough within three months. In group B the blood was from persons who had had the disease at more remote periods, and in group $\mathrm{C}$ from persons, who, so far as they knew, had never had it. Groups B and C were designed as controls to A.

The ages of the children in all groups averaged under three years. In group A, in which convalescent's blood was used, five were in the first half-year of life, one was five weeks old, and one thirty days old. The stage of the disease at which the treatment was given was about the same in the three groups. Dosage was gauged in a rough way to body weight of donnée; this varied between 40 and I $25 \mathrm{cc}$., divided into two, three, or four doses and injected into a muscle (gluteus).

The results of the treatment in the three groups may be summarized; in group A of fifteen children whose average age was twenty-eight months, who received convalescent's blood during the early weeks of whooping cough, there occurred no deaths and no serious complications; the course of the disease was, however, in no definite way different than is usually seen, and was not appreciably influenced by the treatment except in three. This is a very small proportion, and it is more than likely that in fifteen cases three of them might very well run an unexpectedly mild course without attracting a great deal of notice. The blood pictures of two of these, however, were interesting: In case 4, during the third week of the disease, a 50,000 white count came down after $60 \mathrm{cc}$. of convalescent's blood to 18,000 and the mononuclear percentage fell from 74 to $5 \circ$; this drop was coincident with clinical improvement; this was a hand fed baby of five months who weighed but 3,860 gm.

In group B, in case $\mathrm{I}$, in which the blood used was from the mother who had had pertussis twenty years before, quite as satisfactory improvement occurred as in any case in group $\mathrm{A}$; in this group there were two pneumonias which recovered. There were also two pneumonias in group $\mathrm{C}$ with one death, and in this group there was one case which seemed to have been very favorably affected by the injections of normal blood.

\footnotetext{
I Nicolle, C., and Conseil, E.: Ann. de l'Inst. Pasteur, 26, 332. I9I2.

${ }^{2}$ Bleyer, A.: Am.J.M.Sc., 154, 40. I917.
} 
In explanation of these results it may be said that in some diseases injections of normal blood have brought about improvement, and that immunity may be increased at times by injections of normal blood, that this has also occurred after injections of other things, such as protease, lipase, ferments (trypsin), or drugs (kaolin). Hyperleukocytosis, such as occurred in several cases in this series, has assisted immunity in other diseases (arthritis, typhoid), so for lack of consistency in the results obtained in these cases of whooping cough it is not possible to ascribe any of them to a specific action of the blood injected.

\section{USE OF CONVALESCENT AND NORMAL BLOOD IN THE TREATMENT OF SCARLET FEVER}

The development of scarlatinal antitoxin and antibacterial antibodies has largely removed the necessity of using human convalescent serum, but it is still at times useful, especially in cases of sepsis.

The intravenous injection of fairly large quantities (from 2 to $3 \frac{1}{2} \mathrm{oz}$.) of convalescent serum has given good results in the hands of Reiss and Jungman, ${ }^{\mathrm{I}}$ and Koch. ${ }^{2}$ This requires greater skill, and necessitates wasting more than 60 per cent of the blood. These disadvantages are easily avoided by the simple intramuscular injection of whole citrated blood.

Cases of scarlet fever may be divided roughly into two groups for purposes of blood injection:

I. The early toxic or malignant scarlet fever cases which are seen between the first and fourth day of disease, and show the clinical picture of delirium and restlessness, intensive petechial rash, moderate glandular enlargement and severe angina. These cases are suitable, in the absence of antiscarlatinal antitoxin, for the intramuscular injection of convalescent serum or blood taken from second-or third-week convalescent scarlet fever cases. Such convalescents are generally available in the wards of large contagious disease hospitals.

The effect of convalescent blood in these early toxic or malignant cases of scarlet fever is seen in a critical drop in temperature beginning about six hours after the injection and ending in from twenty-four to thirty hours. The temperature often reaches normal and will remain nearly normal in the majority of the cases; in others, especially when complicated with severe tonsillar and faucial exudate, associated with inflamed cervical glands, the temperature may rise again for a few days, but rarely to the same height as at the time of injection. Other results, which are quite as striking, can be seen in the early fading of the rash, the improvement in the circulation and the character of pulse, the general condition, and mental symptoms.

2. In the second group are included the later septic cases, seen from the fifth to the eighth day of disease. The rash may have faded entirely, but the membranous exudate over the fauces and tonsils is severe and extensive, and often appears necrotic. The cervical lymph glands are enlarged and tender. The temperature is high and septic in character, varying between $103^{\circ}$ and $105^{\circ} \mathrm{F}$.

In this group of cases, in the absence of blood from convalescents, I would strong-

${ }^{2}$ Reiss, E., and Jungman, P.: Deutsche Arch.f. klin. Med., I06, 7о. I91 2.

${ }^{2}$ Koch, R.: Miinchen. med. Wchnschr., 60, 26r1. 1913. 
ly recommend the use of fresh normal blood, which can be readily obtained from one or both of the parents, or some other close relative of the patient. The intramuscular injection of $4 \mathrm{oz}$. of blood in children up to the age of four years, of from 6 to $8 \mathrm{oz}$. of blood in older children and adults will give most satisfactory results. These blood injections may have to be repeated once or twice at intervals of four or five days. Such repeated injections are made in the same muscles, since the blood is absorbed with great facility. Not much specific effect is claimed for these injections of fresh normal blood; but the nutritive and stimulating properties and normal antibody content of relatively large amounts of normal blood have shown definite beneficial results in some desperately ill patients, and have convinced us that the treatment in septic cases when the prognosis is doubtful or poor should invariably include the administration of such a harmless and yet frequently efficient remedy.

The method of obtaining the blood, and its injection, are very simple. With a $\mathrm{I}-\mathrm{Oz}$. (30 cc.) record syringe, a Luer syringe, or, in an emergency, an easy working large syringe, and a medium sized needle (preferably a No. I 7 or No. I 8 gauge platinum iridium needle) the necessary quantity of blood is rapidly aspirated from the median cephalic vein of the donor at the bend of the elbow, and immediately citrated by adding the blood to a ro per cent solution of sodium citrate in the proportion of I oz. of blood to each cubic centimeter of the citrate solution. This makes the final dilution of the citrate 0.33 per cent. The needle inserted into the vein of the donor should not be removed; the syringe is detached when full, while an assistant carefully fixes the hub of the needle and attaches a 5 -cc. record syringe containing some I per cent sodium citrate solution to keep the needle free of blood. Three or four syringefuls of blood may thus be obtained before it is necessary to rinse out the larger syringe with a I per cent solution of citrate which is kept ready in a beaker. We prefer to collect the blood in roo-cc. bottles, each of which contains $2 \mathrm{cc}$. of the ro per cent citrate solution. To each bottle $2 \mathrm{oz}$. of blood are added, the bottle being shaken after each addition to distribute the sodium citrate solution. In less than ten minutes the required amount of blood (from 4 to Io oz.) is obtained and is then ready for injection into the patient.

The blood is injected into the following regions: triceps, outer regions of both thighs (vastus externus), the calves (soleus), and both gluteal regions. In young children, $\frac{1}{2}$ oz.; in older children and adults, I oz. is injected into each of these muscles.

Zingher treated twenty-three patients at the Willard Parker Hospital with intramuscular injections of blood. They represent the cases with the poorest prognosis selected out of a total of some nine hundred admissions. Fifteen were treated with convalescent blood, which was citrated in a majority of cases. Nine received pooled blood from two or more donors. The amount injected varied from $2 \frac{1}{2}$ to $8 \mathrm{oz}$. Of the fifteen patients, four died. Of the eleven patients who recovered, only five were of the purely toxic type; the remaining six patients had additional severe septic complications, especially tonsillar and faucial exudates, and inflamed cervical glands.

Eight patients received fresh normal blood; of this group, none died. These cases were far advanced septic cases, several with a poor prognosis. The blood, which was readily obtained from one or both of the parents, was citrated in six. One of the 
patients received two injections of 6 oz. each; another three injections, each 8 oz., at intervals of four or five days. As was to be expected, no striking critical drop but a distinct lowering of the temperature curve was noted, associated with an improvement in the septic complications and the general condition of the patient. The good results in some of these cases are the best proof of the beneficial effect that can be derived from this treatment.

While it is difficult to make any absolute statement or arrive at definite deductions from such a small series of cases, one can only hope that, by continuing this form of treatment, a larger series of cases may finally be collected that will serve as a more definite guide for the value of the intramuscular injections of convalescent and normal blood. The treatment is easily carried out, is quite harmless, and is within the reach of the general practitioner. The beneficial effects noted in the foregoing group of very severe cases were quite distinct. The rational element in the use of fresh normal blood in the septic cases is found, first of all, in clinical observations of its great empiric value; but also to a large extent in a consideration of the great nutritive value of homologous blood, its marked stimulating properties, and its well-known antibody content, which exerts a distinct bactericidal effect on many pathogenic organisms. Even more valuable would be the transfer of the whole blood by transfusion. In this case, one would make available the donor's blood cells as well as his serum with its antibodies.

Convalescent blood or serum has not been used extensively to immunize against scarlet fever. It has the disadvantage that it is far less potent than the horse antitoxin. It has the advantage that it will not produce serum sickness and will not sensitize to horse serum. If the serum is on hand it should have been tested for potency and Icc. should neutralize at least fifty skin-test doses of toxin. 


\title{
CHAP'TER LXXII
}

\section{CONTROL AND STANDARDIZATION OF BIOLOGICAL PRODUCTS}

\author{
G. IV. McCOY \\ Hygienic Laboratory, IVashington, D.C. \\ GENERAL MEASURES OF CONTROL,
}

In certain countries of the world the control of biological products is assigned to governmental agencies separate from those responsible for the purity and safety of drugs in general. This is true in the United States where control of these preparations, in so far as it properly falls under federal jurisdiction, is carried out by the Public Health Service. This agency exercises its functions in this field chiefly through the Hygienic Laboratory. The responsibilities of the federal government are limited in this connection to preparations offered commercially for import, export, or interstate traffic, and to those sold in the District of Columbia.

Foreign countries usually have special control over biological preparations. Germany requires official testing in addition to the tests made by the manufacturer on each lot or batch of certain preparations, notably the antitoxins of diphtheria and tetanus, anti-meningococcus serum and tuberculin; other preparations are for the most part ignored. France and Italy have licensing requirements somewhat similar to those of the United States. England has been without special restrictions until recently when a Therapeutic Substances Act which includes biological products came into force.

Certain products of this group are included in the pharmacopoeias of different countries. In the United States the following are to be found in the tenth revision of the Pharmacopoeia which is official at the present time: diphtheria antitoxin, tetanus antitoxin, and smallpox vaccine.

The field of biological control is one in which there is great activity and in which changes are constantly occurring, chiefly the result of the rapid advances being made by research in infectious diseases.

In the United States the law is interpreted as requiring a license for each product falling in the group of biological products. This at once brings to the fore the question of issuing licenses for preparations which the licensing authorities consider valueless or of doubtful worth. While no hard-and-fast rule can be followed, the practice in general is that of withholding a license unless either experimental or clinical evidence is available at least strongly suggestive that the preparation has prophylactic or therapeutic value. In some cases the licensing authority insists upon considering tests made under its own control prior to reaching a decision on an application for license.

Inspection.-Producing laboratories are inspected prior to the issuing of a license in order that the licensing authority may have full information as to the facilities of the plant as regards personnel, methods, and equipment. Inspections are repeated usually annually, in order that there may be no undetected lapses in any respect which might impair the quality of the products. Inspection discloses such defects as failure 
to keep adequate records of processes, failure to take and keep records of the temperature of horses producing serum, inadequate separation of work on pathogenic sporebearing organisms from other operations, failure to keep horses under immunization constantly protected against tetanus, and inadequate processes of sterilization.

If, as the result of inspection, it is discovered that contaminated or impotent products are being produced, or if such defects are disclosed in any other manner, the license of the manufacturer may be suspended or canceled, or certain lots of products may be withdrawn from trade channels.

Purity.-It is important that extraneous living organisms be excluded during the process of manufacture of most products. This is accomplished by requiring tests for identity and purity of cultures prior to beginning manufacture of a batch of a product. In some cases-e.g., bacterial vaccines - the presence of contaminating organisms may be disclosed by so simple a procedure as the making of smears from finished material. Thus a pertussis vaccine showing gram positive diplococci evidently has been contaminated at some stage of the process of manufacture.

Sterility.-Freedom of the final product from living organisms, excepting in the few cases where these constitute the essential immunizing agents, is assured by the methods of preparing the material, and by tests for sterility. Sterility tests are generally carried out on the bulk preparation prior to filling the trade containers, and upon the contents of the latter as the material is ready for the market. The procedures for sterility testing must take cognizance of the presence of a preservative (if one is present), and must therefore provide for adequate dilution so as to bring about in the culture medium a concentration of the antiseptic sufficiently low to avoid its inhibitory action.

While agar plates and ordinary broth tubes are used to some extent abroad in sterility testing, the most satisfactory means is to combine aerobic and anaerobic tests in one container. This is accomplished by placing the material in flasks of plain broth where the amount of material to be tested is large, or by the use of two-armed fermentation tubes after the design of Theobald Smith when the amount to be tested is small. All the oxygen is driven off from the broth in the long closed arm just before inoculation. Whichever container is used, the proportion of broth to inoculum should be such that the preservative, if phenol or tricresol, shall not exceed I part in 10,000 of the culture medium.

It is desirable to test each lot of broth with an organism that does not grow very readily to determine whether it is satisfactory for the purpose for which it is intended.

Certain special preparations, notably rabies vaccine, may cloud the medium in the fermentation tubes in such manner as to leave one in doubt as to whether contaminants are present. In such a case a transplant is made into a fresh tube of medium, and this transplant should remain free from turbidity or sediment if the material is free of living organisms capable of growing in the medium.

The cultures used in sterility tests are examined after forty-eight hours' incubation, again after four days, and finally at the end of seven days. The two- and fourday examinations are necessary because a contamination may develop and be observable at an earlier reading but disappear before the final observation.

Labeling.--Under the regulations drawn up in accordance with the law of July $\mathrm{r}$, 
I902, which covers the control of biological products, the label is required to show the proper name of the product; the name, address, and license number of the manufacturer; the lot number; a statement as to the potency of the product; and the expiration date. The manufacturer is permitted to utilize any trade name he may see fit to employ in addition to the officially authorized name.

Dating. - The law referred to in the preceding paragraph requires that each package shall be marked with "the date beyond which the contents can not be expected beyond reasonable doubt to yield their specific results." Under this provision of the law somewhat arbitrary requirements have been set up since it is impossible to determine always how long a preparation retains its therapeutic or prophylactic activity. Glycerinated smallpox vaccine is permitted a maximum of three months on the market even under favorable conditions of storage. Dried vaccine virus is permitted six months. The antitoxins for which there are official standards are permitted one year if they are sent out with a 20 per cent excess of potency, and as high as four years if sent out with a 50 per cent excess of potency. Antibacterial sera in general are given one year, anti-meningococcus serum being an exception with but six months from date of manufacture. Bacterial vaccines in general and pollen extracts are given eighteen months.

Standardiation and testing.-The descriptions of procedures given here chiefly will follow the practice of the United States, and theoretical considerations will be omitted. In standardization procedures it is the aim to measure if possible the therapeutically, diagnostically, or prophylactically active elements in the preparation, though there are not at present sufficient data to permit this in every case. This can be done, e.g., in the testing of antitoxins, but in the case of certain other agents-e.g., anti-meningococcus serum-there is no assurance that the immune bodies titratede.g., agglutinins. bacteriotropins, or complement-fixing bodies, depending on the method used-have any important part in the therapeutic activity of the material.

In the United States there are standard units legally established for the antitoxins of diphtheria and tetanus, for those of $\mathrm{Cl}$. perfringens ( $\mathrm{Cl}$. welchii) and the botulinum group and for that of the scarlet fever streptococcus. Control products are distributed by the Hygienic Laboratory for comparison in the testing of anti-dysenteric serum, anti-pneumococcic serum, and anti-meningococcic serum, typhoid and paratyphoid vaccines, scarlet fever streptococcus toxin for the Dick test and for immunization. Standardized methods are prescribed for testing the potency of smallpox vaccine, diphtheria toxin for the Schick test, diphtheria toxin-antitoxin mixture, and diphtheria toxoid.

In the case of other preparations generally, the potency testing and the standardization are left to the discretion of individual manufacturers.

\section{VIRUSES \\ SMALLPOX VACCINE (VACCINE VIRUS)}

Tests are required to be carried out to exclude an excessive number of contaminating organisms in smallpox vaccine and to insure freedom from the tetanus organism. These are as follows:

For the bacterial count poured agar plates are used, the contents of one vaccination out- 
fit being well mixed with the melted agar for each plate. Incubation is at $37^{\circ} \mathrm{C}$. for fortyeight hours. The glycerinated lymph intended for a single vaccination should show not to exceed fifty organisms when this technique is followed. In practice it is rare to find glycerinated lymph which carries more than a dozen contaminating organisms, and those usually clearly of a harmless nature. Many tubes are entirely free from contaminants.

The tests for tetanus are required to be carried out on the harvest from each calf separately. The glycerinated pulp must be tested before any other preservative has been added, and the test must be made after the bacterial count has been reduced to less than fifty per vaccination quantity or after storage for at least seven days at a temperature of $10{ }^{\circ} \mathrm{C}$. Two cc. of the glycerinated vaccine are planted in each of several fermentation tubes, each tube containing not less than $25 \mathrm{cc}$. of meat-infusion broth which, immediately preceding planting, shall have been heated to $100^{\circ} \mathrm{C}$. for thirty minutes, any air bubbles being removed by tipping the tubes. The tubes are incubated at $37^{\circ} \mathrm{C}$. and examined daily. The presence of gas or clouding in the closed arm shall be taken as indication for the inoculation of animals with the culture. Either mice or guinea pigs may be used. The inoculation should be made upon the first observation of growth in the closed arm and again at the end of nine days after the planting. The animals should be kept under observation for at least six days and closely observed for the development of symptoms of tetanus.

There are a number of procedures for testing the potency of smallpox vaccine, all of which are described by Force and Leake. ${ }^{1}$ These authors suggest a method of their own, which is a modification of that proposed by Calmette and Guerin. The modification may be described as follows:

Albino rabbits are employed, preferably mature animals. The hair is removed by clipping, followed by shaving or by plucking. The area utilized extends from the scapular to the iliac region and about $\mathrm{I} 2 \mathrm{~cm}$. on each side of the vertebral column. By means of a sterile plate made from thin galvanized iron the area on each side is marked off into four rectangles of $2.5 \times 5 \mathrm{~cm}$.

The dilutions to be tested are $\mathrm{r}: \mathrm{r}, 000, \mathrm{r}: 3,000, \mathrm{r}: \mathrm{r0}, 000, \mathrm{I}: 30,000$. These dilutions are employed for the vaccine under test and for one of known satisfactory potency which is used as a control.

The inoculations are made by means of glass pipettes, $3-\mathrm{mm}$. inside diameter, which are broken off squarely. With these pipettes charged with about $0.4 \mathrm{cc}$. of diluted vaccine the tightly stretched skin within the rectangle is inoculated. Corresponding areas on the right and left sides of the rabbit are used for the same dilutions of the control vaccine and the vaccine under test.

The readings are made at the end of seven days and a comparison made between corresponding dilutions of the vaccine being tested and the vaccine used for control purposes.

\section{The authors suggest the following criterion of potency:}

A smallpox vaccine of high potency, when diluted $\mathrm{I}$ : I,, 00 should produce a confluent eruption on from 90 per cent to roo per cent of the vaccinated area on the back of a rabbit, and when diluted $\mathrm{r}: 3,000$ the decrease in confluence should not be over 20 per cent. A vaccine satisfying this criterion should produce in all previously unvaccinated human subjects a circular vesicle measuring at least $7 \mathrm{~mm}$. in diameter on the seventh day when applied, undiluted, to a circle of the exposed derma measuring $2 \mathrm{~mm}$. in diameter.

${ }^{x}$ Force, J. N., and Leake, J. P.: IIygienic Laboratory Bull. I.49. 1927. 


\section{RABIES VACCINE}

This product is employed in several different forms, none of wh'ch is satisfactorily standardized. Some vaccines are made of killed virus, others of attenuated virus, and still others of diluted "fixed" virus. The following specific requirements are in force:

Vaccines marketed as killed virus shall be submitted to a test to show the absence of living rabic virus. This is effected by requiring the inoculation subdurally of two rabbits, each with $0.1 \mathrm{cc}$. of the finished emulsion, and keeping the animals under observation for fourteen days-long enough to permit the development of the infection if any of the virus may have escaped the killing agent.

Vaccines prepared by the original Pasteur method and its modifications which utilize living virus are submitted to tests to show that the virus is living in certain of the doses of the preparations as they go on the market. The dried, attenuated virus should be lethal for rabbits when inoculated subdurally with the dose that has been dried three days or less.

When the dilution method is used, the lower dilutions must be capable of infecting rabbits.

\section{ANTITOXINS}

The testing of antitoxins is the most satisfactory and accurate of any potency determination carried on in the control of biological products.

The potency of antitoxins is measured usually in standard units. In this connection, a unit may be defined as the amount of antitoxic strength contained in a stated volume of a standard serum or antitoxin and used as a basis of comparison for measuring the strength of other lots of the antitoxin.

Standard units are distributed by central testing laboratories. For very accurate work, standardized glassware for measuring and carefully selected animals must be used for the tests, and in general conditions made uniform-but for routine testing of commercial sera considerable latitude is permissible.

\section{DIPHTHERIA ANTITOXIN}

In essentials the unit established by Ehrlich (cf. Rosenau) ${ }^{\mathrm{r}}$ is employed practically everywhere in the world.

In preparing to test diphtheria antitoxin, it is necessary to have a well standardized specimen of toxin. The minimum lethal dose of the toxin-i.e., the amount necessary to kill a $250 \mathrm{gm}$. guinea pig on the fourth day-is determined by means of a series of trials with varying doses of toxin. This amount will afford a guide to the doses to be used in determining the test dose.

While the unit of antitoxin is the ultimate basis of standardization, of almost equal importance in practice is the "test dose" of toxin of ten spoken of as the $\mathrm{L}_{+}$dose. To determine this test dose, varying amounts of toxin are mixed with the standard unit of antitoxin, the mixture incubated for one hour at room temperature and injected into test animals. The result of this test-i.e., the death of the animals after a certain period of time - enables one to select the proper test dose of the toxin for the titration of antitoxins of unknown potency.

Rosenau gives the determination of a test dose of a toxin shown in Table $I$.

${ }^{\mathrm{r}}$ Rosenau, M. J.: Hygienic Laboratory Bull. No. 2 I. 1905. 
The protocol shown in Table II illustrates tests on commercial antitoxins.

It is customary to test commercial samples for the exact unitage claimed on the label and for excess potency depending upon the length of time before the date of expiration, hence the fractions above $\mathbf{I}$ unit in the preceding table. Sample A had the unitage claimed, but less than ro per cent excess. Sample B had the unitage claimed,

TABLE I

Tests to Determine the L+ Dose of Toxin No. $74^{*}$

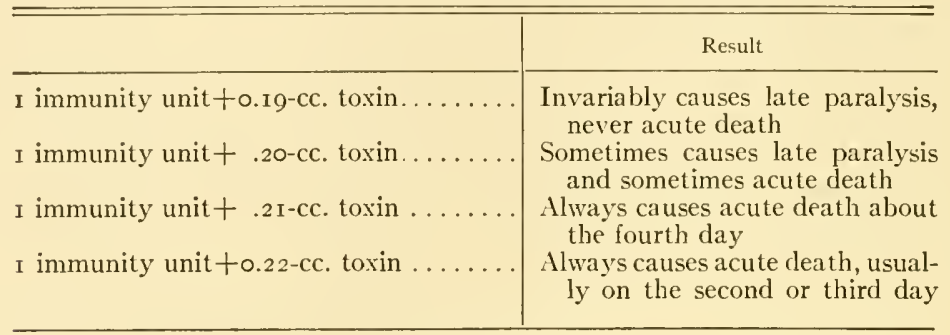

* The $\mathrm{L}_{+}$dose of this toxin is, therefore, just $0.2 \mathrm{I} \mathrm{cc}$.

but less than 30 per cent excess. Sample C was deficient for the unitage claimed, and of course for the excess tested for -5 per cent. The animals on the standard (control) died at the correct time.

Ramon's flocculation method. - Ramon's method of testing the potency of diphtheria antitoxin which eliminates the need for using laboratory animals is employed

TABLE II

\begin{tabular}{|c|c|c|c|c|}
\hline Antitoxin Specimen & $\begin{array}{l}\text { Weight of } \\
\text { Guinea Pig }\end{array}$ & Tested For & $\begin{array}{c}\text { Dose of Toxin } \\
\left(\mathbf{L}_{+}\right)\end{array}$ & Result \\
\hline 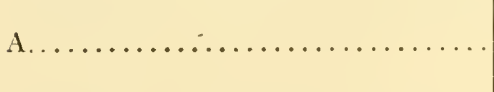 & $\begin{array}{l}260 \\
250\end{array}$ & $\begin{array}{l}\text { Unit } \\
\text { I. O } \\
\text { I. I }\end{array}$ & $\begin{array}{c}\text { cc. } \\
0.165 \\
.165\end{array}$ & $\begin{array}{l}\text { Dead } 7 \text { days, } 22 \text { hr. } \\
\text { Dead } 2 \text { days, I9 hr. }\end{array}$ \\
\hline 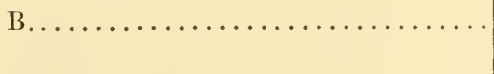 & $\begin{array}{l}260 \\
250\end{array}$ & $\begin{array}{l}\text { I.O } \\
\text { I. } 3\end{array}$ & $\begin{array}{l}.165 \\
.165\end{array}$ & $\begin{array}{l}\text { Discharged i } 7 \text { th day } \\
\text { Dead I day, is hr. }\end{array}$ \\
\hline 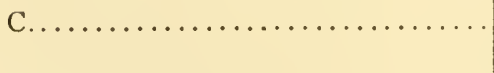 & $\begin{array}{l}270 \\
250\end{array}$ & $\begin{array}{l}\text { I. } 0 \\
\text { I. } 05\end{array}$ & $\begin{array}{l}.165 \\
.165\end{array}$ & $\begin{array}{l}\text { Dead } 2 \text { days, } 9 \text { hr. } \\
\text { Dead } 2 \text { days, } 9 \text { hr. }\end{array}$ \\
\hline 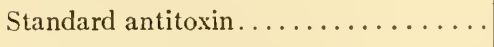 & 260 & I & .165 & Dead 4 days, 20 hr. \\
\hline 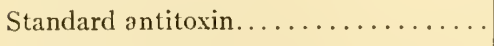 & 250 & & .165 & Dead 4 days \\
\hline Standard antitoxin.... & 380 & & 0.16 .5 & Dead 3 days, I 4 hr. \\
\hline
\end{tabular}

to a considerable extent. In some laboratories abroad the methorl is used for final as well as for preliminary titrations of the serum, but in this country it is employed almost exclusively for the less exacting requirements, such as testing the serum of individual producing animals.

Bayne-Jones ${ }^{2}$ considers that results obtained by Ramon's method agree within 3 per cent of those secured by titrations on animals. The procedure requires first the ti-

See chap. Ivi of this volume.

${ }^{2}$ Bayne-Jones, Stanliope: J. Immunol., 9, 48I. I92ג. 
tration of a toxin to be used in the testing against an antitoxic serum of known potency. When the amount of toxin which flocculates with I unit of antitoxin has been determined, the flocculating value of the toxin expressed as units of antitoxin flocculated per centimeter of toxin is readily ascertained. Fixed amounts of the toxin are then mixed with varying amounts of the antitoxin to be titrated, the mixtures incubated at $55^{\circ} \mathrm{C}$., and the first tube to show flocculation is the indication of the neutralizing value of the serum and from this the unitage is calculated.

\section{TETANUS ANTITOXIN}

The United States unit for this product was originally based ${ }^{\mathrm{r}}$ on a certain amount of a toxin used in the early work, this amount of the toxin being in turn selected as a convenient multiple of its minimal lethal dose. The actual unit ${ }^{2}$ is, as with diphtheria antitoxin, the combining or neutralizing power contained in a

TABLE III

\begin{tabular}{|c|c|c|c|}
\hline Weight of Guinea Pig & $\begin{array}{c}\text { Dose of Toxin } \\
(\mathrm{Gram})\end{array}$ & \begin{tabular}{|} 
Dose of Standard \\
Unit
\end{tabular} & Result \\
\hline 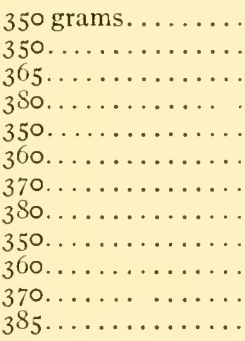 & $\begin{array}{l}0.00078 \\
.00078 \\
.00078 \\
.00078 \\
.00082 \\
.00082 \\
.00082 \\
.00082 \\
.00085 \\
.00085 \\
.00085 \\
0.00085\end{array}$ & $\begin{array}{l}0.1 \\
0.1 \\
0.1 \\
0.1 \\
0.1 \\
0.1 \\
0.1 \\
0.1 \\
0.1 \\
0.1 \\
0.1 \\
0.1\end{array}$ & $\begin{array}{l}\text { Dead } 5 \text { days, } 6 \mathrm{hr} \text {. } \\
\text { Dead } 4 \text { days, I } 8 \mathrm{hr} \text {. } \\
\text { Dead } 4 \text { days, I } 8 \mathrm{hr} \text {. } \\
\text { Dead } 4 \text { days, } 6 \mathrm{hr} \text {. } \\
\text { Dead } 3 \text { days, } 4 \mathrm{hr} \text {. } \\
\text { Dead } 3 \text { days, } 23 \mathrm{hr} \text {. } \\
\text { Dead } 3 \text { days, } 23 \mathrm{hr} \text {. } \\
\text { Dead } 3 \text { days, I } 8 \mathrm{hr} \text {. } \\
\text { Dead } 3 \text { days, I } 5 \mathrm{hr} \text {. } \\
\text { Dead } 2 \text { days, I } \mathrm{hr} \text {. } \\
\text { Dead } 2 \text { days, I } 9 \mathrm{hr} \text {. } \\
\text { Dead } 3 \text { days, } 8 \mathrm{hr} \text {. }\end{array}$ \\
\hline
\end{tabular}

stated volume of the official standard antitoxin which is distributed by the Hygienic Laboratory. A dried toxin is also distributed, with the test dose indicated, and is used for comparing the combining or neutralizing power of the antitoxin to be tested with the standard. As with diphtheria antitoxin, the criterion of exact balance between the doses of toxin and of antitoxin (O.I unit for tetanus) is death at ninety-six hours after injection of the mixture, but heavier guinea pigs are used, weighing about $350-80 \mathrm{gm}$., instead of the 250-80 gm. pigs used for diphtheria.

The test dose of toxin, having been roughly ascertained by preliminary tests, is determined more exactly as in the protocol shown in Table III.

The middle dose $(0.00082 \mathrm{gm}$.) employed is selected as it is as near as is ordinarily practicable to secure to the ideal of one that will kill in just under ninety-six hours. It is better, of course, to err on the safe side by having a dose of toxin slightly in excess rather than to risk one too low which would result in deceptively high titres for serum.

Using the dose selected as above, a test is carried out on commercial samples to be tested as shown in Table IV.

I Rosenau, M. J., and Anderson, J. F.: Hygienic Laboratory Bull. 43. I908.

: United Slates Public Health Service: Miscellaneous Publ. 10, par. 72. x919. 
Sample A contained more than a ro per cent excess of units. Sample B failed. Sample $\mathrm{C}$ had the potency claimed but less than a 25 per cent excess.

The excess for which tests were made should have been present to provide for a maintenance of full, labeled potency up to the expiration date of the product.

In addition to this method of standardization which gives results in terms of what is usually spoken of as the "American" unit, the German unit established originally by Behring is in use to a considerable extent. This unit is approximately sixty-six times the strength of the American unit-in other words, one German unit is the equivalent of approximately sixty-six American units.

Within recent months the Serums Committee of the Health Section of the League of Nations has adopted an international unit which shall be precisely half the strength of the American unit -in other words, two new international units will be the equivalent of one American unit. It is too early to predict the extent to which this unit will come into use.

TABLE IV

\begin{tabular}{|c|c|c|c|c|}
\hline Antitoxin Specimen & $\begin{array}{l}\text { Weight of } \\
\text { Guinea Pig }\end{array}$ & Tested For & $\begin{array}{l}\text { Dose of } \\
\text { Toxin } \\
\left(\mathbf{L}_{+}\right)\end{array}$ & Result \\
\hline 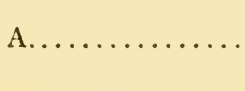 & $\begin{array}{l}345 \\
305\end{array}$ & $\begin{array}{l}\text { Unit } \\
\text { O. I } \\
\text {. I I }\end{array}$ & $\begin{array}{c}\mathrm{gm} . \\
0.00082 \\
.00082\end{array}$ & $\begin{array}{l}\text { Discharged } \\
\text { Discharged }\end{array}$ \\
\hline$B \ldots \ldots \ldots \ldots$ & $\begin{array}{l}340 \\
370\end{array}$ & $\begin{array}{l}. \mathrm{I} \\
\mathrm{I}\end{array}$ & $\begin{array}{l}.00082 \\
.00082\end{array}$ & $\begin{array}{l}\text { Dead I day, I } 8 \mathrm{hr} \text {. } \\
\text { Dead I day, I } 8 \mathrm{hr} \text {. }\end{array}$ \\
\hline$C \ldots \ldots \ldots$ & $\begin{array}{l}390 \\
390\end{array}$ & $\begin{array}{l}\text { I } \\
.125\end{array}$ & $\begin{array}{l}.00082 \\
.00082\end{array}$ & $\begin{array}{l}\text { Dead } 5 \text { days, } 3 \text { hr. } \\
\text { Dead } 2 \text { days, I } 9 \text { hr. }\end{array}$ \\
\hline Standard (control). & $\begin{array}{l}360 \\
410\end{array}$ & $\begin{aligned} . I \\
0 . I\end{aligned}$ & $\begin{array}{r}.00082 \\
0.00082\end{array}$ & $\begin{array}{l}\text { Dead } 3 \text { days, } 18 \mathrm{hr} . \\
\text { Dead } 3 \text { days, I } 8 \mathrm{hr} .\end{array}$ \\
\hline
\end{tabular}

\section{BOTULINUM ANTITOXIN}

There are several immunological types of Clostridium botulimım, designated "A," "B," and "C." The field of usefulness of the antitoxins corresponding to these toxins is not well defined and is very limited.

Bengtson ${ }^{\mathrm{I}}$ has standardized these antitoxins, the method being outlined as follows:

The unit of botulism antitoxin (type A) is a definite amount of the standard antitoxin; for the standard antitoxin in the concentration used at present, and to be sent out to serum establishments which may be licensed for the production of botulism antitoxin, the unit is $0.4 \mathrm{cc}$. of the glycerinated solution. The antitoxin to be tested is compared with the standard unit by means of a test dose of toxin; this test dose of toxin is chosen so that when mixed with one-tenth of a unit of the standard antitoxin and injected subcutaneously into a 250 gram guinea pig, the guinea pig will die in about 96 hours. That amount of the unknown antitoxin which when similarly mixed with the test dose of toxin and injected is just sufficient to protect a 250-gram guinea pig contains one-tenth of a unit. The test dose of toxin will usually be found to contain from 50 to 200 minimal lethal doses. Before injection the doses of toxin and antitoxin are to be thoroughly mixed, and then kept at room temperature for

\footnotetext{
${ }^{x}$ Bengtson, I. A.: Hygienic Laboratory Bull. I36. I924.
} 
one hour to allow combination to take place. The guinea pigs should be between 250 grams and 300 grams in weight, and the doses of toxin and antitoxin are to be increased proportionately to the weight for guinea pigs weighing over 250 grams. With every test of an unknown antitoxin, four control guinea pigs should receive the test dose of toxin mixed with the test dose (one-tenth unit) of standard antitoxin, so that the two antitoxins may be accurately compared and the test dose of toxin increased if necessary in subsequent tests; liquid toxins have been found to decrease gradually in toxicity. In diluting the standard antitoxins, the method indicated on the label should be followed. The first dilution should be made with a capacity pipette, to be washed out in the diluting fluid, on account of the viscosity of the glycerinated serum.

The units of botulism antitoxins (types B and C) are similarly definite amounts of the standard types $\mathrm{B}$ and $\mathrm{C}$ antitoxins to be sent out to serum establishments which may be licensed for the production of these antitoxins, the unit of each being $0.4 \mathrm{cc}$. of the glycerinated solution. Antitoxins to be tested are compared with the standard units by means of test doses of types $\mathrm{B}$ and $\mathrm{C}$ toxins, using the same method as above described for type $\mathrm{A}$.

B. Perfringens ( $\mathrm{Cl}$. welchii) antitoxin.-This antitoxin is designed for use against certain of the "gas gangrene" infections. It is standardized ${ }^{1}$ in accordance with the following:

The unit shall be I cc. of the standard serum which is kept in cold storage. To estimate the potency of a commercial antitoxin, the test toxin shall first be standardized by inoculating pigeons intramuscularly with I/ roo unit of standard serum mixed with varying amounts of toxin to determine the smallest dose of toxin which will overcome this amount of serum and kill the pigeon within 24 hours. This dose of toxin, called the "test dose," is usually somewhat greater than ro minimal lethal doses. The test dose of toxin is then to be mixed with varying amounts of the serum to be tested and injected into a second series of pigeons; that amount of serum which gives protection for 24 hours against the test dose of toxin shall be considered to contain I/ 100 unit. The serum-toxin mixtures are incubated 45 minutes at $37^{\circ} \mathrm{C}$. before injection. Pigeons should weigh preferably between 325 and 375 grams, but the doses of toxin and antitoxin shall be proportioned to the weight, 350 grams being taken as the standard weight.

\section{SCARLET FEVER STREPTOCOCCUS ANTITOXIN}

The unit for measuring the strength of scarlet fever streptococcus antitoxin is defined as the neutralizing power possessed by a definite quantity of anti-streptococcic serum preserved at the Hygienic Laboratory of the United States Public Health Service under special conditions to prevent deterioration. This quantity is now equivalent to ten times the amount of scarlet fever streptococcus antitoxin necessary to neutralize one test dose of the corresponding toxin, each test dose containing five times the amount of toxin necessary to give in the majority of non-immune individuals in twenty-four hours following intracutaneous injection, a skin reaction $\mathrm{I} \mathrm{cm}$. or more in diameter.

The skin-test dose was determined originally by the Drs. Dick ${ }^{2}$ for a certain lot of toxin. Since that time new toxins have been standardized in comparison with the original toxin or against toxins so standardized.

1 Bengtson, I. A.: Hygienic Laboratory Bull. 122. 1920.

${ }^{2}$ Dick, G. F., and G. H.: J.A.M.A., 84, 1477-81. 1925 . 
A standardized toxin and samples of the standard serum are distributed by the Hygienic Laboratory of the United States Public Health Service to all producers of the antitoxin. The neutralizing potency of the manufacturer's antitoxin and the standard serum are then compared.

No laboratory animal, with the possible exception of the goat (to be discussed later), has been found sufficiently susceptible to scarlet fever streptococcus toxin to make it available for testing the toxin or the antitoxin, consequently at present we must resort to the use of human subjects. The proportion of susceptible individuals varies at different ages, but generally about ${ }_{5}$ per cent of adults and a somewhat larger proportion of children are suitable subjects.

The essential parts of the requirements at present in force are given here with a protocol illustrating the results of comparative tests made on six individuals:

The number of units contained in each cubic centimeter of the glycerinated solution of standard serum and the number of test doses of toxin contained in each cubic centimeter of control toxin are stated on the respective labels.

The volume of all injections is o.I cc. and all mixtures of toxin and serum contain one test dose of toxin in the amount injected.

Reactions measuring less than $\mathrm{I} \mathrm{cm}$. in every diameter are considered negative in interpreting results, but are measured and recorded. Reading of reactions is made $20-24$ hours after injection and again $44-48$ hours after injection.

The following points are determined:

a) Reaction to the injection of one skin-test dose of control toxin.

b) Reaction and failure of reaction following the injection of at least two mixtures of the standard serum and control toxin. One of these mixtures must show neutralization and the other mixture must show failure of neutralization. The difference between the amounts of serum in the first mixture and in the second mixture should not be greater than that indicated by the addition of the figure 200 to the denominator of the fraction representing the fraction of a cubic centimeter of serum contained in the first mixture, as shown in the sample protocol given below.

c) Reaction or failure of reaction following the injection of one or more mixtures of the new antitoxin and the control toxin.

d) Reaction or failure of reaction following injection of the standard serum alone. This serum control injection contains the largest amount of serum represented in any of the mixtures of the same serum and toxin.

e) Reaction or failure of reaction following injection of the new antitoxin alone, the potency of which is being tested. This serum control injection contains the largest amount of serum represented in any of the mixtures of the same antitoxin and toxin.

An individual is not considered satisfactory for test purposes if: (a) the reaction to either or both of the serum control injections measures more than $4 \mathrm{~mm}$. in any diameter; $(b)$ the reactions to all the injections of the mixtures of the standard serum and control toxin are positive; $(c)$ there is a negative reaction to the mixture of standard serum and control toxin which contains the least serum; $(d)$ there is a negative reaction to the injection of one skin-test dose of toxin.

Each cubic centimeter of the standard glycerinated serum as distributed at present contains 40 units of antitoxin. In calculating the value of a new antitoxin, the smallest volume of standard serum which neutralizes one test dose of toxin is compared to the volume of the new antitoxin which shows equal neutralizing value. Since the potency of the standard serum is known, the potency of the new antitoxin may be readily determined. For instance, 
if I/400 cc. is the smallest amount of standard serum which, when mixed with one test dose of toxin, results in a negative reaction when tested on a susceptible subject, and a new antitoxin tested at the same time on the same subject results in a negative reaction when $\mathrm{I} / 4,000$ cc. of antitoxin is mixed with one test dose of toxin, the value of the new antitoxin will be considered ten times that of the standard serum, or 400 units per cc.

Cases I and 2 show that $I / 4,000 \mathrm{cc}$. of antitoxin $\mathrm{S}$ is equal in neutralizing power to I/400 cc. of the standard serum $\mathrm{C}$, while case 3 shows that $\mathrm{I} / 6,000 \mathrm{cc}$. of antitoxin $\mathrm{S}$ equals $\mathrm{I} / 600 \mathrm{cc}$. of the standard. In each instance antitoxin $\mathrm{S}$ has ten times the neutralizing power that the

\section{TABLE V}

Potency Test on Scarlet Fever Streptococcus Antitoxin Lot S

\begin{tabular}{|c|c|c|c|c|c|c|c|c|c|c|c|c|c|}
\hline \multirow[b]{2}{*}{ CASE } & \multirow[b]{2}{*}{$\begin{array}{l}0 \\
3 \\
0 \\
0 \\
0 \\
\simeq 1 \\
0 \\
0 \\
0 \\
0 \\
0 \\
0 \\
=\end{array}$} & \multicolumn{5}{|c|}{ Antitoxin S } & \multicolumn{5}{|c|}{$\begin{array}{c}\text { Hygienic Laboratory Standard } \\
\text { SERUM LOT C* }\end{array}$} & \multirow[b]{2}{*}{ 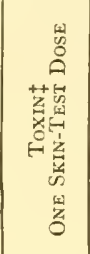 } & \multirow{2}{*}{ 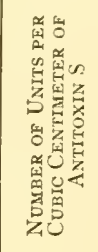 } \\
\hline & & 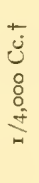 & 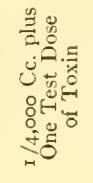 & 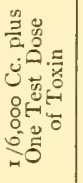 & 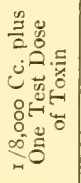 & 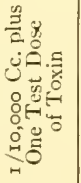 & $\begin{array}{l}+ \\
\dot{u} \\
\dot{8} \\
\dot{+}\end{array}$ & 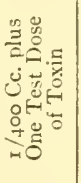 & 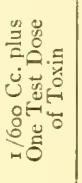 & 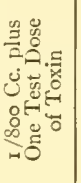 & 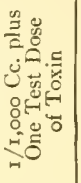 & & \\
\hline & $\left\{\begin{array}{l}24 \\
48\end{array}\right.$ & $\begin{array}{l}0 \\
\circ\end{array}$ & $\begin{array}{l}5 \times 5 \$ \\
8 \times 9\end{array}$ & $\begin{array}{l}4 \times 4 \\
9 \times 10\end{array}$ & $\begin{array}{r}7 \times \\
11\end{array} \times 10$ & $\begin{array}{l}\text { I0 X I I } \\
13 X \text { I I }\end{array}$ & $\begin{array}{l}\circ \\
\circ\end{array}$ & $7 \times 8$ & $\begin{array}{r}4 \times \\
14\end{array}$ & $\begin{array}{r}3 \times \\
1\end{array}$ & $\begin{array}{r}4 \times 4 \\
14 \times 15\end{array}$ & $\left.\begin{array}{c}2 I \times 2 I \\
0\end{array}\right\}$ & 400 \\
\hline $2 \ldots$ & $\left\{\begin{array}{l}24 \\
48\end{array}\right.$ & $\begin{array}{l}0 \\
0\end{array}$ & $4 \stackrel{0}{\times} 5$ & I I $\stackrel{0}{X}$ I I & I $2 \stackrel{0}{\times} I_{4}$ & II $\stackrel{0}{X}$ I $2^{2}$ & $\begin{array}{l}0 \\
0\end{array}$ & $6 \stackrel{0}{\times} 8$ & I $2 \stackrel{0}{X}$ II & $\begin{array}{c}0 \\
9 \times 1\end{array}$ & $\stackrel{\circ}{\mathrm{I}_{2} \mathrm{X}_{\mathrm{I}} 3}$ & $\left.\begin{array}{l}17 \times 21 \\
15 \times 18\end{array}\right\}$ & 400 \\
\hline $3 \ldots$ & $\left\{\begin{array}{l}2.4 \\
48\end{array}\right.$ & $\begin{array}{l}0 \\
0\end{array}$ & $\begin{array}{c}0 \\
5 \times 6\end{array}$ & $\begin{array}{l}6 \times \\
7 \times \\
\end{array}$ & $\begin{array}{r}8 \times I_{0} \\
\text { I } X \text { I I }\end{array}$ & $\begin{array}{r}9 X \text { X } ~ \\
\text { I } 8 \text { X } 8\end{array}$ & $\begin{array}{l}0 \\
0\end{array}$ & $\begin{array}{ll}4 \times & 6 \\
8 \times & 8\end{array}$ & $\begin{array}{l}7 \times 7 \\
7 \times 9\end{array}$ & $\begin{array}{r}7 \times 10 \\
\text { I } 5 \times 15\end{array}$ & $\begin{array}{r}9 \times \mathbf{X I} \\
\text { I } 4 \text { XI } 8\end{array}$ & $\left\{\begin{array}{c}16 \times 18 \\
0\end{array}\right\}$ & 400 \\
\hline $4 \ldots$ & $\left\{\begin{array}{l}2.4 \\
48\end{array}\right.$ & $\begin{array}{l}0 \\
0\end{array}$ & $\begin{array}{l}0 \\
0\end{array}$ & $\begin{array}{l}0 \\
0\end{array}$ & $\begin{array}{l}0 \\
0\end{array}$ & $\begin{array}{l}0 \\
0\end{array}$ & $\begin{array}{l}0 \\
0\end{array}$ & $\begin{array}{l}0 \\
0\end{array}$ & $\begin{array}{l}0 \\
0\end{array}$ & $\begin{array}{l}0 \\
0\end{array}$ & $\begin{array}{l}0 \\
0\end{array}$ & $\left.\begin{array}{l}2 \mathrm{I} \times 2 \mathrm{I} \\
\mathrm{I} 8 \times 2.4\end{array}\right\}$ & $\begin{array}{c}\text { No end- } \\
\text { point }\end{array}$ \\
\hline ;. & $\left\{\begin{array}{l}2.4 \\
4.8\end{array}\right.$ & $\begin{array}{l}0 \\
0\end{array}$ & $\begin{array}{l}14 \times 15 \\
12 \times 13\end{array}$ & $\begin{array}{l}\text { I } 5 \times \text { I } 5 \\
\text { I } 3 \times \text { I } 3\end{array}$ & $\begin{array}{l}\text { I } 6 \times 18 \\
\text { I } 2 X 12\end{array}$ & $\begin{array}{l}20 \times 2 I \\
14 \times 16\end{array}$ & $\begin{array}{l}0 \\
0\end{array}$ & $\begin{array}{l}\text { I } 4 \text { X I } 5 \\
\text { I } 2 \text { X } 12\end{array}$ & $\begin{array}{l}I 6 \times 20 \\
I 3 \times 14\end{array}$ & $\begin{array}{l}25 \times 24 \\
I_{3} \times 13\end{array}$ & $\begin{array}{l}18 \times 18 \\
16 \times 17\end{array}$ & 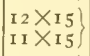 & $\begin{array}{c}\text { No end- } \\
\text { point }\end{array}$ \\
\hline $6 \ldots$ & $\left\{\begin{array}{l}2.4 \\
.8\end{array}\right.$ & $\begin{array}{l}0 \\
0\end{array}$ & $\begin{array}{r}9 \times 11 \\
\text { I6XI9 }\end{array}$ & $\begin{array}{l}\text { I } 3 \times 13 \\
22 \times 24\end{array}$ & $\begin{array}{l}\text { I0 XI4 } \\
21 \times 27\end{array}$ & $\begin{array}{l}\text { I0XI7 } \\
25 \times 27\end{array}$ & $\begin{array}{r}4 X{ }_{4} \\
14\end{array}$ & $\begin{array}{l}14 \times 13 \\
24 \times 27\end{array}$ & $\begin{array}{l}I 8 \times I 7 \\
21 \times 22\end{array}$ & $\begin{array}{l}16 \times 16 \\
23 \times 23\end{array}$ & $\begin{array}{l}18 \times 17 \\
21 \times 22\end{array}$ & $\left.\begin{array}{l}\begin{array}{l}1 \\
2\end{array} \times 15 \\
25 \times 39\end{array}\right\}$ & $\begin{array}{l}\text { Serum } \\
\text { sensitive }\end{array}$ \\
\hline
\end{tabular}

* Standard serum containing 40 units per cubic centimeter.

† Serum control.

† Toxin control.

$\S$ Measurements of two diameters of each reaction are recorded in millimeters. The fraction of a cubic centimeter of antitoxin or serum injected is stated in each instance. Reactions less than $\mathrm{I} \mathrm{cm}$. in diameter are considered negative. Neutralization is considered complete only when reactions to toxin-antitoxin mixtures are negative at both the $2.4-$ and 48 -hour readings.

standard serum possesses, or has a value of 400 units per cubic centimeter. In case 4 the titration of the toxin and antitoxin has not been carried far enough to reach the end-point in this individual. Case 5 shows failure of neutralization of any of the mixtures of toxin and antitoxin tested. Case 6 is serum sensitive.

Kirkbride and Wheeler ${ }^{I}$ have found that among the lower animals tested goats alone proved satisfactory for use in testing scarlet fever streptococcus preparations though ordinarily they are only one-third to one-fourth as susceptible, judged by reaction to intracutaneous injection, as man. About two-thirds of the goats used were found to be sufficiently susceptible to make them available for the testing of toxins and of antitoxins. The authors consider the goat a satisfactory substitute for the human subject in all preliminary tests of toxins and antitoxins. They describe the procedure as follows:

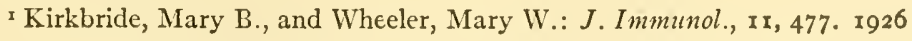


Technique.--The tests were made on the sides and abdomen. At first the hair was clipped and then shaved off but later a barium sulphide and starch depilatory (I volume of barium sulphide to $\mathrm{I} \frac{1}{2}$ volumes of starch) was found more satisfactory than shaving and less irritating to the skin. The toxin dilutions were injected intracutaneously about 3 to $4 \mathrm{~cm}$. apart, the dose being contained in o.I cc. The reactions were read in a bright light after from eighteen to twenty-four hours when they had reached their maximum. At this stage they were usually quite similar to those observed in human subjects. They varied in degree according to the potency of the dilution injected and the susceptibility of the goat, from slightly reddish areas from 1.5 to $2 \mathrm{~cm}$. in diameter, with little or no swelling, to larger reddish areas from 4 to 5 $\mathrm{cm}$. in diameter, with considerable swelling. All reactions faded within from forty-eight to seventy-two hours and there was seldom any pigmentation.

Controls of diluted toxin heated at $100^{\circ} \mathrm{C}$. for one hour, of uninoculated broth containing 0.5 per cent phenol and of mixtures of toxin with scarlet fever antitoxic horse serum, induced no reaction. Normal horse serum, however, did not neutralize the toxin.

A number of other workers have attempted to duplicate this work on goats but generally with results that failed to warrant a continuance of the use of these animals.

\section{ANTI-BACTERIAL SERA}

But three of these-anti-meningococcic, anti-pneumococcic, and anti-dysenteric - are subjected to official tests. In each case the results secured with any given serum are compared with the results secured simultaneously with a control serum distributed by the Hygienic Laboratory. The standardization of these sera is complicated by the existence of several types of the organism which differ immunologically from one another.

\section{ANTI-MENINGOCOCCIC SERUM}

It is to be regretted that there is no satisfactory laboratory test for the therapeutic activity of this valuable preparation. Agglutinins, complement fixing bodies, bacteriotropins, and even protective antibodies have been titrated; but it is generally recognized that none of these tests separately, nor any combination of them, does much more than show that the animals from which the serum has been derived have been immunized more or less intensively against certain strains of the meningococcus. In the United States agglutination tests are very generally used, but occasionally complement fixation is employed. The serum under test, to be reported as satisfactory, must equal the titre of the control serum or at least fall below it by not more than one dilution.

The sera are polyvalent and must contain antibodies for the types of the four principal groups of meningococci. A sample protocol showing two commercial sera slightly superior to the control is shown in Table VI.

\section{ANTI-PNEUMOCOCCIC SERUM}

Official tests in the case of this serum apply only to protection against type I pneumococcus, since this is the only type of infection possibly benefited by the serum, and the clinical evidence of the usefulness of even this type of serum is by no means strong. Mice of about $20-\mathrm{gm}$. weight are the test animals. The test dose of a culture maintained at nearly uniform virulence for mice is not mixed with the serum, but is injected intraperitoneally a few seconds after the serum. The period of observation for the mice after inoculation is ninety-six hours. Two tests of at least four mice apiece 
are required on each lot of serum tested with an interval of at least twenty-four hours between them. The serum under test should at least equal, in percentage of mice protected, the control serum which is distributed for purpose of comparison. This

TABLE VI*

Anti-meningococcic Agglutination Test

\begin{tabular}{|c|c|c|c|c|c|c|c|c|c|c|c|c|c|c|c|c|c|c|c|c|c|c|c|c|c|}
\hline \multirow[b]{3}{*}{ 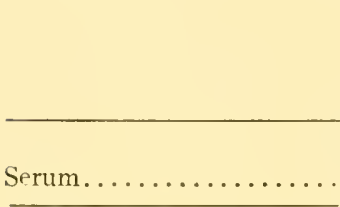 } & \multirow[b]{3}{*}{$\dot{\bar{\theta}}$} & \multirow{2}{*}{\multicolumn{6}{|c|}{$\frac{\text { ANTigen Type } 1}{\text { Culture H.L. I } 23}$}} & \multirow{2}{*}{\multicolumn{6}{|c|}{$\frac{\text { ANTIGEN TyPe II }}{\text { Culture H.L. } 5.5}$}} & \multirow{2}{*}{\multicolumn{6}{|c|}{ Antigen Type $11 \mathrm{II}$}} & \multicolumn{6}{|c|}{ Antigen Type $1 \mathrm{~V}$} \\
\hline & & & & & & & & & & & & & & & & & & & & & ultu & are $\mathrm{I}$ & H.L & 60 & \\
\hline & & 요 & $\stackrel{8}{\circ}$ & 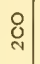 & $\frac{8}{8}$ & 8 & $\begin{array}{l}0 \\
8 \\
0 \\
-1\end{array}$ & 요 & 8 & $\stackrel{4}{8}$ & $\stackrel{8}{8}$ & 8 & $\begin{array}{l}\delta \\
0 \\
= \\
=\end{array}$ & 은 & 8 & 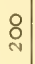 & \& & $\begin{array}{l}8 \\
\infty \\
\infty\end{array}$ & 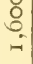 & in & $\stackrel{8}{8}$ & ৪্ & ㅇ. & $\begin{array}{l}0 \\
\infty \\
\infty\end{array}$ & $\stackrel{8}{0}$ \\
\hline 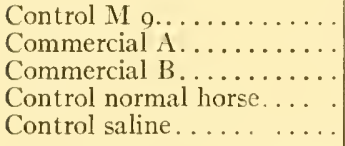 & $\begin{array}{l}\cdots \\
\cdots \\
\cdots \\
\cdots \\
\cdots\end{array}$ & \begin{tabular}{l|l}
4 & \\
4 & \\
4 & \\
0 & \\
0 &
\end{tabular} & $\begin{array}{l}4 \\
4 \\
4 \\
0\end{array}$ & $\begin{array}{l}4 \\
4 \\
4\end{array}$ & $\begin{array}{l}3 \\
4 \\
4\end{array}$ & $\begin{array}{l}1 \\
4 \\
4\end{array}$ & $\begin{array}{l}0 \\
0 \\
2\end{array}$ & $\begin{array}{l}4 \\
4 \\
4 \\
0\end{array}$ & $\begin{array}{l}4 \\
4 \\
4 \\
0\end{array}$ & $\begin{array}{l}4 \\
4 \\
4\end{array}$ & $\begin{array}{l}4 \\
4 \\
4\end{array}$ & $\begin{array}{l}3 \\
4 \\
3\end{array}$ & $\begin{array}{l}0 \\
0 \\
2\end{array}$ & $\begin{array}{l}4 \\
4 \\
4 \\
0 \\
0\end{array}$ & $\begin{array}{l}4 \\
4 \\
4 \\
0\end{array}$ & $\begin{array}{l}4 \\
4 \\
4\end{array}$ & $\begin{array}{l}3 \\
4 \\
4\end{array}$ & $\begin{array}{l}3 \\
3 \\
3\end{array}$ & $\begin{array}{l}2 \\
0 \\
1\end{array}$ & $\begin{array}{l}4 \\
4 \\
4 \\
0 \\
0\end{array}$ & $\begin{array}{l}4 \\
4 \\
4 \\
0\end{array}$ & $\begin{array}{l}4 \\
4 \\
4\end{array}$ & $\begin{array}{l}2 \\
4 \\
4\end{array}$ & $\begin{array}{l}0 \\
3 \\
4\end{array}$ & $\begin{array}{l}\circ \\
0 \\
3\end{array}$ \\
\hline
\end{tabular}

* The figures $1,2,3$, and 4 indicate the degree of agglutination.

control serum is used at the beginning and at the end of each test. The group of eight animals receiving the control serum and the culture should have some mortality, but not complete, at each test, and the control mice which die should show pneumococcus

TABLE VII

Anti-Pneumococcic Serum Test*

\begin{tabular}{|c|c|c|c|c|}
\hline & \multicolumn{4}{|c|}{ Mouse } \\
\hline & 1 & 2 & 3 & 4 \\
\hline \multirow{3}{*}{ 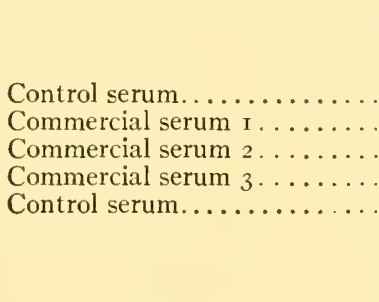 } & \multicolumn{4}{|c|}{ First Test } \\
\hline & $\begin{array}{l}\mathrm{S} \\
\mathrm{S} \\
\mathrm{S} \\
\mathrm{D}-48 \\
\mathrm{~S}\end{array}$ & $\begin{array}{l}\mathrm{D}-36 \\
\mathrm{~S} \\
\mathrm{~S} \\
\mathrm{D}-20 \\
\mathrm{~S}\end{array}$ & $\begin{array}{l}\mathrm{S} \\
\mathrm{S} \\
\mathrm{S} \\
\mathrm{D}-40 \\
\mathrm{~S}\end{array}$ & $\begin{array}{l}\mathrm{D}-40 \\
\mathrm{~S} \\
\mathrm{~S} \\
\mathrm{D}-38 \\
\mathrm{~S}\end{array}$ \\
\hline & \multicolumn{4}{|c|}{ Second Test } \\
\hline $\begin{array}{l}\text { Control serum........... } \\
\text { Commercial serum } 1 \ldots \ldots \ldots \\
\text { Commercial serum } 2 \ldots \ldots \\
\text { Commercial serum } 3 \ldots \ldots \ldots \\
\text { Control serum } \ldots \ldots \ldots\end{array}$ & $\begin{array}{l}\mathrm{S} \\
\mathrm{S} \\
\mathrm{S} \\
\mathrm{D}-36\end{array}$ & $\begin{array}{l}\mathrm{S} \\
\mathrm{S} \\
\mathrm{S} \\
\mathrm{D}-24 \\
\mathrm{~S}\end{array}$ & $\begin{array}{l}\mathrm{S} \\
\mathrm{S} \\
\mathrm{S} \\
\mathrm{D}-30 \\
\mathrm{D}-48\end{array}$ & $\begin{array}{l}\mathrm{S} \\
\mathrm{S} \\
\mathrm{S} \\
\mathrm{D}-22 \\
\mathrm{~S}\end{array}$ \\
\hline
\end{tabular}

$* \mathrm{~S}=$ Survived.

$\mathrm{D}=$ Died-the figure indicating hour of death.

Dose of serum $=0.2 \mathrm{cc}$. intraperitoneally.

Dose of culture $=0.1 \mathrm{cc}$. int raperitonealiy.

Samples $I$ and 2 averaged better than the control, while sample 3 was inert according to this test.

septicemia on necropsy and culture. Animals dying from other causes are considered as if not injected. Two sample protocols covering tests on commercial sera are presented in Table VII. 
ANTI-DYSENTERIC SERUM

At the present time the standardization of this preparation leaves much to be desired. The requirement that the serum shall be at least as active as the control serum in agglutinating the several types of the dysentery organism is recognized as being very untrustworthy. Tests designed to show the antitoxic content of the preparations are more promising though it cannot be said that these are on a substantial basis at present. These latter tests are applicable only to the titration of antisera for the Shiga group of organisms. The subject is complicated by the fact that this organism appears to produce a soluble exotoxin and an endotoxin liberated by lysis of the organism. These toxins act differently, the former affecting chiefly the central nervous system, the latter chiefly the intestinal tract.

Anti-dysenteric serum is being studied at the present time by the Serums Committee of the League of Nations, and it is forecast that in due time a standard antitoxin will be distributed for use as a standard control in toxin-antitoxin neutralization tests.

\section{BACTERIAL VACCINES}

The only preparations of this class subjected to official testing are those made from the typhoid and paratyphoid " $\mathrm{A}$ " and " $\mathrm{B}$ " organisms.

\section{ANTI-ENTERIC VACCINES}

Bacterial vaccines made from the typhoid and the paratyphoid organisms probably are far from uniform as prepared by different producers or by the same producer at different times. It is probable, however, that for practical purposes a sufficiently uniform material is secured when the same procedure is followed in making different batches. Since we do not know the exact mechanism by which typhoid vaccine serves as a prophylactic agent it is not possible to apply any thoroughly satisfactory standardization procedure.

The method in use in the United States ${ }^{\mathrm{I}}$ compares the agglutinin production in rabbits of the products under test with that of a standard control vaccine.

MISCELLANEOUS PREPARATIONS

DIPHTHERLA TOXIN FOR THE SCHICK TEST

This is distributed in packages containing not over one hundred human doses, each human test dose containing I/ 50 or I/40 of a minimum lethal dose for guinea pigs. The human dose is diluted to $0 . \mathrm{I}$ or $0.2 \mathrm{cc}$. for use, according as it contains I/ 50 or I/40 M.L.D. In testing the material the toxin is diluted as for human use, and forty or fifty human doses, depending upon the type of package, are given, subcutaneously, to guinea pigs weighing about $\cdot 250-80 \mathrm{gm}$. At least 75 per cent of these guinea pigs should die between the sixtieth and ninety-sixth hour with the usual lesions of diphtheria toxin poisoning. Table VIII shows a typical protocol of the test on a satisfactory commercial preparation.

\section{TOXIN-ANTITOXIN MIXTURE}

Requirements for this preparation are intended to insure as nearly as possible a preparation sufficiently potent to give immunity when used on human beings but not

`McCoy, G. W.: Hygienic Laborolory Bull. IIO. I9I7. 
sufficiently toxic to be directly harmful to the patient. It is customary to test by giving each of five 300 -gm. guinea pigs a single human dose and each of five of the animals five human doses. The animals on the single human dose should not die acutely but should show paralysis. At least two of the five given five human doses should die acutely-acutely for the purpose of this test being defined as within ten days-and the remainder should die of paralysis.

\section{DIPHTHERIA TOXOID}

This preparation is in use to some extent as a substitute for toxin-antitoxin mixture. It is prepared by treating diphtheria toxin at incubator temperature with dilute formaldehyde solution for about twenty-four hours, and applying the following tests to the finished product:

For toxicity.-Each of four guinea pigs are injected with five human doses of the material. These animals should all remain frec from acute symptoms of diphtheria poisoning.

For potency.-At least 10 guinea pigs, each weighing between 270 and $320 \mathrm{gm}$., receive subcutaneously the initial human dose. At the expiration of thirty days each of these pigs

TABLE VIII

\begin{tabular}{|c|c|c|}
\hline Weight of Guinea Pig & Tested For & Result \\
\hline 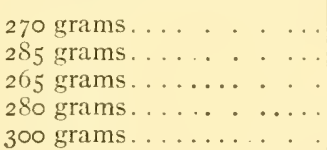 & $\begin{array}{l}\text { I } M . L . D . \\
\text { I M.L.D. } \\
\text { I } M . L . D \text {. } \\
\text { I M.L.D. } \\
\text { I M.L.D. }\end{array}$ & $\begin{array}{l}\text { Dead } 2 \text { days, ig hr. } \\
\text { Dead } 3 \text { days, } 7 \text { hr. } \\
\text { Dead } 3 \text { days, ig hr. } \\
\text { Dead } 3 \text { days, ig hr. } \\
\text { Dead } 4 \text { days, I } 9 \text { hr. }\end{array}$ \\
\hline
\end{tabular}

is injected subcutaneously with 5 M.L.D. of a diphtheria toxin which has been aged sufficiently to reach a stabile toxicity. At least 80 per cent of these pigs must survive for four days.

\section{SCARLET FEVER STREPTOCOCCUS TOXIN FOR THE DICK TEST} (AND FOR IMIMUNIZATION)

The skin-test dose referred to under scarlet fever streptococcus antitoxin is applicable for these preparations. Under present requirements a control toxin is distributed for purpose of comparison when tests are being carried out. The salient points in the testing are as follows:

The dilution of the toxin is determined which will give positive skin reactions by intracutaneous injections in o.I cc. amounts on individuals who are positive to the indicated dose of the control toxin supplied by the Hygienic Laboratory and which will also give a negative reaction on individuals who are negative to the control toxin. The reactions caused by the new toxin on different individuals of varying degrees of susceptibility must be comparable in every way to the reactions produced by the control toxin.

In testing this preparation it is desirable to use at least twenty individuals who are positive to the control toxin-including strongly, moderately, and slightly positive reactors - and at least ten who are negative to it.

The intensity of the reactions as shown by area, color, and swelling produced by the toxin under test must be carefully compared with that due to the control toxin in the same individual. 


\section{TUBERCULIN}

The tests to be mentioned apply to old tuberculin (O.T.) only. There are no satisfactory tests for the various modifications of tuberculin. The tests to be preferred for this preparation, whether intended for diagnostic or therapeutic application, are based on the fact that guinea pigs infected with tuberculosis some weeks earlier react in a specific manner to a subsequent injection of tuberculin. Serological tests have also been employed but are generally considered less satisfactory than those utilizing living animals.

The method longest in use was devised by Koch and depends upon the death of tuberculous guinea pigs when given a sufficient dose of tuberculin at a suitable time after infection. A modification of this method as applied to official standardization by the Bureau of Animal Industry, Department of Agriculture, was described originally by Schroeder and Brett. ${ }^{\text {. }}$ follows:

According to Lewis and Aronson, ${ }^{2}$ the procedure employed more recently is as

A carefully measured minute quantity of tuberculous material, obtained from a tuberculous guinea pig, is injected into a series of animals. About three weeks after infection, the sensitiveness of the guinea pigs to tuberculin is determined by giving I guinea pig daily an intra-abdominal injection of $0.375 \mathrm{gm}$. O.T. per $500 \mathrm{gm}$. body weight. When the sensitiveness of the animals has reached a degree at which this dose causes death within twenty-four hours, 2 guinea pigs are injected daily with $0.25 \mathrm{gm}$. O.T. per $500 \mathrm{gm}$. weight. The day after it is found that this dose kills I at least of the 2 guinea pigs within twenty-four hours, 6 guinea pigs are injected, each with $0.25 \mathrm{gm}$. O.T. per $500 \mathrm{gm}$. weight, and, if 4 or more pigs die in twenty-four hours, the remaining infected pigs are regarded as being sufficiently sensitive for determining the potency of the tuberculin of unknown strength. When the guinea pigs have reached the desired sensitiveness, as determined by the method just described, they are then divided into groups of six. One group is used to each sample of tuberculin the potency of which is to be determined. Each animal of the group receives an intra-abdominal injection of $0.25 \mathrm{gm}$. O.T. to be tested for $500 \mathrm{gm}$. body weight. As a control, another group of 6 guinea pigs is injected, each with an equal amount of the standard tuberculin, and 2 non-tuberculous guinea pigs are injected with the tuberculin which is being tested, in order to make certain that the tuberculin contains no excessive amount of toxic material and no viable tubercle bacilli.

In order to pass the test, it is required that the tuberculin should, within twenty-four hours, kill at least half as many of the test guinea pigs as are killed in the control group injected with the standard tuberculin, and that the animals should show, at autopsy, the characteristic lesions found when tuberculous animals succumb to lethal doses of tuberculin.

Lewis and Aronson modified this method by determining the dose of tuberculin necessary to evoke a cutaneous allergic reaction in tuberculous guinea pigs. The authors give the following instructions for the performance of the titration by the method they proposed:

The reaction to the intracutaneous injection of tuberculin is very definite on the guinea pigs, and one may easily use this method to ascertain the potency of tuberculin by injecting in adjacent places equal amounts of a tuberculin of unknown potency and a standard product.

${ }^{x}$ Schroeder, E. C., and Brett, G. W.: J.A. Vet. M.A. (7th new ser.), 54, 357. I9r9.

${ }^{2}$ Lewis, Paul A., and Aronson, Joseph D.: Am. Rev. Tuberc., 7, 404-I I. I923. 
For this purpose, we have found that light-colored guinea pigs show sharper reactions than do the darker animals.

To remove the hair from the animals, we have employed a depilatory made by mixing barium sulphide with a saturated solution of sodium sulphide, and adding to this mixture the white of egg and water until it assumed a pastelike consistence. The hair over the abdomen and the lower part of the thorax is first cut short with scissors. The depilatory is then applied and permitted to remain for a short time (about one to two minutes) when it is scraped off and the part washed thoroughly with running water. The danger of irritation to the parts is minimized by the addition of egg albumen to the paste and by removal of the depilatory as soon as possible. We have found it advisable to prepare the animals twentyfour to forty-eight hours before injecting them, so that any irritation of the skin will have subsided.

Forty guinea pigs, ranging in weight from 200 to 300 gm., were injected intraperitoneally with o.I mgm. of tubercle bacilli. Virulent cultures of the bovine type were used. Eighteen days later 4 of the animals, when injected intracutaneously with $0.02 \mathrm{mgm}$. of tuberculin of known quality, gave a well-marked reaction which indicated that the animals were sensitive. Three days later, or twenty-one days after infection, the remaining 36 animals were divided into six groups of 6 each, and the potency of a different tuberculin tested on each different group. All 6 pigs in all groups received the following amounts of tuberculin: 0.02 , C.0I, $0.005,0.002,0.00 \mathrm{I} \mathrm{mgm}$., which were brought up to a total volume of $0 . \mathrm{I} \mathrm{cc}$. and injected intracutaneously by means of a tuberculin syringe and a short 26-gauge needle.

Readings were made 24,48 and 72 hours after the injections, but only those made after 48 hours are tabulated, since the reaction reaches its maximum at that time. Degrees of reaction are designated by the figures $\mathrm{I}, 2,3$ and 4 . (4) indicates a very marked reaction, as evidenced by marked redness, edema, necrosis or hemorrhage. (3) and (2) indicate reactions of a less marked redness and edema, depending upon their degree of severity. (I) designates those reactions which show slight redness as well as slight but distinct thickening at the site of injection.

A comparison of the reactions following the employment of the various doses of the standard (control) preparation and of those under test enables one to judge with fair accuracy of the strength of the preparations being tested.

The latter method has recently been studied ${ }^{\mathrm{x}}$ by the Tuberculin Committee of the Health Section of the League of Nations in comparison with the test tube methods of complement fixation and precipitin formation, as well as with the methods using tuberculous persons and tuberculous animals. While the conclusion is not to be regarded as final, it was considered that the method of producing intradermal reactions in sensitized guinea pigs was the most satisfactory of any of those considered, though it was thought preferable to use the same animals for the standard preparation and for the preparations under test. It is desirable to use white skinned guinea pigs of from $45^{\circ}$ to $600 \mathrm{gm}$.

\section{POLLEN EXTRACTS}

Several procedures are in use for the standardization of these preparations, but probably none gives an accurate index of the value of the preparations as applied to clinical use. Noon ${ }^{2}$ used as a unit for pollen extracts the material extracted from

I Calmette, A., and de Potter, F.: Surle Titratedes Tuberculins (Société des nations), Comité d' Hygiene, p. 429. 1926.

${ }^{2}$ Noon, L.: Lancet, 180, 1572. I911. 
I/ 1,000 part of a milligram of pollen. Some manufacturers utilize this for expressing the potency of their preparations.

Clock $^{1}$ employed complement fixation tests. Control antiserum was prepared by immunizing rabbits with a known, potent pollen extract. This serum served as a control in tests of the serum produced by the treatment of rabbits with the extracts under investigation.

Armstrong and Harrison ${ }^{2}$ studied this method and concluded that it might be useful for testing the potency of fresh extracts but that reliable results were not to be secured with old preparations, as old preparations might give a low titre but be quite potent. Perhaps the most frequently used method is that based on the determination of nitrogen in the preparation, though it is obvious that this does not certainly indicate the activity of the material. The technique of Folin and Farmer ${ }^{3}$ is most frequently employed.

None of the methods discussed is regarded as sufficiently satisfactory for adoption as a standard procedure.

TABLE IX

\begin{tabular}{|c|c|c|c|}
\hline Weight of Rabbit & $\begin{array}{l}\text { Venom (Mg. } \\
\text { per Kilo) }\end{array}$ & Dose of Antivenin & Result \\
\hline 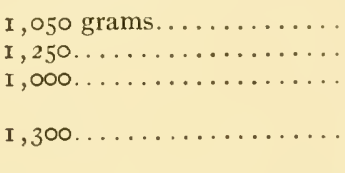 & $\begin{array}{l}4 \cdot 5 \\
6.75 \\
4 \cdot 5 \\
6.75\end{array}$ & $\begin{array}{c}4 \mathrm{cc} . \\
5 \mathrm{cc} . \\
\text { None } \\
\text { (Control) } \\
\text { 5-cc. normal horse } \\
\text { serum (control) }\end{array}$ & $\begin{array}{l}\text { Survived } \\
\text { Survived } \\
\text { Dead I I } \frac{1}{2} \text { hr. } \\
\text { Dead I } 8 \frac{1}{2} \mathrm{hr} \text {. }\end{array}$ \\
\hline
\end{tabular}

\section{ANTIVENIN}

The only antivenin of any importance, so far as concerns North America, is that used against the bites of the Crotalidae (rattlesnakes, moccasins, copperheads). These antisera may be tested in two ways: In one method, doses of the venom surely fatal to experimental animals are mixed with varying amounts of the antivenin, the mixture allowed to stand a short time and then injected into test animals - as in tests with antitoxins. Obviously this gives little indication of the therapeutic value of the serum when we consider the great rapidity with which snake venom acts upon animals. American administrative authorities have therefore insisted that an antivenin should be capable of influencing the action of the poison when injected after the test dose of poison. Consequently the tests are conducted in this manner. The protocol shown in Table IX gives the results of a test of an antivenin against certainly fatal doses of the venom of the North American rattlesnake. The venom was given intramuscularly, and the antivenin about twenty minutes later by the subcutaneous routc. It is obvious that if one wished to determine more accurately the minimum curative dose of the antivenin, smaller quantities than were used in this test would have to be cmployed.

${ }^{x}$ Clock, R. O.: J. Infect. Dis., 22, 80-82. I9r8.

${ }^{2}$ Armstrong, Charles, and Harrison, W. T.: Pub. Heulth Rep., 40, I466. July 10, I925.

3 Folin, Otto, and Farmer, Chester J.: " $\Lambda$ New Method for the Determination of Total Nitrogen in Urine," J. Biol. Chem., II, 493-501. I9I2. 
UNSTANDARDIZED PREPARATIONS

The preparations mentioned are the important ones for which there are standards or standardized methods of testing. There are others for which no method of standardization has been worked out sufficiently to warrant discussion here. There are still others of such doubtful value therapeutically or prophylactically as hardly to warrant the time and effort that would be required to put their standardization on a satisfactory basis. 


\title{
CHAPTER LXXIII
}

\section{ANAPHYLAXIS AND ANAPHYLACTOID REACTIONS}

\author{
HOWARD T. KARSNER
}

School of Medicine, Western Reserve University, Cleveland, Ohio

\section{LIMITATION OF TERMS}

The response of vertebrates to the introduction of foreign substances either enterally through the alimentary canal or parenterally through other channels is variable in species and in the individual. This chapter is concerned principally with quantitative differences in individuals of a species and also in certain measure with differences both qualitative and quantitative in various species.

\section{HYPERSENSITIVENESS}

The usual response in a number of individuals within the species is spoken of as normal, but the normal covers a certain generally accepted range of variability. Individuals who exhibit a greater response than is considered normal are referred to as "hypersensitive." It is well known in pharmacology that certain individuals respond more severely to certain drugs than do others, and thus they are literally hypersensitive. Common experience shows that certain persons become poisoned by contact with poison ivy and sumac whereas others are completely resistant; the former are hypersensitive. Certain individuals respond to inhalation of plant pollens, animal dander, and other organic particulate matter in the atmosphere by exhibiting asthma, coryza, intestinal and other disturbances-materials which to the vast majority are innocuous. When diphtheria antitoxin gained widespread use, it was found that subsequent to injection most individuals develop a more or less severe "serum disease" and that a few are immediately made seriously or even fatally ill. It was noticed that the latter were either naturally hypersensitive or had acquired hypersensitiveness by a previous injection of horse serum. The term "hypersensitiveness" thus covers a variety of forms, some natural and some acquired, some to simple substances, some to such highly organized materials as proteins. In the immunological sense, hypersensitiveness is restricted to that which is inherent in or natural to the individual and that which is acquired. Frequently the hypersensitiveness exhibited in dermatitis venenata is also included in this category, but by common consent that observed in response to drugs and inorganic materials is excluded.

When these abnormal reactions had been studied, Von Pirquet and Schick ${ }^{1}$ suggested the word "allergy" (allos, "altered"; ergia, "reactivity") as a comprehensive term covering the various manifestations, more especially the alteration of reactions in man as observed in the more rapid appearance of the manifestations of serum

${ }^{I}$ von Pirquet, C. E., and Schick, B.: M ünchen. med. Wchnschr., 53, 66. 1906; see also von Pirquet, C. E.: Arch. Int. Mell., 7, 259. 1911. 
disease following a second injection of horse serum. Doerr ${ }^{\mathrm{I}}$ employed the term to indicate protein hypersensitiveness. Others have offered differing definitions. Karsner and Ecker, ${ }^{2}$ in their elementary textbook, decided that the usage is so confusing as to make the term undesirable. $\mathrm{Coca}^{3}$ clearly points out the objections to its use, indicating that etymologically it can include not only increased sensitiveness but also increased resistance. There are, however, certain reactions whose nature is poorly understood, especially as to whether they represent increased sensitiveness or increased resistance, and until these are clarified the term "allergy" might be applied. This means a tentative acceptance of the term to cover literally some alteration in reactions of the organism, whose nature is not as yet specified. The tuberculin reaction at the present time is of this order, and the same applies to the peculiar manifestations of reinfection in tuberculosis, as indicated by Krause and Willis. ${ }^{4}$ Thus, the use of the term "allergy" becomes restricted in contrast to the all-inclusiveness suggested by several writers.

The term "anaphylaxis" has come to have a special meaning. Wells ${ }^{5}$ has furnished a definition with qualifications which meets the views of many authorities. "The term anaphylaxis should be restricted to the condition of hypersensitivity to definitely antigenic substances," with the following limitations:

I. The observed toxicity of the injected material must depend upon the sensitization of the animal; i.e., the substance must not produce similar symptoms in non-sensitized animals.

2. The symptoms produced must be those characteristic of anaphylactic intoxication as observed in the usual reactions with typical soluble proteins, being therefore the same for all antigens with the same test animal, but differing characteristically with each species of animal.

3. It should be possible to demonstrate passive sensitization with the serum of sensitized animals. strip.

4. It should be possible to demonstrate typical reactions in the virgin guinea pig uterus

5. It should be possible to demonstrate amelioration or prevention of the bronchial spasm in guinea pigs by proper use of atropin and epinephrin.

6. The possibility that the observed symptoms are caused by capillary thrombosis or embolism must be excluded.

7. After recovery from anaphylactic shock there should be exhibited a condition of desensitization under proper conditions.

$\mathrm{Coca}^{6}$ objects to No. 5 because of experiments, at that time unpublished, showing that in passively sensitized guinea pigs a maximal dose of epinephrin did not prevent the appearance of shock following a minimal dose of antigen.

The term "atopy" has been devised by Coca "to designate the inherited hyper-

I Doerr, R.: Ergebn. d. Hyg., Bakteriol., u. Immunitätsforsch., I, 257. I914; 5, 71. I922.

${ }^{2}$ Karsner, H. T., and Ecker, E. E.: The Principles of Immunology. Philadelphia and London: Lippincott, I92 I.

${ }^{3}$ Coca, A. F.: Arch. Path. \& Lab. Med., I, 96.1926.

${ }^{4}$ Krause, A., and Willis, H. E.: Am. Rev. Tuberc., 14, 3 I6. I926.

5 Wells, H. G.: The Chemical Aspects of Immunity. New York: Chemical Catalogue Co., I925.

${ }^{6}$ Coca, A. F.: Essentials of Immunology for Medical Students. Baltimore: Williams \& Wilkins, 1925 . 
sensitiveness of human beings. It is a remarkable fact that this sensitiveness, though inherited most probably as a Mendelian dominant character, manifests itself in a number of clinical forms. The recognized forms are hay fever (atopic rhinitis), bronchial asthma, urticaria, eczema, angioneurotic edema and gastro-intestinal disturbance (pains, vomiting, diarrhoea). Duke ${ }^{\mathrm{r}}$ offers strong evidence that atopic sensitiveness can manifest itself also in outspoken bladder symptoms, chiefly pain. . . . . The possibility that other obscure conditions are expressions of hypersensitiveness should be kept in mind" (p. 72). Since it has become established that hypersensitiveness may be produced by enteral absorption of protein the occurrence of atopy as a natural state is now in doubt. In fact, Coca admits that the heritable factor may be regarded as a state of susceptibility to sensitization. Although Cooke and Van der Veer $^{2}$ assumed the factor to be a single Mendelian dominant, and Spain and Cooke ${ }^{3}$ suggested that it may be a multiple factor, neither satisfactorily excluded the assumption of Adkinson ${ }^{4}$ that it is a Mendelian recessive. The fact that in the combined studies of Cooke and Van der Veer and of Spain and Cooke 4r.6 per cent of atopic children show a negative antecedent history is of significance as indicating that at least some of the cases are probably not hereditary. They may have become sensitive in ways not yet clear or by absorption of the "atopen." The arguments are more fully discussed in Coca's chapter in this volume (p. Iо04).

The hypersensitiveness of infection is referred to by Coca as that exhibited in the tuberculin and mallein reactions. That such manifestations, at least the tuberculin test, are truly those of hypersensitiveness is not to be regarded as finally established (Zinsser, ${ }^{5}$ Long and Seyfarth ${ }^{6}$ ). The fact that a patient who has had pneumonia is apparently more susceptible to a subsequent attack is presumably upon a humoral basis, since the lung is anatomically restored to normal in the interim. The incidence of acute endocarditis superimposed on a chronically diseased valve may well be due to alteration of local tissue resistance. Swift and Boots ${ }^{7}$ were unable to sensitize joints to non-hemolytic streptococci. There appears to be no clear experimental evidence as yet to support the conception, in the true sense, of hypersensitiveness to infection. Nevertheless, there are phenomena in infectious disease that strongly suggest this possibility.

\section{ANAPHYLACTOID PHENOMENA}

The parenteral introduction of certain agents, especially into the vascular system, and also by intraperitoneal and subcutaneous routes, leads to certain symptoms and signs which resemble closely anaphylactic shock. When all the features are evaluated, particularly in the light of the criteria offered by Wells, these reactions are not to be regarded as anaphylactic. The resemblance is signified by the term "anaphylactoid

r Duke, W. W.: Allergy-Asthma, II ay Fever, Urticuria, elc. St. Louis: Mosby, I925.

${ }^{2}$ Cooke, R. A., and Van der Veer, A.: J. Immunol., I, 20I. I9I6.

${ }^{3}$ Spain, W. C., and Cooke, R. A.: ibid., 9, 25I. 1924.

${ }_{4}$ Adkinson, J.: Genetics, 5, 363. I920.

s Zinsser, H.: Infection and Resistance (2d ed.), p. 440. New York: Macmillan, I9I8.

${ }^{6}$ Long, E. R., and Seyfarth, Mac H.: Am. Rev. Tuberc., 9, 254. 1924.

${ }^{7}$ Swift, H. F., and Boots, R. II.: J. Exper. M I cl., 38, 573. 1923. 
reactions" or phenomena but this does not indicate necessarily that in their essential nature they are directly related to anaphylaxis.

The original observation of anaphylaxis was with a substance, the poison of the tentacles of actinia, which in itself has toxic effects. Richet and Portier ${ }^{\mathrm{T}}$ in studying this substance found that if a sublethal dose had been injected into a dog and three weeks allowed to supervene a very small second dose proved to be fatal. Eel serum, ox serum, and dog serum are toxic for the guinea pig. Venoms of certain snakes are toxic for a variety of animals, as is true of certain of the substances which produce anaphylactoid symptoms. The substances of protein character usually produce hyperemia, hemorrhage, and edema. They have been grouped by Arthus under the term "congestins." Being of protein character, they may be capable of producing anaphylaxis. As pointed out by Arthus, ${ }^{2}$ and by Karsner ${ }^{3}$ independently, the pathological findings following fatal shock have the special characters of anaphylaxis plus those of the congestins - a congestive type of anaphylaxis, thus a combination of anaphylactic and anaphylactoid phenomena.

\section{ANAPHYLAXIS}

The basic statement of the nature of anaphylaxis is given simply by the discoverer Richet" in the words: "Une substance insuffisante à tuer ou même à rendre malade un animal normal, détermine des accidents foudroyants et mortels chez un animal qui, longtemps auparavant, avait reçue cette même substance." The term "anaphylaxis" (ana, "against"; phylax, "guard"), coined as a result of physiological experiments, came into general use when the more strictly immunological work of Arthus, Otto ${ }^{5}$ and Rosenau and Anderson ${ }^{6}$ gave the key to the mysterious ill effects that sometimes follow the use of horse serum in the treatment of diphtheria. The general reaction is sometimes referred to as the "Theobald Smith phenomenon," the local reaction as the "Arthus phenomenon." The local reaction is practically the same in all animals, but the general reaction varies in different species, and in man shows differences within the species. The special problems deal with sensitization, incubation, intoxication, and the reaction.

\section{ACTIVE SENSITIZATION}

Under this heading must be considered the nature of the sensitizing substance, the mode of introduction into the body, and the size of the dose. Wells, ${ }^{7}$ in his admirable reviews, points out that "anaphylaxis represents an antigen-antibody reaction."

r Richet, C.: L'Anaphylaxie. Paris: Alcan, 1923; Compt. rend. Soc. de biol., 66, 1005. I909; Portier, P., and Richet, C.: ibid., 54, 170. I902.

${ }^{2}$ Arthus, M.: De l'Anaphylaxie à l'immunilê. Paris: Masson, I921.

3 Karsner, H. T.: Zischr.f. Immunitütsforsch.u. exper. Therap., 14, 81. I9I 2.

${ }_{4}^{4}$ Richet, C.: L'Anaphylaxie. Paris: Alcan, I923.

5 Otto, R.: Leuthold Gedenkschrift. Berlin, I906.

${ }^{6}$ Rosenau, M. J., and Anderson, J. F.: Hyg. Lab., U.S. Pub. Health E Marine Hosp. Serv., Bull. 29. Washington, D.C., I906; J. Med. Research, 21, I. I909; Anderson, J. F., and Rosenau, M. J.: Arch. Int. Med., 3, 519. I909; Harvey Lectures, p. I 7. Philadelphia: Lippincott, I908-9.

7 Wells, H. G.: Physiol. Rev., I, 44. I92 I; see also The Chemical Aspects of Immunity. New Xork: Chemical Catalogue Co., I925. 
The antigen may be spoken of as "anaphylactogen" and the antibody as "anaphylactin." There is no doubt that the anaphylactogen is of protein nature, but there remain the questions as to how simple the protein fractions may be and still be antigenic and whether or not non-protein substances may be antigenic.

Whole proteins are not necessarily anaphylactogenic, and even if so there may be certain amazing variations. According to Wells, the proteins which may be spoken of chemically as incomplete, containing a small number of amino acids, are non-antigenic. This is true of protamines and histones and the nucleoproteins and hemoglobins. Starin ${ }^{\mathrm{r}}$ has shown that gelatin, which contains neither tryptophane nor tyrosine and little phenylalanine, is not antigenic. Wells and Osborne ${ }^{2}$ have shown that zein, gliadin, and hordein, poor in diamino acids, are definitely antigenic, whereas protamines, rich in diamino acids, are not antigenic. Wells concludes that lysine and presumably glycine and tryptophane are of no significance, but that "since no protein is known which does not contain either histidine or arginine," nothing can be said definitely about these bodies. According to experiments of TenBroeck, ${ }^{3}$ racemized proteins, which are not attacked by proteolytic enzymes, do not act as anaphylactogens. Gay and Adler ${ }^{4}$ state that the euglobins contain the sensitizing but not the intoxicating substance, but $\mathrm{Kato}^{5}$ maintains that they contain both. After hydrolysis of coagulated egg white with steam under pressure, Fink ${ }^{6}$ found that fractions precipitated by three-fourths and complete saturation with ammonium sulphate were slightly anaphylactogenic and definitely antigenic otherwise. In spite of his controls it is at least possible, especially since the other fractions thrown down with a smaller concentration of ammonium sulphate were non-antigenic, that the reactions were anaphylactoid. The difficulty of interpretation of non-fatal results is becoming more widely recognized, as anaphylaxis and similar phenomena are more fully studied. As Fink points out, some of the earlier results are now subject to changed interpretations. It seems unlikely that protein fractions are antigenic, and as Landsteiner ${ }^{7}$ indicates, it is probable that the antigenic property is a function of the size of the molecule. Trypsin digestion destroys the antigenic property of proteins. In consideration of these facts and especially of the anaphylactoid reactions, as well as certain features of the experiments themselves, Wells is doubtful that the results of Abderhalden ${ }^{8}$ with a synthetic polypeptid and of Zunz ${ }^{9}$ with a simpler polypeptid can be accepted.

Heat or chemicals which render the protein insoluble destroy its sensitizing property, apparently because of the insolubility. Wells points out that the few proteins not made insoluble by heat, casein, ovomucoid, so-called "proteoses of plant

'Starin, W. C.: J. Infect. Dis., 23, I39. I918.

${ }^{2}$ Wells, H. G., and Osborne, T. B.: ibid., 8, 66. rgr r.

${ }^{3}$ TenBroeck, C.: J. Biol. Chem., 17, 369. r914.

${ }_{4}$ Gay, F. P., and Adler, H. M.: J. Med. Reseurch, 13, 433. 1908.

${ }^{5}$ Kato, Y.: Mitt. a.d.med. Fak. d.k. Univ. Tokyo, 18, 109. 1907.

${ }^{6}$ Fink, E. B.: J. Infect. Dis., 25, 97. I919.

'Landsteiner, K.: Biochem. Ztschr., 93, го6. I9I9.

${ }^{8}$ Abderhalden, E.: Ztschr.f. phys. Chemie, 8I, 3I 5 . I9I 2.

${ }^{9}$ Zunz, E.: Arch. internat. de physiol., I5, 179, 192. 1919. 
seeds," and beta nucleoproteins remained antigenic after heating. Besredka ${ }^{\mathrm{I}}$ states that if a coagulable protein be diluted so as to prevent coagulation it is antigenic after heating to $20^{\circ} \mathrm{C}$. Rosenau and Anderson ${ }^{2}$ showed early in their work that if the protein be heated in the dry state and redissolved it will resist temperatures of $170^{\circ} \mathrm{C}$. for ten minutes. Falk and Caulfield ${ }^{3}$ state that a pure protein acts best as a sensitizer when it is on the acid side of its isoelectric point.

Different proteins, ordinarily regarded as antigenic, may under identical conditions not be equally anaphylactogenic. Manwaring ${ }^{4}$ and his colleagues have found that in the dog attempts at sensitization with egg white, goat serum, and horse serum, injected under the same conditions in different individuals, resulted only in sensitization by the horse serum.

The suggestion that lipoids may act as anaphylactogens failed of confirmation in the hands of White, ${ }^{5}$ and no work since then is sufficiently convincing to alter this conclusion. Such reactions as have been elicited were probably due to adsorbed proteins. Although certain polysaccharides are immunologically specific (Heidelberger), ${ }^{6}$ they have not been shown to act as anaphylactogens. The substance prepared by Tomcsik ${ }^{7}$ may be an exception but is not entirely non-nitrogenous.

The various reactions to drugs are apparently the result of idiosyncrasy rather than acquired sensitization. The studies of Pilcher and Sollmann ${ }^{8}$ with morphin show no change in the cutaneous sensitiveness of addicts. The deleterious effects of highly complex arsenicals and mercurials, as well as other drugs given intravenously, are to be regarded as anaphylactoid.

The amount of protein necessary for sensitization is extremely small. Rosenau and Anderson were successful in guinea pigs with $0.00000 \mathrm{I}-\mathrm{cc}$. horse serum and Wells with 0.00000005 -gm. crystallized egg albumin. The sensitization was slight and the shock dose not fatal. Larger doses are necessary with other animals, but the minimum sensitizing dose for man is not known. White and Avery ${ }^{9}$ found that the sensitization increases, up to a certain point, with the size of the sensitizing dose. In a general way, the smaller the sensitizing dose, the larger the minimum intoxicating dose. Besredka states that larger doses increase the length of time for the appearance of sensitization. Lewis ${ }^{\text {Io }}$ has shown that simultaneous injection of large amounts of one serum and small sensitizing doses of another will interfere with sensitization by the latter serum.

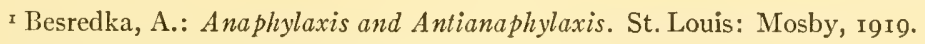

${ }^{2}$ See Rosenau, M. J., and Anderson, J. F.: loc. cit.

3 Falk, I. S., and Caulfield, M. F.: Proc. Soc. Exper. Biol. Eo Med., 20, 199. I923; J. Immunol., 8, 239. I923.

${ }_{4}$ Manwaring, W. H., Marino, H. D., McCleave, T. C., and Boone, T. H.: Proc. Soc. Exper Biol. \& Med., 24, 650. 1927.

5 White, B.: J. Med. Research, 30, 393. I914.

${ }^{6}$ Heidelberger, M.: Chem. Rev., 3, 403. I927.

7 Tomcsik, J.: Proc. Soc. Exper. Biol. \& Med., 24, 81 2. I927.

${ }^{8}$ Pilcher, J. D., and Sollmann, T.: Arch. Int. Med., 33, 516. I924.

9 White, B., and Avery, O. T.: J. Infect. Dis., I3, 103. I913.

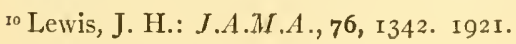


The usual method of sensitization in experimental work is by parenteral introduction. In the guinea pig it is usually a single subcutaneous injection. In the dog it may be two or three subcutaneous injections on successive days, or one subcutaneous injection followed on the next day by an intravenous administration. Intraperitoneal and intrathecal routes have been employed. If sensitization can occur by way of the intestinal canal, it must presumably be due to absorption of native undigested protein. Rosenau and Anderson were able to sensitize guinea pigs by feeding horse serum. Van Alstyne and Grant ${ }^{\mathrm{I}}$ found that by feeding egg white to dogs the native protein could be identified in blood and urine. Using the method of Prausnitz and Küstner, Walzer and Walzer ${ }^{2}$ show that the protein of foods to which patients are passively sensitized may be absorbed through the intestine. The practical importance of these studies is great, but for quantitative work parenteral routes must be employed.

For a period of eight to twelve days after the sensitizing dose, subsequent injections of the homologous protein produce no evidence of hypersusceptibility. If during this period a second injection be given, the animal is more likely to become immune than hypersensitive. The sensitiveness usually reaches its height in about twenty-one days, after which it slowly diminishes but probably persists indefinitely. Extremely minute doses seem to delay the appearance of maximum sensitiveness, as is also true of larger doses. Therefore, there is an optimum sensitizing dose which varies in a fairly wide range.

\section{PASSIVE SENSITIZATION}

The serum (not the cells) of an animal actively sensitized, even before sensitization in that animal can be satisfactorily demonstrated, or even if that animal be in the temporary refractory state called "anti-anaphylaxis," can produce sensitization upon injection into another animal. This passive sensitization can be demonstrated about four hours after intravenous injection, about twenty-four hours after intraperitoneal injection, and about forty-eight hours after subcutaneous injection, persists for three or four days at its height, and gradually disappears in the course of a few days. If a precipitating serum be prepared in an animal against a certain protein and injected into another animal, the latter becomes sensitive to the homologous protein-a fact pointed out by Doerr and Moldovan. ${ }^{3}$ Doerr and Russ, ${ }^{4}$ and Scott, ${ }^{5}$ have shown that the content of precipitin in the serum employed and the intensity of the anaphylactic shock are generally parallel, but exceptions have been noted by Falk and Caulfield. ${ }^{6}$ That the young of sensitive female guinea pigs are also hypersensitive is well established, but it has not been conclusively shown to be by passive sensitization.

\section{ANAPHYLACTIC SHOCK}

When the homologous protein is injected into the sensitized animal, the reaction is in the form of acute (immediate) or subacute (delayed) shock, depending upon the

IVan Alstyne, E. V. N., and Grant, P. A.: J. Med. Reseurch, 25, 399. I9II-I2.

2 Walzer, A. and M.: Am. J. M. Sc., I73, 279. I927.

3 Doerr, R., and Moldovan, J.: Ztschr.f. Immunitätsforsch.u. exper. Therap., 5, т25. I9ro.

4 Doerr, R., and Russ, V. K.: ibid., 3, I8I. I909.

5 Scott, W. W.: J. Path. E Bact., I4, I47. I910.

${ }^{6}$ Falk, I. S., and Caulfield, M. F.: loc. cit. 
degree of sensitiveness, the size of the shock dose, the condition of the protein, and the method of administration. The rapidity and severity of reaction are greatest following intravenous or intracardiac injection and less so following, in order, intrameningeal, intraperitoneal, and subcutaneous injections. Besredka estimates that, with the use of serum, approximately equivalent reactions may be produced by intravenous injections of $0.05-0.1 \mathrm{cc}$, intrathecal injections of $0.066-0.125 \mathrm{cc}$, and intraperitoneal injections of $5.0-6.0 \mathrm{cc}$. Subcutaneous injections in experimental animals rarely produce acute, severe, or fatal reactions. It is obvious that intravenous injections are undesirable when solid proteins, bacteria, and proteins with capacity for direct alteration of the blood are to be employed, because the result may be confused by the occurrence of thrombosis or embolism.

Anaphylactin is subject to the same deteriorative effects as anaphylactogen. Kato's work, quoted above, indicates that the two are not chemically different. The effectiveness of fresh serum decreases during the first ten days by about 50 per cent. The curve flattens thereafter. Besredka found that a serum twenty years old was still effective, and Uhlenhuth and Haendel ${ }^{x}$ state that the same is true of proteins from Egyptian munmies.

Individual variations in animals of the same species make it seem advisable to judge of effectiveness only on the basis of fatal reactions (Karsner and Ecker). ${ }^{2}$ IVells states that blood serum contains so many substances that it can be regarded as an "extract of the animal," an objection that can be overcome only by the use of pure proteins. Much of the reported work is on the basis of serum anaphylaxis, and some of the conclusions may be altered when the use of pure proteins becomes more general. It is said that the size of the minimum intoxicating dose is to the size of the minimum sensitizing dose as 100 to $\mathrm{I}$. In the guinea pig, fatal reactions are rarely obtained with less than 0.025 -cc. serum, and as a rule much larger amounts are required. Wells reports fatal shock with 0.00000 -gm. crystallized egg white.

Features of shock.- These are to be considered under the headings of "Objective Manifestations in the Living Animal," "Morbid Anatomical Changes," and "Functional Disturbances."

The clinical manifestations, essentially the same in both forms, are more severe in acute or immediate shock than in the subacute or delayed shock. In immediate shock in the guinea pig there are ruffling of fur, rubbing of nose, evacuation of urine and feces, spasmodic movements increasing to violent general convulsions, severe dyspnea, cyanosis, and death from asphyxia with the heart still beating. In the dog and the rabbit, the respiratory and convulsive phenomena are less evident, the principal feature being marked fall in blood pressure, violent precordial activity, salivation, vomiting, and diarrhea. Parker and Parker ${ }^{3}$ have found that the white rat behaves much as does the dog. The cat may show pronounced respiratory phenomena but the usual feature is fall of blood pressure (Edmunds). ${ }_{4}^{4}$ In birds, especially fowl and pigeons, the symptoms are generally the same, associated with marked

${ }^{x}$ Uhlenhuth, H., and Haendel, D.: Ztschr. f. Immunitätsforsch. u. exper. Therap., 4, 761. I910 (see p. 774).

${ }^{2}$ Karsner, H. T., and Ecker, E. E.: J. Infect. Dis., 30, 333. 1922.

3 Parker, J. T. and F.: J. Med. Research, 44, 263. 1924.

${ }^{4}$ Edmunds, C. W.: J. Pharmacol. \& Exper. Therap., 5, 518. I9r4;Ztschr. f. Immunitätsforsch. 4. exper. Therap., 22, I8I. I9I4. 
salivation and lacrymation (see Gahringer). In man, the respiratory difficulty may be prominent, but as a rule the circulatory difficulty is more severe and the cause of death. Edema may be observed in lower animals, but is usually demonstrated only by microscopic examination; subcutaneous and pulmonary edema may be severe in man.

The anatomical findings vary with the acuteness and severity of the shock and with the species of animal. Dilatation of the heart, especially of the right side in guinea pigs, is common to all animals. Rigid distention of the lungs, which cannot be compressed except by catheterization of the bronchi, is most marked in the guinea pig in acute shock. Hyperemia, minute hemorrhages, and edema usually observed in both acute and subacute shock in other animals are not severe in the distended lungs of acute shock in guinea pigs but present in delayed shock.

Microscopically, in the rigidly inflated lung there is marked distention of the alveoli, with occasional rupture of their walls, constriction of the bronchi and frequently of the small arteries. The secondary bronchi are likely to show edema in the lumina and in the surrounding tissue. The site of greatest muscular contraction was shown by Schultz and Jordan ${ }^{2}$ to be in the secondary bronchi. The abdominal viscera are the seat of well-marked passive hyperemia. Gay and Southard ${ }^{3}$ describe fatty degeneration of the capillary endothelium near small hemorrhages. Beneke and Steinschneider ${ }^{4}$ found Zenker's hyaline necrosis of skeletal muscles, particularly those of respiration, which Wells believes is due to asphyxia. These changes cannot be regarded as characteristic of anaphylactic shock.

The distention of the guinea pig lung has been studied extensively. This animal has a heavy bronchial and bronchiolar muscle and rather weak extrinsic respiratory muscles. It seems to be more capable of inspiratory than expiratory effect. This may serve to explain why at death the lungs are widely distended. Auer and Lewis ${ }^{5}$ state that the asphyxia is "apparently produced by tetanic contraction" of the bronchiolar musculature. I have confirmed this statement, but in recent studies as yet unpublished have been impressed in addition by the edema described above. Pelz and Jackson ${ }^{6}$ have observed bronchoconstriction in the $\log$, but we have never seen pulmonary distention comparable to that of the guinea pig.

This reaction of smooth muscle has been found to be general. Schultz ${ }^{7}$ demonstrated that isolated intestinal muscle of the guinea pig normally sensitive to fresh serum is more so if the animal has been rendered hypersensitive (anaphylactic). Dale, ${ }^{8}$ subsequently confirmed by $\mathrm{Weil}^{9}$ and others, more conclusively demonstrated the fact in regard to the virgin guinea pig uterus which is not sensitive normally to fresh serum. The same is true of the dog's uterus (Manwaring et al.). ${ }^{10}$ Manwaring and his colleagues have shown that in the dog's alimentary canal the most marked effects are in the colon and rectum. The urinary bladder of the dog and guinea pig reacts strongly, but this is not true of the rabbit's bladder. The blood vessels of the lungs of guinea pigs also react. The question of the reaction of hepatic blood vessels will be discussed subsequently.

I Gahringer, J. E.: J. Immunol., 12, 477. 1926.

${ }^{2}$ Schultz, W. H., and Jordan, H. E.: J. Pharmacol. \& Exper. Therap., 2, 375. I9II.

3 Gay, F. P., and Southard, E. E.: J. Med. Research, 16, I43. 1907.

4 Beneke, R., and Steinschneider, E.: Centralbl.f. allg. Path. u. path. Anat., 23, 529. I9г2.

${ }^{5}$ Auer, J., and Lewis, P.: J. Exper. Med., I2, I51. I9 10.

${ }^{6}$ Pelz, M. D., and Jackson, D. E.: J. Lab. E० Clin. Med., 3, 387. 1918.

${ }^{7}$ Schultz, W. H.: J. Pharmacol. \&o Exper. Therap., I, 549. I909-Io.

${ }^{8}$ Dale, H. H.: ibid., 4, 167. 1912-13.

9 Weil, R.: J. Med. Research, 30, 87, 299. 1914; Proc. Soc. Exper. Biol. \& Med., I I, 86. I914.

ro Manwaring, IV. H., Hosepian, V. M., Enwright, J. R., and Porter, D. F.: J. Immunol., ro, 567. I925; Manwaring, W. II., and Marino, H. D.: ibid., r3, 69. 1927. 
An abrupt fall of blood pressure in anaphylactic shock is common to all animals but is especially pronounced in the dog and cat (Biedl and Kraus, ${ }^{\mathrm{I}}$ Pearce and Eisenbrey, ${ }^{2}$ Schult $z^{3}$ ). The rabbit often shows an evanescent preliminary rise of pressure before the fall. The detailed studies have been principally upon the dog and cat. Biedl and Kraus, and Pearce and Eisenbrey, maintained that the beart is not primarily at fault. The fact that injury to the cat's heart has been reported (Edmunds) 4 loses some of its significance when it is recognized that the non-sensitized heart also responds in the same qualitative way to serum. Cardiac arrythmias in the dog and rabbit (Auer and Robinson, ${ }^{5}$ Robinson and Auer ${ }^{6}$ ) are not assumed by these authors to be the cause of the fall in blood pressure. The vasomotor center is not concerned in the fall (Sollmann). ${ }^{7}$ Peripheral vasodilatation has been assumed to be the cause, but the vessels respond to epinephrin and barium (Sollmann). Manwaring, Chilcote, and Hosepian, ${ }^{8}$ by perfusion experiments, find peripheral vasoconstriction, with occasional vasodilatation in the hind extremities. Increased permeability of capillaries, as demonstrated by Manwaring and his colleagues, ${ }^{9}$ indicated also by the work of Peterson and his collaborators ${ }^{\text {I0 }}$ showing increase in flow and protein content of the lymph, may be in part responsible. Much attention has been given to the return flow of blood to the heart. In general, this is reduced, although in the cat Edmunds ${ }^{1 x}$ found no depression of pulmonary-vein pressure and occasionally an increase. Mautner and Pick ${ }^{\mathrm{x} 2}$ suggested that decreased return is due to contraction of portal vessels. Manwaring ${ }^{13}$ and subsequently Voegtlin and Bernheim $^{\mathrm{I}}$ found that exclusion of the liver prevents the occurrence of anaphylactic shock. Pelz and Jackson ${ }^{15}$ state that exclusion of the entire abdominal circulation does not prevent shock. Simonds, ${ }^{16}$ however, and Manwaring, ${ }^{17}$ continue to assert the significance of the liver, and that view is generally accepted. The explanation of the influence of this organ differs with the two latter investigators. By pressure on the portal veins, Manwaring was able to produce a fall in systemic blood pressure but maintains that the rise in portal pressure is not sufficient to account for the fall in systemic pressure in peptone shock. By compression of the hepatic vein, Simonds ${ }^{18}$ finds that the mechanical disturbance is sufficient to explain the systemic change in peptone and anaphylactic shock. The latter experiment is more in ac-

I Biedl, A., and Kraus, R.: Wien. klin. Wchnschr., 22, 363. 1909.

${ }^{2}$ Pearce, R. M., and Eisenbrey, A. B.: J. Infect. Dis., 7, 565. 1910; Eisenbrey, A. B., and Pearce, R. M.: J. Pharmacol. \& Exper. Therap., 4, 21. I9I2.

${ }^{3}$ Schultz, W. H.: J. Pharmacol. \& Exper. Therap., 3, 299. г9г 2.

${ }^{4}$ Edmunds, C. W.: loc. cit.

${ }^{5}$ Auer, J., and Robinson, G. C.: J. Exper. Med., i8, 450. I913.

${ }^{6}$ Robinson, G. C., and Auer, J.: ibid., p. 556 . I913.

${ }_{7}^{7}$ Sollmann, T.: Mamul of Pharmacology (3d ed.), p. 484. Philadelphia: Saunders, I926.

${ }^{8}$ Manwaring, W. H., Chilcote, R. C., and Hosepian, V. M.: J. Immunol., 8, 233. 1923.

9 Manwaring, W. H., Chilcote, R. C., and Hosepian, V. M.: J.A.MI.A., 80, 303. 1923.

ro Peterson, IV. S., Levinson, S. A., and Hughes, T. P.: J. Immunol., 8, 323. 1923.

II Edmunds, C. W.: loc. cit.

I2 Mautner, H., and Pick, T.: Mïnchen. med. Wchnschr., 62, I14I. I9I5.

${ }^{13}$ Manwaring, W. H.: Ztschr.f. Immunitätsforsch. u. exper. Therap., 8, 1. I910.

${ }^{14}$ Voegtlin, C., and Bernheim, B. M.: J. Pharmacol. Eo Exper. Therap., 2, 507. I9Ix.

${ }^{15}$ See Pelz, M. D., and Jackson, D. E.: loc. cit.

${ }^{16}$ Simonds, J. P.: Am. J. Physiol., 65, 512. 1923; Simonds, J. P., and Brandes, W. W.: ibid., 72, 320. 1925 .

${ }^{17}$ Manwaring, W. H., Brill, S., and Boyd, W. H.: J. Immunol., 8, I21. I923.

${ }^{18}$ Simonds, J. P.: J.A.M.A., 73, 1437. I919. 
cord with the change assumed by Simonds to occur in the intact liver, namely, a constriction of the hepatic veins sufficient to reduce the return flow to the heart. Manwaring ${ }^{1}$ would exclude the factor of vasoconstriction and ascribe the mechanical factor to perivascular edema. More important in his opinion is the explosive formation in the liver of a histaminelike vasodepressor substance.

The blood-cell changes are variable. Bose $\mathrm{e}^{2}$ demonstrated a relative eosinophilia in guinea pigs when hypersensitive. In shock, leukopenia occurs in various animals. It occurs in dogs, apparently due to accumulation of leukocytes in the lung capillaries (Webb), ${ }^{3}$ and is said by Dean and Webb ${ }^{4}$ to be followed by leukocytosis. Richet and his colleagues ${ }^{5}$ tate that in dogs there is an increase in the number of erythroblasts. Zunz $z^{6}$ found thrombopenia in dogs, and Piana ${ }^{7}$ reports large numbers of platelets in the capillaries of the liver.

A decrease in coagulability of the blood is especially marked in dogs and guinea pigs less so in other animals. This has been studied by Biedl and Kraus, ${ }^{8}$ Achard and Aynaud, ${ }^{9}$ Lee and Vincent, ${ }^{\mathrm{I}}$ Pepper and Krumbhaar, ${ }^{\mathrm{II}}$ and others. Gahringer ${ }^{\mathrm{I} 2}$ finds that in pigeons the decrease is preceded by a marked increase. This has been confirmed by Reed and Lamson. ${ }^{13}$ Bulger ${ }^{14}$ concludes that the decrease in coagulability is "due to changes in that stage of the coagulative process at which thrombin is formed through the interaction of prothrombin, calcium, thromboplastin and antithrombin (?). These changes are probably due to variations in thromboplastin."

Gaseous metabolism is increased in the convulsive stage and decreased in the asphyxial stage of shock (Abderhalden and Wertheimer). ${ }^{\text {Is }}$ The body temperature first rises (Richet) and then falls precipitously (Biedl and Kraus). Hirsch and Williams ${ }^{26}$ report a slight increase in blood sugar. McCullough and $\mathrm{O}^{\prime} \mathrm{Neill}^{17}$ demonstrated marked increase in blood sugar and lactic acid. Zeckwer and Goodell ${ }^{18}$ found marked hyperglycemia in bacterial anaphylaxis even when the organisms produced hypoglycemia in normal animals. The asphyxia is also associated with acidosis (Hirsch and Williams) and with a decrease of surface tension (Zunz and La Barre). ${ }^{\text {I9 }}$

I Manwaring, W. H., and Kusama, Y.: J. Immunol., 2, 157. 1917; Manwaring, W. H., and Crowe, H. E.: ibid., 2, 517. I9I7.

${ }^{2}$ Bose, J. P.: Calcutta M. J., 18, 377. I923.

3 Webb, P. A.: J. Path. \& Bact., 27, 79. I924.

${ }_{4}^{4}$ Dean, H. R., and Webb, P. A.: ibid., p. 65. I924.

5 Richet, C., Brodin, P., and St. Girons, F.: Compt.rend. Acad. de sc., r68, 369. I919; see also Brodin, P., and Richet fils: Compt.rend. Soc. de biol., 84, 298. г921.

${ }^{6}$ Zunz, E., and Geerthruyden-Bernard, D.: Inst. d. Therap., p. 79. Bruxelles, 1922.

7 Piana, C.: Riforma med., 39, 702. I923. $\quad{ }^{8}$ Biedl, A., and Kraus, R.: loc. cit.

9 Achard, C., and Aynaud, M.: Compt. rend. Soc. de biol., 67, 83. 1909.

10 Lee, R. I., and Vincent, B.: J. Mled. Research, 32, 445. I915.

"Pepper, O. P. H., and Krumbhaar, E. B.: J. Infect. Dis., I4, 476. I914.

${ }^{12}$ Gahringer, J. E.: loc. cit.

${ }^{13}$ Reed, C. I., and Lamson, R. W.: J. Immunol., r3, 433. 1927.

${ }^{14}$ Bulger, H. A.: J. Infect. Dis., 23, 522. I9I8.

ts Abderhalden, E., and Wertheimer, A.: Pfiuger's Arch.f.d.ges. Physiol., r95, 487. 1922.

${ }^{16}$ Hirsch, E. F., and Williams, J. L.: J. Infect. Dis., 30, 259. 1922.

${ }^{77}$ McCullough, M., and O'Neill, F. I.: ibid., 37, 225. I925.

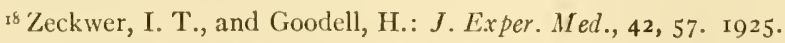

I9 Zunz, E., and la Barre, J.: Compt. rend. Soc. de biol., 90, 658. I924. 
The nitrogen metabolism seems to be variable. The blood shows a slight increase in total non-protein nitrogen (Jobling, Peterson, and Eggstein), ${ }^{\mathrm{r}}$ in urea and creatinine (Major), ${ }^{2}$ and in amino-acids (Zunz and Gyorgy) $;{ }^{3}$ but the lungs (Auer and Van Slyke) ${ }^{4}$ and the liver (Barger and Dale) ${ }^{5}$ show no increase in free amino nitrogen. Wells states that such increases in amino nitrogen, if they occur at all, are not large enough to be significant.

Lumière $^{6}$ has emphasized flocculation of plasma proteins as demonstrated by Dold ${ }^{7}$ and further investigated by use of the nephelometer, tyndallometer, and agglinoscope (see also Kopaczewski). ${ }^{8}$ Zunz and La Barre and Kopaczewski have demonstrated fall in surface tension. The refractive capacity is increased, but not the rotatory. The viscosity of the whole blood is increased and sedimentation speed of corpuscles decreased, together with alteration of colloids so that they are readily precipitated (Wittkower). ${ }^{9}$ Most of these changes are not peculiar to anaphylactic shock and have been demonstrated in certain of the anaphylactoid phenomena.

\section{SPECIFICITY OF ANAPHYLAXIS}

Problems of specificity have been studied in simple fashion by observing shock reactions in animals variously sensitized and variously shocked. Passive sensitization as well as inhibition of shock by injection of proteins has also been employed. Probably the most delicate and certainly more open to quantitative determinations is the guinea pig uterus method of Dale.

By the use of sera and native proteins, the anaphylactic reaction is species specific and shows group reactions just as do the immune reactions. Extracts of organs within the same species show only relative specificity, slight in degree (Pearce, Karsner, and Eisenbrey). ${ }^{10}$ After desensitization with serum there is no organ specificity (Minet and Bruyant). ${ }^{\text {II }}$ The exception to this is the protein of crystalline lens, which shows organ but not species specificity. The experiments of Dale and Hartley ${ }^{12}$ with crystallized egg proteins, employing quantitative methods with the guinea pig uterus, show a certain organ specificity and a presumptive species specificity.

Wells states that "experiments with such hopelessly complex mixtures as are furnished by serum, organ extracts, or even egg white and milk cannot be expected to yield much valuable information concerning specificity." He has utilized purified vegetable proteins and certain animal proteins which resist heat coagulation or are crystallizable. Osborne and Wakeman ${ }^{\mathrm{I} 3}$ have studied in the same manner proteins of

${ }^{2}$ Jobling, J. W., Petersen, W. F., and Eggstein, A. A.: J. Exper. Med., 22, 40I. I9I 5.

${ }^{2}$ Major, R. H.: Deutsche Arch.f. klin. Med., ir6, 248. I9I4.

${ }_{3}$ Zunz, E., and Gyorgy, P.: Ztschr.f. Immunitatsforsch. u. exper. Therap., 23, 402. 1915.

${ }_{4}^{4}$ Auer, J., and Van Slyke, D. D.: J. Exper. Med., 18, 2 I0. I913.

${ }^{5}$ Barger, G., and Dale, H. H.: Biochcm. J., 8, 670. I9I4.

${ }^{6}$ Lumière, A.: Le Probleme de l'anaphylaxie. Paris: Doin, I924.

7Dold, H.: Deutsche med. Wchnschr., 47, 413. I921.

${ }^{8}$ Kopaczewski, W.: Compt.rend. Soc. de biol., 82, 590. I919; Compt.rend. Acad. de sc., 172, 337. I921; Kopaczewski, W., and Roffo, A. H.: ibid., 170, I409. 1920; Compt. rend. Soc. de biol., 83, 857. I920.

9 Wittkower, E.: Klin. Wchnschr., 2, 450. 1923.

${ }^{10}$ Pearce, R. M., Karsner, H. T., and Eisenbrey, A. B.: J. Exper. Med., I4, 44. I9I1.

${ }^{11}$ Minet, J., and Bruyant, L.: Compt.rend. Soc. de biol., 71, I66. I9II.

${ }^{12}$ Dale, H. H., and Hartley, P.: Biochem. J., ro, 408. I916.

r3 Osborne, T. B., and Wakeman, A. J.: J. Biol. Chem., 33, 7, 243. rgr8. 
milk. The essential fact disclosed is that "biological origin is not, per se, the thing that determines the immunological specificity of a given protein, but its chemical composition." The composition and spatial relations of the molecule are the important features. It is, however, possible that certain groups determine the specificity, but what these groups are has not been clearly determined.

\section{INHIBITION OF SHOCK, DESENSITIZATION, ANTI-ANAPHYLAXIS}

Shock may be inhibited in a variety of ways, but subsequently the anaphylactic hypersensitiveness returns. Therefore, it seems unjustified to refer to the temporary refractory state as desensitization (see Coca). ${ }^{\mathrm{I}} \mathrm{A}$ refractory state follows non-fatal shock for a period of several days or several weeks, with subsequent return of hypersensitiveness usually not in the same degree. Besredka has shown that frequently repeated small doses of one protein, not sufficient in themselves to produce clinical manifestations, effectively inhibit or prevent shock. Lewis ${ }^{2}$ has found that by the use of the Woodyatt pump "anaphylactic shock can be prevented in sensitized experimental animals by giving otherwise fatal doses of diluted antigen intravenously at very slow rates." The refractory state appears in a few minutes after intravenous injection, three or four hours after intraperitoneal injection, and about twenty-four hours after subcutaneous injection. Besredka ${ }^{3}$ claims to have desensitized by way of the alimentary canal. If an animal be sensitized to two or more proteins, there may be induced a specific inhibition of shock to one of the proteins without altering the sensitiveness to the others.

There is an extensive literature on inhibition of shock, reviewed by Karsner and Ecker, ${ }^{4}$ who found that heterologous serums inhibit but do not completely prevent shock. Massini ${ }^{5}$ assumes that in sensitization and shock there are two elements, nonspecific and specific.

Drugs which depress smooth muscle excitability, those which depress nerve activity generally, in the group of narcotics and anesthetics, those which tend to maintain blood pressure, are pharmacologically adapted to the inhibition or even prevention of shock. Thus atropin, adrenalin, chloral hydrate, ether, alcohol, atoxyl, and numerous other drugs have been employed. Auer ${ }^{6}$ pointed out the influence of atropin, Mita ${ }^{7}$ stated that it would not protect against a full shocking dose, but Karsner and Nutt ${ }^{8}$ demonstrated a clear quantitative relation. Kyes and Strauser 9 claim that inhibition of anaphylactic shock can be produced in pigeons by the use of heparin, but Hanzlik, Butt and Stockton ${ }^{\mathrm{Io}}$ found no such effect upon the response

\footnotetext{
I Coca, A. F.: loc. cit. ${ }^{2}$ Lewis, J. H.: loc. cit. $\quad{ }^{3}$ Besredka, A.: loc. cit.

${ }^{4}$ Karsner, H. T., and Ecker, E. E.: J. Infect. Dis., 30, 333. 1922.

5 Massini, R.: Zischr.f. Immunitätsforsch. u. exper. Therap., 27, 213. I918.

${ }^{6}$ Auer, J.: J. Exper. Med., 12,638. 1910.

7 Mita, H.: Ztschr.f. Immunitätsforsch. u. exper. Therap., r1, 501. I9 1.

${ }^{8}$ Karsner, H. T., and Nutt, J. B.: J.A.M.A., 57, I023. I91 I.

9 Kyes, P., and Strauser, E. R.: J. Immunol., 1 2, 4 I9. 1926.

ro Hanzlik, P. J., Butt, E. M., and Stockton, A. B.: J. Immunol., 13, 409. I927.
} 
of the pigeon's crop to anaphylactic stimulation. Williams and Van de Carr ${ }^{\mathrm{I}}$ found less clear cut inhibition in the guinea pig, and both $\mathrm{Hyde}^{2}$ and Reed ${ }^{3}$ found nonc at all.

Various agents have been employed to alter the physical state of the bocly. Kopaczewski and Vahram ${ }^{4}$ believe that the inhibitory effect of sodium oleate is due to the lowering of surface tension of the blood. Karsner and Ecker employed various colloids with only slight effect. The inhibitory effect of sodium chloride has been thought by some to be due to alteration of the colloidal state of fluids and tissues, but Richet ${ }^{6}$ believes it to be due to influence on the nerve cells. Hussey found inhibition to result from X-ray exposures, but this did not apply to the uterus when tested by the Dale method. Further discussion is to be found in the review by Longcope. ${ }^{8}$

\section{REVERSE ANAPHYLAXIS}

Forssmann' found that the intravenous injection into guinea pigs of sheep hemolytic immune serum produces anaphylaxis-like symptoms. He interprets this as a union of pre-existing antigen in the guinea pig cells and injected antibody, and gave the phenomenon the name "reverse anaphylaxis." It is more probably an anaphylactoid reaction. More satisfactory are the results of Opie ${ }^{\mathrm{I0}}$ who injected horse serum into rabbits and several hours subsequently a specific precipitating immune serum with production of typical shock. Owing to the toxicity of the immune sera, he could not apply this to the guinea pig. Equally significant are his experiments with reverse local anaphylaxis (Arthus phenomenon) discussed below.

\section{CELLULAR ANAPHYLAXIS}

Investigations of so-called "cellular anaphylaxis" have been concerned principally with erythrocytes. The literature is reviewed by Kritschewsky and Friede, ${ }^{\text {II }}$ who state that they have produced both active and passive anaphylaxis in the dog by the use of sheep erythrocytes. Various reports have appeared concerning the phenomena in other animals. In all cases there is difficulty in excluding hemolytic and hemagglutinative effects since most of the interpretations are based solely on clinical phenomena. Especially is this true regarding passive sensitization and specific inhibition of shock. By work upon dogs, Manwaring, Marino, and Boone ${ }^{12}$ show that the

${ }_{1}$ Williams, O. B., and Van de Carr, R.: Proc. Soc. Exper. Biol. \&o Mcd., 24, 798. 1927.

${ }^{2}$ Hyde, R. R.: Am.J. Hygiene, 7, 614. I927.

${ }^{3}$ Reed, C. I., and Lamson, R. W.: J. Immunol., I3, 433. 1927.

4 Kopaczewski, W., and Vahram, A.: Compt. rend. Acad. de sc., I69, 250. I9I9.

${ }^{5}$ Karsner, H. T., and Ecker, E. E.: J. Infect. Dis., 34, 636. 1924.

${ }^{6}$ Richet, C.: Compt.rend. Acad. de sc., 169, 9. 19r9.

7 Hussey, R.: Proc. Soc. Exper. Biol. \& Med., 19, 22. 192 I.

${ }^{8}$ Longcope, W. T.: Physiol. Rev., 3, 240. 1923.

9 Forssmann, J.: Biochem. Ztschr., I10, I33, I64. 1920; Acta path. et microbiol. Scandinav., 2, 55. $1925 ; 3,749.1926$.

10 Opie, E. L., and Furth, J.: J. Exper.Med., 43, 469. 1926.

${ }^{11}$ Kritschewsky, I. L., and Friede, K. A.: Centralbl. f. Baktcriol., Abt. I, 96, 56, 68. 1925.

${ }^{12}$ Manwaring, W. H., Marino, H. D., and Boone, T. H.: Proc. Soc. Exper. Biol. \& Med., 24, 651. 1927 . 
blood-pressure fall and the increased tone of the bladder occur much sooner than in serum anaphylaxis, and they interpret the result to indicate that the liver plays no part. Much the same questions are raised by the work of Zinsser and Parker, ${ }^{\mathrm{I}}$ and of Sherwood and Stoland, ${ }^{2}$ with bacterial bodies. Hyde ${ }^{3}$ finds that the phenomenon is not due to the carriage of serum by blood cells, and his results favor the conception that the reaction is anaphylactoid.

\section{SITE OF THE REACTION}

Acceptance of the statement that anaphylaxis is an antigen antibody reaction raises the question as to whether the reaction takes place in the circulating fluids (humoral theory) or in the tissue cells (cellular theory).

If the reaction take place in the blood, it should be possible to inject antigen and antibody simultaneously, or nearly so, with the production of shock. Zinsser ${ }^{4}$ quotes experiments of Friedemann, Biedl and Kraus, Briot, Gurd, and himself to support the conception that the meeting of antigen and antibody in the bloodstream, especially of rabbits and certain other animals, may lead to anaphylactic shock, but these results had not the same searching analysis as is now demanded for the distinction between anaphylactic and analphyactoid reactions. By perfusing the rabbit heart, Manwaring, Meinhard, and Denhart ${ }^{5}$ claim to have shown that the reaction is entirely humoral. Subsequent work (Manwaring and Kusama) ${ }^{6}$ with a more suitable organ, the guinea pig lung, demonstrated cellular reaction. By perfusing with antigen and antibody, a reaction occurred which is interpreted as typically anaphylactic and humoral in origin. The work of Hanzlik ${ }^{7}$ and Karsner shows that such reactions may occur under circumstances not to be regarded as truly anaphylactic.

The conception widely held today is that anaphylaxis is a supra- or intracellular phenomenon. Friedberger, in the development of his theory of anaphylatoxins, assumed that the shock is due to the presence of intracellular precipitins ("sessile receptors") which react with injected antigens to stimulate and damage the cells. The work of Schultz and of Dale with isolated smooth muscle in vitro indicated the capacity of cells to react, but was not conclusive because the possible influence of blood in the specimens could not be excluded. At about the same time Pearce and Eisenbrey ${ }^{8}$ transfused the blood of normal dogs into a sensitized dog and that of a sensitized dog into a normal dog. The sensitized dog with normal blood reacted to the shocking dose of serum, whereas the normal dog with "sensitized" blood did not react, clearly indicating that the reaction is cellular. Coca ${ }^{9}$ confirmed this with guinea

I Zinsser, H., and Parker, J. T.: J. Exper. Med., 37, 275. 1923.

2 Sherwood, N. P., and Stoland, O. O.: J. Immunol., 10, 643. 1925.

3 Hyde, R. R.: J. Immunol., I2, 309. 1926.

4 Zinsser, H.: Infection and Resistance (2d ed.), p. 397. New York: Macmillan, 1918.

${ }_{5}$ Manwaring, W. H., Meinhard, A. R., and Denhart, H. L.: Prac. Soc. Exper. Biol. Eo Med., 13, 173,174 . I916.

${ }^{6}$ Manwaring, W. H., and Kusama, Y.: J. Immunol., 2, 157. I9I7.

7 Hanzlik, P. J.: J.A.M.A., 82, 200I. I924.

${ }^{8}$ Pearce, R. MI., and Eisenbrey, A. B.: J. Infect. Dis., 7, 565. 1910.

${ }^{9}$ Coca, A. F.: Ztschr. f. Immunitatsforsch. u. exper. Therap., 20, 662. I914. 
pigs. By the use of perfusion methods, and the uterine strip, both with active and passive anaphylaxis, Weil ${ }^{\mathrm{I}}$ has given most cogent support to the cellular theory of anaphylaxis. He has also shown that the reaction follows in a general way the Danysz effect. It has been shown quantitatively by Coca and by Von Fenyvessy and Freund ${ }^{2}$ that animals react when there is an insufficient quantity of circulating antibodies to account for the phenomenon.

Gay and Southard ${ }^{3}$ were of the opinion that the reaction is principally in the nervous system, a view supported by Besredka ${ }^{4}$ and others. The participation of smooth muscle without the intervention of the central nervous system is unquestioned. Weil ${ }^{5}$ held strongly to the view that the liver is largely concerned. By the use of dye "blockade" of the reticulo-endothelial system, Isaacs ${ }^{6}$ was unable to demonstrate any alteration of the reaction.

\section{TIIEORETICAL CONSIDERATIONS}

PRECIPITINS AND ANAPHYLAXIS

The features of a supposed identity of precipitin and sensitizing substance in anaphylaxis have been referred to above. A precipitating immune serum can passively sensitize an animal to the homologous protein, and the sensitization is generally quantitatively parallel to the precipitin titre. Weil showed that the washed precipitate can confer passive and also active sensitization. Coca was unable to confirm this but did not contradict the underlying assumption. The fact that heating a precipitating serum to $70^{\circ} \mathrm{C}$. for one-half hour completely inlibits its precipitating capacity, but only slightly reduces its sensitizing power, was interpreted by Weil, on the basis of the side-chain theory, to indicate that the special binding group of the precipitin has been destroyed, a group not necessary for sensitization. Nevertheless, this is an irregularity in action, which, together with the work of Falk and Caulfield ${ }^{7}$ showing lack of parallelism between precipitation titre and sensitizing capacity, must indicate that the hypothesis needs further explanation before final acceptance. Kraus ${ }^{8}$ points out that the guinea pig, excellent for work in anaphylaxis, is a poor producer of precipitin, that rabbits may produce a powerful sensitizer without high-titre precipitin, and that goats produce precipitin readily but have a serum incapable of conferring passive sensitization.

Opie, ${ }^{9}$ in extension of the work of others, has studied the relations of antigen and precipitin in vitro and $i n$ vivo and has found that there must be an equilibrium in which

I Weil, R.: J. Med. Research, 27, 497. I912-13; ibid., 30, 87, 199. I914; J. Immunol., 2, 399, 469. I9I 7 .

${ }^{2}$ von Fenyvessy, B., and Freund, J.: Ztschr.f. Immunitütsforsch. u. exper. Therap., 22, 59. I9I4.

3 Gay, F. P., and Southard, E. E.: loc. cit.

4Besredka, A.: loc. cit.

5 Weil, R.: J. Immunol., 2, 525, 57 I. I917.

${ }^{6}$ Isaacs, M. L.: Proc. Soc. Exper. Biol. Eo IIed., 23, I85. 1925.

7 Falk, 1. S., and Caulfield, M. F.: loc. cit.

${ }^{8}$ Kraus, R., and Levaditi, C.: IIandbuch der Technik und Methodik der Immunitätsforschung. Jena: Fischer, Igo8-I I.

${ }^{9}$ Opie, E. L.: J. Immunol., 8, I9, 55. I923. 
a vast excess of immune serum is necessary to produce the reaction. Antigen and precipitin may coexist in serum and in circulating blood; excess of antigen may inhibit precipitation or may dissolve precipitate. It is well known that the actively or passively sensitive animal has a low titre of circulating precipitin, and it is reasonable to suspect that under the conditions of anaphylaxis experiments the necessary equilibrium for the formation of precipitates does not occur. Of further interest is the fact demonstrated by Opie ${ }^{\mathrm{I}}$ that washed specific precipitates produce no obvious injury upon intravenous injections into rabbits.

\section{ANAPHYLACTIC POISONS}

The term is employed to cover a variety of poisonous substances which are assumed to be the cause of shock. Friedberger ${ }^{2}$ produced a poisonous product by incubation of antigen, specific precipitin, and complement, to which he gave the name "anaphylatoxin." Doerr and Russ 3 found that complement was unnecessary to the mixture. Keysser and Wassermann discovered that the interaction of guinea pig serum and kaolin or barium sulphate produced a similar poisonous substance, and Besredka showed the same to be true of serum and peptone agar. Bordet found that serum and dissolved agar react in the same way. Novy and De Kruif ${ }^{4}$ discovered that agar alone and other non-protein colloids produce similar symptoms. Vaughan and Wheeler ${ }^{5}$ demonstrated the intense toxic activity of the split products of protein decomposition and assumed that in anaphylaxis a similar digestion occurs in the animal. That the agar-serun mixtures show an increase of non-protein nitrogen is an indication that in such mixtures digestion occurs. Jobling and his co-workers ${ }^{5}$ showed that mixtures of serum with bacteria or kaolin or agar resulted in adsorption of anti-enzyme with consequent activity of proteases and the production of poisonous products. Dale ${ }^{7}$ pointed out that the immediate response of the uterus strip is difficult to harmonize with the idea that ferment action is involved.

These substances are all effective immediately upon injection intravenously, but not upon intracerebral injection (Besredka). The effect is not specific. No such poison can be demonstrated in the blood in anaphylactic shock. Several of the products have been studied by Hanzlik and Karsner and have been shown to produce anaphylactoid rather than anaphylactic phenomena.

The resemblance of anaphylactic shock to the effects of histamine has been noted by Abel and Kubota, ${ }^{8}$ Dale, Vaughan, and especially by Manwaring and his collaborators. Smith ${ }^{9}$ has emphasized the differences between anaphylaxis and histamine

${ }^{2}$ Opie, E. L.: ibid., 9, 259. I924.

${ }^{2}$ Edrons' Note.-The chapter promised by Dr. Friedberger for this volume was not received.

${ }^{3}$ Doerr, R., and Russ, V. K.: Ztschr. f. Immunitätsforsch. u. exper. Therap., 2, 109. I909; 3, I8 I 1909 .

${ }_{4}^{4}$ Novy, F. G., and de Kruif, P. H.: J.A.M.A., 68, 1524. 1917.

5 Vaughan, V. C., and Wheeler, S. M.: J. Infect. Dis., 4, 476. I907; Vaughan, V. C.: Poisonous Proteins. St. Louis: Mosby, igi 7.

${ }^{6}$ Jobling, J. W., Petersen, W. F., and Eggstein, A. A.: loc. cit. 7 Dale, H. H.: loc. cit.

${ }^{8} \mathrm{Ab} \mathrm{l}, \mathrm{J} . \mathrm{J}$., and Kubota, S.: J. Pharmacol. \& Exper. Therap., 13, 243. I9I9.

${ }^{9}$ Smith, M. I.: J. Immunol., 5, 239. 1920. 
effects. Histamine does not produce the temperature changes nor the alteration of blood coagulability seen in anaphylaxis, nor does it produce a refractory state. Quinine augments anaphylactic shock but has no effect on treatment by histamine. Karsner and Hanzlik have shown that intraperitoneal administration of histamine produces platelet thrombi in pulmonary capillaries, not found in anaphylaxis. De $\mathrm{Eds}^{\mathrm{r}}$ found that in pigeons anaphylactic shock shows no clumping of thrombocytes, whereas negative results are not so clear cut with histamine. Manwaring, Monaco, and Marino $^{2}$ state that the response to histamine is more widespread in the body and more definitely due to edema than is true of anaphylactic shock.

The substance which produces effects most closely simulating anaphylactic shock is Witte peptone. The reactions of smooth muscle, pulmonary distention, fall of blood pressure and of temperature, alteration of coagulation, etc., are practically identical. The assumption that in anaphylactic shock there is formed a protein decomposition product led to the belief that peptone is, or closely resembles, the anaphylactic poison. In the light of our present doubt concerning the action of ferments in anaphylaxis, the work with peptone cannot be regarded as of critical significance in interpreting the reaction. In addition, the fact that peptone does not produce a refractory state is strong evidence against its being the poison of anaphylactic shock. Its effects are due to the peptone and not to a small content of histamine (Hanke and Koessler). ${ }^{3}$

\section{PHYSICAL THEORIES}

The least complex of the physical theories deals with specific precipitation, discussed above. That physical changes occur in anaphylactic shock is beyond question, as has been pointed out earlier in this chapter, but that they are peculiar to this reaction has not been demonstrated. Some of the hypotheses refer only to changes in the circulating fluids without attempt to harmonize with the presumably correct cellular theory. Thus, Doerr ${ }^{4}$ supposed that adsorption of a hypothetical antagonistic fraction of complement by precipitates liberates a toxic element. Kritschewsky ${ }^{5}$ found that the sap of Cotyledon scheiderecki precipitates plasma proteins and agglutinates and hemolyzes erythrocytes. Intravenous or subcutaneous injections produce symptoms resembling anaphylaxis, believed to be due to change in disperseness of the plasma colloids. The anatomical findings lack the uniformity of those of true anaphylaxis. The results and interpretations are similar to those of Doerr and Moldovan ${ }^{6}$ following intravenous injections of water colloidal sols of silicic acid, nucleinic acid, and dialyzed iron hydroxide. Kopaczewski found that the injection of serum rendered toxic by addition of bacterial suspensions or colloid gels reduces the surface tension. The electrical potential of the serum is altered so that with 8 volts, instead of

r de Eds, F.: J. Pharmacol. \& Exper. Therap., 28, 45I. 1926; de Eds, F., and Somerfield, H. A.: Proc. Soc. Exper. Biol. \& IIed., 20, 498. 1923.

${ }^{2}$ Manwaring, W. H., Monaco, R. E., and Marino, D. H.: J. Immunol., 8, 21 7. 1923.

3 Hanke, M. T., and Koessler, K. K.: J. Biol. Chem., 43, 567. 1920.

${ }_{4}$ Doerr, R.: loc.cil.

${ }^{5}$ Kritschewsky, J. L.: J. Infect. Dis., 22, 101. 1918.

${ }^{6}$ Doerr, R., and Moldovan, J.: Ztschr.f. Immunitätsforsch. u. exper. Therap., 5, 125. 1910.

7 Kopaczewski, W.: loc. cit.; Kopaczewski, W., and Roffo, A. H.: loc. cit. 
a collection of precipitate at both poles, it collects alnost entirely at the negative pole. Lumière $^{\mathrm{I}}$ says that mixture of antigen and sensitized serum produces a flocculation, responsible by physical rather than chemical means for anaphylactic shock. The flocculate acting mechanically and abruptly on the endothelium of the vessels of the central nervous system provokes sudden general vasodilatation, the determining factor in the manifestations of shock. Examination of the work of Lumière, Kritschewsky, Doerr and Moldovan, and Kopaczewski, shows that they fail to distinguish between anaphylaxis and anaphylactoid reactions. This, together with obvious objections indicated in discussing the nature of shock and the site of reaction, leaves the hypotheses without secure foundation.

Jobling, Petersen, and Eggstein" demonstrated that anaphylactic shock, "is accompanied by $(a)$ the instantaneous mobilization of a large amount of non-specific protease, $(b)$ a decrease of antiferment, $(c)$ an increase in non-coagulable nitrogen of the serum, $(d)$ an increase in amino-acids, $(e)$ a primary decrease in serum proteoses." They conclude that the "intoxication is brought about by the cleavage of serum proteins (and proteoses) through the peptone stage by a non-specific protease" and that "the specific elements lie in a rapid mobilization of this ferment and the colloidal serum changes which bring about the change in antiferment titer." This refers the matter to the effects of poisonous protein products, discussed above in reference to Vaughan's work. Bronfenbrenner ${ }^{3}$ found that bubbling ether vapor through serum decreases the antitryptic activity, which he believes to be due to an increased dispersion of the colloidal particles. A similar decrease of antitryptic activity occurs when antigen and antibodies are mixed. The explanation is simpler than that of Jobling but based upon the assumption that shock is due to protein split products. Besredka assumes that the shock dose of protein meets with a preformed sensibilisin in the nerve cells and produces there either a liberation or absorption of energy, thermal or otherwise, and that this reaction leads to the phenomena of shock. The work of Opie quoted below indicates that there is no preformed sensibilisin. Besredka supports his hypothesis by the fact that anesthetics inhibit shock. Bronfenbrenner found that certain anesthetics increase antitryptic activity by Ioo per cent or more, and assumes that their inhibitory effect is not due to action on nerve cells but is the result of absence of liberation of proteases.

\section{LOCAL ANAPHYLAXIS (ARTHUS PHENOMENON)}

Described by Arthus ${ }^{4}$ and studied in detail by Arthus and Breton ${ }^{5}$ in the skin, the inflammatory reaction following injection of protein in the skin of sensitized animals is known to occur elsewhere in the body. This is true of either actively or passively sensitized animals. Opie ${ }^{6}$ has found that the involved area shows necrosis, inflammatory exudate of polymorphonuclear leukocytes and other cells, edema, fibrin forma-

\footnotetext{
${ }^{I}$ Lumière, A.: loc. cit.

${ }^{2}$ Jobling, J. W., Petersen, W. F., and Eggstein, A. A.: loc. cit.

3 Bronfenbrenner, J.: Proc. Soc. Exper. Biol. Eo Med., 12, I10. I915; 13, I9. 1915.

${ }^{4}$ Arthus, M.: loc, cit.

${ }^{5}$ Arthus, M., and Breton, M.: Compt. rend. Soc. de biol., 55, I478. 1903.

${ }^{6}$ Opie, E. L.: J. Immunol., 9, 259. 1924
} 
tion, and vascular thrombosis. If washed precipitate be injected, the reaction is milder and no necrosis is observed. Auer ${ }^{\mathrm{I}}$ found that in animals with both antigen and antibody present, the production of local irritation (xylol on the rab)bit's ear) leads to an intense necrotizing inflammation, evidently due to concentration of the two bodies locally. Opie $\mathrm{e}^{2}$ found that if rabbits be given protein intravenously and several hours thereafter intradermal injections of antihorse serum, a local reaction of the same nature occurs, reverse passive local anaphylaxis. As a result of his extensive studies Opie finds that "acute inflammation occurs when antigen and antibody come into contact within the tissue. It is unnecessary to assume that the tissue has undergone any change in the process of 'sensitization.' The combination of antigen and antibody acts as an inflammatory irritant and the usual phenomena of inflammation ensue." The relation of the Arthus phenomenon to human hypersensitiveness is discussed elsewhere in this volume by Coca, ${ }^{3}$ and to the tuberculin reaction by Long. ${ }^{4}$

\section{ANAPHYLACTOID REACTIONS}

Certain agents, when introduced parenterally, produce symptoms and signs which closely simulate those of anaphylactic shock. These have been called "anaphylactoid reactions." Hanzlik and Karsner ${ }^{5}$ have studied the effects, more especially following intravascular and intraperitoneal injections, of non-nitrogenous colloids, colloidal metals and their salts, tissue extracts, sera, nitrogenous colloids and mixtures, microorganisms and vaccines, arsphenamin, coal-tar derivatives, salts of heavy metals, other salts, adsorbents, hypertonic solutions, and certain other materials. The reactions include phenomena which have been described as nitritoid crises, hemoclasis, colloidoclasis, and the responses to certain anaphylatoxins. Our discussion follows closely the admirable review by Hanzlik. ${ }^{6}$

The studies of Hanzlik and Karsner were concerned with fifty-three agents, unrelated physically and chemically, most of which were not protein in nature. Guinea pigs, rabbits, cats, and dogs were employed and little difference in reaction discovered. Hanzlik has done further work with pigeons; symptoms have also been studied in horses, goats, and man. Rats are said to be refractory, but it seems likely that the results of Scott and Thatcher ${ }^{7}$ are of anaphylactoid character.

Intravascular injections, into veins, arteries, or heart, are the most effective and give most uniform results. Forssmann's ${ }^{8}$ carotal complex is probably to be explained on this basis. Intraperitoneal and subcutaneous injections are less effective and less uniform.

In a general way, "the symptoms consist of increased reflex excitability, rapid respiration and dyspnea, sneezing, retching, jerky spasms, slowing or acceleration of

I Auer, J.: J. Exper. Med., 32, 427. 1920.

3 See chap. $1 x x v$ of this volume.

${ }^{2}$ Opie, E. L.: J. Immuno!., 9, 255. I924.

${ }^{4}$ See chap. lxxvi of this volume.

${ }^{5}$ Hanzlik, P. J., and Karsner, H. T.: J. Pharmacol. Eo Exper. Therap., 14, 229, 379, 425, 449, 463, 479. 1919-20.

${ }^{6}$ Hanzlik, P. J.: J.A.M.A., 82, 2001. 1924.

${ }^{7}$ Scott, W. J. M., and Thatcher, H. S.: Arch. Path. E. Lab. Med., 2, So6. 1926.

${ }^{8}$ Forssmann, J.: loc. cit. 
the heart, trembling, expulsion of urine, cyanosis, pupillary relaxation, convulsions, and death with the larger doses."

The morbid anatomy has been studied principally in guinea pigs, although certain of the significant changes are to be seen in other animals. With most of the agents the mode of death is cardiac rather than asphyxial. Consequently, marked dilatation of the heart, particularly of the right side, associated with hyperemia and hemorrhage in various viscera is likely to be striking. Pulmonary distention is not frequent, but local areas of emphysema may be encountered. Usually the lungs show marked hyperemia with gross or only microscopic hemorrhages. The notable exception is peptone, which, as stated above, produces lung changes almost identical with those of anaphylaxis. The same may be true of histamine, agar, and a few other agents.

The microscopic examination of the lungs, following many of the agents, commonly shows some form of thrombosis or embolism in the pulmonary vessels, especially the small arteries and capillaries. The constitution of these varies with different agents but is fairly constant with each. The vessels may be plugged with masses of corpuscles, red or white predominating, more or less conglutinated, and with small amounts of fibrin. With some agents the cells are entirely large mononuclear with little or no fibrin. With others, notably Fuller's earth, the mass may be almost entirely fibrin. Following intraperitoneal injection of histamine, masses of platelets blocking the capillaries are conspicuous. Bronchoconstriction is variable. Perivascular and peribronchial edema are common, and intra-alveolar edema not infrequent.

The physiological alterations are variable. Most of the agents produce an elevation of body temperature, and relatively few any material fall. Increased capillary permeability and edema are common, but such agents as calcium phosphate, barium sulphate, iron phosphate, silicic acid, and gelatin may prevent the edema of mustardoil irritation.

Fall of systemic blood pressure is a common result, principally due to blockage of pulmonary circulation. Abe $\mathrm{x}^{\mathrm{x}}$ has demonstrated increase in pulmonary arterial pressure following use of histamine, peptone, and tissue extracts, and Jackson and Smith ${ }^{2}$ following arsphenamine. Abel and Geiling ${ }^{3}$ have demonstrated peripheral vasodilatation following peptone and histamine administration.

Most of the agents do not excite smooth muscle contractions. "Exceptions are histamine, peptone, serums, tissue extract, congo red and quinine, which tend to increase excitability." Perfusion of guinea pig lungs with agar, toxified agar, peptone, and histamine leads to reduction in caliber of the bronchi, but this is not relieved by atropin and is probably due to vascular changes and edema rather than to muscle contraction. Perfusion with peptone and histamine may lead to block in the liver, but that this is due to smooth muscle contraction is not established. It will be seen that there are few agents which produce smooth muscle contraction and that bronchoconstriction, muscular in origin, is not proved. Certainly there is no such regularity of action on smooth muscle by the anaphylactoid agents as is true in anaphylaxis.

${ }^{1}$ Abe, K.: Tohoku J. Exper. Med., I, 398. 1920.

${ }^{2}$ Jackson, D. E., and Smith, M. I.: J. Pharmacol. \& Exper. Therap., I 2, 22 I. I918.

3 Abel, J. J., and Geiling, E. M. K.: ibid., 23, I. 1924 . 
The physical changes in the blood vary greatly with different agents. Flocculation of plasma colloids may be easily visible or require special instruments such as the agglutinoscope or tyndallometer. Kopaczewski and Gruzewska ${ }^{x}$ report that this will occur also in vitro. The platelets may be increased or decreased, and following the administration of certain agents may agglutinate in the vessels. The latter phenomenon has also been demonstrated in vitro (Dale and Laidlaw, ${ }^{2}$ De Eds and Somerfield ${ }^{3}$ ). We found that hemagglutination occurs in vitro by the use of many of the agents that produce thrombosis and embolism in vivo (shown also by Oliver and his co-workers). ${ }^{4}$ Hanzlik, De Eds, and Tainter ${ }^{5}$ found increased rate of sedimentation of erythrocyte suspensions as the result of treatment with several of the agents. "The physical changes in blood and plasma resulting from the injection of foreign agents, sera, etc., are increase in viscosity and lowered surface tension of the blood, the plasma being unchanged in guinea pigs receiving serum treated with agar (Zunz and La Barre). ${ }^{6}$ Increases in viscosity of the plasma and fibrinogen, decrease in surface tension and changes in the ratio of globulin to albumin (increased globulin) are reported after injections of proteins by Löhr and Löhr."7

Studies of gaseous metabolism, hydrogen-ion concentration, carbohydrate and nitrogen metabolism, and lipin and lipoid constitution have been carried out with numerous agents. Many changes have been noted, but the agents as a group do not produce alterations that can yet be correlated.

It has not been shown that the non-protein agents are capable of rendering animals more sensitized to subsequent injections. There is then no hypersensitization. The agents act immediately without a period of incubation. The blood of injected animals does not render other animals more sensitive; there is no passive sensitization. Desensitization, in the sense of a sort of antianaphylaxis, cannot be produced. If flocculation of proteins or aggregation can be inhibited or prevented, shock may likewise be reduced or prevented. Since circulatory failure is often a cause of death, cardiac and circulatory stimulants may prevent fatal outcome. In other words, successful inhibition of anaphylactoid phenomena depends upon pharmacological rather than upon specific immunological methods.

Although the matter of production of toxic materials in anaphylaxis is an open question, in the anaphylactoid phenomena there is nothing to suggest that a poisonous product is elaborated other than that found in the agents themselves. It is true that their action in many instances is mediated by physical changes produced in the blood. Such effects have been considered above in the discussion of the physical theories of anaphylaxis.

\footnotetext{
${ }^{r}$ Kopaczewski, W.: Compt.rend. Soc. de biol., 88, r39. r923.

${ }^{2}$ Dale, H. H., and Laidlaw, P. P.: J. Physiol., 52, 355. r919.

3 de Eds, F., and Somerfield, H. A.: Proc. Soc. Exper. Biol. Eo Med., 20, 49 S. 1923.

${ }_{4}$ Oliver, J., and Douglas, E.: J. Pharmacol. Eo Exper. Therap., 19, 187. 1922; Oliver, J.s and Yamada, S. S.: ibid., p. 199. 1922.

${ }^{5}$ Hanzlik, P. J., de Eds, F., and Tainter, M. L.: ibid., 21, 217. 1923.

${ }^{6}$ See Zunz, E., and Ia Barre, J.: loc. cit.

7 Löhr, W. and H.: Ztschr.f. d. ges. exper. Med., 29, r39. I922.
} 


\section{RELATION OF ANAPIIYLAXIS AND ANAPH:LACTOID REACTIONS}

Hanzlik supports the idea that the two phenomena have a common basis. The resemblance in the symptoms and the chemical and physical changes in the blood are offered as evidence. The fact that smooth-muscle stimulation is not common to the anaphylactoid reactions is explained by the fact that most of the agents are of large molecular constitution which leads "to a lack of suitable conditions enabling them to diffuse into and penetrate the cell surface and react with the muscle substance." Hanzlik ascribes the differences in gross and microscopical morbid anatomy to difference in degree of the same phenomena.

Against this hypothesis it may be pointed out that the symptoms, in some ways identical, differ in important ways. In the guinea pig, for example, death in anaphylactic shock is practically invariably respiratory, while in anaphylactoid reactions it is commonly circulatory. The physical and chemical changes in the blood in true anaphylactic shock have not been so well studied as in anaphylactoid reactions, and unfortunately those observed in the latter have been assumed to occur in the former. Accepting Hanzlik's explanation of the failure of smooth-muscle reactions in response to many of the anaphylactoid agents, it is presumably safe to say that the reaction in anaphylaxis is within the cell and probably not a surface phenomenon.

Anaphylactoid reactions are for the most part obviously in the circulating fluids, operating secondarily upon various body mechanisms, whereas the reaction in anaphylaxis is independent of changes in the circulating fluids. Against this hypothesis are some of the physical theories of anaphylaxis, which in accordance with our discussion of the matter are not adequately supported.

It is true that anaphylaxis and the various anaphylactoid reactions can be set side by side and arranged in a series so that there might seem to be a gradation from true anaphylaxis through closely similar anaphylactoid reactions to those that diverge widely from true anaphylaxis. No such presentation can overcome the fact that anaphylaxis is an antigen-antibody reaction and that anaphylactoid reactions are not. 


\title{
CHAPTER LXXIV
}

\section{THE TECHNIQUE OF EXPERIMENTATION IN ANAPHYLAXIS}

\author{
W. H. MANWARING \\ Stanford University
}

The technique of non-clinical experimentation in protein hypersensitiveness includes the methods of sensitization and desensitization of laboratory animals and the methods of eliciting and recording anaphylactic responses in intact animals and in isolated organs and tissues from these animals, together with certain serological, chemical, and physico-chemical methods particularly applicable to anaphylactic study. With slight changes in detail, the methods here outlined are also applicable to the study of toxin hypersensitiveness, bacterial hypersensitiveness, and other types of normal and acquired hypersusceptibility.

\section{ACTIVE PROTEIN SENSITIZATION}

a) Guinea pigs.-Under favorable laboratory conditions 200-250-gm. guinea pigs are readily sensitized to a sufficiently high degree to give fatal anaphylaxis with routine test doses by a single subcutaneous injection of $0.0 \mathrm{I}-\mathrm{cc}$. horse serum, or an equivalent amount of other protein-containing fluid. Lethal sensitization, however, may develop after a smaller dose than this, e.g., after $0.00 \mathrm{I}-\mathrm{cc}$. horse serum, or even after $0.000 \mathrm{I} \mathrm{cc}$. Adult guinea pigs and newborn guinea pigs are usually not susceptible to lethal sensitization.

With a subcutaneous injection of o.oI-cc. horse serum, slight specific hypersusceptibility is usually demonstrable as early as the eighth day after the injection, fatal hypersensitiveness to routine test doses about the fourteenth day, and maximum hypersensitiveness about the twenty-first day. After the twenty-first day, the hypersusceptibility rapidly decreases; but for at least three months it usually remains sufficiently high to give fatal anaphylaxis with routine test doses. After the sixth month fatal anaphylaxis usually camnot be elicited with less than two to four times the routine dose. Slight kypersusceptibility to a multiple dose usually persists for the lifetime of the animal and is usually transmitted to the offspring by a hypersensitive mother.

With larger sensitizing doses, e.g., o.I-cc. horse serum, lethal sensitization may not develop before the twenty-first day, and maximum sensitization before the fortieth to the sixtieth day. ${ }^{\mathrm{I}}$ With still larger doses, lethal sensitization may never develop.

Unsuitable food, gastro-intestinal disturbances, unfavorable climatic conditions, rapid changes in temperature or in humidity, intercurrent infections, pregnancy, parturition, physical injury, or the injection or ingestion of toxic substances may inhibit and even completely prevent the development of lethal sensitization of guinea

${ }^{1}$ Thomson, O.: Ztschr.f. Immunitätsforsch. 14. exper. Therap., 26, 213. I9I 7 . 
pigs. ${ }^{\mathrm{T}}$ Among the more important inhibiting substarces are foreign serum, autolytic tissue products, and peptone. An increase in the sensitizing dose and a prolongation of the incubation period will at times ovecome the handicap from unfavorable factors.

b) Rabbits.-Adult rabbits are readily sensitized sufficiently to give recordable anaphylactic reactions, but fatal hypersensitiveness is rarely acquired by full-grown rabbits. Young rabbits (750-900 gm.) occasionally acquire lethal hypersensitiveness following a single subcutaneous injection with $3-5$-cc. horse serum, or an equivalent amount of other protein-containing fluid. A higher percentage of lethal sensitization is obtained with multiple injections. Auer, ${ }^{2}$ for example, made four to eight subcutaneous, intraperitoneal or intravenous injections of $3-5$-cc. horse serum, at fiveto six-day intervals, and tested his animals from four to six weeks after the final sensitizing dose. Drinker and Bronfenbrenner ${ }^{3}$ used daily intravenous injections of $0.5^{-c c}$. sheep serum, in three periods of from three to five days each, with a two- to three-day interval between periods, and tested their animals from ten to fourteen days after the final sensitizing dose. Probably the most dependable method is the method indorsed by Coca, ${ }^{4}$ daily or periodic injections controlled by the precipitin titre, maximum sensitization in rabbits being apparently synchronous with a precipitin titre of $\mathrm{I}: 6,000$ in the rabbit's serum.

Unfavorable food, unsuitable temperature, excessive humidity, pregnancy, intercurrent infections, etc., may inhibit or even completely prevent the development of lethal hypersensitiveness in rabbits.

c) Dogs.-Dogs may be sensitized sufficiently to give recordable anaphylactic reactions by a single subcutaneous injection of $0.3-0.5$-cc. horse serum per kilogram of body weight. Hypersensitiveness, however, usually develops in but two-thirds of the dogs so injected. A higher percentage of sensitization is conferred by Weil's method, ${ }^{5}$ a single subcutaneous injection of $0.3^{-0.5}$-cc.horse serum per kilogram of body weight, followed twenty-four hours later by an intravenous injection with the same dose. About 95 per cent of the dogs so injected become demonstrably hypersensitive, about one-quarter of them sufficiently so as to give lethal anaphylaxis with routine test doses. The height of the sensitization in dogs is apparently reached about the eighteenth to the twenty-first day. After the twenty-fourth day, sensitization gradually decreases. By the seventh week, fully two-thirds of the dogs are completely desensitized.

With certain protein-containing fluids, however, dogs are less readily sensitized. With the same sensitizing doses of goat serum, for example, only about a third of the dogs become hypersensitive, and with equivalent doses of egg white, demonstrable sensitization does not take place. ${ }^{6}$

${ }^{1}$ Wells, H. G.: Physiol. Rev., I, 44. I921 ; Longcope, W. T.: ibid., 3, 240. 1923.

${ }^{2}$ Auer, J.: J. Exper. Med., 14, 476. г9гі.

3 Drinker, C. K., and Bronfenbrenner, J.: J. Immunol., 9, 397. I924.

${ }_{4}$ Coca, A. F.: Essentials of Immunology, pp. I 53, I55. 1925.

5 Weil, R.: J. Imnuınol., 2, 525. r917.

${ }^{6}$ Manwaring, IV. H., Marino, H. D., McCleave, T. C., and Boone, T. H.: Proc. Soc. Exper. Biol. \& Med., 24, 650. 1927 . 
d) Cats.-Cats may be sensitized at times sufficiently to give lethal anaphylaxis, by the doses and methods used in rabbit anaphylaxis. ${ }^{\text {I }}$

c) Rats.-Parker and Parker ${ }^{2}$ have recently sensitized I00-200-gm. white rats by single intraperitoneal injections with $\mathrm{I}$-cc. sheep serum, also by three intraperitoneal injections of 0.5 -cc. sheep serum, given at three-day intervals. Maximum sensitization was observed between seven to eighteen days after the final sensitizing doses. Small rats are apparently incapable of sensitization.

f) Mice.-White mice may be sensitized by repeated intraperitoneal injections with $0.3-0.5-c c$. horse serum. ${ }^{3}$ The height of the sensitization is reached in about twenty-one days.

g) Pigeons.-Gahringer ${ }^{4}$ reports sensitization of pigeons, at times sufficiently high to give lethal anaphylaxis, by a single intravenous injection with 0.25 -cc. dog serum. Slight sensitization was observed as early as the fourth day after the injection, with maximum sensitization about the fourteenth day. After the twentieth day the sensitization gradually decreased, practically disappearing by the seventieth day.

h) Monkeys. - Zinsser ${ }^{5}$ reports slight sensitization of monkeys, following multiple intravenous injections with egg white.

i) Frogs.-Friedberger and $\mathrm{Mita}^{6}$ report lethal sensitization of summer frogs following a single intravenous (abdominal vein) or intralymphatic (dorsal lymph sac) injection of $0.1-0.5$-cc. sheep serum. The frogs were tested after an incubation period of one to four weeks. Winter frogs are apparently not capable of sensitization.

\section{PASSIVE SENSITIZATION}

a) Guinea pigs.--Normal guinea pigs may be passively sensitized sufficiently to give lethal anaphylaxis, by a subcutaneous, intraperitoneal, or intravenous injection with a sufficiently large dose of the serum, defibrinated blood or whole blood of an actively sensitized or an actively immunized guinea pig. Slight hypersensitiveness is usually demonstrable after a latent period of from six to ten hours, and lethal sensitization in about twenty-four hours. Kellaway and Cowell found that maximum sensitization is not reached before the fourth to the sixth day. Homologous passive sensitization usually disappears spontaneously in about thirty days.

Passive sensitization of guinea pigs is at times possible with the serum or tissue products of other animal species. Thus, guinea pigs may be sensitized even sufficiently to give lethal anaphylaxis, ${ }^{8}$ by subcutaneous, intraperitoneal, or intravenous injections with rabbit antiserum. They cannot be sensitized, however, with rat antiserum..$^{9}$

${ }^{x}$ Manwaring, W. H.: Ztschr.f. Immunitätsforsch. u. exper. Therap., 8, I. I9Iо; Schultz, W. H.: J. Pharmacol. \&o Exper. Therap., 3, 299. I9I I; Drinker, C. K., and Bronfenbrenner, J.: loc. cit.

${ }^{2}$ Parker, J. T. and F.: J. Med. Research, 44, 263. I924.

${ }^{3}$ Schiemann, O., and Meyer, H.: Ztschr. f. Ifyg. u. Infektionskrankh., 106, 607. 1926.

4 Gahringer, J. E.: J. Immunol., 12, 477. 1926.

${ }^{5}$ Zinsser, H.: Proc. Soc. Exper. Biol. E० Med., 18, 57. 1920.

${ }^{6}$ Friedberger, E., and Mita, S.: Ztschr. f. Immunitätsforsch. u. exper. Therap., 10, 362. I9ı I.

7 Kellaway, C. H., and Cowell, S. J.: Brit. J. Exper. Path., 4, 255. 1923.

${ }^{8}$ Doerr, R., and Russ, V. K.: Ztschr.f. Immunitütsforsch. u. exper. Therap., 2, Io9. I909.

${ }^{9}$ Parker, J. T. and F.: loc.cit. 
Passive sensitization of guinea pigs may be inhibited and even completely prevented by a previous or simultaneous injection.with certain toxic substances, such as foreign serum, foreign or homologous tissue products, and peptone.

b) Rabbits.-Normal rabbits may be passively sensitized, at times sufficiently to give lethal anaphylaxis, by an intraperitoneal or intravenous injection with a sufficiently large dose of the serum, defibrinated blood, or whole blood of an actively sensitized or an actively immunized rabbit. ${ }^{x}$ Maximum sensitization is apparently not reached before twenty-four hours. Passive sensitization of rabbits is at times possible with the serum or tissue products of other animal species. Tuberculin hypersensitiveness, for example, may be conferred on rabbits by the intraperitoneal injection of muscle juice from tuberculous cattle. ${ }^{2}$

c) Dogs.-Normal dogs may be passively sensitized sufficiently to give recordable anaphylactic reactions, by replacing a third to two-thirds of their total blood volume with the blood of an actively sensitized dog. Slight sensitization is occasionally demonstrable within an hour after this transfusion, ${ }^{3}$ with maximum sensitization in about twenty-four hours. No suggestion of passive sensitization is demonstrable after fractional transfusion with the blood of a dog actively immunized against the same protein. ${ }^{4}$

d.) Rats-Normal rats weighing at least $80 \mathrm{gm}$. may be passively sensitized sufficiently to give severe anaphylactic symptoms by the intraperitoneal injection of a sufficiently large dose of rat antiserum. Maximum sensitization is apparently not reached before the forty-eighth hour. ${ }^{5}$

e) Mice.-Schiemann and Meyer ${ }^{6}$ have recently reported successful passive sensitization of white mice.

\section{DESENSITIZATION \\ SPECIFIC}

Desensitization of actively or passively sensitized animals may be brought about in all animal species thus far tested by the subcutaneous, intraperitoneal, or intravenous injection with a sufficiently large dose of the specific foreign protein used in sensitization. Partial desensitization is usually demonstrable within from five to twenty minutes after this injection, with maximum desensitization in from two to three hours. The animal usually remains relatively or completely desensitized for from twenty-four hours to eight days. Kellaway and Cowell, however, report desensitization of guinea pigs lasting thirty days. ${ }^{7}$

The minimum desensitizing dose varies with the age of the animal and the method of sensitization. ${ }^{8}$ Complete desensitization of horse-serum-sensitized guinea pigs, for example, is usually brought about by the subcutaneous injection of o.or-cc. horse serum. Occasionally, however, I-cc. horse serum produces only partial desensitization.

I Arthus, M.: Arch. internat. de physiol., 15, 164. I919.

${ }^{2}$ Granucci, L.: Clin. vet., 42, 115. I919.

${ }^{3}$ Manwaring, W. H.: loc. cit.

${ }_{4}^{4}$ Manwaring, W. H., Wright, R. W., and Shumaker, P. W.: J.A.M.A., 86, 1271. 1926.

${ }^{5}$ Parker, J. T. and F.: loc.cit.

7 Kellaway, C. H., and Cowell, S. J.: loc. cit.

${ }^{6}$ Schicmann, O., and Meyer, H.: loc, cit.

${ }^{8}$ Thomson, O.: loc. cit. 


\section{NON-SPECIFIC}

Protein hypersensitiveness may.be temporarily reduced or even completely abolished in all animal species thus far studied by unfavorable food, gastro-intestinal disturbances, unfavorable temperatures, rapid changes in temperature, wetting, physical injury, pregnancy, intercurrent infections, and by the injection or ingestion of certain toxic substances. Among the most important desensitizing substances are agar, foreign serum, foreign or homologous tissue products, heparin, ${ }^{\mathrm{T}}$ hirudin, peptone, saccharose, glycerin and alcohol, and even sodium chloride if injected in sufficiently large doses. ${ }^{2}$

Many anesthetics and narcotics act as desensitizing agents. For example, chloral hydrate, ${ }^{3}$ chloroform, ethyl chloride, and cocaine will completely prevent anaphylactic reactions in guinea pigs. Ether given in excessive doses will appreciably reduce the anaphylactic susceptibility in these animals. If used in minimal doses, however, morphine, ether, A-C-E-mixture, urethane, chloral, chloroform, chloretone, paraldehyde, and sodium barbital thave negligible anti-anaphylactic effects with most animal species. 5

\section{PHYSIOLOGICAL REACTIONS}

\section{CIRCULATORY DISTURBANCES}

\section{CARDLAC DISTURBANCES}

Electrocardiographic methods have been applied to anaphylactic phenomena in cats, ${ }^{6}$ rabbits,${ }^{7}$ dogs,${ }^{8}$ and guinea pigs. ${ }^{9}$ Probably the most constant disturbance thus revealed is the development of partial or complete heart-block. Heart-block has also been demonstrated by parallel tracings of arterial and venous blood pressure. ${ }^{\mathrm{I}}{ }^{0}$ In frogs, cardiac disturbances have been studied by means of heart levers attached to the auricle and ventricle. ${ }^{\text {II }}$

The isolated blood-free mammalian heart can be readily tested for its independent anaphylactic potentiality by the simplified perfusion method introduced by Gunn. ${ }^{\mathrm{I2}}$ The heart is removed from the pericardial sac, rinsed in Locke's solution, and the ascending aorta drawn over a cannula delivering perfusion fluid at a constant temperature and pressure. Locke's solution, one of its modern variants, or Locke's solution

${ }^{1}$ Kyes, P., and Strauser, E. R.: J. Immunol., I2, 419. 1926.

${ }^{2}$ Richet, C., Brodin, P., and St. Girons, F.: Compt. rend. Acad. de sc., r69, 9. I919.

3 Banzhaf, E. J., and Famulener, L. W.: J. Infect. Dis., 7, 577. I910.

${ }_{4}^{4}$ Drinker, C. K., and Bronfenbrenner, J.: loc.cit.

5 [ $A$ discussion of the symptomatology and the autopsy findings in various animals has been omitted from this chapter. Cf. a similar presentation in the preceding chapter by Dr. Karsner.EDITORS.]

${ }^{6}$ Lewis, T., and Mathison, G.: Ileart, 2, 47. I910.

7 Auer, J., and Robinson, G. C.: J. Exper.Med., 18, 450. I9I3.

${ }^{8}$ Robinson, G. C., and Auer, J.: ibid., p. 556. I9I3.

${ }_{9}$ Koenigsfeld, H., and Oppenheimer, E.: Klin. U'chnschr., I, 849. 1922.

${ }^{10}$ Auer, J., and Robinson, G. C.: loc.cit.

In Friedberger, E., and Nita, S.: loc.cit.

${ }^{12}$ Gunn, J. A.: J. Physiol., 46, 506. I9r3; Manwaring, W. H., and Williams, T. B.: J. Immunol., 8, 75. 1923 . 
plus I-2 per cent carefully filtered defibrinated blood, may be used as the perfusion fluid. Numerous anaphylactic effects have been reported with this technique. ${ }^{\mathrm{x}}$

Drinker and Bronfenbrenner have recently applied Starling's isolated heart-lungs preparation to anaphylactic study. ${ }^{2}$ In this preparation the systemic circulation is replaced by an outside circulatory resistance connecting the aorta and superior vena cava, the lungs being under artificial respiration. Defibrinated blood is used as the circulating fluid.

\section{ARTERIAL BLOOD PRESSURE}

Changes in blood pressure in the systemic arteries during acute anaphylaxis are readily followed by ordinary kymograph methods. In guinea pigs, for example, there is usually a slight initial rise in the systemic blood pressure, followed by a gradual fall until the death of the animal. ${ }^{3}$ In rabbits, ${ }^{4}$ an initial rise may or may not take place, the typical reaction being a gradual reduction in arterial blood pressure beginning about three minutes after the protein injection and continuing until the death of the animal. In dogs, 5 there is invariably a precipitous fall in the arterial blood pressure. In severe shock the pressure is reduced to about $35-\mathrm{mm}$. Hg. by the end of ninety seconds, with a further gradual decrease to about $25-\mathrm{mm}$. $\mathrm{Hg}$. by the end of twelve minutes. In non-lethal reactions recovery usually sets in about the fifteenth minute, the blood pressure being restored to normal in from one to two hours. In cats ${ }^{6}$ there is often a sharp initial fall in arterial blood pressure, with full recovery in from one to three minutes, due to the normal toxicity of the foreign protein. Recovery is followed by the typical anaphylactic fall in arterial pressure, the minimum being reached in from twenty to forty minutes. In non-lethal shock, recovery is usually complete in from one to two hours.

A simple method of measuring the blood pressure in the pulmonary artery has been devised by Drinker. ${ }^{7}$ In this method, the heart is exposed by means of a smal! window cut in the anterior chest wall. The window is closed by sewing its edges to the opened pericardial sac. The pulmonary blood pressure is recorded by means of a special trocar cannula, inserted into one side of the pulmonary artery. In rabbits and cats, Drinker and Bronfenbrenner ${ }^{8}$ recorded an abrupt rise in the pulmonary blood pressure during the first two minutes of anaphylactic shock. In dogs, this increase did not take place.

× Cesaris-Demel, A.: Gior. A cad.med., Torino, 73, 69. I910; Manwaring, W.H., Meinhard, A. R., and Denhart, H. L.: Proc. Soc. Exper. Biol. $\mathcal{O}^{\circ}$ Med., 13, I74. I916; Leyton, A. S. and H. G., and Sowton, S. C. M.: J. Physiol, 50, 265. 1916.

${ }^{2}$ Drinker, C. K., and Bronfenbrenner, J.: loc. cit.; Starling, E. H.: Principles of II uman Physiology (2d ed.), p. 9i г. I9r5.

3 Auer, J., and Lewis, P. A.: J. Exper. Med., I2, I5I. 1910.

${ }_{4}^{4}$ Arthus, M.: Arch. internat. de physiol., 7, 47 I. I908; Loewit, M.: Arch.f. cxper. Path.u. Pharmakol., 68, 83. 1912; Biedl, A., and Kraus, R.: Wien.klin. Wchnschr., 22, 363. 1909.

5 Biedl, A., and Kraus, R.: loc. cit.; Manwaring, W. H.: loc. cit.

${ }^{6}$ Manwaring, W. H.: loc. cit.; Schultz, W. H.: loc. cil.; Drinker, C. K., and Bronfenbrenner, J.: loc. cit.

7Swift, W. E., Haggart, G. E., and Drinker, C. K.: J. Exper. Aled., 36, 329. 1922.

${ }^{8}$ Drinker, C. K., and Ironfenbrenner, J.: loc cil. 
VASOMOTOR REACTIONS

On account of the explosive edemas of certain tissues in acute anaphylaxis, anaphylactic vasomotor reactions are difficult to study by ordinary plethysomographic methods. Perfusion methods are usually applied to such determinations. Coca, ${ }^{\mathrm{I}}$ for example, demonstrated a marked increase in the perfusion resistance of the pulmonary artery in rabbits dying of anaphylactic shock. Immediately after death, the tip of the heart was cut off, and a cannula tied into the pulmonary artery. The cannula was connected with a pressure bulb filled with warm saline solution. In normal rabbits a pressure of $10-\mathrm{cm} . \mathrm{H}_{2} \mathrm{O}$ is usually sufficient to cause a rapid flow of the perfusion fluid from the left ventricle. After acute anaphylactic shock, a pressure of at least $90-\mathrm{cm} . \mathrm{H}_{2} \mathrm{O}$ is usually necessary to produce a similar flow. Coca believes this increased perfusion resistance is due to vasoconstriction in the pulmonary arterioles.

The relatively blood-free lungs may be tested for their independent anaphylactic potentiality by perfusion methods. Well aerated Locke's solution or one of its modern variants is used as the perfusion fluid. In dogs the most convenient method is to tie the afferent cannula into the superior vena cava, and the efferent cannula into the tip of the left auricle, all collaterals being ligated. ${ }^{2}$ The lungs are then inflated to their mid-respiratory volume, and the trachea clamped. Perfusion pressure, about 25 -mm. $\mathrm{Hg}$. In a typical anaphylactic reaction in blood-free canine lungs, the rate of perfusion flow is usually reduced to about one-quarter the initial flow, within three minutes after adding specific foreign protein to the perfusion fluid. By the end of five minutes, the lungs are remarkably edematous. On releasing the trachial clamp the lungs do not collapse. A large amount of clear frothy fluid escapes from the trachea.

A similar perfusion method applied to the isolated rabbit lungs will show a very marked increase in perfusion resistance on the addition of histamine to the perfusion fluid, ${ }^{3}$ but we have thus far been unable to demonstrate an appreciable change in the perfusion resistance of hypersensitive lungs on the addition of specific foreign protein to the perfusion fluid. ${ }^{4} \mathrm{We}$ are, therefore, inclined to attribute the marked increase in perfusion resistance described by Coca to factors other than vasoconstriction.

One of the most promising perfusion methods thus far applied to anaphylaxis is perfusion of the entire lower half of hypersensitive frogs. The hind quarters are freed from blood plasma and tissue lymph by a twenty-four hour preliminary perfusion with Ringer's solution. Arnoldi and Leschke ${ }^{5}$ report a slight vasoconstriction in normal frogs on adding human serum to the perfusion fluid, with a marked vasodilatation in hypersensitive frogs.

\section{VENOUS BLOOD PRESSURE}

A few studies of anaphylactic changes in the blood pressure in the systemic veins have been made, usually by means of a cannula in the jugular vein. ${ }^{6}$ In dogs, for

r Coca, A. F.: J.Immunol., 4, 2 19. I9I9.

${ }^{2}$ Manwaring, W. H., Chilcote, R. D., and Hosepian, V. M.: ibid., 8, 233. I923.

3 Manwaring, W. H., and Marino, H. D.: ibid., p. 317. 1923.

4 Manwaring, W. H.: unpublished work.

5 Arnoldi, W., and Leschke, E.: Deutsche med. Wchnschr., 46, IоI8. 1920.

${ }^{6}$ Auer, J.: loc. cit. 
example, there is a fall in the venous pressure synchronous with the fall in the arterial blood pressure. ${ }^{\mathrm{I}}$ On account of the absence of valves in the portal vein the portal blood pressure is readily measured by means of a cannula in one of its larger collaterals. In dogs, the portal blood pressure is usually doubled during the first two minutes of acute anaphylaxis. ${ }^{2}$

\section{RATE OF BLOOD FLOW}

Anaphylactic changes in the rate of blood flow in certain organs have been determined by means of a cannula in the efferent vein. In dogs, for example, the normal rate of blood flow through a $10-\mathrm{cm}$. intestinal loop is about $20 \mathrm{cc}$. per minute. This is reduced to about $2 \mathrm{cc}$. per minute by the end of the first ninety seconds of anaphylactic shock, with a further reduction to about $0.2 \mathrm{cc}$. per minute by the end of the third minute. ${ }^{3}$

\section{BLOOD VOLUME}

The only methods of estimating changes in total blood volume thus far applied to anaphylaxis are methods based on observed changes in hemoglobin content, erythrocyte count, and plasma concentration. ${ }^{4}$ Interpretation of such data is difficult on account of the marked reduction in the number of circulating red blood corpuscles due to capillary and sinusoidal stasis, and to possible changes in the amount of circulating protein due to altered capillary and parenchymatous permeability.

\section{CAPILLARY STASIS}

Anaphylactic stasis in the hepatic sinusoids and intestinal capillaries is readily demonstrated by ordinary histological methods. In the canine liver, for example, the sinusoidal plugs, described by Simonds, ${ }^{5}$ sometimes persist for several days after recovery from the shock.

\section{CAPILLARY PERMEABILITY}

The marked edemas of certain organs in acute anaphylaxis suggest possible changes in capillary permeability. Petersen and Levinson ${ }^{6}$ have attempted to demonstrate such changes by determinations of the quantitative changes in thoracic lymph proteins during canine anaphylactic shock. The most convenient organs for the study of such reactions, however, are the isolated canine lungs. Quantitative study of the fluid escaping from the trachea of perfused hypersensitive canine lungs shows that during anaphylactic shock in the blood-free lungs the capillary endothelium offers no demonstrable resistance to the outward passage of serum proteins. ${ }^{7}$ Larger colloidal molecules are held back.

${ }^{x}$ Simonds, J. P.: Am. J. Physiol., 65, 512 . 1923.

${ }^{2}$ Simonds, J. P.: ibid.; Manwaring, WV. H., Brill, S., and Boyd, W. H.: J. Immunol., 8, I 2 I. I923.

3 Manwaring, IV. H.: unpublished data.

${ }^{4}$ Simonds, J. P.: Am. J. Physiol., 72, I. I925.

${ }^{5}$ Simonds, J. P.: J. Infect. Dis., 24, 297. 1919.

${ }^{6}$ Petersen, W. F., and Levinson, S. A.: J. Immunol., 8, 349. 1923.

7 Manwaring, W. H., Hosepian, V. M., and Thomson, W. L.: J.A.1M.A., 82, 542. 1924. 
PARENCHYMATOUS PERMEABILITY

The marked swelling and vacuolization of certain parenchymatous cells during anaphylaxis ${ }^{1}$ suggest anaphylactic changes in parenchymatous permeability. The hepatic parenchyma of dogs, for example, nearly doubles in net weight during the first fifteen minutes of anaphylaxis. ${ }^{2}$ This increase is independent of the apparent increase in hepatic volume due to vasodilatations. Hanzlik and De Erls have attempted to demonstrate an altered hepatic permeability by a determination of the effects of anaphylactic shock on the rate of disappearance of certain dyestuffs from the bloodstream. In dogs, guinea pigs, and pigeons, for example, they report an anaphylactically decreased hepatic permeability to the dyes used in clinical tests of hepatic function.

\section{RESPIRATORY DISTURBANCES}

BRONCHOCONSTRICTION

Lack of parallelism between anaphylactically altered respiratory movements and pulmonary ventilation has been used as an index to anaphylactic bronchoconstriction. Auer and Lewis, ${ }^{4}$ for example, found that in guinea pigs the amount of air entering and leaving the lungs is rapidly decreased during anaphylactic shock, in spite of increased respiratory movements. A cessation of pulmonary ventilation also takes place in the rapidly fatal type of canine anaphylaxis. ${ }^{5}$ In rabbits, Drinker and Bronfenbrenner ${ }^{6}$ found pulmonary ventilation increased. Lack of parallelism between intrathoracic pressure and respiratory movements has also been used as an index to this reaction. ${ }^{7}$ In guinea pigs, for example, under artificial respiration rhythmic changes in intrathoracic pressure cease at the height of the anaphylaxis.

Occluding bronchoconstriction is at times demonstrable by histological methods. Schultz and Jordan, ${ }^{8}$ for example, fixed anaphylactic guinea pig lungs by perfusion through the pulmonary artery with Carnoy's strong solution. ${ }^{9}$ Hanzlik ${ }^{10}$ demonstrated anaphylactic bronchial occlusion in guinea pig lungs by means of celloidin casts of the bronchial tree.

Occluding bronchoconstriction may also be demonstrated by perfusion methods in blood-free organs. In guinea pigs the afferent cannula is most conveniently tied directly into the pulmonary artery. The ductus arteriosus is ligated and the left auricle opened for the escape of perfusion fluid. ${ }^{11}$ Perfusion pressure, about Io-mm.

${ }^{I}$ Weil, R.: loc. cit.; Manwaring, W. H., French, W. O., and Brill, S.: J. Immunol., 8, 2I I. 1923.

2 Manwaring, W. H., Hosepian, H. M., and Beattie, A. C.: ibid., p. 229. I923.

3 Hanzlik, P. J., and de Eds, F.: J. Pharmacol. \& Exper. Therap., 29, 485. 1926.

4 Auer, J., and Lewis, P. A.: J. Exper. Med., 12, I51. 1910.

5 Manwaring, W. H., Chilcote, R. C., and Hosepian, V. M.: Proc. Soc. Exper. Biol. \& Med., 20, 274. 1923.

${ }^{6}$ Drinker, C. K., and Bronfenbrenner, J.: loc.cit.

7 Auer, J., and Lewis, P. A.: loc. cit.

${ }^{8}$ Schultz, W. H., and Jordan, H. E.: J. Pharmacol. Eo Exper. Therap., 2, 375. 1910.

${ }^{9}$ Lee, $\Lambda$. R.: Microtomists, Vude-Mecum (6th ed.), p. 65. 1905.

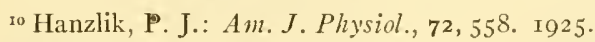

"I Manwaring, W. H., and Kusama, Y.: J. Immunol., 2, 157. I9 7 . 
Hg. If during the perfusion the lungs are alternately inflated and allowed to collapse by their own elasticity, the rate and degree of pulmonary fixation may be readily followed. In hypersensitive lungs, incomplete collapse is usually noted within thirty seconds after adding specific foreign protein to the perfusion fluid, with complete pulmonary immobilization by the end of ninety seconds.

Reactions in the isolated lungs, however, are not always a reliable index to the anaphylactic potentiality of the donor. Typical pulmonary immobilization, for example, may be demonstrated in the isolated lungs of highly immunized guinea pigs.

\section{OXYGEN CAPACITY OF BLOOD}

Quantitative determinations of the oxygen content of anaphylactic blood have shown that there is not only a marked reduction in circulating oxygen due to lessened pulmonary ventilation, but a still further reduction due to anaphylactically decreased oxygen-carrying capacity of the blood. ${ }^{x}$

\section{TISSUE RESPIRATION}

Abderhalden and Wertheimer ${ }^{2}$ have called attention to the possibility of there being an anaphylactically decreased tissue respiration, in addition to the reduced tissue respiration due to decreased circulating oxygen.

\section{SMOOTH MUSCLE REACTIONS}

ISOLATED SMOOTH MUSCLE

The isolated uterus, strips of the urinary bladder, of the intestines, segments of blood vessels, etc., may be tested for their independent anaphylactic potentiality by the Schultz-Dale technique. ${ }^{3}$ The muscle is suspended in Locke's solution or in one of its modern variants, and the changes in tone following the addition of specific foreign protein to the suspension fluid are recorded. The muscle may be obtained in a relatively blood-free condition by a preliminary perfusion of the hind quarters with Locke's solution. In a typical anaphylactic reaction in an isolated guinea pig uterus, for example, contraction usually starts about fifteen seconds after adding specific foreign protein to the suspension fluid, with maximum contraction usually reached by the end of ninety seconds.

Smooth muscle reactions thus recorded, however, are not always reliable indexes to the anaphylactic potentiality of the animal as a whole. The blood-free uterus of highly immunized guinea pigs, for example, will usually give typical anaphylactic contraction with the Schultz-Dale technique. 4 Non-specific desensitization of the entire animal does not always destroy the anaphylactic potentiality of the isolated smooth muscle.

\section{REACTIONS in situ}

The most convenient organ in which to study anaphylactic smooth muscle reactions in intact animals is the urinary bladder. In $\operatorname{dog} s$, a catheter is tied into the

'Houssay, B. A., and Cisneros, H. D.: Compt. rend. Soc. de biol., 93, 886. I925.

${ }^{2}$ Abderhalden, E., and Wertheimer, E.: Arch.f.d.ges. Physiol., 195, 487. 1922.

${ }_{3}$ Dale, H. H.: J. Pharmacol. Eo Exper. Therap., 4, 167. I913; Matthew, W., and Grove, E. F.: J. Immunol., r 0, 498. 1925.

4 Dale, H. H.: loc. cit. 
urethra (perineal incision, males) and the bladder partially inflated with warm saline solution. In smaller animals, the urethra is ligated, the abdomen opened, and a cannula tied into the incised bladder. Changes in intracystic pressure are recorded by means of a mercury manometer. During typical anaphylactic shock in guinea pigs, for example, the intracystic pressure usually increases to about $35-\mathrm{mm}$. $\mathrm{Hg}$. by the end of ninety seconds. ${ }^{.}$In dogs, the intracystic pressure usually increases to about $50-\mathrm{mm}$. Hg. by the end of two and a half minutes. In rabbits, no appreciableanaphylactic bladder contraction takes place. ${ }^{2}$

A similar method is applicable to the intestinal loop, and to the postpartum uterus, the organs being partially inflated with liquid paraffin. ${ }^{3}$ Anaphylactic intestinal and uterine contractions thus recorded are apparently synchronous with anaphylactic contractions in the urinary bladder. Hanzlik ${ }^{4}$ has applied a somewhat similar method to the crop muscle of hypersensitive pigeons, and has demonstrated an anaphylactic contraction of the circular crop muscle, accompanied by a reciprocal relaxation of the longitudinal crop muscle.

\section{SMOOTH MUSCLE TRANSPLANTATIONS}

In dogs, a portion of the descending colon supplied by the inferior mesenteric artery may be readily transferred to a second animal. Cannulae are tied in the right iliac artery and in the mesenteric vein draining the loop, and connected by means of paraffined rubber tubes with the femoral artery and femoral vein of the recipient. All collaterals are now ligated or clamped. The most convenient smooth muscle structure for such transplantation, however, is the urinary bladder (hind quarters). Cannulae are tied in the abdominal aorta and vena cava of the hind quarters to be transplanted, and connected by means of paraffined rubber tubes with the aorta and vena cava of the recipient. A normal urinary bladder thus transplanted into a hypersensitive recipient is thrown into a typical anaphylactic contraction during anaphylactic shock in the recipient. ${ }^{5}$

\section{GLANDULAR REACTIONS}

The rôle of glandular tissues in anaphylaxis is usually studied by determining the effects of surgical removal or toxic destruction of a certain gland on the subsequent development of sensitization, or on the severity of the shock in sensitized animals. Thus, Mautner ${ }^{6}$ reports an inhibition of sensitization in splenectomized dogs. Splenectomy, however, has no demonstrable anti-anaphylactic effect in previously sensitized dogs. The liver is the only glandular structure whose surgical removal or physiological exclusion will prevent anaphylactic shock (dogs).7

I Manwaring, W. H., Hosepian, V. M., Enright, J. R., and Porter, D. F.: J. Immunol., ro, 567 I925.

${ }^{2}$ Manwaring, W. H., and Marino, H. D.: ibid., I3, 69. 1927.

3 Manwaring, W. H., Beattie, A. C., and McBride, R. W.: J.A.M.A., 80, I437. I923.

${ }^{4}$ Hanzlik, P. J., Butt, E. M., and Stockton, A. B.: Proc. Soc. Exper. Biol. \& Med., 24, 327. 1927

5 Manwaring, W. H., Hosepian, V. M., O'Neill, F. I., and Moy, H. B.: J. Immunol., ro, 575 I925.

${ }^{6}$ Mautner, H.: Arch.f. exper. Path. u. Pharmakol., 82, iг6. I917.

7 Manwaring, IV. H.: loc. cit. 


\section{HEPATIC EXCLUSION}

In dogs, hepatic exclusion is best made by the simplified Eck fistula technique described by Dale and Laidlaw. ${ }^{\mathrm{I}}$ In this technique, the portal vein is connected to a renal vein by means of an excised portion of the jugular vein of the same animal, Crile's transfusion cannulae being used to make the connection. This method at times may be simplified by connecting the portal vein directly with the resected abdominal vena cava. In mildly sensitized dogs dehepatization completely abolishes all recordable anaphylactic reactions. In highly sensitized dogs injected with massive doses of specific foreign protein, a slight fall in arterial blood pressure and a slight increase in smooth muscle tone are sometimes noted. ${ }^{2}$ These occasional primary extrahepatic anaphylactic reactions usually begin after a latent period of about five minutes.

\section{EVISCERATION}

A more rapid method of physiological exclusion often applicable to routine dehepatization tests is ordinary physiological evisceration. This is usually performed by ligating or clamping the rectum, esophagus, mesenteric blood vessels, portal vein, and hepatic artery. Physiological evisceration completely prevents demonstrable anaphylactic reactions in mildly sensitized dogs. That the concurrent removal of the gastro-intestinal tract is not the cause of this desensitization is shown by a control gastro-intestinal exclusion without interference with hepatic circulation. This is readily done by connecting the abdominal vena cava with the portal vein by means of a Crile transfusion cannula, the return circulation from the hind quarters replacing the excised portal circulation. Gastro-intestinal removal by this technique does not materially reduce the severity of anaphylactic shock in dogs. ${ }^{3}$

\section{HEPATIC TRANSPLANTATION}

Physiological hepatic transplantation may be made in dogs, by connecting the portal vein and abdominal vena cava of the dog whose liver is to be transplanted, with the carotid artery and the jugular vein, respectively, of the recipient, paraffined rubber tubes being used to make the connections. The hepatic artery and thoracic vena cava are then clamped or ligated. Typical anaphylactic potentiality is conferred upon a normal dog by the transplanted liver of a hypersensitive dog. ${ }^{4}$

\section{HEPATIC PERFUSION}

Perfusion of the isolated liver is readily performed in all laboratory animals. An afferent cannula is tied in the portal vein and an efferent cannula in the abdominal vena cava. The hepatic artery and thoracic vena cava are then ligated. Locke's solution, at $38^{\circ} \mathrm{C}$., is the usual perfusion fluid. Perfusion pressure approximately that of the normal portal pressure in the animal tested. During the operation the liver must

${ }^{2}$ Dale, H. H., and Laidlaw, P. P.: J. Physiol., 52,35r. rgr8.

2 Manwaring, W. H., Hosepian, V. M., Enright, J. R., and Porter, D. F.: loc. cit.

3 Manwaring, W. H., O'Neill, F. I., and McCullough, M.: Proc. Soc. Exper. Biol. Eo Med., 22, 398. I924.

${ }^{4}$ Manwaring, W. H., Hosepian, V. M., O’Neill, F. I., and Moy, H. B..: loc cit. 
be very carefully protected with gauze moistened in warm Locke's solution, otherwise chilling or mechanical injury will cause local stasis.

Hepatic perfusion was first applied to anaphylactic study by Nolf, ${ }^{\mathrm{r}}$ who found that the isolated hypersensitive canine liver, perfused with a mixture of specific foreign protein and uncoagulated blood, is thrown into a marked anaphylactic reaction. This is shown by engorgement, cyanosis, stasis, and reduced rate of perfusion flow. There is a complete loss of the coagulability of the perfusion mixture.

HEPATIC ANAPHYLATOXIN

Toxic products formed or liberated by the hypersensitive liver during the initial stages of acute canine anaphylaxis may be demonstrated by drawing shock blood directly from the ligated inferior vena cava. ${ }^{2}$ This blood injected intravenously into a normal dog produces a typical anaphylactic syndrome. Shock blood drawn from the carotid artery is usually non-toxic.

\section{HEPATIC ANTI-ANAPHYLAXIS}

The anti-anaphylactic function of the hypersensitive liver is usually demonstrated by comparing the ordinary intravenous anaphylactic M.L.D. of the specific foreign protein with the M.L.D. when the injection is made by way of the portal vein. In guinea pigs, for example, from two to four times the ordinary intravenous dose is usually required to produce fatal shock. ${ }^{3}$ In dogs, injection into the portal vein gives a prompter and more pronounced anaphylactic reaction than control injection into the jugular vein.

The detoxicating function of the hypersensitive liver may also be demonstrated by perfusion methods. If a mixture of specific foreign protein and defibrinated ana phylactic blood, for example, is perfused through the liver of a hypersensitive guinea pig, the mixture loses its power to call forth anaphylactic reactions in subsequent perfusions through isolated hypersensitive lungs. ${ }^{4}$

\section{NERVOUS REACTIONS}

Attempts have been made to demonstrate a primary neurological factor in anaphylaxis by applying specific foreign protein directly to hypersensitive nervous tissue. Such tests are usually inconclusive due to constitutional reactions caused by the absorption of the specific foreign protein by the local capillaries. That reactions of the nervous system play an important rôle in the anaphylactic syndrome, however, is readily shown. A partial or complete abolition of reflexes from the skin and mucous surfaces is characteristic of acute anaphylaxis in all animal species thus far tested. Shock animals are also rela tively insusceptible to strychnine, ${ }^{5}$ adrenalin, and electrical

I Nolf, R.: Arch. internat. de physiol., ro, 37. г9го.

${ }^{2}$ Manwaring, W. H., Hosepian, V. M., O'Neill, F. I., and Moy, H. B.: loc. cit.

3 Falls, F. H.: J. Infect. Dis., 22, 83. I918.

${ }^{4}$ Manwaring, W. H., and Crowe, H. E.: J. Immunol., 2, 517. I9I7.

5 Arloing, F., and Langeron, L.: Compt. rend. Soc. de biol., 91, 73. I924. 
stimulation of vasomotor nerves. ${ }^{\mathrm{x}} \mathrm{Kling}^{2}$ has called attention to an increased electrical excitability of motor nerves as a result of sensitization.

\section{LOCAL REACTIONS}

The most pronounced local reaction in hypersensitive animals is the Arthus phenomenon, the local inflammation and subsequent necrosis produced in hypersensitive rabbits by a subcutaneous injection with specific foreign protein. Opie ${ }^{3}$ extracted the inflammatory area with physiological salt solution and showed by quantitative precipitin tests that the injected foreign protein was largely retained in the local area. The Arthus phenomenon, therefore, apparently plays an important defensive rôle by preventing the local absorption of toxic protein. Local reactions have also been studied in internal organs, particularly in the canine liver. ${ }^{4}$

\section{MISCELLANEOUS TECHNICAL METHODS}

Numerous routine technical methods are used to supplement the physiological methods outlined above. Rapid fixation methods, for example, have been applied to the histological study of anaphylactic capillary reactions ${ }^{5}$ and of edemas. ${ }^{6}$ Hematological methods have revealed the characteristic leukopenia, reduced platelet count, ${ }^{7}$ and eosinophilia, as well as changes in the size of the individual red blood corpuscles. ${ }^{8}$ Chemical methods have been used in a study of the anaphylactic reduction in blood coagulability, ${ }^{9}$ to changes in protein and non-protein nitrogen in shock blood ${ }^{10}$ and shock tissues, ${ }^{11}$ and to anaphylactic alterations in such blood components as cholesterol, calcium, ultra-filterable calcium chlorides. ${ }^{12}$ Also to changes in $\mathrm{H}$-ion concentration, alkali reserve, ${ }^{\mathrm{I3}} \mathrm{O}-, \mathrm{CO}_{2^{-}}$, and sugar content, ${ }^{\mathrm{I4}}$ glycogen content, ${ }^{15}$ and in tryptic and antitryptic power. ${ }^{16}$

Physico-chemical methods have revealed changes in the viscosity, surface tension, ${ }^{17}$ and

I Biedl, A., and Kraus, R.: Wien. klin. Wchnschr., 22, 363. I 909; Hoefer, P., and Kohlransch, A.: Klin. Wchnschr., I, I893. 1922.

${ }^{2}$ Kling, C. A.: Ztschr. f. Immunitätsforsch. u. exper. Therap., I 3, 43. I9I 2.

3 Opie, E.: J. Immunol., 9, 259. I924.

${ }_{4}$ Weil, R.: loc. cit.

${ }_{5}^{5}$ Rich, A. R.: J. Exper. Mel., 33, 287. 1921.

${ }^{6}$ Manwaring, W. H., French, W. O., and Brill, S.: J. Immunol., 8, 2 Ir. I923.

${ }^{7}$ Krueger, A. P., and Schultz, E. W.: Proc. Soc. Exper. Biol. Eo Med., 23, r53. 1925.

${ }^{8}$ Zunz, E.: Compt.rend. Soc. de biol., 93, 863. 1925.

${ }^{9}$ Pepper, O. H. P., and Krumbhaar, E. B.: J. Infect. Dis., r4, 476. r9r4.

${ }^{10}$ Hiranobu, K.: Am. J. Physiol., 50, 357. I9I9.

${ }^{1}$ Manwaring, W. H., and Oppenheimer, R.: Proc. Soc. Exper. Biol. \& Med., r 3, 176. rgr6.

ז2 Zunz, E., and la Barre, J.: Compt. rend. Soc. de biol., 91, 802, r 293. 1924; 93, 104.4. I925; Blum, L., Delaville, M., and van Caulaert: ibid., 91, I 289-92. 1924.

${ }^{13}$ Hirsch, E. F., and Williams, J. L.: J. Infect. Dis., 30, 256.1922.

${ }^{14}$ Zunz, E., and la Barre, J.: Compt. rend. Soc. de biol., Ir I, I2r. I924; McCullough, M., and O'Neill, F. I.: J. Infcct. Dis., 37, 225. 1925.

${ }^{15}$ O'Neill, F. I., Moy, H. B., and Manwaring, W. H.: J. Immunol., I o, 583. 1925.

${ }^{16}$ Bronfenbrenner, J.: Proc. Soc. Exper. Biol. \&o Med., 13, 42. I915; J. Exper. Med., 21, 480. I9I 5 .

${ }^{17}$ Zunz, E., and la Barre, J.: Compt. rend. Soc. de biol., 90, 658. 1924. 
freczing point of anaphylactic serum, lymph, and peritoneal fluid, ${ }^{\mathrm{r}}$ also changes in electrical conductivity, index of refraction, and specific rotation, and in the osmotic resistance of erythrocytes. Serological methods have becn used to test the parallelism, or lack of parallelism, between the precipitin content of a serum and its power to produce passive sensitization, ${ }^{2}$ also to a study of the topographical distribution of specific foreign proteins in hypersensitive animals, and to the specific protein affinity of perfused hypersensitive tissues. ${ }^{3} \mathrm{By}$ an electro-osmotic method, Otto and Shirakawa ${ }^{4}$ report the separation of specific sensitizing antibody from specific precipitin.

${ }^{2}$ Fleisher, M. S., and Mayer, L. L.: J. Immunol., 9, 319. 1924.

${ }^{2}$ Lake, G. C., Osborne, J. B., and Wells, H. G.: J. Infect. Dis., I4, 364. I9I4; Weil, R.: op. cit., I, I. I9I6.

3 Manwaring, W. H., Kasama, Y., and Crowe, H. E.: J. Immunol., 2, 5 I1. I9I7.

${ }_{4}^{4}$ Otto, R., and Shirakawa, T.: Ztschr.f. IIyg. u. Infektionskrankh., 103, 426. 1924.

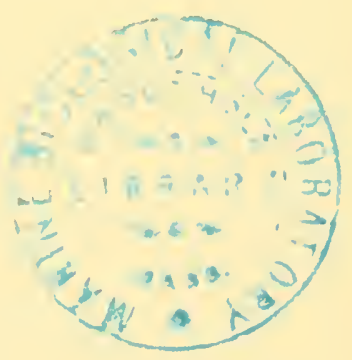




\title{
CHAPTER LXXV
}

\section{ATOPY}

\author{
ARTHUR F. COCA \\ Cornell University Medical College
}

The subject assigned for review to the writer by the editors of this volume does not, in its present state, lend itself to categorical exposition, because its very existence as a pathological-physiological entity, separable from the other phenomena of hypersensitiveness, is generally doubted. The general unwillingness to approve the separation of atopic hypersensitiveness from the other forms is due not only to differences of opinion as to the interpretation of accepted findings, but also, perhaps, to the lack of confirmation of a number of observations that have been used by the writer to characterize the atopic mechanism.

This situation obliges the reviewer to adopt a controversial attitude and also to refer, by way of comparison or contrast, to some other forms of hypersensitiveness, such as anaphylaxis and ordinary serum disease.

\section{DEFINITION}

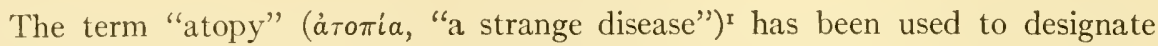
certain clinical forms of hypersensitiveness which occur, so far as is known, only in human beings, and which are subject to inheritance.

The clinical forms included in this category are asthma and hay fever. While it is thought probable that other forms, such as eczema and certain forms of drug and food idiosyncrasy, will ultimately be added to these two, more information is needed before this can be done.

\section{ATOPIC INHERITANCE}

The separation of this group of hypersensitiveness from the others was made possible largely by the studies of Cooke and Vander Veer, ${ }^{2}$ as well as the confirmatory ones of June Adkinson ${ }^{3}$ and of Spain and Cooke. ${ }^{4}$ These investigators have shown in two ways the controlling influence of heredity in the establishment of bronchial asthma and hay fever: First, by the observation that, when one of these conditions occurred in both the paternal and the maternal antecedents, their incidence among the offspring was decidedly greater than it was when only one line was affected, or when the family history on both sides was negative in this respect; and, second, by the observation that the first appearance of the atopic symptoms occurs much earlier among the children subject to a bilateral atopic inheritance than it does among those with a unilateral or negative family history.

${ }^{x}$ Coca, A. F., and Cooke, A. R.: J. Immunol., 8, I63. 1923.

${ }^{2}$ Cooke, R. A., and Vander Veer, A.: ibid., I, 201. 1916.

3 Adkinson, J.: Genetics, 5, 363. 1920.

${ }^{4}$ Spain, W. C., and Cooke, R. A.: J. Immunol., 9, 521. I924. 
Table I (taken from the article by Spain and Cooke) shows the incirlence of the two atopic conditions in the offspring under the three categories of hereditary influence. It is seen that, under a bilateral influence, nearly 70 per cent of the off spring were destined to be affected. This figure approximates the percentage (75) that should be expected if the condition is dependent upon a single Mendelian dominant factor, both parents being assumed to be hybricl with respect to it. 'The percentage incidence found under a unilateral influence approximates the expectation ( $5 \circ$ per cent) on the assumption just mentioned.

In discussing the considerable percentage (about 40) of atopic individuals with negative family history, Spain and Cooke call attention to the fact that these individuals were practically "self-selected from among I I,500 individuals of the general

TABLE I

Showing the Percentage of Atopic Children Estimated to Develop in the Three Classes*

\begin{tabular}{|c|c|c|c|c|c|c|c|c|}
\hline \multirow{2}{*}{ INHERITANCE } & \multirow{2}{*}{ 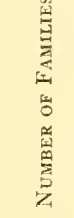 } & \multirow{2}{*}{ 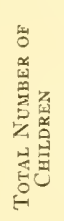 } & \multicolumn{2}{|c|}{$\begin{array}{l}\text { ATOPIC } \\
\text { CHILDREN }\end{array}$} & \multirow{2}{*}{ 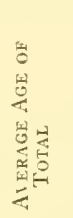 } & \multirow{2}{*}{ 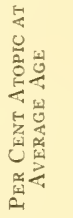 } & \multicolumn{2}{|c|}{$\begin{array}{l}\text { Estimated ATOPIC } \\
\text { CHILDREN }\end{array}$} \\
\hline & & & Number & $\left|\begin{array}{c}\text { Per Cent } \\
\text { of } \\
\text { Total }\end{array}\right|$ & & & Number & $\begin{array}{c}\text { Per Cent } \\
\text { of } \\
\text { Total }\end{array}$ \\
\hline Bilateral: & & & & & & & & \\
\hline Present findings. . . . . . . . . & 32 & 83 & 50 & 60.2 & I 6.8 & 83.9 & $59 \cdot 5$ & 71.6 \\
\hline Cooke and Vander Veer.... & 26 & 70 & 39 & $55 \cdot 7$ & 23.0 & 82.6 & $47 \cdot 2$ & $67 \cdot 5$ \\
\hline Combined findings........ & $5^{8}$ & I 53 & 89 & 58.0 & 19.9 & $8_{3.2}$ & 106.6 & $69 \cdot 5$ \\
\hline Unilateral: & & & & & & & & \\
\hline Present findings. . . . . . . . . & 2 I I & 666 & $32 \mathrm{I}$ & 48.2 & 33.8 & 85.8 & 373.8 & 56.1 \\
\hline Cooke and Vander Veer... & 52 & I 50 & 77 & 5 I. 3 & $27 \cdot 5$ & $84 \cdot 5$ & $9 \mathrm{I} .2$ & 60.0 \\
\hline Combined findings........ & 263 & 816 & 398 & 49.8 & 30.7 & $85 . I$ & $460 . I$ & 58.0 \\
\hline Negative: & & & & & & & & \\
\hline Present findings . . . . . . . . . & So & $2 S_{9}$ & IO4 & 35.9 & 32.2 & $8 \mathbf{r} .8$ & $\mathrm{I} 26.0$ & 43.6 \\
\hline Cooke and Vander Veer.... & I 48 & 631 & 205 & $32 \cdot 4$ & 35.0 & $S_{4} \cdot 0$ & 244.0 & $3^{8.6}$ \\
\hline Combined findings........ & 228 & 920 & 309 & $34 \cdot \mathrm{I}$ & $33 \cdot 6$ & 82.9 & 370.0 & $4 \mathrm{I} . \mathrm{I}$ \\
\hline
\end{tabular}

* From Spain and Cooke.

population." In other words, it would not be permissible to consider the ancestry of this group free from the atopic character, unless I I,500 antecedents had been accessible for examination in this regard-an impossible requirement. However, this idea does not explain why a single dominant character should so often be missing in both parents of the child that exhibits it; and, furthermore, the assumption of a single dominant factor does not easily explain the differences of degree in the inheritance that are revealed in the hereditary influence upon the age of onset.

The influence of heredity upon the age of onset of atopic hypersensitiveness is seen in the characteristic difference in the graphic representation of this feature as it is found under the three categories of atopic inheritance. The three curves plotted by Cooke and Vander Veer and Spain and Cooke, in their study of nearly one thousand cases, are so evidently characteristic that one can doubt the hereditary control of the age of onset only by assuming a gross error in the assembling of the data. These differences can be expressed in part by noting that, by the tenth year of life, atopic 
symptoms have appeared in 72 per cent of the atopic offspring under a bilateral hereditary influence; in 35 per cent of those subject to a unilateral influence, and in only 20 per cent of those with a negative family history; also by noting that all of those subject to bilateral inheritance have shown atopic symptoms by the fortieth year, whereas in the other two groups, the age of onset may be much later - as late as the seventieth year.

It will be shown presently that this clearly demonstrated hereditary control of the age of onset includes the simple corollary that the age of onset in each atopic individual is determined by heredity.

\section{ATOPIC REAGINS}

In the blood of nearly all atopic persons that exhibit a positive cutaneous reaction to the known excitant, specific bodies (atopic reagins) can be found which are capable of sensitizing the human skin (Prausnitz and Küstner, ${ }^{\mathrm{I}}$ De Besche, ${ }^{2}$ and others).

So far as the writer is aware, no means is available for the demonstration of the atopic reagins other than their specific property of sensitizing the human skin. In mixtures of the reagins with the related atopen, no precipitation has been observed nor fixation of complement. Indeed, it seems that the reagins lack even the most characteristic property of antibodies prepared against soluble antigens - the property of rendering the respective antigen specifically inactive. ${ }^{3}$ Thus, antitoxin specifically extinguishes the toxicity and binding power of the related toxin and anaphylactic antibody specifically inhibits the shock-producing property of its related antigen. But atopic reagin seems completely to lack the power to prevent the related atopen from causing its typical reaction in a sensitive human skin.

Coca and Grove ${ }^{4}$ were unable to sensitize the normal human skin with an anti-egg precipitating and sensitizing serum from a rabbit; these wholly negative results have since been confirmed separately by Grove and by the writer, and also in a series of independent experiments by Walzer. ${ }^{5}$ Walzer did obtain some reactions when the injected skin sites were tested within twenty-four hours (less after forty-eight hours); but these were shown to be non-specific, since identical reactions could be obtained with an unrelated antigen (cottonseed extracts). Walzer ${ }^{6}$ has also reported complete failure to sensitize the human skin with an unusually high-titred anti-egg serum from the rabbit, when he tested the injected site by his method of intestinal absorption.

There are some reports of successful passive sensitization of the guinea pig with the serum of idiosyncratic human beings. ${ }^{7}$ These experiments were carried out at a time when the atopic reagins were not known. The more recent experiments of this kind have resulted negatively. Particularly significant are those of Spain, who selected the sera of persons that were atopically sensitive to typical anaphylactogens and who

\footnotetext{
I Prausnitz, L., and Küstner, H.: Centralbl.f. Bakteriol., Orig., 86, r6o. I92 I.

${ }^{2}$ de Besche, A.: Am. J. M. Sc., r66, 265. т923.

3 Levine, Philip, and Coca, A. F.: J. Immunol., I I, 4I I. 1926.

${ }_{4}^{4}$ Coca, A. F., and Grove, E. F.: ibid., ro, 445. 1925.

5 Walzer, M.: personal communication.

6 Walzer, M.: J. Immmnol., 14, I59. 1927.

7 Bruck, C.: Arch.f. Dermat. u. Syph., 96, 241. I909.
} 
determined the presence of atopic reagins in all of them. He also employed the more delicate method of Dale in secking to demonstrate passive sensitization in the guinea pigs. The results of Spain's experiments were wholly negative.

For the present, then, the atopic reagin is identified by its origin in a hypersensitive human being; its property of specifically sensitizing the normal human skin; its inability passively to sensitize the guinea pig's uterus; and its inability to render specifically inactive the related atopen. To these outstanding characteristics may be added the minor ones that the atopic reagin is not a precipitin nor a complementfixing body, and that it is capable, in the passively sensitized skin site, of mediating as many as three successive reactions (wheal and erythema) upon three successive tests with the same quantity of atopen ${ }^{\mathrm{T}}$ - a peculiarity that is lacking in anaphylactic antibodies. ${ }^{2}$

Bodies capable of specifically sensitizing the human skin have been demonstrated by Rackemann ${ }^{3}$ and also by Brunner ${ }^{4}$ in persons that are or that have been harboring intestinal parasites. These bodies have not been studied with respect to the other identifying properties of atopic reagins; but, for the present, they can be separated from the latter by the consideration that the infested individuals do not present any signs of hypersensitiveness to worms.

Sensitizing reagins are not demonstrable in all cases of human hypersensitiveness, particularly in drug idiosyncrasy ${ }^{5}$ exhibited to non-antigenic substances, and in some food idiosyncrasies even to antigenic materials. The reagins seem to be consistently absent in the idiosyncrasies that are expressed in urticaria, angioneurotic edema, and gastro-intestinal symptoms, provided asthmatic symptoms are absent. This principle is illustrated in the two cases of sensitiveness to green pea that were studied by Ella F. Grove with the writer. ${ }^{5}$ One of these subjects presented the symptoms of asthma when green pea was ingested, and exhibited a positive skin reaction to an extract of this vegetable. With the serum of this individual the normal human skin could be locally sensitized to green pea. The second subject suffered no asthma when green pea was ingested, but exhibited angioneurotic edema. The skin test with green pea resulted negatively in this case, and the serum lacked the power of sensitizing the normal human skin. In the considerable group of asthmatics in whom no specific sensitiveness can be demonstrated by means of the skin test, it would seem reasonable to assume that reagins are lacking in the blood. However, the mechanism of the asthmatic attacks in these individuals must, of course, be determined before this question can be finally settled.

Whether the atopic reagins are true antibodies, i.e., developed immunologically under antigenic stimulation, or physiological products like the natural hemagglutinins and hemolysins, is not known. Their increase in the blood during the injections of the excitant (specific treatment) seems to point to an immunological origin. Never-

r Levine, Philip, and Coca, A. F.: op. cit., I I, 4 Ir. I926; Coca, A. F., and Grove, E. F.: loc. cit.

${ }^{2}$ Coca, A. F., and Grove, E. F.: loc. cit.; Levine, Philip, and Coca, A. F.: op.cit., Ir, 4I I. I926.

3 Rackemann, F.: J. Immunol., 13, 389. I927.

4 Brunner, M.: read before the American Association of Immunologists, Rochester, N.Y., April I5, I927. J. Immunol., I5. I928.

5 Coca, A. F., and Grove, E. F.: loc. cit. 
theless, it is strange that the anti-worm reagins (Rackemann, Brunner) can be produced by all persons, presumably at any period of life, under the sole influence of the worm antigen, whereas only the atopic individual can produce reagins to typical atopens, and he only after he has reached a certain age, and then, in some instances, only to a particular substance or group of substances.

\section{ATOPIC SHOCK TISSUES}

The term "shock organ," suggested by Doerr to designate the tissue in which the physiological reaction of anaphylactic shock takes place in the different animals (the bronchial muscle in the guinea pig, the arteriole media in the rabbit, and the hepatic vein in the dog), can be applied also in human hypersensitiveness. In the lower animals, as has just been indicated, the shock organ is different in the different species, but it is the same in all individuals of the same species. In the human being, there are several shock tissues (in the conjunctiva, the nasal mucous membrane, the bronchial mucous membrane, the skin, the subcutaneous and submucous tissue); ${ }^{\mathrm{I}}$ and these are seldom all affected in the same individual; often only one is affected in a single individual. In fact, the same excitant may in one individual excite asthmatic symptoms only, and in another person only those of hay fever.

Thus, in contrast with anaphylactic sensitiveness, which has been demonstrated only in the lower animals, and in which the sensitizing antibodies are physiologically effective always in the same organ, the reagins of atopy may be effective in one person through the bronchial shock tissue (asthma), in another through the nasal shock tissue (hay fever).

The facts just recited clearly indicate that the mere presence of atopic reagins in the blood does not determine the clinical form of the hypersensitiveness (asthma or hay fever) which the individual will exhibit. An atopic state of the shock tissue must be assumed to be necessary for its involvement in the hypersensitive person. This assumption is supported by other evidence.

Baldwin ${ }^{2}$ has shown that specific reagins can be demonstrated in the blood of the offspring of an atopic parent before the child itself has begun to suffer atopic symptoms.

Brunner and Walzer, ${ }^{3}$ in confirming Rackemann's finding of sensitizing reagins in worm-infested persons, observed constitutional reactions (asthma) in three of the individuals whose blood carried the anti-worm reagins, when a certain quantity of worm extract was injected into the skin. These three persons suffered from asthma, but were free of worms. The same quantity of the worm extract was injected into other individuals whose blood contained the anti-worm reagins, but none of these persons exhibited any constitutional reaction. The bronchial shock tissue in these non-atopic persons was not susceptible to the "reaction" between the worm excitant and the reagins; it was not in the atopic state.

In this connection, an explanation may be offered for the success in one case and the failure in the other of passive sensitization in the well-known observations of

\footnotetext{
${ }^{2}$ Coca, A. F.: J. Lab. \& Clin. Med., I2, I135. Sept., I927.

${ }^{2}$ Baldwin, L. B.: J. Immunol., 13, 345. 1927.

3 Brunner, M., and Walzer, M.: to be pullished.
} 
Ramirez. ${ }^{\mathrm{T}}$ A transfusion from the asthmatic donor to one recipient resulted in an asthmatic sensitiveness in one recipient, but not in the second. It seems probable that the bronchial shock tissue in the first recipient was in the atopic state, whereas that in the second recipient, who had received a larger quantity of blood than the first, was normal.

The independence of the several shock tissues from one another seems evident, furthermore, in the difference in the character of the constitutional reactions produced by an overdose of an atopen in different hypersensitive persons. In some, the symptoms are those of asthma only, in others those of hay fever, subcutaneous edema, or urticaria. The atopic state of the same shock tissue exhibits differences in degree in different individuals which are not always parallel with the concentration of the reagins in the blood. One of the numerous mysteries of this subject lies in the fact that the shock tissue may be only slightly susceptible to the natural contact with the atopen, but highly susceptible to the same substance upon its injection, or vice versa. The susceptibility to the injected atopen seems generally to be parallel with the reagin content of the blood. ${ }^{2}$

A shock tissue can be affected by the subcutaneous injection of an atopen which, under the usual conditions of exposure, does not influence it. A case of eczema of Dr. Horace S. Baldwin, of the Cornell Clinic, is one in point. The individual was subject also to plantain pollen hay fever. On two occasions, an overdose of the pollen extract injected in the course of prophylactic treatment caused a decided exacerbation of the eczema.

\section{NON-SPECIFIC INFLUENCE OF THE ATOPIC SHOCK TISSUES}

The anaphylactic shock organs of the guinea pig, the rabbit, and the dog are susceptible to various non-specific influences, such as peptone, histamine, and certain "toxic" foreign sera. As in anaphylactic shock, each individual of the same animal species is affected by these materials in the same way; but the different species are affected in characteristically different manner by the same material. Thus, peptone produces bronchospasm in the guinea pig, but not in the other two animals; it produces the characteristic congestion of the liver in the dog, but not in the guinea pig or rabbit.

It would seem probable that the several atopic shock tissues also are likewise susceptible to non-specific influences. One may look upon the effect of a cold wind or irritating vapors upon some asthmatic subjects or of dust, to which the individual is not specifically sensitive, as illustrations of this kind of influence. Some persons subject to food idiosyncrasy with gastro-intestinal symptoms cannot tolerate "rough foods"; for example, fresh lima beans or green peas can be eaten in unlimited quantities, but the dried beans and peas cause severe symptoms of dyspepsia with headache and vertigo. In many instances, Duke remarks, these non-specific influences may be the primary causes of the reaction of hypersensitiveness. Duke has had occasion to investigate this point in connection with his study of the astonishing cases of specific cutaneous hypersensitiveness to physical agents (heat, cold, light). ${ }^{3}$ The

I Ramirez, M. A.: New York State J. Med., I I2, I I5. 1920.

${ }^{2}$ Levine, Philip, and Coca, A. F.: op. cit., I1, 435. 1926.

${ }_{3}$ Duke, W. W.: Asthma, May Feier, etc., Literature (2d ed.). St. Louis: C. V. Mosby Co., I926. 
primary importance of these physical agents as excitants in his cases is revealed by the demonstrated specificity of the susceptibility in the different individuals to a single one of these agencies. Duke states that there is a distinct family history of atopic hypersensitiveness in his cases.

In this connection may be mentioned the asthmatic symptoms exhibited by practically all individuals coming in sufficient contact with grain infested with the larvae of Pediculoides ventricosus. ${ }^{x}$ The affected individuals suffered also dermatitis, and the infested material possessed immediately irritating properties, as shown by the development of an itching lesion upon first contact with it. It seems at least possible that the asthmatic symptoms in these cases were not an expression of hypersensitiveness, but the result of direct irritation of the bronchial mucous membrane. Reagins could not be found in the blood of the affected individuals. It is not necessary to consider the bronchial shock tissue in these individuals to have been in the atopic state, just as such an assumption is unjustified in the case of normal persons in whom the skin has been passively sensitized to any atopen, and responds, on suitable contact of the excitant, with the local formation of a wheal.

\section{HOW IS ATOPIC HYPERSENSITIVENESS ESTABLISHED?}

With the foregoing considerations in mind, we are in a position to discuss the question as to the manner in which atopic hypersensitiveness is established in an individual under the controlling influence of heredity. ${ }^{2}$ It has been thought that the hypersensitive individuals had inherited an abnormal permeability of the surface membranes to unaltered antigens. This idea is confronted with the fact that many persons became sensitive to only one member of a group of similar excitants (a single pollen atopen or a single animal or vegetable protein). In order to make the theory of abnormal permeability conform with this fact, it would have to be assumed that the abnormal permeability was specific; for this assumption there is no evidence. Moreover, there is ample evidence of a normal permeability of the gastro-intestinal tract to various common proteins (milk, egg, fish, pollen, nut). With the use of his ingenious method of skin sensitization to several proteins, Walzer ${ }^{3}$ has demonstrated a permeability of the gastro-intestinal tract to the unaltered antigens in about 90 per cent of normal individuals. These observations show that practically all people are equally exposed to parenteral and prolonged contact with the atopic excitant, and that this factor cannot therefore be a determining one in the establishment of atopic hypersensitiveness. This fact leads us inevitably to the conclusion that the establishment of the hypersensitiveness is determined wholly by heredity, and this is further supported by the common observation that the subcutaneous or intravenous injection of large quantities of foreign protein (horse serum) does not result in the establishment of an atopic hypersensitiveness. In view of the numerous instances of hypersensitiveness developing to a single substance or group of substances, and, in view of the demonstration by Cooke and his associates ${ }^{4}$ of the controlling influence of heredity upon

x Ancona, G.: Sperimentale, 76, 270. 1922; Grove, E. F.: J.Immunol., 12, 263.1926.

${ }^{2}$ Coca, A. F.: J. Lab. \& Clin. Mled., 12, I135. Sept., 1927.

3 Walzer, M.: J. Immunol., 13, I43. 1927.

${ }^{4}$ See Coca, A. F.: Tice's Practice of Medicine, r, Io7. Hagerstown, Mu.: IV. F. Prior Co., I920; Spain, W. C., and Cooke, R. A.: loc. cit. 
the date of onset of symptoms, it seems necessary to conclude that some persons are destined, by inheritance only, to become sensitive to a certain substance on about a certain date.

It seems reasonable to suppose that the actual state of hypersensitiveness develops only after sufficient contact with the excitant; of course, the exhibition of symptoms is dependent upon such contact.

\section{ANAPIILACTIC ANTIBODIES IN HUMAN BEINGS}

The similarity between anaphylactic and atopic hypersensitiveness has naturally led many writers to look upon these conditions as identical. The chief similarity between these two forms is the existence of the specific mechanism in both, and the identification of the two on this ground could be considered reasonable if reagins were the only specific sensitizing bodies produced by the human being. However, it is known, especially through the valuable researches of Anderson and Schloss, ${ }^{\mathrm{I}}$ that anaphylactic antibodies also are produced by normal human beings, precipitating antibodies having been previously demonstrated in human beings by numerous investigators.

The human being actually is capable of producing the anaphylactic mechanism; yet this mechanism has not been shown to be capable of mediating any physiological reaction in the human being, although it is of course able to sensitize the guinea pig. The normal babies in whose blood Schloss and his co-workers could demonstrate anaphylactic antibodies were not themselves hypersensitive to the respective foreign proteins (milk or egg). Anaphylactic antibodies can sometimes be found in the blood of human beings subject to serum disease. This association is, however, too irregular to permit one to consider the etiological relationship proved.

\section{DANGERS ATTENDING THE INJECTION OF FOREIGN SERUM}

The demonstration of the strict hereditary control of atopic hypersensitiveness carried with it the corollary that, in persons not subject to the atopic hereditary influence, atopy cannot be induced. This conclusion is of course important in connection with the question of the reinjection of a foreign protein such as horse serum. Atopic hypersensitiveness (hay fever or asthma) cannot be induced in a normal human being by injection of horse serum. However, the reinjection of horse serum after the incubation period of serum disease is sometimes followed by an accelerated or immediate reaction of serum disease, which is so severe that it may even endanger life or actually end it. It seems, in some instances of this kind, that the intravenous reinjection is more dangerous than the subcutaneous or intramuscular, although, even after subcutaneous reinjection, symptoms may rarely develop within two or three minutes.

It will be useful to formulate, out of our knowledge of the dangers attending the injection of foreign sera, a reasonable attitude in which the exigencies of the condition requiring serum treatment are weighed properly against the probable risk of serious reaction.

Experience with the injection of the various excitants into atopic persons indicates that the greatest tolerance induced by this means is usually reached only after a pe-

I Anderson, A. F., and Schloss, O. M.: J. Dis. Child., 26, 45 I. I923. 
riod of some weeks; furthermore, this attainable maximum of tolerance is often too low to permit the injection of the usual therapeutic dose of serum. Hence, if the question of a therapeutic or prophylactic injection of antitoxic horse serum is raised with regard to a known horse-asthmatic patient, the physician should refuse to administer the serum and should not attempt what is loosely called "desensitization."

Instances of serious and even fatal reactions following a reinjection of horse serum in non-atopic persons have been reported. The fatal issue for much the greater part followed intravenous or intraspinal reinjection. What percentage of reinjections result in dangerous reactions is not known (exact information upon this question is needed), but it must be small - so small that the risk should be taken without hesitation when the condition of the patient requires the specific treatment. In such cases, the subcutaneous route should be usually preferred, although even this precaution should be waived when the patient's condition urgently requires the more effective intravenous administration.

The observations of Park, ${ }^{\mathrm{I}}$ whose opinion has been stated in the foregoing paragraph, expose the inadequacy of the cutaneous test as a means of determining the individual's susceptibility to a reinjection. In his experience, even a marked skin reaction could be elicited in persons who later tolerated the intravenous injection of 5 or Io cc. of serum.

The procedure of "desensitization," which is ineffective in atopic serum hypersensitiveness, fails also in serum disease. This conclusion has been discussed by the writer $^{2}$ with respect to ordinary serum disease. It is confirmed by the experience of Louis Tuft, about to be reported. The patient, after a three weeks' interval, following the previous administration of serum, was "desensitized" over a period of two and one-half hours by the intramuscular injection of $8.0 \mathrm{cc}$. of serum. Thereupon not more than ro cc. of the serum was slowly injected into the vein. The patient complained suddenly of a burning sensation, and collapsed, dying shortly afterward.

From the foregoing, it seems evident that, where reinjection of therapeutic serum is urgently indicated, it is useless to apply the cutaneous test or the procedure of desensitization as precautionary measures. The physician must be governed in such a situation wholly by the exigencies of the patient's condition.

\section{HYPOSENSITIZATION}

After Wolff-Eisner ${ }^{3}$ and Meltzer ${ }^{4}$ had expressed the view that hay fever and asthma were expressions of hypersensitiveness in human beings, there were several independent attempts made in this country, as well as in Europe, to apply the principle of desensitization in the treatment of these conditions, and it was found by Noon ${ }^{5}$ and his numerous successors ${ }^{6}$ that the procedure applied in hay-fever subjects and

I Park, IV. H.: J. Immunol., 9, I 7. I924.

${ }^{2}$ Coca, A. F.: 'Tice's Practice of Medicine, 1, 107. Hagerstown, Md.: WV. F. Prior Co., Publishers, 1920 .

3 Wolff-Eisner, 1.: Das Ileufieber. igo6.

4 Meltzer, S. J.: J.A.M.A., 55, 1021. 1910.

5 Noon, L.: Lancet, I, I572. 1911 .

'Freeman, J.: ibid., 2, 814. I9ıा; Clowes, G. H. A.: Proc. Soc. Exper. Biol. \$ Mel., 10, 69. I913; Koessler, K. K.: Forscheimer's Therapeusis, 5, 671. 19 I4. 
some asthmatics is actually followed by a relief of the symptoms. Before the atopic reagins were discovered, the protective effect of the injections of the excitant was assumed to be due to a neutralization of the specific mechanism as in anaphylactic desensitization. Some of the early authors reported a disappearance of the cutaneous reaction after such treatment, and Mackenzie ${ }^{\mathrm{r}}$ reported local cutaneous desensitization upon repeated injections of the excitant into the same site. However, Cooke ${ }^{2}$ observed that in no case was the cutaneous reaction extinguished as a result of the injections of pollen extracts, and that in many of them the reaction was quantitatively the same at the end of the course of injections as it had been at the beginning. The reactivity of the conjunctiva also was not in Cooke's experience materially lessened by the course of injections. Upon repeating Mackenzie's experiments, he was able to demonstrate that the local exhaustion which is obtainable by rapidly repeated injection of the excitant was not specific, and was evidently due to a tiring of the physiological functions that take part in the production of the local lesion. Cooke concluded from his observations that the protective effect of specific treatment in hay fever and asthma is not due to a desensitization, and proposed the term "hyposensitization" to designate the establishment of the increased tolerance in atopy by specific treatment.

The discovery of the atopic reagins made it possible to examine this question further, and it could be shown that, in hay fever subjects who had been successfully treated by a course of injections, the atopic reagins were never diminished in the blood, but were sometimes increased two to four times. ${ }^{3}$ This finding completed the proof that the mechanism of hyposensitization in hay fever is different from that of desensitization in anaphylaxis.

Thus, we are left without any conception of the manner in which the injections of the excitant bring about the increased tolerance to the injections, as well as a more or less complete freedom from symptoms upon natural contact. In view of the absence of precipitation in the "neutral" mixtures of atopen with its related reagin, it was thought possible that such mixtures could be different from the mixtures of antigen and its respective precipitating antibody from the rabbit in the development of a "toxic" property. Such mixtures have been tested by three observers in the human skin and in the guinea pig (by intravenous injection), but without positive results. The skin reactions obtained by such mixtures were not greater than those obtained with the same atopic serum mixed with salt solution, instead of the solution of the respective excitant. ${ }^{4}$ More recently, however, positive results have been reported by Gay.5 This disagreement calls for further study.

\section{ATOPY IN LOWER ANIMALS}

Since atopic hypersensitiveness is seen to be subject to heredity and therefore not induced in persons lacking the inherited predisposition, it should not be expected that

I Mackenzie, G. E.: J.A.M.A., 77, г 563. I92 r.

${ }^{2}$ Cooke, R. A.: J. Immunol., 7, 219.1922.

3 Levine, Philip, and Coca, A. F.: op. cit., I I, 435. I926.

${ }^{4}$ Coca, A. F., and Grove, E. F.: loc. cit.; Levine, Philip, and Coca, A. F.: op. cil., I I, 4I I. I926. ${ }_{5}$ See Gay, L. N., and Chant, E.: Bull. Johns Hopkins IIosp., 40, 270.1927. 
it could be found in lower animals. However, there have been some efforts to induce in the guinea pig symptoms that are similar to those of asthma and hay fever, under conditions of contact like those that obtain in these two atopic states.

Guinea pigs that have been made to breathe air in which pollen was suspended have been thought to exhibit signs of hay fever ("sneezing" and lacrimation, without asthma) after a certain period of exposure. ${ }^{\mathrm{I}}$

These experiments, which have not been repeated, must be viewed in the light of the general experience that guinea pigs sensitized with pollen extracts exhibit, upon any mode of testing, symptoms and pathology that are the same as those induced with the usual protein antigens. In other words, the shock organ is always the same in the guinea pig; and it remains the same whether the antigen is administered by injection, by ingestion, or by inhalation. The lacrimation that one occasionally sees in the guinea pig in anaphylactic shock has been likened to hay fever; ${ }^{2}$ but hay fever is more than a lacrimation.

The bronchospasm of anaphylactic shock in the guinea pig has generally been considered identical in its mechanism with the asthmatic attack, and, in guinea pigs sensitized to horse dander, the bronchospasm has been induced by causing the sensitive animal to breathe the antigen in a spray. This mode of administration of the antigen was used in imitation of the natural mode of contact with the excitant in asthma. However, it has been found that, under these conditions, the antigen always reached the bronchial shock organ of the guinea pig through the bloodstream, after being absorbed through the respiratory mucous membrane, exactly as if it had been injected. This could be seen in the fact that, when shock was induced in the sensitive guinea pigs with the inhaled antigen, the uterine muscle was found always to be desensitized. When no shock occurred, the uterus remained sensitive. ${ }^{3}$

More recently an effort has been made to bridge the gap between idiosyncrasy and anaphylaxis by the experimental production of a dermatitis in guinea pigs with nickel salts. ${ }^{4}$ This experiment was suggested by the occurrence of an itching eruption in a considerable percentage of human beings working with nickel. It seems necessary to call attention to the lack of demonstrated connection between atopic hypersensitiveness and the nickel dermatitis. If those that oppose the separation of the hypersensitiveness of atopy from that of anaphylaxis are merely seeking a form of hypersensitiveness that occurs in both human beings and in lower animals, they can most easily find it in the hypersensitiveness of infection (tuberculin-mallein type), which is typically exhibited by the surface membranes. One need not deny the possibility that other forms of hypersensitiveness besides this one affect lower animals as well as human beings, and of all tissues the skin seems most likely to show such common susceptibility. But, if other susceptibilities should be found that are common to man and the lower animals, this will not necessarily remove the many theoretical as well as practically important differences between atopy and anaphylaxis. However, a further study of the nickel dermatitis in guinea pigs would be welcome. E. L. Milford

I Ulrich, Henry L.: J. Immunol., 3, 453. I918.

${ }^{2}$ Doerr, R.: Weichardt's Eirgebn. d. IIyg., 5, 73. I922.

3 Alexander, H. L., Becke, W. G., and Holmes, J. A.: J. Immunol., I I, I 75. I926.

4 Walthard, B.: Scheeis. med. Ifchnschr., 56, 603. I026; Mbstract, J.A.MI.1., 87, 709. 1927. 
and the writer, in unpublished experiments, failed completely to induce dermatitis with I per cent or 2 per cent nickel chloride, painted three times daily over a period of three to four weeks, even when the skin was irritated previous to the application of the nickel with a stiff handbrush.

No one has yet attempted to identify the "urticaria" of lower animals with the totally different human skin lesion of the same name; nor has fagopyrismus in cattle been identified with human food idiosyncrasy. 


\section{CHAPTER LXXVI}

\section{TUBERCULIN AND THE TUBERCULIN REACTION \\ ESMOND R. LONG \\ University of Chicago}

\section{DEFINITION OF TUBERCULIN AND THE TUBERCULIN REACTION}

In I89I Robert $\operatorname{Koch}^{\mathrm{r}}$ announced his discovery of a specific reaction occurring on the introduction of extracts or other products of the tubercle bacillus into the bodies of tuberculous animals. Whole or disintegrated dead bacilli, aqueous and glycerol extracts of them, protein precipitates from such extracts, and the culture medium on which the bacillus had grown, were all effective in producing the reaction. Normal animals failed to respond to the introduction of this material. The tuberculous, on the other hand, reacted locally, focally, and generally, i.e., an acute inflammation developed at the locus of injection, and around all active foci of disease, while the animal exhibited signs of general intoxication with rise in temperature.

The substances eliciting the reaction were grouped under the name "tuberculin," and the specific reaction was designated the "tuberculin reaction." Noting that the tuberculin reaction occurring on the introduction of dead or living tubercle bacilli into the skin of a tuberculous animal led to necrosis with sloughing of the tissue inoculated with bacilli, Koch developed immunological concepts of the tuberculin reaction and introduced tuberculin as a specific therapeutic agent against tuberculosis.

\section{PREPARATIONS USED AND THEIR STANDARDIZATION}

Koch believed a specific active principle was present in all his tuberculin preparations, and made a well-directed effort to isolate it, which will be described below. He advocated two of his many preparations as particularly suitable for therapy. These were his A T (Alt Tuberkulin), or in English O T (Old Tuberculin), and $\mathrm{B} \mathrm{E}^{2}$ (Bacillen Emulsion ["bacillary enulsion"]; new tuberculin). Since Koch's time an enormous number of tuberculin preparations have been described or put on the market. Baldwin, Petroff, and Gardner ${ }^{3}$ list over a hundred such tuberculins and allied products. Of all of these, Koch's Old Tuberculin has been used most extensively. 'This consists of glycerol bouillon on which the tubercle bacillus lias been cultivated, freed from the bacilli by filtration after one hour's boiling of the whole culture, and evaporated to one-tenth of its original volume. His B E consists of a glycerol and water suspension of dried and finely ground bacilli. Both preparations are diluted with salt solution before use.

Koch himself recognized the need for some sort of standardization of these prod-

i Koch, R.: Deulsche med. ITchuschr., I 7, IOI. IS9I.

${ }^{2}$ Koch, R.: ibid., 27, 829. I901.

${ }^{3}$ Baldwin, E. R., Petrof, S. A., and Gardner, L. U.: Tuberulosis. Bacteriology, Palhology, and Laboratory Diagnosis. Philadelphia, 1927. 
ucts. Shortly after his first description of tuberculin, ${ }^{\mathrm{t}}$ he established as a standard of suitable potency a tuberculin of which $0.5 \mathrm{cc}$. or less would kill in from six to thirty hours, with characteristic pathological change, a guinea pig infected one month previously with tuberculosis. Various other standardizing procedures have been devised, which I have reviewed elsewhere. ${ }^{2}$ The ideal standardizing procedure would be quantitative chemical determination of the active principle present. As this is at present impossible in complex preparations, I have developed a biological method with a standard unit, which is accurate, although admittedly cumbersome.

The basis for this method is the guinea pig testicle reaction which I first described in $1924.3^{3}$ This is an allergic reaction of the same biological type as the skin and lethal dose tests, but more specific and more delicate. In experiments on tuberculous infection of the testicle in the guinea pig I found that the normal-appearing testicle of the tuberculous guinea pig is extraordinarily sensitive to tuberculin, minute doses causing profound degeneration and ultimate absorption of the germinal cells, the spermatocyte being the one primarily affected. Doses a thousand times as large are without effect upon the testicle cells of the normal guinea pig. The complete details of this specific test cannot be given here, but the reader is referred to an article ${ }^{2}$ on the assay of tuberculin on the basis of the spermatocyte reaction; and to Figure r, taken from the same article, which illustrates the practical measurement of the spermatocyte unit of tuberculin, i.e., that amount of tuberculin causing complete abolition of spermatogenesis.

This method is on trial in a number of laboratories concerned with large-scale usage of tuberculin. Until its usefulness or that of a better method is demonstrated the view of Baldwin, Petroff, and Gardner may be considered sound, viz., that a constant, standard method of preparation is most important, with recognition of the individual variation of patients in sensitiveness.

TYPES OF TUBERCULIN REACTION IN USE

In practice, tuberculin, which is employed both diagnostically and therapeutically as described below, is used in a great variety of ways. The most common means of administration are: (I) subcutaneous injection, i.e., injection with a hypodermic syringe into the subcutaneous tissue where absorption is rapid; (2) the cutaneous method of von Pirquet, in which a drop of tuberculin is allowed to dry on a freshly scarified point of skin; (3) the intracutaneous test of Mantoux and others, in which a small amount of tuberculin is injected through a fine hypodermic needle ( 26 or 27 gauge, short length) parallel to the surface, lumen of the point up, into the most superficial layer, a white bleb of blanched skin indicating proper injection; (4) the ophthalmic test developed independently by Calmette and by Wolff-Eisner, in which a drop of tuberculin or a disk impregnated with tuberculin is placed on the ocular conjunctiva; and (5) the percutaneous method of Moro, in which a salve made up of tuberculin and lanolin is rubbed into the skin. The first and fifth are commonly used in treatment, and the other three, leading to local reactions at the point selected, in diagnosis.

I Koch, R.: op. cit., I 7, I1 89 . I891.

${ }^{2}$ Long, E. R.: J. Infect. Dis., 37, 368. I925. $\quad 3$ Long, E. R.: Am. Rev. Tuberc., 9, 2 I5. I924.

${ }_{4}$ Baldwin, E. R., Petroff, S. A., and Gardner, L. U.: loc. cit. 
The intracutaneous test is by far the most accurate. Therapeutic administration has been carried out also by inhalation, ingestion, and even absorption through the rectum, urethra, and vagina-methods uncertain, unnecessary, and with no advantages to recommend them. The details concerning these tests may be read in Calmette's book. ${ }^{x}$

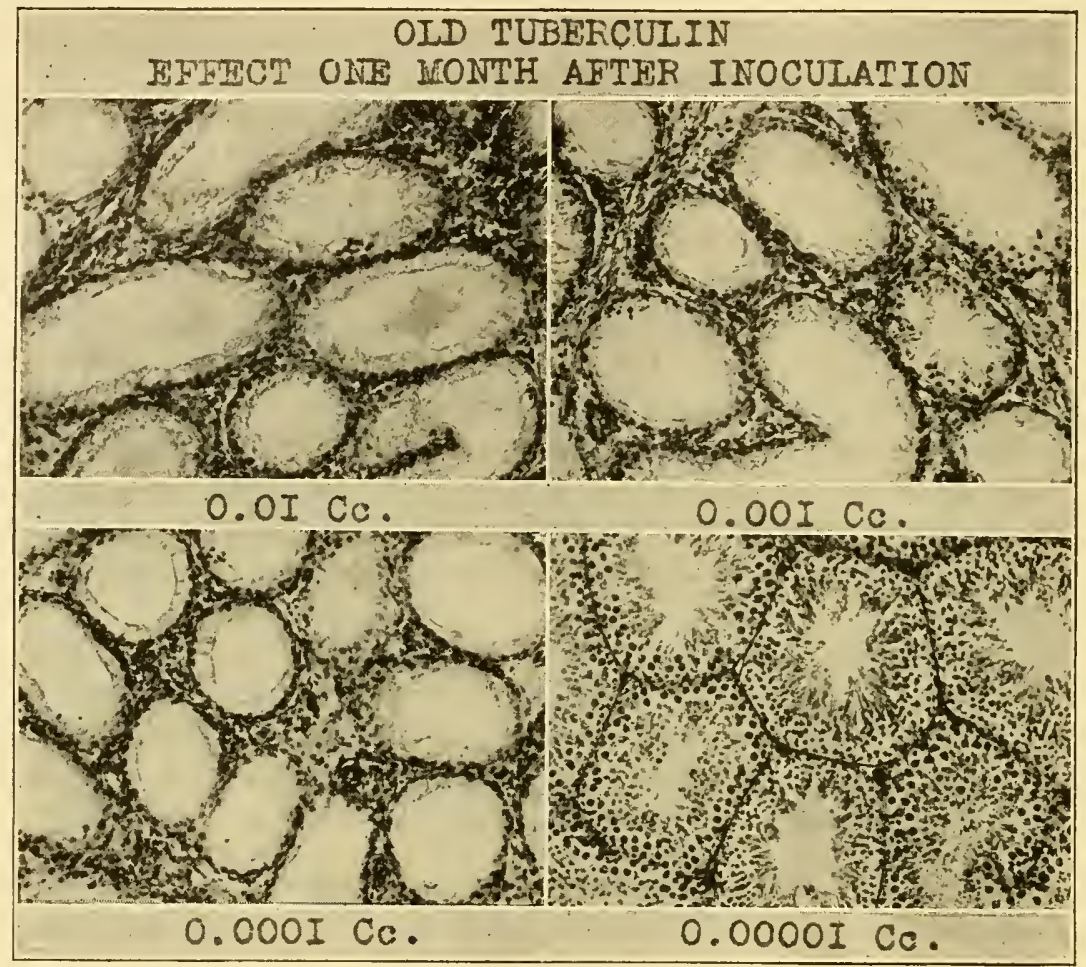

FIG. I.-Old Tuberculin in increasing dilution was injected into the left testis in a series of tuberculous guinea pigs, the fluid volume injected being 0.1 cc. in each case. The animals were killed one month later. In each case the right, control, testis was found to be normal. The illustration shows the condition of the left testis into which injections were made. There is profound atrophy of the tubules of the testes receiving injections with $0.01 \mathrm{cc}$, $0.00 \mathrm{I} \mathrm{cc.,}$ and $0.000 \mathrm{I} \mathrm{cc}$. Spermatocytes, spermatids, and spermatozoa have disappeared. Practically no effect is visible at $0.0000 \mathrm{rcc}$, although the tubules in this case were found to be somewhat smaller than in the right testis into which no injections had been made. The smallest amount causing complete abolition of spermatogenesis was thus $0.000 \mathrm{r} \mathrm{cc}$. On the arbitrary scale here developed this represents I spermatocyte unit, and this sample of tuberculin therefore contains 10,000 spermatocyte units per cubic centimeter.

\section{THE CHEMICAL NATURE OF TUBERCULIN}

KOCH'S ORIGINAL INVESTIGATIONS AND VIEWS

Robert Koch believed, with scarcely questionable correctness, that a chemically as well as biologically specific active principle was responsible for the tuberculin ac-

${ }^{1}$ Calmette, A.: Tubercle Bacillus Infection and Tuberculosis in Man and Animals (trans. by Soper and Smith). Baltimore, I923. 
tivity of all of the many varieties of tubercle bacillus preparations. Koch himself, in his original article, ${ }^{\mathrm{x}}$ stated that the active principle appeared to be related to the proteins. In his second investigation on tuberculin, ${ }^{2}$ he endeavored "das wirksame Prinzip zu isolieren, um es frei von anderen Stoffen anwenden zu können," in the belief that by thus concentrating it he might enhance the therapeutic action of tuberculin and at the same time avoid certain disturbing non-specific manifestations in patients. This work for its time was an exhaustive piece of chemical investigation. Seibert ancl I have reviewed and analyzed his results elsewhere. ${ }^{3}$ Of various methods of precipitation or concentration attempted, precipitation of glycerol extracts of tubercle bacilli with 60 per cent alcohol acted most favorably. This yielded a powder of high tuberculin potency, which he felt to be "vielleicht schön in Wirklichkeit das vollkommen isolierte wirksame Prinzip des Tuberkulins." He called it "purified tuberculin." It was a water-soluble substance, partially coagulable by heat, but stable as regards activity in glycerol-water solution even at temperatures from $130^{\circ}$ to $160^{\circ} \mathrm{C}$. It contained 14 per cent nitrogen and gave all of the common protein reactions. Acetic acid precipitated it with some loss of activity. Alcohol precipitated it well only in the presence of some sodium chloride. His preparations contained $16-20$ per cent ash, however, and he was forced to admit that this high ash content indicated considerable admixture with impurities. In spite of his finding that material with tuberculin activity passed easily and rapidly through dialyzing membranes, he concluded that the active substance belonged to the group of proteins.

\section{OTHER CONCEPTIONS}

Since Koch's time there has been only occasional dissent from the view that the active principle "belongs to the group of proteins." Only one school, that of Hans Much, implicates definite substances other than proteins or their derivatives. This school, with views somewhat apart from the usual immunological concepts, holds that tuberculin activity may be exhibited by lipoid as well as protein substances.

Although most investigators have followed Koch in the belief that the active substance is related to protein, there have been marked differences of opinion as to the type of protein substance involved. I have reviewed in detail elsewhere the various contentions on this subject. ${ }^{4}$ The opinion has not infrequently been held that the active substance is a protein derivative rather than a whole protein. Most of the derivatives of protein recognized in the usual classifications of protein have been considered by one investigator or another to be responsible for tuberculin activity. One of the earliest investigators, Ruppel, ${ }^{5}$ believed a nucleo-protein to be the active principle. Matthes ${ }^{6}$ contended that a proteose was concerned. Löwenstein and Pick ${ }^{7}$

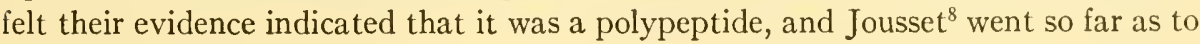

\footnotetext{
${ }^{1}$ Koch, R.: op. cil., I7, I0I. I891.

${ }^{3}$ Long, E. R., and Seibert, F. B.: Tubercle, 8, iा 1926.

${ }^{5}$ Ruppel, W. G.: Ztschr. f. phys. Chemie, 26, 2 I 8. I899.

${ }^{6}$ Matthes, M.: Deulsche Arch.f. klin. Med., 54, 39. I894.

${ }_{7}^{7}$ Löwenstein, E., and Pick, E.: Biochem. Zischr., 31, I42. I9I I.

${ }^{8}$ Jousset, A.: Bull. Acad. de méd., 71, 752. I914.
}

${ }^{2}$ Koch, R.: ibid., p. I189. I891.

+ Wells, H. G., De Witt, Lydia, and Long, E. R.: The Chemistry of Tubcrculosis. Baltimore, I923. 
state that the active principle is made up of substance of low molecular weight corresponding to profound digestion of proteins, viz., the amino acids. A few investigators like Bieling ${ }^{\Upsilon}$ claim to have isolated purified substances quite free from protein and still highly active.

\section{RECENT CHEMICAL STUDIES}

Although it has been known since the earliest report of Koch that the active substance is present both in the bacterial bodies themselves and in the medium on which they have been grown, the latter source has been until recently most commonly used in investigations on the chemistry of the active material. A great step forward was taken with the use of non-protein media on which the tubercle bacillus grows well. After growth of tubercle bacilli these media always become potent tuberculins. It is noteworthy that at the same time protein recognizable by the usual protein tests becomes apparent in these media.

Formulae for a number of media of this character, so-called "synthetic media," have been compiled by Calmette..$^{2}$ I have devised one ${ }^{3}$ similar to a number of these, with certain special advantages. This contains asparagine, ammonium citrate, acid potassium phosphate, sodium carbonate, sodium chloride, magnesium sulphate, ferric ammonium citrate, and glycerol. Acidic and basic radicles are present in such proportion that the resultant mixture is neutral, and in such concentration that the medium is well buffered against changes in reaction resulting from bacillary growth. The medium furnishes a yield of $100-\mathrm{I} 50 \mathrm{gm}$. of moist tubercle bacilli per liter.

In an extensive investigation fostered by the National Tuberculosis Association, Florence B. Seibert and I have studied the active products obtained from this medium after removal of the bacillary growth. The greater part of the chemical study was carried out by Dr. Seibert. Through the generous co-operation of Parke, Davis and Company and the H. K. Mulford Company, larger quantities of material than hitherto possible were made available. We had previously settled quite conclusively that such media invariably contain protein after luxuriant growth of the tubercle bacillus. The results of a number of investigators who had reported tuberculin activity in the absence of protein in media of this type were explained partly by the great delicacy of the tuberculin reaction, as compared with the relatively coarse chemical tests, and partly by our finding ${ }^{4}$ that glycerol, a regular constituent of such media, interferes markedly with the important biuret protein reaction.

Having determined that protein gradually accumulated in media originally protein free, as bacillary growth on the medium progressed and tuberculin activity developed, our next concern was to determine with the unusually large quantity of material available whether the activity resided in whole protein molecules or in products derived from them in the course of autolysis of the bacilli growing upon the medium.

In different experiments the clear, bacteria-free filtrate was subjected to dialysis

I Bieling, R.: Centralbl. f. Bakteriol., Abt. I, Orig., 93, 56, 73. I924.

${ }^{2}$ Calmette, A.: loc. cit.

${ }^{3}$ Long, E. R.: Tubercle, 6, I 29. I924; Long, E. R., and Seibert, F. B.: A m. Rev. Tuberc., I3, 393. I926.

${ }^{4}$ Seibert, F. B., and Long, E. R.: J. Biol. Chcm., 64, 229. I925. 
for varying periods and in different types of apparatus. Precipitation was carried out by acetic acid at the iso-electric point of the bulk of the colloid present, and by all of the protein precipitants commonly used, ammonium sulphate and trichloracetic acid proving the most valuable. In addition to this, cleavage of the protein material present was effected by proteolytic enzymes and acid hydrolysis, in an effort to determine any parallelism between tuberculin activity and the size of protein molecules present. The results of this investigation are briefly summarized in a later article, from which I quote with slight change:

When tubercle bacilli are grown on a non-protein medium of known chemical composition, tuberculin activity develops coincidently with the appearance of protein in the medium, the protein being derived presumably by extraction or autolysis of tubercle bacilli. If tuberculin prepared on this medium is subjected to dialysis, as a general rule the active substance, together with the protein, remains in the dialysing sac, all of the original constituents of the medium, as well as the substances responsible for the characteristic odour and most of the colour, diffusing out. Occasionally traces of substance of protein nature also appear to diffuse, and when this occurs tuberculin activity appears in the diffusate.

The addition of acetic and other acids precipitates from tuberculin prepared on this medium a protein which has moderate tuberculin activity. But precipitation of the protein of the solution is never complete by this method, and, moreover, the protein-containing filtrate from the precipitate retains tuberculin activity. Full saturation with ammonium sulphate, however, precipitates at the same time all of the protein and all of the active substance.

The ammonium sulphate precipitate contains both coagulable and non-coagulable protein and also proteose. When the protein is destroyed by pepsin with little destruction of the proteose, activity is destroyed. When the proteose alone is disintegrated by the action of trypsin in neutral solution or by erepsin, the activity is not affected.

The active principle of tuberculin withstands without loss of activity four hours' heating at $\mathrm{I}_{20} \mathrm{C}$. and $\mathrm{I} 4 \mathrm{-l}$. pressure in the presence of $\mathrm{N} / \mathrm{I}$. hydrochloric acid. Up to this point the protein of the solution remains in such state that it can be precipitated by trichloracetic acid. The precipitate is active. Strengths of acid of N/6 and above hydrolyse the protein so that it no longer precipitates with trichloracetic acid, and at the same time tuberculin activity disappears (see Figs. 2 and 3 ).

The activity of tuberculin thus definitely appears associated with a protein substance. It has not been found possible, by any of a variety of means tried, to separate from this substance any non-protein fraction with tuberculin activity, and therefore it seems doubtful that the close association of activity with protein is merely one of physical absorption. The possibility remains open that in the protein molecule itself activity may be a function of a certain group. The apparent persistence of activity on mild hydrolysis with beginning protein disintegration would support this view. The fact that with further hydrolysis of protein activity disappears, makes it unlikely, however, that an active group can be split off, free from the rest of the protein.

An advance of the first importance which may lead to solution of the whole problem has been made by Dr. Seibert ${ }^{2}$ in the crystallization of the water-soluble protein found in the tuberculin preparations we have used. Fractional coagulation and other methods of concentrating the activity of the water-soluble tuberculin protein failing,

${ }^{2}$ Long, E. R., and Seibert, F. B.: Tubercle, 8, in r. 1926.

${ }^{2}$ Seibert, F. B.: Science, 63, 619. 1926; Tr. 22d Ann. Meeting, Nat. Tuberc. Assoc., p. 274. I926; Tr. 23d Ann. Mceting, Nat. Tubcrc. Assoc., p. 245. 1927; Science, 66, 433. I927. 
she attempted the crystallization of protein present, by methods similar to those which have led to the crystallization and purification of egg albumin. By half saturating

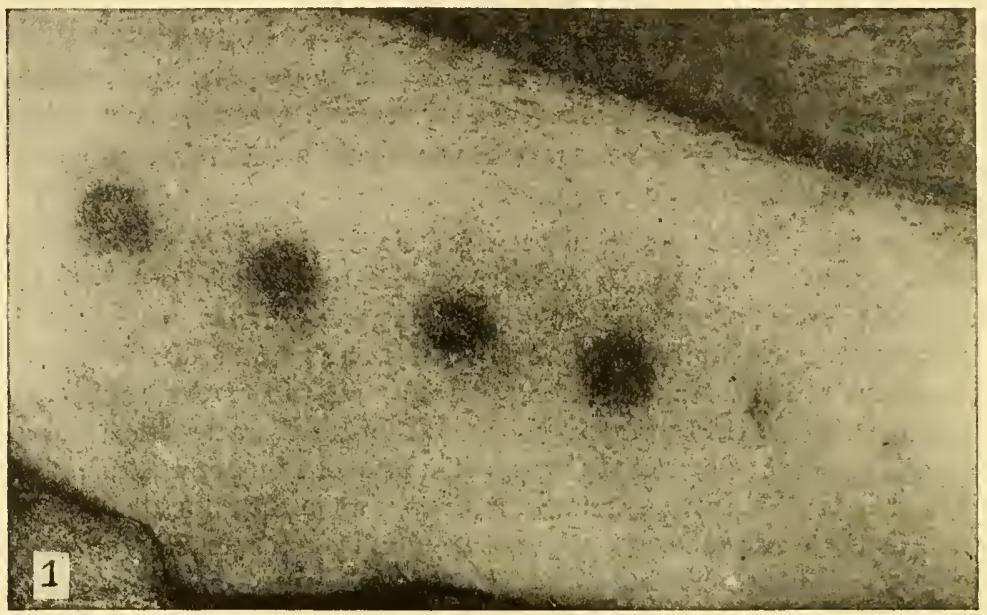

FIG. 2.-Skin reactions in patient sensitive to tuberculin, with samples of tuberculin heated in the autoclave four hours at $120^{\circ} \mathrm{C}$. with $\mathrm{HCl}$. Six samples of the following reactions were autoclaved: No. I, neutral; No. $2, \mathrm{~N} /$ 100; No. $3, \mathrm{~N} / 20$; No. $4, \mathrm{~N} /$ ro; No. $5, \mathrm{~N} / 6$; No. $6 \mathrm{~N} / 3$. After neutralization and adjustment to the same salt concentration and fluid volume, $0.05 \mathrm{cc}$. from each sample was injected intradermally in a tuberculous patient. The figure shows strong reactions with Nos. $1,2,3$, and 4 ; a trace of reaction with No. 5 ; and no reaction with No. 6.

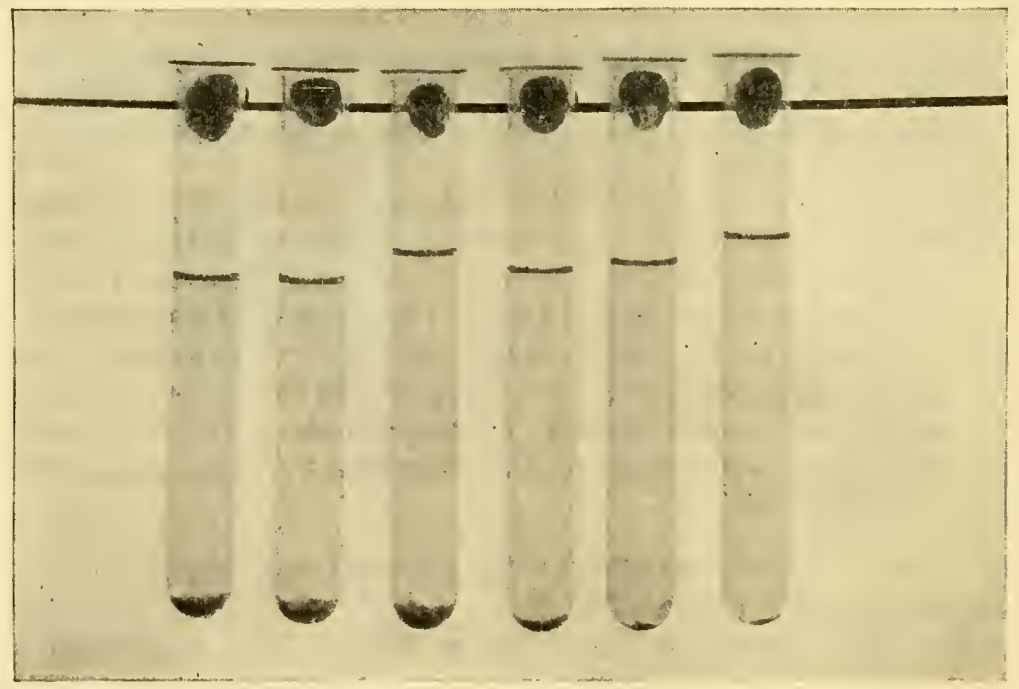

FIG. 3.-Protein precipitates in tuberculin heated with acid. The same samples which were injected as described under Fig. 2 were treated with trichloracetic acid for the precipitation of protein. Precipitation is marked in Nos. I, 2, 3, and 4. A trace of precipitation has occurred in No. 5. No. 6 is practically clear. 
solutions of tuberculin protein with ammonium sulphate and adding acetic acid until a reaction of $\mathrm{pH} 4.8$ was reached, she was able to induce a portion of the protein present to crystallize in needle form. Usually the needles crystallized out in company with amorphous protein. In a few instances they were pure. The crystals obtained took a blue stain with methylene blue, gave the biuret and Millon tests, and were heat coagulable. One of the purest preparations obtained caused a marked skin reaction in tuberculous guinea pigs. The total weight of product, five times recrystallized, from $20 \mathrm{gm}$. of water-soluble protein was only $70 \mathrm{mg}$., but this beginning appears the most hopeful of all the attempts made at purification of the active principle.

An extensive investigation has been carried out by Johnson and his colleagues on the proteins in the body of the tubercle bacillus. Coghill, ${ }^{\mathrm{I}}$ following the analysis outline of Johnson, ${ }^{2}$ prepared an albumin-like protein from tubercle bacilli grown on a synthetic medium, which proved to have marked tuberculin potency. Distinctive chemical features were a high content in basic amino acids and failure to reduce Benedict's solution even after hydrolysis. Inasmuch as no protein precipitation occurred on dialysis of a 5 per cent sodium chloride extract of tubercle bacilli, it was concluded that globulins are practically absent from the structure of the bacillus.

Coghill $^{3}$ found that alkaline extraction of the bacillary mass after removal of the water-soluble protein yielded a protein containing I 4.2 per cent nitrogen and constituting 20 per cent of the dry weight of the bacilli. This proved practically devoid of tuberculin activity.

A preliminary study carried out jointly by Seibert and Coghill ${ }^{+}$brought out the fact that a larger amount of potent water-soluble tuberculin protein is obtainable from the filtered culture medium on which the tubercle bacilli have grown for six weeks than from the bodies of the corresponding bacilli. With shorter periods of growth larger amounts remained in the bacilli, while with incubation of three months most of the activity appeared to have passed in to the medium. The proteins obtained from the two sources were qualitatively similar.

These studies re-emphasize a fact appreciated since Koch's time, viz., a fundamental difference between the active principle of tuberculin and the active principles of the true exotoxins. The latter, as exemplified typically in diphtheria toxin, appear to represent true secretions or excretions from the bacteria elaborating them. Extracts of diphtheria bacilli do not contain appreciable amounts of diphtheria toxin. Extracts of recently grown tubercle bacilli, on the other hand, are rich in the active principle of tuberculin, and the active substance found in the culture medium appears to arise by extraction, possibly associated with autolysis, of the growing bacilli.

\section{THE NATURE OF THE TUBERCULIN REACTION}

ANATOMICAL CHANGES AND EXPLANATIONS OF THEIR CAUSE

When dilute solutions of tuberculin are injected into the skin of a normal animal, no grossly visible change occurs. Microscopically, a transient influx of a few leuko-

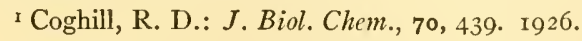

${ }^{2}$ Johnson, T. B.: Am. Rev. Tuberc, , 4 , 164. 1926.

${ }_{3}$ Coghill, R. D.: J. Biol. Chem., 70, 449. 1926.

${ }^{4}$ Seibert, F. B., and Coghill, R. D.: unpublished. 
cytes is observed, but within a few hours there is a complete restoration to normal from this very slight change.

When a similar injection is made into the skin of a tuberculous animal, again no immediate effect is apparent, but within eight to twenty-four hours an acute local inflammation takes place at the site of injection. A red, raised area, with central blanching, $\mathrm{I}-2 \mathrm{~cm}$. in diameter, appears, which is of firmer consistency than the surrounding skin. If the animal is exceptionally sensitive, or the tuberculin solution concentrated

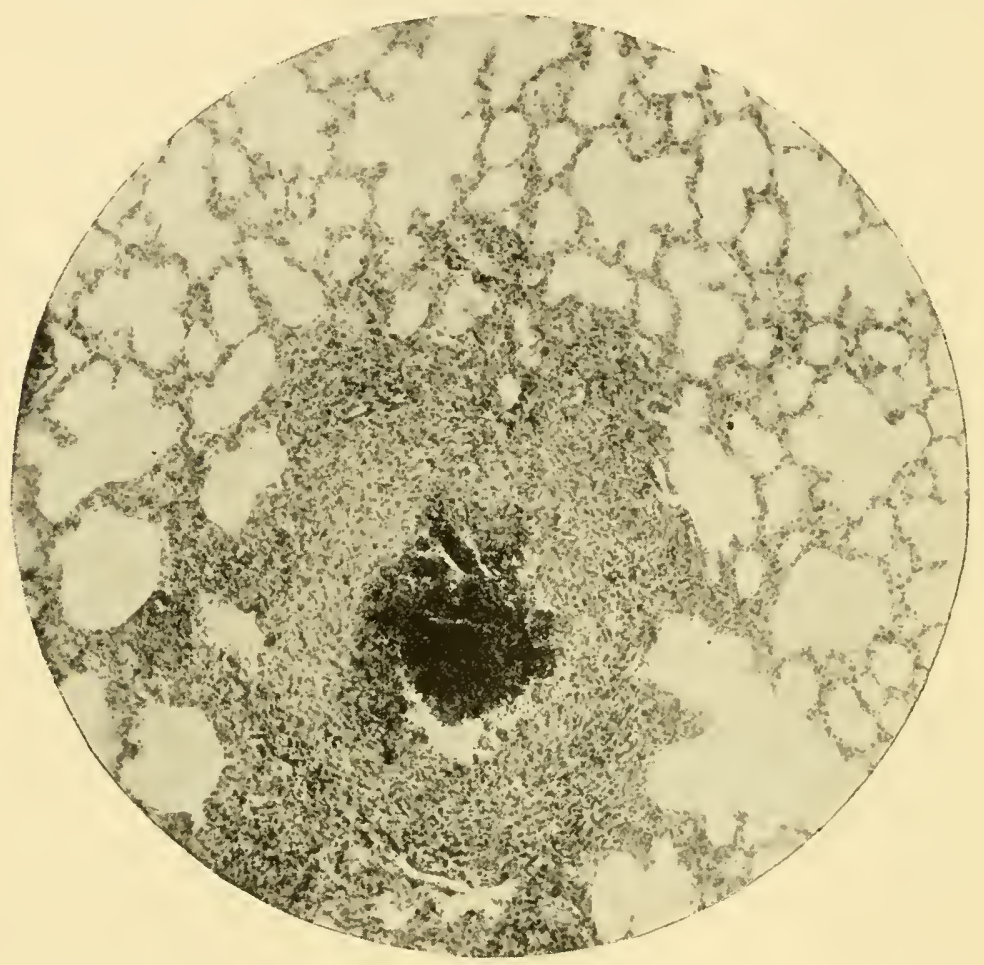

FIG. 4.-Tubercle in lung of guinea pig nct treated with tuberculin. Note sharp border and normality of lung parenchyma around tubercle.

in active principle, central necrosis occurs. Microscopically, the capillaries are seen to be dilated and engorged, and an intense infiltration of large mononuclear and small polynuclear leukocytes is observed. There is an increase in the intercellular fluid, and some coagulated fibrin may be present. When the reaction is carried out in a parenchymatous organ with more specialized cells than those present in the skin, such as the testis, necrosis of the parenchymatous cells is a regular accompaniment of the reaction. Petersen and Levinson ${ }^{\mathrm{r}}$ have demonstrated an increase in capillary permeability in sensitized tissues, which appears to be fundamental to the reaction.

This is obviously an acute inflammation with all of the rubor and tumor and not without the calor and dolor demanded by the ancient definition. The mechanism in-

${ }^{x}$ Petersen, W. F, and Levinson, S. A.: Am. Rev. Tuberc., 8, I22, I923. 
volved in its production, however, although many times and extensively investigated, has never been explained to the satisfaction of all interested.

The most attractive hypothesis, which would simplify our conceptions enormously if it could be properly supported, is the one originally developed by Nicolle ${ }^{\mathrm{r}}$ and Wolff-Eisner, ${ }^{2}$ and more recently elaborated with experimental detail by Zinsser. According to the Nicolle view, lysins exist within the tuberculous body not only for the tubercle bacillus itself, but also for its endotoxin, tuberculin. The products of this

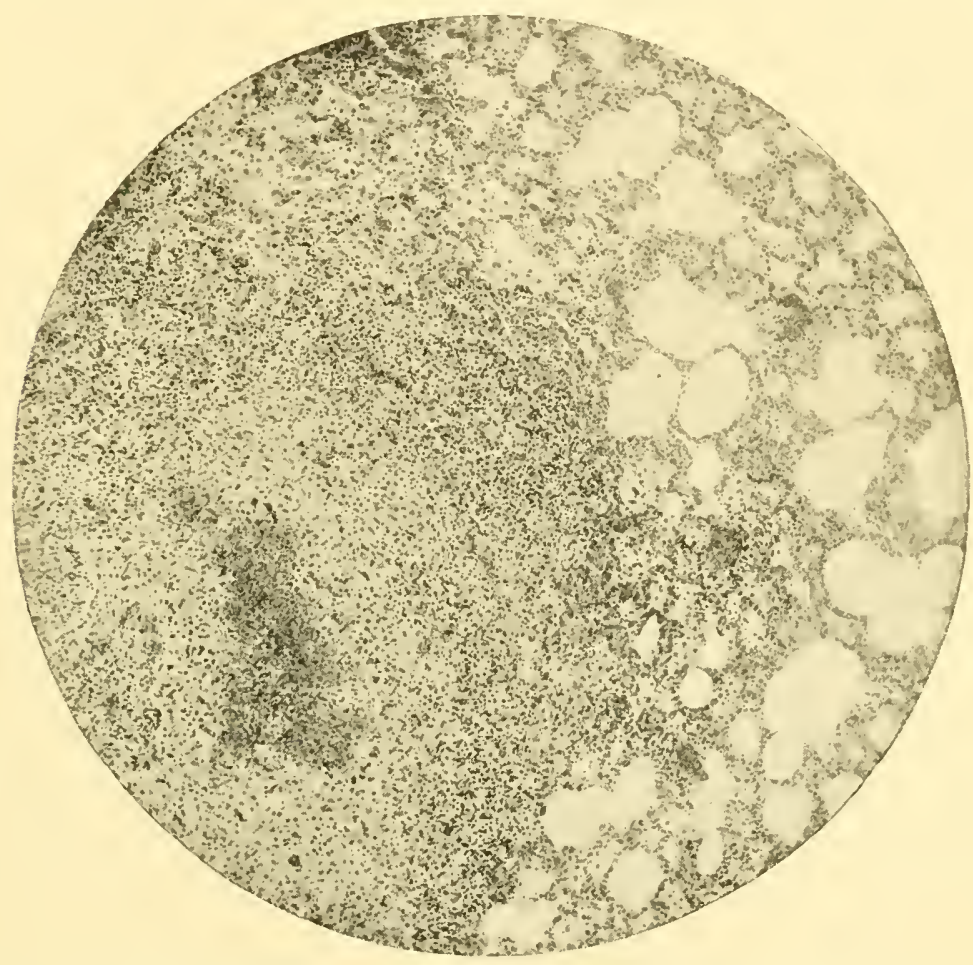

FIG. 5.-Tubercle in lung of guinea pig twenty-four hours after heavy dose of tuberculin. Note poorly defined border and exudation of cells and plasma in surrounding alveoli.

lysis of tuberculin are toxic and capable of inciting acute inflammation. Inasmuch as it is only the tuberculous organism which possesses the necessary lysin, it is only to the tuberculous that tuberculin can be toxic.

A number of results prior to those of Zinsser partially support this view. Bail ${ }^{4}$ claimed in a series of investigations that while tuberculin alone was not toxic for the normal animal, it became so if the crushed tuberculous tissue from a tuberculous ani-

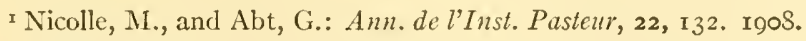

2 Wolff-Eisner, A.: Berl. klin. Wchnschr., 47, 1651. 19ro.

3 Zinsser, H., and Tamiva, T.: J. Exper. Med., 44, 753. 1926.

${ }_{4}$ Bail, O.: Ztschr. f. Immunitätsforsch, u. exper. Therap., r2, 451. I9г2. 
mal was injected into the normal animal simultaneously with the tuberculin. Kraus, Loewenstein and Volk, ${ }^{\mathrm{r}}$ Selter, ${ }^{2}$ Klopstock, ${ }^{3}$ and others could not confirm this.

McJunkin ${ }^{4}$ found that a Berkefeld-filtered extract of the exudate occurring on intraperitoneal injection of tubercle bacilli into a guinea pig with peritoneal tuberculosis would sensitize a normal guinea pig to tuberculin. He reconciled this with the prevalent view that tubercle bacillus infection is required before marked and lasting sensitiveness to tuberculin develops, by the theory that he had accomplished in the peritoneal reaction what takes place spontaneously in the body before sensitization takes place, viz., action of tuberculous tissue on the tubercle bacillus and its products. Lange ${ }^{5}$ secured similar results.

Zinsser and Tamiya ${ }^{6}$ confirmed the results of McJunkin and Lange, without securing strong sensitization, however. They also confirmed the results of the majority of previous investigators that blood serum of tuberculous animals, or of animals inoculated with dead bacilli, does not activate tuberculin $(\mathrm{O} T)$ so that it will produce the tuberculin reaction in normal animals, or neutralize it so that it fails to affect the tuberculous. On the other hand, in a number of trials, although inconstantly and irregularly, they were able to obtain reactions in normal animals with incubated mixtures of Old Tuberculin and tuberculous tissue. Neither the tuberculous tissue (human and guinea pig lung) nor the tuberculin alone caused reaction in the normal animal. Hence the authors concluded that in some manner, perhaps through specific enzyme-like action, the tuberculous tissue had made the tuberculin toxic. The conception is similar to that of Vaughan on toxic and non-toxic fractions of proteins and of Friedberger on anaphylatoxin. Zinsser emphasizes the close association of the phenomenon described with the cells of the tuberculous animal, the blood not being involved.

This result is in apparent contradiction with those which I secured in two previous investigations. ${ }^{7}$ These were based on the fact that the testis of the tuberculous guinea pig is extremely sensitive to tuberculin, necrosis resulting when small amounts are injected into it. If this necrosis is the result of interaction between the testis cells and tuberculin it might be expected that if tuberculin and the crushed testis of a tuberculous animal were intimately mixed for a few hours the tuberculin might be activated by the sensitive tissue so as to become toxic for normal tissue, or be bound or neutralized so as to fail to elicit a tuberculin reaction in a tuberculous guinea pig. As a matter of fact, neither of these results occurred. Tuberculin mixed with ground testis tissue known to be exquisitely sensitive to tuberculin, and tuberculin injected into the living testis of the tuberculous guinea pig in the smallest quantities recognizable by the most delicate method and left there twenty-four hours, failed to undergo

\footnotetext{
${ }^{x}$ Kraus, R., Loewenstein, E., and Volk, R.: Dculsche med. W'chnschr., 37, 389. I9I I.

${ }^{2}$ Selter, H.: Zlschr. f. Immunitätsforsch. u. exper. Therap., 32, 325. $192 \mathrm{I}$.

3 Klopstock, F.: ibit., 40, 27. 1924.

${ }_{4}^{4}$ Mc Junkin, F. A.: J. Exper. Med., 33, 75I. I92I.

${ }^{5}$ Lange, I. B.: J. Med. Research, 44, 293. 1923-24.

${ }^{6}$ Zinsser, H., and Tamiya, T.: loc, cit.

7 Long, E. R., and Seibert, F. B.: J.A.M.A., 85, 650. 1925; Tr. 2 Ist Ann. Merting, Nal. Tulere Assoc, P. 35I. 1925 .
} 
biological alteration. Extracts of the testis after in vitro and in vivo mixture with tuberculin still failed to cause any reaction in the testis or skin of normal guinea pigs, while causing a typical tuberculin reaction in the tuberculous animal. Identical results were secured when the ground sensitive skin of a tuberculous animal was substituted for the testis.

The results were so clear cut in every instance, and the recovery of active principle of tuberculin so nearly quantitative, as determined by the most accurate quantitative method available, as to leave me convinced that the active principle was neither activated nor bound nor neutralized by tuberculin-sensitive tissue. Unlike the experiments of Bail, McJunkin, Lange, and Zinsser, in which the action of truly tuberculous tissue upon tuberculin was concerned, my investigation was confined to the nontuberculous but highly sensitized tissue of the tuberculous animal. And therein probably lies the explanation for the difference in result. As far as the tuberculin reaction in the latter type of tissue is concerned, i.e., tissue known to be suitable for demonstrating the local reaction, I can only say that the active principle is a specific irritant for sensitized cells, eliciting the reaction in high dilution, without itself being altered. This falls far short, obviously, of an explanation of the phenomenon.

\section{SPECIFICITY OF THE TUBERCULIN REACTION}

The tuberculin reaction is commonly considered a highly specific reaction, given only by the animal infected with tuberculosis in response to the parenteral introduction of a specific product of the tubercle bacillus (see Krause ${ }^{\mathrm{I}}$ on the conditions necessary to arouse the state of allergy). It has been proved repeatedly that inoculation with dead tubercle bacilli also produces sensitiveness, and Petroff ${ }^{2}$ has shown that the allergic state thus produced may be more lasting and of higher degree than has commonly been supposed. In either case, however, as Baldwin and Krause have emphasized, the sensitiveness is associated with the presence of anatomic tubercle, dead tubercle bacilli producing a lesion less extensive but histologically similar to that caused by the live micro-organisms. Somewhat less constantly, as Krause, Baldwin, Zinsser, Woolley, and McJunkin have pointed out (see Baldwin, Petroff, and Gardner, op. cit., p. I36), sensitization has been achieved by the simple injection of tuberculoprotein, but the sensitization is more transient and the reactions ordinarily less intense than in tuberculous animals.

As long ago as 1894 the perfect specificity of the tuberculin reaction was questioned, and beginning with 1925 its specificity has been still more seriously doubted. Matthes, ${ }^{3}$ Krehl and Matthes, ${ }^{4}$ and Kircheim and Tuczek (who review the earlier literature $)^{5}$ all claimed that protein derivatives, particularly the deuteroproteoses, from a variety of sources, elicited skin reactions in tuberculous animals. It is noteworthy, however, that much larger amounts of these products were required than of true tuberculin to elicit the reaction.

\footnotetext{
I Krause, A. K.: Am. Rev. Tuberc., I I, 343. 1925.

2 Petroff, S. A.: Tr. 20 th Ann. Mecting, Nat. Tuberc. Assoc., p. 244. I924.

3 Matthes, M.: Deutsches Arch.f. klin. Med., 54, 39. I894.

${ }^{4}$ Krehl, 1., and Matthes, M.: Arch. exper. Path. w. Pharm., 36, 437. I895.

5 Kircheim, L., and 'Tuczek, K.: ibid., 77,387. I9I4.
} 
More recently Selter, ${ }^{\mathrm{I}}$ once a champion of the high specificity of the reaction, has come to the conclusion that it is far less specific than is conventionally supposed. $\mathrm{He}$ has assembled the evidence for this view, ${ }^{2}$ taking the stand that bacterial infections of a variety of types sensitize the skin and other tissues not only to the specific germ of each infection but to other bacterial proteins as well. For example, he and others have repeatedly seen cross-reactions involving the tubercle bacillus, the colon bacillus, the diphtheria bacillus, etc.; and as a consequence he has come to believe that these infections confer an exalted irritability on the tissues rather than specific sensitization.

This view is probably timely for its renewed warning for caution and less empiricism in our interpretation of the tuberculin reaction, but seems to me to exaggerate the importance of the facts observed. In the last analysis it will probably be found that the specificity of the tuberculin reaction lies in its quantitative aspect. The significant fact is not so much that the tuberculous patient has a heightened sensitiveness to a variety of foreign, particularly bacterial, proteins, as that he is extraordinarily sensitive to minute amounts of the active principle of tuberculin. It is not an absolute unspecificity of reaction that is seen, but, as Zinsser and Tamiya ${ }^{3}$ have well pointed out, a simple overlapping of reaction, such as may be seen throughout the whole field of protein immunological reactions.

Non-specific reactions in other fields of immunology are discussed by Wells ${ }^{4}$ who suggests that chemically related proteins as well as identical proteins in different bacterial and animal bodies may be concerned. In view of the fact that the same amino acid building blocks are common to the large group of proteins, which differ chiefly in the arrangement of these blocks, it is not surprising that non-specific biological reactions, dependent in turn upon chemical similarities, occasionally occur.

Until we know more of the chemical composition of the proteins of the tubercle bacillus and other bacteria, speculation on this subject will be idle. In view, however, of the great practical importance attached to the tuberculin test in diagnosis, particularly of cattle tuberculosis, the purest preparations of tuberculin available should be used, and in small quantity. In doing this we are taking the greatest precaution possible against the non-specific element.

RELATION TO OTHER TYPES OF HYPERSENSITIVENESS

Tuberculin hypersensitiveness is one of the types of change coming appropriately under the broad term "allergy," originally introduced by von Pirquet. This term has been used, however, in so many senses that its significance in the individual case is often not clear, and it is best in the case of the tuberculin type of reaction to follow the self-explanatory if somewhat cumbersome designation "hypersensitiveness of infection" used by Coca and Cooke for this type of sensitization, manifested in practice by the skin test with bacterial filtrates.

\footnotetext{
${ }^{x}$ Selter, H., and Tancre, E.: Beitr. z. Klin. Tuberk., 60, 439. 1925.

${ }^{2}$ Selter, H.: Schriften der Königsberger Gelehrlen Gesellschaft, 2, 137. 1926.

3 Zinsser, H., and Tamiya, T.: loc. cit.

4 Wells, H. G.: The Chemical Aspects of Immunity. New York, 1925.

${ }_{5}$ Coca, A. F., and Cooke, R. A.: J. Immunol., 8, 163.1923.
} 
Much discussion has centered on the relation of this type of sensitization to anaphylaxis. Zinsser ${ }^{\mathrm{I}}$ has distinguished quite clearly between the two phenomena, and his colleague Mueller, ${ }^{2}$ and Laidlaw and Dudley, independently have apparently found the antigenic substance concerned in serum reactions and anaplyylactic reactions. This interesting substance is present in the bacilli themselves, and in the filtrates after culture of the bacilli, together with the active principle of the tuberculin reaction. When separated from the latter it does not elicit the tuberculin reaction. It is a carbohydrate gum, analogous to that obtained from pneumococci by Heidelberger and Avery, known to be concerned in pneumococcus serum reactions.

This separation of the substances responsible for the two types of reaction, both of which can be obtained in tuberculous patients and artificially sensitized animals, rules out the serum reactions as previously used as a means of standardizing tuberculin, as Okell, Parish, O'Brien, and their colleagues ${ }^{4}$ have clearly pointed out.

The specificity of the tuberculin reaction, high if not absolute, makes the tuberculin test valuable in the diagnosis of tuberculous infection. This test is used most extensively and reliably in the diagnosis of tuberculosis in cattle. Intracutaneous injection in the caudal fold, subcutaneous injection in the neck and examination for general reaction, and ophthalmic inoculation are the methods commonly used.

In man the usefulness of the test as a means of recognizing active tuberculous disease is limited by the fact that the majority of adults have been infected with tuberculosis, and therefore sensitized to tuberculin, without developing what can be called "clinical disease." Opie and McPhedran ${ }^{5}$ and others distinguisi this latent from pro gressive tuberculosis, however, by using tuberculin in a careful, quantitative fashion.

In the recognition of early tuberculosis of childhood tuberculin is a great aid, although, obviously, the test detects with certainty here, too, only the incidence of infection, which is likely to become latent without setting up clinical disease. Inasmuch as infections in the earliest years of childhood seem more likely to result in clinical disease than those of later years, the tuberculin test applied to young children is of considerable prognostic significance.

In epidemiological studies to determine the incidence of tuberculous infection in large groups of people, tuberculin is invaluable. In fact, it is largely through the use of tuberculin that we have been able to determine the time of life at which the original tuberculous infections occur, to which the healed scars of tuberculosis almost invariably found at necropsy in later years correspond.

\section{THE BASIS FOR TUBERCULIN TREATMENT OF TUBERCULOSIS}

The introduction of tuberculin in the treatment of tuberculosis will long be remembered as one of the most spectacular tragedies in the history of medicine. With the medical profession and patients all over the world waiting trustfully on the man whose lightest word was law in the field of tuberculosis, the discoverer of the tubercle

\footnotetext{
${ }^{1}$ Zinsser, H.: J. Exper. Med., 34, 49.5. 1921.

${ }^{2}$ Mueller, J. H.: ibid., 43, 9. 1926.

3 Laidlaw, P. P., and Dudley, H. W.: Brit. J. Exper. Path., 6, 197. 1925.

4 Okell, C. C., Parish, H. J., O'Brien, R. A., et al: Lancel, p. 433. Feb. 27, 1926.

5 Opie, E. L., and McPhedran, F. M.: Am. Rev. Tuberc., I4, 347. I926.
} 
bacillus was rushed into publishing a premature announcement of the curative virtue of a substance which immediately received almost hysterical reception under the name "Koch's lymph." In the profound disappointment which followed its failure to achieve the cures expected and its actual injury of large numbers of patients, tuberculin was for a time given up and even banned as a poison.

However, in the succeeding years cautious retrials established to the satisfaction of the majority of physicians that, while falling far short of original expectations, tuberculin was of distinct value in cases of tuberculosis of a certain variety. It is usually accepted today that localized tuberculosis of chronic, obstinate type, sluggishly progressive, as seen in bones, lymph nodes, and skin, may be stimulated to scarification and fibrous investment by the proper use of tuberculin. Many ophthalmologists consider it the best treatment for eye tuberculosis.

The technique of its usage is outside the scope of this chapter, and may be read in the standard texts. A rational basis for its administration, which is all that can be touched here, is known today. In the time of Koch the observation leading to its introduction as a cure was empiric. Tuberculin, without effect on the uninfected animal, causes local, focal, and general reactions in the patient with tuberculosis. The local reaction at the site of injection is probably without significance in treatment, except as an index of the patient's sensitiveness. The focal reaction occurring at the site of disease, on the other hand, is of the utmost importance. Mild focal reactions are beneficial, and severe ones detrimental. Marked focal reactions lead to general reactions, presumably from undue absorption of the degenerated tissue at the focus, as Krause ${ }^{\mathrm{I}}$ points out, an event leading to toxemia, fever, loss of weight, and general harm to the patient.

The probable explanation for the focal reaction is exceptional sensitiveness of the cells in the immediate region of tuberculous tissue, surpassing that of all other tissues of the generally sensitized body. Minute amounts of tuberculin, too small to stimulate response from the remote tissues, cause inflammation around tubercles, and if kept within bounds promote walling in of the lesion by phagocytic cells and fibrous tissue.

This condition is illustrated in Figures 4 and 5 , showing pulmonary tubercles in tuberculous guinea pigs, one of which received a large dose of tuberculin, the other not being treated. In the non-treated animal all tubercles were in the condition shown in Figure 4, discrete, sharply marked off from the surrounding lung tissue. The tubercle shown in Figure 5, on the other hand, is poorly defined, the cells of an exudative reaction appearing in the surrounding alveoli. This animal, a mate to the one shown in Figure 4, was killed the day after administration of a heavy dose of tuberculin.

If tuberculin has this effect on artificial administration, it is reasonable to suppose that tuberculin suddenly eliminated into the system from the flaring up of one focus of disease due to any cause might result in a focal reaction around tuberculous regions elsewhere. F. M. Pottenger has shown me Röntgen ray plates indicating that this occurs, and believes such spontaneous tuberculin reactions are important in the natural cure of tuberculosis.

${ }^{r}$ Krause, A. K.: New lork Med. J., April iq, rg22. 
SPONTANEOUS INTERNAL TUBERCULIN REACTIONS AS A CAUSE OF

PATIIOLOGICAL CHANGES IN TUBERCULOSIS

SPECIFIC TUBERCULOUS LESIONS

Reference has just been made to the focal reactions occurring in distant tubercles on flaring up, and, presumably, release of tuberculin, in another tuberculous region. Local spread of tubercle bacilli is likewise accompanied by what may properly be considered a tuberculin reaction. In the chronic bronchiolo-pneumonic reaction or alveolar exudation occurring in the neighborhood of an ulcerating pulmonary lesion, sensitization of the tissue probably plays a part, but the effect of tissue sensitization is much more evident in the acute reactions which follow sudden release of large numbers of tubercle bacilli.

It is known from animal experimentation that in tuberculous animals superinfections in the skin, lung, liver, pleura, peritoneum, meninges, kidney, and testis are acutely inflammatory at the start, instead of more slowly progressive as in the case of the lesion of first infection.

In human pathology analogous phenomena may be observed. It is believed that tuberculous pneumonia, pleuritis, meningitis, arthritis, etc., partake of the nature of tuberculin reactions. They represent the response of sensitized tissue to the specific protein of the tubercle bacillus, followed by the development of anatomical tubercle, in answer to the presence of the multiplying tubercle bacillus.

\section{NON-TUBERCULOUS LESIONS}

If, however, the bacilli which stimulate the acute reaction happen to be dead, as not infrequently must be the case, the acute manifestations, dependent upon the presence of the specific protein in the bacilli may occur, and not be followed by the development of progressive anatomical tuberculosis.

Evidence pointing in this direction is available from animal experiment, and much that appears analogous is seen in human pathology. Figure 6, obviously a late tuberculin reaction in the testis of the type well seen in Figure $I$, is taken from a tuberculous guinea pig, presumably highly sensitive to tuberculin protein. The atrophy of the tubules characteristic of the tuberculin reaction is in this case not the result of deliberate injection of tuberculin, however, as in Figure $\mathrm{I}$, but of spontaneous lodgment of tubercle bacilli in the testis, as shown by the presence of the small tubercle in the center of the field. Hematogenous tuberculosis of the testis is rare in the guinea pig, and in this case the other testis was entirely normal. Dead tubercle bacilli, even disintegrated, as shown by direct experiment, would have been just as effective in causing the atrophy in this sensitized animal. It seems to me not unlikely that some of the testicular atrophies occasionally observed in man, for which no other cause can be assigned, may be explained on this allergic basis, the allergy not being necessarily tuberculous.

I have obtained experimental evidence that even a lesion so far removed anatomically from true tuberculosis as subacute glomerulo-nephritis may be produced by the action of tuberculin protein on the kidney of the tuberculous, and therefore sensitized, animal. If the kidneys of normal anesthetized swine are perfused through the renal artery with tuberculin protein in fine suspension in salt-citrate solution and 
the animals are killed several days to several months later, no significant lesions are found in the perfused kidneys. Similar perfusion, on the other hand, of the kidneys of tuberculous swine leads to pronounced degenerative changes throughout the kidneys, and extensive acute, followed by subacute and chronic, inflammatory manifestations in the glomeruli. In the light of these experimental results a statistical study which I have carried out with Miss Robey on the relation of pulmonary tuberculosis to kidney changes, in which chronic nephritis was found to occur somewhat more

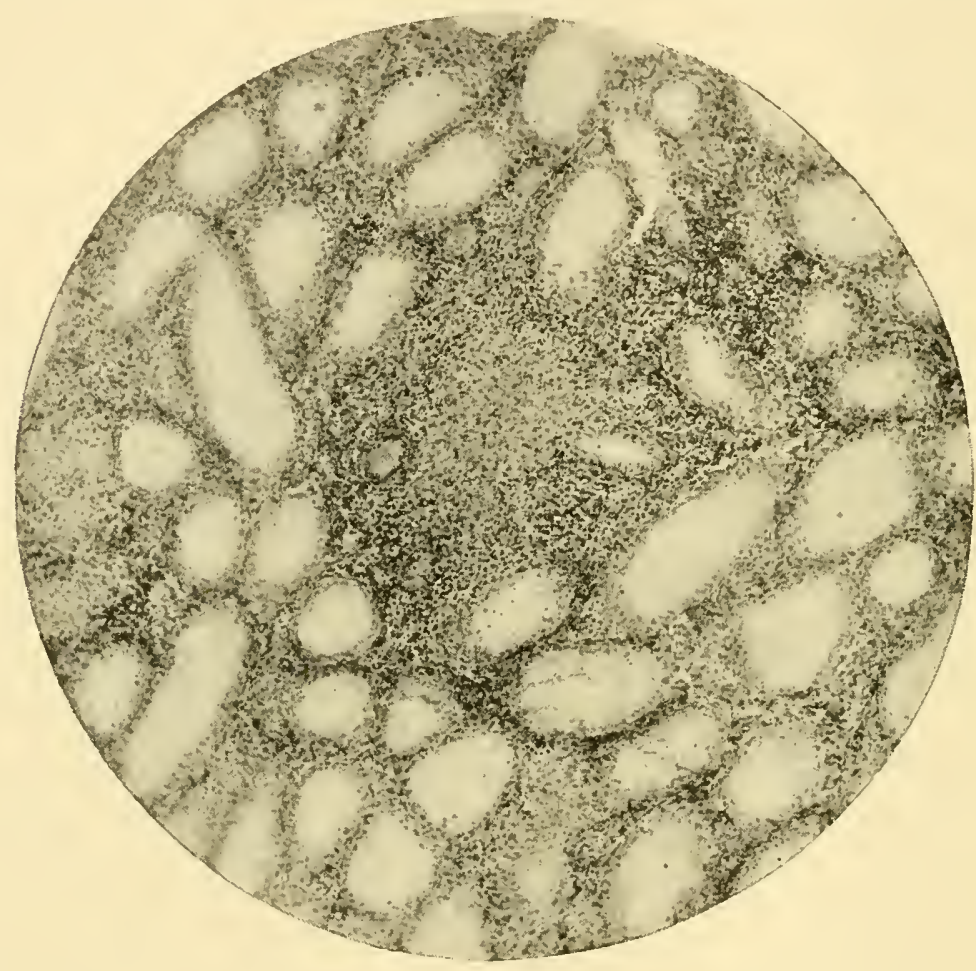

FIG. 6.-Hematogenous tubercle in the testis of a tuberculcus guinea pig with atrophy of the surrounding seminal tubules, which may be interpreted as a tuberculin reaction (cf. Fig. I).

frequently in tuberculous than non-tuberculous bodies within the same age groups, suggests that spontaneous intrarenal tuberculin reactions may be a factor in the production of mild grades of chronic nephritis. Figures 7 and 8 illustrate the types of lesion produced on injection of tuberculin protein into the renal arteries of tuberculous pigs.

\section{RECAPITULATION}

Tuberculin, to use the name originally employed for those preparations from tubercle bacilli which elicit a specific inflammatory reaction in tuberculous animals, in whatever form used, depends for its action upon the presence of a specific active principle. Exhaustive chemical studies bear out the original contention of Koch that this substance is of protein nature. Disintegration of the protein molecule concerned 
destroys the activity of the substance, although possibly slight changes in the molecule can be borne without loss of potency.

The exact mechanism of the tuberculin reaction is not understood. There is some evidence that tuberculous tissue alters tuberculin in such a way as to render it toxic, while normal tissue has no such effect. Thus it is toxic only to the tuberculous host. Careful experiments on highly sensitized, non-tuberculous tissues of the tuberculous animal, viz., the skin and testis, have failed to show that these sensitive tissues have

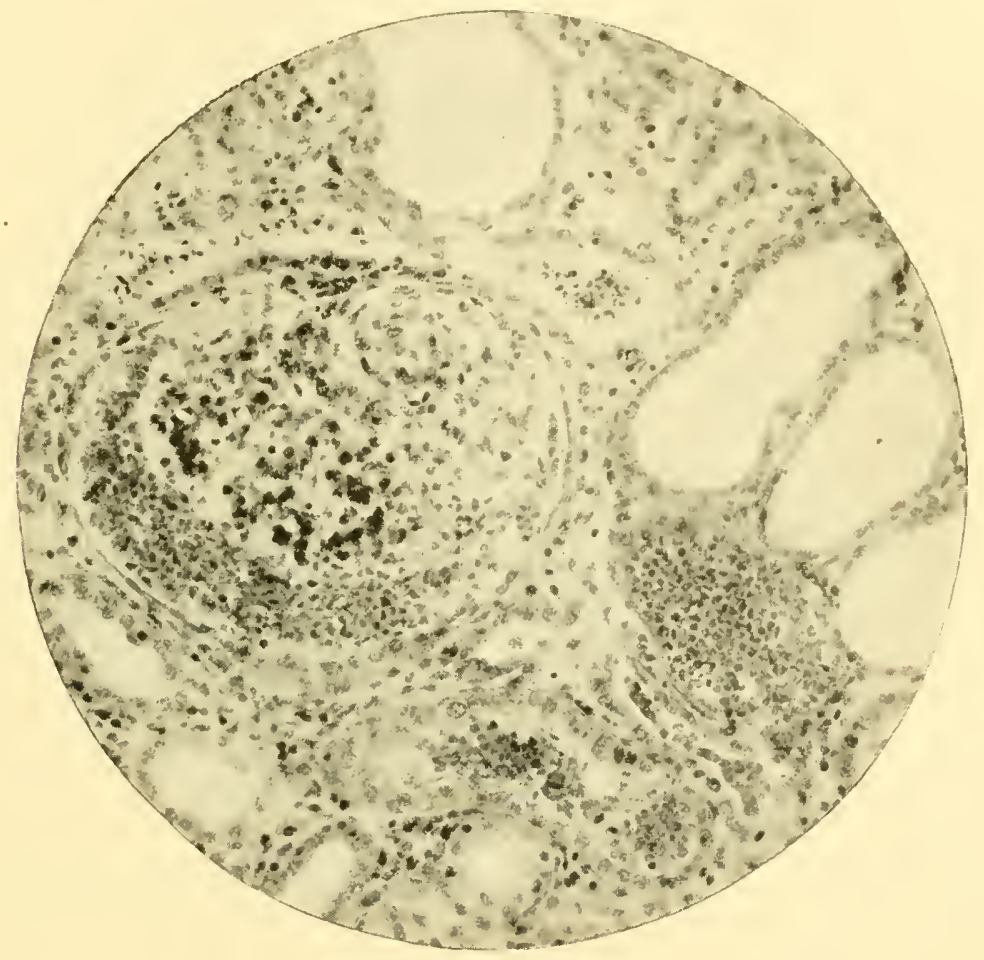

Frg. 7.-Glomerular hemorrhage and exudation in kidney of a tuberculous hog four days after renal perfusion with a suspension of tuberculin protein.

any power whatsoever to alter or neutralize tuberculin. We can only say at present that tuberculin is a specific irritant for these cells.

The tuberculin reaction is of high but not absolute specificity. Anatomically it is characterized by all the features of acute inflammation, in which increased capillary permeability appears to play an important part. The specificity of the reaction is sufficiently high to make the tuberculin reaction reliable in the recognition of tuberculous infection in cattle. The widespread incidence of latent tuberculous infection in adult man, conferring sensitiveness to tuberculin, goes far to nullify the value of tuberculin in the recognition of active tuberculosis, although quantitative tuberculin reactions may be suggestive. Tuberculin is useful in the diagnosis of active tuberculosis in children, and invaluable in epidemiological studies of the incidence of infection in children. 
The basis for tuberculin treatment is believed to lie in the exceptional sensitiveness of the tissue immediately around tuberculous lesions. This may be thrown into focal reaction by the introduction of amounts of tuberculin too small to influence tissues more remote from the lesion. Mild degrees of focal reaction influence healing favorably.

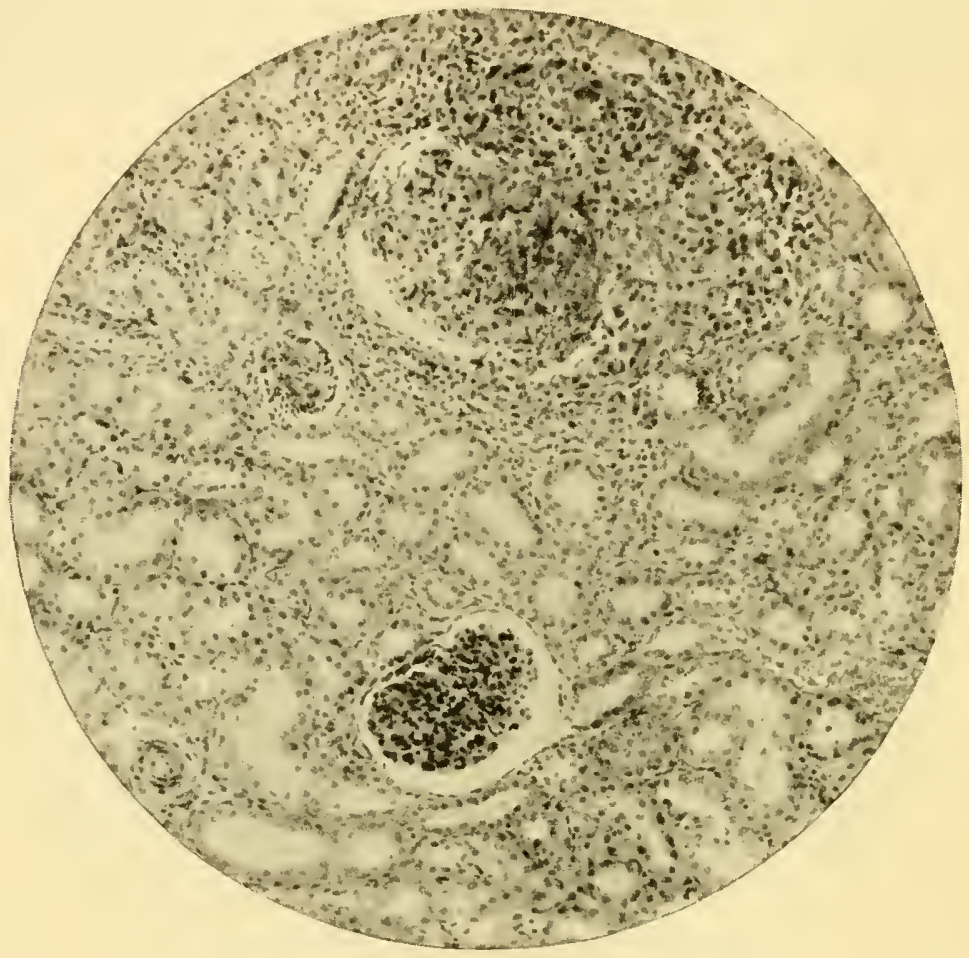

Fig. 8.-Proliferative glomerular lesions in kidney of hog one month after renal perfusion with a suspensicn of tuberculin protein.

Some of the anatomical manifestations of tuberculosis represent allergic reactions of the sensitive tissues of the body to the specific protein of the spreading, sensitizing agent, with the specific anatomical response to the multiplying bacillus superimposed. Other pathological lesions in the tissues, non-tuberculous in character, may represent the effect of the protein from bacilli killed off in the lesions upon more remote but still sensitized tissues elsewhere. 


\section{CHAPTER LXXVII}

\section{ORIGIN OF ANTIBODIES}

\section{KATHARINE M. HOWELL}

\section{Nelson Morris Institute for Medical Research, Michael Reese Hospital, Chicago}

The origin of antibodies has been sought by investigators since Jenner, Pasteur, Behring, and others first perceived that the body had a mechanism of defense against introduced blood-foreign material. It has been proved many times over that when such a foreign material, antigen, is injected into an animal, an antibody is elicited that possesses the power of reacting specifically with the antigen introduced, destroying or modifying its action or neutralizing its poison.

Antibodies have not as yet been isolated in pure state; in fact, it has never been proved that they exist as material objects. Their exact chemical nature, therefore, is unknown; however, they are generally believed to be protein, or so closely attached to one or another protein (euglobulin or pseudoglobulin) of the immune serum that separation has been impossible. A number of investigators have isolated almost pure antibodies. ${ }^{1}$ Wells ${ }^{2}$ has concisely summarized the chemistry of antibodies by stating that like the enzymes they are recognized by what they do rather than by what they are. In 1904 Ehrlich sought to explain the origin of antibodies on the basis of structural chemistry by his ingenious side-chain theory. The consequences of this theory, the experimental evidences, and its present status are adequately discussed in other chapters of this volume.

Experimentally, the origin of antibodies has been approached from both the positive and the negative side. Proof of a positive nature has been offered by examining organ extracts from immune animals, by immunizing various tissue cultures in vitro, by causing the proliferation of special types of cells, or by stimulating the function of particular organs. Negative methods of approach to the problem of antibody formation have been through damaging tissues which possibly produce antibodies with injurious substances; through extirpation of organs; through blocking the reticuloendothelial system with colloids.

\section{ORGAN EXTRACTS}

Extracts of most of the organs and of the tissues of the body have been examined for antibody content to determine whether or not the organ in question might either produce or store antibodies. The titre of the extracts from normal animals has been compared to that of the corresponding organ extracts from immunized animals, and the latter in turn has been compared to the animal's serum. Different criteria have been employed for measuring the antibody content of organ extracts.

Bacteriolysin was detected by Pfeiffer and $\mathrm{Marx}^{3}$ in extracts of spleen, bone

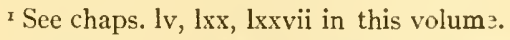

${ }^{2}$ Wells, H. G.: Chemical Aspects of Immunity, chap. iv. New York, 1925.

${ }^{3}$ Pfeiffer, R., and Marx.: Zischr.f. IIyg. u. Infektionskrankh., 27, 272. I898. 
marrow, lymph nodes, and lungs of rabbits injected with cholera spirilla, within two to four days before it was detected in the rabbit serum. After the first few days the serum titre became progressively higher until it equaled and then surpassed that of the organ extracts. Deutsch ${ }^{\mathrm{r}}$ found that, after typhoid organisms were injected into rabbits or guinea pigs, protecting antibodies were higher in the spleen than in the serum, if he made the determination in the first few days of immunization, and higher in the serum if the titre was measured after two to four days. Castellani $^{2}$ among others found protective substances were equal to or greater in the spleen than in the serum.

Hemolysin was detected by Schibayama ${ }^{3}$ in the spleen and lymph nodes of a large number of animals injected with foreign erythrocytes. Tarassevitch ${ }^{4}$ in similar experiments found hemolysin in extracts of omentum, lymph nodes, and spleen, and to a lesser degree in bone marrow and other organs. In the experiments of Motohashi ${ }^{5}$ when suitable doses of erythrocytes were injected, hemolysin was most concentrated in extracts of spleen, unless the animal had been splenectomized. In this case, hemolysin occurred primarily in extracts of bone marrow, lymph nodes, and liver. Cary ${ }^{6}$ immunized rabbits with ox corpuscles until the titre of their serum was high. The. titre was then permitted to run down during a period of one to five months; after this time a large dose of ox corpuscles was injected, and the serum and organ extracts were examined for hemolysin. In most instances the extracts of spleen and liver had a higher titre than that of the serum or other organs. Both Cary and Motohashi agreed with the opinion of $\mathrm{Kyes}^{\gamma}$ that the higher concentration of antibody in the organ extracts was due to the contained hemophages in the tissues.

Agglutinin was demonstrated during the first few days of immunization, by Pfeiffer and $\mathrm{Marx}^{8}$ and by Deutsch, ${ }^{9}$ to be present in extracts of spleen, liver, and lymph nodes, but in a lesser degree than in serum. Van Emden $^{\text {To }}$ injected rabbits with $B$. aerogenes, and after one to two days he detected more agglutinin in spleen than in serum; the liver, kidney, and lung also showed traces of agglutinin. Jatta ${ }^{\text {II }}$ injected rabbits with typhoid vaccine. At the end of twenty-four hours agglutinin for serum was $\circ$, spleen 30 , bone marrow $\circ$, liver $\circ$, kidney $\circ$, lung $\circ$; at the end of one hundred and two hours agglutinin for serum was 300 , spleen 300 , bone marrow roo, liver 30 ; from that time on, the serum had a higher titre than the organs. Castellani ${ }^{12}$ found that agglutinin for dysentery bacilli appeared in the serum and in the spleen at approximately the same time but that it persisted longer in the serum. Jones ${ }^{13}$ recently concluded that agglutinin for hog cholera bacilli was produced by the liver. He con-

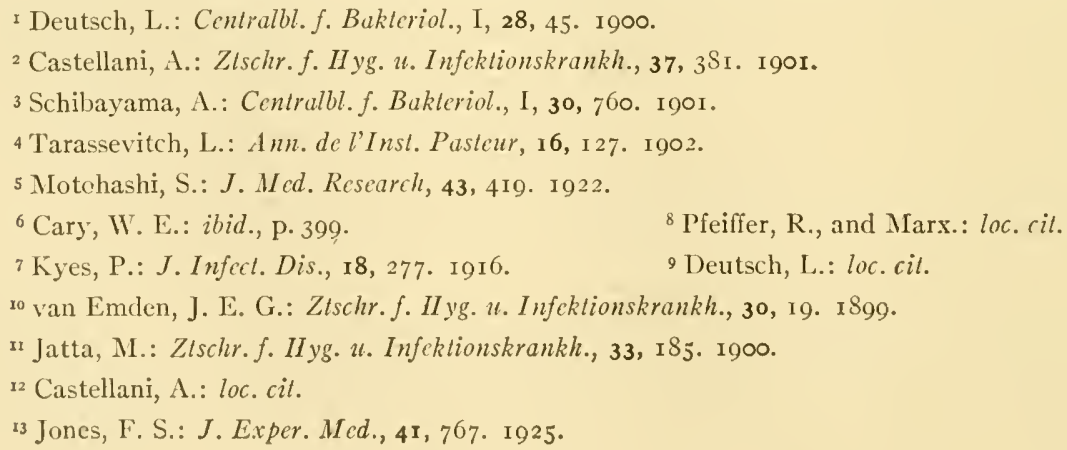


sidered that the bacteria were destroyed by the liver and that in some way during the period of destruction antibodies were formed possibly by the endothelial phagocytes.

Complement fixation antibodies were found by Tsurumi and Kohdla ${ }^{\mathrm{I}}$ in higher concentration in spleens than in sera, twenty-four hours after rabbits were injected with typhoid bacilli. After forty-eight hours the serum gave strongly positive fixation, and later bone marrow and lymph nodes gave slightly positive fixation. Similar experiments on splenectomized rabbits showed that the complement fixation antibodies appeared earlier in the lymph nodes than in the serum.

Precipitin was believed by von Dungern ${ }^{2}$ to be produced by the formed elements. Kraus and Levaditi ${ }^{3}$ demonstrated precipitin in extracts of omentum after intraperitoneal injection of horse serum. The serum and extracts of the other organs did not give this reaction. Kraus and Schiffman ${ }^{4}$ did not obtain precipitin reactions with organ extracts after injection of horse serum either intravenously, intraperitoneally, or subcutaneously. They concluded that possibly precipitin was produced by the vascular endothelium rather than by any single organ. Cantacuzène, ${ }^{5}$ using stronger organ extracts than Kraus and Schiffman, was able to demonstrate precipitin in the extract of spleen within two to three days after injection of horse serum; this precipitin gradually decreased until there was no trace on the seventh day, when precipitin first appeared in the blood.

The experiments cited appear to indicate that primarily the spleen and secondarily the lymph nodes, bone marrow, liver, kidney, lung, and omentum are involved in the production of antibody, since it is present in some or all of these organs before it appears in the serum. The fact that the injection of high titre, immune serum does not increase the antibody content of these organs suggests that they produce rather than store the antibodies. It seems probable that the formation of antibody may depend on some element common to all these organs, such as lymphoid tissue, hematopoietic tissue, or reticulo-endothelial cells. Many investigators, among them $\operatorname{Rath}^{6}$ and Fodor and Rigler, ${ }^{7}$ failed to demonstrate antibodies in organ extracts. In most instances the tests for antibodies were not made in the first days of immunization. Courmont, cited by Rath, ${ }^{6}$ found agglutinin higher in the serum of patients who had died from typhoid fever than in bile or extracts of liver or spleen. Here again the examinations were probably not made early in the disease.

\section{TISSUE CULTURE IMMUNITY}

Shortly after Harrison ${ }^{8}$ in 1907 and Burrows ${ }^{9}$ in I9ro succeeded in growing tissues in vitro, Carrel and Ingebrigtsen ${ }^{\mathrm{Io}}$ recognized that such living tissue culture offered a

I'Tsurumi, M., and Kohda, K.: Ztschr.f. Immunitätsforsch. u. expcr. Therap., 19, 519. I9I3.

${ }^{2}$ von Dungern: ibid., Orig., 21, 326. r914.

3 Kraus, R., and Levaditi, C.: Compt. rend. de l'Acad. de sc., r38, S65. I904.

4 Kraus, R., and Schiffman, J.: Ann. de l'Inst. Pasteur, 20, 225. 1906.

5 Cantacuzène, J.: ibid., 22, 54. I908. $\quad{ }^{6}$ Rath, D.: Ccntralbl.f. Bukteriol., I, 25, 549. I899.

7 Fodor and Rigler: ibid., 23, 930. 1898.

${ }^{8}$ Harrison, R. G.: J. Exper. Zoöl., 9, 787. 1910.

9 Burrows, M. T.: J.A.M.A., 55, 2057. I9I0.

${ }^{30}$ Carrel, A., and Ingebrigtsen, R.: J. Expcr. Med., 15, 287. I9t 2. 
new means of approach to the problem of antibody formation. They demonstrated that tissue living outside of the organism reacts against an antigen by the production of a specific antibody. They cultivated guinea pig bone marrow and lymph nodes in guinea pig plasma and then added goat's erythrocytes; after five days an extract of the tissue culture, plus the goat erythrocytes, was demonstrated to contain a hemolysin for goat's corpuscles. In I9I3 Przygode ${ }^{I}$ produced a specific typhoid agglutinin by inoculating cultures of rabbit spleen with typhoid bacilli, and in I9I4 he $\mathrm{h}^{2}$ obtained a precipitin against the horse serum antigen that he had previously added to cultures of rabbit spleen. These results indicate that bone marrow, lymph node, and spleen may take part in antibody production. Kuczynski, Tenenbaum, and Werthemann, ${ }^{3}$ on the other hand, could detect no hemolysin after inoculating spleen culture with sheep cel's. Bloom ${ }^{4}$ could not demonstrate hemolysin production in rabbit lung cultures inoculated with pigeon's erythrocytes. His experiments showed that, while lung cultures from normal rabbits did not phagocytize pigeon- erythrocytes, a dilute immune serum added to the culture induced rapid phagocytosis of the erythrocytes. Tissue culture from rabbits immunized against pigeon erythrocytes actively phagocytized introduced pigeon erythrocytes. Fischer ${ }^{5}$ immunized a pure culture of fibroblasts against foreign protein (ascitic fluid, dog serum) and found that there was a relation between the amount of antigen, the time of appearance of antibodies (four to five days), and their persistence. Carrel and Ebeling ${ }^{6}$ have found that a foreign protein inoculated into cultures of leukocytes brings about an immediate and nonspecific antibody response, and after four days a specific antibody response. Foot, ${ }^{7}$ Hadda and Rosenthal, ${ }^{8}$ and Lambert and Hanes, ${ }^{9}$ among others have demonstrated that tissue cultures were inhibited in growth when plasma from animals immunized against these particular tissues were used instead of normal plasma.

In vitro tissue culture has indicated a possible solution to the origin of antibody; hemolysin, precipitin, agglutinin, and phagocytosis can be induced in tissue culture. Tissue culture growth can be inhibited by using an immune plasma against the specific tissue in place of normal plasma.

\section{PHYSICAL AND CHEMICAL FACTORS AFFECTING ANTIBODY PRODUCTION}

Various physical and chemical means have been employed to promote antibody production. Hektoen ${ }^{\text {ro }}$ and many others have observed that after massive bleeding

I Przygode, P.: Wien. klin. Wehnschr., 26, 841. I913.

${ }^{2}$ Przygode, P.: ibid., 27, 201. 1914.

${ }^{3}$ Kuczynski, M., Tenenbaum, E., and Werthemann, A.: Virchow's Arch. f. path. Anat., 258, 687. 1925 .

${ }_{4}^{4}$ Bloom, W.: Arch. Path. \&o Lab. Med., 3, 608. I927.

${ }_{5}^{5}$ Fischer, A.: J. Exper. Med., 36, 535. 1922.

${ }^{6}$ Carrel, A., and Ebeling, A. H.: ibid., p. 645. 1922.

7 Foot, N. C.: Centralbl.f. allg. Path. u. path. Anat., 23, 577. I9r 2.

${ }^{8}$ Hadda, S., and Rosenthal, F.: Ztschr. f. Immunitätsforsch. u. exper. Therap., Orig., I6, 524. I9I3.

${ }^{9}$ Lambert, R. A., and Hanes, F. M.: J. Exper. Med., I3, 495. I9II.

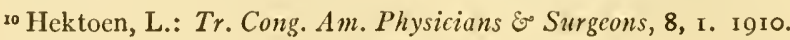


the antibody titre of immunized animals increased, and Hartley ${ }^{\mathrm{I}}$ obtained similar results after repeated small bleedings at short intervals. These results suggest that the stimulated hematopoietic tissues may be instrumental in the increased antibody production. Dry heat was found by Murphy and Sturm ${ }^{2}$ to stimulate antibodies, possibly through the effect on the lymphoid tissues, while exposure to cold, as noted by Foord, ${ }^{3}$ caused no change in hemolysin and only a slight increase in typhoid agglutinin.

By injection of nuclein Bedson ${ }^{4}$ increased the phagocytic activity of leukocytes during a period of hyperleukocytosis, and Tunnicliff ${ }^{5}$ found that leukocytic extract increased phagocytic activity twofold and fourfold. Müller ${ }^{6}$ treated animals with aleuronat during immunization, and found that the serum contained more antibodies and that the leukocyte count was higher than those of control animals. He attributed the two effects to a common action. Such results favor the view of the French school that antibodies are formed in the hematopoietic tissues, or even in the circulatory polymorphonuclear leukocytes - a theory sponsored by Metchnikoff, Bordet, Gruber, Wassermann, Citron, and others. Bachmann ${ }^{7}$ claimed actually to have demonstrated the presence of specific antibodies within leukocytes after he had immunized rabbits with typhoid bacilli. Thompson ${ }^{8}$ showed that tuberculin injected into rabbits prior to the injection of sheep erythrocytes increased the production of specific hemolysin twenty fold. He thought that this result was due to the irritating effect of the tuberculin on the endothelial phagocytes of the liver and spleen, a belief confirmed by the microscopic examination of these tissues.

The simultaneous effect of extracts and active principles of glands on antibody formation has been investigated by many experimenters. Adrenalin, as reported by Také and Marine, ${ }^{9}$ has little effect on specific antibodies. Smith, ${ }^{\text {to }}$ however, has recently noted an antibody response to adrenalin that is most interesting. Intraperitoneal and subcutaneous injections of adrenalin in normal rabbits in doses from 0.5 to $2 \mathrm{cc}$. are followed by increased capacity of the serum to kill bacteria. This reaction is not specific, since it occurs for both B. typhosus and Staphylococcus aureus. It is transitory, lasting only a few hours after antigen injection. Large doses or daily doses diminish the bactericidal action, suggesting either exhaustion of the cells producing antibody or a development of tolerance to adrenalin. Injection of foreign protein has been found by Herrmann ${ }^{12}$ and many others to increase antibody production. This action may be attributed to liberation of bound specific antibodies. Nearly

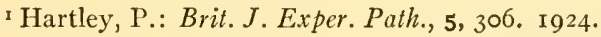

${ }^{2}$ Murphy, J. B., and Sturm, E.: J. Exper. Med., 4I, 245. I925.

3 Foord, A. G.: J. Infect. Dis., 23, 159. I918.

4 Bedson, S. P.: J. Path. E Bact., I9, I9I. I9I4-15.

5 Tunnicliff, R.: J. Infect. Dis., 26, 447. I920. ${ }^{6}$ Müller, P. J.: Arch.f. Hyg., 51, 365. I904.

7 Bachmann, A.: Compt. rend. Soc. de biol., 82, Iо31. 1919.

8 Thompson, H. L.: J. Med. Research, 43, 37. 1922.

9 Také, N. M., and Marine, D.: J. Infect. Dis., 33, 2 I7. 1923.

so Smith, G. H.: J. Immunol., I 2, 20.5. 1926.

ix Herrmann, S. F.: J. Infect. Dis., 23, 457. I9I8. 
every known chemical has been injected into animals, and the simultaneous effect on antibody formation and on various organs and tissues of the body has been observed. Madsen $^{I}$ concluded from his experiments that all inorganic salts stimulated antibody production, a sweeping assertion that many investigators do not confirm, among them McIntosh and Kingsbury, ${ }^{2}$ Swift, ${ }^{3}$ Arkin, ${ }^{4}$ and others. Arkin, ${ }^{4}$ studying immune reactions in rabbits, decided that drugs which stimulate oxidation also stimulate antibody production, while drugs which depress oxidation also depress antibody formation. Horgan ${ }^{5}$ reviewed the reports of the Danish investigators that the use of manganese salts (taken up by the reticulo-endothelial system) injected into horses during immunization increased diphtheria-antitoxin production, but he stated that in his experiments, manganese salts increased the antibody curve in only 50 per cent of the animals. Singer ${ }^{6}$ however, confirmed the work of the earlier investigators when he obtained agglutinin in guinea pigs experimentally infected with typhus fever and injected with manganese chloride, and failed to obtain agglutinin in animals not receiving the drug. Arsenicals have been found by Arkin, ${ }^{7}$ Friedberger, ${ }^{8}$ and others to stimulate antibody production; but Toyama and Kolmer ${ }^{3}$ observed that the larger doses appear to depress hemolysin and agglutinin in immunized rabbits, although small doses were somewhat stimulating. Swift ${ }^{10}$ found that rabbits treated with sodium salicylate before immunization with green producing streptococci and with sheep cells showed diminished complement fixation antibodies, agglutinin, and hemolysin. He concluded that sodium salicylate in some way depressed the power of animals to absorb injected antigen, consequently inhibiting the normal immunity curve. Cook ${ }^{\mathrm{II}}$ discovered that animals treated with sodium citrate formed antibodies more intensely than did controls and those treated with calcium chloride less intensely than control animals. She explained her results by the theory that permeability of cells is increased by increasing the sodium ion and is depressed by increasing the calcium ion and that the rate of antibody formation is dependent on the rate of absorption of antigen.

\section{EFFECTS OF INJURIOUS AGENCIES UPON ANTIBODY PRODUCTION}

Roentgen rays in proper dosage destroy lymphoid and myeloid cells, so that their effect on antibody response may be of especial significance. Murphy and Ellis ${ }^{12}$ observed that exposure to Roentgen rays made both normial and splenectomized mice more susceptible to tuberculosis; they considered that this result was due to the in-

\footnotetext{
${ }^{1}$ Madsen, T.: J. State Med., 31, 5 1. I923 (Harben lecture).

${ }^{2}$ MIcIntosh, J., and Kingsbury, A. N.: Brit. J. Exper. Path., 5, I8. I924.

${ }_{3}$ Swift, H. F.: J. Exper. Med., 36, 735. 1922.

4 Arkin, A.: J. Infect. Dis., I6, 349. I9I5 (b); ibid., 13, 408. I913 (a).

5 Horgan, E. S.: Brit. J. Exper. Palh., 6, 108, 1925.

${ }^{6}$ Singer, E.: Zlschr.f. Immunitätsforsch. u. exper. Therap., 46, 288.1926.

7 Arkin, A.: loc. cit.

${ }^{8}$ Friedberger, E., and Masuda, N.: Therap. Monatschr., 25, 288. 19 Ir.

${ }^{9}$ Toyama, I., and Kolmer, J. A.: J. Immunol., 3, zог. IgI 8.

${ }^{10}$ Swift, H. F.: loc. cit.

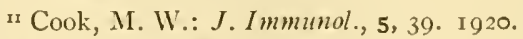

${ }^{12}$ Murphy, J. B., and Ellis, A. W. M.: J. Exper. Med., 20, 397. Igr4.
} 
jury of lymphoid tissue and that lymphocytes were an important factor in antituberculosis defense. When Benjamin and Sluka ${ }^{1}$ exposed rabbits to X-ray before injection of beef serum, precipitin was delayed or absent; but if they did not expose to $\mathrm{X}$-ray until four or five days after injection, the antibody curve was unaffected. Von Heinrich $^{2}$ also found that exposure soon after an injection of antigen in guinea pigs modified the anaphylactic reaction. Hek toen ${ }^{3}$ exposed white rats to $\mathrm{X}$-rays over a period sufficiently long to reduce the leukocytes and to injure the spleen, lymph glands and bone marrow; he then injected sheep corpuscles; the production of hemolysin was restricted, a result in harmony with the conception that the organs mentioned are the site of antibody formation. Dogs and rabbits exposed to $\mathrm{X}$-ray at about the time of injection also had a reduced immunity response. Murphy and Sturm, ${ }^{4}$ by exposing animals to X-ray sufficiently long to injure the lymphoid tissue but not the bone marrow, reduced the antibody response. They thought that this result pointed to lymphoid tissue as the site of antibody production rather than to the reticulo-endothelial system, since the reduction of antibody was out of proportion to the injury of the reticulo-endothelial cells.

In I9I I Selling 5 injected benzene into rabbits and discovered that the bone marrow especially was damaged but that regeneration took place within ten to twenty days. Several years later, Rusk ${ }^{6}$ found that rabljits injected with benzene before or at the time of antigen inoculation showed diminished lysin for sheep corpuscle and reduced precipitin for horse serum. Simons and Jones ${ }^{7}$ injected rabbits with benzene and learned that lysin for dog corpuscles and agglutinin and opsonin were reduced and that the leukocytes, the polymorphonuclear type in particular, were also diminished. Hektoen ${ }^{8}$ ascertained that suitable doses of benzene injected before antigen reduced the lysin response to sheep corpuscles in rabbits and the lysin response in white rats. Simultaneously there were gross bone-marrow lesions and severe leukopenia. Minute doses of benzene in dogs stimulated the production of lysin instead of inhibiting it. Apparently benzene, a drug exerting a destructive action on the bloodforming organs, also depressed antibodies, indicating that there might be some correlation between the blood-forming organs and the antibody response. Thorium, which affects myeloid cells more than lymphocytes and erythrocytes, reduced precipitins. Mustard gas had little effect on antibody production when it was given after antigen injection; but when it was given a few days prior to the injection, the appearance of lysin, agglutinin, and precipitin was delayed. Radium emanations given before or at the same time that sheep corpuscles were injected into rabbits depressed both lysins and precipitins. With all these substances that affect the cells of the bone

I Benjamin, E., and Sluka, E.: W'icn. klin. I'chnschr., 2 I, 3 I I. Igos.

${ }^{2}$ von Heinrich, H.: Ccntralbl. f. Buktcriol., I, 70, 421. I9 I3.

${ }^{3}$ Hektoen, L.: J. Infect. Dis., 17, 415. I915; ibid., 22, 28. 1918; ibid., 27, 23. 1920.

4 Murphy, J. B., and Sturm, E.: loc. cit.

5 Selling, L.: Ziegler's Bcitrage, 51, 576. I9 I1.

${ }^{6}$ Rusk, G.: Univ. Calif. Pub. Path., 2, I39. 1914.

7 Simonds, J. P., and Jones, H. M.: J. Med. Research, 33, I97. I9I5.

${ }^{8}$ Hektoen, L.: J. Infect. Dis., 19, 69. I916; Hektoen, L., and Corper, H. J.: ibill., 26, 330. I920; ibid., 31, 305. I922. 
marrow, spleen, lymph node, or liver, the inhibition of antibody response appears to depend both on the dosage and on the time of administration with reference to the antigen injection.

\section{THE EFFECTS OF EXTIRPATION OF ORGANS}

All the organs of the body that can be removed without destroying life have been extirpated on the chance that antibody production might, be inhibited. Pfeiffer and Marx $^{-1}$ observed no change in the antibody response in splenectomized animals, and they assumed that the rôle of antibody production was quickly taken over by other organs. Deutsch, ${ }^{2}$ Rath, ${ }^{3}$ Hektoen, ${ }^{4}$ and others observed that removing the spleen at about the time of antigen injection delayed antibody response. Extracts of the removed spleens caused the production of antibodies when they were injected into fresh animals. Russ and Kirschner, ${ }^{5}$ reviewing the evidence of the spleen as the site of agglutinin production, concluded that the rôle of the spleen was quickly taken over by other organs when it was removed. Hektoen and Curtis ${ }^{6}$ found that the removal of the stomach or small intestine or thyroid gland had no effect on hemolysin in serum when dogs were operated upon several days before the injection of rat blood. Removal of the pancreas and spleen resulted in an antibody production comparable to that produced by the removal of the spleen alone. The removal of adrenal or half of the liver had no apparent effect on antibody production. Také and Marine, ${ }^{7}$ contrary to the results of Ecker and Rogoff, ${ }^{8}$ Hektoen and Curtis, ${ }^{9}$ and Gates, ${ }^{10}$ discovered that high-grade suprarenalectomy in rabbits increased the titre for hemolysin for sheep cells. They believed that this result was due to the loss of some regulatory and inhibitory influence which the gland normally exerted on the irritability and susceptibility of the body cells. Portis ${ }^{\mathrm{rr}}$ removed the omentum from rabbits, guinea pigs, and dogs; the subsequent production of antibody was reduced in rabbits, but only slightly modified in dogs and guinea pigs, a result due, he thought, to species variation in the structure of the omentum, that of the rabbit containing many clasmatocytes while that of the dog and guinea pig had few or none. Cutler ${ }^{12}$ thought that the endocrine organs might enter into the mechanism of antibody production. He removed part of the hypophysis in guinea pigs and subsequently injected them with B. typhosus and hen erythrocytes. There was practically no reduction in antibody response, and he concluded that the hypophysis in amount necessary for maintaining life had no direct or indirect effect on antibody production.

Launoy and Levy-Bruhl ${ }^{13}$ cite the literature upon the rôle of thyroid in immunity.

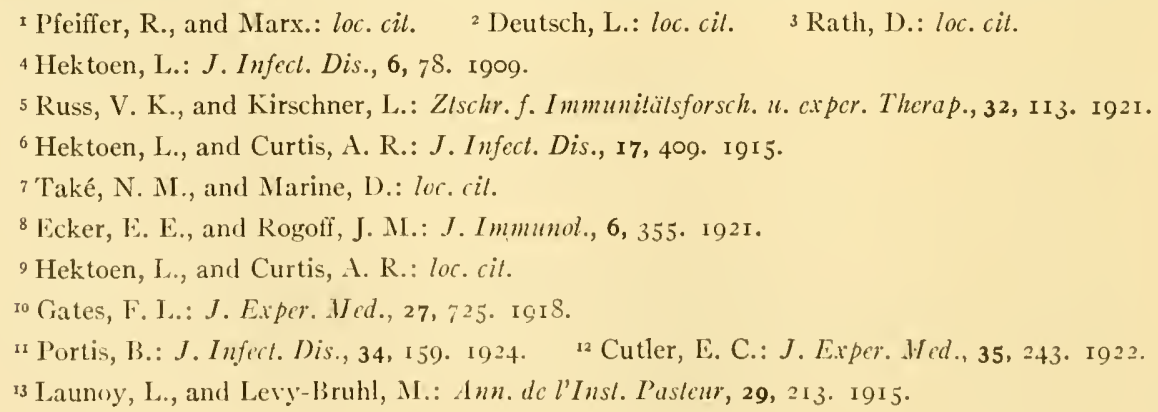


Investigators have obtained diverse results in immunity curves after thyroidectomy. Fassin reported a decrease in hemolysin and bacteriolysin; Marbe, a decrease of opsonin and phagocytic power; Frouin, an increase in tetanus antitoxin; Lerda and Diez, more resistance to intoxications, bacterial and drug; and Fjeldstad, practically no change in antibody formation.

Splenectomy before or soon after the injection of antigen is the only organ extirpation that appears rather uniformly to give a reduced antibody response.

\section{THE RÔLE OF THE RETICULO-ENDOTHELIAL SYSTEM}

Many investigators believe that no one organ can be responsible for so important a function as antibody production, and they consider that many or all the cells in the body may contribute to immunity response. This group of experimenters in the search for antibody formation has considered tissues of widespread distribution, such as the lymphatic tissue, the hematopoietic tissues, or the reticulo-endothelial system of cells. The reticulo-endothelial system of cells has become of increasing interest in the study of immunity response, and a voluminous literature has arisen which has been comprehensively reviewed by Sacks ${ }^{\mathrm{I}}$ and by Kritschewski and Meersohn. ${ }^{2}$ According to Aschoff, ${ }^{3}$ this system of cells has been grouped together because of the common phagocytic properties, and comprises the reticular and endothelial cells of the spleen, the reticulo-endothelial cells of the bone marrow and lymph nodes, the Kupffer cells of the liver, the histiocytes of the general connective tissue, and the monocytes of the circulating blood. Before the cells of the reticulo-endothelial system were definitely grouped together, their ability to phagocytize the red blood cells, bacteria, and other foreign particles had been noted. Cary ${ }^{4}$ had demonstrated that foreign erythrocytes introduced into the circulation of a rabbit were removed by the fixed tissue cells of the liver and spleen and that extracts of spleen and liver from such an animal had a higher hemolytic titre than the corresponding serum. Kyes ${ }^{5}$ also concluded that the fixed tissue phagocytes were active producers of antibody, because pneumococci introduced into the blood of chickens were quickly taken up by the fixed tissue phagocytes, particularly those of the spleen. Motohashi ${ }^{6}$ also found that foreign erythrocytes were taken up by the cells of the spleen, liver, and bone marrow and that after splenectomy the remaining cells of the reticulo-endothelium were more active. Bieling and Isaacs, ${ }^{7}$ splenectomizing mice, noticed little effect on antibody (hemolysin), but after splenectomy and blockade of the reticulo-endothelial system with colloidal iron, hemolysins were inhibited. Siegmund ${ }^{8}$ also found hemolysins and hemagglutinins reduced or totally suppressed in highly blocked and splenectomized rabbits. Neufeld and Meyer $^{9}$ found that after blocking and splenectomizing mice they could not

I Sacks, B.: Physiol. Rev., 6, 504. 1926.

${ }^{2}$ Kritschewski, I. L., and Meersohn, I. S.: Ztschr. f. Immunitätsforsch. u. exper. Therap., 47, 407. 1926.

\footnotetext{
3 Aschoff, L.: Lecture on "Pathology." New York, 1924.

${ }_{4}^{4}$ Cary, II. E.: loc. cit, 5 Kyes, P.: loc. cit. ${ }^{6}$ Motohashi, S.: loc. cit.

7 Bieling, R., and Isaacs, G.: Ztschr.f. d. ges. exper. Hed., 28, i80. I922.

${ }^{8}$ Siegmund, H.: Klin. Wchnschr., I, 2500.1922.

${ }_{9}$ Neufeld, F., and Meyer, H.: Ztschr.f. IIyg., I03, 595. 1925.
} 
be actively immunized against pneumococci. Jungeblut and Berlot ${ }^{1}$ noticed that diphtheria antitoxin formation was suppressed in guinea pigs when large intravenous injections of iron had been previously given. Koboyashi and Shuwatsu (cited by Jungeblut and Berlot) found that blockading the reticulo-endothelial system restricted the typhoid agglutinins. Gay and $\mathrm{Clark}^{2}$ reported that blocking of the system with trypan blue suppressed the production of hemolysins and precipitins. Isaacs ${ }^{3}$ also found that hemolysin and precipitin could be diminished by blockading the system. Freund ${ }^{4}$ discovered that colloidal iron or trypan blue plus splenectomy suppressed the hypersensitiveness of guinea pigs to tuberculin. On the other hand, Rosenthal, Moses, and Petzal ${ }^{5}$ proved an actual increase in the antibody titre after the reticulo-endothelial system was blockaded. Standenath ${ }^{6}$ also noted increased antibody formation after blocking this system. Frankel and Grünenbergi splenectomized rabbits and blockaded the system with iron and then vaccinated with $B$. proteus $\mathrm{X}_{19}$; in comparing with normal rabbits they could detect no difference in the agglutinin titres in the animals. Weiss and Kunze ${ }^{8}$ noted no change in antibody formation after blockade. Lewis and Loomis, ${ }^{9}$ blockading animals with trypan blue, found that the capacity for forming antibody was increased. Paschkis ${ }^{\text {To }}$ discovered that rats lost the power to store vital dyes in the reticulo-endothelial cells of the spleen when they had been previously immunized against streptococci. Singer and Adler, ${ }_{1}^{11}$ immunizing rabbits against pneumococcus type III and then injecting India ink, learned that the rabbits acted as though they were not immune. Singer ${ }^{12}$ injected manganese chloride, a drug taken up by the reticulo-endothelial system, and noted that this resulted in the appearance of agglutinin against $B$. proteus $\mathrm{X}_{19}$ in guinea pigs experimentally infected with typhus fever.

At present the results of reticulo-endothelial blockade are conflicting and confusing; not a surprising fact since the reticulo-endothelial system undergoes constant change because of the rapid proliferation of its cells. Different materials have been used for blockade; the dosage and the period of injection have varied; different experimental animals have been employed; and different antibodies have been investigated.

\section{EFFECTS OF DISEASE}

Disease has not thrown so much light upon the locus of antibody production as might be expected. The immunity response of persons with diseases associated with

I Jungeblut, C. W., and Berlot, J. A.: J. Expcr. Mcd., 43, 613. 1926.

${ }^{2}$ Gay, F. P., and Clark, A. R.: J.A.M.A., 83, I 296. 1924.

3 Isaacs, M. I.: Proc. Soc. Exper. Biol. \& Hed., 23, 185. 1925.

4 Freund, J.: J. Immunol., I1, $3 \AA_{3}$. I926.

5 Rosenthal, I., MIoses, .1., and Petzal, E.: Zlschr.f. d. gcs. expcr. Mcd., 41, 405. 1924.

${ }^{6}$ Standenath, F.: Ztschr.f. Immunilätsforsch. u. cxper. Thcrap., 38, 19. I923.

7 Frankel, F., and Grünenberg, K.: Ztschr.f. d. ges. exper. Med., 41, 5SI. I924.

${ }^{8}$ Weiss, S., and Kunze, J.: Wicn. Arch.f. imn. Med., ro, 45 I. 1925.

9 Lewis, P. A., and Loomis, D.: J. Expcr. Med., 43, 263. 1926.

Io Paschkis, K.: Ztschr.f.d. ges. cxper. Mcd., 43, I75. 1924.

II Singer, E., and Adler, H.: Zischr.f. Immunitätsforsch. u. cxper. Therap., 41, t68. I024.

12 Singer, E.: loc. cit. 
changes in the hematopoietic tissues have been studied by Howell and Schultz. ${ }^{\text {. Five }}$ persons with pernicious anemia, one in remission, two becoming progressively worse, and two in collapse, were injected with a triple vaccine containing $B$. typhosus, $B$. paratyphosus $\mathrm{A}$, and $B$. paratyphosus $\mathrm{B}$. Typhoid immume bodies were produced after approximately the same time and in the same degree as they were in healthy persons; the leukocyte and erythrocyte counts were increased, indicating that the bone-forming organs were stimulated. A case of aplastic anemia did not respond to the stimulus of the vaccine, nor did the blood counts increase in this instance. The immunity response in secondary anemia and polycythemia did not vary from that of healthy persons. Antibodies were not elicited by the injection of the vaccine in persons with lymphatic or myelogenous leukemia. Rotky ${ }^{2}$ also failed to stimulate antibodies by injecting leukemic patients with various bacterial antigens. Moreschi ${ }^{3}$ noted that a patient of his who contracted typhoid fever during the course of a chronic lymphatic leukemia failed to develop specific typhoid agglutinin. This loss of ability of leukemic patients to form antibodies may be due to the alteration in the hematopoictic tissue or it may be due to injury of the reticulo-endothelial tissue.

Hektoen and Corper ${ }^{4}$ found that experimental tuberculosis of rabbits had, except in rare instances, little effect on the production of hemolysin. In acute infectious diseases the immunity curves correspond to those in artificially induced immunity. Experimentally an acute infection, such as pneumonia, has been olsserved by Hektoen ${ }^{5}$ to inhibit the hemolysin response of dogs to foreign corpuscles. Multiple bacterial antigens, according to the experiments of Castellani, ${ }^{6}$ Huntoon and Craig, ${ }^{7}$ Corrigan, ${ }^{8}$ and others, stimulated the production of multiple antibodies, and in amount comparable to the antibody response elicited by each bacterial constituent of the antigen. Animal infection such as spirillosis of chickens, in which there is splenic enlargement, suggested to Launoy and Levy-Bruhl ${ }^{9}$ that the spleen had an important influence on the course of this disease. However, their own experiments showed that in splenectomized chickens the clinical course of the disease was more benign though there was more noticeable septicemia than in chickens without the spleen removed. Rabbits infected with various organisms including anthrax, cholera, and streptococcus gave no evidence that the spleen was more important than any other organ. Bardach ${ }^{10}$ found splenectomized dogs even more resistant than normal dogs to anthrax. In protozoan and spirillar diseases, the majority of investigators found evidence that the infections were more pronounced and more often fatal in splenectomized animals. Apparently, such experiments indicate the importance of the spleen in development

${ }^{x}$ Howell, K. M., and Schultz, O. T.: Proc. of Inst. of Med. of Chicago, 5, 52. 1924; Arch. Int. Med., 26, 706. 1920.

${ }^{2}$ Rotky, H.: Centralbl. f. inn. Med., 35, 953. I914.

${ }^{3}$ Moreschi, C.: Zisclur.f. Immunitätsforsch. u. cxper. Therap., 21, 410. 1914.

${ }_{4}^{4}$ Hektoen, L., and Corper, H. J.: J. Infect. Dis., 37, 82. 1925.

5 Hektoen, L.: loc. cit.

${ }^{6}$ Castellani, A.: J. Trop. Med., I7, 326. 1914.

${ }^{7}$ Huntoon, F. M., and Craig, S.: J. Immunol., 6, 235. I921.

${ }^{8}$ Corrigan, M.: J. Infect. Dis., 37, 549. 1925. $\quad{ }^{9}$ Launoy, L., and Levy-Bruhl, M.: loc. cit.

ro Bardach, M. J.: Ann. de l'Inst. Pasteur, 5, 40. 1891. 
of infection, and the immunity response to infection varies with different animals and with different organisms.

Food deficiencies were investigated by Zilva ${ }^{\mathrm{x}}$ and others. He found that diets low in iron or calcium or potassium or sodium chloride had practically no effect on antibody production. However, those low in phosphorus were associated with a lower agglutinin and hemolysin titre than normal diets. More recently Werkman, Nelson, and Fulmer ${ }^{2}$ have investigated diets low in vitamin $\mathrm{C}$. Although guinea pigs on such a diet were less resistant to anthrax and pneumococcus infections, specific agglutinin to $B$. typhosus did not differ from that of normal animals.

\section{LOCAL IMMUNITY}

Local immunity offers another method of approach to the solution of antibody production. Many immunologists have believed that antibodies are formed at the point of injection of the antigen and that the immunity spreads from this area throughout the body. Local immunity has been investigated by testing the concentration of the antibodies at the site of inoculation to determine whether they are in higher concentration there than elsewhere in the body. These investigators' method of combating disease, therefore, has been to attempt immunization of the specific portal of entry of various injurious antigens. Pasteur prevented anthrax in sheep by skin immunization, believing that sheep contracted anthrax exclusively by that route. Cook and Smith ${ }^{3}$ found that after cutaneous immunization skin, free of blood, would give a precipitin reaction. Rivers, ${ }^{4}$ Gay, ${ }^{5}$ and others have claimed that intracutaneous vaccination protects against erysipelas more effectively than other methods of immunization. Besredka ${ }^{6}$ learned that he could protect the skin from staphylococcus and streptococcus infection by placing a compress soaked in the bacterial filtrate over the skin area twenty-four hours before an inoculation of the organism. Gay claims that he obtained the same results by using a compress soaked in broth; he considered the results in both instances due to stimulation and aggregation of monocytes at the skin surface by the irritating substances. Ledingham ${ }^{8}$ has employed the blockade method, using India ink intradermally, and he has ascertained that a superimposed vaccine either inhibited or decreased antibody response. He explained his results on the theory that the India ink caused the reticulo-endothelium to proliferate and thus enhanced the defense processes at the point of inoculation. Wassermann and Citron ${ }^{9}$ injected typhoid vaccine intraperitoneally, intrapleurally, and intravenously into animals, with the result that each method gave a better agglutinin for its own exudate. Their

r Zilva, S. S.: Chcm. Abs., r3, 2920. r919.

${ }^{2}$ Werkman, C. H., Nelson, V. E., and Fulmer, E. I.: J. Infect. Dis., 34, 447. 1924.

3 Cook, M. W., and Smith, G. H.: J. Immunol., 2, 415. I917.

${ }_{4}^{4}$ Rivers, T. M.: J. Exper. Med., 41, I79. 1925 ; Rivers, T. M., and Tillett, W. S.: ibid., p. I85. 1925.

5 Gay, F. P.: Arch. Path. \& Lab. Med., r, 590. 1926.

${ }^{6}$ Besredka, A.: Ann. de l'Inst. Pasteur, 38, 565. I924.

7 Gay, F. P.: loc. cit.

${ }^{8}$ Ledingham, J. C. G.: Brit. J. Exper. Palh., 8, 12. 1927.

9 Wassermann, A., and Citron, J.: Ztschr.f. IIyg. u. Infektionskrankh., 50, 33r. 1905. 
results have not been confirmed by Hektoen ${ }^{\mathrm{x}}$ and others, who obtained as high or higher antibody titres from the serum as from the other fluids regardless of the method of introducing antigen. Oral immunization to diphtheria and tetanus toxin as shown by Ramon and Zoeller ${ }^{2}$ was less successful than other methods of immunization against these diseases. However, oral vaccination against intestinal organisms, particularly against typhoid bacilli, has been successfully used by Calmette ${ }^{3}$ and Besredka. ${ }^{4}$ Cooper ${ }^{5}$ reports successful immunization against pneumococcus type $I$ in rabbits by introducing pneumococcus type I vaccine into the buccal mucous membrane. Intratracheal inoculations into rabbits with respiratory diseases gave no better protection than intraperitoneal injection, according to the experiments of Jones. ${ }^{6}$ Corneal immunity in rabbits, according to Gay, ${ }^{7}$ is the most convincing evidence that immunity can be restricted to one part. He cites the work of Kraus and Volk, that vaccination of one cornea leads to its protection but not to that of the other cornea. Smith, Orcutt, and Little ${ }^{8}$ concluded that the udders of cows participated in the production of agglutinin; for example, when they experimentally infected one-quarter of the gland, there was an aggregation of leukocytes in that quarter, which was followed by a production of agglutinins.

The principal argument against the theory of the local production of antibody is that the removal of the depot of antigen, whether taking place in a few seconds or after some days, has frequently little effect on antibody production. Hektoen ${ }^{9}$ found that the removal of the vaccinated leg of a dog early in immunization did not modify to any extent the antibody curve of the dog serum. Friedberger (cited by Nasta and Braumer) injected antigen subcutaneously into rabbits' ears; the ears were cut off at intervals varying from ten minutes to one hour after subcutaneous injection; the amount of antibody produced was comparable to that of controls. Nasta and Braum$\mathrm{er}^{\mathrm{I0}}$ and Reitler, ${ }^{11}$ having interrupted the circulation by a ligature at the base of the ear, cut off the ear two to three seconds after subcutaneous injection and failed to inhibit antibody response in the animal. Fernbach and Hepner ${ }^{12}$ cut out the skin at the point of intracutaneous injection of typhoid vaccine; the agglutinin curve elicited was comparable to that obtained from intravenous inoculation, and they concluded that the spleen was the essential organ for antibody production in the defense reaction against typhoid infections. Thus far, experimental work on the local production of specific antibody has not determined the site of antibody formation.

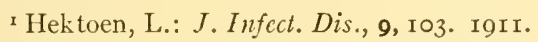

${ }^{2}$ Ramon, G., and Zoeller, C.: Compt. rcnd. Soc. de biol., 95, I409. 1926.

3 Calmette, A.: Ann. de l'Inst. Pastcur, 37, 900. I923.

4 Besredka, A.: Tr. Roy. Soc. Trop. Med. Er IIyg., I7, 346. I924.

5 Cooper, M. L.: J. Infect. Dis., 38, 491. I926.

${ }^{6}$ Jones, F. S.: J. Exper. Med., 37, 789. 1923.

7 Gay, F. P.: loc. cit.

${ }^{8}$ Smith, T., Orcutt, M. L., and Little, R. B.: ibid., p. I53. I923.

${ }^{9}$ Hektoen, L.: loc, cit.

ro Nasta, M., and Braumer, R.: Compt. rend. Soc. de biol., 95, 137. 1926.

II Reitler, R.: Ztschr.f. Immunitätsforsch. u. cxper. Therap., 40, 453. I924.

12 Fernbach, H., and Hepner, F.: Centralbl. f. Baktcriol., roo, 358. 1926. 


\section{CONCLUSION}

In spite of the fact that the origin of antibodies has been investigated by so many methods, both direct and indirect, the site of their formation is still uncertain. Experimentally certain organs, particularly the spleen, and certain cells, such as the tissue phagocytes, seem to be more actively involved in the production of antibody than other tissues. However, from the evidence at hand, it is possible that antibody formation may be a widespread cellular function in which all the cells of the body participate to some degree. 


\section{CHAPTER LXXVIII}

\section{THE ISOLATION OF SUBSTANCES WITH IMMUNE PROPERTIES}

\section{ARTHUR LOCKE' AND EDWIN F. IIIRSCH}

\section{St. Luke's Hospital, Chicago}

The first intensive studies of experimental immunization, marked by the decade of r880-90, suggested that the phenomena of the immunity state might be associated with a production and accumulation, in the tissues of immunized animals, of chemically individual, specific "immune substances." The capacity of the blood serum of an animal experimentally immunized against cholera spirilla, to agglutinate suspensions of that organism appeared, for example, to be referable to a content of specific cholera agglutinin. Concepts of lysins, precipitins, antitoxins, etc., arose and became crystallized to such an extent that Emmerich, in 1887 , made the following comment: "It should be an important task for investigators to seek out these substances which are associated with the immunity state and to ascertain in what chemical grouping they belong." ${ }_{2}$

Chemical examinations of the tissues and tissue fluids of immune and non-immune animals were made, ${ }^{3}$ and the results indicated (I) that immune substances do exist as definite entities; (2) that they are neither dialyzable nor extractable by fat solvents; (3) that they are readily destroyed by agents which denature or hydrolyze proteins; and (4) that they are associated in the serum with some protein fraction, usually the pseudoglobulin or a closely bordering englobulin fraction.

However, this purely analytical method of approach revealed little as regards the true nature of the immune substances. It was apparent that an adsorption of immune substance upon serum globulin might resist dialysis and fat extraction regardless of its nature, and that its inactivation by heat and enzymic digestion might be due simply to changes induced in the protein adsorbent. The protein fractions, upon whose behavior the conclusions as to the nature of the immune substances had been based, probably contained less than 0.2 per cent of the pure immune principle.

Contemporary studies of the physico-chemical relations of antigen and antibody, ${ }^{4}$ undertaken by Landsteiner and by Morgenroth and later by Bordet, were more productive. They demonstrated the existence of a property which completely differentiates the immune principle of an immune serum from the associated serum pro-

${ }^{1}$ Seymour Coman Fellow in Medical Chemistry, Kent Chemical Laboratory, University of Chicago.

${ }^{2}$ Emmerich, R., and Tsuboi, J.: Verhandl.d. XI. Kong.f.inn. Med., p. 202. I892.

3 Tizzoni, G., and Cattani, G.: Centralbl.f. Bakteriol., 9, 685. I891; Pfeiffer, R., and Proskauer, B.: ibid., 19, I91. 1896; Brodie, T. G.: J. Path. \& Bact., 4, 460. 1897; Pick, E. P.: Beitr. s. chem. Phys. u. Path., I, 351. I902.

4 Landsteiner, K.: Mïnchen. med. Wchnschr., 49, 1905. 1902; 50, 764. I903; Morgenroth, J.: ibid., p. 6r. 1903; Bordet, J. (trans. by Gay, F. P.): Studies in Immmity. Wiley \& Sons, rgog. 
teins, namely, a capacity to become selectively adsorbed upon homologous antigen. Sheep erythrocytes, for example, added to chilled, homologous immune rabbit serum not only were agglutinated and flocculated but also removed nearly all of the antisheep immune substances from the serum. The adsorption of the immune substances was demonstrated by centrifugating the agglutinated cells, washing them free of adherent serum, resuspending them in physiological salt solution, and testing their capacity to agglutinate untreated cell suspensions. The agglutinative capacity of the artificial suspension resembled in every way a solution of the corresponding, whole, immune serum. Moreover, a part of the adsorbed immune substances could be recovered from the sedimented cells by extraction with warm, isotonic salt solution.

The recovery of the substances which become adsorbed from immune sera by suspensions of homologous antigen was first accomplished by Hahn and Trommsdorf, ${ }^{I}$ in 1900 . Suspensions of bacteria were left in contact with homologous immune serum until they had become completely agglutinated. The agglutinated sediments were then separated, thoroughly washed with isotonic salt solution, and resuspended in warm $N /$ roo acid. Clear, agglutinin-containing extracts were obtained which gave none of the accepted tests for the presence of protein.

The extreme minuteness of the quantity of protein which may be associated with a physiological effect is perhaps not generally appreciated. Egg albumin produces anaphylactic sensitization in a quantity of less than $0.00005 \mathrm{mg}^{2}$ Purified diphtheria toxin kills guinea pigs in quantities of less than $0.00007 \mathrm{mg} .{ }^{3}$ The protein of the spermatozoön produces specific fertilization and confers the male inheritance in a quantity of $0.0000003 \mathrm{mg} .{ }^{4}$ These quantities are many thousand times smaller than may be recognized by any dependable chemical test for the presence of protein. The substances carrying the immune property in Hahn and Trommsdorf's final extracts were clearly associated with too minute an amount of material to permit of their recognition and identification by any purely chemical method not requiring the concentration of prohibitively large volumes of extract.

The yield of recovered immune substance obtained by the use of a modification of their procedure is considerably augmented when the denaturation consequent to long contact with warm $N /$ roo acid is avoided. ${ }^{5}$ Extraction with cold $N / \mathrm{r}, 000$ acid, dilute alkali, warm water, or warm isotonic salt and saccharose solutions is less injurious. ${ }^{6}$ Concentration of the extracts is effected by precipitation and re-solution in a minimum quantity of dilute alkali or by evaporation in vacuo. Or the extracts

${ }^{x}$ Hahn, M., and Trommsdorf, R.: Mïnchen. med. Wchnschr., 47, 4I3. I900. See also Liebermann, L. von, and Fennyvessy, B. von: Centralbl.f. Bakteriol., 47, 274. I908.

${ }^{2}$ Wells, H. G.: Chemical Aspects of Immunity, p. 28. Chemical Catalog Co., I925.

${ }^{3}$ Locke, A., and Hirsch, E. F.: unpublished observation.

${ }_{4}$ Locke, A.: Proc. Inst. Med., 5, 316. Chicago, 925.

5 Rondoni, P.: Zischr.f. Immunitätsforsch. u. cxper. Therap., 7, 515. rgro.

${ }^{6}$ Locke, A., and Hirsch, E. F.: J. Infect. Dis., 37, 449. 1925; Huntoon, F. M.: J. Immunol., 6, Ir7, 123, I85. 1921; U.S.P., No. 1484038. r924; Ottenberg, R.: Proc. Soc. Exper. Biol. So Med., 21, I4, 303. 1923-24. See also Gay, F. P., and Chickering, H. T.: J. Exper. Mfed., 21, 389. I9I5; Weinstein, I.: J. Immunol., 3, 17. I918; Kosaki, M.: ibid., p. ro9. 1918; Furuhata, T.: Japan Med. World, I, I. I921; Uchicla, S.: J. Infect. Dis., 40, 588. 1927; Locke, A., and Main, E. R.: ibid., 39, 484. 1926. 
may be dialyzed and further purified by a fractional precipitation procedure before concentration. Huntoon has obtained antipneumococcus immune-substance extracts in this way "approximating in antibody content the best immune sera, and containing so low a serum protein content that 5-cc. amounts sensitize guinea pigs only irregularly to subsequent injections of horse serum.' 860 times less nitrogen per immune unit than the immune serum from which they were derived. Ottenberg and Stenbuck have obtained anti-typhoid immune extracts of a similar, remarkable degree of purity. ${ }^{\mathrm{T}}$

The improved extraction procedures which are the essence of this later work and which have made possible the preparation of immune-substance extracts in practicable yields are, unfortunately, by no means selective. They produce no notable dissociation of the antigen-immune substance union represented by the original, washed antigen-immune serum adsorption sediment but create, rather, a disintegration and dispersion of the sediment, out of which a portion of the antigen substances can be removed by direct centrifugation. A further portion of the dispersed antigen material is removed by the various iso-electric separation procedures which have been proposed, but a considerable fraction persists in the final extracts.

However, the antigen-immune substance union may be broken, in the experience of the authors, ${ }^{1}$ by a process of ether extraction, permitting the recovery of immunesubstance preparations which are almost entirely free from contamination with dispersed antigen. The ether extraction causes a shift in the iso-electric range of lipoidcontaining antigen from a value identical with that of the specific immune substance to a value sufficiently removed to permit the loosening of the antigen-immune substance union and the precipitation of the antigen residue. The procedure, as adapted to the purification of an immune hemolysin, is as follows:

Fresh sheep blood is defibrinated, centrifugated, and the cell sediment washed five times with physiological salt solution. Fifteen cc. of the packed, washed cell sediment are equally divided between two centrifuge tubes, and to each portion there is added quickly and with vigorous shaking, $40 \mathrm{cc}$. of perfectly clear, fresh rabbit anti-sheep serum. After two hours, the cells have laked completely and the stroma have flocculated and settled toward the bottom of the tubes. Centrifugation for forty-five minutes completes the separation; 90-99 per cent of the hemolysin originally present has become bound to the stroma sediment. The brilliantly clear, red supernatant liquid is decanted from the sediment and replaced by an equal volume of physiological salt solution. After the stroma is finely suspended in the wash liquor by prolonged shaking, the suspension is allowed to stand for twenty minutes and is then strongly centrifugated for thirty minutes. The washing process is repeated (about six times) until the supernatant liquid has no trace of color and gives no trace of foam when shaken. The stroma obtained is perfectly white and has lost little of the originally bound hemolysin.

The well-washed, hemolysin-saturated stroma is extracted with ether three times. After the removal of the third ether extract, the ether remaining dissolved in the stroma material is removed by centrifugation in a warm centrifuge. The stroma residue material packs at the bottom of the tube, and the salt solution, which made up the cell volume, may be decanted. The residue is washed twice with physiological salt solution and once with distilled water. Considerable hemolysin is lost to the salt solution, but protein impurities, due to surface adsorption, are thereby almost completely removed. The washed residue is extracted re-

${ }^{r}$ See p. ro5o, note 6. 
peatedly with $N /$ I,, 00 sulphuric acid, the extracts are pooled, flocculated by neutralization, and the suspension centrifugated. The flocculation is quantitative as hemolysin is almost insoluble in pure water. The precipitated material is extracted with physiological salt solution and solutions of any desired hemolysin content may be obtained by varying the amount of salt solution used.

The protein content, per unit of hemolysin, of the most avid anti-sheep erythrocyte whole serum purified by this method was approximately $0.07 \mathrm{mg}$. The protein content, per unit of hemolysin, in the final, purified extract, was approximately $0.000044 \mathrm{mg} .{ }^{x}$ representing a purification of 150,000 per cent. The average degree of purification obtained with less avid serums was approximately 10,000 per cent. The minimum degree of purification, obtained with a very poorly avid serum prepared by a procedure of prolonged intraperitoneal immunization, was about 5,000 per cent. It was apparent that the character of the purified preparations yielded by the method was largely dependent upon the character of the immune sera from which they were derived.

The character of an immune serum is largely dependent upon the procedure of its preparation. ${ }^{2}$ Individual, homologous, immune sera differ widely in their titre or immune-substance content, their avidity or combining affinity for specific antigen, their specificity, stability, and their content of associated and of partly denatured immune substances.

A formed antigen such as the stroma of the sheep erythrocyte rarely elicits the production of a single type of immune substance. A variable concentration of hemagglutinin usually accompanies the hemolysins formed, reaching its maximum late in the immunity period when the hemolysin titre is retrogressing from its maximum value. ${ }^{3}$ Rabbits which have been used repeatedly for hemolysin production not only produce sera of inferior hemolysin titre and abnormally high hemagglutinin titre, but these sera may contain a large concentration of "degenerate" or partially denatured immune substances. The hemolysins of a serum prepared as the result of a series of gradually increased injections of sheep cells are frequently less than I 5 per cent adsorbed by a I per cent suspension of that antigen, while the hemolysins of a serum prepared as the result of a single injection occasionally may be more than 90 per cent adsorbed. The latter serum is said to have a greater avidity, per unit of hemolysin content, than the former. If the two sera are aged or heated at identical temperatures (below $60^{\circ} \mathrm{C}$.) for identical periods, the latter undergoes a greater loss in titre and combining capacity than the former, and when the respective capacities of the two sera to combine with heterologous antigens are compared, the latter is found to be the less specific.

The different immune fractions in a hemolysin serum may be separated by successive extractions with small amounts of specific antigen. The most avid, the least stable, and the least specific fractions are adsorbed first, and the least avid, the most stable, and the most specific fractions resist adsorption to the very last. It is apparent

${ }^{2}$ Locke, A., Main, E. R., and Hirsch, E. F.: J. Infect. Dis., 39, I26. 1926.

${ }^{2}$ Locke, A., Main, E. R., and Miller, F. A.: ibid., 41, 32. 1927.

3 Okell, C. C., and Parish, H. J.: Brit. J. Exper. Palh., 5, 355. 1924. 
that not only do different homologous immune sera differ greatly in their character but that the constituent immune substances in a single serum differ in similar ways. ${ }^{x}$ The source of these differences is undoubtedly to be found, in part, in correlated differences in the conditions surrounding the origin of the immune substances in the living tissues during the immunization procedure.

The blood plasma normally contains a small amount of so-called "natural" hemolysins which differ from the true immune hemolysins in that they are less specific and more susceptible to the destructive action of heat. ${ }^{2}$ The content is variable and so readily replaced and augmented after frequent bleedings or injections of substances such as colloidal manganese ${ }^{3}$ as to suggest an adsorption equilibrium in which the natural hemolysin free in the plasma may represent only a small percentage of a larger amount fixed in the tissues. When sheep erythrocytes are injected into a nonimmune rabbit, they adsorb this natural hemolysin so rapidly as to produce a negative phase during which its concentration in the plasma becomes too small to be detected. Adsorption persists until the erythrocyte stroma are saturated and no longer antigenic $^{4}$ nor capable of anchoring to the tissues or, in the excess of antigen, until the animal's supply of pre-antibody substance is exhausted. ${ }^{5}$ The unwieldy antigenantibody aggregates are then withdrawn from the plasma and adsorbed upon the surface of phagocytic cells. ${ }^{6}$

The consequent ingestion and disintegration of the antigen-pre-immune-substance aggregates by the phagocytes ${ }^{7}$ may partially destroy the binding capacity of the antigen in the aggregates, in much the same manner as is accomplished by ether extraction, ${ }^{8}$ leaving the pre-immune substance in the resultant disintegration product with a surplus of binding affinity, rendered specific by the residual inclusion of undigested antigen. ${ }^{9}$

See also Browning, C. H.: Immuno-chemical Studies. Wm. Wood \& Co., I925; Singer, E.: Ztschr.f. Immunitätsforsch.u.exper. Therap., 35, 191. 1922; Thiele, F. H., and Embleton, D.: ibid., 20, I. I9I4; Müller, P. Th.: Kraus-Levaditi's Handb. d. Tech. u. Method.d. Immunitätsforsch. (Ist suppl.), p. I. I9II; Hecht-Johansen, A.: Typhoid-Paratyphoid Group and about Avidity. Copenhagen: Levin \& Munksgaard, 1923; Locke, A., Main, E. R., and Hirsch, E. F.: loc. cit.

${ }^{2}$ Kolmer, J. A.: J. Immunol., 4, 403. I9I g.

3 Mackie, T. J.: J. Hyg., 24, I76. I925.

${ }_{4}^{4}$ Iijima, T.: J. Path. \& Bact., 28, 397. 1925.

5 The duration of the adsorption period is proportional to the size of the animal and to the amount of antigen injected, within certain restrictions (Armstrong, R. R.: Proc. Roy. Soc., B, 98, 525. 1925).

${ }^{6}$ Bloom, W.: Arch. Path. \& Lab. Med., 3, 608. 1927. During this negative or "inductive" phase, when much of the animal's available antibody and pre-antibody substance may be combined with antigen and adsorbed upon the phagocytic surfaces of the reticulo-endothelial system, particu. larly in the liver and spleen, extraction of these organs with water and ether, or water and glycerin, may yield a large amount of immune substance (cf. Cary, W. E.: J. Met. Research, 43, 399. 1922). But the antibodies thus extracted do not necessarily have their entire origin in these tissues. The source of the mobilized pre-antibody or natural immune substances is not yet known. Natural hemolysin is thought to be a secretion of the phagocytic cells by A. Carrel (J.Exper. Med., 36, 655. I922). F. Maltaner believes the hemolysis produced by normal serum to be associated with the mechanism of the blood-coagulation process (J. Immnnol., 6, 27r. 192r).

7 Carrel, A., and Ingebrigtsen, R.: J. Exper. MIed., I5, 287. I9I 2.

${ }^{8}$ See p. I050, note $6 . \quad 9$ Locke, A., Main, E. R, and Hirsch, E. F.: loc. cit. 
According to this mechanism of origin, immune substances are colloidal aggregates, of relatively large dimension, in which an elementary substance, derived from the host, is adsorbed upon nuclei of a substance derived from the specific antigen. Presumably, the amount of pre-antibody substance associated with one immune unit is approximately the same in any rabbit anti-sheep hemolysin preparation which has not been subjected to denaturing actions. But the amount of antigen substance cemented together by one unit of pre-antibody substance may be extremely variable, depending, roughly, among other things, upon the ratio of the total amount of antigen which has been injected (intravenously) during the immunization procedure to the total amount of immune substance which has been produced. The amount of protein associated with one unit of immune substance may be, therefore, a variable, determined by the conditions of origin.

The inclusion of specific antigen, in hemolysin, is of a lipoprotein nature related in structure to that of the whole antigen. In the case of antipneumococcus immune substance, the antigen inclusion may well be of a glycoprotein or polysaccharide nature, ${ }^{1}$ accounting for its extraordinary stability toward the denaturing effect of heat. ${ }^{2}$

The character of the pre-antibody substance is not known. Every one of the purified rabbit hemolysin preparations which the writers have tested has elicited anaphylactic sensitization toward rabbit serum when it has been injected into guinea pigs in sufficient quantity. But it cannot be concluded from these tests, in themselves, whether the apparent serum protein content of the preparations tested may be regarded as an integral part of the immune substances present or as an associated impurity. The writers are of the opinion that the pre-antibody substance is of the same character as the substances which are the precursors of serum globulin. This opinion is based (I) upon a physico-chemical analysis of a series of purified hemolysin preparations obtained from a corresponding series of sera of greatly divergent character, and (2) upon the fact that the most highly purified preparation obtained has elicited anaphylactic sensitization in a nitrogen concentration approaching the threshold concentration at which whole serum just elicits such sensitization.

The specific adsorption method of purification, with the special inclusion of the final ether extraction or other device for the destruction of the combining power of the antigen and the removal of the major part of the dispersed antigen substance, has not been applied with much success, as yet, to the purification of sera of the type of diphtheria antitoxin. The difficulty may lie, in this instance, with the raw material, which is a mixture of antitoxin and precipitin. It contains an enormous proportion of antigen substance, and flocculates with the toxin only in restricted proportions which burden the final fractionation with some 90 per cent of non-serum substances. The most nearly pure preparation of antitoxin which the authors have been able to obtain, at this writing, represents a purification of only ro,000 per cent, as compared with the maximum purification of $\mathrm{r} 50,000$ per cent obtained with hemolysin. ${ }^{3}$

The non-specific methods of purification, based upon iso-electric fractionations

${ }^{1}$ Heidelberger, M.: Physiol. Rev., 7, I07. 1927.

${ }^{2}$ Clowes, G. H. A.: Arch. Path. \& Lab. Med., 2, 456. 1926. $\quad{ }^{3}$ See p. I050, note 6. 
of the whole serum after diminution or augmentation of the electrolyte content, as used by the very early investigators of the isolation problem, have recently been revived for the examination of hemolysin and of antipneumococcus immune sera. ${ }^{\mathrm{I}}$ These methods are of considerable practical value for the cconomical concentration of immune sera intended for therapeutic use, but have not as yet in any way approached the purification obtained by the specific adsorption method.

The demonstration of the isolation and nature of the immune substances awaits the establishment of more perfect criteria of purity rather than the elaboration of more perfect methods of separation. The problem has a considerable practical importance in view of the strong possibility that immune substances can be preparech more economically and with greater therapeutic effectiveness by a synthetic compounding of antigen and pre-antibody substance, in vitro, once the nature and origin of the latter material is established.

r Locke, A., and Hirsch, E. F.: op. cit., 35, 519. 1924; Coulter, C. B.: J. General Physiol., ro, 545. 1927; Felton, L. D.: J. Infect. Dis., 37, 199, 309. 1925. 


\section{CHAPTER LXXIX}

\section{ABDERHALDEN'S DIALYSIS REACTION AND THEORY OF THE SO-CALLED "PROTECTIVE" FERMENTS}

\section{J. BRONFENBRENNER}

Laboratories of the Rockefeller Institute for Medical Research, New York City

The cells of the body are able to assimilate only the substances present in the blood as its normal constituents. The changes brought about in the composition of the blood by the introduction of foreign material lead to more or less pronounced disturbances in the processes of cellular nutrition. The experimental study of the phenomena of disposal of foreign material thus introduced revealed a number of facts pointing to the tendency on the part of the cells to elaborate a special mechanism by means of which they are able to protect themselves in a specific manner against the injurious effects of disturbances of their normal nutrition.

According to Abderhalden, this "protective" mechanism, at least in part, consists in the production of specific ferments, which directly attack the foreign material. These ferments are independent of antibodies which may be produced simultaneously. Indeed, the production of antibodies has been definitely demonstrated only in response to parenteral introduction of complete plant or animal proteins, or their complexes (and that usually on condition that these substances be foreign to the species of the recipient). ${ }^{x}$ The mobilization of protective ferments, on the contrary, has been stated to take place also in response to parenteral introduction of such comparatively simple nitrogenous substances as gelatin, peptone, or even of substances like cane sugar, entirely free from nitrogen. ${ }^{2}$ The group of substances which are capable of acting as antigen and cause the production of antibodies is thus included among the substances capable of stimulating the production of specific ferments, but is only a part of the latter. ${ }^{3}$ Moreover, Abderlalden has found that not only foreign substances, introduced experimentally, ${ }^{4}$ or those penetrating parenterally as a result of infection, but also autogenous tissue cells, or their products, not usually present in the blood (Blutfremd), and finding their way into the bloodstream under special physiological and pathological conditions, may cause the appearance in the blood of specific ferments. ${ }^{5}$ Consequently, by bringing such a blood serum in contact with a suitable

${ }^{1}$ The lens, casein, fibrin, thyroglobulin (Hektoen, L.: J. Immunol., I4, I. I927), and the placenta tissue (Bronfenbrenner, J.: J. Exper. Med., 21, 4So. 1915; Snith, G. H., and Musselman, L. K.: J. Immunol., 12, 7. 1926) represent authenticated exceptions to this rule. They all give rise to tissue-specific and not species-specific antibodies.

${ }^{2}$ Weinland, E.: Zischr. f. Biol., 47, 279. 1907; Abderhalden, E.: Abriehrfermente (4th ed.), p. 58. Berlin, 1914.

3 Weinland, E.: loc. cit.

${ }^{4}$ Abderhalden, E.: op. cil., pp. 77 and 78. Berlin, I9r4; Heilner, E., and Petri, T.: MIïnchen. med. Wchnschr., 60, 1530. 1913.

5 Abderhalden, E.: op. cit., pp. 92 and 93. Berlin, I9r4. 
tissue in vitro one can observe the appearance of products of the digestive activity of these ferments. This observation is the basis of the so-called "Abderhalden reaction."

\section{TIIE PRINCIPLE OF TIIE ABDERHALIEN REACTION}

The principle of the reaction consists in the detection of the products of hydrolysis when a suspected serum is brought in contact with a specially prepared specific substratum. If a given serum exhibits digestive activity in the presence of placenta protein, for instance, the reaction indicates pregnancy; if protein-split products appear in the presence of tumor cells, one concludes that the donor of the serum harbors a tumor, etc.

Although the reliability of the results obtained by means of the Abderhalden reaction has been often questioned, yet there exists in the literature a considerable number of reports indicating that by means of this test helpful information has been secured in a variety of conditions. Such conditions are, for instance, early pregnancy and incipient neoplasms, various disturbances in the activity of the glands of internal secretion, or of the nervous system, as well as syphilis, tuberculosis, and other infectious diseases.

\section{OPTICAL METIIOD}

As stated before, the test consists essentially in the detection of hydrolysis taking place in vitro during the incubation of the suspected serum with a suitable substratum. As many of the products of protein digestion are optically active, their appearance in the solution results in changes of the optical activity of the latter, and thus permits the detection of the digestive process. The test is made by mixing suitable proportions of the suspected serum with the peptone obtained from the corresponding tissue, and at intervals observing the mixture in a polariscope. If the serum contains ferments capable of attacking the given peptone, the plane of rotation undergoes gradual deviation from the initial position. Because of the difficulty of preparing the soluble substrata ${ }^{1}$ and because of the necessity of employing special apparatus, this procedure is not used extensively.

\section{DIALYSIS METHOD}

The procedure adopted by the majority of workers takes advantage of the diffusibility of the products of digestion. If a serum, suspected of containing protective ferments, is placed in a specially selected dialyzing shell, together with tissue (or other substratum) presumably involved, these ferments react with the substratum and the soluble products of the digestion diffuse out of the dialyzing shell into the outer fluid The presence of specific ferments in the serum is thus indicated by the detection of protein-split products in the dialysate by means of a color reaction (the ninhydrin test).

Reagents and apparatus: Serum.-Since the test depends on the appearance during the reaction of dialyzable, ninhydrin-reacting substances, it is essential that such substances should not be present in the serum before its incubation with substratum. In most cases the concentration of such dialyzable substances in the blood is too low to complicate the test, but under certain pathological conditions, in which excessive protein katabolism is involved (as in cases of abscess formation, resorption of exudates or transudates, hemorrhages,

I Abderhalden, E.: Handb. d. biol. Arbeitsmeth., 6, 223. I913. 
etc.), the blood may contain a considerable quantity of such substances. ${ }^{\mathrm{x}}$ On the other hand, the concentration of dialyzable protein constituents of the blood is physiologically increased during, and for some time after, the active digestion of food. To avoid this the blood should be collected as long as possible after the last meal. In pathological conditions in which the amount of dialyzable protein constituents is high and cannot be regulated at will, it is necessary to free the serum of such substances by means of a preliminary dialysis. ${ }^{2}$ Only after the serum has been thus prepared can the Abderhalden test be performed.

Substratum.-The ideal substratum would consist of a pure specific substance. In practice, however, this is possible only in exceptional cases, and mainly in experimental work. ${ }^{3}$ In diagnostic work the substrata are usually represented by tissues, and it is evident that to approach the highest standard the tissues would have to be freed as much as possible of blood, connective tissue, and of non-specific elements in general. ${ }^{4}$ Material thus obtained must be ground to a pulp, quickly coagulated, and extracted repeatedly with boiling water to remove all soluble substances reacting with ninhydrin.

When this has been accomplished, it remains to titrate the substratum, i.e., to ascertain what quantity of substratum is to be used in the test. If the bulk of the tissue representing substratum is composed of specific elements, a much smaller amount is needed for the reaction than is the case when an appreciable portion of the substratum consists of non-specific tissue constituents. An excess of substratum often reacts with normal as well as with specific serum, whereas an insufficient amount may fail to react at all. It is evident that each substratum must be standardized before it can be used in the test. The stock of standardized substratum must be sterilized by boiling and preserved aseptically under a layer of toluol, at low temperature.

Selection of dialyzing thimbles.-Equally important is the selection of suitable dialyzing thimbles. ${ }^{5}$ It is evident that the selective permeability of the thimbles can be relied upon only on the condition that the dialyzing membrane is intact through all the surface of its contact with the solution. If, for instance, there should be even the slightest puncture in a membrane, the unaltered protein will go through and render the reaction valueless. On the other hand, experience has shown that among the membranes used in dialysis there exist wide individual variations in permeability to dialyzable substances. ${ }^{6}$ Thus, for instance, of two apparently identical membranes, one may let through peptones with great ease, whereas the other may let them through very slightly, or sometimes not at all. As the whole outcome of the Abderhalden test depends on the uniform permeability of thimbles, as well as on freedom

r Abderhalden, E.: Mïnchen. med. Wchnschr., 6I, 40 I. I9I4; Abwehrfermente (4th ed.), p. 257. Berlin, I9r4; Lange, C.: Berl. klin. Wchnschr., 51, 785. 1914.

${ }^{2}$ Schlimpert, H., and Issel, E.: M ünchen. med. Wchnschr., 60, I758. I913.

3 Wells, H. G.: Chemical Aspects of Immunity. American Chemical Society, I925; Elsesser, O.: J. Infect. Dis., r9, 655. г916; Smith, G. H., and Cook, M. W.: ibid., r8, r4. 1916; Smith, G. H.: ibid., 16,3 I 3 and 3 I9. I9I5. According to Wells (op. cit.), "There undoubtedly does commonly occur a greater proteolysis with specific antigen than if some other protein is present" (p. 2 ro). However, according to this author, even with a specific antigen the reaction is less sensitive than is the anaphylactic reaction, for instance (p. 176 ).

${ }_{4}$ Lange, C., Biochem. Ztschr., 61, 193. I9r4; Frank, Rosenthal, and Biberstein: cited from Domarus, A., and Barsieck, W.: München. med. Wchnschr., 6r, I553. I914.

5 The dialyzing thimbles used in performing the Abderhalden reaction are especially made for this purpose by Schleicher and Schüll.

${ }^{6}$ Werner, P., and Winiwater, A.: Wien. klin. Wchnschr., 26, I84r. I9г3; Mosbacher, E., and Port, F.: Deutsche med. Wchnschr., 40, r410. I9I4; Abderhalden, E.: Abuehrfermente (4th ed.), pp. 208, 278. Berlin, I9I4. 
from mechanical defects, it is essential to ascertain these qualities in individual thimbles before each test. For this purpose each thimble (Schleicher and Schüll 579-A), whether it be new or used previously, is examined, on the one hand, for its efficiency in holding back the natural protein ( 20 per cent egg white or normal serum), and, on the other hand, for its permeability to diffusible protein derivatives (peptone). The whole procedure must be carried out under conditions of strict asepsis, as even a slight amount of bacterial decomposition taking place during the time allowed for the dialysis will vitiate the results. The thimbles are carefully washed and boiled before each test and again immediately after use. Sterile thimbles are stored in sterile distilled water under toluol, in a closed sterile container.

Ninhydrin test.-The course of the Abderhalden reaction is judged by the appearance of dialyzable protein-split products in the fluid surrounding the thimble. This is ascertained, as stated previously, by a ninhydrin test. The intensity of the color can be used to measure the extent of hydrolysis, provided the concentration of the reagent is strictly uniform in all tests of a given series. For the same reason it is essential to prevent unequal evaporation and consequent concentration of the dialysate, and therefore the contents of the dialyzing thimble, as well as the outer fluid, is overlaid with toluol during the incubation. Even traces of impurity, such as organic particles or free acid or alkali on the glassware, interfere with the test by giving atypical color reactions which may obscure the reading. ${ }^{x}$ Such atypical reactions may, in reality, be due to impurity alone, but at times they may mask a true color test which may be present at the same time. It is therefore necessary to repeat the test in all cases where the color reaction is atypical.

The test.-The necessity of ascertaining the condition of biological reagents makes it imperative to accompany each test, or series of tests, of unknown serum by proper controls. Previous to beginning the test proper, one must ascertain that the substratum has remained free from soluble protein derivatives which by themselves could react with ninhydrin.

A portion of substratum necessary for the performance of the whole test with its controls is removed from the container by means of sterile forceps, placed in a thoroughly clean and dry test tube, and boiled with four or five volumes of sterile distilled water. After about five minutes of boiling, during which care is taken not to burn the tissue, about $5 \mathrm{cc}$. of the water in which the tissue was boiled is filtered through a small piece of hardened filter paper into another thoroughly clean test tube. By adding I cc. of I per cent ninhydrin solution and boiling subsequently, one ascertains whether the tissue is satisfactory. Even the slightest trace of violet color appearing on cooling indicates that the tissue must be re-extracted.

If the tissue is found to be free from soluble ninhydrin-reacting substances, it is washed in sterile distilled water so as to remove any chloroform or toluol which may adhere, and dried between the folds of sterile filter paper. Such tissue (free from an excess of water) is ready for use. In spite of the fact that only substrata free from soluble protein are used for the test, it is still recommended that each be controlled again during the test as follows (Table I, Thimble 7):

Parallel with the test proper (Table I, Thimble 7) one tested thimble receives about 0.5 $\mathrm{gm}$. of the tested substratum. The thimble is held up with sterile forceps and $\mathrm{r} .5 \mathrm{cc}$. of physiological salt solution is introduced, sufficient to cover the substratum. Then the open end of the thimble is closed by means of long forceps, while it is washed with sterile distilled water ${ }^{2}$ to insure the absence of protein on the outside, and placed in an Erlenmeyer flask contain-

r Daetjen, H., and Fraenkel, E.: München. med. Wchnschr., 6r, 466. I914; Abderhalden, E.: Abwehrfermente (4th ed.), p. 289. Berlin, r914.

${ }^{2}$ All the water used must be freshly distilled and sterilized to avoid possible presence of bacterial protein in it. 
ing $20 \mathrm{cc}$. of sterile salt solution. The contents of the thimble, as well as the liquid outside, are covered with a layer of toluol, and the flask covered with a clean, dry watch-glass is placed in the incubator.

After sixteen to eighteen hours of incubation the flask with the thimble is removed from the incubator. The thimble is then carefully withdrawn from the surrounding fluid and placed in a fresh (clean, sterile, and dry) Erlenmeyer flask and the dialysate examined for protein by means of the ninhydrin test. Into a clean, dry test tube of Jena glass is placed $0.2 \mathrm{cc}$. of I per cent ninhydrin solution, Io cc. of dialysate is added, and the mixture is boiled for one minute in an oil bath. ${ }^{x}$ A procedure similar to this is followed in preparing all other dialyzing units.

The second control consists in ascertaining whether the unknown serum is free of dialyzable substances reacting with ninhydrin. This is done by placing $\mathrm{I} .5 \mathrm{cc}$. of the serum into a tested thimble and treating the latter exactly as was done in the case of the one just described. After the period of incubation the dialysate should give a negative ninhydrin test, if the serum is taken with all the precautions mentioned (Table I, Thimble 2).

TABLE I

\begin{tabular}{|c|c|c|c|c|c|}
\hline Thimbles & Character of the Serum Used & $\begin{array}{c}\text { Amount of } \\
\text { Serum }\end{array}$ & $\begin{array}{l}\text { Amount of } \\
\text { Substratum }\end{array}$ & $\begin{array}{c}\text { Amount of } \\
\mathrm{NaCl} \text { Solution }\end{array}$ & $\begin{array}{l}\text { Ninhydrin } \\
\text { Results as } \\
\text { Expected }\end{array}$ \\
\hline $\left.\begin{array}{l}\left.\begin{array}{l}1 \\
2\end{array}\right\} \ldots \ldots \\
3 \\
4\} \\
4 \\
5 \\
5\end{array}\right\} \ldots \ldots$ & $\begin{array}{ll}\text { Unknown } & \left\{\begin{array}{l}\text { Test } \\
\text { Control }\end{array}\right. \\
\text { Known specific }\left\{\begin{array}{l}\text { Test } \\
\text { Control }\end{array}\right. \\
\text { Known normal }\left\{\begin{array}{l}\text { Test } \\
\text { Control }\end{array}\right. \\
\text { No serum }\end{array}$ & $\begin{array}{c}\text { CC. } \\
\text { I. } 5 \\
\text { I. } 5 \\
\text { I. } 5 \\
\text { I. } 5 \\
\text { I. } 5 \\
\text { I. } 5 \\
\ldots \ldots \ldots\end{array}$ & 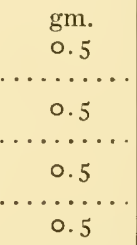 & \begin{tabular}{c}
\multicolumn{1}{c}{ cc. } \\
$\ldots \ldots \ldots$ \\
$\ldots \ldots \ldots$ \\
$\ldots \ldots \ldots$ \\
$\cdots \ldots \ldots$ \\
$\ldots \ldots \ldots$ \\
$\cdots \ldots \ldots$ \\
1.5
\end{tabular} & $\begin{array}{l}\quad \text { ? } \\
\text { Negative } \\
\text { Positive } \\
\text { Negative } \\
\text { Negative } \\
\text { Negative } \\
\text { Negative }\end{array}$ \\
\hline
\end{tabular}

The third control consists in ascertaining whether the substratum has retained its specific properties, namely, while not reacting with normal serum its combination with specific scrum will result in the appearance of dialyzable ninhydrin reacting substances. It is evident that such control consists of two tests-one in which the substratum is incubated with known normal serum (Table I, Thimble 5), and in the other with known specific serum (Table I, Thimble 3). To complete each of these control tests it is necessary to make certain that the known sera used are free from dialyzable split products of protein prior to their incubation with the substratum (Table I, Thimbles 4 and 6).

\section{INTERPRETATION OF RESULTS}

The results of the test are considered reliable only on the condition that all the control tests performed simultaneously have given satisfactory results. As for the outcome of the test of the unknown serum (Table I, Thimbles I and 2), there are, in general, four possibilities:

Case I (see Table II). - The test tube containing the dialysate from the thimble with serum alone, as well as that with serum and substratum, may give negative reactions with ninhydrin. If all the precautions of the test have been followed, this result indicates that there are no protein-split products in the dialysates tested, and this in turn means (according to Abderhalden's conception of the test) that the serum did not contain the ferments necessary to digest the substratum.

${ }^{x}$ Abderhalden, E.: Abwehrfermente (4th ed.), p. 225. Berlin, I9I4. 
Case II (Table II).-The test proper may give a positive ninhydrin reaction and the control with the serum alone negative. If all the controls accompanying the test (see Table I, Thimbles $3,4,5,6$, and 7 ) give expected results, the foregoing outcome of the cxamination of unknown serum means that it contained specific ferment which digested the given substratum.

Case III (Table II).-A third possible outcome of the test may be that the dialysate from the thimble containing the serum alone (control), as well as dialysate from the test proper, in which the thimble contained both the serum and the substratum, both give a positive ninhydrin test. In order to interpret this result correctly, it is necessary to establish the cause of the presence of ninhydrin-reacting substances in the control dialysate, for according to the cause the interpretation may vary.

Assuming that the technique was perfect, so that no protein could have been introduced in the dialysate by faulty manipulation (as, for example, soiling the outside of the thimble with serum while introducing it in the thimble, or contaminating the distilled water in the flask with saliva ${ }^{\mathrm{r}}$ while introducing it by means of a pipette, or depositing organic matter on the outer wall of the thimble by handling it with the fingers instead of with forceps, etc.), there are two other possible reasons for the presence of protein in this dialysate: either the thimble was permeable to unchanged serum or the serum contained dialyzable products before the test.

Although the thimble was supposed to have been tested and found to have been impermeable to natural protein, it is still possible that in further manipulation it might have been punctured. In order to establish this point, it suffices to test the dialysate for the presence of coagulable protein, and if it contains unchanged serum, the thimble must be liscarded and the whole test repeated with a new thimble. If, however, the dialysate does not contain coagulable protein, it means that the thimble was not punctured and that the substances giving the ninhydrin test are of the nature of dialyzable derivatives and must have been present in the serum before the test.

Having established that the ninhydrin test on control dialysate is due to the presence of dialyzable protein-split products in the serum, one must compare the intensity of this reaction with that obtained with dialysate from the thimble containing both serum and substratum. If the latter is decidedly more intense, the outcome of the reaction may still be considered satisfactory. If, however, the intensity of reaction in both cases is nearly equal, the test must be repeated.2

In cases, however, where it is impossible to obtain the specimen of blood free from dialyzable ninhydrin-reacting substances (as, for example, if the patient is having a high temperature, or in general in cases where there is marked active katabolism of protein), it is necessary to resort to a special method suggested by Schlimpert and Issel. ${ }^{3}$ The serum is dialyzed against running salt solution, until free from dialyzable ninhydrin-reacting substances, and only then is it examined according to the usual procedure.

Case IV (Table II).--It is possible, finally, that the test of unknown serum will show a positive ninhydrin reaction with a control dialysate (serum alone), and a negative reaction with dialysate of the test proper (serum and substratum). This result is to be interpreted as follows:

I According to Abderhalden, saliva even in dilution $1: 1,000,000$ in water gives a positive ninhydrin test.

${ }^{2}$ Abderhalden, E.: Abwehrfermente (4th ed.), p. 289. Berlin, 1914; Bisgaard, A., and Korsbjerg, A.: Deutsche med. Wchnschr., 40, 1367 . 19r4.

3 Porchownick, T.B.: Centrabl. f. Gynäk., 37, 1226. I913; Abderhalden, E., and Wildermuth, F.: Miunchen. med. Wchnschr., 61, 862. I9I4. 
It is possible that the failure to obtain the ninhydrin test in the dialysate from the test proper (serum and substratum) could be due to impermeability of the thimble to dialyzable products. This is ascertained by thoroughly washing the thimble and testing it subsequently for permeability to silk peptone. If the thimble is found to be impermeable, the whole test has to be repeated. If the thimble is found permeable, it is evident that digestion did not occur during the incubation period, because the serum did not contain specific ferments.

As to determining the reason for the positive ninhydrin test obtained with the control dialysate, one most proceed as in case III just described, and determine whether the thimble was letting through unchanged serum. If the coagulation test on the dialysate is positive, the thimble should be discarded. If the thimble is not punctured, the ninhydrin test must have been due to the presence in the serum of dialyzable ninhydrin-reacting substances. Such instances in which the serum contains diffusible ninhydrin-reacting substances, which disappear upon the addition of tissue, have been described by several authors and are attributed to the adsorption of such dialyzable substances by the substratum.

TABLE II

\begin{tabular}{|c|c|c|c|}
\hline Case & Character of the Dialysate & \multicolumn{2}{|c|}{ Color Reaction } \\
\hline \multirow{2}{*}{ I $\ldots \ldots \ldots \ldots \ldots \ldots$} & The dialysate from the serum alone (control) & Colorless & (neg.) \\
\hline & The dialysate from serum and substratum (test) & Colorless & (neg.) \\
\hline \multirow[t]{2}{*}{ II. $\ldots \ldots \ldots \ldots \ldots \ldots \ldots$} & The cialysate from serum alone (control) & Colorless & (neg.) \\
\hline & The cialysate from serum and substratum (test) & Blue & (pos.) \\
\hline \multirow[t]{2}{*}{ III $\ldots \ldots \ldots \ldots \ldots \ldots \ldots$} & The dialysate from serum alone (control) & Blue & (pos.) \\
\hline & The dialysate from serum and substratum (test) & Blue & (pos.) \\
\hline \multirow{2}{*}{ IV $\ldots \ldots \ldots \ldots \ldots \ldots \ldots$} & The dialysate from serum alone (control) & Blue & (pos.) \\
\hline & The dialysate from serum and substratum (test) & Colorless & (neg.) \\
\hline
\end{tabular}

\section{MODIFICATIONS OF THE ORIGINAL PROCEDURE}

Experience has shown that if all the necessary precautions and controls are carried out the results secured by the Abderhalden reaction may be of value. However, in spite of all the care taken, certain errors may still occur. ${ }^{\mathrm{x}}$ Thus, for example, one cannot be sure of the fact that thimbles were perfect when used for the test, even though each thimble was presumably tested beforehand, and one should not consider the test finished (or the results obtained final) until all the thimbles used in the test are retested and found satisfactory. For it may happen that boiling a thimble to render it sterile after a preliminary test of its permeability may render such a thimble impermeable to peptone. On the other hand, a thimble found to be perfect in the preliminary test may be rendered permeable to the whole serum through an unobserved mechanical injury, during the subsequent handling in setting up the main test. Another, frequent source of error has been found in the use of ninhydrin as an indicator. ${ }^{2}$ This color test is so easily rendered valueless by various extraneous influences that it was felt desirable to replace it by some other means of detection of cleavage of protein. It is for these reasons that several modifications of the original procedure have been suggested. Thus, Michaelis suggested a means of eliminating the thimbles. ${ }^{3} \mathrm{He}$ recommended placing the serum with its substratum in a glass tube and after a suita-

\footnotetext{
${ }^{r}$ Abderhalden, E.: Mïnchen. med. Wchnschr., 61, 233. I9I4.

${ }^{2}$ Bisgaard, A., and Korsbjerg, A.: loc. cit.

3 Michaelis, L.: Deutsche med. Wchnschr., 40, 429. I9I4.
} 
ble period of incubation and precipitation of the contents with ferric hydroxide, to test the filtrate with ninhydrin. Others have eliminated the ninhydrin test and substituted the colloidal gold test, ${ }^{\mathrm{r}}$ or the amino nitrogen determination in its place. ${ }^{2}$ To take advantage of the changes in physical properties of the solution resulting from the digestion of protein, measuring the changes in the refractive index, the rate of capillary rise, or the conductivity of the solution have been proposed in place of the ninhydrin test. ${ }^{3}$ Finally, it has been found that serum is rendered toxic for homologous animals after incubation with a suitable substratum, ${ }^{4}$ and thus another method of avoiding the use of either thimbles or ninhydrin was made available. ${ }^{5}$ All these methods give approximately similar results when compared with the original procedure. ${ }^{6}$

\section{VALUE OF THE ABDERHALDEN REACTION AS A DIAGNOSTIC TEST}

In considering the value of these modifications attempting to render the test more reliable, it must be remembered that they all are concerned with demonstration of the products of digestion and can be of service only in so far as they eliminate the errors connected with the use of thimbles or of ninhydrin, or both. They cannot be expected to increase the specificity of the reaction, since that portion of the procedure on which the specificity of the reaction rests-namely, the preliminary interaction between the serum and the substratum-is identical in all cases. Different samples of a given substratum differ among themselves in their specific value. Whereas one substratum will react with a given serum in any amount above $0.1 \mathrm{gm}$., another similar preparation may be found suitable only when used in the amounts of $0.3 \mathrm{gm}$. or over. On the other hand, different specific sera react differently with a given substratum. Thus, for instance, in the case of one specific serum, $0.15 \mathrm{gm}$. of the substratum may be sufficient to elicit the reaction, whereas in another similar case $0.4 \mathrm{gm}$. or more of the same substratum may be required. It is thus evident that the amount of substratum to be used in the test must be determined in each instance by extensive preliminary inquiry. This difficulty is further increased by a loss of specificity as more substratum is used, since an excess of substratum tends to react also with normal serum. ${ }^{7}$

The difficulty in establishing the proper dosage of the substratum undoubtedly

${ }^{2}$ Matzkiewitsch, J.: ibid., p. I22 I. I9I4.

2 Abderhalden, E.: Fermentforschung, 6, 230. I922; Abderhalden, E., and Fodor, A.: München. mcd. Wchnschr., 61, 765. I914; Van Slyke, D., Vinograd-Villchur, M., and Losee, J., J. Biol.Chem., 23, 377. 1915 .

3 Abderhalden, E.: Fermentforschung, 6, I 19. 1922; Sellheim, H.: Klin. Wchnschr., 4, 247 and 299. 1925; Blunck, G.: Mïnchen. med. Wchnschr., 69, 1005. 1922.

${ }_{4}$ Bronfenbrenner, J.: Penn. State M. J., I8, 20. Oct., I9I4; Proc. Soc. Exper. Biol. Go Med., I I, 90. 1914 .

${ }^{5}$ Smith, G. H., and Cook, M. IV.: loc. cit.; Smith, G. H.: loc. cit.; Bronfenbrenner, J., Freeland, J. R., and Schlesinger, M. J.: Am.J. Obst. \&o Dis. of Wom. \& Child., 72, 599. I9I5; Bronfenbrenner, J., Mitchell, W. J., and Titus, P.: Biochem. Bull., 4, 86. I9I4; Kolmer, J. A., and Williams, P. F., Am. J. Obst. \& Dis. of Wom. \& Child., 71, 899. I9I5.

${ }^{6}$ Abderhalden, E.: Fermentforschung, 7, 54. I923.

7 Flatow, L.: München. med. Wchnschr., 6r, 468, 608, 1 168. 19r4; Herzfeld, E.: Biochem. Zischr., 64, 103. 1914; Kjaergaard, S.: Ztschr. f. Immunitätsforsch. u. exper. Therap., Orig., 22, 31. 1914; Plaut, F.: Mïnchen. med. Wchnschr., 61, 238. 1914. 
represents the weakest part of the procedure. This point has not been clearly emphasized in the literature, and yet, undoubtedly, it has been the main cause of nonspecific reactions. Until this difficulty has been removed, each test must be preceded by careful titration of substratum. Thus the Abderhalden reaction cannot be adopted as a routine diagnostic procedure, and should be limited to special fields of research, where it will often give valuable information.

\section{VALIDITY OF THE HYPOTHESIS OF "ABWEHRFERMENTE"}

Quite apart from the difficulties arising from the necessity of establishing quantitative relationships between the amounts of substratum and of specific serum in each case, the fact that it is possible in any instance to bring about digestion in the presence of normal serum raises a question as to the validity of the hypothesis of Abderhalden concerning the existence of specific protective ferments (Abwehrfermente). If, according to this hypothesis, the appearance of ninhydrin-reacting substances in the dialysate in the case of pregnancy, for instance, is due to the digestion of the placenta substratum in vitro by the specific ferments present in the sera of pregnant individuals only, how then is it ever possible for a male serum to cause similar changes under any circumstances? Moreover, since normal serum thus may apparently exhibit digestive power in one instance in the presence of an excess of placenta, in another in the presence of carcinomatous tissue, and in a third in the presence of tuberculous lung, etc., as repeatedly reported in the literature, it is necessary to admit either the presence in all of the sera of all sorts of specific ferments, or the oppositethat the apparent digestion in the presence of these various substrata may take place without the co-operation of specific ferments. Besides, the specificity of the ferments responsible for the digestion of the substratum may be questioned also on the basis of the experiments of De Waele, ${ }^{\mathbf{I}}$ who succeeded in demonstrating by means of the Abderhalden reaction the presence in the blood of the ferments a few minutes after the parenteral introduction of the foreign protein into experimental animals, an interval hardly sufficient for the production of new specific ferments. These results corroborate the previous findings of Heilner and Petri, ${ }^{2}$ who concluded from the rapidity with which the ferments appeared in the blood after parenteral introduction of foreign protein that this must be a case not of new formation of such ferments, but of activation (Arteinstellung) of some pre-existing ferment. This is also the conclusion reached by a number of investigators who succeeded in demonstrating the presence of proteolytic ferments in normal sera by submitting the latter to adsorption by various substances, such as kaolin, starch, agar, etc. ${ }^{3}$ However, these findings help to explain only the mechanism by which an excess of substratum might cause activation of the ferments present in a normal serum, ${ }^{4}$ and throw no light on the apparent tendency toward specificity in the Abderhalden reaction.

I de Waele, H.: Ztschr.f. Immunitätsforsch. u. exper. Therap., Orig., 22, 170. 1914.

${ }^{2}$ Heilner, E., and Petri, T.: Mïnchen. med. Wchnschr., 60, 530. I9r3.

3 Flatow, L.: loc. cit.; Herzfeld, E.: Toc. cit.; Kjaergaard, S.: loc. cit.; Peiper, A.: Deutsche mel. Wchnschr., 40, 1467. 1914.

4 Kjaergaard, S.: loc. cit.; Peiper, A.: loc. cit. 
The explanation of this tendency became apparent when the mechanism underlying the Abderhalden reaction was studied by the methods analogous to those used in the study of immunity reactions. This approach was suggested by the observation that a serum giving a characteristic Abderhalden reaction may be rendered inactive upon heating for half an hour at $56^{\circ}-60^{\circ} \mathrm{C}$, and that upon the addition of a suitalle amount of fresh normal serum (complement ?) it regains its activity while retaining its original specificity. ${ }^{\mathrm{I}}$ The experiments of the writer have demonstrated that the elements of the serum which are responsible for its specificity may be removed by adsorption with a corresponding substratum in the manner analogous to that of adsorption of antibodies by the corresponding antigen. ${ }^{2}$ Moreover, it became evident that when the serum is thus exhausted of its specific elements by adsorption with the substratum, the ferments present in the serum become active and digest the protein of the serum itself. ${ }^{3}$ The latter phase of the phenomenon has been questioned by Abderhalden, who suggests that the products of digestion thus appearing in the serum, after its separation from the substratum, originate not in the serum itself, but in the traces of substratum which fail to be completely removed from the serum in centrifugating. ${ }^{4}$ However, the writer has shown that the products of digestion obtained under these circumstances exhibit toxicity for experimental animals entirely independent of the nature of the substratum used, and depending only on the nature of the serum $^{5}$ - thus indicating that the latter undoubtedly is the source of the toxic digestion products. Similar conclusions have been reached by Jobling, Petersen, and Eggstein ${ }^{6}$ who found that the substratum, after contact with the serum, becomes more resistant to the tryptic digestion as a result of having adsorbed the anti-ferment of the serum.

IStephan, R.: Mïnchen. med. Wchnschr., 6r, 801. I914; Hauptmann, A.: ibid., p. 1167. I914; Bettencourt, N., and Menezes, S.: Compt. rend. Soc. de biol., 77, 162. I914; Bronfenbrenner, J.: Proc. Soc. Exper. Biol. \& Med., 12, 3. r914; Abderhalden, E., and Grigorescu, L.: Med. Klin., ro, 728. I9I4; Abderhalden, E.: Abwehrfermente (4th ed.), p. r54. Berlin, I9I4.

${ }^{2}$ Bronfenbrenner, J.: Proc. Soc. Exper. Biol. \& Med., r 2, 3. I9r4.

3 de Waele, H.: loc. cit.; Bronfenbrenner, J.: Proc. Soc. Exper. Biol. E Med., 12, 7. 1914; J. Exper. Med., 21, 22 I. I915.

4 Abderhalden, E.: Fermentforschung, 5, I30. 1921.

5 Bronfenbrenner, J.: Penn. State M.J., 18, 2. Oct., I9 I4; Bronfenbrenner, J., Mitchell, IV. J., and Titus, P.: loc. cit.; Bronfenbrenner, J.: J. Exper. Med., 21, 221. I9I 5.

${ }^{6}$ Jobling, J., Petersen, W., and Eggstein, A.: J. Exper. Med., 22, 40I. I9I 5. 


\section{CHAPTER LXXX}

\section{VENOMS AND ANTIVENINS \\ AFRANIO DO AMARAL \\ Antivenin Institute of America, Glenolden, $\mathrm{Pa}$.}

Venomousness is not peculiar to the ophidians alone. Throughout the animal kingdom there are many species of insects, scorpions, spiders, and other invertebrates and also batrachians, lizards (genus Heloderma), fishes, and even mammals that have venomous qualities. Antitoxic sera or antivenins have likewise been prepared against their venoms. However, snake venoms are those that have been most thoroughly studied, and the corresponding antivenins have been most successfully applied in practice.

\section{POISONOUS SNAKES AND SNAKE VENOMS}

Both from the anatomical and physiological standpoints, practically all snakes should be considered poisonous. As a rule, they have a gland corresponding to the parotid of mammals, and this yields a toxic secretion. From a practical standpoint, however, only the proteroglyphous and solenoglyphous forms must be taken into consideration, because they are the only ones that secrete an amount of venom large enough to cause severe lesions or that inoculate their venom deeply into the tissues. It was, indeed, simply the development of a well-differentiated inoculating apparatus situated on the anterior part of the upper jaw which made biologists first distinguish them from the aglyphous and opisthoglyphous snakes and consider the first two groups as poisonous and the latter as non-poisonous.

Of the proteroglyphous series I recognize two valid and well differentiated families, the Disteiridae and the Elapidae. The former are marine and live in the Pacific and Indian oceans; only one species lives in fresh water. The Elapidae, also proteroglyphous, are all terrestrial and in general are found all over the world except the northern portions of the Holarctic region. They represent by far the greater part of the ophiological fauna of Australia. To this family belong the dreaded "cobra" of Asia and Malay, the "coral snakes" of the Americas, and the "tiger snake" of Australia. The solenoglyphous forms comprise the Viperidae and the Crotalidae. The Viperidae, which are peculiar to the Palaearctic, Ethiopian, and Oriental regions, are represented by the vipers of Europe (genus Vipera), Asia (genus Daboia), and Africa (genera Cobra, Bitis, etc.).

The Crotalidae, also solenoglyphous and distinguished from the preceding family by having a facial pit, live in the eastern Palaearctic but more especially in the Nearctic and Neotropical regions, where they make up the majority of the poisonous species. Their various genera are the following: Trimeresurus Lacépède, which is peculiar to Asia; Bothrops Wagler, which is found chiefly in Central and South America, in the West Indies, and tropical Mexico, its most important representative 
being the "fer-de-lance"; Agkistrodon Beauvois, which is the most widespread of all the Crotalidae, for it occurs in the Palaearctic, Indian, Nearctic, and Neotropical regions, being represented by the "copperhead" and the "cotton-mouth moccasin" in the United States; Sistrurus Garman (main form-the "massasauga"), which is peculiar to the eastern part of the Nearctic and to the extreme northern part of the Neotropical region, and is probably in process of disappearance; Crotalus Linné, which is common to both the Nearctic and the Neotropical regions, being represented by only one species in the American tropics and by about a dozen or so in the United States; and Lachesis Daudin, which, in my opinion, shows the highest degree of differentiation among snakes and occurs in the Neotropical region, its only species, the "bushmaster," L. muta (L), being confined to Central and South America.

\section{POISON APPARATUS}

The venom apparatus consists of a gland, a duct, and one or more fangs located on each side of the head. The venom is the secretion of the external supralabial gland of the ophidians, which is found below and back of the orbit. It receives its blood supply from the "arteria alveolaris inferior ramus glandulae maxillae superioris posterior" of Schlem and is innervated by the "nervus labialis superior" which is one of the terminal branches of the trigeminal.

Histologically, the gland is serous, although its secretion contains also a great deal of mucus. This mucus is excreted both by cells found in the walls of the duct close to its termination, and by a great many alveoli which surround the duct. The size of the gland is usually in proportion to the size of the snake. However, it is relatively larger in the solenoglyphous type than in the proteroglyphous. Among the solenoglyphous, the Brazilian "jararacussú," Bothrops jararacussu Lacerda, the Florida "diamond-back rattler," Crotalus adamanteus Beauvois, and the South American B. alternata Dum \& Bibr., have the largest glands, while the "bushmaster," Lachesis muta (L.), has the longest fangs. According to Boulenger, ${ }^{\mathbf{I}}$ among the proteroglyphous, Doliophis bivirgata Boie has the largest glands, which "instead of being confined to the temporal region, extend along each side of the body for about one-third of its length, gradually thickening and terminating in front of the heart with club-shaped ends." Among solenoglyphous types the species of Causus show a similarly aberrant glandular development. The longest fangs among proteroglyphous snakes are found in Dendraspis antinorii Peters and other African species.

The gland is surrounded by a fibrous capsule which represents a prolongation of the zygomatic ligament and divides it into various segments or lobes receiving fibers from both the anterior and the medium temporal muscles (S. Weir Mitchell).

The duct of solenoglyphous snakes, emerging from the gland, tends upward, then forward, and, finally, turning downward, opens abruptly through a small papilla on the anterior wall of the mucous membrane which involves the base of the fangs. Sometimes the duct, at its end, shows either a few muscular fibers which are connected with the internal pterygoid muscle, as is the case with all the species of rattlesnakes (Crotalus), or a real smooth muscle, as in the sea snakes (Disteiridae). These muscles, which serve as a sphincter to the duct, also insure perfect connection between it and the base of the fang.

\section{VENOM INOCULATION (THE ACT OF BITING)}

Fangs and jawbones are quite movable in the solenoglyphous and occasionally in some aglyphou,, viz., in the genus Ophis (=Xenodon). Apparently, snakes have perfect control

' Boulenger, G. A.: Cat. Sn., Brit. Mis., 3, 400. I896. 
over their fangs, raising and lowering them at will, as may be observed when snakes yawn and stretch their fangs. Introduction of venom is accomplished by the combined action of several muscles which cause the opening of the mouth, the erection of the fangs, and the compression of the glands, thereby forcing the venom through the duct and fangs at the same instant that the snake strikes its victim. At the end of the stroke the fangs are withdrawn from the site of the bite; the mouth closes as the fangs fold back; mechanical compression constricts the duct, and pressure upon the gland is relieved by the relaxation of the involving muscles.

The hollow or grooved fang is firmly ankylosed to the maxillary bone, which is located anteriorly on each side of the upper jaw. There is a pair of functional fangs which are supplemented by one or many more pairs of smaller ones which are not attached to the bone but lie imbedded in the buccal tissues. When, from any cause, one of the larger fangs is broken off, the reserve fang nearest to it moves into its place, adheres to the maxillary bone, and finally becomes the functiona! poisoning fang.

\section{VENOM AMOUNTS}

The quantity of the poison secreted varies according to the species and, as a rule, is proportional to the size of the snake, although there are exceptions to this rule: The Disteiridae secrete very little poison in relation to their size; the Neotropic rattler secretes less and less poison as it grows older, the individuals of medium age yielding the largest amounts. Captivity also exerts a noticeable influence on the secreting activity of the poison glands, retarding or even inhibiting it. In general, however, it may be said that the following is the approximate amount in milligrams of dried poison produced by the medium-size examples of a few species at a bite or obtained at a single extraction.

a) North American species: $\mathrm{Mg}$.

Copperhead (A. mokasen) ............... 45-6o

Water moccasin (A. piscivorus) ............. 90-150

Timber rattler $(C$. horridus $) \ldots \ldots \ldots \ldots \ldots \ldots \ldots$ 60 90

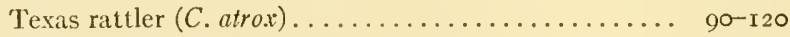

Pacific rattler $(C$. oreganus $) \ldots \ldots \ldots \ldots \ldots \ldots \ldots \ldots \ldots$ 60- 75

Bleached rattler $(C$. mitchellii $) \ldots \ldots \ldots \ldots \ldots \ldots \ldots \ldots$ 70-120

Red rattler $(C$. exsul $) \ldots \ldots \ldots \ldots \ldots \ldots \ldots \ldots \ldots$ 60-120

Florida rattler $(C$. adamante:ts $) \ldots \ldots \ldots \ldots \ldots \ldots .240-350$

b) South and Central American specis:

Rattlesnake $(C$.terrificts $) \ldots \ldots \ldots \ldots \ldots \ldots \ldots \ldots \ldots$. $30-60$

Fer-de-lance $(B$. atrox $) .. \ldots \ldots \ldots \ldots \ldots \ldots \ldots$ 100-180

Jararacussú $($ B. jararacussii $) \ldots \ldots \ldots \ldots \ldots \ldots \ldots \ldots . . \ldots 200-330$

Bushmaster (L. mutu) . . . . . . . . . . . . . . 300-500

c) Australian species:

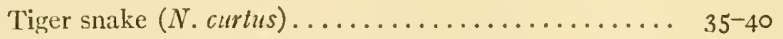

d) Indian species:

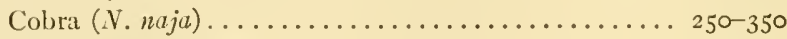

Daboia $(D$. russellii) .................... 200-300

\section{VENOM CHARACTERISTICS}

a) Specificity.-There seems to be some adaptation of the toxicity of venoms to the species of animals on which snakes feed. Venom is one of the snake's digestive juices, and I believe that some differentiation in their action on the tissues of the 
particular animal which the snake is accustomed to capture has taken place during the phylogenetic evolution of the latter. Venoms are also a complex of many toxic and antigenic principles which have especial tropisms for the different animal tissues. It is not unlikely that these principles which probably have gradually appeared in the venoms are specific weapons to which snakes have had recourse in their struggle for life. This remarkable character of the poison is found especially in such snakes as those which have recourse to only one kind of prey. Among the proteroglyphous, Pelamydrus platurus (L.), which feeds on fish, has a venom the M.L.D.of which is as low as $0.00025 \mathrm{gm}$. for the mudfish, according to Rogers, ${ }^{\mathrm{I}}$ and the M.L.D. of the venom of Enhydrina valakadien Boie, another piscivorous sea snake, is $0.0005 \mathrm{gm}$. for the same fish. The Brazilian "coral-snakes" (especially Micrurus frontalis and M. corallinus), which are all ophiophagous, can kill almost instantaneously any snake, either small or large, poisonous or non-poisonous, such as Pseudoboa cloelia (Daudin), Liophis miliarius (L.), and Crotalus terrificus. The most striking example of all is that of Bothrops insularis Amaral (solenoglyphous), ${ }^{2}$ which has become a bird-eater through force of environmental conditions; the M.L.D. of its venom for a pigeon ( $300 \mathrm{gm}$.), by intravenous injection, is $0.000004 \mathrm{gm}$., while it is 0.000050 for a rabbit ( $\mathrm{I} \mathrm{kg}$.) also by intravenous injection.

b) Nature.-Venoms are commonly classified with the proteins on account of their most important reactions and physico-chemical affinities. They lose their toxicity when treated in vitro by such reagents as silver nitrate, sodium and potassium hydroxide, gold chloride, potassium permanganate, etc. They also become atoxic when treated by heat, but at widely different temperatures which may vary from $65^{\circ} \mathrm{C}$. in the case of B. alternata to $120^{\circ} \mathrm{C}$. in the case of Lachesis muta, according to Brazil. ${ }^{3}$ Pancreatic digestion affects the activity of all venoms, making them atoxic; gastric digestion, however, affects only a few, such as those of Crotalus, but not, for instance, those of Vipera, Naja, and Pseudechis. Acids, even in concentrated solution, have different effects, usually destroying only a few of the toxic and antigenic principles of the venom. Glycerin is entirely harmless to venoms, thus serving as their best means of preservation. Some venoms, especially those of Crotalus, change into toxoids by losing their toxophorous group, and perhaps also some of their antigenic principles, when treated by certain chemicals such as calcium hypochlorite, gold chloride, and iodine trichloride. Cold and light have but little effect on the toxicity of dried venoms; electricity and radium, however, gradually destroy them. The venom of $N a j a$ is entirely filterable through porcelain candles; that of Psendechis porphyriaca consists of two substances, one filterable and neurocytolytic and another non-filterable and hemocytolytic. Dialysis has a similar effect. The toxic principles of Vipera aspis (L.) venom are undialyzable; its antigenic principles are dialyzable. Crotalus adamanteus venom loses its hemagglutinin properties but remains hemocytolytic after dialysis. As a rule, the higher the percentage of albuminous material contained in the venom, the more it becomes atoxic by dialysis. Proteroglyphous venoms, which are

${ }^{I}$ Rogers, L.: Proc. Roy. Soc., 76, 48I ff. London, 1902.

2. do Amaral, A.: Anex. Mem. Inst. Butanten, r, 44 and 87. I92r.

3 Brazil, V.: La Défense c. l'ophidisme, p. г30. 1914. 
known to have a low protein content, are practically all dialyzable. However, all the above-mentioned variations are due to the differences existing in the composition of venoms. Each venom is a mixture of special chemical substances, showing peculiar physico-chemical affinities and having different antigenic properties.

c) Composition.-Very little is known concerning the chemical composition and the real nature of the toxic principles of the venom. Faust ${ }^{\mathrm{x}}$ is the only author who claims to have isolated from the venom of Naja naja a non-albuminous substance called "ophiotoxin" which he considered its active principle; and from the venom of Crotalus adamanteus another substance which he called "crotalotoxin." His studies, however, must be repeated by other biochemists before receiving general acceptance. It is still to be proved whether both ophiotoxin and crotalotoxin have antigenic properties and behave like the "antitoxinogens" recently referred to by Zinsser. ${ }^{2}$ Indeed, the main feature of animal substances, such as bufotalin (toad venom), arachnolysin (spider venom), vegetable substances such as abrin, ricin, and crotin, the bacterial leukocidins and hemotoxins, bacterial substances such as diphtheria and tetanus toxin and the toxin of a few other micro-organisms, is their property of eliciting the production of antitoxins in the animal body. Nevertheless, there is some difference between snake venoms and bacterial exotoxins. As a rule, the former do not show any incubation period, that is to say, there usually is no delay in the occurrence of symptoms after their administration as is the case with the latter (exotoxins). But each venom proves to have properties of its own, that of $C$. terrificus, for instance, acting slowly upon the organism, producing perceptible symptoms only after an incubation of a few hours, and never causing even local pain.

In general, crude venom consists of: (a) proteins (albumin, globulin); $(b)$ proteoses and peptones; $(c)$ mucin and mucin-like substances; $(d)$ ferments; $(e)$ fat; $(f)$ detritus (cells, etc.) and micro-organisms-which, as a rule, are not found in properly purified venoms; $(g)$ salts (calcium chloride and calcium, magnesium, and ammonium phosphate).

d) Antigenic principles.-The following principles have thus far been recognized in the various snake venoms:

Proteolysin and histocytolysins are phlogogenous and so cause local symptoms such as pain, swelling, tissue destruction, and necrosis, while all the other principles exert a general or systemic action on the organism. Proteolysin has a very marked potency, either in vitro or in vivo, and is found especially in the venom of the solenoglyphous families (Viperidae and Crotalidae).

Cardiotoxin is responsible for the low blood pressure and heart failure which follow the bite of a few species, namely, Naja naja, Micrurus corallinus, M. frontalis, Daboia russellii, Agkistrodon mokasen and Crotalus atrox.

Hemocytolysins are divided into erythro- and leukocytolysins, first, because some venoms are capable of dissolving white cells after absorption by red cells and vice versa; second, because some venoms, e.g., that of $M$. corallinus, have erythrocytolysin but no leukocytolysin.

Hemorrhagin acts on the endothelial cells of the capillaries, thereby causing

${ }^{1}$ Faust, E.: Arch.f. exp. Palh. u. Pharmakol., 56, 236. 1907; 64, 244. I91r.

${ }^{2}$ Zinsser, H.: Infection and Resistance, P. 4I. I923. 
hemorrhages into the internal organs or through the mucous membranes and skin. This is found in the poisoning of the bothropic type. Histocytolysins in general are prevalent among the solenoglyphous.

Neurocytolysins are found in large quantity in all the proteroglyphous (Disteiridae and Elapidae) and occasionally in a few solenoglyphous (Crotulus terrificus, Agkistrodon spp.). There are three kinds of neurocytolysins: (a) one affecting the bulbar respiratory and circulatory centers, the cranial nerves, and the spinal cord; $(b)$ another the sympathetic nerves of the salivary, lacrimal, and intestinal glands and of the nasal mucosa; $(c)$ and another the neuro-motor apparatus of the diaphragm and the skeletal muscles. It is probable that further work will discover or distinguish other neurolysins.

Chromatolysin acts on the chromatin of the nerve cell nuclei, and its slow action seems to be responsible both for the relapse or recurrence of symptoms and delayed death, which may be observed in the poisoning by Bungarus candidus, Crotalus terrificus, etc.

Antibactericidin prevents the tropism of the leukocytes and other cells having bacteriolytic power, and thus it helps the development of secondary infections. This antibactericidal action of the venoms, discovered by Welch and Ewing, was ${ }^{1}$ interpreted by Flexner and Noguchi ${ }^{2}$ as a fixation or inactivation of the bacteriolytic complement, this fixation being brought about either by the venom amboceptor, according to Noc, ${ }^{3}$ or by the venom proteolytic ferment, according to Noguchi, ${ }^{4}$

Precipitinogen is found in large quantity especially in the venom of Bothrops spp. It should be noted that Lamb, ${ }^{5}$ and Calmette and Massol, ${ }^{6}$ having found that venoms could bring about precipitins in the sera of immune animals, developed a method for antivenin titration based on the precipitin test.

Hemagglutinin is sometimes found in venoms as, for instance, in those of North American species of Crotalus. It can be distinguished from the hemocytolysins in that it is destroyed by heating at $75^{\circ} \mathrm{C}$.

Thrombinogen and anticytozyme, respectively, cause blood coagulation and anticoagulation in the animals bitten by snakes. As a rule, all the proteroglyphous venoms are anticytozymic (anti-coagulant) and the solenoglyphous are thrombinogenic (coagulant). Among the latter, however, those of Daboia russellii, Trimeresurus flavoriridis, Crotalus atrox, and C. adamanteus are anti-coagulant. Houssay and Sordelli, ${ }^{7}$ separately or with Negrette, ${ }^{8}$ who carried out an exhaustive study regarding this point, found that any venom can destroy directly the cytozyme and so inhibit blood coagulation unless it possesses thrombinogenic activity.

Lecithinase is one of the most important antigenic substances because it may be

${ }^{1}$ Welch, W., and Ewing, C.: Tr. Ist Pan-Am. Cong., 1, 354. 1893.

${ }^{2}$ Flexner, S., and Noguchi, H.: J. Exper. Med., 6, 277 . 1902.

3 Noc, F.: Ann. de l'Inst. Pasteur, 19, 209. 1905.

${ }_{4}$ Noguchi, H.: Snake Venoms, etc., p. 2 I8. 1909.

s Lamb, G.: Lancet, Ir, 43I. I902.

${ }^{6}$ Calmette, A., and Massol, L.: Ann. de l'Tnst. Pasteur, 23, I55. I909.

7 Houssay, B., and Sordelli, A.: Arch. Inst. de physiol., I5, 378. I920.

${ }^{8}$ Houssay, B., Sordelli, A., and Negrette, J.: Rev. Inst. Bact. B.A., r, 52 pp. r918. 
responsible for the hemolysis which takes place when a venom is brought into contact with red cells. In 1902, when Kyes, ${ }^{x}$ having taken up the study of the phenomena of venom hemolysis, produced a combination of lecithin and Naja naja venom which he called "cobra lecithid," immunologists thought that his experiments might throw some light upon the chemical constitution of complements. Five years later von Dungern and Coca ${ }^{2}$ attempted to demonstrate that the hemolysis is brought about by a lipoid-splitting ferment contained in "cobra" venom, a ferment which acts on the lecithin, thereby producing a substance which dissolves the red cells. This ferment is the "lecithinase." Delezenne, Ledebt, and Fourneau ${ }^{3}$ were the first who thought that the hemolysis observed by Kyes is the result of the action of a ferment on the serum "phosphatids" from which a hemolytic substance, called "lysocithin" (anhydride of the cholin-palmitophosphoglyceric ether), is formed. Lecithinase may also act directly on a few kinds of red cells, namely, those of the guinea pig, dog, and man, cells which are likely to be as rich in the lysocithinog en phosphatid as the serum itself. Locithinase is a characteristic antigenic substance, for the serum obtained from horses immunized with the whole venom acquires anti-lecithinasic property and so prevents hemolysis. Nevertheless, it remains to be proved whether lecithinase and erythrocytolysin are or are not the same substance.

Snake venoms also have the property of decreasing the globular resistance. This property is entirely independent of their hemolytic activity, as shown recently by Aquino. 4

Lipolysin is another ferment present in a few venoms, namely, those of the different species of Micrurus ("coral snakes"). Besides acting on fatty substances, it is antigenic. Antivenins, when properly prepared, are antilipolytic.

Chymosin is the venom ferment which causes milk coagulation. It is present in considerable quantity in the venom of Agkistrodon mokasen, A. piscivorus, Bothrops atrox, B. neuwiedii, B. jararacussu, and B. alternata.

\section{ANTIVENINS}

Antivenins are the only specific means of neutralizing venoms and arresting their harmful action on the tissues.

Calmette, ${ }^{5}$ to whom therapeutics is indebted for work on the preparation of antivenins, claimed that there is no specificity between antivenins and venoms. He held that any antineurolytic serum could be applied to accidents issuing from any kind of neurolytic snake venom. His theory, however, has proved to be incorrect through studies carried out by Lamb, ${ }^{6}$ Tidswell, ${ }^{7}$ Ishizaka, ${ }^{8}$ Noguchi, ${ }^{9}$ Brazil, ${ }^{\text {Io }}$ Arthus, ${ }^{\text {In }}$

${ }^{\prime}$ Kyes, P.: Berl. klin. Wchnschr., 39, 886 and 918. I902.

${ }^{2}$ von Dungern, E., and Coca, A.: Biochcm. Ztschr., 12, 407. 1908.

3 Delezenne, C., and Ledebt, A.: Compt.rond. Acad.de sc., 155, Iror. r912; Delezenne, C., and Fourneau, E.: Bull. Soc. chim. de France, 15, 421. 1914.

4 Aquino, L.: Rev. Asoc. mel. argent., 22, г I24. 1922.

5 Calmette, A.: Les Venins, etc., p. 263. Paris, 1907.

${ }^{6}$ Lamb, G.: Mem. Off. Med. San. Dept. Gov. India, p. г6. I905.

Tidswell, F.: Reseurch on A ustralian Venoms, p. 35. I906.

${ }^{8}$ Ishizaka, T.: Ztschr.f. exper. Path. u. Therap., 4, 88. I907.

${ }_{9}$ Noguchi, H.: op. cil., pp. 233 ff. r9o9. $\quad$ ro Brazil, V.: Rev. med. S. Paulo, p. 15, Igog.

II Arthus, M.: Compl.rend. Acad. de sc., 153, г504. г9гі. 
Gomes, ${ }^{1}$ Houssay and Negrette, ${ }^{2}$ and myself. ${ }^{3}$ At present we know that for a serum to be really efficacious it must be produced by an animal immunized against the venom of the particular species of snake that has caused the accident or at least that of a closely related species. This means that the principle of the specificity of antitoxins applies to antivenins.

Specificity is due to the fact that we may find a similar antigenic composition only among venoms of snakes closely related to each other from a zoölogical standpoint. Following this principle a number of laboratories have been engaged in the preparation of antivenins against the snakes prevalent in their countries or elsewhere, but unfortunately, not all of them have closely followed the improvements introduced into the technique of serum therapy during the last ten years. Of the various laboratories or investigators that have in the past prepared or are still preparing on a large scale antivenins for curative purposes, the following are the outstanding: Pasteur Institute (Lille and Paris, France) - against the cobra (N. naja) especially; Institute for Infectious Diseases (Tokio, Japan)-against the habu (T. flavoviridis); Butantan Institute (São Paulo, Brazil) - (a) for the rattlesnake (C.terrificus), (b) for coral snakes (now discontinued), (c) for several of the Brazilian species of Bothrops, (d) for Crotalus and several species of Bothrops (polyvalent), besides others for scorpions and spiders; George Lamb (India)-for the cobra and the daboia; Frank Tidswell (Sydney, Australia) - for the tiger snake; finally, the Bacteriological Institute (Buenos Aires, Argentine)-bivalent serum for C. terrificus and B. alternata.

At present the Antivenin Institute of America, Glenolden, Pennsylvania, is preparing two sorts of antivenin: (a) "nearctic crotalidic," for the North American rattler, the copperhead, and the moccasin; $(b)$ "bothropic," for the Central American species of Bothrops.

\section{PREPARATION}

Only four of the various methods which have been developed for the active immunization of animals seem to have fully succeeded in practice. They are the following:

I. Toxoid method.-Calmette in France attempted to destroy the toxophorous group of venom by heat, but was unable to prepare a serum which neutralized even I M.L.D. of the venom used in the immunization. He afterward decided to use as antigens "toxoids" consisting of venom solution plus I : 60 calcium hypochlorite solution which destroys the toxic activity of the venom. It is my opinion, however, that the use of these "toxoids" is responsible for the low activity of Calmette's sera. Indeed, the action of chemicals on the venom is likely not only to destroy its toxic principles but to affect its antigenic composition as well. The same criticism is applicable to Phisalix's technique of immunizing animals with only the filterable or the dialyzable fraction, because filtration and dialysis may remove not only toxic but also antigenic principles of the venom.

2. Mithridatization method,-Brazil, in São Paulo, Brazil, always preferred to immunize horses by gradually increasing doses of venom in natura, that is to say, without any previous physical or chemical treatment. The chief advantage of this

r Gomes, J. F.: Ann. Paulistas med. e cir., 7, r. I920.

${ }^{2}$ Houssay, B., and Negrette, J.: Rev. Asoc. med. argent., 36, 223. I923.

3 Amaral, A.: Bol. Soc. de med. e cir. S. Paulo, 4, I34. I92 I. 
method is that it produces a very marked degree of immunity in the animal which then yields highly antitoxic sera. Nevertheless, it has the disadvantage of taking too much time (at least eight to twelve months).

3. Neutral-mixture method.-In order to overcome this disadvantage, one can resort to the method of neutral mixtures as used by Roux and Babes in France, Kretz, Pawlowsky, and Maksutoff in Germany, Smith and McClintock and Ferry in this country in the immunization against bacterial toxins. When applied to antivenins, this method requires only about three to five months to immunize a horse. The first dose is two hundred to four hundred times as high as that used in the mithridatization. This method, though not economical, because it consumes large amounts of antitoxin, is practically harmless to the animals.

4. Mixed method.-The immunization is begun with neutral mixtures, and the dose of antitoxin is gradually decreased until this becomes so small that it can be omitted and pure venom solution can be injected. By this method a horse can be immunized in about four to six months and with considerable reduction of expense.

\section{ANIMALS USED}

Horses are the best animals for the immunization against snake venoms because of their docility, resistance to venoms, and ready production of antibody in high concentration. Mules may also be used, but they are not so docile and resistant, and do not produce antibodies in such high amounts nor so easily. Cows give antivenins of low antitoxic value. Goats can be used in countries, such as some African districts, where horses, mules, and cows are unavailable. They are quite resistant, but unfortunately they give only about $500 \mathrm{cc}$. of blood at each bleeding, while horses yield at least 6-8 liters, mules $3-4$ liters, and cows $4^{-6}$ liters.

\section{ANTIGEN PREPARATION}

Antigen preparation is one of the most important steps in the immunization technique. In order to produce a highly active and efficient antivenin, the venom must never be treated by any chemical or physical process which may affect its composition. It must retain all of its antigenic principles, and must be prepared in such a way as to remain active indefinitely.

The technique I use in order to accomplish this is as follows: Every fortnight the venom of the specimens of each species is extracted from the glands and collected into a wine glass, as shown in Figure I. After the extraction the venom is desiccated in an incubator and afterward dissolved in equal parts of salt solution and glycerin and used for inoculations. The solution must be about neutral, otherwise the venom will gradually lose its activity. It must also be kept in the icebox about one month in order to become sterile (ripening).

\section{VENOM PURIFICATION (STANDARD VENOM PREPARATION)}

It is known that venoms normally have a definite and invariable activity and, therefore, can be used in the most accurate titrations. For this purpose, however, they must be purified. This is done by centrifugalizing, decanting, and desiccating the supernatant fluid which is pure venom. After drying, the pure venom is kept in a vacuum over calcium chloride or other dehydrating substance. 
BLEEDING

Horses are bled eight to ten days after the final injection. The blood is received in sodium citrate or in sodium oxalate solution to prevent coagulation. The plasma thus obtained is reserved for concentration. Horses are again injected with a few doses of antigen and bled two or three more times until the antitoxic activity of their serum begins to show marked weakening.

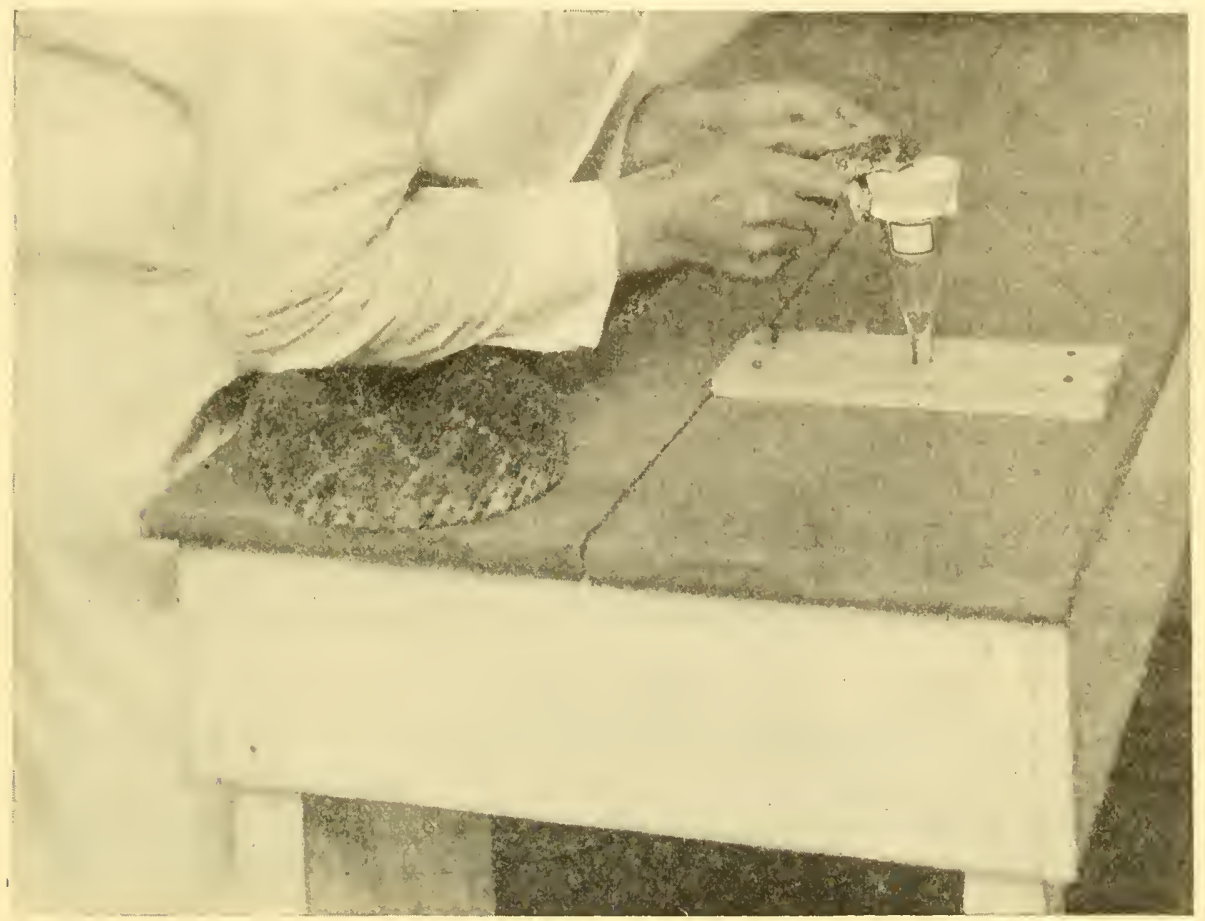

FIG. I.-Venom extraction. (Reprinted from the Bulletin of the Antivenin Institute of America)

ANTIVENIN TITRATION

Of all the methods of antitoxin titration which have been devised, three deserve especial reference:

I. Protection test.-Calmette's method ${ }^{\mathrm{I}}$ consists in determining the protective power of the antivenin on $2-\mathrm{kg}$. rabbits. Antivenin to the amount of $2 \mathrm{cc}$. is given subcutaneously, and two hours later I mg. of the venom is injected by the same channel.

2. Neutralization test.-Brazil's method $^{2}$ consists in determining the neutralizing activity of the venom. Several test tubes are prepared, each containing I cc. of the antivenin plus variable amounts of the standard venom solution, such as $0.2,0.4$, $0.6,0.8, \mathrm{I} .0, \mathrm{I} .5,2.0 \mathrm{cc}$., etc. The tubes are incubated at $37^{\circ} \mathrm{C}$. during one hour to bring about the complete combination of the antitoxin with the toxin (venom). The

I Calmette, A.: op. cit., p. 258. Paris, 1907.

${ }^{2}$ Brazil, V.: Coll. Trabalhos Inst. Butantan, p. 221. I909. 
doses are injected intravenously into adult pigeons (300 gm.). The pigeon which resists the highest dose of venom gives the neutralization index of the antivenin. Pigeons are by far the best animals for this type of work because they may react to differences of even $0.000000 \mathrm{I} \mathrm{gm}$. of venom. They are extremely susceptible to venoms as well as most resistant to intercurrent laboratory infections. I have found that this method can be simplified by keeping the antivenin in contact with the venom during twenty minutes only and at room temperature. The results obtained are comparable to those of the original method. The main disadvantage of the pigeon test lies, in fact, in that it cannot be used with any degree of accuracy to determine the antitoxic potency of sera upon venoms that possess direct blood-coagulant action when injected intravenously.

3. Precipitin test.-Calmette and $\mathrm{Massol}^{2}$ used a precipitin test as well as the above-mentioned protection test for antivenin titration. They tried to determine the amount of antivenin necessary to make a precipitate appear in a solution of $5 \mathrm{mg}$. of venom in saline when in contact for one hour at room temperature. The method was afterward modified by various investigators, but has proved to be inaccurate in many cases. Moreover, it is not applicable to all the venoms and antivenins, as I have verified by repeated experiments.

In my work, it has been found that for an antivenin to be accurately titrated and standardized it is necessary to employ more than one method, the most successful procedure being to associate the neutralization test on pigeons with the determination of both the preventive and the curative potency of the product on white mice and rabbits.

\section{CONCENTRATION}

The last stage in the preparation of antivenins consists in the purification and concentration of the plasma, which is done in more or less the same way as for diphtheria or tetanus antitoxin, with slight variations. The final pseudo-globulin fraction is filtered, tested for sterility, and then standardized before it is sent out for therapeutic application.

\section{TIIERAPEUTIC USE}

Two important factors influence the success of the use of antivenins, viz., early administration and dosage proportional to the amount of poison. In reference to the first factor, it is obvious that the sooner the antivenin is given the better. The subcutaneous or intramuscular methods of injection may serve in the majority of cases of snake poisoning, although preference should be given to the intravenous channel whenever the case is of a serious character. When in doubt as to the condition of the patient, it is best not to hesitate but to give the injection intravenously.

The doses of serum should be proportional, not only to the estimated quantity of poison inoculated by the snake, but also to the toxic activity of the poison and to the relative weight of the patient. As in general the larger the snake the greater the quantity of venom it injects, it is advisable to give more than one dose of antivenin in case of a bite by a large reptile. Also, a large dose of antivenin is advised to counter-

${ }^{1}$ do Amaral, A.: Snake Venoms, elc., p. 55. Thesis, Harvard School of Public Health, I924.

${ }^{2}$ Calmette, A., and Massol, L.: Ann. de l'Inst. Pasteur, 23, I 55. I909. 
act the bite of such snakes as the Neotropic rattler, the cobra, the tiger snake, and the "fer-de-lance," because their venom is very toxic for the tissues of animals. Finally, since the toxicity of the venom is inversely proportional to the borly weight, it is recommended that two or three times as much antivenin be given to a child as to an adult.

By properly applying serum therapy, the death rate from snake bites, which is very high in certain countries, can be considerably reduced. In Brazil, where some twenty years ago the number of persons who died from snake bites every year was estimated to be about five thousand, the increasing use of antivenins has contributed to a material reduction of this figure. In this country where, for the last year, the Antivenin Institute of America has been working with a view to eliminating all cases of death from snake bite, the first results have also been very encouraging. 


\title{
CHAPTER LXXYI
}

\section{A CRITIQUE OF THE EHRLICH THEORY, WITH AN OUTLINE OF THE ENZYME THEORY OF ANTIBODY FORMATION}

\author{
W. H. MANWARING \\ Stanford University
}

The Ehrlich theory of the origin and nature of specific antibodies beautifully explains and co-ordinates all known immunological facts. For three decades this theory has been the accepted basis for immunological deduction and clinical interpretation by the majority of medical workers. Nevertheless, I am convinced there is hardly an element of truth in most of the hypotheses incorporated in the Ehrlich theory, and that for two decades the wide acceptance of the theory has been a serious handicap to medical progress.

\section{THE EHRLICH HYPOTHESES}

Analysis shows that the four major hypotheses formally incorporated or implied in the Ehrlich theory are the assumptions: (a) that specific antibodies are hereditary, preformed, highly specialized, chemical substances, normally present in the animal body; $(b)$ that these substances originate in the fixed and wandering cells of the body, and are by them given off into the body fluids; $(c)$ that the humoral elements thus formed and liberated are purposeful, defensive chemical substances for the body as a whole; and $(d)$ that the formation and liberation of these specific chemical defenses with their subsequent transmission and absorption by other cells are the only specific adaptive mechanisms in the body.

The Ehrlich theory is usually characterized by its minor hypotheses, dealing with the exact method of formation and liberation of these specific defensive internal secretions by the body cells. These hypotheses are based on the specific receptor theory of cell nutrition. This theory pictures each cell of the body as provided with numerous highly specialized superficial atomic groups or side-chains, each side-chain having a highly specialized chemical affinity for a certain food material. By means of these highly specialized side-chains or "receptors" foods are assumed to be selected from the blood stream and brought into chemical union with the living cytoplasm. Ehrlich's minor hypotheses assume: $(e)$ that some of these highly specialized food receptors have accidental affinities for certain antigens; $(f)$ that a receptor having this accidental affinity is permanently injured or denatured by its chemical union with this antigen; $(g)$ that the resulting denatured receptor is eventually cast off by the living cell; and $(h)$ that the cell now regenerates a new receptor of identical chemical structure.

In response to a sufficiently large single dose, or to repeated doses of the same antigen, Ehrlich assumes: (i) that this regeneration is overstimulated, more than one receptor being formed to replace the one denatured and lost; $(j)$ that the superfluous 
receptors thus formed are in time cast off by the living cell; and $(k)$ that these castoff receptors are the specific antibodies with which we deal. To account for their defensive action, Ehrlich assumes: $(l)$ that the cast-off receptors still retain their original accidental affinities for the specific antigen; and $(m)$ that they unite with this antigen in the body fluids, thus preventing its union with the living cells. To explain certain minor phenomena he assumes: $(n)$ that the freed or circulating receptors still retain an affinity for their cells of origin; $(o)$ that receptors are of different types depending upon whether or not they require the co-operation of some normal serum component for their full defensive action against the specific antigen; and $(p)$ that there are differences in the number, avidity, and valence of the same receptors normally present in different cells of the animal body and in corresponding cells of different animal species.

THE EHRLICH HYPOTHESES AS AN EXPLANATION OF KNOWN IMMUNOLOGICAL FACTS

The hypotheses thus formulated explain and co-ordinate all known immunological facts. The high susceptibility of certain animal species for a given antigen, for example, is a logical result of an assumed multiplicity of accidental receptors for this antigen in vital tissues of this species, or of receptors having an assumed unusually high avidity for this antigen. The relative insusceptibility of other animal species is logically explained as a result of an assumed paucity or low avidity of the same receptors in vital tissues, or as a result of an assumed multiplicity or high avidity of these receptors in physiologically inert tissues, thus binding the injected antigen to inert structures, preventing its action on vital parts.

The development of specific hypersusceptibility would be a logical result of an assumed local multiplication of specific receptors in vital tissues, with little or no liberation of these receptors in the body fluids. A subsequent desquamation of the same receptors in to the body fluids would change the hypersusceptibility to an immunity. Passive immunization would be a logical result of a sufficiently large number of freed receptors in the body fluids. Passive sensitization would logically follow a small number of freed receptors, most of the transferred receptors becoming attached to the body cells of the recipient, thus increasing its affinity for the antigen. All specific testtube reactions would be logically explained as a result of direct chemical union between specific antigens and corresponding freed receptors, the details being determined by the number, type, avidity, and valence of these receptors.

\section{VALIDITY OF THE EHRLICH HYPOTHESES}

My conviction that there is something radically wrong in the major assumption of the Ehrlich theory arose from a consideration of the almost universal failure of therapeutic methods based on these assumptions. According to the Ehrlich theory, for example, any specific antibody that can be caused to appear or to increase in the circulating blood as a result of active immunization is a specific defensive chemical substance and represents the only specific immunological adaptation in the body. It is only necessary to obtain this antibody in sufficiently large quantities to have a valuable therapeutic agent. Yet in spite of millions of dollars spent in research and in the commercial exploitation of such antibodies, therapeutic sera have been lamentably unsuccessful except in a small group of relatively unimportant diseases. This 
failure is usually attributed to an assumed impossibility of obtaining antisera of sufficiently high specific titre, or to an assumed antagonism between the human body and foreign antibodies. It is equally logical, however, to assume that the failure is a logical result of an error in our accepted conceptions as to the nature and function of specific antibodies.

That humoral antibodies do not always represent the sole or essential specific defense of the body is readily shown by massive immune blood transfusions. Tubercle bacilli, for example, injected into the peritoneal cavity of a tuberculous guinea pig are rapidly destroyed by a process of extra-cellular lysis. Total blood replacement of a normal guinea pig by transfusion from a tuberculous donor does not confer upon the normal guinea pig the power to produce this intraperitoneal lysis. ${ }^{I}$ This failure cannot be attributed to inadequate dosage, nor to an assumed antagonism to foreign antibodies.

My conviction that there is an error in our currently accepted basic assumptions was strengthened by several years' experience in the study of the quantitative characteristics of serum reactions. ${ }^{2}$ This study led to numerous contradictory and paradoxical results impossible to harmonize with the accepted theory without the necessity of numerous minor hypotheses. ${ }^{3}$ For example, if a relatively large amount of hemolytic amboceptor (heat-inactivated, hemolytic serum) is exposed to red blood corpuscles until sensitization is complete, titration of the residual amboceptor in the supernatant fluid often shows, in place of the expected decrease in amboceptor, a distinct or even marked apparent increase in amboceptor. ${ }^{4}$ This finding can be harmonized with the Ehrlich theory by the assumption that heat-inactivated hemolytic serum contains in addition to hemolytic amboceptor one or more non-hemolytic antibodies capable of binding complement, the non-hemolytic antibodies having a relatively high affinity for the red blood corpuscles. On exposure to corpuscles the relatively greater absorption of the non-hemolytic antibodies would remove from the supernatant fluid a complement-deviating anti-hemolytic agent. This removal might give an apparent increase in hemolytic titre, in spite of a real decrease in amboceptor.

My conviction that there is a radical error in our accepted theory was further strengthened by a study of the so-called "lecithin" activation of cobra venom. Both cobra venom and lecithin are non-hemolytic for certain erythrocytes when tested in ordinary doses. Added to each other, however, they constitute a powerful hemolytic agent. According to the Ehrlich theory, lecithin and cobra venom could co-operate in hemolytic action only by their direct conjugation to form a "cobra-lecithid." Chemical analysis, however, shows that this conjugation does not take place. The active component of cobra venom is a powerful lipase, capable of very rapidly splitting off one fatty acid radical from the lecithin molecule. The resulting "mono-fattyacid-lecithin" is a powerful hemolytic agent, and accounts for the total hemolytic action of a lecithin-venom mixture. ${ }^{5}$

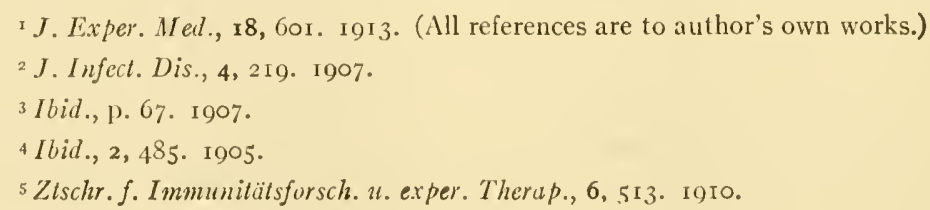


EXPERIMENTAL TESTS OF THE EIIRLICII TIIEORY

It is evidently impossible to prove or disprove the Ehrlich theory by data drawn from test tube experiments, since there is no apparent inconsistency or even paradox in test tube reactions that cannot be harmonized with this theory, or for that matter with any other theory, by a sufficient number of minor hypotheses. Proof or disproof of this theory can come only from physiological studies of hypersensitive and immune tissues.

When one considers the prominent rôle the Ehrlich theory has played in the stimulation and co-ordination of immunological research one marvels at the few attempts thus far made at its physiological verification. During the last twelve years we have been engaged in such work. The physiological reactions used in most of our tests have been: (a) the sharp fall in arterial blood pressure during acute anaphylactic shock in dogs, $(b)$ the accompanying contraction of the urinary bladder, and $(c)$ the marked changes in perfusion resistances in hypersensitive organs, on the addition of specific antigen to the perfusion fluid. The following are some of our typical findings:

a) According to the Ehrlich theory of antibody formation, the blood-free tissues of hypersensitive animals have an increased affinity for the specific antigen due to a local multiplication of "sessile receptors." This increased affinity is the assumed cause of the specific hypersensitiveness. Yet repeated perfusions of blood-free organs of hypersensitive animals with Locke's solution containing even minute quantities of the specific protein used in sensitization give no demonstrable reduction in the concentration of this protein in the perfusate, as determined by specific titration with rabbit precipitin. ${ }^{3}$

b) According to the Ehrlich theory, the blood-free tissues of immune animals are necessarily hypersensitive, at least during the early stages of immunization, due to an increased number of "sessile receptors." Yet the blood-free lungs of protein-immune dogs show no suggestion whatsoever of specific hypersensitiveness on perfusion with Locke's solution containing the specific protein, ${ }^{2}$ nor will the liver of a proteinimmune dog transplanted by blood-vessel anastomosis into a normal dog render the normal dog hypersensitive. ${ }^{3}$ The liver of a hypersensitive dog similarly transplanted renders the recipient typically hypersensitive. ${ }^{4}$

c) The Ehrlich theory assumes that specific immunity is due solely to circulating antibodies serving as portal defenses to the fixed tissues. Yet if a protein hypersensitive dog is exsanguinated as completely as possible, and its blood volume restored to normal by transfusion from a protein-immune donor, the immune blood gives no demonstrable protection to the hypersensitive tissues as determined by an immediate routine anaphylactic test. ${ }^{5}$

d) According to the Ehrlich theory, the only conceivable effect of immune serum on normal tissues is sensitization of these tissues, due to a local "fixation" of specific receptors. Yet the blood of a hypersensitive dog will render a transfused normal dog typically hypersensitive, while fractional transfusion from an immune donor will give

I. Immunol., 2, 511. I9I7.

${ }^{2} J \cdot A \cdot M . A ., 85,1729.1925$.

3 J. Immunol., 13, 59. 1927 .

4 Ibid., 13, 59. 1927.

5 Ibid., 10, 575 . 1925 . 
no suggestion whatsoever of a passive hypersensitiveness. ${ }^{x}$ Moreover, if a proteinhypersensitive dog is exsanguinated as completely as possible and transfused from an immune donor, the hypersensitive tissues are completely desensitized after an incubation period of about forty-eight hours. ${ }^{2}$ Control transfusions from a normal donor give no suggestion of this passive desensitization.

The foregoing typical findings are impossible to harmonize with the Ehrlich theory by any number of minor hypotheses as to the number, type, avidity, and valence of specific receptors. I believe we are forced to the conclusion that the formation of antibodies is quite a different process from the assumed multiplication of preformed sessile receptors, with their subsequent desquamation in to the body fluids.

\section{THE ENZYME THEORY OF ANTIBODY FORMATION}

This conclusion might be acceptable to serologists provided an equally plausible immunological theory were available. I believe such a theory can be readily formulated from our current conceptions of cell nutrition.

Cell nutrition is currently conceived to be mediated largely through enzymes. The main enzymes are of two types: hydrolyzing enzymes and synthesizing enzymes. It is immaterial for our present purpose whether or not hydrolyzing and synthesizing enzymes are considered as separate molecular entities, or as reversible actions of the same entities. These enzymes with activators and inhibitors are demonstrable in both cellular content and body fluids.

According to the enzyme theory of antibody formation, antigens introduced into the animal body are subjected to two main denaturing processes: First, to a series of extracellular and intracelluar hydrolytic cleavages, supplemented by colloidal dissociations, ionic dispersions, and the like, by means of which the antigen is broken down into simpler components. The stages and sequences in this process are presumably similar to those of gastro-intestinal digestion, the details, of course, varying with dosage, portal of entry, and topographical distribution. The diffusability of the antigen or its cleavage products through cellular membranes and its phagocytability would be one of the major determining factors in this topographical distribution. Many of the earlier cleavage products would presumably have the same specificity as the initial antigen, since it is known that complex proteins subjected to artificial tryptic digestion retain their specificity until reduced to relatively simple digestive products. Later cleavage products would presumably be less highly specific or even non-specific. It is even conceivable that some of the cleavage products might have a specificity other than that of the initial antigen, thus setting up a secondary wave of specific immunological adaptations in the body.

The second main denaturing process would be a series of chemical syntheses, coagulations, conjugations, adsorptions, and the like, between the initial antigen or its cleavage products and normal or denatured extracellular and intracellular proteins. Here, also, the details would vary with dosage, portal of entry, and topographical distribution. Many of the earlier synthetic products would presumably have the same specificity as the initial antigen. Later conjugates would presumably be less highly specific, or might even acquire a new specificity. Some of the resulting conjugates or

$$
\text { I J.A.M.A., 86, 1271. 1926. 2 } 2 \text { J. Immunol., 13, 59. I927. }
$$


their dissociation products might conceivably act as specific portal defenses to the fixed tissues. Others might conceivably increase specific susceptibility. Still others might cause non-specific variations in resistance. Many of the conjugates, of course, might be physiologically inert.

PHYSIOLOGICAL DATA IN HARMONY WITI TIIE ENZYME THEORY

During the last two years we have obtained additional physiological data difficult to explain with the specific receptor hypothesis, but readily explained on the enzyme theory. The following are typical findings:

a) If a massive dose of horse serum is injected intravenously into a normal clog, and from one to six hours later a quantitative blood transfusion is made from this dog as donor into a partially exsanguinated hypersensitive recipient, the recipient is thrown into typical anaphylactic shock. The shock, in the transfused recipient, however, is invariably more severe than the shock in a control hypersensitive dog injected intravenously with the calculated amount of horse protein contained in the transferred blood sample. ${ }^{\mathrm{I}}$ Since the donor's blood has no recognizable toxicity on a control transfusion into a normal dog, this increased shock is presumably due to an increased anaphylactic potency of the horse protein contained in the donor's blood. An increased specific toxicity is readily explained under the enzyme theory as a result of the assumed hydrolytic cleavage of the horse protein, with the formation of a larger number of specific protein molecules.

b) If, however, instead of making the transfusion at the end of one to six hours, the transfusion is delayed until the end of four days, the donor's blood is invariably found to be non-toxic for the hypersensitive recipient. ${ }^{\mathrm{I}}$ Specific titration of the donor's blood with rabbit precipitin shows that within the limits of the experimental error there is little or no demonstrable decrease in the amount of horse protein in the donor's blood by the end of four days. This anaphylactic detoxication of the horse protein is readily explained under the enzyme theory as a result of the assumed normal intravenous denaturing of the horse protein, as a result of conjugation with normal serum proteins.

c) If the urinary bladder of a normal dog is transplanted by blood-vessel anastomosis into a horse-serum hypersensitive recipient and the recipient is now thrown into anaphylactic shock, the transplanted normal bladder is thrown into a typical anaphylactic contraction. Physiological analyses have shown that this contraction is due to substances having a histamine-like action on smooth muscle structures, explosively formed or liberated by the hypersensitive liver. ${ }^{2}$ The bladder of an immune dog similarly transplanted is wholly insusceptible to this histamine-like hepatic product. ${ }^{3}$ Moreover, this acquired histamine-like insusceptibility is apparently specific, since a goat-serum immune bladder transplanted into a horse-serum hypersensitive dog shows no suggestion of an acquired insusceptibility to the histamine-like hepatic products formed or liberated during horse-serum anaphylactic shock. ${ }^{4}$ This finding is readily explained under the enzyme theory by the assumption that the histaminelike hepatic product is a secondary specific antigen, formed or liberated during the process of active immunization.
' J. Immunol., 13, 357. 1927 .
3 Ibid., 13, 63. 1927.
${ }^{2}$ J. Immunol., 10, 575. 1925 .
${ }_{4}$ J. Immunol., 13, 319. 1927. 
d) This acquired histamine-like insusceptibility of smooth muscle structures cannot be transferred from an immune dog to a normal dog by even massive immune blood transfusion. ${ }^{\mathrm{I}}$ This finding is readily harmonized with the enzyme theory by the assumption that the histamine-like insusceptibility is due to secondary intracellular antibodies chemically different from the primary humoral antibodies.

THE ENZYME THEORY AS AN EXPLANATION OF KNOWN SEROLOGICAL FACTS

With one minor hypothesis as to the difference in the relative stability and dissociability of earlier and later conjugates, the enzyme theory of antibody formation will explain and co-ordinate all known properties of specific antibodies. Antitoxins, for example, would be pictured as non-toxic dissociation products of slightly dissociable humoral conjugates. During the process of immunization this specific dissociation product would be gradually increased in the body as a result of repeated conjugations of the dissociated toxin with new proteins. This would lead in time to an almost complete suppression of the toxin by the laws of mass action. Toxin added to antitoxic serum would be suppressed by conjugation with the superabundant nontoxic dissociation product. Similar conceptions would apply to agglutinins, precipitins, opsonins, and bacteriolysins. Heat inactivations, reactivation, and "complement deviation" could be explained under this theory without the necessity of the assumption that the only possible method of action of a lytic serum is by a direct chemical union between "amboceptor" and "complement."

Unstable or highly dissociable serum conjugates might readily account for the explosive colloidal readjustments of humoral anaphylaxis. Unstable or highly dissociable cellular conjugates might lead to the explosive increases in cell permeability in cellular anaphylaxis. ${ }^{2}$ Later stable, relatively non-dissociable cellular conjugates might account for the partial or complete disappearance of this increased permeability in immune animals. Passive desensitization of fixed tissues by immune blood transfusion ${ }^{3}$ might be explained as a result of a complete disintegration of the earlier, unstable, highly dissociable anaphylactic cellular conjugates in the presence of more stable and relatively non-dissociable later immune conjugates.

\section{THE ENZYME THEORY IN THE INTERPRETATION OF CLINICAL DATA}

The enzyme theory, however, would necessitate a reinterpretation of much of our accumulated experimental and clinical data. For example, specific serum titrations, though admittedly accurate measures of the specific primary humoral antibodies, could no longer be assumed to be a measure of the specific immunological adaptations of the body as a whole. This might readily account for a puzzling lack of parallelism between serum titre and clinical picture, and between the specific titre of a proposed antiserum and its therapeutic effects. Specific intracellular antibodies chemically different from the humoral antibodies might readily account for the high organ-specificity of certain micro-organisms, so difficult to credit under our present theory. Variations in immunizing power of the same antigen with different methods of injection might be explained on the same basis. Secondary non-specific humoral antibodies might readily account for non-specific serum reactions.
${ }^{1}$ J. Immunol., 13, 59. I927.
2Ibid., 8, 233. 1923.
3 Ibid., 13, 59. 1927 . 
LIMITATIONS OF TIIE ENZYME THEORY

Unlike the Ehrlich theory, however, the enzyme theory would not attempt to explain and co-ordinate all known immunological facts. For example, the enzyme theory would not attempt to explain the variations in natural resistance in different tissues and in different animal species. It would make no assumption as to the physiological rôle of so-called "specific antibodies." Nor would it assume that the formation of these substances is the only specific immunological adaptation in the body. With these limitations, however, the enzyme theory would offer as plausible an explanation of known immunological facts as that offered by the specific receptor hypotheses. I believe the enzyme theory of antibody formation is the most promising sulstitute for the Ehrlich theory, at the present day. 


\title{
CHAPTER LXXXII \\ NON-SPECIFIC PROTEIN THERAPY
}

\author{
WILLIAM F. PETERSEN \\ College of Medicine, University of Illinois \\ Chicago, Illinois
}

INTRODUCTION

The therapeutic procedure to which the name "protein therapy" has been given is of interest to both bacteriologists and immunologists. The immunologist must concern himself with the underlying alterations in the organism when the antigen has entered the body, with the non-specific alterations brought about thereby, with their effects on immunological processes, and with the question how far his specific therapeutic results are masked by wholly non-specific phenomena. The bacteriologist is interested in the sudden termination of an acute infection (a typhoid fever or a puerperal sepsis) or the recovery from the ordinary chronic infections which in some form or other play so large a rôle in human illness.

In its original sense the term "protein therapy" was applied to certain therapeutic procedures whereby a reaction was produced by the injection of protein substancesbacterial suspensions, peptone, or milk. The method was empiric and of diverse origins. One led from the clinical observation that in the treatment of gonococcus infections large intravenous doses of gonococcus vaccine (which according to the Wright theory would be contraindicated) were frequently followed by marked clinical improvement. ${ }^{x}$ Increased fever and accentuation of the presumptive "negative phase" were actually of benefit. The observations of Ichikawa ${ }^{2}$ in Japan, and of Dessy, Grapiolo, and Fossati, ${ }^{3}$ Penna, Kraus, and Cuenca, ${ }^{4}$ that typhoid fever might be promptly cured following an intravenous injection of typhoid or other vaccine, were without doubt the immediate cause of interest in protein therapy. Curiously enough, typhoid fever, for reasons which we shall discuss later, is one of the few acute infectious diseases in which we might anticipate therapeutic effects.

Empiric origin in itself should impose no handicap to scientific interest, for to empiricism we owe many of our most useful therapeutic procedures. The particular clinical experience on which protein therapy was based goes back, indeed, to remotest medical antiquity. Cautery, seton, fontanelle-blister and venesection are its ancient counterparts. All are "alteratives"s in the sense that they may alter the reaction of

I Bruck, C., and Sommer A.: Mïnch. med. Wchr., 60, I I85. I9I3.

${ }^{2}$ Ichikawa, S.: Zeitschr.f. Imm., 23, 32. 1914-15.

3 Dessy, S., Grapiolo, F. L., and Fossati, V.: Semana Medica, Buenos Aires, 21, 357. I9I4.

${ }_{4}$ Penna, J., Kraus, R., and Cuenca, Bonorino: Jour. Conf. Soc. Stud. Am. de hig., I, 8I 5. 1916-I 7.

5 Fundamentally the alteration consists in a lessening of the reaction of the tissue to the irritant. The subject has been discussed in relation to protein therapy by Burmeister (Burmeister, J.: Zeitschr. f. kl. Med., 95, 237. 1922). 
the body to the disease - an ergotropic - and take their place with iodicles, with arsenic, and with phosphorus. The term "protein therapy" must be enlarged to include all the non-specific biological procedures in contradistinction to methods of therapy based on strictly specific concepts.

The establishment of the etiological epoch in the seventies of the last century brought with it an utter disregard of the reaction of the infected host. The simple equation, bacteria + host $=$ disease, sufficed. But finally an impasse was reached; certain facts could no longer be ignored. There were differences in susceptibility; and among the susceptible there were differences in resistance, differences that could not be explained on an immunological basis.

Even during the triumphant bacteriological era competent observers called attention to certain non-specific phenomena-Buchner, Pfeiffer, Rumpf, Matthes, Landerer, might be mentioned-but clinical application never followed these random observations. With I9I4 came a sudden change. A veritable avalanche of clinical reports appeared. Not only was typhoid fever terminated abruptly by intravenous vaccine injection, puerperal fever by coli vaccine, erysipelas and arthritis by milk injections, paresis by tuberculin, skin diseases by autohemotherapy, but even diphtheria was cured by normal instead of antitoxic horse serum.

Whatever may have been the clinical basis for this intense interest, psychologically it probably did represent a protest against the all-too-narrow view of the immunologist concerning the nature of recovery from disease. Specificity and specific antibodies obviously play a rôle in recovery; quite as obviously they are not the sole factors concerned.

Today we seek the solution in biochemical and biophysical alterations. Recent advances have made available methods that were closed to the earlier immunologists. Even now there is danger that the pendulum will swing too far in the other direction. We speak now of "colloidoclysis," of "colloidal instability," of "autonomic unbalance," of "hormone disfunction," and of "bio-polar reactions" as once we spoke of the opsonins, agglutinins, and Abwehrfermente.

Non-specific treatment of paresis, of iritis, and of chronic infections (other than tuberculosis) has become an established procedure, varying in methods and agents and application, it is true, but frequently of great benefit to the patient. Even diseases such as purpura, rickets, asthma, diabetes, and ulcer are influenced by non-specific changes in the organism. For these reasons it may be proper to examine the information that has become available concerning the reaction and the underlying mechanism.

\section{GENERAL THEORIES}

In general we may discern three theoretical lines of explanation for the therapeutic effect:

First, the assumption that a general stimulation of the tissue cells results (plasma activation of Weichardt $)^{\mathrm{r}}$ or, if not a general stimulation, then a stimulation of certain cell groups or organs. Thus $(a)$ preformed antibodies may be released or $(b)$ enzymes mobilized, $(c)$ the polymorphonuclear leukocytes may be changed or $(d)$ the reticulo-endothelium stimulated.

r Weichardt, W.: Münch. med. Wchr., 67, 91. 1920; ibid., 72, 650. I925. 
Second, the "contracoup" theory (ictus morbi) based on the assumption that the summation of disease manifestations in the form of a fastigium will be followed by a rapid reversal to the normal. In its final analysis this rests on the general observance of rhythmicity in biological processes.

Finally, pharmacological and biochemical observations which lead to the belief that most of the phenomena can be explained on definite alterations in the cells and fluids of the body which follow any procedure such as that under analysis. The observations concern the effects on the autonomic nervous system, on the changes in capillary permeability, the liberation of pharmacologically active substances from the tissues, changes in the ionic equilibria and the hormones.

While it is obvious that in a review such as this we cannot be restricted to the examination of theories as such, and shall deal with the experimental evidence, it is well to keep in mind that probably all three are of importance, but not necessarily all operative in any one disease condition. So it seems probable that in typhoid the non-specific mechanism of recovery is built up largely on the intensive splanchnic stimulation (parasympathetic status, with increased lymph flow, transfer of preformed antibodies from the blood stream to lymph channels, bacteriolysis, etc.), that the chronic infectious process is influenced by the focal reaction (increased permeability at the focus), while the effect on asthma and gastric ulcer will be through the autonomic apparatus (muscle tonicity, vascularization, etc.). Presumably, cell stimulation, especially of the reticulo-endothelial system, may influence the more chronic infections such as syphilis, while similar changes (liver) may influence metabolic derangements such as diabetes.

There are qualitative as well as quantitative differences in biological effects which we achieve with protein therapy, the differences depending on the agents, on the dosage, on the patient, and on the disease from which the patient suffers. The reactions may vary from mild changes that are within the range of physiological fluctuation such as may be associated with menstruation, fatigue, digestion, etc.--produced, for instance, by the intracutaneous injection of bland proteins, the ingestion of peptone or glycocoll, or the injection of horse serum - through the range of intramuscular milk injections, nuclein injections, or the innumerable preparations offered in the market, finally to severe "shock" effects following the intravenous injection of typhoid, meningococcus, or gonococcus vaccine. Even with such agents the effect obviously depends on the dosage.

\section{THE BIOLOGICAL REACTION}

Before reviewing the more recent literature dealing with the effects of protein injections on the organism, three factors should be emphasized. In the first place, the reaction produced is distinctly diphasic. A primary stimulation passes over to a secondary refractory (or rest) phase. Unless the investigator keeps this in mind and follows the reaction continuously for a considerable period of time he may reach quite erroneous conclusions.

Second, the reaction elicited will depend on the functional (and pathological) status of the individual organ or tissue. Tissues that are already stimulated or fatigued will respond quite differently from tissues in the resting stage. Therapeutically this means that while nonspecific therapy in broad outline involves general tissue stimulation, actually the agents may be "organotropic" in so far as organs that are the seat of disease may respond in a more 
marked manner than would the normal tissues. P'hysiologically it means that the tissues of the splanchnic region (which are normally functioning at a higher rate than the peripheral tissues) will be stimulated to a greater extent. From the point of view of the constitutional behavior of the individual in response to an injection, we must finally kcep in mind that the individual with an unstable autonomic apparatus will respond in more marked manner than the individual with a stable system. This probably resolves itself into the mechanism of ionic buffering.

Finally, it should be kept in mind that activaling agents may originate in the body either as the result of the non-specific effect on some pathological focus (as in tuberculosis) or following some physiological action (fatigue, digestion, irradiation, etc.). The non-specific agent may therefore initiate a much more profound effect than might be anticipated from control injections in normal individuals.

Effects on the Blood-VÁscular System.-Apart from the obvious clinical observations on temperature, respiration, and pulse-rate that were recorded by the first clinical observers, the changes in the blood have received most attention. It was found that the leukocyte count was greatly altered. A primary leukopenia followed by a later leukocytosis was noted early, ${ }^{\mathrm{I}-4}$ and $\mathrm{Müller}^{5}$ has studied the reaction most thoroughly. He finds that the primary leukopenia is associated with a peripheral sympathetic status during which time the leukocytes accumulate in the splanchnic and thoracic regions. On the reversal of this status, they again appear peripherally, together with those released from the bone marrow. Cowie and Calhoun ${ }^{6}$ and others have studied the differential count, 7,8 which gives evidence of considerable myelotic irritation. The platelet count varies greatly with the dose and agent. $^{6,9,10}$ The erythrocyles, their number, hemoglobin content, and volume and fragility were studied, ${ }^{11},{ }^{12}$ and Stötter ${ }^{13}$ has recently noted that their oxidase content may be increased. The blood proteins have been studied by Berger, ${ }^{14}$ the Löhrs, ${ }^{15}$ and many others. ${ }^{16-25}$

I Jobling, J. IV.: Arch. Int. Mcd., I 7, I042. I9 I7.

${ }^{2}$ Scully, F. T.: Jour. Amer. Med. Assoc., 69, 20. I9 7.

3 Ling, C. Y.: .1rch. Int. Med., 35, 598 and 740. I925.

4 Lange, F. D.: Dent. Arch.f. kl. Med., 94, 552. I908.

5 Müller, E. F.: Arch. Int. Med., 35, 796. I925; Zeitsclir.f. dic ges. cxp. Me1., 32, 120. I923.

${ }^{6}$ Cowie, D. M., and Calhoun, H.: Arch. Int. Med., 23, 64. I919; Jour. Med. Res., 42, 227. I921.

7 Holler, G.: Met. Kl., I 1,639 and 668. I915; ibid., г3, 1038. I917.

${ }^{8}$ Leudke, H.: Berl. kl. II chr., 57, 34. I920.

9 Busson, B.: Wien. kl. W Whr., 35, 45I. I922; Duke, WV. W.: Jour. Amer. Med. Assoc., 65, I60ว. I9I6.

${ }^{10}$ Döllken: Berl. kl. Wchr., 56, 226. I919.

II Schittenhelm, A., and Griesshammer, W.: Zeitschr. f. exp. Path. und Pharm., Io, fiz. I9II.

12 Gruenthal, R. M., and Brown, G. M.: Arch. int. Mel., 30, 99. 1922.

13 Stötter, quoted by Weichardt, loc. cit.

${ }^{14}$ Berger, II.: Zeitschr.f.d. ges. exp. Me1., 28, I. 1922.

${ }_{15}$ Löhr, WV. and H.: ibid., 27, 1. I922; 29, I39. I922.

${ }^{16}$ Starlinger, IV.: Biochem. Zeitscler., 14, I29. I92 I.

17 Lowy, J.: Zgl.f. inn. Mel., 37, 833. 1916.

${ }^{18}$ Van den Velden, R.: Dest. Arch. f. kl. Med., r14, 249. I014.

i9 Moll, H.: Wien. kl. Wichr., I6, I2 I5. I903.

${ }^{20}$ Togeri, C.: quoted by Weichardt, loc. cit.

${ }^{21}$ Petersen, IV. F.: Jour. Immunol., 8, 377. I023. 
Berger followed the changes in the blood proteins of rabbits after serum injection for several months. He found that after a latent period the total amount of protein was first diminished, then followed two waves of increase and finally a return to normal. The globulins, too, followed a similar curve, while the change in the albumin persisted longer than with the other proteins. With my associates, I have followed the changes in the lymph for short periods of time, ${ }^{\mathrm{I}}$ and have reached the conclusion that the change in the globulins is indicative of immediate cellular effect, the larger aggregates being more closely related to cell protein both immunologically and physically. Of great practical importance is the change in the fibrinogen. This is at first diminished, but later greatly increased and plays a rôle in the alteration of the coagulation time. This increase in the larger colloidal aggregates is furthermore of interest because of the marked effect in lessening the colloidal stability of the serum, on the sedimentation time of the red blood corpuscles, and on the viscosity of the plasma. An excellent review is that of Sachs, ${ }^{2}$ and the French investigators, particularly Kapoczweski, ${ }^{3}$ have entered into this phase of the problem in detail. An associated change in the surface tension is of great importance ${ }^{4}$ because it tends to increase osmosis and the penetration of certain substances into the cells.

An increase in proteolytic enzymes, in lipolytic enzymes, and in amylase was noted by Jobling and Petersen, ${ }^{5}$ and has been confirmed repeatedly. ${ }^{6-10}$ The antiferment titer reveals a typical diphasic curve. ${ }^{5}$ Complement has been studied by Malkin, ${ }^{1 \mathrm{r}}$ who has found that there is a direct relationship to the surface tension of the serum, and that the therapeutic effect seems closely related to the increase in complement. The reaction is diphasic, complement being at first diminished.

Coagulation time and sedimentation rate have been studied by Schmidt, ${ }^{12}$ Salomon, ${ }^{13}$ and the Löhrs. ${ }^{14}$ The coagulation time is diminished and the sedimentation rate shortened after non-specific injections.

The chemistry of the blood gives evidence of a primary acidosis, ${ }^{15}$ followed by a rather long-continued alkalosis. The most thorough study is that of Vollmer ${ }^{16}$ He describes a progressive diminution in acid excretion of the urine after a transient increase on the day of the injection, the $\mathrm{H}$-ion concentration shifting to the alkaline side. With this there is an un-

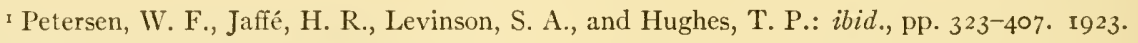

${ }^{2}$ Sachs, H.: Deut. mel. Wchr., 53, 93. 1927.

3 Kapoczweski, A.: Pharmacodynamie c'es Colloids. Paris: G. Doin, 1925.

${ }_{4}^{4}$ Rosenberg, H., and Adelsberger, L.: Zeitschr. f. Imm., 34, 36. 1922.

s Jobling, James W., and Petersen, W. F.: Jour. Lab. and Cl. Med., I, I72, 1915; Jour. Amer. Med. Assoc., 65, 515. I915

${ }^{6}$ Shamberg, J. F., and Brown, H.: Arch. Int. Med., 35, 537. 1925.

7 Wolter, quoted by Malkin, S. J.: Zeitschr. f. Imm., 46, 194. 1926.

${ }^{8}$ Ling, C. Y.: Arch. Int. Med., 35, 752. 1925.

9 Bulger, H. A.: Jour. Infect. Dis., 19, 832. 1916. ${ }^{10}$ Falls, F. H.: ibid., 16, 466.1915.

${ }^{11}$ Malkin, S. J.: Zeitschr. f. Imm., 46, 194. 1926.

${ }^{12}$ Schmidt, R., and Kaznelson, P.: Zeit.f. kl. Med., 83, 79. 1916; Med. Kl., 12, 171. I916.

${ }_{13}$ Salomon, R., and Vey, E.: Arch.f. Gyn., 116, 317. 1922; Monatschr. f. Lab. und Gyn., 9, I 23. 1922 .

${ }^{14}$ Löhr, W., and H.: loc. cit.

${ }^{15}$ Eggstein, A. A.: Jour. Lab. and C7. Med., 6, 48r. 1921; also Gruenthal, R. M., and Brown, (i. M.: loc. cit.

${ }^{16}$ Vollmer, HI: Kl. Wrchr., 2, 529. 1923. 
altered ammonia coefficient, but a reduction in the amount of urine. He concluded that the immediate acidosis was due to cellular irritation, the products of this irritation then effected the oxidative mechanism and an alkalosis resulted - representing an increase in the intermediary oxidative metabolism, the products of oxidation of these intermediary products being alkaloidal in character. ${ }^{\mathrm{T}}$ The results of Vollmer have been repeatedly confirmed, most recently by Lukacs. ${ }^{2}$ In a study which Müller and $I^{3}$ have just completed we have followed the ionic changes of the lymph during the continuous injection of bacteria and have noted that the calcium-potassium ratio shows diphasic alterations. A primary reduction in the calcium-potassium ratio changes later to an increase.

An increase in non-protein nitrogen occurs in the serum, ${ }^{4 \cdot 6}$ here again as a diphasic phenomenon. So, too, there is a primary increase in nitrogen elimination, followed by nitrogen retention. Meyer-Bisch has determined a similar change in the sulphur metabolism. ${ }^{7}$ The non-protein nitrogen of the liver increases after anaphylactic and protein shock ${ }^{8}$ and after fatigue. 9 The basal metabolism has been studied by Du Bois. ${ }^{10}$

IMmUNiTy REACTIONS.-Alterations in the antibody titer of the serum after non-specific reactions have occupied the attention of many investigators. That preformed antibodies might be "shed" into the blood stream after various procedures has been long known. Inasmuch as it is the cell surface that is involved in the specific processes that are initiated with immunization, the probability that antibodies might be altered in such reactions is obvious. A large number of papers ${ }^{\mathrm{II}-29}$ have established that the immunized animal will respond

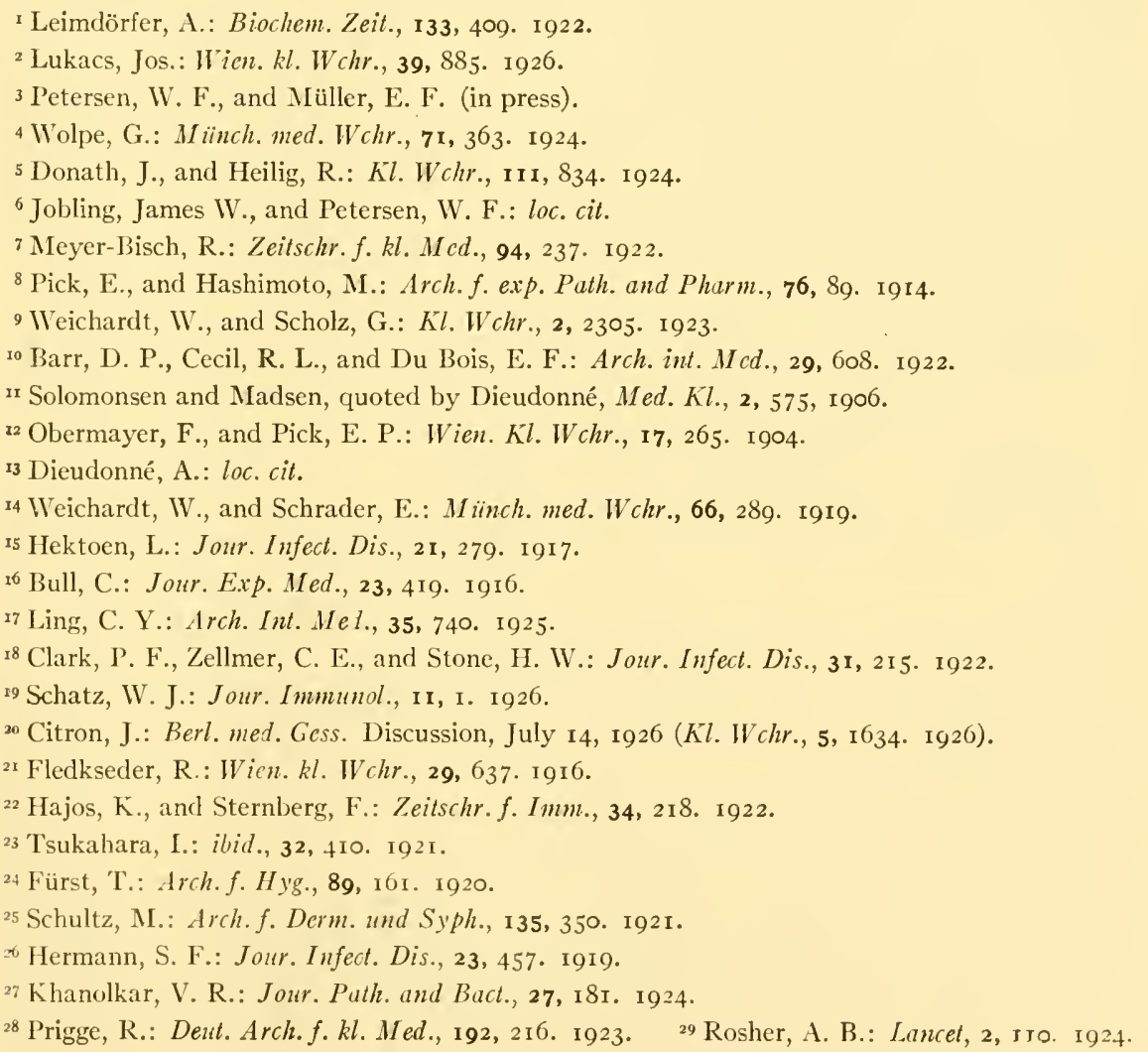


promptly with an increase in antibody titer, variations occurring according to the agent used for stimulation and the particular antibody under investigation. Freund and Dresel have noted the appearance of anthracocidal substances. ${ }^{1}$

Incrcase in protection against infection has been noted ever since the early experiments of Pfeiffer ${ }^{2}$ who studied the effect of non-specific injections on the resistance of guinea pigs to cholera. ${ }^{3}{ }^{6}$ This protection may be effective to approximately ten times the lethal dose, but is of transient character. The incubation time of infections, in humans at least, may be prolonged.7 There is, on the other hand, no clear-cut evidence that non-specific reactions, if initiated at the time of an intoxication or after an intoxication has been established, are in any manner antitoxic. . $^{\text {-10 }}$ It is true that if the animal has been treated before the injection of the toxin (tetanus, diphtheria, ricin, etc.), some evidence for an increase in resistance may be found, but the action is uncertain and erratic.

If we turn now from the consideration of the fluid constituents of the blood to the walls of the capillaries we observe one of the most important tissues in which non-specific reactions take place. And in this connection it is practically impossible to divorce consideration of the autonomic nervous influences, ionic and hormonic changes, and the anatomical peculiarities of the system. From the latter aspect alone we must consider as separate entities the reticuloendothelial system; ;1-13 the endothelium of the splanchnic area (particularly that of the liver, spleen, kidneys, etc.) which is anatomically adopted as a filtering mechanism par excellence (Zimmermann) ${ }^{\mathrm{I} 4}$ and is, in addition, located in organs that normally are functionally very active; and, finally, the ordinary vascular endothelium and the specialized endothelium of the blood and leukocyte-forming tissues. In all cases stimulation is associated with an increased permeability, and this is followed either by a rapid (normal) or slow (fatigue) return to a normal status, which may be overaccentuated. With certain agents (the metallic poisons, etc.) an increased density of the membrane surfaces may become apparent with a diminution of permeability. If severe enough this may, of course, lead to the death of the cell. [For general orientation the monographs of Osterhout, ${ }^{15}$ Lillie, ${ }^{16}$ and Hoeber ${ }^{17}$ should be consulted.] The

I Freund, H., and Dresel, E. G.: Arch.f. exp. Path. und Pharm., 91, 31 7. I92 I.

${ }_{2}$ Pfeiffer, R., and Isaeff: Zeit.f. Hyg., 16, 282 and 287. I894.

3 Kepinow, L.: Comp. rend. de la Soc. Biol., 91, 244. Paris, I924.

4 Harvey, IV. F., and Iyenger, K. R.: Jour. Med. Res., 9, 737. 1922.

5 Bechhold, H.: Münch. med. Wchr., 69, 1447. I922.

${ }^{6}$ Wolff-Eisner, A.: Kl. Wchr., 6, 545. I927.

7 Weichbrodt, R.: Dent. med. Hchr., 51, 1949. I925.

8 Wolff-Eisner, A.: loc. cit.; Hoefer, P. A., and Herzfeld, E.: Arch. f. exp. Path. und Pharm., 99, 380.1923.

9 Herzfeld, E., Meyer-Umhofer, P. and Becker: Deut. med. Wchr., 52, 1464. 1926.

ro Weichardt, W.: Mïnch. med. Wchr., 67, 1085. I920.

II Busson, B.: loc. cit.; Aschoff, L.: Lectures on Pathology. New York: Paul B. Hoeber, I924.

${ }_{12}$ Saxl, P.: Fortschritte und Probleme in der Therapie, innerere Krankheiten. Berlin: Springer, 1926; and Wien. Arch. inn. Med., 13, 7. 1926.

ז3 Mattausch, F.: Med. Kl., 20, 240, 277. 1924.

${ }_{4}$ Zimmermann, K. W.: Der feinere Bau der Blutkapillıren. Miinchen und Berlin: Bergmann and Springer, 1923.

${ }_{15}$ Osterhout, W. J. V.: Injury, Recovery and Death. Philadelphia and London: Lippincott, I922.

${ }^{6}$ Lillie, R. S.: Protoplasmic Action and Nerrous Action. Chicago: University of Chicago Press, 1923.

${ }_{17}$ Hoeber, R.: Physikalisihe Chemie der Zelle und Geivelye (5th ed.). Leipzig, I922. 
changes in capillary permeability following protein therapy have been studied by Müller, ${ }^{\mathrm{t}}$ Starkenstein, ${ }^{2}$ Luithlen, ${ }^{3}$ Petersen, ${ }^{4}$ and many others..$^{5-8}$ An increase in permeability follows the primary reaction, but this increase is largely confined to the splanchnic region. Later there is a reversal to impermeability.

The reticulo-endothelium becomes more active (phagocytosis) and later shows greatly lessened activity. This change is true not only in relation to formed particles and colloids, but seems to hold true for the passage of water. To $\mathrm{Saxl}^{9}$ in particular we are indebted for much information in this connection.

The Nervous System.-Klingro in I91 2 demonstrated the increased irritability of the peripheral nervous system during the course of sensitization. Gamper and Chiari ${ }^{1 r}$ and Weichardt ${ }^{12}$ have confirmed this. Pollitzer and Stolz have studied the effect on the brain centers $^{13}$ for respiration and temperature regulation. Perhaps of far greater importance is the change that takes place in the autonomic nervous apparatus, a subject that has been reviewed by Freund, ${ }^{14}$ Lukacs, ${ }^{15}$ Müller, Myers, and Petersen ${ }^{16}$, and Müller and Petersen. ${ }^{17}$ Freund and Gottlieb ${ }^{18}$ determined that the autonomic apparatus became more susceptible to pilocarpine as well as to epinephrin,,$^{39-2 x}$ but the ultimate effect seems to be a distinct increase toward the sympathetic side. ${ }^{22}$ I shall discuss the subject in greater detail.

\section{PHARMACOLOGICAL ASPECTS}

The pharmacological aspects underlying "protein therapy" have been treated in recent years by $\mathrm{H}$. H. Meyer, ${ }^{23}$ Freund, ${ }^{24}$ Weichardt, ${ }^{25}$ and in this country Hanzlik ${ }^{26}$ has added much to our knowledge. Meyer emphasizes a number of principles

I Müller, quoted by Ebbecke, U.: Deut. med. Wchr., 50, I3I. 1924.

${ }^{2}$ Starkenstein, E.: Minnch. med. Wchr., 66, 205. 1919.

${ }^{3}$ Luithlen, F.: Mled. Kl., 2, 1713. I913.

${ }_{4}^{4}$ Petersen, W. F.: Protein Therapy. Macmillan, 1922; Wien. kl. Wchr., 37, 233-56. I924.

5 Seiffert: Borl. kl. Wchr., 58, 873. 1926.

${ }^{6}$ Siegmund, H.: Kl. Wchr., I, 2566 . 1922.

7 Siegert, quoted by Dollken, Berl. kl. Wchr., 56, 226 and 277. I918.

${ }^{8}$ Embden, G., and Lange, H.: Kl. WV chr., 3, I 29. I924. $\quad 9$ Saxl, P.: loc. cit.

${ }^{\text {10 }}$ Kling, C. A.: Zeilschr. f. Imm., I3, 43. I912.

${ }^{11}$ Gamper, E., and Chiari, O.: Deut. Zeitschr.f. Chirg., I 72, г6г. 1922.

${ }^{2}$ Weichardt, W.: Technik sowie serologische Studien auf dem Ge'sict der exp. Therapie. Stuttgart: Ėnke, I906.

${ }^{13}$ Pollitzer, H., and Stolz, E.: Wien. Arch. inn. Med., I2, I69. I926.

${ }^{14}$ Freund, H.: Deut. med. Wchr., 52, 2187. 1926.

${ }^{15}$ Lukacs, Jos.: Wien. Kl. W'chr., 39, 885. 1926.

${ }^{16}$ Müller, E. F., Myers, C. N., and Petersen, W. F.: Jour. Amer. Med. Assoc., 88, I1 2 S. 1927.

${ }^{17}$ Müller, E. F., and Petersen, W. F.: K7. W'chr., 5, 53. 1926.

${ }^{18}$ Freund, H., and Gottlieb: Arch.f. exp. Palh. und Pharm., 9ז-92, 272. 1922; ibid., 93-94, 92. 1922 .

${ }_{19}$ Klemperer, G.: Berl. med. Gess, June I6, 1926 (Kl. Wchr., 5, 1489. 1926).

${ }^{20}$ Frühlich, A., and Paschkis, K.: Arch.f. exp. Path. und Pharm., I17, 169. 1926.

${ }^{2 x}$ Rosenthal, F., and Holzer, P.: Berl. kl. W'chr., 58, 675. I921.

${ }_{22}$ Adler, A., and Blumenberg, E.: Zeitschr.f. kl. Med., 95, 109. 1922.

${ }^{23}$ Meyer, H. H.: Pharmakologische Grundlagen der Reiskör pertherapie. Wien: Moritz Perles, 1925.

${ }^{24}$ Freund, H.: loc. cil.

${ }_{25}$ Weichardt, W.: loc. cit.

${ }^{26}$ Hanzlik, P. J., Deeds, F., and Tainter, M. L.: Arch. int. Med., 36, 447. I925. 
that should be kept in mind. In the first place, all effects on cells result, in their ultimate analysis, either in an acceleration and augmentation or in a diminution or retardation of their function, and in these alterations the changes of the cell surface are of greatest importance (Straub, ${ }^{\mathrm{T}}$ Heubner, ${ }^{2}$ Schade ${ }^{3}$ ), an increase in activity being associated with an increase in permeability and a decrease in permeability with retardation of function. Ordinary pharmacological agents-the indifferent narcotics, the alkali salts, the alkaloids - produce changes that are readily reversible because they produce only physical changes in the cell membrane (Beutner). ${ }^{4}$ But the nonspecific agents, largely colloidal, and including such agents as sulphur, salvarsan, novasurol, produce changes that are less readily reversible, and the effects may be apparent for a long period of time. ${ }^{5}$ They seem to alter the cell membrane in a much more profound manner. I have pointed out these relations in some detail elsewhere. ${ }^{6}$

In general, the pharmacologist has had difficulty in approaching the problems of non-specific therapy. Apart from typhoid fever, the method is most useful in the more chronic types of infection which occur commonly in man and which can be reproduced with difficulty, if at all, in the lower animals.

There must be considered, too, the fact that most of the agents are practically indifferent when tested by the ordinary experimental methods (muscle and nerve preparations) used in pharmacological technique. Nor are the agents selective poisons in the usual sense of pharmacological activity, i.e., agents which possess affinity for certain cells and for certain organs. Freund ${ }^{7}$ groups the agents into three classes: (I) those which obviously affect certain organs in the living animals, but in which the action differs in the isolated, surviving tissue; (2) those that are inactive when given by mouth, but which seem active when given parenterally; (3) those of the type wherein no direct chemical agent enters the body (irradiation, cautery, venesection, etc.). Without doubt, a basic factor lies in the change of the entire organism after the reaction to the non-specific agent. The Germans use the term Umstimmung to denote that the body reacts differently to a stimulus after the reaction than before the injection. It is a "non-specific desensitization," a state very closely related to specific desensitization or the anti-anaphylactic period that follows in the wake of an anaphylactic shock. Pharmacologically this is novel in that with ordinary drugs an effect is manifest when the drug makes contact with the specific tissue and the effect ceases with the excretion or the neutralization of the agent. Not so with protein therapy. It is the continued effect which becomes manifest that is the basis of the therapeutic effectiveness. I am by no means certain that the pharmacologist is correct in assuming that the original status of the cell is always regained after the ordinary pharmacological effect has apparently subsided; that, however, is a subordinate

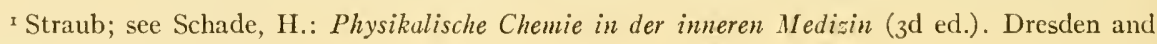
Leipzig: Steinkopff. 1923.

${ }^{2}$ Heubner, W.: ibid.

3 Schade, H., Giesche, T., and Kielholz, S.: Zeit.f. d. ges. Med., 49, 334. 1926.

4 Beutner, R., and Monetoff, A.: Proc. Soc. Exp. Biol. and Med., 24, 462.1927.

s Berger, IV.: loc. cit.

${ }^{6}$ Petersen, W. F.: Jour. Amer. Med. Assoc., 76, 31 2. I921.

7 Freund, H.: loc. cit. 
matter at the moment. Heubner ${ }^{I}$ has used the expression "patho-biosis" for the change in the cell that has at one time been the seat of some toxic (pharmacological) action, the cell remaining altered and the effect being irreversible. As far as the entire organism is concerned, Sachs ${ }^{2}$ finds the expression of this change in an instability of the colloids. A long-continued globulinemia with an increase in non-protein nitrogen and a change in the mineral constituents may persist.

While such alterations result from the general reaction, Freund and Gottlieb ${ }^{3}$ have been able to demonstrate that pharmacologically active substances appear in the blood stream irrespective of the method by which the non-specific reaction is induced. Thus, immediately after the reaction a vasodilating substance can be isolated (a basic-alcohol soluble substance), while later a vasoconstricting principle can be so isolated. Obviously these substances take origin in the body and seem definitely associated with the increased protein destruction apparent early in the reaction. ${ }^{4-\text { ro }}$ Perhaps they are derived from lipoidal metabolism, ${ }^{11}$ for both cholin and cholesterol derivatives may be active on blood vessels. It is possible that they may be histaminlike substances. ${ }^{12}$

That the organism is fundamentally re-oriented is made evident in the change in the autonomic response when aseptic pyretic agents as well as pilocarpine and adrenalin are injected. It is particularly this change in the autonomic status of the organism which accounts for many of the phenomena which are to be oberved with protein therapy (Engel, ${ }^{13}$ Uhlmann, ${ }^{14}$ Klemperer, ${ }^{15}$ and Adler and Blumenberg. ${ }^{16}$ ) It might be well to summarize this concept, with reference to the chain of events that follows the protein injection.

An intravenous injection of bacteria (vaccine) is followed by the rapid fixation of the organisms in the reticulo-endothelial apparatus, largely in the liver and spleen. This causes an immediate stimulation of these cells and, depending on the degree of toxicity, of the parenchymal cells and the vascular and lymphatic endothelium of the region. A parasympathetic status is established (splanchno-peripheral balance). ${ }^{17}$ This involves a sympathetic

I Heubner, W.: loc. cit. ${ }^{2}$ Sachs, H.: loc. cit.

3 Freund, H., and Gottlieb, R.: loc. cit.

${ }_{4}$ Barr, D. P., Cecil, R. L, and Du Bois, E. F.: loc. cit.

5 Amstad, E.: Biochem. Zeit., 145, 168. 1924.

${ }^{6}$ Pick, E., and Hashimoto, M.: Arch.f. exp. Path. und Pharm., 76, 89. igr4.

7 Freund, H., and Rupp, F.: ibid., 99, I37. 1923.

${ }^{8}$ Bieling, R., and Gottschalk, A., and Isaac, S.: Kl. Wchr., I, I560. 1922.

${ }_{9}$ Löhr, H.: Zeit.f. d. ges. exp. Med., 30, I and 6. 1922.

10 Weichardt, W., and Scholz, G.: Kl.Wchr., 2 (No. 2), 2305. 1923.

in Mahnert, A., and Santner, A.: Arch.f. Gyn., Ir6, 98. 1922; also Gruenthal, R. M., and Brown, G. M.: loc. cit.

12 Abelin, J.: Biochem. Zeit., 129, I. 1922.

${ }^{13}$ Engel, D., and Kerekisa, A.: Abs. kl. Wochen., 5, 1689. 1926.

${ }_{14}$ Uhlmann, R.: Zentblatt f. inn. Med., 45, 833. 1924.

is Klemperer, G.: loc. cit.

${ }^{16}$ Adler, A., and Blumenberg, E.: loc. cit.

${ }^{17}$ Müller, E. F., and Petersen, W. F.: Kl. W'chr., 6, 840. 1927. 
status of the periphery and consequently a leukopenia ${ }^{\mathrm{r}}$ and a chill. ${ }^{2} \mathrm{~A}$ rise in temperature takes place because of the relative fixation of the autonomic apparatus (internal parasympathetic, external sympathetic). During this time the glands of internal secretion play a rôle, probably by the liberation of epinephrin (Cannon). The epinephrin may intensify the contraction of the peripheral vessels but causes no contraction of the splanchnic region because here, in stimulated tissue, the reaction is paradoxical.

When the parasympathetic status of the splanchnic region has been established the secretory organs are stimulated. ${ }^{3}$ Thus there is an increased bile flow from the liver, perhaps gall-bladder contraction. ${ }^{4}$ The pancreas secretes insulin as well as its external secretion. ${ }^{5}$ The villi of the intestine exude a thin milky fluid. ${ }^{6}$ The stomach secretes an acid gastric juice and dilates, i.e., assumes a status similar to that after food ingestion (Petersen and Müller) ${ }^{?}$

There is a transient alkalosis (Lukacs), and then the phase of acidosis is initiated. Lymph flow is augmented, the capillaries become more permeable, the calcium-potassium ratio is diminished. All of these changes are associated with the parasympathetic status of the splanchnic region, and the change in organ activity brings with it alterations in the platelet count,${ }^{8}$ in the blood and lymph sugar, ${ }^{10-26}$ in the blood pressure, etc. The periphery is sympathetically oriented, the blood vessels of the skin and muscle contracted, heat loss diminished, glandular activity lessened (Petersen and Müller).

Then follows a reversal of the splanchno-peripheral balance, and the splanchnic region becomes sympathetically and the periphery parasympathetically oriented (leukocytosis, heat loss, etc.). The calcium-potassium ratio is increased, an alkalosis is established, activity of the reticulo-endothelium is greatly reduced ( $\mathrm{Jaffé}$, in unpublished experiments, finds the time of phagocytosis of oleokoniol before the chill between four and five minutes, during the time of chill under three minutes, and after recovery from seven to fifteen minutes, a condition that is maintained for several days), there is positive nitrogen and water balance, etc.

The non-specific reaction autonomically is characterized by its distinct diphasic character, which can be resolved into distinct chemical, endocrine, hormone, and nervous components.

\section{THE REACTION IN TYPHOID FEVER}

Typhoid fever represents an acute infection of peculiar character, peculiar because of the localization in the splanchnic lymphatic apparatus. As I have mentioned,

Müller, E. F.: loc. cil.

${ }^{2}$ Petersen, IV. F., and Müller, E. F.: Arch. Int. Med. (in press).

3 Weichardt, W., and Scholz, G.: loc. cit.

4 Müller, E. F., Petersen, WT. F., and Kast, A. (in press).

s la Barre, J.: Compt. rend. de la Soc. de Biol., 96, I49. Paris, 1927.

${ }^{6}$ King, C. E., Arnold, L., and Church, J. G.: Amer. Jour. Physiol., 6r, So. 1922.

7 Müller, E. F., and Petersen, WV. F.: Mïnch. med. Wchr., 74, 531 and 588 . I927.

${ }^{8}$ Vorschutz, J.: Pflugus Archiv., I89, 4. I921. 9 Stahl, R.: Kl. Hchr., I, 2 I32 I022.

to Gruenthal, R. M., and Brown, G. M.: loc. cil.

${ }^{11}$ Löwy, J.: Berl. kl. II'chr., 54, 989 . I9I7; and Deul. Arch. kl. Med., I20, I31. г9I6.

${ }^{12}$ Löhr, W. and H.: Zeit.f.d.ges. exp. Med., 3 1, 19. 192.3.

${ }^{13}$ Stern, Wr., and Wozak, J.: Monatschr. f. Kinterheilkunde, 29, 490. I924.

I4 Menten, M. L.: Jour. Infect. Dis., 38, 354. I926. 1s P'etersen and Müller (in press).

${ }^{16}$ Fischer, A., and Weiss, H.: Biochem. Zeit., I59, I41. I925. 
typhoid fever is one of the few acute diseases in which protein therapy has yielded particularly interesting results that are of importance for the bacteriologist from the theoretical point of view.

In approximately one-third of the cases after protein therapy, patients recover by crisis. The patient's temperature returns to normal, the spleen becomes small, the rose spots disappear, bacteria disappear from stool and urine. In another third the patients are improved-i.e., the sensorium becomes clearer, appetite is improved, the temperature range lowered. In the other one-third there is practically no change in the clinical condition.

How is an abrupt termination to be explained?

When we inject the non-specific agent into the blood stream the reticulo-endothelial cells immediately fix the formed particles (bacteria) or the foreign colloidal material. Depending on the relative toxicity of the injected substance, the cells are variously stimulated. The vascular endothelium of the splanchnic area participates, and because of its remarkable structure, there is a great increase in the passage of the larger molecular aggregates from the blood to the lymph spaces. Antibodies which have heretofore been concentrated to a greater degree in the blood ${ }^{\mathrm{I}}$ enter the splanchnic lymphatic bed. With them go enzymes and complement. The typhoid bacteria are therefore suddenly subjected to lytic processes and may be destroyed, partly or completely. Their rapid destruction may however entail further production and liberation of endotoxic material. If great in amount, the injury to the patient may be such that death may take place. If only moderate in amount, but with complete destruction of the bacteria, termination of the disease by crisis occurs.

But how can we explain those cases in which crisis does not occur, in which the patient merely gives evidence of improvement? Here evidently the complete destruction of the infectious agent has not taken place, but the reaction of the host has changed. ${ }^{2}$ The same amount of intoxication now no longer brings about an equally intensive response on the part of the body.

It is at once apparent that in typhoid we are dealing with an infection that is peculiar in its localization and particularly favorable for non-specific therapy from the theoretical side. But this does not necessarily hold true for other localized infections (lobar pneumonia, for instance) or a septicemia. Generally, protein therapy in such cases does little good; sometimes it may be harmful. But here again clinical experience has demonstrated that at times a prompt and spectacular recovery may take place after some non-specific injection even in a septicemia.

How are we to explain the mechanism?

A sudden increase in antibody titre in the body fluids? A sudden enhancement of the activity of the reticulo-endothelial system with fixation and digestion? Enzyme mobilization and accelerated digestion of bacterial or tissue toxins? A change in the acid-base equilibrium of the cells, the tissues becoming more alkaline after the primary increase in acidity - with a general sympathetic status of the organism? The accumulation of leukocytes in the splanchnic region with greater destruction of bacteria? The release of fresh leukocytes from the bone marrow? Any and all of them may be

I Becht, F. C., and Luckhart, A.: Amer. Jour. Physiol., 40, 366. I9r6.

2 Burmeister, J.: Zeit.f. kl. Med., 95, 237. 1922. 
involved, and all must be considered when we are dealing with the so-called specific bactericidal effects of the various chemotherapeutic agents which have been prepared in recent years.

\section{THE FOCAL REACTION}

Of greater importance for the bacteriologist is the effect of protein therapy on foci of chronic infection. Whenever an area of chronic irritation exists in the body (chronic bacterial infection, mechanical or chemical irritation, etc.) it is found to be more susceptible to irritation than normal tissue. These irritants may be exogenous or endogenous in character; the effects may become manifest in the pain of an arthritic joint with a change in the barometric pressure, in the activation of a tubercle after remote trauma, in the onset of a psychosis following labor or an alcoholic debauch.

Our knowledge concerning focal reactions has been derived largely from experience with tuberculosis, and because of this, the term "focal reaction" has implied a highly specific tuberculin reaction, although no satisfactory antigen-antibody explanation has ever been presented. ${ }^{\mathrm{I}} \mathrm{A}$ most important factor, namely, that the tissue making up the focus reacts differently to normal as well as to abnormal stimuli because of the previous experience (the patho-biosis of Heubner) has been largely ignored. ${ }^{2}$

The foreign agent injected into the circulation may rapidly pass the capillaries of most tissues without bringing about an alteration and will finally be taken up by the reticuloendothelium. But the endothelium of the capillaries in and about a chronic focus is altered. It is more permeable and responds to stimulation more readily than the unaltered endothelium. As a result, the chronic focus of infection will be subject to diffusion phenomena, increased metabolic activity, with greater penetration of enzymes and antibodies, while at the same time accumulated toxic material may be liberated. The reaction may take several forms. If very severe, the cells may become fatigued, the reversal delayed, and severe injury result to the patient either through digestion and softening (as in a tuberculous focus), through the absorption of toxic material, or through the dissemination of the virus. If only moderate, the reversal of the status of the tissues will initiate a relatively long period of quiescence and ultimate improvement after primary augmentation of the symptomology. If subminimal, the stimulus may bring about general inhibition (rest) of the metabolic processes at the focus without apparent primary injury. This latter would be in agreement with Verworn's theory. Chronic infections have been treated very successfully when these fundamental reactions have been kept in mind.

\section{PARESIS}

One of the most interesting and useful fields of application of protein therapy has been in general paralysis. The method took origin in the work of Wagner von Jauregg, ${ }^{3}$ who has persistently developed the method to the present treatment by malaria and recurrent fever inoculation.

During the course of recent years the factors that modify the course of syphilis

${ }^{x}$ Cf. chap. Ixxv in this volume.

${ }^{2}$ Petersen, W. F., Amer. Rev. of Tuberc., 5, 318. I92I; and 15, 681. 1927.

3 Wagner von Jauregg, J.: Jahrb.f. Psych., 7, 94. I887. 
have been thoroughly studied, in this country particularly by Brown and Pearce. ${ }^{\mathrm{I}}$ The non-specific treatment may either activate or depress the symptoms. Thus Noel found that malarial fever might activate the lesions while $\mathrm{Kyrle}^{3}$ and a number of later clinicians have shown that protein therapy is of material aid in making arsenical or mercury therapy more effective,,$^{4-9}$ and others have altered the course of the disease by protein injections without specific treatment, or by malarial inoculation (Weirauk, Shamberg). ${ }^{\mathrm{ro}, \mathrm{II}}$

In the treatment of syphilis in the secondary and tertiary stages we are obviously ${ }^{2}$ dealing with a focal reaction, the mechanism of which has been discussed in the previous section. Under certain conditions we may activate a lesion. Under such conditions the organisms may be flooded into the blood stream. Scharber ${ }^{12}$ describes the flooding of the blood stream with typhoid bacteria whenever a typhoid carrier was given a non-specific injection. Presumably a similar effect takes place with syphilitic lesions. The Jarisch-Herxheimer reaction is a clinical expression. In the later stages of syphilis the virus seems very susceptible to the destructive agency of the body fluids and seems able to exist only in tissues largely protected from direct contact with the plasma (Finger). ${ }^{13}$

Apparently it is this latter factor which plays a rôle in the treatment of paresis. The meninges become more permeable after a non-specific injection (Kafka-WalterFlatau), ${ }^{\mathrm{I}, \mathrm{r}_{5}}$ and with that increase there is an increased penetration of anti-substances into regions normally deficient. Use is made of this phenomenon in the combined method of treatment whereby the non-specific reaction is immediately followed by the use of arsenicals or mercury.

There seems, furthermore, some important difference in the manner of reaction of the body to the malarial fever and that following a milk injection. Donath and Heiligi have recently shown that there is an increase in amino acids in the serum of patients at the height of the reaction to non-specific therapy but not during the malarial chill.

${ }^{x}$ Brown, Wade, and Pearce, Louise: Arch. Derm. and Syph., Dec., I9zo; Jour. Amer. Med. Assoc., 77, I619. I921.

${ }^{2}$ Noel, P.: Jour. de med. Bordeaux, q1, 515. 1920.

3 Kyrle: Wien. kl. Wchr., 30, 707. 1917; ibid., 37, 1105. 1924.

${ }_{4}^{4}$ Kalberlah, Fr.: Mïnch. med. Wchr., 69, I I4. 1922.

${ }^{5}$ Mulzer: Kl. Wchr., 5, 2347. 1926.

${ }^{6}$ Dujardin, B., and Decapps, N.: Arch. internat. de med. exp., I, 539. Liège, 1925.

7 Greenbaum, S. S., and Wright, C. S.: Arch. Derm. and Syph., 12, 858. 1925.

${ }^{8}$ Ahlswede, E.: ibid., 8, 854. 1923.

9 Bering, F.: Mïnch. med. Wchr., 73, 2016. I926.

to Weirauk, H. V.: Ohio State Med. Jour., 22, 305. 1926.

"I Shamberg, J. F., and Rule: Jour. Amer. Mel. Assoc., 88, I217. I927.

"2 Sharber, G.: Wien. kl. Wchr., 39, 1325. 1926.

I3 Finger: Discussion in the "Foreign Letter," Jour. Amer. Med. Assoc., 88, 1825. 1927.

${ }^{14}$ Flatau, E.: Rev. neurol., 33, 521. Paris, 1926.

${ }^{15}$ Franck: Sch. med. Wchr., 54, 168. 1924.

${ }^{56}$ Donath, J., and Heilig, R.: Wien. kl. Wchr., 39, 353. 1926. 
On the other hand the cerebro-spinal fluid shows such an increase in paresis during the malarial paroxysm, but not following a milk injection. This lends support to the presumption that a focal reaction takes place at the site of the lesion during the malarial reaction, ${ }^{\mathrm{r}, 2}$ and is in accord with the findings of Straussler and Koskinas, ${ }^{3}$ who have demonstrated increased inflammatory reactions during the malarial fever.

\section{SUMMARY}

The parenteral administration of foreign substances (bacteria, serum, milk, etc.) is followed by a diphasic reaction of the organism. This reaction involves a complex biological re-orientation, demonstrable with physical and chemical changes in the blood and tissues, with changes in the reactivity of the central and vegetative nervous system, in secretory glands, in lymph and blood capillaries, in the reticulo-endothelial system, in the leukocytes, and in the antibody mechanism. Usually the injection of a non-specific agent $' s$ followed by a diphasic clinical alteration-first intensification of symptoms followed by diminution. At the site of a local inflammatory lesion a focal reaction takes place. The therapeutic effect of the protein injection is to be sought in definite and long-persisting alterations in certain of the cell membranes of the body, in contradistinction to ordinary pharmacological effects which seem to depend on relatively transient alterations of the physical state of cells or cell groups.

INonne, M.: Med. Kl., 25, I825. I925.

${ }^{2}$ Reese, H., and Peter, K.: ibid., 24, 37. I924.

${ }^{3}$ Straussler, E., and Koskinas, G.: M iinch. med. Wchr., 70, 783. I923. 


\title{
CHAP'TER LXXXIII
}

\section{CHEMOTHERAPY OF BACTERIAL DISEASES ${ }^{\circledR}$}

\author{
JOHN A. KOLMER
}

University of Pennsylvania and Research Institute for Cutaneous Medicine, Philadelphia

"Chemotherapy" may be defined as the prevention and treatment of disease by chemical disinfection or inhibition of the parasitic causes without marked or serious toxic effects. It usually conveys the idea of specific therapy, and properly so, but its practical applications are by no means limited to the treatment of syphilis, malaria, and a few other infectious diseases, as popular impressions would indicate. It is true that most advance has been made in the treatment of these as well as in relapsing fever, frambesia tropica, trypanosomiasis, leishmaniasis, schistosomiasis, amebic and other protozoan and metazoan infestations, but some real progress has been made also in the important field of bacterial diseases and especially when chemotherapy is employed for the treatment of local as well as of systemic infections. In other words, the local disinfection of tissues by chemical agents properly belongs to this new field of therapeutic science and greatly broadens the field of application and usefulness.

It is true, however, that some of the original theories of chemotherapy have begun to show themselves inadequate for our expanding knowledge of the processes concerned and require reconstruction, though without either hasty or wholesale rejection of those principles which served Ehrlich and other early workers so well. But the original idea of chemotherapy, tending to shift the focus of attention from the infected host to the invading parasites by producing chemical agents capable of direct destruction of organisms without the co-operation of the host, has been questioned and especially in relation to the disinfection of the tissues in generalized infections, although this simple mechanism may, and probably does, hold good to a large degree for the disinfection of the tissues by local or topical application of parasiticidal agents.

\section{PARASITOTROPISM, ORGANOTROPISM, AND THE CHEMOTHERAPEUTIC INDEX}

A special and selective affinity of the chemical agent for the parasite designated as "parasitotropism" is the predominant thought in modern chemotherapy. "Organotropism" refers to the affinity and effects of the agent for the blood, lymph, and fixed tissues of the body, and the relation between the two is expressed as the "chemotherapeutic index." In other words, parasitotropism refers to the toxicity of the chemical agent for the parasites, and organotropism to the toxicity for the body cells; the aim of chemotherapeutic research is to discover compounds with a maximum of

$$
\frac{\text { Maximal tolerated dose per kilogram }}{\text { Minimal curative dose per kilogram }}=\text { Chemotherapeutic index. }
$$

A fuller discussion of this subject with complete bibliography will be found in Kolmer, J. A.: Principles and Practice of Chemotherapy with Special Reference to the Treatment of Syphilis. Philadelphia: W. B. Saunders Co., I927. 
the former and a minimum of the latter, i.e., with the highest range or greatest chemotherapeutic index.

The index is subject, however, to wide variations and fluctuations according to the test animal and particular strain of micro-organism employed.

To explain the mechanism of parasitotropism and organotropism Ehrlich evolved his "theory of chemo-receptors," similar to the side-chain theory of immunity, according to which chemical agents cannot act upon either parasites or body cells unless they are first fixed by suitable chemo-receptors (corpora non agunt nisi fixata). Therefore, when arsenic is administered, it is "fixed" by the arseno-receptors of certain cells; mercury in turn is "fixed" by other receptors, and so on through an interminable list. Ehrlich thought that the basic principle of chemotherapy was the possibility of producing chemical substances carrying side-arms capable of being fixed by the receptors of parasites to a much greater extent than by the body cells. Presumably it is not necessary that the whole molecule of a chemical agent possess a combining affinity for certain receptors; if one or more atom groups become attached, it is presumed that it carries with it the remainder of the molecule. Moreover, that atom group which is anchored or is responsible for the anchorage of the entire molecule need not possess any of the properties of the entire molecule or of any part thereof.

But chemotherapy has developed into a far more complex subject since complex chemical and physico-chemical forces are involved in the effects of a chemical agent on both body cells and invading parasites, and the matter of selective destruction of parasites in the tissues would appear to be one of chemical or physico-chemical interaction between the chemical agent and protoplasmic constituents of the parasites, just as the specific immunological processes of the body are being gradually placed on the same basis.

Instead of conceiving a remedy killing invading organisms by direct union through the agency of theoretical chemo-receptors, a broader and more comprehensive conception is required to explain the observed phenomena, as a remedy not acting as such, but in virtue of the formation from it in the body of some directly toxic product, either by a modification of its structure or by its union with some tissue constituent; of modifications of virulence and a reduction of resistance with increased susceptibility to destruction by the body's natural defenses, the mechanism of parasiticidal activity calling into play any one or several of the following factors depending upon circumstances:

I. A direct chemical interaction between the compound or drug as administered or after some transformation in the body, with some protoplasmic constituent of the parasite, resulting in the death or crippling of the latter by interference with its vital processes as those of alternate oxidation and reduction (internal respiration).

2. A physical or physico-chemical interaction with the protoplasmic colloids of parasites involving precipitation, coagulation, changes in electrical charge, etc., sufficient for destruction or crippling.

3. The possible production of new compounds in the tissues capable of chemical or physico-chemical interaction with protoplasmic constituents of invading parasites rather than the production of these effects by the compound direct as administered. 
4. The possible production of antibodies by releasing antigenic substances from the parasites.

5. The stimulation of processes of oxidation; the production of hyperemia and stimulation of reparative processes; the production of leukocytosis and mobilization of proteolytic, lipolytic, and diastatic enzymes capable of interfering with the nutrition or otherwise crippling invading organisms, etc.

And the mechanism of organotropic or toxic effects of chemical agents for the body cells in general or of a particular group is likewise far from being the simple matter of union by chemo-receptors, involving as it does the subjects of absorption, elimination, distribution, fate, and other complex pharmacological and toxicological effects, the production of toxic lesions and symptoms being by one or more of the following processes:

I. By immediate reactions in the blood like agglutination or lysis of erythrocytes, precipitation of plasma proteins or salts, with the production of colloidal shock reactions, embolism, etc.

2. By direct chemical interaction between the compound or drug as administered or after some transformation in the body, with some protoplasmic constituent of the fixed body cells, resulting in the death or crippling of the latter by interference with vital processes. Or by some physical or physico-chemical interaction with the protoplasmic colloids of cells, involving precipitation, coagulation, changes in electrical charge, etc., either mechanism involving the body cells as a whole or particular groups and centers by reason of special affinities or through the function of elimination.

3. By producing excessive hyperemia, cellular infiltrations, and other tissue changes; by promoting excessive enzymic activity with the production of toxic substances from dead or devitalized body cells or parasites; by promoting or stimulating the activities of parasites, as in the Jarisch-Herxheimer reactions of syphilis, etc.

A study of the toxicity of a chemical compound or drug, therefore, in relation to chemotherapy, may be not only a matter of simply determining the minimal lethal or maximal tolerated doses per kilogram of body weight for the lower animals, but under certain circumstances involves a study of its pharmacological and toxicological effects for human beings and especially for sick human beings, which brings into play far more complex processes than are met with in healthy lower animals.

\section{LOCALIZED $v s$. GENERALIZED BACTERIAL INFECTIONS IN RELATION TO CHEMOTHERAPY}

It is readily apparent that the principles and mechanism of the chemotherapy of localized bacterial infections by the direct application of disinfectant substances are quite different from those concerned in generalized or systemic infections requiring the injection of the disinfectant into the blood or its absorption from the gastrointestinal canal, muscles, or subcutaneous tissues. From the standpoint of chemotherapy, a localized infection may be defined as one confined to a certain organ or tissue and accessible by direct or topical application of a chemical disinfectant. It may be a localized infection in the strict sense of being a tissue or organ directly or 
locally infected by an organism, as, for example, the urethra by the gonococcus; but in relation to chemotherapy, it may also include a tissue or organ infected by way of the bloodstream but yet treatable by the local application of a medicament to the infected parts-for example, a joint may be infected by a streptococcus conveyed in the blood from the apical abscess of a tooth but be amenable to treatment by the local injection of a chemical disinfectant. Infections of the skin, the accessory sinuses of the upper respiratory tract, gums, conjunctivae, urethra, bladder, etc., are usually localized infections in the strict sense; but pleuritis, meningitis, and infections of other serous cavities may be also classified as localized infections from the chemotherapeutic standpoint since they may be treated by direct local or topical application or injection of disinfectant substances even though the organisms are brought to the parts by systemic channels. On the other hand, certain infections of other organs as those of the iris, gallbladder, perirenal tissues, etc., may be strictly localized but reachable therapeutically only by way of injection of medicaments directly into the blood or indirectly by absorption from the subcutaneous tissues, muscles, or gastrointestinal tract.

In other words, generalized or systemic infections are those in which organisms occur constantly or intermittently in the blood or lymph streams with one or several localizations in the fixed tissues; usually they are treatable only by way of the lymph or blood streams by direct injection of medicaments into the blood or absorption from the subcutaneous tissues, muscles, or gastro-intestinal tract. Localized infections are those in which the organisms occur in the infected parts as the result of a direct infection or by localization of organisms from the blood; according to accessibility of the part infected they may be treated by the topical application or systemic administration of drugs.

\section{ESSENTIAL PROPERTIES OF A CHEMICAL AGENT FOR THE CHEMOTHERAPY} OF LOCALIZED BACTERIAL INFECTIONS BY TOPICAL APPLICATION

I. They must possess some degree of bactericidal and bacteriostatic activity in the test tube and tissues and especially in the presence of serum, blood, pus, etc. These effects need not be extremely high, and they may be selective, as, for example, gentian violet and other dyes of the triphenylmethane series for gram positive bacteria like staphylococci, streptococci, pneumococci, diphtheria bacilli, etc. While an extremely high bactericidal or bacteriostatic activity in vitro is not essential, yet it is desirable, as such a compound is more likely to retain effective degrees of bacteriostatic activity in vivo, although a chemical agent possessing a high degree of selective bacteriostatic power may fail in the treatment of a mixed infection.

2. In addition they should possess the property of destroying toxins, and especially the toxins of the tetanus and other anaerobic bacilli of infected wounds; in this connection it may be stated that the oxidizing disinfectants, as those of the chlorin group, are believed to have this property.

3. They should not be precipitated or their bactericidal properties neutralized by the proteins or salts of the polybasic acids of the body fuids and tissues with which they come in contact, as by pus and necrotic tissues in wounds or serous cavities, by urine in the pelvis of the kidney or bladder, etc. 
4. They should possess the property of penetrating cells and tissues, and especially phagocytic leukocytes and other cells in which living bacteria may be lurking. This is one of the most important principles of all, and especially in the treatment of chronic infections of mucous membranes in which the bacteria are almost sure to have taken up a position of great strategic strength in the depths of glands and cells.

5. They should be free of local organotropic or toxic effects for the fixed tissues, i.e., free or almost so, of irritating and inflammatory properties; on the contrary, they should be of a soothing or bland nature and preferably possess some degree of analgesic effect.

6. They should not be excessively toxic for the body as a whole in case considerable amounts are absorbed. This is especially important in the irrigation of wounds and much less so in infections of the skin and mucous membranes, the whole subject bearing a direct relation to the question of absorption.

7. They should exert a chemotactic effect, i.e., attract leukocytes about the focus of infection and thereby aid in the walling off of the involved area and possibly aid phagocytosis and the elaboration of cellular proteolytic and lipolytic enzymes. At least they should not repel or destroy leukocytes and thereby favor the extension of the infection.

8. They should not destroy or paralyze the immunological substances like complement, bacteriolysins, and opsonins (bacteriotropins) concerned in the destruction of organisms.

9. They should be rapid in bacteriostatic and bactericidal activity. This is especially important in the treatment of such infected surfaces as the urethra, conjunctivae, etc., where the contact of disinfectant is extremely brief. Indeed, this largely accounts for failure in practice, and it is commonly observed that clinical results are very much better when the disinfectant is retained for some time as, for example, in the case of instillations into the urinary bladder or when it is frequently applied, as in the Carrel method of irrigation of wounds. But whenever the conditions are such that prolonged contact is not possible, rapid penetration and rapid bactericidal effects become of increasing practical importance.

Io. They should possess some degree of dynamogenic activity, i.e., the property of stimulating the fixed tissues to proliferative activity as a further aid in the walling off of lesions followed by cutting off oxygen and blood supply to the organisms and thereby allowing their own acid and waste products to check their growth and eventually destroy them.

\section{METHODS OF TOPICAL TREATMENT OF LOCALIZED INFECTIONS IN RELATION TO CHEMOTHERAPY}

But a compound possessing all or the majority of these essential and desirable properties may yet fail to prove useful in the treatment of localized bacterial infections because it may not be possible to apply it correctly. Indeed, I believe that the essential reason for the skepticism of surgeons regarding the practical value of topical applications of chemical disinfectants in the treatment of infected wounds, serous cavities, sinuses, etc., is due largely to the difficulties or impossibility of correct application. Merely to apply the disinfectant on gauze is usually insufficient, as this may only protect against secondary infection without appreciably influencing the 
existing infection. As so clearly shown by Carrel and his colleagues with the chlorin group of antiseptics in the treatment of infected wounds, it is essential to bring the disinfectant solution into frequent and intimate contact with the infected tissues along with the surgical removal of necrotic tissues when possible and advisable and always the establishment of the best possible drainage. Furthermore, these ends should be attained with the least meddling and disturbance in order to permit granulation and healing to proceed; for these reasons Carrel has contributed his valuable method of irrigation with rubber tubes laid in the wound as a substitute for frequent syringings and packings.

The method of application, therefore, is of considerable importance; indeed, I believe it decidedly outranks in importance the choice of antiseptic solution itself, providing the latter approaches in blandness simple physiological saline or Locke's solutions with a reasonable degree of bactericidal activity.

But, of course, not all localized bacterial infections demand disinfectant treatment. Judgment must be exercised according to the kind of organism present and the duration and extent of the lesion. Ordinary acute staphylococcus abscesses, for example, usually do best with simple incision for adequate drainage; and much handling, squeezing, and syringing are to be avoided. As a general rule, when the incisions of a localized lesion are only large enough for drainage, no attempt should be made to disinfect because of the danger of blocking drainage by additional tubes; but in large lesions, as infected wounds, widely incised staphylococcus and streptococcus abscesses, streptococcus infections of the uterus, etc., where provisions may be made for both drainage and irrigation, I believe that disinfection at short intervals is indicated, and especially in streptococcus infections.

It is true that disinfectant solutions may not reach or influence those bacteria already deeply situated in the tissues and especially in the lymphatics, but the proper kind of disinfectant solution along with drainage may tend to reverse osmosis and thereby reduce the degree of absorption of bacteria and toxins and at least greatly reduce their production, which may be a factor of no little value in streptococcus and pneumococcus infections. Furthermore, some localized bacterial lesions cannot be subjected to frequent disinfection because of anatomical difficulties, but in the treatment of chronic empyema, chronic suppurative arthritis, chronic suppurating sinuses, osteomyelitis, endometritis, etc., the possible advantages from well-directed attempts at disinfection at short intervals should not be overlooked or minimized, and I believe these localized infections constitute a proper field for co-operation between surgeon and chemotherapeutist.

The question of disinfecting a strictly localized bacterial infection by the administration of a compound by intravenous or subcutaneous injection or oral administration is very important, involving the present state of the chemotherapy of bacterial diseases in general, since tuberculosis, bacterial endocarditis, pneumonia, puerperal sepsis, etc., may be localized infections in just the same sense as an abscess at the root of a tooth, a sinusitis, a staphylococcus or streptococcus cellulitis and lymphadenitis. It involves primarily the question of penetration of chemical agents from the blood into the tissues of bacterial infection, but it may be stited that a general answer camnot be given since it would appear that the matter of penetration varies 
according to the infection and the kind of compound administered. For example, it is doubtful if we have available at present a chemotherapeutic agent capable of penetrating tubercles, although some of the dyes may do so; on the other hand, clinical reports indicate that gentian violet and mercurochrome are apparently able to penetrate localized infections caused by $B$. coli, staphylococci, and streptococci, and in severe acute localized bacterial infections it may be possible to reinforce disinfection by local or topical application of disinfecting agents by the systemic administration of a medicament and especially by the administration of mercurochrome.

Related to this subject of specific chemotherapeusis of local bacterial infections by the topical or local application or systemic administration of a disinfectant agent is the possibility of treating infections of the organs of the urinary, biliary, gastrointestinal, respiratory, and cutaneous systems by the administration of compounds largely eliminated in these tracts. Hexamethylamin, for example, apparently owes a part of its beneficial effects in the treatment of infections of the genito-urinary organs to the elimination of small fractions of formaldehyde liberated in acid urine, although it is doubtful if sufficient formaldehyde is liberated in other tissues as in the bile, milk, saliva, cerebrospinal fluid, etc., to produce beneficial results, and hexamethylamin itself is practically free of bactericidal effects. Recently it has been shown that mercurochrome and hexyl-resorcinol administered by ingestion or intravenous injection may be eliminated in the urine in sufficient amounts to render it bactericidal, and the same may be true to a lesser extent of other mercurial compounds. It is well known that arsenic is largely eliminated in the bile, but unfortunately the bactericidal activities are so feeble that curative effects in bacterial infections of the liver, gallbladder, and ducts are not apparent. It is not improbable, however, that mercurial compounds eliminated in the bile may reach bacteriostatic concentrations and even reduce to some extent the bacterial flora of the small intestines. The beneficial effects of creosote, guaiacol, and their derivatives in bronchitis are apparently due in part to their elimination in the bronchial secretions, but I do not know of any drug eliminated by the skin exerting curative activity in bacterial infections of this organ.

But the principle is well established and worthy of much more attention than has been heretofore given this subject. This is especially true of bacterial infections of the genito-urinary organs and biliary passages. It is probable, however, that compounds eliminated in the urine and bile may be so profoundly altered that bactericidal effects are lost; this is apparently true of the organic trivalent arsenicals like arsphenamin and neoarsphenamin, which are largely eliminated in the bile as inert pentavalent compounds, and half- or pseudo-complex compounds of mercury may become dissociated and combine with proteins and thereby lose greatly in bactericidal effects. Unfortunately, the converse of this is apparently an infrequent occurrence; i.e., relatively inert compounds do not acquire bactericidal effects during elimination except in the case of hexamethylamin when traces of formaldehyde are liberated in an acid medium. But it may be that similar effects are more common than we now suppose and thereby explain such beneficial results as those observed, for example, with creosote, guaiacol, and their derivatives in bronchial infections, the salicylates in acute rheumatic fever, etc., which possess but feeble bactericidal effects in vitro. 


\section{ESSENTIAL PROPERTIES OF A CIIEMICAL AGEN'S FOR THE CHEMOTHERAPY} OF BACTERIAL DISEASES BY SYSTEMIC ADMINISTRATION

By systemic medication I refer to the administration of compounds by ingestion or injection (subcutaneous, intramuscular, intravenous, etc.) and their subsequent distribution by the blood. This introduces new problems concerning the fate of the compound before it has had an opportunity for attacking the organisms, and the opportunities and chances for effective disinfection are greatly reduced in comparison with disinfection by direct application. The essential properties of medicaments for this purpose may be summarized as follows:

I. It must be of sufficiently low toxicity for the body cells to permit of the safe administration of adequate amounts without delayed toxic effects or the production of too violent immediate reactions by incompatibility with the blood.

2. It must be sufficiently toxic for the bacteria to exert bactericidal and bacteriostatic effects. For this purpose it must be either sufficiently high in bactericidal activity to be effective in the blood despite high dilution or be a compound convertible in the blood and tissues into a new compound of higher parasitropic activity.

3. It must not enter too rapidly into physical or chemical union with the constituents of the blood or fixed tissues, with the production of inert compounds.

4. From the standpoint of the treatment of tuberculosis and other bacterial infections characterized by the production of pathological exudates and tissues, it must possess the property of penetration sufficient for disinfective purposes by conforming to the physical laws of diffusion or by possessing a chemical affinity for the proteins or other constituents of the organisms according to Ehrlich's conception of parasitropic activity. This, however, is not required for the disinfection of the blood and lymph although an affinity between compound and organism must exist.

5. The compound should not depress immunological processes and especially phagocytosis. On the other hand, it should produce leukocytosis and enhance phagocytosis in both the blood and fixed tissues.

6. In addition to these desirable qualities, the curative activity of a chemotherapeutic agent or drug may be enhanced if it possess some degree of dynamogenic and chemotactic activity, the properties of exciting hyperemia about lesions with serous and cellular exudation and promoting the proliferation of connective tissue which tends to wall off chronic lesions and gradually aid in their disinfection by cutting off oxygen and food supply and allowing the acids of waste products of bacteria and the products of caseation and liquefying necrosis to check and eventually destroy the organisms.

\section{BACTERIAL DISEASES IN RELATION TO CIIEMOTHERAPY}

INFLUENCE OF PATHOLOGICAL TISSUE CHANGES

As would be expected, better results are observed in the chemotherapy of acute than of chronic infections. Probably best results of all are secured in the disinfection of the blood in cases of bacteremia (septicemia). This is true because we have a few drugs like ethylhydrocuprein hydrochlorid, mercurophen, mercurochrome, metaphen, gentian violet, and acriflavin which may be given in sufficient amounts to raise temporarily the bactericidal titre of the blood for effective sterilization although it 
may become reinfected a few hours later by organisms from localized lesions escaping disinfection.

But organisms in the fixed tissues can be reached and destroyed only when sufficient amounts of the compound in an active bactericidal state leave the blood and permeate the lesion. This in turn involves the questions of rapidity of elimination, production of inert combination compounds, the physical laws of diffusion, capillary permeability, etc.; it also involves the degree and kind of pathological tissue changes which may be present and which constitute the theme of the present discussion.

In acute bacterial infections the hyperemia is undoubtedly a favoring factor for bringing the bactericide to the sites of infection, and during the processes of acute cellular and serous exudation a part of it may be swept along into the inflammatory exudates even though the compound itself does not possess the physical characteristics which facilitate its passage through the walls of normal vessels. Permeability is of fundamental importance, but in acute inflammatory lesions it may not be as important as in chronic lesions because of the opportunities, in the former, for the compound's being carried along mechanically into the infected tissues by the processes just mentioned. We may expect, therefore, better results in acute than in chronic infections. But even when compounds like mercurochrome, acriflavin, gentian violet, optochin, etc., leave the capillaries their diffusion may be hindered by the physical characteristics of the edematous fluid and pus as well as by the leukocytes and other cells containing many of the organisms. The problems, therefore, are many and difficult, and particularly good results may not be obtained until we learn more about the laws governing permeability and diffusion under these circumstances and until we succeed in evolving compounds which will accumulate in the lesions to reach bactericidal concentrations. This, I believe, occurs with mercury in the treatment of syphilis and explains its peculiar efficacy in this disease.

In chronic bacterial diseases the lesions may be quite avascular as in the case of tuberculosis; furthermore, many of the blood vessels are likely to be sclerosed, which may amount to occlusion of the smaller vessels. Serous and cellular exudation are reduced to a minimum, and we have therefore a set of circumstances very unfavorable for chemotherapy, especially if our compounds possess but feeble permeability for capillaries at best and a low diffusibility in the products of caseation and liquefying necrosis.

Needless to state, the pathological lesions produced by bacterial infections may also influence the organotropism or toxicity of compounds for the body cells. It is commonly observed that the tolerance of infected animals for a drug is appreciably less than in normal animals, and I believe the same is true of human beings. The bacterial toxins and exudates are always likely to produce albuminous degeneration of the kidneys, heart, and other organs which may proceed to more advanced lesions and interfere with elimination to such an extent as may increase the chances for toxic effects and especially in the case of chronic diseases. On the other hand, just the opposite may occur during the acute stages; for example, the patient with croupous pneumonia has, I believe, a higher tolerance for optochin and quinin compounds in general than normal human beings. This is analogous to the increased tolerance of syphilitics for mercury and of malarial-infected individuals for quinin. In other 
words, the toxins of the disease or some other agencies may have a detoxifying effect upon the drug administered.

\section{INFLUENCE OF IMMUNOLOGICAL FACTORS}

Until we succeed in producing far more satisfactory chemotherapeutic compounds for the treatment of bacterial diseases than available at present, recovery will continue to depend largely upon immunological processes aided in some diseases by biological therapy (vaccines, sera, blood transfusion, etc.). The question naturally arises whether or not it is possible for us to aid immunological processes by the administration of drugs. To be classed in the domain of chemotherapy, must drugs increase antibody production or aid and facilitate phagocytosis in bacterial diseases?

We hear and read a great deal about arsphenamin and its congeners increasing antibody production or aiding immunological processes in syphilis and trypanosomiasis, but the evidence presented so far is not very convincing. In bacterial diseases, however, acquired immunity is developed to a greater degree and plays a far more important part in recovery than in syphilis, and anything that may be safely done to hasten or increase these beneficial changes is to be welcomed.

In the first place, the intravenous administration of mercurochrome, other heavy metals, and some of the dyes, quinin compounds, etc., commonly employed at present in bacterial chemotherapy, may increase the leukocytes of the blood and especially the polymorphonuclear neutrophiles which we regard as playing a very important part in natural and acquired resistance and immunity. The doses required, however, for this result are usually the maximum amounts by intravenous injection. Unquestionably it is reasonable to expect that these leukocytes will prove of aid in overcoming the infection, and the clinical reaction following the intravenous injection of mercurochrome and other heavy metals bears such a close resemblance to the socalled "non-specific protein" reactions produced by the intravenous injection of peptone, vaccines, etc., that one cannot escape the suspicion that a good part of the curative results may be due to the same mechanism as is called into play by the administration of these protein agents.

But I have had so far no clear or conclusive evidence of the increased production of such humoral antibodies as the antitoxins, agglutinins, precipitins, opsonins, and bacteriolysins during the course of experimental or natural bacterial infections, to be ascribed to the influence of such drugs as optochin, gentian violet, acriflavin, and mercurochrome. It may be that such compounds actually stimulate the production of antibodies, since the production of leukocytosis indicates that the bone marrow and the antibody-producing tissues in general may be stimulated, but it is difficult or impossible with present test tube methods to elicit and present the evidence. It is true that the administration of these compounds to normal animals may increase temporarily their resistance to bacterial infection in a manner analogous to the temporary resistance of mice and rats, treated with some of the dyes, to inoculation with pathogenic trypanosomes; but the resistance is of such short duration that one cannot be sure that the results are not due rather to a retention of some of the compound in the blood and tissues than to an increase of immunological resistance. But it may be that some of our chemotherapeutic compounds may actually aid in immunological re- 
sistance and recovery from experimental and natural bacterial diseases of human beings and the lower animals, not so much by the actual stimulation of production of humoral antibodies as by lowering the resistance of bacteria to phagocytosis, by the production of leukocytosis, a febrile reaction, and other changes now so well known clinically as the "non-specific protein reaction."

\section{COMBINATION CHEMOTHERAPY AND BIOLOGICAL THERAPY}

.This raises the question of the practical value of combining chemotherapy with such biological therapy as the administration of immune sera, vaccines, non-specific protein agents, and blood transfusion, in the treatment of bacterial diseases. All of my clinical experience indicates that best results are to be obtained by the judicious use of both kinds of therapy in the treatment of bacterial diseases, and especially the acute infections. Chemoserotherapy, or the combination of chemotherapy with serum therapy, has been before the medical profession for several years and is deserving of the closest study and attention.

After all, we are more interested in the recovery of our patient than in figuring out in exact terms to what recovery may be ascribed, and I rarely depend upon chemotherapy alone in the treatment of the acute bacterial diseases, but always include serum therapy, blood transfusion, or vaccine therapy when indicated. The chemotherapy of bacterial diseases has not progressed by any means to the stage where we can afford to neglect surgery, biological therapy, or any other helpful therapeutic means for winning the battle with infection. For example, I rarely neglect to use antistreptococcus serum in the treatment of acute streptococcus infections with septicemia, along with gentian violet or mercurochrome. I am likewise very partial to transfusions of blood, and especially in the treatment of long-continued and exhaustive infections associated with anemia. Even the administration of vaccines and non-specific agents may be of aid, not only because of their influence upon antibody production and immunological processes, but likewise because their administration may produce mild focal reactions about the diseased parts characterized by hyperemia and serous exudation which may facilitate the penetration of a chemotherapeutic compound in the blood and prove generally useful when due care is exercised in dosage to avoid excessive hyperemia and exudation. I also surmise that judicious tuberculin administration to produce mild focal reactions of this kind may ultimately prove a useful adjuvant to the chemotherapy of tuberculosis, since the hyperemia and exudation may encourage the much-to-be-desired penetration of tubercles by tuberculocidal chemical agents brought to the infected tissues by the blood.

\section{ANILIN DYES IN THE CHEMOTHERAPY OF BACTERIAL DISEASES}

The anilin dyes have long held a place of primary interest and importance in the chemotherapy of bacterial diseases, not only from the standpoint of displaying a direct bactericidal activity themselves, but also as possible carriers of more potent substances to the protoplasm of the organisms. While we now know that mere staining of bacteria does not necessarily indicate their death, yet the idea and hope of utilizing dyestuffs for the disinfection of the tissues have been long entertained and interest in the subject greatly renewed within recent years. 
Practically only the synthetic dyestuffs prepared from coal tar have been employed for the staining of bacteria and for bacteriostatic and bactericidal purposes. Natural dyes, like Indian yellow, quercetin, chrysin, cochineal, carmine, cudbear, hydrastis, etc., have been proved to be without parasiticidal properties and have not been employed for these purposes.

A very large number of dyes have been prepared from coal tar, and their chemistry is exceedingly involved, the exact constitution of not a few being still in dispute and their preparation kept as trade secrets. Furthermore, the various dyes as available are not always in a pure state and sometimes vary greatly in their staining properties, toxicity, bacteriostatic and bactericidal activity. Those mostly employed have belonged to the nitroso, azo, triphenylmethane, diphenylamin, acridin, and pyronin groups.

\section{BACTERIOSTATIC AND BACTERICIDAL ACTIVITY OF THE SYNTHETIC DYES IN RELATION TO CHEMOTHERAPY}

The synthetic dyes vary greatly in bacteriostatic and bactericidal activity. Some dyes, and especially certain members of the triphenylmethane and acridin series, like gentian violet, acid and basic fuchsin, brilliant green, acriflavin, proflavin and rivanol, are very highly bactericidal, while others like methylene blue and fluorescein are relatively feeble and mainly employed in chemotherapy for the purpose of combination with mercury or some other more highly bactericidal substances for aiding penetrability. Gentian violet and magenta may inhibit the growth of staphylococci and streptococci in culture media in dilutions as high as I : I00,000 to I : I, ,, 000 in agar or egg medium and are commonly employed for aiding in the isolation of tubercle bacilli and organisms of the typhoid-colon group.

As a general rule, however, the majority of anilin dyes are relatively feeble in bactericidal activity, and especially in the presence of serum, blood, pus, and other organic matters. Probably gentian violet, acid fuchsin, brilliant green, and acriflavin possess the highest bacteriostatic and bactericidal properties, gentian violet being particularly destructive for gram positive bacteria and the fuchsins for gram negative bacilli; but even dyes of the same series vary greatly in these properties.

Of great interest and importance in relation to the chemotherapy of bacteremia is the question of bactericidal activity and fate of the dyes when introduced into the blood, usually by intravenous injection. This has been studied by injecting the dyes and withdrawing blood at intervals for bactericidal tests and also by producing experimental bacteremias of rabbits and treating with intravenous injections of the dyes. From such experiments it would appear that large doses of gentian violet and acriflavin injected intravenously may at least temporarily increase the bactericidal activity of the blood (for about an hour), but these effects may not be sufficient for materially influencing a severe experimentally produced bacteremia, since the dyes are rapidly absorbed by the tissues.

\section{BACTERICIDAL ACTIVITY OF MIXTURES OF THE DYES}

The question of the bactericidal activity of mixtures of various dyes deserves brief consideration. Gay and Morrison have tested various combinations for streptococci, staphylococci, and typhoid bacilli and found that the final bactericidal dilution 
of such mixtures might reach the limit of the stronger dye, but in case of interaction and precipitation between the two solutions the bactericidal effect was actually decreased. Churchman has observed that mixtures of gentian violet and neutral acriflavin are bactericidal; also mixtures of gentian violet and fuchsin, the gentian violet for gram positive organisms, and the acriflavin and fuchsin for gram negative organisms. In my experiments these observations have been confirmed, and in the mixtures the identity of the dyes appears to be well preserved in that the bactericidal effects of the mixture for gram positive organisms is approximately the same as shown by the gentian violet alone in the same strength, and the bactericidal effect for gram negative organisms is about the same as shown by the acriflavin or fuchsin alone in the same strength. Churchman has advocated, therefore, the use of mixtures of these kinds for the disinfection of tissues infected with both gram positive and gram negative organisms.

\section{INFLUENCE OF HEAT UPON THE BACTERICIDAL ACTIVITY OF THE ANILIN DYES}

Since heat tends to increase the rapidity and rate of chemical reactions, it is to be expected that hot solutions of the anilin dyes will prove more bacteriostatic and bactericidal than cold solutions, and Churchman has furnished definite proof that moderate heating $\left(55^{\circ} \mathrm{C}\right.$. for one hour or $50^{\circ} \mathrm{C}$. for one and one-half hours) increases the penetrative power and bactericidal activity of gentian violet. Working with an impeccable gentian violet-fast strain, he found gentian violet without effect at $22^{\circ}-$ $35^{\circ} \mathrm{C}$., but bactericidal when the solution of dye was heated to $50^{\circ} \mathrm{C}$.; control tests showed that the effects were not due to heat alone or the dye alone, but to the combination of heat plus dye. The phenomenon has not been explained, but the facts established indicate that when the dyes are used for the treatment of localized infections by direct application, the solutions should be comfortably hot in order to secure the enhanced bactericidal effects.

\section{SELECTIVE BACTERIOSTATIC AND BACTERICIDAL ACTIVITY OF THE ANILIN DYES IN RELATION TO CHEMOTHERAPY}

As would be expected, the anilin dyes vary greatly in bactericidal activity and, as shown above, may exert a stronger bacteriostatic and bactericidal activity for some organisms than for others. Most of the anilin dyes in high concentration, say I: 100, will kill practically all organisms irrespective of whether they are cocci or bacilli, gram positive or gram negative, spore-formers or non-spore-formers, virulent or nonvirulent; but some of them show bactericidal and bacteriostatic properties for some organisms in concentrations without effect upon other organisms. This is designated as "selective activity" and bears a very intimate relationship to chemotherapy, since it appears that the chemical constitution of the molecule influences these specific properties.

Churchman has especially contributed a most valuable series of studies bearing upon this important topic of selective activity of the dyes. He has clearly proved that gentian violet, magenta, and other dyes of the triphenylmethane series in general possess a selective bacteriostatic and bactericidal activity for the gram positive organisms, and especially the spore-formers, arbitrarily designated as "normal activity"; curiously enough, however, these dyes may not inhibit the growth of the gram posi- 
tive tubercle bacillus. While Churchman originally believed that the bacteriostatic activity of gentian violet closely followed the gram reaction, i.e., was effective against gram positive organisms but not against $B$. coli and other gram negative bacilli, he has more recently discovered that the process is not so specific, since he found in a culture of the colon bacillus two different strains, one which was restrained by gentian violet and one which was not; Churchman has designated this as "microbic dissociation," and the discovery indicates how involved and complicated is this subject of the selective activity of the dyes.

Furthermore, dyes belonging to the same series may show opposite effects. For example, acidic and basic fuchsins also belong to the triphenylmethane dyes, but yet, as shown by Churchman, these restrain and kill gram negative organisms like $B$. coli, $B$. pyocyaneus, etc., designated as "reverse selective activity," whereas gentian violet kills the gram positives like $B$. diphtheriae, $B$. anthracis, the staphylococci, streptococci, etc. In other words, such closely related dyes as basic triphenylmethanes (magenta) differ in bacteriostatic power from acidic triphenylmethanes (the sulphonated compound, acid fuchsin), since the former is more active than the latter. The subject is of importance in relation to the chemotherapy of bacterial infections, since knowledge already gained indicates that when a dye is to be used for the treatment of a localized infection it should be selected on the basis of greatest bacteriostatic and bactericidal activity in pus for the particular kind of organism present, at least to the extent of selecting gentian violet for gram positive and acid fuchsin or acriflavin for gram negative bacteria. Furthermore, if new compounds of the dyes are to be synthesized, like compounds with mercury, copper, silver, etc., the selection of the dye should be influenced according to the particular group of organisms for which the compound is intended in order that the bactericidal activity of the compound may be enhanced by the intrinsic bactericidal activity of the dye.

\section{CHEMISTRY OF THE ANILIN DYES IN RELATION TO CHEMOTHERAPY}

Unfortunately, but little may be written at the present time upon this very important subject of the relation of chemical constitution of the anilin dyes to their bacteriostatic and bactericidal activities. Experts in the dyeing industries have worked out certain laws governing the dyeing of fabrics in relation to chemical constitution of both dye and fabric, but our information in relation to the bacteria is very meager. It is practically certain, however, as shown by Churchman and others, that staining or dyeing of living bacterial cells does not necessarily kill them, and that the bactericidal portion of a dye may be indeed a colorless portion, these observations indicating that the relation between chemical constitution and disinfection is much more complicated than between chemical constitution and mere protoplasmic staining.

But some information has been gained in this important subject. Browning and Gilmour, for example, working with the triamidotriphenylmethane group of dyes, observed that the unsubstituted triphenylmethane compound (pararosanilin) has only a comparatively weak bactericidal action. The substitution of alkyl groups in the amido-side-chains (hexamethyl- and hexaethyl-violet) leads to the most active bactericidal substances known for staphylococcus, as originally shown by Dreyer, 
Kriegler, and Walker with methyl violet, B. anthracis, and B. diphtheriae. Morgenroth and his colleagues have been especially interested in the chemical constitution of the acridin dyes in relation to bactericidal activity for streptococci, and in tests employing serum-broth dilutions of these dyes found that the structure of the whole molecule may influence the function of the side-arms, but that in the acridins the alkyl groups are the most active therapeutically and the isoamyl groups the least. As a result of these investigations, "rivanol" (2-aethoxy-6, 9-diaminoacridin chlorhydrate) was evolved and found particularly effective in the treatment of mice inoculated subcutaneously with virulent streptococci.

Working with gentian violet and magenta as "normal" triphenylmethanes and with acid fuchsin and the trisulphonic acid salt of rosanilin as representatives of "reverse selective activity," Churchman has shown that the selective activity of these dyes is not to be ascribed to sodium. On the contrary, however, the presence of $\mathrm{SO}_{3}$ radicals appears to account for reverse selective activity. It would appear, therefore, that with this group of dyes, at least, the specific selective activity might depend not so much on the presence of the sulphonic group as on the method of its bonding, though it is exhibited by substances like chromotropic acid, in which the $\mathrm{SO}_{3}$ group is firmly attached, as well as by substances like sulphonic acid, in which the bonding is very weak.

\section{MECHANISM OF THE BACTERIOSTATIC AND BACTERICIDAL ACTIVITY OF THE ANILIN DYES}

Just as we are lacking in sufficient information on the relation of chemical constitution of the anilin dyes to bacteriostatic and bactericidal activities to enable one to establish the highly desirable "laws" for aiding advances in our knowledge of the chemotherapy of these substances by synthesizing new compounds in a more orderly fashion, so are we likewise lacking in accurate information on the mechanism of their bactericidal activities. We ordinarily state that phenol, mercuric chlorid, and other disinfectants kill bacteria by coagulating the proteins of their protoplasm, but the process is probably not so simple and certainly is not in relation to the anilin dyes.

As stated by Churchman, there is a great deal of reason for supposing that bacteriostasis may act by paralyzing the reproductive mechanism of bacteria without otherwise injuring the organisms, due to changes effected by the dye at the surface of the organisms. In his opinion the simplest explanation for the behavior of gentian violet and acid fuchsin toward bacteria is that the whole process is simply one of selective penetration referring not merely to the arrival of the dye within the bacterial membrane, but its arrival at some particular portion of the bacterial body concerned with reproduction.

Furthermore, the method of ionization of the two groups of dyes whose action has been studied by him may explain their behavior. It is suggestive in his opinion that one group is basic and the other acidic, and that if this kind of explanation is the correct one it must be that gram positive spore-bearers and gram negatives differ in their $\mathrm{H}$-ion concentration. He also mentions that since the basic dyes are electro-positive while the acid dyes are electro-negative it is tempting to explain selective activity on the basis of electrical adsorption-an interesting thought deserving of further investigation. 


\section{DYE FASTNESS OF BACTERIA IN RELATION TO CHEMOTHERAPY}

Of considerable interest in relation to the employment of dyes in the chemotherapy of bacterial infections is the question of whether or not some species may acquire or naturally possess a resistance or fastness to the anilin dyes. Simon and Wood believe that some members belonging to dye-susceptible groups of organisms exist in nature which are not inhibited by the dyes in question, and that dye susceptibility may be overcome to a greater or less extent by adaptation or the acquisition of fastness (resistance).

Various spirochetes and trypanosomes, however, have been rendered resistant to anilin dyes, arsenicals, and other substances, and my experience indicates that different strains of staphylococci, streptococci, and colon and typhoid bacilli may exhibit slightly varying natural resistance to the anilin dyes. I have not tried to render any one strain particularly resistant or fast to a particular dye, but am prepared to believe that this is possible by prolonged and careful experiments in which the organisms are exposed to progressively increasing but sub-bactericidal strengths of the dye.

\section{INFLUENCE OF THE ANILIN DYES UPON PHAGOCYTOSIS AND ANTIBODIES}

Numerous questions naturally arise when the dyes are employed for the treatment of local or general bacterial infections in relation to their influence upon phagocytosis, antibodies, and immunological reactions. For example, may these substances applied locally to infected wounds or serous membranes inhibit phagocytosis of the organisms by leukocytes? May they destroy complement and bacteriolysins by local application or intravenous injection? May they increase antibody production upon intravenous injection or absorption from the subcutaneous tissues, muscles, or gastro-intestinal tract? 'These questions are not without interest and some practical importance in relation to the use of the dyes for therapeutic purposes.

Naturally most interest has been commanded by the influence of dyes on phagocytosis since they have been generally employed in the treatment of local infections of wounds and serous cavities, and numerous investigators have shown that it is not advisable to use the dyes in too high concentration for the local treatment of infected wounds, serous cavities, etc. The natural tendency is to employ them in the strongest solutions free of manifest irritation for the tissues, but this practice may tend to defeat their curative activities by preventing the antibacterial properties of phagocytosis and bacteriolysis, the influence of which should not be underestimated.

May the intravenous injection of the dyes increase antibody production? In my experiments I have not found acriflavin, gentian violet, or acid fuchsin, in doses of ○.० Io gm. per kilogram by intravenous injection every five days for four to six doses, to influence appreciably either the complement, or the natural or immune antibodies of the blood of rabbits. It may be that a part of the bactericidal effect of gentian violet and acriflavin injected intravenously in the treatment of bacteremias by streptococci and staphylococci is due to increased leukocytosis as well as the mobilization of various enzymes, but I doubt that the ryes actually increase antibody or complement production by stimulation of the bone marrow and other tissues believed to be concerned in their production. 
TOXICITY AND PHARMACOLOGICAL ACTION OF THE ANILIN DYES IN RELATION TO CHEMOTHERAPY

Since some of the anilin dyes, and especially gentian violet, rivanol, acriflavin, and proflavin, are sometimes injected intravenously in the treatment of streptococcus, staphylococcus, and other bacteremias of human beings, it is important to inquire into the matter of their toxicity and pharmacological action. As would be expected in view of the complicated chemistry and preparation of these substances, toxicity varies considerably with the different dyes and, indeed, with different lots of the same dye prepared in different laboratories. It is imperative in the interests of safety to select for intravenous injection only those dyes especially prepared for intravenous injection by processes of purification to remove the numerous impurities which may be present, and dyes suitable for ordinary bacteriological work may be quite too toxic and unsuitable for intravenous injection.

In my toxicity tests, employing white rats of roo-200 gm. weight, the following results expressed in terms of the maximum tolerated doses over a period of at least two weeks have been usually observed; the solutions varied from 0.5 to I per cent, and were injected at a constant rate of about 2 cc. per minute.

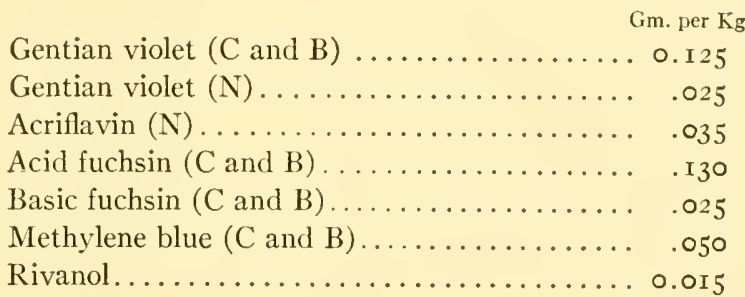

Since gentian violet and acriflavin have been injected into the pleural cavities in the treatment of pleuritis, I have determined the toxicity of these in addition to acidic and basic fuchsin and methylene blue by this route of administration. Normal rats and rabbits were employed, and the general results have been that all of these dyes were approximately 0.5 per cent more toxic by this route than by intravenous injection.

I have also employed some of the dyes in the chemotherapy of experimentally produced streptococcus and staphylococcus meningitis of rabbits; toxicity tests employing normal rabbits injected intracisternally showed that the maximum tolerated dose of gentian violet was from 0.001 to $0.002 \mathrm{gm}$. per kilogram, being at least ten to twenty times more toxic by this route than by intravenous injection. The maximum tolerated dose of acriflavin was between 0.002 to $0.003 \mathrm{gm}$. per kilogram, and methylene blue and acid fuchsin were tolerated in about the same or slightly larger doses. The total amount of fluid injected was about $0.25 \mathrm{cc}$. per kilogram, as this amount of saline solution does not produce untoward effects by pressure and is a convenient amount to inject by this route. Subdural injections in the lumbar region of rabbits and through trephine openings of the skull over the frontal lobes of the cerebrum yielded approximately the same results.

The dose of gentian violet commonly administered by intravenous injection to human beings is $0.005 \mathrm{gm}$. per kilogram, corresponding to $0.3-0.4 \mathrm{gm}$. for an adult of 
average weight (30-40 cc. of a I per cent solution). As a general rule, 20-30 cc. of a I per cent solution has been generally given, and this dose is quite safe, being at least five to ten times less than the maximum tolerated dose for the lower animals. Following the injection, the skin and mucous membranes become a dusky blue in color, suggesting severe cyanosis, but due to the dye, and usually disappearing within a few hours. The breathing and pulse rate may be increased with the slight increase of blood pressure; occasionally the patient experiences a tingling sensation of the skin with abdominal uneasiness, but I have never observed any other untoward effects.

Neutral acriflavin and proflavin are generally administered in doses of $0.005^{-}$ $0.010 \mathrm{gm}$. per kilogram, corresponding to $0.3-0.7 \mathrm{gm}$. for an adult of average weight; usually the dose is $30-50 \mathrm{cc}$. of a I per cent solution $\left(0.3^{-0.5} \mathrm{gm}\right.$.) by slow intravenous injection. The skin may become flushed and especially the face; both cardiac and respiratory rates may be increased for a few minutes, but in the majority of cases there are no other effects. These doses are at least four to five times less than the maximum tolerated doses for the lower animals and thereby within the range of safety, but it is my practice never to inject more than $20 \mathrm{cc}$. of a I per cent solution $(0.2 \mathrm{gm}$.) for the first dose when the patient is profoundly toxic, since resistance is materially reduced by severe bacterial infections.

Methylene blue has been generally administered by ingestion in doses of o. $5 \mathrm{gm}$. $\left(2 \frac{1}{2}\right.$ gr.) without untoward effects.

Rivanol is usually employed by local application to wounds but, according to Morgenroth, may be administered daily intravenously with safety in doses of $30-$ $50 \mathrm{cc}$. of I : I,000 solution for adults, corresponding approximately to $0.0005^{-0.0008}$ gm. per kilogram or about twenty to thirty times less than the maximal tolerated dose.

By local application in solutions of $\mathrm{I}: 500$ to $\mathrm{x}$ : $\mathrm{I}, 000$ strength, the dyes are well borne by wounds and such serous membranes as the pleural, subarachnoid, and joint cavities. All of the dyes appear to be excreted largely in the bile and urine, but the rapidity of elimination varies considerably.

Acriflavin in toxic doses by intravenous injection is said to kill by paralysis of respiration, and I believe that a combination of cardiac and repiratory paralysis accounts for the acute deaths of rabbits and other of the lower animals injected intravenously, intracisternally, or intrapleurally with toxic amounts of the anilin dyes.

Of considerable interest in relation to the mechanism of toxic reactions following intravenous injections of the dyes is the question of their hemolyzing and agglutinating effects upon the erythrocytes. In my experiments acriflavin has proved most agglutinative and gentian violet most hemolytic, but both effects are relatively slight in the presence of serum, and I doubt if these reactions are produced $i n$ vivo by the amounts ordinarily given or exert any important rôle in the production of toxic reactions similar, for example, to hemolysis and hemagglutination in relation to the acute toxic reaction following the intravenous injection of acid solutions of arsphenamin.

ANILIN DYES IN THE CHEMOTHERAPY OF EXPERIMENTALLY PRODUCED LOCAL AND GENERAL BACTERIAL INFECTIONS

But comparatively little work has been done with the anilin dyes in the treatment of experimental bacterial infections of the lower animals except in tuberculosis, al- 
though they have been quite widely employed in the treatment of local and systemic infections of human beings. It is true that experimentally produced infections are usually severe and place a heavy strain upon the curative activities of a medicament, but yet the logical procedure is first to apply studies of this kind whenever possible and before clinical application is made in order to avoid the pitfalls of hasty and erroneous conclusions regarding the practical curative activity of any agent under study.

I have not worked with rivanol, but in so far as gentian violet and acriflavin are concerned, my associates and I have been unable to demonstrate any constant or welldefined curative activities in experimental staphylococcus, streptococcus, and pneumococcus infections, and I believe the same applies to other anilin dyes of the triphenylmethane series. Mention may also be made that Spencer found acriflavin unable to check or control pneumococcus infections of mice, and Churchman has never been able to kill organisms circulating in the blood by intravascular injection of gentian violet.

Naturally a large amount of investigation has been devoted to the subject of the chemotherapy of experimental tuberculosis, infected guinea pigs and rabbits (cornea) being usually employed, but none of the dyes so far tested has shown any definite therapeutic power in experimental tuberculosis in guinea pigs beyond some prolongation of life and some lessening of the severity of the disease, although the penetrating and bactericidal or bacteriostatic powers were sometimes good. It is still possible, however, that some of the easily diffusible dyes may be used as carriers for more efficient therapeutic substances, which may reach in this way the tuberculous tissue which they could not otherwise attain.

Comparatively little work has been reported upon in the chemotherapy of anthrax, tetanus, gas bacillus, and other infections by gram positive bacilli. Since gentian violet and other triphenylmethane dyes show such well marked selective bacteriostasis for gram positive spore-bearing bacilli, it may be expected that these dyes may favorably influence infections produced by these organisms. Dr. Harkins and I have, indeed, observed that gentian violet administered intravenously and intraperitoneally to mice and rabbits infected with virulent anthrax bacilli exerts some degree of curative activity by lessening temporarily the severity of the bacteremia with prolongation of life, but so far we have not been able to cure animals in which the infection was given a twenty-four-hour or longer start.

The dyes have been used in the treatment of experimental $B$. tetani infections, but with negative results; I have not been able to find, however, any records of work devoted to the chemotherapy of experimental infections by $B$. welchii, $B$. maligni edematis, or other gram positive spore-bearers producing wound infections. This would appear to be, however, a field worthy of investigation.

In diseases of human beings the dyes have been mostly employed for the disinfection of wounds and localized infections of mucous membranes. Thus malachite green, brilliant green, gentian violet, fuchsin, acriflavin, proflavin, rivanol, etc., have been employed for the treatment of infected wounds and apparently with some success although thorough preliminary cleansing, and especially with alkaline solutions, is required for the best results. 
Gentian violet, brilliant green, acriflavin, etc., have also been employed for the disinfection of the throat and nose of diphtheria bacilli and meningococci, but with indifferent success, largely due to the difficulty of removing the mucoid secretions sufficiently well to permit the penetration of the dyes into crypts where the organisms are especially likely to persist.

Acriflavin, proflavin, and other dyes have also been employed in the treatment of infections of such mucous membranes as those of the mouth (in gingivitis), the eye, the urethra, and vagina. Success is claimed by some and denied by others, but many of the reports are of a favorable character.

Gentian violet and acriflavin have also been employed for the treatment of pleuritis and infections of the larger joints, and the results have been mainly good when the pus was first removed and the infected tissues cleansed before the injection of the dyes. Morgenroth and others have also reported the successful treatment of furuncles, carbuncles, buboes, suppurative mastitis, pelvic abscesses, etc., with acridin dyes and especially rivanol.

Gentian violet, acriflavin, rivanol, and other dyes have been used with apparent success in the treatment of staphylococcus and streptococcus bacteremias; indeed, these would appear as special diseases calling for chemotherapeutic measures alone or as adjuvants to serum therapy, and some notably successful results have been recorded.

CINCHONA DERIVATIVES IN THE CHEMOTHERAPY OF BACTERIAL DISEASES

While the anilin dyes were among the first substances to be used for selective bacteriostasis and bactericidal activity and especially in vitro in relation to the isolation of organisms, yet to cinchona derivatives belongs the distinction of being first employed for systematic efforts in the development of an efficient chemical disinfection of animals with an experimentally produced generalized bacterial (pneumococcus) infection.

\section{BACTERIOSTATIC AND BACTERICIDAL ACTIVITY OF THE CINCHONA} DERIVATIVES IN RELATION TO CHEMOTHERAPY

Morgenroth and Levy found that ethylhydrocuprein possessed a high and selective degree of pneumococcidal activity in vitro, and this observation has been amply confirmed, although, as would be expected, the pneumococcidal dilutions published by different investigators have varied greatly according to the technique employed. Ethylhydrocuprein hydrochlorid and other quinin compounds, however, act somewhat slowly on organisms, including pneumococci, and demonstrate more clearly their specificity for the pneumococcus in bacteriostatic than in bactericidal tests. In my experiments, when solutions of the drugs were prepared in broth and seeded with pneumococci followed by incubation for eight days, it was found that ethylhydrocuprein hydrochlorid inhibited the multiplication of pneumococci belonging to the first three serological types in dilutions varying from I : I, , o, , with the ordinary quinin salts the inhibiting dilutions ranged from $I: 5000$ to $I: 200$,-

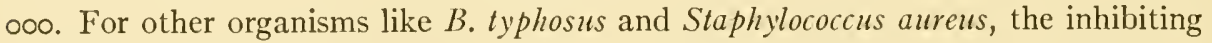
dilutions with all of the quinin compounds varied from I : 500 to I $: 2500$, demonstrating their special and selective effects upon pneumococci. 
Furthermore, while ethylhydrocuprein and practically all cinchona derivatives preserve a high degree of pneumococcidal activity in vitro in a menstruum of serum, it is of special interest in relation to chemotherapy to note that the administration of these compounds to human beings and the lower animals appreciably increases the pneumococcidal activity of the blood. This fact, of course, possesses considerable significance and lends great weight to the claims of Moore and Chesney and others that the internal administration of $\mathrm{I} .5 \mathrm{gm}$. of ethylhydrocuprein per day in divided doses may be of benefit in the treatment of lobar pneumonia.

\section{MECHANISM OF THE BACTERIOSTATIC AND BACTERICIDAL ACTIVITY OF THE CINCHONA DERIVATIVES}

Practically nothing has been yet discovered concerning the mechanism of the bacteriostatic and bactericidal action of ethylhydrocuprein and other cinchona derivatives for pneumococci and other organisms in vitro. Why these compounds possess a high degree of selective activity for pneumococci is likewise unknown. Something in their molecular structure establishes a special affinity for the protoplasm of these

TABLE I

\begin{tabular}{|c|c|c|c|c|c|}
\hline \multirow{2}{*}{ CoMpounds } & \multicolumn{2}{|c|}{ Lethal Dose } & \multicolumn{3}{|c|}{ Tolerated Dose } \\
\hline & Intraven. & Intramus. & Intraven. & Intramus. & Subcut. \\
\hline $\begin{array}{l}\text { Ethylhydrocuprein hydrochlorid . . . . } \\
\text { Quinin and urea hydrochlorid } \ldots \ldots \ldots \ldots \\
\text { Hydroquinin hydrochlorid } \ldots \ldots \ldots \ldots \ldots \\
\text { Quinin hydrochlorid } \ldots \ldots \ldots \ldots \ldots \ldots \ldots\end{array}$ & $\begin{array}{l}0.06 \\
.05^{-0.06} \\
.04^{-} .05 \\
0.05^{-0.06}\end{array}$ & $\begin{array}{l}0.5-0.6 \\
.5 \\
.16-0.25 \\
0.5-0.6\end{array}$ & $\begin{array}{r}0.02-0.04 \\
.02-.07 \\
.02-.06 \\
0.02-0.05\end{array}$ & $\begin{array}{r}0.03^{-0.07} \\
.03^{-} .08 \\
.02-.06 \\
0.03^{-0.07}\end{array}$ & $\begin{array}{l}0.08^{-0} .1 \\
.09^{-} .15 \\
.08^{-} .1 \\
0.09^{-0.18}\end{array}$ \\
\hline
\end{tabular}

organisms, but how they act in producing genesistasis or bacteriostasis and bactericidal effects has not been elucidated. Very probably the mechanism is similar to that concerned in the destructive effects of quinin for the malarial protozoa, but it may be stated, however, that while optochin and ethylhydrocuprein are highly pneumococcidal, they are regarded as inferior in the destruction of malarial parasites, which indicates that they do not possess a pan-therapeutic activity but that their chemical constitution renders them more especially destructive for the pneumococcus.

\section{TOXICITY OF CINCHONA DERIVATIVES}

Cohen, Heist, and myself found the lethal and maximum tolerated doses of ethylhydrocuprein hydrochlorid and some of the salts of quinin approximately as shown in Table I for rabbits and rats per kilogram of weight.

By intraperitoneal injection the maximum tolerated doses have been from 0.150 to $0.200 \mathrm{gm}$. per kilo, by intrapleural injection from 0.020 to $0.040 \mathrm{gm}$. per kilo, and by intracisternal or intraspinal injection about $0.003^{-0.005} \mathrm{gm}$. per kilo.

As a general rule, mice stand slightly larger doses than rabbits. Individual experiments also indicated that young rabbits were more resistant than older and heavier animals, confirming the observations of Moore in this respect.

A few years ago ethylhydrocuprein was rather widely employed in the treatment 
of lobar pneumonia, and toxic effects were sometimes produced. These have generally been transient and have usually disappeared when the drug was discontinued. The principal ones that occur are referable to the eye and the ear. In some cases vomiting has been reported; this may be due in some instances to the bitter taste of the drug, which rivals quinin in that respect.

The symptoms referable to the ear comprise ringing and roaring noises and partial deafness; they are transient and disappear shortly after the drug is discontinued. Those referable to the eyes are more serious. The pupils may be fixed and dilated and may not react to light; oph thalmoscopic examination may show tortuosity of the retinal vessels with some narrowing of the arteries. The amblyopia is usually temporary, but in a few very severe cases atrophy of the optic nerves has resulted.

There is some ground for believing that the visual disturbances which have occurred in about 4-5 per cent of cases of pneumonia may depend to a certain extent on idiosyncrasy indicated by the fact that some patients develop amblyopia after they have had only $2 \mathrm{gm}$. or less of optochin (numoquin) whereas others do not acquire it even after they have received as much as from 10 to $16 \mathrm{gm}$.

CINCHONA DERIVATIVES IN THE CHEMOTHERAPY OF EXPERIMENTAL INFECTIONS

Morgenroth and Levy originally employed mice infected with pneumococci and treated them with subcutaneous injections of optochin (base) suspended in oil. With this method 80--roo per cent of mice were protected against fatal infections, and the specific action of ethylhydrocuprein on the pneumococcus was found to be greater than that of any other compound of the series examined, namely, quinin, hydroquinin (methylhydrocuprein), isopropylhydrocuprein, isobutylhydrocuprein, and isoamylhydrocuprein.

Moore has also confirmed the curative effects of optochin base in pneumococcus infections of mice. Working with 2 per cent suspensions in sterile olive oil and injecting $0.5 \mathrm{cc}$. per $20 \mathrm{gm}$. subcutaneously immediately after infection followed by an equal dose on the second day and $0.4 \mathrm{cc}$. on the third day, he observed a well-marked protective action against many multiples of the minimal lethal dose of all four groups of pneumococci. Cohen, Heist, and myself, however, working with the soluble ethylhydrocuprein hydrochlorid, were not able to elicit as good results as reported by these investigators. Our work was conducted with mice and rabbits infected with ten to one thousand times the minimal lethal dose of types I, II, and III pneumococci by intraperitoneal injection about two hours after the drugs had been administered; in addition to ethylhydrocuprein we also employed quinin and urea bydrochlorid and other cinchonics. Our percentage of "cures" with ethylhydrocuprein hydrochlorid given intravenously within two hours after inoculation with 50 M.L.D. of culture was less than ro per cent. Doses ranging from 0.01 to $0.03 \mathrm{gm}$. per kilo of body weight usually prolonged life beyond that of the controls from one to four days, but ultimately the majority of the mice and rabbits succumbed.

Hydroquinin hydrochlorid in amounts as high as $0.04 \mathrm{gm}$. per kilo of body weight given from one to four hours after infection with 50 M.L.D. of culture did not appreciably prolong life except in a few instances when the animals lived from one to three days longer than the controls. 
The double salt, quinin and urea hydrochlorid, was similarly without curative value in doses as high as $0.04 \mathrm{gm}$. per kilo when given at intervals of an hour or more after inoculation with 50 M.L.D. of culture. When the dose of culture was reduced to Io M.L.D. a distinct effect was evident in the prolongation of life four, five, and six days among animals receiving $0.0 \mathrm{I} \mathrm{gm}$. and more per kilo of body weight.

By intramuscular injection ethylhydrocuprein in doses ranging from 0.02 to 0.06 gm. per kilo of body weight prolonged the life of a small percentage of mice and rabbits when given within two hours after infection with 50 M.L.D. of culture; smaller doses in this time and similar doses given at longer periods were practically without effect. Hydroquinin hydrochlorid, quinin and urea hydrochlorid, and quinin bisulphate in doses ranging as high as $0.04 \mathrm{gm}$. per kilo of body weight were practically without any effect when given within one hour or longer after inoculation with 50 M.L.D. of culture. Three of the cinchona derivatives were administered by repeated intramuscular injections; ethylhydrocuprein hydrochlorid prolonged the lives of a few more mice to the seventh day or longer than did the other cinchonics. It is probable that the death of some of the mice was due to the cumulative effects of the repeated large doses.

Observing that antipneumococcus serum for type II pneumococci was less efficient than the serum for type I, Moore studied the influence of a combined optochin and serum treatment for type II infections among mice, and found that a single dose of optochin suspended in oil and injected subcutaneously, which by itself has practically no protective effect, was capable of increasing the threshold value of type II serum at least fifty times, and that this effect was proportionately many times greater than a simple summation of the protective and curative effects of the two substances separately. Steinfield and myself also observed that ethylhydrocuprein hydrochlorid by subcutaneous injection in doses without protective value usually increased the protective value of antipneumococcus serum type $I$ in a slight but definite manner in severe infections of mice and rats with homologous pneumococci. Several of the commoner compounds of quinin, as quinin and urea hydrochlorid, quinin bromid, and quinin chlorohydrosulphate, given subcutaneously in doses without any appreciable influence on severe and fatal pneumococcus infections, occasionally increase the protective power of antipneumococcus serum but to a lesser extent and less regularly than ethylhydrocuprein hydrochlorid.

Idsumi and I have also observed that the subthecal injection of single doses of ethylhydrocuprein in amounts of $0.5 \mathrm{cc}$. of I : 500 and I : I,000 solutions per kilo of body weight had a distinct beneficial effect on the course of experimental meningitis in rabbits produced by a type I pneumococcus of moderate virulence, when administered not later than four to six hours after the injection of organisms; when given twenty-four hours after the pneumococci this effect was not apparent. With a meningitis produced by a more virulent culture of type II pneumococci, single doses of this drug had but slight or no effect on the duration of the lives of the experimental animals; but in experiments consisting in the repeated subthecal injection of smaller doses of pneumococci followed in a few hours by ethylhydrocuprein, the protective and curative effects of the drug were more apparent.

Lamar has shown in the treatment of experimental meningitis of monkeys that 
sodium oleate in $0.5^{-I}$ per cent solution is destructive for pneumococci, and that weaker solutions, as $0.1-0.00 \mathrm{r}$ per cent, lessen the virulence of pneumococci and increase their susceptibility to the destructive action of antipneumococcus serum, boric acid overcoming the inhibitory influence of the protein constituents. Mixtures of this kind yielded better results than optochin or antipneumococcus serum alone.

Since these experiments on pneumococcus meningitis have indicated that it may be possible to inject enough ethylhydrocuprein into closed sacs to raise the pneumococcidal activity of the exudates without danger of irritation of the serous membranes or the absorption of toxic amounts, Sands and myself have conducted similar experiments on acute suppurative pneumococcus pleuritis induced in guinea pigs and dogs, as having a possible value in the treatment of pneumococcus pleuritis with empyema developing during croupous pneumonia.

The experimental lesions in guinea pigs were very severe, consisting of acute suppurative pleuritis which involved both pleurae and the external portions of the pericardium. The intrapleural administration of ethylhydrocuprein in a dose corresponding to about $2 \mathrm{cc}$. of a $\mathrm{I}: 500$ dilution per kilo of weight in each cavity, a total of 0.008 gm. of drug, had a marked curative influence in the majority of instances unless treatment was delayed longer than twenty-four hours. Such a delay permitted the development of severe bilateral pleuritis, pericarditis, and pneumococcus bacteremia. Multiple injections of $\mathrm{I}$ cc. of a $\mathrm{I}: 500$ solution per kilo into each pleural cavity had an even more pronounced effect upon less severe infections. These results indicate the possibility of chemical disinfection of infected serous cavities with drugs showing a marked and selective bactericidal activity for the infecting bacterium in the presence of pus, as optochin or ethylhydrocuprein hydrochlorid for the pneumococcus, even though sufficient amounts of the drug cannot be administered by mouth, intramuscularly, or intravenously to influence a localized inflammatory process; furthermore, it is highly probable that some of the drug injected into the cavity is absorbed into the blood, where it exerts some restraining influence upon the multiplication of bacteria.

"Vucin" or isocytylhydrocuprein, prepared by Morgenroth, has been found highly bactericidal for staphylococci and streptococci and has been successfully employed in I : 2,000 solution for the treatment of gas-bacillus infection of wounds. Stronger solutions, however, are necrotizing. Solutions as high as I: I2,000 in doses of I cc. per $20 \mathrm{gm}$. have been found curative for mice inoculated subcutaneously with virulent streptococci by Morgenroth's method, and similar results have been observed in staphylococcus infections.

\section{CINCHONA DERIVATIVES IN THE CHEMOTHERAPY OF BACTERIAL DISEASES OF MAN}

Ethylhydrocuprein (optochin) and other cinchonics have been reported by many competent clinicians as possessing some practical therapeutic value in the treatment of pneumococeus bacteremia and pneumonia of human beings. It is true that early expectations have not been realized and that a therapie magna sterilisans has not been secured, but unquestionably it is possible by proper administration to raise the pneumococcidal activity of the blood, as well as probably increasing phagocytosis, and these results are doubtless worth while. 
Ethylhydrocuprein in I per cent solution has also proved efficacious in the treatment of pneumococcus conjunctivitis, and I believe it possesses some curative activity in the local treatment of pneumococcus sinusitis, mastoiditis, and otitis media as well as in pneumococcus meningitis, pleuritis, and for the disinfection of the mouth in carriers.

\section{COMPOUNDS OF MERCURY, ARSENIC, AND OTHER SUBSTANCES IN THE CHEMOTHERAPY OF BACTERIAL DISEASES}

Without doubt the soluble salts of mercury are among the most potent known bacteriostatic and bactericidal agents in vitro in a menstruum of distilled water or simple saline solution. Even in a menstruum of blood, serum, pus, or muscle extract, the majority of mercurial compounds maintain fairly high bactericidal values so that they have long held the attention of chemotherapeutists in relation to the possibility of synthesizing useful compounds for the treatment of local and systemic bacterial infections.

It is hardly necessary, however, to discuss in much detail the bactericidal effects in vitro in water or saline solution of the salts of the heavy metals because these effects are only of value in relation to the sterilization of instruments and other objects. In relation to the chemotherapy of localized and superficial infections of the skin, mucous membranes and wounds by topical application or of systemic infections by subcutaneous or intravenous injection, as well as the disinfection of sputum, feces, urine, pus, etc., only the effects produced in vitro in the presence of blood, serum, or tissue extractives possess interest and practical importance. Possibly an exception may be made to this general statement in dealing with chemical agents primarily designed for treatment of localized infections of the urinary system in which useful tests in vitro may be conducted with a menstruum of sterile urine as employed by Young, White, and Swartz in their studies with mercurochrome.

I have selected a few compounds of the heavy metals for purposes of illustration in the present discussion because of their wide clinical use in the treatment of localized infections of mucous membranes and because in a general manner they serve as examples of the comparative bactericidal activities of the heavy metals represented by them. Working with a blood serum-muscle extract medium seeded with staphylococci and conducting duplicate tests in exactly the same manner and at the same time except that sterile distilled water was substituted for their special medium, I have found that the final concentrations proving completely germicidal at the end of twenty-four hours at $30^{\circ} \mathrm{C}$. were approximately as shown in Table II.

The matter of rapidity of disinfection by compounds of the metals is deserving of mention and especially when they are employed for the treatment of localized bacterial infections. For example, in the treatment of urethritis, conjunctivitis, gingivitis, etc., the disinfectant agent is in contact with the tissue for probably only a matter of a few seconds, and only those agents known to act quickly are likely to prove bactericidal under these conditions; rapidity of sterilization bears a relation to concentration of the chemical agent and its absorption or fixation by cells.

The subject of acquired resistance of bacteria to compounds of mercury and other metals has not commanded much attention since they have not been administered over long periods of time in the treatment of bacterial infections. But the subject is 
not without considerable academic as well as practical interest and especially in relation to the treatment of urethritis and other localized infections by the long-continued application of silver, zinc, and other compounds.

I have experimented with mercurial compounds and cultures of Staphylococcus aureus and $B$. coli with the general result that after a prolonged series of transfers the organisms could be rendered distinctly more resistant, and the same is probably true of compounds of the heavy metals and most bacteria in general, but results in vitro are obtained only after several months of patient work and I doubt if bacteria, like the gonococcus, acquire sufficient resistance in vivo to the silver protein and other bactericidal agents to reduce materially the bactericidal effects of these compounds.

THE SELECTIVE BACTERIOSTASIS OF MERCURIAL AND OTHER COMPOUNDS

In so far as the ordinary organic and inorganic mercurial compounds are concerned, it is my observation that they do not exhibit any noteworthy evidences of

TABLE II

Final Concentration Proving Completely Germicidal After 24 Hours AT $30^{\circ} \mathrm{C}$.

\begin{tabular}{|c|c|c|}
\hline Substances & $\begin{array}{l}\text { Serum-Muscle } \\
\text { Medium }\end{array}$ & Water \\
\hline 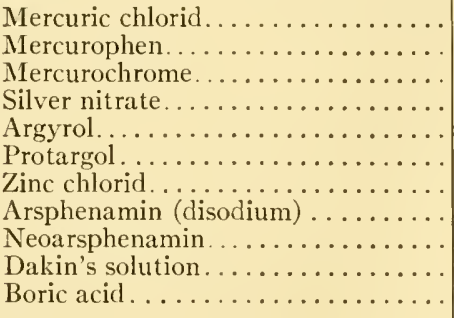 & 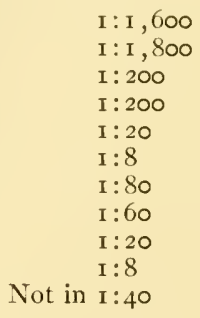 & 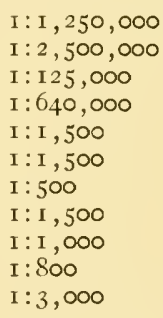 \\
\hline
\end{tabular}

selective bactericidal activities except that as a class they seem to be slightly more effective against gram positive than against gram negative organisms. But nearly all the organic and inorganic salts possess a high degree of bactericidal activity for practically all bacteria, and some like mercurochrome, for example, have been found to influence $B$. coli infections as well as those produced by staphylococci, streptococci, and gonococci. It is true that some organisms are more readily and rapidly killed in vitro than others, but these variations are readily explained by factors other than selective bacteriostasis. But mercurophen, however, does display a certain degree of selective activity for the gram positive cocci (staphylococci, pneumococci, and streptococci), and is likewise somewhat more active for gram positive bacilli (anthrax, subtilis, diphtheria, and tubercle bacilli); but this selective bacteriostasis is not as specific or marked as shown by gentian violet for gram positive organisms. All of the mercurials, and especially mercurophen, are likewise particularly bacteriostatic for spores like those of $B$. anthracis and B. subtilis. Mercurochrome is also somewhat more bactericidal for the gram positive staphylococcus than for the gram negative colon bacillus, and metaphen (4-nitro-3, 5-bisacetoxymercuri-2-cresol), recently described by Raiziss and Severac, has similar properties. 
De Witt has observed that these compounds also display a high bactericidal activity for tubercle bacilli; for example, mercurochrome was observed to inhibit the growth of the tubercle bacillus completely in a dilution of $\mathrm{I}: 5,000$ and partially at I: Io,000; mercurophen inhibited it completely in dilutions of I:50,000. Mercurochrome in a dilution of $\mathrm{I}: 100$, but not in higher dilutions, killed tubercle bacilli in twenty-four hours so that they would not infect guinea pigs. On the other hand, mercurophen, while it did not kill uniformly in less than twenty-four hours, killed in dilutions of $\mathrm{I}: \mathrm{r}, 000$ in twenty-four hours, and the development of the disease in guinea pigs inoculated with the exposed tubercle bacilli was generally delayed much longer than in the controls, even after shorter exposure to dilutions of $\mathrm{r}: 5,000$ and I : 10,000 .

The silver salts appear to be somewhat more selective for the gram negative cocci and especially the gonococcus.

In so far as compounds of copper and gold are concerned, most attention has been devoted to their effects upon tubercle bacilli, and it may be that they possess in slight degree some selective effects for these organisms. But compounds of copper and gold are, as a whole, relatively feeble bactericides and especially in comparison with the compounds of mercury.

Apparently, compounds of tin and zinc have not been studied from the standpoint of selective bacteriostasis, but according to the results of my own limited studies, the ordinary inorganic salts do not display any evidences of this property except that they seem to be slightly more effective against the gram positive cocci like staphylococci, streptococci, and pneumococci than against gram negative bacilli like $B$. coli communis and B. typhosus.

Various organic compounds of arsenic have long been used in the treatment of bacterial infections but more for their possible dynamogenic than bactericidal effects. Arsphenamin and neoarsphenamin display feeble bactericidal activities in vitro and especially in water or saline solutions, and possibly these compounds are somewhat more bactericidal for streptococci than staphylococci; but the differences are only slight and easily accounted for by other factors.

Iodin and compounds of iodin are worthy of brief mention in relation to tuberculosis since they have been long advocated by some physicians in the treatment of this disease, although De Witt and Sherman found that Lugol's solution had very little bactericidal activity, since in full strength the solution was unable to kill all bacilli. Tincture of iodin has a well-marked bactericidal effect upon staphylococci and streptococci, accounting for its popularity as a disinfectant of the skin and for the treatment of local infections by topical application, but its effects in vivo are very much reduced; to the best of my knowledge it does not display any particular selective bacteriostasis and certainly not for Sp. pallida or B. tuberculosis.

The bacteriostatic and bactericidal activity of cresote, guaiacol, and their derivatives in relation to the chemotherapy of tuberculosis of the lungs have long held a place of interest in the treatment of not only this disease but likewise in the treatment of non-tuberculous bronchitis; furthermore, the question of their possible specific bactericidal effects for tubercle bacilli as well as for the organisms of simple bronchitis and secondary infection of tuberculous tissues, such as streptococci, staphylococci, 
pneumococci, etc., becomes one of importance in relation to chemotherapy. But there is practically no evidence of susceptibility of B.tuberculosis to antiseptics of this class in vitro or that they have any favorable influence on the course of tuberculosis in experimental animals.

Interest in the possible selective bacteriostasis of chaulmoogra oil and its derivatives for acid fast bacilli and particularly $B$. tuberculosis has been stimulated by the reports of the curative properties of these substances in leprosy. Walker and Sweeney have found the sodium salts of the total fatty acids highly bactericidal in vitro, but Davis, Jager, and I observed that the oil itself was almost devoid of tuberculocidal properties and without any curative value in experimental tuberculosis of guinea pigs.

The alleged beneficial effects of hexamethylamin in various infectious diseases cannot be explained on the basis of adequate liberation of formaldehyde in the circulation and tissues. The drug itself is not antiseptic, and the hope of securing systemic effects through its use still seems impossible. Its one field of action seems to be in bacterial infections of the urinary tract, and it is necessary that the urine be markedly acid if the drug is to act efficiently.

Jacobs, Heidelberger, Amoss, and Bull, however, have thought it worth while to synthesize new compounds of hexamethylamin, and they have studied these with particular care for evidences of selective bacteriostasis for B. typhosus, streptococcus, meningococcus, and gonococcus with some striking instances of partial specificity. A particularly valuable outcome of their work has been to secure new information bearing upon the important question of chemical constitution of the quaternary salts of hexamethylamin.

It is possible to administer sufficient amounts of some of the new mercurial compounds, and especially mercurophen and mercurochrome, to raise appreciably the bactericidal effects of the blood. It is true that these effects are of short duration because of rapid elimination and fixation of the compounds by the tissues, but yet it is sufficient to lend considerable basis for the hope that it may be possible to reduce appreciably the degree of a bacteremia or dissipate it entirely by the intravenous injection of large but comparatively safe doses of these compounds. In fact, the results of animal experiments have shown that this may occur in some instances.

\section{COMPOUNDS OF MERCURY, ARSENIC, AND OTHER SUBSTANCES IN THE} CHEMOTHERAPY OF EXPERIMENTAL TUBERCULOSIS

With this review of the bactericidal effects of compounds of mercury, arsenic, and other metals in vitro and in relation to the bactericidal properties of the blood in vivo, their influence upon experimental bacterial infections of the lower animals may be now briefly considered. I may state at once that the results have been largely disappointing and of a negative character, but yet within the past few years some compounds belonging to this class have yielded encouraging results. The experimentally produced infections are usually quite severe and of short duration before death is produced, so that it is easily possible to mask or overlook slight but definite and promising curative effects produced by new compounds under study. For this reason it is readily understood that compounds failing to influence appreciably an experimentally pro- 
duced infection may yet show a favorable effect in less severe and fulminating diseases of human beings.

Since tuberculosis was one of the first diseases to be produced experimentally and since the disease is so readily induced in guinea pigs, it is no wonder that the major portion of experimental work in chemotherapy with mercury and other metals was conducted with this infection.

In so far as the ordinary and well-known organic and inorganic salts of mercury are concerned, it may be stated that none has shown any appreciable curative effects upon tuberculosis produced in guinea pigs although De Witt has found that mercurophen and mercurochrome seemed to delay considerably the development of the disease in guinea pigs, although generalized tuberculosis occurred in all the animals that lived long enough.

Although arsenic has long been advocated as beneficial in the treatment of tuberculosis, it has not been demonstrated to have any specific action, and its value in this disease can be attributed only to its favorable influence on metabolism.

Probably no compounds of the heavy metals have commanded as much attention in the treatment of tuberculosis as the salts of gold; indeed, quite a large literature has accumulated upon the subject in so far as the treatment of tuberculosis of human beings is concerned, but relatively few experiments have been conducted with experimental tuberculosis of the lower animals, and the results of these have been largely of a negative character. Within recent years a new gold compound designated as "sanocrysin," produced by Mфllgaard, in Copenhagen, is attracting considerable attention in the treatment of tuberculosis, but a final opinion of its value cannot now be expressed. From our knowledge of the action of gold compounds in general in tuberculosis, I surmise that the toxic reactions produced by this compound are due to foci poisons from the lesions engendered by the tuberculin-like reactions of hyperemia, exudation, and enhanced cellular necrosis. Possibly its curative effects are likewise similar to those produced by these tuberculin-like reactions favoring the laying down of fibrous tissue, without involving the question of direct and marked tuberculocidal properties of the compound itself. Recently Schamberg and Wright have found sodium and gold thiosulphate quite efficacious in the treatment of lupus erythematosis.

Copper is another metal which has attracted considerable attention in the treatment of tuberculosis and especially of human beings, but without much support from the standpoint of its effects in experimental infections. Cyanocuprol has been extensively used in Japan and with apparently good results in some cases but it is now the consensus of opinion that the effects are not specific but due to the focal tuberculin-like reactions which may be produced.

Iodin and iodin preparations have also long held a place of interest in the treatment of tuberculosis and especially, from the chemotherapeutic standpoint, as a carrier of more potent chemical antiseptics into tubercles; but it has as yet given little encouragement in this direction.

The salts of cerium samarium, neodymium, praseodymium, and other rare metals are commanding considerable attention in the chemotherapy of tuberculosis, especially from French physicians, but De Witt states that the results of experimental 
observations do not warrant the amount of clinical enthusiasm that has been aroused.

Creosote, guaiacol, and their derivatives have not given much if any evidence of specific bactericidal effects in vivo, and the results of animal tests have been generally of a negative character.

Cinnamic acid and the cinnamates, bile and the bile salts, fats and fatty acids, and various hydrocarbons have generally yielded negative results in experimental infections, although cod liver oil may owe its curative properties in human tuberculosis to vitamines which bring about an accelerated rate of recalcification and an increase of inorganic phosphorous in the blood in both rickets and tuberculosis in much the same manner as heliotherapy, without involving the question of specific tuberculocidal action from the oil, which is doubtless a very remote and improbable effect.

\section{COMPOUNDS OF ARSENIC, MERCURY, AND OTHER SUBSTANCES IN THE CHEMO- THERAPY OF EXPERIMENTAL STREPTOCOCCUS, PNEUMOCOCCUS, STAPHYLOCOCCUS, AND OTHER INFECTIONS}

Much less investigation has been devoted to the subject of the chemotherapy of experimental pneumococcus, streptococcus, staphylococcus, and other bacterial infections with compounds of the metals, although this is a very important field worthy of intensive study because of the frequency and highly fatal character of these diseases.

Arsphenamin and neoarsphenamin may exert a feeble curative effect upon experimental streptococcus infections, but these effects are not appreciable in the presence of infections sufficiently virulent to maintain the organisms in the blood. Sodium cacodylate has proved without appreciable effect in my experience in doses as high as $0.005 \mathrm{gm}$. per kilo (corresponding to $5 \mathrm{gr}$. per $60 \mathrm{kilos}$ ).

Arsphenamin and neoarsphenamin, especially the former, possess in slight degree an appreciable bactericidal activity for the anthrax bacillus in vivo, but the curative effects are so feeble that it is doubtful if these compounds are capable of favorably influencing anthrax bacteremias in man.

I have also employed arsphenamin, neoarsphenamin, mercurophen, and mercurochrome in experimental pneumococcus infections of mice and rabbits, but with negative results, although occasionally the lives of mice treated simultaneously with inoculation or within two hours thereafter were prolonged from one to three days beyond the controls.

Better results, however, were observed by Harkins and myself with mice infected by subcutaneous injections of hemolytic streptococci, according to Morgenroth's method. As a general rule we inoculated the mice every three days and produced thereby a streptococcic cellulitis with infection of the bloodstream lasting from seven to ten days and more closely duplicating conditions as encountered among human subjects with streptococcus infections. Mercurochrome was given by intraperitoneal injection in doses of $0.005 \mathrm{gm}$. per kilo, at daily intervals and four hours after each inoculation. Controls receiving the drug alone lived indefinitely; untreated controls succumbed with severe streptococcus cellulitis and bacteremias whereas 30 per cent and sometimes more of the treated animals lived indefinitely and showed no local lesions at the site of inoculation. In my experience this technique has proved the most satisfactory of all employed, and I believe that mercurochrome demonstrated unmistakably some degree of curative activity. 
We have had no evidence so far of mercurochrome given by intravenous injection sterilizing staphylococcus abscesses of the internal organs or of the skin following subcutaneous inoculation, but the drug has unmistakably sterilized the blood of experimental animals at least for brief periods of time and thereby prolonged the lives of some animals with less rapid loss of weight and other toxic effects. In our experiments mercurochrome was also found to influence favorably some rather severe experimental B. coli infections.

One of the unsolved mysteries of therapeutics is the mechanism of the beneficial and even curative effects of the salicylates and related substances in acute rheumatic fever. Indeed, these effects are frequently so striking that many physicians have included the salicylates in this disease in the category of the specific chemotherapeutic remedies, but Swift and Boots in an extensive series of experiments with rabbits inoculated intravenously with non-hemolytic streptococci while under the influence of full therapeutic doses of sodium salicylate found that they developed almost as many inflamed joints as the untreated controls similarly inoculated.

\section{THE TOXICITY OF MERCURIAL COMPOUNDS IN RELATION TO THE CHEMOTHERAPY OF BACTERIAL DISEASES}

As a general rule, the maximum tolerated dose of mercurophen and metaphen for white rats by intravenous injection is about $0.008 \mathrm{gm}$. per kilo of weight. These compounds are therefore slightly less toxic than mercuric chlorid which has a maximum tolerated dose of about $0.004^{-0.006} \mathrm{gm}$. per kilo. I have administered both of them to adults by intravenous injection (doses $0.0 \mathrm{I}-0.02 \mathrm{gm}$. or IO-20 cc. of I : I,O00 dilution) without any reactions. Solutions of these compounds are likewise well borne by the skin, and individuals possessing a hypersensitiveness to bichlorid of mercury with the production of dermatitis have used them without reactions. I have also observed that they are well borne by mucous membranes, and may be employed in dilutions of I : I,000 to I:4,000 for the treatment of rhinitis, conjunctivitis, vaginitis, etc., although somewhat higher dilutions like $I: 5,000-1: 8,000$ should be used for irrigation of the male urethra to avoid burning and other symptoms of irritation.

Mercurochrome is less toxic, owing to its lower content of mercury and slower dissociation. White rats ordinarily stand doses as high as $0.030-0.040 \mathrm{gm}$. per kilo without apparent harm although rabbits and dogs are more susceptible. One per cent solutions give no evidence of irritating qualities when used in the conjunctival sac of rabbits. Solutions of this drug in strength from O.I to 5 per cent have been used in the human genito-urinary tract as a local antiseptic. In the kidney pelvis a I per cent solution has been used.

Mercurochrome has been prepared for intravenous administration and is generally injected in doses of $0.005 \mathrm{gm}$. per kilogram of weight dissolved in hot distilled water. The solution does not require heating since it is rapidly self-sterilizing. This dose corresponds to about $0.3 \mathrm{gm}$. or $5 \mathrm{gr}$. for an adult weighing I $30-40 \mathrm{lb}$., and is generally administered in a I per cent solution $(25-30 \mathrm{cc}$.), injected slowly. Smaller doses, like I0-1 $5 \mathrm{Cc}$. of a I per cent solution, may produce practically no reaction at all beyond increased pulse rate and flushing, but the larger dose frequently produces a severe diarrhea in one-half to six hours and the stools are likely to be red in color, due 
to fluorescein, and sometimes mistaken for blood; the urine may be red in color. The patient may complain of nausea and vomit, which may be colored red. A rather severe chill is produced, lasting from ten minutes to an hour but usually not more than twenty minutes, and the temperature is increased by $\mathrm{I}^{\circ}-3^{\circ}$ with increased pulse rate. As a general rule these reactions subside in twelve to twenty-four hours. I have never seen nor heard of a fatal acute reaction of this kind, but several individuals have succumbed to the later toxic effects of mercurochrome. I know of two fatalities due to acute nephritis with almost total suppression of urine. Mercurochrome should not be used promiscuously or too freely and especially if nephritis is present.

\section{MECHANISM OF THE CURATIVE ACTIVITY OF THE MERCURIALS IN BACTERIAL DISEASES}

Unfortunately, but very little has been determined regarding the mechanism of the antibacterial properties in vivo of the compounds of mercury, gold, silver, copper, arsenic, creosote, etc., discussed in this chapter. Whatever direct bactericidal effects they may have in vivo would appear to be due to the metallic ions after dissociation of the compounds. Whether or not the mechanism of curative activity of the mercurials is the same in bacterial diseases as in syphilis cannot be stated. But in view of the well established curative activity of these substances in syphilis and the almost total absence of curative influence in chronic bacterial diseases, it would appear that the ions of mercury have a greater combining affinity for $S p$. pallida or in some other way are most toxic for these parasites.

It is commonly stated that mercury cures syphilis by increasing antibody production, but I have never been able to elicit direct evidence of any such mechanism, and surely such could have no relation to the rapid improvement of acute bacterial infections following intravenous injections of mercurochrome. It may be, however, as discussed above in relation to the chemotherapy of tuberculosis, that various compounds of mercury, gold, arsenic, etc., produce focal reactions of hyperemia with serous and cellular exudation and resulting in increased fibrosis, but the mechanism of these effects, which are so similar to the focal tuberculin reaction in tuberculosis, has never been worked out.

It has also been frequently suggested that the acute mercurochrome reaction and its curative effects in acute bacterial infections are due to a non-specific protein reaction ascribed to the effects of a new compound of mercury with the plasma proteins. It is true that the reaction bears some resemblance to the effects produced by the intravenous injection of vaccines, peptones, and other foreign proteins but the mercurochrome reaction in my opinion is a colloidal phenomenon and similar in its mechanism to the anaphylactoid reactions produced by the intravenous injection of a variety of colloidal preparations of the heavy metals, arsenic, various proteins, etc.

But in truth we do not know how the colloidal reactions are produced nor how bacteria in the blood are destroyed by the mercurials and other compounds mentioned in this chapter; there are still very obscure problems offering great difficulties in elucidation but warranting and urgently demanding investigation for the purpose of placing the chemotherapy of the bacterial diseases upon a more scientific basis and removing it from the realm of its present state of almost pure empiricism. 
COMPOUNDS OF MERCURY, ARSENIC, AND OTHER SUBSTANCES IN THE CHEMOTHERAPY OF BACTERLAL DISEASES OF MAN

It will be apparent from the preceding review of the chemotherapy of bacterial diseases with compounds of mercury, gold, copper, silver, arsenic, creosote, etc., that the subject is still largely, if not almost entirely, in the experimental stage. But enough has been done to show that some bacteria may be removed from the bloodstream at least by some of these compounds although there is much less evidence of curative activity in localized bacterial infections of the fixed tissues.

Practically the only compounds of this group used in the treatment of bacterial diseases of man are the arsphenamins, for anthrax and streptococcus infections; creosote and guaiacol derivatives, for tuberculosis and associated secondary infections; the salicylates, for acute rheumatic fever; sodium cacodylate, for streptococcus endocarditis; mercurophen, metaphen, and mercurochrome, for acute streptococcus, staphylococcus, and B. coli infections. Of course the colloidal silver-protein and various mercurial compounds have also been used in the treatment of localized infections by topical application, and this is likewise a form of chemotherapy, but aside from the examples just mentioned we have as yet made but little advance in the chemotherapy of systemic infections of human beings with these compounds.

But encouraging results have been obtained, and I firmly believe that the whole field of the chemotherapy of bacterial diseases offers much hope for success. No one will deny the importance of continuing investigation in so important a field, especially since the specific treatment of acute and chronic bacterial diseases with biological substances such as sera, vaccines, and similar agents so frequently fail to produce beneficial results. 



\section{AUTHOR INDEX}

Abderhalden, 220, 237, 970, I056-63, 1065

Abderhalden and Fodor, 272, 1063

Abderhalden and Crigorescu, I065

Abderhalden and Wertheimer, 976, $90 \mathrm{~S}$

Abderhalden and Wildermuth, I06I

Abe, 986

Abel and Ford, 7 I 7

Abel and Geiling, 9\$6

Abel and Kubota, 932

Abelin, 1095

Abramow, 778

Abramson, 572

Abt, 2 I6

Abt and Erber, 764, 767, 768

Abt and Loiseau, 216

Achalme, 875 .

Achard and Aynaud, 976

Acton and Knowles, 669, 676

Adam, I65

Adam and Jessop, $\mathrm{S}_{5}$

Adametz, 380, 385, 387

Adami, 653

Adams, 259, 26I

Adamson, 199

Adelheim and Katkin, 885

Adkinson, 968, 1004

Adler and Blumenberg, 1093, 1095

Adolf, 740

Aguilar, 675,676

Agulhon, 376

Ahlberg, 513

Ahlswede, 1099

Aiyer, 329

Akatsu, 474

Albus and Holm, 183

Aldrich, I 23, 134

Alexander, 178

Alexander, Becke, and Holmes, IOI4

Alexander and Thompson, 898

Alivisatos, I I I, I I 5

Allen, 62, $8 \mathbf{I}$

Almquist, $4^{-6}, 85,87-89$

Alstyne, Van, and Grant, 972

Altana, 620

Hvarez, 627

AMARAL, Do, I066-77; 1069, 1073, 1074, 1076

Amato, 35
Ambroz, 39

American Bacteriologists, Society of, I 2 I, I 22, I 27, I 28, I $30-35,845$

American Public Health Association, 286, 302, $303,362,3^{82}, 425,845$

Amoss, 92, 94, 61 2, 632, 931, 932, 942

Amoss and Chesney, 94 I

Amoss and Haselbauer, 633

Amoss and Wollstein, 926

Amstad, 1095

Amzel and Halber, 897

Amzel, Halber, and Hirzfeld, 903

Amzel and Hirzfeld, goI

Ancona, roro

Anderson, 4I 2

Anterson, A. F., and Schloss, IoI I

Anderson, B. G., 25 I, 265, 266, 287

Anderson, J. F., and Leonard, 749, 933

Anderson, J. F., and Rosenau, 969

Andrade, 177

Andrewes, 818-20, 823

Andrewes and Horder, 233, 367

Andrews, 676

Angerer, von, $267,474,4 \delta 7,526,527$

Anigstein, 488, 635

Appelmans, 536, 555

Aquino, 1072

Aristotle, 557

Aristowsky and Hölzer, I I

Arkin, 1040

Arkwright, 92, 96, 97, 542, 550, 551, 570, 797, $814,820,822,823,833$

Arkwright and Goyle, 92 .

Arloing, 5, 37 I, 8I3

Arloing, Cornevin, and Thomas, 285

Arloing and Langeron, rooI

Arloing, Sempré, and Chavanne, 556

Armstrong, C., 415

Armstrong, C., and Harrison, 964

Armstrong, E. F., 233, 235

Armstrong, H. E., 201, 548

Armstrong, R. R., I053

Arneth, 874

Arnold, ro9, 527, 556

Arnold and Weiss, 529

Arnoldi and Leschke, 995

Aronson, 31-33, 221 
Arrhenius, 78, 739, 740

Arthur, 59 r

Arthus, 969, 979, 984, 985, 991, 994, 1002, ro72

Arthus and Breton, 984

Arzt and Kerl, 630

Aschoff, 578, 653,1043

Ashby, 895, 907

Asheshov, 529-3I, 534, 549

Atkin, 93, 728

Atwater, 332

Aubel, 248

Audeieva and Grizevicz, 917

Auer, 978, 985, 990, 995

Auer and Lewis, 974, 994, 997

Auer and Robinson, 975, 993

Auer and Van Slyke, 977

Averill and Meyer, 6r I

Avery, 99, 23I, 272, 702, 727, $93 \mathrm{r}$

Avery and Cullen, 380

Avery and Heidelberger, 702, 7 I4, 723, 730, 731, 834

Avery, Heidelberger, and Goebel, $8, S_{34}$

Avery and Morgan, 214, 723

Avery and Neill, 213

Aycock, 4I6

Ayers, Cook, and Clemmer, 7o, 8I

Ayers and Johnson, 7 I, 81, 289, 403, 422, 427

Ayers, Johnson, and Mudge, 388, 389

Ayers and Mudge, 398

Ayers and Rupp, 216, 225, 248, 380

Ayers, Rupp, and Johnson, I83, 247, 248

Baas-Becking, 325, 327

Baas-Becking and Parks, 322, 325, 328, 329, 33 I Babes, 88

Babes-Ernst, 33

Babtchinski, Iog

Bach, 357

Bach and Sierp, 356

Bach and Warburg, 276

Bachmann, 6r4, 6r 5, ro39

Bächer, Kraus, and Löwenstein, 768

Baermann, 490

Baerthlein, 9r, 92, 98, 570

Baetjer and Sellards, 670, 676

Baginsky, 266, 390, 391, 392

Bail, I I 2, I r 5, 528, 529, 532, 535, 536, 542, 550, 55I, IO25, I027

Bail and Matsumoto, 538

Bail and Watanabe, 535

BAILEY, 802-10; 778, 779, 8r6

Bainbridge, 220, 443

Bainbridge and O'Brien, 445, 609, 6rI

Bainier, 5 I4
Baker and Breed, 38r

Baldwin, E. R., 1027

Baldwin, E. R., Petroff, and Gardner, ror6

Baldwin, H. S., I009

Baldwin, I. L., and Fred, 339

Baldwin, I. L., Fred, and Hastings, 334

Baldwin, L. B., roog

Balfour, Io, 455

Ball and Price-Jones, 637

Balteanu, 92, 94

Bancroft, 782, 79I

Bandini, 206

Bang, 38I, 407

BANzHAF, 745-58; 749, 755, 756

Banzhaf and Famulener, 758, 993

Banzhaf and Greenwald, 749

Barber, 58, 6r, 62, 7r, 8r, 450

Barbier, 109

Barcroft, 25 r

Bardach, I045

Bardsley, 366

Bargen, $5^{8} 3$

Barger and Dale, 977

Barikine, 868

Barker, 65, 645

Barlow and Russell, 359

Barnard and Morgan, 372, 374

Barr, Cecil, and Du Bois, Iogr, I095

Barre, La, rog6

Barrieu, ro4

Barthel, 338

Bary, De, 515, 590, $59 \mathrm{r}$

Bass, 887

Bass and Johns, 696, 697, 698

Bass and Vatson, 8I 8

Basset, $88_{5}$

Bates, Dunn, and St. John, 479, 48I

Battelli, $23 \mathrm{~S}$

Baudet, 623, 625, 634

Bauer, 467, 474, 486

Bauer and Engel, 7 r 2

Bauer-Hecht, 84 I

Baumann, 639

BAUMGÄRTEL, I4-r8; 14

Baumgarten, I32

Bavendamm, 325,328

Bayliss, 200, 276, 326

BAYNE-JoNES, 759-71; 574, 742, 760, 761, 768, $95^{2}$

Bayne-Jones and Van der Lingen, 372, 374

Bayne-Jones and Tuttle, $4 \mathrm{I}$

Bayon, 478, 524

Bazy, 554

Bazzoni, 372,373 
Beans and Eastlake, 787

Beattie and Lewis, 433

Beattie, Yates, and Donaldson, 629

Beaudette, 446

Bechhold, 574, 742, 743, 1092

Bechhold and Villa, 5I9, 520, 526

Becht and Luckhardt, I097

Beck, 62I, 901, 906

Becker, 287

Beckerich and Hauduroy, I 2

Bedson, 1039

Behring, 954, 1035

Behring and Kitasato, 739

Beijerinck, 202, 2I 2, 2 I 4, 2 I 5, 279, 28I, 282, 3 I 3 , $323,325,326,330,33 \mathrm{I}, 35^{2}, 384,387,389$, $390,393 \cdot 520$

Beijerinck and Van Delden, 282, 329

Bell, Hartzell, and Clawson, 780

Belonowsky, 639

Bendix, 230

Benecke, I 32

Beneke and Steinschneider, 974

Bengtson, 288, 447, 954, 955

Ben Harel, 694, 696, 698

Benians, 25-28, 32, 35, 43

Beniasch, 8I 5

Benjamin and Sluka, IO4I

Bennett and Schmidt, 7 I 2, 713

Bensley, 607

Benton, I 8 I

Benton and Albery, 397

Beres. See Ottenberg

Berge, 621

Berger, го89, го9०, 1094

Bergey, I 22, I 25, I 3I-34, 389, 393, 394, 443, $45^{2}, 593,594,626$

Bergstand, 5, 6, 39

Bering, rog9

Berman and Rettger, 220, 223, 224

Bernard, I I9

Bernstein, 894,895 , 9I $3^{-18}$

Berridge, 600,602

Berry, 859

Bertarelli, 475,476

Berthelot, 250

Besche, De, 1006

Besredka, 554, 576, 776, 803, 816, 836, 884-9o, 97 I, 973, 978, 981, 982, 984, 1046, 1047

Besson and De Lavergne, I I3

Bessubetz, 7

Bettencourt and Menezes, 1065

Beutner and Monetoff, Iog4

Bewley and Hutchinson, 338

Bezançon and Etchegoin, 482
Bialosuknia and Hirzfeld, 900

Bialosuknia and Kaczkowski, 903

Biedl and Kraus, 975, 976, 980, 994, I002

Bieling, 2 I 3 , 1020

Bieling and Gottschalk, rog5

Bieling and Isaacs, I043

Bienstock, 204, 205, 222

Bienstock and Gottstein, 3 I, 32

Biernacki, 274, 640

Bigelow, G. H., and Forsbeck, 4 ro

Bigelow, IV. D., 79, 81, 289, 299

Bigelow, W. D., and Esty, 286, 294, 295, 297

Billings, 578

Billitzer, 799

Bilouet, $55^{6}$

Biltz, 74I

Biltz, Much, and Seibert, 740, 74 I

Birge and Juday, 200

Birkhaug, 578, 931, 932

Birt and Lamb, 805

Bisgaard and Korsbjerg, I061, I062

Bjerrum, I 40

Blackfan, 92 I

Blackfan, Petersen, and Conroy, 935

Blair, 539, 540, $55^{\circ}$

Blaizot, 883

Blake, 636

Blanc, Le, 455, 468, 49 I, 7 I 2

Blanchard, I 30, 48I, 483

Blanchard and Lefrou, 490

Blasi, De, 6I4

Blau, 286

Bleyer, 943

Bliss, 8 I 8

Bloedorn and Houghton, 484

Bloom, 887, 1038, 1053

Bloomfield, i io

Blum, Delaville, and Van Caulaert, 1002

Blunck, I063

Bock, 443

Bockhout, 398

Boeck, 676

Boeck and Drbohlav, 676

Boeck and Stiles, 673, 676

Boez, 470

Bofinger, 609

Bogue, 178, 790, 865

Bohr, 137

Bois, Du, IogI

Bolduan, 4I 2

Bolley, 379

Bolton, 557

Bonazzi, 325

Bonne, 468, 488, 635 
Bonomo, 6r 4,8 r 3

Borchardt, 539, 54I

Bordet, 95, I I 2, 519-2 I, 532, 540, 740, 776, 782 , $807,811-16,831,832,9 S_{2}, 1039,1049$

Bordet and Ciuca, 529, 539, 542, 549, $55^{\circ}$

Bordet and Gengou, $831,832,8_{3} 8$

Bordet and Renault, 904

Bordet and Ruelens, 842

Born, 177

Borrel, 453, 495

Bose, 976

Botez, 54 I

Bouchard and Roger, 772

Boullanger and Massol, 357

Bovie, 373

Boxmeyer, 620

Boyd, 694, 698

Boyers, Kofoid, and Swezy, 676

Bragg, 177

Brahn and Schiff, 904

Branch, 614-ig

Brandt, Guth, and Müller 708

BRANHAM, 7I I-20; 447, 7 I 4

Branham and Humphreys, 75, 447

Braun and Cahn-Bronner, 210, 216 , 244, 247

Braunstein and Kepnow 875

Brazil, V., 1069, 1072, 1073, 1075

Bredfeld, 285

Bredig, 797

BREED, 378-94; I 25, I 33, 382

Breed and Breed, $39 \mathrm{I}$

Breed and Conn, I 34

Breed, Conn, and Baker, I27, 128, 130, 132, I34

Breinl, 25

Breinl and Hoder, 549

Breinl and Kinghorn, 467

Breitner, 904

Brem, 904

Brenchley and Thornton, 33 I

Breslaier, 640

Brieger, 228, 236, 772

Brieger and Ehrlich, 878

Briggs, and Shantz, 347

Briot, 980

Brocq-Rousseau and Urbain, 886

Brodie 25 I, 1049

Brodin and Richet, 976

BRONFENBRENNER, 525-56, I056-65; 41, 526, $529,531,544,548,984,1002,1056,1063,1065$

Bronfenbrenner, Freeland, and Schlesinger, 1063

Bronfenbrenner and Hetler, 547

Bronfenbrenner and Korb, 525-2S, 53I, 532, 534, 549, 554

Bronfenbrenner, Mitchell, and Titus, 1063. 1065
Bronfenbrenner and Muckenfuss, 530, 531, 535, 540

Bronfenbrenner, Muckenfuss, and Hetler, 545

Bronfenbrenner, Muckenfuss, and Korb, 536, $549,55 \circ, 554,555$

Bronfenbrenner and Reichert, 531, 532, 574: 742, $753,763,766,768$

Brooks, 73, 8I, 376

Brooks and Hanford, 44r

Brooks, Nain, and Rhodes, 598

Brotzu, 639

Brouardel, 554

Broughton and Alcock, 484

Brown, 783

Brown, H. C., Duncan, and Henry, 247-49

Brown, H. D., 326

Brown, J. G., 603

Brown, J. H., 470

Brown, J. H., Frost, and Shaw, 381, 4I3, 4I4

Brown, N. A., 602

Brown, R. O., $5 S_{3}$

Brown, W. H., 693, 698

Brown, W. H., and Pearce, 468, 476, 1099

Browning, 840, 1053

Browning and Gilmour, II 44

Browning and Gulbransen, 32

Browning and Russ, 372

Bruce, 628

Bruck, 849, 1006

Bruck and Hidaka, 849

Bruck and Sommer, ros6

Bruckner, 888

Brudny, 28

Brumpt, I30, 675, 676

Brunner, 1007 , 1008

Brunner and Walzer, roo8

Brutsaert, II $3,534,538,55^{\circ}$

Bruynoghe, 530, 534, 535, $55^{\circ}$

Bruynoghe and Dubois, 530, $55^{\circ}$

Bruynoghe and Mund, 538

Bryant, 93

Buchanan, G., 486, 488

Buchanan, J. A., 917

Buchanan, R. E., 46-57; 48, 50, 61, 65, 81, I 22 , I $25, \mathrm{I} 28-34,4$ I $3,452,453,475,724$

Buchner, 225, 250, 268, 270, 372, 1087

Buchner, Langard, and Riedlin, 49, 65, 8r

Budd, 362

Budden, 66, $8 \mathrm{r}$

Buder, 327

Buen, De, 48 I

Bürger, 2 I 2

Bürgers, 25

Büscher, 478 
Bugge and Heinke, 62I

Bulger, 976, I090

Bull, 733-38; 630, 805-9, 816, I090

Bull and Bailey, 624

Bull and McKee, 624

Bull and Pritchett, 774

Bulloch and Atkin, $87 \mathrm{I}$

Bulloch and Western, $87 \mathrm{I}$

Bumm, Io8

Bumpus and Meisser, $5^{8} 3$

Bundesen, 425

Bunge, I99

Bunker and Meyers, 900

Bunting, 873

Bunting and Houston, 874

Bunting, Nickerson, and Hard, 648

Burge and Neill, 373

Burgers and Bachman, 527, 539

Burgess, 446

Burke, 25, 28, 30, 288, 29I, 295

Burke and Skinner, 34

Burke, Sprague, and Barnes, 6I, 8I

Burmeister, I086, I097

Burnet, I1 7, 21 7, 542, 543, 550

Burr, 92

Burri and Dügelli, 393

Burri and Hohl, 397

Burri and Stutzer, I03

Burrill, 59 I

Burrill and Hansen, 334, 338

Burrows, 1037

Burton, I7I, I 78

Burton, E. F., $782,783,785,786,788$

Bushnell, 265

Busson, 609, 619, 1089, 1092

Buswell and Neave, 355, 359

Butka, 905

Butler and Peterson, 477

Buxton, 792

Byloff, 6 I 4

Cabot and Crabtree, 779

Cadham, $5^{5} 7$

Cagnetto, 6I4

Cajal, 475

Callow, 214, 215

Calmette, $8_{3} 6,888$, го1 7, I018, 1020, 1047, 1072, 1073,1075

Calmette and Guérin, 950

Calmette, Guérin, Nègre, and Boquet, 877

Calmette and Massol, 742, 759, 764, 768, 834, I071, 1076

Calmette and De Potter, 963

Cameron and Esty, 286
Cameron and Williams, 629

Cameron, Williams, and 'Thompson, 294

Camus and Pagniez, 832

Cannan, 197

Cannan, Cohen, and Clark, 197

Cannon, 637

Cantacuzène, $480,883,1037$

Cantero, 583

Carapelle, 212

Carbo-Noboa, 494

Carega, 22I

Carmichael, I84, I85

Carpano, 619

Carpenter, 38I

Carrel, 1053, I 105, I 106

Carrel and Ebeling, 876, 1038, 1053

Carrel and Ingebrigtsen, 1037

Carrieu and Sollier, 488

Carter, 479

Cary, 1036, 1043, 1053

Cash, 606

Castellani, I15, 133, 477, 482, 484, 815, 883, 1036, 1045

Castellani and Chalmers, I32-34, 390, 393

Castellani and Sturli, 909

Castellani and Taylor, 24I

Castro and Wynter-Blyth, 302

Cavazutti, 899, 900

Cernovodeanu and Henri, 372, 374

Cernovodeanu and Suhatzanu, 885

Certes, $454,457-62$

Césari and Alleaux, 630

Cesaris-Demel, 994

Chalier, Gaté, and Grandmaison, I 10

Chalmers, $4 \circ 9$

Chalmers and $\mathrm{O}^{\prime}$ Farrell, $48 \mathbf{2}$

Chambers, 35, 410

Chapin, 80, 81, 566

Charrin and Roger, 8I $\mathrm{I}$

Chatelier, La, ${ }_{15} 6$

Chauveau, 644

Cheplin, Post, and Wiseman, 647

Chesney, 43, 58, 61, 62, 81, 523

Chester, I32, I34, 387, 390, 392

Chevrel, Ranque, Senez, and Gruat, 6I6, 618

Chick, 9, 70, 72-76, 78, 79, 81

Chick and Martin, 76, 79, 8x

Child, 45

Chilson and Wisler, 430

Cholodny, 328,330

Christiansen, 616, 6I7

Christiansen-Weniger, 337

Christensen, 6I5

Christmanos, 922 
Chudiakow, 202

Church, 515

Churchman, 19-37; 7, I6, 21, 25-27, 29, 34, I I $3-15$, I I I 9

Churchman and Michael, 27, 37

Churchman and Russell, 36

Chvostek, 887

Cipollina, 6r4

Citron, I039, I09I

Ciuca and Manoliu, 554

Clapeyron, 157,158

Clark, G. L., I 77

Clark, H. C., 676

Clark, H. W., and Gage, 74, 35I, 355, 358, 360

Clark, Mrs. J. H., 377

Clark, PaUl F., 38-45

Clark, P. F., and Clark, 554, 555

Clark, P. F., and Ruehl, 3, 4I, 42, 63, 8I

Clark, P. F., Zellmer, and Stone, rogr

CLARK, W. M., 188-97; 197, 211, 238, 40I

Clawson, 578

Clayton and Churchill, 178

Cleland, 484, 908

Clemesha, 367

Cleveland, 26I

Clock, 964

Clowes, I80, I01 2, I054

Coblentz and Fulton, 372-74

CoCA, 1004-15; 745, 899, 906, 967, 968, 978, 980,98 I $, 985,990,995$, го06, го08, гого, Іо 2

Coca and Cooke, roo4, 1028

Coca and Deibert, 908, 913

Coca and Grove, 1006, 1007, 1013

Coca and Klein, 899

Coghill, 1023

Cohen, 73-75, 78, 8I, I97

Cohen and Clark, 6I, 65, 7I, 82

Cohen and Fitzgerald, 17

Cohen and Flemming, 203

Cohen and Markle, 203

Cohendy, 644

Cohn, I, 84, 89, I30, 279, 285, 5IO, 5 I3

Cole, 572

Cole and Kuttner, 522, 523, 62 I

Cole and Lloyd, 68, 82

Colebrook, 106

Coleman, 330

Coleman and Meyer, 449

Coles, 486, 488, 497

Collon, 896

Combiesco, 537

Comes, 591

Commandon, 863

Comrie and Bird, 445
Conant, 197

Conn, 20, 65, 69, 82, 386, 389

Conn, Esten, and Stocking, 389

Connal, 675,676

Connell, 402

Connistein and Ludecke, 504

Conradi and Drigalski, 34

Cook, M. IV., го

Cook, M. IV., and Smith, ro 46

Cooke, Ior 3

Cooke and Van den Veer, 968, 1004, 1005

Coons and Kotila, 6or, 604

Cooper, 880, 1047

Copeman, 520

Coplans, 58, 60, 82, 5 I9

Corbett and Phillips, 94

Corper, 3 I

Corper and Sweany, 275

Corrigan, 1045

Costa Cruz, Da, 529, 530, 538, 543

Couland, 627

Coulter, 797, 1055

Courmont, 805, 1037

Courmont and Durant, 488

Courmont and Rochaix, 370, 772, 773, 781

Couvy, 494

Coventry, 68o, 689, 69I-93, 698

Cowan, 92-94

Cowdry, I2, 631

Cowdry and Nicholson, 631,635

Cowie, 554

Cowie and Calhoun, Io89

Cowie and Chapin, $87 \mathrm{I}$

Craig, I3 I , 669, 676, 845

Craig and Nichols, 835

Cramer, 69, \$2, 218, $23 \mathrm{I}$

Crookshank, 389, 39 I

Crowell, 567

Crozier, 21, 57

Crozier et al., 55

Cumming, 845

Cunningham and Jenkins, 39, 40

Currie, 40I, 512, $5^{\text {I } 5}$

Currie and Thom, 5 I 2

Cutter, IO42

Czapek, 5 Iо

Dack, I05, 449

Daetjen and Fraenkel, 1059

Dairy and Milk Inspectors Association, 434

Dakin, 704

Dakin and Dale, 709

Dale, $974,977,980,982,998$, 1007

Dale and Hartley, 71 2, 716, 977

Dale and Laidlaw, 987, 1000 
Daniels and Armstrong, $6{ }_{3} 6$

Dantec, Le, 484

Danysz, 633, 637, 740, 759, 76r, 768, 98r

Darányi, 9

Darwin, 86

Davaine, 590

Davidsohn, 779

Davis, C. M., Io9

Davis, D. J., 650-59; 482, 653, 658

Davis, D. J., and Pilot, 655

Davis, J. D., 623, 625, 626

Davis, J. S., 907

Davis, J. S., and Ferry, 68, 82

Davison, 507, 508, 537

Dawson and Hume, 488

Day, 6or

Dean, $87 \mathrm{r}$

Dean and Webb, 976

Debye and Hückel, I40, 170, 177, 178

Decastello and Sturli, 892, 895

Degwitz, 938

Delamara, 482

Delépine, 445

Delezenne and Forneau, 1072

Delezenne and Ledebt, 1072

Denys and Le Clef, 870

Dernby, 272, 275

Dernby and Blanc, 275

Dernby and Siwa, 275

Descombey, 762, 767, 768

Desderi, 199

Dessy, 614, 6I5

Dessy, Grapiolo, and Fossati, ro86

Detre, 475,838

Deussen, 26, 27, 29

Deutsch, 883, 1036, 1042

Devereux and Tanner, 68, 82

Dibbelt, $2 \mathrm{r}_{3}$

Dibble, 642

Dick and Dick, 747, 755, 756, 931, 933, 955

Dickson, 199, 295, 297

Dickson and Burke, 297

Dickson, Burke, Beck, and Johnson, 288, 290, 29I, 295, 297

Dickson, Burke, Beck, Johnson, and King, 288

Dienes and Freund, 7 I 4

Dienes and Schoenheit, $7 \mathrm{r} 5$

Diesing, 687, 698

Dieterlen, 609, 6i I

Dietrich, 467

Dieudonné, rogr

Digby, $65 x$

Dimitroff, $467,474,486$

Dimtza, 548
Dingman, $4 \mathrm{I} 6$

Distaso, 620

Distaso and Schiller, 646

Doan, 892, 906

Dobell, 6, 39, 483,663, 676, 686, 698

Dobell and O'Connor, $66_{3}, 676$

Dochez, 23r, 702, 755, 931, 933

Dochez and Avery, 8, 231, 725, 728

Döllken, I089

Doelter, 894,900

Doerr, 524, 538, 540, 542, 545, 967, 983, 1008, Ior 4

Doerr and Berger, 538, 7 I 2, 716, 740

Doerr and Gruninger, 530, 538, 548, 554, 555

Doerr and Hallauer, 709

Doerr and Moldovan, 972, 983, 984

Doerr and Russ, 972, 982, 991

Doerr and Zdansky, 528, 631

Doidge, 594

Dold, 849, 977

Dold and Hanau, 777

Domarus and Bersieck, I058

Dombray, 833

Donaldson, 107, 108, 607

Donath and Heilig, I09I, I099

Donnan, $\mathrm{r}_{52} 2,787$

Donnan and Green, 574

Dooren de Jong, Den, 244, 247

Dopter, 887,922

Dorset, 443

Dossena, 9 r 7

Douglas and Distaso, 7

Douglass, Fleming, and Colebrook, 207

Downes and Blunt, 37 I, 372

Drechsler, 6

Drensky and Hegner, 698

Dreyer, 377

Dreyer, Kriegler, and Walker, rr 14 , I I I 5

Dreyer, Scott, and Walker, 28, 34

Drinker, 994

Drinker and Bronfenbrenner, 990, 99r, 993, 994, 997

Dryer, 88

Dryer and Ward, 849

Duboscq and Lebailly, 453

Dubovsky and Meyer, 199

Duclaux, 37x, 372, 386

Düggeli, 325

Duemling, 859

Duggar and Karrer, 519

Dujardin, rog9

Duke, 968, roo9, гог, ro89

Dulaney, 92, 95, 99

Dulong, 255 
Dumas, Ramon, and Said Bilal, 767,768

Dungern, von, 740, 825, I037

Dungern, von, and Coca, 1072

Dungern, von, and Hirschfeld, 892, 899, 902, 903 , 9I I , 9I 2, 9I4-I 7

Dunham and Schoenlein, 364, 365

Dunlap, 476

Durham, 392, 8I 2, 8 13

Dutton, I I , 540, 550, 553, 859

Dutton and Todd, 469,479

Duval and Harris, 494

Duval and Hibbard, 780

Dyar, 9 I

Dyer, $765,766,768$

Dyke, 894,896

Dyke and Budge, 9 I 7

Eagles, 766,768

Eastcott, 500

Eastwood, 542, 883

Ebbecke, 1093

Eberle-Klein, 648

Eberson, 375

Eberth and Hesse, 636

Eberth and Mandry, 623

Ecker, 447

Ecker and Rogoff, I042

Eckersdorff, 609, 6 I I

Eddy, Kerr, and Williams, 499-50I

Edmunds, 973, 975

Eds, De, 983

Eds, De, and Somerfield, 983, 987

Eggerth, I I4

Eggerth and Bellows, 795, 797

Eggstein, rogo

Eguchi, 526

Ehrenberg, 9, 452, 454, 455, 457-62, 494

Ehrlich, 22, 685, 686, 698, 730, 739, 745, 760, 81 5 , $872,873,88$ I, 883 , 95 I, I035, I IOI, I IO2, I IO8

Ehrlich and Morgenroth, 892, 903

Ehrlich, Roehl, and Gulbransen, 685, 686, 698

Ehrlich and Shiga, 685, 698

Eidam, 5 I4

Eijkman, 9, 273, 548

Einstein, 784

Eisenberg, 24, 25, 28, 29, 85, 9I , 92, 94, 98, 826, 898

Eisenbrey and Pearce, 975

Eisler, 740

Eisler and Kovács, $76_{3}, 768$

Elder and Tanner, 539

Eliava and Pozerski, 549

Eliot, 89 I

Ellenberger, 398
Ellis, 788

Ellis and Wells, 377

Elsesser, I058

Embden and Lange, I093

Emden, Van, I036

Emmerich, 362, 1049

Emmerich and Tsuboi, 1047

Emmerling, 23I

Enderlein, 4, 6, 39, 85, 87-91. I00, I 20, I 2 I, I 29, I34

Engel and Kerekisa, ro95

Engelmann, 202, 328

Engler, I 32, 593

Enlows, I21, I 22, I34, I35, 606, 887, 888

Eoff, Linder, and Beyer, 504

Epstein, I2

Epstein and Fejgin, 536

Epstein and Ottenberg, 910

Erlenmeyer, 648, 850

Ermengem, Van, 37, 288, 444, 448

Ernst, 35, 88

Escherich, 228, 236, 250, 389-93, 484, 639, 640

Esmarch and Jessén, 476

Esten, 386, 389

EsTY, 285-300; 288, 294, 448

Esty and Cathcart, 293

Esty and Meyer, 48, 287, 288, 290-92, 294, 296, 448

Esty and Williams, 9, 296, 297, 299

Eucken and La Mer, I 77

Euler, 269, 272, 277

Evans, 873

Evans, Alice C., 379, 38r, 40 I, 587, 926

Evans, Alice C., and Freeman, 587, 932

Evans, J. S., 589

Ey and Delong, 859

Faber, von, F. C., 605,

Faber, H. K., 779, 780

Faber, H. K., and Murray, 780

Fabian 398

Fabry, II 2, 555

Fahr, 777, 779

Fahraeus, 897

Fair, 356, 377

Fair and Baity, 357

FALK, 565-75; $18,68,75,82,284,566,869$

Falk and Caulfield, 97 I, 972, 98 I

Falk, Gussin, and Jacobson, 570, 599

Falk and Jacobson, 570, 574

Falk, Jacobson, and Gussin, 570

Falk and Matsuda, 57 1, 572, 863

Falk and Powdermaker, 705

Falk, Sharp, and Link, 570, 599, 600 
Falk, Tonney, White, and Jensen, 744

Falk and Winslow, 73, 74, 76, 79, 82

Falls, I00 r, rogo

Fano, Da, 63I

Fantham, 455, 482

Fantham and Thomson, 684, 699

Fasiani, 199

Fassin, 883, I043

Faupel, 859

Faust, ro70

Fay and Olson, 398

Federschmidt, 446

Fejgin, 532, $55^{\circ}$

Felt, 135

Felton, 277, 929, 1055

Felton and Bailey, 573

FENN, 86r-69; 572, 86r, 862, 864-66, 868

Fennel, 8I9

Fenyvessy, von, and Freund, 98I

Fermi, 274, 279

Fermi and Bassu, 202

Fermi and Montesano, 274

Fernbach and Hepner, 1047

Ferran, 209, 210,877

Ferry, 618, 1074

Ferry and Fisher, 7 I 5

Ferry and Hoskins, 623

Fèvre de Arric, Le, 808

Feyerabend, 620, 62I

Fiallow, 24I

Ficker, 74, 82

Fiebiger, 638

Field and Teague, 574, 575, 742-44

Fijin, 5

Fildes, 2 I 7

Findlay and Ludford, 523

Finger, rog9

Fink, 704, 970

Finkelstein, 64I

Finkler and Prior, 89,98

Firtsch, 91, 93, 94, 98

Fischer, I32, 269, 444, 645

Fischer, A., 591, 1038

Fischer, A., and Weiss, Iog6

Fischer, E., 220, 228, 232, 235, 237

Fischer, E., and Thierfelder, 228, 235

Fisher, 505

Fisher, A. B., 697

Fisher and McKinley, 376

Fitch et al., 82I

FitzGerald, 8I 5

FitzGerald and Fraser, 8i r-23

Fjeldstad, ro43

Flack, 922
Flatau, Iog9

Flatow, 1063, 1064

Fledkseder, rogr

Fleisher and Mayer, I0O3

Flexner, 632, 922, 926

Flexner and Amoss, 94I

Flexner and Jobling, 925

Flexner and Lewis, 94 I

Flexner and Noguchi, ro7 I

Flexner and Sweet, 777

Flössner and Kutscher, 764, 769

Flu, 535, 539, 541, $55^{6}$

Flügge, I 23, I32, 386, 389, 39 I

Fodor, 268

Fodor and Rigler, I037

Fokker, 6o, 82

Folin and Farmer, 964

Fontana, 466, 495

Fontes, 4, 5, 89

Foord, I039

Foot, 873,876 , ro3 8

Force and Leake, 950

Ford, 390, 393, 609, 707, 7 I 7

Forssman, 709, 710, 733, 736, 737, 894, 902, 903, 979, 985

Forssman and Folgelgren, 906

Forssman and Hintze 736

Forster and Tomasczewski, 477

Foster, 407

Fowler, $35^{8}$

Fränkel, 224, 389, 39I

Francillon, I05

Francis, 205

Francis el al., $82 \mathrm{I}$

Franck, Io99

Frank, 778

Frank, Moss, and Le Fevre, 435

Frank, Rosenthal, and Biberstein, I05 8

Franke, 685, 699

Frankel, 878

Frankel and Grünenberg, I 044

Frankland, 64, 65, 82

Frankland and Frew, 237, 239

Frankland and Frankland, 82

Frankland and Lumsden, 237

Frankland and Ward, 372

Franzen and Egger, 238

Franzen and Greve, 238

Fraser. See FitzGerald

Fred, 332-40; 336, $35^{\circ}$

Fred and Davenport, 330, 339

Fred and Graul, 337

Fred, Whiting, and Hastings, 334, 338, 605

Freeman, Ior2 
Freeman and Whitehouse, 905

Freudenreich, 379, 387, 394

Freund, 165, 609, 611, 612, 616-20, 624-26, I044, 1093,1094

Freund and Dressel, rog2

Freund and Gottlieb, ro93, ro95

Freund and Rupp, Io95

Freundlich, $178,741-43,782-86,79^{2}, 799,800$

Fricke, 635

Frieber, $25 \mathrm{I}$

Fried, 890

Friedberger, 980, 982, 1026, 1047

Friedberger and Goretti, 736

Friedberger and Hartoch, 736

Friedberger and Masuda, ro4०

Friedburger and Mita, 99 I, 993

Friedburger and Schiff, 607

Friedburger and Scimone, 376

Friedemann, 980

Friedenreich, 898

Frobisher, 183

Frost, W. D., III, 370

Frost, Gumm, and Thomas, $38 \mathrm{I}$

Frothingham, 779

Frouin, I043

Frülich and Paschkis, 1093

Fuchs, 498

Führmann, 85,87

Fürbringer, 639

Fürst, Iogr

Fürth, 744

Fuhrmann, 4, 6, 278

Fukumachi, 908

Fuller, 58, 82

Fulmer, Nelson, and Sherwood, 283

Funck, rog

Funk, 499

Furnihi, 9I7

Furuhata, 917, 1050

Fygin, Epstein, and Funk, 593

Gaarder and Hagem, 325

Gabritschewsky, 469, 480, 747

Gärtner, 444

Gaffsky, 390

Gaffsky and Hesse, 205

Gage and Stoughton, 290

Gahringer, 974, 976, 991

Galeotti, 22 I

Galli-Valerio, 614, 62I

Galthier, 404

Gamaleia, 537, 543

Gamper, 1093

Gardner, 3, 40, 4I, 592, 616
Gardner and Kendrick, 596, 598, 602

Garrow, 8 I 8

Gasperi, De, 645

Gasperi, De, and Sangiorgi, 622

Gasperini, ro8

Gaté and Gardére, $54^{\circ}$

Gaté, Papacostas, and Billa, rog, i ro

Gates, 47 I, 546, 1042

Gates and Olitsky, $47 \mathrm{I}$

GAY, 88I-9I; 729, 82 I, 881, 884, 890, 1046, 1047

Gay and Adler, 970

Gay and Chickering, I05०

Gay and Clark, 89o, 1044

Gay, Clark, and Linton, 887,890

Gay and Morrison, 886, 890, I I 12

Gay and Rhodes, 729

Gay and Rusk, $88_{3}$

Gay and Southard, 974, 981

Gay L. N., and Chant, Ior 3

Gay-Lussac, 227, 237

Gehring, 326

Geiger, 445

Geiger, Dickson, and Meyer, 199, 448

Gengou, 803, 8I 6

Gerdine and Helmholz, $58_{3}$

Gerretsen, Grijns, Sach, and Söhngen, 335, 6oI

Ghiorghiu, 637

Gibbs, 197, 325

Gibson, 749, 756

Gibson and Banzhaf, 756, 757

Gilbert, 838-47

Gilbert and Dominici, 639

Gilbert and Langworthy, 842. 845

Gilbert and Moore, 814

Gilbert and Parsons, 8r 7

Gildemeister and Herzberg, $54^{\circ}$

Gillespie, 197, 602

Giltner, 204

Giltner and Langworthy, 74,82

Giordano, 859

Giordano and Barnes, $5^{8} 3$

Girard and Audubert, 569

Gjфrup, 543, 55०

Glaesner, $74^{\circ}$

Glaue, 625

Glenny, $750,75 \mathrm{I}$

Glenny and Allen, $75^{\circ}$

Glenny and Hopkins, $75^{\circ}$

Glenny, Hopkins, and Pope, $75^{2}$

Glenny and Okell, 760, 761, 769

Glenny, Pope, and Waddington, 750, 762, 769

Glenny, Pope, Waddington, and Wallace, 769

Glenny and Sudmerson, $75^{\circ}$

Glenny and Wallace, 763,769 
Glickhorn, Fürth, and Blüh, 744

Glotoff, 889

Glynn, 8I9

Gmeiner, 632

Godlewsky, 325

Goebel, 7 I 7

Goldworthy, 594

Goldzieher and Peck, 887

Goler, 4 I 2

Gomes, 1073

Gonder, 453

Goodpasture, 63I

Gordon, 232, 244, 524, 926

Gordon and McLeod, I I 7, 244

Gorgas and Guiteras, 49I

Gorini, 276, 379

Gotschlich, 2, 3

Gottstein, 2 I4

Gouy-Debye, 786

Govaerts, 808

Goyle, 92, 822

Gozony and Suranyi, 532

Gräf and Wittneben, 203

Grafe and Graham, 895

Graham-Smith, 26

Gram, 24, 33

Grancher and Ledoux-Lebord, 6I4

Granucci, 992

Gratia, 93, I I 2, 535, 536, 539, 550, 55 I, 886

Gratia and Dath, Io8

Gratia and Doyle, 554

Gratia and Rhodes, ro8

Graves, 476

Greco, 5 I 5

Green, I 79, I82, 329, 779

Green and Halvorson, 799

Green and Stoesser, I 84

Greenbaum and Wright, I099

Greenmann and Dühring, 635, 636

Greenwood, Newbold, Topley, and Wilson, 634

Greenwood and Topley, 632, 634

Greer, 357,367

Griffith, 92, 94, 99

Griffith and Scott, 840,844

Grigoroff, 394

Grijns, 335

Grimbert, 240

Grimme, 39

Grixoni, 206

Groeneweg, I06

Grohmann, 329

Gromakowsky, I I I

Gross, 2, 453, 454, 456-63

Grotenfelt, $385^{-9 I}$
Grove, 899, 908, 1006, гог

Gruber, 85, 804, 8г 2, 8г6, 1039

Gruber and Durham, 8I2, 8I 2

Grünbaum, 8I 2, 8I 3

Gruenthal and Brown, I089, I090, 1095, 1096

Gruenwald, 942

Grumbach and Dimtza, 548-50

Gruner, 328

Günther and Thierfelder, 386

Guérin, 627

Guggenheim, 230

Guillebeau, 380, 38I

Guillemont and Szczawinska, 207

Guilliermond, 39, 40, 498, 499

Guiteras, 49 I

Guldberg and Waage, I4 I

Gundel, 897

Gunn, 993

Gunning, 196, 205

Gurd, 980

Gurd and Denis, 3 I

Gutfield, 735

Guthrie and Huck, 899, 900

Guthrie and Pessel, 906

Guthrie, Pessel, and Huck, 900

Gutstein, 7, 546

Gwatkin, 82 I

Gye, 889

Gyemant, 743

Haan, De, 863, 868

Haber and Klemensiewicz, 786

Hadda and Rosenthal, 1038

Haden, $583,5^{84}$

Haden and Jordan, W. H., 584

Hadley, F., 93, 95

Hadley, P., 84-10 I; 82, 86, 90, 95, 542, 543 , $545,548,550,570,577,596,597$

Haehn, 506

Haehn and Kintoff, 506

Haen, 487

Haffkine, 877

Hahn and Trommsdorf, I050

Hajos, 537, 542

Hajós and Sternberg, rogr

Halber and Hirzfeld, 902, 904

Halberstaedter, 685, 699

Hale, 364, 365

Hale and Melia, 364

HALL, I98-210; I 22, 126, I35, I99

Hall and Ellefson, 365

Hall and Howitt, I99

Hall and Peterson, 205

Hall and Wing, I05 
Hall, von, 604

Hallauer, 706

Haller, 199

Halvorson and Green, I8I, I87

Halvorson and Starkey, 328

Hammer, 389, 397, 398

Hammer and Bailey, 398

Hammer and Baker, 388

Hammerl, 639

Hammerschlag, 3I

Hanke, 7 I 7

Hanke and Koessler, 245, 983

Hansen, 3I, I83, 377

Hanzawa, 5 I 3

Hanzlik, 980, 982, 983, 985, 988, 997

Hanzlik, Butt, and Stockton, 978, 999

Hanzlik and De Eds, 997

Hanzlik, De Eds, and Tainter, 987, 1093

Hanzlik and Karsner, 985

Happ, 895,896

Harass, 206

Harde, 521

Harden, $237,238,248$, 5or

Harden and Walpole, 240

Harden and Young, 237

Harder, 328

Harding and Wilson, 379

Hardy, I 70, 786, 788, 789

HARKINS, I36-78; I77-79, I 81

Harkins and Beeman, 74I

Harkins and Brown, I 78-80

Harkins and Feldman, 864

Harkins and Humphrey, i 78

Harkins and Wilson, I37

Harkins, Young, and Cheng, I74, I78

Harris, 640

Harris, Lockburn, and Anderson, 359

Harrison, 402, 1037

Harrison and Hood, 294

Harrison and Vanderleck, 65,82

Hartley, 752, 753, 764, 769, 1039

Hartman, 694, 699

Hartwell, Mottram, and Mottram, 635

Harvey and Iyenger, Iog2

Harz, 130

Hastings, 380

Hastings, Evans, and Hart, 400

Hastings, Fred, and Carroll, 294

Hata, 2, 207, 476

Hauduroy, 5, 527, 529, 543, 553, 554, 555

Hauduroy and Vandremer, 5

Haughwout, 676

Hauptmann, 1065

Havens, 93I
Hawk, Knowles, Pehfuss, and Clarke, 506-8

Hazen, 888

Hecht, 849

Hecht-Johansen, I053

Hecker and Otto, 467

Hedges, 596

Hegner, 660-78; 672-74, 676, 677

Hegner and Becker, 673,676

Hegner and MacDougall, 696, 699

Hegner and Ratcliffe, 672, 676

Hegner and Taliaferro, 131, 677

Hehewerth, 58, 61, 82

Heidelberger, 231, 572, 702, 714, 726, 727, 97 I, I054

Heidelberger and Avery, 714, 726, 1029

Heidelberger and Goebel, 23 I

Heidelberger, Goebel, and Avery, 714, 727, 834

Heilner and Petri, 1056, I064

Heim, 388, 389

Hein, I32

Heinbecker and Pauli, 908

Heinemann, 6o, 71, 82, 388

Heinrich, von, IO4I

Heintz and Welker, 507

Hektoen, 87 I, 872, 904, 1038, 1041, 1045, 1047, I056, I09I

Hektoen and Corper, I04 I, ro45

Hektoen and Curtis, I042

Hektoen and Schulhof, 7 I 2, 7 I 3

Hektoen and Welker, 7 I 2

Hellriegel, 332, 349

Helmholtz and Lamb, I 70, 786

Helmholtz, Lamb, and von Smoluchowski, 170

Helmholz and Beeler, $5^{83}$

Henningson, I I I

Henri, 375

Henrici, I, 29, 4I, 43, 44, 63, 88

Herelle, d', F., 95, I I6, 519, 520, 525-55, 833

Herelle, d', F., and Eliava, 533

Herelle, d', F., and Hauduroy, 550

Hermann, I039, I09 I

Herter, 645

Herter and Kendall, 645, 648

Hertig and Wolbach, I I, I 2

Herz and Trawinski, 637

Herzenberg, 778

Herzfeld, 1063, 1064

Herzfeld, Meyer-Umhofer, and Becker, Iog2

Hess, 4I 2, 936, 938

Hess and Windaus, 374

Hesse, $219,250,405$

Hesser, 895

Heubner, 1094, 1095, rogs

Heuer, 376 
Heukelekian, 356

Heuser, 633

Hevesy, von, 787

Hibler, von, 199, 206, 287, 288, 290, 29I

Hill, 4I, 673, 677

Hill and Schmidt, 376

Hindle, Io, 455

Hinshaw, 672,677

Hippocrates, 506

Hiranobu, 1002

HrRSCH. See Locke

Hirsch and Williams, 976, 1002

Hirschfeld, 913, 914, 916, 917. See also Hirzfeld

Hirschfeld and Hirschfeld, 907, 908, 913, 917

Hirschfeld, Hirschfeld and Brokman, 91 7, 920

Hirschfelder, 833

Hirschler, 645

Hirzfeld, $892,896,897,908$

Hitchcock, I35, 727, 79 I

Hitchens and Robinson, 926

Hoder, 534, 535, 542, 550, 55 I

Hoder and Suzuki, 539, 550, 55 I

Hoeber, I092

Hoefer and Herzfeld, I092

Hoefer and Kohlransch, 1002

Hölling, 388

Hoen, Tschertlow, and Zipp, 769

Hoff, Van't, 739

Hoff, Van't, and Arrhenius, 55

Hoffmann, E., 474, 482, 483, 487

Hoffmann, E., and von Prowazek, 484

Hoffmann, H., 384

Hoffmann, W. H., 49I

Hofmann, 865

Hogue, 485, 672, 677

Holler, ro89

Holm, Greenbank, and Deysher, 399

Holman, I02-19; 104, II4, II 7, 232, 239, 24I, $563,608,610,616,619$

Holman and Meekison, II3, II5, 366

Holmes, 775

Holst, 566

Hooker, $75 \mathrm{I}$

Hooker and Anderson, 894, 902, 906

Hopkins, 907

Hopkins-Cole, 752

Hopkins and Parker, 808

Hoppe-Seyler, 201

Horgan, I040

Horn, 123

Hort, 39, 40, 4I, 85, 87

Hortock, Shurmann, and Stiner, 376

Horton, 204

Hoskins, 35I
Hoskins and Stout, 636

Hotchkiss, $63,76,82,355$

Hottinger, 25, 28

Houghton, 859

Houghton and Davis, 377

Houssay and Cisneros, 998

Houssay and Negrette, I073

Houssay and Sordelli, ro7 I

Houssay, Sordelli, and Negrette, I071

Houston, 77, 8I, 82, 394

HoweLL, I035-48

Howell and Schultz, 609-11, 6r3, 6r4, I045

Hucker, I 23, I 26, I 27, I 33, I35, 379

Hucker and Marquardt, 379

Huddleson, 38r

Huddleson and Carleson, 857

Hudson, I 4

Hübener, 444, 445, 898

Hübener and Reiter, 467,887

Huelings, Grim, and Horn, 430

Hueppe, I, 385-87, 389-93

Hull, 8I 7, 839

Hull and Rettger, 645, 646

Hunt, 607

Hunter, A., 7 12,7 I 6

Hunter, A. C., 64I

Hunter, O. WV., Io6

Hunter and Cajigas, 859

Huntoon, 926, 928-30, 933, 1050, 1051

Huntoon and Craig, I045

Huntoon and Hutchison, 921-33; 932

Hunwicke and Jephcott, 397

Hussey, 979

Hussey and Northrop, 547

Hutchison. See Huntoon

Hyde, 979, 980

Hygienic Laboratory, 302, 304, 369

Ichikawa, I086

Ido, Hoki, Ito, and Wani, 468,488

Ido, Ito, and Wani, 468, 489

Idsumi and Kollmer, I 23

Iijima, I053

Ikeda, 72,82

Imai, 705

Inada, 497

Inada and Ido, 454, 457-62, 467, 485, 487

Inada, Ido, Hoki, Ito, and Wani, 469, 488

Inada, Ido, Hoki, Kaneko, and Ito, 472, 487, 489

Ingebrigtsen, 907

Ionesco-Mihaiesti, 536, 543, 546

Irwin, 36

Irwin, Haythorn, and Conover, 433

Isaacs, 98I, 1044 
Isaeff and Ivanoff, 8I I

Ishizaka, 1072

Issatschenko, 737

Ito and Matsuzaki, 473

Izar, 5

Jackson, 304, 62 I

Jackson and Smith, 986

Jacobs, 22

Jacobs, Heidelberger, Amoss, and Bull, I 128

Jacobsen, 325

Jacobson and Falk, 570, $57 \mathrm{I}$

Jacobstahl, 849, 855, 856

Jacobsthal, 478

Jagic, 904

Jansky, 892, 902

Jarisch-Herxheimer, I ०99, I I ०3

Jatta, 1036

Jeanneret, 205

Jeimer, 520

Jeney, von, III, II 3,556

Jenner, 479, 877, I035

Jennings, 686, 699

Jensen, C. O., 594, 630

Jensen, H. L., 326

Jensen, L. B., 449, 574

Jephcott, Hunwicke, and Ratcliffe, 397

Jervell, 895, 900, 904, 907, 984

Jobling, 1089

Jobling and Eggstein, 488

Jobling and Petersen, 25, 28, 1090, 109I

Jobling, Petersen, and Eggstein, 977, 982, 984, I065

Jobling and Strause, 876

Jochmann, 774, 875, 925

Jochmann and Kantorowicz, 875

Jochmann and Zeigler, 875

Jötten, 530, 539, $54{ }^{\circ}$

Joffe, 326

Johannesen, 897

Johnson, 360, 859

Johnson, T. B., I023

Johnson, T. B., and Brown, $22 \mathrm{I}$

Johnson, T. L., 689, 699

Jollos, 686, 699

Jona, 776

Jones, A. R., and Glynn, 896, 905

Jones, B. B., 895, 896

Jones, Carmalt, 877

Jones, D. B., and Gersdorff, 709

Jones, D. H., 89, 9 I

Jones, F. E., and Little, 382

Jones, F. S., 636, 797, 1036, 1047

Jones, L. W., 604
Jonescu, 637

Jong, De, $5 \circ 5$

Jong, De, and Hauduroy, 553

JORDAN, E. O., 443-5I; 65, 66, 69, 74, 80, 82, Iо0, $224,275,363,364,369,370,443-45$, $610,629,637,772$

Jordan, E. O., and Dack, 105, 449

Jordan, E. O., and Reith, IO4

Jordan, E. O., Russell, and Zeit, 74, 82, I04, 364,370

Jordan, H. E., 366

Josephson, 272

Jousset, IOI9

Jowett, 632

Juday, 200

Juday and Wagner, 200

Julianelle, 8, 92, 100, 619

Jungano and Distaso, 199, 206

Jungeblut, 890

Jungeblut and Berlot, 890, I044

Kabelik and Kukula, 527

Kabeshima, 542

Kämmerer, 25, 102, 105

Kafka-Walter, I०99

KAHN, 848-6o; 199, 849

Kahn, Landau, and McDermott, 855

Kahn, Nagle, and Kendrick, $85 \mathrm{I}$

Kakehi, 614

Kalberlah, 478, I099

Kanai, 868

Kantorowiecz, 25, 875

KARSNER, 966-88; 969, 980, 982, 983, 993

Karsner, Brittingham, and Richardson, 26I

Karsner and Ecker, 967, 973, 978, 979

Karsner and Nutt, 978

Karsten, 445

Kartulis, 487

Kaserer, 282, 324, 329

Kaspar and Kern, 614, 620

Kato, 970, 973

Kauffmann, II $3,532,548,55^{\circ}$

Kedrowsky, 204, 205

Keegan, 6I6, 6I 8, 635, 937

Keil, 327

Keilin, 215, 2 I 7

Kekulé, I9

Kellaway and Cowell, 99r, 992

Kellerman, 340

Kellerman, McBeth, Scales, and Smith, 274

Kelley and Webber, 4 IO

Kelly, 749

KENDALL, 227-42; IO2, 229, 232, 242

Kendall, Bly, and Haner, 236

Kendall, Cheetham, and Hamilton, 229, 234 
Kendall, Day, and Walker, I99

Kendall, Day, Walker, and Ryan, 233

Kendall and Farmer, 223, 234

Kendall and Schmitt, 230

Kendall and Simonds, 273

Kendall and Yoshida, 235, 236

Kendrick and Kahn, 851

Kennedy, $5^{83}$

Kepinow, 1092

Kessel, 669, 677

Kessel and Svensson, 677

Keyes, 265

Keyes and Gillespie, 265

Keynes, 9I 7

Keysser and Wassermann, 982

Khanolkar, Iog I

Khouvine, гоб

Kilpatrick and Kilpatrick, 525

Kimura, 528, 551

King, Arnold, and Church, Iog6

Kirch, 609

Kircheim and Tuczek, 1027

Kirchensteins, 39

Kirihara, 917

Kirkbride and Dow, 749

Kirkbride and Wheeler, 957

Kitasato, 287

Kite and Wherry, $867,868,872,878$

Kitt, 206, 609, 6I 3

K jaergaard, 1063,1064

Kjerdahl, 648

Klarenbeck, $47^{8}$

Klauder and Kolmer, 847

Klein, 640

Klein, E., Io9, 609, 636

Klein, E., and Houston, 366

Klein, G., and Limberger, 327

Klein, G., and Svolba, 33I

Klein, K., 609

Kleine and Möllers, 687, 699

Klemperer, I093, I095

Klien, 878

Klieneberger, 443

Kliewe and Nagel, 9 I 7

Kligler, I 28, I35

Kligler and Robertson, 472

Kligler and Weitzman, 686, 699

Kline, 482

Kline and Blankenhorn, 482

Kline and Young, 859

Kling, I002, I093

$\mathrm{K}$ linger, 106

Klopstock, A., 707

Klopstock, F., 835, 856, I 026
Kluyver, 2 I4

Kluyver and Donker, 33I, 502, 504

Knapp, Godfrey, and Aycock, 4 I 6

Knorr, 632, 88r, 889

Koboyashi and Shuwatsu, I044

Koch, I, 32, 34, 72, 77, 82, 85, 301, 362, 385, 390, $391,404,590,620,773$, 1016-20, 1023, 1030, IO32

Koch and Gaffky, 624

Koehler, 635

Koenigsfeld and Oppenheimer, 993

Koessler, ror 2

Koessler and Hanke, 230

Kofoid, 669, 677

Kohlbrügge, 640

Kohmann, 203

Kohn, 67, 68, 82

Kolle and Ritz, 468

Kolliker, 628

KOLMER, I I I I-33; 843, 923, 924, IO53, I IOI

Kolmer, Cohen, and Heist, I I 2 I, I I 22

Kolmer, Davis, and Jager, I I 28

Kolmer and Harkins, I I I9, I I 30

Kolmer and Sands, I I 24

Kolmer and Steinfield, I 123

Kolmer and Williarns, 1063

Kolmer, Williams, and Laubaugh, 835

Kondo, 2 I 2

Konradi, 8o, 82

Kopaczewski, 977, 983, 984

Kopaczewski and Gruzewska, 987

Kopaczewski and Roffo, 977, 983

Kopaczewski and Vahram, 979

Kopeloff, 106, 514, 647

Kopetski, 645

Koppanyi, 623

Kosaki, 1050

Koser, 243-49; I I 8, 224, 247, 248, 365, 366, 539

Koser and McClelland, 9

Koser and Rettger, 244, 246

Koser and Skinner, 75, 82

Koshina, Shiozawa, and Kitayama, 468, 493

Kotila and Coons, 599, 601, 604

Kouwenaar, 490

Kozai, 387

Kraft, 905

Kral, 390, 392

Kramár, 8

KRAMER, 557-64; 4I, 2 I 5, 2 I 7

Krasnow, Rivkin, and Rosenberg, 244, 279, 280, 283

Kraus, E., 645

Kraus, R., 524, 622, 824

Kraus, R., and Levaditi, 98I 
Kraus, Loewenstein, and Volk, 1026

Kraus and Schiffmann, 883, I037

Kraus and Uhlenhuth, 607

Krause, 1027,1030

Krause and Willis, 967

Krehl and Matthes, 1027

Kretz, Pawlowsky, and Matsukoff, 1074

Kreussler, 374

Kristensen, II4

Kritschewsky, 983, 984

Kritschewsky and Friede, 979

Kritschewski and Meersohn, I043

Kritchewski and Schwartzmann, 895

Krueger and Schultz, 1002

Krünig and Paul, 72, 82, 29I, 301

Kruif, De, 92, 425, 570

Krumbhaar and Smith, 819

Krumwiede, 34, 445

Krumwiede and Banzhaf, 757

Krumwiede, Cooper, and Provost, 820

Krumwiede, Provost, and Cooper, 446

Krumwiede, Valentine, and Kohn, 610, 6 II

Kruse, I, 8, 25, 278, 386-92, 908

Kruyt, 178

Kruyt and Van Arkel, 800

Kruyt and De Jong, 743

Krylow, 32, 33

Kubanyi, 905

Kuczynski, Tenenbaum, and Werthemann, I038

Kühne, 268

Küster, 644

Kützing, 498

Kuhn, 90, 91, 95, 96, 272

Kuhn and Steiner, 478, 479

Kuisl, 642

Kulo, 647,649

Kunitz, 545

Kurita, 622

Kutchera, 6.35

Kutscher, 632

Kutscher and Meinicke, 888

Kuttner, 537-39, 542

Kyes, 808, 1036, 1043, 1072

Kyes and Strauser, 978, 993

Kyrle, rog9

\section{Lacey, 595,898}

Laidlaw and Dudley, 714, 727, 1029

Laing, 787

Lake, Osborne, and Wells, I003

Lamár, I 123

Lamb, 1070-73

Lamb and Helmholtz, $785,787,789$

Lambert and Hanes, 1038
Lancaster, 89

Lancefield, $723,725,731,834$

Landau, 107

Landerer, 1087

Landsberger, 640

LANDSTEINER, 892-908; 706, 708, $710,718-20$, $723,856,892,895,898,900,902,909,914$, $915,970,1049$

Landsteiner and Berliner, 52 I

Landsteiner and Jablons, 718

Landsteiner and Jagic, 740

Landsteiner and Lampl, 7 18

Landsteiner and Leiner, 895

Landsteiner and Levine, 708, 895, 900-902, 904, 908, 91 I , 917, 919, 920

Landsteiner and Miller, 902, 903

Landsteiner, Müller, and Pötzel, 849

Landsteiner and Pauli, 743

Landsteiner and Richter, 907

Landsteiner and Van der Scheer, 710, 856, 894. 903,904

Landsteiner and Simms, 707, 728, 734

Landsteiner and Stanković, 839

Landsteiner and Witt, 894, 897, 899, 901

Lane-Claypon, 4I, 48, 53, 58, 6I , 65, 7I, 82

Langdon and O'Toole, 833

Lange, 9, 1026, I027

Lange, B., and Yoshioka, 634

Lange, C., 1058

Lange, F. D., 1089

Langmuir, $74 \mathrm{I}$

Lanner, 908

Lantzch, 329

Larkum, 554

LARSON, $179-87$; 184

Larson and Bell, $45^{\circ}$

Larson, Cantwell, and Hartzell, I8I, 209

Larson and Eder, I86

Larson and Evans, I80

Larson, Evans, and Nelson, 746

Larson and Greenfield, 183

Larson, Halvorson, Evans, and Green, I 85

Larson and Larson, I 82

Larson and Montank, 183

Larson and Nelson, 183, 184, 746

Latham, 515

Lattes, 892, 899, 900, 905, 907, 9I3, 9I 7

Launoy and Levy-Bruhl, I042, I045

Laurent, 604

Laven, 625

Laveran, 480, 699

Laveran and Mesnil, 690, 692, 699

Lavoisier, 250, 255

Lawes and Gilbert, 332

Leach, 606 
League of Nations Health Organization, 840 , $841,844,954,960,963$

Learmouth, 917

Lebedev, 329

Lebert, 479

Lederer, 858,859

Ledingham, 92, 775, 809, 863, 868, S9o, 1046

Ledingham and Penfold, 49, 60, 63, 82

Lee, 997

Lee and Vincent, 976

Leger and Ringenbach, $68_{5}, 699$

Legros, 47 I

Lehmann, 378

Lehmann and Neumann, I30, 132, I 35, 380, 384 , $386,388,390$

Lehmann and Sano, 215

Leichmann, 384, 386-88

Leimdörfer, IogI

Leishman, 9, 454, 87 I

Leishman, Adami, Farmer, and Harvey, 40

Leonard, 786

Leone, $6_{3}, 82$

Lepper, 549

Lerda and Diez, 1043

Letts, 358

Leudke, 1089

Leva, 645

Levaditi, 475, 478, 480, 52 I

Levaditi and Landsteiner, 94I

Levaditi and Latapie, 84 I

Levaditi and McIntosh, 686, 699

Levaditi and Maria, 849

Levaditi and Mutermilch, 685, 699

Levaditi, Nicolau, and Schoen, 631

Levin, 45I, 644

Levine, M., 240, 365, 392, 596

Levine, M., Buchanan, and Lease, 74, 82

Levine, M., and Eberson, 4 I3

Levine, M., and Soppeland, 355

Levine, P., 892, 897, 920

Levine, P., and Coca, 1006, 1007, 1009, 1013

Levine, P., and Mabee, 905

Levy, 554, 922

Lévy and Bruns, 287, 813

Lewis, G. N., I45, I 77

Lewis, G. N., and Randall, I46, 177

Lewis, J. H., 705, 97 I, 978

Lewis, J. H., and Wells, 712

Lewis, M. R., and Lewis, 876, 890

Lewis, P. A., 499

Lewis, P. A., and Aronson, 962

Lewis, P. A., and Loomis, ro44

Lewis, T., and Mathison, 993

Lewis, W. C. McC., 783
Leyton, Leyton, and Sowton, 994

Liang, 908

Liebermann, von, 2 I 2, 2 I 3, 548

Liebermann, von, and von Fenyvessy, ro5o

Liebig, 227, 239

Liefmann, 207

Lieske, I08, I99, 326-28, 33 I

Lignières, 443, 623

Lillie, 2 2, 1092

Linden, Turner, and Thom, $45^{\circ}$

Linder and Picton, 792

Lindner, 505, 506

Lindner and Unger, 506

Ling, 504, 1089-9 I

Lingard, 685, 699

LINK, 590-606; 51 2, 570

Link and Hull, 570, 595, 597, 598, 600

Link and Link, 594, 599, 600

Link and Sharp, 570, 594

Link and Taliaferro, 595

Linossier and Lemoine, 826

LIPMAN, 341-50; 350

Lipman and Waksman, 329

Lipschütz, 523

Lisbonne, Boulet, and Carrière, 539

Lisbonne and Carrière, I I 2, 540

Lister, 89, 378, 379, 384-88, 39 I

Litch and Meyer, 629

Literer, 859

Liu and Wang, 908

Liu-Weng-Wang, 913

Lloyd, 68, 82

Locke, 929, 1050

LOCKE AND HIRSCH, r049-55; 1050, 1055

Locke and Main, 764, 769, 1050

Locke, Main, and Hirsch, 1052, 1053

Locke, Main, and Miller, I052

Lockhart, 6r2, 634

Loeb, J., 2 I , $276,786,789,797,798,863$

Loeb, J., and Northrop, 73, 82

Loeb, L., 903

Loeffler, 524, 609, 632, 637, 888

Loeffler and Frosch, 415

Löhnis, 4, 6, 44, 85, 89, 91, I32, 350, 380, 388

Löhnis and Smith, 87, 338

Löhr, I095

Löhr and Löhr, 987, I089, ı 090, Iю96

Lömmel, I 6

Lövgren, 272

Loew, 33 I

Löwenstein, 2 I4

Löwenstein and Pick, rorg

Loewit, 994

Logie, 2 I 2 
Lohwag, 499

LoNG, 1016-34; 32, 221, 244, 245, 985, I OI 7, 1020

Long and Seibert, 714, 1019-21, 1026

Long and Seyfarth, 968

Longcope, 979, 990

Longcope, O'Brien, and Perlzweig, 7 I 3

Lord, 876

Lord and Nye, 876

Lotka, 48, 5I, 53

Lottermoser, 797, 800

Lourens, 89

Lowenstein, 376

Lowy, ro89, Iog6

Lubarsch, 779

Lucas, 500

Lucet, 628

Lucksch, 524

Ludlow, 677

Lübbert, 2 I9

Luithlen, I093

Lukacs, I09I, I093

Lumière, 977, 984

Lunn, I69

Lyman, 373, 374

Lynch, 633, 634

Lyon, 231, 779

Lyon, Fippin, and Buckman, 34I

Lyon and Shackell, 548

Maassen, 2 I I

McBain, 787

MacCallum, 524

McCarthey and Olitsky, 623, 625

McClintock, I074

MacCollum, 499, 634

MacConkey, 240, 364, 614

McCordock and Congdon, 636

МcCoy, 947-65; 926, 928, 960

McCrady, 368

McCullough and O'Neill, 976, I002

MacDonald, 499

MacDougall, 696, 699

Macé, 390, 39 I

McFadyen, 406, 518, 780

McFadyen and Hewlett, 407

McFarland, 8I 7

Macfie, 484,642

M'Gowan, 6i 8

McIntosh, 648, 878

McIntosh and Fildes, $47^{\circ}$

McIntosh and Kingsbury, 1040

McJunkin, 729, 873, 1026, 1027

McKendrick, 869
McKendrick and Pai, 48, 5 r, 53

Mackenzie, 677, roI3

Mackie, 1053

McKinley, 536

McKinley and Larson, I 86

Maclean, $5 \circ 5$

Maclean and Hoffert, 505, 506

MacLean, 859

MCLEOD, 2 I I-I 7

McLeod and Gordon, I I 7, 208, 21 3, 21 4, 2 I 7, 277

McLeod and Govelock, 214

MacNeal, 692, 699

MacNeal and Kerr, 203, 204

MacNeal, Latzer, and Kerr, 639, 648

McNeil, 833

McNeil and Kahn, 275

Mc'Taggart, 786

MclWeeney, 444, 445

Madelung and Born, I 40

Madsen, 754, I040

Madsen and Nyman, 9, 72, 78, 82

Madsen and Schmidt, 762, 764, 769

Magoon, 9, 285, 291, 296

Mahnert and Santner, 1095

Mair, $35^{8}$

Major, 977

Malassez and Vignal, 6I4

Maldovan, 697, 699

Malkin, rogo

Mallmann and Hemstreet, 6or

Mallory, 873, 89o

Mallory and Marble, 886,887

Maltaner, 1053

Malvoz, 8I 4

Manioliu, 539, 554

Mann, 20

Manninger, 540, 543, $55^{\circ}$

Manteufel, 692, 699

MANIAARING, 989-1003, 1078-85; 273, I $_{5}, 88_{3}$, $975,982,991,992,994-96,999,1080-84$

Manwaring, Beattie, and McBride, 997

Manwaring, Brill, and Boyd, 975, 995, 996

Manwaring, Chilcote, and Hosepian, 975, 997

Manwaring and Coe, 808

Manwaring and Crowe, 976, I0о

Manwaring, French, and Brill, 997, 1002

Manwaring and Fritschen, 587

Manwaring, Hosepian, and Beattie, 997

Manwaring, Hosepain, Enwright, and Porter 974, 999, 1000

Manwaring, Hosepain, O'Neill, and Moy, 999IOOI

Manwaring, Hosepian, and Thomson, 996

Manwaring and Kusama, 976, 980,997

Manwaring, Küsama, and Crowe, I003 
Manwaring and Marino, 974, 995, 999

Manwaring, Marino, and Boone, 979

Manwaring, Marino, McCleave, and Boone, 97 I, 990

Manwaring, Meinhard, and Denhart, 980, 994

Manwaring, Monaco, and Marino, 983

Manwaring, O'Neill, and McCullough, I000

Manwaring and Oppenheim, IOO2

Manwaring and Williams, 993

Manwaring, Wright, and Shumaker, 992

Marbe, ro43

Marchoux, 521, 923

Marchoux and Salimbeni, 468, 479, 480

Marcuse, 529, 530, $55^{\circ}$

Marie and Levaditi, $s_{3} 8$

Marie, Levaditi, and Bankowski, 477

Marie, Levaditi, and Banus, 476

Marinesco, 478

Marinesco and Minea, 477

Marmorek, 577

Marpmann, 384-86

Marsh, 4I4

Marshall, 527, 536, 55 I

Martin, 607

Martin and Pettit, 455, 473

Massachusetts, 82

Massaglia, $685,687,700$

Massini, 85, 92, 95, 978

Mast, 86I

Mathews, 202, 219, 234

Matill and Hawk, 639

Mattausch, I092

Matthes, ror9, 1027, 1087

Matthew and Grove, 998

Matsumoto, 535, 536, 5.38, $55^{\circ}$

Mattlet, 5 I 5

Matzkiewitsch, Io63

Matzuchita, 202, 204

Mautner, 999

Mautner and Pick, 975

MAVER, 739-44

Maver and Falk, 575, 743

Maximow, 890

Maxwell, 863

Maynard, 859

Maze, 202

Mazza, 700

Meadow and Hale, 368

Medical Research Committee, I99, 8I 7-19, 840, 844

Meek and Lipman, 325

Meinicke, 849

Meirowsky, I I

Meisser, 582, 586
Meisser and Brock, $5^{83}$

Meissner, 528, 549

Meissner and Baars, 555

Mellon, 4, 6, 32, 39, 85, 87-91, 94, 96, 100, 655, 798,799

Meltzer, Iог 2

Mencl, 39, 40

Mendel, 9 г 2

Mendes-Correa, 9 I9

Mennes, 870

Menton, 629, Io96

Menton and Manning, 629

Merklen and Wolf, 887

Merrill and Wade, I30, I35

Mesnil and Brimont, 700

Mesnil and Leger, 700

Messerschmidt and Keller, 627

Metchnikoff, E., 106, 644, 739, So3, 8I I, 870, $873,875,88 \mathrm{r}, 923$, 1039

Metchnikoff, E., and Besredka, 888

Metchnikoff, E., and Roux, 476

Metchnikoff, O., 644

Meuli, 538, 542, 545

Meyer, 132, 797

Meyer, A., 39, 202, 833

Meyer, E., 272, $52 \mathrm{I}$

Meyer, H. H., I093

Meyer, H., and Ransom, 773

Meyer, K., 234, 714, 734

Meyer, K., and Jacobson, 234

Meyer, K., and Ziskoven, 898

Meyer, K. F., 607-38; 608-I I, 6I 5, 6I 7, 620, $625,626,633 ; 637$

Meyer, K. F., and Batchelder, 6I 4, 6I.5, 620, 6.36

Meyer, K. F., and Matsumara, 637

Meyer, K. F., Neilson, and Feusier, 626

Meyer, N., 890

Meyer, R., 446

Meyer-Bisch, rogI

Meyerhof, 269, 325, 330, 33I

Michaelis, 22, 272, 791, 799, 8I5, 849, 865, I062

Michaelis and Nakahura, 273

Michaelis and Rona, 796

Miehe, 89, 605

Miessner and Trapp, 838

Migula, 282, 380, 387, 389-92, 594

Milford and Coca, Ior4, ror 5

Miller, 39o, 392, 500, 642, 645

Miller, Andrewes, and Swift, 63 I

Millon, 752

Mills, Bartlett, and Kessel, 677

Milner, r 40

Minet and Bruyant, 977

Ministry of Health, 844 
Mino, 896, 899, 900, 917

Miquel, 63, 64, 66, 67, 71 , 82

Mita, 978

Mitchell, 636

Mitchell and Ravenel, 935

Mitchell, S. Weir, I067

Mochtar, 467, 486, 493

M $\varnothing$ llgaard, I I 29

Moench, $5^{83}$

Mohler and Eichhorn, 833

MoHLman, 351-6r; 359

Molisch, 327, 328

Moll, I089

Molliard, 5 I 5

Molliard, Butkewitch, and Currie, $5 \mathbf{1 2}$

Moloney, 769

Moloney and Weld, 750, 753, 763, 764, 769

Moore, 378 , 379, 407, I I 2 I-23

Moore and Carpenter, 38I

Moore and Ward, 379

Moore and Williams, 203, 259, 263

Morax, 640

Moreschi, 1045

Moreschi and Golgi, 776, 777

Morgan, I 79, r80, 392

Morgan and Marshall, 609

Morgenroth, I 049, I I I 5, I I I 8, I I 20, I I 24, I I 30

Morgenroth and Levy, I I 20, I I 22

Morgenroth, Schnitzer, and Berger, 577

Moro, 394, 640-42, roI 7

Morris, Porter, and Meyer, 678

Morrison and Tanner, 285, 286

Mosbacher and Port, 1058

Moser, 747, 755, 756

Moss, 893-95

Motohashi, го36, I043

Much, 4, 33, 88, 89, 706, I0I9

Much and Sartorius, 108

Muckenfuss, 538

Mudd, 4I, 557, 8I 8

Mudd and Mudd, I80, 863

Mühlens, 484

Mühlens and Hartmann, 472

Müller, E. F., 1089, г093, 1096

Müller, E. F., Myers, and Petersen, Io93

Müller, E. F., and Petersen, 1093, 1095, 1096

Müller, E. F., Petersen, and Kast, 1096

Müller, 59-6r, 82, 92, 203, 212, 613, 841, 883

Mueller, J. H. See Zinsser; 714, 727, I029

Mueller, Smith, and Litarczek, 727

Mueller and 'Tomczik, 726

Mueller, Wayman, and Zinsser, 727

Müller, P. J., I039

Müller, P. T., 1053
Münz, 329

Muir and Martin, 809

Mulzer, 484, 1099

Munter and Boenheim, 554

Munter and Rasch, 534

Murlin and Mattell, 506, 507

Murphy, 874

Murphy and Ellis, I040

Murphy and Sturm, I039, I040

Murray, 330

Mutermilch, 686, 700, 841, 903

Mutermilch and Latapie, $84 \mathrm{I}$

Myers, 2I 2

Nabokitch and Lebedeff, 329

Nadson and Konokotina, 505

Naegli, 84, 85, 1 29, 132, 279

Naeslund, I99

Nakahara, 887

Nakamura, 528, 583

Nakanishi, 6, 35, 39

Nakashima, 556

Nasta and Braumer, 1047

Nathansohn, 2 I, 325, 326

National Canners' Association, 442

Neal, 942

Neal and Zingher, 94 I

Needham, 285

Needham and Needham, 197

Nègre and Boquet, 833

Neide, 29

Neill, 208, 214, 845

Neill and Avery, 216

Neill, Fleming, and Gaspari, 722

Neisser, 31, 85, 92, 95, 8I 8

Neisser and Freedemann, 792

Neisser and Wechsberg, 688, 700

Nelis, 746

Nelson, C. I., 7 I4

Nelson, J. B., 6ro

Nelson, J. B., and Smith, 609, 610, 613, 629

Nencki, 103, 205

Nencki and Sieber, 287

Neri, 889

Nernst, 786

Netter, 922, 941

Netter, André, Cesari, and Condoni, I 83

Neuberg, 238, 501, 502, 504

Neuberg and Arinstein, 239

Neuberg and Hirsch, 237

Neuberg and Kobel, 231, 232

Neuberg and Oppenheimer, 269

Neuberg and Reinfurth, 504

Neuberg and Windisch, 239 
Neufeld, 632

Neufeld and Meyer, I043

Neufeld and Rumpau, 87 I

Neumann, 864

Neuschlosz, 700

Neustaedter, Larkin, and Banzhaf, 942

Newburgh, 78I

Newcomer, 372

Neymann and Gager, 844,850

Nichols, $362,477,496$

Nichols and Hough, 476

Nichols and Schmitter, 204

Nichols and Stimmel, 6I 3

Nickel, 583

Nicolas, 886

Nicolle, A., I025

Nicolle, A., and Abt, 1025

Nicolle, C., 537, 542, 543, 71 5

Nicolle, C., and Conseil, 938, 943

Nicolle, C., Blaizot, and Conseil, 468, 479

Nicolle, M., $75^{2}$

Nicolle, M., and Allilaire, 218

Nicolle, M., Cesari, and Debains, 759, 769

Nicolle, M., Debains, and Cesari, $75^{2}$

Nigg, 908

Nigh and Van Ditmar, 244

Nikitinsky, 329

Niklewski, 329

Nissle, I I I, I 2

Nixon, 597, 605

Nobechi, 554

Nobile, De, 445

Noble, 642,857

Noc, $107 \mathrm{I}$

Noel, rog9

Nöller, I 2

Noguchi, 452-97; r99, 453-62, 465, 467-7 I, 474, $476,477,482,483,485,487,488,490,493,521$, $630,835,840,842,850$, 107 I, 1072

Noguchi and Battistini, 5 I9

Noguchi and Kligler, 49I

Noguchi and Moore, 476

Noguchi, Muller, Torres, Silva, Martins, Ribeiro dos Santos, Vianna, and Biāo, 492

Nolf, 7 II, тоO

Nolf and Spehl, 482

Nonne, I 100

Noon, 879,963 , гог 2

Nord, 276,501

North, 408, 435

North and Park, 404, 406, 407

North, Park, Moore, Rosenau, Armstrong, Wadsworth, and Phelps, 406, 425, 430, 433

Northrop, 782-801; 547, 569, 741, 785, 800, 869

Northrop and Cullen, 785
Northrop and Freund, 796

Northrop and De Kruif, 569, 792, 797

Northrop and Kunitz, 785

NoRTON, 362-70, 371-77

Norton and Weight, 366

Nothnagel, 228, 236

Noüy, $\mathrm{Du}, \mathrm{174}, 520$

Novy, 103, 204-8, 219, 259

Novy and Knapp, 89, 467, 480, 48I

Novy and De Kruif, 982

Novy, Roehm, and Soule, 25 I, 254

Novy and Soule, 203, 26I-64

Nowak, 204

Nungester, 93-95

Nuttall, 454, 826, 829

Nuttall and Thierfelder, 644

Nye, 876

Nyfeldt, 35, 36

Obermayer and Pick, 7 I 8,825 , 1091

Obermeier, 479

O'Brien, 609, 61 I

O'Brien, Okell, and Birkhaug, 766, 769

Oettingen, von, 204

Ogata, $540,55^{\circ}$

Okamoto, 609, 61 I , 616, 61 7, 633

Okell and Parish, 1052

Okell, Parish, and O'Brien, I029

Okuda, 535

Olitsky and Gates, I 14, 499

Oliver, 63I

Oliver and Douglas, 987

Oliver and Yamada, 987

Olmstead and Luttinger, 832

Omeliansky, I05

$\mathrm{O}^{\prime}$ Neill, Moy, and Manwaring, 1002

Opie, $774,825,827,875,981,982,984,985,1002$

Opie, Baker, and Dochez, 875

Opie and Furth, 979

Opie and McPhedran, 1029

Oppenheim, 745

Oppenheim and Voigt, 897, 902

Oppenheimer, $237,273,278$

Orcutt, 92

Orcutt and Howe, 275

Ori, 206

Orla-Jensen, I2I, I 25, I26, I28, I3I, I33, I35, 2I 6, 219, 380, 394

Orr, 394

Ørskov, 4I

Osborne, 220

Osborne, Mendel, and Harris, 7 55

Osborne and Wakeman, 713, 977

Oshima, 5I3, 5I5

Oshima and Church, 513 
Osterhout, 548, 1092

Ostertag, 4I 2

Ostwald, $48,5^{2}$

Ostweiler, 645

Osumi, 520, 532, 535

Otsuka, 244

Ottenberg, 844, 892, 907, 908, 91 2, 91 3, 917, 1050

Otrenberg AND Beres, 909-20; 894, 907

Ottenberg and Johnson, $90 \mathrm{I}$

Ottenberg, Kaliski, and Friedman, 904

Ottenberg and Stenbuck, I05I

Ottenberg and Thalhimer, 904

Otto, 969

Otto and Munter, $538,540,54 \mathrm{r}$

Otto, Munter, and Winkler, 530, 536, 539, $54 \mathrm{I}$

Otto and Shirakawa, 1003

Otto and Winkler, $538,540-42$

Ottolenghi and Pabis, 628

Ouchi, 895

Overton, 2I

Owen, 5I5

Owen and Cope, 859

Packard, 200, 202

Paine, 240, 592

Paine and Lacey, 594

Pakes and Jollyman, 238, 24I

Papacostas and Gaté, Io9

Pappenheim, 35

Pappenheimer and von Wedel, 637

Parisi, $35^{8}$

PARK, 934-46; 90, 408, 433, 748, 75I, 925, 928, IOI 2

Park and Banzhaf, 751

Park, Banzhaf, Zingher, and Schroeder, $75^{2}$

Park and Freeman, 940

Park and Williams, 756

Park, Williams, and Krumwiede, 492

Park and Zingher, 939

Parker, 755, 930

Parker and Banzhaf, 930

Parker and Parker, 973, 991, 992

Parkes, 80,83

Parodi, 476

Parr, 642

Parsons and Sturges, 83

Partik, 608

Paschkis, 1044

Passini, 105

Pasteur, 103, 196, 198, 200, 201, 203-5, 227, $228,234,236,239,250,268,285,384,39$ I, 417 $4 \mathrm{I} 8,590,643,724,877,923,95$ I, 1035,1046

Pasteur and Joubert, 198,199

Paul, 78,83

Paul, Birstein, and Reuss, 74,83
Paul and Krönig, 72, 83

Paulson and Andrews, 673,677

Pearce, 62I

Pearce and Eisenbrey, 975, 980

Pearce, Karsner, and Eisenbrey, 977

Pearse and Mohlman, 359

Peck, $35^{8}$

Peiper, 1064

Pelz, 2I 2

Pelz and Jackson, 974, 975

Pemberton, De, 904, 906

Penfold, 58, 6I-63, 7 $1,83,92$

Penfold and Norris, 67,83

Penna, Kraus, and Cuenca, I086

Penzo, 204

Pepper and Krumbhaar, 976, 1002

Pérard, 628, 629

Pereiro da Silva, 488

Pérez-Grovas, 49I

Perkins, 120-35; 240, 390, 392, 393

Perla, 621, 627

Perlzweig and Steffen, 715

Perrín, 483,487

Perrin, $783,784,788$

Perroncito, 62I

Perry and Tidy, 446

Pesch, 95

Pesch and Zschocke, I Io

Peters, 73, 83, 390, 39I

Petersen, 1086-1100; 1089, 1093, 1094, 1098

Petersen, Jaffé, Levinson, and Hughes, rogo

Petersen and Levinson, 996, 1024

Petersen, Levinson, and Hughes, 975

Petersen and Müller, I09I, I096

Petrie, 606

Petrie and O'Brien, 609, 6I1, 613, 614, 621

Petroff, 1027

Petrovanu, 54I

Pfaundler, 809, 81 3

Pfeiffer, 393, 831, 1087

Pfeiffer and Isaeff, I092

Pfeiffer and Marx, 883, 1035, 1036, 1042

Pfeiffer and Proskauer, ro 49

Pfeiler, 6r 5

Phelon, Duthie, and McLeod, 2 I6

Phelps, 72, 73, 75, 78, 79, 83, 361

Philibert, 522, 553

Phillips, 197

Phizalix, 6I9, I073

Piana, 976

Pick, 724, I049

Pick and Hashimoto, I09I, 1095

Pico, 537, 539, 542

Picton and Linder, 790 
Pierret and Bilouet, $55^{2}$

Pilcher and Sollmann, $97 \mathrm{I}$

Pilot, 653,654

Pilot and Davis, 482

Pilot and Pearlman, 654

Pincussen, 375, 377

Pineda, 859

Pirquet, von, 780, 966, 101 7, 1028

Pirquet, von, and Schick, 966

Pittman, 568

Place, Sutton, and Wilner, 415

Plaut, I99, 838 , 1063

Plehn, 904

Plüss, 91 7

Poehl, 645

Pohl, 734

Poisson, I 40

Pollitzer and Stolz, I093

Ponder, 572, 864-66

Pons, 482

Popoff, 640, 9I I

Poppe, 6I4

Porchownick, 106 r

Porges, 795

Porter and Hedges, $7 \mathrm{~S}_{4}$

Portier and Richet, 969

Portis, 1042

Pottenger, I०3०

Potter, De, 5

Potthoff and Heuer, 377

Povitzky, 764-66, 769

Povitzky and Banzhaf, 769

POWELL, 824-30

Powis, 788, 789, 800

Prausnitz, 526, 534, 537, 542

Prausnitz and Firle, 533, 534

Prausnitz and Küstner, 972, 1006

Prausnitz and Lubinski, $49 \circ$

Preger, 896

Preisz, von, 93, 95, 527, 529, 54I-44, 546

Prell, i I I

Prescott, 365, 433

Prescott and Baker, 69, 70, 83

Prescott and Winslow, $83,365,367$

Prévôt, 199

Preyer, 326

Price-Jones, 637

Prigge, I09I

Prillieux, 59I

Pringsheim, I04, I I0

Pritchett, 632, 633

Przygode, 1038

Proca, 205

Proskauer and Beck, 225
Prowazek, II, 483

Prucha, 403

Pryde, 702, 707, 7 10

Przesmycki, 727

Putnam, 419-36; 425

Putnam and Gay, II4

Putter and Vallen, 539-4I

Quastel, 2I3, 2I6, 230, 248, 276

Quastel and Stephenson, 247

Quastel, Stephenson, and Whetham, 213

Quastel and Whetham, 213

Quastel and Wooldridge, 213, 269

Rabinowitsch and Kempner, 690, 700

Rackemann, 1007, 1008

Raebiger, 607, 618, 623, 627

Raebiger and Lerche, 608, 6ro

Rahn, 58, 59, 61, 62, 83

Raistrick, 245

Raistrick and Clark, 245

Raiziss and Severac, i I 26

Raju, 83

Rakusin and Flieher, 742

Ramirez, 1009

Ramon, 614, 615, 705, 719, 742, 745, 750, 751, $754,759-66,768-70,952,1047$

Ramon and Descombey, 767, 769

Ramon and Grasset, 770

Ramsden, 21

Ramsey, 4I3

Rand, 606

Ranke, 476

Raper and Wormall, 272

Ratcliffe, 66r, 662

Rath, I037, I042

Raubischek and Russ, 746

Raulin, 510

REDDISH, 301-9; 302, 303, 306, 307, 647, 649

Redfern, 736

Redfield, 859

Reed, Walter, 49I

Reed and Carroll, 467

Reed and Ettinger, 619

Reed and Lamson, 976, 979

Reed and Orr, 3

Reed and Reynolds, 70, 71, 83

Rees, 674,677

Reese and Peter, II00

Regaud, 453

Regendanz and Kikuth, 686, 691-93, 700

Regendanz and Tropp, 698, 700

Regnault and Rieset, 255

Reich, 628

Reichenow, 628 
Reichert, 527, 530, 534

Reimann, 92, 94

Reimann and Julianelle, 776

Reinders, 866

Reinke and Berthold, 59r

Reis, von der, I IO

Reiss and Jungman, 944

Reiter and Ramme, 472

Reitler, I047

Reitstotter, $74 \mathrm{I}$

Remlinger and Bell, 607

Renaux, 761, 770, 842

Repaci, 47 I

RETTGER, $218-26,639-49 ; 63,83,648$

Rettger and Cheplin, ı०6, 646, 647, 649

Rettger and Horton, 645

Rettger, Hull, and Sturges, 446

Rettger, Reddish, and McAlpine, 507, 508

Rettger, Valley, and Plastridge, 640

Rhein, ro4

Rhumbler, 865

Ribeyro, 488

Ribierre and Kermogant, 482

Rice and Downs, 396

Rich, IOO2

Richards and Carver, 178

Richards and Sawyer, $35^{8}$

Richet, 75, 83, 969, 976, 979

Richet, Brodin, and St. Girons, 976, 993

Richter, 312

Richters, 6I6, 6I 7

Ricketts, I I

Rideal and Walker, 302

Rietz, 9I 7

Riker, 597, 602-4

Ritchie, 32

Ritz, 685, 700

Rivers, 5I 7-24; 532, 931, 932, 1046

Rivers and Tillet, 886, 1046

Rivolta, I30

Roberts, 378

Robertson, 48, 51, 53, 80, 83, Ir 3, 682, 700

Robinson, 445

Robinson and Auer, 975, 993

Robinson and Elliott, $76 \mathrm{I}$

Robinson and Walkden, 597, 603, 604

Roche-Lima, Da, I I

Rockwell, 203, 264

Rockwell and Highberger, 264

Rodet and Vallet, 685,700

Römer, 620, 621, 884, 888

Römisch, 6I4, 627

Roger, ro3

Roger and Weil, 623
Rogers, 395-402; 255, 403, 1069

Rogers, Clark, and Davis, 25 I, 265, 394

Rogers, Clark, and Evans, 240

Rogers and Dahlberg, 367,380

Rolleston, 904

Rolly and Meltzer, 776, 777

Rona, 484

Rondoni, I050

Roos, 620

Root, 677

RosenaU, 403-I8; 403, 406, 407, 95 I

Rosenau and Anderson, 952, 969, 971, 972

Rosenau and McCoy, 410

Rosenberg and Adelsberger, Iogo

Rosenblat, 33

Rosenow, 576-89; II 5, 203, 568, 576-79, 586$89,872,932$

Rosenow and Ashby, $5^{83}$

Rosenow and Meisser, $5^{86}$

Rosenow and Wheeler, $5^{8} 7$

Rosenthal, 108, 207, 209, 210, 542, 543, 685, 686, 700

Rosenthal and Holzer, ro93

Rosenthal, Moses, and Petzal, ro44

Rosher, I09I

Ross, 478

Ross and Hind, 942

Ross and Thomson, 684, 698, 700

Rothe, 627, 632

Rotkay, ro45

Rouget, 700

Rous, 521, 522

Rous and Robertson, 898

Roux, 204, 21 5, 372, 524

Roux and Babes, I074

Roux and Vaillard, 88I

Roux and Yersin, Io8

Rowland, 6I4

Ruchhoft, 366

Rudolf and Marsh, 698,700

Rudolfs, 356,357

Ruehle, 8

Ruhland, 329, 33 I

Rukawischnikoff, I 16

Rumpf, 878 , ro87

Ruppel, 22r, IOI9

Rusk, 883, I04r

Russ and Kirschner, 1042

Russell, E. J., $35^{\circ}$

Russell, E. J., and Hutchinson, 66, 67, 83

Russell, F. F., Nichols, and Stimmel, 82 I

Russell, J., $32 \mathrm{I}$

Russell, R., and Bartow, 359 
Russell and Hastings, 405

Ryle, 488

Rywosch and Rywosch, 2I 4

Sabin, 873

Sabrazès, 484

Saceghem, Van, 614, 615, 693, 700

Sach, 324, 330

Sachs, 1090, 1095

Sachs and Georgi, 849, 856

Sachs and Klopstock, 708, 7 10, 849

Sachs, Klopstock, and Takenomata, 535

Sachs, Klopstock, and Weil, 856

Sachs and Rondoni, 857

Sacks, 1043

Saelhof, 623,626

Sakharoff, 480

Saldanha, 538, 542, 545, 548, 549

Salimbeni, $803,80.4$

Salkowski, 21 2, 542

Salle, 366

Salmonson, 204

Salomon, 6r6

Salomon and Vey, rogo

Salomonsen and Madsen, 88I, rogr

Salthe and Krumwiede, 445, 446

Sambon, 453

Samson, 742

Sanarelli, 485, 522

Sanderson, 535

Sanfelice, 520,548

Sanford, 602

Sani, 885

Sargent and Foley, 479

Sarnowski, von, 629

Sartory, 5 I6

Sasaki, 244

Sasaki and Otsuka, 212

Sato, 639

Savage, $444,445,447,45^{\circ}$

Savage and Forbes, 444

Savage and Gunderson, 445

Savage and Hunwicke, 396

Savage and Read, 633,637

Savage and White, 444-47, 610

Savchenko, 747, 755

Sawtchenko, 868

Sawtchenko and Melbich, 804, 805

Saxl, 1092, I093

Scala, 677

Schade, 238, ro94

Schade, Giesche, and Kielholz, rog4

Schaffer and Tilly, 75, $8_{3}$

Schamberg, I099
Schamberg and Brown, Iogo

Schamberg and Wright, I I 29

Schantyr, 609

Schardinger, 277

Schatz, rog I

Schaudinn, 4, 39, 452-55, 457-62, 475, 477, 494, 66 I

Schaudinn and Hofimann, 454, 475, 483

Scheer, Van der, 856

Scheidegger, 538

Schellack, 10, 479, 480

Schern, 637

Scheurlen, 250

Schibayama, ז036

Schiemann and Meyer, 99I, 992

Schier, 430

Schierge, $27 \mathrm{I}$

Schiff, 892, 894-97, 905, 907, 91 I

Schiff and Adelsberger, 894, 895, 902

Schiff and Halberstaedter, 898

Schiff and Hübener, 896

Schiff and Mendlowicz, 896

Schiff and Ziegler, 908

Schiller, I07, I I 2, 642

Schilling, 685,70 I

Schimmelbusch and Mühsam, 625

Schiötz, Io9

Schittenhelm and Griesshammer, 1089

Schittenhelm and Schroetter, 274

Schlem, 1067

Schlimpert and Issel, 1058, 106 I

Schloss, IOII

Schmidt, H., 707, 761, 764, 770

Schmidt, H., and Scholz, 770

Schmidt, M. B., 779

Schmidt, R., and Kaznelson, rogo

Schmidt, S., 770

Schmidt-Hoensdorf, 6I9

Schneider, I99, 896

Schoen, 276,501

Schoenholz, 66I

Schoenholz and Friedlander, 6r8, 6r9

SCHOENLEIN, 279-84

Scholtz, 204

Scholz, 76 I, $76_{3}, 767,770,77 \mathrm{I}$

Schorer, 406

Schorler, 282

Schottelius, 644

Schottmüller, 636

Schrader, $68,8_{3}$

Schreider, 109

Schroeder, 405

Schroeder and Brett, 962

Schroeder and Cotton, 4I4, 82I 
Schroedinger, 177

Schroeter, 384

Schüffiner, 490,492

Schürmann, 638

Schütz, 640

Schütz and Schubert, 833

Schütze, 445, 476, 822, 899

Schugt, 508

Schultz, 974, 976, 980, 991, 994, ro9 ז

Schultz and Dale, 998

Schultz and Jordan, 974, 997

Schulz, 378

Schulze, 215

Schumacher, 7, 90

Schumm and Cooke, 554

Schwann, 384

Schwartz and Dieckmann, 199

Schwartz, Jobling, and Petersen, 875

Schwartz and McNeil, 926

Schwartzman, 532, 545, 548

Schwer, 623

Sclavo, 923

Scott, J. M., 675, 677

Scott, W. J. M., and Thatcher, 985

Scott, W. M., 446

Scott, W. W., 972

Scully, 1089

Sears and Putnam, Ir5, 242

Sedan and Hermann, 889

Sedgwick and Winslow, 74, 79-8I, 83

Seibert, 219, 705, 715, 1020, 1021

Seibert and Coghill, ro23

Seibert and Long, 1020

Seiffert, 530, 533, 535, 536, 540-42, 554, 555, 1093

Seifried, 62I

Sellards, I 2, 492

Sellards and Thieler, 677

Sellheim, $106_{3}$

Selling, I04I

Selter, 616, 618, 623, 1026, 1028

Selter and Tancre, I028

Senator, 640

Sergent and Sergent, 694, 70r

Settler, 645

Sewerin, 384,388

Sharber, I099

Sharp, G. S., 570, 594, 596-600

Shattock, 898

Shaw, 446

Shaw and Meyer, 6ri, 6r 3

Shaw, Welner, and Meyer, 6 I I

Shawan, 907

Shearer, 569

Sheather, 62I, 628
Shelford, 200

Sherman, 31, 214, 272, 40I

Sherman and Albus, 44, 58, 62, 83, 389

Sherwood and Stoland, 980

Shibley, 574, 796-98

Shunk, 338

Sia, 572

Sickenga, 554

Siegert, I093

Siegmund, 883, ro43, 1093

Siemerling, 478

Sierakowski, 216

Sierakowski and Zajdel, II 7

Sierp, 357, 359

Siler, Hall, and Hitchens, 494

Simon, $5^{20}$

Simon and Wood, 24, 34, III 5

SimondS, 772-8r; i99, 778, 975, 976, 996

Simonds and Baldauf, 775

Simonds and Brandes, 975

Simonds and Jones, 883, I04 I

Sims, 500

Simson, 900

Singer, 1040, 1044, 1053

Singer and Adler, ro44

Slanetz and Rettger, 22.4

Slator, $54,58,83$

Slye, 778

Slyke, Van, 22r, 255

Slyke, Van, Vinogard-Villchur, and Losee, ro63

Small, 578, 933

Smallmann, 609

Smillie, 3, 488

Smith, 1074

Smith, C., 896

Smith, D. E., 8

Smith, D. T., 47 r, 482, 625, 626

Smith, D. T., and Webster, 625, 626

Smith, E. F., 591-98, 600, 603, 604, 606

Smith, E. F., Brown, and Townsend, 597

Smith, E. F., and Quirk, 602

Smith, G. H., 1039, 1058

Smith, G. H., and Cook, 1058

Smith, G. S., and Musselman, 1056

Smith, H. W., 24, 25, 27

Smith, J. H., 73, 83

Smith, M. I., 982

Smith, R. P., III

Smith, T., 8, 93, 202, 205, 206, 21 2, 232, 265, $287,290,405,407,428,469,470,615-18,623$, $625,632,948,969$

Smith, T., and Brown, 38I, 4r 2

Smith, T., and Fabyan, 204

Smith, T., and Florence, 630, $63 \mathrm{I}$ 
Smith, T., and Nelson, 6ri , 6r 2, 6r4, 629

Smith, T., Orcutt, and Little, ro47

Smith, T., and Reagh, 6r3, 8r 4

Smith, T. and D. E., II 2, II3

Smith, T., and Stewart, 609

Smith, T., and Ten Broeck, 446

Smith, 'T., and Tibbets, 633, 635

Smoluchowski, I70, 784, 786, 799, 800

Snijders, 490

Snow, 362

Snyder, 65, 83, 892, 908, 913, 917, 920

Sobernheim, 885,923

Söhngen, 282, 329

Sörensen, 221, 272

Sollmann, 975

Solukha, 645

Sommer, Sommer, and Meyer, 742

Sonnenschein, 529, 548, $55 \mathrm{I}$

Sorauer, 59r

Sordelli and Serpa, 77I

Sorensen, 151

SouLe, 250-67; 93-95, 99, 26r

Spain, 1006, 1007

Spain and Cooke, 968, 1004, 1005, roro

Spallanzani, $25^{\circ}, 285$

Speakman and Phillips, 106

Speer, 478

Spencer, I I 9

Spengler, 2

Sperry and Rettger, 220

Spieckerman, 603, 604

Spray, 444, 445

Stahl, rog6

Standenath, I044

Stapp, 215

Staquet, 917

Starin, 970

Starin and Dack, 449

Starkenstein, 1093

STARKEY, 32I-3I; 320, 326, 330

Starkey and Halvorson, 328

Starling, 994

Starlinger, I089

Stary, 744

Stassano and Beaufort, 526

Stearns and Stearns, 25, 28

Steenbock and Daniels, 374

Stefanski, 6 I 6

Steffan, 689, 70 I

Stein, 368

Steinach, 638

Steiner, 478

Steinmetz and Lerche, 609, 610, 613, 620

Stenstrom, 883
Stephan, I065

Stephenson and Whetham, 247

Stern and Wozak, rog6

Sternberg, 132, 390, 392, 393

Sternberg and Klein, zor

Stessano and Lematte, 375

Stevens, 272, 339, 594

Stevenson, 635

Stiles, I 20, I21, I 23, I 24, I35, 677

Stiles, Peterson, and Fred, 240

Stilling, 34

Stillman, $6 r 6$

Stillman and Bourn, ro4

Stimson, 454, 485, 490, 49r, 496

Stitt, 452, 453, 475

Stockle, 615

Stoddard, $48_{3}$

Stoddard and Woods, 780

Stötter, ro89

Stokes, r69, r 70, 783

Stoklasa, 238

Stoklasa and Doerell, 350

Stolz, 553

Storch, 398

Stovall, Scheid, and Nichols, ro9

Strada and Traina, 6r8, 621

Strasburger, 639, 640, 648

Straub, 1094

Strauss, 627

Straussler and Koskinas, I 100

Streng, 908

Streng and Ryti, 908

Stroganoff, 356,357

Strong, 481-84, 675, 677

Strowd, 335

Stucky and Huntley, 859

Sturges, $58,83,104$

Sturges and Rettger, 205

Sucker, 902

Sucksdorff, 639

Südmersen, 618, 623

Sullivan, 197

Supplee and Ashbaugh, 397

Surányi and Kramár, $55^{2}$

Sustmann, 621, 623, 626, 629

Susuki and Taira, 501

Sutherland, 140

Sutton, 304

Suzuki, 555

Suzuki, Hastings, and Hart, 400

Svedberg, 783

Swellengrebel, 6, r31, 453, 480

Swerew, 883

Swift, ro40 
Swift and Boots, 969

Swift, Haggart, and Drinker, 994

Swithinbank and Newman, 4I3

Symmers, 778

Szczawinska, 210

Szent-Györgi, 8I 5

Szilvasi and Felér, II

Tacke, 349

Tait, 865,866

Takahashi, 513

Takamine, 513,516

Také and Marine, I039, 1042

Takimato, 594

Taliaferro, L. G., 680-83, 694, 695, 70 I

TALIAFERRO, W. H., 679-70I; 680, 682, 689-92, 697,701

Taliaferro, W. H., and Johnson, $687-89$, 7 or

Taliaferro, W. H., and Taliaferro, 682, 683, 689, $690,696,701$

Taliaferro, W. H., Taliaferro, and Fisher, 697, 701

Tamura, 28, 3 I

Tanaka, 623-27

TANNER, 498-508; 499

Tanner, Devereux, and Higgins, 284, 500, $50 \mathrm{I}$

Tanner and McCrea, 289

Tarozzi, 206

Tarrassevitch, 1036

Tartakowsky, 6I6, 618

Taylor, A., 504

Taylor, F. E., 879

Teale, 889

Ten Broeck, 443, 610, 630, 633, 642, 716, So8, 970

Thaysen, 92

Theiler, 92, 480

Theiler and Sellards, 492

Theriault, 353

Thiele and Embleton, 714, 777, 1053

Thiele and Wolf, 372

Thiercelin, 642

Thierfelder, 235

Trom, 437-42, 509-16; $40 \mathrm{I}$

Thom and Church, 509, 510, 514, 516

Thom and Currie, 40I, 516

Thom, Edmondson, and Giltner, 288

Thom and Hunter, 442

Thomas, 238, 609, 610, 790

Thompson, I 16, 1039

Thomsen, 894, 897-99, 9 I 7

Thomsen and Boas, 840

Thomson, 49o, 684, 701, 989, 992

Thornton and Gangulee, 39, 40, 332, 338

Thunberg, 2I 3
Tibbutt and McConnel, 9I3, 9I 7

Tidswell, I072, 1073

Tiedeman, $36 \mathrm{I}$

Tieghem, Van, 512

Tissier, 640-42, 646

Tissier and Martelly, 223

Tizzoni and Cattani, ro47

Tizzoni, Cattani, and Baquis, 206

Toda, 895

Todd, II

Todd and White, 903

Todd and Wolbach, 467

Toenniessen, 7, 8, 724

Togeri, ro89

Tomasczewski, 476

Tomaselli, 5

Tomcsik, 971

Tonney, 4 I 2

Tonney, White, and Danforth, 4I 2

Topley, 61 2, 632-34

Topley and Ayrton, 92, 61 2, 632

Topley, Weir, and Wilson, 633

Topley and Wilson, 554, 632, 634

Topley, Wilson, and Lewis, 554, 633

Torres, 49I

Torrey, 204, 645, 646, 925

Toschiana, 6I 4

Toyama, 486

Toyama and Kolmer, 1040

Traube, 25

Traum, 406, 407

Traum and Hart, 406, 407

Trautmann, 637

Trautwein, 324, 327

Trawinski, 609, 6I I

Trevan, $760,77 \mathrm{I}$

Trevisan, 30, 390, 392

Trou-Hia-Hsü, 735

Truffant and Beyssanoff, 66, 83

Tschernoruzki, 876

Tsukahara, I09I

Tsurumi and Kohda, I037

Tuck, 287

Tulloch, 798,823

Tunnicliff, $89,472,48$ I $, 636,655,872,931$, 1039

Tunnicliff and Nayer, 636

Twort, 520, 524, 530, 537, 543, 63I

Uchida, 6I I, 61 7, 620, 635, 1050

Uhlenhuth, 473

Uhlenhuth and Fromme, 485,486

Uhlenhuth and Haendel, 973

Uhlenhuth and Hübener, 629, 632, 633

Uhlenhuth and Kuhn, 468 
Uhlenhuth and Zuelzer, $484,485,487,489$

Uhlmann, I095

Ulrich, ror 4

Unger, 901, 905, 906

Ungermann, 6 I 6

U.S. Department of Agriculture, 434

U.S. Naval Medical Bulletin, 450, 45I

U.S. Pharmacopoeia, 303, 304

U.S. Public Health Service, 286, 367, 407, 412, $425,433,434,436,635,845,952,956$

Unna, 19, 29

Uschinsky, 279

Vaillard, 287

Vaillard and Rouget, 775

Valassopoulo, 487

Valentine, 2 I 6

Valley and Rettger, 53, 68, 83 , I I 7

Valtes, 5

Vaughan, $776,780,982,984,1026$

Vaughan and Wheeler, 219, 982

Vay, 3

Veillon, 472

Veillon and Zuber, 649

Velde, Van de, $8{ }_{1} 3$

Velden, Van den, Io89

Velu, 849

Verwoort, 473, 489

Verzar and Weszecky, 9o8, 9r8

Vianna and Biāo, 49I

Vianna and Vianna, 49I

Viehöver, 23I, 234

Vignati, I I 2

Viljoen, 294

Vincent, $48,482,483$

Vincent and Bellot, 830

Virchow, I

Voegtlin and Bernheim, 975

Voges and Proskauer, 240

Volhard and Fahr, 780

Volk, 623

Vollmer, I090, I09I

Vorschutz, Iog6

Vries, De, 85,86

Vuillemin, I 29, I32, 474

Waals, Van der, I $_{5} 6$

WADSWORTH, 831-37; 407, 817-I9, 82 I, 843, 845

Wadsworth, Maltaner, and Maltaner, $8_{33}, 8_{34}$

Waele, De, 1064

Wagemans, 530, 534, 535, 554

Wagener, 670, 678

Wagener and Thomson, 670, 678

Wagner, 93
Wagner von Jauregg, 1098

Waite, 605

Wakker, 59I

WAKSMAN, 268-78, 31 0-21; 228, 325, 326, 350

Waksman, Barthel, Cutler, and Bristol-Roach, $32 \mathrm{I}$

Waksman and Davison, 278

Waksman and Joffe, 323, 326

Waksman and Starkey, 216, 326, 330, 33 I

Waldschmidt-Leitz, 278

Walker, 859

Walker, E. L., 675, 678

Walker, E. L., and Sellards, 668, 670, 678

Walker, E. W. A., 85

Walker, M. N., 52 I

Walker, W. F., and Pryer, 374

Walker and Sweeney, I 28

Walthard, IOI4

Walzer, ıо6, го го

Walzer and Walzer, 972

Wang, 849

Warburg, 25I, 330, 547

Warburg and Negelein, 330

Ward, A. R., 65, 83

Ward, A. R., and Gallagher, 607

WARD, H. C., I-I 4

Ward, Marshall, ro3

Ward, M. H., 372

Warden and Schmidt, 831

Warren and Lamb, 450

Warthin, Buffington, and Wanstrom, 630

Wasielewski, von, and Senn, 690, 70I

Wassermann, 832, I039

Wassermann and Bruck, 831, 832

Wassermann and Citron, 884, 1046

Wassermann, Neisser, and Bruck, 475, 831, 838, 849

Wassermann and Tanaka, 88I

Wassilieff, 639

Watanabe, 529

Watson, 822

Watson and Wallace, $75^{2}$

Waworuntu, 628

Wayson, 6r4, 635

Weaver, 609

Webb, 976

Webster, 93, 21 7, 566, 61 2, 61 7, 624-27, 632-34, 790,889

Webster and Burn, 624, 626

Webster and Pritchett, 634

Weech, 935

Wehmer, 509, 512, 514, 5 I6

Weichardt, 1087, 1089, 1092, 1093

Weichardt and Scholz, I09I, I095, I096 
Weichardt and Schrader, IO9I

Weichbrodt, Iog2

Weichselbaum, 925

Weidenreich, 874

Weigert, 3 I

Weil, 710, 974, 98I, 990, 997, 1002, 1003

Weil and Braun, 883

Weil and Felix, 821, 822

Weinberg, 84I, 842

Weinberg and Aznar, 540

Weinberg and Goy, 762, 766, 77I

Weinberg and Otelesco, IO4

Weinberg and Prévôt, 77I

Weinberg, Prévôt, and Goy, 763, 768, 771

Weinberg and Séguin, I99, 874

Weinland, 200, 1056

Weinstein, $105^{\circ}$

Weintraub, 274

Weirauk, Iog9

Weiss, 288, 291, 292, 294, 296, 533, 645

Weiss and Arnold, 529, 543, 546

Weiss and Kunze, ro44

Welch and Ewing, 107 I

Weldin, 392, 393

WeLLS, H. G., 702-I0; 185, 702, 709, 713, 715, $7 \mathrm{I} 6,722-24,74 \mathrm{r}, 782,834,967,970,973,977$, 990, 1028, I035, 1050, 1058

Wells, H. G., De Witt, and Long, rorg

Wells, H. G., Lewis, and Jones, 709

Wells and Osborne, 713, 7I6, 970

Wells, W. F., 368

Wenyon, 452, 473, 475, 678

Werkman, 68, 83, 50 I

Werkman, Nelson, and Fulmer, 1046

Werner, 484

Werner and Winiwater, 1058

Wertemann, 555

Westgren and Reitstötter, 800

Weszeczky, 9 I 7

Weygandt, 838

WHERRY, 870-80; 32, 33, 609, 879

Wherry and Butterfield, 633

Wherry and Ervin, 203, 263

Wherry and Oliver, 203, 204, 579

Wherry and Ray, 203

Whipple, 60, 65, 66, 68, 83

White, 92, 820, $97 \mathrm{I}$

White and Avery, 971

White and Robinson, 749

Whiteman, 500

Whitmore, 696, 701

Whittaker, Archibald, Leete, and Miller, 428

Whitworth, 293

Wichern, 212
Widal, 8I 2

Widal and LeSourd, 832

Widal and Sicard, 81 3,816

Wieland, $2 I_{5}$

Wiener, 784

Wildiers, 68, 83, 283, 499

Wilfarth, 332, 349

Williams, A. W., 747

Williams, H., 4I 3

Williams, O. B., and Van de Carr, 979

Williams, W. C., 895

Willis, 887

Willows and Hatschek, 178

Willstätter, 269, 271, 272

Wiltshire, 8,98

Wilson, J. A., 786

Wilson, J. A., and Daub, 516

Wilson, J. K., 337

Wilson, M. A., 833

Wilson, W. J., 2

Wilson, W. J., and Blair, 367

Wilson, W. J., and Steer, 205

Winkler, 523

Winogradsky, 103, 105, 216, 250, 279, 28I, 312, 3 I3 $323^{-25}, 327,328,330,33$ I 357

Winogradsky and Omeliansky, 330

WinsLow, 58-83; I 23, I 27, I33-35, 445

Winslow and Abramson, 74, 79, 80, 83

Winslow and Brooke, 77,83

Winslow and Cohen, 74, 79, 80, 83

Winslow and Falk, 76, 83

Winslow, Falk, and Caulfield, 572, 786, 787

Winslow and Fleeson, 787

Winslow and Kligler, 80,83

Winslow, Kligler, and Rothberg, 392

Winslow and Lochridge, 75,83

Winslow and Palmer, 367

Winslow and Rogers, 135

Winslow and Sanjiyan, 80,83

Winslow and Shaughnessy, 787

Winslow and Winslow, 135

Winterberg, 648

Winternitz, 645

Winterstein, 23I

Witebsky, 895, 903, 904

Witt, De, I I 27 , I 29

Witt, De, and Sherman, I 27

Wittkower, 977

Wittneben, 616

Wolbach, 524, 642

Wolbach and Binger, $467,485,487$

Wolbach and Schlesinger, I2, I3

Wolbach, Todd, and Palfrey, I2

Wolf, 123 
Wolf, C. G. L., McGill, and Harris, 207

Wolf, E. P., and Lewis, 507

Wolf, K., 888

Wolff, 384, 388, 539

Wolff and Janzen, 536, 541

Wolff-Eisner, I01 2, I0I 7, 1025, 1092

Wolffhügel and Riedel, 68, 83

Wollmann, I I6, 530, 531, 539, 542, 543, 644

Wollmann and Wollmann, 536

Wollstein, 645

Wolman, 368

Wolman and Weaver, 368

Wolpe, Iogr

Wolter, rogo

Woolley, I027

Woronine, 590

Wright, 130

Wright, A. E., 207, 818, 87 г, 872, 877-So, 923

Wright, A. E., and Colebrook, 877,879

Wright, A. E., Colebrook, and Storer, 877

Wright, A. E., and Lamb, 805

Wright, A. E., and Reid, $87 \mathrm{I}, 872$

Wright, H. D., So7

Wright, J. H., and Craighead, 630

Wright, W. H., 339

Wrozek, 206, 207

Yamakami, 895, 920

Yanagisawa, II 4

Yersin, 407

Yorke and Adams, 668, 678

Young and Dawson, 445

Young, White, and Schwartz, I 25
Zajdel and Funk, 500, 50I

Zagari, 6r4

Zdansky, 538, 543, 546, 549, 554-56

Zeissler, 108

Zettnow, 20, 285, 465, 495

Zettnow and Fontana, 494, 495

Ziehl, 32, 34

Ziehl and Neelsen, 24

Zilva, 1046

Zimmermann, Io92

Zingher, 750, 763, 765, 771, 939, 941, 942, 945

Zingher and Soletsky, 889

Zinsser, 204, 568, 703, 704, 714, 723, 725, 730, $968,980,991,1024,1026,1027,1029,1070$

Zinsser and Grinnell, 728

Zinsser, Hopkins, and Gilbert, 47I

ZINSSER AND MUELLER, 721-32; I 7, 572, 703

Zinsser and Parker, 714, 723, 725, 727, 980

Zinsser and Tamiya, 714, 727, 730, 834, 102426,1028

Zlatogoroff, 6I4

Zoeller, 103, I09

Zopf, 131, 385, 389, 39 I

Zuelzer, 452, 454, 455, 475, 486, 494, 630

Zukerman and Minkewitsch, Iro

Zunz, 740, 74I, 970, 1002

Zunz and La Barre, 976, 977, 987, 1002

Zunz and Geerthruyden-Brenard, 976

Zunz and Gyorgy, 977

Zwecker and Goodell, 976

Zwick, 633

Zsigmondy, 800 



\section{SUBJECT INDEX}

ABDERHALDEN'S REACtion, I056-65

diagnosis, value in, $\mathrm{I}_{3} 6_{3}$

dialysis method, I057

interpretation of results, 1060

ninhydrin test, I059

optical method, 1057

principle of, 1057

Abortion, contagious in milk, $38 \mathrm{r}$

Absorbed films from liquids, ${ }^{6} 65$

Abwehrfermente, 1064, 1087

Acceleration growth phase, negative, $47,5^{\text {I }}$

Accessory substances for yeasts, 499

Acetaldehyde, 237

Acetaldehyde-sulphite compound, 237

Acetic acid, 237, 246

fermentation, 239

Acetylmethylcarbinol, 240

Achromatium, 324

Acid

and lag, $6 \mathbf{r}$

in sour milk, 387

Acid fast bacteria, 23, 25

staining of, $3 \mathrm{I}^{-}-33$

Acid fast granules, 5

Acid fastness

loss of, 32

mechanism of, $3 \mathrm{I}$

Acid-forming streptococci, 80

Acid-proteolytic udder cocci, 379

Acidity

of cell sap, 602

of milk, $7 \mathrm{I}$

relations of rood nodule bacteria, 339

Acids, 232

ionization of, in water, 148

toxic effect of, 75

Aciduric group of bacteria, 106, 64r

Acne, use of yeast in treatment of, 506

Actinomyces, I30

in soil, $317,32 \mathrm{r}$

Actinomycelaccae, $\mathrm{I} 30$

Actinomycetales, I30, I32

Acriflavin, I I I 7, I I 8

Activity coefficient, I 76, I 77

calculation from solubility, I46

of electrolytes, 145

formula, I 44

of salts, 146

Adenine, 246

Adenoids, 654

Adipic acid, 246

Adjustment growth phase, $58,6 \mathrm{r}, 7 \mathrm{I}$

Adrenalin, ro39
Adsorbed ion, effect of valence, 17 I

Adsorption

of lytic agent in bacteriophage, 528

theory of Bordet, 740

of toxins and antitoxins, 74I

Aerase, 208

Aeration of soil, 344

Acrobacter aerogenes, 389, 390, 393

Aerobacter cloacae Jordan, 393

Aerobe-anaerobe symbiosis, 203

Aerobes, anaerobes transformed into, 209

Aerobes in wounds, ${ }^{\circ} 4$

Aerobic culture of obligate anaerobes, 205

Aerobic decomposition of sewage, 353

Aerobic spirochetes, 472

Aerobization of anaerobes, 208

African horse sickness, 518

Agalactia, 5 I 8

Agglutinability

inagglutinability, variations in, 570 and P.D., 569

Agglutinable factors, new, in human blood heredity of, 9 I9

Agglutinating sera, effect on potential, 798

Agglutination. See also Isoagglutination and Leukocytes

acid, 8 I 5

within animal body, 803

auto-, 898,899

and bacterial variation, 822

and curative action of sera, $\mathrm{So} 7$

early observations of, 8 I I

electrolytes, effects of, 789

of erythrocytes, 796

factors related to intra vitam, 808

flagella, $8 r_{4}$

formalin-killed organisms in, 8r8

$\mathrm{H}$-ion concentration, 79I

in vivo, experiments on, 805

and leukocytes, 809

Mechanism of, $782-80 \mathrm{I}$

physico-chemical basis of, $\mathrm{SO}_{7}$

in plant pathogens, 599, 600

procedures of, 8 I 6

pseudo-, 897, 899

salt, 8 I 4

salt concentration, effect of, 793

prevented by sodium oleate, 799

spontaneous, 8I 4

test technique, 905

theory of, 8I 6

Agglutinins, $r \circ 36$

absorption of, 8I 5,8 I9

reciprocal, 820

and agglutinogens, 8 I 3 
Bacterial and Their Applications, 81 I-22, 23; 813

cold, 900,921

deficiency of, 899

group, 820

Functronal Rôle of, 802-10; 810

in natural immunity, 806

in infectious disease, 802

isoelectric point, 8 I 5

major and minor, 814

relation to prognosis and recovery, 804

titre of sera, 897

in typhoid fever, 805

Agglutinogens, 8r3

Aggregates, formation of, $78_{5}$

Aggressins, 568

Alastrim, 518

Albumin, 7 I 3

-digesters in sewage, 355

and rabbit septicemia, agglutination of, 79 I

Alcohol, 232, 237

Alcoholic fermentation, 440, 501

Alexin, 740; anti-, 740

Alfalfa nodules, 334

Algae, autotrophic, 3 I 8

Aliphatic Compounds, Utilization of, 243-49

Alkali disease, $4^{1} 4$

Alkalies, action on bacteria, 25

Allantoin, 246

Allelocatalysis, 53

Allergy, 721, 722,966

Aluminium ions, effect on charge of particles, I 7 I

Amanita phalloides, 184, 7 I 7

Amboceptor, venom, I07 I

Amebae. See Intestinal amebae

Amidases, 276

Amine, ethyl, 245

Amino acids, 221, 243-45

complexes, 703

decomposition by bacteria, 22 I

as source of nitrogen, 224

and toxin production, 68

Ammonia

liberation, 222

oxidation, 323

assimilated by soil micro-organisms, 3 I 7

utilization, 225

Ammonium

compounds, 249

salts in fertilizers, 347

Amoebobacter, 324

Amphimixis, 100

Amphoteric antitoxin, 575

Amylase, 274

Anaerase, 208

Anaerobes, 21 2, 642 aerobic culture of, 205 aerobization of, 208 and association of bacteria, I03 devoid of catalase activity, 2 I4

facultative, $20 \mathrm{I}$

gram positive, 26

obligate, I99, 20 I

pathogenicity of transformed, 209

symbiosis with aerobes, 203

in wounds, $\mathrm{IO4}$

Anaerobic conditions, gas metabolism under, 264

Anaerobic extracts of pneumococci, 2 I6

Anaerobic spirochetes, 469

ANaerobiosis, 198-210; I96, 454

biological theories of, 203

mechanism of, $20 \mathrm{I}$

physico-chemical conceptions, 201

use of tissues, 205

and intestinal worms, 200

Anaphylactic antibodies in humans, IOI r

Anaphylactic shock, 972, 1081,1083

blood pressure, 975

cellular, 970

cellular theory of, 980

changes in, 976

features of, 973

findings in, 974

by heterophile antibody, 736

humoral theory of, 980

inhibition of, 978

local, 984

physical theories of, 983

poisons, 982

and precipitins, 98 I

reverse, 979

Anaphylactogens, 970

lipoids, $97 \mathrm{I}$

polysaccharides, 97 I

proteins, 970

ANAPHYLACTOID, 966-68; 988

phenomena, 968

reactions, 985

Anaphylatoxins, 568, I00 I

ANAPHYLAXIS, 966-88

and anaphylactoid reactions, 988

anti-, 978, I00I

and atopy, Ior 4

definition of, 967,969

phenomena

cardiac disturbances, 993-97

arterial disturbances, $994-97$

after evisceration, 1000

glandular, 999

hepatic exclusion, rooo

hepatic transplantation, I000

local, 1002

nervous, I0OI

respiratory

bronchoconstriction, 997

oxygen capacity of blood, 998

tissue respiration, 998

smooth muscle reactions, 998

isolated muscle, 998

in situ, 998

transplantations, 999

specificity, 977 
Technique of Experimentation, 989-1003 and tuberculin, 1029

Anatoxin. See Toxoid

Anemia

of horses, $5 \times 8$

pernicious, $\mathrm{Cl}$. welchii in, 367

Angioblasts, 873

Angioneurotic edema, 987 , 1007

Angstrom units, I 76, 37 I

Anilin dyes. See Dyes

Antagonism, I03

Anthrax. See also B. anthracis

antiserum, 923

bacilli, 567

chemotherapy of, II 33

local immunity to, 885

yeast in treatment, 506

Antibacterial Sera, 921-33. See Sera

Antibodies, 921

in allergies, 722

anaphylactic, IOI I

effect of anilin dyes on, I I 6

charge on, 743

Heterophile, 733-38; 735, 736

in heterophile-immune sera, 737

homologous, 934

humoral, ro80

in idiosyncrasies, 722

immunity without, 809

normal, 934

contained in organ extracts, I035

ORIGIN OF, I035-48; 882

production

and arsphenamin, II IO

diet, effects of, 1046

disease, effects of, I044

drugs, effects of, i i io

factors affecting, 103 8

injurious agencies, IO4O

local, 1047

and local immunity, 1046

organ extirpation, $\mathrm{IO}_{4} 2$

and reticulo-endothelial system, I043

vitamin C, effects of, 1046

sensitive to irradiation, 376

sensitizing, 722

trypanosome resistance to, 686

in trypanosomiasis, 686

Antibody

formation. See Enzyme theory

formation and local immunity, 890

-formers, hematopoietic organs as, $88_{3}$

$\mathrm{pH}$ effect on antibody and bacterial combination, 792

pre-, substance, 1053

ReACTIONS, 72 I-32; 183

antigen-, 838

solution, pneumococcus, 929

Antigen, bacteriophage as, 529

Antigen-antibody

compounds, 722

reactions, 838

Antigenic principles in venoms, 1070-7I
Antigenic properties, 26

of autolysates, 731

OF BACterial Cells, 72I-32

antigens

activity, 704, 705

preparation for antivenins, 1074

charge on, 743

Chesitstry OF, 7 II $^{-20}$

cholesterinized, 846

Complement Fixation with Bacterial, $8_{31}-\mathbf{3 7} ; 8_{32}, 8_{34}$

complex, 708; fractionation of, 7 II

detoxicated, 880

enzymes as, 272

Heterophile, 733-38; 829

for Kahn test, 850

special, 855

standardization of, $85 \mathrm{I}$

suspension for, $85 \mathrm{I}$

lipoids as, 707, 7 I4, 723

molecule, attempts to modify, 718

nature of, 703

non-protein, 706

nucleoprotein, as bacterial, 723

protein, 7I2

pure, 716

AND THEIR SPECIFICITY, 702-10

spirochetal, 835

toxins as, 705

and ultra-violet rays, 375

Wassermann, 83.5

Antiseptic and lag, 62

Antiseptic property of stomach, 640

Antiseptics 66

chlorin group, I 106

in soils, 349

STANDARDIZATION OF, 307-9

tests for antiseptic power, 309

Antiserum inactivation of bacteriophage, 532

Antitoxinogens 722, 1070

Antitoxins, 722

adsorption, 74I

amphoteric, 575

colloidal reactions, 740

electro-colloidal reactions, $74 \mathrm{I}$

electrodialysis, 740

filterability, 562

goats used in testing, 957

neutralization of toxin, 739

Physical Chemistry of, 739-44

preparation, 753-56

Preparation and Purification of, 745-58

protein nature, 744

purification, 742

partial purification, $756-58$

standard units, 949

testing, $95 \mathrm{I}$

botulinum, 954

diphtheria, 95 I

gas gangrene, 955

scarlet fever, 955

tetanus, 953

titration by flocculation method, 759

Antivenins, I072-77; 964. See also Venoms antigen preparation for, 1074 
concentration, ro76

preparation methods (toxoid and mithridatization), I073

therapeutic use of, 1076

titration tests

neutralization, 1075

precipitin, 1076

protective, 1075

Antivirus, 885

Besredka's, 554

Appendicitis, 582

Arginine, 703

Aromatic compounds, 243

Arrhenius' theory, 739

Arsenic in chemotherapy, I I 25 , I I 27

Arsphenamin, I I I o

Arthritic erythema, epidemic, 4 I 5

Arthritis, 582

Arthrospores, I

Arthus' phenomenon, 969, 984

Ascospore production, 514

Aseptic life, 643, 644

Ash in bacterial cells, 218

ASPERGILLI, 509-16

biochemical characteristics of, 510

classification, 510

conidia, 510

diastatic power of, 512

enzymes of, 513

key, artificial, to, 5 I I

pathogenicity of, $5^{\text {I } 4}$

spores, 510

Aspergillosis, 515

Aspergillus niger, 5 I 2

A. okazakii, 514

A. oryzae, 498

Association, BACTERIAL, IO2-I9

alteration by, I I I 5

Asthma, 968, 1007, 1008

Aтомs, ${ }_{136-38}$

arrangement in crystals, 136

arrangement in solids, 136

composition and structure, 136,177

tracks, 137

weight, 137

ATOPY, 1004-15; 967

atopen, 1009

and anaphylaxis, Ior 4

a topic inheritance, $1004-10$

hypersensitiveness, 1010

reagins, 1006

anti-worm, 1008

in worm-infested persons, 1008

shock tissues, 1008, 1009

in lower animals, Ior 3

Ittenuated cultures in immunization, 2 I0

Ittraction between ions, I39

Autocatakinetic growth-curve equation, 52

Autocatakinetic phenomena, 5 I, 53
Autolysates, bacterial, 728

antigenic properties of, $73 \mathbf{I}$

Autolysis, 26, 542, 600

Autotrophic algae, 3 I 8

AUtotropHIC BACTERIA, 322-31; 28I, 318

assimilation of carbon dioxide, 33 I

influence of organic materials on, 330 non-, 282

Autotrophs, 322

obligate, 323

Auxochromes, I9, 20

Avian

diphtheria, 518

paratyphoid strains, 446

Axial filament (Cristispira), $46_{3}$

Azobacter, 104

nitrogen fixation, 316

in soil, 316,319

Azoproteins, 7 I 8

Bacillaceae, 132, I33

Bacillus abortivo-equinus, 446

B. abortus, 82 I

B. acidi paralactici, 388

B. acidophilus, 107 and surface tension, 183

B. actinoides, 636

B. actinomycetum comitans, 106

$B$. aerogenes, action on uric and citric acids, 248

B. aertrycke, II $3,444,6$ I I

in foods, 446

in laboratory animals, 445

in rats, 637

B. amylobacter, nitrogen fixation, 3 I 7

B. analum, 446

B. anthracis, 7-0, 26, 42, I 4 . See also Anthrax heat resistance, 293

nitrogen content, 2 I 8

effects of $\mathrm{pH}$ and heating, 293

$B$. anthracoides, 9

B. botulinus, 48, 105, 781. Sce also Cl. botulinum, Botulism

$B$. bulgaricus and surface tension, $I S_{3}$

B. cereus, 224

B. chauvoei, 103

B. cloacae, 240

B. clostridium pasteurianum, 105

B. coagulans, 397

$B$. cohaerens, 43

B. cylindricus, 286

B. diphtheriae, $3,68,108,229,763$

in disinfection tests, 302, 306

See also Corynebacterium diphtheriae, Diphtheria

B. doederleini, 107

B. duodenale, 393 
B. entcritidis, $2,44,610$

in mice, 632

in rats, 637

toxin, 444

B. fecalis alcaligcnes, 104

B. indicus, $39 \mathrm{I}$

B. infuenzae, 104, II4, 727. See also II. influenzae

oxidizing ferments, 2 I 5

B. mallei, 42,43

B. megatherium, 42, 43, 224

B. mesentericus, 7, 107

B. mycoides, 9, 43 resistance of spores, $29 \mathrm{I}$

$B$. oedematiens, 104

$B$. paralactici, I03

B. paratyphosus, 3, 167, 224

$\mathrm{A}$ and $\mathrm{B}, 444,610$

$B$. pneumasintes, 104

$B$. perfringens, 104, 955

B. pertussis, 42, 214. See also Pertussis

B. pestis, 3, 203

$B$. pestis caviae, 610

and bacteriophage, 554

$B$. prodigiosus, oxidizing ferments, $2 \mathrm{I}_{5}$

$B$. proteus, I04, I05, I I 2,229

in food poisoning, $45^{\circ}$

B. pseudopestis, 6 $\mathrm{I}_{3}, 6 \mathrm{I}_{4}$

B. pseudotuberculosis, $6 \mathbf{I}_{3}, 6 \mathbf{I}_{4}$

B. pullorum, 446

$B$. putrificus verrucosus, $10_{5}$

B. pyocyanetis, I05, II 4,214 oxidizing ferments, 2 I 5

B. radicicola, 8. Sce also Bact. radicicola nitrogen fixation, 3 I 7

B. sanguinarium, 446

B. sporogenes, 104, 105

B. subtilis, 7, 9, 42, 61, 224

B. suipestifer, $42,446,610$

B. suisepticus, 3

B. syncyaneus, $38_{3}$

B. tetani toxin, $78 \mathrm{I}$. See also Cl. tetani.

B. tostus, 286

B. typhi murium, 632

B. tuberculosis. See Mycobacterium tubcrculosis, Tubercle bacillus.

B. volutans, Io6

B. vulgatus, 43

B. welchii 104, 230. See also $\mathrm{Cl}$. welchii

Bacteriaceae, 42, I 32, I33

Bactericidal power of

anilin dyes, 34

mercurial compounds, I I 26

milk, 60

yeast, 508

Bacterioerythrin, 328
Bacteriolysin, 1035

and light rays, 376

Bacteriolysis, complement in, 875

BACTERIOPHAGE, 525-56; 97, I I6, 518

adaptation to phenol, $\mathrm{HgCl}_{2}$, and immune serum, 534

adsorption of lytic agent, 528

as specific antigen, 529

assimilation of substratum, 530

hydrolysis of bacteria by, 547

bacterial origin of , 539

and $B$. pestis caviue, 554

carbon dioxide production by, 53 I

clearing of culture by, 54 I

colonies, 528

filterability, 560

in intestines of fetus, $55^{2}$

inactivation by antiserum, 532

in infectious diseases, $55^{2}$

locomotion, 525

mixed, 536

multiplication in absence of growing bacteria, 530

particulate nature of, 525

in plants, 600, 601

regeneration of, 538

resistance to, $548,55 \mathrm{I}$

resistance to in jury, 533

therapeutic administration, 554 .

See also Phagotherapy

transformations of, 535

and ultra-violet light, 376

unicity (universality), 534

virulence, 535

effect on viscosity, 544

Bacteriophagum intestinale, 525, 527

Bacteriopurpurin, 327

Bacteriostatic effects, 34

of dyes, 25

of mercurial compounds, I I 26

reverse action, 26

Bacteriotropins, 87 I

Bacterium abortus, 203

in udder infection $380,38 \mathrm{I}$

Bact. acidi lactici, 385,39 I

Bact. acrogcnes, 392, 640

Bact. bronchisepticum

guinea pig infections, 6 I 8

rabbit infections, $623,624,626$

rat infections, 636

Bact. citri, 595, 600

Bact. coelicolor, $38_{3}$

Bact. coli, 2, 3, 5, 7, 8, 27, 42, 43, 6I, 70, 74, 78,

$80,106,640$

action on uric and citric acid, 248

anaerogenes, I I I

in stored feces, 370

in ground water, 360

effect of heating, 290

mutabile, 85

nitrogen content, 2 I 8,224

oxidizing ferments, $2 I_{5}$

$\mathrm{pH}, 66$ 
death in dilute salt solution, 73

in sewage, 360

standard for, 367

strains of, 27

tests for, 365,366

viability of, 76

in water, 364,365

Bact. dysenteriae, 5, 42 nitrogen content, 2 I 8 oxidizing ferments, 2 I 5

Bact. güntheri, 386

Bact. lactis, $89,3_{3}$

Bact. lactis-acidi, 70

Bact. lactis aerogenes, 39I

Bact. lepisepticum, 623-26

Bact. malvacearum, 596

Bact. medicaginis var. phaseolicola, 595, 600

Bact. murisepticum, 635

Bact. phaseoli sojense, 595, 599, 600

Bact. radicicola, soil inoculation with, 349 . Sce also $B$. radicicola.

Bact. translucens var. undulosum, liquefaction pits, 596

Bact. tumefaciens, 599, 600, 604 colony variation, 595

Bact. typhi, typhosum, 2, 3, 42, 67, 74, 75, 107 II , 224, 72I. See also Typhoid bacillus death rate of, 77,78

in disinfection tests, 302,303

nitrogen content, 218

oxidizing ferments, 2 I5

Bacteroids, 338

Balantidiosis in man, 675

Balantidium coli, 663, 674

Bartonclla bacilliformis, 474

Bases, ionization of, in water, 148

Beans, cross-inoculation groups, 334. See also Soy bean

Beggiatoa, 324

Benzoic acid, 246, 248

Bernstein's theory for blood groups, 9I4-I9

Besredka's

antivirus, 554

theory on local immunity, 889

Bile

with brilliant green, 365

lactose in presumptive test, 364

soaps, 185

Bimolecular reaction, 73

Biogens and biogenases, 500

Biological Pronucts, Control and StandARDIZATION OF, $947^{-65}$

Bios, 499

Biotypes, 85

Blenorrhea, 5 I8

Blood

normal in treatment of scarlet fever, 944

effect of toxins on, 775
Blood Groups, 892-908, 909-20

agglutination test technique, 905

heredity of, $894,909-20$

agglutinable factors, new, 9 I9

anthropological data, 913

Bernstein's theory, 9I4-I9

genes, 9 r 2

genetic data, 9 Io

linkage, 9 I 9

Mendelian mechanism, or 2

serological basis, $9 \circ 9$

summary, 9 I I

human and animal, 902

racial differences, 903

racial distribution, 907,913

Blue tongue (of sheep), $5 \mathrm{IS}$

Boils, 506

Boltzmann constant, I 76

Bone marrow, I04 I

Bordet-Gengou phenomenon, 831

Bordet's adsorption theory, 740

Borna's disease, 5 r8

Botulinum. Sce Cl. botulinum

Botulism, 449

Bovine tubercle bacilli, resistance to heat, 406

Brain broth, 580

Branching

forms, 3

true, I

Bright's disease, 779

Brilliant green and bile, 365

Brownian movement, I 7 I, 782,784

Buffer

content of synthetic media, $2 \mathrm{~S}_{4}$

tissue as, 207

Bulgarian buttermilk, 395

Butter, 398, 399

air in, 399

aroma, 398

from ripened cream, 399

deterioration of, 399

flavor, 398

salted, 399

starters for, 398

stored, 399

Buttermilk, 395

Butyric

acid, 246

fermentation, 228,239

Calcium

in heated milk, 409

used by soil micro-organisms, 3 I9

Canned foods, botulinum toxin in, 449

Canizzaro

reaction, 239

rearrangements, 502

Canning, 442

$\mathrm{CaO}$, effect on soils, 67

Capillary chemistry, I 78 
Capsulated bacteria, virulence of, 568

Capsules of bacteria, 7, 573

constituents, $23 \mathbf{I}$

formation, 97

structure, 724

Carbohydrases, 273

Carbohydrates

in bacteria, 15

Utilization OF, BY BACTERIA, 227-42

effect on chemical composition of bacteria, $23 \mathrm{I}$

converted into fats, 506

fermentation, 236

for B. coli, 365

by root nodule bacteria, 339

in immunity, 724

influence on metabolism, 273

molecule, effect of configuration of, 234

protein complexes, 7 IO

fraction of proteins, 234

in cultivation of spirochetes, 474

from tubercle bacillus, 7 I4

Carbolic acid coefficient, 75. Sce Phenol coefficient

Carboligase, 23I

Carbon

\section{arc, 372}

from carbon dioxide, $28 \mathrm{I}$

from carbonates, $28 \mathrm{I}$

for growth, 280

in organic form, $28 \mathbf{I}$

phagocy tosis of, 862

requirements, 244

Carbon dioxide

assimilation by autotrophic bacteria, 33I

by oxidation of sulphur, 282

produced by bacteriophage, 531

evolution of, 3 I 5

indispensable to sulphur bacteria, 327

Carbonates, 28I

Carboxydomonas oligocarbophila, 282

Carboxylase, 276, 501

Carboxylation, de-, 245

Carnosine, 246

Carp pox, ${ }_{5}$ I8

Casein, 703

Catalase, II 7, 208, 214, 217,277

activity, 2I4

Cataphoresis, 169,785

cell, 575

of toxin and antitoxin solutions, 742

velocity in, 170

Cataphoretic

potentials, 863

velocity, 171,178

Catarrhal fever of sheep, 518

Cations, effect on bacteria, 76

Cattle

plague, $5 \mathrm{I} 8$

stomatitis, 518

Cell

membrane, $2 \mathrm{I}, 30$

permeability, $2 \mathrm{I}$ structure, 2 I

surface, 25

Cellulose, 23 I

decomposition of, 3 I 5

Cereal grains, 439

Cervix, streptococci from, 579

Chain formation, 43

Charge. See Electrical charge

Chaulmoogra oil in chemotherapy, I 128

Cheese

flavor, 400

gassy, 380

hard, 400

molds in, 401

poisoning, $45 \mathrm{I}$

rennet, 379

varieties, 400,401

Chemical

composition of cell and carbohydrate, 2.31

composition of soils, $34 \mathrm{I}$

disinfectants, 75

factors, influence on enzymes, 27 I

injury to bacteriophage, 533

Structure of BaCteria, I4-I8

Chemistry

classification, I 26

of nitrogen fixation, 337

Chemoceptors, 22

Chemo-receptors, I ro2

Chemotherapeutic index, I IOI

Chemotherapy of Bacterial Diseases, I iol33

acriflavin, I I 8

anilin dyes, I I I . Sce also Dyes

in anthrax, I I 33

arsenic, I I 25 , I 127

and biological therapy, I I I I

chaulmoogra oil, i I 28

chemical agent, essentials for, I I04

cinchona deriva tives, I I 2O, I I 22

copper salts, I 29

creosote, I 133

dye-fastness of bacteria in, $\operatorname{II} 6$

in experimental infections, I I 22, I I 28,1130

in generalized infections, $1 \mathrm{ro3}$

in gonococcus infections, I 127

hexamethylamin, I 28

immunological factors in, I I 0

iodin, I 127

localized infections, I 103, I 105, I I06

Lugol's solution, I 127

in meningitis, 1123

mercurial compounds, I I07, I I 25, I I 31

metallic salts, I I 27

methylene blue, i 1 i 8

in pneumococcus infections, II23, II 30

proflavin, i i 8

in rheumatic fever, acute, I I 33

rivanol, i I 8

sanocrysin, I I 29

in staphylococcus infections, I 130

in streptococcus infections, 1130

tissue changes, r 108

in tuberculosis, I I 27 , I I 28 
Chicken pox, 518, 566. See also Varicella

Chicken sarcoma, 518

Chilling, effect of, on culture growth, 6I

Chilomastix mesnili, 662, 674

Chitin, 23I

Chlamydobacteriales, 132,282

Chlamydozoa, $5 \mathrm{r} 7$

Cholecystitis, $5^{82}$

Chlorin

antiseptics, 1106

needed for growth, 280

Chlorination

of sewage, $36 \mathrm{r}$

of water supplies, 368

Chlorosis, 518

Cholera. See Hog cholera

Cholera vibrio, 2

Cholesterol, $3 \mathbf{I}$

Chromatin, 7, 88, 221, 547

Chromatium, 324

Chromidia, 40

Chromogens, I9

Chromophores, 19

Chromosomes, 542

Ciliates, intestinal, 674

Cinchona in chemotherapy, I 20

ethylhydrocuprein, I I 2 I

in experimental infections, I I 22

toxicity of, II 2 I

Citrate, sodium, 365

Citric acid, 246

produced by $A$. niger, 5 I 2

action of $B$. aerogenes and $B$. coli on, 248

Classification

OF BACTERIA, I 20-35

of bacterial enzymes, 269

chronological, 128

Clones, 85

Clostridium botulinum. See also B, botulinus, Botulism

antitoxin, 449, 755, 766, 954

in food poisoning, 443

heat resistance of spores, 287,448

and $\mathrm{pH}, 292$

toxin, 561, 766, 781

absorption, 449

in canned foods, 449

destroyed by $\mathrm{Cl}$. sporogenes, 449

varieties $A, B$, and $C, 447$

Cl. sporogenes, 449

Cl. tetani, 642. See also B. tetani

destruction of spores, 287

Cl. welchii, 642, 955. See also B. welchii

in gastro-enteritis, 367

in water, $364,366,367$

Clovers, 334

Coagulation

milk, 275

of proteins, by

heat, 703

ultra-violet light, 377

velocity, $800^{\circ}$
Coal-ta: dyes, 19

Cobra-lecithid, I080

Cocaine, filterability of, $5^{6} 3$

Coccaceae, 42, I23, I3I, I32

Cocci, udder, 379

Coccidia, host-parasite relations, 675

Cohesive force, $79^{2}$

nature of, 799

effect of salt solution on, 794

Cold, preserving by, 44I

Colds, 5 r 8

Coli-aerogenes group, $64 \mathrm{I}$ from fecal and non-fecal sources, 365 from water, 364

Colloidal

micellae, 529

particles

gold, 17 I

ionization of, 170

migration of, 575

suspensions

charge of, 785

precipitation, 790

stability of, i 70

systems, surface tension in, I 78

reactions of toxins and antitoxins, 740

Colloids, 178

protective, 77

Colon, 639

Colon-typhoid group, 392

globulins, 7 I 4

in milk, 389,394

in water, 69

Colonies

aberrant, 596

of bacteriophage, 528

pin point, 403

Colony form

as aspect of dissociation, 93

effect of serum, 99

variation, 570

continuous, $57 \mathrm{I}$

in plant pathogens, 595

in Bact. tumefaciens, 595

See also Rough and smooth colony form

Compatibility tests before transfusion, 904

Complement

anticomplementary activities, 836

in bacteriolysins, 875

and ultra-violet rays, 376

Complement fixation antibodies, 1037

Complement Fixation Reaction with BacTERIAL ANTIGENS, $83 \mathrm{I}-37$

antigenic substances for, 834

in leprosy, malaria, pellagra, and pregnancy, 847

TEST FOR SYPHILIS, 838-47

alcoholic extracts for, 839

interpretation of results, 847

methods of, 839-44

and precipitation test, 855

serum of non-syphilitic cases, 847

standardization of technique, 844

diagnosis of tuberculosis, 836 
Composition of bacteria, I $5_{5}$

Congestins, 969

Conidia, 6, 510

Conjugation, 90

Constipation, yeast for, 506

Contact beds, 354

Contamination of FoOd, 437-42

Convalescent Serum, Human, Use of 934-46

in influenzal pneumonia, 942

in lethargic encephalitis, 942

in measles, 936, 938

in mumps, $936-38$

in poliomyelitis, $94 \mathrm{I}$

in scarlet fever, 944

in typhoid fever, 943

in varicella, 935,936

in whooping cough, 943

Copper

in chemotherapy, I I 29

and lag, 6r

Cortex of bacteria, 7

Corynebacteria, 573

C. diphtheriae, 43, 203. See also B. diphtheriae,

Diphtheria, nitrogen content, 219

C. hodgkini, 43

C. hoffmanni, 43

C. rodentium, 614

C. xerosis, 43

Counts, bacterial, for water, 363

Coupled reactions, $24 \mathrm{I}$

Cow pox, 5 I 8

Cream, 409

Creatine, 246

Creatinine, 246

Crenothrix, 324

Creosote in chemotherapy, I 33

Crisis phase, 68, 70, 7 I

Crises, 684

Crista, Io, 463

Cristispira, 454, 457-62

C. balbianii, 454

motility, 463

Crop rotation, 345

Cross-inoculation groups, 334

Crystalline lens of eye, 703

Crystals, I 36 , I 78

Cultural aspects of dissociation, 93

Culture Media, Synthetic, 279-84

Curative action of sera, 807

Curves, Growth, of Bacteria, 46-57

Cycles. See also Life cycles

of growth, 47

of morphology, 44

physiological, 38

of populations, 58

Cyclogeny, 87

in plant pathogens, 596

variations, Ior

Cytoplasm disintegration, 547
Dairy

Products, Bacteria IN, 395-402

wastes, 355

Dandruff, horse, $7 \mathrm{r} 3$

Danysz phenomena, 76I, $98 \mathrm{I}$

Dead ends in pasteurization, 426, 427

Deaminases, 276

Deamination, 245

Death of bacteria

rate, 75

thermal points, 404

Thermal Time, 285-300

variations, 280

of tubercle bacillus, 404,406

velocity constants for, 76

Decrease phase, 72

Decolorizers, 20

Decomposition of organic matter, by soil micro-organisms, 316 of sewage, $35 \mathrm{I}, 353$

Degeneration forms, 85

Dengue 493, 51 8

DETERIORATION OF FOOD, 437-42 of butter, 399

Dextran, I 7

Diabetes, complement fixation test, 847

Diacetyl, 240

Diagnosis by precipitins, 830

Dialysis reaction. See Abderhalden

Diarrhea from milk, 4I 5

Diastase, 274

Dick test, 96r

Dielectric constant, 170

Diet and intestinal flora, 644

Digestion of bacteria by enzymes, 25

Digitalis, 562

Diphtheria. See also B., and

Corynebacterium, diphtheriae

antitoxin, 95I

filterability, 562

flocculation, 765 preparation, 753

avian, 5 I 8

group, io8

from milk, 4I I, 4 I 3

paralysis, post,- 778

toxin, 184,779

flocculation, $76_{5}$

for Schick test, 960

standardization, 746

test dose, 952

toxin-antitoxin, 960

preparation, 748

balanced mixtures, 764

toxoid, 96 I

Disinfectant solutions, I 106

Disinfectants, Standardization of, $30 \mathrm{I}-9$

mercuric chloride, 76

methods, 301, 302

Hygienic Laboratory, 301

Lancet, 302 
Rideal-Walker, zor

thread, 301

test organisms

M. aureus, 302, 305

B. diphtheriae, 302,306

B. tuberculosis, 302,306

B.typhosus, 302,303

Disinfection, 74,75

factors in, 79

mechanism of, 377

process, 72,73

of sewage, $36 \mathrm{r}$

Disintegration, mechanical, 27

Dissociation, 577,598

colonial, cultural, and morphological aspects, 93

and $\mathrm{pH}, 99$

Dissociative Aspects of Bacterial Behavior, $84^{-10}$ I

Dissociative reaction, 86

Distemper of dogs, $5^{1} 8$

Distribution of bacteria, 58

in soil, $3 \mathrm{r} 2$

Donnan equilibrium, 573,786

Dormancy, effect of, on heat resistance, 295

Double layer, electric, I 70, 786

Drainage, 347

Drop weight method for surface tension, 167 , I 73,175

Drugs, influence on antibody production, IIIO

Dry substance of bacteria, 15

Drying of

bacteria, 74

foods, 440

Duodenum ulcers, 582

Dyes. See also Staining anilin

bactericidal power, 34

in chemotherapy, IIII, III 2

selective action, I I I 3

effect on antibodies, I I 16

chemistry of, III4

effect of heat, III3

mechanism of, II I 5

effect of, on phagocytosis, I I 6

toxicity, toxicity tests, I II 7

in staining bacteria, 19

bacterial sensitive to, 37

bacteriostatic and bactericidal activity, 25, III 2

bacteriostatic reverse action, 26

chemistry of, I9

coal tar, I9

for differentiation, 366

dye-fastness of bacteria and chemotherapy, III 6

fat-soluble, staining by, $3 \mathrm{I}$

filterable, 557

for testing filters, $55^{8}$

mixtures, bactericidal activity, I I 2

Systems, Oxidation-Reduction Potentials OF, 188-97
Dysentery (bacillary)

antiserum 924,960

flocculation with toxin and antitoxin, 767

local immunity, 887

from milk, $4 \mathrm{I} 5$

oxidizing ferments, 215

phagotherapy, 553

Eating utensils, 8o

Eberthclla

dysenteriae. See Bact. dysenteriae

parad ysenteriae, 42

typhi. See Bact. typhi

Eczema, 968

Eggs

stored, $44 \mathrm{I}$

white and yolk, 713

Ehrlich's theory, 739

CRITIQUE OF, IO78-IIOO

experimental tests of, 1081

hypotheses, 1078

and immunological facts, 1079

validity of, 1079

Eimeria perforans, 628

Eimeria stiedae, 628

Elective Localization of Bacteria in the Animal Body, 576-89

of streptococci, 578

Electrical characteristics of emulsions and suspensions, 169

Electrical charge, 74r, 863

of antigens and antibodies, 743

of colloidal suspensions, 785

of electron, 176

for microbes, 566

and stability, 788

and specific soluble substances, 572

and virulence, $57^{2}$

Electrical double layer, I7o. See Potential and Potential difference

Electrical forces and phagocytosis, 866

Electrical potential difference. See Potential difference

Electrical repulsion, 170

Electrodialysis of antitoxin, 740

Electro-endosmosis, 785

Electrode potential, 190

Electrolytes, 25

activity coefficient, 145

weak, 148

Electron

charge on, 176

negative, 137

Electrophoresis cell, 575

Electrophoretic charge and agglutinability, 569

Electrophoretic potentials and host resistance, 574 and toxigenicity, 573

and virulence, 596, 599

Embadomonas intestinalis, 674

Emulsification, 178 
Emulsions

electrical characteristics of, 169

stability of, I 73

Encapsulatus pncumoniae, 42. Scc also Friedländer's bacillus

Encephalitis, 5 I 8,587

lethargic, 942

in rabbits, 630

streptococcus antiserum, 932

Encephalitozoön cuniculi, 630

Endamoeba coli, 661, 67 I

Endamoeba gingivalis, $66 \mathrm{I}$

Endamocba histolytica, 66I, 667-70

epidemiology, 667

immunology, 669

sites of infection, 668

host-parasite specificity, 670

symptoms, 669

Endicott experiments on pasteurization, 407, 425,435

Endo medium for Bact. coli, 366

Endolimax nana, 66I

Endosmosis, 785 of toxin and antitoxin, 774

Endothelium, 873. See Reticulo-endothelial

Endotoxins, 447, 568

Energy, I 37

requirements of bacteria, 232

of light to kill bacteria, 373

of surface formation, 162

tensile, I 55

Engineering Aspects of Pasteurization, 419-36

Entamoeba buccalis, 203

Enteric, anti-, vaccines, 960

Enterococcus, 388

Environment of bacteria, 58

Enzyme, anti-, 875

Enzyme Theory of Antibody Formation, 1082-86

Enzymes

of Aspergilli, 513, 514

action on bacteria, 25

OF BACTERIA, 268-78

action measured, $27 \mathrm{I}$

as antigens, 272

classification, 269

formation, 269

nature, 268

occurrence, 273

optimum reaction for, 272

oxidation-reduction by, 276

$\mathrm{pH}, 272$

physical and chemical factors, 27 I

preparation, 270

proteolytic, 275

purification of, 270

secretion, 269

temperature coefficients, 27 I

effect of ultra-violet light, 375

of Bacteriophagum intestinale, 527 of milk (lipase), 399

of monocytes, 875

preparations for jellies, 5 I4

Eosin methylene blue agar for Bact. coli, 366

Epidemics, milk-borne, 4IO, 4I I

Epithelioma, 51 8

Epizoötic of guinea pigs, 5 I 8

Equilibrium

as affected by size of particles, $78_{3}$

membrane, I 52

Erysipelas streptococcus antiserum, 932

Erythema, 4I5, 582

Erythrobacillus prodigiosus, nitrogen content, 2 I $\mathrm{S}$

Erythrocytes, 873 agglutination of, 796

sensitivity of, 896

Escaping tendency, 144

Escherichia acidi lactici, 389, 393

Escherichia coli, communior. Sce Bact. coli

Esterases, 273

Ether extraction and gram behavior, 28

Ethyl amine, 245

Ethylhydrocuprein, 6I 6, I I 21, I I 22

Eubacteriales, I31, I32, I34

Excretion of bacteria, 639

Extractive bodies, 246

Fagopyrismus, IOI 5

Faraday, 176

Fat, 3 I

in bacteria, 15,18

carbohydrates converted into, 506

in tubercle bacillus, I 8

content of yeasts, 506

formation by yeasts, $5 \circ 5$

Fat-soluble dyes, 3 I

Fatty acids, 3I, 505

Fecal and non-fecal sources of coli-aerogenes group, 365

Feces, 639

increase of bacteria in, 66

microscopic appearance, 643

stored, $69,74,80$

Fenugreek, 334

Fermentation, 198, 227

of carbohydrates, 236

for Bact. coli, 365

by root nodule bacteria, 339

glycerol 239,504

lactic acid, 385

miscellaneous, 240

Neuberg equations, 501

pickle, 440

rice, 5 I 3

Fermented milks, 394, 395 therapeutic value of, 396

Ferments, 944. See also Abwehrfermente oxidizing, 2 I 5

protective, 1056

from pneumonic lung, 876 
Fertility. See Soil fertility

Fertilizers, 246, 347

Field and Teague apparatus, 575

Filament, terminal, $46_{3}, 465$

Filamentous bacteria, 324

in activated sludge, 359

in sewage, 359

Filmometer, 165

Films

absorbed from liquids, 164

monomolecular, 163,173

surface, 797

Fil ter-passing stage, 5 sludge, $35^{8}$

Filterability, 557-64

antitoxins, 562

bacterial toxins, 560

bacteriophage, 560

botulinum toxin, $56 \mathrm{I}$

cocaine, 563

digitalis, 562

non-bacterial toxins, 562

opium, 562

spirochetes, 467

strychnine, 563

tetanus toxin, 562

tobacco sap, diseased, 560

tuberculin, 56r

Vibrio percolans, 560

viruses of vaccinia and rabies, 560

Fil terable form of bacteria, 3 yeast, 499

Filterable Viruses, 517-24; 97

cultivation, $52 \mathrm{I}$

diseases from, 517,518

filterability, 5 I 9

in guinea pig diseases, 622

immunity to virus diseases, 523

size, 519

variations of, 520

viability, 520

Filters

Berkefeld, 557

dye tests, $55^{8}$

electrical potential difference for, 637

plaster of Paris, $55^{8}$

gypsum, $55^{8}$

siliceous, 557

trickling, 354

Filtrates, paratyphoid, 7 I 4

Filtration, sand, 354

Fire blight, 59I

Fish, lymphocystic disease of, 5 I 8

Fission, reproduction by simple, I 00

Fixation. See Complement fixation, Nitrogen

Fixed tissue cells, phagocytes, 808

Flagella, 37

Flagella agglutination, 8 I 4

Flagellates. See Intestinal flagellates

Flagellation of root nodule bacteria, 338
Flocculation, I 7 I, 782

Method for Titrating Toxins and AntiTOXINS, 759-7I

botulinum, 766

diphtheria, $76_{5}$

dysentery, 767

miscellaneous, 768

tetanus, 767

Ramon's method, 763, 952

reaction

conditions affecting, $76 \mathbf{I}$

nature of, 763

specificity, 764

technique, 760

value, $172,173,762$

Flood fever, 518

Food

B. aerlrycke in, 446

requirements of bacteria, 67

canning, 442

Contamination and Deterioration of, 437-42

flesh, 439

fruit and sugars, 439,440

handling, $43^{8}$

drying and preserving, 440, 44I

incubation, 438

plant, mobilization of, 348

Poisoning, BACTERIA OF, 443-5I

Cl. botulimum, 443, 447-49

paratyphoid bacilli, 443

$B$. proteus, $45^{\circ}$

staphylococcus, $45^{\circ}$

streptococcus, $45^{\circ}$

typhoid bacilli, 443

Foot and mouth disease, 415, 518

Formiase, 238

Formic acid, 238, 241, 246

Forssman antibodies, 709. See also Heterophile

Fowl plague, 5 I 8

Fowl pox, 5 i 8

Freezing, effects on toxin-antitoxin, 749

Friedländer's bacillus, $2,8,42$, I Io

infections in guinea pigs, 618

specific substance from, 727

Fuchsin, acid, basic, I I 77

Fumaric, 246

Fungi in soil, 3I7, $32 \mathrm{I}$

Fungi imperfecti, 45

Furunculosis, use of yeast in, 506

Fusiform bacilli, 655

Gärtner bacillus, 444

Galaktan, 8

Gallionella, 324

G. ferruginea, 3.30

Gas

constant (Boltzmann), I76, I77

evolution of, 266

gangrene, 955

light, 37 I 
Metabolism of Bacteria, 250-67 under anaerobic conditions, 264 study of, 25 I of tubercle bacillus, 262

-producing power, fluctuations, 443 ratio for Bact. coli, 365

in soil, 343

synergism, I I 5

Gaseous nitrogen, 2 I I

Gelatin, 703

Gelatinase in spore material, 9

Generation spontaneous, 285 time, $47,59,62,63,65,67$

Genes for blood groups, 912

Genotype, I 25

Gentian violet, I 117

German measles 5 I 8

Germicidal

action of light, $37 \mathrm{I}, 374$ properties of milk, 60, 382, 409 region of spectrum, 373

Germicides in soils, 349

Giant cells, 6

Giardia lamblia, 662, 673

Giardiasis, 674

Gibbs' zeta function, 176

Glabrificine, 8I 2

Glanders, 3

Glass

effects of, on heat resistance, 293

Globulin, 7 I 3 of colon-typhoid group, 7 I4

Gloeosporium ampelophagum, 298

Gluconic acid, 235, 5 I 2

Glucosamine, 233, 234

Glucose, 235, 237

brain broth, 580

effect of, on indol production, 229

oxidation of, 322

effect of, on toxin production, 229

Glucoside, 233, 717

Glutaric acid, 246

Glutathione, 2 I 3

Glycerol, 237 fermentation, 239, 504

Glycine, 703

Glycocoll, 248

Glycogen, I 7

Glycollic acid, 246

Goats, use in testing toxins, 957

Gold particles, colloidal, I 7 I

Gonidia, 40, 89, 90

Gonites, 89

Gonococcus, 42, 203, 2 I 4

chemotherapy, I 127

anti-, serum, 925

Grains, 439
Gram stain, 23-30

reaction, 16,23

mechanism, 27,30

reverse, 28,29

behavior reversed by ether extraction, 28

Granulations, 96

Granules

acid fast, 5

nucleus-like, 9 I

in spirochetes, 9, I I

See also Much granules

Granulocyte, 873

Granulose, 17

Grippe, 5 I 8

Growth of bacteria, I, 45. See also Lag acceleration, negative phase, 5 I accessories for influenza bacillus, 657 effect of chilling, 6 I

crisis phase, 68

curves, 46

autokinetic phenomena, 5I, 52

as growth phases, 48

effect of size of inoculum, 53

substrate, 54

temperature, 54,55

cycle, 44

elements

essentials for, 280,28 I

iron, 280, 320

logarithmic phase, $46,49,50,60,67$

changes in morphology during, $38-45$

optimum, 25

rates, 46

effect of temperature, 55

stationary phase, $46-48,5$ I

and surface tension depressants, 182

Guanine, 246

Guinea pig diseases, 608-22

Bact. bronchisepticum, $6 \mathrm{I} 8$

epizoötic, 518

filterable virus infections, 622

Friedländer's bacillus, 6 I 8

paralysis, 5 I 8

paratyphoid, 608-14

pasteurellosis, 6 I 9

pneumococcus, 6r6-18

protozoan infections, 620

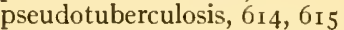

salivary gland disease, 5 I 8

streptococcus, 620

$T$. rhodesiense, 684

tubercle bacillus, 620

Gum

from root nodule bacteria, 339

from tubercle bacillus, 727

Gums, bacteria along, $6_{5} 6$

Habitat of bacteria, 58

as a differential point, 127

Halogens, 25

Haptenes, 708

Hay fever, 968, 1008

Heartwater disease, 518 
Heat

effect of, on B. coli, 290

effect of, on milk, 408, 409

resistance

of bacteria, 289

effect of dormancy, 295

glass, 293

of hydrogen-ion concentration, 292

numbers, 294

oil, 295

$\mathrm{pH}, 292$

salts, 294

of $B$. anthracis, 293

of bovine tubercle bacilli, 406

of spores, $285,287,298$

botulinum, 448

differences, $29 \mathrm{I}$

Helmholtz double layer, 786

Helmholtz-Lamb-Smoluchowski equation, 70

Hemagglutinins, 735

Hematopoietic organs as antibody formers, $88_{3}$

Hemicellulose, 23 I

Hemoglobin, 657

as antigen, 970

Hemoglobinophilic group, I 14

Hemolysin, 275, 735, 1036

anti-, 717

natural, 1053

purification, $105 \mathrm{I}$

reversible oxidation, 214

Hemolysis, 895

venom, I072

Hemolytic antigen, 7 I 7

Hemophage, 808

Hemophilic bacteria, 656

Hemophilus infuenzae, 42. See also Influenza bacillus, Pfeiffer bacillus

H. pertussis. See B. pertussis, Pertussis

Hemopsonins, 872

Hemorrhagic purpura, 776

Herpes zoster, 5 I 8,582

Heterophile. See also Forssman

ANTIGEN, 733-38; 829

ANTIBODIES, 735

and anaphylactic shock, 736

in nature, 733

properties, 734

hemolysin, 735

immune sera, 736,737

substance and lecithin, 735

Heterotrophic

bacteria, 3 I 8

fungi, 318

Heterotrophs, 322

Hexamethylamin in chemotherapy, 1 I 28

Hexyl-resorcinol, I Io7

Hiccup, epidemic of, $5^{87}$

Higher bacteria, 324

$\mathrm{H}$-ion. See Hydrogen-ion

Histamine, 230,983

-like substance from B. welchii, 230
Histidine, 230, 703

Histones, 703

as antigens, 970

Hog cholera, I I3, 5 I 8

Hoppe-Seyler's theory of biological oxidation, 201

Hormones, 542

Horse

African sickness, $5 \mathrm{I} \&$

anemia, 518

dander, guinea pigs sensitized to, Ior 4

dandruff, 713

pox, 518

stomatitis, 518

Host-parasite relations

of Man and His Intestinal Protozoa, 66o-78

of plants with bacteria, 332

localization of parasites in host, $66_{5}$

Host resistance and electrophoretic potential, 574

Hyacinths, $59 \mathrm{I}$

Hydrocuprein, 25

Hydrogen

needed for growth, 280

-ion concentration, I 5 I

and agglutination, 791, 792

and heat resistance, 292

and $\mathrm{pH}, 148$

and ultra-violet action, 374

oxidation, 324,329

peroxide, I I 7

pressure, I 96

sulphide, 21 2, 222

producers in sewage, 355

Hydrogenomonas, 282

Hydrolysis of bacteria, 547

Hygienic Laboratory method for disinfectants, 30 I

phenol coefficient, 302

Hypersensitiveness, 966, I08 I

atopic, IOIO, IOI 2

and tuberculin, 1028

Hypoxanthine, 224, 246

Hysteresis, 9

Ice, 74

Ice cream, 398

Idiosyncrasy, 722

and anaphylaxis, IoI4

drug, $97 \mathrm{I}$

Imhoff tanks, 355

Immune

sera

bacteriophage adaptation to, 534

concentration of, 1055

and potential difference, 574

substances, adsorption of, I050

Immune Properties, Isolation of SubSTANCES WITH, I049-55

Immunity, 565, $72 \mathrm{I}$

without antibodies, So9

of bacteria, $55 \mathrm{I}$ 
carbohydrates in, 724

LOCAL, 88I-9I

antibody formation, 890 , 1046

to anthrax, 885

Besredka's hypothesis, 889

dysentery bacillus, 887

intestinal infections, 887

staphylococcus, 886

streptococcus, 886

natural, 806

Phagocytes and Phagocytosis in, 870-80

reactions in protein therapy, I09I

against spirochetes, 468

Tissue, 88r-9r

tissue cultures, 1037

Immunization

attenuated cultures in, 210

oral, I047

prophylactic and therapeutic, 877

Immunology

in classification, I 28

factors in chemotherapy, I I 10

in plant diseases, $60 \mathrm{r}$

Inactivity of bacteriophage, 538

Increase phase, $6_{3}$

Indicators, oxidation-reduction, I93

Indigo, cross-inoculation groups, 334

Indol production, I I 8, 221, 393

by Bact. coli, 365

effect on glucose, 229

Infantile paralysis from milk, 4I6

See also Poliomyelitis

Infants' digestive tract, 640

Infectious diseases, bacteriophage in, $55^{2}$

Infectious jaundice, 454

and yellow fever, 493

Inflammation, 870

Influenza, 218, 266. See also H. influenzae, Pfeiffer bacillus

bacillus, nutrition of, 657,658

pneumonia, 942

Infra-microbes, 517

Inheritance. See Blood groups atopic, 1004

Injury, relation to lag, $6 \mathrm{r}$

Inoculation groups, cross-, 334

Inoculum, effect of size on growth curve, 53

Insorption, 22

Interfaces

free energy, 865

orientation of molecules, I6I

Interfacial

tension, I 73, I 78 , I 80

zones and antibody reactions, $I 8_{3}$

Intermediate cultures, $57 \mathrm{I}$

Internal structure of bacteria, 6

Intestinal

amebae, 66I, 667, $67 \mathrm{I}$

BACTERIA, 639-49

aciduric group, 64 I

anaerobes, 642 coli-aerogenes group, 641

and diet, 644

micrococci, 642

proteus group, 642

subtilis group, 643

transformation, 646

yeasts and molds, 643

coccidium, 663

flagellates, $662,672,674$

infections

local immunity to, 887

of rabbits, 628

ProtozoA of MAN, 660-78

worms and anaerobic conditions, 200

fate of bakers' yeast, 649

Intoxications. See Sublethal intoxications

Invasiveness, 567

Invertase, 274

Involution forms, 3, 85, 97

Iodin

in chemotherapy, 1127

sensitivity to, 25

Iodamoeba williamsi, $66 \mathrm{r}$

Iogen 17

Ionic strength, I 46

Ionization

acids and bases in water, 148

of colloidal particle, effective, I >o

of salts in solutions, 140,177

of substances not completely ionized, 147

Ions, I38-40; I38. See also Hydrogen, $\mathrm{pH}$, Potentials

adsorbed, effect of, on valence, I 7 I

aluminum, effect of, on charge of particles, I 7 I

attraction between, I 39

effect of, on phagocytosis, 862

in solid salts, I 39

in synthetic media, 284

Iritis, 582

Iron

bacteria, 282, 330

necessary for growth, 280,320

oxidation of, 324,328

Irradiation, 375,376

Irrigation, 347,354

Isoagglutination, 892

elements, development of, 895

human in blood of lower animals, 902

substances in human sperm, 920

test, forensic application, 907

Isoagglutinogens, 892 chemical nature of, 903

Isoelectric

point, I 7 I, $787,788,8 \times 5$

staining range, 25,28

Isohemolysins, 895

Isospora felis, 675

Isospora hominis, 675

Isotopes, I37, 177 
Japanese flood fever, 518

Jaundice of silk worms, 5 I 8. See also Infectious jaundice

Jellies, enzyme preparations, 514

KaHN REACTION, 848-60; 845

antigen for, $850,851,855$

application, 859

interpretation of results, 854,858

procedures, 857,858

serum and antigen suspension, 853

serum, treatment for, 853

Kaolin, 944

Kefir, 395, 396

Keratohyalin, 24

Kinetic energy of molecules, 140

Klossiella muris, 635

Koser test, 366

Kumiss, 395, 396

Laboratory animals

B. aertrycke in, 445

COMMUNICABLE DISEASES OF, 607-38

Lactation, 382

Lactic acid, 237, 246

fermentation, 385

methyl glyoxal changed to, 230

Lactobacillaceae, I33

Lactobacilli

in milk, 394

in souring of vegetables, 440

Lactobacillus acidophilus, 106, 394, 396, 641, 646

L. bifidus, 640

L. bulgaricus, 394-96

L. helveticum, 394

L. odontolyticus-acidophilus, 647

Lactose bile in presumptive test, 364

Lag, 44, 59, 60, 62 phase, 46,49

Lakes, bacteria in, $5^{8}$

Lamb-Helmholtz formula, 785

Lambliasis, 674

Lamprocystis, 324

Lancet method for disinfectants, 301

Lanthanum nitrate, 572

Laxative, yeast as, 506

Leben, 395

Lecithin, 31, 735

Leeches living without oxygen, 200

Leguminous plants

in economic agriculture, 340

cultures for inoculating, 334

Root Nodules OF, 332-40

root tubercles, 590

Lentil, cross-inoculation groups, 334

Leprosy, complement fixation test in, 847

Leptospira sp.?, 490

Leptospira astenalgiae, 493
L. biliohemoglobinuriae, 490

L. hebdomadis, 466,489

L. hemoglobinuriae, 490

L. icterohaemorrhagiae, $466,485,488,821$

L. icteroides, 485

L. interrogans, 454, 466, 490-93

L. pyrogenes, 489

L. trimerodonta, 48I

Leptospiras, 454, 457-62, 485-494

free-living, 485

medium for, 472

motility, $46_{5}$

Leptothrix, 324

Leptothrix innominata, 203

Leptothrix ochracea, 330

Leukocytes, 776

and agglutination, 507

eosinophile, 873,874

-forming organs, 883

in milk, $38 \mathrm{I}$

neutrophile, 873,874

and toxins, 775

count affected by yeast, 507

Leukoprotease, 876

Life cycles of bacteria, 40, 86

Life history of a culture, 4I

Light

action on bacteria, $37 \mathrm{I}$

and bacteriolysins, 376

and coagulation of proteins, 377

gas-, $37 \mathrm{I}$

germicidal action, $37 \mathrm{I}$

and oxidizing reactions, 372

requirement of photosynthetic organisms, 28 I

preservative quality, $37 \mathrm{I}$

sun, 372

action on tubercle bacillus, 373

velocity, $138, \mathrm{r} 76$

vibrations, frequency of, 138

Lime paste, organisms in, 285

Liming, effect of, on soils, 67,345

Linkage in humans, 919

Lipase, 399, 401

Lipins, 25

from tubercle bacillus, $7 \mathrm{I} 4$

Lipoids

as antigens, 707, 714, 723, $97 \mathrm{I}$

anti-, sera, 707

protein-free, 7 I 5

Liquid

junction, I5o, I 5 I

spreading of one on another, r62

surface structure, 160

Litmus, reduction in milk, 389

Localization. See Elective Localization, etc. of parasites in host, 665

Localized infections, chemotherapy of, I 105

Locust, cross-inoculation group, 334

Logarithmic growth phase, 46, 49, 50, 60, 67

Lugol's solution in chemotherapy, I 127 
Lung, pneumonic, ferments from, 876

Lupines, cross-inoculation groups, 334

Lymph nodes, 65

Lymphocyte, 874

Lymphoid tissue and bacteria, $65^{\circ}$

Lysine, 703

Lytic cultures

products of digestion after, 546

resistant individuals in, 549

Lysis

of bacteria, 530

of dead bacteria, 530

active agent of transmissible, 525

Macrocytase, 870

Macronucleus, 7

Macrophages, 870

Magnesium, 25

in soil, 319

Malaria

avian, $68 \mathrm{r}, 696$

asexual and sexual cycle, 68I

reproduction cycle, $68 \mathrm{I}$

and blood sugar, 698

complement fixation test, 847

infections, 694

in treatment of paresis, 698, 1099

organisms in vitro, 697

Maleic acid, 246

Malic acid, 246

Mallein reaction, 968

Malonic acid, 246

Malta fever, 38I, 4I 4, 82 I

Manganese, 282

oxidation of, 324,328

Manures, 346

Mass, 137

law of action, I4, 739

Mast cell, 873

Mastitis milk, 38I

Matzoon, 395

Measles, 518, 566, 936, 938

Medium

optimum, 283

stains in, 34

SyNTHETIC, 279-84

contents, 280

preparation, 284

for bacterial counts of water, 363

Medulla, 7

Megacocci, 2

Meinicke test, 849

Membrane

equilibrium, I 52, 573

limiting, 27

potential, I 53

Mendelian mechanism for blood groups, 9 I 2

Meningitis

cerebrospinal, 922

chemotherapy of, I 123
Meningococcus, 727

anti-, serum, 821, 922, 925, 926, 958

Mercurial compounds in chemotherapy, 1107 , I I 25, I I 26

toxicity of, I I 31

Mercuric chloride, 76

bacteriophage adaptation to, 534

Mercurochrome, I 107, I I 10

Mesohematin, 25

Mesoporphyrin, 25

Metabiosis, 102

Metabolism of bacteria, 542

influence of carbohydrates on, 223

GAS, $250-67$

under anaerobic conditions, 264

Protein, 218-26

toxic products of, 548

Metallic salts in chemotherapy, I I 27

Metastatic effects of toxin, $77 \mathrm{I}$

Methanomonas methanica, 282

Methemoglobin formation by bacteria, 216

Methyl carbinol reaction, 392, 393

Methyl glyoxal changed to lactic acid, 230

Methyl red

test for Bact. coli, $36_{5}$

reaction, 393

Methylene blue, II 7 , I I 8

reduction of, 2 I 2

Mice, diseases of, 632-35

pasteurellosis, 634

pneumonia, 635

pseudotuberculosis, 635

trypanosome infections, $68_{3}$

mouse typhoid, 555, 632-34

Micellae, colloidal, 529

Micrococci, 642

in milk, 379

Micracaccus amylovorus, 59I

M. aureus in disinfection tests, 302, 306

$M$. citricus, 70

MI. freudenreichi, stability of, 26

M. melitensis Bruce, $38 \mathrm{I}$

M. neisseri, 26

Microcytase, 870

Micron, 179

Micronucleus, 7

Microphages, 870

Microplasms, 517

Migration rate, 787

Milk, 6o. See also Pasteurization acid in sour, 387

acidity, $7 \mathrm{I}$

analysis, 68

BACTERIA IN, 378-94; 403

Bact. abortus, 380, 38I

blue milk organism, 382, 383

colon group, 389,394

from extraneous sources, 382

lactobacilli, 394 
micrococci, 379

saprophytic, 382

Streptococcus epidemicus, 4I 3, 4I 4

thermophiles, 403

tubercle bacilli, 407

blood cells in, $38 \mathrm{I}$

calcium in heated, 409

cans, 382

coagulation, 275

condensed, 396

cream volume, 408, 409

creaming ability, 409

diseases borne by, 410-16

enzymes in butter, 399

evaporated, 396

fermented, 394, 396

flat sour, 397

germicidal property, 382,409

heat, effects of, 408, 409

leukocytes in, 38I

litmus reduced, 389

machinery, 423, 430, 431. See also Pasteurizers

mastitis, $38 \mathrm{I}$

Ordinance, Standard, 434

pellicle, 407

powdered, 397

ropy, 395

sickness, 4I 4

souring, $383,387,397$

sterile, 378

taette, 395

Mineral substance of bacteria, 18

Miso, 5I 3

Mitachondria, 12

Mitosis, 40

Molds 643. See also Aspergilli

in cheese, 401

spores, colored, 373

staining of, 24

Molecules

in crystals, 136

kinetic energy of, 140

orientation

in surfaces, 158

in surfaces and interfaces, $16 \mathbf{r}$

theory of, 160

Molisch reaction, 234

Molluscum contagiosum, 518

Mono-amino acids, 244, 245

Monocytes, 873 proteolytic enzymes of, 875

Monomolecular

films, 163

area per mol, I66

stability, I 73

reaction

formula, 72,73

for oxygen in sewage, 354

Monomorphism, doctrine of, 84

Mordants, 20, 28

Morpliology of BaCteria, I-I3; 86

changes in cell, 97

in classification, 121, 125 three conceptions of, 38

cyclical changes in, 44

as aspect of dissociation, 93

ChANGES DURING GROWTH, 38-45

of root nodule bacteria, 340

variation in, 2,3

variations among bacteria resistant to bacteriophage, $55^{\circ}$

Mortality curve (bacterial), 72, 80

Mosaic plant diseases, 518,560

Moth wilts, 5 I 8

Motility, 97,598

in bacteria resistant to bacteriophage, $55^{\circ}$

in spirochetes, 10 mechanism, 462-66

Mouse. See Mice

Mouth. See also Vaccination

spirochetes, 78I

spray pollution, 80

Moving pictures of bacteria, $4 \mathrm{I}$

$\mathrm{Mu}, \mathrm{I} 79$

Much granules, 4, 5, 33, 40, 88

Mucus production, 552

Multiplication

of bacteria, 10, $5^{8}$

in water, 64

of bacteriophage in absence of growing bacteria, 530

of spirochetes, 10

Mumps, 5 I 8, 936

Mutants, 86

Mutation, 85,526

of pneumococci to streptococci, 577

Mycelium, 509

Mycobacteria, senile forms, 44

Mycobacteriaceae, $43, \mathrm{I} 32$

Mycobacterium leprae, 43

Mycobacterium phlei, 43

MIycobacterium smegmalis, 43

Mycobacterium tuberculosis, 4, 107, 203. See also Tubercle bacillus in disinfection tests, 302,306

Mycotic infections, 515

Myelitis, 582

Myositis, 582

Myxobacleriaceae, $\mathbf{1} 32$

Myxobacteriae, $\mathrm{I}_{32}$

Myxobacteriales, 132

Myxomatosis, 518

Nairobi disease of sheep, 578

Necrotizing capacities, 567

Neisser and Wechsberg phenomenon, 698

Neisseria catarrhalis, 42

$N$. gonorrheae. See Gonococcus

$N$. intracellularis, 42

N. mucosis, 42

Nematodes in soil, 321

Nephritis, 779, 780

and tuberculosis, 1032 
Nernst potential, 786

Nervous system, resistance to bacterial invasion, $5^{8} 7$

Neuberg equations, 237,501

Neuralgia, 582

Neuritis, 582

Ninhydrin test (in Abderhalden's reaction), 1059

Nitrates

reduction, $2 \mathrm{II}, 355$

used by soil bacteria, 317,318

Nitrification, 225, 357

Nitrifying bacteria, 281, 355

Nitrites, 3 I 8

oxidation, 323

reduction, 2 I I

Nitrobacter, 132, 225, 281, 31 8,323

Nitrogen

amino acids as source of, 224

atmospheric, utilization of, 225

content of bacteria, 218,219

changes in, $3 \mathrm{I} 6$

compounds, utilization of, 243

fixation by bacteria, 282, $283,319,337,340$

gaseous, 211,283

necessary for growth, 244, 280

inoculation in soy beans, 336

Metabolism of Bacteria, 218-26

non-nitrogenous matter

in bacterial cells, 218

compounds, utilization, 246

nutrition in nodules, 335

oxidation of, 324

purine bases as source of, 224

in soil, transformation of, 316,318

Nitrosococcus, 225, 323

Nitrosomonas, 225, 3 I8, 323

Nocardia, I30

Nucleases, 274

Nuclei, $6,7,39$

changes, 88

material, 221

Nucleoprotein, bacterial, 23, 729, $73 \mathrm{I}$

as antigens, 723,970

dispersion of, 25

of pneumococcus, 702

Nutrition of bacteria, 14

of soil bacteria, 314

nitrogen, in root nodules, 335

O colony forms, 95. See also Rough colony forms

Obligate

anaerobes, 199, 20I, 205

autotrophs, 323

Ocean, bacteria in, 58

Oidium lactis, 70,400

Oil, effect of, on heat resistance, 295

Oligocarbophilous bacteria, $28 \mathrm{I}$

Opium, filterability of, $5^{62}$

Opsonins, 810, $87 \mathrm{I}$

endothelial, 809

index, 878
Optimum

medium, 283

reaction for enzymes, 272

Optochin, I I09, II 22

Organelles, 97

Organic matter

influence on autotrophic bacteria, 330

dry substance of bacteria, I 5

decomposition of, 3 I 5,316

in sewage, 352

and soil fertility, 342

Organotropism, I IOI

Oroya fever, 518

Osmosis, 77

Osmotic pressure, Van't Hoff's law, 739

Otitis of rats, 636

Ovo-vitellin, 7 I 3

Ovomucoid 7 I 3

Oxalic acid, 246, $5^{1} 2$

Oxidase 276

in spore material, 9

Oxidation, bacterial, 215, 21 7

of ammonia, 323

of glucose, 322

Hoppe-Seyler's theory of, $20 \mathrm{I}$

of hydrogen, 324, 329

effect of light on, 372

of nitrite, 323

and $\mathrm{pH}, \mathrm{r} 94$

reversible of hemolysins, 2 I4

in sewage, 357

of sulphur, 282,325

Oxidation reactions of sterile plant tissue, 208

Oxidation-reduction

BACTERIAL, 2 I I-I 7

by enzymes, 276

potentials of dye systems, 188

and anaerobiosis, 196

indicators, 193

and $\mathrm{pH}, 192$

theory, 189

Oxidizing ferments in bacteria, 215

Oxido-reductases, 276

Oxygen

demand, 354

free, 202

necessary for growth, 280

intolerance, modified, 209

leeches living without, 200

pressure, 196

in sewage, 353,354

indispensable for sulphur bacteria, 327

and surface tension, 182

tension, II $7,202,259,26 \mathrm{I}$

tolerance, 202

effect of ultra-violet light, 376

Pancreatitis, 582

Papillae, 96

Pappataci fever, ${ }_{51} 8$

Paralysis, post-diphtheritic, 778 
Parasite-host relations. See Host-parasite

Parasitism, I 33

Parasitotropism, I IOI

Paratyphoid. See also B. aertrycke, B. enteritidis, $B$. paratyphosus

avian, 446

filtrates, 7 I 4

of guinea pigs, 608

of rabbits, 629

of rats, 637

in sewage and sludge, 370

toxins, 447

Paravaccinia, 518

Paresis

malarial treatment, 698, 1099

protein therapy, 1098

Treponema pallidum in, 476

Parotitis, 582, 936. See also Mumps

Particles

carbon and quartz and phagocytosis, 862

size of and phagocytosis, 866

and final equilibrium, 785

and rate of settling, 783

Pasteurella vicida, 42

Pasteurellosis in

guinea pigs, 6 I9

mice, 624

rats, 636

Pasteurization, 403-I8; 4r9-36

commercial, 408

defects, 424, 427, 43 I

Endicott experiments, 407, 425

foam and splash in, 428,430

machinery, 418, 419-29. See also Pasteurizers

methods, 4I 6, 4I9

electropure, 424

flash, 422

holding, 4 I 9

prejudice against, 418

safety factor, 408

standards for, 406,434

temperature, 405, 407, 43I, 432, 434

time, 405,407

tubercle bacilli, effect on, 286, 406, 407, 433

of urban and rural supplies, 4 I 8

Pasteurizers

defects in, 427,43 I

thermometers, 432

types

continuous flow, $420,422,430,43$ I

electric flash, 424

in-bottle, 420

pocket, $420,42 \mathrm{r}, 429$

valveless, 428

vat, 4 Io

valves, leaky, 4 I 9

Pathogenicity, 567

in classification, I 27

P.D., 786. See also Potential difference

Pea plant

cross-inoculation groups, 334

nodules, 353

Pectin, 514
Pellagra, complement fixation test in, 847

Pellicle formation, I 82, 409

Penicillium camembertii, $40 \mathrm{I}$

$P$. roqueforti, 401

Pentatrichomonas, 662

Perihydrase, 276

Permeability of cell, 2 I and lag, 62

Pernicious anemia, Cl. welchii in, 367

Peroxide, 208

production by bacteria, 214, 2 I 6

Pertussis, 943. See also B. pertussis

Pestis-bacteriophage, 536

Pfaundler thread reaction, 809, 8I 3

Pfeiffer

bacillus, 657, 658. See also $H$. influenzae, Influenza bacillus

reaction in yellow fever, 492

Pfeifferella mallei, 42, 43

$\mathrm{pH}, \mathrm{I} 48$

and $B$. anthracis, 293

and $\mathrm{Cl}$. botulinum, 292

and enzymes, 272

and heat resistance, 292

and oxidation, 194

and oxidation-reduction potentials, 192

and sewage, 355,356

and sludge, $35^{6}$

and soils, 352

and ultra-violet action, 374

Phage, 525

Phagocytes

effect of anilin dyes on, I I 6

blockade of, I044

fixed tissue, 808

intracellular digestion by, 875

origin, 872

Phagocytic indices, 572

Phagocytosis, 86r-69; 870-80; 568, 57 I, 8ro

free energy

changes in, $86 \mathrm{I}$

minimum, 863

$\mathrm{H}$-ion effect, 862

IN IMMUNITy, 870-80

MEChanism oF, 86r-69

effect of lanthanum nitrate, 572

of particles of carbon and quartz, 862

and size of particle, 866

surface tension, $86 \mathrm{r}$

temperature and temperature coefficient, 863

virulence and electrical charge, 863

Wright's technique, 877

Phagotherapy, 553, 554

Phenol, 22 I

bacteriophage adaptation to, 534

coefficient, 75, 302

Phosphates in soil, 319

Phosphorus

necessary to growth, 280

in heated milk, 409

Photosynthesis, 322, $32 \mathrm{~S}$ 
Photosynthetic organisms, 28 I

Physiology in classification, 126

Phytobacteriology, 590

Phytophthora infestans, 590

Pickling, 440

Picric acid, r9, 20

Pigment

red bacteria, 327,328

as generic distinction, I 33

Plague, 2

anti-, serum, 927

of fowls, 5 I 8

virulence variations, 566

Plant. See also Cross-inoculation groups, Root nodules

food mobilization, 348

oxidation and reduction reactions, 208

Plant Diseases, Bacteria in, 590-606 control of, 606

bacterial plant pathogens agglutination, 599, 600

classification, 593

colony variation, 595

differentiation, 594

dissemination by insects, 605

distribution, 592

entry, route of, 603

immunology, 60 I

tissue invasion, 603

host relations, 332,570

insect dissemination, 605

mosaic, 5 I 8

Plaques, 525

Plasma cells, $65^{2}$

Plasmodium falciparum, 696

P. malariae, 696

P. praecox, 695

P. vivax, 696

Plasmolysis, 2, 543

Plasmoptysis, 2

Plasteins, 704

Plaster of Paris, 558, 559

Pleomorphism, 2, 44

Pleuropneumonia, 5 I 8

Pneumococci, 8, 62, I I I

anaerobic extracts of, 216

chances of collision, 868

electrical forces, 866

infections in

guinea pigs, 6 I 6

mice, 635 , I 22

nucleoprotein, 702

peroxide production, 216

potential, 799

anti-, sera, 928, 929, $95^{8}$

specific soluble substance, 726

and stickiness of cells, 867

rough and smooth strains, 570

mutations to streptococci, 577

type specificity, 73I

virulence, charge and phagocytosis, 863

virulence for mice, 569
Pneumonia

chemotherapy, I I 23, I 330

influenza, 942

ferments from lung, 876

treatment by ethylhydrocuprein, I 122

Poisoning. See Food

Poisons, anaphylactic, 982

Polar groups, 162

Poliomyelitis, 518, 582, 941, 942. See also Infantile paralysis

streptococcus antiserum, 932

variations in virulence, 566

Pollen extracts, 963

Polluted water, 69,365

Polysaccharides, I 7,834

as antigens, $97 \mathbf{I}$

Populations, Bacterial, 58-83 volume occupied by, 544

Potassium necessary to growth, 280 in soil, 319

Potato blight, 590

Potential difference. See also P.D. and agglutinability, 569

for filters, 557

effect of lanthanum nitrate, 572

for microbes, 566

Potentials, 863 effect of agglutinating sera, 798

critical, of a suspension, 795

electrode, igo

Oxidation-Reduction, of Dye Systems, I 88-97

and pneumococcus, 799

effect of salt solution on, 793

of charged sphere, 169

and stability, I 70

effect on valency, 799

and virulence, 569

zeta, I69

Precipitation

of colloids, 790

and complement fixation, 855

reactions in syphilis, 849

tests for syphilis, 849 . See Kahn reaction

Precipitinogen, 824

precipitin-, combination, 826,827

preparation, 829

Precipitins, 824-29; $92 \mathrm{r}, 1037,1083$

and anaphylaxis, $98 \mathrm{I}$

bacterial, 828

diagnosis by, 830

organ specificity, 826

-precipitinogen combination, 826,827

production of, 828

properties of, 825

species identification by, 829

specificity, 826

test, 8

Pregnancy, complement fixation test in, 847

Preserving of foods, 440,44 I

Presumptive test for coli-aerogenes group, 364 


\section{Proflavin, 1118}

Propionic acid, 246

Protamines as antigens, 970

Protective colloids, 77

Protein

amphoteric, 742

as antigens, 7 I 2, 970

nature of antitoxin, 744

azo-, 718

constituents of bacteria, $15,16,218$

in bacterial cells, 218

carbohydrate complexes, 7 IO

carbohydrate fraction, 234

coagulation by light rays, 377

denaturation, 724

-free lipoid, 7 I 5

heat-coagulated, 703

metabolism of bacteria, 218

molecular weight, 220

pure, 7 I 6

racemized, 704, 970

synthesis by bacteria, 224

tissue, 703

from tubercle bacillus, 7I 4

Protein Therapy, Non-Specific, I086-I 100 blood vessels, permeability of, ro93

changes in, 1089

focal reaction, rogs

immunity reactions, $\log \mathrm{I}$

in paresis, 1098

pharmacological aspects, 1093

in typhoid fever, Iog6

Proteolysis, 22 I

enzymes, 275

inhibition of, 223

and putrefaction, 219

Proteolytic udder cocci, 379, 380

Proteus, 2, 42 group, 642

Proteus vulgaris, nitrogen content, 218

Protista 5 57

Protobios bacteriophagus, 525

Protons, 137

Protozoa

INFECTION BY BLOOD, 679-70I

in guinea pigs, 621

INTESTINAL OF MAN, 660-78

Pseudomonadaceae, 132, 133

Pseudomonas, $\mathrm{I} 32$

Pseudomonas fluorescens, 383

Ps. pyocyaneus, 42, 21 8, 219

Pseudoplasmodial bacteria, 130

Pseudotuberculosis in guinea pigs, 614 mice, 635

Ptomaine poisoning, 222

Ptomaines, 772

Pure lines, 85

Purine bases as source of nitrogen, 224

Purines, 246
Putrefaction, 2 I9

in intestine, 640

in sewage, 353

Pyrimidines, 246

Pyruvic acid, 237, 246, 248

Quantum constant, I3 8

Quartz particles, phagocytosis of, 862

Quinin, 25, 26 compounds, I Ioo

Quotients, respiratory, 255

Rabies virus, 660, $95 \mathrm{I}$

Rabbits, diseases of, 622-32

encephalitis, 630

intestinal infections, 628

myxomatosis, 5 I 8

paratyphoid, 629

respiratory infections, $622-27$

rheumatic fever, 63 I

septicemia, 569, 79I

snuffles, 627

venereal spirochetosis, 630

Virus III infection, 5I 8

Radiations, 373

Ramon's method. See Flocculation

Rat

bite fever, 453

diseases of, $635-38$.

suppurative otitis, $6_{3} 6$

paratyphoid, 637

pasteurellosis, 636

scab, 638

Leptospira icterohaemorrhagiae in wild, 488 viruses, 447

Readjustment phase, 79

Reagins, atopic, 1006

Receptors, 882 analysis, 823 , 1079

Recurrent fevers, 804

Red pigment bacteria, 327,328

Reduction, bacterial, 2I I, 2I 2

agents, particulate nature of, 207

intensity, 194

of litmus in milk, 389

OXIDATION-, 188-97

and anaerobiosis, 196

by enzymes, 276

indicators, 193

potentials and $\mathrm{pH}, \mathrm{I} 92$

theory, 189

reactions of sterile plant tissues, 208 sulphite, 367

Refrigeration, 44I

Relapses, 684

Relapsing fevers, 453,479

complement fixation test in, 847

Rennet, 274, 379

Reproduction

by simple fission, 100

gonidia in, 90

-inhibiting product, $69 \mathrm{r}$ 
Repulsion, electrical, I 70

Residue substance. See Soluble substance

Resistance, 565. See also Heat resistance of bacteria to bacteriophage, $528,548,551$ of bacteriophage, 533 of host and parasite, 665 in lytic cultures, 549 methods of measuring, 679 of spores of B. mycoides, 29 I variants, 8 I

Resorcinol, 640

Respiration of bacterial cells, 2 I9

Respiratory

coefficient, 2 I9

quotients, 255

infections of rabbits, 622

Tract, Bacteria OF, 650-59

spirochetes, $48 \mathrm{r}$

hemolytic streptococci, 653

Reticulo-endothelial system, 890, I043

Rhabdochromatium, 324

Rheumatic fever, 579

chemotherapy, I 133

in rabbits, 630

streptococcus antiserum, 933

Rhinitis, atopic, 968

Rhizobium radicicola, 225

Rhodocapsa, 324

Rhodothece, 325

Rice fermentation, $5 \mathrm{r} 2$

Ricin, antiricin, 740, 744

Ricinoleate, I 80

Rickettsiae, $\mathrm{I} \mathrm{r}-\mathrm{I} 3$

Rickettsia melophagi, 12

R. nipponica, 12

$R$. prowazeki, I I

R. ruminantium, I 2

Rideal-Walker method for disinfectants, 3or

Riensch-Wurl screen, 355

Rinderpest, 5 I 8

Ring method for surface tension, I 74

Rivanol, I I I 7, I I I 8

Rivers, bacteria in, $5^{8}$

Rocky Mountain fever. See Spotted fever

Roentgen rays, 745 , I040

Root, 332

hairs, 332

nodules

a!falfa, 334

BACTERIA IN, 332-40

acidity relations, 339

carbohydrate fermentation, 339

flagellation, 338

gum production, 339

morphology, $33^{8}$

nitrogen fixation, 340

nitrogen nutrition, 335

serological reactions, 339

strain variations, 340

formation, 337 nitrogen nutrition, 335

pea, 353

shape, size, position, 334

soy bean, 333,334

tubercles, 590

Rot, soft, 591

Rough and smooth

colony forms, 94, 95, 597

motility, 598

serological characters, 599

virulence, 598, 599

strains, 822

of pneumococci, 570

Rubella, 518

Sacbrood, 5 I 8

Saccharase, 274

Saccharic acid, 235

Sachs-Georgi reaction, 856

Salicylic acid, 246

Salivary gland disease of guinea pigs, 518

Salmonella acrirycke, 444. See also B. aertrycke

Salmonella enteritidis, 444 See also B.enteritidis

Salmonella group, 610

Salmonella paratyphi, 42, 444. See also B. paratyphosus

Salmonella schottmïlleri, 42, 444

Salmonella suipestifer, 42 See also B. suipestifer

SALTS, I45-5I

activity coefficients, 146

agglutination, 8I4

ammonium in fertilizers, 347

concentration, 76

and cohesive force, 794

and potential and agglutination, 793

effect on heat resistance, 294

ions in solid, I39

ionization in solutions, 140,177

mineral, regulative action, 68

solutions

death of Bact. coli in, 73

water film on, 168

and soil fertility, 342

Sanfoin, cross-inoculation groups, 334

Sanocrysin, I I 29

Saponin, I80

Saprospira, 454, 457-62

Sarcina, oxidizing ferments, 215

Sarcina lutea, 70, $3^{8} 3$

Sarcoma in chickens, 518

Scab, 638

Scarlatinal

antitoxin, 755

toxin standardization, 747

Scarlet fever, 5 I 8

streptococcus antitoxin, 955, 957

toxin, 961

complement fixation test, 847

from milk, 4I I, 4I 3

normal blood in treatment, 944 
Schick test, 748,960

Schizomycetes, I 30

Schizosaccharomyces filtrans, 499

Schlammfieber, 490

Schumann region, 37 I, 373

Sciatica, $5^{82}$

Seasonal changes in numbers of bacteria, $35^{2}$

Secretion of bacterial enzymes, 269

Sedimentation of sewage, 353

Senile forms of Mycobacteria, 44

Sensitivity of red cells, 896

Sensitization, 969

active protein, of laboratory animals, etc., $989-91$

cats, 991

dogs, 990

frogs, 991

guinea pigs, 989

mice, $99 \mathrm{I}$

monkeys, 991

pigeons, 991

rabbits, 990

rats, 99I

desensitization, 978

specific, 992

non-specific, 993, 1012

hyposensitization, 1012

passive, 972; of laboratory animals, 99r, 992

dogs, 992

guinea pigs, 991

mice, 992

rabbits, 992

rats, 992

Septic sore throat, $380,4 \mathrm{II}, 4 \mathrm{I} 3$

Sera. See also Serum

ANTIBACTERIAL, 921-33

anti-anthrax, 923

anti-dysenteric, 924, 960

anti-gonococcic, 925

anti-meningococcic, $925,95^{8}$

anti-plague, 927

anti-pneumococcic, 927,958

anti-staphylococcic, 930

anti-streptococcic, $930-33$

antilipoid, 707

Serological

characteristics and colony form, 599

reactions of root nodule bacteria, 339

Serolysis, 26

Seromucoids, 7 II

Serradella, cross-inoculation groups, 334

Serratia marcescens, 42, 383

Serum

effect of, on colony form, 99

Convalescent, Human, 934-46. See ConVALESCENT SERUM, etc.

disease, 966 , 10 2

foreign, injection of, IOI I

Settling, as affected by size of particles, 783

Seven-day fever, 494
Sewage Treatment, Bacteria in, 35I-6r; 59 albumen digesters in, 355

Bact. coli in, 360

chlorination, $36 \mathrm{I}$

decomposition, $35 \mathrm{I}$; aerobic, $36 \mathrm{I}$

denitrification, 357

disinfection, $61,36 \mathbf{I}$

filamentous bacteria, 359

hydrogen sulphide producers in, 355

monomolecular reaction for oxygen in, 354

nitrate reducers in, 355

nitrification in, 357

nitrifying organisms in, 355

numbers of bacteria, 58,352

organic matter in, $35^{2}$

oxidation in, 357

oxygen requirement, 353

paratyphoid-typhoid group in, 370

$\mathrm{pH}, 355$

putrefaction, 353

removal of bacteria from, 360

seasonal changes in bacteria, $35^{2}$

sedimentation, 353

treatment, 353

Sheathed bacteria, 130

Sheep

blue tongue, 518

Nairobi disease, 518

pox, 518

Shiga bacteriophage, 536

Shock. See Anaphylactic shock, Atopic shock tissues, temperature

Side-chain theory, 815. Sce also Elurlich

Siderocapsa, 324

Sideromonas, 324

Silkworm jaundice, 518

Silver impregnation, 37

Size of bacteria, 42

when multiplying, Io

resistant to bacteriophage, $55^{\circ}$

Skatol, 22 I

Skin

reactions with autolysates, 728

sensitivity, $75 \mathrm{I}$

infections treated with yeast, 506

Skips, 297

Slecping sickness, $68_{3}$

Slime production, $55^{2}$

Sludge

activated, $354,35^{8}$

paratyphoid-typhoid group in, 370

digestion of, $354,356,359$

disposal of, 355

filamentous bacteria in, 359

filter-pressing, $35^{8}$

$\mathrm{pH}, 356$

Smallpox vaccine, 949,950

Smegma, 453

Smooth cclony forms, strains. See Rough colony forms

Snakes and venoms, 1066

Snuffles in rabbits, 627 
Soap, iso

bile, 185

effect of, on toxins, 184,185

Sodium

chloride, space lattice in, 136

citrate, 365

hippurate hydrolized to benzoic acid and glycocoll, 248

ricinoleate, 180

Soil. Ste also Soil fertility, Soil micro-organisms antiseptics in, 66, 349

chemical composition of, $34 \mathrm{I}$

gases, 343

germicides in, 349

inoculation, 349

liming, 67,345

sandy, 58

solution, 343

stored, 60

treatment, 344

Soil Fertility, Micro-ORganisms in, 341-50; 225,320

aeration, 344

nature of, $34 \mathrm{I}$

organic matter, 342

soluble salts, 342

temperature, 343

SOIL MICRO-ORGANISMS, 3 I o-2I

Actinomyces, 317,321

activity, 320

Azobacter, 316, 319

calcium utilized, 3 I 9

cultivation, 313

distribution, 3 I 2

and fertility, 320. See SoIl Fertility, ETC.

fungi, 317,321

functions, 3 I $5^{-20}$

assimilate ammonia, 3 I 7

decompose organic matter, 3 I 5

use nitrates, 3 I 7

effect nitrogen, 316, 3 I 7

isolation, 313

nematodes, 32 I

phosphates, 3 I9

population, 310

potassium, 3 I 9

symbiotic bacteria, 320

Solids in bacterial cells, 2 I 8

Solubility

and activity coefficient, 146

and molecular orientation in surfaces, 1.59 product, I 5 I

Soluble substance, I 7, 570, 572, 724, 731, 834

Friedländer bacillus, 727

pneumococcus, 726

streptococci, 727

yeast, 726

Solutions, theory of, 739

Sorbitol, 235

Souring of

milk, $383,387,397$

vegetables, 440

Soy bean

cross-inoculation groups, 332 nitrogen after inoculation, 336

nodules, 333, 334

Soy sauce, 5 I 3

Space lattice of sodium chloride, 136

Species identification by precipitins, 829

Specific soluble substance. See Soluble substance

Specific substance, 8

Specificity

anaphylaxis, 977

antigens, 702, 708, 709

antivenins, 1073

host-parasite, 666

tuberculin, 1027

type

pneumococci, 73I

polysaccharide, 834

venoms, 1068

Spectral region, effective, 372

Spectrum

germicidal region, 373

visible, 37 I

Sperm, isoagglutinable substances in, 920

Spheres, I3 I potential of charged, 169

Spiral forms, I $3 \mathbf{I}$

Spirella, 453

Spirillaceae, 42, I32 pleomorphism of, 44

Spirochaeta, 452, 454, 457-62

Spirochaeta anserina, 480

S. biflexa, 485

S. eurygyrata, 484

S. gallinarum, I0, 480

$S$. grandis, 454

S. granulosa penctrans, I I

S. (?) interrogans, 454

S. obermeieri, 804

S. phagedenis, 472

S. plicatilis, $452,454,455$

S. pseudoicterogenes, 485

S. stenostrepta, 484

Spirochaetaceae, 455

SPIROCHETES, 452-97; I, 2

aerobic, 472

alterations from prolonged cultivation, 474

anaerobic, 469

antigens, 83.5

carbohydrates in cultivation, 474

classification, $45^{2}$

cultivation, 460

filterability, 467

of genitalia, 483

granules, 9, I I

groups, 475

immunity against, 468

individual, 475

intestinal, 484

motility and its mechanism, 9, I I, 462

of mouth, $48 \mathrm{I}$

multiplication, 9 
in mumps, 518

in respiratory tract, $48 \mathrm{I}, 655$

transmission of, 468

Treponema group, 475-8I

venereal of rabbit, 530

in yellow fever, 518

Spironema, 453

Spironema duttoni, Io

Spironema novyi, ro

Spontaneous generation, 285

Spores, I, 8, 89, I 32

of Aerobacter aerogenes, 390

in animal body, 9

of Aspergilli, 5 Io

of $B$. subtilis, 6r

in boiling water, 286

of $\mathrm{Cl}$. botulinum, 288

of Cl. tetani, 287

rate of destruction, $9,285,287,288$

formation, I, 8, 97, I32

resistance to heat, 285,298

material, 9

of colored mold, 373

resistance of, of $\mathcal{B}$. mycoides, 29I

thermophiles, 286

Sporobolomyces, 498

Spotted fever, Rocky Mountain, 13, 518

Spreading

coefficient, 864

of one liquid on another, 162

Spring waters, 64

Stability, I 70

and electric charge, 788

of emulsion and monomolecular film, I 73

Staining. See also Dyes

of acid fast bacteria, 3I-33

REACTIONS OF BACTERIA, I9-37

of bacteria in media, 34

of bacteria in tissues, 34

of bacterial structure, 34

elective, 24

by fat-soluble dyes, 3 I

history of, I9

isoelectric range, 25

mechanism of, 22

of molds, 24

negative method, 43

polychrome, 20

stains, 19

vital, 35

Standardization of

ANTISEPTICS, 307-9

DISINFECTANTS, $301-7$

Standards

for Bact. coli, 367

for water supplies, 367

Staphylo-bacteriophage, 536

Staphylococcus, 582

in food poisoning, 450

infections

chemotherapy, i i 30

phagotherapy, 553 local immunity, 886

oxidizing ferments, 215

anti-, serum, 930

Stationary phase

initial, 48

maximum, 47, 5 I

Statistical methods applied to bacterial growth studies, 4 I

Sterilizers, ultra-violet, 375

Stokes's law, $78_{3}$

Stomach

antiseptic property of, 640

ulcers, 582

Stomatitis of horses and cattle, 5 I 8

Streams, bacteria in, at different seasons, $35^{2}$

Streptobacillus pseudotuberculosis (rodentium), 6I 4

Streptococci, 80

anti-, sera, polyvalent, 930, 931. See also Encephalitis, Erysipelas, Scarlet fever

in human diseases, 579

in food poisoning, $45^{\circ}$

infections

chemotherapy of, I I 30

in guinea pigs, 620

local immunity, 886

localizing power, 578

peroxide production, 216

mutations to pneumococci, 577

from nasopharynx, 579

in respiratory passages, 653

isolation in rheumatic fever, 579

specific soluble substance, 727

toxin, 184

udder, 380

in water, 69, 364, 367

Streptococcus agalactiae, 380

Str. epidemicus, $38 \mathrm{I}$

in milk, 4I3, 4I4

Str. erysipclatos, 108

Str. fecalis, 642

Str. hemolyticus, 42

Str. kefir Migula, 389

Str. lactis, 379,389

in butter-making, 398

and buttermilk, 395

liquefaciens, 380

Lister, $38_{3}$

Str. mastitis, $38 \mathrm{I}$

Str. paracitrovorus Hammer, 389

Str. tactte, 395

Streptothrix, 130

Strongyloplasms, 5 I 7

Structure

of bacteria, $2 \mathrm{I}, \mathrm{I} 24$

Chemical, I4-I8

internal, 6

staining of, 33

of surface of liquid, 160

Strychnine, filterability of, 563

Sublethal Intoxications with Bacterial Products, 772-8I 
Substrate, effect of, on growth curves, 54

Subtilis group, 643

Succinic acid, 246

Sugar

acids, 728

blood, and malaria, 698

in foods, 440

series, 232

Sulphide, 327

Sulphite reduction, 367

Sulphur

-ous acid and lag, 6r

bacteria, I 30,327

globules of, 327

necessary to growth, 280

oxidation, 282,325

Sunlight, 73,372

SURFACES, $154-68$; 179

of bacterial cell, 23

energy, $154,157,162,178,179$

films, 797

latent heat, 156

orientation of molecules in, $158,161,178$

and solubility, 159

theory of, 160

structure of, 160

tension, $167,173,179,74$ I

effect on bacteria, $179,181,183$

of culture media, 180

depressants, 180

determination of, 178

drop weight method, 173,175

and growth, 182

and dissolved oxygen, 182

in phagocytosis, $86 \mathrm{I}, 864$

ring method, 174

effect on toxins, I 79

Suspensions

colloidal, 170,785

effect of added substances, 797

electrical characteristics of, 169

Swelling of bacteria, $543,545,547$

Swimming pool purification, 375

Swine pox, 518

Symbiotic bacteria, 102, 320

Symplastic structures, 9 I

Synergism, 102, II 5, 24I

Synthesis, protein, by bacteria, 224

Synthetic Culture Media, 279-84

contents, 280

for photosynthetic organisms, 28I

preparation, 284

pseudo-, 279

Syphilis

in apes, 476

Complement Fixation Test, $838-47$

precipitation tests, 849,856 . See also Kahn reaction

Taette milk, 395

Tanks

Imhoff, 355

septic, 354
Tannin solutions, $5 \mathbf{I} 2$

Tartaric acid, 246

Teeth, 656

streptococci from, 579

Temperature

and disinfection, 78

coefficients for enzymes, $27 \mathrm{r}$

effect on growth, 54, 55, 65, $7 \mathrm{x}$

for pasteurization, 405,407

coefficient of phagocytosis, 863

shock, 62

and soil fertility, 343

and survival of bacteria, 77

effect on toxins, 775,776

and ultra-violet light action, 374

Tensile

energy, 155

strength, I 55

Tension. See also Surface tension

interfacial, 173

oxygen, 202, 259, 261

Tetanus

antitoxin, 953, 954

filterability, 562

flocculation titre, 767

preparation, 754

units, 954

toxin, 184,773

filterability, 562

Theobald Smith phenomenon, 969

Therapeutic use of aciduric group, I06 fermented milks, 396

Lact. acidophilus, 396

Lact. bulgaricus, 396 yeast, 506

Therapy, chemo- and biological, II I I

Thermal Death Time, 285-300; 404, 406 of tubercle bacillus, 286,404

Thermal increment, 57

Thermodynamics, 177

Thermophiles, 292, 442

in milk, 403

spores, 286

variations, 289

Thiobacillus, 282, 323-36

Thiobacteriales, 132

Thread

method for disinfectants, 3 or reaction, $809,8 \mathrm{r} 3$

Time necessary to kill bacteria, 374

Tissue. See also Immunity, local use of, in anaerobiosis, 205 as buffer, 207

changes in chemotherapy, 1108

invasion by plant pathogens, 603

staining of bacteria in, 34

sterile plant, oxidation-reduction reactions of, 208

Tobacco, 560

Toluol, 25 
Tonsils, 579,654

streptococci from, 579

Toxic Bacterial Products

effects on blood, 775

effects on capillaries, 775

local effects, 774

SUbleTHAL INTOXICATIONS WITH, 772-81

effects on temperature, 775

Toxicity, 66

test of anilin dyes, I I 6

effect of ions, 284

of mercurial compounds, II 3 I

of metabolic products of bacteria, 518

Toxigenicity, 566 and electrophoretic potential, 573

Toxin-antitoxin mixtures. See also Flocculation effects of freezing, 749

preparation of, 748

substitute for Schick test, 748

skin sensitivity, $75^{\text {I }}$

Toxins use of, 75 I

effect of acids, 75

adsorption, $74 \mathrm{I}$

elaborated in presence of amino acids, 243

amphoteric, 575

as antigens, 705

bacterial, filterability of, $560,56 \mathrm{I}$

non-bacterial, filterability of, $5^{62}$

botulinum, I05, 449

cataphoresis of solutions of toxin and antitoxin, 742

colloidal reactions, 740

diphtheritic, I 84

electro-colloidal reactions, 74I

endo-, 568, 773

endosmosis of solutions of toxin and antitoxin, 744

of B. enteritidis, 444

focal or metastatic effects, 777

and leukocytes, 775

effect of light, 745

effect of Roentgen rays, 745

effect of soaps, 184,185

mouth administration, 773

neutralized by antitoxin, 739

oxidation, effects of, 745

paratyphoid, 447

Physical Chemistry of, 739-44

Preparation and Purification of, 745-58

production, 68,568

effect of glucose, 229

purification, 742

soluble, 772

specificity of, $78 \mathrm{I}$

standardization of, 746

by Ramon's method, 759,760

streptococcic, I 84

tetanus, 184 ; variations with temperature, 773

ultra-violet rays, effect of, 375

Toxoids, I 86

preparation, 745,749

properties of bacterial, 745

purification, 745

ultra-purification, $75^{2}$

use, 749
Treponema, 454, 457-62, 475-84

motility, 464

Treponema buccale, $48 \mathrm{I}, 482$

T. calligyrum, $47 \mathrm{I}, 483$

T. macrodentium, $47 \mathrm{I}, 48 \mathrm{I}, 482$

T. microdentium, $47 \mathrm{I}, 48 \mathrm{I}, 482$

T. mucosum, 47 I , 48 I, 482

T. pallidum, 454, 471, 475-77, 839

T. pcrtcnue, 477

T. refringens, $47 \mathrm{I}, 483$

T. schaudinni, $48 \mathrm{I}, 483$

T. vincenti, $472,48 \mathrm{I}, 483$

Trichomonas buccalis, 672

Trichomonas hominis, 672

Trichomonas intestinalis, 674

Trichomonas vaginalis, 672

Trypanolysis, 685,692

Trypanosoma brucei, 684

Trypanosoma equiperdum, 680, 684

Trypanosoma evansi, 687

Trypanosoma gambiense, 684

Trypanosoma lewisi, 678, 680, 690 variation coefficient, 682

Trypanosoma rhodesiense, $68_{3}, 684$

Trypanosoma togolcnse, 687

Trypanosome infections

resistance to antibody, 686

crises and relapses, 684

lethal and non-lethal, 693

in mouse, $68_{3}$

non-pathogenic, 689

Trypanosomiasis, experimental, antibodies in, 686

Tubercle bacillus, 4. Sce also Mycobacterium tubcrculosis

bovine

heat resistance, 406, 407

in milk, 407

effect of pasteurization, 407

carbohydrate from, 714

extraction of, 32

fat of, 18

filter-passing stage, 5

gas metabolism of, 262

gum from, 727

infections in guinea pigs, 620

destroyed by light, 373

lipin from, 7.14

and pasteurization, 433

protein from, 7I 4

protein-free lipoid from, 7 I 5

thermal death-point of, 286, 404, 406

TUberculin, IOI6-23; 219, 56r, 832

active principle of, 1023

chemical nature of, ror 9

recent chemical studies on, 1020

preparations of, 1016

protein nature of, $102 \mathrm{I}$

skin reactions to, 1022

standardization of, $96 \mathrm{r}, 968,1017$

in treatment of tuberculosis, 1029

and ultra-violet rays, 376 
Tuberculin Reaction, I023-34; 725

and anaphylaxis, I029

anatomical changes, 1023

in diagnosis, 1029

and hypersensitiveness, 1028

and pathological changes in tuberculosis, IO3I

pathology, 1024

specificity, 1027

spontaneous reactions, 1031

Tuberculosis

chemotherapy of, I I 27 , I I 28

diagnosis by complement fixation, $8_{3} 6,8_{47}$

and nephritis, ro32

pathological changes in, IO3I

tuberculin treatment of, IO29

Typhoid bacillus. See also Bact. typhi

from blankets, So

in stored feces, 370

in sewage and sludge, 370

fate in water, 77,369

Typhoid fever, 566, 805, 943

from milk, 4I I, 4I 2

mouse, $555,632-34$

mouth vaccination, 888

protein therapy, rog6

vaccine, $82 \mathrm{I}$

Typhus fever, 5 I 8,82 I

Udder

bacteria, 378,379

cocci, 379

streptococci, 3 So

infection with Bact. abortus, 380

Ulcers of stomach and duodenum, 582

Ultra-sterility, 53 I

ULTRA-VIOLET RAYS, 37I-77

action, mechanism of, 377

animals exposed to, 377

antigens, effect on, 375

bacteriophages, effect on, 376

coagulation of proteins, 377

complement, effect on, 376

enzymes, effect on, 375

and $\mathrm{H}$-ion concentration, 374

and oxygen effect, 376

and $\mathrm{pH}, 374$

residual action, 374

sterilizers, 375

and temperature, 374

toxins, effect on, 375

and tuberculin, 376

absorption by water, 374

Undulant fever, 38 I

Uric acid action on

$B$. aerogenes, 248

Bact. coli, 248, 365

Urine in media, 2

Urticaria, 879, 968, ror 5

Utilization by bacteria of

Aliphatic and Aromatic Compotnds, 243-49

mono-amino acid, 245

nitrogenous compounds, 243

non-nitrogenous compounds, 246
Vaccination, oral, 888,1047

Vaccine virus, 560, 949

Valence, 177

of adsorbed ion, I 7 I

effect of, on potential, 799

Vaporization, heat of, 162

Variants (bacterial), 27, 86, 93

pathological, 85

resistant, 8I

to bacteriophage, $55 \mathrm{I}$

Variations

of bacteria, 2, 3, 39, 44

and agglutination, 822

cyclogenic, IOI

hereditary, 85

impressed, 85, I 23

plain, 85

strain, in root nodule bacteria, 340

of filterable viruses, 520

Varicella, 935, 936. See also Chicken pox

Varioloid, 518

Vegetables, 439, 440

Velocity

in cataphoresis, 170, I 7 I

constants (Bact. typhosum), 76

of light, I 38 , I 76

Venoms, ro66-72. See also Antivenins amounts, 1068

antigenic principles in, $1070-72$

apparatus of snakes, 1067

cobra, 562

composition, 1070

extraction, I075

hemolysis, 1072

inoculation by snakes, 1067

nature, 1069

purification, ro74

specificity, 1068

Verruga peruviana, 518

Vetches, cross-inoculation groups, 332

Viability, 34

of Bact. coli, 76

of filterable viruses, 520

Vibration, 137

Vibrio cholerae, 89

nitrogen content, 2 I 8

V. comma, 42, 43

V. cyanogenus, $38_{3}$

V. finkler-priori, 89

V. metchnikovi, 42

$V$. percolans, 560

V. proteus, 2, 89

V. schuylkilliensis, 42

$V$. septique, 104

$V$. syncyaneus, 383

Vibrion butyrique, 198

VIRULENCE, 565-75; 86, 598

alterations in, 570

of bacteriophage, 535

continuous variations in, 57 I 
and encapsulation, 568

and phagocytosis, 568

for plant hosts, 570

of pneumococci, 569,863

non-toxigenic, and electrophoretic potentials, 569

specific soluble substances and electrical charge, 572

variations in, $565,566,568$

Virulin, 568,872

Viruses, standard, 949. See also Filterable viruses rabies, 660, 95I

ultra-microscopic, 517

vaccine, 949

Viscosity, 170, 544

effect of bacteriophage on, 544

Vitamines, 657 and antibody production, 1046

Voges-Proskauer reaction, 239, 240, 365, 392

Volutin, I6, I7

Vucin in chemotherapy, I 24

Warts, 518

Wassermann. See also Complement fixation antigen, 835

reaction, $838-47,856$

Wastes

dairy, 355

industrial, 359

Water

content of bacteria, 14, 352, 363

BACTERIOLOGY, 362-70

See also Bact. coli

bacterial counts, 363

bacterial species in water, 363

chlorination of water supplies, 368

flavors in water, 369

ground water, 369

standards for water supplies, 368

surface water, flora of, 364

typhoid bacillus in water, 369,370

film on salt solutions, 168

typhoid bacilli survival in, 77

absorption of ultra-violet rays, 374

ultra-violet sterilization of, 375

Waters

drinking, purification of, 374

colored, 374

of lakes and rivers, $5^{8}$

natural, 60

spring, 64

stored, 63,69 toxicity of, 66

turbid, 374

from wells, 369

Waxes of bacteria, i 8

Weight of bacteria, 229

Weil's disease, 487

Wheat, rose-red disease of, $59 \mathrm{r}$

Whooping cough, 943

Widal test, 8 I 7,82 I

Wilt of moths, 5 I 8

Worms and anaerobic conditions, 200

Wounds, I04

Wright's technique for phagocytosis, 877

$\mathrm{X}$-rays, $177,371,1040$

$\mathrm{Y}$-shaped forms, 40

Yaws, 477

complement fixation test in, 847

YEASTS, 498-508; 643, 649

accessory substances, 499

bactericidal effect, $5 \circ 8$

fat content of, 506

fat formation by, $5 \circ 5$

fate of, in intestines, 507

filterability, 499

as laxative, 507

and leukocyte count, 507

morphology, 498

origin, 498

specific soluble substance from, 726

therapeutic use of, 506

Yellow fever, 5 I 8

and dengue, 493

and infectious jaundice, 493

Pfeiffer reaction in, 492

spirochetes in, 490

Yellow trefoil, 334

Yellows (in dogs), 487

Yoghourt, 395

Zeta

function of Gibbs, 176

potential, 169

Zettnow's technique for terminal filaments, 165

Ziehl-Neelsen method of staining, 24

Zone phenomenon, 688, 689

Zygospore formation, 90

Zymase, 276, 277, 50I 


\%

S.

8.

\%

\% \% * * * * * *

\% $\%$ \% $\%$ \%

$\% \times \%$ \% $\%$

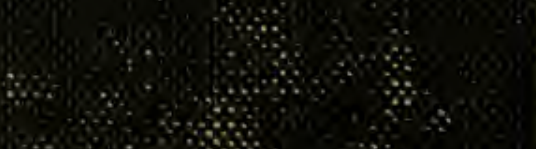

\% $\%$

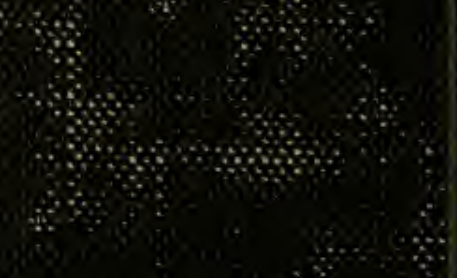

$\therefore$

\% $\%$ \%

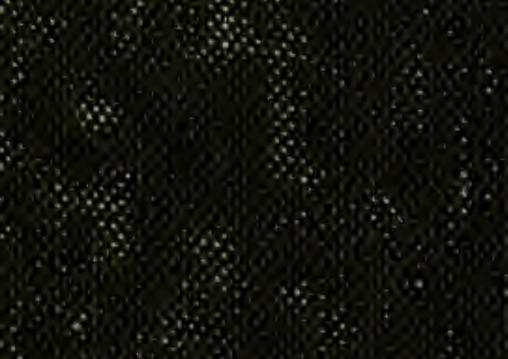

$\alpha$

$\%$

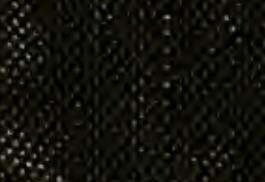

$\%$

\%

$30 \%$

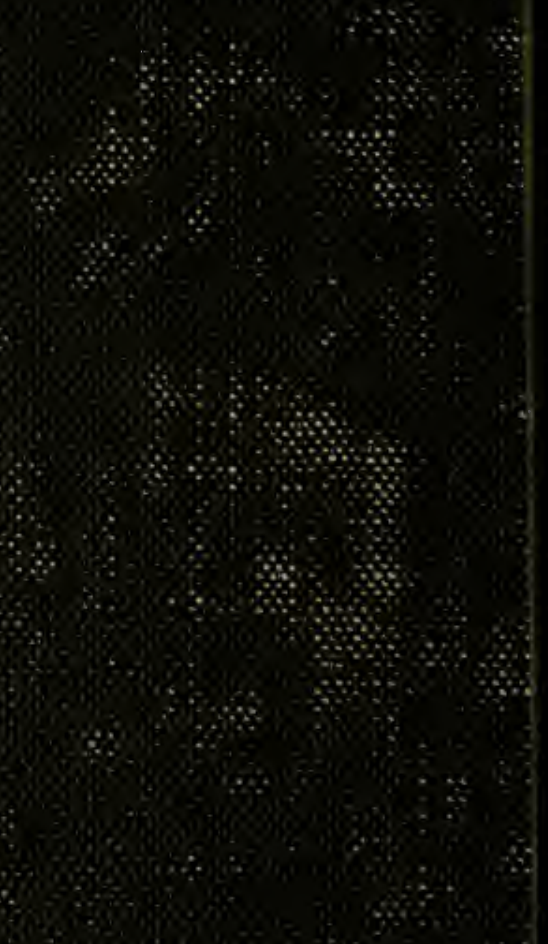

\%

\% $\%$ \%

\% $\%$ \%

10.8\%8\%

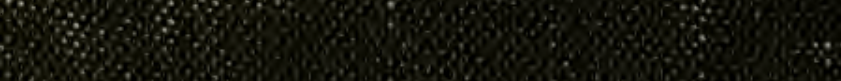

\%

8

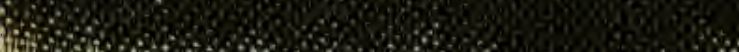

